\title{
The Journal of the
}

\section{Acoustical Society of America}

Vol. 140, No. 4, Pt. 2 of 2, October 2016

www.acousticalsociety.org
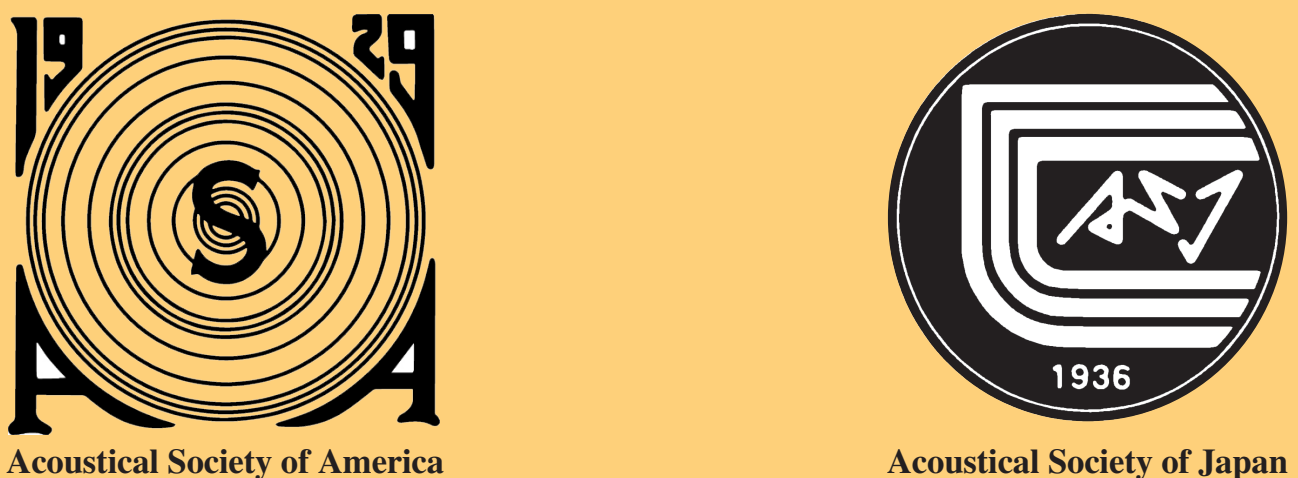

Acoustical Society of Japan

5th Joint Meeting

\section{Hilton Hawaiian Village Waikiki Beach Resort \\ Honolulu, Hawaii \\ 28 November-2 December 2016}

Table of Contents on p. A5

Published by the Acoustical Society of America through AIP Publishing LLC 

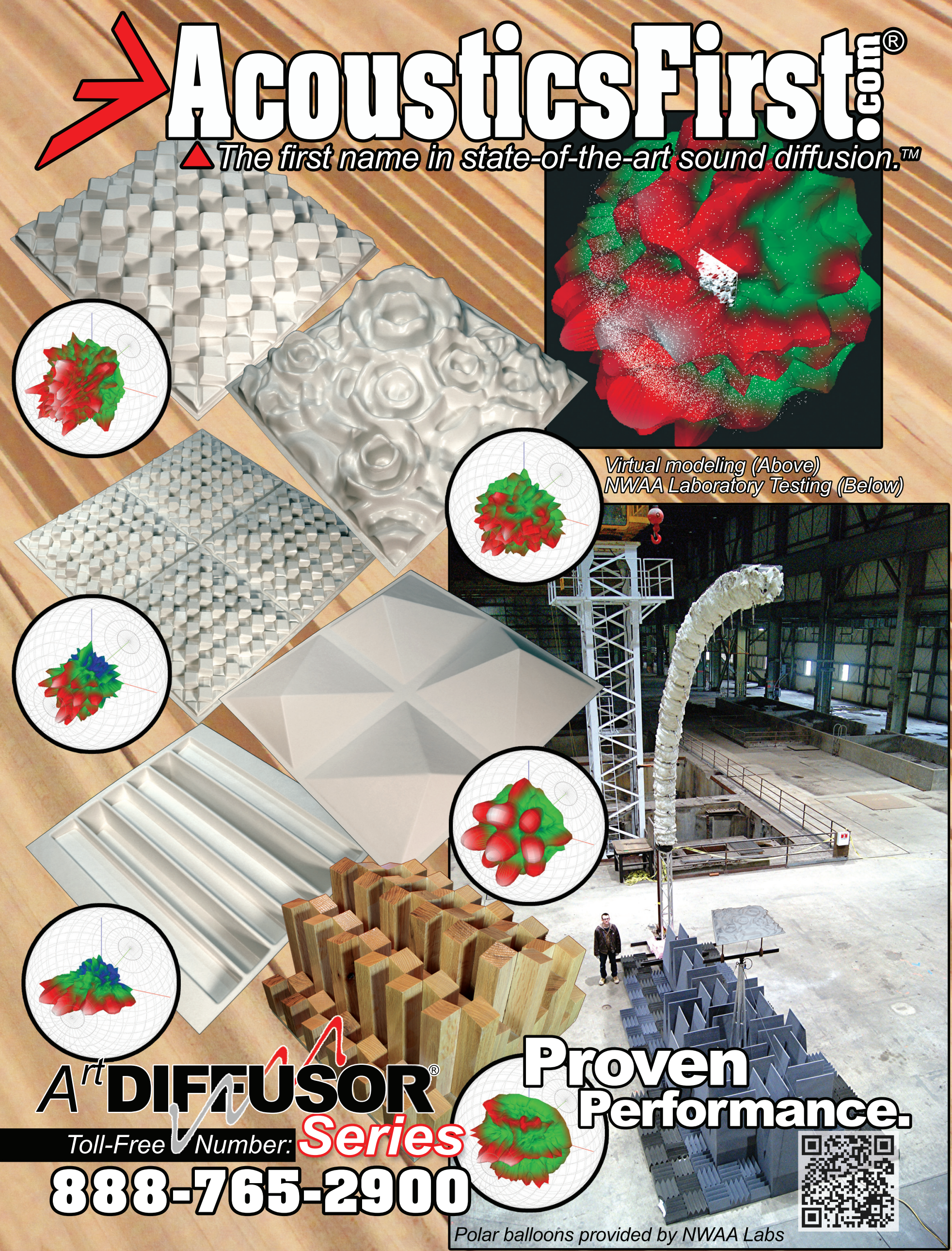


\section{Sound and Vibration Instrumentation Scantek, Inc.}

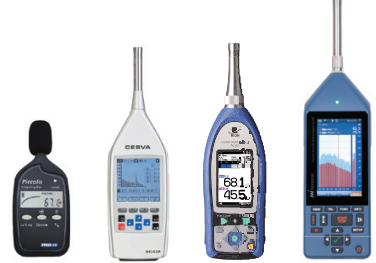

\section{Sound Level Meters}

Selection of sound level meters for simple noise level measurements or advanced acoustical analysis
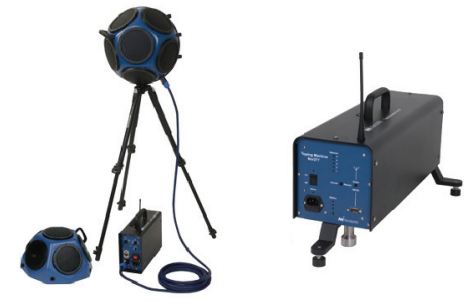

\section{Building Acoustics}

Systems for airborne sound transmission, impact insulation, STIPA, reverberation and other room acoustics measurements

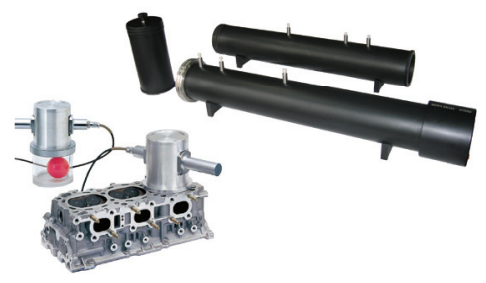

\section{Specialized Test Systems}

Impedance tubes, capacity and volume measurement systems, air-flow resistance measurement devices and calibration systems
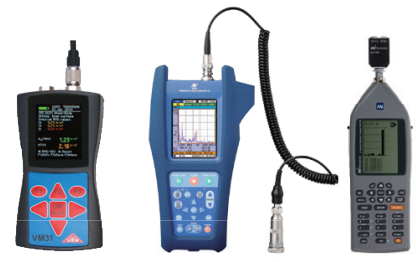

\section{Vibration Meters}

Vibration meters for measuring overall vibration levels, simple to advanced FFT analysis and human exposure to vibration

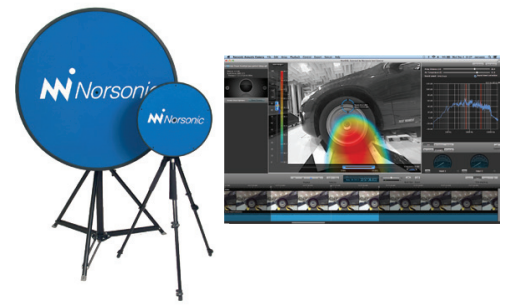

\section{Sound Localization}

Near-field or far-field sound localization and identification using Norsonic's state of the art acoustic camera
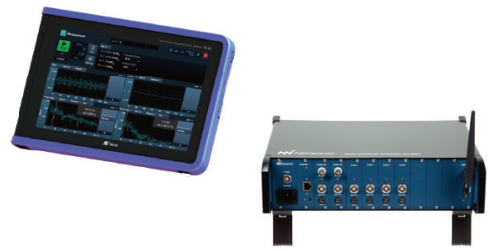

\section{Multi-Channel Systems}

Multi-channel analyzers for sound power, vibration, building acoustics and FFT analysis in the laboratory or in the field

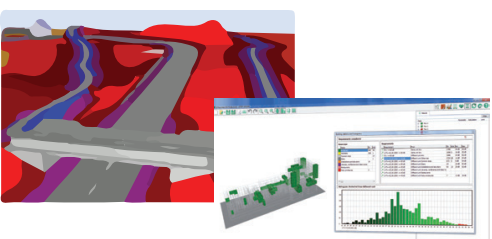

\section{Prediction Software}

Software for prediction of environmental noise, building insulation and room acoustics using the latest standards

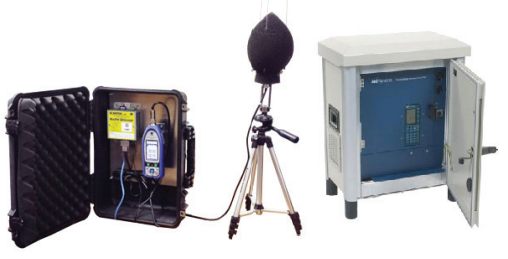

\section{Monitoring}

Temporary or permanent remote monitoring of noise or vibration levels with notifications of exceeded limits
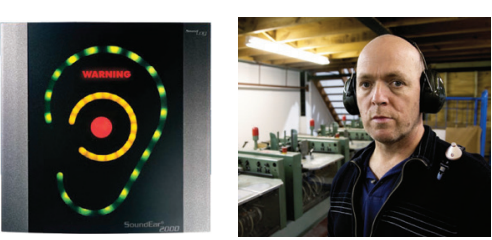

\section{Industrial Hygiene}

Noise alert systems and dosimeters for facility noise monitoring or hearing conservation programs 


\section{INFORMATION REGARDING THE JOURNAL}

Publication of the Journal is jointly financed by the dues of members of the Society, by contributions from Sustaining Members, by nonmember subscriptions, and by publication charges contributed by the authors' institutions. A peer-reviewed archival journal, its actual overall value includes extensive voluntary commitments of time by the Journal 's Associate Editors and reviewers. The Journal has been published continuously since 1929 and is a principal means by which the Acoustical Society seeks to fulfill its stated mission-to increase and diffuse the knowledge of acoustics and to promote its practical applications.

Submission of Manuscripts: Detailed instructions are given in the latest version of the "Information for Contributors" document, which is printed in the January and July issues of the Journal; the most current version can be found online at scitation.aip.org/content/asa/journal/jasa/ info/authors. This document gives explicit instructions regarding the content of the transmittal letter and specifies completed forms that must accompany each submission. All research articles and letters to the editor should be submitted electronically via an online process at the site <http:// jasa.peerx-press.org/>. The uploaded files should include the complete manuscript and the figures. Authors are requested to consult the online listings of JASA Associate Editors and to identify which Associate Editor should handle their manuscript; the decision regarding the acceptability of a manuscript will ordinarily be made by that Associate Editor. The Journal also has special Associate Editors who deal with applied acoustics, education in acoustics, computational acoustics, and mathematical acoustics. Authors may suggest one of these Associate Editors, if doing so is consistent with the content or emphasis of their paper. Review and tutorial articles are ordinarily invited; submission of unsolicited review articles or tutorial articles (other than those which can be construed as papers on education in acoustics) without prior discussion with the Editor-in-Chief is discouraged. Authors are also encouraged to discuss contemplated submissions with appropriate members of the Editorial Board before submission. Submission of papers is open to everyone, and one need not be a member of the Society to submit a paper.

JASA Express Letters: The Journal includes a special section which has a separate submission process than that for the rest of the Journal. Details concerning the nature of this section and information for contributors can be found at the online site http://scitation.aip.org/ content/asa/journal/jasael/info/authors.

Publication Charge: To support the cost of wide dissemination of acoustical information through publication of journal pages and production of a database of articles, the author's institution is requested to pay a page charge of $\$ 80$ per page (with a one-page minimum). Acceptance of a paper for publication is based on its technical merit and not on the acceptance of the page charge. The page charge (if accepted) entitles the author to 100 free reprints. For Errata the minimum page charge is $\$ 10$, with no free reprints. Although regular page charges commonly accepted by authors' institutions are not mandatory for articles that are 12 or fewer pages, payment of the page charges for articles exceeding 12 pages is mandatory. Payment of the publication fee for JASA Express Letters is also mandatory.

Selection of Articles for Publication: All submitted articles are peer reviewed. Responsibility for selection of articles for publication rests with the Associate Editors and with the Editor-in-Chief. Selection is ordinarily based on the following factors: adherence to the stylistic requirements of the Journal, clarity and eloquence of exposition, originality of the contribution, demonstrated understanding of previously published literature pertaining to the subject matter, appropriate discussion of the relationships of the reported research to other current research or applications, appropriateness of the subject matter to the Journal, correctness of the content of the article, completeness of the reporting of results, the reproducibility of the results, and the significance of the contribution. The Journal reserves the right to refuse publication of any submitted article without giving extensively documented reasons. Associate Editors and reviewers are volunteers and, while prompt and rapid processing of submitted manuscripts is of high priority to the Editorial Board and the Society, there is no a priori guarantee that such will be the case for every submission.

Supplemental Material: Authors may submit material that is part supplemental to a paper. Deposits must be in electronic media, and can include text, figures, movies, computer programs, etc. Retrieval instructions are footnoted in the related published paper. Direct requests to the JASA office at jasa@aip.org for additional information, see http://publishing.aip. org/authors.

Role of AIP Publishing: AIP Publishing LLC has been under contract with the Acoustical Society of America (ASA) continuously since 1933 to provide administrative and editorial services. The providing of these services is independent of the fact that the ASA is one of the member societies of AIP Publishing. Services provided in relation to the Journal include production editing, copyediting, composition of the monthly issues of the Journal, and the administration of all financial tasks associated with the Journal. AIP Publishing's administrative services include the billing and collection of nonmember subscriptions, the billing and collection of page charges, and the administration of copyright-related services. In carrying out these services, AIP Publishing acts in accordance with guidelines established by the ASA. All further processing of manuscripts, once they have been selected by the Associate Editors for publication, is handled by AIP Publishing. In the event that a manuscript, in spite of the prior review process, still does not adhere to the stylistic requirements of the Journal, AIP Publishing may notify the authors that processing will be delayed until a suitably revised manuscript is transmitted via the appropriate Associate Editor. If it appears that the nature of the manuscript is such that processing and eventual printing of a manuscript may result in excessive costs, AIP Publishing is authorized to directly bill the authors. Publication of papers is ordinarily delayed until all such charges have been paid.

\section{Copyright 2016, Acoustical Society of America. All rights reserved.}

Copying: Single copies of individual articles may be made for private use or research. Authorization is given to copy articles beyond the free use permitted under Sections 107 and 108 of the U.S. Copyright Law, provided that the copying fee of $\$ 30.00$ per copy per article is paid to the Copyright Clearance Center, 222 Rosewood Drive, Danvers, MA 01923, USA, www.copyright.com. (Note: The ISSN for this journal is 0001-4966.)

Authorization does not extend to systematic or multiple reproduction, to copying for promotional purposes, to electronic storage or distribution, or to republication in any form. In all such cases, specific written permission from AIP Publishing LLC must be obtained.

Note: Copies of individual articles may also be purchased online via scitation.aip.org/content/asa/journal/jasa.

Permission for Other Use: Permission is granted to quote from the Journal with the customary acknowledgment of the source. Republication of an article or portions thereof (e.g., extensive excerpts, figures, tables, etc.) in original form or in translation, as well as other types of reuse (e.g., in course packs) require formal permission from AIP Publishing and may be subject to fees. As a courtesy, the author of the original journal article should be informed of any request for republication/reuse.

Obtaining Permission and Payment of Fees: Using Rightslink ${ }^{\circledR}$ : AIP Publishing has partnered with the Copyright Clearance Center to offer Rightslink, a convenient online service that streamlines the permissions process. Rightslink allows users to instantly obtain permissions and pay any related fees for reuse of copyrighted material, directly from AIP's website. Once licensed, the material may be reused legally, according to the terms and conditions set forth in each unique license agreement.

To use the service, access the article you wish to license on our site and simply click on the Rightslink icon/ "Reprints \& Permissions" link in the abstract. If you have questions about Rightslink, click on the link as described, then click the "Help" button located in the top right-hand corner of the Rightslink page.

Without using Rightslink: Address requests for permission for republication or other reuse of journal articles or portions thereof to: Office of Rights and Permissions, AIP Publishing LLC, 1305 Walt Whitman Road, Suite 300, Melville, NY 117474300, USA; FAX: 516-576-2450; Tel.: 516-576-2268; E-mail: rights@ aip.org 
World Leader in

Noise Control

Solutions

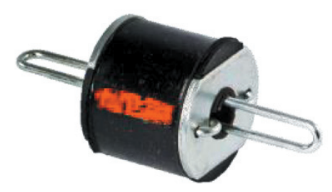

RSIC-WHI

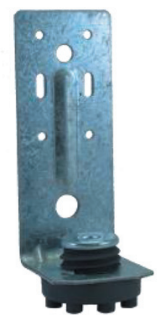

RSIC-DC04

\section{$\underline{\text { RSIC - Products }}$}

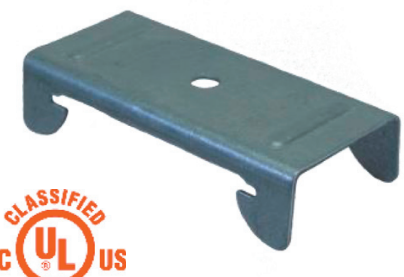

RSIC-V

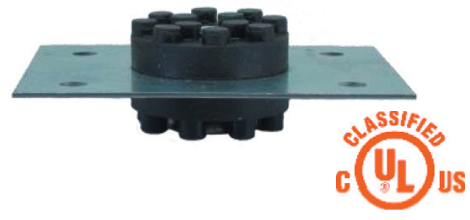

RSIC-U HD
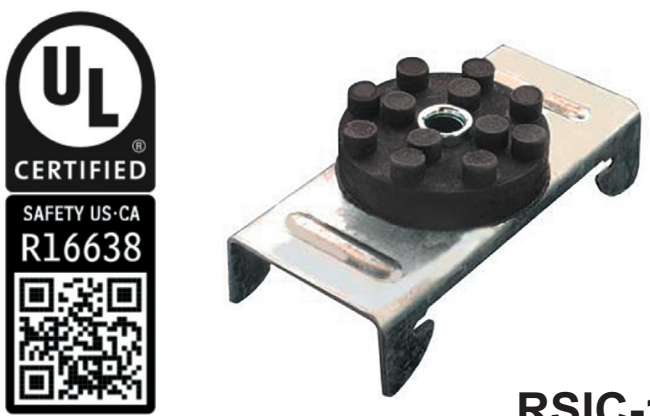

$\underline{\mathrm{RSIC}-1 \otimes}$

The RSIC-1 is the only product ever listed into UL Directory for both Fire and Noise Control.

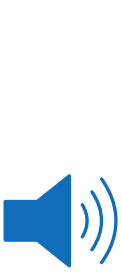

STC: 58
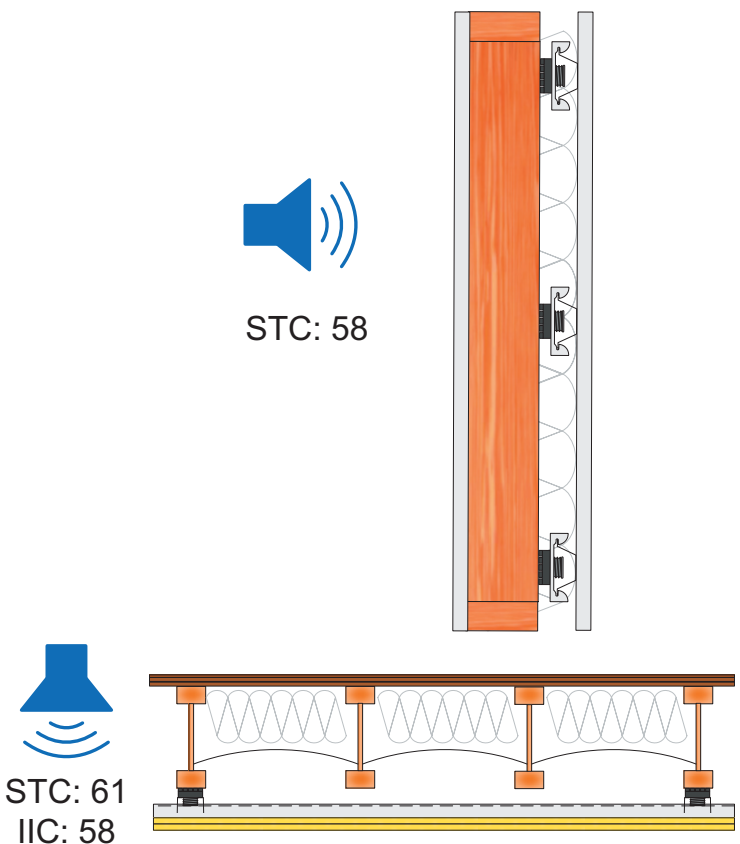

IIC: 58

\section{NEW RC-1 Boost}

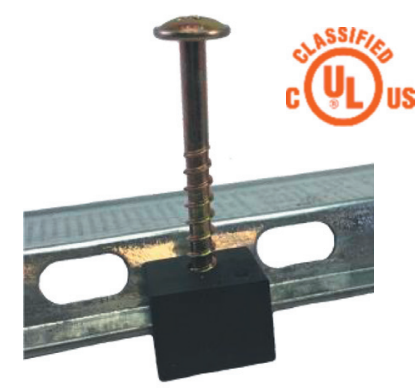

The RC-1 Boost increases the performance of standard RC-1 channel both in IIC and STC. The RC-1 Boost also reduces the chance for a short circuit by moving the channel further away from the framing. The RC-1 Boost is listed in UL.com for use in a one hour floor ceiling assembly. 


\section{Acoustical Society of America Editor-in-Chief: James F. Lynch ASSOCIATE EDITORS OF JASA}

General Linear Acoustics: J.B. Lawrie, Brunel Univ.; A.N. Norris, Rutgers University; A.G. Petculescu, Univ. Louisiana, Lafayette; O. Umnova, Univ. Salford; S.F. Wu, Wayne State Univ.

Nonlinear Acoustics: M. Destrade, Natl. Univ. Ireland, Galway; L. Huang, Univ. of Hong Kong; V.E. Ostashev, Natl. Oceanic and Atmospheric Admin; O.A. Sapozhnikov, Moscow State Univ.

Atmospheric Acoustics and Aeroacoustics: P. Blanc-Benon, Ecole Centrale de Lyon; J.W Posey, NASA Langley Res. Ctr. (ret.); D.K. Wilson, Army Cold Regions Res. Lab.

Underwater Sound: N.P. Chotiros, Univ. of Texas; J.A. Colosi, Naval Postgraduate School; S.E. Dosso, Univ. of Victoria; T.F. Duda, Woods Hole Oceanographic Inst.; B.T. Hefner, Univ. of Washington; A.P. Lyons, Pennsylvania State Univ.; W. Siegmann, Rensselaer Polytech. Inst.; H.C. Song, Scripps Inst. of Oceanography; A.M. Thode, Scripps Inst. of Oceanography

Ultrasonics and Physical Acoustics: M.R. Haberman, Univ. Texas Austin; M.F. Hamilton, Univ. Texas, Austin; V.M. Keppens, Univ. Tennessee, Knoxville; T.G. Leighton, Inst. for Sound and Vibration Res. Southampton; J.D. Maynard, Pennsylvania State Univ.; R. Raspet, Univ. of Mississippi; R.K. Snieder, Colorado School of Mines; J.A. Turner, Univ. of Nebraska-Lincoln; M.D. Verweij, Delft Univ. of Technol.; L. Zhang, Univ. of Mississippi

Transduction, Acoustical Measurements, Instrumentation, Applied Acoustics: M.R. Bai, Natl., Tsinghua Univ.; D.D. Ebenezer, Naval Physical and Oceanographic Lab., India; T.R. Howarth, NAVSEA, Newport; M. Sheplak, Univ. of Florida

Structural Acoustics and Vibration: L. Cheng, Hong Kong Polytechnic Univ.; L.P. Franzoni, Duke Univ.; A.J. Hull, Naval Undersea Warfare Center; N.J. Kessissoglou, UNSW Australia; T. Kundu, Univ. of Arizona; K.M. Li, Purdue Univ.; E.A. Magliula, Naval Undersea Warfare Center; E.G. Williams, Naval Research Lab.

Noise: Its Effects and Control: G. Brambilla, Natl. Center for Research (CNR), Rome; B.S. Cazzolato, Univ. of Adelaide; S. Fidell, Fidell Assoc.; K.V. Horoshenkov, Univ. of Bradford; R.M. Kirby, Brunel Univ.; B. SchulteFortkamp, Technical Univ. of Berlin

Architectural Acoustics: B.F.G. Katz, Computer Science Lab. for Mechanics and Engineering Sciences (LIMSI); F. Sgard, Quebec Occupational Health and Safety Res. Ctr.; J.E. Summers, Appl. Res. Acoust., Washington; M. Vorländer, Univ. Aachen

Acoustic Signal Processing: J. Li, Zhejiang Univ.; Z-H. Michalopoulou, New Jersey Inst. Technol.; K.G. Sabra, Georgia Inst. Tech; K. Wong, Hong Kong Polytech. Univ.
Physiological Acoustics: C. Abdala, House Research Inst.; I.C. Bruce, McMaster Univ.; K. Grosh, Univ. of Michigan; A.K.C. Lee, Univ. of Washington, C.A. Shera, Harvard Medical School; G.C. Stecker, Vanderbilt Univ.

Psychological Acoustics: L.R. Bernstein, Univ. Conn.; V. Best, Natl. Acoust. Lab., Australia; J. Braasch, Rensselaer Polytech. Inst.; V.M. Richards, Univ. California, Irvine; M.A. Stone, Univ. of Cambridge

Speech Production: L.L. Koenig, Long Island Univ. and Haskins Labs.; Z. Zhang, Univ. of California, Los Angeles

Speech Perception: D. Baskent, Univ. Medical Center, Groningen; T. Bent, Indiana Univ.; C.G. Clopper, Ohio State Univ.; S.H. Ferguson, Univ. of Utah; M.S. Sommers, Washington Univ.; B.V. Tucker, Univ. of Alberta

Speech Processing: C.Y. Espy-Wilson, Univ. of Maryland; College Park; M.A. Hasegawa-Johnson, Univ. of Illinois; S.S. Narayanan, Univ. of Southern California

Musical Acoustics: D. Deutsch, Univ. of California, San Diego; T.R. Moore, Rollins College; A. Morrison, Joliet Junior College; J. Wolfe, Univ. of New South Wales

Bioacoustics: C.C. Church, Univ. of Mississippi; R.R. Fay, Loyola Univ., Chicago; G. Haïat, Natl. Ctr. for Scientific Res. (CNRS); D.K. Mellinger, Oregon State Univ.; D.L. Miller, Univ. of Michigan; T.J. Royston, Univ. Illinois, Chicago; K.A. Wear, Food and Drug Admin; S.W. Yoon, Sungkyunkwan Univ.

Animal Bioacoustics: W.W.L. Au, Hawaii Inst. of Marine Biology; M.L. Dent, Univ. at Buffalo; J.J. Finneran, Navy Marine Mammal Program; C.F. Moss, Univ. of Maryland; A.N. Popper, Univ. Maryland; A.M. Simmons, Brown Univ.; J.A. Sisneros, Univ. of Washington; C.E. Taylor, UCLA Ecology and Evol. Biology

Computational Acoustics: D.S. Burnett, Naval Surface Warfare Ctr., Panama City; N.A. Gumerov, Univ. of Maryland; L.L. Thompson, Clemson Univ.

Education in Acoustics: B.E. Anderson, Los Alamos National Lab.; V.W. Sparrow, Pennsylvania State Univ.; P.S. Wilson, Univ. of Texas at Austin

Reviews and Tutorials: J.F. Lynch, Woods Hole Oceanographic Inst.

Forum and Technical Notes: N. Xiang, Rensselaer Polytechnic Univ.

Acoustical News: E. Moran, Acoustical Society of America

Standards News, Standards: N. Stremmel, Acoustical Society of America; C. Struck, CJS Labs

Book Reviews: P.L. Marston, Washington State Univ.

Patent Reviews: S.A. Fulop, California State Univ., Fresno; D.L. Rice, Computalker Consultants (ret.)

\section{ASSOCIATE EDITORS OF JASA EXPRESS LETTERS}

Editor: C.C. Church, Univ. Mississippi

General Linear Acoustics: O.A. Godin, NOAA-Earth System Research Laboratory; S.F. Wu, Wayne State Univ.

Nonlinear Acoustics: M.F. Hamilton, Univ. of Texas at Austin

Aeroacoustics and Atmospheric Sound: V.E. Ostashev, Natl. Oceanic and Atmospheric Admin.

Underwater Sound: P.E. Barbone, Boston Univ.; D. Barclay, Dalhousie Univ.; A.J.M. Davis, Univ. California, San Diego; D.R. Dowling, Univ. of Michigan; W.L. Siegmann, Rensselaer Polytechnic Institute

Ultrasonics, Quantum Acoustics, and Physical Effects of Sound: T.D. Mast, Univ of Cincinatti; J.S. Mobley, Univ. of Mississippi

Transduction: Acoustical Devices for the Generation and Reproduction of Sound; Acoustical Measurements and Instrumentation: M.D. Sheplak, Univ. of Florida

Structural Acoustics and Vibration: J.G. McDaniel, Boston Univ.

Noise: S.K. Lau, National Univ. of Singapore

Architectural Acoustics: N. Xiang, Rensselaer Polytechnic Inst.
Acoustic Signal Processing: D.H. Chambers, Lawrence Livermore Natl. Lab.; C.F. Gaumond, Naval Research Lab.

Physiological Acoustics: B.L. Lonsbury-Martin, Loma Linda VA Medical Ctr. Psychological Acoustics: Q.-J. Fu, House Ear Inst.

Speech Production: A. Lofqvist, Univ. Hospital, Lund, Sweden

Speech Perception: M. Cooke, Univ. of the Basque Country; R. Smiljanic, Univ. of Texas at Austin

Speech Processing and Communication Systems and Speech Perception: D.D. O'Shaughnessy, INRS-Telecommunications

Music and Musical Instruments: D.M. Campbell, Univ. of Edinburgh; D. Deutsch, Univ. of California, San Diego; T.R. Moore, Rollins College

Bioacoustics-Biomedical: C.C. Church, Natl. Ctr. for Physical Acoustics Bioacoustics-Animal: C.F. Moss, Univ. of Maryland

Computational Acoustics: D.S. Burnett, Naval Surface Warfare Ctr., Panama City; L.L. Thompson, Clemson Univ. 


\section{CONTENTS}

$\begin{array}{rr}\text { page } & \text { pas }\end{array}$

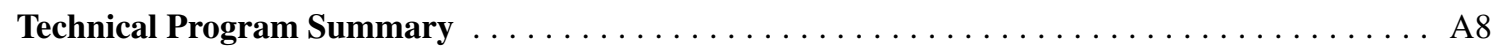

Schedule of Technical Session Starting Times $\ldots \ldots \ldots \ldots \ldots \ldots \ldots \ldots \ldots \ldots \ldots$

Map of Meeting Rooms at the Hilton Hawaiian Village $\ldots \ldots \ldots \ldots \ldots \ldots \ldots \ldots \ldots$

Calendar-Technical Program $\ldots \ldots \ldots \ldots \ldots \ldots \ldots \ldots \ldots \ldots \ldots \ldots \ldots \ldots \ldots \ldots$

Calendar-Other Events $\ldots \ldots \ldots \ldots \ldots \ldots \ldots \ldots \ldots \ldots \ldots \ldots \ldots \ldots \ldots \ldots \ldots \ldots \ldots \ldots$

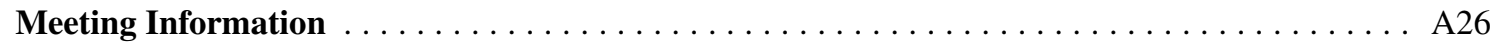

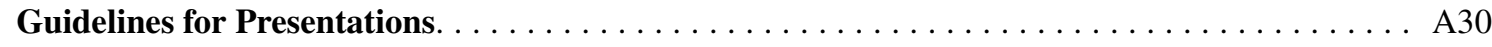

Dates of Future Meetings $\ldots \ldots \ldots \ldots \ldots \ldots \ldots \ldots \ldots \ldots \ldots \ldots \ldots \ldots \ldots \ldots$

Technical Sessions (1a__), Monday Morning. . . . . . . . . . . . . . . . . . . . . . . . . . . . . . 2945

Technical Sessions (1p_, Monday Afternoon . . . . . . . . . . . . . . . . . . . . . . . . 2969

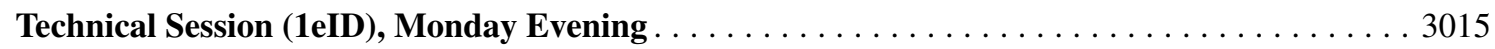

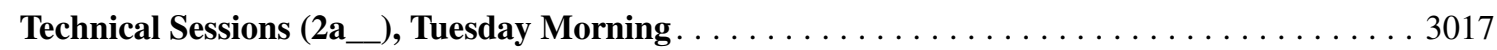

Technical Sessions $\left(2 \mathbf{p}_{\_}\right)$, Tuesday Afternoon $\ldots \ldots \ldots \ldots \ldots \ldots \ldots$

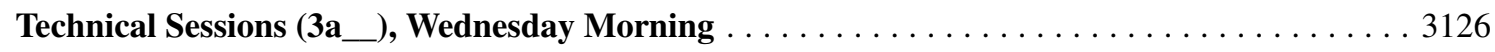

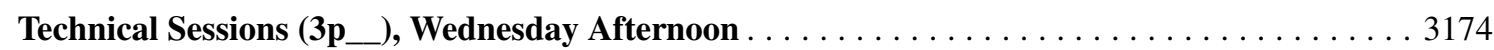

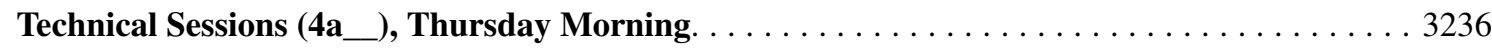

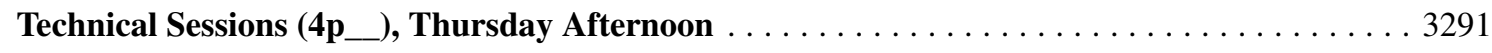

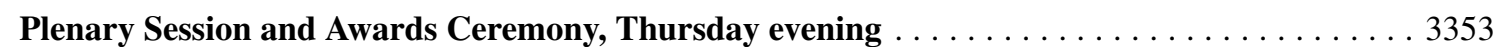

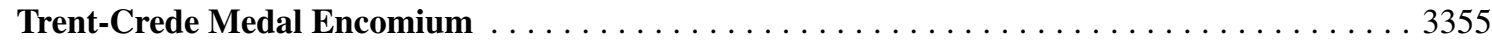

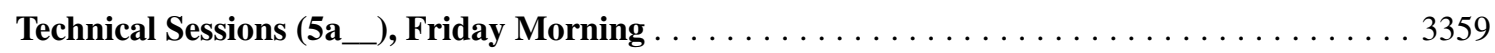

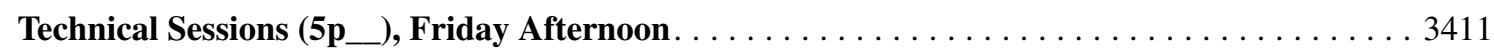

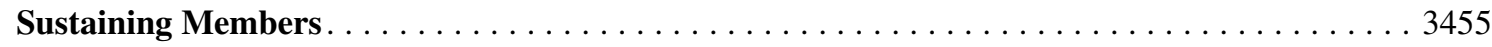

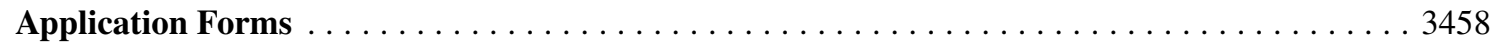

Regional Chapters $\ldots \ldots \ldots \ldots \ldots \ldots \ldots \ldots \ldots \ldots \ldots \ldots \ldots \ldots \ldots$

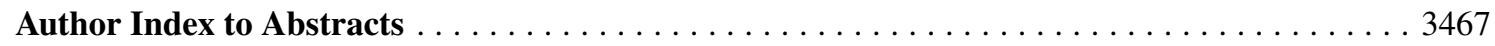


The Acoustical Society of America was founded in 1929 to increase and diffuse the knowledge of acoustics and promote its practical applications. Any person or corporation interested in acoustics is eligible for membership in this Society. Further information concerning membership, together with application forms, may be obtained by addressing Elaine Moran, ASA Office Manager, 1305 Walt Whitman Road, Suite 300, Melville, NY 11747-4300, T: 516-576-2360, F: 631-923-2875; E-mail: elaine@acousticalsociety.org; Web: http://acousticalsociety.org

\section{Officers 2016-2017}

Michael R. Stinson, President Institute for Microstructural Science Nationat National Research Council of Cana Ottawa, ON K1A 0R6, Canada mike.stinson@nrc-cnrc.gc.ca

Marcia J. Isakson, President-Elect Department of Mechanical Engineering University of Texas at Austin Austin, TX 78712-1591 misakson@arlut.utexas.edu
Ronald A. Roy, Vice President

Department of Engineering Science

University of Oxford

Oxford, OX1 3PJ, UK

ronald.roy@ hmc.ox.ac.uk

Michael J. Buckingham, Vice President-Elect Scripps Institution of Oceanography

9500 Gilman Drive, \#0238

La Jolla, CA 92093-0238

mbuckingham@ucsd.edu

David Feit, Treasurer

Acoustical Society of America

1305 Walt Whitman Road, Suite 300

Melville, NY 11747-4300

(516) $576-2360$

dfeit@aip.org

\author{
James F. Lynch, Editor-in-Chief \\ Acoustical Society of America \\ Publications Office \\ P.O. Box 274 \\ West Barnstable, MA 02668 \\ (508) $362-1200$ \\ jasaeditor@acousticalsociety.org \\ Christopher J. Struck, Standards Director \\ CJS Labs. \\ 57 States Street \\ San Francisco, CA 94114-1401 \\ (415) 923-9535 \\ cjs@cjs-labs.com \\ Susan E. Fox, Executive Director \\ Acoustical Society of America \\ 1305 Walt Whitman Road, Suite 300 \\ Melville, NY 11747-4300 \\ (516) 576-2360 \\ sfox@acousticalsociety.org
}

\section{Members of the Executive Council}

Christy K. Holland
University of Cincinnati
231 Albert Sabin Way
Cincinnati, OH 45267-0586
christy.holland@ @uc.edu
Lily M. Wang
Durham School of Architectural
Engineering and Construction
University of Nebraska-Lincoln
Omaha, NE 68182-0816
Iwang4@ @nl.edu
Michael R. Bailey
Applied Physics Laboratory
Center for Industrial and Medical
Ultrasound
1013 N.E. 40th St.
Seattle, WA 98105
(206) 685-8618
bailey @apl.washington.edu

Tessa C. Bent
Indiana University
200 S. Jordan Avenue
Bloomington, IN 47405
tbent@ indiana.edu
Preston S. Wilson
Department of Mechanical Engineering
University of Texas at Austin
Austin, TX 78712-1591
pswilson@ mail.utexas.edu

\author{
Members of the Technical Council \\ R.A. Roy, Vice President \\ M.J. Buckingham, Vice President-Elect \\ L.M. Wang, Past Vice President \\ J.A. Colosi, Acoustical Oceanography \\ C. Erbe, Animal Bioacoustics \\ E.E. Ryherd, Architectural Acoustics \\ N. McDannold, Biomedical Acoustics \\ K.M. Walsh, Engineering Acoustics \\ A.C.H. Morrison, Musical Acoustics \\ W.J. Murphy, Jr., Noise \\ J.R. Gladden, Physical Acoustics \\ M. Wojtczak, Psychological and Physiological Acoustics \\ P.J. Gendron, Signal Processing in Acoustics \\ L. Polka, Speech Communication \\ M.S. Ballard, Underwater Acoustics
}

\section{Organizing Committee}

ASA

Whitlow W.L. Au, Chair

David L. Adams, Cochair

Bruce M. Howe, Technical Program Chair

Gary McAuliffe, Audio/Visual

Gavid L. Adams/Robert Badham, Food/Beverage

Benjamin Reeder, Signs

Benjamin Reeder, Sig
Aude Pacini, Posters

Dorothy Au, Accompanying Persons

Jessica Chen, Student Coordinator

Robert Badham, Meeting Administrator

ASJ

Yoshiaki Watanabe, Cochair

Akio Ando, Cochair

Hiroshi Sato, Vice Chair

Hiroshi Saruwatari, Technical Program Chair

Yasuhiro Oikawa, Secretary

\section{Subscription Prices, 2016}

\begin{tabular}{lrr} 
& U.S.A. \& Poss. & Outside the U.S.A. \\
\hline ASA Members & \multicolumn{2}{c}{ (on membership) } \\
Institutions (print + online) & $\$ 2225.00$ & $\$ 2390.00$ \\
Institutions (online only) & $\$ 2000.00$ & $\$ 2000.00$ \\
\hline
\end{tabular}

The Journal of the Acoustical Society of America (ISSN: 0001-4966) is published monthly by the Acoustical Society of America through the AIP Publishing LLC. POSTMASTER: Send address changes to The Journal of the Acoustical Society of America, 1305 Walt Whitman Road, Suite 300, Melville, NY 117474300. Periodicals postage paid at Huntington Station, NY 11746 and additional mailing offices.

Editions: The Journal of the Acoustical Society of America is published simultaneously in print and online. Journal articles are available online from Volume 1 (1929) to the present at http://asadl.org.

Back Numbers: All back issues of the Journal are available online. Some, but not all, print issues are also available. Prices will be supplied upon request to Elaine Moran, ASA Office Manager, 1305 Walt Whitman Road, Suite 300,
Melville, NY 11747-4300. Telephone: (516) 576-2360; FAX: (631) 923-2875; E-mail: elaine@ acousticalsociety.org.

Subscription, renewals, and address changes should be addressed to AIP Publishing LLC - FMS, 1305 Walt Whitman Road, Suite 300, Melville, NY 117474300. Allow at least six weeks advance notice. For address changes please send both old and new addresses and, if possible, your ASA account number.

Claims, Single Copy Replacement and Back Volumes: Missing issue requests will be honored only if received within six months of publication date (nine months for Australia and Asia). Single copies of a journal may be ordered and back volumes are available. Members-contact AIP Publishing Member Services at (516) 576-2288; (800) 344-6901, membership @ aip.org. Nonmember subscribers-contact AIP Publishing Subscriber Services at (516) 576-2270; (800) 344-6902; E-mail: subs@ @aip.org.

Page Charge and Reprint Billing: Contact: AIP Publishing Publication Page Charge and Reprints-CFD, 1305 Walt Whitman Road, Suite 300, Melville, NY 11747-4300; (516) 576-2234; (800) 344-6909; E-mail: prc@ aip.org.

Document Delivery: Copies of journal articles can be purchased for immediate download at www.asadl.org. 


\section{Acoustics "17 Boston}

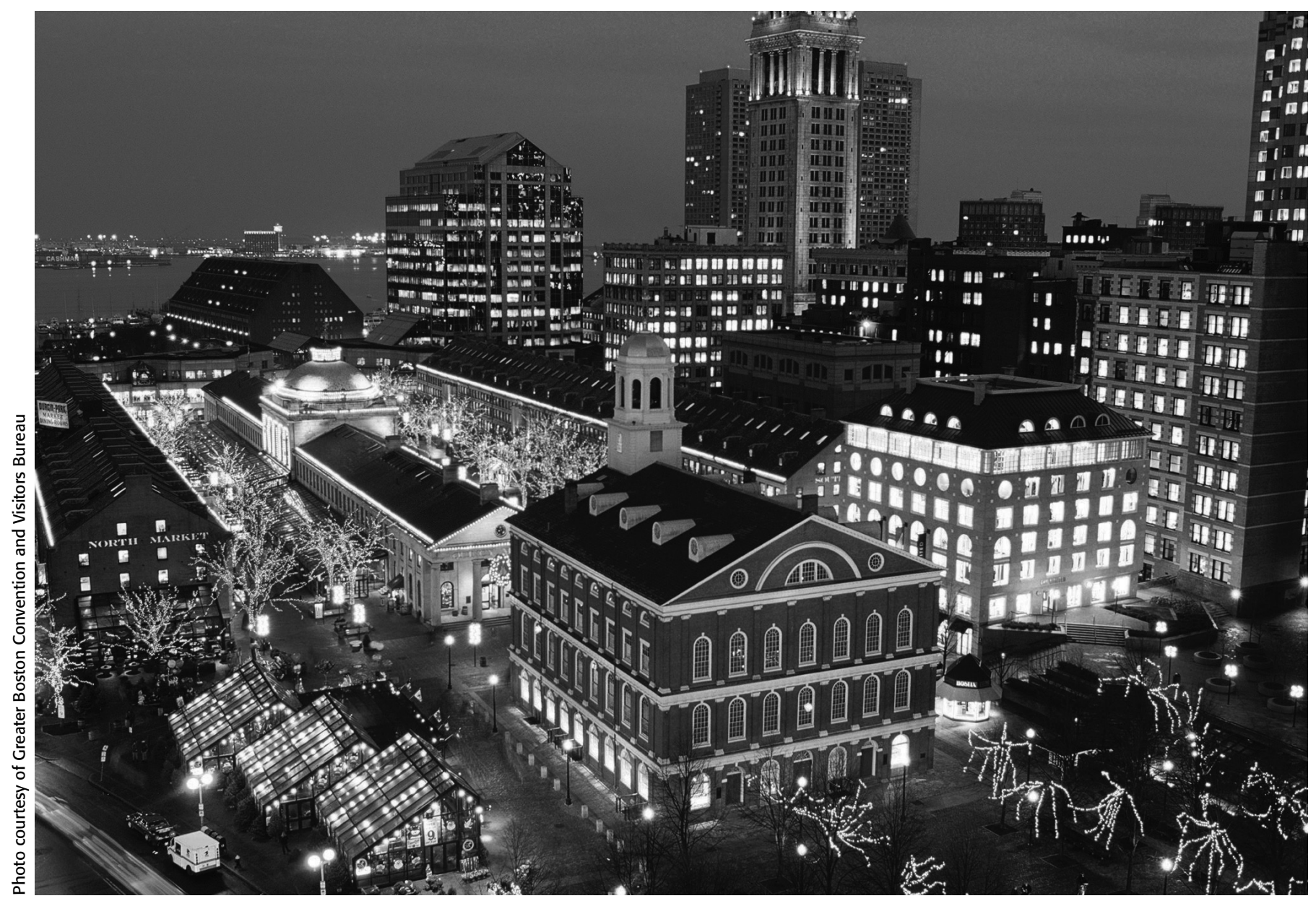

25-29 June 2017 (Sunday-Thursday)

Joint Meeting of the Acoustical Society
of America European Acoustics Association
The Acoustical Society of America (ASA) and the European Acoustics Association (EAA) invite acousticians from around the world to participate in Acoustics '17

Boston to be held 25-29 June 2017 in Boston, Massachusetts, USA. A broad range of topics in acoustics will be covered in technical sessions and keynote lectures. Presentations on emerging topics are especially encouraged. Social events, student events, and an accompanying persons program will be organized. The best features of meetings of both organizations will be combined to offer a premier venue for presenting your work to an international audience.

Boston is the capitol and largest city in Massachusetts and is one of the oldest cities in the United States. It is on the Atlantic coast and is home to many historic sites dating back to the American Revolution, in addition to many other cultural and recreational features. The climate in June is very pleasant and ideal for arranging visits before and after the meeting.

Please join us! 


\section{TECHNICAL PROGRAM SUMMARY \\ *Indicates Special Session}

\section{Monday morning}

*1aAA Acoustics for Children and Pupils I

*1aAB BatEcholocation: New Insights on Biosonar Production, Processing, and Performance from Field and Laboratory Investigations I

1aAO Topics in Acoustical Oceanography I

*1aBA Photoacoustics: Light and Sound I

* $1 \mathrm{aED} \quad$ Attractive Educational Methods and Tools in Acoustics I

1aID Opening Ceremonies, Plenary Lectures

*1aNS Sound Design I

1aPA Thermoacoustics

*1aPP Spatial Hearing and Its Applications I

1aSA General Topics in Structural Acoustics and Vibration I (Poster Session)

1aSC Speech Technology: Synthesis and Interfaces (Poster Session)

1aSP General Topics in Signal Processing I

1aUW Target Scattering

\section{Monday afternoon}

*1pAAa Acoustics for Children and Pupils II

*1pAAb Acoustical Design Metric Comparison Between the US and Japan

*1pAB Bat Echolocation: New Insights on Biosonar Production, Processing, and Performance from Field and Laboratory Investigations II

*1pAO Twenty-Five Years of Acoustical Oceanography in the ASA I

*1pBAa Photoacoustics: Light and Sound II

$1 \mathrm{pBAb}$ Cavitation in Therapeutic Ultrasound I: General

*1pED Attractive Educational Methods and Tools in Acoustics II

*1pMU Music and Sound in Multimedia

*1pNSa Sound Design II

* $1 \mathrm{pNSb}$ Acoustics of Microperforated Materials

*1pPA Advances in Hazards Monitoring with Distributed Infrasound Sensor Networks

*1pPP Spatial Hearing and Its Applications II

1pSA General Topics in Structural Acoustics and Vibration II

*1pSC Speech Robotics

*1pSPa Time Delay Estimation of Acoustic Signals with Applications

$* 1 \mathrm{pSPb} \quad$ Spoken Document Processing

*1pUW Underwater Acoustics Studies in Asian Seas I

\section{Monday evening}

1eMU Special Presentation of the History, Culture, Practice, and Performance of Hawai'ian Music

\section{Tuesday morning}

*2aABa Tropical Bioacoustics I

$* 2 \mathrm{aABb}$ Anthropogenic Transient Noise Sound Field and Its Effects on Animal Communication I

*2aAO Twenty-Five Years of Acoustical Oceanography in the ASA II

2aBAa Cavitation in Therapeutic Ultrasound II: General

$* 2 \mathrm{aBAb}$ High-Frame Rate Imaging with Plane Waves and Diverging Waves

2aBAc Cavitation in Therapeutic Ultrasound III: Tissue Fractionation

2aEA Transducer Systems

*2aMUa Computational Methods of Simulation for Musical Acoustics

*2aMUb Music Signal Processing I

*2aNS Noise Impacts and Soundscapes at Outdoor Gathering Spaces

*2aPA Acoustics of Supersonic Jets: Launch Vehicle and Military Jet Acoustics I

*2aPP Linking Human Physiology with Psychoacoustics-From Brainstem to Cortical Measurements and Beyond

*2aSA Acoustic Metamaterials I

*2aSC Cross-Linguistic Speech Production and Perception

*2aSPa Compressive Sensing in Acoustics I

$2 \mathrm{aSPb}$ General Topics in Signal Processing IV (Poster Session)

*2aUW Underwater Acoustic Studies in Asian Seas II

\section{Tuesday afternoon}

*2pAA At the Intersection of Speech and Architecture I

*2pABa Tropical Bioacoustics II

$* 2 \mathrm{pABb}$ Anthropogenic Transient Noise Sound Field and Its Effects on Animal Communication II
*2pAOa Ocean Acoustic Tomography: Active and Passive, Theory and Experiment I

*2pAOb Acoustical Oceanography Prize Lecture

*2pBAa Bone Sonometry I: Ultrasonic Studies of the Physical Properties of Cortical and Cancellous Bone

$2 \mathrm{pBAb}$ Cavitation in Therapeutic Ultrasound IV: Tissue Fractionation

*2pEAa Acoustic Transduction Material, Sensors, and Array Technologies

$2 \mathrm{pEAb}$ Ocean Acoustics Analysis

*2pMU Music Signal Processing II

*2pNS Community Response to Transportation Noises

*2pPA Acoustics of Supersonic Jets: Launch Vehicle and Military Jet Acoustics II

2pPP Binaural Processing and Localization

*2pSA Acoustic Metamaterials II

2pSC Articulatory and Acoustic Phonetics: Data and Methods (Poster Session)

*2pSPa Compressive Sensing in Acoustics II

$* 2 \mathrm{pSPb}$ Detection, Tracking, and Classification of Unmanned Aircraft and Underwater Vehicles

*2pUW Nonlinear Effects in Underwater Acoustics and Bubbles

\section{Wednesday morning}

*3aAAa At the Intersection of Speech and Architecture II

*3aAAb Advanced Analysis, Simulation, and Auralization in Room Acoustics I

*3aAB Session in Honor of Whitlow Au I

*3aAO Ocean Acoustic Tomography: Active and Passive, Theory and Experiment II

*3aBA Quantitative Ultrasound I: From Micro to In-vivo

3aEA Acoustic Analysis

*3aMU East and Western Instruments: Contrasts and Connections

*3aNS Current Issues in Hearing Protection and Hearing Conservation I

*3aPA Acoustic Micro- and Nanofluidics I

*3aPPa Perspectives of Research in Overlooked Hearing Problems

$3 \mathrm{aPPb}$ From Cochlea to Cortex (Poster Session)

*3aSA Dynamics of Ribbed Structures

*3aSC Double-Weak Theory of Speech Production and Perception: A Session in Honor of Terrance Nearey

*3aSP Signal Processing in Nonlinear Acoustics

*3aUWa Transmission Through the Air-Water Interface

3aUWb Topics in Underwater Acoustics (Poster Session)

3aUWc Inversion, Beam-Forming, and Calibration I

\section{Wednesday afternoon}

*3pAAa Advanced Analysis, Simulation, and Auralization in Room Acoustics II

3pAAb Advanced Analysis, Simulation, and Auralization in Room Acoustics III (Poster Session)

3pAAc Assorted Topics in Architectural Acoustics (Poster Session)

*3pABa Session in Honor of Whitlow Au II

$3 \mathrm{pABb}$ On the Bleeding Edge of Animal Bioacoustics Technology

*3pAO Ocean Acoustic Tomography: Active and Passive, Theory and Experiment III

*3pBAa Quantitative Ultrasound II: From Micro to In-vivo

*3pBAb Bone Sonometry II: Ultrasonic Studies of the Physical Properties of Cortical and Cancellous Bone (Poster Session)

*3pBAc Bone Sonometry III: Ultrasonic Studies of the Physical Properties of Cortical and Cancellous Bone

*3pEA Outdoor Sound Propagation and Outdoor Public Address

*3pEDa Demonstrations and Tools in Acoustics Education

*3pEDb Education in Acoustics for Kids (K-12 students)

*3pNSa Current Issues in Hearing Protection and Hearing Conservation II

$* 3 \mathrm{pNSb}$ Ambient Listening, Communication and Noise Reduction of Special Hearing Protectors

*3pPAa Solid State Acoustics

*3pPAb Acoustic Micro- and Nanofluidics II

*3pPP Recent Progress in Auditory Perceptual Organization Studies

*3pSA Non-Contact Vibration Excitation and Sensing Techniques

3pSC Speech Production and Perception (Poster Session) 
*3pSP Acoustic Communications

3pUWa Inversion, Beam-forming, and Calibration II

$3 p U W b \quad$ Seabed and Sea Surface Interactions

\section{Thursday morning}

4aAA Assorted Topics in Architectural Acoustics I

* 4aAB Sequence Information in Mammalian Vocal Call Production I

* 4aAO Acoustic Scattering by Aquatic Organisms I

* 4aBAa Session in Honor of Floyd Dunn

* 4aBAb Medical Acoustics in Kidney and Liver Disease I

4aEA Simulation and Analysis

*4aID Workshop for Publishing Excellence in JASA

*4aMUa Flute Acoustics Including All "Air-Jet" Instruments

*4aMUb Piano Acoustics and Playing Piano I

*4aNS Prediction of Transportation Noises and Other Topics I

4aPA General Topics in Physical Acoustics

4aPPa Psychological and Physiological Acoustics Potpourri (Poster Session)

$4 \mathrm{aPPb} \quad$ Modelling, Measurement, and Technology (Poster Session)

*4aSA Building Vibration Analysis, Measurement, and Mitigation

*4aSC Second Language Speech Learning and Education

4aSPa Exploitation of Doppler in Acoustic Signal Processing

$* 4 \mathrm{aSPb}$ High Resolution Imaging Sonars Including Real Aperture, Synthetic Aperture, and Tomographic Sonars I

*4aUW Harbor Acoustics I

\section{Thursday afternoon}

4pAAa Assorted Topics in Architectural Acoustics II

*4pAAb It's All About the Details

*4pABa Sequence Information in Mammalian Vocal Call Production II

$* 4 \mathrm{pABb}$ Session in Honor of Whitlow Au III

$4 \mathrm{pABc}$ Animal Bioacoustics Poster Session

*4pAO Acoustic Scattering by Aquatic Organisms II

*4pBA Medical Acoustics in Kidney and Liver Disease II

*4pEA Sound Field Control Techniques

*4pEDa Using Computers for Acoustics Education

*4pEDb Acoustics Education Prize Lecture

*4pMUa Piano Acoustics and Playing Piano II

*4pMUb Musical Instruments from Islands Around the World I

*4pMUc Musical Instruments from Islands around the World II-A Concert

$* 4$ pNSa Prediction of Transportation Noises and Other Topics II

*4pNSb Low Frequency Noise: Measurement, Evaluation, Enforcement, and Policy

*4pPA Nonclassical Nonlinear Acoustics of Solids
*4pPP Gap Detection: New Perspectives from Neuroscience, Perception and Modelling

*4pSA Fluid Flow Induced Vibration and Noise

4pSC Non-Native Speech Production and Perception, Learning, and Adaptation (Poster Session)

*4pSPa High Resolution Imaging Sonars Including Real Aperture, Synthetic Aperture, and Tomographic Sonars II

$* 4 \mathrm{pSPb}$ High Resolution Imaging Sonars Including Real Aperture, Synthetic Aperture, and Tomographic Sonars III

*4pUWa Harbor Acoustics II

$4 \mathrm{pUWb}$ Ocean Ambient Noise: Sources, Variation, and Characterization I

Friday morning

$5 \mathrm{aAB} \quad$ Marine Acoustic Ecology

5aAO Topics in Acoustical Oceanography II

*5aBAa Acoustics for Older Persons with Disabilities

$5 \mathrm{aBAb}$ Biomedical Acoustics Poster Session

5aEA Applied Acoustics I

*5aID Topical Meeting on Data Science and Acoustics I

5aMU General Topics in Musical Acoustics I

*5aNSa Wind Turbine Noise

$* 5 \mathrm{aNSb}$ Innovations in Floor Impact Noise Testing and Evaluation

5aPA Ultrasonics and Non-Destructive Testing

5aPP Pitch and Timbre

5aSC Voice, Tone, Prosody, and Affective and Emotional Qualities of Speech (Poster Session)

5aSP General Topics in Signal Processing II

5aUWa Underwater Acoustic Propagation

$5 \mathrm{aUWb}$ Ocean Ambient Noise: Sources, Variation, and Characterization II

\section{Friday afternoon}

*5pABa Behavioral Response Studies

$5 \mathrm{pABb}$ The Diversity of Bioacoustic Signals

*5pBA Acoustic Imaging

5pEA Applied Acoustics II

*5pID Topical Meeting on Data Science and Acoustics II

5pMU General Topics in Musical Acoustics II (Poster Session)

*5pNSa Design of Noise Control Materials

* 5pNSb Emerging Issues on Quiet Vehicles-A Matter of Soundscape

5pPA Radiation Force and Nonlinear Acoustics

5 pSCa Speech Processing

$5 \mathrm{pSCb}$ Speech Perception and Production by Clinical, Aging, and/or Developing Populations (Poster Session)

$5 \mathrm{pSP} \quad$ General Topics in Signal Processing III 


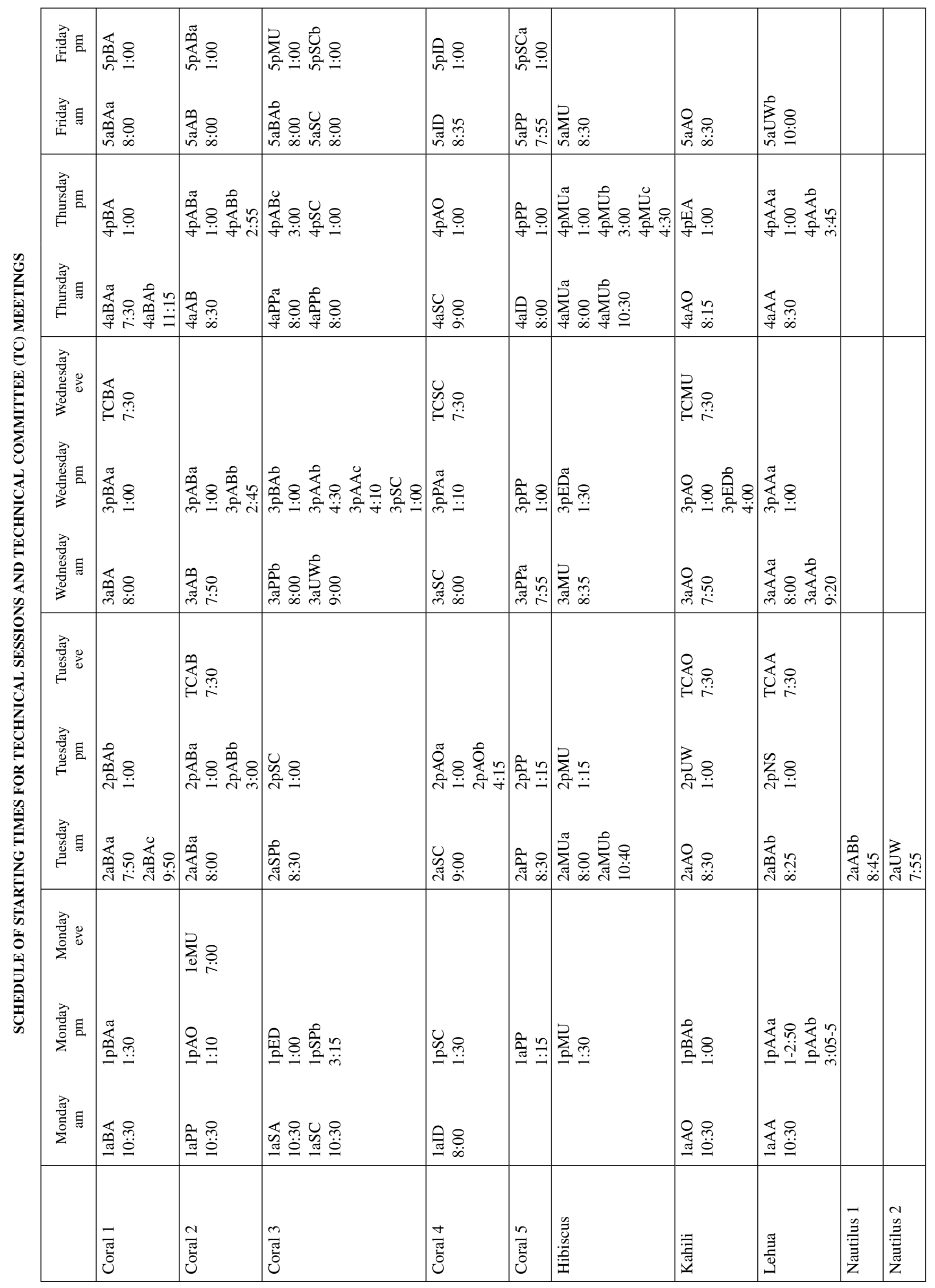




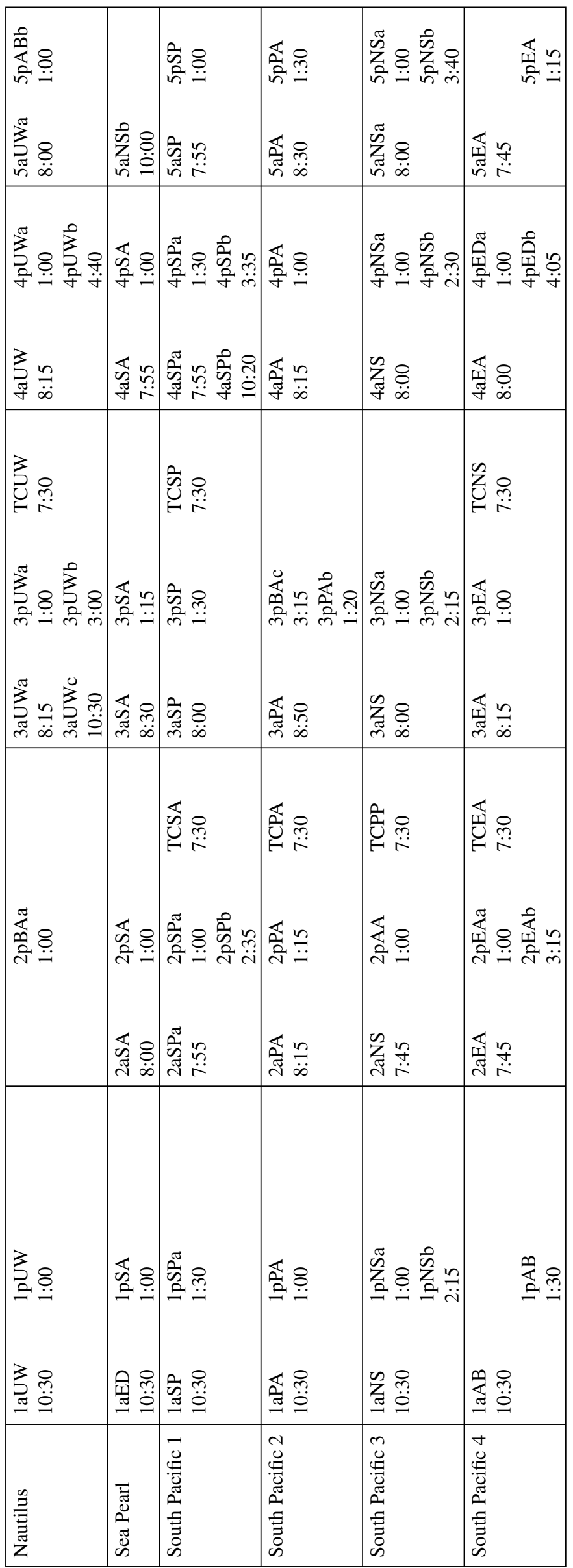


HILTON HAWAIIAN VILLAGE ${ }^{\circledR}$ WAIKIKI BEACH RESORT

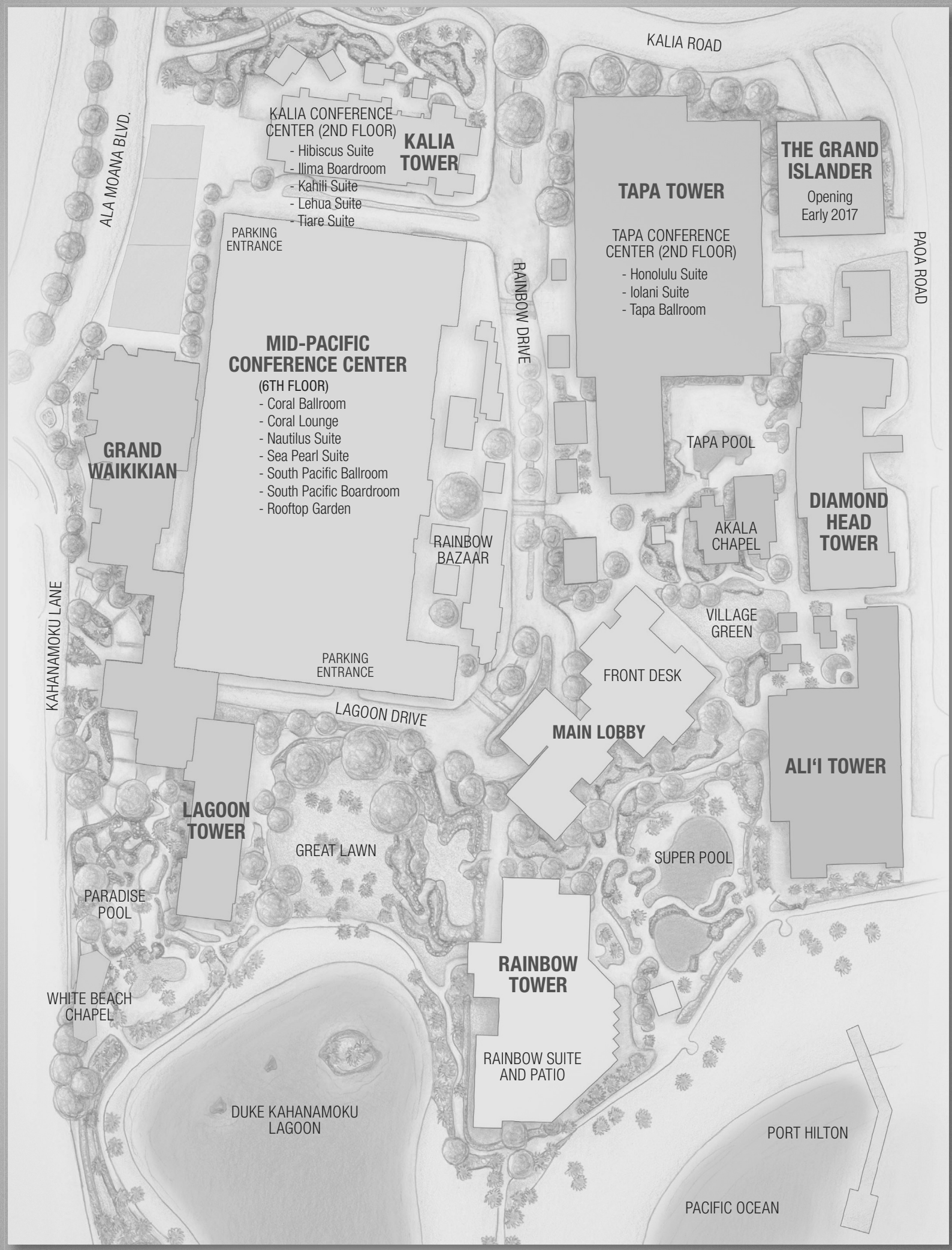




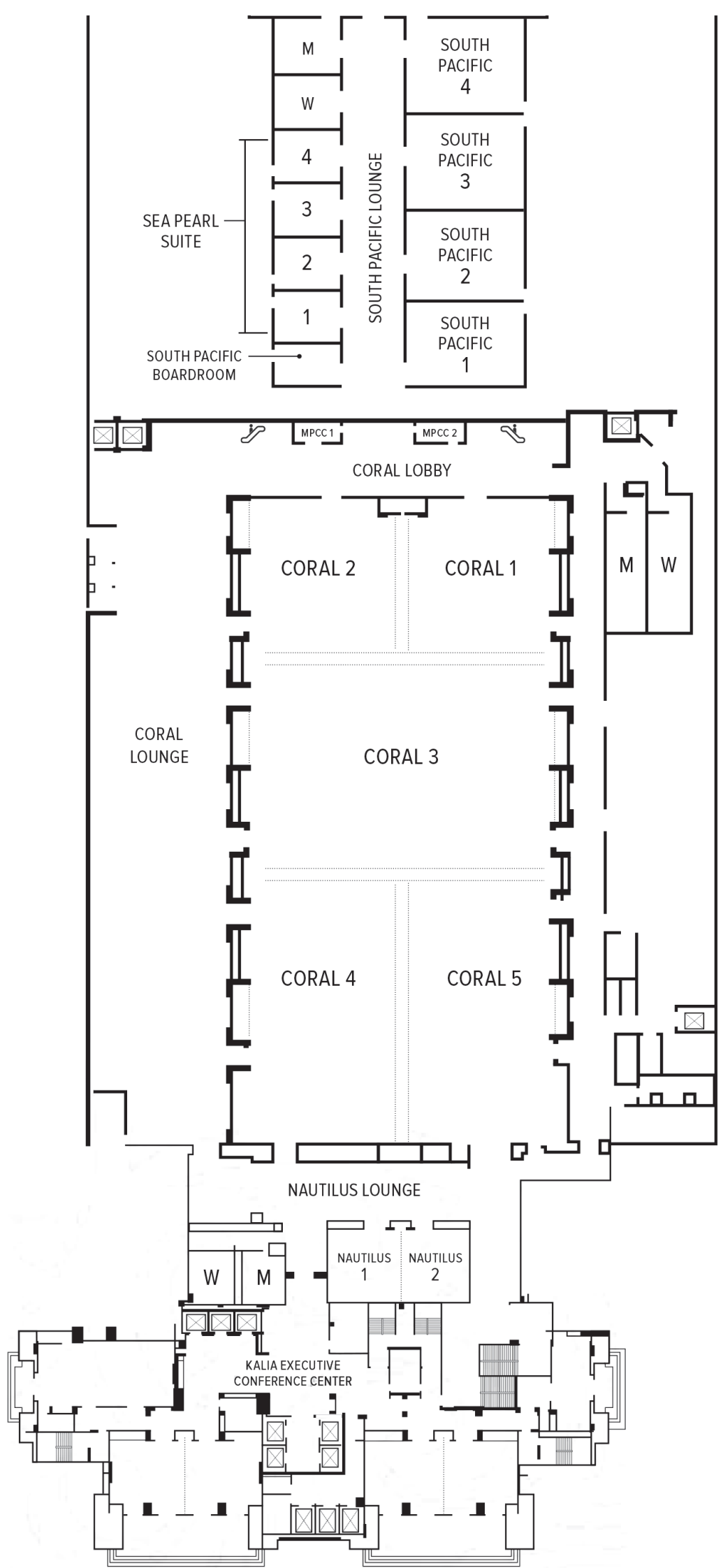




\section{KAHLIA TOWER}

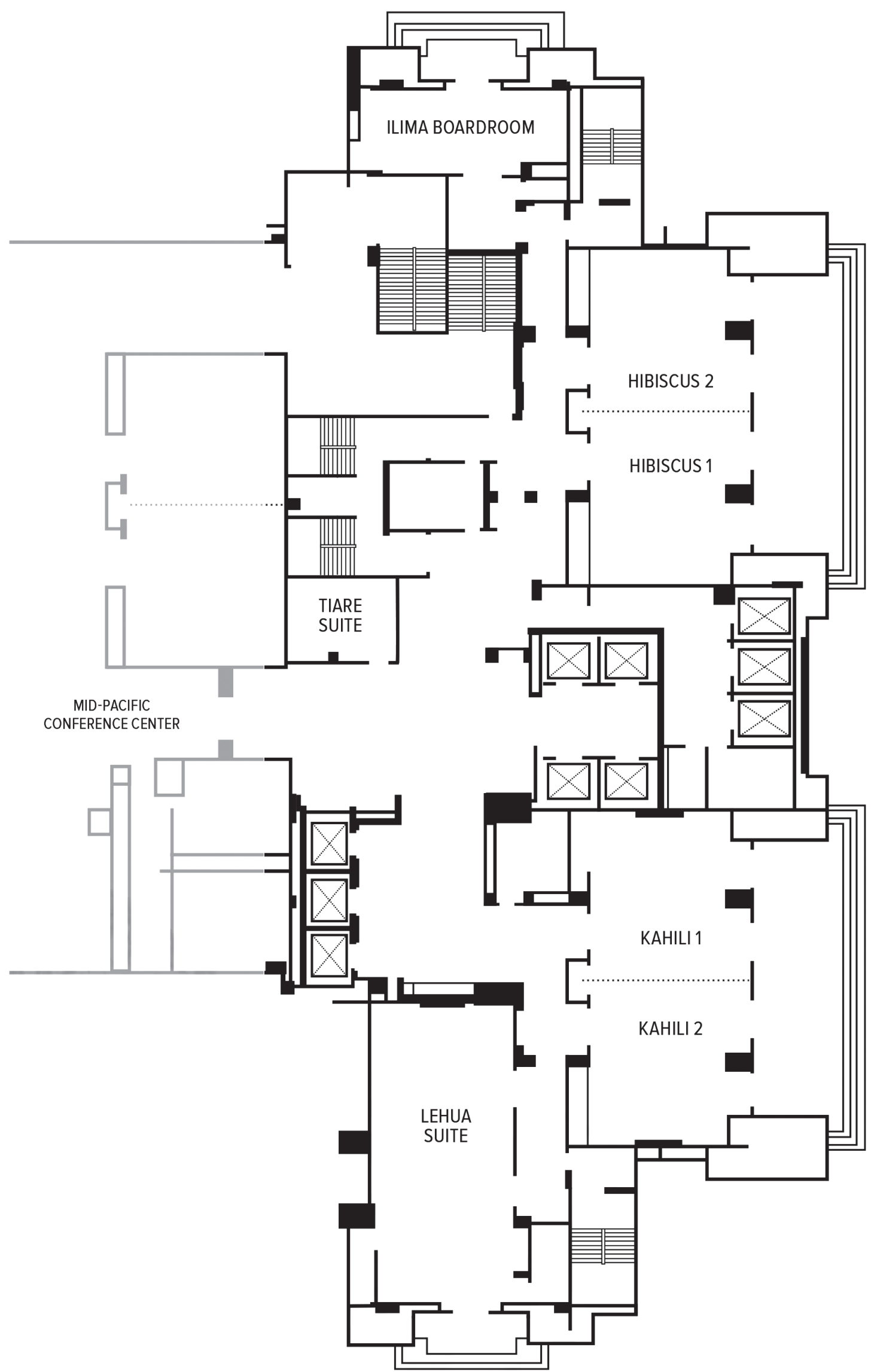




\section{TAPA TOWER}

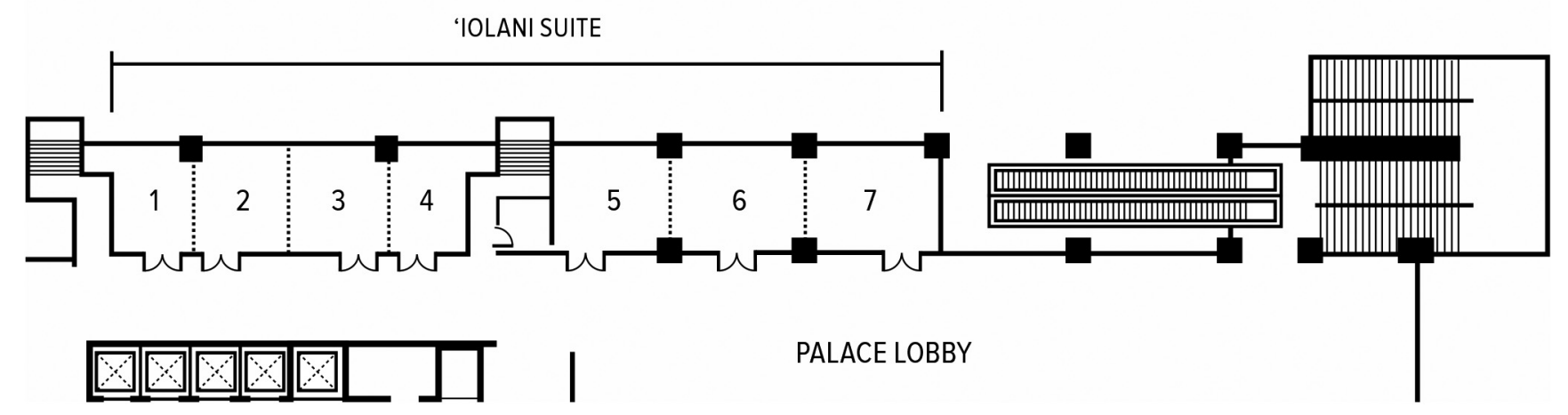

RAINBOW TOWER

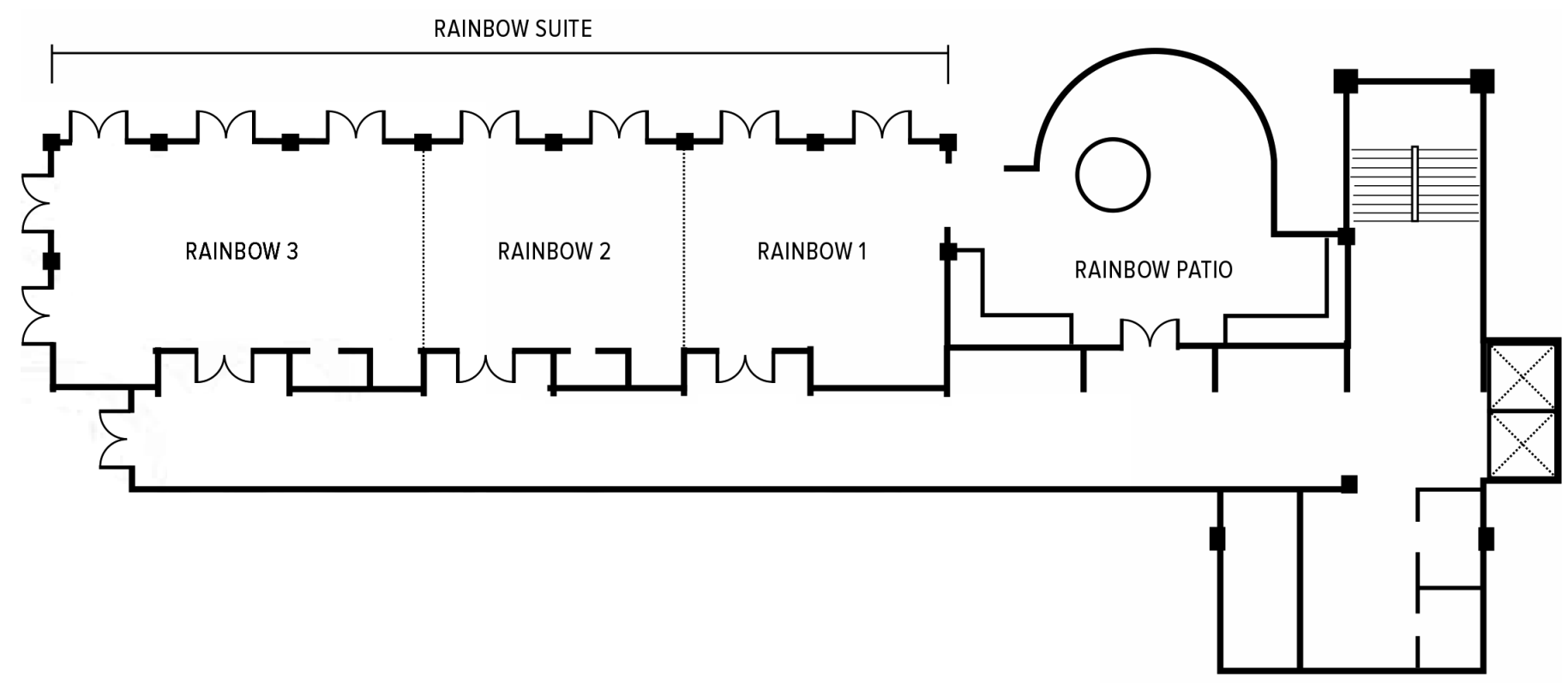




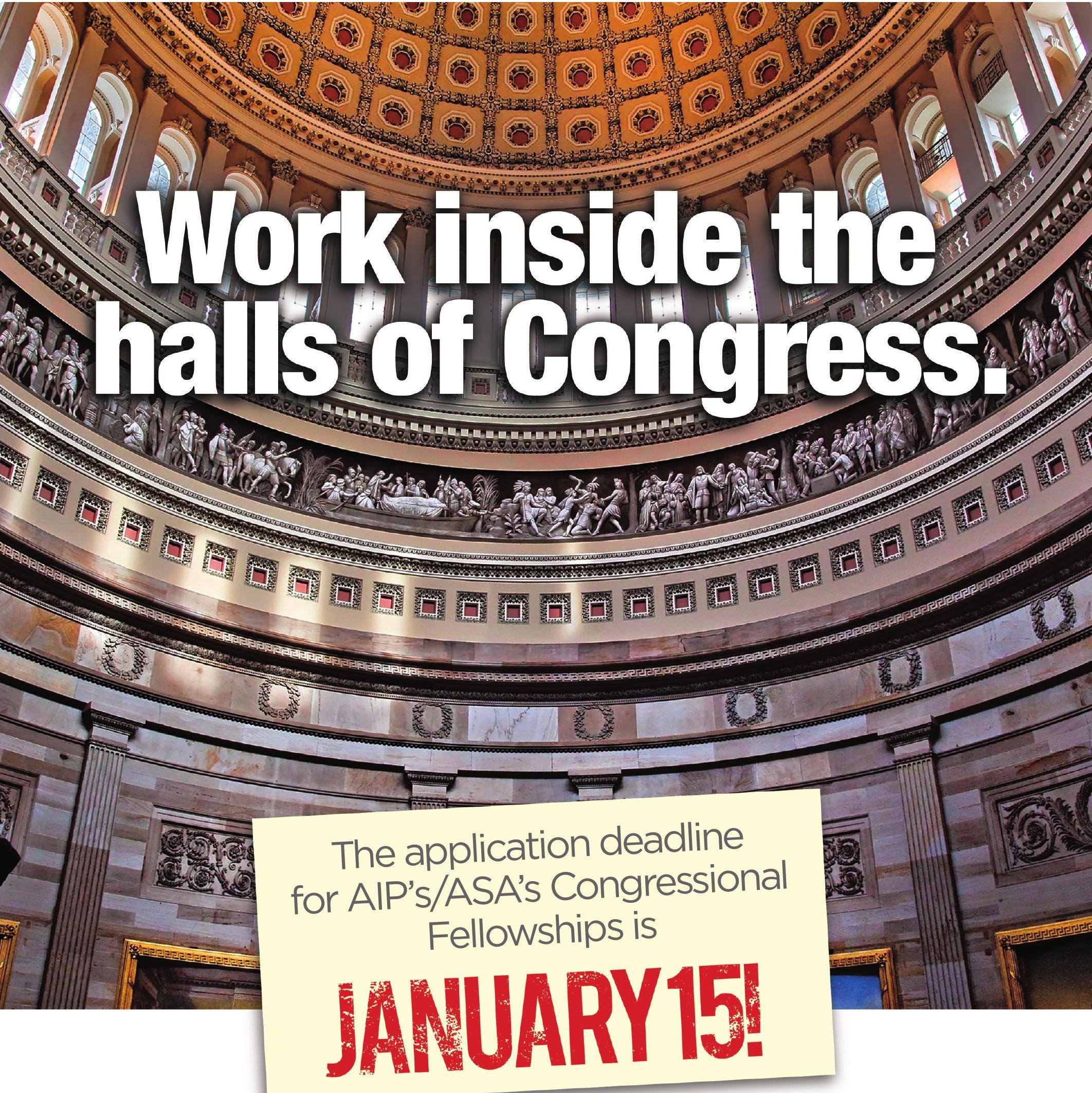

ASA members at all career stages-including mid- and late-career professionals-are invited to apply for AIP/ASA's Congressional Science Policy Fellowships. The annual stipend is $\$ 77,500$ plus benefits.

\section{www.aip.org/policy/fellowships/cf}




\section{TECHNICAL PROGRAM CALENDAR \\ 5th Joint Meeting of the Acoustical Society of America and the Acoustical Society of Japan \\ 28 November-2 December 2016}

\section{MONDAY MORNING}

$\begin{array}{ccl}\text { 8:00 } & \text { 1aID } & \begin{array}{l}\text { Interdisciplinary: Opening Ceremonies, } \\ \text { Plenary Lectures. Coral 4/5 }\end{array} \\ \text { 10:30 } & \text { 1aAA } & \begin{array}{l}\text { Architectural Acoustics: Acoustics for } \\ \text { Children and Pupils I. Lehua }\end{array} \\ \text { 10:30 } & \text { 1aAB } & \begin{array}{l}\text { Animal Bioacoustics: Bat Echolocation: } \\ \text { New Insights on Biosonar Production, }\end{array} \\ & & \text { Processing, and Performance from Field and } \\ & \text { Laboratory Investigations I. South Pacific } 4\end{array}$

10:30 1aAO Acoustical Oceanography: Topics in Acoustical Oceanography I. Kahili

10:30 1aBA Biomedical Acoustics and Physical Acoustics: Photoacoustics: Light and Sound I. Coral 1

10:30 1aED Education in Acoustics: Attractive Educational Methods and Tools in Acoustics I. Sea Pearl

10:30 1aNS

Noise: Sound Design I. South Pacific 3

10:30 1aPA

10:30 1aPP

10:30 1aSA

$10: 30 \quad 1 \mathrm{aSC}$

10:30 1aSP Signal Processing in Acoustics: General

10:30 1aUW Underwater Acoustics: Target Scattering.

Physical Acoustics: Thermoacoustics. South Pacific 2

Psychological and Physiological Acoustics: Spatial Hearing and Its Applications I. Coral 2

Structural Acoustics and Vibration: General Topics in Structural Acoustics and Vibration I (Poster Session). Coral 3

Speech Communication: Speech Technology: Synthesis and Interfaces (Poster Session). Coral 3 Topics in Signal Processing I. South Pacific 1 Nautilus

\section{MONDAY AFTERNOON}

1:00 1pAAa Architectural Acoustics: Acoustics for Children and Pupils II. Lehua

3:05 1pAAb Architectural Acoustics: Acoustical Design Metric Comparison Between the US and Japan. Lehua

1:30 1pAB Animal Bioacoustics: Bat Echolocation: New Insights on Biosonar Production, Processing, and Performance from Field and Laboratory Investigations II. South Pacific 4

1:10 1pAO Acoustical Oceanography: Twenty-Five Years of Acoustical Oceanography in the ASA I. Coral 2
1:30 1pBAa Biomedical Acoustics and Physical Acoustics: Photoacoustics: Light and Sound II. Coral 1

1:00 1pBAb Biomedical Acoustics and Physical Acoustics: Cavitation in Therapeutic Ultrasound I: General. Kahili

1:00 1pED Education in Acoustics: Attractive Educational Methods and Tools in Acoustics II. Coral 3

1:30 1pMU Musical Acoustics: Music and Sound in Multimedia. Hibiscus

1:00 1pNSa Noise: Sound Design II. South Pacific 3

2:15 1pNSb Noise: Acoustics of Microperforated Materials. South Pacific 3

1:00 1pPA Physical Acoustics: Advances in Hazards Monitoring with Distributed Infrasound Sensor Networks. South Pacific 2

1:15 1pPP Psychological and Physiological Acoustics: Spatial Hearing and Its Applications II. Coral 5

1:00 1pSA Structural Acoustics and Vibration: General Topics in Structural Acoustics and Vibration II. Sea Pearl

1:30 1pSC Speech Communication and Signal Processing in Acoustics: Speech Robotics. Coral 4

1:30 $\quad 1 \mathrm{pSPa} \quad$ Signal Processing in Acoustics: Time Delay Estimation of Acoustic Signals with Applications. South Pacific 1

3:15 $1 \mathrm{pSPb}$ Signal Processing in Acoustics and Speech Communication: Spoken Document Processing. Coral 3

1:00 1pUW Underwater Acoustics and Acoustical Oceanography: Underwater Acoustics Studies in Asian Seas I. Nautilus

\section{MONDAY EVENING}

7:00 1eMU Musical Acoustics: Special Presentation of the History, Culture, Practice, and Performance of Hawai'ian Music. Coral 2

\section{TUESDAY MORNING}

8:00 2aABa Animal Bioacoustics: Tropical Bioacoustics I. Coral 2

8:45 $2 \mathrm{aABb}$ Animal Bioacoustics and Signal Processing in Acoustics: Anthropogenic Transient Noise Sound Field and Its Effects on Animal Communication I. Nautilus 1 


\section{Next Generation \\ Headphone Testing}

for better test results

Consumers are demanding a higher definition sound experience. For manufacturers, this has given rise to a number of challenges when it comes to testing their products.

At G.R.A.S. we are proud to introduce the next generation of headphone testing technology.

After all, the better your testing, the better your products.
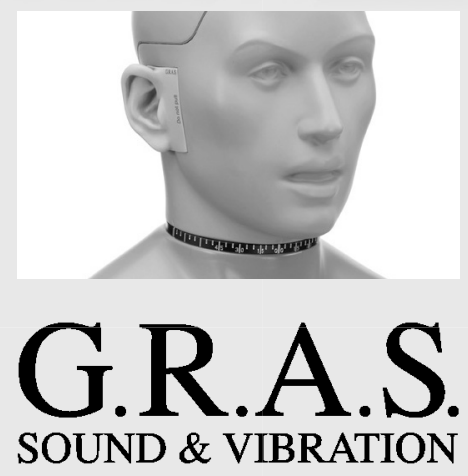
8:30 2aAO Acoustical Oceanography: Twenty-Five Years of Acoustical Oceanography in the ASA II. Kahili

7:50 2aBAa Biomedical Acoustics and Physical Acoustics: Cavitation in Therapeutic Ultrasound II: General. Coral 1

8:25 2aBAb Biomedical Acoustics: High-Frame Rate Imaging with Plane Waves and Diverging Waves. Lehua

9:50 2aBAc Biomedical Acoustics and Physical Acoustics: Cavitation in Therapeutic Ultrasound III: Tissue Fractionation. Coral 1

7:45 2aEA Engineering Acoustics: Transducer Systems. South Pacific 4

8:00 2aMUa Musical Acoustics and Signal Processing in Acoustics: Computational Methods of Simulation for Musical Acoustics. Hibiscus

10:40 2aMUb Musical Acoustics and Signal Processing in Acoustics: Music Signal Processing I. Hibiscus

7:45 2aNS Noise and Architectural Acoustics: Noise Impacts and Soundscapes at Outdoor Gathering Spaces. South Pacific 3

8:15 2aPA Physical Acoustics and Noise: Acoustics of Supersonic Jets: Launch Vehicle and Military Jet Acoustics I. South Pacific 2

8:30 2aPP Psychological and Physiological Acoustics: Linking Human Physiology with Psychoacoustics-From Brainstem to Cortical Measurements and Beyond. Coral 5

8:00 2aSA Structural Acoustics and Vibration and Physical Acoustics: Acoustic Metamaterials I. Sea Pearl

9:00 2aSC Speech Communication: CrossLinguistic Speech Production and Perception. Coral 4

7:55 2aSPa Signal Processing in Acoustics: Compressive Sensing in Acoustics I. South Pacific 1

8:30 $2 \mathrm{aSPb}$ Signal Processing in Acoustics: General Topics in Signal Processing II (Poster Session). Coral 3

7:55 2aUW Underwater Acoustics and Acoustical Oceanography: Underwater Acoustic Studies in Asian Seas II. Nautilus 2

\section{TUESDAY AFTERNOON}

1:00 2pAA Architectural Acoustics and Speech Communication: At the Intersection of Speech and Architecture I. South Pacific 3

1:00 2pABa Animal Bioacoustics: Tropical Bioacoustics II. Coral 2
3:00 $2 \mathrm{pABb}$ Animal Bioacoustics and Signal

Processing in Acoustics: Anthropogenic Transient Noise Sound Field and Its Effects on Animal Communication II. Coral 2

1:00 2pAOa Acoustical Oceanography, Underwater Acoustics, and Signal Processing in Acoustics: Ocean Acoustic Tomography: Active and Passive, Theory and Experiment I. Coral 4

4:15 2pAOb Acoustical Oceanography: Acoustical Oceanography Prize Lecture. Coral 4

1:00 2pBAa Biomedical Acoustics and Signal Processing in Acoustics: Bone Sonometry I: Ultrasonic Studies of the Physical Properties of Cortical and Cancellous Bone. Nautilus

1:00 2pBAb Biomedical Acoustics and Physical Acoustics: Cavitation in Therapeutic Ultrasound IV: Tissue Fractionation. Coral 1

1:00 2pEAa Engineering Acoustics: Acoustic Transduction Material, Sensors, and Array Technologies. South Pacific 4

3:15 2pEAb Engineering Acoustics: Ocean Acoustics Analysis. South Pacific 4

1:15 2pMU Musical Acoustics and Signal Processing in Acoustics: Music Signal Processing II. Hibiscus

1:00 2pNS Noise: Community Response to Transportation Noises. Lehua

1:15 2pPA Physical Acoustics and Noise: Acoustics of Supersonic Jets: Launch Vehicle and Military Jet Acoustics II. South Pacific 2

1:15 2pPP Psychological and Physiological Acoustics: Binaural Processing and Localization. Coral 5

1:00 2pSA Structural Acoustics and Vibration and Physical Acoustics: Acoustic Metamaterials II. Sea Pearl

1:00 2pSC Speech Communication: Articulatory and Acoustic Phonetics: Data and Methods (Poster Session). Coral 3

1:00 $2 \mathrm{pSPa}$ Signal Processing in Acoustics: Compressive Sensing in Acoustics II. South Pacific 1

$2: 35 \quad 2 \mathrm{pSPb}$ Signal Processing in Acoustics and Underwater Acoustics: Detection, Tracking, and Classification of Unmanned Aircraft and Underwater Vehicles. South Pacific 1

1:00 2pUW Underwater Acoustics, Acoustical Oceanography and Physical Acoustics: Nonlinear Effects in Underwater Acoustics and Bubbles. Kahili 


\section{Acoustics 17 Boston Exhibition \\ At the third joint meeting of the Acoustical Society of America and the European Acoustics Association}

25-29 June 2017

John B. Hynes Convention Center

Boston, MA, USA

The 173rd Meeting of the Acoustical Society of America and the 8th Forum Acusticum will bring together experts from all fields of acoustics from the both the USA and Europe, and will provide a forum for the open exchange of scientific information.

Acoustics ' 17 Boston consist of plenary lectures, invited and contributed papers, poster presentations and exhibits.

Acoustics ' 17 Boston will be highlighted by an exhibit featuring displays with instruments, materials, and services for the acoustics and vibration community. The exhibit will be conveniently located near the registration area and meeting rooms and will open with a reception on Monday evening, 26 June, and will close Wednesday, 28 June, at noon.

Morning and afternoon refreshments will be available in the exhibit area.

\begin{tabular}{|c|c|}
\hline For ASA Exhibit information: & $\begin{array}{l}\text { For information on the } \\
\text { ASA Meeting: }\end{array}$ \\
\hline Bob Finnegan, & Acoustical Society of America \\
\hline ASA Exhibits Manager & 1305 Walt Whitman Rd, Suite 300 \\
\hline AIP Publishing LLC & Melville, NY 11747 \\
\hline 1305 Walt Whitman Rd, Suite 300 & Tel: 516-576-2360 \\
\hline Melville, NY 11747 & Fax: 516-576-2377 \\
\hline Tel: 516-576-2433 & Email: asa@aip.org \\
\hline Fax: 516-576-2481 & Web site: \\
\hline Email: rfinnegan@aip.org & http://AcousticalSociety.org/ \\
\hline
\end{tabular}

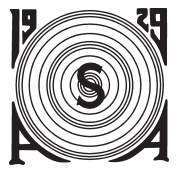

Topics to be covered include:

Acoustical Oceanography

Animal Bioacoustics

Architectural Acoustics

Biomedical Acoustics

Engineering Acoustics

Musical Acoustics

Noise

Physical Acoustics

Physiological Acoustics

Psychological Acoustics

Signal Processing in Acoustics

Speech Communication

Structural Acoustics

and Vibration

Underwater Acoustics 
WEDNESDAY MORNING

8:00 3aAAa Architectural Acoustics and Speech Communication: At the Intersection of Speech and Architecture II. Lehua

9:20 3aAAb Architectural Acoustics and Signal Processing in Acoustics: Advanced Analysis, Simulation, and Auralization in Room Acoustics I. Lehua

7:50 3aAB Animal Bioacoustics: Session in Honor of Whitlow Au I. Coral 2

7:50 3aAO Acoustical Oceanography, Underwater Acoustics, and Signal Processing in Acoustics: Ocean Acoustic Tomography: Active and Passive, Theory and Experiment II. Kahili

8:00 3aBA Biomedical Acoustics: Quantitative Ultrasound I: From Micro to In-vivo. Coral 1

8:15 3aEA Engineering Acoustics: Acoustic Analysis. South Pacific 4

8:35 3aMU Musical Acoustics: East and Western Instruments: Contrasts and Connections. Hibiscus

8:00 3aNS Noise, Psychological and Physiological Acoustics, and ASA Committee on Standards: Current Issues in Hearing Protection and Hearing Conservation I. South Pacific 3

8:50 3aPA Physical Acoustics and Biomedical Acoustics: Acoustic Micro- and Nanofluidics I. South Pacific 2

7:55 3aPPa Psychological and Physiological Acoustics and Speech Communication: Perspectives of Research in Overlooked Hearing Problems. Coral 5

8:00 $3 \mathrm{aPPb}$ Psychological and Physiological Acoustics: From Cochlea to Cortex (Poster Session). Coral 3

8:30 3aSA Structural Acoustics and Vibration: Dynamics of Ribbed Structures. Sea Pearl

8:00 3aSC Speech Communication: DoubleWeak Theory of Speech Production and Perception: A Session in Honor of Terrance Nearey. Coral 4

8:00 3aSP Signal Processing in Acoustics: Signal Processing in Nonlinear Acoustics. South Pacific 1

8:15 3aUWa Underwater Acoustics, Noise and Acoustical Oceanography: Transmission Through the Air-Water Interface. Nautilus

9:00 3aUWb Underwater Acoustics: Topics in Underwater Acoustics (Poster Session). Coral 3

10:30 3aUWc Underwater Acoustics: Inversion, BeamForming, and Calibration I. Nautilus

\section{WEDNESDAY AFTERNOON}

1:00 3pAAa Architectural Acoustics and Signal Processing in Acoustics: Advanced Analysis, Simulation, and Auralization in Room Acoustics II. Lehua

4:30 3pAAb Architectural Acoustics and Signal Processing in Acoustics: Advanced Analysis, Simulation, and Auralization in Room Acoustics III (Poster Session). Coral 3

4:10 3pAAc Architectural Acoustics: Assorted Topics in Architectural Acoustics (Poster Session). Coral 3

1:00 3pABa Animal Bioacoustics: Session in Honor of Whitlow Au II. Coral 2

2:45 3pABb Animal Bioacoustics: On the Bleeding Edge of Animal Bioacoustics Technology. Coral 2

1:00 3pAO Acoustical Oceanography, Underwater Acoustics, and Signal Processing in Acoustics: Ocean Acoustic Tomography: Active and Passive, Theory and Experiment III. Kahili

1:00 3pBAa Biomedical Acoustics: Quantitative Ultrasound II: From Micro to In-vivo. Coral 1

1:00 3pBAb Biomedical Acoustics and Signal Processing in Acoustics: Bone Sonometry II: Ultrasonic Studies of the Physical Properties of Cortical and Cancellous Bone (Poster Session). Coral 3

3:15 3pBAc Biomedical Acoustics and Signal Processing in Acoustics: Bone Sonometry III: Ultrasonic Studies of the Physical Properties of Cortical and Cancellous Bone. South Pacific 2

1:00 3pEA Engineering Acoustics and Physical Acoustics: Outdoor Sound Propagation and Outdoor Public Address. South Pacific 4

1:30 3pEDa Education in Acoustics: Demonstrations and Tools in Acoustics Education. Hibiscus

4:00 3pEDb Education in Acoustics: Education in Acoustics for Kids (K-12 students). Kahili

1:00 3pNSa Noise, Psychological and Physiological Acoustics, and ASA Committee on Standards: Current Issues in Hearing Protection and Hearing Conservation II. South Pacific 3

2:15 3pNSb Noise: Ambient Listening, Communication and Noise Reduction of Special Hearing Protectors. South Pacific 3

1:10 3pPAa Physical Acoustics: Solid State Acoustics. Coral 4

1:20 3pPAb Physical Acoustics and Biomedical Acoustics: Acoustic Micro- and Nanofluidics II. South Pacific 2 
1:00 3pPP

Psychological and Physiological

Acoustics: Recent Progress in Auditory

Perceptual Organization Studies. Coral 5

1:15 3pSA Structural Acoustics and Vibration, Physical Acoustics, Engineering Acoustics, and Signal Processing in Acoustics: NonContact Vibration Excitation and Sensing Techniques. Sea Pearl

1:00 3pSC Speech Communication: Speech Production and Perception (Poster Session). Coral 3

1:30 3pSP Signal Processing in Acoustics: Acoustic Communications. South Pacific 1

1:00 3pUWa Underwater Acoustics: Inversion, Beam-forming, and Calibration II. Nautilus

3:00 3pUWb Underwater Acoustics: Seabed and Sea Surface Interactions. Nautilus

\section{THURSDAY MORNING}

8:30 4aAA Architectural Acoustics: Assorted Topics in Architectural Acoustics I. Lehua

8:30 4aAB Animal Bioacoustics, Signal Processing in Acoustics, and Speech Communication: Sequence Information in Mammalian Vocal Call Production I. Coral 2

8:15 4aAO Acoustical Oceanography, Animal Bioacoustics, and Signal Processing in Acoustics: Acoustic Scattering by Aquatic Organisms I. Kahili

7:30 4aBAa Biomedical Acoustics: Session in Honor of Floyd Dunn. Coral 1

11:15 4aBAb Biomedical Acoustics: Medical Acoustics in Kidney and Liver Disease I. Coral 1

8:00 4aEA Engineering Acoustics: Simulation and Analysis. South Pacific 4

8:00 4aID Interdisciplinary: Workshop for Publishing Excellence in JASA. Coral 5

8:00 4aMUa Musical Acoustics: Flute Acoustics Including All "Air-Jet" Instruments. Hibiscus

10:30 4aMUb Musical Acoustics: Piano Acoustics and Playing Piano I. Hibiscus

8:00 4aNS Noise: Prediction of Transportation Noises and Other Topics I. South Pacific 3

8:15 4aPA Physical Acoustics: General Topics in Physical Acoustics. South Pacific 2

8:00 4aPPa Psychological and Physiological Acoustics: Psychological and Physiological Acoustics Potpourri (Poster Session). Coral 3

8:00 $\quad 4 \mathrm{aPPb} \quad$ Psychological and Physiological Acoustics: Modelling, Measurement, and Technology (Poster Session). Coral 3
7:55 4aSA Structural Acoustics and Vibration and Noise: Building Vibration Analysis, Measurement, and Mitigation. Sea Pearl

9:00 4aSC Speech Communication and Education in Acoustics: Second Language Speech Learning and Education. Coral 4

7:55 4aSPa Signal Processing in Acoustics: Exploitation of Doppler in Acoustic Signal Processing. South Pacific 1

10:20 $4 \mathrm{aSPb} \quad$ Signal Processing in Acoustics: High Resolution Imaging Sonars Including Real Aperture, Synthetic Aperture, and Tomographic Sonars I. South Pacific 1

8:15 4aUW Underwater Acoustics, Signal Processing in Acoustics, and Acoustical Oceanography: Harbor Acoustics I. Nautilus

\section{THURSDAY AFTERNOON}

1:00 4pAAa Architectural Acoustics: Assorted Topics in Architectural Acoustics II. Lehua

3:45 4pAAb Architectural Acoustics: It's All About the Details. Lehua

1:00 4pABa Animal Bioacoustics, Signal Processing in Acoustics, and Speech Communication: Sequence Information in Mammalian Vocal Call Production II. Coral 2

2:55 4pABb Animal Bioacoustics: Session in Honor of Whitlow Au III. Coral 2

3:00 4pABc Animal Bioacoustics: Animal Bioacoustics Poster Session. Coral 3

1:00 4pAO Acoustical Oceanography, Animal Bioacoustics, and Signal Processing in Acoustics: Acoustic Scattering by Aquatic Organisms II. Coral 4

1:00 4pBA Biomedical Acoustics: Medical Acoustics in Kidney and Liver Disease II. Coral 1

1:00 4pEA Engineering Acoustics: Sound Field Control Techniques. Kahili

1:00 4pEDa Education in Acoustics and Signal Processing in Acoustics: Using Computers for Acoustics Education. South Pacific 4

4:05 4pEDb Education in Acoustics: Acoustics Education Prize Lecture. South Pacific 4

1:00 4pMUa Musical Acoustics: Piano Acoustics and Playing Piano II. Hibiscus

3:00 4pMUb Musical Acoustics: Musical Instruments from Islands Around the World I. Hibiscus

4:30 4pMUc Musical Acoustics: Musical Instruments from Islands around the World II-A Concert. Hibiscus 
1:00 4pNSa Noise: Prediction of Transportation Noises and Other Topics II. South Pacific 3

2:30 4pNSb Noise: Low Frequency Noise:

Measurement, Evaluation, Enforcement, and Policy. South Pacific 3

1:00 4pPA Physical Acoustics: Nonclassical Nonlinear Acoustics of Solids. South Pacific 2

1:00 4pPP Psychological and Physiological Acoustics and Speech Communication: Gap Detection: New Perspectives from Neuroscience, Perception and Modelling. Coral 5

1:00 4pSA Structural Acoustics and Vibration, Physical Acoustics, and Noise: Fluid Flow Induced Vibration and Noise. Sea Pearl

1:00 4pSC Speech Communication: Non-Native Speech Production and Perception, Learning, and Adaptation (Poster Session). Coral 3

1:30 4pSPa Signal Processing in Acoustics: High Resolution Imaging Sonars Including Real Aperture, Synthetic Aperture, and Tomographic Sonars II. South Pacific 1

3:35 $4 \mathrm{pSPb}$ Signal Processing in Acoustics: High Resolution Imaging Sonars Including Real Aperture, Synthetic Aperture, and Tomographic Sonars III. South Pacific 1

1:00 4pUWa Underwater Acoustics, Signal Processing in Acoustics, and Acoustical Oceanography: Harbor Acoustics II. Nautilus

4:40 4pUWb Underwater Acoustics: Ocean Ambient Noise: Sources, Variation, and Characterization I. Nautilus

\section{FRIDAY MORNING}

8:00 5aAB Animal Bioacoustics: Marine Acoustic Ecology. Coral 2

8:30 5aAO Acoustical Oceanography: Topics in Acoustical Oceanography II. Kahili

8:00 5aBAa Biomedical Acoustics: Acoustics for Older Persons with Disabilities. Coral 1

8:00 5aBAb Biomedical Acoustics: Biomedical Acoustics Poster Session. Coral 3

7:45 5aEA Engineering Acoustics: Applied Acoustics I. South Pacific 4

8:35 5aID Interdisciplinary: Topical Meeting on Data Science and Acoustics I. Coral 4

8:30 5aMU Musical Acoustics: General Topics in Musical Acoustics I. Hibiscus

8:00 5aNSa Noise: Wind Turbine Noise. South Pacific 3
10:00 5aNSb Noise, ASA Committee on Standards, and Architectural Acoustics: Innovations in Floor Impact Noise Testing and Evaluation. Sea Pearl

8:30 5aPA Physical Acoustics: Ultrasonics and Non-Destructive Testing. South Pacific 2

7:55 5aPP Psychological and Physiological Acoustics: Pitch and Timbre. Coral 5

8:00 5aSC Speech Communication: Voice, Tone, Prosody, and Affective and Emotional Qualities of Speech (Poster Session). Coral 3

7:55 5aSP Signal Processing in Acoustics: General Topics in Signal Processing II. South Pacific 1

8:00 5aUWa Underwater Acoustics: Underwater Acoustic Propagation. Nautilus

10:00 5aUWb Underwater Acoustics: Ocean Ambient Noise: Sources, Variation, and Characterization II. Lehua

\section{FRIDAY AFTERNOON}

1:00 5pABa Animal Bioacoustics: Behavioral Response Studies. Coral 2

1:00 5pABb Animal Bioacoustics: The Diversity of Bioacoustic Signals. Nautilus

1:00 5pBA Biomedical Acoustics, Engineering Acoustics, and Signal Processing in Acoustics: Acoustic Imaging. Coral 1

1:15 5pEA Engineering Acoustics: Applied Acoustics II. South Pacific 4

1:00 5pID Interdisciplinary: Topical Meeting on Data Science and Acoustics II. Coral 4

1:00 5pMU Musical Acoustics: General Topics in Musical Acoustics II (Poster Session). Coral 3

1:00 5pNSa Noise: Design of Noise Control Materials. South Pacific 3

3:40 5pNSb Noise: Emerging Issues on Quiet Vehicles-A Matter of Soundscape. South Pacific 3

1:30 5pPA Physical Acoustics: Radiation Force and Nonlinear Acoustics. South Pacific 2

1:00 5pSCa Speech Communication: Speech Processing. Coral 5

1:00 5pSCb Speech Communication: Speech Perception and Production by Clinical, Aging, and/or Developing Populations (Poster Session). Coral 3

1:00 5pSP Signal Processing in Acoustics: General Topics in Signal Processing III. South Pacific 1 


\section{SCHEDULE OF COMMITTEE MEETINGS AND OTHER EVENTS}

Iolani Suites are located in Tapa Tower/Rainbow Suites are located in Rainbow Tower/All others located in Kaila Tower.

\section{ASA COUNCIL AND ADMINISTRATIVE COMMITTEES}

Sun, 27 Nov, 1:00 p.m. Mon, 28 Nov, 1:00 p.m. Mon, 28 Nov, 3:30 p.m. Tue, 29 Nov, 7:00 a.m. Tue, 29 Nov, 7:30 a.m. Tue, 29 Nov, 11:45 a.m. Tue, 29 Nov, 12:00 noon Tue, 29 Nov, 12:30 p.m. Tue, 29 Nov, 1:30 p.m. Tue, 29 Nov, 4:00 p.m. Tue, 29 Nov, 5:00 p.m. Wed, 30 Nov, 6:45 a.m.

Wed, 30 Nov, 7:00 a.m. Wed, 30 Nov, 7:30 a.m. Wed, 30 Nov, 7:00 a.m. Wed, 30 Nov, 7:00 a.m. Wed, 30 Nov, 11:00 a.m. Wed, 30 Nov, 11:30 a.m. Public Relations Wed, 30 Nov, 12:00 noon Membership Wed, 30 Nov, 1:30 p.m. AS Foundation Board Wed, 30 Nov, 5:30 p.m. Education in Acoustics Wed, 30 Nov, 5:30 p.m.

Thu, 1 Dec, 7:30 a.m. Thu, 1 Dec, 7:30 a.m.

Thu, 1 Dec, 7:30 a.m. Thu, 1 Dec, 2:00 p.m. Thu, 1 Dec, 4:00 p.m. Thu, 1 Dec, 4:30 p.m. Thu, 1 Dec, 4:30 p.m.

Thu, 1 Dec, 4:30 p.m.

Fri, 2 Dec, 7:00 a.m. Fri, 2 Dec, 11:00 a.m. Executive Council Technical Council ASA Books Panel on Public Policy Editorial Board Student Council

Prizes \& Special Fellowships Meetings Newman Fund Advisory Women in Acoustics International Research \& Education

College of Fellows

Finance

Publication Policy

Regional and Student Chapters Rainbow III

Medals and Awards Ilima Boardroom

Iolani $2 / 3$

Iolani 4

Tiari

Lehua

South Pacific

Boardroom

Ilima Boardoom

Tiari Boardroom

Tutorials, Short Courses, Hot Topics

Publishing Services

Strategic Plan Champions

Publishing and Standards

Financial Affairs

Member Engagement and Diversity

Outreach

Technical Council

Executive Council

\section{TECHNICAL COMMITTEEE OPEN MEETINGS}

Tue, 29 Nov, 7:30 p.m. Tue, 29 Nov, 7:30 p.m. Tue, 29 Nov 7:30 p.m. Tue, 29 Nov, 7:30 p.m. Tue, 29 Nov, 7:30 p.m. Tue, 29 Nov, 7:30 p.m.

Tue, 29 Nov, 7:30 p.m.

Wed, 30 Nov, 7:30 p.m. Wed, 30 Nov, 7:30 p.m. Wed, 30 Nov, 7:30 p.m. Wed, 30 Nov, 7:30 p.m.

Wed, 30 Nov, 7:30 p.m. Wed, 30 Nov, 7:30 p.m.
Acoustical Oceanography Animal Bioacoustics Engineering Acoustics Physical Acoustics Psychological and Physiological Acoustics

Structural Acoustics and Vibration

Biomedical Acoustics

Musical Acoustics

Noise Acoustics

Speech Communication

Underwater Acoustics
Signal Processing in
Executive Council Architectural Acoustics
Rainbow III

Rainbow I

Ilima Boardroom

Tiari Boardroom

Rainbow III

South Pacific Boardroom

Rainbow III

Rainbow III

Kahili

Coral 2

Lehua

South Pacific 4

South Pacific 2

South Pacific 3

South Pacific 1

Coral 1

Kahili

South Pacific 4

South Pacific 1

Coral 4

Nautilus

\section{STANDARDS COMMITTEES AND WORKING GROUPS} Level

Mon, 28 Nov, 7:00 p.m. ASACOS Steering South Pacific

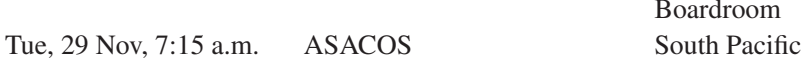

Boardroom

South Pacific

Boardroom

Tue, 29 Nov, 9:00 a.m. $\quad \begin{aligned} & \text { S3/SC1 WG6-To } \\ & \text { Whale Hearing }\end{aligned}$

Thu, 1 Dec, 10:00 a.m. S12/WG11 - Hearing Ilima

Protection

\section{MEETING SERVICES, SPECIAL EVENTS, SOCIAL EVENTS}

Registration

Coral Foyer

1:00 p.m. - 5:00 p.m.

Mon-Fri, 28 Nov - 2 Dec Registration

7:30 a.m. - 5:00 p.m.

Mon-Fri, 28 Nov - 2 Dec, E-mail

7:00 a.m. - 5:00 p.m.

Mon-Fri, 28 Nov - 2 Dec, Internet Zone

7:00 a.m. - 5:00 p.m.

Mon-Thu, 28 Nov - 2 Dec, A/V Preview

7:00 a.m.- 5:00 p.m.

Mon-Fri, 28 Nov - 2 Dec, Accompanying Persons

8:00 a.m. to 10:00 a.m.

Mon-Fri, 28 Nov - 2 Dec, A.M. Coffee Break

9:30 a.m. - 10:45 a.m.

Tue-Thu, 29 Nov - 1 Dec, Resume Help Desk 12:00 p.m. - 1:00 p.m.

Mon, 28 Nov, 8:00 a.m. - 10:00 a.m.

Mon, 28 Nov,

5:00 p.m. - 5:30 p.m.

Mon, $28 \mathrm{Nov}$

5:30 p.m. - 7:00 p.m.

Mon, 28 Nov,

5:30 p.m. - 7:00 p.m.

Tue, 29 Nov,

9:00 a.m. - 5:00 p.m.

Tue, 29 Nov,

2:30 p.m. - 3:45 p.m.

Tue, 29 Nov,

6:00 p.m. - 7:30 p.m.

Wed, 30 Nov,

8:30 a.m. - 12:00 noon

Wed, 30 Nov,

9:00 a.m. - 12:00 noon

Wed, 30 Nov,

11:45 a.m. - 1:00 p.m.

Wed, 30 Nov,

6:00 p.m. - 8:00 p.m.

Wed, 4 Nov

8:00 p.m. - 12:00

midnight

Thu, 1 Dec,

6:00 p.m. - 7:00 p.m.

Thu, 1 Dec,

7:00 p.m. - 8:00 p.m.

Thu, 1 Dec,

8:00 p.m. - 9:00 p.m.

Opening Plenary Session

Coral Foyer

Coral Foyer near

MPCC1

Coral Foyer

MPCC II, Coral

Foyer

Rainbow 1

Coral Foyer

Coral Foyer

Coral 4/5

New Student Orientation

Hibiscus

Student Meet and Greet

Rainbow 1/2

Exhibit Opening Reception

Coral Foyer

Exhibit

Coral Foyer

Afternoon Break

Coral Foyer

Social Hour

Great Lawn

Technical Tour -

Meet in ??? at ???

a.m.

Exhibit

Coral Foyer

Women in Acoustics

Luncheon

Student Reception

ASA Jam

Rainbow 1/2/3

Rainbow $1 / 2$

Irish Rose Saloon (478 Ena Rd.)

Pre-Banquet Reception

Coral Foyer

Banquet

Coral 4/5

Plenary Session/Awards

Ceremony

Coral 4/5

Fri, 2 Dec, 9:15 - 11:15

Workshop for Kids

Coral Foyer
Mon, 28 Nov, 5:00 p.m. S12/WG27-Sound Pressure Ilima 


\section{The Acoustical Society of Japan}

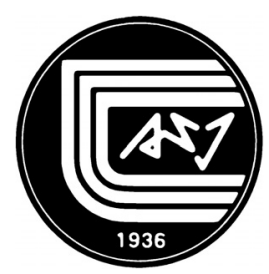

The Acoustical Society of Japan (ASJ) was founded on April 15th, 1936 by 15 members. Therefore, this year, 2016, the Society celebrates its 80th anniversary. As of August 2016, the membership had reached 4,355 including 3,294 Members, 21 Honorary Members, 163 Life Members, and 877 Student Members. There are five Regional Chapters of the ASJ: Kansai, Tohoku, Tokai, Kyushu and Hokuriku.

The ASJ promotes the advancement of the science and technology of acoustics, in all its disciplines, and the exchange of ideas among these various disciplines. The motto of the Society since it was founded is "Acoustics as a discursive, integrated and interdisciplinary science."

\section{EXECUTIVE COUNCIL}

The Executive Council of the ASJ consists of 27 members and meets every month. Under the Council, several committees are formed to work on tasks to promote acoustics, including:

- Administrative Planning • Editorial Board

- Technical Council - Marketing

- Scientific Meetings - Education in Research

- Transition to Electronic Mediums

- Publicity - Publishing

- International Affairs - Special Events

- Membership - Acoustical Standards

\section{TECHNCIAL COMMITEES}

The Society has nine Technical Committees:

- Architectural Acoustics - Electroacoustics

- Musical Acoustics - Noise and Vibration

- Speech

- Acoustic Imaging

- Psychological and Physiological Acoustics

- Ultrasonic and Underwater Acoustics

- Acoustics Education

Each Committee holds a meeting with paper presentations almost every month at various places in Japan.

Other than these technical committees, several temporary Research Committees are formed to study important and timely specific problems. As of August 2016, these include:

- Road Traffic Noise - Acoustic Barrier-free

- Sound Design • Thermoacoustic Technology

- Mass Notification Sound Systems

- Cartilage Conduction Hearing • Bioacoustics

- Human Speech Communication
The ASJ periodically offers fundamental training courses as well as continuing education in various areas, such as psychophysical measurement and signal processing.

\section{PUBLICATIONS}

The Society publishes a monthly journal in Japanese, the Journal of the Acoustical Society of Japan. ASJ also publishes a bimonthly journal in English, the Acoustical Science and Technology, which is available online at no cost (https:// www.jstage.jst.go.jp/browse/ast). These journals include technical papers and review papers. Special issues are occasionally organized and published. The Society also publishes textbooks and reference books to promote acoustics on various topics.

\section{MEETINGS}

Three-day meetings are held in the spring and autumn every year. The meetings cover a wide range of topics in acoustics. At each meeting there are about 600 papers presented and an average of 1,200 participants.

The Society has also hosted or sponsored a large number of International Conferences, including the four ASA-ASJ Joint Meetings in 1978, 1988, 1996 and 2006. Some other major conferences are ICA in 1968 and 2004, Inter-Noise in 1975, 1994 and 2011, ICASSP in 1986, the Intl. Conference on Music Perception and Cognition in 1989, ICSLP in 1990 and 1994, the Intl. Symposium on Active Control of Sound and Vibration in 1991, Intl. Symposium on Simulation, Visualization and Auralization for Acoustic Research and Education in 1997, WESTPRAC VII in 2000 and the ASJ-EAA Joint Symposium in 2002.

\section{PRIZES AND AWARDS}

The Prizes for Distinguished Achievements in Acoustics are given to at most three persons a year. Among them, the Sato Prize is awarded every year for one or two outstanding papers appearing in the ASJ journals to commemorate Dr. Koji Sato, a founding member of ASJ. The Awaya Prize is given every year to around six promising young researchers for outstanding presentations given at the national meetings, to commemorate Dr. Kiyoshi Awaya. The Prize for Outstanding Technological Development in Acoustics is given to about three groups or persons each year.

For more information about the ASJ contact:

The Acoustical Society of Japan

Nakaura 5th-Bldg., 2-18-20 Sotokanda,

Chiyoda-ku, Tokyo 101-0021, Japan

FAX. +81 352561022 URL http://www.asj.gr.jp 
The 5th Joint Meeting of the Acoustical Society of America and the Acoustical Society of Japan will be held Monday through Friday, 28 November-2 December 2016 at the Hilton Hawaiian Village Beach Resort in Honolulu, Hawaii.

\section{SECTION HEADINGS}

1. HOTEL INFORMATION

2. TRANSPORTATION AND TRAVEL

3. STUDENT TRANSPORTATION SUBSIDIES

4. MESSAGES FOR ATTENDEES

5. REGISTRATION

6. TECHNICAL SESSIONS

7. TECHNICAL SESSION DESIGNATIONS

8. OPENING PLENARY SESSION

9. ROSSING PRIZE IN ACOUSTICS EDUCATION AND ACOUSTICS EDUCATION PRIZE LECTURE

10. MEDWIN PRIZE IN ACOUSTICAL OCEANOGRAPHY AND ACOUSTICAL OCEANOGRAPHY PRIZE LECTURE

11. EXHIBIT AND EXHIBIT RECEPTION

12. RESUME HELP DESK

13. TECHNICAL COMMITTEE OPEN MEETINGS

14. TECHNICAL TOUR

15. PLENARY SESSION AND AWARDS CEREMONY

16. ANSI STANDARDS COMMITTEES

17. COFFEE BREAKS

18. A/V PREVIEW ROOM

19. PROCEEDINGS OF MEETINGS ON ACOUSTICS

20. E-MAIL AND INTERNET ZONE

21. SOCIAL

22. BANQUET

23. STUDENTS MEET MEMBERS FOR LUNCH

24. STUDENT EVENTS: NEW STUDENT ORIENTATION, MEET AND GREET, STUDENT RECEPTION

25. WOMEN IN ACOUSTICS LUNCHEON

26. JAM SESSION

27. ACCOMPANYING PERSONS PROGRAM

28. WEATHER

29. TECHNICAL PROGRAM ORGANIZING COMMITTEE

30. MEETING ORGANIZING COMMITTEE

31. PHOTOGRAPHING AND RECORDING

32. ABSTRACT ERRATA

33. GUIDELINES FOR ORAL PRESENTATIONS

34. SUGGESTIONS FOR EFFECTIVE POSTER PRESENTATIONS

35. GUIDELINES FOR USE OF COMPUTER PROJECTION

36. DATES OF FUTURE ASA MEETINGS

\section{HOTEL INFORMATION}

The Hilton Hawaiian Village Beach Resort (2005 Kalia Road, Honolulu, Hawaii 96815) is the headquarters hotel where all meeting events will be held.

\section{TRANSPORTATION AND TRAVEL}

The Honolulu International Airport (http://hawaii.gov/hnl), located on the island of Oahu, is served by 27 international and domestic carriers, three interisland airlines, and four commuter airlines. All major U.S. carriers fly from all major airports in the U.S. to Honolulu. Many Japanese and International carriers have direct flights from Tokyo, Osaka. Sapporo, Nagoya, and Fukuoka to Honolulu.

Airport transportation alternatives include:

Taxi: AMPCO EXPRESS is the managing contractor for the airport's taxi system. Taxis are available at the airport by crossing to the median at Baggage Claim D and G and arranging with a person in a gold-colored shirt with the words TAXI DISPATCHER in black letters on the back. Fare to the hotel is approximately USD \$35-40 one way. For standard-sized baggage there is no charge.

Hele Transportation Services, airport shuttle and taxi: Transportation services all day, every day. Reservations: 1-808-782-1722; Email: hele24hawaii.com; Flat rate: 1 to 3 passengers USD \$24 per vehicle, 4 passengers USD \$32, 5 passengers USD \$38, 6 passengers USD \$44. No charge for standard-sized bags.

Shuttle: Aloha Airport Shuttle, private car service with mini vans. Reservations: 808-538-3355 or toll free: 855-538-3355, call at least 3 days ahead; email: alohaairportshuttle.com Rate: USD \$30 one way, 1-4 passengers.

Car Rental: From Baggage Claim E follow signs to car rental desks for Advantage, Avis, Budget, Hertz, and National. For other rental companies cross to median for rental car shuttle and sites beyond the airport.

Parking: Parking is available at the Hilton Hawaiian Village for USD \$29/day + tax for self-parking and \$36/day + tax for valet parking.

\section{STUDENT TRANSPORTATION SUBSIDIES}

To encourage student participation, limited funds are available to defray partially the cost of travel expenses of students to attend Acoustical Society meetings. Instructions for applying for travel subsidies are given in the Call for Papers which can be found online at http://acousticalsociety. org. The deadline for the present meeting has passed but this information may be useful in the future.

\section{MESSAGES FOR ATTENDEES}

A message board will be located in the Grand Ballroom Foyer near the ASA registration desk. Check the board during the week as messages may be posted by attendees who do not have cell phone numbers of other attendees.

\section{REGISTRATION}

Registration is required for all attendees and accompanying persons. Registration badges must be worn in order to participate in technical sessions and other meeting activities.

Registration will open on Sunday, 27 November, 1:00 p.m. in the Coral Foyer (see floor plan on page A13).

Checks or travelers checks in U.S. funds drawn on U.S. banks and Visa, MasterCard and American Express credit cards will be accepted for payment of registration. Meeting attendees who have pre-registered may pick up their badges and registration materials at the pre-registration desk. 
The registration fees (in USD) are $\$ 570$ for members of the Acoustical Society of America or the Acoustical Society of Japan; \$645 for nonmembers, \$150 for Emeritus members (Emeritus status pre-approved by ASA or ASJ), \$295 for ASA Early Career members (for ASA members within three years of their most recent degrees - proof of date of degree required), $\$ 100$ for ASA Student members, \$135 for students who are not members of ASA, $\$ 0$ for Nonmember Undergraduate Students, and $\$ 150$ for accompanying persons.

One-day registration is available at $\$ 295$ for members and $\$ 360$ for nonmembers (one-day means attending the meeting on only one day either to present a paper and/or to attend sessions). A nonmember who pays the $\$ 645$ nonmember registration fee and simultaneously applies for Associate Membership in the Acoustical Society of America will be given a $\$ 50$ discount off their dues payment for 2017 dues.

Invited speakers who are members of the Acoustical Society of America are expected to pay the registration fee, but nonmember invited speakers who participate in the meeting only on the day of their presentation may register without charge. The registration fee for nonmember invited speakers who wish to participate for more than one day is $\$ 115$ and includes a one-year Associate Membership in the ASA upon completion of an application form.

Special note to students who pre-registered online: You will also be required to show your student id card when picking-up your registration materials at the meeting.

\section{TECHNICAL SESSIONS}

The technical program includes 173 sessions with over 2050 abstracts scheduled for presentation during the meeting.

A floor plan of the Hilton Hotel appears on pages A12. Session Chairs have been instructed to adhere strictly to the printed time schedule, both to be fair to all speakers and to permit attendees to schedule moving from one session to another to hear specific papers. If an author is not present to deliver a lecture-style paper, the Session Chairs have been instructed either to call for additional discussion of papers already given or to declare a short recess so that subsequent papers are not given ahead of the designated times.

Several sessions are scheduled in poster format, with the display times indicated in the program schedule.

\section{TECHNICAL SESSION DESIGNATIONS}

The first character is a number indicating the day the session will be held, as follows:

1-Monday, 28 November

2-Tuesday, 29 November

3-Wednesday, 30 November

4-Thursday, 1 December

5-Friday, 2 December

The second character is a lower case "a" for a.m., "p" for p.m., or "e" for evening corresponding to the time of day the session will take place. The third and fourth characters are capital letters indicating the primary Technical Committee that organized the session using the following abbreviations or codes:

AA Architectural Acoustics

AB Animal Bioacoustics
AO Acoustical Oceanography

BA Biomedical Acoustics

EA Engineering Acoustics

ED Education in Acoustics

ID Interdisciplinary

MU Musical Acoustics

NS Noise

PA Physical Acoustics

PP Psychological and Physiological Acoustics

SA Structural Acoustics and Vibration

SC Speech Communication

SP Signal Processing in Acoustics

UW Underwater Acoustics

In sessions where the same group is the primary organizer of more than one session scheduled in the same morning or afternoon, a fifth character, either lower-case "a," "b," or "c" is used to distinguish the sessions. Each paper within a session is identified by a paper number following the session-designating characters, in conventional manner. As hypothetical examples: paper 2pEA3 would be the third paper in a session on Tuesday afternoon organized by the Engineering Acoustics Technical Committee; 3pSAb5 would be the fifth paper in the second of two sessions on Wednesday afternoon sponsored by the Structural Acoustics and Vibration Technical Committee.

Note that technical sessions are listed both in the calendar and the body of the program in the numerical and alphabetical order of the session designations rather than the order of their starting times. For example, session 3aAA would be listed ahead of session $3 \mathrm{aAO}$ even if the latter session begins earlier in the same morning.

\section{OPENING PLENARY SESSION}

The meeting will begin with an opening plenary session on Monday, 28 November, at 8.00 a.m. in Coral 4/5.

Dr. Hiroshi Hosoi from the ASJ and Nara Medical University will present Cartilage Conduction Hearing-New Sound Conduction Pathway in which he will discuss the discovery of new sound conduction pathways in mammals.

Dr. Peter Narins from the ASA and the University of California Los Angeles, will present Adventures of an Expeditionary Biologist: Neuroethology of Ultrasonic Communication in Amphibians. He will discuss adaptational studies that involve two distantly related organisms: the concave-eared torrent frog (Odorranatormota), calling near fast-flowing mountain streams of Anhui Province, Central China, and the endemic Bornean frog, Huiacavitympanum, living in a very similar riverine habitat in Sarawak, Malaysia.

\section{ROSSING PRIZE IN ACOUSTICS EDUCATION AND ACOUSTICS EDUCATION PRIZE LECTURE}

The 2016 Rossing Prize in Acoustics Education will be presented to Brad H. Story, University of Arizona, at the Plenary Session on Thursday, 1 December. Brad Story will present the Acoustics Education Prize Lecture titled "The role of artificial speech in understanding the acoustic characteristics of spoken communication"on Thursday, 1 December, at 4:05 p.m.in Session 4pEDb in South Pacific 4. 


\section{MEDWIN PRIZE IN ACOUSTICAL OCEANOGRAPHY AND ACOUSTICAL OCEANOGRAPHY PRIZE LECTURE}

The 2016 Medwin Prize in Acoustical Oceanography will be presented to Thomas C. Weber, University of New Hampshire, at the Plenary Session on Thursday, 1 December. Thomas Weber will present the Acoustical Oceanography Prize Lecture titled "Acoustic observations and characterization of oceanic methane gas bubbles rising from the seabed" on Tuesday, 29 November, at 4:15 p.m. in Session 2pAOb in Coral 4.

\section{EXHIBIT AND EXHIBIT RECEPTION}

An instrument and equipment exhibit conveniently located near the registration area and meeting rooms, will be located in the Coral Foyer.

The Exhibit will include computer-based instrumentation, scientific books, sound level meters, sound intensity systems, signal processing systems, devices for noise control and acoustical materials, active noise control systems and other exhibits on acoustics.

The Exhibit will open on Monday with an evening reception with lite snacks and a complimentary drink. Coffee breaks on Tuesday and Wednesday mornings will be held in the exhibit area as well as an afternoon break on Tuesday.

Exhibit hours are Monday, 28 November, 5:30 p.m. to 7:00 p.m., Tuesday, 29 November, 9:00 a.m. to 5:00 p.m., and Wednesday, 30 November, 9:00 a.m. to 12:00 noon.

\section{RESUME HELP DESK}

Are you interested in applying for graduate school, a postdoctoral opportunity, a research scientist position, a faculty opening, or other position involving acoustics? If you are, please stop by the ASA Resume Help Desk in the Coral Foyer near the ASA registration desk. Members of the ASA experienced in hiring will be available review at your $\mathrm{CV}$, cover letter, and research and teaching statements to provide tips and suggestions to help you most effectively present yourself in today's competitive job market. The ASA Resume Help Desk will be staffed on Tuesday, Wednesday, and Thursday during the lunch hour for walk-up meetings. Appointments during these three lunch hours will also be available via a sign-up sheet.

\section{TECHNICAL COMMITTEE OPEN MEETINGS}

Technical Committees will hold open meetings on Tuesday and Wednesday. The meetings on Tuesday will be held in the evening after the social. On Wednesday Technical Committee open meetings will be held starting at 7:30 p.m. The schedule and rooms for each Committee meeting are given on page A10.

These are working, collegial meetings. Much of the work of the Society is accomplished by actions that originate and are taken in these meetings including proposals for special sessions, workshops and technical initiatives. All meeting participants are cordially invited to attend these meetings and to participate actively in the discussions.

\section{TECHNICAL TOUR}

Wednesday, 30 November, 8:30 a.m. to 12 noon - limited to 40 participants - Cost USD\$15

A tour of the Pacific Islands Fisheries Science Center (PIFSC) will be conducted on Wednesday morning with a bus leaving the Hilton Hawaiian Village at 8:30 a.m. and returning before noon. Inquire at the Registration Desk for availability.

PIFSC of the National Marine Fisheries Service (NMFS) is part of the National Oceanic and Atmospheric Administration (NOAA). The Center administers and conducts scientific research and monitoring programs that support the domestic and international conservation and management of living marine resources across the Pacific Islands Region. Key research focus areas include: coral reef ecosystems; fishery and protected species population assessments; marine mammal and sea turtle ecology; life history studies of fish; monitoring of fisheries activity; economics; human dimensions of resource management; development of by catch reduction technologies; health and disease in protected species; fishery interactions with protected species; ecosystem monitoring and integrated assessment; and impacts of climate change on the environment and ecosystems.

Note: All tour participants must submit the following information: place of birth including country, birth date, and passport number (for non-US citizens). Tour registrants will be sent forms requesting this information after they register. The tour registration fee is USD \$15/person and the tour is limited to 40 participants.

\section{PLENARY SESSION AND AWARDS CEREMONY}

A plenary session will be held Thursday, 1 December, immediately following the Banquet in Coral $4 / 5$ at approximately 8:15 p.m.

The Rossing Prize in Acoustics Education will be presented to Brad H. Story. The Medwin Prize in Acoustical Oceanography will be presented to Thomas C. Weber. The Trent-Crede Medal will be presented to Earl G. Williams.

Certificates will be presented to Fellows elected at the Salt Lake City meeting of the Society. See page 3353 for a list of fellows.

All attendees are welcome and encouraged to attend. Please join us to honor and congratulate these medalists and other award recipients.

\section{ANSI STANDARDS COMMITTEES}

Meetings of ANSI Accredited Standards Committees will not be held at the Honolulu meeting.

Meetings of selected advisory working groups are often held in conjunction with Society meetings and are listed in the Schedule of Committee Meetings and Other Events on page A24 or on the standards bulletin board in the registration area, e.g., S12/WGI8-Room Criteria.

People interested in attending and in becoming involved in working group activities should contact the ASA Standards Manager for further information about these groups, or about the ASA Standards Program in general, at the following address: Neil Stremmel, ASA Standards Manager, Standards Secretariat, Acoustical Society of America, 1305 Walt Whitman Road, Suite 300, Melville, NY 11747-4300; T.: 631390-0215; F: 631-923-2875; E: asastds@ acousticalsociety.org

\section{COFFEE BREAKS}

Morning coffee breaks will be held each day from 9:30 a.m. to 10:45 a.m. in the Coral Foyer. Morning breaks on Tuesday 
and Wednesday will be held in the Exhibit area. There will also be an afternoon break on Tuesday from 2:30 p.m. to 3:45 p.m.in the Exhibit area.

\section{A/V PREVIEW ROOM}

MPCC II on the Coral Foyer level will be set up as an A/V preview room for authors' convenience, and will be available on Monday through Friday from 7:00 a.m. to 5:00 p.m.

\section{PROCEEDINGS OF MEETINGS ON ACOUSTICS (POMA)}

The Honolulu meeting will have a published proceedings, and submission is optional. The proceedings will be a separate volume of the online journal, "Proceedings of Meetings on Acoustics" (POMA). This is an open access journal, so that its articles are available in pdf format for downloading without charge to anyone in the world. Authors who are scheduled to present papers at the meeting are encouraged to prepare a suitable version in pdf format that will appear in POMA. It is not necessary to wait until after the meeting to submit one's paper to POMA. Further information regarding POMA can be found at the site http://acousticsauthors.org. Published papers from previous meeting can be seen at the site http://asadl/poma.

\section{E-MAIL AND INTERNET ZONE}

Wi-Fi will be available in all ASA meeting rooms and spaces.

Computers providing e-mail access will be available 7:00 a.m. to 5:00 p.m., Monday to Friday between MPCC I and MPCC II in the Coral Foyer.

Tables with power cords will be set up in the Coral Foyer for attendees to gather and to power-up their electronic devices.

\section{SOCIAL}

A Social will be held on Tuesday evening, 6:00 p.m. to 7:30 p.m. on the Great Lawn at the Hilton Hawaiian Village.

The ASA hosts these social hours to provide a relaxing setting for meeting attendees to meet and mingle with their friends and colleagues as well as an opportunity for new members and first-time attendees to meet and introduce themselves to others in the field. A second goal of the socials is to provide a sufficient meal so that meeting attendees can attend the open meetings of Technical Committees that begin immediately after the social.

\section{BANQUET}

A banquet will be held on Thursday evening, 1 December. Banquet festivities will start at 6:00 p.m. in the Hilton Hawaiian Village Waikiki Beach Resort with a no-host social hour in the Coral Foyer. The banquet, starting at 7:00 p.m. in Coral 4/5, features a Chinese dinner. There will be a special presentation on Hawaiian music as part of the entertainment. The awards ceremony will be held during the banquet.

Banquet tickets are available for USD \$40 each (students USD \$25). Tickets are limited and are not included in the registration fee. Tickets may be purchased at the meeting subject to availability until 5:00 p.m. on Tuesday, 29 November.

\section{STUDENTS MEET MEMBERS FOR LUNCH}

The ASA Education Committee arranges for a student to meet one-on-one with a member of the Acoustical Society over lunch. The purpose is to make it easier for students to meet and interact with members at ASA Meetings. Each lunch pairing is arranged separately. Students who are interested should contact Dr. David Blackstock, University of Texas at Austin, by email dtb@mail.utexas.edu. Please provide your name, university, department, degree you are seeking (BS, MS, or PhD), research field, acoustical interests, your supervisor's name, days you are free for lunch, and abstract number (or title) of any paper(s) you are presenting. The sign-up deadline is 12 days before the start of the Meeting, but an earlier sign-up is strongly encouraged. Each participant pays for his/her own meal.

\section{STUDENT EVENTS: NEW STUDENTS ORIENTATION, MEET AND GREET, STUDENT RECEPTION}

Follow the student twitter throughout the meeting@ ASAStudents.

A New Students Orientation will be held from 5:00 p.m. to 5:30 p.m. on Monday, 28 November, in the Hibiscus room for all students to learn about the activities and opportunities available for students at the Honolulu meeting. This will be followed by the Student Meet and Greet from 5:30 p.m. to 7:00 p.m. in Rainbow I and II. Refreshments and a cash bar will be available.

The Students' Reception will be held on Wednesday, 30 November, from 6:00 p.m. to 8:00 p.m. in Rainbow I and II.This reception, sponsored by the Acoustical Society of America and supported by the National Council of Acoustical Consultants, will provide an opportunity for students to meet informally with fellow students and other members of the Acoustical Society. All students are encouraged to attend, especially students who are first time attendees or those from smaller universities.

Students will find a sticker in their registration envelopes to place on their name tags identifying them as students. Although wearing the sticker is not mandatory, it will allow for easier networking between students and other meeting attendees.

Students are encouraged to refer to the student guide, also found in their envelopes, for important program and meeting information pertaining only to students attending the ASA meeting.

They are also encouraged to visit the official ASA Student Home Page at http://asastudentcouncil.org// to learn more about student involvement in ASA.

\section{WOMEN IN ACOUSTICS LUNCHEON}

The Women in Acoustics luncheon will be held at 11:45 a.m. on Wednesday, 30 November, in the Rainbow Suite. Those who wish to attend must purchase their tickets in advance by 10:00 a.m. on Tuesday, 30 November. The fee is USD \$30 for non-students and USD\$15 for students.

\section{JAM SESSION}

You are invited to Irish Rose Saloon on Wednesday night, 30 November, from 8:00 p.m. to midnight for the ASA Jam. (478 Ena Rd., Honolulu, a 5-minute walk from the Hilton.) Bring your axe, horn, sticks, voice, or anything else that makes music. Musicians and non-musicians are all welcome 
to attend. A full PA system, backline equipment, guitars, bass, keyboard, and drum set will be provided. All attendees will enjoy live music, a cash bar with snacks, and all-around good times. Don't miss out.

\section{ACCOMPANYING PERSONS PROGRAM}

Spouses and other visitors are welcome at the Honolulu meeting. The on-site registration fee for accompanying persons is USD \$150. This entitles access to the accompanying persons room, social on Tuesday evening, the Jam Session. Accompanying Persons may also attend the Thursday evening banquet by purchasing a banquet ticket.

A hospitality room for accompanying persons will be open in Rainbow I at the Hilton from 8:00 a.m. to 10:00 a.m. Monday through Friday.

There will be a short program daily lasting for about 1 hour and will include activities such as lei making, hula demonstration and history, and other presentations of general interest.

Breakfast snacks including beverages will be provided.

\section{WEATHER}

The weather is generally very pleasant in the latter part of November, but cool weather can occur. For the week of the ASA meeting, temperatures are typically in the mid 70-80's during the day, dropping into the lower 60 - 70's at night. Occasional but brief rain showers can occur suddenly so having an umbrella or rain jacket is advisable.

\section{TECHNICAL PROGRAM ORGANIZING COMMITTEE}

Bruce M. Howe, Hiroshi Saruwatari, Technical Program Cochairs; David P. Knobles, Hiroyuki Hachiya, Acoustical Oceanography; Benjamin Taft, Jennifer Miksis-Olds, Hiroyuki Hachiya, Animal Bioacoustics; Damian J. Doria, Ian B. Hoffman, Hayato Sato, Mari Ueda, Architectural Acoustics; Siddhartha Sikdar, Kang Kim, Shin-ichiro Umemura, Hideyuki Nomura, Yasuhiro Oikawa, Biomedical Acoustics; Andrew Piacsek, Eoin King, Akira Nishimura, Education in Acoustics; Kenneth M. Walsh, Yoshifumi Chisaki, Yasuhiro Oikawa, Mari Ueda, Engineering Acoustics; Andrew C.H. Morrison, Masashi Yamada, Musical Acoustics; James E. Phillips, William J. Murphy, Akira Omoto, Noise; Michael R. Haberman, Hideyuki Nomura, Yasuhiro Oikawa, Physical Acoustics; Christopher A. Brown, Masato Akagi, Psychological and Physiological Acoustics; Brian E. Anderson, Mari Ueda, Yoshifumi Chisaki, Signal Processing in Acoustics; Alexander L. Francis, Melissa Baese-Berk, Tuuli Morrill, Norihide Kitaoka, Masato Akagi, Mari Ueda, Speech Communication; Benjamin M. Shafer, Akira Omoto, Structural Acoustics and Vibration; Todd Hefner, Derek Olson, Hiroyuki Hachiya, Underwater Acoustics; Brent Reichman, Kelly L. Whiteford, Student Council.

\section{MEETING ORGANIZING COMMITTEE}

ASA Meeting Committee: Whitlow W.L. Au, Chair; David L. Adams, Cochair; Bruce M. Howe, Technical Program Chair; Gary McAuliffe, Audio/Visual; David L. Adams/Robert Badham, Food/Beverage; Benjamin Reeder, Signs; Aude Pacini. Posters; Dorothy Au, Accompanying Persons; Jessica Chen, Student Coordinator; Robert Badham, Meeting Administrator.
ASJ Meeting Committee: Yoshiaki Watanabe, Cochair; Akio Ando, Cochair; Hiroshi Sato, Vice Chair; Hiroshi Saruwatari, Technical Program Chair; Yasuhiro Oikawa, Secretary.

\section{PHOTOGRAPHING AND RECORDING}

Photographing and recording during regular sessions are not permitted without prior explicit permission of the presenter.

\section{ABSTRACT ERRATA}

This meeting program is Part 2 of the October 2016 issue of The Journal of the Acoustical Society of America. Corrections, for printer's errors only, may be submitted for publication in the Errata section of the Journal.

\section{GUIDELINES FOR ORAL PRESENTATIONS Preparation of Visual Aids}

See the guidelines for computer projection in section 41 below.

- Allow at least one minute of your talk for each slide (e.g., PowerPoint). No more than 12 slides for a 15 -minute talk (with 3 minutes for questions and answers).

- Minimize the number of lines of text on one visual aid. 12 lines of text should be a maximum. Include no more than 2 graphs/plots/figures on a single slide. Generally, too little information is better than too much.

- Presentations should contain simple, legible text that is readable from the back of the room.

- Characters should be at least 0.25 inches $(6.5 \mathrm{~mm})$ in height to be legible when projected. A good rule of thumb is that text should be 20 point or larger (including labels in inserted graphics). Anything smaller is difficult to read.

- Make symbols at least $1 / 3$ the height of a capital letter.

- For computer presentations, use all of the available screen area using landscape orientation with very thin margins. If your institutions logo must be included, place it at the bottom of the slide.

- Sans serif fonts (e.g., Arial, Calibri, and Helvetica) are much easier to read than serif fonts (e.g., Times New Roman) especially from afar. Avoid thin fonts (e.g., the horizontal bar of an e may be lost at low resolution thereby registering as a c.)

- Do not use underlining to emphasize text. It makes the text harder to read.

- All axes on figures should be labeled.

- No more than 3-5 major points per slide.

- Consistency across slides is desirable. Use the same background, font, font size, etc. across all slides.

- Use appropriate colors. Avoid complicated backgrounds and do not exceed four colors per slide. Backgrounds that change from dark to light and back again are difficult to read. Keep it simple.

- If using a dark background (dark blue works best), use white or yellow lettering. If you are preparing slides that may be printed to paper, a dark background is not appropriate.

- If using light backgrounds (white, off-white), use dark blue, dark brown or black lettering.

- DVDs should be in standard format. 


\section{Presentation}

- Organize your talk with introduction, body, and summary or conclusion. Include only ideas, results, and concepts that can be explained adequately in the allotted time. Four elements to include are:

\author{
- Statement of research problem \\ - Research methodology \\ Review of results \\ - Conclusions
}

- Generally, no more than 3-5 key points can be covered adequately in a 15-minute talk so keep it concise.

- Rehearse your talk so you can confidently deliver it in the allotted time. Session Chairs have been instructed to adhere to the time schedule and to stop your presentation if you run over.

- An A/V preview room will be available for viewing computer presentations before your session starts. It is advisable to preview your presentation because in most cases you will be asked to load your presentation onto a computer which may have different software or a different configuration from your own computer.

- Arrive early enough so that you can meet the session chair, load your presentation on the computer provided, and familiarize yourself with the microphone, computer slide controls, laser pointer, and other equipment that you will use during your presentation. There will be many presenters loading their materials just prior to the session so it is very important that you check that all multi-media elements (e.g., sounds or videos) play accurately prior to the day of your session.

- Each time you display a visual aid the audience needs time to interpret it. Describe the abscissa, ordinate, units, and the legend for each figure. If the shape of a curve or some other feature is important, tell the audience what they should observe to grasp the point. They won't have time to figure it out for themselves. A popular myth is that a technical audience requires a lot of technical details. Less can be more.

- Turn off your cell phone prior to your talk and put it away from your body. Cell phones can interfere with the speakers and the wireless microphone.

\section{SUGGESTIONS FOR EFFECTIVE POSTER PRESENTATIONS \\ Content}

The poster should be centered around two or three key points supported by the title, figures, and text. The poster should be able to "stand alone." That is, it should be understandable even when you are not present to explain, discuss, and answer questions. This quality is highly desirable since you may not be present the entire time posters are on display, and when you are engaged in discussion with one person, others may want to study the poster without interrupting an ongoing dialogue.

- To meet the "stand alone" criteria, it is suggested that the poster include the following elements, as appropriate:

- Background

- Objective, purpose, or goal

- Hypotheses

- Methodology

- Results (including data, figures, or tables)
- Discussion

- Implications and future research

- References and Acknowledgment

\section{Design and layout}

- A board approximately $8 \mathrm{ft}$. wide $\times 4 \mathrm{ft}$. high will be provided for the display of each poster. Supplies will be available for attaching the poster to the display board. Each board will be marked with an abstract number.

- Typically posters are arranged from left to right and top to bottom. Numbering sections or placing arrows between sections can help guide the viewer through the poster.

- Centered at the top of the poster, include a section with the abstract number, paper title, and author names and affiliations. An institutional logo may be added. Keep the design relatively simple and uncluttered. Avoid glossy paper.

\section{Lettering and text}

- Font size for the title should be large (e.g., 70-point font)

- Font size for the main elements should be large enough to facilitate readability from 2 yards away (e.g., 32 point font). The font size for other elements, such as references, may be smaller (e.g., 20-24 point font).

- Sans serif fonts (e.g., Arial, Calibri, Helvetica) are much easier to read than serif fonts (e.g., Times New Roman).

- Text should be brief and presented in a bullet-point list as much as possible. Long paragraphs are difficult to read in a poster presentation setting.

\section{Visuals}

- Graphs, photographs, and schematics should be large enough to see from 2 yards (e.g., $8 \times 10$ inches).

- Figure captions or bulleted annotation of major findings next to figures are essential. To ensure that all visual elements are "stand alone," axes should be labeled and all symbols should be explained.

- Tables should be used sparingly and presented in a simplified format.

\section{Presentation}

- Prepare a brief oral summary of your poster and short answers to likely questions in advance.

- The presentation should cover the key points of the poster so that the audience can understand the main findings. Further details of the work should be left for discussion after the initial poster presentation.

- It is recommended that authors practice their poster presentation in front of colleagues before the meeting. Authors should request feedback about the oral presentation as well as poster content and layout.

\section{Other suggestions}

- You may wish to prepare reduced-size copies of the poster (e.g., $81 / 2 \times 11$ sheets) to distribute to interested audience members.

\section{GUIDELINES FOR USE OF COMPUTER PROJECTION}

A PC computer with monaural audio playback capability and projector will be provided in each meeting room on 
which all authors who plan to use computer projection should load their presentations. Authors should bring computer presentations on a CD or USB drive to load onto the provided computer and should arrive at the meeting rooms at least 30 minutes before the start of their sessions. Assistance in loading presentations onto the computers will be provided.

Note that only PC format will be supported so authors using Macs must save their presentations for projection in PC format. Also, authors who plan to play audio during their presentations should insure that their sound files are also saved on the CD or USB drive.

\section{Introduction}

It is essential that each speaker who plans to use his/her own laptop connect to the computer projection system in the $\mathrm{A} / \mathrm{V}$ preview room prior to session start time to verify that the presentation will work properly. Technical assistance is available in the $\mathrm{A} / \mathrm{V}$ preview room at the meeting, but not in session rooms. Presenters whose computers fail to project for any reason will not be granted extra time.

\section{Guidelines}

- Set your computer's screen resolution to $1024 \times 768$ pixels or to the resolution indicated by the AV technical support. If it looks OK, it will probably look OK to your audience during your presentation.

- Remember that graphics can be animated or quickly toggled among several options: Comparisons between figures may be made temporally rather than spatially.

- Animations often run more slowly on laptops connected to computer video projectors than when not so connected. Test the effectiveness of your animations before your assigned presentation time on a similar projection system (e.g., in the $\mathrm{A} / \mathrm{V}$ preview room). Avoid real-time calculations in favor of pre-calculation and saving of images.

- If you will use your own laptop instead of the computer provided, connect your laptop to the projector during the question/answer period of the previous speaker. It is good protocol to initiate your slide show (e.g., run PowerPoint) immediately once connected, so the audience doesn't have to wait. If there are any problems, the session chair will endeavor to assist you, but it is your responsibility to ensure that the technical details have been worked out ahead of time.

- During the presentation have your laptop running with main power instead of using battery power to insure that the laptop is running at full CPU speed. This will also guarantee that your laptop does not run out of power during your presentation.

\section{Specific Hardware Configurations Macintosh}

Older Macs require a special adapter to connect the video output port to the standard 15-pin male DIN connector. Make sure you have one with you.

- Hook everything up before powering anything on. (Connect the computer to the RGB input on the projector).

- Turn the projector on and boot up the Macintosh. If this doesn't work immediately, you should make sure that your monitor resolution is set to $1024 \times 768$ for an XGA projector or at least $640 \times 480$ for an older VGA projector. $(1024 \times 768$ will most always work.). You should also make sure that your monitor controls are set to mirroring. If it's an older PowerBook, it may not have video mirroring, but something called simulscan, which is essentially the same.

- Depending upon the vintage of your Mac, you may have to reboot once it is connected to the computer projector or switcher. Hint: you can reboot while connected to the computer projector in the $\mathrm{A} / \mathrm{V}$ preview room in advance of your presentation, then put your computer to sleep. Macs thus booted will retain the memory of this connection when awakened from sleep.

- Depending upon the vintage of your system software, you may find that the default video mode is a side-by-side configuration of monitor windows (the test for this will be that you see no menus or cursor on your desktop; the cursor will slide from the projected image onto your laptop's screen as it is moved). Go to Control Panels, Monitors, configuration, and drag the larger window onto the smaller one. This produces a mirror-image of the projected image on your laptop's screen.

- Also depending upon your system software, either the Control Panels will automatically detect the video projector's resolution and frame rate, or you will have to set it manually. If it is not set at a commensurable resolution, the projector may not show an image. Experiment ahead of time with resolution and color depth settings in the $\mathrm{A} / \mathrm{V}$ preview room (please don't waste valuable time adjusting the Control Panel settings during your allotted session time).

\section{PC}

- Make sure your computer has the standard female 15-pin DE-15 video output connector. Some computers require an adaptor.

- Once your computer is physically connected, you will need to toggle the video display on. Most PCS use either ALTF5 or F6, as indicated by a little video monitor icon on the appropriate key. Some systems require more elaborate keystroke combinations to activate this feature. Verify your laptop's compatibility with the projector in the $\mathrm{A} / \mathrm{V}$ preview room. Likewise, you may have to set your laptop's resolution and color depth via the monitor's Control Panel to match that of the projector, which settings you should verify prior to your session.

\section{Linux}

- Most Linux laptops have a function key marked CRT/LCD or two symbols representing computer versus projector. Often that key toggles on and off the VGA output of the computer, but in some cases, doing so will cause the computer to crash. One fix for this is to boot up the BIOS and look for a field marked CRT/LCD (or similar). This field can be set to Both, in which case the signal to the laptop is always presented to the VGA output jack on the back of the computer. Once connected to a computer projector, the signal will appear automatically, without toggling the function key. Once you get it working, don't touch it and it should continue to work, even after reboot. 


\section{DATES OF FUTURE ASA MEETINGS}

For further information on any ASA meeting, or to obtain instructions for the preparation and submission of meeting abstracts, contact the Acoustical Society of America, 1305 Walt Whitman Road, Suite 300, Melville, NY 11747-4300; Telephone: 516-576-2360; Fax: 631-923-2875; E-mail: asa@ acousticalsociety.org

173rd Meeting, Boston, Massachusetts, 25-29 June 2017

(The 3rd joint meeting of the Acoustical Society of America and the European Acoustics Association 174th Meeting, New Orleans, Louisiana, 4-8 December 2017 175th Meeting, Minneapolis, Minnesota, 7-11 May 2018 176th Meeting, Victoria, Canada, 6-9 November 2018 177th Meeting, Louisville, Kentucky, 13-17 May 2019 178th Meeting, TBD, fall 2019

179th Meeting, Chicago, Illinois, 11-15 May 2020 180th Meeting, Cancun, Mexico, fall 2020 


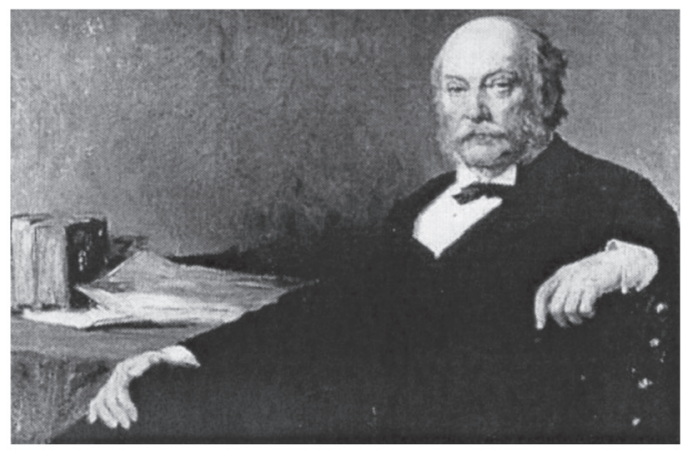

\section{SCIENTIFIC PAPERS Volumes $|-V|$ 1869-1919 Lord Rayleigh (John William Strutt)}

The Scientific Papers of Lord Rayleigh are now available on CD ROM from the Acoustical Society of America. The CD contains over 440 papers covering topics on sound, mathematics, general mechanics, hydrodynamics, optics, and properties of gasses. Files are in pdf format and readable with Adobe Acrobat ${ }^{\circledR}$ reader.

Lord Rayleigh was indisputably the single most significant contributor to the world's literature in acoustics. In addition to his epochal two volume treatise, The Theory of Sound, he wrote some 440 articles on acoustics and related subjects during the fiRy years of his distinguished research career. He is generally regarded as one of the best and clearest writers of scientific articles of his generation, and his papers continue to be read and extensively cited by modem researchers in acoustics.

ISBN 0-9744067-4-0 Price: $\$ 40.00$

\section{ORDER FORM}

1. Payment must accompany order. Payment may be made by check or international money order in U.S. funds drawn on a U.S. bank or by Visa, MasterCard or American Express credit card.

2. Send orders to: Acoustical Society of America Publications, P.O. Box 1020, Sewickley, PA 15143-9998; Tel.: 412-741-1979; Fax: 412-741-0609;

Name

Address

City State Postal Code Country

Tel.: E-mail:

Quantity

Unit Price

Total Cost

copies of the Scientific Papers CD ROM

[ ] Check or money order enclosed for $\$$

[ ] American Express [ ] Visa [ ] Master Card

Account \#

Security Code:

Exp. Date

Signature

Due to security risks and Payment Card Industry (PCI) data security standards e-mail is NOT an acceptable way to transmit credit card information. Please use our secure web page to process your credit card payment (http:// www.abdi-ecommerce10.com/asa) or securely fax this form to (412-741-0609). 


\section{Session 1aID}

\section{Interdisciplinary: Opening Ceremonies, Plenary Lectures}

Whitlow Au, Cochair

University of Hawaii, P.O. Box 1106, Kailua, HI 96734

Akio Ando, Cochair

Electric and Electronics Engineering, Faculty of Engineering, University of Toyama, 3190 Gofuku, Toyama 930-8555, Japan

Chair's Introduction-8:00

\section{Invited Papers}

\section{8:05}

1aID1. Adventures of an expeditionary biologist: Neuroethology of ultrasonic communication in amphibians. Peter M. Narins (Integrative Biology \& Physiol., UCLA, 621 Charles E. Young Dr. S., Los Angeles, CA 90095-1606, pnarins@ucla.edu)

Animal communication occurs when a signal generated by one individual is transmitted through an appropriate channel and results in a behavioral change in a second individual. We have explored specific morphological, physiological, and behavioral adaptations in a wide variety of taxa that appear to have evolved specifically to tailor and sculpt intraspecific communication systems. In this lecture, I will review one of these adaptational studies that involves two distantly related organisms: the concave-eared torrent frog (Odorrana tormota), calling near fast-flowing mountain streams of Anhui Province, Central China, and the endemic Bornean frog, Huia cavitympanum, living in a very similar riverine habitat in Sarawak, Malaysia. In addition to the high-pitched audible components, these species' calls contain previously unreported ultrasonic harmonics. Our studies of these two Asian frogs revealed that they communicate acoustically using ultrasound and that their auditory systems are sensitive up to $34-38 \mathrm{kHz}$. This extraordinary upward extension into the ultrasonic range of both the harmonic content of the advertisement calls and the frogs' hearing sensitivity is likely to have coevolved in response to the intense, predominately low-frequency ambient noise from local streams.

Chair's Introduction-9:00

\section{9:05}

1aID2. Cartilage conduction hearing-The third sound conduction pathway. Hiroshi Hosoi (Nara Medical Univ., 840 Shijo-cho, Kashihara, Nara 6348522, Japan, hosoi@naramed-u.ac.jp)

Hosoi found in 2004 that a clear sound can be heard when a vibration signal is delivered to the aural cartilage from a transducer. This form of signal transmission is referred to as cartilage conduction (CC). Then, we have examined its mechanism, application, and superiority over the well-known air or bone conduction (AC or BC). We have published 11 English articles including JASA by 2016. AC, $\mathrm{BC}$, and $\mathrm{CC}$ are distinguishable according to two points, namely, transmission media and movement of the skull bone. The transmission media are sound in AC, vibration in BC and CC. The movements of the bone are immobile in AC and CC, vibrated in BC. The world first CC hearing aids have been completed. It is very useful for the patients who cannot wear regular hearing aids because of atresia of external auditory canals, for example. More useful applications are cell phones and robots. The CC cell phone has a lot of advantage such as 1) easy volume control without button operation, 2) insulation from outer noise and obtaining the best S/N instantly, 3) no sound leakage to outward, 4) clean liquid crystal display, 5) easy to use even with hearing aids, 6) better suited for very small body type cell phone, and all that. There is no disadvantage compared with the regular phone. Various types of cell phone can be realized using CC. For example, wrist watch type, pen type, eye glass type, finger ring type, etc. Also, CC will give the good method to communication between robots and human beings. 


\title{
Session 1aAA
}

\section{Architectural Acoustics: Acoustics for Children and Pupils I}

\author{
Keiji Kawai, Cochair \\ Kumamoto University, 2-39-1 Kurokami, Kumamoto 860-8555, Japan \\ David S. Woolworth, Cochair \\ Oxford Acoustics, 356 CR 102, Oxford, MS 38655 \\ David Lubman, Cochair \\ DL Acoustics, 14301 Middletown Ln., Westminster, CA 92683-4514
}

\section{Invited Papers}

\section{$10: 35$}

1aAA1. Overview of acoustic environment of child daycare facilities in Japan: Current situation and recent researches. Keiji Kawai (Kumamoto Univ., 2-39-1 Kurokami, Kumamoto 860-8555, Japan, kkawai@kumamoto-u.ac.jp), Kanako Ueno, Saki Noguchi (Meiji Univ., Kawasaki, Kanagawa, Japan), and Hisao Funaba (Iwate Prefectural Univ., Takizawa, Iwate, Japan)

Demands for child daycare facilities are continuously increasing in Japan as the Japanese society is changing with respect to women's social advancement in the workforce. However, since there are virtually no guidelines for the acoustic design of daycare facilities in Japan, many daycare classrooms have been built without considering acoustics or sound insulation. As a result, the rooms tend to be too noisy and too reverberant for children in the process of language and listening acquisition. This is a report both on the present situation and architectural trend of daycare facilities and on the existing researches for the acoustic environment of daycare classrooms, aiming to share the information about daycare facilities in Japan for the discussions to develop guidelines of acoustic design.

\section{$10: 55$}

1aAA2. Statistically defining the construct of "acoustic quality" in K-12 classrooms. Joonhee Lee, Laura C. Brill (Durham School of Architectural Eng., Univ. of Nebraska - Lincoln, 1110 S. 67th St., Omaha, NE 68182-0816, joonhee.lee@huskers.unl.edu), Houston Lester, James Bovaird (The Dept. of Educational Psych., Univ. of Nebraska - Lincoln, Lincoln, NE), and Lily M. Wang (Durham School of Architectural Eng., Univ. of Nebraska - Lincoln, Omaha, NE)

This paper presents preliminary statistical analyses of acoustic measurements taken in over a hundred K-12 classrooms in Nebraska during the 2015-2016 academic year. Noise levels were continuously logged over two consecutive school days, three times seasonally. Other measurements included unoccupied background noise levels and room impulse responses. Equivalent sound pressure levels, percentile levels, reverberation times, and other assorted room acoustic metrics have been calculated from the measured data. Preliminary statistical analyses have been performed to investigate how each metric is related to each other and how the metrics are distributed across classrooms. These quantities have also been statistically analyzed to understand which are most pertinent to a comprehensive construct of "acoustic quality." This "acoustic quality" construct will subsequently be used to investigate how its components are related to the academic performance of students, and its inter-relationships with other environmental conditions such as indoor air quality, thermal, and lighting conditions. [Work supported by the United States Environmental Protection Agency Grant Number R835633.]

\section{1:15}

1aAA3. Exploring correlation between sound levels in active occupied classrooms and unoccupied classrooms. Laura C. Brill, Joonhee Lee, and Lily M. Wang (Durham School of Architectural Eng. and Construction, Univ. of Nebraska-Lincoln, 6349 Cedar Plz, \#222, Omaha, NE 68106, lbrill@huskers.unl.edu)

A large-scale in-situ survey of environmental conditions, including acoustics, is being conducted in over $200 \mathrm{~K}-12$ classrooms in multiple school districts by researchers at the University of Nebraska-Lincoln. Continuing analyses, conducted on logged acoustic sound levels collected seasonally over two occupied class days through Fall 2016, will be presented to determine how unoccupied noise levels are related to levels in the occupied active classroom. Do increased unoccupied noise levels correlate to increased occupied levels, and if so, to what degree? A number of methods are used to explore this relationship including spectral analyses of the logged data, further percentile level analyses, and linking the type of activity in the classroom more clearly to the logged sound levels. [Work supported by the United States Environmental Protection Agency Grant Number R835633.] 
1aAA4. Teachers' accumulation of silence and voicing periods of continuous speech in classroom with different reverberation times. Pasquale Bottalico (Communicative Sci. and Disord., Michigan State Univ., 1026 Red Cedar Rd., Lansing, MI 48910, pb@msu. edu)

The relationship between the distribution of continuous silence and voicing periods accumulated (duration of continuous voiced and unvoiced segments of $0.05 \mathrm{~s}$ ) by teachers teaching in classrooms with different reverberation time (RT) was examined. Understanding the distributions of these accumulations and the relationship with the RT has implications on potential vocal fatigue risk factors and it can be a useful information for clinicians. Twenty-two primary school teachers were monitored over 1 or 2 four-hour workdays with the Ambulatory Phonation Monitor. The RT ranged between $0.58 \mathrm{~s}$ and $1.58 \mathrm{~s}$, with a median equal to $0.9 \mathrm{~s}$. The classrooms were classified as low RT $(0.58 \mathrm{~s}<\mathrm{RT}<0.90 \mathrm{~s})$ and high RT $(0.90 \mathrm{~s} \leq \mathrm{RT}<1.58 \mathrm{~s})$. The values of silence accumulation in low RT classrooms were significantly shorter than the accumulation in high RT classrooms in almost the whole range of accumulation periods $(0.2 \mathrm{~s}-10 \mathrm{~s})$. The difference was negligible for time shorter than $1.5 \mathrm{~s}$ and longer than $8 \mathrm{~s}$. The values of voice accumulation in low RT classrooms are significantly shorter than the accumulation in high RT classrooms in almost the whole range of accumulation periods $(0.2 \mathrm{~s}-1.31 \mathrm{~s})$.

\title{
Session 1aAB
}

\section{Animal Bioacoustics: Bat Echolocation: New Insights on Biosonar Production, Processing, and Performance from Field and Laboratory Investigations I}

\author{
Hiroshi Riquimaroux, Cochair \\ Life and Medical Sciences, Doshisha University, Shandong University, 27 Shanda Nanlu, Jinan 250100, China \\ Laura Kloepper, Cochair \\ Biology, Saint Mary's College, 262 Science Hall, Saint Mary's College, Notre Dame, IN 46556
}

Chair's Introduction-10:30

\section{Invited Papers}

$10: 35$

1aAB1. The model for echolocation of insects by using bat-like sounds. Ikuo Matsuo (Dept. of Information Sci., Tohoku Gakuin Univ., Tenjinzawa 2-1-1, Izumi-ku, Sendai 9813193, Japan, matsuo@mail.tohoku-gakuin.ac.jp)

Bats use frequency-modulated echolocation to identify and capture flying insects in real three dimensional space. Experimental evidence indicates that bats are capable of locating static objects with a range accuracy of less than $1 \mu \mathrm{s}$. A previously introduced model could localize objects in two-dimensional space by accurately estimating the object's range at each timepoint by extracting temporal changes from the time-frequency pattern and interaural range differences while echoes were measured at two receiving points by intermittently emitting LFM sounds. In addition, this model was shown to localize moving objects in two-dimensional space by accurately estimating the object's range under the influences of the Doppler shift, which was dependent on the movements. However, it was not evaluated whether this model could detect and localize small sized objects, e.g., small moths, whose echoes were weak. In this presentation, the echoes were measured from two kinds of moth, Spodoptera litura and Helicoverpa armigera. The accuracies of estimated range and direction were examined while echoes were measured from flying moths by intermittently emitting bat-like sounds.

\section{0:55}

1aAB2. Information-theoretic assessment of the peripheral dynamics in the biosonar system of horseshoe bats. Rolf Müller, Anupam Gupta (Mech. Eng., Virginia Tech, ICTAS Life Sci. District (Mail Code 0917), Virginia Tech, Blacksburg, VA 24061, rolf. mueller@vt.edu), Hongxiao Zhu (Statistics, Virginia Tech, Blacksburg, VA), Mittu Pannala (Mech. Eng., Virginia Tech, Blacksburg, VA), Uzair S. Gillani (Elec. and Comput. Eng., Virginia Tech, Blacksburg, VA), Yanqing Fu (Biomedical Eng. and Mech., Virginia Tech, Blacksburg, VA), Philip Caspers (Mech. Eng., Virginia Tech, Blacksburg, VA), and John R. Buck (Elec. and Comput. Eng., Univ. of Massachusetts Dartmouth, Dartmouth, MA)

Horseshoe bat biosonar is characterized by a pronounced dynamics at the interfaces of ultrasound emission and reception: The bats can alter the shapes of their noseleaves and outer ears (pinnae) through muscular action while they are emitting or receiving biosonar signals. An information-theoretic analysis has been conducted to assess whether these non-rigid baffle motions could affect the ability of 
the bats' biosonar system to encode sensory information. This analysis was based on acoustic characterizations (beampatterns) obtained either through numerical predictions or from measurements of physical models. In total, four time-variant baffle shapes, for emission and reception, numerical and physical each, have been analyzed. The similarity of beampattern across different baffle shape conformations was quantified using normalized mutual information that was found to be $<20 \%$ for distant stages. Hence, the changes in the baffle shapes were capable of providing different views of a biosonar environment. The information added by these views was found to enhance performance bounds for sonar estimation tasks related to target direction. In particular, it was found that the number of resolvable directions and the accuracy of direction-finding (as measured by the Cramer Rao lower bound) increased when beampatterns from across a sequence of shape conformation were combined.

\section{1:15}

1aAB3. Specialized facial muscles support sonar beam-forming by free-tailed bats. Samantha Trent and Michael Smotherman (Biology, Texas A\&M Univ., 3258 TAMU, College Station, TX 77843-3258, smotherman@tamu.edu)

Echolocating bats may be able to manipulate the acoustic projection pattern of their sonar pulse emissions, but the mechanism(s) for this and potential constraints are unexplored. The Mexican free-tailed bat (Tadarida brasiliensis) appears to achieve this by finely adjusting the shape of its mouth cavity (beam-forming) in a behavior akin to supralaryngeal speech motor control by humans. Flying Tadarida raise their nose and lips preceding each echolocation pulse with a hypertrophied set of specialized facial muscles (levator labii complex). We investigated 1) muscle complex activity patterns during sonar performance, 2) whether the muscles tissue displayed necessary fasttwitch specializations to accommodate echolocation, and 3) how manipulations of mouth shape altered 3D beam patterns. First, EMG recordings from awake echolocating bats confirmed the muscles were activated in precise temporal coordination with pulse emissions. Second, we describe the anatomical connectivity of the muscle complex, origin and insertions, and innervation pattern. Histochemical analyses confirmed they were highly aerobic, fast-twitch muscles. Last, direct measures revealed that raising the nose tip alone created a small aperture and wide-angle beam, and simultaneously raising the front and side lips created a wider aperture with narrower beam. These results indicate that Tadarida possesses a specialized neuromuscular apparatus for sonar beam-forming.

\section{1:35}

1aAB4. Neural representation of sonar emissions and echoes in the auditory midbrain of the echolocating bat, Eptesicus fuscus. Michaela Warnecke (Johns Hopkins Univ., Baltimore, MD 21218, warnecke@jhu.edu), Brandon C. Casper (Naval Undersea Warfare Ctr., Newport, RI), Andrea M. Simmons, and James A. Simmons (Brown Univ., Providence, RI)

Local field potentials and multi-unit responses were recorded from the auditory brainstem of the anesthetized big brown bat, Eptesicus fuscus, in response to FM sweeps modeled on those the bat would receive from its broadcasts and also their echoes. Tungsten microelectrodes were inserted at an angle into the inferior colliculus (IC), and responses were recorded at progressively deeper sites in the IC and into the area of the cochlear nucleus $(\mathrm{CN})$. Waveforms of the averaged field potentials varied with depth and recording site, with amplitude and latency of the largest peak in each response consistent with transmission time from the cochlea to the CN and then to the IC. For these responses, values of amplitude-latency trading are consistent with those from previous work. Simulated broadcasts followed by sequences of echoes that mimic reflections from complex sonar scenes evoked sequences of distinct responses. Their latencies were affected by amplitude and also by intersound intervals, creating a dynamic pattern of peaks across time. Latencies tracked the shortening of delay that simulates movement through the scene, providing a data set that can be used as steering inputs to a guidance model. [Work supported by ONR, Capita Foundation.] 


\title{
Session 1aAO
}

\section{Acoustical Oceanography: Topics in Acoustical Oceanography I}

\author{
Darrell Jackson, Cochair \\ Applied Physics, University of Washington, 1013 NE 40th St., Seattle, WA 98105 \\ Carolyn Binder, Cochair \\ Oceanography Department, Dalhousie University, LSC Ocean Wing, 1355 Oxford St., PO Box 15000, Halifax, \\ NS B3H 4R2, Canada
}

\section{Contributed Papers}

$10: 30$

1aAO1. Impacts of environment-dependent acoustic propagation on passive acoustic monitoring of cetaceans. Carolyn Binder (Defence Res. \& Development Canada, LSC Ocean Wing, 1355 Oxford St., PO Box 15000, Halifax, NS B3H 4R2, Canada, carolyn.binder@dal.ca) and Paul C. Hines (Dept. of Elec. and Comput. Eng., Dalhousie Univ., Halifax, NS, Canada)

Significant effort has been made over the last few decades to develop automated passive acoustic monitoring (PAM) systems capable of classifying cetaceans at the species level; however, these systems often require tuning when deployed in different environments. Anecdotal evidence suggests that this requirement to adjust a PAM system's parameters is partially due to differences in the propagation characteristics. The environment-dependent propagation characteristics create variation in how a cetacean vocalization is distorted after it is emitted. If this is not accounted for, it could reduce the accuracy and precision of automated PAM systems. An aural classifier developed at Defence R\&D Canada (DRDC) has successfully been used for inter-species discrimination of cetaceans. It achieves accurate results by using perceptual signal features that model the features employed by the human auditory system. To quantify the impacts of propagation on the perceptual features, an experiment was conducted in which bowhead and humpback whale vocalizations were transmitted over 1-20 km ranges during a two-day sea trial in the Gulf of Mexico. The experimental results will be presented and strategies will be discussed for training the classifier so that it is capable of operating in numerous acoustic environments with minimal adjustment of the classifier's parameters.

\section{0:45}

1aAO2. Measurements of the acoustic properties of crude oil. Scott Loranger (Earth Sci., Univ. of New Hampshire, 24 Colovos Rd., Durham, NH 01950, scottloranger@gmail.com), Christopher Bassett (Alaska Fisheries Sci. Ctr., National Marine Fisheries Service, National Oceanic and Atmospheric Administration, Seattle, WA), and Thomas C. Weber (Ctr. for Coastal and Ocean Mapping, Univ. of New Hampshire, Durham, NH)

The acoustic response of crude oil is dependent on the density and sound speed contrast between the oil and the surrounding media. To detect and quantify crude oil in the marine environment, it is crucial to have accurate measures of the density and sound speed at oceanographically relevant temperatures and pressures. A meta-analysis of currently available sound speed data has found a paucity of sound speed data for crude oil at relevant temperatures $(<298 \mathrm{~K})$ and pressures (0.1-30 MPa). Typical sound speed measurements are confined to reservoir temperatures $(>300 \mathrm{~K})$ and pressures $(>100 \mathrm{MPa})$. In order to evaluate the use of currently available data, timeof-flight measurements in a few different types of crude oil were performed in a purpose-built chamber that simulated oceanographic conditions. These test data were compared with extrapolated sound speed curves from currently available data.
11:00

1aAO3. Efficient sequential Monte Carlo estimation of range-dependent seabed properties. Eric Mandolesi (School of Earth and Ocean Sci., UVIC, 3800 Finnerty Rd., Victoria, BC V8P 5C2, Canada, emand@uvic.ca), Jan Dettmer (GeoSci. Dept., Univ. of Calgary, Calgary, AB, Canada), Stan E. Dosso (School of Earth and Ocean Sci., UVIC, Victoria, BC, Canada), Charles W. Holland, and Sheri Martinelli (Appl. Res. Lab., The Penn State Univ., State College, PA)

Seabed geoacoustic properties play a crucial role in shallow-water sonar applications, including the detection of unexploded ordnance. Our goal is improved efficiency of Bayesian seabed parameter and uncertainty estimation for large data volumes. While Bayesian uncertainty estimation provides important information for sonar applications, the approach is computationally expensive which limits utility for large surveys, where an abundance of range-dependent data can be collected. This work considers the efficiency of a particle filter to quantify information content of multiple data sets along the survey track by considering results from previous data along the track to inform the importance sampling at the current point. Efficiency is improved by tempering the likelihood function of particle subsets and including exchange moves (parallel tempering), and by adapting the proposal distribution for the Markov-chain steps. In particular, perturbations are proposed in principal-component space, with the rotation matrix computed via eigenvector decomposition of the unit-lag parameter covariance matrix. The algorithm is applied to 350 data sets collected along a $13-\mathrm{km}$ track on the Malta Plateau, Central Mediterranean Sea. Improved efficiency from parallel tempering and principal-component proposal densities are studied. [Work supported by the Strategic Environmental Research and Development Program, U.S. Department of Defence.]

\section{1:15}

1aAO4. Toward estimating ocean temperature through passive acoustics. Ana Bela Santos, Paulo Felisberto, and Sérgio M. Jesus (LARSyS, Univ. of Algarve, Campus de Gambelas, Faro P8005-139 Faro, Portugal, Portugal, absantos@ualg.pt)

In this paper, we analyze the noise recorded in two drifting vertical line arrays (VLAs) deployed $1 \mathrm{~km}$ apart, in the area of the Setubal's underwater canyon off the west coast of Portugal during RADAR'07 sea trial. Automatic Information System (AIS) recordings for this period reveal a major tanker passing by the area, whose acoustic signature is clearly seen in the spectrogram. The influence of the underwater canyon in the recorded spectrograms is discussed by simulations. By cross-correlating and spatially filtering (beamforming) the signals received at the VLAs, a wave front structure is obtained with coherent information from propagating paths traversing both VLAs and respective arrival times. This work is a contribution for application in a passive ocean acoustics framework to the estimation of sound speed perturbations in the water column. 
1aAO5. Computing where ocean fluctuations affect the acoustic impulse response. John Spiesberger (Earth and Environ. Sci., Univ. of Pennsylvania, 240 S. 33rd St., Philadelphia, PA 19104-6316, johnsr@sas. upenn.edu) and Dmitry Mikhin (Acacia Res. Pty Ltd., Hendon, SA, Australia)

We compute accurate maps of oceanic fluctuations affecting transient acoustic signals propagating between a source and receiver. The technological advance involves coupling the One-Way Wave Equation (OWWE) propagation model with the theory for the Differential Measure of Influence (DMI). This theory uses two finite-frequency solutions of the linear acoustic wave equation: from source to receiver and vice versa. They must obey reciprocity. OWWE satisfies reciprocity with great accuracy even at basinscales when the field of sound speed varies horizontally and vertically. At infinite frequency, maps of the DMI collapse into infinitely thin rays. Mapping the DMI is useful for understanding measurements of the amplitude and travel time changes of sound at finite frequencies. [Work supported by ONR contracts N00014-12-C0230 and N62909-12-1-7033.]
1aAO6. Applying double-difference methods for fine-scale acoustic tracking using a short aperture vertical array. Ludovic Tenorio-Hallé (ludovictenorio@gmail.com), Aaron M. Thode, Jit Sarkar, Chris Verlinden, Jeffrey Tippmann, William S. Hodgkiss, and William A. Kuperman (Scripps Inst. of Oceanogr., 1044 Loring St., San Diego, CA)

Ray tracing can estimate an acoustic source's depth and range in a waveguide by exploiting multipath arrival information on a vertical array. However, environmental mismatch in the model or array tilt can yield highly scattered trajectories when ray tracing multiple events. "Double-difference" methods have been used to localize earthquakes (Waldhauser and Ellsworth, 2000) and fin whales (Wilcock, 2012) by determining the relative locations of multiple events, rather than their absolute positions. This approach, which exploits changes in relative travel times between events, has recently been reformulated to recover the dive trajectory of a source using a single multihydrophone vertical array, whenever three acoustic rays are available for each event. Here, the method is expanded such that changes in ray elevation angles between events can be used to reduce the number of rays required. This technique is tested on data recorded on a short aperture vertical array off the coast of Southern California in $4 \mathrm{~km}$ deep water. Trajectories from both a controlled towed source and sperm whale dives are examined. [Work supported by Office of Naval Research—Marine Mammals and Biology and Ocean Acoustics Program.]

\title{
Session 1aBA
}

\section{Biomedical Acoustics and Physical Acoustics: Photoacoustics: Light and Sound I}

\author{
Kang Kim, Cochair \\ Medicine, University of Pittsburgh, 950 Scaife Hall, 3550 Terrace Street, Pittsburgh, PA 15261 \\ Parag V. Chitnis, Cochair \\ Department of Bioengineering, George Mason University, 4400 University Drive, 1G5, Fairfax, VA 22032 \\ Yoshifumi Saijo, Cochair \\ Tohoku University, 4-1 Seiryomachi, Aoba-ku, Sendai 980-8575, Japan
}

\section{Invited Papers}

\section{0:30}

1aBA1. Real-time three-dimensional visualization of blood vessels and its condition, based on photoacoustic technology. Takayuki Yagi (ImPACT Program, Japan Sci. and Technol. Agency, K's Gobancho, 7 Gobancho, Chiyoda-ku, Tokyo 102-0076, Japan, takayuki. yagi@jst.go.jp)

In 2014, the Japanese Government created the new innovation funding program, called "ImPACT; Impulsing PAradigm Change through disruptive Technologies" and established in the Council for Science, Technology and Innovation (CSTI), the Cabinet Office. ImPACT consists of 16 programs and its period is for 5 years (JFY2014-2018). One of ImPACT is this program; Innovative Visualization Technology to Lead to Creation of a New Growth Industry. The goal of this program is to develop the technologies for real-time 3D photoacoustic imaging of blood vessels in the human body and the physical properties of substances, and then demonstrate the value of applications in medical, healthcare, and cosmetic fields. The configuration of the program consists of six projects: "Visualization and measurement technology," "Tunable laser technology," "Ultrasound sensor technology," "Wide-field visualization system" and "Microvisualization system" for the achievement of real-time 3D visualization, and "Demonstration of value"for demonstrating the value of applications. Six research institutions and six companies will work together for a common goal. I will present the targets and strategies of the program and show recent research results. 
1aBA2. Ultrasound imaging of extravascular molecular targets with light and optically triggered nanoscale contrast agents. Stanislav Emelianov (School of Elec. and Comput. Eng. and Dept. of Biomedical Eng., Georgia Inst. of Technol., 777 Atlanti Dr., Atlanta, GA 30332-0250, stas@gatech.edu)

To obtain high sensitivity, specificity, and signal/contrast-to-noise ratio imaging of disease, detection of biomarkers or biochemical and cellular processes is often needed. Typically, such information is gathered using imaging contrast agents. Unfortunately, microbubbles-most common ultrasound contrast agent, are too large to escape vascular compartments. Thus, contrast-enhanced molecular ultrasound imaging is often limited to molecular markers on the vascular endothelium and other intravascular targets. Recently, we introduced several high-resolution, high-sensitivity, depth-resolved ultrasound-based molecular imaging techniques augmented with nanometer scale imaging contrast agents capable of visualizing molecular signatures of cells in context of structural and functional properties of tissue. In this presentation, several examples of molecular ultrasound imaging based on synergistic combination of light and sound will be given. Specifically, clinical problems including detection and phenotyping of primary tumor, assessment of micrometastatic lesions in sentinel lymph node, and image-guided cancer cell therapy will be described. Design and synthesis of clinically relevant contrast nanoagents with properties desired for cellular/molecular ultrasound imaging will be discussed. Finally, the presentation will conclude with the analysis of how molecular ultrasound can change both fundamental medical science and the utility of clinical ultrasound imaging in diagnostic and therapeutic applications.

\section{1:10}

1aBA3. Photoacoustic imaging for ultrasound tissue characterization and treatment monitoring. Michael C. Kolios (Phys., Ryerson Univ., 350 Victoria St., Toronto, ON M5B2K3, Canada, mkolios@ryerson.ca)

Photoacoustic imaging relies on generation of ultrasound waves from optically absorbing structures. The ultrasound produced in photoacoustic imaging can be analyzed by methods developed to analyze ultrasound backscatter signals for ultrasound tissue characterization, but the interpretation of the analysis is based on the physics of photoacoustic wave generation. In the absence of exogenous absorbers, blood is one of the dominant optically absorbing tissues. Hemoglobin in red blood cells is the main endogenous chromophore in blood. The spatial distribution of red blood cells in tissue determines the spectral characteristics of the ultrasound signals produced. We are interested in cancer treatment monitoring. Tumor blood vessels have distinct organizational structure compared to normal blood vessels: normal vessel networks are hierarchically organized, with vessels that are evenly distributed to ensure adequate oxygen and nutrient delivery. Tumor vessels are structurally different: they are torturous and hyperpermeable. Therapies that target the vasculature can induce changes in the vascular networks that in principle should be detected using photoacoustic imaging. In this work, we will show how the frequency content of the photoacoustic signals encodes information about the size, concentration, and spatial distribution of non-resolvable blood vessels that can be used to assess treatment response.

\section{1:30}

1aBA4. Dual-wavelength photoacoustic microscopy for cell tracking. Pai-Chi Li (National Taiwan Univ., Dept. of Elec. Eng., National Taiwan Univ., NTU, No.1, Sec. 4, Roosevelt Rd., Taipei 106, Taiwan, paichi@ntu.edu.tw)

Understanding cell interactions can help to develop cell-based therapeutic strategies such as immunotherapy. The ability to track cells thus becomes an important task. 3D in vitro models have become a promising platform for such cell studies. However, conventional optical imaging methods are no longer adequate in 3D models due to limited penetration. To overcome this problem, we developed a dual-wavelength optical-resolution photoacoustic microscope (2L-OR-PAM) to study CD8 + cytotoxic T lymphocytes (CTLs) trafficking in a 3D tumor microenvironment. With the aid of molecular probes, the 2L-OR-PAM system can track both the CTLs and the tumor cells. An Nd:YLF laser was used as a pump laser at $523 \mathrm{~nm}$ passing into a dye laser to generate near-infrared light at $813-\mathrm{nm}$. Two types of AuNPs were used to target the cancer cells and the CTLs. An x-y galvanometer scanner was employed to obtain a high scanning rate. The lateral and the axial resolutions were measured at $1.6 \mu \mathrm{m}$ and $5 \mu \mathrm{m}$, respectively. We successfully showed that the $2 \mathrm{~L}-\mathrm{OR}-$ PAM can map the distribution of CTLs and 3D structures of Hepta1-6 tumor spheres. The 2L-OR-PAM can provide cellular and molecular information and it has the potential for preclinical screening requiring cell tracking. 


\title{
Session 1aED
}

\section{Education in Acoustics: Attractive Educational Methods and Tools in Acoustics I}

\author{
Kazuhiko Kawahara, Cochair \\ Department of Design, Kyushu University, 4-9-1 shiobaru, Minami-ku, Fukuoka 815-8540, Japan \\ Tracianne B. Neilsen, Cochair \\ Brigham Young University, N311 ESC, Provo, UT 84602
}

Invited Papers

10:30

1aED1. Structuring an introductory acoustics course to be a vehicle for improving pre-calculus math skills and recruiting students to technical careers. Andrew A. Piacsek (Phys., Central Washington Univ., 400 E. University Way, Ellensburg, WA 98926, piacsek@cwu.edu)

"Why do I have to take this math course—when will I ever use this stuff?" and "I could never be a scientist—I'm terrible at math." These statements are familiar to educators the world over, much to our frustration... and to the detriment of society's goals of being scientifically and mathematically literate and having a large and diverse population of STEM professionals. The present paper describes elements of a general education course in acoustics at Central Washington University ("Physics of Musical Sound") that serve vital secondary functions of improving math skills and fostering an appreciation of acoustics-related careers, appropriate for the typical demographic of students from diverse academic and social backgrounds, many of whom have not declared a major, that are attracted to the interdisciplinary nature of the course. These secondary goals, as well as the subject matter, are also highly appropriate for high school students. The content, the skills, and the applications are closely linked, leveraging students' native interest in, and familiarity with, music and sound to provide the incentive to develop and hone math concepts. Specific content, applications, and pedagogical approaches will be discussed.

\section{0:50}

1aED2. Using wine glasses, strings, and bars to teach resonance at the undergraduate and graduate levels. Brian E. Anderson (Phys. and Astronomy, Brigham Young Univ., N283 ESC, Provo, UT 84602, bea@byu.edu)

At Brigham Young University, two of the acoustics courses taught in the Physics and Astronomy Department focus on resonance topics. One of the undergraduate courses is a general education, descriptive acoustics course on musical instruments, speech, hearing, and audio. One of the graduate level courses using a differential equations approach to wave motion and resonances on strings, bars, membranes, and plates. In both of these courses, taught at very different levels, the concept of resonance is explored and analyzed. This presentation will discuss various demonstrations used in these classrooms and discuss how they are used in the learning development of the students. Demonstrations include wine glasses, strings, and bars.

\section{1:10}

1aED3. Tools for education in acoustics and phonetics. Takayuki Arai (Dept. of Information and Commun. Sci., Sophia Univ., 7-1 Kioi-cho, Chiyoda-ku, Tokyo 102-8554, Japan, arai@sophia.ac.jp)

We have developed a series of tools for education in acoustics and phonetics. Some of them are in electronic form, while some are physical models. A set of demonstrations and explanations in acoustic phonetics is now available as "Acoustic-Phonetics Demonstrations (APD)" through our WebSite: www.splab.net/APD/. We are adding content and have made YouTube video clips using physical models available on our WebPages. In particular, we use the physical models of the human vocal tract to demonstrate acoustic phenomena and theories. They are useful tools for explaining acoustics in speech production, such as the source-filter theory and the relationship between vocal-tract configurations and vowel quality. Furthermore, the models are effective when teaching articulation/pronunciation of native and non-native languages. Due to the high demand for vocal-tract models of various sounds in multiple languages, we are expanding the range of sounds to include more than Japanese sounds, but also additional vowels and consonants in different languages, including English. We also discuss how to apply three-dimensional printing. 
1aED4. A project-oriented graduate course on ultrasonics. Michael R. Haberman (Appl. Res. Labs. \& Dept. of Mech. Eng., The Univ. of Texas at Austin, 10000 Burnet Rd., Austin, TX 78758, haberman@arlut.utexas.edu)

The study of ultrasonics encompasses a wide range of topics in acoustics. Some topics, like dispersion in elastic waveguides, require mathematical sophistication, while others, like transducer selection and common signal processing techniques, demand detailed technical knowledge. The variety of topics germane to the subject of ultrasonics presents an instructor with a number of challenges that must be addressed through the course design. This talk reports on the current graduate course on ultrasonics in the graduate acoustics program at The University of Texas at Austin. The structure of the course is inspired by the experiential learning model, which emphasizes experimentation and experiences to supplement in-class lectures. This is achieved using numerous in-class demonstrations and an extended team project. Whenever possible, data from in-class demonstrations are used in homework assignments to provide continuity between theory and practice. Specific example course projects will be discussed which illustrate the breadth and depth of course projects resulting from this approach, and perspectives are provided for avenues for improvement using this teaching method for instruction in ultrasonics.

\section{Contributed Paper}

\section{1:50}

1aED5. Comparison of acoustic education in the architecture departments between Turkey and different universities in the world. Filiz B. Kocyigit (Architecture, Acoust. Society of America, Atilim Univ., Faculty of Fine Art Design and Architecture, Kizilcasar Mah. Incek, Ankara 06830, Turkey, filizbk@gmail.com) and Sevgi Lokce (Architecture, TMMOB, Ankara, Turkey)

The aim of this study is to determine the "when and what extent" do architectural acoustics is included in architecture curricula. Additionally, to find out the state of research facilities across academic institutions between Turkey and different countries including Architectural Acoustic. In Turkey, in many architecture programs do not include "Experiences of the Acoustics Laboratory or courses" and also education is not among the objectives of architectural acoustics for their future. On comparing of teaching activities, education objective is to allow professional figures to interpret, define, and govern the processes of maintenance and management with high quality checking in the life cycle of the built systems are researched. It seems that searching a new formation is being established in order to integrate with the EU standards such as European Credit Transfer and Accumulation system (ECTS) and many of the schools have revised their programs according to these new standards. As a consequence of this revision, duration and content of the courses are being re-handled. Curriculum of Architecture, course Group, Percentage of course are compared.

\section{Session 1aNS}

\section{Noise: Sound Design I}

André Fiebig, Cochair

HEAD acoustics GmbH, Ebertstr. 30a, Herzogenrath 52134, Germany

Takeshi Toi, Cochair

Patricia Davies, Cochair

Ray W. Herrick Labs., School of Mechanical Engineering, Purdue University, 177 South Russell Street, West Lafayette, IN 47907-2099

Chair's Introduction-10:30

\section{Invited Papers}

\section{0:35}

1aNS1. Sharpness perception and modeling of stationary and time-varying sounds. Roland Sottek (HEAD Acoust. GmbH, Ebertstr. 30a, Herzogenrath 52134, Germany, roland.sottek@head-acoustics.de)

Psychoacoustic parameters like loudness and sharpness can be used for sound quality assessment and sound design applications. While loudness of stationary sounds has been standardized for decades, standards for sharpness of stationary sounds and loudness of time-varying sounds have only been published since 2009 (DIN 45692:2009-08) and 2010 (DIN 45631/A1:2010-03), respectively. Present calculation methods of sharpness consider the "center of gravity" of the weighted specific loudness patterns as a measure for 
sharpness; thus, their results do not depend on loudness. Only the Aures model includes, to a small extent, the influence of loudness on sharpness by an additional loudness-dependent factor. Until now, mostly stationary synthetic sounds (filtered noise and pure tones) have been used for the derivation of sharpness models. However, when these calculation procedures are applied to generally time-varying technical sounds, the results deviate significantly from the listening test results. This study describes new listening tests performed to investigate systematically the influence of loudness as well as temporal structures on sharpness. The findings from the listening tests should serve as a basis for an improved model of sharpness perception for stationary and time-varying sounds.

\section{0:55}

1aNS2. The role of cross-validation approaches in sound quality metric development. André Fiebig and Fabian Kamp (HEAD Acoust. GmbH, Ebertstr. 30a, Herzogenrath 52134, Germany, andre.fiebig@head-acoustics.de)

The sound of a product is an important feature influencing the perceived quality impression. Thus, product sound is deliberately designed to stand out against competitors. To determine meaningful design targets guaranteeing a perceived sound quality, jury tests are performed and sound quality metrics are developed. Ideally, by means of a developed sound quality metric, the prediction of perceived sound quality of comparable products is possible without the need for further jury testing. However, several aspects influence the reliability and validity of jurors' ratings deteriorating the prediction accuracy of the derived sound quality metric. Moreover, if parameters of a sound quality metric are only estimated in a way to give a best fit with the rating data, the external validity of the metric is possibly reduced. In order to estimate the robustness of a sound quality metric and to avoid overfitting, cross-validation methods and different error measures should be considered. The paper will illustrate the impact of missing robustness analyses on the validity of sound quality metrics and how systematic approaches can be applied to improve the process of metric development. Moreover, general limitations and risks of sound quality metrics based on jury tests will be discussed.

\section{Contributed Papers}

\section{1:15}

1aNS3. Consideration of radiated sound quality for high-grade washing machine through paired comparison test. Junji Yoshida, Rei Yamashita, Shota Yoshida (Mech. Eng., Osaka Inst. of Technol., 5-16-1 Omiya, AsahiKu, Osaka 535-8585, Japan, yoshida@med.oit.ac.jp), Tomohiro Fujii, and Akihiro Hosokawa (Panasonic, Kusatsu, Japan)

In this study, we conducted a sound quality evaluation test to obtain a guide realizing a radiated noise suitable for high-grade washing machines. Twelve sounds at spin drying of drum type washing machines were prepared for paired comparison experiments. Forty eight participants evaluated the presented sound quality about "noisiness," "discomfort," and "luxuriousness." The results indicated that noisiness and discomfort had very high correlation between each other. In addition, loudness values calculated from the recorded sound pressure signal had very high linear relationship with them. On the other hand, evaluation result of luxuriousness showed a different tendency. The relationship between the calculated loudness value and luxuriousness score was high; however, the relationship was not linear but hyperbolic curve. When the loudness of the washing machine was over 10 sone, the luxury feeling of the radiated sound was rarely improved in spite of the loudness reduction. On the contrary, the luxuriousness was found to be expected to increase largely by the noise reduction when the loudness was less than 10 sone. Consequently, decreasing the loudness less than 10 sone was found to be important in any cases to realize a noise suitable for high-grade washing machine.

$$
\text { 11:30 }
$$

1aNS4. Musical mind control: Acoustic convergence to background music in speech production. Ryan G. Podlubny (Linguist, Univ. of Canterbury, 214, Locke Bldg., Christchurch 8011, New Zealand, ryan. podlubny@pg.canterbury.ac.nz)

Talkers adjust their speech to resemble that of their interlocutors-a phenomenon known as speech convergence. Broadly defined, convergence describes automatic synchronization to some external source, and this effect has been recognized in various linguistic domains (e.g., syntactic structuring, morphological choices, and vowel production). Using a speechin-noise reading-task, this paper explores whether or not speech production also converges with non-linguistic signals: Specifically, might a speaker's rhythm, pitch or intensity be influenced by fluctuations in background music? Participants read passages aloud while music was presented at $\sim 45$ $\mathrm{dB}(\mathrm{A})$ via headphones. Music was composed with relatively flat envelopes in the domains of interest, and in test-conditions only Pitch, Tempo, or Intensity was altered. Manipulation was gradual (linear function) and would similarly return to the point of origin after reaching a target (Pitch $=-200$ cents; Amp $=+6 \mathrm{~dB}$; Tempo $=-20$ beats per minute). Control conditions (i.e., no acoustic manipulation) broke up test conditions. Analyzing data from 25 English speakers identifies significant convergence effects in the Pitch condition (tempo and intensity analyses have not yet been conducted). Using Generalized Additive Mixed Models, the predicted Time by Condition interactions were observed, suggesting that environmental noise may constantly be influencing speech production.

\section{$11: 45$}

1aNS5. Controlling smartphone vibration and noise. Inman Jang, Taeyoung Park, Donghyun Kim, Sangbeom Woo, Won-Suk Ohm (School of Mech. Eng., Yonsei Univ., 50 Yonsei-ro, Seodaemun-gu, Seoul 03722, South Korea, kgpbjim@yonsei.ac.kr), and Heungkil Park (Samsung Electro Mech. Co.,Ltd, Seoul, Korea (the Republic of))

Vibration of the internal electronics components and its transmission to the casing has been known as a source of noise radiation that degrades the call quality of a smartphone. Here, we expand upon our previous work [J. Acoust. Soc. Am. 137, 2293 (2015)] that employed the nearfield acoustic holography (NAH) for characterization of the smartphone radiated noise. The NAH, when used in conjunction with a vibration model of the smartphone, is shown to be a powerful tool for controlling the smartphone vibration and noise at the initial design phase. The vibration model is largely based on the classical plate theory and yields concrete guidelines on how to reduce vibration and the subsequent noise. The effectiveness of the technique is demonstrated using some of the smartphones currently available on the market. 


\title{
Session 1aPA
}

\author{
Physical Acoustics: Thermoacoustics
}

\author{
Michael R. Haberman, Chair \\ Applied Research Laboratories, The University of Texas at Austin, 10000 Burnet Rd., Austin, TX 78758
}

\section{Contributed Papers}

10:30

1aPA1. On-off intermittency in coupled chaotic thermoacoustic systems. Rémi Delage, Yusuke Takayama, and Tetsushi Biwa (Mech. Eng., Adv. Mech. Systems and Design, Graduate School of Eng., Tohoku Univ. 6-6-01, Aoba, Aramaki, Aoba-ku, Sendai 980-8579, Japan, delageremi@ hotmail.fr)

We present chaos-chaos synchronization in coupled thermally induced acoustic gas oscillation systems. By introducing a local cross-sectional change in a gas-filled tube subjected to a temperature gradient, chaotic oscillations are found to be achieved through the mode competition. The chaos-chaos synchronization is then obtained by connecting the two thermoacoustic oscillators via a rigid plate with an orifice. Theoretical statistical scaling laws regarding the laminar phases, spectral density, and amplitude probability distribution are found to be satisfied in the coupled thermoacoustic oscillators, as presented from the analysis of pressure fluctuations when the thermoacoustic chaos-chaos synchronization breaks down with the decreased orifice size.

\section{0:45}

1aPA2. Development of a prototype thermoacoustic refrigerator with coaxial geometry. Ayumu Kakuda (Faculty of Sci. and Eng., Doshisha Univ., 1-3 Tatara-miyakodani, Kyotanabe-city, Kyoto, 610-0321 Japan, dup0318@mail4.doshisha.ac.jp), Shin-ichi Sakamoto (Univ. of Shiga Prefecture, Hikone, Shiga, Japan), and Yoshiaki Watanabe (Doshisha Univ., Kyotanabe, Kyoto, Japan)

The thermoacoustic system with coaxial geometry (coaxial-type) is discussed. The coaxial-type has high efficiency because it performs the energy conversion with the traveling wave mode as the same of the looped tube type thermoacoustic system (loop-tube-type). Additionally, the coaxial-type can be realized more downsizing in comparison with the loop-tube-type, and it is also expected to expand the practical use. In this report, the experimental studies of the coaxial-type refrigerator were carried out. A pair of stacks was placed in the experimental setup. Those stacks were employed as a prime mover and a heat pump. In the case of the loop-tube-type, those are usually placed symmetrically. However, it is not practical for the coaxialtype to place stacks symmetrically because of the difficulties for the taking off cold energy; therefore, both of stacks were placed in annular path. As a result, temperature decreases were confirmed, that is, amount of 5 decreases from room temperature were observed at the cooling point of a heat pump. It is thought that the energy conversion with the traveling wave mode is occurring in stacks. These results show that the coaxial-type can be possible to become a new design to take the place of the loop-tube-type.

\section{1:00}

1aPA3. Increasing of work flow on the thermoacoustic system by control of the temperature gradient within the stack. Seiya Fukuda (Elec. Eng. and Electronics, Doshisha Univ., 1-3 Tataramiyakodani, Kyotanabe, Kyoto 610-0321, Japan, dup0305@mail4.doshisha.ac.jp), Shinichi Sakamoto (Univ. of Shiga Prefectural, Hikone, Shiga, Japan), and Yosiaki Watanabe (Doshisha Univ., Kyotanabe, Japan)

A gas column in a tube with a temperature-gradient spontaneously begins to oscillate when air turbulence occurs. The work flow produced in the stack, $\Delta I$, is important for the thermoacoustic oscillation. The amount of $\Delta I$ is determined by the shape of temperature-gradient. Experimental studies on thermoacoustic systems are previously assessed by measurement of temperature ratio, $T_{H} / T_{C}$, of the stack; however, investigation for distribution of temperature within the stack is less discussed. In order to examine the influence of a temperature-gradient within the stack on the thermoacoustic oscillation, we propose that control method for the temperature-gradient under the same $T_{H} / T_{C}$. In particular, the control of the temperature distribution is implemented in actively changing the internal temperature by additional nichrome heater in middle of the stack. The sound pressure in the standingwave thermoacoustic system is measured at several temperature distribution by the proposed method. Furthermore, $\Delta I$ was calculated based on the measured sound pressure. As a result, the work flow by the control of a temperature distribution have increased, and it was qualitatively estimated by the slope of heat flux within the stack from temperature distribution.

\section{1:15}

1aPA4. Amplitude death in delay-coupled thermoacoustic oscillators. Hiroaki Hyodo, Itaru Shinkai, and Tetsushi Biwa (Dept. of Mech. System and Design, Tohoku Univ., 6-6-04 Aoba, Aramaki, Aobaku, Sendai 9808579, Japan, hyodo@amsd.mech.tohoku.ac.jp)

A phenomenon of thermoacoustic oscillation is seen as an undesirable vibration in combustion engineering as it can cause serious damage to the combustor. Lots of methods such as controlling a secondary fuel supply and addition of Helmholtz resonators are proposed to suppress the oscillations, but they often become complicated. As a simple and reliable method, we try to make use of mutual interaction of the coupled thermoacoustic oscillators to stop oscillations completely. It has been demonstrated in the system of coupled electrical circuits that "amplitude death," meaning the stabilization of zero amplitude states, is induced by time-delay coupling even when the oscillators have the same frequency. In this study, we introduce the timedelay coupling in thermoacoustic oscillators by using hollow tubes. In the tube coupling, the sound propagation time through the tube corresponds to the delay time, while the tube diameter controls the coupling strength. The condition of amplitude death is experimentally found for various tube lengths, diameters, and number of tubes. Thus, the tube coupling provides the way to annihilate thermoacoustic oscillations. The results are compared with those of linear stability analysis based on basic equations of hydrodynamics. Both results are qualitatively consistent with each other. 
1aPA5. Thermoacoustics of a T-burner: How to better estimate the combustion response function from a T-burner experiment. Taeyoung Park, Hunki Lee, Won-Suk Ohm (School of Mech. Eng., Yonsei Univ., Eng. Bldg. A391, 50 Yonsei-ro, Seodaemun-gu, Seoul 120-749, South Korea, pty0948@yonsei.ac.kr), and Dohyung Lee (Agency for Defense Development, Seoul, Korea (the Republic of))

The combustion response function, representing the coupling between the pressure oscillation and the combustion rate of a solid propellant, is a key indicator of combustion instability (large unwanted pressure oscillations in a combustion chamber) of a solid rocket motor. To obtain the response function experimentally, a T-burner is often used, in which the lowest longitudinal acoustic resonance of the cylindrical enclosure is excited by the combustion of propellant samples (acting like speakers) at both ends. The existing formula used to derive the combustion response function from a $\mathrm{T}$ burner experiment is based on a series of rather crude assumptions. For example, acoustically rigid boundaries are assumed at both ends, whereas impedance boundary conditions may be more appropriate to reflect the introduction of combustion products from the propellant. In this talk, we propose a few modifications to the current T-burner analysis regime, which could improve the accuracy of the measured response function. The new method is demonstrated using an actual T-burner test. [This work was supported by Defense Acquisition Program Administration and Agency for Defense Development under the contract UD140024GD.]
1aPA6. Numerical study of heat transfer in parallel-plate heat exchangers in thermoacoustic engine. Kazuto Kuzuu and Shinya Hasegawa (Tokai Univ., 4-1-1 Kitakaname, Hiratsuka, Kanagawa 2591292, Japan, kuzuu@tokai-u.jp)

For designing thermoacoustic devices, heat transfer problem of heat exchangers is a key point and is not solved completely yet. In the present study, both computational fluid dynamics (CFD) simulations and numerical analysis based on linear thermoacoustic theory are performed, and heat transfer characteristics, heat fluxes, and Nusselt numbers are investigated from both numerical results. Especially, through parametric studies by linear analysis, the effect of displacement amplitude and thermal penetration depth on heat transfer is discussed. In this simulation, a regenerator is located between hot and cold heat exchangers, and the effect of each channel size is also investigated. Furthermore, simulated results are compared with the conventional heat transfer models for oscillatory flow, and the verifications of those models or simulations are executed. On the other hand, by comparing the CFD results with linear analysis, non-linearity of heat transfer included in thermoacoustic phenomena is also discussed. For this discussion, unsteady behavior of temperature field in heat exchangers is observed in the CFD simulation. Finally, by combining the parametric studies of linear analysis and non-linear effect obtained from the CFD simulation, the heat transfer characteristics for heat exchangers in thermoacoustic engine are comprehensively discussed.

\title{
Session 1aPP
}

\section{Psychological and Physiological Acoustics: Spatial Hearing and Its Applications I}

\author{
Kazuhiro Iida, Cochair \\ Chiba Institute of Technology, 2-17-1, Tsudanuma, Narashino 275-0016, Japan \\ Griffin D. Romigh, Cochair \\ Air Force Research Labs, 2610 Seventh Street, Area B, Building 441, Wright Patterson AFB, OH 45433 \\ Yôiti Suzuki, Cochair \\ Research Inst. Electrical Communication, Tohoku University, Katahira 2-1-1, Aoba-ku, Sendai 981-0942, Japan \\ Douglas Brungart, Cochair \\ Walter Reed NMMC, 8901 Wisconsin Ave., Bethesda, MD 20889 \\ Yukio Iwaya, Cochair \\ Faculty of Engineering, Tohoku Gakuin University, 1-13-1 Chuo, Tagajo 985-8537, Japan
}

\section{Invited Papers}

\section{0:30}

1aPP1. Comparison of localization performance with individualized and non-individualized head-related transfer functions for dynamic listeners. Ravish Mehra, Aaron Nicholls (Res., Oculus, 8747 148th Ave. NE, Redmond, WA 98052, ravish.mehra@oculus. com), Durand Begault (NASA Ames, Moffett Field, CA), and Marina Zannoli (Res., Oculus, Redmond, WA)

The head-related transfer function (HRTF) depends on head, torso, and ear geometry of an individual and typically measured in an anechoic chamber using an HRTF measurement apparatus. This is referred to as the individualized HRTF. In many applications, an HRTF corresponding to an average human head is measured on dummy also called genericHRTFs. Prior research has shown that 
personalized HRTF gives significantly better performance over the generic HRTF for a static listener. However, these studies were limited to only static listeners where the listener's head is fixed during stimuli presentation. In case of dynamic listeners, the head motion enables the listeners to perceive additional spectral cues resulting due to head motion. In the present study, we compare the performance of personalized vs. generic HRTFs for a dynamic listener. We quantify the localization performance of each listener with both individualized and non-individualized HRTFs (virtual conditions) and compare it against their ground truth localization performance. Our data reveals the benefits of personalized HRTF over the generic HRTF for dynamic listeners in virtual environments.

\section{0:50}

1aPP2. A dipole model for estimating frequency versus elevation-angle trajectory of the first spectral notch of head-related transfer functions in the median plane. Hironori Takemoto, Parham Mokhtari, Hiroaki Kato, Ryouichi Nishimura (National Inst. of Information and Communications Technol., 3-5 Hikaridai, Seika-cho, Soraku-gun, Kyoto 619-0289, Japan, hironori.takemoto@p. chibakoudai.jp), and Kazuhiro Iida (Chiba Inst. of Technol., Narashino, Japan)

The first (lowest) spectral notch (N1) of head-related transfer functions is known as a cue for sound localization in the median plane. This may be due to the fact that N1 frequency gradually increases as the sound source approaches the direction above the head. The mechanism of this phenomenon, however, is still unclear. To clarify the mechanism, using finite-difference time-domain simulation, the normalized pressure amplitude patterns were visualized around the head for 12 subjects when the analysis field was excited at various frequencies from a point just outside the blocked meatus. As a result, strong radiations with different phases occurred in the upper and lower directions, while in the intermediate narrow zone, the pressure amplitude became minimum. This zone corresponded to N1 and bent upward with increasing excitation frequency. These facts implied that the two radiated sound waves canceled each other in the zone, where the path difference amounted to a phase difference of half a wavelength. Thus, the shape of the intermediate zone could be modeled by a hyperboloid whose foci were two hypothetical points on the pinna. The model could roughly estimate, for each individual, the frequency versus elevation-angle trajectory of $\mathrm{N} 1$ in the median plane.

\section{1:10}

1aPP3. Improved understanding of head-related transfer functions through sagittal-plane spectral decomposition. Griffin D. Romigh (Air Force Res. Labs, 2610 Seventh St., Area B, Bldg. 441, Wright Patterson AFB, OH 45433, griffin.romigh@us.af.mil), Douglas S. Brungart (Walter Reed NMMC, Bethesda, MD), and Brian D. Simpson (Air Force Res. Labs, WPAFB, OH)

Head-related transfer functions (HRTFs) capture the listener-specific modifications that are imparted on a sound as it travels from a specific location in space, interacts with the listener's head, shoulders, and outer ears, and arrives at the ear drums. HRTFs have often been decomposed into a combination of two parts to aid modeling and analysis: a diffuse-field transfer function (DFTF) that captures non-directional but still listener-specific spectral details of the HRTF, and a directional transfer function (DTF) that captures all of the listener-specific directional information. Because the DTF contains spectral variation along all spatial dimensions, it does not provide a way to separate the spectral cues responsible for lateral and sagittal plane localization judgments, which are largely independent. As an alternative approach, decomposing an HRTF along each sagittal plane (aka along the "cones-of-confusion") provides similar benefits to DTF analysis while providing additional insights into the spectral cues responsible for lateral and vertical localization, independently. This talk will provide a detailed overview of sagittal-plane spectral decomposition along with experimental results showing how the technique can be applied to improve localization with non-individualized HRTFs, separate general and idiosyncratic HRTF features, and cluster listeners' HRTFs based on spectral similarity.

\section{1:30}

1aPP4. Roles of spectral peaks and notches in the head-related transfer functions in the upper median plane for vertical localization. Kazuhiro Iida and Yohji Ishii (Chiba Inst. of Technol., 2-17-1, Tsudanuma, Narashino 275-0016, Japan, kazuhiro.iida@itchiba.ac.jp)

The parametric head-related transfer function (HRTF) recomposed of only a spectral peak (P1) generated by the first resonance of the pinna and two spectral notches (N1 and N2) generated by the first and the second anti-resonances of the pinna has been reported to provide approximately the same localization performance as the measured HRTF for the front and rear directions. However, for the upper directions, the localization performance for some of the subjects decreased. In the present study, we carried out two localization tests with seven target vertical angles in the upper median plane to clarify whether adding a spectral peak (P2) generated by the second resonance of the pinna can resolve this performance decrease. The results suggested the following: (1) N1, N2, and P1 play an important role in vertical localization; (2) localization performance was improved by adding P2 to N1N2P1 at the upper directions; (3) however, a sound image was hardly perceived in the upward direction by reproducing only P2, P1, and P1 + P2; and (4) P2 could be utilized to enhance N1 at the upper direction. 


\title{
Session 1aSA
}

\section{Structural Acoustics and Vibration: General Topics in Structural Acoustics and Vibration I (Poster Session)}

\author{
Benjamin Shafer, Chair \\ Technical Services, PABCO Gypsum, 3905 N 10th St., Tacoma, WA 98406
}

\begin{abstract}
All posters will be on display from 10:30 a.m. to 12:00 noon. To allow contributors in this session to see the other posters, authors of odd-numbered papers will be at their posters from 10:30 a.m. to 11:15 a.m. and authors of even-numbered papers will be at their posters from 11:15 a.m. to 12:00 noon.
\end{abstract}

\section{Contributed Papers}

1aSA1. Evaluation of environmental noise by analyzing faint random vibration in structural health monitoring. Yoshinori Takahashi (Tokyo Metropolitan College of Industrial Technol., 8-17-1, Minamisenju, Arakawaku, Tokyo 116-0003, Japan, yoshinori@ieee.org)

A number of big intermittent aftershocks often follow a great earthquake. Even if a building could avoid collapse by the first main quake, there would be no guarantee that the building can survive from the aftershocks. However, if it is possible to monitor the condition of the building constantly using some faint random vibration caused by environmental noise such as wind, weak earthquakes, and traffic noise, the safety of the victims should be more secured. In a field of machinery diagnostics, although several diagnostic methods using vibration based on environmental noise have been proposed, those methods require that the characteristics of the noise are known since the resonance of the object is shadowed by the environmental noise spectrum. The author has proposed an emphasizing method of hidden resonances in a time-invariant transfer function and has also applied the method to structural health monitoring by analyzing faint random vibration caused by environmental noise. This report presents the results of experiment in an actual building. The results showed that rain showers or temporal thunders gave some influences on the estimation for the resonance of the buildings; however, it was possible to avoid the influences by selecting the analysis parameters correctly.

1aSA2. Modelling of dynamic behaviour of viscoelastic materials in the frequency domain. Tamás Pritz (Budapest Univ. of Technol. and Economics, Múegyetem rkp. 3-9, Budapest 1111, Hungary, tampri@eik.bme.hu)

The modern methods of acoustical and vibration calculus require accurate material models to take properly into account the damping and dynamic elastic properties of the viscoelastic materials used for sound and vibration control. The usual modeling methods (spring-dashpot models, fractional calculus) start out of the stress to strain relations defined in the time-domain, which are then transformed into the frequency domain to find the appropriate material functions. In contrast, the damping and elastic properties are known from measurements made in the frequency domain; therefore, it seems to be reasonable to model the material behavior directly in this domain. The aim of this paper is to show that the typical dynamic behavior of solid viscoelastic materials can efficiently be modeled in the frequency domain. This modeling method is based on simple theoretical considerations, the frequency domain experiences and the causality principle. Two fundamental material models are developed in this way, namely one for the damping increasing with frequency, and the other one for the damping peak, which can be either symmetrical or asymmetrical with respect to the logarithmic frequency. The relation of the frequency domain models to the popular fractional derivative models defined in the time-domain is shown and discussed.
1aSA3. Numerical simulation for low frequency noise from viaduct by the vehicle load. Noboru Kamiakito, Masayuki Shimura (Civil Eng. and Eco-Technol. Consultants Co. Ltd., 2-23-2,Higashi-ikebukuro, Toshima-ku, Tokyo 1700013, Japan, kamiakit@kensetsukankyo.co.jp), Hiroshi Iwabuki (Central Nippon Expressway Co., Ltd., Hachioji-shi, Tokyo, Japan), and Toshikazu Osafune (NEXCO Res. Inst. Co., Ltd., Machida-shi, Tokyo, Japan)

There is the case that the low frequency noise generated complaints of neighborhood residents from the road viaduct. We tried to clarify the source of low frequency noise using the FEM model as one approach for such a problem. First, the 3D-FEM model of the vehicle, bridge, and air was constructed. Then, propagating of the acceleration was calculated by the vehicle traveling on viaduct with road irregularities. Then, the vibration velocity of the viaduct is radiated as sound pressure, and propagating of the sound pressure through the air was carried out by 3D-FEM calculation. Actually, a numerical model for the viaduct that caused the complaint was constructed. The low frequency noise generation mechanism was investigated. The effective measures was considered. As a result, it became clear that a high reduction effect of low frequency noise by performing the measures road irregularities can be obtained in the viaduct. It became clear that there is the acoustic radiation of low frequency noise by the sound insulation wall on the viaduct. By carrying out the parameter study using such a method, we can clarify the occurrence factor of the low frequency noise.

1aSA4. On a fire extinguisher using sound winds. Myungjin Bae and EunYoung Yi (Commun. Eng., Soongsil Univ., 369 Sangdoro, Dongjakgu, Seoul 156-743, South Korea, mjbae@ssu.ac.kr)

Sound fire extinguisher is developed based on principle of quenching fire by lowering its temperature with contacting vibration energy from low frequency sound under $100 \mathrm{~Hz}$ to fire and then blocking inflow of oxygen with a sound wind. The one researched by American students are at very basic level to be commercialized. It concentrates a sound beam through special sound winds and increases sound energy performance 10 times more to put out the fire. This is because it utilizes basic theory of making sound and as sound has a tendency of dispersing, big sound and energy have to be created to extinguish fire. That is, even if $100 \mathrm{~W}$ is produced, it just makes a big noise but only $1 / 10$ of it can be used for putting out fire. This special sound winds has convex shape which concentrates all the sound into one place to increase effectiveness by more than 10 times. Even 1 2 $\mathrm{m}$ away, initial control can be done. As a result, size of battery as well as amplifier can be made small to have minimized size and weight, making it more practical. 
1aSA5. Skull-shaped antenna enables near-field super-resolution in acoustic source localization using elastic waves. Michael Reinwald (Laboratoire d'Imagerie Biomédicale, Université Pierre et Marie Curie, 15 rue de 1'Ecole de Médecine, Laboratoire d'Imagerie Biomédicale, Paris 75006, France, mchlrnwld@gmail.com), Stefan Catheline (LabTAU, Unité U1032, Université Claude Bernard Lyon 1, Lyon, France), Quentin Grimal (Laboratoire d'Imagerie Biomédicale, Université Pierre et Marie Curie, Paris, France), and Lapo Boschi (Institut des Sci. de la Terre de Paris, Université Pierre et Marie Curie, Paris, France)

Internal cues such as bone conducted sound (i.e., long signals involving reverberation within the skull) may be beneficial to acoustic source localization in humans. We investigate the potential of bone conduction for human localization in the near field. Following previous work of the authors, we use a conceptual processing model that translates elastic waves conducted and reverberated in a skull-like object into spatial positioning through a time-reversal analysis. The localization accuracy is tested for one and two piezoelectric receivers glued close to the position of the ears, measuring solely the vibrations of the object. The sound source is placed along the azimuthal and sagittal planes for distances to the skull between 5 and $100 \mathrm{~cm}$. We are able to estimate the source position in the near-field $(<20 \mathrm{~cm})$ with a resolution below the diffraction limit ( $1 / 4$ of a wavelength) for frequencies in the physiological hearing range $(\sim 2 \mathrm{kHz})$. We infer that anatomical details of the skull give rise to complex features of the skull's Green's function enabling super-resolution localization. The role of the skull is presumably particularly relevant in marine animals, owing to the effectiveness of energy transfer from water to bones. We are currently investigating this phenomenon on dolphin skulls.

1aSA6. A sound absorbing metasurface with coupled resonators. Junfei Li, Wenqi Wang, Yangbo Xie, Bogdan Popa, and Steven A. Cummer (ECE, Duke Univ., 101 Sci. Dr., Rm. 3417, FCIEMAS Bldg. Duke Univ, Durham, NC 27708, junfei.li@duke.edu)

An impedance matched surface is able, in principle, to totally absorb the incident sound and yield no reaction, and this is desired in many acoustic applications. Here, we demonstrate a new design of impedance matched sound absorbing surface with a simple construction. By coupling dijerent resonators and generating a hybrid resonance mode, we designed and fabricated a metasurface that is impedance-matched to airborne sound at tunable frequencies with subwavelength scale unit cells. With careful design of the coupled resonators, over $99 \%$ energy absorption at central frequency of 511 $\mathrm{Hz}$ with a $50 \%$ absorption bandwidth of $140 \mathrm{~Hz}$ is achieved experimentally. The proposed design can be easily fabricated and is mechanically stable. The proposed metasurface can be used in many sound absorption applications such as loudspeaker design and architectural acoustics.
1aSA7. Passive structural health monitoring using cross correlation of ambient vibrations in an aluminum plate. Sun Ah Jung (Seoul National Univ., Bldg. 36 212, 1 Gwanak-ro, Gwanak-gu, Seoul 151-742, South Korea, sunj@snu.ac.kr), Keunhwa Lee (Sejong Univ., Seoul, South Korea), and Woojae Seong (Seoul National Univ., Seoul, South Korea)

Green's function extracted from cross correlating ambient diffuse field is used for structural health monitoring. Experimental study was conducted to detect structural damages in an aluminum plate using two accelerometers. Green's function obtained by the cross correlation in an undamaged plate is compared to the signal directly generated by an impact hammer. A good agreement between active (impact hammer) and passive (cross correlation) processing is demonstrated. Various damage scenarios, including mass addition and hole in the plate, are investigated. Different from damage index definition in a recent study which compares the causal and anti-causal part of the Greens' function using the reciprocity theorem, we computed the damage index by comparing the causal part of Green's function of an undamaged plate to that of a damaged plate. Distributions of damage indices, according to various number of noise sources for each type of damage type, are analyzed. Using the support vector machine, we classified the damage index distributions. Error analysis of these classifications is given; it shows that present damage index definition provides better damage diagnosis than previous definitions even when using small number of sources.

1aSA8. Urban seismology: Numerical results and real data observations on the clustering effect of buildings. Andrea Colombi (Mathematics, Imperial College, South Kensington, London SW7 2AZ, United Kingdom, andree. colombi@gmail.com) and Philippe Gueguen (ISTerre, Grenoble, France)

Seismic design parameters for buildings are traditionally based on the seismicity of the area, the so-called site effects, and structural response decoupling with the underlying surface. It is however well known that buildings strongly affect the wavefield interacting in a non-linear way through the foundations and the surrounding soil. In dense urban areas, buildings are very close to each other, and it is reasonable to expect a collective effect known as site-city interaction (SCI). Initially, observations of this phenomenon have been made difficult by the lack of data. Now, the increased availability of continuous records from permanent seismic networks in urban areas or buildings has made SCI an important topic in urban seismology. Here, after presenting some numerical results of SCI in densely built areas, we discuss a recent study on three residential towers located in the city of Grenoble (France) exhibiting an SCI that depends on the strike angle of the seismic energy. Both numerical simulations and recorded data exhibit dynamic coupling within the building cluster that has a non-negligible impact on the building response. The angle dependence helps interpreting the tenants' answers to a survey made right after the Earthquake in each of the tower. 


\title{
Session 1aSC
}

\section{Speech Communication: Speech Technology: Synthesis and Interfaces (Poster Session)}

\author{
Jonathan C. Yip, Chair \\ Department of Linguistics, The University of Hong Kong, Rm. 906, Run Run Shaw Tower, Pokfulam, HK 0000, Hong Kong
}

\begin{abstract}
All posters will be on display from 10:30 a.m. to 12:00 noon. To allow contributors in this session to see the other posters, authors of odd-numbered papers will be at their posters from 10:30 a.m. to 11:15 a.m. and authors of even-numbered papers will be at their posters from 11:15 a.m. to 12:00 noon.
\end{abstract}

\section{Contributed Papers}

\begin{abstract}
1aSC1. Emotional voice conversion system for multiple languages based on three-layered model in dimensional space. Yawen Xue (Information Sci., Japan Adv. Inst. of Sci. and Technol., 1-1 Asahidai, Nomi City, Ishikawa, Japan), Yasuhiro Hamada (Meiji Univ., Tokyo, Japan), and Masato Akagi (Information Sci., Japan Adv. Inst. of Sci. and Technol., Nomi City, Ishikawa, Japan, akagi@jaist.ac.jp)
\end{abstract}

This paper proposes a system to convert neutral speech to emotional one with controlled intensity of emotions for multiple languages. Most of previous researches considering synthesis of emotional voices used statistical or concatenative methods that can synthesize emotions in category. While humans sometimes enhance or relieve emotional states and intensity during daily life, synthesized emotional speech in categories is not enough. A dimensional approach which can represent emotion as a point in a dimensional space can express emotions with continuous intensity. Employing the dimensional approach, a three-layered model to estimate displacement of the acoustic features of the target emotional speech from that of source (neutral) speech and a rule-based conversion method to modify acoustic features of source (neutral) speech to synthesize the target emotional speech are proposed. To convert the source speech freely and easily, Fujisaki model for f0 contour and target prediction model for power envelope are introduced to parameterize dynamic features in prosody. Although this system is trained with Japanese database, neutral speech in two different languages, English and Chinese are tried to convert. Evaluation results show that all converted voices can convey the same impression with satisfactory order of emotional intensity and naturalness as Japanese voices. It means that the emotion voice conversion system can work for other languages with controllable emotion category and intensity although it is built in one language.

1aSC2. Feasibility study on synthesis of English vowels with a vocal tract mapping interface. Riku Aoki (Graduate School of Sci. and Technol., Kumamoto Univ., 2-39-1 Kurokami Chuo-ku Kumamoto, Ogata Lab., Kumamoto 860-8555, Japan, aoki_r@st.cs.kumamoto-u.ac.jp), Kohichi Ogata (Faculty of Sci. and Technol., Kumamoto Univ., Kumamoto, Japan), and Akihiro Taruguchi (Graduate School of Sci. and Technol., Kumamoto Univ., Kumamoto, Japan)

This paper describes the synthesis of English vowels with a vocal tract mapping interface developed in our laboratory. This interface uses five vocal tract shapes that are located at apexes of a pentagonal chart in consideration of Japanese vowels. It allows users to configure the vocal tract shape corresponding to the location of the mouse pointer based on interpolation. Moreover, it has a function of the inverse estimation of the vocal tract from formant frequencies. Although the interface has been developed considering the Japanese vowels, whether English vowels can also be synthesized with this interface is an interesting topic for confirmation of its versatility. Therefore, we investigated the feasibility of producing English vowels ([a], [æ], $\left.[\supset],[\varepsilon],\left[\Im^{\mathrm{c}}\right],[\mathrm{i}],[\mathrm{I}],[\mathrm{u}],[\mathrm{U}],[\Lambda]\right)$ with the mapping interface. As the first step, we investigated that the mapping interface could estimate vocal tract shapes for the English vowels synthesized with the VowelEditor in PRAAT as test sounds. Furthermore, listening tests were conducted to examine whether the re-synthesized vowel sounds with the mapping interface were matched with the original vowel sounds by PRAAT. The experimental results showed that the mapping interface has a potential for producing English vowels.

1aSC3. Speech resynthesis from $1 / \mathbf{f}^{\beta}$ noise. Robert Fuhrman and Eric Vatikiotis-Bateson (UBC, \#402-2588 Alder St., Vancouver, BC V6H4E3, Canada, robert.a.fuhrman@gmail.com)

Speech can be resynthesized from acoustic parameters estimated from the motion of the vocal tract articulators, head, and face. This has shown that speech information is broadly distributed both in space and time across the physiological systems associated with speech production, and correspondingly, it provides a basis for exploring what properties of signal structure are relevant for specifying the relation between acoustics and physiology. In this presentation, we use the resynthesis paradigm to explore the extent to which the acoustic-articulatory relation can be specified in terms of the structure of fluctuations in the speech signal. Specifically, we demonstrate that speech acoustics closely matching the original can be obtained by replacing the vocal tract kinematic signals typically used in the re-synthesis paradigm with surrogate signals having $1 / f^{\beta}$ fractal fluctuation structure-a known (Voss \& Clarke 1975) statistical property of the speech amplitude envelope that holds for low frequencies corresponding to the rate of articulatory motion (<10 Hz: Dudley, 1939). We also address the hypothesis that quantitative descriptors derived from both the fractal and multifractal properties of speech link the time-varying properties of speech acoustics to the cooperative motion of physiological structures involved in speech production.

1aSC4. Singing voice synthesizer with non-filter waveform generation using spectral phase reconstruction. Yasuhiro Hamada (Meiji Univ., Nakano, Nakano 1648525, Japan, hamada@meiji.ac.jp), Nobutaka Ono (National Inst. of Informatics / SOKENDAI, Chiyoda, Japan), and Shigeki Sagayama (Meiji Univ., Nakano, Japan)

This paper discusses a singing voice synthesis system based on non-filter waveform generation using spectral phase reconstruction as an alternative method to replace the conventional source-filter model. Source-filter model has been considered as an essential technique in the long history of speech synthesis as it simulates the human process of speech production consisting of excitation and resonances, even after hidden Markov model was introduced in the 90 s toward statistically trainable speech synthesis. Filter (particularly, recursive filter), however, may cause serious problems in undesired amplitude and long time decay when sharp resonances in recursive filter match harmonics of $\mathrm{F} 0$ of the excitation. As the ultimate purpose of usage of filter is to transfer the spectrogram designed in the TTS system to the listener's hearing organs, an alternative solution can be brought from "phase reconstruction" without using filters. We propose a spectral phase reconstruction, instead of using filter, to generate waveform from the desired 
power spectrogram. We applied this framework to singing voice synthesis where fundamental frequency (F0) was modified according to the target melody. The result of the listening tests showed that the proposed method gives higher quality of naturalness than previous methods with recursive filter.

1aSC5. Analysis of driver workload when using speech interfaces. Daiki Hayashi, Chiyomi Miyajima, and Kazuya Takeda (Graduate School of Information Sci., Nagoya Univ., Furo-cho, Chikusa, Nagoya 4648603, Japan, hayashi.daiki@g.sp.m.is.nagoya-u.ac.jp)

Speech interfaces are useful for improving the accessibility of in-vehicle devices as they are considered to be less distracting than visual interfaces. However, there is still considerable cognitive demand when using a speech interface, which may increase the chance of a traffic accident. This study investigates driver workload induced by various tasks, including a speech interface used for music retrieval. Driving data were collected from 523 drivers operating an instrumented vehicle on urban streets and highways. The drivers were given each of the following secondary tasks while driving: a) repeating random strings of four letters they heard through an earphone, b) interacting with a spoken dialogue system to retrieve and play music, c) being directed to an unfamiliar place by a human navigator on a cell phone, and d) reading aloud words seen on signboards while driving. After driving, each driver subjectively rated the induced workload for each secondary task from 1 (lowest) to 5 (highest). Analysis of the results of their subjective evaluations showed that talking to a navigator on a cell phone resulted in the lowest average workload (about 1.6), which was almost the same as the driving without any secondary task. Repeating random strings of four letters and retrieving music using a speech interface produced the highest average workload (about 2.5).

1aSC6. An Indonesian concatenative speech synthesis system. Toshio Hirai and Ivan Setiawan (Arcadia, Inc., Minoh Sun-plaza 1st Bldg. 7F, 6-31 Minoh, Minoh, Japan, thirai@arcadia.co.jp)

An Indonesian concatenative speech synthesis system was developed that can (1) differentiate between the pronunciation of [e] and [@] (XSAMPA) by utilizing a pronunciation dictionary and pronunciation rules and (2) automatically create derived words that are then inserted into the pronunciation dictionary, which is used in converting text into phonemes. Speech data were recorded from a female native Indonesian speaker. Phrases included proper nouns and text samples from Indonesian textbooks, newspaper articles, and TV scripts, which covered almost all possible Indonesian syllables. There were 4,490 phrases lasting a total of 5.1 hours. The transcription was constructed considering pauses during narration. A forced alignment technique was used to obtain the phoneme boundary of the speech, and each phoneme and its acoustic/linguistic features were added to a database. In the synthesis step, an input text was converted into phonemes. An appropriate segment sequence, which has features similar to the phonemes should have and shows the least concatenative distortion, was selected from the database and was concatenated into a waveform. The synthesized speech was of acceptable quality, but we discovered some problems, such as sound discontinuity. The parameter weights of cost function to evaluate the similarity and the distortion should be optimized further.

1aSC7. Robust text-to-speech duration modelling with a deep neural network. Gustav E. Henter (National Inst. of Informatics, 13F 1313, 2-1-2 Hitotsubashi, Tokyo, Chiyoda-ku 101-8430, Japan, ghenter@inf.ed.ac.uk), Srikanth Ronanki, Oliver Watts, Mirjam Wester, Zhizheng Wu, and Simon King (The Univ. of Edinburgh, Edinburgh, United Kingdom)

Accurate modeling and prediction of speech-sound durations is important for generating more natural synthetic speech. Deep neural networks (DNNs) offer powerful models, and large, found corpora of natural speech are easily acquired for training them. Unfortunately, poor quality control (e.g., transcription errors) and phenomena such as reductions and filled pauses complicate duration modelling from found speech data. To mitigate issues caused by these idiosyncrasies, we propose to incorporate methods from robust statistics into speech synthesis. Robust methods can disregard ill-fitting training-data points-errors or other outliers- to describe the typical case better. For instance, parameter estimation can be made robust by replacing maximum likelihood with a robust estimation criterion based on the density power divergence (a.k.a. the $\beta$-divergence). Alternatively, a standard approximation for output generation with mixture density networks (MDNs) can be interpreted as a robust output generation heuristic. To evaluate the potential benefits of robust techniques, we adapted data from a free online audiobook to build several DNN-based text-to-speech systems, with either conventional or robust duration prediction. Our objective results indicate that robust methods described typical durations better than the baselines. Additionally, listeners significantly preferred synthetic speech generated using the robust methods in a subjective evaluation.

1aSC8. Speech synthesizers controlled by the movements of non-vocal organs. Masashi Ito (Tohoku Inst. of Technol., 35-1 Yagiyama-Kasumicho, Sendai 982-8577, Japan, itojin@tohtech.ac.jp)

A speech-like signal can be synthesized without the text-to-speech conversion if speech parameters, such as loudness, pitch, and phonemic information, are successively determined in the utterance. To control the parameters, human body motions have been utilized in this type of speech synthesis. The speech synthesizers controlled by the movement of non-vocal organs (MNVO) are expected to provide effective assistant tools for speech disabilities. Although there have been extensive studies for the MNVO synthesizers, the optimal conversion strategy from body motions to speech parameters has not been clarified yet. This is because that the optimal MNVO speech synthesizer requires both usability and expressiveness while they have a trade-off relationship in general. In order to address this problem, a refined architecture of the MNVO synthesizer is proposed in the present study. This is characterized by an explicit separation between motion detection and speech synthesis processes, which makes it possible to examine a common conversion strategy regardless of the input devices. Based on this architecture, several MNVO speech synthesizers were realized using different types of the motion sensors. Evaluating the performances of these systems, the effect and limitation of the proposed architecture is discussed.

1aSC9. The UCLA voice synthesizer, version 2.0. Jody Kreiman, Norma Antonanzas-Barroso, and Bruce R. Gerratt (Dept. of Head and Neck Surgery, UCLA School of Medicine, 31-24 Rehab Ctr., Los Angeles, CA 90095-1794,jkreiman@ucla.edu)

Based on our psychoacoustic model of voice quality, the UCLA voice synthesizer allows users to copy synthesize nearly any steady-state voice sample or to create stimuli that systematically vary in specific acoustic dimensions. This new release contains a number of significant improvements from earlier versions. The vocal tract model now includes three spectral zeros with adjustable bandwidths. The precise spectral shape of the harmonic and inharmonic sources can be modified at will, either by adjusting the heights of the harmonics or the noise amplitude in a selected frequency range or by specifying the desired spectral or noise slope in a range. Any number of ranges of any size can be specified. Synthesizer variables can be individually saved and copied between cases. Additional changes increase control of analysis parameters and the ease with which series of stimuli can be created. The synthesizer is fully documented and is freely available for download from headandnecksurgery.ucla.edu/glottalaffairs. Copies will also be available at the conference. [Research supported by NIH.]

1aSC10. Improving Spanish speech synthesis intelligibility under noisy environments. Jaime Lorenzo-Trueba (Speech Technol. Group / NII, 2-1-2 Hitotsubashi, Chiyoda-ku, Tokyo, Tokyo 101-8430, Japan, jaime@nii.ac. jp), Roberto Barra-Chicote (Speech Technol. Group, Madrid, Spain), Junichi Yamagishi (National Inst. of Informatics, Tokyo, Japan), and Juan M. Montero (Speech Technol. Group, Madrid, Spain)

In this paper, we evaluate a newly recorded Spanish Lombard Speech database. This database has been recorded with expressive speech synthesis in mind, and more particularly adaptability to the environment. Real stationary noise recorded inside of a car was used to produce the Lombard speech response in the speaker by means of a headphone. Four different noise levels were used, in steps of $5 \mathrm{~dB}$ and also clean speech to set the clean speech baseline. Finally, a pair of intelligibility evaluations were carried out, one with natural speech that proves the validity of the recorded database by 
showing a $37 \%$ absolute increase in intelligibility in a -10 dB SNR condition when compared to non-Lombard speech. The second evaluation was carried out with synthetic speech, which showed a $10 \%$ absolute increase in intelligibility for both the -10 and $-15 \mathrm{~dB}$ SNR condition.

1aSC11. Example-based spoken chat system which can be customized for each user. Eichi Seto and Norihide Kitaoka (Tokushima Univ., 2-1 Minami-Johsanjima-cho, Tokushima-shi, Tokushima 770-8506, Japan, c501637006@tokushima-u.ac.jp)

There is a need to develop automated dialog systems, such as chatbots, which are capable of engaging in natural conversations with elderly users. We propose an example-based dialog system featuring an adaptation method which customizes the dialog for each user. After retrieving information about the user from the Web using the user's profile, morpheme analysis is applied to the retrieval results and only proper nouns are extracted. Words which appear with high frequencies are considered to be related to the user. We then calculate the similarity between words related to the user and words in example phrases in the dialog system. Cosine similarity between distributed representations of words is calculated using Word2vec. We then generate a phrase which has been adapted to include a topic related to the user by substituting user-related words for highly similar words in the original example phrase. We manually evaluated the naturalness of the generated phrases and found that the system did in fact generate natural-sounding phrases. However, Word2vec sometimes mistook words which were grammatically different parts of speech (POS) for similar words, so we added a POS constraint to the substitution process. As a result, the system achieved higher phrase generation precision.

1aSC12. A DNN-based text-to-speech synthesis system using speaker, gender, and age codes. Hieu Thi Luong (Comput. Sci., VNU - HCM Univ. of Sci., Ho Chi Minh, Viet Nam), Shinji Takaki (Digital Content and Media Sci. Res. Div., National Inst. of Informatics, 2-1-2, Hitotsubashi-cho, Chiyoda-ku, Tokyo 101-8430, Japan, takaki@nii.ac.jp), SangJin Kim (Naver Labs, Naver Corp., Seongnam, South Korea), and Junichi Yamagishi (Digital Content and Media Sci. Res. Div., National Inst. of Informatics, Chiyoda-ku, Tokyo, Japan)

Adaptation and manipulation techniques for creating various characteristics of synthetic speech are important research topics in the speech synthesis field. In this work, we investigate the performance of a DNN-based textto-speech synthesis system that uses speaker, gender, and age codes as well as the text inputs (1) for modeling speaker-independent models called "average voice models," (2) for performing speaker adaptation using a small amount of adaptation data, and also (3) for manipulating characteristics of synthetic speech based on the codes. For these purposes, we extracted a set of studio-quality speech data uttered by 68 males and 70 females, whose age vary between 10 and 80, from our large-scale Japanese corpus and carried out the three experiments: (1) We constructed a DNN-based speaker-independent model using one-hot vectors representing a set of the above speakers. (2) We performed speaker adaptation by estimating a code vector for a new speaker via the back-propagation. (3) We performed manual manipulation of the code vector to modify perceived characteristics, gender, and/or age of synthetic speech. Experimental results showed that high-performance speaker-independent models can be constructed using the proposed code vectors and additionally that adaptation and manipulation using the codes can also be performed effectively.

1aSC13. Speech analysis-synthesis system using principal components of vowel spectra. Tomio Takara, Akichika Higa, Syouki Kaneshiro, and Yuuya Oshiro (Eng., Univ. of Ryukyus, 1 Senbaru, Nishihara-chou, Okinawa-ken 903-0213, Japan, takara@ie.u-ryukyu.ac.jp)

We have studied an effective method using principal components spanning a feature space of isolated vowels. A covariance matrix is calculated from many log-amplitude spectra of isolated vowels uttered by a speaker. An eigen equation of the covariance matrix is solved. The resulting eigenvectors are called principal vectors. In the analysis system, log-amplitude spectrum for each frame of a word uttered by the same speaker is transformed to the components on the principal vectors. In the synthesis system, a log-amplitude spectrum is reconstructed using the components on the principal vectors with the largest eigenvalues and the spoken word is synthesized using the LMA filter. We draw the distribution chart of the first and the second principal components extracted from Japanese vowel data. This figure was very similar to the F1-F2 distribution of vowels and so to the vowel classification map in coordinate axes of the degree of constriction and the tongue hump position. The Listening tests showed that the quality of the word with only 7 parameters per frame synthesized by the proposed method was comparable to the word with 9 to 12 parameters by the cepstral method. Consequently, the effectiveness of the proposed method was shown.

1aSC14. Improved ETSI advanced front-end for automatic speech recognition using quadrature mirror filter synthesis based on robust complex speech analysis. Keita Higa (Toshiba Corp., Tokyo, Japan) and Keiichi Funaki (C\&N Ctr., Univ. of the Ryukyus, Senbaru 1, Nishihara, Okinawa 903-0213, Japan, funaki@cc.u-ryukyu.ac.jp)

An automatic speech recognition (ASR) is commonly used in these days. Current ASR systems perform well in ideal environment; however, it does not perform well in realistic noisy environment. As a robust ASR, ETSI has standardized Advanced Front-End (AFE) that adopts two-stage of iterative Wiener filter (IWF) to realize a speech enhancement as the frontend of ASR. In the ETSI AFE, $16 \mathrm{kHz}$ speech is divided uniformly by QMF (Quadrature Mirror Filter) into lower-band and higher-band signal and only lower-band signal is emphasized by the IWF and MFCC (Mel Frequency Cepstral Coefficient) is extracted from both the second-stage of emphasized lower-band signal and non-emphasized higher-band signal. FFT is used to estimate speech spectrum that designs the Wiener filter. On the other hand, we have already proposed robust complex speech analysis for an analytic signal. It can estimate more robust and more accurate speech spectrum due to the introduced robust criterion and nature of analytic signal. This paper proposes an improved AFE using wide-band robust ELS (Extended Least Square) complex analysis and real-valued analysis instead of FFT. Moreover, the QMF synthesis is also introduced for second-stage of wide-band analysis. The experimental results using the CENSREC-2 speech database demonstrate that the performance is improved.

1aSC15. Spectral and pitch modeling with hybrid approach to singing voice synthesis using hidden semi-Markov model and deep neural network. Kouki Hongo, Takashi Nose, and Akinori Ito (Graduate School of Eng., Tohoku Univ., 6-6-05 Aoba, Aramaki, Aoba-ku, Sendai, Miyagi 9808579, Japan, kouki.hongou.p4@dc.tohoku.ac.jp)

We propose a corpus-based singing voice synthesis system combining the hidden Markov model (HMM) and the Deep Neural Network (DNN). Recently, in the area of text-to-speech synthesis, it was reported that the DNN-based speech synthesis method showed better speech quality than the HMM-based one. However, when we introduced the DNN to statistical singing voice synthesis, it did not improve the synthetic singing voice quality. Thus, we introduced the DNN in the singing voice synthesis in a different way. Instead of modeling the speech spectra, we exploited the DNN to model the difference between the spectra of natural singing voice and synthetic singing voice from the HMM. To do that, we used the DNN to map the input musical information such as lyrics, tones, durations into the difference of output acoustic features between the natural and synthetic singing voice. This allows us to reconstruct the spectral fine structures in singing voice generated by HMMs. Our results proved that the proposed method improved the quality of synthetic singing voice compared to the conventional methods. 
1aSC16. Low delay statistical singing voice conversion with direct waveform modification based on spectral differential considering global variance. Kazuhiro Kobayashi (Information Sci., Nara Inst. of Sci. and Technol., Nakatomigaoka1-4162-1-D06-201, Nara, Nara 6310003, Japan, kazuhiro-k@is.naist.jp), Tomoki Toda (Information Technol. Ctr., Nagoya Univ., Nagoya, Japan), and Satoshi Nakamura (Information Sci., Nara Inst. of Sci. and Technol., Nara, Japan)

This paper presents a low-delay statistical singing voice conversion (SVC) method with direct waveform modification based on spectral differential (DIFFSVC) considering global variance (GV). Statistical SVC based on a Gaussian mixture model (GMM) is a technique to convert singer identity of a source singer's voice into that of a target singer converting several acoustic features. We have successfully improved quality of converted singing voices by proposing the DIFFSVC considering the GV to avoid parameterization errors caused by vocoding process and alleviate over-smoothing effects. On the other hand, this method is based on batch-type conversion processing, and therefore, it is difficult to directly apply it to real-time conversion processing that needs a low delay conversion method. In this paper, to develop a high-quality and real-time SVC system, we propose a lowdelay DIFFSVC method using GV-based post-filtering process. The experimental results have demonstrated that the proposed method makes it possible to improve speech quality of the converted singing voice in low-delay conversion processing. [This work was supported in part by JSPS KAKENHI Grant Number 26280060, Grant-in-Aid for JSPS Research Fellow Number 16J10726, and by the JST OngaCREST project.]

1aSC17. Improvement of quality of voice conversion based on spectral differential filter using STRAIGHT-based Mel-cepstral coefficients. Harunori Koike, Takashi Nose (Tohoku Univ., 6-6-05, Aoba, Aramaki, Aoba-ku, Sendai 980-8579, Japan, harunori.koike.q5@dc.tohoku.ac.jp), Takahiro Shinozaki (Tokyo Inst. of Technol., Yokohama, Japan), and Akinori Ito (Tohoku Univ., Sendai, Japan)

In our previous research, we proposed a technique for converting the speech individuality of speech of an arbitrary input speaker into a specified speaker's one. This technique used the neural network (NN) for many-toone mapping, which was trained from the pairs of multiple source speakers and a target speaker. Using the NN, we design a filter based on the difference of the spectra of the source and the target speech to directly convert the waveform of the input speaker into the target speaker's one. An advantage of the proposed method is that the direct waveform conversion alleviates the quality degradation caused by the F0 extraction error. To improve this proposed method, we use mel-cepstral coefficients extracted by STRAIGHT, the high-quality tool of speech analysis and synthesis. The higher order components of the cepstral coefficients determine the detailed shape of the spectral envelope, but the prediction accuracy of the high-order coefficients might be worse than that of the lower-order ones. Thus, we investigated the effect of the order of cepstral components to find the condition that gave better conversion quality. Additionally, we exploited the dynamic features to reduce the discontinuity of the frame and improve the converted speech quality.

1aSC18. Acquisition and evaluation of a human-robot elderly spoken dialog corpus for developing computerized cognitive assessment systems. Hiroaki Kojima (National Inst. of Adv. Industrial Sci. and Technol. (AIST), 1-1-1, Umezono, Tsukuba, Ibaraki 305-8560, Japan, h. kojima@aist.go.jp), Kana Takaeda (National Rehabilitation Ctr. for Persons with Disabilities, Tokorozawa, Japan), Misato Nihei (The Univ. of Tokyo, Tokyo, Japan), Ken Sadohara (National Inst. of Adv. Industrial Sci. and Technol. (AIST), Tsukuba, Japan), Shinichi Ohnaka (NEC Corp., Kawasaki, Japan), and Takenobu Inoue (National Rehabilitation Ctr. for Persons with Disabilities, Tokorozawa, Japan)

Personal communication robots are expected to assist daily living of elderly people. Aiming at developing computerized cognitive assessment systems, we collected human-robot spoken dialog of a cognitive impairment test scenario based on TICS (Telephone Interview for Cognitive Status) and COGNISTAT (Cognitive Status Examination) in Japanese. For the efficient acquisition of the spoken dialog corpus of this scenario, we implemented a WOz (Wizard of Oz) style spoken dialog system on a commercial personal robot, PaPeRo by NEC. By using this system, we collected 147 dialogs spoken by 48 elderlies whose ages varied about 75-85 and MMSE (Mini Mental State Examination) scores varied around 26.5. Each dialog took about $30 \mathrm{~min}$, and contains around 100 human utterances. In order to evaluate feasibility of automatic assessment, we conducted speech recognition experiments with the speech corpus. In the recognition experiments of the elderly speech of answering to the temporal orientation test of asking today's month and date, the accuracies of discriminating correctness of the answers exceeds $80 \%$ by using speech dictation engines. This preliminary results show potential feasibilities for computerized cognitive assessment systems.

1aSC19. A method to estimate glottal source waves and vocal tract shapes for widely pronounced types using ARX-LF model. Yongwei Li, Daisuke Morikawa, and Masato Akagi (Japan Adv. Inst. of Sci. and Technol., Asahidai 1-1, Nomishi, Ishikawaken 9231211, Japan, yongwei@ jaist.ac.jp)

Accurate estimates of glottal source waves and vocal tract shapes can be used for speech synthesis with high quality. One of source-filter models, ARX-LF model, is widely used to estimate glottal source waves of neutral speech and singing voices. This paper extends the ARX-LF model to accurately estimate glottal source waves and vocal tract shapes with more widely pronounced types. The proposed method mainly contains two steps: the first step is to set initial values of LF parameters and shift glottal closure instant position, and the second step is to save optimum LF parameters and vocal tract coefficients when mean-square equation error is the smallest. The voices are synthesized by different LF parameters and fixed vocal tract filter model, which are used to test whether the proposed method can work well. The results show that the proposed method has ability to estimate LF parameters in more widely pronounced types. The proposed method is further used to estimate glottal source waves and vocal tract shapes of emotional voices. Spectral tilts of estimated glottal waves and vocal tract shapes derived from ARX filters are consistent with other reports and human speech production mechanisms.

1aSC20. Improvement of the noisy speech recognition using method of thresholds and emphasizing wavelet coefficients for clean speech. Yoichi Midorikawa and Masanori Akita (Faculty of Eng., Oita Univ., 700 Dannoharu, Oita, Oita 870-1192, Japan, ymido@oita-u.ac.jp)

In recent years, speech recognition under the noisy environment is one of the very important technologies. This study improves a speech recognition method for the signals under the noisy environment using method of thresholds and emphasizing wavelet coefficients for clean signal data. Noisy speech recognitions are extremely difficult problem. To analyze noise problem, in general, the majority of people have used the Fourier analysis. But the Fourier transform reveals only the frequency information. The general noise filters reduce specific frequency band contained both signal and noise. It is difficult to eliminate only noise component from a signal containing noise components. To overcome this difficulty, we applied the wavelet analysis. In this study, we improve the speech recognition under the noisy environment by bringing it close to reference data and noisy input data by modifying the spectrum by the wavelet transform using thresholds and emphasizing methods for reference clean speech data. We apply this method using wavelet transform to the modification of spectral envelope shape. As a result, noisy speech recognition rate is improved by this method using wavelet transform for clean speech data.

1aSC21. Multimodal control system for autonomous vehicles using speech and gesture recognition. Takuma Nakagawa and Norihide Kitaoka (Tokushima Univ., 2-1 Minami-Johsanjima-cho, Tokushima-shi, Tokushima 770-8506, Japan, c501637005@tokushima-u.ac.jp)

The recent development of autonomous vehicles has attracted much attention, but operating these vehicles may be too complex for average users. Therefore, we propose an intuitive, multimodal interface for the control of autonomous vehicles using speech and gesture recognition to interpret and execute the commands of users. For example, if the user says "turn there" while pointing at a landmark, the vehicle can utilize this behavior to correctly understand and comply with the user's intent. To achieve this, we 
designed a two-part interface consisting of a multimodal understanding component and a dialog control component. Our multimodal understanding and dialog control components can be seen as a concatenation of two separate transducers. One transducer is used for multimodal understanding and the other for a conventional dialog system. We then construct a combined transducer from these two transducers. We developed various scenarios which might arise while operating an autonomous vehicle and displayed these scenes on a monitor. Subjects were then asked to operate a virtual car using speech commands and pointing gestures to control the vehicle while observing the monitor. The questionnaire results show that subjects felt they were able to easily and naturally operate the autonomous vehicle using utterances and gestures.

1aSC22. Experimental study of impression-based statistical mapping between speakers' faces and their voices. Yasuhito Ohsugi, Daisuke Saito, and Nobuaki Minematsu (the Univ. of Tokyo, 7-3-1 Hongo, Bunkyoku, Tokyo 1130033, Japan, yasuhito.ohsugi@gavo.t.u-tokyo.ac.jp)

Recently, many types of voice-user interface (VUI) have been developed and some of them become prevalent. To realize more natural conversation between a user and a machine, one feasible approach is expected to be enhanced personification of the machine. In this study, we attempt to give an adequate face to the voice quality of the speech synthesizer embedded in the machine. A research question here is what kind of face should be provided to a given voice quality? Also, an inverse question is possible when a face is given in advance: what kind of voice quality should be mapped to a given face? To solve a problem of mapping between a set of elements and another set, a statistical mapping is often applied, where a parallel and pairwise corpus of elements and their corresponding ones is required. In this study, GMM-based mapping is adopted as statistical mapping between voice features and face features. Further, a parallel corpus is prepared by asking subjects to select suited voices (faces) for given faces (voices) based on their impressions on the faces and the voices. Experiments showed that, although adequate voices can be automatically generated for given faces, mapping between them was strongly subject-dependent.

1aSC23. A study on acoustic features affecting speaker similarity between recorded voice and synthesized voice. Kenko Ota (Nippon Inst. of Technol., 4-1 Gakuendai, Miyashiro, Minami-saitama 345-8501, Japan, otakenko@nit.ac.jp) and Kohei Yoshida (Nippon Inst. of Technol., Minamisaitama, Japan)

This study focuses on the difference in the audibility between recorded voice and synthesized voice uttered by the same speaker. Voices whose pitch does not fluctuate in an utterance were recorded. Voices are synthesized by STRAIGHT. In this research, recorded voices and synthesized voices are allocated to a three-dimensional space by INDSCAL (Individual Differences Scaling) which is an analysis method of MDS (Multi-dimensional Scaling) in order to investigate the acoustic features which are related to the difference in the audibility between recorded voice and synthesized voice. Subjects compared following three combinations of voices: two recorded voices, two synthesized voices, a recorded voice and a synthesized voice. The acoustic features considered in this research are shown as follows: fundamental frequency, spectral centroid, spectral roll-off, spectral flux, spectral contrast, and spectral intensity. As a result, spectral roll-off and spectral contrast are related to the discrimination whether speakers of recorded voice and synthesized voice with same pitch are same or different. Moreover, fundamental frequency is also related to the discrimination whether speakers of recorded voice and synthesized voice with different pitch are same or different.

1aSC24. An evaluation of the automatically topology-generated autoregressive hidden Markov model with regard to an esophageal voice enhancement task. Akira Sasou (National Inst. of Adv. Industrial Sci. and Technol., AIST, Central1,1-1-1 Umezono, Tsukuba, Ibaraki 305-8568, Japan, a-sasou@aist.go.jp)

An Auto-Regressive eXogenous (ARX) model combined with descriptive models of the glottal source waveform has been adopted to more accurately separate the vocal tract and the voicing source. However, these methods cannot be easily applied to the analysis of voices uttered by different speech production methods, such as esophageal voice. We previously proposed the voicing source hidden Markov model and an accompanying parameter estimation method. We refer to the model combining the HMM with an Auto-Regressive (AR) filter as AR-HMM. The proposed method automatically generates the optimum topology for the HMM using the minimum description length-based successive state splitting algorithm in order to simultaneously and accurately estimate the vocal tract and voicing source based on a voice excited by an unknown, aperiodic voicing source such as an esophageal voice. In this paper, we evaluate the perceived quality of the enhanced esophageal voices, which are synthesized by filtering the voicing source extracted from normal speakers with the AR filter extracted from the esophageal voices.

1aSC25. Evaluation of electrolarynx controlled by real-time statistical F0 prediction. Kou Tanaka (NAIST, 8916-5 Takayama-cho, Augmented Human Commun. Lab. Graduate School of Information Sci. Nara Inst. of Sci. and Technol., Nara, Ikoma 630-0192, Japan, ko-t@is.naist.jp), Tomoki Toda (Nagoya Univ., Aichi, Nagoya, Japan), and Satoshi Nakamura (NAIST, Nara, Japan)

One of the major speaking methods for laryngectomees is a speaking method using an electrolarynx to generate artificial excitation sounds, instead of vocal fold vibration. Although electrolaryngeal (EL) speech is relatively intelligible, its naturalness is quite low owing to the artificial excitation sounds. To make it possible to produce more naturally sounding EL speech, we have proposed an automatic control method of the fundamental frequency (F0) patterns of the excitation sounds generated from the electrolarynx based on real-time statistical F0 prediction. In this method, a vibration of the electrolarynx to generate the excitation sounds is controlled not according to additional signals consciously provided by the laryngectomees but using only their produced EL speech signals. In the previous report, we have developed a prototype system by implementing our proposed method to the electrolarynx and have evaluated its performance objectively through a simulation. In this paper, we evaluate its performance subjectively in terms of naturalness and intelligibility. The experimental results demonstrate that the prototype system can produce more natural EL speech while maintaining its high intelligibility compared to the conventional electrolarynx. [This research was supported in part by JSPS KAKENHI Grant Nos. 15J10727 and 26280060.]

1aSC26. Investigation of using the highway network to predict the F0 trajectory for text-to-speech synthesis. Xin Wang, Shinji Takaki, and Junichi Yamagishi (National Inst. of Informatics, National Inst. of Informatics, 2-1-2 Hitotsubashi, Tokyo, Tokyo 1018430, Japan, wangxin@ nii.ac.jp)

Generation of the F0 trajectory is critical for Text-to-Speech (TTS) synthesis. In most of the recently proposed TTS systems based on neural networks, F0, together with the spectral features of speech, is predicted by a single neural network. However, our recent work, which utilizes the highway network with trainable gates to bypass the non-linear transformation layers, has shown that more accurate F0 can be generated by separating the network for the $\mathrm{F} 0$ and the spectral streams. It is also observed that most of the layers for the F0 stream are inactive. In this work, the inactive network for the F0 stream will be analyzed, and empirical evidence will show that a shallow network as well as a deep network for the F0 stream can perform. Additionally, this work will analyze the sensitivity values of the hidden units in the highway network to identify the uninformative input features for predicting the F0 trajectory, which include the automatically inferred ToBI tags.

1aSC27. Many-to-many voice conversion using hidden Markov modelbased speech recognition and synthesis. Yoshitaka Aizawa, Masaharu Kato, and Tetsuo Kosaka (Yamagata Univ., 4-3-16 Jonan, Yonezawa, Yamagata 9928510, Japan, tnm45806@st.yamagata-u.ac.jp)

This paper describes a many-to-many voice conversion (VC) technique that does not require a parallel training set of source and target speakers. In a previous study, we already proposed many-to-one VC method that consists 
of decoding and synthesis parts, and it does not require a parallel training set. The basic idea of this system is that an input utterance is recognized utilizing the hidden Markov model (HMM) for speech recognition, and the recognized phoneme sequences are used as labels for speech synthesis. The aim of this work is to extend functionality from many-to-one to many-tomany VC. In particular, we focus on the VC of emotional speech. In order to achieve this, we utilize speaker adaptation techniques to adapt HMMs for speech synthesis. By using adaptation techniques, an arbitrary speaker's voice can be produced. In this work, a combination of constrained structural maximum a posteriori linear regression and maximum a posteriori estimation is used for adaptation. In order to evaluate the proposed system, subjective speech intelligibility tests were conducted. In the experiments, the proposed adaptation with 10 utterances was compared with traditional parameter training with 450 utterances. The results showed that speaker adaptation could be carried out without performance degradation.

1aSC28. The effect of training in producing continuous vowels with a data-glove-driven vocal tract configuration tool. Kohichi Ogata (Faculty of Adv. Sci. and Technol., Kumamoto Univ., 2-39-1 Kurokami, Chuo-ku, Kumamoto 860-8555, Japan, ogata@cs.kumamoto-u.ac.jp) and Yusuke Matsuda (Graduate School of Sci. and Technol., Kumamoto Univ., Kumamoto, Japan)

This paper describes vocal tract configuration for continuous vowels by using a data glove. The authors have proposed data-glove-driven vocal tract configuration methods and showed the usefulness of a method with three fingers for producing vowel sounds. In the method, the location of constriction, the degree of constriction, and the lip area are controlled by bending the thumb, middle finger, and index finger, respectively. Moreover, the effectiveness of training for continuous vowels was shown for sequence / aiueo/. Whether any combination of vowels can be synthesized by this method is an interesting topic for confirmation of the effectiveness. To examine the possibility of successful manipulation of the data glove, an experiment for synthesizing various three consecutive vowels was performed with five beginner subjects. Japanese vowel sequences of 60 patterns were used in the experiment. The results proved that the subjects were able to successfully produce the vowel sequences immediately after showing a target sequence through three training sessions for manipulating the data glove.

1aSC29. Generative model of spectra for a word using Fujisaki's model and genetic algorithm. Tomio Takara and Akichika Higa (Information Eng., Univ. of the Ryukyus, 1 Senbaru, Nishihara-cho, Nakagami-gun, Okinawa-ken 903-0213, Japan, k168577@ie.u-ryukyu.ac.jp)

Fujisaki's model is a generative model of fundamental frequency which approximates original time pattern effectively. In this study, we propose a new generative model of spectral sequence using Fujisaki's model. We have already proposed a speech synthesis method using vowel space parameters. The vowel space parameter is defined as the component on principal axis obtained by PCA for log-amplitude spectra of isolated vowels. Log-amplitude spectrum can be reconstructed from linear combination of a few principal vectors with coefficients of the vowel space parameters. Using time sequence of the vowel space parameters, we can synthesize a spoken word. In this study, we apply Fujisaki's model to the time pattern of vowel space parameter. We adopt parameters of Fujisaki's model as genes of the genetic algorithm. Then, we execute the genetic algorithm with the fitness of similarity between time patterns of Fujisaki's model and the pattern generated from the vowel space parameter. We obtained synthesized speech which can be recognized as a spoken word at the first generation of the genetic algorithm. As a result of listening test for vowels showed 45 to $80 \%$ correct rate at the first generation. Consequently, the effectiveness of the proposed method is shown.
1aSC30. Combination method air and bone conducted speech for speaker recognition in i-vector space. Satoru Tsuge (Information Systems, Daido Univ., 10-3 Takiharu-cho, Minami-ku, Nagoya, Aichi 457-8530, Japan, tsuge@daido-it.ac.jp) and Shingo Kuroiwa (Chiba Univ., Chiba, Japan)

Recently, some new sensors, such as bone-conductive microphones, throat microphones, and non-audible murmur (NAM) microphones, besides conventional condenser microphones have been developed for collecting speech data. Accordingly, some researchers began to study speaker and speech recognition using speech data collected by these new sensors. From these new sensors, we focus on a bone-conductive microphone. Recently, the speaker recognition method based on i-vector will be state-of-the-art. Hence, in this paper, first, we report the speaker recognition performance using bone-conducted speech based on i-vector-based speaker recognition system. In addition, we propose a speaker recognition method combined bone-conducted speech with air-conducted speech. In this paper, we investigate three combination methods, which are a distance combination method, an i-vector combination method, and a feature combination method. To evaluate the proposed methods, we conducted the speaker identification experiments. From experimental results, performance of the bone-conducted speech is almost same as that of the air-conducted speech under the condition of the enrolment and the evaluation speech collected on same session. In addition, the experimental results show that all proposed methods are able to improve the speaker recognition performance of air- and bone-conducted speech.

1aSC31. Horseshoe bat inspired reception dynamics embed dynamic features into speech signals. Anupam K. Gupta (IBM T. J. Watson Res. Ctr., 1208 Snyder Ln, Blacksburg, Virginia, anupamkg@vt.edu), Jin P. Han (IBM T. J. Watson Res. Ctr., Yorktown, NY), Philip Caspers (ME, Virginia Tech, Blacksburg, VA), Xiaodong Cui (IBM T. J. Watson Res. Ctr., Yorktown, NY), and Rolf Müller (ME, Virginia Tech, Blacksburg, VA)

Horseshoe bats can alter the shape of noseleaves and outer ears (pinnae) at the time of emission and reception of biosonar pulses. The shape changes are a result of specific muscular action and significantly change the emission and reception beams by a spatial redistribution of energy in mainlobe and sidelobes. Here, we explore the potential of embedding dynamic features into the speech signals by means of bat inspired reception dynamics to improve the speech recognition accuracy and speaker localization. For this analysis, a digit dataset with 11 U.S. English speakers was recorded through a robotic setup that mimics pinna dynamics as observed in bats. The utterances were obtained through open-source speech corpora and amplitude modulated (carrier frequency: $55 \mathrm{kHz}$ ) to shift to ultrasonic band. These utterances were then re-recorded through the dynamic pinna set up and later demodulated to extract the original signal. It was found that reception dynamics have been able to enrich the speech signals with dynamic, directiondependent time-frequency patterns. The next step in this research is to assess whether these patterns can be utilized to improve speech recognition and speaker localization.

1aSC32. Normal-rate to fast-rate speech conversion using non-linear compression maps. Michael D. Fry and Eric Vatikiotis-Bateson (Linguist, Univ. of Br. Columbia, 2329 West Mall, Vancouver, BC V6T 1Z4, Canada, mdfry20@gmail.com)

This paper presents a new technique to convert normal-rate speech into intelligible fast-rate, speeded speech. Speeded speech has long been recognized for its potential to improve spoken media comprehension; however, current tools to significantly speed playback of non-text media are insufficient due to their reliance on inaccurate phoneme analysis. With the ever increasing amount of non-text media online, a method to speed playback that is agnostic of phonemes is needed. Our technique uses spectral and source components of the acoustics to generate a non-linear compression map that characterizes how conversational-rate speech signals are compressed to achieve analogue fast-rate speech signals. A data set containing conversational- and fast-rate speech pairs was processed to determine compression maps corresponding to each pair. A Recursive Neural Network (RNN) was trained on the set of normal-rate speech and the corresponding compression maps. The RNN was then used to generate compression maps for novel normal-rate speech and ultimately output a fast-rate speech signal. 
Elicited fast-rate speech and speeded speech conversions technique are now being compared perceptually for intelligibility and naturalness.

1aSC33. Evaluation of natural language understanding based speech dialog interface's effectiveness regarding car navigation system usability performance. Takahiro Kojima (Graduated School of Information Technol., Aoyamagakuin Univ., Fuchinobe, Chuo-ku, Sagamihara-city, Kanagawa Pre., Sagamihara 251-0038, Japan, tkojima@ wil-aoyama.jp), Atsunobu Kaminuma (Nissan Motor, Astugi, Japan), Naoya Isoyama, and Lopez Guillaume (Aoyamagakuin Univ., Sagamihara, Japan)

Recently, new cell phone services enable taking input by speaking to a quasi-agent using highly accurate speech recognition technologies. However, there are two problems when equipping a vehicle with these technologies. First, we do not understand yet the effect on driver's usability performance of a car navigation system equipped with spoken dialogue interface. Second, the corresponding design techniques to obtain sufficiently good navigation results has not yet been determined. In this study, we first discuss problems involving speech recognition technologies and the types of speech interface used in this study. Second, we experimentally compare in pseudo-driving conditions the effect on driver's physiology of various speech user interfaces: the Command Input (CI) interface, the Natural Language Understanding (NLU) interface, and the NLU/Spoken Dialogue Management (SDM) interface. According to the analysis of physiological signals, parasympathetic nervous system activity index value was higher when using the NLU/SDM interface than when using the CI interface. Furthermore, both subjective assessment result and time satisfaction degree of NLU/SDM interface were higher than others. Therefore, we can conclude that the NLU/SDM-type interface has better usability performance.

\title{
Session 1aSP
}

\section{Signal Processing in Acoustics: General Topics in Signal Processing I}

\author{
R. Lee Culver, Chair \\ ARL, Penn State University, PO Box 30, State College, PA 16804
}

\section{Contributed Papers}

10:30

1aSP1. Noise reduction for sound capturing by creating spatial null sensitivity point. Akio Ando, Kodai Yamauchi, and Kazuyoshi Onishi (Electric and Electronics Eng., Faculty of Eng., Univ. of Toyama, 3190 Gofuku, Toyama 930-8555, Japan, andio@eng.u-toyama.ac.jp)

We had developed a sound source separation system in which two microphones had been arranged in a line toward sound sources to form null sensitivity points at the source positions by solving the weighting coefficients for microphone outputs. The system achieved the sound source separation by excluding non-target sound sources, instead of extracting target sound sources. In this study, we propose the sound capturing system by which the user can set the null sensitivity point at the arbitrary position. The null sensitivity point is formulated as a function of the weight coefficients. The gain function is also defined as a function of a spatial position. The proposed system uses these functions to capture the target sound while suppressing the non-target sound. A computer simulation showed that, instead of a null sensitivity point, the proposed system generated the circular null sensitivity area whose center was at the microphone position.

\section{0:45}

1aSP2. Sound field interpolation in the spatial domain with a rigid spherical microphone array. Cesar D. Salvador, Shuichi Sakamoto, Jorge Trevino, and Yôiti Suzuki (Res. Inst. of Elec. Commun. and Graduate School of Information Sci., Tohoku Univ., 6-6-05, Aza-Aoba, Aramaki, Aoba-ku, Sendai 980-8579, Japan, cdsalv@gmail.com)

Sound field interpolation aims to calculate sound fields at arbitrary points from original measurements at discrete points. Rigid spherical microphone arrays are effective for interpolation because they can capture sound from several directions with uniform resolution. Interpolation from spherical array measurements is typically based on the spherical Fourier transform and assumes no prior knowledge concerning the source positions. The spherical Fourier transform, however, yields results whose accuracy strongly depends on microphone positioning and the inversion method used for its computation. When knowledge of the source positions is available, the pressure generated at any point on the rigid spherical baffle can be estimated with an existing physical model. This model was used in this study to define an analytic transfer function that relates the pressure at two arbitrary points on a rigid sphere. Based on this analytic function, an interpolation method in the spatial domain is presented as an alternative to transformed domain methods. Numerical experiments with a rigid sphere showed that accurate interpolation of magnitude and phase, over the full range of frequencies, is possible on the side of the array ipsilateral to the sound sources. However, on the contralateral side, accuracy systematically decreased as frequency increased.

\section{1:00}

1aSP3. Infinite impulse response inverse filters based on system identification with singular value decomposition. Toshiya Samejima (Faculty of Design, Kyushu Univ., 4-9-1 Shiobaru Minami-ku, Fukuoka, Fukuoka 815-8540, Japan, samejima@design.kyushu-u.ac.jp) and Kento Mitsuyasu (Shimano Inc., Sakai, Osaka, Japan)

The authors propose infinite impulse response (IIR) inverse filters for equalizing non-minimum phase acoustic plants through accurate system identification. System identification of an acoustic plant is an essential and important process in designing a controller for the plant, such as an inverse filter. In the previous paper, one of the authors has introduced the singular value decomposition (SVD) method for system identification of an enclosed sound field. The SVD method, which is the state space model-based system identification technique, involves the characteristics of experimental modal analysis. The author has verified that the SVD method has several advantages, such as high precision of identification. The proposed IIR inverse filter is simple inversion of the transfer function of the accurately identified model of an acoustic plant through the SVD method. The poles outside the unit circle in the $z$-plane, which are originated with the non-minimum phase zeros of the identified plant, are reflected toward the inside of the unit circle. The proposed IIR inverse filter is applied to enclosed sound field 
equalization. It is demonstrated that the proposed IIR inverse filter can achieve lower equalization error or lower order of the inverse filter than conventional inverse filters using the least squares method.

\section{1:15}

1aSP4. Stereo channel music signal separation based on non-negative tensor factorization with cepstrum regularization. Shogo Seki, Kento Ohtani, Tomoki Toda, and Kazuya Takeda (Nagoya Univ., Furo-cho, Chikusa-ku, Nagoya 464-8603, Japan, seki.shogo@g.sp.m.is.nagoya-u.ac.jp)

Music signals are usually generated by mixing many music source signals, such as various instrumental sounds and vocal sounds, and they are often represented as 2-channel signals (i.e., stereo channel signals). Underdetermined source separation for separating the music signals into individual music source signals is a potential technique to develop various applications, such as music transcription, singer discrimination, and vocal extraction. One of the most powerful underdetermined source separation methods is Nonnegative Matrix Factorization (NMF) that models a power spectrogram of an observation signal as a product of two nonnegative matrices; basis and activation matrices. To apply NMF to the stereo channel music signal separation, we have proposed Nonnegative Tensor Factorization (NTF) by further implementing a gain matrix to represent mixing (i.e., panning) information. However, the separation performance of this method is insufficient owing to less prior information to model acoustic characteristics of the individual music source signals. To address this issue, we propose a cepstrum regularization method for NTF to make power spectral envelopes of the separated signals close to those of individual music source signals. We conduct experimental evaluations to investigate the effectiveness of the regularization method and show remaining problems to be addressed.

$$
\text { 11:30 }
$$

1aSP5. Development of unsupervised classifier for beaked whale clicks. Natalia Sidorovskaia and Kun Li (Phys., UL Lafayette, UL BOX 44210, Lafayette, LA 70504-4210, nas@louisiana.edu)

Recent advancements in passive acoustic monitoring lead to the collection of large volumes (hundreds of $\mathrm{Tb}$ ) of acoustic data, which are post- processed to identify various acoustic events. One of the processing goals is the identification of deep diving marine mammal calls, including different species of beaked whales (Gervais', Blainville's, Cuvier's, etc.) Traditional detectors employ a band-energy-based approach to search for acoustic events above baseline level in the click production band. However, such detectors have a large percentage of false positives and require an experienced operator to manually determine the false positive rate and subdivide the class detections (beaked whales) into species specific calls (such as Cuvier's, etc.) Modern supervised and unsupervised machine learning algorithms broadly employed for the analysis of large volumes of data may open opportunities for the development of highly accurate detectors for marine species with minimal operator involvement. Unsurpervised clustering, supervised classification, and neural networks are implemented as second stage detectors for the classification of species-specific beaked whale calls. Algorithm features, optimization, and performance are discussed. [Research supported by GOMRI.]

\section{1:45}

1aSP6. Controlling the wavefront of the de-modulated sound of a parametric loudspeaker isolated from the angle of own beam. Shigeto Takeoka (Shizuoka Inst. of Sci. and Technol., 2200-2, Toyosawa, Fukuroi, Shizuoka 437-8555, Japan, takeoka@ee.sist.ac.jp)

In this report, an array control method for de-modulated wavefront of a parametric loudspeaker is described. Parametric loudspeakers are known for a very sharp directivity due to their ultrasonic carrier wave. A desired audio signal modulated onto this carrier wave is reproduced along the beam by non-linearity in sound propagation. In general, the directivity of the output wave from array speakers is formed by the array factor, determined by the sound source arrangement and the excitation distribution. However, the output sound itself of the parametric loudspeaker is ultrasonic, and the audible sound is de-modulated along the beam secondarily. In this report, by using amplitude modulation, we present an experiment which the ultra directivity sound have individual array factors in the carrier and the modulated signal. The result shows the wavefront of the de-modulated sound is isolated from the angle of the beam.

\title{
Session 1aUW
}

\section{Underwater Acoustics: Target Scattering}

\author{
Kevin Williams, Chair \\ Applied Physics Lab., University of Washington, 1013 NE 40th St., Seattle, WA 98105
}

\section{Contributed Papers}

\section{0:30}

1aUW1. An investigation into bio-inspired sonar search performance. Joseph R. Edwards, Sara Beery, and Kristen E. Railey (MIT Lincoln Lab., 244 Wood St., Lexington, MA 02420, joe.edwards@11.mit.edu)

The ability to detect, localize, and recognize objects on or under the seafloor is a critical capability for missions such as mine countermeasures (MCM), asset recovery, and feature-based navigation. Unmanned undersea vehicles (UUVs) have been increasingly tasked with such missions and are becoming more capable as technology develops. One intended application of developing UUV technology is to replace the dolphins that execute the mine-hunting task in the U.S. Marine Mammal Program. UUV development for MCM has moved toward larger vehicles with more powerful and multi-modal sensors, to the point where next-generation UUVs are significantly larger and have more powerful sensors than the dolphins they are intended to emulate. Dolphins rely on relatively modest sonar to detect and locate bottom and buried mines, but utilize it to great effect. In contrast to rigid UUV search instructions, dolphins employ a flexible approach with respect to both sonar characteristics and search trajectory. In this work, the effectiveness of biomimetic sonar search is investigated through four principal axes of adaptation: click interval, source power, source center frequency, and mobility. It is shown that the introduction of limited flexibility in the sonar search can greatly improve the overall area clearance performance. 


\section{0:45}

1aUW2. Enhanced target detection and classification using two-pulse sonar methods. Nikhil Mistry, Paul White, and Timothy Leighton (Inst. of Sound \& Vib. Res., Southampton Univ., Bldg. 13, Rm. 3049, University Rd., Southampton, Hampshire SO17 1BJ, United Kingdom, nm6g09@ soton.ac.uk)

A number of two-pulse sonar techniques can be employed to separate linear and nonlinear scatterers. Twin Inverted Pulse Sonar (TWIPS) and Biased Pulse Summation Sonar (BiaPSS) are processes that exploit nonlinear bubble dynamics to perform such a classification, with clutter reduction. Consequently, these techniques can be used to enhance target detection in bubbly waters. TWIPS and BiaPSS rely upon bubbles being driven to large nonlinear pulsations, but this is dependent upon availability of a high-amplitude source. Previous studies could only access a narrow-band source and therefore used a low frequency so that, over the rarefaction cycle, most ocean bubbles would expand sufficiently (the size range of near-surface ocean bubbles under waves and wakes being large). However, recent access to a broadband high-power source has allowed exploitation of bubble resonances. This talk presents results of investigations using broadband linear sine sweeps, of which one is based upon the simulation of an Atlantic Bottlenose Dolphin (Tursiops truncatus) echolocation click from Capus et al. (2006). Insonifying a cloud of bubbles with a wide band of frequencies excites as many of those sizes at, or close to, their resonance. As such, the contrast between linear and nonlinear scatter is enhanced, improving the target detection capability.

\section{1:00}

1aUW3. Modeling the acoustic response of elastic targets in a layered medium using the coupled finite element/boundary element method. Ahmad T. Abawi (HLS Res., 3366 North Torrey Pines Court, Ste. 310, La Jolla, CA 92037, abawi@hlsresearch.com), Petr Krysl (Structural Eng., Univ. of California, San Diego, La Jolla, CA), Aubrey Espana, Steve Kargl, Kevin Williams, and Dan Plotnick (Appl. Phys. Lab., Univ. of Washington, Seattle, WA)

The fluid-structure interaction technique provides a paradigm for solving scattering from elastic targets embedded in a fluid by a combination of finite and boundary element methods. In this technique, the finite element method is used to compute the target's elastic response and the boundary element method with the appropriate Green's function is used to compute the field in the exterior medium. The two methods are coupled at the surface of the target by imposing the continuity of pressure and normal displacement. This results in a boundary element equation that can be used to compute the scattered field anywhere in the surrounding environment. This method reduces a finite element problem to a boundary element one with drastic reduction in the number of unknowns, which translates to a significant reduction in numerical cost. In this talk, the derivation of the technique will be outlined; the method will be applied to compute scattering from various targets, including unexploded ordnance (UXO) in complex ocean environments and the results will be compared with measurements.

\section{1:15}

1aUW4. Acoustic response of targets in a shallow water, layered, environment: Experimental observations and simple ray model results. Steven G. Kargl, Aubrey L. España, and Kevin L. Williams (Appl. Phys. Lab., Univ. of Washington, 1013 NE 40th St., Seattle, WA 98105, karg1@ uw.edu)

Synthetic aperture sonar (SAS) data were collected throughout May of 2014 during an experiment in St. Andrew's Bay, Panama City, FL. A brief overview is given of the SAS rail system and procedure used during the "BAYEX14" experiment. BAYEX14 was carried out in a shallow water, layered, environment, where water depth was approximately $8 \mathrm{~m}$ with a thin layer of mud (i.e., 0.15-0.30 m thickness) covering a sandy bottom. All targets settled into the mud and came to rest on the mud-sand interface. Scattering data from an aluminum finite cylinder, an aluminum pipe, and solid replicas of an 100-mm unexploded ordnance will be presented and compared to data taken previously in a sand-only environment and to model results. To model the scattered pressure, the ray paths are assumed to experience negligible refraction in passing through the mud layer to the mud-sand interface. However, the much higher attenuation in mud relative to water must be considered along the portion of the ray path in mud. For targets at long horizontal ranges, ray paths that interact with the water-air interface must be included to model an observed modulation of the target strength. [Research sponsored by ONR and SERDP.]

\section{1:30}

1aUW5. Stability analysis for implementing a distributed-basis transition matrix for acoustic target scattering by highly oblate elastic objects in free-field. Raymond Lim (Naval Surface Warfare Ctr. Panama City Div., 110 Vernon Ave., Code X11, Panama City, FL, FL 32407-7001, raymond.lim@navy.mil)

A variant of Waterman's transition $(\mathrm{T})$ matrix utilizing an ansatz for problematic outgoing basis functions in standard formulations was proposed and demonstrated in a previous meeting to improve the stability of free-field acoustic scattering calculations with highly oblate axisymmetric elastic objects. The ansatz replaced the basis causing instability with a non-local basis consisting of low-order spherical functions made into a complete set by analytically continuing and distributing them along the imaginary axis of the axial coordinate. While this ansatz was found to produce scattering predictions of comparable stability to a full spheroidal-basis implementation of the T matrix, instabilities of unknown origin eventually appeared as higher matrix truncations were used to obtain convergence at higher frequencies. Here, we present a study to uncover the origin of these instabilities and show results of alternative non-local basis ansatzes chosen to attempt remediation. [Work supported by ONR.]

\section{$11: 45$}

1aUW6. Acoustic response of an unexploded ordnance in shallow water, layered, environments: Predictions using hybrid and coupled modeling techniques. Aubrey L. Espana (Acoust. Dept., Appl. Phys. Lab. - Univ. of Washington, 1013 NE 40th St., Box 355640, Seattle, WA 98105, aespana@ apl.washington.edu), Ahmad T. Abawi (HLS Res., Inc., San Diego, CA), Steven G. Kargl, and Kevin L. Williams (Acoust. Dept., Appl. Phys. Lab. Univ. of Washington, Seattle, WA)

Previously, a hybrid, axisymmetric, finite element (FE)—propagation model has shown utility in simulating the response of objects in contact with a flat, undisturbed sand/water interface [Espana et al., J. Acoust. Soc. Am. 136, 190-124, (2014)]. Here, we offer a three-way comparison between data, the hybrid FE/propagation model and a coupled finite element/boundary element model (Coupled FEBE) for the scattering from a replica of a 100-mm unexploded ordnance (UXO) proud, partially buried and fully buried in a sand sediment for geometries where reflection from and refraction into the sand dominate. This comparison offers a more in-depth analysis of the implications of various approximations of the hybrid model, such as the effect of improper fluid loading on the target surface in the partially buried situation. These results will be contrasted to the more complicated mudover-sand layered environment of the BAYEX14 experiment. Preliminary model results will be compared to data, giving further insight into the dominant physical mechanisms affecting the UXO's acoustic response in the more complicated layered environment. [Research sponsored by ONR and SERDP.] 


\title{
Session 1pAAa
}

\section{Architectural Acoustics: Acoustics for Children and Pupils II}

\author{
Keiji Kawai, Cochair \\ Kumamoto University, 2-39-1 Kurokami, Kumamoto 860-8555, Japan \\ David S. Woolworth, Cochair \\ Oxford Acoustics, 356 CR 102, Oxford, MS 38655 \\ David Lubman, Cochair \\ DL Acoustics, 14301 Middletown Ln., Westminster, CA 92683-4514
}

Chair's Introduction-1:00

\section{Invited Papers}

1:05

1pAAa1. Need of good classroom acoustics since the early childhood. Arianna Astolfi, Giuseppina E. Puglisi (Dept. of Energy, Politecnico di Torino, Corso DC degli Abruzzi, 24, Turin 10124, Italy, arianna.astolfi@polito.it), Andrea Prato (National Inst. of Metrological Res., Turin, Italy), Louena Shtrepi (Dept. of Energy, Politecnico di Torino, Turin, Italy), Benedetto Sacchetti, and Tiziana Sacco (Dept. of Neurosci., Univ. of Turin, Turin, Italy)

In everyday lives, the listening experiences often occur in adverse listening environments in which children need to tune out competing sounds to tune into speech. In the early childhood and in the first years of education, a good acoustic environment is mandatory in order to avoid difficulties in literacy development. This is true both for normal children and for children with potential learning disabilities, which can be only diagnosed after this age-period. Whenever children with poor neural processing in speech discrimination are exposed to bad acoustics, they may fall behind their peers in reading development. On the contrary, since the neuroplasticity of the auditory cortex of the human brain is up to about the age of eight years, when at-risk children are trained with learning programs based on sound and visual cues recognition in proper listening environments, the effectiveness of these programs is improved. This work focuses on the evidence of the need of good classroom acoustics by the early stage of education. Preliminary results on a study on the influence of classroom acoustics on the reading skills in the early childhood are reported. The study involved 120 second-grade pupils in three primary schools with different classroom acoustics.

1pAAa2. Relation between children's calmness and sound environment in nursery field: Influence of environmental settings and childcare practice. Saki Noguchi and Kanako Ueno (Meiji Univ., 1-1-1, Higashimita, Kawasaki, Kanagawa 214-8571, Japan, n-saki@ akane.waseda.jp)

In Japanese childcare facilities, there has conventionally been a serious problem regarding too noisy environment in nursery room. This becomes even more serious in an urban area having high population density, particularly because of lack of childcare space. It is concerning that sound environment in childcare facilities, where children live for approximately 8-11 h, has a great influence on the mental and physical development of children. The relation between children's calmness, such as a state of relaxation or concentration on activity, and the sound environment is reported herein, with a view to creating a suitable sound environment for children. We explain this relation in terms of environmental factors and childcare practices using the following cases. (1) Experiment conducted by varying the environmental settings - particularly, spatial reverberation and furniture of play corner; (2) observation of the childcare practice attached great importance to create the calm of sound environment. From the result, we report the influence that sound environment has on children's calmness and discuss methods to ensure that the sound environment supports healthy development of children.

\section{$1: 45$}

1pAAa3. Modeling of venues for music practice and instruction. Ana M. Jaramillo and Bruce C. Olson (Olson Sound Design, LLC, 8717 Humboldt Ave. N, Brooklyn Park, MN 55444, ana.jaramillo@afmg.eu)

Music practice rooms for large groups are spaces with conflicting requirements, especially those that are part of schools. The need for an environment that is similar to the ones where the musicians will be performing (appropriate reverberation for music performance), and on the other hand, the need for music instruction (speech intelligibility for teacher/student and student/student communication) are usually opposing. As part of a renovation of band rooms in two schools, we were called to advise on the room acoustics. The rooms were having floor replacement, from carpet to tile and needed to have the room acoustics reviewed and adjusted for the new floor material with added wall or ceiling absorption to replace what was lost from the floor. 


\section{Contributed Papers}

2:05

1pAAa4. Experimental measurements of word intelligibility among preschool children under differing conditions of reverberation and signalnoise ratio. Kazunori Harada and Keiji Kawai (Graduate School of Sci. and Technol., Kumamoto Univ., 2-39-1 Kurokami, Chuo-ku, Kumamoto 8608555, Japan, kazunori.harada.108@gmail.com)

Many children, from 0 to 5 years old, spend most of their active hours in child day-care centers. Rooms in these centers are often very noisy, due to both the voices of children or sounds of various activities and to the excessive reverberation attributable to the little use of sound-absorbing materials. Some researchers have studied the speech intelligibility of elementary school children, but we were unable to find those of pre-school children. Thus, in this study, an experiment was carried out a word intelligibility test on children from 3 to 5 years old in a classroom of a child day-care center. The procedure was like a game. Each of the presented words was mixed with pink noise at one of two $\mathrm{S} / \mathrm{N}$ ratios, and convolved with the impulse responses of three reverberation times of $0.3,0.9$, and $1.8 \mathrm{~s}$. Children were asked to judge whether the word was food or not, and to raise their hands holding yes or no signs. As a result, the correct answer ratio for 3-year-old children was lower than the ratio for other groups. Also, the correct answer ratio for 4- and 5-year-old children was slightly lower than control groups of elementary school pupils and college students.

$$
\text { 2:20 }
$$

1pAAa5. Effect of design on acoustic performances in classrooms: Case study from Ankara. Filiz B. Kocyigit (Faculty of FAD and Architecture, Dept. of Architecture, Atilim Univ., Kizilcasar Mah. Incek, Ankara 06380, Turkey, filizbk@gmail.com)

Education is one of the most important factors for the development of societies. Efficient education can be possible with a qualified communication. Current spaces are huge factor that effects communications. No doubt that auditory comfort in spaces with completed acoustic development provides better communication. This study is prepared to propound the effects of the acoustic comfort conditions in classrooms which are the educational structure's most important spaces and the material used. Within the scope of this study, two schools which are private and government high school in Ankara situated in Cankaya Municipality have been chosen to make measurements. Selected schools have been researched by the classes' volume in terms of compliance with acoustic and in accordance with the inner surface of equipment. Similar volume classes chosen in both schools and they are measured with acoustic measuring devices, RT, EDT, C80, D50, STI, and RASTI which are significant for the intelligibility of speech has been taken into consideration for evaluation of the measurements taken in the determined classes. The impacts of furniture and surface materials on the acoustic comfort conditions are recovered in renewed or newly designed classrooms for revealing the most ideal level of speech intelligibility in classes.

\section{$2: 35$}

1pAAa6. Effect of music on Turkish medical schools' architectural design in the Seljuk and Ottoman period. Filiz B. Kocyigit (Faculty of Fine Art Design and Architecture, Atilim Univ., Kizilcasar Mah. Incek, Ankara 06380,Turkey, filizbk@gmail.com)

Anatolian people throughout the ages have given importance to art and science. Music has been one of the most important part of life in Ottoman, Anatolian Seljuk Turks. Music therapy has been used with not only traditional medicine but also has been used with modern therapy. It has different historical progresses according to the cultures, nations, and periods, from being a myth to a treatment method. Hospitals' design methods for music therapy have great importance for treatment. Because of the immigration of Turkish people in 11-16th century, different Anatolian works like architectural art and institution affected the other countries. Among these works, we try to state some charity foundations all rendered for the use of the public such as temples, madrasahs, schools, dervish lodges, libraries, and cottage houses. The importance of "Music Therapy" is to show its historical progress, the effects on human being, and to exist the place it takes in psychology science. In this paper, the historical progress of music therapy in Turks is mentioned and information about the hospitals that used "music therapy" in Seljukian and Ottoman era is given, as they were the pioneers for architectural design of a hospital in all over the world. 


\title{
Session 1pAAb
}

\section{Architectural Acoustics: Acoustical Design Metric Comparison Between the U.S. and Japan}

\author{
David Manley, Cochair \\ D.L. Adams Associates, Inc., 1536 Ogden St., Denver, CO 80218 \\ Hayato Sato, Cochair \\ Architecture Engineering, 1-1 Rokkodai, Nada, Kobe 6578501, Japan \\ Chair's Introduction-3:05
}

Invited Papers

3:10

1pAAb1. Metrics for acoustical measurements in North America. Robert A. Hallman (Armstrong World Industries, 2500 Columbia Ave., Lancaster, PA 17604, rahallman@armstrongceilings.com)

The primary source of standards for acoustical measurements in buildings and community noise for North America is ASTM E33 on Building and Environmental Acoustics. This paper focuses on the ASTM E33 standards and the metrics generated. There are six major categories of standards, Sound Absorption, Speech Privacy, Sound Transmission, Application of Materials and Systems, Mechanical and Electrical Noise, and Community Noise. The most familiar metrics include NRC, STC, and IIC, but there is an alphabet soup of metrics including AC, AI, AOITC $(\theta)$, ANISPL, ATL, CNEL, FSTC, NIC, and OINIC. Many of these are variations of the basic measurements targeting a specific measurement environment. This paper focuses on the basic measurements and how the metrics are calculated and then gives a broad overview of the range of variations and specialized test methods available through ASTM Committee E33 standards.

\section{3:30}

1pAAb2. Japanese standards of measurement method and rating for floor impact sound insulation. Atsuo Hiramitsu (National Inst. of Land Infrastructure Management, 1, Tachihara, Tsukuba-City, Ibaraki 305-0802, Japan, hiramitsu-a92ta@nilim.go.jp)

Among the objections and the troubles of buildings in Japan, the floor impact sound is one of the most serious issues. The floor impact sounds are classified in a heavy- and a light-weight floor impact sound by the characteristic of the impact source. One is the floor impact sound caused by human jumping, running, or walking and the other is the floor impact sound caused by falling lightweight object. The measurement and rating method for floor impact sound insulation is standardized in JIS (Japanese Industrial Standard). JIS A 1418-2 is method using standard heavy/soft impact sources, a car-tire source (a bang machine) and a rubber ball source. The heavyweight floor impact sound insulation is started in Japan. JIS A 1418-1, which is corresponded to ISO140-7, is method using standard light impact source, a tapping machine. The evaluation for the floor impact sound insulation is standardized in JIS A 1419-2 which is corresponded to ISO 717-2. This paper summarizes the trends for the floor impact sound insulation in Japan. The single-number quantity " $L_{\mathrm{r}}$ " is usually used for the rating of the floor impact sound insulation from the octave band sound pressure level. Moreover, A-weighted floor impact sound level is used recently.

\section{3:50}

1pAAb3. Comparison of laboratory building acoustics standards from ASTM International, Japanese Industrial Standards Committee, and the International Organization for Standardization. Matthew V. Golden (Pliteq, 616 4th St., NE, Washington, DC 20002,mgolden@pliteq.com)

Testing standards are fundamental to the practice of building acoustics. To that end, ASTM International, the Japanese Industrial Standards (JIS) Committee, and the International Organization for Standardization (ISO) all have standards that cover the laboratory measurement of airborne and impact sound transmission. These standards include ASTM E90 and E492, JIS A 1416, and A 1418 and ISO 10140. This paper will review the theory behind each methods and the derivation of the equations used. It will also discuss each of these standards in detail, including the relative strengths and weaknesses of each as well as the interoperability the resulting data. Previous research comparing the standards will also be reviewed. Finally, a brief overview of the repeatability and reproducibility of all building acoustics standards will be discussed. 
1pAAb4. Test and rating method comparison between Japan and the U.S. for airborne sound insulation in buildings. Kiyoshi Masuda (Technol. Ctr., Taisei Corp., 344-1 Nase-cho, Totsuka-ku, Yokohama, Kanagawa 245-0051, Japan, kiyoshi.masuda@sakura. taisei.co.jp)

The Japanese test methods for laboratory measurement of airborne sound transmission loss for building partitions are basically the same as the American test methods. However, the two countries' field test methods for airborne sound insulation in buildings differ. The octave band sound pressure level difference between two rooms is the most significant value for the field evaluation of sound insulation in Japan, whereas the apparent transmission loss for a partition installed in a building is the most popular value for the field evaluation of sound insulation in the US. Why is the octave band sound pressure level difference popular in Japan? It is the basic way of thinking in Japan that an acoustical engineer should take responsibility for the total spatial sound insulation performance, including sound absorption and flanking transmission from the design stage to completion and the field test and evaluation method as the completion inspection should be simple. If the insulation performance does not meet the specific criterion at the time of its completion, it is necessary for the acoustical engineer to demonstrate the cause of the insulation defect using various measurement techniques, including test methods similar to FTL in ASTM E336.

\title{
Contributed Papers
}

4:30

1pAAb5. Reverberation time versus volume for active acoustic systems. Roger W. Schwenke and Tobi Szuts (Meyer Sound Labs., 2832 San Pablo Ave., Berkeley, CA 94702, rogers@meyersound.com)

If two rooms have the same reverberation time but different cubic volumes, then they will differ in acoustic strength. Many authors have presented recommendations for reverberation time as a function of volume for different purposes. There are many parameters that, if held constant, yield different reverberation times for different cubic volumes: strength, clarity, critical distance, etc. For example, the ITU-R BS.1116 recommendation for reverberation time versus volume corresponds to rooms of constant average absorption per square meter. This paper will review the literature on reverberation time versus volume, show the connection between STI and reverberation versus volume, and propose a recommendation for reverberation versus volume for active acoustic systems.

\section{$4: 45$}

1pAAb6. Acoustic design method of multifunctional small venues. Hideo Miyazaki (YAMAHA Corp., 10-1 Nakazawa-cho, Naka-ku, Hamamatsu 430-8650, Japan, hideo.miyazaki@music.yamaha.com)

Looking at the tendency of resent acoustic design projects in Japan, the number of venues specialized for a certain usage like concert halls is decreasing while the significant differences of the number of multi-purpose venues are not seen between in 1990s and 2000s. On the other hand, regarding the size (seating capacity), the number of over 1,000 seats multi-purpose venues are decreasing and that of below 1000 seats (especially, small venues below 500 seats) tends to be increasing. One of the reasons for this tendency is the finance problem at local governments. Another reason is the background that venues used by local citizens has been focused rather than large venues for touring performances under the strategy to activate the local cities. In this report, the acoustic design examples of multifunctional small venues are introduced while categorized by their design methods.

\section{Session 1pAB}

\section{Animal Bioacoustics: Bat Echolocation: New Insights on Biosonar Production, Processing, and Performance from Field and Laboratory Investigations II}

\author{
Hiroshi Riquimaroux, Cochair \\ Life and Medical Sciences, Doshisha University, Shandong University, 27 Shanda Nanlu, Jinan 250100, China \\ Laura Kloepper, Cochair \\ Biology, Saint Mary's College, 262 Science Hall, Saint Mary's College, Notre Dame, IN 46556
}

\section{Invited Papers}

$1: 30$

1pAB1. Significance of terminal frequency in FM sweeps for bat echolocation. Hiroshi Riquimaroux (SDU-VT Int.. Lab., Shandong Univ., 27 Shanda Nanlu, Jinan, Shandong 250100, China, hiroshi_riquimaroux@brown.edu)

For echolocation of CF-FM bats, the CF component is expected to detect wing-beat of flying insects. However, it also plays an important role to serve as a stable signal carrier from a distant target. Then, once the carrier frequency is removed, echo signal can be easily detected. For this purpose, Doppler-shift compensation and echo amplitude compensation must be conducted to stabilize the signal 
carrier. What about the FM bats? FM bats also appear to utilize a relatively long pseudo-CF component to detect wing-beats of flying insects. They might also utilize pseudo-CF component for an echo signal carrier. However, Doppler-shift compensation in FM bats has not been reported. In the inferior colliculus, majority of neurons are tuned to the pseudo-CF frequency, $20 \mathrm{kHz}$ for Eptesicus fuscus while $40 \mathrm{kHz}$ for Pipistrellus abramus, which are corresponding to the terminal frequencies of downward FM sweep. Overrepresentation of a particular frequency may enhance frequency discrimination, detection of insect wing-beat. Therefore, we may expect majority of cochlear nerve fibers are also tuned to the pseudo-CF frequency. However, overrepresentation of hair cells for a particular frequency does not appear to be shown. [Research supported by MEXT, Japan and Shandong University, China.]

\section{1:50}

1pAB2. Midbrain neurons of the free-flying echolocating bat represent three-dimensional space. Ninad B. Kothari, Melville J. Wohlgemuth, and Cynthia F. Moss (Psychol. and Brain Sci., Johns Hopkins Univ., 3400 N. Charles St., Ames Hall 200B, Baltimore, MD 21218, cynthia.moss@jhu.edu)

Spatial navigation by echolocation in bats is an active and adaptive system: Its success depends upon tight coupling between motor commands for sonar signal production and neural processing that supports spatial perception and attention to objects in the 3D environment. The midbrain superior colliculus (SC) has been implicated in sensorimotor transformations to support adaptive behaviors in the echolocating bat, but the response properties of SC neurons have yet to be studied in free-flying animals engaged in natural spatial navigation behaviors. Using RF telemetry, in combination with a model that computes the direction and arrival time of echoes received at the bat's ears, we have successfully characterized the auditory response profiles of single neurons in the SC of the free-flying big brown bat. Neural data, acquired across intermediate and deep layers of the bat SC, were sorted off-line from multichannel silicon array recordings. Spikes evoked by echo returns were analyzed and used to construct 3D spatial response profiles of over 40 SC neurons in two bats. Echo response profiles showed selectivity to both the direction and delay of sonar returns over biologically relevant ranges. This work opens the door to investigate the contribution of spatial attention to 3D auditory tuning.

\section{2:10}

1pAB3. Signal characteristics and echolocation challenges of Mexican free-tailed bats during high-speed flight. Laura Kloepper (Biology, Saint Mary’s College, 262 Sci. Hall, Saint Mary’s College, Notre Dame, IN 46556, lkloepper@saintmarys.edu) and Meike Linnenschmidt (Div. of Neurobiology, Ludwig-Maximilians-Univ., Munich, Germany)

Female Mexican free-tailed bats (Tadarida brasiliensis) form large maternal colonies numbering up to several million. After their nightly emergence and feeding, they return to the roost from high elevations and speeds nearing $100 \mathrm{~km} / \mathrm{h}$. At these speeds, the bats should experience, among other things, difficulties in object distance encoding due to the large distance travelled before echo returns and significant Doppler shifts to their echoes. Further, they face navigational challenges from nearby conspecifics. In this study, we characterized the acoustics of returning Mexican free-tailed bats and investigated for possible Doppler shift compensation during various flight speeds. Synchronized acoustic and thermal imagery recordings were conducted at several caves across multiple mornings during cave re-entry. Call sequences for individual bats were extracted for different flight speeds, and changes in call parameters were compared within each call sequence. First basic analysis indicates a possible lack of Doppler shift compensation during fast and steep reentry flights. Further, these bats demonstrate a flexible signal design and extreme behavioral maneuvers in flight.

\section{2:30}

1pAB4. Auditory-visual information transfer in echolocating Egyptian fruit bats. Corinna Schilling (Neurobiology, Univ. of Ulm, Ulm, Germany), Alan Grinnell, and Walter Metzner (IBP, UCLA, 621 Ch. E. Young Dr. S., Los Angeles, CA 90095-1606, metzner@ ucla.edu)

Egyptian fruit bats have good vision but can also accurately echolocate relying on their well-developed auditory system. We asked two questions: 1. Can these bats acquire a mental image of an object using vision (or hearing) alone? 2. If such a mental image has been formed, can it be transferred from one sensory modality (e.g., vision) to another (e.g., hearing)? We trained the bats to first localize a rewarded object $(\mathrm{an} X)$ that was presented pseudo-randomly at either the left or right side using either vision (group 1) or hearing (group 2) alone. After only a few weeks, all experimental bats were able to perform these tasks. We then had the bats discriminate between a rewarded object (the X) and an unrewarded object (a circle) using either vision (group 1) or hearing (group 2) alone. Again, all bats mastered this task after only a few weeks of training. Finally, we tested if the same bats could also discriminate the objects using the other sensory modality (group 1: hearing; group 2: vision). After only a few sessions, the bats performed correctly suggesting that the bats can indeed transform a mental image that they obtained using one sensory modality to another modality.

\section{2:50-3:05 Break}




\section{Contributed Papers}

3:05

1pAB5. Dynamic periphery in a biomimetic sonar system introduces time-variant signatures into targets echoes. Joseph Sutlive (Translational Biology, Medicine, and Health, Virginia Polytechnic Inst. and State Univ., ICTAS Life Sci. District (Mail Code 0917), Blacksburg, VA 24061, josephs7@vt.edu), Philip Caspers, and Rolf Müller (Mech. Eng., Virginia Polytechnic Inst. and State Univ., Blacksburg, VA)

Bats have developed unique and refined systems of echolocation throughout the course of their evolutionary history, giving them the ability to navigate and hunt in extremely cluttered environments. While the mechanisms behind many of these abilities remain unknown, it has been observed that the most effective biosonar systems in bats use a variety of dynamic sensing mechanisms. One conspicuous manifestation of this dynamics can be seen in changes to the shapes of the baffle that diffract the emitted biosonar pulses (noseleaves) and the returning echoes (outer ears). Using numerical predictions as well as measurements with biomimetic hardware, our own prior work has established that the dynamics in these baffles can create time-variant emitter and receiver characteristics. However, it has yet to be demonstrated that these time-variant device characteristics have a substantial impact on the received echoes. To address this question, a biomimetic sonar head with dynamic emission and reception baffles was used to ensonify a range of different targets that included simple geometrical shapes (e.g., sphere, cylinder, and cube) as well as natural targets (foliage). The biomimetic dynamics of the sonar head resulted in time-variant signature in the received echoes that could provide a substrate for encoding additional sensory information.

\section{$3: 20$}

1pAB6. Quantitative analysis of rigid and non-rigid motion patterns in rhinolophid and hipposiderid pinnae. Xiaoyan Yin, Peiwen Qiu, Mengna Zhang (Shandong Univ. - Virginia Tech Int.. Lab., Shandong Univ., Shanda South Rd. 27, Jinan, Shandong 250100, China, yinxiaoyan4@gmail.com), and Rolf Müller (Shandong Univ. - Virginia Tech Int.. Lab., Shandong Univ., Blacksburg, VA)

It has long been noted that horseshoe bats (Rhinolophidae) exhibit large ear motions during biosonar behaviors. Some authors have regarded these motions as rigid rotations of the pinna whereas others have pointed out that the animals are capable of non-rigid motions where the shape of the pinna changes. In the current work, we have analyzed pinna motions in greater horseshoe bats (Rhinolophus ferrumequinum) and the related great roundleaf bat (Hipposideros armiger) to investigate the issue of rigid versus non-rigid pinna motions. The bats were found to produce rigid as well as non-rigid motions. The latter were characterized as "open-close" motions where the size of the pinna aperture was changed by varying the distance between the anterior and posterior rims of the pinna. To quantify this, pinna motions were described by three-dimensional tracking of points on the pinna rims using high-speed stereo vision. The point coordinates were used to compute a matrix that contained the maximum change in distance between any pairs of points on the anterior and posterior pinna rims. Dimensionality reduction methods (e.g., linear discriminant analysis) were applied to the vectors containing all elements of these "maximum distance change matrices" to quantify the relationship between rigid and non-rigid motions.

\section{$3: 35$}

1pAB7. Integration of emitter and receiver dynamics in a biomimetic sonar head. Philip Caspers and Rolf Müller (Mech. Eng., Virginia Tech, ICTAS Life Sci. District (Mail Code 0917), Virginia Tech, Blacksburg, VA 24061,pcaspers@vt.edu)

Horseshoe bats (family Rhinolophidae) transmit and receive sonar information about the environment using morphologically complex and dynamic noseleaf and pinnae. Both local shape features and dynamic deformations of the noseleaf and pinnae contribute to properties of the bat biosonar system. This work systematically explores the effect of local shape features on the dynamic properties of emission and reception baffles using a biomimetic robotic system inspired by the horseshoe bat. Parametric models of the greater horseshoe bat (Rhinolophus ferrumequinum) noseleaf and pinnae with selectable combinations of local shape features were developed. Baffles with feature realizations were cast out of flexible silicone and mounted to a platform to dynamically actuate the emission and reception baffle surfaces with motions patterns similar to the greater horseshoe bat. Motions of the baffle surfaces were synchronized to the outgoing and incoming sonar waveform, and the time-frequency properties of the emission and reception baffle realizations were systematically characterized across spatial direction-individually and jointly. Different feature combinations of the noseleaf and pinnae local shape features were ranked for overall dynamic effect. It was found shape and motion patterns impacted the overall dynamics with relative strength over time, frequency, and spatial direction.

\section{3:50}

1pAB8. Challenges of acoustic monitoring of bats-A biosonar perspective. Jens C. Koblitz (BioAcoust. Network, Eichenallee 32 a, Neuss 41469, Germany, Jens.Koblitz@web.de) and Peter Stilz (BioAcoust. Network, Hechingen, Germany)

Acoustic monitoring of bats is increasingly used in biodiversity assessments and population monitoring. Besides accurate species identification, additional factors make it challenging to derive population trends, yetsizes based on acoustic monitoring. Inter- and intra-species as well as individual variation of acoustic parameters and acoustic activity result in varying detection probability. Changes in environmental conditions result in a large changes in the volume monitored by the device. Differences in the devices used for acoustic monitoring make it inherently difficult to compare data collected with different devices. By broadcasting bat echolocation calls from various distances to monitoring devices, the acoustic parameters influencing the successful detection of a call were examined. A microphone array was used to track bats in the vicinity of monitoring devices and the distance between device and bat was measured for each call based on the time of arrival difference. The acoustic detection function, the probability of detecting calls as a function of distance, was then derived for multiple detector types.

\section{4:05}

1pAB9. Do echo flow patterns guide flight behavior of the big brown bat? Michaela Warnecke and Cynthia F. Moss (Dept. of Psychol. and Brain Sci., Johns Hopkins Univ., 3400 N Charles St., Baltimore, MD 21218, warnecke@jhu.edu)

When animals move through the environment, they receive dynamic sensory information from surrounding objects. Past research has demonstrated that visually guided animals rely on optic flow to estimate their relative velocity and distance to objects. More recently, the flight and echolocation behavior of big brown bats was studied in a corridor constructed of poles of variable spacing. When the pole spacing on opposite walls was symmetric, animals centered themselves in the corridor, and when pole spacing was asymmetric, bats steered toward the less echoic wall. This finding raised the question whether bats adjusted their flight paths based on the flow of echoes returning from the corridor walls, or whether the animals steered away from the wall reflecting more intense echoes. To address this question, bats flew through the original corridor with the additional experimental condition of felt coverage on one corridor wall, reducing the reflectivity of pole echoes on that side, while retaining the flow pattern. If the bat's flight path is influenced by the echo intensity profile of a corridor wall, it should deviate toward the felt-covered wall. By contrast, if the bat's behavior is influenced by acoustic flow cues, flight paths should resemble those in the original (no-felt) conditions. 
1pAB10. Jamming avoidance by the echolocating bat during flight with multiple conspecifics. Kazuma Hase, Yukimi Kadoya (Graduate School of Life and Medical Sci., Doshisha Univ., 1-3 Tatara Miyakodani, Kyotanabe, Kyoto 610-0321, Japan, emq1003@mail4.doshisha.ac.jp), Yosuke Maitani (Faculty of Life and Medical Sci., Doshisha Univ., Kyotanabe, Japan), Kohta I. Kobayasi, and Shizuko Hiryu (Graduate School of Life and Medical Sci., Doshisha Univ., Kyotanabe, Japan)

It remains mystery how echolocating bats extract their own echoes from an acoustic jamming caused by vocalizations of multiple conspecifics flying together. Here, we first successfully recorded echolocation pulses of each animal during four-bat group flight by individually mounted on-board microphones, allowing us to assess vocalization in a jamming environment. We used Miniopterus fuliginosus which emit downward frequency-modulated (FM) ultrasounds. The bats experienced both single and group flights in an experimental chamber. As a result, each bat of the group significantly expanded differences in terminal frequency (TF) of pulses from the neighboring bat from $0.6 \pm 0.6$ (mean $\pm \mathrm{SD}$ ) $\mathrm{kHz}$ in single flight to $1.2 \pm 0.6$ $\mathrm{kHz}$ in group flight (Tukey's HSD test, $P<0.05$ ). Although the difference in $\mathrm{TF}$ observed in group flight was much smaller than the bandwidth of pulses (approximately $40 \mathrm{kHz}$ ), computation of cross-correlation providing an index of similarity of two signals demonstrated that the difference is useful to distinguish own signal from others. These results suggest that innate FM signal of bats is jamming-tolerant, and FM bats flying in group highlight differences in $\mathrm{TF}$ to avoid or reduce jamming from conspecifics in a closed space, as implied by previous studies.
1pAB11. Obstacle avoidance navigation by echolocating bat: Compared relationships between acoustic guidance and flight control in unfamiliar space and familiar space. Yuya Yamamoto, Yasufumi Yamada, Kohta I. Kobayasi, and Shizuko Hiryu (Graduate school of Life and medical Sci., Doshisha Univ., 1-3 Tatara Miyakodani, Kyotanabe, Kyoto 610-0321, Japan, dmq1050@mail4.doshisha.ac.jp)

Echolocating bats can fly without colliding with obstacles by synchronizing acoustic guidance and flight control. To research how bats adapt echolocation as they became familiar with the spaces, we made CF-FM bats (Japanese horseshoe bat) exhibit S-sharp flight repeatedly in a chamber arranged acoustically transparent chains. We found (1) flight path was changed continuously to reduce its curvature as the bats repeated the flights, resulting that the maximum flight speed increased from 2.7 to $3.0 \mathrm{~m} / \mathrm{s}$ in the $1 \mathrm{st}$ trial to 3.2 to $3.8 \mathrm{~m} / \mathrm{s}$ in the 12 th (last) trial for all three bats, (2) pulse emission was reduced by $45 \%$ (66/148 pulses) from the first to the last trial, (3) they shifted the pulse direction dynamically relative to their flight direction in the 1st flight whereas the pulse direction was shifted smoothly, the bats emitted more intense pulses toward the intended flight direction as they became familiar with the space. When acrylic boards were arranged, the bats could not pass through the obstacle course until they learned the space. These suggest echolocation is changed between unfamiliar and familiar space flight and the bats adapt their echolocation to feedforward-dominant control for perceiving more at distance for path planning when they have a spatial map.

\title{
Session 1pAO
}

\section{Acoustical Oceanography: Twenty-Five Years of Acoustical Oceanography in the ASA I}

\author{
Andone C. Lavery, Cochair \\ Applied Ocean Physics and Engineering, Woods Hole Oceanographic Institution, 98 Water Street, MS 11, Bigelow 211, \\ Woods Hole, MA 02536 \\ Michael J. Buckingham, Cochair \\ Scripps Institution of Oceanography, University of California, San Diego, 9500 Gilman Drive, La Jolla, CA 92093-0238
}

Chair's Introduction-1:10

\section{Invited Papers}

1pAO1. Twenty five happy years in ocean acoustics. Walter Munk (Scripps Inst. of Oceanogr., Univ. of California, San Diego, 9500 Gilman Dr., La Jolla, CA 92093-0238,wmunk@ucsd.edu)

25 happy years in ocean acoustics

1pAO2. Twenty five years of learning what acoustics can do for oceanographers. David M. Farmer (9860 W. Saanack Rd., 9500 Gilman Dr., La Jolla, California 92093-0238, Farmer.DavidM@gmail.com)

From cracking ice and breaking surface waves to stratified flows and turbulence, the ocean reveals its secrets through underwater sound. Some personal experiences will illustrate the remarkable opportunities that flow from collaboration between oceanographers and ocean acousticians. 
1pAO3. Ocean acoustic tomography: Fortieth anniversary, 1976-2016. Peter F. Worcester and Walter H. Munk (Scripps Inst. of Oceanogr., Univ. of California, San Diego, 9500 Gilman Dr., 0225, La Jolla, CA 92093-0225, pworcester@ucsd.edu)

The year 1976 saw the publication of "Monitoring the ocean acoustically" by Munk and Worcester [Science, Technology, and The Modern Navy, Thirtieth Anniversary 1946-1976, 497-508] and arguably the first ocean acoustic tomography experiment. The field got off to a stormy start when a reviewer of an early proposal wrote that "travel times along ray paths are meaningless in a saturated environment," but a 1978 experiment showed that ray arrivals could be "resolved, identified, and tracked" at $~ 900 \mathrm{~km}$ range, just in time to save the field from an early demise. The need to measure and predict travel times with millisecond accuracy pushed the field of underwater acoustics to develop the requisite instrumentation and mathematical tools to accurately characterize the impulse response of the ocean. Important contributions to our understanding of the oceans include (1) the demonstration that internal tides are coherently generated by surface tides over the Hawaiian submarine ridge (and other similar benthic features) and can be traced for thousands of km to distant regions of dissipation, (2) measurements of oceanic deep convection, and (3) the discovery using trans-Arctic transmissions that the heat content in the Arctic was increasing. In the future, ocean acoustic tomography will likely be one component of multipurpose acoustic systems for acoustic remote sensing, navigation, communications, and passive listening, with special applicability in ice-covered regions.

\section{2:20}

1pAO4. Shallow water acoustics and oceanography at the Woods Hole Oceanographic Institution over the last quarter century. James Lynch, Timothy F. Duda, Ying-tsong Lin, and Arthur Newhall (Woods Hole Oceanographic, MS \# 11, Bigelow 203, Woods Hole Oceanographic, Woods Hole, MA 02543, jlynch@whoi.edu)

Shallow water (coastal) acoustics has been a topic of great interest over quarter century, both from the point of view of learning coastal oceanography and of learning coastal acoustics. Indeed, the two are difficult to separate. In this talk, the research done at the Woods Hole Oceanographic Institution (very often in collaboration with other institutions) over the past 25 years will be discussed, with an emphasis on the major experimental efforts that have been fielded, but also including theoretical and computational efforts. The Barents Sea Polar Front experiment, the Shallow Water Acoustic Random Medium experiment, the Shelfbreak PRIMER experiment, the Asian Seas International Acoustics Experiment, the Shallow Water 2006 experiment, and the Quantifying, Predicting and Exploiting Uncertainty program are some of the at-sea efforts to be discussed. Theoretical advances in shallow water acoustics, as well as computational efforts, such as the Integrated Ocean Dynamics and Acoustics project, will also be treated. Directions for future work will be discussed.

2:40

1pA05. The enigma of the ocean internal wave spectrum: Can acoustics help solve the enigma? John A. Colosi (Dept. of Oceanogr., Naval Postgrad. School, 833 Dyer Rd., Monterey, CA 93943, jacolosi@nps.edu)

In 1979, Carl Wunsch famously asserted "Many sources for the internal wave field have been proposed; but it has not yet been possible to make the kind of statement that can be made about surface waves: namely, when the wind blows surface waves are generated and the larger the fetch and duration, the larger the waves." In spite of decades of work using traditional oceanographic tools (e.g., acoustic doppler current profilers and conductivity, temperature, and depth devices), this statement remains essentially true today and can be equally applied to the question of internal wave sinks (dissipation), and more generally the form of the spectrum. It is therefore possible that acoustic propagation methodologies with their unique sampling properties in frequency-wavenumber space and spatial averaging could shed some light on this enigma. Several ways forward on this problem will be discussed.

\section{3:00}

1pAO6. Inspirations of past leaders in Acoustical Oceanography: Reflections of our roots in AO and bioacoustics. Timothy K. Stanton (Dept. Appl. Ocean. Phys. \& Eng., Woods Hole Oceanographic Inst., Woods Hole, MA 02543, tstanton@whoi.edu)

I had the privilege of knowing the leaders of Acoustical Oceanography (AO) during its early years, first as a topical entity, then when it was eventually formalized as a Technical Committee (TC). I first arrived at the University of Wisconsin in 1980 and did research there with Clarence Clay for eight years as a scientist. The 1977 book, "Acoustical Oceanography” by Clay and (Hank) Medwin, had recently been published and was required reading. At around the same time, Van Holliday, David Farmer, Mike Buckingham, and Medwin, established AO as a formal committee, first as a Specialty Group (Medwin as Chair), and later as a TC in 1991. Buckingham was elected first Chair of the AOTC and, given the anticipated challenges in starting a new TC, I was made Alternate Chair to work alongside Buckingham on many things. Clay and Holliday inspired much of the AO bioacoustics research that I have conducted, including developing acoustic scattering models, physics-based echo statistics, and broadband acoustic methods to use sound to characterize marine organisms such as fish and zooplankton. I will tell some stories and give examples of recent scientific advances that are traceable to Clay and Holliday.

1pA07. Waveguides and whales: A review about using acoustic waveguide theory for localizing whales and inverting for their propagation environment. Aaron Thode (SIO, UCSD, 9500 Gilman Dr., MC 0238, La Jolla, CA 92093-0238, athode@ucsd.edu)

Low-frequency acoustic propagation in shallow-water waveguides is conveniently modeled using sets of normal modes that propagate with different group velocities. Analytical solutions of simple environmental models, such as the Pekeris waveguide, were almost immediately applied to estimate ranges of impulsive sources by measuring the relative arrival times of the modal components, but to the author's knowledge, it was not until the early 1990s that such techniques were first applied to low-frequency baleen whale calls, in a technical report by Gerald D'Spain. Since then waveguide-based methods have been used to track blue whales off Southern California, right whales in the Bering Sea, sei whales in the Atlantic, and bowhead whales in the Chukchi and Beaufort Seas. Some of these 
applications have exploited time-separated modal arrivals on a single hydrophone, while others have used vertical arrays to measure modal interference patterns, and subsequently estimate an animal's range, depth, and local propagation environment. A recent promising development has been the introduction of nonlinear time sampling methods that permit mode isolation without the need for vertical arrays, enabling range estimation of certain whale signals from existing single-hydrophone data, even when the modal arrivals are not cleanly separated in time.

3:40

1pA08. Passive acoustical oceanography. Martin Siderius (ECE Dept., Portland State Univ., P.O. Box 751, Portland, OR 97207, siderius@pdx.edu)

Acoustical Oceanography includes the use of both active and passive acoustic systems to measure various properties of the ocean environment. Active methods use a specifically designed and controlled sound projector while passive methods exploit naturally occurring ocean sounds such as breaking waves, ship noise, or marine mammal vocalizations. Historically, active methods have been the preferred choice when precise propagation times or calibrated sound levels are important. However, passive sensing has a strong appeal due in part to the ease in which listening-only systems can be deployed. There have been a number of advances in passive sensing even for cases where propagation times and sound levels are of interest. For example, it has been shown that surface wave noise can be used to measure vertical propagation times through the seabed and water column. More recently, there is experimental evidence that indicates noise correlations may also be used to determine horizontal propagation times in the seabed. Although replacing controlled projectors with noise sources is an attractive option there are limitations to how and when noise can be used this way. These limitations as well as experimental and modeling results from surface and ship noise will be described in this presentation.

\section{4:00}

1pA09. Studying breaking waves with ambient noise oceanography. Grant B. Deane (Marine Physical Lab., Univ. of California, San Diego, 13003 Slack St., La Jolla, CA 92093-0238, gdeane@ucsd.edu)

Ambient noise oceanography is a powerful tool in the study of breaking ocean waves, yielding insight into the nature of fluid turbulence and air entrainment within whitecaps. Whitecaps limit the height of waves on wind-driven seas, transfer momentum and gasses between the atmosphere and ocean, increase the ocean's albedo, are a source of marine aerosol and generate underwater ambient noise. For such reasons, the relationship between fluid turbulence and the size distribution of bubbles in whitecaps is of considerable interest, but the interior of whitecaps are quite difficult to study in the field. Large extinction rates associated with high air fractions in whitecaps limit the usefulness of optical and acoustical probes, and few measurements from in situ instrumentation have appeared. However, the sound radiated by newly formed bubbles within whitecaps provides a useful signal, one which can be interpreted in terms of bubble creation rates, provided the bubble emission signature and propagation through the breaking wave crest are understood. A physical model for the generation of wave noise and its use to provide insight into the link between fluid turbulence, its saturation in wave crests, and its relationship to bubble formation will be discussed. [Work supported by ONR Ocean Acoustics Division.]

4:20

1pAO10. The depth dependence of ambient noise in deep ocean trenches. David R. Barclay (Dept. of Oceanogr., Dalhousie Univ., PO Box 15000, Halifax, NS B3H 4R2, Canada, dbarclay@dal.ca), Michael J. Buckingham, and Dieter Bevans (Marine Physical Lab, Scripps Inst. of Oceanogr., UCSD, La Jolla, CA)

A series of experiments aimed at measuring the power spectrum and vertical and horizontal noise coherence (directionality) in the deep ocean were carried out between 2009and 2015 using a family of autonomous instrument platforms named "Deep Sound." Deep Sound is a free-falling acoustic recorder designed to descend from the ocean's surface to a pre-assigned depth where it drops an iron weight and returns to the surface under its own buoyancy, while recording pressure time series on four hydrophones with vertical and horizontal spacing over the bandwidth $5 \mathrm{~Hz}-30 \mathrm{kHz}$. The complete vertical noise profile was recorded from the surface to $6 \mathrm{~km}$ in the Philippine Sea, $8.5 \mathrm{~km}$ in the Tonga Trench, and $9 \mathrm{~km}$ in the Serena and Challenger Deeps in the Mariana Trench. Generally, the vertical noise coherence is well described by the Cron \& Sherman surface noise model with some depth-dependence explained by seawater attenuation and local sound speed variations. Below the reciprocal or critical depth (depth at which the sound speed is higher than any sound speed above it), the total noise field statistics depend heavily on the relative contributions of locally generated surface noise and distantly generated propagating noise. Thus, the depth dependence of the sound field varies with local surface conditions, with nearly depth-independent noise power and coherence found at moderate states, while a sharp drop in noise level is found below the critical depth during calm sea states. 


\title{
Session 1pBAa
}

\section{Biomedical Acoustics and Physical Acoustics: Photoacoustics: Light and Sound II}

\author{
Parag V. Chitnis, Cochair \\ Department of Bioengineering, George Mason University, 4400 University Drive, 1G5, Fairfax, VA 22032 \\ Kang Kim, Cochair \\ Medicine, University of Pittsburgh, 950 Scaife Hall, 3550 Terrace Street, Pittsburgh, PA 15261 \\ Yoshifumi Saijo, Cochair \\ Tohoku University, 4-1 Seiryomachi, Aoba-ku, Sendai 980-8575, Japan
}

\section{Invited Papers}

1:30

1pBAa1. Listening to light and seeing through: In vivo multiscale photoacoustic imaging. Chulhong Kim (Creative IT Eng., Pohang Univ. of Sci. and Technol., 328 Bonner Hall, Buffalo, New York 14260-2050, chulhong@buffalo.edu)

High-resolution volumetric optical imaging modalities, such as confocal microscopy, two-photon microscopy, and optical coherence tomography, have become increasing important in biomedical imaging fields. However, due to strong light scattering, the penetration depths of these imaging modalities are limited to the optical transport mean free path $(\sim 1 \mathrm{~mm})$ in biological tissues. Photoacoustic imaging, an emerging hybrid modality that can provide strong endogenous and exogenous optical absorption contrasts with high ultrasonic spatial resolution, has overcome the fundamental depth limitation while keeping the spatial resolution. The image resolution, as well as the maximum imaging depth, is scalable with ultrasonic frequency within the reach of diffuse photons. In biological tissues the imaging depth can be up to a few centimeters deep. In this presentation, the following topics of photoacoustic imaging will be discussed; (1) multi-scale photoacoustic imaging systems (i.e., Photoacoustic Nanoscopy, Optical-Resolution Photoacoustic Microscopy, Fast 2-Axis MEMS based Optical-Resolution Photoacoustic Microscopy, Intravascular Photoacoustic/Ultrasound Catheter, Virtual Intraoperative Surgical Photoacoustic Microscopy, Acoustic-Resolution Photoacoustic Microscopy, and Clinical Photoacoustic/Ultrasound Scanner), (2) morphological, functional, and molecular photoacoustic imaging, (3) potential clinical applications, and (4) contrast agents for photoacoustic imaging.

\section{1:50}

1pBAa2. Development of three-dimensional photoacoustic imaging system using sparse array sensor. Tsuyoshi Shiina, Kengo Kondo (Human Health Sci., Graduate School of Medicine, Kyoto Univ., 53 Kawahara-cho Shogoin, Sakyo-ku, Kyoto, Kyoto 6068507, Japan, shiina.tsuyoshi.6w@kyoto-u.ac.jp), and Masakazu Toi (Dept. of Breast Surgery, Graduate School of Medicine, Kyoto Univ., Kyoto, Japan)

Photoacoustic (PA) imaging offers better spatial resolution of ultrasound for deep imaging with high optical contrast sensitive to physiological parameters such as the oxygen saturation of hemoglobin. We have conducted development of photoacoustic mammography system in Kyoto University/Canon joint research project supported by MEXT. In addition, we are promoting development of 3D-PA technologies to be applicable in wider clinical field and non-medical purposes in ImPACT program supported by cabinet office of Japan from 2014. Three-dimensional imaging is required to trace a vessel structure. However, the number of receiving channels is limited due to hardware restrictions. In such a sparse configuration, artifacts due to grating lobes become dominant. A scanning transducer suppresses grating lobes, which virtually increases the number of channels. However, it degrades temporal resolution and causes motion artifacts. We employ a compressed sensing (CS) technique to reconstruct a PA image from limited channels and bandwidth. Due to the computational cost for large 3D calculations, most CS approaches reconstruct PA images in a 2D slice. In order to reduce the cost while maintaining accuracy, we proposed 3D-PA reconstruction using CS based on k-space algorithm. The feasibility of proposed method was validated by simulation analysis and the experimental results.

\section{2:10}

1pBAa3. Volumetric multi-spectral optoacoustic tomography for high performance structural, functional, and molecular imaging. Daniel Razansky (Tech. University of Munich and Helmholtz Ctr. Munich, Inst. for Biological and Medical Imaging (IBMI), Ingolstaedter Landstrasse 1, Neuherberg 85764, Germany, dr@tum.de)

Optoacoustic (or photoacoustic) imaging is an emerging hybrid modality that can deliver excellent endogenous and exogenous optical absorption contrasts from living tissues with excellent spatio-temporal resolution. Our state-of-the-art implementations of multi-spectral optoacoustic tomography (MSOT) are based on multi-wavelength excitation of tissues to visualize specific molecules within opaque 
tissues. As a result, the MSOT technology can noninvasively deliver structural (i.e., vascular anatomy, solid tumors, and organs), functional (i.e., total hemoglobin concentration, hemoglobin oxygen saturation, blood flow, $\mathrm{pH}$, and metabolic rate of oxygen consumption), and molecular information from living tissues. For highly sensitive molecular optoacoustic imaging, a valuable tool for personalized medicine, exogenous contrast agents (e.g., organic dyes, metallic and nonmetallic nanoparticles, reporter genes, or fluorescence proteins) with biomarkers are commonly utilized. The talk will further introduce the new realm of 5-dimensional (5D) optoacoustic imaging, which enables simultaneous acquisition of information across all the three spatial dimensions, the time and the spectral (optical wavelength) dimensions. Applications are explored in the areas of functional neuro-imaging, fast tracking of agent kinetics and biodistribution, cardiovascular research, monitoring of therapies, and drug efficacy, as well as targeted molecular imaging studies. Clinical translation roadmap will be finally discussed.

\section{2:30}

1pBAa4. Photoacoustic imaging with multiple speckle illumination. Emmanuel Bossy (LIPhy, Université Grenoble Alpes/ CNRS, LIPhy, 140 rue de la Physique, Saint-Martin d'Hères 38402, France, emmanuel.bossy@gmail.com)

In this presentation, I will illustrate how the use of multiple speckle illumination offers new degrees of freedom in photoacoustic imaging. In the acoustic-resolution regime, I will show that speckle illumination can help breaking the acoustic-diffraction limit [1]. I will show how multiple speckle illumination may also be used to perform optical-resolution photoacoustic microscopy, as an alternative to the conventional point scanning approach. [1]. Chaigne, T., Gateau, J., Allain, M., Katz, O., Gigan, S., Sentenac, A., \& Bossy, E. 2016. Super-resolution photoacoustic fluctuation imaging with multiple speckle illumination. Optica, 3(1), 54-57.

$$
\text { 2:50 }
$$

1pBAa5. Real-time, non-contact, non-invasive imaging of elasticity properties in soft tissues using the combination of light and air-coupled ultrasound. Ivan Pelivanov (BioEng., Univ. of Washington, 616 NE Northlake Pl, Seattle, WA 98105, ivan.pelivanov@ gmail.com), Lukasz Ambrozinsky (AGH Univ. of Sci. and Technol., Krakow, Krakow, Poland), Shaozhen Song, Soon Joon Yoon (BioEng., Univ. of Washington, Seattle, WA), David Li (Chemical Eng., Univ. of Washington, Seattle, VA), Tueng Shen (Dept. of Ophthalmology, Univ. of Washington, Seattle, WA), Rikuang Wang, and Matthew O'Donnell (BioEng., Univ. of Washington, Seattle, WA)

A new non-contact method for efficient, non-invasive imaging of elasticity in soft media is presented. It uses transient mechanical waves excited in the object under study from air (i.e., non-contact) using either ultra-violet (UV) pulsed laser radiation (photoacoustic) or air-coupled focused ultrasound (US) and resultant displacements detected a high frame rate, phase-sensitive (PhS)-OCT system. UV laser radiation is absorbed in a thin subsurface region of the tissue creating transient mechanical waves due to thermal expansion, whereas the air-coupled focused US beam reflected from the air/medium interface provides acoustic radiation force (ARF) to the medium surface, launching a transient mechanical wave in the transverse (lateral) direction. Real time tracking/imaging of resultant mechanical wave propagation can be used to reconstruct tissue elastic properties. Tissue mimicking phantoms, porcine eyes, and skin were used in ex-vivoexperiments to prove the concept. Results of these experiments strongly suggest that simple, non-contact excitation holds great promise for non-invasive characterization of soft media, in general, and for elasticity measurements in delicate soft tissues and organs (e.g., in the eye), in particular.

1pBAa6. Skin vasculature imaging by acoustical resolution photoacoustic microscope with parabolic array transducer. Yoshifumi Saijo, Ryo Nagaoka, Israr Ul Haq, Syahril Siregar, Ryo Takagi, Shin Yoshizawa, and ShinIchiro Umemura (Tohoku Univ., 4-1 Seiryomachi, Aoba-ku, Sendai 980-8575, Japan, saijo@idac.tohoku.ac.jp)

Photoacoustic (PA) signal is highly dependent on the structure of the target. In the present study, PA imaging system with parabolic array transducer for multi-angle detection is developed and the skin vasculature is observed. The transducer was consisted of $256 \mathrm{ch} 1$ 3-composite elements with the center frequency of $10 \mathrm{MHz}$. The diameter was $42.4 \mathrm{~mm}$ and the opening angle was 90 degree. A hole was made in the center of the transducer for transmission of the laser. PA signal was acquired by a programmable ultrasound data acquisition system with the sampling frequency of $60 \mathrm{MHz}$. A diode laser with the wavelength of $532 \mathrm{~nm}$, pulse width of $8 \mathrm{~ns}$, pulse energy of $5 \mathrm{~mJ}$, and repetition frequency of $10 \mathrm{~Hz}$ was equipped for generation of PA signal. Three dimensional image was reconstructed by delay-and-sum beamforming of PA signals received by all channels. The frame rate for displaying $x-y, y-z$, and $x-z$ images was $10 \mathrm{~Hz}$, and the full width at half maximum (FWHM) of the $\mathrm{x}-\mathrm{z}$ image was $70 \mu \mathrm{m}$. 3D structure of the skin capillary network of a human hand was successfully observed by the system. The system may contribute to the peripheral blood circulation.

\section{3:30-3:45 Break}




\section{Contributed Papers}

1pBAa7. Changes of masseter muscle in a rat unilateral occlusal model assessed by photoacoustic imaging system. Kouki Hatori (Dept. of Prosthodontics, Matsumoto Dental Univ. School of Dentistry, 1780, Gobara Hirooka, Shiojiri, Nagano 3990781, Japan, khat810@yahoo.co.jp), Yoshifumi Saijo (Dept. of Biomedical Imaging, Tohoku Univ. Graduate School of Biomedical Eng., Sendai, Japan), Masahiro Iikubo (Dept. of Oral Diagnosis, Tohoku Univ. Graduate School of Dentistry, Sendai, Miyagi, Japan), Yoshihiro Hagiwara (Dept. of Orthopaedic Surgery, Tohoku Univ. School of Medicine, Sendai, Miyagi, Japan), Kuniyuki Izumita, Yukihiro Naganuma, and Keiichi Sasaki (Div. of Adv. Prosthetic Dentistry, Tohoku Univ. Graduate School of Dentistry, Sendai, Miyagi, Japan)

Biomedical photoacoustic (PA) imaging system has the unique capability of combining high optical contrast with high ultrasound (US) resolution in a single modality. PA imaging system with $532 \mathrm{~nm}$ laser and $50 \mathrm{MHz}$ US transducer has been developed. When the laser with the wavelength of 532 $\mathrm{nm}$ is used, PA signals are reflected from blood vessels. Patients with unilateral occlusion due to the extraction of unilateral molars are often seen in dental practice. Although the period of the unilateral occlusion induces the changes of masseter muscle (MM), it is not known that how blood vessels and tissue elasticity of MM are affected by the unilateral occlusion. In this study, we aimed to evaluate the sequential changes of MM due to the unilateral occlusion in rats with PA imaging system. PA signals from the MM of the non-occlusal side were significantly lower than that of the occlusal side, and US signals from the MM of the occlusal side were significantly higher than that of the non-occlusal side depending on the period of the unilateral occlusion. Unilateral occlusion affected both the blood vessels and the tissue elasticity. The PA imaging system could evaluate blood circulation and tissue elasticity simultaneously.

\section{4:00}

1pBAa8. All optical frequency domain photoacoustic microscope based on two-wave mixing in a photo-refractive crystal. Deepu George (BioEng., George Mason Univ., 4400 University Dr., MSN 2A1, Krasnow Inst. of Adv. Study, Fairfax, VA 22030, dgeorg10@gmu.edu), Harriet Lloyd, Ronald H. Silverman (Edward S. Harkness Eye Inst., Columbia Univ., New York, NY), and Parag V. Chitnis (Bio-Eng., George Mason Univ., Fairfax, VA)

Optical resolution photoacoustic microscopy (OR-PAM) provides functional and molecular information at the cellular scale. OR-PAM typically employs ultrasonic transducers, which require coupling with tissue, to detect the thermo-elastic waves generated in tissues irradiated by nanosecond laser. Images are acquired by scanning the excitation beam, which is usually focused to a spot size of a few microns, i.e., lateral resolution. However, the axial resolution is constrained to an order of magnitude lower by the bandwidth of the transducer used. Additionally, imaging depth of OR-PAM is constrained to the depth that allows ballistic photons, which is usually less than $1 \mathrm{~mm}$. We present an All Optical Frequency Domain Photoacoustic Microscope where the photoacoustic signal is generated by an amplitude modulated CW laser and detected using a two wave mixing (interferometer) in a photorefractive crystal. Sweeping the amplitude-modulation frequency and using an ultra-broadband optical detector can potentially provide detection bandwidths greater than conventional OR-PAM, e.g., 100-200 MHz. The loss in efficiency of photoacoustic excitation associated with violating the stress-confinement condition is overcome by using a lock-in amplifier. This method promises a high-fidelity microscopy system that does not require acoustic coupling while reducing associated costs and footprint. [Work supported by NIH-NEI 1R21EY023012.]
1pBAa9. Laser-driven resonance of light-absorbing ultrasound contrast microbubbles. Guillaume Lajoinie (Phys. of Fluids group, Univ. of Twente, Postbus 217, Enschede, Netherlands, g.P.R.Lajoinie@utwente.nl), Jeong Y. Lee (Inst. of Biomedical Eng., Univ. of Oxford, Oxford, United Kingdom), Erik Linnartz (Phys. of Fluids group, Univ. of Twente, Enschede, Netherlands), Joshua Owen (Inst. of Biomedical Eng., Univ. of Oxford, Oxford, United Kingdom), Pieter Kruizinga, Nico de Jong, Gijs van Soest (Biomedical Eng., Erasmus MC, Rotterdam, Netherlands), Eleanor Stride (Inst. of Biomedical Eng., Univ. of Oxford, Oxford, United Kingdom), and Michel Versluis (Phys. of Fluids group, Univ. of Twente, Enschede, Netherlands)

The sensitivity of ultrasound imaging is greatly enhanced by the use of microbubble contrast agents through resonant volumetric oscillations. While the increased acoustic contrast is of prime interest for perfusion imaging of organs, microbubbles until now have limited benefit in terms of specificity for ultrasound imaging. Original strategies are required to tackle this difficulty that rely on loading functional targeting ligands onto the microbubble encapsulation. In parallel, laser light offers great specificity in its interaction with tissue. This advantage is put to use in photoacoustic imaging where absorbed laser light is converted into a measurable acoustic signal. Here, we present a novel ultrasound contrast agent designed to make use of the specificity of laser light. The acoustic agent consists of a gas core encapsulated by an oil layer containing an absorbing dye. The resulting laser light absorption can then be used to heat up the gas and drive the system into resonance, thereby generating ultrasound. Combining finite difference simulations and ultra high-speed imaging led to a quantitative physical description of the optical and thermal interactions in the system resulting in the efficient generation of acoustic waves in the $\mathrm{MHz}$ range. A range of physical bubble parameters are investigated, i.e., thickness and composition of the light absorbing oil layer. This new generation of contrast agents will open up new applications in medical diagnostic and therapeutic imaging.

4:30

1pBAa10. Polypyrrole phase-change contrast agents for sonophotoacoustic imaging. David S. Li (Dept. of Chemical Eng., Univ. of Washington, 105 Benson Hall, Box 351750, Seattle, WA 98195, dsli@uw. edu), Soon Joon Yoon (BioEng., Univ. of Washington, Seattle, WA), Thomas J. Matula (Ctr. for Industrial and Medical Ultrasound, Appl. Phys. Lab, Univ. of Washington, Seattle, WA), Matthew O'Donnell (BioEng., Univ. of Washington, Seattle, WA), and Lilo D. Pozzo (Chemical Eng., Univ. of Washington, Seattle, WA)

A new type of light and sound sensitive emulsion-based contrast agent is presented. It features a low boiling point liquid perfluorocarbon core and a polypyrrole (PPy) polymer shell. The PPy coated nanoemulsions can reversibly convert from liquid to gas phase by cavitating the perfluorocarbon core during the negative phase of an acoustic pulse. Alternatively, cavitation can be initiated through heat transfer from light absorption in the PPy shell from a laser pulse. By overlapping transmitted laser and acoustic pulses, activation energies required to cavitate the nanoemulsions are lower than for either source alone. The emulsions are typically produced between 150 and $350 \mathrm{~nm}$ in diameter and PPy has a broad optical absorption covering both the visible and near-infrared wavelengths (peak covers 700-1100 nm). The size, structure, and optical absorption properties of the contrast agents were characterized using dynamic light scattering, ultraviolet-visible spectrophotometry, transmission electron microscopy, and small angle X-ray scattering. The cavitation threshold and signal intensity was measured as a function of both acoustic pressure and laser fluence. We also demonstrate that these agents can be used as a sono-photoacoustic contrast agent, providing a non-linear photoacoustic response much more intense than particlebased contrast agents used in photoacoustic imaging. 
1pBAa11. Reconstruction of vasculature in optical resolution photoacoustic microscopy using wavelet and Hessian based method. Israr Ul Haq, Ryo Nagaoka, Syahril Siregar, and Yoshifumi Saijo (Graduate School of Biomedical Engineeirng, Tohoku Univ., 6-6-05 Aramaki Aza Aoba, Aoba-ku, Sendai, Miyagi 980-8579, Japan, ryo@ecei.tohoku.ac. jp)

Photoacoustic microscopy is a high-resolution and high- contrast function modality where optical absorption is detected by ultrasonic transducer. The noise generated by the detector due to its size, sensitivity make the photoacoustic images difficult to visualize. Denoising and reconstruction of vascular structures is crucial for the early diagnosis and therapy in many medical applications. Therefore, the accurate detection of blood vessels has a great significance to assist the users including radiologists and clinicians by making them visualization of the vascular information in the images. In the proposed method, use of wavelet filtering to enhance the effect of smaller and bigger vessels in Optical Resolution Photoacoustic Microscopy (OR-PAM) is analyzed. The proposed method uses the wavelet to enhance the vasculature and then Hessian based method is applied to classify the vessel-like structures. For the evaluation, the algorithm is tested on photoacoustic images acquired non-invasively from living mouse brain, human finger cuticle, and blood filled tube, which shows appreciable results to enhance vasculature in photo-acoustic imaging.

5:00

1pBAa12. Deconvolution-based approach for super-resolution photoacoustic imaging. Jaesok Yu (BioEng., Univ. of Pittsburgh, 3550 Terrace St., Scaife Hall 958, Pittsburgh, PA 15261, JAY49@pitt.edu) and Kim Kang (Medicine, Univ. of Pittsburgh, Pittsburgh, PA)

Recently, the microbubble-localization technique was able to increase spatial resolution by multifold in ultrasound (US) imaging, overcoming the acoustic diffraction limit. In this study, we applied the super-resolution approach to the photoacoustic (PA) imaging with a mid-frequency medical imaging array transducer. One of the biggest challenges in this application is relatively low PA response from individual contrast agent particles to the limited laser fluence under safety guidelines. The increased concentration of contrast agent enhances PA signal, but it results in convolved and blurred PA signals due to particle clumping. The conventional localization technique likely discards such blurred PA signals and thus requires a large number of imaging frames. Alternatively, a deconvolution method can be utilized to directly identify individual agent particles from the blurred PA signals. In-vitro experiments were performed using the PA sequence programmed US platform with a linear array transducer centered at $5 \mathrm{MHz}$. A pulsed laser of $5 \mathrm{~ns}$ long at $10 \mathrm{~Hz}$ tuned at $800 \mathrm{~nm}$ was synchronized to the US system. Totally 8,000 PA frames were acquired from micron-sized metal beads flowing in a polyethylene tube. Richardson-Lucy deconvolution method successfully identified individual particles from the blurred PA signals generated from clumped beads at high concentration.
1pBAa13. Visualization of ultrasound fields in a transparent medium with ultrasound attenuation using the focused shadowgraphy technique. Yukina Iijima and Nobuki Kudo (Graduate School of Information Sci. and Technol., Hokkaido Univ., N14W9 Kita-ku, Sapporo, Hokkaido 060-0814, Japan, kudo@bme.ist.hokudai.ac.jp)

Knowledge of ultrasound pressure fields of ultrasound inside the human body is essential to confirm the safety of medical ultrasound equipment. Typically, pressure waveforms in the body are estimated by correction of those measured in water considering typical attenuation in biological tissues. However, the correction of frequency-dependent attenuation of a wide band pulse is difficult because biological tissues have a non-linear transmission characteristic. In this study, acoustic fields of pulsed ultrasound propagating in a transparent medium with ultrasound attenuation were visualized using focused shadowgraphy (Kudo, Ultrasound Med. Biol. 41, 2071-2081, 2015). Pressure waveforms of the pulsed ultrasound were also measured using a membrane hydrophone. Brightness curves and their 1st-order integrations derived from the visualized field images were compared with the pressure waves. Experimental results obtained by using castor oil demonstrated that the 1st-order integrations of brightness curves are in better agreement with the pressure waveforms. Furthermore, frequency spectrum analysis showed good agreement of attenuation coefficients derived from the pressure waveforms and integrated brightness curves, indicating the usefulness of the technique not only for visualization of ultrasound fields but also for estimation of pressure waveforms and ultrasound attenuation.

\section{$5: 30$}

1pBAa14. The acoustic noise pressure of sensors with very small apertures. Suzanne M. Leinders, Nico de Jong, and Martin D. Verweij (Acoust. Wavefield Imaging, Delft Univ. of Technol., Lorentzweg 1, Delft 2628 CJ, Netherlands, m.d.verweij@tudelft.nl)

For medical diagnostic modalities like intravascular ultrasound (IVUS) and intravascular photo acoustics (IVPA), it is paramount to have small, sensitive ultrasound elements for detecting the reflected pressure pulses. The development of one and two dimensional arrays for such applications will call for even smaller element sizes and advanced microfabrication techniques. In search for miniature receiving elements we developed an optical ultrasound sensor with an optical strain detector integrated on a thin acoustical membrane [Leinders et al., Sci. Rep. 5, 14328]. To predict the lowest detectable pressure, we wanted to determine the noise level of this sensor. Unlike a piezoelectric sensor, the noise in our sensor is not dominated by the electrical impedance and will only be caused by the thermo-acoustical noise of the sensor's internal mechanical impedance, and the noise caused by thermally agitated medium particles that hit the sensor surface. To expand the existing knowledge, we will analyze both noise mechanisms and show that in thermodynamic equilibrium these give rise to the same noise pressure at the sensor surface. Moreover,we will show that for sensors with vanishing aperture area, the noise pressure will reach a well-defined finite limit, and not go to infinity as predicted by some literature. 


\title{
Session 1pBAb
}

\section{Biomedical Acoustics and Physical Acoustics: Cavitation in Therapeutic Ultrasound I: General}

\author{
Lawrence Crum, Cochair \\ Applied Physics Laboratory, Center for Industrial and Medical Ultrasound, University of Washington, 1013 NE 40 th street, \\ Seattle, WA 98105 \\ Shin-ichiro Umemura, Cochair \\ Graduate School of Biomedical Engineering, Tohoku University, Aoba 6-6-05, Aramaki, Aoba-ku, Sendai 980-8579, Japan \\ Tatiana D. Khokhlova, Cochair \\ University of Washington, 325 9th Ave., Harborview Medical Center, Box 359634, Seattle, WA 98104 \\ Zhen Xu, Cochair \\ Biomedical Engineering, University of Michigan, 2200 Bonisteel Blvd., Rm. 1107 Gerstacker Bldg., Ann Arbor, MI 48109
}

Chair's Introduction-1:00

Invited Paper

1:05

1pBAb1. Cavitation-enhanced diagnostics and therapy in HIFU treatment. Yoichiro Matsumoto (RIKEN, 2-1 Hirosawa, Wako, Saitame 351-0198, Japan, yoichiro.matsumoto@riken.jp)

\begin{abstract}
Medical applications of ultrasound such as High Intensity Focused Ultrasound, Extracorporeal Ultrasound Lithotripsy and Ultrasound-Mediated Gene Transfection have recently been the subject of much interest. In these applications, acoustic cavitation facilitates the medical treatment by improving the image quality and enhancing the therapeutic effects through acoustic emission, localized heating, and erosion. During the ultrasound therapy, it is important to control the focal region on the targeting area, which is moving by the respiratory motion, for the reliable treatment. Ultrasound guide is one of the effective way to track the targeting area compensating the respiratory motion. Cavitation-enhanced diagnostics is useful to obtain the clear image of targeting area utilizing the acoustic emission from the existing micro bubbles during the cavitation-enhanced HIFU where cavitation bubbles are generated at the targeting area to enhance tissue heating. In all these applications, it is essential to understand the cavitation phenomena, including bubble and bubble cloud dynamics. The bubble motion and bubble cloud behavior are strongly influenced by the thermal phenomena inside and outside bubbles and the applied ultrasound wave forms which accelerate therapeutic effects by the cavitation bubbles. In this paper, some fundamental aspects of medical application of microbubbles and acoustic cavitation are highlighted.
\end{abstract}

\section{Contributed Papers}

$1: 25$

1pBAb2. On the formation of bubble clusters and tunnels in tissuemimicking agarose phantoms by focused ultrasound bursts. Pooya Movahed (Mech. Sci. and Eng., Univ. of Illinois at Urbana-Champaign, 305 Coordinated Sci. Lab., 1308 W Main St., Urbana, IL 61801, pooyam@ illinois.edu), Wayne Kreider, Adam D. Maxwell, Michael R. Bailey (Appl. Phys. Lab., Univ. of Washington, Seattle, WA), and Jonathan B. Freund (Mech. Sci. and Eng. and Aerosp. Eng., Univ. of Illinois at UrbanaChampaign, Urbana, IL)

Formation of bubble clusters in soft tissues is a potential injury mechanism in therapeutic ultrasound treatments. To study this phenomenon, transparent tissue-mimicking agarose phantoms were subjected to a series of multiple-cycle ultrasound bursts, using a burst wave lithotripsy (BWL) protocol, and simultaneously imaged with a high-speed camera. The negative pressure in the initial bursts causes preexisting sub-micron bubbles to expand sufficiently to become visible in images ( 200 microns). Additional bubbles appear continuously during the subsequent bursts. A Rayleigh-Plesset-type bubble dynamics model, which is generalized to include elastic resistance and damage mechanisms, is developed and used to explain key observations. It is proposed that material fatigue leads eventually to irreversible fracture-like failure. In addition to isolated, approximately spherical bubbles, long tunnellike features are observed, which seemingly comprise lines of joined bubbles along a possible fracture or defect. Statistics regarding the geometry of these features are reported for agarose phantoms of different stiffness. A mechanism for the formation and observed growth of these long features is proposed, wherein defect/crack growth allows the movement of initially trapped bubbles within the phantom. [Work supported by NIH NIDDK grant DK043881.] 
pressure (p-) are primarily determined by the transducer $F$-number with less focused transducers producing shocks at lower p-. Here, the dependence of inertial cavitation activity on $F$-number was investigated in gel phantoms and ex vivo tissue samples using passive cavitation detection (PCD). Exposures at 1.5 MHz consisted of 60 pulses delivered at $1 \mathrm{~Hz} \mathrm{PRF}$, with each pulse lasting $1 \mathrm{~ms}$ and $\mathrm{p}$ - from 1 to $15 \mathrm{MPa}$. Broadband noise emissions recorded by PCD were batch-processed to extract cavitation probability and persistence. At the same p-, both metrics indicate enhanced cavitation activity at higher $F$-numbers; in agarose phantoms, cavitation probability reached $100 \%$ when shocks formed at the focus, with p-values 5, 9, and 14.5 MPa for respective $F$-numbers $1.5,1$, and 0.75 . These results confirm the impact of nonlinear waveform distortion on inertial cavitation. [Work supported by NIH K01EB015745, K01DK104854, and R01EB7643.]

\section{Invited Papers}

\section{$1: 55$}

1pBAb4. Cavitation-enhanced ultrasonic heating and its monitoring by ultrasound plane wave imaging. Shin Yoshizawa, Ryo Takagi (Communications Eng., Tohoku Univ., 6-6-05 Aoba, Aramaki, Aoba-ku, Sendai 980-8579, Japan, syoshi@ecei.tohoku.ac.jp), Ryosuke Iwasaki, Kentaro Tomiyasu, Kai Suzuki, and Shin-ichiro Umemura (Biomedical Eng., Tohoku Univ., Sendai, Japan)

Cavitation bubbles can accelerate high-intensity focused ultrasound (HIFU) treatments thermally, chemically, and mechanically. Acoustic generation of cavitation bubbles requires extremely high-intensity or long duration time enough to form a standing-wave field. We have proposed and investigated the ultrasound sequence of a high-intensity short pulse, named as trigger pulse, followed by a relatively lower intensity and longer duration burst, named as heating burst, mainly for the cavitation-enhanced ultrasonic heating. Trigger pulses are used to generate cavitation bubbles then form cavitation clouds. The acoustic power of trigger pulses is more than hundreds of $\mathrm{W}$ and the duration is less than $100 \mu$ s to avoid effects of standing-wave components. Cavitation bubbles induced by trigger pulses continuously oscillate with heating bursts. The sequence was repeated 3-15 s. Cavitation clouds formation by trigger pulses was observed with a high-speed camera. The temperature measured by thermocouple and tissue coagulation region showed the effect of heating enhancement. As the cavitation region localization is an essential factor for the safety and efficacy in this method, a real-time monitoring method of bubbles is necessary to be combined. The high-speed pulse inversion images with plane wave transmissions showed its capability for the objective.

\section{2:15}

1pBAb5. Numerical study on cavitation-enhanced thermal and mechanical effects in high-intensity focused ultrasound therapy. Kohei Okita (Mech. Eng., Nihon Univ., 1-2-1 Izumi-cho, Narashino, Chiba 275-8575, Japan, okita.kohei@nihon-u.ac.jp) and Yoichiro Matsumoto (RIKEN, Wako, Saitama, Japan)

Cavitation is becoming increasingly important in therapeutic ultrasound applications such as diagnostic, tumor ablation, and lithotripsy. The behavior of cavitation bubbles is resulted by the complex interactions between bubble oscillation and ultrasound. That depends on not only the exposure parameters of ultrasound but also the physical properties of surrounding tissues and injected microbubbles which are often employed as cavitation nuclei for decreasing cavitation threshold pressure and for controlling cavitation region. An ultrasound simulator treating cavitation/microbubble has been developed for investigating the influences of various parameters in the cavitation-enhanced HIFU therapy. The simulator takes into account the heat generation due to oscillating cavitation bubbles as well as the rectified diffusion which is important role in the initial stage of cavitation bubble growth. Bubble dynamics equation considers the elasticity of the surrounding tissue and the shell of microbubbles, and is strongly coupled with mixture phase to reproduce the interaction between ultrasound and bubble oscillation. Thus, the simulator can reproduce cavitation bubble growth from nuclei around focus. In the present paper, the numerical results for the cavitation/microbubble-enhanced HIFU therapy are shown.

\section{2:35}

1pBAb6. Color-Doppler ultrasound imaging of in situ human kidney stones in a hyperbaric chamber. Julianna C. Simon (Appl. Phys. Lab., Dept. of Mech. Eng. and Ctr. for Industrial and Medical Ultrasound, Appl. Phys. Lab., Univ. of Washington, 1013 NE 40th St., Seattle, WA 98105, jcsimon@uw.edu), Barbrina Dunmire, Bryan W. Cunitz (Ctr. for Industrial and Medical Ultrasound, Appl. Phys. Lab., Univ. of Washington, Seattle, WA), Oleg A. Sapozhnikov (Ctr. for Industrial and Medical Ultrasound, Appl. Phys. Lab., Univ. of Washington, Seattle, WA and Dept. of Acoust., Phys. Faculty, Moscow State Univ., Moscow, Russian Federation), Jeffrey Thiel, Michael R. Bailey (Ctr. for Industrial and Medical Ultrasound, Appl. Phys. Lab., Univ. of Washington, Seattle, WA), and James R. Holm (Div. of Hyperbaric Medicine, Virginia Mason Medical Ctr., Seattle, WA)

Overpressure has been shown to reduce the color-Doppler ultrasound twinkling artifact on ex vivo human kidney stones, leading to the hypothesis that surface crevice microbubbles cause twinkling. For the first time, we investigate the effect of overpressure on in situ human kidney stones. Thus far, five human subjects with kidney stones known to twinkle have been imaged with a Philips/ATL P4-2 transducer and Verasonics ultrasound system for 45 minutes in a hyperbaric chamber. Subjects breathed ambient air while exposed to a maximum pressure of 4 atmospheres absolute (ATA), except for a scheduled decompression stop at 1.6 ATA where subjects breathed pure oxygen. Twinkling was quantified in terms of Doppler power over 2 min intervals before pressurization (baseline), at 4 ATA, and at 1.6 ATA. Preliminary results (averaged over 3 of the 5 subjects) indicate no change in twinkling at 4 ATA compared to baseline levels (ratio of Doppler powers $=1.09 \pm 0.27$ ). Twinkling almost doubled, though, during the pure oxygen stage at 1.6 ATA compared to 
baseline levels (ratio of Doppler powers $=1.85 \pm 0.58$ ). The increase in twinkling associated with breathing pure oxygen continues to support the crevice microbubble hypothesis. Higher pressures than explored in this study may be needed to reduce twinkling on in situ stones, as was often the case in studies using ex vivo human kidney stones. [Work supported by the National Space Biomedical Research Institute through NASA NCC 9-58 and NIH grants DK043881 and DK092197.]

\section{Contributed Papers}

\section{2:55}

1pBAb7. Acoustic cavitation sub-millisecond signal processing for ultrasound surgery. Bjoern Gerold, Jeremie Anquez, and Sylvain Yon (Theraclion, 102 rue Etienne Dolet, Malakoff 92240, France, bjoern. gerold@theraclion.com)

Cavitation in Focused Ultrasound Surgery (FUS) is a stochastic phenomenon that can evolve very rapidly at the high intensities typically employed. Cavitation activity can be monitored analyzing its acoustic signals in the frequency domain. Cavities act as strong scatterers of incident acoustic energy, potentially enhancing different therapeutic effects, e.g., thermal or mechanical, if properly controlled. We report on the development on an instrument capable of controlling acoustic cavitation at sub-millisecond speed. The

\section{3:10-3:25 Break}

$3: 25$

1pBAb8. Elimination of therapeutic ultrasound noise in phase modulated high intensity focused ultrasound treatment. Ryo Takagi (Communications Eng., Tohoku Univ., 6-6-05 Aoba,Aramaki, Aoba-ku, Sendai 9810933, Japan, takagi@ecei.tohoku.ac.jp), Ryosuke Iwasaki (Biomedical Eng., Tohoku Univ., Sendai, Japan), Shin Yoshizawa (Communications Eng., Tohoku Univ., Sendai, Japan), and Shin-ichiro Umemura (Biomedical Eng., Tohoku Univ., Sendai, Japan)

Ultrasound image-guided High Intensity Focused Ultrasound (HIFU) has been become one of the potential surgical techniques for the treatment of the malignant tumor. The ultrasound image during the treatment is severely corrupted by the reflected HIFU wave because HIFU and imaging transducers are simultaneously activated. In our previous study, a new method to selectively eliminate only HIFU noise during the treatment was suggested, but it cannot sufficiently reduce the HIFU noise when the HIFU exposure is phase-modulated with a period less than a few milliseconds. In this study, a new method to selectively eliminate the HIFU noise even when the HIFU exposure is modulated with a certain modulation period. In this study, "multiple HIFU exposure" with a certain modulation period was employed. The focus of HIFU was moved every $25 \mu$ s sequentially for six positions with spacing proper for thermal conduction. the RF signals of the same modulation period were received with (RF2) and without (RF1) transmitting an imaging pulse, respectively. The HIFU noise was eliminated by subtracting RF1 from RF2. As the result of in vitro study, the fundamental to higher harmonic components of the HIFU noise were reduced by 20 to 30 $\mathrm{dB}$ during the HIFU exposure. cavitation noise signal has been recorded and processed by an FPGA coupled with a feedback loop, capable of modulating the driving field every few hundreds of microseconds to maintain an ideal level of cavitation activity and differentiate between several types of cavitation and the onset of boiling. The system has been tested in tissue phantom, ex-vivo tissue and in-vivo and the impact on lesion sizes has been analysed. We also used the experimental data as input for a non-linear simulation of the acoustic field specifically regarding the absorption/scatter ratio in the acoustic focus. [The research leading to these results has received funding from the People Programme (Marie Curie Actions) of the European Union's Seventh Framework Programme FP7/2007-2013/under REA grant agreement n (623608).]

\section{$3: 40$}

1pBAb9. A portable transcranial focused ultrasound system for noninvasive applications in small animals. Pavlos Anastasiadis, Ben Nguyen (Diagnostic Radiology and Nuclear Medicine, Univ. of Maryland School of Medicine, 110 S. Paca St., Rm. 104, Baltimore, MD 21201, panast@som. umaryland.edu), David S. Hersh (Neurosurgery, Univ. of Maryland School of Medicine, Baltimore, MD), Sijia Guo, Rao Gullapalli, and Victor Frenkel (Diagnostic Radiology and Nuclear Medicine, Univ. of Maryland School of Medicine, Baltimore, MD)

Recent studies using focused ultrasound (FUS) have shown significant progress in the realm of brain applications. These transcranial exposures include localized ablation for the treatment of movement disorders and opening of the blood brain barrier to enhance the delivery of therapeutic agents. Whereas these procedures require expensive, MRI-guided systems, one of the newest and most promising applications of FUS being developed involves more portable, hand-held devices for the purpose of neuromodulation. Here, we describe the development of table-top FUS system comprised of a single element FUS transducer $(400-600 \mathrm{kHz})$ specifically for investigations on the stimulation and suppression of neuronal activity in rodents. The transducer assembly includes a cone and extending flexible bolus filled with degassed water for direct coupling to the head of the animals. A custom 3D stage and two diametrically opposed lasers allow for accurate positioning and targeting. Experiments included simulations of the acoustic field using a k-space time-domain method. Localization of the focal zone and its radial and axial dimensions were obtained via thermal exposures in polyacrylamide gel phantoms containing heat sensitive proteins. Last, hydrophone measurements with various sized rodent skulls enabled determination of the expected attenuation to be encountered for future in vivo animal treatments.

\section{Invited Paper}

1pBAb10. High-speed observation for a better understanding of bubble dynamics in an in vivo-like situation of sonoporation. Nobuki Kudo (Graduate School of Information Sci. and Technol., Hokkaido Univ., N14W9 Kita-ku, Sapporo, Hokkaido 060-0814, Japan, kudo@bme.ist.hokudai.ac.jp)

Sonoporation is a technique to temporally increase the permeability of a biological cell membrane by ultrasound exposure and to transduce a foreign gene or drug that normally has no permeability. The mechanisms of sonoporation have not been fully elucidated yet; however, it is widely accepted that microbubbles oscillating adjacent to cells play an important role to induce membrane damage. Dynamics of microbubbles has strong dependence not only on exposure parameters of ultrasound but also on surrounding conditions of the bubbles. To obtain a better understanding of cell-bubble interactions affected by the surrounding conditions, high-speed observation was carried out. A newly designed chamber was used for observation from a lateral direction, enabling visualization of translational 
movements of bubbles beside a scaffold and of cell-bubble interaction without hiding the interacting point by the oscillating bubble itself. It was observed that a bubble on a soft scaffold surface moves translationally in a direction separating from the surface, whereas a bubble on a hard scaffold surface remains on the surface. Adhesion of a bubble to the top of a cell caused a considerable decrease in bubble oscillation amplitude, but membrane damage was confirmed in all of the observed cells with attached bubbles, indicating the importance of taking into account the conditions surrounding a bubble in order to understand the mechanisms of sonoporation.

\section{Contributed Papers}

\section{$4: 15$}

1pBAb11. Toward treatment of abscesses using non-thermal HIFU. Andrew A. Brayman, Anna McClenny, Yak-Nam Wang, Brian MacConaghy, Keith Chan, Wayne Monsky, and Tom Matula (Univ. of Washington, 1013 NE 40th St., Seattle, WA 98105, matula@uw.edu)

Abscesses are infected walled-off collections of pus and bacteria. They can affect any part of the body. Current treatment is typically limited to antibiotics, catheter drainage and hospitalization, or surgical wash-out when inaccessible, loculated or unresponsive to initial care efforts. Although bacteria can develop drug resistance, they remain susceptible to thermal and mechanical damage. High Intensity Focused Ultrasound (HIFU) generates localized heating and cavitation, and represents a potential new noninvasive treatment modality. This talk describes initial experiments in which nonthermal HIFU treatment was used to inactivate small volumes $(100 \mu \mathrm{L}-10$ $\mathrm{mL})$ of Escherichia colisuspensions $\left(\sim 1 \times 10^{9}\right.$ cells $\left./ \mathrm{mL}\right)$ with 1 or $2-\mathrm{MHz}$ transducers. Free-field focal acoustic pressures were as high as 16 (9.9) MPa peak positive (negative). Survival was assessed by coliform counting, and by alamarBlue $\AA$ vital staining. At duty factors of 0.01 or 0.2 , and the highest acoustic pressures, there was no biologically significant heating of the exposed samples. Inactivation was treatment time-dependent, and was well described by a half-life model. There was a well-defined (free field-equivalent) acoustic pressure threshold, with significant cell inactivation above $\sim 7$ MPa peak negative pressure.

\section{$4: 30$}

1pBAb12. Comparative studies on enhancement of cell killing induced by ultrasound in the presence of platinum and gold nanoparticles. Takashi Kondo (Dept. of Radiological Sci., Univ. of Toyama, Sugitani 2630, Toyama 9300194, Japan, kondot@med.u-toyama.ac.jp)

In this study, we report on the potential use of platinum nanoparticles (Pt-NPs), a superoxide dismutase (SOD)/catalase mimetic antioxidant, in combination with $1 \mathrm{MHz}$ ultrasound (US) at an intensity of $0.4 \mathrm{~W} / \mathrm{cm}(2)$, $10 \%$ duty factor, $100 \mathrm{~Hz} \mathrm{PRF}$, for $2 \mathrm{~min}$. Apoptosis induction was assessed by DNA fragmentation assay, cell cycle analysis and Annexin V-FITC/PI staining. Cell killing was confirmed by cell counting and microscopic examination. The mitochondrial and $\mathrm{Ca}(2+)$-dependent pathways were investigated. Caspase- 8 expression and autophagy-related proteins were detected by spectrophotometry and western blot analysis, respectively. Intracellular reactive oxygen species (ROS) elevation was detected by flow cytometry. The results showed that Pt-NPs exerted differential effects depending on their internalization. Pt-NPs functioned as potent free radical scavengers when added immediately before sonication while pre-treatment with Pt-NPs suppressed the induction of apoptosis as well as autophagy AP, and resulted in enhanced cell killing. In addition, we used gold nanoparticles without anti-oxidant activities and will discuss the difference between the two.

\section{Invited Paper}

1pBAb13. Cavitation-mediated extravasation and transtumoral drug delivery by microstreaming: What role do the gas nuclei and the physical properties of the therapeutic play? Constantin Coussios, Rachel Myers, James Kwan, Harriet Lea-Banks, Steven Mo, Megan Grundy, Christophoros Mannaris, Margaret Duffy, Catherine Paverd, Dario Carugo, Christian Coviello, Eleanor Stride, and Robert Carlisle (Dept. of Eng. Sci., Inst. of Biomedical Eng., Univ. of Oxford, Old Rd. Campus Res. Bldg., Oxford OX3 7DQ, United Kingdom, constantin.coussios@eng.ox.ac.uk)

For effective cancer therapy, therapeutic agents must extravasate from the blood stream into the tumour mass, then overcome the elevated intratumoural pressure and dense extracellular matrix to reach each and every cancer cell. Several recent studies have suggested that inertial cavitation in the absence or presence of artificial cavitation nuclei can significantly enhance the delivery, penetration, and distribution of small-molecule or nanoparticulate therapeutic agents into tumors. We first present a comparison of the reported enhancements in delivery achieved for a range of frequencies and therapeutic sizes without or with pre-seeding of cavitation, with particular emphasis on the potential role of cavitation persistence and spatial distribution. With microstreaming hypothesized to be the dominant transport mechanism for drug delivery, the likely benefit of using sub-micron cavitation nucleation agents capable of extravasating alongside the therapeutic, rather than microbubbles confined to the blood pool, is then investigated. Lastly, potential physical and biological modification strategies capable of enhancing the transport of therapeutics by cavitation-microstreaming are discussed, and compared in terms of their relative delivery efficacies in vivo. 


\title{
Contributed Paper
}

5:05

1pBAb14. 1-MHz ultrasound stimulates protective mechanisms in cardiac endothelial cells during oxygen and glucose deprivation. Azzdine Y. Ammi (Knight Cardiovascular Inst., Oregon Health \& Sci. Univ., 3181 SW Sam Jackson Park Rd., UHN-62, Portland, OR 97239, ammia@ohsu.edu), Catherine M. Davis (Anesthesiology \& Perioperative Medicine, Oregon Health \& Sci. Univ., Portland, OR), Igor V. Dykan (Knight Cardiovascular Inst., Oregon Health \& Sci. Univ., Portland, OR), Mohanika Gowda, Nabil J. Alkayed (Anesthesiology \& Perioperative Medicine, Oregon Health \& Sci. Univ., Portland, OR), and Sanjiv Kaul (Knight Cardiovascular Inst., Oregon Health \& Sci. Univ., Portland, OR)

Ultrasound improves myocardial perfusion during coronary occlusion. Our aim was to study the production of vasodilatory compounds by primary mouse cardiac endothelial cells (ECs) exposed to ultrasound after a 2-hour oxygen and glucose deprivation (OGD). A 1.05-MHz transducer was used to insonify ECs with a 50-cycle tone burst at a peak rarefactional pressure of $0.5 \mathrm{MPa}$ and a PRF of $50 \mathrm{~Hz}$. US exposure of ECs after OGD increased the adenosine release to $168 \pm 16 \%$ of control $(n=11, p<0.05)$. It also resulted in an increase in 8,9-, 11,12- and 14,15-EETs reaching $125 \pm 19 \%$, $123 \pm 17 \%$, and $118 \pm 15 \%$ of control $(\mathrm{n}=7, p<0.05)$, respectively. US caused an increase of 5,6-, 8,9-, 11,12-, and 14,15-DHETs to $135 \pm 16 \%$, $138 \pm 17 \%, 133 \pm 15 \%$, and $133 \pm 14 \%$ of control, respectively. It also caused levels of 18-, 19-, and 20-HETEs to be statistically different when compared to control and OGD alone $(\mathrm{n}=7, p<0.05)$. eNOS phosphorylation level was not statistically significant to either control or OGD alone. Higher level of cell viability compared to the ECs not exposed to US was observed $(n=7$, $p<0.05$ ). Pulsed ultrasound at $1.05 \mathrm{MHz}$ has the ability to increase adenosine and eicosanoids production by cardiac ECs after OGD and increase their viability.

\section{Session 1pED}

\section{Education in Acoustics: Attractive Educational Methods and Tools in Acoustics II}

\author{
Kazuhiko Kawahara, Cochair \\ Department of Design, Kyushu University, 4-9-1 shiobaru, Minami-ku, Fukuoka 815-8540, Japan \\ Tracianne B. Neilsen, Cochair \\ Brigham Young University, N311 ESC, Provo, UT 84602
}

\section{Invited Papers}

1:00

1pED1. Advanced technical listening training program at Kyushu University. Kazuhiko Kawahara, Masayuki Takada, and Shinichiro Iwamiya (Faculty of Design, Kyushu Univ., 4-9-1 shiobaru, Minami-ku, Fukuoka 815-8540, Japan, kawahara@design.kyushu-u. ac.jp)

This presentation introduces the Advanced Technical Listening Training (TLT) program at Kyushu University. TLT is a systematic training program designed to improve auditory sensitivity; it consists of discrimination and identification tasks for a wide variety of acoustic features (e.g., frequency, sound pressure level, and spectrum pattern). The TLT II class (i.e., the advanced TLT program) provides training in identification of reverberation time, quantization bit depth, mixing level balance, and spectral slope of harmonic tones. TLT facilitates improved understanding of acoustic theory and phenomena, and psychoacoustics.

\section{$1: 20$}

1pED2. The use of all-in-one sensor devices in the general education acoustics laboratory. Andrew C. Morrison (Natural Sci. Dept., Joliet Junior College, 1215 Houbolt Rd., Joliet, IL 60431, amorrison@jjc.edu)

For a general education acoustics course meeting the physical science laboratory requirement, we have implemented the use of IOLab devices. The IOLab is an all-in-one collection of sensors in a box approximately the size of a graphing calculator. The devices include a microphone, accelerometer, force sensor, light sensor, magnetic field sensor, analog inputs and outputs among other built-in features. Each of the devices communicates wirelessly with laboratory computers. Our acoustics course serves not only students seeking general education credit but also students pursuing careers in music technology and as sonography technicians. The common bond between students in these groups is their shared avoidance of mathematical analysis. In our course, we attempt to build student skills in analytical thinking by scaffolding laboratory activities in a series of mini-labs which make use of the IOLab devices. We discuss the successes and challenges faced using this approach. 
1pED3. Use of smartphones for introductory acoustics education. Kimihiro Sakagami (Environmental Acoust. Lab., Dept. of Architecture, Grad. School of Eng., Kobe Univ., Rokkodai 1-1, Nada, Kobe, Hyogo 657-8501, Japan, saka@kobe-u.ac.jp), Fumiaki Satoh (Dept. of Architecture, Chiba Inst. of Technol., Narashino, Japan), and Akira Omoto (Faculty of Design, Kyushu Univ., Fukuoka, Japan)

In order to propose an effective method using smartphones (including tablets) of acoustics education for introductory courses of architectural and environmental acoustics in architectural studies, the authors have examined some applications which work on smartphones. As the first step, applications measuring sound level and spectrum at reasonable prices are chosen and their precision have been verified. Results showed that the most iOS devices has somewhat reasonable precision, e.g., for SPL measurement, it is similar to Class 2 sound level meter, though some Android devices give lower precision. Then, the authors introduced these tools to student and encouraged them to use these tools to produce a noise map (with dBA only and with both $\mathrm{dBA}$ and sound spectra). Even though these simple tools allow students to understand the relationship between their sensation and physical values. As further studies, the authors also tried to use some more applications which enables students to measure more advanced physical values, such as Band Levels, Leq, Impulse Responses, Reverberation Times, etc. Some examples of the measurement results of them are also presented.

\section{Contributed Papers}

\section{2:00}

1pED4. Visualizing sound directivity via smartphone sensors. Scott $\mathrm{H}$. Hawley (Chemistry \& Physics, Belmont Univ., 1900 Belmont Blvd., Nashville, TN 37212, scott.hawley@belmont.edu) and Robert E. McClain (OmegaLab Studio, Nashville, TN)

We present a fast, simple method for automated data acquisition and visualization of sound directivity suitable for use with introductory acoustics education, made convenient and accessible via a new free smartphone app, "Polar Pattern Plotter." The app synchronizes measurements of sound volume with the phone's angular orientation, obtained from either compass, gyroscope, or accelerometer sensors, and produces a graph and exportable data file. It is generalizable to various sound sources and receivers via the use of an input-jack-adaptor to supplant the smartphone's (omnidirectional) microphone. We show results obtained in a free-field environment for loudspeaker directivity, microphone polar patterns, and two-speaker interference effects. Results provide both a visual and quantitative representation of sound fields and device responses, useful for introductory acoustics experiments and demonstrations.

\section{2:15}

1pED5. Efficacy of a new spatial ear training program for "Ensemble width" and "Individual source width". Hidetaka Imamura (Res. Area of Creativity of Music and Sound, Tokyo Univ. of the Arts, 1-25-1, Senju, Adachi-Ku, Tokyo, Adachi 120-0034, Japan, xiulong1990@gmail.com) and Sungyoung Kim (Elec., TeleCommun., and Comput. Eng. Technol., Rochester Inst. of Technol., Rochester, NY)

The authors have devised a matching-based training paradigm that assists listeners in evaluating spatial width of a reproduced sound field with increased sensitivity and memory. The program uses a learning paradigm of repeated comparison and match to references. All sound sources were horizontally spread using a pairwise constant power panning law over front five loudspeakers located at $+-60,+-30$, and 0 degree. Trainees were asked to adjust a parameter controlling the panning intervals of five-channel sound sources until it matched to the perceived width of a given reference. The program provided visual feedback for isomorphic mapping. To make the training equipment more accessible, the headphone version of the program was also developed using a simulation of listener and sound sources positions in a room. Thirty participants were divided into three groups: a group with loudspeaker-based training, a group with headphone-based training, and a group without training. Participants in the trained groups took 6 days training program with 3 to 5 sessions in a day. The difference between reported and presented intervals before and after the training were measured. The analyzed results showed that regardless of training method (loudspeaker- or headphone-based), trained groups showed improvement in their matching precision.

\section{2:30}

1pED6. Underwater acoustics education at University of Washington Bothell. Shima Abadi (Univ. of Washington, 18115 Campus Way NE, Box 358538, Bothell, WA 98011, abadi@uw.edu)

University of Washington Bothell was founded in 1990 to bring the University of Washington traditions of academic excellence to residents of the Puget Sound region. Mechanical engineering launched as a new undergraduate program in engineering \& mathematics division in fall 2014. Underwater acoustics is one of the main focus areas in ME program which is integrated in several elective courses to provide students with formal training prior to the graduation and prepare them for research, internships, and ocean acoustics job positions. University of Washington Bothell in collaboration with local research institutes and industries supports underwater acoustics capstone projects. In addition to the capstone projects, many students are involved in theoretical and experimental research projects such as marine mammal bioacoustics, acoustical array signal processing, and underwater acoustic sensor design. In this talk, the underwater acoustics emphasis at the University of Washington Bothell will be presented along with the courses offered, research activities, capstone projects, and collaborations with local communities. 


\title{
Session 1pMU
}

\section{Musical Acoustics: Music and Sound in Multimedia}

\author{
Shin-ichiro Iwamiya, Cochair \\ Kyushu University, Shiobaru 4-9-1, Fukuoka 814-8540, Japan \\ Jonas Braasch, Cochair \\ School of Architecture, Rensselaer Polytechnic Institute, 110 8th Street, Troy, NY 12180
}

Invited Papers

$1: 30$

1pMU1. The advanced technique of the counterpoint between music and video in Evangerion: 2.0 You can (not) advance. Masashi Yamada (Dept. of Media Informatics, Kanazawa Inst. of Technol., 3-1 Yatsukaho, Hakusan, Ishikawa 924-0838, Japan, m-yamada@neptune.kanazawa-it.ac.jp) and Riu Yanagida (Graduate School of Eng., Kanazawa Inst. of Technol., Nonoichi, Ishikawa, Japan)

Film director, Akira Kurosawa, used a technique called the counterpoint between music and video, where a part of a film consisted of incongruent features between the music and motion picture, in his films. Hideaki Anno, a director of animated movies, invented an advanced technique of the counterpoint in his film EVENGERION:2.0 YOU CAN (NOT) ADVANCE. In the present study, the emotions of the music and video, and the congruency between them were measured continuously, to reveal the effects of this advanced technique. The results showed that, in the long last scene, the emotional features of the music were incongruent with the video at first. Then, the emotions of the music gradually coincided with the video. Finally, they became congruent. In the present study, various types of music were presented with the original video, and these versions were also used for the continuous measurements. Moreover, overall impressions of these versions, as well as the original version, were measured. The results showed that the original version was perceived as significantly more preferable and enjoyable than the versions where music was consistently congruent with the video.

\section{1:50}

1pMU2. Effects of the tempo of background music on prospective and retrospective duration of a video program. Akira Nishimura (Dept. of Informatics, Tokyo Univ. of Information Sci., 4-1, Onaridai, Wakaba-ku, Chiba 2658501, Japan, akira@ rsch.tuis. ac.jp)

To improve understanding of the effects of music on perception of video productions, subjective duration of a video program was measured in the paired-comparison paradigm using a 10-s video program selected from a 70-s daily news program mixed with a 90 beats-per-minute (bpm) music as a reference. Seven video programs were used for comparison, with actual durations ranging from $8.5 \mathrm{~s}$ to $11.5 \mathrm{~s}$ in steps of $0.5 \mathrm{~s}$. These programs were selected randomly from the same video program and mixed with music played at 50, 90, and $140 \mathrm{bpm}$. Seven participants listened to a pair of video programs and then answered which program of the two was subjectively longer. The psychometric functions of retrospective perception of duration showed that video programs mixed with the fast-tempo music were perceived as short. In addition, five other participants were asked to prospectively produce a 10-s interval on a stopwatch while watching the same video programs. The actual times recorded generally exceeded $10 \mathrm{~s}$, and were significantly long for the program mixed with slow-tempo music. The conflict between the results obtained from the two experiments on subjective duration and its relation to the fixed physical duration is discussed in terms of internal clocks.

2:10

1pMU3. Influence on story prediction of movie by difference in music. Yuki Mito, Ayame Suzuki (Music, Nihon Univ. College of Art, 2-42-1 Asahigaoka Nerima-ku, Tokyo 176-8525, Japan, mito.yuuki@nihon-u.ac.jp), and Hiroshi Kawakami (Music, Nihon Univ. College of Art, Tokyp, Japan)

The music often may assist to predict of movie work that has story line. The interpretation of the movie may sometimes change by music. In fact, there are many movies changing a story by music intentionally. In this way, interpretation of the story changes by using multi-modal. In the preceding research, we have known that the story of the movie can change by music but there are kind of movies used in the research, there is no assumption research of actual television or commercial. In this study, we studied how the difference of the music will influence. We have created four different types of music and silence for the movie, only played by piano as to lose prejudice by using types of other instruments. The short movie which created lasts 30 seconds will ends unfinished in order to match all kinds of stories. As a result, we have found that when the story is vague, prediction will change by the difference of the music after the movie. We hope this study helps producers of television and movies to convey their intentions more appropriately to ordinance. 
1pMU4. Optimum insertion timing of symbolic music to induce laughter in video contents. Ki-Hong Kim (Media \& Information Resources, Surugadai Univ., 698 Azu, Hanno, Saitama 357-8555, Japan, kim.kihong@surugadai.ac.jp), Mikiko Kubo (Hitachi Solutions, Ltd., Tokyo, Tokyo, Japan), and Shin-ichiro Iwamiya (Faculty of Design, Kyushu Univ., Fukuoka, Fukuoka, Japan)

In television variety shows or comedy programs, in addition to real sounds, various sound effects and music are combined with humorous scenes to induce more pronounced laughter from viewer-listeners. Symbolic music whose imagery is associated with special meaning based on past usage is dubbed just after humorous scenes as a sort of "punch line" to emphasize their humorous nature. Rating experiments using a method of paired comparisons were conducted to clarify the best timing for insertion of such music into humorous video contents. In the case of purely comical scene, the optimal insertion time was 0.0 to 0.5 seconds after the target scene. In the case of tragicomic scene (humorous accident), the optimal insertion time was 0.5 to 1.0 seconds after the scene, i.e., a short pause before the music was effective in this case. In both cases, the effects of symbolic music decreased 1.5 to 2.0 seconds after the scenes. The rating experiments showed that optimal timing was associated with highest impressiveness of the videos, highest evaluations, highest congruence between moving pictures and sounds, and inducement of maximum laughter.

1pMU5. Synchronization and periodicity of auditory and visual accents affect perceived congruence between sound and moving pictures. Saki Liu, Fumiyuki Takishita, Jingjing Gao, Kae Ishida, and Shin-ichiro Iwamiya (Kyushu Univ., 4-9-1, Shiobaru, Minamiku, Fukuoka, Japan, sf0530@gmail.com)

Continuous rating experiments focused on the congruence between moving pictures and sounds have shown that synchronization between auditory and visual accents is fundamental to audiovisual congruence. We conducted experiments with snare sounds as auditory accents and changes in the color of a full screen as visual accents. We found that the periodicity of the auditory and visual accents contributed to the audiovisual congruence, in addition to the synchronization between auditory and visual accents. We found the highest level of congruence when the accents were periodic and frequently synchronized. Conversely, the congruence level was lower when the auditory and visual accents were less frequently synchronized. The contribution of auditory periodicity to the perceived congruence was greater than that of visual periodicity. Additionally, when we used stimuli consisting of synchronized and asynchronized audiovisual elements, we observed an effect of context on congruence with respect to continuous ratings. When we presented a stream of asynchronized audiovisual accents after a synchronized stream, the level of high congruence was maintained for a short period. In contrast, just after presentation of a steam of asynchronized accents, we found that the congruence level of a stream of synchronized accents was lower than the original level.

\section{3:10-3:25 Break}

\section{$3: 25$}

1pMU6. Making sense of multiple senses. Barbara Shinn-Cunningham (Boston Univ., 677 Beacon St., Boston, MA 02215-3201, shinn@bu.edu)

Our brains are expert at processing multi-sensory inputs. We automatically "fuse" sounds and sights that come from one source- to the point that we cannot turn off cross-sensory perceptual interactions. Examples like the McGurk effect (in speech perception), the "Flash-Beep illusion" (in temporal perception), and the "Ventriloquism Effect" (in spatial perception) demonstrate how robust and obligatory cross-sensory integration can be. Indeed, these sensory "illusions" are not truly "illusions," but rather examples of how perceptually integrated sensory inputs are in the real world, compared to how we view them in the laboratory. Such cross-modal effects also underscore differences in the kind of information that vision and audition "specialize" in, and what our brain trusts. Specifically, visual inputs convey spatial information precisely, but temporal information poorly. Conversely, auditory inputs convey temporal information precisely, but spatial information poorly. Recent cognitive neuroscience work from our lab gives insight into how the brain handles sensory specialization and cross-sensory coding, revealing distinct cortical networks that support visuo-spatial and auditorytemporal processing. These results will be reviewed, along with their implications for multi-sensory coding in films, video games, and other forms of multimedia.

1pMU7. Telematic systems seen from a music instrument building perspective. Jonas Braasch (School of Architecture, Rensselaer Polytechnic Inst., 110 8th St., Troy, NY 12180, braasj@rpi.edu)

New digital technologies enabled the Virtual Reality movement in the 1990s, which led to the euphoric belief that we could virtually teleport ourselves to a distant location with such great realism that the user would lose the awareness for the enabling technology. Especially, in the particular case of music collaborations, however, it has meanwhile become clear that this goal is unreachable because of the unavoidable transmission latency over long distances and other secondary factors. This presentation explores how we can create new and better audiovisual design goals for telematic music collaborations, by treating telematic systems as a new form of musical instrument. From this viewpoint, the creation of new forms of art for the new medium become a central challenge, and not only the development of better telematic technology. In particular, the new works need to focus on the new affordances that the telematic system brings with it, and not only circumnavigate current system limitations. [Work supported by NSF grant \#12293911.] 
1pMU8. Virtual acoustics in multimedia production-Beyond enhancing the acoustics of concert halls. Wieslaw Woszczyk (Virtual Acoust. Technol. Lab, McGill Univ., Schulich School of Music, 527 Sherbrooke St. West, Montreal, QC H3A1E3, Canada, wieslaw@music.mcgilll.ca)

The paper describes a range of applications of virtual acoustics, the rendering of artificial acoustic spaces, which allow musicians to interact with ambient spaces created in real time. With a number of loudspeakers suitably distributed within a physical enclosure, such a projection system can be used to introduce a range of sound fields, which may effectively transform the acoustic environment to become a creative partner in multimedia production. A necessary component in this system is a low-latency, high-resolution multichannel convolution engine that converts a live audio signal into a structured ambient response, creating a scene in real time. Scenes can be changed to suit various goals of production and sonic narration. A number of techniques have been used to capture and modify impulse responses including temporal segmentation, shaping of magnitude envelope, noise reduction, spectral enrichment, time shifting and alignment, and parallel and sequential convolution. With these methods, artists may interact with novel acoustic responses as if they were musical instruments. Artists claim that many of the familiar as well as novel acoustic responses stimulate their creativity. Flexible variable virtual acoustics opens additional creative possibilities when employed as a component of a recording studio, either directly or when rendered over headphones.

MONDAY AFTERNOON, 28 NOVEMBER 2016

SOUTH PACIFIC 3, 1:00 P.M. TO 2:00 P.M.

\title{
Session 1pNSa
}

\author{
Noise: Sound Design II \\ André Fiebig, Cochair \\ HEAD acoustics GmbH, Ebertstr. 30a, Herzogenrath 52134, Germany \\ Takeshi Toi, Cochair \\ Patricia Davies, Cochair \\ Ray W. Herrick Labs., School of Mechanical Engineering, Purdue University, 177 South Russell Street, West Lafayette, \\ IN 47907-2099
}

\section{Contributed Papers}

1:00

1pNSa1. Influence of rotor eccentricity on electromagnetic vibration and noise in permanent magnet synchronous motor with different slotpole combinations. Fu Lin and Shuguang Zuo (Tongji Univ., Rm. 403B, Clean Energe Automotive Eng. Ctr., No. 4800, Cao'an Rd., Shanghai 201804, China, linfu_911@163.com)

Rotor eccentricity (RE) is a common fault in permanent magnet synchronous motor (PMSM). In this paper, a multiphysics model is proposed to investigate the variation of electromagnetic vibration and noise due to two types of RE including static eccentricity (SE) and dynamic eccentricity (DE). Maxwell stress tensor is first employed to derive the spatial and temporal changes of electromagnetic force from eccentricity, which are validated by 2-D decomposing of radial force calculated by a finite element (FE) model. Then, based on the proposed multiphysics model, the electromagnetic vibration and noise considering RE with different eccentricity levels and types is calculated. The vibration and noise changes with eccentricity are all explained by the force harmonics induced by eccentricity. It is concluded that the influence of RE on vibration and noise is closely related to the slot-pole combination. The vibration and noise in PMSM with higher space order of force is more sensitive to RE. It is also found that, due to the low space order force induced by RE, vibration and noise peaks would transfer to the frequency band close to lower modal frequencies.
$1: 15$

1pNSa2. Investigation of vibration and noise characteristics in axial flux permanent magnet synchronous motor with different magnet shapes. Wenzhe Deng, Shuguang Zuo, Fu Lin, and Shuanglong Wu (Clean Energy Automotive Eng. Ctr., Tongji Univ., No.4800 Cao'an Rd., Jiading District, Shanghai 201804, China, deng_wenzhe@foxmail.com)

This paper investigates the vibration and noise characteristics of axial flux permanent magnet (AFPM) motor with different magnet shapes. A multiphysics model is developed to predict electromagnetic vibration and noise of AFPM motor which contains three steps. In the first step, nodal force exerted on the surface of stator tooth is calculated using 3D finite element method (FEM). Then, structural analysis is performed to investigate the structure natural characteristics and the nodal force calculated is mapped into structural model as the excitation in the second step. In the third step, the vibration and noise of AFPM motor are calculated through modal superposition method (MSM) and boundary element method (BEM) respectively. Vibration and noise characteristics of AFPM motor with different magnet shapes are investigated based on the multiphysics model. The results reveal that AFPM motor with sine-shaped magnet demonstrates lower noise level compared to AFPM motor with sector- or cylinder-shaped magnet. 
1pNSa3. Damping contributions of sound absorbing materials in threedimensional spaces to obtain efficient layouts. Takao Yamaguchi (Mech. Sci. and Technol., Gunma Univ., 1-5-1 Tenjincho, Kiryu, Gunma 376-8515, Japan,yamagme3@gunma-u.ac.jp)

In this research, we have proposed a method for determining an efficient layout of sound absorbing materials in three-dimensional spaces using damping contributions with eigenmodes as weight coefficients. We use the complex effective density and the complex bulk modulus of elasticity for finite elements of sound absorbing material. We formulate discrete equations using the elements. Solutions to the complex eigenvalue problem of the discrete equations are expanded with a small parameter that is related to the damping. By adopting the first order approximation with respect to the small parameter, we derive the dominant equations of motion. By arranging these derived equations, the contribution of each element to modal loss factors can be obtained in consideration of multiple modes. According to these contributions, the sound absorbing materials are filled in the spaces. In this proposed method, the components of eigenvectors at the evaluation points are used as the weight coefficients for the damping contributions. The weight coefficients play an important role in intensively damping the acoustic modes that exert dominant influences on the sound pressure at the

\section{$1: 45$}

1pNSa4. Evaluation of low cost microphone for active noise control in duct. Pedro Henrique R. Lima (Universidade de Brasília - UNB, Rua 138, Aparecida de Goiânia 74946-450, Brazil, cefas_hrl@hotmail.com)

Economic feasibility and cost minimizing of experimental setups is one of the most important factors in many research and this is no different for active noise control (ANC) in ducts. Cheaper acquisition and assembly of equipment is desirable, provided you keep the same efficiency of acquisition, filtering, control, and attenuation. This study aims to compare the performance of low cost microphones with precision microphones, when applied to the acoustic control system in feedforward configuration. This system makes use of a control board with Digital Signal Processor (DSP) in order to identify, analyze, and compare systems of acoustic control in real time. To validate the performance of the sensors, an experimental bench was setup which included a PVC duct and speakers as noise source and actuator. Microphones are the error and reference sensors. The system performance is obtained by a sound pressure meter acting on the outlet duct.

\title{
Session 1pNSb
}

\section{Noise: Acoustics of Microperforated Materials}

\author{
J. S. Bolton, Cochair \\ Ray W. Herrick Laboratories, School of Mechanical Engineering, Purdue University, 177 S. Russell St., West Lafayette, \\ IN 47907-2099 \\ Masahiro Toyoda, Cochair \\ Kansai University, 3-3-35, Yamate-cho, Suita-shi, Osaka 564-8680, Japan
}

Chair's Introduction-2:15

\section{Invited Papers}

1pNSb1. Modeling and formulation of microperforated panels in the finite-difference time-domain method. Daiki Eto, Masahiro Toyoda, and Yasuhito Kawai (Kansai Univ., 3-3-35, Yamate-cho, Suita-shi, Osaka 564-8680, Japan, k832661@kansai-u.ac.jp)

A microperforated panel (MPP) was first developed by Maa and is recently regarded as one of the most promising absorption materials for next generation. Considering the approximation formula suggested by Maa, impedance of the perforations cannot be directly implemented in time-domain calculations because the impedance depends on frequency. Some new ideas are therefore necessary to take into account the effects of an MPP in time-domain analyses. Herein, a method to consider effects of an MPP in the finite-difference time-domain (FDTD) analyses is proposed. An MPP is considered as a boundary condition and the frequency dependence in the calculation is removed by replacing the frequency-dependent terms with constant values which are derived based on the multiple regression analysis. Additionally, the stability condition of the MPP boundary for one-dimensional sound field is developed. Absorption characteristics of MPP absorbers with back air layers of various depths are calculated numerically and compared with analytical ones. Consequently, stable calculations are achieved by satisfying both the CFL condition and the stability condition of the MPP boundary and the high prediction accuracy and wide applicability are confirmed. 
1pNSb2. Computational investigation of microperforated materials: End corrections, thermal effects, and fluid-structure interaction. J. S. Bolton (Ray W. Herrick Labs., School of Mech. Eng., Purdue Univ., 177 S. Russell St., West Lafayette, IN $47907-$ 2099, bolton@purdue.edu), Thomas Herdtle (3M Corporate R \& D, SEMS, Predictive Eng. and Computational Sci., 3M Co., St. Paul, MN), and Nicholas Kim (Ray W. Herrick Labs., School of Mech. Eng., Purdue Univ., West Lafayette, IN)

The concept of microperforated noise control treatments was introduced by Maa 1975; in that theory, the transfer impedance of the microperforated layer was calculated based on oscillatory viscous flow within a small cylinder combined with resistive and reactive end corrections. Recently, new manufacturing procedures have dramatically lowered the cost of these materials, and as a result, there has been renewed interest in studying their properties. Since 1975, Maa's original theory has been widely used. However, in principle, that theory can only be used to describe cylindrical perforation, while in practice, perforations are rarely cylindrical. In addition, there have been questions about the dependence of end corrections on frequency, and on the effect of coupling between the motion of the fluid in the perforations and the solid sheet in which they are formed. Additionally, in his original paper, Maa drew a distinction between the dissipative properties of thermally conducting and adiabatic materials. The latter topic, in particular, has not been considered by any investigators since the idea was introduced. The purpose of this presentation is to introduce numerical tools that can be used to address the open questions mentioned above and to highlight important results obtained by using those tools.

\section{3:00}

1pNSb3. Limp microperforated panel finite elements and its application. Takeshi Okuzono and Kimihiro Sakagami (Architecture, Kobe Univ., 1-1 Rokkodai-cho, Kobe, Hyogo 657-8501, Japan, okuzono@port.kobe-u.ac.jp)

Microperforated panel (MPP) absorbers provide relatively broadband sound absorption with the superior material properties in durability, recyclability, and flexibility of design, and they can be considered as an attractive alternative of conventional sound absorbers. Recently, with the rapid progress of computer technology, numerical methods such as finite element method (FEM) and boundary element method have become powerful design tools for developing MPP absorbers with the consideration of advantage in handling arbitrary geometries, and there exist some computational models of MPP. This paper presents a simple and computationally efficient limp MPP elements to treat MPP absorbers in finite element analysis and the performance is demonstrated in various problems. First, the theory of MPP elements is presented briefly. Then, the validity is shown using impedance tube problems, in which the absorption characteristics of some MPP absorbers calculated using FEM are compared with the theoretical values. Finally, the applicability to room acoustics simulations is demonstrated by calculating transfer functions and impulse responses of rooms with practical dimensions.

3:20

1pNSb4. Sound absorption of micro-perforated panels in complex vibroacoustic environment: Modeling, mechanism, and applications. Li Cheng (Dept. of Mech. Eng., The Hong Kong Polytechnic Univ., Hung Hom, Kowloon 00000, Hong Kong, li.cheng@ polyu.edu.hk)

As an environmental-friendly and broadband sound absorption materials, micro-perforated panels start to find their wide applications in compact mechanical systems featuring complex acoustic behaviors. Evidences show that, under such circumstances, MPPs work quite differently from the way they behave in traditional architectural or building acoustic applications. This paper summarizes some of our recent work on the modelling, analyses and optimizations of mechanical systems with embedded MPP absorbers. It is demonstrated that the complex acoustic environment in which the MPP is put in creates formidable technical challenges in terms of modeling, analyses, and optimization on one hand, but also opportunities for achieving better sound absorption by making use of the vibroacoustic coupling principles for various applications. [This project was supported by the National Natural Science Foundation of China, Grant No. 11272272.]

\section{3:40-3:55 Break}

\section{3:55}

1pNSb5. Optimum duct liners and modal filters. Mats Åbom, Stefan Sack, and Raimo Kabral (The Marcus Wallenberg Lab., KTHThe Royal Inst of Technol., Teknikringen 8, Stockholm 10044, Sweden, matsabom@kth.se)

Two strategies for applying micro-perforated plates (MPP:s) to create duct sections with a high or optimum damping of sound are discussed. The first is based on realizing the so called Cremer impedance by creating a locally reacting wall boundary ("liner") that consists of a MPP backed by an air cavity. This principal can be used to realize the Cremer impedance at a single frequency where typically a damping of several hundred $\mathrm{dB} / \mathrm{m}$ can be achieved. The proposed solution is robust and gives high damping also in a wide band around the target (optimum) frequency. The second strategy is based on MPP plates arranged along pressure nodes (minima) for a given acoustic cross-mode. This means that the MPP will be located at modal velocity maxima, thereby creating a large modal damping. In principle both strategies are optimum for a particular mode and can be seen as a modal filter, i.e., a device that reduces or "kills" a particular incident mode. The proposed strategies have been investigated theoretically as well as numerically and a number of prototypes have been built and tested. In particular, two applications will be discussed: IC-engine compressor noise and aircraft ventilation noise. 
1pNSb6. Three-dimensional space sound absorbers using microperforated panels. Kimihiro Sakagami (Dept. of Architecture, Grad. School of Eng., Kobe Univ., Rokkodai 1-1, Nada, Kobe, Hyogo 657-8501, Japan, saka@kobe-u.ac.jp), Masahiro Toyoda (Dept. of Architecture, Kansai Univ., Suita, Osaka, Japan), Motoki Yairi (Kajima Tech. Res. Inst., Chofu, Tokyo, Japan), and Takeshi Okuzono (Dept. of Architecture, Grad. School of Eng.., Kobe Univ., Kobe, Hyogo, Japan)

A microperforated panel (MPP) is one of the most promising alternatives of the next-generation sound absorbing materials. It has a large potential for various use. However, its conventional use is to place it with a rigid-back wall and an air-cavity in-between. Therefore, its arrangements in a room are limited on to room boundaries, and in some cases, this may cause a problem in room design and its strength: for example, in Japan, it is not allowed by regulation to use it for a wall. Therefore, the authors have been looking at the possibility for using MPPs for space sound absorbers without any backing structure. This can allow MPPs for wider use: a three-dimensional shape MPP space absorbers can be easily produced if a suitable material is used. They can be placed easily in various places: they can be put on the floor or hang from the ceiling, etc. Besides, MPPs can be made of designable, lightweight materials, which can add designability to the absorbers. In this paper, the main results of our studies on three-dimensional MPP space absorbers (mainly cylindrical and rectangular three-dimensional MPP space absorbers) are introduced and summarized.

\section{$4: 35$}

1pNSb7. Equivalent gradient properties of porous material with macro void and panel structures. Fengxian Xin (MOE Key Lab for Multi-functional Mater. and Structures \& State Key Lab for Strength and Vib. of Mech. Structures, Xi'an Jiaotong Univ., Xi'an, China), Changyong Jiang (HKU-ZIRI and Dept. of Mech. Eng., The Univ. of Hong Kong, Haking Wong Bldg., Rm. 704, Hong Kong 00000, Hong Kong), Tianjian Lu (MOE Key Lab for Multi-functional Mater. and Structures \& State Key Lab for Strength and Vib. of Mech. Structures, Xi'an Jiaotong Univ., Xi'an, China), and Lixi Huang (HKU-ZIRI and Dept. of Mech. Eng., The Univ. of Hong Kong, Hong Kong, Hong Kong, lixi@hku.hk)

In order to reduce the interface sound reflection, best sound absorption may be achieved by having a low acoustic impedance at the air-absorber interface and a gradient property is used inside the absorber. This study explores how such gradient properties may be realized by a distribution of voids and/or perforated panel structures. Finite-element simulation for the basic module is conducted to reveal the relationship between the design and equivalent fluid properties. The gradient of various properties are then identified. Genetic algorithm is used to optimize the geometrical parameters for best absorption performance under the constraint of a given thickness and a set of boundary conditions. The optimized design is then considered as parallel-series arrangement of homogeneous layers each with different acoustic impedance. For a chosen practical application, a feasible design is determined and its performance is compared with the traditional absorber of optimized, uniform properties. Gains in broadband sound absorption performance is analyzed with a single-digit descriptor relevant to the application.

\section{4:55}

1pNSb8. Thin low frequency sound absorbers based on shunted loudspeakers. Jiancheng Tao, Chaonan Cong (Key Lab. of Modern Acoust., Inst. of Acoust., Nanjing Univ., Nanjing, China), and Xiaojun Qiu (School of Elec., Mech., and Mechatronic System, Univ. of Technol. Sydney, Sydney, NSW 2007, Australia, xiaojun.qiu@uts.edu.au)

There are two main reasons for using shunted loudspeakers as sound absorbers. One is that the absorbers can be made very thin for the low frequency sound with electronic energy dissipation mechanism and the other is that their absorption performance can be adjusted with the shunt circuit. A shunted loudspeaker typically has high absorption coefficients around its resonance frequencies which are determined by the loudspeaker unit and the shunt circuit together. To extend the effective frequency range of the shunted loudspeaker, one method is constructing more resonant sub-systems in the absorber to have absorption at more resonance frequencies. In this research, two designs based on shunted loudspeakers will be introduced. One is the composite absorber with a micro-perforated panel in front of a shunted loudspeaker, which combines two resonance subsystems to provide broadband absorption. The other is a shunted loudspeaker with a specifically designed shunt circuit for the absorption of three tonal components. Experimental results in an impedance tube will be reported to demonstrate the feasibility of the designs based on the combination concept of multiple resonances. 


\title{
Session 1pPA
}

\section{Physical Acoustics: Advances in Hazards Monitoring with Distributed Infrasound Sensor Networks}

\author{
Milton A. Garces, Cochair \\ HIGP Infrasound Laboratory, University of Hawaii, Manoa, Infrasound Laboratory, 73-4460 Queen Kaahumanu HWY, \#119, \\ Kailua-Kona, HI 96740-2638 \\ Takahiko Murayama, Cochair \\ CTBT National Data Centre - 1, Japan Weather Association, Sunshine 60 Bldg. 55F, 3-1-1 Higashi-Ikebukuro, Toshima-ku, \\ Tokyo 170-6055, Japan
}

Chair's Introduction-1:00

\section{Invited Papers}

1:05

1pPA1. Infrasonic and seismic eruption tremors and their relation to magma discharge rate: A case study for the 2011 eruption of Shinmoe-dake, Japan. Mie Ichihara (Earthquake Res. Inst., Univ. of Tokyo, 1-1-1 Yayoi, Bunkyo-ku, Tokyo 113-0032, Japan, ichihara@eri.u-tokyo.ac.jp)

Infrasound monitoring is a useful tool to capture surface activity of volcanoes. Previous works have attempted to connect observations of infrasonic eruption tremors with magma discharge rate that is an important parameter to characterize an eruption. Power law scaling relations with different power indices have been proposed based on various datasets and models. Independently, different power law relations have been proposed between seismic eruption tremors and magma discharge rate. The 2011 eruption of Shinmoe-dake, Japan, provides an excellent dataset with which to investigate these relationships for a sustained explosive eruption sequence. Magma discharge rates are well constrained by geodetic, geologic, and remote sensing methods. Seismic and infrasonic data were recorded close to the vent. Linear power law relationships are found to fit all pairs of variables (seismic eruption tremor power, infrasonic eruption tremor power, and magma discharge rate) in the quasi-stable or slowly growing stages of the eruption. Existing models do not fully explain the observed relationships. It is proposed that the eruption generated eruption tremors in the ground and atmosphere by successive explosions in the conduit and the linear relation is observed only under the stable condition of eruptive behaviors.

\section{$1: 25$}

1pPA2. Explosion energy estimates based on propagation modeling of observed infrasound airwaves. Keehoon Kim and Arthur Rodgers (Geophysical Monitoring Program, Lawrence Livermore National Lab., 7000 East Ave., L-103, Livermore, CA 94550, kim84@1lnl.gov)

Energetic explosions in the atmosphere produce blast waves, which propagate as infrasound (low-frequency sound waves) and can be detectable at long ranges. Numerous empirical models have been developed to relate explosion energies of events (in terms of trinitrotoluene-equivalent yield) to the metrics of observed infrasonic signals (e.g., peak amplitude, period, and impulse per unit area). However, the existing empirical models often do not take into account variability of atmosphere (e.g., temperature, pressure, and wind) and an empirical model determined in a certain meteorological specification may not be valid in another weather scenario. In this case, unmodeled propagation effects can compromise the accuracy of source energy estimates. We propose another approach to determine explosion energy based on numerical simulations. Infrasound propagation in a given meteorological circumstance is simulated by a full 3-D finite difference method providing accurate Green's functions between the source and receivers. With the numerical Green's functions full-waveform inversion of infrasonic signals is performed to obtain the source time history of the event, and the metrics of the acoustic source time function are related to the energy of explosion source. The technique is applied to controlled chemical explosions and volcanic explosions for verification.

\section{$1: 45$}

1pPA3. Detection of snow avalanche locations using infrasound array data. Nobuo Arai (Nagoya Univ., Furo-cho, Chikusa-ku, Nagoya 464-8601, Japan, arai.nobuo@e.mbox.nagoya-u.ac.jp), Toshiaki Imai, Masaya Otsuki, Yoshihiko Saito (Yuki-ken Snow Eaters Inc., Sapporo, Japan), Takahiko Murayama, and Makiko Iwakuni (Japan Weather Assoc., Tokyo, Japan)

Infrasound observation measures the energy radiated by a snow avalanche into the atmosphere and can be used to detect snow avalanches over large areas. Our research team has conducted infrasound observation in Tokamachi, Niigata Prefecture, Japan, during the last three winter seasons. First, between January and April 2013, we deployed an infrasound sensor in front of a selected slope, obtained visual observations using a web camera, and identified infrasound signals generated by snow avalanches. During the second season 
(2013-2014 winter), we deployed two infrasound sensors approximately $1 \mathrm{~km}$ apart and obtained the attenuation characteristics of infrasound signals. In the 2014-2015 winter season, we deployed three sensors spaced at 1-2 km in a triangular array and attempted automatic extraction of signals associated with snow avalanches from observed raw data using time-domain processing. For the extracted signals, the locations of snow avalanches were estimated with the cross-correlation method. Twelve events were detected and located. The estimated locations were in an area with many steep slopes. An infrasound array monitoring system with real-time processing is capable of providing significant information on snow avalanche activity.

\section{2:05}

1pPA4. ARISE project capabilities for hazards monitoring. Elisabeth Blanc, Nicolas Brachet, Alexis Le Pichon (CEA/DAM/DIF, CEA/DAM/DIF, Arpajon 91297, France, elisabeth.blanc@cea.fr), Emanuele Marchetti (Dept. of Earth Sci., Firenze Univ., Firenze, Italy), Thomas Farges (CEA/DAM/DIF, Arpajon, France), Maurizio Ripepe (Dept. of Earth Sci., Firenze Univ., Firenze, Italy), Pierrick Mialle (Comprehensive Nuclear-Test-Ban Treaty Organization, Vienna, Austria), and Philippe Husson (Meteo France, Toulouse, France)

The ARISE (Atmospheric dynamics InfraStructure in Europe) project funded by the European Union combines the International infrasound monitoring system developed for the CTBT (Comprehensive nuclear-Test-Ban Treaty) verification, the NDACC (Network for the Detection of Atmospheric Composition Changes) lidar network, European observatories at mid latitudes (OHP observatory), tropics (OPAR observatory), high latitudes (ALOMAR), the European infrasound stations and satellites for the study of the dynamics of the atmosphere from ground to thermosphere. The ARISE network is unique by its coverage from equatorial to polar regions and the involved scales both in time (to tens of years) and space (one to thousands of kilometers). One of the major objective of ARISE is the monitoring of extreme events including lightning, earthquakes, tornadoes, and volcanoes. A review of the ARISE results will be presented. Volcano monitoring by the infrasound technology presents a strong interest for aviation safety in case of eruption of distant non instrumented volcanoes in broad coverage in complement to satellite observations, which can be limited by the cloud cover. One of the ARISE objectives is to provide eruption notifications including confidence index representative on propagation conditions and additional analysis results. Such studies interest the VACCs (including VAAC Toulouse) and the IAVWOPSG.

\section{Contributed Papers}

$$
\text { 2:25 }
$$

1pPA5. Past, present, and future infrasound volcanic's explosion detection capability in Southeast Asia. Benoit Taisne, Anna Perttu (Earth Observatory of Singapore, 50 Nanyang Ave., N2-01b-25, Singapore 639798, Singapore, btaisne@ntu.edu.sg), and Milton Garces (Infrasound Lab., Univ. of Hawaii, Manoa, HI)

On August, 26th 1883, meteorologist across Europe, observed an anomaly in the atmospheric pressure measurements, turns out that it was generated by an explosive eruption of Krakatau that happened more than 11,000 $\mathrm{km}$ away. Nevertheless, detection is not easily granted and will be affected by regional to global atmospheric conditions, mainly winds and temperature profile. Other atmospheric phenomena, such as clouds, would not be affecting infrasound, but might reduce the chance of observing the expanding ash cloud from space. Those limitations are of paramount importance when talking about aviation safety. The ever-growing demand for air-traffic is accommodated by increasing the number of travellers in the air at any one time, bringing with it the need to improve early detection of volcanic ash cloud so that planes could be rerouted to safer paths. The issue is especially pertinent in Southeast Asia with the recent boom in air-traffic, and the 749 potentially active volcanoes in the region. In terms of volcanic explosivity index (VEI), the probability of VEI-4 (ash up to $25 \mathrm{~km}$ and spreading for more than 1000 $\mathrm{km}$ ) in any ten years span in SE-Asia is one, while this probability is of 0.630 and 0.135 for VEI-5 and 6, respectively. In this presentation, we will focus on the remote detection capability that we have with today's instruments, and how we can improve it in the future.

\section{2:40}

1pPA6. Acoustic surveillance of hazardous volcanic eruptions (ASHE) in Asia. Maria Ngemaes (Palau National Data Ctr. and National Weather Service, Palau National Weather Service and National Data Ctr., PO Box 520, Koror, Koror 96940, Palau, maria.ngemaes@noaa.gov), Benoit Taisne (Earth Observatory of Singapore, NTU, Singapore, Singapore), Takahiku Murayama (Japan National Data Ctr. and Weather Assoc., Tokyo, Japan), Elisabeth Blanc (Commissariat à l'Energie Atomique et aux Energies Alternatives, Arpajon, France), Andrew Tupper (Bureau of Meteorol., Melbourne, VIC, Australia), Pierrick Mialle (Comprehensive Nuclear-TestBan-Treaty Organization, Vienna, Austria), Hee-Il Lee (Korea National Data Ctr. and Inst. of GeoSci. and Mineral Resources, Daejeon, South Korea), and Milton A. Garces (HIGP Infrasound Lab., Univ. of Hawaii at Manoa and Palau National Data Ctr., Kailua-Kona, HI)

The ASHE Ecuador (2004-2012) collaboration between Ecuador, Canada, and the United States demonstrated the capability to use real-time infrasound to provide low-latency volcanic eruption notifications to the Volcano Ash Advisory Center (VAAC) in Washington DC. The Atmospheric dynamics Research Infrastructure in Europe (ARISE, 2012-2018) supported by the European Commission fosters integrating innovative methods for remote detection and characterization of distant eruptive sources through collaborations with the VAAC Toulouse and the Comprehensive NuclearTest-Ban-Treaty Organization (CTBTO). The ASHE Asia project proposes an international collaboration between the Earth Observatory of Singapore, the VAAC Darwin, National Data Centers in Japan, Korea, and Palau, and will receive the support of ARISE, to provide improved early notification of potentially hazardous eruptions in Asia and the Western Pacific using a combination of established technologies and next-generation mobile sensing systems. The increased availability of open seismo-acoustic data in the ASEAN region as well as recent advances in mobile distributed sensors networks will facilitate unprecedented rapid progress in monitoring remote regions for early detection of hazardous volcanic eruptions and other natural disasters. 
1pPA7. Seven years of infrasound monitoring of volcanic eruptions of Sakurajima. Hee-il Lee, Il-Young Che (Earthquake Res. Ctr., Korea Inst. of GeoSci. and Mineral Resources, 124 Gwahang-no, Yuseong-gu, Daejeon 305-350, South Korea, leel@kigam.re.kr), and Milton Garces (Infrasound Lab., Univ. of Hawaii at Manoa, Kailua-Kona, HI)

Korea Institute of Geoscience and Mineral Resources (KIGAM) is operating an dense infrasound array network which is comprised of eight seismo-acoustic array stations (BRDAR, YPDAR, KMPAR, CHNAR, YAGAR, KSGAR, ULDAR, and TJIAR) and we are continuously monitoring all the natural and artificial infrasonic events occurred in and around Korean peninsula with this network. Sakurajima volcano is one of the most active volcanoes in the world to the point where the Volcanic Ash Advisory Center in Tokyo issued 6,012 warnings over the past 7 years from 2009 to 2015. We have checked and analyzed all the infrasound signals possibly associated with the eruptions chronology of Sakurajima volcano based on the Tokyo VAA (Volcanic Ash Advisories) information, in order to know how daily, seasonal and yearly atmospheric changes affect the detection capability of each infrasound station. The result of preliminary analysis will be presented and how the geographical location of the infrasound network which is fan-shaped to the Sakurajima volcano is affected by stratospheric wind dynamics will be discussed.

\section{3:10-3:25 Break}

\section{$3: 25$}

1pPA8. Characterizing infrasonic ocean ambient noise in Northwest Pacific. Alexis Le Pichon, Julien Vergoz (CEA, DAM, DIF, CEA, DAM, DIF, Arpajon F-91297, France, julien.vergoz@cea.fr), Christoph Pilger, Lars Ceranna (B4.3, BGR, Hannover, Germany), and Fabrice Ardhuin (LPO/CNRS, IFREMER, Brest, France)

The ability of the International Monitoring System (IMS) infrasound network to detect atmospheric explosions and events of interest strongly depends on station specific ambient noise which includes both incoherent wind noise and real coherent infrasonic waves. To characterize the coherent ambient noise, a broadband array processing was performed with IMS continuous waveform archive from 2007 to 2016 using the Progressive MultiChannel Correlation algorithm (PMCC). The processing parameters include a new implementation of adaptive frequency dependent window length and a logarithmic band spacing. Such configuration allows to better discriminate between interfering signals with improved accuracy in the wave parameters estimations. Multi-year comparisons between the observed and modeled directional microbarom amplitude variations at several IMS stations using two-dimensional wave energy spectrum ocean wave products are performed to build of a reference database of infrasound oceanic sources in Northwest Pacific. The expected benefits of such studies concern the use of multi-year complementary data to finely characterize coupling mechanisms at the ocean-atmosphere interface. In return, a better knowledge of the source of the ambient ocean noise opens new perspectives by providing additional integrated constraints on the dynamics of the middle atmosphere and its disturbances where data coverage is sparse.

\section{$3: 40$}

1pPA9. Analysis of explosive sources using a distributed network of infrasound arrays. Philip Blom (Los Alamos National Lab., Los Alamos National Lab., PO Box 1663, Los Alamos, NM 87545, pblom@lanl.gov), Omar Marcillo, Garrett Euler (Los Alamos National Lab., Los Alamos, NM, NM), Fransiska Dannemann, and Junghyun Park (Southern Methodist Univ., Dallas, TX)

Analysis of signatures observed on a distributed network requires a model describing the propagation of the energy between network nodes as well as a statistical framework to quantify confidence in analysis conclusions. For the case of infrasound analysis, the propagation model must account for the inhomogeneous, dynamic nature of the atmosphere and the resulting temporal and spatial variations of propagation effects as well as the anisotropic nature of infrasonic propagation due to the dependence on the directionality of the winds. Association and localization methods have been developed utilizing a Bayesian framework to identify explosive sources from distant, infrasonic observations with possible applications to other infrasonic sources. The confidence of the analysis conclusions is dependent on the accuracy and realism of the likelihood definitions utilized in the method, which are therefore an area of ongoing research. An overview of the association and localization methods will be presented along with several possible definitions of the infrasound likelihoods for comparison using explosive infrasonic signatures.

\section{3:55}

1pPA10. Cosmos supersonic reentry analysis: A case study using traditional and mobile infrasound arrays. Julie Schnurr (HIGP, Univ. of Hawaii Manoa, 1680 East-West Rd., Honolulu, HI 96822, jschnurr@hawaii. edu), Milton Garces (HIGP, Univ. of Hawaii Manoa, Kailua-Kona, HI), and Anthony Christie (ICS, Univ. of Hawaii Manoa, Honolulu, HI)

On August 31, 2015, the fiery reentry of the Soviet Cosmos 1315 was sighted over the Hawaiian Islands. The supersonic reentry of the satellite was detected with both traditional infrasound arrays located on the Big Island, Maui and Kauai, and with iPods using the RedVox infrasound recorder on the Big Island and Oahu. We apply array-processing techniques to reconstruct the trajectory of the satellite. In addition, we have developed a standardized multi-resolution framework that uses the Gabor limit to define a scaled set of frequencies and windows for transient feature extraction. Metrics are evaluated over a set of standardized log-scaled time windows in order to characterize waveforms. I describe a method to detect transients in infrasonic arrays with low signal to noise where beam forming is not possible, and apply it to the analysis of signals produced by the supersonic Cosmos 1315 reentry.

\section{$4: 10$}

1pPA11. Interactions between intermittent gravity waves and infrasounds. Christophe Millet (CEA, DAM, DIF, CEA, DAM, DIF, Arpajon 91297, France, christophe.millet@cea.fr), Bruno Ribstein (CMLA, ENS, Paris, France), and Francois Lott (LMD, ENS, Paris, France)

Infrasound propagation modeling requires accurate wind speed and temperature gradients. Even though the accuracy of atmospheric specifications is constantly improving, it is well-known that the main part of gravity waves is not resolved in the available data. In most infrasound modeling studies, the unresolved gravity wave field is often represented as a deterministic field that is superimposed on a given average background state. Direct observations in the lower stratosphere show, however, that the gravity wave field is very intermittent, and is often dominated by rather well-defined wave packets. These observations suggest that the vertical spectra are likely to result from ensemble averages of quite narrow-banded periodograms. In this study we sample the spectrum by launching few monochromatic waves and choose the gravity-wave properties stochastically to mimic the intermittency. The statistics of acoustic signals are computed by decomposing the original signal into a sum of modal pulses. Owing to the disparity of the gravity and acoustic length scales, the interaction can be described using a multiple-scale analysis and the appropriate amplitude evolution equation involves certain random terms that are related to the gravity wave sources. More specifically, it is shown how the unpredictable low-level small-scale dynamics triggers multiple random stratospheric waveguides in which high frequency infrasound components can propagate efficiently.

\section{$4: 25$}

1pPA12. Bayesian model averaging of infrasound propagation models. Christophe Millet (CEA, DAM, DIF, CEA, DAM, DIF, Arpajon 91297, France, christophe.millet@cea.fr) and Michaël Bertin (CMLA, ENS, Cachan, France)

In infrasound propagation modeling, we often face a large number of potential models with only a limited number of recorded signals to conduct inference from. Prediction of acoustic signals is a complex issue due to constantly changing atmospheric conditions and to the random nature of smallscale flows (turbulence, gravity waves). Thus, the uncertainty over which model to use (both for atmospheric specifications and acoustic propagation) is an important aspect of forecasting and indeed any inference from data. In standard practice data analysts typically select a model from some class of models (linear propagation models, ray tracing techniques,...) and then 
proceed as if the selected model had generated the recorded signal. Such an approach ignores the uncertainty in propagation model selection, leading to over-estimated confidence intervals. In the present work, we use Bayesian Model Averaging (BMA) as a basis for inference about parameters of interest. This approach offers a systematic method for checking the robustness of one's results to alternative model specifications. The method's performance is demonstrated using several data sets from the International Monitoring System (IMS). Our results demonstrate that a Bayesian probabilistic approach together with methods of model reduction to infrasound propagation can improve significantly the posterior distributions from the continuous stream of automatic detections of IMS infrasound stations.

\section{4:40}

1pPA13. A big data pipeline for temporospatial infrasound analysis. Anthony Christe (Infrasound Lab., Univ. of Hawaii at Manoa, 1314 Victoria St., Apt. 1402, Honolulu, HI 96814, achriste@hawaii.edu), Milton Garces (Infrasound Lab., Univ. of Hawaii at Manoa, Kailua-Kona, HI), Julie Schnurr (Infrasound Lab., Univ. of Hawaii at Manoa, Honlulu, HI), and Steven Magana-Zook (Lawrence Livermore National Lab., Livermore, CA)

Smartphone and IoT sensors allow us to build dense distributed sensor networks that supplement traditional networks. We examine next-generation technologies powering the acquisition, analysis, and reporting of infrasound data. With the advent of distributed computing, managing data flow from sensor networks has become increasingly complex. Due to the increased volume, velocity, and variety of data being produced, data acquisition, storage, analysis, and reporting techniques are evolving from single server to distributed computation architectures. In collaboration with Lawrence Livermore National Laboratory, we survey and implement several Big Data technologies to tackle these issues. We implement a system that allows distributed acquisition using Akka actors, a custom time synchronization protocol, intermediate persistent queues with Apache Kafka, long term persistence using a NoSQL database, and real-time analysis and reporting with Apache Spark and Python. We describe how these software components work together to provide acquisition and analysis for recent infrasound signatures of surf, volcanic eruption, and supersonic sources.

\title{
Session 1pPP
}

\section{Psychological and Physiological Acoustics: Spatial Hearing and Its Applications II}

\author{
Kazuhiro Iida, Cochair \\ Chiba Institute of Technology, 2-17-1, Tsudanuma, Narashino 275-0016, Japan \\ Griffin D. Romigh, Cochair \\ Air Force Research Labs, 2610 Seventh Street, Area B, Building 441, Wright Patterson AFB, OH 45433 \\ Yôiti Suzuki, Cochair \\ Research Inst. Electrical Communication, Tohoku University, Katahira 2-1-1, Aoba-ku, Sendai 981-0942, Japan \\ Douglas Brungart, Cochair \\ Walter Reed NMMC, 8901 Wisconsin Ave., Bethesda, MD 20889 \\ Yukio Iwaya, Cochair \\ Faculty of Engineering, Tohoku Gakuin University, 1-13-1 Chuo, Tagajo 985-8537, Japan
}

\section{Invited Papers}

\section{$1: 15$}

1pPP1. Localization-based audiovisual speech-recognition benefit with degraded spatial cues. Sterling W. Sheffield (Walter Reed National Military Medical Ctr., 4954 North Palmer Rd., Bethesda, MD 20889, sterling.sheffield.ctr@mail.mil), Harley J. Wheeler (Walter Reed National Military Medical Ctr., Harrisonburg, VA), and Douglas S. BrungartJoshua G. Bernstein (Walter Reed National Military Medical Ctr., Bethesda, MD)

Degraded sound-localization accuracy is usually associated with reduced spatial awareness, but can also lead to reduced speech intelligibility in cases where audiovisual speech cues are available from a talker in an unknown location. Previous work showed that soundlocalization cues improved speech perception for bilateral cochlear implantees by helping them face the talker of interest in a multitalker environment. This study evaluated the degree of localization accuracy required to obtain an audiovisual benefit. Normal-hearing adults 
used auditory localization cues to orient their gaze toward a video of a target talker. The target-talker location varied randomly among four azimuths spanning $180^{\circ}$. Auditory-localization cues were parametrically degraded with a real-time hearing-loss simulator without affecting auditory speech-recognition performance. Results revealed a maximum audiovisual benefit for speech recognition in noise of 5 $\mathrm{dB}$, diminishing to zero with increasing localization degradation. Head tracking verified the reduction in localization accuracy with increasing degradation. Results were compared to data on traditional broadband-noise localization accuracy. This analysis suggested that listeners with hearing loss, cochlear implants, or wearing hearing protection would need to be able to localize sounds within 35 ( $\sim 50 \%$ of the separation between talkers) to obtain at least some visual benefit from a talker in an unknown location.

\section{$1: 35$}

1pPP2. Head movement in human sound localization. Hayato Sato, Masayuki Morimoto (Environ. Acoust. Lab., Graduate School of Eng., Kobe Univ., 1-1 Rokkodai, Nada, Kobe 6578501, Japan, hayato@kobe-u.ac.jp), and Hiroshi Sato (Dept. of Information Technol. and Human Factors, National Inst. of Adv. Industrial Sci. and Technol., Tsukuba, Japan)

The aim of the present research is to optimize acoustic guide signals for visually impaired persons. The signal must be localized in the correct direction by the impaired person, since they aid their safe movement. Generally speaking, head movements increase localization accuracy of continuous sound. First, we clarified whether or not the effect of head movement appears in sound localization of intermittent sound in place of continuous one. Localization tests showed that effectiveness of head movements persist even if the sound is intermittent rather than continuous. However, investigations have not yet been carried out to make it clear whether or not spontaneous head movements occur at all during natural sound localization, although the previous studies were premised on the movements. If the movements always occur spontaneously, we could expect greater flexibility on designing acoustic guide signals. Second, we discussed directly whether or not spontaneous head movements occur during natural sound localization. Localization tests showed that spontaneous head movements occur during sound localization; however, they do not always occur.

$1: 55$

1pPP3. Factors influencing auditory localization by moving listeners. Douglas S. Brungart, Tricia Kwiatkowski (Walter Reed NMMC, 8901 Wisconsin Ave., Bethesda, MD 20889, douglas.brungart@us.army.mil), Sarah E. Kruger (National Intrepid Ctr. of Excellence, Bethesda, MD), and Thomas Heil (Walter Reed NMMC, Bethesda, MD)

Previous results have shown that observers who are walking at a constant pace on a treadmill are able to localize continuous sound sources and acquire and identify audiovisual targets more quickly than observers who are standing on a stationary platform, with no apparent loss in accuracy. In order to better understand the conditions where self-motion might enhance localization performance, a follow-on experiment was conducted that measured localization accuracy with sounds of three different durations $(250 \mathrm{~ms}, 1000 \mathrm{~ms}$, and continuous) under four different movement conditions (standing on a stationary platform, standing on a moving platform, walking on a treadmill at a self-paced rate, and walking on a treadmill at a constant rate). Localization accuracy was also measured both standing and walking when spatial cues were degraded by the use of a hearing protection device. As expected, the results suggest that short sounds are localized less accurately than long-duration sounds. However, preliminary results also suggest that walking at a constant pace may provide a greater benefit for localization than self-paced walking. The results are discussed in terms of the possible dynamic localization cues listeners might have access to when they are moving relative to the location of a distant sound source. [Work supported by MRMC Grant W81XWH-12-2-0068; The opinions presented are the private views of the authors and are not to be construed as official or as necessarily reflecting the views of the Department of Defense.]

\section{2:15}

1pPP4. Auditory space perception during self-motion. Shuichi Sakamoto (Res. Inst. of Elec. Commun., Tohoku Univ., 2-1-1 Katahira, Aoba-ku, Sendai 980-8577, Japan, saka@ais.riec.tohoku.ac.jp), Wataru Teramoto (Faculty of Letters, Kumamoto Univ., Kumamoto, Japan), Akio Honda (Dept. of Human Sci., Yamanashi-Eiwa College, Kofu, Japan), Yôiti Suzuki (Res. Inst. of Elec. Commun., Tohoku Univ., Sendai, Japan), and Jiro Gyoba (Graduate School of Arts and Letters, Tohoku Univ., Sendai, Japan)

Spatial information inputted to the auditory periphery dramatically changes with a listener's body movements relative to the sound source. Nevertheless, listeners can perceive a stable auditory environment and react appropriately to the sound source. This suggests that the spatial information is reinterpreted in the brain by being integrated with information regarding movement, while it is well known that the motion itself sometimes negatively affects sound localization acuity. We have focused on how people perceive auditory space during movement. A linear-motor-driven chair and a spinning chair were installed in an anechoic room to provide linear and rotatory motion, respectively. The results revealed that the perceived sound position during linear self-motion was displaced compared with the physical sound position. This displacement was observed regardless of the self-motion direction and the inputted sensory information which induces self-motion. Such degradation of sound localization acuity was also observed when listeners were rotated; the detection thresholds for the listener's subjective front were elevated during the motion. Here, we introduce the details of our study and discuss the characteristics of auditory space perception during self-motion. [Work supported by JSPS KAKENHI Grant (26280067).]

\section{2:35}

1pPP5. Relative head angle and eye position drive systematic distortions in the perception of auditory space. W. Owen Brimijoin and Graham Naylor (Inst. of Hearing Res., Medical Res. Council/Chief Scientist Office, MRC/CSO Inst. of Hearing Res., Fl. 3 New Lister Bldg., 16 Alexandra Parade, Glasgow G31 2ER, United Kingdom, owen.brimijoin@nottingham.ac.uk)

The minimum audible movement angle (MAMA) increases as a function of azimuth. This study examined a potential consequence of this: if this change in resolution across azimuth is not perceptually compensated for it would suggest that a sound rotating at a constant angular velocity around the head would not appear to do so but would appear to move faster at the front than at the side. We therefore examined whether the azimuth of two signals affects their point of subjective similarity for motion. We found that equal movement from two different azimuths is not judged as being equivalent: signals centered at $90^{\circ}$ must move at least twice as much as ones centered at $0^{\circ}$ to be judged as moving the same amount. Signals centered at $45^{\circ}$ were also perceived in a proportionally distorted manner. 
Furthermore, in some listeners, this expansion/contraction of auditory space appears to shift systematically with eye position. These interactions suggest that the MAMA may not be simply a measure of acuity, but also reflects an underlying head- and eye-driven nonlinear perceptual organization of space, one we term the "equivalent arc," in which an absolute number of degrees does not correspond to a consistent perceptual unit across azimuth.

\section{2:55-3:15 Break}

$3: 15$

1pPP6. Effects of source- and head-motion on auditory perceptual organization. Hirohito M. Kondo (Human Information Sci. Lab., NTT Commun. Sci. Labs., 3-1 Morinosato Wakamiya, Atsugi, Kanagawa 243-0198, Japan, kondo.hirohito@lab.ntt.co.jp), Daniel Pressnitzer (CNRS UMR 8248 \& ENS, Paris, France), Iwaki Toshima, and Makio Kashino (Human Information Sci. Lab., NTT Commun. Sci. Labs., Atsugi, Japan)

The perceptual organization of auditory scenes needs to parse the incoming flow of acoustic inputs into perceptual streams. It is likely that cues from several modalities are pooled for auditory scene analysis, including sensory-motor cues related to the active exploration of the scene. We show effects of source- and head-motion on auditory streaming. In a streaming paradigm, listeners hear a sequence of repeating tone triplets and indicate their perception of one or two subjective sources called streams. We used a robotic telepresence system, Telehead, to disentangle the effects of head motion: changes in acoustic cues at the ear, subjective location cues, and motor cues. We found that head motion induced perceptual reorganization even when the auditory scene had not changed. We further analyzed the data to probe the time course of sensory-motor integration. Motor cues impacted perceptual organization earlier and for a shorter time than acoustic or subjective location cues, with successive positive and negative contributions to streaming. An additional experiment showed that arm or leg movements did not have an impact on scene analysis. Our results suggest a loose temporal coupling between the different mechanisms involved.

\section{3:35}

1pPP7. Multi-channel loudspeaker reproduction and virtual acoustic environments in the context of hearing aid evaluation. Giso Grimm, Stephan Ewert, and Volker Hohmann (Medizinische Physik and Cluster of Excellence Hearing4all, Universität Oldenburg, Germany, Marie-Curie-Str. 2, Oldenburg 26129, Germany, g.grimm@uni-oldenburg.de)

Virtual acoustic environments reproduced via multiple loudspeakers are increasingly used for evaluating hearing aids in complex acoustic conditions. To further establish the feasibility of this approach, this study investigated the interaction between spatial resolution of different reproduction methods and technical and perceptual hearing aid performance measures using computer simulations. Three spatial audio reproduction methods—discrete speakers, vector base amplitude panning and higher order ambisonics—were compared in regular circular loudspeaker arrays with 4 to 72 channels. The influence of reproduction method and array size on performance measures of multi-microphone hearing aid algorithm classes with spatially distributed microphones and a representative single channel noisereduction algorithm was analyzed. In addition to the analysis of reproduction methods, algorithm performance was tested in a number of different virtual acoustic environments in order to assess the underlying factors of decreased hearing aid performance in complex environments. The results confirm previous findings that spatial complexity has a major impact on hearing aid benefit and demonstrate the potential of virtual acoustic environments for hearing aid evaluation.

3:55

1pPP8. Discrimination of virtual sound fields different in spatial aliasing. Yukio Iwaya (Faculty of Eng., Tohoku Gakuin Univ., 113-1 Chuo, Tagajo, Miyagi 985-8537, Japan, yukio@iwaya-lab.org), Makoto Otani (Kyoto Univ., Kyoto, Kyoto, Japan), and Takao Tsuchiya (Doshisha Univ., KyoTanabe, Kyoto, Japan)

There are theoretical techniques for sound field reproduction with a loudspeaker array. However, physical precision of synthesized sound field is limited in frequency domain. When the distance among loudspeakers is below a half of wavelength, spatial aliasing will occur. The ideal distance among loudspeakers was less than $8.5 \mathrm{~mm} @ 20 \mathrm{kHz}$, and it is impossible to arrange loudspeakers. Therefore, we carried out experiments to know an upper-limit frequency condition, which can give subjective experience identical to an ideal sound field. We assumed a virtual spherical boundary around a listener. Numerous virtual loudspeakers were set on the boundary to simulate a WFS system. Room impulse responses and head-related impulse responses were calculated by computer simulation. Then, binaural impulse responses were synthesized as combinations of RIRs and HRIRs. We picked up the boundary points so that upper-limit frequency without the spatial aliasing of BRIRs was systematically controlled. Listening test was conducted to investigate discrimination of sound fields different in spatial aliasing. We found that the sound field of upper-limit frequency $4 \mathrm{kHz}$ cannot be discriminated from that of $14 \mathrm{kHz}$. Therefore, sound field reproduced without spatial aliasing up to $4 \mathrm{kHz}$ could give same sound experience identical to the ideal sound field.

\section{4:15}

1pPP9. Efficient physics based simulation of spatial audio for virtual and augmented reality. Ramani Duraiswami and Dmitry N. Zotkin (Comput. Sci. \& UMIACS, Univ. of Maryland, A.V. WIlliams Bldg., \#115, College Park, MD 20742, ramani@umiacs.umd.edu)

Many applications in spatial hearing are being enabled by the virtual and augmented reality revolution. Because of these there has been a tremendous improvement in the quality of head mounted displays, and the availability of tracking ( 3 DOF and 6 DOF) in an integrated environment with headphones suitable for binaural rendering. While these devices were initially focused on creating visual representations of the 3D world (in the case of VR) or a visual representation of artificial 3D objects overlaid on the real world (in the case of augmented reality), it was soon realized that unless the audio provided a degree of spatial realism that complemented the visual and was consistent with it, the sense of immersion crucial to maintaining the illusion was lost. In this talk, I will describe the engine for creating virtual simulations in real time that incorporates head motion, environmental reverberation, room materials, as well as generic or individual HRTFs. Trade-offs that allow the engine to work on architectures ranging from single core CPUs, to mobile devices to high end 
multicore devices will be discussed. In contrast to these "object-based" representations of spatial auditory objects, there has also been a revival in approaches based on expanding the sound-field in low order ambisonics representations, and their subsequent binaural rendering. After providing a description of the optimizations made in our engine, we discuss future work.

\title{
$4: 35$
}

1pPP10. Possibility of binaural production by means of bone conduction actuators. Xiuyuan Qin and Tsuyoshi Usagawa (CSEE, Kumamoto Univ., Kumamoto Prefecture, Japan, Kumamoto City 860-0862, Japan, qnini@126.com)

A pair of bone conduction actuators is used for music reproduction as replacement of headphone. Because of strong inter-aural coupling, it is though the stereophonic reproduction is not easy to realize by bone conduction actuators. Haim Sohmera, et al. 2000, reported that "there is no need to vibrate bone in order to obtain 'bone conduction' response." They have shown a fluid pathway from bone to ear. The coupling between left and right side stimuli by bone conduction actuators set at head-of-mandible or mastoid is occurred not only due to bone but also fluid. On the other hand, when we are listening the music by bone conduction actuators, we can enjoy the some extent of special image, at least some extend of lateralization. The authors are examined the possibility of binaural production by means of inter-aural time and intensity trading using a pair of bone conduction actuators. Results of psycho-acoustical experiment indicate that the trading phenomenon between time and intensity is observed around $1000 \mathrm{~Hz}$ even if the results are not stable.

\section{Session 1pSA}

\section{Structural Acoustics and Vibration: General Topics in Structural Acoustics and Vibration II}

\author{
Benjamin Shafer, Chair \\ Technical Services, PABCO Gypsum, 3905 N 10th St., Tacoma, WA 98406
}

\section{Contributed Papers}

1:00

1pSA1. Strong fluid-structure coupling in a two-dimensional waveguide bounded by elastic plates. Jerry H. Ginsberg (School of Mech. Eng., Georgia Inst. of Technol., 5661 Woodsong Dr., Dunwoody, GA 303382854, j.h.ginsberg@comcast.net)

The typical model for compliant walls in a waveguide take them to be locally reacting. The corresponding boundary conditions for the transverse mode functions are Robin-type, which leads to an analysis that is a straightforward modification of the conventional analyses for rigid and pressurerelease walls. The present investigation takes up the case where the walls are elastic plates, and the fluid loading is heavy. The consequence is strong coupling of the fluid and structural responses. The fact that the walls are not rigid obviates the possibility of a planar mode, so it is necessary to consider the transverse dependence of modes at any frequency. The analytically derived characteristic equation for the coupled response vividly displays the interaction of elastodynamics and acoustics. The eigenvalues are the transverse wavenumbers of the various modes at a specified frequency. They can be obtained through numerical methods, but implementing such a procedure to obtain dispersion curves of transverse wavenumber vs. frequency for each mode is cumbersome. An alternative procedure based on contours of the characteristic equation is exceptionally easy to implement, and extensible to other problems. One of the results that follow from the analysis is the observation that there is a minimum frequency below which all modes evanesce.
$1: 15$

1pSA2. Identification the vibroacoustic properties of sandwichcomposite panels from wavenumber measurements. Noureddine Atalla, Raef Cherif (GAUS Mech. Eng., Univ. of Sherbrooke, UdeS, Mech. Eng., 2500 Blvd de 1'Universite, Sherbrooke, QC J1K0A2, Canada, noureddine. atalla@usherbrooke.ca), and Gary Burns (Cabin Interiors \& Structures, Zodiac Aerosp., Huntington Beach, CA)

This paper investigates the identification of the vibroacoustic properties of sandwich composite panels from structural wavenumber measurements over a large frequency band. Several indicators are investigated including the modal density, damping loss factor, radiation efficiency, and sound transmission loss. The accuracy of the identified indicators is studied by comparison with several direct and indirect measurements and analytical predictions. Results show that all identified indicators are in good agreement with measurements and theory for the studied constructions.

\section{$1: 30$}

1pSA3. Sound radiation from unbaffled vibrating plates, using an edgediffraction based approach. Sara R. Martín Román, U. Peter Svensson (Acoust. Res. Ctr., Dept. of Electronics and TeleCommun., Norwegian Univ. of Sci. and Technol., Trondheim, Norway, O.S. Bragstads plass 2a, Trondheim 7034, Norway, sara.martin@ntnu.no), Lauri Savioja (Dept. of Comput. Sci., Aalto Univ., Espoo, Finland), and Julius O. Smith (Ctr. of Comput. Res. in Music and Acoust. (CCRMA), Stanford Univ., Stanford, CA)

The sound radiation from unbaffled vibrating plates is a classical problem that is often solved numerically by coupling a vibration model to the surrounding air with the boundary element method, or the finite differences in the time domain (FDTD). Here, the surrounding air is instead represented 
with Green's functions computed with the edge source integral equation (ESIE) [Asheim and Svensson, JASA 133, pp. 3681-3691, 2013]. Expressions are derived for the analytical edge directivity functions in the ESIE for the special case of a thin flat plate with sources on both sides of the plate, of opposite polarity. Frequency-domain as well as time-domain results are presented, for specified vibration patterns, including an unbaffled piston. The coupling of the Green's functions to an FDTD model of the plate vibration is outlined. The singularity issues that appear with the ESIE for certain radiation directions are handled using a recent hybrid approach [Martín et al., J. Acoust. Soc. Am. 139, 2202 (2016)] where the ESIE is used to calculate the sound pressure on the surface of the radiator, followed by the same propagation stage as in the boundary element method. This approach offers a computationally efficient method, and the time-domain version makes auralization straightforward.

\section{$1: 45$}

1pSA4. Wave dispersion in randomly layered materials. Mieczyslaw Cieszko and Michal Pakula (Inst. of Mech. and Comput. Sci., Kazimierz Wielki Univ. in Bydgoszcz, ul. Chodkiewicza 30, ul. Kopernika 1, Bydgoszcz 85-064, Poland, michalp@ukw.edu.pl)

Wave scattering in materials composed of two kinds of alternating layers with different elastic properties and randomly distributed thicknesses has been modeled. The general form of the dispersion equation is derived for the unbounded layered medium. It defines two basic macroscopic characteristics of the scattered wave: phase velocity and attenuation, which are explicit functions of wave frequency and microscopic parameters of the system: acoustic properties of the layers and stochastic characteristics of their thickness distributions. The analytical expressions are derived for three special cases: for long waves; for a periodic medium composed of layers with constant thicknesses and for random medium with uniform distribution of layer thicknesses. Special attention is paid to the analysis of the frequency dependence of the wave parameters. It was shown that the predictions of the model for long waves and for periodic medium are compatible with the results obtained in the literature. Moreover, comparison of theoretical results for frequency dependent wave parameters with numerical simulations of pulse transmission through the slab of the randomly layered medium shows good qualitative and quantitative agreement in wide frequency range.

\section{2:00}

1pSA5. Vibration analysis of high-speed rotating conical shell with arbitrary boundary conditions. Jinzhao Wang (College of Power and Energy Eng., Harbin Eng. Univ., No.145 Nantong St. Nangang District, Harbin 150001, China, wjinzhao08@163.com), Yipeng Cao (College of Power and Energy Eng., Harbin Eng. Univ., Harbin City, China), and Guang Lin (School of Mech. Eng., Dept. of Mathematics, Purdue Univ., West Lafayette, IN)

Based on the energy method, an analytical model for the vibration characteristics of high speed rotating conical shell with arbitrary boundary is constructed. Effects of centrifugal and Coriolis accelerations as well as initial hoop tension due to rotating are all taken into account. A set of translational and rotational artificial springs to simulate the elastic constraints imposed on the conical shell edges. Under the framework, each of the displacement components of the conical shell is invariantly described by a modified Fourier series, which is composed of a standard Fourier series and closed-form auxiliary functions. All the expansion coefficients are determined by using the familiar Rayleigh-Ritz procedure as the generalized coordinates. To validate the present analysis, a series of comparisons between the present results and those available in the open literature are carried out and very good agreements are obtained. On this basis, some numerical results are given to illustrate the effects of rotating speed and geometric parameters on the frequencies of rotating conical shell. Further, the influence of the boundary springs'stiffness are also discussed.

\section{2:15}

1pSA6. Vibro-acoustics of cylindrical shells with distributed damping liners. Rajendra Singh (Mech. \& Aerosp. Eng., The Ohio State Univ., 201 West 19th Ave., Columbus, OH 43210, singh.3@osu.edu)

Distributed passive damping treatments (such as cardboard liners, and foam or visco-elastic materials) are routinely placed inside the automotive drive shafts to reduce the airborne and structureborne noise over the applicable mid-frequency range. To better understand the underlying mechanism(s), experimental and analytical studies are undertaken. First, a laboratory experiment is designed to measure vibro-acoustic response functions of a thin cylindrical shell with different cardboard liner thicknesses. Second, the properties of a cardboard liner material are determined by conducting an experiment on an elastic plate treated with a cardboard liner. Third, an analytical framework for shell vibration is developed and the relative sliding motion between substructure and liner is explicitly described. The proposed model agrees well with the experiment in terms of natural frequency shifts, modal loss factors, and modal insertion losses. Several damping mechanisms such as material damping, dry friction, and interfacial viscous friction are examined. Finally, various aspects of the problem including unresolved issues will be briefly discussed.

$$
\text { 2:30 }
$$

1pSA7. Quantification of disorder sensitivity of a subordinate oscillator array. John A. Sterling (US Navy, 9500 Macarthur Blvd., Bethesda, MD 20817, jsterling@gmail.com), Joseph Vignola (Mech. Eng., Catholic Univ. of America, College Park, MD), Aldo Glean (Mech. Eng., Catholic Univ. of America, Boston, MA), and Teresa Ryan (Mech. Eng., East Carolina Univ., Greenville, NC)

Recent research has shown that arrays of small dynamic elements attached to a larger, primary structure can be tuned to significantly alter the time and frequency response of the system. Often referred to as "fuzzy structures," such subordinate oscillator arrays have applications including damping, radio frequency filtering, energy harvesting, micro electromechanical systems, and chemical vapor sensing. This work shows that an attached array adds damping and demonstrates a distinct rejection band in the spectral response.The subordinate system here is an array of cantilever beam attachments with a range of isolated natural frequencies that surround a target band. Small errors in the distributions of masses and stiffnesses of the attachments can have a significant degrading effect on desired response. Error on the order of $0.1 \%$ can be significant. This paper discusses experimental verification of this phenomena. In the experiment, small amounts of mass were added to the tip of both individual and combinations of the cantilevers to deliberately deviate from the design. The resultant responses were recorded. A direct relationship between perturbed mass and frequency distributions and response deviation will be shown.

\section{2:45}

1pSA8. Dynamic response of a beam supported with variable gear to base excitations. Hyukju Ham, Deokman Kim, and Junhong Park (Mech. Eng., Hanyang Univ., 222, Wangsimni-ro, Seongdong-gu, Eng. ceneter, 306, Seoul ASIIKRIKS013ISEOUL, South Korea, shadowpl@nate.com)

The beam supported with gear having nonlinearly varying stiffness characteristics is analyzed to study vibration transfer characteristics. The gear attached at the end of the beam was considered as rotational spring and damper having angular dependent variation. The dynamic characteristics of the gear were determined by the contact point between the gear teeth. The displacement-dependent properties of the gear had an effect on vibration transmissibility of the beam system. The computational simulation based on the spectral element method (SEM) was used with several variables (the dynamic characteristics of the gear, the gear design, and excitation level). The validity was verified by comparison with the measured results. The vibration of the beam was strongly influenced by the gear-pair. These results can be utilized to design the optimal gear-pair system for minimizing undesirable vibration.

3:00-3:15 Break 
are analyzed by iterating the simulations at increasing time steps and finer meshes.

1pSA9. Axial force measurement for U-bolt using wave propagation speed monitoring. Gyungmin Toh, Dongki Min, Jaehong Lee, and Junhong Park (Mech. Eng., Hanyang Univ., 222. Wangsimni-ro, Seongdong-gu, Seoul 04763, South Korea, avlrudals@gmail.com)

It is important to inspect clamped parts of a produced vehicle in the manufacturing process non-destructively. In this study, a measuring device of axial force is suggested by utilizing relations between wave propagation characteristics of a beam and a clamping force. Experiments were carried out to determine characteristics of vibration signals for the structure designed for inspection. Neodymium magnet was utilized as the components of the device to attach to the joint without any damage to the products. A rectangular parallelepiped magnet was used to detect the change in the wave propagation speeds in the structure. The change in the wave propagation through the bolt was measured and used in estimation of the axial force. The correlation coefficient was calculated to quantify the change in the wave propagation characteristics. The coefficient was utilized to determine the axial force of the bolted joint by the experiment. The proposed method was applied to actual U-bolts to validate the methodology.

\section{3:30}

1pSA10. Thermoacoustic power sensors: Principles and prediction. Christopher M. Dumm, Jeffrey S. Vipperman (Mech. Eng., Univ. of Pittsburgh, 504 Benedum Hall, 3700 O'Hara St., Pittsburgh, PA 15261, jsv@pitt.edu), Jorge V. Carvajal, Melissa M. Walter, Luke Czerniak, Amy S. Ruane, Paolo Ferroni, and Michael D. Heibel (Westinghouse Electric Co., Cranberry Township, PA)

Thermoacoustic Power Sensor (TAPS) technology can be used to wirelessly measure the temperature and radiation flux conditions in a nuclear reactor core. A TAPS is a self-powered, standing-wave thermoacoustic engine, enclosed in a cylinder, and placed in the reactor core (for example, inside instrumentation tubes). TAPS utilize heat from a radiation-powered heater and cooling from the reactor core coolant to generate acoustic waves; these waves have amplitude proportional to the local radiation flux and frequency proportional to the local coolant temperature. The acoustic waves propagate physically into the reactor coolant and structure, and are detected with receivers (e.g. accelerometers) placed on the outside of the reactor vessel. TAPS signals are interpreted (and the reactor state conditions measured) by comparison of the received signals to a reference generated by predictive modeling. Since TAPS are wireless and self-powered, they offer advantages in safety (e.g., by reducing the required number of vessel penetrations) and in maintenance reduction. Since TAPS contain no internal electronics, they are particularly well-suited to high-temperature, fast radiation environments for which no in-core instrumentation currently exists, such as in sodiumcooled fast reactors (SFRs). A methodology for predictive modeling of TAPS signals is discussed and evaluated experimentally.

\section{$3: 45$}

1pSA11. Estimating mechanical properties of ice on Europa: Impact simulation and sensitivity analysis. Joshua Capozella, Edward Pfaeffle, Martin Ferreira Fernandes, Masataka Okutsu, Joseph Vignola, and Diego Turo (Mech. Eng., The Catholic Univ. of America, 620 Michigan Ave., N.E., Washington, DC 20064, 78pfaeffle@cua.edu)

The primary objective of NASA's Europa Multiple Flyby Mission to Jupiter's moon Europa is to determine the thickness of the ice covering the global subsurface ocean. If the ice proves too thick for the ice penetrating radar, however, an indirect method must be used to estimate the depth of the ice-water boundary. Unfortunately, the properties of Europan ice are not known precisely, with Young's modulus having two orders of magnitude of uncertainty. It has been suggested that seismic response produced by an artificial impact could help estimating the mechanical properties of this ice. By optically measuring the induced surface motion with enough resolution, the dispersion curves can be determined. The associated elastic wave speeds are then used to estimate the moduli and density of the ice. The seismic response of the ice is modeled using a Finite Element Method solver. This presentation describes the sensitivity of the estimated mechanical properties to both temporal and spatial resolutions of the optical measurement. The sensitivities of the elastic constants to the temporal and spatial resolutions
4:00

1pSA12. Study on the exactness of alternative indirect force estimation methods, power dissipation calculation by power or energy flow, and transfer path analysis for structure-borne sound. Akira Inoue (R\&D APL, Hitachi America, Ltd., 34500 Grand River Ave, R\&D-APL, Farmington Hills, MI 48335, akira.inoue@hitachi-automotive.us) and Yosuke Tanabe (Hitachi, Ltd., Hitachinaka, Ibaraki, Japan)

In our presentation, it is first clarified that the matrix inverse method which gives interfacial forces using X/F-type frequency response functions among parallel interfacial points is an exact method derived from Newton's equation of motion. The alternative method using F/X-type frequency response functions with blocked boundaries is next explained as another exact method, though implementation of the blocked boundary may be difficult for real-life systems. Next, as an application of interfacial force, it is shown that the real part of power flow and the imaginary part of energy flow correspond to mechanical power dissipation. When all the power or energy flows into a system are counted, it gives the exact power dissipation of the system. As another application, the exactness of transfer path analysis for structure-borne sound is examined with brief discussion of velocity potential. Computational demonstrations and validations are given for the derived formulas, using a simple finite element model. All the systems are supposed to be linear time-invariant, discretized and in a steady state.

\section{$4: 15$}

1pSA13. Passive defect detection in plate from nonlinear conversion of low-frequency vibrational noise. Emmanuel Moulin, Lynda Chehami, Jamal Assaad (IEMN, UMR CNRS 8520, Univ. of Valenciennes, Le Mont Houy, Valenciennes F-59313, France, emmanuel.moulin@univvalenciennes.fr), Julien de Rosny, Claire Prada (Institut Langevin UMR CNRS 7587, ESPCI ParisTech, Paris, France), Eric Chatelet, Giovanna Lacerra (LaMCoS UMR CNRS 5259, INSA, Univ. of Lyon, Lyon, France), Konstantinos Gryllias (Faculty of Eng., Dept. of Mech. Eng., KU Leuven, Leuven, Belgium), and Francesco Massi (Univ. of Rome La Sapienza, Dept. of Mech. and Aerosp. Eng., Rome, Italy)

It is known that, under the assumption of diffuse noise, the cross-correlation of acoustic signals recorded at two points of a medium allows to passively estimate the impulse response between these points. This principle, associated with coherent array processing, has been successfully applied to defect detection and localization in reverberant plates subject either to distributed noise sources or friction noise. In order to extend the applicability of this principle even in the absence of an adequate ambient noise, we have introduced the concept of secondary noise sources based on the conversion of low-frequency modal vibrations into high-frequency noise by exploiting frictional contact nonlinearities. The device consists of a mass-spring resonator coupled to a flexible beam by a rough frictional interface. The extremity of the beam, attached to the surface of a plate, excites efficiently flexural waves in the plate in the ultrasound range when the primary resonator vibrates around its natural frequency. We have shown that a set of such devices distributed on a plate excited by a shaker at low frequencies is able to produce high-frequency noise suitable for passive defect detection and localization based on noise correlation.

\section{$4: 30$}

1pSA14. The control of resonance curve using the shape modulation of the scattering region in elastic waveguide. Yoshimasa Naito, Hatuhiro Kato, and Takaaki Ishii (Human Oriented Eng., Univ. of Ymanashi, Takeda, Kofu, Ymanashi 400-8511, Japan, g15mh012@yamanashi.ac.jp)

This study focuses on the control of Fano resonance in the elastic plate, which is initially developed in atomic scattering and has been seldom to discuss. Fano resonance is a phenomena involved by the localized/quasi-localized wave, in which the resonance curve becomes asymmetric due to the phase difference between the incident wave and the localized wave. The typical resonance curve available in the system is the frequency dependence of the reflectance under the scattering problem. We will demonstrate that the controllability of the resonance curve using various shapes of the 
scattering region under the simulation and experiment. The experiment system is an elastic waveguide made of aluminium plate and the scattering region is formed by modulating the plate thickness. The localized wave is induced by incident wave in the thinned scattering region and causes the wave tail that penetrates into the lead region of waveguide. The controllability can be realized by the overlap of the wave tail and the incident wave. For analyzing the experiment data, we also develop the method to extract Sparameters. The controllability was already confirmed by the preliminary experiment. Now, we are preparing verify various shapes of the scattering region for detail experiments.

\section{$4: 45$}

1pSA15. Radiation of rail noise regarding surface impedance of ground by using wavenumber domain finite and boundary element. Jungsoo Ryue (School of Naval Architecture and Ocean Eng., Univ. of Ulsan, Rm 309, Bldg. 41, Ulsan 44610, South Korea, jsryue@ulsan.ac.kr) and Seungho Jang (Korea RailRd. Res. Inst., Uiwang, South Korea)

An important source of noise from railways is rolling noise caused by wheel and rail vibrations induced by acoustic roughness at the wheel-rail contact. In conventional approaches to predicting rail noise, the rail is regarded as placed in a free space so that the reflection from the ground is not included. However, in order to predict rail noise close to the rail, the effect of the ground should be contained in the analysis. In this study the rail noise reflected from the ground is investigated using the wavenumber domain finite element and boundary element methods. First, two rail models, one using rail attached to the rigid ground and one using rail located above rigid ground, are considered and examined to determine the rigid ground effect in terms of the radiation efficiency. From this analysis, it was found that the two models give considerably different results, so that the distance between the rail and the ground is an important factor. Second, an impedance condition was set for the ground and the effect of the ground impedance on the rail noise was evaluated for the two rail models.

\section{5:00}

1pSA16. Modeling of multi-jointed structures by complex stiffness based on vibration transfer characteristics. Sangmok Park, Yunsang Kwak, and Junhong Park (Hanyang Univ., 222, Wangsimni-ro, Seongdonggu, Eng. Ctr, 306, Seoul 04763, South Korea, tkdahr619@hanyang.ac.kr)

Complex stiffness representation is proposed to analyze the dynamic characteristics of jointed structures. Measured results for single-jointed specimens were compared with predictions to show the influence of connecting stiffness. The proposed dynamic stiffness representation showed accurate vibration transfer characteristics of the single-jointed structures. For multi-jointed structures, the developed prediction model was extended based on the results of single-jointed structures. For experimentations, multi-jointed specimens having different binding strength and various defected parts were produced. Vibration transfer characteristics were measured. In order to fully represent dynamic characteristics of multi-jointed specimens, the spectral element method was used for theoretical modeling. Complex stiffness allowed modeling of coupling effect by multiple joints. In aspect of wave propagation characteristics, the proposed model was verified by comparison with measured vibrations of the multi-jointed specimens. Locations of defects in specimens were theoretically predicted by the verified model. Binding strength of the multi-jointed structures was quantitatively evaluated using the complex stiffness.

\section{$5: 15$}

1pSA17. You really can hear the corn grow! Acoustic emissions in the growth and breakage of maize. Douglas Cook (Eng., New York Univ. Abu Dhabi, PO Box 903, New York, NY 10276, prof.laji@gmail.com)

Corn (or maize) is the leading grain crop in the United States. More than 350 million metric tons are harvested annually, generating twice as much revenue as any other crop. But a lack of understanding of corn stalk mechanics now hinders further improvement of corn production. The most promising new varieties of corn produce high yields, but are often susceptible to wind-induced failure of the stalk. Failure of this kind is prevalent at two distinct periods: in mid-summer during rapid growth, and after physical maturity, but before harvest. This study utilized acoustic measurements to collect new information about corn growth and failure. Measurements during the growth period were conducted in July 2016 at fields in Nebraska. Late-season stalk experiments involved stalks harvested from South Africa. Flexible, shielded piezo film sensors were used in all measurements (SDT1028K, Measurement Specialties, Hampton, VA). Acoustic emissions were found to occur continuously during corn stalk growth, though typically at levels far below those audible to the human ear. The authors hypothesize that special conditions would be required to render these emissions audible to humans. Acoustic emissions provided valuable information which used to determine modes of stalk failure in late season lodging. 


\title{
Session 1pSC
}

\section{Speech Communication and Signal Processing in Acoustics: Speech Robotics}

\author{
Masakaki Honda, Cochair \\ Philip Rubin, Cochair \\ Haskins Laboratories, 300 George Street, Suite 900, New Haven, CT 06511
}

Chair's Introduction-1:30

Invited Papers

$1: 35$

1pSC1. Talking heads: communication, embodiment, and interaction. Philip Rubin (Haskins Labs., 300 George St., Ste. 900, New Haven, CT 06511, rubin@haskins.yale.edu)

The desire to create robotic speech, including talking human heads, stems back at least several hundred years. This quest continues and presently combines approaches that are computational, cognitive, biological, mechanical, and acoustic, cutting across a wide variety of domains and interests. This presentation provides some highlights of the broad, international effort that includes speech robotics and social robotics, the historical antecedents of this effort, and considers aspects of embodiment and interaction between speakers. By talking heads, we mean models that produce audio-visual speech, either physically or on a computer screen, through the control of parameters derived from physiological, behavioral, or other sources. In addition to their use as scientific tools for exploring speech perception and production, and for simulating physiological systems under normal and adverse conditions, these models can be key components in the synthesis and manipulation of virtual personalities. Avatars in cyberspace environments, facially-realistic animated characters, and virtual personalities on our computer, videogame, video and movie screens, and mobile devices are becoming increasingly common. The realism of these simulations will depend, in part, on the incorporation of the relevant knowledge (including physiological, behavioral, and social) in the models of the wide varieties of cyber-entities that will inhabit our future.

\section{$1: 55$}

1pSC2. A physical figure model of lips for speech production. Makoto J. Hirayama (Faculty of Information Sci. and Technol., Osaka Inst. of Technol., 1-79-1 Kitayama, Hirakata 573-0196, Japan, makoto.hirayama@oit.ac.jp)

A physical figure model of lips were made. In previous studies, tongue models consisting of intrinsic and extrinsic tongue muscles were made using viscoelastic material of urethane rubber gel. At this time, as other important speech articulators, upper and lower lips are modeled using urethane rubber gel. Also, a movable jaw model is made from plastic cray. The lips model is placed on the jaw model. As the lips model is made from viscoelastic material similar to human skin, reshaping and moving lips are possible by pulling or moving lips and jaw by hand to emulate speech articulation of lips and jaw. Orofacial muscles such as orbicularis oris, levator labii superioris, levator anguli oris, depressor anguli oris, depressor labii inferioris, and mentalis are considered for lip motions with the figure model. The proposed model is useful for speech science education or a future speaking robot development based on realistic speech production mechanisms.

2:15

1pSC3. Learning from visual speech. Sarah Taylor (Univ. of East Anglia, School of Computing Sci., Norwich, United Kingdom, sarah.hilder@gmail.com), Taehwan Kim, Yisong Yue (Caltech, Pasadena, CA), Ben Milner (Univ. of East Anglia, Norwich, United Kingdom), and Iain Matthews (Disney Res., Pittsburgh, PA)

Realistic animation of faces has proven to be challenging due to our sensitivity in perceiving visual articulation errors. We study the problem of learning to automatically generate natural speech-related facial motion from audio speech which can be used to drive both CG and robotic talking heads with low bandwidth requirements and low latency. A many-to-one mapping from acoustic phones to lip shapes (i.e., static "visemes") is a poor approximation to the complex, context-dependent relationship visual speech truly has with acoustic speech production. We introduced "dynamic visemes" as data-derived visual-only speech units associated with distributions of phone strings and demonstrated they capture context and co-articulation. Further improvement in predicting visual speech can be achieved using an end-to-end deep learning approach. We train a sliding window deep neural network that learns a mapping from a window of phone labels or acoustic features to a window of visual features. This approach removes the requirement for visual units and can very accurately predict visual speech from phone labels or the audio signal directly. We demonstrate the effectiveness of these approaches on CG face models and discuss their application to animatronic heads. 
1pSC4. A robot-based enjoyable conversation system. Tetsunori Kobayashi (Dept. of Comput. Sci., Waseda Univ., 27 Wasedamachi, Shinjuku-ku, Tokyo 162-0042, Japan, koba@waseda.jp)

A robot-based enjoyable conversation system, SCHEMA, is introduced. The first main function of this robot is acting as a facilitator who keeps the balance of engagement density in a group conversation. In a group conversation, sometimes only a particular pair of participants talk each other, and the other particular side participants lose the opportunities to join in the conversation. In terms of total balance of enjoyment of the conversation, this kind of engagement-density-imbalance should be corrected. The proposed robot can find the participants who feel difficulty in joining in a conversation, and give him a chance to join in without destroying existing conversation. The second characteristic function of this robot is generating subjective opinion. In terms of functionality of conversations, it is enough to reply directly to the question that was explicitly asked. However, in our daily conversations, more informative responses are usually employed in order to hold enjoyable conversations. These responses are usually produced as forms of one's additional opinions, which usually contain their original viewpoints as well as novel means of expression, rather than simple and common responses. The proposed robot can select some messages from WEB review site, arrange them, and generate a message as if it is the opinion of one's own. In this talk, we show these functions successfully utilized to achieve enjoyable conversation.

1pSC5. Spoken and non-verbal interaction experiments with a social robot. Jonas Beskow (Speech Music and Hearing, KTH Royal Inst. of Technol., Lindstedtsvägen 24, 5tr, Stockholm 11428, Sweden, beskow@kth.se)

During recent years, we have witnessed the start of a revolution in personal robotics. Once associated with highly specialized manufacturing tasks, robots are rapidly starting to become part of our everyday lives. The potential of these systems is far-reaching; from coworker robots that operate and collaborate with humans side-by-side to robotic tutors in schools that interact with humans in a shared environment. All of these scenarios require systems that are able to act and react in a social way. Evidence suggests that robots should leverage channels of communication that humans understand—-despite differences in physical form and capabilities. We have developed Furhat-a social robot that is able to convey several important aspects of human face-to-face interaction such as visual speech, facial expression, and eye gaze by means of facial animation that is retro-projected on a physical mask. In this presentation, we cover a series of experiments attempting to quantize the effect of our social robot and how it compares to other interaction modalities. It is shown that a number of functions ranging from low-level audio-visual speech perception to vocabulary learning improve when compared to unimodal (e.g., audio-only) settings or 2D virtual avatars.

\section{$3: 15$}

1pSC6. Infant-caregiver interaction is essential for early vocal development. Minoru Asada (Adaptive Machine Systems, Osaka Univ., 2-1 Yamadaoka, Suite, Osaka 565-0871, Japan, asada@ams.eng.osaka-u.ac.jp)

Vocal interactions between an infant and a caregiver facilitates early vocal development, and one of the issues is how an infant learns to vocalize the caregiver's native language. Explanation of this ability of infant is attempted by many theories which advocate imitation based on acoustic matching. However, the infant's and caregiver's speech are quite different in their acoustic qualities. Therefore, it cannot be fully explained by imitation. In this talk, I assume that the interaction itself may have an important role to play, but the mechanism is still unclear. I review studies addressing this topic based on explicit interaction mechanisms using computer simulations and/or real vocal robots. Modeling approaches are classified into four categories: (a) motor control ability develops through self-monitoring of vocalizations, (b) statistical estimation of caregiver's vowel categories from caregiver's vocalizations, (c) self-organization of shared vowels through imitative interaction, and (d) whole dynamics of interactions. The relationships between them are analyzed after a brief review of the early development of an infant's speech perception and articulation based on observational studies in developmental psychology and a few neuroscientific imaging studies. Finally, future issues related to real infant-caregiver vocal interaction are outlined.

3:35

1pSC7. Coordination as a simplifying factor in human-machine communicative interaction. Eric Vatikiotis-Bateson (Univ. of Br. Columbia, 2613 West Mall, Vancouver, BC V6N2W4, Canada, evb@mail.ubc.ca)

Spoken communication has traditionally been treated as a problem of sending and receiving signals containing elements whose sequential organization specifies meaningful content that is the same for both sender and receiver. Unfortunately, successful communication depends on a host of contextualizing factors that are either not present or impossible to identify in short signal streams (by either humans or machines). This presentation focuses on the crucial role of one such factor, the necessary coordination between perceiver and producer, to suggest that human communicative interaction can be conceived more simply than it has been and thereby improve the likelihood of successful human-machine interaction. For example, humans depend on physiological and cognitive coordination for successful interaction, particularly in signal parsing, alignment, and error correction. However, coordination is loosely constrained under most conditions and can be achieved in human-machine interaction without imbuing machines with human attributes; for example, shared attention. In many other cases, machines outperform humans; for example, in processing multimodal speech signals under adverse acoustic conditions to disambiguate the labial viseme, $/ \mathrm{p}, \mathrm{b}, \mathrm{m} /$. In sum, these and other factors provide texture and coherence, rather than daunting complexity, to our efforts to understand spoken communication.

\section{Contributed Paper}


that most of these ideas can actually be traced back to the 18th and 19th centuries, when they were the dominant perspective. Remarkably, early attempts to create speaking robots considered from the outset, and endeavoured to replicate, not only the physics of sound production in realistic vocal tract geometries, but also the sequencing of those geometries over time. This presentation traces the prehistory of gestural synthesis by using unpublished historical documents to analyze the mechanisms used to create the Abbé Mical's Talking Heads, the first programmable speech synthesizer producing the first conversation between machines, and Joseph Faber's Euphonia, the first mechanical synthesizer to transduce timed sequences of symbols into speech. Both of these mechanisms have direct implications for creating 21 st century speaking robots.
Gestural theories of phonology developed in the latter half of the 20th century have proposed that intrinsically timed sequences of linguistically significant actions of the vocal tract are the natural embodiment of speech, in stark contrast to earlier theories that emphasized the role of pure acoustic, auditory or articulatory representations in speech production and perception. Although these recent theories apparently represent a significant change in viewpoint, and have been highly influential, it has been largely forgotten

\title{
Session 1pSPa
}

\section{Signal Processing in Acoustics: Time Delay Estimation of Acoustic Signals with Applications}

\author{
Brian G. Ferguson, Cochair \\ DSTO, PO Box 44, Pyrmont, NSW 2009, Australia \\ Kenta Niwa, Cochair \\ Media Intelligence Laboratories, NTT, 3-9-11, Midori-Cho, Musashino-Shi 1808585, Japan \\ Cliff Carter, Cochair
}

Chair's Introduction-1:30

\section{Invited Papers}

$1: 35$

1pSPa1. Time delay estimation of broadband acoustic signals and its defense applications. Brian G. Ferguson (Maritime Div., DSTG, PO Box 44, Pyrmont, NSW 2009, Australia, Brian.Ferguson@dsto.defence.gov.au), Michael V. Scanlon (US Army Res. Lab., Adelphi, MD), and Jay W. Chang (US Army RDECOM-ARDEC, Picatinny, NJ)

The measurement of the difference in the times of arrival (or time delay) of an acoustic signal at two spatially separated sensors is fundamental to localizing its source. For continuous broadband signals, the time delay is best estimated by the generalized cross-correlation of a pair of sensor outputs. The lag at which the generalized cross-correlation function reaches its maximum value provides an estimate of the time delay. Generalized cross-correlograms are presented for sensor output data recorded at sea on a submarine. The correct peak is readily identified by cross-correlating the conventional (and also the adaptive) beamformer outputs of a pair of widely-spaced arrays, rather than the outputs of two single hydrophones with the same separation distance $(66.3 \mathrm{~m})$. Similarly, the use of various prefiltering methods is shown to suppress secondary (ambiguous) peaks in the cross-correlogram. This approach is then extended to include mechanical and biological underwater acoustic transient signals with the time delay estimates being used to locate the source using the principle of passive ranging by wavefront curvature. Finally, the role of time delay estimation in the localization of aircraft, ground vehicles, and weapon fire using unattended land-based acoustic surveillance systems is demonstrated using real data.

\section{$1: 55$}

1pSPa2. Real-time time difference of arrival estimation for moving wideband sources. Alexander Sedunov (Stevens Inst. of Technol., 711 Hudson St., Hoboken, NJ 07030, asedunov@stevens.edu)

The modified generalized cross-correlation method is used for finding the time difference of arrival (TDOA) and resolving the angle of arrival toward moving targets in real-time. It addresses the decorrelation due to differential Doppler effect leading to relative time companding (RTC) between sensors. The proposed method efficiently approximates the RTC prior to the computation of cross- 
correlation, thus working faster than methods using maximization of the passive ambiguity function and other approximation methods. The algorithm is based on computing the spectrum of deskewed short-time cross-correlator (DSTC) in the frequency domain by averaging a short-term spectrogram computed on overlapped segments of analysis in a time window. The same spectrogram is used to compute magnitude-squared coherence (MSC), then by maximizing the average MSC in the band of interest, the RTC is found. It is used to compute a single inverse Fourier transform. This method is applied to data collected by the Acoustic Aircraft Detection system that was developed at Stevens and that comprises of a volumetric 5-microphone cluster connected to a data acquisition and an embedded computer that processes the data in real-time. Examples demonstrate the improved TDOA finding for aircraft within an airport at a larger distance by using longer analysis time window and maintenance of tracking at close range, thanks to the RTC compensation. [This work was supported by DHS's S\&T Directorate.]

\section{2:15}

1pSPa3. Phase shifts in passive fathometer processing. Zoi-Heleni Michalopoulou (Mathematical Sci., New Jersey Inst. of Technol., 323 ML King Blvd., Newark, NJ 07102, michalop@njit.edu) and Peter Gerstoft (Scripps Inst. of Oceanogr., La Jolla, CA)

In passive fathometer processing, the presence of wavelets/sincs in the estimate of the medium's Green's function corresponds to the location of reflectors in the seabed; the amplitudes of the wavelets point to properties of the reflecting layers. Particle filtering has been successful in tracking reflectors that define layer interfaces from data from the Boundary 2003 Experiment after both conventional and MVDR processing. Further working on the problem, however, revealed that phase shifts may be present in the wavelets of the Green's function, which hinder accurate layer identification if unresolved. In this work, using a process similar to time delay estimation, we identify and model these shifts and demonstrate the significance of their modeling in accurate reflector identification as well as accurate estimation of reflector strengths. [Work supported by ONR.]

\section{2:35}

1pSPa4. Time delay estimation in the presence of source or receiver motion. Brian G. Ferguson and Kam W. Lo (Maritime Div., DSTG, PO Box 44, Pyrmont, NSW 2009, Australia, Brian.Ferguson@dsto.defence.gov.au)

Generalized cross-correlation of the outputs of two spatially-separated sensors provides an estimate of the time delay (or differential time of arrival) of a broadband acoustic signal at the pair of sensors. The time lag at which the cross-correlation function attains its maximum value corresponds to the time delay. However, if relative motion between the source and the sensors leads to the time scales of the received signals being mismatched, then the time delay will be in error. This mismatch in the time scales is also referred to as the differential Doppler effect. Two practical examples are presented that demonstrate this effect, which is observed when a jet aircraft transits over and along the axis of two widely-spaced microphones or a scuba diver travels past two spatially separated hydrophones. It is shown that the correct time delay and relative time scale can be obtained by implementing wideband cross-correlation with differential Doppler compensation using the continuous wavelet transform. The continuous wavelet transform has the same functional form as the more familiar wideband cross-ambiguity function.

1pSPa5. Huge microphone array to maximize mutual information between many microphones and sound sources. Kenta Niwa (Media Intelligence Labs., NTT, 3-9-11, Midori-Cho, Musashino-Shi, Tokyo 1808585, Japan, niwa.kenta@lab.ntt.co.jp)

Hardware technological evolution makes it possible to capture over 100 signals and apply complex processing for, e.g., source enhancement in real time. Assuming that many microphones are available and we want to emphasize a desired sound source located at a distance in noisy environments, how do we place them? In this talk, the principle of spatial sampling to maximize the mutual information between multiple inputs (microphones) and multiple outputs (sound sources) is discussed. The mutual information is commonly used for measuring how much information related to the sound sources is included in many observed signals. In our previous work, we found that it is possible to increase the mutual information by surrounding the microphones with reflectors. Since the effective spatial cues for identifying a desired sound source are included in observed signals, accurate source enhancement would be achieved by applying appropriate mapping functions. Beamforming with/without post-Wiener filtering is used as an implantation of a mapping function. Experiments for emphasizing a desired sound source located 16.5 meters from the constructed microphone array were conducted. Pinpoint pick up of the desired sound source was achieved.

\section{$3: 15$}

1pSPa6. Acoustic distance measurement based on phase interference between transmitted and reflected waves. Masato Nakayama, Takanobu Nishiura (College of Information Sci. and Eng., Ritsumeikan Univ., 1-1-1 Noji Higashi, Kusatsu, Shiga 525-8577, Japan, mnaka@fc.ritsumei.ac.jp), and Noboru Nakasako (Faculty of Biology-Oriented Sci. and Technol., Kindai Univ., Kinokawa, Japan)

The distance to talkers and objects is very important information for both hands-free speech interfaces and nursing-care robots. However, conventional distance measurement methods cannot measure short distances because the transmitted wave, which has not attenuated sufficiently by the time the reflected waves are received, suppresses the reflected waves for short distances. Therefore, we have proposed an acoustic distance measurement (ADM) method based on interference between the transmitted and reflected waves, which can measure distance even in a short range. In this presentation, we explain principle of the ADM method, and various studies. In the proposed method, the arbitrary audible sound with wideband is emitted as the transmitted wave with a loudspeaker, and the transmitted wave is recorded with a microphone. Thus, we can estimate the distance to a target with single loudspeaker and single microphone. Also, the localization of a target can be achieved using single loudspeaker and multiple microphones on basis of the acoustic imaging with microphone-array. In addition, we have improved the ADM method in order to estimate the target distance in noisy environments. Finally, we confirmed the effectiveness of the proposed method through experiments in real environments. 
1pSPa7. Phase DEMON algorithm for time delay estimation used in small boat tracking. Alexander S. Pollara, Alexander Sutin (Maritime Security Ctr., Stevens Inst. of Technol., 1 Castle Point on Hudson, The Babbio Ctr., Hoboken, NJ 07030, apollara@stevens.edu), Kil W. Chung (Maritime Security Ctr., Stevens Inst. of Technol., Gyeonggi-do, Hwaseong-si, South Korea), and Hady Salloum (Maritime Security Ctr., Stevens Inst. of Technol., Hoboken, NJ)

The Detection of Envelope Modulation on Noise (DEMON) algorithm is a widely used tool in underwater passive acoustics for the detection and classification of vessel sound. The DEMON algorithm extracts the frequencies that modulate the high frequency cavitation noise created by a vessel's propeller. We propose an extension of the DEMON method for time delay estimations of acoustic signals received by two or more hydrophones. This method, based on the phase difference between components in the two DEMON spectra received by different hydrophones, allows the extraction of the Time Difference Of Arrival (TDOA) and direction of arrival of the modulated signals. This method was applied to the acoustic signatures of six small boats collected by Stevens in a large glacial lake in NJ and showed agreement with the traditional cross-correlation method of TDOA estimation and boat GPS tracks. This method allows the separation of several boats TDOA. The DEMON algorithm also provides information of potential use for vessel classification. [This work was supported by DHS's S\&T Directorate.]

\section{4:10}

1pSPa8. Measuring low-frequency ocean acoustic coherence with an estimator-correlator. Matthew Dzieciuch (SIO/UCSD, 9500 Gilman Dr., IGPP-0225, La Jolla, CA 92093-0225,mad@ucsd.edu)

Acoustic signals in the ocean are scattered by a variety of processes, but the end result is a partially coherent signal. The signal processing solution for estimating the travel-time of a partially coherent signal is to use an estimator-correlator (EC). An assumption of the EC is that the coherence time and the coherent bandwidth are known. When used properly, the EC leads to in increase in the SNR of the detected signal as well as reducing the width of the detected peak. An interesting question is "Can the EC be used to estimate the coherence parameters by maximizing the signal SNR and minimizing its width?" This is in contrast to the standard method of estimating the signal coherence from the sample covariance of the matched filter output. A simple model is constructed to test this proposition and is compared to experimental results obtained in the Philippine Sea.

$$
4: 25
$$

1pSPa9. Perceptual thresholds of spatial audio update latency in virtual auditory and audiovisual environments. Narayan Sankaran, James Hillis, Marina Zannoli, and Ravish Mehra (Res., Oculus, 8747 148th Ave. NE, Redmond, WA 98052, ravish.mehra@oculus.com)

When observers in a virtual sound environment are in motion relative to a source, the virtual-auditory display must rapidly track the users head position and update the location-cueing acoustic filters-known as head-related transfer functions (HRTFs) - in order to accurately reflect the source's location relative to the current head orientation and position. The end-to-end spatial audio system latency (SASL) is the time elapsed between the listener assuming a position and sound being delivered to the ears with an HRTF corresponding to that same position. To maintain the stability and plausibility of a virtual sound "object," the SASL must lie below a perceptual temporal threshold. The current study sought to rigorously probe the threshold of SASL detectability in human observers at three different rotational head velocities and at various source locations. These conditions were repeated in an audiovisual experiment in which the sound stimulus was now accompanied by a zero-latency visual stimulus. Thus, we characterized SASL thresholds in environments where cross-modal interactions occur due to visual information accompanying sound as is often the case in virtual reality (VR) applications. Initial results reveal a rich interaction between the contextual factors of the virtual environment and listeners' sensitivity to SASL.
1pSPa10. Self-shape estimation algorithm for a flexible ultrasonic array probe. Yoshiaki Nakajima, Kazuhiro Matsui, Takashi Azuma, Etsuko Kobayashi, and Ichiro Sakuma (The Univ. of Tokyo, 7-3-1 Hongo, Bunkyoku, Tokyo 113-8654, Japan, nakajima@bmpe.t.u-tokyo.ac.jp)

A self-shape estimation algorithm for a flexible ultrasonic array probe was described in the paper. Position information of each element in the array is essentially required to achieve a focal control in ultrasonic imaging process. The purpose of this study is to develop an algorithm to estimate array shape without other sensors. In our proposed algorithm, beam image (BI) was used as an evaluation function in the estimation of the shape. BI is an image representing a transmitted beam profile in the imaging object. BI was obtained by scanning of receive focal points around the transmit focal point. The quality factor of BI was used as an evaluation function and parameters describing assumed shapes were searched. We conducted simulation and gel experiments with commercially available flexible probe unit. The algorithm was evaluated by the quality of an estimated self-shape and reconstructed images using an estimated self-shape. The theoretical lateral and depth resolutions were $0.5 \sim 1 \mathrm{~mm}$ and $0.3 \mathrm{~mm}$ in light of transmitted beam profile. The algorithm could have estimated free curve shapes in error by less than $1 \mathrm{~mm}$ and the sizes of imaged wires were $0.74 \sim 1.5 \mathrm{~mm}$ and $0.625 \sim 1.25 \mathrm{~mm}$ in lateral and depth direction.

\section{4:55}

1pSPa11. Analysis of a baffled circular array to avoid ambiguity in detection of arrival estimation. Fabricio A. Bozzi, William S. Filho (Signal Processing, Brazilian Navy Res. Inst., Rio de Janeiro, Brazil), Fernando P. Monteiro, Fabio O. Silva (Acoust. Instrumentation, Brazilian Navy Res. Inst., Rio de Janeiro, Brazil), and Leonardo M. Barreira (Signal Processing, Brazilian Navy Res. Inst., Rua Ipiru n04, Jd Guanabara, Rio de Janeiro, Brazil, barreira@ipqm.mar.mil.br)

The number of sensors present in an array imply in cost, length, weight, and computational complexity. So, it is desired that an array satisfies its project purpose using the minimum of elements. In this study, we analyze the characteristics of a uniform circular array (UCA). Previous studies show the ambiguity problem when working with only few sensors. The grating lobes and potentials ambiguities in uniform linear array are generally avoided limiting the space between sensors in half of the wavelength. In UCA, these problems are solved also by limiting the space between sensors using an adequate number of sensors for a given radius or changing the radius for a given number of sensors. This study shows that it is possible to avoid the grating lobes controlling the sensor's directivity. The directional gain model is used to represent a realistic baffled vertical stave, three hydrophone each, and the Multiple Signal Classification (MUSIC) is applied to detect sources. Simulation results show the accuracy when using directives sensors. This array is analyzed here, working with experimental data, acquired in an acoustic tank.

\section{$5: 10$}

1pSPa12. Acoustic distance measurement based on the interference between transmitted and reflected waves using cross-spectral method by introducing analytic signal of linear chirp sound. Noboru Nakasako, Shinya Honda (Faculty of Biology-Oriented Sci. \& Tech., Kindai Univ., Nishi-Mitani 930, Kinokawa, Wakayama 649-6493, Japan, nakasako@ waka.kindai.ac.jp), Toshihiro Shinohara (Faculty of Biology-Oriented Sci. \& Tech., Kindai Univ., Kinikawa, Nishi-mitani 930, Wakayama, Japan), Masato Nakayama (College of Info. Sci. \& Eng., Ritsumeikan Univ., Kusatsu, Nojihigashi, 1-1-1, Shiga, Japan), and Tetsuji Uebo (Faculty of Biology-Oriented Sci. \& Tech., Kindai Univ., Kinokawa, Nishi-mitani 930, Wakayama, Japan)

The distance to a target is fundamental information in many engineering applications. Recently, an acoustic distance measurement (ADM) method has been proposed based on the interference between transmitted and reflected waves, but it requires two applications of the Fourier transform. The ADM method in which a linear chirp whose frequency changes linearly 
with lapse of time is adopted as a transmitted sound has been also proposed. However, due to the influence of the measuring system from the loudspeaker to the microphone, the ADM would often estimate the spurious short distance different from true distance. This paper describes a fundamental study on the ADM method by applying the cross-spectral method to observed signals of the adjacent two-channel $(2 \mathrm{ch})$ microphones, adopting a linear chirp as transmitted wave and removing the influence of the measuring system. More concretely, though the linear chirp in a time domain corresponds to the frequency spectrum in a frequency domain, it is not a complex signal but a real signal. To apply the cross-spectral method, the analytic signal of linear chirp is introduced. We confirmed the validity of the chirp-based ADM method by performing a computer simulation and by applying it to an actual sound field.

\title{
Session 1pSPb
}

\section{Signal Processing in Acoustics and Speech Communication: Spoken Document Processing}

\author{
John H. L. Hansen, Cochair \\ Jonsson School of Engineering \& Computer Science, CRSS: Center for Robust Speech Systems; UTDallas, 800 W Campbell \\ Road, The University of Texas at Dallas, Richardson, TX 75080-3021 \\ Norihide Kitaoka, Cochair \\ Tokushima University, 2-1 Minami-Johsanjima-cho, Tokushima 770-8506, Japan
}

\section{Invited Papers}

$3: 15$

1pSPb1. Signal processing challenges for reconstructing NASA's Apollo missions to the moon. Douglas W. Oard (College of Information Studies, Univ. of Maryland, College Park, MD 20740, oard@umd.edu) and John H. Hansen (Univ. of Texas at Dallas, Richardson, TX)

During the Apollo Program, NASA recorded dozens of audio channels to document the coordination activity in the Apollo Mission Control Center. We have digitized these recordings for the entire Apollo 11 mission, but several challenges arise when seeking to use these unique recordings for mission reconstruction. One is that 50 of the 56 recorded channels contain headset audio that documents the mix of radio and intercommunication "loops" that an individual flight controller actually heard. Reconstructing the audio that was available for selection therefore requires multi-channel source separation. The same loop might be recorded on multiple time-synchronized channels, and the some known speakers routinely speak only on certain loops, so a considerable amount of side information is available for this source separation task. Second, several additional audio channels are available from different recorders, notably including recordings made aboard the two Apollo spacecraft). Integrating these additional channels requires content alignment techniques, which can leverage both speech content and incidentally recorded non-speech signals. Third, we have only limited metadata for each channel, indicating (for example) which headset audio was intended to be recorded on that channel and who (by name) might have used that deadest. Signal processing techniques can help to enrich that metadata, helping (for example) to recognize speaker changes associated with shift changes or specific mission events.

\section{3:35}

1pSPb2. Video semantic indexing and localization. Koichi Shinoda (Dept. Comput. Sci., Tokyo Inst. of Technol., 2-12-1 W8-81 Okayama, Meguro-ku Tokyo 152-8552, Japan, shinoda@cs.titech.ac.jp)

Nowadays Internet traffic has been largely occupied by consumer video but most of them are not accompanied with text tags for search. Hence, video semantic indexing, which extracts visual concepts such as objects, scenes, and actions directly from video contents, has been intensively studied. Fundamentally, this task consists of two problems: localization and recognition. While until recently these two problems have been studied independently, emerging end-to-end deep learning techniques using convolutional neural networks (CNNs) and recurrent neural networks (RNNs) offer effective ways to solve them simultaneously. These techniques are deeply related to spoken word detection techniques in the speech field. In this talk, we overview the recent progress in this area and discuss potential directions for future research. 
1pSPb3. Challenges and opportunities in spoken document processing: Examples from keyword search and the use of prosody. Andrew Rosenberg (IBM Watson, IBM TJ Watson Res. Ctr., Yorktown Heights, NY 10598, amrosenb@us.ibm.com)

Spoken document processing presents challenges and opportunities when compared to text processing. Speech transcription contains errors, but speech conveys information beyond the words that are said. To deal with errors, a spoken document should be viewed as a structure of viable hypotheses not an absolute transcription. Also, the manner in which words are spoken, their prosody, can be mined for information about the speaker and his or her intent. This talk will use keyword search as an case study that requires operating under errorful and adverse conditions. It will focus on the efforts of the IBM-led LORELEI Consortium during the IARPA BABEL program over the last four and a half years. This period has been a time of rapid change in automatic speech recognition, and the BABEL program has served as a proving ground for a number of innovations including DNNs for acoustic modeling, multi-lingual acoustic features, graphemic (vs. phonemic) recognition, active learning (and reduced resource conditions), morphological analysis, and so-called "end-toend" speech recognition. The talk will then present areas of opportunity to leverage information communicated via prosody to improve spoken document processing including emotion recognition, dialog act classification, and information extraction on spoken documents.

$4: 15$

1pSPb4. Taking advantage of spontaneous speech for document retrieval: Lessons from the organization of evaluation tasks. Tomoyosi Akiba (Dept. of Comput. Sci. and Eng., Toyohashi Univ. of Technol., 1-1 Hibarigaoka, Tenpaku, Toyohashi, Aichi 441-8580, Japan, akiba@cs.tut.ac.jp)

This work reports our experiments on organizing evaluation tasks aiming at spoken document retrieval (SDR). The NTCIR Project is a series of evaluation workshops designed to enhance research in information access technologies by providing reusable test collections and a forum for researchers. The author's group has proposed the evaluation tasks for SDR at the NTCIR from 2010. They resulted in holding the four successive evaluation tasks called SpokenDoc-1 and 2, and SpokenQuery\&Doc-1 and 2. In the SpokenDoc tasks conducted from 2010 to 2013, we designed and evaluated the SDR tasks targeting spontaneously spoken speech. Based on this data collection, we conducted two subtasks: the spoken term detection subtask and the spoken content retrieval subtask. After two rounds of them, we enhanced our research focus with the query part of information retrieval. In the SpokenQuery\&Doc tasks conducted from 2013 to 2016, we investigated the information retrieval systems that take advantage of a spontaneously spoken query as a medium of expressing one's information need. We have collected spontaneously spoken queries through subjective experiment to construct search topics for information retrieval. All of those experiences has been compiled into the reusable test collections provided at the NTCIR project, which enables researchers to test their ideas anytime on the common testbeds. In this presentation, we will report our task design, methodologies and challenges on constructing our data, and outcomes found through our organization.

\section{Contributed Papers}

\section{$4: 35$}

1pSPb5. Prof-Life-Log: Monitoring and assessment of human speech and acoustics using daily naturalistic audio streams. John H. L. Hansen, Abhijeet Sangwan, Ali Ziaei, Harishchandra Dubey, Lakshmish Kaushik, and Chengzhu Yu (Jonsson School of Eng. \& Comput. Sci., CRSS: Ctr. for Robust Speech Systems; UTDallas, 800 W Campbell Rd., The Univ. of Texas at Dallas, Richardson, TX 75080-3021, john.hansen@utdallas.edu)

Speech technology advancements have progressed significantly in the last decade, yet major research challenges continue to impact effective advancements for diarization in naturalistic environments. Traditional diarization efforts have focused on single audio streams based on telephone communications, broadcast news, and/or scripted speeches or lectures. Limited effort has focused on extended naturalistic data. Here, algorithm advancements are established for an extensive daily audio corpus called Prof-LifeLog, consisting of + 80days of 8-16 hr recordings from an individual's daily life. Advancements include the formulation of (i) an improved thresholdoptimized multiple feature speech activity detector (TO-Combo-SAD), (ii) advanced primary vs. secondary speaker detection, (iii) advanced wordcount system using part-of-speech tagging and bag-of-words construction, (iv) environmental "sniffing" advancements to identify location based on properties of the acoustic space, and (v) diarization interaction analysis which highlights the amount of speech each individual produces along with recipient direction. The diarization advancements are evaluated on CRSSUTDallas Prof-Life-Log. Results show improvements in speech activity detection, word count estimation, and environmental sniffing for naturalistic audio streams. The advancements suggest improved speech/language processing algorithms can address the increased diversity in daily audio for speaker knowledge detection/estimation and summarization.
4:50

1pSPb6. The role of consonants in the perception of vocal attractiveness. Emily Blamire (Linguist, Dept. of Linguist, Univ. of Toronto, Sidney Smith Hall, 4th Fl., 100 St. George St., Toronto, ON M5S 3G3, Canada, emily.blamire@mail.utoronto.ca)

Research on vocal attractiveness has identified several acoustic cues intrinsic to vowels (e.g., pitch) that affect judgements of attractiveness and have shown listeners generally agree in their ratings (e.g., Babel, McGuire, $\&$ King 2014). Research in this area has, however, been largely limited to an examination of vowels. The current study builds on past work by examining an array of acoustic cues intrinsic to consonants to ascertain how they affect judgements of vocal attractiveness. Two native speakers of Canadian English were recorded saying one-syllable words of the form $\mathrm{sCVC}$ or $\mathrm{p} / \mathrm{t} /$ $\mathrm{kVC}$. The acoustic cues of center of gravity and duration of $/ \mathrm{s} /$ and voice onset time of $/ \mathrm{p} /, / \mathrm{t} /$, and $/ \mathrm{k} /$ were manipulated to both increase and decrease the value of each cue. Vowel pitch was also included in the analysis as a baseline for comparison with past research. Participants listened to the original and manipulated tokens and were asked to judge vocal attractiveness using a 7-point Likert scale. The data of 32 people (female, $\mathrm{N}=16$ ) aged 19-32 were analyzed, using mixed effects models. The most interesting finding of the current study was that while not all acoustic cues of consonants played a role in perceptions of vocal attractiveness, /s/ duration did, and for this cue both the speaker and listener sex were important to how the cue was perceived. This suggests that not only are male and female voices judged by different standards as to what is attractive, but that men and women are using different criteria when making these judgements. 
5:05

1pSPb7. A speech pre-processing method to reduce overlap masking in reverberant environments. Julian Grosse and Steven van de Par (Acoust. Group, Cluster of Excellence "Hearing4all", Univ. of Oldenburg, Carl-vonOssietzky-Straße 9-11, Oldenburg 26129, Germany, julian.grosse@unioldenburg.de)

In daily life, we are often exposed to speech that is rendered over loudspeakers in a reverberant acoustical environment (examples are publicaddress systems used in a train station or conference halls). Whereas the early reflections can support speech intelligibility, the late reflections smear the speech in time which will result in an overlap of consecutive speech portions and an effective low-pass filtering of the speech-specific modulation spectrum. This study proposes a perceptually motivated pre-processing approach, based on previous work of Hodoshima et al. [J. Acoust. Soc. 119, 4055-4064 (2006)], which reduces the detrimental effects of reverberation by suppressing steady-state portions and emphasizing potentially inaudible/ masked segments of speech before it is emitted in the reverberant environment. For pre-processing, the impulse response is separated into a direct and a reverberant path to decide whether speech segments are inaudible and can be neglected or should be emphasized. A speech intelligibility prediction model is used to select the optimal pre-processing parameters for each specific acoustical environment. Listening tests showed, that this pre- processing approach is able to partially compensate the detrimental effects of reverberation leading to a reduction in speech reception thresholds of about 2 to $5 \mathrm{~dB}$ measured in speech shaped noise.

\section{$5: 20$}

1pSPb8. An effective speech compression based on syllable division. Yan Zhang, Dong Xiao, Qunyan Ren, Shengming Guo, and Fuyuan Mo (Key Lab. of Underwater Acoust. Environment, Inst. of Acoust., Chinese Acad. of Sci., No. 21,BeiSiHuan XiLu, Beijing 100190, China, yudeyinji512233@126.com)

For medium or long distance acoustic speech communication, transmission rate is relatively low due to various disadvantageous factors such as multipath propagation, under such condition, speech compression methods are necessary for effective transmission. A good method needs to retain the characteristics of the speaker and achieve a good compression rate. Considering that the speech signal is usually redundant, a speech pre-compression algorithm based on syllable division is proposed. It can extract the voiced speech and the silence part of the speech signal, and subsequently delete the redundant part that does not affect the semantic comprehension. Through data processing, this pre-compression algorithm is demonstrated can compress the speech signal up to $80 \%$ and not affect listener differentiating or semantic comprehension.

\title{
Session 1pUW
}

\section{Underwater Acoustics and Acoustical Oceanography: Underwater Acoustics Studies in Asian Seas I}

\author{
Chi-Fang Chen, Cochair \\ Engineering Science and Ocean Engineering, National Taiwan University, No. 1 Roosevelt Road Sec.\#4, Taipei 106, Taiwan \\ Ching-Sang Chiu, Cochair \\ Department of Oceanography, Naval Postgraduate School, 833 Dyder Road, Room 328, Monterey, CA 93943-5193
}

Chair's Introduction-1:00

Invited Papers

1:05

1pUW1. The North Pacific Acoustic Laboratory deep-water ocean acoustics experiments in the Philippine Sea: A review. Peter F. Worcester (Scripps Inst. of Oceanogr., Univ. of California, San Diego, 9500 Gilman Dr., 0225, La Jolla, CA 92093-0225, pworcester@ ucsd.edu)

A series of experiments with participants from many institutions investigated deep-water ocean acoustics in the oceanographically and geologically complex Philippine Sea during 2009-2011. These experiments employed various configurations of a newly developed distributed vertical line array (DVLA) receiver. The time fronts recorded on the DVLAs for transmissions from a variety of acoustic sources, both moored and lowered form shipboard, provide a rich data set on acoustic scattering and variability due to internal waves, internal tides, density-compensated temperature and salinity variations (spice), oceanic fronts, eddies, and bottom interaction. During 2010-2011, a water-column-spanning DVLA was embedded within an ocean acoustic tomography array of six transceivers, enabling the observation of acoustic time fronts and measurement of ambient noise over a 5000-m aperture. The observed travel-time time series were similar to time series computed from ocean state estimates made using an eddy-permitting regional ocean model constrained by non-acoustic data, and the travel-time differences have in turn been successfully used to further constrain the model. Acoustic Seagliders also recorded the tomographic transmissions, and the travel times have been used to test the feasibility and accuracy of long-range acoustic navigation (Underwater GPS). Analyses of the data sets collected during the NPAL Philippine Sea experiments are continuing. 
1pUW2. Single-mode field excitation experiment in shallow water. Juan Zeng, Da Yong Peng, Wen Yao Zhao, Hai Feng Li, and Hai Jun Liu (Inst. of Acoust., CAS, No. 21 Bei Si Huan Xi Lu, Beijing 100190, China, zengjuan_ioa@sina.com)

Single-mode field has advantages in active detection, underwater communication, bottom backscattering measurement, etc. Some of papers have reported on the theory and simulation about the single-mode excitation by open- and closed-loop methods, respectively. The experiment in the tank has also been reported many years ago, but the experiment in the water has not been reported yet. In this paper, an effective closed-loop method for exciting the single-mode field is discussed, which can obtain the optimum estimation of the matrix of the Green's function in the shortest feedback times, with high efficiency of emission of the single-mode signal. The experiments at the different sites in different seasons are reported. The vertical source array with the center frequency at $700 \mathrm{~Hz}$ was used as the source in the experiment. The experimental results show that when the source array spans almost the effective water depth the singlemode field with high quality can be excited. The first three single modes were excited at the sites with the water depth of $14 \mathrm{~m}$ and $36 \mathrm{~m}$ respectively. The experimental results show that more than $97 \%$ of the energy of the received field is concentrated in the single-mode.

\section{$1: 45$}

1pUW3. Shallow-water acoustic variability experiment 2015 (SAVEX15) in the northern East China Sea. Hee-Chun Song (Scripps Inst. of Oceanogr., 9500 Gilman Dr., La Jolla, CA 92093-0238, hcsong@mpl.ucsd.edu), Sea-Moon Kim (Korea Res. Inst. of Ships and Ocean Eng., Dajeon, South Korea), Byoung-Nam Kim (Korea Inst. of Ocean Sci. and Technol., Ansan, South Korea), and SungHyun Nam (Seoul National Univ., Seoul, South Korea)

The SAVEX15 experiment was conducted in shallow water ( 100 m deep) in the Northern East China Sea (ECS), 100 km southwest of Jeju Island, South Korea, over the period 14-28 May 2015. The goal of SAVEX15 was to obtain acoustic and environmental data appropriate for studying the coupling of oceanography, acoustics, and underwater communications in the ECS. Surprisingly, an underwater sound channel (USC) typical in deep water was discovered in this shallow water waveguide with the channel axis at $\sim 40 \mathrm{~m}$, apparently due to the cross-frontal interleaving of water masses carried by the Kuroshio Current and Changjiang river discharge. This talk presents an overview of SAVEX15 including preliminary research results.

\section{2:05}

1pUW4. Acoustic experiments over the South China Sea upper-slope sand dunes. Ching Sang Chiu (Dept. of Oceanogr., Naval Postgrad. School, Monterey, CA 93943, chiu@nps.edu), Linus Y. Chiu (Inst. of Undersea Technol., National Sun Yat-sen Univ., Kaohsiung, Taiwan), Chi-Fang Chen (Eng. Sci. and Ocean Eng., National Taiwan Univ., Taipei, Taiwan), Yiing Jang Yang (Inst. of Oceanogr., National Taiwan Univ., Taipei, Taiwan), Ruey Chang Wei (Inst. of Undersea Technol., National Sun Yat-sen Univ., KaoHsiung, Taiwan), Steven R. Ramp (SOLITON Ocean Services, Inc., Monterey, CA), Chris Miller (Dept. of Oceanogr., Naval Postgrad. School, Monterey, CA), D. Benjamin Reeder (Dept. of Oceanogr., Naval Postgrad. School, Honolulu, Hawaii), and Andrea Y. Chang (Asia-Pacific Ocean Res. Ctr., National Sun Yat-sen Univ., Kaohsiung, Taiwan)

A series of experiments were methodically carried out between 2012 and 2014 to study the impact of large underwater sand dunes and the combined impact of these sand dunes and large-amplitude nonlinear internal waves on sound propagation over the upper continental slope including anisotropic propagation characteristics and focussing/defocussing scattering phenomena. The spatial distribution and scales of the sand dunes were first mapped by two multibeam echo sounding (MBES) surveys in 2012 and 2013. The 2013 experiment also provided some coring and initial acoustic transmission data to give useful knowledge of the geoacoustic properties of the sand dunes based on forward propagation modeling and least-squares fitting to the measured levels. The 2014 experiment was more comprehensive, entailing the deployment of autonomous mobile sources, a towed source and a moored source transmitting signals in different frequency bands and different geometries to a vertical line hydrophone array. Our data analysis results, aided with modeling interpretations, have provided physical insights into the observed spatial and temporal propagation characteristics. These results will be highlighted in this presentation. [The research is jointly sponsored by the Taiwan MOST and the US ONR.]

1pUW5. Nonlinear internal wave characteristics during the Sand Dunes 2014 acoustic propagation experiment. Steven R. Ramp (Soliton Ocean Services, Inc., 691 Country Club Dr., Carmel Valley, CA 93924, sramp@solitonocean.com), Yiing Jang Yang (Inst. of Oceanogr., National Taiwan Univ., Taipei, Taiwan), Ching-Sang Chiu (Dept. of Oceanogr., Naval Postgrad. School, Monterey, CA), and Frederick L. Bahr (Monterey Bay Aquarium Res. Inst., Moss Landing, CA)

The Sand Dunes 2014 field experiment in the South China Sea was located in a region frequented by large amplitude (40-80 m) nonlinear internal waves. Four moorings spanning 386-266 m across the slope near $21^{\circ} 52^{\prime} \mathrm{N}, 117^{\circ} 36.5^{\prime} \mathrm{E}$ observed wave arrival patterns during June 3-19, 2014, that were similar to those previously observed nearby consisting of large (a-waves) arriving diurnally with smaller (b-waves) in between, with all amplitudes modulated by the fortnightly tidal beat. Small waves continued to gain amplitude as they shoaled, whereas large waves maxed out near $342 \mathrm{~m}$ then became smaller between 342 and $266 \mathrm{~m}$. The number of waves/packet increased upslope due to wave dispersion. With just one exception, wave breaking and trapped cores were not observed. This contrasts with previous observations to the southwest near Dongsha Island where the wave energies (550 vs. $300 \mathrm{MJ}$ ) and bottom slopes were larger. Most of the incident waves can be attributed to remote forcing in the Luzon Strait, although some b-waves were formed locally due to convergence of the internal tide. A new result was the arrival of double a-waves two hours apart near spring tide on June 16-19. Some possible forcing scenarios for these double a-waves will be presented. 


\section{Contributed Papers}

1pUW6. Analysis of low frequency ocean ambient noise induced by internal wave in sand dune region of northern South China Sea. RueyChang Wei (Inst. of Undersea Technol., National Sun Yat-sen Univ., 70 Lienhai Rd., Kaohsiung City 804, Taiwan, rcwei@mail.nsysu.edu.tw), KuoFeng Chien, Linus Y.S. Chiu (Inst. of Undersea Technol., National Sun Yat-sen Univ., Kaohsiung City, Taiwan), and Chi-Fang Chen (Eng. Sci. and Ocean Eng., National Taiwan Univ., Taipei, Taiwan)

Most powerful internal waves in oceans were found in South China Sea, which were generated in Luzon Strait and mainly driven by tides. In past decades, researches of internal wave's effects on ocean processes have been concentrated on the slope of continental shelf, where transformations of internal waves were significant, so were the induced phenomena. In recent years, large groups of underwater sand dunes were discovered along the upper continental slope, whose formation was speculated to be related to internal wave's energy. In 2014, acoustical and oceanographic moorings were deployed in the sand dune region to study acoustical fluctuations due to the coupled effects of internal waves and sand dunes. Ocean ambient noise data were collected by four SHRUs (Several Hydrophone Recording Unit) and one VLA (Vertical Line Array) at different depths, analysis, and comparison of noise during internal wave events were presented in this study. Low frequency noises were generated by the passing of internal wave, in forms of agitations of sea surface, dramatic fluctuations of water column, and also strumming of mooring structure. Directivity of induced noise helps to identify the mechanism of source, and in addition spatial variation can show the influences from the topography of sand dune.

\section{3:00-3:15 Break}

\section{$3: 15$}

1pUW7. Acoustic propagation in the South China Sea: Internal waves and sand dunes. D. Benjamin Reeder (Dept. of Oceanogr., Naval Postgrad. School, 73 Hanapepe Loop, Honolulu, HI 96825, dbreeder@nps.edu), Andrea Y. Chang (Inst. of Undersea Technol., National Sun Yat-sen Univ., Kaohsiung, Taiwan), Chi-Fang Chen (Eng. Sci. and Ocean Eng., National Taiwan Univ., Taipei, Taiwan), Ching-Sang Chiu (Dept. of Oceanogr., Naval Postgrad. School, Monterey, California), Linus Y. Chiu (Inst. of Undersea Technol., National Sun Yat-sen Univ., Kaohsiung, Taiwan), Chris W. Miller (Dept. of Oceanogr., Naval Postgrad. School, Monterey, California), Steven R. Ramp (SOLITON Ocean Services, Inc., Carmel Valley, CA), Ruey C. Wei (Inst. of Undersea Technol., National Sun Yatsen Univ., Kaohsiung, Taiwan), and Yiing J. Yang (Inst. of Oceanogr., National Taiwan Univ., Taipei, Taiwan)

Very large subaqueous sand dunes were discovered on the upper continental slope of the northeastern South China Sea (SCS) in the spring of 2007 during the ONR 322OA-funded NLIWI Acoustics field experiment. The dunes' formation mechanism is hypothesized to be the internal solitary waves (ISW) which generate from tidal forcing on the Luzon Ridge on the east side of the SCS, propagate west across the deep basin with amplitudes regularly exceeding $125 \mathrm{~m}$, and dissipate large amounts of energy via turbulent interaction with the continental slope, suspending and redistributing the bottom sediment. These internal waves and sand dunes are important acoustical features, as it is expected that they will cause significant anomalies in the acoustical field. Data analysis and modeling are presented to quantify the degree to which these features impact broadband $(850-1200 \mathrm{~Hz})$ signals propagating along an acoustic transect oriented perpendicular to the internal wave fronts and sand dune crests.
1pUW8. Observations of acoustic propagation and geoacoustic inversion affected by subaqueous sand dunes in the South China Sea. Linus Chiu (Inst. of Undersea Technol., No. 70, Lienhai Rd., Kaohsiung 80424, Taiwan, linus@mail.nsysu.edu.tw), Andrea Chang (Asia-Pacific Ocean Res. Ctr., Kaohsiung, Taiwan), Davis B. Reeder, Ching-Sang Chiu (Naval Postgrad. School, Monterey, CA), Yiing-Jang Yang, Chi-Fang Chen (National Taiwan Univ., Taipei, Taiwan), and Chau-Chang Wang (Inst. of Undersea Technol., Kaohsiung, Taiwan)

The large subaqueous sand dunes are expected to affect underwater acoustic propagation. Very large subaqueous sand dunes on the upper continental slope of the northern SCS were discovered in water depths of $160 \mathrm{~m}$ to $600 \mathrm{~m}$, which composed of fine to medium sand. In this talk, serial acoustic experiments conducted by Taiwan and United States in the South China Sea during 2012-2014 are overviewed and mid-frequency propagation data/ model as well as mid-frequency geo-acoustics are aimed. For mid-frequency propagation, results demonstrate that subaqueous sand dune bedforms fluctuate the distinguishable and dispersive mid-frequency acoustical channel; causing the least distinct arrival patterns in the sand dune area. Numerical simulations using broadband modeling given the adequate initial field and Pade term confirm the observations in the experimental data. This talk also presents experiment results of normal incidence survey tracks, and the errors in reflection coefficient estimation and the resulting sediment properties induced by sand dune bedforms. Results demonstrate that the reflected energy is focused and scattered by different parts of sand dune bedforms, and that they produce significant variation in the estimated reflection coefficients and the inverted geoacoustic properties. [This work was supported by the Taiwan MOST and the US ONR.]

\section{$3: 45$}

1pUW9. Modeling study of underwater acoustic propagation over sand dunes in the South China. Stephanie Yang, Chi-Fang Chen (National Taiwan Univ., No. 1, Sec. 4, Roosevelt Rd., Taipei 10617, Taiwan, r03525010@ntu.edu.tw), Ching-Sang Chiu, and Christopher W. Miller (Dept. of Oceanogr., Naval Postgrad. School, Monterey, CA)

There is an extensive field of underwater sand dunes located at depths between $160 \mathrm{~m}$ and $600 \mathrm{~m}$ in the northeastern South China Sea. The largest amplitude of these sand dunes is about $16 \mathrm{~m}$ with horizontal length scales between 200 and $400 \mathrm{~m}$. Underwater acoustic transmission can be affected by these topographic features. Two-dimensional (2-D) and three-dimensional (3-D) underwater acoustic propagation models, RAM and FOR3D, were employed to simulate the acoustic propagation over these sand dunes from a moored source to a vertical-line hydrophone array deployed in an experiment in 2014. Environmental input to the models were the measured bathymetry and sound speed profiles. To prevent model over initializations at steep angles in an ocean with a high-impedance, low-loss bottom, the generalized Gaussian starter was employed and tuned to match the angular beam-pattern of the moored source to give a proper starting field. Simulation results pertaining to the temporal fluctuations, 2-D and 3-D propagation effects in relation to the sand dunes and the water-column variability are presented and discussed. Simulation results are also compared to the measured transmission loss. [This research was supported by both the Ministry of Science and Technology of Taiwan and the Office of Naval Research of the USA.]

\section{4:00}

1pUW10. Influence of internal wave on the space-time interference patterns of broadband acoustic field. Tao $\mathrm{Hu}$, Shengming Guo, $\mathrm{Li} \mathrm{Ma}$ (Inst. of acousitcs, Chinese Acad. of Sci., Beisihuanxi Rd. 21, Beijing 100190, China, hutao76@aliyun.com), and Wenhua Song (Ocean Univ. of China, Qingdao, China)

The frequency shift of the acoustic interference striations is caused by the depth variation of the thermocline in shallow water waveguide. Based on the perturbation theory, a relationship between the frequency shift of the interference striations and the contoured sound speed profile is established. In a given frequency band, if the modal horizontal wave number difference 
acts as a monotonic function of frequency, the frequency shift of interference striations has a direct correlation with the contoured SSP. This relationship is verified by simulation calculation as well as experiment data. It is found that if the propagation direction of sound is parallel to the wave front of internal wave, the frequency shift of the interference striations has a determined relationship with the contoured SSP that passing through the source; while when sound propagates in a direction perpendicular to the internal wave's wave front, the frequency shift is then associated with the contoured mean SSP in the entire sound propagation path.

\section{4:15}

1pUW11. Successive explosive sources for geoacoustic inversion: An attempt of particle filtering. Qunyan Ren, Licheng Lu, Jiawei Li (Key Lab. of Underwater Acoust. Environment, Inst. of Acoust., Chinese Acad. of Sci., No. 21 North 4th Ring Rd., Haidian District, Beijing 100190, China, renqunyan@mail.ioa.ac.cn), Tianjun Liao (State Key Lab. of Complex System Simulation, Beijing, China), Shengming Guo, and Li Ma (Key Lab. of Underwater Acoust. Environment,Inst. of Acoust., Chinese Acad. of Sci., Beijing, China)

This paper presents the results of sequential geoacoustic characterization of ocean bottom in shallow waters from geoacoustic measurement of successive explosive sources. The recording system is a moored vertical line array (VLA) composed of 32 elements, and the bomb sources were successively deployed from a moving ship. The ship straightly sailed at constant speed of 5 knots, and a total number of 15 bombs were deployed with a time interval of 3-min for adjacent bombs. A particle filtering (PF) technique is adapted here to estimate track the spatial distributions of the geoacoustic parameters by filtering the complex pressure fields recorded on the VLA. As for references, independent batch inversion of conducted by Simulated Annealing (SA) algorithm are applied on bombs at different ranges. The general agreement between PF and SA suggests that, the PF is capable of mapping spatial geoacoustic parameter variability in complex ocean environments.

\section{4:30}

1pUW12. Sequential source localization in coastal water under the circumstances of time-evolving sound speed profiles. Lin Su, Shengming Guo, Qunyan Ren, and Li Ma (Inst. of Acoust., Chinese Acad. of Sci., No. 21 North 4th Ring Rd., Haidian District, Beijing 100190, China, sulin807@ mail.ioa.ac.cn)

The sound speed profile (SSP) in water column is highly variable in time and space and can not be accurately known among measurement periods in coastal water. These factors will lead to the modeling mismatch and influence the performance of source localization of matched filed processor (MFP). The possibility of sequential tracking of source range and depth under the circumstances of time-evolving sound speed profiles is discussed. In this paper, we attempt to describe the time evolution characteristics of SSPs by analyzing the oceanographic dynamics which is the physical reason of the SSP's change. A sequential approach considering the oceanographic dynamics model into the state-space equation is developed and applied. Synthetic simulations are carried out with experimental SSP data and acoustic pressure data from a vertical line array. Result suggests the sequential approach based on oceanographic dynamics model can improve the tracking capabilities.

\section{$4: 45$}

1pUW13. A comparison of finite element and hybrid ray-tracing propagation models for shallow water environments. Marcia J. Isakson (Appl. Res. Labs., The Univ. of Texas at Austin, 10000 Burnet Rd., Austin, TX 78713,misakson@arlut.utexas.edu), Jacob George, and David Harvey (Naval Oceanographic Office, Stennis Space Ctr., MS)

It has been found that the bottom loss can be significantly frequency dependent in shallow water waveguides due to scattering from the rough interface and the volume. This can cause transmission loss to increase with frequency for the same shallow water environment. One way of predicting this frequency dependence is through finite element (FE) propagation modeling which solves the Helmholtz equation exactly within the resolution of the discretization for a completely customizable domain. However, finite element models are difficult to compute for high frequencies and large regions. In this work, two hybrid models are considered and compared with the finite element exact solution. The first model uses a bottom loss calculated using FE for a rough interface as input into a ray based model. The second model uses an even simpler approach by characterizing the bottom loss with the Eckart formulation for use in the ray-based model. The performance of these models as a function of frequency and source and receiver geometry will be assessed. If successful, the simple Eckart formulation could be used to characterize the bottom loss for databases since depends on few parameters. [Work sponsored by the Naval Oceanographic Office and ONR, Ocean Acoustics.]

\section{5:00}

1pUW14. Quantifying the spatial variability of low frequency acoustic propagation in the Northern East China Sea. Sungho Cho, Donhyug Kang, Byoung-Nam Kim, Seom-Kyu Jung (Maritime Security Res. Ctr., Korea Inst. of Ocean \& Sci. Technol., 787 Haean-ro(st), Sangnok-gu, Ansan 426-744, South Korea, shcho@kiost.ac.kr), and Jee Woong Choi (Dept. of Marine Sci. and Convergence Eng., Hanyang Univ., Ansan, Korea (the Republic of))

During a period 14-28 May, 2015, the Shallow-water Acoustic Variability EXperiment 2015 (SAVEX15) was made in the northern East China Sea (ECS) to obtain acoustic and oceanographic data for studying the coupling of physical and geophysical parameters, which could affect the variability of acoustic propagation. A strong underwater sound channel (USC) had been existed at depths ranging from 30 to $50 \mathrm{~m}$ with channel axis at $\sim 40 \mathrm{~m}$ during the SAVEX15 period. Two types of mid-range propagation measurements were conducted in shallow water (nominal water depth of $\sim 100 \mathrm{~m}$ ) using simultaneously both continuous waves superimposed at several fixed frequencies below $1.6 \mathrm{kHz}$ and impulsive broadband signals transmitted by sparker system. A vertical line array composed of temperature and pressure sensors was moored for measuring the acoustic signals and vertical sound speed profiles in time. And a marine geophysical survey using a chirp sonar, sparker system, and sediment cores conducted at the experimental site showed that there was a thin surficial layer of $\mathrm{O}(1 \mathrm{~m})$ overlaying multiple layers under the water-seabed interface. The measurement results of transmission loss are presented and compared to the model predictions using the measured oceanographic data. [KIOST (Project Code No. PE99331).]

\section{$5: 15$}

1pUW15. Range estimation of a broadband source using the array/ waveguide invariant and a vertical array. Hee-Chun Song and Chomgun Cho (Scripps Inst. of Oceanogr., 9500 Gilman Dr., La Jolla, CA $92093-$ 0238,hcsong@mpl.ucsd.edu)

During a recent shallow-water experiment conducted in the northern East China Sea (SAVEX15), a low-frequency (0.5-2 kHz) source (SeaNos) was towed at the mid-depth $(\sim 50 \mathrm{~m})$, being close to the channel axis of the underwater sound channel (USC) observed unexpectedly in a shallow water waveguide environment. It provided a variety of distances and propagation directions between the source and two moored vertical line arrays (VLAs). The channel impulse responses from the SeaNos indicate that the earlier reflected and refracted arrivals from the USC are followed by surface/bottom reflected arrivals. The later reflected arrivals are utilized for source-range estimation using the array/waveguide invariant based on the beam-time migration and the VLAs. 


\section{Exhibit}

The instrument and equipment exhibit is located near the registration area in the Coral Foyer.

The Exhibit will include computer-based instrumentation, scientific books, sound level meters, sound intensity systems, signal processing systems, devices for noise control and acoustical materials, active noise control systems and other exhibits on acoustics.

Exhibit hours are Monday, 28 November, 5:30 p.m. to 7:00 p.m., Tuesday, 29 November, 9:00 a.m. to 5:00 p.m., and Wednesday, 30 November, 9:00 a.m. to 12:00 noon.

Coffee breaks on Tuesday and Wednesday mornings will be held in the exhibit area as well as an afternoon break on Tuesday.

The following companies have registered to participate in the exhibit at the time of this publication:

AIP Publishing: publishing.aip.org/

American Institute of Physics: www.aip.org/

Aqua Sound, Inc.: aqua-sound.com

Echoview Software: www.echoview.com/

Head acoustics GmbH: www.head-acoustics.de/eng/

Mason Industries: www.mason-industries.com/masonind/

Ocean Sonics Ltd.: oceansonics.com/

ODEON A/S: www.odeon.dk/

PAC International: www.pac-intl.com/

RION Co., Ltd: www.rion.co.jp/english/

Sensidyne: www.sensidyne.com/

Springer: www.springer.com/us/

Teledyne RESON Inc.: www.teledyne-reson.com/ 
1eMU

\title{
Musical Acoustics: Special Presentation of the History, Culture, Practice, and Performance of Hawai'ian Music
}

\author{
Andrew C. Morrison, Chair \\ Natural Science Dept., Joliet Junior College, 1215 Houbolt Rd., Joliet, IL 60431
}

Chair's Introduction-7:00

\section{Invited Paper}

7:05

1eMU1. Ho'olono i ka Leo o ka Makani (Hear the Voice of the Wind). Keawe Lopes, Puakea Nogelmeier, Jon Osorio (School of Hawaiian Knowledge, Univ. of Hawaii, Manoa, Spalding Hall, Honolulu, HI 96822, rlopes@hawaii.edu), and Aaran Salā (Univ. of Hawaii, Windward Community College, Kāne‘ohe, HI)

This is a performance of oli, mele, and hula that explores the wide range of chant, song, and dance that Kanaka Maoli have both created and adopted to forge the unique and celebrated music of Hawaii. Scholar/musicians Keawe Lopes, Puakea Nogelmeier, Jon Osorio, and Aaron Sala combine their diverse talents, musical experience and love for the language in this presentation of music, dance, and conversation. Our music is in our nature. Ho'olono i ka leo o ka makani. 


\title{
Session 2aABa
}

\section{Animal Bioacoustics: Tropical Bioacoustics I}

\author{
Thomas F. Norris, Cochair \\ Bio-Waves, Inc., 517 Cornish Dr., Encinitas, CA 92024 \\ Tomonari Akamatsu, Cochair \\ Fisheries Research Agency, 7620-7, Hasaki, Kamisu, Ibaraki 314-0408, Japan
}

Chair's Introduction-8:00

Invited Papers

8:05

2aABa1. Beaked whale species occurrence in the central Pacific and their relation to oceanographic features. Simone BaumannPickering, Anne E. Simonis, Jennifer S. Trickey (Scripps Inst. of Oceanogr., Univ. of California San Diego, 9500 Gilman Dr., La Jolla, CA 92093, sbaumann@ucsd.edu), Marie A. Roch (Dept. of Comput. Sci., San Diego State Univ., San Diego, CA), and Erin M. Oleson (Pacific Islands Fisheries Sci. Ctr., National Oceanic and Atmospheric Administration, Honolulu, HI)

Mesoscale oceanographic features are a major force in structuring the marine environment through processes such as eddy-induced upwelling, and as such effect distribution and aggregation patterns of all organisms along the food chain. It has been shown that top pelagic predators such as cetaceans react to these environmental changes in different ways. We present analysis of frequency-modulated (FM) echolocation pulses of Cuvier's beaked whale (Ziphius cavirostris), Blainville's beaked whale (Mesoplodon densirostis), and an unknown beaked whale species producing FM pulse type "BWC," possibly ginkgo-toothed beaked whale (M. ginkgodens), at five locations in the central Pacific. The recordings were collected at Pearl and Hermes Reef (Northwestern Hawaiian Islands), Kona (Main Hawaiian Islands), Wake Atoll, Tinian, and Saipan (Northern Mariana Islands) between 2008 and 2015, ranging from 4 to 8 years per site. All three beaked whale species were present at all sites in different proportions throughout the recording periods, with a strong nocturnal pattern only for the "BWC" pulse type, yet without seasonal pattern. We examine the varying presence in the context of remotely sensed oceanographic data, including sea surface height deviation, temperature, and salinity, as well as chlorophyll $a$ and derived primary productivity.

2aABa2. Echolocation systems of leaf-nosed bats in Taiwan and Iriomote island, Japan. Hiroshi Riquimaroux (SDU-VT Int. Lab., Shandong Univ., 27 Shanda Nanlu, Jinan, Shandong 250100, China, hiroshi_riquimaroux@brown.edu)

The leaf-nosed bats, Hipposideridae, are one of the most common tropical bats found in Asia. Two species of leaf-nosed bats in Iriomote island, Hepposideros turpis, and Taiwan, Hipposideros terasensis, were compared. They emit short echo location pulses (4-7 ms in duration) consisted of constant frequency (CF) component followed by downward frequency modulated (FM) component. The second harmonics are the most intense frequency component. Comparison lists for two species are shown below. Taiwanese leaf-nosed bats, Hipposiderous terasensis CF2: $70 \mathrm{kHz}(\mathrm{CF} 1: 35 \mathrm{kHz})$ 50-60 g in weight Japanese leaf-nosed bats, Hepposideros turpis CF2: $82 \mathrm{kHz}$ (CF1: $41 \mathrm{kHz}$ ) 20-30 g in weight threatened species (Red list). Both Taiwan and Iriomote island are at around the same North latitude 25 degrees. Findings from H. terasensis and H. turpis are introduced and discussed. [Research supported by MEXT, Japan, and Shandong University, China.]

\section{$8: 45$}

2aABa3. Melody in my head, melody in my genes? Acoustic similarity, individuality, and genetic relatedness in the indris of Eastern Madagascar. Marco Gamba, Valeria Torti, Giovanna Bonadonna (Life Sci. and Systems Biology, Univ. of Torino, Via Accademia Albertina 13, Torino 10123, Italy, marco.gamba@unito.it), Rose M. Randrianarison (Groupe d'Etude et de Recherche sur les Primates de Madagascar, Antananarivo, Madagascar), Olivier Friard, and Cristina Giacoma (Life Sci. and Systems Biology, Univ. of Torino, Torino, Italy)

Indris (Indri indri) are the only singing lemurs and produce different types of songs that can be differentiated according to their temporal patterns. The most distinctive portions of the songs are "descending phrases" consisting of 2-5 units. In our study, indri songs were recorded in the Eastern rainforests of Madagascar from 2005 to 2015. All the recordings were made when the recorder operator was in visual contact with the singing social group and by recognizing individual indris using natural markings. Because the individual songs frequently overlap during the chorus, we extracted the pitch contour of 1084 descending phrases using the program PRAAT. We tested whether the structure of the phrases could provide conspecifics with information about sex and individual identity. We also examined 
whether the structure of the phrases was related to the genetic relatedness of the indris. The results suggest that the songs have consistent sex-, group-, and individual-specific features. We have also found a significant correlation between the genetic distance and the acoustic similarity. The descending phrases may be used by the indris to convey information of sex and identity, and genetic relatedness may play a role in determining song similarity to a larger extent than previously assumed.

\section{9:05}

2aABa4. Recent bioacoustics researches on biosonar, hearing, and noise effect on the Indo-Pacific humpback dolphins (Sousa chinensis). Songhai Li (Inst. of Deep-Sea Sci. and Eng., Chinese Acad. of Sci., 28 Luhuitou Rd., Sanya 572000, China, LISH@IDSSE. AC.CN) and Ding Wang (Inst. of Hydrobiology, Chinese Acad. of Sci., Wuhan, China)

The Indo-Pacific humpback dolphins (Sousa chinensis) are widely distributed in the coastal waters of the tropical and sub-tropical ocean in Asia, including southeast China. Conservation of the humpback dolphins in Chinese waters has been on the agenda of local scientific and conservation communities since the 1980s, despite little research including bioacoustics research has been conducted. Our recent bioacoustics studies indicated that the biosonar clicks of the wild humpback dolphins were short-duration, broadband, ultrasonic pulses, similar to those produced by other whistling dolphins of similar body size. However, their click source levels with an average of around $190 \mathrm{~dB}$ re: $1 \mu \mathrm{Pa}$ in peak-to-peak, appear to be much lower than those of other whistling dolphins. Hearing sensitive frequency range of the humpback dolphins is generally higher than $5 \mathrm{kHz}$ and lower than $120 \mathrm{kHz}$ with possible age-related hearing loss for old dolphins. The humpback dolphin could therefore be characterized as a mid-frequency cetacean, which operates sounds in mid- to highfrequency range. Any sufficiently intense sounds with mid- to high-frequency components could have deleterious effects on the humpback dolphins through interference on animals' behavior and with the animals' ability to detect signals from conspecifics, and echoes of echolocation clicks.

9:25

2aABa5. Acoustic response of Indo-Pacific humpback dolphins to the variability of marine soundscape. Tzu-Hao H. Lin (Res. Ctr. for Information Technol. Innovation, Academia Sinica, No. 1, Sec. 4, Roosevelt Rd., Taipei 10617, Taiwan, schonkopf@gmail.com), Chih-Kai Yang, Lien-Siang Chou (Inst. of Ecology and Evolutionary Biology, National Taiwan Univ., Taipei, Taiwan), Shih-Hau Fang (Dept. of Elec. Eng., Yuan Ze Univ., Taoyuan, Taiwan), and Yu Tsao (Res. Ctr. for Information Technol. Innovation, Academia Sinica, Taipei, Taiwan)

Marine mammals can adjust their vocal behaviors when they encounter anthropogenic noise. The acoustic divergence among different populations has also been considered as the effect of ambient noise. The recent studies discover that the marine soundscape is highly dynamic; however, it remains unclear how marine mammals alter their vocal behaviors under various acoustic environments. In this study, autonomous sound recorders were deployed in western Taiwan waters between 2012 and 2015. Soundscape scenes were unsupervised classified according to acoustic features measured in each $5 \mathrm{~min}$ interval. Non-negative matrix factorization was used to separate different scenes and to inverse the temporal occurrence of each soundscape scene. Echolocation clicks and whistles of Indo-Pacific humpback dolphins, which represent the only marine mammal species occurred in the study area, were automatically detected and analyzed. The preliminary result indicates the soundscape scenes dominated by biological sounds are correlated with the acoustic detection rate of humpback dolphins. Besides, the dolphin whistles are much complex when the prey associated scene is prominent in the local soundscape. In the future, the soundscape information may be used to predict the occurrence and habitat use of marine mammals.

\section{Contributed Papers}

\section{9:45}

2aABa6. Navigating the soundscape: The role of acoustic cues in the settlement behavior of larval reef fishes. Andria K. Salas (Integrative Biology, Univ. of Texas at Austin, Austin, TX), Preston S. Wilson, Megan S. Ballard (Mech. Eng. and Appl. Res. Labs., Univ. of Texas at Austin, 1 University Station C2200, Austin, TX 78713, pswilson@mail.utexas.edu), Andrew H. Altieri (Smithsonian Tropical Res. Inst., Panama City, Panama), and Timothy H. Keitt (Integrative Biology, Univ. of Texas at Austin, Austin, TX)

Nearly all coral reef fishes pass through a pelagic larval stage that concludes when they locate and select appropriate reef habitat. At least some species use the reef soundscape- the collection of sounds produced by reefdwelling organisms - to achieve this task that is necessary for survival. Since larval fishes in the open ocean are inherently difficult to study, investigating their acoustic environment can allow predictions about their behavior in response to these cues. Further, we can predict the roles of different frequency bands in aiding long-distance navigation or short-distance habitat selection. We recorded the soundscapes at four reefs in Caribbean Panama for six weeks to inform the identity and variability of the most common sounds. We next predict the frequency-dependent transmission loss using an acoustic propagation model calibrated with acoustic transects. Including knowledge of source sound levels allows us to elucidate the sound fields created by low frequency fish and high frequency invertebrate sounds originating at the reef. We find that larval fishes are likely to encounter highly complex acoustic environments as a result of frequency-dependent acoustic structure in the water column coupled with temporal and spatial variation in the sources of the potential cues.

\section{0:00-10:15 Break}

\section{0:15}

2aABa7. Macro- and micro-scale spatial variation in the acoustic activity of snapping shrimp on coral reefs in the Pacific. Eden Zang, Marc Lammers (Oceanwide Sci. Inst., 3620 Baldwin Ave, Ste. 206-B, Makawao, HI 96768, ezang@oceanwidescience.org), Max Kaplan, T. A. Mooney (Woods Hole Oceanographic Inst., Woods Hole, MA), Pollyanna FisherPool, and Russell E. Brainard (NOAA Fisheries, Honolulu, HI)

Coral reef soundscapes are increasingly becoming recognized as critical factors in the study of reef dynamics, from the role they play in larval recruitment to the assessment of coral reef biodiversity and ecosystem stability. Snapping shrimp produce the predominant source of sound on most coral reefs at frequencies between 2 and $20 \mathrm{kHz}$. Their activity is influenced by a variety of abiotic factors. As such, coral reef soundscapes are prone to considerable flux and variation. However, this variation is still poorly understood on a variety of spatial and temporal scales, making it difficult to draw meaningful comparisons between the soundscapes of different reefs. We report on an effort to quantify the acoustic activity of snapping shrimp across 12 coral reef sites in the Pacific Ocean separated by distances ranging from hundreds of meters to thousands of kilometers, including reefs across the Hawaiian archipelago, the Northern Mariana Islands, and American 
Samoa. We use data obtained from long-term, bottom-moored acoustic recorders to document the variability in snapping shrimp activity observed on multiple temporal scales and examine factors correlated with this variability at each location.

\section{0:30}

2aABa8. A new baleen whale call recorded in the Mariana Trench Marine National Monument. Sharon L. Nieukirk, Selene Fregosi, David K. Mellinger (Cooperative Inst. for Marine Resources Studies, Oregon State Univ., 2030 SE Marine Sci. Dr., Newport, OR 97365, sharon.nieukirk@oregonstate.edu), and Holger Klinck (BioAcoust. Res. Program, Cornell Lab of Ornithology, Cornell Univ., Ithaca, NY)

In fall 2014 and spring 2015, passive acoustic data were collected via autonomous gliders east of Guam in an area that included the Mariana Trench Marine National Monument. A short (2-4 s), complex, unknown sound was recorded. The call began with a brief $0.4 \mathrm{~s}$ long tone centered at $60 \mathrm{~Hz}$, followed by a $2 \mathrm{~s}$ low-frequency moan that sweep from $44-30 \mathrm{~Hz}$. The moan appeared to have both amplitude modulation and strong harmonics. The moan was followed by two $\sim 60-150 \mathrm{~Hz}$ upsweeps lasting $0.5 \mathrm{~s}$ that often had diffuse energy up to $1000 \mathrm{~Hz}$. The call ended with metallic-sounding upsweeps with most energy between 700 and $800 \mathrm{~Hz}$, but up to $7.5 \mathrm{kHz}$. These sounds were recorded regularly during both fall (326 calls during 38 glider dives) and spring (110 calls during 16 glider dives). Many components were not visible in low signal to noise ratio calls. Calls were typically 5-6 minutes apart and often occurred in long sequences. Aurally, the sound is quite unusual and most resembles the minke whale "Star Wars" call. We propose this sound is biological and is likely produced by a baleen whale.

\section{0:45}

2aABa9. Passive acoustic monitoring of Pygmy blue whales (Balaenoptera musculus brevicauda) in Southern Sri Lanka. Annukka Pekkarinen (Maritime Environ. Res. Group, World Maritime Univ., PO Box 500, Malmö 20124, Sweden, ap@wmu.se), Yeelen Olive (VisioTerra, Champs Sur Marne, Paris, France), and Marianne H. Rasmussen (Univ. of Iceland, Husavik, Iceland)

Two ecological acoustic recorders were deployed in Southern Sri Lanka to study the presence of the assumed resident population of pygmy blue whales. A major shipping lane runs through this feeding area of the whales and it has been recognized by the International Whaling Commission as well as International Maritime Organization as a risk area for ship strikes. To implement any management practices to mitigate ship strikes, a solid scientific basis is needed. Such data must include information on migration and movement patterns of the whale population. Therefore, combining the passive acoustic monitoring data with existing visual transect survey results gives further insight to choosing suitable management practices to address the issue. Passive acoustic monitoring data indicate that at least part of the population is indeed resident in the area throughout the year but there is both seasonal and inter-annual variation in the acoustic activity of the whales. The acoustic data were further combined with data from oceanographic models and the results indicate there is no single parameter explaining the variation, although the acoustic activity has weak correlation with rainfall patterns. The pygmy blue whale call detections also appear to increase during periods of stronger upwelling in Southern Sri Lanka.

\section{1:00}

2aABa10. Spatiotemporal variation in habitat utilization of humpback dolphins (Sousa chinensis) potentially affected by freshwater discharge. Chia-Yun Lee, Tzu-Hao Lin (Inst. of Ecology and Evolutionary Biology, National Taiwan Univ., Taiwan, No. 1, Sec. 4, Roosevelt Rd., Taipei 10617, Taiwan, janiceli0918@gmail.com), Tomonari Akamatsu (Fisheries Res. Agency, National Res. Inst. of Fisheries Sci., Kanagawa, Japan), and LienSiang Chou (Inst. of Ecology and Evolutionary Biology, National Taiwan Univ., Taiwan, Taipei, Taiwan)

Dynamics of habitat utilization of marine mammals are important for the conservation management of coastal ecosystems. Previous studies have shown that seasonal changes of humpback dolphin distribution are associated with seasonal changes of upstream rainfall. However, temporal variations of dolphin behavior at an estuarine habitat remain unclear. In this study, echolocation clicks of humpback dolphins were recorded at the Xinhuwei River Estuary, western Taiwan, between July 2009 and November 2015. Sound source bearing angles to identify different sound sources, intensity, and inter-click intervals (ICIs) as an index of the sensing distance were measured to investigate the sensing and movement of dolphin groups. Humpback dolphins searched for shorter sensing distances and moved back and forth near the estuary during spring and summer, which suggests foraging-related behaviors were much frequently observed during wet seasons. However, humpback dolphins changed to focus on longer detection ranges (ICIs changed from 40 to $50 \mathrm{~ms}$ to 50 to $70 \mathrm{~ms}$ ) and move in straight forward when upstream rainfall increased during this period. The present result indicated the dynamics of habitat utilization of humpback dolphins at an estuary is likely driven by the distribution and abundance of prey resource which is influenced by the temporal change of river runoff.

\section{1:15}

2aABa11. Source level of fin whale calls from the Equatorial Pacific Ocean. Jennifer L. Miksis-Olds (School of Marine Sci. \& Ocean Eng., Univ. of New Hampshire, PO Box 30, Mailstop 3510D, State College, PA 16804,j.miksisolds@unh.edu)

Knowledge of source levels is essential for determining the range of effective fin whale communication and for estimating fin whale density from passive acoustic recordings. Source level calculations were performed on vocalizations automatically detected from spectrograms of Comprehensive Nuclear Test-Ban Treaty Organization data recorded at Wake Island in the Equatorial Pacific Ocean. Received levels were calculated, and transmission loss (TL) was determined using a season- specific OASIS Peregrine parabolic equation model for a $20 \mathrm{~Hz}$ signal. The model incorporated the location of the sensor in the deep sound channel, bathymetry of the area, and local sound speed profiles. TL was modeled for 360 bearings with a $1^{\circ}$ resolution. TL values between the sensor and source were found for individual vocalizations using ranges and bearings calculated through hyperbolic localization. The exact whale depths were unknown but assumed to be 50 $\mathrm{m}$. Over 7500 localizations of the fin whale $20 \mathrm{~Hz}$ call were identified from 2007 to 2009 with an average source level of $184.8 \mathrm{~dB}+/-8.6 \mathrm{~dB}$. When the 7500 calls were subsampled at 6 hour intervals to reduce the bias of loud, persistent singers, the average source level was $168.5 \mathrm{~dB}+/-4.4 \mathrm{~dB}$ $(\mathrm{n}=174)$. [Work supported by ONR.] 


\title{
Session 2aABb
}

\section{Animal Bioacoustics and Signal Processing in Acoustics: Anthropogenic Transient Noise Sound Field and Its Effects on Animal Communication I}

\author{
Shane Guan, Cochair \\ Office of Protected Resources, National Marine Fisheries Service, 1315 East-West Highway, SSMC-3, Suite 13700, Silver \\ Spring, MD 20902 \\ Satoko Kimura, Cochair \\ Field Science Education and Research Center, Kyoto Univ., Kyoto, Japan
}

Chair's Introduction-8:45

Invited Papers

8:50

2aABb1. Quantitative measurements of seismic airgun reverberation in the shallow-water Beaufort Sea. Aaron Thode (SIO, UCSD, 9500 Gilman Dr., MC 0238, La Jolla, CA 92093-0238, athode@ucsd.edu), Melania Guerra (APL, Univ. of Washington, Seattle, WA), Susanna Blackwell (Greeneridge Sci., Santa Barbara, CA), and A. Michael Macrander (Shell Exploration and Production Co., Anchorage, AK)

Shallow-water airgun survey activities off the North Slope of Alaska generate impulsive sounds that have been the focus of much regulatory attention. Reverberation from repetitive airgun shots, however, also increases the background noise levels, which can decrease the detection range of nearby passive acoustic monitoring (PAM) systems and potentially mask communication signals between animals. Typical acoustic metrics for impulsive signals provide no quantitative information about reverberation. Two metrics are suggested here for quantifying reverberation: a "minimum level" metric that measures reverberation levels between airgun pulse arrivals, and a "reverberation metric" that estimates the relative magnitude of reverberation vs. expected ambient levels in the hypothetical absence of airgun activity, using satellite-measured wind data. The metrics were applied to acoustic data measured by autonomous recorders in the Alaskan Beaufort Sea in 2008. They demonstrate how a $3000 \mathrm{cu}$. inch seismic survey in $50 \mathrm{~m}$ deep water can increase the background noise over natural ambient levels by $30-45 \mathrm{~dB}$ within $1 \mathrm{~km}$ of the activity, by $10-25 \mathrm{~dB}$ within $15 \mathrm{~km}$ of the activity, and by a few $\mathrm{dB}$ at $128 \mathrm{~km}$ range. Shallow-water reverberation can thus substantially reduce the performance of PAM systems several kilometers of shallow-water seismic surveys. Other impulsive activities such as pile driving may face similar issues.

9:10

2aABb2. Inter-pulse sound field from a marine seismic survey in the Arctic and its potential effects on marine mammal acoustic masking. Shane Guan (Office of Protected Resources, National Marine Fisheries Service, 1315 East-West Hwy., SSMC-3, Ste. 13700, Silver Spring, MD 20902, shane.guan@noaa.gov) and Joseph F. Vignola (Dept. of Mech. Eng., The Catholic Univ. of America, Washington, DC)

It has been documented that reverberant fields from marine seismic surveys elevate sound levels, and thus has the potential to mask acoustic signals that are important to marine mammals. In this study, we investigated one-third-octave (OTO) bands of airgun inter-pulse sound fields from a shallow water $(<15 \mathrm{~m})$ seismic survey in the Beaufort Sea. The results show that at close distances less than $3 \mathrm{~km}$, peak energy of the OTO band levels were concentrated around $80-110 \mathrm{~Hz}$ and the median sound levels were $40-50 \mathrm{~dB}$ above ambient. The higher frequency components $(>3 \mathrm{kHz}$ ) of inter-pulse sound field at these close distances ranged 5-20 dB above ambient. At distances beyond $3 \mathrm{~km}$, the received acoustic energy becomes more uniform up to $1000 \mathrm{~Hz}$. The median OTO band levels at these distances were approximately 10-20 dB above ambient. For higher frequencies, sound levels at these distances were approximately the same as ambient. These results imply that low-frequency bowhead whales could be affected by acoustic masking from seismic airgun inter-pulse sound fields, especially at relatively close distances. However, potential acoustic masking effects to mid-frequency beluga whale echolocation signals are expected to be less profound. 
2aABb3. Long range airgun inter-pulse noise field in offshore northern Australian waters. Craig McPherson (JASCO Appl. Sci. (Australia) Pty, Ltd., Unit 4, 61-63 Steel St., Capalaba, QLD 4157, Australia, craig.mcpherson@jasco.com), Bruce Martin (JASCO Appl. Sci. (Canada) Ltd., Dartmouth, NS, Canada), Michael Wood (JASCO Appl. Sci. (UK) Ltd., Droxford, Hampshire, United Kingdom), and Alexander MacGillivray (JASCO Appl. Sci. (Canada) Ltd., Victoria, BC, Canada)

Elevated ambient noise levels have been shown to affect marine fauna communication. The effects due to increased noise levels from the multipath propagation and reverberation of airgun pulses over large distances is a relatively new area of interest. This research examines a distant seismic survey opportunistically recorded at two locations during a long-term monitoring program in the Timor Sea, Australia. During the analyzed period, the survey was between 168 and $235 \mathrm{~km}$ from the Autonomous Multichannel Acoustic Recorders (AMAR). The noise levels between airgun pulses were examined using an incremental computation method based on calculating the root-mean-square sound pressure level in $125 \mathrm{~ms}$ sub-intervals. These were compared to ambient noise levels from the month prior to commencement of the seismic survey and during line turns. The received pulse levels and length differences, and therefore inter-pulse noise field, between the recorders were greater than expected given the proportional distance to source difference. The ambient soundscape included biological contributors such as Omura's whale, fish and benthic crustaceans. Propagation modeling was conducted to examine the effect of the propagation path between source and receiver on the received signals.

\section{9:50}

2aABb4. Influence of the ocean-bottom structure in the long-distance propagation by seismic survey source. Toshio Tsuchiya (Japan Agency for Marine-Earth Sci. and Technology/Tokyo Univ. of Marine Sci. and Technol., 2-15 Natsushimacho, Yokosuka, Kanagawa 2370061, Japan, tutiyat@gmail.com), Shinpei Gotoh, Yukino Hirai, and Etsuro Shimizu (Tokyo Univ. of Marine Sci. and Technol., Koto-ku, Tokyo, Japan)

A source of Multi-Channel Seismic survey system (MCS) is the air-gun array which generates a high level and short pulse sound. However, there was anxiety which has an influence on behavior of a marine mammal by very high acoustic pressure. Therefore, JAMSTEC have decided guidance of regulation along a guideline of NOAA (TTS) in safe purpose of a marine mammal. We'll hope for a MCS investigation by a new JAMSTEC R/V "KAIMEI" (5,500GT) in the Hawaii Islands offing jointly with an U.S. research institute by geological interest in the future. So, long-distance propagation (about $100 \mathrm{~km}$ ) from air gun source was calculated by a Parabolic Equation simulation for safety ensuring of marine mammals. The simulation results are as follows: 1) When the ocean-bottom of the long-distance transmission path is basalt, for a reflected pulses to pile up from the bottom in various courses, a received pulse width becomes very long. 2) The received level was lower than the TTS level in the watch possible distance from the marine mammal watch room installed in R/V “KAIMEI." 3) However, when the low restriction level (the behavior obstruction level) was applied for marine mammal protection in the future, we have to lower the air gun transmission level substantially.

\section{0:10-10:25 Break}

\section{0:25}

2aABb5. An attempt to estimate ship noise effect on humpback whales in Japan. Tomonari Akamatsu (National Res. Inst. of Fisheries Sci., Fisheries Res. Agency, 7620-7, Hasaki, Kamisu, Ibaraki 314-0408, Japan, akamatsu@affrc.go.jp), Ryosuke Okamoto (Ogasawara Whale Watching Assoc., Ogasawara, Japan), Kyoichi Mori (Teikyo Univ. of Sci., Uenohara, Yamanashi, Japan), Yoko Mitani, Kouki Tsujii (Hokkaido Univ., Hakodate, Japan), Toshio Tsuchiya (JAMSTEC, Yokosuka, Japan), Takahiro Kijima (Ministry of Land, Infrastructure, Transport and Tourism, Tokyo, Japan), and Naoya Umeda (Osaka Univ., Osaka, Japan)

Ocean noise pollution is getting to be a major issue for the environmental assessment of maritime transportation and engineering. Evidences to estimate the effect of noise on marine creatures are urgently required. A Japanese team consist of government agency, universities, and research institutions launched a new project to observe possible effects of ship noise on humpback whales in Ogasawara archipelago. Radiated noise from a ship was measured according to the ISO standard protocol in deep water. The position and operational conditions of the ship during the daily voyages were precisely monitored onboard. Sound field within $10 \mathrm{~km}$ from the voyage route was calculated by numerical simulation. In parallel, land-based visual observers tracked humpback whales by a theodolite to locate surface position so that the exposure level at the animal can be estimated. Two autonomous stereo recording systems were deployed in the focal area to monitor the phonation behavior of singing whales simultaneously. As the first year result, temporal termination of song sequence was occasionally observed although avoidance behavior from the ship was not quite clear. This project would[NU1] last two more years to provide a scientific evidence of the minimum exposure level to elicit behavioral reaction of the whales.

\section{0:45}

2aABb6. Effects of anthropogenic noise on migrating bowhead whales in the Beaufort Sea. Katherine H. Kim, Susanna B. Blackwell (Greeneridge Sci., Inc., Greeneridge Sci., Inc., 90 Arnold Pl, Ste. D, Santa Barbara, CA 93117, khkim@greeneridge.com), and Aaron M. Thode (Scripps Inst. of Oceanogr., La Jolla, CA)

Greeneridge Sciences, Inc. (Greeneridge), has conducted multi-year, underwater, acoustic measurements at two study areas offshore of the North Slope of Alaska in the Beaufort Sea. The primary objective of these studies was to measure industrial sounds, e.g., those produced by oil production, seismic exploration, and drilling activities, and to assess their potential effects on the behavior of bowhead whales during their annual fall migration. To meet this objective, during open-water season every year from 2001 to 2016 (BP, Hilcorp) and 2007 to 2014 (Shell), Greeneridge deployed passive acoustic recorders equipped with directional sensors (DASARs) along the continental shelf. Over the course of these studies, millions of bowhead calls were localized, while ambient noise and various types of anthropogenic noise, such as tonal sounds associated with machinery and vessels and seismic airgun pulses, were quantified. The localization capabilities of the DASARs, large numbers of observations, and multi-year time series measurements together permitted, with high statistical power, quantitative evaluation of the effects of anthropogenic noise on bowhead behavior. Here, we'll review some of our major findings, including the apparent displacement of calling bowhead whales and changes in their calling rate in response to industrial 
activities, consistency in their call source levels, and a shift in the frequency content of their call repertoire. [Work supported by BP Exploration Alaska, Shell Alaska Venture, and Hilcorp Alaska.]

\section{1:05}

2aABb7. Predicting masking by impulsive noise sources: An experimental evaluation. Jillian M. Sills (Long Marine Lab., Inst. of Marine Sci., Univ. of California at Santa Cruz, 100 Shaffer Rd., Santa Cruz, CA 95050, jmsills@ucsc.edu), Brandon L. Southall (SEA, Inc., Aptos, CA), and Colleen Reichmuth (Long Marine Lab., Inst. of Marine Sci., Univ. of California at Santa Cruz, Santa Cruz, CA)

Impulsive noise sources pose significant challenges in terms of predicting auditory masking using conventional methods. Aside from their obvious time-varying structure, another complicating factor is the influence of propagation on the spectral and temporal characteristics of the noise, especially in water where such sounds may travel considerable distances. To address this, we developed a psychophysical paradigm to quantify masking in Arctic seals during different time intervals of seismic maskers, which were recorded either close to $(1 \mathrm{~km})$ or far from $(30 \mathrm{~km})$ an operational air gun array. Signal-to-noise ratios at threshold (50\% correct detection rate) were obtained behaviorally for trained seals, and were compared to conventional masked threshold predictions based on average noise levels and critical ratio measurements for the same individuals. The experimental data showed that masking predictions were poorest in the time intervals where noise exhibited the greatest amplitude variation. These findings provide insight into whether and how the dynamic sound field surrounding seismic surveys constrains the ability of seals to detect relevant signals, and show how predictive models of masking for transient noise sources can be improved by incorporating time-based analyses of signals and noise. [Work supported by OGP-JIP.]

\section{$11: 25$}

2aABb8. Noise field characterization in the habitat of the East Taiwan Strait Indo-Pacific Humpback Dolphin during the pile driving activity of demonstration offshore wind farm. Chi-Fang Chen (Dept. of Eng. Sci. and Ocean Eng. / Ocean Technol. Res. Ctr., National Taiwan Univ., No. 1 Roosevelt Rd., Sec.\#4, Taipei 106, Taiwan, chifang@ntu.edu.tw), Shane Guan (NOAA/NMFS, Office of Protected Resources, Silver Spring, MD), Lien-Sian Chou (Inst. of Ecology and Evolutionary Biology, National Taiwan Univ., Taipei, Taiwan), Ruey Chang Wei (Inst. of Undersea Technol., National Sun Yat-sen Univ., Kaohsiung, Taiwan), William W. Hu, Jeff C. Wu, Nai-Chang Chen, Wei-Shien Hwang (Dept. of Eng. Sci. and Ocean Eng. / Ocean Technol. Res. Ctr., National Taiwan Univ., Taipei, Taiwan), Sheng-Fong Lin (GEL, Industrial Technol. Res. Inst., Hsin-Chu, Taiwan), and Derrick Lin (Swancor Renewable Energy Co., Ltd., Taipei, Taiwan)

The Eastern Taiwan Strait (ETS) population of Indo-Pacific humpback dolphin (Sousa chinensis) is listed critically endangered in the Red List of Threatened Species by the International Union for Conservation of Nature due to its small population size and narrow distribution. The humpback dolphin habitats off the coast of Miaoli and Changhua are sites selected for future wind farms, therefore, the noise impact of pile driving on this critically endangered population is expected to be serious. This paper presents works done in (1) characterizing the sound field during the test pile driving and associated activities in the humpback dolphin habitat; (2) identifying dominant anthropogenic noise sources of the dolphin habitat during the construction of demonstration wind turbines and associated activities; and (3) examining the implications of the sound field from wind turbine construction and associated activities in relation to humpback dolphins' hearing and communication. The results from the study can provide critical information and conservation recommendations for an environmental impact analysis for the full scale wind farm construction in 2017. 


\title{
Session 2aAO
}

\section{Acoustical Oceanography: Twenty-Five Years of Acoustical Oceanography in the ASA II}

\author{
Andone C. Lavery, Cochair \\ Applied Ocean Physics and Engineering, Woods Hole Oceanographic Institution, 98 Water Street, MS 11, Bigelow 211, \\ Woods Hole, MA 02536 \\ Michael J. Buckingham, Cochair \\ Scripps Institution of Oceanography, University of California, San Diego, 9500 Gilman Drive, La Jolla, CA 92093-0238
}

Invited Papers

8:30

2aA01. A critical review of geoacoustic inversion: What does it really tell us about the ocean bottom? N. Ross Chapman (School Earth and Ocean Sci., Univ. of Victoria, P.O. Box 3065, Victoria, BC V8P 5C2, Canada, chapman@uvic.ca)

Estimation of parameters of geoacoustic models from acoustic field data has been a central theme in acoustical oceanography over the past three decades. Highly efficient numerical techniques based on Bayesian inference have been developed that provide estimates of geoacoustic model parameters and their uncertainties. However, the methods are model-based, requiring accurate knowledge of the acoustic propagation conditions in the ocean to carry out the inversion. More recent research has revealed fundamental limitations of model-based inversion methods in conditions of unknown temporal and spatial variations in the water. In addition, the inversions can generate only effective models of the true structure of the ocean bottom, which are generally highly variable over relatively small spatial scales. There are other questions about the theory for sound propagation in porous sediment media that raise doubt about the validity of inversion results. In most inversions, a visco-elastic theory is used, but is this correct? This paper reviews successes and failures of geoacoustic inversion to understand the limitation of model-based methods. Research directions are suggested in conclusion that show promise for development of new approaches. [Work supported by ONR.]

\section{8:50}

2aAO2. Physics-based inversion of multi-beam echo sounder data for seafloor properties. Darrell Jackson, Brian T. Hefner, and Anatoliy Ivakin (Appl. Phys. Lab., Univ. of Washington, 1013 NE 40th St., Seattle, WA 98105, drj@apl.washington.edu)

This is an extension of work on physics-based seafloor inversion for seafloor properties, pioneered by Pouliquen and Lurton and by Sternlicht and De Moustier. This approach fits model time series for backscattered intensity to data, with the best-fit model parameters constituting the inversion output. In the present case, use of multi-beam sonar provides a large number of time series and consequent stronger constraints on model parameters. The method has been tested at two experiment sites, one with sandy and shelly areas and one with a thin layer of mud over sand. Ground truth data were gathered on seafloor roughness using a laser line scanner, on layering using a conductivity probe, on grain-size distribution using diver cores, and on sediment sound-speed and attenuation using acoustic probes. The inversion process involves three stages. First, a sonar-equation model is used to generate echo intensity time series including scattering by both seafloor roughness and volume heterogeneity. Model-data fit provide estimates of acoustic attenuation, volume scattering strength, and interface scattering strength. Next, physics-based models are fitted to the interface and volume scattering strengths, and finally, regression relations are used to provide a set of geoacoustic parameters sufficient to constrain standard reverberation simulations. [Work supported by SERDP.]

\section{Contributed Papers}

9:10

2aAO3. Acoustic remote sensing of double-diffusive instabilities and intrusions in ocean frontal zones. Timothy F. Duda and Andone C. Lavery (Woods Hole Oceanographic Inst., WHOI AOPE Dept. MS 11, Woods Hole, MA 02543,tduda@whoi.edu)

Small-wavelength sound has been observed to scatter from turbulent microstructure and plankton in the ocean. Additional scattering from salt fingers and diffusive-convective flows that result from double diffusive instabilities (DDI) is also theoretically possible in this same wavelength band, and has been measured in a laboratory setting, though with artificially high gradient parameters. If measurable, small-wavelength backscatter would enable remote sensing of water mass interleaving and mixing from intrusive flows at fronts, which spawn DDI. The factors controlling the visibility of echoes from DDI features is the strength of the features in terms of density and sound-speed anomaly, and the level of masking return from plankton, which may merely interfere or act as passive tracers of these features. Microstructure data collected in double-diffusive instability features are used to compute the expected monostatic backscatter signals. These results suggest that DDI microstructure, from both salt fingering and convection, would be acoustically observable in areas with low to average plankton. The results also suggest that density interfaces (layering structures) associated with DDI would be visible. Acoustic backscatter data show echoes from both the interfaces and DDI microstructure in a frontal zone 
across a range of wavelengths. [Work supported by the Office of Naval Research.]

\section{9:25}

2aA04. Correlation among multipaths for long rang propagation. Arthur B. Baggeroer (Mech. and Elec. Eng., Massachusetts Inst. of Technol., Rm. 5-206, MIT, Cambridge, MA 02139, abb@boreas.mit.edu) and John A. Colosi (Dept. of Oceanogr., Navy Postgraduate School, Monterey, CA)

The multipath arrivals in ocean acoustic tomography are the important observations for the inversion to a sound speed profile and then to temperature. They are also implicit in any matched field processing beamforming. While the covariance of ray path travel times has been examined (Flatte and Stoughton, JGR 91, C6), the correlations among the waveforms of these multipaths has never been determined. There are two conflicting hypotheses: i) the paths radiate from a source, so they must be correlated. ii) Alternatively, the small scale ocean inhomogeneities randomize the paths since they traverse different ocean masses. At short ranges and low frequencies, the paths are correlated, whereas at long ranges and high frequencies, they are uncorrelated. Reality is somewhere in between. We have a power law medium which at large scales correlates paths but decorrelates them at small scales. The key quantity distinguishing the scales is the Fresnel zone extent. We analyze the correlation from two perspectives. A theoretical one based on path integral formulations and a experimental one on numerical Monte Carlo simulations on the massively parallel MIT Lincoln Laboratory LLGrid using adaptive beamforming for path resolution.

\section{9:40}

2aAO5. Reconstruction of the dynamic surface roughness with acoustic imaging technique. Giulio Dolcetti and Anton Krynkin (Mech. Eng., Univ. of Sheffield, Mappin St., Sheffield S1 3JD, United Kingdom, gdolcetti1@ sheffield.ac.uk)

We present a novel technique which recovers the three dimensional profile of the free surface based on the scattering of the narrow-band ultrasonic waves recorded by a two-dimensional array of receivers. The method is based on the inversion of the discretized Kirchhoff integral. The inverse technique implements the Tikhonov regularisation and the generalised cross validation technique in order to obtain solution to an under-determined system of equations. Using airborne ultrasound, the technique has been validated experimentally to reconstruct a two-dimensional composite static surface and numerically to recover the three-dimensional dynamic surface. The application to the typical three dimensional patterns found on the free surface of shallow turbulent flows is illustrated and discussed. The method has important applications for the remote monitoring of natural rivers, where recent studies confirm the direct link between the fundamental hydraulic properties of the flow and the dynamic behavior of the free surface. The potential significance in the oceanic environment is also discussed.

\section{9:55-10:10 Break}

\section{0:10}

2aAO6. Detection of long term trends in underwater noise levels. Claire F. Powell (Cefas, Cefas, Pakefield Rd., Lowestoft NR33 0HT, United Kingdom, claire.powell@cefas.co.uk) and Nathan D. Merchant (Cefas, Lowestoft, Suffolk, United Kingdom)

The risk of adverse impact to marine ecosystems from underwater noise pollution is increasingly recognised by scientists, policymakers, and wider society. Deep water measurements from the Northeast Pacific indicate that ocean noise has increased substantially over recent decades. Policymakers are now considering establishing noise monitoring programs to determine noise levels and trends in their waters. However, the ability of noise monitoring to detect statistically significant trends is a function of the temporal extent, variance, and autocorrelation of the time series. This has implications for the feasibility of evaluating quantitative policy targets within prescribed time frames and hence should inform the formulation of such targets. The present work demonstrates that methods developed in other environmental science disciplines (e.g., atmospheric temperature measurement) to design long-term monitoring networks and assess their statistical power can be applied to noise monitoring programmes. Example datasets are used to show the application of these methods both to assess the significance of long-term trends in ambient noise, and the required monitoring period to detect a given magnitude of trend (e.g., $3 \mathrm{~dB}$ per decade). The implications for the design of noise monitoring networks and target setting for policy purposes are then discussed.

\section{0:25}

2aA07. Lateral diffraction of underwater sounds between Japan and Chile: experimental results and modeling. Kevin D. Heaney (OASIS, Inc., 11006 Clara Barton Dr., Fairfax Station, VA 22039, oceansound04@ yahoo.com), Tomoaki Yamada (Univ. of Tokyo, Tokyo, Japan), Mario Zampolli, and Georgios Haralabus (CTBTO, Vienna, Austria)

One hundred shallow underwater explosions on a $300-\mathrm{km}$ profile along an isoline of $500 \mathrm{~m}$ sea depth above a slope zone of off the coast of Japan are received at a water-column hydrophone triplet with $2-\mathrm{km}$ intervals in Chile. The over 16,000-km propagations from the northwest to the southeast Pacific Ocean are classified into two groups. One is from the southern explosions. The data show high sound pressure levels and normal travel times. On the other hand, data from the northern ones reveal $6.5 \mathrm{~dB}$ lower sound pressure levels and $6.6 \mathrm{~s}$ travel time delays on average. Some islands and seamounts in the central Pacific are located on the halfway from the northern explosions. They affect received sound pressure levels and arrival times, but do not block completely, based on the observation. Modeling of this propagation path, using in-plane 2D modeling, shows nearly complete blockage of the sound by the Northern Hawaiian Island chain. Three dimensional parabolic equation modeling shows that this shadow is filled in from diffraction caused by the Midway Islands. Modeling results quantitatively match the measurements for both energy level and travel time.

10:40-11:10 Panel Discussion 


\title{
Session 2aBAa
}

\section{Biomedical Acoustics and Physical Acoustics: Cavitation in Therapeutic Ultrasound II: General}

\author{
Lawrence Crum, Cochair \\ Center for Industrial and Medical Ultrasound, University of Washington, Applied Physics Laboratory, 1013 NE 40th Street, \\ Seattle, WA 98105 \\ Shin-ichiro Umemura, Cochair \\ Graduate School of Biomedical Engineering, Tohoku University, Aoba 6-6-05, Aramaki, Aoba-ku, Sendai 980-8579, Japan \\ Tatiana D. Khokhlova, Cochair \\ University of Washington, 325 9th Ave., Harborview Medical Center, Box 359634, Seattle, WA 98104 \\ Zhen Xu, Cochair \\ Biomedical Engineering, University of Michigan, 2200 Bonisteel Blvd., Rm 1107 Gerstacker Bldg., Ann Arbor, MI 48109
}

\section{Contributed Papers}

7:50

2aBAa1. Optimisation of ultrasound exposure parameters for extravasation and drug delivery using submicron cavitation nuclei. Christophoros Mannaris, Megan Grundy (Inst. of Biomedical Eng., Eng. Sci., Univ. of Oxford, Old Rd. Campus Res. Bldg. (off Roosevelt Drive), Oxford OX3 7DQ, United Kingdom, christophoros.mannaris@eng.ox.ac.uk), Margaret Duffy, Len W. Seymour (Clinical Pharmacology, Dept. of Oncology, Univ. of Oxford, Oxford, United Kingdom), Robert Carlisle, and Constantin C. Coussios (Inst. of Biomedical Eng., Eng. Sci., Univ. of Oxford, Oxford, United Kingdom)

Sub-micron cavitation nuclei with the ability to passively extravasate through the leaky tumor vasculature have been shown to enhance both extravasation and penetration of pharmaceuticals in tumors. The aim of the present work is to optimize the acoustic parameters and maximize drug delivery mediated by cavitational microstreaming. Either lipid-shell microbubbles, or gas-stabilizing polymeric submicron cups of mean diameter 480 $\mathrm{nm}$ are co-administered with a model drug through a flow channel formed in agarose gel. The effect of ultrasound frequency, pressure amplitude, pulse duration, duty cycle, and pulse repetition frequency on the delivery of the drug is first evaluated, using both a single-layer and a dual-layer flow phantom that better represents the leaky tumor vasculature and dense extracellular matrix. The optimal parameters at $0.5 \mathrm{MHz}$ and $1.6 \mathrm{MHz}$ are then selected and used to deliver an oncolytic virus, genetically modified to express the RFP reporter gene, thus allowing the quantification of the delivery, infection, and subsequent spreading of the virus under fluorescence microscopy. We hypothesize that while inertial cavitation within the vasculature leads to extravasation, enhanced penetration is achieved by subsequent cavitation events of the sub-micron nuclei that passively extravasate and propel the drug deeper into the tumor.

\section{8:05}

2aBAa2. Giant vesicles as cell models to quantify bio-effects in ultrasound mediated drug delivery. Valerio Pereno, Dario Carugo, and Eleanor P. Stride (Univ. of Oxford, Old Rd. Campus Res. Bldg., Oxford OX3 7DQ, United Kingdom, eleanor.stride@eng.ox.ac.uk)

The biophysical mechanisms that underpin the interaction between microbubbles and cells in the context of ultrasound-mediated drug delivery are still poorly understood. To aid the identification of these mechanisms, giant unilamellar vesicles (GUVs) were used as cell models to quantify changes in membrane properties as a result of the interaction with ultrasound (1 MHz, $150 \mathrm{kPa}$, and $60 \mathrm{~s}$ continuous wave) and phospholipidshelled microbubbles (DSPC-PEG40S 9:1 molar ratio), either alone or in combination. The spatial quantification of the vesicle lipid order was performed via spectral microscope imaging, by measuring the contours of generalized polarisation (GP) from the emission spectrum of c-Laurdan, a polarity-sensitive dye. Preliminary data show synergistic mechanical and chemical effects on membranes: ultrasound exposure and shear flow alone generally decrease the vesicle lipid packing, while exposures involving microbubbles reveal contrasting effects depending on the initial vesicle composition and acoustic regime. Results from the present mechanistic study provide an insight into the mechanisms of microbubble-membrane interactions, potentially benefitting the design of effective and predictable microbubble-based ultrasound treatments.

\section{$8: 20$}

2aBAa3. Low intensity pulsed ultrasound and lipid-coated microbubbles enhance chondrogenesis of human mesenchymal stem cells in 3D bioprinted scaffolds. Mitra Aliabouzar, Lijie G. Zhang, and Kausik Sarkar (George Washington Univ., 801 22nd St. NW, Washington, DC 20052, sarkar@gwu.edu)

Annually, over 6 million people visit hospitals in the United States for issues arising from cartilage damages due to osteoarthritis, acute trauma, rheumatoid arthritis, and sports injury. Articular cartilage is a tissue notoriously hard to regenerate. The objective of this study is to investigate the possibility of facilitating cartilage regeneration using low intensity pulsed ultrasound (LIPUS) stimulation and microbubbles. We have investigated effects of lipidcoated microbubbles (MB) prepared in-house along with LIPUS on proliferation and chondrogenic differentiation of human mesenchymal stem cells (hMSCs) in a novel 3D printed poly(ethylene glycol) diacrylate (PEG-DA) hydrogel scaffold. Our results demonstrate for the first time that LIPUS stimulation in the presence of $0.5 \%(\mathrm{v} / \mathrm{v}) \mathrm{MB}$ greatly enhances MSC proliferation for up to $40 \%$ after 5 days of treatment. This value is only $18 \%$ when excited with LIPUS alone. Furthermore, we optimized acoustic parameters such as excitation intensity, frequency and pulse repetition period for chondrogenic differentiation studies. Synthesis of type II collagen and GAG, which are two key cartilage biomarkers, increased $78 \%$ and $17 \%$, respectively, in the presence of LIPUS and MBs, when compared to the controls. 
2aBAa4. Ultrasound-stimulated microbubble enhanced low-dose radiation response. Gregory Czarnota (Sunnybrook Health Sceinces Ctr., 2075 Bayview Ave., Toronto, ON M4N 3M5, Canada, gregory.czarnota@sunnybrook.ca)

We have recently demonstrated that mechanical perturbation of endothelial cells from ultrasound-stimulated microbubbles (USMB) results in enhanced tumor radiosensitivity at low 2 Gy doses of radiation. Our hypothesis is that USMB-based endothelial membrane perturbations produce ceramide via a sphingomyelinase (ASMase) pathway, and act synergistically with radiation to enhance overall tumor response. Here, we investigate the role of the SMase-ceramide pathway on USMB-based endothelial radiosensitization. Experiments were carried out in wild type (C57BL/6) and ASMase knockout mice, implanted with a fibrosarcoma line (MCA-129). Animals were treated with radiation doses varying from 0 to 8 Gy alone, or in combination with ultrasound-stimulated microbubbles. Treatment response was assessed with Doppler ultrasound vascularity index acquired at 3, 24, and 72 hrs using a VEVO770 preclinical ultrasound system. Staining using ISEL, ceramide, and CD31 immunohistochemistry of tumor sections was used to complement results. In contrast to wild type animals, ASMase knockout mice, or wild-type mice receiving S1P, were found to be generally resistant to the anti-vascular effects of radiation and USMB. Minimal cell death and no vascular shutdown was observed following treatments in those experimental groups. Overall conclusions drawn from this work suggest a mechanotransduction-like effect that results in endothelial radiosensitization.

\section{8:50}

2aBAa5. Magnetic targeting of oxygen loaded microbubbles for sonodynamic therapy. Estelle Beguin (Univ. of Oxford, Old Rd. Campus Bldg., Oxford OX3 7DQ, United Kingdom), Jason Sheng, Heather Nesbitt (Ulster Univ., Coleraine, United Kingdom), Joshua Owen (Univ. of Oxford, Oxordf, United Kingdom), Anthony McHale, John Callan (Ulster Univ., Coleraine, United Kingdom), and Eleanor P. Stride (Univ. of Oxford, Oxford, United Kingdom, eleanor.stride@eng.ox.ac.uk)

Previous work has demonstrated that the efficacy of sonodynamic therapy (SDT) can be significantly enhanced by conjugating SDT drugs to oxygen filled microbubbles. For eventual clinical use however, achieving adequate microbubble stability and targeting represents a considerable challenge. This study investigated whether functionalizing microbubbles with magnetic nanoparticles could both improve their longevity and enable localization of the microbubbles to a target site. Phospholipid coated microbubbles containing oxygen and $50 \mathrm{~nm}$ spherical magnetite particles were produced by sonication and conjugated to the SDT drug Rose Bengal via an Avidin-Biotin linker. The addition of the nanoparticles was found to substantially enhance the stability of the microbubbles to changes in size and concentration. Orthotopic tumors were induced in BALB/c SCID mice using the BxPc-3 human pancreatic cell line and exposed to ultrasound for 3.5 $\min \left(3.5 \mathrm{Wcm}^{-2}\right.$ at $1 \mathrm{MHz}$ center frequency, $100 \mathrm{~Hz}$ pulse repetition frequency, and $30 \%$ duty cycle) following intravenous injection of the microbubbles with or without application of a $0.1 \mathrm{~T}$ magnetic field. 38 days postimplantation the tumors treated with both ultrasound and the magnet were $50 \%$ smaller than the control tumors and exhibited a threefold increase in active caspase as a marker of apoptosis. There was no statistically significant effect of ultrasound alone.
2aBAa6. Use of pulse repetition frequency to augment acoustic droplet vaporization in vivo. Robinson Seda, Jonah Harmon (Biomedical Eng., Univ. of Michigan, 2225 Lurie Biomedical Eng., 1101 Beal Ave., Ann Arbor, MI 48105, robseda@umich.edu), Jeffrey B. Fowlkes (Radiology, Univ. of Michigan, Ann Arbor, MI), and Joseph Bull (Biomedical Eng., Univ. of Michigan, Ann Arbor, MI)

Tumor infarction shows promise as a cancer treatment. Gas embolotherapy tries to achieve this by locally occluding blood vessels supplying tumors using bubbles resulting from vaporization of liquid droplets. Sufficient occlusion may be necessary in order to starve tumors to death. The purpose of this study is to increase vaporization of droplets in vivo by changing the pulse repetition frequency (PRF) in order to improve the therapy. One milliliter of a solution containing $3 \times 10^{7}$ lipid-coated perfluorocarbon droplets was injected through the tail vein of a rat at a rate of $0.1 \mathrm{~mL} / \mathrm{min}$. Acoustic droplet vaporization (ADV) was induced in the feeder vessel of the exposed cremaster muscle using a focused, $7.5 \mathrm{MHz}$ transducer and $3 \mathrm{MPa}$ rarefactional pressure. A pulse length of $1 \mu$ s was used, while the PRF was varied from 10 to $1000 \mathrm{~Hz}$. Recordings of ADV showed substantial vaporization in both arterial and venous sides at higher PRFs. Vessel rupture and RBC extravasation were also observed. In other instances, blood vessels experienced a narrowing of the lumen at the site of vaporization (25\% reduction), suggesting an ultrasound/droplet/bubble interaction effect. Bubbles resulting from vaporization at high PRF were more likely to occlude small blood vessels, possibly due to coalescing.

\section{9:20}

2aBAa7. Enhanced chondrocytes growth in 3-D alginate scaffolds with improved porosity and permeability induced by low intensity pulse ultrasound. Juan Tu (Phys., Inst. of Acoust., Nanjing Univ., \#22 Hankou Rd., Nanjing 210093, China, juantu@nju.edu.cn), Xiasheng Guo, Dong Zhang (Phys., Nanjing Univ, Inst. of Acoust., Nanjing, Jiangsu, China), Qingyu Ma, Gepu Guo (Phys., Nanjing Normal Univ., Nanjing, China), and Huan Xu (National Inst. of Metrology, Nanjing, China)

Recent development in applications of new biomaterials and biomedical engineering enable the tissue engineering to become a promising cartilage repair technique. Here, a 3-D alginate scaffold was fabricated by a crosslinked method. Experiments were performed to investigate how the porosity and permeability of the 3-D scaffold, as well as the proliferation rate of seeded cells, were affected by the ultrasound exposure parameters. The scanning electron microscopy and fluorescence imaging were used to examine the micro-structure, porosity, and permeability of the scaffolds, and biochemical analyses were applied to assess the cell growth in the scaffold. The optimum low intensity pulsed ultrasound (LIPU) driving parameters that benefit the enhancement of scaffold porosity and cell proliferation were also explored. The results suggest that, for the scaffold exposed to LIPU, its porosity and permeability could be significantly enhanced by the increasing LIPU amplitude, which might be induced by the microstreaming shear stress generated by ultrasound-driven microbubble oscillations. The assessments of cell proliferation and collagen II expression confirmed that, with appropriately selected LIPUS driving parameters, chondrocytes growth could be effectively promoted in 3-D alginate scaffolds treated by LIPU, because of the improved scaffold porosity and permeability might benefit cell growth space and nutrition supply. 


\title{
Session 2aBAb
}

\section{Biomedical Acoustics: High-Frame Rate Imaging with Plane Waves and Diverging Waves}

\author{
Jeffrey A. Ketterling, Cochair \\ Riverside Research, 156 William St., New York, NY 10038 \\ Alfred Yu, Cochair \\ Electrical and Computer Engineering, University of Waterloo, EIT 4125, Waterloo, ON N2L 3G1, Canada \\ Hideyuki Hasegawa, Cochair \\ University of Toyama, 3190 Gofuku, Toyama 9308555, Japan
}

Chair's Introduction-8:25

\section{Invited Papers}

8:30

2aBAb1. High temporal and spatial resolution ultrasonic imaging and application to cardiovascular imaging. Hideyuki Hasegawa (Univ. of Toyama, 3190 Gofuku, Toyama 9308555, Japan, hasegawa@eng.u-toyama.ac.jp)

Recently, high frame rate ultrasound has become used for various imaging scenarios. It creates multiple scan lines at the same time using unfocused transmit beam to reduce the number of transmissions per image frame. Consequently, high frame rate ultrasound can realize a frame rate of over $1 \mathrm{kHz}$, but the lateral spatial resolution is degraded by using unfocused transmit beams, such as plane and diverging waves. To overcome such a problem, we have developed novel beamforming methods based on adaptive procedures, such as phase coherence imaging and minimum variance beamforming. As a result, high temporal and spatial resolution could be realized simultaneously. The developed high frame rate imaging methods were applied to imaging of cardiovascular dynamics. Blood flow in the carotid artery and cardiac left ventricle were imaged at frame rates of $2500 \mathrm{~Hz}$ and $6250 \mathrm{~Hz}$ with plane wave and diverging wave transmissions, respectively, and vortices in the carotid bifurcation and left ventricle could be observed. The high frame rate imaging method was also applied to tissue motion imaging. In the carotid arterial wall, pulse wave propagation could be visualized clearly even in a very regional segment of about $10 \mathrm{~mm}$. Also, we could also visualize propagation of the shear wave in the liver, which is spontaneously generated by the beating heart and blood vessels, and its propagation speed could be estimated qualitatively. High frame rate ultrasound would be an indispensable technique for measurement of tissue dynamics.

\section{8:50}

2aBAb2. Coherence-based adaptive imaging for high-frame-rate ultrasonic imaging. Pai-Chi Li ( Dept. of Elec. Eng., National Taiwan Univ., No.1, Sec. 4, Roosevelt Rd., Taipei 106, Taiwan, paichi@ntu.edu.tw)

Some success has been demonstrated in the studies of adaptive imaging, but these approaches are generally not suitable for highframe-rate imaging where broad transmit beams are required. This report introduces the signal-to-noise ratio (SNR)-dependent coherence factor (CF), which can be used for adaptive sidelobe suppression in ultrasound (US) imaging. Previous methods employed the minimum-variance-distortionless-response (MVDR)-based CF to achieve remarkable resolution improvement (by MVDR) and to suppress sidelobes (by CF). However, the SNR is often low when using an unfocused acoustic beam, giving such an approach suboptimal performance in these applications since noise also lowers the coherence and thus affects the effectiveness of the sidelobe suppression by these CF-based methods. To overcome this problem, the proposed method takes into account the local SNR in the CF formulation so that the contrast can be restored even when the SNR is low. Simulations show that the proposed method performs well even when the SNR is as low as $-10 \mathrm{~dB}$. Compared to the conventional CF, the contrast (CR) and contrast-to-noise ratio (CNR) in clinical US imaging can be improved, e.g., by an average of $27.2 \%$ in CR and $11.1 \%$ in CNR, with the proposed method.

\section{9:10}

2aBAb3. Two-dimensional blood flow vector obtained with high frame rate acquisition of dual-angle Doppler signal. Yoshifumi Saijo, Osamu Akagawa, Kosuke Fukazu, Naoya Tsugita, Sou Yaegashi, and Ryo Nagaoka (Tohoku Univ., 4-1 Seiryomachi, Aoba-ku, Sendai 980-8575, Japan, saijo@idac.tohoku.ac.jp)

Quantitative measurement of flow in blood vessels is important to assess cardiovascular dynamics. The objective of the present study is to obtain two-dimensional (2D) blood flow vector in some carotid artery models and to validate the method in comparison with particle image velocimetry (PIV). Plane wave ultrasound with the repetition rate of $14 \mathrm{kHz}$ was transmitted and received by a $7.5 \mathrm{MHz}$ linear array transducer and a programmable ultrasound data acquisition system. Compound B-mode images and dual-angle Doppler data with 
the transmission angle of $-10^{\circ}$ and $+10^{\circ}$ were obtained. $2 \mathrm{D}$ flow vector at arbitrary point was calculated by two velocity components along with each angle. Steady and pulsatile flow in normal and stenosed carotid artery models were simultaneously observed by the proposed method and PIV. 2D velocity vectors were successfully obtained in each flow states and the average error was approximately $5 \%$ in comparison with PIV in the carotid artery model. Steady flow in the distal stenosis model showed that the blood flow was influenced by vascular resistance while pulsatile flow seemed to push the blood to the high resistance portion. 2D blood flow obtained with dualangle Doppler signal may provide important information in understanding cardiovascular dynamics.

\section{9:30}

2aBAb4. High frame-rate visualization of blood flow with ultrasound contrast agents. Matthew Bruce, Alex Hannah (Univ. of Washington, 8817 Interlake Ave. N, Seattle, WA 98103, mbruce@uw.edu), Charles Tremblay-Darveau, and Peter Burns (Univ. of Toronto, Toronto, ON, Canada)

Recent breakthroughs in diagnostic ultrasound system architectures have enabled new high frame rate (kHz) capabilities, which are opening new opportunities in blood flow imaging. In order to leverage these increases in temporal resolution broader or unfocused beams are employed. The combination high frame and less focused beams with ultrasound contrast agents introduces both new opportunities and trade-offs. One new opportunity is the ability to combine Doppler based processing to visualize both lower velocity blood flow in the microcirculation and higher velocity flow in larger vasculature not possible with conventional techniques. This can be accomplished by combining nonlinear pulsing sequences to separate microbubble and tissue signals with these less focused beams to separate different microbubble flow velocities. The use of less focused beams to image nonlinear echoes from microbubbles distributes ultrasound energy to microbubbles in temporally and spatially different ways. This work explores the compromises of using these less focused beams to visualize moving microbubbles at higher temporal resolutions. The focus will be on the practical challenges of generating nonlinear signals while balancing microbubble disruption and current system reconstruction capabilities. Both in-vitro and in-vivo results will be presented.

\section{9:50}

2aBAb5. Robust and high-frame-rate visualization of arterial pulse wave propagation dynamics. Alfred C. H. Yu (Elec. and Comput. Eng., Univ. of Waterloo, EIT 4125, Waterloo, ON N2L 3G1, Canada, alfred.yu@uwaterloo.ca), Adrian J. Y. Chee, and Billy Y. S. Yiu (Elec. and Electron. Eng., Univ. of Hong Kong, Pokfulam, Hong Kong)

Crucial to the facilitation of early detection of vascular diseases is the development of new imaging techniques that can track vascular dynamics at sub-millisecond time resolution. Previously, we have designed new high-frame-rate ultrasound imaging solutions that can track complex flow dynamics at $>1000$ fps frame rate, thereby providing hemodynamic insights for vascular diagnostics. Here, we have expanded our suite of vascular diagnostic tools by developing new imaging modes for mapping the structural dynamics of arterial walls. We have particularly devised a novel framework for robust ultrasound-based mapping of the spatiotemporal dynamics of arterial pulse wave (PW) propagation. Our framework involves: 1) plane wave data acquisition; 2) eigen-based data processing; and 3) phasebased motion estimation. The eigen-processing module represents a novel application of singular value decomposition, and its purpose is to isolate the desired forward PW through identifying the corresponding left singular vector through a frequency analysis algorithm, thus making our framework robust against the biasing impact of PW reflections. Also, to visualize PW propagation, positions of PW front were mapped and trailed with its past positions. Using this new eigen-based PW visualization framework, we can now accurately identify differences in PW propagation dynamics in arterial segments of different wall thicknesses and wall elasticity. Corresponding cineloops will be shown at the meeting.

\section{0:10-10:30 Break}

\section{0:30}

2aBAb6. Ultrafast plane-wave imaging of ocular anatomy and blood-flow. Ronald H. Silverman (Ophthalmology, Columbia Univ. Medical Ctr., 635 W 165th St., Rm. 711B, New York, NY 10032, rs3072@cumc.columbia.edu), Jeffrey A. Ketterling (Riverside Res., New York, NY), and Raksha Urs (Ophthalmology, Columbia Univ. Medical Ctr., New York, NY)

Ophthalmic ultrasonography is currently performed virtually exclusively with mechanically scanned, focused single-element transducers. This technology provides B-scan images at approximately $10 \mathrm{frames} / \mathrm{s}$ and does not offer any capacity for imaging or measurement of blood-flow. Conventional linear array systems often exceed FDA guidelines for ophthalmic exposure, especially in Doppler modes. Plane-wave techniques offer both ultrafast imaging and reduced acoustic intensity, since no focusing is performed on transmit. Using a 128-element, $18 \mathrm{MHz}$ linear array, we adapted compound coherent plane-wave techniques for imaging ocular anatomy and blood flow at up to 20,000 frames/s. We measured acoustic intensity at the elevation focus and demonstrated conformance to FDA ophthalmic guidelines. We imaged human subjects and visualized blood flow by acquiring data continuously for at least one cardiac cycle. By applying a singular value decomposition filter to the spatiotemporal covariance matrix, we formed color-flow images and measured flow velocity over the cardiac cycle in the major retrobulbar vessels, choroid and anterior segment, including the iris and ciliary body. Given the major role of blood flow in diseases such as glaucoma, macular degeneration, diabetic retinopathy, and tumors (among others), this technique offers an important new diagnostic capability in ophthalmology not available using current ophthalmic B-scanners or optical coherence tomography. 
2aBAb7. 4-D ultrafast ultrasound imaging: in vivo quantitative imaging of blood flows and tissue viscoelastic properties. Mathieu Pernot, Mafalda Correia, Jean Provost, Clement Papadacci, and Mickael Tanter (Institut Langevin, ESPCI, 1, rue Jussieu, Paris 75004, France, mathieu.pernot@gmail.com)

Very high frame rate ultrasound imaging has enabled the development of novel imaging modalities such as the functional imaging of the brain, cardiac electrophysiology, and the quantitative real-time imaging of the intrinsic mechanical properties of tumors, to name a few. We present here the extension of Ultrafast Ultrasound Imaging in three dimensions based on the use of either diverging or plane waves emanating from a sparse virtual array located behind the probe. High contrast and resolution were achieved while maintaining imaging rates of thousands of volumes per second. A customized portable ultrasound system was developed to sample 1024 independent channels and to drive a 32X32 matrix-array probe. Its capability to track in 3D transient phenomena occurring in the millisecond range within a single ultrafast acquisition was demonstrated for 3-D Shear-Wave Imaging, 4-D Ultrafast Doppler Imaging, and 4D vector flow imaging. These results demonstrate the potential of 4-D Ultrafast Ultrasound Imaging for the volumetric real-time mapping of stiffness, tissue motion and flow in humans in vivo and promises new clinical quantitative applications of ultrasound with reduced intra- and interobserver variability.

\section{1:10}

2aBAb8. Role of high-frame-rate imaging for monitoring high-intensity focused ultrasound treatment. Shin-ichiro Umemura (Graduate School of Biomedical Eng., Tohoku Univ., Aoba 6-6-05, Aramaki, Aoba-ku, Sendai 980-8579, Japan, sumemura@ecei. tohoku.ac.jp), Shin Yoshizawa, and Ryo Takagi (Graduate School of Eng., Tohoku Univ., Sendai, Miyagi, Japan)

During high-intensity focused ultrasound (HIFU) treatment, tissue change, and acoustic cavitation as its precursor, should be monitored both in and outside the HIFU focal zone, to ensure the intended therapeutic effect and to prevent potential adverse effects, respectively. Unlike boiling-induced bubbles, cavitation-generated microbubbles are difficult to detect with conventional B-mode imaging because their life is significantly shorter than the typical image repetition period. An image repetition period less than a few milliseconds is required to detect them. This can only be accomplished by high-frame-rate imaging with plane or diverging waves. In-vivo as well as ex-vivo experimental results evidencing the effectiveness of such imaging to detect cavitation-generated microbubbles will be shown in this paper. A number of researches are underway to ultrasonically detect HIFU-induced tissue change, which occurs at much lower temperature than boiling. During echo data acquisition for detecting such tissue change, HIFU exposure is typically intermitted. High frame-rate-imaging also has a significant advantage there because the tissue motion by being released from the radiation force by HIFU can be ignored if the intermission is within a few milliseconds. Ex-vivo experimental results evidencing the effectiveness to detect HIFU-induced tissue change by high-frame rate imaging will also be shown.

\section{Contributed Papers}

\section{1:30}

2aBAb9. High frequency ultrafast flow and strain imaging in the carotid bifurcation: An ex vivo phantom study. Anne E. Saris, Stein Fekkes, Maartje M. Nillesen, Hendrik H. Hansen (Radiology and Nuclear Medicine, Radboud Univ. Medical Ctr., Music 766, PO Box 9101, Nijmegen 6500HB, Nijmegen, N/A, Netherlands), and Chris L. de Korte (Radiology and Nuclear Medicine, Radboud Univ. Medical Ctr., Netherlands, chris.dekorte@ radboudumc.nl)

In the Western World, the prevalence of plaques in the carotid is high resulting in stroke and transient ischemic events. Quantification of the plaque size and compositions and the remaining flow and velocity profile is crucial for adequate therapy. We developed a high frequency based method for characterization of plaques and flow. A realistic bifurcation phantom with plaque based on CT scans of a patient was constructed. A realistic pulsatile flow was imposed on the phantom resembling a human flow and pressure profile. Using a Verasonics experimental echomachine with a Visualsonics M250 transducer $(\mathrm{Fc}=20 \mathrm{MHz})$, plane wave ultrasound data were acquired at $12.000 \mathrm{fps}$. A cross-correlation based coarse to fine method was applied on the beamformed data to quantify the axial and lateral strain in the arterial wall and to determine the velocity magnitude and direction of the blood. The strain and flow data were presented side by side for 80 frames covering the full pressure cycle. Strain in the non-diseased carotid wall was moderate with respect to the surrounding tissue. The bifurcation shows large lateral strain due to the force of the pulsating blood. The flow profile shows a turbulent region in the systolic phase at the bifurcation.

\section{1:45}

2aBAb10. Plane-wave imaging of in utero mouse embryo at $18 \mathrm{MHz}$. Jeffrey A. Ketterling (Lizzi Ctr. for Biomedical Eng., Riverside Res., 156 William St., New York, NY 10038, jketterling@riversideresearch.org), Orlando Aristizabal (Skirball Inst. of Biomolecular Medicine, NYU School of Medicine, New York, NY), Colin K. L. Phoon (Div. of Pediatric Cardiology, NYU Langome Medical Ctr. and Fink Children's Ctr., New York, NY), Billy Y. S. Yiu (Dept. of Elec. and Electron. Eng., Univ. of Hong Kong, Pokfulam, Hong Kong), and Alfred C. H. Yu (Dept. of Elec. and Comput. Eng., Univ. of Waterloo, Waterloo, ON, Canada)

Plane-wave imaging methods allow for high-speed image capture at a time interval equal to round trip acoustic propagation. Plane-wave imaging is ideally suited for cardiovascular imaging where fine-temporal resolution can reveal important information about cardiac mechanics and blood flow patterns. While plane-wave imaging has been demonstrated in humans for cardiovascular studies, its use in mouse models lags because instrumentation is not yet widely available at appropriate ultrasound frequencies. Thus, the amount of functional information that can be mined from mouse models of cardiovascular disease is limited. Here, an $18-\mathrm{MHz}$ linear-array probe was used to acquire plane-wave data at a frame rate of $10 \mathrm{kHz}$ from an in utero, E14.5 mouse embryo. The probe had 128 elements, $1.5 \mathrm{~mm}$ elevation aperture, and 8 -mm elevation focus. The mother was placed supine on a heated mouse imaging platform, and then, a series of $2 \mathrm{D}+$ time data sequences were captured. The data were beamformed using standard delay-and-sum methods, and then, vector flow estimates were obtained at each pixel location using a least-squares, multi-angle Doppler analysis approach. Although not optimally suited for imaging mouse embryos, the 18- $\mathrm{MHz}$ data clearly revealed blood flow patterns in the liver, heart, and umbilical cord. 


\title{
Session 2aBAc
}

\section{Biomedical Acoustics and Physical Acoustics: Cavitation in Therapeutic Ultrasound III: Tissue Fractionation}

\author{
Tatiana D. Khokhlova, Cochair \\ Harborview Medical Center, University of Washington, 325 9th Ave., Box 359634, Seattle, WA 98104
}

Zhen Xu, Cochair

Biomedical Engineering, University of Michigan, 2200 Bonisteel Blvd., Rm. 1107 Gerstacker Bldg., Ann Arbor, MI 48109

Shin Yoshizawa, Cochair

Communications Engineering, Tohoku University, 6-6-05 Aoba, Aramaki, Aoba-ku, Sendai 980-8579, Japan

\author{
Chair's Introduction-9:50
}

\section{Invited Papers}

9:55

2aBAc1. Histotripsy: Transcranial applications. Charles A. Cain, Jonathan Sukovich, Timothy L. Hall, and Zhen Xu (Biomedical Eng., Univ. of Michigan, 2200 Bonisteel Blvd., Ann Arbor, MI 48109-2099, cain@umich.edu)

Histotripsy produces non-thermal lesions by generating, when an intrinsic threshold is exceeded, dense highly confined energetic bubble clouds that mechanically fractionate tissue. This nonlinear thresholding phenomenon has useful consequences. If only the tip of the waveform (P-) exceeds the intrinsic threshold, small lesions less than the diffraction limit can be generated. Moreover, side lobes from distorting aberrations can be "thresholded-out" wherein part of the relatively undistorted main lobe exceeds the intrinsic threshold producing a clean bubble cloud (and lesion) conferring significant immunity to aberrations. The short very high intensity histrotripsy pulse is significantly backscattered by the cloud it creates, allowing aberration corrections to be made in real time using transmitting elements as receivers. This allows significant increases in peak negative pressures that can be generated through the skull. These same backscattered signals allow a 3D representation of the bubble cloud to be generated providing excellent noninvasive bubble cloud localization. These refinements allow noninvasive transcranial procedures outside of expensive MRI machines.

\section{0:15}

2aBAc2. Pilot in vivo studies on transcutaneous boiling histotripsy in porcine liver and kidney. Vera Khokhlova, George Schade, Tatiana Khokhlova, Yak-Nam Wang, Julianna Simon, Frank Starr, Adam Maxwell, Michael Bailey, and Wayne Kreider (Univ. of Washington, 1013 NE 40th St., Seattle, WA 98105, va.khokhlova@gmail.com)

Boiling histotripsy (BH) uses millisecond-long pulses of focused ultrasound (FUS) waves with shocks to mechanically homogenize tissue. Here we report a pilot in vivo acute study on transcutaneous volumetric $\mathrm{BH}$ ablation of porcine liver and kidney. BH treatment was administered using a $1.5 \mathrm{MHz}$ FUS transducer operating at peak electric power ranging from 0.6 to $4 \mathrm{~kW}$ with B-mode ultrasound guidance using an imaging probe mounted coaxially. Sonication protocols delivered 5-30 pulses of 1-10 ms duration and 1\% duty factor to focal points spaced 1-1.5 mm apart in a rectangular grid with 5-15 mm linear dimensions. Well-demarcated volumetric BH lesions were successfully generated in both liver and kidney without respiratory gating. The treatment was accelerated 6-fold without affecting the quality of tissue homogenization by using shorter duration $\mathrm{BH}$ pulses of larger peak power. These data indicate that transcutaneous volumetric renal and hepatic ablations are feasible in the in vivo porcine model. Studies are ongoing to optimize treatment parameters with the goal of clinical BH ablation of renal and hepatic masses. [This work was supported by NIH R01 EB7643, K01 EB015745, NSBRI through NASA NCC 9-58, RFBR 16-02-00653, and Urology Care Foundation.] 


\section{Contributed Papers}

10:35

2aBAc3. Pilot evaluation of histotripsy treatment for Peyronie's disease. Yak-Nam Wang, Adam D. Maxwell (APL, CIMU, Univ. of Washington, 1013 NE 40th St., Seattle, WA 98105, ynwang@u.washington.edu), Lynda Brady (Dept. of Mech. Eng., Univ. of Washington, Seattle, WA), George R. Schade, Haider Yasser, Franklin Lee (Urology, Univ. of Washington, Seattle, WA), William R. Ledoux (Ctr. for Limb Loss Prevention and Prosthetic Eng., VA Puget Sound, Seattle, WA), Michael R. Bailey (APL, CIMU, Univ. of Washington, Seattle, WA), and Hunter Wessells (Urology, Univ. of Washington, Seattle, WA)

Peyronie's disease (PD) is a debilitating scarring disorder of the penis which can cause significant curvature, pain, and psychological distress. Intra-lesional injection of specialized drugs to break down the fibrous matrix demonstrates modest results and is extremely expensive; surgery remains the gold standard for PD despite possible complications. We evaluated the feasibility of using histotripsy to disrupt the fibrous plaque and alter the mechanical properties as a novel non-invasive treatment. Freshly excised Peyronie's plaques $(\mathrm{n}=5)$ were separated into individual samples for histotripsy treatment or control tissue. Treatments were conducted using a $1.0 \mathrm{MHz}$ custom-built therapy transducer delivering pulses with repetition frequency of $1000 \mathrm{~Hz}$, duty cycle $0.5 \%$, and peak focal pressures of (98/-17 MPa) under ultrasound guidance. Tissue was either formalin fixed for histological evaluation or frozen for mechanical testing. Histotripsy induced disruption of collagen and elastin fibrils was evident in all fibrous portions of plaques. Histotripsy treatment produced decreased mean modulus, ultimate tensile strength, maximum load, and toughness vs. untreated control samples on mechanical testing. Additionally, the displacement to maximum load ratio was greater in treated vs. untreated control samples. [Work supported by
NIH grants K01 DK104854, DK043881, EB007643, NSBRI through NASA NCC 9-58, and VA RR\&D grant A9243C.]

\section{0:50}

2aBAc4. Treatment envelope of transcranial histoptripsy applied without aberration correction. Jonathan R. Sukovich, Zhen Xu, Timothy L. Hall, Steven P. Allen, and Charles A. Cain (Biomedical Eng., Univ. of Michigan, 1410 Traver Rd., Ann Arbor, MI 48105, jsukes@umich.edu)

Due to the characteristically high absorption, attenuation, and aberrating effects of the skull on ultrasound, transcranial HIFU therapies have typically been restricted to targeting brain regions at depths $\geq 2 \mathrm{~cm}$ from the interior surface of the skull. Previous studies using histotripsy, a cavitation based ultrasound therapy relying on short duration ( $<2$ acoustic cycles), high negative pressure amplitude $(\mathrm{P} \leq-30 \mathrm{MPa})$ ultrasound pulses to generate cavitation clouds to fractionate tissue, have shown that targeted bubble clouds can be generated through the skull without aberration correction. Here, we present results from experiments probing the minimum depths with respect to the skull surface at which histotripsy applied without aberration correction can generate lesions. Using a $500 \mathrm{kHz}, 256$-element transducer, histotripsy was applied through an ex vivo human skull to generate lesions in a tissue mimicking phantom embedded within. Lesions were generated at multiple locations within the skull through skull sections with characteristically different geometries. MRI was used to assess lesion generation following treatment. Using histotripsy without aberration correction, we were able to generate lesions at typical depths of between 6 and $12 \mathrm{~mm}$ from the interior skull surface. These results demonstrate the potential of histotripsy to target shallower brain regions than current HIFU therapies.

Invited Papers

\section{1:05}

2aBAc5. Non-invasive thrombolysis using microtripsy for deep vein thrombosis and intracerebral hemorrhage. Zhen Xu, Xi Zhang, Jonathan Sukovich, Tyler I. Gerhardson (Biomedical Eng., Univ. of Michigan, 2200 Bonisteel Blvd., Rm. 1107 Gerstacker Bldg., Ann Arbor, MI 48109, zhenx@umich.edu), Hitinder Gurm (Interventional Cardiology, Univ. of Michigan, Ann Arbor, MI), Gabe Owens (Pediatric Cardiology, Univ. of Michigan, Ann Arbor, MI), Aditya Pandey (Neurosurgery, Univ. of Michigan, Ann Arbor, MI), Tim L. Hall, and Charles A. Cain (Biomedical Eng., Univ. of Michigan, Ann Arbor, MI)

Histotripsy has been demonstrated as a non-invasive, drug-free, image-guided thrombolysis method using cavitation alone. Microtripsy is a new histotripsy approach, where cavitation is generated using 1-cycle ultrasound pulses with negative pressure exceeding a threshold intrinsic to the medium. We investigated microtripsy for treatment of deep vein thrombosis (DVT) and intracerebral hemorrhage (ICH). First, the in vivo feasibility of using microtripsy to treat DVT was investigated. Guided by ultrasound imaging, microtripsy thrombolysis treatment was applied to the clot formed in the femoral vein in 14 pigs using $1 \mu \mathrm{s}$-long pulses at a pulse repetition frequency of $100 \mathrm{~Hz}$ and a peak negative pressure of $30 \mathrm{MPa}$ by a $1 \mathrm{MHz}$ transducer. Blood flow was restored or significantly increased in 13 out of the 14 pigs confirmed by color Doppler. Minor hemolysis was observed. No vessel damage was observed on histology. Second, microtripsy was investigated to liquefy and drain clots through excised human skulls for ICH treatment using a $256-$ element, $500 \mathrm{kHz}$ phased array transducer. Microtripsy generated individual lesions of 0.1 to $1.5 \mathrm{~mm}$ at the geometric location and electronically steered positions out to $20 \mathrm{~mm}$ from the geometric focus. By electronically steering the focus to cover a clot volume, microtripsy liquefied 40 $\mathrm{mL}$ of clot in $25 \mathrm{~min}$, resulting in clot liquefaction rate of $1.6 \mathrm{~mL} / \mathrm{min}$.

\section{1:25}

2aBAc6. A preliminary in-vivo porcine study of long pulse histotripsy for thrombolysis of intraventricular hemorrhagic clot. Thomas Looi (Hospital for Sick Children, 555 University Ave., Burton Wing 7142, Toronto, ON M5G1X8, Canada, thomas.looi@sickkids.ca), Vera Khoklova (Univ. of Washington, Seattle, WA), Kullervo Hynynen (Sunnybrook Res. Inst., Toronto, ON, Canada), and James Drake (Hospital for Sick Children, Toronto, ON, Canada)

Intraventricular hemorrhage is primarily a condition of premature babies where $40 \%$ will develop a form of bleeding that occupies $>50 \%$ of the ventricles in the brain. Due to the fragile nature of patient, there is no treatment except the use of shunts for cerebrospinal fluid accumulation. Long pulse histotripsy (LPH) uses focused ultrasound pulses as a method to non-invasively target and mechanically break up the clot. An in-vivo IVH procine model has been developed and it is used to test the efficacy and safety of using LPH on a Philips Sonalleve. The IVH model has an average clot volume of $3986 \mathrm{~mm}^{3}$ and present in both ventricles. To simulate a neonatal patient, a craniotomy has been performed. The acoustic parameters used are as follows: freq. $1.2 \mathrm{MHz}, 2 \times 6 \mathrm{~mm}$ focus, $10 \mathrm{~ms}$ pulse duration, 10,000 cycles, $1 \%$ duty cycle, and acoustic power from 325 to $400 \mathrm{~W}$. Sonication points were placed at the center of the IVH clot. Pre- 
and post-treatment T1-w, T2-w, and T2*-w MR imaging was completed. Change in the clot volume was measured by segmenting the MR images. The brains were harvested and stained with hematoxylin and eosin for histological examination. Results show that LPH targeted and reduced the clot volume by $28.5-36.7 \%$ with a phase change occurring at target. H\&E staining showed that visible voids were generated in the clots. Based on early data, it appears that LPH can mechanically reduce the volume of IVH clots. Future work includes increasing the study number and conducting chronic studies to determine the changes to recovery.

\title{
Contributed Paper
}

$11: 45$

2aBAc7. Visualizing the histotripsy process: Bubble cloud-cancer cell interactions in a tissue-mimicking environment. Eli Vlaisavljevich (Univ. of Michigan, 1111 Nielsen Ct. Apt. 1, Ann Arbor, MI 48105, evlaisav@ umich.edu), Adam Maxwell (Univ. of Washington, Seattle, WA), Lauren Mancia, Eric Johnsen, Charles Cain, and Zhen Xu (Univ. of Michigan, Ann Arbor, MI)

Histotripsy is an ultrasonic ablation method that uses cavitation to mechanically fractionate tissue into acellular debris. Previous work has led to the hypothesis that the rapid expansion and collapse of histotripsy bubbles fractionate tissue by inducing large strain on the tissue structures immediately adjacent to the bubbles. In this work, the histotripsy fractionation process was visualized at the cellular level for the first time using a custom- built $2 \mathrm{MHz}$ transducer incorporated into a microscope stage. A layer of breast cancer cells were cultured within an optically transparent fibrin-based phantom to mimic cells inside an extracellular matrix environment. The response to single and multiple histotripsy pulses was investigated using high speed optical imaging. Bubbles were generated in the extracellular space, and significant cell displacement/deformation was observed for cells directly adjacent to the bubbles. The largest displacements were observed during collapse for cells immediately adjacent to the bubble, with cells moving more than $150-300 \mu \mathrm{m}$ in less than $100 \mu \mathrm{s}$. Cells often underwent multiple large deformations ( $>150 \%$ strain) over multiple pulses, resulting in the bisection of cells multiple times before complete rupture. These results support our hypothesis and help to explain the formation of the sharp lesions formed in histotripsy therapy.

TUESDAY MORNING, 29 NOVEMBER 2016

SOUTH PACIFIC 4, 7:45 A.M. TO 12:00 NOON

\section{Session 2aEA}

\section{Engineering Acoustics: Transducer Systems}

\author{
Jason E. Gaudette, Cochair \\ NUWC Division Newport, 1176 Howell Street, B1371/3, Newport, RI \\ Yoshinori Takahashi, Cochair \\ Tokyo Metropolitan College of Industrial Technology, 8-17-1, Minamisenju, Arakawaku, Tokyo 116-0003, Japan
}

\section{Contributed Papers}

$7: 45$

2aEA1. Broadband ultrasound emission from a three-layer thermoacoustic transducer. Olivier Y. Burguiere (Graduate School of Life and Medical Sci., Doshisha Univ., 1-3 Tatara Miyakodani, Kyotanabe, Kyoto 610-0321, Japan, dmp5501@mail4.doshisha.ac.jp), Shinji Takayanagi (Graduate School of Eng., Nagoya Inst. of Technol., Nagoya, Aichi, Japan), Mami Matsukawa (Faculty of Sci. and Eng., Doshisha Univ., Kyotanabe, Kyoto, Japan), and Shizuko Hiryu (Graduate School of Life and Medical Sci., Doshisha Univ., Kyotanabe, Kyoto, Japan)

A thermophone generates sound with temporal variation of Joule heat which leads to expansions and contractions of a small volume of air near the surface of the metallic film. Consequently, the SPL of the sound is proportional to the input power and the emitted sound is the second harmonic of the input signal. ${ }^{1}$ Here, the fundamental characteristics of a three-layer thermophone (200-nm-thick Pt film, 5- $\mu$ m-thick glass heat-insulating layer, and $500-\mu \mathrm{m}$-thick Si heat-releasing layer) were investigated. The device $(10 \times 12$ $\mathrm{mm}^{2}, 2.32 \Omega$ ) showed a broadband frequency response up to $140 \mathrm{kHz}$, and its SPL level at $50 \mathrm{~mm}$ was nearly constant $(61.5 \pm 3 \mathrm{~dB})$ from 20 to $140 \mathrm{kHz}$ at $1.8 \mathrm{~W}$ power consumption. The conversion efficiency was $2.68 \times 10^{-5} \%$, which is 150 times the efficiency of the authors' previous prototype, in which the heat release was less efficient (594- $\mu \mathrm{m}$-thick alumina layer). Furthermore, by applying both AC and DC currents to the thermophone, it emitted the fundamental and the second harmonics of a $50-20 \mathrm{kHz}$ FM sound, and hence a broadband (100-20 kHz) compound signal. These results and this device's simple manufacturing process suggest that it may be used as a high resolution ultrasound sensor. ${ }^{1}$ Shinoda et al. (1999), Nature, 400(6747), p.853

\section{8:00}

2aEA2. A circular microphone array beamformer based on spherical harmonics. Gary W. Elko (mh Acoust., 25A Summit Ave., Summit, NJ 07901, gwe@mhacoustics.com) and Jens Meyer (mh Acoust., Fairfax, VT)

A circular microphone array that can easily steer its beampattern(s) to any angle in the horizontal plane around the array would be an attractive microphone array for room audio conferencing. An elegant solution to this problem to design a modal beamformer based on circular harmonics. However, a modal beamformer based on circular harmonics suffers from the loss of control of the beampattern out of the plane of the array. We will present a modified modal beamformer for the circular array that is based on spherical harmonics. This approach allows full control of the beampattern and maintains the efficient steerability known from modal beamformers. Measurements of a specific implementation with 16 sensors will be presented. 
technologies allow the integration of a matrix of piezoelectric elements on

2aEA3. Bio-inspired broadband sonar array prototypes and underwater experiments for two- and three-dimensional acoustic imaging applications. Jason E. Gaudette (Sensors and Sonar Systems, NUWC Div. Newport, 1176 Howell St., B1320, Newport, RI, jason.e.gaudette@navy. mil), Dimitri M. Donskoy (Davidson Lab., Stevens Inst. of Technology, Hoboken, NJ), Caleb J. Martin, Christin T. Murphy (Sensors and Sonar Systems, NUWC Div. Newport, Newport, RI), and James A. Simmons (Dept. of Neurosci., Brown Univ., Providence, RI)

Underwater sonar imaging relies upon the information contained in time correlation delays across numerous channels. Designing higher resolution imaging systems currently requires increasing the array aperture to wavelength ratio $(\mathrm{L} / \lambda)$, where $\mathrm{L}$ is the effective array length and $\lambda$ is the acoustic wavelength in the medium. This fundamental constraint leads engineers down the path of adding a significant number of channels to a sonar design, which in turn increases array complexity and cost. Our research in bioinspired sonar has revealed an approach that circumvents this constraint by exploiting bandwidth in addition to time-delay for the angular imaging process. Presented will be the results of a 2-channel underwater prototype tested in an acoustic tank and design work toward a 3-channel prototype for extending imaging to elevation angles. This work represents ongoing efforts toward developing a compact, low-cost broadband underwater sonar for near- to mid-range imaging and classification. Future integration with an autonomous underwater vehicle will demonstrate this technology for simple obstacle detection and avoidance of complex objects. [Work supported by ONR and internal investments by NUWC Division Newport.]

\section{8:30}

2aEA4. An ultrasound focusing lens design incorporating high transmission and minimum diffraction. Xiasheng Guo (Inst. of Acoust., Nanjing Univ., No.22, Hankou Rd., Nanjing 210093, China, guoxs@nju.edu.cn), Zhou Lin (Inst. of Acoust., Nanjing Univ., Nanjing, Jiangsu, China), Gepu Guo (School of Phys. and Technol., Nanjing Normal Univ., Nanjing, Jiangsu, China), Juan Tu (Inst. of Acoust., Nanjing Univ., Nanjing, Jiangsu, China), Qingyu Ma (School of Phys. and Technol., Nanjing Normal Univ., Nanjing, Jiangsu, China), and Dong Zhang (Inst. of Acoust., Nanjing Univ., Nanjing, Jiangsu, China)

An improved design of ultrasound focusing lens is reported, in which a periodical array of grooves were carved on the surface of a conventional lens. With vibrations produced by a planar wave transducer coupled to the lens's left surface, each groove could be treated as a point source as long as the groove widths are much smaller than the acoustic wavelength. Several uncommon acoustic effects (e.g., collimation and enhanced acoustic transmission) have been incorporated into the designing, providing the benefits ranging from transferring acoustic energies, suppressing the side-lobes, and minimizing shifting of the focal point. It is demonstrated theoretically and experimentally that acoustic focusing achieved by using the lens can suppress the relative side-lobe amplitudes, enhance the focal gain, and minimize the shifting of the focus. The application of the corrugated lens can make a reduction of about $3 \mathrm{~dB}$ in the relative side-lobe amplitudes, a 3.6$\mathrm{dB}$ increase in main-lobe amplitude, and reduction or even elimination of the focus shift which could be enormously valuable in enhancing the safety of noninvasive HIFU therapy. Enlarging the number of grooves could even further enhance the capabilities of improving the focusing efficiency and reducing the relative side-lobe amplitudes.

\section{8:45}

2aEA5. The acoustical performance of an ultrasound matrix transducer directly stacked on a silicon chip. Maysam Shabanimotlagh, Shreyas Raghunathan, Nico d. Jong, and Martin D. Verweij (Imaging Phys., Tech. Univ. of Delft, Rm. D210, Bldg. 20, Lorentzweg 1, Delft 2628CJ, Netherlands,m.shabanimotlagh@tudelft.nl)

Clinical studies have shown a high demand for 3D real time ultrasound imaging for accurate diagnosis. One way to scan a volume with high frame rate is to design a $2 \mathrm{D}$ matrix transducer allowing beamforming in both lateral and elevation directions. Recent developments in manufacturing
Application Specific Integrated Circuit (ASIC). The ASIC is responsible for beamforming, amplification, switching, and significant channel count reduction. Traditionally, the backing material for a linear/phased array is composed of a mixture of epoxy with powder of heavy materials, which causes strong attenuation. This avoids energy being reflected back into to the elements and results in short pulses, high bandwidth, and consequently high axial resolution of the image. However, the ASIC acoustically behaves like a hard and non-absorbing backing material. Therefore, the acoustical energy of the piezoelectric pillar propagates into the chip and affects the performance of the neighbouring elements. In this paper we numerically investigate the effect of the ASIC on the acoustical performance of a transducer. In particular, the transmit and receive performance, crosstalk, and directivity pattern are compared with the results for a traditional backing material.

\section{9:00}

2aEA6. A micromachined low frequency microphone based on a field effect transistor and an electret. Kumjae Shin, Junsoo Kim, Hoonteak Lee, Donghwan Seo (Dept. of Mech. Eng., Pohang Univ. of Sci. and Technology(POSTECH), Pohang, Gyungbuk, South Korea), and Wonkyu Moon (Dept. of Mech. Eng., Pohang Univ. of Sci. and Technology(POSTECH), PIRO 405, POSTECH, San31, Hyoja-dong, Nam-gu, Pohang city, Kyungbuk, South Korea, Pohang, Kyungbuk 790784, South Korea, wkmoon@ postech.ac.kr)

Recently, several Internet of Things (IoT) devices using low-frequency acoustic sound have emerged as promising sensor applications. Unfortunately, the detection of low-frequency sound using miniaturized microphones is restricted due to the low cut-off frequency of capacitive type transduction. To overcome this limitation, a micromachined microphone based on a field-effect transistor (FET) and an electret was reported and its feasibility as a low-frequency microphone was demonstrated in 2015. However, the proposed microphone was realized by bonding two chips mechanically and the FET was embedded in a membrane, which was disadvantageous for sensitivity enhancement. To realize stable highly sensitive modulation, we devised and fabricated a structure in which the electric field due to an electret embedded in the membrane modulates the channel of the FET. The acoustic signal causes the electret mounted on the membrane to vibrate, which changes the distance between the channel of the FET and the electret. The resulting change in the electric field modulates the conductivity of the channel. The use of an electret embedded on the membrane makes it possible to detect the displacement of the membrane directly and enhances the sensitivity of the microphone. Its feasibility as a low-frequency microphone will be examined experimentally.

\section{9:15}

2aEA7. Acoustic lens with exponential horns for selective distant recording. Maho Kobayashi (Tokyo Metropolitan College of Industrial Technol., 2-8-1 Minato-cho, \#1811, Funabashi-shi, Chiba-ken 2730011, Japan, mintgreen070503@gmail.com) and Yoshinori Takahashi (Tokyo Metropolitan College of Industrial Technol., Tokyo, Japan)

Many signal processing techniques for sound source separation using microphone array have been studied as a robust audio recording method to ambient noise. However, the implementation in real-time is still hard to be realized in terms of its large computational cost. As an another approach, a directional microphone has a capability to record sound from a specific direction; however, it is impossible to pick up sound at a specific distance. As a part of research on acoustic transducer, on the other hand, a method to broaden the directivity by using the slant-plate acoustical lenses at the front of loudspeaker has been utilized. In addition, there is a report that a slantplate acoustical lens combined with a conical horn shows a sharper directivity than that by a parabolic microphone. The authors have studied selective distant recording using acoustic lens that synchronously adds the wave fronts after removing the time difference of arrival via the speaking tubes having different lengths. As a result of that the shape of the speaking tube in the acoustic lens was replaced with exponential horn, an improvement in terms of sound quality with a reduction of resonance was obtained. 
cheaper and smaller omnibinaural recording system. In previous research, a

2aEA8. Annular 1-3 piezocomposite high intensity focused ultrasound transducer of a concave geometry. Yongrae Roh, Euna Choi, Seongwon Jang, and Minwoo Jo (School of Mech. Eng., Kyungpook National Univ., 80 Daehakro, Bukgu, Daegu 41566, South Korea, yryong@knu.ac.kr)

For medical therapeutic applications, we have developed a concave annular high intensity focused ultrasound (HIFU) transducer that is made of a 1-3 piezocomposite plate and operates at $3 \mathrm{MHz}$. The transducer has 16 annular channels, and the annuli have been designed to focus at the geometrical center of the sphere. The focal point can be controlled to scan a three dimensional volume by electronic and mechanical means. The focal point is moved along the normal axis of the transducer by electronically steering the sixteen annular channels. The focal point is also rotated back and forth by mechanically wobbling the piezocomposite plate with a kinematic linkage. The kinematic linkage is driven by an electric motor installed inside the transducer. The optimal combination of the number of channels, the width of the kerf between channels, and the width of each channel were determined by means of the OpQuest-Nonlinear programming algorithm and the finite element method. The objective of the optimization was to minimize the side lobe levels while focusing the ultrasound beam to a prescribed position. Based on the design, an experimental prototype of the transducer was fabricated and its performance was measured, which showed excellent agreement with the design.

\section{9:45-10:00 Break}

\section{0:00}

2aEA9. On implantable sensors for totally implantable hearing devices. Diego Calero, Stephan Paul, and Julio A. Cordioli (Mech. Eng. Dept., Federal Univ. of Santa Catarina, Campus Universitário, Trindade, Florianópolis, SC 88040-970, Brazil, diegocal_p@hotmail.com)

Conventional hearing devices, such as cochlear implants and hearing aids, use a microphone as a sensor for capturing the external sound field. Currently, the microphone is located in an external element, which is also responsible for processing the sound signal, but its presence is the cause of problems like discomfort, impossibility of being used during physical activities and sleeping, and social stigma. These limitations have driven studies with the goal of developing totally implantable hearing devices, and the design of an implantable sensor has been of the main issues to be overcome. Different designs of implantable sensors can be found in the literature and on some commercial implantable hearing aids, including different transduction mechanisms (capacitive, piezoelectric, electromagnetic, etc.), configurations (microphones, accelerometers, force sensor, etc.), and locations (subcutaneous or located in the middle ear). In this work, a detail review of such designs is presented and a general classification is proposed. The technical characteristics of the sensors are presented and discussed in view of the main requirements for an implantable sensor for hearing devices, including sensitivity, internal noise, frequency bandwidth, and energy consumption. The implantation feasibility of each sensor is also evaluated and compared.

\section{0:15}

2aEA10. Evaluation of spherical microphone baffle with six hollows for omnibinaural recording. Kento Imamura, Takanori Nishino, Taishi Nakagiri, and Hiroshi Naruse (Graduate School of Information Eng., Mie Univ., 1577 kurimamachiya-cho, Tsu, Mie 514-8507, Japan, imamura@pa.info. mie-u.ac.jp)

A binaural recording system records binaural sounds corresponding to plural listening orientations simultaneously. We devised and evaluated the binaural recording system. A binaural recording is generally performed with a dummy head; however, it can record for single listening orientation only. Moreover, an easy binaural recording system is desired because the dummy head is big, expensive, and difficult to handle. Therefore, we devised a spherical microphone baffle with two hollows was proposed, and the results addressed that it could be considered as a binaural recording system. In this study, a microphone baffle with six hollows was designed and evaluated. The proposed baffle has a spherical shape. Six hollows are located on three orthogonal axes originated from the center of the baffle. The acoustical features were examined by the numerical analysis. The results show that a difference between the previous research and the proposed baffle is small and the proposed baffle can achieve an omnibinaural recording.

\section{$10: 30$}

2aEA11. Piezoelectric low frequency shear horizontal guided wave transduction. Guillaume Boivin, Nicolas Tremblay, Ricardo Zednik, and Pierre Belanger (Mech. Eng., Ecole de technologie superieure, 1100, Notre Dame Ouest, Montreal, QC H3C 1K3, Canada, pierre.belanger@etsmtl. ca)

Ultrasonic guided waves are now routinely used in non-destructive evaluation. In plate-like structures, three fundamental modes can propagate, namely, $\mathrm{A}_{0}, \mathrm{~S}_{0}$, and $\mathrm{SH}_{0}$. Most of the guided wave literature has thus far focused on the use of $\mathrm{A}_{0}$ and/or $\mathrm{S}_{0}$ because these modes are easy to generate in plate-like structures using standard piezoceramic transducers. Yet, at low frequency, $\mathrm{A}_{0}$ and $\mathrm{S}_{0}$ are dispersive. The consequence of dispersion is that signal processing becomes complex for long propagation distances. $\mathrm{SH}_{0}$, on the other hand, has the particularity of being the only non-dispersive guided wave mode. Omnidirectional transduction of $\mathrm{SH}_{0}$ requires a torsional surface stress which cannot be easily generated using standard piezoceramic transducers. This paper compares a transducer concept based on piezoceramic patches assembled to form a discretized circle and a second concept based on a tellurium dioxide disk. The external diameter of the transducers was chosen to be half the $\mathrm{SH}_{0}$ wavelength at $100 \mathrm{kHz}$ in an aluminium plate. Finite element simulations using the Comsol Multiphysics environment showed that in a $1.6 \mathrm{~mm}$ aluminium plate the modal selectivity as well as the omnidirectionality of the tellurium dioxide concept was superior at 100 $\mathrm{kHz}$.

\section{0:45}

2aEA12. Thermal saturation and its suppression in high-power, compact carbon nanotube thin-film thermophones. Timothy $\mathrm{A}$. Brungart, James J. Chatterley, Benjamin S. Beck, Brian L. Kline, and Zachary W. Yoas (Appl. Res. Lab., The Penn State Univ., Appl. Res. Lab., P.O. Box 30, State College, PA 16804-0030, tab7@arl.psu.edu)

Carbon nanotube (CNT) thin-film thermophones, at sufficiently high input power levels, suffer from thermal saturation where an increase in the input power does not result in a corresponding increase in the sound pressure level generated. It is believed that high temperature air, trapped in and around the CNT film, inhibits the ability of the CNT film to cool sufficiently between heating cycles, thus limiting the sound pressure output and increasing in severity with both input power and frequency. Thermal saturation appears to be particularly acute for CNT thermophones designed for compactness or when placed inside a protective or loudspeaker enclosure, where natural convection or heat transfer from the film is inhibited. Fan cooling was integrated into a CNT thermophone and demonstrated to both reduce the temperature of the CNT film and suppress, almost entirely, the effects of thermal saturation.

\section{1:00}

2aEA13. A new standard for transduction materials. Roger M. Logan (Teledyne, 12338 Westella, Houston, TX 77077, rogermlogan@sbcglobal. net)

A proposal has been made to establish a new (ANSI) standard to replace MIL-STD-1376B (cancelled). This presentation will expand on this proposal and provide a rough outline as a possible starting point for such a standard. Feedback and discussion will be encouraged. 


\section{1:15}

2aEA14. Development of anti-cavitation hydrophone using a titanium front plate: Durability test in the high intensity focused ultrasound field. Michihisa Shiiba (Dept. of Clinical Eng., Nihon Inst. of Medical Sci., 1276 Shimogawara, Moroyamamachi Irumagun, Saitama 350-0435, Japan, m-shiiba@nims.ac.jp), Nagaya Okada (Honda Electronics Co., Ltd., Toyohashi, Aichi, Japan), and Shinichi Takeuchi (Toin Univ. of Yokohama, Yokohama, Japan)

Our research group has developed new anti-cavitation hydrophones by depositing a hydrothermally synthesized lead zirconate titanate polycrystalline film with $15 \mu \mathrm{m}$ thickness on the back surface of a titanium front plate with $50 \mu \mathrm{m}$ thickness and $3.5 \mathrm{~mm}$ diameter. A durability test of the anti-cavitation hydrophone was performed when the anti-cavitation hydrophone under test was placed at the focal point of a concave focused ultrasound transducer with $100 \mathrm{~mm}$ diameter and at a resonant frequency of $1.75 \mathrm{MHz}$. The amplified $80 \mathrm{~V}_{\mathrm{p}-\mathrm{p}}$ (calculated electric input power: about $40 \mathrm{~W}$ ) signal was applied to the concave ultrasound transducer at the focal point of the focused ultrasound system and high-intensity ultrasound waves were irradiated in water. The irradiated sound pressure at the focal point was about 4 MPa. Through this research, we will report that the fabricated new anti-cavitation hydrophone was robust and was not damaged easily, even in a high intensity focused ultrasound field with sound pressure of where acoustic cavitation occurred. We will make a durability test by increasing the acoustic power at the focal point of the focused acoustic field with sound pressure higher than $15 \mathrm{MPa}$.

\section{1:30}

2aEA15. Piezoelectric particle counter using the resonance vibration modes of a circular plate. Masatoshi Hayashi, Daisuke Koyama, and Mami Matsukawa (Faculty of Sci. and Eng., Doshisha Univ., 1-3 TataraMiyakodani, Kyotanabe, Kyoto 610-0394, Japan, dup0308@mail4.doshisha.ac.jp)

In the field of environmental measurements, robust and simple sensors with no electric power supply are required. In the river, the size distributions of sand and small stones in river-bed are important factor for river disaster prevention. In this report, a passive piezoelectric sensor for measurement of the particle distribution in flow was investigated. The sensor consists of an aluminum circular plate (diameter: $50 \mathrm{~mm}$; thickness: $2 \mathrm{~mm}$ ) and an annular piezoelectric transducer (inner radius: $5 \mathrm{~mm}$; outer radius: $10 \mathrm{~mm}$ ). Alumina spheres with several diameters $(\varphi=3,5$, and $8 \mathrm{~mm})$ were employed as small particles. When the particles hit the surface of the sensor, the flexural vibration is excited on the circular plate and the electric power is generated through the piezoelectric effect. Two main peaks at 16.3 and $66.7 \mathrm{kHz}$ appeared in the output signal, and the ratio of these peaks depended on the particle size. From the finite element analysis, it was found that these frequencies of 16.3 and $66.7 \mathrm{kHz}$ correspond with the fundamental and second resonance vibration modes. These results indicate that the particle size can be determined from the frequency spectrum of the output signal.

\section{$11: 45$}

2aEA16. Sound recording using optical marker-based motion tracking system: Relation between various constraints. Min-Ho Song and Rolf I. Godøy (Musicology, Univ. of Oslo, Institute for musikkvitenskap ZEBbygningen 2. etg Sem Sælands vei 2, Oslo 0371, Norway, minho.song@ imv.uio.no)

Optical marker-based motion tracking system is the device that can record the motion of moving object using multiple high-speed infrared (IR) cameras. Recent development of the motion capture device enables capturing the detailed motions with high spatial precision of sub-millimeter and high sampling rate up to $10 \mathrm{kHz}$. Currently, the motion tracking cameras can record the local vibrating movement of an acoustic instrument, which makes it possible to retrieve sound from the visual domain. In this study, two constraints in using marker-based motion tracking cameras for the sound recording are discussed. One is the temporal constraint where high sampling rates cause the detectability problem of the retro-reflective marker. When the cameras are operated with high speed, the cameras cannot radiate sufficient IR light and makes it hard to detect. The other is the amplitude constraint where the low camera calibration accuracy increases the signalto-noise ratio. The effect of these constraints is observed using professional motion tracking cameras (Qualisys) and their relations over various physical conditions will be given. 
Session 2aMUa

\title{
Musical Acoustics and Signal Processing in Acoustics: Computational Methods of Simulation for Musical Acoustics
}

\author{
Nicholas Giordano, Cochair \\ Physics, Auburn University, College of Sciences and Mathematics, Auburn, AL 36849 \\ Seiji Adachi, Cochair \\ Department of Acoustics, Fraunhofer Institute for Building Physics, Nobelstr. 12B, Stuttgart 70569, Germany
}

Chair's Introduction-8:00

\section{Invited Papers}

8:05

2aMUa1. Time history theoretical analysis for stick-slip vibration of violin strings. Hideo Utsuno (Mech. Eng., kansai Univ., 3-3-35 Yamate-cho, Suita 564-8680, Japan, utsuno@kansai-u.ac.jp)

The stick-slip vibration of violin string was completely formulated by using CA (Cellular Automaton) method in time history analysis. The local neighborhood rule of the CA method was derived for the travelling wave along the string, both ends of string, and the bow point. The key in success is that the bow point is to be fixed point and also to be velocity excited point at the same time. This means that reflected wave velocity is equal to the incidental wave velocity plus bow velocity. Displacement of arbitrary point of string is calculated and is compared with measured one. Excellent agreement of displacement suggests that CA method can simulate the stick-slip vibration of violin string. Acceleration time of the bow until it reaches a certain speed is also studied to form a complete Helmholtz waves. Step input and acceleration time within fundamental period is not valid to form Helmholtz wave. Acceleration time of more than four times of the fundamental period can substantially form Helmholtz waves.

\section{$8: 25$}

2aMUa2. Accurate time-domain modeling of the bowed string. Hossein Mansour (Music Res., McGill Univ., 555 Sherbrooke St. West, Montreal, QC, Canada), Jim Woodhouse (Eng., Cambridge Univ., Cambridge, United Kingdom), and Gary Scavone (Music Res., McGill Univ., Montreal, QC H3A 1E3, Canada, gary@music.mcgill.ca)

An enhanced time-domain, travelling-wave model of the bowed string is presented. The model includes several new features: realistic damping verified by experimental data, detailed coupling of body modes (derived from experimental data) in both polarizations of string motion, coupling to transverse and longitudinal bow-hair motion, and coupling to vibration of the bow stick. The model is designed to allow the various features to be turned on or off, such that their influence on the results can be assessed independently. Schelleng diagrams are computed using different configurations and are compared based on different metrics to reveal trends of behavior.

8:45

2aMUa3. Time domain simulations of a novel lingual organ pipe construction. Péter Rucz, Nóra M. Nagy (Dept. of Networked Systems and Services, Budapest Univ. of Technol. and Economics, 2 Magyar Tuodósok körútja, Budapest H1117, Hungary, rucz@hit.bme. hu), Judit Angster (Dept. of Acoust., Fraunhofer Inst. for Bldg. Phys., Stuttgart, Baden-Württemberg, Germany), Fülöp Augusztinovicz (Dept. of Networked Systems and Services, Budapest Univ. of Technol. and Economics, Budapest, Hungary), and András Miklós (Steinbeis Transfer Ctr. of Appl. Acoust., Stuttgart, Baden-Württemberg, Germany)

In a traditional pipe organ, the dynamic range of both labial (flue) and lingual (reed) pipe ranks are strictly limited as each rank is tuned and voiced to a nominal windchest pressure. Changing this pressure not only affects the amplitude of the radiated sound but both the pitch and the timbre of the pipes. A new pipe construction with a blown open free tongue was proposed recently to overcome this limitation. Prototype pipes of the new construction were built and measurements were carried out on them at different blowing pressures. It was found that the new construction provides a pleasing stability of the pitch and a broad range of playable amplitudes; however, the timbre of the pipes changes significantly with the blowing pressure. To improve the design in the latter aspect, a physical model of the pipes needs to be established first. In this contribution the sound generation of the experimental pipes is simulated by time domain computations. The resonator and its interaction with the vibration of the tongue are simulated using both the truncated impedance model and the reflection function approach. Results of the temporal simulations and their comparison with measurements are presented. 
2aMUa4. Modeling studies of wind instruments using the Navier-Stokes equations. Nicholas Giordano (Phys., Auburn Univ., College of Sci. and Mathematics, Auburn, AL 36849,njg0003@auburn.edu)

Modeling of musical instruments can be done at different levels of sophistication and realism. Depending on the goal of the simulation, it is sometimes possible to treat components of an instrument in an approximate way. For example, in dealing with a wind instrument it may be possible to consider a free running oscillator coupled to a one dimensional resonator with feedback, and ignore the physical details of the oscillator and feedback. However, in some cases, it is necessary to take a more exact approach and apply the fundamental laws of mechanics and fluid dynamics. For a wind instrument, this means the application of the Navier-Stokes equations, a set of nonlinear partial differential equations that require a numerical solution. In recent years, the available computational power has made it possible to apply the Navier-Stokes equations to instrument geometries that are fairly realistic. This talk reviews some of that work and discusses the kinds of questions that can be addressed with simulations of this kind. [Research supported by NSF grant PHY1513273.]

\section{Contributed Papers}

9:25

2aMUa5. Numerical study for the function of moving pad on tone hole acoustics. Taizo Kobayashi (Teikyo Univ., Misaki-machi, 6-22, Omuta, Fukuoka 836-8505, Japan, tkoba@cc.kyushu-u.ac.jp), Hidetaka Matsuda (The Phys. Labs., Kyushu Inst. of Technol., Iizuka, Fukuoka, Japan), Toshiya Tatami (Oita Univ., Oita, Japan), and Kin'ya Takahashi (The Phys. Labs., Kyushu Inst. of Technol., Iizuka, Fukuoka, Japan)

In this poster, we will discuss numerical methods for calculating a tone hole model with a moving pad of woodwind instrument by using compressible Large Eddy Simulation (LES), i.e., how to treat moving boundary problems. There are three stages of numerical methods to treat a moving pad on a tone hole. The 1st stage is the simplest method in which a dynamical mesh technique is not employed. It is so called "Stop Motion." The numerical conditions of the distance between the pad and the top of the tone hole are set to adequate points to discuss the effect of the distance, e.g., we set it to 0 $\mathrm{mm}$ (i.e., the tone hole is closed by a pad), $0.5 \mathrm{~mm}, 1 \mathrm{~mm}, 2 \mathrm{~mm}, 3 \mathrm{~mm}$, and $5 \mathrm{~mm}$. In the second stage, while a dynamical mesh technique is employed, on the other hand, it does not treat the condition with topological change of the boundary, i.e., in this situation, the distance changes from/into $0 \mathrm{~mm}$. The third stage is a full dynamical mesh technique which provides a method for treating moving boundary problems with topological changes. As the results of calculation, we will also report how the pitch is changed with fingering, and how the fluid field and acoustical field behave around the tone hole when it is changed, comparing with experimental study on the function of tone holes reported by Keefe [1]. Keefe, D.H. "Experiments on the single woodwind tone hole", J. Acoust. Soc. Am., 72 688-699 (1982).

\section{9:40}

2aMUa6. Numerical investigation on the influence of wall vibrations on the behavior of the lip excitation in brass instruments. Vincent Fréour (Res. and Development Div., YAMAHA Corp., Yamaha Corp., 203 Matsunokijima, Iwata 438-0192, Japan, vincent.freour@music.yamaha.com) and Hideyuki Masuda (Res. and Development Div., YAMAHA Corp., Iwata, Shizuoka, Japan)

The influence of wall vibrations in brass instruments has been an object of substantial debate in both musician and scientific communities. The excitation of the instrument wall (from the acoustic field inside the instrument and through the direct mechanical excitation from the lips) may influence the acoustic pressure field inside and outside the instrument, potentially resulting into some noticeable effects on the acoustic input impedance and radiated sound. These effects have been the object of numerical and experimental studies on different families of wind instruments. Furthermore, these vibrations are also likely to induce some perturbations on the excitation mechanism itself (the lips of the player), that may contribute to variations of the produced tone, as well as influence the sensations of the player. In this paper, the effects of the this mechanical coupling between the lips and vibrating wall is studied numerically using a physical model of the lips, coupled acoustically to the air-column of the instrument, as well as mechanically with the instrument wall described by a simplified mechanical admittance. Results from time-domain simulations and linear stability analysis will be presented. Some pathological situations, where a structural mode is close to the playing frequency will be particularly discussed.

\section{9:55}

2aMUa7. Precision harmonics simulation for vacuum-tube electric-guitar pedals and preamplifiers. Kanako Takemoto, Shiori Oshimo, and Toshihiko Hamasaki (Information Systems and Sci., Hiroshima Inst. of Technol., 2-1-1, Miyake, Saki-ku, Hiroshima 731-5193, Japan, md16009@ cc.it-hiroshima.ac.jp)

Recently, vacuum-tube pedals have attracted many electric guitar players, though solid-state circuits or even digital processors are widely accepted. The purpose of this study is to clarify the origin of distorted timbre of the vacuum-tube pedal by circuit simulation with a novel vacuumtube model. In general, the specific sound of vacuum tube for both hi-fi and electric guitar amplifier is attributed to the even number of harmonics originated from the inherent non-linearity. However, significant difference exists in the input signal amplitude between hi-fi and electric guitar system. The simulation with a conventional non-linear model has been limited within the regulated signal swing of hi-fi audio system. The dynamic non-linear phenomena for large signal, such as an input signal modulation, are not considered. Thus, unrealistic harmonics appear in the pedal simulation due to discontinuity of model parameters. The new model is obtained by physical analysis based on enough measurements, considering the actual input condition of pedal and preamplifier as well. The key property is a seamless modulation of non-linearity influenced by circuit operation. As a result, we have succeeded in simulating changes of the dynamic harmonics accurately for the first time.

\section{0:10}

2aMUa8. Electronic cymbals using spectral modeling synthesis with variable timbre by hitting strength and beater hardness. Naoto Wakatsuki and Koichi Mizutani (Univ. of Tsukuba, 1-1-1 Tennodai, Tsukuba, Ibaraki 305-8573, Japan, wakatuki@iit.tsukuba.ac.jp)

Sound source for cymbals in electronic drums based on spectral model synthesis were proposed instead of wavetable based sound sources. In the sound source, the timbre of the synthesized sound can be changed by hitting strength. In the real acoustical cymbals, the timbre change is caused by the coupling among the eigenmodes due to geometrical nonlinearities. In the sound source, it was reproduced using a simple model. As well as the hitting strength, the hardness of beaters hitting a sensor pad are also reflected to the timbre. It was achieved by measuring pulse width in the output analog signal from a commercial sensor pad. The proposed sound source was implemented as a Steinberg's Virtual Studio Technology (VST) plugin. In the talk, the implemented sound source will be simply demonstrated. 


\title{
Session 2aMUb
}

\section{Musical Acoustics and Signal Processing in Acoustics: Music Signal Processing I}

\author{
James W. Beauchamp, Cochair \\ School of Music and Electrical \& Computer Eng., University of Illinois at Urbana-Champaign, 1002 Eliot Dr., Urbana, \\ IL 61801-6824 \\ National Institute of Advanced Industrial Science and Technology (AIST), IT, AIST, 1-1-1 Umezono, Tsukuba 305-8568, \\ Japan
}

Chair's Introduction-10:40

Invited Papers

10:45

2aMUb1. Transcribing piano music in the time domain. Andrea Cogliati, Zhiyao Duan (Elec. and Comput. Eng., Univ. of Rochester, 308 Hopeman, Rochester, NY 14627, zhiyao.duan@rochester.edu), and Brendt Wohlberg (Los Alamos National Lab., Los Alamos, $\mathrm{NM})$

Automatic music transcription is the process of automatically inferring a high-level symbolic representation, such as music notation or piano-roll, from a music performance. It has many applications in music education, content-based music search, musicological analysis of non-notated music, and music enjoyment. Existing approaches often perform in the frequency domain, where the fundamental time-frequency resolution tradeoff prevents them from obtaining satisfactory transcription accuracies. In this project, we develop a novel approach in the time domain for piano transcription using convolutional sparse coding. It models the music waveform as a summation of piano note waveforms (dictionary elements) convolved with their temporal activations (onsets). The piano note waveforms are prerecorded in a context-dependent way, i.e., for the specific piano to be transcribed in the specific environment. During transcription, the note waveforms are fixed, and their temporal activations are estimated and post-processed to obtain the pitch and onset transcription. This approach models temporal evolution of piano notes, and estimates pitches and onsets simultaneously in the same framework. Experiments show that it significantly outperforms a state-of-the-art frequency-domain music transcription method trained in the same context-dependent setting, in both transcription accuracy and time precision, in various scenarios including synthetic, anechoic, noisy, and reverberant environments.

\section{1:05}

2aMUb2. Unsupervised grammar induction from music data. Kazuyoshi Yoshii (Kyoto Univ., Res. Bldg. \#7, Rm. 412, Yoshidahonmachi, Kyoto, Kyoto 606-8501, Japan, yoshii@kuis.kyoto-u.ac.jp)

Music has a lot of similarities to language. Since most languages have clear syntactic structures (e.g., words should be arranged in the SVO order), many linguists have proposed various kinds of grammar theories by carefully and manually investigating language data. The situation is the same with Western music. Although a single musical note (cf. alphabet) has no meaning by itself, a cluster or pattern of multiple musical notes over the quantized time-frequency grid (cf. word) can invoke some impression and such short patterns are concatenated or superimposed (unique to music) to produce more complicated meaning (cf. sentence). We introduce several attempts to discover latent structures underlying music from acoustic or symbolic data (music signals and musical scores) in an unsupervised manner. Integrating statistical acoustic and language models as in speech recognition, for example, it is possible not only to transcribe music but also to discover that particular note combinations can form chords. A key feature of this approach is that both models are trained jointly only from acoustic data. Recently, we have attempted to induce music grammars from polyphonic scores by leveraging the state-of-theart techniques of natural language processing. This would contribute to automatic music transcription and computational musicology.

\section{1:25}

2aMUb3. End-to-end music transcription using a neural network. Paris Smaragdis (CS \& ECE, Univ. of Illinois, 201 N Goodwin Ave., Office 3231, Urbana, IL 61801, paris@illinois.edu)

We present various neural network models that learn to produce music transcriptions directly from audio signals. Instead of employing commonplace processing steps, such as frequency transform front-ends or temporal smoothing, we show that a properly trained neural network can learn such steps on its own while being trained to perform note detection. We demonstrate two models that use raw audio waveforms as input and produce either a probabilistic piano roll output or text in music notation format that can be directly rendered into a score. 
2aMUb4. Multiple-timbre note tracking using linear dynamical systems. Emmanouil Benetos (School of Electron. Eng. and Comput. Sci., Queen Mary Univ. of London, Mile End Rd., London E1 4NS, United Kingdom, emmanouil.benetos@qmul.ac.uk)

This work addresses the problem of multi-pitch detection and note tracking in multiple-instrument polyphonic music recordings. A system is developed that extends probabilistic latent component analysis (PLCA) and supports the use of a five-dimensional dictionary of spectral templates per instrument, pitch, deviation from ideal tuning, and sound state (e.g., attack, sustain, decay). A method based on linear dynamical systems (LDS) is introduced for note tracking, which assumes that the output of the PLCA model is the (noisy) observation in an LDS, with the latent states corresponding to the ideal multi-pitch activation output. The LDS-based process supports the tracking of multiple concurrent pitches and can also be integrated within the PLCA-based model, thus guiding the convergence of the multi-pitch detection process. Experiments performed on several datasets of multiple-instrument polyphonic music demonstrate that the LDS-based method leads to significant improvements in multi-pitch detection as compared to using the frame-based PLCA model alone.

Session 2aNS

\title{
Noise and Architectural Acoustics: Noise Impacts and Soundscapes at Outdoor Gathering Spaces
}

\author{
K. Anthony Hoover, Cochair \\ McKay Conant Hoover, 5655 Lindero Canyon Road, Suite 325, Westlake Village, CA 91362 \\ Brigitte Schulte-Fortkamp, Cochair \\ Institute of Fluid Mechanics and Engineering Acoustics, TU Berlin, Einsteinufer 25, Berlin 101789, Germany
}

Chair's Introduction-7:45

\section{Invited Papers}

\section{$7: 50$}

2aNS1. Nestled in nature but near to noise-The Ford Amphitheatre. K. Anthony Hoover (McKay Conant Hoover, 5655 Lindero Canyon Rd., Ste. 325, Westlake Village, CA 91362, thoover@mchinc.com)

The historic Ford Amphitheatre was relocated in 1920, from what would later become the location of the Hollywood Bowl, across the Hollywood Freeway, into an arroyo with a dramatic natural backdrop. The original wood structure was destroyed by a brush fire in 1929, and rebuilt in concrete in 1931. In the meantime, the freeway has become increasingly noisy. The current renovation to this 1200 seat outdoor amphitheatre includes an expanded "sound wall" that will help to mitigate freeway noise while providing optimal lighting and control positions. The remarkably uniform distribution of ambient noise throughout the seating area, the significantly reverberant character of this outdoor space, and the apparent contributions by the arroyo will be discussed, along with assorted design and construction challenges.

\section{$8: 10$}

2aNS2. Acoustics upstaged: Challenging conundrums at the Starlight Bowl. David A. Conant (McKay Conant Hoover, Inc., 5655 Lindero Canyon Rd., Ste. 325, Westlake Village, CA 91362, dconant@MCHinc.com)

Since its debut in 1935, the 3600-seat Starlight Bowl in San Diego's renowned Balboa Park has been increasingly impacted by environmental noise, principally from aircraft landing at nearby Lindbergh Field. Traditionally, performers would temporarily "freeze" on cue from the director as a jet approached, then resume as though nothing had happened. Although amusing initially, audiences soon were just annoyed. $\mathrm{MCH}$ was retained to identify and rank prospective mitigation measures as the principal tenant was losing audience. In addition to collecting the usual ambient and fly-by noise level data, $\mathrm{MCH}$ examined the efficacy of partial and full enclosure options, and associated implications on audio system planning and operation. Ultimately, the myriad of acoustical/audio noise-mitigating options developed could not be reconciled with the historic venue's name, as the visual impact alone, of large jets flying within $600 \mathrm{ft}$ would forever be up-staging any on-stage performance. We will report on our process, observations, and recommendations. 
2aNS3. "Shhh." and other methods for controlling crowd noise. Shane J. Kanter and Jonathan Laney (Threshold Acoust., 53 W. Jackson Blvd., Ste. 815, Chicago, IL 60604, skanter@thresholdacoustics.com)

As designers of venues for performance, we spend a considerable amount of time and effort on maintaining a low background noise level while the acoustically critical spaces are in use. The typical noise sources, such as mechanical systems and light fixtures, are predictable and controllable. Just as background noise is inferential, audience noise within the common classical music venue is generally understood and under control. Effects of outdoor background noise are explored — such as city noise, road noise, and cicadas at two Chicago outdoor venues: Jay Pritzker Pavilion and Ravinia Festival. Second, because both of these venues have a fixed section of seating near the stage and a large section of general admission law seating, the lawn seating typically fosters a relaxed atmosphere where patrons feel free to converse with one another during performances. Ravinia and the Pritzker Pavilion differ when it comes to how they deal with chatting patrons during performances. Special attention will be paid to the audience noise in both the fixed seating and lawn seating at each venue and through the use of patron interviews, and exploration the effect of audience noise on the performance experience.

\section{8:50}

2aNS4. Amphitheaters: The noise within. Richard H. Talaske (TALASKE I Sound Thinking, 1033 South Blvd., Oak Park, IL 60302, rick@talaske.com)

The continuing success of outdoor performance facilities demonstrates that music and theatre can be enjoyed within environments which are much nosier than the silent indoor concert halls and theaters we know and love. However, there are limits to how much noise is acceptable. Design methods can be implemented to control the noise and significantly improve the listening experience. This presentation offers insight into the design of outdoor music venues and the management of noise as heard by the patron and performers. Numerous amphitheaters will be discussed, including the Jay Pritzker Pavilion, American Players Theatre, and Aurora's River's Edge, thereby offering examples of facilities designed for symphonic music, theatre, and popular music. The importance of acoustic considerations such as signal-to-noise, frequency response, and direction of arrival of sound will be discussed.

\section{9:10}

2aNS5. Overcoming challenges to future outdoor entertainment performances due to disruptive commercial vendor noise intensity and the resulting community outrage. Marlund E. Hale (Adv. Eng. Acoust., 663 Bristol Ave., Simi Valley, CA 93065, mehale@ aol.com)

Outdoor entertainment and concerts are commonplace historically. Many of these public attractions also include commercial, concessions, and other vendor booths. Typically, such events are regulated by local government. During the inaugural performance of a successful music festival, whose sponsors had contractually agreed to the community permitting requirements, a commercial vendor proceeded to generate such loud and intense sounds that the entire festival and closest residential community were bombarded with object vibrating sounds. The public outcry from the local residential community was immediate and so was the reaction of the festival organizers. The commercial vendor was ordered to vacate the venue. However, the local community was so outraged that the festival organizers were put on notice that the festival had violated its contract and would not be permitted again. After many hours of community outreach and public hearings, the festival organizers were given a highly conditional permit to hold the festival again the following year. Strict A-weighted and C-weighted noise limits and heavy violation fines were imposed, as well as a requirement for continuous noise monitoring at multiple sites at the venue and in the residential community. This paper reports the concert performance results of the past few years.

\section{9:30}

2aNS6. Changes to soundscapes at outdoor gathering spaces in Fukushima, Japan, caused by the severe nuclear power plant accident. Koji Nagahata (Fukushima Univ., 1 Kanayagawa, Fukushima 960-1296, Japan, nagahata@sss.fukushima-u.ac.jp)

Five years have passed since the accident at the Fukushima Daiichi Nuclear Power Plant, which caused changes to daily life in the city of Fukushima, Japan. These daily life alterations caused changes to the soundscapes of Fukushima, which are still evolving now. In this study, the soundscape change at Fukushima's outdoor gathering spaces is discussed using field recordings by the author. Shortly after the accident, few human voices could be heard at outdoor gathering spaces, but natural sounds such as birdsongs could be heard as usual per the time of year. Park decontamination started during summer 2011. In some parks where decontamination was successfully completed, people's voices and sounds of children playing returned during spring 2012. However, in other parks where decontamination was done but ineffective, the lack of human voices and artificial sounds continued until radiation levels decreased sufficiently. In this way, soundscape change at outdoor gathering spaces represents people's attitude toward the radioactive contamination; soundscape recording documents not only sonic environments but also people's lives.

\section{9:50-10:05 Break}

\section{0:05}

2aNS7. E-participation in the context of the evaluation of the sonic environment. André Fiebig (HEAD Acoust. GmbH, Ebertstr. 30a, Herzogenrath 52134, Germany, andre.fiebig@head-acoustics.de), Brigitte Schulte-Fortkamp (ISTA Institut für Technische Akustik, Technische Universität Berlin, Berlin, Germany), and Klaus Genuit (HEAD Acoust. GmbH, Herzogenrath, Germany)

E-participation methods are increasingly applied to promote public participation and to achieve greater awareness and involvement by citizen by providing the opportunity to directly report to, e.g., governmental organizations. Within the framework of the German Year of Science 2015, a special initiative, called "Sound of the City," was carried out to encourage the public to report on how their cities typically sound. Participants could upload audio recordings of acoustical environments and had to relate the respective audio files 
to the corresponding places. Moreover, the participants could additionally describe the uploaded sounds. This action lasting several months led to a comprehensive database of audio files including related places, descriptions, and ratings. The database was systematically analyzed with respect to several issues, such as general participation level, hot spot identification, and common features of uploaded sounds. The paper will highlight the benefit and limitations of such actions and initiatives applying e-participation with respect to community noise issues and urban planning. [The "Sound of the City" initiative took place within the framework of the Year of Science 2015 and was organized and supported by the Federal Ministry of Education and Research (BMBF).]

\section{0:25}

2aNS8. Next generation soundscape design using virtual reality technologies. Andy Chung (Smart City Maker, Hong Kong Plaza, Hong Kong HKSAR, Hong Kong, ac@smartcitymaker.com), Wai M. To (Macao Polytechnic Inst., Macao, Macao), and Brigitte Schulte-Fortkamp (TU, Berlin, Germany)

Sound quality has been demanded by the community as part of the smart city initiatives to enjoy a real liveable environment. While a lot of governments pay attention to environmental issues such as air quality and waste, those who care about the sonic environment still rely primarily quantitative levels for either compliance or improvement. Sound quality is most likely ignored. There is at present the international standard ISO 12913-1:2014 providing the definition and the conceptual framework of soundscape. Despite the situation, sound quality is a subjective matter after all and relies very much on human perceptions, as well as the contextual environment. Sound walk, questionnaire, and lab test are common tools used in soundscape studies. These tools allow a good understanding of the perception of the prevailing sonic environment. To figure the sound quality of different design options, though, we can immerse the participants in a virtual, but photorealistic environment, so that they can perceive, compare, and give feedback as if they were in the real environment. A mobile VR application has been developed for iterating soundscape design and fine-tuning. This paper presents this application with case studies.

\section{Contributed Papers}

\section{0:45}

2aNS9. On-site and laboratory soundscape evaluations of three recreational urban spaces. Anna Josefine Sørensen (Dept. of Elec. Eng., Tech. Univ. of Denmark, Ørsteds Plads 352, Kgs. Lyngby 2800, Denmark, annajosefine@gmail.com), Thea Mathilde Larsen, Lærke Cecilie Bjerre (Dept. of Civil Eng., Tech. Univ. of Denmark, Kgs. Lyngby, Denmark), Sébastien Santurette (Hearing Systems, Dept. of Elec. Eng., Tech. Univ. of Denmark, Kgs. Lyngby, Denmark), and Cheol-Ho Jeong (Acoust. Technol., Dept. of Elec. Eng., Tech. Univ. of Denmark, Kgs. Lyngby, Denmark)

Soundscape quality was evaluated using four subjective psychological rating factors in three recreational urban spaces in which water and a variation of other natural and anthropogenic sound sources were present. The noise level was measured at each site during occupant peak flows and recordings for listening experiments were made simultaneously. Listeners answered questionnaires either on site or following playback of the recordings in the laboratory, with or without access to each site's visual context. They rated their perception of loudness, acceptance, stressfulness, and comfort, along with their preference toward eight sound sources. The comfort ratings were negatively correlated with loudness and stressfulness and positively correlated with acceptance. The sound level was found to be a good predictor of these subjective parameters in the laboratory, but not on site. Moreover, the availability of the visual context in the listening experiment had no effect on the ratings. The presence of trees and water was also found to increase on-site comfort. Generally, the participants were more positive towards natural sound sources on-site. Overall, the results suggest that on-site context plays an important role for evaluating acoustic comfort in urban recreational areas.

\section{1:00}

2aNS10. Real-time natural soundscape generation based on current weather conditions for workspace voice-masking. Caitlin Riggs and Jonas Braasch (Rensselaer Polytechnic Inst., 110 8th St., Troy, NY 12180, riggs. cait@gmail.com)

Sound masking in work spaces has been implemented to decrease likelihood of distraction from work tasks and improve speech privacy. Current uses of noise as maskers commonly apply broadband white or pink noise signals due to their perceived neutrality. This research combines work into the restorative properties of exposure to nature/natural sounds and pilot studies of natural sounds as maskers to suggest a noise-masking system of "natural" sounds. This system composes a natural soundscape in real time determined by the current weather and time of day such that the masking audio is aesthetically pleasing and informative about the outside world in addition to providing improved speech privacy and reducing distraction when compared to a setting with no masking system. Currently, there is no experimental foundation to suggest that restoration or slowed attentional fatigue can occur if this type of alternative masking sound is presented during a task. This implementation of a dynamic, immersive soundscape masker begins the investigation into the practical efficacy of such a masking system.

\section{1:15}

2aNS11. Evaluation of the noise disturbance and noise limits of a riding horse shooting competition. Juan C. Montoya (Cross Spectrum Acoust., 41 Churchill St., Springfield, MA 01108, jmontoya@csacoustics.com)

Shooting ranges have turned into an unwanted activity in rural communities due to high levels of impulse sound pressure levels. Permanent complaints from neighbors or neighborhood associations pertaining health concerns and noise pollution around areas nearby shooting ranges are a driving force to create state noise control laws. As such, examining zoning will limit (restricting licenses ) to the use of agricultural or residential land to be used for shooting events. Gun noise is measurable and therefore is able to be quantified and characterized. This study evaluates with field noise measurements the events from a "shooting ranch" which currently operates offering a shooting competition while riding a horse emulating the Old West. The analysis of field data pretends to look at metrics such as LAeq (Aweighted equivalent sound level), L90 (noise level exceeded for $90 \%$ of the measurement period), and Lmax ( maximum level if the measurement period from 50 caliber single action revolver gun sounds while shooter is in movement riding a horse. The field measurements were conducted in different nearby residences. The impulse noise lead to examine instead individual gunshots which exceeded background levels by 14 to 34 decibels. Noise levels from Shooting Ranch events are sometimes more than three times the background noise level. Leq and L90 metrics are applicable within individual events.

\section{1:30-12:00 Panel Discussion}




\title{
Session 2aPA
}

\section{Physical Acoustics and Noise: Acoustics of Supersonic Jets: Launch Vehicle and Military Jet Acoustics I}

\author{
Seiji Tsutsumi, Cochair \\ JEDI Center, JAXA, 3-1-1 Yoshinodai, Chuuou, Sagamihara 252-5210, Japan \\ Kent L. Gee, Cochair \\ Brigham Young University, N243 ESC, Provo, UT 84602 \\ Janice Houston, Cochair \\ Jacobs ESTS Group, 1500 Perimeter Pkwy, Suite 400, Huntsville, AL 35806
}

Chair's Introduction-8:15

\section{Invited Papers}

2aPA1. Influence of launch platform cut-outs on flow and acoustic behavior of rocket exhaust. Karthikeyan Natarajan and Lakshmi Venkatakrishnan (Experimental AeroDynam. Div., CSIR-National Aerosp. Labs., EAD, PB 1779, Old Airport Rd., Bangalore 560017, India, nkarthikeyan@nal.res.in)

A launch vehicle experiences intense acoustic loading in the initial phase of its lift-off which affects the launch vehicle structure, sensitive payloads, and electronics on board. There is immense interest in alleviation of acoustic loads resulting in reduced need for strengthening of the vehicle structure. The effect of jet blast deflector shape on the acoustic loading has been extensively investigated, both computationally and experimentally, by simulating jet(s) impinging on a flat plate. However, contributions from the launch vehicle environment, such as the launch platform, are often ignored. The motivation for this study is that the flow over the launch platform is likely to be significantly influenced by the cut-outs made in the platform for the nozzles. As the nozzles emerge out from the cut-outs during lift-off, the jet exhaust grows and interacts with the launch platform, contributing to the overall acoustic loads experienced by the vehicle. This paper presents an experimental investigation of rocket exhaust interaction with the launch platform using single and twin jets impinging on a flat plate with cut-outs. The measurements include high speed shadowgraphy and microphone measurements in the near and far-field to enable flow and acoustic characterization.

\section{8:40}

2aPA2. A design method of fairing acoustic noise mitigation for Korean space launcher. Soon-Hong Park and Sang-Hyun Seo (Launcher Structures Dept., Korea Aerosp. Res. Inst., 169-84 Gwahangno, Yuseong-gu, Daejeon 305806, South Korea, shpark@kari.re. $\mathrm{kr})$

In this presentation, fairing acoustic protection system (APS) for Korean space launcher is introduced. The design of APS for payload fairing of KSLV-II is summarized. The current APS consists of acoustic blankets for midfrequency range absorption and acoustic resonators for low frequency noise absorption. Detailed design procedures of APS, which include external acoustic loading prediction based on the modified NASA SP-8072 monograph and fairing system vibro-acoustic analysis, are presented. An acoustic test of a cylindrical composite structure with APS is also presented. The effect of spatial correlation of external acoustic excitation on the vibro-acoustic response of fairing structure and its interior volume is discussed. The concept of future APS by using the micro-perforated panel absorber combined with Helmholtz resonators is also introduced. Effects of high pressure environment on the acoustic absorption of micro-perforated panel is discussed.

\section{9:00}

2aPA3. Impact of drift on the vehicle liftoff acoustic environments. Clothilde Giacomoni (All Points Logistics, LLC, M.S. ER42, Bldg. 4203, Huntsville, AL 35812, clothilde.b.giacomoni@nasa.gov) and R. Jeremy Kenny (Marshall Flight Ctr., NASA, Huntsville, $\mathrm{AL})$

During liftoff, a vehicle can drift due to wind, nozzle gimbaling, fly away maneuver, etc. This drift can cause the exhaust plumes to impinge on the deck and cause the noise levels experienced by the vehicle to increase. A small increase in the plume impingement can have a dramatic effect on the noise levels when the vehicle is only a few nozzle diameters from the deck. As the vehicle lifts off the deck the increase in noise levels lessens as the plume impingement increases. Several scale model acoustic tests have been undertaken at Marshall Space Flight Center which had test cases that were used to define the relationship between drift and the noise levels experienced by the vehicle. 
2aPA4. Analysis of nozzle geometry effect on supersonic jet noise using Schlieren. Yuta Ozawa (Tokyo Univ. of Sci., Niijuku 6-3-1, Katsushika-ku, Tokyo 125-8585, Japan, ozawa@flab.isas.jaxa.jp), Akira Oyama (ISAS/JAXA, Sagamihara-shi, Kanagawa, Japan), Masayuki Anyoji (Kyusyu Univ., Kasuga-shi, Fukuoka, Japan), Akira Oyama (ISAS/JAXA, Sagamihara-shi, Kanagawa, Japan), Hiroya Mamori, Naoya Fukushima, Makoto Yamamoto, and Kozo Fujii (Tokyo Univ. of Sci., Katsushika-ku, Tokyo, Japan)

Strong acoustic waves emitted from rocket plume might damage to rocket payloads because rocket payloads consist of fragile structure. Therefore, it is important to predict acoustic directivity and reduce its intensity level. In this study, we conduct experiments of supersonic jet flows and investigate an influence of the nozzle geometry on acoustic waves by means of Schlieren method and microphone measurement. Three different nozzles are examined: a conical nozzle, a convergent-divergent nozzle (referred as C-D nozzle), and a tab-C-D nozzle. Tabs are equipped in the nozzle inside and turbulence is generated in the tab-C-D nozzle case. The Schlieren visualization shows that the strong shock trains are observed in the potential core of the jet for the conical nozzle case, while the shock waves are relatively weak since the nozzles are in the nearly ideal expanded condition in the C-D nozzle and tab-C-D cases. The distribution of near field OASPL (over all sound pressure level) obtained by microphone measurement shows strong directivity in the downstream direction for all the cases. This directivity seems to be the Mach wave radiation. Moreover, conical nozzle cases have strong acoustic intensity level caused by shock associated noise.

\section{9:40}

2aPA5. Visualization movie analysis of acoustic phenomena of a supersonic jet and Its comparison with intensity vectors. Masahito Akamine, Koji Okamoto (Graduate School of Frontier Sci., Univ. of Tokyo, Kashiwanoha 5-1-5, Okamoto lab., Dept. of Adv. Energy, Graduate School of Frontier Sci., Kashiwa, Chiba 277-8561, Japan, akamine@thermo.t.u-tokyo.ac.jp), Kent L. Gee, Tracianne B. Neilsen (Dept. of Phys. and Astronomy, Brigham Young Univ., Provo, UT), Susumu Teramoto, Takeo Okunuki (Graduate School of Eng., Univ. of Tokyo, Bunkyo-ku, Tokyo, Japan), and Seiji Tsutsumi (Japan Aerosp. Exploration Agency, Sagamihara, Kanagawa, Japan)

The authors have studied the acoustic wave from an unheated, Mach 1.8 ideally expanded jet by using the acoustic intensity vector measurement and analysis of high-speed schlieren visualization movies (for example, the Fourier transform and wavelet-based conditional sampling). Both these techniques reveal the characteristics of the acoustic wave, such as the propagation direction and location of a source region. These techniques have their own advantages: the quantitative data can be obtained by using the acoustic intensity vector measurement, whereas the acoustic field including a close region to the jet can be visualized with high spatial resolution by using the Schlieren movie analysis. Therefore, their comparison is a meaningful approach to understand the acoustic phenomenon. This presentation compares these two techniques and describes what can be discussed with their comparison, considering the advantage and disadvantage of each measurement technique.

10:00-10:20 Break

\section{0:20}

2aPA6. Results of scale model acoustic tests using supersonic cold jets for $\mathbf{H 3}$ launch vehicle. Wataru Sarae, Atsushi Sawada, Keita Terashima (JAXA, 2-1-1 Sengen, Ibaraki, Tsukuba 305-8505, Japan, sarae.wataru@jaxa.jp), Takanori Haga, Seiji Tsutsumi (JAXA, Kanagawa, Japan), Tatsuya Ishii (JAXA, Tokyo, Japan), and Tetsuo Hiraiwa (JAXA, Miyagi, Japan)

Acoustic test using Mach 2 cold jet was conducted for the $\mathrm{H} 3$ launch vehicle currently being developed in Japan. Effect of the clustered engines and the newly built movable launcher on the lift-off acoustics was investigated. The overall acoustic level taken by the farfield microphones did not show proportional increase in the number of engines, especially for the angles corresponding to the Mach wave radiation from the free jets. The observation here disagrees with the empirical prediction model. The computational fluid dynamics was also employed to analyze the acoustic mechanism of clustered engines.

\section{0:40}

2aPA7. Comparison of spatial correlation parameters between full and model scale launch vehicles. Clothilde Giacomoni (All Points Logistics, LLC, M.S. ER42, Bldg. 4203, Huntsville, AL 35812, clothilde.b.giacomoni@nasa.gov) and R. Jeremy Kenny (Marshall Flight Ctr., NASA, Huntsville, AL)

The current vibro-acoustic analysis tools require specific spatial correlation parameters as input to define the liftoff acoustic environment experienced by the launch vehicle. Until recently, these parameters have not been very well defined. A comprehensive set of spatial correlation data were obtained during a scale model acoustic test conducted in 2014. From these spatial correlation data, several parameters were calculated: the decay coefficient, the diffuse to propagating ratio, and the angle of incidence. Spatial correlation data were also collected on the EFT-1 flight of the Delta IV vehicle which launched on December 5, 2014. A comparison of the spatial correlation parameters from full scale and model scale data will be presented. 


\section{Contributed Papers}

\section{1:00}

2aPA8. Intensity-based laboratory-scale jet noise source characterization using the phase and amplitude gradient estimator method. Kent $\mathrm{L}$. Gee, Tracianne B. Neilsen, Eric B. Whiting, Darren K. Torrie (Dept. of Phys. and Astronomy, Brigham Young Univ., N243 ESC, Provo, UT 84602, kentgee@byu.edu), Masahito Akamine, Koji Okamoto (Graduate School of Frontier Sci., Univ. of Tokyo, Kashiwa, Chiba, Japan), Susumu Teramoto (Graduate School of Eng., Univ. of Tokyo, Bunkyo-ku, Japan), and Seiji Tsutsumi (Japan Aerosp. Exploration Agency, Sagamihara, Kanagawa, Japan)

A new method for the calculation of vector acoustic intensity from pressure microphone measurements has been applied to the aeroacoustic source characterization of an unheated, Mach 1.8 laboratory-scale jet. Because of the ability to unwrap the phase of the transfer functions between microphone pairs in the measurement of a broadband source, physically meaningful near-field intensity vectors are calculated up to the maximum analysis frequency of $32 \mathrm{kHz}$. This result improves upon the bandwidth of the traditional cross-spectral intensity calculation method by nearly an order of magnitude. The new intensity method is used to obtain a detailed description of the sound energy flow near the jet. The resulting intensity vectors have been used in a ray-tracing technique to identify the dominant source region over a broad range of frequencies. Additional aeroacoustics analyses provide insight into the frequency-dependent characteristics of jet noise radiation, including the nature of the hydrodynamic field and the sharp transition between the Mach wave and sideline radiation.

\section{1:15}

2aPA9. Spatial variation in similarity spectra decompositions of a Mach 1.8 laboratory-scale jet. Aaron B. Vaughn, Tracianne B. Neilsen, Kent L. Gee (Phys. and Astronomy, Brigham Young Univ., Brigham Young University, C-110 ESC, Provo, UT 84602, aaron.burton.vaughn@gmail.com), Koji Okamoto, and Masahito Akamine (Adv. Energy, Univ. of Tokyo, Kashiwa, Japan)

The primary source of noise from supersonic jets is turbulent mixing noise. Tam et al. [AIAA Paper 96-1716 (1996)] proposed similarity spectra for a two-source model of turbulent mixing noise corresponding to noise from omnidirectional fine-scale turbulence structures and directional large- scale turbulent structures. These empirical similarity spectra have been compared with reasonable success to the spectra of both military and laboratoryscale jets. Most application have looked at the variation in angle: fine-scale similarity spectra matches sideline of the jet nozzle, large-scale in the maximum radiation lobe, and a combination needed in between. A similarity spectra decomposition of from an ideally expanded, Mach 1.8 laboratoryscale jet allows for a spatial comparison between the near and far-field spectra. The sound from the convergent-divergent nozzle was collected at the Hypersonic and High-Enthalpy Wind Tunnel at Kashiwa Campus of the University of Tokyo at a variety of near, mid, and far field locations. Comparison of similarity spectra decompositions over distance yield insights into the sound propagation from this supersonic jet. In addition, results from a preliminary wavenumber eduction technique to build a wavepacket-based equivalent source model of the large-scale turbulent mixing noise. [Work supported by Office of Naval Research grant.]

\section{1:30}

2aPA10. Far-field acoustical measurements during QM-2 solid rocket motor static firing. Brent O. Reichman, Tracianne B. Neilsen (Brigham Young Univ., 453 E 1980 N, \#B, Provo, UT 84604, brent.reichman@byu. edu), Won-Suk Ohm (Yonsei Univ., Seoul, South Korea), and Blaine M. Harker (Brigham Young Univ., Provo, UT)

The five-segment Space Launch System solid rocket motor was recently tested at Orbital ATK. Far-field acoustical measurements were performed at angles between $80^{\circ}$ and $120^{\circ}$ relative to the rocket exhaust at a distance of roughly $2500 \mathrm{~m}$ from the rocket, approximately 800 nozzle diameters. The angular aperture allows for evaluating spatial variation in acoustic properties and a comparison with similar tests in the past, including the 2015 test of the same rocket motor. Although terrain variations introduce uncertainty, an approximate $10 \mathrm{~dB}$ change in level is seen throughout the aperture, consistent with previous studies. In addition, at low frequencies a high degree of correlation is seen. Near the peak radiation direction high levels of derivative skewness indicate significant shock content and crackle. This dataset also presents the opportunity to test a new method for processing acoustic vector intensity [Thomas et al., JASA 137, 3366-3376 (2015)]. Comparison with the traditional method shows an increase in usable bandwidth of more than an order of magnitude. 
Session 2aPP

\title{
Psychological and Physiological Acoustics: Linking Human Physiology with Psychoacoustics—From Brainstem to Cortical Measurements and Beyond
}

\author{
G. Christopher Stecker, Cochair \\ Hearing and Speech Sciences, Vanderbilt University, 121521 st Ave. South, Room 8310, Nashville, TN 37232 \\ Adrian KC Lee, Cochair \\ University of Washington, Box 357988, University of Washington, Seattle, WA 98195
}

Chair's Introduction-8:30

Invited Papers

8:35

2aPP1. Pupillary responses signify more than just effort: Windows into processing, prediction, reflection, and uncertainty. Matthew Winn (Speech \& Hearing Sci., Univ. of Washington, 1417 NE 42nd St., Seattle, WA 98105, mwinn83@gmail.com)

Pupil dilation has long been used to index effort or general cognitive arousal in a variety of tasks. Traditionally, the main outcome measure is peak pupil dilation or the mean pupil dilation within some target window. In this talk, we explore how the time course of the growth and decay of pupil dilation can lend further insight into auditory processing. A series of pupillometry experiments revealed elevated listening effort resulting from various influences such as poor spectral resolution, unpredictability of sentence content, selective attention, or the need to reflect on what was heard to produce a creative response. Peak and mean pupillary responses generally confirmed original hypotheses, but more interesting findings emerge upon inspection of unforeseen trends in the data. Specifically, the timing of the pupil decay response can arguably be interpreted as a signature of the completion of sentence prediction, or the completion of processing that relies on memory trace (rather than perception) of the signal. The ultimate goal of this discussion is to unpack a new approach to pupillometry experiments that is aimed at separating processing that stems from perception versus processing that stems from cognitive processing, or reflecting upon a memory trace of the stimulus.

2aPP2. Electrophysiological correlates of auditory object binding with application to autism spectrum disorders. Hari M. Bharadwaj, Sheraz Khan, Matti Hämäläinen (Athinoula A. Martinos Ctr. for Biomedical Imaging, Massachusetts General Hospital, 149 Thirteenth St., Boston, MA 02129, hari@nmr.mgh.harvard.edu), and Tal Kenet (Dept. of Neurology, Massachusetts General Hospital, Boston, MA)

A fundamental problem in auditory neuroscience is to understand how the fragmented physiological representation of sound along the auditory pathway leads to unified percepts of broadband objects and streams. Natural sounds from a single physical source often exhibit strong temporal coherence between their acoustic components. We know from previous psychoacoustic studies that this temporal coherence is a cue for perceptual binding. Using magnetoencephalography (MEG), we measured neural responses to (1) a mixture of modulated tones, and (2) sentences of four-tone sine-wave speech, as we parametrically varied the temporal coherence between their components. We found that in parallel with behavior, MEG responses from the primary auditory cortex varied in with the temporal coherence of the stimulus. Further, cortical oscillatory signatures from the same region also varied with the stimulus coherence. These results suggests that signatures of temporal-coherence-mediated binding are already present at the level of the auditory cortex and may be mediated in-part by oscillatory thalamocortical processes. Finally, when we measured MEG responses in a cohort of children with autism spectrum disorders, we found a reduction in both the evoked responses and the oscillatory activity suggesting anomalous early cortical processing of complex sounds.

9:15

2aPP3. Relating binaural psychophysics to human physiology with functional magnetic resonance imaging: Opportunities and challenges. G. C. Stecker, Nathan C. Higgins, Sandra Da Costa (Hearing and Speech Sci., Vanderbilt Univ., 1215 21st Ave. South, Rm. 8310, Nashville, TN 37232, g.christopher.stecker@vanderbilt.edu), and Susan A. McLaughlin (Univ. of Washington, Seattle, WA)

Functional magnetic resonance imaging (fMRI) has revolutionized the study of human perception in all sensory modalities. While early studies focused on identifying the brain regions involved with various processes ("where"), recent approaches aim to quantify relationships between brain activity and the physical or perceptual features of sensory stimuli ("how"). These modern approaches thus mirror the psychophysical goal of relating sensation to its underlying physical parameters, providing a clear means to connect human psychophysics and brain physiology. This presentation will describe a series of studies investigating the sensitivity of human auditory 
cortex (AC) to binaural features of sounds and their perceptual correlates. Specifically, three questions will be addressed: (1) Does AC activity respond to the physical features or to the perception of binaural sound? (2) Do competing sounds sharpen or broaden the binaural selectivity of AC responses? (3) Does binaural sensitivity in human AC change across spatial, non-spatial, and non-auditory tasks? Questions (1) and (2) assess cortical correlates of psychophysical performance, elucidating the AC processes involved in spatial hearing. Question (3), however, goes a step further by addressing the potential effects of the psychophysical task itself. [Work supported by NIH R01 DC011548.

\section{9:35}

2aPP4. Switching of auditory attention. Adrian K. C. Lee, Susan A. McLaughlin, and Eric Larson (Univ. of Washington, Box 357988, Seattle, WA 98195, akclee@uw.edu)

Active listening in an acoustically crowded environment requires both maintaining and switching attention between auditory objects. General behavioral costs associated with task-switching are well documented in the cognitive literature. Yet there are many unanswered questions about the cost associated with switching between auditory objects relative to the type of feature-based attention employed. For example, do listeners use the same strategy to switch attention between locations as would be used to switch attention between speakers with different pitches? Is it equally difficult to switch attention within the same feature (e.g., attending to the left then the right talker) compared to switching across features (e.g., attending to the left talker then the one with the higher pitch)? Are the neural substrates recruited differentially depending on whether a listener is attending to spatial or non-spatial features of the auditory stimuli? In this talk, evidence from human psychophysical and physiological studies will be presented suggesting that auditory attention switching is not a monolithic construct. The interaction between top-down attention and different degrees of auditory stream degradation will also be discussed.

\section{Contributed Papers}

\section{9:55}

2aPP5. Effects of Auditory Attention on Otoacoustic Emissions. Heivet Hernandez Perez, Catherine McMahon (Linguist, Macquarie Univ., The Australian Hearing Hub, Sydney, NSW 2109, Australia, heivet.hernandezperez@students.mq.edu.au), Sumitrajit Dhar, Sriram Boothalingam (Commun. Sci. and Disord., Northwestern Univ., Chicago, IL), David Poeppel (Dept. of Psych., New York Univ., New York, NY), and Jessica J. Monaghan (Linguist, Macquarie Univ., Sydney, NSW, Australia)

In humans, auditory efferent control of the cochlea has been implicated in sharpening of frequency tuning, improved detection of signals in noise, and the ability to switch the focus of auditory attention. However, it remains unknown whether the modulation of efferent activity during an auditory attention task depends on the degree of task difficulty. This study aimed to compare the suppression of otoacoustic emissions (OAEs), an objective measure of auditory efferent activation, during a lexical decision task with varying degrees of difficulty, compared with passive auditory listening. Ten normal-hearing 18-35 year-olds were assessed using monosyllabic words and non-words presented in a natural or noise-vocoded (less intelligible) condition. The participants were instructed to press a button every time they heard a non-word. Simultaneously, click evoked-OAEs were obtained from the contralateral ear to the speech stimuli with a probe in the external ear canal. Preliminary results showed that OAEs amplitudes were suppressed during the lexical decision tasks relative to passive listening. In addition, an effect of task difficulty was found, whereby the less intelligible condition showed stronger suppression. These data suggest that the auditory efferent system is recruited through auditory attention, and that this may play a role in speech perception.

\section{0:10-10:30 Break}

10:30

2aPP6. Frequency characteristics of neuromagnetic auditory steadystate responses at the supra-threshold level. Asuka Otsuka (Biomedical Res. Inst., National Inst. of Adv. Industrial Sci. and Technol. (AIST), Ikeda, Japan), Masato Yumoto (Graduate School of Medicine, The Univ. of Tokyo, Tokyo, Japan), Shinya Kuriki (Res. Ctr. for Adv. Technologies, Tokyo Denki Univ., Inzai, Japan), and Seiji Nakagawa (Ctr. for Frontier Medical Eng., Chiba Univ., 1-33 Yayoicho, Inage-ku, Chiba, Chiba 263-8522, Japan, s-nakagawa@chiba-u.jp)

Auditory steady-state response (ASSR) is a neuronal electrical component recorded as a continuous sinusoidal signal phase-locked to a modulation frequency of sounds. Since ASSR amplitude shows high correlation with perceptual characteristics at hearing threshold level, the ASSR has been examined as a candidate for an objective index of the human hearing ability per frequency. However, the relationship between ASSR and perception at the supra-threshold level has not been clarified. In this study, characteristics of ASSR magnitude relative to loudness at the supra-threshold level were investigated. Neuromagnetic $40-\mathrm{Hz}$ ASSR was recorded in response to sinusoidally amplitude-modulated sweep tones with carrier frequency covering the frequency range of $0.1-12.5 \mathrm{kHz}$. Sound intensity was equalized at $50-, 60-$, and $70-\mathrm{dB}$ SPL with an accuracy of \pm 0.5 -dB SPL at the phasic peak of the modulation frequency. Corresponding loudness characteristics were modeled by substituting the detected individual hearing thresholds into a standard formula (ISO226:2003(E)). The strength of the ASSR component was maximum at $0.5 \mathrm{kHz}$, and it decreased linearly on logarithmic scale toward lower and higher frequencies, whereas loudness model was plateaued between 0.5 and $4 \mathrm{kHz}$. The results indicated that frequency characteristics of the ASSR were not equivalent to those of SPL and loudness model.

\section{0:45}

2aPP7. Auditory selective attention in cochlear implant users. Inyong Choi, Subong Kim (Dept. of Commun. Sci. and Disord., Univ. of Iowa, 250 Hawkins Dr., Iowa City, IA 52242, inyong-choi@uiowa.edu), and Phillip E. Gander (Dept. of Neurosurgery, Univ. of Iowa, Iowa City, IA)

Selective attention is a fundamental process for our communication in noisy everyday environments. Previous electrophysiologic studies have shown that selective attention modulates the neural representation of the auditory scene, enhancing responses to a target sound while suppressing the background. Given that most cochlear implant (CI) users complain about difficulty understanding speech within background noise, we investigated whether CI users' selective attention performance and underlying neural processes are 1) degraded compared to normal-hearing $(\mathrm{NH})$ listeners and 2) variable among individuals. Using speech stimuli recorded from two competing speakers (male and female), we measured $12 \mathrm{CI}$ and $17 \mathrm{NH}$ listeners' selective attention performance while their cortical neural processes were simultaneously recorded with high-density electroencephalography. While both groups showed above-chance level performance and significantly larger evoked responses to attended stimuli in auditory cortex, the CI group's selective attention performance and neural gain control were poorer than those of NH listeners. Furthermore, a positive correlation was found between the attentional modulation of evoked cortical responses and behavioral performance in the CI group. This result suggests that degraded selective attention processes contribute to an explanation of CI users' difficulty in real-world communication. 
2aPP8. Effects of noise on a person's psychological state under taskloaded condition from the viewpoint of brain function analysis. Takeshi Akita, Naoko Sano (Dept. of Sci. and Technol. for Future Life, Tokyo Denki Univ., 5 Senju-Asahi-cho Adachi-ku, Tokyo 1208551, Japan, akita@cck. dendai.ac.jp), and Ayako Matsuo (Graduate School of Adv. Sci. and Technol., Tokyo Denki Univesity, Tokyo, Japan)

Measurement and analysis of brain function by near infrared spectroscopy (NIRS) obtained from the frontal area of scalp can reveal some aspects of psychological state of human. In the present research, investigation of effects of noise on task-loaded person is attempted using analysis of NIRS. Two experiments are conducted. In the first experiment, brain function of frontal regions is measured under resting and task-loaded condition with back ground noise to confirm that task-loaded person uses their frontal area of brain. The results show that subjects use their frontal region of brain sufficiently while performing their tasks. In the second experiment, subjects carry out their calculating tasks under several noise conditions. Analyses of the data show that activity of frontal brain area diminishes. It is suggested that noise interrupt subjects' thinking activity for carrying out their task. These results suggest that measurement and analysis of NIRS is available for clarifying the negative effects of noise on task-loaded person. Using these methods can lead to create an index of distracting aspects of noise.
2aPP9. An objective measure of temporal fine structure correlates with perception in a speech discrmination task. David McAlpine, Macarena Bowen, and Jaime Undurraga (Linguist, Macquarie Univ., Australian Hearing Hub, 16 University Ave., Sydney, NSW 2109, Australia, david.mcalpine@mq.edu.au)

Sensitivity to the temporal fine structure (TFS) of sounds is considered important for understanding speech, especially in the presence of background noise, but few studies have employed objective measures to assess the relationship between sensitivity to TFS and listening to speech. Here, we used EEG (electroencephalography) to obtain an objective measure of sensitivity to the TFS of low-frequency sounds $(520-\mathrm{Hz}$ amplitude-modulated tones) in listeners who also undertook a speech-discrimination task (discrimination of vowel-consonant-vowel, VCV, sounds in background noise). Two different sound levels (60 and $80 \mathrm{~dB}$ SPL) and three different signal-to-noise ratios (SNRs; -6, 0 and $+6 \mathrm{~dB}$ ) were used. Three symmetric interaural phase modulations (IPMs), were measured: $-60^{\circ} / 60^{\circ},-90^{\circ} / 90^{\circ}$, and $-120^{\circ} / 120^{\circ}$. A fourth condition used an IPM switching between $0^{\circ}$ and $180^{\circ}$ degrees. IPM-FRs were higher for the $0 / 180^{\circ}$ degrees than all other conditions. Employing the ratio of the 3 symmetric to the asymmetric $(0 /$ $180^{\circ}$ ) IPM-FR as a measure of the neural dynamic range of TFS processing, we found that VCV scores increased with age at $60 \mathrm{~dB}$ SPL, but not $80 \mathrm{~dB}$ SPL, and indeed were better at 60 compared to $80 \mathrm{~dB}$ SPL in older listeners, and correlated with a higher ratio of IPM-FRs.

\title{
Session 2aSA
}

\section{Structural Acoustics and Vibration and Physical Acoustics: Acoustic Metamaterials I}

\author{
Christina J. Naify, Cochair \\ Acoustics, Naval Research Lab, 4555 Overlook Ave. SW, Washington, DC 20375 \\ Michael R. Haberman, Cochair \\ Applied Research Laboratories, The University of Texas at Austin, 10000 Burnet Rd., Austin, TX 78758 \\ Jeffrey Cipolla, Cochair \\ Weidlinger Associates, Inc., 1825 K St. NW, Suite 350, Washington, DC 20006
}

\section{Invited Papers}

\begin{abstract}
8:00
2aSA1. Acoustic topological insulators. Romain Fleury (Langevin Inst., ESPCI Paris Tech, 1 University Station C0803, Austin, TX 78712,romain.fleury@utexas.edu)

The recent discovery of topological states in condensed matter physics has spawned a quest for similar effects in classical physics. Topological insulators are bulk insulators supporting bandgap crossing, chiral edge states that are immune to backscattering caused by defects or disorder. Here, we present the acoustic equivalent of topological insulators, obtaining the analog of the Quantum Hall Effect (QHE) and the Quantum Spin-Hall Effect (QSHE) in acoustic metamaterials with, respectively, broken or preserved time-reversal symmetry. We demonstrate how our recent results in the field of non-reciprocal acoustics [cf. Science 343, 516 (2013); Ac. Today. 11, 14 (2015)] can be used to induce an acoustic version of the QHE in active metamaterials with broken time-reversal symmetry. We also put forward resonant and non-resonant schemes to emulate the Kane-Mele Hamiltonian in completely passive acoustic metamaterials, obtaining the acoustic analog of the QSHE. Our work reveals that topological acoustic metamaterials enable fundamentally new ways of manipulating sound, including one-way, chiral propagation of acoustic waves over broad bandwidth and topological protection against geometry imperfections, impedance mismatches, crystal defects, and local disorder.
\end{abstract}


2aSA2. Breaking time reversal symmetry with coriolis mean flow systems. Farhad Farzbod (Dept. of Mech. Eng., Univ. of MS, 201A Carrier Hall, University, MS 38677, farzbod@olemiss.edu) and Michael J. Leamy (Mech. Eng., Georgia Inst. of Technol., Atlanta, GA)

One way to break time reversal symmetry is to have a moving wave propagation medium. If the acoustic wave vector and the moving fluid velocity are collinear, such as the one created by Fluery et al. (Science, 343, 2014) we can use the wave vector shift caused by the fluid flow to break reciprocity. An alternative approach we have taken is to use a fluid velocity field which enters the differential equation of the system as a cross product term with the wave vector. A circular field in which the fluid velocity increases radially has a Coriolis acceleration term. In this system, the acoustic wave enters from the central wall and exits from the perimeter wall. Equations of conservation of mass and momentum, after linearization can be simplified to: $(\partial / \partial \mathrm{t}+\mathbf{V} \cdot \nabla) \mathrm{P}+\rho \mathbf{C}^{2} \nabla \cdot(\mathbf{u}+\mathbf{V})=0, \quad \rho \partial /$ $\partial \mathrm{t}(\mathbf{u}+\mathbf{V})+\rho(\mathbf{V} . \nabla) \mathbf{u}+\rho(\mathbf{u} . \nabla) \mathbf{V}+\rho(\mathbf{V} . \nabla) \mathbf{V}+\nabla \mathbf{P}=0$, in which $\mathbf{u}$ is the particle acoustic velocity and $\mathbf{V}=\mathrm{r} \omega \mathbf{e}_{\theta}$ is the fluid velocity due to the rotational velocity field. It can be shown that the third term in the second equation can be simplified as: $\rho \omega \mathbf{e}_{\mathrm{k}} \times \mathbf{u}$. This term Coriolis acceleration induces nonreciprocity. In this work, we solved the differential equation numerically and investigated the effect of fluid velocity on the nonreciprocity factor.

\section{Contributed Papers}

$8: 40$

2aSA3. Unidirectional wave transmission in a diatomic acoustic metamaterial. Kwek Tze Tan and Bing Li (Mech. Eng., The Univ. of Akron, Mech. Eng. 101 ASEC, Akron, OH 44325, ktan@uakron.edu)

In this study, we propose the use of dual resonators to design a linear diatomic metamaterial, consisting of several small-sized unit cells, to realize large unidirectional wave transmission in low frequency domain (below 1 $\mathrm{kHz}$ ). The asymmetric transmission mechanism is theoretically derived and systematically investigated. Numerical results are verified using both lattice structures and continuum models. This passive system does not require external energy supply or result in any frequency conversion. Various wave transmission band gaps, including passing band, stopping band gap, and asymmetric transmission band, can be theoretically predicted and mathematically controlled, which extends the design concept of unidirectional transmission devices. The asymmetric transmission band can be easily altered by careful and deliberate design of the unit size geometrical parameters and material properties. This proposed idea offers a new design concept in wave energy control through unidirectional systems, and may be applied to practical applications such as ultrasound imaging, directional transducer, and noise control.

\section{$8: 55$}

2aSA4. Loss-induced asymmetric transmission through gradient-index metasurfaces. Chen Shen (Mech. and Aerosp. Eng., North Carolina State Univ., 911 Oval Dr., Eng. Bldg. 3, Campus Box 7910, Raleigh, NC 276957910, cshen5@ncsu.edu), Yong Li (Université de Lorraine, Institut Jean Lamour, Vandœuvre-lès-Nancy, France), and Yun Jing (Mech. and Aerosp. Eng., North Carolina State Univ., Raleigh, NC)

Gradient-index metasurfaces have shown a great potential for wavefront modulation and transmission/reflection control. However, the effect of loss is rarely discussed in acoustic metasurfaces. Here, we study the effect of loss in gradient-index metasurfaces and suggest its application in asymmetric transmission. For a gradient-index metasurface composed of six types of unit cells, theoretical analysis reveals that, when loss is considered, the transmission coefficients for different diffraction modes will have different responses for acoustic waves with oblique incidence. The gradient-index metasurface thus can be utilized to achieve asymmetric transmission. Numerical simulations based on effective medium and real structure are performed to validate the theoretical findings using a single layer of gradientindex metasurface. The transmission contrast can be greater than 20 times in simulations within a certain range of incident angles. This design may be useful in ultrasonic and noise control applications.

\section{9:10}

2aSA5. A transparent broadband metallic lens for focusing underwater sound. Xiaoshi Su and Andrew N. Norris (Mech. and Aerosp. Eng., Rutgers Univ., 98 Brett Rd., Piscataway, NJ 08854, xiaoshi.su@rutgers.edu)

Diffractive lenses usually suffer from spherical aberration. Even though it is possible to minimize aberration by design, the quality of focused sound is still limited owing to the side lobes caused by aperture diffraction. In recent years, hyperbolic secant index profiles have been widely used in gradient index (GRIN) metamaterials design to reduce aberration and suppress side lobes. Here, we introduce a 1D transformation to modify the secant profile for further aberration reduction, and present a GRIN lens comprised of hexagonal unit cells obeying the modified profile for focusing underwater sound in the near field. The transversely isotropic unit cells are designed with water-like acoustic properties using foam mechanics and optimized using a homogenization technique based on finite element method. The inplane shear modulus of the unit cells are minimized to suppress the shear modes in the GRIN lens. Moreover, the impedances of the unit cells are tuned close to water, such that most of the energy carried by the normally incident plane wave propagate across the interface between the lens and water. This lens can focus underwater sound with high efficiency over a broadband $(10 \mathrm{kHz}-40 \mathrm{kHz})$.

\section{9:25}

2aSA6. Experimental validation of the underwater sound focusing properties of a pentamode gradient index metamaterial lens. Colby W. Cushing, Michael R. Haberman, Preston S. Wilson (Appl. Res. Labs., Univ. of Texas at Austin, P.O. Box 8029, Austin, TX 78713-8029, colby.cushing@ utexas.edu), Xiaoshi Su, and Andrew N. Norris (Mech. and Aerosp. Eng., Rutgers Univ., Piscataway, NJ)

A gradient index (GRIN) acoustic lens is a material slab with spatially varying properties that refracts an incident acoustic wave to produce a desired transmitted field. Hyperbolic secant index profiles have widely been used to create focusing lenses with minimal aberration and good sidelobe suppression. This work reports on the underwater experimental characterization of a GRIN lens that employs a modified secant profile for improved aberration reduction when compared to the hyperbolic secant profile. The index profile is produced using a two-dimensional pentamode material created from air-filled aluminum hexagonal unit cells. The lens is fabricated from waterjet cut aluminum plates that are stacked and sealed together. The test apparatus is comprised of a custom plane-wave source, an automated horizontal planar scanner, a pentamode GRIN lens, a custom support structure, and a calibrated hydrophone and associated electronics. The source was tested for amplitude planarity across its aperture at a constant distance to confirm its usability for this system. Experimental results of lens performance over the frequency range of $10-40 \mathrm{kHz}$ are compared with the isolated source and model predictions. [Work supported by ONR.] 
have demonstrated rich nonlinear phenomena, including amplitude-depend-

2aSA7. Spatial acoustic modulator for projecting high-quality holographic image. Bin Liang, Jianchun Cheng, Yifan Zhu (Inst. of Acoust., Nanjing Univ., P. R. China, 22 Hankou Rd., Nanjing, Jiangsu 210093, China, liangbin@nju.edu.cn), Xuefeng Zhu (School of Phys., Huazhong Univ. of Sci. and Technol., Wuhan, China), Xinye Zou, and Jing Yang (Inst. of Acoust., Nanjing Univ., P. R. China, Nanjing, China)

Spatial modulation of acoustic wave has demonstrated theoretical and experimental importance in the field of acoustics, with wide applications such as in medical ultrasound and particle manipulation. However, the existing means for acoustic modulation have to rely on the acoustic metasurfaces providing only pure phase modulation or active element arrays with complicated fabrications and limited spatial resolutions. In this work, we present the design, numerical simulation, and experimental demonstration of a spatial acoustic modulator (SAM) capable of manipulating the phase and amplitude profiles separately and continuously, offering the possibility to address the above challenges by enabling arbitrarily complex acoustical patterns. The proposed structure has a simple configuration with planar profile, high efficiency of spatial modulation, and deep-subwavelength unit scale, ensuring easy and low-cost fabrication and nearly-continuous wavefront manipulation. The designed SAM is used to project high-quality acoustic holograms both theoretically and experimentally to demonstrate its wavesteering functionality. Our metastructure-based holographic projector with simplicity and extreme acoustic performance advances the capability of spatial acoustic modulation, and have various applications in places where the conventional techniques would lead to complexity and limited ability.

\section{9:55-10:10 Break}

\section{0:10}

2aSA8. Quantised acoustic meta-surfaces using metamaterial bricks. Gianluca Memoli (Dept. of Eng., Computing and Design, School of Informatics, Eng. and Design, Univ. of Sussex, Brighton BN1 9RH, United Kingdom, g.memoli@sussex.ac.uk), Mihai Caleap (Eng., Univ. of Bristol, Bristol, United Kingdom), Michihiro Asakawa, Deepak Sahoo (School of Informatics, Eng. and Design, Univ. of Sussex, Brighton, United Kingdom), Bruce W. Drinkwater (Eng., Univ. of Bristol, Bristol, United Kingdom), and Sriram Subramaniam (School of Informatics, Eng. and Design, Univ. of Sussex, Brighton, United Kingdom)

Acoustic meta-surfaces offer an extremely successful way of transforming an input acoustic wave into almost any diffraction-limited field. This is typically achieved by encoding a phase delay function on the unit cells that form the meta-surface, which is then manufactured by rapid prototyping. One of the limits of current technology is that meta-surfaces built in this way are targeted to a single, specific application. In this work, we present a method of building acoustic meta-surfaces using metamaterial bricks, designed to operate at $40 \mathrm{kHz}$ and quantized both in the space and in the phase domains. First, we present finite-elements simulations and experiments to discuss key parameters of our bricks as frequency response, directivity, impedance. Second, our metamaterial bricks are used here to achieve some key beam transformations like steering and focusing, but also more complex fields like an acoustic tractor beam. We use the latter to levitate one or more polystyrene beads and diffraction theory to explain some of the findings. Our method, where the phase in a specific position may be changed by changing the specific brick, may find applications in loudspeaker design, ultrasound imaging/therapy, or acoustic particle manipulation.

\section{0:25}

2aSA9. Nonlinear wave propagation and rotational dynamics In microscale granular media. Samuel Wallen and Nicholas Boechler (Mech. Eng., Univ. of Washington, Mech. Eng., Stevens Way, Box 352600, Seattle, WA 98195,walles@uw.edu)

Granular media have been shown to exhibit complex dynamics stemming from dispersion and nonlinear particulate interactions. Studies of wave propagation in granular materials composed of ordered arrays of spherical particles interacting via Hertzian contact, known as "granular crystals," ent and tunable sound speeds, solitary waves, and discrete breathers. However, most of the past work on granular crystals has considered macroscale spheres, with diameters on the order of centimeters or millimeters. More recent works have explored wave phenomena in microscale granular crystals, having particle diameters of a few microns, which can be fabricated using self-assembly methods, and characterized experimentally using laser ultrasonic techniques. The physics of microscale granular media have been shown to vary drastically from their macroscale counterparts; for example, interparticle adhesion, which is negligible at macroscale, creates an intrinsic static load, which linearizes the contact forces at low amplitude, and can increase the importance of shear interactions and particle rotations. In this work, we present theoretical and computational analyses of nonlinear wave propagation in microscale granular crystals, examining the interplay between nonlinearity and rotational dynamics. In particular, we focus on amplitude-dependent distribution of energy into the translational and rotational modes of a hexagonally close-packed granular crystal.

\section{$10: 40$}

2aSA10. Metamaterials made of granular chains for nondestructive evaluation applications. Piervincenzo Rizzo and Amir Nasrollahi (Civil and Environ. Eng., Univ. of Pittsburgh, 942 Benedum Hall, 3700 O'Hara St., Pittsburgh, PA 15261, pir3@pitt.edu)

In the last two decades it has been demonstrated that highly nonlinear solitary waves (HNSWs) propagating in metamaterials made of chains of granular particles can be used in many physics and engineering applications, including acoustic lenses, impurity detectors, and nondestructive evaluation (NDE). HNSWs are compact nondispersive waves, and in this paper, we demonstrate their application for the nondestructive evaluation of civil, mechanical, and aerospace structures. In particular, this presentation delves with the non-invasive assessment of concrete strength and the evaluation of thermal stress in slender beams. For the concrete application, the NDE method exploits the dynamic interaction between the metamaterial and the concrete, and the hypothesis is that this interaction depends on the stiffness of the specimen being inspected. The results show that the time of flight of the HNSWs is inversely proportional to the modulus of elasticity of the concrete. For the buckling application, we present the coupling mechanism between a beam and the HNSWs propagating along a straight granular chain in contact with the beam. We show that the waves can be used to measure the stress of thermally loaded structures or to infer the neutral temperature, i.e., the temperature at which this stress is null.

\section{$10: 55$}

2aSA11. Enhanced energy dissipation with nonlinear snapping acoustic metamaterial inclusions. Stephanie G. Konarski, Michael R. Haberman, and Mark F. Hamilton (Appl. Res. Labs., The Univ. of Texas at Austin, P.O. Box 8029, Austin, TX 78713-8029, skonarski@utexas.edu)

Through the design of subwavelength structures, nonlinear acoustic metamaterials can be engineered to exhibit regimes of positive and negative incremental stiffness. Negative incremental stiffness leads to large, rapid deformation, commonly called snapping, between local stable configurations. When perturbed by a small external pressure, snapping elements can be exploited to enhance energy absorption of acoustic waves. The present research focuses on a low volume fraction of snapping inclusions, dispersed within a nearly incompressible viscoelastic matrix to create a multiscale nonlinear material. The forced dynamic response of the heterogeneous material is modeled using a generalized Rayleigh-Plesset equation, which couples the dynamic behavior at the micro- and macroscale. We investigate the nonlinear dynamic behavior of the heterogeneous medium for small harmonic perturbations about several pre-strains of the snapping inclusions to demonstrate the influence of the microscale dynamic response on the macroscale response. Of primary interest are energy dissipation capabilities and characteristic acoustic impedance, which change with inclusion pre-strain. The behavior is also compared to heterogeneous media created from conventional inclusions, such as air voids and steel inclusions, to demonstrate the wide range of material behavior obtained using snapping inclusions. [This work was supported by the Office of Naval Research.] 
11:10

2aSA12. Underwater acoustic absorption using a periodically voided soft elastic medium. Gyani Shankar Sharma (School of Mech. and Manufacturing Eng., UNSW Australia, Rm. \#408, Sydney, New South Wales 2052, Australia, gyanishankar.sharma@student.unsw.edu.au), Alex Skvortsov, Ian MacGillivray (Maritime Div., Defence Sci. and Technol. Group, Melbourne, VIC, Australia), and Nicole Kessissoglou (School of Mech. and Manufacturing Eng., UNSW Australia, Sydney, New South Wales, Australia)

A coating comprising periodic cavities in a soft rubber medium can significantly reduce the reflection of acoustic waves from a steel plate. In this work, analytical and numerical models are developed to study acoustic reflection and transmission through a single layer of periodic cylindrical voids embedded in a soft elastic medium with steel backing. The analytical model is based on effective medium approximation theory which allows modeling of a composite material as a homogeneous material using effective material properties. The numerical model is based on the finite element method and developed using commercial finite element code COMSOL Multiphysics. Minimal reflection and transmission and maximal absorption of sound waves in a broad frequency range is attributed to the monopole type resonance of voids. The monopole resonance frequency of voids in an array is shown to be significantly higher compared to the monopole resonance of a single void in an infinite medium due to resonance coupling. An analytical framework to predict the resonance frequency of voids in an array is presented. The effect of strong and weak coupling of void resonances on reflection and transmission characteristics is investigated. Non-dimensionalized results obtained from the analytical and numerical models are compared.

TUESDAY MORNING, 29 NOVEMBER 2016

CORAL 4, 9:00 A.M. TO 12:00 NOON

\title{
Session 2aSC
}

\section{Speech Communication: Cross-Linguistic Speech Production and Perception}

\author{
Katie Drager, Cochair \\ Linguistics, University of Hawaii at Manoa, 1890 East-West Rd., More 569, Honolulu, HI 96822 \\ Timothy Vance, Cochair \\ Dept. of Linguistic Theory and Structure, Natl. Inst. for Japanese Lang. and Ling., Tokyo, Japan
}

Chair's Introduction-9:00

\section{Invited Papers}

9:05

2aSC1. Articulatory settings of high- and low-proficiency second-language speakers. Ian Wilson (CLR Phonet. Lab, Univ. of Aizu, Tsuruga, Ikki-machi, Aizuwakamatsu, Fukushima 965-8580, Japan, wilson@u-aizu.ac.jp)

Articulatory setting, a language-specific underlying position of the articulators during speech, can be measured by using inter-speech posture (ISP) - the speech rest position. In an X-ray study, it was shown to behave like a target posture, in that it has as little variability as /i/, one of the least variable vowels. In an rt-MRI study, it was found to be more mechanically advantageous than absolute rest position. In an ultrasound/Optotrak study, results showed differences across normalized monolingual groups of speakers, and bilinguals who were perceived to be native in each of their languages had a different articulatory setting for each language. In an EMA study, tongue width in the coronal plane during ISP was found to be less efficient in second-language (L2) speakers of English. In recent research using ultrasound and lower-proficiency Japanese-English bilinguals, we were surprised to find differences in their articulatory settings for each of their languages, when it would have been simpler for such a speaker to use the articulatory setting of their dominant language while speaking their non-dominant one. In this talk, a state-of-the-art analysis of research on articulatory setting will be given including recent research on Japanese-English bilinguals.

\section{9:25}

2aSC2. Language contact and accent changes in Japanese. Haruo Kubozono (Theory \& Typology Div., National Inst. for Japanese Lang. and Linguist, NINJAL, 10-2 Midori-cho, Tachikawa 190-8561, Japan, kubozono@ninjal.ac.jp)

This talk discusses how word accent patterns of regional Japanese dialects have changed over the years as they were heavily exposed to standard Tokyo Japanese. A particular attention is paid to the accent changes that have taken place in Kagoshima Japanese, a southern dialect whose accent system is strikingly different from that the standard variety. Original fieldwork studies show that this regional dialect has been heavily influenced by the standard variety with respect to the presence or absence of a pitch fall, while the position of the fall is still determined by its traditional system: e.g., /doo.na.TU/ $\rightarrow$ /doo.NA.tu/ "donut" (Tokyo form: /DOo.na.tu/). Interestingly, the same mechanism is at work when (Tokyo) Japanese borrow words from English as loanwords: the recipient language copies the 
presence or absence of a pitch fall from the host language but it follows its own accent rule with respect to the position of the fall, e.g. / ba'.na.na/ "banana," /wa.shi'n.ton/ "Washington." These observations indicate that native Japanese speakers are highly sensitive to the presence or absence of a pitch fall when listening to a foreign language and a different variety of their native language, but are quite insensitive to the position of the pitch change.

\section{9:45}

2aSC3. Effects of prosodic focus on discourse processing by native speakers versus second language learners. Amy J. Schafer (Dept. of Linguist, Univ. of Hawaii, 1890 East-West Rd., Manoa, Honolulu, HI 96822, amy.schafer@hawaii.edu)

The acquisition of sentence-level prosody and intonation is often challenging for second language learners (L2ers). A speaker's native and second language can differ at several levels of representation involved in the use of prosody, and so multiple new mappings must be acquired for proficiency in the second language's prosodic system. This talk examines prosodic focus in English, Japanese, and Korean, and its effects on discourse processing by native speakers and L2ers. Although all three languages make use of prosodic focus, and draw on some of the same acoustic cues to realize it, there are also substantial differences across the languages in how prosodic focus interfaces with other grammatical components. Results from a series of experiments indicate context-sensitivity in how L2ers respond to prosodic focus, showing native-like responses in one discourse context but quite different responses to the same tonal realization in another context. Such findings point to the importance of testing the perception of prosody across multiple grammatical configurations, particularly when investigating language acquisition.

\section{0:05}

2aSC4. Phonological traits of the Japanese and English spoken by Nikkei speakers in Hawaii. Daniel Long (Japanese Linguist, Tokyo Metropolitan Univ., 1-1 Minami Osawa, Hachioji, Tokyo 1920364, Japan, dlong@tmu.ac.jp)

The Japanese and English language varieties spoken by Nikkei speakers in Hawaii show phonological traits which result from language contact and are uncommon in other varieties. Characteristics of the Japanese spoken by Nikkei speakers which differ from native varieties of the language include the following. Non-geminate and geminate consonants are found in variation ([kappamaki] vs. [tekkamaki], [napa]) and speakers do not hear germination as being phonemically significant. The English labiodental fricative [f] is substituted for the Japanese bilabial fricative [ $\phi]$ ([furo], [furikake]). Vowel lengthening is in inconsistent and not phonemically significant

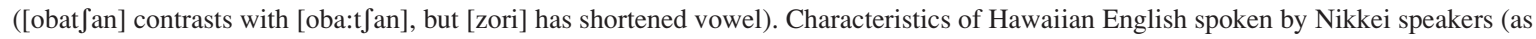
well as other ethnicities) include the following. The alveolar flap /r/ ([karai] 'spicy') is a phoneme contrasted with /l/ and /r/. Phonotactic rules allow for the /ts/ sequence even in the word-initial position (Hawaiian English [tsunami] vs. General American English [sunami]). In this paper, I will use PRAAT acoustical analyses to illustrate these traits.

\section{0:25-10:40 Break}

\section{0:40}

2aSC5. Variation in the second formant: Its role in a creole's coarse-refined contrast. Nala H. Lee (Dept. of English Lang. and Lit., National Univ. of Singapore, Block AS5, 7 Arts Link, Singapore 117570, Singapore, elllhn@nus.edu.sg)

Baba Malay encodes an interesting coarse-refined contrast. Speakers overtly recognize words ending with -al, -ar, and -as (e.g., bakar "burn") as kasar "coarse," while their counterparts ending with - $\varepsilon$ (e.g., bake "burn") are halus "refined." The contrast is neither phonetic nor is it constrained by phonology or morphology. The coarse-refined system may be based on sound symbolism. The frontness of $-\varepsilon$ is associated with a smaller articulatory space in the oral cavity, and hence refinedness, while the more backwards al, -ar, and -as forms, are associated with a larger articulatory space and hence coarseness. A perception experiment, mainly a within-subjects matched guise task, is conducted. Coarse and refined pairs are elicited from speakers. The relevant F2 of both variants are adjusted twice upwards and twice downwards in steps of $100 \mathrm{~Hz}$. Listeners rate these forms on a scale of 1 to 5,5 being most associated with values that are "refined." Results show that the lower F2 is, the more likely listeners are to associate the coarse guise with "coarse" values, and the higher F2 is, the more likely listeners are to associate the refined guise with "refined" values. The kasar-haluscontrast is thus mitigated by sound symbolism operationalized by F2.

\section{1:00}

2aSC6. Hailu Hakka and Taiwan Min tone changes. Ho-Hsien Pan, Shao-Ren Lyu, and Hsiao-Tung Huang (Dept. of Foreign Lang. and Literatures, National Chiao Tung Univ., 1001 University Rd., Hsinchu 300, Taiwan, hhpan@faculty.nctu.edu.tw)

A pitch contour overlap and a tone-sandhi relationship can both contribute to confusion in tone that lead to change in tone. This study investigates the duration, f0, and phonation between Hailu Hakka and Taiwan Min tone pairs that (1) were neither overlapped in pitch contours nor tone-sandhi related, such as Hakka tones 55 and 33, and Min tones 55 and 13; or (2) were overlapped in pitch but not tone-sandhi related, such as Hakka 33 and 11, and Min 13 and 31; or (3) were not overlapped in pitch but tone-sandhi related, such as Hakka and Min tones 3 and 5, or (4) were both overlapped in pitch contour and tone-sandhi related, such as Hakka 24 and 33, and Min tones 31 and 33. The results indicated that Hakka speakers under 30 years of age produced tones 24 and 33 that were overlapped in pitch and tone-sandhi related, and with similar pitch contour; whereas, Min speakers, regardless of age, produced tones 3 an 5 that were not overlapped in pitch but were sandhi-related bi-directionally $(3 \leftrightarrow 5)$ similarly. It is proposed that sound changes occur among sandhi related tones with and without overlap in pitch contours. 
2aSC7. Substrate effects in the phonetic realization of Pidgin vowels. James Grama (Modern Lang., Santa Monica College, 18933 Nashville St., Los Angeles, CA 91326, james.grama@gmail.com)

Hawai'i is home to an English-lexified creole, known locally as Pidgin. While much research has focused on the structural development of Pidgin (e.g., Bickerton \& Odo 1976), little work has investigated acoustic phonetic variation in the Pidgin vowel system. This talk presents results from an analysis of 854 tokens of /a/ produced by 32 speakers of Pidgin from two corpora—one from the 1970s and one from the $2000 \mathrm{~s}$ - and investigates factors that contribute to differences in realizations of this vowel. Results indicate that /a/ in Pidgin exhibits a raised nucleus over real time for female speakers $(\mathrm{p}<0.001)$ but not male speakers. Additionally, analysis reveals that the youngest group of Pidgin speakers in the 2000s corpus realize /a/ in words of Hawaiian origin as higher in the vowel space than words of English origin $(\mathrm{p}<0.0001)$. Possible reasons for the emergence of this fine-grained phonetic difference in the youngest speaker group are discussed, including the role played by Hawaiian language education and the apparent valuation of Hawaiian. Taken together, results suggest that a combination of factors influences the realization of /a/ in Pidgin, including age, gender, and whether a word derives from the superstrate language (=English) or a substrate language (= Hawaiian).

\section{1:40}

2aSC8. Voice onset time and closure duration of word-initial voiceless plosives in Pidgin conversation

. Katie Drager (Linguist, Univ. of Hawaii at Manoa, 1890 East-West Rd., More 569, Honolulu, HI 96822, kdrager@hawaii.edu), James Grama (Santa Monica College, Santa Monica, CA), Lorenzo Gonzalez (Linguist, Univ. of Hawaii at Manoa, Orlando, FL), and Cassidy Copeland (Linguist, Univ. of Hawaii at Manoa, Honolulu, HI)

Hawai' $i$ is home to many languages, including an English-lexified creole, known locally as Pidgin. Despite much work on the development of Pidgin, little is known about the acoustic properties of Pidgin consonants. This talk presents results from an analysis of wordinitial plosives produced by eight speakers of Pidgin across two age groups, investigating what factors influence VOT and closure duration in the plosives. The data were analyzed using linear regression models with by-speaker random intercepts. Results demonstrate that VOT in word-initial plosives tends to be shorter in Pidgin than in English, with mean duration values nearly half those reported for English in Yao (2007). The effects of social and linguistic factors, however, tend to be similar (e.g., shorter VOT for older speakers, VOT for $/ \mathrm{k} /$ is longer than for $/ \mathrm{t} /$ or $/ \mathrm{p} /$ ). Results also indicate that VOT is significantly shorter both for words of Hawaiian origin $(\beta=-0.01$, $\mathrm{t}(289)=-2.0$, and $p<0.05)$ and-in a separate model fit to a subset of the data with only non-Hawaiian words-highly frequent words $(\beta=-0.01, \mathrm{t}(216)=-4.2$, and $p<0.001)$. In contrast, no factors tested significantly predicted closure duration. Taken together, results demonstrate that a combination of linguistic, social, and probabilistic factors influence VOT in Pidgin. 


\title{
Session 2aSPa
}

\section{Signal Processing in Acoustics: Compressive Sensing in Acoustics I}

\author{
Peter Gerstoft, Cochair \\ SIO Marine Phys Lab MC0238, Univ. of California San Diego, 9500 Gilman Drive, La Jolla, CA 92093-0238
}

Jeffrey S. Rogers, Cochair

Acoustics Division, Naval Research Lab, 4555 Overlook Ave. SW, Code 7161, Washington, DC 20375

Yoshifumi Shiraki, Cochair

Communication Science Laboratories, Nippon Telegraph and Telephone Corp., 3-1, Morinosato-Wakamiya, Atsugi-City, Kanazawa pref. 243-0198, Japan

Chair's Introduction-7:55

\section{Invited Papers}

8:00

2aSPa1. Introduction to sparse and compressive sensing. Peter Gerstoft (Scripps Inst. of Oceanogr., Univ. of California San Diego, 9500 Gillman Dr., La Jolla, CA 92093-0238, gerstoft@ucsd.edu)

Compressed sensing or sparse sampling is a signal processing technique for efficiently acquiring and reconstructing a signal, by finding solutions to underdetermined linear systems. I will describe the basic features of compressive sampling that has many opportunities in acoustics.

8:20

2aSPa2. A brief introduction to distributed compressed sensing and its typical reconstruction limits. Yoshifumi Shiraki (Commun. Sci. Labs., Nippon Telegraph and Telephone Corp., 3-1, Morinosato-Wakamiya, Atsugi-City, Kanazawa pref. 243-0198, Japan, shiraki. yoshifumi@lab.ntt.co.jp) and Yoshiyuki Kabashima (Dept. of Computational Intelligence and Systems Sci., Interdisciplinary Graduate School of Sci. and Eng., Tokyo Inst. of Technol., Yokohama, Japan)

A brief introduction to compressed sensing and a discussion of its reconstruction limit analysis are presented. In addition, we briefly introduce the replica method, a technique in statistical mechanics, to show its effectiveness for typical performance analysis on signal processing problems. In this presentation, we especially focus on a signal model called joint sparse model 2 (JSM-2) or the multiple measurement vector problem, in which all sparse signals share their support. The signal model JSM-2 is important for dealing with practical processing problems. We investigate the typical performance of 12,1-norm regularized minimization and the Bayesian optimal reconstruction schemes in terms of mean square error for noiseless and noisy measurement JSM- 2 problems. Employing the replica method, we show that these schemes, which exploit the knowledge that the signal support is shared, can recover the signals more precisely as the number of channels increases. The main focus of our presentation is theoretical analysis; however, we will introduce applications of the signal model JSM-2 for acoustic problems. Most of our presentation is based on papers J. Stat. Mech. (2015) P05029 and J. Stat. Mech. (2016) 063304.

\section{8:40}

2aSPa3. Complex non-negative matrix factorization: Phase-aware sparse representation of audio spectrograms. Hirokazu Kameoka and Hideaki Kagami (NTT Commun. Sci. Labs., Nippon Telegraph and Telephone Corp., 3-1 Morinosato Wakamiya, Atsugi, Kanagawa 243-0198, Japan, kameoka.hirokazu@lab.ntt.co.jp)

Non-negative matrix factorization (NMF) has attracted a lot of attention after being proposed as a powerful approach for audio source separation. Factorizing the magnitude spectrogram of a mixture signal into the product of two non-negative matrices can be interpreted as approximating the observed spectra at each time frame as a linear sum of basis spectra scaled by time-varying amplitudes. One drawback of this approach is that the additivity of magnitude spectra is assumed, which holds only approximately. To address this drawback, we introduce a novel framework called the "Complex NMF," which makes it possible to realize NMF-like signal decompositions in the complex spectrogram domain. We further develop a time-domain extension of this approach, which is noteworthy in that it allows for several extensions that were not possible with the conventional NMF framework. 
2aSPa4. Decomposing guided wavefields with dictionary learning. Joel B. Harley (Dept. of Elec. and Comput. Eng., Univ. of Utah, 50 S Central Campus Dr., Rm. 3104, Salt Lake Cty, UT 84112-9249, joel.harley@utah.edu) and K. Supreet Alguri (Dept. of Elec. and Comput. Eng., Univ. of Utah, Salt Lake City, UT)

Ultrasonic guided waves have become a powerful tool for the structural health monitoring of large structures due to their low attenuation and high damage sensitivity. Yet, one significant challenge of working with guided waves is their complexity. Ultrasonic guided waves demonstrate strong multi-modal and dispersive characteristics and are often used in structures with many boundaries and reflecting elements. In prior work, compressive sensing theory and sparse recovery algorithms have been used to learn the multi-modal and dispersive properties of guided waves based on relatively simple wave models. The algorithms can predict and construct wavefields based on these learned properties. Furthermore, this predictive power can be used to detect and locate damage in a structure. However, these algorithms cannot yet predict and utilize reflections as well as other complexities (such as inhomogeneities) in a structure due to the simplicity of the current model. This paper presents a dictionary learning approach to learn this model directly from simulation or experimental data. This paper focuses on studying the models (i.e., dictionaries) created by dictionary learning under various simulated conditions. We demonstrate that we can use these dictionaries to accurately predict wavefields when waves are distorted by reflections, velocity inhomogeneities, and velocity anisotropy.

\section{9:20}

2aSPa5. Dictionary learning of acoustic sound speed profiles. Michael J. Bianco and Peter Gerstoft (Marine Physical Lab., Univ. of California San Diego, Scripps Inst. of Oceanogr., 6061 La Jolla Hermosa Ave., La Jolla, CA 92037, mbianco@ucsd.edu)

Inversion for true ocean acoustic sound speed profiles (SSPs) is generally a non-linear and underdetermined problem. Traditional regularization methods model SSPs as a summation of leading-order empirical orthogonal functions (EOFs), with a minimum-energy constraint on coefficients. However, this often provides low resolution estimates of ocean SSPs. Sparse processing methods (e.g., compressive sensing) yield high resolution reconstruction of signals from few observations, provided few coefficients (of many) explain the observations. Using dictionary learning techniques, an overcomplete basis or dictionary of shape functions, which represent ocean SSPs using a minimum number of coefficients, is learned from a training set of SSPs. Learned dictionaries, which are not constrained to be orthogonal, optimally fit the distribution of possible ocean SSPs. Thus, each dictionary entry is informative to ocean SSP variability whereas EOFs become less informative for increasing order. It has been found that these learned dictionaries largely explain the variability of ocean SSPs using as few as one coefficient. Thus, in addition to improving the resolution of SSP estimates, learned dictionaries can improve the robustness of SSP inversion by significantly reducing the parameter search space.

\section{9:40}

2aSPa6. Acoustic single-pixel imaging using compressive sensing. Jeffrey S. Rogers (Acoust. Div., Naval Res. Lab, 4555 Overlook Ave. SW, Code 7161, Washington, DC 20375, jeff.rogers@nrl.navy.mil), Christina J. Naify (Jet Propulsion Lab., Pasadena, CA), Charles Rhode, and Geoffrey F. Edelmann (Acoust. Div., Naval Res. Lab, Washington, DC)

The acoustic analog to the single-pixel imager found in the optical and THz imaging literature is presented. Here, the single-pixel detector is replaced with a single omnidirectional acoustic receiver and combined with a series of masks that allow for multiple orthogonal measurements. Acoustic source localization is performed based on the theory of compressive sensing which allows for the resolution of $N$ acoustic sources using $M$ measurements where $M<<N$. A theoretical model for the acoustic single pixel imager is presented and compared to finite element models (FEM) simulated in COMSOL and experimental data taken in an air acoustic 2D waveguide. It is shown that the acoustic single pixel imager is capable of localizing multiple targets using only a single omnidirectional acoustic receiver. [Work supported by the Office of Naval Research.]

\section{0:00-10:20 Break}

\section{0:20}

2aSPa7. The utility of binary-sequence-based signals. James S. Martin (School of Mech. Eng., Georgia Inst. of Technol., 771 Ferst Dr., Love Bldg., Rm. 115, Atlanta, GA 30332-0405, james.martin@me.gatech.edu)

It is frequently implied that there are advantages to the use of signals based on binary sequences for the characterization of linear time invariant (LTI) systems. However, this appears to neglect the role of the interpolation (or reconstruction) filter which is necessary in order to translate discrete sequences into analog signals and to discount the utility of analog signals that are generated by other means. Whereas a discrete sequence may offer desirable or optimal characteristics in comparison with other discrete sequences, these may easily be destroyed by the imposition of the interpolation filter. Similarly, even when these characteristics are substantially preserved in the interrogation signal, they appear consistently to be either mediocre or sub-optimal in comparison to properties of signals that are generated without consideration of binary sequences (e.g., chirps or multi-sine signals). The relative superiority of any signal with respect to the alternatives for interrogation of an LTI system depends on the goals of the characterization and relies on the assumption that some form of nonlinearity will ultimately limit signal amplitude. There does not appear to be any set of goals and limiting nonlinearity that are uniquely suited to binary-sequence-based signals. 
2aSPa8. Compressive beamforming on a cylindrical array. Charles F. Gaumond (Appl. Res. in Acoust. LLC (ARiA), Washington, DC), Jonathan Botts (Appl. Res. in Acoust. LLC (ARiA), Culpeper, VA), and Jason E. Summers (Appl. Res. in Acoust. LLC (ARiA), 1222 4th St. SW, Washington, DC 20024-2302, jason.e.summers@ariacoustics.com)

Previously, we presented subarray compressive beamforming for Doppler sensitive midfrequency active sonar on a cylindrical array, focusing on practical application for mitigating Doppler spreading caused by sidelobes and the width of the main lobe [J. Acoust. Soc. Am. 139, 2050 (2016)]. Here, we describe ongoing work focusing on theoretical challenges of adapting compressive beamforming to a cylindrical array. Unlike linear arrays, for example, the optimal sampling for the sound field incident on a cylinder array is not evident. Formal design guidelines for compressive beamforming on a cylindrical array are discussed, guided by the statistical restricted isometry property (StRIP). Prior work has demonstrated subarray compressive beamforming by combining sparse estimates from arbitrarily chosen subarrays. Here, a formal approach is outlined in which StRIP is used to specify minimal subarrays that can individually reconstruct a sound field of a given sparsity, using a new strategy for combining individual estimates. Finally, differences between the fundamental assumptions and resulting limitations of compressive, conventional, and adaptive beamforming techniques are discussed for the cylindrical array geometry and continuous-wave $(\mathrm{CW})$ waveforms of interest, as demonstrated with high-fidelity simulated data. [Work supported by a NAVSEA Phase I SBIR award.]

\section{1:00}

2aSPa9. Sparse tomographic acoustic imaging for environmental noise-source mapping. Cagdas Tuna, Douglas L. Jones, Shengkui Zhao, and Thi Ngoc Tho Nguyen (Adv. Digital Sci. Ctr., Illinois at Singapore, 1 Fusionopolis Way \#08-10 Connexis North Tower, Singapore 138632, Singapore, cagdas.t@adsc.com.sg)

Environmental noise has become a major problem in large cities, increasing health risks for the urban population. Current methods are overly expensive for city-wide noise-monitoring as they generally require dense deployment of fixed microphones for noise-level measurements. We present here alternative sparse tomographic acoustic imaging techniques using arrays with relatively few number of microphones for large-region acoustic noise mapping. We first demonstrate that the locations and sound pressure levels of fixed noise sources sparsely located in a large field are recovered by collecting acoustic data at multiple locations with a portable microphone array for tomographic reconstruction. We then introduce a nonstationary tomographic imaging approach using fixed microphone arrays, which can also capture the intermittent changes in the acoustic field due to transient and/or moving noise sources. We test both the sparse static and dynamic imaging models with acoustic measurements collected with a circular microphone array mounted on an electric vehicle and two prepositioned circular arrays, respectively. The experimental results show the promise of the proposed methodology for lowcost and time-efficient large-scale noise-monitoring.

\section{1:20}

2aSPa10. Shallow water acoustic channel estimation across fluctuations in channel support. Ananya Sen Gupta (Elec. and Comput. Eng., , 4016 Seamans Ctr. for the Eng. Arts and Sci., Univ. of Iowa, Iowa City, IA 52242, ananya-sengupta@uiowa.edu)

Estimating the shallow water acoustic channel across rapidly varying multipath fluctuations have been an open challenge for decades. Recently, sparse sensing techniques have been employed to take advantage of the heavy-tailed distribution of channel components in the Delay-Doppler domain. Despite noticeable improvement over channel estimator performance by exploiting channel sparsity compared to traditional least-squared based techniques, a key challenge remains. Most sparse recovery techniques are designed to estimate high-amplitude components across static or slowly varying support. Depending on wind and sea conditions, the underlying distribution of the channel components may change unpredictably, leading to fluctuations in the channel support that are difficult to model or track efficiently with high precision. Furthermore, not all channel components are high-energy and therefore, may be unintentionally suppressed by a sparse optimization technique. We will present the current state-of-the-art on applying sparse sensing, particularly mixed norm techniques, to shallow water channel tracking. In particular, we will present extensions to prior work on channel estimation that exploit a non-convex metric to converge faster to the same solution offered by the traditional (and convex) Lasso metric. We will present how non-convex optimization can navigate varying channel sparsity and present results based on experimental field data.

\section{1:40}

2aSPa11. Grid-free channel estimation of acoustic measurement data from SAVEX15. Youngmin Choo, Jungyong Park, Yongsung Park, Minseuk Park (Res. Inst. of Marine Systems Eng., Seoul National Univ., 1, Gwanak-ro, Gwanak-gu, Seoul, Seoul 151 - 744, South Korea, sks655@snu.ac.kr), and Woojae Seong (Naval Architecture and Ocean Eng. and Res. Inst. of Marine Systems Eng., Seoul National Univ., Seoul, South Korea)

Acoustic channel impulse responses (CIRs) consisting of multiple arrivals are extracted from the measured data during SAVEX15 experiment (conducted in the northern East China Sea during May 2015) using compressive sensing, providing high-resolution results due to sparsity promoting objective function. Performance of grid-based compressive channel estimation degrades when basis mismatch occurs because delays of the arrivals do not correspond to delays on the grid. To overcome this, grid-free compressive channel estimation is derived from the formulation of grid-free compressive beamforming [J. Acoust. Soc. Am. 137, 1923-1935 (2015)]. The grid-free compressive channel estimation is applied to signals from SAVEX15; the signals were transmitted from a towed source and received at two separate vertical line arrays (16 elements each); they are used to measure the signals traveling through waveguides. Acoustic structures are observed clearly due to high-resolution CIRs along depth, which are varied by the moving source and internal wave activities. 


\title{
Session 2aSPb
}

\section{Signal Processing in Acoustics: General Topics in Signal Processing II (Poster Session)}

\author{
R. Lee Culver, Cochair \\ ARL, Penn State University, PO Box 30, State College, PA 16804 \\ Brian E. Anderson, Cochair \\ N145 Esc, Brigham Young Univ., MS D446, Provo, UT 84602
}

\begin{abstract}
All posters will be on display from 8:30 a.m. to 11:30 a.m. To allow contributors in this session to see the other posters, authors of odd-numbered papers will be at their posters from 8:30 a.m. to 10:00 a.m. and authors of even-numbered papers will be at their posters from 10:00 a.m. to 11:30 a.m.
\end{abstract}

\section{Contributed Papers}

2aSPb1. Audio spotlight system based on formation of multiple focused sound sources via loudspeakers. Shuji Akamatsu and Yosuke Tatekura (Shizuoka Univ., 3-5-1 Johoku, Hamamatsu, Shizuoka 432-8561, Japan, s-akama@spalab.eng.shizuoka.ac.jp)

We propose a new audio spotlight system which uses focused sound sources arranged in an arc shape, and we evaluate its suppression performance and frequency responses. Audio spotlight systems use ultrasonic waves to produce sharp directional sounds without any external disturbances. However, such systems contain two problems: (1) low efficiency of the electroacoustic conversion and (2) distortion of the reproduced sound. Therefore, we attempted to form an audio spotlight system by using focused sound sources based on wave field synthesis. A single focused sound source is designed to harmonize the phase of the driving signal at an arbitrary position. Thus, the driving signals that correspond to the number of the optional focused sources can be superimposed and reproduced from a loudspeaker array in order to form multiple focused sound sources. By arranging the focused sources in arc shape, we produced the audio spotlight. A numerical simulation revealed that the sound volume difference between the center of an arc and at $1 \mathrm{~m}$ away from the center was approximately $10 \mathrm{~dB}$. Moreover, because a flat frequency response was maintained at the center of the arc, the proposal is expected to be able to control optional sounds with minimal distortion.

2aSPb2. Study on the prevention of Sinkhole in cities through sound analysis. Ik-Soo Ann and Myung-Jin Bae (TeleCommun. \& Information, soolsil Univ., 369 sangdo-ro, Dongjak-gu, Seoul 156-743, South Korea, aisbestman@naver.com)

Sinkhole is a symptom where the surface area collapses due to lack of supporting power and this is caused by erosion of rocks under the ground or washing away of soil. It is very difficult to say that this sinkhole happening in the downtown is natural but rather resulted from development of underground water, water leakage from the old water pipes buried, and subway construction. As this symptom cannot be predicted visually by looking at the surface it happens unexpectedly and thus has a high possibility of causing a lot of damage. However, active research on developing prevention system by using a special laser video or sound has been carried out. In regards to this, this study suggests methods of preventing downtown sinkhole by analyzing sound. The special feature that classifies sound made from tapping the normal surface and surface of the potential sinkhole is the resonance. If we can have a focused control over these surfaces with high potential of sinkhole we will be able to prevent any accidents taking from this.
2aSPb3. Surf infrasound recorded with smartphones during the 2016 Eddie Aikau. Karina A. Asmar (HIGP, Univ. of Hawaii, Manoa, Honolulu, HI 96822, kasmar@hawaii.edu), Milton Garces (HIGP, Univ. of Hawaii, Manoa, Kona, HI), Julie Schnurr (HIGP, Univ. of Hawaii, Manoa, Honolulu, HI), and Anthony Christe (ICS, Univ. of Hawaii, Manoa, Honolulu, $\mathrm{HI})$

Standardization of signal characteristics is a recurring problem in infrasound detection. We are developing a suite of scalable multiresolution algorithms for infrasound data processing that may include unevenly sampled smartphone barometer data. The algorithms include fundamental methods for Lomb-Scargle periodograms and coherent energy estimators. The implementation of these algorithms will facilitate reproducible and transportable characterization of diverse signatures captured by traditional and ubiquitous global infrasound networks. We discuss the application of these algorithms to surf infrasound signals recorded with smartphones during the 25 February 2016 Eddie Aikau Surf Contest held at Waimea Bay, Oahu.

2aSPb4. Directivity control for regular polyhedron loudspeaker array based on weighted least-squares method using adjusted weight in spherical harmonics domain. Makoto Daikohara and Yoichi Haneda (Dept. of informatics, The Univ. of Electro-Communications, 1-5-1 Chofugaoka, Chofu, Tokyo 182-8585, Japan, d1530034@edu.cc.uec.ac.jp)

We propose an adjusted weight least-squares method for directivity control of spherical loudspeaker arrays in the spherical harmonics domain. In previous work, we demonstrated that the sidelobes of the directivity pattern can be suppressed using the weighted least-squares method in the frequency domain. However, the gains of the calculated filter coefficients were very large at low frequencies. Consequently, to overcome this problem, we designed filter coefficients in the spherical harmonic domain. First, we set an ideal directivity pattern using a window function. Next, we obtain the filter based on the weighted least-squares method in the spherical harmonic domain with an initial weighting factor. Then, we update the weighting factor only on the side opposite that being faced based on the difference in sound pressure level between the ideal and the simulated radiation patterns. We then repeat this process until the difference level is satisfied by the condition of the update. The results of comparison of our proposed method with our previous method and the conventional least-squares method indicate that although the beam width in the look direction is wider than our previous method, our proposed method effectively prevents increasing filter coefficients by limiting order at low frequencies. 
2aSPb5. A basic study of acoustic communications between an autonomous surface vehicle and an autonomous underwater vehicle. Mitsuyasu Deguchi, Yukihiro Kida, Koji Meguro, Yoshitaka Watanabe, Takuya Shimura, Hiroshi Ochi (Underwater Acoust. Technol. Group, Japan Agency for Marine-Earth Sci. and Technol., 2-15 Natsushima-cho, Yokosuka, Kanagawa 237-0061, Japan, deguchim@jamstec.go.jp), and Yukinori Akamine (Information Electronics Res. Dept., Res. \& Development Group, Hitachi Ltd., Kokubunji-shi, Japan)

Recently, there has been an increasing interest in submarine resources and demand to survey them. For that purpose, Japan Agency for MarineEarth Science and Technology (JAMSTEC) has developed autonomous underwater vehicles (AUVs). Currently, only one AUV is operated by a research vessel because a mother ship must always follow an AUV. However, to make survey more efficient, the project of multiple AUVs operation, paired with autonomous surface vehicles (ASVs), is being progressed. In this plan, one ASV always monitor status and position of its paired AUV with acoustic communication and localization, and follow it autonomously. The size of an ASV is desired to be as small as possible in terms of operation. Thus, it causes two difficulties. First, such a small ASV rolls and pitches up to a large angle easily. Second, receivers are close to the noise sources, such as thrusters or sea surface. As a basic study, at-sea experiments were demonstrated with various types of modulation using a prototype of an ASV. The results show that OFDM signals are more sensitive to rolling and pitching movement than single carrier modulation (SCM) and that it is possible to achieve demodulation with SCM in spite of noise as mentioned above.

2aSPb6. Separation of individual instrumental sounds in monaural music signals applying a modified Wiener filter on the time-frequency plane. Yukio Fukayama and Masafumi Watanabe (Elec. Systems, Hiroshima Inst. of Technol., 2-1-1 Miyake Saeki-ku, Hiroshima Inst. of Technol., Hiroshima 731-5193, Japan, fukayama@ieee.org)

This research seeks a signal processor for random processes which is suitable for distilling melodies played by a particular instrument from music ensemble. The proposed processor sequentially evaluates statistics of the mixture assuming a model of music sound and employs a modified Wiener filter so as to separate the mixture of time-variant and correlated random processes such as amplitude changing tones and same pitch tones by different instruments. The model of music sounds is assumed to be a weighted mixture of each standard tone, which is a typical tone of every pitch played by a particular instrument with invariant power, where the weight is called the amplitude factor corresponding to the standard tone. Thus, the proposed processor, at first, evaluates the current amplitude factors which are the the coordinates of the mixture where the standard tones form oblique basis. Then, according to evaluated coordinates of the mixture, its current autocorrelation and cross-correlation to each standard tone are calculated for obtaining the modified Wiener filter. Finally, the filter separates the mixture into individual tone, which is a component of a particular pitch played by an instrument with variant power, and combination of which provides a melody.

2aSPb7. Shouted speech detection using hidden markov model with rahmonic and mel-frequency cepstrum coefficients. Takahiro Fukumori, Masato Nakayama, Takanobu Nishiura (Ritsumeikan Univ., 1-1-1, Nojihigashi, Kusatsu 525-8577, Japan, fukumori@fc.ritsumei.ac.jp), and Hiroaki Nanjo (Kyoto Univ., Kyoto, Japan)

In recent years, crime prevention systems have been developed to detect various hazardous situations. In general, the systems utilize the image information recorded by a camera to monitor the situations. It is however difficult to detect them in the blind area. To address the problem, it is required to utilize not only image information but also acoustic information occurred in such situations. Our previous study showed that two acoustic features including rahmonic and mel-frequency cepstrum coefficients (MFCCs) are effective for detecting the shouted speech. Rahmonic shows a subharmonic of fundamental frequency in the cepstrum domain, and MFCCs represent coefficients that collectively make up mel-frequency cepstrum. In this method, a shouted speech model is constructed from these features by using a gaussian mixture model (GMM). However, the previous method with
GMM has difficulty in representing temporal changes of the speech features. In this study, we further expand the previous method using hidden Markov model (HMM) which has state transition to represent the temporal changes. Through objective experiments, the proposed method using HMM could achieve higher detection performance of the shouted speech than the conventional method using GMM.

2aSPb8. Vandermonde transform for complex residual based on complex speech analysis. Taishi Shimabukuro (IIJ Global, Nishihara, Japan) and Keiichi Funaki (C\&N Ctr., Univ. of the Ryukyus, Senbaru 1, Nishihara, Okinawa 903-0213, Japan, funaki@cc.u-ryukyu.ac.jp)

Vandermonde transform using the orthogonal basis is a time-frequency transform method that can describe a speech and audio signal to be uncorrelated and sparse, and can be considered to be also effective in speech processing. In particular, when applied to the residual considered to be effective in a pitch estimation of speech and speaker recognition. On the other hand, we have already proposed Time-Varying Complex AR (TV-CAR) speech analysis for an analytic signal. The TV-CAR analysis can improve spectral estimation accuracy in the low frequencies and it can estimate the complex AR coefficients for each sample. As a result, in the estimated complex residual signal, formant components are more subtracted from speech signal. It can perform better on pitch estimation, or so on. In this paper, we evaluated the time-frequency analysis using the Vandermonde transform based on an Orthogonal Matching Pursuit (OMP) method of the literature using the complex AR residual. The performance is evaluated for several orthogonal basis such as Fourier basis, Cos basis, K-L transform, and Gabor basis. Furthermore, the Ramanujan-sum is also introduced as the basis function to realize time-frequency transform.

2aSPb9. Individual sound image generation by null-space based sound field control under few loudspeakers in real environment. Shinpei Horii and Yosuke Tatekura (Shizuoka Univ., 3-5-1 Johoku, Hamamatsu, Shizuoka 432-8561, Japan, s-horii@spalab.eng.shizuoka.ac.jp)

To achieve an individual sound image generation with fewer loudspeakers based on NBSFC (Null-space Based Sound Field Control) filtering, we attempt to expand NBSFC under underdetermined condition, which means the number of loudspeakers is smaller than the number of control points. NBSFC filtering can suppress the sound pressure only on optional control points. In the conventional NBSFC design, the number of loudspeakers is required to be greater than that of control points. On the other hand, many control points are indispensable to achieve fine individual sound image generation. Thus, NBSFC filters are designed with the maximum control points limited by the conventional method from the transfer functions between the control points and the loudspeakers in various combinations. Also, the expanded NBSFC filters can be designed corresponding to the underdetermined condition by superimposing all conventional NBSFC filters. As the result of numerical simulation with a real environmental data, the sound pressure difference between inside and outside the control area was averaged by $7 \mathrm{~dB}$. This reveals that the control area where sound pressure is suppressed can be realized by locating many control points closely.

2aSPb10. Personal audio reproduction using two wearable end-fire loudspeaker arrays. Kenta Imaizumi and Yoichi Haneda (Dept. of Informatics, The Univ. of Electro-Communications, 1-5-1, Chofugaoka, Chofushi, Tokyo 182-8585, Japan, i1630013@edu.cc.uec.ac.jp)

We propose a wearable personal audio reproduction system that uses two end-fire loudspeaker arrays. The two loudspeaker arrays are attached in front of the listener's breast. Consequently, the system is not close to the ears. Further, because the main beams of the two arrays are directed at each ear, crosstalk between the left and right signals is low. In addition, sound leakage is also low as a result of directivity control. We constructed a prototype of the system using four loudspeaker elements for each array, with intervals of $4 \mathrm{~cm}$ and array length $12 \mathrm{~cm}$. The attenuation level of the area at approximately $50 \mathrm{~cm}$ away from the end-fire arrays was found to be -20 $\mathrm{dB}$ sound pressure level (SPL) relative to the main beam pointed at the ear. In addition, the crosstalk SPL difference was approximately $12 \mathrm{~dB}$. We can control the sound image position by convolving the head-related transfer 
function with the sound source. Further, no crosstalk canceller is needed because the proposed method secures the interaural intensity difference. Improvement or application of the proposed system can facilitate realization of a personal sound field.

2aSPb11. Sound source separation using image signal processing based on sparsity of sound field. Masaaki Ito, Kenji Ozawa, Masanori Morise, Genya Shimizu (Graduate School of Medicine and Eng. Sci. Dept. of Education, Univ. of Yamanashi, 4-3-11 Takeda, Kofu, Yamanashi 400-8511, Japan, g15mk002@yamanashi.ac.jp), and Shuichi Sakamoto (Tohoku Univ., Sendai, Japan)

Microphone arrays have been used to separate sound sources to improve speech recognition in a noisy environment. We propose a method using image signal processing to achieve highly accurate sound source separation. The microphone array is 1-m long and consists of eight microphones. Temporal sequences of the sound pressures obtained from the eight microphones are converted into sequences of luminance. These are arranged in parallel in an image, which is referred to as aspatio-temporal sound pressure distribution image. Sparse modeling using L1 regularization (Lasso) is applied to the image for restoring a high-resolution image. The spatial spectrum of the restored image is obtained using a two-dimensional fast Fourier transform (FFT) algorithm. In this spectrum, the angle of a line through the center denotes the arrival direction of sound, and the distance from the center indicates its frequency. By extracting a line from the spectrum, the sound source can be separated. A computational experiment revealed that high-resolution sound field obtained by a 512-microphone array could be restored using the proposed method. Moreover, SNR was improved by $32.1 \mathrm{~dB}$ when two sounds arrived $45^{\circ}$ apart, indicating sufficient performance to extract a desired sound. [Work supported by JSPS KAKENHI Grant (16K06384).]

2aSPb12. Modified 2nd-order nonlinear infinite impulse response (IIR) filter for compensating sharpness and nonlinear distortions of electrodynamic loudspeakers. Kenta Iwai (Organization for Res. and Development of Innovative Sci. and Technol., Kansai Univ., 3-3-35, Yamate-cho, Suita 564-8680, Japan, kenta1986@gmail.com) and Yoshinobu Kajikawa (Faculty of Eng. Sci., Kansai Univ., Osaka, Japan)

Electrodynamic loudspeakers generate the nonlinear distortions and degrade the sound quality. This is because the nonlinearity of electrodynamic loudspeaker is caused by the large displacement of the diaphragm, in particular, around the lowest resonance frequency. One of the compensation methods for the nonlinear distortions is using mirror filter. This filter is derived from the linear and nonlinear differential equations and designed by second-order nonlinear IIR filter or third-order nonlinear IIR filter. The coefficients of these filters are determined by the physical parameters of the loudspeaker. However, it is difficult to compensate the nonlinear distortions when the sharpness of the loudspeaker is high at the lowest resonance frequency because of large displacement of the diaphragm. Although it is required to compensate the sharpness of the loudspeaker, second-order nonlinear IIR filter cannot compensate the sharpness because of the compensation principle, i.e., this filter adds the nonlinear signal into the input signal and does not include the linear filtering. This paper proposes a modified second-order nonlinear IIR filter that can compensate not only the sharpness but also the lowest resonance frequency. The difference between the conventional and proposed filter structures is only coefficients. Experimental results show the effectiveness of the proposed filter.

2aSPb13. An interleave division multiple access receiver for multi-user underwater acoustic communication. Ning Jia, JianChun Huang, and Dong Xiao (Key Lab. of Underwater Acoust. Environment, Inst. of Acoust. Chinese Acad. of Sci., No. 21 North 4th Ring Rd., Haidian District, 100190 Beijing, China, jianing@mail.ioa.ac.cn)

A downlink interleave division multiple access (IDMA) receiver, employing sparse channel estimation and interference canceller (IC), is proposed for multi-user underwater communications. To eliminate inter-symbol interference (ISI) and multiple access interference (MAI), an equalizer, a decoder, and an interference canceller are implemented in the receiver. The equalizer is used to eliminate ISI. The channel parameters used in the equalizer are estimated by a modified OMP sparse channel estimation algorithm. The performance of channel estimation is further improve ed using the decoder soft output information during the receiver iteration. MAI, reconstructed by the decoder output and channel parameters, can be eliminated in IC section of other users. The receiver's performance is verified in shallow water acoustic channels during a sea-trail in the South China Sea. Three different ranges between the transmitter and the receiver, i.e., 4.5 $\mathrm{km}, 6 \mathrm{~km}$, and $7 \mathrm{~km}$, were investigated with a maximum of four users at the data rate of $500 \mathrm{bps}$ per user. The results demonstrated that the proposed IDMA receiver provides good performance with low bit error rate (BER). Furthermore, the receiver has been applied in a real-time underwater communication network.

2aSPb14. Relationship between speech recognition performance and related spoken document retrieval performance. Noboru Kanedera (Dept. of Electronics and Information Eng., National Inst. of Technol., Ishikawa College, Kitachujou, Tsubata-Machi, Ishikawa 929-0392, Japan, kane@ishikawa-nct.ac.jp)

Along with multimedia information including the speech information having increased, spoken document retrieval has attracted attention. Keywords or query sentences are used for spoken document retrieval. The keywords or query sentences cannot be used when we want to watch the video scenes which are related to a watching video scene. In this report, we propose a method of evaluating spoken document retrieval between video scenes, and report the results of the investigation about the relationship between the speech recognition performance and spoken document retrieval performance by using watching video scene. As a result of simulation experiment, it was confirmed to affect the search performance in order of substituted error, deletion error, insertion error, in the search both by question sentence and by the scene. The search by the scene is easy to be affected by the speech recognition error than the search by the question sentence. It was found that the influence of the insertion error in the search by the scene was bigger than the search by the question sentence.

2aSPb15. Automatic selection of the number of poles for different gender and age groups in steady-state isolated vowels. Thayabaran Kathiresan (Phonet. Lab., Univ. of Zurich, Plattenstrasse 54, Zurich, Zurich 8032, Switzerland, thayabaran.kathiresan@uzh.ch), Dieter Maurer (Inst. for the Performing Arts and Film, Zurich Univ. of the Art, Zurich, Zurich, Switzerland), and Volker Dellwo (Phonet. Lab., Univ. of Zurich, Zurich, Zurich, Switzerland)

Formant frequency estimation using a linear prediction (LPC) algorithm is based on the assumption of age- and gender-specific number of poles. However, when visually crosschecking the calculated formant frequencies along with a spectrogram, investigators often change the parameter because of a lack of correspondence. The misprediction is mainly due to a high variation within the calculated formant tracks, or tracks not matching with spectral peaks, possibly combined with an unexpected low or high number of occurring formants (e.g., formant merging, spurious formants). To solve the problem of changing the number of filter poles, we propose a new method which addresses the first aspect of the constancy of formant tracks. For a given vowel sound, the first three formant frequencies for three different settings (number of poles $=10,12$, and 14 for a frequency range $0-5.5 \mathrm{kHz}$ ) are calculated. The standard deviation of the formant tracks is used to find a Euclidean distance for three settings separately. The algorithm chooses the setting that produces least variability (minimum Euclidean distance) in steady-state vowel nuclei. We tested the method on vowel sounds of standard German /i, y, e, $\varnothing, \varepsilon, \mathrm{a}, \mathrm{o}, \mathrm{u} /$ produced by 14 men, 14 women, and 8 children.

2aSPb16. Performance analysis of passive time reversal communication technique for multi-path interference with synthetic data. Yukihiro Kida, Mitsuyasu Deguchi, Yoshitaka Watanabe, Takuya Shimura, Hiroshi Ochi, and Koji Meguro (Underwater Acoust. Technol. Group, JAMSTEC, 2-15 Natsushima-cho, Yokosuka 2370061, Japan, kiday@jamstec.go.jp)

The interference by time-varying and intense underwater acoustic multipath is one of the most obstructive phenomena in underwater acoustic 
communication. Time-Reversal (TR) communication techniques utilize the reciprocity of acoustic channels to converge the spread signals to the original one, and are considered one of the most effective methods to overcome multi-path interference as shown in many past studies. It is recognized empirically that the large number of multi-path arrival could generally raise the demodulation result of TR. However, its relationship is rarely evaluated quantitatively. In this paper, we investigate the efficiency of the TR acoustic communication techniques for the multi-path interference cancelation by applying our passive TR method to the synthetic datasets of horizontal acoustic communication in shallow water environment. Mainly, we focused on the relation between the Signal to Interference Noise Ratio (SINR) and the output SNR of demodulation result. The result shows: 1) SINR and output SNR has a nearly linear relation up to a limitation, and 2) above the limitation, output SNR reaches its ceiling or slightly decreases.

2aSPb17. Development of an underwater sound monitoring system using two hydrophones for a remotely operated underwater vehicle. Sea-Moon Kim, Sung-Hoon Byun, Hyun-Taek Choi, and Chong-Moo Lee (Korea Res. Inst. of Ships and Ocean Eng., 32 Yuseong-daero 1312beon-gil, Yuseong-gu, Daejeon 34103, South Korea, smkim@kriso.re.kr)

Recently, a sound monitoring system for hearing fishery sound using three hydrophones installed on an ROV (Rountree and Juanes, 2010) has been developed. However, the operation of the system was not successful because of the high level of the self-noise generated by the ROV. Its thrusters had to be turned off to reduce the self-noise and listen to fishery sound clearly. Currently, Korea Research Institute of Ships and Ocean Engineering (KRISO) is developing a light-work class ROV and a similar sound monitoring system was developed for the ROV. The design of the system was focused on the following two strategies: (1) minimization of self-noise effect; (2) reproducing sound for operators to identify source direction. To minimize the self-noise effect, a digital filter with seven filter banks with octave bandwidth was implemented. A time-domain beamforming technique has been applied to analyze the source location. Reproducing sound signals for the two speakers in the control room were generated according to the estimated source location. The performance of the algorithm was verified by using a receiver array signal simulator. Its detail characteristics will be presented in the talk. [This work was financially supported by the research project \# 20130197 funded by KIMST.]

2aSPb18. Study on intelligibility improvement method based on subband waveform processing focusing on dynamic feature of speech. Hiroki Kohara, Hideki Banno, and Kensaku Asahi (Graduate School of Sci. and Technol., Meijo Univ., 1-501 Shiogamaguchi, Tempaku-ku, Nagoya-shi, Aichi-ken 468-8502, Japan, 163430008@ccalumni.meijo-u.ac.jp)

This paper describes intelligibility improvement method for speech signal based on subband waveform processing. Our approach is based on the observation that clear speech has higher delta-cepstrum value in transient parts between phonemes, and emphasizes delta-cepstrum of input speech by a filter in the cepstral domain which amplifies a particular modulation frequency. However, since this approach generates synthetic sound by using an analysis/synthesis system, quality of the generated sound is sometimes degraded. To prevent this degradation, a subband waveform-based method is introduced. This method divides an input signal into several subband signals by a quadrature mirror filter (QMF) which approximately enables perfect reconstruction of input signal from the subband signals, converts an amplification gain sequence in the cepstral domain into that in the subbandwaveform domain, and then multiplies the converted amplification gain sequence to the subband signal on a sample-by-sample basis. Synthetic sounds were generated by the method in the cases where the number of subbands is set to two, four, and eight. We found that the sound that the number of subbands is two includes artificial power fluctuation, and increasing the number of subbands decreases the artificial power fluctuation and makes quality of the generated sound better.
2aSPb19. Stereophonic reproduction based on multi-beam-forming with curved-type parametric array loudspeaker. Shinya Komori, Nana Hasegawa, Takahiro Fukumori, Masato Nakayama, and Takanobu Nishiura (Ritsumeikan Univ., 1-1-1, Noji-higashi, Kusatsu, Shiga 525-8577, Japan, is0116fv@ed.ritsumei.ac.jp)

A parametric array loudspeaker (PAL) has a flat surface arrangement of ultrasonic transducers and achieves a sharper directivity by utilizing an ultrasound wave. It can form a narrow audible space called an "audio spot" that can provide a target sound to a particular listener. In addition, the PAL can achieve a stereophonic reproduction by designing the audio spots for the positions of ears on the basis of delay-and-sum beamforming. However, designing wider audio spots for several listeners is difficult for the conventional PAL because it has overly sharp directivity. To solve this problem, we propose a new stereophonic reproduction with a curved-type PAL that can expand the audio spots. The curved-type PAL consists of ultrasonic transducers arranged on a concave surface. It can form a wider directivity compared with the conventional flat-type PAL. The proposed method steers the acoustic beam by designing the focal point to the positions of ears, and the radius of the curved-type PAL varies the width of the acoustic-beams. In this study, we carried out an objective evaluation experiment to confirm the effectiveness of the proposed method. As a result, we confirmed that the proposed method can design two wider acoustic beams for the stereophonic reproduction.

2aSPb20. High accuracy resampling for acoustic signals using hermite interpolation with pseudo spectral method. Hotaka Maruyama, Kan Okubo, and Norio Tagawa (System Design, Tokyo Metropolitan Univ., 6-6 Asahigaoka, Hino, Tokyo 191-0065, Japan, maruyama-hotaka@ed.tmu.ac.jp)

Interpolation is an essential technique to obtain continuous data from discrete data within a known range of coordinates in many research areas. Especially, resampling acoustic signal is an important application. It is necessary to obtain the value between digitally recorded data using a signal interpolation technology. It is a fundamental method of digital processing operations. In recent years, a $192 \mathrm{kHz}$ high-resolution system is often employed, so that the accurate interpolation method is required to obtain the high resolution signals from the signals measured by the conventional lower sampling rate. In signal resampling using the Hermite interpolation, an interpolated value can be expressed as the convolution form by signal values and their derivatives. By using highly accurate derivatives, accuracy of resampling can be also improved. In this study, we propose a new highly accurate acoustic signal interpolation method combining the Hermite interpolation with the pseudo-spectral method. Moreover, we also propose their efficient computational algorithm employing separation technique. As a result, our evaluation clarified that the proposed method achieved more accurate signal resampling compared to the conventional methods.

2aSPb21. Visualization of one source in a two sources sound field by estimating source positions. Maureen and Yoichi Haneda (Dept. of Informatics, The Univ. of Electro-Communications, 1-5-1 Chofugaoka, Chofu, Tokyo 182-8585, Japan, maureen@uec.ac.jp)

Sound field visualization is important in many applications. Acoustic holography (AH) uses a linear microphone array to visualize sound fields. However, in general, all existing sound sources will appear when AH is used. In this paper, we propose a method that visualizes a single source from two sources in a sound field by estimating source positions and separating them. In the proposed method, sound source positions are estimated based on the microphone input signals' phase components for each frequency. The source positions of all frequencies are placed in a histogram that expresses each position's quantity, which facilitates acquisition of accurate source positions. We determine one source and select frequency bins that indicate the obtained source position. We can also virtually extrapolate the microphone array length by solving the wave propagation model using the least-squares method. The results of sound field visualization experiments conducted considering the depth direction and using the one-dimensional near-field acoustic holography inverse propagation method show that the proposed method can separate a single source by selecting the histogram peak even when the wavefronts of two sources appear simultaneously. Future work will attempt to restore only one source or both two sources to time signal. 
2aSPb22. Utilization of optional instrument sounds for simultaneous measurement of room impulse responses by ensemble music. Yuki Miyauchi and Yosuke Tatekura (Shizuoka Univ., 3-5-1 Johoku, Hamamatsu, Shizuoka 432-8002, Japan, y-miya@spalab.eng.shizuoka.ac.jp)

We describe the influence of the separation accuracy via semi-blind source separation (semi-BSS) upon the measurement accuracy of the room impulse responses. Tatekura et al. had proposed a simultaneous measurement method of the room impulse responses with ensemble music. In this method, the room impulse responses were measured by separating ensemble music into each instrument sound reproduced from each loudspeaker. However, the influence of the separation accuracy via semi-BSS upon the measurement accuracy of the room impulse responses has not been clarified. Therefore, we evaluated the measurement accuracy of the room impulse responses and the separation accuracy by using the different instrument sounds. The room impulse responses were measured between two loudspeakers and a control point with 11 kinds of musical instrument sounds. From the result of the measurement accuracy, the difference between the maximum value and the minimum value was $10 \mathrm{~dB}$. However, from the result of the separation accuracy, most of value held equivalent measurement accuracy. Therefore, under these measurement conditions, this method is expected to be able to measure the room impulse responses by optional instrument sounds.

2aSPb23. An evaluation of speech reconstruction for degraded speech of optical microphone with deep neural network. Tomoyuki Mizuno, Takahiro Fukumori, Masato Nakayama, and Takanobu Nishiura (Ritsumeikan Univ., 1-1-1, Noji-higashi, Kusatsu 525-8577, Japan, is0140kk@ed.ritsumei.ac.jp)

Measuring distant-talking speech with high accuracy is important in detecting criminal activity. Various microphones such as the parabolic and shotgun microphones have been developed for measuring it. However, most of them have difficulty in extracting distant-talking speech at a target position if they are surrounded by noisy sound sources. Therefore, this study focuses on a microphone system to extract the distant-talking speech by vibrating a papery object using laser light. This system is referred to as an optical microphone in this study. The sound quality of the optical microphone is especially degraded at higher frequencies because it utilizes an external diaphragm consisting of various materials as the vibrating papery object. In this study, we therefore propose a reconstruction method for degraded distant-talking speech observed with the optical microphone. The method is realized by using a deep neural network (DNN) that is trained as the system between clean and observed speech signals. In the proposed method, the log-power spectra of 11 frames are used for the input of the DNN. Finally, we confirmed the effectiveness of the proposed system through an evaluation experiment.

2aSPb24. Voice livness detection based on pop-noise detector with phoneme information for speaker verification. Shihono Mochizuki, Sayaka Shiota, and Hitoshi Kiya (Information and Commun. Systems, Tokyo Metropolitan Univ., 6-6, Asahigaoka, Hino-shi, Tokyo 191-0065, Japan, mochizuki-shihono@ed.tmu.ac.jp)

This paper proposes a pop-noise detector using phoneme information for a voice liveness detection (VLD) framework. In recent years, spoofing attacks (e.g., reply, speech synthesis, and voice conversion) have become a serious problem against speaker verification systems. Some techniques have been proposed to protect the speaker verification systems from these spoofing attacks. The VLD framework has been proposed as one of fundamental solutions. The VLD framework identifies that an input sample is uttered by an actual human or played by a loudspeaker. To realize the VLD framework, pop-noise detection methods have been proposed and these methods perform well as the VLD module. However, since pop-noise is a common distortion in speech that occurs when a speaker's breath reaches a microphone, the phenomenon of pop-noise is able to be occurred by winds or attackers arbitrary. It is one problem of the pop-noise detection methods. In order to improve the robustness of the pop-noise detection methods, this paper proposes a pop-noise detector using phoneme information as an evidence of an actual human. From the experimental results, the proposed method increases the robustness of the VLD against spoofing attacks.
2aSPb25. Simultaneous recognition of phone and speaker using threeway restricted Boltzmann machine. Toru Nakashika and Yasuhiro Minami (The Univ. of Electro-Communications, 1-5-1 Chofugaoka, Chofu, Tokyo 182-8585, Japan, nakashika@uec.ac.jp)

In this study, we attempt a simultaneous recognition method of phone and speaker using a single energy-based model, a three-way restricted Boltzmann machine (3WRBM). The proposed model is a probabilistic model that includes three variables: acoustic features, latent phonetic features, and speaker-identity features. The model is trained so that it automatically captures the intensity of relationships among the three variables. Once the training is done, we can apply the model to many speech signal processing tasks because it has an ability to separate phoneme and speaker-related information from the observed speech, and generate a speech signal from the phoneme and speaker-related information on the contrary. Simultaneous phone and speaker recognition is achieved by estimating the latent phonetic features and the speaker-identity features given the input signal. In our experiments, we discuss the effectiveness of the mode lin a speaker recognition and a speech (continuous phone) recognition tasks.

2aSPb26. Segmental conditional random fields audio-to-score alignment distinguishing percussion sounds from other instruments. Ayako Noguchi, Shinji Sako, and Tadashi Kitamura (Nagoya Inst. of Technol., Gokisocho, Showa-ku, Nagoya, Aichi 4668555, Japan, noguchi@mmsp.nitech.ac. jp)

Audio-to-score alignment is useful technique because it can be widely applied to many practical applications for musical performance. However, it is still open problem due to the complexity of audio signal especially in the polyphonic music. Additionally, performing in real-time is also important in practical situation. In this study, we propose a new alignment method based on segmental conditional random fields (SCRFs). The attractive feature of this method is utilizing to distinguish percussion sounds from the other instruments. In general, percussion sounds have a role in managing whole music. Moreover, performers can pick up the percussion sounds from the others by hearing whole sound thanks to their unique features of the sound. In the field of score alignment, hidden Markov models (HMMs) or CRFs was used in previous studies including our previous one. In summary, these methods were formulated as a matching problem of the state sequence of mixed notes with the audio feature sequence. In this study, we extend our previous method by combining an additional state which represents percussion sounds. Furthermore, we introduce the balancing factor to control the importance of classifying feature functions. We confirmed the effectiveness of our method by conducting experiments using RWC music database.

2aSPb27. AI framework to arrange audio objects according to listener preferences. Kento Ohtani (Nagoya Univ., Furo-cho,Chikusa-ku, Nagoya, Aichi 464-8601, Japan, ohtani.kento@g.sp.m.is.nagoya-u.ac.jp), Kenta Niwa (NTT Media Intelligence Labs., Musashino, Tokyo, Japan), and Kazuya Takeda (Nagoya Univ., Nagoya, Japan)

We propose a framework for arranging audio objects in recorded music using artificial intelligence (AI) to anticipate the preferences of individual listeners. The signals of audio objects, such as the tracks of guitars and drums in a piece of music, are re-synthesizes in order to provide the preferred spatial arrangements of each listener. Deep learning-based noise suppression ratio estimation is utilized as a technique for enhancing audio objects from mixed signals. Neural networks are tuned for each audio object in advance, and noise suppression ratios are estimated for each frequency band and time frame. After enhancing each audio object, the objects are resynthesized as stereo sound using the positions of the audio objects and the listener as synthesizing parameters. Each listener supplies simple feedback regarding his/her preferred audio object arrangement using graphical user interface (GUI). Using this listener feedback, the synthesizing parameters are then stochastically optimized in accordance with each listener's preferences. The system recommends dozens of customized synthesizing parameters, and these parameters can then be adjusted by the listener using a single-dimensional slider bar. Several tests were conducted and the proposed framework scored high marks in subjective evaluations as to whether or not the recommended arrangements were indeed preferable. 
2aSPb28. An improved filter employing measured characteristics of diaphragm displacement for compensating loudspeaker nonlinearity. Manabu Omura (Faculty of Eng. Sci., Kansai Univ., 3-3-35, Yamate-cho, Suita, Osaka 564-8680, Japan, k675924@kansai-u.ac.jp), Kenta Iwai (Organization for Res. and Development of Innovative Sci. and Technol., Kansai Univ., Suita, Osaka, Japan), and Yoshinobu Kajikawa (Faculty of Eng. Sci., Kansai Univ., Suita, Osaka, Japan)

In this paper, we propose an improved filter employing measured characteristics of diaphragm displacement for compensating nonlinear distortions in loudspeaker systems. Loudspeaker systems generate nonlinear distortions that degrade the sound quality because of complicated structure of loudspeaker systems. Therefore, we set out to improve the sound quality by employing Mirror filter which compensates nonlinear distortions in electro-dynamic transducer. Mirror filter calculates linear instantaneous displacement of diaphragm from the linear differential equation of the electro-dynamic transducer and generates compensation signals based on the nonlinear differential equation of the electro-dynamic transducer and the calculated displacement of diaphragm. Hence, the compensation performance with Mirror filter is determined by the accuracy of the calculated linear and nonlinear motion based on the differential equations. However the calculated motion of the diaphragm does not agree with the actual motion perfectly because of the errors which come from parameters in the differential equations and modeled error. Therefore, an improved Mirror filter is proposed to exclude these errors. In detail, the improved Mirror filter employs the impulse response, which represents measured characteristics of diaphragm displacement, to calculate instantaneous displacement of diaphragm. In this paper, we demonstrate the validity of the proposed filter through experimental results.

2aSPb29. Amplitude limiters based on phase optimization. Akira Kakitani, Daisuke Saito (The Univ. of Tokyo, Hongo 7-3-1, Bunkyo-ku, Tokyo, Tokyo 1130033, Japan, dsk_saito@gavo.t.u-tokyo.ac.jp), Yasuhiro Kosugi (none, Tokyo, Japan), and Nobuaki Minematsu (The Univ. of Tokyo, Tokyo, Japan)

In order to reduce the peak value of source waveforms without quality degradation, a novel method is proposed. In this method, by exploiting the characteristics of human auditory system, which is often said to be insensitive to phase changes, peak value reduction is made possible. The proposed method is an improved version of our previous study, which manipulated the waveforms mainly based on phase control. In the conventional algorithm, however, amplitude characteristics have to be altered to some degree. The proposed method attempts to minimize the change of amplitude characteristics. Evaluation experiments show that the proposed method outperforms the conventional one in a Perceptual Evaluation of Audio Quality (PEAQ) test but the superiority is not found in a MUltiple Stimuli with Hidden Reference and Anchor (MUSHRA) test.

2aSPb30. Fast multiple moving sound sources localization utilizing sparseness of speech signals. Eiji Sato and Yosuke Tatekura (Shizuoka Univ., 3-5-1 Johoku, Hamamatsu, Shizuoka 432-8561, Japan, e-satoh@spalab.eng.shizuoka.ac.jp)

We propose multiple moving sound sources localization with reduced operation time utilizing the sparseness of speech signals, and we demonstrate the efficacy of proposed method in actual environment experiments. Sound source localization is an essential function for tasks such as robot audition, and particularly for sound sources that often move in the actual environments. Sound source localization requires high resolution and real-time processing because they are utilized for post-processes such as sound source separation. The proposed method is based on MUSIC (MUltiple SIgnal Classification) which is known as a sound source localization method with high resolution. However, MUSIC has a large computational complexity. When the signal has large energy over a wideband in the frequency domain, the operation time increases because MUSIC estimates the direction of the sound source at every frequency. Therefore, we attempted to reduce the operation time by using the sparseness of speech signals in which the sound energies exist sparsely in the time-frequency domain. We chose frequencies with large power from frequency characteristics of the observed signals. Sound source localization was performed in the chosen frequencies. The operation time of the proposed method was about 8.1 times faster than the case of utilizing all frequencies.
2aSPb31. Directivity control of a compact circular loudspeaker array based on selected orders of circular harmonic expansion. Koya Sato and Yoichi Haneda (Dept. of Informatics, The Univ. of Electro-Communications, 1-5-1 Chofugaoka, Chofu-shi, Tokyo 182-8585, Japan, Japan, s1630060@edu.cc.uec.ac.jp)

We investigated directivity control of a compact circular loudspeaker array that can be attached to the arm. The loudspeaker array can be used for various wearable systems, such as personal audio reproduction systems. A personal audio system often requires that sound leakage outside the listening area in three-dimensional space be minimized. Digital filters that control directivity are usually designed using the multi-point control method. However, the multi-point method causes large filter gains at low frequencies, owing to unstable inverse matrix calculation, which results in sound distortion during practical use. Many methods that use a regularization parameter to overcome the unstable inverse matrix have been proposed; however, they are difficult to apply directly. Thus, after studying the relationship between orders of circular harmonic expansion and filter gain, we propose a stable digital filter design based on appropriately selected orders of circular harmonic expansion for each frequency band. Directivity simulations conducted using the measured circular loudspeaker array response based on the proposed method indicate that the proposed method achieves a more stable filter gain than the multi-point control method. Further, it controls threedimensional directivity by sacrificing a narrow directivity at low frequencies.

2aSPb32. Correct phoneme sequence estimation using recurrent neural network for spoken term detection. Naoki Sawada and Hiromitsu Nishizaki (Univ. of Yamanashi, 4-3-11 Takeda, Kofu 400-8511, Japan, sawada@ alps-lab.org)

This paper proposes a correct phoneme sequence estimation method that uses a recurrent neural network (RNN)-based framework for spoken term detection (STD). It is important to reduce automatic speech recognition (ASR) errors to obtain good STD results. Therefore, we use a long shortterm memory (LSTM), which is one of an RNN architecture, for estimating a correct phoneme sequence of an utterance from phoneme-based transcriptions produced by ASR systems in post-processing of ASR. We prepare two types of LSTM-based phoneme estimators: one is trained with a single ASR system's N-best output and the other is trained with multiple ASR systems' 1-best outputs. For an experiment on a correct phoneme estimation task, these LSTM-based estimators could generate better phoneme-based N-best transcriptions rather than the best ASR system's ones. In particular, the estimator trained with multiple ASR systems' outputs worked well on the estimation task. Besides, the STD system with the LSTM estimator drastically improved STD performance compared to our previously proposed STD system with a conditional random field-based phoneme estimator.

2aSPb33. An integration method of multiple search results for spoken term detection. Yoshino Shimizu (Graduate School of Software and Information Sci., Iwate Prefectural Univ., 2-10-37 Honchodori, Morioka-shi, Iwate-ken 020-0015, Japan, g231o015@s.iwate-pu.ac.jp), Eitaro Iwasaki (Iwate Prefectural Univ., Takizawa-shi, Japan), Shi-Wook Lee (National Inst. of Adv. Industrial Sci. and Technol., Tsukuba-shi, Japan), Kazuyo Tanaka (Univ. of Tsukuba, Tsukuba-shi, Japan), Kazunori Kojima, and Yoshiaki Itoh (Iwate Prefectural Univ., Takizawa-shi, Japan)

We propose a new integration method of multiple search results for improving search accuracy of Spoken Term Detection (STD). A usual STD system prepares two types of recognition results of spoken documents. If a query consists of in-vocabulary (IV) terms, the results using word-based recognizer are used, and if a query includes out-of-vocabulary (OOV) terms, the results using subword-based recognizer are used. The paper proposes an integration method of these two search results. Each utterance has a similarity score included in the search results. The scores of two results for an utterance has been integrated linearly using a constant weighting factor so far. Our preliminary experiments showed the search accuracy using the subword-based results was higher for some IV queries. In the same way, that using the word-based results was higher for some OOV queries. In the proposed method, the similarity scores of the two search results are compared for the same utterance and a higher weighing factor is given to the results 
that showed a higher similarity score. The proposed method is evaluated using open test sets, and experimental results demonstrated the search accuracy improved for all test sets.

2aSPb34. The largest Cramer-Rao lower bound for variance estimation of acoustic signals. Hiroki Takahashi (Elec. Eng. Program, Meiji Univ., 11-1 Higashimita, Tama-ku, Kawasaki-shi, Kanagawa 214-8571, Japan, ce51038@meiji.ac.jp) and Takahiro Murakami (Dept. of Electronics andBioinfomatics, Meiji Univ., Kanagawa, Japan)

The Cramer-Rao Lower Bounds (CRLBs) for variance estimation of acoustic signals is discussed. The CRLB expresses the lower limit of the variance of estimation errors of an unbiased estimator. It is empirically known that the accuracy of variance estimation is improved as the signal length is long because the CRLB for variance estimation is inverse proportion to the length of a signal. Therefore, when the variance of estimation errors is desired to be lower than a certain value, the signal length must be longer than the length that is determined by the CRLB. If the distribution of a signal is known, the CRLB can be easily formulated. In practice, however, acoustic signals follow various distributions and they are unknown in advance. In our research, we focus on the distribution that gives the largest CRLB for variance estimation. In order to find such a distribution, we compare the CRLBs for variance estimation using various signal models. Our results conclude that the Gaussian distribution gives the largest CRLB for variance estimation.

2aSPb35. Novel sound logger system for sound source localization using global navigation satellite system. Chikao Takasugi (Elec. Eng. and Comput. Sci., Shibaura Inst. of Technol., Koike Lab., 3-7-5 Toyosu, Kotoh-Ku, Tokyo 135-8548, Japan, ma16065@shibaura-it.ac.jp), Keisuke Takeyama, Takuya Fukaishi, Yoshikazu Koike (Electron. Eng., Shibaura Inst. of Technol., Tokyo, Japan), Harimasa Hojo (SensorComm Co.,Ltd., Tokyo, Japan), and Tatsuo Matsuno (Matsuno Kikaku, Tokyo, Japan)

Accurate sound localization is a very important technology to solve noise problem in the association with air plane, railroad, or wind-power generation and so on. In order to localize the sound source, direction of arrival (DOA) estimations in a couple of positions at a distance of several hundred meters are required. Generally, the measurement start timing is synchronized in each estimation position using 1PPS time signal from the global navigation satellite systems (GNSSs). However, the slight deviation in each measuring instrument occurs at the start time. Because the sampling clock of ADC (analog digital converter) is not synchronized. Therefore, the authors propose the application of time synchronization signal obtained from GNSS module to a sampling clock on an acoustic measurement in a wide range. Current some GNSS modules provide a pulse multiplied by 1PPS, which we call a time-synchronization signal. For the purpose, the authors have developed a logger apparatus which consists of a microphone amplifier, a GNSS, and an ADC module. The developed logger apparatuses are employed to perform sound source localization experiment in the field. As a result, the precision of sound localization within 2 meters is achieved successfully when the microphone sets at a distance of $10 \mathrm{~m}$.

2aSPb36. Evolutionary optimization of long short-term memory neural network language model. Tomohiro Tanaka (Dept. of Information Processing, Tokyo Inst. of Technol., 4259G2-2, Nagatsuta, Midori-ku, Yokohama, Kanagawa 226-8502, Japan, tanaka.t.bh@m.titech.ac.jp), Takafumi Moriya (NTT Media Intelligence Labs., NTT Corp., Yokosuka-Shi, Japan), Takahiro Shinozaki (Dept. of Information and Commun. Eng., School of Eng., Tokyo Inst. of Technol., Yokohama-shi, Japan), Shinji Watanabe, Takaaki Hori (Mitsubishi Electric Res. Laboratries, Cambridge, MA), and Kevin Duh (Johns Hopkins Univ., Baltimore, MD)

Recurrent neural network language models (RNN-LMs) are recently proven to produce better performance than conventional N-gram based language models in various speech recognition tasks. Especially, long shortterm memory recurrent neural network language models (LSTM-LMs) give superior performance for its ability to better modeling word history information. However, LSTM-LMs have complex network structure and training configurations, which are meta-parameters that need to be well tuned to achieve the state-of-the-art performance. The tuning is usually performed manually by humans, but it is not easy because it requires expert knowledge and intensive effort with many trials. In this study, we apply covariance matrix adaptation evolution strategy (CMA-ES) and automate the tuning process. CMA-ES is one of the most efficient global optimization techniques that has demonstrated superior performance in various benchmark tasks. In the experiments, the meta-parameters subject to the tuning included unit types at each network layer (LSTM, RNN, and feed-forward), the number of hidden layers, mini-batch size, and so on. We show that CMA-ES successfully optimizes the meta-parameters of LSTM-LM improving the recognition performance. We also report what are the common characteristics of the well-performing models by analyzing the results of the evolutions.

2aSPb37. Development of a communication system using audio signal using audible frequency bands. Chihiro Terayama, Masahiro Niitsuma, and Yoichi Yamashita (Graduate School of Information Sci. and Eng., Ritsumeikan Univ., Nojihigashi1-1-1, Kusatsu-shi, Shia-ken 525-8577, Japan, terayama-ASJ@slp.is.ritsumei.ac.jp)

This paper proposes a technique of embedding information into audio signal to communicate using loudspeakers and microphones mounted on tablet-type devices. This technique is based on amplitude modulation for high audible frequency bands. The embedded information is represented by existence of sine waves with 11 different frequencies. In consideration of the frequency characteristics of tablet-type devices, two frequency bands to embed information, were chosen: (1) frequencies up to $12 \mathrm{kHz}$; (2) frequencies up to $16 \mathrm{kHz}$. The experimental result showed $85.9 \%$ of correct decoding in the case of (1), and $76.8 \%$ in the case of (2). Moreover, less audible noise was perceived in the case of (2).

2aSPb38. Iterative spectral subtraction based on variable frame processing for musical noise reduction. Yukoh Wakabayashi, Yuki Nagano, Takahiro Fukumori, Masato Nakayama, and Takanobu Nishiura (Ritsumeikan Univ., 1-1-1 Noji-higashi, Kusatsu, Shiga 525-8577, Japan, gr0221ss@ ed.ritsumei.ac.jp)

In recent years, speech communication and speech applications like speech recognition are widely used with the development of information equipment. But a speech corrupted by noises makes fluent communication difficult and causes the poor performance of speech applications. Thus, an accurate noise reduction method is necessary to solve these problems. Spectral subtraction (SS) and iterative spectral subtraction (I-SS) are well-known noise reduction methods that subtract estimated noise from noisy speech in the power spectral domain. However, SS and I-SS generate an artificial noise called musical noise. The noise is non-stationary and has an offensive sound. Miyazaki et al. have proposed a musical-noise-free SS that determines subtraction parameters such that musical noise is not generated. The method effectively reduces the musical noise but requires estimation of the statistical characteristics of the observed noise. Meanwhile, we tackle the musical noise problem by proposing a new I-SS based on variable frame processing. In this processing, variable frame analysis is carried out in each iteration to reduce the musical noise. Evaluation experiments show that the proposed I-SS achieved a high SDR and resulted in less musical noise compared with constant frame processing.

2aSPb39. Effect of individualization of interaural time/level differences with non-individualized head-related transfer functions on sound localization. Kanji Watanabe, Masayuki Nishiguchi, Shouichi Takane, and Koji Abe (Faculty of Systems Sci. and Technol., Akita Prefectural Univ., 84-4 Ebinokuchi, Tsuchiya, Yuri-Honjo, Akita 015-0055, Japan, kwatanabe@ akita-pu.ac.jp)

Head-related transfer functions (HRTFs) are known to include comprehensive auditory cues for sound source positions and show large inter-subject variation. Therefore, individualization of HRTFs is important for highly accurate sound localization systems such as virtual auditory displays. In the present study, we assume that the interaural time deffierence (ITD) and the interaural level difference (ILD) can be estimated from the listener's anthropometric parameters. The individualization of the HRTFs is achieved by replacing the ITDs and ILDs of the non-individualized HRTFs with the 
listener's ones. In this report, the effect of the individualization was evaluated by listening experiment. The non-individual HRTFs were obtained by interpolating magnitude response of the listener's own HRTF and the flat ones. The ratios of the interpolation corresponded to the degrees of individuality. Then, the ITDs and ILDs were added to individualize HRTFs. From the results, the effect of the degree of individuality was insignificant, but the front-back confusions were significantly different between HRTFs on ipsilateral side and contralateral side. The results suggested that the individualization of HRTFs with ITDs/ILDs might be influenced by the spectrum of non-individual HRTFs.

2aSPb40. Projection of a virtual speaker into a vehicle using sound field control. Toshiki Yamamura, Yoshio Ishiguro (Nagoya Univ., Furo-cho, Chikusa-ku, Nagoya, Aichi 464-8601, Japan, yamamura.toshiki@g.sp.m.is. nagoya-u.ac.jp), Takanori Nishino (Mie Univ., Tsu, Japan), and Kazuya Takeda (Nagoya Univ., Nagoya, Japan)

In this study, we propose a system which projects a virtual speaker into a vehicle by controlling the sound field of the interior of the vehicle. The system uses a sound field reproduction technique to reproduce a point source in the vehicle representing a virtual speaker, enabling drivers to communicate with virtual speakers as if they were riding in the same vehicle. The system is based on a sound field reproduction technique known as Wave Field Synthesis (WFS), which can represent the depth of a sound source by reproducing its wavefront precisely. However, since WFS is generally implemented in an ideal environment, the influences of reflection and reverberation are not generally taken into consideration. Therefore, in order to apply WFS to an in-vehicle system, it is necessary to investigate the effects of reflection and reverberation on WFS within a vehicle. To do so, we compare the accuracy of the perception of locations of sound point sources reproduced using WFS with a 32 channel loudspeaker array in both a vehicle and a low reverberation room. We also compare perception accuracy of the proposed system with perception accuracy when a loudspeaker is placed in the same position as the virtual speaker.

2aSPb41. The mathematical paradigm of "copula" to model a faded signal received by multiple sensors. Shih-Hao Huang, Mong-Na L. Huang (Dept. of Appl. Mathematics, National Sun Yat-sen Univ., Kaohsiung, Taiwan), and Kainam T. Wong (Dept. of Electron. \& Information Eng., Hong Kong Polytechnic Univ., Hong Kong Polytechnic University, Hung Hom KLN, Hong Kong, ktwong@iieee.org)

In sonar, radar, or wireless communication that deploys multiple sensors in the receiver for a fading channel, the received signal (at each time instant) may be modeled as a stochastic vector whose elements correspond to the various sensors of the receiver. This random signal's spatial statistics may be described (at each time instant) by a multivariate probability distribution. For this multivariate distribution to have ARBITRARILY different univariate marginal distributions and/or an ARBITRARY spatial correlation function-the mathematical paradigm of "copula" has recently been advanced in Huang, Huang, Wong, and Tseng, "Copula—to model multi-channel fading by correlated but arbitrary Weibull marginal, giving a closed-form outageprobability of selection-combining reception," IET Microwaves, Antennas \& Propagation, December 2015. That seminal work shows how the mathematical paradigm of copula can allow unprecedented flexibility in modeling and can offer unparalleled mathematical simplicity in expressing the receiver's outage probability in a closed and simple mathematical form explicitly in terms of the model parameters. That reference focuses on a particular copula called the survival-Gumbel copula. This paper will explore other copulas.

2aSPb42. Multiple-input multiple-output underwater acoustic communications by using a quasi-orthogonal group space-time architecture. Chen Zhang, FengXiang Ge (Beijing Normal Univ., No. 19, XinJieKouWai St., HaiDian District., Beijing 100875, China, fox@mail.bnu.edu.cn), Lin Dai (Univ. of Hong Kong, Hong Kong, China), and Huiling Zhu (Univ. of Kent, Kent, United Kingdom)

A Quasi-orthogonal Group Space-Time (QoGST) architecture is presented in this paper for better diversity and multiplexing tradeoff gains in Multiple-Input Multiple-Output (MIMO) underwater acoustic (UWA) communications. Both QoGST and Group Layered Space-Time (GLST) are the combination of space-time block coding (STBC) and layered space-time coding. For GLST, the STBC is taken just in each group. For QoGST, however, all groups are encoded together via inter-group STBC, which can effectively suppress the interference between groups with the orthogonal STBC. At the receivers, the STBC decoding is performed and thus we have the higher diversity gains for the group detection. A statistical model of UWA channel which is presented by Milica Stojanovic and contains variations caused by small- and large-scale effects is considered. Simulation results show that the frame error rate (FER) of QoGST is much lower than that of GLST, which means the QoGST has the higher diversity gains. Furthermore, the lower bound diversity and multiplexing tradeoff of QoGST is coincides with that of GLST and the upper bound diversity and multiplexing tradeoff of QoGST is closer to the optimal values.

2aSPb43. Comparing frequency-difference beamforming and delayand-sum beamforming as an estimator of direction-of-arrival. Eldridge Alcantara, Les Atlas (Elec. Eng., Univ. of Washington, Seattle, 3040 17th Ave. West \#404, Seattle, WA 98119, eealcant@uw.edu), and Shima Abadi (Mech. Eng., Univ. of Washington, Bothell, Bothell, WA)

Direction-of-arrival estimation (DOA) is an important and long-studied problem in array signal processing. Existing algorithms such as conventional delay-and-sum beamforming are limited in performance and become inadequate for a high-frequency broadband source and when the receiving linear array is sparse. Abadi, Song, and Dowling (2012), however, showed that DOAS estimation is possible in this case with an algorithm they introduced called frequency-difference beamforming. This was demonstrated empirically in their previous work using a real dataset. In addition, the resemblance between frequency-difference beamforming and delay-and-sum beamforming was briefly discussed. The purpose of this study is to analyze this relationship further and determine how well frequency-difference beamforming compares to delay-and-sum beamforming as an estimator of DOA. The approach taken in this study is to explicitly formulate the estimator equation for frequency-difference beamforming and compare it mathematically to the estimator equation of delay-and-sum beamforming. The equations are compared for the case of one DOA and two DOAs. For both cases, we show the effect of both source frequency and source bandwidth on frequency-difference beamforming. Using these results, we probe the following question: what are the conditions on the source frequency or source bandwidth for which frequency-difference beamforming will outperform delay-and-sum beamforming? 
Session 2aUW

\title{
Underwater Acoustics and Acoustical Oceanography: Underwater Acoustic Studies in Asian Seas II
}

\author{
Chi-Fang Chen, Cochair \\ Engineering Science and Ocean Engineering, National Taiwan University, No. 1 Roosevelt Road Sec.\#4, Taipei 106, Taiwan \\ Ching-Sang Chiu, Cochair \\ Department of Oceanography, Naval Postgraduate School, 833 Dyder Road, Room 328, Monterey, CA 93943-5193
}

Chair's Introduction-7:55

\section{Contributed Papers}

\section{8:00}

2aUW1. Effect of internal waves on acoustic energy structure in the northern East China Sea. Jungyong Park, Sangkyum An (Naval Architecture and Ocean Eng., Seoul National Univ., Bldg. 36, Rm. 212, 1, Gwanakro, Gwanak-gu 151 - 744, Seoul KS013, South Korea, ioflizard@snu.ac.kr), Youngmin Choo, and Woojae Seong (Naval Architecture and Ocean Eng. and Res. Inst. of Marine Systems Eng., Seoul National Univ., Seoul, South Korea)

Relation between depth direction acoustic energy and sound speed fluctuation caused by internal wave activity observed during SAVEX15 experiment is investigated. In the SAVEX15 region (northern East China Sea with water depths around $100 \mathrm{~m}$ ), underwater sound channel was observed during May 2015 along with extensive internal wave activities. When the source is near the sound channel axis, most of the acoustic energy is trapped in the channel. However, internal waves change the sound speed profile, which results in spreading the acoustic energy over depth. Measured data during the experiment is used to investigate the relation between acoustic energy structure and internal wave activity. Parameters reflecting their variabilities are defined. Two main parameters are standard deviation of the acoustic energy integrated over depth and the sound speed gradient at the channel axis, which reflects the internal wave activity. The correlation of two parameters is used to show the effect of internal waves on the changes of acoustic energy distribution.

\section{$8: 15$}

2aUW2. Measurements of mid-frequency bottom interacting signals in Jinhae bay located on the southern coast of Korea. Jee Woong Choi, Hyuckjong Kwon, Su-Uk Son (Dept. of Marine Sci. and Convergence Eng., Hanyang Univ., 55 Hanyangdaehak-ro, Ansan 426-791, South Korea, choijw@hanyang.ac.kr), Sungho Cho (Korea Inst. of Ocean Sci. \& Technol., Ansan, South Korea), Joung-Soo Park, and Jooyoung Hahn (Agency for Defense Development, Changwon, South Korea)

Acoustic bottom interacting measurements were conducted in shallow water (nominal water depth of $60 \mathrm{~m}$ ) in Jinhae bay, southern coast of Korea, using 4 to $12 \mathrm{kHz} \mathrm{CW}$ signals in May 2015 and 2016. The surficial sediment at a site is mainly composed of silty clay with a mean grain size of 8 phi. Since the seafloor is relatively soft, the bottom-bounced path was very weak compared to direct and sea-surface-bounced paths. On the other hand, a strong arrival reflected from the sub-sediment layer was received after the bottom-bounced arrival especially at lower frequencies. The arrival time difference between the arrival reflected from water-sediment interface and that reflected from the second interface is used to estimate the sound speed in the surficial sediment layer. In addition, the bottom loss as a function of grazing angle are estimated using the bottom-bounded path. Finally, the results are compared to the sedimentary structure imaged by chirp sonar survey. [Work supported by Agency for Defense Development, Korea.]

\section{8:30}

2aUW3. Acoustical characteristics of snapping shrimp noise measured during SAVEX 15 and its use as a natural source to estimate sea surface properties. Myungkwon Ko, Dae-Hyuk Lee, Jee Woong Choi (Marine Sci. and Convergence Eng., Hanyang Univ., Hanyangdaehakro 55, Ansan, Gyeonggi do 15588, South Korea, ko.myungkwon@gmail.com), and Sungho Cho (Korea Inst. of Ocean Sci. \& Technol., Ansan, South Korea)

The Shallow-water Acoustic Variability Experiment (SAVEX15) was conducted at a site in the Northern East China Sea (ECS), about $100 \mathrm{~km}$ off the southwest of Jeju Island, Korea in May 2015. The purpose of SAVEX15 was to obtain acoustic and environmental data for studying on oceanography, underwater communication, and acoustic propagation characteristics in the ECS. The experiment utilized a vertical line array (VLA) of 16 elements spanning $56.25 \mathrm{~m}$ of a $100 \mathrm{~m}$ water column. During the experiment, a large number of unintended snapping shrimp noises were recorded by the receiving system. In this talk, the acoustic properties of the snapping shrimp noise are presented. In addition, these noises are used as natural acoustic sources to estimate the sea surface properties from the sea-surface bounced paths. Finally, the results are compared with the sea surface conditions collected by a wave buoy operated during the experimental period. [This work was jointly supported by KRISO, KIOST, MPL, and ONR, and partially supported by ADD, Korea.]

\section{$8: 45$}

2aUW4. Comparison of underwater communication performances in two shallow-water sites with different sediment types located off the coast of Korea. Sunhyo Kim, Kanghoon Choi, Jee Woong Choi (Ocean Sci. and Convergence Eng., Hanyang Univ., Hanyang daehakro 55, Ansan 15588, South Korea, sunhyo4485@hanyang.ac.kr), Joung-Soo Park (Agency for Defense Development, Changwon, South Korea), and SeaMoon Kim (Korea Res. Inst. of Ships and Ocean Eng., Daejeon, South Korea)

Shallow water waveguide characterized by multipath channel produces a significant delay spreading of transmitted signals, which is referred to as inter symbol interference (ISI). Since the ISI results in distortion of communication signals, many studies to reduce the effect of ISI have been conducted. For successful underwater communication, it is important to understand the correlation between the spatial and temporal properties of ISI and communication performance. Underwater acoustic communication experiments were conducted in two different seafloor environments with relatively fine-grained and coarse sediments. The experimental geometries were the same; water depths of both sites were about $40 \mathrm{~m}$ and source 
depths were about $32 \mathrm{~m}$. Communication signals were measured by a fourchannel receiving array, covering waters 5 to $35 \mathrm{~m}$ in depth. Sound speed profiles were measured by CTD casts and the surficial sediment samples were taken by a grab sampler. The received communication signals were demodulated using the time reversal, phase lock loop and decision feedback equalizer techniques. In this talk, the communication performances at two different sites are presented and compared with the channel impulse responses at two sites. [Work supported by ADD(UD140001DD) and KRISO(PMS3310).]

\section{9:00}

2aUW5. Measurements of high frequency acoustic transmission loss during SAVEX15. Su-Uk Son, Jee Woong Choi (Dept. of Marine Sci. and Convergence Eng., Hanyang Univ., 55 Hanyangdaehak-ro, Sangnok-gu Ansan, Gyeonggi-do 15588, South Korea, suuk2@hanyang.ac.kr), Seung Woo Lee, SungHyun Nam (Res. Inst. of Oceanography/School of Earth and Environ. Sci., Seoul National Univ., Seoul, South Korea), and Sungho Cho (Korea Inst. of Ocean Sci. \& Technol., Ansan, South Korea)

Transmission loss measurements in high frequency range along with the ocean environmental measurements were made during the Shallow-water Acoustic Variability EXperiment 2015 (SAVEX15) in the south of Jeju Island, Korea, where the water depth is about $100 \mathrm{~m}$. Two vertical line arrays (VLAs) were moored with a distance of $5.5 \mathrm{~km}$, covering the water column between 24 and $80 \mathrm{~m}$. Continuous waves in a frequency band of 10$25 \mathrm{kHz}$ were transmitted from a vertical source array at depths of 30-45 m. Sound speed and temperature profiles were measured using a standard/stationary conductivity-temperature-depth (CTD), underway CTD, and temperature loggers attached to the two VLAs. The measured sound speed profiles consistently reveal a sound speed minimum layer (SSML) at depths between 30 to $50 \mathrm{~m}$. During the SAVEX15 period, the perturbations in the SSML derived by various kinds of internal waves were observed. In this talk, the temporal fluctuations of the measured transmission loss are presented, and the results are discussed in comparison with the internal wave field in the region. [Work supported by ONR, KIOST, SNU, and ADD.]

\section{9:15}

2aUW6. Sound focusing in shallow water using a vertical phased array. Dayong Peng, Juan Zeng, Haijun Liu, Haifeng Li, and Wenyao Zhao (Inst. of Acoust., Chinese Acad. of Sci., No. 21, North 4th Ring Rd., Haidian District, Beijing 100190, China, pengdayong@mail.ioa.ac.cn)

Vertical phased array is an efficient and complex equipment in underwater acoustics. Single-mode excitation and time-reversal sound focusing are two typical techniques based on vertical phased array, but both of them have some shortcomings. In order to realize sound focusing at variable distance and depth using vertical phased array, two techniques are proposed in this paper. One of them is the Green's function construction of target sound field based on normal mode eigen-function and attenuation coefficient extraction, and the other is controllable sound focusing based on optimal target sound field determination. Simulation and experiment results are presented. This technique indicates a method for objective depth detecting in shallow water.

\section{9:30}

2aUW7. Geoacoustic inversions in the East China Sea for a 3D sediment model. Gopu R. Potty, James H. Miller (Dept. of Ocean Eng., Univ. of Rhode Island, 115 Middleton Bldg., Narragansett, RI 02882, potty@egr.uri. edu), Stan E. Dosso (Univ. of Victoria, Victoria, BC, Canada), Jan Dettmer (Dept. of GeoSci., Univ. of Calgary, Calgary, AB, Canada), and Julien Bonnel (Ensta Bretagne, Brest, France)

Geoacoustic inversions using wide-band acoustic sources (WBS) deployed during the Asian Seas International Acoustic Experiment (ASIAEX) along a circular path of radius $30 \mathrm{~km}$ centered on a vertical hydrophone array will be used to construct a pseudo 3D model of the seabed sediments. The acoustic data used in the inversions consist of modal dispersion as estimated using a warping transform technique. Existing information about the bottom includes historic data, seismic records, and gravity and piston cores which indicate significant spatial variability in the thickness of sediment layers and types of sediment. The geoacoustic inversion approach is based on trans-dimensional Bayesian methodology in which the number of sediment layers is included as unknown in addition to the layer parameters. A sediment-acoustic model, such as viscous grain shearing, will be used to estimate the wave parameters corresponding to the sediment parameters. One-dimensional (depth-dependent) inversions will be applied along the various acoustic propagation paths to construct a pseudo 3D sediment model using interpolation. [Work supported by the Office of Naval research.]

\section{9:45-10:00 Break}

\section{0:00}

2aUW8. Acoustic property of sound production by fat greenling (Hexagrammos otakii) in spawning season. Naoto Matsubara, Hiroyuki Munehara (Hokkaido Univ., 3-1-1, Minato-cho, Hakodate, Hokkaido 041-8611, Japan, naotomatsubaraf91@gmail.com), Ryuzo Takahashi (Japan Fisheries Res. and Education Agency, National Res. Inst. of Fisheries Eng., Kamisu, Japan), Tomonari Akamatsu (Japan Fisheries Res. and Education Agency, National Res. Inst. of Fisheries Sci., Yokohama, Japan), Kazuki Yamato, Ikuo Matsuo (Tohoku Gakuin Univ., Sendai, Japan), Nobuo Kimura, Kazuyoshi Maekawa, and Hiroki Yasuma (Hokkaido Univ., Hakodate, Japan)

Fat greenling (Hexagrammos otakii) is one of the important demersal fishes in Japanese local fisheries. Male fishes come over to neritic zone in spawning season, and make the territory as the spawning bed. It was suggested that fat greenling produces specific sounds during the protection of territory.The objective of this study was to examine acoustic properties of male sound production in spawning season as the basic data for estimating habitat density and activity by the passive acoustic methods. Field recordings were conducted during spawning season between Sept. and Nov. in 2014 and 2015. In each experiments, a specialized recorder was set by a territory throughout the breeding process, including courtship, spawning, and egg care. We extracted the acoustic properties such as the frequency of sound production, pulse duration, pulse number, call duration, and the frequency of sound production from recording data. There were different patterns of sound properties and their occurrence frequencies between before and after spawning. It was suggested that fat greenling changes the frequency and property of sound production depending on the situation like courtship, spawning, and protecting eggs.

\section{0:15}

2aUW9. Acoustic tracking in shallow coastal areas on spawning migrations of pacific herring ( Clupea pallasii) using ultrasonic telemetry. Makoto Tomiyasu (Graduate school of Environ. Sci., Hokkaido Univ., Hakodate-city, Benten-cho, 20-5, Hokkaido 040-0051, Japan, lion-heart@ eis.hokudai.ac.jp), Hokuto Shirakawa (Field Sci. Ctr. for Northern Biosphere, Hokkaido Univ., Hakodate-city, Hokkaido, 20-5 Benten-cho, Japan), Yuki Iino (School of Fisheries Sci., Hokkaido Univesity, Hakodate-city, Hokkaido, Japan), and Kazushi Miyashita (Field Sci. Ctr. for Northern Biosphere, Hokkaido Univ., Hakodate-city, Hokkaido, Japan)

The behavioral ecology of small pelagic fish has been studied by acoustic method for a long time. In particular, on pacific herring (Clupea pallasii), the behavioral ecology in shallow coastal areas which is observed spawning migration should be understood for the resource management and reproduction protection. In this study, supersonic telemetry was used for behavioral tracking of herring at shallow coastal areas. For the tracking, herring of regional group $(\mathrm{n}=23)$ at Akkeshi bay (Depth ave. $5.78 \pm 2.15 \mathrm{~m})$ in east Hokkaido were used. On the horizontal aspect, herring showed two migration patterns (pattern1: staying at the bay, pattern2: migration to Akkeshi lake (ave. $0.84 \pm 0.13 \mathrm{~m}$ ) which is brackish water lake adjacent the bay). The lake is the main spawning grounds for herring, therefore the pattern 2 is indicated to be for spawning, and the pattern1 is before or after spawning. On the vertical aspect, diurnal distribution patterns were shown at the bay (day: ave. $5.41 \pm 3.78 \mathrm{~m}$, night: ave. $3.83 \pm 2.60 \mathrm{~m}$ ). Besides, the rapid horizontal migration was mainly observed at night. From these, the distribution patterns may influence on efficient migration at night without predation risk. Migration span at shallow coastal areas is estimated to be comparatively short, during 4-5 days. 
2aUW10. Regional variations of Saccharina japonica forests distribution around the Tsugaru Strait by acoustic method. Huamei Shao (Graduate school of Environ. Sci., Hokkaido Univ., Bentencho. Hakodate City, Hokkaido, Hakodate, Hokkaido 0400043, Japan, huamshao@ees.hokudai. ac.jp), Kenji Minami (Field Sci. Ctr. for Northern Biosphere, Hokkaido Univ., Hakodate, Hokkaido, Japan), Yoshikazu Fujikawa (Div. of Fisheries Infrastructure Aomori Prefecture, Fisheries Bureau, Aomori, Japan), Takashi Yusa (Aomori Prefectural Industrail Technol. Res., Fisheries Res. Inst., Aomori, Japan), Norishige Yotsukura (Field Sci. Ctr. for Northern Biosphere, Hokakido Univ., Sapporo, Japan), Masahiro Nakaoka (Field Sci. Ctr. for Northern Biosphere, Hokkaido Univ., Akkeshi, Japan), and Kazushi Miyashita (Field Sci. Ctr. for Northern Biosphere, Hokkaido Univ., Hakodate, Japan)

As the global warming, commercially important species Saccharina japonica disappeared, what is called sea desertification occurred in some coastal waters around the Tsugaru Strait recently. For the sustainability of coastal ecosystem and resources use, understanding the distribution and variation of $S$. japonica forests is necessary. Surveys for the distribution of $S$. japonica forests by acoustic method were conducted in four coastal waters: (1) Osatsube, Hakodate, the Pacific Ocean side of Hokkaido; (2) Ishizaki, Hakodate in Tsugaru Strait of Hokkaido; (3) Okoppe, Oma, in Tsugaru Strait of Aomori Prefecture; (4) Shiriyazaki, Higashidori, the Pacific Ocean side of Aomori Prefecture. Thickness and spatial distributions of seaweed were obtained by acoustic method and density of sea urchins by visual observations. In Osatsube coastal water, main species was S. japonicaand which forests were most among survey regions. In Ishizaki and Okkope coastal waters, Sargassum spp. was also luxuriant except S. japonica. In Shiriyazaki coastal water, $S$. japonica dominated and a greater number of sea urchins were observed than other regions. In regions where little benefit from Oyashio Current, Sargassum spp. grew thickly when sea urchins were rare whereas sea urchin barren occurred when sea urchins with high density.

\section{$10: 45$}

2aUW11. Annual change in the distribution of Sargassum horneri using a quantitative echo sounder in Yamada Bay, Iwate, Japan. Kenji Minami (Field Sci. Ctr. for Northern Biosphere, Hokkaido Univ., 20-5 Benten-cho, Hakodate, Hokkaido 0400051, Japan, kminami@fish.hokudai.ac.jp), Chihomi Kita (Graduate school of Environ. Sci., Hokkaido Univ., Hakodate, Hokkaido, Japan), Masayuki Sawai (Sanriku Fisheries Res. Ctr., Iwate Univ., Kamaishi, Iwate, Japan), Hokuto Shirakawa (Field Sci. Ctr. for Northern Biosphere, Hokkaido Univ., Hakodate, Hokkaido, Japan), Huamei Shao, Makoto Tomiyasu (Graduate school of Environ. Sci., Hokkaido Univ., Hakodate, Hokkaido, Japan), Motoki Kobayashi, and Kazushi Miyashita (Field Sci. Ctr. for Northern Biosphere, Hokkaido Univ., Hakodate, Hokkaido, Japan)

Sargassum horneri, a type of seaweed, has attracted attention as a new fisheries resource in Japan because it contains large amounts of functional ingredients. Yamada Bay $\left(16 \mathrm{~km}^{2}\right)$, in Iwate, Tohoku, Japan, was seriously damaged by the 2011 tsunami, and a sustainable new fisheries resources would aid in the reconstruction of local industries. In this study, we estimated annual change in the distribution of $S$. horneri using a quantitative echo sounder for sustainability of $S$. horneri in Yamada Bay. Surveys were conducted in spring (during the growth season) 2013, 2014, and 2015. The acoustic data were obtained using a $120 \mathrm{kHz}$ quantitative echo sounder (KCE300, Sonic Co.). S. horneri were detected on the rope of aquaculture facilities and the rock reef in the coastal sea area. Growth conditions were better in 2013 than those in 2014 and 2015. In 2013, water temperature in Yamada Bay was lower than in 2014 and 2015 because of the influence of cold water from the strong Oyashio Current. It was concluded that primary production of S. horneri in Yamada Bay is influenced by the Oyashio Current, causing their abundance to fluctuate annually.
2aUW12. Accuracy verification of tilt-angle estimation by split-beam echosounder: Comparison with acceleration data-logger data. Hokuto Shirakawa, Kenji Minami (Field Sci. Ctr. for Northern Biosphere, Hokkaido Univ., 20-5 Benten-cho, Hakodate 040-0051, Japan, dipper@fish.hokudai. ac.jp), Yohei Kawauchi (Seikai National Fisheries Res. Inst., Fisheries Res. Agency, Nagasaki, Japan), Makoto Tomiyasu (Graduate School of Environ. Sci., Hokkaido Univ., Hakodate, Japan), Yuichi Tsuda (Bluefin Tuna Resources Div., National Res. Inst. of Far Seas Fisheries, Fisheries Res. Agency, Shizuoka, Japan), Takeru Umetsu, Huamei Shao (Graduate School of Environ. Sci., Hokkaido Univ., Hakodate, Japan), Motoki Kobayashi (Field Sci. Ctr. for Northern Biosphere, Hokkaido Univ., Hakodate, Japan), Yuki Iino (School of Fisheries Sci., Hokkaido Univ., Hakodate, Japan), Masahiro Ogawa (Graduate School of Environ. Sci., Hokkaido Univ., Hakodate, Japan), and Kazushi Miyashita (Field Sci. Ctr. for Northern Biosphere, Hokkaido Univ., Hakodate, Japan)

Fish tilt-angle is one of the most important factors for fish target strength (TS) estimation, because the little change varies TS value dramatically. Split-beam echosounder collects a fish position with 3-D coordinates. Hence, if fish swim within acoustic beam continuously, the tilt-angle can be estimated from the gap between two positions easily. However, the accuracy of the estimation depends on performance of the echosounder. We verified accuracy of the estimated tilt-angle by comparing high-resolution angle from an acceleration data logger. Experiment was conducted at a large experimental tank (W:10m x D:5m x H:6m) in HRCFO. A 2-axis acceleration data logger ( $\varphi 15 \mathrm{~mm}$, L: $53 \mathrm{~mm}$, M190L-D2GT, Little Leonardo, Inc.) was tethered into the tank, and towed at respective angle from -50 to 50 degree (1 degree pitch). Positions of the logger echos (from KCE300, Sonic Co., $120 \mathrm{kHz}$ ) were exported by algorithms of Echoview7.1. After this process, the tilt-angles were calculated. 28 logger echos were identified from the acoustic data, and the estimated tilt-angles was from $-45.59^{\circ}$ to $32.87^{\circ}$. Significant correlation of tilt-angles (logger vs. acoustic data) was found ( $p>$ $\left.0.01, r^{2}=0.90\right)$. This result shows high precision of the estimated tile angle from the split-beam echosounder data.

\section{1:15}

2aUW13. Distributional factors of juvenile Walleye Pollock in the Musashi Bank area, northern Japan Sea. Takeru Umetsu (Graduate School of Environ. Sci., Hokkaido Univ., Bententown 20-5, Hakodate, Hokkaido 040-0051, Japan, ume.take33@gmail.com), Yuichi Tsuda (Bluefin Tuna Resources Div., National Res. Inst. of Far Seas Fisheries, Shizuoka, Japan), Yasushi Ito (The Japanese Inst. of Fisheries Infrastructure and Communities, Chiyoda, Japan), Hokuto Sirakawa, Kenji Minami, and Kazushi Miyashita (Field Sci. Ctr. for Northern Biosphere, Hokkaido Univ., Hakodate, Japan)

Most of juvenile walleye pollock (Gadus chalcogrammus) in the northern Japan Sea migrate to the bottom layer with 150-200 m depth of Musashi bank area in summer. Clarification of distribution factors of nursery area after the migration is important for resource management. We examined the factors of juvenile pollock after migration to bottom in summer 2014 and 2015. The distribution of density of juvenile pollock was obtained by quantitative echo sounder. The distribution of bottom sediment was discriminated by the side scan sonar. The bottom backscattering strength and sediment data were jointed to analyze bottom sediment of quantitative echo sounder survey transect line based on the Roxann method. In the result, bottom sediment was separated into clay, sand, and boulders. Distribution density of juvenile pollock was high in boulders area. Additionally, the density of juvenile pollock was higher at northward upslope than gentle landform and else way upslope in all boulders area. It is considered that northward ocean flow collided with the northward upslope of boulders make upward flowing and stagnate water, and the water forms the retention space of prey plankton that is favorable for juvenile pollock. 
2aUW14. Tracking whales in the Indian Ocean using the comprehensive test ban treaty hydrophone data. David A. Lechner (Mech. Eng., The Catholic Univ. of America, 520 Michigan Ave., NE, Washington, DC 20064,66Lechner@CUA.edu), Shane Guan (Mech. Eng., The Catholic Univ. of America, Silver Spring, MD), and Joseph F. Vignola (Mech. Eng., The Catholic Univ. of America, Washington, DC)

This paper will present preliminary results and status in development of an automated tracking algorithm for whale signals using the Comprehensive Test Ban Treaty (CTBT) sensor network. The data from the CTBT stations is being made available for academic work, and the stations are also detecting bioacoustics sounds as well as seismic activity. We present an approach used to process several channels of the acoustic data collected off Cape Leeuwin, Australia, and automatically search for biologic activity using cross-correlation and Time-Difference-of-Arrival methods. After developing a graph of locations for peak correlation amplitude, the locations are mapped and grouped using location and proximity, in order to develop motion tracks and compare coherence of the signals within a grouping and between groupings, thus identify individual whales based on the differences in location and coherence levels.
2aUW15. Geoacoustic inversion base on modeling ocean-bottom reflection wave. Licheng Lu, Qunyan Ren, and Li Ma (Key Lab. of Underwater Acoust. Environment, Inst. of Acoust., Chinese Acad. of Sci., No. 21 West Rd.,North 4th Ring Rd., Haidian District, Beijing 100190, China, luce_ 1983@sina.com)

Ocean-bottom parameters surveying experiment was conducted at two sites in Chinese South Sea. A vertical array of four elements was used to measure reflection waves from ocean-bottom with the explosive charges. The ocean-bottom reflection waves are processed by analyzing the arriving time to identify the sediment layers, and then, a geoacoustic model with several numbers of homogeneous fluid sediment layers overlying a homogenous substrate half space is constructed. Using the direct arriving wave, an approach that matches the measured and modeled ocean-bottom reflection wave is to invert the bottom velocity and density profile. A low velocity layer is obviously observed at one site and a complex seabed profile at another site is established. The modeled wave form is compared with the measured one and shows a relative agreement.

TUESDAY MORNING, 29 NOVEMBER 2016

CORAL FOYER, 9:00 P.M. TO 5:00 P.M.

\section{Exhibit}

The instrument and equipment exhibit is located near the registration area in the Coral Foyer.

The Exhibit will include computer-based instrumentation, scientific books, sound level meters, sound intensity systems, signal processing systems, devices for noise control and acoustical materials, active noise control systems, and other exhibits on acoustics.

Exhibit hours are Monday, 28 November, 5:30 p.m. to 7:00 p.m., Tuesday, 29 November, 9:00 a.m. to 5:00 p.m., and Wednesday, 30 November, 9:00 a.m. to 12:00 noon.

Coffee breaks on Tuesday and Wednesday mornings will be held in the exhibit area as well as an afternoon break on Tuesday.

The following companies have registered to participate in the exhibit at the time of this publication:

AIP Publishing: publishing.aip.org/

American Institute of Physics: www.aip.org/

Aqua Sound Inc.: aqua-sound.com

Echoview Software: www.echoview.com/

Head acoustics GmbH: www.head-acoustics.de/eng/

Mason Industries: www.mason-industries.com/masonind/

Ocean Sonics Ltd.: oceansonics.com/

ODEON A/S: www.odeon.dk/

PAC International: www.pac-intl.com/

RION Co., Ltd: www.rion.co.jp/english/

Sensidyne: www.sensidyne.com/

Springer: www.springer.com/us/

Teledyne RESON Inc.: www.teledyne-reson.com/ 


\title{
Session 2pAA
}

\section{Architectural Acoustics and Speech Communication: At the Intersection of Speech and Architecture I}

\author{
Kenneth W. Good, Cochair \\ Armstrong, 2500 Columbia Av, Lancaster, PA 17601 \\ Takashi Yamakawa, Cochair \\ Yamaha Corporation, 10-1 Nakazawa-cho, Naka-ku, Hamamatsu 430-8650, Japan \\ Catherine L. Rogers, Cochair \\ Dept. of Communication Sci. and Disorders, Univ. of South Florida, USF, 4202 E. Fowler Ave., PCD1017, Tampa, FL 33620
}

Chair's Introduction-1:00

Invited Papers

1:05

2pAA1. The impact of architecture on both the intelligibility and privacy of speech. Kenneth P. Roy (Bldg. Products Technol. Lab, Armstrong World Industries, 2500 Columbia Ave., Lancaster, PA 17603, kproy@armstrong.com)

Architects design the "visual space" that we can all see, and this we call architecture. Acousticians upon entering this visual space invariably clap their hands, and as such are assessing the resulting "aural space." Acoustics is the result of architecture, and so, speech clarity is determined by architecture-meaning the size, shape, and surface treatments chosen by the architect to deliver the mission for which the space is intended. We can look at (model) or listen to (field evaluate) the impulse response of a room, and from this determine the degree of speech clarity that is projected to any listening location. Designing for good speech intelligibility within a space means providing adequate speech clarity and then protecting this signal with a high signal-to-noise ratio $(\mathrm{S} / \mathrm{N})$ to ensure that the signal can be heard above the ambient noise. If on the other hand we seek to limit the speech intelligibility (privacy) between spaces, we need to design the architecture to limit the intrusion of speech between spaces taking into consideration the architectural choices as barriers to sound transmission via walls, ceilings, and floors. Examples of design approaches in both cases of intelligibility and privacy will be shown.

$1: 25$

2pAA2. Fundamentals of speech privacy in the built-environment. Kenneth W. Good (Armstrong, 2500 Columbia Ave., Lancaster, PA 17601, kwgoodjr@armstrong.com)

Since this session intends to bring together experts in speech, architecture, and hearing, this paper will present the fundamentals of Speech Privacy from the architectural perspective. Considering the room as a transfer-function from the talker to the listener, I will cover the importance of various architectural components, measurements, and design considerations for the built-environment. The intent is sharing practices and perspective across disciplines not necessarily new discoveries in the field.

\section{1:45}

2pAA3. Effects of dominance of the first-arriving sound on speech intelligibility in reverberant sound fields. Hayato Sato, Masayuki Morimoto (Environ. Acoust. Lab., Graduate School of Eng., Kobe Univ., 1-1 Rokkodai, Nada, Kobe 6578501, Japan, hayato@kobe-u.ac.jp), and Yasushi Hoshino (Nippon Sheet Glass Environment Amenity Co., Ltd., Tokyo, Japan)

Generally speaking, the energy of the direct sound exceeds that of reflections in rooms for listening to speech. In this case, earlyreflections enhance loudness of the direct sound. It is well-known that the useful-to-detrimental sound ratio, in which useful is the sum of the direct sound and early-reflections and detrimental is late-reflections, can predict speech intelligibility. On the other hand, considering speech leakage from the adjacent room, the first-arriving sound is attenuated by the boundary wall, and it is sometimes too weak to trigger the temporal integration of loudness. This would change the time structure of the useful energy. In the present study, prediction of speech intelligibility in such particular situations was investigated. Word intelligibility tests were performed in reverberant sound fields varying reverberation time, direct-to-reverberation ratio, and rise time at the onset of reverberation sound. The results demonstrated that prediction accuracy of the useful-to-detrimental ratio was still in a practical range though the parameters systematically affect the prediction error. 
2pAA4. An approach to the design of drama theaters for speech clarity. Gregory A. Miller, John Strong, Carl Giegold (Threshold Acoust., LLC, 53 W. Jackson Blvd., Ste. 815, Chicago, IL 60604, gmiller@thresholdacoustics.com), and Laura C. Brill (Durham School of Architectural Eng. and Construction, Univ. of Nebraska-Lincoln, Omaha, NE)

In drama theaters, the unamplified human voice is used to convey both information and nuanced emotional cues to large groups of listeners. Supportive sound reflective surfaces are relatively distant from the actors, and the inherent (and desirable) reverberance of a larger space run counter to many accepted practices for achieving speech clarity in smaller spaces. Some theater configurations, such as thrust and in-the-round, are even arranged so that actor faces away from large segments of the audience at any given time, offering further challenges to the connection between actor and audience. This paper will present an understanding of the way that people listen to speech in large rooms, and approaches to room design to support the clarity of unamplified speech. A case study will be presented describing the development of theater design from initial concept through computer modeling and finally with three dimensional measurements and visualizations of the acoustic environment in the completed room.

2pAA5. The highly exaggerated attributes of the pop vocal in sound recordings. Alexander U. Case (Sound Recording Technol., Univ. of Massachusetts Lowell, 35 Wilder St., Ste. 3, Lowell, MA 01854, alex@fermata.biz)

The voice in sound recordings must generally reign supreme over the rest of the multitrack arrangement. The loudspeaker mediates its interaction with the architecture. The entire upstream production chain anticipates the challenge. Recording space, microphone choice, and signal processing are carefully coordinated to maximize the audibility of multiple attributes the voice - intelligibility, emotion, musicality, and tonal quality. The highly manipulated approach to speech for sound recording can shed light on approaches taken in the built environment for the talker/listener interface.

\section{2:45}

2pAA6. Basic study based on diffuse sound field model on relationships among architectural conditions, intelligibility, and speech privacy. Takashi Yamakawa (Yamaha Corp., 10-1 Nakazawa-cho, Naka-ku, Hamamatsu, Shizuoka 430-8650, Japan, takashi.yamakawa@music.yamaha.com)

Rates of both speech transmission and speech privacy are usually estimated on the basis of intelligibility score. There are many physical indexes proposed to stand for intelligibility, like STI, AI, U50, SNRuni32, and so on. It will be reported that the results of calculations of intelligibility indexes with various room conditions based on classical diffuse sound field model, which shows relationships between degrees of absorption, sound blocking, background noise, distance between a target source and listeners, and intelligibility indexes. Some graphs of intelligibility versus each architectural condition will be provided. For example, architects could know how much each countermeasure, which includes adjusting absorption, set a barrier, adding masking noise, acquiring space, works for speech privacy to see the graphs. It is expected that the results will give us the basic guideline to develop architecturally oral communication and speech privacy.

\section{3:05-3:20 Break}

3:20

2pAA7. Prediction of speech intelligibility through the use of simulation tools. Bruce C. Olson and Ana M. Jaramillo (Olson Sound Design, LLC, 8717 Humboldt Ave. N, Brooklyn Park, MN 55444, ana.jaramillo@afmg.eu)

In a previous paper, we presented the design of a new sound system for the House of Representatives in the State of Minnesota, with the help of simulations. After the installation was completed, we performed new measurements to compare the predicted speech intelligibility with the resulting one. We will discuss differences between the two sets and how to better use simulation tools for the prediction of speech intelligibility and related metrics in this key project as well as others with similar requirements.

3:40

2pAA8. Controlled minimum background sound, the missing link in acoustic design. Ric Doedens (K.R. Moeller Assoc., Ltd., 1050 Pachino Court, Burlington, ON L7L 6B9, Canada, rdoedens@logison.com)

Current industry standards related to controlling background sound in occupied spaces are all based on "not to exceed" maximum levels. At the other end of the scale, it is assumed that we cannot predict with any degree of certainty how low the background sound level may descend, what its spectral composition is, and when or how it may vary. Given that the "signal to noise ratio" is fundamental to all communication based acoustic design, the absence of this knowledge presents significant challenges. As a consequence, we adapt our acoustic designs to compensate for our inability to control minimum noise levels by over-specifying STC, CAC, and NRC values in order to obtain the desired results. And yet, precise generation of controlled minimum background sound levels is readily available. This session explores the negative impact of traditional compensatory acoustic design practises, the significant value of incorporating predictable/consistent minimum background sound levels into acoustic design planning, and makes a case for establishing minimum background sound level industry standards. 
2pAA9. Experimental studies on speech level and room acoustic condition focusing on speech privacy. Hyojin Lee and Shinichi Sakamoto (Inst. of Industrial Sci., Univ. of Tokyo, Meguro-Ku, Komaba 4-6-1, \#Ce401, Tokyo 1538505, Japan, leehj@iis.u-tokyo.ac. jp)

Recently, concerns about speech privacy to avoid oral information leakage at medical facilities are increasing continuously in Japan. In order to achieve the requirements of the speech privacy, standardization has also been discussed. On discussing the speech privacy, speech level is one of the most important factors. This study investigated the speech levels of Japanese talkers and the relationships between the speech levels and the room acoustic conditions (reverberation time, ratio of direct and reflected sound, and loudness of background noise). Subjective experiments were conducted under three conversation conditions; normal voice level with a partner sitting $1 \mathrm{~m}$ away, raised voice level with the partner sitting $3 \mathrm{~m}$ away, and voice level considering overhearing about the contents by a third party. Acoustic environments were reproduced in an anechoic room with a binaural sound field simulation system. As a result, the speech levels of the three conversation conditions were varied. In the simulated test conditions, it was revealed that the room acoustic conditions affected on the speech levels, but the changes of the levels were small.

4:20

2pAA10. Intelligibility and acoustic analysis of speech production in synthetic and natural reverberant and noisy environments: A case study of Parkinson's disease speech. Mark D. Skowronski (Commun. Sci. and Disord., Univ. of South Florida, 4202 East Fowler Ave., PCD 1017, Tampa, FL 33620, skowronski@usf.edu)

Our understanding of the effects of noise and reverberation on speech production and intelligibility is enhanced by the study of speech from talkers with Parkinson's disease (PD) due to the fact that PD speech spans a wide range of intelligibility in quiet and that talkers with PD also span a wide range of speech motor control for compensation. A previous study on the intelligibility of Parkinson's disease (PD) speech in a synthetic reverberant and noisy environment showed that speech intelligibility suffered primarily due to noise although the effects of reverberation were also significant [Shrivastav, ASHA 2011]. A follow-up study in natural reverberant and noisy environments (allowing for talker compensation) found that the decrease in speech intelligibility as speaking conditions worsened was proportional to dysarthria severity [Kopf et al., ASHA 2013], and an acoustic analysis quantified the range of compensations made by talkers with PD [Shrivastav et al., ASA May 2014]. The current study modeled the intelligibility data from the synthetic environment study and applied the model to the data from the natural environment study to quantify the effects of talker compensation on intelligibility and to relate acoustic measures of speech to talker compensation.

\section{4:40}

2pAA11. Self-fitting hearing aids reveal strong individual preferences in noisy rooms. Peggy B. Nelson and Dianne VanTasell (Univ. of Minnesota, 164 Pillsbury Dr. Se, Minneapolis, MN 55455, peggynelson@umn.edu)

Noisy restaurants are among the most challenging listening situations for listeners with hearing loss, but little data are available to optimize hearing aids for noisy situations. In addition, evidence suggests that listeners are less satisfied with hearing devices in noisy listening environments. New technology from EarMachine(c) allows for individuals with hearing loss to self-adjust hearing aid amplification parameters. Self-adjustment technology can provide data to empirically answer questions about listener preferences in noise. Results from 30 listeners demonstrated self-selected gain profiles that reveal very large individual preferences that are not well predicted by age, gender, or hearing loss. These results indicate that there are strong preferences for listening in noisy rooms that are not yet well understood. [Funding provided by NIDCD R01 DC013267.] 


\title{
Session 2pABa
}

\section{Animal Bioacoustics: Tropical Bioacoustics II}

\author{
Thomas F. Norris, Cochair \\ Bio-Waves, Inc., 517 Cornish Dr., Encinitas, CA 92024 \\ Tomonari Akamatsu, Cochair \\ Fisheries Research Agency, 7620-7, Hasaki, Kamisu, Ibaraki 314-0408, Japan
}

\section{Contributed Papers}

\section{1:00}

2pABa1. The snapping shrimp conundrum: Spatial and temporal complexity of snapping sounds on coral reefs. Ashlee Lillis and T A. Mooney (Woods Hole Oceanographic Inst., 266 Woods Hole Rd., MRF 239, MS\#50, Woods Hole, MA 02543, ashlee@whoi.edu)

Snapping shrimp are abundant crevice-dwelling crustaceans worldwide. The short-duration broadband "snap" generated by the collapse of a cavitation bubble upon the rapid closure of their specialized claw is among the loudest bioacoustic sound in the sea. Because these shrimp form large high density aggregations, their colonies create a pervasive crackling in many coastal environments, and variation in shrimp acoustic activity substantially alters ambient sound levels at a given location or time. Despite their fundamental contribution to the soundscape of coral reefs, and probable influence on sound-receptive organisms, relatively little is known about snapping shrimp sound production patterns, and the underlying behavioural ecology or environmental factors. Recent advances in recording capacity and efforts to sample habitat soundscapes at high spatiotemporal resolution have provided datasets that reveal complex dynamics in snapping shrimp sound production. Our analyses of soundscape data from coral reefs show that snap rates generally exhibit diurnal and crepuscular rhythms, but that these rhythms can vary over short spatial scales (e.g., opposite diurnal patterns between nearby reefs) and shift substantially over time (e.g., daytime versus nighttime dominance during different seasons). These patterns relate to abiotic variables such as temperature, light, and DO, as well as life history processes, but the nature of these relationships and underlying causal mechanisms are only beginning to be explored.

\section{1:15}

2pABa2. Long-term passive acoustic monitoring of Western Australian cockatoos. Shyam Madhusudhana and Christine Erbe (Ctr. for Marine Sci. \& Technol., Curtin Univ., Phys. Bldg. 301 Rm. 136A, Kent St., Bentley, WA 6102, Australia, s.madhusudhana@postgrad.curtin.edu.au)

Carnaby's black cockatoos (Calyptorhynchus latirostris) and forest redtailed black cockatoos (Calyptorhynchus banksii naso) are threatened avian fauna endemic to Southwestern Australia. Both species vocalize during various activities including flight, foraging, feeding, and breeding, and their vocalizations are known to differ by age and gender. In this study, passive acoustic monitoring is employed to assist with ongoing monitoring and recovery efforts. Autonomous acoustic recorders are installed at two locations-an urban setting in the Swan coastal plains and a bushland setting in the Perth hills district. The urban setting is a well-known roosting site for Carnaby's cockatoos. Noise from frequent aircraft activity is a dominant feature at the bushland setting. Call repertoires, established using visual sighting information, are used in developing automatic recognition algorithms for efficient processing of long-term recordings. In addition to providing information on population estimates, distribution, and demographics, the project aims to study the differences in call characteristics between the urban and bushland settings and establish a framework for observing potential seasonal changes in calling behavior.

\section{$1: 30$}

2pABa3. Comparison of whistle classification results for three overlapping false whale populations in the Hawaiian Islands. Yvonne M. Barkley (Hawaii Inst. of Marine Biology, Univ. of Hawaii at Manoa, 1845 Wasp Blvd, Bldg. 176, Honolulu, HI 96818, ybarkley@ hawaii.edu)

The false killer whale (Pseudorca crassidens) is a globally distributed species found in temperate and tropical waters. The Hawaiian Archipelago is home to three genetically distinct populations of $P$. crassidens with overlapping distributions: two insular populations, one within the main Hawaiian Islands (MHI) and the other within the Northwestern Hawaiian Islands (NWHI), and a broadly distributed pelagic population. The mechanisms that created and maintain the separation between these populations are unknown, but previous studies have shown that acoustic diversity may reflect the genetic differences. For this study, whistles from 14 MHI, 11 NWHI, and 7 pelagic groups were extracted and measured to assess whether differences exist between the vocal repertoires of each population. Whistle measurements were tested for statistical differences and then used in two different classification methods: a random forest algorithm and Kullback-Leibler (KL) divergence calculations. Preliminary results suggest that the MHI and pelagic populations are vocally distinct, with up to $100 \%$ correct classification of whistles for some groups. A comparison of the classification results from the random forest and KL divergence studies will be discussed. Understanding the acoustic differences between these populations may open up new acoustic monitoring approaches for a difficult-to-study species.

\section{$1: 45$}

2pABa4. Time frequency analysis of the coconut rhinoceros beetle chirps. John S. Allen, Hans Ramm (Mech. Eng., Univ. of Hawaii-Manoa, 2540 Dole St., Holmes Hall 302, Honolulu, HI 96822, alleniii@hawaii.edu), and Daniel Jenkins (Dept. of Molecular BioSci. and BioEng., Univ. of Hawaii-Manoa, Honolulu, Hawaii)

The Coconut Rhinoceros Beetle attacks the coconut palm trees in the Pacific Region and its foraging has led to the devastion of the palm trees in Guam. The beetle is an invasive species on the island of Oahu, Hawaii, since its latent arrival in 2013. Stridulation sounds produced by the beetles have been reported with respect to mating and aggressive male behavior. Previous studies have proposed the mechanism for the stridulation and reported on some preliminary acoustic recording of the chirp for groups of beetles. However, the chrip characteristics wth respect to different behavior have not been examined extensively and also the time frequency analysis of the chirp sounds specifically or male and female beetles has been limited. In laboratory setting, the chirp characteristics are investigated with respect individual beetles in terms of pulse length and chrip duration. Time frequency analysis is used quantifiy the frequency modulation. An Empirical 
Mode Decompositon (EMD) provides a means of determing the instantaneous frequency and phase. Sounds of wing beats during the beetle's take off and flight are also investigated.

$$
\text { 2:00 }
$$

2pABa5. Preliminary analysis of social calls used by tagged humpback whales in the Los Cabos, México, breeding ground. Kerri Seger (CCOM/ JHC, Univ. of New Hampshire, 9331 Discovery Way, Apt. C, La Jolla, CA 92037, kseger@ucsd.edu), Ann M. Zoidis (Cetos Res. Organization, Bar Harbor, ME), Brooke L. Hawkins (Scripps Inst. of Oceanogr., San Diego, CA), M. Esther Jimenez-Lopez (Universidad Autónoma de Baja California Sur, La Paz, Mexico), Aaron M. Thode (Scripps Inst. of Oceanogr., La Jolla, CA), and Jorge Urban R. (Universidad Autónoma de Baja California Sur, La Paz, Mexico)

Humpback whales produce a large variety of diverse sounds beyond their well-known songs. Mothers, calves, and non-breeding whales may use these "social sounds" to maintain group cohesion, facilitate feeding, and/or increase a calf's safety. To date, social sounds have been studied off Hawaii, Alaska, and Australia. During the 2014-2015 breeding seasons, acoustic tag data were collected from humpback whales off Los Cabos, México. Twenty-one tags successfully recorded data (nine in 2014; 13 in 2015) from three mother/calf pairs, ten mother/calf/escort groups, and eight competitive pods varying in size from four to fourteen individuals. A subsequent manual analysis found 1587 social sounds in 2258 total minutes of data. Currently, there are 42 identified distinct social sounds used by humpback whales in the Los Cabos breeding ground, six of which seem unique to the Cabo region as compared to those published from Australia, Hawaii, and Alaska. Call type usage, call rates, and repertoire diversity (measured using information entropy) vary between mother/calf, mother/calf/escort, and competitive groups. Results suggest high variability in the types of social sounds used by different humpback whale groups within the same geographic area, and some social sounds overlap with repertoires known from other regions.

\title{
Session 2pABb
}

\section{Animal Bioacoustics and Signal Processing in Acoustics: Anthropogenic Transient Noise Sound Field and Its Effects on Animal Communication II}

\author{
Shane Guan, Cochair \\ Office of Protected Resources, National Marine Fisheries Service, 1315 East-West Highway, SSMC-3, Suite 13700, \\ Silver Spring, MD 20902 \\ Satoko Kimura, Cochair \\ Field Science Education and Research Center, Kyoto Univ., Kyoto, Japan
}

Invited Papers

3:00

2pABb1. Time-domain modelling of underwater transient noise for environmental risk assessment. Adrian Farcas (Cefas, Cefas, Pakefield Rd., Lowestoft NR33 0HT, United Kingdom, adrian.farcas@cefas.co.uk), Claire F. Powell, Rebecca C. Faulkner (Cefas, Lowestoft, Suffolk, United Kingdom), Gordon D. Hastie (Univ. of St. Andrews, St. Andrews, United Kingdom), Paul M. Thompson (Univ. of Aberdeen, Cromarty, United Kingdom), and Nathan D. Merchant (Cefas, Lowestoft, Suffolk, United Kingdom)

Underwater transient noise has the potential to cause auditory damage in marine mammals, fish, and invertebrates. The risk of auditory damage is closely linked to the temporal structure of transient signals (e.g., rise time, peak pressure), which is modulated through propagation in the environment. Current models used in environmental risk assessment of underwater noise are based on pulse energy and do not predict the change in temporal structure as the transient propagates away from the source. To address this, the current work presents a time-domain model of transient propagation, based on Fourier synthesis of frequency domain solutions computed using a parabolic equation method. The model outputs are benchmarked first against standard analytical models, then validated against measurements of seismic survey and pile driving noise at various ranges from the source. The results will be discussed in the context of recent regulatory guidelines on the extent of transient acoustic fields and the associated risk of auditory damage to marine mammals.

\section{3:20}

2pABb2. Lost listening area assessment of anthropogenic sounds in the Chukchi Sea. David E. Hannay, Marie-Noel Matthews, Angela Schlesinger (JASCO Appl. Sci., 2305-4464 Markham St., Victoria, BC V8Z 7X8, Canada, David.Hannay@jasco.com), Leila Hatch (Stellwagen Bank Natl. Marine Sanctuary, NOAA, Scituate, MA), and Jolie Harrison (Office of Protected Resources, Natl. Marine Fisheries Service, Silver Spring, MD)

The term listening area, refers to the region of ocean over which sources of sound can be detected by an animal at the center of the space. The lost listening area assessment method has been applied to in-air sounds for a noise effects assessment on birds but not, in our knowledge, previously to the assessment of underwater noise effects on marine mammals. The lost listening area method calculates a 
fractional reduction in listening area due to the addition of anthropogenic noise to ambient noise. It does not provide absolute areas or volumes of space, as does the communication space method; however, a benefit of the lost listening area method is that it does not rely on source levels of the sounds of interest. Instead, the method depends only on the rate of sound transmission loss. We present a preliminary application of the method from an assessment of "cumulative and chronic effects" of noise produced by oil and gas exploration activities used in the National Marine Fisheries Service's Effects of Oil and Gas Exploration in the Arctic Ocean Final Programmatic Environmental Impact Statement.

\section{Contributed Papers}

\section{$3: 40$}

$2 \mathrm{pABb3}$. Characterizing bounded transient sound fields in terms of potential effects on communication in aquatic animals. Mardi C. Hastings (George W. Woodruff School of Mech. Eng., Georgia Inst. of Technol., Atlanta, GA 30332-0405, mardi.hastings@gatech.edu)

Characterization of underwater transient sound fields in laboratory test chambers and shallow water field locations often used for bioacoustic studies is challenging. For example, no closed form solution exists for transient excitation of a water-filled waveguide with elastic walls. Key issues affecting interpretation of effects on animal communication in these situations are spatially dependent initial conditions that create subsequent sound propagation immediately following termination of the source signal; dissipative effects created by interaction of sound with boundaries; and possible excitation of modal resonances within the confined fluid. Therefore, evaluation and interpretation of data require time domain analysis of sound wave propagation and accurate knowledge of the locations of animal receivers within the sound field. Discrepancies in observed effects reported in the literature can often be explained by proper spatial evaluation of the transient sound field. Several examples will be presented along with results of an experimental evaluation of a water-filled steel waveguide excited by pulsed tones.

\section{3:55}

2pABb4. Long range sound propagation modeling for assessing seismic survey noise impacts on marine mammals. Yukino Hirai (Tokyo Univ. of Marine Sci. and Technol., 2-1-6, Etchujima, Koto-ku, Tokyo 135-8533, Japan, hirai.yukino@gmail.com), Toshio Tsuchiya (Japan Agency for Marine-Earth Sci. and Technology/Tokyo Univ. of Marine Sci. and Technol., Yokosuka, Kanagawa, Japan), Shinpei Gotoh, Etsuro Shimizu (Tokyo Univ. of Marine Sci. and Technology/Japan Agency for Marine-Earth Sci. and Technol., Koto-ku, Tokyo, Japan), and Koji Futa (Mitsubishi Precision Co., Ltd., Kamakura, Kanagawa, Japan)

Despite of small land area, Japan has vast EEZ, which is expected to have potential mineral resources. Extensive seismic surveys have been conducted for those targets. However, airguns used for the survey generate intense and low-frequency impulse sound, which could disturb or harm marine mammal behavior and auditory system. In June 2016, NOAA released "Ocean Noise Strategy Roadmap" that recommends modeling of sound propagation in the context of realistic environmental parameters to assess noise impacts on marine creatures. Japan has remarkable seasonal climate changes and complex ocean-bottom topography with trenches and seamounts; therefore, such model is worthwhile. Here, we propose a modeling method utilizing ARGO data includes seasonal climate changes and LEVITUS data, and ocean-bottom topographic configuration data from Google Earth. We calculated long range propagation of airgun sounds in different sound profiles and ocean-bottom topographic configurations around Ogasawara Islands, which is known as a cetacean habitat. Results showed that seasonal climate change and the precision of the ocean-bottom topographic configuration caused significant differences in sound propagation. These findings suggest that the realistic environmental parameters is essentially needed for the modeling of sound propagation and proposed method is a useful tool to assess noise impacts on marine mammals.
$4: 10$

2pABb5. Long-term spatially distributed observations of deep diving marine mammals in the Northern Gulf of Mexico using passive acoustic monitoring. Natalia Sidorovskaia, Kun Li (Phys., UL Lafayette, UL BOX 44210, Lafayette, LA 70504-4210, nas@louisiana.edu), Christopher Tiemann (R2Sonic, Austin, TX), Azmy Ackleh, and Tingting Tang (Mathematics, UL Lafayette, Lafayette, LA)

This paper will present the results of processing long-term passive acoustic monitoring (PAM) data collected July through October 2015 in the Northern Gulf of Mexico in the vicinity of the Deep Water Horizon oil spill site to aid in understanding factors driving the distribution of sperm and beaked whales in the Gulf of Mexico. The Littoral Acoustic Demonstration Center -Gulf Ecological Monitoring and Modeling Consortium (LADCGEMM) deployed five bottom-anchored acoustic moorings (LADC EARS buoys) at 10, 25, and $50 \mathrm{nmi}$ distance from the 2010 oil spill location. Autonomous surface vehicles and a glider were simultaneously operated in the area for the additional collection of PAM data. The daily and monthly activity of three species of beaked whales exhibits spatial and seasonal variability, which appear to be correlated with levels of anthropogenic noise at the monitoring sites. Acoustic detection data are used to estimate abundances at three sites and compare them to the estimates obtained from baseline data collected before (2007) and right after (2010) the oil spill. Long-term abundance trends for both beaked and sperm whales are discussed. [Research supported by GOMRI.]

\section{4:25}

2pABb6. Evaluating underwater ambient noise levels across a changing Arctic environment. Melania Guerra, Kathleen M. Stafford, and Rex K. Andrew (Appl. Phys. Lab., Univ. of Washington, 1013 NE 40th St., Box 355640, Seattle, WA 98105, melania@apl.washington.edu)

As a result of climate change, the Arctic underwater acoustic environment is undergoing an unprecedented transformation of its ambient noise sources, including an expanded wind fetch over open water and an intensification of maritime transportation lanes across Northern routes. This comparative study synthesizes multi-annual passive acoustic monitoring (PAM) data collected at various Alaskan Arctic environments (Bering Strait, Chukchi Plateau, Chukchi, and Beaufort Seas) in order to evaluate the statistics of ambient noise across the changing region. Mean levels at significant frequency ranges are presented, as well as percentile PSD levels in 1-Hz frequency bins. Remote sensing sea ice data is utilized to separate each annual dataset into 3 different regimes: open water, covered, and transition (when ice is forming or breaking up). During each, the dominant large-scale noise sources are characterized and the relationship between noise and local wind speed is evaluated. Through manual inspection, ship transits and seismic surveys have been extracted, allowing ambient level associations on the scale of hours or days. Consistently across all years, the Chukchi Plateau site, which is the most distant from shore, presents the lowest overall ambient noise levels (20-40 dB lower than other locations), potentially indicating a new Arctic ambient noise baseline. 
result of an attempt by the birds to reduce the masking effect of the low rumble of urban noise. In sharp contrast, we report that chiffchaffs reduce song frequency at airports where noise levels are intermittent rather than constant, but at a much higher amplitude level. In addition, we show experimentally that airport birds respond more aggressively to playback of airport songs than the control songs, while a nearby control population does not discriminate between airport and control songs. Potential explanations for these results will be discussed.
In general, it has been found that birds respond to exposure of anthropo-

genic noise by singing at a higher frequency range. This is presumably the
2 Andrew Wolfenden (Conservation, Evolution and Behaviour Res. Group . of Biology and Conservation Ecology, Manchester Metropolitan Univ All Saints Bldg., All Saints, Manchester M15 6BH, United Kingdom, S. Dekort@mmu.ac.uk)

\title{
Session 2pAOa
}

\section{Acoustical Oceanography, Underwater Acoustics, and Signal Processing in Acoustics: Ocean Acoustic Tomography: Active and Passive, Theory and Experiment I}

\author{
Bruce Howe, Cochair \\ Ocean and Resources Engineering, University of Hawaii, 2540 Dole Street, Holmes Hall 402, Honolulu, HI 96822 \\ Arata Kaneko, Cochair \\ Graduate School of Engineering, Hiroshima University, 1-4-1 Kagamiyama, Higashi-Hiroshima 739-8527, Japan
}

Hiroyuki Hachiya, Cochair

Tokyo Institute of Technology, 2-12-1 S5-17, Ookayama, Meguro-ku, Tokyo 152-8550, Japan

\section{Invited Papers}

\section{1:00}

2pAOa1. Passive travel time tomography using ships as acoustic sources of opportunity. Bruce Cornuelle, William A. Kuperman, William S. Hodgkiss, Jeff Tippmann, Jit Sarkar, Chris Verlinden (UCSD-Scripps Inst. of Oceanogr., 9500 Gilman Dr., Dept 0230, La Jolla, CA 92093-0230, bcornuelle@ucsd.edu), and Karim Sabra (College of Mech. Eng., Georgia Inst. of Technol., Atlanta, GA)

Active ocean acoustic tomography transmits known source signals and often includes precise source and receiver positioning. Passive estimation of channel Greens functions has been widely demonstrated using both diffuse and concentrated noise sources, including the estimation of ocean sound speeds and currents. The uncertainty of surface ship positions determined by the Automatic Identification System (AIS) translates to travel time variability that is larger than expected from ocean structure at ranges of a few kilometers, but listening to the same source from spatially-distributed receivers adds more data while maintaining the same source position unknowns and may allow useful estimates of bottom depths from bottom-interacting rays and perhaps ocean structure. A fall 2016 experiment will test these ideas in the Santa Barbara Basin using 4 vertical line receiver arrays recording ship-radiated sound for 10 days. Estimates of the expected performance of the estimates of bottom depth and ocean sound speed have been a part of the design process. A general circulation model will be used to increase the efficiency of information use by parameterizing the four-dimensional ocean sound speed structure in terms of initial conditions, boundary conditions, and surface forcing. We present the design process and some proof-of-concept calculations from experimental observations.

\section{$1: 20$}

2pAOa2. Recent progress in Taiwan on integrating moving vehicles for acoustic tomography applications. Chen-Fen Huang and Naokazu Taniguchi (Inst. of Oceanogr., National Taiwan Univ., No. 1, Sec. 4, Roosevelt Rd., Taipei 10617, Taiwan, chenfen@ntu.edu. tw)

Coastal seas around Taiwan exhibit a wide variety of oceanographic processes associated with the complex bottom topography, tides, winds (monsoons and typhoons), the Kuroshio, and the others. Conventional oceanographic measurements (e.g., acoustic Doppler current profiler) do not provide a synoptic image of the dynamic processes unless a large number of instruments are deployed. Ocean Acoustic Tomography (OAT) is an effective method for mapping the time-evolving spatial distribution of ocean current and temperature. As illustrated by Cornuell et al. (1989), the small spatial scale of ocean features is resolved by incorporating a ship-towed transceiver to the moored transceiver array, referred to as moving ship tomography (MST). The MST technique is applied to the ocean 
current reconstruction in coastal seas around Taiwan and is extended by using an autonomous underwater vehicle (AUV) as an alternative carrier for the transceiver. This talk presents a brief summary of the recent experiments and our upcoming project. Included are 1) a series of current-mapping experiments integrating moving vehicles in shallow-water environments and 2) simulation study of MST for experiment design on mapping the Kuroshio-induced current wakes of Green Island southeast of Taiwan.

\section{1:40}

2pAOa3. Ocean gliders as tools for acoustic tomography. Lora J. Van Uffelen (Ocean Eng., Univ. of Rhode Island, 15 Receiving Rd., Sheets Bldg., Narragansett, RI 02882, loravu@hawaii.edu), Sarah E. Webster, Craig M. Lee, Jason I. Gobat (Appl. Phys. Lab., Univ. of Washington, Seattle, WA), Peter F. Worcester, and Matthew A. Dzieciuch (Scripps Inst. of Oceanogr., Univ. of California, San Diego, La Jolla, CA)

Ocean gliders are capable and increasingly ubiquitous platforms for collecting oceanographic data such as temperature and salinity measurements. These data alone are useful for ocean acoustic tomography applications, but gliders equipped with acoustic receivers can also record transmissions from acoustic sources, potentially providing additional paths for tomographic inversions. There are challenges associated with the use of gliders as tomography receivers, however, notably the uncertainty in underwater glider position, which can lead to ambiguity between sound-speed and glider position. Glider-based acoustic receptions from moored tomography sources can provide range measurements to aid in subsea glider localization. These data can be used in post-processing for precise glider positioning and in real-time for glider navigation. The current state of the art will be reviewed, and preliminary results will be presented from an experiment in the Arctic Ocean, which seeks to use moored acoustic tomography source signals for both subsea glider positioning and real-time navigation, including navigation under ice. [Work sponsored by ONR.]

\section{Contributed Papers}

2:00

2pAOa4. Moving ship tomography experiments in a coastal sea. Naokazu Taniguchi, Yun-Wen Li, and Chen-Fen Huang (Inst. of Oceanogr., National Taiwan Univ., No. 1, Sec. 4, Roosevelt Rd., Taipei 10617, Taiwan, naokazutaniguchi@gmail.com)

This study considers measuring differential travel times (DTT) between moored and ship-towed transceivers in shallow waters as a feasibility study to extend the concept of the moving ship tomography (Cornuelle et al., 1989) to current field reconstructions. The delay-Doppler ambiguity function (AF) method is used to estimate and compensate the waveform distortions induced by the relative motion between transceivers. The Doppler shift (the relative speed between transceivers) is estimated by the peak of the $\mathrm{AF}$, and the delay time series associated with the peak is the Doppler compensated arrival pattern. The DTTs are determined using the compensated arrival patterns in reciprocal directions. For the current estimation the estimated relative speed is used for removing the effect of the transceiver motion from the DTTs. A reciprocal sound transmission experiment was conducted using a ship-towed transceiver and two moored transceivers in the Sizihwan coastal near Kaohsiung, Taiwan. Due to the complicated arrival patterns observed, the DTTs were determined by tracking the peaks of the cross-correlation functions between the compensated reciprocal arrival patterns over the transmissions. The path-averaged currents derived from DTT agreed well with the direct measurement using a bottom-mounted acoustic Doppler current profiler.

\section{2:15}

2pAOa5. Autonomous underwater vehicle navigation using a single tomographic node. Sheng-Wei Huang (Dept. of Eng. Sci. and Ocean Eng., National Taiwan Univ., No. 1, Sec. 4, Roosevelt Rd., Taipei 106, Taiwan, d99525008@ntu.edu.tw), Naokazu Taniguchi, Chen-Fen Huang (Inst. of Oceanogr., National Taiwan Univ., Taipei, Taiwan), and Jenhwa Guo (Dept. of Eng. Sci. and Ocean Eng., National Taiwan Univ., Taipei, Taiwan)

This study investigates AUV navigation using the one-way travel-time from an ocean tomographic sensor node. For the study of ocean acoustic tomography, several tomographic nodes are moored in the study area and broadcast $m$-sequence signals periodically. Each moored node consists of an acoustic transceiver, a GPS module, and a processing unit. The pulse-persecond signal from the GPS module is used to ensure the time synchronization of all the moored nodes. A tomographic sensor is installed on an AUV, serving as a moving node. To obtain highly accurate one-way travel-time between moored nodes and AUV, a chip scale atomic clock is operated on the AUV. Based on dead-reckoning and travel-time measurement, Extended Kalman Filter (EKF) is employed to improve the AUV localization. An experiment was conducted using an AUV and one moored node deployed in a shallow water environment southwest of Taiwan. The measurements from a Doppler velocity logger and a compass are used to calculate the current position of vehicle. The AUV was operated near the surface to obtain the GPS position as the ground truth for the performance evaluation. Preliminary results show the RMS position error between the EKF prediction and ground truth is about $80 \mathrm{~m}$.

\section{2:30}

2pAOa6. Simulation study of moving ship tomography for mapping the current wakes of Green Island southeast of Taiwan. Kai-Fu Chang and Chen-Fen Huang (Inst. of Oceanogr., National Taiwan Univ., No. 1, Sec. 4, Roosevelt Rd., Taipei 10617, Taiwan, r04241107@ntu.edu.tw)

This study examines the design of acoustic arrays using moving ship tomography (MST) applied to the horizontal mapping of the Kuroshioinduced current wakes in the lee of Green Island southeast of Taiwan. The acoustic tomographic array consists of six transceivers; each transceiver can be either fixed at one position or towed by a ship. Numerical tomographic experiments using the synthetic ocean currents from the $8 \mathrm{~km} \mathrm{X} 8 \mathrm{~km}$ study area are conducted for two configurations: five moored transceivers and one shipboard transceiver or four moored transceivers and two shipboard transceivers. Different spatial configurations of moored and shipboard transceivers are considered. For all configurations the ship moved around the periphery of the study area. Compared with the traditional tomographic arrays with only moored transceivers, the current reconstructions using MST show the reduction in residual error from $21 \%$ to $16 \%$. Among all the MST arrays, the smallest residual error variance is obtained for the configuration of the five moored transceivers placed in a pentagon shape and one shipboard transceiver. This study will provide an optimum array design for the coming MST experiment near the Green Island. 


\section{Invited Papers}

2pAOa7. Ocean remote sensing with acoustic daylight: Lessons from experiments in the Florida Straits. Michael G. Brown (RSMAS, Univ. of Miami, 4600 Rickenbacker Cswy, Miami, FL 33149, mbrown@rsmas.miami.edu), Oleg A. Godin (Dept of Phys., Naval Postgrad. School, Monterey, CA), Xiaoqin Zang (RSMAS, Univ. of Miami, Miami, FL), Nikolay A. Zabotin, and Liudmila Y. Zabotina (Cooperative Inst. for Res. in Environ. Sci., Univ. of Colorado, Boulder, CO)

Ambient and shipping noise in the ocean provides acoustic illumination, which can be used, similarly to daylight in the atmosphere, to characterize the environment. Phase information, which is particularly sensitive to sound speed variations and current velocity, can be retrieved from noise observations by the process known as noise interferometry. Approximations to acoustic Green's functions, which describe sound propagation between two locations, are estimated by cross-correlating time series of diffuse noise measured at those locations. Noise-interferometry-based approximations to Green's functions can be used as the basis for a variety of inversion algorithms, thereby providing a purely passive alternative to active-source ocean acoustic remote sensing. This paper gives an overview of results from noise interferometry experiments conducted in the Florida Straits at $100 \mathrm{~m}$ depth in December 2012, and at $600 \mathrm{~m}$ depth in September/October 2013. Under good conditions for noise interferometry, estimates of cross-correlation functions are shown to allow one to perform advanced phase-coherent signal processing techniques to: perform waveform inversions; estimate currents by exploiting nonreciprocity; perform time-reversal/back-propagation calculations; and investigate modal dispersion using time-warping techniques. Conditions which are favorable for noise interferometry are identified and discussed. [Work supported by NSF and ONR.]

\section{3:20}

2pAOa8. Acoustic measurements of vertical velocity and temperature fluctuations of a hydrothermal plume with comparisons to large eddy simulations. Daniela Di Iorio (Dept. of Marine Sci., Univ. of Georgia, 250 Marine Sci. Bldg., Athens, GA 30602, daniela@ uga.edu), J W. Lavelle (none, Seattle, WA), and Ian Adams (Dept. of Marine Sci., Univ. of Georgia, Athens, GA)

The acoustic scintillation method is used to study the hydrothermal plume of Dante within the Main Endeavour vent field (MEF) at the Endeavour segment of the Juan de Fuca Ridge. Forty days of vertical velocity and temperature fluctuations were obtained from the rising plume above the Dante edifice in an environment where the flow is dominated by strong $(5 \mathrm{~cm} / \mathrm{s})$ semi-diurnal tidal currents and a northerly mean residual current $(3 \mathrm{~cm} / \mathrm{s})$. These acoustic measurements provide a window on deep-sea hydrothermal plume dynamics in strong oscillatory cross flows. A large eddy simulation, parameterized with anisotropic mixing coefficients, taking into account ambient stratification and time-dependent background flows and calibrated by the acoustic measurements, yields insight into turbulent processes, entrainment, plume bending, rise height, and, inferentially, mound heat flux. The turbulent dissipation rates for kinetic energy $(\varepsilon)$ and thermal variance $\left(\varepsilon_{\theta}\right)$ is approximated by computing the Reynolds averaged sub-grid scale turbulent production from shear and buoyancy $(\varepsilon=\mathrm{P}-\mathrm{B})$ and from temperature fluxes $\left(\varepsilon_{\theta}=\mathrm{P}_{\theta}\right)$, respectively, which are needed to compare to the acoustic derived turbulence levels. A new cabled observatory reciprocal acoustic scintillation system on the NEPTUNE observatory that will allow real time measurements of mean and turbulent flow of a hydrothermal plume will also be introduced.

\section{$3: 40$}

2pAOa9. Applicability and feasibility studies of coastal acoustic tomography for long-term monitoring of the Indonesian throughflow transport variability. Fadli Syamsudin, Yudi Adityawarman, Reni Sulistyowati (Technol. for Regional Resource Development, Agency for the Assessment and Application of Technol. (BPPT), J1. Salemba Bluntas C216, Jakarta, DKI Jakarta 10430, Indonesia, fadlihiro@yahoo.com), Bayu Sutedjo (Technologyfor Ocean Survey, Agency for the Assessment and Application of Technol. (BPPT), Jakarta, Indonesia), Arata Kaneko, and Noriaki Goda (Graduate School of Eng., Hiroshima Univ., Hiroshima, Japan)

We have been trying to monitor the Indonesian Throughflow (ITF), especially to understand how prominent tides and tidal mixing along the ITF main pathways in the Makassar and Lombok straits could transform the Pacific waters into Indian Oceans in the shorter scale and how the ITF variability in longer time scales affect the climate regimes over the region, using a modern and innovative technology of Coastal Acoustic Tomography (CAT). The first trial will be done in the Lombok sill where two 5-kHz CATs and three tide gauge sensors are put in the sill of depth range 200-300 m, lying between Lombok and Nusa Penida islands in the southern Lombok strait by the end of July 2016. The experimental design is a real-time land-cable CAT system, equipped with a 3G mobile antenna for telemetering CAT data to our Maritime Continent Center of Excellence (MCCOE) monitoring center in Jakarta every 10 minutes interval. Results are expected to verify CAT aplicability and feasibility as a cost effective oceanographic instrument to monitor the ITF transport variability along major straits in the Indonesian seas. This talk will also present the possibility of CAT to study mixing processes as important factors to improve model parametrizations. 


\title{
Session 2pAOb
}

\section{Acoustical Oceanography: Acoustical Oceanography Prize Lecture}

\author{
Andone C. Lavery, Cochair \\ Applied Ocean Physics and Engineering, Woods Hole Oceanographic Institution, 98 Water Street, MS 11, Bigelow 211, \\ Woods Hole, MA 02536 \\ John A. Colosi, Cochair \\ Department of Oceanography, Naval Postgraduate School, 833 Dyer Road, Monterey, CA 93943
}

Chair's Introduction-4:15

Invited Paper

4:20

2pAOb1. Acoustic observations and characterization of oceanic methane gas bubbles rising from the seabed. Thomas C. Weber (Univ. of New Hampshire, 24 Colovos Rd., Durham, NH 03824, tom.weber@unh.edu)

Methane, a potent greenhouse gas, can be found escaping the ocean seabed as gas bubbles in a wide range of geographic locations and water depths. As they rise toward the sea surface, methane gas bubbles undergo a complicated evolution. During ascent, methane gas is transferred into aqueous solution and other dissolved gases are transferred into the bubble. The gas transfer rate-a key factor in determining the ultimate fate of the methane - may be inhibited by hydrate formation in deep, cold water, or potentially by surfactants and adsorbed particulates at any depth. The presence of methane gas bubbles from both natural and anthropogenic sources is often identified using acoustic echo sounders. Beyond simple detection, acoustic techniques can be used to characterize methane bubbles in several ways. For example, narrow-band observations of seep target strength can be used with knowledge of bubble size distributions to estimate methane flux. Similar acoustic observations can be coupled with bubble-evolution models to constrain the fate of bubbles as they rise. Broadband techniques offer the potential to directly observe bubble size and rise speed, and consequently depth-dependent gas flux. The application of these and other techniques for detecting and characterizing methane gas-bubble seeps will be discussed. 


\title{
Session 2pBAa
}

\section{Biomedical Acoustics and Signal Processing in Acoustics: Bone Sonometry I: Ultrasonic Studies of the Physical Properties of Cortical and Cancellous Bone}

\author{
Mami Matsukawa, Cochair \\ Doshisha University, Doshisha University, 1-3 Tatara Miyakodani, Kyotanabe 610-0321, Japan \\ James G. Miller, Cochair \\ Physics, Washington U Saint Louis, Box 1105, 1 Brookings Drive, Saint Louis, MO 63130
}

Chair's Introduction-1:00

\section{Invited Papers}

1:05

2pBAa1. Efficient dispersion analysis of guided waves in cortical bone. Pascal Laugier, Nicolas Bochud, Quentin Vallet, Xiran Cai, Quentin Grimal, and Jean-Gabriel Minonzio (Laboratoire d'Imagerie Biomedicale, Sorbonne Universités, UPMC Univ. Paris 06, CNRS UMR 7371, INSERM UMRS1146, 15 rue de l'ecole de medecine, Paris 75017, France, laugierp@ gmail.fr)

Guided wave propagation is at the heart of the axial transmission techniques designed to assess bone health status. The method involves matching observed and predicted dispersion characteristics of guided waves. The strength of the technique is that it can infer more than one bone property from the measured ultrasonic data, such as cortical thickness, stiffness, or porosity. The suitability of the model chosen for the inversion has recently been debated and the question has been raised whether the physical model must take the soft tissue coating influence into account as well as perhaps other factors such as bone curvature. We present in this talk a series of experiments conducted on bone-mimicking phantoms (plates or tubes) with or without soft tissue-mimicking coating showing evidence $1 /$ that the experimental guided wave branches in the range of $0.4-1.6 \mathrm{MHz}$ mainly exhibit sensitivity to the influence of the solid subsystem (bone) and 2/ that a simple non absorbing transverse isotropic free plate model provides an appropriate inverse model in all investigated cases, i.e., coated or non-coated plates and tubes. Finally, we demonstrate effectiveness of the inversion procedure in characterizing cortical bone using ex vivo and in vivo data.

$1: 25$

2pBAa2. The role of acoustic microscopy in bone research. Kay Raum, Susanne Schrof, Johannes Schneider, Gianluca Iori, Vantte Kilappa, Juan Du, Matthias Pumberger, Michael Putzier (Charité Universitätsmedizin Berlin, Augustenburger Platz 1, Berlin 13353, Germany, kay.raum@charite.de), Jinming Zhou, and Hanna Isaksson (Lund Univ., Lund, Sweden)

Scanning acoustic microscopy (SAM) has been introduced 3 decades ago with the hope to open a new dimension in the microscopic analysis of biological tissues. However, only during the last decade this technology has emerged from a qualitative imaging modality to a quantitative measurement tool that provides fast and nondestructively elastic maps of acoustic and elastic properties with microscale resolution. Particularly, the spatial registration of parameter maps obtained by SAM with those obtained by complementary modalities, e.g., synchrotron microcomputed tomography, Raman spectroscopy, and inelastic micromechanical testing provided unprecedented inside into structure-composition-function relations, tissue changes with respect to adaptation, ageing, pathologies, and healing. Moreover, elastic maps generated by acoustic microscopy can serve as direct input for numerical simulations. This talk will review the key theoretical principles, experimental clues but also the limitations for the reconstruction of calibrated maps of elastic tissue properties and will present recent findings obtained by correlative imaging and SAM-based numerical sound propagation models. In particular, the role of matrix stiffness heterogeneity as a potential indicator for bone brittleness will be discussed.

\section{$1: 45$}

2pBAa3. Recent advances in resonant ultrasound spectroscopy to measure bone stiffness tensor. Quentin Grimal, Xiran Cai, Laura Peralta, Kailiang Xu, Guillaume Marrelec (Biomedical Imaging Lab., Sorbonne Universités - Université Pierre et Marie Curie - CNRS INSERM, 15 rue de l'école de médecine, Paris 75006, France, quentin.grimal@upmc.fr), Hassiba Daoui (Laboratoire de physique des matériaux, Université des Sci. et Technologie Houari Boumediéne d'Alger, Paris, France), and Pascal Laugier (Biomedical Imaging Lab., Sorbonne Universités - Université Pierre et Marie Curie - CNRS - INSERM, Paris, France)

Resonant Ultrasound Spectroscopy (RUS) is a method to measure the elasticity tensor of a material. RUS is particularly advantageous to measure small samples of anisotropic materials. In RUS, resonant frequencies of a sample are measured and computed frequencies of a numerical model of the sample are fitted, yielding the stiffness tensor. RUS was developed in the 1990s, but until recently, it was in practice limited to measure materials with a high quality factor. We have adapted the method to measure bone whose quality 
factor is about 25. Our strategy combines Bayesian methods to retrieve overlapped resonant peaks in the RUS spectra and to solve the inverse problem using a limited number of resonant frequencies. The method allows a quasi-automated processing of RUS spectra where it is not necessary to know a priori the pairing between measured and computed frequencies. In the last years we have extensively used RUS to document the anisotropic elastic properties of human cortical bone and to investigate the determinants of elastic properties. We present in this talk 1) recent advances in signal processing for RUS; 2) a discussion on the precision of bone elasticity measurement; and 3) a new perspective regarding cortical bone elasticity determinants.

\section{2:05}

2pBAa4. Passive twin-layer spatial-temporal phase-interference compensator for improved transcranial ultrasound propagation. Christian M. Langton (Inst. of Health \& Biomedical Innovation, Queensland Univ. of Technol., 60 Musk Ave., Brisbane, QLD 4049, Australia, christian.langton@qut.edu.au)

Transcranial ultrasound wave degradation created by variations in both thickness and tissue composition is a significant impediment to diagnostic and therapeutic interventions of the brain. The current "active" solution is to vary the transmission delay of ultrasound pulses, inherently necessitating electronic control of each individual transducer element. By applying the sonic-ray concept of ultrasound wave propagation, it was hypothesised that wave degradation can be significantly reduced if both the transit-time and propagation pathlength for all sonic-rays are made constant. A computer simulation study was performed to investigate the implications of zero, partial and full spatial-temporal matching. Considering ultrasound propagation through a 20-step acrylic wedge test sample, only full spatialtemporal matching removed phase-interference. A "passive" physical ultrasound phase-interference compensator (UPIC), consisting of two layered materials of variable thickness, was developed and experimentally evaluated using acrylic step-wedge test samples exhibiting varying degrees of transit-time heterogeneity induced phase-interference. Time- and frequency-domain analysis demonstrated that incorporation of the UPIC successfully removed phase-interference in all cases. It is further hypothesised that the UPIC concept may be applied to both pulse-echo mode diagnostic imaging and transmission mode therapeutic interventions, incorporating either a single-element or a multi-element array transducer, of any practicable dimension.

\section{2:25-2:40 Break}

\section{2:40}

2pBAa5. Is there a role for approximations to the Kramers-Kronig relations in understanding the propagation of ultrasound in bone? James G. Miller (Phys., Washington U Saint Louis, Box 1105, 1 Brookings Dr., Saint Louis, MO 63130, james.g.miller@wustl. edu)

Clinical measurements of the speed of sound (SOS) usually exhibit moderately large site-to-site variations within a given individual, leading to rather wide ranges for osteoporosis, osteopenia, and normal. Consequently, the rather modest variation of phase velocity over $200 \mathrm{kHz}$ to $800 \mathrm{kHz}$ predicted by the approximate form of the Kramers-Kronig relations developed in our laboratory would appear to be of limited interest. However, whereas the Kramers-Kronig relations predict a systematic increase in phase velocity with frequency, laboratories in Kyoto, Paris, and Washington, D.C., each reported a systematic decrease in phase velocity with increasing frequency. We suggested that this "anomalous" (negative) decrease in velocity with frequency was actually the consequence of interference between two waves, each of which satisfied the Kramers-Kronig-predicted positive dispersion, with one wave sufficiently stronger than the other that the combination appeared to be only a single wave. Although separation of the fast and slow waves by Bayesian, Prony's, and other methods has provided substantial insight, significant unexplained results remain and are a focus of this presentation. The author wishes to acknowledge significant contributions by former colleagues: C. Anderson, A. Bauer, G. Brandenburger, L. Bretthorst, A. Groopman, J. Hoffman, S. Handley, M. Holland, M. Hughes, E. Jaynes, J. Katz, K. Marutyan, J. Mobley, R. Norberg, M. O’Donnell, R. Trousil, and K. Waters.

2pBAa6. Signal processing methods for through-transmission measurements of fast and slow waves in bovine and equine cancellous bone. Keith A. Wear (Ctr. for Devices and Radiological Health, Food and Drug Administration, Bldg. 62, Rm. 2104, 10903 New Hampshire Ave., Silver Spring, MD 20993, keith.wear@fda.hhs.gov), Amber Groopman, Jonathan Katz (Washington Univ., St. Louis, MO), Mark Holland (Indiana Univ., Indianapolis, IN), Yoshiki Nagatani (Kobe City College of Technol., Kobe, Japan), Katsunori Mizuno (Univ. of Tokyo, Tokyo, Japan), Mami Matsukawa (Doshisha Univ., Kyoto, Japan), and James G. Miller (Washington Univ., St. Louis, MO)

Through-transmission measurements in cancellous bone in vitro often reveal two longitudinal waves. The velocities and amplitudes of fast and slow waves are related to bone microstructure and composition and may provide useful diagnostic information. Phase velocity and attenuation for fast and slow waves were measured as a function of propagation depth in bovine and equine cancellous bone. Ultrasound measurements were performed as the thicknesses of bone samples were systematically reduced from 15 to $6 \mathrm{~mm}$ (bovine) or 12 to $4 \mathrm{~mm}$ (equine) (Nagatani et al., Ultrasonics, 48, 607-612, 2008). Fast and slow wave properties were estimated using a Bayesian method (Marutyan et al., J. Acoust. Soc. Am., 121, EL8-EL15, 2007) and the modified least-squares Prony's method with curve fitting (Wear, J. Acoust. Soc. Am.,133, 2490-2501, 2013). The two methods, although based on different sets of assumptions, showed excellent agreement. This work provides strong support for both algorithms and for the two-wave model for cancellous bone upon which both are based (Marutyan et al., J. Acoust. Soc. Am., 120, EL55-EL61, 2006). 
2pBAa7. Phase cancellation effect on broadband ultrasonic attenuation analysis for human trabecular bone assessment using a 2-D synthetic array. Yi-Xian Qin and Jiqi Cheng (Biomedical Eng., Stony Brook Univ., BioEng. Bldg., Rm. 215, Stony Brook, NY 11794, yi-xian.qin@stonybrook.edu)

Quantitative ultrasound has been developed to evaluate trabecular BMD and structural integrity. The ultrasound parameters, i.e., normalized broadband attenuation (nBUA), have been widely used for bone health status. However, the reproducibility and accuracy of nBUA are influenced by the phenomena of phase cancellation. The objectives of this study have two folds, 1) to accurately investigate the effects of phase cancellation on BUA calculation using an ultra-small receiver (aperture size: $0.2 \mathrm{~mm}$ ) in a newly developed 2-D synthetic array-system, and 2) to evaluate the effects of phase cancellation on human trabecular bones. Most energy was located within a circle with a diameter of $12.70 \mathrm{~mm}$ corresponding to the transmitter aperture size. The tests of ultrasound BUA and micro-CT on the trabecular bone cylinders with a diameter of $25 \mathrm{~mm}$. Both phase sensitive (PS) detection and phase insensitive (PI) detections were performed. The data indicated that the average nBUA is $24.8 \pm 9.5 \mathrm{~dB} / \mathrm{MHz} / \mathrm{cm}$ and $19.2 \pm 5.5 \mathrm{~dB} / \mathrm{MHz} / \mathrm{cm}$ for PS and PI respectively. PS nBUA is $28.5 \%$ higher than PI BUA, which PS-nBUA can explain $81.2 \%$ of the variability in PI nBUA. Both PI-nBUA and PS-nBUA are highly correlated with $\mathrm{BV} / \mathrm{TV}(\mathrm{R}=0.911$ and $\mathrm{R}=0.898, \mathrm{p}<0.0001)$, and the Young's modulus $(\mathrm{R}=0.9061$ and $\mathrm{R}=0.822$ $\mathrm{p}<0.0001)$, respectively.

\section{3:40}

2pBAa8. Structure-function relationship in bone: Anisotropic mechanical properties of trabecular bone determined using poroelastic ultrasound. Luis Cardoso (Biomedical Eng., City College of New York, 160 Convent Ave., Steinman Hall, ST-401, New York, NY10031, cardoso@ccny.cuny.edu)

In the ageing skeleton, trabecular bone adapts its porosity, microarchitecture, and tissue composition in response to its mechanical environment through a complex and well-orchestrated bone remodeling process. Such adaptation process allows bone to be mechanically competent to resist low-trauma fractures. However, the relationship between structure and function in bone is not yet fully known. To take into account the effect of anisotropic architecture on the mechanical properties of porous media, the fabric tensor $\mathbf{F}$ was introduced in bone Biomechanics by Cowin. The fabric tensor $\mathbf{F}$ is a quantitative stereological measure of the degree of structural anisotropy and the mechanical principal orientations of a porous medium. We recently incorporated the fabric tensor into the theory of wave propagation in a fluid saturated porous media, and showed that the fabric tensor is a good predictor of directional-dependent measurements of ultrasound in cancellous bone. In our current study, we demonstrate that the spatial distribution of mass and pore space in trabecular bone (i.e., fabric), when combined with its volume fraction and tissue mineral density, is able to well describe the directional-dependent variability of the anisotropic elastic and yield behavior of bone, which cannot predicted by bone mineral density alone.

\section{4:00}

2pBAa9. Attenuation and dispersion of ultrasound in cancellous bone. Theory and experiment. Michal Pakula (Inst. of Mech. and Comput. Sci., Kazimierz Wielki Univ. in Bydgoszcz, ul. Chodkiewicza 30, ul. Kopernika 1, Bydgoszcz 85-064, Poland, michalp@ukw. edu.pl)

The paper presents theoretical and experimental issues related to modeling of elastic wave propagation in cancellous bone. The Biot's theory, commonly used for modeling of ultrasonic wave propagation in cancellous bone, is discussed in context of its potential applicability for theoretical prediction of wave parameters. Particular attention is focused on the analysis of physical mechanisms of ultrasonic wave propagation in cancellous bone that govern phase velocity and attenuation coefficient as function of frequency and porosity. The analysis of the model is focused on the absorption and scattering mechanisms responsible for attenuation of ultrasonic waves in cancellous bone, which based on the ultrasonic experiments presumably play a predominant role in the total attenuation. The suitability of the model is discussed and verified by comparison of results of sensitivity analysis of the model with experimental ultrasonic data for bone mimicking phantoms and human cancellous bones.

2pBAa10. Ultrasound multiple scattering for the assessment of bone micro-architecture. Mason Gardner (MAE, NCSU, Raleigh, NC), Sylvain Haupert, Guillaume Renaud (Laboratoire d'Imagerie Biomedicale, Paris, France), and Marie M. Muller (MAE, NCSU, 911 oval Dr., Raleigh, NC27695, mmuller2@ncsu.edu)

Retrieving bone micro-architectural parameters non-invasively using ultrasound would enable the monitoring of bone loss, and the follow up of the efficacy of treatments. When ultrasound propagate in bone in the $\mathrm{MHz}$ range, multiple scattering is non-negligible, and we demonstrate that it is possible to take advantage of it. We propose methods based on ultrasound multiple scattering for the quantification of micro-architectural parameters in cortical and trabecular bone. Using linear transducer arrays at 5 and $8 \mathrm{MHz}$, we present both finite differences simulations and experimental results in 3D printed phantoms of trabecular bone, in equine trabecular bone and in human cortical and trabecular bone. We show that the framework of the Independent Scattering Approximation can be used to model the attenuation in both cortical and trabecular bone. We demonstrate that measuring the diffusion constant is feasible in cortical and trabecular bone, and that it is correlated to micro-architectural parameters. Preliminary results indicate a significant correlation between the trabecular anisotropy and the anisotropy of the diffusion constant $(r=0.87, p=7.6 e-8)$. In cortical bone, a significant quadratic relationship was observed between the mean pore size and the diffusion constant. The cortical pore density was highly and significantly correlated to the diffusion constant $(\mathrm{r}=0.98, \mathrm{p}=0.001)$. 
2pBAa11. Ultrasonic backscatter difference measurements of cancellous bone: Relationships with microstructure and bone mineral density. Brent K. Hoffmeister, P. L. Spinolo, Matthew T. Huber, Joshua T. Moore, Ann M. Viano (Dept. of Phys., Rhodes College, 2000 North Parkway, Memphis, TN 38112, hoffmeister@rhodes.edu), and Jinsong Huang (College of Medicine, The Univ. of Tennessee, Memphis, TN)

Background: Backscatter difference techniques are being developed to detect changes in bone caused by osteoporosis. Backscatter difference techniques compare the power in one portion of an ultrasonic backscatter signal to the power in a different portion of the same signal. Goal: Investigate how backscatter difference measurements depend on the density and microstructural characteristics of cancellous bone. Procedure: Ultrasonic backscatter signals were acquired from 30 specimens of bone using a 1 and $5 \mathrm{MHz}$ broadband transducer. The normalized mean backscatter difference (nMBD) was determined by computing the power difference (in dB) between two gated portions of the backscatter signal and dividing by the center to center time separation between gates. Microstructural characteristics of the specimens and bone mineral density (BMD) were determined using high resolution $\mathrm{x}$-ray micro-computed tomography. Results: nMBD demonstrated moderate to strong linear correlations with microstructure and BMD $(0.50 \leq|R| \leq 0.83)$. The measured correlations did not depend strongly on transducer frequency. Conclusions: The backscatter difference parameter nMBD may be sensitive to changes in microstructure and density caused by osteoporosis. [Work supported by NIH/NIAMS R15AR066900.]

\title{
5:00
}

2pBAa12. Fabrication of bone phantoms using 3D printers. Masahiro Ohno (Faculty of Eng., Chiba Inst. of Technol., Tsudanuma 217-1, Narashino, Chiba 275-0016, Japan, ohno.masahiro@p.chibakoudai.jp)

Requests for making physical models of human body parts, or phantoms, are increasing in accordance with the improvement of medical instruments. Advance in 3D technologies such as 3DCAD and 3D printers has facilitated the fabrication of more precise phantoms. In this paper, we report on our trial to make phantoms of bones that can be used in developing ultrasonic methods of osteoporosis diagnosis. We did not aim to make a complete model that mimics some certain diseased bones, but rather aimed to develop models the parameters of which, such as trabecular thickness, pore size, and its orientation, can be set as freely as possible. Multi-sliced X-ray CT images of bovine bones, those of artificial polymer foam, and purely numerically generated images were used as raw data. These images were converted to 3D data using commercial volume data generation software together with some 3DCAD and intermediate software packages, and finally printed by $3 \mathrm{D}$ printers. The bone-volume/total-volume ratio (BV/TV) was controlled by altering the thresholding value in 3D data construction, as well as by air-blast-polishing printed models. Results of ultrasonic transmission experiments are also shown.

\section{Session 2pBAb}

\section{Biomedical Acoustics and Physical Acoustics: Cavitation in Therapeutic Ultrasound IV: Tissue Fractionation}

\author{
Tatiana D. Khokhlova, Cochair \\ University of Washington, 325 9th Ave., Harborview Medical Center, Box 359634, Seattle, WA 98104 \\ Zhen Xu, Cochair \\ Biomedical Engineering, University of Michigan, 2200 Bonisteel Blvd., Rm. 1107, Gerstacker Bldg., Ann Arbor, MI 48109
}

\section{Contributed Papers}

\section{1:00}

2pBAb1. Erosion of soft tissue by focused ultrasound-induced streaming. Adam D. Maxwell (Dept. of Urology, Univ. of Washington School of Medicine, 1013 NE 40th St., Seattle, WA 98105, amax38@u.washington. edu), Oleg A. Sapozhnikov (Phys. Faculty, Moscow State Univ., Moscow, Russian Federation), Michael R. Bailey, Wayne Kreider (Ctr. for Industrial and Medical Ultrasound, Appl. Phys. Lab., Univ. of Washington, Seattle, WA), Tanya D. Khokhlova (Dept. of Gastroenterology, Univ. of Washington Medical Ctr., Seattle, WA), George R. Schade (Dept. of Urology, Univ. of Washington School of Medicine, Seattle, WA), and Vera A. Khokhlova (Phys. Faculty, Moscow State Univ., Moscow, Russian Federation)

Mechanical erosion of soft tissues into subcellular debris has been demonstrated with pulsed high-intensity focused ultrasound, facilitated by either boiling or cavitation bubbles. In this work, we propose acoustic streaming as a primary cause of tissue erosion at a tissue-fluid interface. Bovine liver tissue and polyacrylamide gels were sonicated in a degassed water bath, with the focus positioned at the tissue/fluid interface. Pulses with duration between 1 to $10^{4} \mu$ s and constant duty cycle of $0.5 \%$ were applied from a 1 $\mathrm{MHz}$ transducer generating focal pressures $\left|p_{-}\right| \leq 17 \mathrm{MPa}$ and $p_{+} \leq 90 \mathrm{MPa}$. Results showed a strongly nonlinear change in erosion with pulse duration, being greatest for pulse lengths between 50-500 $\mu$ s. For longer pulses $(>1$ $\mathrm{ms}$ ), high-speed videos showed streaming velocities $>10 \mathrm{~m} / \mathrm{s}$. Moreover, lesions $>1 \mathrm{~cm}$ in depth were produced in tissue phantoms even with a single pulse and tissue disruption was evident where no bubbles were observed at the tissue-fluid interface. Enhancement of erosion was observed with the presence of bubbles not directly adjacent to tissue but along the beam path, possibly due to bubble-enhanced streaming. [Work supported by NIH R01 
EB007643, T32 DK007779, K01 DK104854, P01 DK043881, and NSBRI through NASA NCC 9-58.]

\section{$1: 15$}

2pBAb2. A multimodal evaluation of boiling histotripsy lesion properties in ex vivo bovine liver. Yak-Nam Wang (APL, CIMU, Univ. of Washington, 1013 NE 40th St., Seattle, WA 98105, ynwang@u.washington.edu), Tanya D. Khokhlova (Gastroenterology, Univ. of Washington, Seattle, WA), Adam D. Maxwell (Urology, Univ. of Washington, Seattle, WA), Wayne Kreider (APL, CIMU, Univ. of Washington, Seattle, WA), Ari Partanen (Philips Healthcare, Washington DC, MD), Navid Farr (BioEng., Univ. of Washington, Seattle, WA), George R. Schade (Urology, Univ. of Washington, Seattle, WA), Valeriy P. Chernikov (Res. Instotue of Human Morphology, Moscow, Russian Federation), Sergey V. Buravkov (Ecological and Extreme Medicine, M.V. Lomonosov Moscow State Univ., Moscow, Russian Federation), Michael R. Bailey (APL, CIMU, Univ. of Washington, Seattle, WA), and Vera A. Khokhlova (Dept. of Acoust., M.V. Lomonosov Moscow State Univ., Moscow, Russian Federation)

New types of high intensity focused ultrasound (HIFU) therapy aiming at mechanical homogenization of tissue has shown great promise, namely, cavitation-cloud histotripsy and boiling histotripsy (BH). $\mathrm{BH}$ uses millisecond-long bursts of HIFU waves containing shocks to repeatedly induce boiling at the focus; the interaction of incident HIFU waves with vapor bubbles homogenizes tissue. In this study, degassed ex vivo bovine liver samples were sonicated using a 256-element $1.2 \mathrm{MHz}$ array of a clinical MR-HIFU system. The BH lesions were produced using 10-ms long pulses with 80 $\mathrm{MPa}$ shocks in situ and pulse repetition frequencies (PRFs) of $1-10 \mathrm{~Hz}$ to cover a range of effects from pure mechanical homogenization to thermal ablation. Individual lesions were generated for the multimodal analysis of the lesion including ultrastructure (electron microscopy), molecular (biochemistry), and microstructure (histological) methods. The extent of homogenization and thermal denaturation was evaluated for each lesion. The results of this work showed that the degree of mechanical tissue disruption and the amount of heat generated in large $\mathrm{BH}$ lesions can be tailored to result in a range of desired tissue effects dependent on the target clinical application. [Work supported by NIH EB007643, RFBR 16-02-00653, K01 EB015745, and NSBRI through NASA NCC 9-58.]

\section{$1: 30$}

2pBAb3. Designing a multi-element array transducer for abdominal boiling histotripsy applications. Pavel Rosnitskiy, Petr Yuldashev (M.V. Lomonosov Moscow State Univ., Moscow, Russian Federation), Adam Maxwell, Wayne Kreider, Michael Bailey (Univ. of Washigton, Seattle, WA), Oleg Sapozhnikov (M.V. Lomonosov Moscow State Univ., Moscow, Russian Federation), and Vera Khokhlova (Univ. of Washigton, $1013 \mathrm{NE}$ 40th St., Seattle, WA 98105, va.khokhlova@gmail.com)

The boiling histotripsy (BH) method to mechanically fractionate tissue using high intensity focused ultrasound relies on the presence of high amplitude shocks. Recently, a method of determining single-element transducer parameters to achieve desired shock amplitudes at the focus was developed. It was shown that the transducer F-number is the main parameter that determines the focal pressure level at which fully developed shocks form. Here, a 256-element HIFU array transducer of $1.5 \mathrm{MHz}$ frequency was designed for abdominal $\mathrm{BH}$ applications using the proposed method. An opening was included at the center of the transducer to fit an ultrasound imaging probe and a compact 16-arm spiral layout of uniformly sized elements was chosen for maximizing the transducer power output. The F-number of the array and the diameter of its elements were determined to satisfy technical limitations on the intensity level at the array elements as well as the required shock amplitudes of 90-100 MPa at the focus assuming $9 \mathrm{~dB}$ attenuation losses in tissue. For creating volumetric lesions, the region of safe and efficient steering of the array focus was evaluated using the T-Array software package (www.limu.msu.ru). [This work was supported by RSF 14-12-00974 and NIH R01 EB7643.]

\section{$1: 45$}

2pBAb4. Characterization of high-amplitude fields of an annular array using acoustic holograms of its individual elements. Petr V. Yuldashev (Phys. Faculty, Moscow State Univ., Leninskie Gory, Moscow 119991, Russian Federation, petr@acs366.phys.msu.ru), Martijn Hoogenboom (Dept. of Radiology, RadboudUMC, Nijmegen, Netherlands), Erik Dumont (Image Guided Therapy, Pessac, France), Martijn H. den Brok (Dept. of Tumor Immunology, RadboudUMC, Nijmegen, Netherlands), Pavel B. Rosnitskiy, Oleg A. Sapozhnikov (Phys. Faculty, Moscow State Univ., Moscow, Russian Federation), Jurgen J. Fütterer (Dept. of Radiology, RadboudUMC, Nijmegen, Netherlands), Gosse J. Adema (Dept. of Tumor Immunology, RadboudUMC, Nijmegen, Netherlands), and Vera A. Khokhlova (Phys. Faculty, Moscow State Univ., Moscow, Russian Federation)

High-intensity focused ultrasound system (Image Guided Therapy, Pessac France) with magnetic resonance guidance was developed and used for evaluation of thermal and mechanical ablation methods in mouse tumors. The system comprises a $3 \mathrm{MHz}$ annular array transducer $(48 \mathrm{~mm}$ diameter and $35 \mathrm{~mm}$ radius of curvature) consisting of 16 elements that allow moving the focus electronically along the transducer axis. Experimental characterization of nonlinear acoustic field generated by such high-frequency strongly focused transducer is technically challenging because of the fine spatial structure of the field, small dimensions of the focal lobe, and limited hydrophone bandwidth. Here we evaluated pressure levels and shock-forming conditions for the system using a combination of numerical modeling based on the $3 \mathrm{D}$ Westervelt equation with holographic boundary condition obtained from low-power measurements. The holograms were measured separately for each element and combined together to obtain a boundary condition for the array with all operating elements. Then, nonlinear field simulations were performed at increasing power output levels. It was shown that the transducer is capable to produce focal waveforms with $120 \mathrm{MPa}$ shock amplitude at $110 \mathrm{~W}$ acoustic power and thus is well suited for shockbased ablation therapies in small animals. [Work supported by Radboudumc Ph.D. grant and RSF-14-12-00974.]

\section{Invited Papers}

2pBAb5. Pathology and immune effects of magnetic resonance imaging-guided Boiling Histotripsy in murine tumor models. Gosse J. Adema (Tumor Immunology, RadboudUMC, RIMLS, Geert Grooteplein 26/28, Nijmegen 6525 GA, Netherlands, gosse. adema@radboudumc.nl), Martijn Hoogenboom (Dept. of Radiology, RadboudUMC, RIMLS, Nijmegen, Netherlands), Renske van den Bijgaart, Dylan Eikelenboom (Tumor Immunology, RadboudUMC, RIMLS, Nijmegen, Netherlands), Pieter Wesseling (Dept Pathol., radboudUMC, RIMLS, Nijmegen, Netherlands), Arend Heerschap (Dept. of Radiology, RadboudUMC, RIMLS, Nijmegen, Netherlands), Martijn den Brok (Tumor Immunology, RadboudUMC, RIMLS, Nijmegen, Netherlands), and Jurgen Futterer (Dept. of Radiology, RadboudUMC, RIMLS, Nijmegen, Netherlands)

In situ tumor ablation techniques are successfully applied for the destruction of local, often inoperable tumor masses. Following ablation tumor antigens become instantly available for immune cells, but systemic abscopal effects have only occasionally been reported after ablation monotherapy. Which ablation technique combines optimal local destruction with effective antigen release for induction of anti-tumor immunity is largely unknown. We study non-invasive MRI-guided high intensity focused ultrasound-ablation (MRgHiFU) in 
murine tumor models for local destruction by heating or mechanical disruption using Boiling histotripsy $(\mathrm{BH})$. $\mathrm{BH}$ mechanically fragmentizes soft tissue into submicron fragments that are absorbed as part of a physiological healing response. BH treatment was performed using a MR compatible animal HIFU system (Image Guided Therapy, Pessac, France) with a $3 \mathrm{MHz}$ transducer (focal spot size $0.5 \mathrm{x} 0.5$ x $2.0 \mathrm{~mm}$ ). A 7T animal MR scanner was used for treatment guidance and evaluation. Here, we will present the pathological response and efficacy of BH treatment in three mouse models with different tumor characteristics: a soft-tissue melanoma (B16OVA), a compact growing thymoma (EL4), and a highly vascularized neuroblastoma (9464D) tumor. Furthermore, the impact of the type of ablation on immune cell infiltration and immune cell activation will be discussed.

$2: 20$

2pBAb6. Healing and the immune response following boiling histotripsy ablation of renal carcinoma in the Eker rat. George R. Schade, Yak-Nam Wang, Kayla Gravelle, Stella Whang, Venu Pillarisetty, Joo Ha Hwang, W. Conrad Liles, Vera Khokhlova, Michael R. Bailey, and Tatiana D. Khokhlova (Dept. of Urology, Univ. of Washington, 5555 14th Ave. NW, Apt. 342, Seattle, WA 98107, grschade@uw.edu)

Boiling histotripsy (BH) is an experimental non-invasive focused ultrasound (FUS) technology that uses milliseconds-long ultrasound pulses at low duty cycle to mechanically homogenize targeted tissue. Here, we report the evolution of BH lesions and the resulting immune response to in vivoablation of renal carcinoma (RCC) in a rat model. RCC bearing Eker rats and syngeneic wild-type rats were randomly assigned to transcutaneous $\mathrm{BH}$ or FUS SHAM procedure targeting $\sim 0.5 \mathrm{cc}$ of RCC or non-tumor bearing normal kidney. $\mathrm{BH}$ was delivered with a 1.5 MHz US-guided small animal FUS system (VIFU-2000, Alpinion) operated at duty cycles of 1-2\%, 10-20 ms pulses, and 525-600 W electric power. Rats were survived for up to 56 days post-treatment. BH lesions evolved from sharply demarcated regions of homogenized tissue to small fibrous scars by 56 days. Compared to sham procedure, $\mathrm{BH}$ produced significant alterations in plasma and intrarenal cytokines and tumor/renal infiltrating leukocyte populations. These data describe the immunologic changes and time course of healing following BH ablation in vivo. Future studies will further address tumor control and the impact on metastases. [The Focused Ultrasound Foundation, The Urology Care Foundation, and NIH K01EB015745 and R01CA154451.]

\section{2:40-2:55 Break}

2pBAb7. Bubble-seeded histotripsy for cancer treatment. Ken-ichi Kawabata (Res. \& Development Group, Hitachi, Ltd., 1-280 Higashi-Koigakubo, Kokubunji, Tokyo 185-8601, Japan, kenichi.kawabata.ap@hitachi.com), Takahi Maruoka, Rei Asami, Hideki Yoshikawa (Res. \& Development Group, Hitachi, Ltd., Tokyo, Japan), and Reiko Ashida (Osaka Medical Ctr. for Cancer and Cardiovascular Diseases, Osaka, Japan)

An approach to applying the histotripsy-like mechanical effects of ultrasound for cancer treatments is studied. We investigated the combinational effects of pulsed ultrasound at intensities used for HIFU (high intensity focused ultrasound) therapy and a type of locally injected superheated perfluorocarbon droplet (PCND). The droplets produce microbubbles when exposed to ultrasound. In our approach, chemical agents are also added to obtain combined mechanical and chemical antitumor effects. It was found that, in the presence of locally injected PCND, spatially controlled mechanical effects similar to histotripsy can be achieved with pulsed ultrasound (pHIFU) at several $\mathrm{kW} / \mathrm{cm}^{2}$ ex vivo. Further, it was suggested that even if the target volume is too vast to distribute PCND with a single injection, one injection is enough because PCND particles migrate inside tissues due to pHIFU. Further, the antitumor effects of pHIFU in combination with the local injection of PCND and an antitumor agent (Adriamycin) were investigated in vivo using murine tumors (Colon 26). Experiments were performed under acoustic conditions and with drug doses not severe enough to induce significant antitumor effects. It was found that PCND and Adriamycin were effective at suppressing tumor growth and tumor regrowth after suppression, respectively. The obtained results are very promising for developing novel cancer treatments.

\section{Contributed Papers}

\section{3:15}

2pBAb8. A method for phase aberration correction of high intensity focused ultrasound fields using acoustic nonlinearity. Tatiana Khokhlova, Adam Maxwell, Wayne Kreider, Vera Khokhlova, Matthew O'Donnell, and Oleg Sapozhnikov (Univ. of Washington, 325 9th Ave., Harborview Medical Ctr., Box 359634, Seattle, WA 98104, tdk7@uw.edu)

High intensity focused ultrasound (HIFU) therapies are often affected by aberrations induced by soft tissue heterogeneities. To mitigate these aberrations, phase corrections at different elements of a HIFU transducer array can be introduced, if suitable feedback is available. Existing feedback approaches include maximization of focal tissue displacement induced by acoustic radiation force or scattering from a cavitation bubble nucleated at the focus. Here we propose an aberration correction method based on backscattering of strongly nonlinear HIFU waves that are present only at the focus. A $1.5 \mathrm{MHz}, 12$-element sector array was integrated with a coaxially aligned imaging probe (P7-4). Each element of the array emitted a short, high-amplitude pulse through the aberrative layer toward the focus in tissue, and the imaging probe recorded the backscattered signal at higher frequencies. The focusing gain of each sector element was sufficient to generate a highly nonlinear focal waveform, which allowed localizing the "beacon" for phase correction feedback. The relative transmit time delays for array elements were calculated based on cross-correlations between the nonlinear backscattered signals corresponding to the other elements. The results of hydrophone measurements and ex vivo tissue experiments demonstrate the feasibility of the proposed approach. [Work was supported by NIH K01EB015745 and R01EB7643.]

$$
3: 30
$$

2pBAb9. Histotripsy pulse-reflection for 3D image forming and bubble cloud localization in transcranial applications. Jonathan R. Sukovich, Zhen Xu, Timothy L. Hall, Jonathan J. Macoskey, and Charles A. Cain (Biomedical Eng., Univ. of Michigan, 1410 Traver Rd., Ann Arbor, MI 48105, jsukes@umich.edu)

Here, we present results from experiments using histotripsy pulses scattered off the surface of the skull, as well as bubble clouds generated within, to reconstruct $3 \mathrm{D}$ images of the exterior skull surface and localize bubbles within. These capabilities have the potential to provide image coregistration and real-time ultrasound monitoring for transcranial histotripsy treatment, without the need for MRI guidance. Histotripsy pulses were delivered to an 
ex vivo human skullcap mounted centrally within a $500 \mathrm{kHz}, 256$-element histotripsy transducer with transmit-receive capable elements. Straight-line ray tracing approximations based on the times-of-flight of the emitted pulses and the known geometry of the array were used to calculate points on the skull surface and to localize bubble clouds generated within. Using these methods, we were able to accurately locate and orient the skull within the array and generate a 3D map of its surface for coregistration with an a priori $3 \mathrm{D}$ scan. The points calculated based on the pulse-reflection from the skull were found to be within $1.25 \mathrm{~mm}$ of the surface measured in the a priori 3D scan. Using these signals, the calculated centroid of the generated bubble clouds was likewise found to be within 500 um of the measured focal point of the array through the skull.

\section{Invited Papers}

\section{$3: 45$}

2pBAb10. Monitoring and guidance of histotripsy using ultrafast imaging. Bastien Arnal (Institut Langevin, ESPCI Paris, INSERM U979, CNRS UMR7587, 17 rue Moreau, Paris 75012, France, bastien.arnal@gmail.com), Wei-Ning Lee (Institut Langevin, ESPCI Paris, INSERM U979, CNRS UMR7587, Hong Kong, Hong Kong), Mathieu Pernot, and Mickael Tanter (Institut Langevin, ESPCI Paris, INSERM U979, CNRS UMR7587, Paris, France)

To use histotripsy safely, it is required to visualize the cavitation clouds and the lesion formation in real-time. Conventional ultrasound imaging (USI) is a good candidate for the real-time monitoring of the procedures. However, bubble clouds are not always clearly visible in vivo using USI because of flows, motion or high echogenicity of the target region. Ultrafast imaging, based on plane wave or diverging waves sonications, is able to record a larger amount of data, providing broad information about how signals originating from different sources vary in time. Here, we apply a spatiotemporal singular value decomposition of ultrafast acquisitions that enables the discrimination of cavitation bubbles from moving tissue in vivo. An automation to determine the filtering parameters will be introduced. Moreover, USI depicts the histotripsy lesion only at an advanced stage and can be hindered by the presence of dissolving gas in the tissue. As the tissue microstructure is greatly affected by the treatment, a high stiffness contrast is observed. We will show that quantitative stiffness evaluation allows measuring tissue damage from the beginning of the treatment with a 2 to 6 -fold contrast increase compared to USI.

\section{4:05}

2pBAb11. Comparison of passive cavitation imaging and plane wave B-mode imaging for monitoring histotripsy ablation. Kenneth B. Bader, Kevin J. Haworth (Internal Medicine, Univ. of Cincinnati, 231 Albert Sabin Way, CVC 3935, Cincinnati, OH 452670586, kenneth.bryan.bader@gmail.com), Adam D. Maxwell (Dept. of Urology, Univ. of Washington, Seattle, WA), and Christy K. Holland (Internal Medicine, Univ. of Cincinnati, Cincinnati, $\mathrm{OH}$ )

Histotripsy utilizes the oscillations of bubbles within a cloud for transcutaneous mechanical ablation of tissue and is currently under development to treat benign prostatic hyperplasia. The bubble clouds are hyperechoic, enabling standard B-mode imaging to be used for image guidance. The volumetric oscillations of the bubbles also generate acoustic emissions that can be imaged. The purpose of this study was to demonstrate the feasibility of monitoring histotripsy-induced ablation with passive cavitation images. Prostate tissue phantoms were exposed to mechanically ablative, $1-\mathrm{MHz}$ histotripsy pulses over a range of pulse durations (5-20 ms) and peak negative pressures (12-23 MPa). Bubble clouds generated by the histotripsy pulses were monitored with a linear array using passive cavitation imaging (PCI) and plane wave B-mode imaging. The utility of PCI and B-mode imaging to predict the phantom ablation zone was assessed using receiver operating characteristic (ROC) curve analysis. The area under the ROC curve, accuracy, and sensitivity were greater for PCI relative to B-mode imaging $(p<0.05)$. These results will be discussed in the context of the potential advantages of PCI relative to B-mode imaging for the prediction of histotripsy-induced ablation.

\section{Contributed Papers}

\section{$4: 25$}

2pBAb12. Effects of temperature on the histotripsy intrinsic threshold for cavitation. Eli Vlaisavljevich, Zhen Xu (Univ. of Michigan, 1111 Nielsen Ct. Apt. 1, Ann Arbor, MI 48105, evlaisav@umich.edu), Adam Maxwell (Univ. of Washington, Seattle, WA), Lauren Mancia, Xi Zhang, Kuang-Wei Lin, Alexander Duryea, Jonathan Sukovich, Tim Hall, Eric Johnsen, and Charles Cain (Univ. of Michigan, Ann Arbor, MI)

A histotripsy cavitation cloud can be formed by a single acoustic pulse with one high amplitude negative cycle, when the negative pressure exceeds a threshold intrinsic to the medium. The intrinsic threshold in water-based soft tissues, which is similar to the intrinsic threshold of water, has been experimentally verified in the range of 24-30 MPa over a frequency range of $0.3-3 \mathrm{MHz}$ at $20^{\circ} \mathrm{C}$. In this study, the effects of temperature on the intrinsic threshold was investigated both experimentally and theoretically. Single pulses with one high amplitude negative cycle at $1 \mathrm{MHz}$ were applied to distilled, degassed water at temperatures ranging from $10^{\circ} \mathrm{C}-90^{\circ} \mathrm{C}$. Cavitation was detected and characterized by passive cavitation detection and highspeed photography, from which the probability of cavitation was measured vs. pressure amplitude. The results indicated that the intrinsic threshold significantly decreases with increasing temperature, showing a nearly linear decreasing trend from $29.8 \pm 0.4 \mathrm{MPa}$ at $10^{\circ} \mathrm{C}$ to $14.9 \pm 1.4 \mathrm{MPa}$ at $90^{\circ} \mathrm{C}$. Overall, this study supports our hypothesis that the intrinsic threshold is highly dependent upon the temperature of the medium, which may allow for better predictions of cavitation generation at body temperaturein vivo and at the elevated temperatures commonly seen in high intensity focused ultrasound (HIFU) regimes.

\section{$4: 40$}

2pBAb13. The effects of heat and mass transfer on free oscillations of a bubble in a viscoelastic, tissue-like medium. Eric Johnsen and Carlos Barajas (Univ. of Michigan, 1231 Beal Ave., Ann Arbor, MI 48109, ejohnsen@ umich.edu)

Free oscillations of a bubble in soft tissue is of relevance to a variety of diagnostic and therapeutic ultrasound applications. Heat and mass transfer effects have been explored in the context of bubble oscillations in water; however, the extent to which they influence bubble oscillations in soft materials is presently unknown. Our objective is to use numerical modeling to predict bubble oscillations in viscoelastic, tissue-like media, while 
accounting for heat and mass transfer. We numerically solve the KellerMiksis equation to compute the Rayleigh collapse of a spherical bubble in a Kelvin-Voigt viscoelastic medium with finite-strain elasticity. The energy equation is solved inside and outside the bubble, and a mass conservation equation is solved for the vapor inside the bubble; equilibrium phase change is assumed. Using linear, small-amplitude perturbation theory, we investigate the bubble response. We show how the damping and oscillatory behavior depends on the eigenvalues of the full system; in particular, the time constant does not follow a monotonic relationship with respect to shear modulus. We also identify regimes in which solving the fully partial differential equations for energy and vapor concentration is not necessary. Finally, we interpret our results in the context of ultrasound applications.

\section{$4: 55$}

2pBAb14. Modeling tissue response to a cavitation bubble in histotripsy. Lauren Mancia (Mech. Eng., Univ. of Michigan, 2016 Walter E. Lay Automotive Lab, Ann Arbor, MI 48109-2133, lamancha@umich.edu), Eli Vlaisavljevich, Zhen Xu (Biomedical Eng., Univ. of Michigan, Ann Arbor, MI), and Eric Johnsen (Mech. Eng., Univ. of Michigan, Ann Arbor, MI)

Histotripsy is a noninvasive focused ultrasound procedure that uses cavitation bubbles generated by high-amplitude ultrasound pulses to mechanically homogenize soft tissue. Experimental studies of histotripsy-induced cavitation in tissue phantoms and animal models have shown that tissue mechanical properties such as viscosity and elasticity affect cavitation threshold and bubble behavior. At present, however, the mechanisms responsible for tissue damage observed in histotripsy and other cavitation-inducing ultrasound treatments remain difficult to quantify. In this study, we simulated the dynamics of a single, spherical bubble in a Kelvin-Voigt-based viscoelastic solid, with nonlinear elasticity to better represent nanometer to micron-scale bubble growth. We applied the numerical model to calculate stress, strain, and strain rate distributions produced by a cavitation bubble exposed to forcing representative of a tensile histotripsy cycle. We found that stress and strain in excess of the ultimate tensile strength and fractional strain of most soft tissues occur at the bubble wall and decrease by at least two orders of magnitude within 50 microns from the bubble. Tissue mechanical properties were found to affect the magnitudes of stress and strain developed at different distances from the bubble. We will relate these results to experimentally observed correlates of tissue damage.

\section{5:10}

2pBAb15. Investigation of mechanical and dynamic characteristics for multifunctional contrast agents using atomic force microscopy and acoustic assessments. Gepu Guo, Qingyu Ma (School of Phys. and Technol., Nanjing Normal Univ., 1 Wenyuan Rd., Qixia District, Nanjing, Jiangsu 210023, China, guogepu@njnu.edu.cn), Juan Tu, Dong Zhang (Inst. of Acoust., Nanjing Univ., Nanjing, Jiangsu, China), and Shaotong Feng (School of Phys. andTechnol., Nanjing Normal Univ., Nanjing, Jiangsu, China)

Although multi-parameter fitting algorithms are often used for the characterization of coated-bubbles, it is inevitable to introduce uncertainty into the results. Therefore, it is urgent to develop some improved techniques to analyze the mechanical properties of the multifunctional microbubbles (MBs) accurately and systematically. By combining the measurements of atomic force microscopy, optical and acoustic detection with the simulations of coated-bubble dynamics, a comprehensive technology was developed to determine the size distribution, shell thickness, elasticity, and viscosity of multifunctional MBs precisely. Moreover, the impact of magnetic nanoparticles (MNPs) concentration on the multifunctional MBs' dynamic properties was studied systematically. It is demonstrated that, with the increasing MNPs concentration, the MB mean diameter and shell stiffness increased and ultrasound scattering response and inertial cavitation activity could be significantly enhanced. Although the shell thickness does not depend on the MNPs concentration, the increased MNPs concentration would generally result in enhanced bulk modulus and reduced bulk viscosity. The results of current studies demonstrate that the proposed single-parameter evaluation method could be helpful for further design and development of MB agents in clinic applications. 


\title{
Session 2pEAa
}

\section{Engineering Acoustics: Acoustic Transduction Material, Sensors, and Array Technologies}

\author{
Dehua Huang, Cochair \\ NAVSEANPT, Howell St., Newport, RI 02841 \\ Yasuhiro Oikawa, Cochair \\ Department of Intermedia Art and Science, Waseda University, 3-4-1 Okubo, Shinjuku-ku, Tokyo 169-8555, Japan
}

Invited Papers

1:00

2pEAa1. A nanotube thermophone projector array. Benjamin Dzikowicz, James F. Tressler, and Jeffery W. Baldwin (Naval Res. Lab., 4555 Overlook Ave. SW, Washington, DC 20375, ben.dzikowicz@nrl.navy.mil)

Thermophone projectors fabricated using newly available nanoscale materials as elements hold the promise of a new transducer technology for the Navy. With no moving parts, they can operate over a broad frequency range and can be designed to be lighter and thinner than competing technologies. Scaling arguments and numerical models are explored to understand the parameter space for a gasenclosed thermophone applicable to micro- and nanoscale elements. These models led to the development of an element made from a sparse horizontal array of single-walled nanotubes. Experiments are performed in NRL's Laboratory for Structural Acoustics using a specifically designed enclosure for underwater operations. Results and analysis of experiments using various fill gasses and internal pressures will be presented. [Work supported by NRL.]

$1: 20$

2pEAa2. Investigation of carbon nanotubes for acoustic transduction applications. Thomas R. Howarth, Dehua Huang, Christian R. Schumacher, Nathanael K. Mayo, Donald L. Cox, Jeffrey E. Boisvert (NAVSEA Div. Newport, 1176 Howell St., B1346 R404A, Newport, RI 02841, thomas.howarth@navy.mil), Ali E. Aliev, and Ray H. Baughman (Alan G. MacDiarmid NanoTech Inst., The Univ. of Texas at Dallas, Richardson, TX)

Traditional acoustic transduction sources typically begin with the generation of an electrical excitation pulsed through an amplifier into an electroacoustic material to create a mechanical vibration which is then converted into an acoustic wave to produce sound. The lower the preferred transmitting frequency (and hence, longer acoustic range) desired, the larger the required size of the source. Often this means that for acoustic projectors producing sound at frequencies below a few $\mathrm{kHz}$, that the electroacoustic device will need to be very large in order to produce very long sound waves. This has a limitation for incorporating low frequency sonars on smaller autonomous underwater vehicles (AUVs). The topic of our presentation is an acoustic source technology that relies on the conversion of thermal energy to acoustic energy. Though the concept was first demonstrated in 1917, the recent advent of carbon nanotubes (CNT) now makes it possible to transmit acoustic waves in small and affordable packages using the thermophone approach. The presentation begins with an overview of thermophones and a method for incorporating the CNTs into a useable transduction device. Discussions will include detailing on-going efforts for the encapsulation, packaging and further insight of the thermoacoustic energy conversion process. Recent 2016 experimental data will be shown. New start numerical and analytical modeling efforts and future development thrusts will be discussed.

\section{$1: 40$}

2pEAa3. Thermophone modeling. Donald L. Cox, Thomas R. Howarth, Christian R. Schumacher, and Nathanael K. Mayo (Naval Undersea Warfare Ctr., 1176 Howell St., Newport, RI 02841, donald.1.cox@navy.mil)

The use of carbon nanotube (CNT) films encapsulated within a pouch design pressurized with an inert gas has demonstrated promise for underwater sound projection. To augment this experimental demonstration, a modeling approach has been undertaken to capture the requisite physics and eventually provide a design tool to optimize performance. The modeling approach makes use of the finite element method as programmed in the commercially available COMSOL software. A multi-physics approach is taken where models are coupled that include: electrically stimulated heat, heat transfer to a compressible, viscous fluid, structural response of a thin shell to this computational fluid domain, and external acoustic propagation from the outer surface of the structure. The intent is to capture the physical response of the sensor tested and understand the interaction dynamics of the various media. The multiphysics solution approach taken in this work involves the use of a segregated, time dependent solution of coupled, linear and nonlinear problems. As a result, the process is complicated and computationally intensive. Modeling results will be presented and compared with experimental data. 
2pEAa4. A design of broadband constant beam pattern transducers. Dehua Huang (NAVSEANPT, Howell St., Newport, RI 02841, DHHuang@cox.net)

A constant beam pattern (CBP) transducer is an acoustic transducer, where its beam patterns are independent of frequency. The theory and numerical simulations for constant beam pattern transducer design are introduced. For a hemispherical design, if the radial velocity distribution on the surface of a conventional hemisphere transducer is a single complete Legendre polynomial, the far field angular beam pattern shows the same Legendre polynomial distribution for all frequencies above its cut-off frequency, based on spherical Hankel function asymptotic approximation to the solutions from Helmholtz wave equation. Because of orthogonality, Legendre polynomials form a complete set, per Sturm-Liouville theory that an arbitrary velocity shading function can be expanded by Legendre series. Each polynomial within this Legendre series contributes its share to the far field, such that the converging acoustic beam pattern displays the same shape as the original shading function itself. Numerical simulations with various samples include $(\cos (\text { theta }))^{\wedge} 3$ and Gaussian, a well as equal sidelobe suppression classic Dolph-Chebyshev functions used as shading and achievable as beam patterns for a broadband frequency range.

2pEAa5. Optical sensing of sound fields: Non-contact, quantitative, and single-shot imaging of sound using high-speed polarization camera. Kenji Ishikawa, Kohei Yatabe, Yusuke Ikeda, Yasuhiro Oikawa (Intermedia Art and Sci., Waseda Univ., Ohkubo 3-4-1, Bldg 59, 407-2, Shinjuku-ku, Tokyo 169-8555, Japan, k-ishikawa@fuji.waseda.jp), Takashi Onuma, Hayato Niwa (Photron Ltd., Chiyoda-ku, Tokyo, Japan), and Minoru Yoshii (Kiyohara Optics, Shinjuku-ku, Tokyo, Japan)

Imaging of a sound field aids understanding of the actual behavior of the field. That is useful for obtaining acoustical spatial characteristics of transducers, materials and noise sources. For high spatial resolution imaging, optical measurement methods have been used, thanks to its contactless nature. This paper presents sound field imaging method based on parallel phase-shifting interferometry, which enables to acquire an instantaneous two-dimensional phase distribution of light. Information of sound field is calculated from the phase of light based on the acousto-optic theory. The system consists of a polarization interferometer and high-speed polarization camera, whose measurement points of a single image are $512 \times 512$ and spatial resolution is about $0.2 \mathrm{~mm} \times 0.2 \mathrm{~mm}$. Therefore, the system can image a sound field with much higher spatial resolution compared with conventional imaging methods. The maximum framerate, which is corresponding to the sampling frequency, is $1.55 \mathrm{M}$ frames per second. This paper contains the principle of optical measurement of sound, the description of the system, and several experimental results including imaging of a transducer-generated sound field and propagation and reflection of a sound wave.

\section{2:40}

2pEAa6. Study to fabricate high-quality and portable parametric speakers. Jun Kuroda (Dept. of Intermedia Art and Sci., Waseda Univ., 3-4-1 Ohkubo, Tokyo 169-8555, Japan, jun-kuroda@suou.waseda.jp), Shota Minami, and Yasuhiro Oikawa (Dept. of Intermedia Art and Sci., Waseda Univ., Shinjuku-ku, Tokyo, Japan)

Parametric speakers provide narrow directional audible sounds using modulated ultrasounds. Parametric speakers are employed to build audio communication with privacy protection, which is expected to be installed into various instruments. To respond to this requirement, slim-sized parametric speakers delivering high-quality sound must be fabricated. Ultrasonic transducers usually consist of piezoelectric elements and metal resonators, which have a number of resonant modes. To demodulate audible sounds by the non-linearity of a finite amplitude sonic wave, a large amplitude carrier wave must be emitted. Therefore a predominant resonant mode of an ultrasonic transducer is used to transmit carrier wave. Moreover, frequency band around the predominant resonance is used to transmit modulated Fourier components of signals. While considering these principals of sonic transmission, two issues must be solved to fabricate a slim-sized and high quality parametric speaker, i.e., 1) widening of available bandwidth and improvement of electroacoustic efficiency and 2) optimization of transmitter circuits and modulation method based on characteristics of ultrasound transducers. It is necessary to study parametric speakers as a total system comprised by ultrasound transducers, transmit circuits, and signal processing of modulation, to solve these issues. This paper denotes outline of these issues and the present status of our studies. 


\title{
Session 2pEAb
}

\section{Engineering Acoustics: Ocean Acoustics Analysis}

\author{
Robert Taylor, Cochair \\ Mechanical Engineering, The Catholic University of America, 620 Michigan Ave. NE, Washington, DC 20064 \\ Veronica Koh, Cochair \\ Graduate Program in Acoustics, Pennsylvania State University, 201 Applied Science Building, University Park, PA 16802
}

\section{Contributed Papers}

\section{3:15}

2pEAb1. Numerical investigation on transmission loss of sound above sea surface. Robert Taylor, Diego Turo (Mech. Eng., The Catholic Univ. of America, 620 Michigan Ave. NE, Washington, DC 20064, 45taylor@cua. edu), Teresa Ryan (Mech. Eng., Eastern Carolina Univ., Greenville, NC), and Joseph Vignola (Mech. Eng., The Catholic Univ. of America, Washington, DC)

Determination of the transmission loss of sound travelling over the ocean with various sea states involves a number of variables. One method to approximate the random roughness of the surface is to use the variance spectrum model as proposed by Pierson and Moskowitz. A numerical solution of the wave equation is presented for a small elevation angle over the sea surface at different sea states. This new model has the capability to include both the thermal gradient and wind profile above the sea. The solutions are derived with an implementation of the Crank-Nicolson Parabolic Equation, and the ocean wave roughness profile is introduced into the numerical model by the Generalized Terrain Parabolic Equation method. These results are used to investigate the effect of the sea roughness on the sound transmission loss for different sea states.

\section{3:30}

2pEAb2. Study of two-dimensional underwater particle velocity pickup sensor. Zhao Tianji (College of Underwater Acoust. Eng., Harbin Eng. Univ., No. 145 Nantong St., Nangang District, Harbin, Heilongjiang 150001, China, ztj13613621886@126.com)

Addressing the subject of a suspended co-oscillating vector hydrophone application platform, a new sound wave receiving theory model of underwater particle velocity pickup sensor was established. Based on the study of the inner sound field of an elastic cylinder vibrating freely under the action of sound waves in theory, the influence of material and geometry parameters on the frequency response of particle velocity has been analyzed. According to the results of parameter optimization and taking into account engineering needs, one sample of two-dimensional particle velocity pickup sensor has been designed and tested. By comparing the results of sensitivity and directivity tested in a calibration device, the general rules of theoretical analysis have been verified. The theory and experiments confirmed the feasibility of particle velocity pickup sensors in engineering applications.
$3: 45$

2pEAb3. Adaptive statistical learning models for long-range sound propagation. Carl R. Hart, D. K. Wilson (U.S. Engineer Res. and Development Ctr., 72 Lyme Rd., Hanover, NH 03755, carl.r.hart@usace.army.mil), Chris L. Pettit (Aerosp. Eng. Dept., U.S. Naval Acad., Annapolis, MD), and Edward T. Nykaza (U.S. Engineer Res. and Development Ctr., Champaign, IL)

Uncertainties in source characteristics, meteorological conditions, and topographic variations present formidable challenges for accurately predicting long-range outdoor sound propagation. Numerical propagation models inherently assume perfect knowledge of these uncertain variables and are fixed in a modeling sense. In contrast, statistical learning models can incorporate new observations to update the underlying prediction model. Past work has shown that statistical learning models trained on synthetic data for predicting long-range sound propagation have, at best, an overall rootmean-square error (RMSE) of about $5 \mathrm{~dB}$. This limit appears to be imposed by modeled atmospheric turbulence. It is hypothesized that this prediction limit may be lowered as observational data are incorporated into trained statistical learning models. Furthermore, data are assimilated by a Kalman filtering process for the purpose of updating knowledge of the atmospheric and source characteristics. Within the prediction phase three different statistical learning models are compared: an ensemble neural network, a clusterweighted model, and a random forest regression model. The efficacy of data assimilation is evaluated with respect to each statistical learning model.

\section{4:00}

2pEAb4. Hydrophone calibration system. Alper Biber and Ata C. Corakci (Matererials, Tubitak Mam, Dr. Zeki Acar Cad., Gebze, Kocaeli 41470, Turkey, alper.biber@tubitak.gov.tr)

A system to calibrate underwater electro-acoustic hydrophones by primary method, namely, Reciprocity method, in wide frequency range from few kilohertz to megahertz is presented. The aim is to realize primary level "Hydrophone Calibration System" covering from few kilohertz to upper limit of underwater applications with very limited restrictions in one simple system. This system not only covers wide frequency range of underwater applications but also offers low-cost solution to reach enough uncertainty level required for primary calibration according to standards [1]-[3]. The system is based on very high resolution PC oscilloscope of Pico Technology, namely, PicoScope 4262, used for primary data acquizition and digitizing. Primary signal generator and power amplifier, available in market, are integrated into system for purpose. The detailed description is presented and uncertainty budget is discussed. 
2pEAb5. Design improvements of a low cost underwater projector based on clarinet acoustics. Veronica Koh and Stephen C. Thompson (Graduate Program in Acoust., Penn State Univ., 201 Appl. Sci. Bldg., University Park, PA 16802, vwk102@psu.edu)

Low frequency $(\mathrm{ka}<<1)$ underwater projectors are desired for long range sonar applications and oceanography due to their low absorption losses. However, such low frequency projectors (LFP) tend to be large in size and costly to build. This study aims to assess the feasibility of an underwater clarinet as a low cost LFP. Previous proof-of-concept work on a lab prototype showed stable tone production but with resonance frequencies that were lower than predicted and significant harmonic levels. In this study, a new pressure chamber design for housing the clarinet mouthpiece is built and tested. Improvements in the equivalent circuit model for the underwater clarinet are also made to include the elastic effects of the clarinet wall and the pressure chamber impedance, to better predict its performance. The current measurement results using both PVC and steel resonator for the underwater clarinet will be presented and compared with the improved model. The trade-off considerations between the design parameters of the underwater clarinet and its performance will also be discussed.

\section{4:30}

2pEAb6. Comparison of computational schemes in the design of a large-scale projector array. Eunghwy Noh, Wonjong Chun, Won-Suk Ohm (School of Mech. Eng., Yonsei Univ., Eng. Bldg. A391, 50 Yonsei-ro, Seodaemun-gu, Seoul 120-749, South Korea, hwyya@yonsei.ac.kr), Woosuk Chang, and Hongwoo Youn (Maritime, LIG Nex1, Seongnam, South Korea)

Modeling and simulation for the design of a projector array involves computations in the transducer and acoustic domains. In the transducer domain, each transducer is often described by the two-port, equivalent-circuit model, in which the radiation impedance containing the influence of the neighboring transducers as well as the medium is not known a priori. To obtain the radiation impedance and the subsequent transducer response a set of coupled transducer equations must be solved simultaneously in conjunction with the (computationally expensive) wave-field calculation in the acoustic domain. In this talk, we compare two computational schemes for speed, which differ in the way the mutual interaction of transducers is treated: the first scheme explicitly accounts for the mutual interaction in terms of the mutual impedance, whereas in the second scheme the interaction is reflected implicitly in the iterative solution of the transducer equations. Here, the speed is largely determined by the number of wave-field calculations required for the desired accuracy. Comparison is made for the case of a cylindrical array of 288 tonpilz transducers. [This work was sponsored by LIG Nex1 Co., Ltd.]
2pEAb7. Active control of low-frequency underwater echoes using an array of tile projectors. Wonjong Chun, Eunghwy Noh, Won-Suk Ohm (School of Mech. Eng., Yonsei Univ., 50 Yonsei-ro, Seodaemun-gu, Seoul 120-752, South Korea, wonjong.chun@yonsei.ac.kr), Jaepil Kim, Youna Ji, Youngcheol Park (Comput. \& Telecommunications Eng. Div., Yonsei Univ., Wonju, Gangwon-do, South Korea), and Youngsoo Seo (Agency for Defense Development, Changwon, Gyeongsangnam-do, South Korea)

With the introduction of low-frequency active sonar in anti-submarine warfare, there is a growing need for a novel underwater invisibility device that could replace the existing passive anechoic tiles. In this talk, we describe an experiment on active reduction of underwater echoes at low frequencies using an array of tile-shaped projectors. Each tile projector was designed with the aid of finite-element computations and tested in an acoustic tank for transmit voltage sensitivity and directivity. An array of tile projectors, covering a scale model submarine in a large acoustic tank, were driven by control signals that were intended to produce the impedance match between water and the object. Depending on the frequency of the incident wave, echo reduction as large as $6 \mathrm{~dB}$ was achieved. [This work has been supported by the Low Observable Technology Research Center program of Defense Acquisition Program Administration and Agency for Defense Development.]

\section{5:00}

2pEAb8. Underwater parametric array source transducer composed of PZT rods and thin polymer plate with high power efficiency for wideband sound generation. Hongmin Ahn, Yonghwan Hwang, and Wonkyu Moon (Mech. Eng., POSTECH, Hyoja-dong, Nam-gu, Pohang, Gyeongsangbuk-do 790784, South Korea, idealcircuit@postech.ac.kr)

Nowadays 1-3-type PZT composite transducers are widely used for underwater parametric array (PA) sound source since they can generate highly directional acoustic beam with high intensity, which is the requirements for the PA acoustic phenomenon that results from nonlinearity of a medium. Thanks to the relatively small mechanical quality factor of 1-3 PZT composite, the transducer using it can generate high intensity sounds by relatively low input voltage in the relatively wide frequency band around its resonance frequency. The low mechanical quality factor, however, increase the internal mechanical loss inside the transducer. Consequently, the acoustic radiation efficiency may be lower than expected. In this paper, a dual-resonant-frequency PZT rods are integrated into a thinner polymer plate. Then, the radiation surface increases to enhance radiation power efficiency. The PZT rods with two different lengths, are integrated in the process of molding a polymer plate. The fabricated transducer are operated with the out-of-phase driving method for a dual resonance transducer to generate direct sound beam with a wide frequency bandwidth. The sound pressure levels (SPLs) of the primary waves of the first and second resonant frequencies were $197 \mathrm{~dB}$ and $203 \mathrm{~dB}(\mathrm{re}=1 \mu \mathrm{P})$, respectively, at $9 \mathrm{~m}$. The acoustic radiation efficiencies at $98 \mathrm{kHz}$ and $135 \mathrm{kHz}$ were $50 \%$ and $33 \%$, respectively. The DFW generated by the PA had an SPL of $150 \mathrm{~dB}$ (re = $1 \mu \mathrm{P} @ 30 \mathrm{kHz}$ ) and a high directivity of $3.4^{\circ}$ half-power beam width. 


\title{
Session 2pMU
}

\section{Musical Acoustics and Signal Processing in Acoustics: Music Signal Processing II}

\author{
Masataka Goto, Cochair \\ National Institute of Advanced Industrial Science and Technology (AIST), IT, AIST, 1-1-1 Umezono, Tsukuba 305-8568, \\ Japan \\ James W. Beauchamp, Cochair \\ School of Music and Electrical \& Computer Eng., University of Illinois at Urbana-Champaign, 1002 Eliot Dr., Urbana, \\ IL 61801-6824
}

Invited Papers

$1: 15$

2pMU1. Songle Widget: A web-based development framework for making animation and physical devices synchronized with music. Masataka Goto (National Inst. of Adv. Industrial Sci. and Technol. (AIST), IT, AIST, 1-1-1 Umezono, Tsukuba, Ibaraki 3058568, Japan, m.goto@aist.go.jp), Kazuyoshi Yoshii (Kyoto Univ., Kyoto, Japan), and Tomoyasu Nakano (National Inst. of Adv. Industrial Sci. and Technol. (AIST), Tsukuba, Ibaraki, Japan)

This paper describes a web-based multimedia development framework, Songle Widget (http://widget.songle.jp), that makes it possible to control computer-graphic animation and physical devices such as lighting devices and robots in synchronization with music publicly available on the web. Development of applications featuring rigid synchronization with music playback on the web was difficult because audio signals of the playback are not accessible on a web browser and manual annotation is time-consuming. To overcome such difficulties, Songle Widget makes it easy to develop web-based applications with rigid music synchronization by leveraging musicunderstanding technologies based on signal processing. Songle Widget is implemented by using our public web service called Songle (http://songle.jp) that automatically analyzes songs on the web and annotates four important types of musical elements (music structure, hierarchical beat structure, melody line, and chords). More than 1,000,000 songs on music- or video-sharing services have been analyzed by Songle and can readily be used by music-synchronized applications. Since errors are inevitable when elements are annotated automatically, Songle Widget takes advantage of a Songle's crowdsourcing interface that enables users to correct errors. This is effective when applications require error-free annotation. We made Songle Widget open to the public, and its capabilities and usefulness have been demonstrated.

1:35

2pMU2. Rethinking audio production tools. Bryan Pardo (EECS, Northwestern Univ., 2133 Sheridan Rd., Evanston, IL 60208, pardo@northwestern.edu)

Potential users of audio production software, such as equalizers and reverberators, may be discouraged by the complexity of the interface and a lack of clear affordances in typical interfaces. We seek to simplify interfaces for audio production (e.g., mastering a music album with ProTools), audio tools (e.g., equalizers), and related consumer devices (e.g., hearing aids). Our approach is to center the interaction around user-provided examples, an evaluative paradigm ("I like this sound better than that sound") and descriptive language (e.g., "Make the violin sound "warmer."). To build interfaces that use descriptive language, a system must be able to tell whether the stated goal is appropriate for the selected tool (e.g. making the violin "warmer" with a panning tool does not make sense). If the goal is appropriate for the tool, it must know what actions need to be taken (e.g., add some reverberation). Further, the tool should not impose a vocabulary on users, but rather understand the vocabulary users prefer. In this talk, Prof. Pardo describes recent work in evaluative interfaces, crowdsourcing a vocabulary for language-based production tools, and language-based interfaces for production tools.

\section{$1: 55$}

2pMU3. Smart loop sequencer: An audio-based approach for ease of music creation. Tetsuro Kitahara (Nihon Univ., 3-25-40, Sakurajosui, Setagaya-ku, Tokyo 1568550, Japan, kitahara@chs.nihon-u.ac.jp)

A loop sequencer is expected to be a good tool for non-musicians to compose music. However, it is not easy to appropriately select music loops because a loop sequencer usually has a large scale of loop collection. In this paper, we propose a smart loop sequencer that automatically selects music loops based on the degree of excitement input by the user. The user expresses a temporal evolution of a desired excitement as a continous curve using the mouse of the PC. Then, the system searches for a sequence of music loops that generates the most similar excitement to the user's input. This process is formulated with a hidden Markov model (HMM). A combination of music loops is regarded as a state of the HMM, and an excitement input by the user is regarded as an emission value. Given a sequence of excitement, the most likely combination of music loops for every measure is estimated with the Viterbi algorithm. Experimental results show that non-experts of music easily compose musical pieces with our system. 
2pMU4. Audio scene transformation using informed source separation. Sylvain Marchand (Univ. of La Rochelle, Laboratoire Informatique, Image et Interaction (L3i), Ave. Michel Crepeau, La Rochelle 17042, France, sylvain.marchand@univ-lr.fr)

Giving the listener the freedom to transform the audio scene in real time is a challenging task. An important example of transformation is (re)spatialization: moving sound sources in space. This is equivalent to source separation. Indeed, moving all the sources of the scene but one away from the listener separates that source. And moving separate sources then rendering from them the corresponding scene is easy. But allowing this spatial transformation/source separation with a sufficient quality is a (too) challenging task for classic approaches, since it requires an analysis of the audio scene with inevitable (and often unacceptable) estimation errors. We introduced the informed approach, which consists in inaudibly embedding some additional information in the audio signal. This information, coded with a minimal rate, aims at increasing the precision of the analysis/separation. Thus, the informed approach relies on both estimation and information theories. Since the presentation at the ASA meeting in 2010, several informed source separation methods were proposed. Among the best methods is the one based on spatial filtering (beamforming), with the spectral envelopes of the sources (perceptively coded) as additional information. This allows the manipulation of the sound sources with an unequaled (controllable) quality.

$2: 35$

2pMU5. Evaluation of singing voice by amateur singers from various aspects. Akinori Ito (Graduate School of Eng., Tohoku Univ., 6-6-5 Aramaki-aza-Aoba, Aoba-ku, Sendai, Miyagi 980-8579, Japan, aito@spcom.ecei.tohoku.ac.jp)

Many research works have been conducted for evaluating singing voice so far. Most of the works aimed to investigate the voice of professional vocalists. Besides, evaluation of amateur singers is useful for developing entertainment applications such as scoring of Karaoke. In this presentation, two of such applications are introduced: an evaluation of singing enthusiasm and the detection of mistakes of sung lyrics. The evaluation of singing enthusiasm is based on three kinds of features: the A-weighted power, the vibrato-related feature, and the fall-down feature. Using these features, perceived singing enthusiasm can be estimated relatively accurately. In addition, singers' intention of enthusiasm can also be estimated from the fluctuation of the segmental power of the voice. Detection of mistakes of sung lyrics is based on the dynamic time warping between the input singing voice and the reference singing voice. To normalize the singerdependent variation of voice features, we employed singer normalization based on linear regression. Based on the DTW alignment, frame-by-frame distances between the two voices are obtained. After temporal smoothing of the distances, the lyrics errors are detected based on the peak detection of the distance. High detection accuracy is obtained when the errors are based on phrases.

\section{2:55}

2pMU6. Statistical models for the indirect acquisition of violin bowing controls from audio analysis. Alfonso Perez-Carrillo (Music Technol. Group, Universitat Pompeu Fabra, Roc Boronat 138, Barcelona 08018, Spain, alfonso.perez@upf.edu)

Acoustical studies of sound production in bowed string instruments show that there is a direct relationship between bowing controls and the sound produced. During note sustains the three major bowing parameters that determine the characteristics of the sound are the bowing force (bforce), the bowing distance to the bridge (bbd) and the bowing velocity (bvel). During note attacks the third parameter is acceleration (bacc) rather than velocity. We are interested in understanding this correspondence between bowing controls and sound but in the opposite direction, i.e., mapping sound features extracted from an audio recording to the original bowing controls used. This inverse process is usually called indirect acquisition of gestures and is of great interest in different fields ranging from acoustics and sound synthesis to motor learning or augmented performances. Indirect acquisition is based on the processing of the audio signal and it is usually informed on acoustical or physical properties of the sound or sound production mechanism. In this paper, we present indirect acquisition methods of violin controls from an audio signal based on training of statistical models with a database of multimodal data (control and sound) from violin performances.

\section{3:15-3:30 Break}

\section{3:30}

2pMU7. Adaptive aggregation of regression models for music emotion recognition. Satoru Fukayama and Masataka Goto (National Inst. of Adv. Industrial Sci. and Technol. (AIST), IT, AIST, 1-1-1 Umezono, Tsukuba, Ibaraki 305-8568, Japan, s.fukayama@aist.go.jp)

We present a method for music emotion recognition which adaptively aggregates regression models. Music emotion recognition is a task to estimate how music affects the emotion of a listener. The approach works by mapping acoustic features into space that represents emotions. Previous research has centered on finding effective acoustic features, or applying a multi-stage regression to aggregate the results obtained by using different acoustic features. However, after training regression models, the aggregation happens in a fixed way and cannot be adapted to acoustic signals with different musical properties. We indicate that the most effective feature in estimation differs depending on what kind of emotion we are estimating, and propose a method that adapts the importance of each feature in estimating the emotion. We exploit the variance obtained with Gaussian process regression to measure the confidence of the estimated result from each regression model. The formula for aggregating results from different regression models is derived through the maximum likelihood estimation. We confirmed with an experiment comparing different aggregation approaches that our adaptive aggregation is effective in improving recognition accuracy. 
2pMU8. Experimental modal analysis/synthesis of saxophone input impedances. Esteban Maestre (Music Technol., McGill Univ., Roc Boronat 138, Barcelona 08018, Spain, esteban.maestre@upf.edu) and Gary P. Scavone (Music Technol., McGill Univ., Montreal, QC, Canada)

We present an experimental modal analysis/synthesis framework for the accurate modeling and efficient digital simulation of saxophone input impedances. Starting from impedance measurements, we perform spectral-domain modal analysis by iteratively minimizing the error between measurements and synthetic impedance responses. Synthesized impedances are formulated as passive digital filters in parallel form, enabling the construction of passive digital reflectances with potential use for efficient sound generation via coupling to a reed non-linear model. In this talk, we provide an overview and discuss our ongoing developments, pointing toward future directions in using this technique for real-time sound synthesis.

\section{4:10}

2pMU9. Toward a real-time parametric percussion instrument based on a waveguide mesh. Tamara Smyth and Jennifer Hsu (Music, Univ. of California San Diego, 9500 Gilman Dr. MC 0099, La Jolla, CA 92093-0099, trsmyth@ucsd.edu)

In this work, we aim to create a real-time parametric percussion instrument in which tone quality and note duration may be controlled by the user. Though the well-known waveguide mesh can be used to model two-dimensional wave propagation on drum heads of different size/shape, with variable boundary conditions ultimately influencing rate of sound decay, the prohibitive computational cost of its time-domain implementation makes it unsuitable for real-time performance. Here, the square waveguide mesh, having variable size and parametric boundaries, is replaced by its (exactly) equivalent transfer function — the ratio of the output to the input when excited and tapped at its center. The transfer function is obtained by first generating a system of difference equations describing the output of scattering junction ports - a process that is simplified by the symmetry in the square mesh when excited and tapped at its center. The final transfer function is stored for various mesh sizes, with coefficients being represented symbolically as a function of boundary parameters that can be set (for example, according to desired note duration) during real-time performance.

\section{4:30}

2pMU10. Recent extensions to topological and nonlinear aspects of wave digital filter theory. Kurt J. Werner (CCRMA, Stanford Univ., 223 Ayrshire Farm Ln., Apt. 205, Stanford, CA 94305, kwerner@ccrma.stanford.edu)

The Wave Digital Filter approach can be used to create computational models of lumped reference systems, including rectilinear mechanical, rotational mechanical, acoustical, and electronic systems. When they are applicable, Wave Digital Filters enjoy properties including modularity, accuracy, and guaranteed incremental passivity that make them attractive in the context of musical instrument and audio effect simulation. However, the class of reference systems that can be modeled with Wave Digital Filters has historically been restricted to systems with simple topologies (which can be decomposed entirely into series and parallel connections) and which contain only a single "nonadaptable" element (e.g., a diode, ideal source, or switch). This talk details recent Wave Digital Filter advances from Stanford University's Center for Computer Research in Music and Acoustics (CCRMA), which broaden the applicability of Wave Digital Filters to reference systems with any topology and an arbitrary number of nonlinearities or other nonadaptable elements. These advances have enabled many new and previously intractable musical circuit simulations at CCRMA, including active tone stages and transistor/diode/feedback clipping from guitar distortion pedals, the electromechanical Hammond organ vibrato/chorus system, guitar tube pre-amplifiers and tone stacks, circuits involving operational amplifiers, drum machine circuits, and transistor amplifiers.

2pMU11. Music signal processing by the human brains: Studies on the strategies used by professional pianists to efficiently sightread music. Eriko Aiba (Dept. of Mech. and Intelligent Systems Eng., Univ. of Electro-Communications, 1-5-1 Chofugaoka, Chofu, Tokyo 1828585, Japan, aiba.eriko@uec.ac.jp)

The human brain is an ingenious signal processing system. When musicians play instruments, their brains must process a huge amount and various types of information in parallel. Particularly, the sight-reading of piano music requires the processing of an enormous amount of information as piano music includes many chords and is written on the great staff (or grand staff). Pianists have to read the score, interpret the music, search for the keys to be played while planning the motions of fingers, and control their fingers. In addition, they must adjust the sound intensity and usage of the sustaining pedal according to the output sound. All these are performed simultaneously and successively. The sensory information or signals to be processed include many different modalities, such as visual, auditory, tactile, and motion sensing. In order to complete this complicated task, it is important that the pianists efficiently process this information. In this presentation, I introduce our experimental studies investigating the strategies used by professional pianists to improve the efficiency of information processing while sight-reading music. 


\title{
Session 2pNS
}

\section{Noise: Community Response to Transportation Noises}

\author{
James E. Phillips, Cochair \\ Wilson, Ihrig \& Associates, Inc., 6001 Shellmound St., Suite 400, Emeryville, CA 94608 \\ Takashi Yano, Cochair \\ Architecture, Kumamoto University, Kurokami 2-39-1, Chuo-ku, Kumamoto 860-8555, Japan \\ Chair's Introduction-1:00
}

\section{Invited Papers}

\section{1:05}

2pNS1. Annoyance due to transportation noises in Vietnam. Thulan Nguyen, Takashi Yano (Graduate School of Sci. and Technol., Kumamoto Univ., 2-39-1 Kurokami, Chuo-ku, Kumamoto 8608555, Japan, nguyen@kumamoto-u.ac.jp), Tsuyoshi Nishimura (Graduate School of Eng., Sojo Univ., Kumamoto, Japan), and Tetsumi Sato (Faculty of Eng., Hokkai Gakuen Univ., Sapporo, Japan)

Though understanding differences in the community response to noise among countries is essential to an effective implementation of noise policies, very little noise annoyance data have been accumulated in countries outside Europe and North America. To contribute to Vietnamese and global noise policies, socio-acoustic surveys on the community response to transportation noise have been carried out in Vietnam since 2005. A total of around 9,900 responses were obtained from these surveys. In this paper, a method of drawing exposure-response curves for noise annoyance in Vietnam is presented. Representative exposure-response relationships are proposed for road traffic and aircraft noise annoyance in Vietnam and compared with those of Europe and Korea. The results show that the aircraft noise annoyance curve for Vietnam was slightly higher than the curve obtained by the EU but considerably lower than that for Korea. Vietnamese respondents were less annoyed by road traffic noise than respondents in Europe and Korea. The frequency of use and the attitude to motorbikes and airplanes were found to moderate noise annoyance in Vietnam. This study also shows a method to quantify the difference in the prevalence of annoyance measured by five-point verbal and 11-point numerical scales.

\section{$1: 25$}

2pNS2. Community response to aircraft noise: A step-change study around Hanoi Noi Bai International Airport. Linh T. Nguyen (Sound Traffic Environment Planning INC, Kamikumamoto 3-21-21, Princess Mansion B 402, Kumamoto 8600079, Japan, nguyen. thaolinh0912@gmail.com), Lan T. Nguyen (Graduate School of Sci. and Technol., Kumamoto Univ., Kumamoto, Japan), Takashi Yano, Tsuyoshi Nishimura (Faculty of Information, Sojo Univeristy, Kumamoto, Japan), Tetsumi Sato (Faculty of Eng., Hokkai Gakuen Univ., Sapporo, Japan), Makoto Morinaga (Defense Facilities Environment Improvement Assoc., Tokyo, Japan), and Ichiro Yamada (Aviation Environment Res. Ctr., Tokyo, Japan)

A new terminal building in Hanoi Noi Bai International Airport (Vietnam) opened in December 2014 enhanced the capacity of serving more flights, entailing a step-change in aircraft noise exposure. Three social surveys were conducted around the airport in September 2014, March 2015, and September 2015 to study such a circumstance. Albeit the number of flights increased about 21-24\% after the opening of the new terminal, aircraft noise exposure $\left(L_{\mathrm{den}}\right)$ stayed during the two first surveys $(44,45-66 \mathrm{~dB})$ but increased in the last survey (49-69 dB). Also, noise level at particular sites varied considerably among surveys, especially at sites located near the airport. Curves of exposure-response relationships of three surveys were drawn by using logistic regression. The curve for the second survey fits on the first one with approximately 5\% higher while the last survey's curve is remarkably steeper than others. All three curves of this study located above and notably steeper than the curve of EU's position paper, which means people in Hanoi were more annoyed than people in EU. One of the worth mentioning factors for causing these outcomes was the re-operation of the runways which was closed for maintenance since August to December 2014.

\section{1:45}

2pNS3. Activity disturbances caused by Shinkansen railway noises through the meta-analysis in Japan. Takashi Morihara (National Inst. of Technol., Ishikawa College, Kitachujo, Tsubata, Ishikawa 929-0392, Japan, morihara@ishikawa-nct.ac.jp), Shigenori Yokoshima (Kanagawa Environ. Res. Ctr., Hiratsuka, Japan), and Takashi Yano (Kumamoto Univ., Kumamoto, Japan)

More than 50 years have passed since the Tokaido Shinkansen railway, the first high-speed railway, opened in 1964. The network of Shinkansen lines has been continuously expanded in Japan: the Hokuriku and Hokkaido Shinkansen lines were partially opened in 2015 and 2016, respectively. To preserve the living environment and to protect human health, the Japanese government enacted the Environmental Quality Standards for Shinkansen Superexpress Railway Noise in 1975. Many social surveys on community response to Shinkansen railway noise and vibration have been conducted. A part of these surveys data have been accumulated in Socio-Acoustic Survey 
Data Archive. We reanalyzed data from seven social surveys along six Shinkansen lines carried out from 1995 to 2013 . The number of response to the question of daily activities was from 5441 to 1997 samples. TV/radio listening disturbance was greater than other listening disturbances at the same noise level. The evaluation of awakening exceeded $10 \%$ above the night time noise level of about $45 \mathrm{~dB}$. Through the comparison between the survey, it was indicated that the effect of differences in the number of train service per day.

\section{2:05}

2pNS4. Community response to transportation noises in Japan: Secondary analysis using the Japanese socio-acoustic survey data archive. Shigenori Yokoshima (Kanagawa Environ. Res. Ctr., 1-3-39, Shinomiya, Hiratsuka, Kanagawa 2540014, Japan, yokoshima@k-erc.pref.kanagawa.jp), Makoto Morinaga (Defense Facilities Environment Improvement Assoc., Minato-ku, Japan), Takashi Yano (Kumamoto Univ., Kumamoto, Japan), Takashi Morihara (Ishikawa National College of Technol., Tsubata-town, Japan), and Keiji Kawai (Kumamoto Univ., Kumamoto, Japan)

A number of socio-acoustic surveys on community response to transportation noises have been carried out in Japan since 1970s. The findings from early precious surveys contributed to the establishment of Noise Regulation Law and Environmental Quality Standards regarding noises. However, micro datasets consisting of noise exposure and reactions to noise from recent studies have yet to be accumulated into a unified system. Dose-response relationships obtained through re-analysis of micro-data form the basis of the progress of effective noise policies. Thus, the Japanese Government faces many difficulties in reviewing noise policies. To develop an archive of community responses to noise, we established the Japanese Socio-Acoustic Survey Data Archive (J-SASDA) and have started the secondary analysis since 2011. In this presentation, we introduce the outcomes of our activity. First, we compare exposure-annoyance relationships among the modes of transportation using the J-SASDA and determine the curves per mode of transportation. Next, we focus on the comparison of exposure-annoyance relationships for transportation noises among European and American countries, Vietnam and Japan. Finally, we intend to establish the Asian-SASDA (A-SASDA) in collaboration with researchers from neighboring Asian countries and explain the current status and problem.

2pNS5. Centerline rumble strip sound level evaluation-Comparison of four designs. David Braslau (David Braslau Assoc., Inc., 6603 Queen Ave. S, Ste. N, Richfield, MN 55423, david@braslau.com), Edward Terhaar (Wenck Assoc., Inc., Maple Plain, MN), and Katie Fleming (Minnesota Dept. of Transportation, St. Paul, MN)

Results of sound level monitoring of four alternative centerline sinusoidal rumble strip designs are discussed: a single strip 14 inches wide and a double strip of two 8 inch wide strips 4 inches apart each with depths $-3 / 8$ inch or $1 / 2$ inch. These results supplement those presented at the Pittsburgh 2015 meeting (3aNS5). Tests were performed at $60 \mathrm{mph}$ with three different vehicles-passenger car, pickup truck, and a six-wheel heavy maintenance truck. One-third octave band sound levels were taken 50 and 75 feet from the edge of the roadway, as well as inside the vehicle. Digital audio recordings were also taken. The single 14 inch design with $1 / 2$ inch depth was recommended for implementation by MnDOT since it provided good driver feedback but less exterior noise than the other designs for passenger cars but also good results for pickup trucks, a significant portion of the vehicle fleet. Based upon an evaluation of alternative rumble strips by motorcycles and bicycles at the MnROAD test facility, the single strip was also found more desirable. However, it was recommended that, in areas where there is extreme sensitivity to exterior rumble strip noise, the shallower $3 / 8$ inch design would still provide adequate driver feedback.

2:45

2pNS6. Applications of automatic equipment identification to studies of rail noise and vibration in North America. Eric L. Reuter (Reuter Assoc., LLC, 10 Vaughan Mall, Ste. 201A, Portsmouth, NH 03801, ereuter@reuterassociates.com)

When conducting studies of noise and vibration impacts of existing rail lines, it is useful to identify and log train movements. Since the early 1990s, all railcars and locomotives in North America have been outfitted with automatic equipment identification (AEI) tags. AEI is an RFID system that allows for automated electronic identification of passing equipment with unattended trackside readers. These data reveal the makeup, direction, length, speed, and other useful parameters of each passing train. In order to assess the feasibility of using AEI readers in noise studies, a field study was conducted using a temporary trackside reader and correlated sound and vibration monitors. This paper will present a technical overview of the AEI system and the results of the field study.

\section{3:05-3:20 Break}

\section{Contributed Papers}

3:20

2pNS7. Transportation noise: Nuisance or disability? Abigail Bristow (Loughborough Univ., School of Civil and Bldg. Eng., Loughborough LE11 3TU, United Kingdom, a.1.bristow@lboro.ac.uk)

This paper reviews the state of the art with respect to the valuation of transportation noise nuisance. It considers recent movements away from values based on revealed or stated preferences (SP) to values based on disability weights. Posing the question as to whether the experience of noise nuisance constitutes a disability. Environmental noise imposes amenity losses and adverse health outcomes on the population. As the world becomes more urban, increasingly motorized and aviation growth persists, noise nuisance is likely to affect ever more people. In identifying and prioritising interventions to reduce experienced noise, it is essential to identify the benefits not just in terms of decibel reductions but in terms of the economic benefits of achieving such changes so these may be compared with costs of interventions to ensure best value for money. The values from a recent meta-analysis of SP studies are compared with those derived using the more conventional hedonic pricing (HP) approach including meta-analyses of HP studies of aircraft noise and more recent developments with respect to assessing annoyance in the form of disability adjusted life years (DALYS) and applying a value of a quality adjusted life year (QALY) to this. 
2pNS8. Higway traffic noise effects on annoyance. Aybike Ongel (Civil Eng., Bahcesehir Univ., Ciragan Cad No: 4 Besiktas, Istanbul 34349, Turkey, aybike@gmail.com), Mustafa Ilicali (Istanbul Ticaret Univ., Istanbul, Turkey), Didem Oguz (Baskent Univ., Istanbul, Turkey), Betul Ogutmen (Numune Hospital, Istanbul, Turkey), Tolga Guvenc (Siyami Ersek Hospital, Istanbul, Turkey), and Nurten Sayar (Marmara Univ., Istanbul, Turkey)

Noise pollution has been a major issue in the last decade. Noise was shown to cause adverse health effects including noise-induced hearing impairment, annoyance, sleep disturbance, and cardiovascular diseases. Transportation noise has been the most common one among all noise sources in urban areas. A study has been initiated in Istanbul, Turkey in order to evaluate the effects of highway noise on annoyance and myocardial infarction. A total of 2000 surveys will be conducted in 6 hospitals in Istanbul. The highway noise levels are obtained from the noise maps prepared by Environmental Protection Department of Istanbul Metropolitan Municipality. This manuscript presents the highway noise exposure levels in Istanbul and early findings regarding the annoyance effects of highway traffic noise.

\section{3:50}

2pNS9. Multilevel modeling of recent community noise annoyance surveys. Maurice E. Hayward (Mathematics, College of Williams \& Mary, 200 Ukrop Way, College of William \& Mary Mathematics Dept., Williamsburg, VA 23185, mehayward@email.wm.edu), Jonathan Rathsam (NASA Langley Res. Ctr., Hampton, VA), D. K. Wilson (Cold Regions Res. Eng. Lab., U.S. Army Engineer Res. Dev. Ctr., Hanover, NH), Edward T. Nykaza (ERDC-CERL, US Army Corps of Engineers, Champaign, IL), and Nicole M. Wayant (Geospatial Res. Lab., U.S. Army Engineer Res. Dev. Ctr., Alexandria, VA)

Large scatter in community noise annoyance survey data has recently been linked to systematic variations in noise tolerance. We hypothesize that noise tolerance is related to secondary factors, including environmental (e.g., population density, ambient noise) and attitudinal (e.g., noise sensitivity). If noise tolerance and secondary factors can be linked, the secondary factors provide a means to generalize noise annoyance from surveyed communities to non-surveyed communities. This approach would be useful for generalizing annoyance caused by quiet supersonic flight or military blast noise from a limited number of surveyed communities to other locations. A simple multilevel modeling approach (D.K. Wilson, et al. "Community noise annoyance: Connecting regression methodologies and theoretical models," J. Acoust. Soc. Am. 139(4), 1983 (2016)) offers a useful framework to study the relationship between noise tolerance and secondary factors. The framework has already been applied to historic survey data on transportation noise annoyance. However, the historic surveys contain limited secondary factor information. This presentation applies the multilevel modeling approach to two recent community surveys about which more secondary factors are known. The first survey was conducted on a community exposed to quiet sonic booms. The second survey was conducted on multiple communities exposed to military blast noise.

\section{4:05}

2pNS10. Individual differences in judging the annoyance of the sounds of helicopters. George A. Luz (Luz Social \& Environ. Assoc., Inc., 288 Ninth St. Alley, Ashland, OR 97580, luz_associates@msn.com) and Nicholas P. Miller (HMMH, Burlington, MA)

A rarely used procedure for studying aviation noise annoyance is asking people ro rate indiviidual aircraft events experienced in their homes. Our study employs this procedure with five subjects who self-identified as "highly annoyed" by helicopters. Subjects lived within $400 \mathrm{~m}$ of each other. Flights were recorded at three homes over a 10 day survey period during which subjects registered their judgments on a cell phone app with which a subject initiated a survey by tapping "hear a helicopter." Questions included location (indoors/outdoors), window position (open/closed), annoyance (5 pt scale), loudness ( 3 pt scale), and house rattles (3 pt scale). Recordings corresponding to completed surveys were analyzed for ASEL, CSEL, subjective and objective duration, loudness, loudness level, fluctuation strength, and roughness. Stepwise regression was used to find the most robust predictors of annoyance. The data confirmed the importance of ASEL and/ or measures of loudness as predictors. Compared with in-home observers in a 1983 study at Oklahoma City Airport, these subjects showed a sharp increase in high annoyance when the SEL exceeded $90 \mathrm{dBA}$.

\section{$4: 20$}

2pNS11. Three dimensional noise mapping system with aerial blimp robot. Ryouzi Saitou (Dept. of Intermedia Art and Sci., Waseda Univ., Minamishitaura, 1617-3 308, Miura, Kanagawa 238-0103, Japan, ryouzi5136saitou@fuji.waseda.jp), Yusuke Ikeda, and Yasuhiro Oikawa (Dept. of Intermedia Art and Sci., Waseda Univ., Tokyo, Japan)

Recently, Unmanned Aerial Vehicle (UAV) is used in various situations and research fields, because of low cost and high convenience. For instance, there are many measurement and monitoring systems for taking environmental information by analyzing the image data which is acquired from the air with the UAVs. The UAVs with some propellers, such as quadcopter, always needs to rotate the propellers during flying. The noises of rotating propellers and makes collecting sound information with UAV difficult. In this study, we proposed the environmental sound recording system using a blimp which is filled with helium gas. The system has four propellers in the horizontal direction and two propellers in the vertical direction. This system has also a control board which has a SoC (including both FPGA and CPU) with embedded Linux OS to control six DC motors attaching to the each propeller and to communicate with PC through wireless LAN. The control board can record a sound signal with an A/D converter and an external memory. The experimental results show that it is efficient to make noise maps in horizontal and vertical planes by using the proposed system.

\section{$4: 35$}

2pNS12. Probability of receiving a blast noise complaint based on population density. Dawn A. Morrison (US Army Corps of Engineers, ERDCCERL, 2902 Newmark Dr., Champaign, IL 61826, dawn.a.morrison@ usace.army.mil), Jose M. Gonzalez (Statistics, Univ. of Illinois, UrbanaChampaign, Champaign, IL), and Edward T. Nykaza (US Army Corps of Engineers, ERDC-CERL, Champaign, IL)

This study extends previous work done by Nykaza et al. (2012) on the probability of receiving a blast noise complaint to include population density in the analysis. Nykaza et al. specifically asked, "what is the probability of receiving a blast noise complaint on a given day within $5 \mathrm{~km}$ of a noise monitor?" The original study examined 102,116 blast events and 148 complaints recorded over 641 study days around one military installation. Results from the original study found that while the overall probability of receiving a blast noise complaint was very low, blast noise complaint tolerances around individual noise monitors varied significantly. The current study combines dasymetric mapping techniques-an overlay method for redistributing arbitrarily organized data based on real world circumstances-and regression analysis to examine the different complaint tolerances, and explores what role, if any, characteristics of surrounding population may play in determining the probability of receiving a blast noise complaint. The results indicate that population density has a direct relationship with blast noise complaint tolerance and suggest that population density may be used as a proxy variable in noise complaint forecasting models.

\section{$4: 50$}

2pNS13. Influence of visual information presentation method and sound presentation method on subjective evaluation of boat traffic noise. Suguru Hosono, Kenji Muto (Graduate School of Eng. and Sci., Shibaura Inst. of Technol., 3-7-5 Toyosu, Shibaura Inst. of Technol., Muto lab., Kotoku, Tokyo 135-8548, Japan, ma15072@shibaura-it.ac.jp), and Yasunobu Tokunaga (National Inst. of Technol., Maizuru College, Kyoto, Japan)

Information on the acoustic environment such as the engine noise of cruising boat on the canal is necessary for town planning. Miyagawa has investigated the influence of the visual of a sound source on an impression of the sound. The study has evaluated the shift on the impression of the sound by the visual stimulus. We focus on human subjective evaluation of the noise stimulus of moving source in this study. Our objective is to clarity the influence of visual information on the subjective evaluation of one moving boat noise. In this experiment, we examined the subjective evaluation of 
the moving source comparison between the stimulus of the sound and the stimulus of the sound and visual in the case of moving boat noise. This experiment used a 24-inch monitor, a headphone, or loudspeakers. The sound pressure level of $42-70 \mathrm{~dB}$. As the result, the value of subjective evaluation was decreased by the moving source of loud boat noise, in the case of visual information of the moving boat.

\title{
Session 2pPA
}

\section{Physical Acoustics and Noise: Acoustics of Supersonic Jets: Launch Vehicle and Military Jet Acoustics II}

\author{
Seiji Tsutsumi, Cochair \\ JEDI center, JAXA, 3-1-1 Yoshinodai, Chuuou, Sagamihara 252-5210, Japan \\ Kent L. Gee, Cochair \\ Brigham Young University, N243 ESC, Provo, UT 84602 \\ Alan T. Wall, Cochair \\ Battlespace Acoustics Branch, Air Force Research Laboratory, Bldg. 441, Wright-Patterson AFB, OH 45433 \\ Chair's Introduction-1:15
}

\section{Invited Papers}

$1: 20$

2pPA1. Liftoff and time equivalent duration data evaluation of exploration flight test 1 Orion multi-purpose crew vehicle. Janice Houston (Fluid Dynam., NASA Marshall Space Flight Ctr., Bldg. 4203, Huntsville, AL 35812, janice.d.houston@nasa.gov)

The liftoff phase induces high acoustic loading over a broad frequency range for a launch vehicle. These external acoustic environments are used in the prediction of the internal vibration responses of the vehicle and components. There arises the question about time equivalent (Teq) duration of the liftoff phase and similarity to other launch vehicles. Vibroacoustic engineers require the fatigueweighted time duration values for qualification testing inputs. In order to determine the Teq for the Space Launch System, NASA's newest launch vehicle, the external microphone data from the Exploration Flight Test 1 (EFT-1) flight of the Orion Multi-Purpose Crew Vehicle (MPCV) was evaluated. During that evaluation, a trend was observed in the data, and the origin of that trend is discussed in this paper. Finally, the Teq values for the EFT-1 Orion MPCV are presented.

\section{1:40}

2pPA2. Development of aero-vibro acoustics methods for predicting acoustic environment inside payload fairing at lift-off. Seiji Tsutsumi, Shinichi Maruyama (JEDI Ctr., JAXA, 3-1-1 Yoshinodai, Chuuou, Sagamihara, Kanagawa 252-5210, Japan, tsutsumi.seiji@ jaxa.jp), Wataru Sarae, Keita Terashima (JAXA, Tsukuba, Japan), Tetsuo Hiraiwa (JAXA, Kakuda, Japan), and Tatsuya Ishii (JAXA, Chofu, Japan)

Prediction of harmful acoustic loading to payloads inside launcher fairing due to intense acoustic wave generated from propulsion systems at lift-off is an important design issue. Aero-vibro acoustics method developed in this study for predicting the acoustic loading consists of four elements. Hydrodynamics of jet flowfield generating aeroacoustic wave is computed by the high-fidelity Large-Eddy simulation. Computational aeroacoustics based on the full Euler equations is conducted to simulate propagating acoustic wave from the jet to the payload fairing. Then, finite element method is applied to simulate the structural vibration that radiates acoustic wave inside the fairing. Finally, acoustic behavior inside the payload fairing is also computed by the finite element method. An overview of the methods and recent work for validation and verification will be presented. 
2pPA3. Large eddy simulations of acoustic waves generated from hot and cold supersonic jets at Mach 2.0. Hiroaki Nakano (Mech. Eng., Tokyo Univ. of Sci., 6-3-1,Nijuku, Katsushika, Tokyo 125-8585, Japan, nakano@ flab.isas.jaxa.jp), Taku Nonomura, Akira Oyama (Inst. of Space and Astronautics Sci., JAXA, Sagamihara, Japan), Hiroya Mamori, Naoya Fukushima, and Makoto Yamamoto (Mech. Eng., Tokyo Univ. of Sci., Katsushika, Japan)

Strong acoustic waves are generated from the rocket plumes in the supersonic jet condition. The acoustic waves are significantly affected by temperature of jets. In this study, we perform large eddy simulations of the acoustic waves generated from the hot and cold jets. The temperature ratio of chamber to ambient air is set to be 4.0 and 1.0 for the hot and cold jets, respectively. Mach waves are radiated from the region close to the nozzle exit. In the hot jet case, we confirmed that the shorter potential core length, the larger angle of Mach waves, and the higher sound pressure level, as compared with those in the cold jet case.

2:20

2pPA4. Quantitative evaluation of the acoustic waves generated by a supersonic impinging jet. Daisuke Kato (Aeronautics and Astronautics, Univ. of Tokyo, Yoshinodai, Sagamihara, Kanagawa 2520206, Japan, dkato@flab.isas.jaxa.jp), Taku Nonomura, and Akira Oyama (Japan Aerosp. Exploration Agency, Sagamihara, Japan)

Strong acoustic waves are generated when rocket launch. The accurate prediction of these acoustic waves are important for the design of the rocket launch site because the acoustic waves possibly damage the payload. However, it is difficult for numerical simulation to predict accurately because the numerical simulation overestimatesthe strength of acoustic wave by several decibels compared to that observed in the experiment. The objective the present study is to obtain the knowledge for quantitative evaluation of the acoustic waves generated from supersonic impinging jet. According to the free jet studies, the reason for difference between numerical simulations and experiments in acoustic field is considered to be turbulence intensity in nozzle exit. Thus, the flow inside the nozzle is calculated, together with the turbulent disturbance is added to the boundary layer. In the numerical simulation, three-dimensional compressible Navier-Stokes equations are solved with the high resolution shock capturing scheme (WCNS). The effects of the acoustic field on nozzle internal inflow condition of supersonic impinging jet are maninly discussed.

2pPA5. Modeling the generation of supersonic turbulent jet noise by large-scale coherent structures. Oliver T. Schmidt, Tim Colonius (MCE, California Inst. of Technol., 1200 E California Blvd., MC 104-44, Pasadena, CA 91125, oschmidt@ caltech.edu), and Guillaume A. Brès (Cascade Technologies, Inc., Palo Alto, CA)

Large-scale coherent structures, or wavepackets, are a salient feature of turbulent jets, and the main source of jet mixing noise at aft angles. They are extracted from a high-fidelity Mach 1.5 LES database as spectral POD mode estimates. These most energetic wavepackets obtained via POD and their acoustic far-field radiation patterns are compared to solution to the one-way Euler (OWE) equations recently introduced by Towne \& Colonius (AIAA Paper 2013-2171, 2013; AIAA Paper 2014-2903, 2014). Within the OWE framework, the linearized Euler equations are modified such that all upstream propagating acoustic wave components are removed from the solution. The resulting spatial initial value problem can be solved in a stable and computationally efficient manner by downstream marching the solution. Additionally, the scenario of stochastic forcing of wavepackets by the surrounding turbulence is considered in a resolvent analysis. The resolvent analysis allows for the computation of optimal forcing distributions and corresponding responses. It is based on a singular value decomposition of the transfer function of the governing linear operator. The results of the both methods, OWE and resolvent analysis, are compared to the most energetic POD modes with a special focus on far-field radiation patterns and computational efficiency.

\section{Contributed Papers}

\section{3:00}

2pPA6. Self-similarity of level-based wavepacket modeling of high-performance military aircraft noise. Tracianne B. Neilsen, Kent L. Gee, Blaine M. Harker (Brigham Young Univ., N311 ESC, Provo, UT 84602, tbn@byu.edu), Alan T. Wall (Battlespace Acoust. Branch, Air Force Res. Lab., Wright-Patterson AFB, OH), and Michael M. James (Blue Ridge Res. and Consulting, LLC, Asheville, NC)

Construction of the equivalent acoustic source model for high-performance military aircraft noise presented in this paper begins with a decomposition of sound levels, from ground-based microphones $11.7 \mathrm{~m}$ from a highperformance military aircraft, into portions matching the similarity spectra associated with large-scale and fine-scale turbulent mixing noise (LSS and FSS). [Tam et al. AIAA Paper 96-1716 (1996)]. A Fourier transform of the spatial distribution of the decomposed levels yields frequency-dependent wavenumber spectra [Morris, Int. J. Aeroacoust. 8, 301-316 (2009)]. Each LSS-educed wavenumber spectrum corresponds to a wavepacket, consisting of a spatially varying amplitude distribution with a constant axial phase relationship. This wavepacket model produces a directional, coherent sound field. However, the asymmetry in the LSS-educed wavenumber spectra may indicate that a nonuniform phase relationship is a more physical choice that could be correlated to the axial variation in convective speed. The FSS component is modeled with a line of incoherent monopoles whose amplitude distribution is related to the FSS-educed wavenumber spectrum. While this level-based model is limited, the addition of the FSS-related source yields a better match to the noise environment of a high-performance military aircraft than is found using a single coherent wavepacket. [Work supported by ONR.]

\section{3:15}

2pPA7. Wavepacket source modeling of high-performance military aircraft jet noise from cross-beamforming analysis. Blaine M. Harker, Kent L. Gee, Tracianne B. Neilsen (Dept. Phys. \& Astronomy, Brigham Young Univ., N283 ESC, Provo, UT 84602, blaineharker@gmail.com), and Michael M. James (Blue Ridge Res. and Consulting, LLC, Asheville, NC)

Wavepacket models provide a convenient representation of jet noise source phenomena because of the extended, partially correlated nature of the turbulent mixing noise. When treated as an equivalent source model, they are useful to estimate features of both the radiated noise as well as the source characteristics to assist in jet noise reduction efforts. In this study, advanced cross-beamforming techniques are applied to measurements in the vicinity of a high-performance military aircraft. These results are then decomposed into an azimuthally-averaged multi-wavepacket representation of the data, which can then be treated as an equivalent source. Estimates of 
the field levels and coherence properties using the equivalent source are compared with measurements, and results from the multi-wavepacket model show good agreement with benchmark measurements over a range of frequencies that contribute significantly to the overall radiation. The capabilities and limitations of the model to estimate field properties are quantified with respect to benchmark measurements in the mid and far fields as a function of frequency. Results indicate that the multi-wavepacket representation is an improvement over single-wavepacket models, which do not incorporate spatiotemporal features of the radiation. [Work supported by ONR and USAFRL through ORISE.]

\section{3:30-3:45 Break}

\section{$3: 45$}

2pPA8. Azimuthal coherence of the sound field in the vicinity of a high performance military aircraft. Kevin M. Leete (Phys. and Astronomy, Brigham Young Univ., Provo, UT 84604, kevinmatthewleete@gmail.com), Alan T. Wall (Battlespace Acoust. Branch, U.S. Air Force Rersearch Lab., Dayton, OH), Kent L. Gee, Tracianne B. Neilsen, Blaine M. Harker (Phys. and Astronomy, Brigham Young Univ., Provo, UT), and Michael M. James (Blue Ridge Res. and Consulting LLC, Asheville, NC)

Mixing noise from a jet engine originates from an extended spatial region downstream of the nozzle and is partially correlated both spatially and temporally. Previously, the coherence properties in the downstream (axial) direction of the sound field of a tethered military aircraft were investigated, resulting in the identification of different spatial regions based on coherence length [B. M. Harker et al., AIAA J. 54, 1551-1566 (2016)]. In this study, a vertical array of microphones to the side of the jet plume is used to obtain the azimuthal coherence of the sound field. Although multipath interference effects and a limited angular aperture make coherence length calculation impossible, information about upper and lower bounds can be extracted. The measured azimuthal coherence as a function of downstream distance and frequency is then compared to that predicted by sound field reconstructions using multisource, statistically optimized near-field acoustical holography (M-SONAH) [A. T. Wall et al., J. Acoust. Soc. Am., 139, 1938-1950 (2016)]. This comparison helps to benchmark the performance of a reduced-order M-SONAH algorithm that employs only axisymmetric cylindrical basis functions to represent the direct and image sound fields. [Work supported by USAFRL through ORISE (2016).]

\section{4:00}

2pPA9. In defense of the Morfey-Howell $Q / S$ as a single-point nonlinearity indicator: An impedance-based interpretation. Won-Suk Ohm (School of Mech. Eng., Yonsei Univ., 50 Yonsei-ro, Seodaemun-gu, Seoul 120-749, South Korea, ohm@yonsei.ac.kr), Kent L. Gee, and Brent O. Reichman (Dept. of Phys. and Astronomy, Brigham Young Univ., Provo, UT)

Since the Morfey-Howell Q/S was proposed as a nonlinearity indicator for propagation of intense broadband noise [AIAA J. 19, 986-992 (1981)], there has been considerable debate as to its meaning and utility. Perhaps the most contentious argument against $\mathrm{Q} / \mathrm{S}$ is about its validity as a single-point nonlinearity indicator: the importance of nonlinearity is often judged by observing cumulative effects over some propagation distance, whereas $\mathrm{Q} / \mathrm{S}$ is based on a pressure waveform at a single location. Studies to address these criticisms have emerged over the years, most recently by Reichman et al. [J. Acoust. Soc. Am. 139, 2505-2513 (2016)] in support of Q/S. In this talk we show that the Burgers equation (from which the $\mathrm{Q} / \mathrm{S}$ was originally derived) can be recast in terms of specific impedance, linear absorption and dispersion coefficients, and normalized quadspectral $(\mathrm{Q} / \mathrm{S})$ and cospectral $(\mathrm{C} / \mathrm{S})$ densities. The resulting interpretation is that $\mathrm{Q} / \mathrm{S}$ and $\mathrm{C} / \mathrm{S}$ represent the additional absorption and dispersion, introduced by the passage of a finite-amplitude wave to the existing linear absorption and dispersion. In other words, a nonlinear wave process alters the apparent material properties of the medium, the extent of which can be used as a single-point indicator of the relative strength of nonlinearity.

\section{$4: 15$}

2pPA10. Near-field array measurements of F-35 aircraft noise. Alan T. Wall (Battlespace Acoust. Branch, Air Force Res. Lab., Bldg. 441, WrightPatterson AFB, OH 45433, alantwall@gmail.com), Kent L. Gee, Tracianne B. Neilsen, Kevin M. Leete (Brigham Young Univ., Provo, UT), Michael M. James (Blue Ridge Res. and Consulting, Asheville, NC), and Richard L. McKinley (Battlespace Acoust. Branch, Air Force Res. Lab., Wright-Patterson Air Force Base, OH)

Noise source measurement and modeling efforts are being conducted for current and future generations of high-performance military aircraft. A nearfield microphone array was used in a noise measurement of F-35A and F35B aircraft at Edwards Air Force Base in 2013 in order to extract source models. The two aircraft measured were tethered to the ground on a concrete run-up pad. The array consisted of 76 sensors, spanned $32 \mathrm{~m}$, and was placed on the ground approximately $7 \mathrm{~m}$ from the jet plume. The engines operated over a full range of power settings from idle through full afterburner. In preparation for the execution of source modeling efforts, these near-field data are explored and compared to far-field data published previously [James et al., AIAA Paper 2015-2375]. [Work supported by USAFRL through F-35 JPO.]

\section{$4: 30$}

2pPA11. Noise measurements of the F-35C on an aircraft carrier flight deck. Nourhan K. Abouzahra, Alan T. Wall, Richard L. McKinley, Billy J. Swayne (Air Force Res. Lab., 2610 Seventh St. Bldg. 441, Wright-Patterson AFB, OH 45433, nourhan.abouzahra.ctr@us.af.mil), Michael J. Smith, and Allan C. Aubert (NAVAIR, Patuxent River Naval Air Station, MD)

In 2015, scientists from the Air Force Research Laboratory (AFRL) in collaboration with other teams performed noise measurements of the F-35C aircraft aboard a Nimitz-class carrier. The purpose of the measurements was to quantify the crewmembers' exposure to noise. The measurements were taken using hand-held noise recorder systems, and the recording engineers shadowed actual locations of crew. The near-field noise levels caused by aircraft are reported in the areas experienced by crew on board aircraft carriers. High noise levels can interfere with communications and may pose a risk for hearing loss. These data represent a unique measurement; the previous measurement of aircraft noise from catapult launches and wire arrestments aboard a carrier occurred more than 15 years ago.

\section{4:45}

2pPA12. Nonlinear characteristics of F-35 flyover waveforms. Brent $O$. Reichman, Kent L. Gee, Tracianne B. Neilsen (Brigham Young Univ., 453 E 1980 N, \#B, Provo, UT 84604, brent.reichman@byu.edu), and Sally A. McInerny (Univ. of Louisiana at Lafayette, Lafayette, LA)

Metrics indicating the presence of acoustic shocks are calculated from noise measurements of F-35 aircraft during flyover operations. The sound pressure level, average steepening factor, and skewness of the first time derivative of the pressure waveform are calculated for the A and B variants of the aircraft. Other metrics more specific to flyover measurements are also shown. Comparisons of nonlinear indicators are made between engine thrust conditions, aircraft type, microphone height, microphone size, and sampling rate. Nonlinear indicators are also compared against other full-scale military aircraft. Comparisons are made with similar conditions during ground run-up operations. 


\title{
Session 2pPP
}

\author{
Psychological and Physiological Acoustics: Binaural Processing and Localization \\ Christopher Brown, Chair \\ University of Pittsburgh, 4033 Forbes Tower, 3600 Forbes at Atwood, Pittsburgh, PA 15260
}

\section{Contributed Papers}

\section{1:15}

2pPP1. Coherence influences sound-field localization dominance in a precedence paradigm. Julian Grosse, Steven van de Par (Acoust. Group, Cluster of Excellence "Hearing4all", Univ. of Oldenburg, Carl-vonOssietzky-Straße 9-11, Oldenburg 26129, Germany, julian.grosse@uni-oldenburg.de), and Constantine Trahiotis (Departments of Neurosci. and Surgery (Otolaryngology), Univ. of Connecticut Health Ctr., Farmington, CT)

The ability to localize sound sources in reverberant environments is dependent upon first-arriving information, an outcome commonly termed "the precedence effect." For example, a combination of a leading (direct) sound followed by a lagging (reflected) sound is localized in the direction of the leading sound. We measured how the compactness/diffuseness (i.e., the interaural coherence) of leading and lagging sounds, respectively, influences performance. The compactness/diffuseness of leading or lagging sounds was varied by either presenting a sound from a single loudspeaker or by presenting mutually uncorrelated versions of similar sounds from nine adjacent loudspeakers. The listener pointed to the perceived location of leading and lagging sources of sounds which were 10-ms long, low-pass filtered white noises or 2-second long tokens of speech. The sounds were presented either from speakers located directly in front of the listener or from speakers located $45^{\circ}$ to the right or left. Leading compact/coherent sounds, be they Gaussian noises or tokens of speech, influenced perceived location more so than did leading diffuse/incoherent sounds. The results strongly support the hypothesis that stimulus coherence, often modeled by "straightness" within a cross-correlogram, plays an important role in precedence.

\section{1:30}

2pPP2. Better-ear glimpsing with symmetrically-placed interferers in bilateral cochlear implant users. Hongmei Hu (Medizinische Physik and Cluster of Excellence Hearing4all, Universitat Oldenburg, Carl von Ossietzky-Universitat Oldenburg, Oldenburg 26129, Germany, hongmei. hu@uni-oldenburg.de), Mathias Dietz (National Ctr. for Audiol., Western Univ., London, ON, Canada), and Stephan D. Ewert (Medizinische Physik and Cluster of Excellence Hearing4all, Universitat Oldenburg, Oldenburg, Germany)

For a frontal speaker in spatially symmetrically placed maskers, normal hearing $(\mathrm{NH})$ listeners can use an optimal "better-ear glimpsing" strategy selecting time-frequency segments with favorable signal-to-noise ratio in either ear. It was shown that for a monaural signal, obtained by an ideal monaural better-ear mask (IMBM), NH listeners can reach similar performance as in the binaural condition, but interaural phase differences at low frequencies can further improve performance. In principle, bilateral cochlear implant (BiCI) users could use a glimpsing strategy; however, they cannot exploit (temporal fine structure) interaural phase differences. Here, speech reception thresholds of $\mathrm{NH}$ and $\mathrm{BiCl}$ listeners were measured in two symmetric maskers $\left( \pm 60^{\circ}\right.$; speech-shaped stationaty noise, non-sense speech, single talker) using head-related transfer functions and headphone presentation or direct stimulation in BiCI listeners. Furthermore, a statistically independent masker in each ear, diotic presentation with IMBM processing, and a noise vocoder based on the BiCI electrodogram was used in NH. Results indicate that $\mathrm{NH}$ with vocoder and $\mathrm{BiCI}$ listeners show a strongly reduced binaural benefit in the $\pm 60^{\circ}$ condition relative to the co-located condition when compared to $\mathrm{NH}$. However, both groups greatly benefit from IMBM processing (as part of the CI stimulation strategy). Individual differences are compared.

\section{$1: 45$}

2pPP3. Speech intelligibility by bilateral cochlear implant users when interaural level cues are made more consistent across frequency. Christopher Brown (Univ. of Pittsburgh, 4033 Forbes Tower, 3600 Forbes at Atwood, Pittsburgh, PA 15260, cbrown1@pitt.edu)

Interaural level differences (ILDs) aid speech understanding for listeners with both normal hearing $(\mathrm{NH})$ and bilateral cochlear implants (BCIs). Naturally-occurring ILDs are less than ideal for this outcome; however, as they are generally restricted to frequencies above about $2000 \mathrm{~Hz}$, while the energy in speech is typically concentrated below $2 \mathrm{kHz}$. It has recently been demonstrated that applying larger-than-normal ILDs in the low-frequency region can significantly improve speech intelligibility for BCI users. Despite the benefit, this may result in sound sources that appear to move, depending on where in frequency the speech energy resides at a given moment. The current study examined the effect of applying larger-than-normal ILDs both above and below $2 \mathrm{kHz}$. Speech intelligibility was measured in simulation by 16 individuals with $\mathrm{NH}$, and by eight $\mathrm{BCI}$ users, of a target talker along the midsagittal plane in the presence of symmetrically placed masker talkers on either side. An improvement of about 20 percentage points was observed at a number of masker locations for both populations tested when largerthan-normal ILDs were extended into the high-frequency region, although perceptual movement of sound sources appeared to be more noticeable for the NH group. [Work supported by the NIDCD.]

\section{2:00}

2pPP4. The benefits of cochlear implants for single-sided deafness for sound localization with multiple concurrent sources. Joshua G. Bernstein, Gerald I. Schuchman, Olga A. Stakhovskaya (National Military Audiol. and Speech Pathol. Ctr., Walter Reed National Military Medical Ctr., 4954 N. Palmer Rd., Bethesda, MD 20889, joshua.g.bernstein.civ@ mail.mil), Arnaldo L. Rivera (Otolaryngol. - Head and Neck Surgery, Univ. of Missouri School of Medicine, Columbia, MO), and Douglas S. Brungart (National Military Audiol. and Speech Pathol. Ctr., Walter Reed National Military Medical Ctr., Bethesda, MD)

Individuals with single-sided deafness (SSD) who receive a cochlear implant (CI) realize some benefit for localization and speech perception in noise. Previous studies of SSD-CIs examined simple conditions involving speech perception in the presence of a single noise masker or single-source localization. However, some of the most important benefits of two ears arise in multisource situations where binaural cues and head turning can facilitate the perceptual separation of spatial separated sources. Eight SSD-CI listeners performed a sound-localization task in a multisource environment preimplantation and at least 3 months post-implantation. Environmental sounds were presented from a spherical loudspeaker array. The target sound was presented alone, or concurrently with one or three additional sources. The 
target was cued by being added to or removed from the mixture after a 6-s delay. A head-mounted tracker monitored head movements during the task. The CI substantially improved localization accuracy, reducing the mean azimuthal error by as much as $34 \%$, while reducing the amount of head turning by as much as $44 \%$, even during periods when the target sound was not present. For SSD-CI listeners, bilateral auditory input can improve environmental-sound localization in a complex mixture, while reducing the amount of head-turning effort required. [The opinions and assertions presented are the private views of the authors and are not to be construed as official or as necessarily reflecting the views of the Department of Defense.]

\section{2:15}

2pPP5. The impact of cochlear implantation on spatial hearing and listening effort. Ruth Litovsky, Sara Misurelli, Shelly Godar, Tanvi Thakkar, Alan Kan (Waisman Ctr., Univ. of Wisconsin-Madison, 1500 Highland Ave., Waisman Ctr. Rm. 521, Madison, WI 53705, litovsky@waisman. wisc.edu), and Matthew Winn (Speech and Hearing, Univ. of Washington, Seattle, WA)

We studied the potential benefits of bilateral hearing in cochlear implants (CI) users, and in patients with single-sided deafness (SSD) who receive a $\mathrm{CI}$ in the deaf ear and have normal hearing in the other ear. We hypothesized that listening effort, measured with pupil dilation, can reveal benefits of bilateral hearing that may not be consistently observed when localization or spatial release from masking (SRM) are measured. Result from 12 bilateral CI users showed reduction in listening effort with bilateral hearing compared to the poor ear or better ear alone. In patients with SSD, benefits of adding a CI to a normal hearing ear can emerge over a protracted period of a year or longer. In addition, for at least some of the subjects bilateral hearing (adding a CI to the normal hearing ear) produced release from listening effort even in conditions where SRM was not observed. That is, speech intelligibility did not always improve with spatial separation of target and competing speech, but pupil dilation was reduced, suggesting that pupillometry can reveal subtle aspects of bilateral benefits. The benefits shown with pupillometry are consistent with the patients' subjective reports of improved ability to function in complex, noisy acoustic environments.

\section{2:30}

2pPP6. Uncertainty in binaural hearing linked to inherent envelope fluctuations. Gabrielle O'Brien and Matthew Winn (Speech \& Hearing Sci., Univ. of Washington, 1417 NE 42nd St., Seattle, WA 98105, andronovhopf@gmail.com)

It was recently shown that supra-threshold changes in interaural level differences (ILDs) in 1/3-octave bands of noise were more quickly and reliably perceived when carried by high-frequency $(4000 \mathrm{~Hz})$ rather than lowfrequency $(500 \mathrm{~Hz})$ noise. For fixed-octave ratio noises, however, center frequency differences are accompanied by differences in inherent envelope modulations stemming from differences in absolute bandwidth. We propose that envelope fluctuations (rather than frequency differences) are a more parsimonious explanation of response latency and certainty. In the current study we investigate the hypothesis that for noisier envelopes, there is less certainty that an ILD is a true diversion from noise perceived at center. Differences in inherent envelope modulation strength were controlled using three types of stimuli: low-noise noise (with negligible envelope fluctuations), high-noise noise, and an equal mixture of the two. Perception of ILD changes was measured using anticipatory eye movements elicited by ILD changes. Results confirm that sensitivity and response latency to ILD changes were affected by degree of envelope fluctuation. In addition, highnoise noise provoked more phantom percepts, which were nearly absent for low-noise noise. Finally, we propose a mathematical model of accumulating certainty in this task as a function of time and degree of envelope fluctuation.

\section{$2: 45$}

2pPP7. Front-back sound source localization confusions when sources and listeners are rotated. William Yost (ASU, PO Box 870102, Tempe, AZ 85287,william.yost@asu.edu)

Sound source localization identification judgments were made in the azimuth plane with the goal of determining front-back and back-front confusions. The sounds were filtered $125-\mathrm{ms}$ noise bursts: two and $1 / 10^{\text {th }}$ octave wide at center frequencies of 500 and $4000 \mathrm{~Hz}$, and a wideband $(125-8000 \mathrm{~Hz})$ condition. Sounds were either presented from one of six evenly spaced $\left(60^{\circ}\right)$ random loudspeaker locations (random) or the sound presentation rotated clockwise (rotating) around the six loudspeaker array except on $20 \%$ of the trials when the location of the loudspeaker was randomly determined. Four conditions were tested: Listener Stationary with Eyes Open (L-O), Listener Stationary with Eyes Closed (L-C), Listener Rotating with Eyes Open (R-O), and Listener Rotating with Eyes Closed (R-C). Listener rotation was clockwise at $45^{\circ} / \mathrm{s}$. Localization accuracy and the type of errors, especially front-back and back-front errors, were determined for 18 normal hearing listeners. Sound source localization accuracy and the number of front-back and back-front confusions varied as a function of the different filtered noises, the type of sound source presentation (random or rotating) and the listening condition (L-O,L-C,R-O,R-C). There were very large individual differences, especially in front-back and backfront confusions. [Work supported by Grants from AFOSR and NIH.]

\section{3:00-3:20 Break}

\section{3:20}

2pPP8. Audiometrically-defined "slight" or "hidden" hearing losses can be manifested as changes in binaural detection. Leslie R. Bernstein and Constantine Trahiotis (UConn Health Ctr., UConn Health Ctr., MC3405, 263 Farmington Ave., Farmington, CT 06030, lbernstein@uchc.edu)

We hypothesized that audiometrically-defined "slight" or "hidden" hearing losses might be associated with losses in binaural detection via degradations in precision of coding of interaural temporal disparities (ITDs). Thirtyone listeners were tested using masking configurations that, taken together, allow one to describe how precision of ITD-coding varies as a function of reference ITD and center frequency. Binaural detection thresholds at 500 $\mathrm{Hz}$ and $4 \mathrm{kHz}$ were found to increase magnitude of ITD, consistent with there being a loss of precision of ITD-coding that increases with magnitude of ITD. Binaural detection thresholds were elevated, across all ITDs, for those listeners whose absolute thresholds at $4 \mathrm{kHz}$ exceeded $7.5 \mathrm{~dB} \mathrm{HL}$, but were not greater than $23 \mathrm{~dB}$ HL. No such elevations were observed in conditions having no binaural cues available to aid detection (i.e., "monaural" conditions). Partitioning and analyses of the data revealed the elevated thresholds: 1) were more attributable to hearing level than to age; 2) result from increased levels of internal binaural-processing noise. Our conclusion is that listeners whose high-frequency monaural hearing status would be classified audiometrically as being normal or "slight loss" could still suffer from perceptually meaningful losses of binaural processing.

\section{3:35}

2pPP9. Comparison between individualization methods of spatialization for bone conducted sound in navigation task for walking subjects. Akiko Fujise (Panasonic Corp., Tokyo Fashion Town West, 3-4-10 Ariake, Kotoku, Japan, fujise.akiko@jp.panasonic.com) and Joseph A. Paradiso (MIT Media Lab, Cambridge, MA)

Recent work in human-machine interaction and audiology has indicated the effectiveness of using spatialized bone conduction (BC) hearing in augmented reality (AR) environments. For broader applications, individualization methods are preferred, as they require simpler computation and measurement, despite the fact that spatial hearing for $\mathrm{BC}$ is not yet well investigated at individual levels. Accordingly, this study examines different individualization techniques for spatial audio using $\mathrm{BC}$ applied to a navigation task with a moving virtual sound object in external noise, which simulates a common situation in AR environments. The tested methods include one based on individually measured head-related transfer functions (HRTFs), generic HRTFs, and a modified version based on these. Subjects are instructed to follow a virtual sound source presented from the BC headphones while walking. The virtual sound source is updated so that the source is located ahead of the subject according to the subject's location and head rotation tracked by the motion capture system. The subjects' performance are evaluated as the error of the subjects' actual path from the simulated pathway. Results are discussed on the comparison between individualization methods and in regards to recent reports on sound localization in BC hearing without the listener's body movement. 
2pPP10. Spatial and temporal competitors bias auditory localization of impulse events. Jeremy R. Gaston (US Army Res. Lab., Dept. of the Army, Attn: RDRL-HRS, Aberdeen Proving Ground, MD 21005, jgaston2@gmail. com), Kelly Dickerson, Ashley Foots, Timothy Mermagen, Mark Ericson, and Brandon Perelman (US Army Res. Lab., APG, MD)

Sound events in the real-world rarely occur in isolation and when competing sounds are present, the effect on perception can be significant (e.g., various forms of masking). The present study examines auditory localization for impulse sounds modeled after the real-world sound event of small-arms fire. Small-arms fire consists of two distinct sounds, the muzzle blast and ballistic crack. Across different shooter-observer positions, there can be substantial offsets in spatial and temporal stimulus relationships between the sounds. To examine these relationships, impulse targets were presented across a loudspeaker array in the context of impulse competitors varying across timing and spatial relationships. Results showed that there was a significant increase in absolute localization error as a function of the magnitude of these relationships, and signed error indicated significant bias toward the direction of the competing impulse. These results suggest that listeners integrated information from the competing stimulus into their localization decision.

\section{4:05}

2pPP11. Reverberation enhances the relative potency of onset cues for sound localization. G. C. Stecker (Hearing and Speech Sci., Vanderbilt Univ., 1215 21st Ave. South, Rm. 8310, Nashville, TN 37232, g.christopher.stecker@vanderbilt.edu), Asawer Nihal (Loyola Univ. Chicago, Chicago, IL), Julie M. Stecker, and Travis M. Moore (Hearing and Speech Sci., Vanderbilt Univ., Nashville, TN)

Onset dominance in spatial hearing has been repeatedly demonstrated through studies of the precedence effect, Franssen effect, and temporal weighting in sound localization. Such effects are often cited as evidence that onset dominance enhances spatial perception, particularly in reverberant environments where post-onset cues are degraded by echoes and reverberation. Most studies, however, have presented sounds in anechoic space or via headphones. Thus, the actual effects of reverberation on onset dominance are not well understood. This study adapted the approach of Stecker \& Hafter (2002; JASA 112:1046-57) to measure temporal weighting functions (TWF) for freefield localization of Gabor click trains $(4 \mathrm{kHz} ; 200 \mathrm{~Hz}$ click rate) in the presence or absence of simulated reverberation. Reverberant conditions simulated a $10 \mathrm{~m} \times 10 \mathrm{~m}$ room with modestly absorptive walls $(\alpha=0.5)$. For each potential source location, direct sound and all reflections up to $13^{\text {th }}$ order were synthesized at correct azimuths, intensities, and delays. Anechoic conditions presented only direct sound (i.e., source locations were single loudspeakers). Anechoic TWFs revealed modest onset dominance consistent with Stecker \& Hafter (2002). Reverberant TWFs exhibited significantly stronger onset dominance. The results suggest a greater role of onsets in real-world listening than previously estimated. [Work supported by NIH R01 DC011548.]
2pPP12. Interaural level difference processing as a function of frequency. Matthew J. Goupell and Beth Rosen (Hearing and Speech Sci., Univ. of Maryland, College Park, 0119E Lefrak Hall, MD 20742, goupell@ umd.edu)

To localize in the azimuthal plane, humans utilize interaural time differences and interaural level differences (ILDs). For sounds presented in the free field at different azimuthal locations, ILDs generated by the head are complex functions that are frequency dependent and non-monotonic, and are thought to contribute towards sound localization primarily at frequencies above $1500 \mathrm{~Hz}$. However, previous studies have shown that ILD sensitivity is relatively frequency independent for tones presented over headphones, even at frequencies below $1500 \mathrm{~Hz}$. The purpose of this study was to carefully re-investigate the frequency dependency of ILD sensitivity in normalhearing listeners. Ten normal-hearing listeners were presented ILDs using insert earphones at 31 frequencies between 100 and 11,000 Hz. A 20-dB range of level roving was used to limit listeners' ability to use monaural level cues to perform the task. On average, ILD sensitivity had several high and low sensitivity frequency regions, was best at $7000 \mathrm{~Hz}(1.5 \mathrm{~dB})$ and worst at $1000 \mathrm{~Hz}(2.5 \mathrm{~dB})$. Individuals also showed some unique frequency dependent patterns. These results suggest that ILD sensitivity is frequency dependent and neural models of ILD processing need to accommodate this dependency.

\section{$4: 35$}

2pPP13. Sound image movement of approaching and retreating sounds. Tatsuya Hirahara and Shuhei Okada (Toyama Prefectural Univ., 5180 Kurokawa, Imizu 939-0398, Japan, hirahara@pu-toyama.ac.jp)

We investigated how listeners perceive sound image movements of moving sound sources horizontally approaching and retreating from a listener's head under three conditions: directly listening to approaching and retreating real sound sources, listening to binaurally recorded sounds with headphones, and listening to binaurally synthesized sounds with headphones. White noise was emanated from a small loudspeaker put on a slider. The moving directions of the loudspeaker were eight radial horizontal directions at $45^{\circ}$ intervals, and the moving distance was 5 to $105 \mathrm{~cm}$ from the head. Personal earplug microphones were used for the binaural recording. Personal head-related transfer functions were used to synthesize the binaural signals. Sounds approaching and retreating from listeners' heads in most radial horizontal directions produced straight approaching and retreating sound images. Those in the $45^{\circ}$ and $315^{\circ}$ radial horizontal directions, however, yielded anomalous sound image movement. The sound image moved straight forward, bent to the rear as the sound approached, and then followed the same path in reverse as the sound retreated for the real, binaurally recorded, and binaurally synthesized sounds. The analysis of HRTFs suggests that the growth of the inter-aural level difference very close to the head can be the cause of the anomalous sound image movement. 


\title{
Session 2pSA
}

\section{Structural Acoustics and Vibration and Physical Acoustics: Acoustic Metamaterials II}

\author{
Michael R. Haberman, Cochair \\ Applied Research Laboratories, The University of Texas at Austin, 10000 Burnet Rd., Austin, TX 78758 \\ Christina J. Naify, Cochair \\ Acoustics, Naval Research Lab, 4555 Overlook Ave. SW, Washington, DC 20375 \\ Jeffrey Cipolla, Cochair \\ Weidlinger Associates, Inc., 1825 K St. NW, Suite 350, Washington, DC 20006
}

\section{Invited Papers}

1:00

2pSA1. Superresolution using an acoustic vortex wave antenna. Matthew D. Guild (US Naval Res. Lab., Code 7160, 4555 Overlook Ave. SW, Washington, DC 20375, matthew.guild@nrl.navy.mil), Christina J. Naify (Jet Propulsion Lab., California Inst. of Tech., Pasadena, CA), Theodore P. Martin, Charles A. Rohde, and Gregory J. Orris (US Naval Res. Lab., Code 7160, Washington, DC)

Acoustic waves, like their electromagnetic counterpart, exhibit a diffraction limit, which restricts the ability to resolve or generate fine structures in the pressure field. Previously proposed methods to overcome this limit, and therefore achieve superresolution, have mainly been limited to operating within the near-field region of the aperture. In this work, we describe how far-field superresolution can be achieved using shaped acoustic vortices, created by utilizing the topological diversity of an acoustic vortex wave antenna. Theoretical and numerical results will be presented for the design of an acoustic vortex wave antenna with arbitrary planar arrangement which is capable of generating superresolved far-field features in the radiated acoustic pressure. Variation of the antenna geometry enables different shaped acoustic vortex patterns to be achieved, which propagate from the near-field into the far-field through an arrangement of stable integer mode vortices. Despite the total aperture size being less than a wavelength in diameter, the proposed acoustic vortex wave antenna is shown to achieve far-field superresolution with feature sizes 4-9 times smaller than the resolution limit. Several examples will be presented and discussed in detail. [Work supported by the Office of Naval Research.]

\section{$1: 20$}

2pSA2. The response of an acoustic trace wavelength enhanced array to an isotropic noise field and optimum processing. Ivars $\mathrm{P}$. Kirsteins and Benjamin A. Cray (NUWCDIVNPT, 1176 Howell St., Newport, RI 02841, ivars.kirsteins@navy.mil)

Recently, a new array based on generating Bragg-scattered acoustic wavelengths along the surface of an array was proposed (Enhanced directivity with array grating (J. Acoust. Soc. Am. 136 (2), 2014) and Experimental verification of acoustic trace wavelength enhancement (J. Acoust. Soc. Am. 138 (6), 2015)). The technique, denoted acoustic trace wavelength enhancement, relies on embedding periodic structures within an array, chosen to precisely replicate and shift the incident acoustic wavenumber into higher wavenumber regions. In principle, an important advantage of this array over conventional hydrophone-based arrays of the same size is its greatly improved directivity. A prototype array was built and demonstrated in a test tank under idealized high signal-to-noise ratio conditions. However, two important open questions which remain are how this array responds to ambient noise and how to optimally beamform it when multiple signals are present. Using idealized analytical models for the array's plane wave response, we calculate its spatial wavenumber response to an isotropic noise field and investigate instrumentations for optimum beamforming.

\section{$1: 40$}

2pSA3. Constitutive laws for the design of metallic foam. Elizabeth A. Magliula (NAVSEA Newport, 1176 Howell St., Bldg. 1302, Newport, RI 02841, elizabeth.magliula@navy.mil), James G. McDaniel (Dept. of Mech. Eng., Boston Univ., Boston, MA), and Andrew S. Wixom (Appl. Res. Lab., The Penn State Univ., State College, PA)

Cellular materials have recently found attention in structural applications to buffer impacts and for energy absorption. In order to choose the most suitable cellular material based on its intended application, one must understand its mechanical response either through experimentation and/or modeling. Accurate modeling of the response of a material has many advantages, as it helps avoid extensive experimental laboratory tests and provides a fast and efficient avenue to explore the design space and optimize with a specific intent. The problem solved by the present research is as follows: How does one estimate the foam properties, such as porosity and void fraction, to produce a metallic foam with desired mechanical properties, such as Young's Modulus and Poisson's ratio? The solution to this problem is vitally important as it will yield an entirely new class of materials with tunable mechanical properties and dramatically improved shock and crack resistance. Therefore, the present work seeks to develop constitutive laws for the design of metallic foams. The resulting 
semi-analytical micro-mechanical model is applicable to positive Poisson's ratio (PPR), negative Poisson's ratio (NPR) and zero Poisson's ratio (ZPR) metallic foams. It is developed quasi-analytically, implemented numerically, and compared to experimental data for validation.

\section{Contributed Papers}

2:00

2pSA4. Demonstration of acoustic holographic rendering with twodimensional metamaterial-based passive phased array. Yangbo Xie (Elec. and Comput. Eng., Duke Univ., 3417 CIEMAS, Durham, NC 27705, yx35@duke.edu), Chen Shen, Yun Jing (Mech. and Aerosp. Eng., NC State Univ., Raleigh, NC), and Steven Cummer (Elec. and Comput. Eng., Duke Univ., Durham, NC)

Acoustic holograms in analogy with optical holograms are useful for a variety of applications, such as multi-focal lensing, multiplexed sensing and synthesizing three-dimensional complex sound fields. We previously presented the designs and simulation results of several metamaterial-based acoustic holograms (J. Acoust. Soc. Am. 138, 1751 (2015)). Here, we demonstrate the experimental realization of two of them: one projects a complex pattern on the image plane, while the other focus energy onto multiple circular spots of different sizes. We showcase the holographic reconstruction measurements carried out in an anechoic chamber. The demonstrated passive holograms, without phase-shifting circuitry and transducer arrays, are advantageous for applications where simplicity, robustness, and small footprint are preferred over adaptive control. We also discuss the higher frequency versions of such holograms and their applications in robotic sensing and wireless power transfer. Such metamaterial-based holograms can serve as versatile platforms for various advanced acoustic wave manipulation and signal modulation, leading to new possibilities in acoustic sensing, imaging and energy deposition.

\section{2:15}

2pSA5. A helical-structured acoustic metamaterial. Jie Zhu (Hong Kong Polytechnic Univ., Hong Kong Polytechnic University, Kowloon 00000, Hong Kong, jiezhu@polyu.edu.hk) and Xuefeng Zhu (Huazhong Univ. of Sci. \& Technol., Wuhan, China)

Here, we would like to present a helical-structured acoustic metamaterial that can effectively decelerate sound propagation. Unlike currently avaliable solutions which are mostly dispersive due to the requirement of local resonators., this non-dispersive helical-structured metamaterials is able to provide high effective refractive index. By adjusting the helicity of the acoustic metamaterial structures, we can further modify such refractive index. The performance of this helical-structured acoustic metamaterial has been numerically and experimentally verified. We even designed a lens base on multiple inhomogeneous helical-structured acoustic metamaterial unit cells. This lens can turn normally travelling plane wave into a self-accelerating beam.

\section{2:30}

2pSA6. New frontiers in elastic metamaterials for controlling surface waves. Andrea Colombi (Mathematics, Imperial College, South Kensington Campus, Huxley Bldg., London SW7 2AZ, United Kingdom, andree. colombi@gmail.com), Philippe Roux (ISTerre, Grenoble, France), Daniel Colquitt (Mathematical, Univ. of Liverpool, Liverpool, United Kingdom), Sebastien Guenneau (Institut Fresnel, Marseille, France), Victoriya Ageeva, Matt Clark (Optics and photonics, Univ. of Nottingham, Nottingham, United Kingdom), and Richard Craster (Mathematics, Imperial College, London, United Kingdom)

In this presentation, we show recent results obtained by our research group on two types of elastic metamaterial that are capable of controlling the propagation of surface elastic waves at various physical scales: from tens of meter in geophysics to millimeters in ultrasonic. We begin from the geophysical scale introducing a metamaterial that uses soft soil inclusions in the ground to create a lens for detouring seismic surface waves around an obstacle at frequency $<10 \mathrm{~Hz}$. We then move to the smaller scale presenting a metamaterial that exploits the local resonances in a subwavelength collection of resonators (vertical rods) attached to a finite (plate) or semi-infinite (halfspace) substrate to control either the $\mathrm{A}_{0}$ mode in the plate or Rayleigh waves in the halfspace. We focus on the halfspace case and we illustrate how Rayleigh waves can be (1) mode converted into S-waves or stopped and trapped by a graded array of resonators (seismic rainbow); (2) focused, or detoured using a resonant metalens (Luneburg, Maxwell, and Eaton type) made with the vertical rods. The metamaterial are presented using results from time domain numerical simulations, laboratory and geophysical experiments to highlight the broad applicability of these objects across the wavelength spectrum.

\section{2:45-3:00 Break}

\section{3:00}

2pSA7. A new radial flexible acoustic metamaterial plate. Nansha Gao (School of Marine Sci. and Technol., Northwestern PolyTech. Univ., Xi'an, 710072, China; E-mail:gaonansha@hotmail.com., gaonansha@hotmail. com)

This paper presents the low frequency acoustic properties of a new proposed acoustic metamaterial, which is arranged under axial coordinate. Band structures, transmission spectra, and eigenmode displacement fields are different from previous acoustic metamaterial structures. Numerical calculation results show that first order band gap of radial flexible acoustic metamaterial plate is below $100 \mathrm{~Hz}$. Multiple vibrations coupling mechanism is proposed to explain the low frequency band gaps. Radial flexible acoustic metamaterial plate can restrain low frequency vibration, which can potentially be applied to infrasound protection, generate filters, and design acoustic devices.

\section{3:15}

2pSA8. Experimental measurement of the Willis coupling coefficient in a one-dimensional system. Michael B. Muhlestein (Dept. of Mech. Eng. and Appl. Res. Labs., The Univ. of Texas at Austin, 3201 Duval Rd. \#928, Austin, TX 78759, mimuhle@gmail.com), Caleb F. Sieck (Dept. of Elec. Eng. and Appl. Res. Labs., The Univ. of Texas at Austin, Austin, TX), Preston S. Wilson, and Michael R. Haberman (Appl. Res. Labs. and Dept. of Mech. Eng., The Univ. of Texas at Austin, Austin, TX)

The primary objective of acoustic metamaterial research is to design subwavelength systems that behave as effective materials with novel acoustical properties. Willis coupling is one such property. Initially described by J. R. Willis [Wave Motion 3, pp. 1-11 (1981)], subwavelength structural asymmetry and/or nonlocal effects couple the stress-strain relation to the momentum-velocity relation. This coupling is absent in typical materials. While various theoretical models have predicted the existence of Willis coupling, experimental observation of Willis coupling has not, to our knowledge, been reported in the literature. We present here the first experimental evidence of Willis coupling in a one-dimensional unit cell consisting of a 0.12-mm-thick polyimide membrane under tension, a 5.9-mm-thick layer of air, and a perforated sheet of 0.45 -mm-thick paper (perforation surface fraction 0.48 , circular holes $3.1 \mathrm{~mm}$ in diameter). The properties of the unit cell were extracted using reflection and transmission data measured in a planewave impedance tube operating in the long-wavelength limit relative to the unit cell dimensions. The measured material properties, including the Willis coupling coefficient, are in good agreement with the properties predicted using a transmission line model. [Work supported by ONR.] 
2pSA9. Experimental realization of acoustic double zero index metamaterials. Chengzhi Shi, Marc Dubois, Xuefeng Zhu, Yuan Wang, and Xiang Zhang (Dept. of Mech. Eng., Univ. of California, Berkeley, 3112 Etcheverry Hall, Berkeley, CA 94720, chengzhi.shi@berkeley.edu)

Acoustic double zero index metamaterial with simultaneous zero density and infinite bulk modulus induced by Dirac cone at the Brillouin zone center provide a practical solution for applications. The resulted finite impedance of this metamaterial can be designed to match with surrounding materials. However, such metamaterial consists of scatterers with lower sound speed than the matrix, which is fundamentally challenging for air acoustics because the sound speed in air is among the lowest in nature. Here, we experimentally realize double zero index metamaterial by periodically varying waveguide thickness, which modulates the speed sound of high order waveguide mode. With proper designed dimensions of the scatterers, a Dirac cone at $18.7 \mathrm{kHz}$ formed by the degeneracy of one monopolar and two dipolar modes at the Brillouin zone center is obtain. The monopolar mode modulates the bulk modulus and the dipolar modes modulate the density, resulting in simultaneous zero density and infinite bulk modulus. In our experiment, a point source is mounted at the center of our metamaterial sample. The double zero index metamaterial collimates the wave emitted from the point source to a planar wavefront at $18.7 \mathrm{kHz}$. This point source in a pure waveguide emits cylindrical wave. The experimental result shows that the amplitude of the collimated plane wave is confined within $11 \pm 1$ degrees, closed to the theoretical limit 10.6 degrees.

\section{$3: 45$}

2pSA10. 3D printed membrane-type acoustic metamaterials with structured masses. Alexandre Leblanc and Antoine Lavie (Université Artois, Faculté des Sci. Appliquées, Technoparc Futura, Béthune 62400, France, alexandre.leblanc@univ-artois.fr)

As society evolves, new technologies emerge. They should be considered to address persistent problems such as sound absorption at low frequencies. 3D solid prototyping printers are already used to obtain efficient sound diffusers, but remain marginal for creating acoustic absorbers. Recently, sub-wavelength absorbers have been proposed, particularly in the form of membrane-type acoustic metamaterials. These lasts usually consist on a decorated membrane resonator with tunable weight. In this work, membranetype metamaterials are fabricated by fused deposition modeling, and both the membrane and the added masses are all made by the same flexible material. This allows to study other geometries, while structuring the added masses. Indeed, they can be split to increase peak efficiencies of this metamaterial to obtain an efficient band-stop filter. This application is illustrated with a base decorated membrane, using a divided central platelet and/or an additional split ring. Also, preliminary numerical simulations show that these structured membranes are likely to be more efficient than membranes with rigid inclusions when subjected to a diffuse sound field.

\section{4:00}

2pSA11. Elastic wave dispersion in pre-strained negative stiffness honeycombs. Benjamin M. Goldsberry and Michael R. Haberman (Appl. Res. Labs., Univ. of Texas at Austin, 10000 Burnet Rd., Austin, TX 78758, bmgoldsberry@gmail.com)

A doubly periodic array of curved beams, known as a negative stiffness (NS) honeycomb, has recently been shown to exhibit tunable impact isolation by exploiting nonlinear mechanical behavior [Correa et al., Rapid Prototyping J., 21 (2), 2015]. The primary benefit of NS honeycombs over shape after experiencing an impact. The recoverable nature of their response is a result of the curved beam structure which has a single stable configuration but experiences one or more buckling events when loaded. The complex nonlinear elastic response of NS honeycombs lead to large variations in mechanical stiffness, which makes these materials compelling candidates for tunable control of elastic waves. The present work investigates linear elastic wave propagation of a representative NS honeycomb lattice at varying levels of pre-strain. Eigenfrequency finite element analysis is performed on the unit cell subjected to finite uniaxial deformation. The longitudinal and transverse phase speeds are shown to be anisotropic and highly dependent on uniaxial pre-strain. This is especially true near points of instability, where one observes very different functional dependence of longitudinal and transverse wave motion on pre-strain.

\section{$4: 15$}

2pSA12. Metamaterial ground cloak with anisotropic solid inclusions. Benjamin Beck, Amanda Hanford, and Peter Kerrian (Appl. Res. Lab., Penn State Univ., PO Box 30, MS 300B, State College, PA 16804-0030, benbeck@psu.edu)

There has been much research on the use of metamaterials within ground cloaks to hide objects on a rigid surface. Within the literature, ground cloaks made with metamaterials based on unit cells with solid inclusions have required the use of at least two distinct materials within the unit cell to achieve the necessary anisotropic material properties calculated from a transformation method. Here, we present a method to calculate the material properties of an anisotropic solid inclusion for use in a metamaterial ground cloak. The generated materials properties are then shown to be realized through an additively manufactured anisotropic solid inclusion. Finally, numerical results of cloaking performance of the metamaterial ground cloak are presented.

\section{4:30}

2pSA13. Effect of non-uniform mean flow to the scattering pattern of acoustic cloak. Wonju Jeon (Korea Adv. Inst. of Sci. and Technol. (KAIST), 291 Daehak-ro, Yuseong-gu, Daejeon 34141, South Korea, wonju.jeon@kaist.ac.kr)

During the last decade, most of acoustic cloak researches have been done within a theoretical framework in which the medium is at rest. However, such acoustic cloaks cannot preserve its unique properties or functions in the presence of flow. In this study, we propose a theoretical framework to consider the effect of non-uniform mean flow to acoustic cloak for the purpose of understanding the physics of acoustic cloak within flow and designing a new concept of metamaterial so called aeroacoustic cloak. We formulate a convective wave equation in which the differential operator contains the non-uniform velocity vector coupled with gradient operator and the equivalent source terms due to the mean flow are divided into two terms with their own physical meanings. The performance of existing convective cloak is investigated by using the present formulation in the presence of non-uniform flow. By comparing the existing and present formulations, the polarity and magnitude of equivalent source are compared: the present source shows hexapole pattern whereas the existing source shows quadrupole pattern. The non-uniformity effect was dominant in hexapole pattern. Finally, we found that although the compressibility effect is much smaller than non-uniformity effect in low Mach number range, it is not negligible in high Mach number flow, especially for small Helmholtz number. Understanding the nature of equivalent source is expected to provide a physical insight to design a new acoustic cloak within fluid convection. 


\title{
Session 2pSC
}

\section{Speech Communication: Articulatory and Acoustic Phonetics: Data and Methods (Poster Session)}

\author{
Grant L. McGuire, Chair \\ Linguistics, UC Santa Cruz, 1156 High Street, Stevenson Academic Services, Santa Cruz, CA 95064
}

\begin{abstract}
All posters will be on display from 1:00 p.m. to 5:30 p.m. To allow contributors in this session to see the other posters, authors of odd-numbered papers will be at their posters from 1:00 p.m. to 3:15 p.m. and authors of even-numbered papers will be at their posters from 3:15 p.m. to 5:30 p.m.
\end{abstract}

\section{Contributed Papers}

2pSC1. Acoustic phonetic study of phonemes and tonemes of spoken Punjabi language. Shyam S. Agrawal, Shweta Bansal, Shambhu Saran, and Amritpal Singh (College of Eng., KIIT, Sohna Rd., Near Bhondsi, Gurgaon, Haryana 122102, India, dr.shyamsagrawal@gmail.com)

Punjabi language is one of the important languages among 22 official languages in India. It is spoken by about 105 million people in India. The present paper describes a study and results of detailed acoustic analysis of vowels, consonantal phonemes and tonemes as spoken by the speakers of Malwai dialect of Punjabi language. A database of 1500 words containing all the phonemes and tonemes, selected from a text corpus of 300,000 words were used for the study. These words were recorded and segmented by usingsignal processing tools to analyze the samples of speech. Fundamental frequency, first three formants, and bandwidths for nasal and non-nasal vowels were measured. For the study of consonants, duration of sub-phonemic segments such as occlusion, burst, and VOT have been computed and compared. Special features of tonemes have been studied and compared with non-tonal phonemic segments. Tones are fully phonemic and words with similar spellings are distinguished by varying tones: low, mid, and high and corresponding accent marks. It has been observed that intonation plays a significant role in the discrimination of phonemes and tonemes. These results can be used to create PLS and phonetic dictionary of Punjabi speech.

2pSC2. Intonation contours in Hawai'i Creole and Hawai'i English show influence from Hawaiian. Victoria B. Anderson and M. J. Kirtley (Dept. of Linguist, Univ. of Hawai'i, 569 Moore Hall, 1890 East-West Rd., Honolulu, HI 96822, vanderso@hawaii.edu)

Hawai'i Creole (ISO 639-3 hwc) and Hawai'i English (HE) are speech varieties spoken by local residents of the Hawaiian Islands. Three native speakers of HWC, seven native speakers of HE, and three native speakers of Hawaiian (ISO 639-3 haw) were recorded speaking in casual interviews, participating in map tasks, and saying phonetically controlled sentences. Analysis focused on utterances containing the high (or rise) plus steep fall contour, which is not found in Mainstream American English but is often used for continuations and polar questions in HWC, HE, and Hawaiian (Vanderslice \& Pierson 1967, Anderson 2003, Murphy 2013). Results for all three speech varieties showed alignment of F0 minimums with lexically stressed syllables at the end of an intonation phrase, with F0 peaks occurring on immediately preceding syllables. All three speech varieties show a preponderance of this steep drop in continuations and polar questions, confirming the idea that this is a feature borrowed from Hawaiian (Bickerton and Wilson 1987, Anderson 2003.)
2pSC3. The effect of context on the articulation of harmonic vowels in Assamese: An ultrasound study. Diana Archangeli and Jonathan Yip (Linguist, Univ. of Arizona/Univ. of Hong Kong, Pok Fu Lam Rd., Hong Kong, Hong Kong, darchang@hku.hk)

Tongue root (TR) harmony in Assamese affects high back vowels and mid vowels (Mahanta 2007). The description (based on perception and limited acoustic measurements) is that Assamese shows the following five properties.-Constrast: TR contrasts in monosyllabic words. — [bel] "bell" vs. [bel] "stupid person"-Harmony: Vowels agree with a following vowel, e.g., triggered by suffixes. - [nomori] "not-die.non-finite" vs. [nəməra] "not-die.habitual.non-finite"-CC-blocking: Harmony crosses one consonant only, no harmony in __Ci vs. harmony in _Ci. — [bonti] "lamp" vs. [nomori] "not-die.non-finite"-N-blocking: There is no harmony in

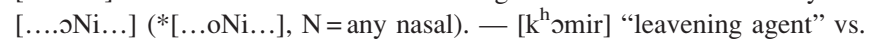
[nomori] "not-die.non-finite"-Idiosyncrasy: /a/, which otherwise does not harmonize, appears as $[\mathrm{e}] /[\mathrm{o}]$ before an idiosyncratic set of suffixes. [kopal] "destiny" vs. [kopolija] "destined." This ultrasound study explores Assamese vowel articulation to determine the nature of tongue position in different harmonic contexts, based on data collected in Assam from 10 subjects. Limitations of ultrasound technology prevented a clear image of the tongue root; indirect measures based on tongue dorsum height and backness are used instead. Results confirm perceptual/acoustic descriptions, showing a contrast in tongue position in all contexts: (i) Contrast, (ii) Harmony, (iii) CC-blocking, (iv) N-blocking, and (v) Idiosyncrasy.

2pSC4. An acoustic analysis of morphological glottalization in Hul'q'umi'num' Salish. Kevin M. Baetscher (Linguist, Univ. of Hawaii at Mānoa, 3239 Huelani Dr., Honolulu, HI 96822, kbaetsch@ hawaii.edu)

Glottalization is lexically contrastive for both obstruents and resonants in Hul'q'umi'num' Salish. Glottalization of resonants is also a morphological process involved in the marking of progressives forms, among others. In these forms, all non-initial resonants (but not obstruents) become glottalized. This study investigates the question of whether the glottalization involved in the formation of progressives is a process that affects resonants locally, or whether glottalization as a feature is applied to the whole word. To this end, the glottal pulse periods of vowels in progressive forms have been measured and compared to modal schwas, to see whether glottalization in these words applies to vowels, as well. The results are negative and thus support the hypothesis that morphological glottalization (at least of progressive forms in Hul'q'umi'num') applies to resonants only, as opaque as this may appear from a phonological point of view. 
2pSC5. Vowel duration before ejective stops. Gašper Beguš (Dept. of Linguist., Harvard Univ., Boylston Hall, 3rd Fl., Cambridge, MA 02138, begus@fas.harvard.edu)

It has been well established that vowels are phonetically longer before voiced than voiceless stops, all else being equal. To my knowledge, however, no studies have measured vowel durations before ejective stops. This paper aims to fill this gap: I present preliminary results from a phonetic experiment with nine speakers of Georgian, establishing for the first time that vowels are (i) significantly longer before ejective stops than before voiceless aspirated stops and (ii) significantly shorter before ejective stops than before voiced stops. While the dependent relationship between vowel duration and obstruent voicing is well known, the cause of this relationship is poorly understood. The results presented in this paper shed new light on our understanding of differences in vowel durations and, in doing so, render several proposed explanations for these differences considerably less likely. In particular, my results show that (i) the voice feature itself likely does not affect vowel duration, (ii) closure duration of the following stop is not inversely correlated with vowel duration, and (iii) perceptual factors likely play no role in determining vowel length. My study shows that laryngeal features are the best predictors of vowel duration, suggesting an articulatory connection between these two factors.

2pSC6. Tenseness in Northern Yi: An ultrasonic and acoustic study. Shuwen Chen (The Dept. of Linguist and Modern Lang., The Chinese Univ. of Hong Kong, G19, Leung Kau Kui Bldg., Hong Kong, Hong Kong, chenshuwen@link.cuhk.edu.hk) and Ziwo Lama (College of Yi Studies, Southwest Univ. for Nationalities, Chengdu, Sichuan, China)

A contrast between tense and lax vowels is a main feature of many Tibeto-Burman (TB) languages spoken in Southwest China. This study investigated the articulation and acoustics of tense-lax contrast in two Northern Yi dialects: Suondi and Yinuo. Both dialects have five pairs of phonologically-defined tense and lax vowels. Previous studies have shown that tense and lax vowels in TB had distinct patterns in phonation types and f0, while the difference in other acoustic dimensions - vowel quality, duration, and intensity are rarely examined, and the difference in the lingual gestures is largely unknown. The current study examined the articulatory and acoustic characteristics of tense-lax contrast using ultrasound imaging and spectrogram analysis. Our preliminary results showed that articulatorily, tense vowels were produced with more retracted tongue root. Acoustically, tense vowels had higher F1 and lower F2, which was caused by the lowered and retracted tongue position. The intensity of tense vowels was lower than that of lax vowels for front vowels. No significant difference was found in duration. Based on the articulatory and acoustic data, as well as the vowel harmony phenomenon in $\mathrm{Yi}$, we propose that the tense-lax distinction in Northern Yi can be better represented as [+RTR] phonologically.

2pSC7. F0 and plosive voicing in Afrikaans. Andries W. Coetzee, Patrice S. Beddor, Dominique Bouavichith, Justin T. Craft (Dept. of Linguist., Univ. of Michigan, 440 Lorch Hall, 611 Tappan St., Ann Arbor, MI 48109, coetzee@umich.edu), and Daan Wissing (North-West Univ., PotchefstRm., North-West, South Africa)

Afrikaans plosives are traditionally described as contrasting in voicing ([b d] vs. [p t]). Coetzee et al. (2014, J. Acoust. Soc. Am. 135, 2421), however, showed that the voicing contrast is collapsing word-initially, with voiced plosives merging with voiceless plosives. They also found that voicing loss does not result in loss of lexical contrast, which is preserved on the following vowel (high f0 after historically voiceless and low f0 after historically voiced plosives). That study investigated word-initial plosives, leaving unanswered whether word-medial plosives are also devoicing. The current study addresses this question. Acoustic analysis of data collected from nine Afrikaans speakers replicated the results of Coetzee et al. for word-initial plosives. For word-medial plosives, a robust voicing contrast was found along with a post-plosive fo difference. The f0 difference in medial position was comparable to that in initial position in both magnitude and duration, and extended throughout the vowel. While lexical contrasts are hence cued by only f0 word initially, they are differentiated by both plosive voicing and the f0 of the following vowel word medially. The relevance of these results for theories of sound change and theories of phonological contrast will be discussed. [Work supported by NSF.]
2pSC8. Contextual place neutralization of nasal codas in Taiwanese Mandarin: An Elecrtomagentic Articulography Study. Yuehchin Chang and Yi Yeh (Inst. of Lingsuit., National Tsing Hua Univ., 101, Sec. II, Kuang Fu Rd., Hsinchu, Taiwan 300, Taiwan, ycchang@mx.nthu.edu.tw)

It has long been noted that /in/ and /in/ are neutralized to either [in] or [in] in Taiwanese Mandarin. This study aims at investigating this phenomenon and more generally, coarticulatory patterns of $\mathrm{VN}$ rimes ([an] and [an] here) from articulatory perspectives. Kinematic data from two speakers were collected using an NDI Wave. Regarding /in/ vs. /in/, a principle components analysis (PCA) was conducted to see if a specific flesh point is more positively (or negatively) related to the distinction of the tongue contours. For the female speaker, Tongue Tip (TT)-height is most positively correlated, whereas Tongue Dorsum (TD)-height is most positively correlated for the male speaker. Regarding the nasal rimes with /a/, TD-height is most positively correlated for the male speaker, while TT-backness is most negatively correlated for the female speaker. The main findings are: (i) the contextual neutralization of place contrasts in nasal codas is not articulatorily confirmed, that is, the phenomenon in question is better characterized as incomplete neutralization, (ii) there is inter-speaker variation in the implementation of articulatory gestures and (iii) "anomalous" tongue shapes are found in the production of [in], suggesting that the actual coarticulatory patterns within VN rimes are more complicated than previously thought. These obtained data will also be compared with the acoustic and airflow data reported in our previous studies.

2pSC9. The vowel system of Nasa Yuwe. Didier Demolin, Angélique Amelot, Lise Crevier-Buchman (LPP Paris 3, 19 rue des Bernardins, Paris 75005, France, ddemolin@univ-paris3.fr), Tulio Rojas, and Esteban Diaz (Universidade de Cauca, Popayan, Colombia)

Nasa Yuwe (sometimes referred as Paes) is a language spoken in Colombia. The vowel system has four vowels timbers [i, e, a, u] that can be oral or nasal (short and long); breathy, breathy nasal; glottal, glottal nasal. This makes a total of 32 contrastive vowels. This study examines the acoustic and articulatory characteristics of each type of vowel. Data come from synchronized acoustic, EGG and fiberscopic measurements. A special focus is given to the acoustic and articulatory features of breathy and glottal vowels. The breathy character appears in the last part of the vowel. This is realized by a slightly wider opening between the vocal folds or by an aperture between the arytenoids. The glottal character of the vowels also appears at the end. The data clearly show that glottal vowels are different from a laryngalized counterpart. Spectrographic and EGG observations show irregularities towards the end glottal vowels. These are followed by a closure or a stricture made either at the glottis or in the epilaryngeal tube. Fiberscopic and EGG data show that there is a glottal closure at the end of these vowels. This closure precedes a closure of the ventricular bands.

2pSC10. Method for recognizing multiple emotions from acoustic features using soft clustering. Joji Uemura, Kazuya Mera, Yoshiaki Kurosawa, and Toshiyuki Takezawa (Graduation School of Information Sci., Hiroshima City Univ., 3-4-1, Ozuka-higashi, Asa-minami-ku, Hiroshima 7313194, Japan,4dai0715@gmail.com)

When a speaker expresses multiple emotions at the same time, emotion recognition methods using multi-class classification cannot recognize all the emotions because they always choose "an" emotion class. We thus propose a method to recognize multiple emotions expressed in parallel by using soft clustering (fuzzy c-means algorithm). Seven support vector machines for regression (SMOreg) calculate element values of the data. The SMOregs calculate probabilities of the following seven emotions: anger, anticipation, disgust, fear, joy, sadness, and surprise. First, 584 pieces of emotional voice data annotated with one of the seven emotions were used to calculate eight cluster centers on the basis of 384 acoustic features. Next, the characteristic of each cluster was defined on the basis of annotated tags of data belonging to the cluster. The characteristic was expressed in terms of valence (pleasure-displeasure) and arousal (high-low). To evaluate whether voice data that have two high membership grades have both emotion characteristics of the highmembership clusters, calculated membership grades were compared with the results of a questionnaire in which 10 subjects gave their impressions of the voices. We will show the experimental results and the comparison in the presentation. 
2pSC11. A nasograph and fibrescopic analysis of nasal diphtongs in Brazilian Portuguese. Rita Demasi, Angélique Amelot, Lise Crevier-Buchman, and Didier Demolin (LPP Paris 3, 19 rue des Bernardins, Paris 75005, France,ddemolin@univ-paris3.fr)

This paper examines velum movements during the production of front and back nasal diphtongs [ẽ̃̃], [ãõ] in Brazilian Portuguese. Velum movements were measured with a female speaker from Sao Paulo using a nasograph and fibrescopic video-recordings synchronized with acoustic recordings. The nasal diphthongs were always situated at the end of a syllable in real words containing nasal diphthongs. Contrasting oral diphthongs were also recorded for comparison. Our results show that there is a nasal appendix the end of the nasal diphthongs. This is made by a contact between the tongue dorsum and the velum. These observation are based on nasograph and fibrescopic data. They confirm previous measurements made by EMA and aerodynamic measurements with the same subject. This appendix is sometimes perceived as a short velar nasal consonant. The duration of nasal appendix is slightly different when front and back nasal diphthongs are compared. Data also show that the lowering of the Velum occurs gradually for a nasal diphthong. This can start at any time during the first part of the diphthong.

2pSC12. For bilinguals, Enxet vowel spaces smaller than Spanish. John Elliott (Linguist, Univ. of Hawai'i at Mānoa, 1117 3rd Ave., Unit C, Honolulu, HI 96816, johnaell@hawaii.edu)

This study compares the vowel spaces of bilingual speakers of Spanish and Enxet Sur (ISO 639-3: enx), a moderately threatened language spoken by around 4000 speakers in the Paraguayan Chaco which purportedly lacks high vowels. The Enxet vowel system has only three phonemic vowel qualities /e,a,o/ and a length distinction. Using recorded natural speech data from two Enxet/Spanish bilinguals, formant values for vowel tokens are used to establish phonetic targets and acoustic vowel clouds for each of the phonemic vowels in both languages. A comparison of these vowel spaces suggests that an individual bilingual speaker uses a smaller vowel space when speaking Enxet (less vowel height, higher minimum F1 frequencies) than when speaking Spanish. Languages like Enxet lacking in true high vowels present problems for the theory of adaptive dispersion in that they do not "take advantage" of maximal distinctiveness in the acoustic and articulatory quality of their vowels. This is the first known phonetic investigation of an Enlhet-Enenlhet language.

2pSC13. Velar flutter does not Occur on Persian radico-uvular /G/ sound: Case reports. Marziye Eshghi (Speech, Lang. and Hearing Sci., Univ. of North Carolina at Chapel Hill, 002 Brauer Hall, Craniofacial Ctr., Chapel Hill, NC 27599, marziye_eshghi@med.unc.edu) and David J. Zajac (Dental Ecology, Univ. of North Carolina at Chapel Hill, Chapel Hill, NC)

Nasal turbulence with velar flutter is an obligatory symptom that occurs during production of high-pressure sounds due to a partially closed velopharyngeal (VP) port. A small VP gap creates air-pressure high enough to set the marginal edges of the velum into vibration. The flutter component is characterized spectrally by low frequencies (Zajac \& Preisser, 2015) and vibratory activity through auto-correlation functions (Eshghi et al., 2015). In this study, two speech-language pathologists independently listened to the audio recordings of stops produced by two Persian-speaking children (1 male, 8 yrs and 1 female, 9 yrs) with repaired cleft palate. Speech samples consisted of Persian plosives including /p, b, t, d, k, g, G/. The two listeners achieved $100 \%$ agreement that velar flutter occurred on all stops except /G/, a voiced radico-uvular sound. Spectral evaluation indicated characteristics of flutter were present during all stops except for /G/. It is hypothesized that tissue vibration was inhibited during the production of $/ \mathrm{G} /$ due to the the tongue making contact with the posterior part of the soft palate and/or uvula. The findings provide cross-linguistic evidence in support of tissue vibration (velar flutter) rather than displacement of mucous (bubbling) as the primary perceptual component of nasal turbulence.
2pSC14. Voiceless vowels in Miyako. Catherine Ford, Benjamin V. Tucker, and Tsuyoshi Ono (Univ. of AB, Unit 204711180 Ave. NW, Edmonton, AB T6B0C7, Canada, cford1@ualberta.ca)

The present study investigates the nature of vowel devoicing in Miyako, a Ryukyuan language spoken on remote Japanese islands near Taiwan. High vowels in Japanese are commonly described as being devoiced after voiceless consonants in two environments: 1) before a voiceless consonant, 2) at the end of a word (Shibatani 1990). Being a Japonic language, Miyako is also described as having a similar phenomenon but devoices all vowels in its phonemic inventory /i, i, a, u/ (Hayashi 2013). Data from several speakers suggests that there may be cases of vowel deletion as well. Current data suggest vowel deletion between two voiceless consonants, creating the potential for consonant clusters in Miyako-something that is said not to occur in Japanese. The acoustic nature of voiceless vowels in Miyako is investigated using both elicited data and discourse data. Data were collected from the Ikema Island community, limiting data to the Ikema dialect. Data were analyzed in PRAAT to determine whether potential voiceless vowels are indeed devoiced or deleted. The present investigation contributes to our overall understanding of the nature of vowel devoicing/deletion in a highly under-documented language Miyako and Ryukyuan languages in general.

2pSC15. A preliminary ultrasound examination of Upper Sorbian rhotics. Phil Howson (The Univ. of Toronto, Sidney Smith Hall, 4th Fl. 100 St. George St., Toronto, ON M5S 3G3, Canada, phil.howson@mail.utoronto.ca)

Upper Sorbian has been influenced tremendously by German, causing it to adopting the uvular rhotic. The current study examines the uvular rhotic and its palatalized counterpart in three vocalic environments (word initial, intervocalic, and word final) with the vowels /a, e, o, i/ using ultrasound. The preliminary results indicate a striking difference between the two phonemes. The plain uvular rhotic was characterized by a retracted and raised tongue dorsum, accompanied by a slightly retroflexed tongue tip. The retroflexed tongue tip is surprising for a uvular rhotic. It could be related to requirements for rhotic production or a remnant of the alveolar rhotic Upper Sorbian had historically. The environments with /i/ were especially striking: the uvular rhotic was palatalized intervocalically; the tongue body was raised near the palatal region, while the dorsum retracted strongly. In other word positions, the rhotic caused a lowering and retraction of /i/. The palatalized rhotic had a raised tongue body, but the tongue dorsum was as retracted as the plain rhotic and less susceptible to environmental influence. The tongue tip was laminal in articulation, rather than retroflexed. The more consistently retracted tongue dorsum may be related to a need to maintain rhotic qualities. [Work supported by SSHRC.]

2pSC16. Temporal organization of onglides in standard Chinese and Taiwanese Mandarin: A cross-dialectal study. Feng-fan Hsieh, Guansheng $\mathrm{Li}$, and Yueh-chin Chang (Inst. of Linguist., National Tsing Hua Univ., 101 Kuang-fu Rd., Sec. 2, Hsinchu 30013, Taiwan, ffhsieh@mx. nthu.edu.tw)

This study examines the temporal organization of prenuclear glide (onglide) gestures within a syllable. Kinematic data were collected from four Standard Chinese (SC) and four Taiwanese Mandarin (TM) speakers using an NDI Wave. Mean relative timing of C/G gestures to a (heterosyllabic) consonantal anchor $/ \mathrm{p} /$ were calculated using the lag between achievement of constriction of the $\mathrm{C} / \mathrm{G}$ and $/ \mathrm{p} /$. By comparing a given subject's mean lags for $\mathrm{Cs} / \mathrm{Gs}$ with CGs, we calculated the subject's leftward (C vs. $\mathrm{CG}$ ) and rightward (G vs. CG) shifts associated with $/ \mathrm{pj} /$ and $/ \mathrm{kw} /$. Results show that in SC, onglides $/ \mathrm{j} /$ and $/ \mathrm{w} /$ don't pattern alike: for $/ \mathrm{kw} /$, all SC speakers show both leftward and rightward shifts (i.e. the C-center effect), except for subject $\mathrm{S} 3$, who fails to show a leftward shift (due to a different regional accent). For $/ \mathrm{pj} /$, only rightward shifts are found for all SC speakers. In contrast, regarding /kw/, only leftward shifts are found for all TM speakers, except for subject S8 (due to a different accent), while for $/ \mathrm{pj} /$, all TM speakers show rightward shifts, again, except for subject S8. Our findings thus suggest a cross-dialectal difference in $/ \mathrm{kw} /$ and confirm the possibility of the rightward shift in gestural coordination. Implications for the phonological status of onglides in Mandarin Chinese will also be discussed. 
2pSC17. Voicing contrast in Punjabi Singletons and Geminates. Qandeel Hussain (Linguist, Macquarie Univ., Australia, 65 Gwen Meredith Loop, Franklin, ACT 2913, Australia, qandeel.hussain@students.mq.edu.au)

Cross-linguistic studies have shown that voiceless geminates are more frequent than voiced geminates (Blevins, 2004). From an articulatory perspective, maintaining voicing and long closure duration in voiced geminates is challenging (Ohala, 1983). Punjabi, an Indo-Aryan language, has been reported to contrast voiceless/voiced singletons and geminates. However, it is not known if voiced geminates in Punjabi are completely voiced and how their durational properties differ from voiceless geminates. The aim of the present study was therefore to investigate the acoustic characteristics of Punjabi voiceless/voiced singleton and geminate stops. Five native speakers of Punjabi participated in the experiment (24-26 years, $\mathrm{M}=24.6)$. The stimuli consisted of nonce words with word-medial $\left(\mathrm{C}_{2}\right)$ voiceless/voiced singleton and geminate stops $\left(\mathrm{C}_{1} \mathrm{~V}_{1} \mathrm{C}_{2} \mathrm{~V}_{2}\right)$. The $\mathrm{C}_{2}$ duration was measured in PRAAT, by using the broadband spectrograms and visual inspection of the waveforms (Ridouane, 2007). The durational analysis of $\mathrm{C}_{2}$ indicated that unlike voiceless singletons/geminates, voiced singletons/geminates showed complete voicing during the closure phase. These findings differ from Tokyo Japanese where voiced geminates are partially devoiced (Kawahara, 2016). However, voiceless singletons/geminates are slightly longer in duration compared to voiced singletons/geminates. The current study raises questions about the cross-linguistic differences in voiceless/voiced singletons and geminates.

2pSC18. Duration of Japanese singleton and geminate stops with devoiced vowel in various speaking rates. Shigeaki Amano (Faculty of Human Informatics, Aichi Shukutoku Univ., 2-9 Katahira, Nagakute, Aichi 480-1197, Japan, psy@asu.aasa.ac.jp), Kimiko Yamakawa (Culture and Lang., Shokei Univ., Kikuchi-gun, Kumamoto, Japan), and Mariko Kondo (School of Int. Liberal Studies, Waseda Univ., Shinjuku-ku, Tokyo, Japan)

Previous studies have shown that a devoiced vowel makes burst duration of a singleton stop consonant longer than a voiced vowel does. However, the effects of devoiced vowels on stop consonants including a geminate have not been fully investigated. To clarify the effects, durations of a burst and a closure in Japanese stops pronounced by 19 native Japanese speakers were analyzed by stop type (singleton or geminate) and speaking rate (fast, normal, or slow). Analysis revealed that at all speaking rates a devoiced vowel makes the burst duration of singleton and geminate stops longer than a voiced vowel does. Analysis also revealed that at normal and fast speaking rates a devoiced vowel has no effect on closure duration in singleton or geminate stops. However, at a slow speaking rate, a devoiced vowel makes the closure duration of a singleton stop longer. These results indicate that a devoiced vowel consistently lengthens the burst duration of singleton and geminate stops but that its effects on closure duration depend on both stop type and speaking rate. [This study was supported by a special-research grant of Aichi Shukutoku University in 2015-2016, and by JSPS KAKENHI Grants Number JP 25284080 and 26370464.]

2pSC19. Oral/nasal airflow during Japanese stop consonants. Masako Fujimoto (Adv. Res. Ctr. for Human Sci., Waseda Univ., 2-579-15, Mikajima, Tokorozawa, Saitama 359-1192, Japan, mfuji@viola.ocn.ne.jp) and Reiko Kataoka (Adv. Res. Ctr. for Human Sci., Waseda Univ., San José, CA)

Voiced obstruents have inherent susceptibility for devoicing due to the Aerodynamic Voicing Constraints (AVC), and the susceptibility is higher for geminate obstruents than singletons. As a way to investigate how Japanese speakers realize the contrast between the [+/-voice] contrast in obstruents, we examined oral and nasal airflow patterns during intervocalic voiced and voiceless stops, in singletons and geminates. The results showed asymmetry between single and geminate stops in realization of the stop voicing contrast. Airflow pattern clearly differentiates voiced vs. voiceless contrast in singletons, but the airflow patterns are similar in geminates. Acoustic signals also shows the same asymmetry between the singletons and geminates. The observed convergence-clear voicing contrast in singletons vs. the lack of the contrast in geminates both in air flow and acoustic signals indicate neutralization of the voiced geminates into voiceless ones. Our results support the idea of phonetic bases in phonological patterning of voicing neutralization in Japanese geminate stops.
2pSC20. Pitch accent and vocal effort in Japanese. Ryota Iwai (Dept. of Korean Studies, Graduate School of Humanities and Sociology, Univ. of Tokyo, 7-3-1, Hongo, Bunkyo-ku, Tokyo 113-0033, Japan, 3181957501@ snu.ac.kr) and Ho-Young Lee (Seoul National Univ., Seoul, South Korea)

It is implicitly assumed that each mora is pronounced with similar vocal effort in Japanese. Our intuition, however, is that the first or second mora is pronounced with the greatest vocal effort. This paper aims to verify this observation by analyzing vocal effort parameters CPP and H1-A3. We prepared two sets of real words and two sets of nonce words, each set consisting of 3 two mora words, 4 three mora words and 5 four mora words, varying the position and existence of pitch accent. Twelve subjects aged 2139 participated in the recording. They read randomized test words 6 times. The result of the H1-A3 measurement shows that the first mora is significantly pronounced with the greatest vocal effort irrespective of the place and existence of pitch accent. The results of the CPP measurement show that the initial accented mora is pronounced with the greatest vocal effort, and that the second mora is pronounced with greater vocal effort than the first one if the first mora is not accented. The non-initial moras of three or four mora words are pronounced with similar vocal effort if the third or fourth mora is accented or a test word is unaccented.

2pSC21. Acoustic characteristics of liquid geminates in Japanese. Maho Morimoto (Linguist, Univ. of California, Santa Cruz, 1156 High St., Santa Cruz, CA 95064, mamorimo@ucsc.edu)

Japanese contrasts consonant length. Much research has been done on the acoustic characteristics of obstruent geminates in Japanese, identifying the constriction duration as the primary acoustic correlate. The ratios of the duration of the consonants and the surrounding vowels have also been investigated, and it was pointed out that the ratios differ from some other languages with distinctive consonant length, such as Italian. The present study examines the acoustics of liquid geminates in Japanese. Phonological phenomena in Japanese such as verb inflection demonstrate that the language dislikes liquid geminates. However, while not as common as obstruent or nasal geminates, liquid geminates are not impossible; they are present in certain loanwords and emphatic expressions. I report on the acoustic correlates of liquid geminates focusing on consonant sonority and consonantvowel ratio, in three environments controlled for vowel quality and pitchaccent position: namely, loanwords (mostly from Italian and Arabic), emphatic forms of adjectives (e.g., karai "spicy" > karrai) and emphatic forms of onomatopoeic expressions (e.g., garagara "expresses emptiness" > garragara), pronounced by three native speakers of Tokyo dialect of Japanese in laboratory settings.

2pSC22. Prosodic analysis of storytelling speech in Japanese fairy tale. Takashi Saito (Appl. Comput. Sci., Shonan Inst. of Technol., 1-1-25, Tsujido Nishikaigan, Fujisawa, Kanagawa 251-8511, Japan, saito@sc.shonanit.ac.jp)

This paper presents a prosodic analysis of storytelling speech in Japanese fairy tale. Recent advances in TTS technologies bring us high quality synthetic speech especially on acoustic aspects. On prosodic aspects, however, there is still room for improvement in the expressiveness since most systems use one-sentence speech synthesis scheme. For instance, storytelling applications expect speech synthesis to be capable of having a control mechanism beyond one sentence. In this paper, a prosodic database is built for real storytelling speech of professional narrators on Japanese fairy tale aiming at investigating the actual storytelling strategies and ultimately reflecting them on the expressiveness of speech synthesis. After conducting a baseline speech segmentation for phones, words, and accentual phrases in a semi-automatic way, a multi-layered prosodic tagging is manually performed to extract information on various changes of "story states" relevant to impersonation, emotional involvement, and scene flow control. Storytelling speech materials of six professional narrators for Japanese fairy tale are prepared to analyze their storytelling strategies. In particular, the dynamics of pitch contours is investigated in terms of expressiveness control beyond sentence considering narrator dependency as well. Based on the findings obtained, pitch control schemes are discussed for storytelling speech synthesis. 
2pSC23. Acoustics of phonation types and tones in Santiago Laxopa Zapotec. Jeffrey M. Adler and Maho Morimoto (Linguist, Univ. of California, Santa Cruz, 1156 High St., Santa Cruz, CA 95064, mamorimo@ucsc. edu)

Zapotec languages are known for their complex tonal and phonation systems. Fieldwork in Santiago Laxopa Zapotec (SLZ), an undocumented language spoken in Oaxaca, Mexico, suggests the implementation of six tones (high, mid, low, high-falling, mid-falling and rising) and four phonation types (modal, creaky, checked and breathy). We report on the reliability of acoustic measures such as $(\mathrm{H} 1-\mathrm{H} 2)$ and $(\mathrm{H} 1-\mathrm{A} 1)$, which have previously been reported to characterize phonation types. Spectral measurements including H1, H2 (first and second harmonics), A1 (amplitude of F1) and A2 (amplitude of F2) are taken from vowels in near minimal pairs controlled for tones and quality. We also report on the relative implementation of tone and phonation. Silverman (1997) argues that languages with contrastive tone and phonation optimize the realization of laryngeal events such that non-modal vowels have a modal portion where the tonal event is implemented. We discuss if Silverman's predictions are borne out in SLZ.

2pSC24. Formant trajectories in the realization of 3 Malayalam rhotics. Alexei Kochetov and Phil Howson (Linguist, Univ. of Toronto, 100 St. George St., Sidney Smith 4076, Toronto, ON M5S 3G3, Canada, al.kochetov@utoronto.ca)

Malayalam has a typologically rare set of three rhotic consonants-an alveolar trill, an alveolar tap, and a retroflex/postalveolar approximant. The first one is described as velarized, while the other two are somewhat palatalized. Previous acoustic work by Punnoose et al. [Phonetica 70, 274-297 (2013)] has established that the contrast can be differentiated by formant values around the constriction, as well as at the midpoint of adjacent vowels. Less clear, however, is the temporal extent of these formant differences and their overall dynamics. In this study, we analyze three rhotics produced by 10 male Malayalam speakers. Measurements of F1-F4 were taken at 10 points throughout the VCV interval and subjected to a series of Smoothing Spline ANOVAs. The results revealed differences in F2 (tap, approximant $>$ trill) and F3 (tap, trill $>$ approximant) among the rhotics extended through most of the preceding and following vowel duration, while differences in F1 (trill > tap, approximant) were less extensive. While similar to the tap in its F2 trajectory, the approximant showed a more abrupt fall during the following vowel, seemingly indicative of a rapid tongue posture change at the release. [Work supported by SSHRC.]

2pSC25. Acoustics of epenthetic vowels in Brazilian Portuguese. Maria Kouneli (Linguist, New York Univ., 10 Washington Pl., New York, NY 10003,mrkouneli@gmail.com)

In this production study involving a task of real words and a task of nonce words, epenthesis in obstruent-obstruent clusters in Brazilian Portuguese was investigated and the acoustic properties of epenthetic and lexical [i] were compared. A number of linguistic factors were found to be significant predictors of the epenthesis rate: voicing of the consonants of the cluster, manner of articulation of the second consonant, place of articulation of both consonants, and position of the cluster with respect to the stressed syllable. Moreover, there was a higher epenthesis rate for nonce words than for real words. As for the quality of the epenthetic vowel, it was found to have the same F1 as the lexical [i] in the language, but it was significantly shorter in duration, and it had a significantly lower F2 in the nonce words, but not in the real words. Finally, we found a relatively high rate of deletion of lexical [i], conditioned by the same factors that conditioned epenthesis rate, but in the opposite direction. It is concluded that epenthesis is a phonological phenomenon in Brazilian Portuguese, but that there is also acoustic reduction in the language that affects both lexical and epenthetic vowels.

2pSC26. Articulatory correlates of /w/ in Korean. Soohyun Kwon (Univ. of Pennsylvania, 3810 Walnut St., Linguist Lab, Philadelphia, PA 19104, suhyunkwon511@gmail.com)

The articulatory properties of / $\mathrm{w} /$ have received relatively little attention compared to those of vowels and consonants. Although lip protrusion as well as dorsum raising and backing have generally been considered the major articulatory correlates of $/ \mathrm{w} /$, there is cross-linguistic evidence that the primary articulation of labiovelars, including /w/, is labial in some languages, while in other languages it is velar ([1]). Partly due to little articulatory data, there is little consensus on what articulatory features are involved in the production of $/ \mathrm{w} /$ in each language. This study used ultrasound to image tongue shapes for $/ \mathrm{w} /$ produced by three native Korean speakers. Also, a video camera was used to capture the movement of lips. The video data of movements of both tongue and lips were subjected to Optical Flow Analysis, a technique used to measure the magnitude of movement (MM) of objects in a video. The results show that there is prominent lip protrusion gestures for $/ w /$ in Korean while there is little movement in tongue dorsum (the average $\mathrm{MM}$ of lip $=1.78$; tongue $=0.48 ; \mathrm{t}(86)=4.02, \mathrm{p}<0.001$ ). This finding indicates that the glide $/ \mathrm{w} /$ in Korean involves labial movements only, contrary to the previous description of Korean glides [2], [3].

2pSC27. Durational and spectral differences in Thai diphthongs and final glides. Phongphat Man-khongdi, Chutamanee Onsuwan (Dept. of English and Linguist, Faculty of Liberal Arts, Thammasat Univ., 2 Phrachan Rd, Phra Boromaharatchawang, Phra Nakhorn, Bangkok 10200, Thailand, phtmankhongdi@gmail.com), and Charturong Tantibundhit (Dept. of Elec. and Comput. Eng., Faculty of Eng., Thammasat Univ., Pathumthani, Thailand)

Acoustic analysis was conducted to compare Thai monophthongs /i, ii, ш, шш, u, uu, a, aa/, diphthongs /ia, ua, ua/, and vowel-to-glides (vowel + / $\mathrm{j} /$ or $/ \mathrm{w} /$ ) in terms of duration, formant frequency, and spectral rate of change $\left(\mathrm{TL}_{\mathrm{roc}}\right)$. Preliminary results from multiple repetitions of 30 target monosyllabic words (from 3 males) show that durational values of long monophthongs are approximately two times as long as the short counterparts. The diphthong onsets (/i/, / $\mathrm{w} /, / \mathrm{u} /)$ and offsets (/a/) as part of diphthongs appear to be more centralized than when they occur in monophthongs. Syllables with final glides appear to be longer than those with other final consonants, reducing length differences between syllables with short and long vowels. Interestingly, not only that Thai diphthongs and vowel-to-glides differ in their articulatory trajectories, but they appear to differ in terms of duration and $\mathrm{TL}_{\mathrm{roc}}$ values. Average duration of diphthongs is shorter than that of vowel-to-glides. $\mathrm{TL}_{\text {roc }}$ of diphthongs is on average higher than that of long-vowel-to-glides, but lower than short-vowel-toglides. Taken together, our preliminary results suggest and agree with the notion that transition of diphthongs is usually slower and more gradual than that of vowel-to-glides.

2 pSC28. Tongue body shape during the production of Irish palatalization and velarization. Grant $\mathrm{L}$. McGuire, Jaye Padgett (Linguist, UC Santa Cruz, 1156 High St., Stevenson Academic Services, Santa Cruz, CA 95064, gmcguir1@ucsc.edu), Ryan Bennett (Linguist, Yale, New Haven, CT), Máire Ní Chiosáin (Univ. College, Dublin, Ireland), and Jennifer Bellik (Linguist, UC Santa Cruz, Santa Cruz, CA)

Irish is an endangered Celtic (Goidelic) language that has a rare phonemic contrast between palatalized and velarized consonants for all places and manners of articulation. Using ultrasound tongue body imaging of 15 native speakers we provide comparative data on the phonetic realization of the contrast, specifically focusing on how place, manner, and vowel context each affect tongue body position during production for each of the three major dialects. Using principal components analysis, we find evidence for the role of tongue root advancement in the contrast independent of the (less surprising) roles of tongue body frontness and raising. This data have consequences for our understanding of the relationship between phonetic and phonological categories as well as the role of perceptual saliency in shaping inventories.

2pSC29. Ultrasound evidence for place of articulation and assimilation behaviors of the Japanese moraic nasal /N/. Ai Mizoguchi, Kevin Roon (The Graduate Ctr., City Univ. of New York, 365 Fifth Ave., Rm. 7304, New York, NY 10016, amizoguchi@gc.cuny.edu), and D. H. Whalen (Haskins Labs., New Haven, CT)

The syllable-final Japanese moraic nasal /N/ is commonly transcribed as velar or uvular or even placeless, but very little articulatory has been 
reported. This study investigated the tongue shape and position for $/ \mathrm{N} /$ in various phonological environments using ultrasound. /N/ assimilates to the place of following segments, so a variety of environments was also examined to assess whether the assimilation occurs categorically or gradually. Tens repetitions of 7 target words with a moraic nasal (/aNCa/, /aNa/, / $\mathrm{aNaN} /$, and $/ \mathrm{uN} /$ ) and 6 control words without a moraic nasal (/aCa/, /aa/) were spoken by 4 native speakers of Japanese. Although there seems to be an oral target for moraic nasal, the place was different for each of our four speakers. The assimilation also varied among speakers, but a gesture for the moraic nasal remained in at least one phonological environment for all the speakers. Assimilation of Japanese moraic nasal to following segments is not always categorical and a gesture for the target of moraic nasal, even though varying among individuals, remains depending on the phonological environments. The ambiguities in transcribing / $\mathrm{N} /$ seem to be reflecting the state of the language accurately: Even with four speakers, four patterns emerged. [Work supported by NIH DC-002717.]

2pSC30. Gradient phonemic contrast in Nanjing Mandarin. Keith Johnson (Linguist, UC Berkeley, 1203 Dwinelle Hall, Berkeley, CA 94720, keithjohnson@berkeley.edu) and Yidan SONG (Nanjing Normal Univ., Nanjing, Jiangsu, China)

The variety of Mandarin Chinese spoken in Nanjing does not have a contrast between [n] and [1]. The standard variety of Mandarin which is now taught in schools in Nanjing does have the contrast. Prior research has shown that listeners' subjective impression of sound similarity is modulated by linguistic experience. When sounds are contrastive in a language they sound more different than when they are not contrastive. We conducted an experiment to test the subjective perceptual consequences of lack of [n]/[1] contrast in Nanjing, and of learning the standard variety of Mandarin in school. The stimuli were seven instances each of [tana] [tala] and [tara]. The listeners were 16 younger $(<45 \mathrm{yrs})$ and 16 older speakers of Nanjing Mandarin, and 35 speakers of American English. The listeners' task was to rate on a 5 point scale their subjective impression of the difference between the words $(1=$ similar, $5=$ different $)$. For American English listeners $[1] /[\mathrm{r}]$ pairs and $[\mathrm{n}] /[\mathrm{r}]$ pairs were rated as different (3.8) while $[\mathrm{n}] /[1]$ pairs were heard as a little more similar than this (3.3). For older Nanjing Mandarin speakers $[1] /[\mathrm{r}]$ and $[\mathrm{n}] /[\mathrm{r}]$ pairs were very different $(4.8)$ and $[\mathrm{n}] /[1]$ pairs were very similar (1.5). Younger Nanjing Mandarin speakers also rated [1]/ $[\mathrm{r}]$ and $[\mathrm{n}] /[\mathrm{r}]$ pairs as very different $(4.6)$, while the $[\mathrm{n}] /[1]$ pairs were not as similar $(2.25)$ (standard errors $<0.1)$. Exposure to a non-native phonological contrast seems to have perceptually enhanced it gradiently.

2pSC31. A classification method for crowded situation using environmental sounds based on Gaussian mixture model-universal background model. Tomoyasu Tanaka, Sunao Hara, and Masanobu Abe (Graduate School of Natural Sci. and Technol., Okayama Univ., 1-1-1 Tsushimanaka Kita-ku, Okayama-shi, Okayama-ken, Okayama, Okayama 700-8530, Japan, pu4o7k17@s.okayama-u.ac.jp)

This paper presents a method to classify a situation of being crowded with people using environmental sounds that are collected by smartphones. A final goal of the research is to estimate "crowd-density" using only environmental sounds collect by smartphones. Advantages of the approach are (1) acoustic singles can be collected and processed at low cost, (2) because many people carry smartphones, crowd-density can be obtained not only from many places, but also at any time. As the first step, in this paper, we tried to classify "a situation of being crowded with people." We collected environmental sounds using smartphones both in residential area and downtown area. The total duration of the collected data is 77,900 seconds. The sound of "crowded with people" is defined as buzz-buzz where more than one person talked at the same time. Two kinds of classifiers were trained on the basis of the GMM-UBM (Gaussian Mixture Model and Universal Background Model) method. The one was trained with acoustic features that are generally used in speech recognition, and the other was trained with additional parameter of sound power. Experiment results showed that the parameter of sound power improves the F-measure to 0.60 from 0.58 .
2pSC32. The phonological preparation unit in the production of English words by Korean learners. Sujin Oh and Jeong-Im Han (English, Konkuk Univ., 120 Neungdong-ro, Gwangjin-gu, Seoul 05029, South Korea, cutmirul@konkuk.ac.kr)

This study investigated how speakers of two languages differing in phonological preparation unit in planning spoken words encode a functional unit of their second language (L2). It was shown that the first phoneme of a word is a functional unit in English, whereas Korean has a strong syllable but not onset phoneme effects. Two groups of native Korean speakers depending on their length of residence in English-speaking countries were asked to name pictures in English, and the names shared the same onsets, rhymes, or had nothing systematically in common. The results showed that 1) no onset effect was shown regardless of length of residence, suggesting that L2 learners' phonological preparation unit is influenced by their native language functional unit; and 2) there was a rhyme interference effect regardless of length of residence, probably due to lexical competition. However, upon close inspection of the results of individual speakers, L2 learners are able to attend to the target language unit if they are extensively exposed to L2 phonology.

2pSC33. Temporal and spectral characteristics of the intervocalic consonant clusters in Korean. Hansang Park (English Education, Hongik Univ., 94 Wausan-ro, Mapo-gu, Seoul 04066, South Korea, phans@hongik. ac.kr)

Park(2008) showed that there is no significant difference in specific segment duration and their temporal structure between $\mathbf{V C C}_{[\text {Asp }]} \mathbf{V}$ and $\mathbf{V C}_{[\text {Asp }]} \mathbf{V}$, and between $\mathbf{V C C}_{[\text {For }]} \mathbf{V}, \mathbf{V C C}_{[\text {Len] }} \mathbf{V}$, and $\mathbf{V C}_{[\text {For }]} \mathbf{V}$, where $\mathbf{V}$ stands for vowel, C for consonant, [Asp] for aspirated, [For] for fortis, and [Len] for lenis. He also showed that there is no significant difference in consonant duration between a singleton and a geminate when the following $\mathrm{C}$ is [For] or [Asp], but a significant difference between them when the following $\mathrm{C}$ is [Len]. On the other hand, Park(2005) showed that there is a significant difference in phonation type index $k$ (PTI $k$ ) across consonant types, where PTI $k$ presents a single and simplified measure of the spectral tilt, which is free from the effects of fundamental frequency and vowel quality. This study, by applying Park(2008)'s and Park(2005)'s methods, investigates temporal and spectral characteristics of the consonant clusters of the other manners and places of articulation. The results showed that temporal characteristics present a similar pattern to those of Park(2008), and that spectral characteristics are associated with the phonation identity of the final consonant of the intervocalic consonant clusters.

2pSC34. Word boundaries attenuate the effects of emphasis in Lebanese Arabic. Andrew N. Pick (Linguist, Univ. of Hawaii Manoa, 1617 Kapiolani Blvd. Apt. 407, Honolulu, HI 96814, pick@ hawaii.edu)

Arabic has a series of pharyngealized consonants, called "emphatics," which are phonologically distinct from their plain counterparts. The strongest acoustic correlate of emphasis is a lowering of the second formant on vowels adjacent to the emphatic consonant. Arabic varieties differ in how far emphasis spreads, and previous work suggests that varieties may differ in whether emphasis can cross word boundaries. This study examines the effect of Lebanese Arabic emphatics on preceding vowels in two environments: within the word and within the phrase. Five native Lebanese speakers were recorded reading a list of phrases containing a target vowel preceding either an emphatic or plain consonant, with or without an intervening word boundary. In both environments, vowels preceding emphatic consonants have a lower F2 than vowels preceding plain consonants. Additionally, F2 is lowered more on vowels that are within the same word as the conditioning emphatic. Overall, these results indicate that emphasis in Lebanese Arabic affects preceding vowels within the domain of the word as well as the domain of the phrase, but this effect is stronger within the word. 
2pSC35. An ultrasound study of the nasal substitution in Javanese. Lang Qin (Dept. of Linguist, The Univ. of Hong Kong, General Office 9.30, Run Run Shaw Tower, The Centennial Campus, Hong Kong 999077, Hong Kong, qinlang@hku.hk)

Nasal substitution in Austronesian languages refers to replacement of voiceless obstruents by homorganic nasals under certain morphological conditions. In Javanese, for example, the active form of verb [tuku] "buy" is [nuku] and that of [t Jakar] "scratch" is [nakar]. However, exception does exist; namely, the sibilant [s] is paired with a palatal nasal [n], e.g., the active form of [sawan] "see" is [naway]. This ultrasound study aims to verify: i) whether the perception of two distinct places of articulation $([\mathrm{s}] /[\mathrm{n}])$ is matched in the articulation, suggesting an abstract relation between the two sounds, ii) or whether the homorganic pattern seen elsewhere in this morphological paradigm is matched in the articulation, suggesting a concrete relation between the two sounds. SS ANOVA results based on data of 8 Javanese speakers reveal that tongue position of /s/ bears more resemblance to $/ \mathrm{t} /$ and its nasal counterpart $/ \mathrm{n} /$, whereas the the tongue position of the nasal counterpart of $/ \mathrm{s} /$ is more posterior and closer to that of $/ \mathrm{t} / /$ and its nasal counterpart $/ \mathrm{n} /$, which supports the abstract relation between [s] and [n].

2pSC36. The Pohnpeian stop contrast between laminal alveolars and apical dentals involves differences in VOT and F2 locus equation intercepts. Bradley Rentz and Victoria Anderson (Linguist, Univ. of Hawai' $i$ at Mānoa, 1890 East-West Rd., 569 Moore, Honolulu, HI 96822, rentzb@ hawaii.edu)

Pohnpeian (ISO639-3 pon) is a phonetically understudied Oceanic language spoken by about 34,000 people in the Federated States of Micronesia, and 12,000 in the United States. Pohnpeian has a typologically uncommon laminal alveolar stop $/ \mathrm{t}_{\curvearrowleft} /$ that contrasts with an apical dental stop $/ \mathrm{t}_{\mathrm{r}} /$. Three male and two female native speakers were recorded in Honolulu, saying phonetically controlled wordlists in sentence frames. VOTs, F2s at vowel onset and steady state, and burst spectral moments (center of gravity and skewness) were measured in PRAAT and analyzed in R. Hierarchical linear modeling showed that $/ \mathrm{t}_{-} /$has a significantly longer VOT than $/ \mathrm{t}_{\mathrm{r}} /$. Linear regressions showed similar F2 locus equation slopes, but a higher intercept for $/ t /$. Neither COG nor skewness exhibited significant differences. The locus equation intercept results, while similar to those of Sussman et al. 1993 for Urdu, have a different cause. Urdu /d/'s higher intercept is due to higher F2 onsets than that of $/ \mathrm{d}_{\mathrm{r}} /$, Pohnpeian's $/ \mathrm{t}_{\mathrm{\sigma}} /$ and $/ \mathrm{t}_{\mathrm{r}} /$ have similar F2 onsets; / $\mathrm{t}_{\mathrm{f}} / \mathrm{s}$ higher intercept is due to vowel allophony: a lower F2 steady state for $/ \mathrm{t}_{\sigma} /$. The burst spectral results remain puzzling in light of source-filter theory, which predicts a higher COG for $/ \mathrm{t} /$ than $/ \mathrm{t} /$.

2 pSC37. Relative fundamental frequency of voiced and voiceless stops and fricatives for different phonation types in normal French speakers. Camille F. Robieux and Christine Meunier (Aix Marseille Univ, CNRS, LPL, Aix-en-Provence, France, 5 Ave. Pasteur, Aix-en-Provence 13100, France, camille.robieux@lpl-aix.fr)

The relative fundamental frequency (RFF) was recently used to assess vocal effort in normal and dysphonic subjects since it reflects the vocal fold tension at the offset and onset of vowels with, in particular, lower values at the edges of voiced consonants compared to their voiceless counterparts. In this study, we explored the RFF variations according to the voicing status and the articulation manner of consonants, in several types of speech production known to involve different levels of vocal effort. Twelve native French speakers ( 6 females) with normal voice were asked to produce 24 trains of eight syllables in seven vocal conditions: spontaneous, low, high, soft, loud, breathy, and pressed voice. We considered the unaspirated voiceless and voiced consonants /p, b, f, v/ with the middle open vowel /a/. As expected, the RFF was lower for voiced than for voiceless consonants. In addition, it was lower for stops than for fricatives. The RFF was also lower for the high, loud, and pressed conditions while it was higher for the low, soft, and breathy conditions in comparison to the values for the spontaneous voice. The RFF measurement seems to be efficient to discern subtle levels of vocal effort in normal subjects.
2pSC38. Preliminary study on physiological contrasts between long and short vowels in Japanese. Ayako Shirose (Tokyo Gakugei Univ., 4-1-1 Nukuikita-machi, Koganei, Tokyo 184-8501, Japan, shirose@u-gakugei.ac. jp), Yukiko Nota (ATR-Promotions, Kyoto, Japan), and Tatsuya Kitamura (Konan Univ., Kobe, Japan)

The purpose of this study is to identify physiological contrasts between long and short vowels in Japanese. Japanese (Tokyo Japanese; TJ) is known as a mora-timed language. Durational length of vowels and consonants, short or long, is used to discriminate between words in Japanese; in one example, kabu, "bottom," and ka:bu, "curve," are distinguished by Japanese speakers. Previous studies have discussed the durational contrasts acoustically and phonologically; however, an articulatory explanation was not taken into account. In order to understand the articulatory process of durational contrasts, we analyzed movements of the lips and tongue using an electromagnetic articulography (the NDI Wave Speech Research System). Two TJ speakers were asked to produce minimal pairs of words including short or long vowels (e.g., kabu-ka:bu, koto-ko:to). The position of six sensors attached on their lips and tongue was tracked during the speech. The sensors were placed on the upper and lower lips, right angular of the lip and the tongue dorsum. Analysis of the data revealed that the lower lip and posterior tongue moved downward more in articulating long vowels than short vowels. This result suggested that long vowels caused not only acoustic and phonological lengthening of syllable duration, but also overlapping of articulatory movements.

2pSC39. Contact effects on voice-onset time in Patagonian Welsh. Morgan Sleeper (Linguist, Univ. of California, Santa Barbara, 712 Bolton Walk, Apt. \#204, Goleta, CA 93117, msleeper@umail.ucsb.edu)

The effects of language contact extend well beyond lexical borrowings and can include morphosyntactic, phonetic, and phonological changes over time (Thomason \& Kaufman 1998). One especially common outcome of long-term contact is phonetic transfer (Matras 2009:222). The Welsh spoken in Patagonia, in close contact with Spanish for the past 150 years, offers one potential example: Jones (1984) observes that younger Patagonian Welsh speakers may be developing unaspirated voiceless stops /p t k/ as a result of Spanish contact. This paper explores this hypothesis through a quantitative study of voice-onset time (VOT) of the Welsh voiceless stops / $\mathrm{p} \mathrm{t} \mathrm{k/} \mathrm{in} \mathrm{con-}$ temporary conversational speech data from Patagonia and Wales, from male and female speakers in three age groups $(0-29,30-59$, and $60+)$. Results show that the tendencies reported by Jones (1984) have held true, and in fact have generalized to become a feature of Patagonian Welsh for speakers of all ages: Patagonian speakers produce the Welsh stops /p t k/ with significantly shorter VOT values than speakers from Wales. These results shed light on an important distinguishing phonetic feature of this understudied variety of Welsh, as well as the dynamics of language contact in action.

2 pSC40. Three-dimensional reduction for three-way contrast: Conversational stops in Korean. Jae-Hyun Sung and Daniel Brenner (Dept. of Linguist, Univ. of AB, Edmonton, AB T6G 2E7, Canada, sung@ualberta. ca)

Phonetic reduction in conversational speech is widely acknowledged across different languages and dialects. Previous experimental studies have reported that spontaneous conversations yield greater variability and reduction in acoustic signals when compared to careful speech. This study investigates the acoustic reduction in intervocalic Korean stops-phonemically lenis, fortis, and aspirated stops-from conversational speech, and adds to the increasing body of reduced speech research. Comparisons of Korean stops from careful and conversational speech confirm greater acoustic variability in conversational speech than in careful speech. Furthermore, conversational stops in Korean show that phonetic reduction occurs in multiple acoustic dimensions. Lenis stops exhibit phonetic reduction represented by both a smaller intensity difference and a higher pitch, fortis stops do so by a higher pitch only, and aspirated stops do so by both a smaller VOT and a higher pitch. Results from this study leave open the possibility of mannerspecific strategies in phonetic reduction within a language, and merit further cross-linguistic examination of conversational speech. 
2pSC41. A preliminary ultrasound study of apical consonants in stressed and unstressed position in Arrernte. Marija Tabain (La Trobe Univ., La Trobe University, Melbourne, VIC 3086, Australia, m.tabain@latrobe.edu.au)

In this study, we present ultrasound data from four female speakers of the Australian language Arrernte. We focus on the apical consonants-alveolar /t n l/ and retroflex /t n V/-in stressed and unstressed position. Previous articulatory results for the Arrernte stops using electropalatography and electro-magnetic articulography (for two of the speakers in the current study) showed that the tongue is most retracted for the retroflex in unstressed prosodic context, but that both apicals have a more advanced tongue position in stressed context. In addition, jaw position was highest for the alveolar in stressed position, and lowest for the retroflex in unstressed position. Current ultrasound results show that the rear-most portion of the tongue is consistently further back for the retroflex in unstressed position. This result confirms our previous conclusion that the most prototypical retroflex is the one found in the weaker prosodic position. By contrast, the stressed retroflex has a more forward position of the rear-most portion of the tongue, while the stressed and unstressed alveolar consonants show varying patterns depending on speaker and on manner class. Results are discussed in light of the variability that is often found for apical articulations in Australian languages.

2pSC42. Implosives in Jakarta Indonesian. Yu Tanaka (Linguist, Univ. of California, Los Angeles, 405 Hilgard Ave., 3125 Campbell Hall, Los Angeles, CA 90095-1543, yutanaka@ucla.edu)

Some Jakarta Indonesian speakers produce implosives as allophones of the voiced stops /b/ and /d/. Implosives are typically characterized by increasing voicing amplitude during closure, shorter closure duration and/or creaky phonation of the following vowel, but their actual realization shows much variation across languages (see Ladefoged \& Maddieson 1996; Lindau 1984). This study investigates the acoustic properties of Indonesian implosives. Thirty-three native speakers of Jakarta Indonesian were recorded. Each speaker produced one hundred words with initial /b/, /d/ or / $\mathrm{p} /$ in different vowel contexts (e.g. /baik/, /biru/) embedded in carrier phrases. A trained phonetician judged whether each case of $/ b /$ and $/ d /$ in their speech was imploded or not. I measured the voicing amplitude and duration of each target stop and the creakiness of the following vowel by means of harmonic amplitude differences such as H1-H2 (Keating et al. 2010). A preliminary analysis reveals that stops judged as implosives generally had more increasing voicing amplitude than those judged as modal plosives. The former also had a shorter duration and their following vowels had relatively low $\mathrm{H} 1-\mathrm{H} 2$ and $\mathrm{H} 1-\mathrm{A} 2$ values, which indicates creakier phonation. The analysis also shows that implosivization is more likely to occur before a non-high back vowel.

2pSC43. Hejazi Saudi Arabic pharyngeal approximant 'Ayn involves 4 of 5 correlates of creak, but not positive H1-H2 spectral tilt. James M. Thorne (None, 1890 East-West Rd., 569 Moore, Honolulu, HI 96822, jamesmathiasthorne@yahoo.com)

Five correlates of creaky phonation in the Arabic pharyngeal approximant 'Ayn - $/ \% /$ - were compared in speech samples of five male native speakers of the Hejazi dialect of Saudi Arabic to determine the degree to which creaky phonation could be a phonetic feature of 'Ayn. Jitter, shimmer, harmonics-to-noise ratio (HNR), $\mathrm{F}_{0}$ lowering, and positive $\mathrm{H}_{1}-\mathrm{H}_{2}$ spectral tilt were compared in 360 (total) speech tokens of $/ \mathrm{a} /, / \mathrm{aa} /$, and $/ \mathrm{a}^{\mathrm{s}} \mathrm{a} /$ vowel sequences. Acoustic analysis indicated significantly increased jitter, increased shimmer, lower HNR, and lower $F_{0}$ values in 'Ayn sequences compared to non-'Ayn sequences, yielding strong evidence of creaky phonation. There was, however, no significant increase in degree of positive $\mathrm{H}_{1}-\mathrm{H}_{2}$ spectral tilt in 'Ayn sequences compared to non-'Ayn sequences. Positive tilt appeared to occur in free variation across vowel sequence types with many non-'Ayn sequences displaying the positive $\mathrm{H}_{1}-\mathrm{H}_{2}$ spectral tilt traditionally associated with creak. If increased jitter, increased shimmer, lower HNR, and local F0 lowering are correlates of creak in these realizations of 'Ayn, we must consider that creak is a key phonetic feature of 'Ayn for these Arabic speakers and that positive $\mathrm{H}_{1}-\mathrm{H}_{2}$ spectral tilt may not be a reliable indicator of creak in Hejazi Arabic.
2pSC44. Changes in Iu-Mien prevoicing: A focus on speakers in the middle. Ela Thurgood (California State Univ., Chico, California State University, Chico, CA 95929, ethurgood@csuchico.edu)

The focus is on speakers who come from families of 8 to 10 children and are in the middle being the $4^{\text {th }}$ or the $5^{\text {th }}$ child in the family. This paper provides an acoustic analysis of syllable-initial Iu Mien stops produced by these speakers, comparing their stops with those of the older siblings. The distinction is important in that the oldest siblings are Iu-Mien native speakers and English non-native speakers, while the youngest siblings are English native speakers with only some passive knowledge of Iu-Mien. Those in the middle constitute a bridge between the other two groups. They are bilingual in both Iu-Mien and English; as the interviews attest, they regularly use IuMien with their parents and older siblings, and English with their younger siblings. This study discusses in detail the differences in the VOT productions of $/ \mathrm{b} \mathrm{p} \mathrm{p} /$ and $/ \mathrm{d} \mathrm{t}^{\mathrm{h}} /$. It shows that the presence or lack of prevoicing divides those in the middle into two groups: those who prevoiced all of the 266 voiced VOTs measured, and those who prevoiced in $25-44 \%$. The percentage of prevoicing is shown to correlate with language competence that influences language transmission.

2pSC45. Voice onset time in Cantonese women across the menstrual cycle. Chi Him Li (Univ. of Hong Kong, Rm. 19B, Block 11, Laguna City, Kowloon, Hong Kong, Hong Kong, jerrylego@ hotmail.com)

This study explores the relationship between voice onset time (VOT) and the menstrual cycle in Cantonese women from Hong Kong. While researchers have investigated how fertility may be related to voice pitch, its connection with VOT is much less understood, let alone that in the non-English speaking population. To fill this gap, a production experiment is conducted to look at VOT in eight Cantonese women at two phases of their menstrual cycle (Day 1-5: low estrogen and progesterone level; Day 1825: high estrogen and progesterone level). Target words contrast in place of articulation $(/ \mathrm{p}, \mathrm{t}, \mathrm{k} /$ ) and tone (High vs. Low). Results show that VOT is longer during the high fertility phase, in line with previous studies. Subsequently, voice quality of the speech data will be analyzed using standard measures (e.g., H1-H2, H1-A1, jitter, shimmer, and harmonicity) to shed light on how longer VOT may be associated with other feminine phonatory strategies. These results are discussed in relation to current theories of vocal attractiveness.

2pSC46. Talker variability and the use of perceptual cues in Hong Kong Cantonese lingual stop codas. Jonathan C. Yip (Linguist, The Univ. of Hong Kong, Rm. 906, Run Run Shaw Tower, Pokfulam, HK 0000, Hong Kong, yipjonat@hku.hk)

Cantonese inaudibly released oral stop codas, whose place cues occur only before constriction and not during or after constriction, appear to be merging among young-adult speakers (Law, Fung, \& Bauer, 2001). Prior work (Yip, 2015) revealed substantial articulatory variation among five talkers, ranging from gestural preservation, to coproduction of lingual-lingual sequences, to gestural loss. Coda contrasts were cued by first- and thirdformant loci (both lower into / $\mathrm{k} /$ than into / $\mathrm{t} /$ ) and preceding vowel duration (longer before $/ \mathrm{t} /$ than before $/ \mathrm{k} /$ ). In the present study, 18 Cantonese-speaking listeners' perception of codas produced by talkers in the prior study was tested in an AXB task in which ambiguous coda productions were judged as more similar to each talker's best coda / $\mathrm{t} /$ or $/ \mathrm{k} /$ production, as assessed with ultrasound tongue imaging. Comparisons of listener sensitivity to cuesbased on LMER estimates for change in accuracy with respect to relevant acoustic response variables-with their accuracy rates for individual talkers indicate that listeners who were sensitive to particular acoustic cues gained a perceptual advantage for talkers who produced those cues. However, greater articulatory achievement in the stimuli did not correlate with better perception, except for productions by talker T4, who produced gestural loss.

2pSC47. Effects of phonetic naturalness in decay of Korean vowel harmony. Hayeun Jang (Univ. of Southern California, 3601 Watt Way, Grace Ford Salvatori 301, Los Angeles, CA 90089-1693, hayeunja@usc.edu)

In this paper, based on Korean vowel qualities measured in the production experiments, I point out that /o/-stems are also actively participating in 
the decay of Korean vowel harmony, and their speed of decay is much faster than /a/-stems' speed. How the late-starter /o/-stems could have the faster speed in the decay of Korean vowel harmony? The role of phonetic naturalness in language learning is the key. Measured vowel qualities show that $/ \mathrm{o} /$ is acoustically much closer to $/ \Lambda /$ as "disharmonic" pair than to $/ \mathrm{a} /$ as "harmonic" pair. In addition, in terms of articulatory, the raising of $/ \mathrm{o} /$ and the backing of $/ \Lambda /$ make them more similar. In the production experiments, 14 Seoul-Korean speakers participated ( 7 males, 7 females). Onset and coda of stems are controlled. For each condition, 1 existing word and 2 nonce words are used. Participants are asked to make a natural conjugation form of a given stem and to produce it three times. Unlike previous corpus and production studies reporting that /o/-stems show no disharmonic forms, the current experimental results show that quality of selected vowels after /o/stems is similar to $/ \mathrm{N} /$, not $/ \mathrm{a} /$. Compared to /a/-stems, /o/-stems' suffix vowel qualities are clearly closer to $/ \Lambda /$.

2pSC48. Acoustic evidence for prosodic units in Navajo. Kayla Palakurthy (Linguist, Univ. of California, Santa Barbara, 714 Gayley Walk, Apt 204, Goleta, CA 93117, kaylaeisman@umail.ucsb.edu)

Although prosody and intonation are generally considered to be linguistic universals, the few prosodic studies of Navajo, a Southern Athabaskan language spoken in the American Southwest, report a lack of meaningful intonation (McDonough 1999; McDonough 2002). This paper revisits the question of Navajo prosody, presenting the results of a quantitative analysis of acoustic correlates of prosodic constituents in personal narratives. Building on previous studies of Athabaskan prosody (Berez 2011; Lovick and Tuttle 2012), the present work employs mixed-effects linear modeling to examine pitch (F0) reset and syllable and pause duration at different prosodic and syntactic boundaries. Results provide evidence for both intonational units marked by final syllable lengthening and also larger prosodic sentences cued by pauses and pitch reset. Furthermore, the demarcation of prosodic units is statistically more robust than clause boundaries, underscoring the orthogonal relationship between prosodic and syntactic structure. These data suggest that Navajo does have systematic prosody, but the cues may only be identifiable in larger stretches of connected speech, rather than individual words or phrases. Results are compared with other work in the small, but growing typological literature on intonational characteristics of large prosodic units.

2 pSC49. A sociophonetic study of the effect of gender on emphatic-plain contrast in Jordanian Arabic. Abdulaziz Alzoubi (Dept. of World Lang. and Cultures, Univ. of Utah, Lang. \& Commun. Bldg. 255 S Central Campus Dr., Rm. 1400, Salt Lake City, UT 84112, Aziz.Alzoubi@Utah.edu)

Mixed results are reported in the literature concerning the status of gender, as social factor, as a predictor of the degree of emphatic-plain contrast in Jordanian Arabic. The present study argues that these mixed results could be an outcome paying no or little attention to demographic factors, more specifically; the original regional dialect subjects belonged to before settling in Jordanian cities. The present study examines the effect of gender, in Amman City, on the realization of emphasis in the emphatic coronals $/ \mathrm{t} / \mathrm{s}$ and $/ \mathrm{s}^{\mathrm{s}} /$ and their plain counterparts $/ \mathrm{t} /$ and $/ \mathrm{s} /$. The study depended on the first three formants of vowels adjacent to the target sounds after normalization for sex differences, Center of Gravity for target sounds, and Voice Onset Time (VOT) for the stops, as the acoustic cues of emphasis. The results from the mixed model, with "speaker" and "word" as random effect, indicated that gender is a reliable predictor of emphasis in Amman City as evident by the results from the first three vowel formants and stops' VOT; while both males and females maintained a significant difference between emphatic and plain sounds, males maintained a greater emphatic-plain contrast than females.

2pSC50. Production and perception of the low vowels in American English. Valerie Fridland (English, Univ. of Nevada, 1664 N. Virginia St., M.S. 0098, Reno, NV 89519, fridland@unr.edu) and Tyler Kendall (Linguist, Univ. of Oregon, Eugene, OR)

Two vowel features are especially pivotal in defining contemporary U.S. dialects: The merger of the low back vowels and the variable realizations of the low front vowel. Several scholars (Bigham 2010, Gordon 2005, Labov, Ash and Boberg 2006) suggest a relationship between the low front and low back vowels such that /æ/ raising and subsequent fronting of /a/ in the North inhibits the tendency toward low back merger. However, little work examines the robustness of this "structural linkage" or whether a similar relationship obtains across different regional varieties. Further, little work examines whether differences in production correlate with differences in regional perception patterns. Here, we compare the low vowel system across U.S. regional dialects and also consider interrelationships (i.e., correlations) between low vowel categories using data from eight fieldsites across the U.S. We then look at what these speakers' perceptions of category shift between the low front and low back vowel tells us about this relationship in production. Our results suggest both that the regional vowel shifts create significantly divergent low vowel systems across regions but also that $/ æ /$ and $/ \mathrm{a} /$ indeed show a structural linkage across regions, while /æ/ and / $/$ / and /a/ and /o/ show no such relationship.

2 pSC51. Individual differences in the relation between perception and production and the mechanisms of phonetic imitation. Donghyun Kim and Meghan Clayards (Linguist, McGill Univ., 1085 Dr. Penfield, Rm. 111, Montreal, QC H3A 1A7, Canada, heydonghyun@gmail.com)

This study uses phonetic imitation to understand more about how individuals perceive and produce speech and to explore the link between the two. We used manipulated stimuli with the goal of more directly probing the link and to test (1) whether individual listeners' perceptual cue weights are related to their patterns of phonetic imitation and (2) the underlying mechanisms of phonetic imitation. Twenty-three native speakers of English completed a $2 \mathrm{AFC}$ identification task followed by a baseline production and a forced imitation task. Perception stimuli were created from productions of head and had recorded by a native speaker of English. Seven steps varying in formant frequency (created with TANDEM-STRAIGHT) were crossed with 7 duration steps (PSOLA in PRAAT). Imitation stimuli were a subset of stimuli from the perception task plus extended and shortened vowel durations. Our results suggest that phonetic imitation is mediated in part by a low-level cognitive process involving a direct link between perception and production as evidenced by imitation of all vowel durations. However, this study also suggests that imitation is mediated by a high-level linguistic component, i.e., phonological contrasts, which is a selective rather than an automatic process as indicated by imitation of phonologically relevant formant frequencies.

2pSC52. Phoneme distribution and syllable structure of entry words in the Carnegie Mellon University Pronouncing Dictionary. Byunggon Yang (English Education, Pusan National Univ., 30 Changjundong Keumjunggu, Pusan 609-735, South Korea, bgyang@pusan.ac.kr)

This study explores the phoneme distribution and syllable structure of entry words in the CMU Pronouncing Dictionary to provide linguists with fundamental phonetic data on English word components. Entry words in the dictionary file were syllabified using an R script and examined to obtain the following results: First, English words preferred consonants to vowels in their word components. In addition, monophthongs occurred much more frequently than diphthongs. When all consonants were categorized by manner and place, the distribution indicated the frequency order of stops, fricatives, and nasals according to manner and that of alveolars, bilabials and velars according to place. Second, from the analysis of syllable structure, two-syllable words were most favored, followed by three- and one-syllable words. Of the words in the dictionary, $92.7 \%$ consisted of one, two, or three syllables. Third, the English words tended to exhibit discord between onset and coda consonants and between adjacent vowels. Dissimilarity between the last onset and the first coda was found in $93.3 \%$ of the syllables, while $91.6 \%$ of the adjacent vowels were different. The author concludes that an analysis of the phonetic symbols in a dictionary may lead to a deeper understanding of English word structures and components. 
2pSC53. Glottalization, reduction, and acoustic variability in function words in American English. Laura Dilley, Meisam K. Arjmandi, Zachary Ireland (Dept. of Communicative Sci., Michigan State Univ., East Lansing, MI 48824, ldilley@msu.edu), Chris Heffner (Univ. of Maryland, College Park, MD), and Mark Pitt (The Ohio State Univ., Columbus, OH)

Function words vary widely in pronunciation; understanding this variation is essential for accurate cognitive modeling of lexical perception and production, as well as for computer speech applications. This study analyzed acoustic variation in pronunciation of vowel-initial words $a$, are, our, and $o r$, as well as her, which has a vowel-initial variant, [x]. Sentences were constructed in which each function word was preceded by a phonetically similar open syllable rhyme, with which the word was expected to sometimes blend spectrally. Twenty-nine participants uttered 100 sentences in a casual speaking style. The degree of clarity of function word onsets was measured, focusing on evidence of onset discontinuities: glottal onset gestures (e.g., low fundamental frequency, F0; amplitude dips) for vowel-initial words $a$, are, our, and or, and, for her, of /h/ (e.g., root-mean-square amplitude, amplitude differences of first and second harmonics). Results showed that a substantial proportion of the time, function words lacked any detectable acoustic onset discontinuity. When discontinuities did occur before vowel-onset function words, there was a high degree of acoustic variability in those realizations. These findings highlight the challenges for speech perception to identify word onsets and/or to overcome variability in recognizing words. [Work supported by NSF Grant BCS-1431063.]

2pSC54. Analysis of acoustic features for speech intelligibility prediction models analysis of acoustic features for speech intelligibility prediction models. Katsuhiko Yamamoto (Graduate School of Systems Eng., Wakayama Univ., Sakaedani 930, Wakayama 640-8510, Japan, s149011@ sys.wakayama-u.ac.jp), Toshio Irino (Faculty of Systems Eng., Wakayama Univ., Wakayama, Japan), Toshie Matsui (Graduate School of Systems Eng., Wakayama Univ., Wakayama, Japan), Shoko Araki, Keisuke Kinoshita, and Tomohiro Nakatani (NTT, Souraku-gun, Japan)

We have developed a new model to predict speech intelligibility of synthetic sounds processed by nonlinear speech enhancement algorithms. The model involves two recent auditory models: the dynamic compressive gammachirp (dcGC) auditory filterbank and the speech envelope power spectrum model (sEPSM). The dcGC-sEPSM was compared with commonly used prediction models based on perceptual intelligibility scores of speech sounds enhanced by classic spectral subtraction and state-of-the-art Wiener filtering. As a result, the dcGC-sEPSM predicted the scores better than the coherence SII (CSII), the short-time objective intelligibility (STOI), and the original sEPSM using the gammatone filterbank. There was, however, still inconsistency between the prediction and data. In this work, we show the analysis of acoustic features used in the prediction models. The CSII calculates the magnitude-squared coherence between clean and processed spectra to derive a signal-to-distortion ratio. The STOI calculates the correlation coefficients between the short-time frame vectors of clean and degraded sound at the output of the one-third octave filterbank. The sEPSM calculates the signal-to-noise ratio in the envelope modulation domain at the output of the auditory filterbank. We summarize the methods and discuss desirable features that improve speech intelligibility predictions.

2pSC55. Ultrasound tongue image denoising for comparison of first and second language tongue trajectories. Shusuke Moriya and Yuichi Yaguchi (Univ. of Aizu, Tsuruga Ikkimachi, Aizuwakamatsu, Fukushima 965-8580, Japan, s1190242@gmail.com)

The main purpose of this research is to specify articulation difference between native and non-native speakers by digitizing tongue motions and analyzing the difference between utterances. Differences in tongue motion directly influence speaker's pronunciation; therefore, it may be possible to improve non-native speaker's efficiency of pronunciation practice with the relevant feedback and visualization. It is necessary for comparison of native and non-native speakers' tongue motions to that end, however, normalization is absolutely necessary to remove the influence of anything except tongue motion before comparison, because every person has a unique shape and size. In our previous research, we proposed normalization methods and some speaking errors were picked up automatically from tongue trajectory in ultrasound tongue image space. However, it is necessary to improve method to extract pure tongue trajectory with accuracy. In this paper, ultrasound tongue images are separated to $5 \times 20$ or $20 \times 5$ strip block. If tongue edge lies on the block, gray scale graduation forming a shape of an arch has occurred on a line of long side of block. We proposed some shape-sensitive filter to cut off an image noise which locates excepting neighborhood of tongue edge. Through our filter, tongue trajectory extracted from ultrasound tongue image could have less noises then previous one. However, some image noises locates on neighborhood of tongue edge are emphasized and inhibited comparison of native and non-native tongue trajectories.

2pSC56. The acoustic counterpart to articulatory resistance and aggressiveness in locus equation metrics and vowel dispersion. Hye-Young Bang (McGill Univ., 1085 Dr. Penfield, Montreal, QC H3A 1A7, Canada, hye-young.bang@mail.mcgill.ca)

Research on locus equation metrics (LEs) tend to take it for granted that vowel space is invariable across consonantal contexts. However, articulation-based studies report a mutual influence between neighboring segments such that segments with greater constraints in dorsal articulation are more resistant to and concurrently more aggressive in coarticulation than those with less constraints (Farnetani, 1990). We examine (1) whether articulatory resistance and aggressiveness can be acoustically captured through LEs and vowel dispersion and (2) how the relationship between LEs and the degree of coarticulation is mediated by vowel dispersion and delay in voicing. These questions are investigated in CV sequences in English -where C is one of $/ \mathrm{p} \mathrm{t} \mathrm{s} /$ / that varies in the articulatory constraints imposed on the tongue dorsum. We manipulated the magnitude of coarticulation and voicing lag by contrastively stressing the target consonants. Our results show that there is a tight relationship between LE slopes and vowel dispersion where articulatory resistance and aggressiveness appear as the mirror image of each other in the acoustic signal. However, effects of hyperspeech simultaneously affect vowel dispersion, voicing delay, and LEs creating confounds. This calls for caution in the use of LE metrics as a measure of coarticulation.

2pSC57. Pitch estimation based on instantaneous robust algorithm for pitch tracking using weighted linear prediction-based complex speech analysis. Wei Shan (Graduate School of Eng. and Sci., Univ. of the Ryukyus, Senbaru 1, Nishihara, Okinawa 903-0213, Japan, funaki@cc.u-ryukyu.ac.jp) and Keiichi Funaki (C\&N Ctr., Univ. of the Ryukyus, Nishihara, Okinawa, Japan)

Pitch estimation plays an important role on speech processing such as speech coding, synthesis, recognition, and so on. Although current pitch estimation method performs well under clean condition, the performance deteriorates significantly in noisy environment. For this reason, robust pitch estimation against additive noise is required. Modified auto-correlation method is commonly used as pitch estimation in which LP residual is used to compute the auto-correlation. We have previously proposed pitch estimation methods based on Time-Varying Complex AR (TV-CAR) analysis whose criterion is the weighted correlation of the complex residual obtained by the TV-CAR analysis, sum of the harmonics for the complex residual spectrum, or so on. On the other hand, Azarov et al. have proposed an improved method of RAPT (Robust Algorithm for Pitch Tracking) using an instantaneous harmonics that is called IRAPT (Instantaneous RAPT). The IRAPT can perform better estimation than RAPT. Since IRAPT uses bandlimited analytic signal to obtain harmonic frequencies, the complex residual signal obtained by the TV-CAR analysis can also be applied to the IRAPT. In this paper, novel pitch estimation method using the instantaneous frequency based on the robust WLP (Weighted Linear Prediction) TV-CAR residual is proposed and evaluated.

2pSC58. The relationship of VOT and F0 contrasts across speakers and words in the German voicing contrast. Hye-Young Bang, Morgan Sonderegger, and Meghan Clayards (McGill Univ., 1085 Dr. Penfield, Montreal, QC H3A 1A7, Canada, hye-young.bang@mail.mcgill.ca)

Recent studies on tonogenesis in progress in Seoul Korean (Kang, 2014; Bang et al., 2015) find that the size of the VOT contrast and the f0 contrast between aspirated and lax stops "trade off" across speakers (e.g., male 
speakers have greater/smaller VOT/f0 contrasts), as well as words (e.g., different frequencies, following vowel heights). We examine whether this parallelism across speakers and words occurs in a language not undergoing tonogenesis by examining the size of the fortis/lenis contrast in VOT and f0 in German, using speech from the PhonDat corpus (Draxler, 1995). Mixedeffect regression models show that the size of the VOT contrast, but not the f0 contrast, is affected by properties of words (e.g., frequency, vowel height), unlike the parallelism observed in Korean. We further investigated whether VOT/f0 parallelism would be observed across speakers by partialing out linguistic factors affecting VOT/f0, and performing one logistic regression per speaker $(n=76)$ of fortis/lenis class as a function of residualized $\mathrm{f} 0$ and VOT. The cue weights of F0 and VOT are negatively correlated across speakers $(\mathrm{r}=-0.213, p=0.0213)$, suggesting parallelism exists across speakers, but not across words.

2 pSC59. Voice activity detection in noisy environment using dynamic changes of speech in a modulation frequency range of 1 to $16 \mathrm{~Hz}$. Suci Dwijayanti and Masato Miyoshi (Graduate School of Natural Sci. and Technol., Kanazawa Univ., Kanazawa, Ishikawa 920-1192, Japan, dwijas@stu. kanazawa-u.ac.jp)

Voice activity detection (VAD) is usually placed at the preprocessing stage of various speech applications. There have been a lot of studies to find acoustic features that are effective in distinguishing speech/non-speech segments on a recorded signal. In this report, we utilize speech characteristics in a modulation frequency range of $1-$ to- $16 \mathrm{~Hz}$, which seem beneficial to avoid misjudgment. The signal amplitudes are evaluated in common logarithms not to miss small changes in speech energy that may have relations with the starting or ending point of an utterance. In order to capture such changes, the first and second derivatives of the signal are calculated. In finding the starting point, the positive second derivatives seem effective. In finding the ending point, the combination of the negative first derivatives and the positive second derivatives seem effective. And these features should be calculated for individual subbands. On these conditions, Deep Neural Network (DNN) is successfully trained to determine the speech/non-speech segments. In this report, we will evaluate this method in comparison with conventional ones for various noisy utterances.

2pSC60. Improvement of sensor for electromagnetic articulograpy to reduce detrimental effects of sensor attachment on articulation. Yukiko Nota (ATR-Promotions, 2-2-2 Hikaridai, Sorakugun Seikacho, Kyoto 6190288, Japan, ynota@atr.jp), Tatsuya Kitamura (Konan Univ., Kobe, Japan), Michiko Hashi (Prefectural Univ. of Hiroshima, Mihara, Japan), and Hiroaki Hatano (ATR Hiroshi Ishiguro Labs., Kyoto, Japan)

Electromagnetic articulography (EMA) tracks the position and direction of small wired sensors attached inside and outside of the mouth during articulation. In this study, we improved a five degree-of-freedom sensor of the NDI Wave speech research system, which is a type of EMA system, to reduce the effects of sensor cable on articulation. This was done because the thickness and stiffness of the original cable appeared to interfere with natural articulation. The new cable has the diameter of $0.1 \mathrm{~mm}$, which is thinner than the original, and is more flexible. The degree of interference due to sensor attachment to the articulators was evaluated by comparing the extent of distortion of speech produced by four elderly speakers with new and original sensor attached to their articulators. The speakers produced a Japanese vowel sequence and VCV sequences with the two types of sensors attached to their articulators, and three Japanese speech language pathologists evaluated their speech for the degree of speech distortion. The results suggest that the new sensors were much less likely to disturb natural articulation. [This research was partly supported by JSPS KAKENHI Grant Nos. 24652085, 25240026, and 25280066.]
2pSC61. Analysis of voice imitation by professional/non-professional impersonators based on Kullback-Leibler divergence between acoustic models. Koji Iwano and Takuto Horihata (Faculty of Informatics, Tokyo City Univ., 3-3-1 Ushikubo-nishi, Tsuzuki-ku, Yokohama, Kanagawa 2248551, Japan, iwano@tcu.ac.jp)

Considering the practical use of speaker verification systems, it is important to investigate the effect of spoofing attacks by professional/non-professional voice imitation. This research proposes an analysis method of voice imitation using the distances between three GMM-based acoustic models trained from cepstral features of "impostor's original voice," "target person's voice," and "impostor's imitated voice." The distance measure is defined by the Kullback-Leibler (KL) divergence. The analysis uses Japanese imitated voice produced by a male professional impersonator and six male non-professional impostors. Each impostor imitated five or six target persons who have never been tried to imitate by the impostors before the experiments. The analysis results show that 1 ) although the non-professional imitators drastically change their voice features by the imitation, the averaged acoustical distance between the imitated and target voice is still large, 2) whereas the professional imitator approaches their voice characteristics towards the target voice; the distance between the imitated and target voice is approximately $70 \%$ of the original distance. The experiments of speaker verification using HMM-UBMbased framework show that the professional imitation certainly yields higher equal error rates than that of non-professional imitation.

2pSC62. Probabilistic simulation for analysis of quantal biomechanicalacoustic relations. Ian Stavness, Francois Roewer-Despres (Comput. Sci., Univ. of SK, 110 Sci. Pl., Saskatoon, SK S7N5C9, Canada, ian.stavness@ usask.ca), and Bryan Gick (Linguist, Univ. of Br. Columbia, Vancouver, BC, Canada)

Acoustic signals that are stable across a range of articulatory parameters have been suggested as an important feature of speech production [Stevens 1989, J. Phonetics, 17, 3-45]. These so-called quantal effects have also been suggested to arise from the biomechanics of the vocal tract [Fujimura 1989, J. Phonetics, 17, 87-90; Gick \& Stavness 2013, Front Psychol 4, 977]. Assessment of potential biomechanical-acoustic quantal relations is hampered by the difficulty of measuring biomechanical parameters, such as muscle excitations, during speech production. Computer modeling has been widely used to probe vocal tract biomechanics, but previous modeling studies have been limited to a small number of deterministic simulations [Gick et al. 2014, CMBBE Imag Vis., 2, 217-22]. We propose a novel probabilistic simulation framework in order to assess how variation in speech motor signals manifests in acoustic variation. We use a detailed 3D biomechanical model of the vocal tract coupled to a source-filter acoustics model [Stavness et al. 2014, Siggraph Asia Tech, 9] in order to generate acoustic output from muscle excitation inputs. Monte Carlo sampling of muscle excitation inputs are used to characterize variation in formant frequencies for vowel production. These large-scale simulations permit us to evaluate the hypothesis that quantal acoustic signals originate from regions of biomechanical stability. If found, quantal biomechanical-acoustic relations would provide a simple, principled mechanism for feedforward control of speech production.

2pSC63. Optimization of topic estimation for the domain adapted neural network language model. Aiko Hagiwara, Hitoshi Ito, Manon Ichiki, Takeshi Mishima (NHK Sci. and Technol. Res. Labs., 1-10-11 Kinuta, Setagaya-ku, Tokyo 157-8510, Japan, hagiwara.a-iy@nhk.or.jp), Akio Kobayashi (NHK Eng. System, Tokyo, Japan), and Shoei Sato (NHK Sci. and Technol. Res. Labs., Tokyo, Japan)

We present a neural network language model adapted for topics fluctuating in broadcast programs. Topic adapted n-gram language models constructed by using latent Dirichlet allocation for topic estimation are widely used. The conventional method estimates topics by separating the corpora into chunks that have few sentences. While the n-gram model uses several preceding words, the recurrent neural network and long short-term memory can learn to store huge amounts of past information in the hidden layers. Consequently, chunks for language models trained by using neural networks may have a longer optimal length than the chunks for language models trained by using the conventional methods. In this paper, the length of chunks and topic estimation process are optimized for the neural network 
language models. For the topic estimation, k-mean clustering, latent Dirichlet allocation, and word2vec were compared. On the basis of the results of comparison, we designed a neural network language model.

2pSC64. Acoustic features of prosodic boundary by Chinese learners of English as a second language. Yuanyuan Zhang and Hongwei Ding (Ctr. for Cross-Linguistic Processing and Cognition, School of Foreign Lang., Shanghai Jiao Tong Univ., 800 Dong Chuan Rd., Minhang District, Shanghai 200240, China, yyuan.zhang@sjtu.edu.cn)

The production and perception of prosodic boundary have been well studied in English, but the research regarding to English as a second language (L2) is sparse. It has been shown that Chinese and English employ different acoustic features to mark prosodic boundary in production. Thus, this study aims to find out whether the Chinese learners of English employ the same strategy to convey prosodic boundary in English as the native speakers do. Ten pairs of syntactically ambiguous utterances composed of two or three food items were designed, with 10 pairs of filler utterances half randomized together. Ten speakers (5 males and 5 females) from Shanghai who passed the annual national examination CET (College English Test) Band 6 were asked to read the utterances. The results showed that, similar to the native speakers, Chinese learners also use pre-boundary lengthening, pause duration and pitch reset to signal the prosodic boundary in English. However, their pitch contour of the pre-boundary syllable is falling under boundary condition, and is rising under non-boundary condition, which is opposite to that of the native English speakers. This result will have an implication in L2 English teaching and learning.

2pSC65. End-to-end neural network modeling for Japanese speech recognition. Hitoshi Ito, Aiko Hagiwara, Manon Ichiki, Takeshi Mishima, Shoei Sato (NHK Sci. and Technol. Res. Labs., 1-10-11 Kinuta, Setagayaku Tokyo, Setagaya-ku 157-8510, Japan, itou.h-ce@nhk.or.jp), and Akio Kobayashi (NHK Eng. System, Tokyo, Japan)

This study proposes end-to-end neural network modeling to adapt direct speech-to-text decoding to Japanese. End-to-end speech recognition systems using deep neural networks (DNNs) are currently being investigated. These systems do not need intermediate phonetic representation. Instead, many of them utilize Recurrent Neural Networks (RNNs) trained by using much more data than ever before. The end-to-end approach makes acoustic models simpler to train. Typically, previous works have dealt with phonogram labels such alphabetic characters. Ideograms such as Kanji, however, make end-to-end speech recognition more complex. A single Kanji can have multiple readings, such as On-yomi (Chinese reading) and Kun-yomi (Japanese reading). In addition, whereas alphabets have at most 100 labels, Japanese has over 2000 labels to predict, such as Kanji, Hiragana, Katakana, the Roman alphabet, digits, and punctuation marks. To resolve this problem, we attempt to make end-to-end neural network modeling allows speech recognition of Japanese without phonetic representation. This method trains RNN and adopts the connectionist temporal classification (CTC) objective function. The proposed method was able to deal with a large amount of character labels. We also analyzed the decoding results and examined ideas for improving the accuracy of word error rate (WER).

2pSC66. Aperiodicity analysis of filled pause in the Corpus of Spontaneous Japanese database. Hideki Kawahara (Wakayama Univ., 930 Sakaedani, 930, Wakayama, Wakayama 640-8510, Japan, kawahara@sys. wakayama-u.ac.jp)

We applied a novel aperiodicity analysis method (Kawahara et al. 2016, SSW9) to CSJ database (Maekawa, 2003, ISCA \& IEEE Workshop). The applied method derives the amount of aperiodicity as a time-frequency map, using three staged procedures. The first stage derives a probability map of the fundamental component witout prior information. The second stage finds the most probable trajectory of the fundamental component based on a state transition model and calculates the initial estimate of FO. The final stage refines this initial estimat using FO adaptive time axis warping and instantaneous frequency of each harmonic component. Recursive application of this refinemet procedure provides simultaneous estimation of FO and aperiodicity map. Classification of filled pause using the refined FO trajectories and the aperiodicity maps will be discussed. [This work was supported by JSPS KAKENHI Grant Number JP26284062.]
2pSC67. Spoken keyword detection using recurrent neural network language model. Shuhei Koike and Akinobu Lee (Dept. of Comput. Sci., Nagoya Inst. of Technol., Gokiso-cho, Showa-ku, Nagoya, Aichi 466-8555, Japan, koike@slp.nitech.ac.jp)

Recently, spoken keyword detection (SKD) systems that listen live audio and tries to capture user's utterances with specific keywords has been extensively studied, in order to realize a truly usable hands-free speech interface in our life: "Okay google" in Google products, "Hey, Siri" on Apple products and "Alexa" on Amazon Alexa / Amazon echo. Since the keyword detectors are typically built from large number of actually spoken keywords, they are irreplaceable and the users of such systems are forced to speak only the keyword they defined. On the other hand, a SKD method based on keyword-filler model using generic phoneme model and garbage filler sequence model is promising in that, since the acoustic pattern of the keyword will can be given as phoneme sequence, it is task-dependent and anyone can use his own keyword. In this study, an improvement of the latter method is studied. Recurrent neural network language model (RNNLM) is introduced as linguistic constraint for both filler-filler and filler-keyword instead of N-gram, and experimental result on actual spoken data for a spoken dialogue system showed that our method can improve the keyword detection performance.

2pSC68. Voice activity detection in movies using multi-class deep neural networks. Ikumi Suga, Ryu Yasuhara, Masashi Inoue, and Tetsuo Kosaka (Yamagata Univ., 4-3-16 Jonan, Yonezawa, Yamagata 9928510, Japan, tkm63745@st.yamagata-u.ac.jp)

For automatic classification of movie genres, detection of the presence or absence of speech is useful. Hence, accurate voice activity detection (VAD) techniques are needed. However, it is difficult to detect speech segments accurately because there are many kinds of noises in movies. Recently, deep learning has received increased attention in the speech processing field. Some VAD techniques based on deep neural networks (DNNs) have been proposed for clean speech conditions and showed better performance than conventional methods. The aim of this study is to improve VAD performance for movies by using DNNs. Generally, VAD is considered to deal with a two-class classification problem, i.e., classification of speech and non-speech segments. However, diverse noises in movies make it difficult. Therefore, it is difficult to detect speech segments accurately by using two-class DNNs. To solve this problem, we propose the use of multi-class DNNs for VAD in movies. In the experiments, we evaluated the proposed method for two types of movies, i.e., conversation-centric (CC) and nonconversation-centric (NCC) movies. The proposed multi-class DNNs showed better performance than conventional DNNs. The results showed that the equal error rates (EERs) for CC and NCC were $7.74 \%$ and $2.94 \%$, respectively.

2pSC69. Automatic estimation of extra-linguistic information in speech and its integration into recurrent neural network-based language models for speech recognition. Shohei Toyama, Daisuke Saito, and Nobuaki Minematsu (The Univ. of Tokyo, 7-3-1, Hongo, Bunkyoku, Tokyo 1138654, Japan, toyama@gavo.t.u-tokyo.ac.jp)

When one talks with others, he/she often changes the way of lexical choice and the speaking style depending on various contextual factors. For example in Japanese, we often use gender-dependent expressions and, when we speak to elderly people, we use polite expressions. It can be said that in any language, formality or informality of speaking changes drastically depending on the situation where speakers are involved. The aim of automatic speech recognition (ASR) is to convert speech signals into a sequence of words and the above fact indicates that the performance of ASR can be improved by taking the various contextual factors into account in the ASR modules. In this study, at first, we attempt to estimate those contextual factors as para-linguistic or extra-linguistic information and then, to integrate the results into language models based on Recurrent Neural Network (RNN) for speech recognition. In experiments, from an input utterance, i-vector and openSMILE features were extracted to represent speaker identity and speaking style. These acoustically driven features were integrated into the reranking process of RNN-based language models. Reductions of perplexity of the language models were shown to be 1 to $2 \%$ relative. 


\title{
Session 2pSPa
}

\section{Signal Processing in Acoustics: Compressive Sensing in Acoustics II}

\author{
Peter Gerstoft, Cochair \\ SIO Marine Phys. Lab. MC0238, Univ. of California San Diego, 9500 Gillman Drive, La Jolla, CA 92093-0238
}

Jeffrey S. Rogers, Cochair

Acoustics Division, Naval Research Lab., 4555 Overlook Ave. SW, Code 7161, Washington, DC 20375

Yoshifumi Shiraki, Cochair

Communication Science Laboratories, Nippon Telegraph and Telephone Corp., 3-1, Morinosato-Wakamiya, Atsugi-City, Kanazawa pref. 243-0198, Japan

\section{Invited Papers}

\section{1:00}

2pSPa1. Super-resolution in sound field recording and reproduction based on sparse representation. Shoichi Koyama, Naoki Murata, and Hiroshi Saruwatari (Graduate School of Information Sci. and Technol., The Univ. of Tokyo, Eng. Bldg. 6-140, 7-3-1 Hongo, Bunkyo-ku, Tokyo 113-8656, Japan, shoichi_koyama@ipc.i.u-tokyo.ac.jp)

Sound field recording and reproduction enables us to construct more realistic audio systems. In practical systems, sound pressure is obtained with microphones in a recording area, and then the sound field is reproduced with loudspeakers in a target area. Therefore, a signal conversion algorithm for obtaining the driving signals of the loudspeakers from the signals received by the microphones is necessary. Most of the current methods are based on the sound field analysis and synthesis in the spatial frequency domain. Although these methods make it possible for stable and efficient signal conversion, artifacts originating from spatial aliasing notably occur, which depends on the intervals of microphones and loudspeakers. We have proposed a signal conversion method based on sparse sound field decomposition, which enables sound field recording and reproduction above the spatial Nyquist frequency. By using sparse decomposition algorithms, the sound pressure distribution can be represented using a small number of fundamental solutions of the Helmholtz equation, such as Green's functions. Group sparse signal models are also required for accurate and robust decomposition. In this presentation, we reports on comparisons between several group sparse signal models and decomposition algorithms as well as their relation to reproduction performances.

\section{$1: 20$}

2pSPa2. Compressive sensing methods for passive localization and tracking of undersea acoustic sources. Pedro A. Forero and Paul Baxley (Maritime Systems Div., SPAWAR Systems Ctr. Pacific, 535, San Diego, CA 92152, pforero@gmail.com)

Matched-field processing techniques can achieve localization of undersea acoustic sources in both range and depth when sufficient environmental information is available. Unfortunately, these techniques are sensitive to environmental mismatch and often fail when localizing multiple acoustic sources. This work presents a family of acoustic source-localization techniques that similarly to matchedfield processing exploit environmental information for localizing acoustic sources in both range and depth. Unique features of these methods are their explicit use of a sparse representation of the source-localization map and ability to model environmental mismatch. Tools from the areas of compressive sensing and mathematical optimization are leveraged for developing computationally tractable solvers that enable fast processing of high-dimensional source-localization maps. These localization techniques are also extended for tracking multiple acoustic sources. In this case, it is possible to exploit the inherent sparsity of the innovations that occur between consecutive source-localization maps to enhance the localization results at a negligible computational cost. Numerical results on experimental data are shown to illustrate the performance of the proposed methods.

\section{1:40}

2pSPa3. Robust multi-frequency sparse Bayesian learning: Theory and simulations. Santosh Nannuru (Marine Physical Lab, SIO, UC, San Diego, 8820 Shellback Way, Spiess Hall, Rm. 452, San Diego, CA 92037, snannuru@ucsd.edu), Kay L. Gemba, William S. Hodgkiss, and Peter Gerstoft (Marine Physical Lab, SIO, UC, San Diego, La Jolla, CA)

Compressive sensing based techniques have recently been applied successfully to underwater acoustics problems such as beamforming and matched field processing. Sparse Bayesian Learning (SBL) is one of the fast compressive sensing methods and is formulated using Bayesian statistics. In the SBL framework, source amplitudes are modeled as complex Gaussian random variables with unknown variance. Evidence maximization is performed to estimate the unknown source amplitude variance and source position. A major advantage of SBL over more commonly used methods such as basis pursuit is that it is computationally faster. In this work, we develop a robust sparse Bayesian learning algorithm that can account for model mismatch leading to errors in the replica vector dictionary. Specifically, the likelihood function is modified so that it takes into account the covariance matrix of error in replica vectors. We also 
extend the SBL algorithm to process observations from multiple frequencies. The derived update rule combines observations from all the frequencies in a holistic manner. We demonstrate the robust SBL algorithm with simulations. A comparison is done with other compressed sensing methods including basis pursuit and orthogonal matching pursuit.

\title{
2:00
}

2pSPa4. Robust multi-frequency sparse Bayesian learning: Data results. Kay L. Gemba, Santosh Nannuru, Edward Richards, William S. Hodgkiss, and Peter Gerstoft (MPL, Scripps Inst. of Oceanogr., Univ. of California, San Diego, 8820 Shellback Way, Spiess Hall, Rm. 446, La Jolla, CA 92037, gemba@ucsd.edu)

Compressive sensing based techniques have been applied successfully to underwater acoustics problems. We present data results using a robust multi-frequency sparse Bayesian learning (SBL) algorithm that can account for model mismatch leading to errors in the replica vector dictionary. We use the SWellEx-96 Event S5 data set to demonstrate SBL capabilities for the beamforming and matched field processing (MFP) application. Beamforming results using the entire 64 element array (design frequency of $400 \mathrm{~Hz}$ ) and source frequencies ranging from 50 to $400 \mathrm{~Hz}$ indicate that multi-frequency SBL outperforms Bartlett and WNC processors in identifying multipath arrivals over the approximately $10 \mathrm{~km}$ source track. MFP results in a multiple-source scenario indicate that SBL offers a degree of robustness in the presence of data-replica mismatch when tracking a quiet source. The data-replica mismatch is especially pronounced at the closest point of approach due to array tilt of approximately 2 degrees. Data results further indicate that the two SBL tuning parameters (diagonal loading of the replica vector covariance matrix and number of sources for the algorithm's noise estimate) do not require excessive calibration.

\section{Session 2pSPb}

\section{Signal Processing in Acoustics and Underwater Acoustics: Detection, Tracking, and Classification of Unmanned Aircraft and Underwater Vehicles}

\author{
Geoffrey H. Goldman, Cochair \\ U.S. Army Research Laboratory, 2800 Powder Mill Road, Adelphi, MD 20783-1197 \\ R. Lee Culver, Cochair \\ ARL, Penn State University, PO Box 30, State College, PA 16804
}

Chair's Introduction-2:35

\section{Invited Papers}

\section{2:40}

2pSPb1. Some comments on detecting unmanned underwater vehicles using passive acoustics. Gerald L. D'Spain (Marine Physical Lab, Scripps Inst. of Oceanogr., 291 Rosecrans St., San Diego, CA 92106, gdspain@ucsd.edu)

Passive methods of detection rely upon the presence of a field radiated by the phenomenon of interest (in this case, unmanned underwater vehicles-UUVs). This talk illustrates the challenges of using passive acoustic methods to detect UUVs. Two types of vehicles are discussed; a 20-ft wingspan, buoyancy-driven, flying wing autonomous underwater glider and a 21-in-diameter, prop-driven UUV. At-sea measurements of the acoustic field radiated by the glider's large (30 liter) buoyancy engine are presented. Whereas a prop-driven propulsion system operates continuously, a buoyancy engine has very low duty cycle: a few percent. Onboard self noise from the glider's internal fluid-based roll control system (which provides control authority near neutral buoyancy) far exceeds that from an aileron-based system. For the 21-in-diameter prop-driven UUV, modifications were made to its propulsion and steering systems to reduce radiated noise by $20-50 \mathrm{~dB}$. The resulting self noise levels recorded at sea by a hull-mounted hydrophone now are below typical background ocean noise levels above $200 \mathrm{~Hz}$, with the noise below $200 \mathrm{~Hz}$ being primarily vibration induced. Highly directional passive acoustic receiving systems are required to detect the transits of these UUVs. [Work supported by the Office of Naval Research and BP.] 
2pSPb2. Acoustic detection, localization, and tracking of tactical autonomous aerial and underwater vehicles. Brian G. Ferguson and Kam W. Lo (Maritime Div., DSTG, PO Box 44, Pyrmont, NSW 2009, Australia, Brian.Ferguson@dsto.defence.gov.au)

By applying single sensor narrowband and multisensor broadband passive acoustic signal processing methods to real microphone data, it is shown that low observable tactical autonomous aerial vehicles can be detected, classified, localized, and tracked by unattended land-based acoustic surveillance systems that exploit a target's acoustic signature. Similarly, the application of passive sonar signal processing methods to real hydrophone data reveals the presence, position and track of an autonomous underwater vehicle by exploiting its radiated noise and its underwater acoustic communication transmissions. A suite of these narrowband and broadband acoustic signal processing methods will be identified and their functional performances quantified.

3:20

2pSPb3. A physical simulation of sound propagation from unmanned aerial vehicles. Z. C. Zheng and Junjian Zhang (Aerosp. Eng., Univ. of Kansas, 2118C Learned Hall, 1530 W 15th St., Lawrence, KS 66045, zzheng@ku.edu)

The study is to investigate sound propagation from UAVs to the environment. The sound propagation is simulated based on the physical time and space, which is a time-domain simulation in a physical spatial domain. The investigation will be focused on the physics that is usually difficult to reveal using non-physical space simulation. The effects to be investigated include source characteristics and motion of UAV noise, influence of local environment such as atmospheric turbulence and wind shear,ground roughness and impedance, diffraction caused by local structures such as terrain, buildings, bushes, and trees. Because of proximity of UAVs to the ground, these effects are more significant for sound propagation from UAVs than from large aircraft that are at higher altitude from the ground. Examples of source recognition using the simulation results will also be discussed.

\section{3:40}

2pSPb4. Spectral broadening of acoustic tones generated by unmanned aerial vehicles in a turbulent atmosphere. Vladimir E. Ostashev, D. K. Wilson (U.S. Army Cold Regions Res. and Eng. Lab., 72 Lyme Rd., Hanover, NH 03755, vladimir.ostashev@noaa. gov), Anthony Finn, Kevin Rogers (Defense and Systems Inst., Univ. of South Australia, Mawson Lakes, SA, Australia), and Emre Barlas (Wind Energy, Tech. Univ. of Denmark, Kongens Lyngby, Denmark)

The acoustic spectrum emitted by unmanned aerial vehicles (UAVs) and other aircraft can be distorted by propagation through atmospheric turbulence. Since most UAVs are propeller-based, they generate a series of acoustic tones and harmonics. In this paper, spectral broadening of these tones due to atmospheric turbulence is studied. The broadening results from the combined Doppler effect of multiply scattered acoustic signals propagating in a non-stationary turbulent atmosphere. It can be assessed as a Fourier transform of the temporal coherence function of a monochromatic signal propagating in an atmosphere with spatial-temporal fluctuations in temperature and wind velocity. This temporal coherence was recently investigated [V. E. Ostashev, D. K. Wilson, S. N. Vecherin, and S. L. Collier, J. Acoust. Soc. Am. 136 (5), 2414-2431 (2014)] for the model of locally frozen turbulence. Based on these results, spectral broadening is calculated and analyzed for typical meteorological regimes of the atmospheric boundary layer and different flight trajectories of UAVs. Experimental results are presented and compared with theoretical predictions. Spectral broadening might also provide a means for remotely sensing atmospheric turbulence.

\section{4:00-4:20 Break}

\section{Contributed Papers}

$4: 20$

2pSPb5. Novel algorithm for acoustic classification of unmanned aircraft systems. Michael Brown, Cory Smith, and Amanda Hanford (Appl. Res. Lab., The Penn State Univ., P.O. Box 30, State College, PA 168040030,mtb5264@psu.edu)

Advances in Unmanned Aircraft System (UAS) technologies have made them smaller and more affordable, causing a proliferation in UAS activity. This increase has led to a rise in UAS-related incidents, exposing the need for stricter UAS regulation. Detection, Localization, and Classification (DLC) of UAS are important techniques for UAS regulation. However, DLC can be challenging, since UAS span a variety of shapes, sizes, speeds, propulsion systems, and operational altitudes; they can be hard to detect with radar; and they can operate in radio silence. Research supporting DLC of UAS has led to the development of novel, passive acoustic signal processing algorithms for real-time classification. In particular, data fusion algorithms combining kinetic and acoustic data into features for classification are being developed. Kinetic data are collected using acoustic signal source tracking algorithms, and spectral content is extracted from frequency-domain analysis. Using free-body modeling and Doppler compensation, classification probabilities can then be calculated for a list of known UAS. Initial classification algorithms have been developed and tested in MATLAB using UAS data collected in operational environments. The test results will also be presented.

\section{$4: 35$}

2pSPb6. Application of cross-correlation methods for passive acoustic unmannded aierial vehicle detection and tracking. Alexander Sedunov (Stevens Inst. of Technol., 711 Hudson St., Hoboken, NJ 07030, asedunov@stevens.edu), Alexander Sutin (Stevens Inst. of Technol., Summit, NJ), and Hady Salloum (Stevens Inst. of Technol., Glen Ridge, NJ)

Stevens Institute of Technology has applied the cross-correlation method for the detection of low flying aircraft using passive acoustics. A modified cross correlation method was tested for finding the time difference of arrival of acoustic signals from various unmanned aerial vehicles (UAV), collected by NASA's Langley Research Center. Cross-correlation was applied for UAV detection and for measurements of the time difference of arrival that is used for estimating the direction of arrival. The microphone separation combined with the high speed of the target had led to a significant differential Doppler effect leading to de-correlation of the received signals. For the compensation of those effects, Stevens applied a modified de-skewed short-time correlator (DSTC) approach. The detection ability of the cross-correlation method also depends on the cross correlation properties of ambient noise. The collection was performed in a relatively quiet area, to estimate the performance of the cross-correlation method in noisy areas the NASA UAV signatures were combined with ambient noise collected by Stevens. We demonstrate how the noise affects the detection distance. Special attention was paid to the influence of correlated components of the ambient noise. [The authors are grateful to Dr. Cabell (NASA's Langley Research Center) for providing the acoustic data. This work was supported by DHS's S\&T Directorate.] 


\section{Invited Paper}

\section{4:50}

2pSPb7. Acoustic detection of small unmanned aerial system using mills cross arrays. Seongil Kim (The 6th R\&D Inst., Agency for Defense Development, PO Box 18, Jinhae, Changwon, Kyung-nam 645-600, South Korea, kim_seongil@yahoo.com)

Two mills cross microphone arrays are used to detect and track a small unmanned aerial system (UAS). Each array consists of two 64-channel line arrays which are perpendicular each other. The cross-shape array is to detect targets approaching from any direction and the two-array system is a minimum number for localizing the target. The microphones of the line array are equally spaced as a design frequency of $2 \mathrm{kHz}$. The received acoustic signal is digitized as a sampling rate of $8 \mathrm{kHz}$ and transferred to a remote signal processor by a radio link in real time. Beam-formed acoustic data are displayed in various ways to detect and localize the moving acoustic target. In this paper, the proto-type acoustic system for UAS detection as well as the experimental results will be discussed. Methods for array beam-forming, tracking and classifying the target and the acoustic characteristics of the target will be briefly mentioned.

\section{Contributed Papers}

2 pSPb8. Acoustic detection results for a small unmanned aircraft system extrapolated over range. Geoffrey H. Goldman (U.S. Army Res. Lab., 2800 Powder Mill Rd., Adelphi, MD 20783-1197, geoffrey.h.goldman.civ@ mail.mil)

Small unmanned aircraft systems (SUAS) are becoming cheaper with more advanced reconnaissance and surveillance capabilities. Low-cost sensors and countermunitions are needed to defeat this asymmetric threat. The first step is to detect SUAS using low-cost sensors such as an array of microphones with robust signal processing algorithms. An analysis of six detection algorithms was performed using acoustic data generated by a class I SUAS at ranges of 50 to $650 \mathrm{~m}$ and measured with a small tetrahedral microphone array with $20-\mathrm{cm}$ length arms. The detection algorithms were based upon the peak power in delay-and-sum, filtered delay-and-sum, and adaptive delayand-sum beamforming algorithms. To test the performance of the algorithms at longer ranges, the measured signal, modeled as target plus additive noise, was modified. First, the signal from the target was attenuated using Bass's model and spherical attenuation, and then, the noise was adjusted to maintain an unbiased estimate of the measured power spectrum density function. Receiver operation characteristics (ROC) curves were generated and the performance of the algorithms as a function of range was evaluated.
$5: 25$

2pSPb9. Stepped FM acoustic signal design. Edmund J. Sullivan (EJS Consultants, 46 Lawton Brook Ln., Portsmouth, RI 02871, bewegungslos@ fastmail.fm)

The linear Frequency Modulated (LFM) signal is a means of providing a broadband signal with a short time duration. One of its main uses is in active sonar, where it provides enhanced target range resolution and improved performance against reverberation. Its generation can be simplified by approximating it using a so-called Stepped Frequency Modulated (SFM) signal, where the pulse is made up of a set of contiguous single frequency pulses. In its simplest form, the frequency of these pulses increases (or decreases) in a linear manner along the time history of the pulse. This paper presents a technique for designing a SFM pulse that best emulates its LFM counterpart in its detection performance. It is shown that if the time-bandwidth product of the LFM is BT, the number of pulses in the SFM that best emulates the LFM is equal to the nearest integer to the square root of BT. Values of N smaller than this reduce the detection performance and values larger than this provide no improvement in detection performance and indeed, can cause some reduction the performance, where this reduction varies erratically as $\mathrm{N}$ increases. 


\title{
Session 2pUW
}

\section{Underwater Acoustics, Acoustical Oceanography, and Physical Acoustics: Nonlinear Effects in Underwater Acoustics and Bubbles}

\author{
Thomas G. Muir, Cochair \\ Applied Research Labotatories, University of Texas at Austin, P/O. Box 8029, Austin, TX 78713 \\ John J. Esplin, Cochair \\ Acoustics, Pennsylvania State University, 201 Applied Science Building, University Park, PA 16802 \\ Tetsuya Kanagawa, Cochair \\ Engineering Mechanics and Energy, University of Tsukuba, 1-1-1 Tennodai, Tsukuba 305-8573, Japan
}

Chair's Introduction-1:00

Invited Papers

1:05

2pUW1. Bulk cavitation extent modeling: An energy-based approach. J. J. Esplin (Acoust., Penn State Univ., 201 Appl. Sci. Bldg., University Park, PA 16802, jje166@psu.edu), Benjamin J. Kim, Michael P. Kinzel (Appl. Res. Lab., Penn State Univ., State College, PA), and R. L. Culver (Appl. Res. Lab., Penn State Univ., Reston, VA)

Bulk cavitation is a phenomenon that occurs when a negative-pressure or tension wave causes a liquid to rupture, or cavitate, over space. It is a process which causes resident microbubbles to grow to many times their original size, forming a bubble cloud. Such bubble clouds are observed in shallow underwater explosions, where negative-pressure waves are formed after shock waves reflect off the water surface; they are also observed in shock wave lithotripsy, shock wave histotripsy, ultrasonic cleaning, and other applications. Models had been developed for predicting the size and shape of such bulk cavitation regions. This work introduces a model that accounts for energy "lost" to bulk cavitation which in turn influences the extent that is dependent on the rate at which the passing negative-pressure wave dissipates. In-laboratory underwater experiments utilizing a spark source for high-amplitude pressure pulse generation, hydrophones and high-speed videography validate the energy transfer from tension wave to bubble cloud formation. These experiments are supplemented by computational fluid dynamics simulations. A cavitation absorption coefficient is introduced and parameterized for accurate prediction of cloud extent.

\section{$1: 25$}

2pUW2. The effects of external acoustic fields on a free-running supercavitating projectile. Peter J. Cameron (School of Mech. Eng., Georgia Inst. of Technol., Cupertino, California), John W. Doane, and Peter H. Rogers (School of Mech. Eng., Georgia Inst. of Technol., Atlanta, GA 30332, peter.rogers@gatech.edu)

Proliferation of supercavitating torpedoes has motivated research on countermeasures against them as well as on the fluid phenomenon which makes them possible. The goal of this research was to investigate an envisaged countermeasure, an acoustic field capable of slowing or diverting the weapon by disrupting the cavitation envelope. The research focused on the interactions between high pressure amplitude sound waves and a supercavity produced by a small free-flying projectile. The flight dynamics and cavity geometry measurements were compared to control experiments and theoretical considerations were made for evaluating the effects. Corrugations on the cavity/water interface caused by the pressure signal have been observed and characterized. Results also show that the accuracy of a supercavitating projectile can be adversely affected by the sound signal. This research concludes with results that indicate that it is acoustic cavitation in the medium surrounding the supercavity, caused by the high pressure amplitude sound, that is responsible for the reduced accuracy. A hypothesis has been presented addressing the means by which the acoustic cavitation could cause this effect. [Much of the content of this paper was published in J. Acoust. Soc. Am. 128, 3381-3392 (2010).] 
2pUW3. An introduction to the applications and bubble dynamics of the combustive sound source. Andrew R. McNeese (Appl. Res. Labs, UT: Austin, 10000 Burnet Rd., Austin, TX 78758, mcneese@arlut.utexas.edu), Preston S. Wilson (Dept. of Mech. Eng. and Appl. Res. Labs, UT: Austin, Austin, TX), and Thomas G. Muir (Appl. Res. Labs, UT: Austin, Austin, TX)

The combustive sound source (CSS) is a versatile underwater sound source with an adjustable output amplitude used in underwater acoustics experiments. Unlike typical impulsive acoustic sources, CSS can maintain a wide bandwidth at low amplitude and can be tuned to operate in a band of interest. The CSS can be used as a source for low-frequency sediment characterization, array calibration, TL measurements, and when deployed on the bottom can produce seismic interface waves. In addition to stationary deployments in the water column, CSS can be deployed in a tow body. The CSS consists of a submersible combustion chamber, open to the water, which is filled with a combustive mixture that is ignited via spark. Upon ignition, the combustive mixture is converted into high temperature combustion byproducts which expand and ultimately collapse back to smaller volume than before ignition. Acoustic pulses are radiated by the bubble activity. Although the far-field acoustic propagation is generally assumed to be linear, the inherent nature of the bubble growth and collapse is a nonlinear phenomenon. Discussion will focus on the latest CSS design including functionality and acoustic output, as well as the nonlinearity of the bubble dynamics associated with a CSS event.

\section{2:05}

2pUW4. Two types of propagations of nonlinear sound beams in nonuniform bubbly liquids. Tetsuya Kanagawa (Eng. Mech. and Energy, Univ. of Tsukuba, 1-1-1 Tennodai, Tsukuba 305-8573, Japan, kanagawa@kz.tsukuba.ac.jp)

Weakly nonlinear propagation of diffracted sound beams in nonuniform bubbly liquids is theoretically examined. The spatial distribution of the number density of the bubbles, initially in a quiescent state, is assumed to be a slowly varying function of the spatial coordinates; the amplitude of variation is assumed to be small compared to the mean number density. Two types of nonlinear wave equations for progressive quasi-plane beams in weakly nonuniform bubbly liquids are then systematically derived via the method of multiple scales. The diffraction effect is incorporated by adding a relation that scales the circular sound source diameter to the wavelength into the original set of scaling relations composed of nondimensional physical parameters. A set of basic equations for bubbly liquids is composed of the averaged equations of mass and momentum in a two-fluid model, the Keller equation for bubble wall, the equation of state for gas and liquid, the mass conservation equation inside the bubble, and the balance equation of normal stresses across the gas-liquid interface. As a result, two types of evolution equations, a nonlinear Schrödinger (NLS) equation including dissipation, diffraction, and nonuniform effects for high-frequency short-wavelength case, and a Khokhlov-Zabolotskaya-Kuznetsov (KZK) equation including dispersion and nonuniform effects for low-frequency long-wavelength case, are derived from the basic set. Finally, numerical and analytical solutions of NLS and KZK equations toward some applications are presented.

\section{Contributed Papers}

\section{2:25}

2pUW5. Low-frequency approximations for the radiation damping of a bubble. Kyle S. Spratt and Mark F. Hamilton (Appl. Res. Labs., The Univ. of Texas at Austin, Austin, TX, sprattkyle@gmail.com)

The recent work by Ainslie and Leighton [J. Acoust. Soc. Am. 126, 2163 (2009)] pointed out that there exist two different expressions in the literature for the scattering cross-section of a spherical gas bubble in liquid. The difference between the two expressions is contained in the term corresponding to the losses due to acoustic radiation. The more common expression, often attributed to R. Wildt ["Acoustic theory of bubbles," in Physics of Sound in the Sea], is based on a low-frequency approximation and is thus only accurate when the wavelength of the incident wave is large compared to the circumference of the bubble. The purpose of the present work is to investigate specifically the accuracy of this low-frequency approximation for various physical parameters and frequencies of interest. An alternative low-frequency approximation is also suggested, which is both simpler and more accurate than the present approximation. Finally the case of a nonspherical bubble is considered, and an expression for the scattering crosssection is given that is based on the same low-frequency assumptions made by Wildt. [Work supported by the IR\&D program at ARL:UT.]

\section{2:40}

2pUW6. Dynamic equilibrium model for stable nano- and micro-bubbles partly covered with hydrophobic material. Kyuichi Yasui, Toru Tuziuti, and Wataru Kanematsu (National Inst. of Adv. Industrial Sci. and Technol. (AIST), 2266-98 Anagahora, Shimoshidami, Moriyama-ku, Nagoya 463-8560, Japan, k.yasui@aist.go.jp)

In the dynamic equilibrium model, a bubble is only partly covered with hydrophobic material in contrast to the skin model that a bubble is completely covered with organic material. On the surface of the hydrophobic material in water, gas is highly concentrated due to the presence of depletion layer. Then, gas diffuses into a bubble near the peripheral edge of the hydrophobic material, which may balance with the gas diffusion out of a bubble from the other part of the uncovered bubble surface due to the higher internal gas pressure than the partial pressure of gas dissolved in the liquid water by the Laplace pressure. In the present study, not only the condition of the mass balance but also that of stability is numerically calculated. The results have indicated that a nanobubble could be stable when the fraction of the surface coverage by the hydrophobic material is more than 0.5 in water both supersaturated and under-saturated with gas (air). In slightly degassed water, a microbubble could be stabilized when the fraction of the surface coverage is on the order of $10^{-4}$ or less. These bubbles could play an important role in underwater acoustics as well as in acoustic cavitation.

\section{2:55}

2pUW7. Phase speed measurements of a bubbly liquid in impedance tubes using a transfer function technique. Stanley A. Cheyne, H O. Thurman, and Cecil M. Tiblin (Dept. of Phys. \& Astronomy, Hampden-Sydney College, Hampden-Sydney, VA 23943, scheyne@hsc.edu)

Two different impedance tube configurations were used to make phase speed measurements of a bubbly liquid. One setup consisted of a vertical water-filled tube with hypodermic needles near the top end to provide a bubbly termination. In this setup, the source was at the bottom of the tube. The second setup was a vertical tube partially filled with water with hypodermic needles at the bottom. In this setup, the source was at the top and the measurements were made in air with the bubbly termination at the bottom of the tube. In both cases, an SRS-785 spectrum analyzer was used to measure and calculate the transfer function. The transfer function technique (IS0 105342-1998) was used to determine the impedance of the bubbly liquid. Once the impedance was found, the phase speed was calculated. Results will be presented with a range of void fractions and bubble radii. 


\section{Invited Papers}

2pUW8. Modes of targets in water excited and identified using the radiation pressure of modulated focused ultrasound. Timothy D. Daniel, Philip L. Marston (Phys., WSU, Phys. and Astronomy Dept., Washington State Univ., Pullman, WA 99164, timothy.daniel@ email.wsu.edu), Ivars Kiersteins (NUWC, Newport, RI), and Ahmad T. Abawi (HLS, Res., La Jolla, CA)

Previously, we excited low frequency flexural modes of a circular plate in water using modulated radiation pressure generated by focused ultrasound [J. Acoust. Soc. Am. 137, 2439 (2015)]. This effect is distinct from excitations associated with parametric array sonar in that static deformation can be produced by radiation pressure as has been measured and analyzed for bubbles [T. J. Asaki and P. L. Marston, J. Acoust. Soc. Am. 97, 2138-2143 (1995)]. In our experiments, solid targets are suspended by lines or supported on sand and the modulated ultrasound is focused on the target's surface. Target sound emissions were recorded and a laser vibrometer was used to measure the surface velocity of the target to give the magnitude of the target response. An improved high-power focused transducer allows us to drive modes of larger more-realistic target models. By varying the modulation frequency and monitoring the target response, resonant frequencies of the target can be found. By scanning the point-like driving force, the target mode shapes can be measured and compared to finite element models. [Work supported by the Office of Naval Research.]

$3: 45$

2pUW9. Improved object detection sonar using nonlinear acoustical effects in bubbly media. Kevin M. Lee, Grace A. Isakson (Appl. Res. Labs., The Univ. of Texas at Austin, 10000 Burnet Rd., Austin, TX 78758, klee@arlut.utexas.edu), and Preston S. Wilson (Dept. of Mech. Eng. and Appl. Res. Labs., Univ. of Texas at Austin, Austin, TX)

Clutter from air bubbles can significantly impact the performance of object detection sonar in shallow water environments where bubbles are present due to breaking waves, wakes, or organic matter. Nonlinear acoustical effects can be excited in bubbly liquids at sound pressures below that required in bubble-free media and hence can be used to discriminate between bubbles and solid objects or to suppress bubble-related clutter altogether. Whereas such effects are widely exploited for ultrasonic biomedical imaging enhancement and it is hypothesized that dolphins exploit them in their biosonar, relatively less attention has been given to their use in naval, commercial, or oceanographic manmade sonar. Here, we describe laboratory tank experiments and modeling efforts aimed at exploiting these effects to improve object detection sonar. A bubble dynamics model was employed to investigate parameter space for nonlinear effects such as subharmonic and higher-order harmonic generation from regions of bubbly liquid and sonar-induced bubble translation. Laboratory experiments were conducted to verify the presence of these effects. Finally, a laboratory demonstration of a nonlinear bubble/object discriminating sonar, in which nonlinear effects are used to place markers on returns from regions of bubbly liquid, will be presented. [Work supported by ONR.]

\section{4:05}

2pUW10. Measurement of the structural waves in a mine and low frequency tomographic imaging using a parametric sonar. Ron J. Wyber (Midspar Systems, 24 Farrer Pl., Oyster Bay, NSW 2225, Australia, rjwyber@ozemail.com.au) and Brian G. Feguson (DSTO, Pyrmont, NSW, Australia)

To investigate the structural response of mines at low frequencies a parametric sonar with a horizontal beamwidth of about 5 degrees and a bandwidth from $1 \mathrm{kHz}$ to $10 \mathrm{kHz}$ was used. Advantage was taken of the wide bandwidth to obtain a high resolution in the impulse response measurements of the targets by transmitting a linear period modulated pulse and applying pulse compression and a Kaiser Bessel shading to the reflected signal. Over the frequency response of the measurements structural waves are excited in the mines which clearly identify them as man-made targets. The structural waves observed include flexural waves propagating around the body of the mine which provide information on the mine construction. Results are presented showing the variation in the structural response as the targets are rotated through 360 degrees. Tomographic imaging is applied to the measured impulse response and it is shown that by gating out the structural waves clear images of the mines can be obtained even at these low frequencies. This provides the opportunity to image buried targets, particularly in muddy areas where burial is most likely. Profiling measurements in Sydney Harbour are presented showing the variation in the bottom penetration with the sediment type.

2pUW11. Parametric sources-From theory to commercial products. Johnny Dybedal (KONGSBERG, Strandveien 1, Stjørdal NO7502, Norway, johnnyd@broadpark.no)

The interest for underwater application of nonlinear acoustic began in the late 1950s and early 1960s with Westervelts idea of using nonlinear effects to generate low frequency sound beams with high directivity; the parametric source. This idea got a lot of attention during the 1960s and 1970s from various scientific institutions. An early experiment with a parametric source was performed by ELAB at the University of Trondheim and Simrad in 1977. In the early 1980s, Bentech started the design of a towed, combined mapping system for bathymetry and detection of buried pipelines. The idea was to do detection while moving along the pipeline, which required a subbottom profiler with steerable beams with high directivity. Due to limited space for transducer, a parametric source was the logical choice. A prototype of the parametric sub-bottom profiler was finished in the late 1980s. Following the prototype, the system was commercialized and additional versions were developed covering applications from shallow water, high resolution work to full ocean depth, high penetration work. Today KONGSBERG is a world leading supplier of parametric sub-bottom profilers. 


\section{Contributed Papers}

$4: 45$

2pUW12. Investigation of nonlinearity parameter $B / A$ of saturated, unconsolidated marine sediments via a statistical approach. Hunki Lee, Eunghwy Noh, Won-Suk Ohm (School of Mech. Eng., Yonsei Univ., 50 Yonsei-ro, Seodaemun-gu, Seoul 120-749, South Korea, ssevi2@yonsei.ac.kr), and Oh-Cho Kwon (The 6th R\&D Inst. Naval System Directorate Principal Researcher, Agency for Defense Development, Changwon, South Korea)

In this talk, we present a theory and the supporting experiment for the nonlinearity parameter $B / A$ of saturated, unconsolidated marine sediments. The model is composed of the quadratic nonlinearity of the equivalent suspension of grains and the interstitial fluid and the nonlinearity resulting from the random grain contacts. To capture the random nature of grain contacts, the number of Hertzian contacts on each grain and the distribution of contact forces between grains are treated statistically. Predictions of $B / A$ are compared with measurements performed with the finite amplitude insertsubstitution (FAIS) method. Dependence of $B / A$ on external compression and the incident sound pressure level is also discussed. [This work was conducted in the Unmanned Technology Research Center (UTRC) sponsored by the Defense Acquisition Program Administration (DAPA) and the Agency for Defense Development (ADD) in the Republic of Korea.]

\section{5:00}

2pUW13. Beam characteristics of the truncated parametric array generated by a panel piston radiator. Desen Yang, Hongdao Li, Jie Shi, Shengguo Shi, Haoyang Zhang, Di Li, and Shiyuan Jin (College of Underwater Acoust. Eng., Harbin Eng. Univ., College of Underwater Acoust. Eng., No. 145, Nantong St., Nangang District, Harbin, Heilongjiang 150001, China, leehongdao@hrbeu.edu.cn)

A theoretical model is presented to describe the difference frequency beam characteristics of the truncated parametric array generated by a panel piston radiator. In this model, a low-pass acoustic filter is used to truncate the virtual-source. The truncated virtual-source can be viewed as a volume distributed cylindrical source and conic source within and beyond the Rayleigh distance, respectively. The difference frequency beam characteristics near the truncated position are significantly changed. Numerical simulation results show that the difference frequency beams near the truncated position are substantially sharpened with the distance between the observation point and the truncated position decreasing. Besides, the difference frequency beamwidth near the truncated position can be used to predict the farfield beamwidth of the truncated parametric array.

\section{$5: 15$}

2pUW14. Research on the influence of dispersion in the shallow water waveguide on the parametric array. Desen Yang, Di Li, Jie Shi, Shengguo Shi, Haoyang Zhang, and Hongdao Li (College of Underwater Acoust. Eng., Harbin Eng. Univ., No.145, Nantong St., Nangang District, Harbin, Heilongjiang 150001, China, donkeysoso@163.com)

In practice, the application of parametric array is limited due to the effect of dispersion in the shallow water waveguide which has a significant impact on the propagation of the difference frequency wave. This paper attempts to provide better understanding of the difference frequency field in the shallow water waveguide by discussing the virtue source of parametric array in such circumstance. In the paper, the Green's function of the shallow water waveguide is employed for calculating the difference frequency field with the help of virtue source in waveguide. It shows that the wavenumber mismatch caused by waveguide dispersion will restrain the accumulation of difference frequency wave and bring about fluctuations during the process of accumulation. Furthermore, this paper discusses the nonlinear interaction with different modes of pump wave based on the former conclusion and reveals pictures to describe the complicated mode structure of the difference frequency field. 


\section{OPEN MEETINGS OF TECHNICAL COMMITTEES}

The Technical Committees of the Acoustical Society of America will hold open meetings on Tuesday, and Wednesday. See the list below for the exact schedule.

These are working, collegial meetings. Much of the work of the Society is accomplished by actions that originate and are taken in these meetings including proposals for special sessions, workshops, and technical initiatives. All meeting participants are cordially invited to attend these meetings and to participate actively in the discussion.

Committees meeting on Tuesday, 29 November

\author{
Committee \\ Acoustical Oceanography \\ Animal Bioacoustics \\ Architectural Acoustics \\ Engineering Acoustics \\ Physical Acoustics \\ Psychological and Physiological \\ Acoustics \\ Structural Acoustics and Vibration
}

$\begin{array}{ll}\text { Start Time } & \text { Room } \\ \text { 7:30 p.m. } & \text { Kahili } \\ \text { 7:30 p.m. } & \text { Coral 2 } \\ \text { 7:30 p.m. } & \text { Lehua } \\ \text { 7:30 p.m. } & \text { South Pacific 4 } \\ \text { 7:30 p.m. } & \text { South Pacific 2 } \\ \text { 7:30 p.m. } & \text { South Pacific 3 } \\ & \\ \text { 7:30 p.m. } & \text { South Pacific 1 }\end{array}$

Committees meeting on Wednesday, 30 November

Committee

Biomedical Acoustics

Musical Acoustics

Noise

Signal Processing in Acoustics

Speech Communication

Underwater Acoustics

\begin{abstract}
Start Time
7:30 p.m.

7:30 p.m.

7:30 p.m.

7:30 p.m.

7:30 p.m.

7:30 p.m.
\end{abstract}

Room

Coral 1

Kahili

South Pacific 4

South Pacific 1

Coral 4

Nautilus 


\title{
Session 3aAAa
}

\section{Architectural Acoustics and Speech Communication: At the Intersection of Speech and Architecture II}

\author{
Kenneth W. Good, Cochair \\ Armstrong, 2500 Columbia Ave., Lancaster, PA 17601 \\ Takashi Yamakawa, Cochair \\ Yamaha Corporation, 10-1 Nakazawa-cho, Naka-ku, Hamamatsu 430-8650, Japan \\ Catherine L. Rogers, Cochair \\ Dept. of Communication Sciences and Disorders, University of South Florida, USF, 4202 E. Fowler Ave., PCD1017, \\ Tampa, FL 33620
}

Chair's Introduction-8:00

\section{Invited Papers}

8:05

3aAAa1. Vocal effort and fatigue in virtual room acoustics. Pasquale Bottalico, Lady C. Cantor Cutiva, and Eric J. Hunter (Communicative Sci. and Disord., Michigan State Univ., 1026 Red Cedar Rd., Lansing, MI 48910, pb@msu.edu)

Vocal effort is a physiological entity that accounts for changes in voice production as vocal loading increases, which can be quantified in terms of Sound Pressure Level (SPL). It may have implications on potential vocal fatigue risk factors. This study investigates how vocal effort is affected by room acoustics. The changes in the acoustic conditions were artificially manipulated. Thirty-nine subjects were recorded while reading a text, 15 out of them used a conversational style while 24 were instructed to read as if they were in a classroom full of children. Each subject was asked to read in three different reverberation time RT ( $0.4 \mathrm{~s}, 0.8 \mathrm{~s}$, and $1.2 \mathrm{~s})$, in two noise conditions (background noise at $25 \mathrm{dBA}$ and Babble noise at $61 \mathrm{dBA})$, in three different auditory feedback levels $(-5 \mathrm{~dB}, 0 \mathrm{~dB}$, and $5 \mathrm{~dB})$, for a total of 18 tasks per subject presented in a random order. The subjects answered questions addressing their perception of vocal fatigue on a visual analog scale. Babble noise and the order of task presentation increased SPL and self-reported fatigue. The SPL increased when the RT and the auditory feedback level decreased further clarifying how vocal effort changes within various conditions.

\section{$8: 25$}

3aAAa2. Relationship of the difference of the speech rate of an announcement at the railway station and the listening impression. Sohei Tsujimura (Structures Technol. Div., Railway Tech. Res. Inst., 2-8-38 Hikari-cho, Kokubunji-shi, Tokyo 185-8540, Japan, tsouhei@rtri.or.jp)

The purpose of this study is to clear the influence of the speech rate of an announcement on the listening impression. In this study, a subjective experiment in which the elderly and the young adults participated was conducted in the simulated station. In this experiment, the speech rate of an announcement was varied from 4.5 to $9.5 \mathrm{mora} / \mathrm{s}$ at $1 \mathrm{mora} / \mathrm{s}$ interval, and subjective evaluations ("Listening difficulty," "Loudness," and "Strangeness") of the announcements were made under three types of background noise conditions $\left(L_{\mathrm{A}} 65\right.$ $\mathrm{dB}, 70 \mathrm{~dB}$, and $75 \mathrm{~dB})$. The relationships of the speech rate of an announcement and the subjective evaluation such as "Listening difficulty," "Loudness," and "Strangeness" were investigated, and the optimum speech rate of an announcement at the railway station was discussed. As a result, it was suggested that "Listening difficulty" has the lowest evaluation value when the speech rate is $6.5 \mathrm{mora} / \mathrm{s}$ or $7.5 \mathrm{mora} / \mathrm{s}$. Furthermore, in this speech rate range, "Strangeness" as an announcement at the railway station was not felt. It was demonstrated that the optimum speech rate of an announcement at the railway station is in the range from $6.5 \mathrm{mora} / \mathrm{s}$ to $7.5 \mathrm{mora} / \mathrm{s}$.

\section{8:45}

3aAAa3. Room acoustics and speech perception: Considerations for the developing child. Lori Leibold (Ctr. for Hearing Res., Boys Town National Res. Hospital, 555 North 30th St., Omaha, NE 68124, lori.leibold@boystown.org)

What children hear in noisy rooms is not the same thing that adults hear. Despite precocious maturation of the peripheral auditory system, the ability to hear and understand speech in the presence of competing backgrounds sounds is not fully developed until adolescence. This talk will review results of behavioral studies of auditory masking in children, with a focus on the development speech perception in complex acoustical environments. Data will be presented that support the hypothesis that children's increased susceptibility to auditory masking relative to adults is related to immature perceptual processing such as sound source segregation and selective attention. Findings from studies investigating the extent to which children benefit from acoustic cues thought to facilitate sound source segregation will be highlighted. 


\title{
Session $3 \mathbf{a A A b}$
}

\section{Architectural Acoustics and Signal Processing in Acoustics: Advanced Analysis, Simulation, and Auralization in Room Acoustics I}

\author{
Michael Vorländer, Cochair \\ ITA, RWTH Aachen University, Kopernikusstr. 5, Aachen 52056, Germany \\ Tetsuya Sakuma, Cochair \\ The University of Tokyo, 5-1-5 Kashiwanoha, Kashiwa 277-8563, Japan \\ Toshiki Hanyu, Cochair \\ Junior College, Department of Architecture and Living Design, Nihon University, 7-24-1, Narashinodai, Funabashi \\ 274-8501, Japan
}

Chair's Introduction-9:20

Invited Papers

9:25

3aAAb1. Can you trust your numeric simulations-How to verify your code and validate your model. Lauri Savioja (Dept. of Comput. Sci., Aalto Univ., PO Box 15500, Aalto FI-00076, Finland, Lauri.Savioja@aalto.fi), Sebastian Prepelită, Pierre Chobeau (Dept. of Comput. Sci., Aalto Univ., Espoo, Finland), and Jonathan Botts (ARiA, Culpeper, VA)

In many fields where numeric simulations are utilized, there are standard practices on how to make sure that the simulation results are of high quality. In room acoustic simulations this seems to be quite rare although there are several factors that affect the accuracy of the simulations. First of all, there should be guarantees that the mathematical model, typically partial differential equations, actually model the physical phenomena under investigation. This can be made sure by validation and it can take place, for example, by comparing the simulation results to some reference solution or to measurement data. This is typically not a concern for the linearized wave equation although adding realistic boundary conditions makes it more challenging. Another essential factor affecting the correctness and reproducibility of the results is the quality of the implementation. Code verification is a procedure that aims to guarantee that an implementation is free of errors. In this paper, we review some common practices of code verification. As a practical example, we show verification studies conducted with our finite-difference time-domain solver. In addition, we show convergence rates obtained with the same solver to demonstrate the order of the accuracy of the underlying models.

\section{9:45}

3aAAb2. Crowd noise simulation system for sound environment evaluation of public spaces. Tetsuya Sakuma (Graduate School of Frontier Sci., The Univ. of Tokyo, 5-1-5 Kashiwanoha, Kashiwa 277-8563, Japan, sakuma@k.u-tokyo.ac.jp) and Yukiko Nishimura (Inst. of Technol., Shimizu Corp., Tokyo, Japan)

Aiming at the prediction and evaluation of sound environment of public spaces, a simulation-based auralization system is developed for reproducing background crowd noise. The system combines room acoustics modeling using a ray tracing method with a six-channel sound field reproduction system in an anechoic room. As sound sources, footsteps and voices of pedestrians moving on a floor, and HVAC noise from a ceiling are modeled. In a preliminary examination simulating several existing public spaces, a general correspondence to real sound field are confirmed in noise level and auditory impressions such as noisiness, liveness, and so on. Next, simulating a variety of imaginary rooms with changing room dimensions, absorption and pedestrian density, a subjective experiment of auditory pairwise comparisons is carried out. The results show that the noisiness fairly corresponds with the noise level, whereas the feeling of reverberation does not clearly related to the reverberation time, which may be due to the continuous overlap of sounds.

\section{0:05-10:20 Break}

\section{0:20}

3aAAb3. Binaural simulation using six channel reproduction based on the finite difference time domain room acoustic analysis. Shinichi Sakamoto (5th Dept., Inst. of Industrial Sci., The Univ. of Tokyo, 4-6-1, Komaba, Meguro-ku, Tokyo 153-8505, Japan, sakamo@iis.u-tokyo.ac.jp), Takatoshi Yokota, and Sakae Yokoyama (Kobayasi Inst. of Physical Res., Tokyo, Japan)

For auralization of room acoustics, various simulation-reproduction systems, such as ray tracing, cone tracing, image source methods, and wave-based numerical analysis for sound field simulation, and transaural system, multi-channel loudspeaker system for sound field reproduction, have been developed. As a sound recording-reproduction system, the six channel system has been developed, in 
which a sound signal is recorded by orthogonally located six directional microphones having a cardioid characteristics and the recorded signals are reproduced by orthogonally arranged six loudspeakers. To obtain binaural signals for auralization with headphones, the six channel reproduction was combined with binaural recording using dummy head microphones. The proposed concept can be efficiently applied to room acoustic simulation. As a room acoustic calculation method, finite-difference time-domain method was employed in this study. The calculated room impulse responses were reproduced by six channel reproduction system, and the binaural signals are recorded using dummy head microphones. In this presentation, the concept of the reproduction system is first stated and some examples on room acoustic problems are introduced.

\section{0:40}

3aAAb4. Evaluation of spatial impression of sound field in a concert hall based on the sound intensity using musical sounds. Toshiki Hanyu (Dept. of Architecture and Living Design, Junior College, Nihon Univ., 7-24-1, Narashinodai, Funabashi, Chiba 274-8501, Japan, hanyu.toshiki@nihon-u.ac.jp), Akiho Matsuo (Graduate School of Sci. and Technol., Dept. of Architecture, Nihon Univ., Funabashi, Chiba, Japan), and Kazuma Hoshi (Dept. of Architecture and Living Design, Junior College, Nihon Univ., Funabashi, Chiba, Japan)

Spatial impression is one of the most important factor for evaluating sound fields in concert halls. Objective indices, such as the lateral energy measures, for evaluating the spatial impression are standardized in the ISO-3382. These indices can be measured by using a figure-of-eight pattern microphone. On the other hand, details of spatial information of sound field can be depicted by measuring instantaneous sound intensity in the sound field. However, thus far, there is no index using the sound intensity. In this study, first the relationship between the existing objective indices and the sound intensity is examined. In particular, the existing objective indices are redefined based on the sound intensity. Secondarily, some objective indices are newly defined by using the sound intensity. These intensity-based indices can basically be calculated by using the sound intensity of impulse responses in a room. Third, potential of measuring the intensity-based indices by using musical sounds is examined. We investigated the intensity-based indices in several sound fields by using both impulse responses and musical sounds. Especially, the dependence of signals used in the measurements on the indices was examined.

\section{1:00}

3aAAb5. Acoustic feedback considerations in simulations of sound systems in rooms. Wolfgang Ahnert and Stefan Feistel (Ahnert Feistel Media Group, Arkonastr. 45-49, Berlin D-13189, Germany, wahnert@ada-amc.eu)

Computers are employed since about 30 years in the simulation of room- and electro-acoustics. Simulation tools have always considered sources, usually loudspeakers, but not microphones, which is the reason that acoustic feedback has not been considered by any simulation tool yet. As a new feature, a database for microphones is introduced analogous to the database of loudspeakers and other general sources. It is possible in this way to simulate the regeneration of sound over the close system loop, and estimate the maximum gain before feedback, and related influence on maximum sound pressure level and speech intelligibility. The paper briefly describes the important features of the new microphone database. Afterwards, the basic mechanism of acoustic feedback is explained, and the dependence of the feedback threshold of different sound sources and receivers as well as the acoustic properties of the room. By calculating the feedback threshold the headroom of the acoustic gain before feedback may be predicted. These threshold values are calculated by simulation and compared with measurements. This will be done for different loudspeaker-microphone arrangements in a selected conference hall.

\section{1:20}

3aAAb6. Sampling the sound field in auditoria using a large scale microphone array. Ingo B. Witew and Michael Vorländer (Inst. of Tech. Acoust., RWTH Aachen Univ., Kopernikusstrasse 5, Aachen 52074, Germany, ingo.witew@akustik.rwth-aachen.de)

Acoustical measurements in auditoria can be laborious with microphones to be placed in large sampling areas that are divided by rows of seating and separated by balconies. As a result, practical studies are often based on measurements of a relatively small number of selected source-receiver combinations. The goal of this paper is to present a new measurement, visualization, and analysis approach for complex wave fields. The measurement apparatus, capable of automatically sampling the sound field in auditoria over a surface of $5.30 \mathrm{~m} \times 8.00 \mathrm{~m}$ in high resolution, is described. Based on data collected with the microphone array, a case study of how sound is reflected and scattered from a concert hall's boundaries is shown. The comparison of repeated measurements with and without the presence of chairs allows a new perspective on grazing sound propagation over theatre seating ("seat dip effect"). The presentation will conclude with the discussion of spatial fluctuations of acoustic properties as a factor of measurement analysis and uncertainty.

\section{1:40}

3aAAb7. Determination of boundary conditions for room acoustics simulation by application of inverse calculation models. Lukas Aspöck, Thomas Maintz, and Michael Vorländer (Inst. of Tech. Acoust., RWTH Aachen, Kopernikusstr. 5, Aachen D-52074, Germany, las@akustik.rwth-aachen.de)

Simulation models for room acoustics simulation usually promise to deliver precise results for a certain frequency range. However, correct results can only be guaranteed if accurate input parameters are provided. Standardized methods for the determination of boundary conditions, the impedance tube and the reverberation chamber, include measurement uncertainties which might lead to different simulation results. These measurement methods also only capture valid results for special situations, e.g., normal or random wave incidence. This insufficient description of the boundary conditions makes it challenging to validate simulation models by comparing them to measured results. Even for rather simple room acoustic situations, state-of-the-art simulations fail to match measurements using in-situmeasurements or textbook values for the boundary conditions. In geometrical acoustics, these deviations can not only explained by insufficient measurement methods for the input data but also by different handling of reflection, scattering, and diffraction. To resolve the mismatch between measured and simulated results, optimization concepts have been proposed to determine input parameter sets for the simulation models. This work discusses two inverse approaches, evaluates their practicability for typical room acoustic scenarios, and presents the application in a comparison of room acoustic simulation software, the international round robin of auralization. 


\title{
Session $3 \mathrm{aAB}$
}

\section{Animal Bioacoustics: Session in Honor of Whitlow Au I}

\author{
Kelly J. Benoit-Bird, Cochair \\ College of Earth, Ocean, and Atmospheric Sciences, Oregon State University, 104 COEAS Admin Bldg., Corvallis, OR 97331 \\ Marc Lammers, Cochair \\ Hawaii Institute of Marine Biology, 46-007 Lilipuna Rd., Kaneohe, HI 96744 \\ Tomonari Akamatsu, Cochair \\ Fisheries Research Agency, 7620-7, Hasaki, Kamisu, Ibaraki 314-0408, Japan
}

Chair's Introduction-7:50

\section{Invited Papers}

$7: 55$

3aAB1. Something just clicked: Whitlow Au and the sonar of dolphins. Robert C. Gisiner (IAGC, 1225 North Loop West, Houston, TX 77008, bob.gisiner@iagc.org)

I suspect that no one, especially not Whit, imagined in 1970 that he would become the world's authority on dolphin biosonar, also known as active echolocation. Since that time Whit has amassed an astonishing list of hundreds of publications on the subject, including the seminal text on marine mammal echolocation, The Sonar of Dolphins, published in 1993. It may surprise many of the younger members of ASA to know that there was a Cold War "arms race" for knowledge about dolphin sonar and that Whit and his U.S. colleagues carried on a lively and mutually enriching correspondence with Soviet colleagues against a background of government intrigues and limitations on travel and conference attendance out of concerns about a "biosonar gap" every bit as worrisome to military leaders as the nuclear arms race (well, maybe not quite as worrisome). Since then Whit has branched out to many other fields of bioacoustics and mentored a diverse array of students at the University of Hawaii, but I will focus on the years at the Navy's marine mammal facility in Kaneohe, Hawaii, where I had the good fortune to work alongside Whit for several years.

\section{8:15}

3aAB2. The engineer in field biologist's clothing: Whitlow Au's academic years. Marc Lammers (Hawaii Inst. of Marine Biology, 46-007 Lilipuna Rd., Kaneohe, HI 96744, lammers@hawaii.edu) and Kelly Benoit-Bird (Monterey Bay Aquarium Res. Inst., Moss Landing, CA)

In 1993, the same year that The Sonar of Dolphins was published, Whitlow Au transitioned from the U.S. Navy's Naval Ocean Systems Center to the Hawaii Institute of Marine Biology. Thus began a second professional act, so to speak, of field-based research and mentoring the next generation of marine bioacousticians. An electrical engineer doing field biology may not seem like the natural order of things at first, but the combination of Whitlow's engineering and acoustic prowess coupled with the enthusiasm and energy of bright young biologists resulted in a powerful research team. Thanks to Whitlow's ingenuity and commitment to advancing the state of the art, Hawaii's coastlines and marine habitats around the world were explored like never before. A lack of funding or the availability of appropriate instrumentation were never an obstacle, but rather became opportunities for creativity and invention. Whitlow and his students' research on dolphins, their prey, snapping shrimp, humpback whales, and other taxa led to many pioneering breakthroughs both in biology and technology. While Whitlow's scientific legacy could be measured by his more than 200 publications, it is the hundreds of lives he influenced though his academic and professional service that will ultimately be his greatest mark.

\section{8:35}

3aAB3. Bioacoustics research in Asia, promoted by Whitlow W. L. Au. Ding Wang, Kexiong Wang (Inst. of Hydrobiology, CAS, 7 Donghu South Rd., Wuhan, Hubei 430072, China, wangd@ihb.ac.cn), SONGHAI LI (Inst. of Deep-Sea Sci. and Eng., Sanya, China), and Tomonari Akamatsu (National Res. Inst. of Fisheries Sci., Fisheries Res. Agency, Japan Fisheries Res. and Education Agency, Kanagawa, Japan)

The sonar of dolphins (1993) written by Whitlow W. L. Au accelerated underwater bioacoustics research, especially in Asian odontocetes. Before this book, very limited ultrasonic recording of odontocetes and bioacoustics research of marine mammals have been done in Asia. The Yangtze freshwater cetaceans provided opportunities for Asian researchers to study biosonar behavior, passive acoustic monitoring methods, and auditory physiology of odontocetes. The above works were accomplished with the Whitlow Au's preceding studies as basic references. The passive acoustic monitoring method developed from the Yangtze finless porpoises has been applied 
internationally in recent years. With encouragement and supports from Whitlow Au, biosonar research, auditory sensitivity measurement, and long term acoustic monitoring have been extensively conducted on Chinese white dolphins and oceanic finless porpoises, which are the key species in coastal environmental assessment, especially for the windmill farm construction area in Asia. In the meantime, Whitlow dispatched students to help our researches and also accommodated Asian students in Hawaii. So far, there are more than five Ph.D. students from Asia in underwater bioacoustics field supervised or partially supervised by Whitlow Au. Some of them have already grown up as leading researchers in marine mammal bioacoustics in Asia.

\section{8:55}

3aAB4. Biosonar detection range of mesopelagic patches by spinner dolphins in Hawaii. Whitlow Au (Univ. of Hawaii, P.O. Box 1106, Kailua, HI 96734, wau@ hawaii.edu), Marc O. Lammers (Univ. of Hawaii, Kaneohe, HI), and Jakob Jung (Bremen Univ. of Appl. Sci., Kaneohe, Hawaii)

Spinner dolphins (Stenella longirostris) in the near-shore waters of the Hawaiian islands forage on the mesopelagic boundary community (mbc) of organisms consisting of myctophids, mid-water shrimp, and small squids. They forage at night in a coordinated fashion swimming parallel to shore hunting for patches of prey that they can encircle and herd into a tight three-dimensional patch. A profiler housing a broadband echo-ranger that projected dolphin-like biosonar signals was used to measure the target strength of the mbc. Echoes consisted of a number of highlights bunched together with target strength between -45 and $-55 \mathrm{~dB}$ based on a dolphin's integration window of $264 \mathrm{~ms}$. Noise values collected by an autonomous acoustic recorder at midnight in the location where the profiler data were obtained were used to estimate the biosonar detection range of spinner dolphins for mesopelagic patches. The receiving directivity index and the width of the auditory filter for Tursiops truncatuswere used to estimate the biosonar detection ranges of Stenella longirostris searching for mbc patches. Using the sonar equation, the biosonar threshold detection range of spinner dolphins was estimated to be approximately 100 plus $\mathrm{m}$, more than sufficient range for the animals to formulate their prey herding behavior.

\section{Contributed Papers}

\section{9:15}

3aAB5. Using acoustics to examine odontocete foraging ecology: Predator-prey dynamics in the mesopelagic. Kelly J. Benoit-Bird (Monterey Bay Aquarium Res. Inst., 104 COEAS Admin Bldg., Corvallis, OR 97331, kbenoit@coas.oregonstate.edu), Brandon Southall (Southall Environ. Assoc., Inc., Aptos, CA), and Mark A. Moline (Univ. of Delaware, Lewes, $\mathrm{DE})$

From his expertise in biosonar, Whitlow Au brought a wealth of ideas on sonar use, design, and context to the study of wild cetaceans, resulting in great contributions to our understanding of odontocete foraging ecology. This contribution follows that foundation, using an integrated approach comprising echosounders deployed in a deep-diving autonomous underwater vehicle, ship based acoustics, visual observations, direct prey sampling, and animal-borne tags to explore the behavior of Risso's dolphins foraging in scattering layers off California. Active acoustic measurements demonstrated that Risso's dolphins dove to discrete prey layers throughout the day and night. Using acoustic data collected from the AUV, we found layers made up of distinct, small patches of animals of similar size and taxonomy adjacent to contrasting patches. Prey formed particularly tight aggregations when Risso's dolphins were present. Squid made up over $70 \%$ of the patches in which dolphins were found and more than $95 \%$ of those at the deepest depths. Squid targeted by dolphins in deep water were also larger, indicating significant benefit from these rare, physically demanding dives. Careful integration of a suite of traditional and novel tools is providing insight into the ecology and dynamics of predator and prey in the mesopelagic.

\section{9:30}

3aAB6. Echolocation behavior of the Icelandic white-beaked (Lagenorhyncus albirostris) dolphins: Now and then. Marianne H. Rasmussen (Husavik Res. Ctr., Univ. of Iceland, Hafnarstett 3, Husavik, Iceland 640, Iceland, mhr@hi.is), Jens Koblitz (BioAcoust. Network, BioAcoust. Network, Neuss, Germany), and Peter Stilz (BioAcoust. Network, BioAcoust. Network, Hechingen, Germany)

First studies of the echolocation behaviour of free-ranging white-beaked dolphins (Lagenorhyncus albirostris) were conducted in Faxafloi Bay in the Southwestern part of Iceland in the years 1997 to 1999. However, the sighting rate of white-beaked dolphins has decreased in that area since then and the current studies were conducted in the Northeastern part of Iceland. The aim of this study was to investigate the difference between normal clicks compared to clicks from buzz sequences. The recordings of the Icelandic white-beaked dolphins were conducted using a vertical linear 16-hydrophone array in Skjalfandi Bay, Northeastern Iceland during August 2015 and June 2016. The hydrophones were connected to NI-Boards and to a laptop computer on board using a sample rate of $1 \mathrm{MHz}$ per channel. The group size of the dolphins varied from three individuals up to 30 animals in the area during the recordings. The dolphin echolocation clicks were recorded and it was possible to track individuals and to estimate beam-pattern from their clicks. Estimated beam pattern from 45 regular clicks gave $-3 \mathrm{~dB}$ BW of 9.6 degrees and maximum source level was $208 \mathrm{~dB}$ re. $1 \mathrm{mPa}$. In addition buzzes with short inter-click-intervals down to $2.5 \mathrm{~ms}$ were recorded on all 16 channels.

\section{9:45-10:00 Break}

\section{0:00}

3aAB7. Echolocation behavior of endangered fish-eating killer whales (Orcinus orca) recorded from digital acoustic recording tags (DTAGs): Insight into subsurface foraging activity. Marla M. Holt, M. Bradley Hanson, Candice K. Emmons (NOAA NMFS NWFSC, 2725 Montlake Blvd East, Seattle, WA 98112, Marla.Holt@noaa.gov), Deborah A. Giles (Wildlife, Fish, \& Conservation Biology, Univ. of California, Davis, Davis, CA), Jeffrey T. Hogan (Cascadia Res. Collective, Olympia, WA), and David Haas (Marine Sci. and Conservation, Duke Univ., Durham, NC)

Killer whales are apex predators with diet specializations that vary among ecotypes. Resident killer whales use broadband echolocation clicks to detect and capture fish prey in their underwater environment. Here, we describe the echolocation behavior of endangered Southern Resident killer whales using DTAGs to determine subsurface foraging activity and to assess the effects of vessel and noise on foraging behavior. We deployed 29 DTAGs on individually-identified killer whales and collected complimentary field data over four seasons in summer habitat. DTAGs had two hydrophones that each recorded sound at sampling rates of 192 or $240 \mathrm{kHz}$, and other sensors to reconstruct whale movement. Prey remains were opportunistically collected during tag deployments to validate feeding. Echolocation signals of the tagged whale were inferred from spectral content and the angle of arrival that corresponded to tag placement. Preliminary results reveal that individuals produced steady click trains during shallow dives then dove to deeper depths while clicking at higher repetition rates that graded into bottom-associated low-level buzzes before ascent occurred. These results, together with movement data, are reliable subsurface foraging cues in this endangered population that can be used to assess vessel effects on foraging behavior. 
Both depth and recording instrumentation influenced the click characteris-

3aAB8. Echolocation parameters of toothed whales measured at sea. Jens C. Koblitz (BioAcoust. Network, Eichenallee 32 a, Neuss 41469, Germany, Jens.Koblitz@web.de), Peter Stilz (BioAcoust. Network, Hechingen, Germany), Lisa Steiner (Whale Watch Azores, Horta, Portugal), and Marianne H. Rassmussen (The Univ. of Iceland's Res. Ctr. in Húsavík, Husavik, Iceland)

Historically, data on toothed whale echolocation parameters and abilities were collected from captive animals. Acoustic parameters under investigation were inter-click-interval, spectral content, source level, directionality, emission direction, including correlation and variation of those parameters. Technological advances over the past decade have allowed collecting data on those parameters from animals at sea using acoustic recording tags or hydrophone arrays. Using a vertical, linear array of 16 hydrophones, echolocation clicks from harbor porpoises, white-beaked dolphins, common dolphins, and bottlenose dolphins were recorded around Iceland and the Azores. The animal's position at click production was computed for each click based on the time of arrival differences. Intensity and spectral differences at the array allowed measuring source levels, beam width, and spectral variation at different angles relative to on-axis. Advancing knowledge on the use and variation of echolocation signals of toothed whales in their natural habitat will allow widespread and effective use of acoustic monitoring.

\section{$10: 30$}

3aAB9. Echolocation detection of fishing hooks and implications for the Hawaiian longline fishery. Aude F. Pacini, Paul E. Nachtigall, Adam B. Smith, Rock Owens, and Stephanie Vlachos (School of Ocean and Earth Sci. and Technol., Hawaii Inst. of Marine Biology, 46-007 Lilipuna Rd., Kaneohe, HI 96744, aude@hawaii.edu)

Interactions between marine mammals and fisheries have a biological and economic impact that is often detrimental to both fishermen and species of concern. False killer whale bycatch in the Hawaii longline fishery has exceeded the potential biological removal (PBR) triggering the designation of a take reduction team under the Marine Mammal Protection Act (MMPA). As an attempt to understand the importance of acoustic cues in depredation events, this study presents preliminary data looking at the echolocation ability of a false killer whale (Pseudorca crassidens) to detect a longline fishing hook at various distances. Using a go/no-go paradigm, the whale was trained to report the presence of the hook at distances varying in $50 \mathrm{~cm}$ increments. A total of 28 sessions of 25 trials each were collected and echolocation signals were recorded using a nine element acoustic array. Number of clicks, acoustic parameters, decision time and performance were recorded. The subject successfully reported the presence of the hook up to 6 $\mathrm{m}$. This work presents evidence that false killer whales can easily detect fishing gear which could influence how they interact with longline fishery.

\section{$10: 45$}

3aAB10. The characteristics of dolphin clicks compared across recording depths and instruments. Marc Lammers (Hawaii Inst. of Marine Biology, 46-007 Lilipuna Rd., Kaneohe, HI 96744, lammers@hawaii.edu), Julie N. Oswald (Bio-Waves, Inc., Encinitas, CA), Anke Kuegler (Marine Biology Graduate Program, Univ. of Hawaii, Honolulu, HI), and Eva M. Nosal (Abakai Int., LLC, Waianae, HI)

The identification of delphinid species on the basis of the characteristics of their acoustic signals is an important aspect of many passive acoustic monitoring efforts. The development of species classifiers relies on the assumption that species-specific signal characteristics will be consistent across different recording scenarios, including depth and instrumentation. However, this assumption has largely remained untested. Here, we report on an effort to examine whether and how the properties of echolocation clicks obtained from different delphinid species vary as a function of recording depth and the instrument employed. Field recordings of seven species of dolphins were obtained off Kona, Hawaii, and San Diego, CA, using a 250 $\mathrm{m}$ vessel-based vertical array composed of five microMARS recorders, two SoundTrap recorders, and four C75 broadband dipping hydrophones (Cetacean Research Technology). The clicks obtained were characterized on the basis of their spectral properties and duration for each recording depth and also compared among different instruments deployed at the same depth. tics observed. However, the click properties of some species varied to a greater degree than others. These results suggest that developing speciesspecific classifiers based on dolphin clicks should be approached with caution and carefully validated.

\section{1:00}

3aAB11. From dolphin sonar to commercial scientific acoustic systems. Lars N. Andersen (Underwater Sci., Simrad, Kongsberg Maritime AS, P.O. Box 111, Horten 3191, Norway, lars.nonboe.andersen@simrad.com)

Dolphins have remarkable sonar systems for underwater navigation, object detection, object inspection, and general understanding of the environment. It is easy to be inspired by these advanced biosonar systems when designing new advanced commercial sonar systems. Similarly, it is also easy to be inspired when discussing bioacoustics with Whitlow Au. His work on dolphin sonar systems has been and still is of significant inspiration for many working with both biological and manmade sonar systems. Personal examples on how Whitlow Au has inspired development of commercial scientific echo sounders will be given. Characteristics of dolphin sonar and commercial scientific acoustic systems will be discussed.

\section{1:15}

3aAB12. The echolocation beam of bottlenose dolphins (Tursiops truncatus): High-resolution measurements of horizontal beam patterns and nearfield/farfield transitions. Jason Mulsow (National Marine Mammal Foundation, 2240 Shelter Island Dr., Ste. 200, San Diego, CA 92106, jason. mulsow@nmmf.org), James J. Finneran (U.S. Navy Marine Mammal Program, San Diego, CA), Brian K. Branstetter, Patrick W. Moore, Cameron Martin, and Dorian S. Houser (National Marine Mammal Foundation, San Diego, CA)

The work of Whitlow Au and colleagues has demonstrated that dolphin biosonar forms a highly directional, forward-facing beam. In our recent studies, we have expanded upon previous work by making biosonar beam measurements using high-resolution hydrophone arrays with up to 48 hydrophones. Bottlenose dolphins were trained to echolocate on both physical targets and phantom echo generators, with clicks simultaneously recorded on all hydrophones at a sampling rate of $2 \mathrm{MHz}$. Target ranges (and simulated target ranges for phantom echoes) were varied in order to examine the resulting effects on the spatial characteristics of the acoustic field. The directivity index of the echolocation beam increased with increasing click level and center frequency, and recordings from extreme off-axis azimuths displayed a two-pulse pattern best explained by internal reflections off of the premaxillary bones. High-density hydrophone arrays placed near echolocating dolphins' heads demonstrated that a transition from the geometric nearfield to the farfield occurs at approximately 0.3 to $0.4 \mathrm{~m}$ from the melon. The results were remarkably similar to the earlier findings of Au and colleagues, and provide further information on the spatial characteristics of the acoustic field associated with dolphin biosonar. [Funding from ONR]

\section{1:30}

3aAB13. Transmission beam characteristics of a spinner dolphin (Stenella longirostris). Adam B. Smith, Aude F. Pacini (Marine Mammal Res. Program, Hawaii Inst. of Marine Biology, 1933 Iwi Way, Honolulu, HI 96816, adambsmi@hawaii.edu), Paul E. Nachtigall (Marine Mammal Res. Program, Hawaii Inst. of Marine Biology, Kailua, HI), Leo Suarez (Philippines Marine Mammal Stranding Network, Subic Bay, Philippines), Lem Aragonez (Inst. of Environ. Sci. and Meteorol., Univ. of the Philippines, Subic Bay, Philippines), Carlo Magno (Ocean Adventure, Subic Bay, Philippines), Gail Laule (Philippines Marine Mammal Stranding Network, Subic Bay, Philippines), and Laura N. Kloepper (Biology, St. Mary's College, South Bend, IN)

Transmission beam characteristics have been described in a small number of odontocete species, providing insight into the biological and ecological factors that have influenced the design of the outgoing echolocation beam. The current study measured the on-axis spectral characteristics and transmission beam pattern of echolocation clicks from a small oceanic delphinid, the spinner dolphin (Stenella longirostis). A formerly stranded individual was rehabilitated in captivity and trained to station underwater in 
front of a 16 element hydrophone array. Preliminary analysis of a subset of recorded clicks showed on-axis spectral characteristics with a mean center frequency of $68 \mathrm{kHz}$ and a mean peak frequency of $52 \mathrm{kHz}$. The dolphin exhibited both a circular beam shape and also varying degrees of a dorsoventrally compressed transmission beam. The mean angular beamwidth for all clicks was 16.6 and 14.3 degrees in the horizontal and vertical planes, respectively, though some clicks exhibited horizontal beamwidths that were almost twice as wide as the vertical beamwidth. The overall beam was generally broader than the beams of previously described species, with a mean directivity index for all clicks of $21.5 \mathrm{~dB}$. These results are the first reports of transmission beam characteristics from a spinner dolphin.

\section{1:45}

3aAB14. Biosonar radiation field on the forehead of a Risso's dolphin during prey capture. Wu-Jung Lee (Appl. Phys. Lab., Univ. of Washington, 1013 NE 40th St., Seattle, WA 98105, wjlee@apl.washington.edu), Hsin-Yi Yu (Inst. for Ecology and Evolutionary Biology, National Taiwan Univ., Taipei, Taiwan), Whitlow W. Au, Adam Smith (Hawaii Inst. of Marine Biology, Univ. of Hawaii, Kaneohe, HI), I-Fan Jen (Farglory Ocean Park, Hualien, Taiwan), Wei-Cheng Yang (Dept. of Veterinary Medicine, National Chiayi Univ., Chiayi, Taiwan), Ying-Ching Fan (Farglory Ocean Park, Hualien, Taiwan), Paul E. Nachtigall (Hawaii Inst. of Marine Biology, Univ. of Hawaii, Kaneohe, HI), and Lien-Siang Chou (Inst. for Ecology and Evolutionary Biology, National Taiwan Univ., Taipei, Taiwan)

On-animal suction cups with embedded hydrophones allow examination of how signals on the forehead of echolocating odontocetes relate to the internal anatomical structure and the transmission beampattern. Risso's dolphin (Grampus griseus) is an interesting species for this investigation due to the presence of a unique vertical groove in the middle of their forehead. In this study, a linear array of six broadband suction cup hydrophones were attached along the forehead groove of an adult female Risso's dolphin trained to catch freshly thawed dead squid in front of an eight-element farfield hydrophone array. The animal's movement was simultaneously observed using an underwater video camera. A total of nine successful prey captures were recorded. During each catch, the animal first emitted regular echolocation clicks, which quickly transitioned into buzzes (clicks with distinctively high repetition rate) until prey capture. The amplitude and relative time of arrival of these signals across all channels were analyzed. For a subset of trials, the relative amplitude distribution across channels vary significantly between regular clicks and buzzes in a manner that may be explained by beampattern changes. These observations were investigated jointly with data from the hydrophone array and interpreted in light of anatomical structure of the melon.

\title{
Session 3aAO
}

\section{Acoustical Oceanography, Underwater Acoustics, and Signal Processing in Acoustics: Ocean Acoustic Tomography: Active and Passive, Theory and Experiment II}

\author{
Bruce Howe, Cochair \\ Ocean and Resources Engineering, University of Hawaii, 2540 Dole Street, Holmes Hall 402, Honolulu, HI 96822 \\ Arata Kaneko, Cochair \\ Graduate School of Engineering, Hiroshima University, 1-4-1 Kagamiyama, Higashi-Hiroshima 739-8527, Japan \\ Hiroyuki Hachiya, Cochair \\ Tokyo Institute of Technology, 2-12-1 S5-17, Ookayama, Meguro-ku, Tokyo 152-8550, Japan
}

Invited Papers

$7: 50$

3aAO1. Recent progress in coastal acoustic tomography in China: Experiments and data assimilation. Xiao-Hua Zhu, Ze-Nan Zhu, Xiaopeng Fan, Wen-Hu Liu, Chuanzheng Zhang, Menghong Dong, Yu Long, and Yun-Long Ma (Second Inst. of Oceanogr., State Oceanic Administration, 36 Baochubei Rd., Hangzhou 310012, China, xhzhu@sio.org.cn)

We will review three recent coastal acoustic tomography (CAT) experiments carried out in China: (1) A 15-day tomography experiment that was carried out for the first time in the Qiongzhou Strait to measure the major tidal current constituents, residual currents, and volume transport; (2) A high precision CAT experiment with 11 CAT systems performed in the winter of 2015 in the Dalian bay, China. The number of successful reciprocal transmission lines reached 51, which may be the highest number in ocean acoustic tomography history. The CAT results showed a very high accuracy of velocity measurements with a root-mean-square difference of $4 \mathrm{~cm} / \mathrm{s} \mathrm{compared}$ with moored ADCP measurements; (3) Rapid sampling CAT measurements were used to map the structure of nonlinear tidal ( $\mathrm{M}_{4}$ and 
$\mathrm{M}_{6}$ ) currents. The results indicate that $\mathrm{M}_{4}$ is predominantly generated by the advection terms, while friction mechanisms are predominant for generating $\mathrm{M}_{6}$. Finally, we will introduce the CAT data assimilated into an unstructured triangular grid ocean model (FVCOM) using the ensemble Kalman filter scheme. The assimilated velocities agreed better with independent ADCP data than those obtained by inversion and simulation, indicating that data assimilation of the CAT data is the optimal method for reconstructing the current field.

\section{8:10}

3aAO2. Real-time monitoring of coastal current and throughflow by ocean acoustical tomography with a time reversal responder. Hong Zheng (Zhejiang Ocean Univ., No. 1 Haida South Rd., Lincheng Changzhi Island, Zhoushan, Zhejiang 316022, China, seahzheng@msn.com) and Arata Kaneko (Hiroshima Univ., Higashi-Hiroshima, Japan)

The conventional Coastal Acoustic Tomography (CAT) system works in a controlled timing of sound transmission from acoustic stations surrounding an observation site. Data received in each station are sent to an integrated data center via communication network and a real-time mapping of current and sound speed is realized. In a new time reversal mirror CAT system (TRM-CAT) proposed, data received at a few land stations by time reversal responders gather all data transferred to the data center. The SNR of received signals is increased and the cost of field experiment is reduced. The TRM-CAT consists of two types of station, land and response stations. The land station is a conventional CAT station that sends coded signals to the response station and receives time reversal signals released. The response station work as a responder, which does not transmit any acoustic signals, until a signals coming from the land station are recorded in a memory, and then reconstructed as a time reversal data to transmit toward the land station at a pre-determined timing. One-way travel time and travel time difference are determined by a matched filter with codes. As a results, the average sound speed and flow velocity can be calculated in land stations. The TRM-CAT is designed and the system simulation is presented. Application to monitoring volume and heat transports through straits and rivers in real time is planned now. Finally, the TRM-CAT serves as a cost effective system for real-time mapping of velocity structures in the coastal sea.

\section{Contributed Papers}

\section{8:30}

3aAO3. An acoustic tomography method for imaging ocean structure with reverberated waves. Robert A. Dunn (Univ. of Hawaii at Manoa, 1680 East-West Rd., Honolulu, HI 96822, dunnr@hawaii.edu)

Seismic wide-angle imaging using ship-towed acoustic sources and networks of ocean-bottom seismographs is a common technique for exploring earth structure beneath oceans. In these studies, the recorded data are dominated by acoustic waves propagating as reverberations in the water column. Ignored by the earth scientist, this data offer an alternative approach for determining the structure of the oceans and advancing understanding of ocean heat content and mixing processes. A method, referred to as ocean acoustic reverberation tomography, is developed that uses the traveltimes of reverberated waves to image ocean acoustic structure. To demonstrate the feasibility of applying this method to existing and future data collected as part of marine seismic studies, a synthetic example is devised and the maximum resolution of the method is explored in the presence of different levels of data noise. Reverberation tomography offers significant improvement over classical ocean tomography in that it can produce images of ocean sound speed over the entire vertical height of the water column and along long survey profiles $(100+\mathrm{km})$, on a scale much finer (100s of meters) than traditional tomography. Variations in acoustic wave speed of $<1 \mathrm{~m} / \mathrm{s}$ are possible to detect with existing technology.

\section{$8: 45$}

3aAO4. Toward underwater channel impulse response estimation using sources of opportunity. Kay L. Gemba, Santosh Nannuru, William Hodgkiss, and Peter Gerstoft (MPL, Scripps Inst. of Oceanogr., Univ. of California, San Diego, 8820 Shellback Way, Spiess Hall, Rm. 446, La Jolla, CA 92037,gemba@ucsd.edu)

Passive acoustic tomography exploits the acoustic energy generated by sources with unknown spectral content such as sources of opportunity (e.g., cargo ships) to study the ocean. However, separating the channel impulse response (CIR) from a possibly moving, random radiator is non-trivial for coherent processing and the amount of unknown variables may leave the problem intractable. Using an incremental approach, we study the sparse
CIR estimation problem for a single receiver. Second, we add multiple receivers, relax source spectrum knowledge, and study processor performance in resolving possibly coherent multipath arrivals across the vertical line array. Third, we discuss the extension to include multiple VLAs and sequential processing for a source with known location. Application of the Noise Correlation 2009 Experiment and the Santa Barbara Channel Experiment 2016 are discussed.

\section{9:00}

3aA05. Deducing environmental mismatch from matched-mode processing ambiguity surface sidelobes. Aaron Thode (SIO, UCSD, 9500 Gilman Dr., MC 0238, La Jolla, CA 92093-0238, athode@ucsd.edu), Julien Bonnel (Lab-STICC(UMR CNRS 6285), ENSTA, Bretagne, France), Chris Verlinden (SIO, UCSD, New London, Connecticut), Margaux Thieury (Lab-STICC(UMR CNRS 6285), ENSTA, Bretagne, France), and Aileen Fagan (U.S. Coast Guard Acad., New London, CT)

The application of non-linear time sampling to broadband acoustic signals propagating in shallow water has made modal filtering possible using a single hydrophone. As a result, incoherent matched-mode processing (MMP) techniques are now practical using only single-hydrophone data. When MMP ambiguity surfaces are constructed from pairs of modes and plotted as a function of range and frequency, both the mainlobe and sidelobes form striations that embed information about the type and amount of environmental mismatch present between the modeled and true environment. Thus the degree of symmetry that a two-mode frequency-averaged ambiguity surface displays around the mainlobe provides a metric for identifying environmental mismatch. Acoustic invariant theory, combined with simulations, demonstrate how mismatched waveguide replicas can be used to (1) estimate the true bottom interface sound speed, (2) determine whether a downward-refracting waterborne profile is present in data, and (3) estimate waterborne sound speed profile slopes. The theory also explains why certain mismatched environments can generate very high MMP correlations, even with broadband signals. In a shallow water environment with a downwardrefracting sound speed profile, taking full advantage of this approach requires reformulation of non-linear time-sampling methods to extract refractive modes. [Work sponsored by the North Pacific Research Board.] 


\section{Invited Papers}

3aAO6. CANAPE-2015 (Canada Basin acoustic propagation experiment): A pilot tomography experiment in the Beaufort Sea. Matthew Dzieciuch (SIO/UCSD, 9500 Gilman Dr., IGPP-0225, La Jolla, CA 92093-0225, mad@ucsd.edu) and Peter F. Worcester (SIO/UCSD, San Diego, CA)

During the summer of 2015, test transmissions were made from a ship-suspended source to a moored vertical line array in the Beaufort Sea. These were carried out at a variety of ranges in order to prepare for a year-long (2016-2017) tomography experiment. A major concern was the expected transmission loss (TL) under the ice for current climatic conditions in the Arctic. A greater proportion of smooth first year ice (rather than rough multi-year ice) was expected due to climate change. This should lead to less scattering and a lower TL. Acoustic propagation in the Artic includes propagation in a weak shallow duct as well as arrivals of deeper turning rays. The TL of these different regimes is compared to that of an experiment in a tropical ocean, the Philippine Sea. The initial analysis shows that the Arctic TL scales with range to the fourth power. This large TL could be because the actual ice conditions in the experimental area had a high proportion of multi-year ice in 2015.

\section{9:35}

3aA07. Capabilities and challenges of ocean acoustic tomography in Fram Strait. Hanne Sagen (Nansen Environ. and Remote Sensing Ctr., Thormøhlensgate 47, Bergen N-5006, Norway, hanne.sagen@nersc.no) and Peter F. Worcester (Scripps Inst. of Oceanogr., Univ. of California, San Diego, La Jolla, CA)

The Fram Strait is of great importance in ocean climate monitoring, as it is the only deep-water connection between the Arctic and Atlantic Oceans. Even though an extensive array of oceanographic moorings has been operated in Fram Strait since 1996 to monitor the transports through the Strait, the small spatial scales of the flow are poorly resolved, leading to large uncertainties. Beginning in the 2005-2010 DAMOCLES (Developing Arctic Modeling and Observing Capabilities for Long-term Environmental Studies) project, underwater acoustic methods were introduced to improve the monitoring of Fram Strait. A 2008-2009 pilot study with a single acoustic path was followed during 2010-2012 in the ACOBAR (ACoustic technology for OBserving the interior of the ARctic Ocean) project by the implementation of a multipurpose acoustic network with a triangle of acoustic transceivers for ocean acoustic tomography, ambient noise, and glider navigation. The measurements were continued during 2014-2016 in UNDER-ICE (Arctic Ocean Under Melting Ice), with eight acoustic paths crisscrossing the Fram Strait at 78-800N. Tomography in Fram Strait is demanding. The sound-speed field has a weak sound channel with little geometric dispersion, making it difficult to resolve and identify individual arrivals. The strong oceanographic variability in space and time reduces the coherence of the received signal and the stability of the arrival pattern. The status of tomography in Fram Strait will be summarized, focusing on the capabilities and challenges.

9:55-10:10 Break

\section{Contributed Papers}

10:10

3aA08. Acoustic noise interferometry and ocean dynamics. Oleg A. Godin (Phys. Dept., Naval Postgrad. School, NPS 833 Dyer Rd., Bldg. 232, Monterey, CA 93943-5216, oagodin@nps.edu)

Noise interferometry relies on averaging long time series to reveal the deterministic features of the noise cross-correlation function, which contain information about the propagation medium. Unlike the solid earth, the ocean exhibits considerable variability at the time scales that are shorter than the necessary noise averaging times of hours and days. This variability is a primary factor that limits useful noise averaging times and underlies the more limited applicability of acoustic noise interferometry than of the better established seismic interferometry. This paper aims to quantify the restrictions, which are imposed on the noise interferometry by the ocean variability, and to identify possible applications of passive acoustic remote sensing to characterizing ocean dynamics. A theory will be presented that quantifies the coherence loss of measured noise cross-correlations due to gravity waves on the ocean surface, tidally induced changes in the water depth, and internal gravity waves. It is found that temporal variations of the ocean parameters lead to frequency-dependent suppression of deterministic features in the noise cross-correlation functions. The coherence loss limits the resolution of deterministic inversions. On the other hand, the passively measured coherence loss can be inverted to statistically characterize ocean dynamics at unresolved spatial and temporal scales.
$10: 25$

3aA09. Inferring salinity microstructure using high-frequency reciprocal acoustic transmission. Andone C. Lavery (Appl. Ocean Phys. and Eng., Woods Hole Oceanographic Inst., 98 Water St., MS 11, Bigelow 211, Woods Hole, MA 02536, alavery@whoi.edu)

The Connecticut River Estuary is highly stratified, strongly influenced by fresh water discharge and tides, and characterized by hydrographic features such as dynamic fronts, plumes, internal waves, shear instability, and the incoming salt-wedge. An experiment was conducted to measure the impact of these hydrographic features on the propagation of high-frequency sound, and to use the path-averaged acoustic fields to infer properties of salinity microstructure. Two $120 \mathrm{kHz}$, 4-element arrays, each element with reciprocal transmission capabilities, were deployed for 5 days mid-watercolumn, spanning multiple tidal cycles and a range of discharge conditions. Extensive environmental data were collected, including ADCP profiles, CTD profiles, salinity microstructure at various depths, and broadband acoustic backscatter (100-600 kHz). Results from this experiment show that 1) shear instabilities dominated the structure of the sound propagation during the ebb tide and were coherent features across the propagation path length, 2) salinity stratification could be deduced from reciprocal transmissions, 3) the slope of the shear instabilities could be inferred by combining the ADCP data and arrival times at different depths, and 4) and the incoming salt-wedge was the dominant hydrographic feature affecting the sound propagation during the flood tide. [This work was funded by the ONR Ocean Acoustics Program.] 
3aAO10. Information content of ocean travel-times in the Philippine Sea. Brian Powell, Sarah Williamson, and Xiaofeng Zhao (Oceanogr., Univ. of Hawaii, 1000 Pope Rd., MSB, Honolulu, HI 96822, powellb@hawaii. edu)

The Philippine Sea is a highly energetic region of varying scales from planetary down to tidal. Significant eddy activity is present throughout the region with enhanced mesoscale activity centered around $22 \mathrm{~N}$. Tidally driven internal waves emit from the Luzon Strait into the Philippine Sea and are the most energetic in the world. These multiple dynamical scales combine to have significant influence on the density structure and sound speed in the region. In 2010, a large deep-water acoustic transmission experiment was conducted to investigate the acoustics in such a dynamic environment. A total of seven transceivers were used for providing significant coverage over the western Philippine Sea. In this talk, we use a dataassimilative numerical ocean model to investigate how travel-times from the acoustic array covary with the ocean. Using both predictions and analyses from the model, we investigate how well the model is capable of resolving acoustic rays and travel-times without the constraint of the acoustic observations. We then work to quantify how the ocean covaries with acoustic travel-times to help us to understand the variability in the ocean that controls the propagation and what the propagation can tell us about the oceanic variability.

\section{$10: 55$}

3aAO11. Deblurring acoustic signals for statistical characterization in applications of ocean acoustic tomography. Viktoria Taroudaki (Biomedical Image Computing Group, Dept. of Pediatrics, Univ. of Washington, 1959 NE Pacific St., Seattle, WA 98195, victtar@uw.edu), Costas Smaragdakis, and Michael Taroudakis (Dept. of Mathematics and Appl. Mathematics \& IACM, Univ. of Crete and FORTH, Heraklion, Greece)

The signal characterization method suggested by Taroudakis et al. (J.Acoust. Soc. Am. 119, 1396-1405 (2006)) based on the statistics of its 1D wavelet transform coefficients and successfully applied for inverting acoustic signals in applications of acoustical oceanography has been proven to be sensitive to noise contamination of the signal, but still, it provides good inversion results if an appropriate denoising strategy is applied. In this work, the statistical signal characterization is applied to signals which are both blurred and noise contaminated. Deblurring of the signal is achieved by means of a technique introduced by Taroudaki and O' Leary (SIAM J. Sci. Comput. 37(6), A2947-A2968 (2015)) for image deblurring, and it is based on a statistical near optimal spectral filtering technique that takes advantage of the singular values of the approximated blurring matrix and the Picard Parameter of the signal that allows for estimation of the additive noise properties and estimation of the error. The study is extended to cases when no accurate knowledge of the blurring mechanism is available. It is shown by typical simulated experimental data that the combination of deblurring and simple denoising strategies provide good results with respect to both signal characterization and subsequent inversions.
3aA012. Acoustic mode travel time variability in the Philippine Sea. Tarun K. Chandrayadula (Ocean Eng., IIT Madras, 109 B Ocean Eng., IIT Madras, Chennai, Tamil Nadu 600036, India, tkchandr@iitm.ac.in), John A. Colosi (Naval Postgrad. School, Monterey, CA), Peter F. Worcester, and Matthew A. Dzieciuch (Scripps Inst. of Oceanogr., San Diego, CA)

During the 2009 NPAL (North Pacific Acoustic Laboratory) PhilSea deep water acoustic propagation experiment, a low frequency source transmitted broadband chirps to a mid-water spanning hydrophone array at a distance of $185 \mathrm{~km}$ every three hours for approximately one month. In addition, transmissions took place every five minutes during some time periods. The source and the receiver array contained conductivity and temperature sensors. The motions of the source and receiver moorings were measured using long-baseline acoustic navigation systems. The experiment site was oceanographically dynamic and contained significant internal tide activity. This work builds a model to compare the acoustic and oceanographic variabilities. The acoustic observations are used to estimate the mode travel times, and then related to the internal tide displacements at the source and the receiver arrays. This paper presents the work in three parts. The first part uses matched subspace detectors to estimate the mode travel times. The second uses the environmental observations to build an internal tide model. The third constructs a linear perturbation model to relate the internal tides to the adiabatic mode travel times. The discrepancies between the model and the observations are attributed to factors such as limitations of the perturbation model for the travel times, range variability not accounted for in the adiabatic mode propagation, and signal processing issues.

\section{1:25}

3aAO13. Deep water sound sources for ocean acoustic tomography and long-range navigation. Andrey K. Morozov and Douglas C. Webb (Teledyne, 49 Edgerton Dr., North Falmouth, MA 02556, amorozov@teledyne.com)

The first test of the tunable organ-pipe was successfully conducted on 11 Sept. 2001. The sound source was efficient, powerful, and had unlimited operational depth, as well as a minimum level of high frequency harmonic content. The projector uses a narrow-band, highly efficient sound resonator, which is tuned to match the frequency and phase of a reference frequencymodulated signal. Since 2001, many deep-water ocean acoustic experiments have used this type of sound source. The source was successfully used for ocean acoustic tomography and long range navigation. Recently, it was shown that a bottom-deployed swept frequency array can be used for highresolution seismic imaging of deep geological formations. The long-term operating experience of the sound sources demands the development of an improved sound source system. A finite-element analysis that shows the structural acoustics of the tunable resonator has been conducted to improve the acoustics of the sound source. The analysis gave a better solution for a tuning mechanism with an octave frequency range. The development of the electric and mechanical systems of the sound source will be discussed. Teledyne Marine Systems continues innovating promising solutions for ocean acoustic tomography, navigation, and seismo-acoustic applications.

\section{Invited Paper}

\section{1:40}

3aAO14. Recent progress in coastal acoustic tomography. Arata Kaneko (Graduate School of Eng., Hiroshima Univ., Hiroshima University, Higashi-Hiroshima 739-8527, Japan, akaneko@hiroshima-u.ac.jp), Fadli Syamsudin, Yudi Adityawarman (Agency for the Assessment and Application of Technol. (BPPT), Jakaruta, Indonesia), Hong Zheng (Zhejiang Ocean Univ., Zhoushan, China), ChenFen Huang, Naokazu Taniguchi (National Taiwan Univ., Taipei, Taiwan), Xiaohua Zhu (State Oceanic Administration, Second Inst. of Oceanogr., Hangzhou, China), Ju Lin (Ocean Univ. of China, Qingdao, China), and Noriaki Gohda (Graduate School of Eng., Hiroshima Univ., Higashi-Hiroshima, Japan)

Coastal acoustic tomography (CAT) which was proposed by Hiroshima University in 1990s as a shallow-sea application of ocean acoustic tomography (OAT) is developed as a mirror-type CAT (MCAT) for measuring deep strait throughflows in Indonesian archipelago seas in real time. MCAT system is composed of a land station (M0) connected to a $100 \mathrm{~m}$ submarine cable edged by a $5 \mathrm{kHz}$ subsurface transceiver and triangular-arrayed bottom-moored stations (M1, M2, and M3). Reciprocal data are first obtained among three station pairs (M1M2, M2M3, and M3M1). Data received at M1 from M2 and M3 are transferred to the land station (M0) by the first 
mirror reflection, resulting in travel time summations $(\mathrm{t} 21+\mathrm{t} 10, \mathrm{t} 31+\mathrm{t} 10)(\mathrm{t} 21$ : travel time from M2 to M1). Data obtained at the offshore stations M2 and M3 are transferred to the nearshore station M1 by the first mirror reflection, resulting in travel time summations $(\mathrm{t} 12+\mathrm{t} 21, \mathrm{t} 13+\mathrm{t} 31, \mathrm{t} 23+\mathrm{t} 31$, and $\mathrm{t} 32+\mathrm{t} 21)$. All the first mirror data at M1 are also transferred to M0 by the second mirror reflection, adding a travel time by $\mathrm{t} 10$ for the above four travel time summations. Finally, travel time differences are calculated for M0M1, M1M2, M2M3, and M3M1 by subtracting the summation data at M0. They are converted to path-average currents along the four transmission lines, succeeding in strait throughflow estimate. The mirror reflection function of MCAT can develop in an underwater transfer system of sound from moving sources by adding a hydrophone.

\title{
Session 3aBA
}

\section{Biomedical Acoustics: Quantitative Ultrasound I: From Micro to In-vivo}

\author{
Jonathan Mamou, Cochair \\ F. L. Lizzi Center for Biomedical Engineering, Riverside Research, 156 William St., 9th Floor, New York, NY 10038 \\ Tadashi Yamaguchi, Cochair \\ Chiba University, 1-33 Yayoicho, Inage, Chiba 2638522, Japan
}

\section{Invited Papers}

\section{8:00}

3aBA1. Emerging quantitative ultrasound applications: From diagnosing disease to monitoring of therapy. Michael L. Oelze (Dept. of Elec. and Comput. Eng., Univ. of Illinois at Urbana-Champaign, 405 N Mathews, Urbana, IL 61801, oelze@uiuc.edu)

Ultrasound has traditionally been considered an imaging modality characterized by high spatial resolution but low contrast. Conventional ultrasonic imaging may be sensitive to the detection of anomalous tissue features, but the ability to classify these tissue features often lacks specificity. However, recent advances in quantitative ultrasound (QUS) have emerged which can improve diagnostics by providing new sources of image contrast and specific numbers tied to tissue state. QUS imaging can encompass a wide variety of techniques including spectral-based parameterization, elastography, flow estimation, and envelope statistics. Spectral-based techniques include the estimation of the backscatter coefficient, estimation of attenuation, and estimation of scatterer properties such as the correlation length associated with an effective scatterer size and the acoustic concentration of scatterers. Envelope statistics include the estimation of the number density of scatterers and quantification of coherent to incoherent signals produced from the tissue. In this talk, successful applications of QUS will be discussed demonstrating the ability of spectral-based QUS and envelope statistics approaches to improve medical diagnostics including cancer detection and classification of solid tumors in the breast or thyroids, grading of fatty liver disease and monitoring and assessment of therapy in solid tumors.

\section{8:20}

3aBA2. Selected quantitative ultrasound successes. Aiguo Han and W. D. O'Brien, Jr. (BioAcoust. Res. Lab., Dept. of Elec. and Comput. Eng., Univ. of Illinois, 405 N. Mathews Ave., Urbana, IL 61801, han51@illinois.edu)

The objective of soft tissue quantitative ultrasound (QUS) is to improve diagnostic ultrasound imaging via quantitative outcomes. Over the past three or so decades, there have been an increasing number of QUS successes. Four QUS successes will be highlighted, from theoretical development to clinical applications. Significant ultrasonic scattering mechanistic details have resulted from the spatial correlation among the scatterers (modeled by the structure function) as well as from the individual scatterers (modeled by the form factor), for which there have been successes with both theory and applications. In collaboration with colleagues at the University of California, San Diego, nonalcoholic fatty liver disease (NAFLD) is being assessed from model-free QUS parameters from which both the backscatter coefficient and the attenuation coefficient show dependencies with the liver fat content in human subjects. Likewise, in collaboration with colleagues at the University of Illinois at Chicago, spontaneous preterm birth is being assessed as early as 20 weeks of gestation in pregnant women from the model-free QUS parameter attenuation coefficient. [NIH Grants NIH R01DK106419 and R37EB002641.] 
3aBA3. Backscatter coefficient estimation in human thyroid carcinoma In vivo. Julien Rouyer (Pontificia Universidad Católica del Perú, Av. Universitaria 1801, San Miguel, Lima 32, Peru,), Rosa Laimes, Claudia Salazar, Joseph Pinto, Jorge Guerrero (Oncosalud, AUNA, Lima, Peru), and Roberto Lavarello (Pontificia Universidad Católica del Perú, San Miguel, Lima 32, Peru, lavarello.rj@pucp.edu.pe)

Spectral-based quantitative ultrasound (QUS) characterizations may provide a potential alternative to invasive gold standard technique-fine-needle aspiration (FNA) — in the diagnostic management of the thyroid cancer. Such potentiality was previously demonstrated in mouse thyroid cancer models ex vivo. Recently, the feasibility of the backscatter coefficient (BSC) estimation of human thyroid in vivo was demonstrated for a controlled group of 20 healthy volunteers ( $24.4 \pm 2.9$ year-old). Among the volunteer, the integrated BSC parameter was found equal to $1.41 \times 10^{-2} \mathrm{sr}^{-1} . \mathrm{cm}^{-1}$ in the 3 to $9 \mathrm{MHz}$ range. The current study focuses on the estimation of the BSC in a clinical context. Preliminary results on patients having malignant lesions are proposed. A research ultrasound imaging system (SonixTouch with an L14-5/38 linear array, Ultrasonix Medical Corp.) was employed by the radiologists of an oncological center first to record radio frequency data of the lesion and second to guide the FNA. The reference phantom technique was used in the same fashion as for the previous healthy volunteer's study. The experimental protocol was approved by an Institutional Review Board, and all enrolled patients provided written informed consent. A controlled group of seven patients with a diagnosed papillary or follicular carcinomas were included in this pilot study to assess the ability of the technique to estimate the BSC and derived parameters in the lesions.

\section{9:00}

3aBA4. Quantitative ultrasound for tumour characterization and a priorichemotherapy response prediction. Gregory Czarnota (Sunnybrook Health Sciences Ctr., 2075 Bayview Ave., Toronto, ON M4N 3M5, Canada, gregory.czarnota@sunnybrook.ca)

Previous studies have demonstrated that quantitative ultrasound (QUS) is an effective tool for monitoring breast cancer patients undergoing neoadjuvant chemotherapy. Here, for the first time, we demonstrate the clinical utility of pre-treatment QUS texture features in predicting the response of breast cancer patients to NAC. Using a $6 \mathrm{MHz}$ center frequency clinical ultrasound imaging system, radiofrequency breast ultrasound data were acquired from 92 locally advanced breast cancer patients prior to their NAC treatment. QUS spectral parameters including mid-band fit, spectral slope, and spectral intercept, and average acoustic concentration and average scatterer diameter were computed from regions of interest in the tumor core and its margin. Subsequently, gray-level co-occurrence matrix textural features were extracted from the parametric images as potential predictive indicators. Results were compared with the clinico-pathological response of each patient determined at their treatment end. A composite QUS feature demonstrated a favourable response prediction with sensitivity, specificity, and AUC of $86 \%, 83 \%$, and 0.77 , respectively, using a Naïve Bayes classifier. The findings here suggest that QUS features of breast tumors are strongly linked to tumor responsiveness. The ability to identify patients that would not benefit from NAC would facilitate clinical management that has minimum patient toxicity and maximum outcome.

9:20

3aBA5. Quantitative ultrasound of the uterine cervix. Timothy J. Hall (Medical Phys., Univ. of Wisconsin, 1005 WIMR, 1111 Highland Ave., Madison, WI 53705, tjhall@wisc.edu), Helen Feltovich (Medical Phys., Univ. of Wisconsin, Provo, UT), Ivan M. RosadoMendez, Lindsey C. Drehfal, Quinton W. Guerrero (Medical Phys., Univ. of Wisconsin, Madison, WI), and Mark L. Palmeri (Biomedical Eng., Duke Univ., Durham, NC)

We are developing methods to objectively describe the acoustic and viscoelastic properties of the in vivo cervix to monitor the structural changes that occur during pregnancy. Clinicians commonly use digital palpation to subjectively judge cervical softness knowing the cervix softens prior to parturition. Premature softening of the cervix is a risk factor for preterm birth which has enormous morbidity and financial consequences. However, this is a challenging tissue for quantitative ultrasound (QUS) investigations because its microstructure is layered and pseudo-aligned within each layer. Also, data are typically acquired with a transducer that is in contact with the outer layer of the cervix, so QUS analysis is necessarily done very close to the transducer surface. To avoid violating assumptions used in QUS algorithm development, we use a prototype transducer that allows us to perform QUS parameter estimation as close as $3 \mathrm{~mm}$ from the transducer surface. Further, we systematically test for various forms of echo signal coherence instead of assuming the backscatter is from diffuse scattering. Preliminary results in humans and non-human primates suggest the cervix significantly softens in early pregnancy, but the underlying collagen microstructure remains intact until very near delivery. Methods for this analysis and results will be briefly described.

\section{9:40-10:00 Break}

\section{0:00}

3aBA6. Compressing and shearing the transplanted kidney. Matthew W. Urban (Dept. of Radiology, Mayo Clinic College of Medicine, 200 First St. SW, Rochester, MN 55905, urban.matthew@mayo.edu), Carolina Amador, and Sara Aristizabal (Dept. of Physiol. and Biomedical Eng., Mayo Clinic College of Medicine, Rochester, MN)

Kidney transplantation is one treatment for end-stage renal disease. Transplant rejection is typically evaluated with an invasive biopsy. Noninvasive methods for evaluating renal transplants would be beneficial for frequent evaluation. We propose the use of shear wave elastography (SWE) to evaluate viscoelastic properties in renal transplants. Further, we also use acoustoelasticity to measure the nonlinear modulus of the renal transplants. Acoustoelasticity is the phenomenon where the shear wave velocity (SWV) in a material varies with applied stress and is quantified by a nonlinear modulus, A. By applying pressure with the ultrasound transducer, stress is applied to the kidney under investigation and B-mode imaging was used to measure induced strain and SWE was used to make measurements of SWV. Using relationships involving the measured strain and shear moduli at different compression levels, we can obtain estimates of A. In our ongoing study, we use a General Electric Logiq E9 to perform SWE measurements in renal transplant patients coming for protocol biopsies. We have studied 63 patients with SWE to quantify viscoelastic properties of the transplanted kidney. We have obtained data from 19 patients for estimation of the nonlinear modulus, A. We compared the measured viscoelastic and nonlinear shear parameters with clinical variables such as the serum creatinine, Banff biopsy scores, and resistive index. We continue to evaluate these methods and their efficacy as indicators for transplant rejection. 
3aBA7. Passive elastography: A seismo-inspired tomography of the human body. Stefan Catheline (INSERM U1032, 151 cours albert thomas, Lyon 69003, France, stefan.catheline@inserm.fr)

Elastography, sometimes referred as seismology of the human body, is an imaging modality recently implemented on medical ultrasound systems. It allows to measure shear waves within soft tissues and gives a tomography reconstruction of the shear elasticity. This elasticity map is useful for early cancer detection. A general overview of this field is given in the first part of the presentation as well as latest developments. The second part, is devoted to the application of noise correlation technique in the field of elastography. The idea, as in seismology, is to take advantage of shear waves naturally present in the human body due to muscles activities, heart beating, and arteries pulsatility to construct shear elasticity map of soft tissues such as the liver, the thyroid, or the brain. It is thus a passive elastography approach since no shear wave sources are used.

\section{0:40}

3aBA8. Elasticity imaging of inhomogeneous media using inverse filtering with multiple shear wave generation. Tsuyoshi Shiina (Human Health Sci., Graduate School of Medicine, Kyoto Univ., 53 Kawahara-cho Shogoin, Sakyo-ku, Kyoto, Kyoto 6068507, Japan, shiina.tsuyoshi.6w@kyoto-u.ac.jp)

Most diseases like cancer cause to change of tissue mechanical properties described by elasticity. Elastography visualizes these changes and aids in early and differential diagnosis of diseases. Shear wave imaging is one of principles of elastography, and using the speed of shear wave generated by mechanical vibration or the acoustic radiation force impulse. Conventional shear wave imaging uses time-of-flight method; however, it causes artifact by reflection and refraction of shear wave. We proposed a method for shear wave elasticity imaging that combines inverse filtering with multiple shear wave sources induced by acoustic radiation force. Shear waves are induced by pushing pulse and recorded by ultrafast imaging, and repeated at multiple pushing points that are sparsely located. An inverse filter can be applied to virtually focus shear waves on an arbitrary point. Shear wave speed is obtained by a measured shear wavelength and its image is constructed by scanning the focal point. An inverse filter can focus a point in a reverberant field. Thus, the proposed method is not based on the assumption of propagating direction, and improved estimation accuracy can be expected. The feasibility of the proposed method was demonstrated in phantom experiments.

\section{1:00}

3aBA9. Quantitative diagnosis method based on model of tissue structure change for liver fibrosis. Hiroyuki Hachiya, Shinnosuke Hirata (Tokyo Inst. of Technol., 2-12-1 S5-17, Ookayama, Meguro-ku, Tokyo 152-8550, Japan, hachiya@ctrl.titech.ac.jp), and Tadashi Yamaguchi (Chiba Univ., Chiba, Japan)

To realize a quantitative diagnosis method of liver fibrosis, we have been developing a modeling method for the probability density function of the echo amplitude. We developed a scatterer distribution model of diseased livers considering the liver lobule structure, because computer simulations are effective for obtaining information on the continuous stage of diseases. We examined relationship between tissue characterization and ultrasonic B-mode image which is simulated by this scatterer distribution model. From these analyses, we proposed a multi-Rayleigh model with three Rayleigh distributions, corresponding to the distribution of the echo amplitude from hypoechoic, normal, and fibrous tissues. We showed quantitatively that the proposed model can model the amplitude distribution of liver fibrosis echo data adequately. We also found that fibrous indices can be estimated stably using the proposed model. To evaluate the liver fibrosis more accurately, each pixel's amplitude value in the clinical echo image was transformed into the fibrotic probabilities by using the multi-Rayleigh model. Clinical echo images of liver fibrosis were analyzed, and the relationship between those probabilities and the stage of liver fibrosis were discussed. We conclude that the proposed method is valid for the quantitative diagnosis of liver fibrosis.

\section{1:20}

3aBA10. Speed of sound, attenuation, and acoustic impedance of hepatic lobule in diseased rat liver observed by scanning acoustic microscopy with 250 MHz. Kenji Yoshida (Ctr. for Frontier Medical Eng., Chibar Univ., 1-33 Yayoicho, Inageku, Chiba, Chiba 263-8522, Japan, kenyoshi1980@chiba-u.jp), Zhihao Deng, Kazuyo Ito (Graduate School of Eng., Chiba Univ., Chiba, Japan), Jonathan Mamou (Lizzi Ctr. for Biomedical Eng., Riverside Res., New York, NY), Hitoshi Maruyama (Dept. of Gastroenterology and Nephrology, Chiba Univ., Chiba, Japan), Hiroyuki Hachiya (Tokyo Inst. of Technol., Tokyo, Japan), and Tadashi Yamaguchi (Ctr. for Frontier Medical Eng., Chibar Univ., Chiba, Japan)

Based on the analysis of echo amplitude envelope and power spectrum of backscattering echo, our group has attempted the characterization of various types of tissue such as liver, lymph node, and skin. For example, the scatterer size estimation of rat liver showed that the estimated size was 30-50 $\mu \mathrm{m}$. However, it was not investigated how the obtained parameters related to the histology in detail. To resolve this problem, it would be invaluable to understand the spatial distribution of acoustic properties, e.g., speed of sound, attenuation, and acoustic impedance in the scale from micro- to millimeter range. Thus, we measure and accumulate the data using ultrasound with center frequency of 1- $500 \mathrm{MHz}$. This report introduces the acoustic properties of specimen prepared from two different types of rats: normal liver and liver fibrosis models. A scanning acoustic microscopy using 250-MHz ultrasound with spatial resolution of $7 \mu \mathrm{m}$ was employed. After the measurement, H\&E staining and Masson trichrome staining were conducted to obtain histology. Image registration of SoS image and histology image enabled to separately evaluate SoS of cell nucleus, cytoplasm, and fibrotic tissue. The result demonstrated that there was no significant difference in SoS among cell nucleus, cytoplasm, and fibrotic tissue. 
3aBA11. Cultured tumor model inspection for anticancer drugs using acoustic impedance microscope. Sachiko Yoshida, Rahma H. Rahayu, Kyoichi Takanashi, Kenta Kishikawa, Hirofumi Kurita, Kazunori Takashima, Naohiro Hozumi (Toyohashi Univ. of Technol., 1-1, Hibarigaoka, Tempaku-cho, Toyohashi 4418580, Japan, syoshida@ens.tut.ac.jp), Kazuto Kobayashi (Honda Electronics Co., Ltd., Toyohashi, Japan), and Seiji Yamamoto (Hamamatsu Univ. School of Medicine, Hamamatsu, Japan)

High-frequency ultrasonic microscope is useful to observe intracellular structure of cultured living cells. We have observed the dynamical change of intracellular actin filaments in several cells, and reported the difference of stabilities between cancer cells and ordinary cells. The dynamics of actin filaments are deeply related to cancer cell viability. In this study, we applied this acoustic microscopy to antitumor drug screening. One of the cultured brain cancer models was established with both normal glial cells and C6 glioma cells. Normal glial cells were labeled by endogenous fluorescent protein to be identified. Both cells were co-cultured on a 75 micrometer-thick polystyrene substrate. Acoustic pulse wave with $320 \mathrm{MHz}$ was focused on the interface between the cell and the substrate, and sent through the substrate. Either classical anticancer drug, cytochalasin B, or novel clinical anticancer drug, temozolomide, was applied to the cultured cancer model, and observed their intracellular acoustic changes using the acoustic microscope. Before and after drug treatment, the cultures were observed with the laser confocal microscopy to confirm survival of each cell. Another of the models was established with fluorescent protein-labeled breast cancer cells. Our model system would be useful to inspect anticancer drugs without whole animals.

WEDNESDAY MORNING, 30 NOVEMBER 2016

SOUTH PACIFIC 4, 8:15 A.M. TO 11:45 A.M.

\section{Session 3aEA}

\section{Engineering Acoustics: Acoustic Analysis}

Rumi Ito, Cochair

Ritsumeikan University, 1-1-1, Noji-Higashi, Kusatsu 525-8577, Japan

Daniel J. Mennitt, Cochair

Electrical and Computer Engineering, Colorado State University, 1201 Oakridge Drive, Suite 100, Fort Collins, CO 80525

\section{Contributed Papers}

\section{8:15}

3aEA1. An evaluation on voice intelligibility for factory noise reduction based on integration of active noise control and auditory masking. Rumi Ito, Takahiro Fukumori, Masato Nakayama, and Takanobu Nishiura (Ritsumeikan Univ., 1-1-1, Noji-Higashi, Kusatsu, Shiga 525-8577, Japan, is0100is@ed.ritsumei.ac.jp)

Factory noise has been focused upon as one of the social problems. Factory workers exposed to loud, continual noises feel strong discomfort by hearing the loud factory noises for a long time. We have previously proposed an integration method of the active noise control (ANC) and the auditory masking for a noise reduction. The ANC reduces the sound pressure level of the noise in a lower-frequency band by emitting the secondary signals, and the auditory masking reduces discomfort which caused the spectral peaks in a higher-frequency band by emitting the secondary signals. We confirmed that the proposed method is effective for reducing discomfort. However, the method might disturb conversations among the workers by emitting secondary signals. In this study, we therefore evaluated the voice intelligibility of the noise-reduced speech obtained by applying the proposed method to the noisy speech recorded in the factory. As a result of the evaluation experiment, we confirmed that the proposed method can keep voice intelligibility almost the same as the intelligibility of the clean speech in the amid factory noise. In addition, we investigated the relationship between the voice intelligibility and each method under the condition that the controlling frequency-bands are set at to $0-500 \mathrm{~Hz}$ for ANC and $500-5500 \mathrm{~Hz}$ for auditory masking.
$8: 30$

3aEA2. Head-related transfer function personalization based on spectral notch estimation with stereo images of pinna. Zhuan Zuo, Taku Yoshimura, Takahiro Fukumori, Masato Nakayama, and Takanobu Nishiura (Ritsumeikan Univ., 1-1-1, Noji-higashi, Kusatsu 525-8577, Japan, is0291hr@ed.ritsumei.ac.jp)

Three-dimensional (3-D) sound field reproduction systems such as surround sound reproduction systems are rapidly being developed in recent few years. However, these systems need a lot of space for the placement of loudspeakers. For this reason, a binaural system has been studied as one of the systems, which controls the sound image localization with the head-related transfer functions (HRTFs) and just by using only a headphones. However, the individual differences in body features would cause the variations of HRTFs which greatly affect the direction perception. For this reason, a method based on the spectral notch estimation from the pinna shape has been studied for the personalization of HRTFs. In this study, we propose a new method, in which the depth information used for the spectral notch estimation is measured with stereo images of the pinna. Two cameras are utilized together as a stereo camera to take the disparate stereo images so that we can use the disparity of them to measure the depth information of the pinna. As a result of an evaluation experiment, we could reduce the cost of actual measurement and the burden to the subjects by the proposed method. 
3aEA3. Arrangement of control points for sound spot generation in the multi-point control method. Kazuya Yasueda (Graduate School of Sci. and Technol., Ryukoku Univ., 1-5 Yokotani, Setaoe-cho, Otsu-shi, Shiga 520-2123, Japan, t16d001@mail.ryukoku.ac.jp), Daisuke Shinjo, and Akitoshi Kataoka (Faculty of Sci. and Technol., Ryukoku Univ., Otsu-shi, Japan)

In this research, we considered control point arrangement by using small-scale linear loudspeaker array. The multi-point control method is proposed for sound reproduction in a limited area/direction. This method is based on boundary surface control principle. The desired signal is reproduced in the target point by the sound pressure control in the control points. According to the MINT (multiple-input/output theorem), the signal is reproduced with accuracy in the target point if the number of sound sources more than the number of control points exists. However, a lot of speakers make it difficult to use in real environment. Therefore, small-scale loudspeaker array is preferable. If the number of control points is greater than the number of sound sources, the filter coefficient is approximated by the least squaresmethod. This approximation affects the sound pressure suppression and sound quality depending on the arrangement of control point. We examined the number of control points arranged. The input signal is the speech signal that has $300-3400 \mathrm{~Hz}$ frequency band. We evaluated some arrangement pattern by sound pressure, SD (Spectral Distortion), and PESQ (Perceptual Evaluation of Speech Quality). As a result, good results were obtained than conventional arrangement.

\section{9:00}

3aEA4. Relation between area of shielding bone-conduction microphone and quality of speech communication in noisy environment. Kojiro Takahashi, Kenji Muto (Graduate School of Eng. and Sci., Shibaura Inst. of Technol., 3-7-5 Toyosu, Muto lab, Koto-ku, Tokyo 135-8548, Japan, ma15051@shibaura-it.ac.jp), and Kazuo Yagi (Tokyo Metropolitan Univ., Arakawa-ku, Tokyo, Japan)

The doctor does not receive the transmitted voice of the patient when the patient becomes unwell in MRI examination, because the voice is faint and MRI acoustical noise is loud. Our purpose is to improve the quality of speech communication of bone-conduction microphone to transmit the patient's faint voice to the doctor. We propose the theoretical formula using the characteristic of vibration propagation for calculating the quality in the case of the shielding microphone. In this report, the improvement level of speech communication quality in the case of the shielding microphone is estimated by the proposed formula in order to discuss the accuracy of the proposed formula. Here, the improvement level of shielding was calculated with a phantom. The phantom consists of a ceramic plate and a silicon rubber representing bones and muscles, respectively. The vibration of vertical direction on the phantom was measured with accelerometers to calculate the improvement level. The results showed that the quality of speech communication is improved by the shielding area, such as an earmuff, to the level of $3 \mathrm{~dB}$.

\section{9:15}

3aEA5. Metasurfaces for perfect transmission of sound from air into water. Eun Bok, Haejin Choi, Jong J. Park, and Sam H. Lee (Phys., College of Sci. 332, 50 Yonsei-ro, Seodaemun-gu, Seoul 120-749, South Korea, skzz430@gmail.com)

Three orders of magnitude difference in acoustic impedance between air and water has so far prevented effective sound transmission through the interface. A conventional method for an antireflection coating is a quaterwave plate. Such a coating would be too thick and bulky because acoustic waves in audible range are long. More importantly, one cannot find a suitable coating material among the naturally occurring materials, the acoustic impedance of which is required to be a geometrical mean of those of air and water. Here, we present fabrication of acoustic metasurfaces of thickness much smaller than the wavelength, which allows perfect transmission of sound through the air/water interface. The metasurface is a two dimensional array of unit cells which are consisting of membrane and cavities. Our work demonstrates usage of metamaterials for impedance matching in extreme conditions.
3aEA6. Gain and directivity of exponential horn receivers. Daniel J. Mennitt (Elec. and Comput. Eng., Colorado State Univ., 1201 Oakridge Dr., Ste. 100, Fort Collins, CO 80525, daniel_mennitt@partner.nps.gov) and Kurt Fristrup (Natural Sounds and Night Skies Div., National Park Service, Fort Collins, CO)

The self-noise of acoustical sensors limits their capacity to monitor extremely quiet environments and measure the subtle, adventitious cues that animals routinely rely upon. Although primarily used in sound production, horns also can amplify sound prior to transduction by a microphone. Given the small size of microelectromechanical microphones, substantial gain can be achieved at wavelengths larger than the horn. An analytical model of an exponential horn embedded in a rigid spherical housing was formulated to describe the gain relative to a free-field receiver as a function of frequency and angle of arrival. Through comparison with experiment and numerical models, the directivity of the horn receiver is shown to be adequately described by the analytical model up to a critical wavelength, beyond which physical assumptions are violated to some degree. Numerical models, based on the equivalent source method, describe the acoustic scattering within and around the horn and provide a means for identifying the mechanisms of deviation in gain at high frequencies and large angles of arrival.

\section{9:45}

3aEA7. Large-scale loudspeaker array system for sound field reproduction using high-speed 1 bit signal processing. Yusei Yamanaka, Daiki Takeuchi, Dai Kuze, Yusuke Ikeda, and Yasuhiro Oikawa (Waseda Univ., 407-2 59-gokan, Okubo Shunjuku-ku, Tokyo 169-8555, Japan, sunset@ akane.waseda.jp)

Recently, many techniques of physical sound field reproduction, such as Wave Field Synthesis, Higher Order Ambisonics and Boundary Surface Control, have been studied. Since the number of loudspeakers generally increases to control with higher spatial resolution and higher frequency, the system becomes too complicated to be constructed. On the other hand, highspeed 1 bit signal processing has been studied widely. The 1 bit audio system has interesting features such as simple circuit implementation, simple signal transmission, and no D/A converter. In this study, we proposed the large-scale loudspeaker array system with high-speed 1 bit signal. The system consists of a master and hub systems with sample rate of $4 \mathrm{MHz}$. The master system reads multi-channel 1 bit signal from SSD and transmits it to each hub system. Each hub system drives 32 loudspeakers by CMOS drivers. The master and each hub system are connected with a LAN cable to implement only hub system near the loudspeakers. It is easy to scale the size of system with flexibility thanks to the simplicity of the circuit and signal flow. We conducted preliminary experiments to control its sound directivity. In the future, we are planning to construct 1536-channel sound reproduction system with our proposed system.

\section{0:00-10:15 Break}

\section{0:15}

3aEA8. Evaluation on overlapping barriers design using SoundPLAN. Sheying Sun, Neil Morozumi, and Richard Patching (Patching Assoc. Acoust. Eng. Ltd., \#20, 2150 - 29th St. NE, Calgary, AB T1Y 7G4, Canada, ssun@patchingassociates.com)

Traffic noise comes along with road developments. To overcome traffic noise problems, noise barriers, low noise pavements, low noise vehicles, traffic control measures, and proper land uses have been proposed. Being considered to have great benefits of easy installation, better noise reduction performance, and ability to soothe annoyed residents, noise barriers have become the most prevalent noise control measures adopted by most agencies. However, any defects in noise barriers may allow unnecessary noise propagation and thus degrade their performance. Consequently, noise barriers should be constructed and maintained with care to uphold their designed noise reduction capability. Overlapping barriers are sometimes necessary for maintenance or community access. There is apparently a need to evaluate the effect of the performance of overlapping barriers. Inappropriate design may cause severity of degradation of acoustical effectiveness. Acquiring the appropriate parameters of the overlapping barrier design will 
serve as a reference for decision makers to properly allocate the gap location and select the sound design. This analysis focuses on the evaluation of barrier overlapping design and receiver regions in the vicinity of an overlap gap using the SoundPLAN software package. The contributions from the various parameters such as materials, barrier height, gap, and overlapping sizes were investigated.

\section{0:30}

3aEA9. The development of wavenumber extended multi-dimensional interpolation techniques for aeroacoustic problems. Seokjong Jang (Seoul National Univ., Daehak-dong, Gwanak-gu, Seoul 151-742, South Korea, clickcoco@snu.ac.kr)

One of the main concerns of computational aeroacoustics is how to solve aeroacoustic problems with complex geometry. The dissipation/dispersion errors should be minimized to obtain accurate acoustic solutions because numerical solutions for aeroacoustic problems are over-susceptible. The wavenumber extended multi-dimensional interpolation techniques were developed in order to satisfy those requirements. The size of a stencil used for standard multi-dimensional interpolation can be determined. Adding the number of stencils with optimized interpolation coefficients enables obtaining the distribution function optimizing the relationship between dispersion and dissipation. Through the optimization process in frequency domains, this technique minimizes the dispersion/dissipation errors generated by the data transfer between multi-grid systems. In addition, a proper limiting process was proposed to remove a numerical instability and oscillations in a discontinuous region. Several problems were numerically simulated by applying the wavenumber extended multi-dimensional interpolation. The results of a advanced technique was validated by being compared with the existing conventional interpolation techniques.

\section{0:45}

3aEA10. Numerical investigation on radiation characteristics of aerointake noise with ground effects. HanAhChim Choung (Mech. and Aerosp. Eng., Seoul National Univ., Daehak-dong, Seoul, Gwanak 151-742, South Korea,good8160@snu.ac.kr)

A hybrid method for the prediction of far-field Aero-intake noise from gas turbine engine with ground effects has been developed. The near-field predictions were obtained by solving the linearized Euler equations with computational aeroacoustic techniques composed of high-order finite difference scheme, non-reflecting boundary condition, overset grids, and parallel computational methods. Kirchhoff integral method was used for the prediction of far-field directivity patterns. In order to predict the noise propagation, ray theory is applied with Geographical Information System (GIS) terrain profile data. Then, the noise level on the ground has been predicted and analyzed, including the effects of air absorption, ground reflection, and diffraction.

\section{1:00}

3aEA11. Determination of the acoustical properties of sisal and coconut fiber samples based on the flow resistivity experimental assessment. Key F. Lima, Nilson Barbieri (Mech. Eng., PUCPR, Imaculada Conceição 1155, Curitiba, Paraná 80215901, Brazil, keyflima@gmail.com), Renato Barbieri (Mech. Eng., UDESC, Joinville, Santa Catarina, Brazil), and Vinicius A. Grossl (Mech. Eng., PUCPR, Curitiba, Paraná, Brazil)

Absorbent materials are fibrous or porous and must have the property of being good acoustic dissipaters. The noise reduction process occurs due to transformation of a part of the sound energy into heat. The attenuation is caused by friction of the viscous air fluid inside the irregular arrangement of fibers during the sound wave propagation. This study assesses the complex wave number and the complex characteristic impedance of two natural fibers with the classical empirical equations of Delany and Bazley (1970). These properties are determined based on experimental evaluation of the flow resistivity of sisal and coconut coir fiber samples. The experimental assessment procedure was performed according to the guidelines of ASTM C522-03 (2009). Samples evaluated have approximately identical densities, different diameters, and thicknesses. The diameters were 62.0, 71.5, and $80.0 \mathrm{~mm}$ and thicknesses were $5,10,15$, and $20 \mathrm{~mm}$. The purpose of testing seven dimensions is to verify the effect of these differences on the flow resistivity experimental measurements. This evaluation is due the standard ASTM does not provide guidelines for the sample dimensions.

\section{1:15}

3aEA12. Multi-physics modeling of conformal, solid-state, and thin-film thermophones. Mahsa Asgarisabet and Andrew R. Barnard (Mech. Eng. Eng. Mech., Michigan Technolog. Univ., 815 R.L. Smith MEEM Bldg., 1400 Townsend Dr., Houghton, MI 49931, arbarnar@mtu.edu)

Thin-film thermophones are thermoacoustic loudspeakers made from new materials such as aligned carbon nanotubes or graphene. They are solid state devices, in that there are no moving parts associated with the sound generation mechanism. Lumped parameter models have been developed for planar thermophones and agree well with experimental results. One benefit of thin-film thermophones is that they can be designed such that the transducer can be conformal to complex geometries. However, the lumped parameter models are not appropriate for complex geometries or electrical/ thermal transients. An electrical-thermal-acoustic multiphysics model of these transducers has been created using COMSOL Multiphysics to address the issues present with the lumped parameter models. Development and validation of the model will be discussed.

\section{$11: 30$}

3aEA13. Study on coupled analysis for compact acoustic systems using finite-difference time-domain method based on Kirchhoff-Love plate theory and elastic wave. Hayato Takimoto, Yoshinobu Kajikawa (Faculty of Eng. Sci., Kansai Univ., 3-3-35, Yamate-cho, Suita, Osaka 564-8680, Japan, k730406@kansai-u.ac.jp), Masahiro Toyoda (Faculty of Environ. and Urban Eng., Kansai Univ., Suita, Osaka, Japan), and Takashi Miyakura (Hosiden Corp., Yao, Osaka, Japan)

In this paper, we propose a coupled analysis method for compact acoustic systems (e.g., micro speakers, electret condenser microphones) using finite-difference time-domain (FDTD) method based on the Kirchhoff-Love plate theory and elastic wave. Acoustic systems are usually analyzed through any numerical analysis technique based on wave propagation. However, an exact frequency response is rarely obtained for compact acoustic systems. This is because the standard FDTD method cannot treat the change of the frequency response due to the air viscosity and cannot also treat the wave propagation in a solid. Thus, we cannot obtain exact frequency responses for compact acoustic systems through the analysis of coupled fluid-solid model based on standard FDTD method. In order to solve these problems, we utilize a coupled analysis method based on Kirchhoff-Love plate theory and elastic wave FDTD method considering air viscosity. The analysis method models diaphragm and voice coil as a two-dimensional plate model based on Kirchhoff-Love plate theory and air as a three-dimensional model based on elastic wave FDTD method considering the air viscosity. In this paper, we demonstrate the effectiveness of the analysis method through some comparisons between calculated and measured frequency responses. 


\title{
Session 3aMU
}

\section{Musical Acoustics: East and Western Instruments: Contrasts and Connections}

\author{
James P. Cottingham, Cochair \\ Physics, Coe College, 1220 First Avenue NE, Cedar Rapids, IA 52402 \\ Toru Kamekawa, Cochair \\ Musical Creativity and the Environment, Tokyo University of the Arts, 1-25-1, Senju, Adachi-ku, Tokyo 120-0034, Japan \\ Chair's Introduction-8:35
}

\section{Invited Papers}

3aMU1. Eastern and Western free reed instruments: Acoustics, history, and culture. James P. Cottingham (Phys., Coe College, 1220 First Ave. NE, Cedar Rapids, IA 52402, jcotting@coe.edu)

There are two families of free reed instruments. Mouth-blown instruments employing free reeds coupled to pipe resonators have been used throughout East and Southeast Asia since ancient times. The sheng, sho, khaen, and bawu are typical examples. Details of the origin and development of these instruments are not known, but are closely connected with the history and prehistory of a multitude of ethnic groups. The instruments of the Western free reed family are of modern origin, originating in Europe around 200 years ago. These include the reed organ, harmonium, harmonica, and the accordion-concertina family. This paper explores significant differences in the acoustical design of the two groups of instruments and surveys historical developments of each, including examples of possible connections and influences between the two families. Interesting developments involving the two families of free reed instruments occurred as elements of their original musical cultures became mixed in the nineteenth and twentieth centuries.

\section{9:00}

3aMU2. Western and non-western approaches to control timbre using multi-resonator/generator systems of wind instruments. Jonas Braasch (School of Architecture, Rensselaer Polytechnic Inst., 110 8th St., Troy, NY 12180, braasj@rpi.edu)

Wind instruments are often modeled as a coupled generator-resonator system, which for the case of the clarinet consists of a reed and a cylindrical pipe. However, the fact that the throat and oral cavities behind the tone generator also play a critical role is often neglected. While the resonance pipe of a wind instrument can be adjusted in length through keyholes or valves, its width is fixed. The opposite can be observed for the human resonance system where the length is fixed but the dimensions of the cross-section are variable. This important fact is routinely used to shape the timbral qualities of tones. In many non-western music cultures, time-varying timbral modifications are more important than melodic aspects, for example, in traditional digeridoo practice. The effects of vocal tract control including Tongue Controlled Embouchure (TCE) will be analyzed and discussed using extended saxophone techniques with adaptive mouthpieces [Braasch, 2014, J. Acoust. Soc. Am. 135, 2245].

\section{9:20}

3aMU3. Finite-difference model of mode shape changes of the Myanmar pat waindrum circle with tuning paste on drum head. Rolf Bader (Univ. of Haburg, Inst. of Systematic Musicology, Neue Rabenstr. 13, Hamburg 20354, Germany, R_Bader@t-online.de)

The tuning process of drums differ considerably between Western and Southeast Asian drums. While Western drums, like tom-toms, snare, or bass drums of a rock or jazz drum kit, are tuned by changing the tension of the drum head, many drums in Southeast Asia are tuned by adding a paste consisting of rice and ash. As an example, the tuning of the Myanmar pat wain, a unique drum circle of a hsain wain orchestra is investigated. The pat wain has 20 pitched drums and is played with great virtuosity. It was investigated by the author during a field trip in northern Myanmar during its tuning process. The drum head is modeled using a time-dependent Finite-Difference Method (FDM) to estimate pitch and mode changes due to adding the paste. The results are compared to the field recordings which show considerable damping and pitch glides with low-pitched drums. Also the tuning of the drum circle is compared to different tuning systems showing similarities to a tuning pattern of Cambodian roneat deikmetalophone. 
3aMU4. Transforming rhythmscapes. Rohan Krishnamurthy (Music, Ohlone College, 544 Sunrise Circle, Kalamazoo, MI 49009, rohan.krishnamurthy@ rochester.edu)

Originating in India over two millennia, the mridangam is one of the most ancient, complex, and versatile hand drums in the world. The pitched nature of the two-sided drum and a complex split finger technique enable the creation of over a dozen unique tones. The acoustics of the mridangam, especially the tonal drumhead, will be discussed. Musical excerpts will demonstrate how the timbres and sound structures of the mridangam have intertwined with diverse musical genres from around the world, including global percussion ensembles, symphony orchestras, jazz ensembles, and Hollywood scores. The recent phenomenon of real-time online music education will also be discussed as a seminal mode of disseminating the mridangam tradition across the globe. The presentation will conclude with a live, interactive performance.

\section{0:00-10:15 Break}

\section{0:15}

3aMU5. String-pressing force of Japanese Koto. Tamaki Ando (Koto Player, 1-3-17 Sakuragaoka, Setagaya-ku 156-0054, Japan, antama@d6.dion.ne.jp), Satoshi Obata, and Eriko Aiba (The Univ. of Electro-Communications, Chofu, Tokyo, Japan)

The koto is a traditional Japanese stringed musical instrument and is similar to the zither. The koto typically has 13 strings and is played by plucking the strings using three fingers (thumb, index, and middle fingers) with picks. Each string is strung over a movable bridge, and the support point can be changed by adjusting the position of the bridge. As the distance between the support point and the right end of the string determines a pitch, the koto is tuned by adjusting the bridge position. In addition, players can change the pitch by pressing the string with their left hand. This technique is called oshide and it is important but difficult to apply accurately for koto players. The subjective difficulties of oshide change depending on the position of the strings (on the near or far side of a player's body), string's material (silk or polyester), condition (old or new) of the string, and so on. In order to ensure good-quality performance, expert koto players modify their string-pressing manner. However, there are no previous studies on this. In this presentation, we present our results of the string-pressing force applied by koto players using oshide under various conditions.

\section{Contributed Papers}

\section{$10: 35$}

3aMU6. Differences between audio engineers and players in the subjective evaluation on the recording position of the Koto (Japanese harp). Shun Saito (Music and PsychoAcoust., Res. Area of Creativity of Music and Sound, Div. of Musicology and Music Studies, Graduate School of Music, Tokyo Univ. of the Arts, Oosumidai1-12-13, Isehara-shi, Kanagawa-ken 259-1105, Japan, s2316907@ms.geidai.ac.jp), Toru Kamekawa, and Atsushi Marui (Music and PsychoAcoust., Res. Area of Creativity of Music and Sound, Div. of Musicology and Music Studies, Graduate School of Music, Tokyo Univ. of the Arts, Tokyo, Japan)

The objective of this study was to examine the appropriate recording position for the Koto (Japanese harp). The sound of the Koto used as experiment stimuli was recorded at 54 different spherical microphone positions. A group of audio engineers and a group of Koto players rated the recorded stimuli on thirteen attribute rating scales. The result of evaluation was performed in an analysis of variance using two factors, namely, the recording position and the evaluator group. In the results, significant differences between the two groups were not found in almost all attributes. However, it should be noted that in the assessment of a few number of attributes, significant differences were observed in interaction among the recording positions and between the groups. For determining suitable factors within the attributes, a factor analysis was performed. The factor scores from the results of the factor analysis showed that the tendencies of each group in regards to the recording positions were different, and results showed that some recording positions had higher scores. It was found that factor one of each group tended to have a higher score in the upward recording position on the players side.

10:50

3aMU7. Modes of vibration of a sheng reed. Thomas W. Kirk (St. Olaf College, St. Olaf College, 1500 St. Olaf Ave., Northfield, MN 55057, kirk@ stolaf.edu), James P. Cottingham (Phys., Coe College, Cedar Rapids, IA), and Eric Sindelar (Phys., Coe College, Cedar Rapids, MN)

The sheng is an Asian mouth organ consisting of a number of bamboo pipes enclosed in a wind chamber with brass free reeds at the end of each pipe inside the chamber. The reed in each pipe typically includes a small wax tuning weight near the free end of the reed tongue. The determination of modes of vibration of these reeds, especially how the tuning weight changes those modes of vibration, is the topic of this paper. Recent research has investigated the acoustics of sheng pipes and compared the measured impedance of sheng pipes augmented with pipe resonators with a theoretical model. This research employs finite element analysis software to construct a theoretical model of sheng reeds and compare that model with measured vibrational analysis data gathered with a laser vibrometer. The primary emphasis is on how well the predicted resonance frequencies compare with measured resonance frequencies of the reed as indicated in a response curve. Also compared are the predicted nodal and antinodal positions along the length of the reed with the measured nodal positions. [Research supported by National Science Foundation REU grant PHY-1004860.]

\section{1:05}

3aMU8. Organologic and acoustic similarities of the European violin and the Chinese $\boldsymbol{e r h} \boldsymbol{u}$. Florian Pfeifle (Univ. of Hamburg, Neue Rabenstrasse 13, Hamburg 20354, Germany, Florian.Pfeifle@uni-hamburg.de)

The Chinese erhu is a bowed spike lute with a recorded history dating back to the Song dynasty (960-1279). It is played in different sociocultural as well as musical settings and in different styles of Chinese music. In modern Chinese orchestras it fills the part of the lead voice in the bowed instruments section, comparable to the role of the European violin in classical orchestras. Even though the design of both instruments differs in several central aspects, they exhibit structural and acoustical similarities. In this work, organologic as well as acoustical similarities of both instruments are highlighted with a special focus on the excitation mechanisms of both instruments. To this end, a set of measurements using high-speed camera recordings of the bowing and the string motion are evaluated and compared. Differences in the resulting "Helmholtz" motion are explained and attributed to the specific string attachment and the structure of the bow respectively. Timbre similarities are shown for several different bowing gestures and the expressive palette of both instruments is compared. Organologic similarities are highlighted through an analysis of the historical evolution of structural components of both instruments again with a strong focus on the bow. 


\title{
Session 3aNS
}

\section{Noise, Psychological and Physiological Acoustics and ASA Committee on Standards: Current Issues in Hearing Protection and Hearing Conservation I}

\author{
Elliott H. Berger, Cochair \\ Personal Safety Division, 3M, 7911, Zionsville Rd., Indianapolis, IN 46268-1650 \\ Jinro Inoue, Cochair \\ Department of Health Policy and Management, University of Occupational and Environmental Health, Japan, 1-1 Iseigaoka, \\ Yahatanishi-ku, Kitakyushu 807-8555, Japan \\ Chair's Introduction-8:00
}

\section{Invited Papers}

8:05

3aNS1. Review of the 2016 Edition of ANSI S12.6 and its place in the panoply of standards on the measurement of real-ear attenuation at threshold since 1957. Elliott H. Berger (Personal Safety Div., 3M, 7911, Zionsville Rd., Indianapolis, IN 46268-1650, elliott.berger@mmm.com)

The American standard specifying the procedure for the measurement of real-ear attenuation at threshold (REAT), often termed the gold standard in measuring hearing protector attenuation, was recently approved as an updated version, ANSI 12.6-2016. REAT was first standardized worldwide in the late 1950s in an American standard ANSI Z24.22-1957 and the method has evolved with time. Changes have affected the electroacoustic requirements for the sound field, instrumentation, audiometric method, and permissible background noise, but more importantly have also improved the specification of how the experimenter works with and fits the test subjects. So too, estimates of uncertainty are now included, and in the 2016 version they have been clarified and brought into harmony with ISO 4869-1. The author, who has been the chair since 1985 of the ANSI working group responsible for S12.6, will compare and contrast the various methods and present representative data as well as a discussion of the expanded uncertainties that are specified. Those values, for the 1/3-octave band test bands from $125 \mathrm{~Hz}$ to $8000 \mathrm{~Hz}$, vary from approximately 1.5-2 dB for earmuffs and 2-3 dB for earplugs for withinlaboratory testing, to 4-6 dB for earmuffs and 6.5-8 dB for earplugs for between laboratory measurements.

\section{$8: 25$}

3aNS2. Field fit testing with E-A-Rfit Dual-Ear validation system in China. Yufei Liu (Person Safety Div., 3M, No.9, Nanxiang Er Rd., Sci. City, Guangzhou, Guangdong 510663, China, sliu9@mmm.com), Wei Gong (Jiangsu Provincial Ctr. for Disease Control and Prevention, Nanjing, China), and Min Yang (Guangdong Province Hospital for Occupational Disease Prevention and Treatment, Guangzhou, China)

This presentation introduces hearing protection fit testing on over 1000 workers in 6 different factories, each with different hearing conservation practices in China. The $3 \mathrm{M}^{\mathrm{TM}}$ E-A-Rfit ${ }^{\mathrm{TM}}$ Dual-Ear validation system was used to measure the Personal Attenuation Rating (PAR), on three different types of Hearing Protection Devices (HPDs), including a foam earplug, a premolded earplug, and a headband earmuff at the work site. A follow up visit to repeat fit-testing was conducted approximately 6 months after the initial visit. Field attenuation of HPDs obtained by workers' and the effects of training toward improving the attenuation were observed. In addition, PARs for the Peltor ${ }^{\mathrm{TM}} \mathrm{X} 4 \mathrm{~A}$ earmuff users were obtained at several points throughout the work shift to capture different fits. Result shows that there is wide variability in PARs and poor agreement between PAR and labelled noise attenuation ratings obtained from laboratory measurement. Hearing protection fit testing is essential to verify the sufficiency of attenuation. Training together with fit testing was shown to improve the PAR. Key words: Hearing Protection Device (HPD), fit testing, Personal Attenuation Rating (PAR), China. 
3aNS3. Personal attenuation ratings reported using Fit Check Solo ${ }^{\text {TM }}$ : Is background noise a concern? Taichi K. Murata (Oak Ridge Inst. for Sci. and Education, WPAFB, OH), Hilary Gallagher (Battlespace Acoust. Branch, Air Force Res. Lab., 2610 Seventh St., Bldg. 441, Wright-Patterson AFB, OH 45433, hilary.gallagher.1@us.af.mil), Elizabeth A. McKenna (SpecPro Tech. Services, WPAFB, OH), Gregory G. Wolff (Air Force Satellite, Defense Health Agency, WPAFB, OH), Sarah C. Sullivan (Hearing Ctr. of Excellence, Dept. of Defense, WPAFB, OH), Matthew R. Ankrom (Oak Ridge Inst. for Sci. and Education, WPAFB, OH), William J. Murphy (National Inst. of Occupational Safety and Health, Cincinnati, OH), Nourhan K. Abouzahra (Oak Ridge Inst. for Sci. and Education, WPAFB, OH), and Quintin A. Hecht (School of Aerosp. Medicine, U.S. Air Force, WPAFB, OH)

It is well known that some personnel, especially Service Members, operate in hazardous noise-environments. To reduce the risk of hearing loss and hearing related disabilities, hearing protection devices (HPDs) are worn to decrease the level of noise at the ear. Noise attenuation provided by HPDs can vary greatly depending on user fit. Fit test systems were developed to measure the noise attenuation performance of HPDs and to report a personal attenuation rating (PAR) based on that particular fit. Elevated levels of background noise in a typical office-type setting (in comparison to a laboratory) could however affect PAR results. A study completed by the Air Force Research Laboratory compared PAR results of 3M EAR Classic earplugs and Howard Leight Airsoft earplugs from data collected using ANSI S12.6 methodology in the laboratory and data collected using Fit-Check Solo ${ }^{\mathrm{TM}}$ in an office environment, a controlled noise office environment, and the laboratory. Results demonstrated that PARs were affected by increased background noise; however, the significance varied based on the number of frequencies used in the PAR calculation. Experimental methodologies, PAR results, and background noise levels will be presented.

9:05

3aNS4. Modeling the effects of hearing protection on speech recognition as function of noise level and hearing status. Christian Giguère (Audiology/SLP Program, Univ. of Ottawa, 451 Smyth Rd., Ottawa, ON K1H8M5, Canada, cgiguere@uottawa.ca) and Elliott H. Berger (Personal Safety Div., 3M, Indianapolis, IN)

The ability to communicate verbally when wearing hearing protectors is an important aspect to consider for compliance in the use of these devices as well as for safety and operational efficiency in the workplace. Speech recognition with hearing protectors is influenced by many factors such as the overall amount of noise reduction, the slope of the attenuation-frequency function and the presence of hearing loss [Giguère and Berger, Int. J. Audiol. 55, S30-S40 (2016)]. Interestingly, results from subjective and modelling studies sometimes show a benefit of using passive hearing protectors on speech recognition in noise compared to unprotected listening, especially with normal-hearing individuals in high-noise levels. The purpose of this modeling study is to further explore the conditions promoting such a facilitative effect of hearing protectors on speech recognition, with a particular attention to the noise level in the environment and the hearing status of the user. This information is important to better guide the selection of the most appropriate hearing protection device, given the characteristics of the user and workplace noise.

\section{9:25}

3aNS5. Evaluation of the improvement in hearing Japanese speech while wearing earplugs advertised as enabling communication. Toshitaka Yokoya, Jinro Inoue, Shinji Kohata, Shoko Kawanami, and Seichi Horie (Health Policy and Management, Industrial Ecological Sci., Iseigaoka 1-1, Yahata-Nishi-Ku, Kitakyushu, Fukuoka 8078555, Japan, yokoya1117@med.uoeh-u.ac.jp)

Workers often refuse to wear earplugs because of the difficulty hearing conversations in noisy workplaces. Little research has been performed to explore whether multiple syllables of the Japanese language are difficult to hear through earplugs. New earplugs recently became available that could be used to communicate clearly in noisy workplaces and simultaneously prevent personal noise exposure. This study aimed to evaluate the hearing of speech using three types of earplugs in a noisy environment. The subjects were 10 people without hearing loss. We conducted this experiment in the anechoic room at the University of Occupational and Environmental Health, Japan. We set up a pink noise environment at $80 \mathrm{dBA}, 85 \mathrm{dBA}$, and $90 \mathrm{dBA}$, and evaluated the hearing of speech with and without earplugs. The subjects were asked to distinguish standard Japanese words comprising two syllables recorded on a CD-ROM (TY-89) established for the evaluation of the fitness of hearing aids. If the speech level required for $90 \%$ intelligibility with those new earplugs was lower than that without earplugs, or lower than that with reference earplugs reported previously, the new earplugs can protect hearing and lead to good communication. A comparison of the new and reference earplugs will be discussed.

\section{9:45-10:00 Break}

\section{0:00}

3aNS6. Uncertainty of hearing protector impulsive attenuation measurements. Cameron J. Fackler, Elliott H. Berger, and Michael E. Stergar (3M Personal Safety Div., 7911 Zionsville Rd., Indianapolis, IN 46268, elliott.berger@mmm.com)

Measurement of the impulsive protection of a hearing protection device (HPD) is currently standardized in ANSI/ASA S12.42-2010 as a measurement of impulse peak insertion loss (IPIL). IPIL is a time-domain metric, determined as the difference between open-ear and occluded-ear peak sound pressure levels of an impulse, measured without and with an HPD in place on an acoustic test fixture. To characterize potential level-dependent protection of an HPD, the IPIL test is repeated at several free-field impulse peak sound pressure levels, typically ranging from approximately $130-170 \mathrm{~dB}$. Based on our experiences measuring IPIL with both a shock tube and a rifle serving as the impulse source, as well as a review of existing literature reporting IPIL studies, we discuss and attempt to quantify the uncertainty of such measurements. Sources of uncertainty include the repeatability of the impulse source, characteristics of the sound field created by the impulse source, spectral characteristics of both the impulse source and the HPD's attenuation, variability between HPD samples and repeat fitting of the HPD to the acoustic test fixture, and variability in the measurement instrumentation and data analysis procedures. Our estimates will be compared to those provided in the uncertainty annex of S12.42. 
3aNS7. Angle dependent effects for impulse noise reduction for hearing protectors. William J. Murphy (Hearing Loss Prevention Team, Centers for Disease Control and Prevention, National Inst. for Occupational Safety and Health, 1090 Tusculum Ave., Mailstop C-27, Cincinnati, OH 45226-1998, wjm4@cdc.gov), Daniel Adams (Dept. Communciation Sci. and Disord., Univ. of Cincinnati, Cincinnati, OH), and Pamela S. Graydon (Hearing Loss Prevention Team, Centers for Disease Control and Prevention, National Inst. for Occupational Safety and Health, Cincinnati, $\mathrm{OH})$

The exposure at the ear in response to a forward-propagating wave depends upon the angle of incidence at the head, the nominal sound pressure level of the impulse and the attenuation of hearing protection (if worn). The unoccluded and occluded responses of an acoustic test fixture equipped with two G.R.A.S. IEC 60711 couplers $1 / 4$ inch microphones were measured in 15 degree increments for impulses with nominal peak sound pressure levels of 150 and 160 decibels. The attenuation was assessed in a variety of ways: Impulse Peak Insertion Loss (IPIL), change in A-weighted Equivalent Energy, and change in the Auditory Hazard Unit. Generally, the $\mathrm{L}_{\mathrm{Aeq}}$ was quite similar to the (IPIL). However, the change in AHUs predicted less attenuation than was actually observed. The lower performance for AHUs may be attributable to the nonlinear hazard growth for the unoccluded ear. These results will be compared with continuous noise assessments of the effect of angle on protection.

\section{0:40}

3aNS8. Middle ear muscle contractions from non-acoustic elicitors. Gregory A. Flamme, Stephen M. Tasko, Kristy K. Deiters (Speech Pathol. and Audiol., Western Michigan Univ., 1903 W. Michigan Ave., MS 5355, Kalamazoo, MI 49008, greg.flamme@ wmich.edu), William A. Ahroon (US Army Aeromedical Res. Lab., Ft. Rucker, AL), and William J. Murphy (Div. of Appl. Res. and Technol., National Inst. for Occupational Safety and Health, Cincinnati, OH)

High-level sounds can elicit middle ear muscle contractions (MEMC), which are commonly known as acoustic reflexes. Tactile stimulation to the face can also elicit MEMC, and it is plausible that MEMC could co-occur with voluntary eye closure gestures. In this paper, we shall present preliminary MEMC results from human volunteers receiving controlled tactile stimulation (nitrogen gas, $10 \mathrm{kPa}$, $200 \mathrm{~ms}$ duration) to four locations on the face and who close the eye ipsilateral to the MEMC detection probe. The MEMC were detected via changes in total energy reflected in the ear using a filtered $(0.2$ to $8 \mathrm{kHz})$ click train. Concomitant muscle activity was measured using electromyography. The morphology and magnitude of the MEMC from these non-acoustic stimuli will be described. If non-acoustic MEMC behaviors do not extinguish and are not highly susceptible to fatigue, they could represent an opportunity to mediate exposure to short-duration noises or help explain between-person differences in noise exposure outcomes.

\section{1:00}

3aNS9. Going global with hearing conservation: Regulations and trends. Laurie Wells (Personal Safety Div., 3M Co., 817 W. 4th St., Loveland, CO 80537, Laurie.Wells@mmm.com)

Noise is recognized universally as an occupational hazard; however, there is not a globally accepted regulatory approach toward protecting the noise-exposed workforce. The regulatory differences for hearing conservation around the world make it challenging for multinational companies to set policies for corporate hearing loss prevention programs. This presentation compares and contrasts selected aspects of various hearing conservation regulations to the United States Occupational Safety and Health Administration requirements. The following jurisdictions are included: Australia/New Zealand, Brazil, China, European Union, India, Japan, and Mexico. Details were collected from English translations of a country regulation as well as by consultation with an experienced, in-country resident whenever possible. Information includes noise exposure limits, noise control, hearing protection device use, standards, and attenuation derating schemes, audiometric testing and hearing shift criteria, and employee training requirements. Studying these criteria will also spark discussion as to best practices and emerging trends in hearing conservation.

\section{1:20}

3aNS10. Introduction of a hearing conservation program in a Japanese enterprise. Naoko Sasaki, Takayuki Ogasawara (Labor Relations and Corporate Services, Mitsubishi Fuso Truck and Bus Corp., 10, Ohkura-cho, Nakahara-ku, Kawasaki, Kanagawa Pref. 2118522, Japan, naoko.sasaki@daimler.com), Ikuo Denda (Personal Safety Tech. Dept., 3M Japan Ltd., Sagamihara, Japan), Jinro Inoue, and Seichi Horie (Dept. of Health Policy and Management, Univ. of Occupational and Environ. Health, Japan, Kitakyushu, Fukuoka Pref., Japan)

To prevent occupational hearing loss, the Ordinance of Industrial Safety and Health in Japan requires employers to measure sound levels in eight hazardous areas in the workplace. In addition, the Guidelines for the Prevention of Noise-Induced Impairments require employers to measure the sound levels in noisy workplaces or provide audiometric testing for employees exposed to occupational noise. However, measurement of personal noise exposure is not a legal requirement in Japan. The Japanese Working Environment Measurement Standards define how to measure occupational noise using a sound level meter (SLM). However, it is difficult to evaluate real noise exposure for employees who use a sound-emitting industrial tool. We measured this exposure using a dosimeter at a Japanese manufacturing enterprise producing transport machinery. The sound levels at some workshops that were evaluated as $85 \mathrm{dBA}$ using a SLM were shown to be more than $90 \mathrm{dBA}$ using a dosimeter. Following these results, we are introducing a Hearing Conservation Program at the enterprise. We would like to report how to develop this new noise management system in Japan and manage personal noise exposure. 


\title{
Contributed Paper
}

11:40

3aNS11. Thoughts on MIL-STD-1474E noise limits. William A. Ahroon (Auditory Protection and Performance Div., U.S. Army Aeromedical Res. Lab., Bldg. 6901, Farrel Rd., Fort Rucker, AL 36362-0577, william.a. ahroon.civ@mail.mil) and Gregory A. Flamme (Dept. of Speech Pathol. and Audiol., Western Michigan Univ., Kalamazoo, MI)

Department of Defense Design Criteria Standard Noise Limits (MILSTD-1474E), published in 2015, is a standard developed for the acquisition of military materiel specifies the maximum permissible noise levels produced by military systems and the test requirements for measuring these levels. It is intended for use by equipment designers and manufacturers, covering typical operational conditions and provides noise limits shall not be exceeded if the materiel is to be acceptable to the procuring activity. It is not intended to be used for health hazard assessments for personnel exposed to impulsive noises. Appendix B established the acquisition requirements for impulsive noise limits. Oddly, the standard allows the use of two different methods, although the U.S. Army must use a method that utilizes the Auditory Hazard Assessment Algorithm for Humans. This paper describes some obstacles in using this appendix for acquisition of weapons systems, including the curious use of two completely different methods for measuring impulsive noise limits, the middle-ear assumptions of the required Army method, and the limits of assumed risk of hearing loss from exposure to impulsive noises.

WEDNESDAY MORNING, 30 NOVEMBER 2016

SOUTH PACIFIC 2, 8:50 A.M. TO 12:00 NOON

\section{Session 3aPA}

\section{Physical Acoustics and Biomedical Acoustics: Acoustic Micro- and Nanofluidics I}

\author{
James Friend, Cochair \\ Mechanical and Aerospace Engineering, University of California, San Diego, 345F Structural and Mechanical Engineering, \\ Mail Stop 411 Gilman Dr, La Jolla, CA 92093
}

Tony Jun Huang, Cochair

Pratt School of Eng., Duke University, Durham, NC 27708

Daisuke Koyama, Cochair

Faculty of Science and Engineering, Doshisha University, 1-3 Tataramiyakodani, Kyotanabe 610-0321, Japan

\section{Invited Paper}

\author{
8:50
}

\begin{abstract}
3aPA1. Controlling sample and particle behaviour in microfluidic systems using acoustic forces. Adrian Neild, Tuncay Alan, Muhsincan Sesen, David Collins, and Jason Brenker (Dept. Mech. \& Aero Eng, Monash Univ., Clayton, VIC 3800, Australia, adrian.neild@ monash.edu)
\end{abstract}

Acoustic forces have been widely used to control microparticles within microfluidic systems. Such acoustofluidic systems have been very successful in tasks such as cell sorting, however, to date efforts have been mostly limited to single phase systems. Just as the contrast in acoustic impedance between a fluid and suspended particle means that acoustic forces can be exerted on the particle, a contrast also exists at the interface between two immiscible fluids. This work explores ways in which such acoustically generated forces can be used in digital (two phase) microfluidic systems. Digital microfluidic systems have gathered significant interest because of the potential of single cell analysis and on-chip chemical reactions. In the paradigm of a lab-on-a-chip, each droplet is analogous to a test tube; however, due to the rapidity of producing large numbers of droplets, reaction based systems can run into difficulties as each test tube contains the same sample. In order to gain control over droplet behavior, acoustically actuated systems have been developed to produce individual droplets, selectively merge droplets, and to steer them at a junction, enabling control of the type of chemical in each droplet in a sequence, chemical reaction initiation and guidance of droplets around a chip.

\section{Contributed Paper}

\section{9:10}

3aPA2. Negative radiation torque using "acoustical sheets". Farid G. Mitri (Area 52 Technol., Chevron, 5 Bisbee Court, Santa Fe, NM 87508, f. g.mitri@ieee.org)

An interesting effect of negative acoustical radiation torque generation and direction reversal of the spinning of an absorptive cylinder is reported
[F.G. Mitri, Wave Motion 66, 31-44 (2016)]. The emergence of the acoustic spin radiation torque experienced by the circular cylinder in a nonviscous host fluid is a consequence of acoustic attenuation inside its absorptive material. Depending on the direction of the shift from the center of a focused Hermite-Gaussian "acoustical-sheet" beam of first-order, the spin torque is negative or positive, causing the rotation of the cylinder in the polar plane in either the clockwise or the anticlockwise direction, respectively. Notice 
that at the center of the beam, the torque vanishes as required by symmetry. In the off-axial configuration, the spin torque sign reversal is dependent on the variation of absorption of both the longitudinal and shear waves inside the viscoelastic material. The effect of changing the non-dimensional size parameter $k a$ (where $k$ is the wavenumber and $a$ is the cylinder radius) has a substantial effect on the negative spin torque generation. Potential applications are in acousto-fluidics, particle manipulation, and handling and imaging microscopy to name a few.

\section{Invited Paper}

3aPA3. Ultrasonic tissue micro-engineering: Making tumor models for optimizing immuno-therapy. Martin Wiklund, Karl Olofsson (Appl. Phys., Royal Inst. of Technol., KTH-Albanova, Stockholm 10691, Sweden, martin@biox.kth.se), Valentina Carannante (Microbiology, Tumor and Cell Biology, Karolinska Inst., Stockholm, Sweden), and Björn Önfelt (Appl. Phys., Royal Inst. of Technol., Stockholm, Sweden)

We demonstrate a method for tissue micro-engineering based on ultrasound-supported three-dimensional (3D) cell culture in a multi-well microplate. The method can be used for producing various tissue-mimicking 3D structures in parallel, and utilizes ultrasonic standing wave trapping forces inside hundred micro-wells in a multi-well microplate fabricated in silicon and glass. We have analyzed and optimized the driving parameters of the ultrasound transducer attached to the microplate, and we demonstrate the production of different micro-engineered tumor models, including micro-scaled 3D solid models of liver, kidney, and skin tumors. The tumor models have been analyzed with flow cytometry in order to investigate the difference between 2D and 3D cultures in expression of protein ligands susceptible to recognition by natural killer (NK) immune cells. We have also designed an image-analysis-based method for quantification of the amount of NK cells needed to defeat a tumor having a certain size, shape, and composition. Our results are important for optimizing future personalized immuno-therapy methods for the treatment of various cancer diseases.

\section{Contributed Paper}

9:45

3aPA4. Neutral core-shell particles under acoustic radiation force. Jose P. Leao (Phys., Federal Univ. of Alagoas, Av. Lourival Melo Mota, sn, Maceio, Alagoas 57035-557, Brazil), Jose H. Lopes (Exact Sci., Federal Univ. of Alagoas, Maceio, Alagoas, Brazil), and Glauber T. Silva (Phys., Federal Univ. of Alagoas, Maceio, Alagoas, Brazil, glauber@pq.cnpq. br)

We theoretically analyze how a core-shell small particle can become nonresponsive under the acoustic radiation force in an ideal fluid. The particle is composed by a fluid core cloaked by an elastic or viscoelastic solid shell with considering its outer radius much smaller than the wavelength, e.g., the so-called Rayleigh scattering limit. By suitably choosing the core volume fraction (the core-to-shell volume ratio) one may suppress the radiation force. In case of a traveling plane wave, radiation force neutrality is related to scattering cancellation by the cloaking shell. However, neutrality is not achieved for absorptive cloaks. The somehow "intuitive" connection between scattering cancellation and radiation force suppression does not happen for a standing plane wave. Also, shell absorption does not change neutrality in this case. It is worth to mention that standing wave is the leit motiv of particle handling in different microfluidics applications. Several examples of radiation force neutrality are illustrated on a fluid core (air, fluorinert, and methanol) with a gold and polyethylene shell. In addition, we show that secondary radiation forces (Bjerknes force) in a many-particle system can also be suppressed by selecting an appropriate core volume fraction.

\section{Invited Papers}

\section{0:00}

3aPA5. Acoustomicrofluidics for micro-object Manipulation. Ghulam Destgeer and Hyung Jin Sung (Dept. of Mech. Eng., KAIST, Rm. 4120, N9, 291 Daehak-ro, Yuseong-gu, Daejeon, Daejeon 34141, Korea (the Republic of), destgeer@kaist.ac.kr)

Interaction of acoustic waves with fluids at a microscale have shown great potential in realizing efficient actuation of liquids for atomization/nebulization, mixing, pumping, gradient generation, etc. It has also enabled researchers working in various fields to dexterously manipulate suspended micro-objects—-microparticles, cells, microorganisms-for concentration, separation, sorting, medium exchange, etc. Surface acoustic waves (SAWs) — travelling as well as standing — have been used to demonstrate several of the above mentioned applications with great success. In particular, standing SAWs along with other bulk acoustic waves-based techniques have been proven to efficiently and continuously separate micro-objects inside a microfluidic channel. Our focus has so far been to explore the unexplored use of travelling SAWs to unravel some of the associated advantages. We have used high frequency travelling SAWs$O(100 \mathrm{MHz})$ - to separate microparticles flowing through a microchannel or suspended within a sessile droplet. Contrary to the formation of pressure nodes in standing SAWs for micro-object manipulation, traveling SAWs generate a strong acoustic streaming flow and impart a direct acoustic radiation force on the suspended particles for efficient manipulation capabilities with all the additional advantages of their non-invasive nature, label-free manipulation, biocompatibility, low costs, and simplicity in operation.

\section{0:20-10:35 Break}




\section{Contributed Paper}

$10: 35$

3aPA6. Temperature stable liquid gigahertz viscosity sensors by combining shear mode piezoelectric ScAIN thin film and AT-cut quartz crystal plate. Ko-hei Sano (Waseda Univ., 3-4-1 Ookubo, Shinjuku-ku, 6306-08B, Tokyo 169-8555, Japan, k-sano@fuji.waseda.jp), Takeshi Mori (Nagoya Inst. of Technol., Nagoya, Japan), Rei Karasawa, and Takahiko Yanagitani (Waseda Univ., Tokyo, Japan)

We here propose the temperature stable liquid $\mathrm{GHz}$ viscosity sensors by combining shear mode piezoelectric thin film and AT-cut quartz crystal plate. In the ultrasonic viscosity sensors, longitudinal wave cannot be used because the longitudinal wave energy leaked into the liquid. Therefore, shear wave is required for the viscosity measurement. We recently reported new large shear wave piezoelectricity in the c-axis tilted ScAlN films. In this study, the resonator type viscosity sensor consisting of c-axis tilted ScAlN films (approximately $7 \mu \mathrm{m}$ ) grown on an AT-cut quartz crystal plate were fabricated. Because the thickness of AT-cut quartz crystal is large enough compared to that of ScAlN film, temperature coefficient of resonant frequency is approximately determined by the extremely temperature stable quartz crystal. This enable robust measurement for the external temperature change. Increase of the viscous penetration depth between the liquid and the resonator interface induces the decrease of resonant frequency. The change of liquid viscosity can be then determined by this frequency shift. We observed strong shear wave excitation at the $403 \mathrm{MHz}$ by using a network analyzer in the air. When the sensor was immersed in the pure water, 50 $\mathrm{kHz}$ decrease of shear wave resonant frequency was clearly observed.

\section{Invited Paper}

\section{0:50}

3aPA7. Pattern deposition on an ultrasonic actuator. Ofer Manor (Chemical Eng., Technion - Israel Inst. of Technol., Technion, Haifa 3200003, Israel, manoro@technion.ac.il)

In recent years, one has observed an increasing interest in the pattern deposition of colloidal particles from an evaporating carrier liquid, a process associated with the coffee ring effect. In particular, various companies employ pattern deposition for the fabrication of medium resolution conducting circuits for various applications from touch screens to antennae. In this talk, I will discuss the pattern deposition of solute particles off an evaporating solution film atop an ultrasonic actuator. We use the actuator to generate a $\mathrm{MHz}$ vibration in the form of a Rayleigh surface acoustic wave (SAW), propagating in the solid substrate and under the solution film at a phase velocity of $4000 \mathrm{~m} / \mathrm{s}$. Transfer of momentum from the SAW to the neighboring solution translates to an acoustic stress, supporting various flow mechanisms, i.e., acoustic streaming, and altering the geometry of the evaporating film. The interplay between the capillary, acoustic, and evaporative stresses within the solution determines the transport dynamics of the solute particles in the solution and the state of pattern deposition on the solid substrate. Using different SAW power levels we alter the dynamic state of the deposition, changing the qualitative geometry of the deposited patterns from dots to stripes and from stripes to solid films.

\section{Contributed Paper}

11:10

3aPA8. Manipulation of microbubbles and targeted single cell sonoporation with by surface acoustic waves in a microfluidic device. Long Meng, Feiyan Cai, Juanjuan Chen, Lili Niu, Hairong Zheng (Paul C. Lauterbur Res. Ctr. for Biomedical Imaging, Inst. of Biomedical and Health Eng., Shenzhen Institutes of Adv. Technol., Chinese Acad. of Sci., ShenZhen, China), and Junru Wu (Phys., Univ. of Vermont, Cook Bldg., Burlington, VT 05405, jwu@uvm.edu)

A microfluidic device was developed to transport the aggregated microbubbles to a targeted cell at arbitrary location in liquid solution by introducing the phase-shift to a planar standing surface acoustic wave (SAW). The device consists of two perpendicular pairs of interdigital transducer (IDTs) and a polydimethyl-siloxane microchannel. By adjusting the relative phase between electric voltages applied to the independent IDTs, the microbubbles can be trapped, continuously moved or rotated due to the translation of pressure nodes. We further demonstrate that a SAW is capable of inducing microbubble cluster destruction at a desired location to achieve a single cell's reparable sonoporation. By controlling the position of the microbubble cluster relative to the targeted cell precisely, the effective size of the collapsing microbubbles is measured to be less than 0.68 times the diameter of microbubble cluster. The sonoporation efficiency and the cell viability are $82.4 \% \pm 6.5 \%$ and $90 \% \pm 8.7 \%$, respectively, when the targeted cell is within the effective microbubble destruction region.

\section{Invited Paper}

\section{1:25}

3aPA9. An emulsification system using a microchannel and a piezoelectric transducer. Takefumi Kanda (Graduate School of Natural Sci. and Technol., Okayama Univ., 3-1-1 Tsushima-naka, Kita-ku, Okayama 700-8530, Japan, kanda@sys.okayama-u.ac.jp)

Ultrasonic emulsification devices have been used in many industrial and scientific fields. Recently, a nanoemulsion which contains small droplets having a diameter of sub-micron scale have attracted attentions. In usual, those emulsions are produced by using ultrasonic transducers oscillated to generate a cavitation field. However, such process has a difficulty to avoid contaminations. For a continuous process, we have generated the nanoemulsion by using microchannel devices and a piezoelectric transducer. The devices are made of stainless steel mainly. The micron-size droplets generated by a Y-type or T-type microchannel device, and are oscillated by a piezoelectric transducer in another microchannel device. The driving frequency of the transducer is over $2.2 \mathrm{MHz}$. We have succeeded in generating sub-micron size droplets which contain anti-cancer drug using this emulsification system. Additionally, we have evaluated the acoustic field in the microchannel by using a micro cavitation sensor. The micro sensor made of a piezoelectric polymer has been installed in the device. The sensor consists of some films deposited and patterned with a photolithography process. Using this sensor, the acoustic state in the microchannel has been evaluated from the acoustic power spectrum. From the results, we can conclude that the cavitation effect is not dominant in the emulsification process by this system. 


\title{
Contributed Paper
}

11:45

3aPA10. Dynamic measurement of microbubble compressibility and interactions in an acoustofluidic device. Gianluca Memoli (Dept. of Eng., Computing and Design, School of Informatics, Eng. and Design, Univ. of Sussex, Brighton BN1 9RH, United Kingdom, g.memoli@sussex.ac.uk), Kate O. Baxter, Christopher R. Fury (Acoust. and Ionising Radiations, National Physical Lab., Teddington, United Kingdom), Philip H. Jones (Phys. and Astronomy, Univ. College London, London, United Kingdom), and Bajram Zeqiri (Acoust. and Ionising Radiations, National Physical Lab., Teddington, United Kingdom)

A key parameter for coated microbubbles in diagnostic and therapeutic applications is the non-linear response of their shell to acoustic pressures. Different measurements of this parameter exist, but most rely on the bubbles to be stuck on a surface or on the absence of acoustic excitation. In this work, we use the dynamic of coated microbubbles in an acoustofluidic device to measure the acoustic forces acting on them and the dynamic response of their shell to an increasing acoustic pressure. The device comprises of a microfluidic glass chip, where acoustical tweezers (operating in the range of $160-180 \mathrm{kHz}$ ) and optical tweezers can be used simultaneously. Comparing results from the calibrated optical tweezers, laser vibrometry and particle tracking allows a precise characterization of the acoustic field as a function of the driving voltage, at fixed frequencies and for pressures up to $4 \mathrm{kPa}$. Building on this result we extend the tracking technique to polymer-coated microbubbles and measure the acoustic forces acting on them over a larger range of pressures and frequencies (acoustic force spectroscopy). As pressure increases, we quantify secondary Bjerknes forces and present a dynamic characterization of microbubble compressibility with acoustic pressure. Finally, we present preliminary measurements on lipidcoated microbubbles.

\section{Session 3aPPa}

\section{Psychological and Physiological Acoustics and Speech Communication: Perspectives of Research in Overlooked Hearing Problems}

\author{
Shigeto Furukawa, Cochair \\ NTT Communication Science Labs, 3-1 Morinosato-Wakamiya, Atsugi 243-0198, Japan
}

Michael G. Heinz, Cochair

Speech, Language, and Hearing Sciences \& Biomedical Engineering, Purdue University, 500 Oval Drive, West Lafayette, IN 47907

Chair's Introduction-7:55

\section{Invited Papers}

8:00

3aPPa1. Predicting effects of hidden hearing loss using signal detection theory. Andrew J. Oxenham and Magdalena Wojtczak (Psych., Univ. of Minnesota, 75 E River Parkway, Minneapolis, MN 55455, oxenham@umn.edu)

Recent physiological studies in animals have shown that noise-induced "temporary threshold shift" can result in a large and permanent loss of synaptic connections between the inner hair cells and the auditory nerve, without any measurable change in absolute thresholds. As audiometric absolute thresholds are the current clinical standard for diagnosing human hearing loss, this form of synaptopathy may remain undiagnosed, or hidden, with its perceptual consequences remaining unclear. Baseline expectations for perceptual consequences can be derived from a simple application of the principles of signal detection theory using a set of simplifying assumptions, such as independent and equal information from each auditory nerve fiber, and optimal integration of information. This approach shows that quite dramatic losses (e.g., 50\%) lead to relatively small changes in predicted behavioral thresholds (1.5 dB in this case), which may not be easily detectable. However, synaptopathy that selectively affects specific neural populations, such as low-spontaneous-rate fibers, may result in larger and more measurable perceptual effects. Preliminary data from our lab also suggest that physiological measures, such as changes in wideband acoustic reflectance via the middle-ear muscle reflex, may provide a sensitive measure of synaptopathy in humans. [Work supported by NIH grant R01DC005216.] 
3aPPa2. Isolating cochlear synaptopathy in people with impaired audiograms: An auditory brainstem and envelope-following response modeling study. Sarah Verhulst, Viacheslav Vasilkov, Anoop Jagadeesh, and Markus Pelz (Oldenburg Univ., Carl-vonOssietzky Strasse 9-11, Oldenburg 26129, Germany, sarah.verhulst@uni-oldenburg.de)

Listeners with impaired audiograms likely suffer from mixtures of peripheral hearing pathologies that include outer-hair-cell loss and the more recently discovered cochlear synaptopathy. These mixed pathologies pose challenges for current audiological practice as objective brainstem EEG metrics might not be able to isolate these different hearing deficits. By simulating different degrees of outerhair-cell and auditory-nerve fiber loss in a functional model of the human auditory periphery, we studied which auditory brainstem $(\mathrm{ABR})$ and envelope-following response (EFR) measures and stimuli are most sensitive to either type of hearing deficit. We validated our model predictions using recordings from 32 listeners with normal or elevated hearing thresholds. The simulations show that high-frequency outer-hair-cell loss steepens the ABR wave-V latency-vs-intensity curve as well as the wave-V amplitude-vs-intensity curve, such that grouping listeners according to the ratio of these metrics (i.e., the ABR growth ratio) offers a way to factor out the outer-haircell aspect of hearing loss. Individual differences within an ABR growth ratio group are sensitive to other aspects of peripheral hearing loss such as cochlear synaptopathy. We furthermore simulated EFRs to pure-tone and broadband stimuli of different modulation depths to study which EFR metrics are sensitive to synaptopathy in hearing impaired listeners.

\section{8:40}

3aPPa3. Characterizing impairments in compression and filter shape to establish their role in hidden hearing loss. Toshio Irino, Toshie Matsui (Wakayama Univ., 930 Sakaedani, Wakayama 640-8510, Japan, irino@sys.wakayama-u.ac.jp), and Roy D. Patterson (Univ. of Cambridge, Cambridge, United Kingdom)

In the study of hidden hearing loss, it is important to characterize an individual's peripheral losses accurately while minimizing measurement time for clinical applications. In this talk, we show how peripheral losses involved in compression and auditory filtering can be estimated simultaneously and accurately using the compressive GammaChirp (cGC) filter and notched noise (NN) maskers. Sets of thresholds were gathered with asymmetric maskers and fitted with the cGC filter model using several constraints associated with cochlear filtering. The slopes of the resultant input-output functions were within the range established with temporal masking curves above $1000 \mathrm{~Hz}$. Studies of the fitting process also revealed how to reduce the number of threshold measurements without unduly increasing estimation error. These improvements led to the extension of the cGC filter model to explain hearing impairment of cochlear origin. The model also led to a hearing impairment simulator which can analyze everyday sounds including speech and music with a given individual's cochlear hearing impairment, and then resythesize the sounds to provide a normal hearing listener with a realistic experience of the sound perception problems facing that hearing impaired individual. By isolating peripheral losses, the simulator will assist the specification of hidden hearing losses.

3aPPa4. Conversion of amplitude modulation to phase modulation on the basilar membrane and its implication in the perceptual consequences of disrupted temporal coding. Sho Otsuka and Shigeto Furukawa (NTT Commun. Sci. Labs., 3-1, Morinosato Wkamiya, Atsugi-shi, Kanagawa Pref. 243-0198, Japan, otsuka.s@lab.ntt.co.jp)

Disrupted fidelity of temporal-structure representation by auditory-nerve (AN) firing is a key component of hidden hearing loss. However, impacts of disturbed temporal processing on everyday listening are yet to be identified. Here we propose a new role of the AN's temporal processing, namely, encoding amplitude modulation (AM). When an AM signal with a constant-frequency carrier is fed to a generic nonlinear amplifier, the carrier phase of the output signal can be modulated, a phenomena referred to as AM-to-PM conversion. By simulating the basilar-membrane (BM) vibration and measuring otoacoustic emissions, we demonstrated that the AM-to-PM conversion takes place on the BM, too. The size of AM-induced PM depends on the stimulus level, which can be accounted for by the characteristics of BM's compressive nonlinearity. Psychoacoustic measurements further indicated the association between the listenerdependent patterns of the level dependence of AM-induced PM and of AM detection performance. These results imply that the AMinduced PM on the BM can be used as a cue for AM processing in the auditory system and that disrupted AN sensitivity to PM should have negative impacts on AM processing.

\section{Contributed Paper}

9:20

3aPPa5. Hidden hearing loss and computational models of the auditory pathway: Predicting speech intelligibility decline. Christopher Smalt, Thomas F. Quatieri (MIT Lincoln Lab., 244 Wood St., Lexington, MA 02420, Christopher.Smalt@11.mit.edu), and Mark A. Parker (Steward St. Elizabeth Medical Ctr., Brighton, MA 02135)

Recent animal studies have shown that noise exposures cause a permanent loss of low spontaneous rate auditory nerve fibers (ANFs) and reduction of auditory brainstem response wave-I amplitudes (Kujawa and Liberman, 2009). This phenomenon is thought to create difficulties understanding speech in noise in humans, although there is currently no established clinical technique to measure cochlear synaptopathy. The goal of this research is to utilize computational models of the auditory periphery and auditory cortex to study the effect of low spontaneous rate ANF loss on the cortical representation of speech intelligibility in noise. The auditory-periphery model of Zilany et al. $(2009,2014)$ is used to make predictions of auditory nerve responses to speech stimuli in noise. The resulting cochlear neurogram, a spectrogram-like output based on ANF outputs, is then used as a foundation for two different but related cortical representations of speech: the Spectro-Temporal Modulation Index (STMI; Elihali et al., 2003) and 2D Fourier Analysis (Wang and Quatieri., 2012). Reducing the number of low spontaneous rate ANFs in the cochlear neurogram was found to cause a blurring of speaker-specific cortical components, increasing the difficulty of speaker separation in the time-frequency modulation domain. Suprathreshold deficits in speech intelligibility, as simulated by auditory pathway models, may be predicting the effect of ANF loss or degradation on listening performance, and could potentially be used as part of a diagnostic tool. 


\section{Invited Papers}

3aPPa6. Effects of hidden hearing loss on the representation of speech-in-noise in the gerbil auditory midbrain. David McAlpine, Jaime Undurraga (Linguist, Macquarie Univ., Australian Hearing Hub, 16 University Ave., Sydney, NSW 2109, Australia, david.mcalpine@mq.edu.au), Jose Garcia-Lazaro, and Roland Schaette (Ear Inst., Univ. College London, London, United Kingdom)

Increasing evidence indicates that noise exposure selectively damages high-threshold auditory nerve fibres, in the absence of damage to sensory hair cells. This "hidden hearing loss (HHL)"—undetected by conventional tests such as audiometry-is suggested to account for undiagnosed difficulties processing speech in background noise. Here, we demonstrate in the midbrain of gerbils exposed to a single, controlled noise insult, and in human listeners, evidence of increased neural gain in the central auditory pathways, indicative of damage to high-threshold ANFs following noise exposure. Neural responses were higher, and discrimination performance for 60-dB SPL "vowel-consonant-vowel" stimulus (VCVs in background noise (speech-shaped, +12 to $-12 \mathrm{~dB}$ signal-to-noise ratio) enhanced, in noise-exposed animals compared to controls. Conversely, for 75-dB SPL VCVs, neural discrimination was better in control, compared to noise-exposed, animals. A similar pattern was evident in human listeners, and was positively correlated with the ratio of wave $\mathrm{V}$ (generated by the midbrain) to wave I (generated by the auditory nerve) of the auditory brainstem response. The data are consistent with the hypothesis that reduced neural output from the cochlea at higher sound levels increases central gain through homeostatic mechanisms that seek to normalize neural activity.

\section{9:55-10:15 Break}

\section{0:15}

3aPPa7. No evidence for hidden hearing loss due to noise exposure in young adults with a normal audiogram. Karolina Kluk, Garreth Prendergast, Hannah Guest, Kevin J. Munro, Agnès Léger (Manchester Ctr. for Audiol. and Deafness (ManCAD), The Univ. of Manchester, Oxford Rd., Manchester m139pl, United Kingdom, karolina.kluk@manchester.ac.uk), Deborah A. Hall (National Inst. for Health Res. (NIHR) Nottingham Hearing Biomedical Res. Unit, Nottingham, United Kingdom), Michael Heinz (Dept. of Speech, Lang., \& Hearing Sci., Purdue Univ., West Lafayette, IN), and Christopher J. Plack (Manchester Ctr. for Audiol. and Deafness (ManCAD), The Univ. of Manchester, Manchester, United Kingdom)

Cochlear synaptopathy, or "hidden hearing loss," refers to a loss of synapses between inner hair cells and auditory nerve fibers, and affects primarily low-spontaneous-rate fibers that encode moderate-to-high intensity sounds. In rodent models, cochlear synaptopathy has been shown to occur as a result of noise exposure even when threshold sensitivity is unaffected. We tested 126 young humans with normal audiograms and a wide range of lifetime noise exposures quantified using a questionnaire. Our electrophysiological and behavioral measures were designed to be sensitive to the contribution of low-spontaneous-rate fibers. We predicted that our test battery would reveal that noise exposure is associated with attenuated electrophysiological responses, and elevated behavioural thresholds, especially at higher levels and frequencies. However, there was no relation between noise exposure history and auditory brainstem response wave I amplitude, nor performance on psychophysical tasks including modulation detection, inter-aural phase difference discrimination, frequency discrimination, and intensity discrimination. There was also no relation between noise exposure and speech-in-noise performance nor self-report listening ability. Only the $16 \mathrm{kHz}$ audiometric thresholds were correlated positively with noise exposure for females, but not for males. Our results suggest that cochlear synaptopathy due to noise exposure is not easily identifiable in young humans with a normal audiogram. [This work was supported by the MRC UK (MR/L003589/1).]

\section{$10: 35$}

3aPPa8. Functional hearing impairments in normal-hearing listeners with a history of deployment-related blast exposure. Douglas S. Brungart (Walter Reed NMMC, 8901 Wisconsin Ave., Bethesda, MD 20889, douglas.brungart@us.army.mil), Lina Kubli (Army Public Health Ctr. (Provisional), Bethesda, MD), Sandeep Phatak, Melissa J. Kokx-Ryan, Lynn M. Bielski, LeeAnn Horvat, and Kenneth W. Grant (Walter Reed NMMC, Bethesda, MD)

Over the past decade, DoD and VA audiologists have reported large numbers of relatively young adult patients who have normalaudiometric thresholds but who report greater than expected difficulty understanding speech in noisy environments. One common theme in the history of these patients is that they self-report having experienced exposure to explosive blast as part of their military service. Recent studies in our laboratory suggest that some blast-exposed patients with normal-hearing thresholds have greater than expected difficulty in tasks that require spatial sound perception (auditory localization and $\mathrm{N}_{0} \mathrm{~S}_{\pi}$ tone detection) or those that involve the perception of time-compressed speech in reverberant environments. They also tend to report more hearing difficulties compared to non-blast exposed patients on a questionnaire that evaluates spatial perception, speech perception, and perceived sound quality. These laboratory results appear to be confirmed by preliminary results from roughly 2000 active-duty service members who participated in a brief hearing test and questionnaire at the time of their annual hearing test. Within this sample, blast-exposed listeners were 3-4 times more likely to experience subjective or objective hearing deficits than those with no blast history. These results suggest a form of hidden hearing loss that may be associated with exposure to blast. [The opinions presented are the private views of the authors and are not to be construed as official or as necessarily reflecting the views of the DoD.] 
3aPPa9. Effects of aging on the absolute pitch judgment and frequency following responses of electroencephalograms. Minoru Tsuzaki, Satomi Tanaka (Kyoto City Univ. of Arts, 13-6 Kutsukake-cho, Oe, Nishikyo-ku, Kyoto 610-1197, Japan, minoru.tsuzaki@ kcua.ac.jp), and Junko Sonoda (none, Weimar, Germany)

Absolute pitch (AP) possessors can name the pitch class of the note simply by hearing a periodic tone. It has been reported that the AP judgment can shift by one or two semitones when AP possessor become old. We confirmed this age-related AP shift by a series of psychophysical experiments with piano sounds as well as synthesized complex tones. AP possessors whose ages ranged from 20s to 50s participated in the experiments. The results showed that the aged participants were likely to assign the higher pitch classes than young participants for the piano sounds. Similar tendencies were observed for the synthesized, complex sounds if they contained lower order harmonics. The hearing levels, OAEs, and the frequency following responses (FFRs) of the EEG were also measured for the same participants. The hearing levels and OAE levels which could be indices in the cochlear mechanical properties could not explain the age-related AP shift. The phase locking values of the FFRs obtained at 98 and $220 \mathrm{~Hz}$ could neither be a good predictor of the AP shift. Although no plausible model to predict it exists, the age-related AP shift might bring another route of overlooked hearing problems.

\section{Contributed Paper}

\section{1:15}

3aPPa10. Discrimination and identification of environmental sounds among people with hearing impairment. Yuuki Yuno, Masaki Matsubara (Library Information and Media Studies, Univ. of Tsukuba, 1-2, Kasuga, Tsukuba, Ibaraki 305-8550, Japan, s1621640@u.tsukuba.ac.jp), Kei Tabaru (Education, Ibaraki Univ., Mito, Japan), Hiroko Terasawa (Library Information and Media Studies, Univ. of Tsukuba, Tsukuba, Japan), and Rumi Hiraga (Industrial Information, Univ. of Technol., Tsukuba, Japan)

We report that people with hearing impairment (HI) can better discriminate environmental sounds than identifying the same sounds. In order to investigate the perception/recognition of environmental sounds by people with HI, researchers often choose identification task as a measurement tool. However, since identification ability is developed upon discrimination ability, the discrimination ability should be studied too. We used 9 environmental sounds as stimuli for both discrimination and identification tasks, and 16 participants with $\mathrm{HI}$ joined the experiment. In the discrimination task, the participants listened to the paired short stimuli, and reported the perceived difference. In the identification task, the participants listened to each stimulus and reported the name of the sound source. The order of discrimination and identification tasks is counterbalanced among the participants, and we also tested 10 people with normal hearing for comparison. The results showed a clear contrast between the discrimination and identification tasks by people with HI. People with HI outperformed in discrimination tasks (97.0\% correct answer on average) than in identification tasks ( $35.5 \%$ correct answer on average). This means that people with HI can discriminate most of the sounds that they cannot identify, suggesting the potential for the acquisition of identification ability.

\section{Invited Paper}

\section{1:30}

3aPPa11. Individualized assessment of suprathreshold hearing and relationship to cochlear synaptopathy. Hari M. Bharadwaj (Athinoula A. Martinos Ctr. for Biomedical Imaging, Massachusetts General Hospital, 149 Thirteenth St., Boston, MA 02129, hari@ nmr.mgh.harvard.edu), Leonard Varghese, Golbarg Mehraei (Ctr. for Computational Neurosci. and Neural Technol., Boston Univ., Boston, MA), Christopher A. Shera (Eaton Peabody Lab. of Auditory Physiol., Massachusetts Eye and Ear Infirmary, Boston, MA), and Barbara G. Shinn-Cunningham (Ctr. for Computational Neurosci. and Neural Technol., Boston Univ., Boston, MA)

Threshold audiometry is currently the foundation of clinical audiology. Yet, many individuals with normal hearing thresholds (NHTs) have difficulty communicating in noisy settings. We previously documented that even among listeners with NHTs and no hearing complaints, large individual differences exist in the ability to perceive subtle temporal features of clearly audible sounds, and to selectively process target speech in the presence of competing sounds. Critically, we find that these suprathreshold differences in individual perceptual sensitivity correlate with physiological measures from the brainstem and auditory nerve. These measures, including brainstem envelope-following responses, auditory brainstem responses, and the middle-ear muscle reflex, show that some perceptually relevant physiological differences arise very early along the auditory pathway. Cochlear synaptopathy—a loss of afferent synapses and nerve terminals innervating the cochlea-is well documented in noise-exposed and aging animals (Kujawa \& Liberman, Hear. Res. 2015). Cochlear synaptopathy in humans may account for both large individual differences and hearing difficulties in human listeners with NHTs. This presentation will summarize updated human evidence consistent with cochlear synaptopathy, discuss some of the challenges that remain with this interpretation, and review the search for clinical markers of such damage. 


\title{
Session 3aPPb
}

\section{Psychological and Physiological Acoustics: From Cochlea to Cortex (Poster Session)}

\author{
Olga Stakhovskaya, Chair \\ Audiology and Speech Center, Walter Reed National Military Medical Center, 4954 North Palmer Road, Bldg. 19, R. 5607, \\ Bethesda, MD 20889
}

\begin{abstract}
All posters will be on display from 8:00 a.m. to 12:00 noon. To allow contributors in this session to see the other posters, authors of odd-numbered papers will be at their posters from 8:00 a.m. to 10:00 a.m. and authors of even-numbered papers will be at their posters from 10:00 a.m. to 12:00 noon.
\end{abstract}

\section{Contributed Papers}

3aPPb1. In-vivo intra-cellular characterization of "OFF" responses in the superior para-olivary nucleus. Tom P. Franken (Univ. of Leuven, Leuven, Belgium), Mark Sayles (Purdue Univ., West Lafayette, IN), Philip H. Smith (Univ. of Wisconsin - Madison, Madison, WI), and Philip X. Joris (Univ. of Leuven, University of Leuven, Leuven, Belgium, philip.joris@ med.kuleuven.be)

The superior para-olivary nucleus (SPN) is an auditory-brainstem OFF channel. Typically, SPN neurons are inhibited during sound presentation and fire at sound offset. Release from sound-induced inhibitory potentials is the hypothesized mechanism, but this has not previously been directly observed in vivo. We obtained in vivo axonal recordings from 33 SPN neurons in the chinchilla, and patch recordings from 17 SPN cell bodies in the Mongolian gerbil. We retrieved several cells anatomically by labeling with biocytin or neurobiotin. Labeled SPN neurons had large dendritic trees, and axons heading into the lateral lemniscus. Their responses typically showed offset spiking to contralateral, and sometimes to ipsilateral stimulation. Spikes in response to amplitude-modulated tones were highly synchronized, up to hundreds of $\mathrm{Hz}$ (vector strength $>0.9$ ). Some SPN neurons were ITD sensitive, either to envelope or low-frequency fine-structure components. Patch-clamp recording allowed us to study sub-threshold potentials. Large IPSPs were generated by contralateral, and often also by ipsilateral sounds, with few EPSPs. Individual phase-locked IPSPs were discernable to lowfrequency tones. High-frequency tones elicited a large onset IPSP, followed by sustained inhibition. For amplitude-modulated tones, IPSPs were locked to the stimulus envelope, with spikes at the offset of inhibitory events.

3aPPb2. Against "broadening with sound level". Mark Sayles (Purdue Univ., Purdue University, West Lafayette, IN 47907, sayles.m@gmail. com), Bertrand Fontaine (Univ. of Leuven, Leuven, Belgium), Philip H. Smith (Univ. of Wisconsin - Madison, Madison, WI), and Philip X. Joris (Univ. of Leuven, Leuven, Belgium)

Spectral resolution is fundamental to audition. Auditory-nerve-fiber responses provide a window onto cochlear tuning. Typically, resolution is quantified using threshold tuning curves. Superficially, these suggest dramatic and systematic filter broadening with increasing intensity, a view often invoked to explain psychoacoustic phenomena. We recorded auditorynerve-fiber spike times from normal-hearing chinchillas in response to 0.01 $20 \mathrm{kHz}$ wideband noise. We gathered data over a wide intensity range from each fiber. Linear filter estimates were obtained at each level by reverse correlation. We characterized their magnitude and phase response. We considered responses from 175 fibers (CF range: [0.1, 3.0] kHz; all SR groups), with noise level varying over a 40 to $100-\mathrm{dB}$ range. $10-\mathrm{dB}$ bandwidth varied minimally with sound level over a 40 to 50 -dB range. Some fibers demonstrated phase pivoting with level; however, most did not follow this simple scheme. Common teaching is "auditory filters broaden with level," usually in reference to threshold tuning curves. Here, gain functions constructed from responses to a fixed-level input show this broadening is not as severe as threshold tuning curves might suggest. Broadening is very subtle over a large range of "low" to "mid" input intensities $(<70-\mathrm{dB}$ SPL), before rapidly increasing at "high" levels ( $>80-\mathrm{dB}$ SPL).

3aPPb3. The efficiency of the cytoarchitecture of the organ of Corti for active cochlear amplification. Hamid Motallebzadeh and Sunil Puria (Eaton Peabody Lab., Harvard Med. School, 243 Charles St., Boston, MA 02114,Hamid_Motallebzadeh@meei.harvard.edu)

A " $Y$ "-shaped structure of cells formed by the basally tilted force generating outer hair cells (OHC) and apically tilted passive phalangeal process $(\mathrm{PhP})$ connected to a Deiter cell (DC) has been hypothesized to be an essential organ of Corti $(\mathrm{OoC})$ building block for cochlear amplification (Yoon et al., 2011). We developed a COMSOL finite-element model of the mouse cochlea, taking into account the spatial arrangement of $Y$-shaped elements and the 3-D fluid-structure interaction. The model was validated by comparison with previously reported basilar membrane (BM) displacement for both passive and active cases (Lee et al., 2015). The baseline model can reproduce an increase of $40 \mathrm{~dB}$ gain of the BM (re stapes velocity) for low level sounds. However, the absence of the $\mathrm{PhP}$ results in an unstable response of the $\mathrm{BM}$ for active cases. The model with the roles of the $\mathrm{PhP}$ and $\mathrm{OHC}$ switched, could not reproduce the active gain. Finally, the model with reversed titling orientation of $\mathrm{OHC}$ and $\mathrm{PhP}$ cells generated significantly lower gains (specially for lower frequencies), but also results in broader resonance peaks. These results demonstrate the efficiency of the natural cytoarchitecture of the OoC. [Work supported by NIH grant R01 DC 07910.]

3aPPb4. Simulating cochlear nonlinearity in an excised, active in vitro segment. Amir Nankali, Aritra Sasmal, and Karl Grosh (ME Dept., Univ. of Michigan, Ann Arbor, MI 48105, nankali@umich.edu)

Hearing relies on a series of coupled acoustical, electrical, and mechanical interactions in the auditory periphery. There exists a distinct nonlinear amplification process inside the cochlea that enables sound processing of a broad range of frequencies and intensities. The precise operating principles of the active mechanics underlying nonlinear cochlear amplifier remains an open question in biophysics. Using the experimental protocol devised by Chan \& Hudspeth of an excised cochlear segment as a model problem, we develop a computational model for studying the active in vitro response of the organ of Corti $(\mathrm{OoC})$ to acoustical stimulation. Both experiment and theory show that there exists a traveling wave even for a very small, $700 \mathrm{~mm}$ cochlear segment. However, we show that the contribution of the traveling wave on the partition dynamics is insignificant in this preparation as the phase accumulation is less than one-tenth of a cycle. This finding enables us to reduce the macroscopic fluid dynamics of the configuration to a loading added mass on the OoC and study the cochlear local dynamics. The two hypothesized active mechanisms of the cochlea (hair bundle motility and somatic electromotility) are included and the contribution of each mechanism on the cochlear amplifier is investigated. It is observed that in our model the $\mathrm{OHC}$ somatic electromotility is sufficient to predict the experimental results. [This work was supported by NIH grants DC-004084 and T32DC-00011.] 
3aPPb5. Evolutionary elongation of the time window of auditory cortical processing: Comparison of effects of stimulus time parameters on human and macaque scalp auditory evoked potentials. Kosuke Itoh (Univ. of Niigata, Asahimachi 1-757, Niigata 951-8585, Japan, itoh@bri. niigata-u.ac.jp), Masafumi Nejime, Naho Konoike, Katsuki Nakamura (Kyoto Univ., Inuyama, Japan), and Tsutomu Nakada (Univ. of Niigata, Niigata, Japan)

Auditory cortex integrates sound signals over time for obtaining neural representations of auditory events, the time window of which affects perception and cognition. Species differences between human and macaque monkey in the time window of auditory cortical processing were investigated by comparing (1) how the cortical auditory evoked potentials (CAEP) increase in amplitude with the duration of a sound $(2-200 \mathrm{~ms})$, and (2) how the CAEPs to a repetitive stimulus train recover from neural adaptation with an increase in interstimulus silent interval $(30-1620 \mathrm{~ms})$. A virtually identical noninvasive scalp recording method was used in the two species for rigorous comparisons. First, the integration windows for the human N1 and N2 were $50 \mathrm{~ms}$ or longer, but the macaque $\mathrm{N} 1(\mathrm{mN} 1)$ and macaque $\mathrm{N} 2(\mathrm{mN} 2)$ were elicited with their full amplitudes by a stimulus as short as $2 \mathrm{~ms}$. Second, the human N1 to a repeated stimulus continued to recover from neural adaptation as the interstimulus interval increased to $1620 \mathrm{~ms}$, and a clear elicitation of $\mathrm{N} 2$ required an interval longer than $500 \mathrm{~ms}$; in the macaque, by contrast, $\mathrm{mN} 1$ fully recovered from adaption by an interval shorter than $1 \mathrm{~s}$, and a clear $\mathrm{mN} 2$ was elicited with a $30 \mathrm{~ms}$ gap. These findings are consistent an evolutionary elongation of the time window of human auditory cortical processing, which enables integrating greater amount of auditory information over time to obtain neural representation of complex auditory features that characterize language and music.

3aPPb6. Role of dopamine in learning beyond reinforcement: Boost of auditory learning and transfer by orally taken or gameplay-generated dopamine. Dinglan Tang (National Key Lab. of Cognit. Neurosci. and Learning, Beijing Normal Univ., Rm. 327, Yingdong Bldg., Beijing 100875, China, dinglantang@sina.com), David R Moore (Commun. Sci. Res. Ctr., Cincinnati Children's Hospital Medical Ctr., Cincinnati, OH), Sygal Amitay (Medical Res. Council-Inst. of Hearing Res., Nottingham, United Kingdom), Jun-Yun Zhang (Psych. Dept., Peking Univ., Beijing, China), and Yu-Xuan Zhang (National Key Lab. of Cognit. Neurosci. and Learning, Beijing Normal Univ., Beijing, China)

We showed here that playing a video game, Tetris, in silence enhanced learning of contiguous auditory perceptual training and its transfer to working memory. The effect could not be explained by game play per seor the visual-spatial training involved, but rather resulted from across-time and modality interaction between game play and target training. The results led us to propose that game play releases reward signals like dopamine in the brain, which serves as a biological marker for significance of perceptual experiences and regulates learning. Consistent with this hypothesis, orally taking Madopar, a dopamine precursor, before training showed similar learning-enhancing effect as Tetris play. Madopar did not influence working memory training, indicating that the dopamine effect was perceptually but not cognitively mediated. Taken together, the results support the learningregulating theory of dopamine, and point to a promising approach for increasing the efficiency and applicability of rehabilitative training with rewarding events like game play.

3aPPb7. Spatial relationship between two sounds in an oddball paradigm affects responses of neurons in the auditory midbrain to the two sounds. Chirag Patel and Huiming Zhang (Dept. of Biological Sci., Univ. of Windsor, 401 Sunset Ave., Windsor, ON N9B3P4, Canada, hzhang@uwindsor.ca)

Neural sensitivity to a novel sound (i.e., an occasionally occurring acoustic event) is important for hearing in a natural acoustic environment. An oddball paradigm is used to study this sensitivity. Such a paradigm is a quick succession of acoustic stimuli, with each stimulus being one of two qualitatively different sounds presented at respective probabilities (i.e., high probability standard sound and low probability deviant sound). We studied action potential discharges in the rat's auditory midbrain in response to oddball paradigms presented from free-field loudspeakers. Our results indicated that many neurons generated a stronger response to a sound presented as a deviant than as a standard stimulus when two sounds in an oddball paradigm were co-localized at the frontal midline. For many neurons, the response to a sound presented at frontal midline (either as a standard or a deviant sound) was enhanced or suppressed by relocating the other sound in an oddball paradigm from frontal midline to an off-midline angle. At extreme angles of separation, the number of neurons showing suppression exceeded that showing enhancement. Thus, a spatial separation between two sounds in an oddball paradigm affects responses to both sounds. Population results suggest that co-localization of two sounds helps maintaining neural sensitivity to both sounds.

3aPPb8. Human electrophysiology of endogenous auditory spatial attention. Erol J. Ozmeral, David A. Eddins, and Ann C. Eddins (Commun. Sci. and Disord., Univ. of South Florida, 3802 Spectrum Blvd., Ste. 210, Tampa, FL33612, eozmeral@usf.edu)

Whereas perceiving the location of a stimulus in space relies on unique bottom-up processing, focusing attention to that location requires the availability and use of separate higher-level processes. It is unclear from previous work how selective attention might mediate and possibly sharpen spatial tuning, as behavioral tasks often lack an unattended comparison, and electrophysiological tasks often lack an attended comparison. We measured evoked responses to changes in the location of continuous noise in the free field when young, normal-hearing listeners were either passively listening or explicitly attending to one speaker location. Stimuli were presented from 1 of 5 frontal loudspeaker locations in the horizontal plane for $1.6 \mathrm{~s}$ before switching to another loudspeaker without pause. To ensure active attention for certain blocks, listeners were directed to press a button at the onset of a sound presented from the target location. Results show evidence of both facilitation for attended locations and suppression for unattended locations when compared to passive listening, and hemispheric distribution of activity was hemifield specific. Together, the data provide electrophysiological measures of a listener's left-right orientation and another step toward cortical models of auditory spatial attention.

3aPPb9. Machine classification of P1-N1-P2 responses elicited with a gated syllable. David J. Morris (Dept. of Nordic Studies and Linguist, Univ. of Copenhagen, Njalsgade120, Copenhagen-S 2300, Denmark, dmorris@hum.ku.dk) and Sigrid Klerke (Ctr. for Lang. Technol., Univ. of Copenhagen, Copenhagen-S, Denmark)

Onsets are generally considered to play an important role in the determination of syllable identity by human listeners. We measured P1-N1-P2 event-related potentials (ERP) from subjects $(n=11)$ in response to the syllable [sdкә]. The complex onset of this syllable was sequentially gated at the consonant boundaries to yield three distinct tokens. Group ERPs to the three tokens were most different within a narrow latency window around $160 \mathrm{~ms}$. This latency corresponded approximately to the lower portion of the ensemble N1-P2 deflection. Single-trial samples from the vertex channel at a range of poststimulus latencies around this point were submitted to multi-class classification by a support vector machine. The three tokens were used as class labels in a one-versus-one machine with a radial basis function kernel. Classification was trained and tested on subsets of the ERP data, and precision was found to be significantly better than chance. As the auditory stimuli used in this experiment differed only in initial consonant, these results indicate that syllable onset information is borne in the N1-P2 vertex response. They also demonstrate that a machine learning algorithm may be useful in retrieving syllable identity from single-trial ERP data.

3aPPb10. Investigation of the processing of ambiguous melodies in the brain by behavioral and neurophysiological experiments. Ryosuke Yuhara (Dept. of Information Environment, Tokyo Denki Univ., 2-1200, Muzaigakuendai, Inzai, Chiba 270-1382, Japan, 15jkm25@ms.dendai.ac. jp)

Melody recognition is not a mere succession of pitch and time perception but must be an active process to a make a meaningful line of tones. To investigate this process, we devised a form of illusory melody resembling but different from the ones used in the studies of streaming. A4 and E5 tones 
are continuously presented while another tone $\mathrm{M}$ (middle tone, for example, C5 or C\#5) between the two continuous tones is presented intermittently ( $0.25 \mathrm{~s}$ presentation and $0.25 \mathrm{~s}$ rest and repeating). One may hear either an illusory continuous melody of repeated $\mathrm{M}_{\mathrm{E} 5}$ or $\mathrm{M}_{\mathrm{A} 4}$ depending on the preceding unambiguous phrase of repetition of $\mathrm{M}_{\mathrm{E} 5}$ or $\mathrm{M}_{\mathrm{A} 4}$. Assuming that this illusion reflects the active process of melody formation, we investigated 1) the transition time from the primary state (either one of the melody forms) fixed by the leading phrase behaviorally, and 2) possible neural correlates by measuring the MEG (magnetoencephalography) responses to the continuous tones separately by amplitude modulating them with different frequencies. MEG signal was filtered for the two modulation frequencies and the ASSR (auditory steady state responses) were obtained for different experimental conditions. The results so far showed wide variance among subjects but seem promising.

3aPPb11. Outer hair cell function in human ultrasonic perception. Tadao Okayasu, Tadashi Nishimura, Akinori Yamashita, Osamu Saito, Toshiaki Yamanaka, Hiroshi Hosoi, and Tadashi Kitahara (OtolaryngologyHead and Neck Surgery, Nara Medical Univ., 840, Shijo-cho, Kashihara, Nara 634-8522, Japan, tokayasu@naramed-u.ac.jp)

Ultrasound can be heard by bone conduction. It is hypothesized that this bone-conducted ultrasound (BCU) is perceived in the basal turn of the cochlea and is a result of direct ultrasonic stimulation of inner hair cells via basilar membrane vibrations without cochlear amplifier. In order to clarify the contribution of outer hair cell function to ultrasonic perception, the present study evaluated changes in hearing sensitivity for BCU and air-conducted audible sound before and after ototoxic treatment. Twenty participants (40 ears) who were undergoing chemoradiation therapy with cisplatin participated in this study. After the treatment, $62.5 \%$ ears were diagnosed with hearing loss according to the criteria of the American Speech-LanguageHearing Association. The reduction of sensitivity to air-conducted audible sound occurred in the high-frequency range, which is thought to be important for ultrasonic perception. Surprisingly, BCU sensitivity was significantly improved after the treatment. These results suggest that direct ultrasonic stimulation of the cochlea, without audible sound generation, induces ultrasonic perception. The improvement of BCU sensitivity following the cisplatin administration may be explained by hypersensitivity associated with outer hair cells' disorder.

3aPPb12. Chord consonance represented in the long-lasting sustained activity in rat auditory cortex. Tomoyo I. Shiramatsu and Hirokazu Takahashi (the Univ. of Tokyo, 4-6-1, Komaba, Meguro-ku, Tokyo 1538904, Japan, isoguchi@brain.imi.i.u-tokyo.ac.jp)

Many sounds in the environment have a rich sound spectrum, which is related to the qualia of the sound such as consonance or dissonance of chords. However, the neural representation of chord consonance is not fully understood. Here, we targeted long-lasting, sustained activities in auditory cortex and investigated whether band-specific power or phase locking value (PLV) represent consonance of the chord consisting of two pure tones. A microelectrode array with 96 recording sites recorded local field potentials (LFPs) in the fourth layer of the auditory cortex of anesthetized rats in response to continuous pure tones and chords. From the LFPs, band-specific powers and PLVs were calculated in 5 bands (theta, 4-8 Hz; alpha, 8-14 $\mathrm{Hz}$; beta, 14-30 Hz; low gamma, 30-40 Hz; high gamma, and 60-80 $\mathrm{Hz}$ ). Then, differences of band-specific power and PLV ( $\Delta$ power and $\triangle \mathrm{PLV}$ ) of a chord with respect to those of the higher-frequency composition tone of the chord were compared between consonance and dissonance. Consequently, $\Delta$ power was significantly smaller, and $\triangle \mathrm{PLV}$ was significantly larger for consonance than dissonance, suggesting that the long-lasting, sustained activity in the auditory cortex is modulated according to the complex sound spectrum, such as consonance of the chords.
3aPPb13. Auditory brainstem responses to anechoic and reverberant two-syllable speech sounds in elderly listeners. Haruna Fujihira (Graduate School of Design, Kyushu Univ., 3-1, Morinosato Wakamiya, Atsugi-shi 243-0198, Japan, fujihira_haruna_a7@lab.ntt.co.jp), Kimio Shiraishi, and Gerard B. Remijn (Faculty of Design, Kyushu Univ., Fukuoka, Japan)

Elderly listeners often complain of difficulty in understanding speech under reverberation. To investigate speech processing in elderly listeners' auditory pathways, some studies have focused on auditory brainstem responses to speech sounds (speech ABRs). In an earlier study, we measured speech ABRs to the speech sound /da/ in elderly listeners and reported that listeners with low word intelligibility under reverberation showed small amplitudes in their speech ABRs to a formants frequency of/da/ (Fujihira \& Shiraishi, 2015). Reverberation is a phenomenon that causes speech sounds to overlap with following speech sounds. Therefore, the effects of reverberation on multiple syllables might be larger than those on one syllable. In present study, we measured the speech ABRs to the two-syllable sound /dada/ in 27 elderly listeners and investigated the difference between the speech ABRs to the first syllable and the second syllable in anechoic and reverberant conditions. The results showed that the amplitudes of speech ABRs to the first syllable decreased compared to those to the second syllable in the anechoic condition, but both amplitudes to the first syllable and the second syllable in the reverberant condition decreased almost equally.

3aPPb14. Investigating the contribution to anti-masking by the inhibitive networks in the dorsal cochlear nucleus: Modeling and simulation. Tzu-Chi Liu and Yi-Wen Liu (Elec. Eng., National Tsing Hua Univ., Rm. 719, Elec. Eng. and Comput. Sci. Bldg., No. 101, Section 2, Kuang-Fu Rd., Hsinchu 30013, Taiwan, s102061556@m102.nthu.edu.tw)

In psychoacoustics, masking happens when the perceptibility of a target sound is influenced by another sound, which is called the masker. The medial olivocochlear (MOC) reflex pathway, which receives information from the cochlear nucleus and gives feedback to the cochlea, has been said to modify the gain function of the cochlea and enhance the audibility of sounds in noise. This enhancement of audibility is referred to anti-masking. To simulate masking and anti-masking, a tone-in-noise stimulus is delivered to an integrated model comprised of a nonlinear model of cochlear mechanics and a leaky integrate-and-fire network describing the dynamics of the MOC pathway. In particular, the T-multipolar (TM) cell in the ventral cochlear nucleus is chosen to transfer signals to the MOC, and the conductance of the outer hair cell is adjusted by the MOC efferent output to simulate anti-masking. Further, the inhibition from the tuberculoventral cell in the dorsal cochlear nucleus to the TM cell is also included in the model so its influence on the auditory nerve rate-level curves can be investigated in the tone-in-noise condition.

3aPPb15. High-pass filtering obscures stimulus encoding characteristics of the auditory brainstem response: Evidence from click and tone stimuli. Alexandra R. Tabachnick (Psychol. and Brain Sci., Univ. of Delaware, McKinley Lab, Newark, PA 19716, atabach@udel.edu) and Joseph C. Toscano (Psych., Villanova Univ., Villanova, PA)

The auditory brainstem response (ABR) is an electrophysiological measure of early auditory processing. While previous work has examined ABRs to clicks, tones, speech, and music, it remains unclear how changes in acoustic properties (e.g., frequency) map onto specific changes in ABR components. This may be partly due to filtering during data processing. High-pass filtering can severely distort cortical and subcortical responses, potentially obfuscating how stimuli are encoded. To address this, we measured ABRs to a wide range of pure tones $(250$ to $8000 \mathrm{~Hz})$ and examined how high-pass filtering affects tone- and click-evoked ABRs. In Experiment 1 , various high-pass filter settings $(0.1-300 \mathrm{~Hz})$ were applied to click-evoked 
ABRs. In Experiment 2, ABRs to brief tones across a six-step frequency continuum were collected, and the same high-pass filter settings were applied. Results indicate that excessive high-pass filtering diminishes the amplitude of ABR components, consistent with previous findings. In addition, filtering can obscure true effects of stimulus frequency. With appropriate filters, we find that the amplitude of wave $\mathrm{V}$ tracks stimulus frequency log-linearly, demonstrating that tonotopic organization is preserved and easily detectable early in processing. Future ABR work should minimize the use of high-pass filters when studying the encoding of acoustic information.

3aPPb16. Level-tolerant duration selectivity in the auditory cortex of the velvety free-tailed bat Molossus molossus. Silvio Macías (Dept. of Animal and Human Biology, Univ. of Havana, Havana, Cuba), Annette Hernández-Abad (Dept. of Animal and Human Biology, Univ. of Havana, Havana, Cuba; Dept. of Physiol., University of AB, Edmonton, AB T6G 2H7, Canada, annette3@ualberta.ca), Julio C. Hechavarría, Manfred Köss1 (Institut für Zellbiologie und Neurowissenschaft, J.W. Goethe Universität Frankfurt, Frankfurt, Germany), and Emanuel C. Mora (Dept. of Animal and Human Biology, Univ. of Havana, Havana, Cuba)

It has been reported previously that in the inferior colliculus of the bat Molossus molossus, neuronal duration tuning is ambiguous because the tuning type of the neurons dramatically changes with the sound level. In the present study, duration tuning was examined in the auditory cortex of $M$. molossus to describe if it is as ambiguous as the collicular tuning. From a population of 174 cortical $104(60 \%)$ neurons did not show duration selectivity (all-pass). Around $5 \%$ (9 units) responded preferentially to stimuli having longer durations showing long-pass duration response functions, 35 $(20 \%)$ responded to a narrow range of stimulus durations showing bandpass duration response functions, $24(14 \%)$ responded most strongly to short stimulus durations showing short-pass duration response functions and two neurons $(1 \%)$ responded best to two different stimulus durations showing a two-peaked duration-response function. The majority of neurons showing short- (16 out of 24) and band-pass (24 out 35) selectivity displayed "O-shaped" duration response areas. In contrast to the inferior colliculus, duration tuning in the auditory cortex of $M$. molossus appears level tolerant. That is, the type of duration selectivity and the stimulus duration eliciting the maximum response were unaffected by changing the sound level.

3aPPb17. Comparing auditory perceptual thresholds in pediatric and adult cochlear implant populations. Gabrielle O'Brien, Mishaela DiNino (Speech and Hearing Sci., Univ. of Washington, 1417 N.E. 42nd St., Box 354875, Seattle, WA 98105-6246, andronovhopf@gmail.com), J. D. Biesheuvel, Jeroen J. Briaire, Johan H. Frijns (Leiden Univ. Medical Ctr., Leiden, Netherlands), and Julie A. Bierer (Speech and Hearing Sci., Univ. of Washington, Seattle, WA)

It is well known that cochlear implant listeners show enormous variability in speech recognition performance, but the individual differences that affect outcomes are still poorly understood. The overall level and variability of psychophysical thresholds obtained with a focused electrode configuration may account for some of the variability in outcomes, as these measures might reflect the quality of the electrode-neuron interface. To date, psychophysical thresholds using direct stimulation have not been assessed in children. In this work, we compare such thresholds between pediatric and adult cochlear implant listeners. Thresholds were collected from 12 pediatric and 34 adult ears using a fast threshold sweeping method with both focused (partial quadrupolar) and broad (monopolar) electrode configurations. On average, both focused and broad thresholds were $4 \mathrm{~dB}$ lower in the pediatric population compared to the adult population. Intra-array variability in thresholds was found to be smaller in pediatric than adult populations for broad but not focused stimulation. Increased threshold observed for focused stimulation compared with broad, quantified as the ratio of focused to broad thresholds, did not differ significantly across populations. These results suggest that there may be differences in the quality of the electrode-neuron interfaces observed in pediatric and adult cochlear implant listeners.
3aPPb18. Interaural-time-difference discrimination as a measure of place of stimulation for cochlear-implant listeners with single-sided deafness. Olga Stakhovskaya, Gerald I. Schuchman (National Military Audiol. and Speech Pathol. Ctr., Walter Reed National Military Medical Ctr., 4954 North Palmer Rd., Bldg. 19, R. 5607, Bethesda, MD 20889, olga. stakhovskaya.ctr@mail.mil), Matthew J. Goupell (Dept. of Hearing and Speech Sci., Univ. of Maryland, College Park, College Park, MD), and Joshua G. Bernstein (National Military Audiol. and Speech Pathol. Ctr., Walter Reed National Military Medical Ctr., Bethesda, MD)

Recently, some patients with single-sided deafness (SSD) have received a cochlear implant (CI), with the goal of improving their spatial-hearing abilities. Because electrode arrays typically do not reach the cochlear apex, standard frequency-to-electrode allocations are likely to create a substantial interaural cochlear place-of-stimulation mismatch, and might not optimize binaural function for SSD-CI listeners. As a first step, we evaluated whether a test of interaural-time-difference (ITD) discrimination could be used to identify the cochlear place of stimulation for individual electrodes. Six SSD-CI listeners were presented with 500-ms bursts of 100-pps electrical pulse trains on a single electrode, and bandlimited pulse trains with variable carrier frequencies in the acoustic ear. Listeners discriminated between two "static" intervals (each containing four bursts with constant ITD) and a "moving" interval (four bursts with variable ITD). For most listeners and electrodes, performance peaked at a particular acoustic carrier frequency, which was on average 0.57 octaves higher (range $=-0.2-1.1$ octaves) than the standard clinically allocated center frequency. These results demonstrate that an ITD-discrimination task can identify the optimal frequency allocation required for a given CI electrode to maximize binaural function for SSD-CI listeners. The opinions and assertions presented are the private views of the authors and are not to be construed as official or as necessarily reflecting the views of the Department of Defense.

3aPPb19. Psychophysical tuning curves as a measure of electrode position in cochlear implant listeners. Lindsay DeVries and Julie A. Bierer (Speech and Hearing Sci., Univ. of Washington, 1417 NE 42nd St., Seattle, WA 98105, lindsdev@uw.edu)

Speech perception abilities vary widely among cochlear implant listeners. A potential source of this variability is electrode position; suboptimal placement has been associated with poorer outcomes. Insight into electrode position can be obtained via postoperative CT imaging, specifically the distance of each electrode from the modiolus. Additional information can be obtained with behavioral measures, which are sensitive to electrode position and neural integrity.Electrode-to-modiolus distance can explain some variability in behavioral thresholds; however, the relationship with psychophysical tuning curves (PTCs) has not been evaluated, which may provide a more detailed assessment of local variations in channel interaction. Four unilaterally implanted adults with the Advanced Bionics HiRes90K device participated. CT scans were obtained and image reconstructions were performed. Using a fast, sweep procedure, PTCs were collected for all available electrodes using the steered quadrupolar configuration within a forward-masking paradigm. Spread of neural excitation was quantified with the equivalent rectangular bandwidth (ERB). Preliminary data show a correlation between electrode-to-modiolus distance and the ERB of PTCs, suggesting poorer electrode placement may cause broader activation. The goal of this research is to develop a fast, non-radiologic method for estimating electrode position, which may lead to improved device programming by reducing unwanted channel interaction.

3aPPb20. Interaural spectral mismatch and binaural fusion in simulated cochlear-implant listening for single sided deafness. Jessica $M$. Wess (Neurosci. and Cognit. Sci. Program, Univ. of Maryland, 4954 N Palmer Rd., Bethesda, MD 20889, jwess@umd.edu), Nathan J. Spencer (Battlespace Acoust., Wright-Patterson AFB, Air Force Res. Lab., Dayton, $\mathrm{OH}$ ), and Joshua G. Bernstein (National Military Audiol. and Speech Pathol. Ctr., Walter Reed National Military Medical Ctr., Bethesda, MD)

Cochlear implants (CIs) can restore some spatial-hearing capabilities for individuals with single-sided deafness (SSD). However, there is a considerable variability in outcomes among SSD-CI listeners. One possible cause is spectral mismatch between the acoustic and CI ears. Vocoder simulations of 
SSD-CI listening were used to investigate the effect of interaural spectral mismatch on subjective fusion for normal-hearing listeners. A virtual cocktail party was created by presenting combinations of 1-6 concurrent talkers to the left, right or both ears. After each trial, listeners were asked how many total voices were heard. If listeners were able to perceptually fuse the voices that were presented to both ears, they should be more likely to report the correct number of total talkers in the scene. If not, they should report more total talkers. Preliminary results show that listeners reported more total talkers when the vocoded speech was processed with a frequency-matched map (simulating a standard CI frequency allocation) then with a placematched map. These data suggest that place-matched CI mapping has the potential to provide SSD-CI listeners with more opportunities for binaural fusion and improved spatial hearing. This method could be applied to SSDCI listeners to evaluate mapping strategies and outcomes after implantation. [The opinions and assertions presented are the private views of the authors and are not to be construed as official or as necessarily reflecting the views of the Department of Defense.]

3aPPb21. Comparison of brainstem frequency-following responses associated with auditory streaming based on spectral and temporal cues. Shimpei Yamagishi (Dept. of Information Processing, Tokyo Inst. of Technol., 4259, Nagatsuta-cho, Midori-ku, Yokohama-shi, Kanagawa-ken 2268503, Japan, yamagishi@u.ip.titech.ac.jp), Sho Otsuka, Shigeto Furukawa, and Makio Kashino (NTT Commun. Sci. Labs., NTT Corp., Kanagawa, Japan)

With the ABA streaming paradigm (van Noorden, 1975), Yamagishi $e t$ al. (2016) used spectrally differing A and B tones and showed that the auditory brainstem frequency-following response (FFR) to the second A tone co-varied with the perceived auditory stream. This study examined whether this effect is specific to the case in which the streaming is based on the spectral cues. We compared stream-perception-related FFRs between ABA stimuli based on spectral and temporal cues. In the spectral condition, the A and B tones differed in frequency ( 315 and $400 \mathrm{~Hz}$, respectively). In the temporal condition, the stimulus consisted of sinusoidally amplitude-modulated 4$\mathrm{kHz}$ tones that had identical long-term excitation patterns but differed in modulation frequency (60 and $170 \mathrm{~Hz}$, respectively). We analyzed the spectral amplitudes of the FFRs at the stimulus frequencies for the spectral condition and those at the stimulus modulation frequencies for the temporal condition. We found that the FFR amplitudes to the second A tone generally tended to be larger in the two-stream percept than in the one-stream one for both the spectral and temporal conditions. This result implies that the mechanism responsible for the stream-perception-related FFR is common across cue domains.

3aPPb22. Sub-clinical auditory neural deficits in patients with type $1 \mathrm{Di}$ abetes Mellitus. Arwa AlJasser, Kai Uus, Richard Baker, and Christopher Plack (School of Psychol. Sci., Univ. of Manchester, Ellen Wilkinson Bldg., Rm. A3.14, Manchester M13 9PL, United Kingdom, arwa.al-jasser@postgrad.manchester.ac.uk)

Diabetes mellitus (DM) is associated with a variety of sensory complications. Very little attention has been given to auditory neuropathic complications in DM. The aim of this study was to determine whether type $1 \mathrm{DM}$ affects neural coding of the rapid temporal fluctuations of sounds, and how any deficits may impact on real-world listening tasks. Participants were 30 young normal-hearing type $1 \mathrm{DM}$ patients, and 30 age-, sex-, and audiogram-matched healthy controls. Tests included non-invasive electrophysiological measures of auditory nerve and brainstem function using the click-evoked auditory brainstem response (ABR), and of brainstem neural temporal coding using the sustained frequency-following response (FFR), as well as behavioural tests of temporal coding (interaural phase difference, IPD, discrimination and the frequency difference limen, FDL) and tests of speech perception in noise. There were no significant differences between DM patients and controls in the ABR. However, the DM group showed significantly lower FFR responses, higher IPD and FDL thresholds, as well as worse speech-in-noise performance. The results suggest that type $1 \mathrm{DM}$ is associated with degraded neural temporal coding in the brainstem in the absence of an elevation in audiometric threshold, and that this deficit may impact on real-world hearing ability.

3aPPb23. Binaural sensitivity in children with bilateral cochlear implants and in normal hearing children. Ruth Litovsky and Erica E. Bennett (Commun. Sci. \& Disord., Univ. of Wisconsin-Madison, 1500 Highland Ave., Waisman Ctr. Rm. 521, Madison, WI 53705, litovsky@ waisman.wisc.edu)

Children with bilateral cochlear implants (CIs), and normal hearing (NH) children were studied. One goal was to understand if sensitivity to interaural time difference (ITD) and interaural level difference (ILD) is attained at young ages with acoustic hearing. A second goal was to determine if children who hear through electrical stimulation of the auditory nerve are also sensitive to ITD and ILD cues. The bilaterally implanted children received stimulation through synchronized research processors, which is unlike the clinical speech processors that they use every day, which lack interaural synchronization. Sensitivity to ILDs was adult-like in NH children, and all children with CIs were able to use ILDs. Sensitivity to ITDs was, on average, adult-like in $\mathrm{NH}$ children, but many of the CI users were unable to use ITDs to determine if a sound was presented to the right or left. This contrasts with prior findings that children with CIs can detect the presence of a stimulus with a binaural cue, regardless of whether they could discriminate ITDs or not. Results underscore the importance of task selection in evaluating binaural sensitivity. Data are interpreted in the context of auditory plasticity and peripheral integrity for the development of binaural coding.

3aPPb24. Dynamic representation of acoustic features in neural responses to continuous speech. Bahar Khalighinejad, Guilherme Da Silva, and Nima Mesgarani (Elec. Eng., Columbia Univ., 500 W. 120th St., Mudd 1310, New York, NY 10027, bk2556@columbia.edu)

Humans are unique in their ability to communicate using spoken language. However, it remains unclear how distinct acoustic features of speech sounds are represented in the auditory pathway over time. In this study, we applied a novel analysis technique to electroencephalography (EEG) signals as subjects listened to continuous speech and characterize the neural representation of acoustic features and the progression of responses over time. We averaged the time-aligned neural responses to phoneme instances and calculated a phoneme-related potential (PRP). We show that phonemes in continuous speech evoke multiple observable responses which are clearly separated in time. These recurrent responses have different scalp distributions, and occur as early as $50 \mathrm{~ms}$, and as late as $400 \mathrm{~ms}$ after the phoneme onset. We show that the responses explicitly represent the acoustic distinctions of phonemes, and that linguistic and non-linguistic information appear at different time intervals. Finally, we show a joint encoding of phonetic and speaker information, where the neural representation of speakers is dependent on phoneme category. This study provides evidence for a dynamic neural transformation of low-level speech features and form an empirical framework to study the representational changes in learning, attention, and speech disorders. 


\title{
Session 3aSA
}

\section{Structural Acoustics and Vibration: Dynamics of Ribbed Structures}

\author{
Elizabeth A. Magliula, Cochair \\ Division Newport, Naval Undersea Warfare Center, 1176 Howell Street, Bldg. 1302, Newport, RI 02841 \\ Andrew J. Hull, Cochair \\ Naval Undersea Warfare Center, 1176 Howell St., Newport, RI 02841 \\ Toru Yamazaki, Cochair \\ Mech Eng., Kanagawa Univ., Yokohama, Japan
}

\section{Invited Papers}

\begin{abstract}
8:30
3aSA1. Acoustic scattering from finite bilaminar composite cylindrical shells with doubly periodic stiffeners-3-D solution. Sabih I. Hayek (Eng Sci. and Mech., Penn State, 953 McCormick Ave, State College, PA 16801-6530, sihesm@engr.psu.edu) and Jeffrey E. Boisvert (NAVSEA Div Newport, Newport, RI)

The acoustic scattering from an insonified finite bilaminar cylindrical shell is analyzed. The shell is reinforced by a two sets of ribstiffeners to form a doubly periodic stiffened shell. The two cylindrical shell laminates are perfectly bonded having the same lateral dimension but have different radii and material properties The two sets of rib-stiffeners have rectangular cross-sections and are perfectly bonded to the inside of the inner shell. One set of stiffeners is heavy and the other set is light, so that a finite number of light stiffeners are set within every pair of heavy stiffeners. The bilaminar stiffened shell is analyzed using the exact theory of three-dimensional elasticity. The stiffeners are analyzed as elastic rings with extensional, torsional, and in-plane and out-of-plane flexural rigidities. The scattered acoustic farfield is evaluated for various incident plane wave frequencies. Shells with different combinations of light to heavy stiffener geometries are analyzed. A uniform steel stiffened shell submerged in water and stiffened with doubly periodic stiffeners was initially analyzed. A second shell made up of an outer elastomer shell bonded to an inner stiffened steel shell was also analyzed. [Work supported by NAVSEA Division Newport under ONR Summer Faculty Program.]
\end{abstract}

\section{8:50}

3aSA2. Reduction of transmitted power on a plane by using mode pair cancellation. Toru Yamazaki (Mech. Eng., Kanagawa Univ., 3-27-1 Rokkakubashi, Kanagawa-ku, Yokohama 221-8686, Japan, toru@kanagawa-u.ac.jp)

Reduction of structure-borne sound is an important issue for industry mechanical products. This paper presents a new idea for controlling structural intensity (SI) on panel structure derived from a cancellation of SI due to mode pairs of multiple roots. At first the modal formulation of SI on a flat plate is summarized. Next we discuss the change of SI on a flat plate by attaching a reinforcement beam cross the plate in one direction. It is shown that this attachment give multiple natural frequencies, and their mode pairs and the pairs of modal components of SI are in same phase in the area of half of the plate and in anti-phase in that of the other half. Then the modes and modal components of SI can be said to be canceled by each modal pairs in the area of half on the plate. Based on this cancellation due to modal pairs, a new method for reducing transmitted power to a given area on the plate has been derived and proposed by attaching a beam on the plate for providing partial symmetry on the plate. It is demonstrated that the partial attachment of a beam on the plate based on the proposed method can reduce the transmitted power to the given area by the cancellation due to the pairs of SI modal components.

\section{9:10}

3aSA3. Influence of stiffeners and internal structures on the vibroacoustic behavior of submerged cylindrical shells under random excitations. Valentin Meyer (DCNS Res., 199 Ave. Pierre-Gilles de Gennes, Ollioules 83500, France, valentin.meyer@dcnsgroup. com), Laurent Maxit (LVA, INSA Lyon, Lyon, France), Christian Audoly, and Ygaäl Renou (DCNS Res., Ollioules, France)

The vibroacoustic behavior of structures excited by random pressure fields such as turbulent boundary layers or diffuse sound fields is of great interest for industrial applications. Many works have been carried out for periodically stiffened plates. In particular, the influence of Bloch-Floquet waves on the panel radiation has been highlighted. However, few studies have investigated more complex structures under random excitations. The present work studies the influence of internal structures on the vibro-acoustic behavior of submerged cylindrical shells. The geometric complexity is successively increased by including periodic, non-periodic stiffeners and various internal frames. The numerical prediction is based on the combination of two methods developed by the authors. The first one is the wavenumber-point $(\mathrm{k}, \mathrm{M})$ reciprocity technique. This method estimates the response of the system at point $\mathrm{M}$ from the shell velocity in the wavenumber space under a point excitation at M. The velocity field is estimated with the second method, called the Condensed 
Transfer Function method. It is a substructuring approach which couples a semi-analytical model of a submerged cylindrical shell with Finite Element models of axisymmetric and non-axisymmetric frames. Numerical results are analyzed to evaluate the influence of the stiffeners and the internal structures on the shell radiation.

3aSA4. Exhaustive optimization of rib-stiffened, layered plate structures for acoustic response. Heather M. Reed (Weidlinger Assoc., Inc., New York, NY) and Jeffrey Cipolla (Weidlinger Assoc., Inc, 1825 K St NW, Ste. 350, Washington, DC 20006, cipolla@ wai.com)

A previously reported structural-acoustic frequency-domain formulation for layered, ribbed structures is used here as the basis for an approach to optimize these systems. We will review a previously reported singular perturbation approach to resolve convergence difficulties in both planar and cylindrical configurations, and discuss verification and validation. The significant successes of topological structural optimization for strength, weight, and efficiency are more challenging to repeat in coupled wave-bearing systems with many modes present. This is due, at least, to the difficulties of defining appropriate cost and regularization functions applicable across frequency ranges of interest and across the orders of magnitude of response characteristic of vibroacoustic systems. We discuss overcoming these difficulties by evading them entirely: the layered ribbed structural-acoustic model we use is sufficiently fast that sophisticated optimization algorithms are not required. To understand the dependence of critical solution performance metrics on the input parameters, we simply sample the design space exhaustively and develop graphical representations of the relative optimality of solutions. By using sensitivity analysis (effected through Fisher Information) and uncertainty quantification (UQ), optimal layered designs are obtained and balanced by performance robustness. In this talk, we will present examples of applying the approach to realistic systems of interest.

\section{9:50-10:05 Break}

\section{0:05}

3aSA5. Active control of bending waves propagating in an orthotropic rectangular panel. Hiroyuki Iwamoto (Seikei Univ., 3-3-1 kichijoji-kitamachi, Musashino, Tokyo 180-8633, Japan, hiroyuki-iwamoto@st.seikei.ac.jp) and Nobuo Tanaka (Tokyo Metropolitan Univ., Tokyo, Japan)

Due to the recent advances in microprocessors, active control of a flexible structure has become a realizable method for suppressing the vibration. Conventional modal-based method encounters difficulties in controlling a distributed parameter structure, since such a structure has an infinite number of vibration modes. To overcome this problem, active wave control has been studied in recent years. This paper presents an active wave control of an orthotropic rectangular panel by expanding the conventional active wave control method for a two-dimensional structural case. First, a transfer matrix method for a rectangular panel is introduced to describe the wave dynamics of the structure. This is followed by the derivation of feedforward control laws for absorbing reflected waves or eliminating transmitted waves. In the proposed method, the control laws are including the modal actuation scheme for uncontrolled direction. Then, from a viewpoint of numerical analyses, basic properties of the proposed method are verified. It is found that the reflected wave absorbing control enables the inactivation of all vibration modes in the controlled direction and the transmitted wave eliminating control enables the generation of an almost vibration-free state.

\section{$10: 25$}

3aSA6. Prediction of reflection and transmission by an elastic barrier with periodic structural discontinuities forced by oblique acoustic waves. Donald B. Bliss, Mauricio Villa, and Linda P. Franzoni (Dept. of Mech. Eng. and Mater. Sci., Duke Univ., Box 90300, Durham, NC 27708-0300, dbb@duke.edu)

An analysis method is developed for acoustic reflection and transmission from an infinite fluid-loaded flexible barrier with spatially periodic discontinuities. The barrier has acoustic fluids on both sides, which can be dissimilar. The structure is excited by an obliquewave incident acoustic field. The fully-coupled structural/acoustic problem is treated by the method of Analytical-Numerical Matching (ANM). The ANM framework separates the problem into a global numerical solution and local analytical solutions. ANM handles rapid spatial around the structural discontinuities, improving the accuracy and convergence rate of reflected and transmitted pressures. Furthermore, the ANM approach offers a way to handle the mathematical difficulties associated with coincidence frequencies. The periodic spatial discontinuities create variations from simple specular-like directivity with multiple reflection and transmission angles, the effect being most pronounced at structural resonances. The periodic discontinuities can be thought of as redirecting a portion of the structural energy into resonant substructures having wavenumbers different from the oblique wave forcing, reradiating reflection and transmission fields with different directivity patterns. Discrete frequency and broadband results are presented. The goal is to develop efficient firstprinciples methods for structural-acoustic reflection and transmission between coupled acoustic spaces and into surrounding media.

\section{0:45}

3aSA7. Sound radiation from a fluid-loaded and elastically coated infinite plate with attached periodically located inhomogeneities. Yanni Zhang, Jie Pan, and Hai Huang (Zhejiang Univ., 38 Zheda Rd., Hangzhou 310027, China, yanni_zhang@zju.edu.cn)

An analytical model is developed for evaluating the sound radiation characteristics of a fluid-loaded and elastically coated infinite plate attached with periodically located inhomogeneities. The vibration and sound responses of such a periodic structure under a local excitation are obtained through wavenumber transformation method. The radiation characteristics of the plate are investigated via comparing with those of a plate with a single inhomogeneity and one without inhomogeneities. Three interesting features of the sound radiation are observed, which are related to plate's global resonant modes, local resonances of the trapped modes formed around each inhomogeneity, and the band-pass characteristics of the periodicity, respectively. These features are explained by the variation in the surface supersonic wavenumber components and the waveguide properties of the fluid-loaded composite plate and the coherent resonance of all inhomogeneities. 
3aSA8. How the sound adjustment process of cymbals affects their vibration and sound radiation characteristics. Fumiyasu Kuratani, Tatsuya Yoshida (Mech. Eng., Univ. of Fukui, 3-9-1 Bunkyo, Fukui 910-8507, Japan, kuratani@mech.u-fukui.ac.jp), Toshio Koide (Koide Works, Ltd. (Koide Cymbals), Osaka, Japan), Taiji Mizuta (Osaka Alloying Works, Co., Ltd., Fukui, Japan), and Kozo Osamura (Res. Inst. for Appl. Sci., Kyoto, Japan)

Cymbals are percussion instruments. They vibrate and radiate sounds when hit with a drumstick or when used in pairs and the sounds depend on the vibration characteristics. Cymbals are made through spin forming, hammering and lathing. The spin forming creates the domed shape of the cymbal, determining its basic vibration characteristics. The hammering and lathing produce specific sound adjustments by changing the vibration characteristics. In this study, we focus on how the hammering affects the vibration and sound radiation characteristics. The hammering produces many shallow dents over the cymbal's surface, generating residual stresses in it. These residual stresses change the vibration characteristics. We perform finite element analysis of the hammered cymbal to obtain its vibration and sound radiation characteristics. In the analysis, we use thermal stress analysis to reproduce the stress distribution and then with this stress distribution we perform vibration analysis. The results show that the effect of thermal load (i.e., hammering) depends on mode: an increase or decrease in the natural frequency. The difference between the modes changes the peak frequencies and their amplitudes in the frequency response. As a result, the deflection shapes and their sound radiation efficiencies at the peak frequencies are changed.

\section{Contributed Paper}

\section{$11: 25$}

3aSA9. Finite-difference time-domain analysis of the vibration characteristics of building structures using a dimension-reduced model. Takumi Asakura (Tokyo Univ. of Sci., 2641 Yamazaki, Noda, Chiba 2788510, Japan, t_asakura@rs.tus.ac.jp), Masahiro Toyoda (Kansai Univ., Osaka, Japan), and Tohru Miyajima (Inst. of Technol., Shimizu Corp., Tokyo, Japan)

In order to accurately predict the vibration characteristics of buildings, wave-based numerical methods are effective from the viewpoint of the modeling accuracy of the physical mechanism and the detailed geometries of the simulated field. However, because of the performance of current PCs, the prediction of real-scale problems remains difficult. In order to address such problems, we herein propose a vibration simulation method for a beam-plate structure using a dimension-reduced modeling method. The target structure is modeled as a composite structure consisting of two-dimensional plate elements and one-dimensional beam elements, which are coupled based on the implicit finite-difference approximation scheme. By applying such a lowdimensional element, a faster simulation that requires less memory, as compared with a three-dimensional discretization scheme, is made available. To validate the method, the vibration characteristics obtained by the proposed scheme are compared to the measured results for model-scale and full-scale structure. The comparison of the measurement and simulation results suggest that the proposed method can be used to accurately simulate a multilayered building structure. 


\title{
Session 3aSC
}

\section{Speech Communication: Double-Weak Theory of Speech Production and Perception: A Session in Honor of Terrance Nearey}

\author{
Michael Kiefte, Cochair \\ Human Communication Disorders, Dalhousie University, 1256 Barrington St., Halifax, NS B3J 1Y6, Canada \\ Hideki Kawahara, Cochair \\ Design Information Sciences, Wakayama University, 930 Sakaedani, 930, Wakayama 640-8510, Japan
}

Chair's Introduction-8:00

\section{Invited Papers}

\section{8:05}

3aSC1. Incongruence in second language vowel perception and production. Ron I. Thomson (Appl. Linguist, Brock Univ., 1812 Sir Isaac Brock Way, St. Catharines, ON L2V 5E6, Canada, rthomson@brocku.ca) and Murray J. Munro (Linguist, Simon Fraser Univ., Burnaby, BC, Canada)

Nearey's work on double-weak theory has brought to the fore the conceptualization of native-language (L1) speech perception and production as autonomous subsystems. In second-language (L2) phonetics research, the perception-production relationship is also a central concern, although the facilitating and hampering effects of a previously learned phonological inventory must be taken into account (Flege, 2003). Some current views of L2 phonetic learning assume that a reorganization of perceptual knowledge normally comes first, and that production eventually falls into line with perceptual representations (Huensch, 2013; Thomson, 2011, 2013). However, the available data point to a number of complexities in the relationship that have yet to be accounted for. In the present report, we synthesize new data on vowel perception and production by English learners from a variety of L1 backgrounds. Among the key findings are that (1) perceptual training on vowels in particular phonetic contexts leads to very limited transfer of learning to new phonetic contexts, (2) perceptual accuracy only weakly predicts production accuracy, especially for cross-linguistically marked vowels, (3) word familiarity and frequency correlate with vowel production accuracy, and (4) elicitation techniques influence production accuracy. These issues are discussed in terms of the problems they pose for models of phonetic learning.

\section{$8: 25$}

3aSC2. Investigating the sliding template model of vowel perception. Santiago Barreda (Linguist, UC Davis, Dept. of Linguist, Edmonton, AB T6G 2E7, Canada, sbarreda@ucdavis.edu)

According to the sliding-template model of vowel perception, vowel quality is specified by the frequencies of the first three formants, and within-category variability in formant-patterns is primarily according to a single scaling-parameter [Nearey, J. Acoust. Soc. Am. 85, 2088-2113, 1989]. In this view, speaker normalization in vowel perception centers on the estimation of the spectral-scaling parameter for a speaker. This process may include consideration of "indirect" evidence such as fundamental frequency (f0), or information about speaker size or sex. This model also suggests the potential integration of vowel perception and speaker-size estimation via shared use of the estimated scaling parameter, which is related to speaker vocal-tract length and height. An experiment is presented where listeners were asked to listen to vowel stimuli whose formant patterns were potentially ambiguous between the /æ/ of a larger speaker and the $/ \Lambda /$ of a smaller speaker. Listeners were asked to make a vowel-category judgment, and to estimate the height of the apparent speaker in feet and inches. Results are consistent with predictions made by the sliding template model: apparent speaker size was predictive of perceived vowel quality independently of the acoustic characteristics of a sound, and f0 appears to affect vowel quality primarily indirectly.

\section{8:45}

3aSC3. Formants in speech perception. Michael Kiefte (Human Commun. Disord., Dalhousie Univ., 1256 Barrington St., Halifax, NS B3J1Y6, Canada, mkiefte@dal.ca)

Formants, or vocal-tract resonances, have played a dominant role in the study of both speech production and perception, particularly with vowels. They form the basis of descriptions of speech in phonetics, speech pathology, speaker verification, sociolinguistics, language acquisition, as well as in many other fields. In contrast, work in engineering applications of speech processing - specifically automatic speech recognition-typically ignores formants in favor of acoustic properties that are significantly easier to extract but which make no assumptions regarding the nature of speech acoustics. This talk describes studies that explore how listeners process formantlike information in speech and how this evidence might relate to speech perception. 
3aSC4. Unusual public voices. Peter F. Assmann (School of Behavioral and Brain Sci., Univ. of Texas at Dallas, MS GR 41, Box 830688, Richardson, TX 75075, assmann@utdallas.edu)

Terry Nearey has a long-standing interest in vocal properties that carry phonetic information and simultaneously contribute to voice quality. He has drawn attention to the perceptual importance of the relationship between time-averaged fundamental frequency (f0) and formant frequencies and shown how combinations of these properties conspire to produce unique and highly identifiable voices, such as that of the late television cook Julia Child, who had atypically low formants but f0 in the expected range; and the cartoon figure Popeye (played by Jack Mercer) who had relatively high formant frequencies but a normal f0 range. This talk will present examples from Terry's collection along with acoustic analyses and a discussion of their perceptual implications.

\section{9:25}

3aSC5. Making decisions about modeling decision-making when analyzing speech perception data. Noah H. Silbert (Commun. Sci. and Disord., Univ. of Cincinnati, French East 344, 3202 Eden Ave., Cincinnati, OH 45267, silbernh@ucmail.uc.edu)

Speech perception researchers are typically interested in, well, perception. But perception is not directly observable. An experimental participant hears (and maybe sees) a stimulus, but the researcher only observes the listener's response (e.g., which button is pushed, how long it takes to push the button). Crucially, a listener's response doesn't directly, or solely, reflect perceptual processing; listeners' responses also reflect what we might call response selection or decision-making. While there is a sizable sub-field in psychology devoted to the study of judgment and decision making, these issues are of more peripheral concern for speech perception researchers. Nonetheless, I will argue that it is important to take response selection into account when analyzing perception data. Terry Nearey is one of a small number of speech researchers who has consistently taken decision-making seriously when analyzing speech perception data. I will discuss a number of mathematical approaches to simultaneously modeling perception and response selection and how changes in modeled perception and decision-making predict distinct observable patterns in data. The importance of decision-making in the study of perception will be discussed and illustrated via a number of Terry Nearey's studies as well as my own.

\section{9:45-10:00 Break}

\section{0:00}

3aSC6. The perception of spontaneous speech: "Lo how complex". Benjamin V. Tucker (Linguist, Univ. of AB, 4-32 Assiniboia Hall, Edmonton, AB T6G 2E7, Canada, bvtucker@ualberta.ca)

While most of the speech that listeners encounter is produced during spontaneous conversation, researchers actually know very little about this type of speech. What we do now is that there is tremendous variability and complexity in spontaneous speech that is not found in laboratory styles of speech. These differences compound and present new challenges for speech researchers, and in this case speech perception. It is possible that the changes found in spontaneous speech are systematic; however, it is also possible that these changes increase the noise in the speech signal. The vast majority of recent research on spontaneous speech has focused on word recognition and has not focused on aspects of perception and how spontaneous speech impacts theories of speech perception. In this presentation, I provide several examples of the variability and complexity found in spontaneous speech. I explore some of the challenges spontaneous speech creates for models of speech perception. I also discuss some perception data on spontaneous speech which sheds some light on future directions in research on speech perception.

\section{$10: 20$}

3aSC7. What does sinewave speech tell us about phonetic perception? James Hillenbrand (Western Michigan Univ., 1903 W Michigan Ave., Kalamazoo, MI 49008, james.hillenbrand@wmich.edu)

It has been 35 years since the striking demonstration that intelligible speech can be created by mixing sinusoids that follow the formants of natural speech [Remez et al. (1981) Science 212, 947-950]. A good deal has been written about what sinewave speech (SWS) might reveal about the mechanisms underlying phonetic perception. However, most of the experimental work on SWS has been carried out using well-formed sentences whose intelligibility depends on many factors other than phonetic identification. Results will be reported for experiments designed to measure SWS intelligibility explicitly at the phonetic level. For example, listeners were asked to identify the vowel in $\mathrm{SW} / \mathrm{hVd}$ / syllables. Intelligibility was far above chance $(55 \%)$ but dramatically lower than that of the original utterances. Further tests showed: (1) intelligibility increased substantially when the SW syllable was preceded by a brief SW carrier phrase (CP); (2) tests that alternated trials with and without the SW CP showed that CP enhancement is a real-time effect; (3) SW vowels can be classified using a template-matching algorithm trained on naturally spokenvowels at rates exceeding those of human listeners. Preliminary results will be reported for consonant recognition using SW nonsense syllables. [Work supported by NIH.]

\section{0:40}

3aSC8. A double-Nearey theory of vowel normalization: Approaching consensus. D. H. Whalen (Haskins Labs., 300 George St. Ste. 900, New Haven, CT 06511, whalen@haskins.yale.edu)

Terry Nearey has provided substantial data and insights into the realm of speech perception throughout his career. A major concern of his is to reconcile seemingly contradictory results. Here, his double-weak theory of perception and his probabilistic sliding template model (PSTM) are examined in relation to vowel normalization. Various algorithms achieve good results, but they must all confront the contradiction in behavior: We normalize the speech of others but we can also tell when they use a (slightly) different vowel than we do. Thus, listeners are (as in double-weak theory) fairly accommodating of different vocal tracts, but, contrarily, they can hear differences as well. PSTM may account for this difference, if deviations from theory-predicted vowel location are just those that speakers identity as being different; this remains to be tested. The various sources of vowel information (lower formants, F0, higher formants, spectral tilt) 
interact in complex ways, as shown in Nearey's work and others'. No theory is comprehensive yet, as acknowledged by Nearey himself. He has also made the observation that our theories of speech perception ought to perform at least as well as automatic speech recognition systems. Although we have not reached that stage, Nearey's work brings us closer.

\section{1:00}

3aSC9. Toward a triple-weak theory of phonology/phonetics. Robert Port (Linguist, Cognit. Sci., Indiana Univ., 5975 S. Handy Rd., Bloomington, IN 47401, port@indiana.edu)

Almost 20 years ago, Nearey proposed the Double-Weak theory of speech production and perception. He pointed to three dominant theories of speech production and perception. All begin by assuming the memory representations of language consist of phonemes made from segmental distinctive features. He called the linguistic approach "double-strong" since it assumed each phoneme has a simple set of articulatory correlates as well as acoustic correlates. The other two theories assume either that the articulatory definitions are simple and strong while the acoustics is messy. Or that acoustics plays the strong role while articulation is messy. However, Nearey's double-weak theory assumed no simple relation of either articulation or acoustics to phonemes was possible. In contrast, my theory agrees with Nearey but further argues there is no fixed, invariant set of discrete, non-overlapping phonemes for spelling words (Port, Lang Sci, 2010). This presentation will also discuss recent attempts to salvage the phoneme noting they include so many caveats and revisions that they basically cede the issue.

\section{1:20}

3aSC10. Vowel dynamics and sentence processing. Diane Kewley-Port (Dept. of Speech and Hearing Sci., Indiana Univ., 5975 S. Handy Rd., Bloomington, IN 47401, kewley@indiana.edu) and Daniel Fogerty (Dept. of Commun. Sci. and Disord., Univ. of South Carolina, Columbia, SC)

Throughout Terrance Nearey's influential academic career, research on the acoustic properties, models and theories of vowels has been a major focus. Studies by Nearey and his colleagues demonstrated the importance of spectral change (VISC) in vowel identification, even for monophthongal vowels. This presentation describes our research on vowel dynamics inspired by VISC research. Starting with syllables, the auditory system can discriminate F1 and F2 formants transition differences very well. Further results using high-fidelity resynthesis with sentences revealed that formant discrimination thresholds are 50\% smaller than differences observed for natural syllables. These results support that human perception is very sensitive to spectral information in dynamically changing vowels in sentences. A second line of research examined the role of vowel information in sentences using noise interruption paradigms. Based on traditional segment boundaries, vowels had a two-to-one perceptual advantage over consonants for recognizing words in sentences. Shifting boundaries to favor consonantal transitions revealed a large advantage for even small proportions of vowel information. Recent research has enhanced understanding of the interdependence between vowel dynamics and suprasegmental contours of F0 and amplitude for accurate speech recognition. Thus, dynamic information in vowels, both VISC and suprasegmental, is essential for sentence intelligibility.

\section{1:40}

3aSC11. Double weak theory as a framework for discovery. Terrance M. Nearey (Linguist, Univ. of AB, 4-32 Assiniboia Hall, Edmonton, AB T6G 0A2, Canada, tnearey@ualberta.ca)

A weak theory is not very attractive, a double-weak one even less so. The double-weak theory of speech perception serves mainly as a cover story for postponing rumination on the deeper principles that ultimately underlie human speech communication. Instead, it adopts minimal assumptions about the relation among symbols, sounds, and gestures that provides a rationale for immediately pursuing some hunches about empirical generalizations, or primitive "laws." It is hoped that some of these may be akin to Kepler's laws of planetary motion or eighteenth century "gas laws" that may serve as grist for later, more deeply explanatory theories. The active search for such empirical laws in speech perception starts from existing hypotheses about the general structure of surface phonological representations (e.g., features, phonemes, syllables) and proceeds by constructing detailed statistical models that accord with the fine structure of human responses in parametrically controlled speech perception experiments. Some examples and possible extensions of existing models are presented. 


\title{
Session 3aSP
}

\section{Signal Processing in Acoustics: Signal Processing in Nonlinear Acoustics}

\author{
Brian E. Anderson, Cochair \\ N145 Esc, Brigham Young Univ., MS D446, Provo, UT 84602 \\ Marcel Remillieux, Cochair \\ Los Alamos National Laboratory, Geophysics Group (EES-17), Mail Stop: D446, Los Alamos, NM 87545 \\ Yoshikazu Ohara, Cochair \\ Department of Materials Processing, Tohoku University, 6-6-02 Aoba, Aramaki-aza, Aoba-ku, Sendai 980-8579, Japan
}

\section{Invited Papers}

\begin{abstract}
8:00
3aSP1. Comparison of various methods of nonlinear signatures extraction. Alexander Sutin (Maritime Security Ctr., Stevens Inst. of Technol., 711 Hudson St., Hoboken, NJ 07030, asutin@stevens.edu)

Nonlinear Acoustic NDE (NA NDE) methods are very sensitive to defect presence and currently number on publications on this matter exceed several thousand. Despite intensive research of NA NDE, these methods are still do not widely used in practice and the goal of this presentation is to give review of various methods of nonlinear signatures extraction and to show advantages on some of them in the comparison with the others. The major part of the NA NDE was applied for characterization the tested part as whole. These methods include: High Harmonic Generation, Nonlinear Resonant Ultrasound Spectroscopy (NRUS), Amplitude Dependent Internal Friction, Nonlinear Wave Modulation Spectroscopy (NWMS) or Vibro Acoustic Modulation (VAM), Slow Dynamics Diagnostics (SDD), Nonlinear Reverberation Spectroscopy (NRS) or Nonlinear Impact Resonance Acoustic Spectroscopy (NRAS), Nonlinear Coda Wave Interferometry, Bi Spectral and High Order Statistic Analysis, Subharmonic and Ultraharmonic Methods. The methods that can provide nonlinear imaging and damage localization include: Nonlinear Time Reversal Acoustics (NTRA) or TR NEWS, Nonlinear Harmonic Imaging, Nonlinear Acoustic Tomography, Pulse Modification of Vibro Acoustic Modulation (VAM), Nonlinear Guided Wave Imaging and Tomography, Subharmonic and Ultraharmonic imaging, Nonlinear Structural Intensity.
\end{abstract}

\section{8:20}

3aSP2. Nonlinear Coda Wave Interferometry: Detecting, quantifying, and locating damage in complex solids. Vincent Tournat (LAUM, CNRS, Université du Maine, Av. O. Messiaen, Le Mans 72085, France, vincent.tournat@univ-lemans.fr), Odile Abraham (IFSTTAR, Nantes, France), Yuxiang Zhang (Université Joseph Fourier, Grenoble, France), Jean-Baptiste Legland, Benoit Hilloulin, Olivier Durand (IFSTTAR, Nantes, France), Stéphane Letourneur, Emmanuel Brasseur, and Mathieu Chekroun (LAUM, CNRS, Université du Maine, Le Mans, France)

In this talk, we report results on the nonlinear interactions of ultrasonic coda waves with lower pump waves, in reverberating or multiple scattering mesoscopic solid media. Using the method of coda wave interferometry (CWI), we analyze the effect of mixing a coda wave with an additional lower frequency pump wave. The extracted CWI parameters, known to be highly sensitive to small geometric or elastic modifications of the tested medium, are shown to be pump-amplitude dependent and to capture finely the results of the nonlinear interactions. Although nonlinear self-action effects with coda waves have been reported in unconsolidated granular media, they are difficult to implement in cracked solids or concrete. Instead, the reported nonlinear CWI class of methods (NCWI) shows robustness, a high sensitivity, and has been applied successfully to various complex media and structures. We show through several examples on « model » media (cracked glass plates) and on concrete structures, that NCWI can be useful for the nondestructive evaluation of complex solids that are strongly scattering at usual probing frequencies. Preliminary results and prospects in nonlinear elastic properties imaging and quantitative evaluation with NCWI are discussed.

\section{8:40}

3aSP3. Evaluation of crack parameters by a nonlinear frequency-mixing laser ultrasonics method. Sylvain Mezil (Faculty of Eng., Div. of Appl. Phys., Hokkaido Univ., N13W8, Kita-Ku, Sapporo 060-8628, Japan, sylvain.mezil@eng.hokudai.ac.jp), Nikolay Chigarev, Vincent Tournat, and Vitalyi Gusev (LAUM, Université du Maine, Le Mans Cedex 9, France)

Nonlinear acoustic methods are commonly used in crack detection because of their high sensitivity in comparison to linear ones. However, the dependence of the nonlinearities on the crack state can not only localize it but also provides information on its characteristics. We present a laser ultrasonic method, based on nonlinear frequency-mixing, to locally evaluate several crack parameters, including some, like the local crack elasticity, which are assessed uniquely by the present technique. Two laser beams, independently intensity 
modulated at two cyclic frequencies $\omega_{\mathrm{H}}$ and $\omega_{\mathrm{L}}\left(\omega_{\mathrm{H}}>>\omega_{\mathrm{L}}\right)$, excite the sample. For a large sinusoidal thermo-elastic stress generated by the low-frequency modulated laser beam, the crack oscillates between closed and open states. In the presence of this variation, nonlinear frequency-mixing ultrasonic components at frequencies $\omega_{\mathrm{H}} \pm \mathrm{n} \omega_{\mathrm{L}}(\mathrm{n}$ is an integer) are detected. By modifying the intensity of the laser beam modulated at $\omega_{\mathrm{L}}$, we can influence the time spent in opened and closed state over a period $\left(2 \pi / \omega_{\mathrm{L}}\right)$. The developed theoretical model demonstrates the dependence of the nonlinear components at $\omega_{\mathrm{H}} \pm \mathrm{n} \omega_{\mathrm{L}}$ on the time spent by the crack in each state. Comparison between theoretical and experimental results offers a way to characterize some local crack properties, including its width and effective rigidity.

\section{9:00}

3aSP4. Quantitative evaluation of strength degradation by using linear-nonlinear ultrasonic techniques. Kyung-Young Jhang (School of Mech. Eng., Hanyang Univ., 204 Eng. Ctr. Annex, 222 Wangsimni-ro, Seongdong-gu, Seoul 04736, South Korea, kyjhang@ hanyang.ac.kr), Jongbeom Kim, and Ju-ho Lee (Dept. of Mech. Convergence Eng., Hanyang Univ., Seoul, South Korea)

The ultrasonic nonlinear parameter, $\beta$, has been known as effective for the evaluation of material degradation, and its correlation with material degradations such as thermal aging, fatigue, creep, and plastic deformation has been reported. However, most studies were limited to the relative measurements that is effective only for the relative comparison and not able to evaluate the degradation in quantitative. To overcome this limit, this study proposes a new algorithm which is able to evaluate the quantitative strength degradation. The proposed method mainly consists of four steps: 1) Measure the linear elastic modulus (E) from the longitudinal and shear wave velocities by using pulse echo method. 2) Measure the absolute ultrasonic nonlinear parameter, $\beta$, by using piezoelectric detection method. 3 ) Construct the stress-strain curve by substituting the measured linear elastic modulus (E) and absolute ultrasonic nonlinear parameter $\beta$ to the nonlinear stress-strain equation. 4) Estimate the $0.01 \%$ offset yield strain and yield strength. The yield strengths estimated by the proposed technique in the heat-treated A16061-T6 and SA508 showed good agreement with the yield strength obtained by tensile test. Consequently, the proposed method makes the quantitative evaluation of strength degradation using ultrasonic measurement possible. [This research was supported by the National Research Foundation of Korea (NRF) grant funded by the Korean government (NRF2013M2A2A9043241).]

3aSP5. Retreiving global nonclassical nonlinearity parameters from local measurements. Martin Lott (Waves and Imaging, CNRSLMA, Marseille, France), Marcel Remillieux (Geophys., Los Alamos National Lab., Los Alamos, NM), Pierre-Yves Le Bas, Timothy J. Ulrich (Detonator Phys., Los Alamos National Lab., Los Alamos National Lab., Geophys. Group, MS D446, Los Alamos, NM 87545, tju@lanl.gov), Vincent Garnier, and Cedric Payan (Waves and Imaging, CNRS-LMA, Marseille, France)

We demonstrate the equivalence between local and global measures of nonclassical nonlinear elasticity in a slender resonant bar of Berea sandstone. Nonlinear effects are first measured globally using Nonlinear Resonance Ultrasound Spectroscopy (NRUS), which monitors the relative shift of the resonance frequency as a function of the maximum dynamic strain in the sample. Subsequently, nonlinear effects are measured locally at various positions along the sample using Dynamic Acousto-Elasticity Testing (DAET). After processing the DAET signals and correcting for three-dimensional strain effects, it is shown that by numerically integrating these corrected data along the length of the sample the NRUS global measures are retrieved almost exactly.

\section{9:40-10:00 Break}

\section{0:00}

3aSP6. Nonlinear ultrasonic phased array for closed crack imaging. Yoshikazu Ohara, Kazushi Yamanaka, and Tsuyoshi Mihara (Dept. of Mater. Processing, Tohoku Univ., 6-6-02 Aoba, Aramaki-aza, Aoba-ku, Sendai 980-8579, Japan, ohara@material.tohoku.ac. jp)

In nondestructive ultrasonic inspection, closed cracks can lead to the underestimation or overlook, resulting in the catastrophic accidents. To solve this problem, nonlinear ultrasonics has been expected as a most promising approach. In nonlinear ultrasonics, by irradiating large amplitude, the nonlinear interaction between closed cracks and ultrasonics generates nonlinear components. Among them, we found that subharmonics has a higher selectivity than the others including higher harmonics. We also found that the subharmonics has higher temporal resolution, providing the high spatial resolution in ultrasonic imaging. Based on these findings, we have developed a novel imaging method, subharmonic phased array for crack evaluation (SPACE), based on the subharmonic generation at closed cracks due to large-amplitude short burst waves input and phased array imaging with frequency filtering. As a result of applying SPACE to closed-crack specimens, SPACE visualized the closed part of cracks, which were not observed in the conventional methods. It was substantiated that SPACE is useful in accurately measuring closed-crack depths.

$10: 20$

3aSP7. How to improve detectability of crack size in nonlinear sub-harmonic imaging. Choon-Su Park, Seunghyun Cho, Dae-Chul Seo (Ctr. for Safety Measurements, Korea Res. Inst. of Standards and Sci., Gajeong-ro 267, Bld. \# 206 / Rm. \# 206, Daejeon 34113, South Korea, choonsu.park@kriss.re.kr), and Jun-Woo Kim (Safety Qualification Technol. Ctr., KHNP Central Res. Inst., Daejeon, South Korea)

Crack growth is regarded as an important monitoring parameter for structural degradation. To precisely estimate crack size, therefore, has been highly demanded to monitor structural healthiness and to assess residual life of various structures from small components to huge plants. Conventional non-destructive inspections have been successfully detected cracks, but some deficiencies for accuracy still remain. Nonlinear sub-harmonic generation was well proved to detect closed cracks that could cause underestimation of crack size. Moreover, sub-harmonic phased array (PA) imaging was proposed to visualize where the closed cracks are. The sub-harmonic PA images, however, has lower spatial resolution than the fundamental frequency PA images due to its longer wavelength, which often prevents from clearly observing closed cracks. Many of signal processing techniques have been developed to improve spatial resolution of 
images for more than half a century, and some resolution enhancing techniques such as deconvolutions and an eigen-analysis based technique are employed to make the resolution of sub-harmonic imaging better. Point spread function issues for deconvolution have been also investigated theoretically and experimentally. In addition, some experiments with CT specimens have been done to prove closedcrack localization by improving spatial resolution of sub-harmonic imaging.

\section{0:40}

3aSP8. Extraction of nonlinear elastic material parameters from single-impact nonlinear ring-down spectroscopy. Parisa Shokouhi, Jiang Jin, and Jacques Riviere (Civil and Environ. Eng., Penn State, 215 Sackett Bldg., University Park, PA 16802, parisa@engr. psu.edu)

Volumetric microcracking is one of the early symptoms of distress in cementitious materials caused by excessive mechanical stress, chemical attacks, and environmental influences. The microcracks widen, coalesce, and develop into larger cracks with the progress of damage. Visible macro-cracks indicate severe damage that often cannot be mitigated. As such, detection of damage at the early stages of development is essential for designing optimal preventive maintenance programs for concrete structures. Nonlinear acoustics-based nondestructive testing techniques have shown great promise in identification of microscopic cracks in diverse materials including concrete. Impact-based alternatives of conventional techniques are gaining popularity for concrete testing mainly due to their field transportability. In this study, we focus on impact-based nonlinear resonance acoustic spectroscopy (INRAS). We compare the results from multi-impact INRAS, where several impacts of increasing intensities are applied, to those from single impact ring-down spectroscopy where only one impact of large intensity is used. Hilbert Huang Transform (HHT) is used to obtain the time-dependent frequency content of the singleimpact ring-down. We propose several models for fitting the entire ring-down in order to extract nonlinear elastic material parameters. We demonstrate that our proposed approach for analyzing single-impact INRAS data yields material parameters compatible with those obtained from conventional testing and analysis.

\section{Contributed Papers}

\section{1:00}

3aSP9. Nonlinear scattering of crossed focused ultrasonic beams in a constricted flow for the detection of a thrombosis. Emily S. Kilen, Theodore R. Johnson, Daniel J. Fisher, and Murray S. Korman (Phys. Dept., U.S. Naval Acad., 572 C Holloway Rd., Annapolis, MD 21402, korman@usna. edu)

Experiments performed at the USNA Acoustics Lab use turbulent flow to generate nonlinear scattering of mutually perpendicular crossed beams ( $f_{1}=1.9 \mathrm{MHz}, \mathrm{f}_{2}=2.1 \mathrm{MHz}, 15 \mathrm{~cm}$ focal lengths) at the combination sum frequency component $\left(f_{+}=4 \mathrm{MHz}\right)$. The object is to simulate blood flow through a constriction, and measure the nonlinear scattering downstream with a receiving circular plane array transducer $(4 \mathrm{MHz})$ that rotates azimuthally in the plane of the transmitted beam axes about the intersection. Angular dependent Doppler shift (about $\mathrm{f}_{+}$), spectral broadening, skewness and kurtosis measurements vs. angle help predict the characteristics of the turbulence. The setup involves a $4 \mathrm{~cm}$ diameter polyethylene $(0.5 \mathrm{~mm}$ thick $)$ tube cut in two sections (22 cm upstream (a), $38 \mathrm{~cm}$ downstream (b)). The sections are mated with a special block union allowing one to insert individual thin orifice plates of different diameters. The far section ends are open but are connected through opposite side ports on (a) and likewise on (b) to in-phase and out of phase synchronized bellows pumps. The apparatus when submerged in a water tank generates pulsating turbulent flow. Nonlinear scattering is measured vs. orifice size. [See S. M. Mock (2013) and D. J. Fisher (2014), USNA Capstones.]

\section{1:15}

3aSP10. Characterizing nonlinear systems with memory while combating and reducing the curse of dimensionality using new volterra expansion technique. Albert H. Nuttall (NUWCDIVNPT (retired), Old Lyme, CT), Derke Hughes, Richard A. Katz, and Robert M. Koch (NUWCDIVNPT, 1176 Howell St., Newport, RI 02841, derke.hughes@verizon. net)

A generalized model for characterizing nonlinear systems was originally proposed by Italian mathematician and physicist Vito Volterra (1860-1940). A further development by American mathematician and MIT Professor Norbert Wiener (1894-1964) was published in 1958. After direct involvement with Norbert Wiener publication, Albert H. Nuttall has recently made new inroads along with his coauthors in applying the Wiener-Volterra model. A general description of a nonlinear system to the third order is termed the Nuttall-Wiener-Volterra model (NWV) after its co-founders. In this formulation, two measurement waveforms on the system are required in order to characterize a specified nonlinear system under consideration: an excitation input, $x(t)$ (the transmitted signal) and a response output, $z(t)$ (the received signal). Given these two measurement waveforms for a given system, a kernel response, $\mathrm{h}=[\mathrm{h} 0, \mathrm{~h} 1, \mathrm{~h} 2, \mathrm{~h} 3]$ between the two measurement points, is computed via a least squares approach that optimizes modeled kernel values by performing a best fit between measured response $z(t)$ and a modeled response $y(t)$. New procedures developed by A. Nuttall are invoked to significantly diminish the exponential growth of the computed number of kernel coefficients with respect to third order and higher orders to combat and reasonably reduce the curse of dimensionality.

\section{$11: 30$}

3aSP11. Superdirective non-linear beamforming with deep neural network. Mitsunori Mizumachi (Dept. of Elec. Eng. and Electronics, Kyushu Inst. of Technol., 1-1 Sensui-cho, Tobata-ku, Kitakyushu 805-8440, Japan, mizumach@ecs.kyutech.ac.jp) and Maya Origuchi (Dept. of Elec. Eng. and Electronics, Kyushu Inst. of Technol., Kitakyushu, Fukuoka, Japan)

Beamforming has been one of important issues in acoustic signal processing, since it can achieve signal enhancement and sound source localization. In general, traditional beamformers are designed by an analytical approach or an adaptive approach. It is, however, difficult to properly optimize the beamformers under the complicated acoustical scene. An alternative non-linear beamforming can be substituted for the linear beamforming. In this study, a flexible framework for optimizing the beamformer is introduced based on a deep neural network. Capturing acoustic signals using a microphone array is regarded as spatial sampling, so that annoying grating lobes appear in beam-pattern when the relationship between the wavelength and the microphone spacing does not satisfy the sampling theorem. The proposed method achieves sub-band beamforming using the non-uniform microphone array with eight nesting microphones, which are carefully designed not to cause spatial aliasing. Feasibility of the proposed method has been confirmed by computer simulation. The proposed non-linear beamformer could successfully achieve superdirectivity compared with conventional beamformers.

\section{1:45}

3aSP12. Defect detection in a concrete bridge by a non-destructive technique. Masato Abe, Toyota Fujioka, and Yoshifumi Nagata (Comput. and Information Sci., Iwate Univ., 4-3-5 Ueda, Morioka 020-8551, Japan, abe@ cis.iwate-u.ac.jp)

Post-tensioned prestressed concrete is a method for overcoming concrete's natural weakness in tension, and it is used widely to produce bridges with a longer span. Because of poor workmanship or quality control during 
construction, however, sometimes the ducts containing the prestressing tendons are not fully filled, leaving voids in the grout where the steel is not protected from corrosion. Therefore, we propose a method to detect such the voids using multiple sensors and an impulse hammer. The sensors pick up the vibration wave caused by the impulse hammer. The experimentally picked-up vibration waves are compared with those simulated by the FiniteDifference Time Domain (FDTD) method, and it is found that these two waves quite similar. It is also found from some experiments for the bridge pier that we can detect (1) the presence or absence of a void and (2) the void position.

\title{
Session 3aUWa
}

\section{Underwater Acoustics, Noise and Acoustical Oceanography: Transmission Through the Air-Water Interface}

\author{
Peter H. Dahl, Cochair \\ Applied Physics Laboratory, University of Washington, Mechanical Engineering, 1013 NE 40th St., Seattle, WA 98105 \\ David R. Dall'Osto, Cochair \\ Acoustics, Applied Physics Laboratory at University of Washington, 1013 N 40th St., Seattle, WA 98105
}

\section{Invited Papers}

\section{8:15}

3aUWa1. Complex acoustic intensity transmission through the air-water interface. David R. Dall'Osto (Acoust., Appl. Phys. Lab. at Univ. of Washington, 1013 N 40th St., Seattle, WA 98105, dallosto@apl.washington.edu) and Peter H. Dahl (Mech. Eng., and Appl. Phys. Lab., Univ. of Washington, Seattle, WA)

Sound transmission through the air-water interface occurs when there is a non-zero normal component of active and reactive acoustic intensity. Transmission occurs for both active intensity (pressure and particle velocity in phase) and reactive intensity (pressure and particle velocity out of phase), and depending on the proximity of the source to the surface these can convert from one form to the other. Intensity transmission through the interface is governed Chapman's Law-which differs slightly from Snell's law but is consistent with the requirement of phase continuity along the surface. Properties of intensity transmission through the air-water interface are examined using three examples: (1) Marine pile driving, where sound generated by the in-air portion of the pile is injected into the water-column, and sound generated underwater leaks into the air. (2) Transmission of far-field active and reactive intensity generated by aircraft, and how Chapman's law or Snell's law describe Doppler shifts. (3) The ability of a fish-catching bat to locate fish underwater with sonar, and how transmitted active intensity resonates with the swim-bladder of the fish generating a near-field that radiates into the air as active intensity. [Research supported by ONR.]

\section{$8: 35$}

3aUWa2. The role of diffraction in sound transmission through air-water interfaces. Oleg A. Godin (Phys. Dept., Naval Postgrad. School, 833 Dyer Rd., Bldg. 232, Monterey, CA 93943-5216, oagodin@nps.edu)

Ray-theoretical modeling and elementary considerations that are based on plane-wave reflection coefficients predict very weak sound transmission through air-water interfaces because of a large contrast in the acoustic impedances of air and water. With ray-type geometric contributions to transmitted acoustic energy being suppressed by the disparities in the sound speeds and densities, wave diffraction often plays the dominant role in coupling acoustic fields in air and water. In particular, acoustic diffraction leads to the phenomenon of anomalous transparency of air-water interfaces for low-frequency sound. Almost all the energy that is radiated by shallow compact underwater sound sources is transmitted into air [O. A. Godin, Phys. Rev. Lett. 97, 164301 (2006)]. Other scenarios, in which diffraction plays a critical role in sound transmission through air-water interfaces, include excitation of Scholte-type surface waves in water by sonic booms and propagation of Lamb waves along the ocean surface. The paper will review the theory and experimental evidence of the anomalous transparency of air-water interfaces for finite-size underwater sound sources and for coupling of acoustic fields in the ocean and atmosphere by Lamb and Scholte-type surface waves. 
3aUWa3. Compiling the air situation picture from a submerged submarine. Brian G. Ferguson and Gary C. Speechley (Maritime Div., DSTG, PO Box 44, Pyrmont, NSW 2009, Australia, Brian.Ferguson@dsto.defence.gov.au)

The transmission of sound through the air-water interface produces an underwater sound field that can have as many as four separate contributions from an airborne acoustic source. These contributions are identified as: direct refraction, one or more seafloor reflections, the evanescent wave, and sound scattered from a rough sea surface. The relative importance of each contribution depends on the horizontal distance of the source from the receiver, the water depth, the depth of the receiver in relation to the wavelength of the signal radiated by the source, and the roughness of the sea surface. This paper demonstrates how a submerged submarine towing an array of hydrophones is able to detect, classify, localize and track maritime patrol aircraft and helicopters, whilst at depth, by exploiting the direct and indirect sound propagation paths of the aircraft's radiated noise (acoustic signature). As the airborne source is in relative motion with respect to the array, the received signal at each hydrophone is Doppler-shifted in frequency. It is shown that the variation with angle of arrival of the observed Doppler-shifted propeller blade passing frequency is in close agreement with that predicted for the direct refraction path. Similar observations are also made for the seafloor reflected propagation paths. Early warning/long range detection of threat aircraft is enabled by one or more reflections from the sea floor.

9:15

3aUWa4. Light aircraft sound for underwater acoustics experiments. Michael J. Buckingham (Scripps Inst. of Oceanogr., Univ. of California, San Diego, 9500 Gilman Dr., La Jolla, CA 92093-0238, mbuckingham@ucsd.edu)

A propeller-driven light aircraft produces sound in the form of a sequence of harmonics from the propeller and from the reciprocating engine. The lowest frequency, from a two-bladed propeller, is around $80 \mathrm{~Hz}$ with detectable harmonics extending up to about $1 \mathrm{kHz}$. When flying over the shallow ocean at low level, some of the aircraft sound penetrates the air-sea interface to undergo multiple reflections between the seabed and the sea surface as it propagates through the channel. On approach to a sub-surface receiver station, the frequency of a given harmonic is Doppler upshifted, whereas on departure it is downshifted. Since these Doppler shifts depend not only on the speed of the aircraft but also on the geo-acoustic properties of the seabed, they provide the basis of a rapid and efficient inversion technique for surveying the seabed. To develop the technique, shallow-water experiments have been performed using various types of light aircraft as the sound source and a single hydrophone as the receiver. It was found that, with the aid of the grain-shearing theory of wave propagation in unconsolidated sediments, it is possible to recover the sediment geo-acoustic parameters from the Doppler-shifted aircraft harmonics. [Research supported by ONR.]

\section{Contributed Papers}

\section{9:35}

3aUWa5. A geoacoustic inversion technique using the low-frequency sound from the main rotor of a Robinson R44 helicopter. Dieter A. Bevans, Michael J. Buckingham (Marine Physical Lab., Scripps Inst. of Oceanogr., 9500 Gilman Dr., La Jolla, CA 92093-0238, dbevans@ucsd.edu), and Paul Hursky (Heat, Light, \& Sound Res. Inc., San Diego, CA)

A series of underwater acoustic experiments utilizing a Robinson R44 helicopter and an underwater receiver station has been conducted in shallow $(16.5 \mathrm{~m})$ water. The receiver station consisted of an 11-element nested hydrophone array with a $12 \mathrm{~m}$ aperture configured as a horizontal line (HLA) $0.5 \mathrm{~m}$ above the seabed. An in-air microphone was located immediately above the surface. The main rotor blades of the helicopter produce low-frequency harmonics, the fundamental frequency being $\sim 13 \mathrm{~Hz}$. The tail rotor produces a sequence of harmonics approximately six times higher in frequency. The first experiment characterized the underwater sound signature of the helicopter with altitude and range. Using analytical and numerical 3-layer (atmosphere-ocean-sediment) acoustic propagation models a sediment geoacoustic inversion technique has been developed. This technique, requiring only knowledge of the relative location of the sensors and sound source (helicopter), uses the cross-correlation between HLA sensor pairs to produce the estimated time delay of the head wave. The results from the simulations and the latest experiment are presented. [Research supported by ONR, SMART(DOD), NAVAIR, and SIO.]
3aUWa6. Model validation of rough surface scattering at a water/air interface using a hybrid parabolic equation model. Kevin B. Smith (Dept. of Phys., Naval Postgrad. School, 833 Dyer Rd., Bldg. 232, Rm. 114, Monterey, CA 93943, kbsmith@nps.edu), Mustafa Aslan (Turkish Naval Acad., Istanbul, Turkey), and Geoffrey Moss (Naval Undersea Warfare Ctr. Div. Newport, Newport, RI)

Traditionally, ocean acoustic propagation models assume the sea surface can be treated as an idealized pressure release boundary. For flat surfaces, this can easily be accomplished through a variety of modeling techniques. Rough surfaces, however, introduce additional complexities in numerical models. For propagation models based on the parabolic equation that utilize split-step Fourier (SSF) algorithms, previous work has involved field transformational techniques to treat the rough surface displacements. Such techniques assume small angle scattering at the interface, which may not produce adequate numerical accuracy. An alternative approach is to model the physical water/air interface, and allow the higher order propagator functions of the parabolic approximation to more accurately model the rough surface scatter. However, the introduction of such large interface discontinuities have been known to introduce phase errors in SSF-based models. In this work, a previously developed hybrid split-step Fourier/finite-difference approach is implemented at the water/air interface. Results are compared with standard SSF smoothing approaches, as well as the pressure release field transformational technique, for simple rough surfaces. A finite element model is utilized to provide a benchmark solution and comparisons are made for both standard Dirichlet and explicit mixed media treatments of the air-water interface. Tradeoffs between accuracy and stability are discussed, as well as transmission across the water/air interface. 


\title{
Session 3aUWb
}

\section{Underwater Acoustics: Topics in Underwater Acoustics (Poster Session)}

\author{
Aubrey L. Espana, Chair \\ Acoustics Dept., Applied Physics Lab. - Univ. of Washington, 1013 NE 40th St., Box 355640, Seattle, WA 98105
}

\begin{abstract}
All posters will be on display from 9:00 a.m. to 12:00 noon. To allow all contributors in this session to see the other posters, authors of odd-numbered papers will be at their posters from 9:00 a.m. to 10:30 a.m. and authors of even-numbered papers will be at their posters from 10:30 a.m. to $12: 00$ noon.
\end{abstract}

\section{Contributed Papers}

3aUWb1. Seabed target discrimination using multistatic acoustic scattering data. Erin M. Fischell and Henrik Schmidt (Mech. Eng., MIT, 77 Massachusetts Ave., 5-204, Cambridge, MA 02139, emf43@mit.edu)

One application for autonomous underwater vehicles (AUVs) is detecting and classifying hazardous objects on the seabed. We have been studying an alternative acoustic approach to this problem in which an acoustic source insonifies seabed targets while receiving AUVs discriminate targets based on features sensed in the resulting 3D scattered fields. The receiving AUVs have entirely passive sensing payloads, and therefore lower power draw and cost. When both the acoustic source and receiver are mobile, multistatic scattering data are collected. Using the OASES-SCATT scattering simulator, we studied how multistatic scattering data collected by AUV-based receivers around targets insonified by AUV-based sources might be used for sphere and cylinder target characterization in terms of target shape, composition, and size. The impact of target geometry on multistatic scattering fields is explored, and a target discrimination approach developed in which the acoustic source and receiver to circle the target with the same radial speed, collecting multistatic scattering data at constant bistatic angles. The frequency components of the multistatic scattering data at different bistatic angles are used to form probabilistic and machine learning models for target characteristics. New data are then classified using these models. [Work supported by ONR and Battelle.]

3aUWb2. A spectral approach to geoacoustic inversion from sparse impulsive sources. Thomas W. Yudichak, Steven A. Stotts, Robert A. Koch, Dan G. Jacobellis, and Daniil Ruditskiy (Appl. Res. Laboratories, The Univ. of Texas at Austin, P.O. Box 8029, Austin, TX 78713-8029, yudi@arlut. utexas.edu)

A common approach to geoacoustic inversion is to match the temporal structure displayed by modeled and measured acoustic data produced at a small number of frequencies by range sampling source emissions over a broad interval between a source and a receiver. An alternative approach is to match spectral structure in modeled and measured acoustic data for a small number of source-receiver separations from a fine frequency sample over a broad bandwidth. The latter approach is demonstrated with Shallow Water '06 experiment data in which the acoustic sources were imploding light bulbs. Geoacoustic parameter values obtained by this approach are compared to values obtained from previous inversions, and the limits of validity of the approach are discussed.

3aUWb3. Reciprocity calibration of underwater acoustic transducer in a reverberation pool. Dajing Shang, Qi Li, and Jundong Sun (Underwater Acoust. Eng. Dept., Harbin Eng. Univ., Nangang District Nantong St., No.145,Harbin City, Heilongjiang Province 150001, China, shangdajing@ hrbeu.edu.cn)

A new underwater acoustic transducer reciprocity calibration method was put forward in this paper. This calibration was finished by the space-averaging in the reverberation pool, and the influence of normal mode interference was eliminated, so the reverberation field acoustic parameters were obtained in the reverberation pool. The calibration can be set up in a small reverberation pool. In the case of the same frequency range acoustic transducer calibration, the reverberation pool for reverberation method reciprocity calibration can be smaller than the free-field pool for free-field reciprocity calibration. The reverberation field reciprocity constant was determined by measuring the reverberation time, the single-frequency sine signal was emission by the sound source higher than the cutoff frequency, the transmitting transducer and receiving transducer was space-averaged in the area of the reverberation, and the corresponding electrical parameters were obtained, so the receiving sensitivity for the reciprocity transducer and the testing hydrophone was calculated. The testing and the results of the reverberation calibration are presented in this paper. The error between the hydrophone calibration by the reverberation method and the factory free field calibration was less than $1.4 \mathrm{~dB}$, and the A kind of measuring uncertainty is less than $0.13 \mathrm{~dB}$. This method can be applied to all kinds of hydrophones and transducer calibration.

3aUWb4. Modeling and simulation technique for development of multibeam echo sounder. Dong Hwan Jung, Jea Soo Kim, and Gi Hoon Byun (Ocean Sci and Technol., Korea Maritime and Ocean Univ., 727 Taejongro, Yeongdo-Gu, 253, Busan 49112, South Korea, ehdghkss104@naver. com)

Multibeam echo sounder (MBES) is commonly used for rapid sea floor mapping. We present a time-domain integrated system simulation technique for MBES development. The simulation and modeling (M\&S) modules consist of four parts: sensor array signal transmission, propagation and backscattering modeling in the ocean environment, beamforming of the received signals, and image processing. Also, the simulation employs a ray-theorybased algorithm to correct the reconstructed bathymetry, which has errors due to the refraction caused by the vertical sound velocity profile. The developed $M \& S$ technique enables design parameter verification and system parameter optimization for MBES. The framework of this technique can also be potentially used to characterize the seabed properties. Finally, typical sea floor images are presented and discussed.

3aUWb5. Prediction of array gain in directional noise field. Jisung Park, Yonghwa Choi, Jeasoo Kim (Ocean Eng., Korea Maritime and Ocean Univ., 727 Taejong-ro, Yeongdo-Gu, Busan 49112, South Korea, pjs840627@gmail.com), Sungho Cho (Maritime Security Res. Ctr., Korea Inst. of Ocean Sci. \& Technol., Ansan, South Korea), and Jungsoo Park (Agency for Defense Development, Jinhae, South Korea)

The Array Gain(AG) is a metric to assess the performance of an array and is dependent on the configuration of array, frequency, as well as on the directionality of noise. In this study, AG is calculated based on the spatial coherence between sensor elements in directional noise environment for a given array shape. The estimated AG is then compared with AG derived from the sea going data based on the signal to noise ratio. The results are presented and discussed. 
3aUWb6. Research for the position detection of the object within the tube liquid using sound wave. Seijun Muto, Katsumi Fukuda (National Inst. of Technol., Tokyo College, School, Hachioji, Kunugidacyo 1220-2, Tokyo, Japan, seijunsu47@yahoo.co.jp), and Yoshihiro Nishimura (National Inst. of Adv. Industrial Sci. and Technol., Tsukuba, Ibaraki, Japan)

The purpose of detecting using sound waves the position of an object in the tube filled with liquid. Experiments were carried out taking into account the frequency and oscillation of the process of the sound. As a result, good results for the position detection of the object to obtain a new knowledge.

3aUWb7. The noise of rock n'roll: Incidental noise characterization of underwater rock placement. Rute Portugal, Sei-Him Cheong (Gardline Environ., Gardline Geosurvey, Ltd., Gardline Environ., Endeavour House, Admiralty Rd., Great Yarmouth NR30 3NG, United Kingdom, rute.portugal@gardline.com), James Brocklehurst (Royal Boskalis Westminster N.V., Papendrecht, Netherlands), and Breanna Evans (Gardline Environ., Gardline Geosurvey Ltd., Great Yarmouth, United Kingdom)

Underwater noise is a growing concern to conservation and stock management efforts to which supra-national organizations (e.g., OSPAR or the European Union) and governments (e.g., USA) are beginning to respond by building catalogues of the noise introduced in the marine environment by human activity. Rock placement is a construction activity for which there is scarcely any data available. In order to fill the knowledge gap, opportunistic recordings were taken while the Gardline Mk 3 hydrophone array was deployed for Passive Acoustic Monitoring and mitigation for marine mammals. The recordings were analysed for their spectral and temporal characteristics, a correlation analysis between the amount of rock placed and the intensity of sound produced was made and the suitability of the hydrophone array for the collection of this type of data was assessed.

3aUWb8. Sound exposure level and energy spectral density of underwater explosions in shallow water over a coral substrate off the southern coast of $\mathrm{O}^{`} \mathbf{a h u}$, Hawai'i. Alexander G. Soloway (Mech. Eng., Univ. of Washington, 1013 NE 40th St., Seattle, WA 98105-6698, soloway@u.washington.edu), Peter H. Dahl (Mech. Eng. and Appl. Phys. Lab, Univ. of Washington, Seattle, WA), and Lee H. Shannon (Naval Facilities Eng. and Expeditionary Warfare Ctr., Pearl Harbor, $\mathrm{HI}$ )

This work presents the sound exposure levels (SEL) and energy spectral densities (ESD) from underwater explosions measured in shallow water (10$18 \mathrm{~m}$ ) at distances of 500 to $1500 \mathrm{~m}$ at the Pu'uloa Underwater Detonation Range off the southern coast of Oahu. Nine explosive charges, with TNTequivalent weights of 2.2 to $8.6 \mathrm{~kg}$, were detonated on a seabed characterized by a thin sand layer over limestone. The ESD of the measurements are characterized by high propagation loss in the frequency ranges 50 to 350 Hz. Previous studies have shown that this is a common characteristic of this environment with the upper and lower frequencies directly related to the geoacoustic properties of the seabed and the waveguide geometry. Unlike measurements collected in sandy environments, where SEL agreed with empirical predictions, the SEL for these measurements differ by up to $30 \mathrm{~dB}$. To understand the mechanisms responsible for these lower than expected levels, a geoacoustic model for the seabed is developed using these frequencies and utilized in broadband modelling simulations. The SEL will also be calculated for these simulations and compared to the measured levels. [Research supported by Pacific Fleet with partial support from the Office of Naval Research.]
3aUWb9. Point measurements of ambient biological noise before, during, and after multiple underwater detonation events over coral substrate off the southern coast of Oahu, Hawai. Dara M. Farrell (Appl. Phys. Lab., Mech. Eng., Univ. of Washington, Henderson Hall, Seattle, WA 98105, daraf@uw.edu), Lee H. Shannon (Marine Resources Assessment Diving Services, Naval Facilities Eng. and Expeditionary Warfare Ctr., Pearl Harbor, Hawaii), Peter H. Dahl (Mech. Eng., Univ. of Washington, Appl. Phys. Lab, Seattle, WA), and David R. Dall'Osto (Appl. Phys. Lab, Seattle, WA)

Measurements were taken of background noise at the U.S. Navy's Pu'uloa Underwater Detonation Range off the south coast of $\mathrm{O}^{\prime}$ ahu during a U.S. Navy underwater explosive training exercise in shallow water (10-18 $\mathrm{m})$ where distance from the detonation site, charge size, and explosive composition were controlled. The autonomous recording unit was deployed approximately $1500 \mathrm{~m}$ from the detonation location. The combined data from the paired low $(-220 \mathrm{~dB}$ re $1 \mathrm{~V} / \mu \mathrm{Pa})$ and high $(-170 \mathrm{~dB}$ re $1 \mathrm{~V} / \mu \mathrm{Pa})$ sensitivity hydrophones gives an approximately $100 \mathrm{~dB}$ dynamic range view of each event. Each trial consisted of three replicate events per charge weight $(2.2,4.5$, and $8.6 \mathrm{~kg})$ for a total of nine explosive events over the coral substrate. An analysis of the background noise environment immediately before and after each replicate is presented using metrics such as spectral probability density. Results are considered in the context of typical coral reef noise including discussion of snapping shrimp noise (one of the dominant features of the soundscape) before and after each event. [Work supported by U.S. Pacific Fleet.]

3aUWb10. Stochasticism in noise generated by an array of marine hydrokinetic devices. Erin C. Hafla (Civil Eng., Montana State Univ., 205 Cobleigh Hall, Bozeman, MT 59717-3900, erinhafla@gmail.com), Erick Johnson (Mech. Eng., Montana State Univ., Bozeman, MT), and Jesse Roberts (Energy \& Climate, Sandia National Labs., Albuquerque, NM)

Marine hydrokinetic (MHK) devices generate electricity from the motion of tidal and ocean currents and ocean waves and provide another source of renewable energy. Additionally, MHK devices are also a new source of anthropogenic noise in the marine ecosystem and must meet regulatory guidelines that mandate a maximum amount of noise that may be generated. In the absence of measured levels from in-situ deployments, a model for predicting the propagation of sound from an array of MHK sources in a real environment needs to be established. A 3D finite-difference, time-domain solution to the governing velocity-pressure equations is used, which permits a finite number of complex sources and spatially varying sound speeds, bathymetry, and bed composition. However, deterministic solutions to these types of problems cannot capture uncertainties in the source profiles that may result from operational changes. This work presents the broadband sound pressure levels from an array of MHK sources as the amplitude and frequency from each source are allowed to vary. This Monte Carlo approach demonstrates that the idealized, deterministic solution, vastly underestimates the compounding uncertainty on the final sound field and that these small variations in the source profile cannot be ignored.

3aUWb11. Acoustic characterization of a wave energy converter. Brian L. Polagye, Paul Murphy (Mech. Eng., Univ. of Washington, Box 352600, Seattle, WA 98195-2600, bpolagye@u.washington.edu), Keith Bethune, Patrick Cross, and Luis Vega (Hawai'i Natural Energy Inst., Univ. of Hawai'i, Manoa, HI)

As progress toward the commercial deployment of wave energy converters accelerates, it is important to ensure that these renewable energy systems do not have unintended, adverse environmental consequences. While the sound from wave energy converters is unlikely to cause acoustic injury to marine animals, it may affect their behavior. Here, we present measurements from a point-absorber wave energy converter at the U.S. Navy Wave Energy Test Site in Kaneohe Bay, HI. Measurements of wave converter sound are obtained for a range of sea states using a combination of freedrifting near-surface measurements and stationary bottom packages. The relative effectiveness of these systems are contrasted and the unique challenges associated with acoustic measurements at energetic sites discussed. For example, fixed measurements are found to be substantially contaminated by flow-noise (non-propagating sound) during long-period ocean 
swell, while free-drifting measurements require significant post-processing to avoid convolving flow-noise or self-noise with wave converter sound. Preliminary results of parabolic equation modeling is also presented and used to interpret spatially distributed measurements.

3aUWb12. Acoustic ground truthing of airgun noise in Chatham Rise, New Zealand. Sei-Him Cheong (Marine Wildlife, Gardline Geosurvey, Endeavour House, Admiralty Rd., Great Yarmouth, Norfolk NR30 3NG, United Kingdom, sei-him.cheong@gardline.com)

Underwater noise is a growing concern to conservation of the marine life. Noise generated by seismic survey in particular is recognised as a significant and pervasive pollutant, with the potential of impacting the marine ecosystem. Between the 1st February and 21st March 2016, a geophysical research survey was conducted in Chatham Rise, New Zealand, to collect seismo-acoustic data using a Sercel Sentinel seismic streamer in order to ground truth the underwater noise impact assessment, conducted according to the Department of Conservation (New Zealand) 2013 Code of Conduct. Data were analyzed in order to determine the received sound level at a distance up to $3 \mathrm{~km}$ from the seismic source array. This paper establishes the method to predict the mitigation impact radii from seismic data in order to validate the results obtained based on predictive noise modelling using Gardline $360 \mathrm{M}$ model. The study was also aimed to provide confidence to the capability of predictive modeling for estimating the acoustic impact zone of a seismic sound source. Data showed that seabed reflection can fluctuate significantly according to sediment topography and other environmental influences; however, a very consistent trend can be obtained from direct propagation to confidently establish mitigation radii. Results show that the employment of a seismic streamer for the establishment of effective mitigation radii is technically feasible and may be used as a tool to ground truth predictive modelling as part of a marine mammal mitigation plan.

\title{
Session 3aUWc
}

\section{Underwater Acoustics: Inversion, Beam-Forming, and Calibration I}

\author{
Steven A. Stotts, Chair \\ Environmental Sciences Laboratory, Applied Research Labs/The University of Texas at Austin, 10000 Burnet Rd., Austin, \\ TX 78759
}

\section{Contributed Papers}

\section{$10: 30$}

3aUWc1. Impact of array tilting on source-range estimation based on the array/waveguide invariant. Chomgun Cho and Hee-Chun Song (Scripps Inst. of Oceanogr., UCSD, 9500 Gilman Dr., La Jolla, CA $92093-$ 0238, chomgun@ucsd.edu)

Recently, the array/waveguide invariant was proposed for robust range estimation of a broadband source in a waveguide environment using a shortaperture vertical array (VA). The approach involves conventional planewave beamforming and exploits the separation of arrivals in beam angle and travel time. The beam angle estimate, however, can be sensitive to array tilting. In this paper, we investigate the impact of array tilting on source-range estimation based on the array/waveguide invariant. Analysis of experimental data indicates that even a small tilt angle $\left(<2^{\circ}\right)$ of a 1.2 -m long VA can result in a relative range error of $20 \%$ or more for a source $(9-17 \mathrm{kHz})$ at $3-$ $\mathrm{km}$ range in $\sim 100$-m deep water. Inversely, the array tilt angle can be estimated for a known source range.

\section{0:45}

3aUWc2. Direction-of-arrival estimation using a three-dimensional cross array equipped underwater glider. Yong-Min Jiang (Res. Dept., NATO-STO-Ctr. for Maritime Res. \& Experimentation, Viale San Bartolomeo 400, La Spezia 19126, Italy, yong-min.jiang@cmre.nato.int)

With the development of autonomous platform and sensor technologies, unmanned underwater vehicles have been more and more involved in maritime intelligent surveillance and reconnaissance missions. The NATOSTO_CMRE has been exploring the capabilities of gliders for monitoring underwater environment by means of passive acoustic sensing. During the CMRE GLISTEN'15 sea trial, an eight-element, three dimensional cross array equipped Webb Slocum glider was deployed to study its capability of providing the direction of arrival (DOA) estimation of the signal of interest. Continuous wave pulses at multiple frequencies $(300-1000 \mathrm{~Hz})$ were transmitted by an acoustic source. The 3D array equipped glider was programmed to glider along a helix curve in the vicinity of the acoustic source. The DOA capability of the glider using the acoustic data collected by the acoustic payload, along with the glider pitch-roll-heading, and position information is evaluated in this study. [Work funded by NATO-Allied Command Transformation.]

\section{1:00}

3aUWc3. Near-field localization of under water noise source based on matrix spatial filter with vector sensor array processing. Wang Xueyan (College of Underwater Acoust. Eng.,Harbin Eng. Univ., Harbin 150001, China, Harbin, Hei Longjiang, China), Shi Shengguo, and Shi Jie (Sci. and Technol. on Underwater Acoust. Lab., Harbin Eng. Univ., Harbin 150001 Harbin, Shuisheng Dept.,College of Underwater Acoust. Lab., Harbin Eng. University, Harbin 150001, China, Harbin, China, shishengguo@ hrbeu.edu.cn)

To detect the weak sources of interest area which are obscured by strong sources, a new algorithm of near-field localization of underwater noise source based on matrix filter is proposed. This algorithm use matrix filter as a pretreatment to filter array data of vector sensor, optimized design method is used to design the matrix filter, and then formulate the problem into a second-order cone programming model to calculate the matrix equation, which can strictly control the attenuation of stop band, and it can avoid increment of noise power by restrain the norm of matrix filter. With this filter matrix, a new covariance matrix is produced for the following beam-forming processing with vector sensor array. Compared with the previous work, this algorithm can accurately locate the weak sources of interest that are obscured by strong sources; in addition, higher location accuracy and spatial resolution, 
better spatial anti-aliasing performance, as well as stronger capability to distinguish the starboard and larboard benefit from using vector sensor array. The simulation and real data show that this algorithm can precisely locate the weak source of interest among some strong sources.

\section{1:15}

3aUWc4. A sequential filtering algorithm for range estimation of a moving ship based on striation geometric features. Qunyan Ren, Li Ma (Key Lab. of Underwater Acoust. Environment, Inst. of Acoust., Chinese Acad. of Sci., No. 21 North 4th Ring Rd., Haidian District, Beijing 100190, China, renqunyan@mail.ioa.ac.cn), Shengchun Piao (Sci. and Technol. on Underwater Acoust. Lab., Harbin Eng. Univ. Harbin, Harbin, China), Shengming Guo (Key Lab. of Underwater Acoust. Environment, Inst. of Acoust., Chinese Acad. of Sci., Beijing, China), and Tianjun Liao (State Key Lab. of Complex System Simulation, Beijing, China)

There exist regular striation pattern in the broad-band noise field excited by a moving ship in shallow waters, whose time (range) frequency structure can be interpreted by the waveguide invariant theory. The striation characteristics have been exploited for underwater acoustic inversion problems, e.g., sediment geoacoustic parameter characterization. In this paper, the geometrical features of specific striations are observed for continuously ship ranging by a sequential algorithm. Comparing to matched-field processing techniques, this approach also has no requirements prior knowledge of environmental properties and burden of forward sound field calculation, which is similar to virtual receiver approach. The technique is tested on the striation fragments extracted from the ship noise data collected in Dalian 2008, and the outputs are in high agreement with in-situ GPS measurements. The results from independent runs with different initial values also suggest that this approach is robust to initial conditions in the sense of all runs can converge to the true values rapidly.

\section{$11: 30$}

3aUWc5. Acoustic source localization in ocean waveguides using principles of Riemannian geometry. Steven Finette and Peter Mignerey (Acoust. Div., Naval Res. Lab.,Washington, DC 20375-5320, steven.finette@nrl. navy.mil)

Source localization in underwater acoustics entails a comparison between acoustic fields involving some measure of correlation, looking for similarity between the acoustic field propagated from the true source location and replica fields propagated from different locations in the waveguide. The uniqueness of the deterministic Green function between pairs of sourcereceiver positions forms the basis for the solution to this inverse problem. We consider a novel approach to source localization based on non-Euclidean geometry, where the "distance" between cross-spectral density matrices (CSDMs) is used to estimate the spatial location of the source. The traditional Euclidean distance is not necessarily appropriate because CSDMs are not arbitrary points in space; rather, they form a manifold constrained by the facts that CSDMs are both Hermitian and positive definite. These properties naturally lead to the interpretation of geodesic distance between CSDMs as a measure of similarity between acoustic fields with this minimum distance, parametrized by replica source location, establishing an estimate of the source position. We discuss the underlying concepts and present simulation results for a waveguide with internal wave-induced ocean variability. Several Riemannian metrics are considered and compared to more traditional approaches to matched-field localization. [Work supported by the Office of Naval Research.]

\section{Exhibit}

The instrument and equipment exhibit is located near the registration area in the Coral Foyer.

The Exhibit will include computer-based instrumentation, scientific books, sound level meters, sound intensity systems, signal processing systems, devices for noise control and acoustical materials, active noise control systems, and other exhibits on acoustics.

Exhibit hours are Monday, 28 November, 5:30 p.m. to 7:00 p.m., Tuesday, 29 November, 9:00 a.m. to 5:00 p.m., and Wednesday, 30 November, 9:00 a.m. to 12:00 noon.

Coffee breaks on Tuesday and Wednesday mornings will be held in the exhibit area as well as an afternoon break on Tuesday.

The following companies have registered to participate in the exhibit at the time of this publication:

AIP Publishing: publishing.aip.org/

American Institute of Physics: https://www.aip.org/

Aqua Sonic, Inc.: aqua-sonic.com

Echoview Software: www.echoview.com/

Head acoustics GmbH: www.head-acoustics.de/eng/

Mason Industries: www.mason-industries.com/masonind/

Ocean Sonics Ltd.: oceansonics.com/

ODEON A/S: www.odeon.dk/

PAC International: www.pac-intl.com/

RION Co., Ltd: www.rion.co.jp/english/

Sensidyne: www.sensidyne.com/

Springer: www.springer.com/us/

Teledyne RESON Inc.: www.teledyne-reson.com/ 


\title{
Session 3pAAa
}

\section{Architectural Acoustics and Signal Processing in Acoustics: Advanced Analysis, Simulation, and Auralization in Room Acoustics II}

\author{
Michael Vorlaender, Cochair \\ ITA, RWTH Aachen University, Kopernikusstr. 5, Aachen 52056, Germany \\ Tetsuya Sakuma, Cochair \\ The University of Tokyo, 5-1-5 Kashiwanoha, Kashiwa 277-8563, Japan \\ Toshiki Hanyu, Cochair \\ Junior College, Department of Architecture and Living Design, Nihon University, 7-24-1, Narashinodai, \\ Funabashi 274-8501, Japan \\ Chair's Introduction-1:00
}

\section{Contributed Papers}

1:05

3pAAa1. A synthesized boundary condition for wave-based room acoustics simulations using ensemble averaged impedance measured in-situ. Toru Otsuru, Reiji Tomiku (Oita Univ., 700 Dannoharu, Oita, Oita 8701192, Japan, otsuru@oita-u.ac.jp), Noriko Okamoto (The Univ. of Kitakyushu, Kitakyushu, Japan), Sakura Saigo, and Saki Yamauchi (Oita Univ., Oita, Japan)

The importance of wave-based room acoustics simulation has been increasing in various fields of researches and design-stages. The problem remained, however, is how to model the rooms' absorptive boundary conditions. It is known that traditional absorption indices like sound absorption coefficient and normal impedance are not always suit for such a fine method like finite element method. Then, the concept and measurement technique of ensemble averaged impedance were presented. In our previous studies, a series of in-situ measurement of ensemble averaged impedances were successfully performed to result excellent reproducibility and repeatability. Herein, the outline of the concept of ensemble averaged impedance including the measurement technique is summarized, first. Second, several example results of practical measurements are given to exhibit that their uncertainties stay within the range for wave-based simulations to keep their resulting uncertainties less than just noticeable difference. Then, a mathematical-physical model of synthesized boundary condition is given using the ensemble averaged impedance in the sound field analysis by finite element method. Finally, the results of sound field simulations of realistic rooms are shown to examine the plausibility of the model.

\section{$1: 20$}

3pAAa2. Absorption characteristics of micro-perforated panels using scale modeling and microflown impedance gun. Stephen Dance, Simon Brown, and Carl Ruegger (Urban Eng., London South Bank Univ., Borough Rd., London SE1 0AA, United Kingdom, dances@1sbu.ac.uk)

A theoretical and experimental comparison of the absorption characteristics of micro-perforated panels was undertaken. The micro-perforated panels were designed using the Sheffield University Java based website, constructed from $0.3 \mathrm{~m}$ by $0.3 \mathrm{~m}$ of acrylic, the thousands of holes were laser cut. The panels were large enough to be tested using the microflown impedance gun as well as a new scale model reverberation chamber based at London South Bank University. The 1:10 scale model reverberation chamber was constructed from acrylic materials and used a 3-D printed dodecahedron sound source. Data acquisition was through a $1 / 4$ " microphone connected through a Nexus preamplifier to a 24 bit $192 \mathrm{kHz}$ sound card. MATLAB code was used to acquire and process the signal in accordance to ISO 354 including air compensation for high frequencies. Results will be presented for theoretical, scale, and impedance based measurement of the absorption coefficients for the micro-perforated panels.

\section{$1: 35$}

3pAAa3. A hybrid impedance measurement method to inversely determine the impedance of finite porous absorber material samples. Rob Opdam, Mark Müller-Giebeler, and Michael Vorländer (Inst. of Tech. Acoust., RWTH Aachen Univ., Kopernikusstr. 5, Aachen 52074, Germany, rob.opdam@akustik.rwth-aachen.de)

The determination of complex angle-dependent reflection factors is not common practice. Mostly, because existing methods are complicated and very time-consuming. This work presents a method that only needs a single sound pressure measurement of a finite porous absorber piece, placed in a semi-anechoic chamber, as input data. The complex pressure data obtained of the measurement are compared with that of a finite element method (FEM) simulation, which is based on the same geometric dimensions of the material sample as in the measurement, but with an arbitrary porous material. With a non-linear fitting algorithm, the simulated complex pressure data are adjusted to fit the measured data iteratively by changing the absorber model parameters (porosity, flow resistance, etc.) in the FEM simulation. When the simulated and measured results are identical within a defined threshold, the characteristic absorber properties are found and with that the angle-dependent complex reflection factors are determined. Applying this inverse approach allows also for a correction of the edge effects of the finite material sample, such that the found impedance is the impedance as measured on an infinite extended material sample and it also determines the individual material properties as porosity and flow resistivity of a porous material. 
frequency range of the directional reproduction, and the results for various

3pAAa4. Finite-difference time-domain analysis in porous materials by using Z-transform theory and infinite impulse response filter design. Jing Zhao, Hyojin Lee, and Shinichi Sakamoto (Inst. of Industrial Sci., Univ. of Tokyo, Japan, Tokyo 153-8505, Japan, zhaojing@iis.u-tokyo.ac. jp)

Porous materials are wildly used to control noise in various places. When simulating sound field containing porous materials by Finite-difference time-domain (FDTD) method, it is necessary to develop FDTD formulations in the porous materials. When the frame of the porous material is motionless, the porous material can be replaced on the macroscopic scale by an equivalent fluid. Based on the equivalent fluid model, FDTD formulations in the porous material are developed. The method combines IIR (Infinite impulse response) filter design and $\mathrm{Z}$ transform theory. The effective bulk modulus and the effective density of the porous material are frequency dependant, which is designed as IIR filter. Wave equations with IIR filter modeled parameters are solved in $\mathrm{Z}$ domain to avoid troublesome convolution integrals in the time domain. At the $\mathrm{Z}$ domain, new parameters are assumed to simplify the wave equations. Finally, wave equations and assumed parameters are returned back to the time domain. The accuracy of the proposed method is verified with the measurement. As two application examples, porous material coved with permeable membrane or non-permeable membrane are simulated and compared with the theory.

\section{2:05}

3pAAa5. Signal processing method for compensating air absorption and phase change in room impulse response. Shinichiro Koyanagi (Takenaka R\&D Inst., 1-5-1, Ohtsuka, Chiba, Inzai-shi 270-1395, Japan, koyanagi.shinichirou@takenaka.co.jp)

It is often required to remove an influence of air absorption effect from a measured impulse response (e.g., absorption coefficient measurements by a reverberation room method). In particular, a room acoustic test in a scale model is greatly influenced by the air absorption and most of the attenuation of the observed impulse response is caused by air absorption, because the test measurement must be carried out in a high-frequency region including ultrasonic band in order to cover a wide frequency audible range in the real scale. Therefore, a high-accuracy compensation technique is needed so as to correctly evaluate the scale model responses. So far, two methods have been proposed, which utilize a subband division or a time-varying filter. In this study, we propose a new signal processing technique to remove the airabsorption effect by using a propagation distance distribution function obtained from the impulse response and a propagation constant. Some practical application results are presented and show that our proposed method can be performed with high accuracy and overcome some disadvantages of the existing methods.

\section{2:20}

3pAAa6. Design of a compact spherical loudspeaker array for simulating accurate instrument directivities for concert hall measurement and auralization. Matthew T. Neal, Molly R. Smallcomb, David A. Dick, and Michelle C. Vigeant (Graduate Program in Acoust., Penn State Univ., 201 Appl. Sci. Bldg., University Park, PA 16802, mtn5048@psu.edu)

An omnidirectional sound source is often used as a repeatable source to measure impulse responses in concert halls to obtain room acoustics metrics. The limitation of using an omnidirectional source is that real sound sources, such as musical instruments, do not exhibit omnidirectional radiation patterns. For an orchestra, many instruments with unique directional radiation patterns are distributed across the stage. To achieve more realistic auralizations, a 20-element compact spherical loudspeaker array was constructed to simulate the frequency-dependent radiation properties of instruments. This loudspeaker array is capable of achieving a truncated thirdorder spherical harmonics representation of an instrument's radiation pattern. Once the source is moved to different instrument locations around stage, frequency-dependent weighting factors for each driver are controlled to match the source's radiation pattern to that instrument. Full three-dimensional impulse response measurements of the source were made to characterize the source's directional performance. From these measurements, frequency-dependent gains were applied to determine the accuracy and instruments will be presented. The implementation of this source into an existing measurement setup for future measurements of U.S. and European concert halls will also be discussed. [Work supported by NSF Award 1302741.]

\section{2:35}

3pAAa7. Musical instrument directivity database for simulation and auralization. Gottfried Behler (Inst. of Tech. Acoust., RWTH Aachen Univ., Kopernikusstraße 5, Aachen D-52074, Germany, gkb@akustik.rwthaachen.de), Noam R. Shabtai (Inst. of Tech. Acoust., RWTH Aachen Univ., Beer Sheva, Israel), Stefan Weinzierl (Audio Commun. Group, Tech. Univ. Berlin, Berlin, Germany), and Michael Vorländer (Inst. of Tech. Acoust., RWTH Aachen Univ., Aachen, Germany)

A study of 41 modern and historical orchestral musical instruments was made recently. All instruments were played in an anechoic chamber and recorded simultaneously with an array of 32 microphones place on a sphere of $4.2 \mathrm{~m}$ diameter. The musicians were placed in the centre of the sphere and instructed to play chromatic scales covering the whole range of each instrument at two levels-very soft and very loud. From these recordings the directivity for steady tones has been derived. The radiation pattern is represented in the 4-th order spherical harmonics domain, using 25 coefficients and 31 third-octave frequency bands. For 7 instruments, acoustic source centering is applied in order to align the acoustic center of the sound source to the physical center of the microphone array using the center-ofmass approach [Ben-Hagai et al., JASA 2011] and using the phase symmetry approach [Shabtai and Vorländer, JASA 2015]. Analysis of the database is found in [Shabtai et al. JASA 2016 (submitted)]. From this analysis, a database has been created that will be available for scientific use soon. This database is recommended to be used in virtual reality applications to improve the sense of realism perceived by the user.

\section{2:50-3:05 Break}

\section{3:05}

3pAAa8. A new metric to predict listener envelopment based on spherical microphone array measurements and ambisonic reproductions. David A. Dick and Michelle C. Vigeant (Graduate Program in Acoust., The Penn State Univ., 201 Appl. Sci. Bldg., University Park, PA 16802, dad325@psu.edu)

The objective of this work is to propose a new metric for listener envelopment (LEV), the sense of being immersed in a sound field. Current LEV metrics, for example, Late Lateral Energy Level $\left(\mathrm{L}_{\mathrm{J}}\right)$, are based only the late sound field. However, recent studies indicate that the late component of the impulse response (IR) alone is inadequate for predicting LEV. For this study, room IR measurements were obtained with a 32-element spherical microphone array in a total of nine halls ranging from $400-2500$ seats. A subset of these IRs were selected for a subjective listening test and processed for 3rd order Ambisonic reproduction over a 30-loudspeaker array. Two sets of modified IRs were also generated for the listening test by combining parts of IRs from different halls and by bandpass filtering to select certain frequency ranges, respectively. The ambisonic IRs were convolved with anechoic orchestral recordings and subjects rated the perceived LEV for each stimuli. The IRs were also analyzed objectively via plane wave decomposition (PWD). Energy from the PWD as a function of time, frequency, and arrival direction was correlated with the subjective LEV ratings to develop the new metric. [Work was supported by NSF Award 1302741.]

\section{$3: 20$}

3pAAa9. Subjective evaluation of sound diffusion in a surround hall. Hyung Suk Jang (Dept. of Architectural Eng., Hanyang Univ., Seoul 133791, South Korea, janghyungs@gmail.com), Hansol Lim, and Jin Y. Jeon (Dept. of Architectural Eng., Hanyang Univ., Seongdong-gu, Seoul, South Korea)

The effects of diffusing elements on sound field diffuseness were investigated by subjective evaluations using binaural impulse responses (BIRs) in seating areas in a surround hall. The room acoustical parameters as well as the number of peaks $(\mathrm{Np})$ and local maxima within $-20 \mathrm{~dB}$ of the amplitude 
of direct sound were investigated to evaluate the diffuseness in the halls. For the listening test, stimuli were selected to explore the combination of acoustic parameters and degree of diffusion. The music signals were convolved with the measured BIRs in different listening positions. Based on the listening test, it was found that positions close to the diffusers with a high scattering coefficient were preferred by the listeners.

\section{$3: 35$}

3pAAa10. The influence of hearing and seeing on aesthetic judgments of performance spaces. Hans-Joachim Maempel and Michael Horn (Staatliches Institut für Musikforschung, Tiergartenstraße 1, Berlin 10785, Germany, horn@sim.spk-berlin.de)

The perception of rooms involves different modalities, particularly hearing and seeing. Fundamental questions of audio-visual room perception have, however, not been answered yet. We investigated to what extent aesthetic judgments on music and speech performances in different spaces are based on hearing, seeing, and their interaction. Meeting methodological criteria such as optoacoustic commensurability and optoacoustic dissociation, and applying a data-based, three-dimensional, high-resolution optoacoustic simulation providing nearly all perceptually relevant physical cues allowed for a valid proportionate quantification of these shares for the first time. BRIRs and panoramic stereoscopic images were acquired in six rooms. Recordings of both a music and a speech performance were put in the rooms by applying dynamic binaural synthesis and chroma-key compositing. The scenes were played back by the use of a linearized extraaural headset and a semi-panoramic stereoscopic projection system. Test participants were asked to rate the pleasantness, powerfulness, and excitingness of the performance, their spatial presence in the virtual scene, and the matching of the acoustical and the optical room. Statistical analyses indicate among others, that the aesthetic judgments mainly relied on acoustic information, whereas the presence and the matching judgment was mainly influenced by the interaction of optical and acoustical room properties.

\section{3:50}

3pAAa11. Study of the perception of warmth in concert halls and correlation with room acoustics metrics. Kristina M. Sorensen and Michelle C. Vigeant (Graduate Program in Acoust., The Penn State Univ., 201 Appl. Sci. Bldg., University Park, PA 16802, kms674@psu.edu)

Challenges in concert hall acoustics research occur in bridging the domain between subjective attributes and objective metrics. Several studies have shown that the primary attributes associated with concert hall acoustic preference ratings are strength, clarity, and timbre. The purpose of this study was to investigate the correlation of warmth, which is closely associated with timbre, with the objective metric of bass ratio (BR), which is a measure of the ratio of acoustic information (such as reverberation time or strength) at low-frequencies to that at mid-frequencies. The stimuli for this study were simulated binaural room impulse responses (BRIRs) obtained in ODEON and convolved with a concise orchestral anechoic motif. The BRIRs were simulated using models of five European halls. A model of the Vienna Musikverein with multiple absorption settings was also used to achieve different values of BR within a single hall geometry. The stimuli were presented to participants over headphones and they were asked to rate warmth, low- and high-frequency reverberance on continuous five-point scales. Participants rated each of the attributes in separate sets to avoid potential bias in the ratings. A repeated measures analysis of variance was conducted to examine the relationship between the subjective ratings and BR.

\section{4:05}

3pAAa12. A fundamental study of evaluating sound fields in performance spaces based on equal tempered scale. Akiho Matsuo (Dept. of Architecture, Graduate School of Sci. and Technol., Nihon Univ., 7-24-1, Narashinodai, Funabashi, Chiba 274-8501, Japan, motu.kepgad@gmail. com), Toshiki Hanyu, and Kazuma Hoshi (Dept. of Architecture and Living Design, Junior College, Nihon Univ., Funabashi, Chiba, Japan)

Generally, the reverberation times, energy decay curves, directional characteristics, and so on are analyzed in 1 octave bands or $1 / 3$ octave bands for evaluating acoustic conditions in performance spaces. The center frequencies such as $1000 \mathrm{~Hz}$ are defined by engineering implication. However, these center frequencies are not always adequate for evaluating performance spaces because music is basically composed of musical scale like the equal tempered scale. Therefore, it is thought that it is reasonable to use center frequencies based on the equal tempered scale in order to evaluate performance spaces. Based on this idea, we calculated reverberation times and energy decay curves for 1/12 octave bands at which center frequencies are set by using the equal tempered scale. Directional characteristic of reflected sounds is an important factor for evaluating spatial impressions in performance spaces. Therefore, we measured the directional characteristics based on sound intensity in the 1/12 octave bands. Moreover, we focused on chord which is a combination of several musical tones, in other words, several $1 / 12$ octave bands. We calculated energy decay curves and directional characteristics focusing on the chord. As a result, characteristic tendencies were obtained on the energy decay curves and the directional characteristics.

\section{$4: 20$}

3pAAa13. The use and mis-use of reactive systems in the design of performance halls. Richard Talaske and Scott Hamilton (TALASKE I Sound Thinking, 1033 South Blvd., Oak Park, IL 60302, rick@talaske.com)

While the "surface of infinite impedance" or "totally absorptive material" makes our acoustic theories understandable and our analytical calculation methods useful, more often than not such conditions are not met in built performance facilities. Since the materiality of a performance hall strongly influences the quality of sound within a space, the physical reality of the reactive impedance of building elements must be managed to achieve quality sound. This paper discusses the practical application of various room acoustic design options, including wall and ceiling material choices, and offers comment on how various reactive systems within performance halls can detract from a room and/or reintroduce sound energy into a space. Discussion will occur regarding how such conditions can be either favorable or detrimental to the aural environment. Examples will be offered which suggest a reactive room surface can offer favorable room acoustic results for impulsive sound events, and unfavorable results with sustained sound events. Observations and subsequent testing suggest that the ability to discern the impact of reactive systems within a hall depends upon the test methods implemented during acoustic measurement sessions. 


\title{
Session 3pAAb
}

\section{Architectural Acoustics and Signal Processing in Acoustics: Advanced Analysis, Simulation, and Auralization in Room Acoustics III (Poster Session)}

\author{
Michael Vorlaender, Cochair \\ ITA, RWTH Aachen University, Kopernikusstr. 5, Aachen 52056, Germany \\ Tetsuya Sakuma, Cochair \\ The University of Tokyo, 5-1-5 Kashiwanoha, Kashiwa 277-8563, Japan \\ Toshiki Hanyu, Cochair \\ Department of Architecture and Living Design, Junior College, Nihon University, 7-24-1, Narashinodai, \\ Funabashi 274-8501, Japan
}

All posters will be on display from 4:30 p.m. to 5:30 p.m.

\section{Contributed Papers}

3pAAb1. Evaluating acoustical features of 3D sound rendering systems by using a visualizing tool of sound intensities. Masataka Nakahara (Onfuture Ltd. / SONA Corp., 2-19-9 Yayoi-cho, Nakano-ku, SONA Corp., Tokyo 164-0013, Japan, nakahara@onftr.com), Takashi Mikami (SONA Corp., Tokyo, Japan), and Akira Omoto (Kyushu Univ. / Onfuture Ltd., Fukuoka, Japan)

The authors have developed a simple measurement/analyzing tool which visualizes $3 \mathrm{D}$ acoustical properties of sound by using sound intensity information. The tool, VSV (Virtual Source Visualizer), has already being used for practical acoustic design works, and the authors have also begun to use it for evaluating acoustical features of 3D sound rendering systems. On the audio industries, various 3D sound reproduction systems have already been commercialized. Therefore, further skill is now required to design acoustic specifications of their production studios. From this point of view, development of a simple method to obtain relationship between loudspeaker positions and source locations rendered by a 3D reproduction system would be helpful. This research introduces analyzing flows of the measurement tool briefly, and shows measured results of physical and/or phantom sound locations detected from sound intensity information in some different 3D reproduction environments including commercial audio strategies such as Dolby Atmos and 22.2ch, and a wave theory based Boundary Surface Control system, BoSC. Verifying differences between target source positions and measured ones, the authors suggest the feasibility of the evaluation method of a rendered 3D sound field by a simple measurement method.
3pAAb2. Evaluating the auralization of a small room in a virtual sound environment using objective room acoustic measures. Axel Ahrens, Márton Marschall, and Torsten Dau (Dept. of Elec. Eng., Hearing Systems group, Tech. Univ. of Denmark, Ørsteds Plads, Bldg. 352, Kgs. Lyngby 2800, Denmark, aahr@elektro.dtu.dk)

To study human auditory perception in realistic environments, loudspeaker-based reproduction techniques have recently become state-of-theart. To evaluate the accuracy of a simulation-based room auralization of a small room, objective measures, such as early-decay-time (EDT), reverberation time, clarity, interaural cross-correlation (IACC), and the speech transmission index were measured in an IEC listening room for 28 sourcereceiver combinations. The room was then modeled in the room acoustics software ODEON, and the same objective measures were also evaluated for the auralized version of the room. The auralizations were generated using the loudspeaker-based room auralization toolbox (LoRA; Favrot and Buchholz, 2010) and reproduced in a 64-channel loudspeaker array, set up in an anechoic chamber. Differences between the objective measures evaluated in the real and the virtual room were within about twice the just-noticeable differences for most measures, and were comparable to the median results of the study by Favrot and Buchholz, who did not consider the contribution of a real reproduction system. However, the EDT showed considerably higher errors than the other measures, even though medians were similar in the real and auralized room. This suggests that fine details in the early part of the room impulse response may be difficult to reproduce accurately. 


\title{
Session 3pAAc
}

\section{Architectural Acoustics: Assorted Topics in Architectural Acoustics (Poster Session)}

\author{
Damian Doria, Chair \\ Stages Consultants LLC, 75 Feather Ln, Guilford, CT 06437-4907
}

\begin{abstract}
All posters will be on display from 4:10 p.m. to 5:30 p.m. To allow all contributors in this session to see other posters, authors of odd-numbered papers will be at their posters from 4:10 p.m. to 4:50 p.m. and authors of even-numbered papers will be at their posters from 4:50 p.m. to 5:30 p.m.
\end{abstract}

\section{Contributed Papers}

3pAAc1. Characterization of the acoustic quality of classrooms in a music education facility. Erasmo F. Vergara (UFSC, Florianópolis, SC, Brazil), Stephan Paul (UFSC, rua Joao Colin, 2700, Joinville 97080-035, Brazil, stephan.paul@ufsc.br), Eric Brandão (Undergraduate program Acoust. Eng., UFSM, Santa Maria, RS, Brazil), and Fernanda Marros (Graduate Program Civil Eng., UFSM, Santa Maria, Brazil)

Classrooms of a music education facility were characterized by the user by means of a questionnaire study and by acoustic impulse response measurements and subsequent calculation of reverberation time, definition, and other room acoustics parameters. The questionnaire study enabled to understand the preferences of students and music teachers regarding the acoustic quality of a total of twenty rooms for music practice and teaching. It was observed that preference for a certain room also depends on the instrument mostly played by the user. Three study rooms and three collective classrooms were the most cited ones and these have been evaluated according to ISO 3382. Opinions of the musicians showed to be coherent with the measurement data as the rooms considered to be dry had reverberation times around 0.3 seconds, and the rooms considered to be reverberant had reverberation times around 1.5 seconds. The six classrooms that were characterized as clear and well defined rooms, showed values for Clarity around $1 \mathrm{~dB}$ for live rooms and 14 to $22 \mathrm{~dB}$ for dry rooms. Definition remained above $43 \%$ for all rooms with Central Time below $24 \mathrm{~ms}$ for clarity rooms and less reverberant, and above $98 \mathrm{~ms}$ for the live rooms.

3pAAc2. Autonomous optimal location of articulation materials using robotics. Masashi Uehara, Shuhei Kawai, Shigeki Okawa (Chiba Inst. of Technol., 2-17-1 Tsudanuma, Narashino-shi, Chiba-ken 275-0016, Japan, s1126017jn@s.chibakoudai.jp), and Manabu Fukushima (Nippon Bunri Univ., Oita-shi, Oita-ken, Japan)

We developed an application that determines optimal places of a robot equipped with articulation materials to improve sound environment. Lots of acoustic devices that incorporated architectural acoustics technology have been developed (e.g., articulation material, sound-absorbing material, etc. ). In many cases, those materials are placed at positions that are effective by professional people. Our purpose is to make an autonomous robot to decide the material's position instead of professionals. We chose "Aural Sonic," the articulation materials to be mounted on the robot. "Aural Sonic" is one of the acoustic devices that is expected effects to improve the hearing. This robot can change the position and angle little by little, to measure the impulse response each time. The application extracts the acoustic features corresponding to the speech clarity from the measured impulse response and calculates the evaluation value. The robot, which gives the position with high evaluation value, could be used for the articulation materials more effectively. We introduce the system that finds the optimal location of the articulation material with the robot.
3pAAc3. Autonomous mobile robot to improve sound environment for speech conversation. Shuhei Kawai, Masashi Uehara, Shigeki Okawa (Chiba Inst. of Technol., 2-17-1 Tsudanuma, Narashino-shi, Chiba-ken 2750016, Japan, s1326035cq@s.chibakoudai.jp), and Manabu Fukushima (Nippon Bunri Univ., Oita-shi, Oita-ken, Japan)

This study pursues to improve sound environment for speech conversation by moving an articulation panel by a robot. We tried to move the articulation panel to behind of the listeners by a small robot to keep fixed distance from the listener. An optimal location of the articulation panel depends on the sound sources such as speech conversation or music appreciation. It is usually decided based on the sense of users and experts. If we use a room for the purpose of speech conversation, the articulation panel is placed behind the listeners. However, moving the articulation panel optimally is almost impossible in terms of economical and physical conditions. In recent years, robot technology for transportation is in progress widely. We attempt resolving these problem by robotics. The articulation panel is framed by wood to have absorption and reflection surfaces, and the robot has omniwheels for ultra-pivot turn performance. It can turn the surface on the sound source by the rotation of the robot. The robot has four-wheel drive because it should have large stable range as to the purpose to carry objects. Several sensors are used such as an ultrasonic sensor and an infrared sensor to have robustness. As a result, we approached to improvement of sound environment by robotics.

3pAAc4. Perceptually plausible acoustics simulation of single and coupled rooms. Torben Wendt, Steven van de Par, and Stephan D. Ewert (Acoust. Group, Medizinische Physik and Cluster of Excellence Hearing4all, Universität Oldenburg, Carl-von-Ossietzky-Straße 9-11, Oldenburg 26111,Germany, torben.wendt@uni-oldenburg.de)

The fast and perceptually plausible room acoustics simulator [RAZR, see Wendt et al., JAES 62, 11 (2014)] has been optimized and extended to simulate the acoustics of connected rooms. RAZR synthesizes binaural room impulse responses (BRIRs) with high computational efficiency using a hybrid approach: early reflections are calculated as image sources for a shoebox-room approximation up to a low order, and the later reverberation is generated by a binaurally extended feedback delay network (FDN). For the extension toward two coupled rooms, validity, visibility, and diffraction of the image sources in the two rooms were taken into account, as well as different reverberation properties and the opening angle of the door to the neighbor room seen from a specific receiver position. The suggested method was evaluated by comparing measured and synthesized BRIRs for several rooms differing in size and reverberation time. For room acoustical parameters such as early decay time, late decay time, definition, and clarity a good agreement in terms of the Pearson correlation coefficient $(0.86-0.99)$ was achieved between measured and simulated BRIRs. Subjective listening tests showed a good agreement of ratings of perceptual attributes (e.g., tonecolor, reverberation time, envelopment, and naturalness) for the measured and synthesized BRIRs. 
3pAAc5. Impact noise insulation performance of commonly used materials when excited by different impact noise sources. Andriele d. Panosso (UCEFF, Rua Benjamin Constant, Santa Maria, Rio Grande do Sul 97050022, Brazil, andrielep@gmail.com) and Stephan Paul (Universidade Federal de Santa Catarina, Joinville, Santa Catarina, Brazil)

This study aimed to verify the performance of different materials used as impact noise insulation when excited by different impact sources. The method applied consisted of several acoustic measurements, following the standards' instructions and using different impact sources, such as the tapping machine and an impact tire. When using the tapping machine results indicate rather big differences in noise insulation performance for the different materials, showing a range from 50 to $76 \mathrm{~dB}$ for the Weighted Standardized Impact Sound Pressure Level while measurements with the tire showed a range of 72 to $82 \mathrm{~dB}$ for the Average Maximum Impact Pressure Level, meaning very similar noise insulation for all materials.

WEDNESDAY AFTERNOON, 30 NOVEMBER 2016

CORAL 2, 1:00 P.M. TO 2:30 P.M.

\title{
Session 3pABa
}

\section{Animal Bioacoustics: Session in Honor of Whitlow Au II}

\author{
Kelly J. Benoit-Bird, Cochair \\ College of Earth, Ocean, and Atmospheric Sciences, Oregon State University, 104 COEAS Admin Bldg., Corvallis, OR 97331 \\ Marc Lammers, Cochair \\ Hawaii Institute of Marine Biology, 46-007 Lilipuna Rd., Kaneohe, HI 96744 \\ Tomonari Akamatsu, Cochair \\ Fisheries Research Agency, 7620-7, Hasaki, Kamisu, Ibaraki 314-0408, Japan
}

\section{Contributed Papers}

\section{1:00}

3pABa1. Interaction of gain-control mechanisms in the sonar of odontocetes. Alexander Supin (Inst. of Ecology and Evolution, 33 Leninsky Prospect, Moscow 119071, Russian Federation, alex_supin@mail.ru) and Paul E. Nachtigall (Hawaii Inst. of Marine Bilogy, Kaneohe, HI)

The sonar of odontocetes processes echo signals within a wide range of echo levels. The level of echoes varies by tens of $\mathrm{dB}$ depending on the the target strength, the distance to the target, and the sound absorption by the media. The sonar of odontocetes has several mechanisms to compensate for the echo-level variation (gain control): (i) variation of emitted sonar pulse levels (the longer the distance to the target, the higher the level of the emitted pulse), (ii) short-term variation of hearing sensitivity based on forward masking of the echo by the preceding self-heard emitted pulse and subsequent release from the masking, and (iii) long-term active control of hearing sensitivity. These three mechanisms not just add to one another but act coordinately and form a united system of gain control. In particular, an increase in the sonar pulse level prolongs the distance of action of the forward-masking mechanisms. Active variation of hearing sensitivity makes the range of action of the forward-masking mechanism fitting the particular target distance and strength. These interactions of the gain-control mechanisms makes the auditory system capable of effectively analysis of echoes within a wide range of target strengths and distances.

\section{1:15}

3pABa2. Beam patterns of the dolphin demonstrate task-dependent dynamics. Dorian S. Houser, Lois Talmadge (National Marine Mammal Foundation, 2240 Shelter Island Dr., San Diego, CA 92106, dorian.houser@ nmmfoundation.org), Josefin Starkhammar (Lund Univ., Lund, Sweden), and Partick Moore (National Marine Mammal Foundation, San Diego, CA)

The echolocation beam of the bottlenose dolphin was first carefully described by Au and colleagues (1978)["Propagation of Atlantic bottlenose dolphin echolocation signals," J. Acoust. Soc. Am. 64, 411-422] using various hydrophone array configurations and targets located in front of the dolphin and along its longitudinal axis. Measured beams were described as vertically elevated with mean vertical and horizontal 3-dB beamwidths of $\sim 10^{\circ}$. The experimental paradigm was later augmented with denser hydrophone arrays, greater spatial coverage of the acoustic field, and the training of target detection with targets presented to the left or right of the dolphin's longitudinal axis. Utilizing two dolphins, beam steering capabilities and beamwidth control were demonstrated. The two dolphins steered the axis of the echolocation beam up to $18^{\circ}$ and $28^{\circ}$ in the horizontal plane. Horizontal beamwidths were bimodally distributed in one dolphin, with peaks at $16^{\circ}$ and $26^{\circ}$. The other dolphin had a unimodal distribution of beamwidths (peak at $\sim 25^{\circ}$ ), likely reflecting a learned strategy for the off-axis detection task. The use of dense hydrophone arrays and challenging echolocation tasks continue to build on the work of Au and colleagues, who provided a paradigm that has greatly contributed to our understanding of dolphin biosonar.

\section{$1: 30$}

3pABa3. Using "phantom" echoes to study dolphin biosonar. James J. Finneran (SSC Pacific Code 71510, US Navy Marine Mammal Program, 53560 Hull St., San Diego, CA 92152, james.finneran@navy.mil), Jason Mulsow, Brian K. Branstetter, Dorian S. Houser, and Patrick W. Moore (National Marine Mammal Foundation, San Diego, CA)

Biosonar studies have greatly benefited from the use of electronic, or "phantom," echoes. In this paradigm, amplitude and timing information are extracted from an emitted biosonar pulse, then a delayed signal is broadcast to the animal to appear as an echo from a more distant target. Phantom echoes provide unique capabilities for studying biosonar, since they allow echo features such as amplitude and delay to be independently manipulated. In 1987, Whit Au and colleagues described a phantom echo system for use with marine mammals [Au et al. (1987). "Phantom electronic target for dolphin sonar research," J. Acoust. Soc. Am. 82, 711-713]. In this system, 
delayed broadcasts of one or more replicas of the dolphin click were triggered by dolphin click emissions. A major improvement in phantom echo systems was later described by Aubauer and Au (1998) ["Phantom echo generation: A new technique for investigating dolphin echolocation," J. Acoust. Soc. Am. 104, 1165-1170], who simulated the impulse response of a physical target, rather than broadcasting a stereotyped waveform. In this talk, phantom echo system concepts are briefly presented and several recent applications of phantom echo systems-inspired by the work of Aubauer and $\mathrm{Au}$ - are discussed. [Work supported by ONR.]

\section{$1: 45$}

3pABa4. Information-seeking in an echolocating dolphin. Heidi E. Harley (Psych., , Div. of Social Sci., New College of Florida, 5800 Bay Shore Rd., Sarasota, FL 34243, harley@ncf.edu), Wendi Fellner (The Seas, Disney's Epcot, Lake Buena Vista, FL), Candice Frances (Psych., New College of Florida, Sarasota, FL), Amber Thomas, Barbara Losch, and David Feuerbach (The Seas, Disney's Epcot, Lake Buena Vista, FL)

Dolphins gain information through echolocation, a publicly accessible sensory system in which dolphins produce clicks to investigate objects. We measured information-seeking behavior by counting clicks that a blindfolded dolphin directed toward the sample object in a three-alternative matching task with object sets that varied in discriminability: Indiscriminable (performance accuracy $\mathrm{M}=33 \%$ ) vs. Easy (performance accuracy M>90\%). The dolphin produced a similar number of clicks when first investigating each set type. Across multiple sessions, however, the dolphin emitted fewer clicks only when investigating indiscriminable (vs. easy) sets. Reduced echoic investigation with indiscriminable, but not easy, object sets was not due to overall motivation: the differential relationship between click number and object set discriminability was maintained when difficult and easy trials were interleaved and when objects from originally difficult sets were grouped with more discriminable objects. Further analyses of the dolphin's click production towards the choice alternatives also revealed that the dolphin produced more clicks towards the chosen (vs. unchosen) alternatives when solving the task. Overall, these data suggest that dolphins calibrate the effort they invest in information seeking in accordance with the information content available in their immediate environment.

2:00-2:30 Panel Discussion

WEDNESDAY AFTERNOON, 30 NOVEMBER 2016

CORAL 2, 2:45 P.M. TO 5:45 P.M.

Session 3pABb

Animal Bioacoustics: On the Bleeding Edge of Animal Bioacoustics Technology

\author{
Selene Fregosi, Chair \\ Oregon State University, Hatfield Marine Science Center, 2030 SE Marine Science Drive, Newport, OR 97365
}

\title{
Contributed Papers
}

\section{2:45}

3pABb1. Acoustic methods of pest detection in agricultural shipments. Alexander Sutin, Timothy Flynn, Nikolay Sedunov, Hady Salloum, Alexander Sedunov (Maritime Security Ctr., Stevens Inst. of Technol., 711 Hudson St., Hoboken, NJ 07030, asutin@stevens.edu), and David Masters (Sci. and Technol. Directorate, Dept. of Homeland Security, Washington, DC)

Stevens Institute of Technology, in cooperation with the DHS Science and Technology Directorate and the U.S. Customs and Border Protection, has been investigating engineering solutions to augment the current inspection process at ports of entry in an effort to minimize the threat posed by invasive species. Stevens has built several sensitive acoustic systems for detection of tiny acoustic/vibrational signals produced by moving insects and tested them in a Laredo, TX port of entry. The system for detection of insects in vegetables and herbs uses a soundproofed case where boxes filled with vegetables or herbs were placed during test. For wood boring insects, sensitive custom-made accelerometers were built. Special algorithms for detection of events connected with insect movement and separating them from the ambient noise were developed. Tests conducted demonstrated reliable detection of Copitarsia larva in a large box of vegetables. Smaller insects similar to Khapra beetles were clearly detected in a relatively small volume of grains (2-4 lbs). [This work was supported by DHS's S\&T Directorate.]
3:00

3pABb2. Experimental audible sonar to model echolocation by the blind. Roman B. Kuc (Elec. Eng., Yale, 15 Prospect St., 511 Becton, New Haven, CT 06511, roman.kuc@yale.edu) and Victor B. Kuc (Comput. Sci., Iona College, New Rochelle, NY)

Some blind humans echolocate by emitting palatal clicks and processing echoes, even when there is temporal emission/echo overlap (EEO). Our previous work indicates that high frequencies in the emission that travel directly to the ears are attenuated and, when high frequencies are heard, they come from target echoes. Binaural processing of these high frequency components in the EEO signals provide target range and bearing. Our experiments with 3D-printed parabolic pinnae and a speaker emitter indicate that while the high frequency components are important for object classification, the low frequencies in the emission provide more robust bearing localization because pinna diffraction effects are reduced. Classifying targets with EEO signals requires analysis in the power spectral domain because of the phase insensitivity of hearing. The power spectrum Fourier inverse estimates the autocorrelation function of the target reflector sequence. Robust autocorrelation estimates occur when the emission and echo contain comparable energies. Our experiments demonstrate that the echo energy varies with target type (e.g., planar or cylindrical) and the optimum range for classification depends on the target and itself forms a target classification feature. 
3pABb3. Simultaneous recordings of marine mammal calls by a glider, float, and cabled hydrophone array. Selene Fregosi (Cooperative Inst. for Marine Resources Studies, Oregon State Univ. and NOAA Pacific Marine Environ. Lab., Hatfield Marine Sci. Ctr., 2030 SE Marine Sci. Dr., Newport, OR 97365, selene.fregosi@oregonstate.edu), Holger Klinck (BioAcoust. Res. Program, Cornell Lab. of Ornithology, Cornell Univ., Ithaca, NY), Haru Matsumoto, Alex Turpin (Cooperative Inst. for Marine Resources Studies, Oregon State Univ. and NOAA Pacific Marine Environ. Lab., Newport, OR), Stephen W. Martin, Brian M. Matsuyama (National Marine Mammal Foundation, San Diego, CA), Tyler A. Helble, E. Elizabeth Henderson (Navy Marine Mammal Program, SPAWAR Systems Ctr. Pacific, San Diego, CA), David J. Moretti, Ronald P. Morrissey (Naval Undersea Warfare Ctr., Newport, RI), and David K. Mellinger (Cooperative Inst. for Marine Resources Studies, Oregon State Univ. and NOAA Pacific Marine Environ. Lab., Newport, OR)

Recent advances in passive acoustic monitoring (PAM) technologies have led to development of mobile autonomous platforms for recording marine mammals. These instruments may allow greater spatial and temporal sampling than traditional towed or bottom moored systems. However, comparison of recording abilities of these instruments to traditional methods has yet to be performed. We deployed two types of commercially available platforms at the Southern California Offshore Range (SCORE) complex in late December 2015 through early January 2016. The QUEphone, based on the APEX float (Teledyne Webb Research, Falmouth, MA, USA), is a buoyancy driven device capable of descending to $2000 \mathrm{~m}$ where it drifts horizontally with the currents. The Seaglider (Kongsberg Underwater Technology, Lynwood, WA, USA) is also buoyancy driven, but dives repeatedly up to 1000 $\mathrm{m}$ following a flight path controlled via satellite. We deployed one glider and two floats, each equipped with identical acoustic sensors developed by Oregon State University, sampling at $125 \mathrm{kHz}$. Each instrument recorded 250-300 hours of data over 13 days. Marine mammal detections included beaked whales, Risso's dolphins, and fin, blue, and humpback whales. We utilized known marine mammal locations derived from the SCORE hydrophone array to compare PAM capabilities of these novel mobile platforms.

\section{3:30}

3pABb4. IRAP: An integrated, real-time, autonomous passive acoustic monitoring system for beaked whale detection, localization, and tracking. Vince Premus, Philip Abbot, Charles Gedney, Russ Christman, Mark Helfrick, Richard Campbell, and Kim Douglas (OASIS, Inc, 5 Militia Dr., Lexington, MA 02421,primus@oasislex.com)

The integration and demonstration of passive sonar hydrophone arrays into autonomous undersea platforms for acoustic marine mammal monitoring has witnessed significant advances in the last few years due to the availability and scalability of low-cost, low-power commercial technology for acoustic remote sensing. In this paper, we will review the at-sea performance of a nine-element Mills Cross high frequency hydrophone array (HFA) integrated into an autonomous undersea vehicle (AUV) for the detection, classification, localization, and tracking (DCLT) of beaked whales during a deployment conducted off leeward Kauai in February, 2016. Using passive sonar equation analysis, we will review the design rationale of the HFA, and quantify system performance using standard signal processing metrics such as measured array gain, tracking accuracy, and detection range on a calibrated high-frequency source transmitting replica beaked whale click trains at a de minimus source level. A candidate autonomous real-time passive sonar processing architecture that combines an adaptive beamformer/matched filter front-end with a false alarm mitigation back-end to balance detection sensitivity, classifier robustness, and processing throughput will also be presented. We conclude with a cost/power/persistence trade-off for some commercially available autonomous platforms. [This work was supported by NAVFAC Living Marine Resources Program.]
3pABb5. Automatic detection and classification of toothed whale echolocation clicks in diverse long term recordings. Scott Lindeneau, Yun Trinh (Comput. Sci., San Diego State Univ., 5500 Campanile Dr., San Diego, CA 92182, slindeneau@gmail.com), Simone Baumann-Pickering (Scripps Inst. of Oceanogr., Univ. of California, San Diego, CA), and Marie Roch (Comput. Sci., San Diego State Univ., San Diego, CA)

We present results of classification of toothed whale echolocation clicks to species for $4 \mathrm{~TB}$ of recordings in the development data of 7 th Intl. DCLDE Workshop. The data span multiple seasons, years, locations, and instruments. Five species were acoustically identified by analysts, and a sixth category was assigned to echolocation clicks that could not be identified to species by analysts. Methods were developed to identify periods of echolocation activity taking into account sporadic false positives that occur throughout the data, and to reduce false positives from both anthropogenic and biologic sources. Dense echolocation activity presented particular challenges for noise removal and long term recordings permitted the targeting of regions for noise estimation outside of echolocation encounters. Extracted features consisted of noise normalized cepstral characterizations of spectra, inter-click interval, and $-3 \mathrm{~dB}$ bandwidth. These features were classified with a Gaussian mixture model (GMM). A robust scoring method was introduced to reduce outlier influence by using a per-click voting scheme within each encounter as opposed to joint likelihood scores. The error rate across 300 Monte Carlo trials on the development data set was 34.5\%. When encounters from the unspecified toothed whales were removed, the error rate dropped to $15.5 \%$.

\section{4:00-4:15 Break}

\section{$4: 15$}

3pABb6. Passive acoustic localization of North Atlantic Right Whales using a modified near-field Bartlett beamformer. Dean L. Hawthorne and Daniel P. Salisbury (Cornell Lab. of Ornithology, Cornell Univ., 159 Sapsucker Woods Rd., Ithaca, NY 14850, dh27@cornell.edu)

Owing to the principle of least commitment, near-field beamformers can produce passive acoustic source localizations which are robust to noise and interference. The power output of a traditional Bartlett near-field beamformer may be formulated in terms of the pairwise cross correlation functions of the sensor signals. We present a modified Bartlett processor wherein the ordinary cross correlation is replaced by generalized cross correlation, generalized in both time and frequency. As an example of the utility of the technique, we present results from a six element pentagonal marine array with a baseline of approximately $15 \mathrm{~km}$ deployed off the coast of Virginia. Localization of synthetic FM sweeps from known source locations within the array yield deviations from the true source location as measured by GPS of less than 100 meters. Localization of actual North Atlantic Right Whale calls provide good alignment of the unknown channel arrivals and acceptably small error ellipses.

\section{$4: 30$}

3pABb7. Prototype of a low-cost towed array with data-logging and short-range telemetry. Eduardo Romero-Vivas, Fernando D. Von Borstel, Francisco Villa Medina (CIBNOR, Av Instituto Politecnico Nacional 195, Playa Palo de Santa Rita Sur, La Paz, Baja California Sur 23096, Mexico, evivas@cibnor.mx), Omar A. Bustamante (Acoust. Lab, ESIME, IPN, IMA, D.F, Mexico), Sergio Beristain (Acoust. Lab, ESIME, IPN, IMA, Mexico City, Mexico), and Joaquin Gutiérrez-Jagüey (CIBNOR, La Paz, Mexico)

Passive Acoustic Monitoring studies have been benefited from open source software initiatives such as PAMGuard and ISHMAEL, but their widespread use is often limited by availability of low cost hardware. This work presents the design of a towed hydrophone array that can be built with off the shelf components. The system comprises a Raspberry Pi computer to $\log$ GPS, three-axis accelerometer, magnetometer, gyroscope, and audio from the hydrophone array $(20 \mathrm{~Hz}-44 \mathrm{kHz})$. Data can be analyzed in PAMGuard, either in real time, through a short range $(10 \mathrm{~m}) \mathrm{RF}$ link, or retrieved later from the data logger. The array has been tested in La Paz bay Mexico, where semi-resident populations of long-beaked common dolphin 
(Delphinus capensis) and bottlenose dolphin (Tursiops truncatus) have been reported. Results and performance of the array in four modalities are presented; as a drift array, as a secondary array deployed from a small vessel, as an array towed by a Kayak, and as an array towed by an Autonomous Surface Vehicle. This array provides a useful tool for fieldwork, and since it is built form off the shelf components on a low budget, it could also be used for educational purposes, such as in PAMGuard Courses.

\section{4:45}

3pABb8. Acoustic cam on a remote operated vehicle for coral reef soundscape recording. Eduardo Romero-Vivas, Joaquín Gutiérrez-Jagüey (CIBNOR, La Paz, Baja California Sur, Mexico), Omar A. Bustamante (Acoust. Lab, ESIME, IPN, IMA, Austral 77 Col Atlanta, Cuahutitlan Izcalli, Mexico City 54740, Mexico, omar.p@hotmail.com), Francisco Villa Médina (CIBNOR, La Paz, Mexico), Sergio Beristain (Acoust. Lab, ESIME, IPN, IMA, Mexico City, Mexico), and Fernando D. Von Borstel (CIBNOR, La Paz, Mexico)

Although the broadband high level sounds of snapping shrimps dominate the soundscape of a coral reef, the spatial, spectral, and temporal variations due to biotic and abiotic factors are indicators of the health of these vulnerable environments; for instance, reefs become louder during new moons of the wet season, when many larval organisms settle, or tends to go quiet as animals abandon them when conditions become less favorable. Moreover, the soundscape is able to provide information on the reef benthic composition and has been identified as a possible driver of reef population dynamics. To study this particular environment, a Remote Operated Vehicle (openROV) is being used to visually identify the biota associated with a shallow coral reef near a major port in La Paz, Mexico. An acoustic linear array has been incorporated, providing directional sound recording capabilities to capture better space variability of the coral soundscape. By combining visual and acoustical clues in an acoustic cam, it is also feasible to associate fish species and acoustic signals. A preliminary assessment of the design for monitoring a shallow coral reef is presented, along with a comparison with traditional diving surveys.

\section{5:00}

3pABb9. A system for acoustic detection, classification, and localization of terrestrial animals in remote locations. Dean L. Hawthorne (Lab. of Ornithology, Cornell Univ., Cornell Lab of Ornithology, 159 Sapsucker Woods Rd., Ithaca, NY 14850, dh27@cornell.edu), William Horn (Coherent Tech. Services, Inc., Lexington Park, MD), and Danny C. Reinke (Environ. Management, U.S. Air Force, Edwards Air Force Base, CA)

Acoustic monitoring of endangered terrestrial animals often involves deploying observation equipment to remote locations which may be difficult or hazardous for human observers to access. We present a system designed for deployment to such locations which is capable of detecting, classifying, and localizing a wide range of vocalizations. The battery/solar powered system can continuously record data for two weeks while sending real-time Wi-Fi detection alerts. Normally, three systems, each composed of a sensor array consisting of a 16 element microphone array, an ancillary processor box, plus ancillary equipment as needed, are deployed allowing detection geolocation. The computer system uses a computationally efficient beamforming algorithm to achieve $12 \mathrm{~dB}$ of gain and locate detections in both azimuth and elevation. Vocalizations are classified using a template matching algorithm in the spectrogram image domain. Localization is achieved by crossing the far-field bearings obtained from the three beamformers. Output is integrated into Cornell's Raven Pro software package. Experimental trials with endangered Mojave Ground Squirrel vocalizations have resulted in successful detection and bearing estimation. [Work sponsored by Air Force SBIR AF112-193 currently in Phase II.]

\section{$5: 15$}

3pABb10. A generic system for the automatic extraction of narrowband signals in underwater audio. Shyam Madhusudhana, Alexander Gavrilov, and Christine Erbe (Ctr. for Marine Sci. \& Technol., Curtin Univ., Phys. Bldg. 301 Rm. 136A, Curtin University - Marine Sci., Kent St., Bentley, WA 6102, Australia, s.madhusudhana@postgrad.curtin.edu.au)

Narrowband acoustic signals occur prominently in underwater environments and are commonly used to monitor or track the sources producing them. Disparate ad hoc systems have previously been developed for the detection or recognition of several forms of narrowband signals produced by specific sources. We present a generic system, based on post-processing of spectrograms, for the automatic extraction of time-frequency contours of narrowband signals produced by any source-biological, anthropogenic, or geophysical. A two-phase approach is proposed where the first phase is based on an image-processing technique for detecting intensity ridges and the second phase is a Bayesian filtering approach for tracing the trajectory of detected ridge apices. The choice of algorithm parameters and conditionals are backed with theoretical motivations and are geared to result in a generic (non-targeted) system. In comparison to an existing method, using publicly available pre-annotated recordings containing four species of dolphins, our system offered improvements of $21 \%$ and $15 \%$ in precision and recall rates, respectively. A streaming-mode implementation in Matlab processed inputs with an average real-time factor of 0.10 on a modest desktop computer, showing that the system is well-suited for both offline and insituapplications.

\section{$5: 30$}

3pABb11. A novel approach to measurement of underwater sound levels in a dangerous tidal fjord using a miniature self-contained acoustic recorder. William C. Burgess (Greeneridge Sci., Inc., 6060 Graham Hill Rd. Stop F, Felton, CA 95018, burgess@greeneridge.com) and Tamara L. McGuire (LGL Alaska Res. Assoc., Inc., Anchorage, AK)

Recent plans for highway construction along the shore of Turnagain Arm, Alaska, raised concerns about acoustic impact on the critically endangered Cook Inlet subpopulation of beluga whales (Delphinapterus leucas). Evaluation of possible impact required estimates of normal background sound levels. However, as a shallow fjord with profound (9-m mean) tides, hazardous currents, tidal bores, and quicksand-like tidal flats, Turnagain Arm has historically defied most vessel operations and thwarted vessel-based sound measurements. In August 2014, the first documented acoustic measurements in Turnagain Arm of which we are aware took place using a novel technique based on a miniature, self-contained acoustic recorder developed as a marine-mammal tag (Acousonde ${ }^{\mathrm{TM}}$, Greeneridge Sciences, Inc.). The recorder's small size allowed it to be suspended from a motorized buoy and towed up to $80 \mathrm{~m}$ from shore. Measurements took place off a point known as Windy Corner at high and low tide as well as near maximum flood and ebb current. Data showed Turnagain Arm to be quieter than expected, with broadband $(40 \mathrm{~Hz}$ to $9.3 \mathrm{kHz})$ sound pressure levels as low as $74 \mathrm{~dB}$ re $1 \mu \mathrm{Pa}$ and below a $125-\mathrm{dB}$ provisional regulatory assumption. [Work supported by Alaska Department of Transportation and Public Facilities.] 


\title{
Session 3pAO
}

\section{Acoustical Oceanography, Underwater Acoustics, and Signal Processing in Acoustics: Ocean Acoustic Tomography: Active and Passive, Theory, and Experiment III}

\author{
Bruce Howe, Cochair \\ Ocean and Resources Engineering, University of Hawaii, 2540 Dole Street, Holmes Hall 402, Honolulu, HI 96822 \\ Arata Kaneko, Cochair \\ Graduate School of Engineering, Hiroshima University, 1-4-1 Kagamiyama, Higashi-Hiroshima 739-8527, Japan \\ Hiroyuki Hachiya, Cochair \\ Tokyo Institute of Technology, 2-12-1 S5-17, Ookayama, Meguro-ku, Tokyo 152-8550, Japan
}

\section{Contributed Papers}

1:00

3pAO1. Application of coastal acoustic tomography to Lake Biwa, Japan. John C. Wells, Yasuaki Aota, Guillaume Auger (Civil Eng., Ritsumeikan Univ., Noji Higashi 1-1-1, Kusatsu, Shiga 525-8577, Japan, jwells@se. ritsumei.ac.jp), Arata Kaneko, and Noriaki Goda (Hiroshima Univ., East Hiroshima, Japan)

We will provide an update on continuing development of coastal acoustic tomography (CAT) in Lake Biwa, Japan, which will provide monitoring data for assimilation into a prototype "nowcast system" for the flow and temperature fields in the lake. Employing $5 \mathrm{kHz}$ transducers, we have achieved a number of successful CAT tests since August 2014 in the North Basin, which has a mean depth of 41 meters. These were performed under both unstratified and stratified conditions, at transmission distances up to 10 $\mathrm{km}$, and included a continuous 2-month deployment in homogenized winter conditions. Due to weaker mixing than coastal seas, stratification-induced refraction in lakes is stronger than normally encountered in CAT. We believe these to be the first successful tests of acoustic tomography ever reported for a lake. Received signals were remarkably different between the nearshore station with bottom depth about $9 \mathrm{~m}$, and offshore of Takeshima island with bottom depths around $35 \mathrm{~m}$. Current efforts aim to improve SNR for reception offshore of Takeshima island.

\section{$1: 15$}

3pAO2. The application of coastal acoustic tomography to a large experimental wave/current basin. Guangming $\mathrm{Li}$, David Ingram (Inst. of Energy Systems,School of Eng., Univ. of Edinburgh, Faraday Bldg., King's Buildings,Colin Maclaurin Rd., Edinburgh, Scotland EH9 3DW, United Kingdom, G.Li@ed.ac.uk), Arata Kaneko, Noriaki Gohda (Graduate School of Eng., Hiroshima Univ., Hiroshima, Japan), and Nick Polydorides (Inst. of Digital Communications, School of Eng., Univ. of Edinburgh, Edinburgh, United Kingdom)

This article describes the use of acoustic tomography to characterize the flow pattern in the Flowave basin. Flowave is a unique circular wave/current basin, $2 \mathrm{~m}$ deep and $25 \mathrm{~m}$ in diameter. Current can be created in any direction at speed of up to $1.2 \mathrm{~ms}^{-1}$. Two coastal acoustic tomography (CAT) stations were used operating at $50 \mathrm{kHz}$ with a M- sequence signal. By combining three different pairs of station positions and seven current directions a network of seven CAT stations has been emulated. Throughout the test, a current speed of $0.8 \mathrm{~ms}^{-1}$ was used. Using the inverse Fourier method, a velocity profile has been obtained. During the experiment, a Nortek vectrino velocimetry was placed between the stations. This paper will present the comparison between the vectrino and CAT measurement.
$1: 30$

3pAO3. 3D assimilation of Hiroshima Bay acoustic tomography data into a Princeton Ocean Circulation Model. Minmo Chen, Arata Kaneko (Graduate School of Eng., Hiroshima Univ., 1-4-1 Kagamiyama, HigashiHiroshima, Hiroshima 739-8527, Japan, d153155@hiroshima-u.ac.jp), Chuangzheng Zhang (State Key Lab. of Satellite Ocean Environment Dynam., Second Inst. of Oceanogr., Hangzhou, Zhejiang, China), and Ju Lin (College of Information Sci. \& Eng., Ocean Univ. of China, Qingdao, Shandong, China)

A baroclinic data assimilation, based on the ensemble Kalman filter (EnKF) method, with coastal acoustic tomography (CAT) data is first applied to Princeton Ocean Model (POM) to elucidate a 3D structure of coastal upwelling which occurred in Hiroshima Bay due to a northerly wind from a typhoon. The CAT experiment with four acoustic stations was performed from Sept. 11 to Sept. 25, 2013, in the northern part of Hiroshima Bay, Japan. Reciprocal data were acquired at three stations and along three transmission lines with a triangular array and travel time were identified every $10 \mathrm{~min}$ for the first and second arrival rays which travel at different depth ranges. As for the EnKF method, the Kalman gain was determined to minimize the observed and simulated travel time differences and summations. The model error covariance was given by adding pseudorandom noises (zero mean value and one variance) with an appropriate multiplication factor to the POM result. The observation error covariance was estimated from the variation of observed travel times. A 3D structure of coastal upwelling which was difficult to be simulated by the POM was reconstructed by data assimilation and compared with the CTD result.

$1: 45$

3pAO4. Feasibility of geoacoustic tomography in shallow water. Altan Turgut, Jeffrey A. Schindall, and Steven L. Means (Naval Res. Lab, Acoust. Div., Code 7160, Washington, DC 20375, altan.turgut@nrl.navy.mil)

In shallow water, spatial and temporal variability of the water column often restricts accurate estimations of bottom properties from long-range acoustic propagation data. Two recent shallow-water experiments, conducted at a sandy site on the New Jersey Shelf and a muddy site on the New England Shelf, showed 5-10 dB acoustic transmission-loss differences between two sites. The New Jersey Shelf experiment was conducted during May 2015 and The New England Shelf experiment was conducted during November 2015, representing summer and winter propagation conditions, respectively. Acoustic modal analysis and pulse-decay tomography methods were used to map the geoacoustic variability of each site covering a $40 \mathrm{~km}$ by $40 \mathrm{~km}$ area. Both numerical simulations and the New England Shelf 
experimental results showed that geoacustic variability can be measured by geoacoustic tomography. Tomographic results from the New Jersey Shelf sites showed less geoacoustic variability that may be due to the presence of a harder bottom. Effects of the water-column variability on the geoacoustic tomography results are also discussed for summer and winter environments. [Work supported by the ONR.]

$$
\text { 2:00 }
$$

3pAO5. Current field tracking based on spatiotemporal evolution model with distributed networked underwater sensor system. Wen $\mathrm{Xu}$, Ying Zhang, Huifang Chen, and T. C. Yang (Zhejiang Univ., No. 38, Zheda Rd., Hangzhou 310027, China,wxu@zju.edu.cn)

Distributed networked underwater sensor (DNUS) system can provide ocean measurements over a wide area with a large number of sensors. This paper studies the estimation of the two-dimensional ocean current field with acoustic travel time difference data observed by a DNUS system. Considering that the current field is correlated in time and space, we present a statistical-based tomography method based on Kalman filter to reconstruct and track the ocean current field. A spatiotemporal autoregressive (AR) model is used to describe the evolution of the current field. In the spatiotemporal AR model, the observation region is divided into sub-triangle grids. The sub-triangles are partitioned into clusters by distance and each cluster is assigned with one AR coefficient. The AR coefficients are updated adaptively with the past estimated current velocities. The proposed method is verified with the synthetic observational data generated by a barotropic ocean model and the tomography experiment conducted near Zhoushan Island, China. Compared with the regular time-independent distributed processing method, the proposed ocean current field tracking achieves a lower root-mean-square error. In addition, the proposed method is robust to the measurement link failure and burst errors commonly occurred in a DNUS system.

\section{2:15}

3pA06. Rip current tomography based on reciprocal sound transmissions using reflective waves. Iwao Nakano (Marine Acoust. Res. Lab. of Japan, 5-12-63-101 Yashio, Shinagawa-Ku, Tokyo 140-0003, Japan, nakano-family@s7.spaaqs.ne.jp), Hiroshi Ishida (Oshima College, National Inst. of Technol., Japan, Suooshima-cho, Japan), and Ichiro Deguchi (Osaka Univ., Japan, Suita, Japan)

In order to monitor the occurrence of rip current in beaches, an acoustic measurement system for rip currents based on passive reciprocal sound transmissions using reflective waves was designed and developed. The offshore current velocity $\mathrm{V}$ is given by $\mathrm{V}=\mathrm{C}_{0}{ }^{2} / \mathrm{L} *\left(\mathrm{t}_{21}-\mathrm{t}_{12}\right) / 2$ where $\mathrm{C}_{0}$ is sound speed, $\mathrm{L}$ is the distance between the transducers, $\mathrm{t}_{21}$ is travel time at onshore transmission, and $t_{12}$ is travel time at offshore transmission. The typical components of the measurement system consisted of one modified dual-frequency sounder, two broad-band acoustic transducers, six sound reflectors, and a data acquisition network. The frequency, source level, and transmission interval of transmitted signal was $50 \mathrm{kHz}, 1 \mathrm{~kW}(\max )$, and $2 \mathrm{~s}$, respectively. The typical monitoring area is $30 \mathrm{~m}$ wide and $90 \mathrm{~m}$ long, and the depth is $1 \mathrm{~m}$. This system simply required the precise position measurement of two transducers and sound reflectors. The sound speed was determined by the direct travel times between the transducers. When the field tests were conducted at Uradome Beach in Tottori Prefecture of Japan in September of 2014, the phases of sound signals were so stable to allow the automatic peak and/or phase tracking. The obtained and reconstructed velocity data were consistent with those by EM current meters.

2:30

3pA07. Multipath propagation of sound in a shallow tidal channel and its implications on tomographic current measurements. Mahdi Razaz, Len Zedel (Physical Oceanogr., Memorial Univ. of NF, 500 Prince Philip Dr., St. John's, NF A1B 3X7, Canada, mrazaz@mun.ca), Alex Hay (Oceanogr., Dalhousie Univ., Halifax, NS, Canada), and Kiyosi Kawanisi (Civil and Environ. Eng., Hiroshima Univ., Higashi-Hiroshima, Hiroshima, Japan)

A pilot observational experiment with Fluvial Acoustic Tomography (FAT) system was conducted in the Grand Passage, Nova Scotia, Canada, in 2014, to assess the capabilities of FAT in continuous monitoring of transport in a tidal channel. To implement the tomographic measurements, two broadband FAT transceivers operated at $7 \mathrm{kHz}$ central frequency were positioned in both sides of the channel emitting a pulse every $30 \mathrm{~s}$ for 4 days. Three coherent arrivals were identified in the acoustic receptions. This paper investigates the influence of physical characteristics of water and current shear on multipath propagation of sound in well-mixed conditions of the channel. At the end, we report the comparison between FAT and reference velocity data collected by a moving-boat ADCP.

\section{2:45}

3pA08. Reliable acoustic path tomography at Aloha Cable Observatory. Vincent J. Varamo and Bruce Howe (Ocean and Resources Eng., Univ. of Hawaii - Manoa, 2540 Dole St., Holmes Hall 402, Honolulu, HI 96822, varamo@hawaii.edu)

Using a mobile ship platform (R/V Kilo Moana) with an acoustic source transmitting to a fixed bottom hydrophone at the ALOHA Cabled Observatory (ACO), we are investigating the feasibility of "RAP tomography" (Reliable Acoustic Path). This will allow the spatial mapping of the path integrated sound speed (temperature) over a $60 \mathrm{~km}$ diameter "teacup" volume of the ocean. This can be considered an extension of an inverted echosounder (from a vertical to near horizontal path) combined with the precise positioning and timing of seafloor geodesy, where noise for the latter, sound speed, is our signal. As a first step, transmissions were sent from a $4 \times 4$ array of transducers at $3.5 \mathrm{kHz}$ when the vessel approached and departed from ACO. The measured travel times were compared with estimated travel times based on CTD measurements. As the next step, we use new instrumentation to ensure exact time of acoustic transmission and to test user-generated signals (LFM sweeps and M-sequences) to improve the time of arrival resolution. Also, to increase the amount of shallow angle energy for the longer ranges, only one transducer with a broader beamwidth will be used. Results from an August 2016 experiment will be presented. [Work supported by ONR.] 


\title{
Session 3pBAa
}

\section{Biomedical Acoustics: Quantitative Ultrasound II: From Micro to In-vivo}

\author{
Jonathan Mamou, Cochair \\ F. L. Lizzi Center for Biomedical Engineering, Riverside Research, 156 William St., 9th Floor, New York, NY 10038 \\ Tadashi Yamaguchi, Cochair \\ Chiba University, 1-33 Yayoicho, Inage, Chiba 2638522, Japan
}

Invited Paper

\begin{abstract}
1:00
3pBAa1. Mutual interpretation between B-mode image and cross-sectional acoustic microscopy. Naohiro Hozumi, Wei-Chean Tan, Sachiko Yoshida (Toyohashi Univ. of Technol., 1-1 Hibarigaoka, Tenpaku, Toyohashi, Aichi 441-8580, Japan, hozumi@icceed. tut.ac.jp), Yuki Ogura (Shiseido, Yokohama, Japan), Kazuto Kobayashi (Honda Electronics, Toyohashi, Japan), and Tadashi Yamaguchi (Chiba Univ., Chiba, Japan)

Acoustic microscopy is a two-dimensional profile of an acoustic parameter such as sound speed and characteristic acoustic impedance. It may be useful to understand how practically important B-mode image looks depending on cross-sectional spatial distribution of acoustic properties. In the presentation, it will be exhibited that B-mode echo-graph can be briefly reproduced from acoustic impedance profile observed by acoustic microscope by a similar process to the time domain reflectometry (TDR), with some simple assumptions. As an example, the acoustic impedance profile of a rat cerebellar tissue was acquired by acoustic impedance microscope that was developed in our lab. The B-mode image reproduced by calculation was compared with an actually acquired B-mode image. A good agreement between these two images was seen. On the other hand, a B-mode profile may be interpreted into cross-sectional distribution of acoustic profile. As it is attributed to inverse problem, several assumptions may be required. In the calculation, strait acoustic beam and no attenuation were assumed. In addition, the B-mode profile with RF waveforms was assumed to be available. In the presentation, it will be exhibited that cross-sectional acoustic impedance profile of a shallow region of human skin can be reproduced from non-invasive B-mode scan.
\end{abstract}

\section{Contributed Papers}

$1: 20$

3pBAa2. Repeatability and reproducibility of ultrasonic attenuation coefficient and backscatter coefficient measures in adults with non-alcoholic fatty liver disease. Aiguo Han (Univ. of Illinois, Urbana, IL), Michael P. Andre, Rohit Loomba, Claude B. Sirlin (Dept. of Radiology, Univ. of California at San Diego, 408 Dickinson St., San Diego, CA 92103, mandre@ucsd.edu), John W. Erdman, Jr., and W. D. O’Brien, Jr. (Univ. of Illinois, Urbana, IL)

The attenuation coefficient (AC) and backscatter coefficient (BSC) are two promising quantitative ultrasound biomarkers, yet their repeatability and reproducibility (R\&R) have never been assessed in human subjects. This study assesses R\&R of the AC and BSC in 30 adults with suspected non-alcoholic fatty liver disease (NAFLD), a wide liver fat content range, age range of 28-71, using a Siemens S3000 scanner. Each subject had two consecutive scans between which the subject was repositioned. In each trial, the $4 \mathrm{C} 1$ and $6 \mathrm{C} 1 \mathrm{HD}$ transducers were used for multiple B-mode/RF acquisitions of the right lobe of the liver. Then, a reference phantom was scanned using the same transducer and machine settings. Two sonographers each scanned 15 patients independently. Two fields of interest (FOIs; with and without visible blood vessels) were drawn for each image. AC and BSC were estimated within the FOIs. AC and log-transformed BSC mean values within 2.6-3.0 MHz were used for the R\&R analysis based on an unweighted sums of squares ANOVA approach. The variabilities of inter-trial, inter-image, inter-FOI, and intertransducer conditions were found to be lower than the inter-patient variability. The inter-transducer variability was the lowest among all analyzed variables. [Support: NIH R01DK106419.]

\section{$1: 35$}

3pBAa3. A method for estimating the 3-D structure function from 2-D histology sections. Aiguo Han (BioAcoust. Res. Lab., Univ. of Illinois, 405 N. Mathews, Urbana, IL 61801, han51@illinois.edu)

Understanding the fundamental scattering mechanism(s) in tissues is critical to the success of Quantitative Ultrasound (QUS) imaging. From a discrete scatterer point of view, the ultrasound scattered power depends not only on the properties of the individual scatterers (modeled by the form factor) but also on the spatial correlation among the scatterers (modeled by the structure function). Previously, the structure function was obtained from the ultrasound backscatter data for dense cell pellet biophantoms. This study introduces a method for obtaining the 3-D structure function from the digitized hematoxylin \& eosin (H\&E) stained histology images. The method starts with calculating the 2-D pair correlation function using the scatterer positions determined from a histology image. Assuming isotropic media with spherical scatterers, the 3-D pair correlation function is then estimated by numerically solving a stereological function that relates the 3-D pair correlation function with the 2-D pair correlation function. The 3-D structure function is finally calculated through a Fourier-Bessel transformation of the 3-D pair correlation function. The proposed method was validated through computer simulations and cell pellet biophantom experiments. Using this method to estimate 3-D structure functions may lead to improved understanding of acoustic scattering in biological media. [Support : R01CA111289 and R01DK106419.] 
BSC. By using the spherical Gaussian model, the effective scatterer diameter (ESD) and acoustic concentration (EAC) are estimated from the BSC. The goal of this study is to compare the LF and QUS parameters to determine how they are correlated and to verify their correlation with ESD and EAC. To do this, RF data were acquired using a Siemens S3000 from 20 patient livers (suspected of Non-Alcoholic Fatty Liver Diseases (NAFLD)) and from these spectra, the LF and QUS parameters were estimated. The liver fat fraction for each patient was estimated using MRI, and ranged from 0.03 to 0.3 . The results show that $\mathrm{LF}$ slope is correlated to $\operatorname{ESD}\left(\mathrm{R}^{2}=0.75\right)$, $L F$ midband to EAC $\left(R^{2}=0.70\right)$, and LF intercept to ESD and EAC. A very high correlation between LF midband and the mean BSC is observed $\left(R^{2}=1.00\right)$, along with the fat fraction $\left(R^{2}=0.63\right)$. [Support: NIH R01DK106419.]

\section{2:35}

rally. The edematous stroma and shrinkage and dedifferentiation of acinar cells, has certain similarities with the morphology in some forms of pancreatic carcinoma. The pancreas' AC and BSC (20-50 MHz) were estimated in vivo and ex vivo at baseline (before cerulein injections) and at $24 \mathrm{~h}, 48 \mathrm{~h}$ and $72 \mathrm{~h}$ after cerulein injections in Sprague-Dawley rats $(\mathrm{N}=24)$. AC and BSC showed the same trends whereby (relative to baseline values) the 24-h AC decreased $\sim 0.3 \mathrm{~dB} / \mathrm{cm}-\mathrm{MHz}$ and BSC decreased $\sim 1-2 \mathrm{~dB}$ with $\mathrm{AC}$ and BSC progressively approaching the baseline values at $48 \mathrm{~h}$ and $72 \mathrm{~h}$, suggesting that as a function of time, the AC and BSC are reflecting a change in the pancreas back to baseline conditions. [Support: NIH Grant R37EB002641.]

\section{2:05}

3pBAa5. Multi-parameter analysis of bladder mechanical properties using ultrasound bladder vibrometry. Matthew T. Huber (Dept. of Phys., Rhodes College, 2000 North Parkway, Rhodes Box 2644, Memphis, TN 38112, hubmt-18@rhodes.edu), Aparna Signh, Mathew Cheong (Dept. of Physiol. and Biomedical Eng., Mayo Clinic College of Medicine, Rochester, MN), Matthew Urban (Dept. of Radiology, Mayo Clinic College of Medicine, Rochester, MN), Mahdi Bayat, and Mostafa Fatemi (Dept. of Physiol. and Biomedical Eng., Mayo Clinic College of Medicine, Rochester, $\mathrm{MN}$ )

Bladder wall mechanical properties are important indicators of bladder compliance. This study compares the effectiveness of the Kelvin-Voigt, Maxwell, and fractional Kelvin-Voigt rheological models in capturing the mechanical properties of normal (compliant) and aberrant (non-compliant) ex-vivo pig bladder walls. Bladders were filled to different volumes, varying their thickness and internal pressure, and excited by the acoustic radiation force. Pulse-echo ultrasound imaged anti-symmetrical Lamb waves traveling along the bladder wall. Two-dimensional Fourier analysis generated velocity-frequency dispersion curves for the Lamb wave propagation. Fitting these experimental curves against theoretical curves from the models yielded values for bladder elasticity and viscosity. Pressure, measured simultaneously, was used as a point of comparison with elasticity. Our findings indicate an increasing trend in both elasticity for the rheological models and recorded pressure with increasing volume. This rate was observed to be significantly higher in aberrant bladders than intact bladders for the Maxwell and fractional Kelvin-Voigt models (p-value of 0.0082). Additionally, all three models showed a similar strong correlation (greater than 0.9) between pressure and elasticity. These findings provide a foundation for selecting an appropriate bladder wall rheology model which can potentially be used in evaluation of human bladder mechanical properties. [Work supported by NIH grant DK99231.]

\section{2:20}

3pBAa6. Comparison of quantitative ultrasound parameters from two approaches on in vivo human fatty liver. Pauline Muleki-Seya, Aiguo Han (Univ. of Illinois, BioAcoust. Res. Lab., 405 N Mathews Ave., Urbana, IL 61801, muleki@illinois.edu), Michael P. Andre, Rohit Loomba, Claude B. Sirlin (Univ. of California at San Diego, San Diego, CA), John W. Erdman, Jr., and William D. O’Brien, Jr. (Univ. of Illinois, Urbana, IL)

Two different quantitative ultrasound (QUS) processing strategies are evaluated and compared. The first method estimates the Lizzi-Feleppa (LF) parameters from the linear fit of the normalized power spectrum, and yields the slope, the intercept, and the midband. The QUS approach relies on the attenuation and BSC versus frequency, and yields the mean attenuation and

3pBAa7. Design of transmission-mode measurements for estimating ultrasound attenuation and nonlinearity in liver. Wayne Kreider, Christopher Hunter (CIMU, Appl. Phys. Lab., Univ. of Washington, 1013 NE 40th St., Seattle, WA 98105, wkreider@uw.edu), Oleg A. Sapozhnikov (Dept. of Acoust., Phys. Faculty, Moscow State Univ., Moscow, Russian Federation), Adam D. Maxwell (Dept. of Urology, Univ. of Washington School of Medicine, Seattle, WA), Vera A. Khokhlova (Dept. of Acoust., Phys. Faculty, Moscow State Univ., Moscow, Russian Federation), Brian MacConaghy, and Yak-Nam Wang (CIMU, Appl. Phys. Lab., Univ. of Washington, Seattle, WA)

Previously, it has been shown that ultrasound measurements of sound speed and nonlinearity can be used to quantify the fatty and non-fatty components of liver tissue. In addition, it has been proposed that ultrasound attenuation measurements can be used to distinguish fatty components comprising either sub-micron lipid droplets (microsteatosis) or much larger cellsized droplets (macrosteatosis). To perform all of these measurements, a caliper device is being developed based on a transmission-mode approach. Design challenges are posed by competing requirements: sub-megahertz frequencies for optimal detection of microsteatosis and nonlinearity estimation based on waveform distortion over relatively short distances. To meet these challenges, a design is proposed in which ultrasound at $667 \mathrm{kHz}$ is generated by a $50 \mathrm{~mm}$ piezoceramic disk and all measurements are made in the planewave regime. Given geometrical and physical constraints, all analysis was performed in the time domain. The nonlinearity coefficient was accurately estimated from a quasi-linear approximation to the lossless Burgers equation for pressure amplitudes less than $1 \mathrm{MPa}$ and propagation distances from 15 to $50 \mathrm{~mm}$. An analogous time-domain approach was implemented for determining attenuation at the fundamental frequency and several odd harmonics. [Work supported by NIH grants EB017857, EB007643, EB016118, and DK104854.]

\section{2:50-3:05 Break}

\section{3:05}

3pBAa8. Characterization of the lung parenchyma using ultrasound multiple scattering. Kaustav Mohanty (Dept. of Mech. and Aerosp. Eng., College of Eng., North Carolina State Univ., 3147 B, 911 Oval Dr., EB-3, Raleigh, NC 27606, kmohant@ncsu.edu), John Blackwell, Thomas Egan (Div. of Cardiothoracic Surgery, Univ. of North Carolina, Chapel Hill, NC), and Marie Muller (Dept. of Mech. and Aerosp. Eng., North Carolina State Univ., Raleigh, NC)

We use multiple scattering of ultrasound waves to characterize the lung micro-architecture in order to differentiate between a healthy lung and a lung suffering from Alveolar Interstitial Lung Diseases. The experimental setup consists of a linear transducer array with an $8 \mathrm{MHz}$ central frequency placed in direct contact of the lung to be assessed. The diffusion constant $\mathrm{D}$ and scattering mean free path $\mathrm{L}^{*}$ of the lung parenchyma are estimated by separating the incoherent and the coherent intensities in the near field. 2D FDTD numerical simulations were carried out on rabbit histology images with varying degrees of lung collapse. Phantom experiments were conducted in melamine sponges to study the variations in $\mathrm{D}$ and $\mathrm{L}^{*}$ with varying air volume fraction. Significant correlations were observed between air volume fraction and $\mathrm{L}^{*}$ in simulation $(\mathrm{r}=-0.9542, \mathrm{p}<0.0117)$ and sponge phantom experiments $(\mathrm{r}=$ $0.9932, \mathrm{p}<0.0068)$. Finally, in vivo measurements were conducted in healthy 
and edematous rat lungs. In the control rat lung, $\mathrm{L}^{*}$ was found equal to $83 \mu \mathrm{m}$ $(+/-14.9)$, whereas in the edematous lung, it was found equal to $260 \mu \mathrm{m}(+/-$ 27). These results are extremely promising for the assessment of lung pathologies using ultrasound.

\section{$3: 20$}

3pBAa9. In-vivo quantitative analysis of the angiogenic microvasculature in tumor-bearing rats using multiple scattering: A preliminary study. Aditya Joshi (Mech. \& Aerosp. Eng., North Carolina State Univ., 2500 Avent Ferry Rd., Apt. No. 203, Raleigh, NC 27606, aajoshi4@ncsu. edu), Sarah Shelton, Virginie Papadopoulou, Brooks Lindsey, Paul Dayton (Biomedical Eng., UNC Chapel Hill, Chapel Hill, NC), and Marie Muller (Mech. \& Aerosp. Eng., North Carolina State Univ., Raleigh, NC)

We propose a method to quantify the vascular density in vascular networks using contrast-enhanced multiple scattering. We measured the diffusion constant $\mathrm{D}$ and transport mean free path $\mathrm{L}^{*}$ from the time evolution of the incoherent intensity in a rat model of cancer. An $8 \mathrm{MHz}$ linear transducer array was used to record the backscattered signals from subcutaneous fibrosarcoma tumors and control tissue. The coherent and incoherent contributions to the backscattered intensity were separated, and the growth rate of the incoherent contribution was measured, giving the $\mathrm{D}$ and $\mathrm{L}^{*}$, knowing the effective speed of sound. By translating the linear array along the tumor, mapping of $\mathrm{L}^{*}$ was achieved. Tumors were implanted in the right flank of four rats, and the contralateral side served as control. Acoustic angiography and measurements of the incoherent intensity were performed. The mean L* values in control and tumor tissue were significantly different $(105.27+/$ 30.96 micron and 41.28+/- 14.23 micron, respectively, $\mathrm{p}=8.4033 \times 10^{-49}$ ). The mean distance between vessels was estimated from acoustic angiography images using Monte-Carlo simulations, and was in agreement with the experimentally calculated values of $\mathrm{L}^{*}\left(\mathrm{r}=0.9507, \mathrm{p}=1.4957 \times 10^{-9}\right)$.

\section{$3: 35$}

3pBAa10. Numerical evaluation of time-domain Green's functions for space-fractional wave equations. Xiaofeng Zhao and Robert McGough (Dept. of Elec. and Comput. Eng., Michigan State Univ., 2120 Eng. Bldg., East Lansing, MI 48824, mcgough@egr.msu.edu)

Ultrasound attenuation in soft tissue follows a power law as a function of the ultrasound frequency. Several different models for the power law attenuation of medical ultrasound have been developed using fractional calculus, where each contains one or more time- or space-fractional derivatives. For certain time-fractional models, exact and approximate timedomain Green's functions have been derived and evaluated numerically, but a similar analysis has not yet been performed on the space-fractional models. To address this deficiency, time-domain Green's functions are numerically calculated here for two space-fractional models, namely the ChenHolm and Treeby-Cox space-fractional wave equations. Numerical results are computed for both of these in breast and liver with power law exponents of 1.5 and 1.139 , respectively. The results show that these two space-fractional wave equations are causal everywhere. Away from the origin, the time-domain Green's function for the dispersive Treeby-Cox space-fractional wave equation is very similar to the time-domain Green's functions calculated for the corresponding time-fractional wave equations, but the time-domain Green's function for the nondispersive Chen-Holm space-fractional wave equation is quite different. To highlight the similarities and differences between these, time-domain Green's functions are compared and evaluated at different distances for both breast and liver.

\section{3:50}

3pBAa11. Fatty-acid species identification by quantitative high-frequency acoustic impedance measurements. Kazuyo Ito (Graduate School of Eng., Chiba Univ., 1-33 Yayoicho, Inage-ku, Chiba, Chiba 2638522, Japan, k_ito@chiba-u.jp), Kenji Yoshida (Ctr. for Frontier Medical Eng., Chiba Univ., Chiba, Chiba, Japan), Hitoshi Maruyama (Graduate School of Medicine, Chiba Univ., Chiba, Chiba, Japan), Jonathan Mamou (Lizzi Ctr. for Biomedical Eng., Riverside Res., New York, NY), and Tadashi Yamaguchi (Ctr. for Frontier Medical Eng., Chiba Univ., Chiba, Chiba, Japan)

Accurate discrimination of non-alcoholic steatohepatitis (NASH) from simple steatosis is a critical issue in current clinical practice because NASH can progress to cirrhosis or even liver cancer. Some studies reported that the free fatty acids (FFA) composition of the liver changes with the progression of NASH. Therefore, an ultrasound-based diagnostic tool could be based on measurements of the acoustical properties of FFAs. This study investigated how FFAs influence the acoustical properties of liver cells at a microscopic level using scanning acoustic microscopy (SAM) with four pathological types of mouse livers, five kinds of FFAs in solution, and five kinds of FFAs in cultured cells. A SAM system based on an $80-\mathrm{MHz}$ center-frequency transducer was employed. The acoustic impedance was computed from the echo amplitude and the pressure-reflection coefficient. One-way ANOVA showed statistically significant differences $(\mathrm{p}<0.01)$ in acoustic impedance among pathological types. NASH was found to have the lowest acoustic impedance. Results obtained in FFA solutions and FFA-containing cultured cells also demonstrated significant differences in acoustic-impedance values $(\mathrm{p}<0.01)$ for all paired tests except one (palmitate and linoleate). Therefore, these initial results suggest that NASH identification and characterization using in-vivo quantitative ultrasound methods may be possible.

\section{4:05}

3pBAa12. 500-MHz scanning acoustic microscopy of cancerous human lymph nodes. Jonathan Mamou, Daniel Rohrbach (F. L. Lizzi Ctr. for Biomedical Eng., Riverside Res., 156 William St., 9th Fl., New York, NY 10038, jmamou@rri-usa.org), Emi Saegusa-Beecorft, and Junji Machi (General Surgery, Univ. of Hawaii and Kuakini Medical Ctr., Honolulu, HI)

Scanning acoustic microscopy (SAM) at $500 \mathrm{MHz}$ permits forming 2D maps of the speed of sound (SOS), attenuation (A) and acoustic impedance (Z) of tissue microstructure with a spatial resolution of $4 \mu \mathrm{m}$. Maps from cancerous lymph nodes (LNs) can improve understanding of ultrasound scattering at $25 \mathrm{MHz}$ and thereby can improve the specificity and sensitivity of quantitative ultrasound (QUS) methods for clinically significant micrometastases. SAM data were acquired from ten fixed LNs from colorectalcancer patients. Deparaffinized, 6- $\mu \mathrm{m}$ thick sections were scanned using a custom-built SAM equipped with a 500-MHz transducer having a $264-\mathrm{MHz}$ bandwidth and a $4-\mu \mathrm{m}$ lateral beamwidth. Reflected signals were digitized at $2.5 \mathrm{GHz}$ and maps of $\mathrm{Z}, \mathrm{A}$, and SOS were generated. Histological thin sections adjacent to the scanned samples were stained using hematoxylin and eosin, and metastatic regions were demarcated on the photomicrograph. The 4- $\mu \mathrm{m}$ spatial resolution permitted distinguishing metastases from other tissue types. The average $\mathrm{Z}$ for metastases was $1.66 \pm 0.06 \mathrm{Mrayl}$, which was significantly larger than the average $Z$ of surrounding tissue. Spatial variations in the $\mathrm{Z}$ maps will translate into new ultrasound-scattering form factors, which are expected to improve QUS approaches for detecting metastases in LNs. [NIH Grant R21EB016117.]

\section{$4: 20$}

3pBAa13. High-frequency ultrasound study of angiogenesis in zebra fish embryos. Dolly A. Sanjinez, Michaelle A. Cadet (Biology, Utah Valley Univ., 496 N 400 E, Orem, UT 84097, dolly.sanjinez@gmail.com), Garrett Wagner (Comput. Eng., Utah Valley Univ., Orem, UT), and Timothy E. Doyle (Phys., Utah Valley Univ., Orem, UT)

Cancer and other prevalent diseases such as heart disease, rheumatoid arthritis, stroke, and diabetic retinopathy are directly associated with either an increase or decrease in levels of angiogenesis in the body. High-frequency (HF) ultrasound (10-100 MHz) is particularly sensitive to small vascular structures that are close in size to the ultrasound wavelength (15-150 $\mu \mathrm{m})$. The ability to rapidly determine the degree of vascularization in the development of small animals in vivo would provide a useful characterization tool for the study of a variety of diseases. The objective of this study was to determine if HF ultrasonic measurements could identify microscopic vessel formation that occurs during the development of zebra fish embryos within 1-3 days post-fertilization (dpf). Casper and P53 Nacre zebra fish embryos were provided by the Stewart Lab at the Huntsman Cancer Institute (Salt Lake City, Utah). The embryos were placed in 12-well cell culture plates, embedded in agarose and egg water, and immobilized with tricaine. Embryos were positioned on their lateral side and tested 1-2 dpf using 50$\mathrm{MHz}$ pulse-echo measurements. The results show that the number of peaks in the ultrasonic spectra correlate with embryo development and may provide a quantitative assessment method for angiogenesis. 
analysis, and interpretation. QIBA profiles also provide specific claims of what can be accomplished by following the QIBA protocol. The intent is to validate the profile across imaging systems in a collaboration between industry, government (NIH, FDA, NIST), Pharma, clinicians, and academics. The RSNA/QIBA effort includes over 850 volunteers worldwide with Biomarker Committees for all major imaging modalities. The effort is expanding with collaborations in Japan (the Japan Radiological Society's QIBA-Japan) and in Europe (European Institute for Biomedical Imaging Research and the European Imaging Biomarker Alliance; EIBIREIBALL). The QIBA ultrasound group began with a committee to investigate and minimize sources of bias and variance in shear wave speed estimates for liver fibrosis assessment. The group has grown to about 200 members and includes committees to advance biomarkers based on measurement of volume blood flow and parameters derived from contrastenhanced ultrasound. The status and implications of these efforts will be presented.

\title{
Session 3pBAb
}

\section{Biomedical Acoustics and Signal Processing in Acoustics: Bone Sonometry II: Ultrasonic Studies of the Physical Properties of Cortical and Cancellous Bone (Poster Session)}

\author{
James G. Miller, Cochair \\ Physics, Washington U Saint Louis, Box 1105, 1 Brookings Drive, Saint Louis, MO 63130 \\ Mami Matsukawa, Cochair \\ Doshisha University, 1-3 Tatara Miyakodani, Kyotanabe 610-0321, Japan
}

\begin{abstract}
All posters will be on display from 1:00 p.m. to 3:00 p.m. To allow contributors in this session to see the other posters, authors of odd-numbered papers will be at their posters from 1:00 p.m. to 2:00 p.m. and authors of even-numbered papers will be at their posters from 2:00 p.m. to 3:00 p.m.
\end{abstract}

\section{Contributed Papers}

3pBAb1. Finite-difference time-domain simulations of piezoelectric effect in bone under ultrasound irradiation. Atsushi Hosokawa (Dept. of Elec. and Comput. Eng., National Inst. of Technol., Akashi College, 679-3 Nishioka, Uozumi, Akashi 674-8501, Japan, hosokawa@akashi.ac.jp)

To understand the piezoelectricity in bone at ultrasound frequencies, numerical simulations of piezoelectric effect under ultrasound irradiation was performed using an elastic finite-difference time-domain method with piezoelectric constitutive equations (PE-FDTD method). First, the effect of piezoelectricity on ultrasound wave propagation in a piezoelectric material was investigated with consideration of conductivity. Ultrasound pulsed waveforms propagating through piezoelectric ceramics, whose piezoelectric parameter values were known, were investigated by the PE-FDTD simulations. In the simulated results, the ultrasound speed in the ceramics increased with piezoelectricity, and the ultrasound attenuation caused by the piezoelectricity increased with conductivity. The simulated waveforms were in good agreement with the experimental waveforms, which demonstrated the validity of the PE-FDTD simulations. Next, the piezoelectric effect on ultrasound wave propagation in cortical bone was simulated. The piezoelectric effect on ultrasound wave propagation in the bone was scarcely observed because of the very low piezoelectricity. Finally, the piezoelectric potential induced in cortical bone by ultrasound irradiation was simulated. The simulated results showed that the piezoelectric potential decreased with conductivity. Moreover, using the cortical bone specimen, on the surfaces of which positive and negative electrodes were deposited, the piezoelectric potential was experimentally observed to compare with the simulated results.
3pBAb2. Effect of intracortical bone properties on the guided wave propagation using low-frequency axial transmission technique: Simulation study. Daniel Pereira (Mech. Eng., École de technologie supérieure, 1100 Rue Notre-Dame O, Montreal, QC H3C1K3, Canada, pereira.ufrgs@ gmail.com), Guillaume Haïat (Laboratoire Modélisation et Simulation Multi Echelle, Paris, France), Julio Fernandes (Ctr. de recherche de l'Hôpital du Sacré-Cœur de Montréal, Montreal, QC, Canada), and Pierre Belanger (Mech. Eng., École de technologie supérieure, Montreal, QC, Canada)

In this work, a semi-analytical finite-element method was on the propagation of acoustic guided wave. The simulations were performed with a realistic bone cross-sectional geometry by considering an axial transmission configuration. A point source excitation was applied using a toneburst centered at $35 \mathrm{kHz}$ as the input signal. The propagating waves were monitored using out-of-plane displacement in the axial direction at 32 positions separated $4 \mathrm{~mm}$ apart. The properties of the endosteal region of cortical bone were varied from healthy to early osteoporotic conditions. The use of the two-dimensional Fourier transform (2DFFT) allowed the measurement of individual mode velocity, which was compared to the velocity typically measured using first arrival signal (FAS). The results have shown that variations in the velocity measured using 2DFFT were mainly associated to a low order flexural-like mode among the eight modes supported by the waveguide. Furthermore, the measured velocity decreased approximately 10/\% due to a degradation of $20 / \%$ of the biomechanical properties in the endosteal region. In contrast, the FAS velocity has shown to be affected by four modes, showing a non-linear variation on the velocity. The flexural mode 
measured using 2DFFT can therefore be considered promising to monitor changes in the endosteal region.

3pBAb3. A fast signal processing technique for characterizing Lamb wave propagation in viscoelastic cortical long bones using one transmitter and two receivers. Shigeaki Okumura (Graduate School of Informatics, Kyoto Univ., Yoshida-Honmachi, Sakyo-ku, Kyoto, Kyoto 6068501, Japan, sokumura@sato-lab.0t0.jp), Vu H. Nguyen (Laboratoire Modélisation et Simulation Multi Echelle, Université Paris-Est, MSME UMR 8208 CNRS, Créteil, France), Hirofumi Taki (Graduate School of Biomedical Eng., Tohoku Univ., Sendai, Miyagi, Japan), and Toru Sato (Graduate School of Informatics, Kyoto Univ., Kyoto, Kyoto, Japan)

Axial transmission technique is useful in assessing bone quality. Most conventional techniques require multiple transmitters and receivers. Decreasing the number of elements and computational complexity are desired for low-cost diagnosis. We propose a technique that characterizes the Lamb wave using one transmitter and two receivers. Because the transfer function of the Lamb wave is mainly determined by the longitudinal wave velocity (LWV), shear wave velocity (SWV), and bone thickness, these parameters may be determined in a fitting procedure using the transfer function. The number of combinations of these parameters is enormous, and we thus select the candidates for these parameters using spatial domain interferometry. The method estimates the phase velocity of zero-th order anti-symmetric mode. We applied the proposed method to numerical simulation data generated by the semi-analytical finite element method. We employed a viscoelastic plate with LWV, SWV, and thickness of $2120 \mathrm{~m} / \mathrm{s}$, $4430 \mathrm{~m} / \mathrm{s}$, and $1.0 \mathrm{~mm}$, respectively. The estimation errors of proposed method for SWV, LWV, and thickness were within $5.0 \mathrm{~m} / \mathrm{s}, 15 \mathrm{~m} / \mathrm{s}$, and $0.05 \mathrm{~mm}$, respectively. The fitting residue was $-18 \mathrm{~dB}$. The calculation time was $3.6 \mathrm{~s}$. These results demonstrate the high potential of the proposed method in the low-cost diagnosis of bone quality.

3pBAb4. Simulation study of ultrasound propagation in anisotropic and heterogeneous cortical bone model. Koki Takano (Doshisha Univ., 1-3 Tatara Miyakodani, Kyotanabe, Kyoto 610-0321, Japan, duq0366@mail4. doshisha.ac.jp), Yoshiki Nagatani (Kobe City College of Technol., Kobe, Japan), and Mami Matsukawa (Doshisha Univ., Kyotanabe, Japan)

Ultrasound propagation in the heterogeneous long cortical bone was studied. First, ring shaped specimens were obtained from the mid-shaft of a bovine radius. The longitudinal wave velocity distribution of each specimen was measured in the MHz range. Then, using the bilinear interpolation and the piecewise cubic Hermite interpolating method, a 3D axial velocity model was created with the resolution of $40 \mu \mathrm{m}$. Assuming the uniaxial anisotropy of the bone, ${ }^{1,2}$ distributions of all elastic moduli of the initial 3D heterogeneous model were estimated. At the surface of the model, the elastic moduli were smaller than those of the inside parts. Elastic finite-difference time-domain method was used to simulate axial ultrasonic wave propagation in the model. The initial model and the thinner model, where the inner part of the cortical bone was removed, were compared. The wave front of the first arriving signal (FAS) depended on the heterogeneity in the model. However, the effects of removal on the arrival time of the FAS was not obvious. 1. Y. Yamato, et al. (2008). Calcif. Tissue Int. 82, 162-169. 2. T. Nakatsuji, et al. (2011). Jpn. J. Appl. Phys. 50, 07HF18.

3pBAb5. Modeling wave propagation through the skull for ultrasonic transcranial Doppler. Shreyank Gupta (École de technologie de supérieure, 8626 rue Waverly, Montreal, QC H2P 2R1, Canada, shreyank. gupta@gmail.com), Guillaume Haïat (CNRS, Laboratoire Modélisation et Simulation Multiéchelle UMR, Creteil, France), Catherine Laporte, and Pierre Bélanger (École de technologie de supérieure, Montreal, QC, Canada)

One problem associated with transcranial Doppler ultrasound (TCD) is the relatively low energy penetrating inside the brain through the skull, seriously limiting the image quality. This may be due to the impedance mismatch at the bone interface and to the bone frequency dependent attenuation. The objective of this paper is to model ultrasonic wave propagation through the skull. To do so, an analytical model was developed based on the estimation of the transmission coefficients inside the brain, leading to frequency dependent overall transmission coefficient for a given skin and bone thickness. Moreover, a finite element model was developed taking into account absorption phenomena. Both methods were validated experimentally by comparing the numerical and analytical results with results obtained from a phantom mimicking the skull having an attenuation coefficient equal to $30 \mathrm{~dB} / \mathrm{cm}$ at $2.25 \mathrm{MHz}$ and a thickness of $4.4 \mathrm{~mm}$. A 2 $\mathrm{mm}$ layer of water mimicked the skin. The difference between the maximum amplitude of normalized received US signals obtained analytically and experimentally was $1 \%$. The average relative difference between them was $0.3 \%$. Thus, a working model is designed which can predict the energy inside the brain. Furthermore, the results show that impedance mismatch plays a major role in transmission loss rather than frequency dependent attenuation

3pBAb6. Comparison between an axial transmission device and high resolution peripheral quantitative computed tomography-A clinical evaluation of ultrasound for the assessment of cortical bone quality. Ryoichi Suetoshi, Dorian Cretin (Furuno Electric Co., Ltd., 9-52 Ashiharacho, Nishinomiya, Hyogo 662-8580, Japan, ryohichi.suetoshi@furuno.co. jp), Ko Chiba (Nagasaki Univ., Nasgasaki, Japan), and Makoto Osaki (Nagasaki Univ., Nagasaki, Japan)

Quantitative ultrasound mainly evaluates cancellous bones, although cortical bone is now thought to be more important to determine bone fragility. Therefore, we developed an axial transmission device ( $\mathrm{f}=3 \mathrm{MHz}$ ) to measure the cortical Speed of Sound (cSOS). The purpose of this study is to investigate the material properties in cortical bone that $\mathrm{cSOS}$ reflects by using High Resolution-peripheral Quantitative CT (HR-pQCT). In 19 patients and healthy volunteers (aged 33-73), the cSOS at the tibia and radius were measured by our ultrasonic device. Cortical bone parameters were measured by HR-pQCT (Scanco). Areal bone mineral density (aBMD) at the lumbar spine and femur were measured by DXA. cSOS had a strong correlation with the cortical volumetric mineral density (Ct.vBMD) measured by HR-pQCT at both the tibia and the radius $(\mathrm{r}=0.83,0.72, \mathrm{p}<0.001)$. A correlation was also found between $\operatorname{cSOS}$ and the cortical porosity at the radius $(r=-0.83, p<0.001)$. No correlation was found between cSOS and $\mathrm{aBMD}$, which makes cSOS unlikely to depend on the bone quantity. The correlation between cSOS and Ct. vBMD may show that cSOS could reflect the bone quality related to mineralization and porosity. cSOS, not being related to DXA but linked to the bone quality, could be useful for the screening of the mineral properties of cortical bone simply and noninvasively.

3pBAb7. Induced electric potential in cortical bone and cartilage by ultrasound irradiation. Shunki Mori, Sayaka Matsukawa, Mami Kawase (Doshisha Univ., 1-3 Tatara Miyakodani, Kyotanabe, Kyoto 6100321, Japan, dmq1028@mail4.doshisha.ac.jp), Shinji Takayanagi (Nagoya Institute of Technol., Nagoya, Aichi, Japan), and Mami Matsukawa (Doshisha Univ., Kyotanabe, Kyoto, Japan)

LIPUS (low intensity pulse ultrasound) can reduce the time of bone fracture healing. The detailed mechanism of ultrasonic effects on bone, however, has not been clearly understood yet. One possible idea seems to be the piezoelectricity of bone. The purpose of this study is to evaluate the piezoelectricity of cortical bone and cartilage. In order to evaluate piezoelectricity of bone in the $\mathrm{MHz}$ range, bone transducers were fabricated. Circular plate cortical bone samples and cartilage samples (diameter; $10.0 \mathrm{~mm}$, thickness; $1.00 \mathrm{~mm}$ ) were used as piezoelectric materials of the transducer to receive ultrasound. The surface of bone plates was normal to the bone axis or the radial axis and that of cartilage plates was normal to bone axis. By irradiating ultrasound in the $\mathrm{MHz}$ range, induced electric potentials were successfully observed in all transducers. The maximum sensitivity of the cartilage transducer was about $93.6 \mathrm{nV} / \mathrm{Pa}$ and that of the bone transducer was about 27.5 $\mathrm{nV} / \mathrm{Pa}$. Induced electric potentials of cartilage transducers were always larger than those of bone transducers. The main part of the cartilage is type collagen. The results may indicate that the piezoelectricity of the cartilage and bone mainly comes from the collagen. 


\title{
Session 3pBAc
}

\section{Biomedical Acoustics and Signal Processing in Acoustics: Bone Sonometry III: Ultrasonic Studies of the Physical Properties of Cortical and Cancellous Bone}

\author{
Mami Matsukawa, Cochair \\ Doshisha University, 1-3 Tatara Miyakodani, Kyotanabe 610-0321, Japan \\ James G. Miller, Cochair \\ Physics, Washington U Saint Louis, Box 1105, 1 Brookings Drive, Saint Louis, MO 63130
}

Invited Papers

3:15

3pBAc1. A novel bone sonometer for estimation of calcaneal bone mineral density. Jonathan J. Kaufman and Gangming Luo (CyberLogic, Inc., 611 Broadway, Ste. 707, New York, NY 10012, jjkaufman@cyberlogic.org)

This study evaluated a new clinical ultrasound device, the $Q R T 250$, in terms of its ability to estimate BMD at the calcaneus. The $Q R T 250$ rests on the floor and permits real-time evaluation of calcaneal BMD. The $Q R T 250$ utilizes a novel heel positioning system that relies on an individual's foot size to locate a specific region-of interest (ROI) in the calcaneus. This feature of the device was designed to account for the relatively high degree of BMD heterogeneity associated with the calcaneus. The device measures in thrutransmission a net time delay (NTD) parameter. NTD has been shown to be highly correlated with bone mass in simulations, in vitro, and in other clinical studies. A linear regression of the NTD is used to provide an estimate of calcaneal BMD that would be measured by DXA. A clinical IRB-approved study was carried out in which 145 adults were measured at the calcaneus using ultrasound and DXA (Lunar GE PIXI; WI, USA). In addition, thirty (30) subjects were each ultrasonically measured two (2) times and the short term precision was evaluated. A linear regression showed that $\mathrm{BMD}_{\mathrm{US}}=0.12 \cdot \mathrm{NTD}-0.11$ and that the linear correlation between $\mathrm{BMD}_{\mathrm{DXA}}$ and $\mathrm{BMD}_{\mathrm{US}}$ was $0.89(\mathrm{P}<0.001)$. We found that calcaneal ultrasound measurements yield results that are very closely associated with those from DXA. In the short-term precision study, we found that the mean percent precision was $1.37 \%$. In summary, the $Q R T 250$ can be used to identify bone loss in osteoporosis and ultimately to reduce the incidence of fragility fractures.

\section{$3: 35$}

3pBAc2. In vivo radius bone evaluation of women in their late teens by two wave apparatus. Mami Matsukawa, Shoko Nakanishi (Doshisha Univ., 1-3 Tatara Miyakodani, Kyotanabe 610-0321, Japan, mmatsuka@mail.doshisha.ac.jp), Isao Mano, Kaoru Horii, Yutaro Yoneda (OYO Electric Co., Ltd., Joyo, Japan), Shiori Umemura, and Etsuko Ozaki (Kyoto Prefectural Univ. of Medicine, Kyoto, Japan)

Making use of the fast and slow wave phenomenon in cancellous bone, an ultrasonic bone measurement system, LD-100 (OYO electric), ${ }^{1}$ has been developed and is now commercially available in Japan. From the measurements of fast, slow, and echo waves, the system gives us cancellous bone density $\left(\mathrm{mg} / \mathrm{cm}^{3}\right)$, cancellous bone elasticity $(\mathrm{GPa})$, and the cortical thickness $(\mathrm{mm})$. The measurement area is the distal $5.5 \%$ site of the radius of the non-dominant hand. In this study, the system was modified for the small radius of teenagers using annular array elements. Under the permission of the ethics committee at Doshisha university, radius bones of 111 high school students were measured (15-17 years old, female). The mean values of cortical thickness of the students were 93.7-97.7\% of the young adult mean (YAM). The cancellous bone densities were 82.6-94.0\% of YAM. The standard deviations of these values were higher than the deviations of YAM. These data possibly indicate that the growth of radius bone depends on the site and the outer cortical shell grows fast. For further discussions, more data should be necessary. 1. H. Sai, et al., Osteoporos Int. (2010) 21, 1781-1790

\section{3:55}

3pBAc3. Ultrasonic backscatter theory, method, and diagnostic instrument for cancellous bone. Dean Ta and Chengcheng Liu (Dept. of Electron. Eng., Fudan Univ., 220 Handan Rd., Shanghai 200433, China, tda@fudan.edu.cn)

Recently, significant progress has been made in the backscatter measurement of cancellous bone. In this work, we will introduce the basic theory and some key techniques for backscatter cancellous bone evaluation. Backscatter measurements in vivo were also carried out with a diagnostic instrument, and the feasibility of using the backscatter for cancellous bone evaluation was investigated. Researchers had reported conflicting associations between backscatter and bone features. Our studies demonstrated that this phenomenon was due to different signal selections. The dense cortical shell obstructs the ultrasonic evaluation of the underlaid cancellous bone in vivo. We determined the influence of the overlying cortex on the backscatter signal and proposed a compensation method for the cortical influence. Using an ultrasonic backscatter bone diagnostic instrument, the measurements were performed on 1226 adults and on 467 neonates in the calcaneus. For the adults, the backscatter was significantly correlated with the bone mineral density (BMD) of the spine and hip. The backscatter parameters were moderately correlated with the gestational age and birthweight in the neonates. The in vivo findings demonstrate the ultrasonic backscatter as a useful adjunct measurement for the multi-feature assessment of cancellous bone. 
3pBAc4. Detection and evaluation of airborne ultrasound passed through a heel for non-contact bone assessment. Shinnosuke Hirata and Hiroyuki Hachiya (Dept. of Systems and Control Eng., Tokyo Inst. of Technol., 2-12-1 Ookayama, S5-17, Meguro, Tokyo 152-8550, Japan, shin@ctrl.titech.ac.jp)

Ultrasonic bone assessment by quantitative ultrasound (QUS), measurement of ultrasonic propagation characteristics, is one of the diagnosis methods of osteoporosis. In typical QUS devices, opposed ultrasonic transducers are brought into contact with tissue surfaces through an ultrasonic gel to effectively propagate ultrasound. We have proposed non-contact measurement of propagation characteristics using airborne ultrasound passed through a heel. Generally, airborne ultrasound which passed through a tissue is extremely attenuated due to large reflections at boundaries between air and the tissue. In the proposed method, therefore, the signal-to-noise ratio of passthrough ultrasound is greatly improved by pulse compression using a higher-order M-sequence. Furthermore, each side of a heel is inclined to the direction of the ultrasonic beam. Pass-through ultrasound cannot fully be received by opposed transducers, because the ultrasonic beam inflects at each surface. Therefore, inclination of each heel side is measured by the pulse-echo method. Then, each transducer is inclined with considering the surface inflection. The propagation speed of ultrasound in the heel is estimated from the time of flight of pass-through ultrasound, the length of the propagation path, and the propagation speed of ultrasound in air.

\section{$4: 35$}

3pBAc5. Ultrasound for bone tissue engineering and regenerative medicine. Frederic Padilla (LabTAU INSERM 1032, French National Inst. of Health and Medical Res. (Inserm) \& Université de Lyon, 151 Cours Albert Thomas, Lyon 69390, France, frederic.padilla@inserm. fr), Mario L. Fabiilli (Dept. of Radiology, Univ. of Michigan Med. School, Ann Arbor, MI), Francisco M. Martín-Saavedra (Hospital Universitario La Paz-IdiPAZ, CIBER de Bioingeniería, Biomateriales y Nanomedicina (CIBER-BBN), Madrid, Spain), Kay Raum, Regina Puts (Berlin-Brandenburg School and Ctr. for Regenerative Therapies, Charité - Universitätsmedizin Berlin, Berlin, Germany), Laurence Vico (Inserm U1059 Lab Biologie intégrée du Tissu Osseux, French National Inst. of Health and Medical Res. (Inserm) \& Université de Lyon, St Etienne, France), and Christopher G. Wilson (Boston University Business Innovation Ctr. · dBMP Program, Bioventus LLC, Boston, MA)

We will review the role of ultrasound in bone tissue engineering and regenerative medicine, for enhancement of fracture healing and reactivation of a failed healing process. These approaches rely typically either on the direct stimulation of the healing process or in the delivery at the injury site of scaffold-embedded biologics such as cells, signaling molecules, and genetic material. Different options are available for ultrasound enhancement of fracture healing such as low intensity pulsed ultrasound (LIPUS) and shock waves, for the delivery of regenerative factors and encoding genes, and for the assembly and patterning of scaffolds. A main emphasis will be put on LIPUS-related bioeffects, the most widespread and studied technique. Pronounced bioeffects on tissue regeneration have been reported employing intensities within a diagnostic range. We will describe the current knowledge of the biological response to LIPUS, involving numerous cell types and molecular pathways, hypothesis about LIPUS biophysics, and our recent findings on mechanosensitive transcriptional response of cells after LIPUS. We will also present the use of focused ultrasound to spatiotemporally control the delivery of growth factors, either by induction of transgene expression controlled by a heat-activated and ligand-dependent gene-switch, or by triggered release using acoustic droplets vaporization within biopolymer scaffolds.

\section{4:55}

3pBAc6. Acoustical estimation of endosseous implant stability: Finite element modeling and experimental validation. Vu-Hieu Nguyen, Romain Vayron, and Guillaume Haiat (Multiscale Modeling and Simulation Lab., CNRS, Laboratoire MSME UMR 8208, fac des Sci., UPEC, 61 av du gal de Gaulle, Creteil, France, vu-hieu.nguyen@u-pec.fr)

Various endosseous implants are widely used in orthopedic and dental surgeries. However, failures are observed, which may have dramatic consequences and are often due to a loss of implant stability. The aim of this presentation is to describe two acoustical methods developed to assess the stability of different implants. First, a quantitative ultrasound method was used to determine the stability of dental implants. A monoelement $10 \mathrm{MHz}$ transducer is screwed into the implant and the signal is processed to obtain an indicator. The method was first validated in implants embedded in biomaterials used as bone substitutes, then in vitro and eventually in vivo by comparison with histology. A 3D FEM was developed to optimize the device conception and carry out sensitivity studies. Second, an impact hammer was used in order to investigate whether the AC implant primary stability can be evaluated using such approach. The method was first validated in vitro and in cadaveric experiments. The results showed that an indicator based on the impact momentum may be correlated to the implant pull-out force. A 3D FEM was also developed to analyze and better understand experimental results. This study shows the potential of acoustical methods to assess non-invasively implant stability.

\section{Contributed Paper}

\section{5:15}

3pBAc7. Acceleration of critical bone defect healing by guided low-intensity ultrasound in a rat tibial model. Yi-Xian Qin, Jingbo Liu, Dongye Zhang, and Xiaofei Li (Biomedical Eng., Stony Brook Univ., BioEng. Bldg., Rm. 215, Stony Brook, NY 11794, yi-xian.qin@stonybrook.edu)

Bone defects, caused by trauma, inflammation, and tumors, may lead to loss of limb functions and substantially affect the quality of patients' life. Lowintensity ultrasound (LIUS) can transmit through and into living tissues as acoustic pressure waves, and generate dynamic acoustic radiation force in the local region to induce stem cell recruitment, and mineralization. The aim of this study was to evaluate the effect of LIUS induced healing in a rat tibial critical defect. A total of 40 skeletally mature female Lewis rats were used in this study with a surgical bone defects of $2 \mathrm{~mm}$ in-diameter at proximal tibia. The defects of the left tibia (LIUS group) received daily treatment of ultrasound, and the defects in the right tibia (Control) received sham stimulation. A modified repetitive frequency at $100 \mathrm{~Hz}$, characteristic frequency of $1 \mathrm{MHz}$ at an intensity of $30 \mathrm{~mW} / \mathrm{cm}^{2}$ were used for the stimulation for 20-min/day. After 2 weeks, the $\mu \mathrm{CT}$ images showed that trabecular bone formed in the defect area of both LIUS and control groups. LIUS treated tibia showed more increased in BV/TV, Cont.D, Tb.N, and BMD, and significant decrease in Tb.Sp compared to control. Four-point bending testing showed LIUS treatment significantly increased the bone stiffness $(\mathrm{kN} / \mathrm{mm})$ relative to control group. The histological analysis and $\mu \mathrm{CT}$ data revealed that LIUS therapy can significantly increase new trabecular bone volume, so as the stiffness and ultimate stress of LIUS treated group with two-week as optimized treatment period for healing. 


\title{
Session 3pEA
}

\section{Engineering Acoustics and Physical Acoustics: Outdoor Sound Propagation and Outdoor Public Address}

\author{
JohnPaul R. Abbott, Cochair \\ Department of Physics and Astronomy, National Center for Physical Acoustics, University of Mississippi, 1 Coliseum Dr., \\ Room 1044, Oxford, MS 38677 \\ Yoshifumi Chisaki, Cochair \\ Chiba Institute of Technology, 2-17-1, Tsudanuma, Narashino 2750026, Japan
}

Chair's Introduction-1:00

Invited Papers

1:05

3pEA1. Irregular qualities of sound sources appeared at the input of the outdoor emergency mass notification sound system. Kiyohiro Kurisu, Yasushi Matsumoto, and Ryota Matsuishi (TOA Corp., 2-1 Takamatsu-cho, Takarazuka, Hyogo 665-0043, Japan, kurisu_kiyohiro@toa.co.jp)

In order to obtain an intelligible speech at a listening point addressed by the mass notification sound system, not only high transmission quality of the system, but also a sufficiently clear speech sound source is needed. However, the qualities of speech sources may not be constant because they are supplied by different types of production and transmission methods, e.g., the variety of speech source production methods causes various specifications and irregular qualities of sources, and some types of transmission methods between the parent station (speech production system) and children stations (acoustic amplification system) cannot convey the source signal with sufficient quality. In this presentation, the authors showed examples of irregular source quality appeared at the input of the acoustic amplification system and discussed the necessity of verifying the signals at several points in the total system (i.e., the system between the speech source and the listening point) to regulate their qualities. Then, the guidelines for the performance verification of the outdoor emergency mass notification sound systems which was published by the Acoustical Society of Japan was introduced.

\section{$1: 25$}

3pEA2. Improvement of signal to noise ratio at outdoor listing position by sound emission timing control over the Internet for mass notification system. Yoshifumi Chisaki (Chiba Inst. of Technol., 2-17-1, Tsudanuma, Narashino, Chiba 2750026, Japan, yoshifumi.chisaki@p.chibakoudai.jp) and Taira Onoguchi (Kumamoto Univ., Kumamoto, Japan)

Mass notification system has multiple nodes, where a node consists of some loudspeakers, in order to cover wide service area. Sound signal is emitted from grouped nodes simultaneously in general. Thus, the simultaneous emission from grouped nodes sometimes makes overlapping of sound signals at a listening point in the service area. Since it affects speech intelligibility, reduction of overlapping is a quite important issue not only for emergency but also reduction of wasteful signal power on mass notification system. Recently,global positioning device and network devices, such as 3G/LTE, become reasonable to be equipped to a node of mass notification system. Therefore, it makes easy to control emission timing at each node intelligently. Development of an intelligent algorithm for mass notification is introduced and a part of the algorithm is implemented on a single board computer, Raspberry PI. Performance on signal to noise ration at all the listing points in service area is presented and discuss it with other evaluation methods.

\section{$1: 45$}

3pEA3. Speech intelligibility prediction method using machine learning for outdoor public address systems. Yosuke Kobayashi (Graduate School of Eng., Muroran Inst. of Technol., 27-1 Mizumoto-cho, Muroran 050-8585, Japan, ykobayashi@csse.muroran-it.ac. jp), Kengo Ohta (Anan College, National Inst. of Technol., Anan, Japan), Kazuhiro Kondo (Graduate School of Sci. and Eng., Yamagata Univ., Yonezawa, Japan), and Shuichi Sakamoto (Res. Inst. of Elec. Commun., Tohoku Univ., Sendai, Japan)

Subjective speech intelligibility assessment is important for the development of outdoor public address system. However, as this assessment is difficult in many cases, we propose an objective speech intelligibility evaluation system that includes a machine learning technique. In this talk, we have proved a subjective evaluation and objective prediction of speech intelligibility using the outdoor public address systems at 10 locations in Sendai City, where impulse responses were recorded after the Great East Japan Earthquake. First, the results of the subjective intelligibility evaluation by different test word lists with the same sound field conditions showed that the root mean squared error (RMSE) was very small, not exceeding 7.0\%. Next, we generated the intelligibility prediction model trained with true/false results of 22 subjects using the support vector machine (SVM). This prediction model extracted the feature vector, using the ITU-T P.563 speech quality feature set of the test speech signal. We evaluated the predictive performance of the prediction model using data that was not used in training, and the RMSE obtained was $4.0 \%$. This result was shown to be highly accurate with a value even less than the subject experiment result. 
3pEA4. Optimization of speech presentation in mass-notification sound systems based on word intelligibility. Shuichi Sakamoto, Zhenglie Cui (Res. Inst. of Elec. Commun., Tohoku Univ., 2-1-1 Katahira, Aoba-ku, Sendai 980-8577, Japan, saka@ais.riec.tohoku.ac. jp), Masayuki Morimoto (Graduate School of Eng., Kobe Univ., Kobe, Japan), and Yôiti Suzuki (Res. Inst. of Elec. Commun., Tohoku Univ., Sendai, Japan)

Outdoor mass-notification sound systems can effectively convey emergency announcements over a wide area without requiring the audience to use any special devices. However, the intelligibility of speech presented via these systems is often severely degraded by long-path echoes. Under such conditions, it is important to consider robust ways of presenting speech information. Various studies have highlighted that there are many factors which affect the speech intelligibility with long-path echoes. We have focused on the effect of word familiarity and inserting pauses between the words in a speech signal. High-familiarity words are easy to understand even when presented under challenging listening conditions. By inserting pauses, speech intelligibility could be improved because more of the speech signal can be received without significant overlapping long-path echoes. In this study, speech materials were modeled based on actual sentences used in emergency notifications given via the Japanese mass-notification system, J-Alert. By using this model, the effects of word familiarity and inserting pauses on word indelibility under long-path echo were examined. The results of the experiments suggest that speech signals can be robustly understood despite long-path echoes by using high-familiarity words with adequate interword pauses.

\section{2:25}

3pEA5. Relationship between speech intelligibility and objective measures in sound fields with a discrete long-path echo. Hayato Sato, Masayuki Morimoto, Yusuke Miyagawa (Environ. Acoust. Lab., Graduate School of Eng., Kobe Univ., 1-1 Rokkodai, Nada, Kobe 6578501, Japan, hayato@kobe-u.ac.jp), and Yôiti Suzuki (Human Information Systems Div., Res. Inst. of Elec. Commun., Tohoku Univ., Sendai, Japan)

The outdoor public address system is one of the important methods to notify evacuation information in disaster situations. However, when speech announcements are simultaneously radiated from a number of dispersedly located loudspeakers, speech intelligibility decreases because of temporal or spatial segregation of speech streams, just like a long-path echo in large closed spaces. The present study is focusing on relationship between speech intelligibility and spatial characteristics of long-path echo. Useful-detrimental ratio $\left(U_{50}\right)$ was tested to predict speech intelligibility scores in sound fields with a discrete long-path echo. A noteworthy feature of $U_{50}$ is that all energy of long-path echo is explicitly counted as detrimental energy. The results of intelligibility tests demonstrated that the feature led to lower intelligibility predictions, that is, predictions on the safe side. Meanwhile, the prediction errors increased when the difference between interaural differences for the direct sound and the long-path echo. The extended $U_{50}$ to consider binaural effects referring to the concept of binaural speech transmission index [van Wijingaarden and Drullman, J. Acoust. Soc. Am. 123, 4514-4523 (2008)] showed a higher correlation with the intelligibility scores relative to the original $\mathrm{U}_{50}$.

\section{2:45}

3pEA6. Subwoofer array modeling and optimization. Wolfgang Ahnert and Stefan Feistel (Ahnert Feistel Media Group, Arkonastr. 45-49, Berlin D-13189, Germany, wahnert@ada-amc.eu)

In the last few years, configuring subwoofer arrays in order to achieve a desired sound radiation pattern has become one of the most discussed topics in the sound reinforcement industry. The directivity of such arrays of low-frequency loudspeakers can typically be controlled by means of delay, gain, and polarity settings applied to the individual elements. A new software solution is presented that allows modeling and optimizing such setups, including various cardioid configurations as well as end-fire and steered setups. Frequencies down to $20 \mathrm{~Hz}$ can be investigated. It is also explained how coherent sound superposition at low frequencies and incoherent interaction of sound waves at high frequencies can be simulated consistently by means of a cross-over frequency band. Finally, an optimization method is presented that allows computing elemental delays automatically based on a user-defined coverage angle for the array.

\section{3:05-3:20 Break}

3pEA7. A framework for providing real-time feedback of environmental noise levels over large areas. Edward T. Nykaza, Michael J. White, Jesse M. Barr, Matthew G. Blevins (ERDC, 2902 Newmark Dr., Champaign, IL 61822, edward.t.nykaza@erdc.dren.mil), Steven L. Bunkley (ERDC, Vicksburg, MS), Nicole M. Wayant (ERDC, Alexandria, VA), and D. Keith Wilson (ERDC, Hanover, NH)

Environmental noise can cause sleep disturbance, annoyance, complaints, and quite possibly adverse health effects. This is true for continuous noise sources such as steady road traffic noise, impulsive noise sources such as blasts or sonic booms, or sources that fall inbetween such as intermittent train and aircraft noise. One way to manage environmental noise is to use noise-monitoring technology to provide both the noise-producers and noise-experiencers feedback on the actual noise environment. Traditional noise-monitoring systems, however, only provide this information at a few locations resulting in an incomplete picture of the noise environment over the entire regions of interest. In this paper, we discuss a framework for providing real-time feedback of the noise environment over a large area $\left(\right.$ e.g., $100 \mathrm{~km}^{2}$ ). We show all the steps that are needed to convert the raw noise-monitor data into noise maps and noise impact maps to help manage environmental noise. We discuss the complexity of the problem and present several different ways to visualize the data. 
3pEA8. Formulation of sound propagation and scattering in forests as a radiative transfer theory. Vladimir E. Ostashev and David K. Wilson (U.S. Army Cold Regions Res. and Eng. Lab., 72 Lyme Rd., Hanover, NH 03755, vladimir.ostashev@noaa.gov)

Several approaches have been used in the literature to characterize sound scattering in forests. In this presentation, an approach based on the radiative transfer equation (RTE) is considered. Although the RTE has been used for other types of scattering problems, it is new to forest acoustics. The RTE is an integro-differential equation for the specific intensity, which is the average power flux density within a unit solid angle in a certain direction of propagation. It describes the processes of sound scattering and absorption by discrete scatterers such as tree trunks, branches, and leaves. RTE is applied to sound propagation in a four-layer forest model (ground, trunks, canopy, and open air). The trunk layer is modelled with vertical cylinders, while the canopy layer is modeled by spheres. The scattering amplitudes and total scattering cross sections of these scatterers are identified. At the interface of the trunk layer and ground, the specific intensity is specularly reflected. Although the RTE is based on a phenomenological theory for propagation of intensities, the mutual coherence function of the sound field (which can be obtained from a Helmholtz equation and the multiple scattering theory) may be expressed in terms of the specific intensity.

4:00

3pEA9. Ubiquitous monitoring of infrasound for early detection of events relevant to disaster. Ryouichi Nishimura, Yasunori Owada, and Shinichi Taira (National Inst. of Information and Communications Technol., 2-1-3 Katahira, Aoba-ku, Sendai, Miyagi 9800812, Japan, ryou@nict.go.jp)

A terrestrial event resulting in natural disaster often radiates infrasound when it occurs. Similar phenomenon can be observed in extreme weathers as well. Infrasound travels through the air approximately at the speed of sound and it is faster than traveling of physical kinetic energy which may sometimes bring about a disastrous impact on human lives. Therefore, early detection and source localization of such events are promising for hazard prevention and damage mitigation. To achieve this purpose, coverage of the land is important in terms of celerity and spatial precision, but facilities suitable for monitoring infrasound are not common. In Japan, one monitoring station, IS30, is in operation under Comprehensive Nuclear-Test-Ban Treaty (CTBT) and a few microbalrometer arrays are experimentally set up by Japan Weather Association (JWA) on some parts of Japanese coast. A group of University of Hawaii developed an app for capturing infrasound on iOS devices, and the captured data can be utilized for further analysis. We developed a similar app for Android and deployed smartphones running it on a wireless network to collect information for 24 hours a day stably. Combining the information gathered, we tried to estimate the source signal and its location.

\section{$4: 20$}

3pEA10. The effects of wind noise on outdoor sound propagation and detection. JohnPaul R. Abbott (National Ctr. for Physical Acoust., Univ. of MS, 100 Bureau Dr. Stop 8361, Gaithersburg, MD 20899, johnpaul.abbott@gmail.com)

Wind noise is a form of pseudo-noise. It is generated and propagated by physical mechanisms that are different from acoustic noise; however, its effect on microphones and similar pressure sensitive devices is indistinguishable from acoustic noise. Wind noise is defined as the pressure fluctuations that are generated and propagated by the turbulent flow of an incompressible fluid. Acoustic noise is generated and propagated by the adiabatic compression and expansion of air. Since the effect of wind noise is the same as acoustic noise, it can have significant effect on the propagation and detection of desired acoustic signals at sonic and infrasonic frequencies. This can lead to adverse impacts on mass and early warning systems. This talk will present an overview of the effects of wind noise on outdoor sound propagation, detection, and efforts to mitigate and account for its influence on sonic and infrasonic acoustic signals. It will also discuss the potential impacts of sonic and infrasonic wind noise on mass and early warning systems.

\section{4:40}

3pEA11. Effects of local and global meteorological data on predicting blast noise levels. Matthew G. Blevins (U.S. Army Engineer Res. and Development Ctr., 2902 Newmark Dr., Champaign, IL 61822, matthew.g.blevins@usace.army.mil), Carl R. Hart (U.S. Army Engineer Res. and Development Ctr., Hanover, NH), Steven L. Bunkley (U.S. Army Engineer Res. and Development Ctr., Vicksburg, MS), and Edward T. Nykaza (U.S. Army Engineer Res. and Development Ctr., Champaign, IL)

Predicting noise levels due to military blasts is important near training installations, since surrounding communities may be adversely affected. Prediction models, however, rely on atmospheric or weather data that is not readily available or easily attainable. In this study, blast noise level predictions 1-8 km from the source are made using two sources of meteorological data: publicly available data derived from weather stations that are tens of kilometers from a detonation site, and near-ground weather stations that are 2-6 km from the source. To predict the blast noise levels, statistical learning regression models are trained on each set of meteorological data and over 1000 measured blast events. Predictions from both meteorological data sources are compared to actual blast noise levels recorded during a field experiment by Valente et al. [2012], which were captured under a wide variety of atmospheric conditions. The root-mean-square errors for each model are compared, and the most important input parameters are identified. 


\title{
Session 3pEDa
}

\section{Education in Acoustics: Demonstrations and Tools in Acoustics Education}

\author{
Tracianne B. Neilsen, Cochair \\ Brigham Young University, N311 ESC, Provo, UT 84602 \\ Akira Nishimura, Cochair \\ Media and Cultural Studies, Tokyo University of Information Sciences, 4-1, Onaridai, Wakaba-ku, Chiba 2658501, Japan
}

\begin{abstract}
This walk-in demonstration session is open to all meeting attendees. Presenters will all be available during the entire session to show and explain their demonstrations, similar to a poster session. In addition to the listed abstracts, additional presenters, who have papers in other Education in Acoustics sessions, are participating. A list of their names with reference to their abstracts is given below.
\end{abstract}

\section{Contributed Papers}

3pEDa1. Applying the cellular automaton model in speech production process simulation. Koji Taka (Math. and Sci. Education Res. Ctr., Kanazawa Inst. of Technol., 7-1 Ohgigaoka, Nonoichi, Kanazawa, Ishikawa 9218501, Japan, taka@neptune.kanazawa-it.ac.jp)

This study investigates the dynamic simulation of the speech production process. When an utterance occurs, the sound pressure and the particle velocity distribution of the vocal tract space changes from word to word. The author examined and visualized this change to illustrate how it happens. The author used the cellular automata as a model to calculate the acoustic characteristics of the vocal tract in each region. This study takes a different approach from the traditional z-transform to the cellular automata to calculate the dynamic sound pressure at each site of the vocal tract space. To simplify the calculation, an acoustic cylindrical tube model was used. Dynamic calculations were made even more manageable by changing the physical model from a three-dimensional to a one-dimensional cellular automaton model. Consequently, this study demonstrates that the relationship between the words and the sound pressure distribution can be examined dynamically using the cellular automata model.

3pEDa2. "A record without (or with) prior acoustic information" and "Given: 1.Manet, 2.Coil-Oscillation from a minimum unit of speaker". Kazuhiro Jo (Faculty of Design, Kyushu Univ. / YCAM, 4-9-1, Shiobaru, Fukuoka, Fukuoka 815-8540, Japan, jo@jp.org) and John Smith (none, Gunma, Japan)

In this demonstration, we'd like to introduce two of our work, "A record without (or with) prior acoustic information" and "Given: 1.Manet, 2.CoilOscillation from a minimum unit of speaker." The first one is a work in which we computationally draw a groove of a record as a vector graphic (with Adobe Illustrator or other tools) by calculating frequencies with a number of zigzags and engrave the groove on diverse materials on diverse material including paper, wood, or acrylic with laser cutter or cutting plotter to produce sounds. Through the demonstration, we show a process of making the record with a cutting plotter on the spot. The result and other examples would be played on a standard analog record player. The second one is a work in which we split a unit of a speaker into a magnet and a coil. We demonstrate an example of the work by Johnsmith as a cartilage conduction hearing with a set of neodymium magnets and a pair of coils with stereo mini jack. Through the demonstration, people could listen the sound from her/his portable audio player by directly vibrating her/his cartilages. Both of the work would present alternate embodiments of matured audio technologies (i.e., vinyl record and headphone) with a help of later technological developments. The demonstration shows basic principles of how audio technologies work. Through our practices at the intersection of media archeology and personal fabrication, we'd like to reconsider the ordinariness of acoustic media technologies.
3pEDa3. Realtime and interactive tools for speech and hearing science education. Hideki Kawahara (Wakayama Univ., 930 Sakaedani, 930, Wakayama, Wakayama 640-8510, Japan, kawahara@sys.wakayama-u.ac. jp)

Recent advances of computational power and software foundations make it possible to introduce interactive and realtime tools in speech and hearing science education with relatively low cost. The tool, SparkNG (Speech Production and Auditory perception Research Kernel, Next Generation) consists of four applications. They are (a) real-time FFT analyzer with interactive spectrogram, (b) real-time display of FFT spectrogram (narrowband and wide-band) and auditory (ERB, Bark and 1/3 octave) spectrogram, (c) real-time vocal tract shape visualizer, and (d) interactive speech production simulator enabling vocal tract shape and transfer function manipulation with aliasing-free L-F model (Kawahara et al., 2015 APSIPA) manipulation. The tool is useful for teachers to demonstrate underlying concepts in an intuitive and attractive manner. It also is useful for researchers to check their ideas quickly. MATLAB source codes of the constituent functions are open to everyone. A compiled MATLAB license-free version is also available. [This work was supported by JSPS KAKENHI Grant Number JP16K12464.]

3pEDa4. Three-dimensional sound-field visualization system using head mounted display and stereo camera. Atsuto Inoue, Yusuke Ikeda, Kohei Yatabe, and Yasuhiro Oikawa (Intermedia Art and Sci., Waseda Univ., 59407-2, 3-4-1, Okubo, Shinjyuku, Tokyo 169-8555, Japan, han-overeem@ toki.waseda.jp)

Visualization of a sound field helps us to intuitively understand various acoustic phenomena in sound design and education. The most straightforward method is to overlap the measured data onto a photographic image. However, in order to understand an entire three-dimensional (3D) sound field by using a conventional two-dimensional screen, it is necessary to move a camera and measure repeatedly. On the other hand, the augmented reality (AR) techniques such as an optical head mounted display (OHMD) have been rapidly developed. In this study, we propose a sound field visualization system using an OHMD and a handheld four-point microphone. This system calculates sound intensity from the four sound signals in real time. Then, the sound intensity distribution is depicted as arrows in the 3D display. The position and angle of the microphones and user's head are acquired via AR markers and a gyro sensor of the OHMD. The system realizes simple and effective visualization of 3D sound field information from the various directions and positions of view. For the experiments, the sound field generated by a two-way loudspeaker was visualized. The results 
suggested that the proposed system can present information of the field in easily recognizable manner.

3pEDa5. Making headphones using plastic bottles and a milk carton. Shinjiro Seguchi (D\&I Creation Dept., Sony/Taiyo Corp., 1402-14, kansui,ohga, Hiji-machi,Hayami-gun, Oita-ken 879-1504, Japan, Shinjiro.Seguchi@jp.sony.com)

The SONY Science Program provides tomorrow's principles and the opportunity for hands-on experience for children. It will hopefully motivate them to acquire skills needed to make a better society by applying the power of science. An inclusion workshop is one of the Science Programs, where children can learn about scientific principles and technology through demonstrations. Making headphones from PET bottles and a milk cartons, organized by technicians from Sony/Taiyo Corporation, is one of the topics of the inclusion workshop. Children learn how the pieces they constructed vibrate and generate sounds. The program is also designed to make the participants aware of the varied individuality of each and every one of them, and to deepen mutual understanding through this experience of diversity and inclusion.
3pEDa6. A modular guitar for teaching musical acoustics. Jeremy Marozeau (Hearing Systems Group, Tech. Univ. of Denmark, HEA Bldg. 352, DTU-ELEKTRO, Lyngby 2800, Denmark, jemaroz@elektro.dtu.dk)

In order to keep students activated in a course on musical acoustics, they were asked to build a modular guitar, designed to be updated throughout the course. In the first stage, dedicated to the physics of strings, a guitar was made out of three strings attached to a long piece of wood. The students measured the effect of the place of plucking on the mode of the vibrations of the strings. The second stage was dedicated to the acoustic resonances. Using a laser cutter, the students built a wooden box that was coupled to their guitar using straps. New acoustical measurements were made to study the effect of the shape of the resonator on the spectrum of the sound. In the third stage, as the different tuning systems were learned, the students built a fingerboard with the appropriated positions of the frets. In the last stage, the students have implemented some digital effects and tested them on their guitar using a piezo-electrical pickup. As nothing was glued, the students were able to easily change each part of the guitar (resonator, sound hole, fret positions, microphone, ...) in order to experience their direct effect and their interactions.

1aED3. Tools for education in acoustics and phonetics — Takayuki Arai

1pED1. Advanced technical listening training program at Kyushu University — Kazuhiko Kawahara

1pED2. The use of all-in-one sensor devices in the general education acoustics laboratory — Andrew Morrison

1pED4. Visualizing sound directivity via smartphone sensors — Scott Hawley

1pED5. Efficacy of a new spatial ear training program for "Ensemble width" and "Individual source width" — Hidetaka Imamura

4pEDa7. Introducing programming through acoustics and audio at Belmont University — Eric Tarr

WEDNESDAY AFTERNOON, 30 NOVEMBER 2016

KAHILI, 4:00 P.M. TO 5:20 P.M.

\title{
Session 3pEDb
}

\author{
Education in Acoustics: Education in Acoustics for Kids (K-12 students) \\ Andrew C. Morrison, Cochair \\ Natural Science Department, Joliet Junior College, 1215 Houbolt Rd., Joliet, IL 60431 \\ Fumiaki Satoh, Cochair \\ Chiba Institute of Technology, Tsudanuma 2-17-1, Narashino 275-0016, Japan
}

\section{Invited Papers}

4:00

3pEDb1. Activities of the research committee on education in acoustics in ASJ for children. Fumiaki Satoh (Architecture, Chiba Inst. of Technol., 2-17-1 Tsudanuma, Narashino-shi, Chiba 275-8588, Japan, fumiaki.satoh@it-chiba.ac.jp), Takayuki Arai (Sophia Univ., Chiyoda-ku, Tokyo, Japan), and Akira Nishimura (Tokyo Univ. of Information Sci., Chiba-shi, Chiba, Japan)

In the Acoustical Society of Japan (ASJ), the Research Committee on Education in Acoustics was established in 1997. Although its history is relatively short in comparison with the Administrative Committee on Education in Acoustics in the Acoustical Society of America (ASA), many activities have been steadily carried out. For example, surveys of syllabi (educational plans of subjects in universities) were made. Demonstration tools used in university classes were also surveyed. The extent of our research was not limited to only 
universities. Textbooks and teaching materials used in elementary schools, junior high schools, and senior high schools were also researched. Furthermore, activities in cooperation with the National Museum of Nature and Science in Japan have been taking place, including an exhibition and a class with acoustic demonstrations and handicrafts for children. We believe that such activities are very important as social contributions of an academic society. In this presentation, some of our activities focusing on the education for children will be introduced.

3pEDb2. Assessment of the Acoustical Society of America educational activity kit: Survey results from K-12 teachers. Michelle C. Vigeant (Graduate Program in Acoust., The Penn State Univ., 201 Appl. Sci. Bldg, University Park, PA 16802, vigeant@engr.psu. edu) and Andrew C. Morrison (Natural Sci. Dept., Joliet Junior College, Joliet, IL)

The Committee on Education in Acoustics within the Acoustical Society of America developed an activity kit for K-12 teachers to use in the instruction of the fundamentals of sound in 2011. The kit includes activities that cover a range of topics, including musical instruments, animal bioacoustics, and hearing loss. Supplies for many of the activities are provided, including tuning forks, a sound level meter, and materials to make instruments. Many electronic resources are also available online, including lesson plans. Since 2011, over 475 kits have been distributed to K-12 teachers across the United States. To evaluate the effectiveness of the kits, a survey was sent out to all kit recipients. The response rate was $23 \%(n=102)$, with $58 \%$ of the respondents being high school teachers. Out of the eight primary activities, the two activities with the highest ratings were (1) striking and listening to the tuning forks, which demonstrates the concepts of frequency and wavelength, and (2) touching a ping pong ball suspended on a string, to a tuning fork, which demonstrates the concepts that sound is a form of vibrational energy and amplitude. The presentation will highlight the key results from the survey and include a sample of the comments.

\section{$4: 40$}

3pEDb3. Descriptions of sound in the textbooks of living environment studies in elementary schools and the development of a life and sound activity programs. Miki Toyomasu (Oita Univ., 5-31-10-403 Chihaya Higashi-ward, Fukuoka, Fukuoka 813-0044, Japan, toyomasu@zd5.so-net.ne.jp), Sayo Suzuki, and Yoshiko Akitake (Univ. of Teacher Education Fukuoka, Munakata, Japan)

In Japan, children learn about the subject Living Environment Studies in the first and second grades at elementary school. Eight companies published the 2015-approved elementary school Living Environment Studies textbooks. In this study, we investigated and analyzed the references to sound in all the Living Environment Studies textbooks and the development of life and sound activity programs. Textbooks were found to include contents relating to nature sounds (e.g., sounds made by insects and the sound of rain); musical sounds; sounds of life (e.g., sound of toys); and sound of voices (e.g., loud screams or shrieks in response to danger seeking help), etc. We developed two activity programs, a quiz on sounds and a competition for loud voices. The purpose of these programs was to learn about enjoying "sound" and understand its role in life. These activity programs conducted in Clubs for After School Activities for Children at F city. A questionnaire survey was conducted after two activity programs. Eight children answered that the quiz on sounds was fun and nine children answered that the loud voice competition was fun $(N=14)$. Further investigation and improvements are required.

\section{5:00}

3pEDb4. Factors influencing students' choice of musical instruments: A case study of Tertiary Institutions in South-Eastern Nigeria, a survey of University of Nigeria, Nsukka, College of Education Eha-Amufu Nsukka and Nnamdi Azikiwe University, Awka. Stephen G. Onwubiko (Music, Univ. of Nigeria, Nsukka Enugu State, Nsukka-Enugu, Nsukka-Enugu 234042, Nigeria, stephen. onwubiko@gmail.com)

The choice of musical instruments is among the most important factors in determining the course of students' music education. Instruments and its/their selection can be a challenging process with attendant factors. Musical acoustics which falls into intrinsic-extrinsic motivating factor and gender stereotyping of instruments are among those factors. The association of musical acoustics with particular instrument can significantly influence students' choice of musical instrument, thereby resulting in numerous negative-hatred consequences, including fewer instrument choices, limited ensemble participation, and peer disapproval. The findings show that musical acoustics reliably predicted the choice of musical instrument. In order for students to make the right instrument choice, musical acoustics should be encouraged, not the family (parents) forcing their children into musical instrument choice careers. Teachers, like parents, work very closely with learners in schools and they know the abilities of their students in various instruments. Therefore, teachers should guide their students in their musical instrument acoustics before their choice and aspirations in line with their abilities in various instruments. Musical acoustic guidance and counselling at tertiary entry level will enable the students learn about and explore careers that ultimately lead to career choice of the musical instrument; questionnaires as a means of data collection. 


\title{
Session 3pNSa
}

\section{Noise, Psychological and Physiological Acoustics and ASA Committee on Standards: Current Issues in Hearing Protection and Hearing Conservation II}

\author{
Elliott H. Berger, Cochair \\ Personal Safety Division, 3M, 7911, Zionsville Rd., Indianapolis, IN 46268-1650 \\ Jinro Inoue, Cochair \\ Department of Health Policy and Management, University of Occupational and Environmental Health, Japan, 1-1 Iseigaoka, \\ Yahatanishi-ku, Kitakyushu 807-8555, Japan
}

Chair's Introduction-1:00

\section{Invited Papers}

\section{1:05}

3pNSa1. Effectiveness of personal noise exposure measurement in the noisy workplace. Koko Takezawa (East Japan Railway Co., Sendai 980-8508, Japan, kokotakahashi@med.uoeh-u.ac.jp), Jinro Inoue (Dept. of Health Policy and Management, Inst. of Industrial Ecological Sci., Univ. of Occupational and Environ. Health, Kitakyushu, Japan), Koki Mori (East Japan Railway Co., Sendai, Japan), Seichi Horie, and Syoko Kawanami (Dept. of Health Policy and Management, Inst. of Industrial Ecological Sci., Univ. of Occupational and Environ. Health, Kitakyushu, Japan)

In Japan, the Working Environment Measurement Standards require employers to measure noise of working environment by two kinds of methods using a sound level meter (SLM) once every 6 months. A-Sampling method is to be measured equivalent continuous sound level for at least $10 \mathrm{~min}$. B-Sampling method is to be performed close to workers at times when the sound level appeared highest. We perform the risk assessment based on the values of the field measurement. In the East Japan Railway Company, workers move around several workshops and use hand tools. Therefore, it is difficult to estimate the personal noise exposure using only an SLM. To compare dose measurement with the Japanese field measurement, we carried out dose measurements for inspection or repair workers using a Personal Noise Dose Meter (Type 4448; Brüel \& Kjær). The results revealed that all sound levels using a dosimeter were less than $85 \mathrm{dBA}$, while the sound levels using an SLM were more than $90 \mathrm{dBA}$. In this case, the risk can be overestimated. Thus, we conclude that the Working Environment Measurement Standards should adopt the dose measurement.

\section{1:25}

3pNSa2. Bacterial attachment and insertion loss of earplugs used for fixed periods in noisy workplaces. Jinro Inoue, Yumi Nakagawa, Aya Nakamura, and Shoko Kawanami (Dept. of Health Policy and Management, Univ. of Occupational and Environ. Health, Japan, 1-1 Iseigaoka, Yahatanishi-ku, Kitakyushu, Fukuoka 807-8555, Japan, j-inoue@med.uoeh-u.ac.jp)

In noisy workplaces, employees often tend to use earplugs for a long time in Japan. We have previously reported conditions related to bacterial attachment and the insertion loss of earplugs using data collected from six companies. In the present study, we distributed different kinds of foam earplugs at five companies and collected them after 1-, 2-, 4-, and 8-week usage $(\mathrm{n}=123)$. We examined the total viable counts and presence of Staphylococcus aureus using 3M Petrifilm. We evaluated the insertion losses by means of the GRAS $45 \mathrm{CB}$ Acoustic Test Fixture. We detected a large number of viable counts in 5\% of the earplugs. We found Staphylococcus aureus in $7 \%$ of the earplugs. We observed a small deterioration in insertion loss after 8-week usage. Neither the condition of bacterial attachment nor the insertion loss correlated with duration of use. We discuss the proper usage of earplugs in terms of industrial health.

\section{1:45}

3pNSa3. Acoustic characteristics of respirators. Takao Sakuma ( JFE Steel Corp., 1 Kawasakicho, Chiba, Chiba 260-0835, Japan, hyakuyen56@gmail.com), Jinro Inoue, Toshitaka Yokoya, and Yukimi Endo (Dept. of Health Policy and Management, Univ. of Occupational and Environ. Health, Japan, Kitakyushu, Fukuoka, Japan)

In some workplaces, employees are obliged to wear a respirator to protect them from harmful substances. However, an acoustical problem with respirator design is that respirators diminish the voice. Frequency analysis of sound through respirators could help tackle the problems associated with this design. We used a mannequin with a loudspeaker attached to its mouth and emitting pink noise. We measured the noise with 59 combinations of respirators and filters. For half-face masks with one or two filters, attenuation of the noise under $1 \mathrm{kHz}$ was relatively small. The attenuations over $1 \mathrm{kHz}$ varied widely among the tested respirators. With full-face masks, the attenuated frequency was lower than with the half-face masks. Respirators with a speaking diaphragm conducted louder sound over $3 \mathrm{kHz}$ than did respirators without a speaking diaphragm. Among disposable masks, the attenuations were smaller than with half-face masks. We discuss the requirements for smooth communication among workers using respirators in light of the attenuation values of respirators at different frequencies. 


\title{
Session 3pNSb
}

\section{Noise: Ambient Listening, Communication and Noise Reduction of Special Hearing Protectors}

\author{
Hilary Gallagher, Cochair \\ Battlespace Acoustics Branch, Air Force Research Laboratory, 2610 Seventh Street, Bldg. 441, Wright-Patterson AFB, \\ $\mathrm{OH} 45433$ \\ Richard L. McKinley, Cochair \\ Battlespace Acoustics, Air Force Research Laboratory, 2610 Seventh Street, AFRL/711HPW/RHCB, Wright-Patterson AFB, \\ OH 45433-7901
}

\section{Invited Papers}

2:15

3pNSb1. Communication and situation awareness needs of hearing protection systems in high noise environments. Kurt Yankaskas (Office of Naval Res., 875 N. Randolph St., Arlington, VA 22203-1995, kurt.d.yankaskas@navy.mil)

The primary mission of aircraft carriers is to launch and recover military aircraft. Critical to the highly choreographed operations on the flight deck of the aircraft carrier is communications between aircraft directors, plane crews, and pilots as well as maintaining situation awareness in a highly dynamic environment. This must occur in an acoustic environment of 110 to $150+\mathrm{dBA}$. These noise levels readily exceed the requirements for double hearing protection. A dilemma is generated in trying to provide adequate hearing protection while maintaining critical communications and situation awareness during flight operations at these noise levels. This paper will review previous studies of hearing protection effectiveness, compliance, factors affecting compliance, and impact on job performance in environments that readily exceed legacy hearing protection. To address the situation awareness shortfalls, a tri-service group (US Army, Air Force and Navy) is developing a standard for detection and localization criteria (commonly known as situation awareness) to assess operational suitability. The standard is intended to drive manufacturers to measure beyond the attenuation a hearing protector provides to address operational needs. New products and techniques are presented which demonstrate a potential solution sets to this critical issue.

\section{$2: 35$}

3pNSb2. Assessing the performance capabilities of tactical hearing protection and communication devices. Hilary Gallagher (Battlespace Acoust. Branch, Air Force Res. Lab., 2610 Seventh St., Bldg. 441, Wright-Patterson AFB, OH 45433, hilary.gallagher.1@us.af. mil), Eric R. Thompson (Battlespace Acoust. Branch, Air Force Res. Lab., WPAFB, OH), and Richard L. McKinley (Oak Ridge Inst. of Sci. and Education, WPAFB, Ohio)

Military personnel work in unpredictable noise environments, which require flexible types of hearing protection (i.e., tactical hearing protection systems) in order to maintain mission effectiveness and situation awareness while reducing the risk of hearing loss. Acquisition decisions need to be made relative to accurate and complete measures of the total performance capabilities of tactical hearing protection systems and their effect on the user. Understanding the noise attenuation performance of tactical hearing protection systems has been a priority in order to protect the user from excessive noise exposure. However, active electronic tactical hearing protection systems have been designed to allow for enhanced communication and situation awareness, while at the same time protecting the auditory system from both impulsive and continuous noise. The Air Force Research Laboratory conducted a multifactorial assessment on currently available tactical hearing protection systems to determine the overall impact of these devices on performance and to enable users to make data-driven, informed acquisition decisions. The assessments included the following measurements: continuous noise attenuation, impulsive peak insertion loss, auditory localization, and speech intelligibility. The methods and results will be presented as well as a discussion on how the results promote an informed device selection.

\section{2:55}

3pNSb3. Noise reduction ratings of active noise cancellation headsets. William J. Murphy (Hearing Loss Prevention Team, Centers for Disease Control and Prevention, National Inst. for Occupational Safety and Health, 1090 Tusculum Ave., Mailstop C-27, Cincinnati, OH 45226-1998, wjm4@cdc.gov), Hilary L. Gallagher, and Richard L. McKinley (Battlespace Acoust. Branch, US Air Force Res. Lab., Wirght Patterson Air Force Base, OH)

The recent promulgation of the MIL-STD 1474 E, "Design Criteria Standard Noise Limits," combines data from the Real Ear Attenuation at Threshold (REAT) and the Microphone in Real Ear (MIRE) measurements to estimate the noise reduction rating of active noise cancellation (ANC) hearing protection devices. This paper will examine the American National Standards Institute standards, S12.6, S12.42, and S12.68 to assess the performance of four aviation headsets measured with REAT and MIRE methods. We will compare the protection estimates for different noises with the Noise Reduction Statistic for A-weighting $\left(\mathrm{NRS}_{\mathrm{A}}\right)$, Noise Reduction Statistic 
for Graphical $\left(\mathrm{NRS}_{\mathrm{G}}\right)$, and Octave Band rating methods. The $\mathrm{NRS}_{\mathrm{G}}$ method provides an efficient and accurate alternative to the Octave Band rating method. In the International Standards Organization, the ISO 4869 standard part 6 is under development to measure the attenuation of ANC protectors. Comparison with the proposed ISO 4869-6 standard will be conducted.

\section{$3: 15$}

3pNSb4. Sound localization performance with level-dependent hearing protection devices. Eric R. Thompson and Griffin D. Romigh (711th Human Performance Wing, Air Force Res. Lab, 2610 7th St., Bldg. 441, Wright Patterson, OH 45433, eric.thompson. 28@us.af.mil)

Level-dependent hearing protection devices (HPDs) offer the promise of the ability to hear low-level sounds while providing protection against dangerous high-level sounds. These devices are useful for people who may be exposed to intermittent, unexpected loud sounds while operating in environments that are otherwise generally quiet. Often, it is important for these users to be able to localize sounds and to maintain auditory situation awareness in these environments while wearing the HPDs. Measurements were made of listeners' abilities to localize quiet and loud sound sources while wearing different level-dependent devices, as well as with unprotected ears. Electroacoustic measurements were also made with the devices on an acoustic test fixture to characterize their input-output functions, effective bandwidths, and directional transfer functions. Sound localization performance was typically worse when wearing the HPDs than with unprotected ears, with more front/back confusions, especially for the devices that have the least variance in their directional transfer functions. Auditory models were used to predict the human localization performance with the electroacoustic measurements as inputs.

\section{3:35-3:50 Break}

3pNSb5. An objective, efficient auditory situation awareness test battery for advanced hearing protectors and tactical communications and protective systems (TCAPS): DRILCOM (detection-recognition/identification-localization-communication). John G. Casali and Kichol Lee (Auditory Systems Lab, Virginia Tech, 250 Durham Hall, Blacksburg, VA 24061, jcasali@vt.edu)

An objective, computer-controlled test battery was developed for measuring the effects of hearing protection devices (HPDs) and TCAPS, on auditory situational awareness (ASA). Four independent ASA tasks with military relevance were tested: Detection-Recognition/Identification-Localization-Communication (DRILCOM). Detection employed threshold-shift with seven 1/3-octave bands, an AK47 burst, and rifle cocking. Detection signals emanated from in front of the subject ( 0 degrees), or at 90, 180, or 270 degrees, to evaluate directionality. The Recognition/Identification task employed 36 signals combined in triads, wherein one "target" sound from 0 or 90 degrees had to be identified, at $\mathrm{S} / \mathrm{N}$ ratios of $-10,0$, and +10 . Localization employed a dissonant pure tone chord, spanning 104-7880 $\mathrm{Hz}$, which provided interaural time and interaural level cuing. Localization entailed 360-degrees of azimuth in 30-degree increments, and 30-degrees in frontal elevation, at a $\mathrm{S} / \mathrm{N}$ of +10 . The Communications task, addressing pass-through communications, utilized QuickSIN messages from 4 directions. In a proof-of-concept experiment on 10 subjects, 2 in-ear TCAPS, 1 earmuff TCAPS, a passive Combat Arms Earplug in "open" setting, and an EB-15LE ${ }^{\mathrm{TM}}$ electronic earplug were tested, as well as the open ear. DRILCOM demonstrated sensitivity to performance differences among devices and the open ear, as well as diagnosticity to within-device performance differences across different ASA tasks. The results have implication for selection and deployment of TCAPS and HPDs.

\section{4:10}

3pNSb6. Standard methods for measuring the effects of special hearing protectors on auditory performance. Richard L. McKinley (Battlespace Acoust., Air Force Res. Lab., 2610 Seventh St., AFRL/711HPW/RHCB, Wright-Patterson AFB, OH 45433-7901, rich3audio@aol.com), Hilary L. Gallagher, and Eric R. Thompson (Battlespace Acoust., Air Force Res. Lab., Dayton, OH)

Traditional hearing protectors attenuate noise via passive and or active technology. The advent of special hearing protectors, i.e., those that provide communications capability, ambient listening/situation awareness function, and/or level dependent attenuation of continuous and/or impulsive noise, has generated additional needs for performance measurement and characterization. ANSI Standards, representing the consensus of the U.S. scientific community, provide accurate, reliable, and repeatable methods for measuring performance. Current standards address the measurement of passive noise attenuation, active and level dependent insertion loss, impulsive peak insertion loss, and speech intelligibility. However, a need exists for a standard measurement method/s to characterize sound localization ability and auditory situation awareness. The presentation will address the application of current standards as well as a framework for a new standard describing three possible methods to measure sound localization performance and auditory situation awareness.

\section{$4: 30$}

3pNSb7. Evaluation of extended-wear earplug technology as a transparent hearing-protection device. Douglas S. Brungart (Walter Reed NMMC, 8901 Wisconsin Ave., Bethesda, MD 20889, douglas.brungart@us.army.mil), LaGuinn Sherlock (Army Public Health Ctr. (Provisional), Bethesda, MD), Nandini Iyer, Elizabeth A. McKenna, Eric R. Thompson (Air Force Res. Lab., WPAFB, OH), and Ashley C. Zaleski (Walter Reed NMMC, Bethesda, MD)

One of the greatest challenges in hearing conservation is providing adequate protection for those listeners who spend most of their time in quiet environments, but are occasionally exposed to high levels of noise that occur at unpredictable times. A classic example of this problem occurs for dismounted soldiers, who rely heavily on their natural hearing acuity to detect threats and opportunities on the battlefield but always face the risk of becoming engaged in a dangerously noisy firefight with little or no warning. One emerging technology that might have application in this domain is the extended-wear hearing aid, which is capable of remaining deep in the ear canal for weeks or months before being removed. Preliminary results suggest that the extended wear earplug can provide protection from blast or continuous noise that is comparable to a conventional earplug or muff, and that it can provide detection and localization performance comparable to that achieved with the open ear. Its form factor also makes it more likely to be compatible with headsets, radios, and personal protective equipment than traditional earplug or earmuff devices. The potential benefits and limitations of the extended-wear 
earplug as a hearing protector will be discussed. [Work supported by MRMC Grant W81XWH-14-1-0254; The opinions and assertions presented are the private views of the authors and are not to be construed as official or as necessarily reflecting the views of the Department of Defense.]

\section{Contributed Papers}

4:50

3pNSb8. Effect of a level-dependent hearing protector on detection thresholds, perceived urgency, and localization performance of reverse alarms. Chantal Laroche, Christian Giguère, Véronique Vaillancourt, Evgenia Shmigol, Mani Rahnama, Anne Gravel, Tanya Vaillancourt, Jérémie Chiasson, and Véronique Rozon-Gauthier (Audiol. and SLP Program, Univ. of Ottawa, 451 Smyth Rd., Ottawa, ON K1H 8M5, Canada, claroche@uottawa.ca)

Previous studies have shown that perception of warning signals in noisy workplaces can be compromised when wearing conventional hearing protectors. Level-dependent hearing protectors have been developed to increase environmental awareness and, by extension, to improve worker safety. A set of studies was conducted to assess the potential benefits of a level-dependent hearing protector operating in its passive and active modes, in quiet and in noise, for a range of listeners with normal hearing listening to reverse alarms. As expected, the detection of reverse alarms in quiet was improved in the active mode compared to the passive mode, but no significant benefit was found in noise. The active mode of the device only marginally helped to restore the sense of urgency conveyed by back-up alarms in noise, which was largely affected by passive attenuation. The localization of reverse alarms was slightly worse when wearing the device compared to unprotected listening, and was not improved in the active mode compared to the passive mode. In summary, when choosing a hearing protector to ensure adequate perception of reverse alarms, for example, different psychoacoustic tasks must be considered, also keeping in mind that level-dependent hearing protection does not necessarily restore performance to unprotected targets.

\section{5:05}

3pNSb9. Speech perception and production with one level-dependent hearing protector. Christian Giguère, Chantal Laroche, Véronique Vaillancourt (Audiology/SLP Program, Univ. of Ottawa, 451 Smyth Rd., Ottawa, ON K1H8M5, Canada, cgiguere@uottawa.ca), Ghazaleh Vaziri, Nicolas N. Ellaham (School of Rehabilitation Sci., Univ. of Ottawa, Ottawa, ON, Canada), and Hilmi R. Dajani (School of Elec. Eng. and Comput. Sci., Univ. of Ottawa, Ottawa, ON, Canada)

Electronic level-dependent hearing protectors aim to enhance communications and situational awareness in the noisy workplace compared to passive hearing protectors. From a listener's perspective, the fixed attenuation provided by passive hearing protection interferes with speech recognition in noise in users with hearing loss. From a talker's perspective, they also disrupt the Lombard reflex and induce an occlusion effect, which leads to a reduced vocal output in noise and compounds the problem. This paper presents laboratory data on speech recognition and production in noise with one level-dependent earmuff device. The device was tested in the level-dependent mode at two different gain settings as well as in a powered OFF condition to simulate passive protection within the same device. Level-dependent hearing protection provided significant speech recognition benefits in noise compared to passive protection, especially for users with hearing loss, and performance often exceeded unprotected listening. Talker speech output levels were also found to be higher in noise when the hearing protector was operated in a level-dependent mode compared to passive protection, but not as high as when unprotected, indicating only a partial recovery of speech production mechanisms.

\section{5:20-5:35 Panel Discussion}




\title{
Session 3pPAa
}

\section{Physical Acoustics and Biomedical Acoustics: Solid State Acoustics}

\author{
Josh R. Gladden, Cochair \\ Physics \& NCPA, University of Mississippi, 108 Lewis Hall, University, MS 38677 \\ Hirotsugu Ogi, Cochair \\ Osaka University, Machikaneyama 1-3, Toyonaka 560-8531, Japan \\ Chair's Introduction-1:10
}

\section{Invited Papers}

\section{1:15}

3pPAa1. Parity-time synthetic phononic media. Johan Christensen (ICMM, Madrid, ICMM, Madrid 28935, Spain, johan.christensen@gmail.com)

Classical systems containing cleverly devised combinations of loss and gain elements constitute extremely rich building units that can mimic non-Hermitian properties, which conventionally are attainable in quantum mechanics only. Parity-time (PT) symmetric media, also referred to as synthetic media, have been devised in many optical systems with the ground breaking potential to create nonreciprocal structures and one-way cloaks of invisibility. Here we demonstrate a feasible approach for the case of sound where the most important ingredients within synthetic materials, loss and gain, are achieved through electrically biased piezoelectric semiconductors. We study first how wave attenuation and amplification can be tuned, and when combined, can give rise to a phononic PT synthetic media with unidirectional suppressed reflectance, a feature directly applicable to evading sonar detection. Nature Rev. Mater. 1, 16001 (2016); Phys. Rev. Lett. 116, 207601 (2016); EPL, 114, 4, 47007 (2016).

\section{$1: 35$}

3pPAa2. Continuous monitoring of fatigue process with surface wave resonance: In the case of rotating bending fatigue. Hirao Masahiko (Osaka Univ., Grad. School of Eng. Sci., Toyonaka, Osaka 560-8531, Japan, hirao@me.es.osaka-u.ac.jp)

Prediction of fatigue failure of metals has not become reality like that of earthquake despite the intensive studies over many decades. But, there is an exception. The accumulating damage and the remaining life of rotating bending fatigue can be known from the ultrasonic observations through the lifetime. Electromagnetic acoustic resonance (EMAR) makes it possible to monitor the evolution of phase velocity, attenuation, and nonlinearity of surface shear wave exited and detected exclusively by electromagnetic acoustic transducer (EMAT), which operates without any contact with the rotating shafts. The probing shear wave travels in the circumferential direction with the axial polarization and penetrates into the metal to the submillimeter depth. Clear indications occur in the attenuation and nonlinearity measurements, and tell the pertinent metallurgical events within the penetration depth. They are caused by the dislocation mobility and restructuring due to cyclic loading, and occur at the specific fractions to the fatigue life, being independent of the bending stress amplitude. Continuous observation with EMAR can only detect such precursors and tell the remaining lifetime of metal components being fatigued. Discussions are given for the mechanism being based on the replication of small cracks and the TEM observation of dislocations at the crack-tip zone.

\section{$1: 55$}

3pPAa3. Structure and Magnetism of $\mathrm{EuTiO}_{3}$. Veerle M. Keppens (Dept. Mater. Sci. and Eng., Univ. of Tennessee, Knoxville, TN 37996,vkeppens@utk.edu)

The tilting of the oxygen octahedra in cubic perovskites is known to induce structural phase transitions, which are often associated with the emergence of intriguing physical phenomena. While $\mathrm{SrTiO}_{3}$ is one of the most extensively studied perovskite oxides for its structural phase transition at $105 \mathrm{~K}$, the discovery of magnetoelectric coupling in isostructural $\mathrm{EuTiO}_{3}$ has triggered many theoretical and experimental studies focused on this compound. I will present resonant ultrasound studies of the extremely subtle cubic to tetragonal structural phase transition at $288 \mathrm{~K}_{\text {in }} \mathrm{EuTiO}_{3}$ single crystals and the evolution of the physical properties upon chemical doping. The collective set of experimental data contributes to a better understanding of the link between lattice, magnetic, and electrical degrees of freedom in the $\mathrm{EuTiO}_{3}$ system, helps evaluate the similarities and differences between $\mathrm{SrTiO}_{3}$ and $\mathrm{EuTiO}_{3}$, and provides insight in the possible origin of the much higher structural transition temperature for $\mathrm{EuTiO}_{3}$ compared to $\mathrm{SrTiO}_{3}$. 
3pPAa4. Watching gigahertz acoustic waves confined in cavities. Oliver B. Wright (Faculty of Eng., Hokkaido Univ., Sapporo, Hokkaido 060-8628, Japan, olly@eng.hokudai.ac.jp)

Surface-acoustic and bulk-acoustic-wave wave devices based on waves in resonators have found extensive application in high-frequency signal processing. In particular, phononic crystals, metamaterials, and micron to sub-micron structures exhibit interesting physical properties, such as omnidirectional stop bands or tight wave confinement, that allow potential improvements to these devices. Here, we present results of real-time imaging and tracking of laser-excited acoustic waves at frequencies from $100 \mathrm{MHz}$ up to $100 \mathrm{GHz}$ in various novel micron-scale cavity geometries: phononic-crystal slab cavity structures, metamaterial extraordinary-transmission structures and sub-micron fibres. Applications include gigahertz sound control and nanoscale acoustic imaging. [1] T. Dehoux et al., Light Sci. Appl. 5, 16082 (2016). [2] O. B. Wright and O. Matsuda, Philos. Trans. Roy. Soc. A 373, 20140364 (2015). [3] J. J. Park et al., Phys. Rev. Lett. 110, 244302-1-5 (2013).

$2: 35$

3pPAa5. From MHz to THz: How ultrasound and inelastic scattering see lattice dynamics. Raphael Hermann (Mater. Sci. and Technol., Oak Ridge National Lab., 1 Bethel Valley Rd. MS 6064, Oak Ridge, TN 37831, hermannrp@ornl.gov)

Resonant ultrasound spectroscopy and direct phonon spectroscopy methods based on inelastic scattering of x-rays or neutrons provide complementary but also sometimes apparently contradicting pictures of the lattice dynamics. In order to resolve these apparent contradictions, the probe energy and length scales must be considered. I will review results for a few classes of energy material, such as bulk [Sergueev et al., Phys. Rev. B 91, 224304 (2015)] and nanostructured [Claudio et al. PCCP16, 25701 (2014); pss B251, 919 (2014); 213, 515 (2016); Klobes et al., Nanoscale8, 856 (2016)] thermoelectric materials, magnetic and magnetocaloric materials [Herlitschke Phys. Rev. B93, 094304 (2016); Klobes Phys. Rev. B92, 014304 (2015)], or rutile oxides [Budai et al., in preparation]. [Work at Oak Ridge National Laboratory was supported by the U.S. Department of Energy, Office of Science, Basic Energy Sciences, Materials Sciences and Engineering Division; work at Forschngszentrum Jülich was supported by the Helmholtz Association VH-NG-407, HRJRG-402, DFG SPP-1386, and BMBF 03X3540. The European Synchrotron Radiation Facility, Advanced Photon Source, Petra III at DESY, and Institut Laue Langevin are acknowledgment for beam time allocation. The invaluable contributions of all collaborators are grateful acknowledged.]

\section{2:55-3:15 Break}

\section{Contributed Papers}

\section{3:15}

3pPAa6. Tuning to a particular acoustic whispering-gallery mode in the GHz range. Sylvain Mezil, Kentaro Fujita, Montonobu Tomoda (Div. of Appl. Phys., Faculty of Eng., Hokkaido Univ., N13W8, Kita-Ku, Sapporo 060-8628, Japan, sylvain.mezil@eng.hokudai.ac.jp), Matt Clark (Faculty of Eng., Div. of Elec. Systems and Optics, Nottingham, United Kingdom), Oliver B. Wright, and Osamu Matsuda (Div. of Appl. Phys., Hokkaido Univ., Sapporo, Japan)

Surface Acoustic Waves (SAWs) generated with sub-picosecond light pulses are commonly used in non-destructive testing. The absorption of pump light by the medium generates SAWs that are detected by delayed probe light pulses. The spatiotemporal evolution of the SAWs can be imaged by scanning the position and time delay of the focused probe light pulses. Commonly used setups show two main constraints: the pump light is focused to a circular spot that generates SAWs in all directions, which does not allow control of directionality, and the laser repetition rate $f_{\text {rep }}$ limits accessible frequencies to $n f_{\text {rep }}(n=1,2, \ldots)$. In the case of laser excitation and detection of whispering-gallery modes (WGM) on a disc, only certain frequencies are detectable, and counter-propagating modes cannot be separately excited. Here, we overcome these limitations in experimental $\mathrm{GHz}$ SAW imaging of WGMs. To access arbitrary frequencies, we periodically modulate in intensity of the pump and probe beams and make use of both in-phase and in-quadrature lock-in detection [1]-[3]. To generate WGMs propagating in a single direction, we make use of a spatial light modulator and computer-generated holograms. These new results extend the possibilities of SAW imaging by allowing fine control of excited surface acoustic modes. [1] S. Kaneko, M. Tomoda, and O. Matsuda, AIP Adv. 4, 017124 (2014); [2] O. Matsuda et al., IEEE Trans. Ultrason. Ferroelectr. Freq. Control 62, 584-595 (2015); [3] S. Mezil et al., Opt. Lett., 40, 2157-2160 (2015).

\section{$3: 30$}

3pPAa7. Discussion on very small temperature dependence of diamond's elastic constants. Akira Nagakubo, Hirotsugu Ogi, and Masahiko Hirao (Graduate School of Eng. Sci., Osaka Univ., 1-3, Machikaneyamacho, Toyonaka, Osaka 560-8531, Japan, akira.nagakubo@abc.me.es.osakau.ac.jp)

Diamond shows high Debye temperature $(2200 \mathrm{~K})$ and small Gruneisen parameter $(\sim 1)$, which represent its small anharmonicity. Therefore, the temperature dependence of elastic constants is very small, preventing accurate measurement. Recently, we measured the temperature dependence of $C_{11}$ of diamond by using picosecond ultrasonics. [A. Nagakubo et al., Appl. Phys. Lett. 108, 221902 (2016).]. Our results indicate that diamond has a further higher Debye temperature than previous measurements, and we found that elastic constants of high-Debye-temperature materials should be measured over a wide temperature range to extract the Debye temperature and the Gruneisen parameter, accurately. We also calculate the temperature dependence of $C_{11}$ by an ab-initio method, which agree with our measurement. In this study, we discuss the temperature dependence of other elastic constants. We found that careful consideration is required for bondbending and bond-stretching resistance to evaluate the temperature dependence of its elastic constants.

\section{$3: 45$}

3pPAa8. Acoustic study of Wigner crystal melting in n-GaAs/AIGaAs at high magnetic fields. Alexey Suslov (NHMFL, Florida State Univ., 1800 E. Paul Dirac Dr., Tallahassee, FL 32310, suslov@magnet.fsu.edu), Irina Drichko, Ivan Smirnov (A. F. Ioffe PTI of RAS, St.-Petersburg, Russian Federation), Loren Pfeiffer (Elec. Eng., Princeton Univ., Princeton, NJ), Ken West (PRISM, Princeton Univ., Princeton, NJ), and Yuri Galperin (Dept. of Phys., Univ. of Oslo, Oslo, Norway)

We have measured absorption and velocity of surface acoustic waves (SAWs) in high-mobility samples $n$-GaAs/AlGaAs in magnetic fields (1218) $\mathrm{T}$ (filling factors $\nu=0.18-0.125)$ at temperatures $\mathrm{T}=(40-340) \mathrm{K}$ and 
SAW frequencies $\mathrm{f}=(30-300) \mathrm{MHz}$. From the measurement data, the complex AC conductance, $\sigma^{A C}(\omega) \equiv \sigma_{1}(\omega)-i \sigma_{2}(\omega)$ and its dependences on frequency, temperature, and the amplitude of the SAW-induced electric field were found. We conclude that in the studied interval of the magnetic field and at $T<200 \mathrm{mK}$, the electronic systems forms pinned Wigner crystal, the so-called Wigner glass. The estimate of the correlation (Larkin) length of the Wigner glass is about $3 \mu \mathrm{m}$, which is much larger than both the distance between the electrons and the magnetic length in the studied field range. At some temperature $T_{m}$, the temperature dependences of both components of the complex conductance get substantially changed: from the dielectric behavior at $\mathrm{T}<\mathrm{T}_{\mathrm{m}}$ to the metallic one at $\mathrm{T}>\mathrm{T}_{\mathrm{m}}$. We ascribed this change of the conduction mechanism to melting of the Wigner crystal and studied the dependence of the so-defined melting temperature on the electron filling factor. [This work was supported by RFBR 14-02-00232, NSF Cooperative Agreement DMR-1157490, the State of Florida, the Gordon and Betty Moore Foundation through the EPiQS initiative Grant GBMF4420, and the NSF MRSEC Grant DMR-1420541.]

\section{4:00}

3pPAa9. Effect of stress wave irradiation on structural change in a colloidal system. Nobutomo Nakamura, Tasuku Okuno, Hirotsugu Ogi, and Masahiko Hirao (Graduate School of Eng. Sci., Osaka Univ., 1-3 Machikaneyama, Toyonaka, Osaka 560-8531, Japan, nobutomo@me.es.osaka-u.ac.jp)

Annealing is a typical method for controlling crystallographic structure of solids, and by increasing the number of phonon modes by heating, structural changes are caused. Considering that stress waves also enhance lattice vibrations, stress-wave irradiation should have similar effect on structural changes. In this study, we investigate the effect of stress-wave irradiation on crystallization of an amorphous solid using a colloidal system. A colloidal system used in this study is a mixture of a solution dyed with a fluorescein sodium salt and silica particles. It shows phases similar to those of atomic systems, and ordered (crystalline) and disordered (amorphous) structures are obtained. Because particle-scale analysis can be performed by using the confocal laser scanning microscopy, it has been used as a model system of atomic materials. We evaluate structural change of a colloidal glass after stress-wave irradiation and find that crystallization is accelerated at a specific condition. In the presentation, details of the results are described, and its origin is discussed.

\section{$4: 15$}

3pPAa10. Resonance-enhanced compact nonlinear acoustic source of low frequency collimated beam for imaging applications in highly attenuating media. Cristian Pantea and Dipen N. Sinha (MPA-11, Los Alamos National Lab., MS D429, Los Alamos, NM 87545, pantea@lanl. gov)

Acoustic imaging in highly attenuating materials requires special acoustic sources that can generate a collimated beam of low frequency. Lower frequencies, in the range of $10-120 \mathrm{kHz}$, have the advantage of deeper penetration in the medium due to lower acoustic attenuation. However, typical acoustic sources at these low frequencies have a large beam spread, resulting in poor lateral resolution. We report on the latest advancements in the development of a very compact source, with approximate dimensions of a cylinder with a diameter of 25-50 millimeters and approximately $10 \mathrm{~mm}$ tall. Low frequency, collimated and steerable acoustic beam source are some of the main characteristics of this source. The newly developed source takes advantage of (1) frequency mixing in an acoustical nonlinear fluid in a cavity to generate the difference frequency of two high frequencies, around $1 \mathrm{MHz}$, and (2) resonance enhancement of the difference frequency in the cavity. An order of magnitude enhancement in amplitude was observed between on- and off-resonance conditions, with a collimation of the beam of approximately 6 degrees. Laboratory experimental data will be presented, and advantages over traditional and other nonlinear acoustic sources will be discussed.

\section{$4: 30$}

3pPAa11. Resonant ultrasound spectroscopy study of novel ceramics designed for high temperature hydrogen fuel cells. Josh R. Gladden, Sumudu Tennakoon, and Ashoka Karunarathne (Phys. \& NCPA, Univ. of MS, 108 Lewis Hall, University, MS 38677, jgladden@olemiss.edu)

Elastic constant measurements, along with temperature and pressure derivatives, are of interest to both the physics and engineering communities. One of the most precise and efficient methods for such measurements is resonant ultrasound spectroscopy (RUS) in which the vibrational resonance spectrum of a sample is used to determine the full elastic tensor of the material. In this talk, we will present the temperature dependent elastic constants of a series of ceramics, including glass ceramics and DMMA, between room temperature and $800 \mathrm{C}$. These ceramics have been designed for use in high temperature fuel cell by LG Fuel Cell Systems. We will discuss the effects of thermal cycling. Some of the ceramics are porous in nature which increases acoustic damping. Damping mechanisms in porous materials and experimental techniques to reduce damping will also be discussed.

\section{4:45}

3pPAa12. Rapid wave velocity measurement by Brillouin scattering using artificially induced phonon. Yoshiaki Shibagaki, Masahiko Kawabe (Doshisha Univ., 1-3 Tatara Miyakodani, Kyotanabe, Kyoto 610-0321, Japan, duq0358@mail4.doshisha.ac.jp), Shinji Takayanagi (Nagoya Institude of Technol., Nagoya, Japan), Kazuma Mori (Doshisha Univ., Kyotanabe, Kyoto, Japan), Takahiko Yanagitani (Waseda Univ., Tokyo, Japan), and Mami Matsukawa (Doshisha Univ., Kyotanabe, Kyoto, Japan)

Brillouin light scattering enables measurements of hypersonic longitudinal and shear velocities in the small area (diameter about $50 \mu \mathrm{m}$ ). One problem of the conventional Brillouin technique is the weak Brillouin scattering peak from thermal phonons and this results in the longer measurement time. To overcome this problem, we have proposed measurements of strong coherent phonons by fabricating a high frequency transducer on the sample surface. However, the fabrication of the transducer is not suitable for simple and nondestructive measurements. In this study, a small glass device with a $\mathrm{ZnO}$ piezoelectric thin film was used as a hypersonic transducer around 1 GHz. By attaching this transducer to the sample with coupling liquid, artificial coherent phonons were successfully induced in a sample. In the case of a quarts sample, strong Brillouin scattering peaks were observed using a tandem Fabry-Perot interferometer. The measured frequency shift of the peaks was equal to the excitation frequency of the $\mathrm{ZnO}$ piezoelectric film (resonance frequency: $822 \mathrm{MHz}$ ). The intensity of Brillouin peak from the induced phonons was about 375 times as strong as the peak from the thermal phonons. The technique of induced phonons can realize nondestructive and rapid velocity measurements in the small area. 


\title{
Session 3pPAb
}

\section{Physical Acoustics and Biomedical Acoustics: Acoustic Micro- and Nanofluidics II}

\author{
James Friend, Cochair \\ Mechanical and Aerospace Engineering, University of California, San Diego, 345F Structural and Mechanical Engineering, \\ Mail Stop 411 Gilman Dr., La Jolla, CA 92093
}

Daisuke Koyama, Cochair

Faculty of Science and Engineering, Doshisha University, 1-3 Tataramiyakodani, Kyotanabe 610-0321, Japan

Tony Jun Huang, Cochair

Duke University

\section{Invited Paper}

$1: 20$

3pPAb1. Actuation and manipulation of nano-confined fluids and particles via room-temperature lithium niobate bonding. Morteza Miansari and James Friend (Mech. and Aerosp. Eng., Univ. of California, San Diego, 345F Structural and Mech. Eng., M.S. 411 Gilman Dr., La Jolla, CA 92093, jfriend@eng.ucsd.edu)

Controlled nanoscale manipulation of fluids and colloids is made exceptionally difficult by the dominance of surface and viscous forces. Acoustic waves have recently been found to overcome similar problems in microfluidics, but their ability to do so at the nanoscale remains curiously unexplored. Here, we show that $20 \mathrm{MHz}$ surface acoustic waves (SAW) can manipulate fluids, fluid droplets, and particles, and drive irregular and chaotic fluid flow within fully transparent, high-aspect ratio 50-250-nm tall nanoslits fabricated via a new direct, room temperature bonding method for lithium niobate (LN). Applied in the same direction, SAW increases the capillary filling rate of the hydrophilic LN nanoslit by 2-5 times. Applied in opposition, the SAW switches the flow direction and drains the channel against $1 \mathrm{MPa}$ capillary pressure, and can be used to controllably manipulate $\sim 10 \mathrm{fL}$ droplets. Finally, entire $10 \mu \mathrm{L}$ droplets can be sieved via SAW through the nanoslit to pass only particles smaller than its height, providing pumpless size exclusion separation.

\section{Contributed Paper}

\section{1:40}

3pPAb2. c-axis tilted ScAIN shear wave acoustic Bragg reflect resonator for gigahertz viscosity measurement. Takeshi Mori (Nagoya Inst. of Technol., Gokiso-cho, Showa-ku, Nagoya, Aichi, 466-8555 Japan, Nagoya 4668555, Japan, t.mori.397@nitech.jp), Yui Yamakawa, Rei Karasawa, Ko-hei Sano (Waseda Univ., Tokyo, Japan), Shinji Takayanagi (Nagoya Inst. of Technol., Nagoya, Japan), and Takahiko Yanagitani (Waseda Univ., Tokyo, Japan)

c-axis tilted ScAlN film is attractive for resonators exciting shear wave ultrasonic. Shear mode resonators are suitable for biomarker sensing and viscosity sensing because shear mode resonators can operate without energy leakage to liquid. These sensors are well known as a QCM (Quartz Crystal Microbalance) consisting of AT-cut quartz crystal which excites shear wave ultrasonic. When the resonator is immersed in liquid, resonant frequency decreases due to the viscosity perturbation layer between the resonator and liquid interface. The rate of resonant frequency decrease depend on the mass ratio of viscosity perturbation layer and the resonator layer. Therefore, thinner high frequency resonator allows high sensitivity viscosity measurement. The maximum resonant frequency of the standard QCM plate is about $30 \mathrm{MHz}$. On the other hand, c-axis tilted ScAlN film can operates over 1 GHz. The sensitivity of the piezoelectric film sensors are much higher than that of the QCM sensors. In this study, we deposited shear wave c-axis tilted ScAlN film on the acoustic Bragg reflector. We clearly observed the shear wave excitation at the $990 \mathrm{MHz}$ by using a network analyzer in air. Apparent resonant frequency shift of $-16 \mathrm{MHz}$ and $-28.5 \mathrm{MHz}$ was observed, when the sensor was immersed in the ethanol and pure water, respectively. 


\section{Invited Paper}

\section{1:55}

3pPAb3. Non-contact acoustic manipulation in microchannel. Teruyuki Kozuka (Elec. and Electronics Eng., Aichi Inst. of Technol., Yakusa-cho, Yachigusa 1247, Toyota, Aichi 470-0392, Japan, kozuka-t@aitech.ac.jp)

Noncontact micromanipulation technique is needed in micromachine technology, biotechnology, and other fields. In the present paper, a standing wave field is generated in a microchannel and a geometric space, and it is possible to trap small objects at nodes of the sound pressure distribution in the medium. A microchannel and a geometric space were made at the center of a glass plate of $50 \mathrm{~mm} x$ $50 \mathrm{~mm} \times 5 \mathrm{~mm}$. In the experiment, when the liquid water containing alumina particles was injected into the microchannel on glass plate irradiated by ultrasound, the particles flowed along several layers in the microchannel. In the geometric space, the particles were trapped in the sound pressure nodes of the standing wave field. By changing the frequency, the geometric pattern of particles aggregation interestingly changed. When the geometric space is a triangular space, the particle moved toward the top from the base of the triangle. When the microchannel is branched at the half circular geometric space, it was able to control the direction of the particle flow by changing the ultrasound frequency in the branched microchannels. Moreover, a sound field was numerically simulated by FEM under the experimental conditions, and the experimental results were discussed.

\section{Contributed Papers}

\section{2:15}

3pPAb4. A study of the limits of microparticle collection over a broad frequency range. Prashant Agrawal, Prasanna S. Gandhi (Indian Inst. of Technol. Bombay, Mumbai, Maharashtra, India), and Adrian Neild (Dept. of Mech \& Aero Eng., Monash Univ., Clayton, VIC 3800, Australia, adrian. neild@monash.edu)

Acoustic fields can be used to collect microparticles over a wide range of frequencies. Within the ultrasonic range acoustic radiation forces cause patterns of particles to form, typically along pressure nodes. At much lower frequencies (in the order of hundreds of Hertz), the collection mechanism is inertial in nature, a particle's inability to follow the fluid motion causes drag forces to act on the particle which are non-zero when integrated across a time cycle due to gradients in the flow field. In both cases, however, the ability to collect particles is limited by acoustic streaming - the steady state fluid flow which results from gradients in the harmonic flow field. In this study, we examine the smallest particle size that can be collected as a function of frequency. For the low frequency vibration, an open fluid chamber is oscillated in the horizontal plane, these conditions are also applied to the ultrasonic case, and the acoustic energy is kept constant across frequencies. It is found that the minimum particle size can be collected at each end of the frequency scale.

3pPAb5. Unconstrained manipulation of micro-particles using phasecontrol of standing ultrasound wave fields. John Greenhall, Fernando Guevara Vasquez, and Bart Raeymaekers (Univ. of Utah, 1495 East 100 South, MEK 1550, Salt Lake City, UT 84112, john.greenhall@utah.edu)

We demonstrate a method of unconstrained manipulation of a spherical microparticle submerged in a fluid medium using a standing ultrasound wave field. The method works by displacing the microparticle in small increments through independent adjustment of the phases and amplitudes of two opposing ultrasound transducers. We model the dynamic behavior of the microparticle during each incremental displacement, taking into account the acoustic radiation force and the time-dependent and time-independent drag force acting on the microparticle. Using this dynamic model, we characterize the transient and steady-state behavior of the fluid-microparticle system during each incremental displacement as a function of the microparticle and fluid medium properties and the phases and amplitudes of the ultrasound transducers. The results show that the displacement time and percent overshoot of the microparticle trajectory are dependent on the ratio of the acoustic radiation force and time-independent damping force. We experimentally demonstrate the method by manipulating the microparticle in an unconstrained manner over multiple wavelengths. In contrast to existing methods that require all acoustic reflections be absorbed, this method takes all reflections into account, removing the need for a complex experimental setup.

$$
\text { 2:45 }
$$

3pPAb6. Micro and nanofluidics of the cochlea: Trade-offs of sensitivity and noise in an active biological system. Aritra Sasmal and Karl Grosh (Mech. Eng., Univ. of Michigan, 2350 Hayward St., Ann Arbor, MI 48109, asasmal@umich.edu)

The cochlea performs an analog time-frequency analysis on the incoming acoustic signal via a coupled electro-mechanical-fluidic response. The ultimate mechanical event that triggers the opening of the sensory mechanically gated ion channels is the fluidic shearing of the stereocilia of the inner hair cells (IHC) of the cochlea. Each IHC is equipped with a tuft of free standing stereocilia which are bathed in a 2-6 micron viscous fluid gap between the reticular lamina and the viscoelastic tectorial membrane. We use thin-film lubrication theory and asymptotics to model and solve a coupled viscous fluid-structure interaction problem to predict HB sensitivity to disturbance. The noise at the HB is due to the dissipation by the viscous forces (as quantified by the fluctuation-dissipation theorem) and stochastic fluctuations in the channel. We used this analysis study the challenges faced by evolution to sense the range of acoustic frequencies important to mammals. Structural acoustic calculations show that the parameters that increase noise may also increase the sensitivity of the system. In this study, the parametric trade-offs between noise and sensitivity of the system has been elucidated. 


\title{
Session 3pPP
}

\section{Psychological and Physiological Acoustics: Recent Progress in Auditory Perceptual Organization Studies}

\author{
Makio Kashino, Cochair \\ Human Information Laboratory, NTT Communication Science Laboratories, 3-1, Morinosato Wakamiya, Atsugi 2430198, \\ Japan \\ Barbara Shinn-Cunningham, Cochair \\ Boston University, 677 Beacon Street, Boston, MA 02215-3201
}

Chair's Introduction-1:00

\section{Invited Papers}

1:05

3pPP1. Perceptual interactions between adjacent time intervals marked by sound bursts. Yoshitaka Nakajima (Dept. of Human Science/Res. Ctr. for Appl. Perceptual Sci., Kyushu Univ., 4-9-1 Shiobaru, Minami-ku, Fukuoka, Fukuoka 815-8540, Japan, nakajima@ design.kyushu-u.ac.jp)

Perceptual interactions take place between adjacent time intervals up to $\sim 600 \mathrm{~ms}$ even in simple contexts. Let us suppose that two adjacent time intervals, T1 and T2 in this order, are marked by sound bursts. Their durations are perceptually assimilated in a bilateral manner if the difference between them is up to $\sim 50 \mathrm{~ms}$. When $\mathrm{T} 1 \lesssim 200 \mathrm{~ms}$ and $\mathrm{T} 1 \lesssim \mathrm{T} 2<\mathrm{T} 1+100 \mathrm{~ms}$, T2 is underestimated systematically, and the underestimation is roughly a function of $\mathrm{T} 2-\mathrm{T} 1$. Except when $\mathrm{T} 1 \simeq \mathrm{T} 2$, this is assimilation of $\mathrm{T} 2$ to $\mathrm{T} 1$, partially in a unilateral manner. This systematic underestimation, time-shrinking, disappears when T1 $>300 \mathrm{~ms}$. When T2 $=100$ or $200 \mathrm{~ms}$ and $\mathrm{T} 1=$ $\mathrm{T} 2+100$ or $\mathrm{T} 2+200 \mathrm{~ms}, \mathrm{~T} 1$ is perceptually contrasted against $\mathrm{T} 2$ : $\mathrm{T} 1$ is overestimated. When $80 \leq \mathrm{T} 1 \leq 280 \mathrm{~ms}$ and $\mathrm{T} 2 \geq \mathrm{T} 1+300$ ms, T2 is contrasted against T1: In this case, T2 is overestimated. Assimilation and contrast are more conspicuous in T2 than in T1. For three adjacent time intervals, $\mathrm{T} 1, \mathrm{~T} 2$, and $\mathrm{T} 3$, the perception of $\mathrm{T} 3$ can be affected by both $\mathrm{T} 1$ and $\mathrm{T} 2$, and the perception of $\mathrm{T} 2$ by $\mathrm{T} 1$.

\section{$1: 25$}

3pPP2. Informational masking of vocal signals in a nonhuman animal. Mark Bee (Ecology, Evolution, and Behavior, Univ. of Minnesota, 140 Gortner Lab., 1479 Gortner Ave., St. Paul, MN 55108, mbee@umn.edu)

Informational masking (IM) interferes with speech perception in the presence of multiple talkers. However, its impact on vocal communication in other animals has received little attention. This talk will present behavioral and neurophysiological evidence-much of it preliminary, circumstantial, or both—suggesting that frogs are also susceptible to IM in the context of vocal communication in noisy social environments. When choosing a mate in a chorus, female treefrogs must extract information related to a male's suitability and quality as a mate from the temporal envelope of his vocalizations. In behavioral experiments, temporally structured maskers constrain a female's ability to process the temporal envelopes of target vocalizations, even when targets and maskers are sufficiently different in frequency to be transduced by different inner ear papillae. (The frog's peripheral auditory system—consisting of multiple sensory papillae that encode different frequency ranges of airborne sound-provides a natural "spectral protection zone" for minimizing the impacts of energetic masking in experimental studies of IM.) In neurophysiological experiments, potential correlates of IM can be observed in the responses of single neurons in the inferior colliculus. Future work will employ a multi-tone masking paradigm to determine how spatial separation, masker uncertainty, and target-masker similarity impact "subcortical" signal processing mechanisms in the context of IM and vocal communication.

\section{$1: 45$}

3pPP3. Uninformative dynamic visual stimuli aid in segregating two similar acoustic stimuli, but not in detecting a single stimulus in noise. Ross K. Maddox (Dept. of Biomedical Eng., Dept. of Neurosci., Univ. of Rochester, 1715 NE Columbia Rd., Box 357988, Seattle, Washington 98195, rkmaddox@uw.edu), Dean A. Pospisil, and Adrian K. Lee (Dept. of Speech and Hearing Sci., Inst. for Learning and Brain Sci., Univ. of Washington, Seattle, WA)

Both the comprehension and detection of speech in noise are improved when the listener sees the talker's mouth. There are multiple reasons for this, from basic physical temporal correlations to higher order linguistic cues; we have recently performed several experiments investigating the former. They were based on artificial stimuli with speech-like dynamics but no linguistic information. Auditory stimuli were a tone or tone complex with randomly modulated amplitude. Visual stimuli were a disc with a randomly modulated radius. We manipulated the correlation between the visual stimulus and each auditory stimulus. In all experiments, the visual stimulus provided no information about the task. In the first study, we presented two competing auditory stimuli and had listeners respond to events in the 
target stimulus (brief pitch or timbre fluctuations). Performance was better when the visual stimulus matched the auditory target than when it matched the masker. The second study employed a two-interval two-alternative forced choice detection task. Despite a range of stimulus variations, no effect of audio-visual coherence on auditory detection was ever observed. Taken together, these results suggest that listening improvements provided by visual stimuli derive from improvements in segregation and scene analysis, more than overcoming simple energetic masking.

3pPP4. Varieties of attention affect auditory perception of scenes. Joel S. Snyder, Breanne Yerkes, Vanessa Irsik, and Christina Vanden Bosch der Nederlanden (Psych., Univ. of Nevada Las Vegas, 4505 Maryland Pkwy, MS 5030, Las Vegas, NV 89154, joel.snyder@ unlv.edu)

Past empirical work on perception of complex auditory scenes has demonstrated striking effects of attention on perception. Here, we present data from recent studies of auditory stream segregation and change deafness, demonstrating that different types of attention influence what is perceived. During auditory stream segregation, we found that the increasing tendency to perceive segregation as more alternations of low and high tones are presented (i.e., buildup) is facilitated by attending to the tone patterns, regardless of whether participants were actively making judgments of segregation. In contrast, performing the segregation task was necessary to enhance the influence of the frequency separation of the immediately prior tone pattern, suggesting a form of task-based (as opposed to stimulusbased) attention. During change detection, we found that cueing participants to which object is likely to be replaced in a scene of multiple objects facilitates successful detection of changes, while providing an invalid cue impairs detection. Furthermore, an object-encoding task performed after each change detection response suggests that attending to objects specifically involved in the change facilitates awareness of changes. These two lines of research together suggest the need to systematically investigate under what conditions various types of attention influence auditory perception.

3pPP5. Stimulus phase locking of cortical oscillation for perceptual organization. Hirokazu Takahashi (Res. Ctr. for Adv. Sci. and Technol., The Univ. of Tokyo, 4-6-1 Komaba, Meguro-ku, Tokyo 1538904, Japan, takahashi@i.u-tokyo.ac.jp)

The phase of cortical oscillations contains rich information and is valuable for encoding sound stimuli. We tested whether and how spatial patterns of oscillatory phase locking within the auditory cortex better predicted perceptual organization than those of response amplitude. A high-density microelectrode array with $10 \times 10$ sites within $4 \times 4 \mathrm{~mm}^{2}$ mapped LFPs at the $4^{\text {th }}$ layer of auditory cortex in rats. First, in response to ABA-sequences with different inter-tone intervals and frequency differences, neurometric functions based on stimulus phase locking in the gamma band could better describe van Noorden's perceptual boundary than the LFP amplitude. Second, when a random tone sequence was switched to a regular tone sequence, the gamma-band phase locking increased immediately after the transition from random to regular sequences. The amplitude of the tone-evoked response, on the other hand, increased with frequency separation with respect to the prior tone, irrespective of putative organization of auditory objects. These results suggest that the evokedresponse amplitude encodes the local order of tones, while the phase locking better predicts the perceptual organization.

3pPP6. Listening in crowded environments: How attention shapes brain responses to unattended sounds. Daniel Bates, Katharine Molloy, Nilli Lavie, and Maria Chait (Univ. College London, 332 Gray, London WC1X 8EE, United Kingdom, m.chait@ucl.ac.uk)

I will review recent work in my lab concerning the effect of attentional focus on brain responses to unattended sounds. The first series of experiments aimed to understand how the neural representations of tracked (attended) and ignored sources change with growing scene size. We used acoustic "scenes," comprised of multiple concurrent tone-pip streams, and a task which required listeners to track one of the streams over a long duration (30 seconds). EEG results show that attention boosts/reduces responses to the tracked/ignored sources, manifested as enhanced/reduced spectral power at its AM rate. The strength of this effect is dependent on the number of competing sources. We also demonstrate increased power coupling between activity in the alpha band and the brain responses to ignored sounds, implicating alpha in the process of active suppression of task-irrelevant information. In the second series of experiments (Molloy et al., 2015), we investigate the effect of focused visual attention on responses to simple sounds (pure tones). High, compared to low, perceptual load led to reduced early $(\sim 100 \mathrm{~ms})$ auditory-evoked activity and later suppression of the P3 "awareness" response. These findings support an account of shared audio-visual resources, which, when depleted under load, result in failure of perception. Reference: K. Molloy, T. D. Griffiths, M. Chait, N. Lavie (2015) “Inattentional deafness—an MEG study,” J Neurosci.35, 16046-54.

\section{3:05-3:25 Break}

\section{$3: 25$}

3pPP7. Auditory scene analysis: Patterns of things to come? Susan Denham (School of Psych., Univ. of Plymouth, Plymouth pl4 8aa, United Kingdom, s.denham@plymouth.ac.uk)

Perception depends on discovering and forming representations of sensory patterns to make sense of the world. Patterns playing out both in time and feature space allow sensory systems to form expectations, and provide a basis for decomposing the world into discrete objects and for detecting meaningfully novel events. Without expectations, the system has no way of autonomously evaluating its understanding of the world. However, patterns come and go. So, the perceptual system needs some way of allowing its operational representations to form and dissolve, dominate and yield, in a way that facilitates veridical perception. In this talk, I will discuss some of the issues of pattern discovery, maintenance, and deletion at different timescales and levels of complexity using exemplar models of SSA and auditory streaming, and new perceptual data. 
3pPP8. Detecting auditory changes by pupillary response. Hsin-I Liao, Makoto Yoneya (Human Information Sci. Lab., NTT Commun. Sci. Labs., 3-1, Morinosato Wakamiya, Atsugi, Kanagawa 243-0198, Japan, liao.hsini@lab.ntt.co.jp), Shunsuke Kidani (Hokuriku Univ., Kanazawa, Japan), Nicolas Barascud (Laboratoire de Sci. Cognitives et Psycholinguistique (LSCP), Ecole Normale Supérieure, Paris, France), Sijia Zhao, Maria Chait (Ear Inst., Univ. College London, London, United Kingdom), Makio Kashino, and Shigeto Furukawa (Human Information Sci. Lab., NTT Commun. Sci. Labs., Atsugi, Japan)

Detecting changes is an essential function that helps the organism to constantly monitor and update the information from the environment. In this talk, I will review our recent work, demonstrating that the human pupillary response can be used as a physiological marker for certain aspects of auditory change detection, in both simple and complex acoustic streams. In study 1, using simple repetitive tones with occasionally presented deviants (oddballs), we found that the oddball induces a pupillary dilation response (PDR), that is modulated by the stimulus properties irrespective of whether attention is directed to the sounds (Liao et al. 2016). In study 2, we examined whether PDR also reflects changes in complex acoustic patterns. We adopted the stimuli in Barascud et al. (2016) which contain transitions between random and regular tone-pip patterns. Results revealed an asymmetry: the PDR was observed only to changes from a regular to a random pattern, but not vice-versa. Overall, the results suggest that the PDR is not simply evoked by any kind of perceptual change per se. Rather, the pattern of results is consistent with the PDR reflecting changes that violate expectation. Possible underlying mechanisms and future directions are discussed.

\section{4:05}

3pPP9. Investigating bottom-up auditory attention. Mounya Elhilali (Elec. and Comput. Eng., Johns Hopkins Univ., 3400 N Charles St. Barton Hall, Baltimore, MD 21218, mounya@jhu.edu)

In everyday life, we are surrounded by a cacophony of sounds that our brain has to sort through in order to focus on important information. The conspicuity of certain sounds allows them to grab our attention and "stand out" relative to the soundscape. In this work, we hypothesize that as sound statistics change over time, variance from these statistics drive bottom-up attention processes that direct our focus to certain objects in the scene. We investigate the perceptual space that renders certain sounds salient relative to their context, and examine neural underpinnings of emergence of these salient sounds using complex acoustic scenes as stimuli. Results reveal adaptive neural representations of the context and salient objects reflecting deviations from the statistical structure of the scene. We speculate that principles of predictive coding could explain a number of observed neural and perceptual findings and discuss relevance of such framework in understanding processes of auditory scene analysis.

\section{$4: 25$}

3pPP10. Difficulty with selective listening in Autism spectrum disorder. Makio Kashino and I-Fan Lin (Human Information Lab., NTT Commun. Sci. Labs., 3-1, Morinosato Wakamiya, Atsugi, Kanagawa 2430198, Japan, kashino.makio@lab.ntt.co.jp)

Individuals with autism spectrum disorder (ASD) often experience difficulty with selective listening in the presence of multiple sounds despite their normal puretone thresholds. We have been conducting a series of studies to uncover neural bases for such difficulty in ASD adults without intellectual disability. First, we compared basic auditory functions between ASD and neurotypical (NT) groups, and found that (1) sensitivities to interaural time and level differences were lower in ASD than NT, and (2) a sub-group of ASD showed low sensitivity to temporal fine structure of acoustic waveforms. These findings suggest deficits in auditory processing in the brainstem of ASD. Second, we compared target detection thresholds between the two groups in the presence of distractors having little energetic masking on the target. Detection performance was dependent on the spectrotemporal coherence of target and distractor components in NT, whereas no such difference was observed in ASD, suggesting that ASD may lack automatic grouping across frequency channels. Additionally, we have observed significant differences between the two groups in behavioral, perceptual, autonomic, and brain responses to speech and/or non-speech stimuli. Taken together, difficulty with selective listening in ASD may involve several different mechanisms in both cortical and sub-cortical neural sites.

3pPP11. How auditory scene understanding can fail in special populations. Barbara Shinn-Cunningham, Scott Bressler, and Le Wang (Boston Univ., 677 Beacon St., Boston, MA 02215-3201, shinn@bu.edu)

One way to gain insight into how the brain normally processes auditory scenes is to explore the perceptual problems in special populations. In our lab, we are studying two types of listeners who, although very different, both have trouble making sense of complex scenes: listeners with autism who are minimally verbal (MVA listeners), and blast-exposed military Veterans. Neither population shows evidence of specific deficits in how well information is encoded subcortically. However, both show deficits when it comes to analyzing sound mixtures with multiple sound sources. Neural responses from MVA listeners suggest that their brains do not automatically organize sound mixtures that typically developing listeners hear as distinct objects, which likely impairs their ability to analyze the content of one sound source in a mixture. In contrast, Veterans exposed to blast appear to have difficulties focusing selective attention on a sound source in order to select it from a mixture, consistent with behavioral deficits when trying to track one sound stream. The problems these two populations have in processing complex scenes supports the idea that successful everyday listening depends both on perceptual organization of the scene and top-down control of attention. 


\title{
Session 3pSA
}

\section{Structural Acoustics and Vibration, Physical Acoustics, Engineering Acoustics, and Signal Processing in Acoustics: Non-Contact Vibration Excitation and Sensing Techniques}

\author{
Brian E. Anderson, Cochair \\ N145 Esc, Brigham Young Univ., MS D446, Provo, UT 84602 \\ Scott D. Sommerfeldt, Cochair \\ Dept. of Physics, Brigham Young Univ., N181 ESC, Provo, UT 84602
}

Chair's Introduction-1:15

\section{Invited Papers}

$1: 20$

3pSA1. Measurement of the structural admittance matrix using external random noise sources. Earl G. Williams (Acoust., Code 7106, Naval Res. Lab., 4555 Overlook Ave., Washington, DC, DC 20375, earl.williams@nrl.navy.mil), Jeffery D. Tippmann (Scripps Inst. of Oceanogr., Univ. of California, LaJolla, CA), Sandrine T. Rakotonarivo (Lab. of Mech. and Acoust., Aix-Marseille Univ., Marseille, France), and W. A. Kuperman (Scripps Inst. of Oceanogr., Univ. of California, LaJolla, CA)

The structural admittance Ys also called the structural Green's function characterizes the vibration, radiation, and scattering physics of a vibrator. When it is known, the vibration of the radiating surface, and hence the acoustic radiation from the surface, is also known for any specified surface load. We demonstrate in this talk that Ys can be constructed when the object is placed in an isotropic random noise field. This construction consists of ensemble averages of cross-correlations of the resulting total normal velocity and total pressure (incident plus scattered) measured over the complete surface of the object. However, the measurement of the surface fields could be prohibitive as the temporal frequency of interest increases. Instead of a surface measurement, one can use a dual conformal surface of microphones placed near to the object together with near-field acoustical holography (NAH) to determine the fields on the surface via the back projection capability of NAH. Although a large number of sensors may be required, the advent of inexpensive MEMS microphones combined with a 3D printing capability to construct the support structure makes this measurement very plausible. [Work supported by the Office of Naval Research.]

\section{1:40}

3pSA2. In-plane wavefield detection in lattice structures via digital image correlation. Massimo Ruzzene, Giuseppe Trainiti, and Marshall Schaeffer (Georgia Inst. of Technol., 270 Ferst Dr., Atlanta, GA 30332, ruzzene@gatech.edu)

We describe an experimental procedure to measure in-plane waves in an hexagonal lattice structure through digital image correlation. Measurement are performed by exciting the structure with a narrowband signal, then recording the evolution of the wavefield by using a single high-speed camera pointed at the lattice. By tracking the position of the lattice intersections, we are able to obtain the time evolution of the interpolated in-plane displacement field, which we use to separate the longitudinal and shear components of motion through Helmholtz decomposition. The proposed approach allows us to investigate the dispersion characteristics of P-and S-modes. For each of these two modes, we focus on the directionality of wave propagation by obtaining experimental dispersion surfaces, which are in excellent agreement with the theoretical ones. We also compare the RMS plots of the experimental displacement field to the components of the group velocity vector for the $\mathrm{P}$ and the $\mathrm{S}$-modes computed theoretically, showing the direction where energy preferentially propagates in the structure. Digital image correlation is a promising tool for investigating in-plane motion in lattice structures, displaying interesting features with respect to analogous techniques, such as the 3D Laser vibrometry.

\section{2:00}

3pSA3. Electronic speckle-pattern interferometry: Techniques for non-contact vibration measurements of musical percussion instruments. Randy Worland (Phys., Univ. of Puget Sound, 1500 N. Warner, Tacoma, WA 98416, worland@pugetsound.edu)

Electronic speckle-pattern interferometry (ESPI) provides a non-contact optical system that can be used to record images showing operating deflection shapes of structures being driven sinusoidally at resonant frequencies. In high-Q systems, these shapes can approximate the normal modes of the test object. Due to the sensitivity of the optical measurement system, non-contact excitation can be achieved acoustically using a speaker. The use of ESPI is described in relation to measurements of musical bars, plates, and membranes under various boundary conditions, though the methods described can be applied to many other types of structures. In addition, a dual-ESPI system that provides simultaneous measurements of two surfaces of an object is illustrated (e.g., two heads of a musical drum). These measurements provide information regarding the degree of coupling and the relative phase of the two vibrating surfaces. Finally, the ESPI techniques are applied to the fluid-structure interaction of an orchestral crotale (a thick metal disk) at various degrees of submersion in water. 
3pSA4. Non-contact source for nonlinear acoustics for crack detection. Pierre-yves Le Bas, T. J. Ulrich, Marcel Remillieux (Geophys. Group, EES-17, Los Alamos National Lab., MS D446, Los Alamos, NM 87545, pylb@lanl.gov), Brian E. Anderson (Brigham Young Univ., Provo, UT), and Lucasz Pieczonka (AGH Univ. of Sci. and Technol., Krakow, Poland)

Over the last decades, nonlinear acoustic techniques have been developed to detect mechanical damage in solids. They have been proven to be far more sensitive to early damage than standard linear acoustic techniques. Unfortunately, they often require high amplitude waves to propagate within the sample. To be practical in industrial applications, signals have to be generated without contact. Here, we will present a novel non contact acoustic source that can produce higher amplitude than standard transducers and how it can be used to image defects into a carbon fiber reinforce plastic plate damaged by an impact. We will show that we can not only image cracks but also separate two overlapping defects according to their orientation. [This work was supported by the US Department of Energy via the used fuel disposition campaign of the nuclear energy program.]

3pSA5. Automated acoustic evaluation of concrete bridge decks. Jinying Zhu (Civil Eng., Univ. of Nebraska-Lincoln, 1110 S 67th St., PKI 205B, Omaha, NE 68182, jyzhu@unl.edu), Suyun Ham (Civil Eng., Univ. of Texas at Arlington, Arlington, TX), and Hongbin Sun (Civil Eng., Univ. of Nebraska-Lincoln, Omaha, NE)

Chain drag testing is commonly used in current practice for bridge deck evaluation due to its low cost and ease of use. However, this method is subjective, and highly depends on the experience of the operators. Ambient noise caused by traffic affects the test speed and accuracy of results. This paper describes a recent research to develop an automated chain drag acoustic scanning system to detect delaminations in concrete structures, including bridge decks. The system consists of an array of chains, a noncontact MEMS microphone sensor array, multi-channel data acquisition device, positioning system, and signal processing schemes. The multi-channel design improves the spatial coverage and resolution of testing. An algorithm for interpreting acoustic signals from the automated chain drag test is developed. This automated system will enable real time visualization of tested areas. Compared to the conventional manual chain drag test, the automated system provides improved accuracy, spatial resolution, repeatability, and practicality.

3:00

3pSA6. Electromagnetic acoustic transducers for nondestructive inspection of dry storage canisters for used nuclear fuel. Hwanjeong Cho, Sungho Choi (Eng. Sci. and Mech., Penn State, 212 EES Bldg, University Park, PA 16802), Matthew Lindsey (Structural Integrity Assoc., State College, PA), and Cliff J. Lissenden (Eng. Sci. and Mech., Penn State, University Park, PA, Lissenden@ psu.edu)

Inspection for stress corrosion cracking in stainless steel canisters that store used nuclear fuel must be done within a harsh environment that requires robotic delivery of the sensing system. The system must tolerate high temperatures and radiation and it must operate in constrained spaces. In order to be delivered robotically noncontact transducers are preferred. Electromagnetic acoustic transducers (EMATs) are noncontact transducers that can be made from materials capable of resisting high temperature and radiation and therefore are an excellent choice for this application. This presentation will describe the requirements of the dry storage environment, the ability of compact EMATs to detect cracks oriented both transverse and parallel to the wave vector using shear horizontal guided waves, and the nondestructive inspection methodology developed for full penetration weld regions. Laboratory experiments indicate that the pulseecho mode is effective at detecting crack-like notches. B-scans constructed from the heat affected zone of welds show both the defects and the welds, which are distinguishable by their time-of-flight.

\section{3:20-3:40 Break}

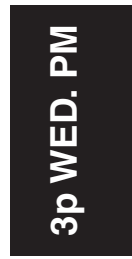

\section{Contributed Papers}

\section{3:40}

3pSA7. Estimation of material properties using vibration magnification. Alyssa T. Liem and J. G. McDaniel (Dept. of Mech. Eng., Boston Univ., Boston, MA 02215,jgm@bu.edu)

This presentation describes the use of video magnification to estimate material properties of a structure within a region of interest. Video magnification is a recently introduced technique that magnifies motions which are mathematically present in a video but often not visible to the human eye. Several recent papers have shown that this technique may be used to derive quantitative information about vibration without contacting the structure. Furthermore, the technique amplifies all components of the three-dimensional vibration field that are present in the plane of the video. In the present work, a mechanical excitation is applied to the center of a region of interest on a structure. The excitation is concentrated in space and time. The response of the structure is captured at high temporal and spatial resolution by a video camera focused on the area of interest. Material properties are then estimated by adjusting values in a model of the region until agreement is obtained throughout the temporal and spatial responses in the video. The present work allows one to understand physical mechanisms and improve numerical models. Use of an impulsive excitation limits the extent of testing to a region of interest. Experimental results will be presented.

\section{$3: 55$}

3pSA8. Extracting vibration characteristics of a guitar using finite element, modal analysis, and digital image correlation techniques. Jiaqi Huang, Kiran Patil, Javad Baqersad (Mech. Eng., Kettering Univ., 1700 University Ave., Flint, MI 48504, jbaqersad@kettering.edu), and Daniel Ludwigsen (Phys., Kettering Univ., Flint, MI)

The sound quality generated by the guitar depends on the vibration characteristics (i.e. natural frequencies and mode shapes) of this instrument. Thus, it is of particular interest to the guitar manufactures to be able to extract global information about the characteristics of the guitar. Traditional sensors can only measure at discrete locations. However, digital image correlation (DIC) can measure full-filed data on the surface of the structure. In the current paper, a finite element (FE) model of a guitar was developed using quadratic shell, and solid elements. An eigensolution was performed on the FE model to extract its natural frequencies and mode shapes. In order to validate the numerical results, a modal impact hammer test was performed on the instrument. Furthermore, a measurement was performed on the guitar using the DIC technique. In this measurement, the guitar was placed in a free-free configuration and was excited using a broadband excitation generated by a sound source. The response of the guitar to the excitation was recorded using a pair of high-speed cameras. The recorded images 
were processed to extract the natural frequencies and mode shape of the guitar. The results show strong correlation between the numerical model and experimental results.

\section{4:10}

3pSA9. Modelling and experimental mapping of the ultrasound pressure field generated from focused ultrasonic transducers using fiber optic acoustic sensors. Songmao Chen, Alessandro Sabato, Christopher Niezrecki, and Peter Avitabile (Structural Dynam. and Acoust. Systems Lab, Univ. of Massachusetts Lowell, One University Ave., Lowell, MA 01854,Songmao_Chen@student.uml.edu)

The dynamic focused ultrasound radiation force has been recently used for excitation of structures with sizes ranging from micro to macro scale having a frequency bandwidth between tens of Hertz and up to $100 \mathrm{kHz}$. Therefore it can potentially be used as an alternative non-contact excitation method for conventional contact type excitations such as modal impact hammer or shaker excitations for experimental modal analysis. However, the dynamic focused ultrasound radiation force remains to be quantified and calibrated. In this paper, we present the results of numerically modeling and experimentally mapping the pressure field generated by a focused ultrasonic transducer (UT) with a radiating diameter of $50 \mathrm{~mm}$ and center frequency of $359 \mathrm{kHz}$. In the simulation, an acoustic model is created using the Rayleigh Integral and the boundary element method. For the experimental testing, a precision microphone and a fiber optic acoustic sensor are used to map the pressure field generated from the UT driven by double sideband suppressed carrier amplitude modulation signals. This novel fiber optic sensor is custom developed with a sensing element having a diameter of $\Phi 125 \mu \mathrm{m}$, enabling high spatial resolution measurement. The simulation and experimental results are compared and are shown to have good agreement.

3pSA10. The non-contact acoustic inspection method for concrete structures using the defect detection algorithm based on the statistic evaluation for a healthy part of concrete. Kazuko Sugimoto, Tsuneyoshi Sugimoto, Nobuaki Kosuge (Graduate School of Eng., Toin Univ. of Yokohama, 1614 Kurogane-cho, Aoba-ku, Yokohama, Kanagawa 225-8503, Japan, kazukosu@toin.ac.jp), Chitose Kuroda, and Noriyuki Utagawa (Satokogyo Co., Ltd., Atsugi, Kanagawa, Japan)

The deterioration of concrete structure becomes a social problem in Japan. Social needs increase as for maintenance, check and renewal of concrete structures such as a tunnel or a bridge. The check of concrete structures has been performed conventionally by a hammering test. Not the inspection depended on a human experience and sense, but the inspection by a quantitative measuring system are demanded. We have developed the technique "non-contact nondestructive acoustic inspection method" to measure the internal defects near the concrete surface to a depth of about $10 \mathrm{~cm}$ using airborne sound and the laser Doppler vibrometer at a distance 5-10 m far from measurement surface. Depending on the surface state (reflectance, dirt, etc.) of concretes, there is a problem to decrease the quantity of the light of the returning laser and there arise optical noise resulting from the leakage of light reception. To remove an abnormal measurement point, we proposed a defect detection algorithm, in which vibrational energy ratio and spectrum entropy are combined. As a result, it enables to distinguish a defect part, healthy part and an abnormal measurement point. However, for a real concrete structure, the gray area exists. We evaluate quantity of acoustic characteristics against a healthy part of concrete statistically. We found the distribution of each acoustic characteristic against a healthy part follow a normal distribution by excluding some outlier. A defective part is separated from a healthy part clearly and visualized vividly.
3pSA11. Study on the measurement speed and signal to noise ratio of multi tone burst wave for high speed non-contact acoustic inspection method. Nobuaki Kosuge, Tsuneyosi Sugimoto, Kazuko Sugimoto (Graduate School of Eng., Toin Univ. of Yokohama, 1614,Kurogane-cho, Aobaku, Yokohama, Kanagawa 2258503, Japan, tm25b10u@ust.toin.ac.jp), Chitose Kuroda, and Noriyuki Utagawa (Tech. Res. Inst., SatoKogyo Co.,LTD., Atsugi, Kanagawa, Japan)

The non-contact acoustic inspection method using an air-borne sound can detect the cavity defect and crack near the measurement surface by using flexural resonance. By this method which uses the conventional single tone burst wave, since only one frequency was used for one sound wave emission, the length of measurement time had become a problem. Therefore, the multi-tone burst wave was devised for high speed improvement. However, measurement time and signal to noise $(\mathrm{S} / \mathrm{N})$ ratio change with the parameters in which a multi-tone burst wave is contained at the time of one sound wave emission, such as the number of frequency, the arranging method, pulse length, and the average number of times. Therefore, the experiment using the concrete test object having the styrofoam which imitated the cavity defect was conducted and a multi-tone burst wave was used, it was investigated how the measurement time and the $\mathrm{S} / \mathrm{N}$ ratio of this method would change. From the experimental result, it became clear that the improvement in the measurement speed of several or more times was realizable maintaining a comparable $\mathrm{S} / \mathrm{N}$ ratio as compared with the conventional single tone burst wave.

\section{$4: 55$}

3pSA12. Remote acoustic sensing of mechanical changes in a plate in an unknown reverberant environment. Tyler J. Flynn and David R. Dowling (Mech. Eng., Univ. of Michigan, Ann Arbor, 1231 Beal Ave., Ann Arbor, MI 48109, tjayflyn@umich.edu)

An experimental method for remote acoustic sensing of changes in radiating structures in an unknown reverberant environment is presented. Acoustic radiation from a mechanical structure due to oscillatory forcing may be described by a frequency response function (FRF) dependent on the structure's material, geometry, and boundary conditions. Mechanical changes may alter the structure's FRF in detectible and potentially predictable ways. However, collecting suitable FRF measurements may be difficult in reverberant environments over the necessary extended measurement times due to the influence of reflected and scattered sound. Here, experimental results are presented for the remote acoustic detection of mechanical changes to a vibrating $0.3-\mathrm{m}$-square by 3 -mm-thick aluminum plate in a reverberant environment. The plate has nominally clamped edges and is subject to swept-frequency base excitation. Sound from the plate with and without a mechanical change is recorded remotely with a 15 microphone linear array. These recordings are then processed using Synthetic Time Reversal to reconstruct a single radiated sound signal that is corrected for the environment's unknown reverberation, and this corrected signal is examined to detect mechanical changes to the test plate. Results from the proposed technique are compared with equivalent results from conventional approaches. [Sponsored by NAVSEA through the NEEC.]

\section{$5: 10$}

3pSA13. High speed non-contact acoustic inspection method using multi tone burst wave. Tsuneyoshi Sugimoto, Kazuko Sugimoto, Nobuaki Kosuge (Graduate School of Eng., Toin Univ. of Yokohama, 1614, Kuroganecho, Aoba-Ku, Yokohama, Kanagawa 2258503, Japan, tsugimot@toin.ac. jp), Chitose Kuroda, and Noriyuki Utagawa (Tech. Res. Ctr., SatoKogyo, Co. Ltd., Atsugi, Kanagawa, Japan)

The non-contact acoustic inspection method using an air-borne sound can detect the cavity defect and crack near the measurement surface by using flexural resonance. By this method using a single tone burst wave, the length of the measurement time of a two-dimensional scan was a practical problem. This is because only one frequency was used for one sound wave emission so that a high signal to noise $(\mathrm{S} / \mathrm{N})$ ratio could be obtained with the laser Doppler vibrometer (LDV) of a weak laser power (e.g., He-Ne 1mW). However, two or more frequency can be used at the time of one sound wave emission (multi tone burst wave) by taking into consideration the difference 
of the propagation velocity of a sound wave and laser light. That is, it becomes possible to perform high-speed measurement as compared with the conventional method, using effectively the time \& frequency gate for raising a $\mathrm{S} / \mathrm{N}$ ratio by terminating measurement before the reflective sound wave reaches a laser head from the measurement surface. The experiment using a concrete test object was carried out and the validity of this multi tone burst wave as high-speed measurement was confirmed from the experimental result.

\title{
Session 3pSC
}

\section{Speech Communication: Speech Production and Perception (Poster Session)}

\author{
Elizabeth A. McCullough, Chair \\ Linguistics, Ohio State University, Box 352425, Seattle, WA 98195
}

\begin{abstract}
All posters will be on display from 1:00 p.m. 5:30 p.m. To allow contributors in this session to see the other posters, authors of odd-numbered papers will be at their posters from 1:00 p.m. to 3:15 p.m. and authors of even-numbered papers will be at their posters from 3:15 p.m. to 5:30 p.m.
\end{abstract}

\section{Contributed Papers}

3pSC1. A data-driven solution to the invariance problem in speech perception. Tasha Nagamine and Nima Mesgarani (Elec. Eng., Columbia Univ., 560 Riverside Dr. 17B, New York, NY 10027, nima@ee.columbia. edu)

A fundamental and unanswered question of speech perception is the ability of a listener to map the highly variable acoustic features of phones to perceptual categories such as phonemes. Several solutions to this problem have been proposed, which include the motor theory, direct realist, distinctive features, and exemplar models. In parallel, the same problem has been studied extensively in algorithmic emulation of speech perception, where the first step is to estimate phonemic categories from the acoustic signal. The recent breakthroughs in machine learning methods, particularly models inspired by neural circuits, have resulted in nearly human-level performance. However, the strategy exploited by the models in these data-driven approaches remains elusive. Here, we analyzed a deep neural network model trained to perform the acoustic-to-phoneme transformation and determined the representational and computational properties of the network as it forms categorical distinctions. We found that the network internally created a representation of phonetic features, such as manner and place of articulation, and explicitly modeled the phone variability. Furthermore, the network selectively and non-linearly warped the acoustic space to separate the more similar acoustic distinctions. This study provides an empirical solution to the invariance problem that can be evaluated in perceptual and neural studies of speech perception.

3pSC2. Mapping the functional pathways to speech intelligibility using neuroimaging techniques: A study of incremental speech perception. Emily Byers (Speech and Hearing Sci., Indiana Univ., 200 S Jordan Ave., Bloomington, IN 47405, elbyers@indiana.edu) and Jaimie Murdock (Cognit. Sci., Indiana Univ., Bloomington, IN)

Speech perception involves a complex feedforward/feedback process of decoding bottom-up auditory input while utilizing top-down cognitive/linguistic knowledge to map stored word forms onto incoming acoustic waves (Kinchla \& Wolfe, 1979; Sloos \& McKeown, 2015). Speech perception in the brain is believed to occur as a result of cortical spreading from the core of the auditory cortex (and peripheral areas), diffusing into non-speech-specific areas of the brain responsible for binding information and memory retrieval. The current study traces the functional pathways between regions associated with speech perception using a combination of fMRI and EEG imaging. Our principle aim is to determine how normal functional connectivity between language-processing areas differs from speech perception where speech ranges from unintelligible (e.g., English phonetic non-word sentences) to intelligible with spontaneous disruptions (code-switched utterances) up to fully intelligible speech. Testing disruption of speech intelligibility in healthy adults using naturally-occurring stimuli recreates the type of breakdown in intelligibility experienced by patients with language disorders as well as typically aging adults. Preliminary results indicate that spontaneous disruption of speech intelligibility due to mixed-language utterances differs from traditional information-masking stimuli both in reaction time to the stimulus change and in the amount of time required to resume online speech processing.

3pSC3. Aspects of generating syntax-free word lists for quantifying speech intelligibility. Stefanie Keller, Werner Hemmert (Bio-Inspired Information Processing, Tech. Univ. of Munich, Garching, Germany), and Florian Völk (WindAcoust. UG, Boltzmannstraße 11, Garching 85748, Germany, florian.voelk@mytum.de)

Several possibilities exist for quantifying speech intelligibility in noise, for example tests with single words or sentences. In the clinical routine, usually a single sentence test is used repeatedly, so that its vocabulary is often known to the patients. Thus, not only speech intelligibility but also long term memory is tested. New speech material is then required to explicitly address intelligibility. With the aim of providing new and optimized material, in this study, semantically, syntactically, and phonetically balanced words were taken from German sentence tests, based on databases for written and spoken German words. The resulting vocabulary consists of 54 words (nouns, adjectives, verbs, and numbers), for which word-reception thresholds were determined for ten normal-hearing subjects. With these thresholds, five-word lists of equal semantic context, equal word intelligibility, and with balanced phonetic cues were built. Non-acoustic advantages of an intelligibility test constructed this way are phonetic balance and semantic coherence without syntactic context, which aim at preventing syntactic and phonetic cues from affecting speech-intelligibility thresholds. As an initial verification, list intelligibilities were measured with the above subject sample. The results show no systematic difference for consonants and vowels. This indicates that the lists were successfully constructed without phonetic biases. 
3pSC4. Eliciting natural conversational Lombard speech in realistic acoustic environments. Timothy Beechey (Linguist, Macquarie Univ., Australian Hearing Hub, 16 University Ave., Macquarie Univ., NSW 2109, Australia, tim.beechey@nal.gov.au), Jorg Buchholz (Linguist, Macquarie Univ., Sydney, NSW, Australia), and Gitte Keidser (National Acoust. Labs., Sydney, NSW, Australia)

Speech produced in noisy environments (Lombard speech) is characterized by a range of acoustic and phonetic changes. These changes stem from increased speaking effort which reflects communicative intent as well as decreased auditory feedback of the speaker's own voice. An accurate understanding of real-world Lombard effects is important in hearing science for the development and assessment of signal processing strategies targeting realistic speech signals. While Lombard effects are well known from the literature, studies of Lombard speech have typically been based on relatively unnatural speaking tasks such as reading from a script and have been measured in simplified acoustic backgrounds such as stationary noise or constructed babble noise. Lombard speech produced under such unnatural conditions may differ significantly from speech produced in real-world settings. This study describes a novel method of eliciting natural conversational speech across five highly realistic everyday acoustic environments. Through the increased realism of both the speaking task and acoustic backgrounds this study aims to provide a more ecologically valid approximation of real-world Lombard speech than has been previously reported. Based on recordings of conversations between 10 pairs of young, normal-hearing people, a continuum of ordered acoustic and phonetic changes in speech is described in relation to changes in acoustic environments and is related to self-reported listening effort ratings across acoustic environments.

3pSC5. Online testing for assessing speech intelligibility. Jonathan E. Peelle, Tracy Zhang, Nisha Patel, Chad S. Rogers (Dept. of Otolaryngol., Washington Univ. in Saint Louis, 660 South Euclid Ave. Box 8115, Saint Louis, MO 63110, peelle@wustl.edu), and Kristin J. Van Engen (Dept. of Psychol. and Brain Sci., Washington Univ. in Saint Louis, Saint Louis, MO)

The use of online testing in the behavioral sciences is increasing due to the potential for lower cost and faster completion than traditional in-person laboratory testing. Online testing presents a special challenge for speech research due to the variety of listeners' acoustic environments. For example, listeners may use headphones or speakers of various types, complete the task in different levels of background noise, or vary in their hearing abilities. Here we presented spoken sentences in speech-shaped noise to participants from the United States recruited online using Amazon Mechanical Turk at SNRs of $-2,-5$, and $-8 \mathrm{~dB}(\mathrm{n} \geq 50$ per SNR). We compared these online results to normative data collected using these same sentences in the lab (SNR of $-5 \mathrm{~dB} ; \mathrm{n}=30$ ). Preliminary results suggest a reasonable correspondence between average intelligibility scores for individual sentences presented using the two methods (Spearman rho $=0.79$ ). Standard deviations suggest greater variability in the online responses (average SD across sentences $=1.24$ ) relative to the lab responses (average $\mathrm{SD}=0.86$ ). We conclude that despite increased variability, online testing can provide reasonable ratings of intelligibility and in many circumstances is a feasible method to supplement or replace laboratory testing.

3pSC6. Variation in normal hearing thresholds predicts word recognition in noise. Kristin Van Engen (Washington Univ. in St. Louis, One Brookings Dr., Campus Box 1125, Saint Louis, MO 63130-4899, kvanengen@wustl.edu)

Analyses of speech identification data collected from young, normalhearing adults often do not include measures of listeners' hearing. Sub-clinical variation in hearing, it is assumed, will not significantly affect the identification of speech signals presented well above threshold levels. This study tested that assumption in the context of an experiment that investigated young adults' recognition of words with few versus many phonological neighbors produced in clear and conversational speaking styles. Words were presented in speech-shaped noise at a $+5 \mathrm{~dB}$ SNR. A mixed effects regression analysis was performed to account for the contributions of lexical difficulty, speaking style, order of word presentation, and listeners' hearing (as measured by pure-tone average (PTA)). This analysis showed, as expected, that correct identification was predicted by word type (easy $>$ hard) and speaking style (clear $>$ conversational). It also showed that identification improved over the course of the experiment. Finally, it showed that PTA was a significant predictor of identification for words produced in conversational speech, even for listeners with normal hearing (PTA range: -3.33$11.66 \mathrm{~dB}$; mean: $2.23 \mathrm{~dB}$ ). These findings show not only that sub-clinical hearing differences can significantly affect speech identification, but also that speaking clearly can mitigate the effect.

3pSC7. Interaction of cognitive load, sentence predictability, and speech degradation in spoken word recognition and memory. Cynthia $R$. Hunter, Caylee Adams, Laura Rund, and David B. Pisoni (Psych., Indiana Univ., 1101 E. 10th St., Bloomington, IN 47405, cynthunt@indiana. edu)

Whether cognitive load and/or listening effort needed to understand degraded speech differentially affect bottom-up and top-down processes is not well understood. The current project examined effects of sentence predictability, speech degradation, and cognitive load on the recognition of sentence-final words and on the recall of short (low-load) or long (high-load) sequences of visual digits presented prior to each spoken sentence. In addition to main effects of sentence predictability and spectral degradation, word recognition in both high- and low-predictability sentences was modulated by cognitive load when accuracy was between 35 and 80 percent. Words were identified more accurately under low load than high load. Digit recall was affected by load, speech degradation, and sentence predictability. Results indicate that cognitive load affects processes used to identify words in both low- and high-predictability sentences, and that listening effort affects memory for visual digits.

3pSC8. Isolating the informational component of speech-on-speech masking. Tim Schoof (Northwestern Univ., 2240 Campus Dr., Evanston, IL 60208, tim.schoof@northwestern.edu), Axelle Calcus (Boston Univ., Boston, MA), Stuart Rosen (Univ. College London, London, United Kingdom), Barbara Shinn-Cunningham (Boston Univ., Boston, MA), and Pamela Souza (Northwestern Univ., Evanston, IL)

Speech perception in the presence of a competing talker involves both energetic (EM) and informational masking (IM). This study aimed to isolate the informational component of speech-on-speech masking. EM can be eliminated by presenting the target and masker to opposite ears (i.e., dichotically). However, this also dramatically reduces the effects of IM by providing listeners with lateralization cues. Previous research using tonal sequences has shown that IM can be isolated by presenting the target and masker dichotically while rapidly switching the two streams across the ears. The question remains whether this technique can also be used for speech materials. Speech reception thresholds (SRTs) were measured for sentences produced by a female talker in the presence of a competing male talker under three conditions: diotic, dichotic, and switching. In the switching condition, target and masker were presented dichotically, but their lateralization was switched after every word in the target sentence. Preliminary data suggests that SRTs for the switching condition are higher than for the dichotic condition but lower than for the diotic condition. This suggests that, contrary to findings for tonal sequences, rapidly switching the target and masker speech across the ears does not fully reintroduce IM. [Work supported by NIH.]

3pSC9. Separation and integration of sound sources in auditory processing. Mio Horigome (Rehabilitation Dept., Okazaki City Hospital, 3-1 Goshoai, Koryuji cho, Okazaki, Aichi 4448553, Japan, horigome.mio@okazakihospital.jp) and Kazuhiko Kakehi (Study of Artificial Interigence, Chukyo Univ., Toyota, Aichi, Japan)

Some perceptual experiments were conducted to elucidate sound source separation processing in speech perception. The phenomenon of phonemic restoration was tested if it occurs in dichotic listening. An original speech is Japanese /VCV/, the stimulus is made by manipulating the /VCV/ as was replaced the consonant part with noise. The duration of replacement was varied at six levels: 10, 20, 40, 60, 80, and $100 \mathrm{~ms}$. There were two hearing modes: in one case, the monaural stimulus was presented to both the ear, and in the other case, the speech part of the stimulus was presented to an ear 
and the noise part to the opposite ear simultaneously. Thirty subjects in the age of twenties participate the listening test. The results show that phonemic restoration effect is clearly observed in both hearing modes. The rate of restoration is $92 \%$, and $95 \%$ even in dichotic listening. There was no feature of speech in the noise has no relation with speech sound. Moreover, the direction of the noise sound was opposite to that of speech sound source, different in 180 degree. Even though the two sound signal are somehow integrated to hear restored speech.

3pSC10. Estimation of binaural speech intelligibility based on the better ear model. Kazuya Taira (Graduate School of Sci. and Eng., Yamagata Univ., 4-3-16 Jonan, Yonezawa, Yamagata 992-8510, Japan, thh04707@st. yamagata-u.ac.jp), Yosuke Kobayashi (Graduate School of Eng., Muroran Inst. of Technol., Muroran, Hokkaido, Japan), and Kazuhiro Kondo (Graduate School of Sci. and Eng., Yamagata Univ., Yonezawa, Yamagata, Japan)

When intelligibility is measured, objective estimation is more convenient than subjective. However, most objective estimation methods estimate monaural intelligibility using monaural signals. Therefore, it is necessary to estimate binaural intelligibility using binaural signals in order to take into account that a person listens using two ears. We evaluated an intelligibility estimation method using the Better Ear Model which selects better value out of the left and right feature values, and found that high estimation accuracy is possible. Accordingly, we proposed and evaluated an extension to this model which divides the signal into critical frequency bands, and takes the better value between left and right channel in each band (Band-Selection Better Ear Model). Furthermore, we tried estimation using machine learning in addition to regression analysis which we applied previously. Neural network and support vector machine were used here. Comparison between the Better Ear Model and the Band-Selection Better Ear Model showed no significant differences in the estimation accuracy. Also, the introduction of machine learning yielded a higher accuracy than that of regression analysis in a closed test. Correlation between subjective and estimated intelligibility increased by 0.1 or more. So far, mixed results are seen in the open test.

3pSC11. Semantic predictability mediates effects of phonetic reduction and social variation on word recognition. Zack Jones, Megan M. Dailey, and Cynthia G. Clopper (Linguist, The Ohio State Univ., 222 Oxley Hall, 1712 Neil Ave., Columbus, OH 43210, jones.5028@osu.edu)

Phonetic reduction due to lexical frequency, phonological neighborhood density, and discourse mention, as well as speaking style and social-indexical variation can constrain listeners' ability to identify spoken single word tokens. In phrasal contexts, however, semantic predictability facilitates word recognition. The aim of the current study was to investigate how semantic predictability influences the intelligibility of words that vary in their lexical, stylistic, and socio-indexical properties. Listeners were presented with auditory English phrases extracted from read passages and were asked to identify each phrase. Phrases were mixed with speech-shaped noise and each contained a target word of interest. Linguistic and social properties of the target words were used to predict listeners' target word recognition accuracy. These factors included semantic predictability, lexical frequency, neighborhood density, speaking style, discourse mention within the passage, talker gender, and talker dialect. The results revealed that greater semantic predictability increased word recognition accuracy, but only for the most phonetically reduced words (e.g., high-frequency words, second mentions, and words in a plain style). These results suggest that the semantic predictability benefit is enhanced primarily for words that might otherwise be difficult to recognize when removed from their semantic context.

3pSC12. Are suffixed words different? Evidence from a modified lexical decision task. Anne Pycha (Linguist, Univ. of Wisconsin, Milwuakee, P.O. Box 413, Milwaukee, WI 53211, pycha@uwm.edu)

We investigated whether listeners process suffixed words differently than prefixed words. We presented primes that combined a clear-speech root plus degraded-speech affix (such as kin- $x$, where $x x$ refers to degraded speech), and measured lexical decision RTs to subsequent clear targets (kinship). Degradation used low-pass filtering $(<500 \mathrm{~Hz})$, such that affixes were speech-like but incomprehensible. Thus, the prime kin-xx sounds like a complete suffixed word, yet it is compatible with the target kin-ship and should not compete with it for activation. The crucial comparison was between prefixed (re-group) versus suffixed (kin-ship) targets, which were matched for frequency, familiarity, probability of phonotactic transition across morpheme boundary, and affix type-parsing ratio (Hay \& Baayen, 2002). On each trial, participants heard a prime, a $1000 \mathrm{~ms}$ ISI, then a target to which they made a speeded decision. Pilot results $(n=5)$ suggest that comparable speech input activated suffixed roots less strongly than prefixed roots. While $x x$-group reduced RTs to re-group by a large amount (-236.65 $\mathrm{ms})$, kin- $x x$ reduced RTs to kin-ship by a much smaller amount $(-133.20$ $\mathrm{ms}$ ). Pseudo-affixed words (resort, worship) did not show a comparable difference, suggesting that the effect arises from morphological constituency.

3pSC13. The effect of semantic predictability on word intelligibility in natural and spectrally degraded sentences. Bahar S. Shahsavarani, Thomas Carrell, and Ashok Samal (Univ. of Nebraska-Lincoln, Commun. Disord., University of Nebraska-Lincoln, Lincoln, NE 68583, bahar@ huskers.unl.edu)

One of the most consistent findings across a wide range of methodologies and stimuli is the positive effect of semantic context and predictability on speech intelligibility. However, there is evidence that this may not be true for a limited range of stimuli. The present study investigated how sentences with spectrally-limited acoustic information constrained the use of semantic predictability in speech perception. This was studied by presenting listeners with spectrally-degraded and natural-speech sentences with highand low-predictability words. The SPIN sentence lists and the associated multi-talker babble was used (Bilger, 1984). The results demonstrated that listeners benefited from high predictability in both cases but the degree of this effect was not equal. High predictability aided the perception of natural and 8-band speech equally. In contrast high predictability had a significantly smaller effect on speech intelligibility for 4-band speech. These findings are of special interest to investigations of speech perception in individuals with poor frequency representation such as those with cochlear implants.

3pSC14. Event-related potential responses reveal simultaneous processing across multiple levels of representation in spoken word recognition. Emma C. Folk and Joseph C. Toscano (Psych., Villanova Univ., 800 E Lancaster Ave., Villanova, PA 19085, efolk@villanova.edu)

A controversial issue in spoken language comprehension concerns whether different sources of information are encapsulated from each other. Do listeners finish processing lower-level information (e.g., encoding acoustic differences) before beginning higher-level processing (e.g., determining the meaning of a word or its grammatical status)? We addressed these questions by examining the time-course of processing using an event-related potential experiment with a component-independent design. Listeners heard voiced/voiceless minimal pairs differing in (1) lexical status, (2) syntactic class (noun/verb distinctions), and (3) semantic content (animate/inanimate distinctions). For each voiced stimulus in a given condition (e.g., lexical status pair TUB/tup), there was a corresponding pair with a voiceless ending (tob/TOP). Stimuli were cross-spliced, allowing us to control for phonological and acoustic differences and examine higher-level effects independently of them. Widespread lexical status effects are observed shortly after the cross-splicing point (i.e., the time when the lexical properties of the word can first be determined) and persist for an extended time. Moreover, there is considerable overlap in the times during which both lexical status and semantic content effects are observed. These results suggest that listeners multiple types of linguistic representations are active simultaneously during spoken word recognition, consistent with parallel processing models.

3pSC15. The perception of speech rate for time manipulated and vocoded sentences. Paul E. Reed, Allen Montgomery, Dan Fogerty, and Charley Adams (Commun. Sci. and Disord., Univ. of South Carolina, Keenan Bldg., Ste. 300, Columbia, SC 29201, reedpe@email.sc.edu)

Naturally produced fast speech reduces certain acoustic-phonetic features that may limit intelligibility relative to linear time compression. However, how reduction affects judgments of speaking rate has not been 
systematically investigated. The purpose of this study was to examine the effects of linear time compression and spectral reduction on judgments of speaking rate. Listeners provided speech rate judgments of sentences. Conditions compared rate perceptions for naturally spoken sentences at slow and fast rates with linearly time-compressed/expanded versions of the same sentence, matched for duration. Conditions also examined rate judgments for noise vocoded sentences that varied in intelligibility and signal-correlated noise that examined rate judgments based only on temporal acoustic features. Our preliminary results demonstrate that linear time-compressed/ expanded sentences were judged as faster than naturally produced sentences. This difference was also found for noise-vocoded versions of the sentences, as well as for signal-correlated noise. Additionally, vocoded stimuli were perceived as faster than naturally produced stimuli. These preliminary results suggest that acoustic-phonetic reductions in naturally produced speech do not appear to increase the perceived speaking rate relative to linear time manipulations. Significantly, temporal properties of speech rhythm appear responsible for coding perceptual aspects of speaking rate independently from factors related to linguistic processing.

3pSC16. Integration and maintenance of gradient acoustic information in spoken language processing. James B. Falandays (Psych., Villanova Univ., 38 North Cliffe Dr., Wilmington, Delaware 19809, jfaland1@villanova.edu), Joseph Toscano (Psych., Villanova Univ., Villanova, PA), and Sarah Brown-Schmidt (Vanderbilt Univ., Nashville, TN)

Models of speech processing seek to explain how continuous acoustic input is mapped onto discrete symbols at various levels of representation, such as phonemes, words, and referents. While recent work has supported models that posit maintenance of fine-grained information, it is not clear how continuous, low-level information in the speech signal is integrated with discrete, higher-level linguistic information. To investigate this, we created acoustic continua between the pronouns "he" and "she" by manipulating the amplitude of frication in the initial phoneme. Using the visual world eye-tracking paradigm, listeners viewed scenes containing male and female referents and heard sentences containing a pronoun, which later disambiguated to a single referent. Measures of eye-gaze revealed immediate sensitivity to both graded acoustic information and discourse-level information. Moreover, when listeners made an initially incorrect interpretation of the referent, recovery time varied as a function of acoustic step along the pronoun continuum, showing that graded acoustic information was maintained over at least a five-word delay. The results suggest that not only are listeners highly sensitive to fine-grained acoustic information in the speech signal but also that non-categorical representations are used to guide linguistic interpretation for extended periods of time.

3pSC17. Prediction of listener perception of reduced, voice stop consonant simulations based on patterns of formant deflections. Megan Willi and Brad Story (Univ. of Arizona, P.O. Box 210071, Tucson, AZ 85721, mkittles@email.arizona.edu)

Previous research on stop consonants found that less than 60 percent of the stops sampled from a speech corpus contained a clearly defined period of silence or prevoicing prior to the plosive release [Crystal \& House, JASA, 1988]. How listeners perceive a reduced form of stop consonants without these cues is not well understood. The purpose of this experiment was to investigate whether recasting typical formant transitions into a measure called a "relative formant deflection pattern" provides a means of predicting listeners' perceptions of approximant-like, voiced stop consonant variants. A computational model of speech production, in which consonant constriction location was varied along the length of the vocal tract, was used to generate place continua of approximate-like, voiced stop consonants imposed on a vowel-to-vowel transition. Stimuli were presented to listeners in three conditions: 1) normal simulated speech, 2) sinewave speech in which three tones replicated the time course of the F1, F2, and F3 contours in the simulated samples, and 3) sinewave speech in which three tones were present, but selected combinations of F1, F2, and F3 were set to a flat contour. Perceptual responses will be compared to the predictions based on relative formant deflection patterns across conditions.
3pSC18. Cue shifting bias between acoustically correlated cues. Meng Yang (Linguist, Univ. of California, Los Angeles, 3125 Campbell Hall, Los Angeles, CA 90095, mengyang@ucla.edu)

Acoustic cues signaling the same phonetic contrast are weighted differently by listeners in sound categorization. From the body of research on perceptual cue weighting, two major views have emerged. On one hand, research has shown that secondary cue weights depend on the distributional informativeness of cues. On the other hand, cues that enhance the percept of other cues have been demonstrated to get a "boost" in cue weight. The current study extends these theoretical claims to cue shifting using breathiness and pitch as cues in a novel sound categorization paradigm that induces changes in cue weights. Two factors affecting cue shifting in adult listeners were tested: distributional learning and perceptual enhancement. While a change in cue distinctiveness did cause listeners to redistribute cue weights to favour the more distinctive cue, cue shifting was not equal in all conditions. The shift was facilitated when the task allowed listeners to make use of the enhancing quality of the two cues. This finding cannot be explained by the distributional learning account. Additionally, listeners found it easier to shift cue weight from pitch onto breathiness than from breathiness onto pitch. This is problematic for both the distributional learning theory and the auditory enhancement theory.

3pSC19. Online perception of coda glottalization in American English. Adam J. Chong (UCLA, 3125 Campbell Hall, Los Angeles, CA 90095, ajchong@ucla.edu) and Marc Garellek (UC San Diego, La Jolla, CA)

In American English, voiceless codas /t, $\mathrm{p} /$ are often realized with glottalization on the preceding vowel. Previous claims suggest that such glottalization can serve to enhance /t/ or, more generally, voicelessness of coda stops. This study examines the timecourse of word recognition to test these claims. 40 American English listeners participated in an eye-tracking study, where they heard synthesized glottalized and non-glottalized versions of CVC English words ending in $/ \mathrm{p}, \mathrm{t}, \mathrm{b}, \mathrm{d} /$ while looking at a display with two words presented orthographically. Target words were presented with a minimal pair differing in place of articulation (e.g., cop-cot), or voicing (e.g., bat-bad, cap-cab). Our results indicate that listeners fixated to target words ending in /t/ marginally faster when they heard the glottalized version and when the competitor presented was a word ending in $/ \mathrm{p} /$. Glottalization did not result in faster fixation to targets for words ending in $/ \mathrm{p} /$. We also found a strong inhibitory effect-lower proportion fixation to targets-for words ending in voiced stops when the preceding vowel was glottalized. Altogether, these results support the claim that glottalization enhances voiceless codas (both $/ \mathrm{t} /$ and $/ \mathrm{p} /$ ), but that the effect is strongest for $/ \mathrm{t} /$.

3 pSC20. The effect of co-occurrence restriction on perception of laryngeal contrast in Seoul Korean and East Kyungsang Korean. Hyun Kyung Hwang (National Inst. for Japanese Lang. and Linguist, Midori-Cho, 10-2, Tachikawa 1908561, Japan, hwang@ninjal.ac.jp)

This study investigates dialect differences between Standard Korean and East Kyungsang Korean with respect to the role of co-occurrence restriction on perception of laryngeal contrast in stops and fricatives. The results obtained from the identification test first confirm that the laryngeal contrast is present only in the stops, but not in the fricatives in East Kyungsang Korean. In order to further explore the role of laryngeal co-occurrence restriction in the two varieties of Korean, identification tests using nonce compounds were conducted. The results provide experimental evidence of the laryngeal co-occurrence restriction; Both Seoul and East Kyungsang speakers's perception of laryngeal contrast in stop consonants is conditioned by laryngeal specification of the preceding consonant. With respect to the perception of laryngeal contrast in fricatives, however, only Seoul Korean speakers exhibit the effect of the co-occurrence restriction. These results lead to the conclusion that the co-occurrence restriction plays a crucial role in the perception of laryngeal contrast both in stops and fricatives in Seoul Korean, while the laryngeal features are phonologically irrelevant for fricatives in East Kyungsang Korean. <img class="UMSRatingIcon" $\mathrm{id}=$ "ums img_tooltip" $_{\text {, }} />$ 
3pSC21. How listeners recognise vowel sounds under highpass or lowpass filtering of vowel-specific frequency ranges. Dieter Maurer (Inst. for the Performing Arts and Film, Zurich Univ. of the Arts, Toni-Areal, Pfingstweidstrasse 96, Zurich 8031, Switzerland, dieter.maurer@zhdk.ch), Thayabaran Kathiresan (Phonet. Lab., Dept. of Comparative Linguist, Univ. of Zurich, Zurich, Switzerland), Heidy Suter (Inst. for the Performing Arts and Film, Zurich Univ. of the Arts, Zurich, Switzerland), and Volker Dellwo (Phonet. Lab., Dept. of Comparative Linguist, Univ. of Zurich, Zurich, Switzerland)

The present paper reports findings of two experiments on filtered sounds of the Standard German vowels /i-y-e- $\varnothing-\varepsilon-\mathrm{a}-\mathrm{o}-\mathrm{u} /$ produced by a female speaker at two fundamental frequencies $f_{o}=220 \mathrm{~Hz}$ and $659 \mathrm{~Hz}$ and a male speaker at $f_{o}=131 \mathrm{~Hz}$ and $523 \mathrm{~Hz}$. High-pitched sounds were included in order to account for a possible impact of the $f_{o}$ level on the perception of filtered vowel sounds. In the first experiment, the frequency region of the first formant of the sounds was highpass filtered, and in the second experiment, the frequency region of the second formant of the sounds was lowpass filtered. Vowel recognition of all sounds was investigated in a listening test. Results revealed shifts in the perceived vowel quality which varied across (i) vowel categories, (ii) $f_{o}$, and (iii) filter cutoff frequencies. Details of the filter parameters and of the perceived vowel quality shifts are given in the paper and implications for the relationship between acoustic cues and vowel recognition are discussed.

3pSC22. Perception of place of articulation of assimilated nasal and oral stops: What do response times and eye fixations tell us? Mercedeh Mohaghegh (Linguist, Univ. of Toronto, 309-15 Gamble Ave., Toronto, ON M4K 2H3, Canada, mercedeh.mohaghegh@mail.utoronto.ca) and Craig Chambers (Psych., Univ. of Toronto at Mississauga, Toronto, ON, Canada)

Two forced-choice identification and two visual world eyetracking experiments examined perception of the place of articulation (PoA) of word-final nasal and oral stops either in canonical (coronal) or assimilated form (coronal-to-labial place assimilation, e.g., 'phone $e^{m} /$ bat $^{p}$ button'). Listeners' response times (RT) and eye fixations were measured as they heard and, using a screen-based paradigm, identified assimilated or unassimilated words presented auditorily either in isolation (excised from recorded sentences) or with the assimilation-triggering context present (next word began with a labial consonant). Listeners were slower to identify isolated words ending in nasals, especially when words were assimilated. When the triggering context was present, RTs were overall faster and no longer different between nasal and oral stops. The eye fixation data further showed an early sensitivity to PoA cues carried by vowel transitions for assimilated oral stops. For words ending in assimilated nasal stops, however, fixation patterns only showed sensitivity to the PoA cues at a later point where the assimilation-triggering context was heard. These findings indicate a distinction in terms of perceptual uptake of acoustic cues between nasal and oral stops. The results also suggest the precise mechanisms involved in compensation for assimilation may vary across sound classes (nasal vs. oral stops).

3pSC23. Affricate-fricative perception in Korean listeners: Evidence for universal and language specific biases. Youngja Nam and Linda Polka (McGill Univ., 8th Fl., 2001 McGill College, Montreal, QC H3A 1G1, Canada, linda.polka@mcgill.ca)

Although language experience has a profound impact on phonetic perception, there is increasing evidence that phonetic perception is also shaped by universal biases which can be revealed as asymmetries in discrimination performance. In the present study, we explore potential perceptual asymmetries in adult Korean perception of four English affricate-fricative contrasts. Korean adults completed a native-language assimilation task and a category-based AX discrimination task with the phonemic contrast $/ \mathrm{t} \mathrm{fa}-\mathrm{sa} /$ and non-phonemic contrasts $/ \mathrm{t} \int \mathrm{a}-\mathrm{\int a} /, / \mathrm{d} z \mathrm{a}-\mathrm{za} / \mathrm{and} / \mathrm{d} 3 \mathrm{a}-\mathrm{za}$ /. Both voiceless contrasts- $/ \mathrm{t} \mathrm{\int a} \mathrm{a} \mathrm{s} /$ and $/ \mathrm{t} \int \mathrm{a}-\mathrm{Ja}$ - were assimilated to distinct Korean affricate and fricative categories and were discriminated very well ( $>90 \%)$; performance revealed no perceptual asymmetries. Both voiced contrasts-/dza-za/ and /dza- $3 \mathrm{a} /$ - were assimilated to the same Korean affricate category $\left(/ \mathrm{t} \int \mathrm{a} /\right)$ and were poorly discriminated (63-65\%); performance was asymmetric on different pairs for both contrasts (fricative-affricate pairs $>$ affricate-fricative pairs) and on same pairs for /dza-3a/ (fricative-fricative pairs $>$ affricateaffricate pairs). These findings, and prior research, show that asymmetrical performance on different pairs is highly uniform and predicted by phone type, pointing to a possible universal bias favoring sharp amplitude onsets. However, asymmetries in same pair performance are predicted by language categorization and thus appear to be shaped by language-specific experience.

3pSC24. Cross-linguistic differences in articulatory timing lag in consonant cluster perception. Harim Kwon, Ioana Chitoran (Université Paris Diderot, 5 rue Thomas Mann, CEDEX 13, Paris 75205, France, harim. kwon@univ-paris-diderot.fr), Marianne Pouplier, Tom Lentz, and Philip Hoole (Ludwig-Maximilians-Universitaet, Muenchen, Germany)

Cross-language research on consonant cluster production has shown that consonant clusters in different languages are produced with different degrees of articulatory timing lags. The present study examines perceptual sensitivity to these cross-linguistic timing differences in consonant clusters. Native German listeners were tested on an AXB similarity judgment test using stimuli including consonant clusters produced by German and Georgian speakers. (German consonant clusters are produced with relatively shorter lag between two consonants than Georgian ones.) Stimuli were /bla, gla, gna/ syllables recorded along with articulatory (EMA) data. Short lag German tokens and long lag Georgian tokens were selected as A and B, with Xs of varying degrees of lag chosen from either Georgian or German recordings. Results showed that German listeners are sensitive to the crosslinguistic differences in articulatory timing lag: when the timing lag of $\mathrm{X}$ was closer to A, participants were more likely to choose A. Moreover, listeners' sensitivity was influenced by the types of clusters: listeners were more sensitive in /bla/ than they were in /gla/ and in /gna/. The effects on the similarity judgment of different measures of articulatory lag, of vocalic releases produced within clusters, and of other sub-phonemic details were investigated. Overall, the results show that the lag differences are salient to German listeners while German and Georgian clusters can differ in a number of respects.

3pSC25. Washback and language change: An investigation of teachers' perception of the Korean vowel length contrast. Goun Lee (Dept. of the English Lang. and Lit., Yonsei Univ., Seoul, South Korea, cconni80@ gmail.com) and Dong-Jin Shin (Hankuk Univ. of Foreign Studies, Seoul, South Korea)

The goal of the current study is to investigate whether the vowel length contrast in Korean is retained in teachers' perception due to a washback effect. Previous studies have demonstrated that the vowel length contrast in Korean has been completely lost in the production of contemporary Seoul Korean (Kang et al., 2015). However, considering that this vowel contrast is still being prescriptively taught in the elementary school curriculum, we hypothesized that teaching linguistically extinct contrasts might influence speech perception. In order to investigate whether Korean teachers have regained the vowel length contrast, we conducted two perception experiments. Twenty-three Korean elementary school teachers and 30 university students (control group) from the Seoul area completed a discrimination task as well as a forced-choice identification test in which vowel length contrasts were manipulated at a ratio of 1:3. The results showed that teachers' perceptual identification accuracy was significantly higher than that of the control group. Interestingly, the control group's identification accuracy also exceeded chance level, indicating that Korean listeners still retained some perceptual sensitivity. These results suggest that the Korean vowel length contrast may not be completely lost in perception, and also that a washback effect from language teaching may inhibit language change.

3pSC26. The N1 event-related potential component as an index of speech sound encoding for multiple phonetic contrasts. Olivia Pereira and Joe Toscano (Psych., Villanova Univ., 800 Lancaster Ave., Villanova, PA 19085, opereira@villanova.edu)

Listeners rely on many phonetic cues to perceive speech, but it is not clear how acoustic and phonological differences are encoded at early stages of perception. Previous work has begun to address this using the event- 
related potential (ERP) technique, demonstrating that the amplitude of the auditory N1 ERP component varies linearly with differences along VOT continua and suggesting that it can serve as an index of cue encoding. However, it is not clear how the N1 varies more generally for other phonetic distinctions. We present ERP data for a large set of naturally-produced word-initial minimal pairs spanning 18 consonants (/b,d,t f, f,g, d $3, \mathrm{k}, \mathrm{l}, \mathrm{m}, \mathrm{n}, \mathrm{p}, \mathrm{I}, \mathrm{s}, \mathrm{f}, \mathrm{t}, \mathrm{v}, \mathrm{w}, \mathrm{z} /$ ), as well as stimuli varying along voice onset time (voicing) and burst frequency (place of articulation) continua for all six stop consonants. The results reveal widespread differences in N1 amplitude for place of articulation and voicing. The N1 is larger for voiced consonants than voiceless consonants. Moreover, N1 amplitude as a function of place of articulation patterns differently for fricatives (larger N1 for alveolar and post-alveolar phonemes) than for stops (smaller N1 for these place of articulation categories). Overall, these results suggest that the N1 serves as a useful index of speech sound encoding across a range of phonetic contrasts.

3 pSC27. Identification of stop consonants produced by an acousticallydriven model of a child-like vocal tract. Brad H. Story and Kate Bunton (Speech, Lang., and Hearing Sci., Univ. of Arizona, 1131 E. 2nd St., P.O. Box 210071, Tucson, AZ 85721, bstory@email.arizona.edu)

A model of a child-like vocal tract has been developed such that the deformation patterns superimposed on a vowel substrate to generate coarticulated consonants are specified by a time-varying set of directional shifts in the first three resonance frequencies. These deflection patterns are denoted as a combination of three numbers each of which can vary between -1 and 1; a negative value implies a downward shift in a resonance frequency whereas an upward shift results for positive value. For example, a "bilabial" consonant specified as $[-1,-1,-1]$ would be transformed via calculations of acoustic sensitivity functions to a time-varying vocal tract shape that presents the expected constriction at the lips, but also modifies other parts of the vocal tract that may be necessary for producing the appropriate formant transitions into and out of the consonant. Using this model, three sets of 30 $\mathrm{VCV}$ utterances were generated in which the values of deflection patterns were set to produce vocal tract shapes that hypothetically produce the stop consonants /b/, /d/, and /g/ embedded in three different vowel-vowel contexts. A perceptual experiment was performed to test their identification by listeners. [Work supported by NIH R01-DC011275 and NSF BCS1145011.]

3pSC28. Asymmetries in the perception of sub-categorical variation. Kuniko Nielsen (Linguist Dept., Oakland Univ., 1025 HHB, Rochester, MI 48309-4401, nielsen@oakland.edu) and Rebecca Scarborough (Linguist Dept., Univ. of Colorado Boulder, Boulder, CO)

Both VOT and vowel nasality show low-level variation in English that may be perceptually motivated (and which, in any case, has perceptual consequences). This study examines the perceptual salience of such variation, investigating in particular a possible perceptual asymmetry between increased vs. decreased degrees of these phonologically relevant features. On each trial of an AXB perceptual task, listeners heard three repetitions of the same word: a token with unchanged phonetic feature $(\mathrm{X})$ and tokens with phonetic features that were artificially increased and decreased to the same degree (A and B). Listeners then had to determine which of the two flanking items (A or B) sounded more similar to the middle item (X). Test words included 18 monosyllabic words with initial /p/ (in simple onsets) and 19 monosyllabic words with nasal codas and were heard by 40 listeners. Based on preliminary work, participants are expected to judge decreasedfeature stimuli of both types (VOT and nasality) as more similar to the natural stimuli, suggesting that increasing these phonetic features is perceptually more salient than decreasing them. These findings demonstrate asymmetrical sub-categorical sensitivity for features known to be perceived categorically (VOT), as well as for features not primary to phonological contrast (vowel nasality).
3pSC29. Perceptual adaptation to nonnative speech by school-aged children. Tessa Bent and Julianne Frye (Dept. of Speech and Hearing Sci., Indiana Univ., 200 S. Jordan Ave., Bloomington, IN 47405, tbent@indiana.edu)

With training, listeners can improve their understanding of speech in adverse listening conditions, including those stemming from the environment (e.g., competing noise) and the talker (e.g., unfamiliar accents). For example, listeners can increase their ability to understand a specific nonnative talker and, when trained with multiple talkers with the same nonnative accent, can generalize their learning to novel talkers with that accent. The current study extends these findings by investigating children's perceptual adaptation to nonnative speech. School-aged children can retune specific speech sound category boundaries, but little is known about their abilities to adapt to naturally produced nonnative speech. To assess children's adaptation to nonnative speech, 6- and 9-year-old children were presented with sentences produced by multiple Mandarin-accented talkers and repeated what they heard (training condition) or were not given training (control condition). A posttest assessed their understanding of sentences produced by a novel Mandarin-accented talker. Performance on the posttest was more accurate for children in the training condition than those in the control condition, which may have resulted from task learning or perceptual adaptation. In addition to phonetic category retuning, the results suggest that children may be able to improve their decoding of nonnative speech.

3pSC30. Acoustic cues underlying the adjustment to talker sex in perception of fricative consonants. Ashley N. Moore and Matthew Winn (Speech \& Hearing Sci., Univ. of Washington, 4217 NE 42nd St., Seattle, WA 98105, anmoore@uw.edu)

Sibilant fricatives are perceived mainly by spectral peak frequency, but are also influenced by surrounding context, which includes the vowel following the fricative. The vowel contains formant structure, formant transitions out of the consonant, and information about talker sex. These factors affect fricative categorization, even if fricative energy remains unchanged. For example, frequency components in voices are lower for men than for women, and the perceived boundary between relatively high ("s") and low ("sh") fricatives is accordingly shifted down for men. In this study, we examined various acoustic cues that underlie accommodation to talker sex, including fundamental frequency (F0), vocal tract length (formant spacing), and breathiness/spectral tilt as they affect fricative perception. Stimuli included a fricative continuum between "s" and "sh" and a variety of vocalic contexts spoken by women and men that were controlled to vary by the aforementioned cues. Listeners identified fricatives in "s"- or "sh"-onset words, and responses were analyzed using binomial logistic regression to measure the effect of contextual cues. Results demonstrate that despite the strength of F0 as a cue for talker sex, formant spacing and spectral tilt contribute most to the context effect, consistent with the importance of spectral contrast in speech perception.

3pSC31. Sociophonetic analysis of vowel variation in African American English in the Southern United States. Yolanda F. Holt (Commun. Sci. and Disord., East Carolina Univ., 300 Moye Bv 3310-X HSB, MS 668, Greenville, NC 27834, holty@ecu.edu)

Sociophonetic analysis is a quantitative method used to measure the dynamic acoustic properties of speech. Sociophonetic analysis pairs the demographic, geographic, and sociopolitical components of sociolinguistic inquiry with instrumental phonetic measurement techniques. This paper applies sociophonetic analysis to the study of regional vowel variation in African American English. Previous research has established robust variation in White American English. Only recently have explorations of dialect variation in African American English been completed. Analyses of vowel space area, vowel fronting, raising, and vowel dispersion reveal both regional alignment and racial/ethnic alignment of vowels produced by African American English speakers in the Southern United States. Regional variation in vowel production in African American English is observed. Findings are discussed with respect to the Southern Vowel Shift, the hypothesized African American vowel system and a proposed omniscient observer effect that directly impacts vowel production in African American English speakers in the United States. The impact of these findings is discussed for their importance in the analysis of theories of speech production and communication. 
3pSC32. Bermudian English: An acoustic analysis of vowel properties with implications for sociophonetic variation. Nicole Holliday (Linguist and Cognit. Sci., Pomona College, Pomona College, Edmunds 185, 185 E Sixth St., Claremont, CA 91711, nicole.holliday@pomona.edu)

Bermudian English is a variety of interest due to its distinctiveness from most varieties of British and American English, but to date, few studies have documented the properties of the dialect's vocalic system (Ayers 1933). This study provides an initial description of the vowel systems of Bermudian English speakers, especially as compared to the systems of speakers of Mainstream U.S. English (MUSE). The study was conducted with seven native Bermudian speakers between the ages of 18 and 21. Subjects participated in a word list task as well as a picture task to elicit naturalistic speech. Results of regression analyses comparing F1, F2, and F3 values of Bermudian speakers with formant values of Mainstream U.S. English speakers from Hillenbrand (1995). indicate that Bermudian speakers differ from MUSE speakers in several striking ways. Bermudian speakers produce significantly fronter /u/ and /o/, and lower /i/ than MUSE speakers. Bermudian speakers also show a near complete merger of $/ \varepsilon /$ and $/ a e /$, though speakers also show some variation between productions in the word list vs. picture tasks, indicating a potential casual/careful distinction. Male Bermduian speakers also show a merger of /i.t/ and / $\varepsilon \mathrm{I} /$ which is a stigmatized production according to speakers, though this does not occur for female speakers. The differences between casual/careful speech in front vowels and the gender differences in the production of prerhotic vowels provide valuable directions for future sociolinguistic and phonetic work on the variety.

3pSC33. Pre-nasal short-a in Northern California: Merged in formant space with $/ \varepsilon /$, but distinct in duration and degree of nasal coarticulation. Georgia Zellou and Renee Kemp (Linguist, UC Davis, 469 Kerr Hall, Davis, CA 95616, gzellou@ucdavis.edu)

Short-a, or /æ/, production varies substantially across American English dialects and is noted to be useful in describing regional pronunciation differences. The current study examines the production and perception of /æ/ in California English, which raises in words with a final nasal coda (both velar and non-velar nasals). We explore both the acoustic characteristics of this potential merger with $/ \varepsilon /$, along with lexical confusability between words with $/ \mathfrak{x} /$ and $/ \varepsilon /$ in prenasal position. Productions of 32 native California speakers reveal that the formant space of prenasal/æ/ indeed overlaps with / $\varepsilon /$ However, these vowels have reliably different patterns of two secondary acoustic features: duration and nasal coarticulation. /æ/ is produced with both a longer duration and a greater degree of nasal coarticulation than $/ \varepsilon /$. Results from a lexical identification task presenting unaltered, as well as duration-neutralized, items to 50 listeners reveal that these vowels are not confusable despite considerable formant overlap. Furthermore, modeling results indicate that greater differences in nasalization between prenasal /æ/-/ع/ pairs lead to more accurate identification. Overall, the results from this study indicate that these secondary features help maintain the perceptual distinctiveness of $/ æ /-/ \varepsilon /$ words where formant merger has taken place.

3pSC34. Understanding the relationship between acoustics and articulation of nasal and oral vowels. Marissa Barlaz, Sarah Johnson, Ryan Shosted (Linguist, Univ. of Illinois at Urbana-Champaign, 707 S. Mathews Ave., MC 168, Urbana, IL 61820, goldrch2@illinois.edu), Christopher Carignan (Western Sydney Univ., Sydney, NSW, Australia), Maojing Fu, ZhiPei Liang, and Brad Sutton (Beckman Inst., Univ. of Illinois at UrbanaChampaign, Urbana, IL)

While real-time magnetic resonance imaging (rt-MRI) provides high spatiotemporal resolution for speech research, the associated audio is noisy, presenting a challenge for research on the relationship between articulation and the acoustic signal and solving the articulatory inversion problem. Using state-of-the-art denoising methods, the current study denoised rt-MRI audio associated with nasal and oral French vowels produced by one speaker, and extracted F1-3 from the midpoints of each vowel for /a, o, e/ and their nasal counterparts. Oblique images were taken of the velopharyngeal port at 25 frames/second, and average pixel intensity (API) in the velopharyngeal region was taken from images corresponding to the vowel midpoint. General additive models showed a significant relationship between API and F1 for oral and nasal vowels. (Lower API indicates a wider velopharyngeal opening.) F1 of /ã/ was lower than /a/, while F1 was higher in the nasal /o/ and /ẽ/than their oral counterparts, all of which are expected effects of naso-pharyngeal coupling. These results show that the acoustic recordings produced during rt-MR imaging can be used to explore the relationship between articulation and acoustics, and can give insight into the articulatory effects of nasality, something difficult to understand through acoustics alone.

3pSC35. The role of visual speech cues in sound change: A study of the cot-caught contrast among Michigan speakers. Jonathan Havenhill (Linguist, Georgetown Univ., 3700 O St. NW, Washington, DC 20057, jeh241@georgetown.edu) and Youngah Do (The Univ. of Hong Kong, Hong Kong, Hong Kong)

Interspeaker articulatory variation has been argued to be a driving force in sound change (Baker et al. 2011), yet the factors governing such variation are not entirely understood. Fronted $/ \mathrm{s} /$ in the Northern Cities Shift is characterized by an increase in F2, and can be produced through either tonguefronting or lip-unrounding (Havenhill 2015), while fronted /u/ in British English is achieved through tongue-fronting alone (Harrington et al. 2011). We investigate the hypothesis that visual speech cues restrict the ways in which articulatory patterns may vary between speakers. Participants were exposed to congruous and incongruous audiovisual nonce word stimuli containing /a/ and $/ \mathrm{s} /$, produced by a Michigan speaker. The perceived vowel was identified by selecting a rhyming English word (e.g., the choices for [zot] were "cot" and "caught"). When paired with visual lip-rounding cues, participants perceived auditory / $/ \mathrm{d} / \mathrm{as} / \mathrm{\rho}$. Yet the same auditory stimulus presented with unround lips was perceived as /a/, suggesting that the visual cue may be sufficient to maintain a contrast, despite acoustic similarity. However, conditions are favorable for merger when speakers produce both / $\mathrm{a} /$ and $/ \mathrm{o} /$ with unround lips. As a result, visibly distinct articulatory variants may be preferred when pressure to maintain a contrast is high.

3pSC36. Mapping vowel categories at high fundamental frequencies using multidimensional scaling of cochlea-scaled spectra. Daniel Friedrichs, Stuart Rosen, Paul Iverson (Dept. of Speech, Hearing and Phonetic Sci., Univ. College London, 2 Wakefield St., London WC1N 1PF, United Kingdom, daniel.friedrichs@gmail.com), Dieter Maurer (Inst. for the Performing Arts and Film, Zurich Univ. of the Arts, Zurich, Switzerland), and Volker Dellwo (Phonet. Lab., Univ. of Zurich, Zurich, Switzerland)

Recent studies have shown that accurate vowel category perception can be maintained at fundamental frequencies $\left(f_{\mathrm{o}}\right)$ up to at least $880 \mathrm{~Hz}$. In such cases, the typical first formant frequency $\left(F_{1}\right)$ of most vowels is exceeded by $f_{\mathrm{o}}$ and the vocal tract transfer function is to a high degree undersampled. It seems therefore unlikely that common formant patterns are the main acoustic features used by listeners to recognize vowels at high $f_{\mathrm{o}}$ s. Here, we present results from multidimensional scaling (MDS) analyses calculated by averaging cochlea-scaled spectra across multiple steady-state vowels $(\mathrm{N}=$ 324; all $250 \mathrm{~ms}$ ) produced by a female native German speaker at nine $f_{\mathrm{o}} \mathrm{s}$ within a range of 220-880 Hz. All vowels (/i, y, e, ø, ع, a, o, u/) were recognized accurately by listeners $(\mathrm{N}=20)$ in a previous study [Friedrichs et al., The phonological function of vowels is maintained at fundamental frequencies up to $880 \mathrm{~Hz}$, J. Acoust. Soc. Am. 138, EL36-EL42 (2015)]. MDS reveals that with increasing $f_{\mathrm{o}}$, the vowel height dimension partially collapses, but the front/back distinction expands, thus allowing the vowels to be distinguished. This indicates that the perceptual space is reorganized and vowel height and frontness are being combined in a correlated way at higher $f_{\mathrm{o}} \mathrm{s}$.

3pSC37. Stability of the main resonance frequency of fricatives despite changes in the first spectral moment. Christine H. Shadle, Wei-rong Chen, and D. H. Whalen (Haskins Labs., 300 George St., New Haven, CT 06511, shadle@haskins.yale.edu)

Spectral moments have been taken as the primary measurements of fricatives, but resonances are evident as well. To contrast the two, the X-ray Microbeam Database (XRMB) was used to investigate acoustic and articulatory behavior in [s] for $10 / \mathrm{sVd} /$ words and $9 / \mathrm{sCV}^{*} /$ words for 24 subjects as in a previous study [Iskarous et al., JASA 129:2, 2011]. Spectral 
parameters were adapted from analysis of an acoustic corpus of adolescents' [s] production [Koenig et al., JSLHR 56, Aug. 2013]. The time series of the frequency of the main resonance $\left(\mathrm{F}_{\mathrm{M}}\right)$ and the first spectral moment (M1) were compared to the constriction location estimated from the most anterior tongue pellet $\mathrm{T} 1 . \mathrm{F}_{\mathrm{M}}$ quickly settles into a constant value largely determined by the position of $\mathrm{T} 1$; vowel context affects $\mathrm{F}_{\mathrm{M}}$ throughout [s] for some subjects. M1 however rises and then falls during [s], matching jaw-raising behavior (as estimated by the y-component of the jaw pellet) and correlated with an estimate of high-frequency energy. The results show that some aspects of the fricative acoustics behave differently over time and by context. It remains to be seen what the perceptual consequences of these differences are. [Work supported by NIH NIDCD-DC-002717.]

3pSC38. Acoustics of speech, articulatory compensation, and dental overjet in Cantonese. Lauretta Cheng, Murray Schellenberg, and Bryan Gick (Linguist, Univ. of Br. Columbia, 2613 West Mall, Vancouver, BC V6T 1Z4, Canada, lauretta.cheng@alumni.ubc.ca)

Studies relating dental anomalies to misarticulations have noted that potential correlations appear to be obscured by articulatory compensation. Accommodation of tongue or mandible positions can help even individuals with severe malocclusion approximate perceptually typical speech [Johnson and Sandy, Angle Orthod. 69, 306-310 (1999)]. However, associations between malocclusion and articulation could surface if examined with acoustic analysis. The present study investigates the acoustic correlates of Cantonese speech as it relates to degree of overjet (horizontal overlap of upper and lower incisors). Production data was collected from native Cantonese-speaking adults, targeting the vowels $/ \mathrm{i}, \mathrm{u}, \mathrm{a} /$, and fricatives /f, s, ts, $\mathrm{ts}^{\mathrm{h}} /$, previously found to be vulnerable phonemes in Cantonese speakers with dentofacial abnormalities [Whitehill et al., J Med Speech Lang Pathol. 9, 177-190 (2001)]. Measures of dental overjet and language background were included as well. Preliminary results from trained listeners show that productions were perceptually typical. Acoustic analysis consisted of spectral moments for fricatives and formant values for vowels. The results improve our understanding of the relationship between malocclusion, compensation and speech production in non-clinical populations.

3pSC39. Reduction of intervocalic coronal stops in colloquial words, formal words and pseudowords in American English: A large-scale production study. Vsevolod Kapatsinski (Dept. of Linguist, Univ. of Oregon, 1290 University of Oregon, Eugene, OR 97403, vkapatsi@uoregon.edu)

Words occurring in reduction-favoring contexts have been hypothesized to become associated with reduced sound variants, reducing more than other words even when the contexts disfavors reduction. In a controlled production study, this paper investigates both this hypothesis and its converse: that words occurring often in reduction-disfavoring contexts become associated with non-reduced sound variants, compared to pseudowords. 130 speakers of American English were recorded reading sentences. Interspersed among fillers, designed to prime either formal or colloquial style, were sentences containing intervocalic $/ \mathrm{t} /$ and $/ \mathrm{d} /$ in the flapping environment. These sentences formed triplets placing different words in the same context, e.g., "She is looking for the \{butter; jitter; witter\}." Words expected to disfavor reduction were overattested in formal registers of the British National Corpus. Those expected to favor it were overattested in American movie subtitles, compared to British ones, and overattested in colloquial registers. Colloquial words exhibited significantly more flap and approximant realizations of the / $\mathrm{t} /$ and /d/ than either formal words or pseudowords. Furthermore, within sound variant (flap vs. stop vs. approximant), colloquial words exhibited shorter closure durations, even with local speech rate controlled. However, pseudowords behaved like formal words, suggesting that full pronunciations are used by default, despite their vanishingly low frequency.

3pSC40. Coronal stop deletion in Hawai'i English. Kavon Hooshiar, Katie Drager, and Cassidy Copeland (Linguist, Univ. of Hawai'i at Mānoa, 1890 East-West Rd., UH 569 Moore, Honolulu, HI 96822, kavon@hawaii. edu)

Coronal Stop Deletion (CSD) is believed to be constrained by the phonological environment and the word's morphological make-up (i.e., monomorphemic, semi-weak past tense, regular past tense) (Guy 1991; Hazen 2011). In this talk, we present results from an analysis of CSD in the speech of four speakers of Hawai'i English. In addition to using a traditional approach (i.e., auditory analysis of CSD in word-final position), we also analyzed CSD in non-final position (e.g., followed by plural -s) and conducted acoustic phonetic analysis of $/ t /$ in tokens where it was realized. The results demonstrate highly significant effects of environment. Additionally, there is no effect of morpheme type; instead, the results provide evidence that deletion is favored in words with a greater number of morphemes, regardless of whether the coronal stop is found in the stem or the affix. Additionally, the acoustic analysis suggests that the effect of following environment is acoustically gradient: tokens followed by a voiceless, non-alveolar consonant (i.e., the environment that most strongly disfavors deletion in our data) are most likely to be realized with complete occlusion in the vocal tract. Implications for models of speech production are discussed.

3pSC41. Temporal structure of repetition disfluencies in American English. Zara Harmon and Vsevolod Kapatsinski (Dept. of Linguist, Univ. of Oregon, 1290 University of Oregon, Eugene, OR 97403, zforough@uoregon.edu)

A repetition disfluency involves an interruption in the flow of speech followed by a restart, leading to repetition of one or more words. We analyzed the temporal structure of one-word, two-word, and three-word repetition disfluencies in the Switchboard Corpus $\left(\mathrm{n}_{\text {one-word }}=30546, \mathrm{n}_{\text {two-word }}=8102\right.$, $\mathrm{n}_{\text {three-word }}=1606$ ). Comparing durations of words preceding an interruption point to their repeated counterparts, we find that repetition is typically accompanied by prolongation, which mainly influences the last word preceding the interruption point. When prolongation does not provide enough time for planning upcoming speech-as there seems to be a temporal limit to prolongation - the speaker repeats parts of the utterance just produced. Our results demonstrate that the number of words repeated is determined both by word duration and by co-occurrence relations between words. Mixed effects logistic regression analysis revealed that longer words are less likely to be repeated $(z=-24.45, p<.0001)$. However, the number of words repeated does not reduce to duration: controlling for word duration, the more frequent a two-word sequence, the less likely it is to be interrupted by a restart $(z=35.43, p<.0001)$. Longer repetitions are produced when a shorter repetition would result in restarting speech production from the middle of a cohesive word sequence.

3pSC42. Inferring velum movements from acoustic features. Hong Zhang (Linguist, Univ. of Pennsylvania, 619 Williams Hall, 255 S 36th St., Philadelphia, PA 19104, zhangho@sas.upenn.edu)

Although acoustical methods have been widely used in the literature on nasality, a direct link between the acoustic features and velum movements during speech production is yet to be established. In this study, we propose a model through which the vertical movements of the velum are inferred from an acoustic feature set. An X-ray Microbeam data set collected at University of Tokyo is used for the modeling. The data recorded the vertical movements of the velum of 11 American English speakers saying both isolated words and sentences. Velum positions are traced by a metal pallet placed on top of the velum. $40 \mathrm{MFCC}$ (Mel-frequency Cepstral Coefficient) features are extracted from the accompanying acoustic signal at each time frame. MFCC features of five frames to both sides of the current frame, together with the current frame, consist of the 440-dimensional feature vector for predicting the velum position of the current frame. The feature vectors are then fitted to a lasso regression model. The inferred velum movements in the validation set are a good fit to the actual observation as judged by the high accuracy in identifying locations of peaks and valleys, and predicting the overall contour shape of the velum trajectory.

3pSC43. Speech adaptation to kinematic recording sensors. Christopher Dromey and Elise Hunter (Commun. Disord., Brigham Young Univ., 133 TLRB, BYU, Provo, UT 84602, dromey@byu.edu)

Measuring articulator movements can yield valuable insights into speech motor control, but attaching sensors to the articulators can affect speech. In order to measure the process of adaptation to the presence of kinematic 
sensors, 20 native English speakers read a sentence before the attachment of sensors to the tongue, lips, and jaw. They read it immediately afterwards, and then 5,10, 15, and 20 minutes later. Participants read aloud continuously between recordings to encourage adaptation. Audio recordings were rated by 20 native English listeners using a visual analog scale with the endpoints labeled as precise and imprecise. Acoustic analysis involved segmenting the fricatives $/ \mathrm{s} /$ and $/ \mathrm{J} /$ from the longer recording and computing spectral center of gravity and spectral standard deviation as well as fricative duration. Results of both perceptual and acoustic analysis revealed a change in speech precision over time, with all post attachment recordings receiving reduced precision ratings. Spectral mean decreased and standard deviation increased following sensor attachment; durations decreased over time. Precision ratings beyond the ten minute recording remained steady. The results show that speakers reached a height of adaptation after 10 minutes but did not at any point return to pre attachment precision levels.

3pSC44. Effect of tongue position in the simplified vocal tract model of sibilant fricatives $/ \mathrm{s} /$ and $/ \mathrm{f} /$. Tsukasa Yoshinaga (Graduate School of Eng. Sci., Osaka Univ., 1-3 Machikaneyama, Toyonaka, Osaka 560-8531, Japan, t.yoshinaga@biomech.me.es.osaka-u.ac.jp), Kazunori Nozaki (Div. of Medical Information, Osaka Univ. Dental Hospital, Suita, Osaka, Japan), and Shigeo Wada (Graduate School of Eng. Sci., Osaka Univ., Toyonaka, Osaka, Japan)

In this study, a simplified vocal tract model which reproduces both sibilant fricatives $/ \mathrm{s} /$ and $/ \mathrm{J} /$ is proposed to investigate the effect of tongue position on flow and sound generation in the vocal tract. The simplified model consists of a tongue with sibilant groove and upper and lower teeth in a rectangular channel. Dimensions of the model were determined based on CT images of the subject pronouncing sibilant /s/. The sound and flow fluctuation generated by the model were measured with a microphone and a hotwire anemometer, respectively. In addition, the large-eddy simulation was conducted to reveal the characteristics of sound source generated by the turbulent flow in the vocal tract. The acoustic measurement showed that the backward shift of the tongue increased the spectral slope value, indicating that the acoustic characteristics were changed from $/ \mathrm{s} /$ to $/ \mathrm{J} /$. The large-eddy simulation showed that the position of sound source downstream of teeth was shifted backward and the magnitude of the sound source was decreased by shifting the tongue backward. These results indicate that both the resonance characteristics and the position of the sound source were changed by shifting the tongue which causes the difference in acoustic characteristics between $/ \mathrm{s} /$ and $/ \mathrm{J} /$.

3pSC45. Tongue root positioning in English voiced obstruents: Effects of manner and vowel context. Suzy Ahn and Lisa Davidson (NYU Dept. of Linguist, New York Univ., 10 Washington Pl., New York, NY 10003, suzy.ahn@nyu.edu)

Previous research on utterance-initial voiced stops in American English (AE) has shown that speakers enlarge the vocal cavity via tongue root advancement whether or not the stop is phonated when compared to voiceless stops (Ahn 2015). The current ultrasound study expands this line of research to examine two further variables: (a) utterance-initial fricatives/ affricates and (b) effect of frontness of the following vowel. Participants included both monolingual $(n=4)$ and simultaneous bilingual $(n=7) \mathrm{AE}$ speakers. Phrase-initial stops (/p,b,t,d,k,g/), fricatives (/f,v,s,z/), and affricates $\left(/ \mathrm{t} \int, \mathrm{d} /\right)$ were followed by /e/ or $/ \mathrm{u} /$. Most productions confirmed that both phonated and unphonated voiced stops/affricates had more tongue root advancement than voiceless ones, but a small proportion showed no difference between phonated, unphonated, and voiceless stops/affricates. Fricative productions were divided between no tongue root difference due to either voicing or phonation, and greater advancement for voiced fricatives regardless of phonation. Tongue root advancement may be less prevalent for fricatives because weakening of the frication can also facilitate the conditions for phonation. A prediction that tongue root advancement for voicing before /e/ might be limited by the positioning requirements of the front vowel was weakly supported, especially for fricatives. The potential effects of bilingual speakers are also addressed.
3pSC46. An ultrasound study of tongue position during Hindi laryngeal contrasts. Suzy Ahn (Dept. of Linguist, New York Univ., 10 Washington Pl., New York, NY 10003, suzy.ahn@nyu.edu)

Initiating or maintaining phonation during stop closure involves several adjustments, including tongue root advancement to enlarge oral cavity volume (Westbury 1983). Ultrasound imaging shows that in English, the tongue is more advanced for phonologically voiced stops, whether phonated or unphonated (Ahn 2015). The current study uses ultrasound to examine tongue positioning during Hindi stops. Hindi has a unique four-way laryngeal contrast: voiced, murmured, voiceless unaspirated, and voiceless aspirated. Eight native Hindi speakers recorded phrase-initial stops at three places of articulation (labial, dental, velar) followed by the low vowel /a/. Results show a clear distinction in tongue position between voiced and voiceless unaspirated/aspirated stops. The tongue root is advanced for voiced stops in comparison to voiceless stops. However, there is no difference in tongue root position between voiceless unaspirated and aspirated stops. Murmured stops showed variation among speakers in comparison to other stops, while the majority of speakers show more advanced tongue root compared to voiceless stops. The results suggest that tongue root advancement facilitates phonation in Hindi. Thus, in Hindi, tongue root position corresponds to a phonological distinction in phonation most notably between voiced unaspirated stops and voiceless stops, whereas in English, tongue root position reflects a more abstract phonological distinction in voicing that does not correspond to phonation.

3pSC47. Articulatory and acoustic realization of French and German /R/. Cedric Gendrot, Barbara Kuhnert, and Didier Demolin (Linguist, Université Sorbonne Nouvelle, Lab. of Phonet. and Phonology, 19 rue des bernardins, Paris 75005, France, cgendrot@univ-paris3.fr)

French uvular / $\mathrm{\zeta} /$ is usually considered as problematic due to its variability, especially in positions such as word initial and word final. In this study, an articulatory (EMA) and aerodynamic experiment allowed us to determine its major axes of variation. We show that the degree of constriction between the tongue and the palate is related to the voicing of the consonant and we validate the use of a harmonic-to-noise ratio for the measurement of variation of $/ \mathrm{B} /$. Aerodynamic data also show that the variation of Nasal Airflow is not significant but a ratio between Oral Airflow and Intra-Oral Pressure significantly varies according to the different realizations of $/ \mathrm{B} /$. Subglottic Pressure only plays a significant role in specific cases. These results allow us to propose a new acoustic measurement for the variation in uvular/b/ production. An acoustic study is then presented on large corpora of continuous speech, so as to test the variability of French / $\mathrm{b} /$ in terms of the aforementioned results, and we show the importance of prosodic factors. Finally, a parallel with a German similar corpus of articulatory and acoustic data is drawn, we suggest that German /в/ shows less variation than French / $\mathrm{B} /$ due to phonological reasons.

3pSC48. Articulatory setting as global coarticulation: Simulation, acoustics, and perception. Bryan Gick, Chenhao Chiu (Linguist, Univ. of Br. Columbia, 2613 West Mall, Vancouver, BC V6T1Z4, Canada, gick@ mail.ubc.ca), Francois Roewer-Despres (Comput. Sci., Univ. of SK, Saskatoon, SK, Canada), Murray Schellenberg (Linguist, Univ. of Br. Columbia, Vancouver, BC, Canada), and Ian Stavness (Comput. Sci., Univ. of SK, Saskatoon, SK, Canada)

Articulatory settings, language-specific default postures of the speech articulators, have been difficult to distinguish from segmental speech content [see Gick et al. 2004, Phonetica 61, 220-233]. The simplest construal of articulatory setting is as a constantly maintained set of tonic muscle activations that coarticulates globally with all segmental content. In his early Overlapping Innervation Wave theory, Joos [1948, Language Monogr. 23] postulated that all coarticulation can be understood as simple overlap, or superposition [Bizzi et al. 1991, Science 253, 287-291], of muscle activation patterns. The present paper describes an implementation of Joos' proposals within a modular neuromuscular framework [see Gick \& Stavness 2013, Front. Psych. 4, 977]. Results of a simulation and perception study will be reported in which muscle activations corresponding to English-like and French-like articulatory settings are simulated and superposed on activations for language-neutral vowels using the ArtiSynth biomechanical modeling 
toolset (www.artisynth.org). Simulated visible and acoustic outputs presented to perceivers familiar with both languages speak to the question of whether overlapping muscle activations generate outputs that look and sound language-appropriate to perceivers, testing a unified, context-independent model for both coarticulation and articulatory setting. [Research funded by NIH Grant DC-02717 and NSERC.]

3pSC49. Articulatory settings of English-French bilinguals reanalyzed by SS-ANOVA. Yuki Iguro, Ian Wilson, and Julian Villegas (Univ. of Aizu, Tsuruga, Ikki-machi, Aizuwakamatsu, Fukushima 965-8580, Japan, s1200249@gmail.com)

To improve the skill of speaking a second language (L2), one good way may be to be aware of the underlying tongue position for a language. We focused on such underlying position differences between English and French, particularly when pausing for a short time between speaking; something called inter-speech posture (ISP). In past research, Wilson and Gick investigated ISP between English and French spoken by bilinguals. In that research, bilinguals had distinct articulatory settings for each language, mostly in the lips. However, their tongue data was for only 4 points of articulatory settings: distance from the ultrasound probe to tongue root, tongue dorsum, tongue body, and tongue tip, but not overall shape. Furthermore, to measure tongue tip position, past research relied on the alveolar ridge, which is unclear to see: possibly making the results inaccurate for tongue tip. In this study, we analyzed the whole shape of the tongue and made models of them using SS-ANOVA in R so that we could compare the difference from past research using a different measurement method. Our results showed that bilinguals who are perceived as native in both languages have a different ISP in the posterior half of the tongue.

3pSC50. F3 variability in allophones of /1/: Acoustic-articulatory relations. Anisia Popescu (Univ. Paris Diderot, 54 Ave. d'Italie, Paris 75013, France, anisiapopescu358@gmail.com)

F3 is know to exhibit higher values in darker varieties of /1/. This contrast has been attributed to differences in closure fronting and front cavity configuration. In this paper we propose a possible alternative explanation for higher F3 in dark /1/, based on sensitivity functions. Both allophones have an apical gesture, but differ in their tongue dorsum gestures: clear /1/ has a raising and fronting gesture, while dark /1/ has postdorsum retraction towards the uvular region. We propose that the gestural composition of dark /1/ creates two constriction sites in the vocal tract that correspond to the two maxima of the F3 sensitivity function, thus resulting in higher values for dark /1/. To address these predictions we analyzed both acoustic and articulatory data from 6 speakers of the Wisconsin XRMB database. Articulatory strategies vary both inter and intra-speakers. High F3 values are obtained with both elevated tongue tip and retracted postdorsum, while low F3 values, have small values of postdorsum retraction. Overall results show that, despite inter-speaker variability in vocal tract shape and articulatory strategies, F3 is positively correlated with tongue dorsum retraction.

3pSC51. Gestural coordination and blending among liquid consonants and vowels in American English. Rachel Walker (Dept. of Linguist, Univ. of Southern California, GFS 301, 3601 Watt Way, USC, Los Angeles, CA 90089-1693, rwalker@usc.edu) and Michael Proctor (Dept. of Linguist, Macquarie Univ., Sydney, NSW, Australia)

In General American English (GAE), tense/lax vowel contrasts occur before syllables ending in /l/ (e.g., peel, pill), but not those ending in / $\mathrm{I} /$ (e.g., peer; no $[\mathrm{i}] /[\mathrm{I}]$ contrast). No such restrictions occur after a syllable-initial liquid consonant (e.g., lip, leap, rip, reap). This study investigates the hypothesis that these phonotactic asymmetries arise from the interaction of differences in gestural coordination between nuclear vowels and liquid consonants in syllable onset versus coda position (Browman \& Goldstein 1995) and differences in blending parameter strength for GAE laterals versus rhotics. A study of speech production by four GAE speakers was conducted using real-time Magnetic Resonance Imaging (Narayanan et al. 2004). Liquid consonants were produced in various vocalic environments and syllable positions (syllable-final, syllable-initial: word-initial and intervocalic). Analysis using a center of gravity metric defined by midsagittal lingual outlines points to greater articulatory stability in rhotics versus laterals across different vocalic contexts and less coarticulatory displacement of pre-lateral vowels than pre-rhotic vowels. Further analysis of spatial and temporal effects in the data will be used to test hypotheses about coordination of vowel and liquid consonant gestures in different syllable positions and their relation to phonotactic patterns. [Research supported by NIH and ARC.]

3pSC52. Revisiting articulatory positions of Japanese vowels as a function of duration on the basis of analysis of large-scale speech corpora. Takayuki Kagomiya (Ctr. for Frontier Medical Eng., Chiba Univ., 1-33, Yayoicho, Chiba, Chiba 263-8522, Japan, kagomiya@chiba-u.jp)

The purpose of this study is to illustrate certain features of the place of articulation of Japanese vowels on the basis of formant frequency. For this purpose, two types of relationship between vowel duration and formant frequency were examined. The first of these relationships was the correlation between speaking rate and formant frequency. Speech materials are obtained from two large-scale speech corpora: Electrotechnical Laboratory Spoken Word database (ETL-WD) and The corpus of spontaneous Japanese (CSJ). The results of analyses showed that as speaking rate slows, articulations get more distinct: open vowels increase in openness, close vowels increase in closeness, front vowels increase in frontness, and back vowels increase in backness. The second relationship was between formant frequencies and vowel length. The result of this analysis showed that place of articulation is more distinct in long vowels than in short vowels. The exception was the back vowel /u/, all of which did not show greater backness in long vowels. This result supports previous arguments that Japanese / $\mathrm{u} /$ is not a typical back vowel.

3pSC53. Articulatory movement in consonant clusters: A contrastive study of Japanese and English. Seiya Funatsu (Prefectural Univ. of Hiroshima, 1-1-71 Ujinahigashi Minami-ku, Hiroshima 734-8558, Japan, funatsu@pu-hiroshima.ac.jp) and Masako Fujimoto (Waseda Univ., Tokyo, Japan)

We investigated the articulation of consonant clusters with Japanese and English speakers using the WAVE system (NDI Corp.). Until now we measured the displacement of tongue tip only, but in this study we also measured the displacement of the mandible. The subjects were three Japanese and two English speakers. The speech samples were 4 words: blat, bnat, plat, pnat. All these second consonants, $/ \mathrm{l}, \mathrm{n} /$ are articulated with tongue tip placed behind alveolar ridge. These words were embedded in a carrier sentence, "Say X.," and these sentences were uttered 5 times each in random order. The articulation time from first consonants, /b/, /p/, to second consonants, /l/ , /n/, were measured. Specifically, the time from the highest point of the mandible to that of the tongue tip was measured. Overall average time was $131.2 \mathrm{~ms}$ (Japanese) and $48.1 \mathrm{~ms}$ (English). Two-way ANOVA (Language vs. Cluster) showed a significant main effect in Language $(\mathrm{p}<0.0001)$. This result suggested that for English speakers, the first and second consonants are strongly co-articulated, while in Japanese speakers, they are not coarticulated or weakly co-articulated. The measurement of mandibular displacement more clearly showed the difference of the strength of co-articulation between Japanese and English.

3pSC54. Co-articulation of unreleased final stops to preceding vowels in Cantonese: An ultrasound study. Suki Yiu (Linguist, Univ. of Hong Kong, School of Humanities (Linguistics), University of Hong Kong, Pokfulam Rd., Hong Kong, Hong Kong, syutji@hku.hk)

Unreleased final stops are more susceptible to reduction than their released counterparts, thus co-articulatory information on the preceding vowel is important to signal place contrasts of post-vocalic stops. This ultrasound study examines the gestural co-ordination of a VC sequence of monosyllabic words in Cantonese, a language which has preserved phonemic contrasts of six unreleased final stops $/ \mathrm{k}, \mathrm{t}, \mathrm{p}, \mathrm{ng}, \mathrm{n}, \mathrm{m} /$ to form a VC sequence with three vowels /a, $\mathrm{e}, \mathrm{\jmath} / \mathrm{We}$ expect properties of /t, k/ to be (i) only immediately present at the vowel offsets (i.e. minimal co-articulation), or (ii) present from vowel midpoints to offsets (i.e. gradual co-articulation). If the $/ \mathrm{t}, \mathrm{k} /$ contrast is (iii) consistently present throughout the vowel from 
onset to offset, it indicates phonologization of the $/ \mathrm{t}, \mathrm{k} /$ contrast from the stops to the preceding vowels. Preliminary SS ANOVA results show that the lingual contours at vowel midpoints have early lingual raising into final stop constrictions, and the vowel offsets look more like the upcoming stops than the preceding vowels. This agrees with Khouw \& Ciocca's 2006 acoustic study, that unreleased final stops use $\mathrm{C}$-to- $\mathrm{V}$ co-articulation to strengthen the place contrast.

3pSC55. Source properties of dorsal fricatives. Charles Redmon and Allard Jongman (Linguist, Univ. of Kansas, 1541 Lilac Ln., Rm. 427, Lawrence, KS 66046, redmon@ku.edu)

Present models of voiceless fricative acoustics assume excitation of the vocal cavity anterior to a narrow constriction by an aperiodic source generated from turbulence within that constriction (or in the case of dipole sources from interaction of the jet with an obstacle downstream). This model has been applied successfully to anterior places of articulation (labial to palatal) but for dorsal fricatives, i.e., velar $/ \mathrm{x} /$ and uvular $|\chi|$, there is evidence to suggest the assumption of a line source may not hold. Rather, preliminary data from our lab and from studies such as Zeroual (ICPhS, 2003) and Shosted \& Chikovani (JIPA, 2006) indicates intermittent presence of a mixed source due to passive vibration of the uvula, yet no study to date has directly addressed this element of the sound source in dorsal fricative production. Acoustic and aerodynamic data on dorsal fricatives produced by four speakers each of Arabic, Persian, and Spanish are presented to validate the nature of the dorsal fricative source component and the degree of crosslinguistic variation in production of these sounds. Measures of periodicity duration and cycle amplitude/shape are employed to estimate the oscillatory pattern of the uvula and model its contribution to the radiated acoustic signal.

3pSC56. Investigating patterns of vowel production using real-time magnetic resonance imaging. Michael I. Proctor (Linguist, Macquarie Univ., 16 University Ave., NSW 2019, Australia, michael.proctor@mq.edu. au)

Characteristic tongue postures of 21,478 vowels produced by four speakers of General American English were compared in lexically stressed and unstressed positions to examine patterns of articulation and reduction. Image frames capturing midsagittal vocal tract configurations were reconstructed at the acoustic midpoint of each vowel produced in a 3,450 word forced-aligned transcribed speech corpus (Narayanan et al. 2014). Sixty image sets were constructed, consisting of all exemplars of each contrastive vowel quality of American English, as produced by each speaker: up to 1049 tokens of the most frequent vowel /i/, and 48 tokens of the least frequent vowel /oI/. Image frames were cropped to a region of interest centred on the tongue dorsum, and linearized into one-dimensional vectors in which pixel intensity corresponds to presence of soft tissue. Principle components analysis was applied to each set of image vectors to examine the distribution and variability of tongue tissue associated with each vowel posture. "Eigentongues" were constructed from the most significant components in each image set (Sirovich \& Kirby 1987; Hueber et al. 2007), resulting in a set of images characterizing each speakers' vowel space, produced in both lexically stressed and unstressed positions.

3pSC57. Physiological features of geminate depending on the speaking rate. Ken-Ichi Sakakibara (Health Sci. Univ. Hokkaido, 2jo-5chome, Ainosato, Kita-ku, Sapporo 002-8072, Japan, kis@hoku-iryo-u.ac.jp), Kimiko Yamakawa (Shokei Univ., Kumamoto, Japan), Hiroshi Imagawa, Takao Goto (Univ. Tokyo, Tokyo, Japan), Akihito Yamauchi (National Ctr. for Global Health and Medicine, Tokyo, Japan), Katsuhiro Maki, and Shigeaki Amano (Aichi Shukutoku Univ., Nagakute, Japan)

In this study, we examined the physiological and acoustic characteristics of plosive- and fricative-type geminates, three each of minimal pairs for the plosive- and fricative-type geminates were recorded in different speaking rates, with there ranges are from 3 to 13 mora /s. Physiological analysis showed that (1) an opened duration of vocal folds depends on a speaking rate; (2) a closing duration of vocal folds depends on a speaking rate but an opening duration does not. Acoustical analysis showed that, when a speaking rate increases, (1) logarithm of closure and fricative durations linearly decrease; and (2) a ratio of the duration of a singleton to a geminate decreases.

3pSC58. A preliminary acoustic analysis of three-dimensional shape of the human nasal cavity and paranasal sinuses extracted from conebeam CT. Tatsuya Kitamura (Faculty of Intelligence and Informatics, Konan Univ., 8-9-1, Okamoto, Higashinada, Kobe, Hyogo 658-8501, Japan, t-kitamu@konan-u.ac.jp), Hironori Takemoto (Chiba Inst. of Technol., Narashino, Chiba, Japan), Hisanori Makinae (National Res. Inst. of Police Sci., Kashiwa, Chiba, Japan), Tetsutaro Yamaguchi, and Kotaro Maki (Showa Univ., Shinagawa, Tokyo, Japan)

The shape of the nasal cavity and paranasal sinuses is complex and varies between individuals. Because the shape is almost stable during speech, the acoustic properties could constantly provide speaker specific information to speech sounds, that is, speaker individuality. In this preliminary analysis, the shape was extracted from cone-beam CT data for a subject using a machine learning technique and its acoustic properties were examined using finite-difference time-domain simulation. The transfer function from the glottis to the nostrils was calculated and the distribution pattern of the pressure anti-nodes was visualized at frequencies of major spectral peaks and dips. In addition, transfer functions were calculated when each of the paranasal sinuses other than the ethmoidal ones was occluded to identify which sinus caused which dip. As a result, the longitudinal resonance in the right or left half of the nasal cavity generated each peak, while the transverse resonance in the pharyngeal cavity caused the major dips. The right maxillary, sphenoidal, and frontal sinuses generated dips below 1 $\mathrm{kHz}$. The left maxillary sinus and ethmoidal sinuses, however, contributed to generating not only dips but also peaks. [This research was partly supported by JSPS KAKENHI Grant Numbers 15K00263, 25280066, and 25240026.]

3pSC59. Modeling the effect of palate shape on the articulatory-acoustics mapping. Sarah Bakst and Keith Johnson (Linguist, Univ. of California Berkeley, 1203 Dwinelle Hall, Berkeley, CA 94720, bakst@berkeley. edu)

Previous research [Brunner et al. (2009, JASA 125(6), 3936-3949) and Bakst \& Lin (ICPhS 2015)] shows that articulatory variability is reduced for people with flatter palates. Brunner et al. (2009) hypothesized that this is because the mapping between articulation and acoustics is more linear for flatter than for more domed palates; in order to maintain similar levels of acoustic consistency, people with flatter palates must be more articulatorily precise than people with more domed palates. A revised version of the Maeda synthesizer was used to model how vocal tract anatomy influences the mapping of articulation onto acoustics, using American English / $\mathrm{r} / \mathrm{as}$ a test case. A retroflex-able tongue tip was added to the synthesizer. Two additional palate shapes were also added to the synthesizer, and a script was used to search the articulatory-acoustic space for vocal tract configurations that resulted in a low F3 (the hallmark acoustic cue for /r/) for each palate. Palate shape influences not only the overall sensitivity of the articulatoryacoustic mapping, but also the effect of each individual articulatory parameter on F3.

3pSC60. Acoustic effects of phonetic conditions and laryngeal specification on phonation in English voiceless obstruents. Lisa Davidson (Linguist, New York Univ., 10 Washington Pl., New York, NY 10003, lisa. davidson@nyu.edu)

A detailed acoustic analysis of phonation in voiceless obstruents in American English (AE) is presented to investigate potential acoustic outcomes of the laryngeal coordination relationships that have been reported in the literature. The current study examines the realization of phonation in voiceless obstruents in a corpus of read speech (37 AE speakers). Similar to phonation in voiced obstruents (Davidson 2016), linguistic factors such as phrase and word position, stress, and the preceding phoneme condition the presence and degree of phonation during the constriction period of stops and fricatives. The amount of phonation present is further analyzed by characterizing where in the constriction interval phonation appears. Carryover 
phonation (or "bleed") from a preceding sonorant is most common for stops, while a "trough" pattern (phonation that dies out and then begins again before the end of the closure) is more prevalent for fricatives. The linguistic conditioning factors, and the overall lower rate of partial voicing and proportion of phonation in the constriction as compared to voiced obstruents, illustrate the acoustic and aerodynamic consequences of the various laryngeal coordination patterns that have been proposed for English voiceless obstruents in different prosodic environments.

3pSC61. Visuomotor pursuit tracking accuracy for intraoral tongue movement. Vedad Fazel and William F. Katz (Commun. Sci. \& Disord., The Univ. of Texas at Dallas, The University of Texas at Dallas/Callier Ctr., 1966 Inwood Rd., Dallas, TX 75235, vxf130030@utdallas.edu)

Visuomotor pursuit tracking (VMT) tasks are used to study the movement of the articulators in order to better understand the processes involved in speech motor planning and execution. Because most of this research has focused on lip/jaw tracking of sinusoids visually presented on a screen, little is known about the tracking capabilities of the tongue, or for visual targets placed in the oral cavity. The present study used a novel technique for measuring tongue (and jaw) VMT based on a 3D electromagnetic articulography (EMA) system. Streaming EMA data from oral sensors were used to construct a real-time avatar of the tongue which was shown to subjects on a computer monitor. Subjects also viewed a (virtual) intra-oral spherical target that they were required to "hit" with the tongue tip sensor. The target was programmed to move in a sinusoidal direction and was varied in frequency $(\mathrm{Hz})$, direction, and predictability. Preliminary results suggest that tracking accuracy is inversely related to target frequency and is higher for vertical and horizontal motion than for lateral motion. The role of target predictability will also be described.

3pSC62. Performance and function meet structure: A white matter brain connection tuned for motor speech control. John J. Sidtis (Nathan Kline Inst., 6 Prairie Ave., Suffern, NY 10901, john.sidtis@nyu.edu), Muhammad A. Mubeen, Ali Asaei, Babak Ardekani (Nathan Kline Inst., Orangeburg, NY), and Diana Sidtis (New York Univ., Orangeburg, NY)

Despite bilateral brain activation during speech tasks, we have shown that performance is predicted by a blood flow increase in the left inferior frontal region and decrease in the right caudate, consistent with classic lesion studies. This study characterized the structural connections between these brain areas using diffusion tensor imaging and examined their relationships with measures of motor speech control. Probabilistic tractography estimated the connection strength between these two brain structures, both ipsilaterally and contralaterally, in 25 normal subjects. Speech was recorded at a separate evaluation. The majority of fiber connections were ipsilateral, but contralateral connections were also present. The relative connection strength between the right caudate and the left inferior frontal region was significantly associated with acoustic measures of stability for frequency (correlations for the repetition of $/ \mathrm{pa} /=0.46 ; / \mathrm{ta} /=0.5 ; / \mathrm{ka} /=0.46 ; / \mathrm{pataka} /$ $=0.52)$ and amplitude $(/ \mathrm{pa} /=0.43 ; / \mathrm{ta} /=0.5 ; / \mathrm{ka} /=0.49 ; / \mathrm{pataka} /=0.49)$, echoing the predictive value of blood flow in these regions during speech. This was not observed for other connections. These results suggest that white matter connections share functional specialization with the structures they connect in the motor-speech system.

3pSC63. On the link between the ability to reproduce rhythm and reading speed: Effects of visual grouping marks. Antonin Rossier-Bisaillon and Victor Boucher (Linguistique et traduction, Université de Montréal, 3150, rue Jean-Brillant, Montréal, QC H3T 1N8, Canada, antonin.rossierbisaillon@umontreal.ca)

Studies have revealed a link between a deficient ability to reproduce rhythm and dyslexia. Moreover, a normal ability to reproduce rhythm has been shown to correlate with reading speed. Some associate these findings to effects of neural oscillations and a visual parsing of text input (Vidyasagar, 2013). The present study aimed to clarify the properties of the visual stimuli that support a correlation between rhythm reproduction and reading speed. The experiments were partly based on Tierney and Kraus (2014). Thirty participants were asked to reproduce heard regular $(2$ beats/sec) and irregular (1-3 beats/sec) rhythms by tapping on a keyboard. Then, the participants had to read out loud, at a fast rate, visually displayed sequences of words and non-words. The sequences contained either no spaces between items (baseline condition) or spaces marking regular and irregular groups. Mean reading speeds were calculated on accurately decoded sequences. Among the significant findings, strong correlations were observed between mean reading speeds of texts containing spaces and the reproduction of certain rhythm patterns. However, no significant correlations appeared for text where there were no spaces separating words and non-words. This supports the view that the ability to reproduce rhythm can link to a visual parsing.

3pSC64. Post-focus compression: All or nothing? Ting Huang (Dept. of Linguist \& Philosophy, Massachusetts Inst. of Technol., 77 Massachusetts Ave., Cambridge, MA 02139, funting.huang@gmail.com) and Feng-fan Hsieh (Graduate Inst. of Linguist, National Tsing Hua Univ., Hsinchu, Taiwan)

Post-focus compression (PFC) has been claimed to be a "hard-toevolve," inherent prosodic feature that may have a single historical origin (Proto-Nostratic). This study explored the distribution of PFC in two subdialects of Malaysian Hokkien (Southern Min Chinese), i.e., Penang Hokkien (PH) and Melaka Hokkien (MH), using novel experimental designs and statistical techniques (SS ANOVA). Specifically, in addition to the conventional nasal onset-only contexts, stop and fricative onset-only contexts were taken into consideration. We analyzed F0 and duration of the constituents in question under three focus conditions (Initial vs. Medial vs. Final focus in the Subject-Verb-Object frame). The results are (a) the presence of PFC may be dependent on different segmental contexts: in MH, PFC is attested in the stop onset-only environments but absent elsewhere and (b) the absence of PFC "induces" Post-focus shortening in the nasal and fricativeonly contexts in $\mathrm{MH}$ and in all data in $\mathrm{PH}$. The findings suggest that PFC may not be an "inherent" feature because $\mathrm{MH}$ and $\mathrm{PH}$ are closely related sub-dialects and both have had language contact with the same language, Malay. Finally, global F0 raising is found for the first time in Final focus (Object) in both $\mathrm{MH}$ and $\mathrm{PH}$.

3pSC65. Tone sequences in lexical processing of Beijing Mandarin. Isabelle Lin (Dept. of Linguist, Univ. of California Los Angeles, 3125 Campbell Hall, UCLA, Los Angeles, CA 90095-1543, isabellelin@ucla.edu)

Previous research on the role of tone information in lexical access in Mandarin Chinese has focused on monosyllabic words. Using corpus data, we show that tone is more informative on Mandarin disyllables than monosyllables. Next, we investigate the role of tone sequences in disambiguating two segmentally identical disyllabic word candidates that differed only in tone. Using a priming paradigm, we show that tone frequency biases lexical decisions. Listeners were presented with a disyllabic sequence that is tonally ambiguous between two lexical entries. When word frequency and tone frequency did not favour the same candidate, a tonally-matched prime reduced the likelihood of picking the candidate with the matching tone. Preliminary results suggest that listeners are sensitive to the overall likelihood of encountering a given sequence of tones in running speech, instead of just tone sequences within disyllabic words.

3pSC66. Surface phonetic or underlying phonological representations: A mismatch negativity study of Mandarin tone assimilation. $\mathrm{Yu}-\mathrm{Fu}$ Chien (Linguist, Shanghai Int. Studies Univ., Number 550, DaLian West Rd., HongKou District, Shanghai, China, y078c178@ku.edu), Robert Fiorentino, Xiao Yang, and Joan A. Sereno (Linguist, The Univ. of Kansas, Lawrence, KS)

Phonological alternation, in which a sound changes depending on its phonological environment, poses challenges to spoken word recognition models. Mandarin T3 sandhi is such a phenomenon in which a tone 3 changes into a tone 2 when followed by another T3, which raises questions regarding whether the human brain processes the surface acoustic-phonetic representation or the underlying linguistic representation of Mandarin T3 sandhi words. We conducted a mismatch negativity (MMN) study examining this issue. Participants passively listened to a T2 word [tşu2 je4] /tşu2 je4/, a T3 word [țu3 żən4] /tşu3 z.ən4/, a Sandhi word [tşu2 jen3] /tşu3 
jen3/, or a mix of $\mathrm{T} 3$ and sandhi words in an odd-ball paradigm. All were interspersed with a T2 word [tsu2] (deviant). Results showed an MMN only in the T2 and T3 condition but not in the Sandhi or Mix condition. The fact that the surface acoustic information in the $\mathrm{T} 2$ and Sandhi conditions was identical, yet yielding disparate MMN results suggests that Mandarin speakers process T2 and Sandhi words differently. These data provide evidence for a stored linguistic representation, either an underspecified or underlying tone 3 syllable, which must be operative when listeners map the signal during spoken word processing.

3pSC67. Effects of aero-tactile stimuli on continuous speech perception. Donald Derrick, Greg A. O'beirne, Tom De Rybel, Jennifer Hay, and Romain Fiasson (NZILBB, Univ. of Canterbury, Private Bag 4800, Christchurch 8140, New Zealand, donald.derrick@canterbury.ac.nz)

We follow up on research demonstrating that aerotactile information can enhance accurate identification of stop- and fricative-onset syllables in twoway forced-choice experiments (Derrick, et al., 2014) to include open-set identification tasks. We recorded audio and speech airflow simultaneously from the lips of two New Zealand English (NZE) speakers (one female, one male), and used these recordings to produce an auditory/aero-tactile matrix sentence test. The airflow signal is used to drive a piezoelectric air pump that delivers airflow to the right temple simultaneously with presentation of noise-degraded auditory recordings. Participants (including native NZE speakers with and without hearing impairment, and normal-hearing native non-NZE and non-native English speakers) listen to and repeat 5-word sentences presented in noise with and without simultaneous airflow. Their open-set responses are scored by the researchers. Custom-written software identifies the SNRs for $20 \%$ and $80 \%$ word identification accuracy using a dual-track adaptive algorithm, and these data are fitted to psychometric curves relating SNR to speech intelligibility. Psychometric curves for airflow and no-airflow conditions will be compared in order to identify the influence of airflow on continuous speech perception. Data collection is in progress, and the results will be presented at the ASA conference.

3pSC68. Just noticeable differences for pitch height and pitch contour for Chinese and American listeners. Allard Jongman, Zhen Qin, Jie Zhang, and Joan Sereno (Linguist, Univ. of Kansas, 1541 Lilac Ln., Lawrence, KS 66045, jongman@ku.edu)

Previous studies suggest that Chinese listeners may be more sensitive to pitch direction while American listeners primarily attend to pitch height. The present study sought to establish JNDs for pitch discrimination using a three-interval, forced-choice procedure with a two-down, one-up staircase design (cf. Liu, JASA 2013). We manipulated a high rising and a high falling Mandarin tone produced by a female speaker. Specifically, for pitch height, a standard contour rising from $200 \mathrm{~Hz}$ at tone onset to $230 \mathrm{~Hz}$ at tone offset was paired with deviants that were parallel to the standard but started (and ended) at lower and higher f0 values (onsets ranging from 185 to $215 \mathrm{~Hz}$ in $1 \mathrm{~Hz}$ steps). Likewise, a standard contour falling from $200 \mathrm{~Hz}$ at tone onset to $170 \mathrm{~Hz}$ at tone offset was paired with parallel deviants whose onsets ranged from 215 to $185 \mathrm{~Hz}$. For pitch direction, standard rising and falling contours were paired with deviants with either steeper or shallower slopes, again varying in $1 \mathrm{~Hz}$ steps. Results indicate that, overall, Chinese listeners are more sensitive to pitch direction and American listeners to pitch height. However, these effects are modulated by both the direction (falling/rising) and steepness of the contours.

3pSC69. The articulation of $/ \mathrm{x} /$ in New Zealand English. Matthias Heyne, Xuan Wang, Kieran Dorreen, Donald Derrick, and Kevin Watson (NZILBB, Univ. of Canterbury, Private Bag 4800, Christchurch, 8140, New Zealand, donald.derrick@canterbury.ac.nz)

A large number of studies have investigated the articulation of approximant /.I/ in American English (AE) (e.g., Delattre \& Freeman, 1968). This research has found that a low third formant (F3), the main acoustic cue signaling rhoticity, can be achieved using many different tongue configurations; the two main tongue shapes used for /I/ are "tip-down" ("bunched") and "tip-up" ("retroflex") (cf. Hagiwara, 1994). While speakers likely employ various "trading relationships" to maintain a constantly low F3 across production strategies (Guenther et al., 1999), they have access to a pool of variation, which some use to form complex and idiosyncratic patterns of allophony (Mielke et al., 2016). Such patterns may arise during speech acquisition (Magloughlin, 2016). This study focuses on a non-rhotic dialect, New Zealand English (NZE), to test whether dialect rhoticity constrains idiosyncratic allophony. Ultrasound video was collected for 63 speakers articulating 13 words containing tokens of $/ \mathrm{x} /$ in different phonetic environments. Analysis aims to determine whether NZE speakers utilize the same tongue gestures as seen in $\mathrm{AE}$, and whether they display similar patterns of allophonic variation. The data include productions from 12 children (under 10) and 13 youth (11-18), allowing examination of /x/ during childhood development.

3pSC70. Spatial congruence in multimodal speech perception. Megan Keough, Murray Schellenberg, and Bryan Gick (Linguist, Univ. of Br. Columbia, 2613 West Mall, Vancouver, BC V6T 1Z4, Canada, mkeough@ alumni.ubc.ca)

A growing literature provides evidence for the importance of synchronicity of cross-modal information in speech perception [e.g., audio-visual, Munhall et al. 1996, Perception \& Psychophysics 58: 351-362; audio-aerotactile, Gick et al. 2010, JASA 128: 342-346; visual-aerotactile, Bicevskis et al. submitted $\mathrm{ms}$ ]. While considerable work has investigated this role of temporal congruence, no research has directly explored the role of spatial congruence (i.e., co-directionality) of stimulus sources. If perceivers are picking up a localized distal speech event [e.g., Fowler 1986, Status Report of Speech Research: 139-169] cross-modal sources of information are predicted to be more likely to integrate when presented codirectionally than contradirectionally. An audio-aerotactile pairing lends itself well to this question as both modalities can easily be presented laterally. The current study draws on methodology from previous work [Gick \& Derrick 2009, Nature 462: 502-504] to ask whether cross-modal integration persists when cross-modal cues are spatially incongruent. Native English perceivers were presented with syllables contrasting in aspiration and embedded in noise, with some tokens accompanied by inaudible air puffs applied to the neck; aerotactile source locations either matched or opposed the spatial direction of the acoustic signal. Implications of results for multimodal integration theories will be discussed. [Funded by NIH Grant DC-02717 and NSERC.]

3pSC71. Cross-modal association between auditory and visual-spatial information in Mandarin tone perception. Beverly Hannah, Yue Wang (Linguist, Simon Fraser Univ., 9213 Robert C. Brown Bldg., 8888 University Dr., Burnaby, BC, Canada, yuew@sfu.ca), Allard Jongman, and Joan A. Sereno (Linguist, Univ. of Kansas, Lawrence, KS)

Speech perception involves multiple input modalities. Research has indicated that perceivers may establish a cross-modal association between auditory and visual-spatial events to aid perception. Such intermodal relations can be particularly beneficial for non-native perceivers who need additional resources to process challenging new sounds. This study examines how co-speech hand gestures mimicking pitch contours in space affect nonnative Mandarin tone perception. Native English as well as Mandarin perceivers identified tones with either congruent or incongruent auditory-facial and gestural $(\mathrm{AF} / \mathrm{G})$ input. Perceivers also identified congruent and incongruent auditory-facial (A/F) stimuli. Native Mandarin results showed the expected ceiling-level performance in the congruent $\mathrm{A} / \mathrm{F}$ and $\mathrm{AF} / \mathrm{G}$ conditions. In the incongruent conditions, while $\mathrm{A} / \mathrm{F}$ identification was primarily auditory-based, AF/G identification was partially based on gestures, demonstrating the use of gestures as valid cues in tone identification. The English perceivers' performance was poor in the congruent $\mathrm{A} / \mathrm{F}$ condition, but improved significantly in $\mathrm{AF} / \mathrm{G}$. While the incongruent $\mathrm{A} / \mathrm{F}$ identification showed some reliance on facial information, incongruent $\mathrm{AF} / \mathrm{G}$ identification relied more on gestural than auditory-facial information. These results indicate positive effects of facial and especially gestural input on non-native tone perception, suggesting that cross-modal (visual-spatial) resources can be recruited to aid auditory perception when phonetic demands are high. 
3pSC72. Worse than the dentist: Effects of simultaneous acoustic and somatosensory feedback degradation on speech. Elliot A. Pollack, Mary C. Rowley, and Elizabeth D. Casserly (Psych., Trinity College, 300 Summit St., Life Sci. Ctr., Hartford, CT 06106, elizabeth.casserly@trincoll.edu)

Speakers use their sensory feedback to monitor real-time speech production; when unexpected changes to that feedback occur, they alter their articulation in immediate, perturbation-specific ways. However, it is not currently known how the feedback from different sensory systems is integrated across contexts. This study varied the reliability of feedback in the auditory and somatosensory domains and examined vowel acoustic phonetics for changes as a result of the manipulation. Acoustic feedback was degraded via eight-channel real-time cochlear implant simulation, while an over-the-counter oral anesthetic (benzocaine) was applied to speakers' tongues and lips to degrade somatosensory feedback. Speakers $(\mathrm{N}=18)$ produced 139 isolated English words under both baseline and feedbackdegraded conditions. F1 and F2 measurements were taken from stressed tokens of eight vowels [i, I, $\varepsilon, æ, a, \Lambda, U, u]$. Significant differences in speakers' responses to feedback degradation were observed both across vowels and across individuals. [u]-fronting was the most consistent response observed, along with isolated classes of vowels (commonly, front or back) being hyper- or hypo-articulated. The variability suggests that the speech motor control system lacks a single, automatic consequence for global feedback degradation.

3pSC73. Plasticity of the internal model for speech sounds: Articulatory changes during intensive visual biofeedback treatment. Jennell C. Vick, Rebecca L. Mental (Psychol. Sci., Case Western Reserve Univ., 11635 Euclid Ave., Cleveland, OH 44106, jennell@case.edu), Holle Carey (Vulintus, Dallas, TX), Nolan T. Schreiber (Elec. Eng. Comput. Sci., Case Western Reserve Univ., Cleveland, OH), Andrew Barnes (Psychol. Sci., Case Western Reserve Univ., Cleveland, OH), and Gregory S. Lee (Elec. Eng. Comput. Sci., Case Western Reserve Univ., Cleveland, OH)

Visual biofeedback, commonly delivered by a display of ultrasound of tongue position, has demonstrated effectiveness for treating residual speech errors in older children and adults. It can be challenging to make kinematic measures during treatment without the use of a head stabilizing system, however, and it is likewise not possible to measure the changes in speech motor control that accompany improvements in speech sound production. Opti-Speech, a visual biofeedback treatment software that uses EMA to provide positional data, has the benefit of providing a steady stream of position data throughout baseline and treatment sessions. In this study, Opti-Speech treatment was provided with an intensive schedule ( $2 x /$ day for 5 days) to two adolescent males with persistent speech errors (i.e., lateralized /s/). Marked improvements in $/ \mathrm{s} /$ accuracy and quality were noted over the course of treatment and are reported in another paper (Mental et al., this meeting). Kinematic measures were made of tongue position throughout the course of the three baseline and 10 treatment sessions, including peak velocity, duration, and measures of token-to-token variability. The covariance of changes in these measures with increasing accuracy and quality of /s/ productions will be reported.

3pSC74. Acoustic modification across speaking modes in English and Korean. Yunjung Kim and Hyunju Chung (Louisiana State Univ., 86 Hatcher Hall, Baton Rouge, LA 70803, ykim6@1su.edu)

Previous studies have reported temporal and spectral modification of acoustic signals of speech according to varying speaking modes (i.e. clear, loud speech) in several languages (Krause \& Braida, 2004; Lam \& Tjaden, 2015). Interestingly, some acoustic characteristics such as vowel contrasts, inferred from the size of the corner vowel space, have been discussed to exhibit common, equivalent change between languages despite the different inventory sizes (Smiljanic \& Bradlow, 2005). The current study, as an expansion of this line of research, seeks to examine the effect of native language on selected acoustic measures when speakers voluntarily switch speaking modes among conversational, clear, and slow speech. 24 female adults (12 English-speaking, 12 Korean-speaking) were asked to read CVCV structures in the three aforementioned conditions. English and Korean were chosen mainly because of their differences in vowel and stop inventories. Vowel duration, formant structure (F1-F2), and VOT were measured from the target syllable $(\mathrm{CVCV})$, while intensity and $\mathrm{F}_{0}$ were analyzed for the entire utterance to extend the observations of the non-segmental aspects of speaking modification. Findings are expected to reflect both language-independent and -dependent effects as well as to call for the need to consider the given language in behavioral management of diverse speech disorders.

3pSC75. Fricatives in conversational vs. read speech in mid-Western American English. Daniel Brenner (Univ. of AB, Edmonton, AB, Canada), Viktor Kharlamov (Florida Atlantic Univ., 777 Glades Rd., CU-97, Ste 280, Boca Raton, FL 85721, vkharlamov@fau.edu), and Benjamin V. Tucker (Univ. of AB, Edmonton, AB, Canada)

The present study examines the acoustic characteristics of fricatives across two corpora from mid-Western American English. Our goal is to determine (i) the range of acoustic variation in the production of fricatives in conversational vs. read speech, and (ii) the type and extent of individual variation schemes demonstrated by the speakers. The study surveys over 100,000 fricative tokens from the conversational Buckeye Corpus (Pitt et al. 2005) and the read speech TIMIT Corpus (Zue and Seneff 1988). We test an extensive list of acoustic measures compiled on the basis of prior work with laboratory data (incl. duration, center of gravity, skew, kurtosis, peak power, etc.) and explore the implications of the findings for our understanding of the group and individual patterning of speech production processes across the two different speech styles.

3pSC76. Effects of phonetic reduction and regional variation on lexical access. Ellen Dossey and Cynthia G. Clopper (The Ohio State Univ., 108A Ohio Stadium East, 1961 Tuttle Park Pl., Columbus, OH 43210, dossey.1@ osu.edu)

This study investigates the impact of acoustic variability on speech processing using a cross-modal priming experiment. Participants heard an audio prime consisting of a single lexical item followed by a printed word on the computer screen and were asked to indicate whether or not the word they heard was the same as the word on the screen. Auditory primes were balanced for linguistic factors that contribute to phonetic reduction, including lexical neighborhood density and whether the token was the first or second mention of the word in the larger context from which it was extracted. Primes were also balanced for social factors which contribute to phonetic variation, including regional dialect. To analyze the effect of these factors on speech processing, reaction times to matching prime-target pairs were analyzed. The results show that reaction times for Midland listeners were significantly faster following Midland primes than Northern primes, suggesting a native dialect benefit. However, the effect of dialect was smaller for words with a lower neighborhood density and for second mention primes relative to first mention primes. Together, these results suggest that dialect variation may have a smaller impact on speech processing in contexts that favor phonetic reduction.

3pSC77. Assessment of areas of the English-Japanese bilingual brain by using functional MRI. Keiko Asano (School of Medicine, Juntendo Univ., 1-11-2905, Kinko-cho, Kanagawa-ku, Yokohama-city 221-0056, Japan, kiasano@uu.em-net.ne.jp), Hidenori Sugano, Hajime Arai, Madoka Nakajima, and Shigeki Aoki (School of Medicine, Juntendo Univ., Tokyo, Japan)

Bilinguals are often determined by how early they are exposed to L2 and the length of their immersion in the L2 environment, both of which affect factors that are important for acquiring language. To analyze the relationship between these, the language function of English-Japanese bilinguals was assessed using fMRI. In order to aim to observe differences in the brain, language function areas were activated by tasks specific to L2 usage. There were 24 English-Japanese bilingual speakers, average age of 25, categorized into four groups according to the age of exposure (before or after age 8), and the length of exposure (longer or shorter than 5years):Early Long (EL), Early Short (ES), Late Long (LL) and Late Short (LS). A PHILIPS Achieva 3.0T TX MRI machine was used to scan the subjects. Tasks consisted of hearing a short story in Japanese (Issunboshi) and English (Peter Rabbit) and their reverse plays by comparison of BOLD oxygenation levels. Focus was given to Broca's area, Wernicke's area and the premotor cortex. This study showed that broad areas of the premotor cortex were 
activated in ES and LS. EL and LL scans showed localization in Wernicke's area. Since function in Broca's area and the premotor cortex were consistent in ES and LS, it was assumed that these areas are necessary for proficiency and plasticity of language.

3pSC78. Effects of attention on lexically-informed perceptual learning for speech and letter perception. Julia R. Drouin, Nicholas R. Monto, Stephen Graham, Jacqueline Ose, and Rachel M. Theodore (Speech, Lang., and Hearing Sci., Univ. of Connecticut, 850 Bolton Rd., Unit 1085, Storrs, CT, nicholas.monto@uconn.edu)

Listeners use lexical information to adjust the mapping to prelexical representations in both the auditory and visual modalities, a phenomenon referred to as lexically-informed perceptual learning (LIPL). Recent research points to attention mechanisms as potential constraints on this type of learning; learning is attenuated when listeners explicitly note anomalies in the speech input, and learning is negatively correlated with performance on tasks of attention-switching control. Here we examine whether graded lexical recruitment as modulated by attention influences LIPL in speech (Experiment 1) and print (Experiment 2). All participants completed a training phase where they were exposed to an ambiguous sound (midway between $/ \mathrm{s} /$ and $/ \mathrm{J} /$ ) or an ambiguous grapheme (midway between $\mathrm{H}$ and $\mathrm{N}$ ). Attention was manipulated during exposure by asking participants to make a lexical decision, a syntactic decision, or a decision regarding surface variation (i.e., amplitude, color). All participants then completed a categorization task to assess LIPL. The results to date indicate that LIPL is influenced by attention; the magnitude of the learning effect was not equivalent among the exposure conditions for either modality. However, differences between the two modalities were observed, which are considered with respect to the neural systems that support spoken and written language.

3pSC79. Bi-dialectal homophone effects in Kansai Japanese lexical decision tasks. Karen Tsai, Nicholas Lester, and Fermín M. del Prado Martín (Dept. of Linguist, Univ. of California, Santa Barbara, Santa Barbara, CA 93106,karentsai@umail.ucsb.edu)

Many Japanese varieties, including Tokyo Japanese, have lexical pitch accent patterns that can result in homophones that vary across dialects, such as ame HL 'rain (Tokyo)' and ame LH 'candy (Tokyo), rain (Kansai)'. Experimental research on lexical pitch accent in Japanese has been limited, however, to Tokyo Japanese and accentless varieties (Utsugi, Koizumi \& Mazuka, 2011). This study explores homophone effects of minimal accent pairs for speakers of Kansai Japanese, a dialect that, unlike Tokyo Japanese, has base tone melodies, HL or LHL (Haraguchi, 1977), in addition to lexical pitch accent. Unlike previous studies on processing effects of Japanese homophones (Cutler \& Otake, 1999; Minematsu \& Hirose, 1995) that focus on visual recognition in Tokyo Japanese (Hino, Kusunose, Lupker \& Jared, 2013; Sekiguchi, 2006), the current experiment measures reaction times to auditory stimuli in a lexical decision task comparing Tokyo Japanese with Kansai Japanese, whose speakers are typically bi-dialectal speakers of Tokyo Japanese as well. Results of the study will shed light on the issue of how pitch accent affects spoken word recognition in two different pitch accent varieties of Japanese.

$3 p S C 80$. The native language benefit for voice recognition is not contingent on lexical access. Nicholas R. Monto, Rachel M. Theodore (Speech, Lang., and Hearing Sci., Univ. of Connecticut, 850 Bolton Rd., Unit 1085, Storrs, CT, nicholas.monto@uconn.edu), Adriel J. Orena, and Linda Polka (School of Commun. Sci. and Disord., McGill, Montreal, QC, Canada)

Listeners show heightened talker recognition for native compared to nonnative speech, formalized as the language familiarity effect (LFE) for voice recognition. Some findings suggest that language comprehension is the locus of the LFE, while others implicate expertise with the linguistic sound structure. These hypotheses yield different predictions for the LFE with time-reversed speech, a manipulation that precludes lexical access but preserves some indexical and phonetic properties. Research to date shows discrepant results for the LFE with this impoverished signal. Here we reconcile this discrepancy by examining how the amount of exposure to talkers' voices influences the LFE for time-reversed speech. Three experiments were conducted. In all, two groups of English monolinguals were trained and then tested on the identification of four English talkers and four French talkers; one group heard natural speech and the other group heard timereversed speech. Across the experiments, we manipulated exposure to the voices in terms of number of training trials and duration of the talkers' sentences. A robust LFE emerged in all cases, though the magnitude was attenuated as the amount of exposure decreased. These results are consistent with the account that the LFE for talker identification is linked to the sound structure of language.

3pSC81. Language familiarity mediates identification of bilingual talkers across languages. Adriel John Orena, Linda Polka (School of Commun. Sci. and Disord., McGill Univ., 2001 McGill College, 8th Fl., Montréal, QC H3A 1G1, Canada, adriel.orena@mail.mcgill.ca), and Rachel M. Theodore (Dept. of Speech, Lang., and Hearing Sci., Univ. of Connecticut, Storrs, CT)

Many studies show that listeners are more accurate at identifying talkers in their native language than in an unfamiliar language; yet, little is known about the nature of this language familiarity effect in bilingual speech. Here, we investigate the links between language and talker processing further by assessing listeners' ability to identify bilingual talkers across languages. Two groups were recruited: English monolinguals and English-French bilinguals. Participants learned to identify bilinguals speaking in only one language (English); they were then tested on their ability to identify the same talkers speaking in the trained language (same language context: English) and in their other language (different language context: French). Both monolinguals and bilinguals showed above chance performance in identifying talkers in both language contexts at test, confirming that there is sufficient information in bilingual speech to generalize across languages. Moreover, the results showed a language context effect that was facilitated by language familiarity: monolinguals showed a substantial decrease in performance between the same and different language contexts, whereas the bilinguals — who understood both languages at test—showed only a slight decrease in performance between the two language contexts. These results indicate that language familiarity affects talker encoding and retrieval, even for bilingual speech.

3pSC82. Interspeech posture in Spanish-English bilingual adults. Merrily R. Shary, Kyna S. Betancourt, Stefan A. Frisch, and Nathan D. Maxfield (Commun. Sci. and Disord., Univ. of South Florida, 4202 E. Fowler Ave., PCD 1017, Tampa, FL 33620, merrily@usf.edu)

Interspeech posture (ISP) is a term used to define the position of a person's articulators when preparing to speak. Wilson and Gick (2014) found French-English bilinguals used different tongue posture during ISP depending on which language they were speaking. The authors suggested that ISP may be representative of a speaker's phonological knowledge in a particular language but recommended other languages be examined. The purpose of this study was to replicate Wilson and Gick (2014) using Spanish-English bilingual speakers. To this end, bilingual Spanish-English adults were asked to produce sentences while speaking in monolingual and bilingual modes. While they were speaking, ultrasound images of the oral cavity were obtained and tongue tip height during each interutterance pause was measured. Additionally, monolingual English speakers rated the accentedness of each bilingual's speech in English as a behavioral correlate of language proficiency. Overall results of this study supported Wilson and Gick (2014); bilingual Spanish-English speakers judged to be native sounding in English only utilized similar postures in monolingual and bilingual speaking modes. Different measures of ISP and speaker variability will also be completed to determine if there is a better method for measuring ISP in Spanish-English bilinguals. 
3pSC83. A preliminary investigation of velar-vowel coarticulation in Spanish-English Bilinguals. Stefan Frisch, Marsha Allen, Kyna Betancourt, and Nathan Maxfield (Commun. Sci. and Disord., Univ. of South Florida, 4202 E Fowler Ave., PCD1017, Tampa, FL 33620, sfrisch@usf. edu)

Coarticulation, the influence of one speech sound on another in running speech, has been suggested as an index of speech motor control. Therefore, proficient and stable coarticulation could be a diagnostic tool in language assessment and speech-language pathology. In the present study coarticulation was investigated in Spanish-English bilingual adults to determine whether velar-vowel coarticulation patterns differ in English versus Spanish. Velar-vowel coarticulation was measured in six Spanish-English bilingual university students. Participants recited sentences in blocks of English, Spanish, and a mix of both languages. Each sentence included a target word starting with $/ \mathrm{k} /+$ vowel. Tongue movement was recorded using a sub-mental ultrasound probe held in a stabilizing helmet. Ultrasound images were analyzed to identify tongue posture following procedures of Frisch \& Wodzinski (2016, Journal of Phonetics, doi: 10.1016/j.wocn.2016.01.001). The results indicate a pattern similar to that found for Interspeech Posture in French-English bilinguals (Wilson \& Gick, 2014, Journal of Speech, Language, Hearing Research, doi: 10.1044/2013 JSLHR S-12-0345). Some bilinguals showed a single pattern for both languages while others produced distinct velar postures between English and Spanish.

3pSC84. Speech sound naturalness alters compensation in response to transformed auditory feedback. Sadao Hiroya and Takemi Mochida (NTT Commun. Sci. Labs., 3-1 Morinosato-Wakamiya, Atsugi, Kanagawa 2430198, Japan, hiroya.sadao@lab.ntt.co.jp)

Articulatory compensations in response to real-time formant perturbation have revealed that auditory feedback plays an important role in speech production. However, these compensatory responses were at most $40 \%$ for formant shifts and varied depending on vowel type and subjects. Although previous formant perturbation studies have been done using linear predictive coding (LPC), it is known that the estimation accuracy for low vowels and female speech would be degraded due to a glottal source-vocal tract interaction. To improve the accuracy, we have developed a real-time robust formant tracking system using phase equalization-based autoregressive exogenous (PEAR) model which utilizes the glottal source signals measured by electroglottography. In this study, we compared compensatory responses to real-time formant perturbation using PEAR and LPC. Eleven Japanese subjects (seven females) read a Japanese mora (/hi/ or /he/) with headphones. The first two formant frequencies were altered. Results showed that compensatory responses using PEAR were significantly larger than LPC. Moreover, naturalness of altered speech sounds was improved by PEAR. This indicates that improving speech sound naturalness by PEAR led to larger compensatory responses. Therefore, our system would be useful to understand the auditory feedback mechanisms in more detail.

3pSC85. Speech stability as a characteristic of the individual. Stefan Frisch, Jessica Massey, and Nathan Maxfield (Commun. Sci. and Disord., Univ. of South Florida, 4202 E Fowler Ave., PCD1017, Tampa, FL 33620, sfrisch@usf.edu)

Coarticulation, the influence of one speech sound on another in running speech, has been suggested as an index of speech motor control. Therefore, proficient and stable coarticulation could be a diagnostic tool in language assessment and speech-language pathology. In the present study, stability in the production of velar-vowel coarticulation was measured within speakers across experimental sessions to determine whether differences in stability can be attributed to individual differences in speech production ability. Velar-vowel coarticulation was recorded in children aged 8-12 in three sessions roughly one month apart. Participants recited sentences that included a target word starting with $/ \mathrm{k} /$ + vowel. Ultrasound images were analyzed to identify tongue posture and stability in posture across repetitions following procedures of Frisch, Maxfield, \& Belmont (2016, Clinical Linguistics \& Phonetics, doi:10.3109/02699206.2015.1137632). Preliminary analysis of two participants suggests there is less variation in stability within a participant across sessions than there is between participants. Analysis of the remaining participants is ongoing. This suggests that speech stability may indeed be a property of an individual speaker. If this finding holds, there are implications for models of speech production that include individual differences, including differences in language proficiency or clinical diagnosis.

3pSC86. Event related speech readiness potentials to spoken words and CV's. Silas Smith, Robert Sears, and Al Yonovitz (The Univ. of Montana, Dept. of Communicative Sci. and Disord., Missoula, MT 59812, al.yonovitz@umontana.edu)

This study investigated the temporal and dynamics of brain activity to speech and motor preparation. Previous EEG studies have identified a slow negativity wave that occurs previous to the onset of speech. Brain electrical potentials were obtained in subjects prior to initiation of speech in a using a single vertex electrode. Both a repeated single syllable word and CV's were used. The sample rate permitted an analysis of both slow negative waves and faster neurogenic signals. The purpose of this research was to establish a real-time event related brain electrical potential system. This research uses a vocal signal as the marking point, and displays in real time the eventrelated potential. Each time epoch was sampled at $25600 \mathrm{samples} / \mathrm{sec}$. One second of these signals were averaged for 100 trials just prior to initiation of the word "pool" or a randomly presented CV. The inter-trial interval was approximately 25 seconds. For the 100 trials, each trial was saved. Separate odd and even event potentials were averaged. The obtained waveform (prespeech) was consistent and reliable between and within subjects. The microstructure of the waveform was also obtained showing specific waveform morphology. The reliability was greater for the $\mathrm{CV}$ productions.

3pSC87. What the $f^{* * *}$ : An acoustic-pragmatic analysis of meaning in The Wire. Erica Gold and Dan McIntyre (Dept. of Linguist and Modern Lang., Univ. of Huddersfield, Queensgate, Huddersfield HD1 3DH, United Kingdom, e.gold@hud.ac.uk)

The award-winning television drama, The Wire, contains a famous scene in which Detectives Moreland and McNulty discuss the crime scene they are investigating using only variations of a single expletive-fuck. Despite limited vocabulary, viewers are able to extract meaning and interpret the scene. This study considers all of the expletives produced in the scene, and carries out an acoustic analysis of / $\Lambda$ / - F1 F3 (midpoint and averages) and vowel duration. These measurements are then used in combination with Gricean (Grice 1975) and neo-Gricean (e.g. Horn 1984) pragmatic analysis in an attempt to categorize the intended meaning of fuck as: intensification, confusion, dissatisfaction or suspicion (Fairman 2009). Vowel measurements are considered for Moreland and McNulty individually, and vowel normalization is carried out in order to determine pragmatic categorizations across both detectives. This paper argues that acoustic-phonetic analysis can augment Gricean and neo-Gricean pragmatic analysis by providing a means of determining whether what appears at a lexical level to be conversationally implicated meaning is in fact conventional implicature conveyed at the phonetic level.

3pSC88. Historical, current, and legal bases for forensic speaker identification. Al Yonovitz (Dept. of Communicative Sci. and Disord., The Univ. of Montana, Missoula, MT 59812, al.yonovitz@umontana.edu), Herbert Joe, and Joshua Yonovitz (Yonovitz \& Joe, LLP, Dallas, TX)

It is of great interest to the legal system to identify individuals by their voice. Controversy in this area has continued for nearly seven decades with states divided with regard to the merits and standards used for forensic voice identification. Forensic speaker identification and admissibility of expert testimony has always been questionable, and is meeting more legal and scientific criticism. A number of methodologies have evolved that provide different approaches to speaker identification. These methods will be contrasted with a systematic review. In addition, the legal qualifications to testify as an expert witness in state or Federal courts-regardless of specialty, including in civil or criminal cases have evolved since the landmark 1923 Frye case, as well as the more sweeping 1993 Daubert case, and continue to evolve. This presentation discusses the history of the qualifications, an overview of different jurisdictional requirements, and the legal requirements of a speaker identification voice expert. The enigma of speaker identification for use in forensic applications will be illustrated by utilizing actual cases applying various methods of speaker identification or elimination. 


\title{
Session 3pSP
}

\section{Signal Processing in Acoustics: Acoustic Communications}

\author{
Grace A. Clark, Cochair \\ Grace Clark Signal Sciences, 532 Alden Lane, Livermore, CA 94550
}

Henry A. Scarton, Cochair

Mechanical, Aerpspace, and Nuclear Engineering, Rensselaer Polytechnic Institute, Rensselaer Polytechnic Institute, 110 Eight Street, JEC 4008, Troy, NY 12180

Tadashi Ebihara, Cochair

Faculty of Engineering, Information and Systems, University of Tsukuba, 1-1-1 Tennodai, Tsukuba 3058573, Japan

Chair's Introduction-1:30

\section{Invited Papers}

\author{
$1: 35$
}

3pSP1. Digital acoustic communications through solids. Henry A. Scarton (Mech., Aerpspace, and Nuclear Eng., Rensselaer Polytechnic Inst., Rensselaer Polytechnic Inst., 110 Eight St., JEC 4008, Troy, NY 12180, scarton@rpi.edu), Gary Saulnier (Elec. Comput. and Systems Eng., Rensselaer Polytechnic Inst., Troy, NY), Kyle Wilt (Mech., Aerpspace, and Nuclear Eng., Rensselaer Polytechnic Inst., Troy, NY), and Michael Cunningham (Elec. Comput. and Systems Eng., Rensselaer Polytechnic Inst., Troy, NY)

Digital acoustic communication solid channels make it possible to send data through metallic barriers with up to very high rates while also transferring power and with lower complexity, lower size and power. This paper presents different techniques for achieving this including data transfer rates up to sever kilobits per second (kbps) using simple switching technique to many megabits per second through thick steel. Modulation techniques include Chirp-On-Off Keying (C-O-OK) to Orthogonal Frequency Division Multiplexing (OFDM) and even Multiple-Input Multiple -Output (MIMO) approaching $700 \mathrm{Mbps}$. Frequencies up to $4 \mathrm{MHz}$ are used with piezoelectric elements propagating longitudinal waves or transversely polarized shear waves serving as the sending and receiver sensors.

\section{$1: 55$}

3pSP2. Characterizing ocean acoustic channel through databases: Issues, requirements, and methods. Tsih C. Yang (College of Information Sci. and Electron. Eng., Zhejiang Univ., 38 Zhe Da Rd., Bldg. of Information Sci. \& Elec. Eng., Xihu District, Hangzhou 310058, China, tsihyang@gmail.com) and San Ho Huang (Ocean College, Zhejiang Univ., Zhoushan, Zhejiang, China)

There is a growing interest in underwater acoustic telemetry. This calls for methods to evaluate the performance of various (proposed) modulation and equalization algorithms under the same channel conditions. An ideal solution is to develop acoustic channel simulators. However, this effort has not been fruitful since acoustic channel simulations (at practical communication frequencies) so far have failed to capture the characteristics of real ocean channels. Currently, acoustic communications still rely on field experiments for evaluation of the algorithm performance. The problem with this approach is that each experiment is usually tailored to a particular modulation scheme and the cost prohibits testing of many different algorithms under the same ocean conditions. Some data-based channel simulators have been proposed where realizations of the channel impulse response (CIR) functions are generated based on the scattering function estimated from real data but it is still not adequate. A scheme to build ocean channel database is proposed in this paper, where CIR is deduced from data on a fractional symbol-by-symbol basis with negligibly small channel estimation error (using the surrogate signal predication error as an indicator) so that the developers can test the performance of different algorithms under same, realistic ocean conditions.

\section{2:15}

3pSP3. Harbor trial of underwater acoustic communication using Doppler-resilient orthogonal signal division multiplexing. Tadashi Ebihara (Faculty of Eng., Information and Systems, Univ. of Tsukuba, 1-1-1 Tennodai, Tsukuba, Ibaraki 305-8573, Japan, ebihara@iit.tsukuba.ac.jp), Geert Leus (Faculty of Elec. Eng., Mathematics and Comput. Sci., Delft Univ. of Technol., Delft, Netherlands), and Hanako Ogasawara (Dept. of Earth and Ocean Sci., National Defense Acad., Yokosuka, Kanagawa, Japan)

Underwater acoustic channels are characterized by a severe spread in time and frequency. To provide a highly reliable communication environment in doubly spread channels for UWA communication, we have proposed Doppler-resilient orthogonal signal-division multiplexing (D-OSDM). D-OSDM is a combination of the OSDM technique and orthogonal multiple access, and it preserves orthogonality among data streams even after propagation through doubly spread channels. We have conducted test tank experiments and 
simulations, and have found that D-OSDM could cope well with time- and frequency-spread channels and that it achieves a good communication quality [1]. In this talk, we will show some results of our first sea trial of D-OSDM, that was performed on 21 June, 2016 at Hashirimizu port, Kanagawa, Japan. In this trial, the transmitter is fixed on the harbor quay, and the receiver is fixed on a remotely-operated survey boat. Acoustic communication was performed by moving the boat to generate a severe spread in frequency. The experiments show that D-OSDM achieves a good communication quality even if the boat moves at a speed of about 1.5 kt. [1] Tadashi Ebihara and Geert Leus: Doppler-Resilient Orthogonal Signal-Division Multiplexing for Underwater Acoustic Communication, IEEE J. Ocean. Eng. 41 (2016) 408-427.

\section{$2: 35$}

3pSP4. Multiuser and multiple-input-multiple-output underwater acoustic communication using time reversal. Takuya Shimura, Yukihiro Kida, Mitsuyasu Deguchi, Kohji Meguro, Yoshitaka Watanabe, and Hiroshi Ochi (Maine Tech. Development Dept., JAMSTEC, 2-15 Natsushima-cho, Yokosuka, Kanagawa 237-0061, Japan, shimurat@jamstec.go.jp)

Time reversal is an effective method of channel equalization for underwater acoustic communication. Its temporal and spatial focusing effect collects long time spread signals due to a rich multipath environment inherent in underwater acoustics. Additionally, it is easy to compensate Doppler frequency shift after time reversal focusing. Recently, demands for multiuser or multiple-input-multiple-output (MIMO) communication have increased widely for multiple autonomous underwater vehicles (AUVs) or high rate underwater acoustic network communication. Similarly, in Japan Agency Marine-Earth Science and Technology (JAMSTEC), a plan of multiple AUVs operation is being progressed. Time reversal is a promising solution also for such multiuser or MIMO communication by realizing space division multiplexing (SDM) based on its spatial focusing and nulling effect without sacrificing data transmission rate. In this study, the performance of time reversal is analyzed with simulation or real data comparing with orthogonal frequency-division multiplexing (OFDM) as a conventional or commonly used method. As results, the ability of time reversal to suppress multiuser or multistream interferences is much better than those of maximum ratio combining (MRC), zero forcing (ZF) or minimum mean-square error (MMSE) OFDM, in both vertical and horizontal communication.

\section{2:55}

3pSP5. Differential frequency hopping performance in doubly spread underwater acoustic communication channels. Geoffrey S. Edelson (Maritime Systems \& Technol., BAE Systems, MER15-2350, P.O. Box 868, Nashua, NH 03061-0868, geoffrey.s.edelson@baesystems.com) and Luca Cazzanti (Machine Learning and Data Sci., Ctr. for Maritime Res. and Experimentation, La Spezia, Italy)

Underwater acoustic communication requires waveforms that are robust to the signal distortions caused by the channel. Many waveforms used for this purpose require the transmission of training symbols that span the inter-symbol interference (ISI) to compensate for these channel effects. Differential frequency hopping (DFH) is a fast frequency hopping, digital signaling technology that requires minimal information at the transmitter to communicate in the underwater channel. The relationships between the parameters of Doppler spread-inducing underwater environments and DFH bit-error performance are characterized. Wind speed determines the nature of the effect that the water surface imposes on acoustic DFH waveforms with low wind speeds resulting in an essentially flat, low-absorption sea surface. In this case, strong surface reflections and little frequency spreading make ISI the dominant effect on the received waveforms. At high wind speeds, air bubbles in the surface layer absorb almost all energy incident on the surface, resulting in no surface reflections reaching the receiver so that the surface has little effect on the received signal. The intermediate ranges of wind speed, with a mix of ISI and surface-induced Doppler spread, pose the greatest challenge. Simulations and at-sea experiments show that DFH performance is robust across environmental conditions.

\section{3:15-3:30 Break}

\section{Contributed Papers}

\section{3:30}

3pSP6. Waveform designs for computationally efficient acoustic response estimation. Jacob L. Silva (ECE Dept., Univ. of Massachusetts Dartmouth, Darmouth, MA) and Paul J. Gendron (ECE Dept., Univ. of Massachusetts Dartmouth, 285 Old Westport Rd., North Dartmouth, MA 02747, pgendron@umassd.edu)

Broadband source waveforms are constructed that allow for the minimum mean square error (MMSE) estimation of an observed acoustic response function with minimal computational effort at the receiver. The waveforms offers near perfect auto and cross correlation properties over the limited propagation delay spread of the acoustic response allowing for least squares estimation of the whitened response with simple matched filtering. MMSE estimation of the whitened response can be accomplished in many cases from this least squares estimate. We compare the approach to conventional maximal length sequences as well as Gold sequence to determine the merits of the approach we further demonstrate the approach for communications at low signal to noise ratios.
$3: 45$

3pSP7. A covert underwater acoustic communication method based on spread spectrum digital watermarking. Shaofan Yang, Zhongyuan Guo, Qunyan Ren, and Shengming Guo (Inst. of Acoust., Chinese Acad. of Sci., No. 21 North Fourth Ring Rd., Beijing 100190, China, 1172966054@qq.com)

The covert underwater acoustic communication is necessary under some circumstances when the contents of transmission or location of transmitter are required to be concealed. In this paper, the signal transmitter and the information detector are designed, which hides the spread spectrum digital watermark (SSDW) into the log-spectrum of dolphin whistles that are as a carrier and detects SSDW by matched filtering in the same log-spectrum. Various factors of noise, carrier and multipath in channels may degrade the performance of the method. In order to analyze their effects, corresponding channel models in log-spectrum domain are derived from the additive Gaussian noise channel and the underwater time-varying acoustic multipath channel. Results suggests that the effects of channel noise and the carrier are equivalent to that of two additive Gaussian noise terms attached to the correct decision and the proposed method also has a good multipath extension tolerance performance. Preliminary results obtained form at-sea experiments showed that the data rate can achieve 6 bps and bit error rate (BER) under $10^{-3}$ in the distance of $5 \mathrm{~km}$. 
BER as a parameter. Significantly, the approach avoids resampling and the need for buffering received data packets. This method also reduces computational complexity significantly.

\section{$4: 15$}

3pSP9. Multichannel equalization method based on vector time-reversal and decision feedback equalizer technology. Xueli Sheng, Yewu Ruan, Dian Lu, JIngwei Yin, and Longxiang Guo (College of Underwater Acoust. Eng., Harbin Eng. Univ., Harbin, Heilongjiang 150001, China, shengxueli@ hrbeu.edu.cn)

A kind of underwater communication receiver combined with Vector Time-Reversal algorithm and multichannel decision feedback equalizer (VTR-MDFE) is studied, which can address the problem of inter-symbol interference and waveform distortion, resulting from time-varying multipath channel. Impacts of environmental noise can be suppressed by use of vector sensor's combined directivity and Corresponding vector signal processing technology. Meanwhile, error rate is reduced while system capacity is enhanced by time-reversal algorithm's anti-multipath ability. The performance of VTR-MDFE are proved by simulated results in the communication channel provided by a ray-based acoustic model, for different ocean conditions and source-receiver geometries.

\title{
Session 3pUWa
}

\section{Underwater Acoustics: Inversion, Beam-forming, and Calibration II}

\author{
Steven A. Stotts, Chair \\ Environmental Sciences Laboratory, Applied Research Labs/The University of Texas at Austin, 10000 Burnet Rd., \\ Austin, TX 78759
}

\section{Contributed Papers}

1:00

3pUWa1. Application of maximum entropy to statistical inference for inversion of data from a single track segment. Steven A. Stotts and Robert A. Koch (Environ. Sci. Lab., Appl. Res. Labs/The Univ. of Texas at Austin, 10000 Burnet Rd., Austin, TX 78759, stotts@arlut.utexas.edu)

An approach is presented for statistical inference, based on maximum entropy (ME) with inversion data from a single source track segment, to account for model mismatch. A previous approach requires data from multiple track segments to set the, otherwise undetermined, ME constraint value specifying the posterior probability density (PPD). One effect of mismatch is that the lowest cost inversion solutions for some parameter values, e.g., source track parameter values obtained from GPS measurements or source levels from towed sources, may be well outside a relatively well known, narrow, uncertainty interval. The basis for the new approach is that the ME constraint value is determined by requiring for such a parameter value that the inferred uncertainty interval encompass the entire uncertainty interval comprising its prior information. Motivating this approach is the hypothesis that the proposed constraint determination, applied to the PPD for a model space broader than that parameter value's prior, could account for the effect of mismatch on the inferred, but a priori less well defined, values for other parameters.
$1: 15$

3pUWa2. Wave physics of the frequency difference autoproduct: Helmholtz equation analysis. David R. Dowling (Mech. Eng., Univ. of Michigan, 1231 Beal Ave., Ann Arbor, MI, drd@umich.edu) and Brian M. Worthmann (Appl. Phys., Univ. of Michigan, Ann Arbor, MI)

The frequency-difference autoproduct $(\Delta f-\mathrm{AP})$ is a quadratic product of solutions of the inhomogenous Helmholtz equation that differ only in frequency, and may be easily constructed from the Fourier transform of measured time-domain signals. Recent findings involving beamforming (Abadi et al., 2012, JASA 132, 3018-3029) and matched field processing (Worthmann et al., 2015, JASA 138, 3549-3562) suggest that the $\Delta$-AP is similar to an acoustic field at the difference frequency, even when the difference frequency lies below the recorded signal's bandwidth. This presentation provides mathematical analysis that supports this interpretation and indicates its limitations, along with examples from a Lloyd's mirror environment where a frequency sweep from $1-2 \mathrm{kHz}$ was broadcast from $100 \mathrm{~m}$ below the reflecting surface out to a range of $2 \mathrm{~km}$. In particular, the $\Delta f$-AP in time-independent, inhomogeneous, multipath environments should be locally indistinguishable from an acoustic field at the difference frequency when: $(i)$ the in-band acoustic propagation is well described by a ray-path sum, (ii) the inband field gradient is dominated by phase (not amplitude) variations, and 
(iii) the products of the signal bandwidth and ray travel-time differences are large enough so that a signal-bandwidth average suppresses ray-path cross terms from the quadratic product. [Sponsored by ONR and NSF.]

\section{$1: 30$}

3pUWa3. Wave physics of the frequency difference autoproduct: Bilinear time-frequency analysis. Brian M. Worthmann (Appl. Phys., Univ. of Michigan, 1231 Beal Ave., Ann Arbor, MI 48109, bworthma@umich.edu) and David R. Dowling (Mech. Eng., Univ. of Michigan, Ann Arbor, MI)

Frequency difference beamforming (Abadi et al., 2012, JASA, 132, 3018-3029) and matched field processing (Worthmann et al., 2015, JASA, $138,3549-3562$ ) are array signal processing techniques that determine acoustic ray-path directions and source locations, respectively, from sparse array recordings by shifting the signal processing to a lower, out-of-band, difference-frequency bandwidth. This frequency down-shift is accomplished through the use of the frequency difference autoproduct ( $\Delta f$-AP), a quadratic product of complex frequency-domain acoustic field amplitudes at two different frequencies. In this presentation, the physical and mathematical underpinnings of this quadratic product are discussed. Specifically, in the branch of applied mathematics termed bilinear time-frequency analysis, this $\Delta f$-AP is actually one of four quadratic time-frequency functions, each interrelated through forward and inverse Fourier transforms. The other three functions are the time-domain autoproduct, the Wigner distribution, and the ambiguity function. These four functions and several of their interesting properties are reviewed, including their relationship to the Wiener-Khintchine Theorem, and the spatial and spectral coherence and correlation functions. Additionally, the behavior of these functions when evaluated from simulated acoustic recordings from ideal single-path and multi-path acoustic environments is shown and compared. [Sponsored by ONR and NSF.]

\section{$1: 45$}

3pUWa4. Low frequency beamforming in shallow water environments. Shima Abadi, Cole Amaratunge, Gavin Boyd, and Matthew Daniels (Univ. of Washington, 18115 Campus Way NE, Box 358538, Bothell, WA 98011, abadi@uw.edu)

Ray theory is a high frequency approximation of the wave equation solution. Beamforming is a spatial filtering method that uses the ray theory to estimate the arrival angle from the array recordings. However, the ray theory is restricted to situations where water column is much deeper than the propagated wavelength and fails when water depth is smaller compared to the acoustic wavelength (i.e., in shallow-water low-frequency propagation). In this situation, the underwater sound propagation is modeled by normal-modes theory, the exact solution of the wave equation. In the theory of normal modes, each mode propagates at a different incident angle and the total field is composed of a discrete sum of the propagation modes excited at the broadcast frequency. In this situation, the beamforming performance degrades because of the interference between individual mode incident angles. In this presentation, the performance of the conventional beamforming technique is investigated when low frequency signals are propagated in shallow water environments and recorded by either a vertical or a horizontal linear array of hydrophones. Simulations are undertaken to understand how the resolved angle from the conventional beamforming method is related to the bearing angle and the incident angle of each mode.

\section{2:00}

3pUWa5. The investigation of the method for measuring the low-frequency radiated sound power in a reverberation tank. Yi-ming Zhang, Rui Tang, Qi Li, and Da-jing Shang (Harbin Eng. Univ., Nantong Str. Nangang Dist. No.145, Harbin 150001, China, tangrui@hrbeu.edu.cn)

In order to measure the low-frequency radiated sound power of the underwater sound source in a reverberation tank, the measuring method based on the characteristic acquisition of the testing sound field is proposed. Based on the normal-mode theory, the spatial averaging properties of the reverberation sound field with the ideal boundaries is firstly derived, of which the sound field transfer relationship from the reverberation field to the free field is obtained. In addition, for the purpose of obtaining the characteristic of the testing sound field with the general boundaries, the numerical method based on the ACTRAN software is utilized. According to obtaining the characteristic of the testing sound field in advance, the lowfrequency radiated sound power can be got by measuring the square pressure in a reverberation tank. The proposed method is checked by the experiment measurement. The test results show that by utilizing the proposed method, the low-frequency radiated sound power of the underwater sound source has been accurately measured in the glass tank below the Schroeder cut-off frequency, of which the radiated sound power level deviation is less than $2 \mathrm{~dB}$ compared with the free field.

\section{2:15}

3pUWa6. Calibration of the matched filter beam output from the triplet portion of the Five Octave Research Array. Dale D. Ellis (Phys., Mount Allison Univ., 18 Hugh Allen Dr., Dartmouth, NS B2W 2K8, Canada, daledellis@gmail.com) and Jie Yang (Appl. Phys. Lab., Univ. of Washington, Seattle, WA)

The Five Octave Research Array (FORA) [K.M. Becker and J. R. Preston, Proceedings Oceans 2003, 2607-2610] includes a triplet section in which the elements are three omnidirectional sensors. The elements can be combined as a standard linear array to produce conical beam patterns, or the triplets can be used to form directional beams that allow for "left-right" discrimination. The beam output can then be matched filtered to produce high-temporal-resolution beams over the full $360^{\circ}$ azimuth, and identify scattering features in reverberation data. To extract scattering strengths, the calibration of the beams and the matched filter output must be known accurately. Here we look at results from the 2013 Target and Reverberation Experiment (TREX13), where data were received on the FORA array, then beamformed and matched filtered by various procedures that had different normalizations. The broadside beam and several natural beams of the linear array can readily be calibrated against a simple "exact" procedure. No simple procedure was obtained for the directional beams, but comparisons of the peaks of the features in the left and right triplet beams compare well with the peaks of the linear beams.

\section{2:30}

3pUWa7. Source localization in underwater waveguides using machine learning. Haiqiang Niu and Peter Gerstoft (Marine Physical Lab., Scripps Inst. of Oceanogr., 9500 Gilman Dr., San Diego, CA 92093-0238, hniu@ ucsd.edu)

The problem of underwater acoustic source localization is solved using an artificial neural network (ANN) under the machine learning framework. Source localization in a waveguide is posed as a classification problem by training a feed-forward neural network (FFN) with one hidden layer. In this paper, the acoustic pressure signals received by a vertical linear array are preprocessed by constructing the normalized cross-spectral density matrices (CSDM), which are used as input of the FFN. Each unit of the output layer represents one possible range (Here, for simplicity, source range is the only parameter that needs to be determined). Different from model-based localization methods such as matched field processing (MFP), ANN as a datadriven method can learn features from real acoustic data, thereby bypassing the sound propagation modeling step completely. Simulations show that FFN achieves a good performance in determining the source ranges even with deficient training data samples and low signal-to-noise ratios (SNRs). The validity of FFN in source localization is further demonstrated with vertical array data from Noise09 experiment where the ship is located successfully using FFN. 


\title{
Session 3pUWb
}

\section{Underwater Acoustics: Seabed and Sea Surface Interactions}

\author{
Charles W. Holland, Cochair \\ Applied Research Laboratory, The Pennsylvania State University, P.O. Box 30, State College, PA 16804 \\ Megan S. Ballard, Cochair \\ Applied Research Laboratories at the University of Texas at Austin, P.O. Box 8029, Austin, TX 78758
}

\section{Contributed Papers}

3:00

3pUWb1. A comparison between direct measurements and inference results from an acoustic propagation experiment in Currituck Sound. Megan S. Ballard, Jason D. Sagers, Kevin M. Lee, Andrew R. McNeese (Appl. Res. Labs. at the Univ. of Texas at Austin, P.O. Box 8029, Austin, TX 78758, meganb@arlut.utexas.edu), Preston S. Wilson (Mech. Eng. and Appl. Res. Labs., Univ. of Texas at Austin, Austin, TX), Thomas G. Muir (Appl. Res. Labs. at the Univ. of Texas at Austin, Austin, TX), and R. D. Costley (GeoTech. and Structures Lab., U.S. Army Engineer Res. \& Development Ctr., Vicksburg, MS)

An acoustic propagation experiment was conducted in Currituck Sound to characterize low frequency propagation in a very shallow-water environment with water depths of only a few meters. Signals from a Combustive Sound Source (CSS) were recorded on bottom mounted geophones and a vertical array of hydrophones. The CSS produces a broadband signal with significant low frequency energy, and the analysis presented in this paper focuses on frequencies below $2.5 \mathrm{kHz}$. A statistical inference method was applied to obtain an estimate of the sediment sound-speed profile as a function of depth in the seabed. During the experiment, in situ measurements of compressional and shear wave speed and attenuation were also collected 30 $\mathrm{cm}$ below the sediment-water interface. Bimorph bender elements were employed to generate and receive horizontally polarized shear waves in the frequency range from $200 \mathrm{~Hz}$ to $1 \mathrm{kHz}$. Compressional waves were measured using cylindrically shaped piezoelectric elements operated in the $5 \mathrm{kHz}$ to $100 \mathrm{kHz}$ frequency band. Sediment acoustics models were fit to the in situ wave speed and attenuation measurements to enable comparison with the inferred low frequency sound speeds. [Work supported by ERDC, ARL:UT, and ONR.]

\section{$3: 15$}

3pUWb2. On the dependence of the nonlinearity coefficient of marine silica sands on grain size, shape, and packing density. William Sanders, Allen Reed, and Warren Wood (Naval Res. Lab., Code 7433 Bldg. 1005, Stennis Space Ctr., MS 39529, wsanders@nrlssc.navy.mil)

Several applications of parametric sonars rely on utilizing nonlinear propagation of sound in marine sediments. Whereas it has been speculated that the nonlinearity coefficient for granular materials is expected to be relatively high, few measurements have been made to support the notion, nor has any theory been used to satisfactorily predict the parameter. Moreover, unlike fluids, the nonlinearity coefficient of granular materials does not follow from an equation of state, but is likely the composite effect of multiple mechanisms. Hence, we aim to compile a comprehensive set of measurements of the nonlinearity coefficient for a variety of sediments and conditions. This effort reports on the initial set of observations on unconsolidated quartz silica sands in water with grain sizes ranging from 0.05 to $1 \mathrm{~mm}$, a variety of shapes (angular, sub-rounded, and rounded), and a variation in packing density. [This work was supported by ONR.]
3pUWb3. Near-field measurements of a parametric source. William Sanders (Naval Res. Lab., Code 7433 Bldg. 1005, Stennis Space Ctr., LA 39529, wsanders@nrlssc.navy.mil), Allen Reed, and Warren Wood (Naval Res. Lab., Stennis Space Ctr., MS)

In order to characterize nonlinear interactions within granular materials, measurements were made in the near-field of a $50 \mathrm{kHz}$ transducer (an ITC 5270). High-resolution beam patterns were observed which reveal significant diffraction effects. Observed beam patterns were used to predict the difference frequency signal and this resulted in more than a $10 \mathrm{~dB}$ difference from that predicted by assuming constant amplitudes. This points to an inadequacy of the typical treatment of the parametric field as that due to collimated beams of constant amplitude in the near field, e.g. as in the Moffet and Mellen model. [This work was supported by ONR.]

\section{$3: 45$}

3pUWb4. Estimating seabed structure from perturbed layer resonance fringes. Charles W. Holland (Appl. Res. Lab., The Penn State Univ., P.O. Box 30, State College, PA 16804, cwh10@psu.edu)

Waves reflecting from layered media give rise to constructive and destructive interference fringes. These well-known fringes occur at integer multiples of half- and quarter-wavelength conditions respectively. When any of the layer boundaries are rough, the fringe pattern is perturbed. One class of observed perturbations is when the when the fringe exhibits a stairstep pattern in frequency and angle, instead of the smooth behavior predicted by theory. These perturbations can lead to difficulties for statistical inference when using the flat layer assumption. First, the physics behind the stair-step perturbations are described. Then, from the physics we show how to estimate or bound small-scale roughness parameters from the stair-step perturbations. Both simulations and measured data are employed to probe the information content of reflection data for small-scale roughness in layered media. [Research supported by the ONR Ocean Acoustics Program.]

\section{4:00}

3pUWb5. Volume scattering from turbidite sequences. Derek R. Olson (Appl. Res. Lab., Penn State Univ., 201 Appl. Sci. Bldg, University Park, PA 16802, olson.derek.r@gmail.com) and Charles W. Holland (Appl. Res. Lab., Penn State Univ., State College, PA)

The seabed environment near continental margins is commonly composed of turbidite sequences, which are alternating layers of silt/sand and pelagic clay (mud). Scattering can occur both from interface roughness structure, as well as volume heterogeneities within each layer. A modeling technique is detailed that takes into account the layering structure of a turbidite for propagation into and out of the sediment, and uses the Born approximation to model the scattering. This model properly accounts for effects of the layering structure on the incident field (instead of using an effective 
medium approximation), which is expected to be important particularly at high frequencies (relative to the scales of the layering). Model results from several types of environments will be shown, and also compared to bottom scattering data collected in the Ionian sea.

\section{4:15}

3pUWb6. Numerical study of three-dimensional sound reflection from corrugated surface. Youngmin Choo (Res. Inst. of Marine System Eng., Seoul National Univ., 1, Gwanak-ro, Gwanak-gu, Seoul 151 - 744, South Korea, sks655@snu.ac.kr), Heechun Song (Scripps Inst. of Oceanogr., San Diego, CA), and Woojae Seong (Naval Architecture and Ocean Eng. and Res. Inst. of Marine Systems Eng., Seoul National Univ., Seoul, South Korea)

When a sound wave propagates in a water medium bounded by a smooth surface wave, reflection from a wave crest can lead to focusing and result in rapid variation of the received waveform as the surface wave moves [J. Acoust. Soc. Am. 125, 66-72 (2009)]. In prior work, propagation paths have been constrained to be in a plane parallel to the direction of corrugated surface waves, i.e., a two-dimensional (2-D) propagation problem. In this paper, the azimuthal dependence of sound propagation as a three-dimensional (3-D) problem is investigated using an efficient, time-domain HelmholtzKirchhoff integral formulation. When the source and receiver are in the plane orthogonal to the surface wave direction, the surface wave curvature vanishes in conventional 2-D treatments and the flat surface simply moves up and down, resulting in minimal temporal variation of the reflected signal intensity. On the other hand, the 3-D propagation analysis reveals that a focusing phenomenon occurs in the reflected signal due to the surface wave curvature formed along the orthogonal plane, i.e., out-of-plane scattering.

$$
4: 30
$$

3pUWb7. Measurements and predictions of surface Doppler and arrival time spread in a variety of wind conditions. Sean Walstead (Naval Undersea Warfare Ctr., 1176 Howell St., Newport, RI 02841, sean.walstead@ navy.mil)

Measurements from a bistatic surface scattering experiment are presented. Acoustic waveforms in the $200 \mathrm{kHz}-400 \mathrm{kHz}$ (VHF) frequency regime were transmitted and received in a variable speed wind wave channel at the Scripps Institution of Oceanography. Physics based analytic predictions for Doppler shift and arrival time spread are compared to experimental measurements. The effect of source/receiver depth and surface wave shape on acoustic properties of the surface multipath are quantified as a function of wind speed. The importance of transmit waveform Doppler sensitivity of common underwater communications signals is quantified for various surface scattering regimes. This work has direct application to the improved performance of phase coherent underwater acoustic communications systems and the remote sensing of gravity-capillary waves.

\section{$4: 45$}

3pUWb8. Perturbation theory for mode coupling over a sloping penetrable bottom. Feilong Zhu (State Key Lab. of Acoust., Inst. of Acoust., Chinese Acad. of Sci., No.21, Bei-Si-Huan-Xi Rd., Haidian District, Beijing 100190, China, zhufl@mail.ioa.ac.cn) and Eric I. Thorsos (Appl. Phys. Lab., Univ. of Washington, Seattle, WA)

In environments with water depth variations, one-way modal solutions involve mode coupling. One approach is to model the depth variation as a series of steps, and use a new set of modes at each step (Evans, JASA, 1983). Higham and Tindle (JASA, 2003) developed an accurate and faster approach using perturbation theory to locally determine the change in mode functions at steps and limit the required number of new sets of mode functions. The method of Higham and Tindle is limited to low frequency $(<250$ $\mathrm{Hz}$ ). We have extended the coupled mode perturbation method so it can apply to higher frequencies. The approach will be described and some examples shown.

\section{5:00}

3pUWb9. Toward a test of the existence of the Biot slow wave in sand using layer resonances. Anthony L. Bonomo, Gabriel R. Venegas, Marcia J. Isakson, and Preston S. Wilson (Appl. Res. Labs., The Univ. of Texas at Austin, P.O. Box 8029, Austin, TX 78713, abonomo@arlut.utexas.edu)

Geoacoustic models based on Biot's theory of poroelasticity have found some success in predicting the interaction of underwater sound with sandy sediments. One of the unique features of Biot theory is its prediction that poroelastic media can support two types of compressional waves- the conventional compressional wave, referred to as the "fast wave," and an additional, slower compressional wave due to the out-of-phase motion of the saturating fluid with respect to the solid frame dubbed the "slow wave." While Biot-based sand models have been shown to accurately fit measured compressional sound speed and attenuation data, shear wave sound speed and attenuation data, bottom loss data, and backscattering strength data, the so-called slow wave has not been directly measured in sand. Rather than attempt to directly measure the slow wave, it is the goal of this work to confirm or disprove the slow wave's existence from the resonances it causes in thin sand layers. It is expected that these resonances would be apparent in measurements of normal-incidence bottom loss. Finite element models are used to demonstrate the possible effectiveness of this approach. Experimental design and preliminary measurements are also considered. [Work supported by ONR, Ocean Acoustics.] 


\section{OPEN MEETINGS OF TECHNICAL COMMITTEES}

The Technical Committees of the Acoustical Society of America will hold open meetings on Tuesday, and Wednesday. See the list below for the exact schedule.

These are working, collegial meetings. Much of the work of the Society is accomplished by actions that originate and are taken in these meetings including proposals for special sessions, workshops, and technical initiatives. All meeting participants are cordially invited to attend these meetings and to participate actively in the discussion.

Committees meeting on Tuesday, 29 November

Committee

Acoustical Oceanography

Animal Bioacoustics

Architectural Acoustics

Engineering Acoustics

Physical Acoustics

Psychological and Physiological

Acoustics

Structural Acoustics and Vibration
Start Time

7:30 p.m.

7:30 p.m.

7:30 p.m.

7:30 p.m.

7:30 p.m.

7:30 p.m.

7:30 p.m.
Room

Kahili

Coral 2

Lehua

South Pacific 4

South Pacific 2

South Pacific 3

South Pacific 1

Committees meeting on Wednesday, 30 November

Committee

Biomedical Acoustics

Musical Acoustics

Noise

Signal Processing in Acoustics

Speech Communication

Underwater Acoustics

\begin{abstract}
Start Time
7:30 p.m.

7:30 p.m.

7:30 p.m.

7:30 p.m.

7:30 p.m.

7:30 p.m.
\end{abstract}

Room

Coral 1

Kahili

South Pacific 4

South Pacific 1

Coral 4

Nautilus 


\title{
Session 4aAA
}

\section{Architectural Acoustics: Assorted Topics in Architectural Acoustics I}

\author{
Shinsuke Nakanishi, Cochair \\ Department of Engineering, Hiroshima International University, 5-1-1, Hiro-koshingai, Kure 737-0112, Japan \\ David S. Woolworth, Cochair \\ Oxford Acoustics, 356 CR 102, Oxford, MS 38655
}

\section{Contributed Papers}

8:30

4aAA1. Design, construction, and evaluation of an omni-directional loudspeaker (Dodecahedron). Maryam Landi, Vahid Naderyan (Dept. of Phys. and Astronomy \& National Ctr. for Phys. Acoust., Univ. of MS, NCPA, 145 Hill Dr., University, MS 38677, mlandi@go.olemiss.edu), and David S. Woolworth (Roland, Woolworth \& Assoc., Oxford, MS)

Dodecahedron loudspeaker (Dodec) is an omni-directional sound source in the shape of a 12-sided loudspeaker with each side being a pentagon. The omni-directionality of this sound source makes it mainly applicable in room acoustical and sound insulation measurements and research as it can excite and saturate the room as much as possible. Dodec is a good approximation of a point sound source. Commercially available omni-directional loudspeakers are not economically efficient for some purposes. This paper outlines a simple and inexpensive Dodec loudspeaker designed, constructed, and evaluated using ISO 140 and ISO 3382 standards as reference as part of an independent coursework. The Dodec was evaluated in an anechoic chamber. The measured directivity of this Dodec meets the standards for omnidirectionality at all frequencies.

\section{$8: 45$}

4aAA2. Room acoustic measurement techniques for large venues (greater than 10,000 cubic meters) in 2016. Jason R. Duty, Jeremy L. Decker, and Peter K. Holst (Charles Salter Assoc., 130 Sutter St., Fl. 5, San Francisco, CA 94104, jason.duty@cmsalter.com)

From sine sweeps to maximum-length sequence (MLS) type signals, balloon explosions to the yachting cannon, options to measure room acoustics in large venues have increased over the past couple of decades. There are new developments by academia and equipment manufacturers, as well as improvements on older methods. What are these new methods? What acoustical characteristics can one measure with each method? This presentation describes the different source options to measure room acoustical characteristics in larger spaces.

\section{9:00}

4aAA3. Acoustical energy relations in auditoriums. Jianliang Gao, ShiuKeung Tang (Bldg. Services Eng., The Hong Kong Polytechnic Univ., Rm. ZS801, 8/F, Block Z, 181 Chatham Rd. South, Hunghom, Kowloon 999077, Hong Kong, jianliang.gao@connect.polyu.hk), and Yuezhe Zhao (State Key Lab. of Subtropical Bldg. Sci., South China Univ. of Technol., Guangzhou, Guangdong, China)

Extensive objective energy-based parameters have been measured in three auditoriums ( 2 scale models and 1 site survey) in the present study. Mono-aural measurements in the frequency range from $125 \mathrm{~Hz}$ to $4000 \mathrm{~Hz}$ (octave band) were conducted in unoccupied auditoriums according to the standard ISO 3382-1: 2009. Acoustical parameters, namely, the clarity $C_{80}$, the definition $D_{50}$, the early sound level $L e$, the late sound level $L l$, the sound strength $G$ and the center time $T s$, have been estimated through analyzing the impulse responses measured in each auditorium. These parameters were spectrally averaged according to the most accepted criteria for sound quality evaluation in auditoriums and were expressed as a function of source-receiver distance. The experimental results were compared with predictions by classical and existing theoretical models proposed for concert halls and churches. A semi-empirical model based on the measured values of early and late sound levels is proposed in this work. The good agreement between predicted values and experimental data of clarity, definition, sound strength, and center time in the auditoriums analyzed indicates that the newly proposed model can be used for design predictions with reasonable accuracy.

\section{9:15}

4aAA4. Elucidation of a mechanism of acoustic impedance technique using two cardioid microphones and ensemble averaging method. Kazuma Hoshi and Toshiki Hanyu (Dept. of Architecture and Living Design, Nihon Univ., 7-24-1, Narashinodai, Funabashi, Chiba 2748501, Japan, hoshi@arch.jcn.nihon-u.ac.jp)

Recently, there is growing need for in-situ impedance measurement of several materials. We propose using two cardioid microphones (called C-C sensor) for it. C-C sensor has many advantages against two omnidirectional microphones (called P-P sensor) and one omnidirectional microphone and sound velocity (called P-U sensor). I reported the possibility of using C-C sensor for measuring acoustic impedance on 12th Western Pacific Acoustics Conference 2015 (WESPAC2015) and International Congress on Acoustics 2016 (ICA2016). When measuring some porous materials, C-C method can measure acoustic impedance as same as using P-P method, and C-C method can do it from $100 \mathrm{~Hz}$ to over $10 \mathrm{kHz}$. Next, we tried to elucidate the mechanism of using C-C sensor and Ensemble Averaging (EA) method using numerical experiments. As a result, the range in application of C-C sensor for measuring acoustic impedance was clarified. In this presentation, we'll introduce the measuring technique and mechanism.

\section{9:30}

4aAA5. Absorption coefficient: Dead or alive? Results of the "ASTM C423 Inter Laboratory Study". Ronald Sauro (NWAA Labs, Inc., 90 Tower Blvd., Elma, WA 98541, audio_ron@msn.com)

In 2009 and 2011, papers were presented questioning the procedures used to measure absorption and the conversion to an "Absorption Coefficient." Part of the problem was the results of these conversions resulted in coefficients above 1.0. These results indicated a problem with the process or with the conversion to an "Absorption Coefficient." Since that time enough data have been presented to the ASTM to result in an Inter Laboratory Study (ILS) on the efficacy of ASTM C-423 and other similar worldwide standards. Many corporate and independent laboratories took part in this study and the results of this ILS will be presented in this paper. These results 
helped show that the process of measuring absorption and the calculation of the "Absorption Coefficient" has had a basic flaw in it ever since it was first proposed in the 1920s. Proposed changes are discussed as well as proposed new calculations for use in calculating absorption in rooms. This should result in more accurate and consistent calculations of reverberation times for acoustic consultants. It is hoped that these results will result in a new standard for these measurements.

\section{9:45}

4aAA6. Sound absorption of Helmholtz resonators included a neck extension built in surface panel. Shinsuke Nakanishi (Dept. of Eng., Hiroshima Int. Univ., 5-1-1, Hiro-koshingai, Kure 737-0112, Japan, s-nakani@ it.hirokoku-u.ac.jp)

Acoustic resonant absorber like a perforated panel or a Helmholtz resonator can be tuned at a low frequency by extending its neck or enlarging cavity volume. However, a total size of the resonators is often quite large when the neck or the cavity is simply extended for tuning at a low frequency. Previous researchers have studied Helmholtz resonator shortened in its size by subsided neck into back air cavity, and confirm that this resonator is tuned at low frequency without a deep cavity. The author has studied the effects of a winding built-in neck extension to sound absorption of perforated panels, which shows same effects as the subsided neck into back cavity. This study obtains sound absorption coefficient by measuring surface acoustic impedance at Helmholtz resonator, and discusses sound absorption of the resonator included various neck extensions built in a surface panel. Discussions in this paper focus effects of path length, patterns, or number of turns of the winding neck extension and cavity volume to the sound absorption of the Helmholtz resonator.

\section{0:00-10:15 Break}

\section{0:15}

4aAA7. A case for modular partitions in healthcare facilities. Basel Jurdy and Kathleen Gray (Acoust., Stantec, 4100 194th St. SW Ste. 400, Lynnwood, WA 98036, kathleen.gray@stantec.com)

In healthcare, good acoustic separation between rooms is critical for speech privacy and user comfort. While there are multiple factors that contribute to acoustic separation, this is usually achieved by increasing the acoustical performance of the separating architecture between rooms. This is done by running a wall of a specific Sound Transmission Class (STC) to structure to minimize flanking paths. However, running a modular wall up to or slightly above the ceiling tile can reduce the cost of construction and improve programmatic flexibility. In situations where the wall runs only to the ceiling, the acoustic ceiling tile (ACT) and plenum condition begins to play a large role in the acoustic performance of the separating architecture. Given a high-performing ACT, a high-STC modular wall, and partially open plenum, is there a condition where good speech privacy can be achieved between rooms without running walls to structure? This was examined with a mock-up for EvergreenHealth in Kirkland, WA. The results show that given certain plenum conditions and a CAC-40 tile, the separating architecture using a modular partition can perform at the minimum STC-45 separation between patient rooms set by the 2014 Facility Guidelines Institute (FGI) for healthcare facilities.

10:30

4aAA8. Objective acoustical quality in healthcare office facilities. Murray Hodgson (UBC, 2206 East Mall, Vancouver, BC V6T1Z3, Canada, murray.hodgson@ubc.ca)

Health-care facilities include many non-clinical office spaces for administrative staff; the role of acoustics in these spaces has been underexplored. This paper discusses the acoustical part of a study of indoor environmental quality (IEQ) in 17 healthcare office facilities. Physical acoustical measurements were made in six types of rooms, some with sound-masking systems, to determine the acoustical characteristics, assess their quality, relate them to the building designs, and develop prediction models. Background-noise levels were measured in the occupied buildings. In the unoccupied buildings, measurements were made of reverberation times, and "speech" levels needed to calculate speech intelligibility indices for speech intelligibility and speech privacy. In open offices, sound-level reductions per distance doubling (DL2) were measured. Noise isolations of internal partitions of different designs (double-plasterboard construction, modular or built in-situ, rising to the suspended ceiling or to the floor-ceiling slab, without and with doors, different amounts of glass) were measured. The acoustical characteristics were compared to design criteria to evaluate their acceptability. The results are presented, and are related to room type and partition design. An empirical model for predicting partition noise isolation, developed using regression techniques, is discussed. The knowledge gained from this study informs the decision-making of designers and facilities management for upgrades and future design projects.

\section{0:45}

4aAA9. Subjective acoustical quality in healthcare office facilities. Murray Hodgson (UBC, 2206 East Mall, Vancouver, BC V6T1Z3, Canada, murray.hodgson@ubc.ca)

Health-care facilities include many non-clinical office spaces for administrative staff; the role of acoustics in these spaces has been underexplored. This paper discusses the acoustical part of a study of indoor environmental quality (IEQ) in 17 healthcare office facilities. A subjective survey assessed office worker perceptions of their environments in general, and satisfaction with the acoustics. Self-reported productivity, well-being, and health outcomes were also captured. Satisfaction was lower with acoustics than with other aspects of IEQ. Satisfaction results were related to room type (e.g., open plan vs. shared vs. private office) and the absence or presence of a sound-masking system. Acoustics was the most important aspect of IEQ in predicting occupant satisfaction and well-being. Regression models were developed to predict workplace satisfaction, well-being, and job satisfaction from survey responses. Results of physical acoustical measurements showed very low correlations with subjective responses. The knowledge gained from this study informs the decision-making of designers and facilities management for upgrades and future design projects.

\section{1:00}

4aAA10. Airport Cooperative Research Project 02-51: Evaluating methods for determining interior aircraft noise levels. Randy Waldeck (CSDA Design Group, 475 Sansome St., Ste. 800, San Francisco, CA 94111, rwaldeck@csdadesigngroup.com), Paul Schomer (Schomer \& Assoc., Champaign, IL), and John Freytag (Freytag \& Assoc., LLC, Newport Beach, CA)

This paper documents the results of a study of acoustical testing methods used to quantify facade noise reduction measurements for airport sound insulation programs. The research was performed for the National Academy of Sciences as Airport Cooperative Research Project (ACRP) 02-51. Field measurements, in combination with acoustical calculations, were used to determine which methods are best suited for airport sound insulation programs. Loudspeaker (i.e., exterior elevated, exterior ground level, and interior), flyover, and sound intensity measurements were conducted throughout the United States. In addition, air infiltration measurements and noise reduction calculations were made. Results from each method were analyzed and compared. An analysis of each method's margin of error is presented, along with Best Practices for each method. Finally, a decision matrix for selecting the appropriate acoustical testing method is included.

\section{1:15}

4aAA11. Low frequency analysis of acoustical parameters of emotional speech for use with functional magnetic resonance imaging. Peter M. Moriarty (Graduate Program in Acoust., Penn State Univ., 201 Appl. Sci. Bldg., University Park, PA 16802, moriarty@psu.edu), Michelle C. Vigeant (Graduate Program in Acoust., Penn State Univ., State College, PA), Pan Liu, Rick Gilmore, Rachel Wolf, and Pamela M. Cole (Psych., The Penn State Univ., State College, PA)

A mother's voice plays a pivotal role in the social development of her child. Extreme exposure to vocalized anger in the home environment can weaken a child's ability to manage their emotions and potentially compromise basic societal opportunities. It is therefore critical to understand the vehicle of the emotional content in speech, known as prosody, and specifically 
the neurological interactions of a mother's voice with her child. Functional magnetic resonance imaging (fMRI) was used to measure children's brain activity while stimulated by recorded sounds of mothers speaking with an angry, happy, sad, and neutral affect. Activity was measured in response to prosodic (e.g., pitch period variations, speech rate) and voice quality features (e.g., spectral distribution, jitter). Block-style fMRI analysis often uses mean values of these acoustic features taken over the length of each utterance, usually lasting many seconds or repetition time (TR) multiples. In an effort to retain some of the time-varying prosodic information of the mothers's speech, this study included utterance-level spectral analysis of these acoustic features below the Nyquist frequency of the MRI. Preliminary results support the utility of such low-frequency analysis as a method for generating affect-differentiable information. [Work supported by NIH R21 104547.]

\title{
Session $4 \mathrm{AAB}$
}

\section{Animal Bioacoustics, Signal Processing in Acoustics, and Speech Communication: Sequence Information in Mammalian Vocal Call Production I}

\author{
Marie A. Roch, Cochair \\ Dept. of Computer Science, San Diego State Univ., 5500 Campanile Dr., San Diego, CA 92182-7720 \\ Tadamichi Morisaka, Cochair \\ School of Marine Science and Technology, Tokai University, 3-20-1, Orido, Shimizu-ku, Shizuoka 4248610, Japan
}

Chair's Introduction-8:30

\section{Invited Papers}

\section{$8: 35$}

4aAB1. Examining the temporal structure and information entropy of leopard seal calling bouts. John R. Buck (ECE Dept., UMass Dartmouth, 285 Old Westport Rd., Dartmouth, MA 02747, johnbuck@ieee.org) and Tracey L. Rogers (School of BEES, Univ. of New South Wales, Sydney, NSW, Australia)

Leopard seals (Hydrurga leptonyx) produce sequences of stereotyped sounds, or bouts, during their breeding season. The seals share common sounds but combine them in individually distinctive sequences. This study examines the underlying structure of the calling bouts by estimating the information entropy of the sound sequences with three entropy estimators. The independent identically distributed (IID) model estimates entropy from the simple frequencies of each sound. The Markov model estimates entropy from the frequency of pairs of sounds. Finally, the nonparametric sliding window match length (SWML) estimator exploits a relationship between the information entropy and the average subsequence match length. A better model for a given sequence achieves a lower entropy estimate. This study analyzed the calling bouts of 35 leopard seals recorded during the 1992-1994 and 1997-1998 Antarctic field seasons. The decrease of entropy estimates between the IID and Markov models for all seals analyzed confirmed the presence of temporal structures in the bouts. For twenty-one of thirty-five seals, the SWML entropy estimate was not significantly less than the Markov estimate, suggesting that a first-order Markov model accurately represents the structure of their bouts. [Work supported by the Australian-American Fulbright Commission and the Australian Research Council.]

\section{8:55}

4aAB2. Sequential calls and associated behavior in captive bearded seals (Erignathus barbatus). Daisuke Mizuguchi (Hokkaido National Fisheries Res. Inst., National Res. and Development Agency, Japan Fisheries Res. and Education Agency, 116 Katsurakoi, Kushiro, Hokkaido 085-0802, Japan, mizgangan@gmail.com), Masatoshi Tsunokawa, Mamoru Kawamoto (Otaru Aquarium, Otaru, Hokkaido, Japan), and Shiro Kohshima (Wildlife Res. Ctr., Kyoto Univ., Kyoto, Japan)

Bearded seals produce complex underwater sounds suggested to function as territorial and/or courtship signals. However, little is known about behavioral context of the vocalization, mainly because direct observation is difficult in the wild. In this study, we recorded underwater sounds and behaviors of three captive bearded seals (an adult male and two adult females) in Otaru aquarium, Japan, between March 2012 and April 2016, in order to identify the caller and to record association between vocalization and behaviors. The male continuously vocalized from December until April with a peak in March, equivalent to breeding season in the wild. In contrast, the females vocalized only 1-2 weeks in March or April. The male and the females produced 3 and 5 call types, respectively, sharing 2 call types. The 3 call types of the male occurred in a regularly ordered sequence that was stable during this study. The females responded to the male sequential calls by nuzzling against the throat of vocalizing male during the breeding season, when the females also actively vocalized. Our results suggest that underwater vocalization of bearded seals might be used as a courtship signal between male and female possibly to advertise their reproductive status. 
4aAB3. Temporal patterning differences in contact calls among odontocetes' species. Tadamichi Morisaka (School of Marine Sci. and Technol., Tokai Univ., 3-20-1, Orido, Shimizu-ku, Shizuoka 4248610, Japan, chaka@tokai-u.jp)

Contact calls in odontocetes (toothed whales) have information of individual identity or group identity. Sound types used for contact calls, however, differ among species. Signature whistles in bottlenose dolphins, which are thought to function as "names" in human, are tonal sounds, whereas PS1 calls in beluga whales are pulsed sounds. Both signature whistles and PS1 calls have usually exchange pattern that calls are followed by calls by other individuals within around 1 second. Dolphins tend to produce next call when no individuals call back. Contact calls are usually produced repeatedly in a bout. Moreover, signature whistles often include multiple loops, sometimes with breaks between loops. The duration of breaks between loops is different between bottlenose dolphins and Indo-Pacific bottlenose dolphins. These results suggest that temporal patterning in contact calls in odontocetes is important for species identity.

\section{Contributed Papers}

9:35

4aAB4. Intra-click time-frequency patterns across the echolocation beam of a beluga whale. Josefin Starkhammar (Biomedical Eng., Lund Univ., Faculty of Eng., Hoby 371, LUND 225 91, Sweden, josefin.starkhammar@bme.lth.se), Isabella Reinhold (Mathematical Statistics, Ctr. for Mathematical Sci., Lund Univ., Faculty of Eng., Lund, Sweden), Patrick Moore, Dorian Houser (National Marine Mammal Foundation, San Diego, CA), and Maria Sandsten (Mathematical Statistics, Ctr. for Mathematical Sci., Lund Univ., Faculty of Eng., Lund, Sweden)

The echolocation beam of toothed whales has been studied ever since it was first discovered in 1960. Recent studies have focused on the frequency distributions across the cross sections of the beams. Other studies have focussed on describing the entire acoustic field around the animal. However, no one has yet described the timing of each frequency component in the main lobe beam in relation to the other frequency components. Even though the echolocation clicks of broadband click species like the beluga whales (Delphinapterus leucas) are short in time (around $70 \mu \mathrm{s}$ ), previous results have shown indications on a frequency dependence with time, within each click. Little is known about the details of how the signal is generated and transmitted into the water. Investigations of when in time the frequency components occur within each click would give us further knowledge to how the signals are generated. This study takes a closer look at these intraclick time-frequency patterns across the echolocation beam of a beluga whale. This is done by analyzing echolocation clicks with a novel reassigned spectrogram method, developed especially for separating and determining time and frequency locations of multi-component transient signals.
9:50

4aAB5. Nonlinearities in the vocalizations of Stenella species in the Southwest Atlantic Ocean. Juliana R. Moron, Franciele R. de Castro, and Artur Andriolo (Laboratório de Ecologia Comportamental e Bioacústica LABEC, Universidade Federal de Juiz de Fora, Rua José Lourenço Kelmer, Juiz de Fora, Minas Gerais, Brazil, julianamoron@hotmail.com)

Some complex features found in tonal emissions may be the result of nonlinear phenomena in sound production systems, which may result in individually acoustic signals highly variable and complex. From the analysis of five opportunistic acoustic records of distinct groups, two of spinner (Stenella longirostris) and three of Atlantic spotted dolphins (Stenella frontalis), we registered the emission of six different types of nonlinearities. Spinner dolphins had less events presenting mostly biphonation in the form of nonparallel bands and subharmonics. Atlantic spotted dolphins presented a higher rate of these features presenting mostly biphonation and screams on their vocalizations. Although the exact function of these processes is still being investigated, recent documentation in several species suggests that they may play a communicative role. Describing the occurrence of these characteristics can indicate their level of importance, as well as its structure may be indicative of its function. This is the first record of these events in Stenella species in the Southwest Atlantic Ocean. Future studies that allow to associate the behavior of these species while emitting these signals would greatly increase the understanding of these events. This study was funded by Chevron.

10:05-10:20 Break

Invited Papers

$10: 20$

4aAB6. Music in the brain: The neuronal control of bird song. Michale Fee (BCS, MIT, 77 Massachusetts Ave., 46-5133, Cambridge, MA 02139, fee@mit.edu)

Whether we are speaking, swimming, or playing the piano, we are crucially dependent on our brain's capacity to reliably step through a learned sequence of states. Songbirds provide a marvelous animal model in which to study this phenomenon. Using newly developed technologies for monitoring and manipulating the activity of neurons in the brain of singing bird, we have identified circuits in the songbird brain that perform key functions of vocal production and learning. One of these circuits produces a highly precise representation of time in the brain—essentially a clock. Another circuit produces highly variable output that drives large fluctuations in the songs of juvenile birds, allowing them to creatively explore different vocal patterns during learning. I will combine these observations to propose a simple theory of how brains learn and perform such sophisticated behaviors as speech and music. 
4aAB7. Neural-inspired segmentation of audio streams into phone-like units. Marie A. Roch (Dept. of Comput. Sci., San Diego State Univ., 5500 Campanile Dr., San Diego, CA 92182-7720, marie.roch@sdsu.edu)

Recent work has shown that the human preauditory and/or auditory cortex is likely to play a role in acoustic landmark processing, such as the recognition of syllable and phoneme boundaries. Neurons appear to track acoustic envelopes with neural activity corresponding well with acoustic landmarks. These structures have also been observed in non-human primates, suggesting that acoustic landmark processing could be present in non-human primates and have an evolutionary role. Should landmark processing occur in non-humans, it could provide new methods for approaching animal communication. In this work, we use a simplified computer model to examine envelopes of acoustic signals that were filtered through a series of octave spaced band-pass filters. Peaks are detected in the signal envelopes with closely spaced peaks suppressed for a period of time after the first detection. Preliminary work shows an ability to detect human phoneme boundaries with a recall of $45 \%$ and a precision of $72 \%$ on the TIMIT speech corpus. The TIMIT corpus contains phonemelevel transcriptions and successful matches were defined as detecting landmarks within $20 \mathrm{~ms}$ of the over quarter million TIMIT phoneme boundaries. We also show qualitative results of the system for segmenting non-human primate calls into phone-like units.

\section{1:00}

4aAB8. Call concatenation in non-primate mammals. Marta B. Manser (Animal Behaviour, Univ. of Zurich, Winterthurerstr. 190, Zurich, Zurich 8057, Switzerland, marta.manser@ieu.uzh.ch)

Animals produce a variety of call sequences, from simple repetitions of the same acoustic unit to the combination of different, meaningful acoustic units resulting in a new meaning. On the example of several highly vocal mongoose species, including meerkats (Suricata suricatta), and banded mongoose (Mungos mungo) I identify the composition of different acoustic combinations and relate them to their function and meaning. I discuss this in regards to the underlying cognitive mechanisms and the differences and similarities in relation to other combinatorial communication systems in birds, but particularly shown for primates, and also to human language.

\section{Contributed Paper}

\section{1:20}

4aAB9. Stereotypy and variability differ between humpback whale (Megaptera novaeangliae) phrase types offering structural support for the hypothesis that song is multi-message display. Anita Murray, Rebecca A. Dunlop (Cetacean Ecology and Acoust. Lab., The Univ. of Queensland, Gatton, QLD 4343, Australia, anita.murray1@uqconnect.edu.au), Anne W. Goldizen (Behavioural Ecology Res. Group, The Univ. of Queensland, St Lucia, QLD, Australia), and Michael J. Noad (Cetacean Ecology and Acoust. Lab., The Univ. of Queensland, Gatton, QLD, Australia)

Humpback whale studies support inter- and/or intrasexual song functions, suggesting dual functionality. Some animals produce multi-message displays with dual functionality. Stereotyped components, for mate attraction and/or male-male interactions, convey species/group membership. Variable components, for courtship, convey male quality. If humpback whale song is a multi-message display, then song should include both stereotyped and variable components. Recordings of east Australian males (2004:8, 2011:9) were used to investigate this hypothesis. Song comprises "units" arranged into sequences ("phrases") that are repeated ("themes"). Themes are characterized by different phrase types. Variation among phrases repeated within a theme ("phrase variants") is mostly overlooked by other studies. Whether some phrase types are more variable than stereotyped is unknown. To assess this, repertoire size (number of unit types and phrase variants) was determined per phrase type. Whether phrase variants were unique to individuals or shared were also determined. Unit types were defined by self-organizing maps. Levenshtein distances between phrases with cluster analyses determined phrase types and phrase variants illustrating a novel application of these methods. Stereotypic phrase types contained shared phrase variants and limited repertoire. Variable phrase types contained unique phrase variants and large repertoire. These results support the hypothesis that song is a multi-message display. 


\title{
Session 4aAO
}

\section{Acoustical Oceanography, Animal Bioacoustics, and Signal Processing in Acoustics: Acoustic Scattering by Aquatic Organisms I}

\author{
Kelly J. Benoit-Bird, Cochair \\ College of Earth, Ocean, and Atmospheric Sciences, Oregon State University, 104 COEAS Admin Bldg., Corvallis, OR 97331
}

Kouichi Sawada, Cochair

Fisheries Technology, National Research Institute of Fisheries Engineering, FRA, 7620-7, Hasaki,

Kamisu 3140408, Japan

Timothy K. Stanton, Cochair

Woods Hole Oceanographic Institution, Dept. Appl. Ocean. Phys. \& Eng., Woods Hole Oceanographic Institution, Woods Hole, MA 02543

Chair's Introduction-8:15

\section{Invited Papers}

8:20

4aAO1. Emergent pattern and underlying process: The what, why, and how of fish schooling. Julia K. Parrish (School of Aquatic and Fisheries Sci., Univ. of Washington, 1492 NE Boat St., Seattle, WA 98195, jparrish@uw.edu)

Over half of the world's fish aggregate at some point in their lives. As larvae, juveniles and/or adults, fish assemble into dense threedimensional groups that last hours to lifetimes. The largest single stock fisheries in the world-anchoveta in Peru and pollock in the Bering Sea - are schooling fish. At several times the entire world's fish catch, the myctophid aggregations making up the deep scattering layer form the world's largest school. But are all schools alike? Are the "rules of fish schooling" at the scale of the individual the same across all species? Do different rules produce similar emergent pattern scaled up to the group, population, and ecosystem? Could differences across these scales from individual behavior to population response be determined acoustically, allowing technology to inform behavior, ecology, and conservation? This talk explores the diversity of ways that fish assemble, fiss and fuse, and disaggregate on a daily, seasonal, and annual basis through three related lens: the patterns of schooling, reasons why fish school, and analysis of how fish maintain the physical structure of the school.

\section{8:40}

4aAO2. Coastal observation systems to monitor physical, chemical, and biological parameters. Hidekatsu Yamazaki (Dept. of Ocean Sci., Tokyo Univ. of Marine Sci. and Technol., 4-5-7 Konan, Minato, Tokyo 108-8477, Japan, hide@kaiyodai.ac.jp), Eiji Masunaga (Ibaraki Univ., Ibaraki, Japan), Scott Gallager (Woods Hole Oceanogr. Institution, Woods Hole, MA), Mamoru Tanaka, Marika Takeuchi, Kazuo Amakasu (Dept. of Ocean Sci., Tokyo Univ. of Marine Sci. and Technol., Tokyo, Japan), and Hayato Kondo (Dept. of Maritime System Eng., Tokyo Univ. of Marine Sci. and Technol., Tokyo, Japan)

We have developed a free-fall multi-parameter profiler (YODA Profiler) to measure various physical and biological parameters in coastal ocean. We found internal bores create a strong mixing event. Sediment resuspension is associated with the mixing event and also AZFP detected fish school at the front of bore. We have deployed a cable observatory system (Oshima Coastal Environment data Acquisition Network System, OCEANS) in a coastal area to monitor coastal ecosystem continuously. OCEANS can measure various physical, chemical and biological parameters simultaneously, and operates a plankton imaging system (Continuous Plankton Imaging and Classification System, CPICS). Based on acquired images of phytoplankton and zooplankton, we are investigating how planktonic biodiversity is affected by multi-scale physical processes, such as Kuroshio and internal waves. We are developing a technique to predict the biodiversity of plankton from three-dimensional hydrodynamic model using a newly developed plankton ecosystem model. We are also developing a AUV (MEMO-pen) that carries CPICS as well as microstructure profiler (TurboMAP) in order to simultaneously observe turbulence and plankton. We will introduce the most recent results from these observation systems. 


\section{Contributed Papers}

9:00

4aAO3. Seasonal and interannual changes in the sound scattering layer at deep-sea floor in the Amundsen Sea, Antarctica. Hyoung Sul La, Keyhong Park (Korea Polar Res. Inst., Songdo-miraero 26, Yeonsu-gu, Incheon 406-840, South Korea, hsla@kopri.re.kr), Anna Wåhlin (Univ. of Gothenburg, Gothenburg, Sweden), Ho Kyung Ha (Inha Univ., Incheon, South Korea), Angus Atkinson (Plymouth Marine Lab., Plymouth, United Kingdom), Sophie Fielding (Br. Antarctic Survey, Cambridge, United Kingdom), Dong Sun Kim (Korea Inst. of Ocean Sci. and Technol., Ansan, South Korea), Eun Jin Yang, Tae Wan Kim, SangHoon Lee (Korea Polar Res. Inst., Incheon, South Korea), JungHo Im (Ulsan National Inst. of Sci. and Technol., Ulsan, South Korea), and Hyoung Chul Shin (Korea Polar Res. Inst., Incheon, South Korea)

Vertical migration of zooplankton is ubiquitous behavior in marine plankton community; however, seasonal and interannual behavior are little observed in the deep sea under seasonal varying sea ice. Here, the first evidence that sound scattering layers of zooplankton can support the knowledge for understanding the effect of climate change is presented, based on four-years acoustic backscattering strengths in the Amundsen Sea, Antarctica. Amundsen Sea is a biological hotspot region with the rapid oceanic melting of the ice shelf as well as the most productive (per unit area) in the Antarctic. High-temporal resolution profiles of acoustic backscattering strength collected from a bottom-moored, upward looking Acoustic Doppler Current Profiler were examined to describe the temporal variation of sound scattering layers. Our observations show that sound scattering layers exhibited clear diel, seasonal, and interannual pattern associated with solar radiation, sea ice concentration, and phytoplankton biomass. The timing of seasonal variation is also closely related with climate index for Southern Annular Mode and El Niño Southern Oscillation. This observation points out that acoustic signals from sound scattering layers could be a proxy to understand zooplankton ecosystem response to climate shifts.

\section{9:15}

4aAO4. Development and field application of early warning system for harmful algal blooms (red-tide) early warning using ultrasound wave, Korea. Donhyug Kang, Hansoo Kim, Seungwon Jung, Mira Kim (Korea Inst. of Ocean Sci. \& Technol., 787 Haeanro, Ansan, Seoul 15627, South Korea, dhkang@kiost.ac.kr), and Byoung-Kweon Kim (Syscore Inc., Seoul, South Korea)

The harmful algal blooms (HABs) are affecting the ecosystems, coastal fisheries, and social economics in Korea. In the case of 2013 summer season, massive damage due to HABs (species: Cochlodinium polykrikoides) was reached to \$23 million during August, 2013 in Korea. The most important things for minimizing of HABs damage is rapidly detection in initial stage. For this purpose, we have developed a real-time HABs detection system using ultrasound. The integrated system is composed by several sensors (3.5 MHz frequency, temperature and salinity, Chlorophyll, Dissolved oxygen), power supply, network communication, and remote control modules. For evaluating performance of the developed system, several experiments were intensively implemented in the laboratory and in-situ environments during summer seasons, 2013-2016. As a result, both of the performance tests are confirming the acoustic backscattering signal increases with the number of $C$. polykrikoides. Furthermore, the integrated system shows successfully in-situ performance for real-time red-tide detection and corresponding environmental data.

\section{9:30}

4aAO5. Comparison of the volume backscattering strength measured by EK60 and EK80. Tohru Mukai (Faculty of Fisheries Sci., Hokkaido Univ., 3-1-1 Minato, Hakodate, Hokkaido 0418611, Japan, mukai@fish. hokudai.ac.jp) and Kazuo Amakasu (Tokyo Univ. of Marine Sci. and Technol., Tokyo, Japan)

Walleye pollack (Gadus chalcogrammus) resources are abundant around Hokkaido in Japan. The acoustic survey of the walleye pollack using the quantitative echo-sounder has been performed around Hokkaido since 1995.
The quantitative echo-sounder that is mainly used for this survey is the EK60 system consisting of GPT + ER60 software made by Simrad. On the other hand, EK80 system using the WBT has been released in 2015, and the acoustic survey will be conducted by EK80 system from now. Therefore, it is necessary to examine whether the acoustic data measured by the EK60 and EK80 systems are identical. In this study, in the area where sound scattering layer was seen, these two systems were used alternately on the same track line to examine whether there are differences in the acoustic data to be measured. Systems used in this study were GPT in frequency 38,120 , and $200 \mathrm{kHz}$, controlled by ER60 (Ver.2.4.3) and WBT in CW mode at 120, $200 \mathrm{kHz}$ controlled by EK80 (Ver.1.8.3). The acoustic data of the same transect were collected by both systems. The difference of SV between both systems will be discussed.

\section{9:45}

4aA06. Size estimation of walleye pollack (Theragra chalcogramma) by using a broadband split-beam system. Tomohito Imaizumi (National Research Inst. of Fisheries Eng., Japan Fisheries Res. and Education Agency, 7620-7 Hasaki, Kamisu-shi 314-0408, Japan, imat@affrc.go.jp), Kazuhiro Sadayasu (Marine Fisheries Res. and Development Ctr., Japan Fisheries Res. and Education Agency, Yokohama-shi, Japan), and Tomonari Akamatsu (National Res. Inst. of Fisheries Sci., Japan Fisheries Res. and Education Agency, Yokohama-shi, Japan)

Recently, broadband quantitative echo sounders have been developed. These systems can measure single echo traces from individual fish with high range resolution. They can be used for the accurate size distribution by measuring the target strength. On the other hand, walleye pollack is one of the important species for stuck management in Japan. They form a school at juvenile stage. We compared the captured fish size using bottom trawl net with the acoustically estimated values. We conducted the survey in July 2015 in east side of off Hokkaido, Japan, using the fisheries research vessel Kaiyo-maru No. 5 (495 tons), which is equipped with narrowband echo sounder transducers (EK-60, Kongsberg, 38, 70, 120, and $200 \mathrm{kHz}$ ). The broadband split-beam transducer (FSS-SBX, Furuno) was deployed at the ship sideboard. All of the clock of the system were synchronized for comparison of data. More single echo traces could be measured using the broadband system than those measured by the narrowband system. Fork lengths were estimated at $3 \sim 70 \mathrm{~cm}$ based on the maximum normalized target strength value: $-60 \mathrm{~dB}$. We confirmed that good agreement of size distribution between catch and acoustic estimation. The broadband systems may be helpful tool for estimating body size of fish.

\section{0:00}

4aA07. Potential for broadband acoustics to improve stock assessment surveys of midwater fishes. Christopher Bassett (NOAA Fisheries, Alaska Fisheries Sci. Ctr., National Marine Fisheries Service, Alaska Fisheries Sci. Ctr., Resource Assessment and Conservation Eng., 7600 Sand Point Way NE, Seattle, WA 98115, chris.bassett@noaa.gov), Thomas C. Weber (Ctr. for Coastal and Ocean Mapping, Univ. of New Hampshire, Durham, NH), Chris Wilson, and Alex De Robertis (NOAA Fisheries, Alaska Fisheries Sci. Ctr., Seattle, WA)

Acoustic-trawl surveys rely on a combination of backscatter measured with echosounders and species composition data from trawls to apportion the backscatter to different species and size classes. Narrowband echosounders have been widely used in this context for decades. Multi-frequency analysis of narrowband echosounder data has been shown to be effective for discriminating between diverse taxa (e.g., euphausiids vs. swimbladdered fishes) but distinguishing morphologically similar species (e.g., swimbladdered fishes) remains a major challenge. Previous work indicates that broadband backscatter techniques have the potential to improve such acoustic target characterizations by exploiting nearly continuous frequency spectra, but these methods have not been widely applied in fisheries surveys. The recent commercial availability of broadband transceivers is accelerating the evaluation of this technology. We present operational data from two broadband acoustic scattering systems: (1) $14-160 \mathrm{kHz}$ and (2) $3-10 \mathrm{kHz}$ used during surveys of walleye pollock (Gadus chalcogrammus) in the Gulf of 
Alaska and Eastern Bering Sea. The presentation focuses on the potential for: 1) discrimination among common species in the area and 2) utilization of swimbladder resonance to estimate the dominant size class in single-species fish aggregations. The implications for fish stock assessment surveys are considered.

\section{0:15-10:30 Break}

\section{0:30}

4aAO8. Broadband acoustics: A viable tool for quantification of cod egg density and distribution? Gavin Macaulay, Egil Ona, Rokas Kubilius, and Olav Rune Godø (Marine Ecosystem Acoust., Inst. of Marine Res., C Sundts Gate 64, Bergen 5817, Norway, gavin.macaulay@imr.no)

Fish eggs are ichthyoplankton with a similar behavior in the water column as small zooplankton. Their specific density relative to sea water determines their vertical distribution and spread; horizontal transport of the layers will then determine their fate and geographical position during the hatching of the larvae. Acoustic techniques are routinely used to assess the quantities and distribution of fish populations. In comparison to fish, zooplankton and ichthyoplankton are very weak acoustic reflectors and this makes the application of acoustic techniques challenging. We assessed the potential feasibility of using broadband acoustics to detect cod eggs in a two stage process: first, we carried out small-scale tank experiments to measure the acoustic reflectivity of individual and grouped cod eggs over the 170 to $360 \mathrm{kHz}$ frequency range. These experiments showed that natural densities of cod eggs could be detected above the background acoustic noise level. This lead to the second stage whereby we conducted a field test in an area with known concentrations of cod eggs, using a combined acoustic-optical towed and moored system. We postulate that the high spatial resolution, yet rapid wide area coverage possible with acoustics will lead to a better description of egg distributions and subsequent larval distributions and help to understand the extremely variable recruitment success in sub-arctic and arctic ecosystems.

\section{0:45}

4aA09. Mid-frequency clutter and reverberation characteristics of fish in a shallow ocean waveguide. Wu-Jung Lee, Dajun Tang, and Eric I. Thorsos (Appl. Phys. Lab., Univ. of Washington, 1013 NE 40th St., Seattle, WA 98105,wjlee@apl.washington.edu)

Horizontal-looking, mid-frequency sonar systems allow synoptic underwater observation over kilometer scales and are useful for a wide range of applications. However, quantitative assessment of fish aggregations using such systems in a shallow water environment is challenging due to the complexity of sound interactions with the surface and seafloor. Based on data collected during TREX13 (Target and Reverberation Experiment 2013), this study investigates methods to reliably distinguish fish echoes from background contributions to reverberation. The data were collected on a fixed, horizontal receiving line array from a source transmitting linear frequencymodulated signals in the band between 1.8 and $3.6 \mathrm{kHz}$. The experimental site was nearly isodepth (approximately $20 \mathrm{~m}$ ) and characterized by a wellmixed, isothermal water column. Fish echoes were ubiquitously found in the data, with noticeable differences between day and night. In a particularly interesting case, a large aggregation of fish was observed emerging from a shipwreck, evolving in space, obscuring the wreck echoes temporarily, and eventually dispersing and disappearing into the overall reverberation background. Using a physics-based approach, the scattering level, statistical features, and spatial characteristics of both the fish echoes and background reverberation in this data set were analyzed and modeled. [Work supported by the APL postdoctoral fellowship and ONR.]

\section{1:00}

4aAO10. Heterogeneity of deep scattering layer shapes the Bahamian mesopelagic ecosystem. Mei Sato and Kelly J. Benoit-Bird (Oregon State Univ., 104 CEOAS Admin Bldg., Corvallis, OR 97331, msato@coas.oregonstate.edu)

We explored deep scattering layers off the Bahamas where the beaked whales have been historically observed. By comparing the habitats frequently used by the beaked whales with the ones rarely utilized, we examined the differences in mesopelagic ecosystem potentially driving the whales' prey habitat through bottom-up control. Using ship-based multifrequency echosounders and direct net sampling, we identified common layer structures characterized by the diffuse, broad layers ( $>100 \mathrm{~m}$ in thickness) observed across the study site. Within those diffuse layers, we occasionally observed distinctively bounded intense layers ( $20 \mathrm{~m}$ in thickness) located at the upper edges. Instead of a specific layer structure dominating the habitat characteristics, we found that heterogeneity of the layer structures shaped the preferred habitat of beaked whales. Frequently used habitat was characterized by evenly distributed diffuse layers alone, or combined with patchy thin layers. Less-used habitat showed diverse characteristics such as diffuse layers combined with highly variable patchiness of the thin layers. The layer depth, its thickness, and frequency response were not related to the preference of the habitats. Heterogeneity of the layer structures reveals the dynamics of the mesopelagic ecosystem, potentially impacting higher trophic levels.

\section{1:15}

4aA011. Comparison of zooplankton density estimated by acoustic inversion method and net sampling. Kouichi Sawada (Fisheries Technol., National Res. Inst. of Fisheries Eng., FRA, 7620-7, Hasaki, Kamisu, Ibaraki 3140408, Japan, ksawada@fra.affrc.go.jp), Tohru Mukai (Faculty of Fisheries Sci., Hokkaido Univ., Hakodate, Hokkaido, Japan), Yoshiaki Fukuda (Bluefin Tuna Resources, National Res. Inst. of Far Seas Fisheries, FRA, Shizuoka, Shizuoka, Japan), and Tomohiko Matsuura (Fisheries Technol., National Res. Inst. of Fisheries Eng., FRA, Kamisu, Ibaraki, Japan)

A multi-frequency acoustic zooplankton fish profiler (AZFP, ASL Environmental Sciences) with temperature-salinity and depth sensor was moored at $10 \mathrm{~m}$ from the bottom (ca. $30 \mathrm{~m}$ ) at the mouth of Yamada Bay in Iwate Prefecture, Japan, from 14 January to 16 June 2015 in order to measure volume backscattering strength profiles from the transducer surface to the water surface at four frequencies $(125,200,455$, and $769 \mathrm{kHz})$. Zooplankton density by size was estimated from the measured volume backscattering strength at four frequencies by the inversion method. Monitoring period was selected to cover the period when juvenile salmon stay in the bay before leaving for oceans. Zooplankton sampling was conducted periodically (once a week or two weeks, day and night) using a Kitahara Net (30 cm dia., 335 çm mesh) and a Norpac Net (45 cm dia., $335 \mathrm{~cm}$ mesh) at near the moored position. These observations revealed that large zooplankton, which juvenile salmon prefers, arrived with the Oyashio Current, and the zooplankton density increased from late April to the middle of May. Acoustically estimated variation pattern in zooplankton density over time was similar to that obtained by net sampling. [This work was supported by AFFRC, Japan.]

\section{1:30}

4aAO12. Effects of target strength variability on estimates of abundance: The case of Atlantic mackerel (Scomber scombrus). Ben Scoulding (Echoview, GPO Box 1387, Hobart, TAS 7001, Australia, ben. scoulding@echoview.com), Sven Gastauer (Ctr. for Marine Sci. and Technology, Curtin Univ., Perth, WA, Australia), David MacLennan (Inst. of Biological and Environ. Sci., Aberdeen Univ., Aberdeen, United Kingdom), Sascha Fässler (IMARES, IJmuiden, Netherlands), Phillip Copland (Marine Scotland Sci., Aberdeen, United Kingdom), and Paul Fernandes (Inst. of Biological and Environ. Sci., Aberdeen Univ., Aberdeen, United Kingdom)

Atlantic mackerel is a small pelagic, migratory fish which supports valuable commercial fisheries. Despite the fact that these fish school in massive numbers, and are readily detected using echosounders, fishery-independent estimates of the abundance of mackerel in the Northeast Atlantic do not yet consider acoustic data. Echo-integration surveys could provide annual estimates of abundance, with additional scope for studying mackerel distributions throughout the year. However, as in all acoustic surveys, this requires accurate estimates of target strength (TS). The present study provides in situ TS estimates for mackerel from measurements made at sea with a multi-frequency split-beam echosounder. Empirical results suggest mean TS of $51.22 \mathrm{~dB}$ at $18 \mathrm{kHz},-59.83 \mathrm{~dB}$ at $38 \mathrm{kHz},-55.51 \mathrm{~dB}$ at $120 \mathrm{kHz}$, and -53.43 $\mathrm{dB}$ at $200 \mathrm{kHz}$, for a mean fish length of $33.3 \mathrm{~cm}$. These differ significantly from the values currently used in acoustic surveys. The sensitivity of mackerel abundance estimates to variations in TS estimates was investigated 
using data from a dedicated mackerel acoustic survey. Total stock biomass estimates at 38 and $200 \mathrm{kHz}$ were in very good agreement with each other (to within $2.2 \%$ ) and were in the range of values from an independent (i.e., non-acoustic) mackerel stock assessment. It is recommended that future acoustic estimates of mackerel are based on integration at $200 \mathrm{kHz}$.

\section{1:45}

4aA013. Using echoview software to characterise acoustic scattering by aquatic organisms: The case of Atlantic mackerel (Scomber scombrus). Toby Jarvis and Ben Scoulding (Echoview, GPO Box 1387, Hobart, TAS 7001, Australia, toby.jarvis@echoview.com)

In a separate presentation, Scoulding et al. describe the rationale and foundation for regular acoustic surveys of Atlantic mackerel (Scomber scombrus) in the northeast Atlantic Ocean. Two key aims of this work were (1) to obtain a good knowledge of the in situ target strength of these weak scatterers, and (2) to settle on a robust and efficient workflow for the processing of mackerel acoustic survey data. To achieve these aims, dedicated mackerel acoustic surveys were carried out in 2012 and 2014 around the Shetland Islands, Scotland, and the data were processed using Echoview software. In this software-focused presentation, we provide details on how the multifrequency single-beam echosounder data were processed, and we discuss this in the context of a general workflow for acoustic data processing that can be considered for any type of echosounder and application. The aim is to provide specific usage examples of some of the powerful features of Echoview and to provide general guidance for those who use or who are considering using Echoview to characterise acoustic scattering by aquatic organisms.

THURSDAY MORNING, 1 DECEMBER 2016

CORAL 1, 7:30 A.M. TO 11:00 A.M.

\title{
Session 4aBAa
}

\section{Biomedical Acoustics: Session in Honor of Floyd Dunn}

\author{
W. D. O'Brien, Jr., Cochair \\ University of Illinois, 405 N. Mathews, Urbana, IL 61801 \\ Yoshifumi Saijo, Cochair \\ Tohoku University, 4-1 Seiryomachi, Aoba-ku, Sendai 980-8575, Japan
}

Chair's Introduction-7:30

\section{Invited Papers}

\section{$7: 35$}

4aBAa1. Celebration of Floyd Dunn: Some remarks about his life, career, and accomplishments. W. D. O'Brien, Jr. (Univ. of Illinois, 405 N. Mathews, Urbana, IL 61801, wdo@uiuc.edu)

No Celebration of Floyd Dunn would be complete without some remarks about the life, career, and accomplishments of this remarkable scientist/engineer. 1946 is a key year in the history when Floyd arrived at the University of Illinois as an undergraduate student, having served in the European Theater during World War II. The same year, William J. (Bill) Fry and Francis J. (Frank) Fry were hired by the University of Illinois, and the Bioacoustics Research Laboratory was established (70 years ago!). Floyd had made significant scholarly contributions in six general themes. The six ultrasonic biophysics themes include: absorptive processes, nonlinear phenomena, application in living systems, toxicity, measurement techniques, and ultrasonic microscopy. Floyd has been recognized for his accomplishments by being the recipient of most the important national and international awards including: National Academy of Sciences, National Academy of Engineering, ASA's Gold Medal and Silver Medal in Bioresponse to Vibration, Acoustical Society of Japan's Medal of Special Merit, IEEE's Edison Medal, and election to Fellowship in six professional societies.

\section{$7: 55$}

4aBAa2. History of biomedical ultrasound microscope in Japan. Yoshifumi Saijo (Tohoku Univ., 4-1 Seiryomachi, Aoba-ku, Sendai 980-8575, Japan, saijo@idac.tohoku.ac.jp)

Floyd Dunn greatly contributed to the history of biomedical ultrasound microscope (USM) in Japan. Ten years after the concept of ultrasound microscope was proposed by Sokolov of Soviet Union, he realized the ultrasound absorption microscope in 1959. During the time he directed the Bioacoustics Research Laboratory at the University of Illinois, many Japanese researches stayed in his laboratory. Especially, the researchers from Tohoku University learned both technology and philosophy of USM and they started development of scanning acoustic microscopy for medicine and biology in 1982. The USM system had been used for nearly 30 years until the Great East Japan Earthquake destroyed the system. Not only USM, other high frequency ultrasound imaging was also developed in 1990s. Especially, clinical application of intravascular ultrasound filled a gap between several MHz ultrasound used in conventional B-mode and $100 \mathrm{MHz}$ used in USM. High frequency technology and computer science helped developing new generation USM such as ultrasound impedance microscope and 3D ultrasound microscope. Now the application area is spread and Biomedical Ultrasound Microscope Conference is biannually held in Japan. Now, we thank Floyd again for the fruitful activity of USM in Japan. 
4aBAa3. Non-invasive in-situ quantitative observation of biological cells by acoustic microscopy. Naohiro Hozumi, Kyoichi Takanashi, Mamoru Washiya, Sachiko Yoshida (Toyohashi Univ. of Technol., 1-1 Hibarigaoka, Tenpaku, Toyohashi, Aichi 441-8580, Japan, hozumi@icceed.tut.ac.jp), Kazuto Kobayashi (Honda Electronics, Tohohashi, Japan), and Yoshifumi Saijo (Tohoku Univ., Sendai, Japan)

Several ways of quantitatively observing biological cells will be exhibited. Cells are cultured on a thin plastic film in order to make is possible to access by high-frequency acoustic beam focused onto the cell. Reflection waveform has a spectrum briefly spreading from 100 to $400 \mathrm{MHz}$. Mechanical scan makes it possible to acquire a 2-D profile. Cells in contact with the substrate can be characterized by means of characteristic acoustic impedance. In most of observations, buffer liquid that is simultaneously observed with cells may be employed as the reference material for calibration. In the process of cell culture, some of the cells may be delaminated from the substrate. In such a case significant error in acoustic impedance measurement may take place. In order to avoid such an error waveform separation is performed. As a result of such a waveform analysis, delamination distance may be calculated as well as the acoustic impedance of the cell that is slightly distant from the substrate. As an example $\mathrm{C} 2 \mathrm{C} 12$ cells that develop into muscle tissue were observed after differentiation-inducing. Finally, feasibility of cross-sectional observation of cells will be discussed by showing some examples.

\section{$8: 35$}

4aBAa4. Evolution of acoustic microscopy in the commercial marketplace. Lawrence W. Kessler (Sonoscan Inc., 2149 E. Pratt Blvd., Elk Grove Village, IL 60007, 1kessler@sonoscan.com)

The promise of Acoustic Microscopy from the 1970s, - to image features and structures of samples from a micromechanical aspect-has found a few niche markets in industry, and, in particular, defect detection in manufactured products. The hope for its widespread application in medicine and pathology has not yet been reached in the commercial market, though there are several pockets of significant biomedical research that employ acoustic microscopy methods. This paper will give this author's perspective on the field from his 40 years of experience at Sonoscan, Inc., a company originally founded to explore commercial applications, and whose primary activities have found to be serving the semiconductor and microelectronics industries. Based on the original Ultrasonic Absorption Microscope paper by Dunn and Fry in 1959, the early commercial technique substituted a scanning laser as an acoustic detector for their thermocouple probe to produce two dimensional acoustic images.

\section{8:55}

4aBAa5. At the Intersection: Science, fatherhood, and historical events. Unscientific remarks about Floyd Dunn through the lens of his career at the meeting point of historical events, fatherhood, and possibility. Roo Dunn (None, 57 Green St., Bath, ME 04530, dunnroo@gmail.com)

Floyd's 60 years of scientific contributions to ultrasonic biophysics and his life experiences created, and crossed with, many of the thrilling and tumultuous events of the 20th and early 21 st centuries. We will consider national and global events in economics, war, geopolitics, cosmology, and black holes. This paper will look at these topics through conversations spanning some 40 years. We will examine how they meshed with his life, family and the hypotheticals that were frequently discussed which might have drastically altered the outcome. The material is based on informal talks between father, son, and youngest grandson.

\section{9:15}

4aBAa6. Application of scanning acoustic and photoacoustic microscopes in orthopedic surgery. Yoshihiro Hagiwara (Dept. of Orthopaedic Surgery, Tohoku Univ. School of Medicine, 2-1 Seiryo-machi, Aoba-ku, Sendai 980-8574, Japan, hagi@med.tohoku.ac.jp) and Yoshifumi Saijo (Dept. of Biomedical Imaging, Graduate School of Biomedical Engineering, Tohoku Univ., Sendai, Japan)

Demands for orthopedic surgery, decreasing pain and disabilities, are increasing in developed countries with older nations. Together with increasing number of patients, its cost is now rising. Among modalities for noninvasive diagnosing techniques, such as plain Xrays, computed tomography, magnetic resonance imaging (MRI), and ultrasonography (US), MRI is a powerful tool for evaluating anatomical abnormalities. However, location and economic limitations for routine use remain. US is quick and inexpensive with a higher resolution than MRI. US plays an important role in assessing musculoskeletal soft tissues. A scanning acoustic microscope (SAM) characterizes biological tissues by estimating the elastic parameters based on sound speed. Biomedical photoacoustic (PA) imaging has the unique capability of combining high optical contrast and high ultrasound resolution in a single modality. Osteoarthritis and frozen shoulder are the major problems in musculoskeletal diseases. Osteoarthritis is a degenerative joint disorder characterized by the progressive degeneration of articular cartilage, osteophyte formation, and subsequent joint space narrowing. Further, frozen shoulder is characterized with severe pain and a decrease in shoulder motion, which is caused by joint capsular stiffness. We applied SAM and PA to animal models for assessing.

\section{9:35-9:50 Break}

\section{9:50}

4aBAa7. Low-energy extracorporeal shock wave therapy, preclinical and clinical studies. James F. Greenleaf, Carolina A. Carrascal, Matthew W. Urban, Randy Kinnick, Amir Lerman, and lilach Lerman (Biomedical Eng., Mayo, 200, First ST SW, Rochester, MN 55901,jfg@mayo.edu)

Floyd Dunn's extensive research career contributed significantly to safety and efficacy of diagnostic and therapeutic ultrasound. Positive effects of low-energy shock wave therapy (SWT) show preclinical promise in renal disease in pigs and in cardiovascular disease in humans. Preclinical: a set of 26 pigs were divided into four groups, normal + SWT, atherosclerotic renal artery stenosis (ARAS) + SWT, 
and Normal and ARAS pigs. After three weeks of ARAS or sham, SWT was applied twice a week for three weeks. SWT after ARAS decreased blood pressure, recovered the stenotic microvascular density, improved renal blood flow and glomerular filtration rate, and decreased fibrosis. No noticeable kidney injury was observed. Low-energy SWT recovers the stenotic kidney toward normal function by preserving intra-renal microcirculation and may also alleviate renovascular hypertension. 72 patients with refractory angina, 43 were treated with low-energy SWT in the area peripheral to their infarct and 29 patients were in the control group. No adverse effects were seen in the treated group. At six months post-treatment angina class score was decreased $(p=0.0002)$, nitroglycerine use was reduced $(\mathrm{p}=0.03)$, and hospitalization rate was reduced $(\mathrm{p}=0.03)$. Low-energy shockwave therapy may be a tool for treating diseases caused by ischemia.

\section{0:10}

4aBAa8. Cavitation in therapeutic ultrasound: To be avoided or to be utilized. Shin-ichiro Umemura (Graduate School of Biomedical Eng., Tohoku Univ., Aoba 6-6-05, Aramaki, Aoba-ku, Sendai 980-8579, Japan, sumemura@ecei.tohoku.ac.jp), Shin Yoshizawa, and Ryo Takagi (Graduate School of Eng., Tohoku Univ., Sendai, Miyagi, Japan)

Nearly three decades ago, I spent a kind of sabbatical year in Bioacoustic Research Laboratory, University of Illinois, which was supervised by Professor Floyd Dunn. Human knowledge and technologies on acoustic cavitation were significantly limited at that time compared to now. As the result, acoustic cavitation was considered to be avoided even in therapeutic applications of ultrasound. However, I was thinking that it should be utilized and had discussions with him on it, who was the expert on bioeffects of ultrasound including those induced by cavitation. Human technologies on acoustic cavitation have been advanced since then mainly in two aspects: 1) ultrasonic as well as optical observation of cavitation microbubbles at a high frame rate, and 2) generation of a short ultrasonic pulse at extremely high amplitude. A high speed camera had made possible to observe that a short extremely high-amplitude pulse can generate cavitation microbubbles localized near the ultrasonic focal point without inducing their chaotic generations at least in water. High frame rate ultrasonic imaging have made possible to confirm the localization in biological tissue during the therapeutic focused ultrasound treatment. In-vivo as well as ex-vivo experimental results evidencing those will be shown and discussed in the paper.

\section{Contributed Papers}

\section{$10: 30$}

4aBAa9. Cumulative effect in the chronotropic effect in rat hearts caused by pulsed ultrasound. Olivia C. Coiado (Donald P. Shiley School of Eng., Univ. of Portland, 5000 N Wilamette Blvd., Portland, OR 97203, coiado@up.edu) and William D. O'Brien, Jr. (Univ. of Illinois, Urbana, IL)

This study investigated the cumulative effect of a decreasing sequence of pulse repetition frequencies (PRFs) on the chronotropic effect via the application of 3.5-MHz pulsed ultrasound (US) on the rat heart. Two 3-moold female rat groups $(\mathrm{n}=4$ ea) were studied: control (US off) and PRF decrease. Rats were exposed to transthoracic ultrasonic pulses at $\sim 0.25 \%$ duty factor at 2.0-MPa peak rarefactional pressure amplitude. Three PRF sequences were applied. The PRF started greater than that of the rat's heart rate and was decreased sequentially in 1-Hz steps every $10 \mathrm{~s}$ (i.e., 6,5 and 4 $\mathrm{Hz}$-one sequence) for a total duration of $30 \mathrm{~s}$. The total US exposure was $90 \mathrm{~s}$ (3-sequences). For the PRF decrease group, the ultrasound application resulted in an $\sim 8 \%$ significant negative chronotropic effect after the first PRF sequence, $\sim 9 \%$ significant negative chronotropic effect after the second PRF sequence, and $\sim 10 \%$ significant negative chronotropic effect after the third PRF sequence. No significant changes were observed for the control group. The ultrasound application caused a negative chronotropic effect after US exposure for the PRF decrease group and a slight cumulative effect was observed. [Support: NIH Grant R37EB002641.]

\section{0:45}

4aBAa10. The interaction of pulsed ultrasound with mammalian lung. Douglas Miller (Radiology, Univ Michigan, 3240A Medical Science I, 1301 Katherine St., Ann Arbor, MI 48109-5667, douglm@umich.edu)

The impedance difference for a water-air interface yields virtually complete reflection for ultrasound, which coincidentally delivers an acoustical radiation surface pressure (ARSP). ARSP may be a physical mechanism for pulmonary capillary hemorrhage induced by diagnostic ultrasound. Fundamental tissue parameters in biomedical acoustics are the density $\rho$, speed of sound $\mathbf{c}$, and the resulting acoustical impedance $\mathbf{z}=\rho \mathbf{c}$. Many tissues are similar to water ( $\mathbf{z}=1,628 \mathrm{krayl})$, but not lung. The gas content of lung imparts an extreme heterogeneity with air having $\mathbf{z}=0.424$ krayl. If the lung surface is modeled simply as a water-air interface, then the ARSP is maximal. During a diagnostic ultrasound pulse, ARSP can be comparable to the pulmonary capillary blood pressure, thus adding stress to capillaries. However, the bulk properties of the lung are different from air. One of the few reports on acoustical properties of lung was by Floyd Dunn (JASA 1986;80:1248). Consideration of these bulk properties of lung tissue yields a much higher $\mathbf{z}$ $\approx 336 \mathrm{krayl}$ at $2.8 \mathrm{MHz}$, and indicates substantial transmission of ultrasound through the pleura (O’Brien et al. JASA 2000;108:1290). This model suggests a reduced ARSP, and the potential involvement of additional mechanisms for capillary injury within the pulmonary interior. 


\title{
Session 4aBAb
}

\section{Biomedical Acoustics: Medical Acoustics in Kidney and Liver Disease I}

\author{
Norihiro Koizumi, Cochair \\ Graduate School of Informatics and Engineering, The University of Electro-Communications (UEC), 1-5-1 Chofugaoka, \\ Chofu, Tokyo 182-8585 JAPAN, Chofu 182-8585, Japan \\ Michael Bailey, Cochair \\ Center for Industrial and Medical Ultrasound, Applied Physics Lab., University of Washington, 1013 NE 40th Street, \\ Seattle, WA 98105 \\ Hiroyuki Fukuda, Cochair \\ Yokohama City Univ. Medical Center, Yokohama, Japan
}

Adam D. Maxwell, Cochair

University of Washington, 1013 NE 40th St., Seattle, WA 98105

Chair's Introduction-11:15

\section{Contributed Paper}

11:20

4aBAb1. Quantitative fibrotic imaging based on multi-Rayleigh model for ultrasound B-mode image of liver fibrosis. Shohei Mori, Shinnosuke Hirata (Systems and Control Eng., Tokyo Inst. of Technol., 2-12-1 S5-17, Ookayama, Meguro-ku, Tokyo 152-8552, Japan, mori@us.ctrl.titech.ac.jp), Tadashi Yamaguchi (Ctr. for Frontier Medical Eng., Chiba Univ., Chiba, Japan), and Hiroyuki Hachiya (Systems and Control Eng., Tokyo Inst. of Technol., Tokyo, Japan)

A quantitative diagnostic method for liver fibrosis using ultrasound Bmode image is highly required. To quantitatively evaluate liver fibrosis using ultrasound B-mode image, we have been focusing on the echo amplitude distribution properties. A probability density function (PDF) of echo envelope from a normal liver can be approximated by a Rayleigh distribution; however, the PDF of echo envelope from a fibrotic liver deviates from the Rayleigh distribution. To approximate the PDF of liver fibrosis, we have been proposing the multi-Rayleigh model which is a combination of Rayleigh distributions with different variances. By using the multi-Rayleigh model, the ultrasound B-mode image of liver fibrosis could be converted to the quantitative fibrotic probability image. In this study, we applied the fibrotic probability imaging method to clinical data which is classified into F0 (normal liver), F1 (early-stage hepatitis), F2 (moderate hepatitis), and F3 (late hepatitis) in accordance with a new Inuyama classification. First, a region of interest (ROI) was automatically selected based on the amplitude distribution property. Then, removal method of nonspeckle signals was used to improve the accuracy of the multi-Rayleigh model approximation. Finally, the ultrasound B-mode image in ROI was converted to the fibrotic probability image based on the multi-Rayleigh model.

\section{Invited Paper}

$11: 35$

4aBAb2. Ultrasound and microbubbles induced extravasation in a machine-perfused pig liver. Michalakis A. Averkiou, Christina P. Keravnou, and Ine De Cock (BioEng., Univ. of Washington, William H. Foege Bldg., 3720 15th Ave. NE, Seattle, WA 98195, maverk@uw.edu)

Ultrasound driven microbubbles interact with capillaries and cells and have been proposed to be beneficial for localized drug delivery and uptake. Some of the mechanisms involved is transient cell membrane permeability alteration and vessel poration which increase particle extravasation. Ex vivo machine perfusion of human-sized organs is a technique that provides an ideal environment for preclinical investigations with high physiological relevance not possible with in vitro experiments. In this work, ex vivo machine-perfused pig livers combined with an image-guided therapy system were used to investigate extravasation induced by ultrasound driven microbubbles. Local microvascular flow changes (measured by contrast enhanced ultrasound) and leakage of Evans blue dye in liver parenchyma were used to assess the degree of extravasation. 1-4 MPa peak negative pressure and 20-1000 cycles were considered. Liver areas that were exposed to long pulses (1000 cycles) and peak negative pressure $>2.5 \mathrm{MPa}$ showed a detectable perfusion change. Two fold increase in Evans blue concentration was observed at areas treated with peak negative pressure above 1 MPa and 500 cycles, indicating that extravasation still occurs in lower pressures where flow changes cannot be detected with contrast enhanced ultrasound quantification. 


\title{
Contributed Paper
}

11:50

4aBAb3. Enhanced high rate shockwave lithotripsy stone comminution in an in-vivo porcine model using acoustic bubble coalescence. Hedieh Alavi Tamaddoni, William W. Roberts (Univ. of Michigan, 2131 Carl A. Gerstacker Bldg., 2200 Bonisteel Blvd., Ann Arbor, MI 48109, alavi@ umich.edu), Alexander P. Duryea (HistoSonics, Inc., Ann Arbor, MI), and Timothy L. Hall (Univ. of Michigan, Ann Arbor, MI)

While cavitation on the surface of urinary stones has been shown to aid stone fragmentation, bubbles along the propagation path away from the stone may attenuate shockwaves. We have shown applying low amplitude acoustic waves after each shockwave can force bubble coalescence enhancing lithotripsy efficacy in vitro. For the present study, we demonstrate the feasibility of applying acoustic bubble coalescence (ABC) in vivo. Artificial model stones were percutaneously implanted in the right kidney of ten $50 \mathrm{~kg}$ female pigs and treated with 2500 shockwaves at $120 \mathrm{SW} / \mathrm{min}$ with or without $\mathrm{ABC}$. $\mathrm{ABC}$ sequences consisted $1 \mathrm{MPa}$ tone bursts at 500 $\mathrm{kHz}$ for a total duration of $16 \mathrm{~ms}$. After treatment, kidneys were removed and dissected. Remaining fragments in the kidney were retrieved and filtered for size. Comparing size distributions, a significant improvement was observed when $\mathrm{ABC}$ was used. Without $\mathrm{ABC}$, only $25 \%$ of the remnant mass of fragments was less than $2 \mathrm{~mm}$. With $\mathrm{ABC}, 75 \%$ of the mass was less than $2 \mathrm{~mm}$. These results suggest $\mathrm{ABC}$ can reduce the shielding of cavitation bubbles resulting in a more efficient SWL treatment.

THURSDAY MORNING, 1 DECEMBER 2016

SOUTH PACIFIC 4, 8:00 A.M. TO 12:00 NOON

\section{Session 4aEA}

\section{Engineering Acoustics: Simulation and Analysis}

\author{
Colin J. Novak, Cochair \\ Mechanical, Automotive and Materials Engineering, Department of Mechanical Engineering, University of Windsor, \\ 401 Sunset Ave., Windsor, ON N9B 3P4, Canada \\ Shouichi Takane, Cochair \\ Department of Electronics and Information Systems, Akita Prefectural University, 84-4 Ebinokuchi, Tsuchiya, \\ Yurihonjo 015-0055, Japan
}

\section{Contributed Papers}

\section{8:00}

4aEA1. Integrated simulation model for prediction of acoustic environment of launch vehicle. Seoryong Park, Kiseop Yoon, Jeongwoo Ko, HanAhChim Choung, Seokjong Jang (Aerosp. Eng., Seoul National Univ., Republic of Korea, 1, Gwanak-ro, Gwanak-gu, Seoul, Korea, Seoul 151744, South Korea, tjfyd11@snu.ac.kr), and Soogab Lee (Ctr. for Environ. Noise and Vib. Res., Eng. Res. Inst. College of Eng., Seoul National Univ., Seoul, South Korea)

During a lift-off of a launch vehicle, a large amount of acoustic wave is generated by jet plumes. Acoustic waves lead to pressure fluctuations on the surface of the vehicle in the form of acoustic loads and these are transmitted to the internal payload. In order to evaluate the effects of acoustic loads, we developed the integrated simulation model. The model is mainly divided into two parts, prediction and reduction parts. First, the external acoustic loads are predicted empirically based on the Distributing Source Method-II (DSM-II) of NASA SP-8072. We developed the improved method able to consider the additional effects of surrounding structures. We perform a next prediction process for the internal acoustic environment by using Statistical Energy Analysis (SEA). For the Korean Sounding Rocket-III (KSR-III), we predict the internal acoustic environment and evaluate the stability of the payload in the fairing. After the prediction process, we perform the noise reduction simulation. With an active noise control (ANC) simulation, we could confirm the possibility and reliability of the noise reduction system.
$8: 15$

4aEA2. Micro load theory of surface acoustic wave gravimetric sensors. Li-Feng Ge (School of Elec. Eng. and Automation, Anhui Univ., 111 Jiulong Rd., Hefei 230601, China, lf_ge@hotmail.com)

Surface acoustic wave (SAW) gravimetric sensors have been widely applied in the field of chemical and biological detections for decades. However, it is noticed that the operating mechanism of this type of sensors is not understood well. A perturbation theory commonly adopted has been used to predict the mass sensitivity of the SAW sensors, but, as indicated also, "there is a significant and systematic quantitative discrepancy" between the theoretical and experimental results [Wohltjen, et al. IEEE UFFC, 1987]. In this paper, a micro load theory is presented. The coating film with biochemical material adsorbed is not regarded as a perturbation source, but a micro mechanical load. Then, it is revealed that the fractional frequency shift caused by the load is minus and half of the fractional area density change, where the unloaded area density is a product of the substrate material's density and SAW wavelength. Also, it is interesting to note that the expression is the same with that for Lamb wave sensors if SAW wavelength is replaced by the plate thickness [Li-Feng Ge, POMA, ASA, 2009], which shows a general character of wave-based gravimetric sensors. [The work was supported by NSFC, Grant Nos. 60374044 and 60774053]. 
valuable for stable and low-dispersive computation of acoustic Doppler effect. In present study, we first perform some numerical simulations by the

4aEA3. Development of a high presence virtual reality system by binaural sound field rendering with head mount display. Kyosuke Sugiura, Shingo Iino, and Takao Tsuchiya (Sci. and Eng., Doshisha Univ., 1-3 Tatara-Miyakodani, Kyotanabe, Kyoto 610-0394, Japan, dup0154@mail4. doshisha.ac.jp)

In this study, a high presence virtual reality (VR) system which integrates head mount display (HMD) and the binaural sound field rendering is developed. To realize a high presence VR system, it is necessary to provide interactive visual and sound environments in real-time. Recent VR systems can provide interactive visual environment by HMD; however, interactive sound environment is not enough, because enormous binaural room impulse responses (BRIRs) are required to obtain HRTFs at various room positions. So, we calculate BRIRs numerically using the CE-FDTD method with the GPU cluster system. For BRIR calculation, a room model including dummy-head scanned by 3D scanner is developed. In this system, it is possible to render binaural-sound interactively by convolving calculated BRIRs. The developed VR system consists of visual and sound part which is respectively processed by Unity and MAX. Position and head rotation of listener are obtained from tracking sensor by Unity, and the data are sent to MAX by OSC. MAX convolves calculated BRIRs changing by cross-fade. Listener can move their head in virtual room, and can feel sound field differences. It is confirmed that the system can produce the high presence virtual room.

\section{$8: 45$}

4aEA4. Three-dimensional numerical simulation of parametric speaker by compact explicit finite-difference time-domain method. Takao Tsuchiya (Dept. of Information Systems Design, Doshisha Univ., 1-3 TataraMiyakodani, Kytanabe City, Kyoto 610-0321, Japan, ttsuchiy@mail.doshisha.ac.jp) and Hideyuki Nomura (Commun. Eng. and Informatics, Univ. of Electro-Communications, Chofu, Tokyo, Japan)

In this study, the three-dimensional numerical simulation of a parametric speaker is carried out. To simulate the secondary sound field of difference frequency generated by the high intense ultrasonic wave, the Westervelt equation is numerically solved by the compact explicit finite-difference time-domain (CE-FDTD) method which is a high-accuracy version of the standard FDTD method. In the simulation, the interpolated wideband (IWB) scheme that is one of the most accurate scheme of the CE-FDTD method and whose cut-off frequency is in agreement with the Nyquist frequency, is chosen to reduce computer resources. The IWB scheme is then implemented on a graphics processing cluster system. Three-dimensional numerical models whose size is $1.5 \mathrm{~m} \times 1.5 \mathrm{~m} \times 8 \mathrm{~m}$ are developed with cell sizes of $1 \mathrm{~mm}$. Some demonstrations are made for the nonlinear sound wave propagation. The propagation characteristic and the directivity of the parametric speaker are obtained with reasonable accuracy.

\section{9:00}

4aEA5. A novel numerical simulation of acoustic Doppler effect using CIP-MOC method. Takuro Sonobe, Kan Okubo, Norio Tagawa (Graduate School of System Design, Tokyo Metropolitan Univ., 6-6 Asahigaoka, Hino, Tokyo 191-0065, Japan, sonobe-takuro@ed.tmu.ac.jp), and Takao Tsuchiya (Faculty of Sci. and Eng., Doshisha Univ., Kyotanabe, Kyoto, Japan)

Utilizing the Doppler effect (the Doppler frequency shift) is an effective method to detect the speed of the moving object; in particular, the measurement of the acoustic Doppler effect might be an important for recognition of the surrounding environment in the automatic operation and robot technology. To date, many researchers investigated acoustic numerical simulation methods. However, a numerical simulation considering acoustic Doppler effect has not been sufficiently developed yet. In this study, we employ the constrained interpolation profile (CIP) method, one of the methods of characteristics (MOC). A noticeable feature of the CIP method is that it allows analyses not only of sound fields on grid points, but also analyses of their spatial derivatives on grid points, whereas the CIP-MOC method adopts the co-located grid system. That is, sound field components (air pressure and vertical velocity) and their derivatives are located on the same grid, so that we can model accurate interface between different media. This feature is CIP-MOC method for sound propagation involving the Doppler effect and then demonstrate the effectiveness of this scheme.

\section{9:15}

4aEA6. Comparative study of a low frequency emergency siren to traditional siren technology. Frank Angione, Colin Novak, Chris Imeson, Ashley Lehman, Ben Merwin, Tom Pagliarella, Nikolina Samardzic, Peter D'Angela, and Helen Ule (Univ. of Windsor, 401 Sunset Ave., Windsor, ON, Canada,novak1@uwindsor.ca)

This study measures and compares the acoustic characteristics of a traditional electronic emergency siren and an innovative low frequency Rumbler siren technology. The Rumbler siren's low frequency emissions can travel further and have a greater ability to penetrate and induce structure-bone excitation in nearby vehicle cabins compared to other siren technology. The result is a better ability to warn both nearby vehicles and pedestrians thus lessening the potential of emergency vehicle collisions. The siren technologies were evaluated using three measurement scenarios to determine acoustic localization characteristics, drive-by effectiveness, and the ability to overcome the problem shadowing phenomenon typical at congested intersections. A comparative analysis of the acoustical characteristics of the standalone siren and the addition of The Rumbler system provided insight in regards to the relative effectiveness of each siren mode. The siren system equipped with The Rumbler technology gave a noticeable increase in sound pressure level for each of the three test scenarios. Through analysis of the measured data, the overall performance of the emergency siren system with the Rumbler technology was shown to be a more effective emergency notification device compared to the standalone electronic siren.

\section{9:30}

4aEA7. Study of acoustic localization of an emergency siren. Frank Angione, Colin Novak, Peter D'Angela, and Helen Ule (Univ. of Windsor, 2958 Conservation, Windsor, ON n8w5j3, Canada, angionef@uwindsor. ca)

Having the ability to adequately detect the direction of an approaching emergency siren is critical to the effectiveness of the emergency system. Having this ability allows both pedestrians and drivers of nearby vehicles to more quickly and safely react to an approaching emergency vehicle. This study considers a typical electronic siren system that is currently being used by the Windsor Fire \& Rescue Services Department. This siren has two fundamental settings: the standard siren signal and the air horn mode, which is typically used when the emergency vehicle is approaching roadway intersections, as these pose the most danger to occupants of both emergency vehicle and general public. Siren and air horn signals were recorded at specific distances from the driver's position at $45^{\circ}$ radial increments. From these, the recorded signals and sound pressure levels measured inside the cabin of the vehicle at the various approach angles were used to prepare a subjective jury evaluation to determine the localization characteristics under simulated roadway intersections conditions. Using the outcomes from this study, valuable knowledge was learned which can be applied to future improvements to enhance the localization characteristics of emergency siren systems.

\section{9:45-10:00 Break}

\section{0:00}

4aEA8. Analysis of the electrostatic ultrasonic airborne actuator using the finite-difference time-domain method. Ryosuke Igai, Takefumi Shibamata, and Minoru K. Kurosawa (Dept. of Elec. and Electron. Eng., Tokyo Inst. of Technol., 4259 Nagatsuta-cho, Midori-ku, Kanagawa, Yokohama 226-8503, Japan, igai.r.aa@m.titech.ac.jp)

We have investigated the structure of an electrostatic airborne ultrasonic actuator by using the FDTD method. The actuator structure has low-mass pleat-fold diaphragms. The pleat designed $10 \mathrm{~mm}$ deep and $2 \mathrm{~mm}$ wide, vibrate at a frequency range of $10 \mathrm{kHz}$ to $200 \mathrm{kHz}$. The diaphragms of the pleat vibrate and carry out high power and wide directivity ultrasonic sound waves from a narrow output opening end. We have found the correlation between the depth of the pleat and the wavelength that the sound pressure 
level had peak and dip depending on the frequency. Using numerical simulation by the FDTD method, the interference in the pleat animated and described visibly. Instead of the plane wave source that caused the interference of the sound source in the pleats, progressive wave sources have mitigated interference and gained higher outputs. In the FDTD simulation, progressive wave source at $200 \mathrm{kHz}$ generated $73 \mathrm{dBSPL}$ and about +30 dBSPL higher compared to the plane wave when the vibration amplitude and velocity were 33 micron and $41 \mathrm{~mm} / \mathrm{s}$ at the diaphragm surface. The particle velocity at output point compared to the sound source gained 4.9 times faster at $20 \mathrm{kHz}$ and 7.3 times faster at $200 \mathrm{kHz}$.

\section{0:15}

4aEA9. Some investigations on properties of spatial principal components analysis of individual head-related transfer functions. Shouichi Takane (Dept. of Electronics and Information Systems, Akita Prefectural Univ., 84-4 Ebinokuchi, Tsuchiya, Yurihonjo, Akita 015-0055, Japan, takane@akita-pu.ac.jp)

Analysis for compact representation of spatial change of Head-Related Transfer Functions (HRTFs) based on principal components analysis (PCA), called Spatial PCA (SPCA), is investigated in this report. Although the SPCA of HRTFs has been researched for about 30 years, there exist some questions left unclear. In this report, the author tries to answer the following two questions: (1) how much amount of data (number of subjects and/or directions) is enough for generation of principal components, and (2) which domain (impulse response, frequency spectrum, etc.) is the best for compact representation of individual HRTFs based on the SPCA. As for (1), the amount of data may be relatively small as a result of the preliminary investigation, meaning that the HRTFs of a certain subject can be reconstructed by using the SPCA with the HRTFs of the other subjects of relatively small numbers. As for (2), the author concluded that the complex frequency spectrum brings about the most compact representation as the results of the SPCA of a single Head-and-Torso Simulator (HATS) [S. Takane, Appl. Acoust. 101, 64-77(2016)]. In this report, whether the same results are obtained or not is examined via comparison of the reconstruction accuracy among the individual subjects. [A part of this research was supported by JSPS KAKENHI (25330202).]

\section{0:30}

4aEA10. Numerical simulations of detecting perforated slim hole length in formation with an acoustic method. Shubo Yang, Xiaohua Che, and Wenxiao Qiao (China Univ. of Petroleum, No. 18, Fuxue Rd., Changping District, Beijing 102249, China, yangshubo-cup@foxmail.com)

Water-jet deep penetrating perforation, which utilizes high pressure water-jet to penetrate reservoirs, is a new well interference technology for increasing deliverability. The length of water-jet hole is a key parameter for evaluating water-jet effect. We simulated and compared acoustic fields both inside and outside water-jet hole in different conditions with the time-domain finite difference method. The water-jet hole in the modeling is $3 \mathrm{~cm}$ in diameter and $10 \mathrm{~m}$ in length. Based on the simulation results, we analyzed the feasibility to detect the length of water-jet hole mainly by adopting low frequency source. Simulation results show that the high frequency source can excite multiple waves inside the water-jet hole, including refraction $\mathrm{P}$ and $\mathrm{S}$ waves, pseudo-Rayleigh waves, and Stoneley waves. Thus, the signals received in borehole are complex. On the contrary, the low frequency source excites few modes inside the water-jet hole. Specifically, there are only Stoneley waves when the source frequency is lower than $1 \mathrm{kHz}$. The Stoneley wave velocity is approximately equal to the $\mathrm{P}$ wave velocity of the fluid. As a result, we can utilize the arrival time and the velocity of Stoneley waves to calculate the length of water-jet hole.

\section{0:45}

4aEA11. Multi-dimensional numerical simulation of sound propagation with background flow using constrained interpolation profile method. Akihiro Fukuda, Kan Okubo (Graduate School of System Design, Tokyo Metropolitan Univ., 6-6 Asahigaoka, HIno, Tokyo 191-0065, Japan, fukudaakihiro@ed.tmu.ac.jp), Takuya Oshima (Niigata Univ., Niigata, Niigata, Japan), Takao Tsuchiya (Doshisha Univ., Kyotanabe, Kyoto, Japan), and Masashi Kanamori (JAXA, Chofu, Tokyo, Japan)

To date, numerical simulation for sound wave propagation has been widely investigated as a result of advances in computer technology. For acoustic imaging and prediction, the development of accurate and effective analysis methods in time domain has become an important technical issue. The constrained interpolation profile (CIP) method, a novel low-dispersive numerical scheme, is a type of method of characteristics (MOC) employing a co-located grid system. In our past study, we have applied the CIP method to solving the linearized sound propagation equation. MOC is the calculation method which transforms governing equations of acoustic field into advection equations to solve the acoustic fields. Therefore, the method should be suitable for solving sound propagation considering the background flow effect, e.g., prediction of sound noise environment generated from a propulsion system of aircrafts or rockets. In the study, we proposed and examined the calculation method of sound propagation in an arbitrary background flow using CIP method. We made a numerical comparison between proposed method and the linearized Euler equations (LEE) method. The presentation has clarified that the CIP method is more accurate and effective for solving sound propagation in a strong and/or heterogeneous background flow.

\section{1:00}

4aEA12. Numerical study of mean flow effect on sound propagation through an aerosol. Ersen Arslan, Yusuf Özyöruk, and Mehmet Caliskan (Middle East Tech. Univ., Dept. of Mech. Eng., G-308, Ankara 06800, Turkey, caliskan@metu.edu.tr)

Previous studies on the sound attenuation and dispersion in an aerosol consisting of air and water droplets have been extended to accommodate mean flow effects. A numerical methodology based on solution of one dimensional, linearized Euler equations in the frequency domain has been developed. The interactions between the fluid and particle phases have been considered to be through Stokesian drag force. First, the verification of the numerical code has been shown for the zero mean flow case with comparative evaluation of results available in literature. Next, the attenuation and dispersion results of both upstream and downstream flows have been presented. The comparison of results suggests that significant deviations exist for attenuation values between the stationary and the mean flow cases at the same frequencies. Keywords: Suspension Acoustics, Finite Difference in Frequency Domain (FDFD), Mean Flow Effect

\section{1:15}

4aEA13. Thévenin acoustics. Randall P. Williams and Neal A. Hall (Dept. of Elec. and Comput. Eng., The Univ. of Texas at Austin, 10100 Burnet Rd., Bldg. 160, Rm 1.108, Austin, TX 78758, randy.williams@utexas. edu)

Thévenin's theorem can be applied to the analyses of acoustic systems, without limitation to systems that have been reduced to analogous circuit models. The method is particularly convenient in the analysis of acoustic scattering when the scattering object is mobile. In this presentation, the method is illustrated through an alternative derivation of the well-known "mass law" for transmission through a partition and is also applied to the case of acoustic scattering from a rigid, mobile cylinder of arbitrary size in an ideal plane progressive wave. Differences between the conventional solution approach for such problems and the Thévenin-inspired method are discussed, along with the potential benefits of taking such an approach for the simplification of other problems in physical acoustics. 
$11: 30$

4aEA14. Evaluation of dependence on calculation parameters in interface settings for finite-difference time-domain analyses of acoustic fields. Chihiro Ogawa, Kan Okubo (Graduate School of System Design, Tokyo Metropolitan Univ., 6-6 Asahigaoka, Rm. 4-312, Hino-shi, Tokyo 1910065, Japan, ogawa-chihiro@ed.tmu.ac.jp), and Takao Tsuchiya (Faculty of Sci. and Eng. Dept. of Information Systems Design, Doshisha Univ. Graduate School, Kyotanabe-shi, Kyoto-shi, Japan)

To date, numerical analysis for sound wave propagation in time domain has been investigated widely as a result of advances in computer technology. The finite difference time domain (FDTD) methods are very widely used for time domain numerical analysis. The following is a family of FDTD method, for example, the standard FDTD method based on Yee's algorithm, wave equation FDTD (WE-FDTD) method, the $\operatorname{FDTD}(2,4)$ method, and so on. The FDTD and WE-FDTD methods cause numerical dispersion error due to using second order finite difference (FD) approximation. To overcome this problem, the $\operatorname{FDTD}(2,4)$ method using higher order spatial FDs have been proposed. On the other hand, the settings of the interface between different media are important issue to solve acoustic wave propagation in non-uniform media. In this study, we examine the dependence on calculation parameters in the settings of interface for some FDTD methods. The present study shows dependence on the CFL number and ratio of sound velocity and impedance between the boundaries. We demonstrate that accuracy of the interface between different media strongly depend on calculation parameters and the ratio of sound velocity. Moreover, the proposal settings of the interface for the $\operatorname{FDTD}(2,4)$ methods are more accurate.

\section{$11: 45$}

4aEA15. Localization of acoustic source in windy environment using ray tracing. Yeong-Ju Go, Donghun Choi, Jaehyung Lee, and Jong-Soo Choi (Chungnam National Univ., 99 Daehak-ro, Yuseong-gu, Daejeon 34134, South Korea, yjgo@cnu.ac.kr)

Position estimation of sound source using ray tracing method is introduced based on wind field measurement. TDOA (time difference of arrival) estimate error occurs during outdoor sound measurement due to the atmospheric condition such as wind, temperature, humidity and so on. Such condition can possibly change the trajectories of rays. It produces erroneous propagation time information accordingly. In this study, simplex method is used to estimate the source position using ray tracing. The basic concept is to find the estimation point which generates smallest mean square error of propagation distances or times from the candidate points. At first the estimation area is set around the first estimate which is calculated from the TDOA estimation. The simplex method is then applied to each point in the estimation area. Nelder-Mead simplex method is used to find the best position that produces the minimum error in travelling distances. In order to apply the ray tracing, the wind distribution is interpolated based on the wind measurements around the boundary. Simulation and experiments are conducted to verify the performance of the algorithm.

\title{
Session 4aID
}

\section{Interdisciplinary: Workshop for Publishing Excellence in JASA}

\author{
Ning Xiang, Cochair \\ School of Architecture, Rensselaer Polytechnic Institute, Greene Building, 110 8th Street, Troy, NY 12180 \\ James Lynch, Cochair \\ Woods Hole Oceanographic, MS \# 11, Bigelow 203, Woods Hole Oceanographic, Woods Hole, MA 02543
}

Chair's Introduction-8:00

Invited Papers

8:05

4aID1. Choosing venues (including e-mail, internet posting, and archival journals) for the dissemination, discussion, and publication of research. Allan D. Pierce (Retired, PO Box 339, 399 Quaker Meeting House Rd., East Sandwich, MA 02537, allanpierce@verizon.net)

This paper is intended for researchers desiring that their papers get the attention of relevant research community members and will remain widely accessible for the indefinite future. It is suggested that authors send preprints of their works as electronic attachments to all those colleagues they suspect of having possible interest in the work. They should also consider the possibility of posting their preprints on personal and institutional web sites and on e-print servers such as arXiv. They may also want to consider establishing accounts on social networking sites such as ResearchGate so they can make their publications available to all other account holders (worldwide) who explicitly choose (and are enticed by ResearchGate) to follow their work. Before selecting a journal for submission of any paper, they should familiarize themselves with the terms of the copyright agreement and decide whether the scope of authors' rights is sufficient for their desires. Various factors important for the selection of a journal are discussed: relevance of previously published papers to the author's field of research, its likelihood of perpetual existence, the familiarity of the editors with the field, the quality reputation of the past published papers, and the timeliness of the processing of submitted articles. 
4aID2. The publication process as seen from various angles. James Lynch (Woods Hole Oceanographic, MS \# 11, Bigelow 203, Woods Hole Oceanographic, Woods Hole, MA 02543, jlynch@whoi.edu)

The process of publishing a paper in JASA involves many people and several process components. The authors, reviewers, Associate Editors, Publication Office personnel, and our AIPP publishers all play their parts and interact to produce any paper that is eventually published. In this talk, the various components of the publication process will be examined, making an effort to present the tasks and viewpoints of the various participants. The merits and deficiencies of various parts of the publication process will also be discussed, with the goal of both promoting understanding of the system and also improving it.

\section{$8: 45$}

4aID3. Preparing and submitting manuscripts to the Journal of the Acoustical Society of America-Express Letters. Charles C. Church (NCPA, Univ. of MS, 1 Coliseum Dr., University, MS 38677, cchurch@olemiss.edu)

The general process for preparing a manuscript for the Journal of the Acoustical Society of America-Express Letters (JASA-EL) is the same as for any other scientific publication. First, have something new and interesting to say to your colleagues. Second, say it clearly, without ambiguity, and with a minimum of unnecessary literary flourish. Finally, and most importantly for JASA-EL, be brief. Failure to follow any of these simple rules may produce lengthy delays in publication. In extreme cases, it may result in rejection of the manuscript even after the review process has been completed! Once a manuscript has been completed, it is very important to follow the formatting requirements for the journal, both for the length and the appearance of the text and tables of the manuscript itself but also for the file types accepted by our publisher, AIP (American Institute of Physics) Publishing. The submission procedure is relatively simple. For example, neither a cover letter nor a list of suggested reviewers is currently required (although this may change in the future!). However, there are a few characteristics of the process that authors should consider before beginning. These and other areas that are frequently sources of difficulty will be discussed.

\section{9:05}

4aID4. Instructions for prospective authors to submit acceptable manuscripts to the Journal of the Acoustical Society of America. Ning Xiang (School of Architecture, Rensselaer Polytechnic Inst., Greene Bldg., 110 8th St., Troy, NY 12180, xiangn@rpi.edu)

It is a common perception that the Journal of Acoustical Society of America (JASA) is considered as one of leading journals which has been serving the acoustics community for over 85 years. The JASA encourages authors to submit papers for JASA publication and at the same time practices a rigorous peer-review process to meet its aspirations for a higher degree of publishing excellence. For prospective authors a better understanding of JASA's peer-review process is critically important. To increase the chance for submission acceptance, this talk will shed light to its peer-review process of the JASA, draw audience's attention to some detailed, updated guidance and instructions regularly published by the JASA editorial board. As a frequent reviewer, an Associate Editor, and particularly a nonnative author of the JASA papers, this author will share his own experience to analyze possible reasons for successful and unsuccessful publication effort using some disguised examples.

\section{9:25}

4aID5. Best practices for publishing in scientific journals. Christy K. Holland (Internal Medicine, Div. of Cardiovascular Diseases and Biomedical Eng. Program, Univ. of Cincinnati, Cardiovascular Ctr. Rm. 3935, 231 Albert Sabin Way, Cincinnati, OH 45267-0586, Christy.Holland@uc.edu)

A string of impressive publications can be the lifeline for promotion and success for an academic. Once excellent data is produced from your innovative research program, how do you communicate the results as clearly as possible? As the editor in chief of Ultrasound in Medicine and Biology, I have witnessed first hand a range of successful strategies to get papers published. Making sure that your manuscript is aimed at the appropriate journal at the outset can save a great deal of effort and can get your work into print faster. Editors and Associate Editors seek advice from reviewers who are knowledgeable in the general subject of the paper, and the reviewers give opinions on various aspects of the work, including whether the work is original and correct. The editor and reviewers who examine each manuscript are the authors' peers: persons with comparable standing in the same research field as the authors themselves. The composition of a concise cover letter, the creation of an effective response to the reviewers' critiques, and strategies for polite communication will be outlined and discussed. The good news is that editors want to publish your paper and appreciate individuals who create the scientific content that makes a journal thrive.

\section{9:45-10:25 Panel Discussion}




\title{
Session 4aMUa
}

\section{Musical Acoustics: Flute Acoustics Including All “Air-Jet” Instruments}

\author{
Naoto Wakatsuki, Cochair \\ University of Tsukuba, 1-1-1 Tennodai, Tsukuba 305-8573, Japan \\ Nicholas Giordano, Cochair \\ Physics, Auburn University, College of Sciences and Mathematics, Auburn, AL 36849 \\ Chair's Introduction-8:00
}

Invited Papers

8:05

4aMUa1. How well do lumped-element models describe acoustic amplification in the recorder? John C. Price (Phys., Univ. of Colorado, 390 UCB, Boulder, CO 80309, john.price@colorado.edu)

Nearly 50 years have passed since Cremer and Ising [Acustica 19, 142-153 (1967)] established lumped-element feedback models as the dominate paradigm for understanding flute-family musical instruments. Modern versions of these models include one feedback path through the resonant acoustic flow in the pipe, and a second path that accounts for edge-tone phenomena. They also incorporate results of later experimental and theoretical work on jet formation, jet deflection, and growth of instabilities along the jet. In this work, we test a lumped model of the recorder air-jet amplifier by replacing the recorder body with a waveguide reflectometer. When the mean flow from the air-jet into the waveguide is not blocked, the air-jet amplifier is unstable to edge-tone oscillations. When it is blocked, the airjet is deflected somewhat outward, gain is reduced, and the system becomes stable. It is then possible to measure the reflection coefficient of the air-jet amplifier versus blowing pressure and acoustic frequency under linear response conditions, avoiding the complication of gain saturation. The results provide a revealing test of lumped flute drive models under simple conditions and with few unknown parameters. The strengths and weaknesses of modern flute drive models are discussed.

\section{$8: 25$}

4aMUa2. Direct aeroacoustic simulations and measurements of flow and acoustic fields around a recorder with tone holes. Hiroshi Yokoyama (Mech. Eng., Toyohashi Univ. of Technol., 1-1,Hibarigaoka,Tempaku-cho, Toyohashi, Aichi 4418580, Japan, h-yokoyama@me.tut.ac.jp), Akira Miki (Res. \& Development Div., Yamaha Corp., Shizuoka, Japan), Ryoma Hamasuna (Mech. Eng., Toyohashi Univ. of Technol., Toyohashi, Aichi, Japan), Hirofumi Onitsuka (Res. \& Development Div., Yamaha Corp., Shizuoka, Japan), and Akiyoshi Iida (Mech. Eng., Toyohashi Univ. of Technol., Toyohashi, Aichi, Japan)

Direct aeroacoustic simulations and experiments were performed regarding the flow and acoustic fields around a recorder, which is one of air-reed instruments. The present simulations reproduced the jet oscillations and acoustic radiation around the recorder with tone holes. The effects of conditions of the tone holes and the jet velocity on the flow and acoustic fields are discussed.

\section{8:45}

4aMUa3. Acoustic energy generation of "air-jet" instruments: Energy transfer between jet oscillation and acoustic field. Kin'ya Takahashi (The Phys. Labs., Kyushu Inst. of Technol., Kawazu 680-4, Iizuka, Fukuoka 820-8502, Japan, takahasi@mse.kyutech.ac.jp), Sho Iwagami (Mech. Information Sci., Kyushu Inst. of Technol., Iizuka, Fukuoka, Japan), Taizo Kobayashi (Faculty of Fukuoka Medical Technol., Teikyo Univ., Omuta, Fukuoka, Japan), and Toshiya Takami (Comput. Sci. and Intelligent Systems, Oita Univ., Oita, Oita, Japan)

In this talk, we discuss how to estimate the acoustic energy generation of "air-jet" instruments with numerical simulation. To attack this problem, we use Howe's energy corollary, with which we can estimate energy transfer between unsteady flow, i.e., oscillating jet and acoustic field. To calculate Howe's formula, we need solenoidal velocity of the flow and its vorticity together with acoustic particle velocity separated from the whole velocity of compressible fluid. Recently, a method, which allows us to approximately calculate Howe's formula, was developed in experiments by Bamberger and Yoshikawa et al., and it can be applied for the numerical calculation. We apply the method for the numerical calculation of a flue organ pipe model. We also introduce a toy model of the oscillating jet to investigate the mechanism of sound generation from the oscillating jet in detail. The acoustic energy is mainly generated in the downstream of the oscillating jet near the edge of the mouth opening, but it is consumed in the upstream near the flue exit to synchronize the jet motion with it. Our results are in good agreement with the experimental result by Yoshikawa et al. as well as Howe's theoretical prediction. 
4aMUa4. Simulation studies of the flute. Nicholas Giordano (Phys., Auburn Univ., College of Sci. and Mathematics, Auburn, AL 36849,njg0003@auburn.edu)

Simulations of the transverse flute using direct numerical solutions of the Navier-Stokes equations are described. Constructing a numerical model of the instrument itself is straightforward, but making a semi-realistic model of the player's lips is more challenging. One solution to this problem is described and used to explore the kinds of tones that are possible. Conditions are found for which the model produces a well-behaved musical tone for a range of blowing speeds. When the blowing speed is increased beyond this range the behavior becomes complex and is appears to be chaotic. An analysis of this behavior in terms of the sensitivity to initial conditions and a route to chaos involving Hopf bifurcations is described. The relevance of these results to real instruments played by real players is also discussed. [Research supported by NSF grant PHY1513273.]

\section{9:25}

4aMUa5. Study on the principle of sound resonance in human whistling using physical models of a human vocal tract. Mikio Mori (Univ. of Fukui, 3-9-1 Bunkyo, Fukui City, Fukui 910-8507, Japan, mikoi@u-fukui.ac.jp)

The principle behind sound production in human whistling is relatively unknown. A good understanding of this principle would be beneficial to both trainer and trainee. Rayleigh identified whistling frequency as being determined by the mouth cavity and pointed out that earlier ideas that relate the sound-producing mechanism to the vibration of the lips are inaccurate. Wilson et al. reported that the human whistling resonant frequency is close to the Helmholtz resonant frequency, and through some physical-model-based experiments, they determined that the resonator can be excited by a flow through the smooth-edged orifices bounding the resonant cavity. Thus, the principles of whistling in terms of articulation have been reported to be based on the Helmholtz resonance. However, some whistlers can produce high-pitched sounds by blowing harder without changing the capacity of the resonance chamber, which is similar to a highpitched sound produced by a wind instrument by air-column resonance. This work studies the principle of sound production during human whistling using physical models of a human vocal tract, which is obtained from an X-ray computed tomography image during whistling. We have demonstrated that the principle of resonance in human whistling includes not only the Helmholtz resonance but also an air-column resonance.

\section{Contributed Papers}

\section{9:45}

4aMUa6. Numerical study on acoustic intensity of edge tone with change of jet velocity. Iwagami Sho (Mech. Information Sci. and Technol., Kyushu Inst. of Technol., Kawazu 680-4, Iizuka, Fukuoka 820-8502, Japan, iwagami@chaos.mse.kyutech.ac.jp), Taizo Kobayashi (Faculty of Fukuoka Medical Technol., Teikyo Univ., Omuta, Fukuoka, Japan), Kin'ya Takahashi (Mech. Information Sci. and Technol., Kyushu Inst. of Technol., Iizuka, Fukuoka, Japan), and Yuji Hattori (Inst. of Fluid Sci., Tohoku Univ., Sendai, Miyagi, Japan)

To understand the sounding mechanism of "air-jet" instruments we numerically study the acoustic intensity of edge tone, i.e., the sound source of "air-jet" instruments, with compressible LES and DNS comparing with experimental results. The edge tone is aerodynamic sound and we need to investigate the sound generation based on Lighthill's theory. According to Lighthill's theory, the acoustic intensity increases the eighth power of fluid velocity in transonic and subsonic ranges, but it may obey different rules in a range less than the subsonic. Indeed, we find with compressible LES that the edge tone has three stages, in each of which a different increasing rate is observed. In particular, the fundamental and second modes obey the second-power law in the second stage. The same result was obtained by an experiment provided by YAMAHA Corporation. To clarify the radiation mechanism of the edge tone, which exhibits the three stage, we have to calculate Lighthill's sound source with high accuracy. So, we will show our effort in this direction: improving the calculation with compressible LES together with DNS, which gives very accurate results at low Reynolds numbers.

\section{0:00}

4aMUa7. Numerical study of the influence of the mouth-flue-foot geometry on sounding mechanism of an "air-jet" instrument model. Shuhei Tateishi, Genki Tsutsumi (Mech. Information Sci. and Technol., Kyushu Inst. of Technol., Kawazu 680-4, Iizuka, Fukuoka 820-8502, Japan, shuuhei.tateishi@gmail.com), Taizo Kobayashi (Facalty of Fukuoka Medical Technol., Teikyo Univ., Omuta, Fukuoka, Japan), Toshiya Takami (Comput. Sci. and Intelligent Systems, Oita Univ., Oita, Oita, Japan), and Kin'ya Takahashi (Mech. Information Sci. and Technol., Kyushu Inst. of Technol., Fukuoka, Fukuoka, Japan)

The geometry of flue and foot influences the stability of jet oscillation, which products aerodynamic sound as the sound source of "air-jet instruments. Thus, we numerically study the influence of mouth-flue-foot geometry on the stability of jet oscillation with compressible LES. We introduce a 2D flue organ pipe model terminated with a closed end. We investigate the influence of the length of the flue, the existence of chamfers at the flue exit and the volume of the foot. As a result, eliminating the chamfer makes the jet oscillation unstable, while adding the chamfer stabilizes the jet oscillation and sound generation. A long flue makes the jet motion stable and robust, so that it spends a long time to reach a stable oscillation and cannot response quickly to the change of the air supply. Thus a relatively short flue with the chamfers is suitable for a performance. This result qualitatively agrees with the experimental result reported by Segoufin et al.. We also found that the existence of foot stabilizes the sound oscillation in the pipe: the pressure oscillation in the foot synchronizes with that in the pipe with a phase delay, but eliminating the foot rather makes the sound oscillation unstable. 


\title{
Session 4aMUb
}

\section{Musical Acoustics: Piano Acoustics and Playing Piano I}

\author{
Isoharu Nishiguchi, Cochair \\ Information Media, Kanagawa Institute of Technology, 1030, Shimo-ogino, Atsugi 243-0292, Japan \\ Nicholas Giordano, Cochair \\ Physics, Auburn University, College of Sciences and Mathematics, Auburn, AL 36849
}

Chair's Introduction-10:30

\section{Invited Papers}

$10: 35$

4aMUb1. Measurement and numerical simulation of two-dimensional vibration of a single piano string. Daisuke Naganuma (Tokyo Int. Univ., 1-13-1, Matobakita, Kawagoe, Saitama 350-1197, Japan, naganuma@tiu.ac.jp), Hideyuki Nomura, and Tomoo Kamakura (The Univ. of Electro-Communications, Chofu, Tokyo, Japan)

A piano string begins to vibrate vertically as soon as a hammer strikes it. After the vertical vibration has begun, the string then starts to rotate owing to the generation of horizontal vibration. The rotation direction changes several seconds later, which suggests that the frequencies of the vertical and horizontal components of the vibration are slightly different. We previously reported an equivalent mechanical circuit model for physically elucidating such two-dimensional movements of the string. In this presentation, we show the verification and validation of the equivalent model by comparing with experiments. The movements of a string in low frequencies were observed using a high-speed camera and a mirror. In order to increase the measurement accuracy of displacement, interpolation technique was applied to the correlation of recorded images. It was noted that the frequency of a partial vibration of the string in the vertical direction is higher or lower than that in the horizontal direction to the soundboard. The rotation directions and the rotation change periods suggest how the soundboard acts on the generation of the partial vibration as mass-dominated or stiffness-dominated control at the string end, and to what extent does the soundboard affect the partial vibration of the string.

\section{0:55}

4aMUb2. Simulation of three-dimensional motion of a piano string using detailed and simplified computational models. Isoharu Nishiguchi (Information Media, Kanagawa Inst. of Technol., 1030, Shimo-ogino, Atsugi, Kanagawa 243-0292, Japan, nishiguc@sd. kanagawa-it.ac.jp)

It is well known that the decay curve of the piano sound is steep for the first few seconds and followed by slower decay at longer time. The causes of this double decay are also known: the effect of polarization of a string which is considered to be caused by the motion of the bridge which is connected to the string by two bridge pins and interaction among multiple strings for a note. As for the later, it is explained that multiple strings for a note tend to vibrate out of phase each other when they have slightly different frequencies and this suppresses the energy transferred to the soundboard through the bridge. However, to make quantitative evaluations of these causes, it is indispensable to develop a physical model of the piano in which contributions of each factor of these causes can be simulated with a necessary degree of accuracy. In this study, the finite element method and the mode superposition method are employed to simulate the three-dimensional motion of a piano string in which interaction with a bridge on a soundboard is taken into account. The polarization and the longitudinal vibration of a piano string were measured to assess the simulation.

\section{1:15}

4aMUb3. Localization in the piano soundboard. Gautier Lefebvre, Marcel Filoche, and Xavier Boutillon (Physique de la Matière Condensée, École polytechnique, CNRS, Université Paris-Saclay, Laboratoire de Mécanique des Solides, Aile 3, École polytechnique, Palaiseau 91128, France, lefebvre@lms.polytechnique.fr)

The soundboard is the complex plane structure that radiates the piano sound. We focus on its structural vibrations. The arrangement of the stiffeners (parallel bars) displays some disorder (height, separation distance) that has been shown responsible for the localization of the vibrations above a precise frequency (Ege 2013, JSV; Chaigne 2013, JASA) By means of a finite-element method, we investigate the localization properties of the eigenmodes of a simplified soundboard model. The localization occurs close to the band-edge of the first Brillouin zone, indicating the importance of a slightly disordered structure, similarly to photonic or phononic crystals with defects (John 1987, PRL). For structural parameters typical of a piano soundboard, this phenomenon seems to force the soundboard to remain in the subsonic regime in a very large frequency range. Moreover, by the adaptation of the theory of the localization landscape (Filoche 2012 PNAS), we compute a dual landscape (Lyra 2015 EPL) for the high-frequency modes of a stiffened orthotropic thin plate. This enables to predict the position of localized eigenmodes. [Work supported by the ANR grant ANR-14-CE07-0014.] 
4aMUb4. Real-time physical model of a grand piano soundboard on field programmable gate array hardware. Florian Pfeifle and Rolf Bader (Univ. of Hamburg, Neue Rabenstrasse 13, Hamburg 20354, Germany, Florian.Pfeifle@uni-hamburg.de)

A modern grand piano consists of over 10,000 singular parts and has a production cycle of approximately one year. This leads to the predicament that researching and changing acoustical features resulting from variable material properties of the instrument cannot be realized in a straightforward manner. The inevitable time delay as well as the variability of parameters makes a controlled research very hard, if not impossible. In this work, a system is presented that enables piano makers to change, simulate as well as auralise acoustical vibrations of a grand piano soundboard in real time using physical modelling methods on field programmable gate array (FPGA) hardware. This specific implementation focusses on synthesizing and simulating the vibrations of a geometrically complete model of a concert grand piano soundboard, including the ribs, the bass, and main bridge, realistic material parameters as well as coupled strings. It can be parametrized and controlled from a software GUI running on a personal computer. This implementation is part of a larger project aiming at modeling a complete grand piano in real-time for research, instrument making as well as purely musical applications.

THURSDAY MORNING, 1 DECEMBER 2016

SOUTH PACIFIC 3, 8:00 A.M. TO 11:50 A.M.

\title{
Session 4aNS
}

\section{Noise: Prediction of Transportation Noises and Other Topics I}

\author{
James E. Phillips, Cochair \\ Wilson, Ihrig \& Associates, Inc., 6001 Shellmound St., Suite 400, Emeryville, CA 94608 \\ Shinichi Sakamoto, Cochair \\ 5th Department, Institute of Industrial Science, The University of Tokyo, 4-6-1, Komaba, Meguro-ku, Tokyo 153-8505, Japan
}

Chair's Introduction-8:00

Invited Papers

8:05

4aNS1. Evaluation of lateral attenuation for aircraft flyover and ground operation noise in airport noise modeling. Naoaki Shinohara (Aviation Environment Res. Ctr., AirportI Environment Improvement Foundation, Ryuuen Bldg., 1-3-1, Shiba-Koen, Minato-ku, Tokyo 150-0011, Japan, n-shinohara@aeif.or.jp), Kazuyuki Hanaka (Narita Int. Airport Fromotion Foundation, Shibayama, Chiba, Japan), and Ichiro Yamada (Aviation Environment Res. Ctr., Airport Environment Improvement Foundation, Minato-ku, Tokyo, Japan)

At the side of flight path, especially on the side of runway, noise level observed greatly changes dependent on the effects of excess ground attenuation and meteorological conditions (in particular, vector wind and temperature gradient). Thus, taking account of appropriate adjustment for lateral attenuation in airport noise modeling leads to an increase in the reliability of predictions. This paper makes a review of equations for lateral attenuation such as SAE/AIR 1751, SAE/AIR 5662, and our equation 1751M, which is a modified equation we proposed based on AIR/1751 and is applicable under various meteorological conditions. It is also described with further issues related to the examining of lateral attenuation for aircraft flyover noise. In Japan, it is obligatory to take into account noise contributions of aircraft ground operations such as APU operation, taxiing, and engine run-up in airport noise modeling. However, we have not yet fully examined whether we can evaluate lateral attenuation of such ground noise similarly to flyover noise. It is not yet solved how to calculate excess ground attenuation under the effects of sound shielding by barriers or embankment. This paper also discusses how to evaluate lateral attenuation for noise due to aircraft ground operations.

\section{$8: 25$}

4aNS2. Study of lateral attenuation for aircraft noise modeling based on field measurements near the airport. Kazuyuki Hanaka (Narita Int. Airport Promotion Foundation, 76 Amazutsumi Kayamashinden Shibayama-Town,Chiba, Shibayama, Chiba 289-1601, Japan, hanaka@napf.or.jp), Naoaki Shinohara, and Hisashi Yoshioka (Airport Environment Improvement Foundation, Tokyo, Japan)

It is essential to appropriately evaluate effects of lateral attenuation in predicting aircraft noise at the side of flight path near the airport. About fifteen years ago, we examined the validity of existing equations of lateral attenuation, but noise characteristics of aircraft and engines have changed very much. Especially, in case of horizontal sound propagation over ground such as aircraft rolling on the runway, it is necessary to take into account that meteorological conditions may strongly affect lateral attenuation. Thus, we carried out field measurements of aircraft noise repeatedly on the side of runway at several airports including Narita Airport and examined the actual 
situation of lateral attenuation. As a result, it was found that lateral attenuation greatly changes dependent on meteorological conditions and that calculations from existing equations of lateral attenuation did not match measurements of lateral attenuation. Additionally, considering actual situation of land use around the airport, it is not always realistic to assume that the ground surface is flat and uniform as well as acoustically soft. It is sometimes necessary to assume that acoustic property of the ground changes. It is also necessary to evaluate lateral attenuation on conditions that the sound propagates over urbanized city area.

\section{8:45}

4aNS3. Evaluating aircraft ground operation noise in airport noise modeling and monitoring in Japan. Ichiro Yamada (Airport Environment Improvement Foundation, Ryuuen Bldg. 1-3-1, Shiba-Koen, Minato-Ku, Tokyo 105-0011, Japan, i-yamada@aeif.or.jp)

The current Japanese noise guideline "Environment Quality Standard for Aircraft Noise" requires considering noise of aircraft ground operation, which we call ground noise here, like APU operation, taxiing, and engine run-up, if necessary. It has inevitably required us not only to take account of ground noise contributions in the Japanese airport noise model JCAB2, but also to include techniques to identify or discriminate ground noise from the ambient noise floor when fulfilling airport noise monitoring. Ground noise sometimes lasts very long and is usually low level compared with flyover noise. First, this paper makes a brief review of the way to evaluate ground noise in airport noise modeling and monitoring in Japan. Second, it discusses further issues to improve the reliability in the evaluation of ground noise under the influences of excess ground attenuation, meteorological condition, sound shielding by obstructions like building structures and embankment, as well as contamination by noise events.

\section{9:05}

4aNS4. An aircraft noise prediction model considering meteorological effects on noise propagation developed for air traffic optimization. Hirokazu Ishii, Adriana Andreeva-Mori (Japan Aerosp. Exploration Agency, 6-13-1 Osawa, Mitaka, Tokyo 181-0015, Japan, ishii.hirokazu@jaxa.jp), Takatoshi Yokota, Koichi Makino, and Toshio Matsumoto (Kobayasi Inst. of Physical Res., Tokyo, Japan)

With the growing transportation demand, the volume of air traffic is already approaching and will soon exceed the capacity limit posed by current air traffic control. Japan Aerospace Exploration Agency (JAXA) has conducted a research project called DREAMS, aiming to develop technologies for future air traffic management system. One of the research topics examined by the project is noise abatement flight technology. It optimizes approach paths considering the meteorological effects on noise propagation. Its key element is an aircraft noise prediction model which can take into account the effect of meteorological conditions on noise propagation. The effect is computed by Green's Function-Parabolic Equation method and verified by field experiment using an elevated sound source. Aircraft noise is measured around approach flight path for overall verification, and the results show the average and the standard deviations of the prediction error are $-0.66 \mathrm{~dB}$ and $1.55 \mathrm{~dB}$, respectively. The presentation provides an overview of the development and the verification of the noise prediction model, followed by a comparison between noise footprints calculated with JAXA's model and the Integrated Noise Model (INM). Application of the noise prediction model to descent flight path optimizations for noise abatement is also shown.

9:25

4aNS5. A field survey on sound power level and spectrum of Japanese road vehicles. Miki Yonemura (School of Eng., The Univ. of Tokyo, Komaba 4-6-1, Inst. of Industrial Sci., Ce402, Meguro-ku, Tokyo 153-8505, Japan, m-yone@iis.u-tokyo.ac.jp), Hyojin Lee, and Shinichi Sakamoto (Inst. of Industrial Sci., The Univ. of Tokyo, Meguro-ku, Tokyo, Japan)

For precise prediction and appropriate countermeasure of road traffic noise, to grasp the sound power level and power spectrum of running vehicles is important. In Japan, there exists a road traffic noise prediction model "ASJ RTN-Model 2013," which provides a simple sound source modeling and simple calculation methods of the noise propagation. Recently, vehicles with good fuel-efficiency such as hybrid vehicles are increasing in the category of passenger car. Such vehicles may have less noise emission than previous gasoline engine vehicles. The authors conducted field surveys to grasp the noise of the current road vehicles especially in the category of passenger cars. As a result of that, in terms of A-weighted sound power levels, which can be expressed as a function of running speed, the difference between gasoline engine vehicles is quantitatively discussed. As for the power spectrum, there is no appreciable difference between gasoline engine vehicles and hybrid vehicles. However, both types of vehicles have more or less different spectral characteristics from ASJ RTN-Model 2013.

\section{Contributed Paper}

\section{9:45}

4aNS6. Effect of accuracy of digital map data on predicting road traffic noise in Japan. Yasuhiro Hiraguri (Dept. of Civil Eng. and Architecture, National Inst. of Technol., Tokuyama College, Gakuen-dai, Shunan, Yamaguchi 7458585, Japan, hiraguri@tokuyama.ac.jp) and Kazutoshi Fujimoto (Kyushu Univ., Fukuoka, Japan)

Use of GIS for evaluating the Environmental Quality Standards for Noise is established at local authorities in Japan. A digital map on a scale of $1 / 2,500$ is generally used. However, it has not been clarified yet whether the digital map with such a scale has an accuracy to apply for the estimation of environmental noise. A ground plan of buildings on the digital map is a roof plan, because the digital map data are made on a basis of an aerial photograph. It is feared that the noise estimated by using such a map may have some differences in the effects of buildings on the noise propagation from reality. In spite of the fact that the propagation of road traffic noise is highly affected by whether a road is visible from receiving point or not, when the roof plan is used as the floor plan, the road is sometimes visible from the receiving point on the digital map, though it is invisible in practice. The aim of this study is, therefore, to reveal the effect of accuracy of the digital map in GIS on the estimation of road traffic noise for evaluation of the Environmental Quality Standards for Noise. 


\section{Invited Paper}

\section{$10: 15$}

4aNS7. Modeling noise from motocross facility with complex terrain. Leisa Nalls and Richard Carman (Wilson Ihrig, 6001 Shellmound, Ste.400, Emeryville, CA 94608, Inalls@wiai.com)

The authors prepared a study for an existing motocross facility under new ownership. The study was required by the county for a continued use permit. The facility is in a rural setting and will host weekend races and practice activity. The terrain of the motocross track is complex with a hill and flat section with jumps. To conduct the study, an empirical noise model was constructed for future track activity using noise measurements performed at selected locations at the facility's property line during simulated race and practice activity. A model of the future dirt bike noise was created by scaling the measured race and practice activity based on the level of future bike activity anticipated by the owner. The data were scaled using the number of bike rider hours to account for the time riders spend riding, the number of riders per activity, and the number of different activities per day. Long-term noise was measured at the same locations to obtain typical ambient levels during hours with no bike activity. The expected Ldn noise exposure at the property line was obtained by combining the projected race and practice activity noise for different scenarios with the typical ambient noise.

\section{Contributed Papers}

$10: 35$

4aNS8. Four methods of estimating population exposure to transportation noise. Enda Murphy and Owen Douglas (Univ. College Dublin, Dublin, Dublin 1, Ireland, enda.murphy@ucd.ie)

This paper assesses four methods for estimating population exposure to road traffic noise within the context of the EU Environmental Noise Directive (END). Employing a case study in central Dublin, Ireland, the methods-MAX, MIN, VBEB/CNOSSOS and AVE—are tested utilising the $\mathrm{L}_{\mathrm{den}}$ and $\mathrm{L}_{\text {night }}$ indicators. The study first investigates the extent to which exposure estimates may vary depending on the method utilised while controlling for the noise calculation method. Second, it investigates how estimates of exposure vary depending on the calculation method used; in this case, CRTN and NMPB. The results show that controlling for noise calculation method and employing the same input data, estimations of population exposure differ substantially depending on the exposure method employed. Furthermore, the potential variability in estimated night-time exposure and the potential for under or over estimation of the health effects of environmental noise on a given population when different methods are utilised is clearly demonstrated. The results also show that the method of noise calculation employed has an effect on estimated exposure, particularly for $\mathrm{L}_{\mathrm{den}}$ measures. Values for $\mathrm{L}_{\text {night }}$ were found to be very similar regardless of the calculation method employed.

\section{0:50}

4aNS9. Sound source identification on automotive muffler surface using single explosion excitation and near-field acoustic holography method. Ryota Matsumoto, Takehiko Seo, Masato Mikami (Mech. Eng., Yamaguchi Univ., 2-16-1 Tokiwadai, Ube, Yamaguchi 755-8611, Japan, v051ve@ yamaguchi-u.ac.jp), and Takashi Esaki (Sango Co., Ltd., Miyoshi, Japan)

Recent automobiles tend to have larger interior space, resulting in the smaller space for arranging the muffler. Flat shape mufflers are required for this reason but cause relatively large radiation noise from the surface. In order to improve understanding of radiation noise generation mechanism of flat shape muffler, we tried to identify the noise sources using the near-field acoustic holography (NAH) method on muffler surface excited by a single explosion pulse, which simulates a single pulsation in the exhaust system,. Two types of mufflers were tested with different shapes. Results show that some areas had large vibration velocity at specific frequencies depending on the muffler shape. The flat muffler tends to generate large radiation noise and vibration velocity as compared with a conventional one. In the front and rear outer plates of the muffler, large vibration velocity exists within a certain range of frequency. Within the same frequency range, the flat muffler has more number of the peak of radiation noise as compared with the conventional one.

\section{1:05}

4aNS10. Robustness of the extended delayed-X harmonics synthesizer algorithm applied to engine exhaust noise control. Sergej Jukkert and Delf Sachau (Mechatronics, Helmut-Schmidt-Univ. Hamburg, Holstenhofweg 85, Hamburg, Hamburg 22043, Germany, jukkerts@hsu-hh.de)

Exhaust noise of a combustion engine contains several harmonic components of the fundamental frequency that depends on the engine speed. Active noise cancellation (ANC) in the exhaust line of a diesel-electric drive faces serious challenges such as high temperature, high sound pressure levels, standing waves phenomena, a time-varying environment, and slightly varying disturbance frequencies. In the case of a non-acoustic reference sensor, also an offset or a mismatch in the estimation of the disturbance frequencies negatively affects the performance. The extended delayed-X harmonics synthesizer (DXHS) is known from several researches to be appropriate for control of periodic noise with multiple harmonic components. The algorithm is robust against observation noise and fluctuation of the plant under control. In this paper, the extended DXHS is implemented in an ANC-system for a diesel-electric drive and experimentally investigated in laboratory experiments. A modified update rule, a convergence factor regulation, and the pre-filtering of the error signal are implemented to improve the DXHS. Performance and robustness are tested in a mockup of an exhaust line in which realistic fluctuations of temperature and gas flow as well as varying frequencies and amplitudes of the exhaust noise are simulated.

\section{1:20}

4aNS11. A study on transient noise-generation characteristics in a diesel engine with rapid premixed-charge compression ignition combustion. Shodai Sagara, Yoshiki Sumida, Takehiko Seo, and Masato Mikami (Mech. Eng., Yamaguchi Univ., 2-16-1 Tokiwadai, ube, Yamaguchi, Ube, Yamaguchi 755-8611, Japan, v014ve@yamaguchi-u.ac.jp)

This study investigates time-frequency characteristics of noise generation in a diesel engine with rapid PCCI (premixed-charge compression ignition) combustion. We increased the pilot injection quantity from a base PCCI condition to simulate rapid PCCI combustion. We measured the incylinder pressure and sound pressures and analyzed them using fast Fourier transformation and wavelet transformation. The main cause of the engine noise is the combustion impact on the combustion chamber. We clarified the process that the combustion impact vibrates the engine structure and then lead to sound radiation. We identified main frequencies, whose noise components largely affect the overall level of engine noise. Almost all engine noise components at the main frequencies were found to be strongly affected by the combustion impact. We researched the noise source and transmission path of each noise component by using a sound field visualization device and measuring accelerations on the engine surface. 
$11: 35$

4aNS12. Proposal of suspended floor structure for reducing interior noise of railway vehicle. Tomonori Goto and Katsuya Yamamoto (Vehicle Structure Technol. Div., Railway Tech. Res. Institute, 2-8-38, Hikari-cho, Kokubunji-shi, Tokyo 185-8540, Japan, goto.tomonori.98@rtri.or.jp)

In this study, we propose a new floor structure "suspended floor" as one of the countermeasures against the interior noise of a railway vehicle. The interior noise of a railway vehicle is mainly composed of the transmitted noise and the structure-borne noise from floor panels, where the vibration of the bogie is propagated. Although floor panels are generally supported on joists mounted on the floor structural panels, it is possible to reduce the vibration of floor panels by suspending them from less vibrating points. We investigated the vibrational characteristics of the car body and clarified that the vertical vibration of the side structural panel is less than that of the floor structural panel. Therefore, it was expected that the floor structure in which floor panels are suspended from side structural panels is effective in reducing the vibration of floor panels. The floor structure prototype was fabricated in the test car, and experiments have been made to verify the vibrational reduction effect. As a result, the sound power radiated from the floor panel was reduced by 5-10 dB. In conclusion, the suspended floor structure is effective in reducing structure-borne noise in a railway vehicle.

THURSDAY MORNING, 1 DECEMBER 2016

SOUTH PACIFIC 2, 8:15 A.M. TO 11:45 A.M.

\title{
Session 4aPA
}

\section{Physical Acoustics: General Topics in Physical Acoustics}

\author{
Michael R. Haberman, Cochair \\ Applied Research Laboratories, The University of Texas at Austin, 10000 Burnet Rd., Austin, TX 78758 \\ Kevin M. Lee, Cochair \\ Applied Research Laboratories, The University of Texas at Austin, 10000 Burnet Road, Austin, TX 78758
}

\section{Contributed Papers}

$8: 15$

4aPA1. Stick and slip mechanisms in the acoustic emissions of ice fracture. Rintaro Hayashi, Melisa Yashinski, and John S. Allen (Mech. Eng., Univ. of Hawaii-Manoa, 2540 Dole St., Holmes Hall 302, Honolulu, HI 96822, alleniii@hawaii.edu)

Arctic ice noise has been qualitatively described in terms of crashing and cracking noises. Ships navigating through icy waters and colliding with icebergs have reported distinctive cracking sounds characteristic to the specific environmental conditions and the location. Many previous field studies of ice acoustics have been done with measurements from isolated, single hydrophones. These have provided useful physical insights into the ambient noise characteristics of ice, but the underlying mechanisms of the sounds are still not well understood. The mechanical properties of ice have been extensively studied in laboratory settings, but few studies have examined the acoustics emissions during fracture with respect these properties. The salinity content of sea ice can significantly influence fracture. The acoustics of ice fracture in water and air is investigated in laboratory using simultaneous acoustic and high speed optical measurements. Also, the mechanical properties of ice samples as a function of salinity were studied with three point bending. A stick and slip mechanism found in fracture of low salinity ice is supported by an Empirical Mode Decomposition of the acoustic signals.
8:30

4aPA2. Determining average flow speed in a scale model smokestack by measuring correlated noise through a long-wavelength acoustic flow meter. JohnPaul R. Abbott (National Ctr. for Physical Acoust., Univ. of MS, 100 Bureau Dr. Stop 8361, Gaithersburg, MD, johnpaul.abbott@gmail. com), Keith A. Gillis, Michael R. Moldover (National Inst. of Standards and Technol., Gaithersburg, MD), and Lee J. Gorny (None, Mountain View, CA)

As part of its Greenhouse Gas and Climate Science Measurements Program, the National Institute of Standards and Technology (NIST) has successfully developed long-wavelength acoustic flowmeters (LWAFs) to measure the average flow speed, $V$, and the speed of sound, $c$, for a fluid passing through 1:100 scale test model of a coal-burning power plant smokestack with standard measurement uncertainties of less than $1 \%$ and $0.1 \%$, respectively. This improves upon the estimated $5-20 \%$ standard measurement uncertainty for existing technologies. With this success NIST has constructed a 1:10 scale test model for further investigation. Current LWAF measurement techniques are reliant on a good signal-to-noise ratios (SNR), which may be problematic for the 1:10 scale test model since the amount of turbulence and difficulty generating high-amplitude low-frequency excitation signals at larger scales increases. Anticipating poorer SNRs for larger scale test models, NIST is investigating the use of the LWAF to correlate low-frequency acoustic noise, flow noise, and other excitation signals as an alternative technique to measure $V$ and $c$ with the goal of obtaining the same standard measurement uncertainties of less than $1 \%$ and $0.1 \%$, respectively. The latest results of this ongoing investigation are presented. 
4aPA3. Cut-off frequency criterion for wind noise contributions for wind noise reduction enclosures. JohnPaul R. Abbott (National Ctr. for Physical Acoust., Univ. of MS, 100 Bureau Dr. Stop 8361, Gaithersburg, MD 20899, johnpaul.abbott@gmail.com), Richard Raspet (Dept. of Phys. and Astronomy, National Ctr. for Physical Acoust., Univ. of MS, Oxford, MS), Sandra Collier, W. C. K. Alberts, and John Noble (U.S. Army Res. Labs., Adelphi, MD)

A cut-off frequency criterion for contributions for the wind noise generated from the undisturbed and surface regions for a wind noise reduction device, such as wind fence enclosures and semi-porous fabric domes, is given. The calculations of the measured wind noise inside an enclosure assume that contributions from the undisturbed region and the surface region dominate and are physically restricted to turbulence wavelengths that are larger than the enclosure and the same size or smaller than the enclosure, respectively. The cut-off frequency criterion defines the wavelength where the dominant source of wind noise transitions from interactions in the undisturbed region to the surface region. The transition point is determined by considering when a volume enclosed by a wavelength, $\mathrm{V}_{\lambda}$, is equal to 100 times the volume enclosed by the reduction device, $\mathrm{V}_{\mathrm{E}}$. Wind noise calculations following this criterion are presented for a set of semi-porous fabric domes that are $4 \mathrm{~m}$ wide and $2.1 \mathrm{~m}$ tall, and three cylindrical wind fences that are $5 \mathrm{~m}$ wide and $2.9 \mathrm{~m}$ tall, $5 \mathrm{~m}$ wide and $5.8 \mathrm{~m}$ tall, and $10 \mathrm{~m}$ wide and $2.9 \mathrm{~m}$ tall.

\section{9:00}

4aPA4. Numerical determination of parameter sensitivity of transmission loss in atmospheric sound propagation above a general ground surface. Seth D. Hubbard, David Lechner, Joseph F. Vignola (Dept. of Mech. Eng., Catholic Univ. of America, 620 Michigan Ave., Washington, DC 20064, 22hubbard@cua.edu), Teresa J. Ryan, Melissa Hall (Dept. of Eng., East Carolina Univ., Greenville, NC), John A. Judge, and Diego Turo (Dept. of Mech. Eng., Catholic Univ. of America, Washington, DC)

A transmission loss model, similar to the sonar equation, is used to study the transmission loss in atmospheric sound propagation over ground. The model takes into account contributing factors from spreading loss, absorption, wind, temperature gradient, and ground impedance. Numerical predictions obtained with the Crank-Nicolson Parabolic Equation implementation are used to quantify the effects of these factors. The transmission loss sensitivity to these parameters will be presented. These sensitivity findings will be compared to a set of field measurements. An acoustic source is placed in an open field and six horizontally aligned microphones are used to record the signal. Microphones were spaced at regular intervals up to approximately $400 \mathrm{~m}$ away from the source and 1 meter above the ground. Temperature, atmospheric pressure, and relative humidity measurements were made concurrent with the acoustic recordings.

\section{9:15}

4aPA5. Measurement of atmospheric sound propagation and meteorological parameters to support development of an acoustic propagation model. Melissa A. Hall, Teresa Ryan (Dept. of Eng., East Carolina Univ., 248 Slay Hall, Greenville, NC 27858, hallme14@students.ecu.edu), Seth Hubbard, Joseph F. Vignola, John A. Judge, and Diego Turo (Catholic Univ. of America, N.E. Washington, DC)

Acoustic propagation over long distances is affected by many conditions including atmospheric properties, topography, vegetation, source characteristics, and frequency content. This work presents the results of a set of measurements of acoustic propagation of impulsive sound in the low kilohertz frequency range. These measurements are made over flat ground with limited vegetation at propagation ranges of up to $400 \mathrm{~m}$. At multiple locations along the propagation path, the temperature, pressure, and relative humidity, along with altitude and GPS position are recorded to provide high spatial resolution characterization of the air column in the propagation path. A mast-mounted weather station at the acoustic source provides a record of wind speed and direction at ground level. These measurements are intended to inform related efforts developing a model of acoustic propagation that uses a Crank-Nicolson Parabolic Equation implementation. The measured transmission loss will be compared to preliminary results from the numerical model.
4aPA6. De-Dopplerization of acoustic signatures. Frank S. Mobley (Human Effectiveness Directorate, U.S. Air Force, 2610 Seventh St., Bldg. 441, Wright-Patterson AFB, OH 45433, frank.mobley.1@us.af.mil) and Brian S. Davis (CDO Technologies Inc., WPAFB, OH)

Shifting energy within and between adjacent fractional octave bands removes Doppler shifting in overflight measurements of acoustic signatures without complex resampling techniques and stationary requirements. Analyzing acoustic measurements with Fourier transforms to obtain spectral information requires complex resampling to overcome stationary signal requirements. A simple shifting of band energy obtained from fractional octave digital filters generates a de-Dopplerized spectrum without complex algorithms. A numerical equation is developed based on the fractional octave center frequency to define the amount of energy shifting required. This equation is applied to a numerical simulation and an overflight measurement to remove the Doppler effect in spectral data. The de-Dopplerization through application of energy shifting accurately removes vehicle motion effects from acoustic measurements without complex resampling.

\section{9:45-10:00 Break}

\section{0:00}

4aPA7. Focus control of a nematic liquid crystal cell using ultrasound vibration. Yuki Shimizu, Daisuke Koyama (Graduate School of Sci. and Eng., Graduate School of Doshisha Univ., Tataramiyakodani1-3, Kyotanabe, Kyoto 610-0321, Japan, duq0360@mail4.doshisha.ac.jp), Satoki Taniguchi (Faculty of Life and Medical Sci., Doshisha Univ., Kyotanabe, Kyoto, Japan), Akira Emoto (Graduate School of Sci. and Eng., Graduate School of Doshisha Univ., Kyotanabe, Kyoto, Japan), Kentaro Nakamura (Lab. for Future Interdisciplinary Res. of Sci. and Technol., Tokyo Inst. of Technol., Yokohama Midori-ku, Kanagawa, Japan), and Mami Matsukawa (Graduate School of Sci. and Eng., Graduate School of Doshisha Univ., Kyotanabe, Japan)

Nematic liquid crystals used in optical devices such as liquid crystal displays are controlled by the electric fields. Although indium tin oxide (ITO) is generally employed as the transparent electrode material to apply the electric field, it includes the rare metal indium and needs complicated processes such as sputtering deposition method. Our group has been investigating control techniques of molecular orientation of liquid crystals using ultrasound. In this study, we have applied these techniques to optical variable-focus lenses and the optical characteristics were examined. The ultrasonic liquid crystal cell has a simple structure with no transparent electrodes; it consists of has an annular ultrasound PZT transducer (inner diameter: $30 \mathrm{~mm}$; thickness: $2 \mathrm{~mm}$ ) and a liquid crystal cell in which a nematic liquid crystal layer (thickness: $25 \mu \mathrm{m}$ ) was formed between two glass substrates (thickness: 0.7 $\mathrm{mm}$ ) and orientational films. The concentric flexural vibration modes could be generated on the cell at the several resonance frequencies, and the liquid crystal molecular orientation was changed by the acoustic radiation force. The transmitted light through the liquid crystal cell was focused, and the focal length could be controlled by the input signal.

\section{0:15}

4aPA8. Standing shear waves in nonlinear gel-like media under static shear stress. Timofey Krit, Shamil Asfandiyarov, and Valery Andreev (Dept. of Acoust., Faculty of Phys., Moscow State Univ., Leninskie Gory, Bldg. 1/2, Moscow, Moscow 119991, Russian Federation, timofey@acs366. phys.msu.ru)

Standing shear waves in a resonator in the form of layer of gel-like medium placed between two rigid plates are studied. The bottom plate is fixed to the vibrator and oscillates in the horizontal direction with a preset amplitude. Two rubber threads attached to the upper plate can displace the plate by a specified value in the horizontal direction. The change in the tension of the threads creates an additional static deformation of the elastic layer resulting in the effective shear elasticity increase. The measured static stress-strain dependence of the elastic layer can be described by the cubic parabola. We measured the dependences of the resonance frequency on the static deformation of the layer. For static deformations of the layer less than $0.3 \mathrm{~h}$ ( $\mathrm{h}$ - is the layer thickness), the resonance frequency increases linearly, 
that can be explained by a linear growth of the elastic force of rubber threads. In the deformation range of 0.3-1 h, an additional shift of the resonance frequency caused by the nonlinearity of a gel-like medium appears. The method allows the dynamic nonlinear parameter measurement in gellike media at the low frequency range.

\section{$10: 30$}

4aPA9. Acoustic-vortex interaction in reacting wakes near the global hydrodynamic stability limit. Benjamin Emerson and Tim Lieuwen (Aerosp. Eng., Georgia Inst. of Technol., 635 Strong St., Atlanta, GA 30318, bemerson@gatech.edu)

This study experimentally characterizes the effect of acoustics on the vortex dynamics in reacting wakes. This work is motivated by the problem of combustion instabilities, where the natural combustor acoustics excite hydrodynamic instabilities of the flow that, in turn, induce heat release oscillations. Time resolved PIV, Mie scattering, and chemiluminescence imaging measurements are obtained in a reacting wake facility with acoustic excitation. Measurements and analysis are performed where the acoustic frequency is varied relative to the global mode frequency. We observe that the acoustic waves excite a varicose mode of vortex shedding, which is locked into the acoustic frequency. When the acoustic frequency is close to the natural global hydrodynamic frequency of the wake, we observe resonance, which manifests as very rapid amplification of these shed vortices as they convect downstream. Interestingly, in the resonant case, the vortices stagger from the varicose structure deposited by the acoustic waves and assume a sinuous structure that resembles the hydrodynamic global mode. A linear, local stability analysis, together with a nonlinear analysis, help elucidate the physics that govern the vortex staggering. The study counterintuitively concludes that the resonance, due to the change from varicose to sinuous structure, may help alleviate combustion instability.

\section{0:45}

4aPA10. A second-order, perfectly matched layer formulation to model 3D transient wave propagation in anisotropic elastic media. Hisham Assi and Richard S. Cobbold (Inst. of BioMater. \& Biomedical Eng., Univ. of Toronto, IBBME, 164 College St., Toronto, ON M5S 3G9, Canada, hisham.assi@utoronto.ca)

Perfectly matched layers (PML) are a well-developed method for simulating wave propagation in unbounded media enabling the use of a reduced computational domain without having to worry about spurious boundary reflections. Using this approach, a compact three-dimensional (3D) formulation is proposed for time-domain modeling of elastic wave propagation in an unbounded general anisotropic medium. The formulation is based on a second-order approach that has the advantages of well-posedness, physical relationship to the underlying equations, and amenability to be implemented in common numerical schemes. However, many auxiliary variables are usually need to described second-order PML formulations. The problem becomes more complex for the $3 \mathrm{D}$ case modeling which would explain the dearth of compact second-order formulations in 3D. Using finite element method (FEM), 3D numerical results are presented to demonstrate the applicability of our formulation, including a highly anisotropic medium example. Extension of the PML formulation to model the case of a KelvinVoigt viscoelastic medium is also provided. In addition, we will present the extension of this formulation to model the transient wave propagation in an unbounded fluid-solid 3D medium in a similar way to that presented by the authors (JASA, 2016) for the 2D case.

\section{1:00}

4aPA11. Performance analysis of finite-difference time-domain schemes for acoustic simulation implemented on multi-core and many-core processor architectures. Ryo Imai, Yukihisa Suzuki (Graduate School of Sci. and Eng., Tokyo Metropolitan Univ., 1-1 Minami-Osawa, Hachioji, Tokyo 192-0397, Japan, imai-ryou@ed.tmu.ac.jp), Kan Okubo, Yuta Katori (Graduate School of System Design, Tokyo Metropolitan Univ., Hino, Japan), and Naoki Kawada (ELSA Japan Inc., Hino, Japan)

There have been many discussions about hardware-accelerations for the large-scale finite-difference time-domain (FDTD) method to deal with various wave dynamics in scientific and engineering computations. Recently, there is growing interest in many-core based parallel computing with graphics processing unit (GPU) or many integrated Core (MIC) accelerators. Therefore, it is worth applying those to large-scale and long-time acoustic simulation for FDTD methods. In this paper, performance analyses are performed for three types of acoustic FDTD schemes, which are $\operatorname{FDTD}(2,2)$, $\operatorname{FDTD}(2,4)$ and wave equation FDTD (WE-FDTD) $(2,2)$, implemented on GPU, Intel MIC, and multi-core central processing unit (CPU). Here, $\operatorname{FDTD}(T, S)$ denotes Tth-order accuracy for the time derivative and Sthorder accuracy for the spatial derivative, respectively. The parallelized FDTD schemes are implemented with Compute Unified Device Architecture (CUDA) and OpenACC, respectively, on GPU, and OpenMP for MIC and multi-core CPU. As a method for performance analysis, we employ the modified roofline model which adds the concept of cache hit ratio to operational intensity. The analysis provides performance comparison results of attainable performance and measured performance for each FDTD scheme and each processor. We performed software optimizations for MIC based on the performance analysis and achieved $78 \%-91 \%$ of attainable performance on MIC.

\section{1:15}

4aPA12. Extended optical theorem in elastic solids: Applications to Bessel beam scattering. J. H. Lopes (Exact Sci., Federal Univ. of Alagoas, Arapiraca, Alagoas, Brazil), J. P. Leao, A. L. Baggio, and Glauber T. Silva (Phys., Federal Univ. of Alagoas, Av. Lourival Melo Mota, sn, Maceio, Alagoas 57035-557, Brazil, glauber@pq.cnpq.br)

We derive the extended version of the optical theorem for the scattering of an elastic wave with arbitrary wavefront by an inclusion of any shape embedded in a solid medium. The inclusion can be made of an elastic, viscoelastic, fluid, and rigid material or be an empty cavity. The extended optical theorem relates the extinction cross-section, which is defined as the timeaveraged power extracted from the incident wave per incident intensity, in terms of the coefficients of the partial-wave series representation for the incident and scattered waves. With this formalism, the optical theorem for a longitudinal Bessel beam scattering is obtained in a solid for the first time. Additionally, we established the connection between the optical theorem and radiation pressure exerted on a spherical inclusion by a longitudinal and shear plane wave. In conclusion, the developed framework might be useful for beamforming in ultrasound nondestructive test and ultrasound imaging of biological tissue.

\section{1:30}

4aPA13. Chaos in a stadium-shaped acoustic cavity. Roger Yu (Phys., New York Inst. of Technol., 1885, New York, NY 10023, ryu@nyit. edu)

A numerical scheme has been developed to solve wave equations for chaotic systems such as stadium-shaped cavity. The same numerical method can also be used for finding wave properties of rectangle cavities with randomly placed obstacles. About 30,000 eigenvalues have been obtained accurately on a normal circumstance. For comparison, we also initiated an experimental study which determines both eigenfrequencies and eigenfunctions of a stadium-shaped cavity using pulse and normal mode analyzing techniques. The acoustic cavity was made adjustable so that the transition from nonchaotic (circle) to chaotic (stadium) waves can be investigated. 


\title{
Session 4aPPa
}

\section{Psychological and Physiological Acoustics: Psychological and Physiological Acoustics Potpourri (Poster Session)}

\author{
Frederic Apoux, Chair \\ Otolaryngology - Head \& Neck Surgery, The Ohio State University, 915 Olentangy River Road, Columbus, OH 43212
}

\begin{abstract}
All posters will be on display from 8:00 a.m. to 12:00 noon. To allow contributors in this session to see the other posters, authors of odd-numbered papers will be at their posters from 8:00 a.m. to 10:00 a.m. and authors of even-numbered papers will be at their posters from 10:00 a.m. to 12:00 noon.
\end{abstract}

\section{Contributed Papers}

4aPPa1. Behavioral and electrophysiological evidence for backward masking. Robert Sears, Silas Smith, Brittany Galvin, and Al Yonovitz (Dept. of Communicative Sci. and Disord., Univ. of Montana, Missoula, MT 59812, al.yonovitz@umontana.edu)

Backward Masking (BM) functions have been shown to relate to age, lead toxicity, and are differentiated in children with language disorders. These functions may be indicative of auditory processing deficits. This study compared BM functions obtained from Evoked Potentials (EP) to behaviorally obtained functions. A stimulus followed by an ISI and a noise masker were presented. All were studied individually in the appropriate temporal alignment. The design of this study allowed observation of the early, middle, and late auditory evoked potentials. For the behavioral task, stimuli were presented with a method of adjustment. This study randomly presented four different stimulus conditions: 1) stimulus alone, 2) noise alone, 3) stimulus and noise, and 4) silence. With a long inter-trial interval $(1 \mathrm{~s})$ and high sample rate $(25,600 \mathrm{~Hz})$ EP's were obtained for 4000 trials. The stimuli were pure-tones $(1000 \mathrm{~Hz}, 10 \mathrm{~ms}$ duration with a Blackman function and brief 100 microsecond clicks of varying intensity and varied ISI. Older and younger subjects were tested. Results indicated that EP's could be arithmetically combined to observe the differential electrophysiological responses and were highly correlated with the behaviorally obtained BM functions.

4aPPa2. Backward masking abilities for speech and tones in persons who stutter. Shriya Basu, Robert S. Schlauch, and Jayanthi Sasisekaran (Dept. of Speech Lang. Hearing Sci., Univ. of Minnesota, 115 SHevlin Hall, Minneapolis, MN 55455, basux045@umn.edu)

We examined eight persons who stutter (PWS) and eight carefully matched adult, control participants for their ability to identify vowel-consonant (VC) syllables and to detect tones in a backward masking paradigm. Speech scores (percent correct) were obtained in quiet and in conditions with a fixed-level broadband masker with a delay of $0 \mathrm{~ms}$ and $300 \mathrm{~ms}$. Tonal thresholds $(1000 \mathrm{~Hz})$ were obtained using an adaptive procedure in quiet and with the same masker delays as in the speech conditions. The results revealed significantly poorer performance for PWS for all of the speech conditions, including the quiet condition. Tonal thresholds in quiet and for the condition with a $300 \mathrm{~ms}$ delay were identical for the two groups, but, on average, the trend was for higher masked thresholds for PWS than for the control group in the $0 \mathrm{~ms}$ condition. The poorer speech results are consistent with the idea that PWS may have less distinct representations of phonemic categories that are revealed in a VC task that lacks a language context.
4aPPa3. Within- and between-frequency gap duration discrimination as a function of the standard duration. Hyunsoo Cho (Graduate school of Information Sci. and Elec. Eng., Kyushu Univ., 744 Motooka Nishi-ku, Fukuoka, Fukuoka, Japan, choh@cog.inf.kyushu-u.ac.jp), Kazuhito Ito, Nobuyuki Hirose, and Shuji Mori (Faculty of Information Sci. and Elec. Eng., Kyushu Univ., Fukuoka, Japan)

It is well known that individuals exhibit worse performance during between-frequency gap detection than during within-frequency gap detection. A similar performance difference is also found between within- and between-frequency gap duration discrimination involving undetectably short standard durations. This is reasonable because such discrimination could be considered to be a variant of gap detection. However, because of the paucity of existing data, it is unclear whether frequency differences affects gap discrimination tasks involving longer standard durations. In this study, we measured within- and between-frequency discrimination thresholds in the presence of various standard durations from 1 to $100 \mathrm{~ms}$. As a result, it was shown that the within-frequency thresholds were significantly lower than the between-frequency thresholds when a standard duration of 1-ms was employed, whereas there were no significant differences between them when the standard duration was $>50 \mathrm{~ms}$. This implies that the within- and between-frequency gap discrimination processes become equivalent as the standard duration increases.

4aPPa4. Why does gap detection performance in cochlear implant users differs between free-field and direct-stimulation? Etienne Gaudrain (Auditory Cognition and PsychoAcoust., CNRS UMR 5292, Lyon Neurosci. Res. Ctr., Université Lyon 1, UMCG, KNO, Huispostcode BB20, PO box 30.001, Groningen 9700 RB, Netherlands, etienne.gaudrain@cnrs.fr), Pranesh Bhargava, and Deniz Başkent (Dept. of Otorhinolaryngology, Univ. of Groningen, Univ. Medical Ctr. Groningen, Groningen, Netherlands)

Gap detection (GD) is used to measure temporal resolution in the auditory system. Previous studies have shown that GD performance in cochlear implant (CI) users is similar to, or sometimes better than, that observed in normal hearing $(\mathrm{NH})$ listeners when simple stimuli are used (e.g., tones, noise). In a recent study employing more ecological speech stimuli, we observed that $\mathrm{CI}$ listeners only performed comparably to $\mathrm{NH}$ listeners when bypassing the clinical processor and stimulating the implant directly from a research interface. When the stimuli were presented in free field, i.e., through the participants' clinical processor, their GD performance was markedly worse than that of $\mathrm{NH}$ listeners. To determine the origin of the discrepancy between direct stimulation and clinical processor results on GD, in the present study, we compared the electrodograms delivered by the implant in the direct stimulation condition to those delivered by the clinical processors of the participants. Our results show that certain aspects of the processing in the implant, likely related to automatic gain control, do reduce the depth of the gap, which is consistent with a reduction in gap detectability. We suggest that GD with natural stimuli could be used to better tune the implant. 
4aPPa5. Relationship between the frequency asymmetry of across-frequency gap detection and the temporal asymmetry of cochlear responses. Akihide Takamura (Graduate School of Information Sci. and Elec. Eng., Kyushu Univ., 744 Motooka, Nishi-ku, Fukuoka, Fukuoka 8190395, Japan, takamua@cog.inf.kyushu-u.ac.jp), Kazuhito Ito, and Shuji Mori (Faculty of Information Sci. and Elec. Eng., Kyushu Univ., Fukuoka, Japan)

Gap detection is often used to estimate the temporal resolution of the human auditory system. Gap detection performance becomes worse when the frequency difference between the leading and trailing markers that delimit a silent gap gets larger. In addition, the across-frequency gap detection threshold is not always constant when the presentation order of the two markers is altered, even if the frequency difference remains unchanged. We suspect that these inconsistencies in gap detection performance are partly caused by cochlear delay; i.e., low frequency signals reach the responding areas of the cochlea later than higher frequency signals. To examine whether across-frequency gap detection thresholds increase when the leading marker is higher in frequency than the trailing marker, as would be expected if the above mentioned hypothesis is correct, we conducted across-frequency gap detection tasks by altering the presentation order of the two markers (marker frequency: from 250 to $8000 \mathrm{~Hz}$ ). The gap detection thresholds appeared to vary irrespective of the presentation order of the two markers, which does not support the cochlear delay hypothesis.

4aPPa6. Influence of noise on sensitivity to ripple shift and spacing in rippled-spectrum signals. Dmitry Nechaev, Olga Milekhina, and Alexander Supin (Inst. of Ecology and Evolution, 33 Leninsky Prospect, Moscow 119071, Russian Federation, dm.nechaev@yandex.ru)

The influence of noise on hearing thresholds of ripple shift and ripple spacing in rippled-spectrum signals was investigated in humans. The bandlimited noises with rippled spectra were used as signals. The signal spectrum band was 1 octave wide, centered at $2 \mathrm{kHz}$, the ripple spacing was frequency proportional. With the ripple-shift test, the ripple density was 3.5 cycles per octave and ripple-shift threshold was measured. With the ripple-spacing test, ripple-spacing threshold was measured using a ripple reversal paradigm. A two-alternative forced-choice procedure was combined with adaptive stimulation procedure. Signal levels were 50 or $80 \mathrm{~dB}$ SPL. Masker levels varied from 30 to $100 \mathrm{~dB}$ SPL. Masked thresholds were obtained for five maskers centered at 0 (on-frequency masker), $0.5,0.75,1$, and 1.25 oct below the signal (low-frequency maskers). Both on- and low-frequency maskers increased thresholds of both ripple shifts and ripple spacing. The effect of the on-frequency masker depended more on masker/signal ratio than on masker or signal SPL. Effects of low-frequency maskers decreased with increasing the frequency distance between the signal and masker, and its effects became more dependent on masker SPL than on masker/signal ratio.

4aPPa7. Effects of frequency separation and fundamental frequency on perception of simultaneity of the tones. Satoshi Okazaki (Adv. Integration Sci., Chiba Univ., 1-33 Yayoi-cho, Inage-ku, Chiba 263-8522, Japan, okazaki@chiba-u.jp) and Makoto Ichikawa (Letters, Chiba Univ., Inage-ku, Chiba, Japan)

Present study investigated the range of perceptual simultaneity, within which listeners perceive two asynchronous tones as simultaneous, as a function of frequency separation and fundamental frequency of the tones. This function was obtained with frequency separations ranging from 0.03 to 5.00 octaves, and fundamental frequencies ranging from 100 to $800 \mathrm{~Hz}$. Listener's task was to judge whether the two asynchronous pure tones were perceptually simultaneous or not. Results showed a V-shaped function for the perceptual simultaneity range against the frequency separation regardless of fundamental frequency. The perceptual simultaneity range steeply decreased and then gradually increased with the increment of frequency separation. The breakpoint of the V-shaped function was appeared at around the critical bandwidth. This breakpoint was best predicted by CB (Critical Bandwidth) scale, rather than by ERB (Equivalent Rectangular Bandwidth) or octave scales. These findings supported the notion (Okazaki \& Ichikawa, 2015, Proc. Acoust. Soc. Jpn.) that the perception of simultaneity involves two different processes. These two processes would be explained respectively by the mechanics of cochlear basilar membrane motion, and by phase locking of the auditory nerve fibers.
4aPPa8. Threshold shifts when water was injected into the ear canal reveal the difference among air-, bone-, and cartilage conductions. Tadashi Nishimura, Hiroshi Hosoi (Otolaryngology-Head \& Neck Surgery, Nara Medical Univ., 840 Shijo-cho, Kashihara, Nara 634-8522, Japan, t-nishim@naramed-u.ac.jp), Ryota Shimokura (Area of Architecture and Production Design Eng., Shimane Univ., Matsue, Japan), and Tadashi Kitahara (Otolaryngology-Head \& Neck Surgery, Nara Medical Univ., Kashihara, Japan)

Cartilage conduction (CC) is a new form of sound transmission which is induced by a transducer being placed on the aural cartilage. To clarify the difference between $\mathrm{CC}$ and air or bone conduction ( $\mathrm{AC}$ or $\mathrm{BC}$ ), the vibrations of the cartilaginous portion were evaluated by the threshold shifts when water was injected into the ear canal. Seven volunteers with normal hearing participated in this experiment. $\mathrm{AC}, \mathrm{BC}$, and $\mathrm{CC}$ thresholds at $0.5-4$ $\mathrm{kHz}$ were measured in the $0 \%-, 40 \%$, and $80 \%$-water injection conditions. The water injection elevated the AC thresholds by $22.6-53.3 \mathrm{~dB}$, and the threshold shifts for $\mathrm{BC}$ were within $14.9 \mathrm{~dB}$. For $\mathrm{CC}$, when the water was filled within the bony portion, the thresholds were elevated to the same degree as AC. When the water was additionally injected to reach the cartilaginous portion, the thresholds at 0.5 and $1 \mathrm{kHz}$ dramatically decreased by 27.4 and $27.5 \mathrm{~dB}$, respectively. In addition, despite blocking $\mathrm{AC}$ by the injected water, the $\mathrm{CC}$ thresholds in force level were remarkably lower than those for $\mathrm{BC}$. The current findings suggest that $\mathrm{CC}$ is not a hybrid of $\mathrm{AC}$ and $\mathrm{BC}$. CC generates airborne sound in the canal more efficiently than $\mathrm{BC}$.

4aPPa9. Predicting the characteristics of wideband absorbance in ears with middle-ear pathologies. Joseph Kei (Audiol. Div., School of Health and Rehabilitation Sci., Univ. of Queensland, Brisbane, QLD 4072, Australia,k.kei@uq.edu.au), Venkatesh Aithal, Sreedevi Aithal (Dept. of Audiol., Townsville Hospital and Health Service, Townsville, QLD, Australia), Shane Anderson, and David Wright (ENT Dept., Townsville Hospital and Health Service, Townsville, QLD, Australia)

Wideband absorbance (WBA), a measure of the proportion of energy absorbed by the middle ear, is an emerging technology to detect middle-ear dysfunction. WBA results in display absorbance as a function of frequency. WBA characteristics in adults with normal middle-ear function show a broad peak between 2 and $4 \mathrm{kHz}$. While changes in WBA results in patients with middle-ear dysfunction have been described previously, the acoustical properties accounting for those changes have not been investigated. This study investigated the wideband acoustical properties of the middle ear using a spring-mass-friction mechanical system modeling technique. When the system undergoes simple harmonic motion with damping, its frequency of vibration is given by $\mathrm{f}=1 / 2 \pi,(\mathrm{k} / \mathrm{m})$, where $\mathrm{k}$ and $\mathrm{m}$ represent stiffness and mass of the system, respectively. In the presence of middle-ear dysfunction, the $\mathrm{k}$ to $\mathrm{m}$ ratio changes, resulting in reduced WBA amplitude and a change of the frequency of vibration as shown by a shift of the broad peak of WBA. This study demonstrated the applicability of this modelling technique to account for the changes in acoustical properties of the middle ear in the presence of middle ear dysfunction arising from Eustachian tube dysfunction, otosclerosis, ossicular-chain discontinuity, and cholesteatoma.

4aPPa10. Flexibility of ossicular joints reduces damaging impulse peaks. Peter K. Gottlieb, Yona Vaisbuch (Stanford Univ., Stanford, CA), and Sunil Puria (Eaton Peabody Lab., Harvard Med. School, 243 Charles St., Boston, MA 02114,Sunil_Puria@meei.harvard.edu)

The presence of three distinct middle-ear ossicles is a uniquely mammalian trait whose benefits are still debated. One hypothesis is that the flexibility afforded by the two synovial joints connecting the ossicles provides a protective advantage by dispersing potentially damaging impulsive stimuli before they reach sensitive cochlear structures. The 3D velocity of points along the ossicular chain in unaltered cadaveric human temporal bones $(\mathrm{N}=9)$, stimulated with acoustic impulses, was measured in the time domain using a Polytec CLV-3D laser Doppler vibrometer. The measurements were then repeated after fusing one or both of the ossicular joints. Sound transmission was characterized by measuring the amplitude, width, and delay of the impulsive velocity profile as it traveled from the eardrum to the cochlea. In most cases, a delay was noted across either or both of the ossicular joints, and the velocity profile at the stapes was lower in amplitude and broader 
than at the umbo. Typically, fusing the incus-stapes joint had a small effect, while fusing the incus-malleus joint both significantly increased the amplitude and decreased the width of the velocity profile at the stapes relative to the unaltered case. [Work supported by NIH grants R01 DC 05960 and F31 DC 013943A.]

4aPPa11. Can resonance frequency of the outer ear be measured in neonates using wideband tympanometry? Joseph Kei, Joshua Myers (Div. of Audiol., School of Health and Rehabilitation Sci., Univ. of Queensland, Brisbane, QLD 4072, Australia, k.kei@uq.edu.au), Sreedevi Aithal, Venkatesh Aithal, and Alehandrea Manuel (Dept. of Audiol., Townsville Hospital and Health Service, Townsville, QLD, Australia)

This study investigated the feasibility of measuring the resonance frequency (RF) in healthy neonates' ears using wideband tympanometry (WBT). WBT measures admittance and absorbance as a function of frequency and ear canal pressure. Clinically, RF is the frequency at which the susceptance, the vertical component of admittance, equals zero. In this study, RF was successfully measured in 154 ears (114 newborns) out of 297 ears (182 newborns), which passed a battery of tests including automated auditory brainstem response, $1000-\mathrm{Hz}$ tympanometry and distortion product otoacoustic emissions. The success rate of measuring RF in healthy neonates was $51.9 \%$. The normative data revealed a mean $\mathrm{RF}$ of $323 \mathrm{~Hz}(\mathrm{SD}=$ $67 \mathrm{~Hz}$; range $=240-595 \mathrm{~Hz}$; median $=313.5 \mathrm{~Hz} ; 90 \%$ range $=246-440 \mathrm{~Hz}$ ). No significant gender or ear effects were found. The results of the present study are consistent with the resonance frequency of the outer ear (i.e., elastic ear canal wall) in neonates using a sweep frequency impedance technique (Murakoshi et al., 2013). In conclusion, while it is feasible to measure $\mathrm{RF}$ of the outer ear in neonates using WBT, the success rate is low.

4aPPa12. Relationship between voicing perception and auditory brainstem responses to stop consonants. Shunsuke Tamura, Kazuhito Ito, Nobuyuki Hirose, and Shuji Mori (Kyushu Univ., 744 Motooka, Nishi-ku, Fukuoka, Japan, Rm. 827, 8th Fl., West Zone II Bldg., Fukuoka 819-0395, Japan, tamuras@cog.inf.kyushu-u.ac.jp)

A number of psychophysical studies have suggested that auditory temporal resolution is related to the voicing boundaries of stop consonants. For example, hearing-impaired listeners with poor temporal resolution have difficulty perceiving voiceless stop consonants. However, there is little neurophysiological evidence for the existence of a relationship between voicing boundaries and auditory temporal resolution. The auditory brainstem response $(A B R)$ is suited to investigations of this relationship because it has proved useful in assessments of auditory temporal processing and speech processing. We conducted a speech identification task using synthesized /da/-/ta/ stimuli in which we varied the voice onset time from 3 to $15 \mathrm{~ms}$ in $2 \mathrm{~ms}$ steps. There were two types of /da/-/ta/ stimuli whose aspiration noise exhibited high or low power. ABR were measured for the some stimuli across voicing boundaries. The aspiration noises with higher power displayed shorter voicing boundaries and longer peak-to-peak latency between ABR onset responses to the leading noise and vowel parts. This finding suggests that the perception of voicing reflects subcortical temporal processing. [Work supported by JSPS KAKENHI Grant No. 25240023.]

4aPPa13. Comparison of $226 \mathrm{~Hz}$ and $1000 \mathrm{~Hz}$ tympanometry results in infants. Monika-Maria Oster and Lynne A. Werner (Speech and Hearing Sci., Univ. of Washington, 1417 North East 42nd St., Seattle, WA 98105, mmoster@uw.edu)

Tympanometry with a $226 \mathrm{~Hz}$ probe tone is a reliable indicator of middle ear disease in adults and older children. However, it is reported to produce a high rate of false-negatives in young infants. Tympanometry with a $1000 \mathrm{~Hz}$ probe tone has been reported to better indicate middle ear dysfunction in infants. However, age-graded norms for $1000 \mathrm{~Hz}$ tympanometry are currently not available. Furthermore, the results of $226 \mathrm{~Hz}$ and $1000 \mathrm{~Hz}$ tympanometry have not been compared in large numbers of infants of different ages. Tympanograms with 226 and $1000 \mathrm{~Hz}$ probes were analyzed from 18797 - to 40 -week-old infants to determine whether tympanometric compliance changes with age during infancy, whether $226 \mathrm{~Hz}$ compliance predicts $1000 \mathrm{~Hz}$ compliance and whether tympanometric screening results obtained with these probe frequencies agree. Preliminary results indicate that infants with $226 \mathrm{~Hz}$ compliance of $0.1 \mathrm{mmh}$ had low $1000 \mathrm{~Hz}$ compliance. For infants with mean $(0.3 \mathrm{mmh})$ or high $(0.5 \mathrm{mmh}) 226 \mathrm{~Hz}$ compliance, $1000 \mathrm{~Hz}$ compliance increased with age. Controlling for age, $226 \mathrm{~Hz}$ compliance predicted $1000 \mathrm{~Hz}$ compliance. For infants younger than 24 weeks, screening results nearly always agreed. For infants older than 24 weeks, $29.3 \%$ of infants passed screening at $226 \mathrm{~Hz}$ but failed at $1000 \mathrm{~Hz}$, while almost all who passed, passed at both frequencies.

4aPPa14. Identifying conductive conditions in neonates using wideband acoustic immittance. Joshua Myers (Dept. of Audiol., Townsville Hospital and Health Service, 100 Angus Smith Dr., Douglas, QLD 4814, Australia, myers.josh@gmail.com), Joseph Kei, Alicja N. Malicka (School of Health and Rehabilitation Sci., Univ. of Queensland, Brisbane, QLD, Australia), Sreedevi Aithal, Venkatesh Aithal (Dept. of Audiol., Townsville Hospital and Health Service, Townsville, QLD, Australia), Carlie Driscoll, Asaduzzaman Khan (School of Health and Rehabilitation Sci., Univ. of Queensland, Brisbane, QLD, Australia), Alehandrea Manuel (Dept. of Audiol., Townsville Hospital and Health Service, Townsville, QLD, Australia), and Anjali Joseph (School of Health and Rehabilitation Sci., Univ. of Queensland, Townsville, QLD, Australia)

Wideband acoustic immittance (WAI) is an innovative method of middle ear assessment with significant advantages over currently available clinical tests. Previous large-scale studies in neonates have assessed accuracy against evoked otoacoustic emissions but further research is needed using a more stringent gold standard. The aim of this study was to evaluate the test performance of WAI in neonates against a composite reference standard consisting of distortion-product otoacoustic emissions (DPOAEs) and highfrequency tympanometry (HFT). Five hundred and five neonates were recruited from the maternity ward of the Townsville Hospital to participate in the study. DPOAEs and HFT were performed on each neonate to assess outer and middle ear function. Wideband absorbance and complex admittance (magnitude and phase) were measured from 226 to $8000 \mathrm{~Hz}$ in each neonate at ambient pressure using a click stimulus. Best separation between groups that passed and failed the reference standard occurred at frequencies from 1500 to $3000 \mathrm{~Hz}$ for absorbance, 1000-2000 Hz for admittance magnitude, and $2000-4000 \mathrm{~Hz}$ for admittance phase. The WAI response was modelled using multivariate logistic regression and the model validated with bootstrap resampling. The WAI model accurately identified conductive conditions with an area under the receiver operating characteristic curve of 0.89 .

4aPPa15. On the influence of sensorineural hearing loss on the pitch strength of bandpass noise. Maria Horbach, Jesko L. Verhey, and Jan Hots (Dept. of Experimental Audiol., Otto von Guericke Univ. Magdeburg, Leipziger Straße 44, 39120 Magdeburg, Germany, Leipziger Str. 44, Magdeburg 39120, Germany, maria.horbach@st.ovgu.de)

In the perception of environmental noises and in the field of speech and music, tonality plays a crucial role. A tonal character can help the listeners to identify the sound source. However, environmental noises with a tonal character are also considered as especially annoying sounds. The psychoacoustic measure "pitch strength" describes the strength of the tonal sensation evoked by the sound on a scale from weak to strong. For normalhearing listeners, it was shown in the literature that the pitch strength of bandpass noises decreased with increasing bandwidth when the center frequency was fixed and increased with increasing center frequency when the noise bandwidth was fixed. These results are presumably linked to the frequency selectivity of the auditory system. Since cochlear damage leads to a pathological widening of the auditory filters in the inner ear, an altered perception of pitch strength seems possible. In our study, we investigated whether and to which extent pitch strength is influenced by cochlear damage. For this, we measured the pitch strength of bandpass noise for subjects with sensorineural hearing loss via an absolute scaling method and compared the results to own results and results in the literature of normal-hearing subjects. 
4aPPa16. Bandwidth discrimination in normal-hearing listeners. Yuan $\mathrm{He}$ and Jennifer Lentz (Dept. of Speech and Hearing Sci., Indiana Univ., 200 South Jordan Ave., Bloomington, IN 47405, heyuan@indiana.edu)

People's ability to discriminate between different noise bandwidths has not been fully studied. Only a few terms such as "less fluctuating" or "less noisy" are used to describe perceptual changes caused by bandwidth differences. This current study was designed to examine the discrimination threshold of bandwidth in band-pass white noises in normal-hearing listeners. In three experiments, we explored whether center frequency region and base bandwidth would affect discrimination performances, whether discriminating a broader bandwidth is easier than a narrower one, and whether bandwidth discrimination relies on additional cues provided by fixed center frequencies. Thresholds were estimated in a three-interval-three-alternative forced choice task using a three-down one-up adaptive procedure and an oddball paradigm. Visual feedback was given to aid learning about oddball stimulus. We varied the intensity level for sounds within and across trials to avoid the use of intensity cues in all experiments. We also varied the center frequency from interval to interval in one of the experiments to investigate the center frequency cues. In general, we found that normal-hearing listeners may not be very good at discriminating noise bandwidths, especially without the assistance of fixed center frequencies. Large individual differences were observed in all three experiments.

4aPPa17. Context effects in pitch discrimination. Dorothée Arzounian and Alain de Cheveigné (Laboratoire des Systèmes Perceptifs, CNRS, UMR 8342, 29 rue d'Ulm, Paris 75005, France, dorothee.arzounian@ens.fr)

The perceived direction of pitch change between a pair of tones depends on their frequencies, but may also depend on the prior history of stimulation or behavioral response. To quantify the contribution of such context effects, we used a recently developed pitch change discrimination paradigm in which subjects judge the direction of change after each tone of a continuous sequence of tones. Responses were determined primarily by the frequency change of the last pair of tones, but could also be significantly influenced by the frequency of the tone preceding the pair, as well as by the latest response. The magnitude and sign of the effects were subject-dependent, some subjects showing no measurable effect, while in others the weight of the previous stimulus in the decision can go up to half of the weight of the ongoing stimulus. Taking into account these contributions allows for a better estimation of the slope of the psychometric function relating response to frequency change, and thus a better estimate of sensory sensitivity.

4aPPa18. Frequency modulation detection for Mandarin congenital amusics and tone agnosics. Mingshuang Li, Chang Liu (Dept. of Commun. Sci. and Disord., Univ. of Texas at Austin, 1 University Station A1100, Austin, TX 78712, limingshuang@utexas.edu), Yun Nan, Wei Tang, Wenjing Wang, and Qi Dong (State Key Lab. of Cognit. Neurosci. and Learning \& IDG/McGovern Inst. for Brain Res., Beijing Normal Univ., Beijing, China)

Previous studies showed that congenital amusia affected music pitch and lexical tone processing, as well as the pitch contour discrimination. The current study aimed at exploring whether the deficit in musical and/or tone processing also influenced frequency modulation (FM) detection. Thresholds of frequency modulation detection were examined among three Mandarin Chinese-native listeners groups: tone agnosics (amusics with deficits in lexical tone processing), pure amusics (with normal tone processing) and normal controls. Three acoustic parameters were manipulated: duration $(250$ and $500 \mathrm{~ms})$, carrier frequency $(250$ and $1000 \mathrm{~Hz})$, and modulation frequency $(2,8$, and $32 \mathrm{~Hz}$ ). Preliminary results showed that thresholds were higher for pure amusics and tone agnosics than for the control group in most conditions. Auditory model related to the musical and tonal processing for these listener groups will be discussed.
4aPPa19. Tone adjustments, or how an added tone makes a noise less pleasant. Jan Hots and Jesko L. Verhey (Dept. of Experimental Audiol., Otto von Guericke Univ. Magdeburg, Leipziger Straße 44, Magdeburg 39120, Germany,jan.hots@med.ovgu.de)

Several technical sounds contain tonal components which usually arise from rotating parts. Typical examples are sounds originating from turbines or electric engines. These sounds are commonly less pleasant than equallevel sounds without tonal components. To consider this effect on noise pollution, several standards include sections dedicated to tonal components. The standards have in common that they estimate the magnitude of the tones relative to the noise (e.g., tone-to-noise ratio, prominence ratio). Some standards include a second step where a few decibles are added to the measured sound levels, referred to as tone adjustment, to account for the reduction in pleasantness if tonal components are present. The aim of the present study was to measure the tone adjustment. First, the individual masked thresholds of three tonal components were determined. Then, the tonal components were added to the noise at three different levels above the individual masked threshold and a noise alone was adjusted in an adaptive matching experiment to elicit the same loudness or preference as the noise containing the tone. The results are compared to the tone adjustments calculated on the basis of the German standard 45681 .

4aPPa20. Effects of age and modality on children's perception of musical meter. Jessica Nave-Blodgett, Erin Hannon, and Joel Snyder (Psych., Univ. of Nevada, Las Vegas, 4505 Maryland Parkway \#455030, Las Vegas, NV 89154, erin.hannon@unlv.edu)

When listening to music, experienced adults perceive a metrical hierarchy of stronger and weaker events. Children can tap along with the main beat of music. However, it is unclear whether children can perceive more nuanced and hierarchical aspects of musical meter, such as the greater prominence of events on the downbeat of the measure. We asked 5- to 10-yearold children to provide ratings of fit between musical excerpts and auditory or visual metronomes. Metronomes could match 1) both the beat and measure, 2) the beat but not the measure, 3) the measure but not the beat, or 4) neither the beat nor the measure. Children at all ages gave higher fit ratings to all beat-matching auditory metronomes. For visual metronomes, the same pattern was observed among older children, but younger children's ratings did not vary across conditions. At no age did children give higher ratings to metronomes that matched the measure level, suggesting that, unlike adults, children perceive a main beat but not a hierarchy of beats. These results suggest that children's beat perception develops earlier in the auditory modality than in the visual modality, and listeners' sensitivity to metrical hierarchies may emerge after age 10 .

4aPPa21. Effect of temporal asynchrony on children's detection performance in a random-frequency, multi-tonal masking task. Mary M. Flaherty (Boys Town National Res. Hospital, 555 N. 30th St., Omaha, NE 68131, mary.flaherty@boystown.org), Emily Buss (Univ. of North Carolina, Chapel Hill, Chapel Hill, NC), and Lori J. Leibold (Boys Town National Res. Hospital, Omaha, NE)

This study examined the degree to which children can use temporal onset/offset asynchronies to improve performance in a random-frequency, multi-tonal masking task. Normal-hearing school-age children (5-10 years) and adults were tested in two experiments. In Experiment 1, masked thresholds were measured in a 3IFC adaptive procedure. A $1000-\mathrm{Hz}$ pure-tone signal was presented with a random-frequency, 4-tone masker played at $60 \mathrm{~dB}$ SPL. Onset/offset asynchrony was manipulated across conditions by fixing the signal duration at $200 \mathrm{~ms}$ and examining performance for masker durations of 200, 280, and $400 \mathrm{~ms}$. The signal, when present, was temporally centered in the masker, resulting in asynchronies of 0,40 and $100 \mathrm{~ms}$. In Experiment 2, asynchrony was manipulated by holding masker duration constant and varying signal duration to be 200,320 , or $400 \mathrm{~ms}$. Preliminary results suggest that children can use temporal onset/offset asynchrony to segregate a fixed-frequency signal tone from a tonal masker. Potential developmental differences in the ability to use a variety of temporal asynchronies in the presence of competing sounds will be discussed. 
4aPPa22. What ability is measured in "infant psychophysics"? Lance Nizami (Independent Res. Scholar, Wilkie Way, Palo Alto, CA 94306, nizamii2@att.net)

The magazine American Scientist recently celebrated Davida Teller's "forced-choice preferential looking," a psychophysical method that yields a detection/discrimination function for human infant vision. The method was soon adopted and modified for auditory detection/discrimination. Eventually, sufficient data accumulated for a retrospective ("Human auditory development," L. Werner et al.). In the auditory trials, an assistant holds an infant exposed to auditory stimuli, and a hidden observer records the infant's behavior, from which an analyst constructs a psychometric function or other quantifier of detection/discrimination. But whose detection/discrimination is quantified, exactly? Consider the roles of the laboratory personnel. The analyst (a receptor) interprets the data (stimulus) supplied by the hidden observer, who is an interpreter (receptor) of the behavior (stimulus) of the infant/infant-holder duo, who constitute a probe (receptor) of the acoustical environment. Altogether, the detection/discrimination ability being inferred is that of the entire Laboratory. In particular, infants' apparent capabilities should improve with the observer's ability to interpret infant behavior, and/ or with the observer's expectations, both of which may increase with infant age. Substitute methods, e.g., some already-employed non-invasive physiological recordings such as electro-encephalograms, likewise represent the ability of the Laboratory to probe the environment by way of the infant.

4aPPa23. Womb-like sounds: An intervention for apnea of prematurity. Joanna J. Parga (Neonatology, Univ. of California, Los Angeles, Div. of Neonatal-Perinatal Medicine 10833 Le Conte Ave., MDCC B2-375, Los Angeles, CA 90095, jparga@mednet.ucla.edu), Ravi R. Bhatt (Pediatric Pain and Palliative Care, Univ. of California, Los Angeles, Los Angeles, CA), Kalpashri Kesavan, Myung-Shin Sim (Neonatology, Univ. of California, Los Angeles, Los Angeles, CA), Harvey Karp (Pediatrics, Univ. of Southern California, Los Angeles, CA), Ronald M. Harper (Neurobiology, Univ. of California, Los Angeles, Los Angeles, CA), and Lonnie K. Zeltzer (Pediatric Pain and Palliative Care, Univ. of California, Los Angeles, Los Angeles, CA)

We hypothesized that premature infants exposed to womb-like sounds would show decreased apneic, hypoxemic, and bradycardic events. We believed these findings could be explained by the auditory system exerting influence over the autonomic system and potentially enhancing parasympathetic tone in neonates. Twenty premature infants without comorbidities, 32 to 37 weeks corrected, were their own controls. Infants were exposed to four 6-hour blocks of alternating womb-like sounds with intervening silence. Continuous ECG, respiratory, and oxygen saturation data were collected. Cortisol and alpha amylase salivary samples were obtained at study onset and offset during the 24-hour study period. Intermittent hypoxemic episodes significantly decreased from study onset $(p=0.03)$, along with bradycardic episodes $(\mathrm{p}=0.05)$. No reductions in apneic events, cortisol levels, alpha amylase levels, or changes in heart rate variability were found. Exposing premature infants to womb-like sounds reduces hypoxemic and bradycardic events, indicating the potential influence of familiar auditory input on breathing and autonomic output. The sound exposure may provide a lowcost, non-invasive intervention for apnea of prematurity.

4aPPa24. Effects of meaningful or meaningless noise on psychological impression for annoyance and selective attention to stimuli during intellectual task. Takahiro Tamesue and Tetsuro Saeki (Yamaguchi Univ., 216-1, Tokiwadai, Ube 755-8611, Japan, tamesue@yamaguchi-u.ac.jp)

The presence of noise during the performance of cognitive task, commonly causes a subjective experience of annoyance, which can lead to a decline in performance. This tendency is stronger for meaningful noise such as a conversation than for meaningless noise such as heating, ventilating, and air-conditioning noise. This paper first focus on the degree of meaningfulness of noise, then discuss the psychological impression of annoyance during auditory or visual cognitive tasks under the meaningful noise or meaningless noise. In addition, we considered how the brain responds on transient event-related potentials (ERPs), elicited by external auditory or visual stimuli, can be measured using electroencephalography (EEG). The present experiment was designed to determine the effects of meaningfulness of the noise on selective attention to stimuli under the odd-ball paradigm. To this end, we examined differences in the N100 and P300 ERPs of these components. The principal component analysis (PCA) was adopted to define a set of components. Our results suggested that degree of meaningfulness of noise has a strong influence on not only the psychological impression of annoyance but also the selective attention to stimuli in cognitive tasks.

4aPPa25. Toward an evidence-based taxonomy of everyday sounds. Oliver C. Bones, Trevor J. Cox, and William J. Davies (Computing, Sci. and Eng., Univ. of Salford, 119 Newton Bldg., Acoust. Res. Ctr., Salford, Greater Manchester M5 4WT, United Kingdom, o.c.bones@salford.ac.uk)

An organizing account of everyday sounds could greatly simplify the management of audio data. The job of an audio database manager will typically involve assigning a combination of textual descriptors to audio data, and perhaps allocation to a predefined category. Retrieval is likely achieved by matching the descriptor to keyword search terms or by browsing through categories. While classification of musical instruments using this type of approach is relatively simple by virtue of the fact that a predefined taxonomy can follow a signal-related hierarchy, non-musical sounds do not necessarily follow such a hierarchy. In addition, classification is made more problematic by the ambiguity of words used to describe everyday sounds. Another area in which the issue of establishing a taxonomy of everyday sounds is particularly pertinent is that of soundscape research; research into soundscapes - acoustic environments as they are perceived - is a relatively new and multidisciplinary area, and as such descriptive terms for everyday sounds are currently used inconsistently. Many existing attempts to taxonomize everyday sounds prescribe categories that are not mutually exclusive, in that everyday sounds could exist in a number of categories; moreover, many are not based on empirical evidence. Here, we present a robust method for creating an evidence-based taxonomy of everyday sounds, involving hierarchical clustering from dimensions generated by correspondence analysis of data from a simple card-sorting and naming procedure.

4aPPa26. Auditory and tactile senses for perceiving the hardness of wood board. Yuri Amimoto and Takayuki Arai (Sophia Univ., 7-1 Kioicho, Sophia University, 4-295C, Arai Lab., Chiyoda, Tokyo 102-8554, Japan, amim0t0.u.ri@gmail.com)

The purpose of the present research is to explore the relationship between auditory and tactile senses on hardness perception. To testify this relationship, this research used a comparative judgment on knocking between two wood boards which have different levels of hardness. The participants were asked to knock the pair of wood boards, and compare the hardness in two conditions: knock with or without auditory information. In the condition with auditory information, sounds were heard directly, or through a sound system, which consists of the frequency filters and soundproof box. The other condition without auditory information masks the knocking with white noise. In short, the participants compared hardness through these conditions, and the correctness of their judgement were used to measure the contribution of auditory and tactile senses toward perceiving hardness. Consequently, there are differences in judgement between with and without the knocking sound. This suggests the possibility of auditory information affects tactile perception.

4aPPa27. Perception of the weight of a falling ball. Kenji Torii and Hiroshi Hasegawa (Graduate School of Eng., Utsunomiya Univ., 7-2-1 Yoto, Utsunomiya-shi, Tochigi-ken 321-8585, Japan, kenji_torii@n.t.rd.honda.co.jp)

Among perceived qualities, perceived weight is connected with the feeling of luxury. Many studies of methods for controlling the perception of weight have been reported; most concern visual and haptic effects, but auditory effects have not been considered. This presentation reports analysis of how the perception of weight changes with changes of visual and auditory stimuli in videos in which a falling ball strikes a flat plate. We made 6 balls and 4 plates of different materials with the same size but different weights. We made 24 sets ( 6 balls $\times 4$ plates) and recorded scenes in which each ball struck each plate. Eleven subjects evaluated the perception of the weight of the ball on a scale of 1 to 7 in all 552 pairs of video comparisons $(24 \times 23)$. Scheffé's paired comparison test revealed that not only visual stimulus but also auditory stimulus can affect the perception of weight. 
4aPPa28. A study on the summer forest sound acoustic characteristics using psychoacoustics parameters in South Korea. Seonggeon Bae (Div. of Comput. Media Information Eng., Kangnam Univ., 40, Kangnam-ro, Giheong-gu, Youngin-si, Gyeonggi-do, Korea, Youngin 446-702, South Korea, sgbae@kangnam.ac.kr) and Myungjin Bae (School of Electron. Eng., Soongsil Univ., Seoul, South Korea)

In this paper, summer forest sound acoustic characteristics has been analyzed through psychoacoustics Parameters. Forest sound is closely related to Psychological factors of the human which are analyzed variously. Forest sound is applied to the improvement of depression and anxiety of psychology characteristics. Thus, we study efficient method to apply acoustic properties of forest sound using psychoacoustics parameters.

4aPPa29. Auditory bubbles reveal sparse time-frequency cues subserving identification of musical voices and instruments. Vincent Isnard (IRCAM, 1 Pl. Igor-Stravinsky, Paris 75004, France), Clara Suied (IRBA, Brétigny-sur-Orge, France), and Guillaume Lemaitre (IRCAM, Paris 75004, France, GuillaumeJLemaitre@gmail.com)

Classical timbre studies have modeled timbre as the integration of a limited number of auditory dimensions and proposed acoustic correlates to these dimensions to explain sound identification. Here, the goal was to highlight time-frequency patterns subserving identification of musical voices and instruments, without making any assumption about these patterns. We adapted a "random search method" proposed in vision. The method consists of synthesizing sounds by randomly selecting "auditory bubbles" (small time-frequency glimpses) from the original sounds' spectrograms, and then inverting the resulting sparsified representation. For each bubble selection, a decision procedure categorizes the resulting sound as a voice or an instrument. After hundreds of trials, the whole time-frequency space is explored, and adding together the correct answers reveals the relevant time-frequency patterns for each category. We used this method with two decision procedures: human listeners and a decision algorithm using auditory distances based on spectro-temporal excitation patterns (STEPs). The patterns were strikingly similar for the two procedures: they highlighted higher frequencies (i.e., formants) for the voices, whereas instrument identification was based on lower frequencies (particularly during the onset). Altogether these results show that timbre can be analyzed as time-frequency weighted patterns corresponding to the important cues subserving sound identification.

4aPPa30. A research on understanding of piano players' control of performance behavior in brushing up processes of music piece using Nearinfrared spectroscopy. Ayako Matsuo (Graduate School of Adv. Sci. and Technol., Tokyo Denki Univ., 5 Asahi-cho Senju Adachi-ku, Tokyo 1208551, Japan, matsuoaya.dendai@gmail.com), Takeshi Akita, and Naoko Sano (School of Sci. and Technol. for Future Life, Tokyo Denki Univ., Tokyo, Japan)

In this paper, we are studying about the method in which we could catch the relationship between players' perception of sonic field environment and the following control of playing behavior characteristically. Based on the above way of thinking, we made some measuring experiments on some actual performances in brushing up processes, and analyzed and considered about the difference between experimental results from the view point of the perception and behavior control. Already, it has been shown that measuring the change in frontal lobe brain blood flow using Near-infrared spectroscopy (NIRS) Brain-measuring-apparatus and comparative-analyzing the measured data was different by a variety of psychological conditions. In this paper, adopting the above method, we measured subjects' frontal lobe brain blood flow on two actual performance of the early stage "performance of playing at sight" and the final stage "performance of automated levels" in proficiency-process. After some supplementary interviewing to subjects about their measured practices, we analyzed about control of performance behaviors in some sonic environment. The analysed results show that we could measure two each status of "the perception and behavior control" on actual performance of "performance of playing at sight" and "performance of automated levels" in proficiency-process characteristically.
4aPPa31. Investigating the effect of selective attention and the spatial relationships of competing auditory stimuli on the sound-induced flash illusion. Lindsey R. Kishline, Adrian K. C. Lee (Univ. of Washington, Portage Bay Bldg., Rm. 204, Seattle, WA 98195, lrk4@uw.edu), and Ross K. Maddox (Univ. of Rochester, Seattle, Washington)

The sound-induced flash illusion (SIFI) provides a way of testing multisensory integration through perceptual illusion. Studies disagree on the influence of auditory-visual spatial (in)congruence on SIFI. To better assess the possible influence of spatial proximity, we manipulated the spatial congruence of competing auditory stimuli. Study participants were presented with two timbrally distinct concurrent auditory stimuli of one or two beeps and a visual stimulus composed of one or two flashes. One auditory stimulus always matched the number of visual flashes, and the other did not. The auditorymatching stimulus was manipulated to either be spatially congruent or not with the visual flashes, which could occur centrally or to the left or right. Participants were instructed to report the number of flashes they saw. For half of each session participant's attention was not directed and had no knowledge of flash location on the coming trial, while in the other half there was a visual spatial cue prior to the trial to the location of the visual flashes. We compare and contrast the results with and without spatial attention cueing in order to examine the effect of spatial attention on this multisensory illusion.

4aPPa32. How loudness affects everyday sounds recognition? Patrick Susini, Olivier Houix, Lou Seropian, and Guillaume Lemaitre (STMS IrcamCNRS-UPMC, 1 Pl. Igor Stravinsky, Paris 75004, France, susini@ircam.fr)

It has been recently shown that loudness affects the perceptual representation of sounds. Following this idea, the present experiment examines whether recognition of everyday sounds is sensitive to study-to-test changes in sound pressure level. In addition, the experiment tests the hypothesis that the sound pressure level changes could hinder recognition more strongly when an encoding task focuses participants on sensory properties of the sounds (e.g., loudness) during the study phase. The study phase used three encoding tasks: sensory (participants rated loudness), semantic (participants categorized sounds in three categories), and control tasks. The test phase measured recognition scores and response times for a list of targets (sounds presented in the study phase) mixed with distractors, both presented at two different levels (L1: the level of the phase study; L2: $15 \mathrm{~dB}$ higher for half of the sounds, and $15 \mathrm{~dB}$ lower for the other half). Results reveal a significant effect of the level change on recognition scores; recognition is more accurate for sounds at L1. As predicted, recognition is weaker with the sensory encoding task, though the effect is not significant. Those results suggest that loudness is encoded together with semantic attributes in the memory representations of sounds.

4aPPa33. Auditory guided adjustment shows robust loudness constancy. Akio Honda (Dept. of Human Sci. and Cultural Studies, Yamanashi-Eiwa College, 888 Yokone-machi, Kofu, Yamanashi 400-8555, Japan, honda@ yamanashi-eiwa.ac.jp), Ayumi Yasukouchi, and Yoichi Sugita (Dept. of Psych., Waseda Univ., Tokyo, Japan)

Perceptual constancy refers to a tendency to perceive an object as having a constant shape, size, and brightness despite apparent changes in these stimulus features. Few studies have systematically and critically examined loudness constancy. We use two adjustment methods to examine it in a natural environment: "auditory guided adjustment", by which listeners play a musical instrument as loud as a model player; and "volume adjustment," by which listeners adjust the loudness of the sound produced by a loudspeaker. The experiments were conducted in the Yamanashi Eiwa College gymnasium, where dark noise was 32-35 dB(A). Stimuli were 2-s musical sounds with G4 $(393 \mathrm{~Hz})$ pitch produced by the actual musical instrument performance. The distances separating the model performer and the participant were $2 \mathrm{~m}, 8 \mathrm{~m}$, and $32 \mathrm{~m}$. Sound pressure levels of the stimuli were set as small, medium, and large corresponding to 60,75 , and $86 \mathrm{~dB}(\mathrm{~A})$. Participants were asked to produce the sound of the same loudness. It was found that auditory guided adjustment showed more robust loudness constancy than volume adjustment. Transfer to learning through action observation and action-specific perception were discussed. 
4aPPa34. Influence of simultaneous grouping mechanisms in the loudness judgment of a multi-tone mixture. Michaël Vannier (Lab. STMS Ircam-CNRS-UPMC, 1, Pl. Igor Stravinsky, Paris 75004, France, michael. vannier@ircam.fr), Nicolas Grimault (CNRS UMR 5292 CRNL, Univ Lyon 1, Lyon Cedex 06, France), Nicolas Misdariis, and Patrick Susini (Lab. STMS Ircam-CNRS-UPMC, Paris, France)

Our main concern is to understand how the perceptual organization of an acoustical mixture into distinct auditory objects (known as an ASA mechanism) can affect the judgments of loudness. Three main questions have been raised: Is the loudness of one auditory object influenced by concurrent objects? How the partial loudness of different auditory objects are combined into one global loudness percept? Does global loudness depend on the perceptual organization of the mixture? In the present experiment, the mixtures consisted of frequency-modulated harmonic complexes. The same or different modulation frequencies have been applied to the odd and even harmonics to ensure that participants perceived either one or two auditory objects, respectively. As a first result, when two objects were well segregated (without spectral masking), the loudness of one object in the mixture equaled the loudness of the same object presented alone. It suggests that spectral grouping mechanisms prevail over loudness. Then, the global loudness pattern obtained for two auditory objects as a function of their partial loudness will be drawn up. Global loudness modeling for two auditory objects will be discussed with regards to the predictions given by classical loudness models. [Research supported by the chair "MOUVIE", carried by the UPMC.]

4aPPa35. Effects of spectral gaps on the contribution of specific frequency bands to the loudness of broadband sounds. Walt Jesteadt, Marcin Wróblewski, and Katie Thrailkill (Boys Town National Res. Hospital, 555 N. 30th St., Omaha, NE 68131, walt.jesteadt@boystown.org)

Contributions of individual frequency bands to judgments of total loudness can be assessed by varying the level of each band independently from one presentation to the next and determining the relation between the change in level of each band and the loudness judgment. Recent studies using stimuli consisting of 15 bands of noise, each two critical bands wide, have shown that frequencies near the lower and upper edges of a broadband noise make a greater contribution to overall loudness than frequencies in the center of the spectrum. In the present study, eight listeners with normal hearing compared the loudness of two 15-band stimuli with average levels of $59 \mathrm{~dB}$ SPL per band and of stimuli where 1,3,5, or 7 bands were missing from the center of the spectrum. Listeners placed greater weight on the bands at the edges of the spectral gap and the effect was larger for greater gap widths. Edge bands received more weight regardless of absolute frequency and the high-frequency edge of the low bands received greater weight than the low-frequency edge of the high bands. These effects are not predicted by loudness models. [Work supported by NIH.]

4aPPa36. Interindividual differences in directional loudness. Sabine Meunier, Sophie Savel, Jacques Chatron, and Guy Rabau (LMA, CNRS, UPR 7051, Aix Marseille Univ., Centrale Marseille, 4, Impasse Nikola TESLA, CS 40006, Marseille Cedex 13 F-13453, France, meunier@lma. cnrs-mrs.fr)

Sensibility of loudness to sound direction was measured in a two-intervals, $2 \mathrm{AFC}$ interleaved-adaptive procedure. We measured the level (at the center of the listener head) of a test sound matching the loudness of a reference fixed at $0^{\circ}$ and $65 \mathrm{~dB}$ SPL (at the center of the listener head). Azimuths of the test sound varied from 0 to $180^{\circ}$ by steps of $15^{\circ}$. Elevations were $15^{\circ}$, $30^{\circ}, 45^{\circ}, 60^{\circ}$, and $90^{\circ}$. Both test and reference sounds were wide-band noise. The difference between the levels of reference and test sounds at the point of loudness equality defined the Directional Loudness Sensibility (DLS). A group of 24 listeners showed up large differences in DLS. For the majority, the highest DLSs were around $-4 \mathrm{~dB}$. For a few of them DLSs were as large as $-9 \mathrm{~dB}$. Some listeners did not exhibit any DLS and could even achieve positive DLSs. The sound pressure levels, measured at the entrance of the ear canal, were related to the DLSs to see whether the interindividual differences in DLS were exclusively due to the filtering by the head or if extra-acoustics factors (such as binaural loudness constancy) might be involved in directional loudness sensitivity.
4aPPa37. Role of static and dynamic interaural differences in the localization of spatially segregated sounds. Daisuke Morikawa (Japan Adv. Inst. of Sci. and Technol., 1-1 Asahidai, Nomi, Ishikawa 923-1292, Japan, morikawa@jaist.ac.jp)

We clarify the role of interaural time difference (ITD) and interaural level difference (ILD) in the localization of spatially segregated sounds. Listeners were asked to distinguish between two sources of white noise having various ITDs/ILDs under head-still and head-rotating conditions. For the former, the segregated rate reached $80 \%$ for an ITD of $0.3 \mathrm{~ms}$, when the ITD of other sound source corresponded to the other side. The segregated rate also reached $80 \%$ when the ILD between the two noise sources corresponded to an angular difference of $30^{\circ}$. Under head-rotating conditions, the segregated rate reached almost $100 \%$, regardless of the ITDs of the two noise sources. This was because when only one white noise source was used, it was perceived as separate high and low sound images. In confirmations with low- and high-pass noises, the lower-frequency sound image was contained for $<1.7 \mathrm{kHz}$ and the higher-frequency one was contained for $>1.2 \mathrm{kHz}$. In addition, the segregated rate was essentially the same for the ILD under head-still conditions when the ILDs of two noise sources corresponded to the separate side. The sound image was integrated into a single one when the ILDs of two noise sources corresponded to the same side.

4aPPa38. Binaural processing in adults with a history of traumatic brain injury. Christina M. Roup and Julie Powell (Speech and Hearing Sci., Ohio State Univ., 110 Pressey Hall, 1070 Carmack Rd., Columbus, OH 43210,roup.2@osu.edu)

An auditory processing deficit is a perceptual issue affecting how the central auditory nervous system understands and makes use of auditory information. Auditory processing deficits are typically associated with the pediatric population; however, anecdotal clinical evidence and the recent focus on central auditory effects of traumatic brain injury (TBI) suggests that auditory processing deficits may be prevalent among adults with a history of TBI. Individuals with symptoms of an auditory processing deficit often score within the normal range on standard clinical assessments. The objectiveof the current study, therefore, was to target measures of binaural processing to identify and characterize auditory processing deficits among adults with a history of TBI. Two groups were recruited: adults with a history of TBI and a control group. Binaural processing was measured with: 1) the Revised Speech Perception in Noise test measured in soundfield; 2) dichotic word recognition without and with low-pass filtering measured in the free recall and directed recall conditions; 3 ) the 500-Hz masking level difference; and 4) the Listening in Spatialized Noise Sentences Test. Results suggest that individuals with a history of TBI have lower than normal binaural performance.

4aPPa39. Influence of sound source arrangement on the precedence effect. Koji Abe (Akita Prefectural Univ., 84-4 Ebinokuchi Tsutiya, YuriHonjyo 015-0055, Japan, koji@akita-pu.ac.jp), Shouichi Takane, Masayuki Nishiguchi, and Kanji Watanabe (Akita Prefectural Univ., Yuri-Honjo, Japan)

As such robot audition, problem of estimating the direction of the sound image from the physical sound signal has high necessity. In order to develop an estimation system of the direction of sound image corresponding to the multiple sound sources, it is necessary to know the behavior of the precedence effect in comprehensive sound source arrangement. So, we investigated the influence of the sound source arrangement on the precedence effect experimentally. The minimum angle between the directions of two sound sources is 30 degrees. Experimental method is a three Alternative Forced Choice Task. The three choices are in the direction of two sound sources and its intermediate. In the case of a bilaterally symmetrical arrangement of sound sources, our experimental results were in good agreement with the findings obtained in previous studies. However, our experimental results show that deviation of the localized direction of sound image is dependent on the sound image arrangement. Particularly, in the case of left-right asymmetrical and front-back symmetrical arrangement, shift of a sound image direction with respect to time difference had a characteristic trend. 
4aPPa40. Buildup of contextual plasticity in sound localization. Norbert Kopco and Gabriela Andrejkova (Inst. of Comput. Sci., P. J. Safarik Univ. in Kosice, Jesenna 5, Kosice, Slovakia, norbert.kopco@upjs.sk)

Contextual plasticity (CP) is a form of plasticity in sound localization induced by preceding stimulation. $\mathrm{CP}$ is observed as shifts in responses to a click stimulus when, on interleaved trials, the target click is preceded by an identical distractor click coming from a known location. This study examines temporal properties of $\mathrm{CP}$ by analyzing behavioral data from two previous experiments [Kopčo, Best, Shinn-Cunningham (2005), ARO Abstract \#965; Tomoriová, Kopčo and Andoga (2010), ARO Abstract \#827]. In the experiments, the distractor type (single click, train of 8 clicks, or noise), consistency (distractor type fixed within block vs. varying from trial to trial), and location (frontal vs. lateral) were manipulated. The results show that contextual plasticity buildup duration depends on the distractor location. Also, the buildup was stronger with 8-click distractor than 1-click distractor. When distractor type varied, the context type on immediately preceding trial influenced performance in the middle of the run, such that trials following an 8-click context exhibited shifts up to $5^{\circ}$ larger than the trials following a 1-click context. No such bi-stable percept was observed in other parts of the run. These results show that $\mathrm{CP}$ has complex temporal profile on time scales of seconds to minutes. [Work supported by APVV-045212.]

4aPPa41. The role of skull resonance on localization. Michael S. Gordon (Psych., William Paterson Univ., 300 Pompton Rd., Wayne, NJ 07470, gordonm10@wpunj.edu) and Jitwipar Suwangbutra (Psych., William Paterson Univ., New Milford, NJ)

The skull is a dense resonant body that may filter sounds affecting many auditory tasks. Its possible influence has been minimally investigated with localization-a paradigm directly addressed in the current study. Unique spectral information was extracted from the skull recordings of 27 participants. The major lower frequency resonances $(93.75-656.25 \mathrm{~Hz})$ and higher frequency resonances $(984.38-4781.25 \mathrm{~Hz})$ from each skull were used to filter a set of narrowband noises that were enharmonic with the skulls in this sample. The $75 \mathrm{~ms}$ narrowband noises were presented concurrently to both ears or manipulated by adding a $2 \mathrm{~ms}$ delay in the left or right channel. Listeners were presented with the stimuli over headphones and were asked to determine its location (left, center, or right). Results indicated that listeners were more accurate localizing low-frequency filtered sounds that were enharmonic with their skull than high frequency peaks. In addition, participants were more accurate localizing to the left and had the most difficulty localizing sounds in the center. These results may be used to suggest the influence of the skull's resonance on localization. Specifically, the resonance of the skull may cause listeners to be more sensitive to sounds of enharmonic spectra.

4aPPa42. Using pupillometry to investigate the better ear advantage. Alan Kan (Univ. of Wisconsin-Madison, 1500 Highland Ave., Madison, WI 53705, ahkan@waisman.wisc.edu) and Matthew B. Winn (Univ. of Washington, Seattle, WA)

The dichotic digit span (DDS) task involves listening to and repeating back strings of different digits that are presented to the right and left ears. Listeners with normal hearing typically show better recall of the digits that are presented to the right ear (called the ear advantage, or EA), for reasons that are not fully understood. Conventional measures of intelligibility are sometimes not sensitive enough to reveal a right EA, making it difficult to explore potential explanations for the effect (e.g., whether it is driven by attention or anatomical differences, etc.). In this study, we used pupillometry to investigate the EA because it has been shown to be able to detect subtle differences in speech processing (or listening effort) in cases that are not well distinguished by intelligibility scores. Pupillary responses to various conditions of the DDS test were measured in a group of people with no suspected hearing loss to establish baseline data. Results were at times variable within listeners, which opposes a strict anatomical basis for the EA. Interestingly, the responses showed a sustained period of effort after the end of the stimuli, suggesting the EA could involve working memory rather than the perceptual auditory system alone.
4aPPa43. Behavioral and neural sensitivity to changes in large interaural level differences: Implications for sound localization. Andrew D. Brown, Victor Benichoux (Physiol. \& Biophys., Univ. of Colorado School of Medicine, 12800 East 19th Ave., RC1-N, Rm. 7401G, M.S. 8307, Aurora, CO, Andrew.D.Brown@ucdenver.edu), Heath G. Jones (U.S. Army Aeromedical Res. Lab., Fort Rucker, AL), and Daniel J. Tollin (Physiol. \& Biophys., Univ. of Colorado School of Medicine, Aurora, CO)

Sound localization acuity depends on source azimuth: minimum audible angles (MAAs) are smallest for sources near the frontal midline. The azimuthal dependence of MAAs can be partially accounted for by the decreasing rate of change in the acoustic cues to sound location (interaural time and level differences [ITDs and ILDs]) with increasing azimuth. Previous work has shown that changes in low-frequency MAAs across azimuth are wellaccounted for by changes in physical ITD alone. Here we consider whether acoustical constraints are sufficient to account for the azimuthal dependence of MAAs for high-frequency tones, which are localized on the basis of ILDs. Psychophysical data were obtained from human subjects tested in two different headphone ILD tasks (discrimination and lateralization) and compared to previously published measurements of psychophysical MAAs and acoustical ILDs across azimuth. We conclude that acoustic factors alone are not sufficient to account for the azimuthal dependence of high-frequency MAAs: rather, the increase in MAAs for off-midline positions arises from a combination of reduced ILD information and reduced precision of behavioral ILD sensitivity at large reference ILDs. Further, we show that this psychophysical effect is consistent with the responses of ILD-sensitive neurons, which tend to be weakly modulated at large ILDs.

4aPPa44. Effects of a listener's very slow rotation on sound localization accuracy. Sayaka Tsunokake (Res. Inst. of Elec. Commun., Tohoku Univ., 980-8577, Sendai, Miyagi 2-1-1, Katahira, Aoba-ku, Japan, tsuno@ais.riec. tohoku.ac.jp), Akio Honda (Faculty of Human Sci. and Cultural Studies, Yamanashi Eiwa Collage, Kofu, Yamanashi, Japan), Yôiti Suzuki, and Shuichi Sakamoto (Res. Inst. of Elec. Commun., Tohoku Univ., Sendai, Miyagi, Japan)

A listener's head movement is effectively used to accurately localize sounds. By contrast, when a sound stimulus is presented during head rotation, sound localization acuity decreases. Previous studies have shown that sound localization accuracy is degraded even during slow head rotation at $50 / \mathrm{s}$. In this study, we investigated the sound localization accuracy during very slow head rotation from $0.6250 / \mathrm{s}$ to $50 / \mathrm{s}$. We measured the detection thresholds (DTs) at the listener's subjective front. The experiment consisted of static and rotation conditions. Listeners were asked to report whether a $30 \mathrm{~ms}$ noise burst was presented from the left or right of the subjective front (2 Alternative Forced Choice Task). In the results, the DTs in the rotation condition were larger than that in the static condition. Moreover, DTs seem almost independent of the rotation speed. This suggests that the sound localization resolution at the subjective front is degraded by a listener's passive rotation irrespective of the rotation speed. This is very interesting because a listener only rotates 0.150 during stimulus presentation, which is much less than the DTs in the static condition. Therefore, we investigated the origin of this phenomenon by changing the experimental parameters, including stimulus duration.

4aPPa45. Auditory training and subsequent generalization with speech and non-speech stimuli. Nathaniel Spencer (Wright-Patterson Air Force Base, 711th Human Performance Wing, Wright-Patterson AFB, OH, spencernj80@gmail.com), Eric R. Thompson, Matthew G. Wisniewski, Brian D. Simpson, and Nandini Iyer (Wright-Patterson Air Force Base, Dayton, $\mathrm{OH})$

Auditory training studies have varied in degree-of-similarity between the training and testing conditions. Some focused testing on the trained task (e.g., Sweetow and Henderson Sabes, 2007), while others addressed whether the training effects generalize to other tasks. The current study used speech and non-speech stimuli to explore how much training in a specific analytical listening task would generalize to performance improvements for another. Naïve listeners participated. Percent correct was measured as a function of signal-to-noise ratio (SNR) in pre- and post-tests with the "multiple-bursts 
same" (MBS) paradigm (Kidd et al., 1994) and the speech-target/speechmasker Coordinate Response Measure (CRM) paradigm. There were three training groups in a between-groups design: 1) training in a masked speech identification task (the CRM) with the competitors varying in $\mathrm{f}_{0}$ separation (Darwin et al., 2003); 2) training in the MBS task with the protected-region (frequency region with no maskers) bandwidth around the target varying; and 3) no training. Preliminary data describe individual-subject variability in pre-test performance and indicate performance improvements with auditory training. The degree of generalization will be explored for the trained sub-groups. 1) Int J. Audiol. 46. 374. 2) J. Acoust. Soc. Am. 114, 2913. 3) J. Acoust. Soc. Am. 95, 962.

4aPPa46. Talker identification: Effects of masking, hearing loss, and age. Virginia Best (Dept. of Speech, Lang. \& Hearing Sci., Boston Univ., Boston, MA 02215, ginbest@bu.edu), Jayne B. Ahlstrom (Dept. of Otolaryngology-Head and Neck Surgery, Medical Univ. of South Carolina, Charleston, SC), Christine R. Mason, Elin Roverud, Tyler K. Perrachione, Gerald Kidd (Dept. of Speech, Lang. \& Hearing Sci., Boston Univ., Boston, MA), and Judy R. Dubno (Dept. of Otolaryngology-Head and Neck Surgery, Medical Univ. of South Carolina, Charleston, SC)

The ability to identify voices was examined under both quiet and masked conditions. Subjects were grouped by hearing status (normal hearing/sensorineural hearing loss) and age (younger/older adults). Listeners first learned to identify the voices of four same-sex "target" talkers in quiet, with a fixed amount of training. On each trial subjects identified which of the four target talkers was asking a brief question (e.g., "What is 2 plus 3?"), and response feedback was given. At test, subjects identified voices (1) in quiet, (2) masked by speech-shaped noise, and (3) masked by a single, unfamiliar same-sex talker. Masked conditions included a range of target-tomasker ratios. The psychometric functions obtained in the speech masker were shallower than those in the noise masker, analogous to typical findings for the task of sentence identification/intelligibility. Both younger and older adults with hearing loss, as well as older adults with normal hearing, generally performed more poorly than younger adults with normal hearing, although large individual differences were observed in all conditions. These preliminary findings suggest that both hearing loss and age may affect the recall of who spoke in conversations, which may contribute to listeners' difficulty in solving the "cocktail party problem." [Work supported by NIH/ NIDCD.]

4aPPa47. On the color of speech: The effect of speaking styles on speech recognition by cochlear-implant users. Terrin N. Tamati (Dept. of Otorhinolaryngology/Head and Neck Surgery, Univ. Medical Ctr. Groningen, P.O. Box 30.001, Groningen 9700RB, Netherlands), Esther Janse (Radboud Univ. Nijmegen, Ctr. for Lang. Studies, Nijmegen, Netherlands), and Deniz Başkent (Dept. of Otorhinolaryngology/Head and Neck Surgery, Univ. Medical Ctr. Groningen, Groningen, Netherlands, d.baskent@UMCG. NL)

Speaking styles are a common source of real-life variability that may affect cochlear implant (CI) speech communication. Speech transmitted via a CI is heavily degraded in acoustic-phonetic cues. Therefore, the extent to which (and for what conditions) individual CI users benefit from carefully articulated speech (or are hindered by casually articulated/reduced speech) is unclear. First, we examined the impact of speaking styles on CI speech recognition in favorable listening conditions. CI users and normal-hearing (NH) listeners tested with 8- and 4-channel acoustic CI simulations completed a sentence recognition task with casual and careful sentences (with similar lexical and semantic content) in the quiet. Second, we explored individual performance on this task and its relation with standard speech recognition scores and self-reported real-life hearing abilities. Listeners were overall more accurate on careful sentences than the casual sentences, but casual sentences were disproportionately more difficult than careful sentences for 4-channel stimulations (simulated "poor" CI users) and some CI users. Additionally, CI users with lower self-reported real-life hearing abilities and with lower standard sentence recognition scores were also less accurate on the casual sentences. The results suggest that speaking styles can challenge CI speech recognition, especially for listeners with poor overall recognition skills.
4aPPa48. Minimal local signal-to-noise ratio for glimpsing. Frederic Apoux (Otolaryngol. - Head \& Neck Surgery, The Ohio State Univ., 1070 Carmack Rd., Columbus, OH 43210, fred.apoux@gmail.com), Brittney L. Carter, and Eric W. Healy (Speech and Hearing Sci., Ohio State Univ., Columbus, $\mathrm{OH})$

Glimpsing models assert that speech recognition in noise relies on the ability to detect and combine time-frequency (T-F) units with "usable" speech information. A factor typically used to determine the usability of a unit is the local signal-to-noise ratio (SNR). Determining the SNR below which a unit stops contributing to overall intelligibility, however, is challenging and most approaches have consisted of varying the local SNR criterion. Here we implemented a new approach in which all the units exhibited the same local SNR. In order to prevent glimpsing within a T-F unit, their time and frequency span was lower than the subjects' resolution. Stimuli were created by mixing two sentences at a given overall SNR and retaining only those units with the desired local SNR. This procedure was iterated at various overall SNRs with the same two sentences until we were able to reconstruct $50 \%$ of the mixture with only T-F units at the desired local SNR. Sentence recognition was measured for mixtures with a variable (VAR) or constant (CON) local SNR. Our results showed a systematic drop in performance in $\mathrm{CON}$ of up to $40 \%$ points. More importantly, they suggest a limited contribution of T-F units below $-3 \mathrm{~dB}$.

4aPPa49. Comparing speaking fundamental frequency in bilingual and L2-accented speech. Ye-Jee Jung, Soo Bin Lim, Goun Lee, and Seok-Chae Rhee (Yonsei Univ., Yonsei-ro, Seoul 50, South Korea, yj71877@gmail. com)

This study investigates how speaking fundamental frequency (SFF) represents linguistic communities (Korean vs. English) between bilingual and monolingual speakers, and whether speech mode influences SFF. Twelve Korean-English sequential bilingual speakers (6 males, mean age $=26$, $\mathrm{SD}=4)$ and twelve Korean L2 learners of English (6 males, mean age $=26$, $\mathrm{SD}=3$ ) participated in this study. In the controlled speech mode, subjects were recoded producing 22 declarative sentences as well as a short fable; in the spontaneous speech mode, subjects were asked to elaborate on wellknown tales. Mean F0 and F0 range were measured for each sentence. The findings established a significant cross-linguistic difference in mean F0, with higher F0 in Korean than in English. We also found that the F0 range is influenced by speech mode, with lower mean F0 and greater F0 range in spontaneous mode. Interestingly, the F0 range differences between the two speech modes were greater in Korean than in English productions only for bilingual speakers. Regarding the L2 effect, inconsistently with the previous findings (Zimmer et al., 2016) we did not find a compressed F0 range in L2. These findings confirm that pitch profile can characterize language identity and demonstrate the effect of speech mode on bilingualism.

4aPPa50. Effect of age and hearing loss on spectral integration and speech identification. Mini N. Shrivastav (Commun. Sci. and Special Education, Univ. of Georgia, 570 Aderhold Hall, Athens, GA 30602, mnshriv@ uga.edu) and David Eddins (Commun. Sci. and Disord., Univ. of South Florida, Tampa, FL)

The goals of the project were to determine how advancing age and hearing loss affect the integration of acoustic features across the audio-frequency spectrum. Several experiments were designed to test listener's abilities to either combine or compare intensive and spectral information in non-speech and speech stimuli across frequency channels, and the association between spectral integration and speech recognition. Listeners included were 16 young normal-hearing, 16 older normal-hearing, and 16 older hearingimpaired individuals. The results indicate that age and/or hearing loss impact aspects of feature encoding as well as the ability to combine intensive information across channels. Also, age-related but not hearing-lossrelated deficits were observed in spectral integration of temporal information with deficits in encoding as well as the ability to compare temporal information across channels. Taken together, the results are consistent with the notion that age- and hearing-loss-related deficits in spectral integration have both peripheral and central components. Results also indicate that the ability to finely discriminate intensive and temporal changes across frequency may not be strongly associated with the ability to recognize 
meaningful speech, even though speech is also comprised of dynamic changes in intensive and temporal features.

4aPPa51. Speech-on-speech recognition in children and adults: Effect of whispered speech. Margaret K. Miller, Lori J. Leibold (Boys Town National Res. Hospital, 555 N. 30th St., Omaha, NE 68131, margaret. miller@boystown.org), Lauren Calandruccio (Dept. of Psychol. Sci., Case Western Reserve Univ., Cleveland, OH), and Emily Buss (Dept. of Otolaryngology/Head and Neck Surgery, The Univ. of North Carolina at Chapel Hill, Chapel Hill, NC)

The harmonic structure of speech appears to provide adults with a robust auditory grouping cue, improving speech-on-speech recognition performance. This study evaluated the effect of harmonicity on speech-on-speech recognition in normal-hearing children (5-7 yrs) and adults (19-35 yrs). Masked word recognition was evaluated using a $3 \mathrm{AFC}$ procedure with a picture-pointing response. Targets were one-syllable words that lent themselves to illustration, and maskers were samples of two-talker speech. All stimuli were recorded by female talkers in two styles: whispered and natural. Thresholds corresponding to $70.7 \%$ correct word recognition were estimated adaptively by adjusting SNR while holding the overall level of the signalplus-masker at $60 \mathrm{~dB}$ SPL throughout testing. Performance was evaluated for whispered target words presented in a whispered two-talker masker and for naturally produced target words presented in a naturally produced twotalker masker. Consistent with previous reports, adults required a more advantageous SNR to achieve criterion performance using whispered compared with natural speech. Children performed more poorly than adults in all conditions, and tended to show a smaller effect of speaking style than adults. These results suggest that children may be less adept than adults at using the harmonic structure of speech to segregate simultaneously presented voices.

4aPPa52. Recognition of vocal emotion in noise-vocoded speech by normal hearing and cochlear implant listeners. Zhi Zhu, Ryota Miyauchi (School of Information Sci., Japan Adv. Inst. of Sci. and Technol., 1-1 Asahidai, Nomi, Ishikawa 9231292, Japan, zhuzhi@jaist.ac.jp), Yukiko Araki (School of Humanities, Kanazawa Univ., Kanazawa, Ishikawa, Japan), and Masashi Unoki (School of Information Sci., Japan Adv. Inst. of Sci. and Technol., Nomi, Ishikawa, Japan)

Chatterjee et al. (2015) reported that cochlear implant (CI) listeners have difficulty recognizing vocal emotions due to the limited spectral cues provided by CI devices. Researches on vocal emotion perception of CI listeners have been studying ways to simulate responses of CI listeners by using noise-vocoded speech (NVS) as CI simulations with normal-hearing $(\mathrm{NH})$ listeners. However, it is still unclear whether the results of CI simulations with $\mathrm{NH}$ listeners are reliable with regards to CI listeners. This study aims to clarify whether CI listeners can perceive vocal emotion the same way as NH listeners with NVS do. Vocal-emotion recognition experiments were carried out by having both $\mathrm{NH}$ and CI listeners listen to original emotional speech and its NVS. The results for CI listeners revealed that they recognized sadness and hot anger more easily than joy and cold anger in both original emotional speech and NVS conditions. Moreover, the results for NH listeners with NVS showed the same trend. The results suggested that the vocal-emotion recognition paradigm using NVS can be used to investigate vocal emotion recognition by CI listeners. [Work supported by the Mitsubishi foundation and Grant in Aid for Scientific Research Innovative Areas (No. 16H01669) from MEXT, Japan.] 


\title{
Session $4 \mathrm{aPPb}$
}

\section{Psychological and Physiological Acoustics: Modelling, Measurement, and Technology (Poster Session)}

\author{
Mathias Dietz, Chair \\ National Centre for Audiology, Western University, 1201 Western Road, London, ON N6G 1H1, Canada
}

\begin{abstract}
All posters will be on display from 8:00 a.m. to 12:00 noon. To allow contributors in this session to see the other posters, authors of odd-numbered papers will be at their posters from 8:00 a.m. to 10:00 a.m. and authors of even-numbered papers will be at their posters from 10:00 a.m. to 12:00 noon.
\end{abstract}

\section{Contributed Papers}

\begin{abstract}
4aPPb1. Using Markov chain Monte Carlo algorithms to estimate auditory perceptual abilities. Jennifer Lentz (Dept. of Speech and Hearing Sci., Indiana Univ., 200 S. Jordan Ave., Bloomington, IN 47405, jjlentz@indiana.edu) and Noah Silbert (Dept. of Commun. Sci. and Disord., Univ. of Cincinnati, Cincinnati, OH)
\end{abstract}

The development of methods to rapidly estimate perceptual abilities has always been a focus of sensory systems research, as many methods can be very time consuming and fairly tedious to the observer. One method recently developed in cognitive psychology is the application of Markov Chain Monte Carlo algorithms to measure perception of stimuli with multiple dimensions, abbreviated MCMCP where the $\mathrm{P}$ stands for people. Our original interest was to apply these methods to assess and characterize the tinnitus percept. However, validation of these methods for tinnitus assessment is imperative and development of the algorithm to measure perception of acoustic stimuli could have great advantages for psychoacoustic measurement as well. Here, we present data in which we have applied MCMCP to simultaneously measure the ability to perceive the frequency and bandwidth of a variety of noise bands with different center frequencies and bandwidths. Data suggest that the algorithm converges rapidly on the frequency dimension (typically within 10-15 trials) but not as quickly or reliably on the bandwidth dimension. Our data suggest that it is critical to validate methods used for tinnitus assessment as only certain perceptual dimensions may be wellsuited for using such algorithms. These algorithms may also have limitations for the measurement of auditory perceptual abilities.

4aPPb2. A framework for auditory model comparability and applicability. Mathias Dietz (National Ctr. for Audiol., Western Univ., 1201 Western Rd., London, ON N6G 1H1, Canada, mdietz@uwo.ca), Piotr Majdak (Institut für Schallforschung, Österreichische Akademie der Wissenschaften, Vienna, Austria), Richard M. Stern (Carnegie Mellon Univ., Pittsburgh, PA), Torsten Marquardt (Ear Inst., Univ. College London, London, United Kingdom), William M. Hartmann (Michigan State Univ., East Lansing, MI), Dan F. Goodman (Dept. of Elec. and Electron. Eng., Imperial College London, London, United Kingdom), and Stephan D. Ewert (Medizinische Physik und Akustik, Universität Oldenburg, Oldenburg, Germany)

Many computational models of the auditory system exist, most of which can predict a variety of psychoacoustical, physiological, or other experimental data. However, it is often challenging to apply existing third party models to own experimental paradigms, even if the model code is available. It will be demonstrated that model applicability is increased by providing a framework where the model acts as artificial observer performing exactly the same task as the subject (e.g., adaptive staircase procedure). A possible separation of the actual auditory processing of the model from the decision making stage will be discussed, which allows for testing the auditory processing of one model in a variety of experimental paradigms. The framework will consist of a citable data repository providing the required data for the models as well as toolboxes implementing both the auditory models and a variety of experimental paradigms. The model framework will be demonstrated with exemplary binaural models applied to the three most common binaural psychoacoustic paradigms: just noticeable difference (e.g., interaural time difference), tone in noise detection (e.g., binaural masking level difference), and absolute judgment (e.g., sound source localization). Further development of the framework will be discussed.

4aPPb3. Predicting masked thresholds based on physiological models of auditory nerve and inferior colliculus population responses. Laurel $\mathrm{H}$. Carney (Univ. of Rochester, 601 Elmwood Ave., Box 603, Rochester, NY 14642, Laurel.Carney@Rochester.edu) and Virginia M. Richards (Dept. of Cognit. Sci., Univ. of California, Irvine, CA)

Acoustic cues for detection of tones in background noise include envelope, fine-structure, and energy. These cues are transformed in the process of neural coding and are represented in fundamentally different ways by different types of neurons. Here, to study the detection of a tone masked by noise population responses were derived from model auditory-nerve (AN) fibers with low or high spontaneous rates (HSR or LSR) and inferior colliculus (IC) neurons [M. S. A. Zilany, I. C. Bruce, and L. H. Carney, J. Acoust. Soc. Am. 135, 283-286 (2014) and J. Mao, A. Vosoughi, and L. H. Carney, J. Acoust. Soc. Am. 134, 396-406 (2013)]. Initial analyses focused on average response rates. Percent correct on two-interval forced-choice tasks was estimated based on the distance between responses to each stimulus interval and a template based on noise-alone responses. Although HSR AN fibers had saturated rates, the addition of a tone reduced low-frequency fluctuations of the time-varying rate, a change encoded by modulation-sensitive model IC cells. This HSR-based code was robust to the roving-level paradigm. While the non-saturated LSR AN fibers responded to added tones with an increase in average rate, in the roving-level paradigm these fibers did not provide reliable information to the model IC cell.

4aPPb4. A neural network model for top-down regulation of sensory plasticity. Yu-Xuan Zhang, Dinglan Tang (National Key Lab. of Cognit. Neurosci. and Learning, Beijing Normal Univ., Yingdong Bldg., Beijing 100875, China, zhangyuxuan@bnu.edu.cn), Ying-Zi Xiong, and Cong Yu (Psych. Dept., Peking Univ., Beijing, China)

Auditory cortex displays remarkable plasticity, but how such plasticity relates to human perceptual learning remains unclear. Here, we present a neural network model that attempts to link the two. The model consists of an encoding layer, a decoding/decision layer, and an interneuron that receives both top-down regulation and bottom-up stimulation. The interneuron, once activated, implements a neural learning mechanism such as reorganization of the tonotopic map or lateral inhibition. During active training, top-down regulation sensitizes the interneuron and initiates the learning process. Depending on the relative influence of top-down regulation and bottom-up stimulation, learning ranges from partially to completely stimulus specific, consistent with empirical observations. For stimulus-specific learning, we have recently shown that passive exposure to an untrained 
frequency after, but not before active training induces transfer to that frequency. The model displays similar behaviors regardless of the specific neural learning mechanism (remapping or inhibition) implemented. Overall, the simulation results indicate that auditory cortical plasticity is subject to topdown regulation and that the plasticity, once unlocked, can afford perceptual learning with bottom-up stimulation only.

4aPPb5. The position-variable model at 40. Richard M. Stern (Dept. of Elec. and Comput. Eng., Carnegie Mellon Univ., 5000 Forbes Ave., Pittsburgh,PA15213,rms@cs.cmu.edu)

The position-variable model was first described in 1976 as a means to characterize and predict a variety of binaural lateralization, discrimination, and detection phenomena. The model was motivated by a desire for a more complete understanding of the putative mechanisms by which interaural time and intensity differences were combined, as well as the extent to which results in interaural discrimination and binaural detection experiments are mediated by cues based on subjective lateral position. Predictions of the model were based on the patterns of activity of the display of information proposed earlier by Jeffress and Colburn, based on the auditory-nerve response to the experimental stimuli. The model was originally limited to predictions for a limited set of experiments at $500 \mathrm{~Hz}$. While various extensions of the model have been proposed over the years, they frequently were developed based on incomplete or inconsistent model formulations. This paper describes the capabilities of an extended implementation of the model that addresses a wider variety of experimental phenomena over an extended range of frequencies, all with a single set of assumptions. An implementation of the model will be integrated into the framework for evaluating auditory models described by Dietz et al. at this meeting.

4aPPb6. Modeling the effect of olivocochlear efferents on the subcortical envelope following response in humans. Christopher J. Smalt (MIT Lincoln Lab., 244 Wood St., Lexington, MA 02420, Christopher.Smalt@11. mit.edu), Michael G. Heinz, and Elizabeth A. Strickland (Speech, Lang., \& Hearing Sci., Purdue Univ., W Lafayette, IN)

The medial olivocochlear reflex (MOCR), a feedback mechanism that controls outer-hair-cell gain, is thought to provide protection and signal enhancement for a listener in background noise. This listening advantage can be attributed to reduced auditory-nerve firing to noise, which results in an increased dynamic range for encoding signals of interest. The computational model presented here updates the modeling work of Smalt et al. (2014) by adding dynamic MOCR characteristics to the more recent "humanized" model version (Zilany, et al., 2014). This MOCR implementation effectively reduces outer-hair-cell gain depending on the stimulus frequency, level, and timing. Human Envelope Following Responses (EFRs; Bharadwaj, 2015) were compared to outputs of the Nelson and Carney (2004) Inferior-Colliculus (IC) model combined with the MOCR auditorynerve model. Adding simultaneous contralateral noise did not significantly affect the model IC modulation-filter energy at the stimulus frequency, while a 500-ms contralateral noise precursor increased the output by $\sim 3 \mathrm{~dB}$ consistent with the human data. Further study of the auditory-nerve model with the MOCR may help to understand human susceptibility to noiseinduced hearing loss, the impact of damage to low-spontaneous rate auditory-nerve fibers, as well as to develop new algorithms for hearing aids to restore normal speech perception in noise.

4aPPb7. Testing a computational model for detection of "real-world" sounds. Evelyn M. Hoglund, Niall A. Klyn (Speech and Hearing Sci., Ohio State Univ., 1070 Carmack Rd., 110 Pressey Hall, Columbus, OH 43210, hoglund.1@osu.edu), Karl Lerud (Psychol. Sci., Univ. of Connecticut, Storrs, CT), Yonghee Oh (Otolaryngology-Head and Neck Surgery, Oregon Health \& Sci. Univ., Portland, OR), Edward Large (Psychol. Sci., Univ. of Connecticut, Storrs, CT), and Lawrence L. Feth (Speech and Hearing Sci., Ohio State Univ., Columbus, $\mathrm{OH}$ )

Hoglund (et al., 2010) reported the ability of listeners to detect a recorded signal masked by nine different ambient sounds. Signals were selected from 410 recordings of two different helicopters; maskers were selected from nine outdoor field recordings from three different locations. Signal and masker levels were randomized from trial to trial in a 2I,2AFC adaptive procedure to produce psychometric functions. Signal-to-masker ratios (SNR) ranged from -22 to $-4 \mathrm{dBA}$ for $70 \%$ correct detections. The Hoglund et al. results were used to tune a network of non-linear oscillators to optimize its performance. To evaluate the model, recordings of three fixed-wing aircraft were obtained for use as signals in the same nine environmental backgrounds. Signal and masker recordings were pre-processed to ensure that overall RMS voltage measures reflected the sound pressure level measured in a flat-plate coupler at the headphone used by the listeners. Model predictions for complete psychometric functions were obtained before experimental results were available for comparison. This poster presents details of the pre-processing of signals and maskers, and of the adaptive, single-interval procedure used to obtain the SNR for $\mathrm{P}(\mathrm{C})=$ $66.5 \%, 75 \%$, and $87.5 \%$ by 16 listeners. Good agreement was found between model predictions and listener performance. [Work supported by a SBIR grant from AFOSR.]

4aPPb8. Modelling the effect of pulse-rate on coding of interaural time differences in listeners with cochlear implants. Suyash N. Joshi and Bastian Epp (Hearing Systems, Tech. Univ. of Denmark, Tech. University of Denmark, Bldg. 352, Ørsteds Plads, Kgs. Lyngby 2800, Denmark, suyashnjoshi@gmail.com)

CIs stimulate the auditory nerve fibers (ANF) with a train of amplitude modulated current pulses. Depending on polarity, the pulses can generate spikes at different sites along the ANF. The latency difference between spikes generated at the central and the peripheral axons was found to be up to $200 \mu \mathrm{s}$ in cats and up to $450 \mu \mathrm{s}$ in humans. These timing differences could be the reason underlying the poor performance of CI listeners in ITD perception. A model of ANF responses to electrical stimulation (Joshi et al., 2016), which includes two sites of spike generation along the ANF was used to simulate the ANF responses to constant-amplitude and modulated pulse trains for different pulse-rates. The fidelity of the temporal coding was quantified by calculating the phase-locking value. The results show that an increase in pulse-rate leads to higher uncertainty in the site of spike generation, reduction in phase-locking, and increase in variance of its distribution. This may account for impaired ITD thresholds observed at high pulse-rates. The simulated monaural spike trains are then used predict the ITD discrimination thresholds by imposing delays between the two pulse trains and to identify the factors that affect ITD coding in CI listeners.

4aPPb9. Assessment of the middle-ear assumption of the auditory hazard assessment algorithm for humans. Heath G. Jones, Nathaniel T. Greene, Bankole K. Fasanya, Sean A. Hollonbeck, and William A. Ahroon (Auditory Protection and Performance Div., U.S. Army Aeromedical Res. Lab., Bldg. 6901, Fort Rucker, AL 36362, heath.g.jones2.ctr@mail.mil)

The Auditory Hazard Assessment Algorithm for Humans (AHAAH) is an electrical equivalence model of the human ear designed to reproduce sound transmission from the outer into the inner ear in order to predict potential injury from a given sound exposure. This model was generated and validated using a (mostly) feline model, thus several key assumptions may not hold when adapted to the human. The current project aims to test some of these assumptions, such as the effects of middle-ear muscle contraction (MEMC) on sound transmission and whether the MEMC can be elicited by a conditioning stimulus prior to sound exposure (i.e., a "warned" response). In order to quantify the MEMC, we use laser-Doppler vibrometry to measure tympanic membrane motion in response to a reflex-eliciting acoustic impulse. After verifying the MEMC, we will attempt to classically condition the response by pairing the reflex-eliciting acoustic impulse (unconditioned stimulus, UCS) with a light from a LED display (conditioned stimulus, CS). Changes in the magnitude and/or time-course of the MEMC following repeated UCS-CS pairings will be evidence of MEMC conditioning. Validation of these effects will allow updates to Damage-Risk Criteria to better protect the hearing of Soldiers and civilians exposed to high-level impulsive noises. 
4aPPb10. Dimensional interactions in the perception of frequency and bandwidth. Noah H. Silbert (Commun. Sci. and Disord., Univ. of Cincinnati, French East 344, 3202 Eden Ave., Cincinnati, OH 45267, silbernh@ ucmail.uc.edu) and Jennifer Lentz (Speech and Hearing Sci., Indiana Univ., Bloomington, IN)

As part of a project to develop efficient techniques for measuring the characteristics of tinnitus percepts, multiple listeners exhibited difficulties accurately perceiving the bandwidth of stimuli. Frequency perception seems to dominate bandwidth perception and relative bandwidth judgments seem to interact with frequency judgments in complex ways. In the present work, we use general recognition theory (GRT) to directly probe perceptual interactions between center frequency and bandwidth in noise stimuli. GRT is a multidimensional generalization of signal detection theory, and it allows us to simultaneously model within-category and between-category interactions between dimensions, as well as dimension-specific patterns of response bias. The most common stimulus set for GRT experiments consists of the factorial combination of two levels on each of two dimensions. In order to more fully investigate the perception of frequency and bandwidth, we extend this standard approach to encompass multiple factorial sets of stimuli spanning a larger region of frequency-by-bandwidth space. We will present multilevel Bayesian GRT models fit to frequency-by-bandwidth perception data.

4aPPb11. Measurement of tone-burst-evoked oto-acoustic emissions under various band-noise conditions. Shunsuke Kidani (Hokuriku Univ., 1-1 Taiyogaoka, Kanazawa, Ishikawa 920-1180, Japan, s-kidani@hokurikuu.ac.jp), Masashi Unoki, and Ryota Miyauchi (Japan Adv. Inst. Sci. and Technol., Nomi, Ishikawa, Japan)

Oto-acoustic emission (OAE), which is reflected by the outer hair cells (OHCs) function, varies depending on the presence of noise. Because it is unclear under what noise conditions the OAE varies, this study investigates the amplitude of the OAEs under various noise conditions. Tone-burstevoked (TBE)-OAEs were measured at probe frequency with/without band noise in which the bandwidth was $4 \mathrm{ERB}_{\mathrm{N}}$. The noise was presented at either high or low frequency side of the probe to study how the frequency area affects the TBE-OAEs. In addition, the difference between the noise and the probe frequency was varied to investigate how the presence of the noise inside or outside $1 / 4 \mathrm{ERB}_{\mathrm{N}}$ from the probe frequency affects the TBEOAEs. The results indicated that the amplitude of TBE-OAEs decreased when noise was presented to the higher side of the probe frequency and the inside of $1 / 4 \mathrm{ERB}_{\mathrm{N}}$ from the probe frequency. Interestingly, these results suggest that the amplitude of TBE-OAEs was varied when the noise was presented at the frequency area that has many efferent pathways to the OHCs. [Work supported by JSPS KAKENHI (15K13158).]

4aPPb12. Estimation of auditory compression and filter shape of elderly listeners using notched noise masking. Toshie Matsui (Graduate School of Systems Eng., Wakayama Univ., Sakaedani 930, Wakayama, Wakayama 640-8510, Japan, tmatsui@sys.wakayama-u.ac.jp), Toshio Irino, Hazuki Inabe (Faculty of Systems Eng., Wakayama Univ., Wakayama, Japan), Yuri Nishimura (Graduate School of Systems Eng., Wakayama Univ., Wakayama, Japan), and Roy D. Patterson (Dept. of Physiol., Development and Neurosci., Univ. of Cambridge, Cambridge, United Kingdom)

Notched-noise (NN) masking and the compressive GammaChirp (cGC) auditory filter have recently been used to estimate auditory compression and filter shape simultaneously with young normal-hearing (NH) listeners. This paper reports an extension of the paradigm to measure compression and filter shape in elderly listeners with a range of hearing losses. Six elderly listeners (65-75 year old, male) participated in a compact $\mathrm{NN}$ experiment with one probe frequency, $2.0 \mathrm{kHz}$. There were $21 \mathrm{NN}$ conditions chosen to maximize sensitivity to compression in the mid-level range. The listeners' hearing levels (HLs) at $2.0 \mathrm{kHz}$ ranged from 10 to $50 \mathrm{~dB}$. The cGC filter was fitted to the threshold data to derive level-dependent filter shapes of individuals and their cochlear compression functions. The slope of the central portion of the compression function was normal for all of the listeners except the one with the $50 \mathrm{~dB} \mathrm{HL}$, whose slope was close to unity $(>0.8 \mathrm{~dB} / \mathrm{dB})$. The filter bandwidths were marginally wider than those of young NH listeners. Gathering thresholds in the $21 \mathrm{NN}$ conditions took approximately 3 hours per listener. This is acceptable for laboratory studies with the elderly but still somewhat long for clinical applications.

4aPPb13. Experimental examination of acoustic sensing using super-directivity speaker and super-resolution signal processing with pulse compression technique. Yuya Asakura, Kan Okubo, and Norio Tagawa (Graduate School of System Design, Tokyo Metropolitan Univ., 6-6 Asahigaoka, Hino, Tokyo 191-0065, Japan, asakura.wad3@gmail.com)

Acoustic sensing technology in the air is a promising method to acquire shapes and/or position of a target object. Universal and smart technology in Acoustic sensing, however, cannot be sufficiently established because of various acoustic environmental noises, attenuation effect, etc. Normally, pulse transmission using higher frequency cannot obtain high SNR images due to the atmospheric attenuation, whereas the spatial resolution becomes lower by use of signals in lower frequency range. That is, there is the tradeoff between frequency and spatial resolution of transmission signal. In recent years, with the development of computer technologies, a single processing method becomes important for the real-time sensing in air. To overcome these problems, therefore, in this study, we propose and examine acoustic sensing in the air using the super-directivity speaker, super-resolution signal processing, and pulse compression technique (PCT). By applying the super-resolution signal processing and called SCM-MUSIC (FM-chirp based Super resolution Correlation Method-MUltiple SIgnal Classification) method, we can improve the SN ratio and spatial resolution in acoustic sensing. Our experimental results suggest that combination of super-directivity speaker and super-resolution signal processing with PCT enables high-resolution acoustic imaging. Additionally, we discuss the feasibility of far-field high resolution imaging by the proposed method.

4aPPb14. Development of cartilage conduction receiver available in noisy condition. Ryota Shimokura (Shimane Univ., Nishikawatsu-cho 1060, Matsue, Shimane 690-8504, Japan, rshimo@riko.shimane-u.ac.jp), Hiroshi Hosoi, and Tadashi Nishimura (Nara Medical Univ., Kashihara, Japan)

Sound information is known to travel to the cochlea via either air or bone conduction. However, a vibration signal, delivered to the aural cartilage via a transducer, can also produce a clearly audible sound. This type of conduction has been termed "cartilage conduction." The aural cartilage forms the outer ear and is distributed around the exterior half of the external auditory canal. In cartilage conduction, the cartilage and transducer play the roles of a diaphragm and voice coil of a loudspeaker, respectively. A benefit of using the cartilage conduction is to be able to amplify the sound in the canal by pushing the transducer on the cartilage more strongly. And when the pushed ear tragus obscures the canal, the sound pressure in the canal reaches a maximum and the environmental noise is blocked at the same time. In our study, participants could catch a speech clearly in this way in different kinds of noise condition (speech noise, road noise, station noise, and cleaner noise in 50,60,70, and $80 \mathrm{~dB}$ ). This benefit can be applied for a smartphone for workers who have to answer the phone no matter what noisy condition they are in.

4aPPb15. Computer-controlled audiometer's application as an earplug fit-testing tool. J. R. W. Stefanson and William A. Ahroon (Auditory Protection and Performance Div., US Army Aeromedical Res. Laboratory/ Hearing Ctr. of Excellence, 6901 Farrel Rd., Fort Rucker, AL 36362, earl.w. stefanson.ctr@mail.mil)

Clinical audiometers are usually coupled with a calibrated pair of TDH39 headphones, supra-aural earphones that rest on a person's ears. Hearing protector testing with these types of earphones is problematic due to a couple of reasons; the earplug may protrude from the ear canal and come in contact with the earphone thereby alerting the individual that the earplug is likely not inserted adequately or the earphone may impend on the position and affect the seal of the earplug and thereby void any measurement data. Deeper supraural ear-cup cushions were 3D printed for the TDH-39 headphones in order to allow for an insert earplug to be fit-tested without interference. The 3D printed set of ear-cups was calibrated to the computercontrolled audiometer and 20 subjects were tested in the unoccluded and 
occluded conditions. The differences between the two measurements at each of the seven frequencies tested (i.e., 500, 1000, 2000, 3000, 4000, 6000, and $8000 \mathrm{~Hz}$ ) were averaged across subjects, personal attenuation ratings computed. Results are compared with a commercially available hearing protective device field attenuation estimation system (i.e., fit-check or fit-test system).

4aPPb16. Audio quality in everyday listening situations. Michael Oehler, Thomas Wildenburg (Inst. of Sound and Vib. Eng. (ISAVE), Univ. of Appl. Sci. Dusseldorf, Josef-Gockeln-Str. 9, Dusseldorf 40474, Germany, michael.oehler@hs-duesseldorf.de), and Christoph Reuter (Musicological Dept., Univ. of Vienna, Vienna, Austria)

The quality of digital audio which uses a form of lossy data compression is in the focus of several studies. The experimental focus of these studies includes the fundamental difference between objective and subjective measurement methods (Pocta \& Beerends, 2015), test standards (Breebaart, 2015), or signal processing (Khaldi et al., 2013; Jung et al., 2016). But there are only few studies concerning the impact of perceived quality differences in everyday listening situations (ELS). Short excerpts of recent popular music recordings were encoded with different bit rates based on codecs that are commonly used by popular music streaming services. While the encoded files were presented via standard Samsung in-ear headphones, the everyday listening situation was simulated by using binaural recordings of a shopping mall soundscape that was simultaneously presented via Stax electrostatic headphones. In a control condition no soundscape was used. The stimuli were rated by 30 participants in a MUSHRA listening test (ITU-R BS.1534). A repeated measures ANOVA showed that there are significant differences for both conditions concerning the different stimuli (ELS: $\mathrm{F}(10,20)=25.237, \quad p=.0001, \quad \mu^{2}=.927 ; \quad$ no ELS: $F(10,20)=51.073$, $\left.p=.0001, \mu^{2}=.962\right)$, but the effect sizes are consistently smaller in the condition with simulated everyday listening situation. The difference between both conditions gets smaller, the lower the bit rates are. A follow-up study additionally includes binaural audio that is currently used by radio stations.

4aPPb17. Noise suppression based on loudness management for hearing aid. Takeru Hiramoto (Dept. of Control and Information Systems Eng., National Inst. of Technol., Kumamoto College, Suya 2659-2, Koshi, Kumamoto 8611102, Japan, ae16hiramoto@g.kumamoto-nct.ac.jp), Nobuhiko Hiruma (Hearing aid and audiological equipment Eng., RION Co., Ltd., Kokubunji, Tokyo, Japan), Hidetoshi Nakashima (Dept. of Control and Information Systems Eng., National Inst. of Technol., Kumamoto College, Koshi, Kumamoto, Japan), and Yoh-ichi. Fujisaka (Hearing Aid and Audiological Equipment Eng., RION Co., Ltd., Kokubunji, Tokyo, Japan)

It is well known that speech intelligibility does not improve even if SNR is improved and noise annoyance decreases by noise suppression for hearing aids, since the noise suppression decreases the level of the desired signal as well as the one of the noise. One solution for the problem is to minimize the speech distortion as much as possible even if a large quantity of residual noise exists. In this study, we propose a noise suppression algorithm based on loudness management to improve speech intelligibility for hearing aid wearer. Our algorithm not only minimizes the speech distortion but also maintains the speech loudness. In the algorithm, the speech spectrum is estimated from the observed signal first, and then the loudness of the estimated speech spectrum is calculated. Simultaneously, the loudness of the observed signal is also calculated and the gains in each frequency band are controlled in order to correspond between these two loudness. In our loudness management, even if the bands are almost filled by noise component, it is not suppressed as much as possible not to reduce barely audible speech component. As results, the noise suppressed signal with low speech distortion is obtained and speech intelligibility is improved comparing with commonly used method.
4aPPb18. Real rooms vs. artificial reverberation: An evaluation of actual source audio vs. artificial ambience in the rear height channels of immersive audio systems. Richard L. King (Music Res., McGIll Univ., 555 Sherbrooke West, Montreal, QC H3A 1E3, Canada, richard.king@mcgill.ca), Brett Leonard (School of Communications, Webster Univ., Webster Groves, MO), and Will Howie (Music Res., McGIll Univ., Montreal, QC, Canada)

Many spatial audio researchers and content producers agree that the best source material for height channels in immersive audio is provided by the capture of actual elevated channels in the room. Particularly for music recording, this technique is preferred as opposed to signal processing, providing a more natural and realistic impression of immersion. While previous work has proven this to be the case in the front channels of various 3D playback systems such as 22.2, the content of the rear height channels has not been specifically evaluated in this respect. Multichannel audio recording, specifically $3 \mathrm{D}$ recording, can be a cumbersome task as the channel counts expand-and so the question arises-is it really necessary to capture discrete rear height information? This research compares four height channel capture points are compared to two capture points applied to the front height channels in conjunction with artificial reverberation in the rear channels. A two-part study is employed - the first is a simple ABX test to determine discriminability between the real rooms and the artificially generated version. Part two is a preference test, based on several standard acoustic/perceptual descriptors, revealing the subtle differences between real and artificial rear height channel information.

4aPPb19. Low latency dereverberation with considering speech naturalness for hearing aids. Kotoyo Nozaki, Yusuke Ikeda, Yasuhiro Oikawa (Waseda Univ., 59-407-2, 3-4-1, Ohkubo, Shinjuku, Tokyo 169-8555, Japan, kotokoto3726@asagi.waseda.jp), Yoh-ichi Fujisaka, and Masahiro Sunohara (RION Co., Ltd., Tokyo, Japan)

It is well known that long reverberation makes speech unclear especially for hearing impaired. For a long time, a lot of researches on dereverberation have been studied. However, the computational cost of most of those methods are too expensive for hearing aids. Therefore, we have proposed the simplified dereverberation method, which can estimate reverberation energy from observed signal. In addition, it is also necessary implementing the processing with low latency to avoid an annoyance for hearing aid wearer. Processed signal have to be natural as much as possible to reduce listening effort, although there is performance limit because of simplicity. In this study, we make two proposals in our previous method. First, we apply Frequency-Warped Filterbank (FWF) in analysis and synthesis systems in order to make frequency bands as Bark scaled alignment, and to take the tolerable latency all over frequency for hearing. Second, we introduce the gain control in each frequency to maintain naturalness of processed speech, because reverberation energy varies depending on frequency bands in actual reverberant environments. From subjective experiments, there was a tendency that they prefer this proposal to the previous method.

$4 \mathrm{aPPb20}$. A study of a voice evacuation guidance system using a precedence effect. Miyu Hashimoto (Elec. Eng., Nihon Univ., 5-6-18, Hino, Kohnan-ku, Yokohama-shi, Kanagawa-ken 234-0051, Japan, csmy16031@ g.nihon-u.ac.jp), Ayumu Osumi, and Youichi Ito (Elec. Eng., Nihon Univ., Chiyoda-ku, Tokyo-to, Japan)

We have developed a voice evacuation guidance system using a precedence effect when a fire breaks out in a building in order to guide refugees to safe place efficiently. This system has already applied to an underground shopping center which has a space like a straight path as an aggressive evacuation guidance system. Further, a method for adapting this system to large interior space is examined and the effectiveness of this system have been identified basically. As a goal of this research, it was intended to develop a highly accurate acoustic design method for designing the voice evacuation guidance system. For realizing this, it is necessary to clarify relationship between acoustic conditions and a sound direction recognition in this system and create a database of these relationship. In this report, as one of important factors of developing this system, we performed an experimental measurement in relation to a recognition time of a composed sound by a preceding sound and a secondary sound. It was studied in particular detail for the secondary sound coming from various angles relative to the preceding sound. 
4aPPb21. Characterizing real-world auditory scenes using $360^{\circ}$ audiovisual capture. Ashley Foots (Army Res. Lab., P.O. Box 531, Rising Sun, MD 21911, ashleynicolefoots@gmail.com), Kelly Dickerson, and Jeremy Gaston (Army Res. Lab., Aberdeen Proving Ground, MD)

Accurate sound identification is a critical aspect of situational awareness. Environmental sound identification is influenced by physical stimulus dimensions and subjective factors, such as pleasantness or familiarity. Ecological frequency, the prevalence of sounds' occurrences in the environment, may also play an important role; however, accurately capturing this information for real-world sounds is difficult. Due to limited information about ecological frequency and the difficulty of accurately characterizing acoustic characteristics of real-world sounds studies do not typically account for this information during stimulus selection. The present study evaluates ecological frequency for environmental sounds collected using a RealSpace Camera, a spherical array of 64 microphones and 5 HD cameras. Environmental sound recordings were captured in various urban and rural settings. Samples were recorded for 30 seconds at five to ten minute intervals for one hour (resulting in up to six samples per session). These multichannel, shortinterval recordings were augmented by hour-long single channel samples to enable comparison between long and short term spectro-temporal changes associated with the captured scenes. Human raters then evaluated recorded scenes with and without video confirmation to estimate ecological frequency. This effort is the first step in characterizing the real-world sensory environment and its influence on perceptual performance.

4aPPb22. Externalization of sounds with non-individualized headrelated transfer functions and without visual information. Thibaud Leclère, Mathieu Lavandier (LGCB, ENTPE - Université de Lyon, rue Maurice Audin, Vaulx-en-Velin, Rhône 69518, France, thibaud.leclere@ entpe.fr), Kevin Perreaut (LGCB, ENTPE - Université de Lyon, Lyon, France), and Fabien Perrin (Auditory Cognition and PsychoAcoust. team, Lyon NeuroSci. Res. Ctr., UCBL - CNRS UMR 5292 - Inserm U1028, Lyon, Rhône, France)

A sound produced by a real source is perceived as "externalized," i.e., outside the head, meaning that it is part of the sound environment of the listener. Conversely, when listening to sounds over headphones, they are generally perceived as "internalized," i.e., inside the head. In the framework of a research project dealing with the evaluation of comatose patients using sounds reproduced over headphones, it is aimed to investigate the influence of the realism of auditory stimulation through some degree of externalization. Experiments were conducted with non-patients (normal-hearing, awake) while conserving the constraints related to the clinical evaluation (non-individualized head-related transfer functions and no visual reference). A first experiment investigated sound externalization using four types of signals (pink noise, music, speech, and bottles clinging) convolved with different non-individualized binaural room impulse responses measured at different azimuths in different rooms. Diotic reference sounds were also tested. A second experiment investigated the influence of direct-to-reverberant ratio and interaural differences by varying these parameters artificially. The results indicated that externalization was affected by both binaural information and reverberation, suggesting the implication of a temporal binaural mechanism. The data were used to seek an acoustical correlate for the degree of non-individualized externalization.

4aPPb23. What is the impact of individualizing head-related transfer functions used for sound externalization? Thibaud Leclère, Mathieu Lavandier (LGCB, ENTPE - Université de Lyon, rue Maurice Audin, Vaulx-enVelin, Rhône 69518, France, thibaud.leclere@entpe.fr), and Fabien Perrin (Auditory Cognition and PsychoAcoust. Team, Lyon NeuroSci. Res. Ctr., UCBL - CNRS UMR 5292 - Inserm U1028, Lyon, France)

Natural listening can be reproduced via headphones by introducing head-related transfer functions (HRTFs) which represent the temporal and spectral modifications occurring when a sound travels from the source to the listener's ears. HRTFs are individualized, they differ from one listener to the other and strongly depend on their morphology. For practical reasons, it might be complicated to measure HRTFs from each listener and, as a result, non-individualized HRTFs (e.g., measured using a manikin) are sometimes preferred. This study investigated the consequences of this non- individualization on perceived sound externalization. First, individualized HRTFs were measured for a set of listeners in different rooms for sources at different azimuths. Non-individualized HRTFs were also measured at the same positions using a manikin. In a first experiment, listeners evaluated the perceived degree of externalization of sounds convolved with their own HRTFs (individualized condition) or with those measured using the manikin (non-individualized condition). In a second experiment, the effect of equalizing the frequency-response of the headphones was investigated by using either the headphones (equalized or not) or (non-equalized) earphones to deliver the convolved signals. The results of this study are used for a preliminary quantification of the differences between individualized and non-individualized sound externalization.

4aPPb24. Auditory evaluation of head-related transfer functions from multiple databases. Xiao-li Zhong (School of Phys. and Optoelectronics, South China Univ. of Technol., Bldg. No. 18, Wu Shan Rd. No. 381, Guangzhou, Guangdong 510641, China, xlzhong@scut.edu.cn)

In order to rationalize the combined utilization of multiple databases of head-related transfer functions (HRTFs), this work investigates the influence of different HRTF measuring conditions on auditory perception. First, the HRTFs of KEMAR artificial head from five databases were selected, and the diffusion-field equalization was applied to the original HRTFs as a preprocessing method to reduce the differences from different measuring conditions. Then, the spectral deviations among the equalized HRTFs were calculated to objectively evaluate the HRTF's differences from different measuring conditions. Finally, a series of listening experiments (including localization and discrimination experiments) were conducted to subjectively evaluate different HRTF measuring conditions on auditory perception. Results indicate that the different measuring conditions has less influence on the localization performance of HRTFs, suggesting the auditory consistency among multiple HRTF databases in terms of localization; however, only part of HRTF databases are consistent in terms of indiscernibility which is a stricter metric. [Work supported by National Natural Science Foundation of China, No.11474103.]

4aPPb25. A high-resolution head-related transfer function dataset and 3D ear model database. Janina Fels and Ramona Bomhardt (Inst. of Tech. Acoust., Medical Acoust. Group, RWTH Aachen Univ., Kopernikusstraße 5, Aachen 52072, Germany, rbo@akustik.rwth-aachen.de)

Binaural reproduction techniques, which use head-related transfer functions (HRTFs), become more and more popular. Human localization performance using binaural synthesis is, however, often poor due to nonindividual HRTFs. To investigate the physical relationship between the ear geometry and the directional HRTFs, the ears and the HRTF datasets of 48 subjects are measured. The detailed ear models are obtained from magnetic resonance imaging scans of the subjects. Two rough scans series of the head and a detailed horizontal scan series, which covers both ears, are taken. The 3D model meshes, which are generated from the MRI scans, allow a very precise determination of anthropometric dimensions which can be used for the individualization of the HRTFs. Additionally, the relationship between the anthropometric dimensions and monaural cues of the HRTFs is studied by the boundary element method. These simulations show which parts of the pinna are involved in case of resonances or destructive interferences.

4aPPb26. Head-related transfer function range extrapolation and nearfield compensated high-order Ambisonics. Bo-sun Xie (Acoust. Lab., School of Phys. and Optoelectronics, South China Univ. of Technol., Guangzhou 510641, China, phbsxie@scut.edu.cn)

Head-related transfer function range extrapolation (HRTF-RE) predicts HRTFs at various source distances from the known HRTFs at a fixed source distance and discrete directional grid. Near-field-compensated higher order Ambisonics (NFC-HOA) recreates virtual source at various distances by using a set of loudspeakers arranged on a spherical surface with fixed radius. HRTF-RE and NFC-HOA seem to be two different fields and were studied individually in previous works. The present work analyzes the relationship between HRTF-RE and NFC-HOA. Based on the spherical harmonics-Bessel expansion of sound field, it is proved that HRTF-RE and NFC-HOA are 
mathematically equivalent and therefore share some common characters and limitations. Based on the equivalence, the interchanging some of the methods used by the two fields is discussed. [This work was supported by the Guangzhou Science and Technology Plan Projects under No. 2014Y200021.]

4aPPb27. Individualization of head-related transfer functions by the principle component analysis based on anthropometric measurements. Ramona Bomhardt and Janina Fels (Inst. of Tech. Acoust., Medical Acoust. Group, RWTH Aachen Univ., Kopernikusstraße 5, Aachen 52072, Germany, rbo@akustik.rwth-aachen.de)

A binaural reproduction either uses individual or generic head-related functions (HRTFs). The assessment of individual spatial high-resolution HRTF datasets is very time-consuming and requires a special measurement setup. On the other hand, a generic HRTF dataset from a dummy head can lead to localization errors such as front-back confusions and in-head localizations. In the present work, the generation of individualized HRTF datasets using the principle component analysis and multiple regression analysis from a generic HRTF dataset is discussed. The individualized HRTFs reduce the localization errors by taking individual anthropometric dimensions of the head and pinna into account. The method is applied to 48 individual HRTF datasets to determine the principal components and their weights. The anthropometric dimensions are obtained from the 3D magnetic resonance imaging scans of the 48 subjects. The focus of the work is on the number of necessary principle components with respect to the measurement grid and redundancy of anthropometric dimensions.

4aPPb28. Emphasizing interaural level difference corrects the shift of perceived direction of sound for consumer-grade bone-conduction headphones. Mamoru Iwaki and Yoshiki Chigira (Graduate School of Sci. and Technol., Niigata Univ., 8050 Ikarashi-2-nocho, Niigata 950-2181, Japan, iwaki@eng.niigata-u.ac.jp)

Sounds are perceived not only in air-conduction (AC) manner but also in bone-conduction (BC) one. According to our investigation [Autumn Mtg Acoust. Soc. Japan, 3-1-18, pp. 865-866, 2013; IEEE Int'l Conf. GCCE, pp. 194-197, 2014] about the discrepancy of perceived directions of sound through a BC headphone (TEAC Filltune HP-F200) and an AC headphone (SONY MDR-Z900), the perceived direction through a BC headphone inclined toward the center. In this report, we examined a correction of the shift of perceived direction of sound by emphasizing interaural level difference (ILD). Seven students with normal audibility participated in our experiment. It was carried out in a sound proof room. Sound stimuli were pure-tones of $500 \mathrm{~Hz}, 1000 \mathrm{~Hz}, 2000 \mathrm{~Hz}$, and $4000 \mathrm{~Hz}$, with 11 different directions for each stimulus. The sound level through AC headphone was 70 $\mathrm{dB}$. That through $\mathrm{BC}$ headphone was adjusted to have the same loudness, for each participant. As a result, the perceived direction through $\mathrm{BC}$ headphones was improved. Although the $\mathrm{BC}$ headphone used in our experiment produced about $20 \mathrm{~dB}$ of ILD in the external auditory meatus, it is difficult to explain that in terms of cross hearing and trans-cranial attenuation, but cartilage conduction hearing. Emphasizing ITD had little improvement of perceived direction.

4aPPb29. Effect of characteristics compensation between air-conduction and bone-conduction headphones on sound localization in an anechoic chamber. Satoki Ogiso (Ph.D. Program in Empowerment Informatics, Univ. of Tsukuba, Tennodai 1-1-1, Tsukuba, Ibaraki 305-8573, Japan, ogiso@aclab.esys.tsukuba.ac.jp), Koichi Mizutani, Naoto Wakatsuki, Keiichi Zempo, and Yuka Maeda (Faculty of Eng., Information and Systems, Univ. of Tsukuba, Tsukuba, Japan)

Bone-conduction (BC) headphones are used when users need to hear provided sounds as well as environmental sounds since they do not occlude the environmental sounds. Sound localization characteristics of the BC sounds are usually worse than that of the air-conduction (AC) sounds. If the frequency characteristics of $\mathrm{AC}$ sounds can be reproduced with $\mathrm{BC}$ head- phones, better sound localization with $\mathrm{BC}$ headphones can be achieved. To compensate the frequency characteristics difference between these sounds, a method to measure the frequency characteristics difference is proposed. Also, compensated BC sound is examined by localizing sounds. At first, the difference between $\mathrm{AC}$ and $\mathrm{BC}$ sounds are measured by tuning their amplitudes and phases to cancel at perceiving sound. The measurements were conducted for 30 frequencies in the audible range. Second, localization characteristics are measured in an anechoic chamber. The sounds are presented from $\mathrm{AC}$ and $\mathrm{BC}$ headphones using the corresponding head related transfer functions in the horizontal plane. Especially for the $\mathrm{BC}$ headphone, the characteristics difference between $\mathrm{AC}$ and $\mathrm{BC}$ are compensated. Based on single-user experiment, characteristic peaks were confirmed in the frequency characteristics difference. The more detailed experimental results with multiple number of users and discussions will also be provided.

4aPPb30. Investigating integration between auditory and visual location information presented in an augmented-reality device. Hiraku Okumura (R\&D Div., Yamaha, 203 Matsunokijima, Iwata, Shizuoka 438-0192, Japan, hiraku.okumura@music.yamaha.com), Khiwadkar Sushrut, and Sungyoung Kim (Rochester Inst. of Technol., Rochester, NY)

Effective rendering technique of auditory information for an augmented reality (AR) device has been investigated. Researchers and industries are searching for new applications incorporating AR and Virtual Reality (VR) technologies, which provide enhanced user-experience of interactivity and intimacy. To utilize these benefits best, homogeneous integration between visual and auditory information is important. To date, the binaural technology based on head-related transfer function (HRTF) has been used to create immersive and three-dimensional audio objects for VR and AR devices. In this study, we compared the HRTF method with a stereophonic panning method that only controls Inter-aural Level Difference (ILD) for an AR device (a smart see-through glass) and investigated the precision of auditory information required for a coherent representation with a target visual image at the locations of 0 degree, -5 degrees, and -10 degrees in a counter clockwise. Auditory stimuli were rendered to have target locations in the horizontal plane from +45 degrees to -45 degrees with a 5-degree interval. In the subjective evaluation, each participant answered whether a randomly given auditory location was matched to a target location of the visual image. The results showed that two audio rendering methods did not produce significant difference in creating integrated perception in an AR device.

4aPPb31. Subjective attributes of realistic sound fields: What makes acoustic environments complex? Adam Weisser and Jorg M. Buchholz (Linguist, Macquarie Univ., National Acoust. Labs., Australian Hearing, Level 4, 16 University Ave., NSW 2109, Australia, adam.weisser@nal.gov. au)

Real-life acoustic environments are commonly considered to be complex, because they contain multiple sound sources in different locations, room reverberation, and movement-all at continuously varying levels. In comparison, most laboratory-generated sound fields in hearing research are much simpler. However, the continuum between simple and complex fields is likely to be a function of multiple instrumental as well as perceptual factors. Although most of the applied knowledge has been gathered about simple, controlled, and synthetic acoustic environments, inferring listeners' performance and perception from that to realistic scenarios is non-trivial. In order to clarify some of these relationships, subjective responses to twelve virtualized acoustic environments were examined using exploratory questionnaires with both descriptive and rating questions. These were specifically designed for the purpose of identifying attributes that may correlate with perceived acoustic complexity. Environments were recorded and processed using higher-order ambisonics, and reproduced in a 41-loudspeaker array in an anechoic chamber. Eleven environments spanned from a quiet library to a loud food court and diffuse noise was used as a control environment. Attributes included questions about busyness, variability, reverberance, annoyance, and others. The results enable us to break down the perceived complexity to its constituent factors. 
4aPPb32. Simulation of frequency characteristics of bone conduction by own speech. Takayoshi Nakai and Kenta Suzuki (Faculty of Eng., Shizuoka Univ., 3-5-1 Johoku, Naka-ku, Hamamatsu, Shizuoka 432-8561, Japan, nakai.takayoshi@shizuoka.ac.jp)

When a speaker himself/herself hear his/her own speech, it is different from recorded speech. It is known that it is due to bone conduction. But we measured sound at the entrance of the ear, and it is shown that speech heard by speaker himself/herself is almost agreement with sound at the entrance of the ear at more than $1 \mathrm{kHz}$. This time, we simulate frequency characteristics of bone conduction. The vocal tract have loss factor of air viscosity and heat conduction of the wall, and it is assumed that the ear drum is vibrated by vibration of the vocal tract. These results are reported.

4aPPb33. Control of a sound image to the rearward direction using band-stop filters and laterally located loudspeakers. Tomomi Hasegawa, Satoshi Oode, Kazuho Ono (NHK STRL, 1-10-11, Kinuta, Tokyo, Setagaya-ku 157-8510, Japan, hasegawa.t-eo@nhk.or.jp), and Kazuhiro Iida (Chiba Inst. of Technol. / NHK STRL, Chiba, Japan)

The ability to control the sound image to the rearward direction using front or lateral loudspeakers is an important step to popularize multichannel 3D audio systems in the home environment. Rear "directional bands" were found for narrow-band signals which are perceived only in the rearward direction, but any kind of directional bands for wide-band signals, which are perceived only in the rearward direction, have not been reported. It is known that spectral notches in head-related transfer functions are important cues for a sound image localization in the median plane. In the present study, the possibility of sound image control to the rearward direction was investigated using wide-band noise of which frequency components adjacent to the rear directional bands were eliminated. A total of 56 kinds of band-stop filters were used to eliminate one to the ten consecutive $1 / 3$-octave bands with center frequencies of 1.6 to $12.5 \mathrm{kHz}$. In-phase stimuli were emitted from a pair of loudspeakers located at azimuth angles of $\pm 90^{\circ}$ in an anechoic room. The results showed that the probability of perceiving a sound image to be in the rearward direction was $95 \%$ when consecutive frequency bands, which were customized to individual subject, were eliminated.

4aPPb34. Psychophysical investigation of binaural spatial-audio music perception with varying degrees of realism. Craig T. Jin (School of Elec. and Information Eng., Univ. of Sydney, Maze Crescent, Bldg. J03, Sydney, NSW 2006, Australia, craig.jin@sydney.edu.au), Nicolas Epain (Inst. of Res. and Technol., B<>Com, Cesson-Sévigné, Rennes, France), and Mengyao Zhu (Commun. and Information Eng., Shanghai Univ., Shanghai, China)

A psychoacoustic listening test is conducted to explore the influence of varying degrees of realism on binaural spatial-audio music perception. The specific factors that are varied in combination are: (1) whether or not headtracking is used; (2) whether or not individualized Head-Related Impulse Response filters are used; (3) the number of sound-track channels: mono, stereo, and 5.2 surround; and (4) whether or not reverberation is used. Experiments are repeated a number of times with listeners requested to make judgement comparisons regarding: externalization, quality of the frontal image, timbre, and preference. Open headphones (AKG 1000) are used so that listeners can compare the various binaural spatial-audio rendering conditions with standard non-binaural headphone presentation and also loudspeaker presentation. The results indicate the impact of varying degrees of realism on various aspects of binaural spatial-audio music perception. 


\title{
Session 4aSA
}

\section{Structural Acoustics and Vibration and Noise: Building Vibration Analysis, Measurement, and Mitigation}

\author{
James E. Phillips, Cochair \\ Wilson, Ihrig \& Associates, Inc., 6001 Shellmound St., Suite 400, Emeryville, CA 94608 \\ Hasson M. Tavossi, Cochair \\ Physics, Astronomy, and Geosciences, Valdosta State University, 1500 N. Patterson St., Spring Valley Cir, Valdosta, \\ GA 31698-0055 \\ Yoshihiro Hirao, Cochair \\ Noise \& Vibration Division, Kobayasi Institute of Physical Research, 3-20-41, Higashi Motomachi, Kokubunji 185-0022, \\ Japan \\ Chair's Introduction-7:55
}

\section{Invited Papers}

4aSA1. INCE/J Vibration Measurement Manual for Buildings-Part 1: A commentary on the manual for vibration measurement. Shigenori Yokoshima (Kanagawa Environ. Res. Ctr., 1-3-39, Shinomiya, Hiratsuka, Kanagawa 2540014, Japan, yokoshima@kerc.pref.kanagawa.jp)

Vibration Regulation Law in Japan has regulated vertical ground vibration caused by road traffic, operation of a factory machine, and construction work since 1976. However, inhabitants perceive vibrations not on the ground but in buildings which are residential spaces. For the purpose of assessing building vibrations, technical committee on Environmental Vibration Evaluation in the Institute of Noise Control Engineering (INCE/J) organized Vibration Manual for Buildings. The manual is desired to provide technical materials contributing to effective measures for the vibrations to be solved. To achieve the aim, the manual concerns the method of measurement of the following vibration characteristics: propagation of ground vibrations, transmission of house vibrations, and vibration exposures to inhabitants according to vibration sources. In addition, the accumulation of detailed measurement data contributing to the revision of vibration index in the future is an important issue. Specifically, items of the manual are the following: scope, measurement (measurement unit, instrument, location direction, time zone, and notices) and calculation of measurements. For example, measurement unit is based on one-third octave band analysis. The vibration shall be measured at the location in building where the inhabitants perceive highest magnitude of the vibration.

\section{8:20}

4aSA2. INCE/J Vibration Measurement Manual for Buildings-Part 2: On the assessment based on the vibration. Yasunao Matsumoto (Dept. of Civil and Environ. Eng., Saitama Univ., 255 Shimo-Okubo, Sakura, Saitama 338-8570, Japan, ymatsu@mail.saitama-u.ac.jp)

This paper discusses the assessment of building vibration in terms of human responses based on vibration measurements specified by the Vibration Measurement Manual for Buildings developed by the Technical Subcommittee on Environmental Vibration Evaluation of the Institute of Noise Control Engineering of Japan. As stated in some of the current international and national standards, building vibration could cause adverse comments from occupants when the vibration magnitude is in excess of human perception only slightly. For the assessment of building vibration based on the measurement manual, therefore, a range of human vibration perception threshold was derived from the previous studies that determined the perception thresholds in experiment involving human subjects. The vibration perception threshold range implies a range of vibration magnitude for different frequencies that may correspond to lowest vibration magnitudes people with different sensitivities to vibration can detect. The vibration magnitude within or in excess of the perception range may be assessed as a magnitude that can be perceived by occupant at different possibilities. The assessment with the vibration perception threshold range does not necessarily show if the building vibration to be assessed has a magnitude high enough to cause adverse comment. 
4aSA3. INCE/J Vibration Measurement Manual for Buildings—Part 3: Measurement example of building vibrations. Yoshihiro Hirao (Noise \& Vib. Div., Kobayasi Inst. of Physical Res., 3-20-41, Higashi Motomachi, Kokubunji, Tokyo 185-0022, Japan, hirao@ kobayasi-riken.or.jp)

The environmental impact on humans in buildings is caused by ground-borne vibration from rail transit systems, road traffic, construction sites, and industrial plants. To estimate the effect of vibration on humans, it is necessary to measure the tri-axial vibrations in the vertical and horizontal directions in buildings specified in ISO 2631-2:2003 or "Vibration Measurement Manual for Buildings" publicized by the Technical Subcommittees on Environmental Vibration Evaluation of The Institute of Noise Control Engineering of Japan. In this measurement example of building vibrations, vibration measurement was carried out for eight hours in a three-story, wooden detached house adjacent to a construction site. The residents perceived discomfort or annoyance from the vibration caused by the demolition work at the construction site. Five vibration measurement devices recorded simultaneously the vibration accelerations on the floor of each story, the concrete substructure and the asphalt pavement surface near the substructure. The 1/3 octave band vibration accelerations of each floor were estimated by the vibration perception threshold range of the vibration measurement manual. The vibration amplifications caused by building structural resonance was evaluated as ratios and level differences in the vibration accelerations measured at the ground near the substructure and floors in the houses.

\section{9:00}

4aSA4. INCE/J Vibration Measurement Manual for Buildings_Part 4: Measurement of ground propagation characteristic and long-term measurement in building for road traffic vibration. Ryosuke Kazama, Kodai Yamashita, Hiromitsu Umayahara, and Toshiyuki Hasumi (RION Co., Ltd., 3-20-41 Higashimotomachi, Kokubunji, Tokyo 185-8533, Japan, r-kazama@rion.co.jp)

Vibration Measurement Manual for Buildings, issued by Technical Subcommittees on Environmental Vibration Evaluation of The Institute of Noise Control Engineering of Japan, describes how to measure and evaluate vibration. According to the manual, vibration level and $1 / 3$ octave band vibration acceleration levels, which are calculated from tri-axial acceleration waveforms, are used as measurement values. For the values of each axis, we extracted the 10 maximum values of each band level around the time when vibration levels reached maximum by vibration occurrences. The final measurement result is arithmetic average of the maximum 10 data. Some measuring instruments including vibration level meters, data recorders and waveform analysis software are necessary to obtain the result. To show how to measure vibration and how to operate measuring instruments based on the manual, we report two measurement examples about road traffic vibration which is easily perceptible. First, we measured a ground propagation characteristic at multi points along straight line from vibration source. Second, we conducted a long-term vibration measurement in a building, assuming low occurrence of vibration which caused complaints. As a result, we found that the level of certain frequency bands in vertical direction reached the vibration perception threshold range.

4aSA5. INCE/J Vibration Measurement Manual for Buildings-Part 5: Mounting method for vibration pick-up on groundborne vibration measurements. Kozo Yamamoto (Tech. Res. Inst., Toyo Construction, 1033-1, ukeryo, Miho-mura, Inashiki-gun, Ibaraki 300-0424, Japan, yamamoto-kozo@toyo-const.co.jp), Chikara Oishi (Architectural Environ. Res. and Eng. Inc., Yokohama, Kanagawa, Japan), and Yoshihiro Hirao (Noise and Vib. Lab., Kobayasi Inst. of Physical Res., Kokubunji, Tokyo, Japan)

In order to avoid the negative effect of contact resonance caused by the vibration system consisting of the vibration damping spring (ground surface) and the mass (vibration pick-up) in ground-borne vibration measurements, the vibration pick-up is put on the hard ground surface. However, when it must measure on the soft ground, after removing dry soil and grass, it is necessary to fully tread on the ground surface. In addition, the mounting method using the vibration pick-up stand with quad-peg is recommended in "The Vibration Measurement Manual for Buildings" proposed by the Technical Subcommittee of Environmental Vibration Evaluation of the Institute of Noise Control Engineering of Japan. We verified that new single-peg type vibration pick-up stand was a practical and useful tool via comparison with quad-peg type vibration pick-up stand. Moreover, we verified that the suitable hardness treading on the ground surface was acquired from the support strength of ground surface.

\section{9:40}

4aSA6. Vertical sensory evaluation on residential floor by traffic vibration. Toru Matsuda (Daiwa House Industry, 6-6-2, Sakyo, Nara, Nara 631-0801, Japan, toru.matsuda@daiwahouse.jp), Katsuo Inoue, and Ryuta Tomita (Nihon Univ., Chiyoda, Tokyo, Japan)

If vibration failures have occurred in building caused by traffic, in Japan, it is often evaluated by "Guidelines for the evaluation of habitability to building vibration." This evaluation method has to be collated acceleration maximum value of $1 / 3$ octave band analysis on the evaluation curve, to evaluate the response acceleration of adjacent frequency bands to reference curve. This performance evaluation curves are presented from experimental results based on sine vibration, under real environment, random vibration containing multiple frequency components. Therefore, it is necessary to consider the influence of the frequency components other than determination frequency, because it is considered to affect the vibration sense. Therefore, in this paper, as a basic study, we examined the good evaluation physical quantity of corresponding to vibration sense from relationship of the vibration sense and vibration response physical quantity for traffic vibration of railway and road. As a result, in the range of more than feeling the vibration, in the case of the narrowband vibration response, which is equal to broadband vibration response, the broadband vibration response has been suggested the increase in perception and discomfort. That is, the frequency characteristics other than the determination frequency have been suggested as one of the factors affecting vibration sense.

10:00-10:15 Break 
4aSA7. Spectral analysis of absorption characteristics of particulate dampers to mitigate building vibration. Hasson M. Tavossi (Phys., Astronomy, and GeoSci., Valdosta State Univ., 1500 N. Patterson St., Spring Valley Cir, Valdosta, GA 31698-0055, htavossi@ valdosta.edu)

Particulate medium can act as efficient damper for building internal and external vibrations. Mechanical properties of the particulate material, their shape and size distributions determine the vibration absorption characteristics of these dampers. It has been shown that an unconsolidated particulate damper act as a bandpass filter for vibration frequencies of mechanical vibrations transmitted through them. The particulate dampers can also have frequency bandgap structure, where transmitted mechanical vibration of a particular frequency is strongly attenuated by absorption and scattering. The samples of non-consolidated uniform fused-silica spherical particulates are subjected to mechanical vibrations of different frequencies and intensities to determine their absorption characteristics as a function of frequency, intensity, and direction of propagation. The effects of a particulate diameter and layer thickness on absorption characteristics are determined experimentally, at low and at high frequency ranges encountered in building vibration. The goal of this investigation is to find the required absorption characteristics for particulate damper for applications to mitigate building vibration.

\section{0:35}

4aSA8. Measurements of a wave barrier trench for isolation of rail vibration. David W. Dong and John LoVerde (Veneklasen Assoc., 1711 16th St., Santa Monica, CA 90404, wdong@veneklasen.com)

Where buildings are adjacent to railroad tracks, one option for reducing levels of ground vibration is to dig a trench between the tracks and the project to act as a wave barrier. The application of this method has been rare within the United States, and consequently, practical experience is very limited. On a recent project of the authors, a wave barrier trench was constructed [Dong and LoVerde, JASA 139, 2159 (2016)]. Measurements have been performed is various conditions both before and after construction of the trench. The vibration insertion loss was measured and compared to theory, and recommendations based on the experience are provided. This paper extends the analysis with additional information and data.

\section{0:55}

4aSA9. Isolation of residential buildings from ground vibration from railroad. John LoVerde and David W. Dong (Veneklasen Assoc., 1711 16th St., Santa Monica, CA 90404, jloverde@veneklasen.com)

For residential developments near railroad tracks, ground vibration due to train movement is often a concern. Although there are usually no code requirements for vibration levels, ground vibration levels are often compared to the criteria recommended by the FTA; these criteria are sometimes explicitly included as part of the Conditions of Approval or other governing documents required for the development of the site. These criteria are often exceeded when the development is in proximity to the tracks, but experience has provided limited practical applications for reducing the vibration. Some of the methods suggested include isolating the building on elastomeric bearings, digging a wave barrier trench, increasing the mass of the foundation, and stiffening the joist structure. However, these measures have been rarely employed, and little data are available on the objective effects of the mitigation, correlated with the benefit to occupants. Despite this, such methods are sometimes required on a project without analysis of efficacy or even an understanding of the anticipated result. The authors have compiled the results of recent measurements on several buildings that employed some these measures, and discuss the benefits of the mitigation measures and their application to building construction strategy.

\section{Contributed Papers}

\section{1:15}

4aSA10. Flanking sound transmission in connected panels of cross-laminated-timber at low frequencies. Blasius Buchegger, Heinz Ferk (Lab. for Bldg. Sci., Graz Univ. of Technol., Inffeldgasse 24, Graz 8010, Austria, buchegger@tugraz.at), and Martin Schanz (Inst. of Appl. Mech., Graz Univ. of Technol., Graz, Austria)

The sound transmission between adjacent rooms can be dominated by the structure-borne sound transmission in the flanking walls. A wall can be defined as a subsystem of the overall system based on two coupled rooms. These subsystems show local modes, if they are not connected to each other. In contrast, connected subsystems result in global modes showing a high response of both subsystems, if the comparable local modes have similar eigenfrequencies. The flanking sound transmission especially at low frequencies is reduced, if a generation of such global modes can be suppressed. As a consequence, the flanking sound transmission in buildings can be mitigated significantly using a proper design of the walls (geometry, material, etc.). The principles and the impact of this effect are studied in this paper using numerical models based on the finite element method. Selected constructions are implemented in real size 2D- and 3D-models of two adjacent rooms. Panels of cross-laminated-timber are used because the variable composition of the single layers of a panel allows an extensive variation of the vibration response of the single walls. The results confirm the applicability of the described effect for common practical situations.

\section{1:30}

4aSA11. Vibration isolation of large fan array air handling units in healthcare. Edward Logsdon (D. L. Adams Assoc., Inc., 1536 Ogden St., Denver, CO 80218, elogsdon@dlaa.com)

A new hospital facility constructed in Denver, CO, has all the mechanical equipment that is typically on the roof of the building located directly over all the Operating Rooms (ORs). Many of the ORs are used for procedures involving equipment found in new facilities like high powered microscopes for laser eye surgery or neurosurgery, ceiling mounted highdefinition video displays and robotic surgical systems using high resolution cameras. All of which are vibration sensitive. Also, in the future the client wanted flexibility in how the operating rooms are used as the equipment and procedures will change. Because "fan array" mechanical units, produce less vibration than traditional air handling units, they were selected by the design team. The air handling units varied in size from medium with six supply and six return fans to very large with 22 supply and 16 return fans. Because of their size and location in the building, the units were loaded into the facility in multiple modular sections that were then bolted together. This required the development of a vibration isolation solution that allowed for the easy assembly of the mechanical units on site. The rational for the location of the mechanical rooms above the operating rooms will be discussed along with the vibration criteria, analysis, and details of the isolation system. This includes vibration measurements of the final results. 


\title{
Session 4aSC
}

\section{Speech Communication and Education in Acoustics: Second Language Speech Learning and Education}

\author{
Tessa Bent, Cochair \\ Department of Speech and Hearing Sciences, Indiana University, 200 S. Jordan Ave., Bloomington, IN 47405 \\ Keiichi Tajima, Cochair \\ Research Laboratory of Electronics, Massachusetts Institute of Technology, 36-511, 77 Massachusetts Ave., Cambridge,
} MA 02139

Chair's Introduction-9:00

\section{Invited Papers}

9:05

4aSC1. Predicting and explaining second language speech through individual differences: Insights from testing and extending the Second Language Linguistic Perception model. Paola Escudero (The MARCS Inst., Western Sydney Univ., Locked Bag 1797, Penrith, NSW 2751, Australia, paola.escudero@westernsydney.edu.au)

In this talk, I will present five studies aimed at demonstrating that individual native production and perception abilities predict and explain success in L2 sound perception and word recognition. These studies tested and extended the theoretical and computational framework of the Second Language Linguistic Perception (L2LP) model was tested and extended. The model's main claim states that accurate predictions for L2 success should be based on individual learners' native production and perception as well as their responsiveness to rapid perception training. In Study 1, we show how differences in how listeners produce native vowel sounds are predictive of their discrimination accuracy when perceiving L2 vowel contrasts. The predictive power of individual native and L2 vowel perception on the learning of novel L2 words are reported in Studies 2 and 3. Study 4 shows that distributional training, based on the frequency distributions of difficult non-native contrasts, leads to short and long term improvement of difficult non-native contrasts and whether this improvement generalizes the contrasts produced in different contexts and by multiples speakers. Finally, Study 5 tests whether the perception training in Study 4 has short and long term impact on the L2 word learning. A discussion of the findings and their implications for the L2LP proposal and that of other models of L2 speech will be presented.

\section{9:25}

4aSC2. Perception of American English alveolar stops and flaps by Japanese learners of English: Does allophonic variation matter? Mafuyu Kitahara (Waseda Univ./Sophia Univ., 1-6-1 Nishiwaseda, Shinjuku, Tokyo 169-8050, Japan, kirahara@waseda.jp), Keiichi Tajima (Hosei Univ., Chiyoda, Tokyo, Japan), and Kiyoko Yoneyama (Daito Bunka Univ., Itabashi, Tokyo, Japan)

A lexical decision experiment was conducted with Japanese learners of English with relatively low English proficiency to investigate whether second-language (L2) learners utilize allophonic variation when recognizing words in L2. The stimuli consisted of 36 isolated bisyllabic words containing word-medial /t/, half of which were flap-favored words (e.g., better, city) and the other half were [t]-favored words (e.g., faster, custom). All stimuli were recorded with two surface forms: /t/ as a flap (e.g., better with a flap) or as [t] (e.g., better with [t]). The stimuli were counterbalanced in the lists using a Latin Square design, so that participants only heard one of the two surface forms. The accuracy data indicated that flap-favored words pronounced with a flap (e.g, city with a flap) were recognized significantly less accurately than [t]-favored words with a flap (e.g., faster with a flap) and [t]-favored words with [t] (e.g., faster with [t]). These results suggest that Japanese learners prefer canonical forms over allophonic variants, a result which is inconsistent with Pitt et al.'s (2011) study with native American English listeners. The results' implications for the role of allophonic variation in L2 learning will be discussed. [Work supported by JSPS KAKENHI 26370508, 15K02492, and 16K02646.]

\section{9:45}

4aSC3. Goodness evaluation by L1 Spanish speakers of various allophones, made by human and by vocal tract models, of the Spanish phonemes /// and /r/. Takuya Kimura (Dept. of Spanish Lang. and Lit., Seisen Univ., 3-16-21 Higashi Gotanda, Shinagawaku, Tokyo 1418642, Japan, kimura@seisen-u.ac.jp) and Takayuki Arai (Dept. of Information and Commun. Sci., Sophia Univ., Tokyo, Japan)

The present study reports the results of two perceptive experiments, in which L1 speakers of Peninsular Spanish were asked to listen to a series of sound stimuli and to evaluate the goodness of each stimulus as a realization of Spanish consonant /l/ or /r/. The stimuli were made of natural human voice in the first experiment, and with a set of vocal tract models in the second experiment. The results were similar for the two experiments. The lateral approximant stimuli were highly evaluated as allophones of /1/. Some lateral 
approximant stimuli were longer than others, but the duration difference caused little effect on the evaluation. The flap stimuli obtained low scores as /1/. Conversely, for the phoneme /r/, only the flap stimuli acquired high evaluations, while all the lateral approximant stimuli obtained extremely low scores. The retroflex approximant stimuli, similar to English /r/, obtained low evaluations for both /1/ and /r/ in the first experiment using a human voice, while they were evaluated slightly higher as / $/$ than as /r/ in the second experiment using the vocal tract models. These results are expected to provide L2 Spanish learners with useful knowledge in their pronunciation learning.

\section{0:05}

4aSC4. Interactions between perception and production during speech sound learning. Melissa M. Baese-Berk (Dept. of Linguist, Univ. of Oregon, 1290 University of Oregon, Eugene, OR 97403, mbaesebe@uoregon.edu)

It is frequently assumed that perception and production develop in tandem during non-native speech sound acquisition. However, several previous studies have suggested that simply producing tokens during training can disrupt perceptual learning (e.g., Leach \& Samuel, 2009). Here, I present several experiments examining some sources of such a disruption. Further, I ask how learning in production progresses when listeners fail to learn in perception. In the first set of experiments, I examine performance on a discrimination task after training in perception alone, or training perception + production, in which listeners repeat tokens on every trial. In follow up experiments, rather than repeating the training tokens, listeners read an unrelated letter aloud between each perceptual training trial or respond to the unrelated letter with a button press, rather than reading the letter aloud. In a second set of experiments I examine how production learning progresses when learners produce tokens during training. The critical factor examined is whether listeners are able to differentiate between tokens in perception and whether this ability correlates with learning in production. Taken together, the results of these studies suggest that the relationship between perception and production is complex, especially during learning.

\section{0:25-10:40 Break}

10:40

4aSC5. Asymmetrical interpretation of English liquid consonants by Japanese speakers. Mariko Kondo (School of Int. Liberal Studies, Waseda Univ., SILS, 1-6-1, Nishi-Waseda, Shinjuku-ku, Tokyo 169-8050, Japan, mkondo@waseda.jp)

Japanese speakers have difficulty in differentiating English /1/ and /r/ because they are not contrastive. Actually, variations of both /1/ and $/ \mathrm{r} /$ occur in Japanese speech. The most common realization of Japanese $/ \mathrm{r} /$ is alveolar tap $[\mathrm{r}]$ and all variants of both $/ \mathrm{l} /$ and $/ \mathrm{r} /$ are considered as allophones of /r/. Previously, it was found that Japanese speakers have more problems with /l/ than with /r/ in their English production. So, this study investigates why these differences occur. Analysis of Japanese speakers' mimicry speech of (a) American English and (b) English accented Japanese suggested that Japanese speakers are aware of acoustic and articulatory features of English approximant [I]. Japanese speakers overused approximant $[\mathrm{I}]$ and r-colored vowels in their mimicries of both (a) and (b). Further articulatory analysis of Japanese and English consonants showed that the English approximant [I] is quite distinct from Japanese consonants, all of which lack lip rounding. The results of these studies suggest that Japanese speakers may not be able to recognize English /1/ and / r/ as separate phonemes, but that they can hear the approximant $[\mathrm{I}]$ as it forms a new sound category, whereas they interpret $/ \mathrm{l} / \mathrm{as} / \mathrm{r} /$.

\section{1:00}

4aSC6. Consolidation during sleep stabilizes non-native speech sound learning. Emily Myers (Speech, Lang., and Hearing Sci., Univ. of Connecticut, 850 Bolton Rd., Storrs Mansfield, CT 06269, emilybmyers@gmail.com)

Learning to perceive non-native speech sound categories is a challenge for many adult listeners. Even after successful learning, individuals vary in the degree to which they can successfully retain and consolidate learned perceptual categories in memory. In this talk, I describe a recent line of work from our lab showing that sleep plays a greater role in perceptual learning of non-native speech sounds than previously supposed. Specifically, we find that consolidation processes during overnight sleep protect learned phonetic information from interference from similar sounds from one's native language. I will discuss the potential sources of native language interference by reviewing a series of studies that test the low-level acoustic and abstract phonological contributions to interference. Finally, overnight gains in performance can be specifically linked to the duration and quality of sleep, suggesting a key role for sleep, per se, in the retention of non-native speech categories over multiple sessions. Taken together, this line of research suggests that individual variability in non-native speech sound learning may in part be linked to individual differences in sleep behavior and differences in the degree of presleep exposure to competing native-language phonetic contrasts.

\section{1:20}

4aSC7. Neurobiological constraints on speech learning: Individual differences and plasticity. Bharath Chandrasekaran (Univ. of Texas at Austin, 1 University Station, Austin, TX 78712, bchandra@austin.utexas.edu)

A primary goal of auditory neuroscience is to understand how conspecific sounds are represented, learned, and mapped to constructs. Speech signals, conspecific to humans, are multidimensional, acoustically variable, and temporally ephemeral. A significant computational challenge in speech perception (and more broadly, audition) is categorization, that is, mapping continuous, multidimensional, and variable acoustic signals into discrete, behavioral equivalence classes. Despite the enormity of this computational challenge, native speech perception is rapid and automatic. In contrast, learning novel speech categories is effortful, and considered one of the most challenging categorization tasks for the mature brain. I will discuss three lines of ongoing research using multimodal neuroimaging, computational modeling, and behavioral training approaches: a) examine the multiple neural systems underlying successful L2 speech category learning in adulthood, b) assess sources of individual differences in L2 speech category learning, and c) design optimal, neurobiologically constrained training paradigms that reduce inter-individual differences in L2 speech category learning. These studies will provide insights into these fundamental questions: consistent with prior work on visual category learning, are multiple neural systems involved in speech categorization? What is the role of emerging expertise and individual differences in mediating neural and computational processes involved in speech category learning? 
4aSC8. Individual-based accent clustering of World Englishes using the Speech Accent Archive. Nobuaki Minematsu (The Univ. of Tokyo, 7-3-1, Hongo, Bunkyo-ku, Tokyo 113-8656, Japan, mine@gavo.t.u-tokyo.ac.jp)

In a language class of English, one specific type of pronunciation is often adopted as model pronunciation and learners try to imitate that pronunciation. Even though they acquire native-like pronunciation successfully, once they go out to the real world of spoken English, they surely encounter the pronunciation diversity of English and feel the necessity to survive it. As is well-known, English pronunciation is altered variously depending on the language background of speakers, called accents. This talk describes a technical attempt of automatic and individual-based clustering of these accents using the Speech Accent Archive. This technique may be used to realize a really individual-based map of World Englishes, which can be used to introduce learners to the real state of English. For clustering, the accent gap has to be quantitatively estimated between any two speakers of the archive, where non-linguistic factors such as age and gender have to be adequately cancelled in estimation. Pronunciation structure analysis is used for cancellation and experiments show that the estimated gaps have a better correlation to IPA-based manually defined gaps than phoneme-based gaps have to the IPA-based gaps.

\title{
Session 4aSPa
}

\section{Signal Processing in Acoustics: Exploitation of Doppler in Acoustic Signal Processing}

\author{
Paul Hursky, Cochair \\ HLS Research Inc., 12625 High Bluff Drive, Suite 211, San Diego, CA 92130 \\ Shinnosuke Hirata, Cochair \\ Department of Systems and Control Engineering, Tokyo Institute of Technology, 2-12-1 Ookayama, S5-17, Meguro 152-8550, \\ Japan
}

Chair's Introduction-7:55

Invited Papers

8:00

4aSPa1. Doppler spectral moments of multibeam echosounder data to infer the ascent velocity of methane bubbles emanating from natural seafloor seeps. Christian de Moustier (10dBx LLC, PO Box 81777, San Diego, CA 92138, cpm@ieee.org)

A multibeam echosounder operating at $500 \mathrm{kHz}$ was deployed on a remotely operated vehicle to detect methane bubble streams emanating from natural seafloor seeps in 1400 - $\mathrm{m}$ water depths. The sonar transmitted 10 -ms tone bursts every $100 \mathrm{~ms}$ over a $7^{\circ} \times 120^{\circ}$ beam, and formed 128 receive beams regularly spaced $0.94^{\circ}$ apart over the $120^{\circ}$ sector. The sonar head faced upward and signals backscattered from ascending bubbles were sampled at about $6 \mathrm{kHz}$ between $10 \mathrm{~m}$ and $50 \mathrm{~m}$ from the head. Doppler spectral moments were computed for each ping with the conventional data autocovariance method. For unimodal and symmetric Doppler spectra, the mean power is related to the backscattering strength of the sampled volume, the mean Doppler frequency yields the mean radial velocity of the bubbles, and the spectral width measures the mean velocity uncertainty with some indication of bubble oscillation. Mean ascent velocities of 0.2 $\mathrm{m} / \mathrm{s}$ matched those obtained from concurrent optical measurements. However, beams free of bubbles showed mean descent velocities of about $1.5 \mathrm{~cm} / \mathrm{s}$, most likely due to marine snow, which could bias mean ascent velocity estimates for bubbles at greater distance from the sonar because the corresponding Doppler spectra might no longer be unimodal.

\section{8:20}

4aSPa2. A personal history of Doppler sonar development at the Marine Physical Laboratory, SIO. Rob Pinkel (MPL, SIO, Scripps Inst. of Oceanogr., 0213, San Diego, CA 92093-0213, rpinkel@ucsd.edu)

In the early 1970s, the possibility of remotely measuring oceanic and atmospheric flows was becoming appreciated. By then, techniques for information extraction from sets of discrete targets were well advanced. Inferring fluid motion from the echo off a random cloud of independent scatterers was a different issue, as illustrated by the abject failure of contemporary matched-filter technology. Conceptual as well as hardware challenges were addressed over time, leading to the variety of Doppler sonars and sodars available commercially today. In this talk, I focus on systems developed at the Marine Physical Laboratory of Scripps over the past 42 years. Both pulse-to-pulse coherent and incoherent backscatter systems are reviewed with an emphasis on real-world problems encountered. Presently, under- 
exploited techniques based on sea-surface scattering or bi-static geometries are discussed. While many quality Doppler sonar manufacturers are now active, a large gap exists between what is technically possible and what is commercially available. The continuing vitality of university-based development groups that create scientifically significant (but not necessarily commercially profitable) systems is critical to advancing the state of the art.

\section{$8: 40$}

4aSPa3. Some effects of ocean surface roughness on high and low duty cycle, broadband waveforms. Paul C. Hines (Dept. of Elec. and Comput. Eng., Dalhousie Univ., PO Box 15000, Halifax, NS B3H 4R2, Canada, phines50@gmail.com), Stefan M. Murphy (Defence R\&D Canada, Dartmouth, NS, Canada), Douglas A. Abraham (CausaSci LLC, Ellicott City, MD), and Grant B. Deane (Scripps Inst. of Oceanogr., La Jolla, CA)

In this paper, data from TREX13 are used to compare the impact of the environment on High Duty Cycle (HDC) sonar and Pulsed Active Sonar (PAS). This paper presents the results of an examination of short-range single surface-reflection echoes, and longer-range target echoes from an air hose. Measurements showed that for an $18 \mathrm{~s} \mathrm{HDC}$ pulse, the mean (coherent) component of the specular arrival decreased with increasing rms roughness whereas $0.5 \mathrm{~s}$ PAS pulse echoes showed no correlation with roughness. The standard deviations of the mean echo levels are used to examine the incoherent (scattered) component of the specular arrivals. The incoherent component of the specular arrival increased with the product of the surface correlation length and the square of the rms roughness, for both HDC and PAS, with the PAS data having a $1 \mathrm{~dB}$ higher standard deviation. A normal mode propagation model and a rough surface scattering model used in conjunction with a simple model that accounts for motion-induced coherence loss from the matched filter, are used to interpret the results. [Work sponsored by ONR Code 32.]

\section{Contributed Paper}

9:00

4aSPa4. Testing the feasibility of a concurrent comparison of continuous and pulsed active sonar. Stefan M. Murphy (DRDC, 9 Grove St., Dartmouth, NS B2Y 3Z7, Canada, stefan.murphy@drdc-rddc.gc.ca), Matthew Coffin (Geospectrum Technologies, Inc., Dartmouth, NS, Canada), Paul C. Hines (Elec. and Comput. Eng., Dalhousie Univ., Dartmouth, NS, Canada), and Diana F. McCammon (McCammon Acoust. Consulting, Black Rock, NS, Canada)

The performance of continuous active sonar (CAS) was compared to conventional pulsed active sonar (PAS) during the TREX13 (Target and Reverberation Experiment 2013) sea trial. The approach was to execute a one-hour CAS run followed closely by a one-hour PAS run to limit the environmental variability between runs and allow a fair comparison. This approach was possible because the sonar source and receiver were fixed to the seabed. A different approach was required for a more recent sea trial, LCAS15 (Littoral Continuous Active Sonar 2015), where the source and receiver were towed from a ship. Ship motion increases variability in sonar performance, therefore a simultaneous comparison of CAS and PAS was desired so that any motion effects were the same for both waveforms. The approach of transmitting CAS and PAS concurrently in two separate frequency bands was taken. The risk with this approach is that potential differences in propagation in the two bands could bias the comparison. A run in which equivalent CAS waveforms were transmitted simultaneously in the two bands was therefore performed to establish a baseline for the performance difference between the bands. Doppler-corrected replicas were required in all of the correlation processing due to the high Doppler sensitivity of the long-duration, wideband CAS waveforms. Preliminary results from the LCAS15 sea trial will be presented.

\section{Invited Paper}

4aSPa5. Active control of passive acoustic fields: Passive synthetic aperture/Doppler beamforming with data from an autonomous underwater vehicle. Gerald L. D'Spain, Eric Terrill, C. David Chadwell, Jerome A. Smith, and Stephen D. Lynch (Marine Physical Lab, Scripps Inst. of Oceanogr., 291 Rosecrans St., San Diego, CA 92106, gdspain@ucsd.edu)

Autonomous underwater vehicles (AUVs) equipped with hull-mounted arrays can actively modify received acoustic fields to optimize extraction of information. Ocean acoustic data collected by an AUV-mounted two-dimensional hydrophone array, with overall dimension one-tenth wavelength at $200-500 \mathrm{~Hz}$, demonstrate aspects of this control through vehicle motion. Source localization is performed using Doppler shifts measured at a set of receiver velocities by both single elements and a physical array. Results show that a source in the presence of a 10-dB higher-level interferer having exactly the same frequency content, as measured by a stationary receiver, is properly localized and that white-noise-constrained adaptive beamforming applied to the physical aperture data in combination with Doppler beamforming provides greater spatial resolution than physical-aperture-alone beamforming and significantly lower sidelobes than single element Doppler beamforming. A new broadband beamformer that adjusts for variations in vehicle velocity on sample by sample basis is demonstrated with data collected during a high-acceleration maneuver. The importance of including the cost of energy expenditure in determining optimal vehicle motion is demonstrated through simulation, further illustrating how vehicle characteristics are an integral part of the signal/array processing structure. [Work sponsored by the Office of Naval Research.] 


\title{
Contributed Papers
}

9:35

4aSPa6. Posterior inference of two closely spaced wave vectors for inference regarding the state of an acoustic target. Abner $\mathrm{C}$. Barros (ECE Dept., Univ. of Massachusetts Dartmouth, Darmouth, MA), David Anchieta (Elec. and Comput., Universidade Federal do Pará, Belém, Brazil), and Paul J. Gendron (ECE Dept., Univ. of Massachusetts Dartmouth, 285 Old Westport Rd., North Dartmouth, MA 02747, pgendron@umassd.edu)

A bistatic sonar arrangement is employed to infer the depth, speed, and range of an oncoming submerged acoustic target from a continuous wave transmission. Surface and direct interacting paths associated with closely spaced angles and frequencies from the returned acoustic wave fronts are resolved. A Gibbs sampler is employed to construct the posterior joint density of all parameters taking full advantage of the analytic tractability of the amplitude and phase of the wave fronts while the ordered wave vectors, their angles and frequencies are sampled numerically by two-dimensional quantile function. The inferred density of target depth, range, and speed is accomplished by constructing a numerical inverse-transformation of the forward propagation model. Simulations are used to demonstrate the potential of sub-Rayleigh resolution accuracy at less than $-10 \mathrm{~dB}$ received SNR with an 9 element vertical array.

$$
\text { 9:50 }
$$

4aSPa7. Processing of aircraft signatures with significant Doppler, simultaneously observed on microphones and distributed underwater sensors. Paul Hursky (HLS Res. Inc., 12625 High Bluff Dr., Ste. 211, San Diego, CA 92130, paul.hursky@hlsresearch.com)

An experiment was performed with a source mounted on an aircraft with a microphone array rigidly mounted on a research vessel, and with sonobuoys and a vertical line array deployed in the ocean underneath. Programmed waveforms, including tones, linearly frequency modulated tones, and $\mathrm{m}$-sequences, were transmitted from the airplane. The Doppler in this configuration was substantial, due to the sound speed in air being $1 / 5$ that of water, with much of the acoustic path passing through the air. We will present results of matched filtering the transmitted waveforms, using various combinations of observed signals, including using replicas augmented by modeling, to compensate for the Doppler in order to enhance the coherent integration gain in this configuration.

\section{Session $4 \mathrm{aSPb}$}

\section{Signal Processing in Acoustics: High Resolution Imaging Sonars Including Real Aperture, Synthetic Aperture, and Tomographic Sonars I}

\author{
Brian G. Ferguson, Cochair \\ DSTO, PO Box 44, Pyrmont, NSW 2009, Australia \\ Timothy Marston, Cochair \\ APL-UW, 1013 NE 40th Street, Seattle, WA 98105
}

Chair's Introduction-10:20

\section{Invited Papers}

\section{$10: 25$}

4aSPb1. High resolution systems: The resolution argument. Yan Pailhas and Yvan Petillot (Heriot-Watt Univ., Riccardo Campus, School of EPS, Edinburgh EH14 4AS, United Kingdom, Y.Pailhas@hw.ac.uk)

Resolution comes from the Latin word resolvo, which literally means "untie a knot." The meaning evolved, in optics, toward the capability to distinguish two scattering points. For the MCM (Mine Counter Measure) problem, the introduction of synthetic aperture systems, and the resulting drastic increase in resolution, has been a game changer, as the useful information shifts from shadows to highlights. If SAS resolution has been well studied, new SAS reacquisition patterns have emerged, in particular circular acquisitions known as CSAS. In this talk, we will derive the theoretical resolution of such systems based on a PSF (point spread function) energy leakage interpretation rather than the traditional Rayleigh resolution criteria. We will compare the CSAS resolution capability with the newly developed MIMO paradigm. The statistical framework of MIMO systems greatly differs from the deterministic approach of synthetic aperture systems, and offers certain advantages in term of resolution. All these coherent systems approach the imaging problem without any priors, other than the presence of scatterers. Going back to the original meaning of resolvo however, we will show that, by introducing priors such as target shape, wideband sonars shift the analysis into the phase domain and are able to outperform imagery. 
4aSPb2. Moving beyond side-looking imaging with synthetic aperture sonar. James Prater and Jonathan King (NSWC PCD, 110 Vernon Ave., Panama City, FL 32407, jonathan.1.king1@navy.mil)

The desire to provide long-range high-resolution side-scanning sonar imagery necessitates the use of lower frequencies to overcome the relative high attenuation of high frequency sound waves and long apertures in order to achieve high resolution. This is typically accomplished through the application of synthetic apertures which collect wideband widebeam sonar data over time and post-process the data to produce high resolution imagery. While many of these systems were initially designed with high resolution long range imagery in mind, the wideband widebeam and temporal nature of the data provides the potential for signal processing and analysis to produce information about the imaged area beyond image intensity. This paper will discuss recent advances in SAS signal processing that exploit the wideband, widebeam, and temporal features of unbeamformed SAS data to extract additional information about the imaged area. Specifically, techniques that exploit aspect dependence (from widebeam data), frequency dependence (from wideband data), and temporal phenomena will be discussed.

\section{1:05}

4aSPb3. Effect of low complexity adaptive beamforming on seafloor backscatter statistics. Tor I. Lønmo (Dept. of Informatics, Univ. of Oslo, P.O.Box 111, Horten 3191, Norway, toribi@ifi.uio.no) and Anthony Lyons (Ctr. for Coastal and Ocean Mapping, Univ. of New Hampshire, Durham, NH)

In contrast with the common delay-and-sum (DAS) beamformer, adaptive beamformers can be used to increase the resolution of acoustic measurement systems without sacrificing sidelobe levels. The non-linear nature of adaptive beamforming methods may cause changes to measured scattered amplitude distributions. For example, large differences in speckle statistics have been observed in some cases when using the adaptive minimum variance distortionless response (MVDR) beamformer in medical ultrasound, unless preventative measures were implemented. Low complexity adaptive (LCA) beamforming techniques have shown similar, but smaller, differences. Recently, experiments have been conducted in the Oslofjord, Norway, to determine whether low-complexity adaptive (LCA) beamforming techniques can improve the performance of bathymetric echosounders. When using LCA with these types of sonar systems, it is important to understand any changes to the backscatter statistics, as these changes may affect, e.g., seafloor classification methods. In this talk we will present comparisons between backscatter statistics estimated using a weighted delay-and-sum beamformer and those estimated using a low complexity adaptive beamformer. We will discuss any differences seen, possible mechanisms causing these differences and potential remedies. [Research supported by The Norwegian Research Council and Kongsberg Maritime. The first author is employed in Kongsberg Maritime.]

\section{Contributed Papers}

\section{$11: 25$}

4aSPb4. Paradigm shift of underwater echo sounding technology IPrinciple and algorithm. Ikuo Matsuo (Dept. of Information Sci., Tohoku Gakuin Univ., Tenjinzawa 2-1-1, Izumi-ku, Sendai 9813193, Japan, matsuo@mail.tohoku-gakuin.ac.jp) and Toyoki Sasakura (FUSION INC., Tokyo, Japan)

Underwater echo sounding technology was developed 65 years ago and has been applied in various systems such as fishfinder, bathymetry, sonar, side scanning sonar, multi beam echo sounding system, and synthetic aperture sonar. Here, we proudly suggest a new concept that may change the history of underwater echo sounding technology, the "Paradigm Shift." In the conventional system, the transmitting signal is transmitted after receiving the target signal, e.g., a bottom echo. Therefore, the transmission intervals are always longer than the time of the round trip distance to the target divided by the underwater sound velocity. On the other hand, by adapting our new "Paradigm Shift" into the system, the transmission intervals can be decided without depending on a target and it will be possible to conduct a bathymetry survey that transmits 100 times per second. The system was tested and confirmed in the actual sea trial. This new concept, the "Paradigm-Shift-Echosounder" ( PSE ), utilizes the $7^{\text {th }}$ order and 127 kinds of Gold code sequence signals. Different code of Gold code signals will be transmitted in each transmission, and receiving signals will be identified by the correlation of the 127 kinds of Gold code sequences of different transmission signals.

\section{1:40}

4aSPb5. Paradigm shift of underwater echo sounding technology IIExperiment and actual sea trial. Toyoki Sasakura (FUSION INC., 1-1-1806, Daiba, Minatoku, Tokyo 1350091, Japan, sasakura@fusion-jp.biz) and Ikuo Matsuo (Dept. of Information Sci., Tohoku Gakuin Univ., Sendai, Japan)

The newly suggested "Paradigm-Shift-Echosounder" (PSE) for bathymetry survey was tested in a sea trial experiment using a prototype. Experiment was conducted at the depth of $30-80 \mathrm{~m}$ using $200 \mathrm{kHz}$ signals transmitted 100 times per second and succeeded to obtain continuous bottom echo data. We also conducted a side scanning sonar experiment using $88 \mathrm{kHz}$ signals, at a site with a depth more than $500 \mathrm{~m}$ and succeeded to obtain the echo of a $2 \mathrm{~m} \times 5 \mathrm{~m}$ artificial reef moored at the depth of $50 \mathrm{~m}$. Correlation processing was done and we managed to reconstruct a high-resolution echogram of the artificial reef. The prototype used in the trial experiments utilizes the $7^{\text {th }}$ order Gold code sequence. Four cycles of carrier signals as 1 bit, the transmission signals are all phase-modulated. The pulse length of transmission signal is $2.54 \mathrm{~ms}$ for $200 \mathrm{kHz}$. As there are $127 \mathrm{Gold}$ codes, transmission signals of the same code are repeated every $1.28 \mathrm{sec}-$ onds. The receiving signals are then processed by the correlator, with a shift register length of 4064 stages by 8-times sampling frequency for carrier frequency. There are 127 kinds of replicas of correlator, and the numbers of "Exclusive OR + Summation" circuits are also 127. 


\title{
Session 4aUW
}

\section{Underwater Acoustics, Signal Processing in Acoustics, and Acoustical Oceanography: Harbor Acoustics I}

\author{
Peter J. Stein, Cochair \\ Scientific Solutions, Inc., 99 Perimeter Road, Nashua, NH 03063 \\ David L. Bradley, Cochair \\ Penn State University, PO Box 30, State College, PA 16870
}

Chair's Introduction-8:15

\section{Invited Papers}

$8: 25$

4aUW1. Physical oceanographic modeling of the inner Continental Shelf. Alan F. Blumberg (Stevens Inst. of Technol., Stevens Inst. of Technol., 1 Castle Point, Hoboken, NJ 07030, ablumberg@stevens.edu)

The nowcast and forecast of the marine environment in the shallow waters $(0-20 \mathrm{~m}$ depth) adjacent to the coast, the inner shelf, is important for the development of acoustic surveillance systems. These waters are among the most challenging marine environments in the world because they are subject to the combined geometrical constraints of irregular coastlines and highly variable bathymetry, and are forced both by a complex array of tidal, wind, and buoyancy forces on a broad range of space/time scales. Their proximity to the deeper waters flowing offshore introduces another array of complex sets of forcing. The resulting circulation patterns include both persistent and time-variable fronts, intense currents with strong spatial (offshore and/or vertical) dependence, waves, internally generated variability, large horizontal water mass contrasts, strong vertical stratification, and regions of intense turbulent mixing in both surface and bottom boundary layers. Frequently, the interplay between these multiple forcing mechanisms, geographic detail, stratification, and nonlinear dynamics, is significant, and this demands that a physically sophisticated coastal ocean model be capable of representing a comprehensive suite of dynamical processes. Drawing on the operational shallow water New York Harbor Observing and Prediction System (NYHOPS), we illustrate, by example, the breadth of dynamical processes that influence near coastal ocean circulation.

\section{$8: 45$}

4aUW2. Three-dimensional acoustic modeling of a complex harbor environment. Michael B. Porter (Heat, Light, and Sound Res., 3366 N. Torrey Pines Ct., Ste. 310, La Jolla, CA 92037, mikeporter@hlsresearch.com), Laurel J. Henderson (Heat, Light, and Sound Res., San Diego, CA), Timothy F. Duda, and Arthur E. Newhall (Woods Hole Oceanographic Inst., Woods Hole, MA)

Typically, we model sound propagation in the ocean viewing it as an "open" environment where the sound propagates outwards from the source. Of course, targets are reflectors, and other features such as islands and seamounts have been of interest as false targets. However, the standard acoustic models based on rays, normal modes, spectral integrals, and parabolic equations generally consider outgoing energy confined to a single bearing line. In contrast, constrained or partially closed environments such as harbors introduce special issues. Obviously, the sound can be reflected back by seawalls and ship hulls, to mention just a couple of features of harbors environments. Considering coastal environments more broadly, sound reflections also may be caused by wharves, docks, piers, boat landings, quays, floats, groins, and jetties as well as natural features such as submarine canyons. In short, we have sound refracted in the latitude/ longitude plane and reflected by a variety of features. In terms of computational ocean acoustics there are few options for modeling such problems. We discuss a comprehensive 3D beam tracing approach implemented in a model called BELLHOP3D that can handle 3D effects including complete reversal of the sound. It is also capable of handling narrowband waveforms (tonals) as well as full broadband timeseries. We will demonstrate and test the model using an example location that is expected to become the site of a future sea test.

\section{9:05}

4aUW3. Eigenray complexity in harbor environments. Ruth E. Keenan (Appl. Res. Laboratory/UT, 10000 Burnet Rd., Austin, TX 78758, rkeenan@arlut.utexas.edu)

Acoustic eigenray complexity patterns will be demonstrated in a simulated harbor environment. Using bistatic surface and bottom scattering strength models, reverberation levels will be estimated for the same environmental parameterization. Eigenrays are the basis of any acoustic simulation, especially an active acoustics simulation, that requires high fidelity arrival time and arrival angle information. The sensitivity of cross harbor acoustic links, ranges 1 to $5 \mathrm{~km}$, will be examined for their response to fresh water intrusions (as evidenced by sharp temperature and salinity changes due to river outflow and rain events) as well as the diurnal surface heating and wind effects. The optimal acoustic frequency will be examined for its' sensitivity to the temperature and salinity changes which can significantly influence the volume attenuation. Harbors are typically very shallow water environments and the effect of the tidal cycle on the propagation will be examined as well. The spatial bathymetry and sediment composition variability over the acoustic links will also be examined. 
4aUW4. Underwater sound propagation and scattering in Ports and Harbors. Mohsen Badiey (College of Earth, Ocean, and Environment, Univ. of Delaware, 261 S. College Ave., Robinson Hall, Newark, DE 19716, badiey@udel.edu)

Ports and Harbors are crucial life lines in our economy and commerce. To ensure safe and efficient utilization of these important facilities, a thorough understanding of underwater sound propagation and scattering in very shallow water regions is critical. These regions however, present a challenging waveguide for underwater acoustics. Weather the goal is active acoustics (such as underwater communication) or passive acoustics (such as source detection and localization), detailed understanding of channel response function is necessary in order to develop and apply proper security measures. Several studies in recent years have addressed interesting results in acoustic signal propagation and scattering. These include effects of sound speed profile in water column, sea surface dynamic behavior, and seafloor attenuation. Signal coherence, and spatial and temporal variabilities due to environmental parameters have all been examined and to some extent modeled. Deterministic features due to interaction of broadband acoustic signals with the dynamic environment are shown. These results have been incorporated in development of robust underwater communications that can also be utilized for Harbor security implementation. In this paper, we present results of several recent experiments and modeling using Ray and Parabolic Equation approaches to understand this problem. [Work supported by ONR 322 OA.]

\section{9:45}

4aUW5. Building a practical model for acoustic harbor surveillance. David R. Dall'Osto, Eric I. Thorsos, and D. J. Tang (Acoust., Appl. Phys. Lab. at Univ. of Washington, 1013 N 40th St., Seattle, WA 98105, dallosto@apl.washington.edu)

The ability to simulate acoustic field in very shallow and confined environments is essential for both design and for successful operation of harbor protection sonar systems. In order to predict the acoustic field, which includes scattering from targets and the environment, a model has been developed to simulate active sonar operations. This paper uses this model to investigate the effects of environmental features common to most harbors, including sloped bathymetry from dredged areas, a variable sound-speed profile, and the effect of tides. These features are discussed in terms of their effect on detection of targets of interest, and the model is used to predict the best remedy for the adverse features. Guidelines for adequate planning of a harbor security sonar system are provided in response to specific harbor features. These include what sonar signals, beam-patterns, and multi-static deployment geometry will provide optimal detection.

\section{Contributed Paper}

\section{0:05}

4aUW6. 3D modeling of low-to-mid-frequency acoustic propagation through an estuarine salt wedge. D. Benjamin Reeder (Dept. of Oceanogr., Naval Postgrad. School, 833 Dyer Rd., Bldg. 232, SP-311B, Monterey, CA 93943, dbreeder@nps.edu) and Ying-Tsong Lin (Appl. Ocean Phys. \& Eng., Woods Hole Oceanographic Inst., Woods Hole, MA)

An estuary is an example of a constrained environment which often hosts a salt wedge, the stratification of which is a function of the tide's range and speed of advance, river discharge volumetric flow rate, and river mouth morphology. A field experiment was carried out in the Columbia River to test the hypothesis that the estuarine salt wedge is acoustically observable in terms of low-to-mid-frequency acoustic propagation. Linear frequencymodulated (LFM) acoustic signals in the $500-2000 \mathrm{~Hz}$ band were collected during the advance and retreat of the salt wedge. Data-model comparisons demonstrate the degree to which acoustic propagation in this constrained environment is controlled by horizontal refraction and out-of-plan scattering by bedforms along the acoustic transect.

\section{0:20-10:35 Break}

\section{Invited Papers}

\section{0:35}

4aUW7. Bioacoustics of fishes (and invertebrates) in harbors. Arthur N. Popper (Univ. of Maryland, Biology/Psych. Bldg., College Park, MD 20742, apopper@umd.edu)

Harbors can be noisy places. While some sounds are natural, there is often a cacophony of transient and continuous man-made sound from boating, shipping, construction, bridge traffic, near-by roadways, tunnels, etc. The question is whether these sounds potentially interfere with the behavior of resident and transient fish and invertebrate populations. The greatest effect may result from acoustic masking which would shorten the distances over which animals can communicate and detect sounds of biological importance such as from potential predators and prey. In order to understand potential effects of man-made sounds on harbor animals, much needs to be known about such sounds. However, the nature and extent of man-made sound has rarely been measured in harbors. Even when studied, focus has often been either on frequencies outside of the hearing range of the fishes and invertebrates and/or done for very short time periods so that a real sample of the daily and seasonal cycle of sounds is not examined. Finally, studies are usually limited to sound pressure, whereas most fishes and invertebrate primarily detect particle motion. This paper will discuss the man-made sounds of harbors and relate these to potential effects on the aquatic inhabitants. 
4aUW8. Marine mammal hearing and the effects of noise. Brandon L. Southall (SEA, Inc., 9099 Soquel Dr., Ste. 8, Aptos, CA 95003, Brandon.Southall@sea-inc.net)

Marine mammals use sound for critical life functions through passive listening and, in some species, active sonar. Major recent advances have been made in understanding what sounds they make, how they hear, and how noise affects their hearing, behavior, and physiology. While data are lacking for whole groups, notably baleen whales, direct hearing measurements are available for most taxa. Some species, such as odontocete cetaceans, hear extremely broad frequency ranges, well into ultrasonic biosonar frequencies. Pinnipeds have more limited frequency ranges but are adapted for aerial and underwater hearing. Indirect data suggest whales use and are more sensitive to low frequencies. For most species, quite high received sound levels are required to temporarily or permanently affect hearing. Direct experimental measurements of behavioral responses have revealed strong species and context dependencies, with factors such as proximity, novelty, and spatial relationships influencing response probability as well as relative loudness. For active surveillance sonar systems operating in the 30-200 kHz range, whales and otariid pinnipeds are of much less concern in terms of audibility and potential behavioral disturbance than phocid pinniped and odontocete cetaceans, notably porpoises. Hearing loss or other injuries from such systems are quite unlikely in almost all practical scenarios.

\section{1:15}

4aUW9. Characteristics of surface scattering of very high frequency acoustic signals. James Preisig (JPAnalytics LLC, 638 Brick Kiln Rd., Falmouth, MA 02540, jpreisig@jpanalytics.com) and Grant Deane (Scripps Inst. of Oceanogr., La Jolla, CA)

Many applications of acoustics in harbors are characterized by relatively shallow sources and receivers and a sound speed profile that does not strongly refract signals away from the surface. Therefore, surface scattering is an important factor in determining the characteristics of propagating signals in harbor environments. In addition, applications often involve relatively short propagation distances so very high frequency (VHF) acoustic signals, defined here to be signals above $100 \mathrm{kHz}$ in frequency, can be important components of acoustic systems in harbor environments. These VHF signals have very short wavelengths (approximately $2.7 \mathrm{~mm}$ at a frequency of 550 $\mathrm{kHz})$ so surface waves as small as capillary waves can have a significant impact on the surface scattered signals. Thus, even in light wind and wave conditions, the surface scattering of VHF can be highly dynamic. A test of the surface scattering of acoustic signals between $350 \mathrm{kHz}$ and $750 \mathrm{kHz}$ by wind-driven waves was conducted in a wave tank with the received signals being recorded at a vertical line array. This talk will present the analysis of the characteristics of the surface scattered signals including their spatial structure and Doppler spreading and the periodicity of their temporal fluctuations.

\section{1:35}

4aUW10. Acoustic monitoring of the bottom characteristics of a harbor. Christian de Moustier (10dBx LLC, PO Box 81777, San Diego, CA92138, cpm@ieee.org)

Acoustic monitoring of the underwater environment typically found in a harbor requires knowledge of the spatial and temporal variability of the noise, the water properties, the currents, and the bottom characteristics. For safety of navigation, repeat bottom surveys must be performed at regular intervals to monitor changes in water depth along navigation channels due to accumulation or depletion of sediments, and to detect potential hazards on or near the bottom. Imaging side-looking sonars, particularly those with synthetic aperture capabilities, are most effective for hazard detection but their performance in waters 5-10 $\mathrm{m}$ deep can be hampered by multipath and by changes in water properties at the scale of the scene being imaged. Their inherent spatial filtering characteristics make multibeam echosounders effective tools for synchronous bathymetry and acoustic backscatter imagery of the water column and the bottom. In harbor surveys, such echosounders achieve decimetric spatial resolution by operating at several hundred kilohertz, but their measurements are sensitive to spatial and temporal changes in water properties at the scale of the survey swath, potentially reducing lateral coverage. The corresponding variations in acoustic absorption affect acoustic backscatter imagery and yield increased uncertainty in the outcome of a repeat harbor survey. 


\title{
Session 4pAAa
}

\section{Architectural Acoustics: Assorted Topics in Architectural Acoustics II}

\author{
Niels W. Adelman-Larsen, Cochair \\ Flex Acoustics, Diplomvej 377, Kgs. Lyngby 2800, Denmark \\ Reiji Tomiku, Cochair \\ Department of Architecture, Oita University, Dannoharu 700, Oita 8701151, Japan
}

\section{Contributed Papers}

\section{1:00}

4pAAa1. Acoustics for amplified music and a new, variable acoustics technology. Niels W. Adelman-Larsen (Flex Acoust., Diplomvej 377, Kgs. Lyngby 2800, Denmark, nwl@flexac.com)

Surveys among professional musicians and sound engineers reveal that a long reverberation time at low frequencies in halls during concerts of reinforced music such as pop and rock is a common cause for an unacceptable sounding event. Mid- and high-frequency sound is seldom a reason for lack of clarity and definition due to a 6 times higher absorption by audience compared to low frequencies, and a higher directivity of speakers at these frequencies. Lower frequency sounds are, within the genre of popular music, rhythmically very active and loud, and a long reverberation leads to a situation where the various notes and sounds including vocals cannot be clearly distinguished. This reverberant bass sound rumble often partially masks even the direct higher pitched sounds. A new technology of inflatable, thin plastic membranes presents a solution to this challenge of needed low-frequency control. It is equally suitable for multipurpose concert halls that need to adjust their acoustics by the push of a button and for halls and arenas that only occasionally present amplified music and need to be treated just for the event. The technology, permanently mounted, is being projected in various concert halls around the world and is being installed in the new Dubai Opera and the Sheik Jaber Al Ahmad Cultural Center, Kuwait during spring 2016. This paper presents the authors' research of also large venues as well as the technology showing applications including on/off measurements of reverberation time versus frequency.

\section{$1: 15$}

4pAAa2. Proposed design schemes for vineyard concert halls with subjective test of solo singing. Weihwa Chiang, Yi-Run Chen, and Melisa Rosiana Tanadi (College of Design, National Taiwan Univ. of Sci. and Technol., 43 Keelung Rd., Section 4, Taipei 106, Taiwan, whch@mail. ntust.edu.tw)

Although a vineyard hall has both acoustical and architectural merits associated with its short source-receiver distance, attention to acoustical design needs to be placed upon the terrace seating that primarily governs the energy distribution in such a hall, in particular when listening to the vocal sound where both acoustic and visual deprivation exists. Design schemes were developed sequentially with an emphasis on seating division and evaluated by computer modeling using Odeon 9.2 acoustic software for a hall with approximately $1 / 4$ of the seats surrounding the stage. The effect of solo singing was evaluations by listeners representing musicians and the general public based on pair comparison. Anechoic soprano and baritone singing segments with calibration information were recorded. Side terraces, preferably stepped back or splayed toward both ends of the hall, are essential for controlling the overall energy distribution. 3-dB high-frequency energy compensation can be provided for the terrace seats surrounding the stage by introducing a frontal terrace and terraces near the corners of the hall. The improvement in objective measures was verified by subjective evaluation where the perceived quality at a terrace seat surrounding the stage was not significantly less than the ones facing the stage.

\section{$1: 30$}

4pAAa3. Electronic shell-Improvement of room acoustics without orchestra shell utilizing active field control. Takayuki Watanabe and Hideo Miyazaki (Yamaha Corp., 10-1 Nakazawa-cho, Nakaku, Hamamatsu 4308650, Japan, takayuki.watanabe@music.yamaha.com)

This paper introduces an example of Electronic Shell acoustic enhancement system that was installed in a multi-purpose hall without an orchestra shell. The system is based on the concept of Active Field Control using electro-acoustic means. The three objectives of this system were 1) the enhancement of early reflection for performers, 2) the extension of the reverberation time and the enhancement of the total sound energy on stage, and 3) the enhancement of early reflection in the audience area. The application of this system showed an improvement of about 1 to $2 \mathrm{~dB}$ in STearly and more than $2 \mathrm{~dB}$ in $\mathrm{G}$ in the audience area, which is equivalent or better performance than simple mobile type orchestra shell. Regarding the spatial sound structure and the time domain characteristics between simple mobile type shell and the Electronic Shell system, there are differences since the mobile type shell mainly takes care of lateral reflections; on the other hand, the Electronic Shell system takes care of overhead reflections, and it cannot provide some early reflections after the direct sound arrives. It was clarified the relationship between the differences in physical features and the subjective improvement of the musical performance using a temporal system installed in a rehearsal hall.

\section{$1: 45$}

4pAAa4. Stadia acoustic atmosphere and spectator experience: Quantifying crowd noise with architectural form. Elizabeth Valmont, Matthew Wilkinson, and Ashwin Thomas (Acoust., Arup, 12777 West Jefferson Boulevard, Bldg. D, Ste. 100, Los Angeles, CA 90066, elizabeth.valmont@ arup.com)

Very little reliable data exist on noise levels and associated crowd atmosphere in stadia. Discussed frequently in the media, several stadia are often portrayed as having the "best" and/or "noisiest" atmosphere. However, there is no accurate basis or measurement for these claims. This poses challenges when designing new stadiums with the intent to improve spectator experience. Architectural elements such as overhangs and vertical sound reflecting surfaces are well known to redirect acoustic energy and improve the overall noise level. However, the direct impact of these elements on crowd behavior and overall atmosphere is not well documented and limits quantifying drivers for new designs. Noise level data were gathered in this study from various stadia and correlated to architectural form using acoustic modeling. The paper details the data gathered at football games played at Autzen Stadium (partial roof overhang) and the Los Angeles Memorial 
Coliseum (open bowl architecture). The measured statistical data are analyzed and linked to the results of computer acoustic models of each stadium. Using the known dimensions of each stadium, the effect of architectural elements on the direct and reverberant noise level in the stands is presented.

\section{2:00}

4pAAa5. Diffusion unwrapped, new testing that reveals real data. Richard L. Lenz (RealAcoustix LLC, 2637 N. Washington Blvd., \#125, N. Ogden, UT 84414, RL@RealAcoustix.com)

The concept of acoustic diffusion and devices that create diffuse properties has been around for well over 40 years. While there have been a number of test methods that have purported to show diffusion from these devices, the evidence of this is questionable at best. The author, along with others, has developed a new test method that can differentiate between specular and diffuse reflections as well as show many other characteristics desirable for acousticians and designers. This test method, being developed in coordination with ASTM, allows the user to see many different components of diffusion including polar responses, frequency response, attenuation, and phase characteristics, a key component of understanding a diffuse reflections. At the same time, the test method also shows the character of specular reflections, which difference is quite clear in the output data. The paper will also show how devices, from quadratic residue diffusers to geometric shapes, do not necessarily have the frequency response or diffuse reflections that we have assumed for many years. Many different examples will be presented as well as the different data outputs required to see how a diffuser actually performs.

\section{2:15}

4pAAa6. Diffusion: Better ways of measuring it, displaying the data and using it in room simulations. Ronald Sauro (NWAA Labs, Inc., 90 Tower Blvd., Elma, WA 98541, audio_ron@msn.com)

This paper will present and discuss diffusion and the many problems with trying to measure it, displaying the data and using this data in ways that allow us to better predict room responses. We will quickly discuss prior standards, their strengths and weaknesses and how a new proposed ASTM standard will correct those weaknesses. We will show that methods used in other areas of acoustics and electro-acoustics can be used to measure diffusion and display it in ways that allow directivity, magnitude, and phase to be displayed. These data sets can then be used in simulation programs, such as EASE. Odeon and CATT Acoustics, to better predict room responses for use in the design of rooms. We will present examples of those uses and the advantages in these proposals.

\section{2:30}

4pAAa7. Designing of variable Schroeder diffusers using digital architecture techniques. Hassan Azad (Architecture, Univ. of Florida, 3527 SW, 20th Ave., 1132B, Gainesville, FL 32607, h.azad@ufl.edu) and Gary Siebein (Siebein Assoc., Inc., Gainesville, FL)

Schroeder diffusers consist of a series of wells of the same width and different depths. The wells are separated by thin fins. The depths of the wells are determined by a mathematical number sequence, such as the quadratic residue sequence. The sequence number for the $n^{\text {th }}$ well, $S_{n}$, is given by ${ }^{1}: S_{n}=n^{2}$ modulo $\mathrm{N}$ where modulo indicates the least non-negative remainder and is often written as mod for short. $\mathrm{N}$ is the number generator which in this case is also a prime and the number of wells per period. The well width follow the simple equation as follow in which $\lambda_{\min }$ is the minimum wavelength before cross-modes in the wells appear ${ }^{1}: \mathrm{W}=\lambda_{\min } / 2 \mathrm{In}$ this paper it is intended to build a physical model of a $\mathrm{N}=7$ diffuser and make the depth of each well separately variable by means of digital architecture tools. The tools which is referred to would be Arduino kit micro controller and 7 stepper motors and 7 drivers. The idea behind this practice is twofold. First is to come up with a variable diffuser which works in several (preferably 5) design frequencies and hence their integer multiples. The user can make this adjustment via a simple computer program. Second, to try other mathematical number sequence and observe the diffusion characteristics of new products using the analyses of acoustic measurement results. 1. Acoustic Absorbers and Diffusers Theory, Design and Application, T. J. Cox and P. D'Antonio, $2^{\text {nd }}$ ed.

\section{2:45}

4pAAa8. Finite element sound field analysis for investigation on measurement mechanism of reverberation time in non-diffuse sound field. Reiji Tomiku, Toru Otsuru (Dept. of Architecture, Oita Univ., Dannoharu 700, Oita 8701151, Japan, tomiku-reiji@oita-u.ac.jp), Noriko Okamoto (Dept. of Architecture, The Univ. of Kitakyushu, Oita, Japan), Takeshi Okuzono (Dept. of Architecture, Kobe Univ., Oita, Japan), and Youtarou Kimura (Dept. of Architecture, Oita Univ., Oita, Japan)

As well known, the reverberation time is essential to evaluate room acoustics; however, it is not so easy to predict in a practical room where the assumption of diffuse sound field is not satisfied. On the other hand, the numerical analyses based on the wave equation have been intensively used to explore many kinds of acoustic problems. In this study, 15 kinds of rooms with different absorption surfaces are analyzed by time domain finite element method. This study shows effectiveness of the analysis for investigation on measurement mechanism of the reverberation time of sound field in rooms where the assumption of diffuse sound field is not satisfied. To evaluate a sound field in a room for the measurement, the ratio of incident sound energy to a part of boundary in those to all boundary of a sound field in a room is calculated from results of the time domain finite element analysis. At first, the calculation method of the ratio of incident sound energy to a part of boundary in those to all boundary of a sound field in a room is described, and the ratios are calculated in the 15 kinds of rooms at each frequency. Finally, relationship among the ratios, mean absorption coefficients, reverberation times calculated from the results of TDFE analysis and those from Sabin's formula is investigated.

\section{3:00}

4pAAa9. Spatialized sound reproduction for telematic music performances in an immersive virtual environment. Samuel Chabot (Rensselaer Polytechnic Inst., 40 3rd St., Troy, NY 12180, chabos2@rpi.edu)

Telematic performances connect musicians and artists at remote locations to form a single cohesive piece. As these performances become more ubiquitous as more people have access to very high-speed Internet connections, a variety of new technologies will enable the artists and musicians to create brand new styles of works. The development of the immersive virtual environment, including Rensselaer Polytechnic Institute's own Collaborative-Research Augmented Immersive Virtual Environment Laboratory, sets the stage for these original pieces. The ability to properly spatialize sound within these environments is important for having a complete set of tools. This project uses a local installation to exemplify the techniques and protocols that make this possible. Using the visual coding environment MaxMSP as a receiving client, patches are created to parse incoming commands and coordinate information for engaging sound sources. Their spatialization is done in conjunction with the Virtual Microphone Control system, which is then mapped to loudspeakers through a patch portable to various immersive environment setups.

\section{$3: 15$}

4pAAa10. A simplified approach to virtual reality and auralizations for architectural acoustics. Christopher Buckley (Omny Acoust., LLC, PO Box 757, Port Austin, MI 48467, info@omnyacoustics.com) and Giuliano Bernardi (none, Leuven, Belgium)

The use of auralizations to convey important architectural acoustics design decisions is well known, but the use of accurate auralizations paired with virtual reality (VR) are typically limited to new, large-budget constructions. Smallerbudget projects often cannot afford the expensive software packages necessary to perform such auralizations, or are limited by design time or computational resources. In order to provide accurate VR-enabled auralizations for a larger variety of projects, this paper demonstrates a simplified method which can be used with a pre-calibrated combination of a smartphone and headphones. The presented method allows for modeling room-to-room sound transmission in addition to 3D spatialization cues from early reflections and reverberation. The room-toroom sound transmission is handled by simple filtering operations, which simulate the transmission loss of the demising partition, while the 3D spatialization cues are generated from one of the many freely available spatialization engines such as the Oculus Native Spatializer or Google VR Spatial Audio. This simplified method does not intend to replace existing more robust solutions, but instead allows for a more wide-spread usage of VR-enabled auralizations in cases where a high performance computer or detailed 3D model are not available. 


\title{
Session 4pAAb
}

\section{Architectural Acoustics: It's All About the Details}

\author{
Shane J. Kanter, Cochair \\ Threshold Acoustics, 53 W. Jackson Blvd., Suite 815, Chicago, IL 60604 \\ John T. Strong, Cochair \\ Threshold Acoustics, 53 W. Jackson Blvd., Suite 815, Chicago, IL 60604 \\ Kimihiro Sakagami, Cochair \\ Dept. of Architecture, Grad. School of Engrg., Kobe University, Rokkodai 1-1, Nada, Kobe 657-8501, Japan \\ Motoki Yairi, Cochair \\ Kajima Technical Research Institute, Chofu, Tokyo 182-0036, Japan
}

Chair's Introduction-3:45

\section{Invited Papers}

3:50

4pAAb1. Numerical study on the absorption performance of MPP sound absorber installed in small rectangular rooms. Takeshi Okuzono and Kimihiro Sakagami (Architecture, Kobe Univ., 1-1 Rokkodai-cho, Kobe, Hyogo 657-8501, Japan, okuzono@port.kobe-u. ac.jp)

Sound absorbers using microperforated panel (MPP) are one of the most promising alternatives of the next-generation sound absorbing materials, thanks to the superior material performances in durability, weatherability, recyclability, and flexibility of design, as well as the attractive broadband sound absorption characteristics. Many previous researches focus on the development of absorbers itself. However, in order to bring out the absorption performance sufficiently or to use it appropriately in room acoustics applications, it is important to understand the absorption effect of MPP absorbers on sound fields in rooms. For the purpose, recently, highly accurate wavebased acoustics simulation techniques such as FEM can be used to analyze sound fields in practical sized rooms with MPP absorbers. This paper presents the absorption performance of MPP absorbers installed in a small rectangular room of $91 \mathrm{~m}^{3}$ using 3D-FEM in frequency domain. Both the steady-state and the unsteady-state sound fields are calculated up to $1 \mathrm{kHz}$ under the various absorber locations. The unsteady-state sound fields are calculated using inverse Fourier transform of transfer functions of rooms. The results are compared to explore the optimum sound absorber locations.

4:10

4pAAb2. Effect of the mass-spring resonance of a slab covered with carpets on the sound insulation performance. Motoki Yairi, Atsuo Minemura, and Takashi Koga (Kajima Tech. Res. Inst., Chofu, Tokyo 182-0036, Japan, yairi@kajima.com)

It is well known that the overall sound insulation performance between two adjacent rooms in actual buildings is not so much increased even if a high spec wall is used for the partition because of several flanking sound transmission paths. In recent years, the phenomena that a slab covered with carpets increases the sound transmission through the floor have been found. This is caused by the resonance with the mass of the carpets and the spring of those underlying materials, and consequently the sound insulation performance significantly decreases in the mid-low frequency range. The resonance frequency can be shifted to higher frequencies using formed plastic sheets of higher stiffness for the underlying materials. Although this would usually be one of countermeasures to increase the overall sound insulation performance, it is not a fundamental solution for noise control engineering. In the present work, in order to control the mass-spring resonance itself, a method of focusing on permeability of the carpets is proposed. The resonance is expected to be damped with the flow resistance, which is caused by setting an appropriate permeability. It is theoretically investigated using a simple model, and an experimental validation is also carried out. 
4pAAb3. Essential details in performing art facilities' practice rooms. Jennifer Nelson Smid (Threshold Acoust., 141 W Jackson Blvd, Ste. 2080, Chicago, IL 60604, jnelson@thresholdacoustics.com)

Limited space and multi-use programming often leaves practice rooms working for several instrumental and vocal groups within performing arts facilities. During programming, communication about expected performance of the space and adjacencies, as well as design team collaboration will help to keep the project in scope and on budget. Special details for multiple trades are required to design a well isolated and multi-functioning practice room for various quantities and ranges of instruments and voice. The details are important not only throughout the design phases but must be carefully observed during build-out so that special isolation requirements are incorporated correctly. The entire process from design, detailing, and into construction is presented with emphasis on the various communication processes required to arrive at successful outcomes. Specific examples are provided highlighting the importance of each step in the process.

\section{4:50}

4pAAb4. Increasing liveness and clarity in a multipurpose civic center. Hans Michel and Joseph Myers (Acoust., Kirkegaard Assoc., 801 W. Adams 8th Fl., Chicago, IL 60607, hmichel@kirkegaard.com)

The Des Moines Civic center recently underwent a renovation primarily for symphonic uses, to improve hearing conditions on stage and in the house. This renovation included a new movable orchestra shell, rebuilt rear wall, and new finishes at sidewalls. The original shell did not adequately support bass or project sound to the audience, and musicians reported communication problems on stage. The rear wall was typically covered by a curtain, and returned confusing echoes when the curtain was withdrawn. The most unusual aspect of the room was the design of its sidewalls, broken into convex, down-leaning pylons, a signature of Paul Veneklasen, the original acoustician. KA analyzed the reflection pattern off the side walls and devised a treatment that maintained the beneficial aspects of the original design while dramatically improving clarity and presence. This paper focuses on the testing and modeling methods used to confirm our hypotheses about the problems caused by side wall reflections; the selection of appropriate materials based on in situ measurements and subjective listening; and architectural coordination.

\section{Contributed Papers}

\section{5:10}

4pAAb5. Acoustical considerations in shipping container architecture. Anat Grant (CSDA Design Group, 4061 Glencoe Ave., Ste. B, Marina del Rey, CA 90292, agrant@csdadesigngroup.com) and Randy Waldeck (CSDA Design Group, San Francisco, CA)

The inherent strength, wide availability, and relatively low expense of intermodal freight containers have led to an increase in their popularity as framing elements for modular construction. In school expansion and renovation projects, modular construction can reliably achieve sustainability and energy efficiency targets, often with shorter construction schedules. Residential housing, offices, commercial buildings and other building types are also constructed using shipping containers. While shipping container architecture is a green alternative with many advantages, it can also present acoustical challenges in buildings occupied by people. Low ceiling heights often limit acoustical treatment possibilities, and acoustical test data on sound isolation is limited. Case studies and measurement data presented will include an elementary school with large divisible classrooms, a community education center, an after-school youth education and classical music program in an economically struggling Los Angeles community, and a multifamily apartment building in Berkeley, CA.

\section{$5: 25$}

4pAAb6. Silent Room: A (non)sensory interactive art installation. Matthew C. Zeh, Fiona Cheung (Mech. Eng. Dept., Univ. of Texas at Austin, 204 East Dean Keeton St., Austin, TX 78712-1591, mzeh@utexas.edu), Simon Heijdens (Artist/Creator of Silent Rm., Studio Simon Heijdens Ltd., London, United Kingdom), and Preston S. Wilson (Mech. Eng. Dept., Univ. of Texas at Austin, Austin, TX)

Simon Heijdens, a London-based Dutch artist, was commissioned by South by Southwest (SXSW), an annual film, media, and music festival in Austin, TX, to create an installation artwork to premiere at SXSW in March 2016. In reaction to the sensory overload of the festival, he created Silent Room. Students and professors from the University of Texas at Austin worked alongside Mr. Heijdens to design a portable, self-sufficient, reusable space providing sound isolation and absorption from outside and inside the chamber, respectively. Housing the space in a shipping container, Silent Room was successfully built and showcased at SXSW in downtown Austin to hundreds of visitors, achieving a minimum sound reduction of $27 \mathrm{dBA}$ at $40 \mathrm{~Hz}$ and a maximum reduction of $55 \mathrm{dBA}$ at $1.45 \mathrm{kHz}$. These results were achieved using a suspended floor and decoupled wall/ceiling construction of Auralex rubber U-Boats, 2" x 4" wood studs, mineral fiber insulation, plywood, Auralex SheetBlok vinyl, and acoustically treated gypsum board. After initial acoustical measurements, more U-Boats were added, decoupling the container underside from the street and isolating it from ground-borne vibration. However, the room performance worsened after this change, possibly due to exposure to a new air-borne path for noise ingress. 


\title{
Session 4pABa
}

\section{Animal Bioacoustics, Signal Processing in Acoustics, and Speech Communication: Sequence Information in Mammalian Vocal Call Production II}

\author{
Marie A. Roch, Cochair \\ Dept. of Computer Science, San Diego State Univ., 5500 Campanile Dr., San Diego, CA 92182-7720 \\ Tadamichi Morisaka, Cochair \\ School of Marine Science and Technology, Tokai University, 3-20-1, Orido, Shimizu-ku, Shizuoka 4248610, Japan
}

\section{Invited Papers}

1:00

4pABa1. Fin whale call sequence analysis from tracked fin whales on the Southern California Offshore Range. Glenn Ierley (Scripps Inst. of Oceanogr., Houghton, MI) and Tyler A. Helble (SSC-PAC, 2622 Lincoln Ave., San Diego, CA 92104, tyler.helble@ gmail.com)

Time difference of arrival (TDOA) methods were utilized to localize and track fin whales on the Southern California Offshore Range (SCORE). In several instances two whales were shown to converge at the same location on the range. Analysis of $20 \mathrm{~Hz}$ call sequences revealed changes in call type and intercall-interval coinciding with changes in swim kinematics of the nearby transiting whales. Call sequence analysis may provide some insight on the ability of fin whales to convey information to conspecifics on location and bearing of travel.

$1: 20$

4pABa2. Blue whale song sequencing off Southern California. Leah A. Lewis (Scripps Inst. of Oceanogr., Univ. of California, San Diego, 9500 Gilman Dr., La Jolla, CA 92093-0205, lalewis@ucsd.edu), John Calambokidis (Cascadia Res. Collective, Olympia, WA), John A. Hildebrand, and Ana Širović (Scripps Inst. of Oceanogr., Univ. of California, San Diego, La Jolla, CA)

Blue whales off Southern California produce a unique song consisting of pulsed A unit and tonal B unit calls. These units are either sequenced in an alternating $\mathrm{AB}$ pattern, or a single $\mathrm{A}$ unit is followed by multiple $\mathrm{B}$ units (here denoted $\mathrm{ABB}$ ). To investigate whether there is geographic variation in the occurrence of these different sequence types, data were analyzed from four High-frequency Acoustic Recording Packages deployed at two sites each inshore and offshore of the Channel Islands from September 2009 to June 2010. Additionally, fine-scale behavior associated with song and its sequencing was analyzed from acoustic tags deployed on blue whales in Southern California since 2000. A higher proportion of song bouts detected in offshore recordings were type ABB, whereas the dominant song type observed inshore was $\mathrm{AB}$. This pattern may indicate geographic variability in song function. Most song units recorded on tags were produced during surface-travel dives, with the whale less than $30 \mathrm{~m}$ deep. Song bouts detected on tags occurred primarily at night, and whales did not interrupt their calling sequence with breathing intervals. The observed differences in calling and song preference off Southern California may be useful in identifying regionally distinct behavioral contexts for blue whales.

\section{Contributed Papers}

1:40

4pABa3. Central and western Pacific blue whale song and occurrence. Pollyanna I. Fisher-Pool, Erin Oleson (NOAA Fisheries, Pacific Islands Fisheries Sci. Ctr., 1845 Wasp Blvd., Bldg. 176, Honolulu, HI 96818, pollyanna.fisher-pool@noaa.gov), and Ana Širović (Scripps Inst. of Oceanogr., La Jolla, CA)

Blue whale (Balenoptera musculus) occurrence in the central and western Pacific is not fully understood. However, passive acoustics offer an effective way to monitor remote sites. Blue whale songs are regionally distinct, and their stereotyped characteristics may be used to distinguish populations. Most blue whale song consists of multiple, pulsed and tonal units. However, song in the central and western Pacific consists of only simple tonal units. We investigated song variability of Hawaii, Wake Atoll, Palmyra, and Tinian in the Mariana Islands Archipelago, and highlight differences between blue whale songs amongst sites. Song calls of two different frequencies were recorded by High-frequency Acoustic Recording Packages. Detailed measures of call frequency were taken along the contours of tonal calls and patterning of song sequences was evaluated for recordings from the four locations during 2012 and 2013. The spatial and temporal occurrence of these patterns is discussed.

\section{$1: 55$}

4pABa4. Stereotyped, repetitive gunshot call patterns produced by the North Pacific right whale, Eubalaena japonica. Jessica Crance and Catherine Berchok (AFSC/NMFS/NOAA, National Marine Mammal Lab., 7600 Sand Point Way NE, Seattle, WA 98115, Jessica.Crance@noaa.gov)

The Marine Mammal Laboratory has deployed long-term passive acoustic recorders along the $50 \mathrm{~m}$ and $70 \mathrm{~m}$ isobaths throughout the Bering Sea since 2007. Instruments recorded at either $4 \mathrm{kHz}$ on a $\sim 7-11 \%$ duty cycle, or $8 \mathrm{kHz}$ on a $30-45 \%$ duty cycle. In addition, directional sonobuoys were 
deployed during field surveys to allow real-time monitoring for large whale presence. During the 2010 survey, a stereotyped, repetitive gunshot call pattern was acoustically detected on sonobuoys. This same call pattern was then detected on two different long-term moored recorders in two separate years. Since then, six different stereotyped, repetitive patterns have been documented, three of which have been analyzed. Preliminary results show that each pattern has a minimum of 30 iterations repeated over several hours. Furthermore, in several cases, these patterns are repeated throughout the season, in consecutive years, and in one instance, in non-consecutive years. While male North Atlantic right whales produce long gunshot bouts similar to the reproductive advertisement known in other species, right whales are not known to produce any type of repeated stereotyped pattern. This represents the first study to document stereotyped repetitive gunshot patterning in right whales. [Work funded by the Bureau of Ocean Energy Management.]

\section{2:10}

4pABa5. Fin whale song patterns in Southern California and the Gulf of California. Ana Sirovic (Scripps Inst. of Oceanogr., 9500 Gilman Dr. MC 0205, La Jolla, CA 92093-0205, asirovic@ucsd.edu), Erin M. Oleson (Pacific Islands Fisheries Sci. Ctr., Honolulu, HI), Jasmine S. Buccowich, and Ally Rice (Scripps Inst. of Oceanogr., San Diego, CA)

The most common fin whale calls, $20 \mathrm{~Hz}$ pulses, are often produced in regular, stereotypic sequences termed songs. The main variability in songs comes from the differences in the duration of the interval between successive pulses, the interpulse interval (IPI). Data recorded between 2000 and 2012 in Southern California and 2004 to 2010 in the Gulf of California were analyzed for temporal and geographic patterns in fin whale songs. During this time, fin whales in Southern California and the Gulf of California produced four song types with distinct IPI sequences. Two common songs in Southern California were the short doublet and the long doublet and they were detected year-round. The IPIs in the short doublet song have been increasing over the long term, while the IPIs in the long doublet song showed no long-term trends but were seasonally variable. In the Gulf of California, the most common songs were the short triplet and the long triplet. These songs were detected in Gulf of California year-round, although their occurrence decreased August through October. There was some exchange of sons between the two areas that might point to seasonal movement of parts of these populations.

\section{$2: 25$}

4pABa6. Fin whale occurrence and population structure in the central and western Pacific through detection and characterization of song patterns. Erin M. Oleson (Protected Species Div., NOAA Fisheries, Pacific Islands Fisheries Sci. Ctr., 1845 Wasp Blvd. B176, Honolulu, HI 96818, erin.oleson@noaa.gov), Ana Širović, Ally Rice, and Leah M. Varga (Scripps Inst. of Oceanogr., Univ. of California, San Diego, La Jolla, CA)

Fin whale songs comprise series of $20 \mathrm{~Hz}$ pulses and song structure can be characterized by patterns of inter-pulse intervals (IPIs). Recent studies in the northeast Pacific indicate the occurrence of a broadly distributed, seasonally changing, but annually stable IPI pattern, heard from the Bering Sea to southern California and west to Hawaii. Other geographically localized song patterns are also known in the Gulf of California, the Bering Sea, and off southern California. We expanded the assessment of fin whale song across the Pacific, analyzing occurrence and song patterns from multi-year passive acoustic data collected at several sites in the central and western Pacific, from Palmyra Atoll to the Northwestern Hawaiian Islands and west including Wake Atoll and the Mariana Archipelago. Fin whales are relatively uncommon in these tropical and sub-tropical waters, though they are detected in all seasons at some sites. Song IPI patterns suggest multiple and potentially annually variable fin whale song structures throughout the region. The common northeast Pacific song is heard at Hawaii and Wake Atoll sites, with different song types at Palmyra and in the Mariana Archipelago. Long-term temporal and geographic patterns of song occurrence across the region will be discussed. 


\title{
Session 4pABb
}

\author{
Animal Bioacoustics: Session in Honor of Whitlow Au III
}

\author{
Kerri Seger, Chair \\ Scripps Institution of Oceanography, 9331 Discovery Way, Apt. C, La Jolla, CA 92037
}

\section{Contributed Papers}

\section{2:55}

$4 p A B b 1$. Comparison of three Stenella spp. audiograms measured using auditory evoked potentials and behavioral methods. Danielle R. Greenhow (School of Ocean Sci. and Technol., Univ. of Southern MS, $2012 \mathrm{~W}$ 2nd St., Apt. 320, Long Beach, MS 39560, danielle.greenhow@usm.edu), Adrienne Cardwell (Mote Marine Lab., Clearwater, FL), Jessica R. Powell (Mote Marine Lab., Saint Petersburg, FL), Mandy L. Hill-Cook (Dept. of Biology, Portland State Univ., Portland, OR), M. Andrew Stamper (Mote Marine Lab., Orlando, FL), Lynne Byrd (Mote Marine Lab., Sarasota, FL), Charles A. Manire (Mote Marine Lab., Juno Beach, FL), Micah C. Brodsky (Micah Brodsky, V.M.D. Consulting, Miami Shores, FL), Gordon B. Bauer (Div. of Social Sci., New College of Florida, Sarasota, FL), and David A. Mann (College of Marine Sci., Univ. of South Florida, Sarasota, FL)

The oceanic dolphin genus Stenella is underrepresented in cetacean hearing data. In this study the hearing of three Stenella spp. dolphins was measured using auditory evoked potential (AEP) methods. A single male juvenile Atlantic spotted dolphin (Stenella frontalis) was rehabilitated in Key Largo, Florida, after being sighted alone and emaciated. The Atlantic spotted dolphin's greatest sensitivity was at $40 \mathrm{kHz}$, with functional hearing up to $128 \mathrm{kHz}$. A female spinner dolphin (Stenella longirostris), housed at Mote Marine Laboratory, had peak sensitivity at $40 \mathrm{kHz}$ and functional hearing up to $120 \mathrm{kHz}$, the highest frequency tested. The sensitive high frequency hearing of the spinner and Atlantic spotted dolphin is similar to that found in other oceanic odontocetes. A pantropical spotted dolphin (Stenella attenuata) was housed at Mote Marine Laboratory and hearing thresholds were determined using AEP and behavioral methods. The pantropical spotted dolphin had a peak sensitivity at $10 \mathrm{kHz}$, with a cutoff frequency between 14 and $20 \mathrm{kHz}$. The source of the dramatic high-frequency hearing loss is not known; possible causes include congenital hearing loss (present at birth) or hair cell death due to treatment with ototoxic drugs during rehabilitation.

\section{3:10}

$4 \mathrm{pABb2}$. Functional optical imaging from bat inferior colliculus using a micro-endoscope. Hidetaka Yashiro (Doshisha Univ., 1-3, Miyakodani, Tatara, Kyotanabe, Kyoto 6100321, Japan, hidetaka.yashiro@gmail.com), Kazuo Funabiki (Inst. of Biomedical Res. and Innovation, Kobe, Japan), Andrea M. Simmons, James A. Simmons (Brown Univ., Providence, RI), and Hiroshi Riquimaroux (Shandong Univ., Tokyo, Japan)

We developed a confocal micro-endoscopic system that enables simultaneous recording of electrical neuronal responses-local field potentials (LFPs) and multi-unit activities, (MUA) — and fluorescence changes reflecting calcium activation derived from Oregon green dye. We recorded these responses from different depths of the inferior colliculus (IC) in two species of bats (Carollia perspicillata and Eptesicus fuscus) to tone bursts, noise bursts, and FM sweeps. Electrical responses were fast and registered the occurrence of sounds with submillisecond and millisecond precision. LFPs contained strong onset responses and weaker, extended oscillations that persisted for much of the sound's duration. In contrast, calcium responses were slow, building up gradually over the course of 50-100 ms, and declined slowly following sound offset. Full horizontal-vertical video image scanning of the entire fiber optic bundle's face was limited to 18.9 frames/s by the scanning mirror, but isolation and recording of successive individual horizontal scans, done by freezing the vertical scan, yielded 7,550 lines/s, or 132 microseconds for 1 line. Line-scanning revealed that the calcium responses indeed were slow, in the 50-100 ms range. They also were spatially diffuse, suggesting a distributed dendritic origin. [Work supported by JSPS, MEXT Japan, Shandong University, ONR, Capita Foundation.]

\section{$3: 25$}

4pABb3. Human listening experiments provide insight into cetacean auditory perception. Caroline M. DeLong (Psych., Rochester Inst. of Tech., 18 Lomb Memorial Dr., Rochester, NY 14623, cmdgsh@rit.edu)

Odontocete cetaceans produce an array of sounds including echolocation clicks that can be used for object recognition and whistles used primarily for communication. Echoes and whistles contain a multitude of acoustic features, and the salient features used by animals when identifying objects or in social contexts are often difficult to isolate. One method for detecting salient acoustic features is to compare the performance of cetaceans and humans on the same auditory perception tasks. Human listeners can be presented with echoes produced with simulated cetacean clicks or whistle-like stimuli and provide verbal feedback on discriminatory cues. An early human listening study was performed by Au and Martin (1989). Seven studies performed over the past 15 years show that humans perform as well or better than cetacean subjects in a variety of tasks: echoic discrimination of objects varying in size, shape, material, texture, or wall thickness; echoic recognition of objects from various aspect angles; echoic object discrimination using clicks from different cetacean species; and recognition of frequency-modulated whistle-like sounds. Analyzing the error patterns of humans and cetaceans alongside the reported cues reveals processing mechanisms and decision strategies cetaceans may use. This comparative approach sheds light on how cetaceans perceive and represent sounds.

\section{3:40}

4pABb4. Acoustic processes in an echolocating bottlenose dolphin's (Tursiops aduncus) head using a finite element model. Chong Wei (College of Ocean and Earth Sci., Hawaii Inst. of Marine Biology, Xiamen Univ., Lilipuna Rd., Xiamen, Fujian 361102, China, weichong3310@foxmail.com), Whitlow W. Au (Hawaii Inst. of Marine Biology, Kaneohe, HI), Zhongchang Song (College of Ocean and Earth Sci., Xiamen Univ., Xiamen, Fujian, China), Darlene R. Ketten (Woods Hole Oceanographic Inst., Woods Hole, MA), and Yu Zhang (College of Ocean and Earth Sci., Xiamen Univ., Xiamen, Fujian, China)

Bottlenose dolphins (Tursiops aduncus) are a well-known species using broadband echolocation signals for searching prey and spatial orientation. In this study, the computed tomography (CT) scan data were obtained to set up a two-dimensional finite element model. In the vertical plane, the acoustic field on the animal's forehead and the far field transmission beam pattern of an echolocating dolphin were calculated. The simulation results and prior measurement results were consistent qualitatively. The role of the main structures on the sound propagation pathway such as air sacs, melon, skull, 
and connective tissues was investigated. Furthermore, the signal at the source excitation was investigated. It suggested that the broadband echolocation dolphins may not have the same driving signals at the source excitation as the narrowband echolocation dolphins. The results can help us gain further understanding of the acoustic processes in dolphin's biosonar.

\section{3:55}

4pABb5. Ultra-high-frequency hearing in seals and sea lions in the context of other secondarily aquatic mammals. Colleen Reichmuth (Inst. of Marine Sci., Long Marine Lab., Univ. of California Santa Cruz, 100 Shaffer Rd., Santa Cruz, CA 95060, coll@ucsc.edu), Jason Mulsow (National Marine Mammal Foundation, San Diego, CA), Jillian M. Sills, Brandon L. Southall, and Kane Cunningham (Inst. of Marine Sci., Long Marine Lab., Univ. of California, Santa Cruz, CA)

The underwater hearing abilities of marine mammals near the upper frequency limit of hearing are poorly understood. While the maximum frequency of hearing is often identified following a sharp roll-off in sensitivity, it is apparent that many marine mammals can perceive high-amplitude tonal sounds above this frequency region. We recently measured underwater detection thresholds in quiet conditions in the $50-180 \mathrm{kHz}$ range for one California sea lion, one spotted seal, and one harbor seal. Absolute sensitivity curves from these subjects exhibited two distinct slope regions at frequencies near the high-frequency hearing limit. The first region is characterized by an initial, rapid decrease in sensitivity with increasing frequency - i.e. a steep slope-while the second shows a much less rapid sensitivity decrease-i.e. a shallower slope. An additional masking study conducted with one of the seals suggested that the initial, rapid decrease in sensitivity is not due to cochlear constraints, as has been proposed, but rather due to constraints on the underlying bone-conduction mechanism. These results can be viewed in the context of the selective pressures influencing ultra-high-frequency hearing in other aquatic mammals, including those capable of specialized echolocation.

\section{4:10}

4pABb6. Killer whale (Orcinus orca) audiograms. Brian K. Branstetter (National Marine Mammal Foundation, 2240 Shelter Island Dr. \#200, San Diego, CA 92106, brian.branstetter@nmmpfoundation.org), Doug Acton (Sea World San Antonio, 10500 Sea World Dr., San Antonio, CA), John Stewart (Sea World San Diego, 500 Sea World Dr., San Diego, CA), Dorian Houser (National Marine Mammal Foundation, San Diego, CA), Judy St. Ledger (Sea World San Diego, 500 Sea World Dr., San Diego, CA), James Finneran, and Keith Jenkins (US Navy Marine Mammal Program, SSC Pacific, Code 17519, 53560 Hull St., San Diego, CA)

Historically, understanding of killer whale (Orcinus orca) hearing was based on behavioral and evoked potential data from three animals, one of which had significant hearing loss. For the other two whales, the mean detection threshold at $20 \mathrm{kHz}$ was $36 \mathrm{~dB}$ re $1 \mu \mathrm{Pa}$; this is the lowest underwater behavioral detection threshold of any marine mammal tested, suggesting that it may be an outlier. The current study measured the behavioral audiograms of eight killer whales at two different facilities. Hearing sensitivity was measured from $100 \mathrm{~Hz}$ to $160 \mathrm{kHz}$ in animals ranging in age from 12 to 52 years. Two whales had hearing loss consistent with presbycusis and a third displayed atypical low-frequency hearing loss (but normal high frequency hearing); previously measured low thresholds at $20 \mathrm{kHz}$ were not replicated in any animal. Hearing in the killer whales was generally similar to other delphinids, with lowest threshold ( $49 \mathrm{~dB}$ re $1 \mu \mathrm{Pa}$ ) at approximately $40 \mathrm{kHz}$, good hearing (i.e., within $20 \mathrm{~dB}$ of best sensitivity) from 5-80 $\mathrm{kHz}$, and low- and high-frequency hearing cut offs $(>100 \mathrm{~dB}$ re $\mu \mathrm{Pa})$ of $600 \mathrm{~Hz}$ and $114 \mathrm{kHz}$, respectively.
$4 p A B b 7$. Control ultrasound beam by tissues in the head of finless porpoise acting as a tunable gradient index material. Yu Zhang, Zhongchang Song, Chong Wei (Dept. of Appl. Phys. and Eng., College of Ocean and Earth Sci., Xiamen Univ.,Rm. C3-209, Xiping Bldg., Xiangan Campus of Xiamen Univ., Xiangan South Rd., Xiangan District, Xiamen, Fujian 361102, China, yuzhang@xmu.edu.cn), Whitlow W. Au (Hawaii Inst. of Marine Biology, Univ. of Hawaii, Kaneohe, HI), Wenwu Cao (Dept. of Mathematics and Mater. Res. Inst., The Penn State Univ., Pennsylvania, PA), and Xianyan Wang (Lab. of Marine Biology and Ecology, Third Inst. of Oceanogr., State Oceanic Administration, Xiamen, Fujian, China)

Porpoises are well known to emit directional ultrasound beams for detecting and tracking preys; however, how they produce and manipulate directional beams are challenging. Here, we investigated physical mechanism of ultrasound beam formation and control of finless porpoise (N. a. sunameri) by using an integrated scheme of computed tomography, tissue and field measurements, and numerical modeling. The results showed that complex acoustic structures in the porpoise's forehead contributed to producing directional acoustic field. Furthermore, we demonstrated that the skull, air sacs, connective tissue, muscle, and melon constituted a gradient index (GRIN) structure whose density and sound velocity are positively correlated, and thus regulated the directional beam. The removal or compression deformation of the forehead tissues decentralizes energy and widens sound beam, indicating that the forehead tissues as a tunable natural GRIN material significantly impact beam patterns of the finless porpoise. The results might be valuable for understanding control mechanism of acoustic beam of other toothed whales.

\section{$4: 40$}

4pABb8. Multi-species Baleen Whale audiogram modelling. Darlene R. Ketten (Otology and Laryngology, Harvard Med. School, Woods Hole Oceanographic Inst., Woods Hole, MA 02543, dketten@whoi.edu), Aleks Zosuls, and Andrew Tubelli (Biomedical Eng., Boston Univ., Boston, MA)

In this research, we produced model audiograms for two Mysticetes (baleen whales) that are among the species most likely to be subject to impacts from common lower frequency anthropogenic sound sources deployed in the oceans. These models are needed for species-specific risk assessments for hearing impacts, for determining optimal signals for playback experiments, and for determining effective electrode and sound source placements for auditory brainstem response (ABR) measures in live stranded whales. We employed micro and UHRCT, dissection, and histology of minke (Balaenoptera acutorostrata) and humpback (Megaptera novaeangliae) heads and ears to calculate inner ear frequency maps for determining total hearing range, the frequency of peak sensitivity, and the probable frequency of NIHL liability ("notch"). The anatomically derived data were then combined with direct measures via nanoindentation of middle ear stiffness, Young's modulus, frequency response, and inner ear stiffness to determine the middle ear transfer function and basilar membrane stiffness gradients. FEM simulations were employed to further explore the responsivity of the tympanum and middle ear ossicular chain. In order to test the validity of the model techniques, model audiograms were produced also for two odontocetes, the bottlenose dolphin and the harbor porpoise, and compared with well-established behaviorally obtained audiograms for these species. [This research was supported by the LMR and JIP marine research programs.]

\section{4:55}

4pABb9. Acoustic properties reconstruction of forehead tissues in an Indo-Pacific humpback dolphin (Sousa chinensis), with investigation on temperature effects on the species tissues sound velocity and beam. Zhongchang Song, Yu Zhang, Chong Wei (Dept. of Appl. Phys. and Eng., College of Ocean and Earth Sci., Xiamen Univ.,Rm. C3-210, Xiping Bldg., Xiangan Campus of Xiamen Univ., Xiangan South Rd., Xiangan District, Xiamen, Fujian 361102, China, songzhongchang@foxmail.com), and Per Berggren (Dove Marine Lab. and School of Marine Sci. and Technol., Newcastle Univ., Newcastle, United Kingdom)

Computed tomography (CT) imaging and ultrasound experimental measurements were used to reconstruct the acoustic properties (density, 
velocity, and impedance) of forehead tissues from a deceased Indo-Pacific humpback dolphin (Sousa chinensis). The nonlinear regression methods were used to demonstrate the relationships between the sound velocity and temperature in melon, muscle and connective tissue. The obtained nonlinear equations were then combined with the original CT scanning results and sound velocity distributions reconstructed at room temperature $25^{\circ} \mathrm{C}$ to reconstruct the dolphin head's sound velocity distribution at temperature $37^{\circ} \mathrm{C}$. The beam formation and beam properties between two temperatures $37^{\circ} \mathrm{C}$ and $25^{\circ} \mathrm{C}$ were then compared and discussed. The results could provide important information for understanding the species' bioacoustic characteristics and the acoustic data can be used for investigation of biosonar beam formation of this species.

\section{$5: 10$}

4pABb10. Say what? Hearing abilities and subsequent variability for a wild population of Beluga Whales (Delphinapterus leucas). T. Aran Mooney (Biology Dept., Woods Hole Oceanographic Inst., 266 Woods Hole Rd., Woods Hole, MA 02543, amooney@whoi.edu), Manuel Castellote (Alaska Fisheries Sci. Ctr., National Marine Mammal Lab., NMFS, Seattle, WA), Lori Quakenbush (Alaska Dept. of Fish and Game, Fairbanks, AK), Roderick Hobbs (Alaska Fisheries Sci. Ctr., National Marine Mammal Lab., NMFS, Seattle, WA), Caroline Goertz (Alaska SeaLife Ctr., Seward, $\mathrm{AK}$ ), and Eric Gaglione (Georgia Aquarium, Atlanta, Georgia)

Odontocetes use sound for vital biological functions and hearing is considered their primary sensory modality. To understand and predict noise impacts, we need to know natural hearing ability at the population-level. Yet we have no wild population-level hearing data for any cetacean. Here, we measured the audiograms (4-150 kHz) of wild beluga whales from 2012-2016 using auditory evoked potential methods. The goal of this work was to establish the mean audiogram and variance of wild beluga whales to define what this population hears. Audiograms were collected from 26 belugas during capture-release events in Bristol Bay, AK. In general, belugas showed excellent sensitivity $(<60 \mathrm{~dB})$ from 45 to $100 \mathrm{kHz}$ and most $(76 \%)$ heard up to at least $120 \mathrm{kHz}$. Hearing thresholds at lower frequencies $4-11 \mathrm{kHz}$ tracked the low environmental noise levels $(70 \mathrm{~dB} / \mathrm{Hz})$, suggesting that quiet habitats may allow substantial hearing sensitivity. Beluga audiograms show substantial (20-40 dB) variation but less than that of bottlenose dolphins suggesting possible differences between populations and the need for comparative data. Further, the hearing variability among belugas suggests multiple measures best describe the maximum sensitivity and population variance. These audiograms quadruple the data for beluga whales as a species and provide the beginning of population-level auditory data for a wild odontocete.

\title{
Session 4pABc
}

\section{Animal Bioacoustics: Animal Bioacoustics Poster Session}

\author{
Benjamin N. Taft, Chair \\ Landmark Acoustics LLC, 1301 Cleveland Ave., Racine, WI 53405
}

\begin{abstract}
All posters will be on display from 3:00 p.m. to 5:30 p.m. To allow contributors in this session to see the other posters, authors of odd-numbered papers will be at their posters from 3:00 p.m. to 4:15 p.m. and authors of even-numbered papers will be at their posters from 4:15 p.m. to 5:30 p.m.
\end{abstract}

\section{Contributed Papers}

4pABc1. Ultrasonic vocalizations in laboratory mice. Kali Burke, Laurel A. Screven, and Micheal L. Dent (Psych., Univ. at Buffalo, SUNY, 246 Park Hall, Buffalo, NY 14260, kaliburk@buffalo.edu)

Mice produce diverse ultrasonic vocalizations (USVs), which vary in spectrotemporal parameters including frequency, intensity, and duration. Although it is currently unclear whether mice are using these USVs for communication, researchers have attempted to parse USVs into categories based upon their spectrotemporal characteristics. There is recent evidence that shows that mice are producing context-specific vocalizations, but it is still unclear if USVs have any of the language-like characteristics that researchers would like to impose on them. The current literature clearly indicates a need for a comprehensive catalog of the USVs mice are producing in order to better understand if and how these animals are using their USVs for communication. Here, 20 male and 20 female mice were exposed to a same sex or opposite sex stimulus mouse for 1 hour and recorded immediately following separation for $3 \mathrm{~min}$. All vocalizations were categorized based on their spectrotemporal characteristics. This experiment provides a catalog of the vocal repertoire of laboratory mice, adding to the existing knowledge of how they are using their USVs.
4pABc2. Discrimination of natural from synthetic ultrasonic vocalizations by laboratory mice. Anastasiya Kobrina, Laurel Screven, and Micheal Dent (Psych., SUNY Univ. at Buffalo, B23 Park Hall, Amherst, NY 14261, akobrina@buffalo.edu)

Mice produce spectrotemporally complex ultrasonic vocalizations (USVs) which are thought to be important for mate attraction, courtship, and other social interactions. Despite this assertion, little is known about how mice perceive or use these auditory signals. USVs have been categorized based on a variety of spectrotemporal parameters, including frequency, amplitude, and duration. Previous studies have established that mice are capable of detecting and discriminating natural, synthetic, and altered USVs using physiological and behavioral methodologies. The current research examined whether mice are capable of discriminating natural USVs from their synthetic analogs. Discrimination performance was tested in six adult mice using operant conditioning procedures with positive reinforcement. Discrimination was high for some USV categories but low for others, suggesting that more than simple spectrotemporal similarity is involved in the discrimination of USVs. 
4pABc3. Acoustic multi-tasking in beaked whales: The use of alternating echolocation regimes during the descent phase of deep foraging dives. Jennifer S. Trickey, Simone Baumann-Pickering, and John A. Hildebrand (Scripps Inst. of Oceanogr., Univ. of California, San Diego, 9500 Gilman Dr., La Jolla, CA 92093, jtrickey@ucsd.edu)

Beaked whales use echolocation to navigate and hunt at extreme depths, and the timing of their echolocation clicks while actively searching for prey is generally characterized by a stable inter-click interval (ICI). However, analysis of autonomous, long-term passive acoustic data revealed a markedly different pattern during the vocal portion of their descent towards the seafloor. Diving beaked whales were found to alternate between two ICI regimes, while also gradually increasing their overall click rate. This strategy is presumably used to simultaneously monitor two different target ranges as they approach their preferred foraging depth. One ICI regime likely corresponds to the two-way-travel time of sound to the seafloor, and by examining the rate at which the time interval between clicks decreased in this seafloor-tracking ICI regime, we calculated estimates of dive descent rate. The second ICI regime was consistently more rapid, and represents a shorter search range that is likely used by the whale to inspect the nearby water column for the presence of prey and other features. This unique echolocation behavior was identified in acoustic encounters of four different species of beaked whales recorded at a variety of sites in the North Pacific Ocean and the Gulf of Mexico.

4pABc4. Detection of marine mammal vocalizations off Cape Hatteras, modulated by the Gulf Stream. Stephen B. Lockhart (Marine Sci., Univ. of North Carolina, CB\#3300, Chapel Hill, NC 27599-3300, sblockhart.z0@ gmail.com), Lindsay L. Dubbs, Michael Muglia, Patterson Taylor (Univ. of North Carolina Coastal Studies Inst., Wanchese, NC), and Robert E. Todd (Woods Hole Oceanographic Inst., Woods Hole, MA)

To assess the ecological impact of extracting energy from the Gulf Stream, the University of North Carolina Coastal Studies Institute has deployed a mooring on the continental slope off Cape Hatteras at a depth of $230 \mathrm{~m}$, equipped with an ADCP, a CTD, and a hydrophone. Analyzing data from the first deployment, we automatically detected marine mammal vocalizations. We found a strong correlation between the amount of vocalizations and the position of the Gulf Stream, with vocalizations increasing when the Gulf Stream was farther offshore. We then derived sound speed profiles for the region around the mooring using temperature and salinity profiles collected by WHOI Spray gliders. These sound speed profiles were fed into a propagation modeling tool. When the Gulf Stream was far offshore with respect to the mooring position, the model showed a set of rays that propagate down the slope in an acoustic channel along the bottom. When the Gulf Stream was nearly over the mooring, the range of angles that could propagate down the slope was reduced by a factor of six. Therefore, the propagation models suggest that the observed correlation is due to a propagation effect, resulting from the varying position of the Gulf Stream.

4pABc5. Emergence density and stream frequency spectra of Tadarida brasiliensis. Stephanie Dreessen, Cassi Mardis, and Laura Kloepper (Biology, Saint Mary's College, 262 Sci. Hall, Saint Mary's College, Notre Dame, IN 46556, sdrees01@saintmarys.edu)

Bats depend on echolocation to maneuver through their environment. Mexican Free-Tailed bats (Tadarida brasiliensis) form large maternal colony roosts and emerge in large, dense groups. Previous work found that the $T$. brasilensis bats adjust their call structure during emergence, which may help the bats avoid flight collisions. The limited physical space between bats in the emerging stream is not only problematic with flight collisions, but also with echolocation interference. In this study, we tested how the density of emergence affects echolocation. Specifically, we tested the hypothesis that the density of flying bats affects echolocation characteristics of the entire stream. We determined six emergence density categories from thermal imagery, and randomly selected $20,500 \mathrm{~ms}$ audio samples corresponding to each density. For each audio sample, we calculated the frequency spectrum, and analyzed how the stream frequency spectrum changed according to population density. Across densities, the stream frequency spectra all have the same shape and peak frequencies, but the variance for certain frequencies differs. This change in variance suggests that these bats are attempting to avoid jamming in dense emerging streams by altering their frequencies from bat-to-bat, irrespective of density.

4pABc6. A first look at the Ocean Noise Reference Station Network: The soundscape of Stellwagen Bank National Marine Sanctuary. Samara M. Haver (Cooperative Inst. for Marine Resources Studies, Oregon State Univ. and NOAA Pacific Marine Environ. Lab., 2030 SE Marine Sci. Dr., Newport, OR 97365, samara.haver@oregonstate.edu), Holger Klinck (BioAcoust. Res. Program, Cornell Lab of Ornithology, Cornell Univ., Ithaca, NY), Sofie Van Parijs (NOAA Northeast Fisheries Sci. Ctr., Woods Hole, MA), Haruyoshi Matsumato, Robert P. Dziak (NOAA Pacific Marine Environ. Lab., Newport, OR), Jason Gedamke (Office of Sci. and Technol., NOAA Fisheries, Silver Spring, MD), and Leila T. Hatch (Gerry E. Studds Stellwagen Bank National Marine Sanctuary, NOAA Office of National Marine Sanctuaries, Scituate, MA)

In order to compare long-term changes and trends in soundscapes around the United States, a multi-year network of identical autonomous passive acoustic recording systems, the Ocean Noise Reference Station (NRS) Network, has been established. In partnership with the National Oceanic and Atmospheric Administration (NOAA) Office of Oceanic and Atmospheric Research, NOAA Pacific Marine Environmental Laboratory, NOAA National Marine Fisheries Service, NOAA Office of National Marine Sanctuaries, and the National Park Service, hydrophone moorings were deployed in 12 discrete soundscapes in the Northeast Pacific and Northwest Atlantic oceans to record underwater ambient sound levels in the 10 to $2200 \mathrm{~Hz}$ frequency range. This initial analysis utilized the first year of available data from the NRS deployed in Stellwagen Bank National Marine Sanctuary (SBNMS), a busy area for both natural and anthropogenic activity. Preliminary results indicate that: (1) broadband (10-2200 Hz) ambient sound levels at SBNMS are stable year round in association with constant vessel activity in the nearby shipping lane to Boston, MA, (2) surface wind speed is positively correlated with broadband noise levels, (3) intensity of low-frequency baleen whale calling activity varies seasonally, but signals are acoustically detectable yearround. Future analyses will compare the soundscapes of all 12 NRS sites.

4pABc7. Analysis of fin whale vocalizations south of Rhode Island. Jennifer L. Giard, Makio Tazawa, James H. Miller, Gopu R. Potty (Ocean Eng. Dept., Univ. of Rhode Island, 215 S Ferry Rd., Narragansett, RI 02882, jennifer_giard@my.uri.edu), Ying-Tsong Lin, Arthur Newhall, and Mark Baumgartner (Woods Hole Oceanographic Inst., Woods Hole, MA)

Fin whale vocalizations were recorded south of Rhode Island during late summer through early fall of 2015 using a number of underwater recording systems. These systems were deployed to monitor broadband noise, including pile driving, from construction of the Block Island Wind Farm. Two vertical hydrophone array moorings were deployed in approximately 40 meters of water each with four hydrophones. Additionally, a tetrahedral array was deployed in about 30 meters of water just above the seabed. The tetrahedral array consisted of four hydrophones spaced 0.5 meters apart. The spacing between each of these recording systems was approximately $7.5 \mathrm{~km} .20-\mathrm{Hz}$ fin whale vocalizations were recorded numerous times on all of the sensors both during and after construction was completed. An analysis and localization effort of these signals was performed to estimate the source level, directionality, and the track of the whale over the period of the vocalizations. The results of this analysis will be discussed. [Work supported by the BOEM.]

4 ABC8. Detection and localization of whale vocalizations in archived data of seafloor cabled observatories in eastern Japan. Ryoichi Iwase (CEAT, JAMSTEC, 3173-25 Showa-machi, Kanazawa-ku, Yokohama, Kanagawa 236-0001, Japan, iwaser@jamstec.go.jp)

Through the examination of archived OBS (Ocean Bottom Seismometer) waveform data of seafloor cabled observatories in the eastern Japan, fin whale vocalizations were detected. Most of those vocalizations were observed with a single OBS because of the sparseness of the OBS deployment. At the observatory off Kushiro-Tokachi in Hokkaido, the fin whale vocalizations were localized using waveform data of both OBS and hydrophone at the same site (Iwase, 2015). At the observatory off Kamaishi in Tohoku District composed of OBSs without a hydrophone, seismic wave velocities in sediment, which are necessary parameters in estimating 
horizontal range of sound source from apparent emergence angle of transmitted wave at seafloor, were estimated in situ based on air gun signal observation (Iwase, 2016). By using those results, the fin whale vocalizations were localized. At the observatory off Hatsushima Island in Sagami Bay, sperm whale vocalizations were detected in the audible sound signals of a single hydrophone which were recorded on the soundtrack of video tapes with seafloor images. To date, more than 7000 video tapes recorded since 1993 have been archived, and the result of analysis in progress will be shown at the presentation. References: Iwase (2015), DOI: 10.7567/ JJAP.54.07HG03. Iwase (2016), DOI: 10.7567/JJAP.55.07KG01.

4pABc9. Improvements to using inter-click intervals to separate odontocete click trains from multiple animals. Jeremy Young, Anders HøstMadsen (Dept. of Elec. Eng., Univ. of Hawaii at Manoa, 2540 Dole St., Holmes Hall 483, Honolulu, HI 96822, jbyoung@hawaii.edu), and EvaMarie Nosal (Dept. of Ocean \& Resources Eng., Univ. of Hawaii at Manoa, Honolulu, HI)

At the December 2013 ASA meeting, we introduced a method to separate click trains from multiple odontocetes that relies on click timing only. The method assumes that ICIs are slowly-varying according to some distribution. Click trains are classified by sequentially going through the clicks and maximizing the likelihood based on these distributions. Since then, additions have been made to improve performance and make it more practical including a source number estimation method, a refinement to the algorithm when the ICI distributions are unknown, and a way to incorporate information from the shape of clicks. We will present the results of these new methods on real and simulated datasets.

4pABc10. Investigating the occurrence of spinner dolphins around Maui Nui, Hawaii. Megan M. McElligott (Marine Biology Graduate Program, Univ. of Hawaii at Manoa, Hawaii Inst. of Marine Biology, 46-007 Lilipuna Rd., Kaneohe, HI 96744, meganmce@hawaii.edu), Marc O. Lammers (Hawaii Inst. of Marine Biology, Univ. of Hawaii at Manoa, Honolulu, HI), and Pollyanna Fisher-Pool (Cetacean Res. Program, NOAA Pacific Islands Fisheries Sci. Ctr., Ford Island, HI)

Spinner dolphins (Stenella longirostris) occur commonly along Hawaii's sloping coastlines where they follow a daily behavioral routine of foraging offshore at night on vertically migrating mesopelagic and epipelagic prey and then return to shallower waters to rest during the day. Populations of spinner dolphins have been well documented off Hawaii island and Oahu, but much less so off the other islands. In the Maui Nui region (Maui, Molokai, Lanai, and Kahoolawe), spinner dolphins are sighted regularly, but little is known about their use of the nearshore habitat, which is characterized by much shallower bathymetry than Hawaii island and Oahu. As a result, spinner dolphins in Maui Nui offer a unique opportunity to examine the adaptability of the species to a habitat that would not be considered ideal for their life history needs. To better understand the habitat-use patterns of spinner dolphins in Maui Nui, bottom moored acoustic recorders are being used to examine spinner dolphin presence off west Maui and southeast Lanai. These data are compared to similar data previously obtained from a well-studied spinner dolphin population off west Oahu. Preliminary results from these analyses are presented in combination with data on habitat characteristics and anthropogenic impact.

4pABc11. Sound localization of fish calls recorded by two stereo-underwater-recorders. Kazuki Yamato, Ikuo Matsuo (Tohoku Gakuin Univ., 21-1 Tenjinzawa, Izumi-ku, Sendai 981-3193, Japan, kyamato@mail.tohokugakuin.ac.jp), Ryuzo Takahashi (Japan Fisheries Res. and Education Agency, Kamisu, Japan), Naoto Matsubara, and Hiroki Yasuma (Hokkaido Univ., Hakodate, Japan)

Some fish produce sounds during the spawning and ramping behaviors These sounds have been used for discrimination of fish species. Recently, the method was proposed to estimate the direction of fish call by using the arrival time difference between two hydrophones. However, it was difficult to estimate sound localization in the case of using the measured data from two hydrophones, that is, one stereo recorder. In this presentation, we used two stereo recorders, including four hydrophones for sound localization. The sound data were measured during spawning at fish, Hexagrammos otakii, in the littoral region of Usujiri-cho, Hakodate, Hokkaido, Japan (N41 ${ }^{\circ} 56^{\prime} 11$, E $\left.140^{\circ} 56^{\prime} 41\right)$. First, the fish calls were automatically detected by using the sound features including duration, max frequency, and amplitude. These features were determined by analyzing the sound data manually. Second, the arrival directions of detected fish calls were estimated in terms of each stereo recorder. Then, the fish calls were localized by using two arrival directions. As a result, it was clear that there were fishes producing call sounds around the spawning bed.

4 pABc12. Source levels and calling rates for humpback whale (Megaptera novaeangliae) non-song vocalizations in Glacier Bay Alaska. Michelle Fournet (Fisheries and Wildlife, Oregon State Univ.- Hatfield Marine Sci. Ctr., 425 SE Bridgeway Ave., Corvallis, OR 97333, mbellalady@ gmail.com), Holger Klinck (BioAcoust. Res. Program, Cornell Lab of Ornithology, Cornell Univ., Ithaca, NY), and Christine Gabriele (Humpback Whale Monitoring Program, Glacier Bay National Park, Gustavus, AK)

Marine resource managers in Glacier Bay National Park, Alaska, have been tasked with assessing the impact of vessel noise on marine mammal species. Humpback whales (Megaptera novaeangliae) are a highly vocal baleen whale that forage between spring and fall in Park waters. While calling rates and source levels for this species have been described on breeding grounds and migratory corridors, these fundamental acoustic parameters have not been thoroughly described on foraging grounds, and have never been empirically measured in the North Pacific. Using a three-element hydrophone array deployed in the Beardslee Island complex of Glacier Bay during the 2015 summer foraging season humpback whale "whup" and "growl" calls were localized and source levels were calculated. Array recordings were paired with shore based abundance estimates from the same period and calling rates (calls per whale per hour) were estimated. Known calling rates and source levels are essential for developing detection algorithms, masking metrics, and determining communication space on this historically important foraging ground.

$4 p A B c 13$. Source levels of harbor seal underwater reproductive advertisement displays. Leanna P. Matthews (Biology Dept., Syracuse Univ., 114 Life Sci. Complex, 107 College Pl., Syracuse, NY 13244, lematthe@ syr.edu), Jamie Womble (Glacier Bay National Park and Preserve, Juneau, AK), Christine Gabriele (Glacier Bay National Park and Preserve, Gustavus, AK), Holger Klinck (BioAcoust. Res. Program, Cornell Lab of Ornithology, Cornell Univ., Ithaca, NY), and Susan Parks (Biology Dept., Syracuse Univ., Syracuse, NY)

Harbor seals (Phoca vitulina), along with the majority of other phocid species, mate underwater. During the breeding season, male harbor seals set up underwater territories and use acoustic cues, known as roars, to defend these areas against intruder males and possibly to attract females. Vocalizations are low in frequency, predominately around $60-100 \mathrm{~Hz}$, with some broadband components reaching up to $5 \mathrm{kHz}$, and range from 4-10 seconds in duration. Previously, source levels of vocalizations have only been estimated. Here we present a method for measuring vocalizations to estimate source levels of harbor seal roars. We used a three-element hydrophone array in Glacier Bay National Park to record harbor seal vocalizations during the breeding season (June-July 2015). Using the array, we localized individual vocalizing animals and calculated source levels of calls in the 40$500 \mathrm{~Hz}$ range. Knowledge of source levels for vocalizations of harbor seals is important for understanding how anthropogenic forces, such as noise from vessels, may impact the acoustic behavior and communication range of these animals. 
4pABc14. Unsupervised clustering of toothed whale species from echolocation clicks. Yun Trinh, Scott Lindeneau (Comput. Sci., San Diego State Univ., 5500 Campanile Dr., San Diego, CA 92182, ytrinh@rohan.sdsu.edu), Maya Ackerman (Comput. Sci., San Jose State Univ., San Jose, CA), Simone Baumann-Pickering (Scripps Inst. of Oceanogr., La Jolla, CA), and Marie Roch (Comput. Sci., San Diego State Univ., San Diego, CA)

Supervised learning of categories related to animal signals requires labeled data that are used to train a classifier. In areas where species assemblages are poorly understood, such labeled data are usually unavailable. We show that unsupervised learning techniques may be used to provide an initial assessment of an area. We demonstrate that clustering techniques can be used to group sets of echolocation clicks from different species into clusters that tend to contain a single species without any labeled examples. Results are presented on the 2015 Detection Classification Localization and Density Estimation (DCLDE) toothed-whale development dataset, which contains analyst labels for several species of odontocetes as well as an unknown category. Echolocation clicks were detected and grouped into acoustic encounters from which spectral and temporal features were extracted. We used a simplifying assumption of single species encounters and estimated distributions of features in each encounter. Dendrograms were constructed by average-linkage clustering using a symmetric Kullback-Leibler similarity metric. Different algorithms were used to partition the dendrogram, with partition quality estimated by the silhouette algorithm. Comparison of the best machine-generated partitioning with that produced by analysts yielded an adjusted Rand statistic of 0.66 , demonstrating a good degree of concurrence.

$4 p A B c 15$. Monitoring of swine sneezing using time-frequency analysis for detecting diseases. Takuji Kawagishi (Graduate School of Systems and Information Eng., Univ. of Tsukuba, Tennodai1-1-1, Acoust. Lab. of Program in Intelligent Interaction Technologies, Tsukuba, Ibaraki 305-8571, Japan, kawagishi@aclab.esys.tsukuba.ac.jp), Koichi Mizutani, Keiichi Zempo, Naoto Wakatsuki (Faculty of Eng., Information and Systems, Univ. of Tsukuba, Tsukuba, Japan), Nobuhiro Takemae, and Takehiko Saito (Div. of Transboundary Animal Disease, National Inst. of Animal Health, NARO, Tsukuba, Japan)

This research mentions a detection method of swine sneezing in noisy swine house. While it is important to prevent swine infectious disease expansion in early stage, it is difficult to detect an infection in early stage because we cannot judge an infection from appearance of swine. However, it is a well-known fact that a swine with an infection tends to sneeze much more than healthy swine. The aim of this research is to achieve a detection of diseased swine in an automated way using the sound in the swine house and signal processing. We found that the sound of swine sneeze includes high frequency components $5-30 \mathrm{kHz}$ and a sound feature of spectra, compared to the environmental noise in swine house. Based on these features of sneezing sound, we propose the detecting technique of sneezing sound based on the composition template of a frequency feature by a time-frequency analysis which is calculated from a lot of sneezing sounds. This method calculates the difference between frequency values of templates and recorded sounds. That could judge the recorded sounds as sneezing if sounds are smaller than the threshold. As a result, the method demonstrated effectiveness in a detection of swine sneezing.

4pABc16. Age-related hearing loss in TrpV1 knockout mice. Hongzhe Li (VA Loma Linda Healthcare System, 11201 Benton St., Res. Service, Loma Linda, CA 92357, Hongzhe.Li@va.gov)

Several transient receptor potential (TRP) channels including TRPV1 are located on hair cell membranes. The gating mechanisms of these channels are associated with cellular stress, inflammation, and cytoplasmic uptake of aminoglycosides. Thus, TRPV1 channel may serve as a functional target that links acoustic trauma and enhanced aminoglycoside trafficking. TrpV1 mutant mice have no apparent hearing loss as young adults. Here, we assessed the hearing sensitivity of TrpV1 mutant mice (B6.129X1$\operatorname{Trpv} 1^{\mathrm{tm} 1 \mathrm{Jul}} / \mathrm{J}$, stock \#3770) by auditory brainstem responses to a broad frequency range, from 4 to $48 \mathrm{kHz}$. At 7 weeks of age, the auditory sensitivity in TrpV1 mutant mice was comparable to their heterozygous littermates. By 11 weeks of age, mutant TrpV1 mice exhibited evident high frequency hearing loss, with 30-to-40 dB elevated thresholds between 24 and $48 \mathrm{kHz}$, compared to littermate controls. At 18 weeks of age, high frequency hearing sensitivity worsened in both groups of mice, and this age-related progressive hearing loss was further expedited in TrpV1 mutant mice compared to littermate controls. Further research is required to determine the otoprotective role of TRPV1 channel in maintaining auditory sensitivity as mice age, and whether TrpV1 functionality exacerbates aminoglycoside-induced ototoxicity.

4pABc17. Demonstration of limited infrasonic sensitivity in the mallard duck (Anas platyrhynchos). Evan M. Hill (Psych., Univ. of Nebraska-Kearney, 2507 11th Ave., COPH - Psych., Kearney, NE 68849, hillem@unk. edu)

The purpose of this study was to determine the hearing range and sensitivity of the mallard duck, for the comparative purposes of identifying if the detection of infrasound (i.e., frequencies below $20 \mathrm{~Hz}$ ) is a common feature of the avian auditory system. Prior to this work, only two species have had their low-frequency sensitivity fully assessed: the domestic pigeon (Columba livia), and the domestic chicken (Gallus gallus domsticus). Both species showed sensitivity to infrasonic acoustic signals as low as $2 \mathrm{~Hz}$. The results of this study found the mallard to have a limited ability to detect infrasound, but inability to detect signals below $16 \mathrm{~Hz}$. Given the lack of sensitivity to infrasound relative to what was observed in other species, this ability likely has limited functional value for this species. Future research on the topic should focus on developing a comprehensive theory that would allow for the selection of avian species for testing, to determine the origin and purpose of infrasonic sensitivity in birds.

4pABc18. Tissue physical property in the head of small toothed whales: Effect on the clicks propagation and directivity. Mika Kuroda (Hokkaido Univ., 3-1-1, Minato-cho, Hakodate, Hokkaido 041-8611, Japan, mika.kuroda@fish.hokudai.ac.jp), Motoki Sasaki (Obihiro Univ. of Agriculture and Veterinary Medicine, Obihiro, Japan), Kazutaka Yamada (Azabu Univ., Sagamihara, Japan), Nobuhiro Miki (Future Univ. Hakodate, Hakodate, Japan), Masao Amano (Nagasaki Univ., Nagasaki, Japan), Tadasu K. Yamada (National Museum of Nature and Sci., Tokyo, Tsukuba, Japan), and Takashi Matsuishi (Hokkaido Univ., Hakodate, Japan)

In the current study, the distribution of acoustic impedance in the head of harbor porpoise and striped dolphin were reported and the transmission factor in the head was calculated. Clicks properties are considered to be closely connected to the structure of sound producing organ, however, few effective investigations have been carried out to reveal the cause of the species diversity of clicks properties. Clicks of toothed whales has been confirmed to be produced at the dorsal barsae (DB) and propagated to the melon where the clicks' beam is progressively focused, and emitted into the seawater from the emitting surface (ES), a circular aperture at the frontal part of the melon. In our observation of the melon, a continuous gradients were observed from DB to ES for three species. At the ES, acoustic impedance of melon was matched with that of seawater. This mechanism would enable efficient click propagation and emission. The estimated directivity of clicks only from the transmission factor was much weaker than observed directivity, which suggests that the directivity of clicks is strongly affected by the refraction in the melon than a reflection and attenuation in the head tissue.

$4 p A B c 19$. Cicada sound impacts avian dawn chorus in a subtropical forest of Taiwan. Yi C. Chen and Tzung S. Ding (School of Forestry and Resources Conservation, National Taiwan Univ., No. 1, Sec. 4, Roosevelt Rd., Taipei, 10617 Taiwan, Taipei 10617, Taiwan, r04625003@ntu.edu.tw)

Acoustic space is a limited resource for animal communications. To reduce signal competition, animals could differentiate their acoustic frequency range or time to produce sound. Many birds call or sing at dawn for higher signal transmission efficiency. However, in tropical and subtropical forests, the dawn chorus were frequently interferenced by cicadas. In order to examine the effect of cicadas on the avian dawn chorus, we used automated acoustic recorders at subtropical forest of Yangmingshan National Park, Taiwan, to monitor acoustic signals of eight bird species and three cicada species. We found that all bird species postponed their acoustic 
activities during cicada's breeding season. Meanwhile, we also found frequency partitioning of acoustic signals between birds and cicadas when their acoustic signals overlapped temporally. The results show that cicadas could play a crucial role in the avian dawn chorus. Birds would delay their signal producing time and acoustic frequency to partition the acoustic niche.

$4 \mathrm{pABc20}$. Variation in frequency-modulated call type for different flight densities in Mexican Free-tailed bats. Cassi Mardis, Stephanie Dreessen, and Laura Kloepper (Biology, Saint Mary's College, 262 Sci. Hall, Saint Mary's College, Notre Dame, IN 46556, cmardi01@saintmarys.edu)

Mexican free-tailed bats (Tadarida brasiliensis) adapt their call type depending on multiple factors. These bats have two general types of echolocation sounds, frequency-modulated (FM) and constant frequency (CF). CF calls are generally produced during the search phase of foraging, and FM calls are used during approach, prey capture, and roost emergence. In this study, we examined how Mexican free-tailed bats adapt their calls during emergence to avoid echo interference. We extracted individual calls from within a 500-ms sample that corresponded to five different emergence density categories. We found that these bats produce eight different FM call types during emergence. For each call within the sample, we characterized the call shape and calculated different parameters (duration, start frequency, peak frequency, end frequency). We compared the acoustic parameters across density categories and call shape, and compared the distribution of call shape across density categories. The results indicate that the call parameters and call shape vary from bat to bat, but there is no clear trend that this variance is due to the bat density. Therefore, these bats are likely changing their call shape and frequency characteristics to distinguish their own echoes from those of other bats.

$4 p A B c 21$. The relationships between vocalizing bearded seals and sea ice extent in the southern Chukchi Sea. Mina Jimbo (Environ. Sci., Hokkaido Univ., Benten, 20-5, Hakodate, Hokkaido 040-0051, Japan, jimbo. min56@gmail.com), Daisuke Mizuguchi (Japan Fisheries Res. and Education Agency, Kushiro, Hokkaido, Japan), Koki Tsujii (Environ. Sci., Hokkaido Univ., Hakodate, Hokkaido, Japan), Kazushi Miyashita, and Yoko Mitani (Field Sci. Ctr. for Northern Biosphere, Hokkaido Univ., Hakodate, Hokkaido, Japan)

Bearded seal Erignathus barbatus is one of ice-obligate species, which breeds on seasonal sea-ice in the Arctic and sub-Arctic during March-May. They are threatened by the rapid changes in the Arctic environment due to recent climate change, which causes sea-ice reduction or transition of their prey biomass. To understand the seasonal distribution of bearded seals and assess the impacts of climate change, we deployed the underwater sound recorder at the Southern Chukchi Hotspot $(\mathrm{SCH})\left(67.72^{\circ} \mathrm{N}, 168.83^{\circ} \mathrm{W}\right)$ during July 2012-October 2015. Calls of bearded seals were manually detected from September before sea-ice formation started, temporarily decreased during late-November-early-December when sea-ice were formed and increased again from January to the end of our recording periods (March 2013, May 2014 or June 2015) when the SCH were freezing. Combined with previous study, our results indicate that bearded seals come to the $\mathrm{SCH}$ from the northern part (i.e., Beaufort Sea) in September possibly to forage, and some move southerly to their breeding area (i.e., Bering Sea) coincident with sea-ice formation. These results suggest that seasonal distribution and vocal activity of bearded seals at the $\mathrm{SCH}$, which are tied with sea-ice, might be changed by the Arctic sea-ice decline in the future.

$4 p A B c 22$. Localization of acoustic windows in the beluga by acoustic delays from different sound-source positions. Evgeniya Sysueva, Alexander Supin, Vladimir Popov, Dmitry Nechaev (Inst. of Ecology and Evolution, 33 Leninsky Prospect, Moscow 119071, Russian Federation, evgeniasysueva@gmail.com), Alena Lemazina (Lomonosov Moscow State Univ., Moscow, Russian Federation), and Mikhail Tarakanov (Inst. of Ecology and Evolution, Moscow, Russian Federation)

Acoustic windows were localized by acoustic delays from different sound-source positions in a beluga whale (Delphinapterus leucas). The delays were measured by recording the earlies component of the auditory evoked potential (AEPs) to short tone pips. This component recorded from the lateral head surface reflects the activity of the auditory nerve and features true monaural properties. Monaural nature of the component allows to localize an acoustic window with a better precision than binaural components. The acoustic delays were measured from a sound source at different positions at frequencies from 22.5 to $90 \mathrm{kHz}$ (half-octave steps).A common sound-receiving region was identified for all these frequencies. The relation of the results to hypotheses of sound conduction in odontocetes is discussed. The use and care of the animals were adhered to the guidelines of the Russian Academy of Sciences for Research Involving Human and Non-Human Animals.

$4 p A B c 23$. Intraspecific variation in the swimbladder occurrence and possibility of acoustic discrimination of the dominant mesopelagic fish Diaphus garmaniioff western Kyushu, Japan. Hiroki Yasuma, Shinya Ohshima (Graduate School of Fisheries Sci., Hokkaido Univ., 3-1-1 Minato, Hakodate, Hokkaido 0418611, Japan, yasuma@fish.hokudai.ac.jp), Tohya Yasuda (Seikai National Fisheries Res. Inst., Nagasaki, Japan), Masa-aki Fukuwaka (Hokkaido National Fisheries Res. Inst., Hokkaido, Japan), and Koki Abe (National Res. Inst. of Fisheries Eng., Ibaraki, Japan)

Diaphus garmanii (Family, Mictophidae) is the most abundant mesopelagic fish in the continental slope off western Kyushu. For the application of acoustic monitoring, we have observed the swimbladder morphology of this species to estimate the target strength (TS). In this session, we show the intraspecific variation in the occurrences and shapes of swimbladder in $D$. garmanii, and propose the acoustic discrimination of the swimbladder morphology for accuracy improvement in biomass estimates. Acoustic data and biological samples were collected in the summer of 2012 to 2014. Both of fishes with $(30 \%$ in all samples) and without $(70 \%)$ swimbladder were observed by the soft X-ray method, and frequencies of the swimbladder occurrence were significantly different between male and female fishes. Theoretical scattering models showed that the TS difference between 38 $\mathrm{kHz}$ and $70 \mathrm{kHz}$ was the most preferable parameter to discriminate the swimbladder occurrence of $D$. garmaniiin field acoustic data. Field acoustic data analyses implied different patterns of vertical distribution between swimbladder and swimbladderless (male and female) fishes. 


\title{
Session 4 pAO
}

\section{Acoustical Oceanography, Animal Bioacoustics, and Signal Processing in Acoustics: Acoustic Scattering by Aquatic Organisms II}

\author{
Kelly J. Benoit-Bird, Cochair \\ College of Earth, Ocean, and Atmospheric Sciences, Oregon State University, 104 COEAS Admin Bldg., Corvallis, \\ OR 97331 \\ Kouichi Sawada, Cochair \\ Fisheries Technology, National Research Institute of Fisheries Engineering, FRA, 7620-7, Hasaki, Kamisu 3140408, \\ Japan \\ Timothy K. Stanton, Cochair \\ Woods Hole Oceanographic Institution, Dept. Appl. Ocean. Phys. \& Eng., Woods Hole Oceanographic Institution, \\ Woods Hole, MA 02543
}

\section{Contributed Papers}

\section{1:00}

4pA01. Three dimensional observation of pelagic fish schools by multibeam echosounder ME70. Koki Abe, Tomohiko Matuura (National Res. Inst. of Fisheries Eng., Japan Fisheries Res. and Education Agency, 7620-7, Hasaki, Kamisu, Ibaraki 314-0408, Japan, abec@fra.affrc.go.jp), Tohya Yasuda, and Yohei Kawauchi (Seikai National Fisheries Res. Inst., Japan Fisheries Res. and Education Agency, Nagasaki, Nagasaki, Japan)

In this study, three-dimensional observation of pelagic fish using a multibeam echosounder with a quantitative echosounder are discussed. Multibeam echosounder originally has been developed for bathymetry survey, transmits and receives fan-shaped acoustic beam, and has extensive search area compared with single beam conventional echosounder. SIMRAD ME70 used in this study was developed specifically for fisheries research while maintaining the characteristics of fan-shaped wide multibeam. Because the calibration software is built in the ME70 for quantitative analysis of the acoustic beams, the output data can be compared with that from the quantitative echosounder like the EK60. Therefore, the ME70 and the EK60 are installed in the R/V YOKO-MARU used in alternate transmission, and carried out the observation for the same fish schools. Echograms were obtained from both echosounders and compared in terms of differences in characteristics of these echograms. Multibeam echosounder covered a widespread area to the side way of the vessel that the conventional echosounder could not observe and allowed to express fish schools in three dimensions.

\section{$1: 15$}

4pAO2. Size estimation of a giant jellyfish Nemopilema nomurai using quantitative echosounder in the East China Sea. Tomohiko Matsuura, Koki Abe (Fisheries Technol., National Res. Inst. of Fisheries Eng., Hasaki 7620-7, firmheights \#202, Kamisu-shi, Ibaraki 314-0408, Japan, mtsr@fra. affrc.go.jp), Haruya Yamada, and Yoko Kiyomoto (Fisheries management and Oceanogr., Seikai National Fisheries Res. Inst., Nagasaki, Japan)

The maximum bell-diameter of giant jellyfish Nemopilema nomurai is over one meter. They are transported by the Tsushima current to the Sea of Japan and are sufficient to seriously damage coastal fisheries. It is necessary to monitor the population and the distribution of them for reduction of the damage. Therefore, acoustic-optical surveys of the giant jellyfish have been conducted around the Tsushima Strait by a visual observation, a quantitative echosounder (EK60, SIMRAD) and an underwater video camera from 2009 to 2014. The giant jellyfish's size estimation method using an echotrace height was developed on the surveys. Using the method, the habitat and size distribution were obtained around the East China Sea in June and July 2015 to confirm the appearance of them earlier. The giant jellyfish were distributed from surface to about $40 \mathrm{~m}$ depth as results of the quantitative echosounder and the visual observation. The measured bell-diameter range was from 30 to $80 \mathrm{~cm}$ and giant jellyfish about $60 \mathrm{~cm}$ was most detected by the visual observation. The estimated bell-diameter range using the quantitative echosounder was from 10 to $80 \mathrm{~cm}$. The value of highest frequency was $53 \mathrm{~cm}$ and agreed with the result of the visual observation.

$1: 30$

4pAO3. Statistics of the backscattered returns from random aggregations of omnidirectional point scatterers and comparisons to fish schools measurements. Adaleena Mookerjee, David R. Dowling (Mech. Eng., Univ. of Michigan, 1231 Beal Ave., 2010 Autolab, Ann Arbor, MI 48109, adaleena@umich.edu), and Kelly Benoit-Bird (Monterey Bay Aquarium Res. Inst., Moss Landing, CA)

Remote assessment of scattering objects in the ocean by analyzing the statistics of backscattered returns has been of interest for decades. This presentation describes how simulated and measured backscatter results may be combined to assess the natural variability of fish density in extended schools. The simulations were based on numerical solution of Foldy's (1945) equations for harmonic illumination of spherical and spheroidal aggregations of randomly-placed omnidirectional point scatterers. The aggregations contained hundreds to thousands of scatterers, and varied in size from $12 \leq k a \leq 32$, where $k$ is the acoustic wave number and $a$ is the aggregation's volume-equivalent radius. Based on thousands of realizations, simulated backscattered returns were found to be Rayleigh distributed independently of the scatterers' and aggregations' characteristics. However, the distribution of volumetric scattering strengths from echo-sounder measurements from natural fish schools is broader. When taken together, the two results suggest that backscattering from natural aggregations of fish might be well described by a weighted superposition of Rayleigh distributions with the weighting function being a natural aggregation characteristic that can be remotely monitored. Results from simulations and measurements at $38 \mathrm{kHz}$ and $120 \mathrm{kHz}$ are shown and compared. [Work supported by ONR and UM Advanced Research Computing.] 
4pAO4. Broadband target strength measurements of juvenile walleye pollock (Theragra chalcogramma). Kazuo Amakasu (Tokyo Univ. of Marine Sci. and Technol., 4-5-7 Konan, Minato-ku, Tokyo 108-8477, Japan, amakasu@kaiyodai.ac.jp), Tohru Mukai, Jun Yamamoto, Hiroki Hayashi, and Kohji Iida (Hokkaido Univ., Hokkaido, Japan)

Walleye pollock (Theragra chalcogramma) is one of very important fisheries resources in Japan. The acoustic-net surveys have been conducted around Hokkaido since 1995. To improve the present resource management, the estimation of the standing stock of juvenile walleye pollock is necessary. The target strengths of juvenile walleye pollock at $38 \mathrm{kHz}$ have been wellstudied through measurement. However, there are little knowledge of the target strengths at frequencies other than $38 \mathrm{kHz}$. In this study, therefore, the target strengths at other frequencies were measured. The acoustic system was consist of a broadband transducer and commercially available equipment. The calibration of the system was performed by using a 20.6-mm-diameter tungsten carbide sphere. The transmitting signal was $20-160 \mathrm{kHz}$ linear frequency modulated signal. The target strengths of nine specimens were measured and their fork lengths were ranged from 6.9 to $12.1 \mathrm{~cm}$. Each individual specimen was anesthetized and tethered in a tank $(5 \mathrm{~m} \mathrm{x} 5$ $\mathrm{m} \times 5 \mathrm{~m}$ ) with a thin fishing line. Orientation angles of the specimen were varied between -50 to 50 degrees and the target strengths were measured at each angle. Orientation and length dependences of the target strengths are presented and discussed.

\section{2:00}

4pAO5. Modeling target strength of individual herring (Clupea harengus) at any aspect as a function of pressure and frequency. Geir Pedersen (Instrumentation, Christian Michelsen Res. AS, P.O. Box 6031, Bergen 5892, Norway, geir.pedersen@cmr.no), Gavin J. Macalay, Hector Peña (Inst. of Marine Res., Bergen, Norway), and Sascha M. Fässler (Wageningen Inst. for Marine Resources and Ecosystem Studies, Ijmuiden, Netherlands)

Fish target strength is primarily dependent on the physical dimensions of the fish, the acoustic frequency, and the orientation of the fish. In traditional vertically observing echosounder surveys, fish are insonified in the dorsal aspect with fairly limited tilt angle variation. In oblique-angled sonar surveys, however, fish may be insonified at other aspects. For herring, target strength is also depth dependent as they cannot refill the swimbladder at depth. Understanding the depth dependent target strength from several insonification angles is thus required for quantitative measurements with sonar. The dataset used in this study consists of seven herring, imaged using magnetic resonance imaging (MRI). The herring were placed in a pressure chamber inside the MRI, and subjected to different pressures corresponding to water depths of $0,20,40$, and $60 \mathrm{~m}$. Images were acquired of each specimen at each pressure. The swimbladders were segmented and 3D models of the swimbladders for each fish and pressure were constructed. These models were then used for computing the directivity pattern of the swimbladder at any angle as a function of frequency using the finite element method. Modeling results are also compared with measured dorsal and side aspect TS at different depths. The broadband backscattering $(30-200 \mathrm{kHz})$ is evaluated for the potential for species and size discrimination and the effect including the fish body is discussed.

\section{2:15-2:30 Break}

\section{2:30}

4pAO6. Acoustic scattering by tube-building worms (Polychaeta: Maldanidae) of the New England Mud Patch. Matthew C. Zeh, Preston S. Wilson, Kevin M. Lee, Megan S. Ballard (Dept. of Mech. Eng. and Appl. Res. Labs., Univ. of Texas at Austin, 204 East Dean Keeton St., Austin, TX 78712-1591, mzeh@utexas.edu), and Kelly M. Dorgan (Dauphin Island Sea Lab, Dauphin, AL)

The acoustic effects of biological activity within shallow water ocean environments are not well understood, yet are of increasing importance in some sonar applications. These environments remain insufficiently characterized in part due to the presence of benthic organisms within and near the sediment and water-sediment interface. Among the most prevalent infauna
Maldanidae). The presence of and bioturbation caused by these organisms can affect the acoustical properties of the ocean bottom sediment. These effects are being studied in experiments being conducted in the New England Mud Patch, on the continental shelf south of Martha's Vinyard, MA. To begin to quantify these effects, laboratory acoustic scattering measurements of naturally collected worms and worm tubes were completed over a range of frequencies $(50 \mathrm{kHz}$ to $1 \mathrm{MHz})$ and incidence angles. Predictive models by Faran [J. Acoust. Soc. Am. 23, 405 (1951)] approximating the worms as elastic cylinders, and Doolittle [J. Acoust. Soc. Am. 39, 272 (1966)] approximating the worm tubes as cylindrical shells, were used to interpret the measured results. Measurements and model comparisons will be discussed along with implications relating to effective bulk sediment properties. [Work supported by ONR.]

\section{$2: 45$}

4pA07. Modelling macroalgae scattering (Ecklonia radiata). Jean-Pierre Hermand (LISA - Environ. HydroAcoust. Lab, Université libre de Bruxelles, av. F.D. Roosevelt 50, CP165/57, Brussels, Brussels Capital 1050, Belgium, jhermand@ulb.ac.be)

This paper will discuss the numerical modelling of acoustic scattering by macroalgae. Using earlier measurement of tissue acoustic properties, the scattering function of a thalus of Ecklonia radiata species is estimated by means of finite elements. Results will be presented for frequencies up to 20 kHz. [Work supported by ONR and ONR Global.]

\section{3:00}

4pA08. An inter-individual communication biotelemetry system using Pseudo-random noise (Gold code). Yoshinori Minamoto (Tokyo Univ. of Marine Sci. and Technol., 4-5-7 Konan, Minato, Tokyo 108-8477, Japan, miyamoto@kaiyodai.ac.jp), Nobuaki Arai (Field Sci. Education and Res. Ctr., Kyoto Univ., Kyoto, Japan), Hiromichi Mitamura (Graduate School of Informatics, Kyoto Univ., Kyoto, Japan), Keiichi Unhid (Tokyo Univ. of Marine Sci. and Technol., Tokyo, Japan), Satoko S. Kimura (Field Sci. Education and Res. Ctr., Kyoto Univ., Kyoto, Japan), Kazushi Miyashita, Hokuto Shirakawa, Yuichi Tsuda (Field Sci. Ctr. for Northern Biosphere, Hokkaido Univ., Hakodate, Japan), Takashi Kitagawa (Atmosphere and Ocean Res. Inst., The Univ. of Tokyo, Kashiwa, Japan), and Minlee Yap (Tokyo Univ. of Marine Sci. and Technol., Tokyo, Japan)

Recently, bio-logging techniques involving electronic data-storage tags (data-logger) and acoustic transmitters have been increasingly used to understand migratory fish movements and behaviors. The monitoring of migratory fish can provide important insights into the marine ecosystem dynamics. The number of tags data-loggers used, however, is normally limited due to cost and the data-logger recovery rate is usually low. Therefore, we developed a new system that combines the acoustic communication and data logger. In other words, a data-logger that can share data with each other. One of the challenges is to develop an inter-individual communication biotelemetry system based on hydro-acoustic methods. In this system, we used the phase modulation of pseudo-random noise (Gold code). At the final stage of development, we will be doing conduct a field test of this inter-individual communication biotelemetry system on wild herrings and bonitos in the open sea. We expect this new technology to help overcome the bottleneck of the conventional bio-logging and bio-telemetry, and lead to a breakthrough in marine ecosystem studies.

\section{$3: 15$}

4pA09. Global access to sonar data: Where can it take you? Carrie C. Wall, Charles Anderson (Cooperative Inst. for Res. in Environ. Sci., Univ. of Colorado at Boulder, 216 UCB, Boulder, CO 80309, carrie.bell@colorado.edu), and Michael Jech (NEFSC, NMFS, Woods Hole, MA)

Scientific echosounders aboard NOAA fishery survey vessels are used to estimate biomass, measure fish school morphology, and characterize habitat. These surveys produce large volumes of data that are stored locally and difficult to access. Data that are easily discoverable and accessible provide valuable information beyond their original collection purpose. NOAA's National Centers for Environmental Information, in partnership with the National Marine Fisheries Service and the University of Colorado, created a 
national archive for the stewardship and distribution of water column sonar data. A data access web page allows users to query the metadata and access the raw sonar data. Visualization products allow researchers and the public to understand the quality and content of large volumes of archived data. Such products transform the complex raw data into a digestible image and are highly valuable for a broad audience of varying backgrounds.
Concurrently collected oceanographic and bathymetric data are being integrated into the data access page to provide an ecosystem-wide understanding of the area surveyed. Benefits of the archive include global access to an unprecedented nationwide dataset and the increased potential for researchers to address new questions to advance the field of marine ecosystem acoustics.

\title{
Session 4pBA
}

\section{Biomedical Acoustics: Medical Acoustics in Kidney and Liver Disease II}

\author{
Norihiro Koizumi, Cochair \\ Graduate School of Informatics and Engineering, The University of Electro-Communications (UEC), 1-5-1 Chofugaoka, \\ Chofu, Tokyo 182-8585 JAPAN, Chofu 182-8585, Japan \\ Michael Bailey, Cochair \\ Center for Industrial and Medical Ultrasound, Applied Physics Lab, University of Washington, 1013 NE 40th Street, \\ Seattle, WA 98105
}

Hiroyuki Fukuda, Cochair

Yokohama City Univ. Medical Center, Yokohama, Japan

Adam D. Maxwell, Cochair

University of Washington, 1013 NE 40th St., Seattle, WA 98105

\section{Contributed Papers}

1:00

4pBA1. Kidney stone pushing and trapping using focused ultrasound beams of different structure. Oleg A. Sapozhnikov, Anastasia V. Nikolaeva, Marina E. Terzi, Sergey A. Tsysar (Phys. Faculty, Moscow State Univ., Leninskie Gory, Phys. Faculty, Moscow State University, Moscow 119991, Russian Federation, oleg@acs366.phys.msu.ru), Adam D. Maxwell, Bryan W. Cunitz, and Michael R. Bailey (Ctr. for Industrial and Medical Ultrasound, Appl. Phys. Lab., Univ. of Washington, Seattle, WA)

A technology to reposition kidney stones with radiation force was recently proposed by our team and already used to transcutaneously facilitate passage of small stones. While successful, the trial revealed a need for optimization of the ultrasound beam structure, frequency, and intensity to make it more effective. In the current work, the effect of the ultrasonic beam diameter vs. stone size using a quasi-Gaussian beam model was numerically investigated. Radiation force on a kidney stone was found to be strongest when the beam width was slightly wider than the stone diameter. This can be explained by more effective generation of shear waves inside the stone resulting from their effective coupling with the acoustic waves in liquid at the stone edges. In another study, the possibility of using vortex beams to trap the stones in the lateral direction was investigated. Both theoretical modeling and experiments were performed using two systems: a single-element transducer combined with a sector-shaped phase plate and a 12element sector array. Human stones approximately $3-5 \mathrm{~mm}$, as well as glass and styrofoam beads, were controllably translated along the surface transverse to the beam. [This work was supported by RBBR 14-02-00426, NIH NIDDK DK43881, DK104854, and DK092197, and NSBRI through NASA NCC 9-58.]
$1: 15$

4pBA2. Robust servoing method for renal stones/tumors for the noninvasive ultrasound theragnostic system. Atsushi Kayasuga (The Univ. of Tokyo, 8-14-6, Goko, Matsudo-shi, Chiba 270-2213, Japan, kayasuga04@ gmail.com), Norihiro Koizumi, Kyohei Tomita, Yu Nishiyama (The Univ. of Electro-Communications, Tokyo, Japan), Hiroyuki Tsukihara (The Univ. of Tokyo, Tokyo, Japan), Hiroyuki Fukuda (The Univ. of Yokohama City, Tokyo, Japan), Kiyoshi Yoshinaka (National Inst. of Adv. Industrial Sci. and Technol., Tokyo, Japan), Takashi Azuma, Hideyo Miyazaki, Naohiko Sugita (The Univ. of Tokyo, Tokyo, Japan), Kazushi Numata (The Univ. of Yokohama City, Tokyo, Japan), Yukio Honma, Yoichiro Matsumoto, and Mamoru Mitsuishi (The Univ. of Tokyo, Tokyo, Japan)

The main problem on HIFU (High Intensity Focused Ultrasound) therapy is the difficulty to locate HIFU focus precisely onto the focal lesion, which is located in the moving organ such as livers/kidneys, due to the deformation and rotation, which is caused by respiration. Furthermore, rib bones frequently block the acoustic path to the lesion. The acoustic shadow, which is generated by the rib bone, is observed in the ultrasound images and the lesion is hidden in the shadow. To cope with this problem, we have developed a novel method to track, follow, and monitor the lesion by utilizing the contour information of the organ, which incorporate the lesion under those difficult conditions. As for the tracking method, the contour of the organ, which is deformed and rotated in accordance with respiration, is extracted automatically in 2-D ultrasound images. The missing contour information in the acoustic shadow area is estimated and compensated by the surrounding contours, which are successfully extracted. To confirm the effectiveness of the proposed method, we compared the proposed method 
with the conventional method in terms of the tracking performance of the stone. As a result, we achieved the tracking performance (the average servo error is $2.3 \mathrm{~mm}$ ) by the proposed method, while the average servo error is $3.57 \mathrm{~mm}$ by the conventional method. This shows the validity of our novel proposed method.

\section{$1: 30$}

4pBA3. Combining burst wave lithotripsy and ultrasonic propulsion for enhanced kidney stone comminution. Theresa A. Zwaschka, Bryan W. Cunitz, Michael R. Bailey, Barbrina Dunmire (Ctr. for Industrial and Medical Ultrasound, Appl. Phys. Lab., Univ. of Washington, 1013 NE 40th St., Seattle, WA 98105, azwaschka@ucla.edu), and Adam D. Maxwell (Dept. of Urology, Univ. of Washington School of Medicine, Seattle, WA)

Burst wave lithotripsy (BWL) and ultrasonic propulsion (UP) are two new focused ultrasound technologies for noninvasively treating kidney stones. BWL applies short, focused bursts of ultrasound to fracture stones. UP employs long-duration bursts of ultrasound to reposition stones and has been successfully tested on humans. We hypothesize that application of lowlevel UP can improve comminution by reorienting the stone and dislodging intervening fragments from the stone surface. Experiments were performed in a degassed water bath with a polyvinyl chloride tissue phantom mimicking a kidney calyx. An artificial calcite stone was placed in the phantom and aligned with the focus of a $330-\mathrm{kHz}$ BWL transducer with a co-axial P4-2 imaging probe used for UP. Fragmentation size was compared between stones treated with a fixed 5-minute BWL exposure using 20-cycle bursts at 40 bursts/sec, with or without interleaved UP. The results showed improved fragmentation with the combined exposure, with 3 UP bursts/minute resulting in $45 \%$ of stone debris $<2 \mathrm{~mm}$ versus $23 \%$ without UP ( $p=0.011)$. Stones treated with UP alone did not fragment. These results suggest UP can improve stone fragmentation by BWL. [Work supported by NIH K01 DK104854 and P01 DK043881, and NSBRI through NASA NCC 9-58.]

\section{1:45}

4pBA4. Clinical experience of intra-operative high intensity focused ultrasound in patients with colorectal liver metastases. Results of a Phase II study. David Melodelima (LabTAU - INSERM U1032, 151 cours Albert Thomas, Lyon 69003, France, David.Melodelima@inserm.fr), Aurelien Dupre, David Perol, Yao Chen (Dept. of Surgery, Ctr. Leon Berard, Lyon, France), Jeremy Vincenot, Anthony Kocot (LabTAU - INSERM U1032, Lyon, France), and Michel Rivoire (Dept. of Surgery, Ctr. Leon Berard, Lyon, France)

The aim of this study was to assess the feasibility, safety and accuracy of HIFU ablation in patients with liver metastases in a prospective, phase III trial. The transducer has a toroidal shape (diameter: $70 \mathrm{~mm}$, radius of curvature: $70 \mathrm{~mm}$ ) and was divided into 32 ring-shaped emitters operating at 3 MHz. Twenty-eight patients were included. HIFU ablations were created to ablate metastases (20 mm maximal diameter) with safety margins in all directions. The exposure time varied from 40 seconds to 370 seconds according to the diameter of the metastases to be treated. One metastasis of $10 \mathrm{~mm}$ in diameter was ablated in 40 seconds with safety margins. Using electronic focusing metastases of $2 \mathrm{~cm}$ in diameter were ablated with safety margins ( $>3 \mathrm{~mm}$ in all directions) in 370 seconds. The dimensions of these HIFU ablations were a diameter of $48 \pm 4.9 \mathrm{~mm}$ and a long axis of $51 \pm$
$3.4 \mathrm{~mm}$. No damage occurred to neighboring tissues. This HIFU device safely achieved ablations of small metastases $(<20 \mathrm{~mm})$ with planned safety margins of at least $3 \mathrm{~mm}$ in all directions. This study is the first clinical use of intra-operative HIFU in patients with liver metastases.

\section{2:00}

4pBA5. Generation of different types of surface acoustic waves by shock wave-Stone interaction. Ying Zhang, Chen Yang, and Pei Zhong (Mech. Eng. and Material Sci., Duke Univ., Hudson 229, Durham, NC 27708, zhang.ying@duke.edu)

The generation of different types of surface acoustic waves (SAWs) in lithotripsy is investigated by numerical simulations using COMSOL, considering either the case of a weakly focused shock wave as an incident plane wave (in the focal plane) in shock wave lithotripsy (SWL) or a spherically divergent shock wave produced by a spark discharge in Nano Pulse Lithotripsy (NPL). The interaction of these two types of shock waves with an artificial kidney stone immersed in water may generate three different types of SAWs: namely, Scholte wave, acoustic surface evanescent wave (ASEW), and leaky Rayleigh wave (LRW). In particular, we examined the generation of SAWs on a flat, cylindrical, or spherical surface under different incident angles. It was found that the geometry and acoustic properties of the stone could significantly influence the types of SAWs generated and the resultant peak tensile stress produced at the stone-water boundary. Comparison with experimental measurements (i.e., Schlieren and photoelastic imaging) and stone fracture will also be made. [Work supported by NIH through 5R37DK052985-20.]

\section{2:15}

4pBA6. Modeling and experimental analysis of acoustic cavitation bubble clouds for burst-wave lithotripsy. Kazuki Maeda, Tim Colonius (California Inst. of Technol., 1200 E California Blvd., Pasadena, CA 91125, maeda@caltech.edu), Wayne Kreider, Adam Maxwell (Univ. of Washington, Seattle, WA), and Michael Bailey (Univ. of Washington, Seattle, CA)

Understanding the dynamics of cavitation bubble clouds formed inside a human body is critical for the design of burst-wave lithotripsy (BWL), a newly proposed method that uses focused ultrasound pulses with amplitude of $O(10) \mathrm{MPa}$ and frequency of $O(0.1) \mathrm{MHz}$ to fragment kidney stones. We present modeling and three-dimensional direct numerical simulations of interactions between bubble clouds and ultrasound pulses in water. We study two configurations: isolated clouds in a free field, and clouds near a rigid surface. In the modeling, we solve for the bubble radius evolution and continuous flow field using a WENO-based compressible flow solver. In the solver, Lagrangian bubbles are coupled with the continuous phase, defined on an Eulerian grid, at the sub-grid scale using volume averaging techniques. Correlations between the initial void fraction and the maximum collapse pressure in the cloud are discussed. We demonstrate acoustic imaging of the bubbles by post-processing simulated pressure signals at particular sensor locations indicating waves scattered by the clouds. Finally, we compare the simulation results with experimental results including high-speed imaging and hydrophone measurements. The time evolution of the cloud void fraction and the scattered acoustic field in the simulation agree with the experimental results. [Funding supported by NIH 2P01-DK043881.] 
4pBA7. A training phantom for ultrasound-guided boiling histotripsy therapy. Barbrina Dunmire, John C. Kucewicz, Wayne Kreider (Appl. Phys. Lab, Univ. of Washington, 1013 NE 40th St., Seattle, WA 98105, mrbean@uw.edu), Tanya D. Khokhlova (Medicine - Gastroenterology, Univ. of Washington, Seattle, WA), George R. Schade, Adam D. Maxwell (Urology, Univ. of Washington, Seattle, WA), Oleg A. Sapozhnikov (Appl. Phys. Lab, Univ. of Washington, Seattle, Washington, U.S. and Dept. of Acoust., Moscow State Univ., Seattle, Washington), Larry A. Crum (Appl. Phys. Lab, Univ. of Washington, Seattle, WA), and Vera A. Khokhlova (Appl. Phys. Lab, Univ. of Washington, Seattle, Washington, U.S. and Dept. of Acoust., Moscow State Univ., Seattle, Washington)

Boiling histotripsy $(\mathrm{BH})$ uses millisecond-long focused ultrasound pulses with shocks to mechanically disrupt targeted tissue under real-time ultrasound monitoring. However, adipose tissue and ribs can interfere with $\mathrm{BH}$ therapy through aberration, absorption, and diffraction. Here we introduce a robust abdominal wall phantom that includes fat, muscle, and rib layers for demonstrating the use of $\mathrm{BH}$ and investigating the impact of anatomic structures on treatment success. The skin is a silicone sheet; the fat and muscle layers are polyvinyl alcohol phantoms with irregular-shaped walls; the ribs are 3D-printed sections from a human model anatomically relevant to liver or kidney treatments. The target is a transparent alginate or polyacrylamide gel that allows visualization of the lesion. The pieces are assembled in a water-filled container providing coupling between layers and allowing components to be shifted in position relative to the transducer. A BH transducer $(1.2 \mathrm{MHz}, 12.5 \mathrm{~cm}$ focal length, $\mathrm{f}=1$ ) containing an imaging probe in its central opening was used for initial phantom assessment. Preliminary results show that each layer impacts the beam in ways similar to observations from in vivo and ex vivo experiments. [Work supported by NSBRI through NASA NCC 9-58, NIH RO1EB007643, K01EB015745, and K01DK104854.]

\section{Contributed Paper}

\section{2:45}

4pBA8. Broad beam for more effective ultrasonic propulsion of kidney stones. Michael R. Bailey, Bryan W. Cunitz, Barbrina Dunmire, Brian MacConaghy, Yak-Nam Wang (Ctr. for Industrial and Medical Ultrasound, Appl. Phys. Lab, Univ. of Washington, 1013 NE 40th St., Seattle, WA 98105, mike.bailey.apl@gmail.com), Karmon Janssen, Timothy C. Brand (Dept. of Urology, Madigan Health Care System, Tacoma, WA), Doug Corl, Oren Levy (SonoMotion, Inc., San Francisco, CA), Mathew D. Sorensen (Dept. of Urology, VA Puget Sound Health Care System, Seattle, WA), Jonathan D. Harper, and Adam D. Maxwell (Dept. of Urology, Univ. of Washington, Seattle, WA)

Ultrasonic propulsion uses focused bursts of ultrasound to generate radiation force to non-invasively reposition stones. Ultrasonic propulsion has been implemented on a C5-2 curvilinear array and shown to be safe and effective for expelling small stones and fragments in a human feasibility study. Here, we evaluated the efficacy of three different focal beam patterns in moving stones: 1) the existing approach exciting all 128 elements of the C5-2 transducer at $2 \mathrm{MHz}, 2$ ) exciting only 40 elements, and 3) implementing a separate single element transducer at $350 \mathrm{kHz}$. The capability of each method to lift clusters of 1-2 mm and 3-4 mm calcium oxalate stone fragments in a pipette was measured, filmed and compared by calculating the sum of the product of fragment area and distance moved for all fragments. For the same peak pressure, the alternative approaches (2 and 3) lifted greater stone mass than the original output. With 1-2 mm fragments, improvement with the three methods was $14 \%$ and $500 \%$. For $3-4 \mathrm{~mm}$, improvement was $45 \%$ and $178 \%$. [Work supported by NIH NIDDK grants DK043881, DK107094, DK104854, and DK092197, the NSBRI through NASA NCC 9-58, and resources from the VA Puget Sound Health Care System.]

\section{3:00-3:15 Break}

\section{Invited Paper}

3:15

4pBA9. HIFU-induced cloud cavitation control for kidney stone treatment. Teiichiro Ikeda (Res. and Development Group, Hitachi Ltd., 1-280, Higashi-koigakubo, Kokubunji, Tokyo 1858601, Japan, teiichiro.ikeda.hv@hitachi.com), Shin Yoshizawa (Dept. of Elec. and Commun. Eng., Tohoku Univ., Miyagi, Japan), and Yoichiro Matsumoto (RIKEN, Saitama, Japan)

The shock wave lithotripter uses an order of microsecond pulse durations and up to a $100 \mathrm{MPa}$ pressure spike triggered at approximately $0.5-2 \mathrm{~Hz}$ to fragment kidney stones through mechanical mechanisms. One important mechanism is cavitation. We proposed an alternative type of lithotripsy method that maximizes cavitation activity to disintegrate kidney stones using high-intensity focused ultrasound (HIFU). We designed a two-frequency wave (cavitation control (C-C) waveform); a high-frequency ultrasound pulse to create a cavitation cloud, and a low-frequency pulse following the high-frequency pulse to force the cloud into collapse. High-speed photography showed cavitation collapse on a kidney stone and shock wave emission from the cloud. We also conducted in-vitro erosion tests of model and natural stones. For the model stones, the erosion rate of the C-C waveform showed a distinct advantage with the combined high- and low-frequency waves over either wave alone. Natural stones were eroded and most of the resulting fragments were less than 1 $\mathrm{mm}$ in diameter. The small fragments were small enough to pass through the urethra. The results demonstrate that, with the precise control of cavitation activity, focused ultrasound has the potential to be used to develop a less invasive and more controllable lithotripsy system. 


\section{Contributed Paper}

3:30

4pBA10. Liver tracking system utilizing template matching and energy function in high intensity focused ultrasound/radio frequency ablation therapy. Kyohei Tomita, Norihiro Koizumi, Ryosuke Kondo (Graduate School of Informatics and Eng., The Univ. of Electro-Communications, 1-51 Chofugaoka, Chofu, Tokyo 182-8585, Japan, tomitakyohei@uec.ac.jp), Atsushi Kayasuga (The Univ. of Tokyo, Bunkyo-ku, Japan), Yu Nishiyama (Graduate school of Informatics and Engineerling, The Univ. of ElectroCommunications, Chofu, Japan), Hiroyuki Tsukihara (The Univ. of Tokyo, Bunkyo-ku, Jersey), Hiroyuki Fukuda, Kazushi Numata (Yokohama City Univ., Yokohama, Japan), Yoichiro Matsumoto (RIKEN, Wako, Japan), and Mamoru Mitsuishi (The Univ. of Tokyo, Bunkyo-ku, Japan)

Monitoring and evaluating the therapeutic effects, in ultrasound images during HIFU (High Intensity Focused Ultrasound) and RFA (Radio Frequency Ablation) therapies, are important. However, the common problem is the difficulty to monitor the progress and identify the positions of the focal lesion precisely in accordance with the progress of the treatment. This problem is caused by the movement of organs and the change of the textures information in ultrasound images. To overcome those problems, we have developed a novel method to track, follow, and monitor the focal lesion by the combination of the energy function method and the template matching method. The template matching method uses the characteristic texture information of the organ near the focal lesion. The energy function is implemented in order to reinforce the robustness of the tracking performance of the template matching method. Particularly, we evaluate the existing probability of the focal lesion considering the continuity of the movement and the relative distance from the surface of the liver. The estimated position of the focal lesion is obtained by minimizing the above mentioned energy function. The effectiveness of the proposed method is confirmed by comparing the novel proposed method and the conventional template matching method in terms of the tracking performance for the liver tumor during the real RFA treatments.

\section{Invited Paper}

$3: 45$

4pBA11. Construction methodology for non-invasive ultrasound theragnostic system by medical digitalization. Norihiro Koizumi (Graduate School of Informatics and Eng., The Univ. of Electro-Communications (UEC), 1-5-1 Chofugaoka, Chofu, Tokyo 182-8585, Japan, nkoizumi@ieee.org), Hiroyuki Tsukihara (The Univ. of Tokyo, Bunkyo-ku, Japan), Kiyoshi Yoshinaka (National Inst. of Adv. Industrial Sci. and Technol., Tsukuba, Japan), Hideyo Miyazaki (The Univ. of Tokyo, Bunkyo-ku, Japan), Hiroyuki Fukuda (Yokohama City Univ., Yokohama, Japan), Kazushi Numata (The Univ. of Tokyo, Yokohama, Japan), Yukio Homma (The Univ. of Tokyo, Bunkyo-ku, Japan), Yoichiro Matsumoto (RIKEN, Bunkyo-ku, Japan), and Mamoru Mitsuishi (The Univ. of Tokyo, Bunkyo-ku, Japan)

We propose a noninvasive ultrasound theragnostic system (NIUTS) that compensates for movement by tracking and following the area to be treated by using stereo ultrasound imaging while irradiating the focal lesion with HIFU. Theragnostics involves therapeutics and diagnostics. Our proposed system uses focused ultrasound to destroy tumors and stones without damaging the surrounding healthy tissue. This is achieved by tracking and following the focal lesion (stones/tumors), in order to compensate for movement due to respiration, heartbeat, etc. In the overall research project, we aim to enhance the focal lesion servo (FLS) performances based on our original medical support system construction methodology, which is called "Me-DigIT (medical digitalization by IT technology, which includes especially robot technology)." In the present report, we illustrate the mechanisms, controllers, and robot vision core technology to enhance the focal lesion tracking and following performance of the NIUTS. As a result, with the constructed NIUTS, tracking performance within $2.5 \mathrm{~mm}$ was achieved for a healthy human kidney. The standard deviation of the position of the servo target was $1.92 \mathrm{~mm}$ with FLS, while it was $13.2 \mathrm{~mm}$ without FLS. In other words, $86 \%$ of body movement could be compensated for by using NIUTS.

\section{Contributed Papers}

\section{4:00}

4pBA12. An ultrasound guided monitoring system for high intensity focused ultrasound and radio frequency ablation therapies. Ryosuke Kondo, Norihiro Koizumi, Kyohei Tomita (Graduate School of Informatics and Eng., The Univ. of Electro-Communications, 1-5-1 Chofugaoka, Chofu, Tokyo 182-8585, Japan, k1312058@edu.cc.uec.ac.jp), Atsushi Kayasuga (The Univ. of Tokyo, Bunkyo-ku, Japan), Yu Nishiyama (Graduate school of Informatics and Engineerling, The Univ. of Electro-Communications, Chofu, Japan), Hiroyuki Tsukihara (The Univ. of Tokyo, Bunkyo-Ku, Japan), Hiroyuki Fukuda, Kazushi Numata (Yokohama city Univ., Yokohama, Japan), Yoichiro Matsumoto (RIKEN, Wako, Japan), and Mamoru Mitsuishi (The Univ. of Tokyo, Bunkyo-ku, Japan)

In accordance with the progress of the HIFU (High Intensity Focused Ultrasound) and RFA (Radio Frequency Ablation) treatments, the intensity of the focal lesion and the surrounding marginal area changes little by little. Here, it should be noted that the human eyes are very weak when the image takes a long time to change even if the image change from the start of the ablation treatment is large. To cope with those problems, we propose an ultrasound guided monitoring system to evaluate the progress of the ablation therapy quantitatively in order to secure the certain level of the monitoring during the HIFU and RFA therapies by reinforcing the lack of experience of medical doctors. Particularly, we propose a system to monitor and evaluate the intensity of the focal lesion and the intensity of the surrounding marginal area by tracking the position of the characteristic texture area near the focal lesion, which moves in accordance with the respiration. Our method to overlay the focal lesion and the surrounding marginal area on the ultrasound image is also effective in order not to miss the area to be treated. The experimental results during RFA ablation therapy shows the effectiveness of our constructed system. 
repetition frequency, representing a rate of energy delivery well in excess of

4pBA13. Modeling shock-wave fields generated by a diagnostic-type transducer. Maria M. Karzova, Pavel B. Rosnitskiy, Petr V. Yuldashev, Oleg A. Sapozhnikov (Phys. Faculty, Moscow State Univ., Moscow 119991, Russian Federation, masha@acs366.phys.msu.ru), Wayne Kreider, Bryan W. Cunitz, Michael R. Bailey (Ctr. for Industrial and Medical Ultrasound, Appl. Phys. Lab., Univ. of Washington, Seattle, WA), and Vera A. Khokhlova (Phys. Faculty, Moscow State Univ., Moscow, Russian Federation)

New applications of ultrasound imaging may benefit from increased in situ pressure levels. However strong nonlinear propagation effects have not been studied in detail for fields generated by diagnostic transducers. Here we compare two modeling approaches for predicting shock formation in fields generated by a curvilinear imaging probe (C5-2). The field was simulated in two ways: 1) a 3D full-diffraction model based on the Westervelt equation, with a boundary condition defined on a cylindrical surface; 2) an axially symmetric parabolic model based on the KZK equation to define a flat, circular equivalent source. For both models, boundary conditions are adjusted to match low-power axial pressure measurements in the focal lobe of the beam. Simulations and measurements were performed for operation of 40,64, and 128 central probe elements, and a wide range of clinically relevant output levels was considered. Comparison of focal waveforms shows that the KZK model can predict the amplitudes of fully developed shocks within 5\% for 64 and 128 active elements, and within $15 \%$ for 40 elements. The full 3D calculations provide better agreement with experiments (within 3\%) but require significantly more computational resources. [Work supported by RSF 14-12-00974 and a scholarship of the president of Russia.]

\section{$4: 30$}

4pBA14. Scattered shear wave contribution to acoustic radiation force on spheres in soft tissue. Benjamin C. Treweek, Yurii A. Ilinskii, Evgenia A. Zabolotskaya, and Mark F. Hamilton (Appl. Res. Labs., Univ. of Texas at Austin, 10000 Burnet Rd., Austin, TX 78758, btreweek@utexas.edu)

A theory for acoustic radiation force on a sphere in soft tissue was developed for arbitrary incident compressional wave fields [Ilinskii et al., Proc. Meet. Acoust. 19, 045004 (2013)]. This theory includes two contributions to the radiation force. The first depends only on the incident and scattered compressional waves, whereas the second depends on the scattered shear waves as well. Each contribution in turn has two parts, one due to direct integration of the time-averaged Piola-Kirchhoff stress tensor over the surface of the sphere, and the other due to the irrotational component of the body force on the sphere. While both parts are known analytically for the compressional waves, only the first part has been obtained analytically for the contribution involving shear waves. The irrotational portion associated with shear waves and its effect on the total radiation force is the subject of this presentation. The analysis is conducted via Helmholtz decomposition of the body force associated with shear waves and subsequent integration of the irrotational portion over the surface of the sphere. Simplifying analytical approximations based on numerical calculations are examined for various elastic properties of the sphere and soft tissue. [Work supported by the ARL:UT McKinney Fellowship in Acoustics.]

\section{$4: 45$}

4pBA15. Thresholds for sustained bubble cloud generation in burst wave lithotripsy. Christopher Hunter (CIMU, Appl. Phys. Lab., Univ. of Washington, 1013 NE 40th St., Seattle, WA 98105), Adam D. Maxwell (Dept. of Urology, Univ. of Washington School of Medicine, Seattle, WA), Bryan W. Cunitz, Barbrina Dunmire, and Wayne Kreider (CIMU, Appl. Phys. Lab., Univ. of Washington, Seattle, WA, wkreider@uw.edu)

Burst wave lithotripsy (BWL) is a new non-invasive approach for disintegrating kidney stones using tone bursts of sub-megahertz ultrasound. An important advantage of BWL compared to shock wave lithotripsy (SWL) is the rate at which acoustic energy can be delivered without causing cavitation that injures functional renal tissue. BWL treatments capable of breaking stones have been safely delivered to pig kidneys in vivo at a $40 \mathrm{~Hz}$ burst that used in SWL. To facilitate the design of BWL treatments that safely and optimally break stones, this work focuses on understanding how treatment parameters affect the generation of sustained bubble clouds. Highspeed photography and ultrasound imaging were used to characterize the presence and persistence of cavitation activity in vitro. Experiments were conducted for BWL treatments at $335 \mathrm{kHz}$ to evaluate the impact of treatment rate, dissolved gas concentration, volumetric confinement (representing kidney collecting space), and presence/absence of a stone. Threshold pressures for generating cavitation clouds were observed to vary with each of these variables. [Funding support by NIH P01-DK043881, R01DK092197, K01-DK104854, and NSBRI through NASA NCC 9-58.]

\section{5:00}

4pBA16. Evaluation of a model-based poroelastography algorithm for edema quantification. John J. Pitre (Biomedical Eng., Univ. of Michigan, 2125 Lurie Biomedical Eng. Bldg., 1101 Beal Ave., Ann Arbor, MI 48109, jpitre@umich.edu), William F. Weitzel (Dept. of Veterans Affairs Medical Ctr., Ann Arbor, MI), and Joseph L. Bull (Biomedical Eng., Univ. of Michigan, Ann Arbor, MI)

A critical component of end stage renal disease treatment is optimal fluid management. Patients with reduced kidney function risk developing fluid overload, and many dialysis patients exceed recommended levels of fluid retention, leading to frequent clinical intervention and higher mortality. Despite this, current clinical standards of care rely on reactive semi-quantitative tests to grade fluid overload and peripheral edema. Poroelastography has been proposed as a quantitative means of estimating fluid overload in peripheral edema, but clinical studies have met limited success, in part because of measurement noise. We developed a new poroelastography method based on an inverse problem formulation to address the shortcomings of current methods. Our technique iteratively minimizes the error between ultrasound displacement measurements and the solution of a Biot poroelastic model. This formulation does not depend on noise-amplifying derivatives and ratios. We tested our algorithm in a simulation study using synthetic displacement data with Gaussian noise and speckle tracking measurements from simulated sonograms. Our algorithm accurately estimated the Poisson's ratio at all noise levels tested. Reconstruction errors for all cases were less than $10 \%$ and outperformed traditional methods. Although our method required 4-12 hours to run, it is easily parallelized. Future work will focus on benchtop validation.

\section{$5: 15$}

4pBA17. Computer-aided B-mode ultrasound diagnosis of hepatic steatosis. Gert Weijers, Johan Thijssen (Radiology and Nuclear Medicine, Radboud Univ. Medical Ctr., Nijmegen, Netherlands), Geert Wanten, Joost Drenth (Gastroenterology, Radboud Univ. Medical Ctr., Nijmegen, N/A, Netherlands), Marinette van der Graaf, and Chris L. de Korte (Radiology and Nuclear Medicine, Radboud Univ. Medical Ctr., MUSIC 766, PO Box 9101, Nijmegen 6500HB, Netherlands, chris.dekorte@radboudumc.nl)

A computer aided ultrasound (CAUS) protocol was developed for the diagnosis and staging of hepatic steatosis. Calibration of the fixed imaging preset enables expression of the US parameters relative to the tissue mimicking phantom used and consequently a vendor independent comparison. The CAUS protocol was validated on high yielding dairy cows $(n=151)$, which severed as a reference model for human non-alcoholic fatty liver disease. The study showed high values for: area under the curve $\left(A_{R O C}\right): 0.94$; sensitivity: $87 \%$; specificity: $83 \%$. A pilot study in patients on Home Parenteral Nutrition (HPN, $n=14$ ) and a more extensive study in an obese cohort $(n=116)$ was performed. Validation was performed by Magnetic Resonance Spectroscopy (MRS). Both studies showed that of all parameters, the residual attenuation coefficient (RAC, the depth dependent mean echo level corrected for beam profile and normal attenuation in liver tissue) has the highest correlation to the reference (MRS fat percentage). For the HPN cohort, a correlation of 0.91 was found. In the obese population, this value was only 0.64 ; however, an $\mathrm{A}_{\mathrm{ROC}}$ of 0.86 , a sensitivity of $75 \%$, and a specificity of $84 \%$ were obtained. In conclusion, CAUS shows promising results for non-invasive quantification of liver steatosis. 


\title{
Session 4pEA
}

\section{Engineering Acoustics: Sound Field Control Techniques}

\author{
Stephen Elliott, Cochair \\ Inst. of Sound and Vibration Res., Univ. of Southampton, Southampton, United Kingdom \\ Yoshinobu Kajikawa, Cochair \\ Kansai University, 3-3-35, Yamate-cho, Suita, Osaka 564-8680, Japan
}

Invited Papers

1:00

4pEA1. Controlling 3D sound field generated by a 2D surface from its 1D boundary. Xiaojun Qiu (School of Elec., Mech., and Mechatronic System, Univ. of Technol. Sydney, Sydney, NSW 2007, Australia, xiaojun.qiu@uts.edu.au), Shuping Wang, and Jiancheng Tao (Key Lab. of Modern Acoust., Inst. of Acoust., Nanjing Univ., Nanjing, China)

As described by the Kirchhoff-Helmholtz integral equation, for a 3D space without internal sources, the inside sound field is completely determined by the sound pressure and its normal gradient on the boundary, so if only a portion of the boundary surface vibrates as the primary sound source while all the remaining portion of the surface is acoustically rigid, a sufficiently large number of secondary sound sources can be located evenly on the primary sound surface to control the 3D sound field completely. The question to be investigated in this research is whether it is possible to control the 3D sound field by only applying secondary sound sources along the 1D boundary of the 2D primary sound surface, for example, controlling the sound transmission from a door with secondary sound sources only on its frame. Simulation and experimental results will be presented to demonstrate the feasibility of the idea.

\section{1:20}

4pEA2. Analysis and synthesis of spatial sound fields with room response modeling using an integrated microphone and loudspeaker array system. Mingsian R. Bai, Yi Li, and Yi-Hao Chiang (Power Mech. Eng., National Tsing Hua Univ., No. 101, Section 2, Kuang-Fu Rd., Hsinchu, Taiwan 30013, Taiwan, msbai63@gmail.com)

A unified framework is proposed for analysis and synthesis of spatial sound fields. In the sound field analysis (SFA) phase, an unbaffled 24-element circular microphone array (CMA) is utilized to "encode" the sound field based on the plane-wave decomposition, whereas in the sound field synthesis (SFS) phase a 32-element rectangular loudspeaker array is employed to "decode" the target sound field using pressure matching technique. Depending on the sparsity of the sound sources, the SFA stage can be implemented in two ways. For the sparse-source scenario, a two-stage algorithm is utilized to estimate the source bearings using the minimum power distortionless response (MPDR) and the associated amplitude coefficients of plane waves using the Tikhonov regularization (TIKR) algorithm. Alternatively, a one-stage algorithm based on compressive sensing (CS) algorithm can be used. For the nonsparse-source scenario, a one-stage TIKR is utilized to solve for the amplitude coefficients for plane-wave components uniformly distributed in the angular domain. The SFA technique for the non-sparse source scenario is also useful in establishing the room response model, as required in the pressure matching step of the SFS phase. The integrated acoustic array system is validated with localization and listening tests.

\section{1:40}

4pEA3. Three-dimensional sound field reproduction based on spatial fusion of carrier and sideband waves with parametric array loudspeaker. Masato Nakayama and Takanobu Nishiura (College of Information Sci. and Eng., Ritsumeikan Univ., 1-1-1 Noji Higashi, Kusatsu, Shiga 525-8577, Japan, mnaka@fc.ritsumei.ac.jp)

It is very important to provide a personal audible space (audio-spot) to listeners. Parametric array loudspeaker (PAL) has been proposed in order to provide an audio-spot because it has super directivity. It however has difficulty in reproducing the audible sound only to the particular space because the acoustic beam with the PAL is reflected and intercepted. Therefore, we have proposed the threedimensional sound field reproduction based on spatial fusion of carrier and sideband waves with the PAL. In addition, we have proposed the method of near-sound-field reproduction based on spatial fusion of the carrier and sideband waves. In this presentation, we explain principle of the proposed method, and its applications. In the proposed method, we utilize principle which the intense ultrasound selfdemodulates to the audible sound by nonlinear interaction in the air. More specifically, the intense ultrasound consists of the carrier and sideband waves, and the audible sound is demodulated as the different tone between carrier and sideband waves. The proposed method forms the audio-spot by fusing the carrier and sideband waves in the particular space. Finally, we evaluate the effectiveness of the proposed method through the evaluation experiments. 
4pEA4. Perceptual optimization of sound field reproduction system and sound field rendering with software/hardware-based acoustic simulation. Makoto Otani (Graduate School of Eng., Kyoto Univ., Kyoto daigaku-Katsura, Nishikyo-ku, Kyoto 615-8540, Japan, otani@archi.kyoto-u.ac.jp), Takao Tsuchiya (Faculty of Sci. and Technol., Doshisha Univ., Kyotanabe, Japan), Yukio Iwaya (Faculty of Eng., Tohoku Gakuin Univ., Tagajo, Japan), and Yasushi Inoguchi (Ctr. for Information Sci., Japan Adv. Inst. of Sci. and Technol., Nomi, Japan)

The performance of sound field reproduction based on integral equations is restricted by interval of boundary surface discretization, which would cause spatial aliasing when it is not sufficiently fine. Physically accurate reproduction without spatial aliasing throughout an entire audible frequency range would require impractically large number of discretization points. However, the requirement would be relaxed if perceptually satisfactory reproduction is possible even with spatial aliasing. In this presentation, first, we introduce results of perceptual evaluation of effects of spatial aliasing in the sound field reproduction on auditory perception in order to find out a reasonable requirement for realization of perceptually satisfactory system. Second, we introduce development of sound field rendering system consisting of the sound field reproduction system and FDTD (Finite Difference Time Domain) method as an acoustic simulation solver. Finally, we also introduce our attempt to develop a real-time sound field rendering system that utilizes hardware-based acoustic simulation using DHM (Digital Huygens Model) implemented in ASIC (Application Specific Integrated Circuit).

\section{2:20}

4pEA5. Sound field reproduction system with active reverberation. Akira Omoto (Faculty of Design, Kyushu Univ., Shiobaru 4-9-1, Minami-ku, Fukuoka 815-8540, Japan, omoto@design.kyushu-u.ac.jp)

Sound field reproduction system can be categorized roughly into two types. The first is physically assured system which reproduce the wavefront or another physical parameters by engineering means. Typical examples are the boundary surface control system, the wave field synthesis, the ambisonics, and so on. The second type is a psychological system that reproduces phantom images one sound at any desired position by artificial manipulation with rather simple technique such as volume based amplitude panning. Examples are the two channel stereo system and the surround system using 5.1 or more loudspeakers. The latter can be recognized as somewhat "artistic" method, because it requires norms based on artistic sense. The present study aims to fuse the engineering and artistic systems mentioned above. The boundary surface control system, which consists of 24-channel narrow directional microphones and 24 channel loudspeakers, was used as the platform of research. Based on the conventional control technique using inverse filtering, additional manipulation such as simple reproduction scheme for high frequency was introduced. Also, the concept of variable reflection acoustic wall system, which adds the artificial reverberation to the sounds that listener makes, was involved in the reproduction system for the direction of the presence.

\section{2:40}

4pEA6. Directivity control of a graphene loudspeaker array using elementwise switching technique. Dong-Hwan Kim and JungWoo Choi (School of Elec. Eng., Korea Adv. Inst. of Sci. and Technol., 291, Daehak-ro, Yuseong-gu, Daejeon 34141, South Korea, dh. kim92@kaist.ac.kr)

Graphene is a promising material for thermoacoustic transducers because of its low heat capacity per unit area and easy fabrication process. In contrast to the conventional voice-coil loudspeaker, a small thermoacoustic loudspeaker can produce omnidirectional radiation even without enclosures. In addition, the sound level from a thermoacoustic loudspeaker is less sensitive to its radiating surface area, which makes it an ideal omnidirectional sound source in high frequency region. In this work, we present an elementwise switching control of a graphene loudspeaker array utilizing the advantages of thermoacoustic loudspeakers. With an array of 3D graphene transducers, a directivity is controlled by polarity switching of each element according to a pre-determined pattern. A single channel input signal is modulated into two different channel signals using a polarity inverter. Each graphene element is driven by the same signal with different sign, and the polarization pattern of the array forms a designated beam pattern. The proposed technique simplifies the control of beam patterns without using large number of independent channels, and hence, enables the construction of a high frequency driver with variable-directivity.

\section{3:00-3:15 Break}

\section{$3: 15$}

4pEA7. Cancellation, reproduction, and cloaking using sound field control. Jordan Cheer (Inst. of Sound and Vib. Res., Univ. of Southampton, University Rd., Highfield, Southampton, Hampshire SO17 2LG, United Kingdom, j.cheer@soton.ac.uk)

The active control of sound fields has been applied in active noise cancellation, sound field reproduction, and active acoustic cloaking; however, relatively few studies have considered the relationship between these three areas of research. This paper investigates the physical limitations on active noise control, sound field reproduction, and active acoustic cloaking in terms of the controllability and observability of the sound field. This has been achieved through a series of simulations that consider the control of the sound field in the presence of a scattering object. In terms of the controllability problem, it is shown that while active noise control and sound field reproduction are most effective when considering the interior control problem, active acoustic cloaking is most effective when facing the exterior control problem. Different sensing strategies are then considered for the cancellation, reproduction, and cloaking problems and their impact on the observability of the control problem is discussed. 
4pEA8. Parallel feedback architecture for ambisonics based active noise control. Anshuman Ganguly and Issa Panahi (Elec. Eng., The Univ. of Texas at Dallas, 800 W Campbell Rd., Richardson, TX 75080, axg124330@utdallas.edu)

Active noise control(ANC) has been widely studied for smaller acoustic cavities, like cars and small enclosures, over past several years. One of the key challenges in implementing ANC systems for larger acoustic environments lies in extending the target "quiet" zone for maximizing the noise cancellation. Traditional ANC systems use two "omnidirectional" microphones to access reference noise signal and "residual" error signal at target zone. The target zone around the error microphone remains to be small. Feedback ANC with Ambisonics is aimed to extend the quiet zone by employing specialized encoding and decoding of the noise signal accessed using a single Soundfield microphone. In this paper, a parallel Feedback ANC architecture is fused with the Ambisonics encoding to improve the noise cancellation performance. The proposed ANC system also features an adaptive signal decomposition technique to effectively handle noise types with dominant predictable components, like machinery noise. Noise attenuation level (NAL) is used to compare and quantify the advantage of the proposed method. In this paper, an attempt has been made to explore an improved ANC system for realtime noise control in larger acoustic enclosure such as museums and concert halls.

\section{3:55}

4pEA9. Open loop active control of noise through open windows. Chuang Shi, Tatsuya Murao, Dongyuan Shi, Bhan Lam, and Woon-Seng Gan (DSP Lab., School of Elec. \& Electron. Eng., Nanyang Technolog. Univ., 50 Nanyang Ave., S2-B4a-03, Singapore 639798, Singapore, shichuang@ntu.edu.sg)

Noise propagating through open windows is a big concern of liveability in metropolises, where residential buildings and main roads are built densely. Active noise control (ANC) is a convincing technique to improve the current situation. Classic ANC systems adopt the close loop control. The quietness after control is continuously monitored by error microphones. However, ANC systems that attenuate noise propagating through open windows should ideally avoid the use of error microphones for the ease of implementation and maintenance. Furthermore, as open windows are commonly large as compared to the wavelength of noise, multichannel ANC systems are necessary to be carried out. They result in huge computational burdens that cannot be handled by low-cost hardware platforms. This paper investigates acoustic characteristics of open windows and formulates the control strategies as a spatial sampling and reconstruction problem. This theoretical development prompts the fact that controlling noise propagating through open windows essentially leads to global noise reduction. Both centralized and decentralized open loop control are feasible to deal with broadband noise. The upper bound of the effective band is limited by the spacing between channels, while the lower bound is dependent on the frequency response of secondary sources.

\section{4:15}

4pEA10. Virtual sensing technique for feedforward active noise control. Shoma Edamoto (Kansai Univ., 3-3-35, Yamate-cho, Suita, Osaka 5648680, Japan, umaumasound@gmail.com), Chuang Shi (Nanyang Technolog. Univ., Singapore, Singapore), and Yoshinobu Kajikawa (Kansai Univ., Osaka, Japan)

Active noise control (ANC) is one of techniques to reduce unwanted acoustic noise and is based on the superposition property of acoustic waves. If an anti-noise wave is exactly generated to have the same amplitude and inverse phase of the unwanted acoustic noise wave, ANC can reduce the noise level at the desired location, where an error microphone is placed to monitor the error signal. In the case when the error microphone cannot be placed at the desired noise reduction location, virtual sensing techniques are useful. In this paper, we examine one of the virtual sensing techniques in an experiment setup using the parametric array loudspeaker as the secondary source. Experiment results show that improved noise reduction can be obtained at the virtual microphone location. 


\title{
Session 4pEDa
}

\section{Education in Acoustics and Signal Processing in Acoustics: Using Computers for Acoustics Education}

\author{
Hiroshi Suda, Cochair \\ Dept. of Information and Network Science, Chiba Institute of Technology, 2-17-1, Tsudanuma, Narashino 275-0016, Japan
}

Victor Sparrow, Cochair

Grad. Program in Acoustics, Penn State, 201 Applied Science Bldg., University Park, PA 16802

Chair's Introduction-1:00

\section{Invited Papers}

\section{1:05}

4pEDa1. Physics girl experience-Using YouTube to teach acoustics and wave physics. Dianna L. Cowern (PBS, PO Box 9281, San Diego, CA 92169, dianna@physicsgirl.org)

YouTube is usually considered an entertainment medium to watch cats, gaming, and music videos. But educational channels have been gaining momentum on the platform, some garnering millions of subscribers and billions of views. The Physics Girl YouTube channel has focused solely on physics education. Using this channel, we can examine the ways that educational channels grow, their demographical reach, how they are utilized, and how online media and technology can facilitate good and bad learning. YouTube provides unique access to a wide audience. The videos can vary in length and purpose-from tutorials to classroom supplements. In addition, the online world provides the ability to organize and serve educational communities, like PBS Learning Media. With all these tools available, Physics Girl aims to introduce physics as related to real-world examples and interesting questions that are not always covered in traditional teaching. While the goals of each channel vary, each aims to contribute to better STEM learning.

\section{$1: 25$}

4pEDa2. Interactive simulators for acoustic education. Hiroshi Suda (Dept. of Information and Network Sci., Chiba Inst. of Technol., 2-17-1, Tsudanuma, Narashino 275-0016, Japan, suda@net.it-chiba.ac.jp)

In the field of acoustic education, there are many complex theories and invisible phenomena. In the lecture of acoustics, the lecturer places much value on forming and expanding mathematical expressions, using textbooks and the blackboard. Students often have difficulty and want new teaching/learning methods and materials. New learning materials can help a student understand a phenomenon; for example, with a short movie describing an actual phenomenon or an environment of experiments using limited models. Now, the calculating power of computers, tablets and smartphones has enough to easily simulate acoustic phenomena. Here distributed web-based interactive simulators for acoustic education are presented.

\section{$1: 45$}

4pEDa3. Use of the student edition of ACTRAN in acoustics education. Victor Sparrow (Grad. Program in Acoust., Penn State, 201 Appl. Sci. Bldg., University Park, PA 16802, vws1@psu.edu), Jean-Louis Migeot, and Jean-Pierre Coyette (Free Field Technologies, Mont-Saint-Guibert, Belgium)

The power of computation has changed the way problems are solved in acoustics, and students should be trained to have an adequate background in modern techniques and software packages to simulate noise and vibration. Universities often cannot provide industrialgrade packages for their students due to the cost limitations. But recently a student version of a modern acoustics computation package was released, Student Edition of ACTRAN. This tool lets students solve a wide variety of problems using finite elements and infinite elements. This talk will describe the software and how it was used in a graduate level course in computational acoustics. Two examples that will be shown include finding the modes of an irregularly shaped room and computing the acoustic radiation from a gearbox. But it is clear that this software can be used in many different ways, for both undergraduate and graduate acoustics education. Instructors can demonstrate acoustical phenomena for students not adept at using the software themselves, easily visualizing the physics involved in acoustics with the Student Edition of ACTRAN. The software is free for downloading from http://www.mscsoftware.com/page/actranstudent-edition. 
4pEDa4. An interactive speech synthesizer for acoustic education developed with pure data. Naofumi Aoki (Graduate School of Information Sci. and Technol., Hokkaido Univ., N14 W9, Kita-ku, Sapporo 060-0814, Japan, aoki@ime.ist.hokudai.ac.jp)

Developing real-time sound systems that allow interactive controls requires advanced skills of sound programming. It often takes time even for experienced programmers to develop such systems. This paper shows an approach for rapid prototyping of such systems by using Pure Data. Especially, this paper focuses on the techniques of interactive controls by combining personal computers and some other peripheral devices such as a smart phone with Touch OSC. This paper describes its possibilities through showing demonstrations. As an example, this paper shows an interactive speech synthesizer. It allows users to control formants by pointing the F1-F2 diagram on the touch screen of a smart phone. Since this system allows time varying controls of the formants, it can synthesize semi-vowel sound as well as vowel sound. In addition, it also allows users to synthesize some other phrases by controlling appropriate movement of the formants by pointing the F1-F2 diagram. These experiences may potentially be effective for students who learn acoustics to be speech therapists. With this system, students can understand the mechanism of speech production in an experimental way. This paper mentions its availability for acoustics education.

\section{2:25-2:40 Break}

\section{Contributed Papers}

\section{2:40}

4pEDa5. Computing the curriculum-Embedding computation in undergraduate acoustics. Matthew C. Wright (ISVR, Univ. of Southampton, Southampton SO17 1BJ, United Kingdom, mcmw@isvr.soton.ac. uk)

I will describe an approach to teaching acoustics to undergraduate engineering students that integrates mathematics, coding, numerical computation, and physical measurement. This approach developed and continues to develop according to my experience as Director of taught acoustical programmes at the Institute of Sound and Vibration Research (ISVR) in the Faculty of Engineering and the Environment at the University of Southampton, UK. Our program is somewhat unusual in that it teaches acoustics in depth to undergraduate students, whose understanding of the physics must develop in parallel with their proficiency in mathematical analysis and coding. I will explore how both numerical computation and coding can be integrated into an acoustics curriculum from the very start, how their inclusion can enhance learning, and how the curriculum can be restructured in order to make best use of these elements, highlighting the features of acoustics as a subject that make it particularly suited to this approach. I will also discuss how the choice of programming languages and environments, and modelling software affect the curriculum and vice versa. I will present examples from my teaching using Jupyter (formerly IPython) notebooks and discuss how to use these effectively.

\section{2:55}

4pEDa6. Computer-based acoustics projects for audiology and speechlanguage pathology students. Jennifer Lentz (Dept. of Speech and Hearing Sci., Indiana Univ., 200 S. Jordan Ave., Bloomington, IN 47405, jjlentz@ indiana.edu)

Graduate and undergraduate students studying audiology and speechlanguage pathology are often required to have a basic working knowledge of acoustics. However, convincing these students the necessity of this knowledge is a common challenge for the acoustics instructors. Consequently, motivating students to be enthusiastic about the material may be facilitated by including methods that replace or supplement traditional teaching methodologies. Computer-based projects that reinforce acoustic concepts learned in the classroom provide an outstanding hands-on opportunity to expose students to the principles of acoustics. Importantly, they also offer clear examples of how knowledge of acoustics is core to the fields of audiology and speech-language pathology. Here, I will illustrate a number of Matlab-based projects that apply acoustics to the topics of hearing, hearing loss, speech production, and speech disorders. These projects are designed to be discipline-specific and also target specifically the abilities and needs of students in audiology and speech-language pathology.
3:10

4pEDa7. Introducing programming through acoustics and audio at Belmont University. Eric W. Tarr (Audio Eng. Technol., Belmont Univ., 1900 Belmont Blvd., Nashville, TN 37212, eric.tarr@belmont.edu)

A course in computer programming has been offered within the department of Audio Engineering Technology at Belmont University to provide an introduction to audio and physics students interested in learning about programming. This course is based on a foundation of acoustics and audio concepts, without requiring previous programming experience. The pedagogical approach has been to expose students to the application of programming within their discipline of interest as a motivation for further exploration. Topics in the course introduce programming concepts for specific signal processing methods. Programming concepts include: variables, operators, data types, as well as basic control structures like loops, conditional statements and functions. While learning programming concepts, students perform various signal processing methods such as: changing a signal's amplitude, signal synthesis, stereo panning functions, mid-side processing, amplitude fades, amplitude modulation, soft/hard clipping, rectification, bit reduction, echo effects, convolution, algorithmic reverberation, expander/compressor dynamic-range processing, and various spectral filters. Course lessons for a "flipped classroom" are freely and publically available at http://www.hackaudio.com/. Since the initial offering of this course, the number of students in the audio engineering program who double-major or minor in computer science has quadrupled.

\section{3:25}

4pEDa8. Teaching Mathematica ${ }^{\circledR}$ based computer computations for support of laboratory experiments, special projects, and research for undergraduates taking acoustics and for mentorship students. Murray S. Korman (Phys. Dept., U.S. Naval Acad., 572 C Holloway Rd., Chauvenet Hall Rm. 295, Annapolis, MD 21402, korman@usna.edu)

Undergraduate senior level physics majors taking Acoustics and oceanography majors taking Underwater Acoustics and Sonar learn how to use Mathematica ${ }^{\circledR}$ for doing standard laboratory experiments, special projects, solving homework problems requiring detailed computations or graphics. Students in electrical engineering, economics, mechanical engineering and ocean engineering (along with physics majors) have enrolled in a one credit course-Mathematica ${ }^{\circledR} 10$ for Scientists and Techies. The feedback from students using a computer language in their course work for the first time is very encouraging and the teaching experience quite rewarding. High school mentorships volunteering to do research after school use Mathematica ${ }^{\circledR}$ for doing projects in the Physical Acoustics Laboratory. Some undergraduate lab projects involve Fourier analysis, measuring sound speed vs. temperature in water, and plotting theoretical and experimental Bessel mode shapes (or cosine shapes) for standing wave resonance in a cylindrical cavity. Here, a long slender tube mounted on the microphone probe translates along the radial or axial directions. Students enjoy generating their own data on a 
spectrum analyzer, transferring files to Mathematica ${ }^{\circledR}$ and plotting tuning curves vs. frequency in Helmholtz resonators. One student got involved in nonlinear drum vibration while another became interested in synthetic aperture imaging, for senior research projects.

THURSDAY AFTERNOON, 1 DECEMBER 2016

SOUTH PACIFIC 4, 4:05 P.M. TO 5:00 P.M.

\title{
Session 4 pEDb
}

\section{Education in Acoustics: Acoustics Education Prize Lecture}

\author{
Robin Samlan, Chair \\ Speech, Language, \& Hearing Sciences, University of Arizona, P.O. Box 210071, Tucson, AZ 85721
}

Chair's Introduction-4:05

Invited Paper

4:10

4pEDb1. The role of artificial speech in understanding the acoustic characteristics of spoken communication. Brad H. Story (Speech, Lang., and Hearing Sci., Univ. of Arizona, 1131 E. 2nd St., P.O. Box 210071, Tucson, AZ 85721, bstory@email.arizona.edu)

Models have long been used to understand the relation of anatomical structure and articulatory movement to the acoustics and perception of speech. Realized as speech synthesizers or artificial talkers, such models simplify and emulate the speech production system. One type of simplification is to view speech production as a set of simultaneously imposed modulations of the airway system. Specifically, the vibratory motion of the vocal folds modulates the glottal airspace, while slower movements of the tongue, jaw, lips, and velum modulate the shape of the pharyngeal and oral cavities, and coupling to the nasal system. The precise timing of these modulations produces an acoustic wave from which listeners extract phonetic and talker-specific information. The first aim of the presentation will be to review two historical models of speech production that exemplify a system in which structure is modulated with movement to produce intelligible speech. The second aim is to describe theoretical aspects of a computational model that allows for simulation of speech based on precise spatio-temporal modulations of an airway structure. The result is a type of artificial talker that can be used to study various aspects of how sound is generated by a speaker and perceived by a listener. 


\title{
Session 4pMUa
}

\section{Musical Acoustics: Piano Acoustics and Playing Piano II}

\author{
Isoharu Nishiguchi, Cochair \\ Information Media, Kanagawa Institute of Technology, 1030, Shimo-ogino, Atsugi 243-0292, Japan \\ Nicholas Giordano, Cochair \\ Physics, Auburn University, College of Sciences and Mathematics, Auburn, AL 36849
}

\section{Invited Papers}

1:00

4pMUa1. Microphone array measurements of concert grand piano soundboards in different stages of production. Niko Plath, Florian Pfeifle, Christian Koehn, and Rolf Bader (Inst. of Systematic Musicology, Univ. of Hamburg, Germany, Neue Rabenstr. 13, Hamburg, Hamburg 20354, Germany, niko.plath@uni-hamburg.de)

A series of measurements is taken on two concert grand pianos in seven different stages of production, starting with the glue-laminated soundboard planks and ending with the completely assembled piano in concert tuned state. Due to the large size of the soundboard as well as its irregular shape, measuring deflection shapes is a nontrivial task. Common measurement tools such as piezoelectric accelerometers can affect the acoustic vibrations of the soundboard due to the added mass. To this end, a noninvasive microphone array method is utilized for the present work. The array consists of 105 microphones successively placed parallel to the soundboard, resulting in a total number of 1289 microphones covering the entire surface. The Soundboard is excited using an acoustic vibrator at 15 positions associated with string termination points on the bass and main bridge. Impulse responses are obtained using the SineSweep technique. The measured sound pressure can be back-propagated to the radiating soundboard surface using a minimum energy method. Based on the measured vibrational and acoustical data a set of signal features is derived which cover direct physical behavior (e.g., driving point mobility, damping, radiation efficiency) as well as perceptive parameters (e.g. attack time, spectral centroid). The empirical findings will contribute to a software tool (based on a real time physical model) to help piano makers estimate the impact of design changes on the generated sound.

$1: 20$

4pMUa2. Analysis of piano performance using an eigenperformance. Masanobu Miura (Dept. of Media Informatics, Ryukoku Univ., 1-5, Seta, Oe-cho, Yokotani, Otsu 5202194, Japan, miura@mail.ryukoku.ac.jp)

Analysis of piano performance has been a great task for musicologists, in particular the "artistic deviation" on performance has been intensively discussed. Introduced here is a method to clarify what occurs on music performance by observing the eigenvector of obtained several piano playings, "Eigenperformance." The eigenperformance is obtained for MIDI-piano playing from the tendency of onset, MIDI-velocity, duration, and instantaneous tempo curves. Eigenperformance is possible to indicate tendencies for a set of piano playing. By using eigenperformance, here investigates 1) the differences in evaluation criteria between pianists and non-pianists, and 2) the differences of deviations due to player's skill. For 1), results of simple scale performance show that pianists evaluate short and sudden tempo changes with taking long time in ending notes along with dynamic and temporal expressions based on phrases on sheet music as good, whereas non-pianists never provide such evaluation. For 2), piano etude "For Elise" is dealt with to be investigated by four classes of proficiency. The eigenperformance revealed that the proficient performance shows same patterns of dynamics among first and second sections in sheet music, whereas the novice performance does not show any analogous on corresponding parts in sheet music.

\section{$1: 40$}

4pMUa3. Visualization of Kansei causality for the digital piano sound-Kansei modeling for musical instruments. Rento Tanase and Hideki Sakanashi (Res. \& Development Div., Yamaha Corp., 203 Matsunokijima, Iwata, Shizuoka 4380192, Japan, rento.tanase@ music.yamaha.com)

Kansei-causality-model (structural causality model of subjective impression) for the digital piano sound and some considerations are presented. At first, the directed graph of hypothetical causality is figured from the report about the performance tests of musical instruments and the interview with the sound engineers. Second, the quantitative Kansei-data acquired by subjective test with semantic-differential method is associated with the directed graph. Then, the Kansei-causality-model are estimated from the directed graph and Kanseidata by SEM (Structural Equation Modeling) / Covariance Structural Analysis. The result suggests structurally the meanings of the words (for example "hiki-gotae (japanese)" and "high grade feeling) for the evaluation of the digital piano sound. The different causality flow represented as two passes on the structural causality model suggests the polysemy of "Noisy. Moreover, the result of CFA (Check Factor Analysis) for the same data indicates the two factors, such as must-be quality and attractive quality, in the sound engineering criterion for the digital piano design. 


\title{
Contributed Papers
}

4pMUa4. The role of real-time acquisition of visual and auditory information in the performance of scale and arpeggio tasks in pianists. Chie Ohsawa (Faculty of Music, Kyoto City Univ. of Arts, 13-6 Kutsukake-Cho, Oe, Nishikyo-Ku, Kyoto 610-1197, Japan, ohsawachie@gmail.com), Kenichi Sawai (Graduate Schools for Law and Politics, Univ. of Tokyo, Tokyo, Japan), and Minoru Tsuzaki (Faculty of Music, Kyoto City Univ. of Arts, Kyoto, Japan)

We previously found that the spatial memory of piano keyboard was not accurate enough to play without external spatial cues even in trained pianists. Therefore, the real-time acquisition of spatial information should be essential for piano performance. The aim of the present study was to test how and when the online acquisition of the visual and auditory information contribute to the performance of pianists. Seven undergraduate and graduate students majoring piano performance at a university participated in our experiment. They were asked to play the task sequences, which consisted of scale, arpeggio of triads, alternating fourth and fifth, and alternating fourth, fifth and one octave leap, on an electric piano. Each pianist played in four conditions: "Ordinary," "No-sound," "No-vision," and "No-sound \& Novision." The locations of the pressed keys were assigned to the locations of the keys to be pressed with dynamic programming after the midi note numbers were converted to the "location numbers." We analysed the error size and the alignment costs. The results indicated that the visual information clearly contributed for touching accurate place in arpeggio tasks, while the contribution of the auditory information was limited in any task type.
$2: 15$

4pMUa5. Acoustical properties of the front-duplex system in a grand piano. Florian Pfeifle (Univ. of Hamburg, Neue Rabenstrasse 13, Hamburg 20354, Germany, Florian.Pfeifle@uni-hamburg.de)

The duplex scala, patented by Theodore Steinway in 1872, has been subject to several disputes since it found its first application in grand pianos. Not without good reason because, as was shown in the work of Öberg \& Askenfeldt (2012), the physical effects explained in the patent can not be substantiated by measurements mainly because the tune of the duplex strings deviate considerably from the suggested theoretical values. Nonetheless, as published in the same work, the perceptual relevance of the rearand more so of the front-duplex is stressed. As is shown there, even untrained musicians are able to perceive the influence of both duplex scalae. In this treatise, physical properties of front-duplex strings are considered and an explanation of the acoustical importance regarding the string vibration of the main string is given. To this end, a series of high-speed camera measurements of several duplex strings are presented. Due to the small deflection of front-duplex strings, the recorded data is processed using motion magnification methods showing the influence of different modes of vibration in different frequency bands. A physical model of a grand piano string chorus is proposed, aiding in characterizing the main influence of the front duplex string.

\section{Session 4pMUb}

\section{Musical Acoustics: Musical Instruments from Islands Around the World}

\author{
Peter L. Hoekje, Chair \\ Physics and Astronomy, Baldwin Wallace University, 275 Eastland Rd., Berea, OH 44017
}

\section{Invited Papers}

4pMUb1. Voices of Hawai'i's rhythmic world. Marques H. Marzan (Cultural Resources, Bernice Pauahi Bishop Museum, 1525 Bernice St., Honolulu, HI 96817, marques@bishopmuseum.org)

Hawai'i is home to a myriad of musical instruments made from a variety of materials, which include stone, wood, gourd, and shell. The resonance that each produces, complements the transmission of knowledge within the Hawaiian culture, through storytelling, dance, and song. Both the secular and spiritual worlds harness the energy these instruments produce, infusing ceremony and daily life with the voices of the uncountable manifestations of akua, the gods. The material choices used in the creation of Hawaiian instruments connect the realm of man to the realm of the gods, as all animate and inanimate materials represent a kinolau, the physical manifestation of the pantheon of Hawaiian gods. The relationships that are forged with our surroundings, through these tools, allow us a glimpse into a world of endless possibilities. 
4pMUb2. Sado Island: Student cultural activities-The drum performance. Shuji Morishita (Music Education, Niigata Univ., 8050, Ikarashi-2-no-cho, Nishi-ku, Niigata, Niigata 9502181, Japan, morishta@ed.niigata-u.ac.jp)

Sado is an island in the Sea of Japan. The population of the island is 57,114 (October 1, 2015). Sado had a gold mine and has a rich natural environment. It was also rich economically and many people came to Sado from many other places in Japan. People brought many entertainment styles to Sado. The traditional arts are still strong, such as Noh drama and Oni-Daiko (ogredrum performances). We have been active in Oni-Daiko performances in Toyooka village since 2009. The festival in Toyooka is held on the first or second Sunday of April every year. It begins in the Shrine of the enshrined deity of the village. We go around the houses in the village and come back to the shrine. It is performed in front of shrines and houses. The students perform it in public at the end of their one-week lesson. We perform it from 7 a.m. to 8 p.m. with the village people. Students mature after taking part in these activities. As the population of Sado begins to decline, we do not know how long we will be able to continue this activity. However, we want to continue carrying out this activity as long as possible.

3:40

4pMUb3. Managing matagia: Instruments and aesthetics in contemporary Tokelau performance. Candice E. Steiner (Univ. of Hawai'i at Mānoa, 1890 East-West Rd., Moore 212, Honolulu, HI 96822, candice.steiner@gmail.com)

The hallmark of contemporary Tokelau performances is a significant building of energy over the course of a song and dance, which is driven by gradual tempo increases, upward key modulations, increases in volume, intensification of dance movements, and oneness among performers. The powerful pōkihi (box drum) and accompanying instruments lead the entire group through this unified move from subdued to exhilarating delivery, and when the technical skills of the performers and the cultural meanings of the performance combine in a way that fully engages the performers and audience alike, the resulting energy is palpable. In this presentation, I describe the key musical instruments used in contemporary Tokelau performances, summarize previous research on the instruments' history and use, and discuss the role of the instruments in upholding the Tokelau aesthetics described above.

\section{Contributed Papers}

4:00

4pMUb4. Construction and modernization of Kundun Pentatonic xylophone. Tobi E. Kemewerigha (Music, College of Education Akakpa, College of Education Akakpa Cross-River, Calabar State, Nigeria, Akakpa CrossRiver, Akakpa 234, Nigeria, kemewerighatobi@yahoo.com) and Stephen G. Onwubiko (Music, Univ. Of Nigeria, Enugu, Nsukka, Nigeria)

This thesis is all about the constructional techniques and modernization of the resonant qualities of Kundun musical instrument from Birom in Plateau State of Nigeria. A discussion of the traditional Xylophone based on the field work conducted in Birom ethnic group understanding a kundun instrument maker, was made. The structure of this Xylophone highlights both the tone qualities and also the use of the cow-horn as pipes which amplifies the resonance of the slab. The construction and methods used here embodied traditional technology together with modern innovations, with the aim of improving the tone and aesthetic qualities of the instrument. This report also offered guides on the correct choice of wood and other materials for construction. Special attention was given to the acoustic behavior of kundun and the particular problems of the tropical climate conditions, to which the instrument could be exposed and to the overall tone quality of the instrument during performance. Some scientific approaches, in terms of the accurate measurements and amplification of sound, were taken into cognizance so as to enhance the knowledge of the construction of kundun musical instruments and its aesthetic quality.

\section{$4: 15$}

4pMUb5. Calculating scale tunings of Polynesian nose flutes. Peter L. Hoekje (Phys. and Astronomy, Baldwin Wallace Univ., 275 Eastland Rd., Berea, OH 44017, phoekje@bw.edu)

The Polynesian nose flute is found in two versions that are blown in a similar manner but fingered and tuned differently. The Eastern Polynesian flute, known in Hawai'i as 'ohe hano ihu, but is found also to Tahiti and Niue. The tube is closed by a segment node at one end but with a hole for blowing and open at the other end. It typically has two or three finger holes along the tube and three or four playable notes. Being similar to other flutes, the scale tunings can be calculated from hole and tube dimensions by analytic expressions. In Fiji, New Zealand, Samoa, and Tonga, where it is known as fangufangu, both ends of the Western Polynesian flute are closed by segment nodes. There are five holes are placed along the top, usually evenly spaced from one end to the other, with a sixth hole in the middle underneath, giving a variety of fingering options with four to six notes and two registers. The scale tunings are determined by first calculating the frequency response using a lumped-element method. 


\title{
Session 4pMUc
}

\section{Musical Acoustics: Musical Instruments from Islands around the World II-A Concert}

\author{
Peter L. Hoekje, Chair \\ Physics and Astronomy, Baldwin Wallace University, 275 Eastland Rd., Berea, OH 44017
}

\begin{abstract}
In Hawai'i, hula complements Hawaiian storytelling presented in song form. Various instruments are also used in accompaniment of these songs, most often by ipu, gourd drums, pahu, wooden drums, and kāla'au, dance sticks, either used by the dancer or the chanter. Various instruments help to emphasize the stories, through tone and cadence.
\end{abstract}

\section{Session 4pNSa}

\section{Noise: Prediction of Transportation Noises and Other Topics II}

\author{
James E. Phillips, Cochair \\ Wilson, Ihrig \& Associates, Inc., 6001 Shellmound St., Suite 400, Emeryville, CA 94608 \\ Shinichi Sakamoto, Cochair \\ 5th Department, Institute of Industrial Science, The University of Tokyo, 4-6-1, Komaba, Meguro-ku, Tokyo 153-8505, Japan
}

\section{Contributed Papers}

\section{1:00}

4pNSa1. Experimental contribution analysis of external noise components to interior noise of automobile. Seongil Hwang (Mech. Eng., Texas A\&M Univ., 3123 TAMU, College Station, TX 77843), Myunghan Lee, Kang Duck Ih (Hyundai Motor Co., Hyundai Motor Co., Hwaseong, Geongi-do, South Korea), and Yong-Joe Kim (Mech. Eng., Texas A\&M Univ., College Station, TX, joekim@tamu.edu)

The contribution analysis of various noise sources to interior noise is important, enabling to design an automobile with a low interior noise level. Here, using a modified Cholesky Decomposition (CD), it is proposed to decompose interior noise spectra into multiple spectra, each represents the contribution of a specific noise source to the interior noise. Through an experiment with the two speakers driven by two independent white noise signals, it is shown that the measured noise spectrum can successfully be decomposed into two contributions, each associated with noise radiated from one of the two speakers. Then, an automobile was tested on a road at the speeds of $65 \mathrm{mph}$ and $80 \mathrm{mph}$. In this experiment, 64 external and 4 interior microphones were used to measure external noise source signals and interior noise signals, respectively. The contribution results obtained by processing the measured microphone signals show the highest contributions of the aeroacoustic sources on the windows and the hood to the interior noise. It was also observed that the contribution of the windows increased as the speed increased and that the higher contribution to the interior noise at a specific seat could be obtained from the window closer to the seat.
$1: 15$

4pNSa2. Noise produced within spaces adjacent to high performance jet operations on large amphibious assault ships. Ronald Hughes, Steven Antonides, and Chandrasekhar Kannepalli (NSWCCD, 9500 Macarthur Blvd., West Bethesda, MD 20817, ronald.g.hughes@navy.mil)

Airborne noise levels have been measured in spaces adjacent to F-35B operations in proximity to the flight deck on Large Amphibious Assault Class ships (L Class). Noise levels observed in some manned spaces are hearing hazardous. The noise produced during high performance jet operations is intermittent to steady state in duration. Results reported here are only for vertical landings (VL) and do not include short takeoffs (STO); however, noise due to both VL and STO exhibit similar characteristics. Observed VL noise features include two prominent types of noise sources during aircraft decent as well as source directionality. These features are compared to LES CFD predictions for a supersonic jet impinging on a plate. Qualitative features observed in data have been included in a noise model to predict noise levels in Gallery deck compartments. Predicted noise levels are used to ascertain noise treatments and hearing protection on L Class ships during F-35B air operations. Motivations for controlling noise levels are to prevent or reduce hearing threshold shifts in crew members and to promote hearing recovery and communication intelligibility. Noise characteristics of F-35B landings and diagrams of predicted noise levels that address noise-induced hearing loss, hearing recovery, and communication intelligibility are shown. 
$1: 30$

4pNSa3. Detached eddy simulation of a highlift airfoil noise with spectral difference method. Junhui Gao and Xiaodong Li (Beihang Univ., New Main Bldg., D401, Beijing 100191, China, gaojhui@buaa.edu.cn)

In this study the noise generated by the $30 \mathrm{P} 30 \mathrm{~N}$ highlift airfoil is simulated with a high order computational aeroacoustics code. To handle the complex geometry of the airfoil, the high order spectral difference method based on unstructured mesh is used for spatial discretization. The multitime-step method based on Adam-Bashforth scheme is utilized for time marching, and a speed-up ratio greater than 10 is obtained comparing with the single time step method. The detached eddy simulation method is conducted for turbulence modeling. Radiation and outflow non-reflective boundary conditions are used in the far field and downstream. In this simulation, the inflow Mach number is 0.17 , and the Reynolds number based on the inflow velocity and the chord length of the airfoil is $1.7 \mathrm{e} 6$. Three attack angles, which are 4, 5.5, and 8.5 degrees, respectively, are considered in this study. The dynamic pressure on the surface of the slat is sampled and compared with the experimental data by other researchers, and a good agreement is obtained. The far field noise, computed with the permeable Ffowcs Williams-Hawkings (FW-H) integration method, is analyzed and compared with the experimental data. The noise source is analyzed and identified with a correlation analysis of the dynamic pressure on the slat and the near field noise.

\section{1:45}

4pNSa4. Three-dimensional computational fluid dynamics prediction of mild surge in a turbocharger compression system vs. experiments. Rick Dehner, Ahmet Selamet, and Emel Selamet (Mech. Eng., The Ohio State Univ., 930 Kinnear Rd., Columbus, OH 43212, dehner.10@osu.edu)

The flow instabilities in a turbocharger compression system are studied numerically by employing a compressible, three-dimensional computational fluid dynamics code. The model represents the full compression system of a turbocharger test stand, consisting of a compressor inlet duct breathing from ambient, a centrifugal compressor, an exit duct connected to a plenum, followed by another duct which incorporates a valve restriction. The detailed compressor model includes the full rotating impeller and resolution of the clearance gap between the impeller blades and shroud. The simulation begins at a converged, stable operating point, where the flow rate at the outlet boundary is gradually reduced and maintained at the final value. Characteristics of mild surge are captured as the mass flow rate is reduced below the stability limit, including a discrete low frequency sound peak near the Helmholtz resonance of the compression system. The predictions are then compared with experimental results obtained from the turbocharger stand placed in a hemianechoic room. The computational results are shown to be capable of reproducing a number of key experimental observations, including the details of low-frequency pressure fluctuations in the compressor ducts and plenum, and the transition from stable operation to oscillating mild surge.

\section{2:00}

4pNSa5. Numerical Simulation of time domain wall impedance on circular and annular ducts using the immersed boundary method. Braulio Gutierrez Pimenta (Mech. Eng. Dept., Univ. of Brasília, Universidade de Brasília, Campus Universitário Darcy Ribeiro, Faculdade de Tecnologia, Departamento de Engenharia Mecânica, Brasília, Distrito Federal 70910900, Brazil, braulio.pimenta@gmail.com), Ana Luisa P. Maldonado (RollsRoyce University Technol. Ctr., Univ. of Southampton, Southampton, United Kingdom), and Roberto F. Bobenrieth Miserda (Mech. Eng. Dept., Univ. of Brasília, Brasília, Brazil)

In this work, time domain boundary conditions of wall impedance are simulated on circular and annular ducts of constant cross section. The discretization technique used here is the immersed boundary method in the framework of finite volume formulation. It has fourth order of precision in space and uses a third order Runge-Kutta method for time stepping. Several boundary conditions for wall impedance are tested and then compared with the analytical results of the one-dimensional resonating chamber for code validation. The impedance boundary conditions are simulated on a circular duct with wall treatment and with a synthetic sound source. Mean flow conditions include constant axial velocity and swirling flows of rigid body rotation and potential flow. The results are then compared with a fourth order eigensystem method of linearized governing equations. The results of the immersed boundary methodology are assessed along with the wall impedance boundary condition models and their linear validity is compared with the results of the eigensystem method. 


\title{
Session 4pNSb
}

\section{Noise: Low Frequency Noise: Measurement, Evaluation, Enforcement, and Policy}

\author{
David S. Woolworth, Cochair \\ Oxford Acoustics, 356 CR 102, Oxford, MS 38655 \\ Yukio Takahashi, Cochair \\ National Institute of Occupational Safety and Health, Japan, 6-21-1 Nagao Tama-ku, Kawasaki 214-8585, Japan
}

\section{Invited Papers}

2:30

4pNSb1. The state of low frequency noise regulation in the United States. Leslie D. Blomberg (Noise Pollution Clearinghouse, Box 1137, Montpelier, VT 05601-1147, les@nonoise.org) and David S. Woolworth (Oxford Acoust., Oxford, MS)

This paper surveys state and local regulation of low frequency noise in the United States. Included in the survey are the noise regulations of the 500 largest cities in the United States, state regulations from the dozen states with reasonably comprehensive noise regulations, and various other regulations, usually related to mineral extraction or energy production. The prevalence of low frequency criteria in noise regulations as well as the metrics and criteria used are discussed and evaluated.

\section{2:50}

4pNSb2. Case report of a low-frequency noise complaint settled through adjudication by the Environmental Dispute Coordination Commission. Yukio Takahashi (National Inst. of Occupational Safety and Health, Japan, 6-21-1 Nagao Tama-ku, Kawasaki, Kanagawa 214-8585, Japan, takahay@h.jniosh.johas.go.jp)

In Japan, complaints about environmental low-frequency noise have been increasing in recent 20 years. The Environmental Dispute Coordination Commission is a subsection of the Ministry of Internal Affairs and Communications and one of its main duties is to resolve environmental disputes promptly and appropriately through adjudication, mediation, and other ways. The Commission treats some cases of complaint about low-frequency noise every year. This presentation outlines the activity of the Commission and reports a case of a low-frequency noise complaint settled through adjudication by the Commission. The author took part in the adjudicating process as an external technical expert, and supported on-site measurement of the noise and evaluation of its effect. The source of the noise in the case was air compressors located at a supermarket adjacent to the complainant's house. In evaluating the effect of the noise, much importance was given not to merely the sound pressure level of the noise but to the correspondence between the sound pressure level of the noise and subjective ratings of the complainant's annoyance. Thanks to a good correspondence found between them, the complainant and the supermarket arrived at compromise.

\section{3:10}

4pNSb3. Characterization of low frequency sounds emanating from entertainment venues. David S. Woolworth (Roland, Woolworth \& Assoc., 356 CR 102, Oxford, MS 38655, dave@oxfordacoustics.com)

The advent of inexpensive low frequency reproduction for audio has changed the way modern popular music is composed and recorded; these inexpensive systems are more common than ever in clubs (and vehicles), which has a direct effect on the soundscape resulting from high sound level playback and limited sound attenuation for low frequencies in typical building structures. This paper characterizes the low frequency content of sounds propagated intra-building, inter-building, and to the outside from various entertainment venues from the perspective of both frequency and time. Additionally, the paper will discuss suggested techniques for testing venues under controlled situations with pre-recorded and live sound sources to augment testing based on standards.

\section{$3: 30$}

4pNSb4. Psychological and physiological response of low frequency noise of ordinary persons and complainants. Shinji Yamada (The Univ. of Yamanashi, 1-9-3 Shiobe, Kofu 400-0026, Japan, shinji-yamada@ae.auone-net.jp), Yukio Inukai, Tsutae Sebayashi (Non Profitable Organization on Noise, Vib. and Low Frequency Noise, Kofu, Japan), and Toshiya Kitamura (The Univ. of Yamanashi, Kofu, Japan)

In Japan, there are some complainants of low frequency noise. There are two sorts of complains on low frequency noise. One complain is the phenomenon of rattling noise. Light windows or doors are vibrated and rattled by the pressure of low frequency noise. Sound sources are a big diesel engine, pass by of a heavy truck on a highway bridge, a gas turbine engine generator etc. The other complaints are the direct hearing or detection of low frequency noise. Sound sources are a boiler, a cooling tower, etc., near neighbors, or solid borne noise inside a room. But recently, there are some complains at very low level and the sound source cannot be detected. In these 
cases, there may be much influences by mass media and Internet knowledge. In this paper we explain the mechanism of complains on low frequency noise as bellows. Hearing thresholds of ordinary persons and complainants. Physiological response of ordinary persons and complainants. Time history of annoyance of complainants according to on-and-off of sound source. Recent complains against low frequency noise. Guideline on low frequency noise: Mechanism of complaint in brain to wind turbine low frequency noise.

\section{3:50-4:05 Break}

\section{Contributed Papers}

4:05

4pNSb5. Numerical analysis of low frequency noise propagation from outdoor into house. Dai Hashimoto (Yamaha Corp., 10-1, Nakazawa-cho, Naka-ku, Hamamatsu, Shizuoka 430-8650, Japan, dai.hashimoto@music. yamaha.com), Naohisa Inoue, and Tetsuya Sakuma (Graduate School of Frontier Sci., Univ. of Tokyo, Kashiwa, Chiba, Japan)

There have been increasing environmental problems caused by low-frequency noise: window rattle, human annoyance and so on. Sound transmission from outdoor into a house in the low-frequency range is a considerably complex phenomenon because the house behaves as a total vibrating system composed of windows, walls, frames, ventilation holes, and so on. This paper presents a finite element model of a simple house in order to investigate the low-frequency sound transmission. The features of the model are as follows: 1) a rigid hollow cube with an aperture on its one side is placed on a rigid plane, 2) a windowpane mounted on the aperture is modeled as a limp membrane or an elastic plate, 3) the outdoor domain is truncated by perfectly matched layers in order to simulate a semi-free field, and 4) a noise source is supposed to be an air conditioning outdoor unit placed near the house. It is confirmed that the indoor SPL gains mainly due to two resonance systems: one is the global motion composed of indoor air tightness and mass around the aperture, and the other is the series of normal modes excited in a cubic room.

\section{$4: 20$}

4pNSb6. Influence on human body of audibility amplitude modulation low-frequency noise in residential environment. Hiroshi Matsuda and Nobuo Machida (College of Sci. and Technol., Nihon Univ., 7-24-1 Narashinodai, Funabashi, Chiba 2748501, Japan, matsuda@eme.cst.nihon-u.ac.jp)

Low-frequency noise of about $100 \mathrm{~Hz}$ including audible frequency is positioned as one of the factors of environmental problem. First, this paper describes countermeasures of psychological complaint by low-frequency noise generated by residential environment in Japan published in the Ministry of the Environment. Next, we experimentally examined the human body influence of audibility amplitude modulation low frequency noise (AMLFN) on the psychological and physiological response measured by whole-body exposure. The AMLFN used by this research fluctuated the fluctuation cycle of the carrier wave and the sound pressure level periodically by the amplitude modulation. The measuring objects of the psychological response are an annoyance by the psychological questionnaire and an impression evaluation by the semantic differential method. The measuring objects of the physiological response are a salivary amylase activity, a R-R interval, and a power spectrum of heart rate variability. The stress reaction by AMLFN exposure was examined from these measurement results. From these experimental results, the stress reactions during AMLFN exposure were tendency increased with an increase of the modulation depth of the sound pressure level and a decrease of the fluctuation cycle, and the annoyance was tendency increased with a decrease of the carrier frequency.

\section{$4: 35$}

4pNSb7. Wind-induced noise in a convective boundary layer. Carl R. Hart and D. K. Wilson (U.S. Engineer Res. and Development Ctr., 72 Lyme Rd., Hanover, NH 03755, carl.r.hart@usace.army.mil)

Wind-induced noise, as sensed by a screened microphone in the atmosphere, produces a highly varying noise floor. At low frequencies, this noise source may dominate the ambient noise spectrum and present a challenge for signal detection. The sensed noise is affected by several factors in the atmospheric convective boundary layer: the mean flow at the height of the microphone, turbulent eddy interaction with the windscreen, and pressure fluctuations within the turbulent flow. Recently, van den Berg (2006) compared measured wind noise to modeled spectra. In that study, several meteorological variables were inferred from fits of modeled wind noise spectra rather than from meteorological observations. If additional meteorological observations are collected, then it may be possible to conduct model validation. In addition to temperature, pressure, and wind velocity, the surface roughness length for momentum and the vertical temperature flux must be observed. Recent models of turbulent wind velocity, in the convective boundary layer, suggest that the planetary boundary layer height may also be an important variable for wind-induced noise. This study analyzes data collected in the Southwestern United States during a 2007 long-range sound propagation experiment. Wind noise data are compared against concurrent meteorological data and several wind-induced noise models.

\section{4:50}

4pNSb8. Low frequency sound sources, regulations, and the wind noise evaluation for appropriate procedure against complaints. Masayuki Shimura (INCE/Japan, 2-23-2, Higashi-ikebukuro Toshima-ku, Tokyo 1700013, Japan, shimura@kensetsukankyo.co.jp)

There are many kinds of low frequency noise sources, including cannon, tunnel-blasts in construction, the Shinkansen, arch dams, elevated roads, wind farms, and so on. For example, low frequency noise by bombardment practice of the Ministry of Defense is dominant in the $8-16 \mathrm{~Hz}$ bands in the emission area nearby dwelling houses. Local regulations for soundproofing are applied in such cases. In the sound emission problems by drainage from the dam, we have to discuss the noise abatement of low frequency sound. However, there is neither existing regulation nor environmental assessment. Individual correspondence has been done for each noise source based on the complaints from dwellers. There are not unified countermeasures for low frequency sources in Japan. Evaluation of low frequency noise is very difficult because the measuring results are influenced by wind pressure fluctuation in natural wind. The physiological and psychological influence by such noise is also not clear. In this presentation, a new method to measure low frequency sound in natural wind is proposed. 


\title{
Session 4pPA
}

\section{Physical Acoustics: Nonclassical Nonlinear Acoustics of Solids}

\author{
Lev A. Ostrovsky, Cochair \\ PSD, NOAA ESRL, 325 Broadway, R/PSD99, Boulder, CO 80305 \\ Paul A. Johnson, Cochair \\ Geophysics, LANL, MS D443, Los Alamos, NM 87545 \\ Yoshikazu Ohara, Cochair \\ Department of Materials Processing, Tohoku University, 6-6-02 Aoba, Aramaki-aza, Aoba-ku, Sendai 980-8579, Japan
}

\section{Invited Papers}

1:00

4pPA1. Propagation of a finite-amplitude pulse in a bar of berea sandstone: The mechanisms of classical nonlinearity, conditioning, and hysteresis. Marcel C. Remillieux, James A. Ten Cate, Pierre-Yves Le Bas, and T. J. Ulrich (Los Alamos National Lab., Geophys. Group (EES-17), Mail Stop: D446, Los Alamos, NM 87545, mcr1@lanl.gov)

We study the propagation of a finite-amplitude pulse in a slender bar of Berea sandstone. The center frequency of the pulse and aspect ratio of the bar are such that the problem can be adequately described by the propagation of a longitudinal wave in a 1D system. The evolution of the three Cartesian components of the particle velocity on the surface of the bar as functions of the propagation distance and source amplitude is carefully monitored without contact using a 3D laser Doppler vibrometer. In these experiments, we evidence simultaneously the effects from classical nonlinearity, hysteresis, and conditioning (i.e., elastic softening) in the impulsive waveforms, as the pulse propagates away from the source. Traditionally, this type of experiments has been conducted to quantify only classical nonlinearity, through the parameter $\beta$, based on the amplitude growth of the second harmonic as a function of the propagation distance. In this work, we also use these experiments to quantify conditioning, through the parameter of nonclassical nonlinearity $\alpha$, based on the relative change in the arrival time of the pulse as a function of strain and distance from the source.

\section{$1: 20$}

4pPA2. Creep-induced nonlinear ultrasonic change in an austenitic stainless steel. Toshihiro Ohtani, Yutaka Ishii (Mech. Eng., Shoan Inst. of Technol., 1-1-25, Tsujido-nishikaigan, Fujisawa, Kanagawa 2518511, Japan, ohtani@mech.shonan-it.ac.jp), Masaaki Tabuchi, Kota Sawada, and Hiromichi Hongo (Creep Resistant Mater. Group, National Inst. for Mater. Sci., Tsukuba, Ibaraki, Japan)

We investigated the evolutions of two nonlinear acoustic characterizations: resonant frequency shift and three-wave mixing, with electromagnetic acoustic resonance (EMAR) throughout the creep life in an austenitic stainless steel, JIS-SUS 304. EMAR was a combination of the resonant acoustic technique with a non-contact electromagnetic acoustic transducer (EMAT). We used bulk- shear-wave EMAT, which transmits and receives shear wave propagating in thickness direction of a plate specimen. Creep tests with plate specimens were carried out at $973 \mathrm{~K}$, and $100 \mathrm{MPa}$ and interrupted at several time steps. Two nonlinear acoustic parameters showed peas at $40 \%$ of creep life. After that they deceased, they increased from $60 \%$ of creep life to rupture. We interpreted these phenomena in terms of dislocation recovery, recrystallization, and restructuring and the initiation and growth of creep void, with support from the SEM and TEM observation.

\section{Contributed Papers}

1:40

4pPA3. Nonclassical nonlinearity and crystal defects in lithium niobate. Chandrima Chatterjee, Lucien Cremaldi (Phys. and Astronomy, Univ. of MS, Lewis Hall, Rm. 108, University, Oxford, MS 38677), and Igor Ostrovskii (Phys. and Astronomny, Univ. of MS, University, MS, iostrov@phy. olemiss.edu)

Nonclassical nonlinearity (NN) in ultrasound attenuation and acoustic memory in $\mathrm{LiNbO}_{3}$.may be connected to crystal defects. The optical spectra of photoluminescence (PL) and absorption taken from 350-900 nm reveal multiple point defects and impurities such as F-center, $\mathrm{Nb}_{\mathrm{Li}}{ }^{+}, \mathrm{Fe}, \mathrm{Ba}, \mathrm{Ar}, \mathrm{Xe}$ and others. The spectra are taken at room temperature from the Czochralski grown bulk crystals and wafers. The samples are scanned by taking PL spectra from different locations along the $\mathrm{z}$ - and $\mathrm{y}$-axes. The data from several samples reveal a nonuniform distribution of the defects along the crystallographic axes. The dependencies of PL peaks versus position have a sine-like component on top of some constant intensity. For instance, a scan for F-center has a period (P) from 0.27 to $0.75 \mathrm{~mm}$ and for $\mathrm{Ba}$ impurity from 0.25 to $0.75 \mathrm{~mm}$ in bulk crystals. In the wafers, $\mathrm{P}$ may be smaller, that is $0.1 \mathrm{~mm}$ and smaller. However, a ratio of maxima to minima in PL peaks is higher for bigger $\mathrm{P}$. The overall spread of $\mathrm{P}$ is roughly from $<0.1 \mathrm{~mm}$ to about 1 $\mathrm{mm}$. Some defects are electrically charged, and may influence the 
piezoelectric properties. Consequently, a real crystal has some constants which are nonuniform along its axes. The measured periods $\mathrm{P}$ correspond to ultrasonic wavelengths in the Megahertz frequency range. The comparison of the periods $\mathrm{P}$ in defects distributions with the frequencies of NN phenomena points to considering crystalline defects as those responsible for the NN observed in $\mathrm{LiNbO}_{3}$.

\section{1:55}

4pPA4. Ultrasonic array imaging of contact-acoustic nonlinearity. Jack Potter, Jingwei Chen, Bruce Drinkwater, and Anthony Croxford (Mech. Eng., Univ. of Bristol, Queen's Bldg., University Walk, Bristol BS8 1TR, United Kingdom, jack.potter@bristol.ac.uk)

Classically, ultrasonic arrays achieved physical beam-forming through the application of phase delays to the parallel transmission of elements. Alternatively, beam-forming may be emulated through post-processing of sequentially transmitted array data. Though exactly equivalent for linear systems, these parallel and sequential fields differ in their nonlinear propagation. Information pertaining to elastic nonlinearity is encoded onto the relative parallel/sequential field by interference between the field components of individual element transmissions. A family of nonlinear imaging techniques has been developed which exploit differences between parallel and sequential fields in order to image elastic nonlinearity. Diffuse energy and coherent scattering variants of the technique are demonstrated for the imaging and characterisation of contact-acoustic nonlinearity in application to the detection and sizing of closed fatigue cracks.

\section{2:10}

4pPA5. Simulation of coupled structural-acoustic response with dynamic damage evolution. Jonathan S. Pitt (The Penn State Univ., Appl. Res. Lab, PO Box 30, Mailstop 3320B, State College, PA 16804, jonathan. pitt@psu.edu)

A novel time-domain method for simulating dynamic damage evolution in a coupled structural-acoustic system is presented. The system is derived via the theory of continuum damage mechanics, and incorporates standard damage evolution models, but is readily extendible to more exotic formulations. The overall solution method is staggered, solving for the dynamic damage evolution first with an explicit step, and then using the new values in the coupled computation of the structural-acoustic system. The spatial domain is discretized using a mixed finite element method, and the temporal domain is discretized with a higher-order implicit time discretization scheme. Efforts toward fully coupled verification of the solution algorithm are presented, as are validation studies for cases without evolving damage. Applications with evolving damage are presented, and present a first principles study of changes in the structural acoustic response to dynamically evolving damage in the structure. Special attention is given to brittle fracture. Examples of downstream usage of the evolving structural response are discussed in the concluding remarks.

\section{2:25}

4pPA6. Evaluation of fatigue damage in a pure copper with nonlinear three-wave interaction. Yutaka Ishii (Graduate School of Eng., Shonan Inst. of Technol., 1-1-25, Tsujido-nishikaigan, Fujisawa, Kanagawa 2518511, Japan, 11t1501@sit.shonan-it.ac.jp), Toshihiro Ohtani (Mech. Eng., Shoan Inst. of Technol., Fujisawa, Kanagawa, Japan), Masayoshi Nakaniwa (Nippon Steel \& Sumikin Technol., Amagasaki, Hyogo, Japan), and Masayuki Kamaya (Inst. of Nuclear Safety System, Inc., Mikata-gun, Fukui, Japan)

We investigated nonlinear ultrasonic characterization, nonlinear threewave interaction, of the pure copper during fatigue with EMAR (Electro- magnetic Acoustic Resonance), which was the combination with ultrasonic resonance and non-contacting transducer, EMAT (Electromagnetic Acoustic Transducer). In nonlinear three-wave interaction method, two intersecting ultrasonic waves produced a scattered wave when the resonance condition was satisfied. The amplitude in resonant scattering wave was measured. Nonlinear three-wave interaction method exhibited high sensitivity to micro-structural change of the damaged material. It rapidly increased from $50 \%$ of fatigue life to the fracture. TEM (Transmission Electron Microscope) and EBSD (Electron Backscatter Diffraction) observations supported this phenomenon caused by dislocation movement. The sensitivity in threewave interaction method was higher than that in linear methods. The noncontact resonance-EMAT measurement can monitor the evolution of nonlinearity throughout the fatigue life and has a potential to assess the damage advance and to predict the fatigue life of metals.

\section{2:40}

4pPA7. Resonant acoustic nonlinearity of defects: Non-classical manifestations and applications for diagnostic imaging. Igor Solodov (Inst. for Polymer Technol., Univ. of Stuttgart, 32 Pfaffenwaldring, Stuttgart 70569, Germany, igor.solodov@ikt.uni-stuttgart.de)

Acoustic nonlinearity of non-bonded interface (Contact Acoustic Nonlinearity $(\mathrm{CAN})$ ) is a type of non-classical nonlinearity perspective for applications in non-destructive evaluation of cracked defects. The presence of such defects also leads to a local decrease in rigidity and manifests in a characteristic frequency of the defect. A frequency match between the driving acoustic wave and this frequency provides a Local Defect Resonance (LDR) and an efficient energy delivery from the wave into the defect. A combination of CAN and LDR, therefore, manifests a profound nonlinearity even at moderate acoustic excitation level. Under resonance conditions, a strong enhancement of the higher harmonic (HH) amplitudes is observed. A high quality factor of LDR is also used as a filter/amplifier/resonator for efficient frequency mixing. These "conventional" nonlinear effects are not the only dynamic scenario of nonlinear phenomena for resonant defects. A combined effect of LDR and CAN also results in qualitatively new features characteristic of nonlinear and parametric resonances. Due to parametric instability, nearly total input energy at fundamental frequency can be converted into $\mathrm{HH}$ or subharmonic vibrations of the defects. Both super- and subharmonic LDR are strongly localized in the defect area that provides a background for highly-sensitive defect-selective imaging.

\section{2:55}

4pPA8. In situ nonlinear ultrasonic probing during plastic deformation. Carolina Espinoza, Vicente Salinas, Nicolás Mujica, and Fernando Lund (Phys., Universidad de Chile, Av. Blanco Encalada 2008, Santiago 837.0415, Chile, deplasticoverde@gmail.com)

In is well known that nonlinear ultrasound is sensitive to micro-structural changes in materials. Here, we present recent results obtained during tensile tests of aluminum samples. Using continuous ultrasonic waves, we study the second harmonic generation and its relationship with dislocation proliferation during plastic deformation. The experimental setup is based on two transducers, an emitter and a receiver, both coupled face to face to a probe mounted on a standard tensile test machine. We measure both the first and second harmonic received amplitudes, $\mathrm{A}_{\omega}$ and $\mathrm{A}_{2 \omega}$ respectively, for several amplitudes of the emitted signal. These are related by a nonlinear parameter $\beta$, through $\mathrm{A}_{2 \omega}=\beta \mathrm{A}_{\omega}{ }^{2}$. We observe that $\beta$ is independent of the applied stress during the elastic regime, whereas it presents strong variations once plasticity occurs, presenting a pronounced maximum before fracture. This maximum can be considered as a signature, a warning, of a fracture that is soon to come.

\section{3:10-3:25 Break}




\section{Invited Papers}

4pPA9. Fast and slow nonlinear elastic response of disparate rocks and the influence of moisture. Jacques Riviere (GeoSci., Penn State Univ., Deike 510, University Park, PA, jvr5626@psu.edu), Parisa Shokouhi (Civil Eng., Penn State Univ., University Park, PA), Robert A. Guyer, and Paul A. Johnson (EES-17, Los Alamos National Lab., Los Alamos, NM)

We study nonlinear elastic phenomena in rocks at the laboratory scale, with the goal of characterizing and understanding observations at crustal scales, for instance, during strong ground motion and earthquake slip processes. A dynamic perturbation of microstrain amplitude in rocks results in a transient elastic softening followed by a $\log (\mathrm{t})$-type relaxation back to the initial unperturbed elastic modulus as soon as the excitation is removed. Here we use Dynamic Acousto-Elastic Testing (DAET) to investigate the relaxation behavior over 7 orders of magnitude in time (from $10^{-4} \mathrm{~s}$ to more than $10^{3} \mathrm{~s}$ ). We find that relaxation starts for all samples between $10^{-3}$ and $10^{-2} \mathrm{~s}$. Some samples then exhibit a nearly perfect $\log (\mathrm{t})$-relaxation, implying that no characteristic time can be extracted, while some other samples show a preferential relaxation around $0.1 \mathrm{~s} / 1 \mathrm{~s}$. Such features appear insensitive to the amplitude of the dynamic perturbation and to the moisture content within the sample. The full nonlinear elastic response (fast dynamics) is also extracted at all amplitudes and moisture content. Adsorption of water on the grains strongly increases the elastic softening during the dynamic perturbation and the non-classical nonlinear features, whereas the classical features seem rather unaffected.

\section{$3: 45$}

4pPA10. Steady-state analysis of nonlinear ultrasonic waves caused by crack face friction. Taizo Maruyama and Terumi Touhei (Civil Eng., Tokyo Univ. of Sci., 4th Fl., 5th Bldg., Noda Campus, 2641 Yamazaki, Noda City, Chiba Pref. 278-8510, Japan, taizo_maruyama@rs.tus.ac.jp)

In general, the detection of closed cracks is difficult by using the conventional linear ultrasonic testing. Therefore, the nonlinear ultrasonic testing (NLUT) based on the contact acoustic nonlinearity (CAN) is expected to be an effective technique for the problem. Several studies have reported the presence of nonlinear ultrasonic waves (higher- and sub-harmonic waves) due to the CAN. However, the theoretical explanation of the physical phenomena is not sufficient at present. For accurate NLUT, theoretical investigation of the nonlinear vibration phenomena of crack faces is desirable as a basic research. It is true that the time-domain analysis is becoming possible for the purpose. However, it is difficult to investigate in detail steady-state vibration of crack faces, which is supposed to affect behavior of nonlinear ultrasonic waves significantly. In this study, a novel numerical method is developed for the NLUT by coupling the boundary element method (BEM) and harmonic balance method (HBM) in order to investigate the above steady-state vibration. The scattering model is for the 2-D SH wave field that causes the crack face friction. Several numerical examples are presented in order to demonstrate validity and effectiveness of the proposed method.

\section{Contributed Papers}

\section{4:05}

4pPA11. The soil plate oscillator: Measuring nonlinear mesoscopic elasticity of granular medium in flexural vibration. Emily V. Santos and Murray S. Korman (Phys. Dept., U.S. Naval Acad., 572 C Holloway Rd., Chauvenet Hall Rm. 295, Annapolis, MD 21402, korman@usna.edu)

The soil plate oscillator apparatus (SPO) consists of two circular flanges (5 inch ID, 8 inch OD, 2.5 inch thick) sandwiching and clamping a thin (1/8 inch) circular elastic acrylic plate. Granular media (soil, masonry sand, glass beads, or even edible granular material) is supported at the bottom by the plate and side walls of the upper flange. A magnetic disk $(1 \mathrm{~cm})$ fastened below the plate (on center) is driven by a swept sinusoidal current in a coil placed below the magnet. This electrodynamic SPO, placed in a Wheatstone bridge, was used to measure the on-axis motional electrical impedance $Z_{\text {mot }}$ with and without granular loading. Nonlinear tuning curves near resonance exhibit softening with increasing drive amplitude. Measurements of Real\{$\left.Z_{\text {mot }}\right\}$ and $\operatorname{Imag}\left\{Z_{\text {mot }}\right\}$ were used to predict the nonlinear mechanical point impedance $\left(Z_{\text {mech }}=\right.$ constant $\left./ Z_{\text {mot }}\right)$ behavior of the SPO system. A lumped element model (granular media and elastic plate system) help determine the mesoscopic nonlinear properties of the granular media alone. Nonlinear tuning curves of the magnet's acceleration were also measured. A bilinear hysteresis model fits the tuning curve results. See I. T. Perez-Miravete et al., JASA 125, 1302 (2009) for a nonlinear mesoscopic analysis of a flexural bar.
$4: 20$

4pPA12. Second harmonic ultrasonic waves detection using a doublelayered piezoelectric transducer. Makoto Fukuda and Kazuhiko Imano (Dept. of Mathematical Sci. and Electrical-Electronic-Comput. Eng., Graduate School of Eng. and Resource Sci., Akita Univ., 1-1 Tegata Gakuenmachi, Akita 010-8502, Japan, mfukuda@gipc.akita-u.ac.jp)

A real-time detection system for second harmonic ultrasonic pulse waves using a double-layered piezoelectric transducer (DLPT) has been constructed. We also analyzed the effective detection of the second-harmonic ultrasonic pulse waves generated by closed-cracks and/or contact surfaces of solids (CAN: contact acoustic nonlinearity) using DLPT and the pulse inversion averaging (PIA) method. Lamb waves were also detected in a double-layered piezoelectric transducer (DLPT). The second harmonic components generated from a closed crack in the glass plate were detected in the pulse-echo method. The second harmonic components in the received waveform in the closed crack area were approximately $6 \mathrm{~dB}$ higher than that in the crack-free area. To confirm the origin of the second harmonic components, vibration velocities were detected by using laser Doppler vibrometry. As a result, the threshold amplitude for the generation of the second harmonic components of the Lamb wave was confirmed. This result indicates the existence of the closed cracks in the glass plate. 
methods. Two examples from the application to concrete and stabilized soil are presented. In addition quantitative values of the hysteretic nonlinear parameter from stabilized soil samples are compared with the measured ultimate compressive strength of each sample.

\section{$5: 05$}

4pPA15. Using elastic nonlinear modulation of sound by sound to detect fracture orientation in reservoir rocks. James A. TenCate (Geophys. Group, Los Alamos National Lab, M.S. D446, EES-17 Earth and Environ. Sci., Los Alamos, NM 87545, tencate@lanl.gov), Alison E. Malcolm (Earth Sci., Memorial Univ., St John's, NF, Canada), and Michael C. Fehler (Earth, Atmospheric and Planetary Sci., Massachusetts Inst. of Technol., Cambridge, MA)

Cracks play a key role in our ability to produce oil and gas or monitor water resources, from microscale cracks that enable permeability in tight formations to larger faults and fractures that compartmentalize reservoirs. Our ability to sense and understand cracks is thus of key importance. We explore the role that cracks play in the nonlinear interaction of propagating waves using an elastic version of modulation of sound by sound. We present a laboratory experiment in which a strong S-wave slightly changes the velocity of a lower amplitude P-wave, and use a rock sample with aligned fractures to demonstrate that this signal is strongly dependent on fracture orientation. We build on the linear slip theory to show that the propagating $\mathrm{S}$-wave is indeed able to open the cracks that the P-wave velocity will be most sensitive to. This gives firm, direct evidence that cracks are a controlling factor in the nonlinear elastic properties of rocks, and opens up the possibility of using such signals to remotely map fracture orientations.

\section{$5: 20$}

4pPA14. Impact non-linear reverberation spectroscopy applied to nondestructive testing of building materials. Nils Ryden (Biomedical Eng., Faculty of Eng., Lund Univ., Box 118, Lund 22100, Sweden, nils.ryden@tg. 1th.se), Unn Dahlen (Mathematical Statistics, Faculty of Eng., Lund Univ., Lund, Sweden), Per Lindh (Swedish GeoTech. Inst., Linköping, Sweden), and Andreas Jakobsson (Mathematical Statistics, Faculty of Eng., Lund Univ., Lund, Sweden)

The development of non-destructive testing techniques to detect early micro damage in concrete and other building materials is important for efficient maintenance and management of existing infrastructure. Nonlinear acoustic methods have shown potential for the identification of early damage in brittle materials such as concrete. Commonly, these methods evaluate relative nonlinearity parameters from multiple resonance tests at different amplitudes. We demonstrate a recently developed alternative method, Impact Nonlinear Reverberation Spectroscopy (INRS), where quantitative nonlinearity parameters are evaluated from a single impact resonance test. The recorded reverberation of the measured signal is matched to a synthetic nonlinear damped signal. The proposed model allows instantaneous true physical amplitude, frequency, and damping of each mode to be characterized as a function of time, allowing for quantitative information of the nonlinear parameters. The hysteretic material nonlinearity can be quantitatively characterized over a notably wider dynamic range compared to conventional
4pPA16. How does the impact strength affect slow time relaxation in rock. Lev A. Ostrovsky (Appl. Mathematics, Univ. of Colorado, 325 Broadway, R/PSD99, Boulder, CO 80305, lev.a.ostrovsky@noaa.gov) and Andrey Lebedev (Geophys., Inst. of Appl. Phys., Nizhniy Novgorod, Russian Federation)

Earlier we suggested a model for slow time relaxation in rock which is based on the existence of metastable contacts which slowly return to the basic state due to thermal processes. This model allows to explain the logarithmic law of relaxation and estimate the characteristic scale of metastable contacts. In this presentation, along with a brief outline of the problem, we make the next step, namely, describe the dependence of the process on the amplitude of the initial impact. For this, the distribution function of the contacts' strength is used. Based on the known data, we use the lognormal distribution for the thresholds of contact breakups. In the physically evident case of small percentage of breaking contacts, the excited states' concentration is shown to linearly depend the impact stress. The latter is in agreement with the known laboratory experiments. We also used the data from the Parkfield earthquake to evaluate the initial strain level (of the order of 10-5) preceding the relaxation. This estimation seems reasonable as compared with data for earthquakes having the magnitude of M6. 


\title{
Session 4pPP
}

\section{Psychological and Physiological Acoustics and Speech Communication: Gap Detection: New Perspectives from Neuroscience, Perception, and Modeling}

\author{
Shuji Mori, Cochair \\ Department of Informatics, Kyushu University, 744 Motooka Nishi-ku, Fukuoka 819-0395, Japan \\ Willy Wong, Cochair \\ Electrical and Computer Engineering, University of Toronto, 10 King's College Rd., Toronto, ON M5S3G4, Canada
}

Chair's Introduction-1:00

\section{Invited Papers}

1:05

4pPP1. What underlies across-channel gap detection: Overview. Shuji Mori (Dept. of Informatics, Kyushu Univ., 744 Motooka Nishi-ku, Fukuoka, Fukuoka 819-0395, Japan, mori@inf.kyushu-u.ac.jp)

Gap detection, which measures a person's ability to hear gaps in tones and noises, is commonly used to assess auditory temporal resolution. There are a variety of ways to measure the minimum detectable gap duration, or gap threshold, including across-frequency gap detection (detecting a silent gap between two spectrally different markers) and between-ear gap detection (detecting silence between a leading and trailing maker delivered to separate ears). A topic of particular interest concerns across-frequency gap detection, where the gap threshold has been found to be an order of magnitude larger than that obtained for a within-frequency task. The between-ear task has also been demonstrated to yield much longer gap thresholds than the within-frequency task. A number of studies have attempted to resolve the discrepancies between within-frequency and between-frequency/ear tasks (termed jointly as across-channel tasks), and research is still ongoing with a number of different approaches and avenues: psychoacoustic experimentation, electrophysiological measurements at the brainstem and cortical levels, as well as mathematical modeling. In this talk, I will provide an overview of research on across-channel gap detection, including the studies conducted at our laboratory.

\section{$1: 25$}

4pPP2. Auditory across-channel processing as assessed by monaural and between-ear temporal gap detection tasks. Ito Kazuhito (Faculty of Information Sci. and Elec. Eng., Kyushu Univ., 744 Motooka Nishi-ku, Fukuoka, Fukuoka 819-0395, Japan, ito@inf. kyushu-u.ac.jp), Takamura Akihide (Graduate School of Information Sci. and Elec. Eng., Kyushu Univ., Fukuoka, Japan), and Shuji Mori (Faculty of Information Sci. and Elec. Eng., Kyushu Univ., Fukuoka, Japan)

Gap detection is a measure of auditory temporal resolution. Hearing people exhibit acute sensitivity to silent gaps between leading and trailing markers when the two markers are identical or similar in frequency. However, sensitivity to such gaps declines when the two markers are of dissimilar frequencies. To examine the hypothesis that auditory gap detection performance declines during temporal comparisons of activity between different perceptual channels, we conducted monaural and between-ear temporal gap detection tasks. In the latter task, the leading and trailing sinusoidal markers delimiting the gap were presented to separate ears, which are regarded as independent channels, at least up to the superior olivary complex in the auditory brainstem. The between-ear gap detection thresholds were increased, even when the two markers were identical in frequency, and elevated gradually as the frequency difference between the two markers became greater than an octave. Furthermore, the patterns of the across-ear gap detection thresholds were roughly comparable with those of the monaural gap detection thresholds, except when the two markers were identical in frequency. These findings suggest that the different "across-channel" processes assessed by monaural and between-ear gap detection tasks share a common central mechanism for managing temporal information originating from different channels.

\section{1:45}

4pPP3. Marking time: The precise measurement of auditory gap detection across the lifespan. Jennifer J. Lister (Commun. Sci. and Disord., Univ. of South Florida, 4202 E. Fowler Ave., PCD 1017, Tampa, FL 33620, jlister@usf.edu)

Deficits of temporal resolution are thought to contribute to speech understanding in noise difficulties and may be documented using auditory gap detection thresholds (GDTs). It is important to establish the appropriate methods to measure GDTs clinically. We have established GDTs for a variety of stimuli, ages (7-90 years), equipment, degrees of hearing loss, psychophysical paradigms, neurophysiological paradigms, marker relationships (within-channel, across-channel), time points, and presentation ears (left, right, diotic). A number important findings will be discussed: 1 . Best stimulus for measurement of GDTs is narrow-band noise. 2. GDTs improve from ages 7 to 9 , stabilize between ages 9 and 40, and deteriorate with age thereafter. 3. GDTs may be measured reliably using a variety of 
equipment. 4. Hearing loss has a minor impact on GDTs. 5. A 2-interval psychophysical paradigm is best for measuring GDTs. 6. GDTs may be documented using the P1-N1-P2 auditory evoked potential. 7. Across-channel GDTs provide different information than withinchannel GDTs. 8. GDTs are reliable within and across test sessions. 9. GDTs do not differ across ear conditions. A stable and sensitive measure of temporal resolution that may be used in a clinical setting to assess temporal resolution will be recommended.

4pPP4. Auditory evoked fields elicited by repetitive gaps embedded within a continuous tone. Hidehiko Okamoto (Dept. of Integrative Physiol., National Inst. for Physiological Sci., 38 Nishigo-Naka, Myodaiji, Okazaki 4448585, Japan, hokamoto@nips.ac.jp)

The N1m response, which is a prominent auditory evoked component with a latency of around $100 \mathrm{~ms}$, elicited by sound onset is known to decrease with the repetition of identical sound stimuli. Previous studies demonstrated that a silent gap embedded within a continuous sound could also elicit the N1m responses; however, it remains elusive whether the N1m decrement occurs with a repetition of silent gaps. In the present study, we investigated the decrement of $\mathrm{N} 1 \mathrm{~m}$ responses elicited by five repetitive pure tones presented in silence and by five repetitive silent gaps embedded within a continuous tone using magnetoencephalography. The results obtained demonstrated that the $\mathrm{N} 1 \mathrm{~m}$ responses elicited by the repetitive pure tones decreased from the $1 \mathrm{st}$ to the 2 nd and remained constant from the 2nd to the 5th, whereas those elicited by the repetitive gaps in a continuous tone gradually decreased from the 1 st to the 5 th. Those results indicate that neural refractoriness appears to mainly contribute to the decrement of the $\mathrm{N} 1 \mathrm{~m}$ responses elicited by repetitive pure tones, while habituation may play an additional role in the decrement of the N1m responses elicited by repetitive gaps in a continuous tone.

\section{2:25}

4pPP5. Temporal asynchrony and rhythm perception: Neural bases of perceptual asymmetries. Andrew J. Oxenham and Magdalena Wojtczak (Psych., Univ. of Minnesota, 75 E River Parkway, Minneapolis, MN 55455, oxenham@umn.edu)

Temporal gap detection has been proposed as a perceptual and neural measure of temporal resolution. This measure has not shown strong frequency dependence but, if anything, high frequencies have been found to produce slightly lower (better) thresholds than very low frequencies. In apparent contradiction to these findings, a recent study claimed superior time perception for lower frequencies and proposed the finding as an explanation for why low-frequency instruments typically carry the rhythm in music [Hove et al. (2014), Proc. Natl. Acad. Sci. U.S.A. 111, 10383-10388]. The present study reviewed those findings and replicated them, but found that the results in fact reflect an asymmetry in asynchrony detection, reported earlier, whereby asynchronies are more readily detected when the lower-frequency tone is leading than when the higher-frequency tone is leading [Wojtczak et al., (2012). J. Acoust. Soc. Am. 131, 363-377]. New results using behavioral and EEG approaches reconcile the data from these different studies and confirm that low frequencies do not provide superior temporal resolution. Instead they suggest an asymmetry in how onset asynchronies are perceived, which may have its roots in the acoustic properties of natural sounds and in the mechanics of cochlear filtering. [Work supported by NIH grant R01DC005216.]

\section{2:45}

4pPP6. A peripheral model of gap detection. Willy Wong and Hugo Lepage (Elec. and Comput. Eng., Univ. of Toronto, 10 King's College Rd., Toronto, ON M5S3G4, Canada, willy.wong@utoronto.ca)

Gap detection is an important measure of the temporal resolution of the auditory system. We present a model of gap detection involving the statistics of peripheral auditory neural activity: detecting a gap involves the detection of an interval that is different from the usual interspike interval found in the auditory spike train. The model is capable of resolving a number of important observations in empirical gap research including why across-channel (or across-frequency) gap detection is an order of magnitude larger than gap detection performed in channel (i.e., using the same frequency). We achieve this by recognizing that in-channel detection involves a different strategy than across-channel detection. The model also provides an explanation for why the intensity dependence of gap detection is limited to a $40 \mathrm{~dB}$ range, and why critical bands are crucial towards defining the notion of a channel in gap detection. Specific predictions can be made about the shapes of the psychometric functions.

3:05-3:25 Panel Discussion 


\title{
Session 4pSA
}

\section{Structural Acoustics and Vibration, Physical Acoustics, and Noise: Fluid Flow Induced Vibration and Noise}

\author{
Robert M. Koch, Cochair \\ Chief Technology Office, Naval Undersea Warfare Center, Code 1176 Howell Street, Bldg. 1346/4, Code 01CTO, Newport, \\ RI $02841-1708$
}

Kuangcheng $\mathrm{Wu}$, Cochair

Ship Survivability, Newport News Shipbuild, 4101 Washington Ave., Newport News, VA 23693

Chair's Introduction-1:00

\section{Invited Papers}

1:05

4pSA1. A trial application of acoustic excitation for jet noise reduction. Shigehiko Kaneko (Dept. of Mech. Eng., The Univ. of Tokyo, 7-3-1, Hongo, Bunkyo-ku, Tokyo 113-8656, Japan, kaneko@mech.t.u-tokyo.ac.jp) and Tatsuya Ishii (Aeronoutical Technol. Directorate, Japan Aerosp. Exploration Agency, Tokyo, Japan)

Jet noise, one of the dominant noise components from an aircraft jet engine is known as the result of developed vortices produced during jet mixing process. In last couple of years, a method for jet noise reduction which acoustically controls the jet mixing layer using a sound excitation is proposed and investigated under the collaboration between The University of Tokyo and JAXA. In this paper, the main idea, experimental apparatus, experimental condition and experimental results will be explained. The experiment was conducted under an unheated and subsonic jet condition. The results show that low frequency sound excitation results in an increase in low frequency noise and a decrease in high frequency noise. The low frequency sound excitation is considered to excite the instability of jet flow and collapse the jet plume. On the other hand, the high frequency sound excitation resulted in a slight increase of high frequency noise and a slight decrease of low frequency noise $(0.5 \mathrm{~dB}$ reduction in maximum) similar to the effect of Chevron nozzle. In addition, LES simulation was conducted to investigate the effect of mixing process caused by sound excitation.

\section{$1: 25$}

4pSA2. Simultaneously modeling of multiple fluid-structure interaction subsystems of the human vocal folds. Ryan M. Oakey, Eduardo J. Alvarez (Mech. Eng., Brigham Young University-Idaho, Rexburg, ID), and Scott L. Thomson (Mech. Eng., Brigham Young Univ., 435 CTB, Provo, UT 84602, thomson@byu.edu)

Voice production is a direct result of vocal fold flow-induced vibration. Air from the lungs dynamically interacts with vocal fold tissue to generate self-sustained vocal fold oscillation, which in turn creates a fluctuating pressure field that is the source of sound for voice and speech. In addition to this primary air-tissue interaction that drives vocal fold vibration, other fluid-structure interactions (FSIs) occur within the interior regions of the vocal folds. For example, during voicing, the vocal fold vasculature is subjected to a time-varying displacement field as the tissue vibrates, thereby potentially affecting blood flow dynamics. Additionally, in benign vocal fold lesions comprised of fluid-filled pockets within the vocal folds, known as polyps, there are significant interactions between the fluid within the polyps and the surrounding tissues. The vocal fold system, therefore, potentially consists of multiple subsystems with different types of fluid-structure interactions that are distinct, yet coupled. In this presentation, experimental and computational studies that have focused on simultaneous modeling of these multi-FSI subsystems will be discussed. Methodologies for modeling the various FSI components will be described, sample results will be presented, and current capabilities and limitations of the various approaches will be summarized.

\section{$1: 45$}

4pSA3. Acoustic emission due to partial cavity shedding on a hydrofoil. Juliana Wu (Naval Architecture and Marine Eng., Univ. of Michigan, 2010 Beal Ave., Ann Arbor, MI 48109, wuju@umich.edu), Harish Ganesh (Mech. Eng., Univ. of Michigan, Ann Arbor, MI), and Steven Ceccio (Naval Architecture and Marine Eng., Univ. of Michigan, Ann Arbor, MI)

The partial cavity shedding dynamics on a NACA 0015 hydrofoil were examined at angles of attack of 7 and 10 degrees over a range of cavitation numbers. Hydrophone measurements in conjunction with high-speed videos and time resolved X-ray densitometry were used to acquire time synchronous measurements of the void fraction field of the cavity and the emitted noise. Cavitation dynamics changed significantly with the reduction of the inlet cavitation number. At higher cavitation numbers, small stable partial cavities were observed. Upon reduction of cavitation number, the cavities grew in length and pinched off from the rear of the cavity, due to a liquid re-entrant jet. Upon further reduction of inlet cavitation number, the dynamics changed significantly with the cloud collapse of the shed 
vapor inhibiting the cavity growth. X-ray densitometry measurements revealed the presence of a propagation bubbly shockwave as a mechanism of shedding at the lowest cavitation numbers. This process caused complex, multi-step cavity dynamics. Spectral analysis of the acoustic signal showed there were multiple acoustic frequency peaks, instead of a single well-defined shedding frequency. Spectral content of the acoustic measurements was then analyzed to identify different flow processes responsible for their generation.

\section{2:05}

4pSA4. Experimental investigation of compliant wall deformation in a turbulent channel flow. Cao Zhang, Jin Wang, and Joseph Katz (Dept. of Mech. Eng., Johns Hopkins Univ., 3400 N. Charles St., Baltimore, MD 21218, katz@jhu.edu)

Interaction of a compliant wall with a turbulent channel flow is investigated by simultaneously measuring the time-resolved, threedimensional flow field using tomographic PIV and the two-dimensional surface deformation using Mach-Zehnder interferometry. The friction Reynolds number is $R e_{\tau}=2300$, and the Young's Modulus of the wall is $0.93 \mathrm{MPa}$, resulting in a ratio of shear speed to centerline velocity $\left(U_{0}\right)$ of 6.8 . The wavenumber-frequency spectra of deformation contain a non-advected low-frequency component and advected modes, some traveling at $U_{0}$ and others at $0.72 U_{0}$. The wall dynamics is elucidated by correlating the deformation with flow variables, including the 3D pressure distribution. The pressure-deformation correlations peak at $y / h \sim 0.12$ ( $h$ is half channel height), the elevation of Reynolds stress maximum in the log-layer. Streamwise lagging of the deformation behind the pressure is caused in part by phase-lag of the pressure with decreasing elevation, and in part by material damping predicted by the Chase (1991) model. Positive deformations (bumps) are preferentially associated with ejections involving spanwise vortices downstream and quasi-streamwise vortices with spanwise offset, consistent with presence of hairpin-like structures. The negative deformations (dents) are preferentially associated with a positive pressure fluctuation at the sweep-ejection transition. [Sponsored by ONR.]

\section{Contributed Papers}

$$
\text { 2:25 }
$$

4pSA5. Structural vibration of an elastically supported plate due to excitation of a turbulent boundary layer. Jonmarcos Diaz, Kevin Maki, and Nickolas Vlahopoulos (Naval Architecture and Marine Eng., Univ. of Michigan, 2600 Draper Dr., Ann Arbor, MI 48109-2145, jonmarco@umich.edu)

High-Reynolds number turbulent boundary layers are an important source for noise and structural vibration. Indeed small features of a structure can have important influence on the resulting noise and vibration. In this work we develop a new method to couple a high-fidelity fluid solver with a dynamic-finite-element solver for the structure. The flow solver is based on the OpenFOAM opensource CFD toolkit. The fluid solver uses the LargeEddy Simulation closure for the unresolved turbulence. Specifically, a local and dynamic one-equation eddy viscosity model is employed. The fluid pressure fluctuation on the structure is mapped to the dynamic finite element model with a suitable interpolation routine. A modal decomposition of the dynamic finite-element model is used to reduce the number of degrees-offreedom of the numerical structural model. The numerical method is validated for the turbulent flow over a flat plate. The plate is elastically supported along its edges, and the turbulence is excited upstream of the plate by the means of a solid obstruction. Results for the structural vibration on the plate will be compared with previously published experimental measurements. Furthermore, the results will be compared with other numerical predictions in which the fluid forcing is determined using statistical methods

\section{2:40}

4pSA6. Effects of vocal tract inertance on the glottal flow. Charles P. Farbos de Luzan, Liran Oren (Dept. of Otolaryngol. - HNS, Univ. of Cincinnati, Medical Sci. Bldg., Rm. 6303B, Cincinnati, OH 45267-0528, farboscs@ mail.uc.edu), Ephraim Gutmark (Dept. of Aerosp. Eng., CEAS - Univ. of Cincinnati, Cincinnati, OH), and Sid Khosla (Dept. of Otolaryngol. - HNS, Univ. of Cincinnati, Cincinnati, $\mathrm{OH}$ )

Compressible large eddy simulation is employed to numerically investigate the laryngeal flow. One static model of the canine larynx with a divergent glottis is considered, with the presence of false vocal folds (FVFs). This computational model is developed from empirical data, and compared to similar configurations that do not involve the subject-specific geometrical features. Due to the high enough Reynolds number, the flow is unsteady and develops asymmetric states downstream of the glottis. The intra-glottal vortex structures are formed on the divergent wall of the glottis, immediately downstream of the separation point. The vortices are then convected downstream and characterized by a significant negative static pressure. The FVFs are a main factor in the generation of stronger vortices, and thus on the closure of the TVFs. Models with and without divergent vocal folds are investigated and linked to the existence of vortices. The direct link between the FVFs geometry and the motion of the TVFs, and by extension to the voice production, is of interest for medical applications as well as future research works.

\section{2:55}

4pSA7. Noise source identification and noise reduction in a multi-state, centrifugal air compressor system. Je-Heon Han (Mech. Eng., Korea Polytechnic Univ., 237. Sangidaehak-ro, Siheung-si, Gyeonggi-do 15073, South Korea, jeep2000@kpu.ac.kr), Yaying Niu (Dept. of Mech. Eng., Texas A\&M Univ., College Station, TX), Changwoo Lim (R\&D Ctr., Hanwha Techwin, Seongnam-si, South Korea), and Yong-Joe Kim (Dept. of Mech. Eng., Texas A\&M Univ., College Station, TX)

The objective of this research is to recommend practical noise control schemes for Hanwha Techwin's multi-stage, centrifugal air compressors. For purpose of achieving this objective, far- and near-field noise measurements, sound intensity measurements, and structural vibration measurements were performed first to identify the acoustic characteristics of a compressor. Through these measurements, it was shown that the most dominant noise and vibration components were at the blade passing frequencies (BPFs) of the compression stage impellers. Thus, by suppressing these tonal BPF noise components, the overall noise level was reduced effectively. Here, Helmholtz resonators, dynamic dampers, noise insulation materials, and an enclosure were recommended to reduce these tonal noise components. The Helmholtz resonators could be used to reduce the overall SPL up to $3.5 \mathrm{dBA}$. By applying the mass dampers, $2.7 \mathrm{dBA}$ reduction at $7 \mathrm{kHz}$ and $1.5 \mathrm{dBA}$ reduction at $10 \mathrm{kHz}$ could be achieved. The installation of the sound insulation material could achieve $8 \mathrm{dBA}$ overall SPL reduction. By installing the enclosure around the compressor, $4.3 \mathrm{~dB}$ reduction in the overall SPL could be achieved. To achieve a higher noise reduction using the enclosure, the size of leakage holes between the compressor pipe and the enclosure is recommended to be reduced. 


\title{
Session $4 \mathrm{pSC}$
}

\section{Speech Communication: Non-Native Speech Production and Perception, Learning, and Adaptation (Poster Session)}

\author{
Nicole Holliday, Chair \\ Linguistics and Cognitive Science, Pomona College, Pomona College, Edmunds 185, 185 E Sixth St., Claremont, CA 91711
}

\begin{abstract}
All posters will be on display from 1:00 p.m. to 5:30 p.m. To allow contributors in this session to see the other posters, authors of odd-numbered papers will be at their posters from 1:00 p.m. to 3:15 p.m. and authors of even-numbered papers will be at their posters from 3:15 p.m. to 5:30 p.m.
\end{abstract}

\section{Contributed Papers}

4pSC1. The influence of long-term exposure on the perception of foreign-accented speech. Hayk Abrahamyan (Psych., SUNY at Buffalo, Univ. at Buffalo, Park Hall 204, Buffalo, NY 14260, hayk@buffalo.edu)

We recognize spoken words in our native language with relative ease. Yet, difficulties arise when we encounter a talker with an accent. Short-term lab exposure leads to observable improvements in comprehension of foreign-accented speech. Life-long exposure markedly improves perception of within language variability (i.e. dialects) and refines listeners' representations. Less is known about the benefits of long-term exposure to foreignaccented speech. We used cross-modal identity priming to investigate if long-term exposure to Chinese-accented English by native speakers of American English would modulate their processing of Chinese-accented speech. We predicted that listeners with long-term exposure to the foreign accent, assumed based on long-term residence in the New York City area, should be faster and more accurate at recognizing Chinese-accented words than listeners without long-term exposure, assumed based on long-term residence in less linguistically diverse areas. Our preliminary results present a surprising finding indicating that the assumed long-term exposure had the opposite effect, where the listeners with long-term exposure to a foreign accent are unexpectedly slower and less accurate than the listeners without long-term exposure. Our results suggest that long-term exposure does affect listeners' abilities to recognize and represent foreign-accented spoken words.

4pSC2. Segmental versus prosodic predictors of perceived accentedness. Elizabeth A. McCullough and Richard Wright (Linguist, Univ. of Washington, Box 352425, Seattle, WA 98195, emccullo@uw.edu)

Previous work has identified both segmental and prosodic influences on the perceived foreign accentedness of non-native speakers, but their relative contributions remain poorly understood, in part because they are often investigated independently. In this study, both segmental and prosodic properties of word-length stimuli were assessed. Native English-speaking listeners rated the accentedness of trochaic English words produced by talkers from four language backgrounds: Hindi, Korean, Mandarin, and Spanish. Each token's deviation from native English productions was quantified for two segmental properties (VOT and vowel quality) and three prosodic properties (ratios of duration, intensity, and f0 across the two syllables). For each language background, a linear mixed-effects regression model was created to predict accentedness ratings from the phonetic deviations, and the significance of each fixed effect was examined. In each model, the significant predictors included both segmental and prosodic properties. For L1 Hindi and L1 Spanish talkers, the most influential predictor was segmental; however, for L1 Korean and L1 Mandarin talkers, the most influential predictor was prosodic. Thus, even for short stimuli, both segmental and prosodic information must be considered in accounting for accentedness judgments. Listeners are sensitive to different ways that foreign accent may be manifested across different non-native backgrounds.
4pSC3. Recognition of foreign-accented words in isolation. Mirrah Maziyah Mohamed (Univ. at Buffalo, Singapore, Singapore, Singapore) and Hayk Abrahamyan (Univ. at Buffalo, 245 Park Hall, Buffalo, NY 14260, hayk@buffalo.edu)

Foreign-accented speech presents a challenge for native speakers of a given language. When presented with foreign-accented speech, native speakers tend to rely heavily on context and other lexical cues. When processing isolated spoken words, participants do not have access to contextual cues and have to rely solely on acoustic cues. Thus, processing accented spoken words in isolation might be a bigger challenge. We tested the hypothesis that identification accuracies by native speakers of American English of two foreign-accented speakers with different accent strengths will not differ in isolated spoken word recognition task. Ten native speakers of American English rated the accent of a Chineseaccented (heavy accent) and a Korean-accented (moderate accent) speakers after hearing a small passage read by both speakers. Different set of native speakers of American English heard a list of isolated words spoken by both speakers and had to type what they had heard. Overall, participants did not differ in their accuracy in the identification task even though the speakers differed in the strength of their accent, while there was no significant difference in participants rating of the strength of the speakers' accents. Implication of the results on the processing of foreign-accented speech will be discussed.

4pSC4. Speaker similarity, acoustic properties, and perceptual learning with non-native speech. Hanyong Park (Dept. of Linguist, Univ. of Wisconsin-Milwaukee, Johnston Hall 123, P.O. Box 413, 2522 East Hartford Ave., Milwaukee, WI 53211, park27@uwm.edu) and Noah H. Silbert (Dept. of Commun. Sci. \& Disord., Univ. of Cincinnati, Cincinnati, OH)

Variability in speech signals plays an important role in perceptual learning of non-native speech. Between-talker differences are a major source of variability. The present work aims to quantify within- and between-talker variability, following the approach of Silbert \& Park (2014, JASA, 136, 2714) to quantify variation within and across Korean stop categories. We analyze 20 (10 male and 10 female) native speakers' repeated productions of the Korean CVC nonword /p'ap/, using statistical techniques for dimensionality reduction to map multidimensional acoustic space to lower-dimensional talker-similarity space. We then compare the modeled similarity space to speaker discrimination data. Data were collected in an oddball task, where the listeners choose the oddball after listening to a set of triplets consisting of /p'ap/s spoken by two speakers (two distinct tokens from one speaker, one token from the other). The modeled similarity space allows us to automatically select tokens based on talker similarity and provides a rigorous quantitative method for assessing relationship between multidimensional acoustic properties and perceived speaker similarity. This approach promises to provide a rigorous, automated method for quantitatively defining and manipulating variability in perceptual learning experiments. 
4pSC5. The role of vowel quality in the perception of English lexical stress for native speakers of Japanese. Sayoko Eguchi (Kobe Univ., ATR, Nada-ku tsurukabuto 1-2-1, Kobe-shi, Hyougo-ken 657-8501, Japan, syk. eguchi@gmail.com)

Vowel quality has been hypothesized to be a cue in determining the location of stress in English words. This study examined the role vowel quality plays in perception by native speakers of Japanese and English using synthesized English words: conflict, contract, and content. Suprasegmental (SP) parameters-F0, duration, and intensity - and vowel quality (VQ) parameters - the frequencies of the first two formants, were independently manipulated to make varying versions of the stimuli. Results of the perception experiment showed that native speakers of Japanese identified the stress location more accurately in the SP-driven stimuli, in which the stress pattern was generated using SP parameters, than in the VQ-driven stimuli, in which the stress pattern was generated using VQ parameters. In contrast, native speakers of English did not differentiate between SP-driven stimuli and VQdriven stimuli. Furthermore, for the stimuli that SP parameters and VQ parameters indicated incongruent syllable, Japanese speakers identified the stress as located on the SP-driven syllable more often than English speakers did. These results demonstrated that native speakers of Japanese do not use VQ as a cue to perceive stress location whereas native speakers of English do use VQ as a cue. [Work supported by JSPS KAKENHI 23242032.]

4pSC6. Enlglish-as-a-second-language speakers' perception of personality in English speech. Bin Li, Yingting Cui, Yan Dou (Dept. of Linguist and Translation, City Univ. of Hong Kong, Kowloon Tong 000, Hong Kong, binli2@cityu.edu.hk), and Yan Liu (Computing, the Hong Kong Polytechnic Univ., Hong Kong, Hong Kong)

We make inferences on speaker's personality and emotion from their voices. This study aims at English-as-a-second-language (ESL) speakers' perception of personality as reflected in public speech online given by native English speakers. Phonetic parameters such as speaking rate and pitch are manipulated and combined in different ways to examine their effects on perceptual judgement of personality and content comprehension. Our ESL listeners are 50 university students who are native speakers of Cantonese and fluent users of English. They answer questions on the content of the speech samples, and also make judgement on various traits of the speaker' personality. The correct percentage and the judgement scores are recorded and examined for correlation between manipulation types and listening comprehension as well as that between phonetic parameters and perception of personality. Modification to the speaker's pitch seem in general improve listeners' comprehension accuracy as well as results in higher scores of personality perception, whereas that to the speaking rate does not show positive influence on speech comprehension or personality judgment. Our findings will provide insights to general phonetics and speech communication, but also pedagogical implications to academic English in nonnative settings.

4pSC7. Perception of speech rate in speech rate perception. Yahya Aldholmi and Hanyong Park (Dept. of Linguist, Univ. of Wisconsin-Milwaukee, Johnston Hall, Rm. 117, 2522 East Hartford Ave., Milwaukee, WI 53211,aldholmi@uwm.edu)

This study investigates what influences a listener's perception of speech rate. Three factors were investigated: pause type, actual speech rate, and utterance type (native, accented, and unfamiliar). Using manually accelerated vs. decelerated but structurally-identical sentences with three-type pauses (long, short, and filled) in accented, native, and unfamiliar language (Spanish) utterances, a group of Arabic speakers judged whether the sentences they heard were slow or fast, and rated the speed on a 1-7 scale $(1=$ slow, $7=$ fast). The findings show that long pauses triggered lower ratings, while filled pauses achieved ratings higher than both long and short pauses. Unfamiliar language received the highest ratings while the accented speech received the lowest. This suggests that, regardless of actual speech rate, silent pauses affect our perception of speech rate, and that when the duration of silence differs, our perception of speech rate will differ accordingly. The results also show that we tend to think of accented speech as being slower and of unfamiliar speech as being faster even when the two speech samples have the same length and pause types.
4pSC8. English listeners' judgments of Japanese-accented English vowel productions: Relation to acoustic and kinematic characteristics. Sonya Mehta and William F. Katz (The Univ. of Texas at Dallas, Callier Ctr., 1966 Inwood Rd., Dallas, TX 75235,naya@utdallas.edu)

The present study investigates how monolingual English listeners categorize foreign (Japanese) productions of English tense and lax vowels with the aim of relating vowel identification to the acoustic and kinematic vowel space. American English front vowels (/i/, /I/, /e/, / / /, and /æ/) were elicited in $/ \mathrm{hVd} /$ context from ten Japanese adults who learned English as a second language (ESL). Listeners categorized these vowel productions in a six-alternative, forced-choice paradigm (including the choice $/ \Lambda /$ ). Vowels produced by two native English speakers were included in the perceptual task for comparison. Preliminary results showed higher perceptual accuracy for tense vowels than for lax vowels. As expected, vowels from English talkers were more accurately identified than those from Japanese ESL speakers. To determine whether listeners' perceptual categorization corresponded with acoustic and kinematic patterns, perceptual data for contrasting vowel categories were compared with measured differences in formant (F1/F2) and articulatory space. Pilot data suggest a strong relation between acoustic and kinematic measures, but a weaker correspondence between these two data sources and listeners' perceptual judgments. Additional factors, including the roles of duration, talker and listener variability, and vowel similarity between languages, will be explored.

4pSC9. Non-native speakers perform poorer than native speakers in Japanese speech recognition in reverberation. Eri Osawa, Takayuki Arai (Sophia Univ., 7-1, Kioi-cho, Chiyoda-ku, Tokyo 102-8554, Japan, eri1989. 16.11@gmail.com), Nao Hodoshima (Tokai Univ., Tokyo, Japan), and Takako Igeta (Sophia Univ., Tokyo, Japan)

Reverberation affects perceptual performance, especially non-native listeners, elderly people, and people with hearing impairments. Native Japanese speakers confused some Japanese consonants and they failed to distinguish Japanese length contrast in reverberation [Arai et al., Proc. Autumn Meet. Acoust. Soc. Jpn., 2016]. The current study examined degradation degree of non-native speakers (native English speakers) in Japanese speech recognition in reverberation, using the same experiment settings with the previous study. There were two sets of stimulus: 1) Japanese consonant-vowel (CV) syllables, 2) non-words varying in duration of a certain vowel/consonant along durational continuum. In the results, although nonnative speakers confused consonants even in non-reverberant condition, the number of their confusion in reverberation was larger than that in non-reverberant condition. Confusion of non-native speakers in reverberation was much more than that of native speakers. In addition, the results indicated that non-native speakers could hardly distinguish the length contrast of Japanese in reverberation. The proportion of "long" responses did not change sharply in the perception of vowel continuum, while native speakers changed their responses at some points on the continuum. The results suggest that learners need training for listening to speech sounds in reverberation.

4pSC10. Transferring cue-weighting from second language into first language: Group trends and individual differences. Olga Dmitrieva, Jenna Conklin (Purdue Univ., 640 Oval Dr., Stanley Coulter 166, West Lafayette, IN 47907, odmitrie@purdue.edu), and Yulia Nigmatulina (SaintPetersburg State Univ., Saint-Petersburg, Russian Federation)

Second language learning has been shown to affect first language production (Flege, 1987; Major, 1992; Sancier \& Fowler, 1997, inter alia). Less is known about whether and how experience with another language may affect first language perception. The present study examines the use of preceding vowel duration vs. voicing during closure as cues to word-final and word-medial stop voicing by three groups of listeners: native speakers of American English (34), native speakers of Russian (34), and Russian expatriates in the United States (24). The results suggest that bilingual listeners are transferring a greater reliance on vowel duration, characteristic of English perceptual mode, into native speech categorization. The transfer occurred in word-final condition where vowel duration as a correlate of voicing is of very different importance in the two languages. Individual trends revealed that bilinguals' reliance on vowel duration may even be 
exaggerated in native, compared to non-native, speech perception. The use of vowel duration in bilinguals was positively correlated with such individual differences as length of residence in the United States and average amount of speaking English per week. The triggers and possible benefits of such perceptual transfers are discussed in the context of language use in the expatriate communities.

4pSC11. Categorical perception of Mandarin tones by Chinese-native, English-native and Chinese-as-a-second-language English listeners. Wenyi Ling, Theres Grüter (Second Lang. Studies, Univ. of Hawaii at Manoa, 1711 East West Rd., 614B, Honolulu, HI 96848, wenyi9@hawaii. edu), and Amy Schafer (Linguist, Univ. of Hawaii at Manoa, Honolulu, $\mathrm{HI})$

Previous research found that native-Chinese listeners perceive tones more categorically than listeners with no knowledge of a tonal language (naïve listeners; Hallé et al., 2004). This study examined tone perception by 26 native-English adult learners of Mandarin (L2ers) in comparison to 30 naïve and 30 native-Chinese listeners. Identification and AXB discrimination tasks tested monosyllables (/pi/, /pa/) with 9-step F0 continua between all possible Mandarin tone pairs. Native listeners showed more categorical identification (steeper slopes) than naive listeners. The L2 group showed significantly shallower identification slopes than native listeners $(p<0.01)$ and steeper slopes than naive listeners $(\mathrm{p}<0.01)$. L2 proficiency (listening test plus self-report) positively correlated with identification performance $(\mathrm{r}=0.36, \mathrm{z}=-2.56, \mathrm{p}<0.01)$, suggesting higher proficiency may lead to more native-like tone identification. However, although L2ers' discrimination accuracy $(0.90)$ was significantly higher than native listeners' $(0.82 ; z=-4.76$, $\mathrm{p}<.001)$, it did not differ significantly from naïve listeners' $(0.90 ; \mathrm{z}=-0.61$, $\mathrm{p}=0.54)$, suggesting that the non-native groups discriminated tone pair continua in a similar way. Tone pairs involving level tones (e.g., T1-T3) showed more categorical patterns than those involving two contour tones (e.g., T2-T3) in each group, consistent with the Anchor Hypothesis (Xu et al., 2006).

4pSC12. Reaction time of Japanese listeners to retroflex and bunched /r/ pronunciation by native English speakers. Mai Gunji (Univ. of Aizu, Tsuruga, Ikki-mati, Aizuwakamatsu, Fukushima 965-8580, Japan, s1210154@u-aizu.ac.jp), Ian Wilson, and Jeremy Perkins (Univ. of Aizu, Aiduwakamatu, Japan)

In this study, we focus on Japanese learners' reaction time (RT) to retroflex and bunched pronunciation of $/ r /$ in English words spoken by native English speakers. In junior high school, Japanese students generally learn only retroflex pronunciation of $/ \mathrm{r} /$. If there is a strong link between production and perception, we would expect those students to be able to perceive retroflex /r/ faster than bunched /r/. We carried out a forced-choice RT experiment for 30 native Japanese listeners and 4 native English controls. This experiment used 2 speakers' voices (both Canadian English) and 9 minimal pairs of /r/ and /1/ words. Stimuli were spoken words and picturepairs (two simultaneously presented in each trial). Listeners had to identify the spoken word by choosing the left or right picture. As a result, we could measure whether it is easier to perceive sounds pronounced the same way you speak or not. From the results, we found that the RTs for retroflex and bunched pronunciation of English words spoken by native speakers were not significantly different, even for native listeners. In addition, overall accuracy rates were very low among the Japanese speaking participants (66.3\%, compared to $99.7 \%$ for the English speaking participants).

4pSC13. Phonotactic knowledge and phonetic details in the perception of non-native consonant sequences: Evidence from Mandarin Chinese. Qianwen Guan and Harim Kwon (Université Paris Diderot, Bâtiment Olympe de Gouges 8 Pl. Paul-Ricoeur, Paris 75013, France, qianwen_ guan@hotmail.com)

Previous research has shown that non-native consonant sequence perception is influenced by both the phonotactic knowledge of native language and the phonetic details of the stimuli. To further examine the effects of these two factors, we conducted two perception experiments with monolingual Mandarin listeners. In experiment 1 , the listeners determined whether they heard a vowel between two consonants. Stimuli were $\mathrm{VC}_{1} \mathrm{C}_{2} \mathrm{~V}$ with various intervocalic consonant sequences produced by a Russian speaker. The listeners reported hearing a vowel between two consonants (56\%), providing evidence for the effects of native phonotactics. We also found that duration of $\mathrm{C} 1$ release influenced the perception of epenthetic vowel. In experiment 2 , the same participants heard the non-native sequences in word-initial and intervocalic positions and transcribed them in Pinyin. 75\% of the transcription data showed a vowel epenthesis. In addition, different strategies were observed in different positions in a word: word-initial position showed more $\mathrm{C} 1$ deletion presumably due to weak acoustic cues of $\mathrm{C} 1$ and intervocalic position showed more $\mathrm{C} 2$ deletion. Taken together, these findings indicate that while the native phonotactics play a dominant role in Mandarin listeners' perception of non-native consonant sequences, the effects of phonetic details of the stimuli are not negligible.

4pSC14. Lexical age of acquisition effects on native and non-native speech perception. Renee Kemp (Linguist, Univ. of California, Davis, 469 Kerr Hall, UC Davis, Davis, CA 95616, rlkemp@ucdavis.edu)

Lexical difficulty has been shown to play a role in both speech production and perception. The relative difficulty of a word can be determined based on lexicon properties such as usage frequency, neighborhood density, or lexical Age of Acquisition (AoA). These factors have been shown to predict a range of different acoustic modifications such as hyperarticulation and increased duration. The current study investigates the effect of AoA-conditioned phonetic variation in both plain and foreigner-directed speech conditions on both native and non-native English speech perception. 60 total subjects completed a lexical decision task. Investigating these two populations allows for an examination of the role of lexical difficulty in language acquisition. The data show that lexical AoA influences the accuracy of lexical decision responses. Specifically, native speakers were more accurate in their identification of words with a low AoA rating compared to a high AoA rating (i.e. more lexically difficult items). Interactions between AoA and speech condition were also observed. The findings of this study suggest acoustic modifications can serve, at least in part, to facilitate the perception of lexically difficult words.

4pSC15. Comparative acoustic analyses of non-native English: The search for systematic variation. Rebecca Laturnus (Linguist, New York Univ., 10 Washington Pl., New York, NY 10003, rc1309@nyu.edu)

Previous research has shown that exposure to multiple foreign accents facilitates adaptation to an untrained novel accent (Baese-Berk et al. 2013). The explanation offered is that $\mathrm{L} 2$ speech varies systematically, such that there are commonalities in the productions of non-native speakers, regardless of their language background (e.g. L2 speech is slower and many of English's contrasts are difficult for speakers of other languages). The current work conducted a systematic acoustic comparison between two native English speakers and six non-native accents that closely matched those used in Baese-Berk et al. (2013). All talkers, taken from the Wildcat Corpus, were male, and the non-native voices had comparable foreign accentedness ratings (Van Engen et al. 2010). VOT and formant values of stressed and unstressed vowels were analyzed, comparing each non-native accent to the native English talkers. Additionally, pairwise variability indices were calculated for vocalic and consonantal intervals as measures of rhythm. The results for each measure show substantial variability across speakers, reflecting phonetic transfer from individual L1s, rather than commonalities in their productions. The data are therefore more consistent with a hypothesis of accent attunement wherein variability causes listeners to relax their expectations of what constitutes a good category exemplar.

4pSC16. The effects of prenasal raising of American English /æ/ on the identification of American English vowels by native Japanese and Korean listeners. Takeshi Nozawa (Lang. Education Ctr., Ritsumeikan Univ., 1-1-1 Nojihigashi, Kusatsu 525-8577, Japan, t-nozawa@ec.ritsumei.ac.jp) and Sang Yee Cheon (Dept. of East Asian Lang. \& Literatures, Univ. of Hawaii at Mānoa, Honolulu, HI)

This study attempts to investigate the effect of prenasal raising of American English /æ/ on the identification of the vowel by native Japanese and 
Korean listeners by comparing the identification accuracy of /æ/ in preplosive and prenasal context by these two listener groups. Native Japanese speakers generally equate /æ/ with Japanese low vowel /a/ whereas native Korean speakers identify /æ/ with Korean mid front vowel /e/, and /æ/-/e/ is one of the most difficult English vowel pairs for Korean speakers to discriminate (Ingram \& Park 1997, Frieda \& Nozawa 2007). It was expected that Korean listeners would identify raised /æ/ in prenasal context as /E/ or /eI/,

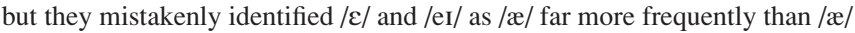
as $/ \varepsilon /$ or /eI/. The results revealed that while Japanese listeners' identification accuracy of /æ/ is significantly lower in prenasal context than in preplosive context, Korean listeners' identification accuracy of /æ/ is unaffected by the raising of /æ/. Korean listeners far more often mistakenly identify $/ \varepsilon /$ as $/ \mathfrak{x} /$ in preplosive context than the other way around, and this tendency stays the same even when $/ \mathfrak{x} /$ is raised in prenasal context.

4pSC17. The effects of second language experience on the perception of native language prosody. Grace E. Oh (English Lit. and Lang., Konkuk Univ., 120 Neungdong-ro, Gwangjin-gu, Seoul 05029, South Korea, gracey1980@yahoo.com)

The effects of second language experience on adult learners' perception of native language prosody were investigated. A total of 39 sentences produced in Korean, Mandarin, Japanese by three female speakers were lowpass filtered and presented to three groups of 10 participants with different language backgrounds: Native Korean speakers with no L2 experience (NK group), native Mandarin speakers (NM group) and Korean learners of Chinese (KC group) with an average LOR in China of 1 year. The participants were instructed to listen to each stimulus and decide whether it was Korean or not based on the suprasegmental features. Overall, the NK group (76\%) revealed significantly higher discrimination accuracy than the $\mathrm{KC}(65 \%)$ or the NM group (57\%). However, the NM participants with extensive Korean experience showed significant improvement in accuracy for both Korean $(75 \%)$ and Chinese $(84 \%)$. The results, on the one hand, support the significant effect of L2 experience on the acquisition of L2 prosody. The KC group's lower accuracy for Korean, on the other hand, suggests that too much attention may have been directed towards acoustically salient L2 cues (e.g., F0 range) in the perception of L1 prosody.

4pSC18. English spoken word recognition for Japanese native speakers: A comparison with online and offline processing. Yoko Sakamoto (Premedical Sci., Dokkyo Medical Univ., 880 Kitakobayashi, Mibu, Shimotsugagun, Tochigi 3210293, Japan, y-saka@dokkyomed.ac.jp)

The aim of this study was to investigate the mechanisms of English (L2) spoken word recognition for Japanese native speakers by two experiments using a cloze test. In Experiment 1, 90 Japanese university students were divided into three groups based on their listening level. Then they took a listening test of a CNN material. The correct rates and error patterns were analyzed. The result showed that the correct rates for three groups were less than $50 \%$, however, the upper group took the highest correct rate and the lowest number of blanks, extra phoneme insertions and phoneme recognition errors. In Experiment 2, 32 Japanese university students were divided into two groups, and one group took a cloze test without listening, and the other took the cloze test with listening. The results of correct number showed that the similar tendency between two groups. This may indicate that listening to L2 is difficult, as much research has suggested, but if Japanese speakers continue to learn English listening, they can better develop recognition of the spoken word more accurately and the inferring skill from top down information such as grammar and semantics may play a role for listening in the second language.

4pSC19. Cross-linguistic perception of continuous speech: Neural entrainment to the speech amplitude envelope. Jieun Song and Paul Iverson (Speech, Hearing and Phonetic Sci., Univ. College London, Rm. 326, Chandler House, 2 Wakefield St., London WC1N 1PF, United Kingdom, jieun.song@ucl.ac.uk)

Brain oscillations in the auditory cortex become entrained to slow amplitude fluctuations in the speech signal (i.e., amplitude envelope) during speech comprehension. Previous research has suggested that this cortical activity plays an important role in speech perception by aligning neuronal excitability to parts of the incoming speech signal that contain linguistic information such as syllables. However, it remains unclear how cortical entrainment to the speech envelope relates to higher-level linguistic processes during speech comprehension. The aim of the present study was to see if cortical entrainment to the amplitude envelope varies depending on whether or not the listener understands the linguistic content of the speech signal. To this end, the phase-locking between neural oscillations and amplitude envelopes of speech were measured in EEG recordings from listeners with different linguistic backgrounds (i.e., native English and Korean speakers) while they heard continuous speech in three languages (i.e., English, Korean, and Spanish). The results demonstrate that auditory-cortical tracking of the envelope is not affected by whether or not listeners understand the speech, supporting the view that envelope-tracking activity is mainly independent of higher linguistic processes.

4pSC20. Cross-linguistic perception of clearly spoken English tense and lax vowels based on auditory, visual, and auditory-visual information. Keith K. Leung (Linguist, Simon Fraser Univ., Robert C. Brown Hall Bldg., Rm. 9201, 8888 University Dr., Burnaby, BC V5A 1S6, Canada, kw123@sfu. ca), Charles Redmon (Linguist, The Univ. of Kansas, Lawrence, KS), Yue Wang (Linguist, Simon Fraser Univ., Burnaby, BC, Canada), Allard Jongman, and Joan Sereno (Linguist, The Univ. of Kansas, Lawrence, KS)

Studies on acoustic and visual characteristics of English tense and lax vowels show consistent enhancement of tensity contrasts in clear speech. However, the degree to which listeners utilize these enhancements in speech perception remains unclear. The present study addresses this issue by testing speech style effects on tense and lax vowel perception by 23 native English and 30 non-native Mandarin-Chinese listeners in audio-only (AO), audiovisual (AV), and visual-only (VO) stimulus modes. English and Chinese listeners showed similar relative differences in performance by mode $(\mathrm{VO}<$ $\mathrm{AO}<\mathrm{AV}$ ) and style (plain $<$ clear). However, the two groups differed in the nature of the interaction between tensity, style, and stimulus mode. English listeners showed advantages for clear speech for both tense and lax vowels in all but VO stimuli, whereas Chinese listeners showed a clear speech advantage only for tense vowels, while clear lax vowels showed no improvement in AO and reduced accuracy in AV and VO. While temporal and spectral acoustic cues may coordinate to preserve or improve tense-lax category identity in clear speech, non-native listeners may not be attending to both dimensions. Further, Chinese listeners' greater reliance on visual information may account for their less accurate lax vowel identification.

4pSC21. Functional load of fundamental frequency in the native language predicts learning and use of these cues in second-language speech segmentation. Annie Tremblay (Linguist, Univ. of Kansas, 1541 Lilac Ln., Blake Hall, Rm. 427, Lawrence, KS 60045-3129, atrembla@ku.edu), Mirjam Broersma (Ctr. for Lang. Studies, Radboud Univ., Nijmegen, Netherlands), Caitlin E. Coughlin (Linguist, Univ. of Kansas, Lawrence, KS), and Monica Wagner (Donders Inst. for Brain, Cognition and Behaviour, Ctr. for Cognition, Radboud Univ., Nijmegen, Netherlands)

This study investigates whether second-language (L2) learners make greater use of prosodic cues to word boundaries if these cues have a higher functional load in the native language (L1). It examines the use of fundamental-frequency (F0) rise in the segmentation of French speech by English- and Dutch-speaking L2 learners of French matched in French proficiency and experience (and native French listeners). In both English and Dutch, an F0 rise tends to signal word-initial boundaries in pitchaccented words with initial stress, but English has more vowel reduction than Dutch, and thus full vowels also signal word-initial boundaries in English. By contrast, in French, an F0 rise tends to signal word-final boundaries. Participants completed an eye-tracking experiment: They heard sentences where the monosyllabic target ended or did not end with an F0 rise (duration being held constant), and saw four orthographic words in the display, including the target (bal "ball") and a disyllabic competitor (balcon "balcony"). Growth curve analyses on participants' fixations revealed that Dutch listeners made earlier and greater use of F0 cues than English (and French) listeners. These results suggest that the functional load of F0 in the $\mathrm{L} 1$ predicts the learning and use of these cues in the L2. 
4pSC22. The effects of acoustic and lexical factors on perception of accented speech. Christina Y. Tzeng (Emory Univ., 36 Eagle Row, Atlanta, GA 30322, ctzeng@emory.edu), Jessica E.D. Alexander (Centenary College of Louisiana, Shreveport, LA), and Lynne C. Nygaard (Emory Univ., Atlanta, GA)

Foreign-accented speech differs from native pronunciation on a variety of spectral and temporal properties that affect both accentedness and intelligibility. The specific acoustic correlates, however, as well as the role of signal-independent lexical characteristics on accentedness judgments and intelligibility, remain unclear. The current study examined the potential contribution of particular acoustic (F1, F2, duration) and lexical (frequency, neighborhood density) factors to native speakers' ratings of accentedness and assessments of intelligibility for English words spoken by native Spanish speakers. Previous research has found that in native English speech, hard words (low frequency, high neighborhood density) have more dispersed vowel spaces relative to easy words (Pardo et al., 2013). Preliminary findings in the current study indicate the opposite pattern such that foreignaccented easy words exhibited more vowel dispersion than hard words. Further, vowel dispersion measures for easy, but not hard, words predicted accentedness and intelligibility such that as speakers' vowel spaces became more dispersed, the more accented and less intelligible their utterances were rated. Together, these findings suggest that signal-dependent and signal-independent factors have differential effects on the production and perception of accented versus native speech, implying a complex relationship between acoustic and lexical properties that is unique to foreign-accented speech.

4pSC23. Effects of native language on the use of segmental and suprasegmental cues to stress in English word recognition: An eye-tracking study. Katrina Connell (Linguist, Univ. of Kansas, 541 Lilac Ln. Blake Hall, Rm. 427, Lawrence, KS 60045-3129), Simone Hüls (Speech-Language-Hearing: Sci. \& Disord., Univ. of Kansas, Lawrence, KS), Maria Teresa Martínez-García (Modern Lang., Fort Hays State Univ., Hays, KS), Zhen Qin, Seulgi Shin (Linguist, Univ. of Kansas, Lawrence, KS), Hanbo Yan (Shanghai Int. Studies Univ., Shanghai, China), and Annie Tremblay (Linguist, Univ. of Kansas, Lawrence, KS, atrembla.illinois@gmail. com)

This study investigates whether the presence of lexical stress in the native language (L1) determines second-language (L2) learners' ability to use stress in L2 lexical access. It focuses on (standard Mandarin) Chinese and (Seoul) Korean listeners' (and native English listeners') use of segmental and suprasegmental cues to stress in English word recognition. Stress placement in English is signaled by segmental (vowel reduction) and suprasegmental (fundamental frequency, duration, and intensity) cues. Chinese has full-full and full-reduced words that differ in stress placement, with segmental and suprasegmental cues signaling stress. By contrast, Korean does not have lexical stress. Participants completed an eye-tracking experiment. They heard stimuli containing a target word with initial stress (parrot), and saw four orthographic words in the display, including the target and one of two competitors (stress match: parish; stress mismatch: parade). The first syllable of the target and stress-mismatch competitor differed in both segmental and suprasegmental information (parrot-parade) or only in suprasegmental information (mystic-mistake). Growth-curve analyses on fixations revealed that only Chinese and English listeners used stress to recognize English words, confirming L1 effects on the use of stress in L2 lexical access. Furthermore, only English listeners made greater use of stress in the presence of vowel reduction.

4pSC24. The examination of acoustic feature of English obstruent coda by Mandarin and Cantonese speakers. Lu Wanling (Linguist, Univ. of Hong Kong, R4, F5, Block D, Kwan Yick Phase Two, Des Voeux Rd., Hong Kong Island, Hong Kong 999077, Hong Kong, katielu@hku.hk)

Mandarin and Cantonese, though are both Chinese, exhibit differences in L2 English pronunciation, especially in obstruent codas. For the reason that only nasals -n, -m and -ng can be found at coda in Mandarin whereas apart from nasals, unreleased consonants - $\mathrm{p}-\mathrm{t}$ and $-\mathrm{k}$ exits as well in Cantonese, it is reasonable to believe that $\mathrm{L} 1$ transfer makes influences on modifying codas for L2 learners. This present study aims at comparing the strategies used by Mandarin and Cantonese speakers in coda modification and resyllabification. By embedding the stimuli in a natural conversation, room-cleaning task, data are elicited and analyzed by Praat. Results indicates that up to $93 \%$ of codas are released by Mandarin speakers and $41 \%$ of those are unreleased by Cantonese speakers. Further, instead of epenthesizing vocalic elements, Mandarin speakers are found to strongly aspirate the codas with $58 \%$, which contradicts most of the previous studies claiming that epenthesis is the main modification used among Mandarin L2 learners.

4pSC25. Do listeners' expectations about the source of a speaker's accent affect intelligibility? Charlotte Vaughn (Linguist, Univ. of Oregon, 1290 University of Oregon, Eugene, OR 97403-1290, cvaughn@uoregon. edu)

When encountering accented speakers, listeners initially show a processing cost but are able to adapt to the accent within minutes (Bradlow \& Bent, 2008; Floccia et al., 2009). However, the extent to which processing a foreign accent (L2-accented) is similar to processing a within-language accent (L1-accented) is still an open question (cf. Cristia et al., 2012). This project considers whether intelligibility is affected by listeners' expectations about the source of the accent, namely, whether the speaker is believed to be an L1 or an L2 speaker. In this study, native English listeners transcribed English sentences in noise spoken by a bilingual Spanish/English speaker. Expectations about the source of the speaker's accent were manipulated using a matched guise design: Some listeners received no information about the L1 status of the speaker, others were told the speaker was an L1-Spanish speaker, and others were told the speaker was a Hispanic American whose L1 was English (Latino English, e.g., Fought, 2003). Transcription accuracy is compared across conditions to explore whether listeners are sensitive to the kinds of acoustic properties differentiating L1-accented vs. L2-accented speech, and whether listeners' expectations about the source of a speaker's accent affects the intelligibility of that speaker.

4pSC26. The effects of visual and production components in training the perception and production of English consonant contrasts by Mandarin speakers. Janice Wing Sze Wong (Dept. of Commun. Studies, Hong Kong Baptist Univ., 9/F, Commun. and Visual Arts Bldg., 5 Hereford Rd., Kowloon Tong, Hong Kong, Hong Kong, janicewong@hkbu.edu.hk)

Studies have shown the effectiveness of auditory-only training in improving the perception and/or production of non-native contrasts. This study investigated whether providing additional visual and production components are more useful than auditory-only training. The contrasts $/ \delta /-/ z /$ and $/ \theta / / / \mathrm{s} /$ were chosen since studies reported these common confusions among Mandarin EFL learners. Forty-five participants were divided into five groups receiving 10 training sessions: auditory-only (A), audio-visual (AV), auditory and explicit production (AP), visual-only (V) or explicit production-only (P). All auditory and visual stimuli were produced by six native General American speakers while the production training involved a native GA speaker giving immediate feedback. Word-level identification and reading pre/post-tests were used to measure their perception and production performance. Robust perceptual improvement of the two consonant pairs was found in A, AV, AP, and P although they did not differ significantly. Their production only improved significantly when they were trained under $\mathrm{A}, \mathrm{AV}, \mathrm{AP}$, and $\mathrm{P}$, whereas $\mathrm{AP}$ and $\mathrm{P}$ groups performed significantly better than other groups. These results showed auditory-only training could already improve the perception and production of difficult contrasts, while visual and production components did not make a significant difference in perception improvement. Only direct production training was more beneficial in training participants' production. 
4 pSC27. Contextual analysis on geminate/singleton identification difficulties for L2 learners of Japanese based on perceptual features. Yanlong Zhang (GITS, Waseda Univ., Rm. 702, Bldg. 66, Nishi Waseda Campus, Okubo 3-14-9, Shinjuku, Tokyo 169-8555, Japan, chouenryu0903@ruri.waseda.jp), Hideharu Nakajima (NTT Media Intelligence Labs., NTT Corp., Tokyo, Japan), Mee Sonu (College of Humanities and Sci., Nihon Univ., Tokyo, Japan), Hiroaki Kato (NICT, Kyoto, Japan), and Yoshinori Sagisaka (GITS / Dept. of Appl. Mathematics. FSE, Waseda Univ., Tokyo, Japan)

It is widely known that Japanese geminate/singleton consonant identification is one of the biggest problems for L2 learners. We have been analyzing identification error characteristics based on their perceptually motivated features. In this presentation, after briefly introducing observed remarkable identification error chracteristics on phonetic context differences with speech rate variations, we have tried to analyze and quantify the difficulties using identification error rates by Japanese beginners of Korean natives. 36 geminate/singleton pairs in 2-5 mora with three speech rates were used for identification experiments. To understand the difficulties quantitatively, objectively measurable acoustic variables such as duration length, loudness and its jumps of the corresponding geminate/singleton consonants were employed to carry out prediction analysis for L2's identification error rates. Correlation between predicted error rates and observed ones has turned out to be around 0.6 with the speech rates where more identification errors existed. Through these analyses, we could have confirmed that perceptually motivated parameters could provide reasonable and effective explanation in contextual differences of identification errors by L2 learners and its quantification. These results suggest the possibility of effective L2 learning based on perceptual difficulties.

4pSC28. Vowel and tone identification of Mandarin Chinese: Comparison between native Chinese and Korean listeners. Hui Zhang, Lilong Xu, Can Xu, Xiaohu Yang, and Hongwei Ding (School of Foreign Lang., Speech, Lang. and Hearing Ctr., Ctr. for Cross-Linguistic Processing and Cognition, 800, Dongchuan Rd., Minhang District, Shanghai 200240, China, zhanghui_Helen@126.com)

Speech in tone languages requires listeners to identify not only phonemes but also tones. As non-tone language users, Korean-native speakers may have difficulty in identifying tones and vowels and processing both tonal and phonemic information at the same time, which are not presented in their mother tongue. The goal of the present study was to investigate the identification of Mandarin Chinese vowels and tones for both native Mandarin Chinese and native Korean speakers with medium and high Chinese proficiency. Results showed that for the identification of Mandarin vowelplus-tone, vowel, and tone, there was no difference between Chinese- and Korean-native listeners with high Chinese proficiency, whereas Chinese listeners significantly outperformed Korean listeners with medium Chinese proficiency, particularly for tones 1,3 , and 4 and vowel /y/. For Korean listeners, there was no significant correlation between the onset age of Chinese learning and identification scores, while the identification performance significantly correlated with the length of Chinese learning. Moreover, for both Chinese and Korean listeners, identification scores of vowel-plus-tone, tone and vowel are comparable, suggesting that both groups may process dual cues (vowel and tone) as efficiently as individual cues (vowel or tone).

4pSC29. Informational masking of six-talker babble on Mandarin Chinese vowel and tone identification: Comparison between native Chinese and Korean listeners. Can $\mathrm{Xu}$, Hui Zhang, Yuxia Wang, and Hongwei Ding (Speech, Lang. and Hearing Ctr., Ctr. for Cross-linguistic Processing and Cognition, School of Foreign Lang., Shanghai Jiao Tong Univ., 800 Dongchuan Rd., Minhang District, Shanghai, Shanghai 200240, China, 8023xc@sjtu.edu.cn)

The goal of this study was to investigate the informational masking (IM) of Mandarin six-talker babble on Mandarin vowel and tone perception for native Chinese and Korean speakers with high and medium Mandarin proficiency. Mandarin Chinese speech sounds (vowel plus tone) were presented in quiet and two noises: six-talker babble and babble-modulated noise, which was spectrally and temporally matched with babble. The IM was computed as the difference in the identification scores between the two types of noises. Overall, all the four factors (listener group, noise type, vowel-plus-tone category, and SNR) significantly affected participants' identification of Mandarin stimuli. Particularly, in both quiet and noisy conditions, Chinese listeners had comparable performance with Korean listeners with high Mandarin proficiency, while Chinese listeners significantly outperformed Korean listeners with medium Mandarin proficiency. However, no significant difference was found in IM among the listener groups. In conclusion, at the syllabic level, highly-proficient Korean listeners had native-like performance of Mandarin vowel and tone identification in quiet and noise, whereas Korean listeners with medium proficiency had greater difficulty, specifically in noise, likely due to their lower capacity to process phonemic and tonal information, rather than the IM of babble.

4pSC30. Modeling native phonology and non-native speech perception using electroencephalography signals. Daniel McCloy and Adrian K. Lee (Inst. for Learning and Brain Sci., Univ. of Washington, Box 357988, Seattle, WA98115-7988,drmccloy@uw.edu)

Studies of language learning in adulthood show that learners' native language phonologies shape their non-native perception and production abilities. Nonetheless, listeners are able to learn to perceive new speech sound contrasts given training. The present study attempts to model how foreign consonants are perceived prior to training or second language study. Brain responses were recorded during passive listening using electroencephalography (EEG), using twelve monolingual English-native listeners and isolated consonant-vowel (CV) syllables read by speakers of several languages (Dutch, English, Hungarian, Hindi, Swahili). To model native-language phonology, EEG responses to native (English) syllables were used to train classifiers based on phonological feature labels for the consonants in each syllable. The trained classifiers were then applied to the EEG responses to foreign syllables, and the classifier outputs used to generate confusion probabilities between each pair of foreign and native consonants for each of the four foreign languages. Confusion matrices based on the EEG classifiers compare favorably to confusion matrices based solely on number of phonological feature mismatches. Ongoing work investigates whether optimal phonological features can be learned from the EEG data using clustering algorithms.

4pSC31. Perception of the Japanese moraic-nasal (/N/) by Korean native speakers: Concerning /N/ followed by vowels. Heesun Han (Graduate School of Lang. and Culture, Osaka Univ., 1-8 Machikaneyama, Toyonaka, Osaka 560-0043, Japan, kenkyuhhs@gmail.com)

This study examines the perception of the Japanese Moraic-Nasals /N/ by Korean native speakers. In the case of the /N/ followed by vowels (/N/+/ $\mathrm{a}, \mathrm{i}, \mathrm{u}, \mathrm{e}, \mathrm{o} /$ ), there is a tendency towards pronunciation as various nasal vowels. The actual sound of each $/ \mathrm{N} /$ is, however, prone to change according to the speech style. Korean language has three nasal codas (/m, n, y/). The Korean nasal codas are pronounced [m], [n], [n] respectively, regardless of speech styles. Therefore, if Korean native speakers consider Japanese /N/ from the perspective of the Korean nasal coda, it is possible that they will misjudge Japanese $/ \mathrm{N} /$. A perception test was conducted using 6 minimal pairs consisting of "/N/+ vowel (eg. /saNin/)" and "vowel + vowel (eg. / saiin/)". The test words were recorded in "common," "emphasized," and "unclear" speech styles. The results showed that native Japanese speakers recognized $/ \mathrm{N} /$ in most cases. On the other hand, Korean speakers with no experience in learning Japanese were unable to recognize the Japanese $/ \mathrm{N} /$, and perceived $/ \mathrm{N} /$ as vowel. Korean Native speakers with advanced Japanese proficiency had results similar to the native Japanese participants. Interestingly, in some cases they showed more accurate judgement than that of the Japanese. This, therefore, suggests that, as language learning progresses, the Japanese / $\mathrm{N} /$ is acquired. Advanced learners, however, tended to pay greater attention to the $/ \mathrm{N} /$ sounds rather than the contextual factors on which Japanese were more likely to rely on. 
4pSC32. Acoustic analysis of oral and nasal Hindi vowels spoken by native and non-native speakers. Shyam S. Agrawal, Shweta Bansal, Shambhu Sharan, and Minakshi Mahajan (College of Eng., KIIT, Sohna Rd., Near Bhondsi, Gurgaon, Haryana 122102, India, dr.shyamsagrawal@ gmail.com)

This paper presents acoustic analysis of Hindi oral and nasal vowels as spoken by the native Hindi speakers and non-native speakers belonging to Punjabi and Nepali linguistic backgrounds. The objective of the study is to determine the measurable acoustic parameters of Hindi vowel sounds which can be used to distinguish oral and nasal vowels by Hindi speakers on one hand and the effect on these parameters due to non-native speakers on the other hand. The database consists of 150 phonetically rich sentences uttered by 10 speakers of each language in a sound treated room. These were analysed using PRATT and Wave Surfer software tools. Five long Hindi vowels (oral as well as nasalized) i.e. $/ \mathrm{i} /, / \mathrm{\varepsilon} /, / \mathrm{a} /, / \mathrm{o} /$ and $/ \mathrm{u} /$ were selected for this study and segmented from the sentence utterances. The parameters such as frequencies, amplitude of formants and the pitch and intensity values at the steadiest portion of the vowels were computed. The results show several parameters which distinguishes oral vowel from Nasal vowels as well as the effect of languages of Non-native speakers on Hindi Vowels. Significant observations and the results of the study have been discussed in detail in this paper.

4pSC33. Pitch accents in the Spanish of Japanese-Spanish bilinguals. Tanya Flores (Lang. and Lit., Univ. of Utah, 255 S Central Campus Dr., LNCO 1400, Salt Lake City, UT 84109, Tanya.Flores@utah.edu)

This study examines supra-segmental traits in the Spanish speech productions of thirteen Japanese L1-Spanish L2 speakers living in Valencia, Spain. The analysis presented here will focus on the use of pitch accents and final syllable lengthening. The data in this study shows speakers using Japanese pitch accent patterns in Spanish words, often as the only phonetic indicator of second language speech. The goal of this study is to identify specific Japanese pitch accent patterns used and determine if and how they are systematically transferred into Spanish. Following the autosegmentalmetric model ( $\mathrm{Sp}_{\text {ToвI }}$ ), pitch accents will be measured using PRAAT. Findings will contribute to the currently limited acoustic research on supra-segmental or prosodic traits of L2 speech in language contact situations.

4pSC34. Bidirectional prosodic effects of code-switching in SpanishBasque bilinguals. Ann M. Aly (Linguist, UCLA, 3125 Campbell Hall, Los Angeles, CA 90095, ann.m.aly@ucla.edu)

This study investigates the prosody of code-switching in Spanish-Basque bilinguals, which has not been previously documented. Peninsular Spanish and Basque have several prosodic differences, including peak alignment in pitch accents. Spanish has delayed peaks (Estebas Plana \& Prieto (2008)), while Basque has early peaks (Elordieta \& Hualde (2015)). The current study investigates whether code-switched words are conditioned by prosodic position and whether the peak alignment and f0 maximum of a pitch accent differ between monolingual context (Spanish only) and codeswitched contexts. Bilinguals (aged 21-31) from a Basque-dominant region (Lekeitio, $n=3$ ) and a Spanish-dominant region (Bilbao, $n=4$ ) participated in the present study. The data was coded for the following predictor variables: prosodic position, peak alignment, and fo maximum in pitch accents. Results reveal that code-switches occur almost equally in phrase-medial and final positions. For peak alignment, Lekeitio speakers produced early peaks in both code-switching contexts (Spanish-Basque, Basque-Spanish), which matches monolingual norms for Lekeitio varieties of Spanish and Basque. However, Spanish-dominant speakers also produced early peaks in both code-switching contexts, despite delayed peak norms for Peninsular Spanish. Finally, preliminary results reveal greater fo during code-switching contexts for Lekeitio speakers only. Further results and implications will be discussed.
4pSC35. Japanese singleton and geminate stops mispronounced by nonnative speakers. Kimiko Yamakawa (Culture and Lang., Shokei Univ., 2-81 Musashigaoka-kita, Kikuyo, Kikuchi, Kumamoto 8618538, Japan, jin@ shokei-gakuen.ac.jp) and Shigeaki Amano (Aichi Shukutoku Univ., Nagakute, Aichi, Japan)

Non-native Japanese speakers often mispronounce Japanese singleton and geminate stops. However, it has been unclear how durations of speech segments in their mispronunciation differ from those of correct pronunciation by native Japanese speakers. This study analyzed mispronunciation of singleton and geminate stops by non-native speakers (8 English, 12 French, and 10 each of Korean, Taiwanese, Thai, and Vietnamese). Non-native speakers' mispronunciations were identified based on 10 native Japanese speakers' production boundary between singleton and geminate stops. It was revealed that when non-native speakers mispronounce a singleton stop as a geminate stop, stop closure is longer whereas its previous mora is shorter than in native speakers' correct pronunciation. Meanwhile, when non-native speakers mispronounce a geminate stop as a singleton stop, stop closure is shorter whereas its following mora is longer. These findings indicate that non-native speakers' mispronunciations are caused not only by the durations of closure but also by the lengths of the previous/following mora. [This study was supported by JSPS KAKENHI Grants Number JP $25284080,26370464,15 \mathrm{H} 03207$, and 16K13221. It was also supported by a special-research grant of Aichi Shukutoku University in 2015-2016.]

4pSC36. Production and reproduction of Japanese pitch-accent by German, Italian, Vietnam, and Swahili speakers. Ryoko Hayashi (Graduate School of Intercultural Studies, Kobe Univ., Nada-ku Tsurukabuto 1-2-1, Kobe, Hyogo 6578501, Japan, rhayashi@kobe-u.ac.jp), Kazuhiro Isomura (The Japan Foundation Japanese-Lang. Inst., Tokyo, Japan), Makiko Matsuda (Kanazawa Univ., Kanazawa, Japan), Natsuya Yoshida (Hokkaido Bunkyo Univ., Sapporo, Japan), and Motoko Ueyama (Univ. of Bologna, Forli', Italy)

Japanese pitch accent is lexically distinctive. JFL learners should know the position of the accent to pronounce the words correctly, which is said to be very difficult. In our experiment, we investigate the effectiveness of showing the accent mark to help learners' pronunciations. We compared the ability of perception, production and reproduction of pitch accent by JFLs of four different languages. 42 Japanese words were presented on a PC screen and 30 subjects participated in three tasks of the experiments. Task 1 was to pronounce the words without any suggestion, task 2 was to imitate the model pronunciation with seeing the accent marks. Task 3 was to pronounce by just seeing the accent marks. The results of task 1 show the percentage of correct accent position was only around $40 \%$, but it rose up to around $90 \%$ in task 2 and fell again in task 3 but still significantly higher than in task 1 . The results of task 1 showed different patterns of phonetic interference, task 2 showed that the subjects could easily perceive the position of accent and imitate the pitch pattern. Task 3 implied the effectiveness of showing the accent mark.

4pSC37. Tonal realization of English intonation and register by Japanese learners of English as a foreign language compared to English speakers. Toshiko Isei-Jaakkola (College of Humanities, Chubu Univ., Matsumoto 1,200, Kasugai, Aichi 487-8501, Japan, tiseij@isc.chubu.ac.jp) and Keiko Ochi (National Inst. of Informatics, Chiyoda-ku, Tokyo, Japan)

Theoretically, there are four levels of tone in English intonation, whereas in standard Japanese, phonologically, there are two levels (high and low) and phonetically, there are three levels of in lexical tone. The Japanese said to be a mora-timed and pitch-accented language and English to be a stress-timed. The Japanese language sounds monotonous. Further, English speakers (EL1) seem to have a wider register than Japanese learners of English as a foreign language (JEFL). There is only one kind of interrogative sentence in Japanese, while there are two kinds in English. Moreover, there is a difference in the position of the nucleus between Japanese and English. This study aims to examine whether JEFL can realize the four levels of tone in English, and the degree of difference in register between JEFL and EL1 when producing a sample English intonation pattern. We conducted experiments to investigate JEFL's realization of English intonation in one wordsentences, affirmative sentences, and interrogative sentences with rising, 
falling, and rising-falling tones. We found that JEFL could produce intonational contours resembling the model English sentences but with insufficient nuclei (weak intensity), and that EL1 have a wider register than JEFL in both minimum F0 and maximum F0 as presumed.

4pSC38. Speech rhythm characteristics of L2 English produced by Japanese. Saya Kawase, Jeesun Kim, and Chris Davis (MARCS Inst., Western Sydney Univ., Locked Bag 1797, Penrith, NSW 2751, Australia, S. Kawase@westernsydney.edu.au)

This study investigated the durational rhythm characteristics of L2 English produced by L1 Japanese talkers with a particular focus on the interaction between L1 and L2 speech rhythm and the role of L2 experience. More specifically, for English sentences $(\mathrm{N}=40)$ spoken by native Japanese $(\mathrm{N}=$ 10) and native Australian English ( $\mathrm{N}=10)$ talkers, we examined (1) mean consonant and vowel durations and durational variability and (2) vowel and consonant timing patterns within consonant clusters. Half of the Japanese talkers had more experience in L2 English. L2 productions had longer consonant and vowel duration and less variability compared to native English ones. The degree of L2 experience played a role: inexperienced L2 talkers produced less variable vowel durations than the experienced ones. The analyses of speech timing patterns in consonant cluster productions showed that inexperienced Japanese talkers produced significantly shorter second consonants in consonant clusters compared to native English productions, possibly compensating for the difficulty in producing nonnative consonant clusters. Additional analyses of the pattern of consonant cluster production by experienced $\mathrm{L} 2$ talkers will also be reported.

4pSC39. Types of errors in English prosody made by native speakers of Korean. Heeju Lee and Sun-Ah Jun (UCLA, 290 Royce Hall, Box 951540, Los Angeles, CA 90095-1540, heejulee@ucla.edu)

This study investigates the types of errors that are made by speakers of L1 Korean in the production of L2 English prosody. English and Korean differ in prosodic and rhythmic structure and in the ways word prominence and semantic/pragmatic meaning are marked. In English, a prominent word is marked by a pitch accent on its stressed syllable, and semantic/pragmatic information is delivered by pitch accent types (e.g., $\mathrm{H}^{*}, \mathrm{~L}+\mathrm{H}^{*}$ ) and boundary tones. In Korean, a language without lexical stress, a word's prominence is marked by its location at the beginning of a phrase, and semantic/pragmatic information is delivered by prosodic phrasing and boundary tones. Data were selected from recordings of the UCLA TOP (Test of Oral Proficiency) exam taken by Korean students. The present study analyzes the part where students (twelve) present information from the same syllabus in front of raters and questioners acting as students. Control data from three native English speakers was collected in the same format. Prominence, phrasing, and intonation patterns were marked following English ToBI conventions. Preliminary results indicate that the types of errors can be distinguished in terms of prosodic phrasing, location and types of pitch accent, and realization of pitch accents and stressed/unstressed syllables.

4pSC40. Oral gestural reduction of English nasal coda produced by Mandarin English-as-a-foreign-language learners. Yadong Liu (Linguist, The Univ. of Hong Kong, Run Run Shaw Tower, Hong Kong, Hong Kong, yadong@connect.hku.hk)

Mandarin post-vocalic nasal production studies focus on vowel-to-nasal formant transitions (e.g. Jian and Le [(2014), Information Technology Journal, 13(11), 1793-1799] show the vowel-to-nasal boundary was absent in the context of VN.V). However, it remains unclear whether this feature of Mandarin speakers' L1 extends to their L2 productions. The present study investigates five Mandarin EFL learners' articulation of English postvocalic nasal codas / $\mathrm{n}, \mathrm{y} /$ and homorganic oral codas / $\mathrm{d}, \mathrm{g} /$ in monosyllabic words in pre-vocalic contexts. Articulatory data from ultrasound-imaging were analyzed for lingual gesture achievement (in terms of aperture), where a reduced oral constriction corresponds to absence of a stop. The results show reduction of the oral constriction of both $/ \mathrm{n} /$ and $/ \mathrm{n} /$ relative to their oral counterparts. Further, the reduction varied by place, with greater reduction in $/ \mathrm{y} /$ compared to $/ \mathrm{n} /$. Additionally, the height of the preceding vowel affects oral constriction reduction of both nasals, with low vowels eliciting more reduction than high vowels. This research studies nasal provides evidence of oral constriction reduction during Mandarin EFL learners' English nasal coda production from an articulatory perceptive, consistent with the L1 nasal closure reduction shown in Jian and Le (2014).

4pSC41. Adaptation to foreign-accented speech with decreased spectral resolution. Michelle R. Kapolowicz, Vahid Montazeri, James F. Kuang, and Peter F. Assmann (Behavioral and Brain Sci., The Univ. of Texas at Dallas, 800 West Campbell GR 4.1, Richardson, TX 75080, mrk092020@ utdallas.edu)

Listening to foreign-accented speech requires greater effort, and intelligibility is lower than for native speech. We have recently shown that speech processed by a vocoder to simulate reduced spectral resolution imposes a substantial additional cost to intelligibility. The present study investigated perceptual adaptation to foreign-accented speech in conditions with reduced spectral resolution using a 9-channel tone vocoder compared to unprocessed speech. Preliminary results suggest that listeners can quickly adapt to foreign-accented speech with decreased spectral resolution. Native American English listeners achieved performance levels similar to those obtained with initial exposure to the same unprocessed speech from multiple talkers of Mandarin-accented English. However, with increased exposure, performance did not improve to the same degree found for unprocessed foreignaccented speech. Results indicate that the adaptation process is negatively impacted by the interaction of foreign accent and reduced spectral resolution, and suggests that special consideration should be given to the difficulties experienced by listeners with cochlear implants (whose devices provide limited spectral resolution) when they communicate with speakers who have a foreign accent.

4pSC42. Japanese English learners use a variety of articulation strategies to produce English liquids. Jeffrey Moore (Sophia Univ., Chiyodaku, Kioi-cho, 6-2, Tokyo, Tokyo 102-0094, Japan, jeffmoore@sophia.ac. jp), Jason Shaw (Yale Univ., New Haven, CT), Shigeto Kawahara (Keio Univ., Tokyo, Japan), and Takayuki Arai (Sophia Univ., Tokyo, Japan)

This study examines the production of English liquids/r/ and /l/ by Japanese speakers. Six speakers of varying levels of proficiency were recorded using an NDI Wave Electromagnetic Articulograph. They produced words containing $/ r /$ and $/ l /$ in initial singletons and initial clusters. Two native speakers of English were also recorded for comparison. The resulting articulatory data are analyzed for spatial targets, gestural timing, and the amount of variation between utterances. Results indicate a variety of strategies employed by speakers to achieve the non-native targets; however, more native-like, though distinctly Japanese-accented production, among advanced speakers was found. Lower-proficiency and intermediate speakers showed relatively large amounts of within-speaker variation, indicating the ongoing development of articulation strategies. These findings suggest that the acquisition of foreign language sounds is not merely a straight path from L1 transfer to target-like pronunciation, but a complex process involving many paths, hypothesis testing, and experimentation to find a suitable articulation.

4pSC43. Articulatory variations of Mandarin retroflex consonants produced by second language speakers: An electromagnetic articulograph study. Haruka Saito (School of Commun. Sci. and Disord., McGill Univ., 522 Ave. des Pins, Rm. \#5, Montreal, QC H2W 1S6, Canada, haruka. saito2@mail.mcgill.ca)

This study investigated articulatory variations in tongue positions and shapes during the production of Mandarin post-alveolar retroflex consonants produced by speakers with varying Mandarin proficiency (native Mandarin speakers, Japanese L2 speakers of Mandarin, and Japanese monolinguals with no knowledge of Mandarin). We aimed to examine (1) whether there are articulatory variations for Mandarin retroflex consonants and (2) if preferred variations differ across groups. Speakers either read aloud or imitated the consonants after hearing them and their tongue positions and shapes were measured by electromagnetic articulography (WAVE, NDI). Results showed that there are multiple articulatory variations for Mandarin retroflex consonants. Native Mandarin speakers produced a concave or convex 
tongue shape; all Japanese L2 speakers of Mandarin produced a convex tongue shape. In contrast, the majority of Japanese monolinguals imitated the sounds with an entirely different tongue position: bunching their tongues in the middle, which somewhat resembled the "bunched" rhotic in American English. Despite these articulatory variations, productions by most L2 speakers and several monolinguals were successfully identified as retroflex consonants by native Mandarin listeners. These results suggest that L2 speakers may prefer certain articulatory variations and the preference may change depending on proficiency.

4pSC44. A limited role of hand gestures and head nods in native English speakers' production of Mandarin tones. Yukari Hirata (Ctr. for Lang. and Brain, Colgate Univ., 13 Oak Dr., Hamilton, NY 13346, yhirata@colgate.edu), Annie Zheng (Psych., Univ. of Texas at Austin, Austin, TX), and Spencer D. Kelly (Ctr. for Lang. and Brain, Colgate Univ., Hamilton, NY)

Theories of embodied cognition have emphasized the importance of the whole body in human communication (Lakoff \& Johnson, 1999), and numerous empirical studies have shown that bodily actions affect multiple aspects of speech production and perception. This study examined the roles that hand and head gestures play in production of Mandarin tones by native English speakers. Twenty-four native English speakers were asked to imitate the four Mandarin tones from a model across three conditions: (1) audio-only, (2) audio and hand gestures, and (3) audio and head nods. For (2) and (3), participants watched videos in which a native Mandarin speaker visually depicted the tones with her hand and head, respectively. The participants' production was then presented to seven native Mandarin listeners in an identification test to determine percent correctly produced. A 3 (conditions) x 4 (tones) ANOVA revealed a significant main effect of tone $(1>3$, $4>2$ ) but no main effect or interaction of condition, indicating that hand and head gestures did not help learners accurately produce Mandarin tones. The results corroborate earlier acoustic analysis of the production data, confirming a limited role of hand and head gestures in phonetic and phonological aspects of L2 learning.

4pSC45. Collocational patterns of disfluencies in native and non-native speech: Evidence from the Wildcat Corpus. Misaki Kato, Tyler Kendall, and Melissa Baese-Berk (Linguist, Univ. of Oregon, 270 Straub Hall, 1290 University of Oregon, Eugene, OR 97403, misaki@uoregon.edu)

While disfluencies are generally considered a natural part of spontaneous speech, patterns of disfluencies in non-native (NN) speech could contribute to making that speech sound less proficient or less "fluent". NN speech has been described as having more frequent and longer pauses, pauses at within-clause boundaries, shorter mean length of runs, and slower speech rate, compared to native (N) speech (e.g., Riggenbach, 1991; Trofimovich \& Baker, 2006). However, in order to understand what makes NN disfluency patterns unique, it is important to examine such phenomena locally by examining the contexts of different types of disfluency. In the present study, we describe what features collocate with disfluencies in spontaneous English dialog. We examine speech from the Wildcat Corpus (Van Engen et al., 2010) which includes English dialogue produced by various pairings of $\mathrm{N}$ and $\mathrm{NN}$ speakers (N-N, N-NN, or NN-NN). Specifically, we extract turn-internal disfluencies of several types (e.g., silent pause, filled pause, repetition), and examine lexical (e.g., part-of-speech) and phonetic (e.g., vowel length) features of speech surrounding these disfluencies. Further, we examine whether these disfluency patterns change depending on speaker pairings. Differences between $\mathrm{N}$ and $\mathrm{NN}$ disfluency patterns are discussed in terms of their potential sources (e.g., speaker- vs. listeneroriented).

4pSC46. Transfer of $\mathrm{L} 1$ tone knowledge to $\mathrm{L} 2$ vowel quantity contrasts as a secondary cue: The case of Cantonese learners of Japanese. Chun Yin Liu (The Univ. of Hong Kong, Pok Fu Lam Rd., Hong Kong, Hong Kong, yin2013@hku.hk) and Yukari Hirata (Ctr. for Lang. and Brain, Colgate Univ., Hamilton, NY)

Distinguishing vowel quantities in Japanese is problematic for Cantonese-speaking learners. Vowel length is lexically contrastive in Japanese but not in Cantonese. Apart from duration, fundamental frequency (F0) patterns are secondary cues to vowel length distinction. As tone is lexically contrastive in Cantonese (particularly rising vs. high level), learners would likely be able to transfer this phonological knowledge to help themselves acquire vowel length contrast in Japanese. A perception experiment was conducted in which learners listened to Japanese disyllabic words. The first syllable of which was lengthened according to the within-word vowel duration ratio in eight steps. A level or dynamic (rising or falling) F0 pattern was then imposed on the first vowel. Similar to other L2 learners, Cantonese-speaking learners did not perceive the long vs. short contrast categorically, but gradiently. While both dynamic F0 patterns signal a long vowel, they only exploited the rising pattern to recognise a long vowel. Our study suggests that learners have assimilated the falling contour to high level, both of which belong to the same tonal category in Cantonese. The theoretical implications of these results are discussed.

4pSC47. Native listeners' evaluation of natural and synthesized prosody in Mandarin of American learners. Ying Chen, Xueqin Zhao, and Li Liu (School of Foreign Studies, Nanjing Univ. of Sci. and Technol., 200 Xiaolingwei St., Nanjing, Jiangsu 210094, China, ychen@njust.edu.cn)

Compared to duration and intensity, F0 was found the most difficult acoustic parameter to acquire in L2 prosody, especially post-focus compression of F0 (Chen, 2016). This study examined three groups' Mandarin production of prosodic focus: native Beijing speakers, early American learners and late American learners. PENTAtrainer2 (Xu \& Prom-on, 2014), a datadriven system for prosody analysis and synthesis, was used to model and synthesize F0 contours based on speaker groups and layered annotations of communicative functions: lexical, sentential and focal. Native Mandarin speakers were recruited to identify focus status (neutral, initial, medial, or final focus) and rate the naturalness (1-5 scale) of original and synthesized speech. Results reveal that natural speech was recognized and rated better than synthesized speech, early learners' speech better than late learners' speech, focused sentences better than no-focus sentences, and initial focus and medial focus better than final focus. Tones of focused words interacted with focus status of the sentence and speaker group. Future work will involve pairwise shape comparisons, root-mean-square error, and Pearson's correlation coefficient comparing between natural and synthesized F0 contours. [This work was supported by the National Science Foundation of China 61573187 and Fundamental Research Funds for the Central Universities in China NJUSTWGY14001.]

4pSC48. Articulatory habit versus adaptive flexibility in L2 phone learning. Matthew Faytak (Linguist, Univ. of California, Berkeley, 2632 San Pablo Ave., Apt. A, Berkeley, CA 94702, mf@berkeley.edu)

Learning a motor task is thought to be facilitated when it can be achieved within a set of thoroughly-learned motor patterns, here termed articulatory habit. Learners of novel phonological categories in an L2 may first attempt to produce a novel sound by working over the possibility space of acoustic outputs defined by well-known articulatory dimensions. A side effect of this neuromuscular habituation to L1 may be a limit to the adaptive flexibility of articulatory resources to the L2. To evaluate the role of L1 habit in individuals' L2 category productions, 18 L1 English learners of French repeated French stimuli from a model talker while ultrasound and video of lip movement were recorded. Stimuli include the front rounded vowels $/ y ø /$, which are outside of the subjects' articulatory habit in inter-articulator coordination and the particulars of tongue position. A second phase of the study captured L1 English articulatory habit by eliciting a range of English words and recording articulator activity in the same way. Principal components analysis was performed on raw image data of both phases in order to gauge L1-L2 articulatory similarity and to highlight any dimensions not exploited in L1 but observed in L2 or vice-versa.

4pSC49. Age and consonantal context effects on second language speech perception of French vowels. Andrew Lee (McGill Univ., 3700 McTavish St., Montreal, QC H3A 1Y2, Canada, andrew.lee@mcgill.ca)

This study investigated the effects of age of acquisition (AOA) and consonantal contexts on second language (L2) speech perception of French 
vowel pairs $/ \mathrm{i} /-/ \mathrm{y} /$ and $/ \mathrm{y} /-/ \mathrm{u} /$. A total of 60 Korean learners of French participated in either an Early-AOA group (under 16 years), a Mid-AOA group (16-26 years), or a Late-AOA group (over 26 years). To measure their perceptual accuracy, AX categorical discrimination tasks were employed in which the two target pairs, in addition to a control pair/i/-/u/, were provided with three different consonants (i.e., $/ \mathrm{p} /, / \mathrm{t} /$, and $/ \mathrm{k} /$ ) in a consonantal-vowelconsonantal structure. Overall, the Korean participants had more difficulty discriminating the pair /i/-/y/than the pair /y/-/u/. In particular, the Korean participants revealed more difficulty with the former pair in the $/ \mathrm{p} /$ context, whereas they showed more difficulty with the latter pair in the /p/ and /t/ contexts. While those in the Early-AOA group significantly outperformed those in the Mid- and Late-AOA groups, those in the former group perceived the two target pairs in a way that was less affected by the consonantal contexts. Accordingly, this paper will conclude by highlighting the importance of AOA and consonantal contexts in L2 speech learning.

4pSC50. Effects of language bias and proficiency on cross-language activation: Evidence from eye-tracking. Maria Teresa Martinez-Garcia (Modern Lang., Fort Hays State Univ., 600 Park St., Hays, KS 67601-4099, mtmg87@gmail.com) and Annie Tremblay (Linguist, Univ. of Kansas, Lawrence, KS)

Language bias and proficiency have been proposed to modulate crosslanguage activation, but it is unclear how they operate and whether they interact. This study sheds further light on this by investigating whether stress differences between Spanish-English cognates (material, final-syllable stress in Spanish) affect how native-English second-language-Spanish bilinguals recognize Spanish words (materia "subject/matter," second-syllable stress in Spanish). In a Spanish-English eye-tracking experiment, participants heard trisyllabic Spanish targets with second-syllable stress (materia) and saw four orthographic words, including the target and a Spanish-English cognate competitor. Cross-language activation was examined by manipulating the stress of the cognate in English; English cognates with the same stress as the Spanish target (target: materia, competitor: material) were predicted to cause more cross-language interference than English cognates with a different stress (target: litera "bunk bed," competitor: literal). Participants were assigned to a Spanish-bias condition, with 20\% of English (filler) items, or an English-bias condition, with $65 \%$ of English (filler) items. Spanish proficiency was assessed with a cloze test and LexTALE. Results indicate that English cognates with the same stress as the Spanish target interfered with the recognition of the Spanish target only in the English-bias condition, and bilinguals better controlled this cross-language interference with increasing Spanish proficiency.

4pSC51. The novel Ventriloquist paradigm: Studying L2 phonetic learning in dialogue with experimental control over phonetic detail. Mirjam Broersma (Ctr. for Lang. Studies, Radboud Univ., P.O. Box 9103, Nijmegen 6500 HD, Netherlands, m.broersma@let.ru.nl)

The novel Ventriloquist paradigm enables the study of phonetic accommodation and perceptual learning in dialogue, while allowing full control over the phonetic detail of the input that participants are exposed to. It is being developed to investigate L2 speech learning for production and perception in an ecologically valid yet maximally controlled way. Participants take part in a dialog which they believe to be genuine; in fact, however, their (real-life) interlocutor is a confederate whose speech is not just "scripted" (as in the confederate scripting task), but fully prerecorded. This guarantees control over all characteristics of the speech input, e.g., precluding that a confederate accommodates his/her pronunciation to the participant. The setup is fully tuned to upholding the illusion that the confederate is actually speaking with the participant. The confederate sits opposite the participant, face briefly hidden when he/she "speaks." Participants hear the prerecorded speech over closed headphones. In addition to the standard input, to facilitate a smooth flow of the conversation, the confederate can play prerecorded non-verbal interaction markers and stop-gap replies to any unanticipated remarks or questions from the participant. The new paradigm thus reconciles ecological validity with experimental control for the study of (L2) phonetic processing in dialog.
4pSC52. Non-native perception and production of lexical tones before and after high-variability training. Yan Chen (Linguist Dept., Univ. of Arizona, Tucson, AZ 85721, yanchen@email.arizona.edu)

This study investigates non-native perception and production of lexical tones. 26 English speakers received 3 hours of high-variability AX training to perceive tonal contrasts in Cantonese. Half of them were trained with additional iconic tone marks (AV) and half of them were not (AO). Pre-test and post-test were conducted and both of them consisted of high-variability AXB followed by delayed repetition using the stimuli from AXB. Preliminary results showed that T2 (high-rising) vs. T5 (low-rising) and T3 (midlevel) vs. T6 (low-level) were the most difficult pairs in AXB, with about $70 \%$ correct for T2-T5 and slightly above chance performance for T3-T6 in post-test. AV listeners did not achieve significantly higher accuracy than AO listeners. In delayed repetition, both groups produced distinct contours for T2 and T5 and for T3 and T6 even in pre-test, which did not differ significantly from post-test. Exposure to pre-test AXB might have facilitated talker normalization and tone production in the delayed repetition task. In addition, AV subjects had slightly more semitone difference in the second half of rhyme (offset- $50 \%$ of rhyme duration) for T2 in post-test than AO subjects, but this effect of iconic visual information is limited.

4pSC53. Neural changes accompanying overnight nonnative phonetic learning. F. S. Earle and Emily B. Myers (Dept. of Speech, Lang. and Hearing Sci., Univ. of Connecticut, 850 Bolton Rd., Unit 1085, Storrs, CT 06268,frances.earle@uconn.edu)

Sleep is important in the consolidation of learning, including the perceptual learning of non-native speech sounds. Previous work investigating the role of post-training sleep on nonnative phonetic learning has demonstrated that sleep significantly improves identification for a trained talker in the absence of further training. In addition, sleep appears to facilitate generalization of learning to non-native tokens produced by an untrained talker. We investigated the neural correlates of these behavioral observations using fMRI. Participants were trained in the Hindi dental-retroflex contrast in the evening on Day 1. Immediately following training, participants performed the category identification task in the scanner, and then again 12 hours later. Sleep duration was monitored via wrist Actigraphs. On Day 1, significant differences in activation for the Trained vs. Untrained Talker were identified in the supramaginal gyrus and adjacent parietal areas, whereas on Day 2, activation for both talkers was observed to move into the temporal lobes bilaterally. After sleep, and in the absence of further training, decreases in activation were observed in right superior temporal gyrus, right precentral gyrus, and left prefrontal cortex. Based on these findings, we argue that newly acquired nonnative sounds are processed in classic (i.e., more native-like) speech regions following sleep.

4pSC54. Effects of perceptual training on the perception of Korean boundary tones by Chinese learners. Ho-Young Lee, Hyosung Hwang (Linguist, Seoul National Univ., 1, Gwanak-ro, Gwanak-gu, Seoul 08826, South Korea, hshwang415@gmail.com), and Eunkyung Yoon (Daegu Cyber Univ., Daegu, South Korea)

Previous studies have proven that High Variability Phonetic Training (HVPT) is an effective method for training non-native listeners to discriminate L2 phoneme contrasts or prosodic contrasts, such as Chinese tone and Japanese segment length. However, it still remains uninvestigated whether this training method can be successfully applied to the perceptual training of nondistinctive L2 intonation. This study aims to prove that the HVPT method is also effective in training Chinese learners of Korean to distinguish Korean boundary tones. Korean boundary tones have three important grammatical and pragmatic functions: distinction of declarative and interrogative sentences, distinction of wh- and yes-no questions, and distinction of ambiguous meanings of some terminal endings. Our experiment is designed to train Chinese learners to discriminate these three functions using the HVPT method. 40 Chinese learners participated in 10 online sessions. They were exposed to 276 sentences delivered by different speakers each session. The subjects showed significant improvements in discriminating all three intonational functions in the posttest. Lower proficiency learners showed greater improvements. A notable change occurred in the development of categorical perception. The category boundaries between declarative and interrogative sentences shifted closer to the native speakers' after the training. 
4pSC55. Relationship between learners' attention and perception training effects of a foreign language: Based on questionnaire study to young learners. Yuko Ikuma (English Education, Osaka Kyoiku Univ., 4-1-1-801, Bingocho, Nada-ward, Kobe, Hyogo 657-0037, Japan, yyikuma13@gmail.com)

Many previous practices studied learners' attitude in the field of the second language (L2) research. They observed the effectiveness of the improvement of learners' motivation or instruction for enhancing learners' self-efficacy. From the author's series of the preceding training research targeting at over one thousand young students who utilized self-study-typed computer-assisted language learning (CALL) system, the author has found that the continuous perceptual training be important in order to maintain the ability. This paper focused on the Japanese-speaking young learner's attitude and its progress towards L2 speech perception training mainly based on the questionnaire response after longitudinal training period using CALL materials. The result showed that the accuracy rates for phoneme and rhythm perception tasks improved; that tendency was observed slightly strong on the students who were interested in "phoneme discrimination" or "conversation" from the questionnaire response. It was also demonstrated that higher proficiency students showed interest in broader perspective like "conversation," while lower students in rather narrower like "discrimination," or "sound." These results suggest that learners who got attention toward phoneme might improve their perception, however, to promote their proficiency, they need some instruction to have attention to wider perspective of speech. [Work supported by JSPS 26780498.]

4pSC56. Preliminary analysis of training non-native sounds in noise. Hinako Masuda (Faculty of Sci. and Technol., Seikei Univ., 3-3-1 Kichijoji Kitamachi, Tokyo, Musashino-shi 180-8633, Japan, h-masuda@st.seikei.ac. jp)

Past research has repeatedly shown that perceiving speech in non-laboratory environments is challenging for both native and non-native listeners, even if they are able to perform well in laboratory (quiet) environment. However, whether the challenge of non-native listeners' perception of non-native sounds in noise can be overcome with training has not been fully investigated. The present study reports preliminary analysis of training Japanese learners of English, focusing on the identification of English voiceless fricatives and approximants in multispeaker babble noise, as Japanese learners of English are well-known for having difficulty in accurate perception of such sounds (e.g., Lambacher et al., 2001; Masuda 2016 among others), especially in noisy listening environments. The experiment consists of three sessions: 1) pre-test, 2) training sessions in noise, and 3) post-test. Identification rates of 1) pre- and 3) post-tests will be compared to investigate the effect of 2) training in noise. The effect of English learning experience will also be discussed.

4pSC57. Noninvasive brain stimulation to facilitate foreign language speech-sound learning in low-aptitude learners. Tyler K. Perrachione, Sara C. Dougherty, Ja Young Choi, and Elly R. Hu (Dept. of Speech, Lang., and Hearing Sci., Boston Univ., 635 Commonwealth Ave., Boston, MA 02215, tkp@bu.edu)

Native English speakers with poor pitch-contour identification abilities also have a low aptitude for learning lexical tones, as in Mandarin Chinese. In a four-day training paradigm, native English-speaking young adults with low pitch-contour perception abilities learned a vocabulary incorporating minimal contrasts based on lexical tones (with level, rising, and falling contours), in which they matched spoken words to photographs of objects. Participants were assigned to two groups: The "stimulation" group received anodal transcranial direct current stimulation (tDCS) over left inferior frontal lobe at $2 \mathrm{~mA}$ during training; the "sham" group underwent an identical set-up procedure each session, but did not receive brain stimulation during training. Participants' learning trajectories, learning attainment, and ability to generalize word recognition to novel talkers were compared between groups and to previously published training data from both low- and high-aptitude learners. Active brain stimulation improved learning for some, but not all, low-aptitude learners. Some low-aptitude learners who received brain stimulation performed as well as high-aptitude learners in previous studies. Low-aptitude learners who received sham stimulation learned no better than in previous studies. These results suggest noninvasive brain stimulation may help recover speech-sound learning abilities in listeners with low pre-training perceptual aptitude.
4pSC58. Lexical training using accented speech improves non-native contrast discrimination. Navin Viswanathan and Annie J. Olmstead (Speech-Language-Hearing, Univ. of Kansas, 1000 Sunnyside Ave., Lawrence, KS 66045, navin@ku.edu)

Native American-English (AE) listeners have difficulty distinguishing between dental and retroflex stop consonants. For example, when asked to classify voiceless retroflex and dental stops, AE listeners typically label both items as the alveolar/t/. While AE lacks the dental-retroflex distinction, varieties of Indian English (IE) use this contrast to instantiate the AE / $\mathrm{t} /-/ \mathrm{d} /$ distinction (tanks vs. thanks). Specifically, the dental $/ \mathrm{t}_{n} / \mathrm{is}$ used in instances where $/ \mathrm{d} /$ would be used in AE while the retroflex $/ \mathrm{t} /$ is used in place of $/ t /$. This situation provides us with the opportunity to present the dental-retroflex difference in naturally produced tokens of English. In the current set of experiments, we examine the effect of exposure to IE tokens on AE listeners' discrimination of / $/ \mathrm{d} / \mathrm{and} / \mathrm{t} /$. Participants transcribed English words spoken by an IE speaker. The critical items were words that began with either /t/ (e.g., take), /// (e.g., thorough), or were /t/-/\%/ minimal pairs (tanks vs. thanks). An AXB discrimination task performed before and after the transcription showed that AE speakers improved in their discrimination of $/ \mathrm{t} /$ and $/ \mathrm{t} /$ after transcription. Subsequent studies examine this learning effect to investigate the role of specific lexical contexts and of speaker-specific information.

4pSC59. Sleep facilitates talker generalization of accent adaptation. Xin Xie, F. Sayako Earle, and Emily B. Myers (Dept. of Speech, Lang. and Hearing Sci., Univ. of Connecticut, 850 Bolton Rd., Storrs, CT 06269, xin. xie@uconn.edu)

Lexically-guided phonetic retuning helps listeners adapt to non-canonical productions produced by a given speaker (e.g., a foreign-accented speaker). Our previous work shows that when tested immediately after training on one accented talker, listeners generalized phonetic retuning only to accented talkers that were acoustically similar to the trained talker. In the present study, we tested whether sleep-mediated consolidation promotes talker generalization to a novel talker who is not acoustically similar to a trained talker in their phonetic realizations. Focusing on word-final alveolar stops, we examined native-English listeners' 1) adaptation to a trained Mandarin-accented talker and 2) generalization to another untrained Mandarin talker. Participants were tested in a categorization task immediately after training in either the morning or evening and again after twelve hours. Morning and evening groups had comparable performance for the trained talker during the second test, suggesting maintenance of talker-specific learning. Importantly, only evening-trained participants, who had slept before the second test, gained an advantage for the novel talker, whereas morning-trained participants did not improve over the 12 hours in wake state. It is argued that sleep helped listeners to abstract away from specific acoustic properties of the trained talker and thereby facilitated generalization to the novel accented talker.

4pSC60. The effect of training of poetry recitation on English rhythm of Cantonese native speakers. Angel L. Chui (Dept. of Linguist, The Univ. of Hong Kong, Hong Kong, Hong Kong, u3518907@hku.hk)

English rhythm, although reported a hurdle to many ESL students, remains one of the least taught aspects of pronunciation. Despite the key role rhythm plays in facilitating comprehension and reducing foreign accentedness, systematic training in the ESL classroom is rare. To promote the teaching of rhythm in the local curriculum, this study explores the effect of poetry recitation training on advanced learners' acquisition of English rhythm. Two groups of advanced Cantonese-speaking English learners were recorded reading poems of different foot patterns as well as non-poetic materials (BKB sentences and a fable), each on a separate occasion. As reflected from rhythmic metrics, the group trained in recitation manifest nativeness better despite the similar proficiency level of the two. I, therefore, argue that poem recitation is an effective means to help ESL learners acquire native-like rhythm in English. 
4pSC61. Development of a visual app for improving learner's pronunciation with ultrasound and the speech accent archive. Kyori Suzuki and Ian Wilson (Univ. of Aizu, Tturuga, Ikki-machi, Aizuwakamatsu, Fukushima 965-8580, Japan, s1210136@gmail.com)

Although many language learners desire to improve their pronunciation of a foreign language, there are not many apps to help them do so. Most commercial apps for pronunciation evaluation and training focus on only the acoustic signal. However, few systems give visual movements of native speakers' lips, tongue, and jaw. In this paper, we describe the ongoing development of and app that is programmed in Swift for iPhone. The app incorporates and links together different kinds of phonetic data for the pronunciation learner-for example, recorded frontal and side videos of a native speaker's face during pronunciation with an ultrasound movie of the tongue moving in the mouth overlaid. The training text is a paragraph from the Speech Accent Archive. The initial version of this app has two systems. First, listening to English sentences by a native speaker and checking tongue movement with ultrasound. Second, this app has buttons that play from user-chosen words and it also plays in slow motion. The method of these systems may help people to learn a second language more easily and accurately by letting them shadow the audiovisual speech of a native speaker. This app will be demonstrated in front of the poster.

4pSC62. Analysis of the effects on pronunciation of training by using song or native speech. Saori Nemoto, Ian Wilson, and Jeremy Perkins (Univ. of Aizu, Tsuruga, Ikki-machi, Aizuwakamatsu, Fukushima 9658580, Japan,hy3148tay@gmail.com)

This research is an investigation of whether Japanese speakers' English pronunciation improves more after training on sung or spoken speech. The stimulus was a 14-word sentence taken from one English song's lyrics, and it had some words that are difficult to pronounce for most Japanese English learners. Thirty Japanese learners of English were recorded before training. Then, half of them trained by listening to the English song and singing it, and the other half trained by listening to a native speaker speaking the lyrics. Each group was allowed to train individually for 10 minutes, and then were recorded again. Then, 15 native or near-native English speakers at an American university and 100 native English speakers from Amazon Mechanical Turk evaluated those randomly-presented recordings. Listeners gave points for various phrases' pronunciation, the overall accent and the overall intonation. As a result, we found out that training by using the music condition resulted in generally worse results than the regular speech training. In addition, perhaps surprisingly, intonation of the whole sentence had an additional significant negative effect following music training. These results seem to show that training by using regular speech is more effective for English learners than training by using songs.

4pSC63. Developing ultrasound overlay videos for SENĆOTEN language learners. Heather Bliss, Sonya Bird (Linguist, Univ. of Victoria, PO Box 1700 STN CSC, Victoria, BC V8W 2Y2, Canada, hbliss@uvic.ca), and Bryan Gick (Linguist, Univ. of Br. Columbia, Vancouver, BC, Canada)

This paper reports on an initiative to develop a pronunciation training tool for learners of SENĆOTEN (Salish: Canada). In acquiring new speech sounds, second language learners rely on both acoustic and articulatory information. Ultrasound has been demonstrated to be effective for pronunciation instruction, as it allows learners to visualize tongue movements during speech. However, ultrasound-based instruction can be difficult to implement with large groups or with learners wanting to learn independently. To address these limitations, we developed a technique for creating ultrasound overlay videos which combine ultrasound images of tongue movements with external profile views of a speaker's head. Ultrasound is particularly useful in SENĆOFEN, as its inventory includes many lingual articulations that are difficult to distinguish without visual cues, such as velar/uvular contrasts, and a complex coronal series (e.g., $\left.\mathfrak{t}, \mathfrak{t}^{\theta}, \mathfrak{t}, \int, \lambda, t, 1, \mathrm{l}\right)$. SENĆOFEN is critically endangered, but efforts are underway to reawaken the language. Children participate in immersion programs, but parents need languagelearning opportunities that are adaptable to their schedules and needs. We developed a series of videos for distribution to community members, in particular parents, giving them opportunities to improve their pronunciation to better communicate with their children in their heritage language.
4pSC64. Influence of age-related factors on English / $/ \mathrm{/} / \mathrm{/l} / \mathrm{l}$ audiovisual training for Japanese speakers. Yasuaki Shinohara (Faculty of Sci. and Eng., Waseda Univ., Bldg. 51, 5th Fl., Rm. 08, 3-4-1, Okubo, Shinjuku-ku, Tokyo 169-8555, Japan, y.shinohara@aoni.waseda.jp)

Audiovisual training has proven successful for improving Japanese adults' identification accuracy of the English /r/-/l/ contrast. However, its effects on Japanese children have not been investigated yet. In the present study, 10 sessions of audiovisual English /r/-/l/ identification training were given to Japanese adults and children, and the age effects on learning the contrast in three testing conditions (audio-only, visual-only and audiovisual) were examined. It was hypothesized that children would improve their visual perception more than adults and they would show more audiovisual advantage than adults (i.e., higher improvement in the audiovisual condition than in the audio-only and visual-only conditions). The results demonstrated that both adults and children improved their identification accuracy in all three conditions, and the increase of the identification accuracy in the audiovisual condition was higher than that in the audio-only condition. However, there was no significant difference in improvement between adults and children. Due to a possible ceiling effect, it was unclear whether children had an advantage over adults in integrating visual information to auditory perception.

4pSC65. Acoustic analysis on before- and after-training English speeches by a male Japanese high-school student. Kikuyo Ito (National Inst. of Technol., Kagawa College, 355 Chokushi-cho, Takamatsu, Kagawa 761-8058, Japan, kikuyoito@hotmail.com)

Two sets of a 7-minute English speech by a 15-year-old Japanese male speaker, one made before two-month intensive training (approx. 2 hours per day, 3 to 4 days per week) and the other made after the training, were recorded and acoustically analyzed. The speech manuscript was constructed for a national English speech competition for students of the National Institutes of Technology in Japan, with ages ranging from 15 to 22 years. The intelligibility ratings of the two speeches evaluated by native speakers of American English (AE) indicated that the post-training speech was much more intelligible than the pre-training speech. Preliminary acoustic analysis focusing on the most frequently occurring words "traffic accidents" indicated that the temporal alignments of the consonant-vowel sequences of the words, along with the intensity and the duration of the stressed and unstressed vowels, were evidently different between the two speeches, with the tokens in the post-training speech closer to those in the speech by a native $\mathrm{AE}$ speaker than those in the pre-training speech. To identify the acoustic properties that make non-native speech "better sounding," threeway comparisons between the pre-training speech, the post-training speech, and the native speech are discussed in detail.

4pSC66. Auditory and acoustic-phonetic mechanisms of adaptation in the perception of sibilant fricatives. Eleanor Chodroff and Colin Wilson (Cognit. Sci., Johns Hopkins Univ., Krieger Hall 237, 3400 N. Charles St., Baltimore, MD 21218, chodroff@cogsci.jhu.edu)

Previous research has demonstrated that speech perception is highly dependent on preceding acoustic context (e.g., Ladefoged \& Broadbent, 1957), and suggested that this reflects adaptation to the long-term average spectrum (LTAS; e.g., Holt, 2006). The present study examined whether adaptation is further enhanced by acoustic-phonetic covariation among speech sounds within the natural class of sibilants. In each trial of Experiment 1, listeners were exposed to a [z]-initial CVC syllable and then categorized one member of an $[\mathrm{s}]-\left[\int\right]$ continuum. The distribution of the spectral center of gravity (COG) of $[\mathrm{z}]$ in the adaptation stimuli was manipulated (high vs. low). In Experiment 2, the [z]-initial syllables were replaced by LTASmatched white noise. Listeners adjusted the $[\mathrm{s}]-\left[\int\right]$ boundary in accordance with the exposure conditions, showing contrastive adaptation in both experiments; however, the adaptation effect was significantly stronger with the speech precursor. Furthermore, in Experiment 1, fricative COG provided a better quantitative account of responses than COG of the full syllable ( $\triangle$ BIC: 24$)$. These findings indicate that while general auditory processes can give rise to adaptation, listeners also exploit acoustic-phonetic relations among sounds: here, high correlations among fricative COG means observed in a large multi-talker corpus ([s]-[z], $r=0.62$, [s]-[ $], r=0.53$ ). 
4pSC67. Adaptation and aftereffects in response to bite block perturbation. Brett Myers and Antje Mefferd (Dept. of Hearing and Speech Sci., Vanderbilt Univ. Medical Ctr., 8310 Medical Ctr. East, Nashville, TN, brett. myers@vanderbilt.edu)

Although speakers can instantly adapt to the presence of a bite block (BB), they also refine their compensatory behavior over time. However, the extent to which $\mathrm{BB}$ perturbations elicit aftereffects and the mechanisms that contribute to refinement and aftereffects remain unknown. In this study, speakers belonging to either a practice or a no-practice group produced sentence repetitions under five conditions: PRE-BB (jaw-free), BB1 (jaw-fixed, initial BB exposure), BB2 (jaw-fixed, after 20 minutes of BB exposure), POST1 (jaw-free, immediately after BB removal), POST2 (jaw-free, one minute after BB removal). All speakers held a $10 \mathrm{~mm}$ BB in place for 20 minutes with the practice group reading aloud and the no-practice group sitting quietly. Jaw and posterior tongue kinematics were examined during the vowel /i/ embedded in the word "please" using electromagnetic articulography. Preliminary findings (14 speakers) revealed a significant main effect of condition only. That is, relative to PRE-BB, tongue height did not significantly change during BB1 and BB2 (indicating successful adaptation), but significantly increased during POST1 and POST2 (indicating an aftereffect). Jaw height remained unchanged from PRE-BB to POST1 and POST2 (indicating no aftereffect). Findings will be discussed with regard to internal models and the roles of proprioceptive and acoustic feedback.

4pSC68. Generalization in VOT imitation: Feature adaptation or acoustic-phonetic covariation? Colin Wilson, Eleanor Chodroff (Dept. of Cognit. Sci., Johns Hopkins Univ., Baltimore, MD), and Kuniko Nielsen (Linguist Dept., Oakland Univ., 1025 HHB, Rochester, MI 48309-4401, nielsen@oakland.edu)

After hearing instances of a word initial voiceless stop with lengthened VOT (e.g., long-[ $\left.\mathrm{p}^{\mathrm{h}}\right]$ ), speakers lengthen their VOTs in unheard words beginning with the same stop $\left(\left[\mathrm{p}^{\mathrm{h}}\right]\right)$ and, critically, in words beginning with a different stop $\left(\left[\mathrm{k}^{\mathrm{h}}\right]\right.$; Nielsen, 2011). This pattern of generalized phonetic imitation has previously been attributed to talker adaptation at the level of distinctive features or gestural organization. Implicit in these accounts is the assumption that talkers implement the same feature or gesture in a uniform way: generalization from long- $\left[\mathrm{p}^{\mathrm{h}}\right]$ to long- $\left[\mathrm{k}^{\mathrm{h}}\right]$ is an effective and rational adaptation strategy only if VOT values for different stops covary across talkers. Building on previous findings (e.g., Theodore, 2009; Chodroff \& Wilson, 2015), we establish that covariation is robust in the laboratory speech of Nielsen (2011). In baseline productions, the mean VOTs of $\left[\mathrm{p}^{\mathrm{h}}\right]$ and $\left[\mathrm{k}^{\mathrm{h}}\right]$ were almost perfectly correlated across participants $(r=0.97, p<.001)$. Following familiarization the correlation remained strong but was somewhat reduced $(r=0.87, p<.001)$, reflecting clear but imperfect generalization of VOT lengthening. These results are consistent with abstract adaptation accounts targeting features or gestures, but suggest a novel model of VOT adaptation that directly exploits the empirical correlations between stops.

4pSC69. Phonetic convergence in an immersive game-based task. Tifani M. Biro, Joseph Toscano (Psych. Dept., Villanova Univ., Tolentine Hall, 800 E Lancaster Ave., Villanova, PA 19085, tbiro@villanova.edu), and Navin Viswanathan (Speech-Language-Hearing: Sci. \& Disord., Univ. of Kansas, Lawrence, KS)

Phonetic convergence occurs when talkers change the acoustic-phonetic characteristics of their speech to be more similar to a conversational partner. It is typically studied using laboratory tasks, but the extent to which talkers converge varies considerably across studies. One aspect of these tasks that differs from real-world settings is how engaging they are. Highly contrived tasks may fail to elicit natural speech production, which could influence whether or not talkers converge. We address this issue by comparing the extent to which interlocutors converge in a repetitive, unengaging task versus an immersive video game-based task. Both tasks were designed to elicit production of specific words. Thirty word-initial voicing minimal pairs were used as stimuli, and we measured the degree to which phonetic cues (e.g., voice onset time; VOT) changed over the course of the experiment. For the more engaging task, participants' VOT values for voiceless tokens trended towards convergence (i.e., they gradually shifted their VOTs to be more similar to their partner's speech). In contrast, we found no clear evidence for convergence in the less engaging task. These results suggest that engaging, naturalistic tasks may yield results that more accurately reflect real-world conversational speech and phonetic variation than traditional laboratory experiments.

4pSC70. Acoustic and articulatory measures of prosodic convergence in a cooperative maze navigation task. Yoonjeong Lee, Samantha Gordon Danner (Linguist, Univ. of Southern California, 3601 Watt Way, Grace Ford Salvatori 301, Los Angeles, CA 90089, yoonjeol@usc.edu), Benjamin Parrell (Linguist, Univ. of Delaware, Newark, DE), Sungbok Lee, Louis Goldstein, and Dani Byrd (Linguist, Univ. of Southern California, Los Angeles, CA)

Convergence effects have been found in many aspects of communication between speakers in conversation. This study examines how phonetic properties of each speaker's prosodic structure differ before, during and after participating in a cooperative maze task. Using two NDI electromagnetic articulatory tracking systems simultaneously, we recorded speakers of American English in dyads (one male dyad \& one female dyad). Acoustic measurements included: 1) sentence durations, 2) phrase-final lengthening, 3) occurrence frequency of sentence-final boundary tones (percentage $\mathrm{H} \%$ ), and 4) peak F0 values in sentence-final words. Articulatory boundary strength measures for word-final consonant(s) articulations included: 1) movement durations, 2) displacement, and 3) time-to-peak velocity in phrase-medial and phrase-final positions. Speakers exhibited distinct phonetic patterns before the cooperative maze task. However, conversing speakers showed convergence effects in prosodic boundary strength, choice of boundary tone, and quantitative characteristics of boundary tone. Convergence effects persisted in the speech of one or both members of each dyad even after conversation has ended. Our findings raise the possibility that qualitative and quantitative aspects of prosody can be modeled as arising from a single system of control and that this system of prosodic structuring can show coupling effects across speakers. [Work supported by NIH.]

4pSC71. Acoustic correlates of vocal alignment to accented speech. Eva M. Lewandowski (Psych., Emory Univ., 36 Eagle Row, Dept. of Psych., Ste. 270, PAIS Bldg., Atlanta, GA 30306, elewand@emory.edu) and Lynne C. Nygaard (Psych., Emory Univ., Alanta, GA)

Language users can vocally align to the speech characteristics of other talkers both within and across accent groups. Although perceptual assessments of vocal alignment indicate convergence to accented speech, the acoustic properties of the speech signal that contribute to these perceptual judgments are less clear. The present investigation examined the acoustic correlates of vocal alignment to foreign-accented and native speech and related acoustic to perceptual measures of convergence. Native speakers of American English shadowed utterances produced by four model talkers; male and female native speakers of English and Spanish. Baseline utterances were obtained from visual naming blocks and shadowed utterances were compared to both baseline and model talker utterances. Fundamental frequency (F0), utterance duration, and vowel spectra (F1, F2) served as acoustic indices of vocal alignment. The results showed that different patterns of alignment emerged across the three measures. Utterance duration, but not fundamental frequency or vowel spectra, demonstrated convergence. However, both duration and vowel spectra measures significantly predicted perceptual assessments of vocal alignment. The results suggest that perceptual judgments of alignment behavior depend on multiple acoustic properties and that the dimensions of acoustic change during vocal alignment appear similar across accented and native speech.

4pSC72. Explicit imitation and working memory. Miranda R. Morris (Linguist, UC Davis, 1 Shields Ave., Davis, CA 95616, mirmor@ucdavis.edu)

Much research a has been conducted in regards to implicit imitation, that is, phonetic imitation that occurs largely without a speaker's knowledge. However, explicit imitation, which occurs intentionally, has received far less attention. While it is observed that individuals vary in explicit imitative abilities, i.e., some individuals' speech shift more towards a given target voice than others, the factors that contribute to these differences have yet to be identified. The current study investigates individual differences in explicit imitation by exploring the correlation between explicit imitation and working memory, the memory involved in short term conscious processing. Subjects 
participated in a baseline word reading task as well as an imitation task which involved repeating the same words aloud after hearing prerecorded stimuli from a Texan speaker. Additionally, subjects completed a reading Span task in order to measure working memory capacity and determine the effect of working memory on degree of explicit phonetic imitation. The degree of proportional phonetic shift towards the target speaker was correlated with Span scores in order to determine what link exists between explicit imitation and working memory on the level of the individual.

4pSC73. An experimental investigation of acoustic task-based carryover effects. Rachael Tatman (Linguist, Univ. of Washington, Guggenheim Hall, 3940-2425 Benton Ln., Seattle, WA 98195, rctatman@uw.edu)

Carryover effects, where conditions in one experimental task affect behavior in subsequent tasks, have not received much attention in linguistics. This is worrying, given that linguistic elicitation tasks do affect talkers' production (Labov et al. 1972, Warner et al. 2012). This study used before and after within-subjects design. 16 talkers completed three tasks: a memory task, one of a set of intermediate tasks (passage reading, word reading or an interview), and a second memory task.Pitch, intensity and duration of stressed vowels were measured for productions from each task. Nested mixed linear effects models were constructed for each measure. In all models, acoustic measures were used as the response variable and subject and token as random intercepts. For data from the intermediate tasks, the inclusion of task identity significantly improved model fit for pitch $(\mathrm{p}<0.05)$, intensity $(\mathrm{p}<0.001)$ and duration $(\mathrm{p}<0.001)$. In other words, there were very strong task effects. However, when comparing data from the first and second memory task, including the intermediate task did not improve model fit for pitch $(\mathrm{p}=0.71)$, intensity $(\mathrm{p}=0.78)$ or duration $(\mathrm{p}=0.70)$; these strong task effects did not carry over.

\title{
Session 4pSPa
}

\section{Signal Processing in Acoustics: High Resolution Imaging Sonars Including Real Aperture, Synthetic Aperture, and Tomographic Sonars II}

\author{
Brian G. Ferguson, Cochair \\ DSTO, PO Box 44, Pyrmont, NSW 2009, Australia \\ Timothy Marston, Cochair \\ APL-UW, 1013 NE 40th Street, Seattle, WA 98105
}

Invited Papers

$1: 30$

4pSPa1. Tomographic sonar imaging of underwater objects and the excitation of structural waves. Brian G. Ferguson (Maritime Div., DSTG, PO Box 44, Pyrmont, NSW 2009, Australia, Brian.Ferguson@dsto.defence.gov.au) and Ron J. Wyber (Midspar Systems, Oyster Bay, NSW, Australia)

Tomographic sonar imaging (or image reconstruction from projections) requires insonifying the object to be imaged with a wideband monostatic sonar over a complete set $\left(360^{\circ}\right)$ of look angles (or aspect angles). The tomographic sonar image that is reconstructed from the projection data (variation with aspect angle of the sonar impulse response) represents the projection of the object's three-dimensional acoustic reflectivity function projected on the imaging plane. The tomographic reconstruction of the backscattered sonar signals reveals the geometrical shape of the object, together with any prominent features on the object like lifting lugs. However, the formation of the tomographic image can be adversely affected by the presence of structural waves that can be excited by the insonification of the object at certain aspect angles. It is shown that a variety of these structural waves are readily observed in the projection data space where they are resolved in both time and aspect angle. The mapping of these structural waves to particular featues in the tomographic sonar image is demonstrated. The presence of these structural waves provides another means of classifying the object that complements sonar imagery.

\section{1:50}

4pSPa2. Circular synthetic aperture sonar images and aspect dependent spectral properties of solid elastic cubes. Viktor Bollen, Philip L. Marston (Phys. and Astronomy Dept., Washington State Univ., Pullman, WA 99164-2814, viktor.bollen@wsu.edu), and Daniel S. Plotnick (Acoust., Appl. Res. Lab, Seattle, WA)

While cubes may not be commonly found underwater, studying their aspect and material-dependent backscattering mechanisms may be useful in leads to understanding the scattering physics of other objects. Two solid cubes, made of either steel and or brass, were insonified in water using a circular synthetic aperture sonar (CSAS) system; Fourier based techniques were used to reconstruct target images. Identifiable backscattering mechanisms include edge-diffraction and elastic responses. Rayleigh waves, a class of surface elastic waves, are excited near a material specific incident angle (Rayleigh angle) that depends on the material of the cube. Those waves may 
retroreflect from the edges of the cube's facee edges of the cube's face in such a way as to enhance backscattering [K. Gipson and P. L. Marston, J. Acoust. Soc. Am. 105, 700-710 (1998)]. The steel cube displayed a strong Rayleigh wave response, easily visible in CSAS images; for the smaller brass cube that said mechanism was relatively subtle. Aspect dependent spectral properties can bewere extracted from the images or directly from the time-domain records. When the cube's top ridge is slanted vertically, an aspect angle dependent splitting in the low frequency spectrum can bewas observed and associated with edge diffraction. The experimental results were compared to Kirchhoff-Integration based simulations, which did notthat don't include elastic responses. [Work supported by ONR.]

\section{2:10}

4pSPa3. Sonar image reconstruction of objects near a reflective boundary. Daniel Plotnick (Appl. Phys. Lab., Univ. of Washington, 1510 NW Turner Dr., Apt. 4, Pullman, WA 99163, dsplotnick@gmail.com), Philip L. Marston (Washington State Univ., Pullman, WA), and Timothy M. Marston (Appl. Phys. Lab., Univ. of Washington, Seattle, WA)

The properties of reconstructed acoustic images, created from data obtained using backscattering synthetic aperture sonar (SAS) systems, have been studied for objects in the free field. The presence of a nearby reflecting boundary, such as the seafloor or surface, greatly increases the number of mechanisms or raypaths by which sound may be backscattered to the sonar system. Some of these raypaths involve one or more interactions with the reflecting boundary (multipaths), and others involve sound scattering multiple times from the same target (multiple scattering). Experiments were conducted using an air-water interface and simple cylindrical targets in order to examine the properties of the reconstructed circular SAS (CSAS) images, including effects due to vertical obliquity of the target [D. Plotnick et al., J. Acoust. Soc. Am. 137, 470-480 (2015)]. The CSAS image is by definition a two-dimensional representation of threedimensional scattering; the image is thus highly dependent on the object's 3-D shape, position, and orientation; many of these image properties may be understood. An additional experiment was conducted using a linear SAS system for two closely spaced cylinders in the free field, allowing the effects of multiple scattering to be examined. [Work supported by ONR.]

\section{2:30}

4pSPa4. Quality assessment of acoustic color signatures. Daniel A. Cook (Georgia Tech Res. Inst., 7220 Richardson Rd., Smyrna, GA 30080, dan.cook@gtri.gatech.edu), J. D. Park (The Penn State Univ. Appl. Res. Lab., State College, PA), and Alan J. Hunter (Univ. of Bath, Bath, Somerset, United Kingdom)

Recent years have witnessed increasing research by the minehunting community into the use of wideband, widebeam, low frequency sonar, typically below $50 \mathrm{kHz}$, whose purpose is to excite and observe an object's structural response. These signatures exhibit uniqueness allowing mines to be more clearly distinguished from clutter objects. Considerable effort has been directed toward studying the scattering physics, but less work has been done to optimize the signal processing used for extracting signatures from data collected at sea under realistic conditions. Specifically, the tradeoff between signals, sources of noise, and signal processing parameters is not well understood. For example, a long observation interval can capture structural acoustic effects occurring later in time than the geometric scattering from an object, at the expense of increasing the interference from sea floor reverberation. Relationships such as this are described, and metrics are suggested for choosing the most appropriate data collection and signal processing strategies. 


\title{
Session $4 \mathrm{pSPb}$
}

\section{Signal Processing in Acoustics: High Resolution Imaging Sonars Including Real Aperture, Synthetic Aperture, and Tomographic Sonars III}

\author{
Brian G. Ferguson, Cochair \\ DSTO, PO Box 44, Pyrmont, NSW 2009, Australia \\ Timothy Marston, Cochair \\ APL-UW, 1013 NE 40th Street, Seattle, WA 98105
}

\section{Invited Papers}

$3: 35$

4pSPb1. Three-dimensional image reconstruction of objects using synthetic aperture sonar. Daniel Plotnick (Appl. Phys. Lab., Univ. of Washington, 1510 NW Turner Dr., Apt. 4, Pullman, WA 99163, dsplotnick@gmail.com) and Timothy M. Marston (Appl. Phys. Lab., Univ. of Washington, Seattle, WA)

Synthetic aperture sonar imaging is typically considered for the case of linear or circular scans, where the reconstructed acoustic image is a two-dimensional representation of the true three-dimensional scattering from some object. However, a multi-dimensional aperture may be used to create a 3-D or volume image reconstruction of the acoustic scattering by that object. The 3-D image reconstruction may be performed using a delay-and-sum algorithm, or using comparatively fast wavenumber based methods. Simulated point scatterers will be used to initially demonstrate the algorithms, followed by a demonstration involving a simple object near a reflecting boundary. For objects that are located close to a reflecting boundary there exists a rich 3-D scattering structure. When imaged in two dimensions, object features becomes distorted due to the projection of scattering loci (e.g. the broadside reflection from a cylindrical target) that may be located outside of the image plane [Plotnick et al., J. Acoust. Soc. Am. 139, 2053 (2016)]. Volume images of such targets move these scattering loci to new locations in 3-D space, which may aid in understanding the target's shape and orientation. The point spread function and 3-D sampling requirements will also be considered.

\section{3:55}

4pSPb2. Scan geometries for three dimensional synthetic aperture sonar tomography. Timothy Marston (APL-UW, 1013 NE 40th St., Seattle, WA 98105, marston@apl.washington.edu) and Jermaine L. Kennedy (NSWC-PCD, Panama City Beach, FL)

Synthetic aperture sonar (SAS) exploits vehicle motion and coherent multi-ping signal integration to enhance image resolution. SAS is typically used to generate high-resolution 2D sonar imagery; however the generation of high-resolution, voxel-based 3D images of targets has also been demonstrated by synthesizing multi-dimensional apertures from scans conducted at diverse grazing-angles. In the latter case, the geometry of the multi-dimensional synthetic aperture can have significant ramifications for all stages of the beamforming process, ranging from navigation refinement and beamformer design to very practical experimental issues, such as the time necessary to scan a target. In this paper, the effects that different multi-dimensional scans have on three-dimensional SAS are considered. Results of field tests for different scan geometries are shown and interpreted in light of their various benefits and detriments.

4pSPb3. The Advanced Synthetic Aperture Sonar Imaging eNgine (ASASIN), a time-domain backprojection beamformer using graphics processing units. Isaac Gerg (Signal Processing, Penn State Appl. Res. Lab, 120 Forest Glen Circle, Port Matilda, PA 16870, idg101@arl.psu.edu)

We present a synthetic aperture sonar beamforming engine called ASASIN. ASASIN is a graphics processing unit based time-domain backprojection beamformer for Windows and Linux utilizing the NVIDIA architecture. It supports arbitrary array geometries and has a modular data input system. ASASIN uses one or more graphics processing units to beamform synthetic aperture sonar data faster than real-time. Its interface is suitable for operators and research scientists. In this talk we discuss ASASINs algorithms and computational performance along with the choices confronted (both algorithmic and computational) in its design. Finally, we discuss its computational performance across a variety of NVIDIA graphics processing units including their embedded models. 
4pSPb4. Moving away from the phase center approximation in micronavigation for synthetic aperture sonar. Isaac Gerg (Commun., Information, \& Navigation Office, Penn State Appl. Res. Lab, 120 Forest Glen Circle, Port Matilda, PA 16870, idg101@arl.psu. edu)

In many respects, the difficult problem of synthetic aperture sonar is motion estimation of the platform since there exists no reasonably priced inertial measurement unit which can meet the location accurately requirements needed to generate high resolution imagery. Many beamforming codes estimate their motion using a displaced phase center technique. This technique is popular but makes some approximations that break down in long range systems and in significant motion environments. In this paper, we discuss the motion estimation algorithm used in ASASIN, a time domain back projection beamformer developed at Penn State Applied Research Laboratory. Our motion estimator assumes displaced phase centers but moves away from the phase center approximation. Rather than estimating the vehicle position delta ping-to-ping independently, we estimate the vehicle's velocity and acceleration through all pings in 3 space. We use Google's Ceres solver to solve the resulting non-linear equations and additionally add a regularization term to deal with missing and bad data samples. Finally, we show imagery formed using our algorithm.

\title{
Contributed Paper
}

\section{$4: 55$}

4pSPb5. Noncontact measurement of body position and vital information using airborne ultrasound. Kotaro Hoshiba (Dept. of Systems and Control Eng., Tokyo Inst. of Technol., W8-30 2-12-1 Ookayama, Meguroku, Tokyo 152-8552, Japan, hoshiba@cyb.mei.titech.ac.jp), Kazuhiro Nakadai (Dept. of Systems and Control Eng., Tokyo Inst. of Technol., Honda Res. Inst. Japan Co., Ltd., Meguro-ku, Tokyo, Japan), Shinnosuke Hirata, and Hiroyuki Hachiya (Dept. of Systems and Control Eng., Tokyo Inst. of Technol., Meguro-ku, Tokyo, Japan)

Because of a growing need for unconstrained medical monitoring for unobtrusively observing individuals in a living space, we have been studied about non-contact measurement of vital information such as respiration and heartbeat using airborne ultrasound. In previous study, the measurement system of small displacement using the M-sequence-modulated signal and tracking phase difference of reflected signals from the target has been proposed. The measurement of respiration and heartbeat of the target in a standing position using a pair of the loudspeaker and the microphone has also been performed. However, body position cannot be measured using a pair of the loudspeaker and the microphone because the system can measure the distance to the body only. In this paper, we describe a basic study of the measurement of body position, respiration, and heartbeat. In the system, using microphone array, body position was estimated by synthetic aperture processing. In addition, small body-surface velocity by respiration and heartbeat were measured by tracking phase difference of reflected signals.

\section{Session 4pUWa}

\section{Underwater Acoustics, Signal Processing in Acoustics, and Acoustical Oceanography: Harbor Acoustics II}

\author{
Peter J. Stein, Cochair \\ Scientific Solutions, Inc., 99 Perimeter Road, Nashua, NH 03063 \\ David L. Bradley, Cochair \\ Penn State University, PO Box 30, State College, PA 16870
}

Chair's Introduction-1:00

\section{Invited Paper}

1:05

\begin{abstract}
4pUWa1. High-frequency seafloor scattering in a dynamic harbor environment: Observations of change over time scales of seconds to seasons. Thomas C. Weber and Larry Ward (Univ. of New Hampshire, 24 Colovos Rd., Durham, NH 03824, tom.weber@unh. edu)

Predicting sonar performance in a harbor environment can be made challenging by the dynamics of the upper and lower surface boundaries. In this talk we examine measurements of the seabed in Portsmouth Harbor, NH. Tidally influenced currents in the Piscataqua River make Portsmouth Harbor one of the most challenging commercial ports to navigate in the northeastern United States. The level of
\end{abstract}


interaction between these currents and the harbor floor is a function of the substrate type. Stereo-camera observations in a sand-wave field near the harbor entrance show fluctuations in microscale roughness and optical reflectance at time scales of seconds during periods of high current. Despite the observed microscale dynamics in the sand-wave field, acoustic observations of both high-frequency seabed scattering strength and mesoscale topography in the same area appear stationary over time scales up to seasons. The low-level of observed fluctuations in scattering strength from the sand-wave field are commensurate with the gravel (and presumably less mobile) river thalweg. Nearby bedrock and sand seafloors show similarly low fluctuations over large time scales, despite seasonal variations in benthic fauna.

\section{Contributed Paper}

4pUWa2. Laboratory measurements of a phase shift in the reflection from a water-mud interface due to water column variability. Gabriel $R$. Venegas and Preston S. Wilson (Dept. of Mech. Eng. and Appl. Res. Labs., The Univ. of Texas at Austin, 204 E Dean Keeton St., Austin, TX 787121591,gvenegas@utexas.edu)

Harbor basins and certain continental shelf environments experience significant variability due to tides, surface heating, currents, and other oceanographic processes. Dynamic processes such as these can cause substantial sound speed and density fluctuations in the water column over short time periods, which are often unknown to sonar users. Such variations are not critical over high-velocity bottoms such as sand, but can produce significant changes in how sound interacts with low-velocity fine-grained sediments. High levels of variability and a low-velocity bottom, such as mud, can therefore present challenges in applications including mine detection, port protection and shallow water sonar. At certain penetration angles, temporal variations in the water-to-sediment sound speed ratio can cause 180 degree phase shifts in the reflected field, which in turn produces waveguide propagation and target scattering field variability. To begin to understand these processes, laboratory measurements of plane wave reflection were obtained from a water-mud interface while varying salinity and temperature of the water. Results indicate that the dynamic nature of sound speed ratio at the ocean bottom can cause significant effects in shallow water environments with muddy bottoms. [Work supported by ONR.]

\section{Invited Papers}

\section{$1: 40$}

4pUWa3. Two-stage active sonar network track-before-detect processing in a high clutter harbor environment. Geoffrey S. Edelson (Maritime Systems \& Technol., BAE Systems, MER15-2350, P.O. Box 868, Nashua, NH 03061-0868, geoffrey.s.edelson@baesystems.com)

Reliable active acoustic detection and tracking of small targets while minimizing the number of false alerts is challenging in a shallow, multipath-inducing, high-clutter harbor environment. These targets often exhibit low target strength and the acoustic clutter fields can be dense and highly dynamic. One approach to detect and track targets in this environment involves a two-stage tracker due to the processing gain required to continually track a weak target in such a significant clutter field. The first stage uses more than a single frame of observations to not only initiate tracks but also to contribute to track maintenance. The second tracking stage is initiated by the tentative target tracks extracted from the first stage. Track segment association algorithms are employed to combat periods or locations track intermittency that inhibit detection and cause tracks to fail and restart. This paper describes the results of this two-stage approach to track-before-detect processing applied to a networked harbor surveillance active acoustic detection and tracking system that consists of a large number of relatively simple nodes, employs a windowed Hough-Transform tracker at the beam level, and a Kalman-based, multi-object tracker at the single- and multi-node levels.

\section{2:00}

4pUWa4. Underwater threat detection and tracking using multiple sensors and advanced processing. Timothy W. Acker (BioSonics, Inc., 4027 Leary Way NW, Seattle, WA 98107, tacker@biosonicsinc.com) and Andy Meecham (Northern Defense Industries, Alexandria, VA)

The vulnerability of military installations and critical infrastructure sites from underwater threats is now well accepted and, in order to combat these security weaknesses, there has been growing interest in — and adoption of — sonar technology. The well known challenges of the underwater environment, particularly in a harbor or port setting, can lead to a high Nuisance Alarm Rate (NAR). This, in turn, can lead to a lack of confidence from end users and a possibility that "real" alerts are incorrectly dismissed. In the past, this has been addressed by increasing the capability of individual sensors, leading to ever-increasing sensor complexity, however, the relationship between sensor performance and complexity/cost is highly non-linear. Even with the most complex and capable sensors, the fundamental limit to performance is often limited by acoustics, not sensor capability. In this paper, we describe an alternative approach to reducing NAR and improving detection of difficult targets (e.g., UUV's), through intelligent combination and fusion of outputs from multiple sensors and data/signal processing algorithms. We describe the statistical basis for this approach, as well as techniques, methodologies and architectures for implementation. We describe the approach taken in our prototype algorithms/system, as well as quantitative and qualitative results from testing in a real-world environment. These results show a significant reduction in NAR and increase in classification/alert range. 
4pUWa5. Use of integrated instrumentation to detect and classify targets in shallow water. Emma D. Cotter, Paul Murphy (Mech. Eng., Univ. of Washington, Mech. Eng., UW Mailbox 352600, Seattle, WA 98195-2600, ecotter@uw.edu), James Joslin (Appl. Phys. Lab., Univ. of Washington, Seattle, WA), Steven Brunton (Mech. Eng., Univ. of Washington, Seattle, WA), Andrew Stewart (Appl. Phys. Lab., Univ. of Washington, Seattle, WA), and Brian L. Polagye (Mech. Eng., Univ. of Washington, Seattle, WA)

The detection and classification of targets in shallow water environments (e.g., depth $<20 \mathrm{~m}$ ) is of increasing interest, both in security applications and more general environmental studies. Here, we report the ability of a cabled integrated instrumentation system to detect and classify targets in a shallow (15 $\mathrm{m}$ deep), narrow (150 m wide) channel influenced by moderate tidal currents (peak currents $<2 \mathrm{~m} / \mathrm{s}$ ). The instrumentation system incorporates a passive acoustic array, multibeam sonar, acoustic camera, stereo-optical camera, and acoustic Doppler current profiler. The system effectiveness is benchmarked using autonomous "cooperative" targets (e.g., drifters with known position and acoustic characteristics), divers, and opportunistic observations of fish and marine mammals. By fusing data streams from multiple sensors, detection and classification rates are improved, particularly for smaller targets. Approaches to data management (data rates exceed $80 \mathrm{MB} / \mathrm{s}$ ), real-time classification, and de-noising of acoustic imagery are also discussed.

\section{2:40-2:55 Break}

2:55

4pUWa6. Harbor passive acoustic monitoring systems. Philip Abbot, Vince Premus, Charles Gedney, and Mark Helfrick (OASIS, Inc, 5 Militia Dr., Lexington, MA 02421, helfrick@oasislex.com)

The feasibility of detecting quiet moving targets (e.g., unmanned undersea vehicles) in harsh harbor environments by using passive acoustic bottom-mounted hydrophone arrays, combined with advanced signal processing detection systems tuned to the target's radiated noise signature characteristics is presented. The harsh shallow water environment is in Narragansett Bay, Rhode Island where UUV operations have been conducted. The sonar equation study considers the use of high resolution, multi-channel hydrophone arrays, measured source levels of representative UUVs (they are quiet), measured noise levels in the harbor (can exhibit high levels), and corresponding array gain. The resulting detection ranges for quiet targets are presented along with estimated false alarm rates. It is shown that passive sonar systems can be a viable component or modality for an underwater harbor defense monitoring system.

\section{3:15}

4pUWa7. Stevens acoustic research in the Hudson River. Alexander Sutin and Hady Salloum (Maritime Security Ctr., Stevens Inst. of Technol., 711 Hudson St., Hoboken, NJ 07030, asutin@stevens.edu)

Stevens Institute of Technology conducted numerous acoustic tests in the Hudson River. The main instrument was Stevens Passive Acoustic Detection System (SPADES) that consists of a simple array with 5 hydrophones and that was initially developed for diver detection. Later, we collected a large library of acoustic signatures of various vessels and developed numerous algorithms for detection, tracking and classification. We present a brief review investigating acoustic applications in the Hudson River, including: Acoustic tomography tests were conducted for measuring the river currents. The difference of time of flight to emitter and receiver placed near the opposite bank of the River was used for calculating the current. Numerous tests were conducted for estimating the Impulse Response and its variation that can be used for prediction of reliability and operating distances for underwater acoustic communication. Noise measurements from passing boats were used for estimating the acoustic attenuation in a wide frequency band. Several other possible future researches may include: Time Reversal Acoustic Focusing for diver deterrence. Active/Passive acoustic fence for diver detection. Bubble curtain for the suppression of underwater noise from a pile drive.

\section{3:35}

4pUWa8. Passive and active sonar signal processing methods for port infrastructure protection and harbor security. Brian G. Ferguson and Kam W. Lo (Maritime Div., DSTG, PO Box 44, Pyrmont, NSW 2009, Australia, Brian.Ferguson@dsto.defence.gov.au)

High frequency passive and active sonar signal processing methods can be used in cluttered shallow water complex acoustic harbor environments for the detection, classification, localization and tracking of asymmetric threats including fast inshore attack craft, divers, underwater vehicles, and sea mines. For fast inshore attack craft, the event duration is short (tens of seconds) which necessitates the automation of the functions of a high frequency high resolution active sonar. The sonar also cues effectors that enable a layered defense capability to counter the threat. For wide area passive undersea surveillance, single hydrophones can be used for multipath passive ranging, a pair of hydrophones for direction finding, and an array of hydrophones for estimation of a complete set of target motion parameters. Also, it will be shown that the measurement of the time delays of the direct path and multipath arrivals at a single pair of hydrophones enables estimation of a complete set of target motion parameters for a surface vessel. 
4pUWa9. Deep learning approach to passive monitoring of the underwater acoustic environment. Eric L. Ferguson, Rishi Ramakrishnan, Stefan B. Williams (Australian Ctr. for Field Robotics, Univ. of Sydney, Rose St. Bldg., Sydney, NSW 2006, Australia, e.ferguson@acfr.usyd.edu.au), and Craig T. Jin (Computing and Audio Res. Lab., Univ. of Sydney, Sydney, NSW, Australia)

A cost effective approach to remote monitoring of protected areas such as marine reserves, harbors and restricted naval waters, is to use passive sonar to detect, classify, localize, and track marine vessel activity (including small boats and autonomous underwater vehicles). This paper uses data from a single hydrophone mounted above the sea floor to compare a conventional cepstral analysis method with a deep learning approach for monitoring marine vessel activity. Cepstral analysis of the underwater acoustic data enables the time delay between the direct path arrival and the first multipath to be measured, which in turn enables estimation of the instantaneous range of the source (a small boat). However, this conventional method is limited to ranges where the Lloyd's mirror effect (interference pattern formed between the direct and first multipath arrivals) is discernible. It is shown that a Convolutional Neural Network (CNN) operating on cepstrum data with a regression output improves the single sensor ranging performance by considering additional multipath arrivals. To demonstrate the effectiveness of the approach, a surface vessel is ranged using the $\mathrm{CNN}$ and the results compared with the conventional method.
4pUWa10. Multi-agent acoustic noise field characterization using optimal control. Caitlin Bogdan (Boston Univ., 110 Cummington Mall, Boston, MA 02215, cbogdan@bu.edu), Andrew Wixom (Structural Acoust., Penn State Univ., Appl. Res. Lab., State College, PA), and J. G. McDaniel (Boston Univ., Boston, MA)

Investigations of acoustic-driven control schemes have stayed away from traditional Optimal Control methods because of the computational load and lack of direct solution methods. The method presented here overcomes these challenges and learns a spatially varying noise field by applying the Pontryagin Maximum Principle (PMP) to solve for optimal trajectories over a changing estimate of the noise field. The resulting control drives the agents to the regions of the estimated field with the most uncertainty. This optimization is conducted with multiple agents interested in the noise field for a single frequency as well as for multiple agents simultaneously optimizing over multiple frequencies of interest. The challenges of finding a solution to the mixed boundary value problems is achieved through an eigenvalue decomposition method. The selected cost function is designed to minimize time and thus bound error introduced by the open loop control derived through the PMP methodology. Simulations and preliminary experimental results are presented to demonstrate the effectiveness of this method. The resulting information from utilizing this method are useful in optimized sensor location problems, which are especially relevant for the large dynamic array system used to do the initial sensing. [Research supported by NAVSEA and Raytheon Company.]

\title{
Session $4 p U W b$
}

\author{
Underwater Acoustics: Ocean Ambient Noise: Sources, Variation, and Characterization I \\ D. Benjamin Reeder, Chair \\ Oceanography, Naval Postgraduate School, 833 Dyer Rd., Bldg. 232, SP-311B, Monterey, CA 93943
}

\section{Contributed Papers}

\section{$4: 40$}

4pUWb1. Spatial variation of ocean ambient noise spectrum in offshore China. Guoli Song (Key Lab. of Underwater Acoust. Environment, Inst. of Acoust.,Chinese Acad. of Sci., North Fourth Ring Rd., Haidian District, No. 21, Beijing 100190, China, songguoli13@mails.ucas.ac.cn), Xinyi Guo, Li Ma, and Qianchu Zhang (Key Lab. of Underwater Acoust. Environment, Inst. of Acoust.,Chinese Acad. of Sci., Beijing, China)

Ocean ambient noise always presents spatial difference because of the diversity of the noise source and the propagation condition. This paper analyzes the noise spectrum level (NL) of two stations measured in offshore China, and finds that the difference of the two NL is more than $10 \mathrm{~dB}$ with different variation for per octave. Through analyze the NL and the transmission loss level (TL) from the noise source to the measuring station, a correlation is found between the noise level difference (NLD) and transmission loss difference (TLD), with a correlation coefficient of 0.78 at the band of $50 \mathrm{~Hz} \sim 500 \mathrm{~Hz}$. This is a strong proof to connect the spatial variation of NL with the varied propagation channel, and also suggests that at this band the ambient noise is closely related to the ship noise sources. In addition, the difference of the ship noise source level coincides with the number of vessels before and after the fishing moratorium period in China, which further explains the difference between the NL of two experimental stations are highly related to ships.

\section{4:55}

4 pUWb2. Spatial dependence of very low frequency wind-generated noise in the sound channel. Stephen M. Nichols and David L. Bradley (Appl. Res. Lab., The Penn State Univ., 201 Appl. Sci. Bldg., State College, PA16802, smn5198@psu.edu)

Storm-driven non-linear interaction of ocean waves excites Very Low Frequency (VLF) $(<5 \mathrm{~Hz})$ sounds locally. As the surface wind speed increases, the slope of the VLF acoustic spectrum approaches approximately $20 \mathrm{~dB} /$ octave, corresponding to a similar behavior observed in the surface wave spectrum. The amplitude of the measured acoustic spectrum in this frequency region is strongly correlated with local surface wind speeds [J. 
Acoust. Soc. Am. 139(3), 1110 (2016)]. Due to the sound propagation conditions in the open ocean, it is expected that sound from distant storms will also provide a significant contribution to the measured sound field. The impact of distant meteorological activity on the local acoustic spectrum is examined by comparing the ambient noise recorded by the Comprehensive Nuclear-Test Ban Treaty Organization's (CTBTO) hydro-acoustic monitoring system to global records of surface wind speed, remotely sensed via satellite.

\section{5:10}

4pUWb3. Characterization of very low frequency ambient noise by principal component analysis. Stephen M. Nichols and David L. Bradley (Appl. Res. Lab., The Penn State Univ., 201 Appl. Sci. Bldg., State College, PA 16802,smn5198@psu.edu)

Long-term acoustic monitoring datasets compose a dynamic mixture of many source types, creating a unique noise field in each measurement location and for each recording time. Principal Component Analysis (PCA), and the correlation matrix from which the Principal Components are derived, offer an effective means of estimating the fundamental acoustic contributors, and their portion of the noise field. An improved characterization of ambient noise data sets is discussed, by representing the sound field in a smaller parameter space of principal components, each component reflecting the spectral characteristics of a specific sound source (i.e. fin whale vocalizations). This procedure leads to a definitive parsing of the contributing source types in the measured spectra. The datasets used are recordings from the Comprehensive Nuclear-Test Ban Treaty Organization's (CTBTO) hydro-acoustic monitoring system.
$5: 25$

4pUWb4. Comparison of scattering mechanisms for excitation of axial modes by surface sources. Mehdi Farrokhrooz and Kathleen E. Wage (George Mason Univ., 4450 Rivanna River Way PMB3740, Fairfax, VA 22030,mfarrokh@masonlive.gmu.edu)

The vertical spectrum of low-frequency ambient noise in deep water contains significant contributions from distant shipping. Distant noise is concentrated at angles around broadside that are associated with the low order modes. Near-surface sources cannot excite the low modes directly. Dashen and Munk suggest three mechanisms for transferring energy from high modes to low modes: internal wave (IW) scattering, downslope conversion, and excitation at high latitudes where the sound channel intersects the surface. Dashen and Munk conclude that slope conversion is the most likely due to the weakness of IW scattering and the lack of shipping traffic at high latitudes. Commenting on their IW analysis, Dashen and Munk note that they are not totally convinced by their theoretical results and admit that IW scattering may have a significant effect. This talk compares the IW scattering and downslope conversion mechanisms for transferring surface source energy to the low modes. IW scattering is modeled using transport theory [Colosi and Morozov, JASA 2009] and compared to parabolic equation and coupled mode simulations. Results show that IW scattering from a large number of ships can produce vertical spectra comparable to those obtained through downslope conversion. [Work supported by ONR.] 


\author{
Plenary Session and Awards Ceremony \\ Michael R. Stinson, Chair \\ President, Acoustical Society of America \\ Kentaro Nakamura \\ President, Acoustical Society of Japan
}

Presentation of Certificates to New Fellows

G. Clifford Carter - For contributions to time delay estimation in the presence of source or receiver motion

Francesco L. di Scalea - For contributions to the theory and applications of ultrasonic guided waves

Truls T. Gjestland - For contributions to research and standards development on transportation noise effects on communities

Nathan J. McDannold - For contributions to therapeutic ultrasound

Robert J. McGough - For contributions to numerical modeling in medical ultrasound

Jennifer L. Miksis-Olds - For contributions to underwater acoustic noise research and the integration of acoustics into marine ecology

Joseph A. Sisneros - For contributions to the understanding of fish hearing

James A. TenCate - For contributions to nonlinear acoustics of earth materials

Blake S. Wilson - For the development and enhancement of cochlear implants

\title{
Introduction of Award Recipients and Presentation of Awards
}

Medwin Prize in Acoustical Oceanography to Thomas C. Weber

Rossing Prize in Acoustics Education to Brad H. Story

Trent-Crede Medal to Earl G. Williams

Recognition of ASJ Organizers

Recognition of ASA Organizers 


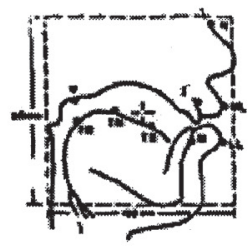

\section{NOW AVAILABLE ON DVD}

\section{MEASURING SPEECH PRODUCTION: VIDEO DEMONSTRATIONS OF SPEECH INSTRUMENTATION}

This series of demonstrations, for use in teaching courses on speech acoustics, physiology and instrumentation are now available on DVD from the Acoustical Society of America. The DVD contains thirteen video demonstrations of equipment and techniques used in speech research. The demonstrations are categorized into three areas: (1) Respiration, phonation and aerodynamics; (2) Indirect articulatory measurements; (3) Direct articulatory measurements. A pdf file on the DVD describes the demonstrations and lists additional readings that are updated from the original videotape.

PART ONE - RESPIRATION, PHONATION AND AERODYNAMICS

1. The whole body plethysmograph in speech research. John J. Ohala

2. Aerodynamic end respiratory kinematic measures during speech. Elaine T. Stathopoulos

3. Physiologically based models of phonation. Ingo R. Titze

4. Use of the electroglottograph in the laboratory and clinic. James J. Mahshie

5. Endoscopy, stroboscopy, and transillumination in speech research. Anders Lofqvist, Kiyoshi Oshima

PART TWO - INDIRECT ARTICULATORY MEASUREMENTS

6. Magnetic resonance imaging (MRI) in speech research. Carol Gracco, Mark Tiede

7. Imaging the tongue with ultrasound. Maureen Stone

8. Estimating articulatory movement from acoustic data. Kenneth N. Stevens

9. Electromyography in speech research, Kiyoshi Oshima. Katherine S. Harris, Fredericka Bell-Berti

PART THREE - DIRECT ARTICULATORY MEASUREMENTS

10. The rise and fall of the soft palate: The Velotrace. Fredericka Bell-Berti, Rena A. Krakow, Dorothy Ross, Satoshi Horiguchi

11. Dynamic electropalatography. William J. Hardcastle, Fiona Gibbon

12. Measuring articulatory movements with an electromagnetic midsagittal articulometer (EMMA) system. Joseph S. Perkell, Mario A. Svirsky, Melanie L. Matthies, Joyce Manzella

13. Optoelectronic measurement of orofacial motions during speech production. Eric Vatikiotis-Bateton, Kevin Munhall, David Ostry

Each demonstration displays the instrument and how it is used; what the data look like; how data are analyzed and their applications for speech pathology, linguistics and speech processing. Anyone at any level interested in speech production and speech physiology will find these demonstrations useful. Price: $\$ 52.00$

\section{ORDER FORM}

-Payment by Visa, MasterCard or American Express, or check or money order in US funds on US bank must accompany the order.

-Send orders to: Acoustical Society Publications, P.O. Box 1020, Sewickley PA 15143-9998; Tel: 412-741-1979; Fax: 412-741-0609

-Postage and handling: U.S. orders--\$6 plus \$2 for each additional DVD; Non-US orders: \$10 plus \$4 for each additional DVD.

-Returns are not accepted

Name [ ] ASA member [ ] Nonmember

Address

City State/Prov Zip Country

Tel.: Fax: Email:

Quantity

Measuring Speech Production DVD Shipping and Handling

\begin{tabular}{ll} 
Unit Price & Total \\
$\$$ & $\$$ \\
$\$$ & $\$$ \\
\hline Total Remitted & $\$$
\end{tabular}

[ ] Check or money order enclosed for $\$$ (U.S. funds/drawn on U.S. bank)
[ ] American Express
[ ] VISA
[ ] MasterCard

Account number: Security Code: Exp. Date:

Signature:

Due to security risks and Payment Card Industry (PCI) data security standards e-mail is NOT an acceptable way to transmit credit card information. Please use our secure web page to process your credit card payment (http://www.abdi-ecommerce10.com/asa) or securely fax this form to (412-741-0609). 


\section{ACOUSTICAL SOCIETY OF AMERICA TRENT-CREDE MEDAL}

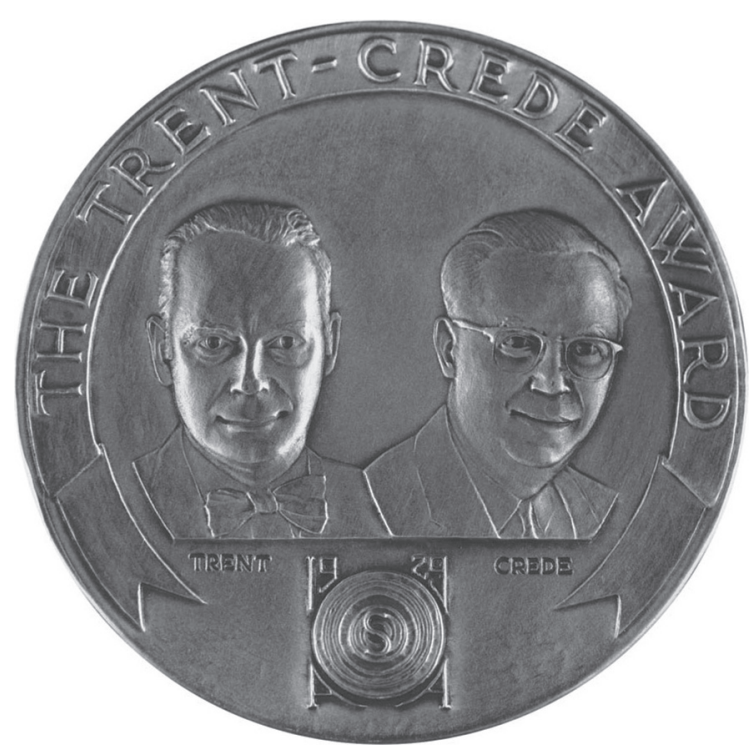

\section{Earl G. Williams}

\section{6}

The Trent-Crede Medal is presented to an individual, irrespective of nationality, age, or society affiliation, who has made an outstanding contribution to the science of mechanical vibration and shock, as evidenced by publication of research results in professional journals or by other accomplishments in the field.

\section{PREVIOUS RECIPIENTS}

Carl Irwin Vigness

Raymond D. Mindlin

Elias Klein

J. P. Den Hartog

Stephen H. Crandall

John C. Snowdon

Eric E. Ungar
1969

1971

1973

1975

1978

1980

1983
Miguel C. Junger

1987

Gideon Maidanik

Preston W. Smith, Jr.

David Feit

Sabih I. Hayek

Jerry H. Ginsberg

Peter R. Stepanishen 


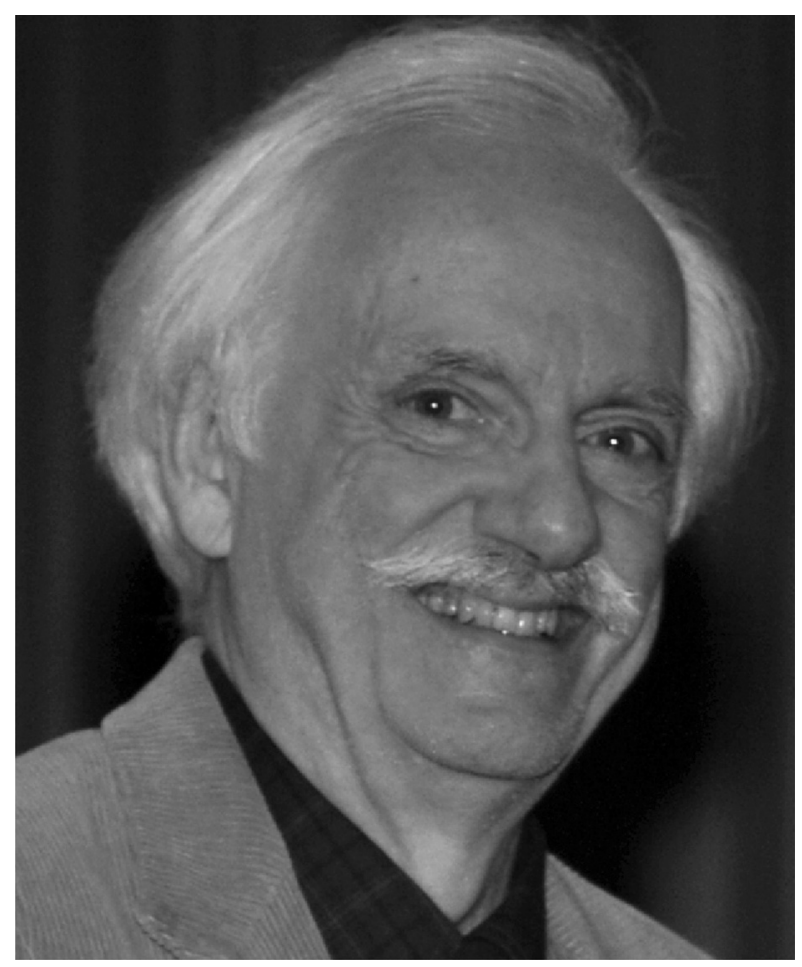




\section{CITATION FOR EARL G. WILLIAMS}

“... for development and application of near-field acoustical holography”

HONOLULU, HAWAII • 1 DECEMBER 2016

Earl Williams' interest in acoustics is not incidental; he is an accomplished cellist, and his curiosity as to how the cello delivers the best sound to an audience led to a true passion for understanding the radiation of sound from a vibration structure. Earl received a Bachelor's degree in Electrical Engineering from the University of Pennsylvania in 1967 and a Master's degree from Harvard University in 1968, studying acoustics under the supervision of Ted Hunt. Earl continued his studies in acoustics with Eugen Skudrzyk at The Pennsylvania State University where he was awarded a Ph.D. in Acoustics in 1979. The title of his thesis was "Vibrations of Plates of Various Geometries."

After receiving his Ph.D., Earl remained at Penn State as a postdoc for J. D. Maynard. This collaboration led to the development of an array of microphones for measuring an acoustic "hologram", and data-processing algorithms, which demonstrated the remarkable power of nearfield acoustic holography (NAH) for measuring the sound radiation with an image resolution previously assumed to be impossible. The results of this research were published in Physical Review Letters (Vol. 45:554-57, 1980) and, with a more detailed description, in the Journal of the Acoustical Society of America (JASA) (Vol. 78:1395-1413, 1978); this latter paper is perhaps the most cited paper in the now extensive field of nearfield sound measurement.

At Penn State there was considerable NAH activity, with measurements on test sources, plain plates, plates with structure, musical instruments including Earl's cello, etc. Earl demonstrated a remarkable and rare ability: when confronted with a particular problem, he could identify the real crux of the problem, design and conduct appropriate experimental measurements and, having targeted the most important aspect, arrive at a comprehensive and valid understanding of the problem. This ability should be evident in the discussion of Earl's research accomplishments below.

With his Postdoctoral Scholar position having a finite duration, Earl sought a position elsewhere and received a number of job offers. Earl decided that a position with the Naval Research Laboratory (NRL) in Washington, DC, would provide the best opportunity for pursuing research; however, he probably stipulated that he would not be making sound radiation measurements on his priceless cello underwater. Earl began research at NRL in 1982, and it was there that he made some of the most important developments in sound radiation measurement.

The remarkable power of NAH arises because measurements in the nearfield of a source permit the detection of sound from modes which very weakly radiate from the source; such modes typically reveal the details of how sound is ultimately propagated from the source. A fundamental problem is that some modes are so weak that even in the nearfield their signals fall into the noise of the measurement system. This problem has two aspects: 1) identifying the weakly radiating modes, and 2) reducing the effects of the noise in the reconstruction process. In 1989, W.A. Veronesi showed that the first problem could be solved with singular value decomposition; this was followed by a flurry of activity trying to solve the second problem. Ideally one would want a standard, one-size-fits-all solution, but unfortunately there was an overabundance of solutions. Earl addressed this issue by undertaking a thorough, exhaustive survey of all the solutions and in 2001 published a paper in JASA (Vol. 110:1976-88), which could be used to identify the appropriate solution; this is perhaps the second most cited paper in the field.

Typically sound radiation studies are performed for structures which radiate outward, and one may assume the boundary condition at infinity of outward propagation only. However, there is also the important problem of sound radiation into the interior of a vibrating structure, such as the cabin of an automobile or aircraft. This is very difficult problem, but 
Earl showed that useful measurements were possible, and published landmark results in JASA (Vol. 108:1451-63) in 2000.

A fundamental problem with measuring sources which radiate outward is that, theoretically, the measurement surface should enclose the entire vibrating structure in order to capture all of the radiated sound. Needless to say, this is a substantial problem for practical field measurements. Earl discovered that this theoretical restriction could be relaxed far more than one might expect and found that the measurement surface could in fact be considerably smaller than the size of the vibrating surface. Earl's crucial insight was that when measuring close to a vibrating (source) surface, the signal received near the center of the measurement surface would be dominated by the sources directly below. For the radiated field beyond the perimeter of the measurement surface one could make an educated approximation, and the reconstruction of the source surface just below the center of the measurement surface would probably be quite accurate. One could then use this reconstructed source to propagate out to get a new estimate of the field beyond the perimeter of the measurement surface, and this process could be repeated iteratively. This method, referred to as "patch" holography, is one of the most important practical extensions of nearfield measurement.

In 1999, Earl published a book, Fourier Acoustics: Sound Radiation and Nearfield Acoustic Holography, which made the theory and practice of NAH accessible throughout the acoustics community. Earl has made many more innovations other than the few discussed above, and his achievements have been recognized throughout the world as well as within NRL. No one has done more to make NAH a "household acronym" in the sound radiation and noise communities.

Earl has been recognized for his achievements by many organizations. He is a Fellow of the Acoustical Society of America (ASA) and an Associate Editor of JASA in Structural Acoustics. He has presented over 70 invited lectures at international meetings, many of them keynote lectures, and has presented 26 invited lectures and organized five special sessions at meetings of the ASA. His NAH research was formally recognized by NRL as one of the Navy's most innovative technologies over a period of 75 years. In 2002, Earl was awarded the distinguished rank of Senior Technologist at NRL. In 2003 he was invited to present the Rayleigh Lecture at a meeting of the American Society of Mechanical Engineers (ASME), and in 2009 was awarded the Per Bruel Gold Medal for Noise Control and Acoustics by the ASME.

It is most appropriate that Earl Williams be honored with the Trent-Crede Medal of the Acoustical Society of America.

\section{J. D. MAYNARD \\ DAVID FEIT}




\title{
Session $5 \mathbf{a A B}$
}

\section{Animal Bioacoustics: Marine Acoustic Ecology}

\author{
Jennifer L. Miksis-Olds, Chair \\ Center for Coastal and Ocean Mapping, Univ. of New Hampshire, 24 Colovos Rd., Durham, NC 03824
}

\section{Contributed Papers}

8:00

5aAB1. A multi-year time series of marine mammal distribution in the Alaskan Arctic. Jessica Crance and Catherine Berchok (AFSC/NMFS/ NOAA, National Marine Mammal Lab., 7600 Sand Point Way NE, Seattle, WA 98115, Jessica.Crance@noaa.gov)

The Marine Mammal Laboratory has deployed long-term, moored, passive acoustic recorders at numerous locations in the Alaskan Arctic since the BOEM-funded Chukchi Acoustics, Oceanography, and Zooplankton (CHAOZ) study began in 2010. These instruments, often collocated with oceanographic instruments, collect year-round passive acoustic data (16 $\mathrm{kHz}$ sampling rate, $\sim 30 \%$ duty cycle). All acoustic recordings (100\%) were analyzed using an in-house Matlab-based manual analysis program for 12 different Arctic and sub-Arctic cetacean and pinniped species, as well as for vessel, seismic airgun, and ice noise. Bowhead and beluga distributions showed similar patterns, with bimodal temporal distributions representing the fall and spring migrations. Infrequent gray whale detections (isolated on nearshore recorders) followed a similar, albeit less defined, bimodal distribution. Walrus and bearded seal calls were detected almost year-round, with peak bearded seal calling from April through June. Detections of sub-Arctic species (e.g., humpback, fin, killer, and minke whales) were rare north of $70^{\circ} \mathrm{N}$. Correlation of marine mammal distribution with concurrent oceanographic parameters revealed strong associations with oceanographic and prey variables, more specifically, ice concentration and thickness, chlorophyll, wind speed, and currents. This study illustrates the importance of collecting concurrent long-term passive acoustic and oceanographic data in a rapidly changing environment.

\section{$8: 15$}

5aAB2. A preliminary acoustic survey of echolocating marine mammals in the Bering Sea. Kerri Seger (CCOM, Univ. of New Hampshire, 9331 Discovery Way, Apt. C, La Jolla, CA 92037, kseger@ucsd.edu), Jennifer Miksis-Olds (CCOM, Univ. of New Hampshire, Durham, NH), and Bruce Martin (JASCO Res., Ltd., Victoria, BC, Canada)

As the Arctic seas rapidly change with increased ocean temperatures and decreased sea ice extent, traditional Arctic marine mammal distributions will be altered, and non-traditionally Arctic species may shift poleward. Arctic species typically include sperm, bowhead, humpback, right, gray, fin, and blue whales; odontocetes, specifically killer and beluga whales; and several pinnipeds species. Their acoustic presence has been documented because they produce relatively low-frequency sounds that are detectable by many common, remotely-deployed recording platforms. Until recently, however, recording constraints of power and storage limited higher sampling rates and prevented the detection of many high-frequency-producing species in the Arctic seas. Such species likely include Baird's, Cuvier's, and Stejneger's beaked whales, as well as Northern right whale and Pacific white-sided dolphins. Using one of the first long-term data sets to record relatively high frequencies in the Bering Sea, signal types similar to those of Baird's and Cuvier's beaked whales, and Pacific white-sided and Northern right whale dolphins have been detected. Their relative acoustic presence from the last several years will be presented, as well as the acoustic presence of other species, like Risso's dolphin, that may be shifting northward into the Arctic seas. [Work supported by ONR.]
5aAB3. Did humpback whales go missing off Maui, Hawaii? A comparison of song activity between the 2014/15 and 2015/16 breeding seasons. Anke Kügler (Marine Biology Graduate Program, Univ. of Hawaii at Manoa, 2525 Correa Rd. HIG 132, Honolulu, HI 96822, akuegler@hawaii. edu), Marc O. Lammers (Hawaii Inst. of Marine Biology, Univ. of Hawaii at Manoa, Kaneohe, HI), Eden J. Zang (Oceanwide Sci. Inst., Honolulu, HI), Maxwell B. Kaplan, and T A. Mooney (Woods Hole Oceanographic Inst., Woods Hole, MA)

Each winter, thousands of humpback whales (Megaptera novaeangliae) migrate from their high latitude feeding grounds in Alaska to mate and calve in the shallow tropical waters around the Main Hawaiian Islands. Population estimates suggest that up to 10,000 animals winter in Hawaiian waters, making up more than half of the total North Pacific stock. However, in the 2015/16 season, anecdotal reports from commercial operators and researchers tell of an unusually low number of whales compared to previous years off the island of Maui. To examine this issue, data from long-term passive acoustic monitoring with autonomous Ecological Acoustic Recorders (EARs) during the 2014/15 and 2015/16 seasons off the west coast of Maui were analyzed using male chorusing levels as a proxy for relative whale abundance. Rootmean-square sound pressure levels (SPLs) were calculated to compare low frequency acoustic energy $(0-1.56 \mathrm{kHz})$ between both seasons. The data showed that chorusing levels dropped in early January 2016. Although levels subsequently increased again until they peaked in February as expected, SPLs remained lower than in 2014/15 throughout the remaining season. These results suggest that the number of singing males was indeed lower during the 2015/16 season off west Maui and that future monitoring is warranted.

8:45

5aAB4. How reliable is song as a cue for acoustic surveys of humpback whales (Megaptera novaeangliae) with changing population size and density? Michael J. Noad, Rebecca A. Dunlop, and Amelia Mack (School of Veterinary Sci., The Univ. of Queensland, Gatton, QLD 4343, Australia, mnoad@uq.edu.au)

Acoustic surveys of marine mammals have several advantages over traditional visual surveys. For an acoustic survey to be accurate, however, there needs to be a predictable relationship between vocal behavior and density. Humpback whale's song, produced by males primarily during the breeding season, is potentially a good candidate for such surveys. The function of song, however, remains enigmatic and so its usefulness as a predictor of population density is still not known, particularly when population density changes. We examined aspects of singing behavior during migration off eastern Australia between 1997 and 2015 including the proportion of whales that sang, the spacing between singers, and the use of song by males when joining other whales. Over this period, there was a six-fold increase in the population but this had little effect on the proportion of whales singing, and singers spaced randomly, implying that it could be useful as a survey tool. However, the proportion of singers that continued to sing while joining other whales decreased, showing some density effects on singing behavior. Altogether, while singing behavior can be used as a reasonable proxy of population size at the densities observed, behavioral strategies may start to confound this relationship at higher densities. 
a source at one or more underwater hydrophones. This estimation task can

5aAB5. The correlation between the local environment and gray whale behavior as tracked using a sparse hydrophone array in Monterey Bay National Marine Sanctuary. Regina A. Guazzo, John A. Hildebrand, Sean M. Wiggins, Gerald L. D’Spain (Scripps Inst. of Oceanogr., Univ. of California, San Diego, UCSD/SIO/0205, 9500 Gilman Dr., La Jolla, CA $92093-$ 0205, rguazzo@ucsd.edu), and David W. Weller (Southwest Fisheries Sci. Ctr., NOAA, La Jolla, CA)

Gray whales (Eschrichtius robustus) make one of the longest annual migrations of any marine mammal. While the seasonal periodicity of the migration and related travel corridors have been well-studied, little is known about how these whales use acoustics while migrating. Acoustic array processing is a powerful tool to localize and track vocalizing whales without disrupting their behavior. Concurrent with the NOAA Southwest Fisheries Science Center visual and infrared gray whale census, four hydrophone-recorder packages with sites separated by approximately $2 \mathrm{~km}$ in 58 to $110 \mathrm{~m}$ water in Monterey Bay National Marine Sanctuary offshore of Granite Canyon, California, recorded from November 2014 to June 2015. An automatic call detector identified gray whale M3 calls in the dataset. Spectrogram cross-correlation was used to measure the time-difference-of-arrival of these calls and estimate the location of the calling animal. These localizations provide gray whale swimming and calling behavior and indicate changes on diel and seasonal time scales. Results from 15 high-quality tracks show calling gray whales swimming at a mean speed of $1.97 \mathrm{~m} / \mathrm{s}$ with a range of 1.18 to $2.54 \mathrm{~m} / \mathrm{s}$. Acoustic detections and tracks are compared with environmental parameters to investigate how gray whale behavior relates to their physical environment. These results are necessary for understanding the cues gray whales use to navigate from their feeding grounds offshore of Alaska to their breeding grounds in Baja California.

\section{9:15}

5aAB6. Vocalization source level distributions and pulse compression gains of diverse baleen whale species in the Gulf of Maine. Delin Wang, Wei Huang, Heriberto Garcia (Elec. and Comput. Eng., Northeastern Univ., 006 Hayden Hall, 370 Huntington Ave., Boston, MA 02115, wang.del@ husky.neu.edu), Nicholas C. Makris (Mech. Eng., Massachusetts Inst. of Technol., Cambridge, MA), and Purnima Ratilal (Elec. and Comput. Eng., Northeastern Univ., Boston, MA)

The vocalization source level distributions and pulse compression gains are estimated for four distinct baleen whale species in the Gulf of Maine: blue, fin, minke, and sei. The vocalizations were received on a large-aperture densely sampled coherent hydrophone array system deployed to monitor marine mammals over instantaneous wide areas using the passive ocean acoustic waveguide remote sensing technique. For each baleen whale species, between 400 to over 1400 measured vocalizations with significantly high signal-to-noise ratios ( $\mathrm{SNR}>10 \mathrm{~dB}$ ) after coherent beamforming and localized with high accuracies ( $<10 \%$ localization errors) over ranges spanning roughly $1 \mathrm{~km}$ to $38 \mathrm{~km}$ are included in the analysis. The whale vocalization received pressure levels are corrected for broadband transmission losses modelled using a calibrated parabolic equation based acoustic propagation model for a random range-dependent ocean waveguide. The broadband vocalization equivalent pulse-compression gains are found to be $2.5 \pm 1.1$ for fin, $24 \pm 10$ for blue, and $69 \pm 23$ for sei. The source level distributions and pulse compression gains are essential for determining signal-tonoise ratios and hence detection regions for baleen whale vocalizations received passively on underwater acoustic sensing systems, as well as for assessing communication ranges and potential for echolocation in baleen whales.

\section{9:30}

5aAB7. Blind channel estimation of time-varying underwater acoustic waveguide impulse responses. Brendan P. Rideout, Eva-Marie Nosal (Dept. of Ocean and Resources Eng., Univ. of Hawaii at Manoa, 2540 Dole St., Holmes Hall 402, Honolulu, HI 96822, bprideou@hawaii.edu), and Anders Host-Madsen (Elec. Eng., Univ. of Hawaii at Manoa, Honolulu, $\mathrm{HI})$

Some techniques for underwater passive acoustic localization make use of estimates for the direct and/or interface-reflected acoustic arrival times of be difficult for non-impulsive sources (e.g., humpback whales) due to early arrivals masking later ones. In linear system analysis, the impulse response (IR) of a system is the system output when the input is an impulse (i.e., a short duration, large bandwidth signal), and expresses how a source signal interacts with the environment to yield the output waveform (e.g., that recorded by a hydrophone). Recordings of a relatively impulsive vocalization (e.g., a walrus knock), given that they approximate the IR between the walrus and hydrophone, may facilitate arrival time estimation. IR estimation for unknown, non-impulsive calls is more challenging, particularly for timevarying channels. Blind channel estimation is the process of estimating the set of IRs between a single (unknown) source and multiple receivers, and can potentially help estimate direct and interface-reflected arrival times for non-impulsive marine mammal vocalizations since the IRs can be analyzed rather than waveforms/spectra. In this paper, we use simulations to explore the importance of ocean surface gravity waves on blind estimation of acoustic IRs.

\section{9:45-10:00 Break}

\section{0:00}

5aAB8. Low-frequency propagation and noise in the intertidal zone. James S. Martin, Peter H. Rogers (School of Mech. Eng., Georgia Inst. of Technol., 771 Ferst Dr., Love Bldg., Rm. 115, Atlanta, GA 30332-0405, james.martin@me.gatech.edu), Ashwin Bhandiwad, Orphal Colleye, and Joseph Sisneros (Dept. of Psych., Univ. of Washington, Seattle, WA)

Measurements of ambient noise and short-range propagation $(<2 \mathrm{~m})$ were made in the intertidal zone with water depths ranging from 50 to 80 $\mathrm{cm}$ using vector sensors. The sites selected for the measurements were known nesting areas for plainfin midshipman (Porichthys notatus). Males of this species produce continuous acoustic advertisement calls with fundamental frequencies in the 70 to $100 \mathrm{~Hz}$ range. The temporal and spectral structure of these calls must convey information regarding the source's range, direction, and/or fitness over the maximum detectable range in a complex environment. Both fundamental and harmonic components appear to be above a rapid low-frequency increase in the noise floor due to local wave motion and below the frequency range of several observed noise transients at times with modest wind speeds $(<5 \mathrm{mph})$ and small wave heights $(<20 \mathrm{~cm})$. There is little evidence of significant propagation differences between specific frequencies within the band of interest.

\section{0:15}

5aAB9. Passive acoustic monitoring of fishes and a crustacean using a towed hydrophone in Tateyama bay. Ryuzo Takahashi (Fisheries Technol. Dept., Japan Fisheries Res. and Education Agency, 7620-7, Hasaki, Kamisu-shi, Ibaraki-ken 314-0408, Japan, takahashiryuzo@affrc.go.jp), Tomonari Akamatsu (Japan Fisheries Res. and Education Agency, Yokohama-shi, kanagawa-ken, Japan), Tomohito Imaizumi (Fisheries Technol. Dept., Japan Fisheries Res. and Education Agency, Kamisu-shi, Ibarakiken, Japan), and Ikuo Matsuo (Tohoku Gakuin Univ., Sendai-shi, Miyagiken, Japan)

Passive acoustic monitoring is useful to observe presence of marine organisms such as marine mammals, fishes and crustaceans. We aimed to estimate the distribution of fishes and crustaceans using a towed hydrophone. A range-wide passive acoustic monitoring was conducted in Tateyama bay in Japan using R/V Takamaru (61t) on July, September and November in 2015 of day and night. The towed hydrophone (Towed Aquafeeler, AquaSound Inc., Japan) was used for the observation. The vessel cruised 5 knot on 5 lines to cover the focal survey area (N $35^{\circ} 020^{\prime}-\mathrm{N}$ $35^{\circ} 02^{\prime}$ and $\mathrm{E} 139^{\circ} 46-\mathrm{E} 139^{\circ} 51$ ). Recorded data with frequency from $100 \mathrm{~Hz}$ to $20 \mathrm{kHz}$ were analyzed. Recorded fish sounds have dominant frequency between $200 \mathrm{~Hz}$ and $1500 \mathrm{~Hz}$ and duration between $0.1 \mathrm{~s}$ and 2.1 s. Crustacean sound shows dominant frequency as $3 \mathrm{kHz}-20 \mathrm{kHz}$ with the duration of $10 \mathrm{~ms}-20 \mathrm{~ms}$. Using these features, number of fish sounds was counted automatically using custom-made MATLAB program. Large number of the sounds were recorded in the nighttime than in the daytime. Most frequently received month was July. Both of the fish and crustacean sounds were observed in shallow water $(<30 \mathrm{~m})$ showing acoustic presence map. 
10:30

$5 \mathrm{aAB10}$. Seasonal acoustic presence of fin and bowhead whales in relation to prey abundance and oceanographic environments in the southern Chukchi Sea. Koki Tsujii, Mayuko Otsuki (Graduate School of Environ. Sci., Hokkaido Univ., 20-5 Benten-cho, Hakodate, Hokkaido 0400051, Japan, kou1114t@gmail.com), Tomonari Akamatsu (National Res. Inst. of Fisheries Sci., Fisheries Res. Agency, Yokohama, Japan), Ikuo Matsuo (Tohoku Gakuin Univ., Sendai, Japan), Kazuo Amakasu (Tokyo Univ. of Marine Sci. and Technol., Konan, Japan), Minoru Kitamura, Takashi Kikuchi (Japan Agency for Marine-Earth Sci. and Technol., Yokosuka, Japan), Kazushi Miyashita, and Yoko Mitani (Field Sci. Ctr. for Northern Biosphere, Hokkaido Univ., Hakodate, Japan)

To assess how seasonal and ice-associated whales differently respond to the Arctic climate changes, understanding the relationships among their presence and environmental conditions is necessary. We examined the seasonal acoustic presence of fin Balaenoptera physalus and bowhead whales Balaena mysticetus in relation to prey abundance and oceanographic conditions in the southern Chukchi Sea from July 2012 to July 2014 using passive and active acoustic methods. Oceanographic conditions obtained were water temperature, salinity and sea ice concentrations. Fin whale calls were detected from summer to fall, only in ice-free and high prey abundance period. Moreover, the call-detected period was longer in 2013 than 2012. In contrast, bowhead whale calls were mainly detected from fall to winter and in spring, during ice-free and ice-covered periods. The call presence of bowhead whales overlapped less frequently with that of fin whales in fall 2013 than 2012. The reason for the differences in their acoustic presence between these two years may be caused by higher water temperature and late sea-ice formation in 2013 compared to those in 2012. These results suggest that annual variations in the oceanographic conditions possibly affect the distribution of both species in the southern Chukchi Sea.

\section{0:45}

5aAB11. Presence of ribbon seal vocalizations are related to sea ice extent in the Nemuro Strait, the Okhotsk Sea. Mayuko Otsuki (Graduate School of Environ. Sci., Hokkaido Univ., 20-5 Benten, Hakodate, Hokkaido 040-0051, Japan, motsuki@ees.hokudai.ac.jp), Tomonari Akamatsu (National Res. Inst. of Fisheries Sci., Japan Fisheries Res. and Education Agency, Yokohama, Japan), Takahiro Nobetsu (Shiretoko Nature Foundation, Rausu, Japan), and Yoko Mitani (Field Sci. Ctr. for Northern Biosphere, Hokkaido Univ., Hakodate, Japan)

Vocalizations of ice-breeding ribbon seals Histriophoca fasciata were recorded using underwater passive acoustic methods from November 2012 to March 2014 off the Nemuro Strait, Japan. Seal presence in the strait was examined in relation to the sea ice extent in the Okhotsk Sea. Ribbon seal downsweeps were only detected when sea ice was present in the strait (February and March), with more detections in March leading up to the spring breeding season. Since ribbon seals require ice for breeding, underwater communication for breeding could be needed during the sea ice presence in this strait. Northeasterly winds were another indirect driver of ribbon seal occurrence, since winds from this direction likely transport sea ice from the central Okhotsk Sea into the Nemuro Strait. Downsweep detections decreased in the middle of the day, which is consistent with observations that seals hauled out on the ice during this time, and thus were producing fewer underwater vocalizations. Our results suggest that a decrease in the sea ice extent in the Okhotsk Sea may change the distribution of ribbon seals and impact their breeding behaviour since the Nemuro Strait region of the Okhotsk Sea is likely the southern limit of their breeding range.

\section{1:00}

5aAB12. Comparison between trawl volume and composition and acoustic backscatter. Adrienne M. Copeland (Univ. of Hawaii at Manoa, P.O. Box 1346, Kailua, HI 96734, acopelan@hawaii.edu), Whitlow W. Au (Hawaii Inst. of Marine Biology, Kailua, HI), and Jeffrey Polovina (PIFSC, NOAA, Honolulu, HI)

Active acoustics is an important tool to quantitatively assess the densities of pelagic organisms. While it is an important tool, it is hard to determine the organism composition recorded acoustically especially in oligotrophic waters where mixed organism assemblages are common. One such mixed stock in the Hawaii Islands is the Deep Scattering Layers (DSL). These layers are made up of micronekton (small squid, fish, and crustaceans) which may be an important component linking primary producers with higher trophic levels. Trawling can identify the composition in these layers but this tool can be costly and unable to sample as much area as active acoustics. Many organisms in the DSL diurnally migrate to the surface between 0 and $200 \mathrm{~m}$ to feed. We surveyed these organisms using active acoustics and trawling collected in June 2013 and March 2014 on the R/V Oscar Elton Sette. We compared the composition of organisms, volume by functional group, and total volume in the trawl with the acoustic backscatter to determine the relationship between trawl and active acoustic data. We determined that the acoustic densities changed as a function of the trawl composition and volume potentially allowing for the ability to exclusively use acoustics in the future.

\section{1:15}

5aAB13. Studying deep diving odontocetes potential prey fields' densities and size structure in Hawaii using a DIDSON imaging sonar. Giacomo Giorli and Whitlow W. Au (Hawaii Inst. of Marine Biology, Univ. of Hawaii at Manoa, PO BOX 1306, Kaneohe, HI 96744, giacomog@hawaii.edu)

Deep diving odontocetesodontocetes feed in the deep ocean on mesopelagic squid and fish. The distribution of these prey are poorly known but does affect the distribution of deep diving odontocete predators. Hence, it is important to collect data on the density and composition of potential prey to identify deep sea habitats that attract deep diving odontocetes. We used a DIDSON imaging sonar to investigate the density and size structure of mesopelagic animals along the Kona coast of Hawaii, where beaked, sperm and pilot whales are regularly seen. Data were collected at multiple stations during three research cruises by lowering the sonar in the water column to sample at different depths and monitoring the mesopelagic biomass with an EK60 $38 \mathrm{kHz}$ echosounder. Foraging activity of whales was monitored at few stations using passive acoustic recorders and a towed array of hydrophones. We were able to count and size 7068 pelagic animals. Density and size varied in space, with higher densities and bigger animals at the offshore stations. The DIDSON was effective at detecting targets bigger than $10 \mathrm{~cm}$, a size range that includes potential prey of deep diving odontocetes. Preliminary data suggest differences may exists in habitat selection by each deep diving odontocetes.

\section{1:30}

5aAB14. Snapping shrimp: Changes in environmental noise in Laguna de Términos, Campeche, México, after hurricanes. Carmen Bazúa Durán (UNAM, Facultad de Ciencias, Circuito Exterior s/n, Ciudad Universitaria, México, D.F. 04510, Mexico, bazua@unam.mx), Pamela A. Azamar Reyes (FES Iztacala, UNAM, Tlalnepantla, Edo. de México, Mexico), and Whitlow W. Au (MMRP, HIMB, Univ. of Hawaii, Kaneohe, HI)

Laguna de Términos, Campeche, México, is one of the largest coastal lagoons in Mexico where snapping shrimp is the main source of biological sound. We measured the environmental noise from 2004 to 2008 in stations distributed homogeneously in this lagoon during 17 sampling periods using a digital audio tape recorder sampling at $48 \mathrm{kHz}$ with 16 bits for 1 minute at each station. The environmental noise in the recordings was measured with a semi-automatic MATLAB routine designed for this purpose. Results indicate that environmental noise in the lagoon changes from one sampling to another, but it changed drastically after the hurricanes of 2005 in some of the stations. This changes in submarine environmental noise helps us in understanding the effects of natural phenomena in the distribution and abundance of marine wildlife, such as snapping shrimps and bottlenose dolphins. [Work supported by CONACyT-Campeche and PAPIT\&\&ASPA-UNAM.] 


\title{
Session 5aAO
}

\section{Acoustical Oceanography: Topics in Acoustical Oceanography II}

\author{
Boris Katsnelson, Cochair \\ Marine Geosciences, University of Haifa, Mt. Carmel, Haifa 31905, Israel
}

\section{Contributed Papers}

8:30

5aAO1. Effect of sand and silt particles on the attenuation of compressional waves in marine mud sediments. Allan D. Pierce (Retired, PO Box 339, 399 Quaker Meeting House Rd., East Sandwich, MA 02537, allanpierce@verizon.net), William L. Siegmann, and Elisabeth Brown (Mathematical Sci., Rensselaer Polytechnic Inst., Troy, NY)

Mud in marine sediments is a mixture of clay, sand, and silt particles. Present paper follows up on a suggestion by Holland and Dosso (JASA, 2013) that the variability of the measured frequency-dependent compressional wave attenuation may be caused by the variability of the amounts of sand and silt particles. The premise is that the porosity for the mud is high and that the sand and silt particles are in suspension. They do not settle out to the bottom of the layer because the card-house fabric of the clay particles tends to hold them in place. This supposition leads to a theory where the clay configuration gives a base-line attenuation, and the contribution from the individual sand and silt particles is additive. The estimation of the latter is distinguished from the existing theories of attenuation of sound in sandy/ silty sediments in that the particles are presumed not to touch each other. Particles are assumed to be spherical and there is no slip between particle surfaces and the surrounding water. Earlier theories of attenuation in suspensions by Lamb (Hydrodynamics), Urick (JASA. 1948), and others are criticized because of their assumption that vorticity in fluid is zero. The present theory predicts that the attenuation contribution from a given category of particle is proportional to the square of the frequency and to square of particle diameter, and inversely proportional to the viscosity, in the limit of low frequencies. It approaches a frequency-independent constant at higher frequencies. [Work supported by ONR.]

\section{8:45}

5aAO2. Acoustic wave propagation analysis for detecting a sensor robot in heat transfer pipe. Yoshihiro Nishimura, Yoshinobu Kawagoe, and Yoshihisa Harada (AMRI, AIST, 2-1-2, Namiki, Tsukuba, Ibaraki 3053564 , Japan, ykawagoe7@gmail.com)

Ultrasonic Testings of heat transfer pipes in electric power plant are important. Those pipes, which are over $60 \mathrm{~m}$ and whose inner diameters are $25 \mathrm{~mm}, 50 \mathrm{~mm}$, and $75 \mathrm{~mm}$, have more than 20 curves and are located at directly unaccessible place. We developed a cableless robot for measuring thickness of pipe in a pipe filled with water but it is difficult to know where our robot is in the pipe at real time. We developed a projector to propagate an intensive single-mode wave into the pipes and examined the relations between frequencies-intenseness and wave propagation $(3 \mathrm{kHz}-50 \mathrm{kHz})$. Attenuations of acoustic waves in the pipe filled with water were much lager than those in the pie filled with air and examined for metal pipes and plastic pipes. Acoustic wave tunneling through pipe-wall was calculated and compared with experimental results. Acoustic wave tunneling through the pipewall was found to be the main reason why acoustic wave could not propagate longer in the pipe filed with water than that with air. We derived useful data to develop a system for measurement of where our robot is.
9:00

5aA03. On using multi-look synthetic aperture sonar analysis for the investigation of scattering mechanisms. Anthony $\mathrm{P}$. Lyons (Ctr. for Coastal and Ocean Mapping, Univ. of New Hampshire, University of New Hampshire, Durham, NH 03824, anthony.lyons@ccom.unh.edu) and Daniel C. Brown (Appl. Res. Lab., Penn State Univ., State College, PA)

Multi-look coherence explores the information content of synthetic aperture sonar (SAS) images by splitting the total angle and frequency spectral bandwidth of a complex SAS image into sub-bands. In the presence of a random distribution of surface or volume scatterers, the spectral coherence is proportional to the degree of overlap in sub-bands of the SAS image. Consequently, for a perfectly random distribution of scatterers, the spectral coherence should be zero when the sub-bands do not overlap. If the scatterer distribution deviates from this basic random distribution, the spectral coherence may be non-zero for special cases that would be related to scattering from specific structures such as points or facets. For that reason it appears to be possible to separate coherent features from the random scattered background signal using spectral coherence, suggesting possibilities for detecting and/or characterizing objects (especially in low signal-to-reverberation situations). In this talk, we will discuss the concept and the utility of using the complex coherence between a series of sub-look SAS images to investigate different scattering mechanisms as well as show examples of the technique using real and modeled SAS data. [Work performed under ONR Grants N00014-16-1-2469 and N00014-16-1-2313.]

\section{9:15}

5aAO4. Multi target positioning system using Gold code pingers applied to Japanese squid in Japan Sea. Keiichi Uchida (Tokyo Univ. of Marine Sci. and Technol., 5-3-6, ko-nan, Minato-ku, Tokyo 108-8477, Japan, kuchida@kaiyodai.ac.jp), Toyoki Sasakura (AquaSound, Inc., Tokyo, Japan), Takashi Kamoshida (AquaSound, Inc., Kobe, Japan), Aki Miyagi (AquaSound, Inc., Tokyo, Japan), Yasuo Fujikawa (NICHIA Corp., Yokohama, Japan), Keiichi Kamiya (HAMADE Co., Ltd., Hakodate, Japan), Hiroshi Inada, and Yoshinori Miyamoto (Tokyo Univ. of Marine Sci. and Technol., Tokyo, Japan)

To study the effect of light on Japanese squid (Todarodes pacificus) behavior, we developed a new ultrasonic biotelemetry system that can simultaneously observe and measure multiple targets. We tested the system on 40 squids in open water. Each squid was attached with a Gold code pinger (AquaSound Inc.) that transmits single frequency signals of multiple codes (IDs). By using the combination of 32 kinds of Gold code sequences, we succeeded to develop a receiving system that can simultaneously track 40 pingers. In the field test, 40 squids attached with the Gold code pingers were released simultaneously into open water and signals were recorded from three fishing boats for a maximum of six hours. The underwater positioning of the squids was calculated based on the "reverse cross-bearing" method by using the cross points from each direction against the target obtained by two boats. With the addition of depth data obtained by the pressure sensor installed in each Gold code pinger, we succeeded to measure the 3D positions of all 40 target squids. Considering the movement of boats by wind and tide, the positions of all three boats were measured every second using the GNSS (FURUNO Electronics Co., Ltd.). 
5aA05. Acoustic characterization of the new Arctic during Ice Exercise 2016. John E. Joseph, D. B. Reeder, and Mitchell S. Nelson (Oceanogr., Naval Postgrad. School, 833 Dyer Rd., Monterey, CA 93943, jejoseph@ nps.edu)

The Naval Postgraduate School (NPS) participated in Ice Exercise 2016 (ICEX-16), a multi-national naval exercise conducted in the Beaufort Sea during March 2016. Operating at a remote ice camp, NPS deployed several conductivity, temperature and depth (CTD) sensors to capture oceanographic variability to $500 \mathrm{~m}$ depth while performing a series of propagation tests. Mobile and dipped mid-frequency sources transmitted signals to a pair of vertical line array receivers positioned in the field to investigate depth, range, angular and specular characteristics of acoustic propagation and their correlation to variability in oceanographic structure and under-ice conditions. CTD data indicate there was significant variability in sound speed at $50 \mathrm{~m}$ depth where cold, fresh mixed-layer water interfaces with contrasting warm, saline Pacific Summer Water (PSW) that lays immediately below it. The data also show a persistent and stable subsurface sound channel existed as a result of the PSW with peak temperature at $80 \mathrm{~m}$ situated above colder Pacific Winter Water (PWW), resulting in a sound channel axis near $140 \mathrm{~m}$ depth. Both features have important implications on sonar performance in the Arctic. Modeled and measured transmission loss are compared to quantify the effects.

\section{9:45}

5aAO6. Modeling reflection and scattering by deep-ocean turbidites. Darrell Jackson and Dajun Tang (Appl. Phys. Lab., Univ. of Washington, 1013 NE 40th St., Seattle, WA 98105, drj@apl.washington.edu)

Turbidites are commonly found in deep ocean sediments and are composed of multiple thin layers of fine and coarser sediments, the coarser sediments being deposited by turbidity currents. Although acoustics is a unique means to quantitatively probe the physical and geophysical properties of turbidites, research on this subject is still limited. The work of Gilbert and of Holland and Muncill has shown that this layering can cause acoustic reflection to be very strong, with bottom loss less than that expected for hard seafloors. We examine this problem further, looking at the effect of layering on penetration into the seafloor and also considering scattering by roughness of the inter-layer interfaces. It is found that penetration can be severely limited in some cases, and that this behavior can be understood as a form of Anderson localization. Prospects for inversion of broadband, vertical-incidence data are considered. [Work supported by ONR.]

\section{0:00-10:15 Break}

\section{0:15}

5aA07. Doppler sonar measurements of bedload sediment transport: Yes there's a signal, but can it be quantified? Len Zedel (Phys. and Physical Oceanogr., Memorial Univ. of NF, Chemistry-Phys. Bldg., St. John's, NF A1B 3X7, Canada, zedel@mun.ca), Jenna Hare, Alex Hay, and Greg Wilson (Oceanogr., Dalhousie Univ., Halifax, NS, Canada)

It has been demonstrated that Doppler sonar measurements of apparent bottom velocity in rivers is correlated with bedload transport. We explore the degree to which this measurement can be quantified using a field deployable multi-frequency (1.2-2.2 MHz), bistatic Doppler sonar system that provides three-component velocity profiles over a $\sim 30 \mathrm{~cm}$ interval with $\sim 5 \mathrm{~mm}$ resolution at a rate of 50 profiles/sec. Model simulations of system performance demonstrate that the estimates of bed movement are proportional to sediment flux but scaling to actual sediment flux would require an empirical fit. In order to explore the system capability under actual field conditions, a series of experiments was undertaken at the St. Anthony Falls Laboratory (SAFL). The SAFL facility provides a $1.8 \mathrm{~m}$ deep, $2.75 \mathrm{~m}$ wide flume tank that allows flow rates of order $1 \mathrm{~m} / \mathrm{s}$ over a mobile bed and where the bedload transport can be measured by sediment traps built into the flume system. We report on preliminary results from trials made at SAFL.
5aAO8. Sound intensity fluctuations as evidence of mode coupling due to moving nonlinear internal waves in shallow water. Boris Katsnelson (Marine GeoSci., Univ. of Haifa, 199 Abba Khouchy Ave., Haifa 3498838, Israel, bkatsnels@univ.haifa.ac.il) and Yun Ren (State Key Lab, Inst. of Acoust., CAS, Beijing, China)

One of manifestation of mode coupling produced by moving nonlinear internal (NIW) waves is temporal fluctuations of intensity or amplitude. In spectrum of fluctuations measured during a few hours there are maximums at frequencies, corresponding to periodical (in time) interaction of moving NIW with periodical (in range) interference structure of the sound field. If velocity of NIW along acoustic track is $v$, then peaks are placed at frequencies $F_{m n} \sim v\left(k_{m}-k_{n}\right)$, where $k_{m}$ is propagation constant of mode $m$. Due to a few remaining modes in a long range propagation, spectrum should have peaks at a few predominating frequencies. In given work on the base of experimental data of ASIAEX 01 and the corresponding theoretical analysis, it is shown existence of predominating frequencies in spectrum of the sound intensity fluctuations in the presence of NIW moving along acoustic track of the length $\sim 35 \mathrm{~km}$. Both speed of NIW and scales of interference beating determine mentioned frequencies. Note that acoustic track consists of two parts with different depths: $\sim 250 \mathrm{~m}$ (NIW are moving during time interval considered) and $120 \mathrm{~m}$ (position of receiving array). So, spectrum of fluctuations depends on modal structure both parts of this acoustic track. [Work was supported by ISF and NSFC.]

\section{0:45}

5aA09. Mapping the acoustical properties of the upper sedimentary layer in Lake Kinneret. Boris Katsnelson (Marine GeoSci., Univ. of Haifa, Mt. Carmel, Haifa 31905, Israel, bkatsnels@univ.haifa.ac.il), Andrey Lunkov (General Phys. Inst., RAS, Moscow, Russian Federation), Regina Katsman (Marine GeoSci., Univ. of Haifa, Haifa, Israel), and Ilia Ostrovsky (Igal Alon Kinneret Lab, IOLR, Haifa, Israel)

A series of field experiments were conducted to estimate the effective acoustical properties of the bottom in Lake Kinneret (Israel). A wideband sound source $(300 \mathrm{~Hz}$ to $2 \mathrm{kHz}$ ) was positioned at different ranges $(1 \mathrm{~m}$ to 6 $\mathrm{km})$ from a single hydrophone. Both local and long-range measurements of the sound field were carried out. All the acoustic data demonstrated a high reflectivity of the lake bottom at all grazing angles that is specific feature of gas-bearing sediments. Experimental sound pulses were compared with the simulated ones to obtain the values of effective sound speed in the sedimentary layer at different locations in the lake. Using a basic three component sediment's model (water, rigid particles, and gas bubbles), the gas fraction in the bottom was estimated. A high correlation between the calculated gas fraction and previously measured organic matter concentration was observed. [Work was supported by ISF.]

\section{1:00}

5aA010. Model-based design of multibeam sonar system. Jun Kuroda (Radio Application, Guidance and Electro-Optics Div., NEC Corp., 10, Nisshincho 1-chome, Fuchu City, Tokyo 211-8666, Japan, j-kuroda@bq.jp.nec. com) and Motohiro Kaname (Maritime Sensor Eng. Div., NEC Network and Sensor Systems, Ltd., Fuchu City, Tokyo, Japan)

Multibeam sonar is a technique used to many applications in offshore activities, e.g. oceanographic survey, fish finder and sound navigation in maritime. Sonar consists of integrated system of components, which are acoustic transducers, electrical transmitter receivers, and signal processing circuits. Numerical prototyping by model-based design (MBD) is widely employed to develop of various instruments, which are also useful to design algorithm and configuration of sonar. A sonar system is divided roughly into two system categories, that is to say, category 1: acoustic transducers and acoustic fields, and category 2: electrical transmitters, receivers, and signal processing circuits. Design of the category 1 needs numerical simulation of sonic waves reflected by targets. One issue for MBD is to study a high speed and low-cost computation method to simulate echo signals. Numerical computation methods, such as finite-element method (FEM), boundary-element method (BEM) and finite-difference-time-domain method (FDTD), require lots of computation resources to solve large acoustic field. Multibeam sonar is usually used to targets which are away from transducers. 
Therefore, equations to simulate transmitted and received sonic wave can be simplified by the far field approximation. This paper proposes a numerical simulation method of acoustic echo signals between transducers and targets for MBD.

\section{1:15}

5aAO11. Ultrasonic characterization of seagrass leaf blades (Posidonia oceanica). Jay R. Johnson, Gabriel R. Venegas, Preston S. Wilson (Mech. Eng., The Univ. of Texas at Austin, 1 University Station, C2200, Austin, TX 78712, johnson.jayrichard@utexas.edu), Jean-Pierre Hermand (LISA Environ. HydroAcoust. Lab, Université libre de Bruxelles (ULB), Brussels, Brussels Capital, Belgium), and Thibault Urban (NDT Dept., Vinçotte, Vilvoorde, Belgium)

It has been shown that simple mixture theory models are inadequate to describe low-frequency sound propagation through seagrass meadows and further understanding of the acoustic properties of seagrass tissue is necessary. To that end, we present two ultrasonic sound speed measurements on the Mediterranean seagrass Posidonia oceanica. First, a transit time measurement through a stack of 120 leaf blades at $2.25 \mathrm{MHz}$ are compared to similar measurements made on macroalgae Ecklonia radiata. Sound speed differences are related to the gas content within the aerenchyma of seagrass and other tissue characteristics. Second, ultrasonic sound speed measurements through a suspension of finely divided $P$. oceanica leaf blades at frequencies between 1 and $4 \mathrm{MHz}$ are compared to low-frequency (1-8 kHz) sound speed obtained on the same blade suspension by means of a resonance chamber.

\section{$11: 30$}

5aAO12. Simulation of acoustic backscattering from bubbles and droplets under different shape regimes with implications for underwater detection of leakages using active acoustic sensors. Geir Pedersen (Instrumentation, Christian Michelsen Res. AS, P.O. Box 6031, Bergen 5892, Norway, geir.pedersen@cmr.no)

Safe subsea production of oil and gas, as well as storage of $\mathrm{CO}_{2}$ in geological formations subsea, require rapid detection of accidental releases, eliminating potential harm to the environment. Monitoring of natural gas seeps are important in a climate and environmental aspect. Previous studies demonstrate the potential of sonars for detection and quantification of oil and gas releases of natural or anthropogenic origin. Simulations of acoustic backscattering from plumes is an important tool for design of leakage detection systems and in situ leakage quantification. Backscattering from subsea leakages are often simulated assuming spherical shapes using, e.g., effective medium theory, however in real world situations bubble and droplets are not spherical. In this study the backscattering from single bubbles $\left(\mathrm{CO}_{2}, \mathrm{CH}_{4}\right.$, and air) and droplets (light and heavy oil) are simulated under different shape regimes (i.e. as a function of Reynolds and Eötvös number). The shapes are extracted from time dependent Computational Fluid Dynamics simulations and the backscattering is modeled using adaptive cross approximation accelerated Boundary Element Method (BEM). The backscattering from single bubbles and droplets are used as input to effective medium theory simulations of plumes. Simulations using BEM and effective medium theory with spherical and non-spherical bubbles and droplets are compared with controlled in situ measurements of backscattering with concurrent shape measurements.

\section{$11: 45$}

5aA013. Determination of acoustic waveguide invariant using ships as sources of opportunity in a shallow water marine environment. Christopher M. Verlinden, Jit Sarkar (Marine Physical Lab., Scripps Inst. of Oceanogr., Univ. of California, San Diego, 9500 Gilman Dr., La Jolla, CA 92093-0701, cmverlin@ucsd.edu), Bruce Cornuelle, and William A. Kuperman (Marine Physical Lab., Scripps Inst. of Oceanogr., Univ. of California, San Diego, San Diego, CA)

The waveguide invariant (WGI) is a property that can be used to localize acoustic radiators and extract information about the environment. Here, the WGI is determined using ships as sources of opportunity, tracked using the Automatic Identification System (AIS). Using a single hydrophone the acoustic intensity as a function of range and angle is measured in the presence of ships at a variety of ranges and angles. The relationship between range, intensity, and frequency is used to determine the WGI parameter $\beta$ for ships in a variety of positions. These Beta values are interpolated and a map of $\beta$ is created for the environment in question. The method is demonstrated using data collected on a single hydrophone in a shallow water environment off the coast of Southern California. 


\title{
Session 5aBAa
}

\section{Biomedical Acoustics: Acoustics for Older Persons with Disabilities}

\author{
Takayuki Arai, Cochair \\ Information and Communication Sciences, Sophia University, 7-1 Kioi-cho, Chiyoda-ku, Tokyo 102-8554, Japan \\ Mari Ueda, Cochair \\ Department of Design Strategy, Kyushu University, 4-9-1 Shiobaru, Fukuoka 815-8540, Japan
}

\section{Invited Papers}

8:00

5aBAa1. The activities of the research committee on "Oto barrier-free" of the Acoustical Society of Japan. Kimio Shiraishi (Faculty of Design, Kyushu Univ., 4-9-1 Shiobaru, Minami-ku, Fukuoka 815-8540, Japan, kimio@design.kyushu-u.ac.jp), Kentaro Nakamura (Inst. of Innovative Res., Tokyo Inst. of Technol., Tokyo, Japan), Yasuhiro Oikawa (Faculty of Sci. and Eng., Waseda Univ., Tokyo, Japan), and Mari Ueda (Faculty of Design, Kyushu Univ., Fukuoka, Japan)

The number of the persons above 65 years old has reached 26\% of the population in Japan. Consequently, the number of persons with hearing and/or visual impairments has increased very rapidly. It is important to support communication or sound information compensation for these persons. From this background, the research committee on "Oto barrier-free" of the Acoustical Society of Japan has started in 2006. The "Oto barrier-free" committee intends to resolve problems related to improving accessibility in daily life for persons with disabilities or elderly people. The research fields are very diverse and include speech communication, electro- acoustics, noise and vibration, architectural acoustics, and laws for disabled persons. The research activities for persons with hearing impairments or elderly people aim to improve their listening environment in indoor spaces, such as homes for the aged or station buildings, by testing the use of sound absorbing materials and hearing assistive devices. The research assists or complements the use of aids such as voice guidance devices for persons with a visual impairment, and will be useful for foreigners visiting the Tokyo Olympics and Paralympics Games in 2020 too.

$8: 20$

5aBAa2. Relationship between auditory degradation and fricatives/affricates production and perception in elderly listeners. Keiichi Yasu (National Rehabilitation Ctr. for Persons with Disabilities, 1, Namiki 4-chome, Tokorozawa 359-8555, Japan, keiichi.yasu@ gmail.com), Takayuki Arai (Sophia Univ., Tokyo, Japan), Kei Kobayashi (The Univ. of Auckland, Auckland, New Zealand), and Mitsuko Shindo (Sophia Univ., Tokyo, Japan)

Elderly people often complain that they struggle with consonant identification when listening to spoken language. In previous studies, we conducted several experiments, including identification tests for young and elderly listeners using /shi/-/chi/ (CV) and /ishi/-/ ichi/ (VCV) continua. We also recorded production of such CV and VCV syllables. For the CV stimuli, confusion of /shi/ as /chi/ increased when the frication had a long rise time. The degree of confusion increased when auditory property degradation was observed such as threshold elevation in high frequency. In the VCV stimuli, confusion of /ichi/ as /ishi/ occurred for a long silent interval between the first $\mathrm{V}$ and $\mathrm{C}$ with auditory property degradation. In the present study, we analyzed the utterances of those CV and VCV syllables and measured the duration of frication and silent interval. The direction of the boundary shift in the perception of fricatives and affricates by auditory degradation was consistent with that of production. We found that degradation of auditory properties affects both perception and production of fricatives and affricates in elderly people.

\section{8:40}

5aBAa3. A speech synthesizer of Japanese saves patients' voices. Takayuki Arai (Sophia Univ., 7-1 Kioi-cho, Chiyoda-ku, Tokyo 102-8554, Japan, arai@sophia.ac.jp), Shigeto Kawahara (The Keio Inst. of Culture and Linguistic Studies, Tokyo, Japan), Musashi Homma (Tokyo Metropolitan Neurological Hospital, Tokyo, Japan), and Takaki Yoshimura (Pasobora, Sasebo, Japan)

"MyVoice," free speech synthesis software for Japanese, developed by one of the authors, is widely used to save many patients' voices in Japan. We lose our voice for various reasons. Amyotrophic Lateral Sclerosis, or ALS, is an example. When an ALS patient has difficulty breathing, Tracheotomy becomes an option, and the patient loses the ability to speak. Laryngectomy is another example. By using "MyVoice," patients can keep communicating using their own voice even after losing that ability. To facilitate this, patients record utterances with their voice before surgery. The recording is designed to minimize time and load of patients as much as possible, by including Japanese basic mora set so that potentially any word can be concatenated from the recorded sounds. The graphical user interface of this software is well-designed so that therapists as well as the patients' family and friends can also use it with a minimum of effort. In fact, we often hear heart-warming stories of making "MyVoice." "MyVoice" continues to rescue patients' voices and we will continue making improvements to it in the future. 
5aBAa4. Audio service for elderly people in broadcasting. Tomoyasu Komori, Takehiro Sugimoro, and Atsushi Imai (Sci. \& Technol. Res. Labs., NHK (Japan broadcasting corporation), 1-10-11 Kinuta Setagaya-ku, Tokyo 1578510, Japan, komori.t-bw@nhk.or.jp)

Elderly viewers sometimes feel that the background sound (music and sound effects) in television programs is too loud or that the narration or speech is too fast to understand. We attempted to solve these problems by two methods: improvement of the receiver system and the development of a new broadcasting system. We prototyped a receiver system in which loudness balance between the dialog (narration or speech) and background sound can be adjusted, as well as the speech rate. The results of subjective evaluation experiments with elderly viewers showed that the use of this system could significantly facilitate listening to television sound. In 2016, we started test broadcasting of $8 \mathrm{~K}$ Super Hi-Vision ( $8 \mathrm{~K} \mathrm{SHV}$ ) programs that have highly immersive surround sound produced through 22.2 multichannel $(22.2 \mathrm{ch})$. With the aim of improving the functionality of $8 \mathrm{~K}$ SHV sound as a broadcasting service, a domestic standard has been established for the dialogue control functions, including the dialog enhancement and a dialog replacement function. In this presentation, we show these two types of audio service for elderly people.

9:20

5aBAa5. Use of data hiding in aerial sounds for accessibility. Akira Nishimura (Dept. of Informatics, Tokyo Univ. of Information Sci., 4-1, Onaridai, Wakaba-ku, Chiba 2658501, Japan, akira@rsch.tuis.ac.jp)

This article reviews previous technologies for aerial acoustic data transmission, namely, audio data hiding, acoustic modems, and a hybrid of the two. These technologies can provide an information channel to convey index data for static text messages or data for text messages to assist people who are deaf or hard of hearing. The payload bitrate of these technologies ranges from several bits per second for long distances $(>100 \mathrm{~m})$ to hundreds of bits per second for short distances $(<1 \mathrm{~m})$. Previous studies have mainly focused on developing methods for embedding and encoding information into aerial sounds. However, few works have evaluated actual aerial transmission in the presence of various disturbances, such as reverberation and reflections, transfer functions of loudspeakers and microphones, nonlinear distortions in loudspeakers, background noise, and Doppler effects. Therefore, in this study, evaluation methods for these acoustic data transmission technologies are proposed and discussed. In addition, practical applications are discussed regarding the use of the technologies as a means of accessibility for people with speech and language disabilities.

9:40

5aBAa6. AcousessMap: Smartphone-based collaborative tool to facilitate assessing acoustical accessibility conditions for visually impaired people. Mari Ueda (Dept. of Design Strategy, Kyushu Univ., 4-9-1 Shiobaru, Fukuoka, Minami-ku 815-8540, Japan, mueda@design.kyushu-u.ac.jp), Takahiro Miura, Ken-ichiro Yabu (Univ. Tokyo, Tokyo, Japan), Takashi Morihara (INCT, Kanazawa, Japan), and Yoshio Tsuchida (K I T, Kanazawa, Japan)

Assistive instruments such as textured paving blocks and acoustical traffic signals are installed for helping visually impaired people to walk comfortably and avoid traffic accidents, who have any inconvenience to move outside. Though these precipitous situations of accessibility progress affect their migration pathway for their destination, up-to-date accessibility information is difficult to gain quickly because of local information disclosure. Thus it is necessary to develop a comprehensive system that appropriately acquires and arranges scattered accessibility information, and then presents this information intuitively. However, these systems present volunteers with difficulties when they are gathering accessibility conditions and then organizing them. Also, most of the volunteers do not know the current situations of acoustical support systems for the visually impaired. In this study, our final goal is to establish an efficient scheme to share the conditions and places of the acoustical support systems by many volunteers. Particularly, in this report, we aimed to check the feasibility to share the acoustical conditions to the web by persons without any knowledge of the support systems. We first developed a smartphone-based application for sharing accessibility conditions and carried out events to share accessibility conditions as a part of a lecture course for technical college students. 


\title{
Session 5aBAb
}

\section{Biomedical Acoustics: Biomedical Acoustics Poster Session}

\author{
Kang Kim, Chair \\ Medicine, University of Pittsburgh, 950 Scaife Hall, 3550 Terrace Street, Pittsburgh, PA 15261
}

\begin{abstract}
All posters will be on display from 8:00 a.m. to 12:00 noon. To allow contributors in this session to see the other posters, authors of oddnumbered papers will be at their posters from 8:00 a.m. to 10:00 a.m. and authors of even-numbered papers will be at their posters from 10:00 a.m. to 12:00 noon.
\end{abstract}

\section{Contributed Papers}

5aBAb1. Tissue boundary roughness index for evaluating malignancy of cancer in ultrasound-computed tomography system. Kazuhiro Yamanaka, Takahide Terada, Yushi Tsubota, Wenjing Wu, Atsuro Suzuki, Takashi Maruoka, and Ken-ichi Kawabata (Res. \& Development Group, Hitachi, Ltd., 1-280, Higashi-Koigakubo, Kokubinji, Tokyo 185-8601, Japan, kazuhiro.yamanaka.uu@hitachi.com)

Ultrasound-computed tomography (USCT) is a promising candidate for a radiation free, painless, and quantitative modality for breast cancer examination. We developed a USCT prototype with a ring-shaped transducer array (frequency: $1.5 \mathrm{MHz}$ ) that moves along the ring axis for scanning the entire breast. Distributions of speed and attenuation of ultrasound are computed from ultrasound transmitted through tissue. Although prior research shows that there is a correlation between malignancy and the speed of sound and attenuation values, in some cases both benign and malignant tumors have similar values. In this study, we propose to quantify the boundary roughness of a tumor from the spatial power distributions of reflected ultrasounds that are transmitted from different apertures of the ring transducer. In conventional ultrasound echography, the boundary roughness of a tumor is an important indicator of malignancy. However, roughness is judged by radiologists based on B-mode images and is a subjective index. We simulated the ultrasound reflection signal from boundaries of different roughness. The simulation result indicates that a spatial power concentration has a significant correlation with boundary roughness. We confirmed through gel phantom experiments that the index distinguishes rough and smooth boundaries, which is not evident in B-mode images.

5aBAb2. Tissue lesion formation induced by high intensity focused ultrasound along a spiral pathway. Dong Zhang, Juan Tu, Xiasheng Guo (Phys., Inst. of Acoust., Nanjing Univ, 22 Hankou Rd., Nanjing 210093, China, dzhang@nju.edu.cn), Qingyu Ma, and Gepu Guo (Phys., Nanjing Normal Univ, Nanjing, China)

Both theoretical and experimental studies were performed here to investigate the lesion formation induced by high-intensity focused ultrasound (HIFU) operating in continuous scanning mode along a spiral pathway. The Khokhlov-Zabolotskaya-Kuznetsov equation and bio-heat equation were combined in the current model to predict HIFU-induced temperature distribution and lesion formation. The shape of lesion and treatment efficiency were assessed for a given scanning speed at two different grid spacing (3 $\mathrm{mm}$ and $4 \mathrm{~mm}$ ) in both the gel phantom and ex vivo studies. The results show that uniform lesions can be generated by the homogenization of thermal diffusion along the spiral scanning pathway. The complete coverage of the entire treated volume can be achieved as long as the spacing grid of the spiral pathway is smaller than a critical value that right matches the maximum thermal diffusion dimension, and the treatment efficiency can be optimized by selecting an appropriate scanning speed. This study can provide guidance for further optimization of the treatment efficiency of HIFU therapy.
5aBAb3. Relative impedance variation based temperature monitoring and dose control for high intensity focused ultrasound therapy. Qingyu Ma, Gepu Guo (School of Phys. andTechnol., Nanjing Normal Univ., 1 Wenyuan Rd., Nanjing, Jiangsu 210023, China, maqingyu@njnu.edu.cn), Juan Tu, Dong Zhang (Inst. of Acoust., Nanjing Univ., Nanjing, Jiangsu, China), and Shaotong Feng (School of Phys. andTechnol., Nanjing Normal Univ., Nanjing, Jiangsu, China)

High intensity focused ultrasound (HIFU) can be used as a non-invasive and efficient therapy approach to treat tumors by heat coagulation with less metastasis, although it is still limited by inaccurate temperature monitoring and efficacy evaluation. Based on the relationship between temperature and electrical impedance, a model of impedance measurement during HIFU therapy was established. Numerical studies on the distributions of acoustic pressure, temperature and conductivity were simulated, and the impedances in the model were calculated with the electrical potential and current density. It is proved that, for a fixed acoustic power in the focal region, the relative impedance variation (RIV) linearly increases with the increasing treatment time and is inversely proportional to acoustic power, while the relative impedance variation rate (RIVR) shows a linear relationship with the acoustic power. The treatment time that is required for the temperature in the focal region to reach 70 degree is inversely proportional to the square of acoustic power. The experimental results of RIV and RIVR measured at different powers and treatment times are in good agreements with the simulated data. The results suggest that the RIV can be used as a useful indicator for the non-invasive temperature monitoring and efficacy evaluation, and might provide new strategy for accurate dose control of HIFU therapy.

5aBAb4. A high-frequency ultrasound method for testing skin cancer margins ex vivo. Zachary A. Coffman (Biology, Utah Valley Univ., 2750 W. Spaulding Ln., West Jordan, UT 84088, zachary.a.coffman@gmail. com), Caitlin Carter (BioTechnol., Utah Valley Univ., Orem, UT), Dolly A. Sanjinez (Biology, Utah Valley Univ., Orem, UT), Garrett Wagner (Comput. Eng., Utah Valley Univ., Orem, UT), Robyn K. Omer (Botany, Utah Valley Univ., Orem, UT), and Timothy E. Doyle (Phys., Utah Valley Univ., Orem, UT)

Mohs surgery is the standard surgical treatment for basal cell carcinoma and several melanomas. It involves resecting successive layers of the skin cancer and surrounding tissue (margins), analyzing the tissue in a pathology laboratory, and using the results to determine whether all of the cancer has been removed or whether another layer needs to be resected. Although resection of each layer only takes 5-10 minutes, procedure times for Mohs surgery are approximately 4 hours since the pathology analysis is very time consuming. A rapid specimen assessment technique for detecting skin cancer in surgical margins would therefore be of significant benefit to dermatology surgeons and their patients. A high-frequency ultrasound method has been developed to test Mohs surgical specimens without the need for direct immersion or transducer contact on the specimens. The ultrasound system 
uses a $50-\mathrm{MHz}$, narrow-beam $(1.5 \mathrm{~mm})$ pachymeter probe; a $50-\mathrm{MHz}$, broad-beam $(6.25 \mathrm{~mm})$ transducer; a high-frequency pulser-receiver; a 1$\mathrm{GHz}$ digital oscilloscope; and an aluminum test stage to hold and scan the specimen. Pitch-catch and pulse-echo measurements are collected from each test position on the specimen to determine the position's thickness and ultrasonic wave speed. Other parameters calculated from the data include attenuation and spectral peak density.

5aBAb5. Transducer influence on magnetoacoustic tomography with magnetic induction based on 3D equivalent source analysis for tissues. Qingyu Ma, Shaotong Feng, Gepu Guo (School of Phys. andTechnol., Nanjing Normal Univ., 1 Wenyuan Rd., Qixia District, Nanjing, Jiangsu 210023, China, maqingyu@njnu.edu.cn), Juan Tu, and Dong Zhang (Inst. of Acoust., Nanjing Univ., Nanjing, Jiangsu, China)

As a noninvasive imaging modality based on the coupling of magnetic and acoustic fields, magnetoacoustic tomography with magnetic induction (MAT-MI) has been demonstrated to have the capability of imaging the variation of conductivity distribution inside the object. However, the image resolution is still limited by the parameters of the receiver. Based on the theory of acoustic dipole, $3 \mathrm{D}$ equivalent source analysis was used to simulate transducer detected pressures and waveforms for MAT-MI. The influence of transducer was studied both theoretically and experimentally for a cylindrical model. It is demonstrated that large-radius transducer with strong reception pattern can detect the acoustic signals transmitted along its normal direction with sharp pressure peaks, reflecting the divergence of the induced Lorentz force. By considering the effect of acoustic attenuation and the accuracy of image reconstruction, the acoustic pressure with acceptable peak pressure ratio and improved SNR can be detected when the scanning distance is 5-10 times the radius of the object. Wide bandwidth transducer should also be selected to reduce the boundary width of borderline stripes and improve the spatial resolution of reconstructed images. The favorable results confirm the influence of transducer on MAT-MI and also provide the fundamentals for transducer selection in further study to improve the accuracy of electrical impedance reconstruction.

5aBAb6. Optimization of sphingosylphosphorylcholine concentration for detection of cytoskeletal changes in malignant pancreatic cells using high-frequency ultrasound. Caitlin Carter (BioTechnol., Utah Valley Univ., 886 E. Old English Rd., Draper, UT 84020, caitlin.carter03@gmail. com), Dolly Sanjinez (Biology, Utah Valley Univ., Orem, UT), Mandy Marvel (BioTechnol., Utah Valley Univ., Orem, UT), and Timothy E. Doyle (Phys., Utah Valley Univ., Orem, UT)

The biomechanical properties of cells can be greatly influenced by modifications of the cytoskeleton of the cell. Cytoskeletal modifications can often be linked to disease and disease pathways. Cancer metastasis has been previously associated with the cytoskeletal modifications that are induced by the addition of sphingosylphosphorylcholine (SPC) to panc-1 cells. SPC is a bioactive lipid that reorganizes keratin filaments in the cytoskeleton into a perinuclear shape that enables cell mobility. The maximum effect of SPC on the keratin reorganization has been previously observed as being concentration dependent, with $5-10 \mu \mathrm{M}$ concentration being optimal as observed through keratin immunostaining. In this work, high frequency ultrasound was used to observe the SPC induced keratin reorganization through an ultrasound signature. Ultrasound measurements were obtained using $10 \mu \mathrm{M}$ SPC and $\sim 17 \mu \mathrm{M}$ SPC concentrations. Significant time-dependent changes in the ultrasound measurements were observed in the higher concentration of SPC as compared to control cell cultures that were not treated with SPC. In contrast, the lower concentration of SPC did not show visible changes in the ultrasound measurements taken. Future work will include optimizing a more specific concentration of SPC for observing changes in the ultrasound measurements taken of panc- 1 cells.
5aBAb7. Modular arrays for transpinal ultrasound application. Shan Qiao, Constantin Coussios, and Robin Cleveland (Dept. of Eng. Sci., University of Oxford, Inst. of Biomedical Eng., Old Rd. Campus Res. Bldg., Headington, Oxford, Oxford OX3 7DQ, United Kingdom, shan.qiao03@ gmail.com)

Lower back pain is one of the most common health problems in developed countries, and is normally caused by the degeneration of intervertebral discs. High intensity focused ultrasound can be used to mechanically fractionate degenerate disc tissue by inertial cavitation. Due to the complexity of the spine structure, delivering sufficient focused acoustic energy to the target zone without damaging surrounding tissue is challenging and further exacerbated by patient-to-patient variability. Here we propose the design of modular arrays, each consisting of 32 elements at $0.5 \mathrm{MHz}$, which can be configured to optimize delivery for a specific patient by the means of timereversal using the patient geometry derived from CT scans. Initial tests were carried out in water and compared with simulations. Using four different configurations of four modules, focusing was accomplished using a needle hydrophone at the target location to determine the transmit phase for each element. In each of the four configurations a focal gain of approximately 35 was achieved and steering range of the focus was $+/-30 \mathrm{~mm}$ in azimuth, +/$5 \mathrm{~mm}$ in elevation. These results confirm that the numerical model accurately predicts the acoustic field of the modular array and can be used for treatment planning in a disc. [Work supported by EPSRC, UK.]

5aBAb8. Dynamics of an acoustically driven liquid-gas interface. Brandon Patterson and Eric Johnsen (Univ. of Michigan, 1231 Beal Ave., Rm. 2016, Ann Arbor, MI 48109, awesome@umich.edu)

Interactions between acoustic waves and liquid-gas interfaces occur in a variety of applications, including diagnostic ultrasound of the lung. We hypothesize that, because of the sharp density gradient at the fluid discontinuity, acoustic waves may generate sufficient baroclinic vorticity to appreciably deform liquid-gas interfaces. This nonlinear effect has been studied for shock-accelerated interfaces, i.e., the Rictmyer-Meshkov instability; however, it is not describable through traditional, linear acoustic methods and is not well studied for acoustic waves. To investigate our hypothesis, we simulate an inviscid, compressible fluid system with acoustic waves impinging from water into air. We find that acoustic waves capable of deforming the interface during the wave-interface interaction period will deposit lasting baroclinic vorticity along the interface, capable of driving deformation that continues long after the passage of the wave. We use dimensional analysis to describe the dependence of the late-time interface growth on the deposited vorticity. Lastly, we show results to demonstrate that for acoustic waves with properties relevant to diagnostic ultrasound encountering a water-air interface, nearly all of the vorticity is generated in gas-dominated fluid. We explain this using an order of magnitude analysis.

5aBAb9. Parametric array for tissue harmonic imaging. Christina $P$. Keravnou and Michalakis A. Averkiou (BioEng., Univ. of Washington, William H. Foege Bldg., 3720 15th Ave. NE, Seattle, WA 98195, maverk@uw. edu)

More than $50 \%$ of abdominal scans are performed on technically difficult patients due to worldwide increase of obesity. Tissue harmonic imaging has shown some improvements on obese patients but the problem remains unsolved. The use of dual frequency pulses allows the generation of sum and difference frequencies in tissue. We hypothesize that the difference frequency ( $\Delta f$, parametric array) can survive larger penetration depths and increase SNR while maintaining the spatial characteristics of the primary wave. Our objective was to optimize the parameters for efficient generation of the parametric array and study its spatial characteristics for imaging obese patients. Numerical simulations using KZK equation and measurements in tissue-like media were performed to study parametric arrays. Pulses with two frequencies $\left(f_{1}, f_{2}\right)$ were considered where $0.1 f_{1} \leq \Delta f \leq f_{1}$. Pulse inversion was utilized to isolate even harmonics, including $\Delta f$. The amplitude of $\Delta f$ was maximized at $\Delta f=f_{1}$. The axial resolution is not seriously compromised by the lower frequency of $\Delta f$ when using PI. For $0.5 \mathrm{~dB} / \mathrm{cm}$ attenuation difference between $\Delta f$ and $2 f 1, \Delta f$ echoes from $20 \mathrm{~cm}$ deep were greater by 10 dB. Application of the parametric array on larger technically difficult patients would offer similar benefits as those in sonar applications. 
5aBAb10. Vascular vector flow mapping for ultrasonic two-dimensional flow estimation. Rei Asami, Tomohiko Tanaka, Motochika Shimizu, Yoshinori Seki, Tomohide Nishiyama, Hajime Sakashita, and Takashi Okada (Hitachi, Ltd., 1-280 HIgashi Koigakubo, Kokubunji, Tokyo 185-0003, Japan, rei.asami.fq@hitachi.com)

Vector flow mapping (VFM) is an echocardiographic approach for visualizing two-dimensional cardiac flow dynamics by estimating the radial component of flow from the Doppler velocities and wall motion velocities using mass-conservation equations. Although VFM provides two-dimensional flow in a clinically suitable, fast, and easy manner, it is only applicable to bounded regions such as ventricles. In this study, the VFM algorithm is modified so that the velocities are estimated regardless of the flow geometry. The proposed method, vascular VFM, operates on the same principle as VFM but with additional boundary conditions created by using Doppler velocities measured at a different steering angle. The method was optimized and validated using a common carotid artery phantom and particle-imagevelocimetry (PIV). The results indicated that the optimal angle of an additional Doppler beam ranged from 10 to 20 degrees. Under this optimalbeam-angle condition, the standard deviation (SD) of total vascular VFM error, normalized by maximum velocity, is reasonably small (8.1 to $16.3 \%$ ). The error is predominantly from input Doppler velocities, which has error of up to $12.7 \%$. When calculated with ideal inputs, the algorithm error ranges from 1.9 to $4.3 \%$. These results indicate that vascular VFM algorithm estimates two-dimensional flow accurately in the non-bound region and may be clinically significant in cases such as arteriosclerosis diagnosis.

5aBAb11. Quantitative evaluation of hemolysis induced by pulsed ultrasound exposure. Kentaro Tani, Masato Imura (Graduate of Sci. and Eng., Doshisha Univ., 1-3 Miyakodani,Tatara610-0349, Kyotanabe, Kyotanabeshi 610-0349, Japan, duq0369@mail4.doshisha.ac.jp), Daisuke Koyama (Faculty of Sci. and Eng., Doshisha Univ., Kyotanabe, Japan), and Yoshiaki Watanabe (Faculty of Life and Medical Sci., Doshisha Univ., Kyotanabe, Japan)

To establish safety criteria of ultrasound for blood, we examined hemolysis induced by low-intensity pulsed ultrasound and performed in vitro experiments using bovine blood. Quantitative evaluation by microscopic observations and the relationship between the hemolysis and cavitation microbubbles were discussed. Hemolysis was evaluated by red blood ghost cell. The presence of red blood ghost cells in the plasma component implies that the cell membrane was broken and the internal hemoglobin flowed out to surrounding blood plasma. Pulsed ultrasound at $1 \mathrm{MHz}$ with the maximum sound pressure amplitude of $200 \mathrm{kPa}$ was irradiated for one minute, and the ratio of non-exposure time $(0-80 \%)$ was changed. After ultrasound exposure, the blood samples were centrifuged and divided into two layers: blood cells and plasma components. $10 \mu \mathrm{L}$ of the plasma components was sampled and the number of the red blood ghost cells was counted. The number of red blood ghost cells increased gradually with the decrease in the ratio of non-exposure time and decreased again at $0 \%$ (continuation wave). This result implies that the hemolysis is mainly caused by microbubble cavitation and the non-exposure time of ultrasound is one of the important factors for hemolysis in such low-intensity ultrasound field.

5aBAb12. Controlled nanoparticle release through microbubble microstreaming: Physical insight and applications. Guillaume Lajoinie (Phys. of Fluids Group, Univ. of Twente, P.O. Box 217, Enschede 7500 AE, Netherlands), Ying Luan (Biomedical Eng., Erasmus MC, Rotterdam, Netherlands), Erik C. Gelderblom (Phys. of Fluids Group, Univ. of Twente, Enschede, Netherlands), Benjamin Dollet (Institut de Physique de Rennes, Rennes, France), Heleen Dewitte, Ine Lentacker (Faculty of Pharmaceutical Sci., Ghent Res. Group on Nanomedicines, Ghent, Belgium), and Michel Versluis (Phys. of Fluids Group, Univ. of Twente, Enschede, Netherlands, m.versluis@utwente.nl)

Microbubbles driven by ultrasound are used in a number of applications including surface cleaning, ultrasound imaging, and as a vehicle for local drug delivery. To prolong the microbubble lifetime, its gas core is coated with a stabilizing shell, typically consisting of phospholipids. The coating can also be used to attach a payload of functional nanoparticles. Interestingly, upon ultrasound irradiation at several hundreds of $\mathrm{kPa}$, the payload was observed to be released in a highly controlled way. This release carries great potential for using microbubbles as drug delivery agents in the context of personalized medical therapy. However, until now, limited experimental observations of the phenomenon are available. Here, we study using ultra high-speed and fluorescence imaging techniques in top and in side-view the underlying mechanisms of the release. We also developed a model on the basis of a Rayleigh-Plesset-type equation that reveals that non-spherical bubble behavior is key to the release mechanism. We also quantified the streamlines and acoustic streaming velocity responsible for the microfluidic transport of the material and propose for the first time a complete physical description of the controlled release of ultrasound actuated microbubbles.

5aBAb13. All-in one small modular transducer system for ultrasound therapy. Kiyoshi Yoshinaka, Hideki Takeuchi, Shingo Toyoda, Akira Sasaki (Health Res. Inst., AIST, AIST East-Build, 1-2-1, Namiki, Tsukuba, Ibaraki 3058564, Japan, k.yoshinaka@aist.go.jp), Takashi Azuma, and Shu Takagi (Univ. of Tokyo, Tokyo, Japan)

We developed new concept all-in one small modular transducer system for ultrasound therapy. Focus position control of HIFU (High Intensity Focused Ultrasound) with multi-elements phase delay control is very popular in clinical application. However, multi-elements driving amplifiers are very large size like a large refrigerator. So, we adopted a direct drive amplifier system for the multi-elements modular transducer. The modular system has some advantages compared to conventional systems. It can be reduced the energy loss of the connecting cable between the transducer element and the amplifier. Other advantages are below. Free placement of module transducer elements like a patient specific body line. Easy maintenance of each element. Easy design change to reduce the manufacturing time. The modular transducer system is composed of four blocks. The first module is multichannel transducer elements. The second module is multi-channel direct drive amplifier system. The third module is a name-card size multi-channel phase delay signal generator with the phase delay signal set memory bank. The fourth module is a phase delay signal set generating software with a personal computer. It can transmit the signal set to the third module. We manufactured the prototype very small all-in one modular transducer system for ultrasound therapy.

5aBAb14. Detection of tumoral netrin-1 protein levels using ultrasound microbubble contrast agents: In vitro validation. Jennifer Wischhusen (LabTAU IINSERM 1032, French National Inst. of Health and Medical Res. (Inserm) \& Université de Lyon, 151 Cours Albert Thomas, Lyon 69390, France), Jean-Guy Delcros, Patrick Mehlen (Inserm U1032, Res. Cancer Ctr. of Lyon, Lyon, France), and Frederic Padilla (LabTAU IINSERM 1032, French National Inst. of Health and Medical Res. (Inserm) \& Université de Lyon, Lyon, France, frederic.padilla@inserm.fr)

Netrin-1 is a protein secreted by tumor cells in breast cancers. It triggers survival and proliferation through its interaction with the dependence receptor UNC5B. Netrin-1 is the target for a therapy under development. To identify patients who can benefit from treatment, a companion diagnostic is required. Our aim is to characterize netrin-1 as a target for ultrasound molecular imaging and to validate netrin-1-targeted microbubbles. Netrin-1 interactions with the extracellular matrix were analyzed by flow cytometry and Western blot. Microbubbles were functionalized and binding under static and dynamic conditions on purified netrin-1 or netrin-1-expressing cells was assessed by light microscopy. Netrin-1 interacted with heparin sulfate proteoglycans on the surface of the cells and with the cell surface receptor UNC5B. Microbubbles specifically and dose-dependently bound to netrin-1 protein and netrin-1-expressing cells, in absence and presence of UNC5B. Our findings confirmed that netrin-1, although being a secreted protein, is a potential target for ultrasound molecular imaging using microbubbles that specifically attach to netrin-1 presented on the cell surface. Currently, netrin-1 presentation on the luminal side of the vascular endothelium and ultrasound molecular imaging in the living animal are assessed. 
5aBAb15. Surgeon assessment versus laboratory measurement: brain tissue mechanics quantified by expert opinion. Sheronica L. James (Chemical and Biomedical Eng., Cleveland State Univ., 2121 Euclid Ave., Cleveland, OH 44115, sheronicajames@gmail.com), Mark Howell, Qi Wang, and Gregory T. Clement (Biomedical Eng., Cleveland Clinic, Cleveland, $\mathrm{OH}$ )

Transcranial ultrasound (tUS) is a plausible means of measuring motion of the brain due to traumatic force or structural abnormalities. However, limited data in the literature on the mechanical properties of human brain tissue make it practically impossible to develop a mechanically realistic ultrasound phantom that can mimic the brain under these conditions. The present study aimed to determine, by indirect qualitative assessment, the stiffness of in-vivo brain tissue. Six surgeon examiners under blinded conditions physically examined 16 gelatin-based tissue phantoms (0.020-0.095 $\mathrm{g} / \mathrm{mL}$ ) to determine which sample best matched the stiffness of brain tissue in-vivo. Ten cylindrical phantom samples, acoustically matched to human brain tissue, were prepared based on the examiners' responses $(0.030 \pm 0.008 \mathrm{~g} / \mathrm{mL})$, and stress-strain behavior was characterized under compression up to $50 \%$ strain. The average compressive stress at $50 \%$ strain was $10.3 \pm 1.4 \mathrm{kPa}$. These results correlate well with those previously reported in the literature, and add a practical measure of brain stiffness to the paucity of information available on human brain tissue mechanics. Furthermore, it is potentially an initial step in developing mechanically realistic models of structural disorders of the brain and traumatic brain injury to evaluate theories on the brain's mechanical behavior under these circumstances.

5aBAb16. Bio-acoustic levitational assembly of heterocellular multilayer constructs for tissue engineering. Charlene Bouyer (LabTAU INSERM 1032, French National Inst. of Health and Medical Res. (Inserm) \& Université de Lyon, 151 Cours Albert Thomas, Lyon 69390, France), Pu Chen, Utkan Demirci (Bio-Acoust. MEMS in Medicine (BAMM) Lab, School of Medicine, Stanford Univ., Stanford, CA), and Frederic Padilla (LabTAU INSERM 1032, French National Inst. of Health and Medical Res. (Inserm) \& Université de Lyon, Lyon, France, frederic.padilla@inserm.fr)

Many tissues are comprised of multiple heterocellular layers, where interlayer cell communications between heterogeneous cell populations are essential to sustain normal tissue functions. Mimicking such natural organization presents a challenge to tissue engineering, and few methods are available to bioengineer heterocellular multilayer constructs in vitro. We have developed a bulk acoustic levitation (BAL) technique to assemble multilayer 3D neuronal constructs from homogenous neural progenitor ${ }^{1}$. We present here a novel approach to build heterocellular multilayer tissues by combining BAL and layer-by-layer assembly. Cells are levitated into several layers within fibrin prepolymer solution by bulk acoustic waves and immobilized in a fibrin hydrogel layer via gelation. Several hydrogel units can then be stacked subsequently, each unit with a distinct cell type, to form a single hydrogel construct. We demonstrate the bioengineering of a heterogenous multilayer construct composed of three different cell types (wildtype-mCherry and eGFP HeLa cells) spatially distributed in a single 3D hydrogel. This novel approach enables rapid formation 3D multilayer structures in minutes with tunable interlayer spacing, layer composition and thickness, and is potentially useful to bioengineer homogenous and heterogeneous multilayer cell organization for tissue engineering. ${ }^{1}$ Bouyer $e t$ al. (2015) Adv. Mater. 28:161-7

5aBAb17. Locally-concentrated sonicated microbubbles: Potential as an antibiotic. Mark Howell, Qi Wang (Biomedical Eng., Cleveland Clinic, 9500 Euclid Ave., ND20, Cleveland, OH 44195, howellm2@ccf.org), Shota Shimizu (Eng., Mie Univ., Ikoma-gun, Nara-ken, Japan), Albert Feeny (Lerner College of Medicine, Cleveland Clinic, Cleveland, $\mathrm{OH}$ ), and Greg Clement (Biomedical Eng., Cleveland Clinic, Cleveland, $\mathrm{OH}$ )

While the antimicrobial effects of ultrasound have been well established, application as a bactericide in medicine has been hindered in part by significant variation in reported efficacy. Non-thermal mechanisms of cell death are generally attributed to cavitation, prompting us to investigate the potential for locally applied microbubbles to serve as a catalyst for reliably increasing bacterial death, while sparing surrounding cells. This preliminary study was limited to gram-negative bacteria $(E$-coli $)$ suspended in buffered saline. Preliminary work at $40 \mathrm{kHz}$ continuous-wave sonication established a threshold time for our setup of 15 minutes, below which negligible cell death was observed relative to control samples. Experiments were then performed with and without the introduction of octofluropropane-filled lipid shells microbubbles, sonicated over the 15 minute period. Thermometry and passive cavitation monitoring (PCD) were both conducted. Analysis by dilution and plating indicate a $65-80 \%$ reduction in bacteria relative to controls, while microscopy, cavitation detection, and a negligible temperature rise all point to cavitation as the mechanism of death. Results motivate further investigation into the utility of microbubbles in combination with ultrasound for the treatment of certain non-perfusing infections such as chronic wounds and abscesses. Further work is necessary to confirm similar effects for gram-positive bacteria.

5aBAb18. Numerical investigation of the nonlinear dynamics of interacting microbubbles. Amin Jafari Sojahrood, Hossein Haghi, Raffi Karshafian, and Michael C. Kolios (Dept. of Phys., Ryerson Univ., 350 Victoria St., Toronto, ON M5B2K3, Canada, amin.jafarisojahrood@ryerson.ca)

The successful application of MBs requires detailed understanding of the nonlinear behavior of microbubbles (MBs), especially in polydisperse clouds, where the dynamic of every individual MB affects the other MBs. However, there is not enough information on how the nonlinear oscillations of one MB influences the other and vice versa. In this work the dynamics of 2 and 3 interacting MBs of initial radii of $1 \mu \mathrm{m}<\mathrm{R} 0<4 \mu \mathrm{m}$ are studied by investigating the pressure dependent resonance curves and the bifurcation diagrams of the MBs $(1 \mathrm{MHz}<\mathrm{f}<15 \mathrm{MHz}, 1 \mathrm{kPa}<\mathrm{p}<1 \mathrm{MPa})$ for cases of no interaction and interaction with varying $\mathrm{MB}-\mathrm{MB}$ distances. Results show that, for small enough distances, the pressure dependent resonance frequencies (fr) and the pressure threshold for harmonic and SH fr decreases. The larger MB may force new peaks in the resonance curves of the smaller MB at fr, harmonic fr and SH fr of the larger MB. The larger MB can control the dynamics of the smaller MB and force the smaller MB to exhibit the same nonlinear behavior (e.g., $1 / 2,1 / 3,1 / 4 \mathrm{SH}$ ) as of the larger MB. The dynamics of a cloud of interacting MBs maybe controlled by controlling the dynamics of the larger $\mathrm{MB}$ in the population.

5aBAb19. High-throughput production of microbubble contrast agents using a sonofluidic device. Eleanor P. Stride, Richard Browning, Paul Rademeyer, and Dario Carugo (Univ. of Oxford, Old Rd. Campus Res. Bldg., Oxford OX3 7DQ, United Kingdom, eleanor.stride@eng.ox.ac. uk)

The response of microbubbles to a given sound field is determined by their size and coating. These, in turn, depend on their chemical formulation and the production technique. Sonication is the most commonly employed method and can generate high concentrations of microbubbles rapidly but with a broad size distribution and poor reproducibility. Microfluidic devices provide excellent control over size, but the small-scale architectures required are often challenging to manufacture, offer low production rates, and are prone to clogging. Microbubbles may also have inferior surface characteristics and stability compared to those produced by sonication. In this study we investigate a hybrid technique in which monodisperse microbubbles of $\sim 200 \mu \mathrm{m}$ diameter are produced at high flow rates in a simple Tjunction and then undergo controlled fragmentation by exposure to ultrasound via an integrated transducer operating between $71-73 \mathrm{kHz}$. Microbubbles were prepared using the device or a standard sonication protocol and compared in terms of their size, size distribution, concentration, stability, acoustic response, and surface molecular concentration using quantitative fluorescence microscopy. The characteristics of the microbubbles produced by the device were found to be equivalent in terms of production rate, stability and acoustic response but with a narrower size distribution and tunable mean size. 
5aBAb20. Propagation of low amplitude focused ultrasound waves in a bubbly medium: Finite element simulation and theoretical considerations for optimization of the treatment parameters. Amin Jafari Sojahrood, Hossein Haghi, Raffi Karshafian, and Michael C. Kolios (Dept. of Phys., Ryerson Univ., 350 Victoria St., Toronto, ON M5B2K3, Canada, amin.jafarisojahrood@ryerson.ca)

Administration of microbubbles (MBs) increases the attenuation and changes the sound speed (Cs) of the medium. These phenomena are nonlinear and depend on MB size frequency and acoustic pressure. The increased attenuation can shield the focal region and reduce the efficacy of the application (e.g., drug delivery). Detailed information on the attenuation and $\mathrm{Cs}$ in the medium is needed to optimize these applications. In this study, using our recently developed nonlinear model, the pressure dependent (PD) attenuation and Cs of a bubbly medium are calculated and classified in terms of the resonance frequency (fr) of the MBs for $(0.5 \mathrm{fr}<\mathrm{fr}<4 \mathrm{fr})$ and for pressures between $1 \mathrm{kPa}<\mathrm{P}<2 \mathrm{MPa}$. The propagation of the focused waves was simulated for each classified case using FEM simulation of the Helmholtz model. Results show that the presence of the MBs may shilled the target and eliminate the focal zone especially for frequencies close to fr. We show that, by choosing the optimal frequency (e.g., PD fr), the attenuation of the pre-focal region can be minimized while the attenuation at the focus maximized. This restores the focal pressure with minimum loss and decreases the pre and post-focal MB activity which may result in a better imaging or treatment efficacy.

5aBAb21. Study on ultrasound irradiating conditions for apoptosis induction in the rat glioma cell line C6. Sugamata Hiroaki, Satou Takaaki, and Takeuchi Shinichi (Dept. School of Eng., Toin Univ. of Yokohama, 1614 Kurogane-cho, Aoba-ku, Yokohama, Kanagawa 225-8503, Japan, b24c027o@ust.toin.ac.jp)

We're paying attention on ultrasound irradiation the cancer treatment method using apoptosis of a cell self-destruction program. We think that our method can be expected as a new effective and safety treatment method, if the cells were induced into apoptosis by using ultrasound. In this experiment, ultrasound irradiation experiments, to rat glioma cell line C6 were performed. The experiments were performed by using the standing wave type ultrasound irradiation system at frequency of $150 \mathrm{kHz}$. Also, we estimated the apoptosis induction after ultrasound irradiation by using Annexin V Assay Kit. We examined apoptosis induction of the cells after 8 hours of ultrasound irradiation. The fluorescence of FITC and PI was observed in the same cells, when ultrasound was irradiated to the cells by applying the continuous sinusoidal wave with voltage of $150 \mathrm{~V}_{\mathrm{pp}}$. It was suggested that the cells wave in the late stage of apoptosis and the stage of necrosis. When irradiated with applied voltage of $213 \mathrm{~V}_{\mathrm{pp}}$, only the fluorescence of FITC could be observed in the same cells. It was suggested an early stage of apoptosis. We're planning to perform the experiment, using the focused ultrasound irradiation system at frequency of $1.75 \mathrm{MHz}$ in near future.

5aBAb22. Time delay spectrometry methods for broadband characterization of plastics and tissue-mimicking materials for high intensity therapeutic ultrasound. Subha Maruvada, Yunbo Liu (U.S. Food and Drug Administration, 10903 New Hampshire Ave., Bldg. WO 62-2222, Silver Spring, MD 20993, subha.maruvada@fda.hhs.gov), Paul Gammell (Gammell Appl. Technologies, Exmore, VA), and Keith Wear (U.S. Food and Drug Administration, Silver Spring, MD)

Because of the nonlinear nature of High Intensity Therapeutic Ultrasound (HITU), tissues and tissue-mimicking materials must be characterized over a broad frequency band. Time Delay Spectrometry (TDS) measures the frequency response of a system or sample by applying a swept frequency source and using a tracking receiver to select only those through-transmission signals that have the desired time delay. Due to the greater time-bandwidth product, TDS provides considerable signal-to-noise improvement over Fourier transform techniques. This system has been used previously to provide broadband measurements of complex hydrophone sensitivity. We have further refined our TDS system to provide frequency-dependent attenuation and phase velocity characterization of materials over the range from 1 $\mathrm{MHz}$ to $19 \mathrm{MHz}$. We have compared the results to predicted dispersion curves based on the Kramers-Kronig relations. The measurements of lowdensity polyethylene (LDPE) and a HITU-compatible tissue-mimicking material agree to within $1 \%$ for both TDS and PR acquisition methods, but the TDS measurements provide superior bandwidth over the PR. Measured phase velocity agrees to within $1 \%$ with Kramers-Kronig-based dispersion curves from $1 \mathrm{MHz}$ to $19 \mathrm{MHz}$.

5aBAb23. Targeted phase-change contrast agents for intracellular delivery to breast cancer tumors: An In vitro study in MDA-MB-231 breast cancer cells. Kyle P. Hadinger, Joseph P. Marshalek (Medical Imaging, Univ. of Arizona, Tucson, AZ), Paul S. Sheeran (Medical Biophys., Univ. of Toronto Stonybrook, Toronto, ON, Canada), Pier Ingram, Russell S. Witte (Medical Imaging, Univ. of Arizona, Tucson, AZ), Paul A. Dayton (Biomedical Eng., Univ. of North Carolina, Chapel Hill, NC), and Terry O. Matsunaga (Medical Imaging, Univ. of Arizona, PO Box 245067, 1609 N. Warren St., Tucson, AZ 85724, tmatsunaga@radiology.arizona.edu)

We have found that folate-targeted, ultrasound-activated phase-change contrast agents (PCCAs) composed of volatile perfluorocarbons are able to bind and internalize within breast cancer cells prior to vaporization into intracellular microbubbles. We here report studies assessing internalization, stability to thermal activation, and ultrasound activation of PCCAs formed by condensation of dodecafluoropentane (DDFP), decafluorobutane (DFB), and octafluoropropane (OFP). Using a Zonare "z.one-ultra" ultrasound system and L14-5sp probe operating in B-mode at $9 \mathrm{MHz}$ (frame rate $13 \mathrm{~Hz}$ ) to activate PCCAs and imaging with a Leica confocal microscope, we studied delivery in cultured MDA-MB-231 breast cancer cells with folate-targeted PCCAs. The number of internalized bubbles pre- and post- insonation were measured as a function of: 1) PCCA incubation times (i.e 10 mins, 60 mins); 2) ultrasound exposure (4 passes vs. 12 passes); and 3) mixtures of OFP, DFB, and DDFP. DFB:DDFP PCCAs, 60 minute incubations, and 12 passes offered a promising combination of stability and activation, with post-ultrasound to pre-ultrasound microbubble ratios approximating 3.4 (microbubbles pre-ultrasound per cell $=0.68+/-0.49$, microbubbles postultrasound per cell $=2.35+/-0.79$ ). Results indicate that intracellular delivery of targeted-PCCAs can potentially offer a new methodology for clinical application with ultrasound-mediated intracellular imaging of breast cancer.

5aBAb24. Micro ultrasound motor with coiled stator for rotationally driving of ultrasound beam of Intravascular ultrasound imaging system. Shinichi Takeuchi (Dept. of Biomedical Eng., Toin Univ. of Yokohama, 1614 Kurogane-cho, Aoba-ku, Yokohama 225-8503, Japan, shin1@toin.ac. jp), Keisuke Kurita, Chouyuu Uehara (Dept. of Biomedical Eng., Toin Univ. of Yokohama, Yokohama, Kanagawa, Japan), and Seiya Ozeki (Dept. of Medical Care Technol., Tsukuba Int. Univ., Tsuchiura, Ibaraki, Japan)

For the purpose of performing radial scanning by rotating ultrasound beam in intravascular ultrasound imaging system for diagnosing the state of thrombus and stenosis in a blood vessel such as the coronary arteries of the heart, we are developing micro Coiled Stator UltraSound Motor (CS-USM). Three types of coiled stators with materials of titanium, stainless steel or copper were prepared. These coiled stators were 5 wound acoustic waveguide with $0.5 \mathrm{~mm}$ width and $0.1 \mathrm{~mm}$ thickness. Size of coiled stator is 0.9 $\mathrm{mm}$ diameter and $3 \mathrm{~mm}$ length. PZT ceramic vibrator or hydrothermal PZT film vibrator was employed. When two vibrators-type inner rotor CS-USM having stainless steel coiled stator were driven by voltage of $30 \mathrm{~V}_{\mathrm{pp}}$ at 311 $\mathrm{kHz}$, rotational speed was $3500 \mathrm{rpm}$, torque was $0.34 \mu \mathrm{Nm}$. Maximum rotational speed of one vibrator-type inner rotor CS-USM having hydrothermal PZT film was $1100 \mathrm{rpm}$. Maximum rotational speed of one vibrator-type inner rotor CS-USM with titanium or stainless steel coiled stator was 2400 rpm. Rotational speed of the one vibrator-type inner rotor CS-USM with copper coiled stator driven by same conditions was 3200rpm. Maximum rotational speed of two vibrator-type outer rotor CS-USM with stainless steel coiled stator and PZT ceramic vibrators was $1400 \mathrm{rpm}$. 
5aBAb25. Acoustic characterization of stethoscopes using auscultation sounds with synchronized electrocardiography recordings as test signals. Lukasz J. Nowak (Inst. of Fundamental Technolog. Res., Polish Acad. of Sci., ul. Pawinskiego 5B, Warszawa 02-106, Poland, 1nowak@ippt.pan. pl) and Karolina M. Nowak (Dept. of Endocrinology, Ctr. of Postgraduate Medical Education, Warsaw, Poland)

The modern stethoscopes implement different technologies and features in order to better transmit the sounds from the inside of a body of a patient to the doctor's ears. However, the objective evaluation of the actual influence of various solutions on the acoustic parameters of the diagnostic device is difficult. This is due to the fact, that the acoustic coupling between the body of an auscultated patient and the chest piece of a stethoscope can significantly alter the determined parameters, and due to the variable and noisy nature of the auscultation signals. The present study introduces a detailed methodology for acoustic characterization of the stethoscopes using the auscultation sounds as test signals. Information obtained from additional, synchronized electrocardiography measurements is used to extract short, specific fragments of recordings, defined as acoustic events. Analysis of the spectral characteristics of many acoustic events allows to compare the acoustic properties of various stethoscopes and to estimate the measurement uncertainty. The determined statistical acoustic parameters of the auscultation signals are presented, showing relatively large variation even within a single patient examination. The example results concerning various commonly used acoustic stethoscopes are introduced, showing discrepancies between the declared and the actual acoustic properties.

5aBAb26. Discrepancies in the reproduction of the spectral characteristics of the acoustic stethoscopes by the electronic stethoscopes and potential diagnostic consequences. Karolina M. Nowak (Dept. of Endocrinology, Ctr. of Postgraduate Medical Education, Ceglowska 80 St., Warsaw 01-809, Poland, karolina.nowak@ippt.pan.pl) and Lukasz J. Nowak (Inst. of Fundamental Technolog. Res., Polish Acad. of Sci., Warsaw, Poland)

The electronic stethoscopes implement selectable low- and high-pass digital filters in order to mimic the declared acoustic characteristics of the bell and diaphragm chest pieces of the acoustic stethoscopes. However, the question arises if such a simple approach can exactly reproduce the actual, complex spectral characteristics of the acoustic stethoscopes, which are strongly affected by the acoustic coupling between the body of a patient and the chest piece? The present study introduces the results of the relevant experimental investigations. The acoustic characteristics of four different electronic stethoscopes operating in bell and diaphragm modes were determined during the heart auscultation examinations combined with the synchronized ECG recordings. The heart sounds were recorded using a microphone placed in one of the earpieces, while the ECG signals were used to extract interference-free fragments corresponding to single heartbeats. The averaged spectra of a large numbers of such fragments were used to determine the acoustic parameters of the investigated devices. The obtained results are compared to the corresponding results obtained for the acoustic stethoscopes, showing significant discrepancies. The potential diagnostic consequences of such discrepancies are discussed based on the available auscultation guidelines for physicians, which do not take into account the described effects.

5aBAb27. Study on measurement technique for acoustic cavitation using cavitation bubble signals. Takeyoshi Uchida, Masahiro Yoshioka, Youichi Matsuda, and Ryuzo Horiuchi (NMIJ, AIST, AIST Tsukuba Central3 1-1-1 Umezono, Tsukuba, Ibaraki 305-8563, Japan, takeyoshi. uchida@aist.go.jp)

Acoustic cavitation shows an excellent effect on ultrasonic cleaning and it also has prospects of application to cancer treatment. However, further study is essential to solve the problems such as low yield ratio and safety for human body because too much cavitation can damage the target. In this respect, quantitative evaluation of the generated cavitation is required. The mechanical index has been used so far but it does not reflect the quantity of the generated cavitation. Thus, we have been studying a new measurement technique by using the signal generated from cavitation bubbles, frequency spectrum of which is composed of broadband noise and subharmonics. We have reported that broadband noise has potential as an index for the amount of the generated cavitation. In this presentation, we experimentally investigated the level change of both broadband noise and subharmonics by increasing the acoustic pressure. We found that these two components are observed at the different level of acoustic pressures, probably corresponding to the different kinetic state of the cavitation bubbles.

5aBAb28. Measurement of volumetric heat capacity of biological tissues heated by ultrasound exposure. Mariko Sugiyama, Hiroaki Kanayama, and Iwaki Akiyama (Medical Ultrasound Res. Ctr., Doshisha Univ., 1-3 Tatara-Miyakodani, Kyotanabe, Kyoto 610-0394, Japan, dmp1029@mail4. doshisha.ac.jp)

The authors have proposed a simple method of measuring volumetric heat capacity $\mathrm{Cv}$ from the small temperature rise of biological tissues heated by a short time ultrasound exposure. In this method, $\mathrm{Cv}$ is estimated by substituting the measured values of temperature rise speed and the acoustic parameters of specimen to the bio-heat transfer equation. The feasibility of the proposed method was studied by a block of porcine muscle as a specimen. In the experiments, it was heated by ultrasound exposure by the focusing concave transducer of diameter of $25 \mathrm{~mm}$ and focal length of $32.5 \mathrm{~mm}$ with a central hole of $2.0 \mathrm{~mm}$ in diameter. The temperature rise was measured by a type $\mathrm{K}$ thermocouple which was inserted into the hole through the transducer. The measured value of temperature rise speed was $0.7^{\circ} / \mathrm{s}$, by an ultrasonic exposure of $2 \mathrm{MHz}$ in frequency and $8.0 \mathrm{~W} / \mathrm{cm}^{2}$ in intensity. As a result, the value of $\mathrm{Cv}$ was measured as $3.6 \mathrm{~J} /{ }^{\circ} \mathrm{C}^{\circ} \mathrm{cm}^{3}$ which was approximately agreed with the value of $\mathrm{Cv}$ of porcine muscle referred as 3.7 $\mathrm{J} /{ }^{\circ} \mathrm{C} \mathrm{cm}^{3}$. [This study was supported by MEXT-Supported Program for the Strategic Research Foundation at Private Universities, 2013-2017.]

5aBAb29. Intracellular $\mathrm{Ca}^{2+}$ increase in endothelial cells induced by shock wave irradiation. Toru Takahashi (Graduate School of Sci. and Eng., National Defense Acad. of Japan, Hashirimizu 1-10-20, Yokosuka, Kanagawa 239-8686, Japan, em54026@nda.ac.jp), Keiichi Nakagawa (Graduate School of Eng., The Univ. of Tokyo, Bunkyo-ku, Japan), Tada Shigeru, and Akira Tsukamoto (Graduate School of Sci. and Eng., National Defense Acad. of Japan, Yokosuka, Japan)

Shock wave irradiations induce bioeffects including angiogenesis and bone healing. However, physical mechanisms underlying those bioeffects remain elusive. Extracorporeal shock wave treatment (ESWT) is one of well-known shock wave therapies. In ESWT, shock waves destroy kidney stones with cavitation and negative pressures. Energy flux density of shock waves in ESWT is around $0.1-1 \mathrm{~mJ} / \mathrm{mm}^{2}$. In this study, shock waves with energy flux density of $0.001 \mathrm{~mJ} / \mathrm{mm}^{2}$, around $1 / 100$ of ESWT, were irradiated on endothelial cells. In those cells, intracellular $\mathrm{Ca}^{2+}$ increase, i.e. a typical physiological response, was observed. Along the intracellular $\mathrm{Ca}^{2+}$ increase, extracellular fluid flow was not present. As well, both plasma membrane permeabilization and cell detachment were not detected. On the other hand, actin cytoskeletons, which link extracellular substrates and cellular components, were suggested to involve in the intracellular $\mathrm{Ca}^{2+}$ increase. Thus, it was supposed that shock waves initiated intracellular $\mathrm{Ca}^{2+}$ increase by loading forces on actin cytoskeletons. Although some previous studies have suggested that extracellular fluid flow could involve in shock wave induced bioeffects, intracellular $\mathrm{Ca}^{2+}$ increase seems to rely on extracellular fluid flow.

5aBAb30. Multimodal measurement of acoustic properties in cartilage tissue. Naotaka Nitta, Akio Kaya, Masaki Misawa, Koji Hyodo (National Inst. of Adv. Industrial Sci. and Technol. (AIST), 1-2-1 Namiki, Tsukuba 305-8564, Japan,n.nitta@aist.go.jp), and Tomokazu Numano (Tokyo Metropolitan Univ., Tokyo, Japan)

In order to detect the disease and degeneration of cartilage early, evaluations of acoustic properties are expected, but the physical meaning should be clarified. On the other hand, we have proposed a multimodal method using ultrasound (US) and magnetic resonance (MR) images for in vivo SOS measurement. In this study, the method was applied for the tissue-engineered cartilage, and the SOS was compared with T1, T2, apparent diffusion coefficient (ADC), attenuation coefficient (ATT), and Young's modulus (YM). All procedures were approved by the Animal Care and Use Committee of our institution. 12-week-old male rats were used, and five different 
types of tissue-engineered cartilage was subcutaneously implanted in the back of rat $(n=30)$. Under anesthesia, MR and US images including same cross-sections were acquired separately, by using a MR imager of 2 Tesla and a US device of $13 \mathrm{MHz}$. At this time, T1, T2, ADC were also measured. The SOS was determined by the thickness measurement using the MR image and the time-of-flight measurement using the US image. After that, the ATT and YM were measured by using the extracted samples of tissueengineered cartilage. As a result, the SOS exhibits negative correlations with $\mathrm{T} 1, \mathrm{~T} 2$, and $\mathrm{ADC}\left(\mathrm{R}^{2}=0.30,0.93,0.82\right)$, and positive correlations with ATT and YM $\left(\mathrm{R}^{2}=0.98,0.87\right)$. These results suggest the SOS of tissue-engineered cartilage strongly reflects the elasticity. Consequently, the physical meaning of SOS was clarified through the multimodal measurement.

5aBAb31. A novel visualization method of bubble cavitation caused by infinitesimal amount of microbubbles. Ren Koda, Yoshiki Yamakoshi, Takumu Origasa, Toshitaka Nakajima (Grad. School of Sci. and Technol., Gunma Univ., 1-5-1 Tenjin-cho, Kiryu-shi, Gunma 376-8515, Japan, koda@gunma-u.ac.jp), and Takahito Nakajima (Grad. School of Medicine, Gunma Univ., Maebashi-shi, Japan)

Microbubbles modified with targeting ligands have a potential in early diagnosis by ultrasound because the targeted-bubble has both a selective property for accumulation in diseased area and a high degree of echogenicity. However, conventional ultrasonic imaging methods, such as B-mode and ultrasound color Doppler method, do not have enough sensitivity for microbubbles when only very small amount of microbubbles exists in ROI. In order to detect infinitesimal amount of microbubbles, we proposed a novel visualization of bubble cavitation signal (BCS) induced by high intensity US irradiation. In this method, high intensity US (h-US) is irradiated to microbubble with a fixed time delay after introducing an imaging US (i-US) and BCS is detected by power Doppler imaging unit. Due to the time delay of two waves, BCS appears on power Doppler image as a vertical line image (T-image) whose intensity is modulated by the amplitude of BCS. By this method, BCS measurement which is free both for direct propagated h-US and Doppler signal caused by bubble motion can be achieved. We detected the infinitesimal amount of bubbles (Sonazoid) with the concentration of $8 \times 10^{-8} \mu \mathrm{L} / \mathrm{ml}$, which is 100 times lower than minimum detectable sensitivity of B-mode observation.

5aBAb32. Focused ultrasound therapy of cervical lymph nodes in rats for alleviating multiple sclerosis symptoms. Anthony Podkowa, Rita J. Miller (Dept. of Elec. and Comput. Eng., Univ. of Illinois at Urbana-Champaign, 405 N Mathews, Urbana, IL 61801, tpodkow2@illinois.edu), Robert W. Motl (Kinesiology and Community Health, Univ. of Illinois at UrbanaChampaign, Urbana, IL), and Michael L. Oelze (Dept. of Elec. and Comput. Eng., Univ. of Illinois at Urbana-Champaign, Urbana, IL)

Multiple sclerosis (MS) is a prevalent neurological disease among adults worldwide. Disease modifying therapies are only modestly effective for slowing long-term progression of pathological and disability outcomes. One approach to treating MS is to reduce the burden of lymphocytes entering the central nervous system. We hypothesized that using focused ultrasound (FUS) to heat the cervical lymph nodes in rats with EAE, an animal model of MS, would alleviate symptoms of EAE by reducing lymphocyte burden. EAE was induced in rats through injection of myelin oligodendrocyte glycoprotein (MOG) and EAE disability scores were recorded over 21 days post injection. The cervical lymph nodes of rats with EAE were treated at day 9 and day 12 post MOG injection using FUS to elevate the temperature to 43$44{ }^{\circ} \mathrm{C}$ for 20 minutes. On average the EAE remittance score for FUS treated rats was $1.14 \pm 0.48$ versus $0.33 \pm 0.27$ for sham treated rats. These differences were statistically significant $(p=0.037)$. Remittance of the EAE disability scores were highly correlated with the last therapy application. Therefore, FUS treatment of cervical lymph nodes in rats with EAE resulted in a significant reduction in EAE score, which was not observed in sham treated rats.
5aBAb33. Shear wave elastography reveals dispersion regimes in porous materials. Johannes Aichele, Chadi Zemzemi, Maxime Lescanne, Stefan Catheline (Labtau, INSERM, Cours Albert Thomas 152, Lyon 69003, France, johannes.aichele@inserm.fr), and Philippe Roux (ISTERRE, GIÈRES, France)

Ultrasound shear wave elastography is a well established tool for characterization of biologic tissues. While it has found application in various medical disciplines such as oncology and urology its feasibility for pneumology still has to be shown. We provide experimental results of ultrasound shear wave elastography of porous materials in phantoms and ex-vivo lung tissue. Phantom foams immersed in water show a strong phase velocity dispersion with increasing frequency. Two regimes can be identified in the dispersion curves in the investigated frequency range from 50 to $700 \mathrm{~Hz}$. These experimental results are in reasonable agreement with the theory of porous materials. The results from water-filled phantom foams are compared to gelatinfilled phantom foams. Finally, the phantom study is compared to surface waves dispersion curves of porcine lungs obtained with an ultra-fast optical camera and the first results of ex-vivo shear wave elastography in porcine lung. Ultrasound shear wave elastography is standard in non-porous organs such as muscle, liver, and breast tissue. Its application to porous materials could offer a noninvasive and nonionizing alternative for lung characterization.

5aBAb34. Reconstruction of sound speed distributions from pulse-echo data. Anthony Podkowa, Mert Hidayetoglu, Chunxia Yang, Michael Oelze, and Weng C. Chew (Elec. and Comput. Eng., Univ. of Illinois at UrbanaChampaign, 1009 W. Clark St. Apt. 205, Urbana, IL 61801, tpodkow2@illinois.edu)

Ultrasonic imaging is perhaps the most ubiquitous form of biomedical imaging currently employed in the clinical setting due its safety, affordability, and real-time imaging capabilities. However, the poor image quality of standard brightness mode (B-Mode) images has inspired research into alternative acoustic imaging modalities, such as sound speed imaging. Historically, sound speed tomography has been employed in transmission-mode because of the limited k-space coverage available in backscattered data. However, such imaging techniques require specialized hardware configurations, and are often only limited to tissue such as the breast where full angular coverage is available. A recent approach [Jaeger et al., UMB, 41: 235-250, 2015] has demonstrated the feasibility of sound speed imaging using only pulse-echo data, which only requires a single array probe, commonly available in a clinical setting. This approach allows for complementary k-space coverage by utilizing relative temporal shifts between successive steered plane wave transmissions. Motivated by these successes, we present simulated and experimental pulse-echo sound speed reconstructions using three different methods: a modified Jaeger-like approach, the Born iterative method (BIM), and the distorted Born iterative method (DBIM).

5aBAb35. Experimental and theoretical studies on acoustic droplet vaporization. Krishna N. Kumar, Mitra Aliabouzar, and Kausik Sarkar (George Washington Univ., 801 22nd St. NW, Washington, DC 20052, sarkar@gwu.edu)

Phase shift nanodroplets are better alternatives to microbubbles due to their enhanced stability and smaller size distribution. These nanodroplets undergo phase transition from liquid to highly echogenic gaseous state under acoustic excitation through a process termed acoustic droplet vaporization (ADV). In this study, we synthesized lipid-coated perfluoropentane (PFP)-filled nanodroplets via sonication and mechanical agitation methods. We investigated the ADV threshold of these nanodroplets as a function of acoustic parameters such as excitation frequency and pulse repetition period (PRP). Experiments were performed at frequencies 2.25, 5, and $10 \mathrm{MHz}$. The acoustic signature of droplet vaporization was observed to be a broadband signal at all the studied frequencies. The ADV threshold was studied by increasing the excitation pressure in steps of $200 \mathrm{kPa}$. The ADV threshold at 2.25 and $5 \mathrm{MHz}$ was found to be 1.8 and $2.2 \mathrm{MPa}$, respectively. The scattered response from droplets were studied at different PRPs of 500 çs, 1 $\mathrm{ms}$, and $10 \mathrm{~ms}$; the results indicate that the scattered response is PRP-independent. Classical nucleation theory (CNT) was employed to determine the dependence of $\mathrm{ADV}$ threshold on parameters such as surface tension of the 
liquid, droplet size, degree of superheat of the droplet liquid, and excitation frequency.

5aBAb36. High frequency and high intensity ultrasonic transducers for medical applications using oriented piezoelectric thick films by hydrothermal method. Mutsuo Ishikawa (Medical Eng., Toin Univ. of Yokohama, 1614, Kurogane, Aobaku, Yokohama 2258503, Japan, m.ishikawa@ toin.ac.jp), Yosuke Uchida (Medical Eng., Toin Univ. of Yokohama, Yokohama, Kanagawa, Japan), Marie Tabaru, Minoru Kurosawa (Tokyo Inst. of Technol., Yokohama, Kanagawa, Japan), Nobuyuki Kosuge, and Hideto Sugiyama (Medical Eng., Toin Univ. of Yokohama, Yokohama, Kanagawa, Japan)

Recently, high-frequency ultrasonic transducers are proposed for medical applications using piezoelectric crystal. In this case, it is known that the high piezoelectric constant and the higher limitation of vibration velocity are important for high power application. Normally, the piezoelectric ceramics has limitation due to maximum vibration velocity. Thus it is very difficult to radiate for high intensity ultrasound with nonlinear acoustics due to the limitation of maximum vibration velocity. Therefore, we tried to develop a very high intensity and high frequency ultrasonic transducer using hydrothermal $\mathrm{KNbO}_{3}$ thick film. The piezoelectric constant was over 80 $\mathrm{pm} / \mathrm{V}$ and the vibration velocity of thickness mode of $\mathrm{KNbO}_{3}$ thick film was $2.5 \mathrm{~m} / \mathrm{s}$ or over at $20 \mathrm{MHz}$. This maximum vibration velocity is twice of reported piezoelectric materials. Then, a radiation sound-pressure from the prototype ultrasonic transducer with $\mathrm{KNbO}_{3}$ thick film was measured. The result of radiation ultrasound was $4 \mathrm{MPa}$ or over in a water. It is very high intensity at high frequency. Consequently the hydrothermal $\mathrm{KNbO}_{3}$ thick film is usefulness for medical applications. The reason for why it is able to radiate for high intensity ultrasound at high frequency will be discussed in this presentation.

5aBAb37. Sensitivity study of high-frequency ultrasound to skin cancer using micro-tumor mimicking phantoms. Nicole Cowan (BioTechnol., Utah Valley Univ., 1294 Parkside Dr., Saratoga Springs, UT 84045, ncowan18@gmail.com), Zachary A. Coffman (Biology, Utah Valley Univ., West Jordan, UT), Robyn K. Omer (Botany, Utah Valley Univ., Orem, UT), Caitlin Carter (BioTechnol., Utah Valley Univ., Orem, UT), and Timothy E. Doyle (Phys., Utah Valley Univ., Orem, UT)

Skin cancer is the most common cancer in the United States. An estimated one in five Americans will be diagnosed with skin cancer in their lifetime with approximately 145,000 new cases each year. Mohs surgery (Mohs micrographic surgery) is a surgical technique used to treat skin cancer. During Mohs surgery, thin layers of skin tissue are excised from the cancer site and examined until only cancer-free tissue remains. The examination of excised layers is tedious and time-consuming (4-5 hours). A rapid assessment tool for detecting cancerous tissue in excised margins would prove beneficial for both dermatologists and their patients. High-frequency $(\mathrm{HF})$ ultrasound (20-80 MHz) has been explored as a rapid assessment tool for such cases. The purpose of this study was to test the sensitivity of HF ultrasound to micro-tumors present in skin cancer margins using histology mimicking phantoms. Phantoms were created from distilled water, agarose powder, 10X TBE stock solution, and polyethylene microspheres to simulate micro-tumors. Pitch-catch and pulse-echo measurements were acquired using a high-frequency ultrasound system with glycerol as the coupling agent. The phantom results show consistent increases in attenuation when testing positions containing micro-tumors, confirming the sensitivity of HF ultrasound to skin cancer pathologies.

FRIDAY MORNING, 2 DECEMBER 2016

SOUTH PACIFIC 4, 7:45 A.M. TO 12:00 NOON

\title{
Session 5aEA
}

\section{Engineering Acoustics: Applied Acoustics I}

\author{
Bernhard R. Tittmann, Cochair \\ Eng. Sci. \& Mechanics, Penn State University, 212 EES Bldg., University Park, PA 16802 \\ Masatonakayama, Cochair \\ Ritsumeikan Univ., Kyoto, Japan
}

\section{Contributed Papers}

\section{$7: 45$}

5aEA1. Ultrasound for nuclear reactors. Bernhard R. Tittmann, Brian Reinhardt, and Andrew Suprock (Eng. Sci. \& Mech., Penn State Univ., 212 EES Bldg., University Park, PA 16802, brt4@psu.edu)

Ultrasonic methods offer the potential for Structural Health Monitoring of critical components in nuclear reactors. These efforts have been limited by ultrasonic transducers incapable of performance under high temperatures and/or irradiation conditions. Here we report on piezoelectric transducers designed, fabricated, tested and optimized to perform in harsh environments. Test capsules with piezoelectric transducers were fabricated with Aluminum Nitride (AlN), Zinc Oxide (ZnO), and Bismuth Titanate (BiTi) as the active elements. Measurements were performed in the MIT Reactor for 18 months. The transducers experienced an integrated neutron fluence of approximately $8.68 \mathrm{E}+20 \mathrm{n} / \mathrm{cm}^{2}$ for $\mathrm{n}>1 \mathrm{MeV}$, temperatures in excess of $420{ }^{\circ} \mathrm{C}$, and a gamma fluence of $7.23 \mathrm{~Gy} / \mathrm{cm}^{2}$. The AlN transducer acoustically coupled to a Kovar cylinder gave acceptable pulse-echo data throughout the test. We show a summary of the test results. Thus the feasibility of ultrasonic transducers in a nuclear reactor has been established and opens the door to leave-in-place sensors for in-reactor conditions and materials. [The authors gratefully acknowledge support from the Department of Energy under the ATR-NSUF program.]

\section{8:00}

5aEA2. Writer recognition with a sound in hand-writing. Daichi Asakura, Takanori Nishino, and Hiroshi Naruse (Graduate School of Information Eng., Mie Univ., 1577 Kurimamachiya-cho, Tsu, Mie 514-8507, Japan, asakura@pa.info.mie-u.ac.jp)

Hand writing sound is one of noises existing in the real environment. Generally speaking, noises are meaningless; however, some noises, e.g., footsteps and a road noise, are effective to recognize circumstances. Hand 
writing sound also has some information to recognize a writing character. Previous study addressed the recognition of handwritten numeric characters by using writing sound, and the average recognition rate was $88.4 \%$. We advanced previous research and examined the possibility of writer recognition from writing sound. We asked our subjects to write three target names. When subjects wrote on paper by a ballpoint pen, writing sounds were recorded. Three subjects chosen as the target name also wrote their own names under the different conditions, e.g., writing slowly and writing in another day. In our experiments, writer recognition was performed by the hidden Markov model. Acoustic features were mel-frequency cepstrum coefficients (MFCC), delta coefficients, and delta-delta (acceleration) coefficients. Experimental results showed that the writer recognition is possible; however, problems still exist such as how to deal with the difference between the writing conditions. In future works, it is necessary to increase learning data and to improve the recognition method.

\section{$8: 15$}

5aEA3. Effect of the horizontal panning on sense of presence in threedimensional audio system based on multiple vertical panning. Toshiyuki Kimura (Faculty of Eng., Tohoku Gakuin Univ., 1-13-1, Chuo, Tagajo, Miyagi 985-8537, Japan, t-kimura@m.ieice.org) and Hiroshi Ando (Ctr. for Information and Neural Networks, National Inst. of Information and Communications Technol., Suita, Osaka, Japan)

We previously proposed a three-dimensional (3D) audio system using the multiple vertical panning (MVP) method to develop a 3D audio system that matches a multi-view 3D video display system (REI display). In this paper, in order to apply our proposed method to the teleconference system, we performed two audio-visual psychological experiments and evaluated the effect of the horizontal panning on the sense of presence in our proposed method. We found that the performance is maintained when four loudspeakers were placed at the position except the edges of the display.

5aEA4. Design of a bullet beam pattern of an ultrasound transducer with shaded electrodes and a multifocal lens. Yongrae Roh, Seongwon Jang, Euna Choi, and Yeonsue Park (School of Mech. Eng., Kyungpook National Univ., 80 Daehakro, Bukgu, Daegu 41566, South Korea, yryong@ knu.ac.kr)

Ultrasonic imaging transducer is required to compose a beam pattern of a low sidelobe level and a small beam width to achieve good image resolution. Normal ultrasound transducers have many channels along its azimuth, which allows easy formation of the sound beam. However, they have no control of the beam pattern along their elevation. In this work, a new method is proposed to manipulate the beam pattern by using an acoustic multifocal lens and shaded electrodes in the transducer. The shading technique splits an initially uniform electrode into several segments. For a given elevation width and frequency, the optimal pattern of the split electrodes was determined to achieve the lowest sidelobe level. The requirement to achieve a small beam width with a long focal region was satisfied by employing an acoustic lens of three multiple focuses. The geometry of the multifocal lens was also optimized. For the optimization, a new index was devised to evaluate the on-axis response: focal region ratio $=$ focal region/ minimum beam width. The larger was the focal region ratio, the better was the beam pattern. The validity of the design has been verified through fabricating and characterizing an experimental prototype of the transducer.

\section{$8: 45$}

5aEA5. Fast measurement of the temperature distribution in a blast furnace using impulse response measurement. Gottfried Behler, Jan-Gerrit Richter (Inst. of Tech. Acoust., RWTH Aachen Univ., Kopernikusstraße 5, Aachen D-52074, Germany, gkb@akustik.rwth-aachen.de), and Sebastian Buzga (Z\&J, IMI Critical Eng., Düren, Germany)

The temperature profile on top of a blast furnace gives meaningful information about the heat distribution and the activity of the ballast below. Since a direct measurement using temperature probes is both complicated due to the filling procedures and expensive due to the repeated damage of the sensors. An indirect measurement of the temperature is given by the measurement of the propagation delay for sound which by a known distance reveals the average temperature of the gas in between source and receiver position. The temperature distribution is then derived by a tomographic approach in one plain above the blast furnace with different source and receiver positions. Basically, this method is not new and it is used with stochastic (pneumatic excitation) signals in many applications. The typical measurement time for a single temperature profile takes about 20-30 seconds. It is obvious that the temperature distribution will not be stable for such a long time; hence, the measurement results are not very much reliable. The background noise in the blast furnace and the excitation signal are of the same type (noise), hence the correlation of the measured signal very often fails. In this paper, a new electroacoustic method using interleaved swept sine excitation will be described.

\section{9:00}

5aEA6. Defect position and size estimation in billet by ultrasonic transmission method using a small number of transducers. Ryusuke Miyamoto (Graduate School of Systems and Information Eng., Univ. of Tsukuba, 1-1-1 Tennodai, Tsukuba, Ibaraki 305-8577, Japan, miyamoto@aclab.esys.tsukuba. ac.jp), Koichi Mizutani, Tadashi Ebihara, and Naoto Wakatsuki (Faculty of Eng., Information and Systems, Univ. of Tsukuba, Tsukuba, Japan)

For a steel billets inspection, we have proposed defect detection and size estimation from time-of-flight (TOF) profile by ultrasonic transmission method with linear scanning. In this method, a defect is detected by deviation of TOF of transmitted wave caused by diffraction at the defect. In addition to defect detection, defect size can be estimated from deviation of TOF because TOF is affected by defect size. However, deviation of TOF is also affected by the depth of the defect, which cannot be precisely estimated only by linear scanning of a pair of transducers. In this study, we propose defect position and size estimation using multiple pairs of transducers and validity of the method was evaluated. By using multiple pairs of transducers, TOF of inclined paths, where transducers is not facing each other are also obtained as well as parallel paths, so that the position of defect can be estimated. As a result, the defect position could be estimated by using two pairs of transducers and defect size could also be estimated correctly compared with conventional linear scanning method.

\section{9:15}

5aEA7. Shape optimization of reactive mufflers using threshold acceptance and finite element methods. Abdelkader Khamchane (Laboratoire de Mécanique Matériaux et Energétique (L2ME), Université Abderahman Mira de Bejaia, Rte. de Targa Ouzemour, Béjaia 06000, Algeria, abdelkader.khamchane@yahoo.fr), Youcef Khelfaoui, and Brahim Hamtache (Laboratoire de Mécanique Matériaux et Energétique (L2ME), Université Abderahman Mira de Bejaia, Bejaia, Bejaia, Algeria)

Recently, research on the acoustic performance of reactive mufflers under space constraint becomes important. The attenuation performance of single and double expansion-chambers under space constraint is presented in this paper. A shape optimization analysis is performed using a novel scheme called Threshold Acceptance (TA), the best design obtained by the shape optimization method are analysed by Finite Element Method (FEM). The acoustical ability of the mufflers obtained is than assessed by comparing the FEM solution with the analytical method. Results show that the maximal STL is precisely located at the desired targeted tone. In addition, the acoustical performance of mufflers with double expansion-chamber is found to be superiors to the other one. Consequently, this approach provides a quick and novel scheme for the shape optimization of reactive mufflers.

\section{9:30}

5aEA8. A mixed-order ambisonic scheme to improve performance for sound sources on the horizontal plane. Jiho Chang (Korea Res. Inst. of Standards and Sci., 267 Gajeong-ro, Yuseong-gu, Daejeon 34113, South Korea, chang.jiho@gmail.com)

This paper proposes a mixed-order ambisonic scheme called \#P\#L that improves performance in terms of estimation of the coefficient of spherical harmonics expansion, reconstruction of sound fields, and spatial resolution for sound sources located on the horizontal plane. Mixed-order ambisonics (MOA) selects appropriate components of spherical harmonics expansion instead of truncating up to a certain order as in higher-order ambisonics 
(HOA). Several schemes of MOA including the proposed scheme and HOA are compared each other in several ways. The results show that the proposed scheme has several advantages compared with the other MOA schemes. This scheme can be useful to further improve performance of microphone arrays if the number of the microphones is greater than what is required for HOA schemes or a non-uniform layout of microphones is used.

\section{9:45-10:00 Break}

\section{0:00}

5aEA9. Force feedback microelectromechanical microphones for high performance applications. Owain L. Boorman (ISVR, Univ. of Southampton, Bldg. 13, Highfield Campus, University Rd., Southampton, Hampshire SO17 1BJ, United Kingdom, olb1g11@soton.ac.uk), Nicholas Harris (ECS, Univ. of Southampton, Southampton, United Kingdom), and Matthew C. Wright (ISVR, Univ. of Southampton, Southampton, United Kingdom)

Microelectromechanical System (MEMS) condenser microphones are widely used because of their low cost, small size, high sensitivity, and wide bandwidth. For certain specialist applications, however, they are still outperformed by the best conventional condenser microphones, which have greater bandwidth and dynamic range, but at higher cost and larger size. The sensitivity, and hence, signal-to-noise ratio of smaller MEMS microphones can be increased by using two perforated back-plates instead of one. The maximum amplitude is limited by membrane excursion, which leads to nonlinearity and, ultimately, failure. The use of force feedback holds the promise of avoiding these problems by holding the membrane at its equilibrium position while measuring the force required to do so. Previous attempts to accomplish this using a Sigma-Delta modulator have had only limited success in terms of signal-to-noise ratio, bandwidth and stability. Instead we propose to use an Electro-Mechanical Phase Locked Loop (EMPLL) to overcome these limitations. We will present lumped-parameter and Finite Element models of the performance of such a microphone, and discuss the challenges associated with its fabrication. [This work was supported by Roke Manor Research Limited.]

\section{0:15}

5aEA10. Personalization of head-related transfer functions in the median plane based on spectral correction with pinna angle. Sakiko Mishima, Hajime Komatsu, Takahiro Fukumori, Masato Nakayama, and Takanobu Nishiura (Ritsumeikan Univ., 1-1-1, Noji-higashi, Kusatsu, Shiga 525-8577, Japan, is0188if@ed.ritsumei.ac.jp)

Head-related transfer functions (HRTFs) are studied to realize high-precision sound field reproduction systems. These systems usually utilize the HRTFs of other listeners because self-measurement of one's own HRTFs is a heavy burden. The localized sound accuracy of the listener, especially in the median plane, is degraded when the systems utilize the HRTFs of other listeners, which have different characteristics from the listener's own ones. Thus, simple personalization of HRTFs is necessary for solving this problem. In this research, we focus on personalization of HRTFs in the median plane on the basis of spectral correction with pinna angles as anthropometric parameters. We first made analyses of the relationship between pinna angles and the spectral envelopes of HRTFs in the median plane through an experiment with a dummy head with a replicated pinna made from silicone. From these analyses, the spectral envelopes were confirmed to be affected by pinna angles. We then proposed a method for personalization of HRTFs on the basis of spectral correction with the pinna angles. Objective and subjective evaluation experiments were carried out in order to demonstrate the effectiveness of the proposed method. As a result, we confirmed improvement in the performance of sound image localization.

\section{$10: 30$}

5aEA11. Design of distributed mode loudspeaker through evolutionary structural optimization. Goki Shirouzu (Graduate School of Design, Kyushu Univ., 4-9-1 Shiobaru Minami-ku, Fukuoka, Fukuoka 815-8540, Japan, shirouzu.g.111@s.kyushu-u.ac.jp) and Toshiya Samejima (Faculty of Design, Kyushu Univ., Fukuoka, Fukuoka, Japan)

The distributed mode loudspeaker (DML) is a flat panel loudspeaker that consists of a thin plate as a diaphragm and several driving exciters. In designing the DML, a number of flexural resonance modes are encouraged to be excited. Thereby, complex vibration is produced in the diaphragm, and the sound field around the DML is diffuse at high frequencies. In the DML design procedures, choosing proper aspect ratio of the diaphragm, driving points, and suspension points are important to deliver flexural resonance modes as many as possible. In this research, the authors focus on the shape of diaphragm. The authors try to optimize the diaphragm shape that delivers more equalized interval between the natural frequencies of the diaphragm so that it does not have many degenerated modes. To achieve this, Evolutionary Structural Optimization (ESO) method, which is one of structural topology optimization methods, is adopted. The scheme is based on the idea that, by gradually removing inefficient materials, the structure shape evolves toward optimum one. This method enables us to select the best shape of the diaphragm from shapes given in the process. Trial designs demonstrate that the ESO method is effective in designing the diaphragm of the DML.

\section{0:45}

5aEA12. Spatial post-filter estimation for speech enhancement in the specific area using a pair of microphone arrays. Takuto Yoshimizu (Graduate School of Sci. and Technol., Ryukoku Univ., 1-5, Seta Oe-cho Yokotani, Otsu-shi, Shiga-ken 520-2194, Japan, t15m083@mail.ryukoku. ac.jp) and Akitoshi Kataoka (Faculty of Sci. and Technol., Ryukoku Univ., Otsu-shi, Shiga-ken, Japan)

Suppression methods using the beamformer and the Wiener post-filter by microphone array has been proposed. However, these methods are hard to suppress noise on the environment, such as the desired source is surrounded by noise. We propose the suppression method using a pair of microphone arrays arranged in different positions on the environment, such as the desired source is surrounded by noise. We can get different information of sound by using two microphone arrays. We make the post-filter using its different information of sound. This method enhance the desired source to be the sound source in the specific area. Therefore, we divide the room into nine areas by using two microphone arrays. By estimating the cross spectrum between two microphone arrays of each partition area, we make the post-filter to noise suppression. By the experiment results under actual environment, we confirmed that this method was higher in noise suppression performance than MVDR using one microphone array that is general beamformer.

\section{1:00}

5aEA13. Noise impact assessment on residential area near highway and noise control measures development. Ilya E. Tsukernikov, Igor L. Shubin, Leonid A. Tikhomirov, Natalia E. Schurova (Res. Inst. of Bldg. Phys., Odoevskogo proezd, h.7, korp.2, fl. 179, 21 Lokomotovny pr., Moscow 117574, Russian Federation, 3342488@mail.ru), and Ilya O. Tsukernikov (Moscow State Univ. of Printing Arts, Moscow, Russian Federation)

Currently, from 30 to $50 \%$ of urban population of the Russian Federation it is affected by the increased traffic noise. A similar situation is observed not only in big cities, but also in rather small settlements. Instrumental and calculated assessment of existing noise levels in the residential area "Zarechie" adjacent to a site of the Moscow Ring Road and having a soundproofing barrier on the border of the territory is performed in the paper. Acoustic situation on the territory without existing soundproofing barrier is modeled with use of the program "ARM Acoustics," developed by Russian Company "TECHNOPROEKT" and the effectiveness of the noise reducing by the screen is evaluated. A calculation for feasibility of a further increase in the height of the existing barrier is carried out. Noise maps are built which allowed to identify the main sources of acoustic discomfort in the residential area. The analysis of the impact on the acoustic environment of the building being built is conducted. Against this background options for protecting the area from noise are proposed.

\section{1:15}

5aEA14. Wideband audio systems for hearing aid devices. Michael Salameh (Starkey Hearing Technologies, 6600 Washington Ave. S., Eden Prairie, MN 55344, michael_salameh@starkey.com)

Multi-loudspeaker design has been one of the most widely used methods for improving the sound quality of audio systems. Some of the 
electroacoustic design concepts defined for typical earphones can be used for other wideband miniature audio systems as well. However, wideband audio for hearing aid devices and smart earphones introduce new design factors. Efficiency and transducer size are among the factors that become important in the electroacoustic design. In this paper, the electroacoustic design factors for woofer-tweeter audio systems are revisited and discussed for hearing aid devices. Simulation and measurement results are presented and analyzed.

\section{1:30}

5aEA15. A broadband acoustic absorber based on phase-modulating reflective metasurfaces. Wenqi Wang, Junfei Li, Bogdan-Ioan Popa, and Steven Cummer (Elec. and Comput. Eng., Duke Univ., 3417 FCIEMAS, 101 Sci. Dr, Durham, NC 27708, wenqi.wang@duke.edu)

Most high performance acoustic absorbers exhibit outstanding absorption capabilities at resonant frequencies, thus their bandwidth are significantly limited. Acoustic metasurfaces enabled unprecedented wave manipulation with their planar profiles, subwavelength thicknesses, and large degree of design freedom. However, studies have been primarily focused on extraordinary wavefront shaping functionalities, and little explorations has been made on manipulating transmitted/reflected power of diffracted beams. By carefully tailoring the design and arrangement of acoustic metamaterial cells, enhanced absorption can be achieved for a broad range of frequencies. Building on our previous works on transmissive and reflective metasurfaces with exotic properties, including anomalous refraction, surface mode conversion, and extraordinary beam-steering (Xie et al. Nat. Commun. 2014), we present in this work a broadband acoustic absorber built with an expanded library of labyrinthine acoustic metamaterials. We demonstrate that by engineering the phase modulation profile of phase-modulating metasurfaces, incident waves can be effectively converted to surface modes that propagate along the air-metasurface interface, thus minimal reflection is observed in the far field. Our work extends functionalities of acoustic metasurfaces, demonstrates an alternative route to the design of broadband acoustic absorbers, and can be potentially applicable to noise control.

\section{1:45}

5aEA16. Intracochlear sound sensor-electrode system for fully implantable cochlear implant. Chuming Zhao, Katherine E. Knisely (Mech. Eng., Univ. of Michigan, 2350 Hayward, Ann Arbor, MI 48109, chumingz@ umich.edu), Deborah J. Colesa, Bryan E. Pfingst, Yehoash Raphael (Kresge Hearing Res. Inst., Dept. of Otolaryngology-Head and Neck Surgery, Univ. of Michigan, Ann Arbor, MI), and Karl Grosh (Mech. Eng., Univ. of Michigan, Ann Arbor, MI)

We designed, fabricated, and tested an intracochlear sound sensor-electrode system consisting of an intracochlear sound sensor (ISS) and a $50 \mu \mathrm{m}$ Pt-Ir wire electrode. This system was designed to sense acoustic signals and transmit electrical stimuli inside the cochlea. Potential applications include acting as the front end of a fully implantable cochlear implant to treat sensorineural deafness or as a transducer in cochlear mechanics experiments. The ISS was comprised of an array of piezoelectric cantilevers and was built using micro-electrical-mechanical-system (MEMS) techniques. The ISS was tested in air and underwater to compare its functionalities to the numerical predictions. Later, the ISS was implanted in an anesthetized guinea pig and tested in vivo. A 90-100dB SPL pure tone acoustic excitation over 1$30 \mathrm{kHz}$ was delivered into the ear canal of the guinea pig, and $0.1-30 \mu \mathrm{V}$ was measured by the ISS. The sensed signal was linear, repeatable, and immune to the electrical interference from the extracochlear and intracochlear environment. When using the electrode, electrical auditory brainstem response (eABR) measurements were performed by sending a $25 \mu$ s single pulse stimulus to the electrode. Stimulus amplitudes ranging in $220-400 \mu \mathrm{A}$ were found to evoke an eABR. These results show that the concept of sensing acoustic signals and transmitting electrical stimulation inside the living cochlea using one device is feasible. 


\title{
Session 5aID
}

\section{Interdisciplinary: Topical Meeting on Data Science and Acoustics I}

\author{
Matthew G. Blevins, Cochair \\ U.S. Army Engineer Research and Development Center, 2902 Newmark Drive, Champaign, IL 61822 \\ Andrew Christian, Cochair \\ National Institute of Aerospace, 100 Exploration Way, Hampton, VA 23666 \\ Joonhee Lee, Cochair \\ Durham School of Architectural Engineering, University of Nebraska - Lincoln, 1110 S. 67th Street, Omaha, NE 68182-0816 \\ Hiroshi Sato, Cochair \\ Dept. of Information Technology and Human Factors, Natl. Inst. of Advanced Industrial Sci. and Tech., Tsukuba, Japan
}

Chair's Introduction-8:35

\section{Invited Papers}

8:40

5aID1. High performance and robust audio search. Avery Wang (Shazam, 2114 Broadway St., Redwood City, CA 94063, avery. wang@shazam.com)

In this talk, I will give an overview of the Shazam audio recognition technology. The Shazam service takes a query comprised of a short sample of ambient audio (as little as 2 seconds) from a microphone and searches a massive database of recordings comprising up to 40 million songs. The query audio may be distorted with significant additive noise ( $<0 \mathrm{~dB}$ SNR), environmental acoustics, as well as nonlinear distortions. The computational scaling is such that a query may cost as little as a millisecond of processing time. Previous algorithms could index hundreds of items, required seconds of processing time, and were less tolerant to noise and distortion by 20-30 dB SNR. In aggregate, the Shazam algorithm represented a leap of more than $1 \mathrm{E}+9$ in efficiency. I will discuss the various innovations leading to this result.

5aID2. Data visualization methods. Claudio Silva (New York Univ., 2 MetroTech Ctr., Rm 10.093, New York, NY 11201, csilva@ nyu.edu)

Future advances in science, engineering, and medicine depend on the ability to comprehend the vast amounts of data being produced and acquired. Data visualization is a key enabling technology in this endeavor: it helps people explore and explain data through software systems that provide a static or interactive visual representation. Despite the promise that visualization can serve as an effective enabler of advances in other disciplines, the application of visualization technology is non-trivial. The design of effective visualizations is a complex process that requires understanding of existing techniques and how they relate to human cognition. For a visualization to be insightful, it needs to be both effective and efficient. This requires a combination of design and science to reveal information that is otherwise obscured. In this talk, we will give a general background for data visualization, and present some work on interactive visualization techniques and tools for a variety of analysis purposes, including signal processing, some of which can be repurposed for the analysis of audio data.

\section{0:10-10:30 Break}

\section{0:30}

5aID3. Primitives in time series mining: Algorithms and applications. Abdullah A. Mueen (Comput. Sci., Univ. of New Mexico, 1 University of New Mexico, Albuquerque, NM 87131, mueen@unm.edu)

Time series patterns are waveforms with properties useful for various data mining tasks such as summarization, classification and anomaly detection. In this talk, I present three types of time series patterns: Motifs, Shapelets, and Discords. Motifs are repeating patterns that repeat in seemingly random time series data; Shapelets are small segments of long time series characterizing their sources; Discords are anomalous waveforms in long time series that do not repeat anywhere else. I briefly discuss efficient algorithms to discover these patterns and present cases in mining data from robots, humans and social media. Cases include activity classification using accelerometer data, correlated clusters in social media data, and anomaly in astronomical data. 
5aID4. The promises and realities of machine learning. Dan Valente (Chartbeat, 826 Broadway, 6th Fl., New York, NY 10003, dan@ chartbeat.com)

These days, anyone with a laptop, Python, and a free afternoon can build an intelligent machine. In fact, if you have a bunch of data, go ahead and throw it into a deep neural net: your worries will be over! Machine learning will save us all! Unfortunately, those of us who spend our days fighting with these systems know that this is far from the truth. As anything created by humans, these algorithms have limitations, and there is no algorithmic substitute for careful thought. The gory details of techniques like Support Vector Machines, Neural Networks, and K-means clustering can be found in innumerable blog posts, texts, and online courses; we won't discuss the details here. Instead, this talk will focus on the conceptual and mathematical framework under which all machine learning algorithms fall. We'll briefly discuss the workings of a few of the most popular modern techniques (\#DeepLearning, anyone?) and how they fit into this framework. More importantly, we'll discuss some often-overlooked considerations that go into the design of these systems: human biases, transparency, and maintainability. Ultimately, we hope to provide a solid understanding of what these algorithms can do, what they can't do, and when they should or should not be used.

FRIDAY MORNING, 2 DECEMBER 2016

HIBISCUS, 8:30 A.M. TO 11:30 A.M.

\title{
Session 5aMU
}

\section{Musical Acoustics: General Topics in Musical Acoustics I}

\author{
James P. Cottingham, Chair \\ Physics, Coe College, 1220 First Avenue NE, Cedar Rapids, IA 52402
}

\section{Contributed Papers}

\section{8:30}

5aMU1. Analysis of the relationship between muscle activity and acoustic features during trumpet play and the construction of a myoelectric visual feedback system. Megumi Satou (Library, Information and Media Studies, Univ. of Tsukuba, 1-2, Kasuga, Tsukuba, Ibaraki 305-8550, Japan, megumi@slis.tsukuba.ac.jp), Tetsuro Kitahara (Information Sci., Nihon Univ., Tokyo, Japan), Hiroko Terasawa, and Masaki Matsubara (Library, Information and Media Studies, Univ. of Tsukuba, Tsukuba, Ibaraki, Japan)

Breath control and lip vibration are crucial for a stable performance while playing the trumpet. We analyzed the differences in abdominal and orofacial muscle activity in acoustic features such as pitch (Bb2, F3, Bb3, $\mathrm{F} 4, \mathrm{~B} b 4)$, intensity (pp, $\mathrm{mf}, \mathrm{ff})$, and duration $(0.75,6 \mathrm{~s})$ during the preparation and sustain periods using surface electromyography in 11 amateur trumpeters. When the pitch was high, the activity of both muscles increased in both the preparation and sustain periods. However, when the intensity was high, the activity of both muscles increased only during the sustain period. Orofacial muscle activity was lower after tone production and abdominal muscle activity was higher after tone production than before tone production. In addition, we developed a visual feedback system that displays the muscle activities and acoustic features related to the produced sound as biofeedback can make learning performing technique efficient (LeVine, 1984). This system enables the player to objectively recognize the acoustic features and muscle activities related to sound. We aim to enhance performing technique by determining the relationship between the use of the body during a performance and the produced sound.

\section{8:45}

5aMU2. Sound has size: Stages of concert halls. James B. Lee (None, 6016 S. E. Mitchell, Portland, OR 97206, cadwal@macforcego.com)

Sabine characterized whole concert halls by ratio of volume to absorption, which devolves into a "reverberation time" of logarithmic decay of intensity of sound. These times, on the order of seconds, are generic but indeterminate functions of architecture. The critical time for human perception, the aural integration period, where perception of time (intervals) passes over to inverse time (frequencies), is two orders of magnitude shorter, and can be used to scale and define the "orchestral region" of a hall. In $25 \mathrm{~ms}$ sound travels $8.5 \mathrm{~m}$, and $8.5 \mathrm{~m}$ is the wavelength of $40 \mathrm{~Hz}$, near low E, the lowest orchestral note. If, by analogy with optics, $8.5 \mathrm{~m}$ is thought of as a "coherence length," it affects the time domain by promoting ensemble and the frequency domain by enhancing bass tones through proximity effects. A primary property of human hearing so scales the stages of concert halls.

\section{9:00}

5aMU3. Suppression of wolf-tone based on equivalent circuit model. Kei Ogura (Graduate School of Systems and Information Eng., Univ. of Tsukuba, 1-1-1 Tennodai, Tsukuba, Ibaraki 305-8577, Japan, ogura@aclab. esys.tsukuba.ac.jp), Koichi Mizutani, and Naoto Wakatsuki (Faculty of Eng., Information and Systems, Univ. of Tsukuba, Tsukuba, Japan)

Large size bowed stringed instruments, such as cello can pose undesired phenomenon called "wolf-tone" when you play at the specific pitch. When it occurs, a body strongly vibrates and a bow leaps on a string continuously. So, we are trying to figure out the cause of the wolf-tone and control it effectively. Thus, we devised "body-string coupled model" where body and string are coupled at bridge, and "bowed-string model" which expresses the relation between frictional force and relative speed, as it is called "stick and slip motion." We simulated using finite-difference time-domain method and figured out the result that reproduces particular string motion called "Helmholz motion." In addition, we set two parameters, speed of bow and pressure of bow, and calculated sound wave, oscillation mode, and the number of stick and slip. Then, we got the result of some existence ranges for wolf-tone. On this simulation, we added a small resonator in order to suppress the specific resonance mode which provides wolf-tone and observed wave forms. Based on these simulation results, we verified its effect experimentally on a real instrument. 
5aMU4. Investigations of the coupling of doubled mandolin strings using high-speed video. Steve Tufte, Zach Rose, and Karlie Schwartzwald (Dept. of Phys., Lewis \& Clark College, MSC 15, 0615 SW Palatine Hill Rd., Portland, OR 97219, tufte@1clark.edu)

The mandolin has four courses of doubled strings and in each pair the strings are tuned in unison. By using a macro lens on a quality high-speed camera, we are able to study the bridge-coupled motions of the strings in exquisite detail. We use an angled mirror to allow monitoring of the vertical and horizontal motions of both strings in a pair. With small colored tags on each string, we have automated the amplitude vs. time measurements using the Tracker Video Analysis tool. We observe clear evidence of coupling including the exchange of energy between the four modes. There is a strong tendency for the two strings to anti-correlate, while the vertical and horizontal modes of one string usually differ in phase by 90 degrees. We clearly observe the double-decay phenomena noted in Weinreich's study of piano strings. The coupling thus serves to add richness to mandolin tones and also increases the sustain. We compare the experimental results to a Runge-Kutta coupled oscillator model implemented in Matlab. Matching the observations requires the model damping constants to be slightly larger than the coupling constants and a slight detuning of the strings is necessary to produce the observed anti-correlated behavior.

5aMU5. Higher-order frequency locking of an organ pipe. Masahiro Okada and Tokihiko Kaburagi (Graduate School of Design, Kyushu Univ., 4-9-1 Shiobaru, Minami-ku, Fukuoka 815-8540, Japan, 3DS16004N@s. kyushu-u.ac.jp)

To investigate frequency locking of an organ pipe, Abel et al. forced a sounding pipe with a pure tone (J. Acoust. Soc. Am. 119, 2467-2475, 2006). They showed that when the frequency of the pure tone approaches that of the pipe's fundamental, frequency locking can take place, whereby both frequencies are identical; that is, they are synchronized with a 1:1 frequency ratio. To gain a deeper understanding of organ pipes from the viewpoint of nonlinear oscillators, we searched for frequency locking at different ratios of the pure tone to the pipe's fundamental (pipe : pure tone $=m: n$ ). First, experiments were carried out for $\mathrm{m}: \mathrm{n}=1: 2,1: 3,2: 1$, and $2: 3$. We demonstrated that frequency locking occurs only if $\mathrm{m}=1$; that is, when the pure tone approaches the second or third harmonic of the pipe sound. We then analyzed our observations using synchronization theory, which describes frequency locking of a limit cycle oscillator. We found that $\mathrm{m}$ should be one when the external signal is a pure tone. The behavior of the organ pipe agrees well with that predicted according to synchronization theory; hence, the organ pipe can be treated as a nonlinear oscillator.

\section{9:45}

5aMU6. The effect of harmonic overtones in relation to "sharpness" for perceived brightness of distorted guitar timbre. Koji Tsumoto, Atsushi Marui, and Toru Kamekawa (Tokyo Univ. of the Arts, 1-25-1 Senju, Adachi-ku, Tokyo 120-0034, Japan, tsumoto@tcn-catv.ne.jp)

Perceived timbral brightness is often predicted by "sharpness," which is the conventional predictor described by von Bismarck. Perceived brightness and the predicted "sharpness" value increases when the energy of harmonic overtones in high-frequency range increases. Studies regarding the relationship between "sharpness" and harmonic overtones are relatively rare. "Sharpness" and harmonic overtones may have the synergistic effect or the canceling effect over perceived brightness. The electric guitar is one of the representative musical instruments which controls its timbre by adjusting the harmonic overtones. Under the assumption that there is a specific canceling effect, the stimuli generated by non-linear distortion processor for the electric guitar were compared for the first experiment. The values of the "sharpness" were adjusted by shelving filters. The onset of the each stimulus was deleted for the second experiment, since any variables other than "sharpness" and the harmonic overtones needed to be eliminated. The result indicated that "sharpness" and the amount of harmonic overtones had a canceling effect. A high amount of harmonic overtones decreased the perceived brightness. This result provokes the question of "why did the harmonic overtones cancel the perceived brightness predicted by sharpness?" This canceling effect was discussed by observing acoustic features.
10:00-10:15 Break

\section{0:15}

5aMU7. Acoustic design of timpani using vibro-acoustic numerical analysis. Yozo Araki (Graduate School of Design, Kyushu Univ., 4-9-1 Shiobaru, Minami-ku, Fukuoka 815-8540, Japan, araki.yozo.105@s. kyushu-u.ac.jp) and Toshiya Samejima (Faculty of Design, Kyushu Univ., Fukuoka, Japan)

A vibro-acoustic analysis method of timpani reveals several conditions taking account of the interdependency among design parameters of timpani for delivering harmonic overtones. The head of a timpani is analyzed with the modal expansion type of the theoretical solution for an ideal circular membrane. The sound field around the timpani is analyzed with the normal derivative form of boundary element method. The theoretical solution of the membrane is included into the boundary element method as a characteristic matrix through proper vibro-acoustic coupling conditions. Therefore, the vibration displacement of the membrane is not included as unknowns in the equation to be solved. Frequency response functions of a timpani calculated with the analysis method show good agreement with measured ones. A timpani is designed based on the analysis method such that it has a strong sense of pitch. Tensions and surface densities of heads and kettle shapes that shift eigenfrequencies of the timpani into nearly harmonic ratios are presented. It is concluded that the consideration of the interdependency among such design parameters of timpani is important for composing harmonic overtones.

\section{0:30}

5aMU8. The effect of adaptive music playing system on emotion regulation. Wei-Chun Wang (Dept. of Humanities and Social Sci., National Taiwan Univ. of Sci. and Technol., No. 43, Sec. 4, Keelung Rd., Taipei 10607, Taiwan,vgnwang@hotmail.com)

We live in an industrially polluted environment, surrounded with stress and negative emotions. Since music has the marvelous power of arousing different moods and regulating emotions depending on the features of musical elements, people could be prescribed specific kinds of music to help alleviate their symptoms. Therefore, refer to entrainment, iso, and diversion principles of music therapy stated by Campbell, the investigator developed an Adaptive Music Playing System base on the two-dimensional arousal-valence emotion model. The purposes of this study were: (1) to explore the two-dimensional emotional responses of subjects when listening to specific music; (2) to survey the effects of gender, music preference on their perception of musical emotions; (3) to compare the relativities of various musical elements with musical emotions; (4) to detect the healing effect of adaptive music playing system. 56 college students were recruited as subjects in this study. Subjects imported their instant mood value, and the Adaptive Music Playing System would automatically play three music pieces. The results of this study showed that Adaptive music playing system could successfully lead the subjects in a better physical and mental condition. Gender effect wasn't significant for subjects' perception. Music preference affected subjects' perception.

\section{0:45}

5aMU9. Sixteen types' chord label estimation from acoustic signal of electric guitar. Nozomiko Yasui (National Inst. of Technol., Matsue College, 14-4 Nishi-ikuma-cho, Matsue, Shimane 690-8518, Japan, n_yasui@ matsue-ct.jp) and Masanobu Miura (Faculty of Sci. and Technol., Ryukoku Univ., Otsu, Shiga, Japan)

It's difficult to determine an chord label for acoustical signal of musical playing, in particular when playing chords with "omitting", "inversions," or "tension voicing" on the guitar. Additionally, "enharmonic equivalence" produces multiple possibilities. This study developed a chord estimation system that deals with an audio signal output from electric guitars considering such techniques. All of the chord types employed in this study are the sixteen patterns frequently used in guitar chord playing. Chord labels are estimated by combination of salient pitch classes (or chroma), and some of them are dealt with as "performed notes" assumed as the member of played chord. Obtained performed notes are input to the "search tree for chord 
labels" so as to search possible chord labels, by referring chord progression patterns included in the "chord progression database." Sixteen chord types are triad such as major, minor, aug, dim and sus4, with four-note chords such as 6th, 7th, Maj7, aug7, 7sus4, add9, min6, $\min 7, \operatorname{minMaj} 7, \min 7(\mathrm{~b} 5)$, and $\operatorname{dim} 7$. This study investigated appropriate threshold and type of filter used in the judgment of performed note. Results found that chord labels are estimated as $89 \%$ of accuracy when using three types of electric guitars.

\section{1:00}

5aMU10. Effect of music experience on perception of tempo change. Mayuko Yamashita, Masuzo Yanagida (Doshisha Univ., 1-3 Tatara Miyakodani, \#221 Kochikan, Kyotanabe, Kyoto 610-0394, Japan, duq0171@mail4. doshisha.ac.jp), Ichiro Umata (KDDI R\&D Labs., Tokyo, Japan), Tsuneo Kato, and Seiichi Yamamoto (Doshisha Univ., Kyotanabe, Kyoto, Japan)

Tempo is one of the basic factors in music expression and perception. Although there have been studies on the perception of tempo change, little is known about how the type of music experience affects the sensitivity to this change. We analyze the effects of music experience on the perception of tempo change to contribute to music education. Our analysis focuses on sensitivity to tempo change. Participants were classified into three groups according to their musical experience: (A) inexperienced in any musical instrument, (B) players majoring in the piano, and (C) amateur players belonging to brass bands. We performed experiments in which monotone piano sequences that gradually change tempo from the initial inter-onset interval (IOI) to the target IOI were used. We manipulated three tempo change patterns, namely, (I) linear, (II) exponential, and (III) the average of (I) and (II). We compared the point of tempo change perception among the three groups with the assumption that the sensitivity would be higher in (B) and (C) than in (A). The results, however, showed that the sensitivity was lowest in (B), except for one player who often plays with other players. These results suggest that ensemble experience may affect sensitivity to tempo change.

\section{1:15}

5aMU11. Automatic arrangement for ensemble music by estimating playing difficulty on instrumentalists. Nozomiko Yasui, Shiori Nabara (National Inst. of Technol., Matsue College, 14-4 Nishi-ikuma-cho, Matsue, Shimane 690-8518, Japan, n_yasui@matsue-ct.jp), and Masanobu Miura (Faculty of Sci. and Technol., Ryukoku Univ., Otsu, Shiga, Japan)

Since arrangement of sheet music for orchestral ensemble requires a certain musical knowledge and expertise, amateur orchestral musicians often have difficulties when arranging. An automatic arrangement method was proposed by employing Eigenmusic which is a set of eigenvector for many music excerpts, in order to evaluate the similarity among phrases. The difficulty in playing the arranged sheet music, however, was not evaluated; so, whether players feel difficulties on playing, or playability, was out of discussion. This study proposes a method to evaluate the degree how easy to play the phrases on sheet music. This method generates phrases for woodwind instruments using a MIDI database of backing phrases. Each musical phrases selected from the database are allocated to instrumentalists. In turn, the phrases' playability is evaluated based on instrumentalist's individual ratings for playing features such as cross-fingering, jumping notes, and so forth. Finally, phrases evaluated as the easiest are conjoined in each instrumentalist. We conducted an experiment to investigate the playability of generated sheet music by musician's rating. Experimental results showed that the evaluation of playability by proposed method is effective so that proposed method generates easier phrases to play than our previous method. 


\title{
Session 5aNSa
}

\author{
Noise: Wind Turbine Noise \\ Yasuaki Okada, Cochair \\ Meijo University, 1-501 Shiogamaguchi, Tempaku-ku, Nagoya 468-8502, Japan \\ Paul D. Schomer, Cochair \\ Schomer and Associates Inc., 2117 Robert Drive, Champaign, IL 61821 \\ Nancy S. Timmerman, Cochair \\ Nancy S. Timmerman, P.E., 25 Upton Street, Boston, MA 02118
}

\section{Invited Papers}

\section{8:00}

5aNSa1. On translating minimum siting distances into percentages of receiving properties meeting a stated dB(A) criterion. Paul D. Schomer and Pranav K. Pamidighantam (Schomer and Assoc. Inc., 2117 Robert Dr., Champaign, IL 61821, schomer@SchomerAndAssociates.com)

Around the world, minimum siting distances are used by regulators and developers to limit the effects of wind turbine noise on people. Acousticians know that the proper calculation is equal sound level contours, but customers, in this case the communities, developers, and regulators, all want simpler solutions. This study makes use of data collected at over 1200 dwelling units as a part of the Health Canada Study. This paper provides a method to determine minimum siting distances based on predicted percentages of exceedances of $\mathrm{dB}(\mathrm{A})$ criterion at dwellings.

5aNSa2. Can inaudible and audible low level Infrasound and low frequency noise be an acoustic trigger of the startle reflex? Steven E. Cooper (The Acoust. Group, 22 Fred St., Lilyfield, NSW 2040, Australia, drnoise@acoustics.com.au)

Modulation of audible low frequency ventilation fan noise reproduced in a laboratory has been shown to trigger the startle reflex in people sensitised to that noise. Using a range of low frequency and infrasound noise signals to sensitised and unsensitized subjects can show a causal relationship between an acoustic trigger and a physiological stress response, which engineers call "annoyance" or "noise annoyance" symptoms, and which biologists recognize as the "startle reflex." Utilizing low level amplitude and frequency modulation as the source triggers a "startle reflex" response for comparison with the typical "startle reflex" to high level noise impulses.

\section{8:40}

5aNSa3. Wind farm infrasound-Are we measuring what is actually there of something else-(Part 3). Steven E. Cooper (The Acoust. Group, 22 Fred St., Lilyfield, NSW 2040, Australia, drnoise@acoustics.com.au)

With the method of analysis demonstrated in part 2 of the subject title, and the requirement for accurate reproduction of the signal (presented in Salt Lake City) the hypothesis as to infrasound contributions is revised in light of further investigations with test subjects for both inaudible ILFN and audible LFN to $50 \mathrm{~dB}(\mathrm{~A})$. The work ties in with the investigations into the startle reflex using wind turbines as the source of the acoustic trigger. The ramifications as to suitable criteria for the protection of the community are discussed.

\section{9:00}

5aNSa4. Measures of sway as perceptual response to infrasound. Peggy B. Nelson, Michael Sullivan, Meredith Adams, and Andrew Byrne (Ctr. for Applied/Translational Sensory Sci., Univ. of Minnesota, 164 Pillsbury Dr. Se, Minneapolis, MN 55455, peggynelson@ umn.edu)

Some reports suggest possible negative human perceptual responses to infrasound and audible noise from wind turbines. Recordings were made in the field of turbine noise (audible and infrasound) at 300 meters. In our multisensory laboratory (Center for Applied and Translational Sensory Science at the University of Minnesota) we are re-creating these natural stimuli in a controlled environment. Infrasound stimuli were presented at approximately $85 \mathrm{~dB}$ SPL; audible stimuli were $55 \mathrm{~dB}$ SPL. Stimulus configurations were combinations of audible and infrasound emissions, testing the effects of these stimuli individually and in combination. We measured sway using an AMTI AccuSway Optimized force plate. Preliminary data suggest that measures of sway are stable and reproducible, and that some persons may show changes in stance in the presence of combinations of audible and infrasound stimuli. [Funding provided by Xcel Energy RD-14 to the University of Minnesota.] 
5aNSa5. Observations of vibration velocity on wind turbine with propagated sound to surroundings. Teruo Iwase (Niigata Univ., 8050 Igarashi 2 Nocho Nishi-ku, Niigata 950-2181, Japan, iwase@cc.niigata-u.ac.jp), Hideo Uchida (NS Environ. Sci. Consultant, Saitama, Japan), Hiroyasu Kurono (Faculty of Eng., Meijo Univ., Niigata, Japan), Yasuaki Okada, and Koichi Yoshihisa (Faculty of Sci. and Technol., Meijo Univ., Nagoya, Japan)

The authors newly tried measurements of the excited vibration on many parts of wind turbine such as outside surface of nacelle storing power generation system and tall tower by using a laser Doppler vibration meter. Observation and analysis of the natural vibration frequencies of blade in the stopping were done. Observations on sound in surroundings of wind turbine were also done. FFT analyses on them with high resolution to obtain detailed frequency characteristics and to know the relations between vibration velocity and propagated sound to surroundings were done in addition to ordinal spectral analyses. Sharp spectra at near $1 \mathrm{~Hz}$ as slightly lower or higher were appeared in the analysed results on blade and tower. In both the analyzed results of vibration velocity on wind turbine and propagated sound, a lot of sharp spectral peaks were recognized in wide frequency range from very low frequency to several hundreds of hertz with high coherencies between vibration velocity and propagated sound at their peak spectral frequencies. Certain values of coherency were remained even for the case of sound received in about $200 \mathrm{~m}$ distant from a wind turbine. These observed results would be effective for understanding key parts to make sound radiation lower.

\section{9:40}

5aNSa6. Experimental study on noise radiation from wind turbines. Yasuaki Okada, Yui Mizutani, Koichi Yoshihisa (Meijo Univ., 1-501 Shiogamaguchi, Tempaku-ku, Nagoya, Aichi 468-8502, Japan, okada@meijo-u.ac.jp), and Teruo Iwase (Niigata Univ., Niigata, Japan)

Noise emitted from wind turbines is composed of aerodynamic and mechanical sound and has directional radiation characteristics. To investigate the horizontal sound directivity around a wind turbine under various wind conditions, field measurements of noise generated from two different wind turbines have been performed over long periods. Wind turbine operational data such as the nacelle direction and rotor rotational speed were collected at $1 \mathrm{~s}$ intervals along with corresponding acoustic data. An empirical formula for the directivity correction was derived from the A-weighted sound pressure levels measured at the several receiving points around the wind turbine. We also focused on the amplitude modulation components of wind turbine noise in emission areas and compared the estimated rotor speeds by using measured sound pressure levels with actual values. The results showed that the directivity pattern of the Aweighted sound pressure level for two different wind turbines is almost the same, whereas the frequency dependence of the sound directivity is different for the individual wind turbines. Additionally, the radiation characteristics of wind turbine noise depend strongly on the rotor rotational speed, which can be estimated from the blade-passing-frequency detected by using the amplitude modulation components of wind turbine noise.

10:00-10:15 Break

\section{Contributed Papers}

10:15

5aNSa7. The influence of periodic wind turbine noise on infrasound array measurements. Christoph Pilger and Lars Ceranna (BGR Hannover, Stilleweg 2, Hannover 30655, Germany, christoph.pilger@bgr.de)

Aerodynamic noise from the continuously growing number of wind turbines in Germany creates increasing problems for infrasound array measurements recording acoustic signals at frequencies below $20 \mathrm{~Hz}$. Ten years of continuous data (2006-2015) from the 4-element infrasound array IGADE in Northern Germany are analysed to quantify the influence of wind turbine noise in terms of enhanced amplitude modulations. Furthermore, a theoretical model is derived and validated by a field experiment with mobile microbarometers. Fieldwork was carried out to measure the infrasonic pressure level of a single horizontal-axis $200 \mathrm{~kW}$ wind turbine and to extrapolate the noise effect for turbines with higher electric powers and for a larger number of collocated wind turbines. The model estimates the generated sound pressure level of wind turbines and thus enables for specifying the minimum allowable distance between wind turbines and infrasound stations for undisturbed recording. This aspect is particularly important to guarantee the monitoring performance of the German infrasound stations I26DE in the Bavarian Forest and I27DE in Antarctica. These stations are part of the International Monitoring System (IMS) verifying compliance with the Comprehensive Nuclear-Test-Ban Treaty (CTBT), and thus have to meet stringent specifications with respect to infrasonic background noise.
$10: 30$

5aNSa8. A numerical study of inflow turbulence distortion in the vicinity of blade leading edges. Thomas Hainaut, Gwenael Gabard, and Vincent Clair (Inst. of Sound and Vib. Res., Univ. of Southampton, Bldg. 13 R2009, University Rd., Southampton SO171BJ, United Kingdom, t.hainaut@soton.ac.uk)

The interaction of the rotor with inflow turbulence is a source of broadband noise, dominant at high wind speeds. In the vicinity of the leading edge of realistic blades, the mean flow distorts the turbulence, resulting in an attenuation of the high-frequency part of the radiated noise compared to zero-thickness blades. In this paper, to study this turbulence distortion by the mean flow, two different numerical approaches are considered. First, the linearized Euler equations are solved in the time-domain using a finite difference code to model the response of an isolated blade interacting with synthetic turbulence. Second, a vorticity approach is applied, using the BiotSavart law combined with a vortex panel method. Using these approaches on multiple configurations, the up-wash velocity fluctuations along a streamline which goes to the stagnation point, show a decrease of the turbulent levels, from a threshold distance independent of the wavenumber. This decay is then inverted for low frequencies after a wavenumber dependent threshold distance. The turbulence characteristics are found to have no effect on the distortion, whereas the geometry forward the position of the maximum thickness has an effect on this distortion. The full paper will contain more physical insights responsible for this distortion. 


\title{
Session 5aNSb
}

\section{Noise, ASA Committee on Standards, and Architectural Acoustics: Innovations in Floor Impact Noise Testing and Evaluation}

\author{
David Lubman, Cochair \\ DL Acoustics, 14301 Middletown Ln., Westminster, CA 92683-4514 \\ John LoVerde, Cochair \\ Veneklasen Associates, 1711 16th St., Santa Monica, CA 90404 \\ Manabu Tanaka, Cochair \\ General Building Research Corporation of Japan, Fujishiro-dai 5-8-1, Suita, Osaka 5650873, Japan \\ Chair's Introduction-10:00
}

Invited Papers

10:05

5aNSb1. Acoustic characterization of in-room footfall noise. Samantha B. Rawlings (Veneklasen Assoc., 1711 16th St., Santa Monica, CA 90404, srawlings@veneklasen.com), Joshua A. Magee (Lawrence Livermore National Lab., Livermore, CA), Robert M. Tanen (Shen Milsom Wilke, New York City, NY), Jonathan C. Silver (none, Tel Aviv, Israel), and Robert D. Celmer (Acoust. Program \& Lab, Univ. of Hartford, West Hartford, CT)

Existing literature concerning footfalls is primarily focused on its transmission between spaces, such as a floor/ceiling's Impact Isolation Class. Studies at the University of Hartford measured in-room sound power spectra produced by footfalls on twelve different floor surfaces using human subjects and a standard tapping machine. Within a qualified reverberation room, fourteen male and female subjects walked on the floor surfaces while wearing three different types of footwear: leather-soled shoes (hard), rubber-soled shoes (medium), and sneakers (soft). Sound power spectra and vibratory signatures were measured in 1/3 octaves according to the ISO 3741 standard. A tapping machine was also used on each floor profile using both standard drop weights and with cored samples of the same shoe soles attached to the bottom of each weight. The data for each floor profile produced averages by shoe type along with corresponding $95 \%$ confidence intervals. Correlations between sound power and vibratory spectra produced with human walkers versus tapping machine (both with and without "shoes") were investigated, resulting in correction factors to model machine tapping as human footfalls. [Work supported by The Paul S. Veneklasen Research Foundation.]

\section{0:25}

5aNSb2. American Society of Testing Materials draft standard for rating in-room floor impact noise. Jerry G. Lilly (JGL Acoust., Inc., 5266 NW Village Park Dr., Issaquah, WA 98027, jerry@jglacoustics.com)

In 2010 an ASTM subcommittee was formed to assess the possibility of rating the noisiness of different floor surfaces from impacts such as footfalls and dropped objects. Since that time the subcommittee has been meeting biannually to discuss potential impact noise sources and measurement techniques that could be used in a future standard. Different impact sources were evaluated including footfalls with different shoe types, golf balls, and the standard mechanical tapping machine. Both field and laboratory measurements were considered. This paper presents some of the preliminary test results that were obtained and the current status of the proposed laboratory standard.

\section{0:45}

5aNSb3. Lateral impact noise isolation: motivation, methods, and mitigation. John LoVerde and David W. Dong (Veneklasen Assoc., 1711 16th St., Santa Monica, CA 90404, jloverde@veneklasen.com)

Impact noise isolation measurements and evaluation are traditionally defined and considered for vertical adjacencies exclusively, where the impact source is located in the space above the receiving room. ASTM E1007 has specific protocols, procedures and methods for the measurements and analysis of the data collected. However, impact noise transmission into the receiving room laterally adjacent to the source room (i.e., on the same floor) is important in some conditions (e.g., condominiums, apartments, and hotels). The existing standard provides no guidance for performing a lateral impact noise isolation measurement. The authors have developed methods and practices for performing this measurement. These are presented along with a selection of results demonstrating potential issues, criteria, and mitigation. 
5aNSb4. Difference in subjective magnitude between heavy-weight floor impact sources using auditory experiment. Jongkwan Ryu and Minju Jeong (Chonnam Univ., School of Architecture, Chonnam National University, 77 Yongbong-ro, Buk-gu, Gwangju 61186, South Korea, jkryu@jnu.ac.kr)

Difference in subjective magnitude between heavy-weight floor impact sources was investigated using two auditory experiments. In the first experiment, subjective magnitude to various level (41 56 dB, L'i,Fmax,Aw) of heavy-weight floor impact sound by bang machine, impact ball and children's jumping was investigated using rating scale method (7 points scale). And then, point of subjective equality (PSE) of floor impact sounds by bang, ball and children's jumping was drawn using relative annoyance judgement method in second experiment. Stimuli were pairs consisting of the reference (fixed sound level in $50 \mathrm{~dB}, \mathrm{~L}$ 'i,Fmax,Aw) and test stimulus (varied sound level), and the subjects rated the relative annoyance of test stimuli compared with a reference stimulus using a numerical scale $(-3 \sim 0 \sim+3)$. Results showed that floor impact sound level by bang machine was $3 \sim 4 \mathrm{~dB}$ lower than that of impact sound by impact ball in the PSE. It was also found that subjective magnitude of floor impact sound by ball was quite similar as impact sound by children jumping.

\section{Contributed Paper}

\section{1:25}

5aNSb5. High-sound-insulation double floor structure with Helmholtz resonators: Experimental study on effect of resonator specifications. Yosuke Yasuda, Syunpei Hirose, Hidehisa Sekine (Dept. of Architecture, Faculty of Eng., Kanagawa Univ., Rokkakubashi 3-27-1, Kanagawa-ku, Yokohama-shi, Kanagawa 221-8686, Japan, yyasuda@kanagawa-u.ac.jp), and Mitsuru Yabushita (YAB Corp., Yokohama, Kanagawa, Japan)

We have developed a high-sound-insulation double floor structure using Helmholtz resonators. The main targets of this structure are a lot of old apartment buildings that should be renovated, which have thin slabs and cannot sufficiently insulate floor impact sound. The proposed double floor structure greatly improved the insulation performance for heavy-weight floor impact sound in a real old apartment building with 110-mm slab: about $10 \mathrm{~dB}$ for $63-\mathrm{Hz}$ and $125-\mathrm{Hz}$ octave bands. In this presentation, experimental results are shown using a small unit structure partially taken out from the whole double floor structure to thoroughly grasp the actual vibration phenomenon. The results are compared to calculation results obtained from a two-particle system theory. Main conclusions are summarized as follows: (i) A sufficiently large rigidity of the resonator walls and a large number of resonator necks are required for obtaining a clear dip in the vibration transmissibility as calculated from the two-particle system theory. (ii) The horizontal and vertical displacement of the resonator necks hardly affects the vibration transmissibility. (iii) The effects of other factors such as the neck length and neck number are qualitatively predictable by the two-particle system theory. 


\title{
Session 5aPA
}

\section{Physical Acoustics: Ultrasonics and Non-Destructive Testing}

\author{
Jinying Zhu, Chair \\ Civil Engineering, University of Nebraska-Lincoln, 1110 S 67th St, PKI 204C, Omaha, NE 68182
}

\section{Contributed Papers}

\section{$8: 30$}

5aPA1. A non-contact detection of the internal defect in solid material by aerial ultrasonic beam. Yuriko Mukaiyama (Elec. Eng., Nihon Univ., 4-34-11, Ohizumimachi, Nerima-ku, Tokyo-to 178-0062, Japan, csyu16035@g.nihon-u.ac.jp), Ayumu Osumi, and Youichi Ito (Elec. Eng., Nihon Univ., Chiyoda-ku, Tokyo-to, Japan)

Measurement method using aerial sound waves and an optical equipment is utilized in a non-contact and non-destructive testing. Technique that we have been conventionally proposed, uses high-intensity aerial ultrasonic waves $(20 \mathrm{kHz})$. Specifically, a surface of an object is vibrated by high-intensity ultrasonic waves with a point focus. A vibration generated on the surface of the object is measured by laser Doppler vibration galvanometer. In general, the vibration of defect area is larger than that of defect-free area when the sound waves were irradiated to the object. Therefore, by performing the measurement over a target area, it is possible to image the defect area from the vibration distribution. However, this method needs a long time to measure the object with the defect. Therefore, we adopted a method of irradiating high-intensity ultrasonic to a wide range on the surface of the object using an aerial ultrasonic waves focused into a beam. In this report, we describe the details of the experimental results to detect defects in the object by using this technique.

\section{$8: 45$}

5aPA2. Non-contact method for detecting crack in shallow layer of solid materials by using very high-intensity aerial ultrasonic wave and optical equipment. Ayumu Osumi (Nihon Univ., 1-8,KandaSurugadai, Chiyoda 101-8308, Japan, oosumi@ele.cst.nihon-u.ac.jp), Masashi Ogita, and Youichi Ito (Nihon Univ., Chiyoda-ku, Japan)

We have studied a noncontact and nondestructive method of imaging by using a high-intensity aerial ultrasonic waves and an optical equipment. This non-contact method detects a defect in solid materials by measuring a vibration velocity distribution on an object surface continuously irradiated by the aerial ultrasonic waves. In previous study, it was confirmed to image a relatively large peeling and defect in size by proposed method. Meanwhile, one of nondestructive testing target is a crack. It is difficult to vibrate the crack by this method has a lack of sound pressure of irradiating sound wave because the crack is very smaller than peeling. Therefore, in this study, we developed a new very high-power aerial ultrasonic sound source and attempted to detect the crack. In this report, we investigated to detect a crack in shallow layer of solid materials by this ultrasonic sound source. As a result, it is confirmed to image the crack around the shallow layer of object and the micro crack for extending to front surface of object by proposed method.

\section{9:00}

5aPA3. Acoustic resonances greatly improve detection of leaks from large pressure vessels. Keith A. Gillis and Michael R. Moldover (Sensor Sci. Div., National Inst. of Standards and Technol., 100 Bureau Dr., Mailstop 8360, Gaithersburg, MD 20899-8360, keith.gillis@nist.gov)

We measured the pressure $p$ and the acoustic resonance frequency $f_{\mathrm{a}}$ of argon gas in a $300 \mathrm{~L}$ tank. We then used the ratio $p / f_{\mathrm{a}}^{2}$ (proportional to the mass $M$ of gas) to determine the fractional leak rate $(\mathrm{d} M / \mathrm{d} t) / M \approx$ $-13 \times 10^{-6} / \mathrm{hr}$ of gas from the tank. The tank was filled with argon at 450 $\mathrm{kPa}$ and was exposed to sunshine-driven temperature and pressure fluctuations as large as $(\mathrm{d} T / \mathrm{d} t) / T \approx(\mathrm{d} p / \mathrm{d} t) / p \approx 5 \times 10^{-2} \mathrm{~h}^{-1}$ (i.e., $1000 \times$ larger) in a 24-hour record. This leak could not be detected in a 72-hour record of $p / T$. Theory predicts that the resonance frequencies are insensitive to linear temperature gradients; however, we do not have a detailed understanding of why the acoustic frequencies are insensitive to asymmetric, time-dependent heating of the tank. From auxiliary measurements and information from the literature, we believe that convective currents near the tank's walls carry most of the heat between the hot and cool regions, leaving a linear temperature gradient in the majority of the gas's volume. We plan to report preliminary measurements for a much larger $(1800 \mathrm{~L})$ tank. We expect that the results of this investigation will lead to effective acoustic methods for flow calibrations and leak detection in large volumes.

\section{9:15}

5aPA4. Bonding characterization of three layers metal/adhesive/metal using Lamb waves. Camille Gauthier (LOMC, Univ. of Le Havre, France, Le Havre, France), Mihai Predoï (Univ. Politechnica of Bucarest, Bucarest, Romania), Ech-Cherif El-Kettani Mounsif (LOMC, Univ. of Le Havre, France, IUT, Pl. Robert Schuman, B.P. 4006, Le Havre 76610, France, elkettani@univ-lehavre.fr), Jocelyne Galy (IMP INSA LYON, Lyon, France), Damien Leduc, and Jean-Louis Izbicki (LOMC, Univ. of Le Havre, France, LE HAVRE, France)

The studied samples are three layer Aluminum/Epoxy/Aluminum structures, realized with the cooperation of Physico-Chemists, to obtain samples of controlled adhesion properties, and acousticians for ultrasounds characterization. Different mechanical (roughness) and/or chemical (silanisation) surface treatments at the interface substrate/adhesive are performed. A numerical FEM model is achieved to solve a rheological description of the interface as springs distribution. This model allows the study of the sensitivity of Lamb waves to the interface and the determination of their energy in the cross section of the structure, depending on the stiffness values of the springs. The sensitive modes are then looked for to be excited in the experimental study, particularly the vertical modes that are indicative of a more or less coupling in the three layer structure. Experimental results show different adhesion levels, depending on the surface treatments, and correspond stiffness springs value to each sample.

\section{9:30}

5aPA5. Modeling the scattering of elastic waves from defects in buried pipes. Ray Kirby (Mech. Eng., Brunel Univ., Uxbridge, Middlesex UB8 3PH, United Kingdom, ray.kirby@brunel.ac.uk) and Wenbo Duan (Mech. Eng., Brunel Univ., London, United Kingdom)

Long Range Ultrasonic Testing (LRUT) is a popular non-destructive evaluation technique for identifying defects in pipelines. The method sends an elastic wave down the walls of a pipe and then monitors echoes that arise if the wave is scattered by a defect. The technical challenge of LRUT lies in separating out, and interpreting from coherent and random background noise, those signals that belong to a defect. This becomes particularly challenging when a pipe is buried, because energy in an elastic wave is known 
to leak out of the pipe walls when it is surrounded by materials such as soil, concrete, or sand. To address this problem it is necessary to develop a better understanding of how elastic waves propagate in buried structures, and so, a finite element based model is introduced here that seeks to analyze the scattering from a defect in a buried pipe in both the frequency and time domain. Results from the implementation of this numerical model for the torsional $\mathrm{T}(0,1)$ mode are presented, and the effects of the burying material on the LRUT time domain response are discussed.

\section{9:45-10:00 Break}

10:00

5aPA6. Characterization of a local scatterer in a plate from reverberation of flexural waves. Emmanuel Moulin (IEMN, UMR CNRS 8520, Univ. of Valenciennes, Le Mont Houy, Valenciennes F-59313, France, emmanuel.moulin@univ-valenciennes.fr), Hossep Achdjian (GREMAN, INSA Ctr. Val de Loire, Université François Rabelais, Blois, France), Farouk Benmeddour, Lynda Chehami, Jamal Assaad, and Lucie Dupont (IEMN, UMR CNRS 8520, Univ. of Valenciennes, Valenciennes, France)

In media where acoustic waves are subject to multiple propagation paths (scattering or reverberation), recorded acoustic signals present a random aspect. Still, such signals carry some information about the medium, and ensemble averaging can give access to the estimation of a number of useful parameters. A well-known example, in room acoustics, is the estimation of absorption coefficients of walls from average decrease of the reverberation envelopes. In the work reported here, we have proposed a statistical model allowing to relate the scattering properties of a local heterogeneity (defect) to the average properties of reverberated acoustic signals in a solid plate. A theoretical expression of the averaged envelope of signals produced by scattered and reverberated flexural waves has been derived and both numerically and experimentally validated. A simple curve-fitting procedure applied to signals recorded on a few receivers then allows an experimental estimation of the scattering cross-section of the heterogeneity.

\section{0:15}

5aPA7. Integrity evaluation of adhesive anchors using electromagnetic acoustic transducer. Kazuhiko Hasebe, Yosuke Mizuno, and Kentaro Nakamura (Lab. for Future Interdisciplinary Res. of Sci. and Technol., Tokyo Inst. of Technol., 4159-R2-26 Nagatsuta-cho, Midori-ku, Kanagawaken 226-8503, Japan, khasebe@sonic.pi.titech.ac.jp)

The aging of social infrastructures has recently become a serious problem. The high efficiency of the testing is required to handle increased inspection demand. There is interest in testing the soundness of adhesive anchors. Bolts are connected to a concrete using holes filled with adhesive materials. Since anchor bolts is inspected by skilled workers manually, it is difficult to improve the efficiency. Several sensing methods have been proposed, but these measurements require a precision alignment or contact. We propose a non-contact evaluation method of adhesive anchors using an electromagnetic acoustic transducer (EMAT). An EMAT is a transducer for non-contact vibration generation and reception based on electromagnetic mechanisms. A neodymium ring magnet and two coils were used as an EMAT. We used $100 \mathrm{~mm}$ long M8 and M12 steel bolts mounted with epoxy resin in $70 \mathrm{~mm}$ depth holes in concrete. The EMAT which was placed around the bolt excited longitudinal vibrations of bolt by burst waves. Vibration signals were received after the excitation. The relationships between the resin amount and the resonance characteristics of the vibrations are evaluated for pursuing the applicability of this method to the on-site inspection. Resonance frequencies and quality factors are utilized for health monitoring of adhesive anchors.
5aPA8. Liquid loading characteristics of multiple SH-wave roundtrip in c-axis parallel oriented $\mathbf{Z n O}$ film/silica glass pipe. Shinji Takayanagi (Nagoya Inst. of Technol., Gokiso-cho, Syowa-ku, Nagoya 466-8555, Japan, takayanagi.shinji@nitech.ac.jp), Shoko Hiyama, Mami Matsukawa (Doshisha Univ., Kyotanabe, Japan), and Takahiko Yanagitani (Waseda Univ., Shinjyuku, Japan)

Shear waves with in-plane displacement are suitable for liquid sensors because they can propagate without the energy leakage into liquid. In previous study, we have demonstrated the SH-SAW excitation with (11-20) oriented $\mathrm{ZnO}$ film whose c-axis is parallel to the substrate plane [1]. For a high sensitive sensor, the long propagation path is needed to detect small changes of the velocity and amplitude. Multiple wave roundtrips on a pipe surface realizes the long propagation path. In this study, we fabricated IDT/c-axis parallel oriented $\mathrm{ZnO}$ film/silica glass pipe structure. The fourth roundtrip was observed by the time response measurement using a network analyzer. The insertion loss of the first lap consisted of two frequency components at $131 \mathrm{MHz}$ and 160-350 MHz. We also measured the liquid loading characteristics by immersing the inside pipe surface. Compared with the unloaded characteristics, insertion losses with pure water increased $0.6 \mathrm{~dB}$ at $131 \mathrm{MHz}$ and $1.1 \mathrm{~dB}$ at 236 $\mathrm{MHz}$. Because of small increases in the insertion losses, we can conclude that SH-type plate wave was excited. Viscosity measurements with the inside pipe surface are expected. [1] A. Tanaka, et al., IEEE TUFFC, 55, 2709 (2008).

\section{0:45}

5aPA9. Transmission and irradiation of high-intensity aerial focused ultrasonic waves using pipe. Norifumi Suzuki (Elec. Eng., Nihon Univ., 2-217, Imai, Chuo-ku, Dia Palace Keiyo Soga II \#1205, Chiba-shi, Chiba-ken 260-0834, Japan, csno16021@g.nihon-u.ac.jp), Ayumu Osumi, and Youichi Ito (Elec. Eng., Nihon Univ., Chiyoda-ku, Tokyo-to, Japan)

In this study, we have developed a method that high-intensity aerial ultrasonic waves (sound pressure level: $180 \mathrm{~dB}$ ) is transmitted to a far place and irradiate on optional and specified place without attenuation. To irradiate on the place that is optional and specified with high-intensity aerial ultrasonic waves, it is necessary to move the sound source. If any obstacle is placed between the specified place and the sound source, however, it will be difficult to irradiate to that place. In addition, there will also be a problem that ultrasonic waves attenuate during propagation in air. If these problems can be solved, it will be expected that more effective irradiation with ultrasonic waves may be realized and the range of applications may be widened. To solve the problem above, we proposed to use a rigid wall pipe whose inner diameter is similar to a wavelength to transmit the sound waves and attempted to simulate and experiment on the problem. In this report, we confirmed that the high-intensity aerial focused ultrasonic waves (frequency: 20 $\mathrm{kHz}$ ) can be transmitted by using the pipe.

\section{1:00}

5aPA10. Non-contact heating of surface on the object using by high-intensity aerial ultrasonic waves. Hirotaka Sato (Elec. Eng., Nihon Univ., 22-1, Omotechou, Sakura-shi, Chiba-ken 285-0811, Japan, cshi15015@g. nihon-u.ac.jp), Ayumu Osumi, and Youichi Ito (Elec. Eng., Nihon Univ., Chiyoda-ku, Tokyo-to, Japan)

In the present study, the authors investigate a method of rising a temperature of an object's surface for an instant without contact by using high-intensity aerial ultrasonic waves (frequency 20 to $50 \mathrm{kHz}$ ). In recent years, much attention has been directed at a thermal effect technology which utilizes ultrasonic energy such as HIFU and MRgFUS treatment. These technologies are realized for heating by using ultrasonic source which contact with a target. It is expected to realize the same heat effect by aerial ultrasonic waves, but a research of using ultrasonic waves to heat objects without contact has never been done. The authors propose a new method to use a very high-intensity aerial ultrasonic waves (intensity is about $25 \mathrm{kPa}$ ) which are generated by a point-converging acoustic source with a stripe-mode vibration plate and attempt to establish a technique. Recently, the authors reported that a temperature of silicone rubber material rise about $15^{\circ} \mathrm{C}$ after 1 second from the start of ultrasonic irradiation. With the realization of the technology, it is able to be a new underlying technology and is expected to be applied to a development of heating technique and non-destructive inspection. 


\section{1:15}

5aPA11. A novel high-volume separation technology base on acoustophoresis for treatment of produced water in the oil and gas industry. Jason P. Dionne (FloDesign Sonics, Inc., 499 Bushy Hill Rd., Simsbury, CT 06070, j.dionne@fdsonics.com) and Bart Lipkens (FloDesign Sonics, Inc., Wilbraham, MA)

A novel emulsion splitting separation technology has been developed using acoustic radiation forces to recover the dispersed oil phase from an oil water emulsion. Current separation technologies suffer from high energy costs, use of consumables, fouling, and limited efficiency in separation of micron-sized particles. Multi-dimensional ultrasonic standing waves are used to trap a dispersed phase in a fluid. The action of the acoustic forces results in clustering and coalescence of droplets. Upon reaching a critical size, they are continuously separated through enhanced buoyancy. A second mode of operation uses acoustic radiation forces to increase the average droplet size and reduce the sub-20 micron droplet concentration. Earlier work was presented at ICA 2013(Dionne [1]). New results are shown for prototypes with a $1 \times 2,3 \times 4$ and $6 \times 6$ inch flow chamber driven by $2 \& 3$ MHz PZT transducers operating at flowrates of $1 \mathrm{~L} / \mathrm{h}, 30 \mathrm{~L} / \mathrm{h}$, and $227-1136$ $\mathrm{L} / \mathrm{h}$, tested with produced water samples from four US locations. Measured separation efficiencies of over $90 \%$ have been documented as well as particle size shifts of $>100$ micron. The particle size shift is particularly appealing as it acts as a complimentary technology to existing oil \& gas separation technology. [1] J. Dionne, B. McCarthy, B. Ross-Johnsrud, L. Masi, and B. Lipkens, "Large volume flow rate acoustophoretic phase separator for oil water emulsion splitting," J. Acoust. Soc. Am., Vol. 133, No. 5, Pt. 2, May 2013, pp. 3237. [Work supported by NSF SBIR IIP- 1330287.]

11:30

5aPA12. Injection of liquid into ultrasonic standing wave fields by exciting flexural vibrations on needle. Haruna Tadakoshi, Hiroki Tanaka, Yosuke Mizuno, and Kentaro Nakamura (Lab. for Future Interdisciplinary Res. of Sci. and Technol., Tokyo Inst. of Technol., 4259-R2-26 Nagatuta, Midoriku, Yokohama, Kanagawa 226-8503, Japan, htadakoshi@sonic.pi. titech.ac.jp)

There is a large need to handle droplets without contact in the development of new drug and materials. We have been trying to utilize ultrasonic levitation for this aim. It is needed to inject a droplet of precise volume into required location of the levitation device. But it is difficult to control the droplet volume using a syringe due to the influence of surface tension. In this study, by exciting ultrasonic vibrations on a needle, we investigated the control method of droplet volume and ejection timing. Exciting flexural vibrations on a needle for 0.2 seconds, a water droplet of $18-28 \mathrm{mg}$ was dropped with a good controllability and the timing of inject can be regulated. To elucidate this dropping mechanism, we observed the behavior of the droplet. Capillary waves were excited on the droplet, and the force of 0.7 times of gravity exerted by vibration was applied on the droplet according to our estimation. Next, we examined the effect of droplet volume on the resonance frequency of flexural vibrations of the needle, and we confirmed that there was a little influence between them. This means that there is no need to change the frequency in accordance with the volume of droplets.

\section{1:45}

5aPA13. Behavior of liquid in a thin type vessel irradiated with high-intensity aerial ultrasonic waves. Taichi Urakami (Elec. Eng., Nihon Univ., 1336-7, Nakao, Midori-ku, Saitama-shi, Saitama-ken 336-0932, Japan, cstt15003@g.nihon-u.ac.jp), Ayumu Osumi, and Youichi Ito (Elec. Eng., Nihon Univ., Chiyoda-ku, Tokyo-to, Japan)

A high-intensity aerial ultrasonic waves can remove liquid that has penetrated into the pores instantaneously. Further, tiny particles adhered to a solid surface is removed from the surface of object by radiation of the ultrasonic waves instantaneously. These techniques are implemented to a target by an action aerial ultrasonic waves directly. If these operations can be realized similarly even when the ultrasonic waves pass through the object, the field of application is expected to spread extremely. For example, if it is possible to exert a force to the liquid injected into a small container from the outside of the container by irradiating the aerial ultrasonic waves, various applications can be greatly expected. We have investigated the observations of liquid behavior when the high-intensity aerial ultrasonic waves were irradiated to the very small vessel filled with liquid. As a result, tiny bubbles were generated from the vicinity irradiated with the aerial ultrasonic waves, and the bubbles had a fierce tiny vibration. Further, in the observation of the liquid in the vessel by using ink, the ink is greatly diffused throughout the vessel and the behavior of the bubbles was confirmed to contribute to the diffusion of the ink extensively.

FRIDAY MORNING, 2 DECEMBER 2016

CORAL 5, 7:55 A.M. TO 12:00 NOON

\title{
Session 5aPP
}

\section{Psychological and Physiological Acoustics: Pitch and Timbre}

\author{
Xin Luo, Chair \\ Speech and Hearing Science, Arizona State University, 975 S. Myrtle Ave., Tempe, AZ 85287
}

\section{Contributed Papers}

$7: 55$

5aPP1. Pitch model as interspike interval periodicity present at all active Bark bands during harmonic sound perception. Rolf Bader (Inst. of Systematic Musicology, Univ. of Haburg, Neue Rabenstr. 13, Hamburg 20354, Germany, R_Bader@t-online.de)

A Finite-Difference Time Domain (FDTD) physical model of the basilar membrane $(\mathrm{BM})$ is implemented including the transition of mechanical energy on the BM into spike excitation. The spike train caused by this transition shows energy at all Bark bands of a harmonic input sound. Still higher partials are often not able to produce a regular interspike interval (IS) firing pattern all through one periodicity of the fundamental frequency. Instead drop-outs occur, where one or several spikes are missing. This produces higher ISI periodicities and therefore lower frequencies as the critical frequency of the respective Bark bands. These lower frequencies are integer subharmonics of the critical frequency. The lowest of these frequencies is the fundamental frequency of the input sound. Therefore, the model shows these fundamental frequencies in all Bark banks of active sound input 
frequency. The fundamental frequency of a harmonic sound is therefore present at many cochlea fibers and may be the reason for pitch perception. This mechanism also works for residual pitches. Compared to other pitch models which need further calculation in following neural nuclei, like autocorrelations, the pitch model proposed here has pitch intrinsically present right at the cochlea output with no need of further computations. From this view, a pitchness can be defined as the strength of a frequency present at many Bark bands, in accordance with the strength of a pitch perception.

\section{8:10}

5aPP2. More about monaural noise edge pitch. William M. Hartmann and Aimee Shore (Phys. and Astronomy, Michigan State Univ., 567 Wilson Rd., East Lansing, MI 48824, hartmann@pa.msu.edu)

Lowpass noise with a sharp spectral cutoff generates a monaural noise edge pitch (MNEP) that listeners match with a sine tone having a frequency somewhat below the noise cutoff frequency (negative pitch shift). If the MNEP is a proper musical pitch, listeners should be able to identify musical intervals made using only the MNEP stimulus. Open-set MNEP experiments in the edge-frequency range of $600-2400 \mathrm{~Hz}$ found that four out of four listeners with musical training correctly identified octaves, fifths, fourths, and major thirds, proving that the MNEP is a musical pitch. The MNEP can be heard for edge frequencies at least as low as $100 \mathrm{~Hz}$, but the negative pitch shift becomes larger than a semitone, consistent with a timing model of the MNEP having a fixed integration time. If the MNEP reflects the expected quasi-periodic timing response of the auditory system to a sharp spectral edge, the pitch should become inaudible if the edge frequency becomes too high for neural synchrony - expected to be about $5000 \mathrm{~Hz}$. Sine tone pitch matching experiments showed that such a limit clearly applies for most listeners, but less clearly for others. [Work supported by the USAFOSR.]

\section{$8: 25$}

5aPP3. The effect of pitch change direction on melodic interval ranking. Xin Luo (Speech and Hearing Sci., Arizona State Univ., 975 S. Myrtle Ave., Tempe, AZ 85287, xinluo@asu.edu)

Our previous study showed that listeners had significantly lower ascending interval ranking thresholds than descending ones. Experiment 1 tested whether the effect on interval ranking of local pitch change direction within intervals relies on the global pitch change direction between intervals. Both ascending and descending intervals were ranked in both the 2nd-intervalhigher and lower conditions. Ascending interval ranking thresholds were significantly lower than descending ones in the 2nd-interval-higher conditions, but similar to or higher than descending ones in the 2nd-interval-lower conditions. Interval ranking was distracted when the global pitch change between intervals and local pitch change within intervals were in the opposite direction. To better simulate music listening, Experiment 2 measured ascending and descending interval ranking in the 3-tone conditions, where the three tones forming two intervals were played back to back in time. The 4-tone conditions with a 1-sec temporal gap between intervals were also tested. Similar ascending and descending interval ranking thresholds were observed when the global pitch change between intervals and local pitch change within intervals had the same direction. Interval ranking thresholds were significantly lower in the 4-tone conditions than in the 3-tone conditions, indicating that interval ranking was enhanced by the temporal gap between intervals.

\section{8:40}

5aPP4. Amplitude modulation masking for frequency modulation detection. Andrew J. King (Laboratoire des systemes perceptifs, CNRS, Ecole Normale Superieure, Paris Sci. et Lettres Res. Univ.,UMR CNRS 8248, Departement d'Etudes Cognitives, Ecole normale superieure, 29 rue d'Ulm, Paris 75005, France, andrew.king@ens.fr) and Christian Lorenzi (Laboratoire des systemes perceptifs, CNRS, Ecole normale superieure, Paris Sci. et Lettres Res. Univ., Paris, Ile de France, France)

It is generally assumed that frequency modulation (FM) is detected as amplitude modulation (AM) for fast FM rates with low carrier frequencies, and for all FM rates with high carrier frequencies. If this is the case, then FM detection in the presence of an AM masker should exhibit the main features of AM masking: tuning, dependency on AM masker depth, negative masking, beating effects and phase effects. We explored the masking effects produced by sinusoidal AM on detection thresholds of sinusoidal FM for normal-hearing listeners. FM rates ranged between 2 and $64 \mathrm{~Hz}$. The carrier was either a $500-\mathrm{Hz}$ or a $5000-\mathrm{Hz}$ pure tone that was either unmodulated in amplitude or modulated in amplitude at 2 or $16 \mathrm{~Hz}$. The masker AM depth was fixed to either $50 \%$ or $25 \%$, and stimulus duration was set to either 500 ms or $1 \mathrm{sec}$. Additionally, detection thresholds were tested as a function of the phase relationship between a $2-\mathrm{Hz}$ FM target and a $2-$ or $4-\mathrm{Hz}$ AM masker, and between a $16-\mathrm{Hz}$ FM target and an $8-, 16-$, or $32-\mathrm{Hz} \mathrm{AM}$ masker. The data will be discussed in light of previous studies on AM masking and the modulation filter-bank concept.

\section{8:55}

5aPP5. Effect of harmonic structure of notes on categorical chord perception. Chiaki Utsugi, Mariko Hamamura, and Kiyoaki Aikawa (School of Media Sci., Tokyo Univ. of Technol., 1404-1 Katakuracho, Hachioji, Tokyo 192-0982, Japan, mariko@hamamura.biz)

This paper reports distinct differences among the harmonic structures of notes on chord perception. Experimental results clarified that the existence of harmonics greatly improved chord perception accuracy. Subjective tests were conducted for 20 combinations of three notes from a minor chord to a major chord. The fundamental notes and the highest notes were fixed. The fundamental frequency of the middle note was shifted at an equal interval on the log scale. The harmonic structure was based on saw-tooth wave. The categorical perception accuracies were compared between pure tone and complex tone including five harmonics. The fundamental notes were $\mathrm{C} 5$ $(523.3 \mathrm{~Hz}), \mathrm{A} 5(880 \mathrm{~Hz})$, and C7 $(2093 \mathrm{~Hz})$. The stimuli were diotically presented at $60 \mathrm{~dB}(\mathrm{SPL})$ through electro-static headphones. The subjects were requested to answer major or minor with the $2 \mathrm{AFC}$ method. The categorical perception accuracy was extremely low in case of $\mathrm{C} 7$ without harmonics. The percent corrects were as low as $46.7 \%$ and $54.8 \%$ for $\mathrm{Cm}$ and $\mathrm{C}$, respectively. The $\mathrm{Cm}$ and $\mathrm{C}$ percent accuracies with harmonics were as high as 77.3\% and $79.5 \%$. The $\mathrm{Cm}$ and $\mathrm{C}$ accuracies were $97.7 \%$ and $93.2 \%$ for $\mathrm{C} 5$. The perceived categories turned from minor to major in the region between 8 th and 15 th note combinations.

\section{9:10}

5aPP6. Every advantage has its disadvantage: Pitch perception of tone language speakers is better at low frequencies but poorer at high frequencies. Yu-Xuan Zhang, Xiang Gao, Dinglan Tang, Ting Huang (National Key Lab. of Cognit. Neurosci. and Learning, Beijing Normal Univ., Rm. 330, Yingdong Bldg., Beijing 100875, China, zhangyuxuan@ bnu.edu.cn), and Chang Liu (Dept. Commun. Sci. \& Disord., Univ. Texas Austin, Austin, TX)

Tone language experience has been associated with more accurate and stronger phase locking of neural responses in the brainstem to auditory stimuli. However, whether this neurophysiological advantage translates to better pitch perception remains under debate. Previous studies have yielded controversial results, possibly due to variations in methods and large individual variability. Here we examined tone frequency discrimination (FD) over the frequency range of $250 \mathrm{~Hz}$ to $8 \mathrm{kHz}$ in Chinese and English speakers using relatively large sample sizes $(\mathrm{N}>50)$. For both brief $(7.5-\mathrm{ms})$ and steady (100-ms) tones, FD threshold showed an interaction between group and frequency: Chinese speakers outperformed English speakers up to $4 \mathrm{kHz}$, and the advantage was reversed at higher frequencies. The only exception was the $7.5-\mathrm{ms} 250-\mathrm{Hz}$ condition, where the stimuli were too short $(<2$ cycles $)$ for pitch extraction by phase locking and performance was worse in Chinese than English speakers. Overall, the pattern of FD differences between Chinese and English speakers indicates that tone language experience enhances temporal coding of pitch at the cost of place coding. 
sounds. Further, listeners produced more false alarms for the more pleasant sounds. These results suggest that listeners are biased to report recalling more pleasant sound regardless if they had been heard previously.

\section{0:30}

5aPP10. Comparing identification of vocal imitations and computational sketches of everyday sounds. Guillaume Lemaitre, Olivier Houix, Frédéric Voisin, Nicolas Misdariis, and Patrick Susini (IRCAM, 1 Pl. stravinsky, Paris 75004, France, GuillaumeJLemaitre@gmail.com)

Sounds are notably difficult to describe. It is thus not surprising that human speakers often use many imitative vocalizations to communicate about sounds. In practice, vocal imitations of non-speech everyday sounds (e.g., the sound of a car passing by) are very effective: listeners identify sounds better with vocal imitations than with verbal descriptions, despite the fact that vocal imitations are often inaccurate, constrained by the human vocal apparatus. The present study investigated the semantic representations evoked by vocal imitations by experimentally quantifying how well listeners could match sounds to category labels. It compared two different types of sounds: human vocal imitations, and computational auditory sketches (created by algorithmic computations), both based on easily identifiable sounds (sounds of human actions and manufactured products). The results show that performance with the best vocal imitations was similar to the best auditory sketches for most categories of sounds. More detailed analyses showed that the acoustic distance between vocal imitations and referent sounds is not sufficient to account for such performance. They suggested that instead of reproducing the acoustic properties of the referent sound as accurately as vocally possible, vocal imitations focus on a few important features dependent on each particular sound category.

\section{$10: 45$} Most research has separately examined perception of pitch patterning in the musical and linguistic domains; however, less is known about how listeners flexibly recruit musical and linguistic knowledge to interpret similar pitch patterns across different contexts, nor how these abilities develop with age. We asked children and adults to detect pitch changes to vocal stimuli that were presented in separate blocks as music (part of a "musical") or speech (part of a "play"). Stimuli were overtly sung (in the musical) or spoken (in the play) but matched in pitch range and contour, and across both contexts we occasionally presented ambiguous vocalizations (speech-to-song illusions). Pitch changes were created by subjecting stimuli to a pitch range expansion which preserved the pitch contour but distorted pitch interval relationships. Adults showed greater detection accuracy for overtly sung than spoken utterances; however, children showed comparable performance for speech and song. No difference was observed for ambiguous vocalizations at any age. Implications for the development of domain-specificity of pitch processing will be discussed.

\section{9:55-10:15 Break}

\section{0:15}

5aPP9. The relationship between perceived pleasantness and memory for environmental sounds. Kelly Dickerson (Army Res. Lab., 131 Waldon Rd., Abingdon, MD 21009, dickersonkelly23@gmail.com), Laura Sherry (Audiol., Speech-Lang. Pathol. and Deaf Studies, Towson Univ., Towson, MD), and Jeremy Gaston (Army Res. Lab., Aberdeen Proving Ground, MD)

The everyday auditory environment contains many sounds that can be influenced by semantic information and listener experiences. Some sounds are more memorable than others and there is limited work examining the role of specific semantic or subjective attributes on retrieval success for environmental sounds. The present study examines the relationship between pleasantness ratings and memory performance on a cued-recall task. A group of listeners were presented with 36 environmental sounds and asked to rate each for perceived pleasantness to provide baseline evaluations for each sound. Based on the ratings, stimuli were sorted into two pleasantnessbalanced groups. A second group of listeners evaluated half of the stimuli for pleasantness during the "study" phase, which was followed by a cued recall test. At test, half of the items were "old" (from the study phase), and half were "new" (from the set of 36 sounds). The results demonstrate that listener's recalled more pleasant sounds more accurately than unpleasant
5aPP11. The perceptual effects of tonewood choice for the back/side plates of the steel-string acoustic guitar. Samuele Carcagno (Dept. of Psych., Lancaster Univ., Lancaster LA1 4YF, United Kingdom, s.carcagno@lancaster.ac.uk), Roger Bucknall (Fylde Guitars, Penrith, United Kingdom), Jim Woodhouse (Dept. of Eng., Univ. of Cambridge, Cambridge, United Kingdom), and Christopher J. Plack (Dept. of Psych., Lancaster Univ., Lancaster, United Kingdom)

The sound qualities of different tonewoods used for the back/side plates of acoustic guitars are the subject of intense debate among guitarists. However, comparisons between different guitars are rarely performed under blinded conditions. We asked a sample of professional, semi-professional, and amateur guitarists $(n=52)$ to rate the sound quality of six steel-string acoustic guitars, built to be as similar as possible except for the woods used for the back/side plates, which were: Brazilian rosewood, Indian rosewood, mahogany, maple, sapele, and walnut. The guitarists played the guitars in a dimly lit room while wearing welder's goggles to prevent visual identification. The sound quality ratings did not differ significantly between guitars. Test-retest correlations, measured for a subset of guitarists $(n=34)$, were low, suggesting that the lack of significant rating differences was not due to averaging out contrasting individual preferences. The results of a blinded cate that guitarists could not easily distinguish the guitars by their sound or feel. Overall, the results suggest that the species of wood used for the back/ side plates of a steel-string acoustic guitar may play a lesser role in perceived sound quality than is commonly believed.

\section{1:00}

5aPP12. Psychoacoustic assessment of auditory impression of the coolness and warmness induced by HVAC noise. Seiji Nakagawa (Ctr. for Frontier Medical Enineering, Chiba Univ., 1-33 Yayoicho, Inage-ku, Chiba, Chiba 263-8522, Japan, s-nakagawa@chiba-u.jp), Takuya Hotehama (Biomedical Res. Inst., National Inst. of Adv. Industrial Sci. and Technol. (AIST), Ikeda, Osaka, Japan), and Masaru Kamiya (DENSO Corp., Kariya, Aichi, Japan)

Noise induced by a heating, ventilation and air conditioning (HVAC) system in a vehicle is an important factor that affects the comfort of the interior of a car cabin. To date, much effort has been devoted to reduce noise levels. However, for example, silence sometimes causes a loss of operation ABX discrimination test, performed by a subset of guitarists $(n=31)$, indi- 
feeling-we recognize the HVAC doesn't work enough in case the air flow sound is too quiet, even if it works well actually. Therefore, there is a need for a new sound design that addresses the noise problem from a different point of view in addition to the reduction of noise levels. In this study, focusing on the auditory impression of automotive HVAC noise concerning coolness and warmness, listening tests were performed using a paired comparison technique under various conditions of room temperature. Five stimuli were synthesized by stretching the spectral envelopes of recorded automotive HVAC noise to assess the effect of the spectral centroid, and were presented through headphones at $70 \mathrm{dBA}$. Results show that the spectral centroid significantly affects the auditory impression concerning coolness and warmness; a higher spectral centroid induces a cooler auditory impression regardless of the room temperature.

\section{1:15}

5aPP13. On the color of voices: Does good perception of vocal differences relate to better speech intelligibility in cocktail-party settings? Nawal El Boghdady, Deniz Başkent (Dept. of Otorhinolaryngology, Univ. of Groningen, Univ. Medical Ctr. Groningen, Hanzeplein 1, Groningen, Groningen 9700RB, Netherlands, n.el.boghdady@umcg.nl), and Etienne Gaudrain (Auditory Cognition and PsychoAcoust., CNRS UMR5292, Lyon Neurosci. Res. Ctr., Université Lyon 1, Groningen, Netherlands)

Understanding speech in cocktail-party settings poses a challenge for cochlear implant (CI) users. Distortions of vocal cues as they are perceived through the implant have been hypothesized to partially contribute to this difficulty. Vocal cues can be defined along two orthogonal dimensions: the fundamental frequency, F0, related to the pitch, and the vocal tract length (VTL), correlated with speaker size. While deficiency in F0 perception in CI users has been long known, recent research has shown a large deficit in VTL perception. How this contributes to speech intelligibility in the presence of a competing talker (speech-on-speech; SOS) remains unknown. In this study, we investigated the relationship between SOS intelligibility and the sensitivity to F0 and VTL differences (JNDs) in 18 CI listeners using two separate speech tasks. Results indicate a strong correlation between voice cue sensitivity and SOS intelligibility: participants who are more sensitive to small differences in F0 and VTL tend to perform better on the two SOS tasks compared to those who are less sensitive. These results are consistent with the hypothesis that degraded voice cue sensitivity contributes to SOS perception deficits, but other potential explanations, like differences in cognitive processing and attention, cannot yet be ruled out. [Funding: The University Medical Center Groningen (UMCG), Advanced Bionics, Netherlands Organization for Scientific Research (NWO).]
11:30

5aPP14. Can a pitch be "sharp," "bright," "large," "narrow," and "high?" Questioning the automaticity of audiovisual correspondences. Laura Getz and Michael Kubovy (Univ. of Virginia, PO Box 400400, Charlottesville, VA 22904, laura.getz@villanova.edu)

Previous research has found that there is an inherent association between auditory and visual dimensions such as the height a pitch and the size of an object. From this, researchers have assumed that such audiovisual correspondences must result solely from bottom-up processing. In a series of studies, we sought to separate bottom-up and top-down effects in the correspondence between pitch and visual size, elevation, spatial frequency, brightness, and sharpness. Using a modified speeded classification task, we asked participants to pair audiovisual dimensions in "compatible" (e.g., high pitch/small circle) and "incompatible" (e.g., high pitch/large circle) conditions. We compared their reaction times across conditions and found that in most cases participants can pair the dimensions in either direction with similar speed and accuracy. We conclude that top-down effects such as task instructions and language knowledge do influence the strength of audiovisual associations. We thus strongly question the assumption of automaticity prevalent in the cross-modal correspondence literature.

\section{1:45}

5aPP15. Comparing preferred spectral balances of listeners from four countries. Sungyoung Kim (ECT Eng. Technol., RIT, ENT-2151, 78 Lomb Memorial Dr., Rochester, NY 14623, sungyoungk@gmail.com), Ron Bakker (Commercial Audio, Yamaha Music Europe, Vianen, Netherlands), and Masahiro Ikeda (System Platform Group, Yamaha Corp., Hamamatsu, Shizuoka, Japan)

An influence of cultural background in timbre perception was investigated. The authors have conducted a listening test in the United States, Japan, Korea, and the Netherlands. In the test, each participant was asked to adjust gains of a three-bands equalizer and generate spectral balances of five musical excerpts (four popular songs, one each from four countries and one orchestral music) according to one's preference. All listeners used a same type headphone and audio interface, and repeated the entire process twice. The authors analyzed the consistency of each participant by measuring a total spectral difference (TSD) value between the two trials. Subsequently the data of listeners with less than $45 \mathrm{~dB}$ TSD were analyzed through a mixed-design ANOVA. The results showed that the MUSIC factor was not significantly differentiated the reported data $(F(4,86.1)=2.087, p=0.086)$, while a significant interaction existed between GROUP and MUSIC $(\mathrm{F}(12,319.3)=2.58, \mathrm{p}=0.004)$. In addition, the frequency BAND factor $(\mathrm{F}(2,1472)=28.154, \mathrm{p}=0)$ and its interaction with GROUP were significant $(\mathrm{F}(6,404.3)=2.578, \mathrm{p}=0.026)$. The results implicate that when asked to actively render a preferred timbre balance, the cultural background starts to significantly interact with other variables. 


\title{
Session 5aSC
}

\section{Speech Communication: Voice, Tone, Prosody, and Affective and Emotional Qualities of Speech (Poster Session)}

\author{
Tanya Flores, Chair \\ Languages and Literature, University of Utah, 255 S Central Campus Dr., LNCO 1400, Salt Lake City, UT 84109
}

\begin{abstract}
All posters will be on display from 8:00 a.m. to 12:00 noon. To allow contributors in this session to see the other posters, authors of oddnumbered papers will be at their posters from 8:00 a.m. to 10:00 a.m. and authors of even-numbered papers will be at their posters from 10:00 a.m. to 12:00 noon.
\end{abstract}

\section{Contributed Papers}

5aSC1. Effects of perception and vocabulary training of Mandarin tones for native speakers of Japanese: Pre-, post-, and retention test comparison. Shuyi Yang (Graduate School of Intercultural Studies, Kobe Univ., Hyōgo-ken, Kōbe-shi, Nada-ku, Tsurukabuto, 1 Chome-2-1, Kobe 6570815, Japan, syuiyang@gmail.com) and Reiko Akahane-Yamada (ATR, Soraku-gun, Kyoto, Japan)

Native speakers of Japanese were trained to perceive four Mandarin tones, and to semantically and phonetically distinguish Chinese monosyllabic word contrasting in tones. Various tests were administered not only before and after the training period but also forty days after the completion of training. Participants were divided into three groups. First group received perception training first and vocabulary training later. Second group received vocabulary training first and perception training later. The last group, which was the control group, received no training and participated only in testing sessions. The result showed that the accuracy in perceptionrelated-tests improved by perception training, and the accuracy in vocabulary-related-tests improved by vocabulary training. In addition, the effect of training had retained even 40 days after the completion of training. More importantly, the group which received perception training first showed significantly larger improvement from pre-test to post-test than in the group which received vocabulary training first. Note that two groups received the same amount of equivalent trainings in total. Preset results demonstrated the existence of the order effect in foreign language learning. [Work supported by JSPS KAKENHI 23242032.]

5aSC2. What ranges of two-mass model parameters should be used in subject-specific and population-based modeling studies? Douglas Cook (Eng., New York Univ. Abu Dhabi, PO Box 903, New York, NY 10276, prof.laji@gmail.com)

Two-mass models have been used in voice research for over 40 years. It is therefore both surprising and somewhat troubling that there is no firm consensus regarding the values of model parameters that should be used to represent human phonation. A knowledge of the parameter ranges that can (or should) be used is essential for scientifically valid studies involving population-based or subject-specific modeling. In this study, four techniques were used to examine the ranges of two-mass model parameter values that produce behavior representative of human phonation. The first approach involved a review of values that have been used in previous modeling studies. The second approach utilized unrestricted Monte Carlo sampling to examine which ranges can be used to simulate human phonation. The third approach also utilized Monte Carlo sampling, but parameters were restricted based on physical features of the vocal folds. Finally, a reduction or order technique was developed that allows the determination of two-mass model parameters from the physical features of human vocal folds. Finally, results from each of the four methods were compared and contrasted to provide a better understanding of parameter ranges for two-mass models.
5aSC3. A measurement study on voice instabilities during the register transition. Yasufumi Uezu and Tokihiko Kaburagi (Kyushu Univ., 4-9-1, Shiobaru, Minami-ku, Fukuoka-shi, Fukuoka 815-8540, Japan, 3DS14006W@s.kyushu-u.ac.jp)

When one of the dominant harmonics, the fundamental frequency and its harmonic components, is close to the first formant frequency, the effect of the source-filter interaction can induce voice register transition, in which the vocal-fold vibration becomes unstable and the pitch jumps drastically. In this study, we investigated the relationship between the dominant harmonics and the first formant frequency in the modal-falsetto transition to clarify the effect of source-filter interaction. While five subjects performed rising glissandi with /a/ and /i/ vowels, we simultaneously measured their vocal-fold vibration by using electroglottography and the acoustic response from the vocal tract by using the external acoustic excitation method. We analysed temporal patterns of the fundamental and the first formant frequencies in the transition section. We found that the fundamental frequency was regularly in the vicinity of the first formant frequency for the /i/ vowel. Additionally, for the /a/ vowel, it was occasionally observed that the second or third harmonic component was close to the first formant frequency in the transition. Our results indicate that the source-filter interaction is a common factor of the modal-falsetto transition for the participants.

5aSC4. Rhythm segment constitution showing regular periodicity. Shizuka Nakamura (Graduate School of Informatics, Kyoto Univ., 36-1 Yoshida-Honmachi, Sakyo-ku, Kyoto 606-8501, Japan, shizuka@sap.ist.i.kyotou.ac.jp)

To verify the possibility of regular periodicity of English rhythm, each sentence was divided into respective rhythm segments and the properties of its durations were analyzed. Rhythm segment (RhySeg) was defined as a segment including one syllable with a primary/secondary stress to which an adjacent unstressed syllable(s). The following locations of a stressed syllable in RhySeg were compared: forward, semi-forward, middle, semi-back, and back. To reflect the perceptual effect to the RhySeg constitution, the following factors to equally compress all of the unstressed syllables were compared: $0.1-1.0$ at an interval of 0.1 . To find the RhySeg constitution showing regular periodicity, not only the degree of concentration of the distribution, but the degree of closeness between RhySeg with a secondary stress and 1/2 of that with a primary stress, whose engagement on regular periodicity was indicated in previous studies, was applied as a criterion. Comparative experiments showed the best when the stressed syllable located semi-forward or forward, and the factor was 0.7. Furthermore, the result of harmonic analysis and resynthesis applied to the time function of the average duration of syllables in a sentence indicated periodicity is consisted of the combination of the fundamental and its second harmonic components. 
5aSC5. Laryngeal dynamics and vocal fold tissue engineering in a rabbit model. Michael Döllinger (Dept. of Otorhinolaryngology, Head and Neck Surgery, Div. of Phoniatrics and Pediatric Audiol., Univ. Hospital Erlangen, Bohlenplatz, 21, Erlangen, Bavaraia 91054, Germany, michael. doellinger@uk-erlangen.de), Veronika Birk, Stefan Kniesburges (Dept. of Otorhinolaryngology, Head and Neck Surgery, Div. of Phoniatrics and Pediatric Audiol., Univ. Hospital Erlangen, Erlangen, Bavaria, Germany), Christoph Alexiou (Dept. of Otorhinolaryngology, Head and Neck Surgery, Section of Experimental Oncology and Nanomedicine, Univ. Hospital Erlangen, Erlangen, Bavaria, Germany), Olaf Wendler (Dept. of Otorhinolaryngology, Head and Neck Surgery, Experimental ENT Res. Lab. I, Univ. Hospital Erlangen, Erlangen, Germany), Marina Pöttler (Dept. of Otorhinolaryngology, Head and Neck Surgery, Section of Experimental Oncology and Nanomedicine, Univ. Hospital Erlangen, Erlangen, Germany), Anne Schützenberger, and Stefan Dürr (Dept. of Otorhinolaryngology, Head and Neck Surgery, Div. of Phoniatrics and Pediatric Audiol., Univ. Hospital Erlangen, Erlangen, Germany)

Vocal fold surgery, especially due to cancer treatment, yields reduced voice quality and consequently reduced quality of life for patients. Hence, the development of vocal fold implants, to restore missing vocal fold tissue after surgery, is an urgent clinical need. To achieve this, a rabbit model is applied as a first step. Ex-vivo dynamic experiments were performed on twelve rabbit larynges providing normative phonatory data. The larynges were phonated at sustained phonation for different elongation levels at varying subglottal pressures. Laryngeal vibrations, airflow, and acoustics were recorded. Subsequently, for each larynx, a defined area of one vocal fold was resected, simulating the surgical intervention, and were phonated again with the same stimulations. The untreated larynges showed expected behavior regarding flow-pressure relation, acoustics and dynamics. In contrast, the phonatory quality of the resected larynges was significantly reduced showing, as expected, highly disturbed dynamics and acoustics. Parallel, vocal fold fibroblasts were isolated from rabbit larynges and cultured. These cells were treated with superparamagnetic iron oxide nanoparticles enabling their magnetic control. By magnetic tissue engineering three dimensional structures were designed. Next, the applicability of this tissue engineered implant will be tested in the dynamic ex-vivo rabbit model to compare the phonatory outcome.

5aSC6. A study of reliability parameters extracted through voice analysis. Hyung Woo Park (IT, SoongSil Univ., 1212 Hyungham Eng. Bldg. 369 Snagdo-Ro, Dongjak-Gu, Seoul, Seoul 06978, South Korea, pphw@ssu.ac. kr) and Sangmin Lee (Business Administration, SoongSil Univ., Seoul, South Korea)

The human voice is one of the easiest methods for the information transmission between human beings. The characteristics of the voice can be varied by different people and different situations, such as utterance speed, pitch tone, vocal organ features, and the gender. Moreover, the voice can be used as a factor for deciding the personal credit rating scores. The reliable parameters of speech signal can be extracted from different characteristics of the spoken information. In this paper, we collected the voices from people who has relatively high personal credit scores, and analyzed them.

5aSC7. Acoustic similarities among female voices. Patricia Keating and Jody Kreiman (Dept. of Linguist, UCLA, Los Angeles, CA 90095-1543, keating@humnet.ucla.edu)

Little is known about how to characterize normal variability in voice quality within and across utterances from normal speakers. Given a standard set of acoustic measures of voice, how similar are samples of 50 women's voices? Fifty women, all native speakers of English, read 5 sentences twice on 3 days-30 sentences per speaker. The VoiceSauce analysis program estimated many acoustic parameters for the vowels and approximant consonants in each sentence, including F0, harmonic amplitude differences, harmonic-to-noise ratios, formant frequencies. Each sentence was then characterized by the mean and standard deviation of each measure. Linear discriminant analysis tested how well each speaker's set of 30 sentences could be acoustically distinguished from all other speakers' sentences. Initial work testing just 3 speakers from this sample found that the speakers could be completely discriminated (classified) by these measures, and largely discriminated by just 2 of them. Such a simple result is not expected for the larger sample of speakers. We will present results concerning how successfully speakers can be discriminated, how well different numbers of discriminant functions do, and which acoustic measures do the most work. Implications for recognition by listening will be discussed. [Work supported by NSF and NIH.]

5aSC8. A study on prediction of end-of-utterance by prosodic features and phrase-dependency structure in spontaneous speech. Yuichi Ishimoto (Ctr. for Res. Resources, National Inst. for Japanese Lang. and Linguist, 10-2 Midoricho, Tachikawa, Tokyo 190-8561, Japan, yishi@ninjal.ac. jp), Takehiro Teraoka, and Mika Enomoto (School of Media Sci., Tokyo Univ. of Technol., Hachioji, Tokyo, Japan)

This study is aimed at predicting the end of utterance by prosodic features and syntactic structure for spontaneous speech. In spontaneous everyday conversation, participants must predict the ends of utterances of a speaker to perform smooth turn-taking. We consider that they utilize not only syntactic factors but also prosodic factors for the end-of-utterance prediction because of the difficulty of prediction of a syntactic completion point in spontaneous Japanese speech. In previous studies, it was observed that prosodic factors changed such that the general fundamental frequency of utterance declined gradually toward the end of an utterance, and the intensity decreased significantly in the final accentual phrase. However, it is not clear what prosodic features support the prediction. We focused on dependency structure among bunsetsu-phrases as the syntactic factor and investigated the relation between the phrase-dependency and prosodic features based on a spontaneous Japanese conversation corpus. The results showed that the average fundamental frequency and the average intensity for accentual phrases did not decline until the modified phrase appeared. This suggests the possibility that prosodic changes and phrase-dependency relations inform the hearer that the utterance is approaching its end.

5aSC9. Working memory facilitates the detection and correction of feedback errors in vocal pitch regulation. Hanjun Liu, Zhiqiang Guo, and Xiuqin Wu (Rehabilitation Medicine, The First Affiliated Hospital of Sun Yat-sen Univ., 58 Zhongshan 2nd Rd., Guangzhou, Guangdong 510080, China, 1hanjun@mail.sysu.edu.cn)

In speech processing, information related to the speech motor command and sensory re-afference can be stored in working memory (WM) within a feedback circuit and recalled when needed to adjust the motor activity. Whether WM facilitates the online monitoring of speech motor control, however, remains unclear. The present event-related potential study sought to examine the impact of WM on the auditory-motor processing of pitch feedback errors. Participants sustained a vowel phonation while hearing their voice pitch-shifted +200 or +500 cents five times. In the WM task, participants were asked to determine whether the sequence of 5 pitch shifts was different or not between two consecutive vocalizations. In the control task, they did nothing but maintaining their vocalizations steady when exposed to pitch-shifted auditory feedback. The behavioral results revealed a significant increase of vocal responses in the WM task as compared to the control task. At the cortical level, the WM task elicited significantly larger $\mathrm{N} 1$ responses and smaller P2 responses than the control task. Taken together, these findings demonstrate the influence of WM on the neurobehavioral responses to pitch-shifted voice auditory feedback, indicating that WM can facilitate the detection and correction of pitch feedback errors in vocal motor control.

5aSC10. Effect of level difference between left and right vocal folds on phonation: Physical experiment and theoretical study. Ryo Shimamura (Graduate School of Sci. and Eng., Ritsumeikan Univ., Noji-higashi, 1-1-1, Kusatsu, Siga 525-0058, Japan, rt0021hf@ed.ritsumei.ac.jp) and Isao T. Tokuda (Graduate School of Sci. and Eng., Ritsumeikan Univ., Kusatsu, Shiga, Japan)

The vocal folds, which are constituted by muscles covered with a mucous membrane, generate a primary sound called the voice source, as airflow passes them. In some voice disorders, asymmetry between left and right vocal folds was observed. We focus on level difference, which is defined as the distance between the upper surfaces of the bilateral vocal 
folds in the inferior-superior direction and is caused by symptom of such disorders. Physical models of the vocal folds were utilized to study the effect of the level difference on the phonation threshold pressure. For three types of different self-oscillating synthetic models, our experiments reveal that the phonation threshold pressure increases significantly as the level difference is extended. Furthermore, based upon a small amplitude approximation of the vocal fold oscillations, a theoretical formula was derived for the phonation threshold pressure. Our theory was in good agreement with the experiments, especially when the phase difference between the left and right vocal folds is not too large. From these results, we conclude that the level difference affects voice production, therefor the effect of the vocal fold geometry needs to be taken into account for the observation of voice disorders.

5aSC11. Psychoacoustic roughness as creaky voice predictor. Julián Villegas (Univ. of Aizu, The University of Aizu, Tsuruga, ikki-machi, Aizu Wakamtsu, Fukushima 965-8580, Japan, julian@u-aizu.ac.jp), Jeremy Perkins (Univ. of Aizu, Aizu-Wakamatsu, Japan), and Seunghun J. Lee (Int. Christian Univ., Tokyo, Japan)

The use of psychoacoustic roughness as a predictor of creaky voice is reported. Roughness, a prothetic sensation elicited by rapid changes in the temporal envelop of a sound $(15-300 \mathrm{~Hz})$, shares qualitative similarities with a kind of phonation known as vocal fry or creakiness. When a creakiness classification made by trained linguists was used as a reference, a classifier based on an objective temporal roughness model yielded results similar to an artificial neural network-based predictor of creakiness, but the former classifier tended to produce more type I errors. We also compare the results of the roughness-based prediction with those predicted by samples of three populations who use creakiness contrastively in different degrees: Japanese (where creakiness is not systematically used for phonetic contrast), Mandarin (where creakiness is used as a secondary cue), and Vietnamese (where creakiness is used as a phonetic contrast between tones). The roughness-based classification seems to better agree with classifications made by the untrained listeners. Our findings suggest that extreme roughness values ( $>4$ asper) in combination with local prominences on the roughness temporal profile of vocalic segments could be used for classification of creaky intervals in running speech.

5aSC12. A study on the intonation of Japanese modal particles "ne" and "yo". Haixia Zhang and Ayako Shirose (Graduate School of Education, Tokyo Gakugei Univ., 4-1-1,Nukuikita-machi, Koganei, Tokyo 184-8501, Japan,wsbxk544@yahoo.co.jp)

The Japanese sentence-final particles "ne" and "yo" represent modality. These particles are assumed to have several meanings, but the definition of the meanings of them has been an open question. In particular, the relationship between the meaning of these particles and their intonation contours remains unresolved. Therefore, to clarify this, we investigated the meaning of the particles "ne" and "yo" in actual utterances using a questionnaire and analyzed the pitch contours of the utterances acoustically. A Standard Japanese speaker was asked to produce 36 utterances with "ne" or "yo" particles controlling three types of pitch contours, falling, rising and flat. First, we asked 44 Japanese university students (aged 18-24) to judge the "naturalness" of the obtained utterances and to describe their meaning. The results revealed that the particle "ne" tended to have a negative connotation in the case of the falling intonation. Second, the pitch contours of final two syllables of the utterances were analyzed acoustically. The results indicated that the intonation contours differed between the two particles; the rising range was wider in "ne" than "yo". This was assumed to reflect the different meaning between the two particles. Speech corpus data will be examined to verify above results.
5aSC13. Mandarin tone recognition based on unsupervised feature learning from spectrograms. Charles Chen, Razvan Bunescu (Elec. Eng. and Comput. Sci., Ohio Univ., 1 Ohio University, Athens, OH 45701, 1c971015@ohio.edu), Li Xu (Commun. Sci. and Disord., Ohio Univ., Athens, $\mathrm{OH}$ ), and Chang Liu (Elec. Eng. and Comput. Sci., Ohio Univ., Athens, $\mathrm{OH})$

In tone languages, such as Mandarin Chinese, a syllable with different tones conveys different meanings. Previously, Mandarin tone recognition based on Mel-frequency cepstral coefficients (MFCCs) and Convolutional Neural Networks (CNN) was examined and the results outperformed the model of conventional neural network using manually edited F0 data. In the present study, Mandarin tone recognition based on spectrograms, instead of MFCCs, was explored. Unsupervised feature learning was applied to the unlabeled spectrograms directly with a denoising autoencoder (dAE). Then, the model convolved the labeled spectrograms with the learnt "sound features" and produced a set of feature maps. A dataset that consisted of 4500 monosyllabic words collected from 125 children was used to evaluate the recognition performance. Compared with methods based on MFCCs, there are more parameters to train in the new approach based on spectrograms. As a result, the new model might better capture the statistical distribution in the original data. Therefore, the new approach, with unsupervised feature learning, could perform even better than previous methods based on MFCCs or those based on the extracted F0 information. The advantages and shortcomings of various approaches for lexical tone recognition will be discussed. [Work supported in part by the NIH NIDCD Grant No. R15DC014587.]

5aSC14. Distribution of accentual phrase medial tones in Seoul Korean. Hyun Ji Yoo and Sun-Ah Jun (Linguist, UCLA, University of California, Los Angeles, Los Angeles, CA 90095, yhj8290@ucla.edu)

According to the model of Korean (Seoul) intonation (Jun 1993, 2007), the underlying tonal pattern of an Accentual Phrase (AP) is either HHLH (if the AP-initial segment is aspirated or tense) or LHLH (elsewhere). The second tone, $\mathrm{H}$ (= + H in K-ToBI; Jun 2000), or the third tone, L (= L + in K$\mathrm{ToBI}$ ), or both are known to be often undershot when the AP is shorter than 4 syllables, but so far factors determining the distribution of these medial tones have not been investigated. This study aims to find predictors of APmedial tone realization based on K-ToBI labeled speech of various speech styles. Preliminary results show that in general $\mathrm{L}+$ is more frequently undershot than $+\mathrm{H}$ regardless of the AP size. This pattern was clearer in L-initial APs than H-initial APs. Furthermore, the type of the AP-medial tone (when IP-final) showed an interaction with the type of immediately following Intonational Phrase boundary tones: $\mathrm{L}+$ was more often undershot than $+\mathrm{H}$ before $\mathrm{L} \%$, but the opposite was true before $\mathrm{H}(\mathrm{L}) \%$. The results suggest that the tonal context adjacent to the AP-medial tone plays an important role in the distribution of AP-medial tones. More predictors will be discussed at the meeting.

5aSC15. On the necessity of using 3D imaging of vocal fold vibration. David Berry (Surgery, UCLA, 31-24 Rehab, Los Angeles, CA 90095-1794, daberry@ucla.edu)

Physical mechanisms of regular and irregular vocal fold vibration were first studied using a computational model of vocal fold vibration. Later physical mechanisms of vocal fold vibration were studied in laboratory hemilarynx studies, where the medial surface of the vocal folds were imaged. While the method was also extended to clinical studies of vocal vibration, the method exhibited significantly less interpretive power in the clinical applications in which the superior surface of the vocal folds was imaged in 2D. Our hypothesis is that the interpretive power of the method of empirical eigenfunctions in studying the superior surface dynamics of the vocal folds will be significantly increased if one performs 3D imaging of the dynamics versus the standard $2 \mathrm{D}$ imaging. To test this hypothesis, the method of empirical eigenfunctions was employed on the same finite element model used in previous computational experiments. Our results confirm the hypothesis that the interpretive power of the method of empirical eigenfunctions was significantly increased in studying the superior surface dynamics of the vocal folds when 3D imaging was employed. 
5aSC16. Relations on prosody of Japanese back-channeling word "hai" and listener's impression: An investigation with synthesized voices. Tetsu Tanahashi and Tetsuro Kitahara (Graduate School of Integrated Basic Sci., Nihon Univ., 3-25-40, Sakurajosui Setagaya-ku, Tokyo 156-8550, Japan, tanahashi@kthrlab.jp)

The back-channeling word "hai" in Japanese is basically used in positive contexts, but it may give a negative impression to the listener if an inappropriate prosody is used. The same thing will occur in human-system dialogues using synthesized voices. Therefore, we investigated the relations on the prosody of synthesized voices of "hai" and the listener's impression. An experiment was conducted using the semantic differential method. Synthesized voices of "hai" were presented with different speaking rates (slow and fast) and different fundamental frequencies (low and high). 16 participants (university students, 8 males and 8 females) rated them on 28 adjective scales. The results were analyzed with the factor analysis, so that four factors were extracted. For the first factor, the loadings of "definite", "calm", "active",etc., were higher than 0.5 , so the first factor is considered to represent positiveness. For the second factor, the loadings of "definite," "violent," "cold," and etc., were higher than 0.5 , so the second factor is considered to represent unfriendliness. The first factor tends to be higher as the fundamental frequency is lower. The second factor tends to be lower as the speaking rate is higher.

5aSC17. The nature of tone sandhi in Sixian Hakka. Jie Zhang (Linguist, The Univ. of Kansas, 1541 Lilac Ln., Blake Hall, Rm. 427, Lawrence, KS 66045, zhang@ku.edu), Hanbo Yan (Shanghai Int. Studies Univ., Shanghai, China), Yuwen Lai, and Shao-ren Lyu (National Chiao-Tung Univ., Hsinchu, Taiwan)

Sixian Hakka is a Hakka dialect spoken in Taiwan. The language has four contrastive tones on non-checked syllables: 24, 11, 31, and 55. But before 24 or 55 , a tone sandhi pattern changes 24 to 11 , neutralizing the tonal contrast to three. We report two experiments that tap into the nature of this tone sandhi pattern in this paper. The first is a nonce-probe test, in which participants produced novel words that met the sandhi environment (/24-24/, /24-55/), and the f0 of the first syllable was compared to the f0 produced in real words to test the productivity of the sandhi. The second is an auditory lexical decision experiment with auditory priming, which tested whether disyllabic tone sandhi words were primed by monosyllables that shared the base tone or sandhi tone of the first syllable. Both experiments were conduced in Miaoli, Taiwan. We will report data from 15 participants for the first experiment and 32 for the second experiments. Together with experimental studies on various types of tone sandhi patterns elsewhere (e.g., Mandarin, Taiwanese), the results here will shed further light on how speakers internalize complex tonal alternation. The study also provides experimental data on an understudied and disenfranchised language.

5aSC18. A computational study of vocal fold dehydration during phonation. Liang Wu and Zhaoyan Zhang (Dept. of Head and Neck Surgery, Univ. of California, Los Angeles, 1000 Veteran Ave, 31-24 Rehab Ctr., Los Angeles, CA 90095, zyzhang@ucla.edu)

Vocal fold dehydration during phonation is investigated in a continuum model of the vocal folds. Based on the linear poroelastic theory, the model simulates water movement inside the vocal folds during phonation, water exchange between the vocal folds and the surface mucosal layer through the epithelium layer, surface water accumulation and loss to the glottal airway, and water resupply from blood through the lateral boundary. Parametric studies are conducted to investigate water loss within the vocal folds after 5 minutes of phonation at different voice conditions. The results show that with normal water resupply from the blood, water loss within the vocal folds increases with greater vibration amplitude and higher epithelium permeability. At very large vibration amplitudes, the water loss within the vocal folds can be as high as $3 \%$, which may severely affect body functions and possibly phonation characteristics and vocal effort. Reduced water resupply from blood further increases the degree of dehydration. In contrast, water loss in the surface mucosal layer is an order of magnitude higher than water loss within the vocal folds, indicating the surface dehydration level is likely not a good indicator of the degree of dehydration within the vocal folds.
5aSC19. Where is the beat? Lauryn Zipse (Inst. for Health ProfessionsMGH, Boston, MA), Olivia Murton, Katie Dunn, and Stefanie ShattuckHufnagel (Speech Commun. Group, MIT, 36-511 MIT, 77 Mass Ave., Cambridge, MA 02139, sshuf@mit.edu)

The ability to find the beat in a sequence of auditory events may be linked to the ability to learn vocal communication, raising the question of how beat structure in speech events relates to that in other event sequences. We conducted a series of entrainment experiments designed to compare spoken syllable repetition with tapping. Producing taps to a periodic string of auditorily-presented spoken /pa/ syllables resulted in the tap falling between the release burst for the $/ \mathrm{p} /$ and the onset of voicing for the vowel. This is consistent with participants intending to align their taps with the vowel onsets but exhibiting the well-documented Negative Mean Asynchrony (NMA) effect, such that the taps precede their "target." The finding of alignment with the voicing onset is reminiscent of a large body of work on the Pcenter in repeated spoken syllables. In contrast, producing repeated utterances of the syllable /pa/ to an auditorily-presented click train resulted in coincident occurrence of the release burst with the click, putting the voicing onset after the stimulus (inconsistent with a voicing-onset target with associated NMA). These findings indicate that the location of the beat may differ across tasks involving speech perception and various types of speech production.

5aSC20. An empirical model linking intrinsic laryngeal muscle activation to vocal fold geometry and stiffness. Zhaoyan Zhang, Dinesh K. Chhetri, and Andrew M. Vahabzadeh-Hagh (Dept. of Head and Neck Surgery, Univ. of California, Los Angeles, 1000 Veteran Ave, 31-24 Rehab Ctr., Los Angeles, CA 90095, zyzhang@ucla.edu)

Although vocal fold vibration and the resulting voice production depend on the geometry and mechanical properties of the vocal folds, control of voice is achieved through laryngeal muscle activation, which postures the vocal folds into desired geometry and mechanical properties. Such posturing process is known to cause simultaneous changes in vocal fold position, geometry, stiffness, and tension, which has been hypothesized to be a possible origin of vocal registers. Understanding such co-variations in vocal fold geometry and mechanical properties is thus critical to our understanding of human voice production and control, and the development of muscularly controlled, computationally efficient models of phonation. In this study, an empirical model is developed to link the glottal gap, vocal fold length, vocal fold vertical thickness, and the mechanical properties of the vocal fold to the activation levels of the cricothyroid, thyroarytenoid, lateral cricoarytenoid, interarytenoid, and posterior cricoarytenoid muscles, based on data from in vivo larynx experiments. Combined with a continuum model of phonation, this model is used to investigate muscular control of pitch and vocal registers and the preliminary results are discussed.

5aSC21. Empirical relationship in excised canine larynx. Sid M. Khosla, Liran Oren (Otolaryngol., Univ. of Cincinnati Academic Health Ctr., 231 Albert Sabin Way, 0528, Cincinnati, OH 45208, khoslasm@uc.edu), and ephraim gutrmark (Aerosp. Eng., Univ. of Cincinnati, Cincinnati, OH)

Our lab uses particle imaging velocimetry to measure intraglottal pressure and geometry. Although we have described relationships between maximum divergence angle (MDA) and maximum negative pressure during closin (MNP) for individual experiments, we have not yet looked at the combined data for over 15 larynges without a vocal tract. In this presentation, plots will be shown for the following relationships: MDA vs MNP, MNP vs vortex strength, MNP vs maximum closing speed and maximum vocal fold closing speed vertical stiffness gradient vs divergent angle. Correlation coefficients and generalized empirical relationships will be shown. This will allow approximations for finding variable that are hard to calculate computationally. For example, it is much easier to calculate divergence angle than vortex strength. Comparison of empirical and computational relationships will be described. 
5aSC22. Tone-intonation interaction in context in Thai. Amber B. Camp (Linguist, Univ. of Hawaii at Manoa, 1890 East West Rd., Moore Hall \#569, Honolulu, HI 96822, acamp@ hawaii.edu)

The acoustic realization of lexical tone is influenced by sentence-level intonation. For example, F0 measurements show that a phonologically falling tone in Thai is warped when overlaid with a falling intonational contour, causing F0 to fall further and to begin its decline at an earlier timepoint than when influenced by other contours. This categorical perception study, which includes both identification and discrimination tasks, uses a nine-step continuum of target words created with naturally produced lexical tone endpoints, presented within two different naturally produced sentence frames. Specifically, these tasks investigate the perception of high and falling tones in sentence-medial and sentence-final positions in Thai. This test of stimulus continua within natural sentence contexts reveals the listeners' contextmediated perception of deviation from a canonical lexical tone, shedding light on the interaction and relative weights of both tone and intonation perception in natural speech, and also offering insight into the mechanism with which language users process suprasegmental information.

5aSC23. Acoustic properties of stress in Kubeo. Matthew K. Gordon (Dept. of Linguist, UC Santa Barbara, UC Santa Barbara, Santa Barbara, CA 93106, mgordon@linguistics.ucsb.edu), Thiago C. Chacon (Univ. of Brasilia, Brasilia, Brazil), Kaveh Varjoy (Linguist, UC Santa Barbara, Santa Barbara, CA), and Jullie A. Ferreira (Univ. of Brasilia, Brasilia, Brazil)

This paper explores the realization of stress in Kubeo, a Tukanoan language from the Northwest Amazonia with both stress and tone (H vs. HL). The first $\mathrm{H}$ tone of a word docks on the primary stressed syllable, which by default is the second syllable except in words with lexical initial stress. Secondary stress occurs every two syllables to the right of the primary stress. F0, duration, intensity and the first two formants were measured for vowels in a corpus of 150 words ranging from one to six syllables. Results from two speakers indicate that primary stress is associated with the greatest duration and most peripheral vowel qualities. Unstressed pre-tonic syllables are phonetically more prominent than their post-tonic counterparts along all dimensions except F0, which depends on lexical tone. In post-tonic unstressed syllables, duration, intensity, and vowel quality are all dependent on lexical tone. Secondary stress is less salient due to interference from lexical tones but appears to be associated with longer duration and more peripheral vowels relative to unstressed syllables. Our results enrich typological knowledge of both the acoustic realization of stress in tone languages and the interplay of tonal and metrical phonology in languages with complex word level prosody.

5aSC24. An intonational investigation of five sentence types in Kannada. Kirsten Helgeson (Linguist, Univ. of Hawaii at Mānoa, 1890 EastWest Rd., 569 Moore, Honolulu, HI 96822, kjhelg@hawaii.edu)

Kannada (ISO 639-3 kan) is a Dravidian language spoken by almost 40 million people, primarily in Karnataka state in southern India. It is the official language of Karnataka and one of the scheduled languages of India. Despite its prominence, there is little published in English on the language's intonation systems. This project furthers knowledge of Kannada intonation that is available to speakers of English, with implications for pedagogy and cross-cultural communication. Two female and two male native speakers were recorded in dyads, participating in a scripted conversation containing five types of sentences. F0 contours were examined to determine intonational tunes for different sentence types.

5aSC25. Cue weighting in the tonal register contrast of Jiashan Wu. Bing'er Jiang and Meghan Clayards (McGill Univ., 1085 Dr. Penfield, Montreal, QC H3A 1A7, Canada, binger.jiang@mail.mcgill.ca)

Chinese $\mathrm{Wu}$ dialects are known to have two tonal registers, where the lower register is realized with lower pitch and breathy phonation and the upper register is realized with higher pitch and modal phonation. In Jiashan $\mathrm{Wu}$, the falling tone is realized differently in the two registers: the pitch contour of the upper register is slightly steeper than the lower register. This study investigates how speakers of Jiashan Wu weight the three cues (i.e., breathiness, pitch height, pitch contour) in the register contrast. We recorded two words $/ \mathrm{ka} /$ from the upper and lower register and created stimuli varying in both dimensions ( 5 steps pitch height $\mathrm{x} 5$ step breathiness $=25$ stimuli) and imposed the two contours on all stimuli. 28 native listeners performed a forced-choice categorization task on 5 repetitions of each stimuli in random order. A mixed effect logistic model shows that all three factors affect categorization, and that pitch contour is the most important cue and breathiness the least. Moreover, the effect of breathiness was smaller with higher pitches and a steep contour, and the effect of pitch height is smaller with a steep contour. Data are being collected comparing Jiashan and Shanghai dialects.

5aSC26. Acoustic correlates of prominence in Besemah (Malayic, Indonesia). Bradley McDonnell (Dept. of Linguist, University of Hawai'i at Mānoa, 1890 East-West Rd., 569 Moore, Honolulu, HI 96822, bradley. mcdonnell@gmail.com)

This paper examines the acoustic realization of word-level stress and phrase-level prominence (i.e., pitch-accents, boundary tones) in Besemah, a little-described Malayic language of southwest Sumatra, Indonesia. There has been much disagreement over the status of word-level stress in the languages of western Indonesia, particularly with regards to well-known varieties of Malay (i.e., Standard Malay-Indonesian). Utilizing acoustic cues, word-level stress has been claimed to be present by some (e.g., AdisasmitoSmith \& Cohn 1996), though studies involving perception experiments have questioned this position (e.g., van Zanten \& van Heuven 2004). All of these studies, however, are complicated by significant influence from substrate languages like Javanese, which apparently lack word-level stress (Goedemans \& van Zanten 2007). As a follow up to McDonnell (forthcoming), which found that word-level stress in Besemah falls on the final syllable of the word and is cued by increased intensity, the present study reports the results of a more complex sentence completion task where six speakers of Besemah ( 3 male, 3 female) uttered target words in different frames that vary along two dimensions: [ \pm focus] and [ \pm final position in utterance]. The study aims to show the acoustic correlates of prominence in Besemah, teasing apart word-level stress, pitch-accent, and boundary tones.

5aSC27. Modeling the cross-linguistic variations of tonal systems. Jianjing Kuang (Linguist, UPenn, 255 S 36th St., Philadelphia, PA 19104, kuangj@sas.upenn.edu)

This study aims to simulate the cross-linguistic variations of tonal systems with low dimensional models. Individual syllables of Mandarin, Yoruba, southern Yi, and Hmong were retrieved from existing speech corpora. Voice quality measures as well as f0 were extracted for all data. For each language, Functional Principal Component Analysis (FPCA) is used to parameterize the f0 contours of individual syllables. It is found that three FPCs are sufficient to account for more than $90 \%$ of the variance for pitch contours for all languages. Moreover, the same FPCs are shared among tonal languages and appear to be phonologically meaningful: FPC1 is related to the pitch range; FPC2 is related to the direction of the contour (e.g. rising or falling); FPC3 is related to more complex contours such as dipping and convex. Tone classification models were built for each language, and both voice quality measures and f0 PCs were fitted into the models. Voice quality cues significantly improve the accuracy of the predictions, but different languages vary in the relative importance of voice quality cues. This study provides a new effective way to computationally model the cross-linguistic variation of tonal systems, and has practical implications for speech synthesis.

5aSC28. Distinguishing ambiguity: Cantonese speakers using prosody to disambiguate surface identity in syntax. Elaine Lau (Linguist, Univ. of Hawai'i at Manoa, 7E, Block 3, Site 4, Whampoa Garden, Kowloon 00000, Hong Kong, elau@hawaii.edu)

This paper explores how prosodic and syntactic structures correspond in Cantonese. We consider how native speakers disambiguate object relative clauses (ORC, 1a) and main clauses (MC, 1b), which have a surface identity due to the homonymy between the relative clause marker $(\mathrm{go}+\mathrm{CL})$ and the demonstrative go2+CL 'that'. (1) a. [RC naam4zai2 sek3gan2, go2 go3 neoi5zai2 boy kiss-PROG that CL girl "the girl that the boy is kissing" $b$. 
naam4zai2 sek3gan2 go2 go3 neoi5zai2 boy kiss-PROG that CL girl "The boy is kissing that girl." Given fundamental frequency $\left(\mathrm{F}_{0}\right)$ is used to mark lexical contrasts in the language, it is interesting what other prosodic cues may be used for different parses of the identical string of constituents. Six speakers are recruited for an elicited production study. Pictures designed to elicit ORC vs. MC were presented in random order. Results of acoustic analyses revealed that Cantonese speakers mainly use duration, rather than $\mathrm{F}_{0}$, to disambiguate the two constructions, reminiscent of focus prosody where $F_{0}$ is found to play a limited role. The theoretical implications of these results in relation to the interplay between syntax and prosody are discussed.

5aSC29. Production of neutral tones in three Mandarin dialects. Yang Li (Univ. of Cambridge, Cambridge, United Kingdom) and Arthur L. Thompson (Dept. of Linguist, The Univ. of Hong Kong, Hong Kong, Hong Kong, arthurlewisthompson@gmail.com)

Neutral tone (NT) in Mandarin refers to syllables with shorter duration and whose pitch realisations depend on preceding syllables (Chao 1968; Lee and Zee 2008). There are few acoustic studies on NT in Mandarin dialects outside the standard, and on how NT interacts with sentence modality and prosodic position. We report results from a reading task with 3 speakers each from Chongqing, Kunming, and Nanjing $(\mathrm{N}=9)$. A set of 8 syllables with potential NT and control lexical tones are placed in phrase-medial and final positions and in statements and echo questions. Pitch contours of entire utterances are analysed to determine the variation in pitch height, slope and tonal coarticulatory influence on target syllables. We find NT-like behaviour in Chongqing and Kunming, despite the longstanding claim of their absence (Luo and Wang 1981; Zhai 1996): e.g., in Chongqing, NT syllables are high-falling medially, but undergo more pitch reduction than control and exhibit obligatory tonal spreading from preceding tone regardless of speech rate. Nanjing NT interacts strongly with modality: statement-final NT is almost invariably realised as strong creak if preceding tone is mid-falling. We also report hitherto unreported variation and hypothesise on the impact of standard Mandarin on NT in these dialects.

5aSC30. Two types of intermediate phrase boundary tones in Korean. Hyunah Ahn (Univ. of Hawaii at Manoa, 1890 East-West Rd., \#570, Honolulu, HI 96822, hyunah.ahn@hawaii.edu)

The current study shows that there are two types of Intermediate Phrase (ip) boundary tones, distinctively used for focus marking and syntactic phrasing in spoken Korean. Jun (2011) argued that focused ip (ipF) are characterized by raised initial high $(+\mathrm{H})$ tone and dephrasing of subsequent materials and syntactic ip(ipS) by a phrasal juncture (larger than AP but small than IP) on the phrase-final boundary and partial dephrasing of the following phrase. To empirically test the claim, an experiment was conducted where participants were given an ambiguous sentence to compose a story with. The ambiguous construction was made up of an overt NP, a VP and a covert NP. The ambiguity depended on where the covert NP goes. If the word order is a. "overt NP + covert NP + VP," the syntactic juncture between the overt NP and the VP is larger than when the covert NP is placed before the overt NP (b. covert NP+ overt NP+VP). In stories where the syntax of the sentence was a, the likelihood of ipS was significantly larger than when the syntax was $b(p<.05)$. The distribution of ipF was related to the information structure of the story (operationalized as the location of the sentence within the story) $(\mathrm{p}<.01)$. The results show that the two different types of intermediate phrase (ip) boundary tones are used in real-time production of spoken Korean. The findings provide empirical evidence for Jun's (2011) claim for the Korean prosodic hierarchy.

5aSC31. Vocal fry in realistic speech: Acoustic characteristics and perceptions of vocal fry in spontaneously produced and read speech. Sarah T. Irons and Jessica E. Alexander (Psych., Centenary College of Louisiana, 2911 Centenary Blvd., Shreveport, LA 71104, jalexander@centenary. edu)

There has been a great deal of recent interest in vocal fry, both in production and perception. However, much of the scientific literature that has used naturally produced fry has focused on speech elicited through reading, rather than spontaneous speech. The current study compares reading with spontaneous speech elicited in various ways for both male and female speakers, recorded in dyads. The speakers were asked to teach their partner information, give instructions, and describe their qualifications for a scholarship, with reading controls for each task. Surprisingly, we found more vocal fry (in both proportion of words and words/duration) for men than women. Men differed in the amount of fry across task types. Interactions of speaker sex, task type, and fry were examined for measures of F0, pitch range, intensity level, jitter, shimmer, and HNR. Listener perceptions of speech with and without vocal fry were also obtained. Task type affected listener perceptions of speech with vocal fry. Vocal fry in spontaneously produced speech seems to differ from fry in speech produced during reading, in quantity, acoustics, and listener perceptions. Additionally, acoustic measures suggest that vocal fry may be intimately tied to decreased vocal effort across task types and speaker sex.

5aSC32. A longitudinal study of within-individual variation in human voice pitch. Katarzyna Pisanski (Mammal Vocal Commun. \& Cognition Res. Group, Psych., Univ. of Sussex, School of Psych., Pevensey Bldg. Rm. 2A13, Falmer, Brighton BN1 9QH, United Kingdom, K.Pisanski@sussex. ac.uk), Meddy Fouquet (Mammal Vocal Commun. \& Cognition Res. Group, Psych., Univ. of Sussex, Saint-Etienne, France), Nicolas Mathevon (Equipe Neuro-Ethologie Sensorielle, ENES/Neuro-PSI CNRS UMR 9197, Univ. of Lyon/Saint-Etienne, Saint-Etienne, France), and David Reby (Mammal Vocal Commun. \& Cognition Res. Group, Psych., Univ. of Sussex, Brighton, United Kingdom)

Individual differences in human voice pitch (fundamental frequency, $F 0$ ) have evolutionary relevance. Fundamental frequency indicates the sex, age, and even dominance of the speaker, and influences a host of social assessments including mate preferences. Yet, due to the almost exclusive utilization of cross-sectional designs in previous work, it remains unknown whether individual differences in $F 0$ emerge before or after sexual maturation, and whether $F 0$ remains stable throughout a person's lifetime. In our study, we tracked within-individual variation in the $F 0$ s of male and female speakers whose voices were recorded from childhood into adulthood. Voice recordings were extracted from digital archives. Our results corroborate those of earlier cross-sectional studies indicating a sharp decrease in male $F 0$ at puberty resulting in the emergence of sexual dimorphism in adult $F 0$. Critically, our results further revealed that men's pre-pubertal $F 0$ strongly predicted their $F 0$ at every subsequent adult age, and that $F 0$ remained remarkably stable within-individuals throughout their adulthood. These findings suggest that adult men's voice pitch may be linked to pre-natal/prepubertal androgen exposure and may function as a reliable and stable signal of mate quality, with implications for our understanding of the developmental mechanisms, adaptive functions, and social perception of human voice pitch.

5aSC33. Surface categorical variation In the prosodic marking of English focus. Adam J. Royer and Sun-Ah Jun (Linguist, UCLA, 3125 Campbell Hall, Los Angeles, CA 90095-1543, ajroyer@ucla.edu)

This study investigates the variability of focus prosody in English. In intonational phonology of English, focus is known to be marked with a $\mathrm{L}+\mathrm{H}^{*}$ pitch accent on a focused word followed by deaccenting $(\varnothing)$ postfocus words (Beckman \& Pierrehumbert 1986). 80 speakers participated in a picture naming task where they named images (e.g., blue ball, yellow chair) they saw using the phrase "There was a Adjectivel Nounl, but now there's a Adjective 2 Noun2." Contrastive focus (CF) trials elicited narrow focus on the Adjective2 and Non-contrastive (NC) ones did not. For "a Adjective2 Noun2", f0 contours were ToBI annotated and various acoustic measurements were taken. Preliminary results indicate that in the CF condition, Adjective 2 was most often marked by $\mathrm{L}+\mathrm{H}^{*}$, but it is used only $40 \%$ of the time. In the other cases, Adjective 2 carried either $\mathrm{H}^{*}, \mathrm{H}+! \mathrm{H}^{*}$, $\mathrm{L}+! \mathrm{H}^{*}$, or ! $\mathrm{H}^{*}$. The tune used on Adjective 2 Noun2 was mainly $\mathrm{L}+\mathrm{H}^{*} \varnothing$, but $\mathrm{L}+\mathrm{H}^{*} ! \mathrm{H}^{*}, \mathrm{H}^{*} \varnothing$, and $\mathrm{H}+! \mathrm{H}^{*} \varnothing$ also occurred. These results raise questions about previous claims on phonological marking of focus and the level of tonal representation: what is the phonological status of the various pitch accent types used to mark focus? Are they distinctive or allotones of $\mathrm{L}+\mathrm{H}^{*}$ ? 
5aSC34. Aeroacoustic sound generation during phonation in a synthetic larynx model. Stefan Kniesburges (Dept. of Otorhinolaryngology, Head and Neck Surgery, Div. of Phoniatrics and Pediatric Audiol., Univerity Hospital Erlangen, Cauerstrasse 4, Erlangen, Bavaria 91058, Germany, stefan. kniesburges@uk-erlangen.de), Alexander Lodermeyer, Matthias Tautz, Stefan Becker (Dept. of Process Machinery and Systems Eng., FriedrichAlexander Univ. Erlangen-Nürnberg, Erlangen, Germany), and Michael Döllinger (Dept. of Otorhinolaryngology, Head and Neck Surgery, Div. of Phoniatrics and Pediatric Audiol., Univerity Hospital Erlangen, Erlangen, Germany)

The fluid flow within the human larynx plays an essential role in the fluid-structure-acoustic interaction during voice production. This study addresses the supraglottal flow field downstream of aerodynamically driven, synthetic vocal folds based on the M5 model. The larynx replica is designed to provide full optical access to the flow region. Two different approaches based on Particle-Image-Velocimetry (PIV) were applied for measuring the flow: Phase-averaged (PA) and High-speed (HS) PIV. Beside a comparison of the supraglottal flow field, the acoustic sources were calculated based on both PIV approaches. Furthermore, the simulated far-field sound based on the HS-PIV data is compared to experimental results. Within both PIV approaches, the typical asymmetric jet flow was detected. However, transient flow field characteristics as high vorticity and maximal velocities peaks could only be observed in HS-PIV data. A strong aeroacoustic source was found immediately downstream of the glottis for both PIV approaches. However, the sources in the jet region could only be observed in the HSPIV data owing to the averaging procedure for PA-PIV. The comparison between simulated and measured sound spectra revealed good agreement. In contrast, subharmonic tones could not be detected hinting to an additional non-aeroacoustic mechanism of sound generation.

5aSC35. The duration effect in categorical perception of pitch directions and its interactions with vowel quality and language experience. $\mathrm{Si}$ Chen (Chinese and Bilingual Studies, The Hong Kong Polytechnic Univ., Hong Kong Polytechnic University, Hong Kong 00000, Hong Kong, qinxi3@gmail.com), Yiqing Zhu, Ratree Wayland, and Edith Kaan (Dept. of Linguist, Univ. of Florida, Gainesville, FL)

It is rarely studied how duration affects categorical perception of pitch directions, and whether this effect interacts with vowel quality or groups of listeners. We created a continuum of fundamental frequency on different vowels and duration values for identification and discrimination tasks. Fifteen native Mandarin speakers and fifteen native English speakers were recruited from the Hong Kong Polytechnic University and the University of Florida. The main effects of vowel quality, groups, pitch directions and duration as well as their interactions significantly contributed to identification rate. The sharpness of the categorical boundary differs significantly when a duration difference reaches $40 \mathrm{~ms}$, and it increases first and then decreases as the duration becomes shorter. Tonal listeners exhibit significantly sharper categorical boundary than non-tonal listeners, when a stimuli exceeds $60 \mathrm{~ms}$. Moreover, the sensitivity of between- and within-category discrimination differs by groups and duration, whereas vowel quality reaches marginal significance. Duration also affects the peakedness of discrimination significantly. These findings may help explain the observed differences in perceiving segments like vowels and consonants with different length, and shed light on the interaction between the perception of tones and vowel quality due to compensation mechanism in perceiving the intrinsic F0 effect.

5aSC36. Psychometric measurement of vocal strain using a matching task with sustained vowel stimuli. David A. Eddins, Mark D. Skowronski, Supraja Anand (Commun. Sci. \& Disord., Univ. of South Florida, 4202 E. Fowler Ave., PCD 1017, Tampa, FL 33620, deddins@usf.edu), and Rahul Shrivastav (Vice President for Instruction, Univ. of Georgia, Athens, GA)

Psychometric measurement of voice quality is important for quantitative assessment of dysphonia. Previous work established the efficacy of a singlevariable matching task to index vocal breathiness and roughness using appropriately designed comparison stimuli, providing a context-independent perceptual task that improves the validity and accuracy of perceptual data compared to magnitude estimation or rating tasks. Physiologically, vocal strain is characterized by excessive vocal fold adduction during phonation, resulting in a glottal pulse with a low duty cycle. The narrowing of the glottal pulse causes the excitation spectrum roll-off to decrease, resulting in a flattening of the spectrum and significant changes in spectral skewness and kurtosis [Moore et al., 1997]. Analogous to comparison stimuli for breathy and rough measurement, a set of noisy sawtooth waveforms was created using modified step filters that varied in spectral slope $1 \mathrm{~dB} /$ octave steps, producing a wide range of perceived strain in the comparison stimuli. The comparison stimuli were used in a listening experiment to measure the perceived strain of a set of 15 samples of /a/ selected from the Kay-PENTAX Disordered Voice Database using stratified sampling. The results validate the strain comparison stimuli and extend the matching task paradigm to the three primary dimensions of voice quality perception.

5aSC37. Focus perception in Japanese: Effects of focus location and accent condition. Albert Lee (Dept. of Linguist, Univ. of Hong Kong, Rm. 9.23 Run Run Shaw Tower, Pokfulam HK, Hong Kong, albertlee@hku.hk), Faith Chiu, and Yi Xu (Dept. of Speech, Hearing and Phonetic Sci., Univ. College London, London, United Kingdom)

This study explores the contexts in which native Japanese listeners have difficulty identifying prosodic focus. Theories of intonational phonology, syntax, and phonetics make different predictions as to which focus location would be the most challenging to the native listener. Lexical pitch accent further complicates this picture. In a sentence with mixed pitch accent conditions (e.g., U-A-U), the lexical accent would naturally stand out as more prominent than the unaccented words $(\mathrm{U})$ in terms of modifications to the F0 contour, thus potentially resembling focus. A focus identification task was conducted with 16 native listeners from the Greater Tokyo area. Natural and synthetic stimuli were played to the listeners who then chose which word of the sentence was under focus. Neutral focus (or no focus) was also an option. Stimuli contrasted in accent condition and focus location. Results showed a highly complex interplay between these two factors. For example, accented narrow foci were always more correctly identified (51\%) than unaccented ones $(28 \%)$, whereas the identification rate for final focus was the lowest (31\%) among all focus locations. These results are discussed with reference to the research literature on focus production and formal representation of intonation.

5aSC38. Measurement of vocal breathiness perception with a matching task for sustained phonation and running speech. Rahul Shrivastav (Vice President for Instruction, Univ. of Georgia, Athens, GA), Mark D. Skowronski, Supraja Anand, and David A. Eddins (Commun. Sci. \& Disord., Univ. of South Florida, 4202 E. Fowler Ave., PCD 1017, Tampa, FL 33620, deddins@usf.edu)

Laboratory measurements of the perception of vocal breathiness typically employ recordings of sustained vowel phonations. Sustained vowels are thought to provide a good representation of the underlying vocal disorder and are relatively easy to produce, analyze, and synthesize. Clinical assessments of voice quality are sometimes based on the production of speech utterances that are more ecologically valid than sustained vowel phonation and that capture the dynamics of laryngeal function, breath support, and a variety of complex cognitive and neuromuscular challenges typically involved in natural speech. As a result, voice quality associated with running speech may correspond more closely with perceived handicap and may represent more relevant treatment targets than sustained vowels. In a listening experiment with naive listeners, breathy voice quality was evaluated for exemplars of sustained /a/ production as well as read speech from a selection of talkers that vary widely in terms of their vocal breathiness. A singlevariable matching task was used to index perceived breathiness for both stimulus types. Listener reliability was high, and matching thresholds for the sustained phonation and speech stimuli were highly correlated, demonstrating the efficacy of the matching task for measuring vocal breathiness from speech utterances. 
5aSC39. Revisiting English word stress and rhythm in the post-nuclear domain. Jonathan Howell (Montclair State Univ., 1 Normal Ave., Montclair, NJ 07043, howellj@montclair.edu)

In English, grammatical category and word stress are often highly correlated: disyllabic nouns and verbs frequently occur with trochaic and iambic stress, respectively. Kelly and colleagues $(1988,1992)$ suggest that this relationship was shaped by rhythm but may also be subverted by rhythm. We revisit a 1978 production study by Huss, who argued that the usual stress correlation is neutralized in postnuclear position and that acoustic differences are subject to effects of sentence rhythm. Twelve pairs of homophones were embedded in full sentences preceded by a possessive or plural noun with ultimate or penultimate stress: e.g. farms produce, farm's produce, farmers produce, farmer's produce. A postnuclear environment was elicited by prepending a contrastive sentence (e.g., Some say that only supermarket produce may vary in quality but also a farmer's produce may vary in quality.) Results suggest, contra Huss, that prosodic differences between nouns and verbs persist in the postnuclear environment, despite effects of rhythm. In a mixed-effects logistic regression $(\mathrm{N}=18)$, acoustic cues of syllable duration and maximum intensity were significant predictors of part of speech in nuclear and postnuclear position. We also discuss results of a perception experiment in progress using the production stimuli.

5aSC40. Perception and analysis of "Moe "voice. Sayoko Takano (Kanazawa Institute of Technol., 7-1 Ohgigaoka, Nonoichi 921 - 8501, Japan, tsayoko@neptune.kanazawa-it.ac.jp) and Masashi Yamada (Kanazawa Institue of Technol., K, Ishikawa, Japan)

The term "Moe" is used in Japanese animation and cartoons. "Moe" mainly refers to cute young girls, but it does include direct sexual desire. We investigated the "Moe" voice using "onichan CD" which includes 1200 different utterance from 12 voice actress. "Onichan" means brother in Japanese, and the voice actress says the word with happiness, anger, sadness, adding up to 100 different situations. In experiment one, the impressions of the "Moe" voice was examined using Semantic Differential Method. The subjects answer revealed that Moe is not related to the emotions such as happiness or sadness, but "Moe" is comfort to the listener. Furthermore, "Moe" is mostly associated with the voice actress. This indicates that "Moe" voice is mainly related to the personality. In experiment two, the speech voice was analyzed to measure the acoustic characters. In experiment three, the voice was resynthesized using TANDEM-STRAIGHT. All the results showed that the "Moe" voice is associated with the young girl as small, energetic hypertension.

5aSC41. Cross-cultural differences in the development of emotion perception from face and voice between Japanese and Dutch speakers. Akihiro Tanaka (Psych., Tokyo Woman's Christian Univ., 2-6-1 Zempukuji, Suginami-ku, Tokyo 167-8585, Japan, akihiro@lab.twcu.ac.jp), Disa Sauter (Univ. of Amsterdam, Amsterdam, Netherlands), and Misako Kawahara (Psych., Tokyo Woman's Christian Univ., Tokyo, Japan)

Recent studies have demonstrated cultural differences in multisensory emotion perception from faces and voices. Tanaka et al. (2010, Psychol. Sci.) showed that Japanese people are more tuned than Dutch people to vocal processing in adults. The current study investigated how such a cultural difference develops in children and adults. In the experiment, Japanese and Dutch participants observed affective expressions of both Japanese and Dutch actors. A face and a voice, expressing either congruent or incongruent emotions, were presented simultaneously on each trial. Participants judged whether the person is happy or angry. Results in incongruent trials showed that the rate of vocal responses was higher in Japanese than Dutch participants in adults, especially when in-group speakers expressed a happy face with an angry voice. The rate of vocal responses was very low and not significantly different between Japanese and Dutch 5-6-year-olds. However, it increased over age in Japanese participants, while it remained the same in Dutch participants. These results reveal the developmental onset of cultural differences in multisensory emotion perception.
5aSC42. Effects of discrepancy between vocal emotion and the emotional meaning of speech on identifying the speaker's emotions. Sumi Shigeno (Psych., Aoyama Gakuin Univ., 4-4-25 Shibuya, Shibuya-ku, Tokyo 150-8366, Japan, sshigeno@ephs.aoyama.ac.jp)

This study investigates the effects of a discrepancy between vocal emotion and emotion contained in the literal meaning of speech on the identification of speaker's emotions. Except measuring response time, few studies have examined this discrepancy between the emotional meaning of speech and vocal emotion. In the current study, two stimulus conditions were prepared: the "congruent condition," where the emotional meaning of speech was compatible with the tone of voice and the "incongruent condition," where the emotional meaning of speech was discrepant to the vocal emotions. Four native Japanese actors (two males and two females) spoke short sentences emotionally. We used a repeated-measures design with the emotional meaning of speech (happiness/sadness) and the tone of voice (neutral/ happy/sad) as two factors. Using a 5-point scale, 31 participants were required to identify the speaker's emotions in a forced-choice task with seven alternatives and report the degree of their confidence for every response. Results indicated that identification of speaker's emotion was successful even in the incongruent condition and that the degree of confidence differed little except the identification of sad voices. The results suggest that Japanese participants infer the speaker's intended emotion even if it is incongruent to the emotional meaning of speech.

5aSC43. Appropriateness of acoustic characteristics on perception of disaster warnings. Naomi Ogasawara (Int. Commun., Gunma Prefectural Women's Univ., 1395-1 Kaminote, Tamamura, Gumma 370-1193, Japan, naomi-o@mail.gpwu.ac.jp), Kenta Ofuji, and Akari Harada (Comput. Sci. \& Eng., Univ. of Aizu, Aizuwakamatsu, Japan)

The appropriateness of acoustic characteristics of speech warnings for natural disasters was examined in this study. Three acoustic parameters (voice gender, speech rate, and pitch) were tested in order to find out the best combination of the acoustics which enhances perceived intelligibility, credibility, and urgency by listeners. A hundred native speakers of Japanese listened to short verbal warnings spoken by a male and a female Japanese speaker with normal pitch, $20 \mathrm{~Hz}$ higher, or $20 \mathrm{~Hz}$ lower pitch; normal speed, $20 \%$ faster, or $20 \%$ slower speed. Participants rated each stimulus on 1-to-5-scale. A single effect analysis shows that 1) female voice was perceived with higher credibility and urgency; 2) normal pitch was rated best in the three criteria; and 3) normal speed was perceived best except that faster speed was perceived with higher urgency. A mixed effect analysis found that warnings with the combination of female voice, normal speed, and higher pitch most raised credibility and urgency. Faster speed also raised these two criteria although intelligibility was a little sacrificed. Based on the results, we suggest that disaster warnings with female voice and higher pitch would be effective with the appropriate speaking rate to keep good intelligibility.

5aSC44. The relationship between perception of cuteness and duration of voices. Ryohei Ohno (Nihon Univ., 3-25-40, Sacurajosui Setegaya-ku, Tokyo, Japan, Setagaya-ku 156-8550, Japan, ryouhei@kthrlab.jp), Masanori Morise (Univ. of Yamanashi, Kofu, Japan), and Tetsuro Kitahara (Nihon Univ., Tokyo, Japan)

The "cuteness" is one of the most important features in female voices. In fact, voice actresses who are known as cute voice holders are very popular in Japan, particularly for Japanese anime freaks. We have therefore been investigating the relationship between the perception of the cuteness of voices and their acoustic features. Here, we focus on the duration of voices. We hypothesized that it is difficult to perceive cuteness when the voice is short, and we conducted a listening test using voice stimuli with various durations. Given voices (around $1 \mathrm{sec}$ ) saying "oniichan" (older brother) by 12 actresses, we extracted 75-ms, 150-ms, 300-ms, and 600-ms parts from them. Participants listened to each stimulus three times and rated their cuteness on a scale of 1 to 5. Experimental results show the following two findings: 1) The stability of ratings for the same voice (i.e., the difference between the highest and lowest ratings for the same voice within each participant) tends to become lower as the voice is shorter. 2) Regarding actresses whose full-version voices are rated 4 or higher by each participant, the participant also rated more than half of the shortened voices (even 75 -ms ones) 4 or higher. 
5aSC45. Perceptual impressions and acoustical characteristics of simulated "feminine" voices. Maki Waragai and Ayako Shirose (Graduate School of Education, Tokyo Gakugei Univ., 4-1-1 Nukuikita-machi, Koganei, Tokyo 184-8501, Japan, m151423x@st.u-gakugei.ac.jp)

This study aims to clarify characteristics of voice quality that give an impression of femininity. With regard to female voices and gender variation of voices, previous research has studied their acoustical features. Klatt \& Klatt (1990) examined male and female voices acoustically and showed that female voices were more breathy than male voices. In order to reveal typical characteristics of female voices, this study investigated perceptual impressions and acoustic features of simulated female voices. Analyzes data were utterances produced by twelve speakers ( 6 females and 6 males, aged 20-22). The speakers were asked to simulate a female "feminine" voice. Firstly, we carried out an impression rating experiment on the obtained data. Twenty-two participants were asked to evaluate the utterances according to various rating scales. The scales included "clear," "thin," "feminine" and so on (Kido and Kasuya, 1999; 2001). Analysis showed that a significant correlation with "feminine" was found in "high," "clear," "thin." Second, /a/ vowels extracted from the utterances were analyzed acoustically. Acoustic parameters were F0, the amplitude gap between $\mathrm{H} 1-\mathrm{H} 2$, and CPP. The relation between these acoustic parameters and the impression ratings of voice quality will be discussed.

5aSC46. Japanese "street seller's voice". Toshiyuki Sadanobu (Graduate School of Intercultural Studies, Kobe Univ., 3-3-18, Nigawa-cho, Nishinomiya-shi 662-0811, Japan, sadanobu@kobe-u.ac.jp), Chunyue Zhu (School of Lang. and Commun., Kobe Univ., Kobe, Japan), Donna Erickson (Kanazawa Medical Univ., Kanazawa, Japan), and Kerrie Obert (Ohio Univ., Athens, $\mathrm{OH}$ )

The term "Street Seller's Voice" (henceforth SSV) refers to a category of voice quality which can be uttered only by, but not all, Japanese young girls, especially selling something cute and fashionable. We can hear this voice at cake shops, but never at "wagashi" (i.e., Japanese cake) shops, since "wagashi" is not cute and fashionable, unlike western cake which for Japanese people is cute. In order to catch the nature of SSV we recorded voices of several street sellers, and also conducted MRI experiments with 3 subjects. The results suggest that SSV has a twang quality [e.g., Estill et al. 1983, Proc. Stockholm Music Acoustics Conference, 157-174; Honda et al. 1995, Vocal Fold Physiology; Voice Quality Control, 23-38], which is manifested in the acoustic signal by, among other things, sustained high energy in the upper frequency regions, and may possibly be produced with high larynx and change in pharyngeal cavity size. One advantage to this type of voice production is it is easily heard in a noisy environment and puts minimum strain on the vocal folds [Estill, personal communication]. [This work was supported by the following grants: JSPS Grants A \#15H02605 and A \#25240026.]

5aSC47. Effect of temporal fluctuation in speech on perception of humanness of synthesized speech. Fumiya Yokomori, Masanori Morise, and Kenji Ozawa (Graduate School of Medicine and Eng. Sci. Dept. of Education, Univ. of yamanashi, 4-3-11 Takeda, Kofu, Yamanashi 400-8511, Japan,g15mk019@yamanashi.ac.jp)

The sound quality of speech synthesized using modern speech synthesis systems is expected to be approximated to human speech. We investigated the effect of temporal fluctuation of speech on the perception of humanness. Speech stimuli used in the evaluation were generated by voice morphing. The morphing source (morphing rate of $0 \%$ ) and target (morphing rate of $100 \%$ ) were speech without temporal fluctuation and original speech, respectively. There were three kinds of morphing factors: fundamental frequency (F0), spectral envelope (SP), and both (F0+SP). We used MUSHRA as the evaluation method involving two speakers (one male and one female), two phonemes (/a/ and /i/), and two F0s (high and low). Nine morphing rates (every $25 \%$ from -100 to $100 \%$ ) were used, and ten subjects with normal hearing participated in the evaluation. The results show that the stimuli with a morphing rate of $0 \%$ scored the lowest humanness for all factors. The stimuli with morphing rates of $-100 \%$ scored significantly lower than those with a morphing rate of $100 \%$ in SP and F0 + SP. The most dominant factor was $\mathrm{F} 0+\mathrm{SP}$, and the effect of $\mathrm{F} 0$ was the smallest of all factors. [Work supported by JSPS KAKENHI Grant Numbers 15H02726, 16H05899, 16H01734.]
5aSC48. A framework for systematic studies of attitudes in speech. Hiroya Fujisaki (The Univ. of Tokyo, 3-31-12 Ebisu, Tokyo 150-0013, Japan, fujisaki@alum.mit.edu), Sumio Ohno (Tokyo Univ. of Eng., Tokyo, Japan), and Wentao Gu (Nanjing Normal Univ., Nanjing, China)

We present here a framework for a systematic study of attitudes expressed by speech. Although the term "attitude" is commonly used to refer to phenomena at several different levels, we shall assign separate terms to each of these levels for the sake of clarity. The term " attitude" can refer both to a person and an object (either concrete or abstract), here we shall be concerned only with personal relationships. Stances: Those (attitudes) related to official relationships, often influenced by social factors together with long-term personal factors (such as commanding, subordinate, etc.) Attitudes (in the narrow sense): Those referring to private but mid- to long-term relationships, often involving the whole personality (such as kind, friendly, remote, etc.) Manners: Those (attitudes) referring to short-term but somewhat consistent individual behaviors/acts (such as polite, rude, abrupt, etc.) For example, when a teacher tries to call the attention of a young child to an imminent danger, his/ her utterance will have commanding stance, kind attitude, and abrupt manner simultaneously. Although in real life these distinctions are not always clearcut, our data collection is based on this principle. The result of a preliminary analysis supports the validity of our approach. [This work was funded by the NSSFC major program 13\&ZD189.]

5aSC49. Automatic recognition of negative emotion in speech using support vector machine. Yuto Yamamoto, Masahiro Niitsuma, and Yoichi Yamashita (Graduate School of Information Sci. and Eng., Ritsumeikan Univ., 1-1-1 Noji-higashi, Kusatsu Shiga 525-8577, Kusatsu, Shiga-Pre 525-8577, Japan, yamamoto-ASJ@slp.is.ritsumei.ac.jp)

This paper addresses negative emotion recognition using paralinguistic information in speech for speech dialogue system. Speech conveys not only linguistic information but also paralinguistic and non-linguistic information such as the emotions, attitudes, and intentions. This easily perceivable information plays a key role in a spoken dialog system. However, most of previous speech recognition systems fail to consider this significant information, focusing only on linguistic information, thus hindering the development of more natural speech dialog systems. In order to utilize these significant information for speech dialog systems, this paper focuses on negative emotion recognition from Japanese utterances. 6552-dimensional acoustic features were extracted from 6300 Japanese utterances of 50 people in three emotional state: negative; positive; and neutral. Negative emotion includes anger, sad and dislike. While positive emotion includes favor, joy, and relief. They were classified by SVM and evaluated by a 10 -fold cross validation. The experimental result showed the recognition rate of $93.4 \%$ for the classification of negative and positive and $95.0 \%$ for the classification of negative and neutral.

5aSC50. Natural language dialog system considering speaker's emotion for open-ended conversation. Takumi Takahashi, Kazuya Mera, Yoshiaki Kurosawa, and Toshiyuki Takezawa (Graduate School of Information Sci., Hiroshima City Univ., 3-4-1, Ozuka-higashi, Asaminami-ku, Hiroshima 731-3194, Japan, takahashi@1s.info.hiroshima-cu.ac.jp)

To respond appropriately to an utterance, human-like communication system, should consider not only words in the utterance but also the speaker's emotion. We thus proposed a natural language dialog system that can estimate the user's emotion from utterances and respond on the basis of the estimated emotion. To estimate a speaker's emotion (positive, negative, or neutral), 384 acoustic features extracted from an utterance are utilized by a Support Vector Machine (SVM). Artificial Intelligence Markup Language (AIML)-based response generating rules are expanded so that the speaker's emotion can be considered as a condition of these rules. Two experiments were carried out to compare impressions of a dialog agent that considered emotion (proposed system) with those of an agent that did not (previous system). In the first experiment, 10 subjects evaluated the impressions after watch four conversation videos (no emotion estimation, correct emotion estimation, inadequate emotion estimation, and imperfect emotion estimation). In the second experiment, another 10 subjects evaluated the impressions after talk with both dialog agents. These experimental results and a demonstration of the proposed system will be shown in the presentation. 
5aSC51. Universal vs. language-specific aspects in human vocal attractiveness: An investigation towards Japanese native listeners' perceptual pattern. Anqi Xu and Shue-sum Leung (Dept. of Linguist, The Univ. of Hong Kong, Flat A, 16F, Federate Bldg., No. 292-298 Queen's Rd. West, Sai Ying Pun, Hong Kong 999077, Hong Kong, xuanqi@hku.hk)

Studies on Western societies show that male voices with acoustic parameters encoding a big body size (low F0, narrow formant dispersion, and F0 range) were considered to be attractive, while the opposite was true for female voices (e.g., [Xu et al., 2013, PLoS ONE, 8(4), e62397]). The present work investigates whether Japanese native listeners are guided by the same principles in assessing the voices of the opposite sex. We replicated the design in Xu et al. (2013) with the added parameter of creaky voice, which is prevalent in North America nowadays and hotly debated in terms of attractiveness. Thirty-four heterosexual participants ( 16 female) rated the attractiveness of synthetic stimuli controlling for F0 height, formant distribution, F0 range and voice quality. Results indicate that their preferences for voice quality are similar with studies on Western societies (breathy $>$ modal >creaky > pressed/tensed). Additionally, low-pitched male voice with narrow formant dispersion and high-pitched female voices were also favorable. Interestingly, a narrow F0 range significantly lowered the attractiveness ratings, regardless of the gender of the voice, which contradicts $\mathrm{Xu}$ et al. (2013). These various results are discussed in light of the cross-linguistic/cross-ethnic divergences in vocal attractiveness.

5aSC52. Modeling uncertainty in spontaneous speech: Lexical and acoustic features. Griffin Romigh (Air Force Res. Lab., Dayton, OH), Clayton Rothwell, Brandon Greenwell (Infoscitex Corp., 4027 Colonel Glenn Hwy, Ste. 210, Dayton, OH 45431, crothwell@infoscitex.com), and Meagan Newman (Wright State Univ., Dayton, OH)

Previously, spoken uncertainty has been analyzed using either lexical or acoustic features, but few, if any, studies have used both feature types in combination. Therefore, it is unknown to what extent these feature types provide redundant information. Additionally, prior research has focused on the study of acoustical features of only single words, and it is unclear if those results can generalize to perceived uncertainty in spontaneous speech. The current study elicited spontaneous speech through a team dialogue task in which two people worked together to locate street-level pictures of different houses on an overhead map. The communications were recorded, transcribed, broken into utterances, and presented to 10 individuals who rated each utterance on a 5-pt Likert scale from 1 (very uncertain) to 5 (very certain). A large number of acoustic and lexical features from the literature were calculated for each utterance. Random forest classification (Breiman, 2001) was used to select features and then investigate feature importance individually and also at the aggregate level of feature type. Results indicate that lexical features were much more important than acoustic features and suggest that previous findings using acoustic features might not generalize to spontaneous speech. Additional acoustic features are explored to improve performance.

5aSC53. Assessing the effect of phonetic distance on accommodation. Stephen Tobin (Dept. Linguistik, Universität Potsdam, 406 Babbidge Rd., Storrs, CT 06269-1020, stephen.tobin@uconn.edu) and Adamantios Gafos (Dept. Linguistik, Universität Potsdam, Potsdam, Brandenburg, Germany)

In investigations of phonetic accommodation, convergence is the most frequently reported finding (e.g., Babel, 2012). However, divergence is also attested under some circumstances (Giles, Coupland \& Coupland 1991). On the basis of observations and modeling of Tobin and Nam (2009), Tobin, Nam and Fowler (under review) and Kopecz and Schöner (1995), we asked whether and how phonetic distance along some relevant phonetic dimension would modulate accommodation. Following Roon and Gafos (2014) and Gallantucci, Fowler and Goldstein (2009), who report systematic perceptuomotor effects in speech, we used a cue-distractor paradigm to assess the effect of distance. Participants were visually cued to produce a syllable ( $t a$ or $\mathrm{ka}$ ). $150 \mathrm{~ms}$ later they heard a distractor syllable from a 5-step ta or $\mathrm{ka}$ VOT-continuum. Participants were assigned continua either near or distant from their baseline VOT. An ordinal logistic regression of data from 12 participants in the near condition indicated patterns of divergence from the mean distractor VOT among those closest to the distractor, convergence among those farthest, and maintenance at intermediate distances. Pending analysis of data from the distant condition, we conclude that targets must fall within a range neither too close to nor too far from speakers' baseline to induce convergence.

5aSC54. A study on judgement of intoxication using pitch alteration of speech signal in VHF environment. Won-Hee Lee and Myung-Jin Bae (SoongSil Univ., Hyungnam Eng. Buliding 1212, Soongsil University 369 Sangdo-Ro, Dongjak-Gu, Seoul 156-743, South Korea, vbluelovev@ssu.ac. $\mathrm{kr}$ )

In this study, speech characteristics from before and after alcohol intoxication has been comparatively analyzed though speech analysis to obtain the degree of intoxication along with its parameters in VHF environment. It is studying speech characteristics of alcohol intoxicated person as pitch and formant and changing of sound levels, but it cannot analyze about before and after alcohol intoxication using these speech characteristics because it is sensitive about environmental changing so we need finding more precise parameters. Especially, speech signal is distorted from the frequency carrier characteristics in VHF environment. Thus, we study speech characteristics from before and after alcohol intoxication using pitch alteration in VHF environment.

5aSC55. Perceived vocal attractiveness by gay listeners in Hong Kong. Shue-sum Leung and Anqi Xu (Univ. of Hong Kong, 2/F, 263 Hang Ha Po, Tai Po, N.T., Hong Kong, Hong Kong, rory@hku.hk)

This study investigates how Cantonese-speaking homosexual males perceive vocal attractiveness; namely, how different phonetic properties of voice influence their judgment. To this end, the present project follows the methodology in Xu et al's (2013) study of vocal attractiveness and emotion in English, and recruited 14 Cantonese-speaking gay men in Hong Kong to rate the attractiveness of digitally synthesised stimuli. The stimuli varied in voice quality (breathy / modal / pressed), fundamental frequency and formant dispersion, all of which were means to project the speaker's intended body size. The attractiveness ratings show that homosexual male subjects prefer male voices with low pitch, breathy voice quality and normal (neither dense nor sparse) distribution of formants. Interestingly, these preferences resemble the ratings by female English subjects in Xu et al's (2013) study with one exception-the females favoured male voices with densely distributed formants, which signal a large body. This divergence needs to be further investigated by follow-up studies targeting different language and sexuality groups, in order to distinguish factors that are universal and language-specific.

5aSC56. Importance of F0 for predicting vocal emotion categorization. Margaret K. Pichora-Fuller, Kate Dupuis (Psych., Univ. of Toronto, 3359 Mississauga Rd., Mississauga, ON L5L 1C6, Canada, k.pichora.fuller@ utoronto.ca), and Pascal Van Lieshout (Speech Pathol., Univ. of Toronto, Toronto, ON, Canada)

Affective prosody is used to produce and express specific emotions to conversation partners. While pitch has been identified as a crucial cue for differentiating between emotions, there has been significant variation in the stimuli used by different groups of researchers to examine the acoustic cues necessary for the perception of vocal emotions. The Toronto Emotional Speech Set consists of 2800 items: 200 sentences (carrier phrase "say the word" followed by a target word) spoken by two adult female actors (one younger and one older) to portray seven emotions (anger, disgust, fear, sadness, happiness, pleasant surprise, neutral). In the current study, these stimuli were analyzed to determine which acoustical cues accounted for the most variance in categorizing stimuli into one of the seven pre-determined emotional conditions. The acoustical characteristics of mean duration, mean intensity, mean F0, mean range of intensity, and mean range of F0 were analyzed using a customized Praat script for each of the 2800 stimuli. For both talkers, mean F0 was the most important acoustical cue for accurately categorizing the TESS stimuli into the different emotional conditions. The second most important cue was F0 range for the younger talker and duration for the older talker. 
5aSC57. Judgments of American English male talkers who are perceived to sound gay or heterosexual: Certain personality traits are associated with each group of talkers. Erik C. Tracy (Psych., Univ. of North Carolina Pembroke, PO Box 1510, Pembroke, NC 28372, erik.tracy@uncp. edu)

Prior research (Campbell-Kibler, 2011; Gaudio, 1994) discovered that some personality traits (e.g., emotional and intelligent) are associated with gay male talkers and other traits (e.g., masculine and reserved) are associated with heterosexual male talkers. The present investigation examined additional traits listeners associated with four groups of talkers: gay talkers who were perceived as sounding gay, gay talkers who were perceived as sounding heterosexual, heterosexual talkers who were perceived as sounding gay, and heterosexual talkers who were perceived as sounding heterosexual (Tracy, Bainter, \& Satariano, 2015). After hearing a spoken utterance, listeners rated talkers along eight traits: bored, confident, intelligent, mad, old, outgoing, sad, and stuck-up (Tracy \& Charlton, 2016). During the experiment, the talkers' self-identified and perceived sexual orientation were not referenced. The results demonstrated that the gay and heterosexual talkers who were perceived as sounding gay were rated as being significantly more confident, mad, stuck-up, and outgoing; the gay and heterosexual talkers who were perceived as sounding heterosexual were rated as being significantly more bored, sad, and older. There were no significant rating differences for intelligence. It was concluded that a talker's perceived sexual orientation, rather than their self-identified sexual orientation, influenced which personality traits listeners associated with them.

5aSC58. Phonological structure of toasting practices in Japan. Yoko Sakamoto (Premedical Sci., Dokkyo Medical Univ., 880 Kitakobayashi, Mibu, Shimotsugagun, Tochigi 3210293, Japan, y-saka@dokkyomed.ac. jp)

The aim of this study was to investigate toasting practices in Japan from the point of view of phonological structure. In Japan, toasting practices means calls or songs to encourage someone to drink alcohol at one stroke. This habit has lasted for 30 years among university students and company young employees in Japan, although many campaigns have been held against toasting practices. The background of this habit may have complex factors, such as social, psychological, etc., but one of the factors may be in the calls and songs themselves. Thus, in the present study, calls of songs of toasting practices were collected as database, and the phonological structure (foot, syllable, mora, phoneme) and the tempo was analyzed. The result showed that the calls and songs of toasting practices in Japan are categorized into three groups; calls, parody songs and others (such as famous phrases), but these calls and songs have common phonological structure. They tend to have 4 morae 2 feet structures and combinations of Motherese syllable structures. The tempos of calls and songs were 120-140, which were the double speed of adult's normal heartbeat. Thus, it has been concluded that toasting practices in Japan have regularity in phonological structure.

5aSC59. Constructing boyishness: A sociophonetic analysis of Japanese anime voice actresses performing male roles. Ryan C. Redmond (Linguist, Univ. of California Davis, 2505 5th St. Apt. 117, Davis, CA 95618, rcredmond@ucdavis.edu)

The present study investigates the performance of different gendered roles by Japanese female seiyuu [voice actors] through analysis of fundamental frequency (f0). Ten voice actresses who have been known for commonly voicing male characters were sampled for analysis. For each actress, two characters from their repertoire were chosen (one male, one female), and from each character 75 intonation phrases were sampled, for a total of 1500 utterances. For each of these samples, pitch data were extracted and analyzed. Results for both male and female role performances display an average fo that generally falls inside the category previously defined as hegemonically "feminine" in Japanese. While male role measurements were on average lower than female role measurements, only occasionally did any actress produce pitches in the average expected range for a Japanese man. However, because the female role measurements were significantly higher than that of average Japanese women, the differences between the "male role" and "female role" categories proved statistically significant in terms of minimum pitch, maximum pitch, mean pitch, and pitch range. A theory is proposed that the hyper-femininity of many female roles is what necessitates this difference. 


\title{
Session 5aSP
}

\section{Signal Processing in Acoustics: General Topics in Signal Processing II}

\author{
Ning Xiang, Chair \\ School of Architecture, Rensselaer Polytechnic Institute, Greene Building, 110 8th Street, Troy, NY 12180
}

\section{Contributed Papers}

\section{$7: 55$}

5aSP1. Sound quality improvement based on weighted AM/FM modulations for parametric loudspeaker. Kirara Ariyoshi, Takuya Kimura, Takahiro Fukumori, Masato Nakayama, and Takanobu Nishiura (Ritsumeikan Univ., 1-1-1, Noji-higashi, Kusatsu, Shiga 525-8577, Japan, is0152hv@ed. ritsumei.ac.jp)

A parametric loudspeaker has high-directivity, and it utilizes a modulated wave that is designed by modulating an ultrasound wave with an audible sound wave. The conventional modulation methods, amplitude modulation (AM) and frequency modulation (FM), have been proposed. Furthermore, an AM/FM method has been proposed that can improve the sound pressure level (SPL). However, its sound quality is inferior to the AM method. The AM/FM method utilizes constant modulation parameters in all frequency bands. However, the power of audible sound demodulated by AM and FM modulations is different at each frequency band. Therefore, we propose a new AM/FM method that utilizes the weighted modulation parameters in each frequency band and can improve the sound quality. The proposed method has comparable sound quality to the AM method and a comparable SPL to the FM method. In this proposed method, we divided all frequency bands into three frequency bands and utilized the optimum weighted modulation parameters in the three frequency bands on the basis of the results of the preliminary experiments. As a result of evaluation experiments, we confirmed the effectiveness of the proposed method compared with the conventional methods.

\section{8:10}

5aSP2. MATLAB visualization program for analysis of C3D motion capture data of speech and language. Andrew Barnes, Rebecca Mental, Brooke Macnamara, and Jennell Vick (Dept. of Psychol. Sci., Case Western Reserve Univ., 11635 Euclid Ave., Rm. 308, Cleveland, OH 44106, acb48@case.edu)

Investigation of the underlying physiology of speech and language motor control often includes analyses of kinematic parameters derived from fleshpoint tracking. A MATLAB program has been developed to visualize, boundary mark, and analyze aspects of motion capture data in a C3D file format. C3D is the biomechanics data standard for binary 3D data that is widely used in many industries and motion research. Included in the format is marker names, arrays of position data, and analog inputs. While this format is highly versatile, it is difficult to assess the comparison of the markers positions without a visualization program. The developed program allows for flexible comparison of any fleshpoint markers, in any set of repetitions. It also allows for easy visualization of the differences between varying data conditions. Data analysis is done on-the-fly for information such as joint angles through three markers, distance between any two markers, and variability analyses of any selected data. Data are output in a format suitable for additional statistical analyses. Recent analyses using this program were completed on highly complex data streams with over 30 fleshpoint markers. The program provides an incredibly useful tool for exploration of these data and quick analyses of coordination and variability.
$8: 25$

5aSP3. Development of a 3D impulse response interpretation algorithm. Federico N. Cacavelos, Alejandro Bidondo, and Augusto Bonelli Toro (Engineer, Universidad Nacional de Tres de Febrero, Mosconi 2736, CABA, Saenz Peña 1674, Argentina, fnahuelc@gmail.com)

The development of software 3D interpretation impulse responses is presented. This tool allows to identify the spatial provenance for time windows of an impulse response recorded in a soundfield microphone. In this way, it is possible to discern between the direct sound and reflections both temporarily and spatially. To understand the basics of this method, a brief introduction to all concepts involved is presented. Alternatively, different types of procedures are shown for data evaluation. Finally, system applications are shown in controlled acoustical environments so evaluating the precision and the potential of this tool.

\section{8:40}

5aSP4. The acoustical features analysis of Indo-Pacific humpback dolphins' whistle and modeling research. Siyuan Cang, Xueli Sheng, Jintao Sun, Dian Lu, Jingwei Yin, and Longxiang Guo (Harbin Eng. Univ., College of Underwater Acoust. Eng., Harbin Eng. University, Harbin, Heilongjiang 150001, China, cangsiyuan@hrbeu.edu.cn)

Depending on species-specific characteristics of echolocation and recognition,dolphin could travel in groups without collision, and they could also prey and defend mutually. As a multi-harmonics, frequency-modulated signal, the variety of whistles revealed the rich group identification and location information of free-ranging Indo-Pacific humpback dolphins (Sousa chinensis). This paper introduces the features of whistle on the basis of Joint time-frequency distribution, dealing with recording data collected in the area of Guangxi and Zhanjiang seacoast, in China. Then the ability of individual identification is discussed from the correlation of whistles, we will get the knowledge of dolphins how to cognize the communication information in the complex environment. The transmission loss function will be depicted using the BELLHOP at last. By extracting the acoustical features, the modeling of whistle signal is implemented based on the Sinusoids Model (Buck, 2000). Specifically, the validity of mathematical model is proved according to comparison with the recording data.

\section{8:55}

5aSP5. Comparisons of conventional and frequency-difference beamforming. Alexander S. Douglass and David R. Dowling (Mech. Eng., Univ. of Michigan, 2010 AL, 1231 Beal, Ann Arbor, MI 48109, asdougl@umich. edu)

Conventional beamforming can isolate ray arrival directions from transducer array recordings, provided the array element spacing does not significantly exceed half of a signal wavelength. However, when the signal frequency is high and the array elements are many wavelengths apart, conventional beamforming may break down due to spatial aliasing. Frequency difference beamforming avoids this limitation by utilizing a quadratic product of complex signal amplitudes at different frequencies to extract wave propagation information at a difference frequency chosen low enough to avoid aliasing. For this presentation, the performance of frequency 
difference beamforming was evaluated two ways. First, simulations of acoustic propagation in a 106-m-deep shallow-ocean sound channel using the BELLHOP ray-tracing code were completed with frequency-sweep signals from $11 \mathrm{kHz}$ to $33 \mathrm{kHz}$ and $1100 \mathrm{~Hz}$ to $3300 \mathrm{~Hz}$ broadcast from a single source to a 56-m-long 16-element vertical array. Second, lab experiments in a 1.07 -m-diameter 0.8 -m-deep cylindrical water tank were conducted with pulse signals having center-frequencies of $165 \mathrm{kHz}$ and 15 $\mathrm{kHz}$ broadcast from a single projector to a $0.7-\mathrm{m}$-long 14 -element horizontal array. In both cases, frequency difference beamforming utilizing the higher frequency signal produced results comparable to conventional methods applied to the lower frequency signal. [Sponsored by ONR.]

\section{9:10}

5aSP6. A novel method to detect rising and setting tones. Suci Dwijayanti and Masato Miyoshi (Graduate School of Natural Sci. and Technol., Kanazawa Univ., Kanazawa, Ishikawa 920-1192, Japan, dwijas@stu.kanazawa-u.ac.jp)

Rising or setting tone is a natural phenomenon caused by the chorus emission, which has a discrete frequency time structure and increasing or decreasing frequencies. It usually happens in the Very Low Frequency (VLF) range and sounds like bird tweeting. Since the chorus emission seems to have a clue to space research for safe and secure satellite operations, there is a strong demand to detect such tweeting tone. To this end, we develop a new signal processing tool wherein the running spectra of a tone are first treated as the signals received with individual sensors in a linear senorarray. Then, the estimation of signal parameter via rotational invariance techniques (ESPRIT) algorithm is applied to estimate the rising or setting speed of the tone. In order to evaluate the proposed tool, we conduct simulations using chirp signals of different rising or setting speeds. The simulation results show that the rising or setting speed of a tone can accurately be estimated with the proposed tool.

\section{9:25}

5aSP7. An analysis of methods for the acoustic localization of unattended aerial vehicles. David Grasing (ARDC U.S. Army, Bldg. 407, Picatinny, NJ 07806, david.b.grasing.civ@mail.mil)

In this paper methods, models, and difficulties associated with the acoustical detection and tracking of Unattended Aerial Vehicles (UAVs) are presented. The chief advantages of acoustics include passive sensing, the ability to operate in day or night, and relatively low cost. Acoustics also presents several notable challenges including low signal to noise, and issues arising from ground reflections. It is the presence of ground reflections that is explored in particular detail as it presents one of the more unique challenges to this problem. Issues arise because the differences in path length between the line-of-sight and ground reflection arrival of the source are of the same order as the wavelengths of the lower frequencies we are able to detect. Methods for bearing estimation will also be presented, explored, and evaluated in terms of their efficacy. The harmonic structure created by the UAV's propellers and engines are extracted. Bearings are estimated using various beamforming methods including CB, MVDR, and MUSIC. A black box optimization method based on the Nelder-Mead method on the surface of sphere is presented and used to evaluate different bearing estimation criteria. Finally, bearing estimates at different frequencies are combined via coherent and incoherent methods.

\section{9:40-10:00 Break}

\section{0:00}

5aSP8. Application of scanning acoustic and photoacoustic microscopes in orthopedic surgery. Yoshihiro Hagiwara (Dept. of Orthopaedic Surgery, Tohoku Univ. School of Medicine, 2-1 Seiryo-machi, Aoba-ku, Sendai 9808574, Japan, hagi@med.tohoku.ac.jp) and Yoshifumi Sajo (Dept. of Biomedical Imaging, Graduate School of Biomedical Eng., Tohoku Univ., Sendai, Japan)

Demands for orthopedic surgery, decreasing pain and disabilities, is increasing in developed countries with older nations. Together with increasing number of patients, its cost is now rising. Among modalities for noninvasive diagnosing techniques, such as plain X-rays, computed tomography, magnetic resonance imaging (MRI) and ultrasonography (US), MRI is a powerful tool for evaluating anatomical abnormalities. However, location and economic limitations for routine use remain. US is quick and inexpensive with a higher resolution than MRI. US plays an important role in assessing musculoskeletal soft tissues. A scanning acoustic microscope (SAM) characterizes biological tissues by estimating the elastic parameters based on sound speed. Biomedical photoacoustic (PA) imaging has the unique capability of combining high optical contrast and high ultrasound resolution in a single modality. Osteoarthritis and frozen shoulder are the major problems in musculoskeletal diseases. Osteoarthritis is a degenerative joint disorder characterized by the progressive degeneration of articular cartilage, osteophyte formation, and subsequent joint space narrowing. Further, frozen shoulder is characterized with severe pain and a decrease in shoulder motion, which is caused by joint capsular stiffness. We applied SAM and PA to animal models for assessing osteoarthritis and human samples with frozen shoulder.

\section{0:15}

5aSP9. Convolutional bidirectional long short-term memory hidden Markov model hybrid system for polyphonic sound event detection. Tomoki Hayashi (Nagoya Univ., Furo-cho, Chikusa-ku, Nagoya 464-8603, Japan, hayashi.tomoki@g.sp.m.is.nagoya-u.ac.jp), Shinji Watanabe (Mitsubishi Electric Res. Labs. (MERL), Cambridge, MA), Tomoki Toda (Nagoya Univ., Nagoya, Japan), Takaaki Tori, Jonathan L. Roux (Mitsubishi Electric Res. Labs. (MERL), Cambridge, MA), and Kazuya Takeda (Nagoya Univ., Nagoya, Japan)

In this study, we propose a polyphonic sound event detection method based on a hybrid system of Convolutional Bidirectional Long Short-Term Memory Recurrent Neural Network and Hidden Markov Model (CBLSTMHMM). Inspired by the state-of-the-art approach to integrating neural networks to HMM in speech recognition, the proposed method develops the hybrid system using CBLSTM to estimate the HMM state output probability, making it possible to model sequential data while handling its duration change. The proposed hybrid system is capable of detecting a segment of each sound event without post-processing, such as a smoothing process of detection results over multiple frames, usually required in the frame-wise detection methods. Moreover, we can easily apply it to a multi-label classification problem to achieve polyphonic sound event detection. We conduct experimental evaluations using the DCASE2016 task two dataset to compare the performance of the proposed method to that of the conventional methods, such as non-negative matrix factorization (NMF) and standard BLSTM-RNN, and also investigate the effect of network structures on the detection performance.

\section{0:30}

5aSP10. Chatter diagnosis using Mel frequency cepstral coefficient of vibrational signal for various operating conditions. KwangHun Jeong, JeWon Lee, and Junhong Park (Mech. Eng., Hanyang Univ., 222, Wangsimni-ro, Seongdong-gu, Eng. Ctr., 306, Seoul 04763, South Korea, zjavbfk@hanyang.ac.kr)

The chatter reduces quality of the machined parts and induces vibration and sound that is different from those under normal cutting process. A variety of cutting conditions are significantly correlated with the occurrence of chatter. After changing of the cutting conditions such as rotational speeds and depths of machine tools blade, the vibration signals was measured using vibrational accelerometers. The chatter diagnosis algorithm using Mel Frequency Cepstral Coefficient (MFCC) and Deep belief network (DBN), one of the Deep learning algorithm is proposed. Deep belief network is effective on classifying complicated signals as it represents the hierarchical cognitive process of a human brain. To acquire features from the distinctive noise and vibration under normal and chatter status, the MFCCs was used. Chatter diagnosis algorithm, DBN using MFCCs as input features, is suggested and verified. 


\section{0:45}

5aSP11. Application of acoustic contrast control for out door music performance: Feasibility study and experiments. Yang-Hann Kim (KAIST, Dept. of M.E., Sci. Town, Daejon-shi 305-703, South Korea, yanghann@ kaist.ac.kr), Wanjung Kim, Hwan Kim, Jueseok Kim, Sang-Hyeon Kim (SQand, Daejeon, South Korea), Jungwoo Choi (KAIST, Daejeon, South Korea), and Jong-Hwa Lee (SQand, Daejeon, South Korea)

Ideal outdoor music hall will be what has invisible but acoustically hard wall. This rather impossible objective can be achieved, at least, in some extent, for example allowing quite acceptable sound level in certain zone or zones where most residence lives and hear unwanted sound. This challenging problem can be tackled by finding the solutions that determines the magnitudes and phases of speakers, which surround the ideal hall's wall, that minimizes acoustic contrast between the listeners zone (bright zone) and residence zone (dark zone). It is interesting to find that acoustic contrast control essentially builds up acoustically hard wall, in other words, it makes large impedance mismatch on the wall. This bright observation, however, has been essentially based on two dimensional simulation and experiment. Next step has to go how well this can be extended to general 3D cases. We attempted to have another layer of speaker arrays, and requiring bright zone inside of the double array with certain height, therefore making a cylindrical volume of bright zone, and dark zone having certain angle, between two lines: one connects between the center and the bottom array and another one is the line between the center and upper array, rotating these two lines makes a diverging volume outside of the wall that has certain angle. Simulations and experiments demonstrated how well it can be practically applied and what are its limitations.

\section{1:00}

5aSP12. Modified DCTNet for audio signals classification. Yin Xian, Yunchen Pu, Zhe Gan (Duke Univ., 111 Blue Crest Ln., Durham, NC 27705, poline3939@gmail.com), Liang Lu (Univ. of Edinburgh, Edinburgh, United Kingdom), and Andrew Thompson (Oxford Univ., Oxford, United Kingdom)

We introduced the use of modified DCTNet for audio signals feature extraction. The modified DCTNet is a development of DCTNet, with its center frequencies of filterbanks geometrically spaced. The modified DCTNet is adaptive to different acoustic scales, and it can better capture low frequency acoustic information that is sensitive to human audio perception. We use features extracted by the modified DCTNet and put them to classifiers. Experimental results on bird song classification, music genre classification, and artist identification show that the modified DCTNet and RNN improve classification rate, and achieve state of the art performance.

\section{1:15}

5aSP13. A nested beamforming approach to sound source separation using an array-of-arrays recording system. Jorge A. Trevino Lopez, Shuichi Sakamoto, and Yôiti Suzuki (Graduate School of Information Sci. and Res. Inst. of Elec. Commun., Tohoku Univ., 2-1-1 Katahira, Aoba-ku, Sendai, Miyagi 9808577, Japan, jorge@ais.riec.tohoku.ac.jp)

Beamforming is a technique used with microphone arrays to selectively isolate sounds according to their direction of incidence. However, conventional methods struggle to separate sound sources if the spatial resolution of the recording system is not enough to define a recording zone containing only the target. In this presentation, a sound source separation method using multiple beamforming arrays is introduced. The proposal consists of three stages. The first stage applies modal beamforming methods to individual spherical microphone arrays; this results in a virtual array of directional microphones. The second stage distributes the recordings from the virtual microphones onto a new angular coordinate system. This stage transforms the radial coordinates for the different sound sources into pairs of parallax angles between them and the spherical microphone arrays. Finally, the third stage uses a least-squares optimized beamforming method defined on the parallax-angle coordinate system. The combination of these three stages takes advantage of parallax differences between spherical microphone arrays and allows for the isolation of sound sources at specified positions. The new method is evaluated using a computer simulation of two 64-channel spherical microphone arrays. The proposal shows satisfactory results even in the presence of aligned interfering sources, which hinder separation using conventional beamforming methods.

\section{1:30}

5aSP14. Sound enhancement system using selective binary filtering. Tomomi Suzuki (Graduate School of Information Sci., Nagoya Univ., Furocho, Chikusa-ku, Nagoya, Aichi 464-8601, Japan, suzuki.tomomi@g.sp.m. is.nagoya-u.ac.jp), Takanori Nishino (Graduate School of Eng., Mie Univ., Tsu, Mie, Japan), Yoshio Ishigiro (Inst. of Innovation for Future Society, Nagoya Univ., Nagoya, Aichi, Japan), and Kazuya Takeda (Graduate School of Information Sci., Nagoya Univ., Nagoya, Aichi, Japan)

Our proposed system allows users to selectively focus on particular sounds by choosing from various filtering options. Our goal is to develop a sound enhancement system which increases human auditory performance, especially through selective listening. To manage multiple filtering options numerically, we propose a series of listening patterns consisting of sets of binary indicators which show whether or not signals from a particular source will be presented to the listener. We then calculate the similarity of each pattern and order these patterns on the basis of their similarity. We conducted an experiment to evaluate our proposed method. Subjects listened to a sound recording using the various patterns in the order determined using the proposed method, as well as listening in an order determined using a comparative method. Subjects were asked to evaluate the naturalness of the transitions from pattern to pattern using each method, to select a listening pattern corresponding to one which was described to them, and to evaluate ease of use when searching for a desired listening pattern. Results support the feasibility of the proposed sound enhancement system. One issue which needs to be resolved is how to deal with complex environments with numerous sounds as possible targets. 


\title{
Session 5aUWa
}

\section{Underwater Acoustics: Underwater Acoustic Propagation}

\author{
Ahmad T. Abawi, Chair \\ HLS Research, 3366 North Torrey Pines Court, Suite 310, La Jolla, CA 92037
}

\section{Contributed Papers}

\section{8:00}

5aUWa1. Long range propagation and refraction of acoustic signals measured on the Comprehensive Nuclear Test Ban Treaty hydroacoustic network in the Indian Ocean. David R. Dall'Osto (Acoust., Appl. Phys. Lab. at Univ. of Washington, 1013 N 40th St., Seattle, WA 98105 , dallosto@apl.washington.edu)

This paper investigates 3 days data collected on the Comprehensive Nuclear Test Ban Treaty hydro-acoustic network in the Indian Ocean. This data includes that from March 8, 2014, the day the Malaysian airliner MH370 went missing - presumably crashing into the Indian Ocean as evidenced by found aircraft debris. This presentation focuses on the earthquakes and other anthropogenic and natural sounds recorded at South Station of Diego Garcia [7.662 S, 72.613 E] and Cape Leeuwin located $\sim 5,000 \mathrm{~km}$ away off of SW Australia [34.981 S, 114.144 E]. These measurements show that "hydroacoustic blockage," shadowing caused by a land mass located between the source and the receiving station, is incomplete and that acoustic signals can be detected on the far side of an island. The land mass significantly alters the time and frequency dependence of the signal, in comparison to an unblocked path. Acoustic models are used to investigate this propagation phenomena, and suggest capability for localizing sound sources using "blocked" stations. The presentation also includes analysis of a conspicuous signal, localized to an area in the West Indian Ocean. The source of this signal is unknown, although it originated shortly after the last satellite last transmission from the missing airliner.

\section{$8: 15$}

5aUWa2. Bottom-diffracted surface-reflected arrivals in the North Pacific. Ralph A. Stephen (Woods Hole Oceanographic Inst., 360 Woods Hole Rd., Woods Hole, MA 02543-1592, rstephen@whoi.edu), Peter F. Worcester (Scripps Inst. of Oceanogr., La Jolla, CA), S. T. Bolmer (Woods Hole Oceanographic Inst., Woods Hole, MA), Ilya A. Udovydchenkov (The MITRE Corp., Bedford, MA), and Matthew A. Dzieciuch (Scripps Inst. of Oceanogr., La Jolla, CA)

Bottom-diffracted surface-reflected (BDSR) arrivals were first identified in the 2004 Long-range Ocean Acoustic Propagation Experiment (LOAPEX) in the North Pacific (Stephen et al., 2013, JASA, 134, 3307-3317). In deep water, ambient noise and PE predicted arrivals are sufficiently quiet that BDSR arrivals, scattered from small seamounts, can be the largest amplitude arrivals observed. The Ocean Bottom Seismometer Augmentation in the North Pacific (OBSANP) Experiment in June-July 2013 studied BDSRs at the same site in detail. They are most readily identified by their move-out on lines of transmissions and are clearest on the vertical component channel. There appear to be two classes of diffractors. The diffraction point for Type 1 BDSRs occurs on the side of small seamounts and is substantially out of the source-receiver sagittal plane (for example, the BDSR observed on NPAL04). The diffraction point for Type 2 BDSRs is essentially in the source-receiver sagittal plane and occurs on the relatively featureless abyssal plain. In at least one case the same BDSR is observed for 77.5, 155 and $310 \mathrm{~Hz}$ M-sequence transmissions. Other characteristics of BDSRs will be discussed. [Work supported by ONR.]
$8: 30$

5aUWa3. ACOustic keyhole. Rhett Butler, Frederick K. Duennebier, and Bruce M. Howe (School of Ocean and Earth Sci. and Technol., Univ. of Hawaii at Manoa, 1680 East-West Rd., Post 602, Honolulu, HI 96822, rgb@hawaii.edu)

The Aloha Cabled Observatory (ACO) is located on the seafloor about $100 \mathrm{~km}$ north of O'ahu at $4800 \mathrm{~m}$ depth. Seismoacoustic T-waves generated by earthquakes in the South Pacific from Tonga-Kermadec to the Solomon Islands travel $40-70^{\circ} \Delta$, passing through an acoustic keyhole-the Ka'ie'ie Waho channel-between $\mathrm{O}^{\prime}$ ahu and Kaua'i before reaching ACO. In contrast to ACO and the Pacific seafloor at depths greater than $4500 \mathrm{~m}$, the channel shallows to 1 and $3 \mathrm{~km}$ in depth, blocking deeper SOFAR ray/mode paths. T-waves from $>50$ earthquakes ( $\mathrm{Mw} 6.1$ to 8 ) are reviewed, correcting apparent velocities for near-source P-wave path effects. We examine Twaves crossing the Ka'ie'ie Waho channel and descending to ACO, comparing and contrasting with T-waves propagating unhindered across the North Pacific from events in offshore Japan (Tohoku aftershocks), Kuril Islands, Aleutian Islands, and offshore Alaska. Preliminary review indicates that the T-waves exhibit seismoacoustic modal-coupling to the seafloor, exhibiting a structure observed previously* at the Hawai ' $\mathrm{i}-2$ Observatory $(\mathrm{H} 2 \mathrm{O})$ at $5 \mathrm{~km}$ depth between Hawai'i and California. *R. Butler (2006), J. Acoust. Soc. Am. 120(6), 3599-3606. *R. Butler and C. Lomnitz (2002), Geophys. Res. Lett. 29(10), 57 1-4.

\section{$8: 45$}

5aUWa4. Acoustic features of sharp and continuous three-dimensional curved coastal fronts. Brendan J. DeCourcy (Mathematical Sci., Rensselaer Polytechnic Inst., 110 8th St., Troy, NY 12180, decoub@rpi.edu), Ying-Tsong Lin (Appl. Ocean Phys. \& Eng., Woods Hole Oceanographic Inst., Woods Hole, MA), and William L. Siegmann (Mathematical Sci., Rensselaer Polytechnic Inst., Troy, NY)

Three-dimensional curved shelf-slope fronts have been modeled with sharp [Lin and Lynch, J. Acoust. Soc. Am. (2012)] and continuous [DeCourcy et al., J. Acoust. Soc. Am. (2016)] sound speed changes. For the sharp front, expressions were found for determining dependence of acoustic quantities such as modal speeds on feature-model parameters. Asymptotic approximations are used to simplify terms in the dispersion relation for the horizontal wavenumber, and further analysis leads to convenient and accurate formulas for the parameter dependence. Corresponding results will be presented for the continuous front, which possesses a different class of acoustic modes known as tunneling modes that carry larger amounts of energy across and along the front than other modes. Results for both frontal models will be discussed, comparing and contrasting characteristics including wavenumber distributions, mode types, and parameter dependences. An important goal is to examine the similarities and differences for sharp or continuous fronts in the sensitivity of acoustic quantities to feature parameters, such as front location and width, bottom slope angle, and source frequency. This would permit identification of situations where the more convenient sharp front model is appropriate. [Work supported by ONR Grants N00014-14-1-0372 and N00014-11-1-0701.] 
5aUWa5. Analysis of sound propagation in the front region off the east of Tsugaru straits. Yoshiaki Tsurugaya (Sanyo P. T., 11-14 Ooi 5-Chome, Shinagawa-Pref., Tokyo 140-0014, Japan, tsul@mvb.biglobe.ne.jp), Toshiaki Kikuchi (National Defence Acad., Ooyabe, Kanagawa-pref., Japan), and Koichi Mizutani (Univ. of Tsukuba, Tsukuba, Ibaraki-pref., Japan)

Off the east of Tsugaru straits, located in the north of the main-island, there are three big water masses. One is the Oyashio (Kuril current) that is a cold water mass. Other water masses are the warm water masses: the Tsugaru warm current, and Kurosio extension. And, the Tsugaru warm eddy is made from the Tsugaru warm current on the Oyashio. Therefore, the front is generated in the region to which those water masses bound. The temperature structure changes rapidly in that region. In addition, the bottom depth is changed too. Then, bi-directional sound propagation is analyzed on the line of a right-angled direction to the front that Tsugaru warm eddy is bounded to the Oyashio. It is considered about the sound propagation of $220 \mathrm{~km}$ that passes the front from the Tsugaru warm eddy to the Oyashio cold water mass, and the opposite path. In the front region, the sound propagation is changed, and suffers the impact of the bottom at the same time. Therefore, the propagation configuration is different in the bi-directional propagation. The transmission loss in the Tsugaru warm eddy was large when the sound source crosses the front from the Oyashio.

\section{9:15}

5aUWa6. Influence of source frequency spreading on transmission loss patterns. Elisabeth M. Brown (Mathematical Sci., Rensselaer Polytechnic Inst., Troy, NY 12180, browne6@rpi.edu), Allan D. Pierce (Boston Univ., East Sandwich, MA), and William L. Siegmann (Mathematical Sci., Rensselaer Polytechnic Inst., Troy, NY)

A classical experiment [Wood and Weston, Acustica 14 (1964)], conducted in Emsworth Harbor at low tide, examined propagation in a $1 \mathrm{~m}$ mud layer overlying gravel. Hydrophones on the mud bottom transmitted six source frequencies between 4 and $72 \mathrm{kHz}$ and received signals at ranges up to $50 \mathrm{~m}$. Signal levels show modal fluctuations at lower frequencies but not at higher frequencies, even though the authors did not perform data smoothing. This behavior is hypothesized to arise from a frequency-dependent spread of transmitted frequencies. A Pekeris two-layer model of mud and gravel with a pressure release mud surface is used to idealize the waveguide. Transmission loss is calculated using a weighted frequency average of timeaveraged intensity, with a Lorentzian function (Cauchy distribution) modeling the frequency spread. Appropriate values for the Q-factor of the distribution are examined. Compressional wave attenuation values from a recent inversion of the data [Pierce, et al., POMA, accepted (2015)] are incorporated into the calculations. Computational results demonstrate the same behavior in intensity fluctuations as in the data with identifiable modal interference at the three lower frequencies and smoothed patterns at the three higher. [Work supported by the ONR.]

\section{9:30}

5aUWa7. Analytical formulas for incoherent transmission loss in shallow water based on effective approximations of seafloor depth and reflectivity. Zhi Y. Zhang (Defence Sci. and Technol. Group, West Ave., DSTG/MD, Edinburgh, SA 5111, Australia, YongZhang.work@gmail. com)

We derive analytical formulas for modelling incoherently averaged transmission losses in shallow water environments by taking into account the effects of energy spreading, in-water absorption, sea surface scattering, and leakage into the seafloor. The formulas are based on the effective depth approximation to include the effect of sound penetration into the seafloor and use effective two-parameter representations of seafloor reflectivity. Comparison with published measurement data at several shallow water sites worldwide shows that the formulas are capable of reproducing the main features of shallow water sound propagation in environments with different types of sediments. The formulas are useful for quick assessments of incoherent transmission losses in generic yet representative underwater environments where detailed environmental knowledge is lacking or the fine details of underwater sound propagation is not required.
5aUWa8. Modeling surface scatter in the channel impulse response with a Helmholtz-Kirchhoff integral. Edward Richards, William Hodgkiss, and Heechun Song (Scripps Inst. of Oceanogr., Univ. of California, San Diego, 9500 Gilman Dr., La Jolla, CA 92093, edwardlrichards@ucsd. edu)

Several experimental measurements of channel impulse responses (CIRs) using underwater communication bandwidths have shown distinct striation features arriving up to 10 milliseconds after the main surface bounce, e.g. Badiely et al. [J. Acoust. Soc. Am. 132, EL290 (2012)]. Choo et al. [J. Acoust. Soc. Am. 136, 1046 (2014)] used an intuitive geometric ray model to argue that the source of these arrivals was the reflection from a wave trough near the receiver. This presentation extends Choo's approach and focuses on a quadrature solution of the time domain Helmholtz-Kirchhoff $(\mathrm{HK})$ integral in a half space. This approach provides the same intuitive geometric ray perspective on the surface scattering, but additionally models arrival amplitudes near caustics and higher order catastrophe phenomena induced by surface waves. It is shown that a simple shadowing correction of the HK result produces all of the major features of the full Helmholtz scattering integral solution. The HK integral is computationally tractable, accurate, and can show the path from the surface that contributes to each CIR arrival.

\section{0:00-10:15 Break}

\section{0:15}

5aUWa9. Random matrix theory simulation of propagation through internal waves. Kathleen E. Wage (Elec. and Comput. Eng., George Mason Univ., 4400 University Dr., MSN 1G5, Fairfax, VA 22030, kwage@gmu. edu) and Lora J. Van Uffelen (Ocean Eng., Univ. of Rhode Island, Narragansett, RI)

Hegewisch and Tomsovic proposed using random matrix theory (RMT) to simulate the effect of internal wave scattering on long-range propagation in deep water [JASA, 2013]. They model mode propagation as the multiplication of a series of unitary propagator matrices whose statistics are derived using perturbation theory. A simplifying assumption facilitates the generation of broadband time series. One potential advantage of the RMT approach is that it requires fewer computations than a full wave simulation to produce a large set of statistical realizations of the received time series [Hegewisch \& Tomosovic, Europhys. Lett. 2012]. Efficient simulation techniques are especially important for applications that require predictions of the timefront and/or its statistics at a large number of possible receiver ranges. For example, simulating data sets for acoustic gliders operating within a tomographic array is computationally intensive. While Hegewisch and Tomsovic illustrated the performance of their approach for one deep water simulation environment, they also highlighted the need for additional research. This talk investigates the feasibility of using RMT to efficiently simulate broadband time series for use in analysis of glider data. The underlying assumptions of the RMT approach will be assessed based on results of prior experimental work. [Work sponsored by ONR.]

\section{0:30}

5aUWa10. The optimal weight vectors research on the far-field sound field control of the low-frequency phased array. Haifeng Lee (Inst. of Acoust., CAS, No. 21,West Rd. of Beishihuang, Haidian, Beijing 100190, China, cust77@mail.ioa.ac.cn) and Li Ma (Inst. of Acoust., CAS, Peking, China)

The theory and simulation studies were developed to analyze the farfield sound field control effect of the low-frequency phased array emitting the different weight vectors. First, the relationship between the weight vector and the mean square deviation of time-delay estimation were set up theoretically. Second, using the relationship, the optimal weight vector was solved and discussed. Finally, under the same energy input conditions, the several weight vectors were used to compare the far-field sound field control effects of low-frequency phased array. From the simulating results and the mean square deviation of time-delay estimation exited by the weight vectors, it was showed and proved that the phased array emitting the No.1 normal mode was not always the optimum in the far-field sound field control. 


\section{0:45}

5aUWa11. Spatial diversity of ambient noise in the new Arctic. Henrik Schmidt, Scott Carper, Thomas Howe (Mech. Eng., Massachusetts Inst. of Technol., 77 Massachusetts Ave., Rm. 5-204, Cambridge, MA 02139, henrik@mit.edu), and Andrew Poulsen (Appl. Physical Sci., Lexington, MA)

The Arctic Ocean is undergoing dramatic changes, the most apparent being the rapidly reducing extent and thickness of the summer ice cover. As has been well established over prior decades, the environmental acoustics of the ice-covered Arctic is dominated by two major effects: the highly inhomogeneous ice cover, and the monotonically upward refracting sound speed profile, the combination of which forces all sound paths to be exposed to strong scattering loss and the associated loss of coherence. In some portions of the Arctic Ocean, however, inflow of warm Pacific water has created the so-called "Beaufort Lens," a neutrally buoyant high sound velocity layer at 70-80 meter depth, which has dramatically altered the acoustic environment, creating a strong acoustic duct between approximately 100 and $300 \mathrm{~m}$ depth. This duct has the potential of trapping sound out to significant ranges (80$100 \mathrm{~km}$ ) without interacting with the ice cover, resulting in much higher coherence and signal preservation. Acoustic noise measurement results collected with a vertically suspended array during ICEX 2016 illustrate the spatial and temporal noise properties in the presence of this acoustic duct at different depths. Comparisons of the ICEX 2016 data are also made with modeled Arctic noise data. [Work supported by ONR and DARPA.]

\section{1:00}

5aUWa12. Arctic ambient noise measurement comparisons in the Beaufort Sea. Andrew Poulsen (Appl. Physical Sci., 49 Waltham St., Lexington, MA 02421, poulsen@alum.mit.edu) and Henrik Schmidt (Massachusetts Inst. of Technol., Cambridge, MA)

During ICEX 2016, an MIT autonomous underwater vehicle (AUV) was deployed in the Beaufort Sea region of the Arctic Ocean with the technical objectives of demonstrating the deployment, operation and recovery of an AUV with a towed array under extreme under-ice Arctic conditions, and the scientific objective of characterizing the acoustic environment. Part of this effort included suspending the AUV from a hydro-hole with the acoustic array hanging in a vertical configuration. This new data set created a choice opportunity to reprocess similar vertical array data from the same region of the Beaufort Sea collected by MIT during SIMI 1994. In the intervening twenty-two years between these two ice camps, significant changes have occurred in the Arctic, including rapidly reducing extent and thickness of the summer ice cover. Furthermore, a persistent inflow of a shallow "tongue" of warm Pacific water has recently been discovered in the Beaufort Sea, creating a strong acoustic duct between approximately 100 and $200 \mathrm{~m}$ depth. This paper presents noise properties measured more than two decades apart in a region of rapid and significant change. [Work supported by ONR and DARPA.]

\section{1:15}

5aUWa13. Noise background levels and noise event tracking/characterization under the Arctic Ice Pack: Experiment, data analysis, and modeling. Kevin Williams, Michael L. Boyd, Alexander G. Soloway, Eric I. Thorsos, Steven G. Kargl, and Robert I. Odom (Appl. Phys. Lab., Univ. of Washington, 1013 NE 40th St., Seattle, WA 98105, williams@apl.washington.edu)

In March of 2014 an Arctic Line Arrays System (ALAS) was deployed as part of an experiment in the Beaufort Sea. The background noise levels in the frequency range from $1 \mathrm{~Hz}$ to $25 \mathrm{kHz}$ were measured. The goal was to have a $3 \mathrm{~d}$ sparse array that would allow determination of the direction of sound sources out to $100 \mathrm{~s}$ of $\mathrm{km}$ and both direction and range of sound sources out to 1-2 km from the center of the array. ALAS started recording data at 02:12 on March 10, 2014 (UTC). It recorded data almost continuously at a sample rate of $50 \mathrm{kHz}$ until 11:04 on March 24, 2014. Background noise spectral levels are presented for low and high floe-drift conditions. Tracking/characterization results for ice cracking events, including the initiation of an open lead and a seismic event are presented. Results from simple modeling indicate that the signature of a lead formation may be a combination of both previously hypothesized physics and enhanced emissions near the ice plate critical frequency. The seismic T-wave arrival time indicates that a significant amount of energy coupled to T-wave energy somewhere along the path between the earthquake and ALAS.

\section{1:30}

5aUWa14. Environmentally adaptive autonomy for acoustic communication and navigation in the new Arctic. Henrik Schmidt (Mech. Eng., Massachusetts Inst. of Technol., 77 Massachusetts Ave., Rm 5-204, Cambridge, MA 02139, henrik@mit.edu) and Toby Schneider (Gobysoft LLD, Woods Hole, MA)

The particular sensitivity of the Arctic to climate change is well established. and the significance to undersea operations can be dramatic. As part of the recent ICEX16 US Navy Exercise in the Beaufort Sea, MIT deployed an autonomous underwater vehicle with a towed hydrophone array below the ice cover for assessing the climate induced changes to the undersea ambient noise environment. The safe underwater operation depended on navigation updates from the submarine tracking range being communicated to the vehicle for fusion with the onboard inertial navigation. However, the changes in the environment severely deteriorated the tracking performance compared to previous deployments. The reason was clearly associated with a previously observed neutrally buoyant layer of warm Pacific water persistently spreading throughout the Beaufort Sea, which severely alters the acoustic environment with dramatic effects for both long and short range acoustic sensing, communication and navigation. This paper describes the effects observed and discusses how robust acoustic connectivity in this environment makes it paramount that the manned or unmanned undersea platforms are capable of adapting to the environment for sensing, communication, and navigation. [Work supported by the Office of Naval Research.]

\section{$11: 45$}

5aUWa15. Detection and communication in the "Beaufort Lens". Arthur B. Baggeroer, Henrik Schmidt, Scott A. Carper (Mech. and Elec. Eng., Massachusetts Inst. of Technol., Rm. 5-206, MIT, Cambridge, MA 02139, abb@boreas.mit.edu), Jon Collis, and Jordan Rosenthal (MIT Lincoln Lab., Lexington, MA)

The WHOI Ice Tethered Profilers in the Beaufort has confirmed a "sound speed duct" at depths between 100 to $250 \mathrm{~m}$ labeled as the "Beaufort Lens." It is thought to be caused by warm water intrusion from the Bering Strait. A significant consequence of the duct is the prediction of $10 \mathrm{~dB}$ lower transmission losses at ranges of $100 \mathrm{~km}$ for sources and receivers both within the duct. We examine how the properties of the duct impact detection and communication. Transmission loss is strongly affected by frequency. Ducted propagation is not supported below a modal cutoff and absorption losses become consequential at higher frequencies. For active sonar, where wideband waveforms are employed, dispersion effects and multipath become important. The shape of the duct, especially boundary gradients, impacts this dispersion. While a vertical line array spanning the duct can potentially exploit the multipath ray/mode coherence and the resolution of the array impacts whether coherent or incoherent combination should be employed to achieve desired gains. Data for validating and verifying long range transmission loss in the duct were acquired by two submarines participating in ICEX-16. This presentation examines the signal processing for detection and communication within the so called "Beaufort Lens." 


\title{
Session 5aUWb
}

\author{
Underwater Acoustics: Ocean Ambient Noise: Sources, Variation, and Characterization II \\ D. Benjamin Reeder, Chair \\ Oceanography, Naval Postgraduate School, 833 Dyer Rd., Bldg. 232, SP-311B, Monterey, CA 93943
}

\section{Contributed Papers}

10:00

5aUWb1. Study for computation/measurement of underwater ship radiated noise toward marine environmental protection in Japan. Hikaru Kamiirisa, Nobuaki Sakamoto, Akiko Sakurada (Fluids Eng. \& Hull Design Dept., National Maritime Res. Inst., Japan, 6-38-1 Shinkawa, Mitaka, Tokyo 181-0004, Japan, kamiirisa@nmri.go.jp), takahiro kijima (mlit, Tokyo, Japan), yoshihito tominaga (JSTRA, Tokyo, Japan), and naoya umeda (Osaka Univ., Osaka, Japan)

USRN has been a large interest among maritime industries in terms of mariners' comfort as well as environmental protection. This paper presents the characteristics of the measured USRN in full scale as well as the near field propeller cavitation noise estimated by viscous computational fluid dynamics (CFD) simulation. The USRN measurement was carried out according to the ISO standard protocol in deep water. The results show that the characteristics of USRN spectrum, differences of SPL in tonal noise depending on broadside and engine load are successfully captured for which the level of background noise is low enough to distinguish target noise and background noise. For the CFD simulation, two methodologies have been tested to predict frequencies and sound pressure level (SPL) of near field propeller cavitation noise, i.e., 1) direct estimation and 2) indirect estimation. The results are encouraging in that the present CFD simulation is capable of capturing sheet cavitation and resultant tonal noise. The estimation of broadband noise is still challenging by the present CFD simulation yet its application in conjunction with the semi-empirical formula is likely to be effective for predicting the upper bound level of broadband noise.

\section{0:15}

5aUWb2. The design and implementation of ocean ambient noise acquisition system based on the underwater glider. Lu Liu (Institute of Acoust.,Chinese Acad. of Sci., No. 21 North Sihuan West Rd., Haidian District, Beijing 100190, China, 13398625258@163.com)

Ocean ambient noise (OAN) is the background noise of the ocean,which is considered as the interference for the underwater acoustic equipment,on the other hand, it can be used to invert the parameters of the ocean environment. It is important to acquire and study the spatial temporal characteristics of ocean ambient noise. As a new type of underwater detection platform, underwater glider's performance is suitable to acquire the ocean ambient noise. This paper designed and realized an OAN acquisition system (OANAS), which is mounted on the Underwater glider. The system mainly includes two parts, the deepwater hydrophone array and the acquisition system. This design uses the ADI's BF518,the AD73360 and the uClinux to make the acquisition system have high speed,low power consumption and low noise abilities, it can also sample eighteen underwater signal roads synchronously and meet the requirement of the system. The data memorized is proved effective,the system is proved to be stable and meet the design requirement through the test which the OANAS mounted on the Hybrid Underwater Glider in the National Ocean Technology Center.
10:30

$5 a U W b 3$. Eigenvalues of the sample covariance matrix for a vertical array in shallow water. Jie Li and Peter Gerstoft (Marine Physical Lab., Scripps Inst. of Oceanogr., 9500 Gilman Dr. La Jolla, San Diego, CA 92093, ji1004@ucsd.edu)

The eigenvalue spectrum of the sample covariance matrix (SCM) contains valuable information about the environment and the source and noise generation. Random Matrix Theory (RMT) combined with physical models is used to explain the eigenvalue distribution. For conventional three-dimensional and two-dimensional free space isotropic noise models, there is a jump in the eigenvalue spectrum of SCM which only depends on array properties (element spacing to wavelength ratio). This paper investigates the eigenvalue distribution of SCM for a vertical array in shallow water noise environment using Harrison's noise model [Harrison, J. Acoust. Soc. Am. 99, 2055-2066 (1996)]. Results show that when bottom is considered, the jump location depends on both array and bottom properties. RMT is applied to study the eigenvalue spectrum estimation with different sound speed and attenuation in the bottom. Shallow water vertical array data are analyzed and it is demonstrated that the eigenvalues of the SCM compare well with theory.

\section{0:45}

5aUWb4. Investigating flow noise on underwater gliders acoustic data. Francisco A. dos Santos, Pedro M. São Thiago, André Luis S. de Oliveira, Rafael Barmak (PROOCEANO, Av. Rio Branco, 311/1205, Centro, Rio de Janeiro, RJ 20040-009, Brazil, francisco@prooceano.com.br), José Antônio M. Lima (CENPES, PETROBRAS, Rio de Janeiro, RJ, Brazil), Fernando G. de Almeida (UO-BS, PETROBRAS, Santos, SP, Brazil), and Thiago P. Paula (CENPES, PETROBRAS, Rio de Janeiro, RJ, Brazil)

Since November 2015, two underwater gliders equipped with external hydrophones were deployed in the South Brazilian Bight in order characterize the area's soundscape. Contrasting to standard fixed mooring systems (where flow noise is generated by currents passing by the hydrophones), gliders are subject to noise generated by its own downward and upward motion, which may compromise soundscape characterizations if not properly evaluated. In order to investigate induced flow noise on the hydrophone and its characteristics, 563 hours of acoustic data from the gliders were correlated to the navigation settings. Results can be comparable to previous flow noise descriptions for fixed systems. A high correlation was observed between the glider speed (both vertical and total) and 1/3 octave band levels centered at frequencies below $20 \mathrm{~Hz}$. Estimates of the broadband sound pressure level were accomplished with different lower frequency limits and found to be uncorrelated to the glider speed above $40 \mathrm{~Hz}$. 


\section{1:00}

5aUWb5. Acoustic data quality assessment tools and findings for ocean observing systems. Thomas Dakin, John Dorocicz, Ben Biffard (Ocean Networks Canada, TEF-128A 2300 McKenzie Ave, University of Victoria, Victoria, BC V8W2Y2, Canada, tdakin@uvic.ca), Nathan D. Merchant (Ctr. for Environment, Fisheries and Aquaculture Sci., Lowestoft, United Kingdom), David Hannay (JASCO Applied Sci., Victoria, BC, Canada), Steve Mihaly, Marlene Jeffries, and Jeannette Bedard (Ocean Networks Canada, Victoria, BC, Canada)

Ocean Networks Canada (ONC) operates long time series, ocean observatories in the Pacific and Arctic. These include the large VENUS and NEPTUNE observatories, many small community based observatories and the Underwater Listening Station (ULS) for the Vancouver Fraser Port Authority. Passive acoustic monitoring systems are a component of all ONC observatories and passive acoustic data quality is therefore a concern. All the observing systems have multiple underwater electronics and sensor types, many of which can negatively impact the passive acoustic sensor data. Hydrophone sensitivity degradation due to time, water absorption, and biofouling need to be assessed to ensure accurate ambient noise measurements and accurate vessel underwater radiated noise level measurements. The performance and suitability of the hydrophones for specific areas also needs to be assessed so the acoustic analysts can be aware of the hydrophone induced data limitations. ONC has been examining the use of in situ calibration verifications, spectral probability density (SPD) plots, spectrograms, and wave data as tools to assess the passive acoustic data quality. The preliminary findings on the impact of all of the above acoustic error sources are presented.

\section{1:15}

$5 a U W b 6$. Some new observations on the underwater sound field from impact pile driving. Peter H. Dahl and David R. Dall'Osto (Appl. Phys. Lab. and Mech. Eng., Univ. of Washington, 1013 NE 40th St., Seattle, WA 98105, dahl@apl.washington.edu)

The underwater sound field from impact pile driving has been studied in recent years, motivated largely by the high levels and impulsive character of the sound and potential impacts on marine life. Here we report on new observations made on a vertical line array (VLA) and neutrally buoyant geophone (vector sensor) both positioned $120 \mathrm{~m}$ from the pile, to inform future modeling and mitigation efforts. These observations include: (1) a groundwave precursor of amplitude $200 \mathrm{~Pa}$, or $10 \%$ of peak pressure (2) delayed arrival structure in the impulsive time series due to injection of airborne sound from the hammering process, and (3) properties of the Scholte wave as a function of receiver depth. The study originates from Puget Sound, WA, and involves a $30-\mathrm{cm}$ diameter steel pile installed with impact hammer energy $\sim 200 \mathrm{~kJ}$. Water depth at pile was $9 \mathrm{~m}$, gradually increasing to $17 \mathrm{~m}$ at the VLA range. Pile depth was recorded over the sequence of 300 pile strikes, and this knowledge is essential to interpret these observations as the pile was driven $12 \mathrm{~m}$ into the sediment. [Research supported by Washington State Dept. of Transportation with partial support from ONR.]

\section{1:30}

5aUWb7. Optical detection of mach shock wave cones in water using refracto-vibrometry. Matthew T. Huber (Dept. of Phys., Rhodes College, 2000 North Parkway, Rhodes Box 2644, Memphis, TN 38112, hubmt-18@ rhodes.edu), Nathan Huber, and Thomas Huber (Dept. of Phys., Gustavus Adolphus College, St. Peter, MN)

Recently, a hydrophone array was used to demonstrate Mach shock wave cone formation in water when $1 \mathrm{~m}$ diameter steel pile rods for civil structures were driven into the ground by massive impact hammers. These shock wavefronts are of concern for marine mammals and fish. In the current project, Mach shockwave cones were directly imaged using refractovibrometry. A Polytec PSV-400 laser Doppler vibrometer was directed through a water tank towards a stationary retroreflective surface. The density variations of acoustic wavefronts which pass through the laser cause variations in the optical path length between the laser and retroreflector. This results in a time-varying modulation of the laser signal returning to the vibrometer, enabling optical detection of the acoustic wavefronts. A $35 \mathrm{~mm}$ diameter rod (steel or other metal) immersed in a water tank was repeatedly "impacted" by narrow pulses from a $1 \mathrm{MHz}$ ultrasound transducer. The vibrometer sampled numerous scan points to generate videos of the time evolution of a Mach cone wavefronts launched as the compressional and shear waves travel through the rod. The angle of the Mach cone produced is consistent with the speed of waves in the rod.

\section{1:45}

5aUWb8. Statistical wavelet filtering for impulsive noise mitigation. Jit Sarkar, Christopher M. Verlinden, Jeffery D. Tippmann, William S. Hodgkiss, and William A. Kuperman (Marine Physical Lab., Scripps Inst. of Oceanogr., 9500 Gilman Dr., Mail Code 0238, La Jolla, CA 92093-0238, bsarkar@ucsd.edu)

Impulsive noise sources in acoustic data can represent a significant amount of contamination that is not easily addressed by spectral methods alone. In ocean acoustics, such noise sources can be biological in nature, e.g., marine mammal localization clicks, or even mechanical noise induced on an instrument itself. While the broadband character of an impulse does not lend itself to frequency domain filtering, the wavelet domain is more appropriate and has been used to analyze impulsive sources of interest in the past. We present a method for filtering unknown nuisance impulses using basic statistics of the continuous wavelet transform (CWT), with minimal disturbance to the remaining signal(s) of interest. Examples from both simulation and collected ocean acoustic data are presented. 


\title{
Session 5pABa
}

\section{Animal Bioacoustics: Behavioral Response Studies}

\author{
Rebecca Dunlop, Cochair \\ School of Veterinary Science, University of Queensland, Cetacean Ecology and Acoustics Lab, Gatton QLD 4343, Australia \\ Kotaro Ichikawa, Cochair \\ Field Science Education and Research Center, Kyoto University, Kitashirakawa Oiwake-cho, Sakyo-ku, Kyoto 606-8502, \\ Japan
}

Chair's Introduction-1:00

\section{Invited Papers}

1:05

5pABa1. Southern California behavioral response study-Overview and synthesis of results to date. Brandon L. Southall (SEA, Inc., 9099 Soquel Dr., Ste. 8, Aptos, CA 95003, Brandon.Southall@sea-inc.net), John Calambokidis (Cascada Res., Olympia, WA), Ari Friedlaender (Oregon State Univ., Newport, OR), Stacy DeRuiter (Calvin College, Grand Rapids, MI), Alison Stimpert (Moss Landing Marine Lab., Moss Landing, CA), Jeremy Goldbogen (Hopkins Marine Station, Stanford Univ., Monterey, CA), Elliott Hazen (UC Santa Cruz, Santa Cruz, CA), David Moretti (Naval Undersea Warfare Ctr., Newport, RI), Ann Allen, Glenn Gailey, Annie Douglas, Greg Schorr, Erin Falcone (Cascada Res., Olympia, WA), Dave Cade (Hopkins Marine Station, Stanford Univ., Monterey, CA), Fleur Visser (Kelp Marine Res., Amsterdam, Netherlands), and Jay Barlow (NMFS Southwest Fisheries Sci. Ctr., NOAA, LaJolla, CA)

The Southern California Behavioral Response Study (SOCAL-BRS) is an interdisciplinary, multi-team collaboration that uses highresolution, multi-sensor tags to document behavioral responses of cetaceans to Navy mid-frequency (2.5-5 kHz) active sonar (MFAS). Individual animals are monitored before, during, and after controlled exposure experiments (CEEs) using either simulated or [the firstever use of] actual U.S. Navy ship-based MFAS. Over 175 tags have been deployed on individuals of ten cetacean species and over 80 CEEs have been conducted. Results including baseline behavioral data, as well as behavioral responses to sound exposure have been published in more than a dozen scientific publications. Behavioral responses to MFAS and other mid-frequency sounds have been documented in several species with changes in diving and feeding behavior and avoidance of sound sources being the most common responses observed, though some individuals did not respond despite relatively high received sound levels. Responses appear to depend on species, exposure context (e.g., behavioral state, prey distribution, proximity to sound sources), and source type (simulated versus real). These experiments provide controlled, scientific measurements of response, or lack of response, that are directly applicable to improving Navy environmental compliance assessments of behavioral response to MFAS.

\section{$1: 25$}

5pABa2. Baleen whale calling behavior and response to anthropogenic sound. Alison K. Stimpert (Moss Landing Marine Labs., 8272 Moss Landing Rd., Moss Landing, CA 95039, alison.stimpert@gmail.com), Stacy L. DeRuiter (Calvin College, Grand Rapids, MI), Erin A. Falcone (Marecotel, Seabeck, WA), John Joseph, Tetyana Margolina (Naval Postgrad. School, Monterey, CA), David Moretti (Naval Undersea Warfare Ctr., Newport, RI), Selene Fregosi, Ari S. Friedlaender (Oregon State Univ., Newport, OR), John Calambokidis (Cascadia Res. Collective, Olympia, WA), Peter L. Tyack (Scottish Oceans Inst., Univ. of St. Andrews, St. Andrews, Scotland, United Kingdom), Jeremy A. Goldbogen (Dept. of Biology/Hopkins Marine Station, Stanford Univ., Pacific Grove, CA), and Brandon L. Southall (Southall Environ. Assoc., Aptos, CA)

Without a means of studying large whales in a controlled experimental environment, less is understood about their sound production mechanisms than is understood about those of smaller odontocetes. To describe call production behavior in fin whales, we used a recent technique that correlates fast-sampling accelerometer signals from tags with concurrently recorded acoustic signals to identify calls produced by the tagged animal. We tagged 18 fin whales as part of the Southern California Behavioral Response Study (SOCAL BRS), of which four were confirmed to be calling. We were then able to describe their kinematic and social behavior in relation to call production. Behaviors associated with elevated call rates included shallow maximum dive depths, little body movement, and negative pitch in body orientation, similar to some other calling baleen whale species. These are the first descriptions of body orientation and dive depths at which fin whales are most likely to call. We also describe calling responses (or lack thereof) from blue and fin whales exposed to simulated mid-frequency active sonar. The call behavior characterizations presented here will help with predicting calling behavior from surface behavior, informing interpretation of passive acoustic data, and further investigating effects of anthropogenic sound on baleen whales. 
5pABa3. The behavioral response of humpback whales to seismic air gun noise. Rebecca Dunlop, Michael J. Noad (School of Veterinary Sci., Univ. of Queensland, Cetacean Ecology and Acoust. Lab., Gatton, QLD QLD 4343, Australia, r.dunlop@uq.edu.au), Robert McCauley (Curtin Univ., Perth, WA, Australia), and Douglas Cato (Univ. of Sydney, Sydney, NSW, Australia)

Four major experiments have been conducted off Australia to quantify the behavioral response of migrating humpback whales to various seismic air gun arrays. The first, using a $20 \mathrm{in}^{3}$ air gun, was used to develop the analysis framework, which was then applied to later experiments. The following two experiments tested a 4-step "ramp-up" procedure (20, 60, 140, and $440 \mathrm{in}^{3}$ array sequence) and a 140 $\mathrm{in}^{3}$ array. Both studies found a change in movement behavior in response to the air guns, where groups deviated more from their course and made less progress towards the source. There was no evidence of any change in surface behaviors, including respiration rates. The final experiment involved ramp-up to a 3130 in full commercial array. In response, whales decreased their dive time and displayed an elevated respiration rate, more so to the full array phase. In this phase, they also changed their surface behavior, in that breaching rates were elevated but tail and pectoral slapping behaviors were reduced. Consistent with previous studies, whales also changed their movement behavior, more so during ramp-up. Therefore the full array elicited a greater variety and magnitude of behavioral changes than observed with the smaller air gun arrays.

5pABa4. Vocal response of dugongs (Dugong dugon) to playbacks of conspecific calls suggest ranging function of chirps. Kotaro Ichikawa, Nobuaki Arai (Field Sci. Education and Res. Ctr., Kyoto Univ., Kitashirakawa Oiwake-cho, Sakyo-ku, Kyoto, Kyoto 6068502, Japan, ichikawa.kotaro.5r@kyoto-u.ac.jp), and Kongkiat Kittiwattanawong (Phuket Marine Biological Ctr., Phuket, Thailand)

Dugongs vocalize bird-like calls, such as chirps and trills but the functional definitions of the calls are yet to be clarified. A series of playback experiments was conducted in Thai waters to investigate their call-back behavior. The population was exposed to 4 different playback stimuli; a recorded wild dugong's chirp, a synthesized down-sweep sound having similar frequencies to the dugong chirp, a synthesized constant-frequency sound, and no sound as a control. Vocalizing dugongs were localized using an array of stereo-underwater-recording systems (AUSOMS-Ds). Total of 4068 calls were observed in reaction to the stimuli. Wild dugongs vocalized more frequently after the playback of dugong chirps $(2.8$ calls $/ \mathrm{min})$ than those of constant-frequency $(0.55$ calls $/ \mathrm{min})$ and control $(0.2 \mathrm{calls} / \mathrm{min})$. Ratio of the dugong chirps to all of the call type increased during the playback period. Dugongs were localized on 52 occasions within $25 \mathrm{~m}$ range from the playback source. Source level and duration of the chirps from wild dugongs responding to the playback showed positive correlation with distances between the caller and the playback speaker. These results suggest that dugongs can advertise their relative locations by exchanging chirps. Frequency-modulated chirps may have facilitated ranging between individuals.

2:25

5pABa5. Response to playback test in the captive Amazonian manatees (Trichechus inunguis) in Brazil. Mumi Kikuchi (Kyoto Univ., Wildlife Res. Ctr., 2-24 Tanaka-Sekiden-cho, Sakyo-ku, Kyoto 606-8203, Japan, mumikomo@gmail.com), Diogo de Souza, and Vera M. da Silva (National Inst. of Amazonian Res. (INPA), Aquatic Mammals Lab. (LMA), Manaus, Brazil)

Previous studies suggested that manatee calls were primarily for communication and not for navigational purposes. In this study, the vocal response of captive Amazonian manatees to playbacks of several acoustic stimuli was investigated. Experiments were conducted using nine captive Amazonian manatees at the National Institute of Amazonian Research (INPA), Brazil, in 2014. All manatees, except one, are orphan calves rescued from the illegal hunting or incidental catch. They were kept in the outdoor pool with a group of 2-3 individuals, which are considered to be related. Manatees were exposed to five different playback stimuli: a recorded vocal from a related manatee, a recorded vocal from an unrelated manatee, a synthesized constant frequency based on the fundamental frequency of a related manatee vocal, a synthesized sound which entirely different from manatee vocal, and silence. A total of 58 playback sessions was conducted and 22,590 calls were recorded. While manatees showed inter-individual variability in the response to the playback stimuli, they tended to produce more vocalization during the playback of the related manatee vocal. They also tended to vocalize more to the playback of the constant frequency sound. Some individual showed strong reactions; touching or staying near the speaker during the experiment.

\section{Contributed Papers}

\section{2:45}

5pABa6. Baleen whale responses to a high frequency active pinger: Implications for upper frequency hearing limits. E. E. Henderson (Navy Marine Mammal Program, SSC Pacific, 53560 Hull St., Bayside Bldg Rm. 205, San Diego, CA 92152-5001, elizabeth.e.henderson@navy.mil), Alison Stimpert (Moss Landing Marine Lab., Santa Cruz, CA), and Amanda Debich (Scripps Inst. of Oceanogr., San Diego, CA)

In order to test the possibility of using high frequency pinger tags to track baleen whales on Navy instrumented ranges, three blue (Balaenoptera musculus) and one humpback (Megaptera novaeangliaea) whales were exposed to two high frequency pingers. The pinger frequencies were 37 and $45 \mathrm{kHz}$, with estimated received levels between 106 and $134 \mathrm{~dB}$ re $1 \mu \mathrm{Pa}$. The whales were monitored prior to the exposure in order to establish their behavioral state and to acclimate them to the presence of the boat. Two of the blue whales were deep feeding, while the third blue whale and the humpback whale were traveling with intermittent bouts of possible foraging near the surface. Each exposure lasted approximately 30-40 minutes, and the whales were observed for an additional two to three surfacing intervals post-exposure. None of the blue whales demonstrated any behavioral response. The humpback whale's response was inconclusive, as other foraging animals entered the area at the same time; however, it is likely there was no response. These data may begin to provide information on the upper frequency limits of baleen whale hearing, as these species have responded to Navy sonar-like sounds at lower frequencies but similar received levels and behavioral states.

\section{3:00-3:15 Break}

\section{$3: 15$}

5pABa7. New parametric acoustic alarm proves effective at alerting wild manatees of approaching boats. Edmund R. Gerstein (Psych., Florida Atlantic Univ., 777 Glades Rd., Boca Raton, FL 33486, gerstein2@aol.com) and Laura Gerstein (Levaithan Legacy Inc., Boca Raton, FL)

The efficacy of a bow mounted parametric acoustic alarm for alerting manatees of approaching boats was demonstrated with wild manatees in Florida. Two experimental conditions were tested; (1) boat approaches with 
the alarm-on, and (2) approaches with the alarm-off. Synchronized underwater acoustic buoys and aerial video recorders documented manatee behavior and acoustic conditions prior to, during, and after controlled boat approaches. $95 \%$ of the manatees during alarm-on trials elicited overt changes in behavior (avoidance reactions) prior to the boat reaching the focal manatee. In contrast, only $5 \%$ of manatees during alarm-off trials elicited any change in behavior prior to the vessel having to veer off. Changes in behavior observed prior to the boat reaching focal manatees was significantly greater $(\mathrm{F}=471.9, \mathrm{df}=1, \mathrm{p}<0.01)$ during alarm-on trials. The mean distance at which manatees reacted to boat approaches during alarm-on trials was also $20 \mathrm{~m}$ compared to only $6 \mathrm{~m}$ for alarm-off trials $(\mathrm{F}=218.4 \mathrm{df}=1$, $\mathrm{p}<0.01$ ). Manatees responded when received acoustic levels exceeded 12 $\mathrm{dB}$ of their critical ratios (behavioral threshold). Counter-intuitive to manatee protection laws, slow boats can be very difficult for manatees to detect and locate. Shallow water attenuates the dominant lower frequencies of slower moving propellers. The directional alarm assures manatees will be able to detect and locate boats at levels \& distances sufficient to avoid injury. [Funded by DOD Legacy Natural Resource Management Program, USFWS Permit MA063561-4.]

\section{3:30}

5pABa8. Time-frequency analysis of bird songs and neurobehavioral study of the "Catbird Dumetella carolinensis". Babak Badiey (Newark Charter School, 20 Mcintire Dr., Newark, DE 19711, babak.badiey@gmail. com)

There is a commonality between the acquisition of spoken language in human infants and the acquisition of bird songs at the behavioral, neural, genetic, and cognitive levels. Similarities between how birds learn their language and how humans learn is believed to be fundamentally related to the way the brain works. Although the effects of the environment on hearing sensations, and hence the behaviors seems intuitive, it is not yet fully understood. In this paper, we use common digital recording systems to study bird songs in their natural habitat. Then by utilizing the principals of signal processing and spectral analysis individual syllabi are identified and studied for various songs. With the knowledge of the environment from direct measurement and the change in background noise level, the behavior of birds can be studied and documented. This technique was applied to recordings of Dumetella carolinensis (Gray Catbird) in the North East United States. It is shown that in spite of the natural instinct, the catbirds do not easily change their behavior at the onset of loud transient industrial sound. This may be related to the fact that the birds have already been exposed and have learned to live in noisy cosmopolitan environments.

\section{$3: 45$}

5pABa9. Effects of industrial tones on bowhead whale calling rates. Susanna B. Blackwell (Greeneridge Sci., Inc., 90 Arnold Pl., Ste. D, Santa Barbara, CA 93117, susanna@greeneridge.com), Christopher S. Nations, Mandy E. Kauffman (WEST, Inc., Cheyenne, WY), Aaron M. Thode (Scripps Inst. of Oceanogr., San Diego, CA), Robert G. Norman, and Katherine H. Kim (Greeneridge Sci., Inc., Santa Barbara, CA)

In summer 2012, exploratory drilling was performed by Shell at Sivulliq, a lease holding in the Beaufort Sea, located within the autumn migration corridor of bowhead whales. The drilling operation involved a number of vessels performing various activities, such as towing the drill rig, anchor handling, and drilling. We aimed to assess the effect of sounds from these activities on bowhead whale calling rates. Acoustic data were collected with six arrays of directional recorders (DASARs) deployed on the seafloor over $\sim 7$ weeks in Aug-Oct. Industrial sound was quantified with the use of indices, that measured the presence and amplitude of tones from machinery, or the presence of airgun pulses. For each 10-min period of data collected at each of the 40 recorders, the number of whale calls detected was matched with the "dose" of industrial sound received, and the relationship between calling rates and industrial sound was modeled using negative binomial regression. The analysis showed that with increasing tone levels, bowhead whale calling rates initially increased, peaked, and then decreased. This dual behavioral response is similar to that previously described for bowhead whales and airgun pulses. [Work supported by Shell Exploration and Production Company.]
5pABa10. Opportunistic behavioral-response studies of baleen whales in response to US Navy Sonar Training off Kauai, Hawaii. Stephen W. Martin, Cameron R. Martin, Brian Matsuyama (National Marine Mammal Foundation, 2240 Shelter Island Dr., Ste. 200, San Diego, CA 92106, steve. martin@nmmpfoundation.org), Elizabeth E. Henderson, and Tyler Helble (SPAWAR Systems Ctr. Pacific, San Diego, CA)

Opportunistic behavioral responses of baleen whales to disturbances from US Navy mid-frequency active sonar (MFAS) training at the Pacific Missile Range Facility, Kauai, Hawai'i, are being studied utilizing passive acoustic recordings. Automated passive acoustic detection, classification, localization, and tracking analyses of the data have shown a behavioral response in terms of a reduction, or cessation, of minke whale "boing" calling in response to US Navy training during the month of February 2011, 2012, and 2013 over a study area of $3,780 \mathrm{~km} 2$. The reduced calling is expressed as reduced minimum densities in the study area by utilizing acoustically localized individual whale counts. In February 2011, the density before sonar training was 3.64 whales while the density during sonar training was 0.69 whales (95\% confidence intervals of 3.31-4.01 and 0.271.8 whales, respectively). Individual ship-whale encounters have been observed to show cessation of calling from ship approaches without MFAS activity as well as ship approaches with MFAS. Sound Pressure Levels and Cumulative Sound Exposure Levels animals are exposed to are being estimated for evaluation of dose-response relationships. Tracking individual whales allows investigation of kinematics coupled with acoustic call details to establish baseline behaviors for comparison with observations during US Navy training.

\section{$4: 15$}

5pABa11. Mid-frequency active sonar and beaked whale acoustic activity in the Northern Mariana Islands. Anne Simonis, Bruce Thayre (Scripps Inst. of Oceanogr., 9500 Gilman Dr., La Jolla, CA 92037-0205, asimonis@ucsd.edu), Erin Oleson (Pacific Islands Fisheries Sci. Ctr., National Oceanic and Atmospheric Administration, Honolulu, HI), and Simone Baumann-Pickering (Scripps Inst. of Oceanogr., La Jolla, CA)

Mid-frequency active (MFA) sonar has been associated with multiple mass stranding events of beaked whales around the world. A recent increase in military training exercises in the Mariana Archipelago corresponds with the presence of MFA sonar in the surrounding waters. We provide a quantitative report on MFA sonar and beaked whale acoustic activity detected on two autonomous acoustic recording packages deployed near Saipan and Tinian from March 2010 through December 2013. There were no detections of MFA sonar at Saipan during the 5-month deployment in 2010. On August 21, 2011, MFA sonar was detected near Saipan concurrent with a stranding event involving two Cuvier's beaked whales (Ziphius cavirostris). After one observed day of MFA sonar activity in Saipan and Tinian in 2011, observations increased to 1 month of ongoing activity at Saipan and nearly 3 months ongoing activity at Tinian in 2012. In 2013, MFA sonar events were observed during one day at Saipan and zero days at Tinian. Received levels, sound exposure levels, and temporal descriptions of the MFA sonar events are reported along with detections of beaked whale acoustic activity. Here, we highlight the importance of ongoing passive acoustic monitoring, especially for species like beaked whales that are difficult to visually detect at sea.

\section{$4: 30$}

5pABa12. Marine mammal passive acoustics applied to the monitoring of long-term trends in beaked whale abundance and to the derivation of a behavioral risk function for exposure to mid-frequency active sonar. David Moretti (NUWC, 71 Woodmark Way, Wakefield, RI 02879, morettidj@hotmail.com), Tiago Marques (Univ. of St. Andrews, Lisbon, Portugal), Len Thomas (Univ. of St. Andrews, Saint Andrews, United Kingdom), Stephanie Watwood, Nancy DiMarzio, Karin Dolan, Ronald Morrissey, Jessica Shaffer, Joao F. Monteiro, and Susan Jarvis (NUWC, Newport, RI)

Knowledge of Cuvier's (Ziphius cavirostris) and Blainville's (Mesoplodon densirostris) beaked whales' distinct dive and vocal behavior has allowed for the development of multiple methods of passive acoustic abundance and density estimation (Marques et al., 2009, Moretti et al., 2010). 
These methods are being applied to multiple years of data to estimate longterm trends in abundance for Blainville's beaked whales at the Atlantic Undersea Test and Evaluation Center (AUTEC) in the Bahamas and at the Pacific Missile Range Facility (PMRF) in Hawaii, and for Cuvier's beaked whales at the Southern California Offshore Range (SCORE). These passive acoustic beaked whale dive data were combined with sonar and Range shiptrack data to derive a behavioral risk function for Blainville's beaked whales at AUTEC, and are being extended to Cuvier's beaked whales at SCORE and Blainville's beaked whales at PMRF. The behavioral risk function maps the probability of beaked whale dive disruption as a function of the receive level of mid-frequency active sonar. The risk function and long-term trend analysis will help inform environmental policy going forward.

\section{$4: 45$}

5pABa13. The effects of wind turbine noise on male Greater PrairieChicken vocal output during the breeding season. Cara Whalen, Mary B. Brown (School of Natural Resources, Univ. of Nebraska, Lincoln, NE), JoAnn McGee (Developmental Auditory Physiol. Lab, Boys Town National Res. Hospital, 555 North 30th St., Omaha, NE 68131), Larkin Powell (School of Natural Resources, Univ. of Nebraska, Lincoln, NE), and Edward J. Walsh (Developmental Auditory Physiol. Lab, Boys Town National Res. Hospital, Omaha, NE, edward.walsh@boystown.org)

At a previous meeting of the ASA, we reported that the Greater PrairieChicken (Tympanuchus cupido pinnatus) is, in all probability, able to detect noise produced by wind turbine installations, a finding based on turbine noise output and auditory brainstem response threshold measurements (Walsh et al., 2015). That investigation was conducted in concert with an effort to determine if noise generated by a wind turbine farm located near Ainsworth, Nebraska affects the vocal output of male prairie chickens occupying breeding grounds (i.e., leks) where they perform elaborate physical and vocal mating displays. Whalen et al. (2014) paved the way to address this question by characterizing the acoustic properties of booms, cackles, whoops and whines, the primary call types produced by male birds, at sites remote from the turbine farm. At lek sites located relatively close $(<1 \mathrm{~km})$ to the wind farm installation, boom and whoop production levels were higher, boom durations were shorter, whine fundamental frequency was higher, and cackle biphonation occurred less frequently than among birds occupying leks located at remote locations. Although differences in vocal characteristics were statistically significant, vocal adjustments were relatively minor and the significance of wind turbine farm noise on call acoustics will be discussed. [Funded in part by USFWS-WSFR Wildlife Restoration Project W-99-R administered by the NGPC.]

5:00-5:15 Panel Discussion

FRIDAY AFTERNOON, 2 DECEMBER 2016

NAUTILUS, 1:00 P.M. TO 4:30 P.M.

\title{
Session 5pABb
}

\section{Animal Bioacoustics: The Diversity of Bioacoustic Signals}

\author{
T. Aran Mooney, Chair \\ Biology Department, Woods Hole Oceanographic Institution, 266 Woods Hole Rd., Woods Hole, MA 02543
}

\section{Contributed Papers}

1:00

5pABb1. Prusten and the acoustic character of socializing tigers. Adam Smith, JoAnn McGee (Developmental Auditory Physiol. Lab, Boys Town National Res. Hospital, 555 North 30th St., Omaha, NE 68131), Douglas Armstrong (Henry Doorly Zoo and Aquarium, Omaha, NE), and Edward J. Walsh (Developmental Auditory Physiol. Lab, Boys Town National Res. Hospital, Omaha, NE, edward.walsh@boystown.org)

Although tigers are generally solitary animals, acoustic communication among conspecifics plays an important role in their biology and behavior. This investigation was undertaken to extend understanding of prusten, one of the most common vocalizations produced by Panthera tigris. Prusten is a low-level, social vocalization uttered in close proximity of conspecifics. Calls were recorded from a group of nine captive adult tigers, representing the Amur ( $P$. tigris altaica), Bengal ( $P$. tigris tigris) and Malayan subspecies (P. tigris jacksoni). As described previously, the acoustic character of prusten consists of three to nine brief rhythmic pulses emitted at a mean rate of 11.5 pulses per second with a total mean duration of 0.5 seconds. Calls were generally low level, with a mean of $71 \mathrm{~dB}$ SPL re $20 \mu \mathrm{Pa}$ at 1 meter from the source. Spectral energy was broadband, extending from below 20 $\mathrm{Hz}$ to above $22 \mathrm{kHz}$, although low frequency energy dominated the call with mean peak and centroid frequencies of 130 and $987 \mathrm{~Hz}$, respectively. A small subset of calls contained infrasonic energy. This study affirms and extends findings from previously published descriptions and is the first report of the production of infrasound in this low level, pulsatile vocalization. [Work supported in part by NSF Grant Award \#0823417.]

\section{1:15}

$5 \mathrm{pABb2}$. Using CT to predict vocal tract resonances in mammal vocalizations: Are calls nasalized? David Reby (School of Psych., Univ. of Sussex, University of Sussex, Brighton BN2 9TJ, United Kingdom, reby@ sussex.ac.uk), Megan T. Wyman (Dept. of Evolutionary Biology and Environ. Studies, Univ. of Zurich, Zurich, Switzerland), Roland Frey (Leibniz Inst. for Zoo and Wildlife Res. (IZW), Berlin, Germany), and Joel Gilbert (Laboratoire d'Acoustique de l'Université du Maine - UMR CNRS, Université du Maine, Le Mans, France)

Males of several species of deer have a descended larynx, which gives them an unusually long vocal tract (VT). They can extend their VT by further lowering their larynx during the production of their sexual loudcalls. Formant frequencies are lowered as the vocal tract is extended, as predicted when approximating the vocal tract as a uniform quarter wavelength resonator. However, formant frequencies in polygynous deer follow uneven distribution patterns, suggesting that the vocal tract shape is in fact rather complex. We CT-scanned the artificially extended vocal tract of two adult fallow deer, and measured the cross-sectional area of the supralyngeal vocal 
tract along the oral and nasal pathways. We used this data to model resonances patterns produced by these VT including the oral pathway, the nasal pathway, or both. We found that the combined oral/nasal VT produced a formant pattern that more closely matches that observed in fallow deer groans and enables a better estimation of VT length from formant patterns. This clear indication that the nasal cavity and oral cavity are both simultaneously involved in the vocal production of a nonhuman mammal suggest that the potential for partial nasalization of putative oral loud calls should be carefully considered.

$1: 30$

5pABb3. Restoring dueting behavior in a mated pair of buffy cheeked gibbons after exposure to construction noise at a zoo through playbacks of their own sounds. Jeanette A. Thomas, Brett Friel, and Sarah Yegge (Biological Sci., Western Illinois Univ.-Quad Cities, WIUQC, 3300 River Dr., Moline, IL 61265, jeanettethomaswiu@gmail.com)

There is concern about anthropogenic noise effecting zoo animals. Gibbons are known for duets exhibited by a mated pair. During a 6-month period in 2009, Niabi Zoo conducted construction (sewer/water lines, and paving) within $100 \mathrm{~m}$ of the buffy cheeked gibbon exhibit. Prior to construction, a male/female pair (plus their 4-year old son) performed loud, elaborate duets each day. Friel collected behavior data using focal animal and instantaneous scan-sampling for 10 days; 5 times per day prior to construction and for 20 days during construction. SEL measurements were taken during both periods. In general, the immature gibbon vocalized little; the female vocalized most. Gibbons vocalized less during construction noise. After construction, the subadult male became ill and was euthanized; thereafter the pair ceased vocalizing. Two years later, Yegge played back sounds to encourage dueting: 1) their own duet, 2) the duet of wild gibbons, 3) rock music, and 4) a silent control. Their behavior was collected for 3, 15-min playback sessions per day for 30 days. The pair's own playback elicited a duet even during the first session. The female vocalized significantly more than the male, especially during the wild gibbon duet. Currently, the pair continues dueting on their own.

\section{$1: 45$}

5pABb4. Baby's first words: Vocal behavior and ontogeny of Northern right whales in the southeast critical habitat. Edmund R. Gerstein (Psych., Florida Atlantic Univ., 777 Glades Rd., Boca Raton, FL 33486, gerstein2@aol.com), Vasilis Tyrgonis (Univ. of the Agean, Lesvos, Greece), and James Moir (Marine Resource Council of Florida, Palm Bay, FL)

North Atlantic right whales are one of the most endangered of the great whales. A remnant population of $\sim 500$ inhabits the eastern seaboard of North America. A small fraction (2\%) travels south to their critical calving habitat along the Florida and Georgia coast. By late November and through the winter, right whales give birth and nurse their calves in these shallow waters before departing in early spring to their northern habitats. In the southeast critical habitat mother-calf pairs remain generally isolated from other whales, presenting a unique platform to study vocal development and learning in large whales. Using small boats, GPS-instrumented, free-drifting autonomous acoustic buoys were deployed in close proximity to 44 photoidentified mother-calf pairs over 7 calving seasons. Surface video and synchronized underwater recordings documented their social and vocal behavior. With the exception of some low-energy gunshot sounds, mothers, and their calves, remained predominantly silent during the first 4 weeks. This might be due to calf maturation, and or a strategy to avoid harassment by other whales or potential predators. Over 100 calls have been analyzed from 15 different calves. Some of these calves were resampled at different stages at $<1$ week up to 12 weeks of age. Evidence of age-related variance and changes in call structure, complexity, power, rates, as well as vocal mimicry and learning are presented. [Funding: HBOI Florida PFW License Plate Fund, The Harry Richter Foundation and IBM, NOAA Permit \#14233.]
2:00

5pABb5. Dwarf sperm whale (Kogia sima) echolocation clicks from Guam (Western North Pacific Ocean). Karlina Merkens (CRP, NOAA/ PIFCS (Lynker Tech.), 1845 Wasp Blvd., Bldg. 176, Honolulu, HI 96818, karlina.merkens@noaa.gov), Yvonne Barkley, Marie Hill (CRP, NOAA/ PIFSC (JIMAR), Honolulu, HI), and Erin Oleson (CRP, NOAA/PIFSC, Honolulu, HI)

The cryptic species of the genus Kogia, including the dwarf sperm whale (Kogia sima) and the pygmy sperm whale (Kogia breviceps), are very difficult to observe in any but the most calm sea conditions. However, recordings of signals from wild and captive animals reveal that they echolocate at high frequencies (peak frequencies $>100 \mathrm{kHz}$ ) which makes passive acoustic monitoring (PAM) a possibility. We present details from a recent encounter with K. sima in the wild near the island of Guam (Western North Pacific Ocean). Three individuals were observed during a small-boat, visual survey in May 2016, and recordings were collected using a Compact Acoustic Recording Buoy (CARB). These clicks, with mean peak frequency of $126 \mathrm{kHz}(+/-4.3 \mathrm{kHz})$, mean click duration of $72 \mathrm{us}(+/-21 \mathrm{us})$, and $-3 \mathrm{~dB}$ bandwidth $5.5 \mathrm{kHz}(+/-1.6 \mathrm{kHz})$, had similar properties to recordings of wild $K$. sima from the Bahamas (Atlantic Ocean), and also appear similar to published recordings from $K$. breviceps (Madsen et al. 2005). Available data and analyses to date do not allow for absolute determination between the two Kogia species at this time, but recordings such as these bring us closer to definitive species classification.

\section{2:15}

5pABb6. Inter and intra specific variation in echolocation signals among cetacean species in Hawaii, the northwest Atlantic and the temperate specific. Tina M. Yack, Kerry Dunleavy, Julie N. Oswald (BioWaves, Inc., 364 2nd St., Ste. \#3, Encinitas, CA 92024, tina.yack@biowaves.net), and Danielle Cholewiak (NOAA Northeast Fisheries Sci. Ctr., Woods Hole, MA)

Odontocete species use echolocation signals (clicks) to forage and navigate. The aim of this study is to explore inter- and intra-specific variation in clicks among odontocete species in the Northwest Atlantic, Temperate Pacific, and Hawaii. Clicks were examined for seven species of delphinids in the western North Atlantic; common dolphin, Risso's dolphin, pilot whale, rough-toothed dolphin, striped dolphin, Atlantic spotted dolphin, and bottlenose dolphin. Newly developed PAMGuard tools were used to automatically measure a suite of click parameters. Five parameters were compared between species; duration, center frequency, peak frequency, sweep rate, and number of zero crossings. Significant differences in duration, center and peak frequency were evident between species within regions (Dunn's test with Bonferroni adjustment $\mathrm{p}<0.05$ ). Geographic variation in click parameters between the three study regions was compared for five species; bottlenose dolphin, common dolphin, striped dolphin, pilot whale, and Cuvier's beaked whale. Significant differences in several parameters were found for all species between the regions (Dunn's test with Bonferroni adjustment $\mathrm{p}<0.05$ ). These results suggest that there are species specific differences in clicks among delphinids and that geographic variation exists for multiple species. The ecological significance of these findings will be discussed along with implications for classifier development.

\section{2:30-2:45 Break}

\section{2:45}

5pABb7. Determining killer whale (Orcinus orca) call variability from passive acoustic monitoring in the Chukchi and Bering Sea, Alaska. Brijonnay C. Madrigal (Vertebrate Ecology Lab., Moss Landing Marine Lab., 8272 Moss Landing Rd., Moss Landing, CA 95039, bmadrigal@mlml.calstate.edu), Catherine L. Berchok, Jessica L. Crance (Marine Mammal Lab., Alaska Fisheries Sci. Center/NMFS/NOAA, Seattle, WA), and Alison K. Stimpert (Vertebrate Ecology Lab., Moss Landing Marine Lab, Moss Landing, CA)

Killer whales (Orcinus orca) are a highly vocal species that produce three types of vocalizations; pulsed calls, whistles, and clicks. Unlike the Northern and Southern Resident populations of western Canada and the Pacific Northwest, little is known regarding the acoustic behavior of resident 
and transient killer whale populations north of the Aleutian Islands in the Bering and Chukchi Seas. Acoustic data were analyzed from moored recorders deployed by the Marine Mammal Laboratory at two sites each in the Bering and Chukchi Seas (BOEM-funded). The recorders sampled at 4 $\mathrm{kHz}$ on a $7 \%$ duty cycle (Bering) or $16 \mathrm{kHz}$ on a $28 \%$ duty cycle (Chukchi). Over 1100 calls were identified, and discrete call classification was conducted using an alphanumerical system that distinguished calls by location, general contour, and segment variation. Parameters analyzed included call duration, start/end frequency, and delta frequency/time; periods of call repetition were common. These results will help determine if resident populations occur in the Chukchi Sea, and identify which transient populations are present. This initial work classifying killer whale sounds in the Arctic and along the Bering Sea shelf will also facilitate comparisons of call types within and among transient and resident killer whale populations.

\section{3:00}

5pABb8. Whistle characteristics of newly defined species, the Burrunan dolphin (Tursiops australis), in coastal Victorian waters in Australia. Rebecca Wellard (Ctr. for Marine Sci. and Technol., Curtin Univ., GPO Box U1987, Perth, WA 6845, Australia, becwellard@gmail.com), Kate Charlton-Robb (Australian Marine Mammal Conservation Foundation, Hampton East, VIC, Australia), Christine Erbe (Ctr. for Marine Sci. and Technol., Curtin Univ., Perth, WA, Australia), and Bob Wong (School of Biological Sci., Monash Univ., Clayton, VIC, Australia)

A newly defined species, the Burrunan dolphin (Tursiops australis), was described in 2011 by Charlton-Robb et al., and is endemic to southern and south-eastern Australian coastal waters. This species' distribution is characterized by small, isolated, and genetically distinct populations. With only two known populations in Victoria, the species is now listed as "Threatened" under the Victorian Flora and Fauna Guarantee Act. Describing and quantifying the vocal repertoire of a species is critical for subsequent analysis of signal functionality, geographic variation, social relevance, and identifying threats associated with anthropogenic noise. Here, we present the first quantitative analysis of whistle characteristics for the species, undertaken on these endemic Victorian populations. Vocalizations of T. australis were recorded during population based surveys in 2007 and 2014 across the Gippsland Lakes and Port Phillip Bay, Victoria. Acoustic parameters of whistles were measured including minimum frequency (fmin), maximum frequency (fmax), start frequency (sf), end frequency (ef), delta frequency $(\mathrm{df}=\mathrm{fmax}$-fmin), duration, number of extrema, number of inflection points, and number of steps. We review and compare T. australis whistle features to the two other bottlenose dolphins, $T$. truncatus and $T$. aduncus, to assess the similarity and/or differences between the sounds of the three species of bottlenose dolphins.

\section{3:15}

5pABb9. Visual acoustic analysis techniques for North Atlantic right whale sounds. James A. Theriault, Gary Inglis (Underwater Sensing, Defence Res. and Development Canada - Atlantic Res. Ctr., 1 Challenger Dr., Dartmouth, NS B2Y 4A2, Canada, jim@theriault-family.ca), and Hilary B. Moors-Murphy (Ocean and Ecosystem Sci. Div., Maritimes Region Fisheries and Oceans Canada, Dartmouth, NS, Canada)

Passive acoustic monitoring (PAM) is becoming a more widely accepted tool in mitigating the potential impact of man-made noise on marine mammals. Many marine mammals, and in particular cetaceans (whales and dolphins), use sound to communicate, navigate, forage, and avoid predators. Automated vocalization detectors have been in development for many years. Validation is achieved through aural/visual verification by analysts examining acoustic data in both the time and frequency domains. However, many factors also influence the ability of the analyst to properly detect and classify marine mammal sounds. First, a priori knowledge of the acoustic signature and the analysts experience are critical factors. Assuming the a priori knowledge exists, the ability to correctly assess the presence of call is dependent on the signal processing and displays available. Frequency resolution, temporal integration, and normalization either enhance or inhibit the ability to make the assessment. The signal processing parameterization varies between species and vocalization types. Six types of vocalizations have been previously described for the North Atlantic right whale (Eubalaena glacialis); upcalls, gunshots, screams, downcalls, blows, and warbles. The parameterization required to optimally assess each of the vocalization types will be examined.

\section{3:30}

5pABb10. Song learning in humpback whales: Lessons from song hybridization events during revolutionary song change. Ellen C. Garland, Luke Rendell (School of Biology, Univ. of St. Andrews, St. Andrews, Fife KY16 9TH, United Kingdom, ecg5@st-andrews.ac.uk), M. Michael Poole (Marine Mammal Res. Program, Maharepa, French Polynesia), and Michael J. Noad (Cetacean Ecology and Acoust. Lab., School of Veterinary Sci., The Univ. of Queensland, Gatton, QLD, Australia)

Humpback whale songs are one of the most startling examples of transmission of a cultural trait and social learning in any non-human animal. Here, we investigate extremely rare cases of song hybridization, where parts of an existing song are spliced with a novel, revolutionary song, to understand how songs are learnt. Song unit sequences were extracted from over 800 phrases recorded during a song revolution (French Polynesia 2005), to allow fine-scale analysis of composition and sequencing. Clustering of song sequences (i.e., phrases) using the Levenshtein distance indicated songs clustered into three song types; a single hybrid phrase was identified representing the transition of one singer between two of these song types. A predictive model was fitted to the data and tested against the only other known recordings of humpback song hybridization: the eastern Australia 1996-97 song revolution. Songs change during revolutions through combining multiple complete phrases and themes from one song type, before transitioning through a hybrid phrase into the phrases and themes of a second song type. These extremely rare snapshots of song change represent the only known examples of song learning in over 20 years of data, spanning five populations of humpback whales were song revolutions occur.

\section{$3: 45$}

$5 p A B b 11$. Creation of an acoustic dictionary to classify the vocal repertoire of humpback whale song. Jennifer Allen (School of Veterinary Sci., Univ. of Queensland, Bldg. 8106, University of Queensland Gatton Campus, Gatton, QLD 4343, Australia, j.allen3@uq.edu.au), Ellen Garland (Univ. of St. Andrews, St. Andrews, United Kingdom), Anita Murray (School of Veterinary Sci., Univ. of Queensland, Brisbane, QLD, Australia), Michael Noad, and Rebecca Dunlop (School of Veterinary Sci., Univ. of Queensland, Gatton, QLD, Australia)

Animal vocal repertoires can be varied and complex, and it is often difficult to obtain a comprehensive picture. One of the primary reasons for this difficulty stems from the classification of acoustic signals, which has typically relied on qualitative and subjective methods. Vocal signals (units) were measured from east Australian humpback whale song from 2002-2014 and classified using self-organising maps (SOM). Humpback song was used here to provide an example of a particularly complex display of vocal signals. The use of SOM methodology takes an important step towards a more objective classification system. The resulting classifications were used to create a set of 149 hypothetical "exemplar" units to serve as an acoustic dictionary, representing the song's repertoire. The intent is to use this dictionary to determine vocal repertoire, as well as to facilitate the transcription and sequential analysis of large numbers of recordings with only a small representative subset of units requiring intensive acoustic feature measurement. Acoustic dictionaries are integral to studies of information content, microstructure, and syntax, and this methodology is thus applicable across the vocalization research community.

\section{4:00}

5pABb12. Acoustic characteristics of humpback whale (Megaptera novaeangliae) mother and calf vocalizations in the Hawaiian wintering grounds. Jessica Chen (Hawaii Inst. of Marine Biology, Univ. of Hawaii at Manoa, 46-007 Lilipuna Rd., Kaneohe, HI 96744, jchen2@hawaii.edu), Adam A. Pack (Departments of Psych. and Biology, Univ. of Hawaii at Hilo, Hilo, HI), Alison K. Stimpert (Moss Landing Marine Labs., Moss Landing, CA), and Whitlow W. Au (Hawaii Inst. of Marine Biology, Univ. of Hawaii at Manoa, Kaneohe, HI)

Humpback whale calves in the winter breeding grounds vocalize and the spectral characteristics of some of these vocalizations have been described, 
but sound levels of calf vocalizations have not been investigated. There is also a lack of general information on vocalizations from mothers. To address these issues, we deployed suction cup acoustic and movement recording tags on humpback whale calves, mothers and a lone post-yearling stage female on the Hawaiian breeding grounds to record vocalizations and percussive sounds. Deployments took place in waters off West Maui over four winter seasons. Tags were deployed on 7 humpback whale mothers, 6 calves, and one lone female for approximately 57 hours of recordings. Calling rates of tagged animals were relatively low compared to song, with individual's means ranging from 0 to 16 vocalizations per hour. However, most calls occurred singly or in bouts, with long periods of silence before the next vocalization or set of vocalizations. These included sounds resembling non-calf social sounds as well as single song units, with durations up to 2 seconds and fundamental frequencies below $1.5 \mathrm{kHz}$. Our findings provide information that is critical to understanding vocal development in humpback whale calves and the sounds produced by adult females.

\section{$4: 15$}

5pABb13. Loud and clear: Characterization of particle motion in Humpback whale song and its potential role in communication. T. Aran Mooney, Maxwell B. Kaplan (Biology Dept., Woods Hole Oceanographic Inst., 266 Woods Hole Rd., Woods Hole, MA 02543, amooney@whoi.edu), and Marc O. Lammers (Hawaii Inst. of Marine Biology, Univ. of Hawaii, Kaneoho, HI)

Acoustic communication can rapidly transfer a substantial amount of information, yet emitted signals must be conveyed with enough clarity to allow appropriate responses. Many mysticete calls such as humpback whale (Megaptera novaeangliae) song can be detected over large distances as a result of the propagating acoustic pressure wave, yet little is known regarding the particle motion component of these signals. To explore the particle velocity of humpback whale song, three singing whales were recorded from a vessel off Maui, HI in March 2015, using a sensor that contained a digital magnetometer, tri-axial accelerometer, and an omnidirectional hydrophone. The median magnitude of the acoustic particle velocity signal was substantial $(64.5 \mathrm{~dB}$ re: $1 \mathrm{~m} / \mathrm{s})$ for song components with a median pressure of 135.4 $\mathrm{dB}$ re: $1 \mu \mathrm{Pa}$. As vessel and sensor gradually drifted away or toward the whales, acoustic particle velocity and sound pressure correspondingly decreased or increased (range: $49.3-77.9 \mathrm{~dB}$ re: $1 \mathrm{~m} / \mathrm{s}$ and 118.4-148.1 dB re: $1 \mu \mathrm{Pa}$ ). The particle velocity signals were high even at $200 \mathrm{~m}$ from the whale indicating this cue is substantial well into the far-field. There were predictable trends in the acoustic particle motion signal component and this component of song is a cue available to nearby animals.

\title{
Session 5pBA
}

\section{Biomedical Acoustics, Engineering Acoustics and Signal Processing in Acoustics: Acoustic Imaging}

\author{
Yasuhiro Oikawa, Cochair \\ Department of Intermedia Art and Science, Waseda University, 3-4-1 Okubo, Shinjuku-ku, Tokyo 169-8555, Japan \\ Hideyuki Hasegawa, Cochair \\ University of Toyama, 3190 Gofuku, Toyama 9308555, Japan
}

\section{Invited Papers}

1:00

5pBA1. Signal processing for optical sound field measurement and visualization. Kohei Yatabe, Kenji Ishikawa, and Yasuhiro Oikawa (Intermedia Art and Sci., Waseda Univ., 59-407 3-4-1 Okubo, Shinjuku-ku, Tokyo 169-8555, Japan, k.yatabe@asagi.waseda.jp)

Accurately measuring sound pressure is not an easy task because every microphone has its own mechanical and electrical characteristics. Moreover, the existence of a measuring instrument inside the field causes reflection and diffraction which deform the wavefront of sound to be measured. Ideally, a sensing device should not have any characteristic nor exist inside a measuring region. Although it may sound unrealistic, optical measurement methods are able to realize such ideal situation. Optical devices can be placed outside the sound field, and some of the sensing techniques, which decode information of sound from the phase of light, are able to cancel optical and electrical characteristics. Thus, optical sound measurement methods have possibility of achieving higher accuracy than ordinary sound measurement in principle. However, they have two main drawbacks that have prevented their applications in acoustics: (1) pointwise information cannot be obtained directly because observed signal is spatially integrated along the optical path; and (2) increasing signal-to-noise ratio is difficult because optical measurement of less than a nanometer order is typically required. To overcome the above difficulties, we have proposed several signal processing methods. Here, those methods are introduced with the physical principle of optical sound measurement. 
5pBA2. Position and velocity measurement for moving objects by pulse compression using M-sequence-coded ultrasound. Shinnosuke Hirata and Hiroyuki Hachiya (Dept. of Systems and Control Eng., Tokyo Inst. of Technol., 2-12-1 Ookayama, S5-17, Meguro, Tokyo 152-8550, Japan, shin@ctrl.titech.ac.jp)

The pulse-echo method is one of the typical methods for ultrasonic distance measurement. Pulse compression using an M-sequence can improve the SNR of the echo reflected from an object and distance resolution in the pulse-echo method by the cross correlation between the received signal and the reference signal which correspond to the transmitted M-sequence-coded signal. In the case of a moving object, however, the echo is modulated due to the Doppler effect. The Doppler-shifted M-sequence-coded signal cannot be correlated with the original reference signal. Therefore, Doppler velocity estimation by autocorrelation of cyclic M-sequence-coded signal and cross correlations with Doppler-shifted reference signals which correspond to estimated Doppler velocities has been proposed. In this paper, measurement of position and velocity of the static and moving objects by the proposed method using the loud speaker and the microphone array are described. First, the Doppler velocity of the moving object is estimated in each array element. Second, received signals are correlated with the original reference signal for the static object. Then, received signals are correlated with Doppler-shifted reference signals for the moving object. Finally, positions of both objects are determined from the B-mode image formed by the synthetic aperture focusing technique.

\section{1:40}

5pBA3. A trial on the measurement of the acoustic properties by using a parametric loudspeaker. Akiko Sugahara (Eng., the Univ. of Tokyo, 4-6-1, Komaba, Meguro, Tokyo 153-8505, Japan, sugahara@iis.u-tokyo.ac.jp), Hyojin Lee, Shinichi Sakamoto (Inst. of Industrial Sci., the Univ. of Tokyo, Meguro, Tokyo, Japan), and Shigeto Takeoka (Shizuoka Inst. of Sci. and Technol., Fukuroi-shi, Shizuoka, Japan)

Measurement of acoustic properties of materials, such as absorption and reflection coefficient in a free field, has the results largely affected by diffraction by the sample edge. In order to minimize this, the use of a parametric loudspeaker, which enables to produce a directional audible sound by using the nonlinear effect of sound beams based on the parametric array theory, is investigated. At first, the directivity and the distance attenuation were measured as fundamental characteristics of the sound source. Then, the directivity of reflected sound from a surface, flat and diffuser plate, under oblique incidence conditions was measured in an anechoic chamber. The data were compared with those obtained by an experiment using a conventional loudspeaker, and with a wave-based numerical analysis using the mode matching method. The results show that the narrow sound directivity of the parametric loudspeaker is capable of obtaining the directivity properties of reflection sound from the plates more accurately than a conventional loudspeaker.

\section{Contributed Papers}

\section{2:00}

5pBA4. Non-contact acoustic imaging using single element transducer applied by time reversal focusing. Hideyuki Nomura and Hirotaka Saitoh (The Univ. of Electro-Communications, 1-5-1 Chofugaoka, Chofu-shi 1828585, Japan, h.nomura@uec.ac.jp)

A large number of transducers have been used for the Time reversal (TR) processing to focus sound at a specific position and a specific time. In this study, TR focusing of ultrasound in space and time domains was experimentally confirmed in air using sound waves radiated from single element transducer. Experiments were carried out using a piezoelectric transducer glued on inner wall of a cavity for the reverberations of sounds. A microphone indicated that time reversed ultrasounds at about $50 \mathrm{kHz}$ radiated from the hole of the cavity were focused at a specific time and a specific position outside of the cavity. This proposed TR focusing was applied to acoustic imaging for a defect on a brass plate with $0.3 \mathrm{~mm}$ in thickness. Time reversed ultrasound was focused at arbitrary position on the plate, and the laser Doppler vibrometry measured the vibration of the plate. Twodimensional distribution of the vibration obtained by TR focusing indicated the presence of added small brass piece on the plate as a defect. These results suggest that the feasibility of single element TR focusing of ultrasound and an application of that on non-contact acoustic imaging.

\section{2:15}

5pBA5. Design of a low profile conformal array for transcranial ultrasound imaging. Aref Smiley, Mark Howell, Aaron Fleischman, Qi Wang, and Gregory T. Clement (Biomedical Eng., Cleveland Clinic, 9500 Euclid Ave., ND20, Cleveland, OH 44195, aref.smiley@gmail.com)

In our ongoing work toward transskull ultrasound brain imaging, we investigate an optically-registered "belt-type" conformal transducer designed to maximize energy transfer while maintaining precise phase control. Previous simulations determined that approximately 500 channels is sufficient for two-dimensional brain imaging by utilizing a tomographic approach valid on irregular boundaries [Clement, Inverse Problems30 125010 (2014)]. The present design is based around an assembly of rigid planar sub-arrays with flexible interconnects. Each piezo-composite subarray $(500 \mathrm{kHz}, 1-3$ random-fiber, $20 \mathrm{~mm} \mathrm{X} 20 \mathrm{~mm})$ is formed by electroding both faces of the composite before partially dicing the rear surface to produce 19 signal electrode terminals (width $0.85 \mathrm{~mm}$, height $20 \mathrm{~mm}$, and kerf $\sim 0.18 \mathrm{~mm}$ ) with a shared ground face. Sub-arrays are bonded to a custom printed circuit boards and wired to shielded ribbon cable. The full transducer is then created by UV bonding the electrodes to a flexible band, forming the front transducer surface. Length may be customized $\sim 500$ elements for an average-sized head. Element performance is assessed by underwater scanned hydrophone characterization. Measurements numerically back-projected to the transducer face indicate good element isolation. Signals through an anatomically-correct full head phantom will be presented as well as the methodology for registering the array to the head using a 3D optical scanner.

\section{2:30}

5pBA6. Simulation of shear wave elastography imaging using the toolbox “k-wave". Fabrice Prieur (Dept. of Informatics, Univ. of Oslo, P.O Box 1080, Blindern, OSLO 0316, Norway, fabrice@ifi.uio.no), Bradley Treeby (Dept. of Medical Phys. and BioEng., Univ. College London, LONDON, United Kingdom), and Stefan Catheline (INSERM, LabTau, U1032, LYON, France)

Elastography is used to map the local elasticity of tissue. It can detect areas inside the body with a different elasticity from that of surrounding tissue indicative of pathologies like tumors. Shear wave elastography imaging produces an elasticity map by analyzing the propagation of shear waves. One source of shear wave is the acoustic radiation force produced by ultrasound. The prediction of the acoustic radiation force and of the shape and amplitude of the ensuing shear displacement is crucial. In this study, we present simulations of the radiation force produced by an ultrasound transducer and of the shear displacement it produces using the software package "k-wave." Results from simulations in a homogeneous and isotropic medium are compared against analytical solutions and results from a finite element modeling software. The obtained shear displacements from $\mathrm{k}$-wave are very similar to the analytical solution with a root mean square error around 
the focal zone below $9 \%$ and $21 \%$ for short and large propagation times, respectively. K-wave appears to be an accurate and efficient tool for simulation of acoustic radiation force and shear wave propagation. It combines the simplicity of finite time differences methods with the flexibility to simulate in any heterogeneous medium.

\section{$2: 45$}

5pBA7. Ultrasound-based closed-loop control of implantable devices. Kostyantyn Shcherbina, Parag V. Chitnis (Dept. of BioEng., George Mason Univ., 4400 University Dr., 1G5, Fairfax, VA 22032, pchitnis@gmu.edu), and Samuel Sia (Biomedical Eng., Columbia Univ., New York, NY)

Implantable devices have a large potential to impact healthcare, but they are often made of biofouling materials that necessitate special coatings, rely on electrical connections for external communication, and require a continuous power source. We present an alternative platform, where an implanted biocompatible capsule can be wirelessly controlled by ultrasound to trigger the release of compounds. The capsule prototype was approximately $1 \mathrm{~mm}$ thick and consisted of a PDMS shell containing a co-polymer gel (NiPAAm-co-AAm). The co-polymer gel formulation was optimized so that it contracts when heated above body temperature (i.e., at 450C) to release compounds through an opening in the PDMS shell. This gel-containing capsule is free of toxic electronic or battery components. Ultrasound was employed for visualization (B-mode imaging), actuation (localized heating) and closed-loop control (ultrasound-based thermometry). This was achieved by integrating a diagnostic transducer coaxially within the central aperture of an spherically sectioned focused ultrasound (FUS) transducer. The combination of this gel-based capsule and compact ultrasound hardware can serve as a platform for triggering local release of compounds, including potentially in deep tissue, to achieve personalized and localized drug delivery. Illustrative results that show the ability to tailor release kinetics will be presented. [Work supported by NSF 1509748.]

\section{3:00-3:15 Break}

\section{$3: 15$}

5pBA8. Development of an ultrasound tomography system: Preliminary results. Pedram Mojabi and Joe LoVetri (Elec. and Comput. Eng., Univ. of MB, 75 Chancellor's Circle, Winnipeg, MB R3T5V6, Canada, pedram. mojabi@gmail.com)

We are currently developing an ultrasound tomography system at the University of Manitoba. This system consists of eight circular rings of transducers so as to provide the potential to create two- and three-dimensional images. Thirty-two individual piezo-electric transducers are mounted in each ring, and each transducer can both transmit and receive ultrasonic waves. We utilize a frequency-domain inverse scattering framework to invert the measured ultrasound data so as to create quantitative images of the ultrasonic properties of the object being imaged. To this end, the measured data obtained from this system are calibrated before being inverted. For the calibration step, we discuss methods to accurately find the transducers' positions, properly convert the measured time-domain data into the frequency domain, and then minimize the discrepancy between the actual system and the simulated one through the use of calibration coefficients. Once this process is done, the calibrated frequency-domain data is processed by a frequency-domain inverse scattering algorithm. We utilize the Born iterative method (BIM) to invert this calibrated measured data to create twodimensional images corresponding to ultrasonic properties of the object of interest [Mojabi \& LoVetri, JASA, 2015]. Preliminary results of this BIM inversion are then presented and discussed.

\section{3:30}

5pBA9. Proposal of ultrasonic imaging of thermophysical property distribution in vivo by ultrasonic heating. Mai Morimoto, Yukako Tsujimoto, and Iwaki Akiyama (Medical Ultrasound Res. Ctr., Doshisha Univ., 1-3 Tatara-Miyakodani, Kyotanabe 610-0394, Japan, dmp1018@mail4.doshisha.ac.jp)

This study proposes a novel ultrasonic imaging of sound velocity change distribution by a short time ultrasonic heating. According to the bio-heat transfer equation, the sound velocity change due to ultrasonic heating depends on the volumetric heat capacity, attenuation coefficient, and the temperature dependence of the sound velocity. Thus, the resultant image is correspondent to the thermophysical property distribution. The sound velocity change is estimated by the time difference of echoes from the tissue between before and after heating. The feasibility of the proposed technique was studied by using a block of porcine fat and muscle exposed to ultrasound. A concave circular transducer of $5.2 \mathrm{MHz}$ in resonance frequency was used for the measurement of sound velocity change. Ultrasound waves of $3.2 \mathrm{MHz}$ in frequency was transmitted from a coaxial arranged concave ring transducer for heating. As a result, the sound velocity change of the porcine muscle is $1.3 \mathrm{~m} / \mathrm{s}$ and the porcine fat is $-2.3 \mathrm{~m} / \mathrm{s}$. Therefore, it is possible to identify the region of porcine muscle and fat, respectively. [This study was supported by MEXT-Supported Program for the Strategic Research Foundation at Priviate Universities, 2013-2017.]

\section{$3: 45$}

5pBA10. Temperature measurement using backscattered ultrasonic power for non-invasive thermometry during HIFU ablation. David Melodelima and Victor Barrere (LabTAU - INSERM U1032, 151 cours Albert Thomas, Lyon 69003, France, David.Melodelima@inserm.fr)

The temperature dependence of ultrasonic tissue parameters has been reported extensively. In this work, the relationship between changes in ultrasound backscattered power and temperature during HIFU treatments was studied. An analytical model was developed based on attenuation, backscattered coefficient, velocity and thermal expansion to predict temperature changes. In vitro and in vivo tests were conducted in liver to confirm simulations. HIFU treatments were performed using a focused transducer working at $3 \mathrm{MHz}$. The radius of curvature and the diameter were both $70 \mathrm{~mm}$. An ultrasound imaging probe working at $7.5 \mathrm{MHz}$ was placed in the center of the HIFU transducer. Long exposure time (120 seconds) was used to observe smooth temperature increase from 37 to $70^{\circ} \mathrm{C}$. Simulations predicted that backscattered power increased nearly logarithmically with temperature over the range from $37^{\circ} \mathrm{C}$ to $70^{\circ} \mathrm{C}$. The model predicted a linear increase $10 \mathrm{~dB}$. A linear increase $8 \mathrm{~dB}$ was measured in ultrasound backscattered power during experiments. The tissue temperature increase estimated using backscattered energy correlated well $(\mathrm{r}=0.79)$ with temperature measurements performed using thermocouples. This linear relationship between changes in the backscattered energy and actual temperature was observed up to $70^{\circ} \mathrm{C}$. Successful temperature estimation may allowed creating 2D temperature maps during HIFU treatments.

\section{4:00}

5pBA11. Color Doppler shear wave imaging and its application to breast imaging. Yoshiki Yamakoshi (Graduate School of Sci. and Technol., Gunma Univ., 1-5-1 Tenjin, Kiryu 376-8515, Japan, yamakoshi@gunma-u. ac.jp), Takahito Nakajima (Graduate School of Medicine, Gunma Univ., Maebashi, Japan), Mayuko Yamazaki, Ren Koda, and Naoki Sunaguchi (Graduate School of Sci. and Technol., Gunma Univ., Kiryu, Japan)

Imaging of continuous shear wave (CSW) induced by a small mechanical vibrator attached on tissue surface is low-cost and easy-to-use elastography. A problem of CSW is shear wave's reflection and refraction at tissue boundary, which degrade the accuracy of shear wave velocity estimation. We propose a novel shear wave elastography of CSW (Color Doppler Shear Wave Imaging: CD-SWI). Shear wave's propagation in soft tissue is reconstructed directly without adding any function to conventional color flow imaging instrument. Two conditions for shear wave frequency and displacement amplitude are required to obtain maps. However, they are not severe restrictions because frequency is chosen among several frequencies suited for elastography and the required displacement amplitude is a few tens of microns. Both Fourier analysis along time axis and a directional filter on wave-number vector space improve image quality. Accuracy and image quality of CD-SWI are evaluated by comparing with ARFI method. CDSWI is applied to breast in vivo and shear wave propagation in breast $(\mathrm{f}=297 \mathrm{~Hz})$ is observed. Inherent shear wave propagation in soft tissue reconstructed by CD-SWI, such as diffraction and refraction around tissue with different stiffness as well as shear wave velocity, may give novel information in tissue characterization by shear wave. 
5pBA12. Dynamic contrast specific ultrasound tomography. Libertario Demi, Ruud van Sloun, Hessel Wijkstra, and Massimo Mischi (Elec. Eng., Eindhoven Univ. of Technol., Den Dolech 2, Eindhoven 5612 AZ, Netherlands,1.demi@tue.nl)

Cumulative phase delay imaging (CPDI) is a modality recently introduced for contrast-specific ultrasound tomography. CPDI has already shown capable of imaging ultrasound contrast agents (UCAs) when working at pressures $(0.05 \leq \mathrm{MI} \leq 0.2)$ and frequencies $(2.5-3 \mathrm{MHz})$ of interest for clinical applications. However, its ability of capturing UCA-kinetics was never shown. To this end, we imaged the passage of UCA-boluses through a dedicated gelatin flow-phantom. Each bolus resulted from a 5-mL injection with a $240-\mu \mathrm{L} / \mathrm{L}$ SonoVue ${ }^{\circledR}$ dilution. For imaging, the ULA-OP ultrasound research platform and an Esaote LA332 linear-array probe were employed. A frequency of $2.5 \mathrm{MHz}$ and mechanical index (MI) equal to 0.07 were used. CPDI and Harmonic Imaging (HI) were simultaneously applied to each bolus-passage (in tomographic and echo-mode, respectively) to compare the two methods. Features commonly used to quantify UCA-kinetics were evaluated and used for the comparison: full-width-half-maximum (FWHM), wash-in-time (WIT), arrival-time (AR), and peak-time (PT). The obtained median absolute differences were equal to $0.625 \mathrm{~s}, 0.25 \mathrm{~s}, 0.1875 \mathrm{~s}$, and $0.06 \mathrm{~s}$ for FWHM, AT, WIT, and PT, respectively. These results are encouraging, and open the way for the development of dynamic contrastspecific ultrasound tomography, possibly adding important features to multi-parametric ultrasound tomography of the breast, and improving breast cancer localization.

\section{4:30}

5pBA13. Null subtraction imaging: achieving super resolution by imaging with beam nulls. Jonathan Reeg and Michael L. Oelze (Dept. of Elec. and Comput. Eng., Univ. of Illinois at Urbana-Champaign, 405 N Mathews, Urbana, IL 61801, oelze@uiuc.edu)

Null subtraction imaging (NSI) uses multiple apodizations on receive to create images with low side lobes and super lateral resolution. The first apodization weight has a zero mean when summed across the aperture, placing a null in the beam at broadside. The second apodization simply adds a small DC offset to the first. The third apodization is the transpose of the second. An image created with the zero-mean apodization is then subtracted from the mean of images created with the second and third apodizations. Imaging tasks were performed using a 9L4 array connected to an Ultrasonix RP/SonixDAQ system on an ATS539 phantom containing wire, anechoic and hyperechoic targets. Images were constructed using NSI and compared with rectangular apodization. Image quality was assessed by estimating lateral resolution (-3-dB receive beamwidth), the mainlobe to sidelobe ratio (MSR) in $\mathrm{dB}$ and the contrast-to-noise ratio (CNR). NSI provided lateral resolution better than 25 times that of rectangular apodization. The MSR decreased by an average of $29 \mathrm{~dB}$ with NSI. However, because the speckle of NSI images become point like, the contrast of the contrast targets were reduced using NSI (e.g., CNR of hyperechoic targets was 1.35 and 0.36 for rectangular versus NSI).
5pBA14. Ultrasonic imaging of sum frequency components by crossed beam contrast echo method. Ayane Kihara, Kazumasa Kanai, and Iwaki Akiyama (Biomedical Information, Doshisha Univ., 1-3 Miyakodani, Tatara, Kyotanabe, Kyoto 610-0394, Japan,dmp1009@mail4.doshisha.ac. jp)

In a breast cancer diagnosis, the clinical usefulness of the contrast echo harmonic imaging has been reported. The authors proposed a contrast echo method by crossing the two ultrasonic beams to improve the accuracy. The microbubbles in the crossed region re-emit the secondary wave including the sum frequency components. It is capable of discriminating between the echo from microbubbles and the echo from tissue without microbubbles, since the sum frequency are not generated during the nonlinear propagation of primary wave outside the crossed region. Thus the contrast of the resultant image is improved. This study measured the sum frequency components in the echoes from the microbubbles of Sonazoid ${ }^{\circledR}$ in degassed water by using the ultrasonic imaging system with 64 channels. The ultrasonic wave of $5 \mathrm{MHz}$ was transmitted from the array probe of central frequency of 6 $\mathrm{MHz}$ equipped with the imaging system. Since the beam formed by the array probe crossed the other beam of $2 \mathrm{MHz}$ at the position $8 \mathrm{~cm}$ apart from the probe at 90 degrees, the value of sum frequency is the same as 7 MHz. $7 \mathrm{MHz}$ components in the echo from the microbubbles in the crossed region was observed as $20 \mathrm{~dB}$ increased.

\section{5:00}

5pBA15. 3-D ultrafast ultrasound strain imaging to improve breast cancer detection. Gijs A. Hendriks, Chuan Chen, Hendrik H. Hansen, and Chris L. de Korte (Radiology and Nuclear Medicine, Radboud Univ. Medical Ctr., MUSIC 766, PO Box 9101, Nijmegen 6500HB, Netherlands, chris. dekorte@radboudumc.nl)

The automated breast volume scanner (ABVS) is used for breast cancer detection and consists of a linear transducer translating over the breast while collecting ultrasound data to reconstruct a breast volume. Although the ABVS shows high sensitivity, specificity remains a limitation resulting in high recall-rates. To improve ABVS specificity, we verified if it is feasible to implement 3-D strain imaging to discriminate between malignant (firmlybound and stiff) and benign lesions (loosely bound and less stiff) based on lesion-to-surrounding-tissue connectivity (shear strain) and on stiffness (axial strain). Three phantoms containing loosely- (1) and firmly-bound (2) three times stiffer lesions were scanned twice by the ABVS, pre- and postdeformation $(0.5 \mathrm{~mm})$. Data was collected by plane-wave imaging to reduce acquisition times below one breath-hold. Displacements were calculated by cross-correlation from which axial and octahedral-shear strains were derived. Shear strains were distributed tightly and globally around the lesion for phantom (1) and (2), respectively. Using axial strain, the lesions could be clearly differentiated from the surrounding tissue in both phantoms $\left(\mathrm{CNR}_{\mathrm{e}} 30 ; \mathrm{SNR}_{\mathrm{e}} 19 \mathrm{~dB}\right)$. Therefore, we can conclude that it is feasible to implement 3-D strain imaging in the ABVS, and to improve its specificity by discriminating lesions by elastic properties. 


\title{
Session 5pEA
}

\section{Engineering Acoustics: Applied Acoustics II}

\author{
Michael S. McBeth, Cochair \\ SSC Atlantic, SSC Atlantic/NASA Langley Research Center, 11 West Taylor Street, Mail Stop 207, Hampton, VA 23681 \\ Hikaru Aono, Cochair \\ Mechanical Engineering, Tokyo University of Science, 6-3-1 Niijyuku, Katsushika-ku, Tokyo 125-8585, Japan
}

\section{Contributed Papers}

\section{1:15}

5pEA1. Acoustic field measurement of high-intensity ultrasound source system using acoustic waveguide for calibration of hydrophone. Shigeru Igarashi (Polytechnic Univ., 2-32-1 Ogawa-nishimachi, Kodaira-shi, Tokyo 187-0035, Japan, s-igarashi@uitec.ac.jp), Takeshi Morishita, and Shinichi Takeuchi (Toin Univ. of Yokohama, Yokohama, Kanagawa, Japan)

In recent years, high-intensity ultrasound equipments have been used. It is expected that nonlinear calibration and evaluation will be needed more often. It is necessary to develop a high-intensity ultrasound source that transmit a plane wave in far-field for calibration of hydrophone in order to evaluate high intensity acoustic field. We proposed an ultrasound source system using a cylindrical type acoustic waveguide and a concave type piezoelectric transducer. The focused ultrasound wave is emitted from the concave type transducer, propagated through the acoustic waveguide, and the output from that forms high-intensity acoustic beam. We simulated the output acoustic pressure of the proposed source system using axisymmetric 3D acoustic field simulation based finite element method, compared with that of a flat disk type transducer with the same aperture size as the acoustic waveguide, and the simulated data showed the acoustic pressure of our proposed system was higher than that of the flat disk type transducer with the same main beam width. We fabricated the experimental source system using the concave type piezoelectric transducer and the acoustic waveguide using closed cell sponge, and measured acoustic pressure distribution. The obtained results were nearly coincident with the simulated data

\section{$1: 30$}

5pEA2. Effects of wing flexibility on sound characteristics of a fourwing flapping wing micro air vehicle. Hikaru Aono (Mech. Eng., Tokyo Univ. of Sci., 6-3-1 Niijyuku, Katsushika-ku, Tokyo 125-8585, Japan, aono@rs.tus.ac.jp), Yuta Ozawa (Graduate School of Eng., Tokyo Univ. of Sci., Tokyo, Japan), Makoto Yamamoto, Hitoshi Ishikawa (Mech. Eng., Tokyo Univ. of Sci., Tokyo, Japan), Chang-kwon Kang (Mech. and Aerosp. Eng., Univ. of Alabama, Huntsville, AL), Taku Nonomura (Inst. of Space and Astronautical Sci., Japan Aerosp. Exploration Agency, Sagamihara, Japan), and Hao Liu (Graduate School of Eng., Chiba Univ., Chiba, Japan)

The objective of this study was to understand the sound generation of flapping wings. In particular, the structural properties of the wings were varied to assess the effects of wing flexibility on the sound generation of flapping wings. A four-wing, hummingbird-inspired flapping wing micro air vehicle (FMAV) was considered. The half wing span was approximately six centimeters and the flapping frequency was approximately $25 \mathrm{~Hz}$. The sound produced by the FMAV was measured using a $1 / 2$ inches diameter microphone in an acoustic chamber under the quiescent flow condition. Time-averaged aerodynamic forces were measured by the force and torque sensor Nano17 (ATI Industrial Automation). Results revealed the characteristics of flapping-wing sound in terms of directivity, frequency response, and attenuation. Moreover, the degree of wing deformation led to a variation in the frequency response corresponding to the maximum sound pressure level as well as time-averaged aerodynamic force, even when the flapping frequency was held constant in all the cases. All outcomes of this study would be helpful to control the sound generation of FMAVs and to develop new, silent FMAVs.

\section{1:45}

5pEA3. Battery outage and portable video recorders. Al Yonovitz (The Univ. of Montana, Dept. of Communicative Sci. and Disord., Missoula, MT 59812, al.yonovitz@umontana.edu), Joshua Yonovitz, Herbert Joe (Yonovitz \& Joe, LLP, Dallas, Texas), and Brittany Galvin (The Univ. of Montana, Missoula, MT)

The advent of user-friendly compact video recorders with large storage capacities has made them ideal for recording investigations and interviews by law enforcement agencies. In addition, they are also useful for surreptitious recordings both by citizens and government services. When these recorders are powered by an internal battery, the battery recording may be interrupted when the battery discharges. The analysis of this premature stoppage of the recorded video file may be important in the determination of the authenticity of the recording for courtroom admissibility. The differentiation between a purposeful edit and the normal shutdown process was investigated with a series of such recordings using a variety of modern recorders and common smartphones. Recordings were made with the battery at 80 percent charge and allowed to deplete. The recorder was then immediately restarted as well as restarted while being immediately attached to permanent power. The resultant recordings were compared and analyzed for any extraneous information related to the shutdown process.

\section{2:00}

5pEA4. Detection of low intensity sound with laser Doppler anemometry. Michael S. McBeth (Space and Naval Warfare Systems Ctr. Atlantic, SSC Atlantic/NASA Langley Res. Ctr., 11 West Taylor St., M.S. 207, Hampton, VA 23681, m.s.mcbeth@ieee.org) and Robert Younts (Organic and Carbon Electronics Labs and Dept. of Phys., North Carolina State Univ., Raleigh, NC)

Laser Doppler anemometry (LDA) sound measurement systems have traditionally been designed to achieve point measurements of a sound field so they keep the laser path lengths through the water short to minimize the signal contribution from the acousto-optic effect and use relatively large beam separation angles to minimize the interference fringe volume. While the traditional LDA approaches provide reasonable sensitivity to the Doppler shifts from microparticles oscillating in a low frequency sound field, at high frequencies the sound measurement sensitivity depends on the optical path length difference (OPD). OPD increases due to the acousto-optic effect as the sound pressure is integrated over the entire path length. An LDA system using a small beam separation angle and long laser light paths through the water is shown to be capable of detecting low intensity sound pressure signals. Theoretical and experimental results are presented. 
5pEA5. Optical displacement detection using multi-level diffraction gratings having no zeroth-order reflection. Randall P. Williams, Samuel K. Hord, and Neal A. Hall (Dept. of Elec. and Comput. Eng., The Univ. of Texas at Austin, 10100 Burnet Rd., Bldg. 160, Rm. 1.108, Austin, TX 78758,randy.williams@utexas.edu)

Displacement detection using optical interferometric techniques allows for low minimum detectable displacements which are unmatched by other displacement measurement methods. However, diffraction gratings commonly used in such applications can generate zeroth-order reflected beams which result in reduced sensor performance, packaging limitations, and laser instability. A new grating concept has been fabricated and tested which has the effect of eliminating the zeroth-order component by imparting a halfwavelength phase shift to a portion of the reflected light. The design criteria for zeroth-order beam elimination is illustrated using a simple model based on phasor arithmetic. Fabrication methods for producing these optical gratings, as well as comparisons between experimental test results and models, will be reported.

\section{2:30}

5pEA6. Sound source positioning using microphone array installed on a flying drone. Seongho Oh, Yeong-Ju Go, Jaehyung Lee, and Jong-Soo Choi (Aerosp. Eng., Chungnam National Univ., 99, Daehakro, Yuseong-gu, Daejeon 305-764, South Korea, osh09072@cnu.ac.kr)

This study presents an application of acoustic source localization using microphone array installed on a drone. Although recent technology of drone enables to record audio and video, there are still acoustic problems such as engine noise, propeller-induced flow noise, microphone directivity, etc. Such unwanted noise shows strong tonal characteristics with broad band noise contents. To acquire usable acoustic signal from array measurements while a drone is flying, we implemented acoustic signal conditioning and array processing. The approach to the problem is to adapt beamforming and TDOA techniques that provide localization capability of multiple sources and the position or direction of arrival of source, respectively. The array configuration and angle resolution are adjusted depending on the application requirements. We limit our attention to stable hovering condition to rule out position and heading error occurred by the drone motion. The experimental results of position estimation under a various noise conditions are discussed in the study.

\section{2:45}

5pEA7. Experimental evaluation of noise damping performance of induct perforated orifices with different geometric shapes. Dan Zhao (Aerosp. Eng. Div., Nanyang Technolog. Univ., Singapore, Singapore, Singapore), Chenzhen Ji, Nuomin Han (Aerosp. Eng. Div., Nanyang Technolog. Univ., School of Mech. and Aerosp. Eng., Singapore, Singapore, czji1000@gmail.com), and He Zhao (School of Energy and Power Eng., Jiangsu Univ. of Sci. and Technol., Zhenjiang, China)

In this work, 10 in-duct perforated plates are experimentally tested in a cold-flow pipe. These plates have the same porosities but different number $\mathrm{N}$ and geometric shaped orifices: 1) triangle, 2) square, 3) pentagon, 4) hexagon, and 5) star. The damping effect of these orifices is characterized by power absorption coefficient $\Delta$ and reflection one $\mathrm{R}$ from 100 to $1000 \mathrm{~Hz}$. It is found that the orifice shape has little influence on $\Delta$ and $\mathrm{R}$ at lower frequency $\left(\omega_{\mathrm{a}} / 2 \pi \leq 700 \mathrm{~Hz}\right)$. However, as $\omega_{\mathrm{a}}$ is increased, star-shaped orifice is shown to be with much lower $\Delta$ in comparison with that of other shaped orifices. For the in-duct plates with the same shaped orifices, increasing $\mathrm{N}$ does not lead to an increase of $\Delta$ at lower frequency $\left(\omega_{\mathrm{a}} / 2 \pi \leq 900 \mathrm{~Hz}\right)$. However, the orifice with the same shape and porosity but larger $\mathrm{N}$ is found to be associated with $20 \%$ more power absorption at approximately $970 \mathrm{~Hz} . \Delta$ max is approximately $85 \%$ around $190 \mathrm{~Hz}$, as $\mathrm{Ma} \approx 0.29$. The optimum Ma depends on the orifice shape. The present parametric measurements shed light on the roles of the number and geometric shapes of orifices and the flow parameters on its noise damping performance.
5pEA8. Omni-binaural recording system for $360^{\circ}$ video. Takanori Nishino (Graduate School of Eng., Mie Univ., 1577 Kurimamachiya-cho, Tsu, Mie 5148507, Japan, nishino@pa.info.mie-u.ac.jp) and Kenta Niwa (Media Intelligence Labs., NTT, Musashino, Tokyo, Japan)

A binaural recording system for $360^{\circ}$ video is developed and evaluated. The $360^{\circ}$ images can be stitched by using multiple cameras. A binaural recording generally uses a dummy head microphone or binaural recording microphones; however, the orientation of the dummy head needs to be changed depending on the selected view. Binaural recording corresponding to multiple orientations of a dummy head is very difficult and sometimes impossible. Therefore, we propose a binaural recording system that can simultaneously record the sound for the entire corresponding $360^{\circ}$ video. The proposed equipment is cylindrical in shape and has four hollows that correspond to the cavity of the concha on the side. Binaural signals corresponding to a desired direction are generated by a weighted average of the recorded signals. Three types of weights, i.e., linear, spline, and optimum weights, were examined. The degree of suitability between the video and generated binaural signals was evaluated using subjective tests. Nine subjects answered that all the weight conditions were acceptable and the proposed system was very interesting and entertaining.

\section{3:15-3:30 Break}

\section{$3: 30$}

5pEA9. Acoustical design of the ELA4 launch pad for the Ariane 6 launcher. Hadrien Lambaré (CNES, CNES Direction des lanceurs 52 rue Jacques Hillairet, Paris 75612, France, hadrien.lambare@cnes.fr)

A new launch pad, ELA4, is going to be built in Kourou (French Guyana) for the future launcher Ariane 6. As the A64 version of the launcher will be more powerful at lift-off than Ariane 5, the exhaust ducts and the water injection systems have to be carefully designed, in order to be as effective as possible, and to reduce the acoustics on the payloads and the launcher. This has been done using the experience acquired and Ariane 5 and VEGA launch pads, as well as the understanding of the complex phenomena obtained by research activities. The general design have been validated and optimized by subscale test campaigns at the MARTEL test bench. Numerous configurations and parameters have been tested, including the geometry of the ducts and the launch table, the altitude of the launcher, the position and the mass flow rate and the spray type of the water injection systems. [These works have been done in collaboration between ESA, CNES, ASL, ONERA and PPRIME.]

\section{$3: 45$}

5pEA10. Head mounted device for gesture recognition using acoustic time of flight sensor and 2D camera. Minchul Shin, Stephen Zeigler, Anton Gomez, and Christopher Reid (Mech. Eng., Georgia Southern Univ., Po Box 8045, Statesboro, GA 30465, mshin@georgiasouthern.edu)

The goal of this work is to design a 3D imaging system with acoustical transducers, 2D cameras and real-time signal processing to enable gesture sensing for a Head Mounted Device (HMD) design. The system offers enhanced user interfaces using vivid spatial information for use on mobile platforms such as wearable electronics. Through an intuitive user interface, hand gestures and finger shapes can provide easy acquisition of instruction as a sign language between devices and users. To achieve this intuitive gesture-based communication, key characteristics are required from imaging systems: light insensitivity, and high spatial density (high depth rate and $x-y$ plane resolution). For gesture recognition, a variety of sensing methods using infrared, laser, and acoustics have been developed, but they have a critical issue: light disturbance. This device overcomes sunlight interference that limits 3D camera performance outdoors. Multiple microphones with resonant frequency $40 \mathrm{kHz}$ are used as a depth sensor for 3D rendering or localization by using the TOF method. Eventually, motion tracking and localization via this $3 \mathrm{D}$ imaging system integrated with acoustic TOF sensor and RGB cameras allows communication using the dynamic and agile movements of users' hands and fingers and will serve as a major step toward expanding human-computer interactions. 
individual vibration when using the vibration source with diagonal slits. In order to solve this problem, we have developed an ultrasonic longitudinaltorsional vibration source consisting of two transducers in which the longitudinal-torsional vibration can be controlled. The developed vibration source is consists of a longitudinal transducer and a torsional transducer attached to the both ends of a stepped horn with the length of two wavelength of the propagating vibration. This vibration source can generate planar vibration locus of substantially square. In this study, ultrasonic metal welding of dissimilar metals was studied by using the vibration source consisting of two transducers. As a result, high weld strength was obtained in the case of welding of the planar locus than welding of only longitudinal or torsional vibration loci.

\title{
Session 5pID
}

\section{Interdisciplinary: Topical Meeting on Data Science and Acoustics II}

\author{
Matthew G. Blevins, Cochair \\ U.S. Army Engineer Research and Development Center, 2902 Newmark Drive, Champaign, IL 61822 \\ Andrew Christian, Cochair \\ National Institute of Aerospace, 100 Exploration Way, Hampton, VA 23666 \\ Hiroshi Sato, Cochair \\ Dept. of Information Technology and Human Factors, Natl. Inst. of Advanced Industrial Sci. and Tech., Tsukuba, Japan
}

\section{Invited Papers}

\section{1:00}

5pID1. Deep neural networks for learning classification features and generative models from synthetic aperture sonar big data. Johnny L. Chen (Appl. Res. in Acoust. LLC (ARiA), Culpeper, VA) and Jason E. Summers (Appl. Res. in Acoust. LLC (ARiA), 1222 4th St. SW, Washington, DC 20024-2302, jason.e.summers@ariacoustics.com)

Autonomous synthetic aperture sonar (SAS) imaging by unmanned underwater vehicles (UUVs) provides an abundance of high-resolution acoustic imagery useful for studying the seafloor and identifying targets of interest (e.g., unexploded ordnance or mines). Unaided manual processing is cumbersome as the amount of data gathered by UUVs can be enormous. Computer-vision and machinelearning techniques have helped to automate classification and object-recognition tasks, but often rely on hand-built features that fail to generalize. Deep-learning algorithms facilitated by emergence of graphics-processing unit (GPU) hardware and highly optimized neural-network implementations have recently enabled great improvements in computer vision. Autoencoders allow for deep unsupervised learning of features based on a reconstruction objective. Here, we present unsupervised feature learning applied to seafloor classification of SAS images. Deep architectures are also capable generative models. We illustrate this with generative networks that are capable of generating realistic SAS images of different seafloor bottom types. Deep models allow us to construct algorithms that learn hierarchical and higher-order SAS features, which promise to improve automatic target recognition (ATR) and aid operators in processing the large data volumes generated by UUV based SAS imaging. [Work supported by the Office of Naval Research.]

$1: 20$

5pID2. Acoustic and seismic time series analysis using ensemble empirical mode decomposition. Gopu R. Potty and James H. Miller (Dept. of Ocean Eng., Univ. of Rhode Island, 115 Middleton Bldg., Narragansett, RI 02882, potty@egr.uri.edu)

Empirical mode decomposition (EMD) is an adaptive or data-driven time series analysis technique ideally suited to investigate non-stationary signals. EMD decomposes the signal into fast and slow oscillations called intrinsic mode functions (IMFs). Ensemble EMD (EEMD) methods have been developed to alleviate the mode-mixing phenomenon present in the EMD technique. Various real signals will be analyzed using these methods to investigate whether the IMFs correlate to acoustic modes. These real signals include underwater acoustic signals from broadband sources, Scholte and Rayleigh wave signals and music signals. These underwater acoustic signals and the seismic signals exhibit multi-modal structure. In addition these modes are also dispersive in nature. Accurate resolution of these modes in the time-frequency plane is critical for the estimation of medium properties via inverse schemes. Finally, the EEMD technique will be used to 
investigate the complex dynamic structure of the pitching structure in South Indian Classical music. In all these cases investigated the mapping of the signal into the time-frequency plane reveal distinct features representing the modal dispersion and pitching structure, respectively. EEMD will be used to extract these features accurately with high resolution. [Work supported by Office of Naval Research.]

\section{$1: 40$}

5pID3. Deep learning for unsupervised feature extraction in audio signals: Monaural source separation. Edward T. Nykaza (ERDC, 2902 Newmark Dr., Champaign, IL 61822, edward.t.nykaza@erdc.dren.mil), Arnold P. Boedihardjo (ERDC, Alexandria, Virginia), Zhiguang Wang, Tim Oates (Comput. Sci. and Elec. Eng., UMBC, Baltimore, MD), Anton Netchaev, Steven L. Bunkley (ERDC, Vicksburg, MS), and Matthew G. Blevins (ERDC, Champaign, IL)

Deep learning is becoming ubiquitous; it is the underlying and driving force behind many heavily embedded technologies in society (e.g., search engines, fraud detection warning systems, and social-media facial recognition algorithms). Over the past few years there has been a steady increase in the number of audio related applications of deep learning. Recently, Nykaza et al. presented a pedagogical approach to understanding how the hidden layers recreate, separate, and classify environmental noise signals. That work presented some feature extraction examples using simple pure tone, chord, and environmental noise datasets. In this paper, we build upon this recent analysis and expand the datasets to include more realistic representations of those datasets with the inclusion of noise and overlapping signals. Additionally, we consider other related architectures (e.g., variant-autoencoders, recurrent neural networks, and fixing hidden nodes/layers), explore their advantages/drawbacks, and provide insights on each technique.

\section{2:00}

5pID4. Comparison between multilevel and traditional logistic regression analysis of laboratory indoor annoyance caused by shaped sonic booms. Jonathan Rathsam (NASA Langley Res. Ctr., Hampton, VA), Noah H. Silbert (Commun. Sci. and Disord., Univ. of Cincinnati, French East 344, 3202 Eden Ave., Cincinnati, OH 45267, silbernh@ucmail.uc.edu), Kathryn Ballard (NASA Langley Res. Ctr., Hampton, VA), and Jasme Lee (Dept. of Statistics and Biostatistics, Rutgers Univ., Piscataway, NJ)

In the 1970s commercial supersonic flight was forbidden over communities because conventional N-wave sonic booms caused excessive annoyance. Through recent advances in aircraft design techniques, conventional sonic booms are being modified into quieter, shaped sonic booms. As noise regulators consider allowing shaped booms over communities, NASA is providing data and expertise. Recently, a laboratory study quantified the additional annoyance caused by directly felt structural vibrations (J. Rathsam and J. Klos, "Vibration penalty estimates for indoor annoyance caused by sonic boom," J. Acoust. Soc. Am. 139 (4), 2007 (2016)). To confirm experimental results and complement the traditional logistic regression analysis, a multilevel logistic regression model was fit to individuallevel data. The multilevel analysis allows us to simultaneously model individual- and group-level factors. As a result, it is straightforward to test for relationships between annoyance and individual-level factors such as noise sensitivity.

\section{2:20-2:40 Break}

\section{2:40}

5pID5. Anomaly detection and other practical considerations for estimating acoustical metrics from time series data. Daniel J. Mennitt (Elec. and Comput. Eng., Colorado State Univ., 1201 Oakridge Dr., Ste. 100, Fort Collins, CO 80525, daniel_mennitt@partner. nps.gov) and Kurt Fristrup (Natural Sounds and Night Skies Div., National Park Service, Fort Collins, CO)

The scope of environmental acoustic monitoring and rate of data collection are growing rapidly. These increases in the quantity of information have elevated the necessity of detecting anomalous data and the difficulty of doing so. Analysis of contaminated data leads to incorrect results, including biased parameter estimation and flawed model selection. Censoring of data requires strong justification, as the loss of information can lead to these same problems. For acoustics, this issue is compounded by the widespread use of time-average sound levels $\left(\mathrm{L}_{\mathrm{eq}}\right)$, which are especially sensitive to anomalous measurements. This talk will discuss applications of anomaly detection to acoustic pressure time series data for the calculation of long-term metrics. There is no single universally applicable or generic approach to data cleaning and preparation, which often consumes a disproportionate amount of time and effort relative to interpretation of results. Examples drawn from terrestrial and aquatic acoustical monitoring data sets of varying resolution and associated metadata will provide context for issues that compromise data quality, anomaly detection methods, and mitigation efforts.

\section{3:00}

5pID6. The effect of sample rate reduction and audio compression on noise metric accuracy and statistical learning classifier performance. Matthew G. Blevins, Edward T. Nykaza (U.S. Army Engineer Res. and Development Ctr., 2902 Newmark Dr., Champaign, IL 61822, matthew.g.blevins@usace.army.mil), and Anton Netchaev (U.S. Army Engineer Res. and Development Ctr., Vicksburg, MS)

With the increasing availability of low-cost and portable noise monitors, the amount of captured data can reasonably be predicted to grow exponentially. The financial and computational expense of portable data storage, processing hardware, and wireless or cellular upload bandwidth, however, necessitate data compression techniques to ensure feasible continuous operation. Sample rate reduction causes a loss of high frequency components while compressed formats (such as mp3) remove parts of the signal that are inaudible to humans, but may be important to statistical learning algorithms. The degree to which these data reduction techniques degrade the accuracy of metrics calculated from the signals (e.g. peak level, equivalent level, sound exposure level, etc.) and the performance of statistical learning classifiers that use the metrics and/or band levels is examined. Over 20,000 recordings from noise monitors located on military installations are systematically down-sampled and compressed to use in the analysis. The root-mean-square error for each of over 40 noise metrics and the mean decrease in accuracy for a statistical learning classifier are presented.

\section{Contributed Papers}


classified into clusters located apart while segments with multiple bird calls and/or noise will lie down on the clusters in the middle. The classified segments are then labelled by measuring the distance between the feature vec-

5pID7. Facilitation of bird call classification using self-organising mapsA pilot study on bird calls in New Zealand. Tsutomu Miyoshi (Dept. of Media Informatics, Faculty of Sci. and Eng., Ryukoku Univ., Yokotani 1-5, Seta Oe-cho, Otsu-shi, Shiga 520-2194, Japan, mijosxi@rins.ryukoku.ac.jp) and Yusuke Hioka (Mech. Eng., Univ. of Auckland, Auckland, New Zealand)

Automatic detection and classification of bird calls from audio recordings attract interests of various users from amateur bird watching hobbyists to professional biologists and park rangers studying biodiversity and conservation. Previous studies utilize machine learning techniques many of which require supervised learning; therefore, they cannot detect bird calls that are not included in the supervisory data. Their performance also severely degrade when a segment of a bird call is overlapped by other birds' calls or contaminated with various ambient noise. This study proposes an alternative approach for bird call classification, which is facilitated by an algorithm based on the self-organising maps (SOM). Since the SOM is an unsupervised learning algorithm, which classifies similar input features into neighboring clusters, it is expected that segments of different bird species will be tors in each cluster and that of known bird call segments given a priori. The performance of the proposed method is evaluated by applying the algorithm to data recorded in a national park in New Zealand.

$$
\text { 3:35 }
$$

5pID8. Noise injection for acoustics fault sample expansion. linke zhang (School of Energy and Power Eng., Wuhan Univ. of Technol., 1178 Heping Ave., Wuhan, Hubei 430063, China, lincol_zhang@126.com) and NA Wei (Inst. of Acoust., Nanjing Univ., Nanjing, China)

Sample expansion is an effective approach to resolve the incomplete samples problem in acoustics fault source identification. Noise injection is applied to acoustics fault sample expansion by mixing noise signals with different signal-to-noise ratio in real samples to obtain expansion samples, which enhances training data. Experimental results demonstrate the effectiveness of this proposed approach.

3:50-4:30 Panel Discussion

FRIDAY AFTERNOON, 2 DECEMBER 2016

CORAL 3, 1:00 P.M. TO 4:00 P.M.

\title{
Session 5pMU
}

\section{Musical Acoustics: General Topics in Musical Acoustics II (Poster Session)}

\author{
Andrew C. Morrison, Chair \\ Natural Science Department, Joliet Junior College, 1215 Houbolt Rd., Joliet, IL 60431
}

\begin{abstract}
All posters will be on display from 1:00 p.m. to 4:00 p.m. To allow contributors in this session to see the other posters, authors of oddnumbered papers will be at their posters from 1:00 p.m. to 2:30 p.m. and authors of even-numbered papers will be at their posters from 2:30 p.m. to 4:00 p.m.
\end{abstract}

\section{Contributed Papers}

5pMU1. Real-time gaze-tracking performance under synchronous and asynchronous auditory feedback conditions. Andrew Lucila (Psych., William Paterson Univ., 780 Allwood Rd., Clifton, NJ 07012, Drewlucila@ gmail.com), Franklin Roque (Psych., William Paterson Univ., Paramus, NJ), Michael Morgan, and Michael S. Gordon (Psych., William Paterson Univ., Wayne, NJ)

Visual attention and manual coordination were tested in pianists and other instrumentalists tasked with reading baroque music. Musicians were assessed in their ability to perform a single melodic line relative to a two-clef, three voice score with bimanual coordination (an extension from past research). In this study, musicians were asked to read and perform music under three auditory feedback conditions: synchronous, delayed (one-beat behind), and prelayed (one-beat ahead). Real-time gaze-tracking analysis was conducted, in addition to measures of performance, under each auditory condition. Results from these analyses indicated several trends. Gaze-tracking measures were taken from visual fixations and regressions during performance. These measures indicated that musicians had more difficulty in the asynchronous auditory feedback conditions relative to the synchronous condition. Particularly the prelay auditory feedback condition tended to cause an increase in the number of visual fixations per measure and required more effortful gaze. This effect was found to be consistent across different instrumental performances. In addition, indications of cognitive effort increased in the bimanual conditions. These results indicate the challenge musicians face when reading music in conditions of higher cognitive and manual load.
5pMU2. A study on acoustic feature representing breathiness of singing voice based on vocal-fold vibration modeling. Masahiro Itou, Hideki Banno, and Kensaku Asahi (Graduate School of Sci. and Technol., Meijo Univ., 1-501 Shiogamaguchi, Tempaku-ku, Nagoya-shi, Aichi-ken 4680073, Japan,153430003@ccalumni.meijo-u.ac.jp)

There are some techniques expressing emotion in singing. One of the techniques used by some professional singers is controlling breathiness of uttered voice intentionally. However, this breathiness control can make it difficult for an amateurish singer to keep pitch and pressure of uttered voice steady. In this publication, we verify what kind of feature is appropriate for representing breathiness of singing voice by converting the feature of normal voice into that of breathy voice. Extracted features for conversion are aperiodicity index and STRAIGHT spectrum ("voice quality parameters" below) obtained through STRAIGHT analysis of recorded vowel singing whose pitch, pressure and breathiness are constant and under several conditions. We have tested two conversion methods; one compensates voice quality parameters of normal voice statically so that the averaged parameters of generated voice are equivalent to those of high-breathiness voice, another exchanges voice quality parameters of normal voice totally with those of high-breathiness voice. It was found that the generated sound by the latter method is perceived to have higher quality than that by the former method. This result implies that features to represent breathiness properly must include not only static information but also dynamic information of breathy voice. 
5pMU3. An application of multiple-stage non-negative matrix factorization to music analysis. Kenko Ota, Takuya Ichigo, and Takahiro Shirasawa (Nippon Inst. of Technol., 4-1 Gakuendai, Miyashiro, Minami-saitama 3458501, Japan, otakenko@nit.ac.jp)

Non-negative matrix factorization (NMF) is an analysis technique for matrix with non-negative elements. NMF decomposes a sound spectrogram $\mathrm{X}$ obtained by a short-time Fourier transform into the product of two nonnegative matrices $\mathrm{H}$ and $\mathrm{U}$. The matrix $\mathrm{H}$ represents the bases of $\mathrm{X}$ and the matrix $U$ represents the activation gains of $\mathrm{H}$. As an extension of NMF, multiple-stage NMF has been proposed in order to represent the hierarchy of data. This research focuses on the hierarchy of music data, and attempts to apply multiple-stage NMF to music analysis. For simplicity, sounds treated in this research are assumed as follows: single tone consists of fundamental tone and harmonic overtones and a chord consists of some single tones. According to this assumption, observed sound spectrogram can be decomposed into three stages. In the first stage, the basis matrix is approximated by a Gaussian distribution in order to represent a spectrum of fundamental tone and harmonic overtones. In the second stage, the basis matrix consists of the set of single tone with fundamental tone and harmonic overtones. And in the third stage, the basis matrix consists of the set of chord with some single tones. Evaluation was carried out by MIDI data.

5pMU4. An acoustic analysis of Arirang, a representative folk song of Korea. Sang-Bum Park (Dept. of Information and Technol. Eng., Soongsil Univ., Seoul, South Korea), Myung-Sook Kim (Dept. of English, Soongsil Univ., Sando-ro 369, Dongjak-gu, Seoul, Korea, Seoul 06978, South Korea, kimm@ssu.ac.kr), and Myung-Jin Bae (Dept. of Information and Technol. Eng., Soongsil Univ., Seoul, South Korea)

Arirang, a representative folk song of Korea, became inscribed on the Representative List of the Intangible Cultural Heritage of Humanity program by UNESCO in 2012. Since then, the Korean government as well as some Korean private sector companies have been promoting the song and its unique feelings in melody and lyrics drawn from Korea's own historical backgrounds. This study presents an acoustic analysis of Arirang from a psychoacoustic point of view, first concerning its rhythm/tempo and second concerning its acoustic characteristics found in certain vowels and consonants in lyrics. Since Arirang has so many versions according to different regional origins in Korea, Arirang can produce contradictory feelings to its listeners depending on its version as well as its tempo. In general, when played slowly, the listeners may feel sad and compassionate, but when played fast the listeners may feel cheerful and even excited. As far as its lyrics are concerned, certain vowels and consonants evoke special feelings: first, the vowel /a/, which appears so many times in the song as in its title, has higher fundamental frequency in Milyang Arirang $(200 \mathrm{~Hz})$ than in Jindo Arirang $(400 \mathrm{~Hz})$, evoking a variety of emotions; second, the consonant $/ \mathrm{r} /$, as in its title, covers a wide range in amplitude with many variable frequency bandwidths than other consonants and thus produces a cheerful feeing.

5pMU5. Recognizing musical melodies: Effects of audio and visual representations of emotion. Alejandro L. Ataucusi (Psych., William Paterson Univ., 909 Florida Grove Rd., Keasbey, NJ 08832, ataucusia@student. wpunj.edu) and Michael S. Gordon (Psych., William Paterson Univ., Wayne, NJ)

Memory for music was tested with a set of emotionally valenced melodies encoded with and without an accompanying visual scene. The music consisted of original melodies that were normed for their emotional valence. During the training phase, participants were presented a set of happy, neutral, and sad musical melodies. Half of the melodies were presented with an affective, visual stimulus, depicting a scene consistent with emotional expression of the melody. After a distraction period, participants were tested in an audio-only recognition task. The recognition task included a mix of melodies from the training phase (with equal representation of audiovisual and audio conditions and each of the affective conditions) and emotionallymatched novel solo guitar melodies. Using signal detection analyses participants were found to be more sensitive to stimuli for which they had been trained in the Auditory-only conditions than in the Audiovisual conditions. Sensitivity also tended to be highest with the neutral affective melodies and lowest with the sad melodies. These findings show the potential for distraction with visual imagery to musical memory formation-despite the consistency of that emotional content to the melody. Moreover, less emotionally valenced music tended to be more effectively encoded.

5pMU6. Acoustic analysis on vocal voice of Lee Nan-Young, a legendary singer of Korea. Myungsook Kim (English, Soongsil Univ., Sangdo-ro 369, Seoul 06978, South Korea, kimm@ssu.ac.kr) and Myung-Jin Bae (Information and Technol. Eng., Soongsil Univ., Seoul, South Korea)

Lee Nan-Young, a legendary female popular singer of Korea, made her debut in 1934 and enjoyed a tremendous popularity among Korean people in the 1930-40s. She was a special comfort to Korean people who were suffering from colonial exploitations by the Japanese government during that time. To celebrate her 100th birthday, we analyzed her vocal recordings in order to find its unique characteristics that set her apart. Findings can be summarized as follows: first, her voice has harmonics found around 4500 $\mathrm{Hz}$ and even higher, which is three times higher than average; second, she changes tones from $250 \mathrm{~Hz}$ to $1500 \mathrm{~Hz}$ (2.5 octaves), producing an attractive plaintive voice; third, she gives many short cut-offs in low tones but produces rich resonant voice in high tones, practicing a variety of singing methods; fourth, she has a command of producing all-frequency-ranged voice including low, middle, and high frequency and makes rich vibrations resulting in deep and touching voice; last, her voice naturally and smoothly links with a variety of musical instruments accompanying her songs. Based on these findings, we conclude that she deserves to be recognized as one of the best popular singers who have ever performed.

5pMU7. Study on specific musical instrument sound extraction by clustering of nonnegative matrix factorization bases with linear predictive coding mel-cepstrum. Saki Umeda, Hideki Banno, and Kensaku Asahi (Meijo Univ., TenpakukuShiogamaguchi1-501, Nagoya 468-8502, Japan, 163430005@ccalumni.meijo-u.ac.jp)

Nonnegative matrix factorization (NMF) is one of a technique for audio source separation. It separates input audio signal into a set of basis spectra and a set of intensity trajectories of the basis spectra. We utilize this technique to extract particular musical instrument sound from musical audio signal. However, the resultant separation by NMF is known to be unrelated to instrumentation of the audio signal. Therefore, clustering technique by using a spectral distance measure is applied to the basis spectra so that the clustered signal corresponds to the audio signal of particular musical instrument. In this study, we investigate the optimal number of bases and the optimal distance measure for clustering. Experiments using audio signals that include two musical instrument sounds and employing mel-cepstrum, linear predictive coding (LPC) cepstrum and LPC mel-cepstrum as a distance measure, showed that LPC mel-cepstrum tends to classify more properly than the others do. This tendency is caused by the fact that the resultant spectrum obtained by LPC mel-cepstrum keeps some important peaks to classify musical instrument sounds properly. Future work includes integrating LPC mel-cepstrum into a distance measure used in NMF algorithm inside to fit with extraction of particular musical instrument sound.

5pMU8. A simple model of cross-fingering explaining both pitch flattening and sharpening. Seiji Adachi (Dept. of Acoust., Fraunhofer Inst. for Bldg. Phys., Nobelstr. 12B, Stuttgart 70569, Germany, seiji.adachi@ibp. fraunhofer.de)

Cross-fingering is a technique of playing woodwind instruments in which one or more tone holes are closed below the first open hole. It usually yields a pitch lower than that played with normal fingering. There is, however, an exception resulting in pitch sharpening. This paper proposes understanding these pitch bending phenomena in a unified manner with a model of two coupled mechanical oscillators, each of which represents a bore above or below the open hole. This coupled system has two resonance frequencies $\omega_{ \pm}$, which are respectively higher and lower than those of the upper and lower bores $\omega_{U}$ and $\omega_{L}$. The $\omega_{ \pm}$differ even if $\omega_{U}=\omega_{L}$. The normal effect of cross-fingering, i.e., pitch flattening, corresponds to excitation of the $\omega_{-}$-mode, which occurs when $\omega_{L} \geq \omega_{U}$ and the admittance peak of the $\omega_{-}$-mode is higher than or as high as that of the $\omega_{+}$-mode. Excitation of 
the $\omega_{+}$-mode yields pitch sharpening. This occurs when $\omega_{L} \leq \omega_{U}$ and the peak of the $\omega_{+}$-mode becomes sufficiently high. With an extended model having three degrees of freedom, pitch bending of the recorder played with cross-fingering in the second register can also be successfully explained.

5pMU9. Relation between violin timbre and harmony overtone. Masao Yokoyama, Yoshiki Awahara (Information Sci., Meisei Univ., 2-1-1 Hodokubo, Hino 191-8506, Japan, masao.yokoyama@meisei-u.ac.jp), and Genki Yagawa (Tokyo Univ., Tokyo, Tokyo, Japan)

The timbre of violins has been studied by several researchers from various points of view including the structure, the acoustic characteristic, the chemical composition of the varnish and the acoustic radiation. Although many of them have mentioned that the Stradivari's violin gives the most beautiful timbre, the reasons of which have not been clarified yet. We have studied the timbre of about 30 violins from old ones to new ones, namely, the relation between the harmonic overtones and the expression words, which the audience receives from the sound of violin. But, we have not been successful in clarifying how the structure of overtone is related with the feeling of the listeners of sound such as "rich", "blight" and "soft" as the timbre of violin. In this paper, we have analyzed the change of overtone structure with the difference of violinist's performance. For instance, the power $(\mathrm{dB})$ of sound at the frequency area over $2000 \mathrm{~Hz}$ played in fortissimo or near a bridge like sul ponticello was approximately $10 \%$ to $20 \%$ higher than that played in pianissimo or on finger board such as sul tast. We also found that the power of non-harmonic overtone of the sound which was played in the "rich" or "soft" expression was high at the frequency area over $2000 \mathrm{~Hz}$ and the kurtosis of the peak of harmonic overtone was small.

5pMU10. Effect of hardening piano hammer felt on piano sound. Shingo Takaku (Hamamatsu Gakuin Univ. / Shizuoka Univ., 3-2-3 Nunohashi,Naka-ku, Hamamatsu 432-8012, Japan, takaku@hgu.ac.jp) and Yosuke Tatekura (Shizuoka Univ., Shizuoka, Japan)

To clarify the effect of hardening the hammer felt of a piano on its sound, experiments evaluating the sound quality of the piano were performed using a new piano hammer. The adjustment of a piano hammer is necessary to obtain the desired tone. The elasticity and hardness of the hammer are also recognized as indicators of the condition of the instrument. To maintain the condition of piano hammers, piano craftsmen make adjustments using various tools and chemicals while listening to the resulting change in the sound after each alteration. Hammer adjustment is often performed based on intuition and experience. In this study, the quantity of the hardening agent was gradually increased, and a sound quality experiment was performed using a new Renner hammer. A low frequency of $58.27 \mathrm{~Hz}$ (A\#1) was selected as the piano note investigated in this study because it is more affected than other frequencies by the hardening of the hammer. The results of this study indicate that the optimal amount of hardening agent needed to achieve a desired tone and desired tone should be determined before the agent is applied to the hammer felt.

5pMU11. Warbling in steadily bowed viol strings. D. Murray Campbell (School of Phys. and Astronomy, Univ. of Edinburgh, James Clerk Maxwell Bldg., Mayfield Rd., Edinburgh EH9 3JZ, United Kingdom, d.m.campbell@ ed.ac.uk), Patsy Campbell (Edinburgh College of Art, Univ. of Edinburgh, Edinburgh, United Kingdom), and Jim Woodhouse (Eng. Dept., Cambridge Univ., Cambridge, United Kingdom)

The timbre of a note played on a bowed string instrument depends on a number of performance parameters, including bow pressure, bow speed, and distance of the bowing point from the bridge. When these parameters are held constant during the bowing of a note played without vibrato the radiated sound usually has a stable timbre, with a frequency spectrum which is unchanging apart from small random fluctuations. For certain combinations of instrument and bow this timbral stability is disrupted, leading to a periodic modulation of the timbre which can be described as a "warble." The warbling effect was first noticed on a large bass viol, but it can also be found on other bowed string instruments including the cello. Unlike the well known "wolf" phenomenon, the warbling effect is not associated with a particular played pitch on the instrument, but occurs over a wide range of pitches. Experimental studies on bass viols have shown that the warble is a modulation of upper harmonics of the played note, with a modulation frequency of a few hertz. Possible mechanisms which could explain the generation of this modulation are discussed.

5pMU12. The effect of attack time and release time of the sound source on the sense of listener envelopment. Toru Kamekawa and Atsushi Marui (Musical Creativity and the Environment, Tokyo Univ. of the Arts, 1-25-1, Senju, Adachi-ku, Tokyo 120-0034, Japan, kamekawa@ms.geidai.ac.jp)

The effect of attack time and release time of the sound source on the sense of Listener Envelopment (LEV) was investigated. From the results of a previous experiment, it was found that there were at least two kinds of sense in LEV, namely, spatial continuity between front and back, and expanse of lateral direction. In this experiment, authors conducted pairwise comparison tests for each sense using simple sine wave stimuli changing four kind of attack time and three kind of release time. Furthermore these short sine waves were convolved with four channel room impulse responses and played back form five channel loud speakers. The result of pairwise comparison showed that the sense of spatial continuity between front and back was related to first attack time and another sense of expanse of lateral direction was related to slower attack time.

5pMU13. Acoustic and perceptual differences between novice and professional music theatre singers. Lynn M. Maxfield (National Ctr. for Voice and Speech, Univ. of Utah, 136 S Main St., Ste \#320, Salt Lake City, UT 84101, lynn.maxfield@utah.edu) and Brian Manternach (Dept. of Theatre, Univ. of Utah, Salt Lake City, UT)

Research examining contemporary commercial music (CCM) styles of singing has increased significantly over the last ten years. While acoustic analysis has helped define which characteristics define various vocal genres, discrepancy still exists in how those acoustic characteristics are perceived, described, and evaluated. The current study recorded novice and professional musical theatre singers performing belt, legit, and mix vocal samples. Three acoustic analyses were applied to the excerpted recordings from each singer using the Praat voice and speech analysis software: The spectral slope of the long term average spectrum (LTAS) was calculated, as well as the noise to harmonic ratio (NHR), and the dominant harmonic $\left(1^{\text {st }}, 2^{\text {nd }}\right.$, or $\left.3^{\text {rd }}\right)$ was noted for each sample. Results were compared across pitch, style (belt v. legit), and training level (professional v. novice). Finally, raters listened to the recordings and rated each sample on the basis of style (belt v. legit), roughness (rough v smooth), and tone quality (brassy v flutey, and bright $\mathrm{v}$ dark). A $120 \mathrm{~mm}$ liechert scale was provided to each rater for each of the rating variables. Results of the perceptual assessments were compared with acoustic measures to elucidate discrepancies in how ccm characteristics are perceived.

5pMU14. Vowel duration dependent hidden Markov model for automatic lyrics recognition. Shohei Awata, Shinji Sako, and Tadashi Kitamura (Comput. Sci., Nagoya Inst. of Technol., Gokisocho,Showa-ku Nagoyashi,Aichi, Nagoya 466-8555, Japan, awata@mmsp.nitech.ac.jp)

Recently, due to the spread of music distribution service, a large amount of music is available on the Internet. Accordingly, it is generally increasing the demand of music information retrieval (MIR). In the field of MIR research, there are several researches to extract meaningful information from music audio signals. However, automatic lyrics recognition is still a challenging problem because the variation of singing voice is much larger than that of speaking voice and a large database of singing voice is not available. In the relevant study, lyrics recognition was performed by extending the framework of speech recognition using hidden Markov model (HMM). However, accuracy rate was not sufficient. To recognize singing voice precisely, one promising approach is utilizing musical features. This study considers the task of recognizing syllable from a cappella singing voice. To respond to the variation of the length of a phoneme, we construct the duration dependent HMM. A large database of singing voice is essential for training the acoustic model. We use synthetic singing voice by HMM based singing voice synthesis system to solve the lack of the database of a cappella singing voice. We confirmed the effectiveness of our method. 
5pMU15. Sound source separation and synthesis for audio enhancement based on spectral amplitudes of two-channel stereo signals. Masayuki Nishiguchi, Ayumu Morikawa, Kanji Watanabe, Koji Abe, and Shoichi Takane (Dept. of Electronics and Information Systems, Akita Prefectural Univ., 84-4 Ebinokuchi, Tsuchiya, Yurihonjo, Akita 015 0055, Japan, nishiguchi@akita-pu.ac.jp)

A sound source separation algorithm based on the spectral amplitudes of 2-channel signals has been developed for the up-mixing playback of 2-channel stereo. Short-term Fourier transforms (STFT) of the signals on the left and right channels are first calculated. The coefficients of the discrete Fourier transform (DFT) are used to calculate the ratio of the spectral amplitudes of the left and right channels, which is termed the channel level difference (CLD). The DFT coefficients are then divided into multiple groups on the basis of the CLD, with each group representing a separated sound source. The signal-to-distortion ratio (SDR)is used to evaluate the signal separation performance. It was found that a rough estimate of the CLD threshold yielding the best SDR could be obtained by cross-correlating the separated sounds. For playback on a headset, each separated signal is convoluted with head-related transfer functions (HRTF) that represent the direction of that particular sound source. Subjective listening tests showed that the sound synthesized by this method is more realistic than that synthesized with HRTFs that represent only left and right speakers.

5pMU16. Study on relationship between subjective reproducibility of individuality and distance measure for vibrato of singing voice. Chifumi Suzuki, Hideki Banno, Kensaku Asahi (Graduate School of Sci. and Technol., Graduate School of Meijo Univ., 1-301 Shiogamaguchi Tenpaku-ku, Nagoya 468-8502, Japan, 123441501@ccalumni.meijo-u.ac.jp), and Masanori Morise (Univ. of Yamanashi, Koufu, Japan)

In this research, a distance measure between two vibratos had been developed to evaluate singing style. This paper verifies whether the measure properly represents subjective distance by the following two subjective experiments. In these experiments, synthetic sounds whose vibratory amplitude computed from a target singer's singing is variable were used, and listeners were asked to answer reproducibility of individuality of the target singer for the synthetic sound. In the first experiment, listeners were asked to remember vibrato of the target singer by listening to the singing. As a result, it was found that not only synthetic sounds showing small distance to the target singer in the distance measure but also those not showing small distance produced higher subjective reproducibility, as long as the sound whose vibratory amplitude is large, i.e., includes deformation in the F0 trajectory. To confirm whether this tendency is caused by the experimental procedure, the second experiment that listeners were compared with the reference synthetic sound was conducted. As a result, it was found that the results were mostly the same as those of the first experiment, and the vibrato rate affects the subjective reproducibility more strongly, as the magnitude of vibratory amplitude becomes large.

5pMU17. Tempo estimation for acoustic signal of tempo variation music. Sota Okada (Dept. of Media Informatics, Graduate School of Ryukoku Univ., 1-5, Yokoe, Oe-Cho, Otsu, Shiga 520-2153, Japan, xkre3x@ gmail.com) and Masanobu Miura (Dept. of Media Informatics, Ryukoku Univ., Otsu, Shiga, Japan)

This study proposes a tempo estimation method for acoustic signal of tempo variation music. Previous studies observe the power spectrum of envelope curve for its acoustic signal, where its length must be sixty seconds or longer for the purpose of keeping the tempo resolution as $1 \mathrm{bpm}$. The method then picked the highest power up as estimated tempo. The music with tempo variation that is occurred within the sixty seconds (for example ten seconds) were, however, hard to estimate. In order to estimate tempo for each time of such tempo variation music, the conventional zero-padding method is carried out when performing FFT. Tempo value is therefore estimated under high accuracy yet the estimation errors have not discussed so far. Accordingly, the optimum ratio of zero-padding was investigated. As a result, the optimized ratio of acoustic signal and 0 in the zero-padding is.125-.875\%. Furthermore, tempo value on each one second is estimated for tempo variation music that has orchestral rubato expression. The estimated accuracy of presented method was evaluated by comparing estimated with hand-labeled tempo.

5pMU18. Drum sound onset detection based on class separation using deep neural network. Takahiro Kuriwaki, Takanori Nishino, and Hiroshi Naruse (Graduate School of information Eng., Mie Univ., 1577 kurimamachiya-cho, Tsu, Mie 514-8507, Japan, kuriwaki@pa.info.mie-u.ac.jp)

This study reports an onset detection method for the snare drum sounds for music signals constructed with plural instruments. We propose a method which detects an onset with a class separation using a deep neural network (DNN). To train the network, frames which include the waveform of the snare drum were extracted from music signal, and the log mel-filter bank channel outputs were calculated for each frame. The DNN was trained by using these feature parameters. The inputs were the calculated filter bank outputs, and the teaching signals were binary information whether to include the waveform of a snare drum or not. To detect onsets, candidate frames of the onset time were found based on changing in the power of the target music signal, and feature values were extracted from the candidate frames. Obtained values were used as the input data for the trained DNN, and detection of whether or not to include a waveform of a snare drum can be achieved. Experiments were conducted for 52 popular songs included in the music database, and an average F-measure was 0.73 .

5pMU19. Computational estimation of released decades for Japanese women idol music from musical audio. Sota Okada (Dept. of Media Informatics, Graduate School of Ryukoku Univ., 1-5, Yokoe, Oe-Cho, Otsu, Shiga 520-2153, Japan, xkre3x@gmail.com) and Masanobu Miura (Dept. of Media Informatics, Ryukoku Univ., Otsu, Shiga, Japan)

This study realizes computational estimation of released decades for Japanese women idol (or diva) music from musical audio. Even though the released year of music is explicitly rebelled on the music, the feeling of decade, for example " 1980 's, or old-fashioned music" is completely subjective so it is thought as difficult for computers to evaluate, yet people may feel it when listening to music. Such feeling is thought to be derived from musical arrangement, instruments, and so forth, yet previous study clarified the possibility to estimate based on acoustic feature of music. If such estimation is realized, it could contribute to enhance conventional recommendation system of music by giving a query: "newly released but old-fashion music." This study conducted to establish explicit criteria for audio parameters by using logistic regression. Proposed system uses 25 types of acoustic parameters for each musical audio as training data set, and estimates single decades by simple logistic under a 10-fold cross validation scheme. As a result, estimation result of accuracy rate is $58.56 \%$ and kappa statistic is 0.48 , being almost comparative to that of subjective evaluation. The estimation system is then implemented in a lap-top compute, which will be demonstrated at presentation.

5pMU20. Differences of characteristics of music singing between in usual Karaoke and in Hitokara. Junko Matsumoto (Nagano College of Nursing, 1694, akaho, Komagane, Nagano 399-4117, Japan, matsumoto@ nagano-nurs.ac.jp)

Recently some of people in Japan go to Karaoke by oneself. This is called Hitokara (solo Karaoke). We investigated the differences of characteristics of music singing between in usual Karaoke and in Hitokara. Participants were 103 university students. They were asked to fulfill a questionnaire about characteristics of music singing both in usual Karaoke and in Hitkoara. As a result, about one third of participants had at least once experience of Hitokara singing, and they preferred both usual Karaoke singing and Hitokara singing. However, they preferred the music with characteristics of elation or lightness in usual Karaoke singing, in Hitokara singing they preferred the music with various characteristics. In Hitokara singing, people sing actively and they would be able to cope well with various moods by singing various music. 
5pMU21. An acoustic and perceptual evaluation of four historic recordings of Chinese narrative singing. Shawn L. Nissen (Commun. Disord., Brigham Young Univ., 128 TLRB, Provo, UT 84602, shawn_nissen@byu. edu) and Francesca R. Lawson (Comparative Arts and Letters, Brigham Young Univ., Provo, UT)

This study is an acoustic and perceptual musicological evaluation of four recordings of vocal renditions from two schools of Chinese narrative singing that were differentiated by gender during the first half of the twentieth century. The analyses were based on four renditions of "At Break of Day" by Liu Baoquan (1869-1942) and Xiao Lanyun (1923-1992) of the "male school", as well as Luo Yusheng (1914-2002) and Lu Yiqin (1933-) of the "female school." The musicological analysis was based on transcriptions of the recordings, comparing melody, rhythm, ornamentation, and tonality. The acoustic analyses involved measures of central tendency and variability for duration and fundamental frequency (F0) using Praat acoustic analysis software. All four vocalists exhibited a similar mean F0, however differences in relative duration, F0 range, F0 variation, and F0 slope were found across singers at both the phrase and syllable level. Differences within schools of singing (male and female) and between instructor and student (i.e., Liu Baoquan and Xiao Lanyun) were also noted. These acoustic differences provide additional insights for ethnomusicologists that may be unattainable through traditional methods of perceptual analysis.

5pMU22. Effects of swing position in one measure on the feeling of musical rhythm: Examination using the rhythm dividing every beat. Shimpei Ikegami and Sumi Shigeno (College of Education, Psych. and Human Studies, Aoyama Gakuin Univ., 4-4-25 Shibuya, Shibuya-ku, Tokyo 1508366, Japan, s-ikegami@ephs.aoyama.ac.jp)

Swing is an expression technique used in the performance of popular music. Swing causes the feeling of wanting to move the body in accordance with the musical rhythm. Swing is expressed by dividing one beat into two temporally unequal parts (e.g., 2:1). This study examined the effects of a swing's position in one measure on the feeling of musical rhythm. Every beat of the duple meter rhythm was divided into two parts. Four conditions of the swing's position were prepared: the "every-beat swing," the "oddbeat swing" (the swing's position being the first beat), the "even-beat swing" (the swing's position being the second beat), and the "no swing" conditions. This study had 114 participants listening to musical rhythms presented with Scheffés paired-comparison method and rated the degree of "wanting to move the body" and "pleasantness." The results showed that the "every-beat swing" condition got the highest rating scores for a feeling of "wanting to move the body." We consider that the partial presence of swing, irrespective of the swing's position, might hardly allow listeners to attune to the rhythm of duple meter music.

5pMU23. An electromyographic study of left hand between experts and novices in violin playing. Satoshi Obata and Eriko Aiba (The Univ. of Electro-Communications, 1-5-1 Chofugaoka, Chofu, Tokyo 182-8585, Japan, s-obata@hi.is.uec.ac.jp)

We recently recorded string clamping force on the violin during simple (without vibrato) tone production by expert violinists. The results of our studies showed that the peak force exceeded $4.5 \mathrm{~N}$ at slow tempi, which decreased to $1.7 \mathrm{~N}$ at fast tempi. However, subjective assessment of playing effort indicated an opposite trend; the players felt that playing at faster tempi was more strenuous. We also measured muscular activity along with finger kinematics. We found that the mean left forearm muscle activity increased with tempo. The findings suggest that the speed of finger movement is more related to the arm and hand discomfort when playing a violin, as compared to clamping force. In the present study, we investigated the expert-novice difference in the nature of the string clamping force and related muscles in the left hand and arm while playing a violin. Information regarding the string clamping force and left hand muscle activity can help in teaching novices about the magnitude and timing of the appropriate string stabilizing action during sound production. Surface electromyography (EMG) and string clamping force were measured for this purpose.
5pMU24. Estimation of tempo, timing, and melody for piano practice support systems. Shota Asahi, Satoshi Tamara, Satoru Hayamizu (Eng., Gifu Univ., 1-1, Yanagido, Gifu-shi, Gifu, Gifu 501-1112, Japan, asahi@ asr.info.gifu-u.ac.jp), and Yuko Sugiyama (Chubu Gakuin College, Seki, Japan)

In piano learning as voluntary practice, it is difficult especially for beginners to judge whether their musical performances are appropriate in terms of timing and melody. Therefore, we have studied piano learning support systems for beginners. In this study, we introduce a method for the system, to estimate tempo, timing and melody of performances, and to visualize the data for piano learners and instructors. In our method, a tempo for each musical bar is firstly estimated by measuring first notes in current and incoming bars. Subsequently, a timing of each musical note in the bar is obtained based on the estimated tempo. A chroma vector is then computed for each note, in order to compare it with the vector obtained from a corresponding instructor's performance, and finally to detect misplayed keys. In order to confirm effectiveness of our method, we conducted experiments. We collected musical performances (No.12 Bayer) from seven subjects. Assuming voluntary practice, the subjects were asked to listen to music and watch corresponding movies in which an instructor played piano with musical scores and instructions, before they made performances. We analyzed the performance data and conducted questionnaire to the subjects, to confirm the effectiveness.

5pMU25. Mouthpiece pressing force for pitch and loudness control in playing the French horn. Takeshi Hirano (J. F. Oberlin Univ., 3758 Tokiwa-machi, Machida-shi, Tokyo 194-0294, Japan, hira.take.713@gmail. com) and Hiroshi Kinoshita (Osaka Aoyama Univ., Osaka, Japan)

Using a force-sensing French horn mouthpiece developed by the present authors, lip pressing force was examined during pre-attack and post-attack phases of 2 -sec sustained tone production at varied pitches $(87,174,349$, 466, and $698 \mathrm{~Hz})$ and dynamics $(95,100,105,110$, and $115 \mathrm{~dB})$ performed by 12 highly trained horn players. Dependent variables examined were: force at the onset of sound, a peak of the force rate before the sound onset, and mean and SD forces during steady state tone production. All of these force variables increased nearly linearly with pitch. They also increased lightly with louder dynamics. The pitch-related and dynamic-related increase of the force varied largely among the players. These findings indicate that skilled horn performances are supported by pre-attacking the mouthpiece pressure properly to prepare for a target pitch at target dynamics. The notable inter-player difference in force production in skilled players suggests a proper level of mouthpiece pressures witch differs among players due possibly to congenital physical properties of their lips.

5pMU26. Spatial acoustic-radiation characteristic of a violin captured in the vicinity of a violinist. Katuhiro Maki (Faculty of Human Informatics, Aichi Syukutoku Univ., 2-9 Katahira, Nagakute, Aichi 480-1197, Japan, maki-ns@umin.ac.jp)

The spatial characteristic of the sound of a violin is thought to affect the timbre of a violin in a reverberant space such as a concert hall. Therefore, the spatial characteristic is one of important key to clarify the timber of the violin. In this study, the spatial characteristic was investigated by placing 42 small microphones around a violinist and recording the sound of the violin being played. The experiment-targeting nine modern violins-was performed in an anechoic chamber. The professional violinists were asked to perform musical scales and compositions. According to the results of an analysis of the sound, in the horizontal plane, the sound is radiated roughly toward diagonally forward left of the violinist; in the sagittal plane, sounds with frequency components lower than around $1 \mathrm{kHz}$ are radiated downwards, and sounds with frequency components higher than $1 \mathrm{kHz}$ are radiated upwards. Moreover, it was observed that the directionality of the radiated sound of the violin is particularly strong around $1 \mathrm{kHz}$ and $3 \mathrm{kHz}$, and somewhat strong near $350 \mathrm{~Hz}$. It was also observed that during the performances of the compositions, the direction of the sound radiation at each analyzed frequency varies significantly with time. 


\title{
Session 5pNSa
}

\section{Noise: Design of Noise Control Materials}

\author{
J. S. Bolton, Cochair \\ Ray W. Herrick Laboratories, School of Mechanical Engineering, Purdue University, Ray W. Herrick Laboratories, 177 S. \\ Russell St., West Lafayette, IN 47907-2099 \\ Takashi Yamamoto, Cochair \\ Mechanical Engineering, Kogakuin University, 2665-1 Nakano, Tokyo 1920015, Japan
}

Chair's Introduction-1:00

\section{Invited Papers}

\section{1:05}

5pNSa1. A level set-based topology optimization for fluid-structure coupled problems by using two-phase material model. Takashi Yamamoto (Mech. Eng., Kogakuin Univ., 2665-1 Nakano, Tokyo, Hachioji 1920015, Japan, takashi_yamamoto@cc.kogakuin.ac. jp), Yuki Noguchi, Takayuki Yamada, Kazuhiro Izui, and Shinji Nishiwaki (Graduate School of Eng., Kyoto Univ., Kyoto, Japan)

In this study, we develop a two-phase material model mixed with a solid and fluid phases to represent an elastic material and an acoustic material in a unified set of governing equations. By applying the model, boundary conditions at boundaries between different two-phase materials are satisfied naturally, and the boundaries do not have to be expressed explicitly. This characteristic is effective for topology optimization of fluid-structure coupled problems by using finite element method. Consequently, we formulate an optimization method utilizing the two-phase material model and a level set method to express boundaries between different two-phase materials. An approximate method to obtain topological derivative is also developed by applying the variational analysis and the adjoint variable method. Topological derivative of an objective functional is considered to be proportional to the derivative of the level set functions with respect to time and the values of the level set functions are updated by solving time evolutionary reaction diffusion equation with a regularization term. After the verifications of the two-phase model, we present several numerical demonstrations for directional acoustic cloaking of a cylindrical obstacle placed in unbounded air medium.

\section{$1: 25$}

5pNSa2. Level set-based topology optimization for the design of acoustic metamaterial using two-phase material model. Yuki Noguchi (Dept. of Mech. Eng. and Sci., Kyoto Univ., Kyoto daigaku-katsura C3, Nishikyo-ku, Kyoto 615-8540, Japan, noguchi.yuuki. 34s@st.kyoto-u.ac.jp), Takashi Yamamoto (Dept. of Mech. Eng., Kogakuin Univ., Tokyo, Japan), Takayuki Yamada, Kazuhiro Izui, and Shinji Nishiwaki (Dept. of Mech. Eng. and Sci., Kyoto Univ., Kyoto, Japan)

Acoustic metamaterials are artificial materials composed of periodic structures made of elastic and fluid material. They could be used for noise reduction problems and efficient sound propagation control by their unusual properties, such as negative bulk modulus, mass density, and refractive index. The performance of acoustic metamaterial is strongly affected by its designs rather than its material properties. Therefore, topology optimization method could be used to obtain a desired property of acoustic metamaterial. To model acoustic-elastic coupled system, we introduce a two-phase material model where the solid and fluid phases are mixed. While coupling boundary conditions are required in a usual acoustic-elastic coupled analysis, this model allows us to analyze the system without imposing such boundary conditions since their conditions are satisfied naturally. In this research, we propose a level set-based topology optimization method for the design of acoustic metamaterial. First, we introduce the two-phase material model and level set-based topology optimization method. An optimization problem for an acoustic metamaterial that has negative mass density is formulated and sensitivity analysis is performed based on the concept of topological derivative. After verifying the topological derivative by comparing it to numerical differences, we present two-dimensional numerical examples for negative mass acoustic metamaterial.

\section{1:45}

5pNSa3. Acoustic media with pore size distribution and assignment of the pore characteristic lengths and permeabilities. Kirill V. Horoshenkov (Univ. of Sheffield, Mappin St., Sheffield S1 3JD, United Kingdom, k.horoshenkov@sheffield.ac.uk), Matti Niskanen, Jean-Philippe Groby, Olivier Dazel, and Aroune Duclos (de l'Universitée du Maine, de l’Universitée du Maine, Le Mans, France)

The model by Champoux and Allard [J. Appl. Phys. 70(4), 1975-1979 (1991)] is used extensively for research and development of new porous media solutions and for predicting sound propagation in the presence of porous media. Four key non-acoustical parameters in this model which are rarely measured non-acoustically are: the viscous and thermal characteristic lengths, thermal permeability and Pride parameter Pride et al [Phys. Rev. B 47, 4964-4978 (1993)]. In this work we show how these parameters unambiguously relate to the pore size distribution which is a characteristic measured nonacoustically and more routinely. We compare the predictions by this model against the log-normal pore size distribution model by Horoshenkov et al. [J. Acoust. Soc. Am. 104, 1198-1209 (1998)] and show that these two models provide very close predictions when the four non-acoustic parameters are expressed through the mean pore size 
and its standard deviation. We also confirm these results through a 4-microphone impedance tube experiment in which we determine the dynamic density and complex bulk modulus of a range of porous media. We also study the values of the viscous and thermal characteristic lengths and viscous and thermal permeabilities which fit our acoustic data.

5pNSa4. Effect of membranes at microscopic polygonal faces of polyurethane foam by using homogenization method. Takashi Yamamoto and Yuki Imae (Mech. Eng., Kogakuin Univ., 2665-1 Nakano, Hachioji, Tokyo 1920015, Japan, takashi_yamamoto@cc. kogakuin.ac.jp)

Sound absorption coefficient in poroelastic media depends on the geometries in the microscopic scale. In some polyurethane foams that exhibit high sound absorption coefficients in the middle frequency range around $1 \mathrm{kHz}$, the faces of polygonal shape of microscopic structure are covered by thin membranes. Moreover, a hole is frequently found in some membranes. This type of microscopic structure is considered to contribute to the high sound absorption performance. Multi-scale analysis for sound-absorbing poroelastic media with periodic microscopic geometries has been recently proposed by one of the authors. In this method, the homogenization method based on the asymptotic expansions are utilized and further extended to express the viscous dissipation in the vicinity of the boundary between the solid and fluid phases, thermal dissipation from the fluid phase to the solid phase. Analysis in the microscopic scale is first performed by using a unit cell of the periodic structure and macroscopic properties are derived. The properties are then applied to calculate macroscopic response such as sound absorption coefficient. In this paper, we apply this multi-scale analysis and investigate numerically the relationship between the size of the hole in membranes and sound absorption performance.

\section{Contributed Papers}

5pNSa5. Design and optimization of membrane-type acoustic metamaterials using genetic algorithms. Matthew G. Blevins (Durham School of Architectural Eng. and Construction, Univ. of Nebraska - Lincoln, 408 Ginger Bend Dr. Apt. 207, Champaign, IL 61822, matthew.g.blevins@usace. army.mil), Siu-Kit Lau (Dept. of Architecture, National Univ. of Singapore, Singapore, Singapore), and Lily M. Wang (Durham School of Architectural Eng. and Construction, Univ. of Nebraska - Lincoln, Omaha, NE)

One of the most difficult problems in noise control is the attenuation of low frequency noise. Typical solutions require barriers with high density and/ or thickness. Membrane-type acoustic metamaterials are a novel type of engineered material capable of high low-frequency transmission loss despite their small thickness and light weight. These materials are ideally suited to applications with strict size and weight limitations such as aircraft, automobiles, and buildings. This paper reviews computationally efficient dynamic models for designing membrane-type acoustic metamaterials that have been proposed by the authors, based on the impedance-mobility approach. The computational efficiency of the impedance-mobility models compared to finite element methods enables implementation in design tools based on a graphical user interface and in optimization schemes. Genetic algorithms are used to optimize the unit cell design for a variety of noise reduction goals, including maximizing transmission loss for broadband, narrow-band, and tonal noise sources. The degree to which the optimal configurations extend to systems of multiple unit cells and unit cells of different shapes is explored.

\section{2:40}

5pNSa6. An application of a parametric transducer to measure the acoustical properties of a living green wall. Anna Romanova (Eng. \& Sci., Univ. of Greenwich, Central Ave., Chatham Maritime, Chatham, Kent ME4 4TB, United Kingdom, a.romashk@gmail.com) and Kirill V. Horoshenkov (Mech. Eng., Univ. of Sheffield, Sheffield, United Kingdom)

Greening of urban spaces provides a number of environmental benefits. Living Green Walls (LGW) is a most typical example of greening which is also known for its ability to absorb unwanted noise. However, this ability of LGW to absorb noise is rather hard to quantify, because there is a lack of reliable experimental methods to measure it in-situ. This work reports on a new method to measure the absorption coefficient of LGW which makes use of a highly directional parametric transducer and acoustic intensity method. This method is tested under controlled laboratory conditions and in a typical street environment. The results of these experiments demonstrate the ability of the method to measure the absorption of a LGW. It also enables us to quantify the effects of the plant type and moisture content in the soil on the ability of the LGW to absorb sound. The proposed method has certain benefits over ISO354-2003 and CEN/TS 1793-5:2003 standard methods.
5pNSa7. Adaptive algorithms for combined feedforward-feedback controllers. Jacob Bean, Chris Fuller (Aerosp. Eng., Virginia Tech, 100 Exploration Way, Hampton, VA 23666, bjacob09@vt.edu), and Noah Schiller (Structural Acoust. Branch, NASA Langley Res. Ctr., Hampton, VA)

Traditional approaches to feedback and hybrid active noise and vibration control are generally based on adaptive gradient search methods using estimates of the instantaneous gradient. Most of these algorithms employ internal model control and are derivatives of the least mean squares algorithm commonly used in adaptive filtering. In these algorithms, the gradient calculation neglects the presence of a feedback path. To avoid potential algorithm divergence due to the presence of the feedback path, it is common to update the filter conservatively by including a leakage term, which is in general a trial and error procedure. This paper presents mathematical derivations for the updating mechanisms in feedback and hybrid controllers that make no assumptions regarding the accuracy of the plant model. Rather than deriving update equations assuming an ideal plant model and then modifying the adaptation process to be less sensitive to model errors, the reverse approach is taken. It will be seen that the presence of the feedback loop can be accounted for directly in the update equation. This allows either hard or probabilistic bounds on the uncertainty to be set at the outset of design. Computer simulations are presented to validate the algorithms.

\section{3:10}

5pNSa8. Characteristic analysis of screeching noise using $24 \mathrm{bit} / 192 \mathrm{kHz}$ high-resolution measurement system in anechoic room. Shuya Ogino and Kan Okubo (Graduate School of System Design, Tokyo Metropolitan Univ., Asahigaoka 6-6, Hinosi, Tokyo 191-0065, Japan, shuwashuwa555@gmail.com)

Many researches on the screeching sound have been investigated for some decades. Halpern et al.(1986) reported acoustic evaluation of "chilling sound" as a pioneering study. The screeching sound makes us feel so-called hair-raising, bloodcurdling chills, which is generated by scratching a blackboard and frosted glass with a fingernail, metal, and so on. This might be common sensation shared by the world. Although Halpern et al. (1986) employed a tape recorder of up to $20.0 \mathrm{kHz}$ as a recording equipment, in recent years researchers can use a $24 \mathrm{bit} / 192 \mathrm{kHz}$ high-resolution measurement system. The frequency range of screeching sound can't be not fundamentally limited up to $20.0 \mathrm{kHz}$. Scratching should cause the sound above the human audible range; it is known as "Hypersonic effect." In this study, therefore, we try to record and analyze the screeching noise at the frequency range of up to $96 \mathrm{kHz}$. We employ $24 \mathrm{bit} / 192 \mathrm{kHz}$ high-resolution measurement system in anechoic room as a recording equipment. This presentation reports the findings from characteristic analysis of screeching sound obtained by high-resolution measurement. We demonstrate that scratching generates the sound above $20.0 \mathrm{kHz}$, and that so-called screeching noise have many overtone sounds above $20.0 \mathrm{kHz}$ and remarkable features of like sawtooth wave. 


\title{
Session 5pNSb
}

\section{Noise: Emerging Issues on Quiet Vehicles-A Matter of Soundscape}

\author{
Brigitte Schulte-Fortkamp, Cochair \\ Institute of Fluid Mechanics and Engineering Acoustics, TU Berlin, Einsteinufer 25, Berlin 101789, Germany \\ Klaus Genuit, Cochair \\ HEAD acoustics GmbH, Ebertstr. 30a, Herzogenrath 52134, Germany \\ Katsuya Yamauchi, Cochair \\ Faculty of Design, Kyushu University, Shiobaru 4-9-1, Fukuoka 815-8540, Japan
}

Chair's Introduction-3:40

Invited Papers

$3: 45$

5pNSb1. E-vehicle: A positive impact on Soundscape? Klaus Genuit (HEAD Acoust. GmbH, Ebertstr. 30a, Herzogenrath, NRW 52134, Germany, Klaus.Genuit@head-acoustics.de)

The future will see more and more electric cars on the road as stronger exhaust emission regulations are put into effect. A byproduct of this shift is a broad reduction of noise: no combustion-, exhaust- or intake-noise in addition to reduced tire-road noise. On the other hand, concerns have been raised with respect to pedestrian safety and electric vehicles being too quiet. The significant impact on the soundscape manifested by electric vehicles will be described in this paper, as well as efforts to address safety concerns. The request to compensate the low noise level of electrical vehicles by acoustical warning signals should be discussed very carefully, as even low level warning signals could increase perceived annoyance. This scenario will be explained by several examples.

\section{4:05}

5pNSb2. Relationship between the use of portable audio devices and taking notice of the approach informing sound of quiet vehicles. Mariko Hamamura, Shotaro Takamaki, and Yasunari Obuchi (School of Media Sci., Tokyo Univ. of Technol., 1404-1 Katakuracho, Hachioji, Tokyo 192-0982, Japan, mariko@hamamura.biz)

Lively discussion about the approach informing sound of quiet vehicles is being conducted; however, people often listen to media on portable audio devices in various environments and may not notice the approach informing sound. To clarify the relationship between the use of portable audio devices and the experience of encountering dangers associated with the approach of quiet vehicles, a questionnaire survey was conducted with 158 university students. Portable audio device ownership was $99.3 \%$; only one respondent reported not owning such a device. Twenty-two respondents (15.5\%) had encountered dangerous situations while using these devices outdoors. Thirteen respondents reported that they had experienced near-misses with vehicles while walking or biking, and seven reported that the vehicles were quiet vehicles. Multiple correspondence analysis revealed some differences in the usage habits of portable audio devices between the users who had and had not encountered dangerous situations. To improve notification effectiveness of quiet vehicles, usage habits related to portable audio devices, especially concerning young people, should be considered. In addition, means of preventing portable audio device users' tendency to "tune out" other sounds should be addressed.

5pNSb3. Computational analysis of aeroacoustic noise generated from a rotating tire with a longitudinal groove. Daisuke Kato, Gonghwi Lee (Aeronautics and Astronautics, Univ. of Tokyo, Yoshinodai,Sgamihara,Kanagawa,Japan, Sagamihara, Kanagawa 2520206, Japan, dkato@flab.isas.jaxa.jp), Yoshiaki Abe (Tokyo Inst. of Technol., Yokohama, Japan), Taku Nonomura, Akira Oyama (Japan Aerosp. Exploration Agency Inst. of Space and Astronautical Sci., Sagamihara, Kanagawa, Japan), Kozo Fujii (Tokyo Univ. of Sci., Katsushika, Japan), Toshiyuki Ikeda, and Masataka Koishi (The Yokohama Rubber, Hiratsuka, Japan)

It is known that acoustic waves which is higher than $1000 \mathrm{~Hz}$ are generated from a rotating tire, and one possibility of their generation mechanism is aeroacoustic effect. The main objective of this study is to understand the generation mechanism and acoustic wave characteristic for realization of low noise tire. For this main objective, the effects of a longitudinal groove shape on acoustic field are investigated in the present study. Three cases, without a groove case (slick tire), a small longitudinal groove and large longitudinal groove are analyzed by large eddy simulations. The result shows that compression or expansion of the vortex is alleviated and weaken sound source by attaching a groove on tire surface. On the other hand, we also confirm that a groove contributes generation of new fluctuation and affects the spread of fluctuations. It can be considered that acoustic fields of front side of tire are affected by a tradeoff between the effect of compression or expansion of the vortex and those of the spread of fluctuation. 
5pNSb4. Effectiveness of additional warning sounds for quiet vehicles in urban noise environment. Katsuya Yamauchi (Faculty of Design, Kyushu Univ., Shiobaru 4-9-1, Fukuoka 815-8540, Japan, yamauchi@design.kyushu-u.ac.jp)

Relative quietness of electric or hybrid electric vehicles is one of the most important topics in environmental acoustics. Although reduced vehicle noise is eligible for urban sound environment, it is also a matter of pedestrians' safety concern. Hence, regulations regarding additional warning sounds for the quiet vehicles have been developing in Japan as well as in global. Recently the UN-regulation regarding the warning system to compensate their quietness by additional warning device, Acoustic Vehicle Alerting System (AVAS) according to the regulation, has been approved. Those kind of measures should be carefully discussed. Several studies have been conducted in different institutions to examine the feasible sound design for the warning to be detected in urban noise environment. This paper provides a cross-cutting perspective on the detectability of the AVAS sound compared to the background noise levels, which illustrates that the AVAS could solve problems only in limited scenarios.

\title{
$5: 05$
}

5pNSb5. The move toward quiet vehicles-A paradigm shift. Brigitte Schulte-Fortkamp (Inst. of Fluid Mech. and Eng. Acoust., TU Berlin, Einsteinufer 25, Berlin 101789, Germany, b.schulte-fortkamp@tu-berlin.de)

The introduction of so-called quiet vehicles is not simply a matter of acoustics. It also presents a major paradigm shift in both the car industry and daily life as a whole. A remaining consideration is how adequate public communication can support the acceptance of quiet vehicles. Soundscape is a construct of human perception, influenced by one's socio-cultural background as well as the acoustic environment in context. Among many concerns, the meanings of sound, the composition of diverse sound sources, the listener's attitude, and expectations towards the acoustic environment are particularly important in one's perception of a soundscape. Previous experiences with internal-combustion engines in vehicles significantly inform people's different perceptions and assessments of the environment, and likewise are important factors when considering the introduction of quiet cars in society. The discussion in this paper will show how the soundscape approach can be used to involve people in assessing the needs and uses of these "new cars."

5:25-5:45 Panel Discussion

FRIDAY AFTERNOON, 2 DECEMBER 2016

SOUTH PACIFIC 2, 1:30 P.M. TO 4:00 P.M.

\section{Session 5pPA}

Physical Acoustics: Radiation Force and Nonlinear Acoustics

\author{
Kyle S. Spratt, Chair \\ Applied Research Laboratories, The University of Texas at Austin, Austin, TX 78713
}

\section{Contributed Papers}

1:30

5pPA1. Acoustic radiation force on non-spherical objects in a liquid. Bart Lipkens (FloDesign Sonics, 1215 Wilbraham Rd., Box S-5024, Springfield, MA 01119, blipkens@wne.edu), Yurii A. Ilinskii, and Evgenia A. Zabolotskaya (FloDesign Sonics, Austin, Texas)

Macro-scale acoustophoretic separation has applications in oil/water separation and biopharmaceutical cell clarification. Interesting phenomena include deformation of oil droplets and clustering of cells into cylindrical shapes. Particle and cluster size are not necessarily small relative to the wavelength. A general computational framework is needed to calculate the acoustic radiation force on particles, clusters, and cells of various shapes. Ilinskii et al. presented a method to evaluate an acoustic radiation force on spherical objects, based on expansion of incident and scattered field in spherical waves. The acoustic force is given by the incident wave amplitudes and dimensionless scattering coefficients. This method is generalized for particle shapes which are not spherical. The incident acoustic and scattered field are expanded with respect to spherical waves. An evaluation of the scattering amplitudes for non-spherical objects can be done numerically. Analytically, scattering amplitudes are calculated from boundary conditions for spheroidal functions that are solutions of a wave equation in spheroidal coordinates $\xi, \eta, \varphi$. Boundary conditions are defined at surface $\xi=$ constant, i.e. on an ellipsoid surface. Solutions for prolate and oblate ellipsoids have been estimated. The asymptotic values of spheroidal solutions are determined for $\xi$ large enough to obtain required amplitudes of scattered field. Ilinskii et al. POMA, 19 (2013).

\section{$1: 45$}

5pPA2. Negative pulling acoustic radiation force on spheroids in Bessel vortex beams. Farid G. Mitri (Area 52 Technol., Chevron, 5 Bisbee Court, Santa Fe, NM 87508, f.g.mitri@ieee.org)

The emergence of a negative pulling acoustic radiation force on rigid spheroids is examined, with the use of acoustical Bessel vortex (helicoidal) "tractor" beams. Numerical predictions based on the acoustical scattering phenomenon [F. G. Mitri, IEEE Trans. Ultrason. Ferroelectr. Freq. Control 62, 1809-1818 (2015); Ann. Phys. (NY) 363, 262-274 (2015)] that is extended to calculate the longitudinal acoustic radiation force (i.e., acting along the direction of wave propagation) illustrate the theory, with particular emphasis on the aspect ratio of the spheroid, the half-cone angle and order of the beam, as well as the dimensionless frequency parameter. It is shown that the Bessel vortex beam composed of progressive (i.e., traveling) waves 
is capable of generating a negative pulling force acting in opposite direction of the forward linear momentum density flux associated with the incident waves [F. G. Mitri, Europhys. Lett. 112, 34002 (2015)]. The scope of the analysis is further expanded to include the cases of standing and quasistanding Bessel beam tweezers [F. G. Mitri, Wave Motion 57, 231-238 (2015)]. The results potentially suggest the use of Bessel "tractor" vortex beams for pulling, propelling, or manipulating arbitrary-shaped objects deviating from the spherical geometry for applications in remote sampling, particle manipulation, and handling, to name a few areas.

\section{2:00}

5pPA3. Study on the non-contact trapping of a small object using a pipe radiating travelling waves and a reflective rod-The effect of the shape of reflector surface on the trapping state. Manabu Aoyagi (Muroran Inst. of Technol., 27-1, Mizumoto, Muroran, Hokkaido 050-8585, Japan, maoyagi@mmm.muroran-it.ac.jp), Hideki Tamura, and Takehiro Takano (Tohoku Inst. of Technol., Sendai, Japan)

Flexural damped travelling waves can be excited in a lossy transmission pipe. The waves generate travelling acoustic flow in the inside of pipe and near the pipe end and the acoustic flow serves as travelling acoustic waves. It has been confirmed that a non-contact transportation of a small object can be performed in the pipe, and additionally, a small object can be trapped at a nodal point of the standing wave formed by placing a reflector rod near the pipe end. In the experiment, a polycarbonate pipe (Outer dia.: $13 \mathrm{~mm}$, Inner dia.: $9 \mathrm{~mm}$, Length:160 mm) for the lossy ultrasonic transmission pipe that a piezo-ceramic annular plate vibrating in the first radial vibration mode is adhered at the pipe end was tried and the study on the effect of the shape of reflector surface on the trapping state is considered. The reflector is made of an acrylic rod (Dia.:13 mm) and the reflective surface is formed in to the conical shape. It was confirmed that the conical shape to be adapted for the trapping exists. To improve in the trapping force of the device, we considered the pipe attaching a reflective ring at the pipe end. The apparatus and distinctive characteristics are shown.

\section{2:15}

5pPA4. Trapping and manipulation of a small object in the space between two pipes placed to the counter direction. Takehiro Takano, Hideki Tamura (Tohoku Inst. of Technol., 35-1 Kasumi-cho, Yagiyama, Sendai 982-8577, Japan, ktakano@tohtech.ac.jp), and Manabu Aoyagi (Muroran Inst. of Technol., Muroran, Japan)

A small object can be trapped in the space between two pipes which are placed to the counter direction and radiated traveling acoustic waves from the pipe ends. In the study, two pipes vibrating in axisymmetric flexural damped traveling wave to generate acoustic flow in the pipe inside are used. The flow propagates in the pipe inside and is radiated for traveling acoustic waves from the pipe end. The pipe made of polycarbonate is used for ultrasonic transmission pipe and two piezo-ceramic annular plates are adhered at the each pipe end for exciting the vibration modes. The first radial vibration mode of the annular plate is used and its resonance frequency is about 59 $\mathrm{kHz}$. When the two vibrators are driven in opposite phase, a small object can be trapped at intermediate point in the space between two pipe ends. The gap of the space is about $10 \mathrm{~mm}$. The trapping object can be shifted its position corresponding to the phase difference of the two driving voltages. That is, the object can be moved on non-contact manipulation in the space between the two pipe ends. These results are confirmed experimentally and by simulation according to FEA.

$$
\text { 2:30 }
$$

5pPA5. Non contact pick-up of drop of liquid from micro-well with megahertz ultrasound. Hiroki Tanaka, Yosuke Mizuno, and Kentaro Nakamura (Lab. for Future Interdisciplinary Res. of Sci. and Technol., Tokyo Inst. of Technol., R2-26, 4259 Nagatsuta, Yokohama, Kanagawa 226-8503, Japan, htanaka@sonic.pi.titech.ac.jp)

In recent years, considerable attention has been paid to the noncontact transport of small objects and droplets as an important technology in pharmacy industries, and new material science/engineering. Our aim in this report is to develop a noncontact pipette, and we focused on the method to pick-up a single droplet from micro-well plate without contact. A focused immersion transducer is placed below a micro-well plate. By emitting burst waves at $1.5 \mathrm{MHz}$, a droplet is ejected from the well and caught by the pressure nodal plane in the standing wave field. First, by observing the deformation of the ejected droplet and the surface of liquid in the micro-well plate with a high-speed video camera, we discussed the principle of droplet pickup. Next, in order to pick-up any type liquid, we investigated the effect of surface tension and viscosity on the noncontact ejection of droplet for water, ethanol, and silicone oil. In this experiment, we found a linear relationship between the ejected droplet volume and the surface tension. In addition, we discuss the ratio of Ohnesorge number to Reynolds number in association with the stability of drop-on-demand inkjet printing operations, and determine the condition for droplet jetting.

\section{2:45-3:00 Break}

3:00

5pPA6. Shape and rotation analysis on an ultrasonically levitated droplet using distributed point source method and least square moving particle semi-implicit method. Yuji Wada, Kohei Yuge (Faculty of Sci. and Technol., Seikei Univ., 3-3-1 Kichijoji-kitamachi, Musashino, Tokyo 1808633, Japan, yuji.wada@st.seikei.ac.jp), Hiroki Tanaka, and Kentaro Nakamura (Lab. for Future Interdisciplinary Res. of Sci. and Technol., Tokyo Inst. of Technol., Yokohama, Kanagawa, Japan)

Ultrasonic droplet levitation has recently been drawing attention as a way of non-contact transportation. Many experiment revealed that the shape changes or streaming field on the levitating droplets, however, quite small number of numerical simulation have discussed this phenomenon including fluid dynamics within the droplet. In this paper, coupled analysis using the distributed point source method (DPSM) and the least square moving particle semi-implicit (LS-MPS) method, both of which do not require grids or meshes to handle the moving boundary with ease, is suggested. The acoustic radiation force including viscoacoustic torque, which emerges from the viscous boundary layer, is calculated from the distributed point source method result using the idea of boundary layer normal velocity and input to the LSMPS surface particles. A droplet levitated in an acoustic chamber is simulated using the proposed calculation method. The droplet is vertically supported by a plane standing wave from an ultrasonic driver and subjected to a rotating sound field excited by two acoustic sources on the side wall with different phases. The rotation of the droplet is successfully reproduced numerically and its acceleration is discussed and compared with those in the literature.

\section{$3: 15$}

5pPA7. Pressure dependence of the attenuation and sound speed in a bubbly medium: Theory and experiment. Amin Jafari Sojahrood (Dept. of Phys., Ryerson Univ., 350 Victoria St., Toronto, ON M5B2K3, Canada, amin.jafarisojahrood@ryerson.ca), Qian Li (Biomedical Eng., Boston Univ., Boston, MA), Hossein Haghi, Raffi Karshafian (Dept. of Phys., Ryerson Univ., Toronto, ON, Canada), Tyrone M. Porter (Biomedical Eng., Boston Univ., Boston, MA), and Michael C. Kolios (Dept. of Phys., Ryerson Univ., Toronto, ON, Canada)

Existence of bubbles in a medium changes the attenuation and sound speed (Cs) of the medium. The relationship between the nonlinear oscillations of the MBs and acoustical parameters of the medium is not fully understood. In this work, monodisperse solutions of lipid coated bubbles with mean diameter of 5.2 micron and peak concentration of (5000 microbubbles/ml) were sonicated with a broadband pulse with center frequency of $2.25 \mathrm{MHz}$. The attenuation and Cs, were measured over a pressure range of 10-100 kPa. Using our recent nonlinear model and by solving the Marmattont Model, the attenuation and Cs of the solution were numerically analyzed. Experimental results showed that as the pressure increased, the attenuation peak increased by $\sim 7-8 \mathrm{~dB}$ while its frequency decreased from 2.05 to $1.55 \mathrm{MHz}$. The maximum Cs of the medium increased with pressure (1521 to $1529 \mathrm{~m} / \mathrm{s}$ ) and shifted towards lower frequencies (2.34 to 1.95 $\mathrm{MHz}$ ). At a fixed frequency (e.g., $1.65 \mathrm{MHz}$ ) the Cs increased by $\sim 14 \%$. The Cs of the bubbly medium remained the same as the Cs in the absence of bubbles at the frequency of the attenuation peaks. Numerical simulations were in good agreement with experimental observations and confirm the experimentally observed phenomenon. 
5pPA8. Evaluation of nonlinear effects at the focus of a hemispherical acoustic source. Oleg A. Sapozhnikov, Pavel B. Rosnitskiy, and Vera A. Khokhlova (Phys. Faculty, Moscow State Univ., Leninskie Gory, Moscow 119991, Russian Federation, oleg@acs366.phys.msu.ru)

Several theoretical models exist for simulating nonlinear generation of harmonics for weakly or moderately focused waves. Under these conditions, the acoustic field can be considered as a directed beam. In the case of hemispherical sources, this assumption is no longer valid, so development of different models is needed. Here, a theoretical approach is presented for evaluating the amplitude level of the second harmonic generated in hemispherically focused fields. The approach is based on the successive approximation method and includes several steps. First, the linear acoustic problem is solved for a hemispherical source using a spherical harmonics series expansion. Next, the volume sources for the second harmonic generation are calculated using the solution to the linear problem. Finally, these sources are used for obtaining an analytic solution for the second harmonic amplitude at the focus. The developed method was applied to study the acoustic field of the ExAblate 3000 clinical transducer that has a hemispherical shape of $30 \mathrm{~cm}$ diameter, operating frequency of $670 \mathrm{kHz}$, and acoustic power of several hundred watts. Calculations predict that nonlinear effects are weak even for the free-field focusing in water at acoustic power levels up to 1 kW. [Work supported by RSF 14-15-00665.]
5pPA9. Introducing Hugonions: Particle-like shocks that travel, interact, and annihilate. Jae-Wan Lee and Won-Suk Ohm (School of Mech. Eng., Yonsei Univ., Yonsei University, 50 Yonsei-ro, Seodaemun-gu, Seoul 03722, South Korea, fantalex@yonsei.ac.kr)

This talk presents a novel computational method for 1-D wave processes in fluids, the applicability of which extends to strongly-nonlinear waves of arbitrary strength. A common approach to modeling waves of arbitrary strength in fluid dynamics is the Riemann solver of the Euler equations, requiring enormous computation time and resources. In this study, we expand upon our previous work [Lee et al., AIP Conf. Proc. 1685, 070011 (2015)], in which the propagation of a strongly-nonlinear wave is described by a collection of forward-traveling, particle-like shocks, dubbed "Hugonions" for their adherence to the Rankine-Hugoniot relations. To extend the applicability of Hugonions to compound waves, the Riemann solution of an arbitrary discontinuity is augmented to the defining characteristics of Hugonions. This way, Hugonions behave like particles, which travel, interact with each other, and annihilate if certain conditions are met. Because a computational mesh is not necessary (i.e., the method simply keeps track of Hugonions), and most importantly Hugonions will reduce in numbers following successive interactions, our computational method leads to a few orders-of-magnitude reduction in computation time compared to the existing Riemann solver-based schemes. Verification of the method against the Riemann solver is performed for a number of well-known benchmark problems. 


\title{
Session 5pSCa
}

\section{Speech Communication: Speech Processing}

\author{
Matthew Goupell, Chair \\ Hearing and Speech Sciences, University of Maryland, College Park, 0119E Lefrak Hall, MD 20742
}

\section{Contributed Papers}

\section{1:00}

5pSCa1. Better-ear glimpsing in Japanese adult bilateral cochlear implant users. Baljeet Rana, Jörg M. Buchholz (National Acoust. Labs. (NAL), Level 5, Australian Hearing Hub 16 University Ave., Sydney, NSW 2109, Australia, baljeet.rana@nal.gov.au), Catherine Morgan (Cochlear Asia pacific, Sydney, NSW, Australia), Mridula Sharma (Dept. of Linguist (Audiol. Section), Macquarie Univ., Sydney, NSW, Australia), Tobias Weller (National Acoust. Labs. (NAL), Sydney, NSW, Australia), Shivali A. Konganda (Dept. of Linguist (Audiol. Section), Macquarie Univ., Sydney, NSW, Australia), Kyoko Shirai, and Atsushi Kawano (Dept. of Otolaryngol., Tokyo Medical Univ., Tokyo, Japan)

Better-ear glimpsing (BEG) is a phenomenon that helps understanding speech in the presence of fluctuating, spatially separated distractors. This phenomenon has been studied in normal-hearing $(\mathrm{NH})$ and hearing-impaired listeners but remains untapped in adults with cochlear implants (CIs). Further, it has not been investigated how far providing CIs in both ears can improve BEG over providing a single CI in one ear. In the current study, seven Japanese adult bilateral CI users with post-lingual deafness were recruited. Male and female speech distractors were presented from $+90^{\circ}$ and $-90^{\circ}$ and target questions taken from the Helen sentence test (translated into Japanese, spoken by a native Japanese female speaker) were presented from the front. Speech comprehension thresholds (SCTs) were measured in both a co-located and spatially separated condition with CIs in both ears and only in one ear. BEG was calculated as the difference in SCTs between the co-located and spatially separated condition. BEG noted with bilateral CIs was about $2 \mathrm{~dB}$ and with unilateral $\mathrm{CI}$ was about $0.3 \mathrm{~dB}$, which is significantly smaller than found in $\mathrm{NH}$ listeners. The bilateral benefit noted was about $3 \mathrm{~dB}$ indicating the advantage of having CIs in both the ears.

\section{$1: 15$}

5pSCa2. Challenges in understanding and modeling speech perception. Adelbert W. Bronkhorst (Human Factors, TNO, Kampweg 5, Soesterberg 3769DE, Netherlands, adelbert.bronkhorst@tno.nl)

Speech perception performance is one of the most widely used measures in audiology and auditory research. Nevertheless, our understanding of how speech is processed by the auditory system is limited. This is not only due to the subject-the complexity of the speech signal itself and the intricacy of disentangling target speech from interfering sounds-but also to the way it is approached: different aspects of speech perception are studied by largely separate research communities. Also when only early processing of speech is considered, excluding psycholinguistic aspects and effects of memory and learning, at least three relevant research fields can be discriminated: audibility and masking, auditory scene analysis, and attention. Although there is increasing interest in the crosslinks between these fields, approaches are still insufficiently integrated and important questions remain unanswered. Furthermore, modeling efforts are lagging behind and are only partly driven by advances in neurophysiological research. After reviewing progress in the three fields, a conceptual model will be presented that attempts to tie results together. Finally, knowledge gaps and open research issues will be highlighted.
$1: 30$

5pSCa3. Unusual auditory/speech processing in autism spectrum disorder revealed by verbal transformation effects. Chihiro Itoi (Chuo Univ., 742-1 Higashinakano Hachioji-shi, Tokyo 192-0393, Japan, itoi.chihiro@ gmail.com), Nobumasa Kato (Medical Inst. of Developmental Disabilities Res., Showa Univ., Tokyo, Japan), and Makio Kashino (NTT Commun. Sci. Labs., Nippon Telegraph and Telephone Corp., Kanagawa, Japan)

Autism spectrum disorder (ASD) is a neurodevelopmental disorder characterized by an impaired ability to communicate and restricted and repetitive patterns of behavior. Sensory abnormality, a trait included in restricted patterns of behavior, is often observed in individuals with ASD. This study used an auditory illusion called "verbal transformation (VT)" to examine abnormal auditory/speech processing in ASD. In VT, a series of subjective perceptual changes occurs when subjects listen to a repeating word without a pause. The characteristic of VT is that, physically, the stimulus is the same repeated word but the perceptual change differs for each person. In this point, VT could be useful for demonstrating certain ASD traits such as favoring repetition. Twenty-four ASD adults and 24 neurotypical controls listened to three samples of repeated Japanese words for five minutes, and reported their percepts verbally whenever they experienced perceptual changes. As for the number of perceptual changes and that of reported forms, we found no significant difference between the ASD group and controls. However, the phonetic variations of form changes were different, and ASD group showed more phonetic variation than controls. We interpret the results from the viewpoint of unusual adaptation in auditory and/or phonetic processing.

\section{$1: 45$}

5pSCa4. Listening effort and accent in competing-talker speech recognition by native and non-native listeners: Electrophysiological measures of auditory and lexical processing. Paul Iverson, Emma Brint, Jieun Song, and Mengfan Wu (Univ. College London, 2 Wakefield St, London WC1N 1PF, United Kingdom, p.iverson@ucl.ac.uk)

Listening effort modulates auditory processing for speech, such that attention to a target talker in the presence of a distractor increases the entrainment of cortical activity to the target speech amplitude envelope. Similarly, lexical-processing effort can be measured in EEG recordings, with more extensive lexical search being measured as a more negative N400. The present study compared effort at both levels for native speakers of southern British and Glaswegian English, and Chinese second-language English speakers. Listeners heard sentences spoken in southern British and Glaswegian accents, either with single or competing talkers. The results demonstrate that competing talkers and difficult accents have similar effects at a lexical level, increasing the amount of lexical search for native listeners and disrupting lexical search for non-native listeners. In contrast, corticalacoustic entrainment was higher for targets than distractors in competingtalker conditions, but was not modulated by accent; entrainment was also similar for different language groups in the present study, although language-background differences in entrainment can be found in easier conditions with spatial cues to segregation. Accent may thus have little role in the 
auditory segregation of competing talkers, despite the fact that accent and listener background affects intelligibility and lexical access.

$$
\text { 2:00 }
$$

5pSCa5. Adaptive speech modifications and its effect on communication effectiveness in complex acoustic environments. Nandini Iyer, Eric Thompson, Kelly Stillwagon, Zachariah Ennis, Abbey Willis, and Brian Simpson (Air Force Res. Lab., 2610 Seventh St., Bldg 441, Wpafb, OH 45433, Nandini.Iyer.2@us.af.mil)

Previous studies of communication in noisy environment have shown that interlocutors, when residing in the same acoustic environment, continuously adapt their speech patterns in order to overcome communication difficulties (Cooke et al., 2014; Lindblom, 1990). However, little is known about the impact on communication when individuals are in disparate acoustic environments and if/how these individuals would adapt their speech to support effective communication. In this study, eight pairs of interlocutors were required to completed the "spot-the-difference" Diapix task (Van Engel et al., 2010) while being exposed to one of 2 acoustic environments presented at $85 \mathrm{~dB}$ SPL over headphones: 1) sparse (2-talker) babble; or 2) dense (8-talker) babble. In some conditions, both individuals were in similar acoustic environments while in others they were in different acoustic environments. A control condition, where both interlocutors communicated in quiet environments was also included. Preliminary results showed that the time for task completion increased when listeners communicated in a dense rather than sparse babble, thus indicating decreased communication efficiency. Acoustic characteristics of the speech produced in these adverse environments suggests that talkers modulate their speech for their own listening environment and not necessarily to meet the needs of their communication partners

2:15

5pSCa6. The contribution of acoustic complexity to speech-specific processing. Faith Chiu and Jyrki Tuomainen (Speech, Hearing and Phonetic Sci., Univ. College London, Rm. 326, Chandler House, 2 Wakefield St., London WC1N 1PF, United Kingdom, Faith.Chiu.11@ucl.ac.uk)

Speech segments are delivered in an acoustically complex signal. This paper examines the role of acoustic complexity in speech-specific processing in the brain. Mismatch Negativity (MMN) is a deviance detection eventrelated response elicited by both speech and non-speech stimuli. Speechspecific MMN can further be sensitive to segment-driven categorisation processes (Nääänen et al., 1997). Existing research on the neural basis of speech processing often employs only pure tones as a non-speech control (Schofield et al, 2009). We investigate the specific contribution of acoustic complexity to speech-specific processing using a passive oddball task to elicit MMN in three conditions: speech, spectrally rotated speech, and pure tones. EEG event-related potentials were examined for scalp-based observations and source connectivity; source activity at the level of pyramidal cells in neuronal groups was modeled as dynamic states (Kiebel et al., 2007). Results indicate left lateralization only for acoustically complex sounds; both speech and rotated speech show within-region cortical coupling in left Heschl's gyrus. However, an increase in feedforward activity towards left superior temporal gyrus is only observed in speech but not rotated speech. Pure tone stimuli do not elicit left lateralization. We conclude that acoustic complexity is a necessary but insufficient condition for speech-specific processing.

$$
\text { 2:30 }
$$

5pSCa7. A study on the effect by adding reverberation to speech-like masking sound. Jen W. Tang, Jacky Wan, and Yusuke Hioka (Dept. of Mech. Eng., Univ. of Auckland, Privatebag 92019, Auckland 1142, New Zealand, jtan160@aucklanduni.ac.nz)

Problems arising from the acoustical privacy point of view in public spaces have been known to be an issue. The lack of acoustical privacy has been known to affect the human's health both physically and psychologically, thus keeping the acoustical privacy in public spaces will significantly reduce social loss. Masking is the most commonly practically used technique to make a target speech unintelligible to the unintended listeners without needing to install any physical structures. Time-reversed speech has been known to effectively mask information for speech privacy applications; however, the annoyance and distraction caused by the time-reversed speech is known to be higher than other masking sound. This study explores a solution to compromise the suggested problem by adding a reverberant effect to a time-reversed speech. Subjective listening tests have been conducted to measure the intelligibility of target speech, annoyance and distraction caused by the masking sound. The experimental results suggest that adding artificial reverberation to a time-reversed speech has a significant effect to reduce the annoyance level while maintaining the masking effectiveness of the original time-reversed speech. A trend was also observed that the addition of artificial reverberation could reduce the level of distraction caused by the masking sound.

$$
\text { 2:45 }
$$

5pSCa8. Estimating realistic speech levels for virtual acoustic environments. Jorg M. Buchholz (Australian Hearing, National Acoust. Labs., 126 Greville St., Chatswood, NSW 2067, Australia, jorg.buchholz@nal.gov.au), Adam Weisser (Linguist, Audiol., Macquarie Univ., NSW, Australia), Tim Beechey, James Galloway, Gitte Keidser, and Katrina Freeston (Australian Hearing, National Acoust. Labs., Macquarie University, NSW, Australia)

Speech intelligibility is commonly assessed in rather unrealistic acoustic environments at negative signal-to-noise ratios (SNRs). As a consequence, the results seem unlikely to reflect the subjects' experience in the real world. To improve the ecological validity of speech tests, different sound reproduction techniques have been used by researchers to recreate field-recorded acoustic environments in the laboratory. Whereas the real-world sound pressure levels of these environments are usually known, this is not necessarily the case for the level of the target speech (and therefore the SNR). In this study, a two-talker conversation task is used to derive realistic target speech levels for given virtual acoustic environments. The talkers communicate with each other while listening to binaural recordings of the environments using highly open headphones. During the conversation their speech is recorded using close-talk microphones. Conversations between ten pairs of young normal-hearing talkers were recorded in this way in 12 different environments and the corresponding speech levels were derived. In this presentation, the methods are introduced and the derived speech levels are compared to results from the literature as well as from real sound-field recordings. The possibility of using this technique to generate environmentspecific speech material with realistic vocal effort is discussed.

\section{3:00-3:20 Break}

$3: 20$

5 pSCa9. Effect of audibility, frequency region, and hearing loss on better-ear glimpsing. Baljeet Rana and Jörg M. Buchholz (National Acoust. Labs. (NAL), Level 5, Australian Hearing Hub 16 Univ. Ave., Sydney, NSW 2109, Australia, baljeet.rana@nal.gov.au)

Better-ear glimpsing (BEG), which utilises short-term interaural level difference cues (ILDs), helps understanding speech in noise. Since ILDs are mainly available at high frequencies where hearing loss is usually strongest, hearing-impaired (HI) listeners cannot take full advantage of BEG. One possible solution is to provide artificially generated ILDs at low and mid frequencies where hearing loss is usually less pronounced. Rana and Buchholz [JASA, 2016] showed that HI listeners can take advantage of such artificial ILDs, but their received benefit in speech intelligibility was still smaller than for normal-hearing (NH) listeners. To understand how far this difference can be explained by differences in audibility, speech recognition thresholds (SRTs) were measured in four frequency regions (low, mid, high, and broadband) at four audibility levels $(0,10,20$, and $30 \mathrm{dBSL})$ in $10 \mathrm{NH}$ and $10 \mathrm{HI}$ listeners. SRTs were measured for BKB like sentences in the presence of vocoded speech distractors. The difference between co-located and spatially separated SRTs was used as a measure of BEG. Results revealed that providing equal audibility removed the performance differences between groups. Moreover, the effect of BEG was roughly frequency independent and increased by about $2 \mathrm{~dB}$ for each $10 \mathrm{~dB}$ increase in sensation level. 
thresholds across the array has recently been validated in adults. Here, the findings are extended to children who were bilaterally implanted sequentially. These children have different etiologies than the typically studied adults and different durations of auditory deprivation and implant use between their two ears. By comparing within listeners, the role of cognitive or linguistic factors is minimized. Within ears, the confusions listeners made were consistent between conditions of quiet and 4-talker babble background noise at a $+10 \mathrm{~dB}$ signal-to-noise ratio. For individual children, however, confusion patterns differed between the ears, suggesting that time with the implant and the electrode-neuron interface may indeed play a role in vowel confusions, as opposed to more global perception factors. Finally, the pattern of high-threshold regions corresponds to predictable vowel confusions, similar to those observed in adults.

ation along a continuum of signal modulation characteristics. Sentence stimuli were combined with either 1-, 2-, or 6-talker modulated noise at a range of SNRs. Speech-in-noise signals were processed at a range of reverberation times with the speech and noise signals co-located. Stimuli were then processed at a range of compression release times (RT) using a hearing aid simulation which mimicked six-channel hearing aid processing. Signals were acoustically analyzed using a digital inversion method which employed phase cancellation to separate speech and noise signals at the output of the compressor. This enabled us to quantify changes to SNR as a result of compression. Results indicated that shorter RTs altered SNR more than longer RTs; however, reverberation reduced or eliminated the differences among RTs. Additionally, less modulated maskers lead to poorer output SNRs than modulated maskers. [Work supported by NIH.]

\section{3:50}

5pSCa11. Real-time simulation of hearing impairment: Application to speech-in noise intelligibility. Nicolas Grimault (Univ. Lyon 1, CNRS UMR 5292 CRNL, UMR CNRS 5292 CRNL, Univ. Lyon 150 av T Garnier, Lyon cedex 07 69366, France, nicolas.grimault@cnrs.fr), Etienne Parizet (INSA-Lyon, Laboratoire Vibrations Acoustique, Villeurbanne, France), Alexandra Corneyllie (Univ. Lyon 1, CNRS UMR 5292 CRNL, Lyon, France), Laurent Brocolini (INSA-Lyon, Laboratoire Vibrations Acoustique, Villeurbanne, France), Richard Weyn, and Samuel Garcia (Univ. Lyon 1, CNRS UMR 5292 CRNL, Lyon, France)

Accurately simulating cochlear hearing loss in real time is of paramount importance for various applications, including easier testing of the impact of cochlear damage on auditory perception in real-life situations, evaluating sound qualities as perceived by hearing-impaired listeners, and pedagogical demonstrations. Following an idea originally proposed by Irino and colleagues, a real-time hearing-loss simulator was developed. Using an inverse compressive gammarchirp filters analysis, the simulator aims to replicate the increase of hearing thresholds, the level-dependent widening of auditory filters, and modification of the loudness curve (recruitment). The characteristics of the simulator, and basic psychoacoustical measurements performed with and without the simulator (absolute thresholds, auditory filters estimated with notch-noise data, cochlear input/output functions estimated with temporal masking curves) will be presented. Finally, an experiment comparing the intelligibility of speech in noise for real and simulated hearingimpaired listeners with a French version of the Four Alternative Auditory Feature Test (FAAF) will be presented. On average, the results of normalhearing listeners using the simulator were found to be similar to the results obtained in individuals with hearing loss of presumed cochlear origin, suggesting that the simulator is valid.

\section{4:05}

5pSCa12. Within-listener comparisons of vowel confusions in pediatric, bilateral cochlear implantees. Julie A. Bierer (Speech and Hearing Sci., Univ. of Washington, 1417 NE 42nd St., Box 354875, Seattle, WA 98105 , jbierer@uw.edu) and Mishaela DiNino (Neurosci., Univ. of Washing, Seattle, WA)

Tremendous variability in speech perception abilities exists in children and adults who wear cochlear implants (CIs). Preliminary data in adults suggests that variability in the interface between CI channels and auditory neurons can influence spectral resolution and thus vowel perception. One method for assessing the electrode-neuron interface is by using focused electrical fields to measure thresholds. A quick method for measuring

5pSCa13. Cochlear implant listener vowel identification performance and confusion patterns with selective channel activation programs. Mishaela DiNino, Matthew B. Winn, and Julie A. Bierer (Dept. of Speech and Hearing Sci., Univ. of Washington, 1417 NE 42nd St., Seattle, WA 98105 , MDiNino@u.washington.edu)

Cochlear implants (CIs) restore auditory perception to profoundly deaf individuals, yet identification of speech sounds remains limited by degraded or warped spectral representations. The present study aimed to measure the ways in which the perception of vowels (distinguished primarily by their spectra) is affected by selective activation of high or low quality electrode channels. Individualized experimental processing programs were created for eight CI users by selectively deactivating channels with poor electrode-neuron interfaces (identified by high auditory perception thresholds with focused stimulation), and reallocating frequencies to remaining electrodes ("High Off"). A contrary program in which channels with better electrodeneuron interfaces were deactivated ("Low Off") was created for each participant for comparison. A program with all channels activated ("All") served as a control. CI users performed a vowel recognition task with each experimental program. Overall percent correct did not change significantly across programs. However, perceptual distance and perceptual vowel space analyses indicated large differences in vowel confusion patterns between listening with "All," "High Off," and "Low Off" programs within individual CI users. These results suggest that vowel perception is dramatically altered by CI channel deactivation and frequency reallocation, which is not evident solely from average identification performance.

\section{4:35}

5pSCa14. Perception of low-pass filtered speech in hearing-impaired children, with and without cochlear dead regions and children with normal hearing. Alicja N. Malicka (School of Health and Rehabilitation Sci., The Univ. of Queensland, St. Lucia, QLD 4067, Australia, a.malicka@uq. edu.au), Kevin J. Munro (Manchester Ctr. for Audiol. and Deafness, The Univ. of Manchester, Manchester, United Kingdom), Thomas Baer (Dept. of Experimental Psych., Univ. of Cambridge, Cambridge, United Kingdom), Richard J. Baker (Manchester Ctr. for Audiol. and Deafness, The Univ. of Manchester, Manchester, United Kingdom), and Brian C. Moore (Dept. of Experimental Psych., Univ. of Cambridge, Cambridge, United Kingdom)

There are reports that adults with high-frequency cochlear dead regions (CDRs) exhibit an enhanced ability to use audible low-frequency acoustic information. This results in better performance of participants with CDRs compared to those without CDRs on tasks where the speech is low-pass filtered with a cutoff frequency near the edge frequency of the CDR (Moore and Vinay 2009). This enhanced ability to use low-frequency information may be related to cortical plasticity induced by the presence of a CDR and may be stronger in children due to maximal plasticity of the central auditory pathways. The aim of this study was to determine if children (aged 7-15 years) with a high-frequency congenital hearing loss and CDRs also show enhanced ability to use low frequency information. Vowel-consonant-vowel nonsense speech stimuli were low-pass filtered at various frequencies, amplified to correct for any hearing loss and presented via headphones. The percentage of correctly identified consonants for each low-pass filtering condition were measured in each ear separately. The performance of children with CDRs was compared against performance of age matched children 
without CDR, children with normal hearing and against the Speech Intelligibility Index prediction. Preliminary data do not support the hypothesis that children with high-frequency CDRs use low-frequency information more effectively than their no-CDR counterparts or normal hearing children.

\section{4:50}

5pSCa15. For some bilateral cochlear implantees, a second implant can cause interference rather than improved speech understanding in the presence of competing speech. Matthew Goupell, Olga Stakhovskaya (Hearing and Speech Sci., Univ. of Maryland, College Park, 0119E Lefrak Hall, College Park, MD 20742, goupell@umd.edu), and Joshua G. Bernstein (Walter Reed National Military Medical Ctr., Bethesda, MD)

The goal of bilateral cochlear implantation is to provide binaural-processing benefits, such as improved sound localization and speech understanding in background noise. A recent study [Bernstein et al., Ear Hear. 37, 282-288] showed that bilateral cochlear-implant (BICI) listeners could receive up to $5 \mathrm{~dB}$ of binaural speech-understanding benefit when listening with two ears compared to one. However, one outlier demonstrated interference rather than improvement from the second ear, which warranted further investigation. We recruited nine BICI listeners with pre- or peri-lingual onset of deafness and/or a long duration of deafness in at least one ear. We hypothesized these listeners might also demonstrate interference in binaural speech understanding. They listened to a male target and a male interferer, both taken from the Coordinate Response Measure corpus, in monaural (target and interferer in one ear) and binaural (monaural target, diotic interferer) listening configurations. On average, listeners received $9 \mathrm{~dB}$ of interference from their second ear. For listeners with a much longer duration of deafness in one ear, interference patterns were asymmetrical (little interference in the first implanted ear, extreme interference in the second). These data demonstrate that certain spatial listening configurations may hinder the advantages of bilateral implants for some users.

\section{5:05}

5pSCa16. Can spectral centroid explain voice pitch and vocal-tract length perception in normal-hearing and cochlear implant listeners? Etienne Gaudrain (Auditory Cognition and PsychoAcoust., CNRS UMR 5292, Lyon Neurosci. Res. Ctr., Université Lyon 1, UMCG, KNO, Huispostcode BB20, PO Box 30.001, Groningen 9700 RB, Netherlands, etienne. gaudrain@cnrs.fr)

Voice pitch (F0) and vocal-tract length (VTL) are two principal voice characteristics that play a major role in segregating voices in cocktail party situations. Cochlear implant (CI) listeners struggle with taking advantage of voice differences in such situations. Recent studies show that they have difficulties discriminating voices on the basis of these two cues. Yet, the mechanisms underlying perception of these cues in CI users, but also in normal hearing $(\mathrm{NH})$ listeners, remain largely unknown. In CIs, F0 can be coded temporally and spectrally in the lower frequency channels, but some recent studies have suggested that spectral centroid (SC) could be used instead. VTL could be perceived through its effect on individual formants, but is also often likened to timbre, and as such, it has been argued that VTL perception might rely on SC. However, these assumptions tend to overlook the SC variability occurring in natural speech. In this study, it is shown how this variability would influence F0 and VTL JNDs in NH and CI listeners if they were based on SC, using a signal detection theory model. The results suggest that $\mathrm{SC}$ is unlikely to be a reliable cue for vocal F0 and VTL perception in natural speech.

\title{
Session 5pSCb
}

\section{Speech Communication: Speech Perception and Production by Clinical, Aging, and/or Developing Populations (Poster Session)}

\author{
Jessica Alexander, Chair \\ Psychology, Centenary College of Louisiana, 2911 Centenary Blvd, Shreveport, LA 71104
}

\begin{abstract}
All posters will be on display from 1:00 p.m. to 5:30 p.m. To allow contributors in this session to see the other posters, authors of oddnumbered papers will be at their posters from 1:00 p.m. to 3:15 p.m. and authors of even-numbered papers will be at their posters from 3:15 p.m. to 5:30 p.m.
\end{abstract}

\section{Contributed Papers}

5pSCb1. Perception of geminate consonants and devoiced vowels in Japanese by elderly and young listeners. Eri Iwagami, Takayuki Arai (Sophia Univ., 7-1, Kioi-cho, Chiyoda-ku, Tokyo 102-8554, Japan, alice. erieri@gmail.com), Keiichi Yasu (Res. Inst. of National Rehabilitation Ctr. for People with Disabilities, Tokyo, Japan), Emi Yanagisawa (Meiji Univ., Tokyo, Japan), and Kei Kobayashi (The Univ. of Auckland, Auckland, New Zealand)

The goal of this study is to compare the perception of geminate consonants and devoiced vowels by elderly and young Japanese speakers. This study consisted of two perceptual experiments (Exp. 1and Exp. 2). In Exp.1, materials were nonsense words "ata" and "atta" having the structures: ' $\mathrm{V}_{1} \mathrm{CV}_{2}$ ', ' $\mathrm{V}_{1} \mathrm{C}: \mathrm{V}_{2}$ ', and the following three parameters were changed: 1) formant transition of $\left.\mathrm{V}_{1}(\mathrm{FT}), 2\right) \mathrm{V}_{1}$ duration, and 3) closure duration. In Exp. 2, materials were 27 Japanese words whose vowels can be devoiced. The results of Exp. 1 showed that compared with young listeners the perceptual boundary of geminate for elderly listeners shifted towards longer closure duration in the absence of FT. Moreover, the results of Exp. 2 showed that the misperception rate of young listeners was low with and without devoicing, whereas the rate was high with devoicing and low without devoicing in elderly listeners.

5 pSCb2. Cognitive predictors of older adults' perception of difficult speech. Erin Ingvalson, Kaitlin Lansford, Valeriya Federova, and Gabriel Fernandez (School of Commun. Sci. and Disord., Florida State Univ., 201 W Bloxham St., Tallahassee, FL 32306, eingvalson@fsu.edu)

Recent work suggests that receptive vocabulary, over working memory capacity, may predict older adults' ability to perceive disordered speech, particularly when hearing acuity is poor (McAuliffe et al., 2013). Further work in younger adults suggests that both receptive vocabulary and 
cognitive flexibility may predict listeners' ability to perceive accented speech (Baese-Berk, et al., 2015). Fifteen older adults transcribed phrases produced by five non-native English speakers and four talkers with dysarthria and completed measures on receptive vocabulary, auditory working memory, cognitive flexibility, and inhibitory control. Listeners were more accurate identifying speech produced by talkers with dysarthria than by talkers with foreign accents $(t(14)=11.08, p<.001)$. Mixed effect models indicated that hearing acuity, auditory working memory, and inhibitory control were significant predictors of both difficult speech conditions; cognitive flexibility was an additional predictor of older adults' ability to perceive dysarthric speech. In contrast to earlier work, receptive vocabulary was not a significant predictor of listeners' accuracy in either condition, nor were listeners' perception of the accented speech and the dysarthric speech correlated. These data add to the growing picture that difficult speech perception is a cognitively demanding task for older adults, regardless of hearing ability.

5pSCb3. Articulation rates of people who do and do not stutter during oral reading and speech shadowing. Rongna A (Res. Inst., National Rehabilitation Ctr. for Persons with Disabilities, 4-1 Namiki, Tokorozawa, Saitama 3598555, Japan, hohchahar-aruna@rehab.go.jp), Keiko Ochi (National Inst. of Informatics, Tokyo, Japan), Keiichi Yasu, Naomi Sakai, and Koichi Mori (Res. Inst., National Rehabilitation Ctr. for Persons with Disabilities, Tokorozawa, Saitama, Japan)

Purpose: Previous studies indicate that people who stutter (PWS) speak more slowly than people who do not stutter (PWNS), even in the fluent utterances. The present study compared the articulation rates of PWS and PWNS in two different conditions: oral reading and speech shadowing in order to elucidate the factor that affect the speech rate in PWS. Method: All participants were instructed to read aloud a text and to shadow a model speech without seeing its transcript. The articulation rate (mora per second) was analyzed with an open-source speech recognition engine "Julius" version 4.3.1 (https://github.com/julius-speech/julius). The pauses and disfluencies were excluded from the calculation of the articulation rate in the present study. Results: The mean articulation rate of PWS was significantly lower than that of PWNS only in oral reading, but not in speech shadowing. PWS showed a significantly faster articulation rate, comparable to that of the model speech, in shadowing than in oral reading, while PWNS did not change the articulation rate depending on the task. Conclusion: The group and task comparisons indicate that the ability of PWS to rapidly articulate is not impaired but somehow impeded during oral reading.

5pSCb4. Acoustics of palilalia: A case study. Christina C. Akbari (Commun. Disord., Arkansas State Univ., 4305 Bekah Dr. \#8, Jonesboro, AR 72404, cakbari@astate.edu) and Amy Shollenbarger (Commun. Disord., Arkansas State Univ., State University, AR)

Palilalia is a rather rare speech disorder associated with a variety of etiologies including Parkinson's disease, stroke, epilepsy, and others. According to the literature, palilalia is characterized by repetitions of words, phrases, and/or sentences produced with an increasing rate and decreasing intensity. Because of the rare nature of this disorder, literature involving the characteristics is limited and somewhat contradictive. Although the original definition involves increasing rate and decreasing intensity, some researchers (i.e., Kent \& Lapointe, 1982) have found different characteristics such as decreasing rate and increasing intensity when examining individual cases. This study adds to the literature regarding characteristics of palilalic speech in terms of change in rate and fundamental frequency from the original utterance to the first repeated train. This study utilized a single-subject design involving a 42 year old male who suffered an anoxic brain injury. Spontaneous speech samples were collected and analyzed. The results showed significant differences in terms of rate and fundamental frequency with the first repeated train in comparison to the original utterance. The rate was significantly faster and the fundamental frequency was significantly higher in the repeated utterance.
5 pSCb5. Influence of clear speech on voice onset time and intelligibility of word-initial stop consonants in electrolaryngeal speech. Steven R. Cox, Lawrence Raphael (Commun. Sci. and Disord., Adelphi Univ., Hy Weinberg Ctr. Rm. 136, Adelphi University, Garden City, NY 11530, scox@adelphi.edu), and Philip C. Doyle (Health and Rehabilitation Sci., Western Univ., London, ON, Canada)

This study addressed the influence of clear speech (CS) on voice onset time (VOT) and intelligibility of word-initial (WI) stops in electrolaryngeal (EL) speech. Eighteen consonant-vowel-consonant words containing /p/, /t/, $/ \mathrm{k} /, / \mathrm{b} /, / \mathrm{d} /$, and $/ \mathrm{g} /$ in WI position were spoken by 10 laryngectomees in both habitual speech (HS) and clear speech (CS) conditions. Twelve, naive listeners transcribed a total of 4,320 words across HS and CS conditions of which 720 words (containing WI stops) were analyzed. Results indicate that when using CS, EL speakers produced stops with $\sim 4$ milliseconds of greater lag time compared to HS. Further, the intelligibility of WI stops improved $3.8 \%$ during CS. Finally, listeners omitted $2.22 \%$ more stops in WI position when using HS compared to CS. However, repeated measures analysis of variance revealed no significant effect of speaking condition on VOT or the intelligibility of stops. Collectively, these findings provide initial evidence that volitional attempts to make EL speech clearer via CS does not necessarily facilitate improved VOT or intelligibility for EL speakers.

5pSCb6. Patterns in speech errors among children with auditory processing disorder. Brooke DeVore (Dept. of Linguist and Cognit. Sci., Univ. of Delaware, 210 South College Ave., Newark, DE 19716, bdevore@udel. edu), Kyoko Nagao, Olivia Pereira (Ctr. for Pediatric Auditory and Speech Sci., Nemours/Alfred I. duPont Hospital for Children, Wilmington, DE), Julianne Nemith, Rachele Sklar (Dept. of Linguist and Cognit. Sci., Univ. of Delaware, Newark, DE), Emily Deeves (Dept. of Speech, Lang., and Hearing Sci., Univ. of Colorado Boulder, Boulder, CO), Emily Kish (Dept. of Commun. Sci. and Disord., West Chester Univ., West Chester, PA), Kelsey Welsh (Dept. of Commun. Sci. and Disord., Montclair State Univ., Montclair, NJ), and Thierry Morlet (Ctr. for Pediatric Auditory and Speech Sci., Nemours/Alfred I. duPont Hospital for Children, Wilmington, DE)

Auditory Processing Disorder (APD), a neurological hearing impairment, often leads to delays in speech development when diagnosed in childhood. It is unclear whether children with APD make articulation errors due to auditory processing deficits. The purpose of this study was to analyze the articulation error patterns of children diagnosed with APD. We examined speech samples from 26 children diagnosed with APD who participated in a previous research study involving speech perception testing. During this task, each child repeated each of 50 monosyllabic words presented to them in quiet. For each incorrect response, we examined the errors by type of articulation error (substitution, deletion, insertion, or distortion) and the syllable position (onset or coda). Speech perception scores were normal in quiet. However, 22 subjects responded incorrectly for one or more words, with substitution errors (40-50\%) being the most prevalent type of errors. Articulation errors in the coda position $(81 \%)$ were more common than errors in the onset position (19\%). These results imply that children with APD, though generally performing well on speech tests in quiet, tend to display articulation issues not typically seen in normally developing children in the same age group. [Work supported by NIH COBRE Grants 2P20RR020173-06A1 and P30GM114736.]

5pSCb7. Exploiting the Ganong effect to probe for phonetic uncertainty resulting from hearing loss. Steven P. Gianakas and Matthew Winn (Speech \& Hearing Sci., Univ. of Washington, 4217 NE 42nd St., Seattle, WA98105, spgia5@uw.edu)

The "Ganong effect" is the tendency to perceive an ambiguous speech sound as a phoneme that would complete a real word, rather than completing a nonsense/fake word. For example, a sound that could be heard as either $/ \mathrm{g} /$ or $/ \mathrm{k} /$ is perceived as $/ \mathrm{g} /$ when followed by "ift" but perceived as $/ \mathrm{k} /$ when followed by "iss." Because the target speech sound $(/ \mathrm{g} /$ or $/ \mathrm{k} /$ ) is the 
same across both environments, this effect demonstrates the influence of top-down processing rather than simply bottom-up processing in speech perception. We hypothesized that degradations in the auditory system (including simulations of hearing loss or cochlear implantation), speech stimuli will be rendered more ambiguous, and an increased Ganong effect should be observed. Participants heard three speech continua that varied by spectral cues of varying speeds, including fast (stop formant transitions), medium (fricative spectra), and slow (vowel formants). Stimuli were presented with clear spectral quality, or with varying amounts of spectral degradation using a noise vocoder. Responses were analyzed within stimulus pairs using binomial logistic regression, revealing increased Ganong effect with degraded speech. This test is proposed as a simple measure of how phonetic uncertainty can lead to an individual's increased reliance on top-down processing.

5pSCb8. Target-word identification in noise with formant enhancement for hearing-impaired listeners. Jingjing Guan and Chang Liu (Univ. of Texas at Austin, 1 University Station A1100, Austin, TX 78712, jane. guan@utexas.edu)

Degraded speech intelligibility in background noise is a common complaint of most listeners with hearing loss. The purpose of the current study is to investigate the effect of spectral enhancement of F2 on target-word identification in noise for the old listeners with hearing loss (HL) and with normal hearing $(\mathrm{NH})$. Target words (e.g., name, color, and digit) were selected and presented based on the paradigm of coordinate response measure $(\mathrm{CRM})$ corpus of English and Chinese versions. Identification tasks with original and F2-enhanced speech in the 2-talker and 6-talker babbles were designed for the two groups (HL and $\mathrm{NH})$. Thresholds of word identification was measured by using adaptive up-down methods. Results showed that listeners with $\mathrm{NH}$ had better performance on word identification in noise than listeners with HL in almost all listening conditions. More importantly, thresholds of both groups were improved for enhanced speech signals. Compared with NH group, listeners with HL gained significantly greater benefits in the most challenging condition. These preliminary results suggested that speech sounds with F2 enhancement might improve listeners' ordinary speech perception in noise.

5pSCb9. Ideal binary masking based noise reduction and interleaved processing for bilateral cochlear implant users. Shaikat Hossain (Univ. of Texas at Dallas, 7220 Mccallum Blvd. \#307, \#307, Dallas, TX 75252, shossa3@gmail.com), Vahid Montazeri, and Peter F. Assmann (Univ. of Texas at Dallas, Plano, TX)

The present study investigates the interaction between ideal binary masking (IdBM) noise reduction and interleaved processing for bilateral cochlear implant $(\mathrm{CI})$ users and normal-hearing $(\mathrm{NH})$ listeners attending to vocoder simulations. IdBM decomposes a signal into time-frequency (T-F) bins and retains only regions where the target is higher than a local threshold (LC). IdBM benefits in CI users have been found to be limited due to factors such as current spread from neighboring electrodes. Interleaving channels across ears in bilateral CI users has been one approach to mitigating the effects of current spread. In the present experiments we tested NH listeners attending to vocoder simulations and CI users with IEEE sentences presented from different azimuths in speech-shaped noise at $5 \mathrm{~dB}$ signal-tonoise ratio and IdBM processing with LC values of 5 and $-10 \mathrm{~dB}$. Speech intelligibility was poorer for the interleaved condition in the presence of noise. As predicted, however, greater IdBM benefits were found for interleaved compared to non-interleaved processing for both $\mathrm{NH}$ and $\mathrm{CI}$ listeners. For CI users, the LC value of $5 \mathrm{~dB}$ led to greater improvements whereas the NH listeners had comparable benefits for both LC values. Findings have relevance for the clinical application of interleaved processing.

5pSCb10. Effect of dimension-switch ability in children with high-functioning Autism's perception of emotional prosody. Chieh Kao and FengMing Tsao (National Taiwan Univ., No. 1, Sec. 4, Roosevelt Rd., Taipei 106, Taiwan, chiehkao.ckao@gmail.com)

Children with High-Functioning Autism's (HFA) sensitivity to emotional prosody in speech sounds, was long under debate. The stiffness prohibiting them to switch between diverse features might be one of the factors that make them difficult to understand emotional prosody in daily communications. Present study explored how dimension-switch ability in identifying lexical tone would modulate children's perception of emotional prosody, since both prosody and lexical tone are conveyed by pitch height and contour. Mandarin-speaking HFA $(n=22)$ and typically developing (TD, $\mathrm{n}=16$ ) children aged 7 to 12 first classified emotions of 24 emotionprosody congruent/incongruent words. Second, they identified lexical tone in a categorical perception task, and then received a dimension switch task, which asked them to choose the wrong answer. Two groups of children performed similarly in these tasks. HFA children identified basic emotions well as TD children even under the incongruent word pairs, which could be explained by their even performances in dimension switch task. Despite the similarity, there was a correlation between the accuracy rate of emotion classification and lexical tone perception in HFA children, which was not seen in TD children. This showed that HFA children used correlated mechanisms in understanding emotional prosody and lexical tone.

5pSCb11. Auditory frequency discrimination in preschool and schoolage children with specific language impairment. Huei-Mei Liu (Special Education, National Taiwan Normal Univ., 162 Ho-Ping East Rd., Sec. 1, Taipei 106, Taiwan, liumei@ntnu.edu.tw) and Feng-Ming Tsao (Psych., National Taiwan Univ., Taipei, Taiwan)

The view of "perceptual processing deficits" in children with specific language impairment (SLI) emphasized that the detection and discrimination of basic acoustic components contributed to their difficulties in higher linguistic process. The purpose of this study was to examine the auditory frequency discrimination in Mandarin-speaking children with SLI across ages. Eighteen 5-year-old SLI and 18 age-matched controls, 20 8-year-old SLI and 20 controls participated. All the participants were assessed by the standardized intelligence and language tests. In the computerized auditory frequency discrimination task, children were asked to do AX discrimination of $1000-1030 \mathrm{~Hz}$ and $1000-1070 \mathrm{~Hz}$ in conditions with and without noise masking. A mixed ANOVA was used to examine the effects of group, frequency difference, and masking conditions. The results showed that both preschool and school-age children with SLI performed significantly poorer on the frequency discrimination compared to the controls, especially in the masking condition. The preschoolers performed poorer on the frequency difference of $30 \mathrm{~Hz}$ than $70 \mathrm{~Hz}$, but the school-age children performed similarly on both frequency differences. Individuals' frequency discrimination were positively correlated with their language performance across two ages. These results suggest that deficits in frequency discrimination in children with SLI are important to their language difficulties.

5pSCb12. Do clear-speech consonants always provide the best intelligibility for hearing-impaired listeners? Matthew J. Makashay (Army Hearing Program, Army Public Health Ctr. (Provisional), 5158 Blackhawk Rd., Aberdeen Proving Ground, MD 21010, matthew.j.makashay.civ@mail.mil), Nancy P. Solomon, and Van Summers (Audiol. and Speech Pathol. Ctr., Walter Reed National Military Medical Ctr., Bethesda, MD)

Hearing-aid amplification enhances speech intelligibility for many hearing-impaired (HI) listeners in quiet, but often provides no clear benefit in noise. Requesting that talkers use clear speech is one strategy to overcome these listening challenges. Paradoxically, one feature of clear speech is a shift to higher frequencies, which may move speech energy into a frequency range that is inaudible or has more distortion for certain HI listeners. Conversely, casual conversational speech may shift speech energy into a lower frequency range that is more audible or has less distortion. This study examined the intelligibility of 21 casually- and clearly-spoken American English coda consonants in nonsense syllables for 9 aided normal-hearing and 18 aided HI listeners. As expected, most clear-speech consonants yielded higher recognition scores. However, certain phonological processes common in casual speech, namely affrication and palatalization, generated significantly higher scores than their clear counterparts for some HI listeners in noise. These results have implications for coaching conversational partners of aided HI listeners. [The views expressed in this abstract are those of the authors and do not reflect the official policy or position of the Department of the Army, Department of Defense, or the U.S. Government.] 
5pSCb13. Bite block effects on vowel acoustics in talkers with amyotrophic lateral Sclerosis and Parkinson's Disease. Antje Mefferd and Mary Bissmeyer (Dept. of Hearing and Speech Sci., Vanderbilt Univ. Medical Ctr., 8310 Medical Ctr. East, Nashville, TN 37232, antje.mefferd@vanderbilt.edu)

Amytrophic Lateral Sclerosis (ALS) affects predominantly the tongue and leaves the jaw relatively spared. By contrast, Parkinson's disease (PD) is thought to affect the motor system globally with minimal jaw movements potentially further limiting tongue articulatory movements. However, currently only few studies have directly investigated articulator-specific contributions to deviant speech acoustics in talkers with dysarthria. In this study, a $10 \mathrm{~mm}$ bite block was used to decouple tongue and jaw and delineate their specific contributions on vowel acoustics in talkers with distinctly different impairment profiles. Acoustic vowel contrast was examined during sentence repetitions produced by talkers with ALS, PD, and controls under jaw-free and jaw-fixed conditions. Preliminary findings of seven speakers per group revealed that acoustic vowel contrast was significantly lower during the jaw-fixed than the jaw-free condition in ALS. However, in PD acoustic vowel contrast tended to be greater when the jaw was fixed. For controls, no difference was found between the two conditions. Further, compared to controls acoustic vowel contrast was significantly reduced during the jaw-fixed condition in ALS and during the jaw-free condition in PD. Outcomes suggest that the jaw promotes acoustic vowel contrast in ALS but may hinder it in PD.

5pSCb14. Changes in lateralized /s/ production after treatment with opti-speech visual biofeedback. Rebecca L. Mental (Case Western Reserve Univ., 2744 Euclid Heights Blvd., Apt. 3, Cleveland Heights, OH 44106, rlm142@case.edu), Holle Carey (Vulintus, Dallas, TX), Nolan Schreiber, Andrew Barnes, Gregory S. Lee, and Jennell Vick (Case Western Reserve Univ., Cleveland, $\mathrm{OH}$ )

Opti-Speech is a visual biofeedback software for the treatment of speech sound placement errors. It utilizes Northern Digital Wave platform for electromagnetic articulography. Opti-Speech tracks the movement of five sensors on a talker's tongue to animate a 3D tongue avatar that moves in real time with the talker's own tongue. The avatar is viewable from multiple angles, and virtual targets can be created to guide the participant's speech movements. Opti-Speech has found initial success in small feasibility studies (Katz and Mehta, 2015; Vick et al., 2016). The two participants described in this study are part of a larger scale clinical trial to evaluate efficacy. Both adolescent males presented with the lateralized /s/ speech error. Their intensive biofeedback treatment schedule included two one-hour sessions per day over five sequential days. Data analyses include acoustic measures (spectral mean and kurtosis of the /s/) and trained perceptual judgments of speech sound accuracy on a 16 item probe list from baseline and across all treatment sessions. Overall impressions of the utility of this biofeedback system as a treatment tool will be discussed from the perspective of both the treating clinician and the participants.

5pSCb15. Phonological deficits in Mandarin-speaking congenital amusics. Yun Nan, Wei Tang, and Qi Dong (State Key Lab. of Cognit. Neurosci. and Learning, Beijing Normal Univ., 204 Office, Brain Imaging Ctr., 19 Xin-Wai St., Hai-Dian District, Beijing 100875, China, nany@bnu.edu. cn)

Congenital amusia is a neurodevelopmental disorder that mainly affects pitch processing in music and speech. Recent research reveals possible phonological deficits among amusic individuals from non-tonal language speakers. In a tone language such as Mandarin, only a subgroup of amusic individuals showed speech tone deficits. In the present study, we examined whether a similar phonological deficit exists in Mandarin amusics and whether the phonological deficits are confined to the subgroup of amusics who showed speech tone deficits. An simple identification task targeted at either vowel, tone, or a combination of vowel and tone for 20 monosyllabic words was administered to three groups of Mandarin speakers: amusics with (tone agnosics) and without tone deficits (pure amusics), and matched controls. Tone agnosics lagged significantly behind both the pure amusics and controls when identifying tone and the combination of vowel and tone, but performed similarly to the latter two groups when identifying vowel. However, pure amusics performed comparable with the control group for identification of vowel, tone, and the combination of vowel and tone. These results suggest that only the tone agnosics were impaired in phonological processing and their phonological difficulties are mainly due to the speech tone deficits.

5pSCb16. Acoustic measures versus crowdsourced listeners' ratings: Evaluating the efficacy of treatment for hypokinetic dysarthria. Christopher Nightingale (Speech-Lang. Pathol., Hawaii State Dept. of Education, 2450 Prince Edward St., Apt. 204A, Honolulu, HI 96815, cn883@nyu.edu), Michelle T. Swartz (Communicative Sci. \& Disord., New York Univ., Beesleys Point, NJ), Lorraine A. Ramig (Speech, Lang., and Hearing Sci., Univ. of Colorado-Boulder, Boulder, CO), and Tara McAllister Byun (Communicative Sci. \& Disord., New York Univ., New York, NY)

Clinical interventions for speech disorders aim to produce changes that are not only acoustically measurable or perceptible to trained professionals, but are also apparent to naïve listeners. Due to the difficulty of collecting ratings from a suitably large sample, few researchers evaluate speech interventions relative to this criterion. Crowdsourcing technologies could enable a shift toward a more naturalistic standard to evaluate speech interventions. This project compared 35 naive crowdsourced listeners' ratings against acoustic measures of speech samples collected from patients with hypokinetic dysarthria secondary to Parkinson's Disease. The data come from a published efficacy study (Sapir et al., 2007) that documented significant acoustic changes after a period of intensive treatment, Lee Silverman Voice Treatment. Specifically, the ratio of mean F2 in /i/ versus /u/, calculated for each subject, was greater in post-treatment relative to pre-treatment samples. Mixed-effects logistic regression indicated that words elicited post-treatment, presented in randomly ordered pairs with words elicited pre-treatment, were significantly more likely to be rated "more clear" (beta $=1.24, \mathrm{SE}=.46, \mathrm{p}<.01)$. This result supports the original conclusion of Sapir et al. (2007); it also supports the validity of crowdsourcing as a means to obtain ratings of disordered speech data.

5pSCb17. Prosodic changes in Parkinson's disease. Sona Patel (SpeechLang. Pathol., Seton Hall Univ., 400 South Orange Ave., South Plainfield, NJ 07080, sona.patel@shu.edu), Sabiha Parveen (Commun. Sci. and Disord., Oklahoma State Univ., Stillwater, OK), and Supraja Anand (Commun. Sci. and Disord., West Chester Univ., West Chester, PA)

Parkinson's disease often results in a hypokinetic dysarthria, causing well-known effects on speech and voice. Speech from individuals with Parkinson's is typically studied through listening tasks (i.e., by family members and naïve listeners) and is known to affect intelligibility. However, the exact speech changes causing degradations in speech quality have not been defined. Previous investigations have focused on standard measures of mean fundamental frequency, intensity, and rate. We hypothesize that speech changes are present at the prosodic level in addition to at the acoustic level. To test this hypothesis, we collected spontaneous conversational speech samples from 15 individuals with Parkinson's and 13 age-and-gender matched neurologically healthy speakers (Control group). Several parameters were extracted from the speech signal to describe rate, phrasing, and stress in addition to fundamental frequency and intensity. These measures were compared with listener ratings of intelligibility performed by 12 naïve listeners. Listeners rated the speech samples using a visual analog scale. Preliminary findings suggest that prosody is impaired in Parkinson's disease. Statistical analysis of variance between the speaker groups (Parkinson's vs. Controls) are reported in addition to linear regressions with listener ratings. Our findings can facilitate in early diagnosis and the development of targeted speech therapy for individuals with Parkinson's. 
5pSCb18. Effect of F0 on the intelligibility of emotional speech in noise for younger and older listeners. Jana Besser (Dept. of OtolaryngologyHead and Neck Surgery, Section Ear \& Hearing and EMGO Inst. for Health and Care Res., VU Univ. Medical Ctr., Zurich, Switzerland), Margaret K. Pichora-Fuller (Psych., Univ. of Toronto, 3359 Mississauga Rd., Mississauga, ON L5L 1C6, Canada, k.pichora.fuller@utoronto.ca), S T. Goverts, Sophia E. Kramer, and Joost M. Festen (Dept. of Otolaryngology-Head and Neck Surgery, Section Ear \& Hearing and EMGO Inst. for Health and Care Res., VU Univ. Medical Ctr., Amsterdam, Netherlands)

This study investigated if F0 variability could explain why intelligibility in noise is better for speech spoken to portray fear compared to emotionally neutral speech. Word recognition accuracy was measured for stimuli produced with neutral vocal emotion, for intact stimuli portraying fear, and for six versions of the fear stimuli with varying reductions in F0 variability. Younger and older adults were tested in speech-spectrum noise and twotalker babble. Younger adults outperformed older adults. As F0 variability in the fear stimuli was reduced, performance in speech-spectrum noise for both age groups decreased and approached performance for the neutral stimuli. In the two-talker babble, even when F0 variability was most reduced, performance remained higher for the fear stimuli than the neutral stimuli, especially for older adults. The mean $\mathrm{F}_{0}$ for the fear stimuli was higher than for the neutral stimuli, while mean F0 of the neutral stimuli and the twotalker masker were similar. Thus, although the greater F0 variability in the fear stimuli confers an advantage to both age groups in speech-spectrum noise, F0 mean and variability both contribute to the effect of emotion on intelligibility in two-talker babble, especially for older listeners.

5pSCb19. Vocal fold vibration and voice quality in the elderly. Robin A. Samlan (Speech, Lang., \& Hearing Sci., Univ. of Arizona, P.O. Box 210071, Tucson, AZ 85721, rsamlan@email.arizona.edu), Melda Kunduk (Commun. Sci. \& Disord., Louisiana State Univ., Baton Rouge, LA), and Takeshi Ikuma (Otolaryngol., LSU Health Sci. Ctr., New Orleans, LA)

The vocal fold vibration patterns of incomplete glottal closure, aperiodicity, and asymmetry are characteristic of older adults with normal voice quality. Given that these patterns are also reported in older adults with voice concerns (i.e., age-related dysphonia, presbylarygis, or presbyphonia), the question of whether they relate to voice quality becomes relevant to providers making evaluation and management decisions. METHOD: Thirtyeight adults aged 70 years and older underwent laryngeal high-speed video examination at 4000 frames per second. Analysis included measurements of the glottal closure over time (i.e., open quotient, relative glottal gap), spectral noise (i.e., harmonics-to-deterministic-noise ratio, harmonics-to-noise ratio), and symmetry (i.e., amplitude symmetry, phase symmetry). Multiple regression will be used to compare the high-speed measures to cepstral peak prominence of the acoustic signal and perceived voice quality. Results will be interpreted in terms of how glottal closure and vibratory periodicity and symmetry affect voice quality in elderly speakers. Implications for clinical management will be discussed.

5pSCb20. Perceptual differentiation of African American and European American children: Accuracy, confidence, and reaction time. Emily Simmons, Dalila Salas, Nicole Marsh, Julia Licata, and Sonja TrentBrown (Hope College, 35 E 12th St., Holland, MI 49423, emily.simmons@ hope.edu)

Previous research has demonstrated that adult speakers can be perceptually differentiated with respect to gender and ethnicity (Thomas \& Reaser, 2004). We examine how age, gender, and ethnicity of child speakers, ages $8-12$, affect the perceptual accuracy of adult listeners, determining the point at which perceptual accuracy meets the level for adult speakers. Undergraduates listened to forward and reversed recordings of European American and African American children producing /h-vowel-d/ words (/ $\mathrm{i}, \mathrm{I}, \mathrm{e}, \varepsilon, \mathfrak{x}, \Lambda, \mathrm{a}, \mathrm{\supset}, \mathrm{o}, \mathrm{U}, \mathrm{u}, \mathrm{x}^{\mathrm{C}} /$ ) and sentences ("I hear the sound of $/ h-V-d /$ some more") in General American English. Listeners identified speaker ethnicity and gender and rated their confidence. Reaction times were also measured for identification and confidence ratings. We expect greater accuracy, faster reaction times, and higher median ratings for forward blocks, sentences, and female speakers. We do not expect there to be a difference in identification of European American voices versus African American voices. We also anticipate that accuracy of identification will improve as the age of the speaker increases. Implications of this work can be extended for individuals who work with children and in speech-related professions to reduce biases that occur as a result of personal language experience.

5 pSCb21. The use of ultrasound to determine the most effective placement cues for /s/ during articulation therapy. Kathleen Siren (SpeechLanguage-Hearing Sci., Loyola Univ. Maryland, 4501 North Charles St., Baltimore, MD 21210, ksiren@loyola.edu)

Although two distinct normal tongue configurations for /s/ have been documented for many years (Borden \& Gay, 1978), estimates of the frequency of tongue tip up vs. tongue tip down /s/ articulation in the general population vary. Therapy procedures often focus exclusively on one or the other of the two tongue postures. Given that /s/ is one of the most frequently misarticulated sounds in English, clinicians may need guidance in determining which tongue placement is most natural for each client. In recent years, portable ultrasound devices have become more widely accessible for use in speech therapy. In such cases, ultrasound has generally been used as a visual feedback technique for clients (Bernhardt, Gick, Bacsfalvi \& Adler-Bock, 2005; Cleland, Scobbie \& Wrench, 2015). The current study investigates the use of ultrasound imaging as a preliminary information gathering tool for clinicians to guide decisions regarding /s/ therapy techniques and procedures. In this investigation, students utilized ultrasound to attempt to determine tongue tip configuration during /s/ production for various individuals. Results indicate that ultrasound can provide clinicians with enough initial information to determine the most natural tongue configuration for $/ \mathrm{s} /$ production for most clients, thus potentially reducing therapy time and increasing therapy effectiveness.

5pSCb22. Effect of speech tasks on cepstral measures of articulation. Supraja Anand, Mark D. Skowronski (USF, 4202 East Fowler Ave., PCD 1017, Tampa, FL 33620, supraja1984@gmail.com), and Rahul Shrivastav (UGA, Athens, GA)

Hypokinesia in articulatory movements is one of the predominant characteristics in individuals with Parkinson's disease (PD). Past research quantified such hypokinesias through measures of formant frequencies (F1 and F2) and/or derivative measures such as vowel space area. Extractions of these acoustic measures are labor intensive, time consuming and require phonetic segmentation. In this study, we characterize acoustic measures of articulatory range, rate and acceleration using human factor cepstral coefficients (HFCCs) across speech tasks (e.g. syllable repetition, sentence reading and monologues). Cepstral measures of articulation can be readily derived from entire sentences in an automated, time/computationally efficient method. We hypothesize that these cepstral measures will show a significant effect of group and speech task. Twelve individuals with clinical diagnosis of idiopathic PD and age/gender matched healthy controls repeated syllables at a fast rate, read some sentences, and provided monologue samples. Articulatory range, rate and acceleration were studied using cepstral coefficient standard deviation sum (CC SDS), delta $(\Delta)$, and delta delta $(\Delta \Delta)$ coefficients, respectively. Preliminary findings suggest that temporal derivatives differentiated the groups better in the syllable repetition task and CC SDS revealed trends in the reading and monologue tasks, showing promise for differential and early diagnosis.

5pSCb23. Mathematical error in the American Medical Association and American Academy of Otolaryngology-Head and Neck Surgery percent binaural hearing impairment calculation. Ashley N. Clark and Ron Leavitt (Audiol., Corvallis Hearing Ctr., 1025 NW Ninth St. Ste. D, Corvallis, OR 97330, researchchc@gmail.com)

Percent hearing loss calculation forms the basis of computation for those whose hearing has been damaged in industry or in the military. As such medical professionals are admonished to use the best science available when making these important calculations. Unfortunately, current computational methods do not meet current high standards of scientific evidence and are riddled with mathematical and psychoacoustic errors. A number of these errors will be discussed in this poster session. 
5pSCb24. Temporal gap and amplitude modulation detection evaluated using the electrically evoked auditory change complex in patients with auditory brainstem implants. Shuman He, Tyler C. McFayden, Bahar S. Shahsavarani, and Katherine E. Gill (Ctr. for Hearing Res., Boys Town National Res. Hospital, 555 N. 30th St., Omaha, NE 68131, Shuman.He@ boystown.org)

This study aims to objectively evaluate temporal processing capabilities using the electrically evoked auditory change complex (eACC) in patients with auditory brainstem implants (ABIs). Study participants include seven Cochlear Nucleus 24M ABI recipients (six children and one adult). All children received $\mathrm{ABIs}$ due to cochlear nerve deficiency and the adult recipient was diagnosed with neurofibromatosis type II (NF-2). The speech processor was bypassed and electrical stimulus was a biphasic pulse train directly sent through individual electrodes of the ABI. Gap durations tested in this study ranged from 4 to $256 \mathrm{~ms}$. Amplitude modulation frequency was $10 \mathrm{~Hz}$. Modulation depths tested in this study ranged from $1 \%$ to $99 \%$. Behavioral gap detection thresholds were also obtained for two participants. The eACCs were evoked by temporal gaps and amplitude modulations. The shortest gap duration and the smallest amplitude modulation depth that can be used to evoke the eACC vary across study participants and across stimulating electrodes within individual participants. These preliminary data also suggest that the temporal gap detection primarily depends on subcortical auditory neural structures. The eACC can be used to objectively evaluate temporal processing capabilities in both adult and pediatric $\mathrm{ABI}$ recipients.

5pSCb25. An event related potentials method for assessing audiovisual speech integration: Implications for children with autism spectrum disorders. Julia Irwin, Lawrence Brancazio, Jacqueline Turcios, Trey Avery, and Nicole Landi (Haskins Labs., 300 George St., Ste. 900, New Haven, CT 06511, julia.irwin@yale.edu)

Perceptual studies of children with autism spectrum disorders (ASD) strongly implicate deficits in processing of audiovisual (AV) speech. Previous research with AV stimuli has typically been conducted in the context of auditory noise or with mismatched auditory and visual ("McGurk") stimuli. Although both types of stimuli are well-established methods for testing typically developing (TD) participants, they may create additional processing problems for children with ASD. To more precisely examine audiovisual (AV) speech perception in children with ASD, we developed a novel measure of AV processing that involves neither noise nor AV cross-category conflict. The speech stimuli include clear exemplars of the syllable /ba/ and a modified version of $/ \mathrm{ba} /$ in which the consonant is substantially weakened so that the syllable is heard as " $/ \mathrm{a} /$ ". These are dubbed with a video of the speaker saying /ba/. Audiovisual integration should result in the visual information effectively "restoring" the weakened auditory "/a/" cues so that the stimulus is perceived as /ba/. Using event related potentials (ERP), we will present evidence from typically developing adults and preliminary results from children with ASD and TD to examine whether children with ASD are weaker in $\mathrm{AV}$ speech integration.

5pSCb26. Naming practice app on tablets and communication robots for people with aphasia. Shingo Kuroiwa, Yasuo Horiuchi (Chiba Univ., 1-33, Yayoi-cho, Inage-ku, Chiba, Chiba 2600033, Japan, kuroiwa@ faculty. chiba-u.jp), Sachiyo Muranishi, Daisuke Furukawa (Kimitsu Chuo Hospital, Kisarazu, Chiba, Japan), Kyohei Ishihata, Akihiko Morimoto (Robocure Inc., Tokyo, Japan), and Hiroji Suzuki (Escor Inc., Kisarazu, Japan)

Aphasia is a language disorder that negatively affects a person's ability to understand and generate language. Although it has been said that patients in the chronic phase are not likely to recover their ability, a number of recent studies report that highly frequent and continuous training enables recovery. Therefore, we have started a project that aims to improve the environment in which people with aphasia can continue language learning at home. In this presentation, we demonstrate naming practice apps, using speech recognition technology for people with aphasia. They can check whether their utterances are accurate, through the use of speech recognition and they can listen to the correct pronunciation of words through the use of speech synthesis. Field trials using the app for tablets have been conducted since Dec. 2014, and for the communication robot, Pepper, since Dec. 2015. Although the average of speech recognition accuracy was $61 \%$ (false acceptance: $2 \%$, false rejection: $37 \%$ ), the participants accepted the poor robustness of the speech recognition, and tried to speak clearly. Some participants felt as if the robot was somewhat similar to a child, and others understood its inflexibility, and so in consequence they both accepted its low speech recognition ability.

5pSCb27. Suprasegmental characteristics of spontaneous speech produced in good and challenging communicative conditions by younger and older adults. Outi Tuomainen and Valerie Hazan (Speech Hearing and Phonetic Sci., Univ. College London, 2 Wakefield St., London WC1N 1PF, United Kingdom, o.tuomainen@ucl.ac.uk)

Our study investigates strategies used to clarify our speech and compensate for masking effects when communicating in challenging listening conditions in older and younger adults. A total of 50 older (OA, 65-85 years, 30 $\mathrm{F}$ ) and 23 younger adults (YA, 18-35 years, $14 \mathrm{~F}$ ) were recorded (in "Talker A" role) while they completed the problem-solving diapix task with a young adult ("Talker B") in four listening conditions: with no interference (NORM), when Talker B had a simulated hearing loss (HLS), Talker B heard babble (BAB1) or both heard babble (BAB2) noise. We measured articulation rate, fundamental frequency (f0) median and range, energy in 1$3 \mathrm{kHz}$ band reflecting spectral tilt (ME1-3 kHz) for Talker A. In NORM, OAs were slower speakers and had lower ME1-3 kHz and wider f0 range than YAs. Median f0 also converged for men and women in OA talkers. In adverse conditions, YAs slowed down their speech (HLS) and increased the f0 range (BAB1 and $\mathrm{BAB} 2$ ) more than OAs, and OAs raised their median f0 more than YAs (BAB2). These results indicate that there are age-related changes in suprasegmental characteristics of spontaneous speech, and that OA talkers use different strategies than YA talkers to clarify their speech.

5pSCb28. Fathers' and mothers' differential talk to sons and daughters with hearing loss. Mark VanDam, Carsen Jessup (Elson Floyd Coll. Med., Dept. Speech \& Hear Sci., Washington State Univ., PO Box 1495, Spokane, WA 99202, mark.vandam@wsu.edu), and Tracy Tully (Com Sci Disord, Eastern Washington Univ., Spokane, WA)

In a natural family environment, fathers have been shown to talk less than mothers, and fathers may talk more to sons than to daughters. There is conflicting evidence showing mothers' word use increases talking both to sons and to daughters. Further, preschoolers who are hard-of-hearing have been shown to encounter a similar number of parent utterances as compared with their typically-developing peers. This work looks at fathers and mothers talking to their preschool boys and girls and explores whether child hearing loss affects parent word use. Here we used all-day recordings from a wearable child audio recorder, analyzed using automatic speech processing algorithms to determine estimated number of syllables spoken by fathers, mothers, boys, and girls. About half of the families included children with hearing loss. Results showed that mothers talked more than fathers, parents offered about the same amount of talk to boys or girls who were typically developing, fathers talked more to their boys who were hard-of-hearing, and mothers talked more their girls who were hard-of-hearing. The asymmetry of fathers talking more to boys with hearing loss and mothers talking more to girls with hearing loss is considered in terms of the differential experience hypothesis.

5 pSCb29. Comparison of speech rate and intelligibility between children with cerebral palsy and typically developing children in Taiwan. Chu-Yin Wu, Li-mei Chen (Foreign Lang., National Cheng Kung Univ., 1 University Rd., Tainan 701, Taiwan,wu25689@gmail.com), Yu Ching Lin (Physical Medicine and Rehabilitation, National Cheng Kung Univ., Tainan, Taiwan), Katherine C. Hustad, and Raymond D. Kent (Waisman Ctr., Madison, WI)

Due to the defect of speech motor control, cerebral palsied children (CP) with dysarthria often show slower, weaker, and imprecise articulation with poor coordination, which have profound impact on speech intelligibility (Hodge \& Gotzke, 2014; Hustad, Gorton, \& Lee, 2010). It was reported that $\mathrm{CP}$ children had lower speech rate and intelligibility than typically developing (TD) children. However, little research discussed the application of this finding in languages other than English. The present study investigated the 
correlations between speech rate and intelligibility in 4 Mandarin-speaking CP children aged 4-8 and 4 TD children aged 5. Speech data were collected from the repetition tasks of sentences ranging from 3 to 8 words. Speech rate were calculated through WPM (words per minute) and IWPM (intelligible words per minute), and speech intelligibility of each child was judged by three listeners. The results showed that: 1) TD had significantly higher mean intelligibility and slightly higher IWPM than the CP group; 2) Mean WPM of CP was higher than TD; 3) Higher WPM in CP did not correspond to higher intelligibility. Contrary to the previous findings, in the present study, CP showed higher WPM but lower intelligibility. This discrepancy requires further examinations in future studies.

5pSCb30. A comparative study of Mandarin compound vowels produced by prelingually deafened children with cochlear implants and normal hearing peers. Jing Yang (Commun. Sci. and Disord., Univ. of Central Arkansas, CSD/ Speech Lang. Hearing Ctr., 201 Donaghey Ave., UCA Box 4985, Conway, AR 72035, jyang@uca.edu) and Li Xu (Commun. Sci. and Disord., Ohio Univ., Athens, $\mathrm{OH}$ )

The present study examined the dynamic features of compound vowels in native Mandarin-speaking children with cochlear implants (CIs). Fourteen prelingually deafened children with CIs (aged 2.9-8.3 years) and 14 age-matched, normal-hearing $(\mathrm{NH})$ children produced monosyllables containing six Mandarin vowels (/aI/, /av/, /uo/, /ic/, /iav/, /iov/). The frequency values of the first two formants were measured at nine equidistant time points (10-20-30-40-50-60-70-80-90\%) over the course of vowel duration. All formant frequency values were normalized and then used to calculate vowel trajectory length, vowel section length, overall spectral rate of change, and vowel section rate of change. The results revealed that the CI children produced significantly longer durations for all six compound vowels. They clearly showed positional deviation from the NH children on vowels /aI/, /uo/ and /ie/. The CI children's ability to produce formant movement for the compound vowels varied considerably. Some CI children produced relatively static formant trajectories for certain diphthongs whereas others produced certain vowels with greater formant movement than did the $\mathrm{NH}$ children. As a group, the CI children roughly followed the $\mathrm{NH}$ children on patterns of vowel dynamic spectral change, but they moved the articulators with a slower rate of change than did the $\mathrm{NH}$ children.

5pSCb31. Age estimation in Japanese speech based on feature selection. Atsushi Morimoto, Masahiro Niitsuma, and Yoichi Yamashita (Graduate School of Information Sci. and Eng., Ritsumeikan, 1-1-1 Nojihigashi, Kusatsu-shi 525-8577, Japan, morimoto-ASJ@slp.is.ritsumei.ac.jp)

This paper addresses age estimation of Japanese speech utterance, using paralinguistic information in speech. Our speech conveys not only linguistic information but also paralinguistic and non-linguistic information. Nonlinguistic information includes potentially valuable social information such as personal parameters, body conditions, gender and ages. This significant information, however, has not been investigated enough. The main purpose of our study was to develop less-invasive methods to extract this information, by proposing a method to classify speakers' age into five groups $(20 \mathrm{~s}, 30 \mathrm{~s}$, $40 \mathrm{~s}, 50 \mathrm{~s}$, and 60s). 6375-dimentional audio features were extracted from 1579 samples of 913 male speakers extracted from CSJ (Corpus of Spontaneous Japanese) and feature selection was conducted based on Fisher's Ratio. Naïve Bayes with top 20 parameters of Fischer's ratio, yields highest accuracy rate of $51.2 \%$.

$5 \mathrm{pSCb32}$. The articulation of direct bone conducted sound in noisy environment. Hidehiko Maeda (Dept. of Rehabilitation Sci., Health Sci. Univ. of Hokkaido, 1757, Kanazawa, Toubetsucho-Ishikarigun, Hokkaido 0610293, Japan, maehide@hoku-iryo-u.ac.jp) and Kiyoshi Yonemoto (Dept. of Social welfare, Iwate Prefectural Univ., Takizawa, Iwate, Japan)

We have constructed the direct bone conducted sound (DBC) detection system which is connected with piezo electronic accelerometer and the titanium implant of bone-anchored hearing aid (Baha) implanted on the temporal bone. Our previous study has reported that the sound distortion of the DBC was less than that of conventional bone conducted sound (BC) due to its property of outputting the DBC without going through the skin. This study reports the articulation of the DBC in noisy environment. The subject who output the DBC wears a Baha on his left side for hearing loss. He pronounced 50 monosyllables and words in quiet and extremely noisy environment. His voices were output simultaneously throughout three pathways, DBC, BC, and air conducted sound (AC). Sixteen participants with normal hearing heard those voices and answered how those were heard. In the quiet environment, the percentage of collect answers of the AC showed statistically significance $(<.01)$ than those of the DBC and the BC. However, in extremely noisy environment, those of the DBC showed statistically significances $(<.01)$ than those of the $\mathrm{AC}$ and the $\mathrm{BC}$. This study clarified that the DBC kept the articulation without changing it in extremely noisy environment.

5pSCb33. The perceptual and acoustic characteristics of Japanese elderly speech-The relationship between cognition and speech motor performance. Takako Yoshimura, Makoto Kariyasu, Akie Saito, Minoru Toyama (Speech and hearing Sci. and Disord., Kyoto Gakuen Univ., 18, Gotanda-cho, Yamanouchi, Ukyo-ku, Kyoto 615-8577, Japan, takakoy@ kyotogakuen.ac.jp), and Mariko Osaka (Ctr. for Information and Neural Networks, Osaka, Japan)

Recent studies showed the evidences that language complexity or cognitive load may deteriorate the speed and accuracy of speech. No study was found to explore the relationship between cognitive load and speech movement directly from the perspective of working memory (WM) capacity. In Reading Span Test (RST), WM capacity would be reduced for speech production as the number of sentences and target words increased. The aim of our study is to reveal perceptual and acoustic characteristics of the elderly speech in relation to WM capacity. It was hypothesized that slower speech rate and/or slurred speech would be observed for elderly adults with reduced WM capacity or low cognitive function. Twelve elderly adult speakers of Japanese participated in this study. The scores of Montreal Cognitive Assessment Japanese version was used to divide the participants into high and low cognitive groups. For RST, each participant was asked to read visually presented sentence(s) aloud and to recall target word(s). Reading performances were recorded by a microphone being set in front of each participant for later acoustic evaluations. The reading performances of two cognitive groups were compared and results regarding on the presence of slower speech rate and slurred speech, judged by experienced speech language pathologists, were presented. We will discuss the relationship between the cognitive load and speech movement in the elderly based on the acoustic evaluation.

5pSCb34. Effect of denture adhesives on enhancement pronunciation accuracy of denture wearers. Hyung Woo Park and Sang hwi Jee (IT, SoongSil Univ., 1212 Hyungham Eng. Bldg. 369 Snagdo-Ro, Dongjak-Gu, Seoul, Seoul 06978, South Korea, pphw@ssu.ac.kr)

People who have lost their natural teeth for any reason need to wear dentures (false teeth in other words) to eat and talk. And the teeth chewing role, and also has an important role in having the pronunciation. However, when people wear a denture, it causes some problems such as aesthetic or pronunciation. Among denture users, pronunciation errors may occur depending on how bonded and which location of false teeth inside of our mouth. In this paper, a comparative analysis on the bandwidth of formant frequency found in pronunciation errors is utilized in order to analyze pronunciation errors caused by different positions of dentures and thus evaluate the accuracy of pronunciation for each position. In the experiment result, that show the pronunciation accuracy enhancing around 5\%, when using adhesives, who use denture correctly. And accuracy of pronunciation is evaluated differently depending on the position of dentures.

5pSCb35. The effects of deep brain stimulation on conversational and repeated speech. Diana V. Sidtis (New York Univ., 665 Broadway, Rm. 936, New York, NY 10012, diana.sidtis@nyu.edu) and John J. Sidtis (Geriatrics, Nathan Kline Inst., Orangeburg, NY)

The effects of the DBS on speech remain controversial. This approach used intelligibility by listeners and acoustic parameters as measures of conversation and repeated speech. Persons with DBS-STN provided 
conversational speech samples and then repeated portions of their conversation in both on and off stimulation. Excerpts were arranged for a listening protocol adjusted for the Parkinsonian hypophonia. Dependent measures were intelligibility and difficulty ratings by 30 healthy listeners and acoustic measures of voice. Fewer words were correctly transcribed from conversation compared to repetition $[\mathrm{F}(1,29)=14.06 ; \mathrm{p}=0.001]$. Similarly, intelligibility for STN-DBS on worse than off $[F(1,29)=9.462 ; p=0.005]$. More words were correctly transcribed from repetition than from conversation with STN-DBS on $[\mathrm{t}(29)=-2.342 ; \mathrm{p}=0.026]$ and off $[\mathrm{t}(29)=-3.619 ; \mathrm{p}=$ 0.001]. Comparisons of conversation versus repetition and DBS state revealed significant effects of both task and DBS on intelligibility. Repetition yielded better voice quality than conversation and DBS improved voice. DBS is mildly disruptive to conversational speech, but the effect of task is greater. The external example inherent in repetition may reduce the burden for speech, as is seen for gait and arm reach.

5pSCb36. Ideal binary masking and the integration of spectro-temporal glimpses in cochlear implant simulations. Vahid Montazeri, Shaikat Hossain, and Peter F. Assmann (School of Behavioral and Brain Sci., Univ. of Texas at Dallas, GR 41, Richardson, TX 75080, vahid.montazeri@utdallas. edu)

Ideal binary masking (IdBM) can improve listeners' speech recognition scores in the presence of background noise. It retains the time-frequency (TF) regions where the target dominates the noise while discarding the remaining regions. Li and Loizou (J. Acoust. Soc. Am. 123, 1673-1682, 2008) suggested that IdBM is effective because the pattern of target-dominated T-F units (IdBM pattern) directs the listener's attention to target glimpses. This study extends the above explanation and investigates listeners' abilities to integrate target glimpses when the IdBM pattern is held constant. Speechshaped noise maskers were added to IEEE target sentences at a signal-tonoise ratio of $-5 \mathrm{~dB}$ and the mixture was processed with IdBM. The IdBMprocessed mixture was then decomposed into the IdBM-processed target and masker. The IdBM-processed target/IdBM-processed masker power ratio (TMR) was then varied to produce an altered IdBM-processed mixture. These processed signals were either sent to an eight-channel tone-vocoder or kept intact, and were presented to normal hearing listeners. Results showed that IdBM benefit heavily depends on TMR, especially in the vocoder condition. Overall, the results suggest that IdBM benefit is mediated by listeners' ability to integrate the target glimpses, despite the IdBM pattern being constant.

5pSCb37. Support system for pronunciation instruction and practice in special education classes for language-disabled children enrolled in conventional schools. Ikuyo Masuda-Katsuse (Kindai Univ., 11-6 kayanomori, Iizuka 820-8555, Japan, katsuse@fuk.kindai.ac.jp)

We developed two types of support systems for pronunciation instruction and practice in special education classes for language-disabled children: a stand-alone system and a web application system. The aims of the systems were (1) to encourage students to practice the pronunciation they learned in their classes repeatedly and (2) to promote cooperation between teachers and the speech-language-hearing therapists (STs) who support the teachers. The teachers registered practice words and their probable error pronunciations into the system, taking into account individual pronunciation needs, and the students were encouraged to read these practice words aloud. Three speech evaluation methods were prepared: automatic speech recognition, phonemic discrimination between the correct and probable error pronunciation of a consonant period, and articulation tests from STs. The web application system simplified access to the students' speech and exercise records for the STs. Practical field test results demonstrated that all of the teachers found that our system helped improve their students' pronunciation, the STs felt that it simplified not only articulation tests, but also observation of the students' pronunciation improvement process, and the STs and teachers believed that it would promote their mutual cooperation for more effective instruction.
5pSCb38. Levels of prominence in Spanish infant-directed speech. Ann M. Aly (Linguist, UCLA, 3125 Campbell Hall, Los Angeles, CA 90095, ann.m.aly@ucla.edu), Stephanie Jacobson (Palomar Health, Escondido, CA), and Megha Sundara (Linguist, UCLA, Los Angeles, CA)

Research on Spanish prosody in adult-directed speech (ADS) (OrtegaLlebaria \& Prieto 2005, Ortega-Llebaria \& Prieto 2011) shows evidence for three acoustic levels of prominence: stressed and focused, stressed and unfocused, and unstressed and unfocused. We tested whether Spanish infantdirected speech (IDS) contains the same levels of prosodic prominence as ADS. In order to test this hypothesis, 30-minute play sessions between adult Spanish-speaking females of Latin American origin $(n=12)$ and 12-month old infants were recorded. We extracted target words from the recordings containing stressed and unstressed /a/, /e/, /i/, and /o/ in non-final position. Voicesauce (Shue et al 2011) was then used to obtain acoustic correlates of stress in Spanish: pitch, duration, intensity. We also labeled pitch accents using $\mathrm{Sp}_{\mathrm{ToBI}}$ conventions (Estebas Vilaplana \& Prieto 2009). Latent class analyses to be performed on the data will determine the number of distinct prominence levels present in Spanish IDS as realized by Latin American speakers.

5pSCb39. Developmental progressions in the harmonic structure of infant-directed speech. Jhonelle Bailey (Marcus Autism Ctr., Children's Healthcare of Atlanta, Marcus Autism Ctr., 1920 Briarcliff Rd. NE, Atlanta, GA 30329, jbail22@emory.edu), Shweta Ghai, and Gordon Ramsay (Dept. of Pediatrics, Emory Univ. School of Medicine, Atlanta, GA)

Caregivers speak differently to infants than to adults early in life. Previous studies of this special register, "motherese," have sought to identify key differences in voice quality between infant-directed and adult-directed speech. Acoustic properties most salient to infants appear to reside in exaggerations of prosodic structure, but these findings are limited by speech analysis techniques that do not fully capture the acoustic structure of the maternal voice or the way motherese changes over the course of development. In this study, multitaper analysis was used to measure developmental progressions in the complete harmonic structure of the maternal voice. Samples of adult- and infant-directed speech were extracted from home audio recordings of 20 mothers collected monthly from 0-24 months using LENA technology. Multitaper analysis was used to calculate the time-varying amplitude and phase of every harmonic component, the residual noise component, and the spectral envelope, permitting a complete statistical analysis of source and resonance properties. Key differences and developmental changes between adult- and infant-directed registers were found not only in the fundamental frequency, but in the overall structure of the harmonic spectrum, indicating that manipulations of the whole voice source may be employed to create motherese.

5 pSCb40. Acoustic correlates to word order in Infant- and adult directed speech: A cross-linguistic study. Irene de la Cruz-Pavía, Judit Gervain (CNRS-Université Paris Descartes, Laboratoire Psychologie de la Percept., 45, rue des Saints-Pères, Paris 75006, France, idelacruzpavia@ gmail.com), Michael McAuliffe (McGill Univ., Montreal, QC, Canada), Eric Vatikiotis-Bateson, and Janet F. Werker (Univ. of Br. Columbia, Vancouver, BC, Canada)

The acoustic realization of phrasal prominence correlates systematically with the order of Verbs and Objects in natural languages. Prominence is realized as a durational contrast in $\mathrm{V}(\mathrm{erb})-\mathrm{O}$ (bject) languages (English: short-long, to [Ro]me), and as a pitch/intensity contrast in O(bject)-V(erb) languages (Japanese: high-low, ['To]kyo hi). Seven-month-old infants can use phrasal prominence to segment unknown artificial languages. This information might thus allow prelexical infants to learn the basic word order of their native language(s). The present study investigates whether this differing realization of phrasal prominence is also found in I(nfant) D(irected) $\mathrm{S}$ (peech), previously unexamined speech style. We recorded 15 adult native talkers of languages with opposite word orders producing target phrases 
(English: VO, e.g., behind furniture, Japanese: OV, e.g., jisho niwa) embedded in carrier sentences, both in A(dult) D(irected) S(peech) and IDS, and conducted acoustic analysis of the phrases (i.e., pitch maximum, mean pitch and intensity, duration). The results revealed the expected contrast in pitch in Japanese IDS and a similar trend in ADS. In English, the predicted durational contrast was found in ADS, additionally accompanied by a pitch contrast. Interestingly, a pitch contrast but no durational contrast was found in IDS.

5pSCb41. Phonological development is affected by speech discrimination skills in typically developing children. Eija M. Aalto (Faculty of Humanistics/LogopeDC, Univ. of Oulu, Univ. of Oulu, P.O. Box 1000, Oulu 90014, Finland, eijaalto@gmail.com), Katri Saaristo-Helin, and Suvi Stolt (LogopeDC, Univ. of Helsinki, Helsinki, Finland)

In early language development accurate auditory discrimination is necessary for building phonological representations to mental lexicon. However, little is known about the connections between phonological development and auditory discrimination in noise in children at the age of $3 ; 6$ years. The aim of this study is to clarify the associations between phonological skills and auditory perception of 3;6 aged children $(\mathrm{N}=65)$ acquiring Finnish. Phonological skills were assessed using Finnish Phonology Test (FPT) that has been normed previously. Auditory discrimination skills were assessed using a recognizing task which was created for the present study (ADT). In the task, children recognize and point from pictures (6-8 pictures in 5 subtasks) phonological neighbour words in babble noise (signal-tonoise ratio $13 \mathrm{~dB}$ ). The results show that FPT and ADT scores correlated moderately (Spearman, $r=0.32, p=0.01$ ). Results support the view that auditory discrimination skills and phonological skills are associated in typically developed Finnish children. Thus, even typically developing children may benefit from decreased noise levels in their learning environment.

5pSCb42. Longitudinal profiling of speech development in bilingual infants at risk of autism. Shweta Ghai (Dept. of Pediatrics, Emory Univ. School of Medicine, 1920 Briarcliff Rd., Atlanta, GA 30329, shweta.ghai@ emory.edu) and Jhonelle Bailey (Marcus Autism Ctr., Children's Healthcare of Atlanta, Atlanta, GA)

Bilingualism is common and increasingly prevalent worldwide, as are the autism spectrum disorders (ASD). However, there is little information on the impact of bilingual exposure on the development of language and communication skills in children with ASD. In the literature, only a few cross-sectional studies have analyzed language skills in both monolingual and bilingual toddlers with ASD, and have reported similar language outcomes using qualitative assessment instruments. Although outcomes may appear to be similar, it is not yet known if both groups follow the same developmental trajectories during infancy. To resolve this issue, this longitudinal study tracks speech development in monolingual and bilingual infants at low and high risk of autism using day-long audio recordings collected in the home with LENA technology from birth to 24 months. The relation between quantitative measures of speech development (adult volubility, child volubility, and conversational turns) over this period and qualitative language outcomes at 24 months is evaluated. The results of this study will help to determine the pace and patterns of language growth in bilingual infants relative to monolinguals at risk of autism, and thus provide insight into what may be alternative bases for the language acquisition process in bilinguals with ASD.

5pSCb43. Vowel final noise in child coda /s/. Helen M. Hanson (ECE Dept., Union College, 807 Union St., Schenectady, NY 12308, helen.hanson@alum.mit.edu), Stefanie Shattuck-Hufnagel (Res. Lab. of Electronics, Massachusetts Inst. of Technol., Cambridge, MA), and Jackson Doughty (ECE Dept., Union College, Schenectady, NY)

Child speakers of American English in the age range 2;6-3;6 often produce vowel final noise (VFN), or preaspiration, for [-voice] labial and velar stops in coda position of monosyllabic words [e.g., Shattuck-Hufnagel, Hanson, and Zhao, "Feature-cue-based processing of speech: A developmental perspective," in Proc. ICPhS 2015]. In contrast, the female caregivers of these children produce VFN less often than the children, with significantly shorter durations. The current work extends the earlier work by studying a fricative, /s/, in coda position. Data from the same nine child/adult pairs are used. While the coda stops were all preceded by the vowel $/ \Lambda /$, the corpus includes two words with coda /s/, Gus and goose, allowing the observation of possible vowel effects. Preliminary analysis of a subset of the data shows that, as for coda stops, children are more likely than adults to produce VFN, with longer durations. However, adults show a vowel effect, being much more likely to produce VFN when /s/ follows / $\mathrm{N}$ than when it follows / $\mathrm{u} /$. A vowel effect is not observed for the child subjects.

5 pSCb44. Cluster duration in a nonword repetition study of Russianspeaking children. Darya Kavitskaya (Slavic Lang. and Literatures, Univ. of California Berkeley, 6303 Dwinelle Hall, Berkeley, CA 94720, dkavitskaya@berkeley.edu) and Sharon Inkelas (Linguist, Univ. of California Berkeley, Berkeley, CA)

This paper reports results from a nonword repetition study of typically developing Russian-speaking children aged 7-10, finding that consonant cluster duration correlates with whether or not the cluster is lexically attested in Russian. Stimuli consisted of 144 nonwords, repeated once each. 36 nonwords began with biconsonantal (CC) clusters; 18 with triconsonantal (CCC) clusters. None of CCC clusters are attested in Russian. Half of the $\mathrm{CC}$ clusters are attested. The CCC clusters were frequently produced as CC, with deletion/omission of one of the three consonants. This study compares the durations of CCC clusters which were reduced to CC (example: stimulus ptkoka, produced as tkoka) to the CC clusters which were produced as intended. The main finding is that the mean duration of intended CC clusters (153 ms) is shorter than that of corresponding reduced (CCC to CC) clusters $(215 \mathrm{~ms})(\mathrm{n}=14, \mathrm{p}<.01)$. Lexical attestedness also played a role. The mean duration of produced clusters which are lexically attested in Russian also tended to be shorter than that of lexically unattested clusters (159 ms vs. $214 \mathrm{~ms}$ ). These findings suggest that both past production experience (the effect of attestedness) and planning complexity (the effect of reduction) affect cluster duration.

5pSCb45. Infant auditory and audiovisual speech discrimination. Kaylah Lalonde and Lynne A. Werner (Speech and Hearing Sci., Univ. of Washington, 1417 NE 42nd St., Seattle, WA 98105, klalonde@uw.edu)

Although infants are sensitive to the correspondence between auditory and visual speech cues, previous studies have not investigated whether infants can use this correspondence to improve speech perception in noise. This experiment used a modified observer-based psychoacoustic procedure to measure audiovisual benefit to speech discrimination in 6- to 8-month-old infants. Infants heard a single standard CV syllable repeatedly in a $65 \mathrm{~dB}$ A steady-state speech spectrum noise at $17 \mathrm{~dB} \mathrm{nHL}$ (re: adult thresholds). Infants were trained to respond when they heard a new CV syllable. Some infants were tested with a vowel contrast; others were tested with a consonant contrast. Each infant completed either the auditory-only or audiovisual condition; when possible, they completed both conditions. In the auditoryonly condition, a still image of the female talker remained on the screen throughout testing. In the audiovisual condition, a synchronous, congruent video of the talker played throughout testing. Preliminary results from 30 infants show a trend for better discrimination of audiovisual syllables than auditory-only syllables, suggesting visual speech improves infants' perception of speech in noise and could be useful in speech and language learning. Differences in results for vowel contrasts, consonant contrasts, between-subjects analysis, and within-subjects analysis will be discussed.

5pSCb46. The development of gendered/s/ perception in children and adolescents. Fangfang Li (Psych., Univ. of Lethbridge, 4401 University Dr., Lethbridge, AB T1K 3M4, Canada, fangfang.li@uleth.ca)

The sound /s/ in English is gender differentiating: women tend to produce a variant of /s/ with higher spectral mean frequency than men (Fox \& Nissen, 2005; Stuart-Smith et al., 2007). The gender marking cue inherent in /s/ frication is perceptually salient (Munson et al., 2006; Strand \& Johnson, 1996), but prior research has focused exclusively on adults. The current study aims to identify the developmental course of the perception of speaker gender when hearing isolated segments of fricative /s/. Sixty children and 
adolescents from three age groups (7 8 years; 10-11 years; and 13-14 years) participated in the study. They were asked to listen to a series of $/ \mathrm{s} / \mathrm{seg}$ ments and identity speakers' gender for each sound by clicking on a female's or a male's face on the screen. Our results indicate the developmental change in adolescents in the accuracy of gender perception and their association with the primary gender-marking cue in /s/ frication when comparing with the two younger groups of children. This study suggests that speech perception, particularly the type of perceptual knowledge related to socio-indexical aspects, can continue to develop beyond school years.

5pSCb47. Acoustic variation among African American and European American children: Age, gender, and ethnicity. Dalila Salas, Emily Simmons, Nicole Marsh, Julia Licata, and Sonja Trent-Brown (Psych., Hope College, 35 East 12th St., Holland, MI 49423, dalila.salas@hope.edu)

Previous research has shown vowels are acoustically specified based on their formant frequencies (Peterson \& Barney, 1952). Hillenbrand et al. (1995) replicated and extended this study. While both studies included children, neither included variation across children's gender or ethnicity. The present study explores variations in phonemic production for children across age, gender, and ethnicity. Children were presented with a list of /h-vowel$\mathrm{d} /$ words and sentences ("I hear the sound of $/ h-V-d /$ some more") contain-

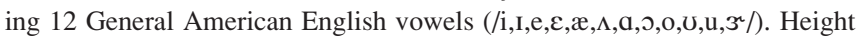
and weight were also measured. We hypothesize that fundamental and formant frequencies will be higher for 8 to 9 year olds than for 10 to 12 year olds and higher for girls (by age 10). For ethnicity, we anticipate no significant differences in frequency measures between European-American and African-American children. This study contributes to our knowledge of developmental trajectories for specified acoustic parameters. As gender and ethnicity are vital cues for adult speakers, it is important to investigate how salient acoustic parameters are for child speakers and at what ages child parameters begin to approximate adult measures. Results will have implications for audiologists, speech language pathologists, developmental scientists, and others in the field of communication sciences and disorders.

$5 \mathrm{pSCb48}$. The role of auditory working memory In the development of glimpsing skills in children. Jessica R. Sullivan (Commun. Sci. and Professional Counseling, Univ. of West Georgia, 1601 Maple St., Carrolton, GA 30118, jreneesullivan@gmail.com), Erin C. Schafer (Univ. of North Texas, Denton, TX), and Homira Osman (Univ. of Washington, Seattle, WA)

Glimpsing is a complex process involving lower-and higher level auditory skills required for speech understanding in noise. However, little is know the role of working memory as glimpsing develops in children. The present study aimed to determine the extent to which auditory working memory contributes across the continuum of auditory perception skills in children. This study investigated the role working memory plays in speech recognition and auditory comprehension task in the presences of noise. We measured working memory, speech recognition and auditory comprehension performance with noise in 20 seven-to-nine year old children with normal hearing. Backward digit recall was used to assess working memory. Speech recognition in noise performance was measure using the Hearing In Noise Test. Comprehension was measured by a child's ability to answer five questions associated to auditorily-presented stories. Each question evaluated a different level of comprehension. In general, there are no relationships between the speech recognition in noise performance and working memory. However, the relationship between working memory and comprehension was stronger. These results suggest that working memory contributes more when the auditory task is more complex especially in the presence of noise.

5pSCb49. English-learning infants' perception of boundary tones. Megha Sundara (Dept. of Linguist, UCLA, 3125 Campbell Hall, Los Angeles, CA 90095-1543, megha.sundara@humnet.ucla.edu), Monika Molnar (Basque Ctr. on Cognition, Brain and Lang., Donostia, Spain), and Sonia Frota (Dept. of Linguist, Faculdade de Letras da Universidade de Lisboa, Lisbon, Portugal)

Like consonants and vowels, infants' ability to discriminate lexical pitch becomes language-specific with age (Mattock \& Burnham, 2006). We know less about when infants perceive pitch marking of prosodic units. We tested English-learning infants on Portuguese bisyllabic stimuli where statements and yes/no questions are segmentally identical but distinguished by a fall versus a rise on the final syllable. Using the visual habituation paradigm, we found that unlike Portuguese infants (Frota et al., 2014), only English 8-, but not 4-month-olds are able to distinguish final rise from fall in the presence of segmental variability. Besides having a rising pitch, the final syllable in Portuguese questions is also longer. We are currently testing English 8-month-olds with resynthesized Portuguese stimuli that maintain the pitch difference, but neutralize duration. Together, the results will help us reconcile the previously reported failure of English-learning infants to distinguish English statements from questions (Soderstrom et al., 2011; Geffen, 2014), with their precocious sensitivity to prosody (Jusczyk, 1997).

5pSCb50. Developmental trends of perceiving happy voice in preverbal infants. Feng-Ming Tsao, Yu-Hsin Hu, Chieh Kao (Psych., National Taiwan Univ., No. 1, Sec. 4, Roosevelt Rd., Taipei 106, Taiwan, tsaosph@ mail2000.com.tw), and huei-mei liu (Special Education, National Taiwan Normal Univ., Taipei, Taiwan)

Perception of emotional prosody is essential for children to identify emotions of communication partners from utterances. Newborns prefer listening to happy talks but children start to weight emotional prosody over lexical meanings to judge emotions in speech around middle childhood. Happy voice is expressed with higher fundamental frequency (F0) and F0 variations in syllables manifest lexical tones in Mandarin. Would lexical tones affect happy prosody perception development in preverbal infants? The goal of this study was to explore whether the ability of perceiving happy prosody varies with lexical tones. Mandarin-learning children master the production of Tone 2 earlier than Tone 3 , and development trend of lexical tone production might reflect perceptual trends of lexical tones. Thirtynine Mandarin-learning 7- and 11-month-old infants were tested with two prosody (happy vs. neutral prosody) contrasts in a speech discrimination task. For each contrast, speech stimuli were monosyllabic words /ji/, varied with emotional prosody. Tonal information (Tone 2 or Tone 3 ) varied between contrasts and remained the same within a contrast. Results showed that older infants were more accurate than younger infants to distinguish happy prosody and the pitch contours of lexical tones did not greatly shift the developmental trend.

5pSCb51. Analysis of children's high front vowel area function using three-dimensional ultrasound imaging. Gary Yeung (Dept. of Elec. Eng., Univ. of California, Los Angeles, 420 Westwood Plaza, 56-125B Eng. IV Bldg., Los Angeles, CA 90095, garyyeung@g.ucla.edu), Steven M. Lulich (Dept. of Speech and Hearing Sci., Indiana Univ. Bloomington, Bloomington, IN), Asterios Toutios (Signal Anal. and Interpretation Lab., Univ. of Southern California, Los Angeles, CA), Abeer Alwan, and Amber Afshan (Dept. of Elec. Eng., Univ. of California, Los Angeles, Los Angeles, CA)

Understanding children's speech production can follow from an analysis of children's vocal tract area functions. While some studies have used imaging techniques on children to measure tongue contours, to our knowledge, no vocal tract area functions of children have been examined. We attempt to measure the oral cavity vocal tract area functions of children's vowels using three-dimensional ultrasound imaging recordings of the tongue and palate. In this investigation, we model the oral cavity area function of the vowel [i] of children and compare it to that of an adult oral cavity area function measured using the same ultrasound technique. We show evidence that the tongue positioning and articulation of the vowel [i] is dramatically different for children and adults. Specifically, we focus on how the different tongue positioning of children and adults affects the vocal tract transfer function of [i]. Some theories of children's speech, either mechanically or perceptually based, can be inferred from these findings and will be compared to previous studies. [Work supported in part by NSF Grant 1551113, NSF Grant 1551131, NSF Grant 1514544, and NIH Grant R01DC007124.] 
5pSCb52. The time course of top-down processing during speech perception by normal hearing and cochlear-implant users. Anita E. Wagner (KNO, UMCG, Hanzeplein 1, Groningen 9713 GZ, Netherlands, a.wagner@umcg.nl)

Cochlear implant (CI) users need to rely on top-down processes, such as integration of semantic information, to facilitate perception of CI speech, degraded in acoustic-phonetic details. This paper investigates the timecourse of semantic integration for normal-hearing $(\mathrm{NH})$ listeners, when processing natural and CI-simulated speech, as well as for experienced CI users. In eye-tracking experiments, we recorded listeners' gaze fixationsan online measure of lexical decision-making, and time-locked pupil dilation-a measure of the mental effort involved. Participants' ocular responses were measured while listening to sentences and simultaneously performing a search among pictures that displayed objects referred to in the sentence. The stimuli contained a target word (baby) that was either preceded or followed by disambiguating context (crawl). The display showed next to the target also a phonological competitor (bay), a word that was semantically viable given the sentential context (worm), and an unrelated distractor. For natural speech, gaze fixations show a fast integration of sentential context, and pupil dilations reveal a release from mental effort when sematic information is integrated. Degraded signals delay semantic integration, and further constrain listeners' ability to compensate for inaudible information. We will discuss sources of individual variation among CI users.
5pSCb53. Aging effect on categorical perception of Mandarin level-rising and level-falling tones. Lilong Xu (Univ. of Cambridge, University of Cambridge, Cambridge CB21TN, United Kingdom, babybear747@hotmail. com), Xiaohu Yang (Speech, Lang., and hearing Ctr., Ctr. for Cross-Linguistic Processing and Cognition, Shanghai Jiao Tong Univ., Shanghai, China), Chang Liu (Dept. of Commun. Sci. and Disord., Univ. of Texas at Austin, Austin, TX), and Yuxia Wang (Speech, Lang., and hearing Ctr., Ctr. for Cross-Linguistic Processing and Cognition, School of Foreign Lang., Shanghai Jiao Tong Univ., Shanghai, China)

The primary purpose of the study was to investigate the aging effect on categorical perception of Mandarin Chinese tone 1 (level F0 pitch contour) and tone 2 (rising F0 pitch contour), as well as tone 1 and tone 4 (falling F0 pitch contour). Using level/rising and level/falling fundamental frequency continuum, both tone identification and discrimination was measured for older and younger native Mandarin listeners. Moreover, the stimuli duration was manipulated at 100,200 , and $400 \mathrm{~ms}$ with a purpose to examine whether longer duration made tone perception easier for the older listeners. Results reported reduced catgoricality in terms of shallower identification slope and smaller discrimination peakedness for the older listeners compared with their younger counterparts. Meanwhile, longer duration was observed with enhanced categorical perception of lexical tones for the older listeners. These results were interpreted as the decline of aging-related temporal/spectral processing and inhibitory control in the central auditory system.

FRIDAY AFTERNOON, 2 DECEMBER 2016

SOUTH PACIFIC 1, 1:00 P.M. TO 5:05 P.M.

\title{
Session 5pSP
}

\author{
Signal Processing in Acoustics: General Topics in Signal Processing III
}

\author{
Trevor W. Jerome, Chair \\ Department of Acoustics, The Pennsylvania State University, GTWT Water Tunnel, State College, PA 16804
}

\section{Contributed Papers}

1:00

5pSP1. Strong direct path interference suppression based on spatial filtering. Xueli Sheng (Underwater Acoust., Harbin Eng. Univ., No. 145 Nantong St., Shuisheng Bldg. 803, Harbin, China, shengxueli@aliyun.com), Xiaoyi Sun, Yali Shi, Longxiang Guo, and Dian Lu (Underwater Acoust., Harbin Eng. Univ., Harbin, Heilongjiang, China)

Strong direct path interference suppression is one of the problems to be solved in AUVs' cluster system which has the advantages of concealment, precision, anti-jamming capability and function distance. In order to estimate the target direction, 3 methods of beamforming, including covariance matrix taper(CMT) condition, null-constrained condition and second-order cone programing(SOCP) constraint condition, are used to restrain direct path interference suppression separately in this paper. Comparing the 3 methods with simulation, SOCP gives the weights taking main lobe response, sidelobe level, depth, and width of zeros into consideration; nullconstrained condition has a smaller amount of calculate and better anti-interference ability. But when the array disturbance exists, all the methods' performances are not good. Then, an improved algorithm is proposed to improve the tolerance of array disturbances.
$1: 15$

5pSP2. Comparison of the multiple maximum length sequences and multiple exponential sweeps method for parallel measurements of impulse responses. Shoma Kuroda (Graduate School of Eng., Oita Univ., 700 Dannoharu, Oita City 870-1192, Japan, v15e3010@oita-u.ac.jp), Shingo Uenohara, Keisuke Nishijima, and Ken'ichi Furuya (Faculty of Eng., Oita Univ., Oita, Japan)

The measurement of room impulse responses is a central problem in audio signal processing, particularly for spatial audio rendering and sound field reproduction applications. The calibration of modern rendering systems requires the knowledge of the room impulse responses between the loudspeakers and several possible listener positions, in order to compensate for the characteristics of the loudspeakers and the room. The parallel measurement of multiple loudspeakers can be efficiently performed using the multiple maximum length sequence (MMLS) or the multiple exponential sweep (MES) method. This study evaluates the MMLS and MES methods for performing parallel measurements of multi-channel acoustic systems. Moreover, we apply noise reduction to improve their measurement accuracies. Experiments were conducted to evaluate the accuracy, measurement duration, and computing time for the two methods. The results show that the MMLS method was as accurate as the MES method. Although the measurement duration was shorter for the MMLS method compared with the MES method, the computing time was longer. 
5pSP3. Nonstationary signals, holograms, and concert halls. James B. Lee (None, 6016 S. E. Mitchell, Portland, OR 97206, cadwal@macforcego. com)

Sabine's analysis of sound in rooms involves a "mean-free-path," 4V/S, scaling linearly; for concert halls this measure is about $30 \mathrm{~m}$, implying that perception in the time domain will be an issue. It is not possible to average signals over the space within a concert hall, for it is not possible to average speech and music over time. Speech and music are nonstationary signals, therefore concert halls cannot be ergodic systems. The necessary and sufficient condition for proper acoustics in large spaces is that every listener receive the same full information at some time. It is fulfilled if the space is a time-varying diffuse volumetric hologram with direct sound from the orchestral region acting as reference beam. If the space contains specularly reflected components those will be heard as echoes, representing information about size and shape of the hall; this "architectural information" develops at the expense of "musical information." Therefore, diffuse reflection is essential in the body of a concert hall.

\section{$1: 45$}

5pSP4. Passive imaging of scatterers in an acoustic daylight imaging configuration. Jie Li, Peter Gerstoft (Marine Physical Lab., Scripps Inst. of Oceanogr., 9500 Gilman Dr. La Jolla, San Diego, CA 92093, ji1004@ucsd. edu), Dazhi Gao (Ocean Univ. of China, Qingdao, Shandong, China), Guofu Li (Ocean Univ. of China, Tianjin, China), and Ning Wang (Ocean Univ. of China, Qingdao, China)

The arrival-time structure of Green's functions is extracted by crosscorrelating one-sided aeroacoustic surf noise in an acoustic daylight imaging configuration, where 14 microphones (separation 0.08-3.7 m) are located between the noise and a cylinder. The cylinder is a hollow PVC pipe (radius $20 \mathrm{~cm}$, height $2 \mathrm{~m}) 2.5 \mathrm{~m}$ landside of a microphone array. The frequency band for computing the cross correlation function is $400-2000 \mathrm{~Hz}$, and total averaging time is $\mathrm{T}_{\mathrm{ave}}=3 \mathrm{~min}$. Results show that arrivals corresponding to the scattered (travel-time for source-scatterer-receiver path) waves and the time-difference (travel-time difference between two scatterer-receiver paths) waves emerge in the crosscorrelation functions for an acoustic daylight imaging configuration. Only one microphone pair is needed to locate the scatterer passively with the scattered and time-difference traveltime. We show imaging of 20 crosscorrelation functions with clear time-difference and scattered waves. Each estimated location is along the seaside boundary of cylinder, and the average location is close to the origin of the seaside half circle of cylinder.

\section{2:00}

5pSP5. Extracting elastic target information from acoustic scattering data of underwater munitions. David E. Malphurs (Naval Surface Warfare Ctr. Panama City Div., 110 Vernon Ave., Panama City, FL 32407, david. malphurs@navy.mil)

Synthetic Aperture Sonar (SAS) acoustic scattering data from a variety of underwater munitions was collected in the Target and Reverberation Experiment 2013 (TREX13), Bay Experiment 2014 (BayEX14) and from the Acoustic Test Facility at Naval Surface Warfare Center Panama City Division. One data product from these tests is two dimensional templates of back scattered acoustic intensity as a function of aspect angle and frequency. A common method of target classification utilizes normalized template cross-correlations as the input into classification algorithms. This is typically done in a manner agnostic to observable physics-based scattering phenomena derived from the physical size, material composition, and geometry of the targets that might help improve classification performance. This presentation discusses methods explored to process the available data to make target elastic phenomena easier to recognize so that associated information can be extracted and used as target classifier input. The methods explored include: lower dimensional representations of the data that preserve the physics-based phenomena, analysis of the data in time-frequency space, and using phase information in template correlations to help isolate resonance related information. [Work supported by ONR.]
5pSP6. A method for real-time, multiple-source localization using spherical harmonic decomposition. Jonathan Mathews and Jonas Braasch (Architectural Sci. - Architectural Acoust., Rensselaer Polytechnic Inst., 110 8th St, Greene Bldg., Troy, NY 12180, mathej4@rpi.edu)

By taking advantage of the spherical harmonic transform, spherical microphone arrays are able to decompose three-dimensional sound fields into their basic components, which enables detailed analysis and efficient spatial filtering algorithms. In recent years, methods for determining relative source positions around an array have been studied. Weighting functions can be generated to create a steerable beam which attenuates a sound field except in a specific direction. This research describes a real-time framework for multiple-source detection, which can be implemented using these methods. By finding the energy-sum distribution across a matrix of weighted harmonic signals, directional information may be simultaneously and efficiently obtained for multiple sources over time.

\section{2:30}

5pSP7. Multi-channel non-negative matrix factorization with binary mask initialization for automatic speech recognition. Iori Miura (Graduate School of Eng., Oita Univ., 700 Dannoharu, Oita, Oita 870-1192, Japan, v16e3019@oita-u.ac.jp), Yuuki Tachioka, Tomohiro Narita, Jun Ishii (Mitsubishi Electric, Kanagawa, Japan), Fuminori Yoshiyama (Graduate School of Eng., Oita Univ., Oita, Japan), Shingo Uenohara, and Ken'ichi Furuya (Faculty of Eng., Oita Univ., Oita, Japan)

Non-negative Matrix Factorization (NMF) factorizes a non-negative matrix into two non-negative matrices. In the field of acoustics, multichannel expansion has been proposed to consider spatial information for sound source separation. Conventional multi-channel NMF has a difficulty in an initial-value dependency of the separation performance due to local minima. This paper proposes initial value settings by using binary masking based sound source separation whose masks on the time frequency domain are calculated from the time difference of arrival of each source. The proposed method calculates initial spatial correlation matrices using separated sources by binary masking. The music separation experiments confirmed that the separation performance of the proposed method was better than that of the conventional method. In addition, we evaluated initial value settings by using binary masking for automatic speech recognition (ASR) tasks in noisy environments. The ASR experiments confirmed that appropriate initial value settings were effective because initializations starting from previously wellestimated spatial correlation matrices achieved better ASR performances than random initializations.

\section{2:45}

5pSP8. Performance improvement of snore activity detection in a noisy environment. Keisuke Nishijima, Shingo Uenohara, and Ken'ichi Furuya (Comput. Sci. and Intelligent Systems, Oita Univ., Dannoharu 700, Oita-shi, Oita 870-1192, Japan, k-nisijima@oita-u.ac.jp)

Health promotion and maintenance is becoming increasingly important and depends on three elements: nutrition, exercise, and rest (sleep). In the present work, focusing on sleep, we develop a smartphone-based system based on snore activity detection to investigate day-to-day variations in the sleep state, which does not require dedicated hardware. Snore activity detection is performed by machine learning using a support vector machine (SVM) with a linear kernel; the SVM is trained by labeled acoustic features, and the trained SVM models are used to detect snore activity. As acoustic features, The sound pressure level and mel-frequency cepstrum coefficients are calculated from sleep sound data obtained using a smartphone. In this paper, we investigated the effects of adding sleep environment noise recorded before sleep to the training set in snore activity detection, and we considered ways to improve detection performance. Performance comparison among the conventional method of SVM and the proposed method was presented. The performance comparison was evaluated by the cross-validation. The detection performance was improved by adding sleep environment noise recorded before sleep to the training set.

3:00-3:20 Break 
symmetry ambiguities in the localization. We investigate this relation for

5pSP9. MRI noise suppression using weighted spectral subtraction based on noise estimation with long-and-short terms. Sayaka Okayasu, Maiko Yoshiura, Takahiro Fukumori, Masato Nakayama, and Takanobu Nishiura (Ritsumeikan Univ., 1-1-1 Noji-higashi, Kusatsu, Shiga 525-8577, Japan, is0159ee@ed.ritsumei.ac.jp)

MRI devices are often utilized as surgery support systems. However, communication among medical staff during surgery is difficult because conversations can be disturbed by loud noise emitted from devices. In this study, we propose a MRI noise suppression method using a weighted spectral subtraction to address the problem. Spectral subtraction is generally utilized for suppressing stationary noise. However, directly applying this method to non-stationary noise such as MRI noise, which has frequency and amplitude fluctuations in the long-and-short terms, may be difficult. In the proposed method, the noise from a MRI device is estimated on the basis of the frequency fluctuation in the short term. The estimated noise is then subtracted by processing the weighted spectral subtraction whose coefficients are calculated on the basis of the amplitude fluctuation in the long term. Objective evaluation experiments were carried out to evaluate the quantities of noise reduction and sound distortion. As a result, we confirmed the effectiveness of the proposed method.

\section{3:35}

5pSP10. Feature extraction for acoustic signatures of small boats. Alexander S. Pollara, Alexander Sutin, and Hady Salloum (Maritime Security Ctr., Stevens Inst. of Technol., 1 Castle Point on Hudson, Babbio Ctr., Hoboken, NJ 07030, apollara@stevens.edu)

Small boats represent a particular challenge to maritime surveillance due to their ubiquity, low radar cross-section, and absence of AIS transmission. We present a review of previously developed acoustic classification methods and demonstrate the importance of both the engine, and propeller as the two sources of small vessel noise. The features used for classification are extracted using various signal processing methods including signal spectra, spectra of Detection of Envelope Modulation on Noise (DEMON), and Cepstrum. The normalization of the features allowing to remove the dependence of the parameters on the distance to a boat and its speed was conducted. Acoustic signatures of six small boats including a panga, a jetski, an electrical boat, a go fast boat and two speed boats of the type common in the United States were collected by Stevens in a large glacial lake in NJ. The sound sources of various tonal components in the signal spectra are discussed. The program for automated extraction of classification features is presented and the identification of each of the 6 small boats with these features is demonstrated. [This work was supported by DHS's S\&T Directorate.]

\section{$3: 50$}

5pSP11. Numerical simulations of sound source localization with twodimensional bio-inspired antennas of varying geometric complexities. Michael Reinwald (Laboratoire d'Imagerie Biomédicale, Université Pierre et Marie Curie, 15 rue de l'Ecole de Médecine, Laboratoire d'Imagerie Biomédicale, Paris 75006, France, mchlrnwld@gmail.com), Lapo Boschi (Institut des Sci. de la Terre de Paris, Université Pierre et Marie Curie, Paris, France), Stefan Catheline (LabTAU, Unité U1032, Université Claude Bernard Lyon 1, Lyon, France), and Quentin Grimal (Laboratoire d'Imagerie Biomédicale, Université Pierre et Marie Curie, Paris, France)

Many animals use audition as their primary tool to navigate and hunt. The accuracy with which they complete those tasks suggests that they effectively harness detailed waveform information. In addition to inter-aural time (ITD) or level differences (ILD) they are likely to extract information from sound conducted by the skull. It is well established that an object of complex shape, in which elastic waves propagate (such as the skull of an animal), can be used as an antenna to locate the position of an acoustic source, even using a single receiver. We hypothesize that complexities in the shape of mammalian skulls enhance source localization performance. We evaluate the localization precision achieved with two-dimensional skull-shaped antennas of varying complexity, generating synthetic data by time-domain numerical simulations and "inverting" them via signal processing techniques such as ITD, ILD and time-reversal. Full waveform algorithms (e.g., time-reversal) benefit from increasing geometric complexity of the antenna, e.g., removing aforementioned algorithms and combinations thereof. Our simulations are a first step towards experimental and numerical investigations of the role of bone conduction in source localization using skulls of different mammals.

\section{4:05}

5pSP12. Applying the unit circle constraint to the diagonal loaded minimum variance distortionless response beamformer. Colin Ryan (Elec. and Comput. Eng., Univ. of Massachusetts Dartmouth, 679 Main St., Harwich, MA 02645, cryan2@umassd.edu) and John R. Buck (Elec. and Comput. Eng., Univ. of Massachusetts Dartmouth, Dartmouth, MA)

Estimating the direction of arrival of a narrowband planewave using a uniform line array is a common array processing problem. Capon's minimum variance distortionless response (MVDR) adaptive beamformer (ABF) suppresses interferers by steering nulls in their directions while maintaining unity gain in the desired look direction. Practical ABFs replace the ensemble covariance matrix $(\mathrm{ECM})$ with a sample covariance matrix (SCM) estimated from array observations, or snapshots. When the $\mathrm{ABF}$ cannot average enough snapshots, the SCM is a poor estimate of the ECM, and may be rank deficient. Applying diagonal loading (DL) to the SCM improves the ABF's performance by limiting the white noise power, but may also limit interferer suppression. Projecting the zeros of the beamformer's array polynomial onto the unit circle (UC) provides deeper nulls to suppress interferers but exhibits worse white noise behavior than DL. The UCDL MVDR beamformer combines the UC constraint with the DL MVDR weights to achieve better SINR than either the UC or DL MVDR alone. Even when the UCDL beamformer suffers mismatch on the DL level, the UCDL SINR rivals or betters the DL MVDR with the optimal DL level. [Research funded by ONR grant N00014-15-1-2238.]

\section{$4: 20$}

5pSP13. Sound source localization based on sparse estimation and convex clustering. Tomoya Tachikawa, Kohei Yatabe, Yusuke Ikeda, and Yasuhiro Oikawa (intermedia art and Sci., Waseda Univ., 59-407-2,3-4-1, Okubo, Shinjuku, Tokyo 169-8555, Japan, 14919320tt611@toki.waseda.jp)

Sound source localization techniques in array signal processing have been interested for many years. A lot of direction-of-arrival (DOA) estimation methods have been proposed. Most of them use plane waves as model. On the other hand, 3D sound source localization techniques are important for many applications. They are required to estimate the DOA and the distance of sound sources. In the case of the techniques targeted to estimate the distances of sound sources, monopoles must be used for the model. The positions of monopoles are candidates for the sound source positions. However, it is difficult to handle a large number of monopoles placed at various distances. Moreover, it is difficult to decide the number of sound sources to be estimated from the candidates. The proposed method uses sparse estimation with a monopole dictionary in frequency domain, as is well known that the effectiveness of sparse representation for DOA estimation has been in late years. After the sparse estimation, the proposed method uses convex clustering to estimate the DOA and the distance of sound sources. In addition, the proposed method includes the idea that decides the number of all sound sources without a priori information about the number of sound sources.

\section{$4: 35$}

5pSP14. Context recognition system for smartphone applications with acoustic data. Miho Tateishi (School of Sci. for Open and Environment Systems, Keio Univ. Graduate School of Sci. and Technol., Hiyoshi 3-14-1, Kohoku-ku, Yokohama 223-8522, Japan, m.tateishi-3303@keio.jp)

This paper aims to create an original context recognition system on smartphones by using acoustic data. In this paper, the context represents that how the smartphone users move or how their contiguous environment is. In current systems, the context refers to the place where they are. It means that it is difficult to recognize the context when they are in a new place for which the system does not have the data. Our system simply categorizes the situation: "Train" when the user on the train, "Quiet Work Place" when they in a place like a library, PC room, or laboratory, and so on. This system features sound because it would be containing a lot of information. From volume to spectrum, we analyze the data from a built-in microphone and abstract multiple feature values. In addition to this, 
accelerator and luminance data are also used as feature values. Then these feature values are classified into context categories. This situation based categorizing system realizes that wherever the user goes, the context can be understood. It would be a flexible platform for smartphone applications which need the context recognition system.

\section{4:50}

5pSP15. Identification of phonocardiographic signals using noise suppression based on wavelet transform. Shinya Kudo, Keisuke Nishijima, Shingo Uenohara, and Ken'ichi Furuya (Comput. Sci. and Intelligent Systems, Oita Univ., 700 Dannnoharu, Oita 870-1192, Japan, v15e3009@oita-u.ac.jp)

While heart disease is one of the three major diseases, only well-qualified doctors can evaluate phonocardiographic signals. However, phonocardiographic signals are not always used in healthcare because only a few professionals are experts in evaluating phonocardiographic signals. We need to develop an easily available system that can automatically diagnose phonocardiographic signals. In previous study, phonocardiographic signals are typically analyzed using wavelet transform to match and extract the characteristics of known normal and abnormal phonocardiographic signals. However, everyday noises such as lung and breath sounds, environmental noises, and blood flow noises may contaminate these signals and hinder analysis. We have previously proposed noise suppression using wavelet transform for phonocardiographic signals. In this study, we compare the proposed method and spectral subtraction to identify the kind of phonocardiographic signals. The experiment results show that the proposed method provides better identification performance compared with the spectral subtraction method. 


\section{ETHICAL PRINCIPLES OF THE ACOUSTICAL SOCIETY OF AMERICA FOR RESEARCH INVOLVING HUMAN AND NON-HUMAN ANIMALS IN RESEARCH AND PUBLISHING AND PRESENTATIONS}

The Acoustical Society of America (ASA) has endorsed the following ethical principles associated with the use of human and non-human animals in research, and for publishing and presentations. The principles endorsed by the Society follow the form of those adopted by the American Psychological Association (APA), along with excerpts borrowed from the Council for International Organizations of Medical Sciences (CIOMS). The ASA acknowledges the difficulty in making ethical judgments, but the ASA wishes to set minimum socially accepted ethical standards for publishing in its journals and presenting at its meetings. These Ethical Principles are based on the principle that the individual author or presenter bears the responsibility for the ethical conduct of their research and is publication or presentation.

Authors of manuscripts submitted for publication in a journal of the Acoustical Society of America or presenting a paper at a meeting of the Society are obligated to follow the ethical principles of the Society. Failure to accept the ethical principles of the ASA shall result in the immediate rejection of manuscripts and/or proposals for publication or presentation. False indications of having followed the Ethical Principles of the ASA may be brought to the Ethics and Grievances Committee of the ASA.

\section{APPROVAL BY APPROPRIATE GOVERNING AUTHORITY}

The ASA requires all authors to abide by the principles of ethical research as a prerequisite for participation in Society-wide activities (e.g., publication of papers, presentations at meetings, etc.). Furthermore, the Society endorses the view that all research involving human and non-human vertebrate animals requires approval by the appropriate governing authority (e.g., institutional review board [IRB], or institutional animal care and use committee [IACUC], Health Insurance Portability and Accountability Act [HIPAA], or by other governing authorities used in many countries) and adopts the requirement that all research must be conducted in accordance with an approved research protocol as a precondition for participation in ASA programs. If no such governing authority exists, then the intent of the ASA Ethical Principles described in this document must be met. All research involving the use of human or non-human animals must have met the ASA Ethical Principles prior to the materials being submitted to the ASA for publication or presentation.

\section{USE OF HUMAN SUBJECTS IN RESEARCH-Applicable when human subjects are used in the research}

Research involving the use of human subjects should have been approved by an existing appropriate governing authority (e.g., an institutional review board [IRB]) whose policies are consistent with the Ethical Principles of the ASA or the research should have met the following criteria:

\section{Informed Consent}

When obtaining informed consent from prospective participants in a research protocol that has been approved by the appropriate and responsiblegoverning body, authors must have clearly and simply specified to the participants beforehand:

1. The purpose of the research, the expected duration of the study, and all procedures that were to be used.

2. The right of participants to decline to participate and to withdraw from the research in question after participation began.

3. The foreseeable consequences of declining or withdrawing from a study.

4. Anticipated factors that may have influenced a prospective participant's willingness to participate in a research project, such as potential risks, discomfort, or adverse effects.

5. All prospective research benefits.

6. The limits of confidentially.

7. Incentives for participation.

8. Whom to contact for questions about the research and the rights of research participants. The office/person must have willingly provided an atmosphere in which prospective participants were able to ask questions and receive answers.

Authors conducting intervention research involving the use of experimental treatments must have clarified, for each prospective participant, the following issues at the outset of the research:

1. The experimental nature of the treatment;

2. The services that were or were not to be available to the control group(s) if appropriate;
3. The means by which assignment to treatment and control groups were made;

4. Available treatment alternatives if an individual did not wish to participate in the research or wished to withdraw once a study had begun; and

5. Compensation for expenses incurred as a result of participating in a study including, if appropriate, whether reimbursement from the participant or a third-party payer was sought.

\section{Informed Consent for Recording Voices and Images in Research}

Authors must have obtained informed consent from research participants prior to recording their voices or images for data collection unless:

1. The research consisted solely of naturalistic observations in public places, and it was not anticipated that the recording would be used in a manner that could have caused personal identification or harm, or

2. The research design included deception. If deceptive tactics were a necessary component of the research design, consent for the use of recordings was obtained during the debriefing session.

\section{Client/Patient, Student, and Subordinate Research Participants}

When authors conduct research with clients/patients, students, or subordinates as participants, they must have taken steps to protect the prospective participants from adverse consequences of declining or withdrawing from participation.

\section{Dispensing With Informed Consent for Research}

Authors may have dispensed with the requirement to obtain informed consent when:

1. It was reasonable to assume that the research protocol in question did not create distress or harm to the participant and involves:

a. The study of normal educational practices, curricula, or classroom management methods that were conducted in educational settings

b. Anonymous questionnaires, naturalistic observations, or archival research for which disclosure of responses would not place participants at risk of criminal or civil liability or damage their financial standing, employability, or reputation, and confidentiality

c. The study of factors related to job or organization effectiveness conducted in organizational settings for which there was no risk to participants' employability, and confidentiality.

2. Dispensation is permitted by law.

3. The research involved the collection or study of existing data, documents, records, pathological specimens, or diagnostic specimens, if these sources are publicly available or if the information is recorded by the investigator in such a manner that subjects cannot be identified, directly or through identifiers linked to the subjects.

\section{Offering Inducements for Research Participation}

(a) Authors must not have made excessive or inappropriate financial or other inducements for research participation when such inducements are likely to coerce participation. 
(b) When offering professional services as an inducement for research participation, authors must have clarified the nature of the services, as well as the risks, obligations, and limitations.

\section{Deception in Research}

(a) Authors must not have conducted a study involving deception unless they had determined that the use of deceptive techniques was justified by the study's significant prospective scientific, educational, or applied value and that effective non-deceptive alternative procedures were not feasible.

(b) Authors must not have deceived prospective participants about research that is reasonably expected to cause physical pain or severe emotional distress.

(c) Authors must have explained any deception that was an integral feature of the design and conduct of an experiment to participants as early as was feasible, preferably at the conclusion of their participation, but no later than at the conclusion of the data collection period, and participants were freely permitted to withdraw their data.

\section{Debriefing}

(a) Authors must have provided a prompt opportunity for participants to obtain appropriate information about the nature, results, and conclusions of the research project for which they were a part, and they must have taken reasonable steps to correct any misconceptions that participants may have had of which the experimenters were aware.

(b) If scientific or humane values justified delaying or withholding relevant information, authors must have taken reasonable measures to reduce the risk of harm.

(c) If authors were aware that research procedures had harmed a participant, they must have taken reasonable steps to have minimized the harm.

\section{HUMANE CARE AND USE OF NON-HUMAN VERTEBRATE ANIMALS IN RESEARCH-Applicable when non-human vertebrate animals are used in the research}

The advancement of science and the development of improved means to protect the health and well being both of human and non-human vertebrate animals often require the use of intact individuals representing a wide variety of species in experiments designed to address reasonable scientific questions. Vertebrate animal experiments should have been undertaken only after due consideration of the relevance for health, conservation, and the advancement of scientific knowledge. (Modified from the Council for International Organizations of Medical Science (CIOMS) document: "International Guiding Principles for Biomedical Research Involving Animals1985”). Research involving the use of vertebrate animals should have been approved by an existing appropriate governing authority (e.g., an institutional animal care and use committee [IACUC]) whose policies are consistent with the Ethical Principles of the ASA or the research should have met the following criteria:

The proper and humane treatment of vertebrate animals in research demands that investigators:

1. Acquired, cared for, used, interacted with, observed, and disposed of animals in compliance with all current federal, state, and local laws and regulations, and with professional standards.

2. Are knowledgeable of applicable research methods and are experienced in the care of laboratory animals, supervised all procedures involving animals, and assumed responsibility for the comfort, health, and humane treatment of experimental animals under all circumstances.

3. Have insured that the current research is not repetitive of previously published work.

4. Should have used alternatives (e.g., mathematical models, computer simulations, etc.) when possible and reasonable.

5. Must have performed surgical procedures that were under appropriate anesthesia and followed techniques that avoided infection and minimized pain during and after surgery.

6. Have ensured that all subordinates who use animals as a part of their employment or education received instruction in research methods and in the care, maintenance, and handling of the species that were used, commensurate with the nature of their role as a member of the research team.
7. Must have made all reasonable efforts to minimize the number of vertebrate animals used, the discomfort, the illness, and the pain of all animal subjects.

8. Must have made all reasonable efforts to minimize any harm to the environment necessary for the safety and well being of animals that were observed or may have been affective as part of a research study.

9. Must have made all reasonable efforts to have monitored and then mitigated any possible adverse affects to animals that were observed as a function of the experimental protocol.

10. Who have used a procedure subjecting animals to pain, stress, or privation may have done so only when an alternative procedure was unavailable; the goal was justified by its prospective scientific, educational, or applied value; and the protocol had been approved by an appropriate review board.

11. Proceeded rapidly to humanely terminate an animal's life when it was necessary and appropriate, always minimizing pain and always in accordance with accepted procedures as determined by an appropriate review board.

\section{PUBLICATION and PRESENTATION ETHICS-For publications in ASA journals and presentations at ASA sponsored meetings Plagiarism}

Authors must not have presented portions of another's work or data as their own under any circumstances.

\section{Publication Credit}

Authors have taken responsibility and credit, including authorship credit, only for work they have actually performed or to which they have substantially contributed. Principal authorship and other publication credits accurately reflect the relative scientific or professional contributions of the individuals involved, regardless of their relative status. Mere possession of an institutional position, such as a department chair, does not justify authorship credit. Minor contributions to the research or to the writing of the paper should have been acknowledged appropriately, such as in footnotes or in an introductory statement.

\section{Duplicate Publication of Data}

Authors did not publish, as original data, findings that have been previously published. This does not preclude the republication of data when they are accompanied by proper acknowledgment as defined by the publication policies of the ASA.

\section{Reporting Research Results}

If authors discover significant errors in published data, reasonable steps must be made in as timely a manner as possible to rectify such errors. Errors can be rectified by a correction, retraction, erratum, or other appropriate publication means.

\section{DISCLOSURE OF CONFLICTS OF INTEREST}

If the publication or presentation of the work could directly benefit the author(s), especially financially, then the author(s) must disclose the nature of the conflict:

1) The complete affiliation(s) of each author and sources of funding for the published or presented research should be clearly described in the paper or publication abstract.

2) If the publication or presentation of the research would directly lead to the financial gain of the authors(s), then a statement to this effect must appear in the acknowledgment section of the paper or presentation abstract or in a footnote of a paper.

3) If the research that is to be published or presented is in a controversial area and the publication or presentation presents only one view in regard to the controversy, then the existence of the controversy and this view must be provided in the acknowledgment section of the paper or presentation abstract or in a footnote of a paper. It is the responsibility of the author to determine if the paper or presentation is in a controversial area and if the person is expressing a singular view regarding the controversy. 


\section{Sustaining Members of the Acoustical Society of America}

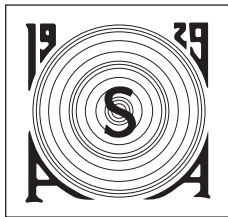

The Acoustical Society is grateful for the financial assistance being given by the Sustaining Members listed below and invites applications for sustaining membership from other individuals or corporations who are interested in the welfare of the Society.

Application for membership may be made to the Executive Director of the Society and is subject to the approval of the Executive Council. Dues of $\$ 1000.00$ for small businesses (annual gross below $\$ 100$ million) and $\$ 2000.00$ for large businesses (annual gross above $\$ 100$ million or staff of commensurate size) include a subscription to the Journal as well as a yearly membership certificate suitable for framing. Small businesses may choose not to receive a subscription to the Journal at reduced dues of \$500/year.

Additional information and application forms may be obtained from Elaine Moran, Office Manager, Acoustical Society of America, 1305 Walt Whitman Road, Suite 300, Melville, NY 11747-4300. Telephone: (516) 576-2360; E-mail: elaine@acousticalsociety.org

\section{Acentech Incorporated}

www.acentech.com

Cambridge, Massachusetts

Consultants in Acoustics, Audiovisual and Vibration

ACO Pacific Inc.

www.acopacific.com

Belmont, California

Measurement Microphones, the ACOustic Interface ${ }^{\mathrm{TM}}$ System

\section{American Institute of Physics}

www.aip.org

College Park, Maryland

Career resources, undergraduate education, science policy, and history

\section{Applied Physical Sciences Corp.}

www.aphysci.com

Groton, Connecticut

Acoustics, Hydrodynamics, Electromagnetics, Manufacturing

\section{BBN Technologies}

www.bbn.com

Cambridge, Massachusetts

R\&D company providing custom advanced research based solutions

\section{D’Addario \& Company, Inc.}

www.daddario.com

Farmingdale, New York

D'Addario strings for musical instruments, Evans drumheads, Rico woodwind reeds and Planet Waves accessories

\section{G.R.A.S.}

Sound \& Vibration ApS

www.gras. $d k$

Vedbaek, Denmark

Measurement microphones, Intensity probes, Calibrators

\section{InfoComm International Standards}

www.infocomm.org

Fairfax, Virginia

Advancing Audiovisual Communications Globally

Knowles Electronics, Inc.

www.knowles.com

Itasca, Illinois

Manufacturing Engineers: Microphones, Recording, and Special

Audio Products

\section{Massa Products Corporation}

www.massa.com

Hingham, Massachusetts

Design and Manufacture of Sonar and Ultrasonic Transducers

Computer-Controlled OEM Systems

\section{Meyer Sound Laboratories, Inc.}

www.meyersound.com

Berkeley, California

Manufacture Loudspeakers and Acoustical Test Equipment

\section{National Council of Acoustical Consultants \\ www.ncac.com \\ Indianapolis, Indiana \\ An Association of Independent Firms Consulting in Acoustics}

\author{
Raytheon Company \\ Integrated Defense Systems \\ www.raytheon.com \\ Portsmouth, Rhode Island \\ Sonar Systems and Oceanographic Instrumentation: R\&D \\ in Underwater Sound Propagation and Signal Processing
}

\section{ROXUL, Inc. - Core Solutions (OEM) \\ www.roxul.com}

Milton, ON, Canada

Offers a variety of insulation products ranging in density and dimension to meet any production requirements. Products are successfully used in numerous acoustical OEM applications providing solutions for a number of industries

\section{Shure Incorporated}

www.shure.com

Niles, Illinois

Design, development, and manufacture of cabled and wireless microphones for broadcasting, professional recording, sound reinforcement, mobile communications, and voice input-output applications; audio circuitry equipment; high fidelity phonograph cartridges and styli: automatic mixing systems; and related audio components and accessories. The firm was founded in 1925 .

\section{Thales Underwater Systems}

www.thales-naval.com

Somerset, United Kingdom

Prime contract management, customer support services, sonar design and production, masts and communications systems design and production

\section{M Occupational Health \& Environmental Safety Division}

www.3m.com/occsafety

Minneapolis, Minnesota

Products for personal and environmental safety, featuring E.A.R and Peltor brand hearing protection and fit testing, Quest measurement instrumentation, audiological devices, materials for control of noise, vibration, and mechanical energy, and the E.A.RCALSM laboratory for research, development, and education, NVLAP-accredited since 1992.

Hearing conservation resource center

www.e-a-r.com/hearingconservation

\section{Wenger Corporation}

www.wengercorp.com

Owatonna, Minnesota

Design and Manufacturing of Architectural

Acoustical Products including Absorbers, Diffusers, Modular Sound

Isolating Practice Rooms, Acoustical Shells and Clouds for Music

Rehearsal and Performance Spaces

\section{Wyle Laboratories}

www.wyle.com

Arlington, Virginia

The Wyle Acoustics Group provides a wide range of professional services focused on acoustics, vibration, and their allied technologies, including services to the aviation industry 


\section{ACOUSTICAL · SOCIETY · OF · AMERICA}

\section{APPLICATION FOR SUSTAINING MEMBERSHIP}

The Bylaws provide that any person, corporation, or organization contributing annual dues as fixed by the Executive Council shall be eligible for election to Sustaining Membership in the Society.

Dues have been fixed by the Executive Council as follows: $\$ 1000$ for small businesses (annual gross below $\$ 100$ million); $\$ 2000$ for large businesses (annual gross above $\$ 100$ million or staff of commensurate size). Dues include one year subscription to The Journal of the Acoustical Society of America and programs of Meetings of the Society. Please do not send dues with application. Small businesses may choose not to receive a subscription to the Journal at reduced dues of $\$ 500 /$ year. If elected, you will be billed.

Name of Company

Address

Telephone: Fax:

E-mail: WWW:

Size of Business:

$\square$ Small business

$\square$ Small business—No Journal

Large business

Type of Business

\section{Please enclose a copy of your organization's brochure.}

In listing of Sustaining Members in the Journal and on the ASA homepage we should like to indicate our products or services as follows:

(please do not exceed fifty characters)

Name of company representative to whom journal should be sent:

It is understood that a Sustaining Member will not use the membership for promotional purposes.

Signature of company representatives making application:

Please send completed applications to: Executive Director, Acoustical Society of America, 1305 Walt Whitman Road, Suite 300, Melville, NY 11747-4300, (516) 576-2360, asa@ acousticalsociety.org 


\section{Now Available on CD ROM \\ Technical Memoranda issued by \\ Acoustics Research Laboratory - Harvard University 1947-1971}

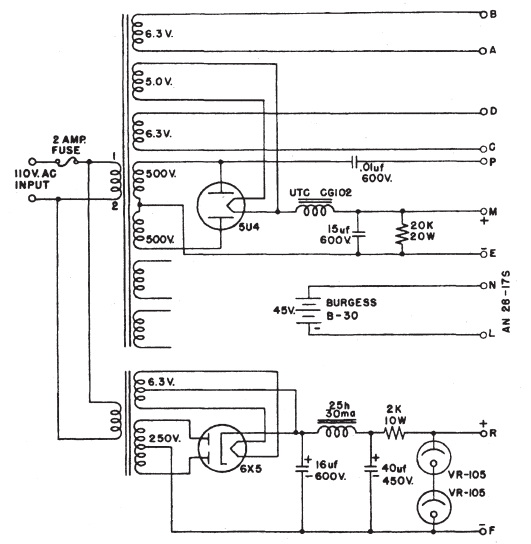
a single research group, the topics represented by the TMs fall mainly in the following categories: radiation, propagation, and scattering; bubbles and cavitation; acoustical instruments; electroacoustic transducers; and properties of solids, liquids, and gases.
The Harvard Acoustics Research Laboratory was established in 1946 to support basic research in acoustics. Research results were disseminated formally by means of reports called technical memoranda (TMs). This CD includes the 61 reports issued between 1946 and 1971, when the contract with the Office of Naval Research was completed.

About half the TMs are doctoral theses in report form though some incorporate substantial additions. Most of the other half represent output by the postdoctoral fellows. The collection is introduced by David T. Blackstock of the University of Texas at Austin and brief bios for all of the TM authors are included.

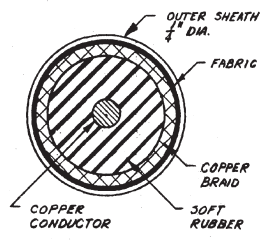

Fig.5. Schematic section

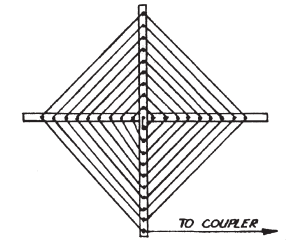

Pig.6. Configuration of the
spirai mounting for the cable.

\section{ORDER FORM}

1. Price: $\$ 25$ (includes shipping and handling)

2. Payment by check in U.S. funds/U.S. bank or VISA, MasterCard or American Express credit card must accompany all orders,

3. Send orders to: Acoustical Society of America, 1305 Walt Whitman Road, Suite 300, Melville, NY 11747-4300; Tel: 516-5762360; Fax: 631-923-2875.

Name

Address

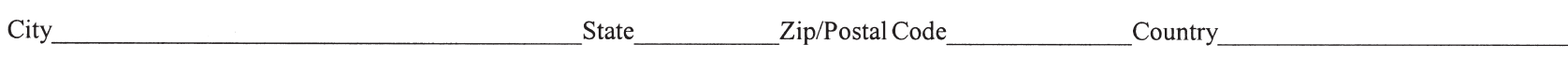

Tel:

Fax: Email:

PLEASE SEND ME:

$\begin{array}{ll}\text { Quantity } & \text { Publication Title } \\ & \text { Harvard Technical Memoranda CD ROM }\end{array}$

Due to security risks and Payment Card Industry (PCI) data security standards e-mail is NOT an acceptable way to transmit credit card information. Please use our secure web page to process your credit card payment (http://www.abdi-ecommerce10.com/asa) or securely fax this form to (631-923-2875). 


\section{MEMBERSHIP INFORMATION AND APPLICATION INSTRUCTIONS}

Applicants may apply for one of four grades of membership, depending on their qualifications: Student Member, Associate Member, Corresponding Electronic Associate Member or full Member. To apply for Student Membership, fill out Parts I and II of the application; to apply for Associate, Corresponding Electronic Associate, or full Membership, or to transfer to these grades, fill out Parts I and III.

\begin{tabular}{|c|c|c|c|c|}
\hline BENEFITS OF MEMBERSHIP & Full Member & Associate & ce-Associate & Student \\
\hline JASA Online-Vol. 1 (1929) to present & $*$ & $*$ & $*$ & $*$ \\
\hline JASA tables of contents e-mail alerts & $*$ & $*$ & $*$ & $*$ \\
\hline$J A S A$, printed or CD ROM & $*$ & $*$ & & \\
\hline JASA Express Letters-online & $*$ & $*$ & $*$ & $*$ \\
\hline Acoustics Today-the quarterly magazine & $*$ & $*$ & $*$ & $*$ \\
\hline Proceedings of Meetings on Acoustics & $*$ & $*$ & $*$ & $*$ \\
\hline $\begin{array}{l}\text { Noise Control and Sound, It's Uses and Control- } \\
\text { online archival magazines }\end{array}$ & * & $*$ & * & $*$ \\
\hline $\begin{array}{l}\text { Acoustics Research Letters Online (ARLO)- } \\
\text { online archive }\end{array}$ & $*$ & $*$ & $*$ & * \\
\hline Programs for Meetings & Online & Online & Online & Online \\
\hline Meeting Calls for Papers & Online & Online & Online & Online \\
\hline Reduced Meeting Registration Fees & * & $*$ & & $*$ \\
\hline 5 Free ASA standards per year-download only & $*$ & $*$ & & $*$ \\
\hline Standards Discounts & $*$ & $*$ & & $*$ \\
\hline Society Membership Directory & Online & Online & Online & Online \\
\hline Electronic Announcements & $*$ & $*$ & $*$ & $*$ \\
\hline Physics Today & $*$ & $*$ & $*$ & $*$ \\
\hline Eligibility to vote and hold office in ASA & $*$ & & & \\
\hline Eligibility to be elected Fellow & $*$ & $*$ & & \\
\hline Participation in ASA Committees & $*$ & $*$ & $*$ & $*$ \\
\hline
\end{tabular}

\section{QUALIFICATIONS FOR EACH GRADE OF MEMBERSHIP AND ANNUAL DUES}

Student: Any student interested in acoustics who is enrolled in an accredited college or university for half time or more (at least eight semester hours). Dues: \$45 per year.

Associate: Any individual interested in acoustics. Dues: $\$ 95$ per year. After five years, the dues of an Associate increase to that of a full Member.

Corresponding Electronic Associate: Any individual residing in a developing country who wishes to have access to ASA's online publications only including The Journal of the Acoustical Society of America and Meeting Programs [see http://acousticalsociety.org/ membership/membership_and_benefits]. Dues $\$ 45$ per year.

Member: Any person active in acoustics, who has an academic degree in acoustics or in a closely related field or who has had the equivalent of an academic degree in scientific or professional experience in acoustics, shall be eligible for election to Membership in the Society. A nonmember applying for full Membership will automatically be made an interim Associate Member, and must submit \$95 with the application for the first year's dues. Election to full Membership may require six months or more for processing; dues as a full Member will be billed for subsequent years.

\section{JOURNAL OPTIONS AND COSTS FOR FULL MEMBERS AND ASSOCIATE MEMBERS ONLY}

- ONLINE JOURNAL. All members will receive access to the The Journal of the Acoustical Society of America (JASA) at no charge in addition to dues.

- PRINT JOURNAL. Twelve monthly issues of The Journal of the Acoustical Society of America. Cost: \$35 in addition to dues.

- CD-ROM. The CD ROM mailed bimonthly. This option includes all of the material published in the Journal on CD ROM. Cost: \$35 in addition to dues.

- COMBINATION OF THE CD-ROM AND PRINTED JOURNAL. The CD-ROM mailed bimonthly and the printed journal mailed monthly. Cost: $\$ 70$ in addition to dues.

- EFFECTIVE DATE OF MEMBERSHIP. If your application for membership and dues payment are received by 15 September, your membership and Journal subscription will begin during the current year and you will receive all back issues for the year. If you select the print journal option. If your application is received after 15 September, however, your dues payment will be applied to the following year and your Journal subscription will begin the following year.

\section{OVERSEAS AIR DELIVERY OF JOURNALS}

Members outside North, South, and Central America can choose to have print journals sent by air freight at a cost of $\$ 165$ in addition to dues. JASA on CD-ROM is sent by air mail at no charge in addition to dues. 


\section{ACOUSTICAL SOCIETY OF AMERICA \\ 1305 Walt Whitman Road, Suite 300, Melville, NY 11747-4300, asa@aip.org}

\section{APPLICATION FOR MEMBERSHIP}

Applicants may apply for one of four grades of membership, depending on their qualifications: Student Member, Associate Member, Corresponding Electronic Associate Member or full Member. To apply for Student Membership, fill out Parts I and II of this form; to apply for Associate, Corresponding Electronic Associate, or full Membership, or to transfer to these grades, fill out Parts I and III.

PART I. TO BE COMPLETED BY ALL APPLICANTS (Please print or type all entries)

\begin{tabular}{llll}
\hline CHECK ONE BOX & $\square$ NON-MEMBER APPLYING FOR: & $\square$ STUDENT MEMBERSHIP & Note that your choice of \\
IN EACH COLUMN & $\square$ MEMBER REQUESTING TRANSFER TO: & $\square$ ASSOCIATE MEMBERSHIP & journal option may in- \\
ON THE RIGHT & & $\square$ CORRESPONDING ELECTRONIC & crease or decrease the \\
& & ASSOCIATE MEMBERSHIP & amount you must remit.
\end{tabular}

\section{SELECT JOURNAL OPTION:}

Student members will automatically receive access to The Journal of the Acoustical Society of America online at no charge in addition to dues. Remit \$45. (Note: Student members may also receive the Journal on CD ROM at an additional charge of \$35.)

Corresponding Electronic Associate Members will automatically receive access to The Journal of the Acoustical Society of America and Meeting Programs online at no charge in addition to dues. Remit $\$ 45$.

Applicants for Associate or full Membership must select one Journal option from those listed below. Note that your selection of journal option determines the amount you must remit.

[ ] Online access only-\$95

[ ] Online access plus print Journal $\$ 130$

[ ] Online access plus CD ROM- $\$ 130$

[ ] Online access plus print Journal and CD ROM combination-\$165

Applications received after 15 September: Membership and Journal subscriptions begin the following year.

OPTIONAL AIR DELIVERY: Applicants from outside North, South, and Central America may choose air freight delivery of print journals for an additional charge of $\$ 165$. If you wish to receive journals by air, remit the additional amount owed with your dues. JASA on CD-ROM is sent by air mail at no charge in addition to dues.

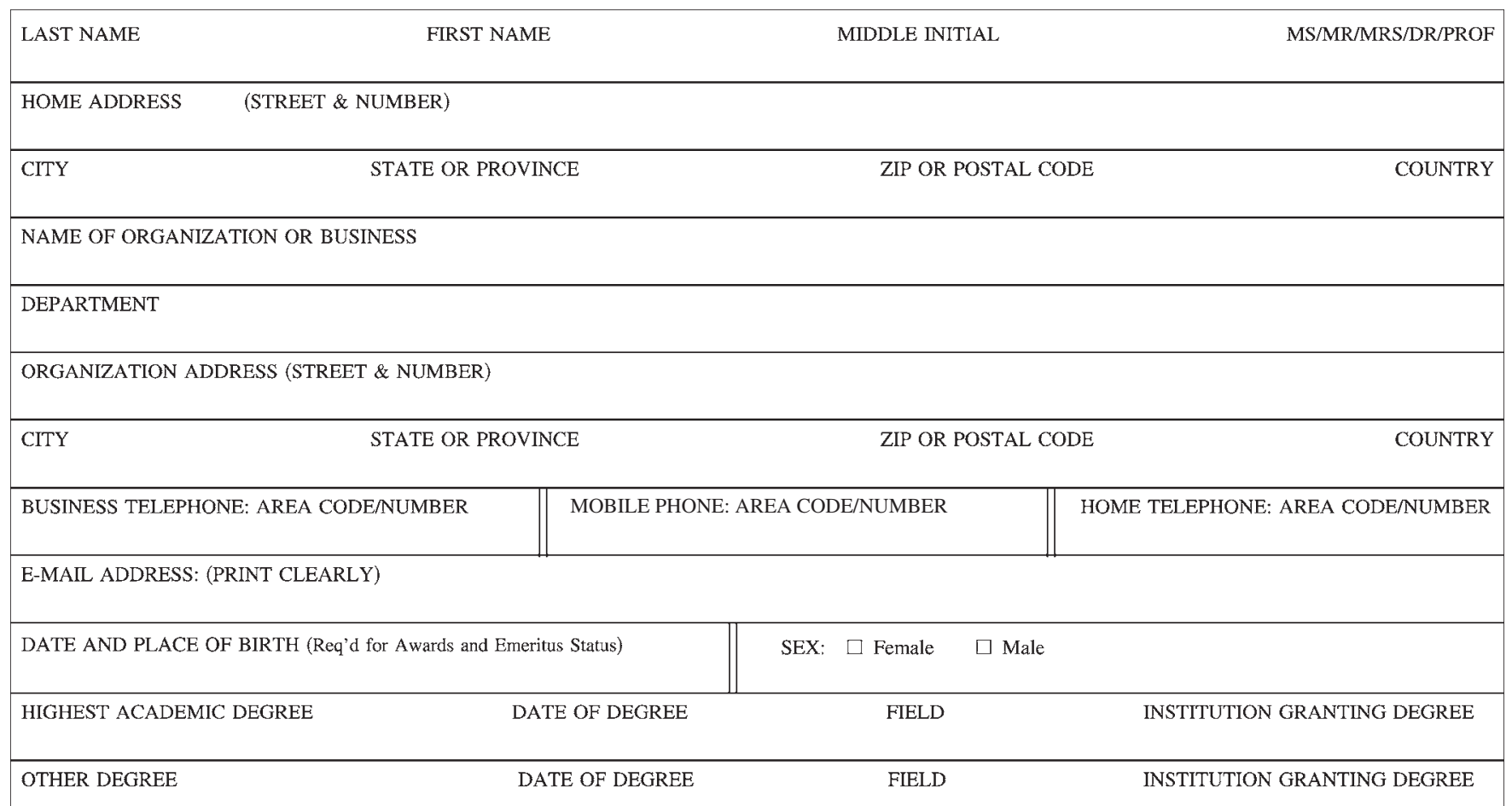

HOME

\section{ORGANIZATION}


PART I CONTINUED: ACOUSTICAL AREAS OF INTEREST TO APPLICANT. Indicate your three main areas of interest below, using 1 for your main interest, 2 for your second, and 3 for your third interest. (DO NOT USE CHECK MARKS.)
$\square$ ACOUSTICAL OCEANOGRAPHY M
$\square$ MUSICAL ACOUSTICS C
$\square$ ANIMAL BIOACOUSTICS L
$\square$ ARCHITECTURAL ACOUSTICS A
NOISE \& NOISE CONTROL D
SIGNAL PROCESSING IN ACOUSTICS N
$\square$ PHYSICAL ACOUSTICS E
SPEECH COMMUNICATION H
$\square$ PSYCHOLOGICAL \&
STRUCTURAL ACOUSTICS
\& VIBRATION G
$\square$ BIOMEDICAL ACOUSTICS K
PHYSIOLOGICAL ACOUSTICS F
UNDERWATER ACOUSTICS J

\section{PART II: APPLICATION FOR STUDENT MEMBERSHIP}

\begin{tabular}{|l|r|}
\hline \multicolumn{2}{|l}{ NAME AND ADDRESS OF COLLEGE OR UNIVERSITY WHERE PRESENTLY ENROLLED } \\
\hline DEGREE EXPECTED & MONTH \& YEAR DEGREE EXPECTED \\
\hline PRINT NAMES \& E-MAIL ADDRESSES OF TWO FACULTY MEMBERS CERTIFYING THAT YOU ARE REGISTERED FOR AT LEAST ONE-HALF OF FULL TIME \\
\hline SIGNATURES OF THE TWO FACULTY MEMBERS LISTED ABOVE CERTIFYING THAT YOU ARE REGISTERED AT LEAST HALF TIME \\
\hline SIGNATURE OF APPLICANT
\end{tabular}

PART III: APPLICATION FOR ASSOCIATE MEMBERSHIP, CORRESPONDING ELECTRONIC ASSOCIATE MEMBERSHIP OR FULL MEMBERSHIP (and interim Associate Membership)

SUMMARIZE YOUR MAJOR PROFESSIONAL EXPERIENCE on the lines below: list employers, duties and position titles, and dates, beginning with your present position. Attach additional sheets if more space is required.

CONTRIBUTIONS TO ACOUSTICS: LIST MAIN PUBLICATIONS, PATENTS, ETC. Attach separate sheets if required.

SPONSORS AND REFERENCES: An application for full Membership requires the names, addresses, and signatures of two references who must be full Members or Fellows of the Acoustical Society. Names and signatures are NOT required for Associate Membership, Corresponding Electronic Associate Membership or Student Membership applications.

\begin{tabular}{|l|l|}
\hline PRINT NAME OF REFERENCE (required for Full Member applications only) & PRINT NAME OF SECOND REFERENCE (required for Full Member applications only) \\
\hline ADDRESS OF REFERENCE & ADDRESS OF SECOND REFERENCE \\
\hline SIGNATURE OF REFERENCE (required for Full Member applications only) & SIGNATURE OF SECOND REFERENCE (required for Full Member applications only) \\
\hline SIGNATURE OF APPLICANT & DATE \\
\hline
\end{tabular}

MAIL THIS COMPLETED APPLICATION, WITH APPROPRIATE PAYMENT TO: ACOUSTICAL SOCIETY OF AMERICA, 1305 WALT WHITMAN ROAD, SUITE 300, MELVILLE, NY 11747-4300.

\section{METHOD OF PAYMENT}

$\square$ Check or money order enclosed for \$ (U.S. funds/drawn on U.S. bank)

$\square$ American Express $\square$ VISA $\square$ MasterCard Signature

Account Number

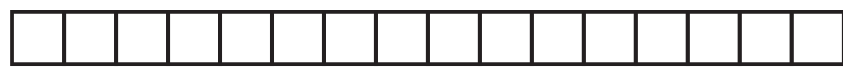

(Credit card orders must be signed) Expiration Date
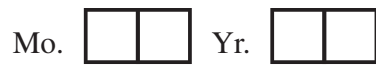

Security Code

Due to security risks and Payment Card Industry (PCI) data security standards e-mail is NOT an acceptable way to transmit credit card information. Please return this form by Fax (631-923-2875) or by postal mail. 


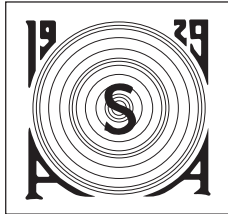

\section{Regional Chapters and Student Chapters}

Anyone interested in becoming a member of a regional chapter or in learning if a meeting of the chapter will be held while he/she is in the local area of the chapter, either permanently or on travel, is welcome to contact the appropriate chapter representative. Contact information is listed below for each chapter representative.

Anyone interested in organizing a regional chapter in an area not covered by any of the chapters below is invited to contact the Cochairs of the Committee on Regional Chapters for information and assistance: Evelyn M. Hoglund, Ohio State University, Columbus, OH 43204, hoglund1@osu.edu and Sandra Guzman, Columbia College Chicago, Chicago, IL 60605, sguzman@colum.edu

\author{
AUSTIN STUDENT CHAPTER \\ Benjamin C. Treweek \\ 10000 Burnet Rd. \\ Austin, TX 78758 \\ Email: austinacousticalsociety@gmail.com
}

\section{BRIGHAM YOUNG UNIVERSITY STUDENT CHAPTER}

Kent L. Gee

Dept. of Physics \& Astronomy

Brigham Young Univ.

N283 ESC

Provo, UT 84602

Email: kentgee@byu.edu

www.acoustics.byu.edu

\section{CASCADIA REGIONAL CHAPTER}

David Dall'osto

Applied Physics Lab.

Univ. of Washington

Seattle, WA 98105

Email: dallosto@apl.washington.edu

\section{CHICAGO}

Shane Kanter

Threshold Acoustics LLC

141 W. Jackson Blvd.

Chicago, IL 60604

Email: skanter@thresholdacoustics.com

\section{UNIVERSITY OF CINCINNATI STUDENT CHAPTER}

\section{Kyle T. Rich}

Biomedical Engineering

Univ. of Cincinnati

231 Albert Sabin Way

Cincinnati, OH 45267

Email: richkt@mail.uc.edu

\section{COLUMBIA COLLEGE CHICAGO STUDENT CHAPTER}

Lauren Ronsse

Dept. of Audio Arts and Acoustics

Columbia College Chicago

33 E. Congress Pkwy., Rm. 6010

Chicago, IL 60605

Email: 1ronsse@colum.edu

\section{FLORIDA}

Richard J. Morris

Communication Science and Disorders

Florida State Univ.

201 W. Bloxham

Tallahassee, FL 32306-1200

Email: richard.morris@cci.fsu.edu

\section{GEORGIA INSTITUTE OF TECH- NOLOGY STUDENT CHAPTER \\ Charlise Lemons \\ Georgia Institute of Technology \\ Atlanta, GA 30332-0405 \\ Email: clemons3@gatech.edu}

\section{GREATER BOSTON}

Eric Reuter

Reuter Associates, LLC

10 Vaughan Mall, Ste. 201A

Portsmouth, NH 03801

Email: ereuter@reuterassociates.com

\section{UNIVERSITY OF HARTFORD STUDENT CHAPTER}

\section{Robert Celmer}

Mechanical Engineering Dept., UT-205

Univ. of Hartford

200 Bloomfield Ave.

West Hartford, CT 06117

Email: celmer@hartford.edu

\section{UNIVERSITY OF KANSAS \\ STUDENT CHAPTER}

Robert C. Coffeen

Univ. of Kansas

School of Architecture, Design, and Planning

Marvin Hall

1465 Jayhawk Blvd.

Lawrence, KS 66045

Email: coffeen@ku.edu

\section{LOS ANGELES}

Neil A. Shaw

www.asala.org

\section{MID-SOUTH}

Tiffany Gray

NCPA

Univ. of Mississippi

University, MS 38677

Email: midsouthASAchapter@gmail.com

\section{NARRAGANSETT}

David A. Brown

Univ, of Massachusetts, Dartmouth

151 Martime St.

Fall River, MA 02723

Email: dbacoustics@cox.net

UNIVERSITY OF NEBRASKA
STUDENT CHAPTER
Matt Blevins
Architectural Engineering
Univ. of Nebraska
Peter Kiewit Institute
1110 S. 67th St.
Omaha, NE 68182-0681
Email: mblevins@ huskers.unl.edu

NORTH CAROLINA

Noral Stewart

Stewart Acoustical Consultants

7330 Chapel Hill Rd., Ste.101

Rayleigh, NC

Email:noral@sacnc.com

\section{NORTH TEXAS}

Peter F. Assmann

School of Behavioral and Brain Sciences

Univ. of Texas-Dallas

Box 830688 GR 4.1

Richardson, TX 75083

Email: assmann@utdallas.edu

\section{NORTHEASTERN UNIVERSITY STUDENT CHAPTER \\ Victoria Suha \\ Email: northeasternasa@gmail.com}

\author{
OHIO STATE UNIVERSITY \\ STUDENT CHAPTER \\ Joey Hribar \\ The Ohio State Univ. \\ Columbus, OH 43210 \\ Email: hribar.11@osu.edu
}

\section{PENNSYLVANIA STATE \\ Martin Lawless \\ Pennsylvania State Univ. \\ University Park, PA 16802 \\ Email: ms1224@psu.edu \\ www.psuasa.org} UNIVERSITY STUDENT CHAPTER

\section{PHILADELPHIA}

Kenneth W. Good, Jr.

Armstrong World Industries, Inc.

2500 Columbia Ave.

Lancaster, PA 17603

Email: kwgoodjr@armstrong.com

\section{PURDUE UNIVERSITY STUDENT CHAPTER}

Kai Ming Li

Purdue Univ.

585 Purdue Mall

West Lafayette, IN 47907

Email:mmkmli@purdue.edu

Email:purdueASA@gmail.com 


\section{RENSSELAER POLYTECHNIC INSTITUTE STUDENT CHAPTER}

Erica Hoffman

Email: hoffme2@rpi.edu
UPPER MIDWEST

David Braslau

David Braslau Associates, Inc.

6603 Queen Ave. South, Ste. N

Richfield, MN 55423

Email: david@braslau.com
WASHINGTON, DC

Shane Guan

National Marine Fisheries Service

Office of Protected Resources

1315 East-West Hwy., Ste. 13826

Silver Spring, MD 20910

Email: Shane.guan@noaa.gov 


\section{ACOUSTICAL SOCIETY OF AMERICA • BOOKS, CDS, DVD, VIDEOS ON ACOUSTICS Order online at http://www.abdi-ecommerce10.com/asa}

ACOUSTICAL DESIGN OF MUSIC EDUCATION FACILITIES. Edward R. McCue and Richard H. Talaske, Eds. Plans, photographs, and descriptions of 50 facilities with explanatory text and essays on the design process. 236 pp, paper, 1990. Price: \$23. Item \# 0-88318-8104

ACOUSTICAL DESIGN OF THEATERS FOR DRAMA PERFORMANCE: 1985-2010. David T. Bradley, Erica E. Ryherd, \& Michelle C. Vigeant, Eds. Descriptions, color images, and technical and acoustical data of 130 drama theatres from around the world, with an acoustics overview, glossary, and essays reflecting on the theatre design process. 334 pp, hardcover 2010. Price: \$45. Item \# 978-0-9846084-5-4

ACOUSTICAL DESIGNING IN ARCHITECTURE. Vern O. Knudsen and Cyril M. Harris. Comprehensive, non-mathematical treatment of architectural acoustics; general principles of acoustical designing. 408 pp, paper, 1980 (original published 1950). Price: \$23. Item \# 0-88318-267X

ACOUSTICAL MEASUREMENTS. Leo L. Beranek. Classic text with more than half revised or rewritten. 841 pp, hardcover 1989 (original published 1948). Available on Amazon.com

ACOUSTICS. Leo L. Beranek. Source of practical acoustical concepts and theory, with information on microphones, loudspeakers and speaker enclosures, and room acoustics. $491 \mathrm{pp}$, hardcover 1986 (original published 1954). Available on Amazon.com

\section{ACOUSTICS-AN INTRODUCTION TO ITS PHYSICAL} PRINCIPLES AND APPLICATIONS. Allan D. Pierce. Textbook introducing the physical principles and theoretical basis of acoustics, concentrating on concepts and points of view that have proven useful in applications such as noise control, underwater sound, architectural acoustics, audio engineering, nondestructive testing, remote sensing, and medical ultrasonics. Includes problems and answers. 678 pp, hardcover 1989 (original published 1981). Price: \$33. Item \# 0-88318-6128

ACOUSTICS, ELASTICITY AND THERMODYNAMICS OF POROUS MEDIA: TWENTY-ONE PAPERS BY M. A. BIOT. Ivan Tolstoy, Ed. Presents Biot's theory of porous media with applications to acoustic wave propagation, geophysics, seismology, soil mechanics, strength of porous materials, and viscoelasticity. 272 pp, hardcover 1991. Price: \$28. Item \# 1-56396-0141

ACOUSTICS OF AUDITORIUMS IN PUBLIC BUILDINGS. Leonid I. Makrinenko, John S. Bradley, Ed. Presents developments resulting from studies of building physics. 172 pp, hardcover 1994 (original published 1986). Price: \$38. Item \# 1-56396-3604
ACOUSTICS OF WORSHIP SPACES. David Lubman and Ewart A. Wetherill, Eds. Drawings, photographs, and accompanying data of worship houses provide information on the acoustical design of chapels, churches, mosques, temples, and synagogues. $91 \mathrm{pp}$, paper 1985. Price: \$23. OUT-OF-PRINT

ASA EDITION OF SPEECH AND HEARING IN COMMUNICATION. Harvey Fletcher; Jont B. Allen, Ed. A summary of Harvey Fletcher's 33 years of acoustics work at Bell Labs. A new introduction, index, and complete bibliography of Fletcher's work are important additions to this classic volume. 487 pp, hardcover 1995 (original published 1953). Price: \$40. Item \# 1-56396-3930

AEROACOUSTICS OF FLIGHT VEHICLES: THEORY AND PRACTICE. Harvey H. Hubbard, Ed. Two-volumes oriented toward flight vehicles emphasizing the underlying concepts of noise generation, propagation, predicting and control. Vol. 1589 pp/Vol. 2 426 pp, hardcover 1994 (original published 1991). Price per 2-vol. set: \$58. Item \# 1-56396-404X

COLLECTED PAPERS ON ACOUSTICS. Wallace Clement Sabine. Classic work on acoustics for architects and acousticians. 304 pp, hardcover 1993 (originally published 1921). Price: \$28. Item \# 0-932146-600

CONCERT HALLS AND OPERA HOUSES. Leo L. Beranek. Over 200 photos and architectural drawings of 100 concert halls and opera houses in 31 countries with rank-ordering of 79 halls and houses according to acoustical quality. 653 pp. hardcover 2003. Price: \$50. OUT-OF-PRINT

CRYSTAL ACOUSTICS. M.J.P. Musgrave. For physicists and engineers who study stress-wave propagation in anisotropic media and crystals. 406 pp. hardcover (originally published 1970). Price: \$34. Item \# 0-9744067-0-8

DEAF ARCHITECTS \& BLIND ACOUSTICIANS? Robert E. Apfel. A primer for the student, the architect and the planner. $105 \mathrm{pp}$. paper 1998. Price: \$22. Item \#0-9663331-0-1

THE EAR AS A COMMUNICATION RECEIVER. Eberhard Zwicker \& Richard Feldtkeller. Translated by Hannes Müsch, Søren Buus, Mary Florentine. Translation of the classic Das Ohr Als Nachrichtenempfänger. Aimed at communication engineers and sensory psychologists. Comprehensive coverage of the excitation pattern model and loudness calculation schemes. $297 \mathrm{pp}$, hardcover 1999 (original published 1967). Price: \$50. OUT-OF-PRINT 
ELECTROACOUSTICS: THE ANALYSIS OF TRANSDUCTION, AND ITS HISTORICAL BACKGROUND. Frederick V. Hunt. Analysis of the conceptual development of electroacoustics including origins of echo ranging, the crystal oscillator, evolution of the dynamic loudspeaker, and electromechanical coupling, 260 pp, paper 1982 (original published 1954). Available on Amazon.com

ELEMENTS OF ACOUSTICS. Samuel Temkin. Treatment of acoustics as a branch of fluid mechanics. Main topics include propagation in uniform fluids at rest, trans-mission and reflection phenomena, attenuation and dispersion, and emission. $515 \mathrm{pp}$. hardcover 2001 (original published 1981). Price: \$30. Item \# 1-56396-997-1

EXPERIMENTS IN HEARING. Georg von Békésy. Classic on hearing containing vital roots of contemporary auditory knowledge. 760 pp, paper 1989 (original published 1960). Price: \$23. Item \# 0-88318-6306

FOUNDATIONS OF ACOUSTICS. Eugen Skudrzyk. An advanced treatment of the mathematical and physical foundations of acoustics. Topics include integral transforms and Fourier analysis, signal processing, probability and statistics, solutions to the wave equation, radiation and diffraction of sound. 790 pp. hardcover 2008 (originally published 1971). Price: \$60. Item \# 3-211-80988-0

HALLS FOR MUSIC PERFORMANCE: TWO DECADES OF EXPERIENCE, 1962-1982. Richard H. Talaske, Ewart A. Wetherill, and William J. Cavanaugh, Eds. Drawings, photos, and technical and physical data on 80 halls; examines standards of quality and technical capabilities of performing arts facilities. 192 pp, paper 1982. Price: \$23. OUT-OF-PRINT

HALLS FOR MUSIC PERFORMANCE: ANOTHER TWO DECADES OF EXPERIENCE 1982-2002. Ian Hoffman, Christopher Storch, and Timothy Foulkes, Eds. Drawings, color photos, technical and physical data on 142 halls. $301 \mathrm{pp}$, hardcover 2003. Price: \$56. Item \# 0-9744067-2-4

HANDBOOK OF ACOUSTICAL MEASUREMENTS AND NOISE CONTROL, THIRD EDITION. Cyril M. Harris. Comprehensive coverage of noise control and measuring instruments containing over 50 chapters written by top experts in the field. 1024 pp, hardcover 1998 (original published 1991). OUT-OF-PRINT

HEARING: ITS PSYCHOLOGY AND PHYSIOLOGY. Stanley Smith Stevens \& Hallowell Davis. Volume leads readers from the fundamentals of the psycho-physiology of hearing to a complete understanding of the anatomy and physiology of the ear. $512 \mathrm{pp}$, paper 1983 (originally published 1938). OUT-OF-PRINT

NONLINEAR ACOUSTICS. Mark F. Hamilton and David T. Blackstock. Research monograph and reference for scientists and engineers, and textbook for a graduate course in nonlinear acoustics. 15 chapters written by leading experts in the field. $455 \mathrm{pp}$, hardcover, 2008 (originally published in 1996). Price: $\$ 45$. Item \# 0-97440-6759

NONLINEAR ACOUSTICS. Robert T. Beyer. A concise overview of the depth and breadth of nonlinear acoustics with an appendix containing references to new developments. $452 \mathrm{pp}$, hardcover 1997 (originally published 1974). Price: \$45. Item \# 1-56396-724-3

NONLINEAR UNDERWATER ACOUSTICS. B. K. Novikov, O. V. Rudenko, V. I. Timoshenko. Translated by Robert T. Beyer. Applies the basic theory of nonlinear acoustic propagation to directional sound sources and receivers, including design nomographs and construction details of parametric arrays. 272 pp., paper 1987. Price: \$34. Item \# 0-88318-5229

OCEAN ACOUSTICS. Ivan Tolstoy and Clarence S. Clay. Presents the theory of sound propagation in the ocean and compares the theoretical predictions with experimental data. Updated with reprints of papers by the authors supplementing and clarifying the material in the original edition. 381 pp, paper 1987 (original published 1966). OUT-OF-PRINT

ORIGINS IN ACOUSTICS. Frederick V. Hunt. History of acoustics from antiquity to the time of Isaac Newton. 224 pp, hardcover 1992. Price: \$19. OUT-OF-PRINT

PAPERS IN SPEECH COMMUNICATION. Papers charting four decades of progress in understanding the nature of human speech production, and in applying this knowledge to problems of speech processing. Contains papers from a wide range of journals from such fields as engineering, physics, psychology, and speech and hearing science. 1991, hardcover.

Speech Perception, Joanne L. Miller, Raymond D. Kent, Bishnu S. Atal, Eds. 764 pp. OUT-OF-PRINT

Speech Production, Raymond D. Kent, Bishnu S. Atal, Joanne L. Miller, Eds. 880 pp. Item \# 0-88318-958-5

Speech Processing, Bishnu S. Atal, Raymond D. Kent, Joanne L. Miller, Eds. 672 pp. Price: \$38. Item \# 0-88318-9607

PROPAGATION OF SOUND IN THE OCEAN. Contains papers on explosive sounds in shallow water and long-range sound transmission by J. Lamar Worzel, C. L. Pekeris, and Maurice Ewing. hardcover 2000 (original published 1948). Price: \$37. Item \#1-563969688

RESEARCH PAPERS IN VIOLIN ACOUSTICS 1975-1993. Carleen M. Hutchins, Ed., Virginia Benade, Assoc. Ed. Contains 120 research papers with an annotated bibliography of over 400 references. Introductory essay relates the development of the violin to the scientific advances from the early 15 th Century to the present. Vol. 1, $656 \mathrm{pp}$; Vol. 2, 656 pp. hardcover 1996. Price: $\$ 120$ for the two-volume set. Item \# 1-56396-6093

RIDING THE WAVES. Leo L. Beranek. A life in sound, science, and industry. 312 pp. hardcover 2008. OUT-OF-PRINT

THE SABINES AT RIVERBANK. John W. Kopec. History of Riverbank Laboratories and the role of the Sabines (Wallace Clement, Paul Earls, and Hale Johnson) in the science of architectural acoustics. 210 pp. hardcover 1997. Price:\$19. Item \# 0-932146-61-9 
SONICS, TECHNIQUES FOR THE USE OF SOUND AND ULTRASOUND IN ENGINEERING AND SCIENCE. Theodor F. Hueter and Richard H. Bolt. Work encompassing the analysis, testing, and processing of materials and products by the use of mechanical vibratory energy. $456 \mathrm{pp}$, hardcover 2000 (original published 1954). Price: \$30. Item \# 1-56396-9556

SOUND IDEAS, Deborah Melone and Eric W. Wood. Early days of Bolt Beranek and Newman Inc. to the rise of Acentech Inc. 363 pp. hardcover 2005. Price: \$25. Item \# 200-692-0681

SOUND, STRUCTURES, AND THEIR INTERACTION, Miguel C. Junger and David Feit. Theoretical acoustics, structural vibrations, and interaction of elastic structures with an ambient acoustic medium. $451 \mathrm{pp}$, hardcover 1993 (original published 1972). Price: \$23. Item \# 0-262-100347
THEATRES FOR DRAMA PERFORMANCE: RECENT EXPERIENCE IN ACOUSTICAL DESIGN, Richard H. Talaske and Richard E. Boner, Eds. Plans, photos, and descriptions of theatre designs, supplemented by essays on theatre design and an extensive bibliography. 167 pp, paper 1987. Price: \$23. Item \# 0-88318-5164

THERMOACOUSTICS, Gregory W. Swift. A unifying thermoacoustic perspective to heat engines and refrigerators. $300 \mathrm{pp}$, paper, 2002. Price: \$50. Item \# 0-7354-0065-2

VIBRATION AND SOUND, Philip M. Morse. Covers the broad spectrum of acoustics theory, including wave motion, radiation problems, propagation of sound waves, and transient phenomena. 468 pp, hardcover 1981 (originally published 1936). Price: \$28. Item \# 0-88318-2874

VIBRATION OF PLATES, Arthur W. Leissa. 353 pp, hardcover 1993 (original published 1969). OUT-OF-PRINT

VIBRATION OF SHELLS, Arthur W. Leissa. 428 pp, hardcover 1993 (original published 1973). Item \# 1-56396-2934

\section{CDs, DVD, VIDEOS, STANDARDS}

Auditory Demonstrations (CD). Teaching adjunct for lectures or courses on hearing and auditory effects. Provides signals for teaching laboratories. Contains 39 sections demonstrating various characteristics of hearing. Includes booklet containing introductions and narrations of each topic and bibliographies for additional information. Issued in1989. Price: \$23. Item \# AD-CD-BK

Measuring Speech Production (DVD). Demonstrations for use in teaching courses on speech acoustics, physiology, and instrumentation. Includes booklet describing the demonstrations and bibliographies for more information. Issued 1993. Price: \$52. Item \# MS-DVD

Scientific Papers of Lord Rayleigh (CD ROM). Over 440 papers covering topics on sounds, mathematics, general mechanics, hydrodynamics, optics and properties of gasses by Lord Rayleigh (John William Strutt) the author of the Theory of Sound. Price: \$40. Item \#09744067-4-0

Proceedings of the Sabine Centennial Symposium (CD ROM). Held June 1994. Price: \$50. Item \# INCE25-CD

Fifty Years of Speech Communication (VHS). Lectures presented by distinguished researchers at the ASA/ICA meeting in June 1998 covering development of the field of Speech Communication. Lecturers: G. Fant, K.N. Stevens, J.L. Flanagan, A.M. Liberman, L.A. Chistovich--presented by R.J. Porter, Jr., K.S. Harris, P. Ladefoged, and V. Fromkin. Issued in 2000. Price: \$30. Item \# VID-Halfcent

Speech Perception (VHS). Presented by Patricia K. Kuhl. Segments include: I. General introduction to speech/language processing; Spoken language processing; II. Classic issues in speech perception; III. Phonetic perception; IV. Model of developmental speech perception; V. Cross-modal speech perception: Links to production; VI. Biology and neuroscience connections. Issued 1997. Price: \$30. Item \# SP-VID

Standards on Acoustics. Visit the ASA Store (https://global.ihs.com/home_page_asa.cfm?\&rid=ASA) to purchase or download National (ANSI) and International (ISO) Standards on topics ranging from measuring environmental sound to standards for calibrating microphones .

Order the following from ASA, 1305 Walt Whitman Road, Suite 300, Melville, NY 11747-4300; asa@aip.org; Fax: 631-923-2875 Telephone orders not accepted. Prepayment required by check (drawn on US bank) or by VISA, MasterCard, American Express.

Study of Speech and Hearing at Bell Telephone Laboratories (CD). Nearly 10,000 pages of internal documents from AT\&T archives including historical documents, correspondence files, and laboratory notebooks on topics from equipment requisitions to discussions of project plans, and experimental results. Price: \$20 (postage included).

Collected Works of Distinguished Acousticians CD - Isadore Rudnick (CD + DVD). 3 disc set includes reprints of papers by Isadore Rudnick from scientific journals, a montage of photographs with colleagues and family, and video recordings of the Memorial Session held at the 135 th meeting of the ASA. Price $\$ 50$ (postage included).

Technical Memoranda issued by Acoustics Research Laboratory-Harvard University (CD). The Harvard Research Laboratory was established in 1946 to support basic research in acoustics. Includes 61 reports issued between 1946 and 1971 on topics such as radiation, propagation, scattering, bubbles, cavitation, and properties of solids, liquids, and gasses. Price $\$ 25.00$ (postage included). 


\section{ORDER FORM FOR ASA BOOKS, CDS, DVD, VIDEOS}

Place your order online at http://www.abdi-ecommerce10.com/asa/ for faster processing.

1. Payment must accompany order. Payment may be made by check or international money order in U.S. funds drawn on U.S. bank or by VISA, MasterCard, or American Express credit card.

2. Send orders to: Acoustical Society of America, Publications, P. O. Box 1020, Sewickley, PA 15143-9998; Tel.: 412-741-1979; Fax: 412-741-0609.
3. A $10 \%$ discount applies on orders of 5 or more copies of the same title only.

4. Returns are not accepted.

\begin{tabular}{|c|c|c|c|}
\hline Item \# & Quantity & Price & Total \\
\hline & & & \\
\hline & & & \\
\hline & & & \\
\hline & & & \\
\hline & & & \\
\hline & & & \\
\hline & & & \\
\hline & & & \\
\hline & & Subtotal & \\
\hline & & $\begin{array}{l}\text { Shipping Costs (all orders) based on weight and distance. For quote call } \\
412-741-1979, \quad \text { visit http://www.abdi-ecommerce10.com/asa, or email } \\
\text { asapubs@abdintl.com }\end{array}$ & \\
\hline & & $10 \%$ discount of orders of 5 or more of the same title & \\
\hline & & Total & \\
\hline
\end{tabular}

Name

Address

City State ZIP Country

Tel.: Fax: Email:

\section{Method of Payment}

[ ] Check or money order enclosed for $\$$ (U.S. funds/drawn on U.S. bank made payable to the Acoustical Society of America)

[ ] American Express [ ] VISA [ ] MasterCard

Cardholders signature

$$
\text { (Credit card orders must be signed) }
$$

Account \#

Expires Mo. Yr.

Due to security risks and Payment Card Industry (PCI) data security standards email is NOT an acceptable way to transmit credit card information. Please use our secure webpage to process your credit card payment (http://www.abdi-ecommerce10.com/asa/) or securely fax this form to (631-923-2875). 


\section{AUTHOR INDEX}

to papers presented at

\section{5th Joint Meeting ASA/ASJ}

\section{A, Rongna -3440}

Aalto, Eija M.-3447

Abadi, Shima-2987, 3063, 3232

Abawi, Ahmad T.-2968, 3123, Chair Session 5aUWa (3406)

Abbot, Philip-3181, 3350

Abbott, JohnPaul R.-3194, 3259 , 3260, Cochair Session 3pEA (3192)

Abe, Koji-3062, 3268, 3428

Abe, Koki-3303, 3304

Abe, Masanobu-3110

Abe, Masato-3167

Abe, Yoshiaki-3432

Åbom, Mats-2992

Abouzahra, Nourhan K.-3098, 3145

Abraham, Douglas A.-3285

Abraham, Odile-3165

Abrahamyan, Hayk-3332

Achdjian, Hossep-3387

Acker, Timothy W.-3349

Ackerman, Maya-3302

Ackleh, Azmy-3073

Acton, Doug- 3298

Adachi, Seiji-3426, Cochair Session 2aMUa (3036)

Adams, Caylee-3214

Adams, Charley-3215

Adams, Daniel-3146

Adams, Ian-3076

Adams, Meredith-3382

Adelman-Larsen, Niels W.-3291,

Cochair Session 4pAAa (3291)

Adema, Gosse J.-3082

Adityawarman, Yudi-3076, 3135

Adler, Jeffrey M.-3109

Afshan, Amber-3448

Ageeva, Victoriya-3103

Agrawal, Prashant-3206

Agrawal, Shyam S.-3105, 3338

Ahlstrom, Jayne B.-3270

Ahn, Hongmin-3089

Ahn, Hyunah-3397

Ahn, Suzy-3221

Ahnert, Wolfgang-3128, 3193

Ahrens, Axel-3177

Ahroon, William A.-3146, 3147, 3273,3274

Aiba, Eriko-3092, 3143, 3429

Aichele, Johannes-3373

Aikawa, Kiyoaki-3389

Aithal, Sreedevi-3263, 3264

Aithal, Venkatesh-3263, 3264

Aizawa, Yoshitaka-2964

Akagawa, Osamu-3027

Akagi, Masato-2960, 2963

Akahane-Yamada, Reiko-3392

Akamatsu, Shuji-3056

Akamatsu, Tomonari-3019, 3021, $3065,3129,3242,3360,3361$, Cochair Session 2aABa (3017), Cochair Session 2pABa (3071),
Cochair Session $3 \mathrm{aAB}$ (3129),

Cochair Session 3pABa (3179)

Akamine, Masahito-3043, 3044

Akamine, Yukinori-3057

Akbari, Christina C.-3440

Akiba, Tomoyosi-3010

Akihide, Takamura-3328

Akita, Masanori-2963

Akita, Takeshi-3047, 3267

Akitake, Yoshiko-3197

Akiyama, Iwaki-3372, 3419, 3420

Alan, Tuncay-3147

Alavi Tamaddoni, Hedieh-3248

Alberts, W. C. K.-3260

Alcantara, Eldridge-3063

Aldholmi, Yahya-3333

Alexander, Jessica-Chair Session $5 \mathrm{pSCb}(3439)$

Alexander, Jessica E.-3397

Alexiou, Christoph-3393

Alguri, K. Supreet-3054

Aliabouzar, Mitra-3025, 3373

Aliev, Ali E.-3086

AlJasser, Arwa-3158

Alkayed, Nabil J.-2986

Allen, Ann-3411

Allen, Jennifer-3416

Allen, John S.-3071, 3259

Allen, Marsha-3228

Allen, Steven P.-3031

Altieri, Andrew H.-3018

Alvarez, Eduardo J.-3330

Alwan, Abeer-3448

Aly, Ann M.-3338, 3446

Alzoubi, Abdulaziz-3113

Amador, Carolina-3137

Amakasu, Kazuo-3241, 3242, 3305, 3361

Amano, Masao-3302

Amano, Shigeaki-3108, 3223, 3338

Amaratunge, Cole-3232

Ambrozinsky, Lukasz-2979

Amelot, Angélique-3106, 3107

Amimoto, Yuri-3266

Amitay, Sygal-3155

Ammi, Azzdine Y.-2986

An, Sangkyum-3064

Anand, Supraja-3398, 3442, 3443

Anastasiadis, Pavlos-2984

Anchieta, David-3286

Andersen, Lars N.-3131

Anderson, Brian E.-2952, 3211, Cochair Session 2aSPb (3056), Cochair Session 3aSP (3165),

Cochair Session 3pSA (3210)

Anderson, Charles-3305

Anderson, Shane-3263

Anderson, Victoria-3111

Anderson, Victoria B.-3105

Ando, Akio-2966, Cochair Session 1aID (2945)

Ando, Hiroshi-3375

Ando, Tamaki-3143
Andre, Michael P.-3185, 3186

Andreev, Valery-3260

Andreeva-Mori, Adriana-3257

Andrejkova, Gabriela-3269

Andrew, Rex K.-3073

Andriolo, Artur-3239

Angione, Frank-3249

Angster, Judit-3036

Ankrom, Matthew R.-3145

Ann, Ik-Soo-3056

Anquez, Jeremie-2984

Antonanzas-Barroso, Norma-2961

Antonides, Steven-3320

Anyoji, Masayuki-3043

Aoki, Naofumi-3315

Aoki, Riku-2960

Aoki, Shigeki-3226

Aono, Hikaru-3421, Cochair Session 5 pEA (3421)

Aota, Yasuaki-3183

Aoyagi, Manabu-3434

Apoux, Frederic-3270, Chair

Session $4 \mathrm{aPPa}(3262)$

Aragonez, Lem-3131

Arai, Hajime- 3226

Arai, Nobuaki-3305, 3412

Arai, Nobuo-2994

Arai, Takayuki-2952, 3196, 3266, $3282,3333,3339,3365,3439$,

Cochair Session 5aBAa (3365)

Araki, Shoko-3114

Araki, Yozo-3380

Araki, Yukiko-3271

Archangeli, Diana-3105

Ardekani, Babak-3224

Ardhuin, Fabrice-2996

Aristizabal, Orlando-3029

Aristizabal, Sara-3137

Ariyoshi, Kirara-3403

Arjmandi, Meisam K.-3114

Armstrong, Douglas-3414

Arnal, Bastien-3084

Arslan, Ersen-3250

Arzounian, Dorothée-3265

Asada, Minoru-3005

Asaei, Ali-3224

Asahi, Kensaku-3059, 3425, 3426, 3428

Asahi, Shota-3429

Asakawa, Michihiro-3049

Asakura, Daichi-3374

Asakura, Takumi-3161

Asakura, Yuya-3274

Asami, Rei-3083, 3369

Asami, Takuya-3423

Asano, Keiko-3226

Asfandiyarov, Shamil-3260

Asgarisabet, Mahsa-3141

Ashida, Reiko-3083

Aslan, Mustafa-3169

Asmar, Karina A.-3056

Aspöck, Lukas-3128

Assaad, Jamal-3002, 3387
Assi, Hisham-3261

Assmann, Peter F.-3163, 3339 3441, 3446

Astolfi, Arianna-2969

Atalla, Noureddine-3000

Ataucusi, Alejandro L.-3426

Atkinson, Angus-3242

Atlas, Les-3063

$\mathrm{Au}$, Whitlow-3130, Cochair Session 1aID (2945)

Au, Whitlow W.-3132, 3297, 3298 , 3361,3416

Aubert, Allan C.-3098

Audoly, Christian-3159

Auger, Guillaume-3183

Augusztinovicz, Fülöp-3036

Averkiou, Michalakis A.-3247, 3368

Averkiou, Mike-3188

Avery, Trey-3444

Avitabile, Peter-3212

Awahara, Yoshiki-3427

Awata, Shohei-3427

Azad, Hassan-3292

Azamar Reyes, Pamela A.-3361

Azuma, Takashi-3008, 3306, 3369

Bader, Kenneth B.-3084

Bader, Rolf-3142, 3256, 3317, 3388

Badiey, Babak-3413

Badiey, Mohsen-3289

Bae, Myung-Jin-2958, 3056, 3267, 3401, 3426

Bae, Seonggeon-3267

Baer, Thomas-3438

Baese-Berk, Melissa-3340

Baese-Berk, Melissa M.-3283

Baetscher, Kevin M.-3105

Baggeroer, Arthur B.-3024, 3408

Baggio, A. L.-3261

Bahr, Frederick L.-3012

Bai, Mingsian R.-3311

Bailey, Jhonelle-3446, 3447

Bailey, Michael-3030, 3082, 3307, Cochair Session 4aBAb (3247),

Cochair Session 4pBA (3306)

Bailey, Michael R.-2982, 2983, 3031, 3081, 3082, 3083, 3306, 3307, 3308, 3310

Baker, Richard-3158

Baker, Richard J.-3438

Bakker, Ron-3391

Bakst, Sarah-3223

Baldwin, Jeffery W.-3086

Ballard, Kathryn-3424

Ballard, Megan S.-3018, 3233 3305, Cochair Session 3pUWb (3233)

Bang, Hye-Young-3114

Banno, Hideki-3059, 3425, 3426, 3428

Bansal, Shweta-3105, 3338

Baqersad, Javad-3211

Barajas, Carlos-3084

Barascud, Nicolas-3209 
Barbieri, Nilson-3141

Barbieri, Renato-3141

Barclay, David R.-2977

Barkley, Yvonne-3415

Barkley, Yvonne M.-3071

Barlas, Emre-3119

Barlaz, Marissa-3219

Barlow, Jay-3411

Barmak, Rafael-3409

Barnard, Andrew R.-3141

Barnes, Andrew-3226, 3403, 3442

Barr, Jesse M.-3193

Barr, Richard G.-3188

Barra-Chicote, Roberto-2961

Barreda, Santiago-3162

Barreira, Leonardo M.-3008

Barrere, Victor-3419

Barros, Abner C.-3286

Baskent, Deniz-3262, 3270, 3391

Bassett, Christopher-2949, 3242

Basu, Shriya-3262

Bates, Daniel-3208

Bauer, Gordon B.-3297

Baughman, Ray H.-3086

Baumann-Pickering, Simone-3017, 3181, 3300, 3302, 3413

Baumgartner, Mark-3300

Baxley, Paul-3117

Baxter, Kate O.-3150

Bayat, Mahdi-3186

Bazúa Durán, Carmen-3361

Bean, Jacob-3431

Beauchamp, James W.-Cochair Session 2aMUb (3038), Cochair Session 2pMU (3090)

Beck, Benjamin-3104

Beck, Benjamin S.-3034

Becker, Stefan-3398

Bedard, Jeannette-3410

Beddor, Patrice S.-3106

Bee, Mark-3207

Beechey, Tim-3437

Beechey, Timothy-3214

Beery, Sara-2967

Begault, Durand-2956

Beguin, Estelle-3026

Beguš, Gašper-3106

Behler, Gottfried-3175, 3375

Bélanger, Pierre-3189

Belanger, Pierre-3034, 3188

Bellik, Jennifer-3109

Benetos, Emmanouil-3039

Benichoux, Victor-3269

Benmeddour, Farouk-3387

Bennett, Erica E.-3158

Bennett, Ryan-3109

Benoit-Bird, Kelly-3129, 3304

Benoit-Bird, Kelly J.-3130, 3243,

Cochair Session 3aAB (3129),

Cochair Session 3pABa (3179),

Cochair Session $4 \mathrm{aAO}$ (3241),

Cochair Session 4pAO (3304)

Bent, Tessa-3218, Cochair Session 4aSC (3282)

Berchok, Catherine-3295, 3359

Berchok, Catherine L.-3415

Berger, Elliott H.-3144, 3145,

Cochair Session 3aNS (3144),

Cochair Session 3pNSa (3198)

Berggren, Per-3298

Beristain, Sergio-3181, 3182
Bernardi, Giuliano-3292

Bernstein, Joshua G.-3099, 3157 , 3439

Bernstein, Leslie R.-3100

Berry, David-3394

Bertin, Michaël-2996

Beskow, Jonas-3005

Besser, Jana-3443

Best, Virginia-3270

Betancourt, Kyna-3228

Betancourt, Kyna S.-3227

Bethune, Keith-3171

Bevans, Dieter-2977

Bevans, Dieter A.-3169

Bhandiwad, Ashwin-3360

Bharadwaj, Hari M.-3045, 3153

Bhargava, Pranesh-3262

Bhatt, Ravi R.-3266

Bianco, Michael J.-3054

Biber, Alper-3088

Bidondo, Alejandro-3403

Bielski, Lynn M.-3152

Bierer, Julie A.-3157, 3438

Biesheuvel, J. D.-3157

Biffard, Ben-3410

Binder, Carolyn-2949, Cochair Session 1aAO (2949)

Bird, Sonya-3343

Birk, Veronika-3393

Biro, Tifani M.-3344

Bissmeyer, Mary-3442

Biwa, Tetsushi-2955

Bjerre, Lærke Cecilie-3041

Blackwell, John-3186

Blackwell, Susanna-3020

Blackwell, Susanna B.-3021, 3413

Blamire, Emily-3010

Blanc, Elisabeth-2995

Blevins, Matthew G.-3193, 3194, 3424, 3431, Cochair Session 5aID (3378), Cochair Session 5pID (3423)

Bliss, Donald B. -3160

Bliss, Heather-3343

Blom, Philip-2996

Blomberg, Leslie D.-3322

Blumberg, Alan F.-3288

Bobenrieth Miserda, Roberto F. -3321

Bochud, Nicolas-3078

Boechler, Nicholas-3049

Boedihardjo, Arnold P.-3424

Bogdan, Caitlin-3351

Boisvert, Jeffrey E.-3086, 3159

Boivin, Guillaume-3034

Bok, Eun-3140

Bollen, Viktor-3345

Bolmer, S. T.-3406

Bolton, J. S.-2992, Cochair Session 1pNSb (2991), Cochair Session 5 pNSa (3430)

Bomhardt, Ramona-3276, 3277

Bonadonna, Giovanna-3017

Bonelli Toro, Augusto-3403

Bones, Oliver C.-3266

Bonnel, Julien-3065, 3133

Bonomo, Anthony L.-3234

Boorman, Owain L.-3376

Boothalingam, Sriram-3046

Boschi, Lapo-2959, 3451

Bossy, Emmanuel-2979
Bottalico, Pasquale-2947, 3126

Botts, Jonathan-3055, 3127

Bouavichith, Dominique-3106

Boucher, Victor-3224

Boutillon, Xavier-3255

Bouyer, Charlene-3370

Bovaird, James-2946

Bowen, Macarena-3047

Boyd, Gavin-3232

Boyd, Michael L. -3408

Bozzi, Fabricio A.-3008

Braasch, Jonas-2989, 3041, 3142, 3450 , Cochair Session 1pMU (2988)

Brachet, Nicolas-2995

Bradley, David L.-3351, 3352, Cochair Session 4aUW (3288), Cochair Session 4pUWa (3348)

Brady, Lynda-3031

Brainard, Russell E.-3018

Brancazio, Lawrence-3444

Brand, Timothy C. -3308

Brandão, Eric-3178

Branstetter, Brian K.-3131, 3179, 3298

Braslau, David-3094

Brasseur, Emmanuel-3165

Brayman, Andrew A.-2985

Brenker, Jason-3147

Brenner, Daniel-3111, 3226

Brès, Guillaume A.-3097

Bressler, Scott-3209

Briaire, Jeroen J.-3157

Brill, Laura C.-2946, 3069

Brimijoin, W. Owen-2998

Brint, Emma-3436

Bristow, Abigail-3094

Brocklehurst, James-3171

Brocolini, Laurent-3438

Brodsky, Micah C.-3297

Broersma, Mirjam-3335, 3341

Bronkhorst, Adelbert W.-3436

Brown, Andrew D.-3269

Brown, Christopher-3099, Chair Session 2pPP (3099)

Brown, Daniel C.-3362

Brown, Elisabeth-3362

Brown, Elisabeth M.-3407

Brown, Mary B.-3414

Brown, Michael-3119

Brown, Michael G.-3076

Brown, Simon-3174

Browning, Richard-3370

Brown-Schmidt, Sarah-3216

Bruce, Matthew-3028

Brungart, Douglas-Cochair Session 1aPP (2956), Cochair Session $1 \mathrm{pPP}(2997)$

Brungart, Douglas S.-2957, 2997. 2998, 3099, 3152, 3200

Brungart, Timothy A.-3034

Brunton, Steven-3350

Buccowich, Jasmine S.-3296

Buchegger, Blasius-3281

Buchholz, Jorg-3214

Buchholz, Jorg M.-3277, 3437

Buchholz, Jörg M.-3436, 3437

Buck, John R.-2947, 3238, 3451

Buckingham, Michael J.-2977, 3169, Cochair Session 1pAO
(2975), Cochair Session 2aAO (3023)

Buckley, Christopher-3292

Bucknall, Roger-3390

Bull, Joseph-3026

Bull, Joseph L.-3310

Bunescu, Razvan-3394

Bunkley, Steven L.-3193, 3194, 3424

Bunton, Kate-3218

Buravkov, Sergey V.-3082

Burgess, William C. -3182

Burguiere, Olivier Y.-3032

Burke, Kali-3299

Burns, Gary-3000

Burns, Peter-3028

Buss, Emily-3265, 3271

Bustamante, Omar A.-3181, 3182

Butler, Rhett-3406

Buzga, Sebastian-3375

Byers, Emily-3213

Byrd, Dani-3344

Byrd, Lynne-3297

Byrne, Andrew-3382

Byun, Gi Hoon-3170

Byun, Sung-Hoon-3059

Cacavelos, Federico N.-3403

Cade, Dave-3411

Cadet, Michaelle A.-3187

Cai, Feiyan-3149

Cai, Xiran-3078

Cain, Charles-3032, 3084

Cain, Charles A.-3030, 3031, 3083

Calambokidis, John-3295, 3411

Calandruccio, Lauren-3271

Calcus, Axelle-3214

Caleap, Mihai-3049

Calero, Diego-3034

Caliskan, Mehmet-3250

Callan, John-3026

Cameron, Peter J.-3121

Camp, Amber B.-3396

Campbell, D. Murray-3427

Campbell, Patsy-3427

Campbell, Richard-3181

Cang, Siyuan-3403

Cantor Cutiva, Lady C. -3126

Cao, Wenwu-3298

Cao, Yipeng-3001

Capozella, Joshua-3002

Carannante, Valentina-3148

Carcagno, Samuele-3390

Cardoso, Luis-3080

Cardwell, Adrienne-3297

Carey, Holle-3226, 3442

Carignan, Christopher-3219

Carlisle, Robert-2985, 3025

Carman, Richard-3258

Carney, Laurel H.-3272

Carper, Scott-3408

Carper, Scott A.-3408

Carrascal, Carolina A.-3245

Carrell, Thomas -3215

Carson, Paul L. -3188

Carter, Brittney L.-3270

Carter, Caitlin-3367, 3368, 3374

Carter, Cliff-Cochair Session 1pSPa (3006)

Carugo, Dario-2985, 3025, 3370

Carvajal, Jorge V.-3002

Casali, John G.-3200 
Case, Alexander U.-3069

Casper, Brandon C.-2948

Caspers, Philip-2947, 2965, 2974

Casserly, Elizabeth D.-3226

Castellote, Manuel-3299

Catheline, Stefan-2959, 3138, 3373, 3418,3451

Cato, Douglas-3412

Cazzanti, Luca-3230

Ceccio, Steven-3330

Celmer, Robert D.-3384

Ceranna, Lars-2996, 3383

Chabot, Samuel-3292

Chacon, Thiago C.-3396

Chadwell, C. David-3285

Chait, Maria-3208, 3209

Chambers, Craig-3217

Chan, Keith-2985

Chandrasekaran, Bharath-3283

Chandrayadula, Tarun K.-3135

Chang, Andrea-3013

Chang, Andrea Y.-3012, 3013

Chang, Jay W.-3006

Chang, Jiho-3375

Chang, Kai-Fu-3075

Chang, Woosuk-3089

Chang, Yuehchin-3106

Chang, Yueh-chin-3107

Chapman, N. Ross-3023

Charlton-Robb, Kate-3416

Chatelet, Eric-3002

Chatron, Jacques-3268

Chatterjee, Chandrima-3324

Chatterley, James J.-3034

Che, Il-Young-2996

Che, Xiaohua-3250

Chee, Adrian J. Y.-3028

Cheer, Jordan-3312

Chehami, Lynda-3002, 3387

Chekroun, Mathieu-3165

Chen, Charles-3394

Chen, Chi-Fang-3013, 3022,

Cochair Session 1pUW (3011),

Cochair Session 2aUW (3064), 3012, 3013

Chen, Chuan-3420

Chen, Huifang-3184

Chen, Jessica-3416

Chen, Jingwei-3325

Chen, Johnny L.-3423

Chen, Juanjuan-3149

Chen, Li-mei-3444

Chen, Minmo-3183

Chen, Nai-Chang-3022

Chen, $\mathrm{Pu}-3370$

Chen, Shuwen-3106

Chen, Si-3398

Chen, Songmao-3212

Chen, Wei-rong-3219

Chen, Yan-3341

Chen, Yao-3307

Chen, Yi C. -3302

Chen, Ying-3340

Chen, Yi-Run-3291

Cheng, Jianchun-3049

Cheng, Jiqi-3080

Cheng, Lauretta-3220

Cheng, Li-2992

Cheon, Sang Yee-3334

Cheong, Mathew-3186

Cheong, Sei-Him-3171, 3172
Cherif, Raef-3000

Chernikov, Valeriy P.-3082

Cheung, Fiona-3294

Chew, Weng C.-3373

Cheyne, Stanley A.-3122

Chhetri, Dinesh K.-3395

Chiang, Weihwa-3291

Chiang, Yi-Hao-3311

Chiasson, Jérémie-3201

Chiba, Ko-3189

Chien, Kuo-Feng-3013

Chien, Yu-Fu-3224

Chigarev, Nikolay-3165

Chigira, Yoshiki-3277

Chisaki, Yoshifumi-3192, Cochair Session 3pEA (3192)

Chitnis, Parag V.-2980, 3419, Cochair Session 1aBA (2950), Cochair Session 1pBAa (2978)

Chitoran, Ioana-3217

Chiu, Chenhao-3221

Chiu, Ching-Sang-3012, 3013, Cochair Session 1pUW (3011), Cochair Session 2aUW (3064)

Chiu, Faith-3398, 3437

Chiu, Linus-3013

Chiu, Linus Y.-3012, 3013

Chiu, Linus Y.S.-3013

Cho, Chomgun-3014, 3172

Cho, Hwanjeong-3211

Cho, Hyunsoo-3262

Cho, Seunghyun-3166

Cho, Sungho-3014, 3064, 3065, 3170

Chobeau, Pierre-3127

Chodroff, Eleanor-3343, 3344

Choi, Donghun-3251

Choi, Euna-3034, 3375

Choi, Haejin-3140

Choi, Hyun-Taek-3059

Choi, Inyong-3046

Choi, Ja Young-3342

Choi, Jee Woong-3014, 3064, 3065

Choi, Jong-Soo-3251, 3422

Choi, Jung-Woo-3312

Choi, Jungwoo-3405

Choi, Kanghoon-3064

Choi, Sungho-3211

Choi, Yonghwa-3170

Cholewiak, Danielle-3415

Chong, Adam J.-3216

Choo, Youngmin-3055, 3064, 3234

Chou, Lien-Sian-3022

Chou, Lien-Siang-3018, 3019, 3132

Choung, HanAhChim-3141, 3248

Christe, Anthony-2997, 3056

Christensen, Johan-3202

Christian, Andrew-Cochair Session 5aID (3378), Cochair Session 5pID (3423)

Christie, Anthony-2996

Christman, Russ-3181

Chui, Angel L.-3342

Chun, Wonjong-3089

Chung, Andy-3041

Chung, Hyunju-3226

Chung, Kil W.-3008

Church, Charles C. -3252

Cieszko, Mieczyslaw-3001

Cipolla, Jeffrey-3160, Cochair Session 2aSA (3047), Cochair Session 2pSA (3102)
Clair, Vincent-3383

Clark, Ashley N.-3443

Clark, Grace A.-Cochair Session 3pSP (3229)

Clark, Matt-3103, 3203

Clayards, Meghan-3113, 3114, 3396

Clement, Greg-3370

Clement, Gregory T.-3370, 3418

Cleveland, Robin-3368

Clopper, Cynthia G.-3215, 3226

Cobbold, Richard S.-3261

Coetzee, Andries W.-3106

Coffin, Matthew-3285

Coffman, Zachary A.-3367, 3374

Cogliati, Andrea-3038

Coiado, Olivia C. -3246

Cole, Pamela M.-3237

Colesa, Deborah J.-3377

Colleye, Orphal-3360

Collier, Sandra-3260

Collins, David-3147

Collis, Jon-3408

Colombi, Andrea-2959, 3103

Colonius, Tim-3097, 3307

Colosi, John A.-2976, 3024, 3135, Cochair Session 2pAOb (3077)

Colquitt, Daniel-3103

Conant, David A.-3039

Cong, Chaonan-2993

Conklin, Jenna-3333

Connell, Katrina-3336

Cook, Daniel A. -3346

Cook, Douglas-3003, 3392

Cooper, Steven E.-3382

Copeland, Adrienne M.-3361

Copeland, Cassidy-3052, 3220

Copland, Phillip-3243

Corakci, Ata C. -3088

Cordioli, Julio A.-3034

Corl, Doug-3308

Cormack, John M.-3327

Corneyllie, Alexandra-3438

Cornuelle, Bruce-3074, 3364

Correia, Mafalda-3029

Costley, R. D.-3233

Cotter, Emma D.-3350

Cottingham, James P.-3142, 3143, Chair Session 5aMU (3379), Cochair Session 3aMU (3142)

Coughlin, Caitlin E.-3335

Coussios, Constantin-2985, 3368

Coussios, Constantin C.-3025

Coviello, Christian-2985

Cowan, Nicole-3374

Cowern, Dianna L.-3314

Cox, Donald L.-3086

Cox, Steven R.-3440

Cox, Trevor J.-3266

Coyette, Jean-Pierre-3314

Craft, Justin T.-3106

Crance, Jessica-3295, 3359

Crance, Jessica L. -3415

Craster, Richard-3103

Cray, Benjamin A.-3102

Cremaldi, Lucien-3324

Cretin, Dorian-3189

Crevier-Buchman, Lise-3106, 3107

Cross, Patrick-3171

Croxford, Anthony-3325

Crum, Larry A. -3308
Crum, Lawrence-Cochair Session $1 \mathrm{pBAb}$ (2982), Cochair Session 2aBAa (3025)

Cui, Xiaodong-2965

Cui, Yingting-3333

Cui, Zhenglie-3193

Culver, R. L. -3121

Culver, R. Lee-Chair Session 1aSP (2966), Cochair Session $2 \mathrm{aSPb}$ (3056), Cochair Session 2pSPb (3118)

Cummer, Steven-3103, 3377

Cummer, Steven A.-2959

Cunitz, Bryan W.-2983, 3306, 3307, 3308, 3310

Cunningham, Kane-3298

Cunningham, Michael-3229

Cushing, Colby W.-3048

Czarnota, Gregory-3026, 3137

Czerniak, Luke-3002

Da Costa, Sandra-3045

Dahl, Peter H.-3168, 3171, 3410, Cochair Session 3aUWa (3168)

Dahlen, Unn-3327

Dai, Lin-3063

Daikohara, Makoto-3056

Dailey, Megan M.-3215

Dajani, Hilmi R.-3201

Dakin, Thomas-3410

Dall'Osto, David R.-3168, 3171, $3289,3406,3410$

Dall'Osto, David R.-Cochair Session 3aUWa (3168)

Dance, Stephen-3174

D'Angela, Peter-3249

Daniel, Timothy D.-3123

Daniels, Matthew-3232

Dannemann, Fransiska-2996

Daoui, Hassiba-3078

Da Silva, Guilherme-3158

da Silva, Vera M.-3412

Dau, Torsten-3177

Davidson, Lisa-3221, 3223

Davies, Patricia-Cochair Session 1aNS (2953), Cochair Session $1 \mathrm{pNSa}(2990)$

Davies, William J.-3266

Davis, Brian S.-3260

Davis, Catherine M.-2986

Davis, Chris-3339

Dayton, Paul-3187

Dayton, Paul A.-3371

Dazel, Olivier-3430

de Almeida, Fernando G.-3409

de Castro, Franciele R.-3239

de Cheveigné, Alain-3265

De Cock, Ine-3247

de Jong, Nico-2980, 2981

de Kort, Selvino R.-3074

de Korte, Chris L.-3029, 3310, 3420

de la Cruz-Pavía, Irene-3446

de Moustier, Christian-3284, 3290

de Oliveira, André Luis S.-3409

De Robertis, Alex-3242

de Rosny, Julien-3002

De Rybel, Tom-3225

de Souza, Diogo-3412

Deane, Grant-3290

Deane, Grant B.-2977, 3285

Debich, Amanda-3412

Decker, Jeremy L.-3236 
DeCourcy, Brendan J.-3406

Deeves, Emily-3440

Deguchi, Ichiro-3184

Deguchi, Mitsuyasu-3057, 3058, 3230

Dehner, Rick-3321

Deiters, Kristy K.-3146

del Prado Martín, Fermín M.-3227

Delage, Rémi-2955

Delcros, Jean-Guy-3369

Dellwo, Volker-3058, 3217, 3219

DeLong, Caroline M.-3297

Demasi, Rita-3107

Demi, Libertario-3420

Demirci, Utkan-3370

Demolin, Didier-3106, 3107, 3221

den Brok, Martijn-3082

den Brok, Martijn H.-3082

Denda, Ikuo-3146

Deng, Wenzhe-2990

Deng, Zhihao-3138

Denham, Susan-3208

Dent, Micheal-3299

Dent, Micheal L.-3299

Derrick, Donald-3225

DeRuiter, Stacy-3411

DeRuiter, Stacy L.-3411

Destgeer, Ghulam-3148

Dettmer, Jan-2949, 3065

DeVore, Brooke-3440

DeVries, Lindsay-3157

Dewitte, Heleen-3369

Dhar, Sumitrajit-3046

Di Iorio, Daniela-3076

Diaz, Esteban-3106

Diaz, Jonmarcos-3331

Dick, David A.-3175

Dickerson, Kelly-3101, 3276, 3390

Dietz, Mathias-3099, 3272, Chair

Session $4 \mathrm{aPPb}(3272)$

Dilley, Laura-3114

DiMarzio, Nancy-3413

Ding, Hongwei-3116, 3337

Ding, Tzung S. -3302

DiNino, Mishaela-3157, 3438

Dionne, Jason P.-3388

Dmitrieva, Olga-3333

Do, Youngah-3219

Doane, John W.-3121

Doedens, Ric-3069

Dolan, Karin-3413

Dolcetti, Giulio-3024

Dollet, Benjamin-3369

Döllinger, Michael-3393, 3398

Dong, David W.-3281, 3384

Dong, Menghong-3132

Dong, Qi-3265, 3442

Donskoy, Dimitri M.-3033

Dorgan, Kelly M.-3305

Doria, Damian-Chair Session 3pAAc (3178)

Dorocicz, John-3410

Dorreen, Kieran-3225

dos Santos, Francisco A.-3409

Dossey, Ellen-3226

Dosso, Stan E.-2949, 3065

Dou, Yan-3333

Dougherty, Sara C.-3342

Doughty, Jackson-3447

Douglas, Annie-3411

Douglas, Kim-3181
Douglas, Owen-3258

Douglass, Alexander S.-3403

Dowling, David R.-3212, 3231, 3232, 3304, 3403

Doyle, Philip C. -3440

Doyle, Timothy E.-3187, 3367, 3368,3374

Drager, Katie-3052, 3220, Cochair Session 2aSC (3050)

Drake, James-3031

Dreessen, Stephanie-3300, 3303

Drehfal, Lindsey C.-3137

Drenth, Joost-3310

Drichko, Irina-3203

Drinkwater, Bruce-3325

Drinkwater, Bruce W.-3049

Driscoll, Carlie-3264

Dromey, Christopher-3220

Drouin, Julia R.-3227

D'Spain, Gerald L.-3118, 3285, 3360

Du, Juan-3078

Duan, Wenbo-3386

Duan, Zhiyao-3038

Dubbs, Lindsay L. -3300

Dubey, Harishchandra-3010

Dubno, Judy R.-3270

Dubois, Marc-3104

Duclos, Aroune-3430

Duda, Timothy F.-2976, 3023, 3288

Duennebier, Frederick K.-3406

Duffy, Margaret-2985, 3025

Duh, Kevin-3062

Dumm, Christopher M.-3002

Dumont, Erik-3082

Dunleavy, Kerry-3415

Dunlop, Rebecca-3412, 3416, Cochair Session 5pABa (3411)

Dunlop, Rebecca A.-3240, 3359

Dunmire, Barbrina-2983, 3307, 3308,3310

Dunn, Katie-3395

Dunn, Robert A.-3133

Dunn, Roo-3245

Dupont, Lucie-3387

Dupre, Aurelien-3307

Dupuis, Kate-3401

Duraiswami, Ramani-2999

Durand, Olivier-3165

Dürr, Stefan-3393

Duryea, Alexander-3084

Duryea, Alexander P.-3248

Duty, Jason R.-3236

Dwijayanti, Suci-3115, 3404

Dybedal, Johnny-3123

Dykan, Igor V.-2986

Dziak, Robert P.-3300

Dzieciuch, Matthew-3008, 3134

Dzieciuch, Matthew A.-3075, 3135, 3406

Dzikowicz, Benjamin-3086

Earle, F. S. -3341

Earle, F. Sayako-3342

Ebihara, Tadashi-3229, 3375, Cochair Session 3pSP (3229)

E.D. Alexander, Jessica-3336

Edamoto, Shoma-3313

Eddins, Ann C. -3155

Eddins, David-3270

Eddins, David A.-3155, 3398

Edelmann, Geoffrey F.-3054
Edelson, Geoffrey S.-3230, 3349

Edwards, Joseph R.-2967

Egan, Thomas-3186

Eguchi, Sayoko-3333

Eikelenboom, Dylan-3082

El Boghdady, Nawal-3391

Elhilali, Mounya-3209

Elko, Gary W.-3032

Ellaham, Nicolas N.-3201

Elliott, John-3107

Elliott, Stephen-Cochair Session 4pEA (3311)

Ellis, Dale D.-3232

Emelianov, Stanislav-2951

Emerson, Benjamin-3261

Emmons, Candice K.-3130

Emoto, Akira-3260

Endo, Yukimi-3198

Ennis, Zachariah-3437

Enomoto, Mika-3393

Epain, Nicolas-3278

Epp, Bastian-3273

Erbe, Christine-3071, 3182, 3416

Erdman, Jr., John W.-3185, 3186

Erickson, Donna-3400

Ericson, Mark-3101

Esaki, Takashi-3258

Escudero, Paola-3282

Eshghi, Marziye-3107

Espana, Aubrey-2968

Espana, Aubrey L.-2968, Chair Session 3aUWb (3170)

España, Aubrey L.-2968

Espinoza, Carolina-3325

Esplin, J. J.-3121

Esplin, John J.-Cochair Session 2pUW (3121)

Eto, Daiki-2991

Euler, Garrett-2996

Evans, Breanna-3171

Ewert, Stephan-2999

Ewert, Stephan D.-3099, 3178, 3272

Fabiilli, Mario L.-3191

Fackler, Cameron J.-3145

Fagan, Aileen-3133

Falandays, James B.-3216

Falcone, Erin-3411

Falcone, Erin A.-3411

Fan, Xiaopeng-3132

Fan, Ying-Ching-3132

Fang, Shih-Hau-3018

Farbos de Luzan, Charles P.-3331

Farcas, Adrian-3072

Farges, Thomas-2995

Farmer, David M.-2975

Farr, Navid-3082

Farrell, Dara M.-3171

Farrokhrooz, Mehdi-3352

Farzbod, Farhad-3048

Fasanya, Bankole K.-3273

Fässler, Sascha-3243

Fässler, Sascha M.-3305

Fatemi, Mostafa-3186

Faulkner, Rebecca C.-3072

Faytak, Matthew-3340

Fazel, Vedad-3224

Federova, Valeriya-3439

Fee, Michale-3239

Feeny, Albert-3370

Feguson, Brian G.-3123

Fehler, Michael C.-3327
Feistel, Stefan-3128, 3193

Fekkes, Stein-3029

Felisberto, Paulo-2949

Fellner, Wendi-3180

Fels, Janina-3276, 3277

Feltovich, Helen-3137

Feng, Shaotong-3085, 3367, 3368

Ferguson, Brian G.-3006, 3007,

3119, 3169, 3345, 3350, Cochair

Session 1pSPa (3006), Cochair

Session 4aSPb (3286), Cochair

Session 4pSPa (3345), Cochair

Session 4pSPb (3347)

Ferguson, Eric L.-3351

Ferk, Heinz-3281

Fernandes, Julio-3188

Fernandes, Paul-3243

Fernandez, Gabriel-3439

Ferreira, Jullie A.-3396

Ferreira Fernandes, Martin-3002

Ferroni, Paolo-3002

Festen, Joost M.-3443

Feth, Lawrence L. -3273

Feuerbach, David-3180

Fiasson, Romain-3225

Fiebig, André-2954, 3040, Cochair Session 1aNS (2953), Cochair

Session 1pNSa (2990)

Fielding, Sophie-3242

Filho, William S.-3008

Filoche, Marcel-3255

Finette, Steven-3173

Finn, Anthony-3119

Finneran, James-3298

Finneran, James J.-3131, 3179

Fiorentino, Robert-3224

Fischell, Erin M.-3170

Fisher, Daniel J.-3167

Fisher-Pool, Pollyanna-3018, 3301

Fisher-Pool, Pollyanna I.-3295

Flaherty, Mary M.-3265

Flamme, Gregory A.-3146, 3147

Fleischman, Aaron-3418

Fleming, Katie-3094

Fleury, Romain-3047

Flores, Tanya-3338, Chair Session 5aSC (3392)

Flynn, Timothy-3180

Flynn, Tyler J.-3212

Fogerty, Dan-3215

Fogerty, Daniel-3164

Folk, Emma C.-3215

Fontaine, Bertrand-3154

Foots, Ashley-3101, 3276

Ford, Catherine-3107

Forero, Pedro A.-3117

Fouquet, Meddy-3397

Fournet, Michelle-3301

Fowlkes, J B.-3188

Fowlkes, Jeffrey B.-3026

Frances, Candice-3180

Franken, Tom P.-3154

Franzoni, Linda P.-3160

Freeston, Katrina-3437

Fregosi, Selene-3019, 3181, 3411, Chair Session 3pABb (3180)

Frenkel, Victor-2984

Fréour, Vincent-3037

Freund, Jonathan B.-2982

Frey, Roland-3414

Freytag, John-3237 
Friard, Olivier-3017

Fridland, Valerie-3113

Friedlaender, Ari-3411

Friedlaender, Ari S.-3411

Friedrichs, Daniel-3219

Friel, Brett-3415

Friend, James-3205, Cochair Session

3aPA (3147), Cochair Session

3 pPAb (3205)

Frijns, Johan H.-3157

Frisch, Stefan-3228

Frisch, Stefan A.-3227

Fristrup, Kurt-3140, 3424

Frota, Sonia-3448

Fry, Michael D.-2965

Frye, Julianne-3218

Fu, Maojing-3219

Fu, Yanqing-2947

Fuhrman, Robert-2960

Fujihira, Haruna-3156

Fujii, Kozo-3043, 3432

Fujii, Tomohiro-2954

Fujikawa, Yasuo-3362

Fujikawa, Yoshikazu-3066

Fujimoto, Kazutoshi-3257

Fujimoto, Masako-3108, 3222

Fujioka, Toyota-3167

Fujisaka, Yoh-ichi-3275

Fujisaka, Yoh-ichi.-3275

Fujisaki, Hiroya-3400

Fujise, Akiko-3100

Fujita, Kentaro-3203

Fukaishi, Takuya-3062

Fukayama, Satoru-3091

Fukayama, Yukio-3057

Fukazu, Kosuke-3027

Fukuda, Akihiro-3250

Fukuda, Hiroyuki-3306, 3309,

Cochair Session 4aBAb (3247),

Cochair Session 4pBA (3306)

Fukuda, Katsumi-3171

Fukuda, Makoto-3326

Fukuda, Seiya-2955

Fukuda, Yoshiaki-3243

Fukumori, Takahiro-3057, 3059,

3060, 3062, 3139, 3376, 3403, 3451

Fukushima, Manabu-3178

Fukushima, Naoya-3043, 3097

Fukuwaka, Masa-aki-3303

Fuller, Chris-3431

Funaba, Hisao-2946

Funabiki, Kazuo-3297

Funaki, Keiichi-2962, 3057, 3114

Funatsu, Seiya-3222

Furukawa, Daisuke-3444

Furukawa, Shigeto-3151, 3158,

3209, Cochair Session $3 \mathrm{aPPa}$ (3150)

Furuya, Ken'ichi-3449, 3450, 3452

Fury, Christopher R.-3150

Futa, Koji-3073

Futterer, Jurgen-3082

Fütterer, Jurgen J.-3082

Gabard, Gwenael-3383

Gabriele, Christine-3301

Gafos, Adamantios-3401

Gaglione, Eric-3299

Gailey, Glenn-3411

Gallager, Scott-3241
Gallagher, Hilary-3145, 3199,

Cochair Session 3pNSb (3199)

Gallagher, Hilary L.-3199, 3200

Galloway, James-3437

Galperin, Yuri-3203

Galvin, Brittany-3262, 3421

Galy, Jocelyne-3386

Gamba, Marco-3017

Gammell, Paul-3371

Gan, Woon-Seng-3313

Gan, Zhe-3405

Gander, Phillip E.-3046

Gandhi, Prasanna S.-3206

Ganesh, Harish-3330

Ganguly, Anshuman-3313

Gao, Dazhi-3450

Gao, Jianliang-3236

Gao, Jingjing-2989

Gao, Junhui-3321

Gao, Nansha-3103

Gao, Xiang-3389, 3390

Garces, Milton-2995, 2996, 2997, 3056

Garces, Milton A.-2995, Cochair Session 1pPA (2994)

Garcia, Heriberto-3360

Garcia, Samuel-3438

Garcia-Lazaro, Jose-3152

Gardner, Mason-3080

Garellek, Marc-3216

Garland, Ellen-3416

Garland, Ellen C.-3416

Garnier, Vincent-3166

Garra, Brian S. -3188

Gastauer, Sven-3243

Gaston, Jeremy-3276, 3390

Gaston, Jeremy R.-3101

Gaudette, Jason E.-3033, Cochair Session 2aEA (3032)

Gaudrain, Etienne-3262, 3391, 3439

Gaumond, Charles F.-3055

Gauthier, Camille-3386

Gavrilov, Alexander-3182

Ge, FengXiang-3063

Ge, Li-Feng-3248

Gedamke, Jason-3300

Gedney, Charles-3181, 3350

Gee, Kent L.-3043, 3044, 3097, 3098, Cochair Session 2aPA (3042), Cochair Session 2pPA (3096)

Gelderblom, Erik C.-3369

Gemba, Kay L.-3117, 3118, 3133

Gendron, Paul J.-3230, 3286

Gendrot, Cedric-3221

Genuit, Klaus-3040, 3432, Cochair Session $5 \mathrm{pNSb}(3432)$

George, Deepu-2980

George, Jacob-3014

Gerg, Isaac-3347, 3348

Gerhardson, Tyler I.-3031

Gerold, Bjoern-2984

Gerratt, Bruce R.-2961

Gerstein, Edmund R.-3412, 3415

Gerstein, Laura-3412

Gerstoft, Peter-3007, 3053, 3054, $3117,3118,3133,3232,3409$, 3450 , Cochair Session $2 \mathrm{aSPa}$ (3053), Cochair Session 2pSPa (3117)

Gervain, Judit-3446
Getz, Laura-3391

Ghai, Shweta-3446, 3447

Giacoma, Cristina-3017

Giacomoni, Clothilde-3042, 3043

Gianakas, Steven P. -3440

Giard, Jennifer L. -3300

Gick, Bryan-3115, 3220, 3221, 3225,3343

Giegold, Carl-3069

Giguère, Christian-3145, 3201

Gilbert, Joel-3414

Giles, Deborah A.-3130

Gill, Katherine E.-3444

Gillani, Uzair S.-2947

Gillis, Keith A.-3259, 3386

Gilmore, Rick-3237

Ginsberg, Jerry H.-3000

Giordano, Nicholas-3037, 3254, Cochair Session 2aMUa (3036), Cochair Session 4aMUa (3253), Cochair Session 4aMUb (3255),

Cochair Session 4pMUa (3317)

Giorli, Giacomo-3361

Gisiner, Robert C.-3129

Gladden, Josh R.-3204, Cochair Session 3pPAa (3202)

Glean, Aldo-3001

Go, Yeong-Ju-3251, 3422

Gobat, Jason I.-3075

Goda, Noriaki-3076, 3183

Godar, Shelly-3100

Godin, Oleg A.-3076, 3134, 3168

Godø, Olav Rune-3243

Godøy, Rolf I.-3035

Goertz, Caroline-3299

Gohda, Noriaki-3135, 3183

Gold, Erica-3228

Goldbogen, Jeremy-3411

Goldbogen, Jeremy A.-3411

Golden, Matthew V.-2971

Goldizen, Anne W.-3240

Goldman, Geoffrey H.-3120, Cochair Session 2pSPb (3118)

Goldsberry, Benjamin M.-3104

Goldstein, Louis-3344

Gomez, Anton-3422

Gong, Wei-3144

Gonzalez, Jose M.-3095

Gonzalez, Lorenzo-3052

Good, Kenneth W.-3068, Cochair Session 2pAA (3068), Cochair Session 3aAAa (3126)

Goodman, Dan F.-3272

Gordon, Matthew K.-3396

Gordon, Michael S.-3269, 3425, 3426

Gordon Danner, Samantha-3344

Gorny, Lee J.-3259

Goto, Masataka-3090, 3091, Cochair Session 2aMUb (3038), Cochair Session 2pMU (3090)

Goto, Takao-3223

Goto, Tomonori-3259

Gotoh, Shinpei-3021, 3073

Gottlieb, Peter K.-3263

Goupell, Matthew-3439, Chair Session 5pSCa (3436)

Goupell, Matthew J.-3101, 3157

Goverts, S T. -3443

Gowda, Mohanika-2986

Graham, Stephen-3227
Grama, James-3052

Grant, Anat-3294

Grant, Kenneth W.-3152

Grasing, David-3404

Gravel, Anne-3201

Gravelle, Kayla-3083

Gray, Kathleen-3237

Graydon, Pamela S.-3146

Greene, Nathaniel T.-3273

Greenhall, John-3206

Greenhow, Danielle R.-3297

Greenleaf, James F.-3245

Greenwell, Brandon-3401

Grimal, Quentin-2959, 3078, 3451

Grimault, Nicolas-3268, 3438

Grimm, Giso-2999

Grinnell, Alan-2973

Groby, Jean-Philippe-3430

Groopman, Amber-3079

Grosh, Karl-3154, 3206, 3377

Grosse, Julian-3011, 3099

Grossl, Vinicius A.-3141

Grundy, Megan-2985, 3025

Grüter, Theres-3334

Gryllias, Konstantinos-3002

Gu, Wentao-3400

Guan, Jingjing-3441

Guan, Qianwen-3334

Guan, Shane-3020, 3022, 3067,

Cochair Session 2aABb (3020),

Cochair Session 2pABb (3072)

Guazzo, Regina A.-3360

Gueguen, Philippe-2959

Guenneau, Sebastien-3103

Guerra, Melania-3020, 3073

Guerrero, Jorge-3137

Guerrero, Quinton W.-3137

Guest, Hannah-3152

Guevara Vasquez, Fernando-3206

Guild, Matthew D.-3102

Guillaume, Lopez-2966

Gullapalli, Rao-2984

Gunji, Mai-3334

Guo, Gepu-3026, 3033, 3085, 3367, 3368

Guo, Jenhwa-3075

Guo, Longxiang-3231, 3403, 3449

Guo, Shengming-3011, 3013, 3014, 3173,3230

Guo, Sijia-2984

Guo, Xiasheng-3026, 3033, 3367

Guo, Xinyi-3351

Guo, Zhiqiang-3393

Guo, Zhongyuan-3230

Gupta, Anupam-2947

Gupta, Anupam K.-2965

Gupta, Shreyank-3189

Gurm, Hitinder-3031

Gusev, Vitalyi-3165

Gutierrez Pimenta, Braulio-3321

Gutiérrez-Jagüey, Joaquin-3181

Gutiérrez-Jagüey, Joaquín-3182

Gutmark, Ephraim-3331

Gutrmark, Ephraim-3395

Guvenc, Tolga-3095

Guyer, Robert A.-3326

Gyoba, Jiro-2998

Ha, Ho Kyung-3242

Haas, David-3130

Haberman, Michael R.-2953, 3048, 3049, 3103, 3104, Chair Session 
1aPA (2955), Cochair Session 2aSA (3047), Cochair Session 2pSA (3102), Cochair Session 4aPA (3259)

Hachiya, Hiroyuki-3138, 3191, 3247,3348 , 3418, Cochair Session 2pAOa (3074), Cochair Session 3aAO (3132), Cochair Session 3pAO (3183)

Hadinger, Kyle P.-3371

Hafla, Erin C.-3171

Haga, Takanori-3043

Haghi, Hossein-3370, 3371, 3434

Hagiwara, Aiko-3115, 3116

Hagiwara, Yoshihiro-2980, 3245, 3404

Hahn, Jooyoung-3064

Haiat, Guillaume-3191

Haïat, Guillaume-3188, 3189

Hainaut, Thomas-3383

Hale, Marlund E. -3040

Hall, Deborah A.-3152

Hall, Melissa-3260

Hall, Melissa A.-3260

Hall, Neal A.-3250, 3422

Hall, Tim-3084

Hall, Tim L.-3031

Hall, Timothy J.-3137, 3188

Hall, Timothy L.-3030, 3031, 3083, 3248

Hallman, Robert A.-2971

Ham, Hyukju-3001

Ham, Suyun-3211

Hamada, Yasuhiro-2960

Hämäläinen, Matti-3045

Hamamura, Mariko-3389, 3432

Hamasaki, Toshihiko-3037

Hamasuna, Ryoma-3253

Hamilton, Mark F.-3049, 3122, 3310,3327

Hamilton, Scott-3176

Hamtache, Brahim-3375

Han, Aiguo-3136, 3185, 3186

Han, Heesun-3337

Han, Je-Heon-3331

Han, Jeong-Im-3110

Han, Jin P.-2965

Han, Nuomin-3422

Hanaka, Kazuyuki-3256

Haneda, Yoichi-3056, 3057, 3059. 3061

Hanford, Amanda-3104, 3119

Hannah, Alex-3028

Hannah, Beverly-3225

Hannay, David-3410

Hannay, David E.-3072

Hannon, Erin-3265, 3390

Hansen, Hendrik H.-3029, 3420

Hansen, John H.-3009

Hansen, John H. L.-3010, Cochair Session 1pSPb (3009)

Hanson, Helen M.-3447

Hanson, M. Bradley-3130

Hanyu, Toshiki-3128, 3176, 3236, Cochair Session 3aAAb (3127), Cochair Session 3pAAa (3174), Cochair Session 3pAAb (3177)

Hara, Sunao-3110

Harada, Akari-3399

Harada, Kazunori-2970

Harada, Yoshihisa-3362
Haralabus, Georgios-3024

Hare, Jenna-3363

Harker, Blaine M.-3044, 3097, 3098

Harley, Heidi E.-3180

Harley, Joel B.-3054

Harmon, Jonah-3026

Harmon, Zara-3220

Harper, Jonathan D.-3308

Harper, Ronald M.-3266

Harris, Nicholas-3376

Harrison, Jolie-3072

Hart, Carl R.-3088, 3194, 3323

Hartmann, William M.-3272, 3389

Harvey, David-3014

Hase, Kazuma-2975

Hasebe, Kazuhiko-3387

Hasegawa, Hideyuki-3027, Cochair Session 2aBAb (3027), Cochair Session 5pBA (3417)

Hasegawa, Hiroshi-3266

Hasegawa, Nana-3059

Hasegawa, Shinya-2956

Hasegawa, Tomomi-3278

Hashi, Michiko-3115

Hashimoto, Dai-3323

Hashimoto, Miyu-3275

Hastie, Gordon D.-3072

Hastings, Mardi C. -3073

Hasumi, Toshiyuki-3280

Hatano, Hiroaki-3115

Hatch, Leila-3072

Hatch, Leila T. -3300

Hatori, Kouki-2980

Hattori, Yuji-3254

Haupert, Sylvain-3080

Havenhill, Jonathan-3219

Haver, Samara M.-3300

Hawkins, Brooke L.-3072

Hawley, Scott H.-2987

Haworth, Kevin J.-3084

Hawthorne, Dean L.-3181, 3182

Hay, Alex-3184, 3363

Hay, Jennifer-3225

Hayamizu, Satoru-3429

Hayashi, Daiki-2961

Hayashi, Hiroki-3305

Hayashi, Masatoshi-3035

Hayashi, Rintaro-3259

Hayashi, Ryoko-3338

Hayashi, Tomoki-3404

Hayek, Sabih I.-3159

Hayward, Maurice E.-3095

Hazan, Valerie-3444

Hazen, Elliott-3411

He, Shuman-3444

He, Yuan-3265

Healy, Eric W.-3270

Heaney, Kevin D.-3024

Hechavarría, Julio C.-3157

Hecht, Quintin A.-3145

Heerschap, Arend-3082

Heffner, Chris-3114

Hefner, Brian T.-3023

Heibel, Michael D.-3002

Heijdens, Simon-3294

Heil, Thomas-2998

Heinz, Michael-3152

Heinz, Michael G.-3273, Cochair Session 3aPPa (3150)

Helble, Tyler-3413

Helble, Tyler A.-3181, 3295
Helfrick, Mark-3181, 3350

Helgeson, Kirsten-3396

Hemmert, Werner-3213

Henderson, E. E.-3412

Henderson, E. Elizabeth-3181

Henderson, Elizabeth E.-3413

Henderson, Laurel J.-3288

Hendriks, Gijs A.-3420

Henter, Gustav E.-2961

Herdtle, Thomas-2992

Hermand, Jean-Pierre-3305, 3364

Hermann, Raphael-3203

Hernandez Perez, Heivet-3046

Hernández-Abad, Annette-3157

Hersh, David S.-2984

Heyne, Matthias-3225

Hidayetoglu, Mert-3373

Higa, Akichika-2962, 2965

Higa, Keita-2962

Higgins, Nathan C.-3045

Higuchi, Yusuke-3423

Hildebrand, John A.-3295, 3300, 3360

Hill, Evan M.-3302

Hill, Marie-3415

Hill-Cook, Mandy L.-3297

Hillenbrand, James-3163

Hillis, James-3008

Hilloulin, Benoit-3165

Hines, Paul C.-2949, 3285

Hioka, Yusuke-3425, 3437

Hiraga, Rumi-3153

Hiraguri, Yasuhiro-3257

Hirahara, Tatsuya-3101

Hirai, Toshio-2961

Hirai, Yukino-3021, 3073

Hiraiwa, Tetsuo-3043, 3096

Hiramitsu, Atsuo-2971

Hiramoto, Takeru-3275

Hirano, Takeshi-3429

Hirao, Masahiko-3203, 3204

Hirao, Yoshihiro-3280, Cochair Session 4aSA (3279)

Hirata, Shinnosuke-3138, 3191, 3247, 3348, 3418, Cochair Session 4aSPa (3284)

Hirata, Yukari-3340

Hirayama, Makoto J.-3004

Hiroaki, Sugamata-3371

Hirose, Nobuyuki-3262, 3264

Hirose, Syunpei-3385

Hiroya, Sadao-3228

Hiruma, Nobuhiko-3275

Hiryu, Shizuko-2975, 3032, 2975

Hiyama, Shoko-3387

Hobbs, Roderick-3299

Hodgkiss, William-3133, 3407

Hodgkiss, William S.-2950, 3074, $3117,3118,3410$

Hodgson, Murray-3237

Hodoshima, Nao-3333

Hoekje, Peter L.-3319, Chair Session 4pMUb (3318), Chair Session 4pMUc (3320)

Hoffmeister, Brent K.-3081

Hogan, Jeffrey T.-3130

Hoglund, Evelyn M.-3273

Hohmann, Volker-2999

Hojo, Harimasa-3062

Holland, Charles W.-2949, 3233, Cochair Session 3pUWb (3233)
Holland, Christy K.-3084, 3252

Holland, Mark-3079

Holliday, Nicole-3219, Chair

Session 4pSC (3332)

Hollonbeck, Sean A.-3273

Holm, James R.-2983

Holst, Peter K.-3236

Holt, Marla M.-3130

Holt, Yolanda F.-3218

Homma, Musashi-3365

Homma, Yukio-3309

Honda, Akio-2998, 3267, 3269

Honda, Masakaki-Cochair Session 1pSC (3004)

Honda, Shinya-3008

Hongo, Hiromichi-3324

Hongo, Kouki-2962

Honma, Yukio-3306

Hoogenboom, Martijn-3082

Hoole, Philip-3217

Hooshiar, Kavon-3220

Hoover, K. Anthony-3039, Cochair Session 2aNS (3039)

Horbach, Maria-3264

Hord, Samuel K.-3422

Hori, Takaaki-3062

Horie, Seichi-3145, 3146, 3198

Horigome, Mio-3214

Horihata, Takuto-3115

Horii, Kaoru-3190

Horii, Shinpei-3057

Horiuchi, Ryuzo-3372

Horiuchi, Yasuo-3444

Horn, Michael-3176

Horn, William-3182

Horoshenkov, Kirill V.-3430, 3431

Horvat, LeeAnn-3152

Hoshi, Kazuma-3128, 3176, 3236

Hoshiba, Kotaro-3348

Hoshino, Yasushi-3068

Hosoi, Hiroshi-2945, 3156, 3263, 3274

Hosokawa, Akihiro-2954

Hosokawa, Atsushi-3188

Hosono, Suguru-3095

Hossain, Shaikat-3441, 3446

Høst-Madsen, Anders-3301

Host-Madsen, Anders-3360

Hotehama, Takuya-3390

Hots, Jan-3264, 3265

Houix, Olivier-3267, 3390

Houser, Dorian-3239, 3298

Houser, Dorian S.-3131, 3179

Houston, Janice-3096, Cochair Session 2aPA (3042)

Howarth, Thomas R.-3086

Howe, Bruce-3184, Cochair Session 2pAOa (3074), Cochair Session 3aAO (3132), Cochair Session 3pAO (3183)

Howe, Bruce M.-3406

Howe, Thomas -3408

Howell, Jonathan-3399

Howell, Mark-3370, 3418

Howie, Will-3275

Howson, Phil-3107, 3109

Hozumi, Naohiro-3139, 3185, 3245

Hsieh, Feng-fan-3107, 3224

Hsu, Jennifer-3092

Hu, Elly R.-3342

Hu, Hongmei-3099 
Hu, Tao-3013

Hu, William W.-3022

Hu, Yu-Hsin-3448

Huang, Chen-Fen-3074, 3075, 3135

Huang, Dehua-3086, 3087, Cochair

Session 2pEAa (3086)

Huang, Hai-3160

Huang, Hsiao-Tung-3051

Huang, JianChun-3058

Huang, Jiaqi-3211

Huang, Jinsong-3081

Huang, Lixi-2993

Huang, Mong-Na L.-3063

Huang, San Ho-3229

Huang, Sheng-Wei-3075

Huang, Shih-Hao-3063

Huang, Ting-3224, 3389

Huang, Tony Jun-Cochair Session

3aPA (3147), Cochair Session $3 p P A b(3205)$

Huang, Wei-3360

Hubbard, Seth-3260

Hubbard, Seth D.-3260

Huber, Matthew T.-3081, 3186, 3410

Huber, Nathan-3410

Huber, Thomas -3410

Hughes, Derke-3167

Hughes, Ronald-3320

Hull, Andrew J.-Cochair Session 3aSA (3159)

Hüls, Simone-3336

Hunter, Alan J.-3346

Hunter, Christopher-2983, 3186, 3310

Hunter, Cynthia R.-3214

Hunter, Elise-3220

Hunter, Eric J.-3126

Hursky, Paul-3169, 3286, Cochair

Session 4aSPa (3284)

Hussain, Qandeel-3108

Husson, Philippe-2995

Hustad, Katherine C.-3444

Hwang, Hyosung-3341

Hwang, Hyun Kyung-3216

Hwang, Joo Ha-3083

Hwang, Seongil-3320

Hwang, Wei-Shien-3022

Hwang, Yonghwan-3089

Hynynen, Kullervo-3031

Hyodo, Hiroaki-2955

Hyodo, Koji-3372

Ichigo, Takuya-3426

Ichihara, Mie-2994

Ichikawa, Kotaro-3412, Cochair

Session 5pABa (3411)

Ichikawa, Makoto-3263

Ichiki, Manon-3115, 3116

Ierley, Glenn-3295

Igai, Ryosuke-3249

Igarashi, Shigeru-3421

Igeta, Takako-3333

Iguro, Yuki-3222

Ih, Kang Duck-3320

Iida, Akiyoshi-3253

Iida, Kazuhiro-2957, 3278, Cochair

Session 1aPP (2956), Cochair

Session 1pPP (2997)

Iida, Kohji-3305

Iijima, Yukina-2981

Iikubo, Masahiro-2980
Iino, Shingo-3249

Iino, Yuki-3065, 3066

Ikeda, Masahiro-3391

Ikeda, Teiichiro-3308

Ikeda, Toshiyuki-3432

Ikeda, Yusuke-3087, 3095, 3140, $3195,3275,3451$

Ikegami, Shimpei-3429

Ikuma, Takeshi-3443

Ikuma, Yuko-3342

Ilicali, Mustafa-3095

Ilinskii, Yurii A.-3310, 3433

Im, JungHo-3242

Imae, Yuki-3431

Imagawa, Hiroshi-3223

Imai, Atsushi-3366

Imai, Ryo-3261

Imai, Toshiaki-2994

Imaizumi, Kenta-3057

Imaizumi, Tomohito-3242, 3360

Imamura, Hidetaka-2987

Imamura, Kento-3034

Imano, Kazuhiko-3326

Imeson, Chris-3249

Imura, Masato-3369

Inabe, Hazuki-3274

Inada, Hiroshi-3362

Inglis, Gary-3416

Ingram, David-3183

Ingram, Pier-3371

Ingvalson, Erin-3439

Inkelas, Sharon-3447

Inoguchi, Yasushi-3312

Inoue, Akira-3002

Inoue, Atsuto-3195

Inoue, Jinro-3145, 3146, 3198 , Cochair Session 3aNS (3144), Cochair Session 3pNSa (3198)

Inoue, Katsuo-3280

Inoue, Masashi-3116

Inoue, Naohisa-3323

Inoue, Takenobu-2963

Inukai, Yukio-3322

Iori, Gianluca-3078

Ireland, Zachary-3114

Irino, Toshio-3114, 3151, 3274

Irons, Sarah T. -3397

Irsik, Vanessa-3208

Irwin, Julia-3444

Isakson, Grace A.-3123

Isakson, Marcia J.-3014, 3234

Isaksson, Hanna-3078

Isei-Jaakkola, Toshiko-3338

Ishida, Hiroshi-3184

Ishida, Kae-2989

Ishigiro, Yoshio-3405

Ishiguro, Yoshio-3063

Ishihata, Kyohei-3444

Ishii, Hirokazu-3257

Ishii, Jun-3450

Ishii, Takaaki-3002

Ishii, Tatsuya-3043, 3096, 3330

Ishii, Yohji-2957

Ishii, Yutaka-3324, 3325

Ishikawa, Hitoshi-3421

Ishikawa, Kenji-3087, 3417

Ishikawa, Mutsuo-3374

Ishimoto, Yuichi-3393

Isnard, Vincent-3267

Isomura, Kazuhiro-3338

Isoyama, Naoya-2966
Ito, Akinori-2962, 2963, 3091

Ito, Hitoshi-3115, 3116

Ito, Kazuhito-3262, 3263, 3264

Ito, Kazuyo-3138, 3187

Ito, Kikuyo-3343

Ito, Masaaki-3058

Ito, Masashi-2961

Ito, Rumi-3139, Cochair Session 3aEA (3139)

Ito, Yasushi-3066

Ito, Youichi-3275, 3386, 3387, 3388

Itoh, Kosuke-3155

Itoh, Yoshiaki-3061

Itoi, Chihiro-3436

Itou, Masahiro-3425

Ivakin, Anatoliy-3023

Iverson, Paul-3219, 3335, 3436

Iwabuki, Hiroshi-2958

Iwagami, Eri-3439

Iwagami, Sho-3253

Iwai, Kenta-3058, 3061

Iwai, Ryota-3108

Iwaki, Mamoru-3277

Iwakuni, Makiko-2994

Iwamiya, Shin-ichiro-2986, 2989,

Cochair Session 1pMU (2988)

Iwamoto, Hiroyuki-3160

Iwano, Koji-3115

Iwasaki, Eitaro-3061

Iwasaki, Ryosuke-2983, 2984

Iwase, Ryoichi-3300

Iwase, Teruo-3383

Iwaya, Yukio-2999, 3312, Cochair

Session 1aPP (2956), Cochair

Session 1pPP (2997)

Iyer, Nandini-3200, 3269, 3437

Izbicki, Jean-Louis-3386

Izui, Kazuhiro-3430

Izumita, Kuniyuki-2980

Jackson, Darrell-3023, 3363,

Cochair Session 1aAO (2949)

Jacobellis, Dan G.-3170

Jacobson, Stephanie-3446

Jafari Sojahrood, Amin-3370, 3371, 3434

Jagadeesh, Anoop-3151

Jakobsson, Andreas-3327

James, Michael M.-3097, 3098

James, Sheronica L. -3370

Jang, Hayeun-3112

Jang, Hyung Suk-3175

Jang, Inman-2954

Jang, Seokjong-3141, 3248

Jang, Seongwon-3034, 3375

Jang, Seungho-3003

Janse, Esther-3270

Janssen, Karmon-3308

Jaramillo, Ana M.-2969, 3069

Jarvis, Susan-3413

Jarvis, Toby-3244

Jech, Michael-3305

Jee, Sang hwi-3445

Jeffries, Marlene-3410

Jen, I-Fan-3132

Jenkins, Daniel-3071

Jenkins, Keith-3298

Jeon, Jin Y.-3175

Jeon, Wonju-3104

Jeong, Cheol-Ho-3041

Jeong, KwangHun-3404

Jeong, Minju-3385
Jerome, Trevor W.-Chair Session $5 \mathrm{pSP}$ (3449)

Jessup, Carsen-3444

Jesteadt, Walt-3268

Jesus, Sérgio M.-2949

Jhang, Kyung-Young-3166

Ji, Chenzhen-3422

Ji, Youna-3089

Jia, Ning-3058

Jiang, Bing'er-3396

Jiang, Changyong-2993

Jiang, Yong-Min-3172

Jie, Shi-3172

Jimbo, Mina-3303

Jimenez-Lopez, M. Esther-3072

Jin, Craig T.-3278, 3351

Jin, Jiang-3167

Jin, Shiyuan-3124

Jing, Yun-3048, 3103

Jo, Kazuhiro-3195

Jo, Minwoo-3034

Joe, Herbert-3228, 3421

Johnsen, Eric-3032, 3084, 3085, 3368

Johnson, Erick-3171

Johnson, Jay R.-3364

Johnson, Keith-3110, 3223

Johnson, Paul A.-3326, Cochair

Session 4pPA (3324)

Johnson, Sarah-3219

Johnson, Theodore R.-3167

Jones, Douglas L.-3055

Jones, Heath G.-3269, 3273

Jones, Philip H.-3150

Jones, Zack-3215

Jong, Nico d.-3033

Jongman, Allard-3223, 3225, 3335

Joris, Philip X.-3154

Joseph, Anjali-3264

Joseph, John-3411

Joseph, John E.-3363

Joshi, Aditya-3187

Joshi, Suyash N.-3273

Joslin, James-3350

Judge, John A.-3260

Jukkert, Sergej-3258

Jun, Sun-Ah-3339, 3394, 3397

Jung, Dong Hwan-3170

Jung, Jakob-3130

Jung, Seom-Kyu-3014

Jung, Seungwon-3242

Jung, Sun Ah-2959

Jung, Ye-Jee-3270

Jurdy, Basel-3237

Kaan, Edith-3398

Kabashima, Yoshiyuki-3053

Kabral, Raimo-2992

Kaburagi, Tokihiko-3380, 3392

Kadoya, Yukimi-2975

Kagami, Hideaki-3053

Kagomiya, Takayuki-3222

Kajikawa, Yoshinobu-3058, 3061,

3141, 3313, Cochair Session 4pEA (3311)

Kakehi, Kazuhiko-3214

Kakitani, Akira-3061

Kakuda, Ayumu-2955

Kamakura, Tomoo-3255

Kamaya, Masayuki-3325

Kamekawa, Toru-3143, 3380, 3427, Cochair Session 3aMU (3142) 
Kameoka, Hirokazu-3053

Kamiakito, Noboru-2958

Kamiirisa, Hikaru-3409

Kaminuma, Atsunobu-2966

Kamiya, Keiichi-3362

Kamiya, Masaru-3390

Kamoshida, Takashi-3362

Kamp, Fabian-2954

Kan, Alan-3100, 3269

Kanagawa, Tetsuya-3122, Cochair Session 2pUW (3121)

Kanai, Kazumasa-3420

Kaname, Motohiro-3363

Kanamori, Masashi-3250

Kanayama, Hiroaki-3372

Kanda, Takefumi-3149

Kanedera, Noboru-3058

Kaneko, Arata-3076, 3133, 3135, 3183, Cochair Session 2pAOa (3074), Cochair Session $3 \mathrm{aAO}$ (3132), Cochair Session 3pAO (3183)

Kaneko, Shigehiko-3330

Kanematsu, Wataru-3122

Kaneshiro, Syouki-2962

Kang, Chang-kwon-3421

Kang, Donhyug-3014, 3242

Kang, Kim-2981

Kannepalli, Chandrasekhar-3320

Kanter, Shane J.-3040, Cochair Session 4pAAb (3293)

Kao, Chieh-3441, 3448

Kapatsinski, Vsevolod-3220

Kaplan, Max-3018

Kaplan, Maxwell B.-3359, 3417

Kapolowicz, Michelle R.-3339

Karanam, Saraswathi-3231

Karasawa, Rei-3149, 3205

Kargl, Steve-2968

Kargl, Steven G.-2968, 3408

Kariyasu, Makoto-3445

Karp, Harvey-3266

Karshafian, Raffi-3370, 3371, 3434

Karunarathne, Ashoka-3204

Karzova, Maria M.-3310

Kashino, Makio-2999, 3158, 3209, 3436, Cochair Session 3pPP (3207)

Kataoka, Akitoshi-3140, 3376

Kataoka, Reiko-3108

Kathiresan, Thayabaran-3058, 3217

Kato, Daisuke-3097, 3432

Kato, Hatuhiro-3002

Kato, Hiroaki-2957, 3337

Kato, Masaharu-2964

Kato, Misaki-3340

Kato, Nobumasa-3436

Kato, Tsuneo-3381

Katori, Yuta-3261

Katsman, Regina-3363

Katsnelson, Boris-3363, Cochair Session 5aAO (3362)

Katz, Jonathan-3079

Katz, Joseph-3331

Katz, Richard A.-3167

Katz, William F.-3224, 3333

Kauffman, Mandy E.-3413

Kaufman, Jonathan J.-3190

Kaul, Sanjiv-2986

Kaushik, Lakshmish-3010

Kavitskaya, Darya-3447
Kawabata, Ken-ichi-3083, 3367

Kawabe, Masahiko-3204

Kawada, Naoki-3261

Kawagishi, Takuji-3302

Kawagoe, Yoshinobu-3362

Kawahara, Hideki-3116, 3195, Cochair Session 3aSC (3162)

Kawahara, Kazuhiko-2986, Cochair Session 1aED (2952), Cochair Session 1pED (2986)

Kawahara, Misako-3399

Kawahara, Shigeto-3339, 3365

Kawai, Keiji-2946, 2970, 3094, Cochair Session 1aAA (2946), Cochair Session 1pAAa (2969)

Kawai, Shuhei-3178

Kawai, Yasuhito-2991

Kawakami, Hiroshi-2988

Kawamoto, Mamoru-3238

Kawanami, Shoko-3145, 3198

Kawanami, Syoko-3198

Kawanisi, Kiyosi-3184

Kawano, Atsushi-3436

Kawase, Mami-3189

Kawase, Saya-3339

Kawauchi, Yohei-3066, 3304

Kaya, Akio-3372

Kayasuga, Atsushi-3306, 3309

Kazama, Ryosuke-3280

Kazuhito, Ito-3328

Keating, Patricia-3393

Keenan, Ruth E.-3288

Kei, Joseph-3263, 3264

Keidser, Gitte-3214, 3437

Keitt, Timothy H.-3018

Keller, Stefanie-3213

Kelly, Spencer D.-3340

Kemewerigha, Tobi E.-3319

Kemp, Renee-3219, 3334

Kendall, Tyler-3113, 3340

Kenet, Tal-3045

Kennedy, Jermaine L.-3347

Kenny, R. Jeremy-3042, 3043

Kent, Raymond D.-3444

Keough, Megan-3225

Keppens, Veerle M.-3202

Keravnou, Christina P.-3247, 3368

Kerrian, Peter-3104

Kesavan, Kalpashri-3266

Kessissoglou, Nicole-3050

Kessler, Lawrence W.-3245

Ketten, Darlene R.-3297, 3298

Ketterling, Jeffrey A.-3028, 3029, Cochair Session 2aBAb (3027)

Kewley-Port, Diane-3164

Khalighinejad, Bahar-3158

Khamchane, Abdelkader-3375

Khan, Asaduzzaman-3264

Khan, Sheraz-3045

Kharlamov, Viktor-3226

Khelfaoui, Youcef-3375

Khokhlova, Tanya D.-3081, 3082, 3308

Khokhlova, Tatiana-2983, 3030, 3083

Khokhlova, Tatiana D.-3083, Cochair Session 1pBAb (2982), Cochair Session 2aBAa (3025), Cochair Session 2aBAc (3030), Cochair Session 2pBAb (3081)
Khokhlova, Vera-2983, 3030, 3082, 3083

Khokhlova, Vera A.-3081, 3082, 3186, 3308, 3310, 3435

Khoklova, Vera-3031

Khosla, Sid-3331

Khosla, Sid M.-3395

Kida, Yukihiro-3057, 3058, 3230

Kidani, Shunsuke-3209, 3274

Kidd, Gerald-3270

Kiefte, Michael-3162, Cochair Session 3aSC (3162)

Kiersteins, Ivars-3123

Kihara, Ayane-3420

Kijima, Takahiro-3021, 3409

Kikuchi, Mumi-3412

Kikuchi, Takashi-3361

Kikuchi, Toshiaki-3407

Kilappa, Vantte-3078

Kilen, Emily S.-3167

Kim, Benjamin J.-3121

Kim, Byoung-Kweon-3242

Kim, Byoung-Nam-3012, 3014

Kim, Chulhong-2978

Kim, Deokman-3001

Kim, Dong Sun-3242

Kim, Dong-Hwan-3312

Kim, Donghyun-2954, 3113

Kim, Hansoo-3242

Kim, Hwan-3405

Kim, Jaepil-3089

Kim, Jea Soo-3170

Kim, Jeasoo-3170

Kim, Jeesun-3339

Kim, Jongbeom-3166

Kim, Jueseok-3405

Kim, Junsoo-3033

Kim, Jun-Woo-3166

Kim, Kang-Chair Session 5aBAb (3367), Cochair Session 1aBA (2950), Cochair Session 1pBAa (2978)

Kim, Katherine H.-3021, 3413

Kim, Keehoon-2994

Kim, Ki-Hong-2989

Kim, Mira-3242

Kim, Myungsook-3426

Kim, Myung-Sook-3426

Kim, Nicholas-2992

Kim, Sang-Hyeon-3405

Kim, SangJin-2962

Kim, Sea-Moon-3012, 3059, 3064

Kim, Seongil-3120

Kim, Subong-3046

Kim, Sungyoung-2987, 3277, 3391

Kim, Sunhyo-3064

Kim, Tae Wan-3242

Kim, Taehwan-3004

Kim, Wanjung-3405

Kim, Yang-Hann-3405

Kim, Yong-Joe-3320, 3331

Kim, Yunjung-3226

Kimura, Nobuo-3065

Kimura, Satoko-Cochair Session $2 \mathrm{aABb}$ (3020), Cochair Session $2 \mathrm{pABb}(3072)$

Kimura, Satoko S.-3305

Kimura, Takuya-3282, 3403

Kimura, Toshiyuki-3375

Kimura, Youtarou-3292

King, Andrew J.-3389
King, Jonathan-3287

King, Richard L. -3275

King, Simon-2961

Kinnick, Randy-3245

Kinoshita, Hiroshi-3429

Kinoshita, Keisuke-3114

Kinzel, Michael P.-3121

Kirby, Ray-3386

Kirk, Thomas W.-3143

Kirsteins, Ivars P.-3102

Kirtley, M. J.-3105

Kish, Emily-3440

Kishikawa, Kenta-3139

Kishline, Lindsey R.-3267

Kita, Chihomi-3066

Kitagawa, Takashi-3305

Kitahara, Mafuyu-3282

Kitahara, Tadashi-3156, 3263

Kitahara, Tetsuro-3090, 3379, 3395, 3399

Kitamura, Minoru-3361

Kitamura, Tadashi-3060, 3427

Kitamura, Tatsuya-3111, 3115, 3223

Kitamura, Toshiya-3322

Kitaoka, Norihide-2962, 2963, Cochair Session 1pSPb (3009)

Kittiwattanawong, Kongkiat-3412

Kiya, Hitoshi-3060

Kiyomoto, Yoko-3304

Klerke, Sigrid-3155

Klinck, Holger-3019, 3181, 3300 , 3301

Kline, Brian L.-3034

Kloepper, Laura-2973, 3300, 3303, Cochair Session 1aAB (2947),

Cochair Session 1pAB (2972)

Kloepper, Laura N.-3131

Kluk, Karolina-3152

Klyn, Niall A.-3273

Kniesburges, Stefan-3393, 3398

Knisely, Katherine E.-3377

Ko, Jeongwoo-3248

Ko, Myungkwon-3064

Kobayashi, Akio-3115, 3116

Kobayashi, Etsuko-3008

Kobayashi, Kazuhiro-2963

Kobayashi, Kazuto-3139, 3185, 3245

Kobayashi, Kei-3365, 3439

Kobayashi, Maho-3033

Kobayashi, Motoki-3066

Kobayashi, Taizo-3037, 3253, 3254

Kobayashi, Tetsunori-3005

Kobayashi, Yosuke-3192, 3215

Kobayasi, Kohta I.-2975

Koblitz, Jens-3130

Koblitz, Jens C.-2974, 3131

Kobrina, Anastasiya-3299

Koch, Robert A.-3170, 3231

Koch, Robert M.-3167, Cochair Session 4pSA (3330)

Kochetov, Alexei-3109

Kocot, Anthony-3307

Kocyigit, Filiz B.-2953, 2970

Koda, Ren-3373, 3419

Koehn, Christian-3317

Koga, Takashi-3293

Koh, Veronica-3089, Cochair Session 2pEAb (3088)

Kohara, Hiroki-3059

Kohata, Shinji-3145 
Kohshima, Shiro-3238

Koide, Toshio-3161

Koike, Harunori-2963

Koike, Shuhei-3116

Koike, Yoshikazu-3062

Koishi, Masataka-3432

Koizumi, Norihiro-3306, 3309,

Cochair Session 4aBAb (3247),

Cochair Session 4pBA (3306)

Kojima, Hiroaki-2963

Kojima, Kazunori-3061

Kojima, Takahiro-2966

Kokx-Ryan, Melissa J.-3152

Kolios, Michael C.-2951, 3370, 3371, 3434

Komatsu, Hajime-3376

Komori, Shinya-3059

Komori, Tomoyasu-3366

Konarski, Stephanie G.-3049

Kondo, Hayato-3241

Kondo, Hirohito M.-2999

Kondo, Kazuhiro-3192, 3215

Kondo, Kengo-2978

Kondo, Mariko-3108, 3283

Kondo, Ryosuke-3309

Kondo, Takashi-2985

Konganda, Shivali A.-3436

Konoike, Naho-3155

Kopco, Norbert-3269

Korman, Murray S.-3167, 3315, 3326

Kosaka, Tetsuo-2964, 3116

Kössl, Manfred-3157

Kosuge, Nobuaki-3212

Kosuge, Nobuyuki-3374

Kosugi, Yasuhiro-3061

Kothari, Ninad B.-2973

Kouneli, Maria-3109

Koyama, Daisuke-3035, 3260, 3369, Cochair Session 3aPA (3147),

Cochair Session 3pPAb (3205)

Koyama, Shoichi-3117

Koyanagi, Shinichiro-3175

Kozuka, Teruyuki-3206

Kramer, Sophia E.-3443

Kreider, Wayne-2982, 2983, 3030, 3081, 3082, 3083, 3186, 3307, 3308,3310

Kreiman, Jody-2961, 3393

Kripfgans, Oliver- -3188

Krishnamurthy, Rohan-3143

Krit, Timofey-3260

Kruger, Sarah E.-2998

Kruizinga, Pieter-2980

Krynkin, Anton-3024

Krysl, Petr-2968

Kuang, James F.-3339

Kuang, Jianjing-3396

Kubilius, Rokas-3243

Kubli, Lina-3152

Kubo, Mikiko-2989

Kubovy, Michael-3391

Kubozono, Haruo-3050

Kuc, Roman B. -3180

Kuc, Victor B.-3180

Kucewicz, John C.-3308

Kudo, Nobuki-2981, 2984

Kudo, Shinya-3452

Kuegler, Anke-3131

Kügler, Anke-3359

Kuhnert, Barbara-3221
Kumar, Krishna N.-3373

Kunduk, Melda-3443

Kuperman, W. A.-3210

Kuperman, William A.-2950, 3074, 3364,3410

Kuratani, Fumiyasu-3161

Kuriki, Shinya-3046

Kurisu, Kiyohiro-3192

Kurita, Hirofumi-3139

Kurita, Keisuke-3371

Kuriwaki, Takahiro-3428

Kuroda, Chitose-3212

Kuroda, Jun-3087, 3363

Kuroda, Mika-3302

Kuroda, Shoma-3449

Kuroiwa, Shingo-2965, 3444

Kurono, Hiroyasu-3383

Kurosawa, Minoru-3374

Kurosawa, Minoru K.-3249

Kurosawa, Yoshiaki-3106, 3400

Kuze, Dai-3140

Kuzuu, Kazuto-2956

Kwak, Yunsang-3003

Kwan, James-2985

Kwiatkowski, Tricia-2998

Kwon, Harim-3217, 3334

Kwon, Hyuckjong-3064

Kwon, Oh-Cho-3124

Kwon, Soohyun-3109

La, Hyoung Sul-3242

Lacerra, Giovanna-3002

Lai, Yuwen-3395

Laimes, Rosa-3137

Lajoinie, Guillaume-2980, 3369

Lalonde, Kaylah-3447

Lam, Bhan-3313

Lama, Ziwo-3106

Lambaré, Hadrien-3422

Lammers, Marc-3018, 3129, 3131, Cochair Session 3aAB (3129),

Cochair Session 3pABa (3179)

Lammers, Marc O.-3130, 3301, 3359,3417

Landi, Maryam-3236

Landi, Nicole-3444

Laney, Jonathan-3040

Langton, Christian M.-3079

Lansford, Kaitlin-3439

Laporte, Catherine-3189

Large, Edward-3273

Laroche, Chantal-3201

Larsen, Thea Mathilde-3041

Larson, Eric-3046

Laturnus, Rebecca-3334

Lau, Elaine-3396

Lau, Siu-Kit-3431

Laugier, Pascal-3078

Laule, Gail-3131

Lavandier, Mathieu-3276

Lavarello, Roberto-3137

Lavelle, J W.-3076

Lavery, Andone C.-3023, 3134, Cochair Session 1pAO (2975), Cochair Session 2aAO (3023), Cochair Session 2pAOb (3077)

Lavie, Antoine-3104

Lavie, Nilli-3208

Lawson, Francesca R.-3429

Le Bas, Pierre-Yves-3166, 3211, 3324

Le Pichon, Alexis-2995, 2996
Lea-Banks, Harriet-2985

Leamy, Michael J.-3048

Leao, J. P.-3261

Leao, Jose P.-3148

Leavitt, Ron-3443

Lebedev, Andrey-3327

Leblanc, Alexandre-3104

Lechner, David-3260

Lechner, David A.-3067

Leclère, Thibaud-3276

Ledoux, William R.-3031

Leduc, Damien-3386

Lee, Adrian K.-3207, 3337

Lee, Adrian K. C.-3046, 3267

Lee, Adrian KC-Cochair Session 2aPP (3045)

Lee, Akinobu-3116

Lee, Albert-3398

Lee, Andrew-3340

Lee, Chia-Yun-3019

Lee, Chong-Moo-3059

Lee, Craig M.-3075

Lee, Dae-Hyuk-3064

Lee, Dohyung-2956

Lee, Franklin-3031

Lee, Gonghwi-3432

Lee, Goun-3217, 3270

Lee, Gregory S.-3226, 3442

Lee, Haifeng-3407

Lee, Hee-Il-2995, 2996

Lee, Heeju-3339

Lee, Hoonteak-3033

Lee, Ho-Young-3108, 3341

Lee, Hunki-2956, 3124

Lee, Hyojin-3070, 3175, 3257, 3418

Lee, Jaehong-3002

Lee, Jaehyung-3251, 3422

Lee, Jae-Wan-3435

Lee, James B.-3379, 3450

Lee, Jasme-3424

Lee, Jeong Y.-2980

Lee, JeWon-3404

Lee, Jong-Hwa-3405

Lee, Joonhee-2946, Cochair Session 5aID (3378)

Lee, Ju-ho-3166

Lee, Keunhwa-2959

Lee, Kevin M.-3123, 3233, 3305,

Cochair Session 4aPA (3259)

Lee, Kichol-3200

Lee, Myunghan-3320

Lee, Nala H.-3051

Lee, Sam H.-3140

Lee, SangHoon-3242

Lee, Sangmin-3393

Lee, Seung Woo-3065

Lee, Seunghun J.-3394

Lee, Shi-Wook-3061

Lee, Soogab-3248

Lee, Sungbok-3344

Lee, Wei-Ning-3084

Lee, Won-Hee-3401

Lee, Wu-Jung-3132, 3243

Lee, Yoonjeong-3344

Leete, Kevin M.-3098

Lefebvre, Gautier-3255

Léger, Agnès-3152

Legland, Jean-Baptiste-3165

Lehman, Ashley-3249

Leibold, Lori-3126

Leibold, Lori J.-3265, 3271
Leighton, Timothy-2968

Leinders, Suzanne M.-2981

Lemaitre, Guillaume-3267, 3390

Lemazina, Alena-3303

Lentacker, Ine-3369

Lentz, Jennifer-3265, 3272, 3274, 3315

Lentz, Tom-3217

Lenz, Richard L.-3292

Leonard, Brett-3275

Lepage, Hugo-3329

Lerman, Amir-3245

Lerman, lilach-3245

Lerud, Karl-3273

Lescanne, Maxime-3373

Lester, Houston-2946

Lester, Nicholas-3227

Letourneur, Stéphane-3165

Leung, Keith K.-3335

Leung, Shue-sum-3401

Leus, Geert-3229

Levy, Oren-3308

Lewandowski, Eva M.-3344

Lewis, Leah A.-3295

Li, Bin-3333

Li, Bing-3048

Li, Chi Him-3112

Li, David-2979

Li, David S.-2980

Li, Di-3124

Li, Fangfang-3447

Li, Guangming-3183

Li, Guan-sheng-3107

Li, Guofu-3450

Li, Hai Feng-3012

Li, Haifeng-3065

Li, Hongdao-3124

Li, Hongzhe- 3302

Li, Jiawei-3014

Li, Jie-3409, 3450

Li, Junfei-2959, 3377

Li, Kun-2967, 3073

Li, Mingshuang-3265

Li, Pai-Chi-2951, 3027

Li, Qi-3170, 3232

Li, Qian-3434

Li, Songhai-3018, 3129

Li, Xiaodong-3321

Li, Xiaofei-3191

Li, Yang-3397

Li, Yi-3311

Li, Yong-3048

Li, Yongwei-2963

Li, Yun-Wen-3075

Liang, Bin-3049

Liang, Zhi-Pei-3219

Liao, Hsin-I-3209

Liao, Tianjun-3014, 3173

Licata, Julia-3443, 3448

Liem, Alyssa T.-3211

Lieuwen, Tim-3261

Liles, W. Conrad-3083

Lillis, Ashlee-3071

Lilly, Jerry G.-3384

Lim, Changwoo-3331

Lim, Hansol-3175

Lim, Raymond-2968

Lim, Soo Bin-3270

Lima, José Antônio M.-3409

Lima, Key F.-3141

Lima, Pedro Henrique R.-2991 
Lin, Derrick-3022

Lin, Fu-2990

Lin, Guang-3001

Lin, I-Fan-3209

Lin, Isabelle-3224

Lin, Ju-3135, 3183

Lin, Kuang-Wei-3084

Lin, Sheng-Fong-3022

Lin, Tzu-Hao-3019

Lin, Tzu-Hao H.-3018

Lin, Ying-tsong-2976, 3289, 3300, 3406

Lin, Yu Ching-3444

Lin, Zhou-3033

Lindeneau, Scott-3181, 3302

Lindh, Per-3327

Lindsey, Brooks-3187

Lindsey, Matthew-3211

Ling, Wenyi-3334

Linnartz, Erik-2980

Linnenschmidt, Meike-2973

Lipkens, Bart-3388, 3433

Lissenden, Cliff J.-3211

Lister, Jennifer J.-3328

Litovsky, Ruth-3100, 3158

Liu, Chang-3265, 3389, 3394, 3441, 3449

Liu, Chengcheng-3190

Liu, Chun Yin-3340

Liu, Hai Jun-3012

Liu, Haijun-3065

Liu, Hanjun-3393

Liu, Hao-3421

Liu, Huei-Mei-3441, 3448

Liu, Jingbo-3191

Liu, Li-3340

Liu, Lu-3409

Liu, Pan-3237

Liu, Saki-2989

Liu, Tzu-Chi-3156

Liu, Wen-Hu-3132

Liu, Yadong-3339

Liu, Yan-3333

Liu, Yi-Wen-3156

Liu, Yufei-3144

Liu, Yunbo-3371

Lloyd, Harriet-2980

Lo, Kam W.-3007, 3119, 3350

Lockhart, Stephen B.-3300

Lodermeyer, Alexander-3398

Logan, Roger M.-3034

Logsdon, Edward-3281

Lokce, Sevgi-2953

Long, Daniel-3051

Long, Yu-3132

Lønmo, Tor I.-3287

Looi, Thomas-3031

Loomba, Rohit-3185, 3186

Lopes, J. H.-3261

Lopes, Jose H.-3148

Lopes, Keawe-3016

Loranger, Scott-2949

Lorenzi, Christian-3389

Lorenzo-Trueba, Jaime-2961

Losch, Barbara-3180

Lott, Francois-2996

Lott, Martin-3166

LoVerde, John-3281, 3384, Cochair

Session 5aNSb (3384)

LoVetri, Joe-3419

Lu, Dian-3231, 3403, 3449
Lu, Liang-3405

Lu, Licheng-3014, 3067

Lu, Tianjian-2993

Luan, Ying-3369

Lubman, David-Cochair Session 1aAA (2946), Cochair Session 1pAAa (2969), Cochair Session 5 aNSb (3384)

Lucila, Andrew-3425

Ludwigsen, Daniel-3211

Lulich, Steven M.-3448

Lund, Fernando-3325

Lunkov, Andrey-3363

Luo, Gangming-3190

Luo, Xin-3389, Chair Session 5aPP (3388)

Luong, Hieu Thi-2962

Luz, George A.-3095

Lynch, James-2976, 3252, Cochair Session 4aID (3251)

Lynch, Stephen D.-3285

Lyons, Anthony-3287

Lyons, Anthony P.-3362

Lyu, Shao-Ren-3051, 3395

Ma, Li-3013, 3014, 3067, 3173, 3351,3407

Ma, Qingyu-3026, 3033, 3085, 3367,3368

Ma, Yun-Long-3132

Macalay, Gavin J.-3305

Macaulay, Gavin-3243

MacConaghy, Brian-2985, 3186, 3308

MacGillivray, Alexander-3021

MacGillivray, Ian-3050

Machi, Junji-3187

Machida, Nobuo-3323

Macías, Silvio-3157

Mack, Amelia-3359

MacLennan, David-3243

Macnamara, Brooke-3403

Macoskey, Jonathan J.-3083

Macrander, A. Michael-3020

Maddox, Ross K.-3207, 3267

Madhusudhana, Shyam-3071, 3182

Madrigal, Brijonnay C.-3415

Maeda, Hidehiko-3445

Maeda, Kazuki-3307

Maeda, Yuka-3277

Maekawa, Kazuyoshi-3065

Maempel, Hans-Joachim-3176

Maestre, Esteban-3092

Magana-Zook, Steven-2997

Magee, Joshua A.-3384

Magliula, Elizabeth A.-3102, Cochair Session 3aSA (3159)

Magno, Carlo-3131

Mahajan, Minakshi-3338

Maintz, Thomas-3128

Maitani, Yosuke-2975

Majdak, Piotr-3272

Makashay, Matthew J.-3441

Maki, Katsuhiro-3223

Maki, Katuhiro-3429

Maki, Kevin-3331

Maki, Kotaro-3223

Makinae, Hisanori-3223

Makino, Koichi-3257

Makris, Nicholas C. -3360

Malcolm, Alison E. -3327

Maldonado, Ana Luisa P.-3321
Malicka, Alicja N.-3264, 3438

Malphurs, David E.-3450

Mamori, Hiroya-3043, 3097

Mamou, Jonathan-3138, 3187 ,

Cochair Session 3aBA (3136),

Cochair Session 3pBAa (3185)

Mancia, Lauren-3032, 3084, 3085

Mandolesi, Eric-2949

Manire, Charles A.-3297

Man-khongdi, Phongphat-3109

Manley, David-Cochair Session $1 \mathrm{pAAb}(2971)$

Mann, David A.-3297

Mannaris, Christophoros-2985, 3025

Mano, Isao-3190

Manor, Ofer-3149

Manser, Marta B.-3240

Mansour, Hossein-3036

Manternach, Brian-3427

Manuel, Alehandrea-3264

Marchand, Sylvain-3091

Marchetti, Emanuele-2995

Marcillo, Omar-2996

Mardis, Cassi-3300, 3303

Margolina, Tetyana-3411

Marozeau, Jeremy-3196

Marquardt, Torsten-3272

Marques, Tiago-3413

Marrelec, Guillaume-3078

Marros, Fernanda-3178

Marschall, Márton-3177

Marsh, Nicole-3443, 3448

Marshalek, Joseph P.-3371

Marston, Philip L.-3123, 3345, 3346

Marston, Timothy-3347, Cochair

Session 4aSPb (3286), Cochair

Session 4pSPa (3345), Cochair Session $4 \mathrm{pSPb}$ (3347)

Marston, Timothy M.-3346, 3347

Martin, Bruce-3021, 3359

Martin, Caleb J.-3033

Martin, Cameron-3131

Martin, Cameron R.-3413

Martin, James S.-3054, 3360

Martin, Stephen W.-3181, 3413

Martin, Theodore P.-3102

Martín Román, Sara R.-3000

Martinelli, Sheri-2949

Martínez-García, Maria Teresa-3336

Martinez-Garcia, Maria Teresa-3341

Martín-Saavedra, Francisco M.-3191

Marui, Atsushi-3143, 3380, 3427

Maruoka, Takahi-3083

Maruoka, Takashi-3367

Maruvada, Subha-3371

Maruyama, Hitoshi-3138, 3187

Maruyama, Hotaka-3059

Maruyama, Shinichi-3096

Maruyama, Taizo-3326

Marvel, Mandy-3368

Marzan, Marques H.-3318

Masahiko, Hirao-3202

Masatonakayama-Cochair Session 5aEA (3374)

Mason, Christine R.-3270

Massey, Jessica-3228

Massi, Francesco-3002

Masters, David-3180

Masuda, Hideyuki-3037

Masuda, Hinako-3342

Masuda, Kiyoshi-2972
Masuda-Katsuse, Ikuyo-3446

Masunaga, Eiji-3241

Mathevon, Nicolas-3397

Mathews, Jonathan-3450

Matsubara, Masaki-3153, 3379

Matsubara, Naoto-3065, 3301

Matsuda, Hidetaka-3037

Matsuda, Hiroshi-3323

Matsuda, Makiko-3338

Matsuda, Osamu-3203

Matsuda, Toru-3280

Matsuda, Youichi-3372

Matsuda, Yusuke-2965

Matsui, Kazuhiro-3008

Matsui, Toshie-3114, 3151, 3274

Matsuishi, Ryota-3192

Matsuishi, Takashi-3302

Matsukawa, Mami-3032, 3035, 3079, 3189, 3190, 3204, 3260, 3387, Cochair Session 2pBAa (3078), Cochair Session 3pBAb (3188), Cochair Session 3pBAc (3190)

Matsukawa, Sayaka-3189

Matsumato, Haruyoshi-3300

Matsumoto, Haru-3181

Matsumoto, Junko-3428

Matsumoto, Ryota-3258

Matsumoto, Toshio-3257

Matsumoto, Yasunao-3279

Matsumoto, Yasushi-3192

Matsumoto, Yoichiro-2982, 2983, 3306, 3308, 3309

Matsunaga, Terry O.-3371

Matsuno, Tatsuo-3062

Matsuo, Akiho-3128, 3176

Matsuo, Ayako-3047, 3267

Matsuo, Ikuo-2947, 3065, 3287, 3301, 3360, 3361

Matsuura, Tomohiko-3243, 3304

Matsuyama, Brian-3413

Matsuyama, Brian M.-3181

Matthews, Iain-3004

Matthews, Leanna P.-3301

Matthews, Marie-Noel-3072

Matula, Thomas J.-2980

Matula, Tom-2985

Matuura, Tomohiko-3304

Maureen-3059

Maurer, Dieter-3058, 3217, 3219

Maxfield, Lynn M.-3427

Maxfield, Nathan-3228

Maxfield, Nathan D.-3227

Maxit, Laurent-3159

Maxwell, Adam-2983, 3030, 3032, 3082, 3083, 3084, 3307

Maxwell, Adam D.-2982, 3031, 3081, 3082, 3084, 3186, 3306, 3307, 3308, 3310, Cochair Session 4aBAb (3247), Cochair Session 4pBA (3306)

Mayo, Nathanael K.-3086

McAllister Byun, Tara-3442

McAlpine, David-3047, 3152

McAuliffe, Michael-3446

McBeth, Michael S.-3421, Cochair Session 5pEA (3421)

McCammon, Diana F.-3285

McCauley, Robert-3412

McClain, Robert E.-2987

McClenny, Anna-2985 
McCloy, Daniel-3337

McCullough, Elizabeth A.-3332,

Chair Session 3pSC (3213)

McDaniel, J. G.-3211, 3351

McDaniel, James G.-3102

McDonnell, Bradley-3396

McElligott, Megan M.-3301

McFayden, Tyler C.-3444

McGee, JoAnn-3414

McGough, Robert-3187

McGuire, Grant L.-3109, Chair Session 2pSC (3105)

McGuire, Tamara L.-3182

McHale, Anthony-3026

McInerny, Sally A.-3098

McIntyre, Dan-3228

McKenna, Elizabeth A.-3145, 3200

McKinley, Richard L.-3098, 3199, 3200 , Cochair Session 3pNSb (3199)

McLaughlin, Susan A.-3045, 3046

McMahon, Catherine-3046

McNeese, Andrew R.-3122, 3233

McPherson, Craig-3021

Means, Steven L. -3183

Meecham, Andy-3349

Mefferd, Antje-3344, 3442

Meguro, Kohji-3230

Meguro, Koji-3057, 3058

Mehlen, Patrick-3369

Mehra, Ravish-2956, 3008

Mehraei, Golbarg-3153

Mehta, Sonya-3333

Mellinger, David K.-3019, 3181

Melodelima, David-3307, 3419

Memoli, Gianluca-3049, 3150

Meng, Long-3149

Mennitt, Daniel J.-3140, 3424,

Cochair Session 3aEA (3139)

Mental, Rebecca-3403

Mental, Rebecca L.-3226, 3442

Mera, Kazuya-3106, 3400

Merchant, Nathan D.-3024, 3072, 3410

Merkens, Karlina-3415

Mermagen, Timothy-3101

Merwin, Ben-3249

Mesgarani, Nima-3158, 3213

Metzner, Walter-2973

Meunier, Christine-3111

Meunier, Sabine-3268

Meyer, Jens-3032

Meyer, Valentin-3159

Mezil, Sylvain-3165, 3203

Mialle, Pierrick-2995

Miansari, Morteza-3205

Michalopoulou, Zoi-Heleni-3007

Michel, Hans-3294

Midorikawa, Yoichi-2963

Migeot, Jean-Louis-3314

Mignerey, Peter-3173

Mihaly, Steve- -3410

Mihara, Tsuyoshi-3166

Mikami, Masato-3258

Mikami, Takashi-3177

Mikhin, Dmitry-2950

Miki, Akira-3253

Miki, Nobuhiro-3302

Miklós, András-3036

Miksis-Olds, Jennifer-3359
Miksis-Olds, Jennifer L.-3019, Chair Mo, Fuyuan-3011 Session 5aAB (3359)

Milekhina, Olga-3263

Milkowski, Andy-3188

Miller, Chris-3012

Miller, Chris W.-3013

Miller, Christopher W.-3013

Miller, Douglas -3246

Miller, Gregory A.-3069

Miller, James G.-3079, Cochair Session 2pBAa (3078), Cochair Session 3pBAb (3188), Cochair Session 3pBAc (3190)

Miller, James H.-3065, 3300, 3423

Miller, Margaret K.-3271

Miller, Nicholas P.-3095

Miller, Rita J.-3186, 3373

Millet, Christophe-2996

Milner, Ben-3004

Min, Dongki-3002

Minami, Kenji-3066

Minami, Shota-3087

Minami, Yasuhiro-3060

Minamoto, Yoshinori-3305

Minematsu, Nobuaki-2964, 3061, 3116, 3284

Minemura, Atsuo-3293

Minonzio, Jean-Gabriel-3078

Misawa, Masaki-3372

Mischi, Massimo-3420

Misdariis, Nicolas-3268, 3390

Mishima, Sakiko-3376

Mishima, Takeshi-3115, 3116

Mistry, Nikhil-2968

Misurelli, Sara-3100

Mitamura, Hiromichi-3305

Mitani, Yoko-3021, 3303, 3361

Mito, Yuki-2988

Mitri, Farid G.-3147, 3433

Mitsuishi, Mamoru-3306, 3309

Mitsuyasu, Kento-2966

Miura, Hikaru-3423

Miura, Iori-3450

Miura, Masanobu-3317, 3380, 3381, 3428

Miura, Takahiro-3366

Miyagawa, Yusuke-3193

Miyagi, Aki-3362

Miyajima, Chiyomi-2961

Miyajima, Tohru-3161

Miyakura, Takashi-3141

Miyamoto, Ryusuke-3375

Miyamoto, Yoshinori-3362

Miyashita, Kazushi-3065, 3066, 3303, 3305, 3361

Miyauchi, Ryota-3271, 3274

Miyauchi, Yuki-3060

Miyazaki, Hideo-2972, 3291

Miyazaki, Hideyo-3306, 3309

Miyoshi, Masato-3115, 3404

Miyoshi, Tsutomu-3425

Mizoguchi, Ai-3109

Mizuguchi, Daisuke-3238, 3303

Mizumachi, Mitsunori-3167

Mizuno, Katsunori-3079

Mizuno, Tomoyuki-3060

Mizuno, Yosuke-3387, 3388, 3434

Mizuta, Taiji-3161

Mizutani, Koichi-3037, 3277, 3302, 3375, 3379, 3407

Mizutani, Yui-3383
Mo, Steven-2985

Mobley, Frank S.-3260

Mochida, Takemi-3228

Mochizuki, Shihono-3060

Mohaghegh, Mercedeh-3217

Mohamed, Mirrah Maziyah-3332

Mohanty, Kaustav-3186

Moir, James-3415

Mojabi, Pedram-3419

Mokhtari, Parham-2957

Moldover, Michael R.-3259, 3386

Moline, Mark A.-3130

Molloy, Katharine-3208

Molnar, Monika-3448

Monaghan, Jessica J.-3046

Monsky, Wayne-2985

Montazeri, Vahid-3339, 3441, 3446

Monteiro, Fernando P.-3008

Monteiro, Joao F.-3413

Montero, Juan M.-2961

Montgomery, Allen-3215

Monto, Nicholas R.-3227

Montoya, Juan C.-3041

Mookerjee, Adaleena-3304

Moon, Wonkyu-3033, 3089

Mooney, T. A.-3018

Mooney, T. Aran-3299, 3417, Chair Session 5pABb (3414)

Mooney, T A.-3071, 3359

Moore, Ashley N.-3218

Moore, Brian C. -3438

Moore, David R-3155

Moore, Jeffrey-3339

Moore, Joshua T.-3081

Moore, Partick-3179

Moore, Patrick-3239

Moore, Patrick W.-3131, 3179

Moore, Travis M.-3101

Moors-Murphy, Hilary B.-3416

Mora, Emanuel C.-3157

Moretti, David-3411, 3413

Moretti, David J.-3181

Morgan, Catherine-3436

Morgan, Michael-3425

Mori, Kazuma-3204

Mori, Koichi-3440

Mori, Koki-3198

Mori, Kyoichi-3021

Mori, Mikio-3254

Mori, Shohei-3247

Mori, Shuji-3262, 3263, 3264, 3328, Cochair Session 4pPP (3328)

Mori, Shunki-3189

Mori, Takeshi-3149, 3205

Moriarty, Peter M.-3237

Morihara, Takashi-3093, 3094, 3366

Morikawa, Ayumu-3428

Morikawa, Daisuke-2963, 3268

Morimoto, Akihiko-3444

Morimoto, Atsushi-3445

Morimoto, Maho-3108, 3109

Morimoto, Mai-3419

Morimoto, Masayuki-2998, 3068, 3193

Morinaga, Makoto-3093, 3094

Morisaka, Tadamichi-3239, Cochair Session 4aAB (3238), Cochair Session 4pABa (3295)

Morise, Masanori-3058, 3399, 3400, 3428
Morishita, Shuji-3319

Morishita, Takeshi-3421

Moriya, Shusuke-3114

Moriya, Takafumi-3062

Morlet, Thierry-3440

Moron, Juliana R.-3239

Morozov, Andrey K.-3135

Morozumi, Neil-3140

Morris, David J.-3155

Morris, Miranda R.-3344

Morrison, Andrew C.-2986, 3197, Chair Session 1eMU (3016), Chair Session 5pMU (3425), Cochair Session 3pEDb (3196)

Morrison, Dawn A.-3095

Morrissey, Ronald-3413

Morrissey, Ronald P.-3181

Moss, Cynthia F.-2973, 2974

Moss, Geoffrey-3169

Motallebzadeh, Hamid-3154

Motl, Robert W.-3373

Moulin, Emmanuel-3002, 3387

Mounsif, Ech-Cherif ElKettani-3386

Movahed, Pooya-2982

Mubeen, Muhammad A.-3224

Mueen, Abdullah A.-3378

Muglia, Michael-3300

Muhlestein, Michael B.-3103

Muir, Thomas G.-3122, 3233, Cochair Session 2pUW (3121)

Mujica, Nicolás-3325

Mukai, Tohru-3242, 3243, 3305

Mukaiyama, Yuriko-3386

Muleki-Seya, Pauline-3186

Muller, Marie-3186, 3187

Muller, Marie M.-3080

Müller, Rolf-2947, 2965, 2974

Müller-Giebeler, Mark-3174

Mulsow, Jason-3131, 3179, 3298

Munehara, Hiroyuki-3065

Munk, Walter-2975

Munk, Walter H.-2976

Munro, Kevin J.-3152, 3438

Munro, Murray J.-3162

Murakami, Takahiro-3062

Muranishi, Sachiyo-3444

Murao, Tatsuya-3313

Murata, Naoki-3117

Murata, Taichi K.-3145

Murayama, Takahiko-2994, Cochair

Session 1pPA (2994)

Murayama, Takahiku-2995

Murdock, Jaimie-3213

Murphy, Christin T.-3033

Murphy, Enda-3258

Murphy, Paul-3171, 3350

Murphy, Stefan M.-3285

Murphy, William J.-3145, 3146, 3199

Murray, Anita-3240, 3416

Murton, Olivia-3395

Muto, Kenji-3095, 3140

Muto, Seijun-3171

Myers, Brett-3344

Myers, Emily-3283

Myers, Emily B.-3341, 3342

Myers, Joseph-3294

Myers, Joshua-3264

Myers, Rachel-2985

Nabara, Shiori-3381 
Nachtigall, Paul E.-3131, 3132, 3179

Naderyan, Vahid-3236

Nagahata, Koji-3040

Nagakubo, Akira-3203

Nagamine, Tasha-3213

Nagano, Yuki-3062

Naganuma, Daisuke-3255

Naganuma, Yukihiro-2980

Nagao, Kyoko-3440

Nagaoka, Ryo-2979, 2981, 3027

Nagata, Yoshifumi-3167

Nagatani, Yoshiki-3079, 3189

Nagy, Nóra M.-3036

Naify, Christina J.-3054, 3102,

Cochair Session 2aSA (3047),

Cochair Session 2pSA (3102)

Naito, Yoshimasa-3002

Nakada, Tsutomu-3155

Nakadai, Kazuhiro-3348

Nakagawa, Keiichi-3372

Nakagawa, Seiji-3046, 3390

Nakagawa, Takuma-2963

Nakagawa, Yumi-3198

Nakagiri, Taishi-3034

Nakahara, Masataka-3177

Nakai, Takayoshi-3278

Nakajima, Hideharu-3337

Nakajima, Madoka-3226

Nakajima, Takahito-3373, 3419

Nakajima, Toshitaka-3373

Nakajima, Yoshiaki-3008

Nakajima, Yoshitaka-3207

Nakamura, Aya-3198

Nakamura, Katsuki-3155

Nakamura, Kentaro-3260, 3365,

3387, 3388, 3434, Chair Session (3353)

Nakamura, Nobutomo-3204

Nakamura, Satoshi-2963, 2964

Nakamura, Shizuka-3392

Nakanishi, Shinsuke-3237, Cochair Session 4aAA (3236)

Nakanishi, Shoko-3190

Nakaniwa, Masayoshi-3325

Nakano, Hiroaki-3097

Nakano, Iwao-3184

Nakano, Tomoyasu-3090

Nakaoka, Masahiro-3066

Nakasako, Noboru-3007, 3008

Nakashika, Toru-3060

Nakashima, Hidetoshi-3275

Nakatani, Tomohiro-3114

Nakayama, Masato-3007, 3008,

$3057,3059,3060,3062,3139$,

$3311,3376,3403,3451$

Nalls, Leisa-3258

Nam, SungHyun-3012, 3065

Nam, Youngja-3217

Nan, Yun-3265, 3442

Nanjo, Hiroaki-3057

Nankali, Amir-3154

Nannuru, Santosh-3117, 3118, 3133

Narins, Peter M.-2945

Narita, Tomohiro-3450

Naruse, Hiroshi-3034, 3374, 3428

Nasrollahi, Amir-3049

Natarajan, Karthikeyan-3042

Nations, Christopher S.-3413

Nave-Blodgett, Jessica-3265

Naylor, Graham-2998
Neal, Matthew T.-3175

Nearey, Terrance M.-3164

Nechaev, Dmitry-3263, 3303

Neild, Adrian-3147, 3206

Neilsen, Tracianne B.-3043, 3044, 3097, 3098, Cochair Session 1aED (2952), Cochair Session 1pED (2986), Cochair Session 3pEDa (3195)

Nejime, Masafumi-3155

Nelson, Mitchell S.-3363

Nelson, Peggy B.-3070, 3382

Nelson Smid, Jennifer-3294

Nemith, Julianne-3440

Nemoto, Saori-3343

Nesbitt, Heather-3026

Netchaev, Anton-3424

Newhall, Arthur-2976, 3300

Newhall, Arthur E.-3288

Newman, Meagan-3401

Ngemaes, Maria-2995

Nguyen, Ben-2984

Nguyen, Lan T.-3093

Nguyen, Linh T.-3093

Nguyen, Thi Ngoc Tho-3055

Nguyen, Thulan-3093

Nguyen, Vu H.-3189

Nguyen, Vu-Hieu-3191

Ní Chiosáin, Máire-3109

Nicholls, Aaron-2956

Nichols, Stephen M.-3351, 3352

Nielsen, Kuniko-3218, 3344

Nieukirk, Sharon L.-3019

Niezrecki, Christopher-3212

Nightingale, Christopher-3442

Nigmatulina, Yulia-3333

Nihal, Asawer-3101

Nihei, Misato-2963

Niitsuma, Masahiro-3062, 3400, 3445

Nikolaeva, Anastasia V.-3306

Nillesen, Maartje M.-3029

Nishiguchi, Isoharu-3255, Cochair Session 4aMUb (3255), Cochair Session 4pMUa (3317)

Nishiguchi, Masayuki-3062, 3268, 3428

Nishijima, Keisuke-3449, 3450, 3452

Nishimura, Akira-2988, 3196, 3366 , Cochair Session 3pEDa (3195)

Nishimura, Ryouichi-2957, 3194

Nishimura, Tadashi-3156, 3263, 3274

Nishimura, Tsuyoshi-3093

Nishimura, Yoshihiro-3171, 3362

Nishimura, Yukiko-3127

Nishimura, Yuri-3274

Nishino, Takanori-3034, 3063, 3374, Oehler, Michael-3275 $3405,3422,3428$

Nishiura, Takanobu-3007, 3057, 3059, 3060, 3062, 3139, 3311, $3376,3403,3451$

Nishiwaki, Shinji-3430

Nishiyama, Tomohide-3369

Nishiyama, Yu-3306, 3309

Nishizaki, Hiromitsu-3061

Niskanen, Matti-3430

Nissen, Shawn L.-3429

Nitta, Naotaka-3372

Niu, Haiqiang-3232
Niu, Lili-3149

Niu, Yaying-3331

Niwa, Hayato-3087

Niwa, Kenta-3007, 3060, 3422,

Cochair Session 1pSPa (3006)

Nizami, Lance-3266

Noad, Michael-3416

Noad, Michael J.-3240, 3359, 3412, 3416

Nobetsu, Takahiro-3361

Noble, John-3260

Nogelmeier, Puakea-3016

Noguchi, Ayako-3060

Noguchi, Saki-2946, 2969

Noguchi, Yuki-3430

Noh, Eunghwy-3089, 3124

Nomura, Hideyuki-3249, 3255, 3418

Nonomura, Taku-3097, 3421, 3432

Norman, Robert G.-3413

Norris, Andrew N.-3048

Norris, Thomas F.-Cochair Session 2aABa (3017), Cochair Session 2pABa (3071)

Nosal, Eva M.-3131

Nosal, Eva-Marie-3301, 3360

Nose, Takashi-2962, 2963

Nota, Yukiko-3111, 3115

Novak, Colin-3249

Novak, Colin J.-Cochair Session 4aEA (3248)

Nowak, Karolina M.-3372

Nowak, Lukasz J.-3372

Nozaki, Kazunori-3221

Nozaki, Kotoyo-3275

Nozawa, Takeshi-3334

Numano, Tomokazu-3372

Numata, Kazushi-3306, 3309

Nuttall, Albert H.-3167

Nygaard, Lynne C.-3336, 3344

Nykaza, Edward T.-3088, 3095, 3193, 3194, 3424

Oakey, Ryan M.-3330

Oard, Douglas W.-3009

Oates, Tim-3424

Obata, Satoshi-3143, 3429

O'beirne, Greg A.-3225

Obert, Kerrie-3400

O'Brien, Gabrielle-3100, 3157

O'Brien, Jr., W. D.-3136, 3185, 3244, Cochair Session 4aBAa (3244)

O’Brien, Jr., William D.-3186, 3246

Obuchi, Yasunari-3432

Ochi, Hiroshi-3057, 3058, 3230

Ochi, Keiko-3338, 3440

Odom, Robert I.-3408

O’Donnell, Matthew-2979, 2980, 3083

Oehler, Michael-3275

Oelze, Michael L.-3136, 3373, 3420

Ofuji, Kenta-3399

Ogasawara, Hanako-3229

Ogasawara, Naomi-3399

Ogasawara, Takayuki-3146

Ogata, Kohichi-2960, 2965

Ogawa, Chihiro-3251

Ogawa, Masahiro-3066

Ogi, Hirotsugu-3203, 3204, Cochair Session 3pPAa (3202)

Ogino, Shuya-3431
Ogiso, Satoki-3277

Ogita, Masashi-3386

Ogura, Kei-3379

Ogura, Yuki-3185

Ogutmen, Betul-3095

Oguz, Didem-3095

Oh, Grace E.-3335

Oh, Seongho-3422

Oh, Sujin-3110

Oh, Yonghee-3273

Ohara, Yoshikazu-3166, Cochair Session 3aSP (3165), Cochair Session 4pPA (3324)

Ohm, Won-Suk-2954, 2956, 3044. 3089, 3098, 3124, 3435

Ohnaka, Shinichi-2963

Ohno, Masahiro-3081

Ohno, Ryohei-3399

Ohno, Sumio-3400

Ohsawa, Chie-3318

Ohshima, Shinya-3303

Ohsugi, Yasuhito-2964

Ohta, Kengo-3192

Ohtani, Kento-2967, 3060

Ohtani, Toshihiro-3324, 3325

Oikawa, Yasuhiro-3087, 3095, 3140,

$3195,3275,3365,3417,3451$,

Cochair Session 2pEAa (3086),

Cochair Session 5pBA (3417)

Oishi, Chikara-3280

Okada, Masahiro-3380

Okada, Nagaya-3035

Okada, Shuhei-3101

Okada, Sota-3428

Okada, Takashi-3369

Okada, Yasuaki-3383, Cochair Session 5aNSa (3382)

Okamoto, Hidehiko-3329

Okamoto, Koji-3043, 3044

Okamoto, Noriko-3174, 3292

Okamoto, Ryosuke-3021

Okawa, Shigeki-3178

Okayasu, Sayaka-3451

Okayasu, Tadao-3156

Okazaki, Satoshi-3263

Okita, Kohei-2983

Okubo, Kan-3059, 3249, 3250, $3251,3261,3274,3431$

Okumura, Hiraku-3277

Okumura, Shigeaki-3189

Okuno, Tasuku-3204

Okunuki, Takeo-3043

Okutsu, Masataka-3002

Okuzono, Takeshi-2992, 2993, 3292, 3293

Oleson, Erin-3295, 3413, 3415

Oleson, Erin M.-3017, 3296

Olive, Yeelen-3019

Olmstead, Annie J.-3342

Olofsson, Karl-3148

Olson, Bruce C.-2969, 3069

Olson, Derek R.-3233

Omer, Robyn K.-3367, 3374

Omoto, Akira-2987, 3177, 3312

Omura, Manabu-3061

Ona, Egil-3243

Önfelt, Björn-3148

Ongel, Aybike-3095

Onishi, Kazuyoshi-2966

Onitsuka, Hirofumi-3253

Ono, Kazuho-3278 
Ono, Nobutaka-2960

Ono, Tsuyoshi-3107

Onoguchi, Taira-3192

Onsuwan, Chutamanee-3109

Onuma, Takashi-3087

Onwubiko, Stephen G.-3197, 3319

Oode, Satoshi-3278

Opdam, Rob-3174

Oren, Liran-3331, 3395

Orena, Adriel J.-3227

Orena, Adriel John-3227

Origasa, Takumu-3373

Origuchi, Maya-3167

Orris, Gregory J.-3102

Osafune, Toshikazu-2958

Osaka, Mariko-3445

Osaki, Makoto-3189

Osamura, Kozo-3161

Osawa, Eri-3333

Ose, Jacqueline-3227

Oshima, Takuya-3250

Oshimo, Shiori-3037

Oshiro, Yuuya-2962

Osman, Homira-3448

Osorio, Jon-3016

Ostashev, Vladimir E.-3119, 3194

Oster, Monika-Maria-3264

Ostrovskii, Igor-3324

Ostrovsky, Ilia-3363

Ostrovsky, Lev A.-3327, Cochair Session 4pPA (3324)

Osumi, Ayumu-3275, 3386, 3387, 3388

Oswald, Julie N.-3131, 3415

Ota, Kenko-2964, 3426

Otani, Makoto-2999, 3312

Otsuka, Asuka-3046

Otsuka, Sho-3151, 3158

Otsuki, Masaya-2994

Otsuki, Mayuko-3361

Otsuru, Toru-3174, 3292

Owada, Yasunori-3194

Owen, Joshua-2980, 3026

Owens, Gabe-3031

Owens, Rock-3131

Oxenham, Andrew J.-3150, 3329

Oyama, Akira-3043, 3097, 3432

Ozaki, Etsuko-3190

Ozawa, Kenji-3058, 3400

Ozawa, Yuta-3043, 3421

Ozeki, Seiya-3371

Ozmeral, Erol J.-3155

Özyöruk, Yusuf-3250

Pacini, Aude F.-3131

Pack, Adam A.-3416

Padgett, Jaye-3109

Padilla, Frederic-3191, 3369, 3370

Pagliarella, Tom-3249

Pailhas, Yan-3286

Pakula, Michal-3001, 3080

Palakurthy, Kayla-3113

Palmeri, Mark L.-3137

Pamidighantam, Pranav K.-3382

Pan, Ho-Hsien-3051

Pan, Jie-3160

Panahi, Issa-3313

Pandey, Aditya-3031

Pannala, Mittu-2947

Panosso, Andriele d.-3179

Pantea, Cristian-3204

Papadacci, Clement-3029
Papadopoulou, Virginie-3187

Paradiso, Joseph A.-3100

Pardo, Bryan-3090

Parga, Joanna J.-3266

Parizet, Etienne-3438

Park, Choon-Su-3166

Park, Hansang-3110

Park, Hanyong-3332, 3333

Park, Heungkil-2954

Park, Hyung Woo-3393, 3445

Park, J. D.-3346

Park, Jisung-3170

Park, Jong J.-3140

Park, Joung-Soo-3064

Park, Junghyun-2996

Park, Jungsoo-3170

Park, Jungyong-3055, 3064

Park, Junhong-3001, 3002, 3003, 3404

Park, Keyhong-3242

Park, Minseuk-3055

Park, Sang-Bum-3426

Park, Sangmok-3003

Park, Seoryong-3248

Park, Soon-Hong-3042

Park, Taeyoung-2954, 2956

Park, Yeonsue-3375

Park, Yongsung-3055

Park, Youngcheol-3089

Parker, Mark A.-3151

Parks, Susan-3301

Parrell, Benjamin-3344

Parrish, Julia K.-3241

Partanen, Ari-3082

Parveen, Sabiha-3442

Patching, Richard-3140

Patel, Chirag-3155

Patel, Nisha-3214

Patel, Sona-3442

Patil, Kiran-3211

Patterson, Brandon-3368

Patterson, Roy D.-3151, 3274

Paul, Stephan-3034, 3178, 3179

Paula, Thiago P.-3409

Paverd, Catherine-2985

Payan, Cedric-3166

Pedersen, Geir-3305, 3364

Peelle, Jonathan E.-3214

Pekkarinen, Annukka-3019

Pelivanov, Ivan-2979

Pelz, Markus-3151

Peña, Hector-3305

Peng, Da Yong-3012

Peng, Dayong-3065

Peralta, Laura-3078

Pereira, Daniel-3188

Pereira, Olivia-3217, 3440

Perelman, Brandon-3101

Pereno, Valerio-3025

Perez-Carrillo, Alfonso-3091

Perkins, Jeremy-3334, 3343, 3394

Pernot, Mathieu-3029, 3084

Perol, David-3307

Perrachione, Tyler K.-3270, 3342

Perreaut, Kevin-3276

Perrin, Fabien-3276

Perttu, Anna-2995

Petillot, Yvan-3286

Pettit, Chris L.-3088

Pfaeffle, Edward-3002

Pfeiffer, Loren-3203
Pfeifle, Florian-3143, 3256, 3317 , 3318

Pfingst, Bryan E.-3377

Phatak, Sandeep-3152

Phillips, James E.-Cochair Session 2pNS (3093), Cochair Session 4aNS (3256), Cochair Session 4aSA (3279), Cochair Session $4 \mathrm{pNSa}(3320)$

Phoon, Colin K. L.-3029

Piacsek, Andrew A.-2952

Piao, Shengchun-3173

Pichora-Fuller, Margaret K.-3401, 3443

Pick, Andrew N.-3110

Pieczonka, Lucasz-3211

Pierce, Allan D.-3251, 3362, 3407

Pilger, Christoph-2996, 3383

Pillarisetty, Venu-3083

Pinkel, Rob-3284

Pinto, Joseph-3137

Pisanski, Katarzyna-3397

Pisoni, David B.-3214

Pitre, John J.-3310

Pitt, Jonathan S.-3325

Pitt, Mark-3114

Plack, Christopher-3158

Plack, Christopher J.-3152, 3390

Plath, Niko-3317

Plotnick, Dan-2968

Plotnick, Daniel-3346, 3347

Plotnick, Daniel S.-3345

Podkowa, Anthony-3373

Podlubny, Ryan G.-2954

Poeppel, David-3046

Polagye, Brian L.-3171, 3350

Polka, Linda-3217, 3227

Pollack, Elliot A.-3226

Pollara, Alexander S.-3008, 3451

Polovina, Jeffrey-3361

Polydorides, Nick-3183

Poole, M. Michael-3416

Popa, Bogdan-2959

Popa, Bogdan-Ioan-3377

Popescu, Anisia-3222

Popov, Vladimir-3303

Popper, Arthur N.-3289

Port, Robert-3164

Porter, Michael B. -3288

Porter, Tyrone M.-3434

Portugal, Rute- 3171

Pospisil, Dean A.-3207

Potter, Jack-3325

Pöttler, Marina-3393

Potty, Gopu R.-3065, 3300, 3423

Poulsen, Andrew-3408

Pouplier, Marianne-3217

Powell, Brian-3135

Powell, Claire F.-3024, 3072

Powell, Jessica R.-3297

Powell, Julie-3268

Powell, Larkin-3414

Pozzo, Lilo D.-2980

Prada, Claire-3002

Prater, James-3287

Prato, Andrea-2969

Predoï, Mihai-3386

Preisig, James-3290

Premus, Vince-3181, 3350

Prendergast, Garreth-3152

Prepelită, Sebastian-3127
Pressnitzer, Daniel-2999

Price, John C.-3253

Prieur, Fabrice-3418

Pritz, Tamás-2958

Proctor, Michael-3222

Proctor, Michael I.-3223

Provost, Jean-3029

$\mathrm{Pu}$, Yunchen-3405

Puglisi, Giuseppina E.-2969

Pumberger, Matthias-3078

Puria, Sunil-3154, 3263

Puts, Regina-3191

Putzier, Michael-3078

Pycha, Anne-3215

Qiao, Shan-3368

Qiao, Wenxiao-3250

Qin, Lang-3111

Qin, Xiuyuan-3000

Qin, Yi-Xian-3080, 3191

Qin, Zhen-3225, 3336

Qiu, Peiwen-2974

Qiu, Xiaojun-2993, 3311

Quakenbush, Lori-3299

Quatieri, Thomas F.-3151

Rabau, Guy-3268

Rademeyer, Paul-3370

Raeymaekers, Bart-3206

Raghunathan, Shreyas-3033

Rahayu, Rahma H.-3139

Rahnama, Mani-3201

Railey, Kristen E.-2967

Rakotonarivo, Sandrine T.-3210

Ramakrishnan, Rishi-3351

Ramig, Lorraine A.-3442

Ramm, Hans -3071

Ramp, Steven R.-3012, 3013

Ramsay, Gordon-3006, 3446

Rana, Baljeet-3436, 3437

Randrianarison, Rose M.-3017

Raphael, Lawrence-3440

Raphael, Yehoash-3377

Rasmussen, Marianne H.-3019, 3130

Raspet, Richard-3260

Rassmussen, Marianne H.-3131

Rathsam, Jonathan-3095, 3424

Ratilal, Purnima-3360

Raum, Kay-3078, 3191

Rawlings, Samantha B.-3384

Razansky, Daniel-2978

Razaz, Mahdi-3184

Reby, David-3397, 3414

Redmon, Charles-3223, 3335

Redmond, Ryan C.-3402

Reed, Allen-3233

Reed, Heather M.-3160

Reed, Paul E. -3215

Reeder, D. B.-3363

Reeder, D. Benjamin-3012, 3013, 3289, Chair Session 4pUWb (3351), Chair Session 5aUWb (3409)

Reeder, Davis B.-3013

Reeg, Jonathan-3420

Reichman, Brent O.-3044, 3098

Reichmuth, Colleen-3022, 3298

Reid, Christopher-3422

Reinhardt, Brian-3374

Reinhart, Paul-3438

Reinhold, Isabella-3239

Reinke, Danny C. -3182 
Reinwald, Michael-2959, 3451

Remijn, Gerard B.-3156

Remillieux, Marcel-3166, 3211, Cochair Session 3aSP (3165)

Remillieux, Marcel C.-3324

Ren, Qunyan-3011, 3014, 3067, 3173, 3230

Ren, Yun-3363

Renaud, Guillaume-3080

Rendell, Luke-3416

Renou, Ygaäl-3159

Rentz, Bradley-3111

Reuter, Christoph-3275

Reuter, Eric L.-3094

Rhee, Seok-Chae-3270

Rhode, Charles-3054

Ribstein, Bruno-2996

Rice, Ally-3296

Richards, Edward-3118, 3407

Richards, Virginia M.-3272

Richter, Jan-Gerrit-3375

Rideout, Brendan P.-3360

Riggs, Caitlin-3041

Ripepe, Maurizio-2995

Riquimaroux, Hiroshi-2972, 3017, 3297, Cochair Session 1aAB (2947), Cochair Session 1pAB (2972)

Rivera, Arnaldo L.-3099

Riviere, Jacques-3167, 3326

Rivoire, Michel-3307

Rizzo, Piervincenzo-3049

Roberts, Jesse- -3171

Roberts, William W.-3248

Robieux, Camille F.-3111

Roch, Marie-3181, 3302

Roch, Marie A.-3017, 3240, Cochair Session 4aAB (3238), Cochair Session 4pABa (3295)

Rodgers, Arthur-2994

Roewer-Despres, Francois-3115, 3221

Rogers, Catherine L.-Cochair Session 2pAA (3068), Cochair Session 3aAAa (3126)

Rogers, Chad S.-3214

Rogers, Jeffrey S.-3054, Cochair Session 2aSPa (3053), Cochair Session 2pSPa (3117)

Rogers, Kevin-3119

Rogers, Peter H.-3121, 3360

Rogers, Tracey L.-3238

Roh, Yongrae-3034, 3375

Rohde, Charles A.-3102

Rohrbach, Daniel-3187

Rojas, Tulio-3106

Romanova, Anna-3431

Romero-Vivas, Eduardo-3181, 3182

Romigh, Griffin-3401

Romigh, Griffin D.-2957, 3200,

Cochair Session 1aPP (2956),

Cochair Session 1pPP (2997)

Ronanki, Srikanth-2961

Roon, Kevin-3109

Roque, Franklin-3425

Rosado-Mendez, Ivan M.-3137

Rose, Zach-3380

Rosen, Beth-3101

Rosen, Stuart-3214, 3219

Rosenberg, Andrew-3010

Rosenthal, Jordan-3408
Rosnitskiy, Pavel-3082

Rosnitskiy, Pavel B.-3082, 3310, 3435

Rossier-Bisaillon, Antonin-3224

Rothwell, Clayton-3401

Roup, Christina M.-3268

Roux, Jonathan L.-3404

Roux, Philippe-3103, 3373

Rouyer, Julien-3137

Roverud, Elin-3270

Rowley, Mary C.-3226

Roy, Kenneth P.-3068

Royer, Adam J.-3397

Rozon-Gauthier, Véronique-3201

Ruan, Yewu-3231

Ruane, Amy S.-3002

Rubin, Philip-3004, Cochair Session $1 \mathrm{pSC}$ (3004)

Rucz, Péter-3036

Ruditskiy, Daniil-3170

Ruegger, Carl-3174

Rund, Laura-3214

Ruzzene, Massimo-3210

Ryan, Colin-3451

Ryan, Teresa-3001, 3088, 3260

Ryan, Teresa J.-3260

Ryden, Nils-3327

Ryu, Jongkwan-3385

Ryue, Jungsoo-3003

S, Ravi Shankar-3231

Saaristo-Helin, Katri-3447

Sabato, Alessandro-3212

Sabra, Karim-3074

Sacchetti, Benedetto-2969

Sacco, Tiziana-2969

Sachau, Delf-3258

Sack, Stefan-2992

Sadanobu, Toshiyuki-3400

Sadayasu, Kazuhiro-3242

Sadohara, Ken-2963

Saegusa-Beecorft, Emi-3187

Saeki, Tetsuro-3266

Sagara, Shodai-3258

Sagayama, Shigeki-2960

Sagen, Hanne-3134

Sagers, Jason D.-3233

Sagisaka, Yoshinori-3337

Sahoo, Deepak-3049

Saigo, Sakura-3174

Saijo, Yoshifumi-2979, 2980, 2981, 3027, 3244, 3245, Cochair Session 1aBA (2950), Cochair Session 1pBAa (2978), Cochair Session 4aBAa (3244)

Saito, Akie-3445

Saito, Daisuke-2964, 3061, 3116

Saito, Haruka-3339

Saito, Osamu-3156

Saito, Shun-3143

Saito, Takashi-3108

Saito, Takehiko-3302

Saito, Yoshihiko-2994

Saitoh, Hirotaka-3418

Saitou, Ryouzi-3095

Sajo, Yoshifumi-3404

Sakagami, Kimihiro-2987, 2992, 2993, 3293, Cochair Session 4pAAb (3293)

Sakai, Naomi-3440

Sakakibara, Ken-Ichi-3223

Sakamoto, Nobuaki-3409
Sakamoto, Shin-ichi-2955

Sakamoto, Shinichi-3070, 3127, $3175,3257,3418$, Cochair Session 4aNS (3256), Cochair Session 4pNSa (3320)

Sakamoto, Shuichi-2966, 2998, 3058, 3192, 3193, 3269, 3405

Sakamoto, Yoko-3335, 3402

Sakanashi, Hideki-3317

Sakashita, Hajime-3369

Sako, Shinji-3060, 3427

Sakuma, Ichiro-3008

Sakuma, Takao-3198

Sakuma, Tetsuya-3127, 3323, Cochair Session 3aAAb (3127), Cochair Session 3pAAa (3174),

Cochair Session 3pAAb (3177)

Sakurada, Akiko-3409

Salā, Aaran-3016

Salameh, Michael-3376

Salas, Andria K.-3018

Salas, Dalila-3443, 3448

Salazar, Claudia-3137

Salinas, Vicente-3325

Salisbury, Daniel P.-3181

Salloum, Hady-3008, 3119, 3180, 3350,3451

Salvador, Cesar D.-2966

Samal, Ashok-3215

Samardzic, Nikolina-3249

Samejima, Toshiya-2966, 3376, 3380

Samlan, Robin-Chair Session $4 \mathrm{pEDb}$ (3316)

Samlan, Robin A.-3443

Sanders, William-3233

Sandsten, Maria-3239

Sangwan, Abhijeet-3010

Sanjinez, Dolly-3368

Sanjinez, Dolly A.-3187, 3367

Sankaran, Narayan-3008

Sano, Ko-hei-3149, 3205

Sano, Naoko-3047, 3267

Santos, Ana Bela-2949

Santos, Emily V.-3326

Santurette, Sébastien-3041

São Thiago, Pedro M.-3409

Sapozhnikov, Oleg-2983, 3082, 3083

Sapozhnikov, Oleg A.-2983, 3081, 3082, 3186, 3306, 3308, 3310, 3435

Sarae, Wataru-3043, 3096

Saran, Shambhu-3105

Saris, Anne E.-3029

Sarkar, Jit-2950, 3074, 3364, 3410

Sarkar, Kausik-3025, 3373

Saruwatari, Hiroshi-3117

Sasaki, Akira-3369

Sasaki, Keiichi-2980

Sasaki, Motoki-3302

Sasaki, Naoko-3146

Sasakura, Toyoki-3287, 3362

Sasisekaran, Jayanthi-3262

Sasmal, Aritra-3154, 3206

Sasou, Akira-2964

Sato, Eiji-3061

Sato, Hayato-2998, 3068, 3193, Cochair Session 1pAAb (2971)

Sato, Hiroshi-2998, Cochair Session 5aID (3378), Cochair Session 5pID (3423)
Sato, Hirotaka-3387

Sato, Koya-3061

Sato, Mei-3243

Sato, Shoei-3115, 3116

Sato, Tetsumi-3093

Sato, Toru-3189

Satoh, Fumiaki-2987, 3196, Cochair Session 3pEDb (3196)

Satou, Megumi-3379

Saulnier, Gary-3229

Sauro, Ronald-3236, 3292

Sauter, Disa-3399

Savel, Sophie-3268

Savioja, Lauri-3000, 3127

Sawada, Atsushi-3043

Sawada, Kota-3324

Sawada, Kouichi-3243, Cochair Session 4aAO (3241), Cochair Session 4pAO (3304)

Sawada, Naoki-3061

Sawai, Ken-ichi-3318

Sawai, Masayuki-3066

Sayar, Nurten-3095

Sayles, Mark-3154

Scanlon, Michael V.-3006

Scarborough, Rebecca-3218

Scarton, Henry A.-3229, Cochair Session 3pSP (3229)

Scavone, Gary-3036

Scavone, Gary P.-3092

Schade, George-3030

Schade, George R.-3031, 3081, 3082, 3083, 3308

Schaeffer, Marshall-3210

Schaette, Roland-3152

Schafer, Amy-3334

Schafer, Amy J.-3051

Schafer, Erin C.-3448

Schanz, Martin-3281

Schellenberg, Murray-3220, 3221, 3225

Schiller, Noah-3431

Schilling, Corinna-2973

Schindall, Jeffrey A.-3183

Schlauch, Robert S. -3262

Schlesinger, Angela-3072

Schmidt, Henrik-3170, 3408

Schmidt, Oliver T.-3097

Schneider, Johannes-3078

Schneider, Toby-3408

Schnurr, Julie-2996, 2997, 3056

Schomer, Paul-3237

Schomer, Paul D.-3382, Cochair Session 5aNSa (3382)

Schoof, Tim-3214

Schorr, Greg-3411

Schreiber, Nolan-3442

Schreiber, Nolan T.-3226

Schrof, Susanne-3078

Schuchman, Gerald I.-3099, 3157

Schulte-Fortkamp, Brigitte-3040,

3041, 3433, Cochair Session

2aNS (3039), Cochair Session $5 \mathrm{pNSb}(3432)$

Schumacher, Christian R.-3086

Schurova, Natalia E. -3376

Schützenberger, Anne-3393

Schwartzwald, Karlie-3380

Schwenke, Roger W.-2972

Scoulding, Ben-3243, 3244

Screven, Laurel-3299 
Screven, Laurel A.-3299

Sears, Robert-3228, 3262

Sebayashi, Tsutae-3322

Seda, Robinson-3026

Sedunov, Alexander-3006, 3119, 3180

Sedunov, Nikolay-3180

Seger, Kerri-3072, 3359, Chair

Session $4 \mathrm{pABb}$ (3297)

Seguchi, Shinjiro-3196

Seki, Shogo-2967

Seki, Yoshinori-3369

Sekine, Hidehisa-3385

Selamet, Ahmet-3321

Selamet, Emel-3321

Sen Gupta, Ananya-3055

Seo, Dae-Chul-3166

Seo, Donghwan-3033

Seo, Sang-Hyun-3042

Seo, Takehiko-3258

Seo, Youngsoo-3089

Seong, Woojae-2959, 3055, 3064, 3234

Sereno, Joan-3225, 3335

Sereno, Joan A.-3224, 3225

Seropian, Lou-3267

Sesen, Muhsincan-3147

Setiawan, Ivan-2961

Seto, Eichi-2962

Seymour, Len W.-3025

Shabanimotlagh, Maysam-3033

Shabtai, Noam R.-3175

Shadle, Christine H.-3219

Shafer, Benjamin-Chair Session 1aSA (2958), Chair Session $1 \mathrm{pSA}(3000)$

Shaffer, Jessica-3413

Shahsavarani, Bahar S.-3215, 3444

Shan, Wei-3114

Shang, Dajing-3170

Shang, Da-jing-3232

Shannon, Lee H.-3171

Shao, Huamei-3066

Sharan, Shambhu-3338

Sharma, Gyani Shankar-3050

Sharma, Mridula-3436

Shary, Merrily R.-3227

Shattuck-Hufnagel, Stefanie-3395, 3447

Shaw, Jason-3339

Shcherbina, Kostyantyn-3419

Sheeran, Paul S.-3371

Sheffield, Sterling W.-2997

Shelton, Sarah-3187

Shen, Chen-3048, 3103

Shen, Tueng-2979

Sheng, Jason-3026

Sheng, Xueli-3231, 3403, 3449

Shengguo, Shi-3172

Shera, Christopher A.-3153

Sherlock, LaGuinn-3200

Sherry, Laura-3390

Shi, Chengzhi-3104

Shi, Chuang-3313

Shi, Dongyuan-3313

Shi, Jie-3124

Shi, Shengguo-3124

Shi, Yali-3449

Shibagaki, Yoshiaki-3204

Shibamata, Takefumi-3249

Shigeno, Sumi-3399, 3429
Shigeru, Tada-3372

Shiiba, Michihisa-3035

Shiina, Tsuyoshi-2978, 3138

Shimabukuro, Taishi-3057

Shimamura, Ryo-3393

Shimizu, Etsuro-3021, 3073

Shimizu, Genya-3058

Shimizu, Motochika-3369

Shimizu, Shota-3370

Shimizu, Yoshino-3061

Shimizu, Yuki-3260

Shimokura, Ryota-3263, 3274

Shimura, Masayuki-2958, 3323

Shimura, Takuya-3057, 3058, 3230

Shin, Dong-Jin-3217

Shin, Hyoung Chul-3242

Shin, Kumjae-3033

Shin, Minchul-3422

Shin, Seulgi-3336

Shindo, Mitsuko-3365

Shinichi, Takeuchi-3371

Shinjo, Daisuke-3140

Shinkai, Itaru-2955

Shinn-Cunningham, Barbara-2989, 3209, 3214, Cochair Session 3pPP (3207)

Shinn-Cunningham, Barbara G. -3153

Shinoda, Koichi-3009

Shinohara, Naoaki-3256

Shinohara, Toshihiro-3008

Shinohara, Yasuaki-3343

Shinozaki, Takahiro-2963, 3062

Shiota, Sayaka-3060

Shirai, Kyoko-3436

Shiraishi, Kimio-3156, 3365

Shirakawa, Hokuto-3065, 3066, 3305

Shiraki, Yoshifumi-3053, Cochair Session 2aSPa (3053), Cochair Session 2pSPa (3117)

Shiramatsu, Tomoyo I.-3156

Shirasawa, Takahiro-3426

Shirose, Ayako-3111, 3394, 3400

Shirouzu, Goki-3376

Shmigol, Evgenia-3201

Sho, Iwagami-3254

Shokouhi, Parisa-3167, 3326

Shollenbarger, Amy-3440

Shore, Aimee-3389

Shosted, Ryan-3219

Shrivastav, Mini N.-3270

Shrivastav, Rahul-3398, 3443

Shtrepi, Louena-2969

Shubin, Igor L.-3376

Sia, Samuel-3419

Siderius, Martin-2977

Sidorovskaia, Natalia-2967, 3073

Sidtis, Diana-3224

Sidtis, Diana V.-3445

Sidtis, John J.-3224, 3445

Siebein, Gary-3292

Sieck, Caleb F. -3103

Siegmann, William L.-3362, 3406, 3407

Signh, Aparna-3186

Silbert, Noah-3272

Silbert, Noah H.-3163, 3274, 3332, 3424

Sills, Jillian M.-3022, 3298

Silva, Claudio-3378
Silva, Fabio O.-3008

Silva, Glauber T.-3148, 3261

Silva, Jacob L. -3230

Silver, Jonathan C.-3384

Silverman, Ronald H.-2980, 3028

Sim, Myung-Shin-3266

Simmons, Andrea M.-2948, 3297

Simmons, Emily-3443, 3448

Simmons, James A.-2948, 3033, 3297

Simon, Julianna-3030

Simon, Julianna C.-2983

Simonis, Anne-3413

Simonis, Anne E.-3017

Simpson, Brian-3437

Simpson, Brian D.-2957, 3269

Sindelar, Eric-3143

Singh, Amritpal-3105

Singh, Rajendra-3001

Sinha, Dipen N.-3204

Sirakawa, Hokuto-3066

Siregar, Syahril-2979, 2981

Siren, Kathleen-3443

Sirlin, Claude B.-3185, 3186

Sirović, Ana-3295, 3296

Sirovic, Ana-3296

Sisneros, Joseph-3360

Sklar, Rachele-3440

Skowronski, Mark D.-3070, 3398, 3443

Skvortsov, Alex-3050

Sleeper, Morgan-3111

Smallcomb, Molly R.-3175

Smalt, Christopher-3151

Smalt, Christopher J.-3273

Smaragdakis, Costas-3135

Smaragdis, Paris-3038

Smiley, Aref-3418

Smirnov, Ivan-3203

Smith, Adam-3132, 3414

Smith, Adam B.-3131

Smith, Cory-3119

Smith, Jerome A. -3285

Smith, John-3195

Smith, Julius O.-3000

Smith, Kevin B.-3169

Smith, Michael J.-3098

Smith, Philip H.-3154

Smith, Silas-3228, 3262

Smotherman, Michael-2948

Smyth, Tamara-3092

Snyder, Joel-3265, 3390

Snyder, Joel S.-3208

Solodov, Igor-3325

Solomon, Nancy P.-3441

Soloway, Alexander G.-3171, 3408

Sommerfeldt, Scott D.-Cochair Session 3pSA (3210)

Son, Su-Uk-3064, 3065

Sonderegger, Morgan-3114

Song, Guoli-3351

Song, Hee-Chun-3012, 3014, 3172

Song, Heechun-3234, 3407

Song, Jieun-3335, 3436

Song, Min-Ho-3035

Song, Shaozhen-2979

Song, Wenhua-3013

SONG, Yidan-3110

Song, Zhongchang-3297, 3298

Sonobe, Takuro-3249
Sonoda, Junko-3153

Sonu, Mee-3337

Sørensen, Anna Josefine-3041

Sorensen, Kristina M.-3176

Sorensen, Mathew D.-3308

Sottek, Roland-2953

Southall, Brandon-3130

Southall, Brandon L.-3022, 3290, 3298, 3411

Souza, Pamela-3214, 3438

Sparrow, Victor-3314, Cochair Session 4pEDa (3314)

Speechley, Gary C.-3169

Spencer, Nathan J.-3157

Spencer, Nathaniel-3269

Spiesberger, John-2950

Spinolo, P. L.-3081

Spratt, Kyle S.-3122, Chair Session 5pPA (3433)

Stafford, Kathleen M.-3073

Stakhovskaya, Olga-3157, 3439,

Chair Session $3 \mathrm{aPPb}$ (3154)

Stakhovskaya, Olga A.-3099

Stamper, M. Andrew-3297

Stanton, Timothy K.-2976, Cochair Session 4aAO (3241), Cochair Session 4pAO (3304)

Starkhammar, Josefin-3179, 3239

Starr, Frank-3030

Stavness, Ian-3115, 3221

Stecker, G. C.-3045, 3101

Stecker, G. Christopher-Cochair Session 2aPP (3045)

Stecker, Julie M.-3101

Stefanson, J. R. W.-3274

Stein, Peter J.-Cochair Session 4aUW (3288), Cochair Session 4pUWa (3348)

Steiner, Candice E.-3319

Steiner, Lisa-3131

Stephen, Ralph A.-3406

Stergar, Michael E.-3145

Sterling, John A.-3001

Stern, Richard M.-3272, 3273

Stewart, Andrew-3350

Stewart, John-3298

Stillwagon, Kelly-3437

Stilz, Peter-2974, 3130, 3131

Stimpert, Alison-3411, 3412

Stimpert, Alison K.-3411, 3415, 3416

Stinson, Michael R.-Chair Session (3353)

St. Ledger, Judy-3298

Stolt, Suvi-3447

Story, Brad-3216

Story, Brad H.-3218, 3316

Stotts, Steven A.-3170, 3231, Chair Session 3aUWc (3172), Chair Session 3pUWa (3231)

Strickland, Elizabeth A.-3273

Stride, Eleanor-2980, 2985

Stride, Eleanor P.-3025, 3026, 3370

Strong, John-3069

Strong, John T.-Cochair Session 4 pAAb (3293)

Su, Lin-3014

Su, Xiaoshi-3048

Suarez, Leo-3131

Subramaniam, Sriram-3049 
Suda, Hiroshi-3314, Cochair Session 4 EDDa (3314)

Suetoshi, Ryoichi-3189

Suga, Ikumi-3116

Sugahara, Akiko-3418

Sugano, Hidenori-3226

Sugimoro, Takehiro-3366

Sugimoto, Kazuko-3212

Sugimoto, Tsuneyoshi-3212

Sugimoto, Tsuneyosi-3212

Sugita, Naohiko-3306

Sugita, Yoichi-3267

Sugiura, Kyosuke-3249

Sugiyama, Hideto-3374

Sugiyama, Mariko-3372

Sugiyama, Yuko-3429

Suied, Clara-3267

Sukovich, Jonathan-3030, 3031, 3084

Sukovich, Jonathan R.-3031, 3083

Sulistyowati, Reni-3076

Sullivan, Edmund J.-3120

Sullivan, Jessica R.-3448

Sullivan, Michael-3382

Sullivan, Sarah C.-3145

Sumida, Yoshiki-3258

Summers, Jason E.-3055, 3423

Summers, Van-3441

Sun, Hongbin-3211

Sun, Jintao-3403

Sun, Jundong-3170

Sun, Sheying-3140

Sun, Xiaoyi-3449

Sunaguchi, Naoki-3419

Sundara, Megha-3446, 3448

Sung, Hyung Jin-3148

Sung, Jae-Hyun-3111

Sunohara, Masahiro-3275

Supin, Alexander-3179, 3263, 3303

Suprock, Andrew-3374

Sushrut, Khiwadkar-3277

Susini, Patrick-3267, 3268, 3390

Suslov, Alexey-3203

Sutedjo, Bayu-3076

Suter, Heidy-3217

Sutin, Alexander-3008, 3119, 3165,

$3180,3350,3451$

Sutlive, Joseph-2974

Sutton, Brad-3219

Suwangbutra, Jitwipar-3269

Suzuki, Atsuro-3367

Suzuki, Ayame-2988

Suzuki, Chifumi-3428

Suzuki, Hiroji-3444

Suzuki, Kai-2983

Suzuki, Kenta-3278

Suzuki, Kyori-3343

Suzuki, Norifumi-3387

Suzuki, Sayo-3197

Suzuki, Tomomi-3405

Suzuki, Yôiti-2966, 2998, 3193,

3269, 3405, Cochair Session

1aPP (2956), Cochair Session $1 \mathrm{pPP}(2997)$

Suzuki, Yukihisa-3261

Svensson, U. Peter-3000

Swartz, Michelle T.-3442

Swayne, Billy J.-3098

Syamsudin, Fadli-3076, 3135

Sysueva, Evgeniya-3303

Szuts, Tobi-2972
Ta, Dean-3190

Tabachnick, Alexandra R.-3156

Tabain, Marija-3112

Tabaru, Kei-3153

Tabaru, Marie-3374

Tabuchi, Masaaki-3324

Tachikawa, Tomoya-3451

Tachioka, Yuuki-3450

Tadakoshi, Haruna-3388

Taft, Benjamin N.-Chair Session $4 \mathrm{pABc}$ (3299)

Tagawa, Norio-3059, 3249, 3274

Taira, Kazuya-3215

Taira, Shinichi-3194

Taisne, Benoit-2995

Tajima, Keiichi-3282, Cochair Session 4aSC (3282)

Taka, Koji-3195

Takaaki, Satou-3371

Takada, Masayuki-2986

Takaeda, Kana-2963

Takagi, Ryo-2979, 2983, 2984, 3029, 3246

Takagi, Shu-3369

Takahashi, Hirokazu-3156, 3208

Takahashi, Hiroki-3062

Takahashi, Kin'ya-3037, 3253

Takahashi, Kin'ya-3254

Takahashi, Kojiro-3140

Takahashi, Ryuzo-3065, 3301, 3360

Takahashi, Takumi-3400

Takahashi, Toru-3372

Takahashi, Yoshinori-2958, 3033, Cochair Session 2aEA (3032)

Takahashi, Yukio-3322, Cochair Session $4 \mathrm{pNSb}$ (3322)

Takaki, Shinji-2962, 2964

Takaku, Shingo-3427

Takamaki, Shotaro-3432

Takami, Toshiya-3253, 3254

Takamura, Akihide- 3263

Takanashi, Kyoichi-3139, 3245

Takane, Shoichi-3428

Takane, Shouichi-3062, 3250, 3268, Cochair Session 4aEA (3248)

Takano, Koki-3189

Takano, Sayoko-3399

Takano, Takehiro-3434

Takara, Tomio-2962, 2965

Takashima, Kazunori-3139

Takasugi, Chikao-3062

Takayama, Yusuke-2955

Takayanagi, Shinji-3032, 3189, 3204, 3205, 3387

Takeda, Kazuya-2961, 2967, 3060, 3063, 3404, 3405

Takemae, Nobuhiro-3302

Takemoto, Hironori-2957, 3223

Takemoto, Kanako-3037

Takeoka, Shigeto-2967, 3418

Takeuchi, Daiki-3140

Takeuchi, Hideki-3369

Takeuchi, Marika-3241

Takeuchi, Shinichi-3035, 3371, 3421

Takeyama, Keisuke-3062

Takezawa, Koko-3198

Takezawa, Toshiyuki-3106, 3400

Taki, Hirofumi-3189

Takimoto, Hayato-3141

Takishita, Fumiyuki-2989
Talaske, Richard-3176

Talaske, Richard H.-3040

Talmadge, Lois-3179

Tamara, Satoshi-3429

Tamati, Terrin N.-3270

Tamesue, Takahiro-3266

Tamura, Hideki-3434

Tamura, Shunsuke-3264

Tan, Kwek Tze-3048

Tan, Wei-Chean-3185

Tanabe, Yosuke-3002

Tanadi, Melisa Rosiana-3291

Tanahashi, Tetsu-3395

Tanaka, Akihiro-3399

Tanaka, Hiroki-3388, 3434

Tanaka, Kazuyo-3061

Tanaka, Kou-2964

Tanaka, Mamoru-3241

Tanaka, Manabu-Cochair Session $5 \mathrm{aNSb}(3384)$

Tanaka, Nobuo-3160

Tanaka, Satomi-3153

Tanaka, Tomohiko-3369

Tanaka, Tomohiro-3062

Tanaka, Tomoyasu-3110

Tanaka, Yu-3112

Tanase, Rento-3317

Tanen, Robert M.-3384

Tang, D. J.-3289

Tang, Dajun-3243, 3363

Tang, Dinglan-3155, 3272, 3389

Tang, Jen W.-3437

Tang, Rui-3232

Tang, Shiu-Keung-3236

Tang, Tingting-3073

Tang, Wei-3265, 3442

Tani, Kentaro-3369

Taniguchi, Naokazu-3074, 3075, 3135

Taniguchi, Satoki-3260

Tanter, Mickael-3029, 3084

Tantibundhit, Charturong-3109

Tao, Jiancheng-2993, 3311

Tarakanov, Mikhail-3303

Taroudaki, Viktoria-3135

Taroudakis, Michael-3135

Tarr, Eric W.-3315

Taruguchi, Akihiro-2960

Tasko, Stephen M.-3146

Tatami, Toshiya-3037

Tateishi, Miho-3451

Tateishi, Shuhei-3254

Tatekura, Yosuke-3056, 3057, 3060, 3061, 3427

Tatman, Rachael-3345

Tautz, Matthias-3398

Tavossi, Hasson M.-3281, Cochair Session 4aSA (3279)

Taylor, Patterson-3300

Taylor, Robert-3088, Cochair Session 2pEAb (3088)

Taylor, Sarah-3004

Tazawa, Makio-3300

Ten Cate, James A.-3324

TenCate, James A.-3327

Tennakoon, Sumudu-3204

Tenorio-Hallé, Ludovic-2950

Terada, Takahide-3367

Teramoto, Susumu-3043, 3044

Teramoto, Wataru-2998

Teraoka, Takehiro-3393
Terasawa, Hiroko-3153, 3379

Terashima, Keita-3043, 3096

Terayama, Chihiro-3062

Terhaar, Edward-3094

Terrill, Eric-3285

Terzi, Marina E.-3306

Thakkar, Tanvi-3100

Thayre, Bruce-3413

Theodore, Rachel M.-3227

Theriault, James A.-3416

Thiel, Jeffrey-2983

Thieury, Margaux -3133

Thijssen, Johan-3310

Thode, Aaron-2976, 3020, 3133

Thode, Aaron M.-2950, 3021, 3072, 3413

Thomas, Amber-3180

Thomas, Ashwin-3291

Thomas, Jeanette A.-3415

Thomas, Len-3413

Thompson, Andrew-3405

Thompson, Arthur L.-3397

Thompson, Eric-3437

Thompson, Eric R.-3199, 3200, 3269

Thompson, Paul M.-3072

Thompson, Stephen C.-3089

Thomson, Ron I.-3162

Thomson, Scott L.-3330

Thorne, James M.-3112

Thorsos, Eric I.-3234, 3243, 3289, 3408

Thrailkill, Katie-3268

Thurgood, Ela-3112

Thurman, H O.-3122

Tianji, Zhao-3088

Tiblin, Cecil M.-3122

Tiemann, Christopher-3073

Tikhomirov, Leonid A.-3376

Timmerman, Nancy S.-Cochair Session 5aNSa (3382)

Tippmann, Jeff-3074

Tippmann, Jeffery D.-3210, 3410

Tippmann, Jeffrey-2950

Tittmann, Bernhard R.-3374, Cochair Session 5aEA (3374)

To, Wai M.-3041

Tobin, Stephen-3401

Toda, Tomoki-2963, 2964, 2967, 3404

Todd, Robert E. -3300

Toh, Gyungmin-3002

Toi, Masakazu-2978

Toi, Takeshi-Cochair Session 1aNS (2953), Cochair Session 1pNSa (2990)

Tokuda, Isao T.-3393

Tokunaga, Yasunobu-3095

Tollin, Daniel J.-3269

Tomiku, Reiji-3174, 3292, Cochair Session 4pAAa (3291)

tominaga, yoshihito-3409

Tomita, Kyohei-3306, 3309

Tomita, Ryuta-3280

Tomiyasu, Kentaro-2983

Tomiyasu, Makoto-3065, 3066

Tomoda, Montonobu-3203

Tori, Takaaki-3404

Torii, Kenji-3266

Torrie, Darren K.-3044

Torti, Valeria-3017 
Toscano, Joe-3217

Toscano, Joseph-3216, 3344

Toscano, Joseph C.-3156, 3215

Toshima, Iwaki-2999

Touhei, Terumi-3326

Tournat, Vincent-3165

Toutios, Asterios-3448

Toyama, Minoru-3445

Toyama, Shohei-3116

Toyoda, Masahiro-2991, 2993, 3141, 3161, Cochair Session $1 \mathrm{pNSb}(2991)$

Toyoda, Shingo-3369

Toyomasu, Miki-3197

Tracy, Erik C.-3402

Trahiotis, Constantine-3099, 3100

Trainiti, Giuseppe-3210

Treeby, Bradley-3418

Tremblay, Annie-3335, 3336, 3341

Tremblay, Nicolas-3034

Tremblay-Darveau, Charles-3028

Trent, Samantha-2948

Trent-Brown, Sonja-3443, 3448

Tressler, James F.-3086

Trevino, Jorge-2966

Trevino Lopez, Jorge A.-3405

Treweek, Benjamin C.-3310

Trickey, Jennifer S.-3017, 3300

Trinh, Yun-3181, 3302

Tsai, Karen-3227

Tsao, Feng-Ming-3441, 3448

Tsao, Yu-3018

Tsubota, Yushi-3367

Tsuchida, Yoshio-3366

Tsuchiya, Takao-2999, 3249, 3250, 3251, 3312

Tsuchiya, Toshio-3021, 3073

Tsuda, Yuichi-3066, 3305

Tsuge, Satoru-2965

Tsugita, Naoya-3027

Tsujii, Koki-3303, 3361

Tsujii, Kouki-3021

Tsujimoto, Yukako-3419

Tsujimura, Sohei-3126

Tsukamoto, Akira-3372

Tsukernikov, Ilya E.-3376

Tsukernikov, Ilya O.-3376

Tsukihara, Hiroyuki-3306, 3309

Tsumoto, Koji-3380

Tsunokake, Sayaka-3269

Tsunokawa, Masatoshi-3238

Tsurugaya, Yoshiaki-3407

Tsutsumi, Genki-3254

Tsutsumi, Seiji-3043, 3044, 3096, Cochair Session 2aPA (3042), Cochair Session 2pPA (3096)

Tsuzaki, Minoru-3153, 3318

Tsysar, Sergey A.-3306

Tu, Juan-3026, 3033, 3085, 3367, 3368

Tubelli, Andrew-3298

Tucker, Benjamin V.-3107, 3163, 3226

Tufte, Steve-3380

Tully, Tracy-3444

Tuna, Cagdas-3055

Tuomainen, Jyrki-3437

Tuomainen, Outi-3444

Tupper, Andrew-2995

Turcios, Jacqueline-3444

Turgut, Altan-3183
Turo, Diego-3002, 3088, 3260

Turpin, Alex-3181

Tuziuti, Toru-3122

Tyack, Peter L. -3411

Tyrgonis, Vasilis-3415

Tzeng, Christina Y.-3336

Uchida, Hideo-3383

Uchida, Keiichi-3362

Uchida, Takeyoshi-3372

Uchida, Yosuke-3374

Udovydchenkov, Ilya A.-3406

Uebo, Tetsuji-3008

Ueda, Mari-3365, 3366, Cochair Session 5aBAa (3365)

Uehara, Chouyuu-3371

Uehara, Masashi-3178

Uemura, Joji-3106

Ueno, Kanako-2946, 2969

Uenohara, Shingo-3449, 3450, 3452

Ueyama, Motoko-3338

Uezu, Yasufumi-3392

U1 Haq, Israr-2979, 2981

Ule, Helen-3249

Ulrich, T. J.-3211, 3324

Ulrich, Timothy J.-3166

Umata, Ichiro-3381

Umayahara, Hiromitsu-3280

Umeda, Naoya-3021, 3409

Umeda, Saki-3426

Umemura, ShinIchiro-2979

Umemura, Shin-ichiro-2983, 2984, 3029, 3246, Cochair Session 1pBAb (2982), Cochair Session 2aBAa (3025)

Umemura, Shiori-3190

Umetsu, Takeru-3066

Undurraga, Jaime-3047, 3152

Unhid, Keiichi-3305

Unoki, Masashi-3271, 3274

Urakami, Taichi-3388

Urban, Matthew-3186

Urban, Matthew W.-3137, 3245

Urban, Thibault-3364

Urban R., Jorge-3072

Urs, Raksha-3028

Usagawa, Tsuyoshi-3000

Utagawa, Noriyuki-3212

Utsugi, Chiaki-3389

Utsuno, Hideo-3036

Uus, Kai-3158

Vahabzadeh-Hagh, Andrew M.-3395

Vaillancourt, Tanya-3201

Vaillancourt, Véronique-3201

Vaisbuch, Yona-3263

Valente, Dan-3379

Vallet, Quentin-3078

Valmont, Elizabeth-3291

van de Par, Steven-3011, 3099, 3178

van den Bijgaart, Renske-3082

van der Graaf, Marinette-3310

Van Engen, Kristin-3214

Van Engen, Kristin J.-3214

Van Lieshout, Pascal-3401

Van Parijs, Sofie-3300

van Sloun, Ruud-3420

van Soest, Gijs-2980

Van Uffelen, Lora J.-3075, 3407

Vance, Timothy-Cochair Session 2aSC (3050)

VanDam, Mark-3444
Vanden Bosch der Nederlanden, Christina-3208, 3390

Vannier, Michaël-3268

VanTasell, Dianne-3070

Varamo, Vincent J.-3184

Varga, Leah M.-3296

Varghese, Leonard-3153

Varjoy, Kaveh-3396

Vasilkov, Viacheslav-3151

Vatikiotis-Bateson, Eric-2960, 2965, 3005, 3446

Vaughn, Aaron B.-3044

Vaughn, Charlotte-3336

Vayron, Romain-3191

Vaziri, Ghazaleh-3201

Vega, Luis-3171

Venegas, Gabriel R.-3234, 3349, 3364

Venkatakrishnan, Lakshmi-3042

Vergara, Erasmo F.-3178

Vergoz, Julien-2996

Verhey, Jesko L.-3264, 3265

Verhulst, Sarah-3151

Verlinden, Chris-3074, 3133, 2950

Verlinden, Christopher M.-3364, 3410

Versluis, Michel-2980, 3369

Verweij, Martin D.-2981, 3033

Viano, Ann M.-3081

Vick, Jennell-3403, 3442

Vick, Jennell C.-3226

Vico, Laurence-3191

Vigeant, Michelle C.-3175, 3176, 3197, 3237

Vignola, Joseph-3001, 3002, 3088

Vignola, Joseph F.-3020, 3067 , 3260

Villa, Mauricio-3160

Villa Medina, Francisco-3181

Villa Médina, Francisco-3182

Villegas, Julian-3222

Villegas, Julián-3394

Vincenot, Jeremy-3307

Vipperman, Jeffrey S.-3002

Visser, Fleur-3411

Viswanathan, Navin-3342, 3344

Vlachos, Stephanie-3131

Vlahopoulos, Nickolas-3331

Vlaisavljevich, Eli-3032, 3084, 3085

Voisin, Frédéric-3390

Völk, Florian-3213

Von Borstel, Fernando D.-3181, 3182

Vorlaender, Michael-Cochair Session 3pAAa (3174), Cochair Session 3pAAb (3177)

Vorländer, Michael-3128, 3174, 3175, Cochair Session 3aAAb (3127)

Wada, Shigeo-3221

Wada, Yuji-3434

Wage, Kathleen E.-3352, 3407

Wagner, Anita E.-3449

Wagner, Garrett-3187, 3367

Wagner, Monica-3335

Wåhlin, Anna-3242

Wakabayashi, Yukoh-3062

Wakatsuki, Naoto-3037, 3277, 3302, 3375, 3379, Cochair Session 4aMUa (3253)

Waldeck, Randy-3237, 3294
Walker, Rachel-3222

Wall, Alan T.-3097, 3098, Cochair Session 2pPA (3096)

Wall, Carrie C.-3305

Wallen, Samuel-3049

Wallig, Matthew A.-3186

Walsh, Edward J.-3414

Walstead, Sean-3234

Walter, Melissa M.-3002

Wan, Jacky-3437

Wang, Avery-3378

Wang, Chau-Chang-3013

Wang, Delin-3360

Wang, Ding-3018, 3129

Wang, Jin-3331

Wang, Jinzhao-3001

Wang, Kexiong-3129

Wang, Le-3209

Wang, Lily M.-2946, 3431

Wang, Ning-3450

Wang, Qi-3370, 3418

Wang, Rikuang-2979

Wang, Shuping-3311

Wang, Wei-Chun-3380

Wang, Wenjing-3265

Wang, Wenqi-2959, 3377

Wang, Xianyan-3298

Wang, Xin-2964

Wang, Xuan-3225

Wang, Yak-Nam-2985, 3030, 3031, 3082, 3083, 3186, 3308

Wang, Yuan-3104

Wang, Yue-3225, 3335

Wang, Yuxia-3337, 3449

Wang, Zhiguang-3424

Wanling, Lu-3336

Wanten, Geert-3310

Waragai, Maki-3400

Ward, Larry-3348

Warnecke, Michaela-2948, 2974

Washiya, Mamoru-3245

Watanabe, Kanji-3062, 3268, 3428

Watanabe, Masafumi-3057

Watanabe, Shinji-3062, 3404

Watanabe, Takayuki-3291

Watanabe, Yoshiaki-2955, 3369

Watanabe, Yoshitaka-3057, 3058, 3230

Watanabe, Yosiaki-2955

Watson, Kevin-3225

Watts, Oliver-2961

Watwood, Stephanie-3413

Wayant, Nicole M.-3095, 3193

Wayland, Ratree-3398

Wear, Keith-3371

Wear, Keith A.-3079

Webb, Douglas C.-3135

Weber, Thomas C.-2949, 3077, 3242, 3348

Webster, Sarah E.-3075

Wei, Chong-3297, 3298

Wei, NA-3425

Wei, Ruey C.-3013

Wei, Ruey Chang-3012, 3022

Wei, Ruey-Chang-3013

Weijers, Gert-3310

Weinzierl, Stefan-3175

Weisser, Adam-3277, 3437

Weitzel, William F.-3310

Wellard, Rebecca-3416

Weller, David W.-3360 
Weller, Tobias-3436

Wells, John C. -3183

Wells, Laurie-3146

Welsh, Kelsey-3440

Wendler, Olaf-3393

Wendt, Torben-3178

Werker, Janet F.-3446

Werner, Kurt J.-3092

Werner, Lynne A.-3264, 3447

Wess, Jessica M.-3157

Wesseling, Pieter-3082

Wessells, Hunter-3031

West, Ken-3203

Wester, Mirjam-2961

Weyn, Richard-3438

Whalen, Cara-3414

Whalen, D. H.-3109, 3163, 3219

Whang, Stella-3083

Wheeler, Harley J.-2997

White, Michael J.-3193

White, Paul-2968

Whiting, Eric B.-3044

Wiggins, Sean M.-3360

Wijkstra, Hessel-3420

Wiklund, Martin-3148

Wildenburg, Thomas-3275

Wilkinson, Matthew-3291

Willi, Megan-3216

Williams, Earl G.-3210

Williams, Kevin-3408, Chair

Session 1aUW (2967), 2968

Williams, Kevin L.-2968

Williams, Randall P.-3250, 3422

Williams, Stefan B.-3351

Williamson, Sarah-3135

Willis, Abbey-3437

Wilson, Chris-3242

Wilson, Christopher G.-3191

Wilson, Colin-3343, 3344

Wilson, D. K.-3088, 3095, 3119, 3323

Wilson, D. Keith-3193

Wilson, David K.-3194

Wilson, Greg-3363

Wilson, Ian-3050, 3222, 3334, 3343

Wilson, Preston S.-3018, 3048, $3103,3122,3123,3233,3234$, 3294, 3305, 3349, 3364

Wilt, Kyle-3229

Winn, Matthew-3045, 3100, 3218, 3440

Winn, Matthew B.-3269, 3438

Wischhusen, Jennifer-3369

Wisniewski, Matthew G.-3269

Wissing, Daan-3106

Witew, Ingo B.-3128

Witte, Russell S.-3371

Wixom, Andrew-3351

Wixom, Andrew S.-3102

Wohlberg, Brendt-3038

Wohlgemuth, Melville J.-2973

Wojtczak, Magdalena-3150, 3329

Wolf, Rachel-3237

Wolfenden, Andrew-3074

Wolff, Gregory G.-3145

Womble, Jamie-3301

Wong, Bob-3416

Wong, Janice Wing Sze-3336

Wong, Kainam T.-3063

Wong, Willy-3329, Cochair Session 4pPP (3328)
Woo, Sangbeom-2954

Wood, Michael-3021

Wood, Warren-3233

Woodhouse, Jim-3036, 3390, 3427

Woolworth, David S.-3236, 3322,

Cochair Session 1aAA (2946),

Cochair Session 1pAAa (2969),

Cochair Session 4aAA (3236),

Cochair Session 4pNSb (3322)

Worcester, Peter F.-2976, 3011, 3075, 3134, 3135, 3406

Worland, Randy-3210

Worthmann, Brian M.-3231, 3232

Woszczyk, Wieslaw-2990

Wright, David-3263

Wright, Matthew C.-3315, 3376

Wright, Oliver B.-3203

Wright, Richard-3332

Wróblewski, Marcin-3268

Wu, Chu-Yin-3444

Wu, Jeff C. -3022

Wu, Juliana-3330

Wu, Junru-3149

Wu, Kuangcheng-Cochair Session 4pSA (3330)

Wu, Liang-3395

Wu, Mengfan-3436

Wu, Shuanglong-2990

Wu, Wenjing-3367

Wu, Xiuqin-3393

Wu, Zhizheng-2961

Wyber, Ron J.-3123, 3345

Wyman, Megan T.-3414

Xian, Yin-3405

Xiang, Ning-3252, Chair Session 5aSP (3403), Cochair Session 4aID (3251)

Xiao, Dong-3011, 3058

Xie, Bo-sun-3276

Xie, Xin-3342

Xie, Yangbo-2959, 3103

Xin, Fengxian-2993

Xiong, Ying-Zi-3272

$\mathrm{Xu}$, Anqi-3401

Xu, Can-3337

Xu, Huan-3026

Xu, Kailiang-3078

Xu, Li-3394, 3445

Xu, Lilong-3337, 3449

Xu, Wen-3184

Xu, Yi-3398

Xu, Zhen-3030, 3031, 3032, 3083, 3084, 3085, Cochair Session 1pBAb (2982), Cochair Session 2aBAa (3025), Cochair Session 2aBAc (3030), Cochair Session $2 \mathrm{pBAb}(3081)$

Xue, Yawen-2960

Xueyan, Wang-3172

Yabu, Ken-ichiro-3366

Yabushita, Mitsuru-3385

Yack, Tina M.-3415

Yaegashi, Sou-3027

Yagawa, Genki-3427

Yagi, Kazuo-3140

Yagi, Takayuki-2950

Yaguchi, Yuichi-3114

Yairi, Motoki-2993, 3293, Cochair Session 4pAAb (3293)

Yamada, Haruya-3304

Yamada, Ichiro-3093, 3256, 3257
Yamada, Kazutaka-3302

Yamada, Masashi-2988, 3399

Yamada, Shinji-3322

Yamada, Tadasu K.-3302

Yamada, Takayuki-3430

Yamada, Tomoaki-3024

Yamada, Yasufumi-2975

Yamagishi, Junichi-2961, 2962, 2964

Yamagishi, Shimpei-3158

Yamaguchi, Tadashi-3138, 3185, 3187, 3247, Cochair Session 3aBA (3136), Cochair Session 3pBAa (3185)

Yamaguchi, Takao-2991

Yamaguchi, Tetsutaro-3223

Yamakawa, Kimiko-3108, 3223, 3338

Yamakawa, Takashi-3069, Cochair Session 2pAA (3068), Cochair Session 3aAAa (3126)

Yamakawa, Yui-3205

Yamakoshi, Yoshiki-3373, 3419

Yamamoto, Jun-3305

Yamamoto, Katsuhiko-3114

Yamamoto, Katsuya-3259

Yamamoto, Kozo-3280

Yamamoto, Makoto-3043, 3097, 3421

Yamamoto, Seiichi-3381

Yamamoto, Seiji-3139

Yamamoto, Takashi-3430, 3431, Cochair Session 5pNSa (3430)

Yamamoto, Yuto-3400

Yamamoto, Yuya-2975

Yamamura, Toshiki-3063

Yamanaka, Kazuhiro-3367

Yamanaka, Kazushi-3166

Yamanaka, Toshiaki-3156

Yamanaka, Yusei-3140

Yamashita, Akinori-3156

Yamashita, Kodai-3280

Yamashita, Mayuko-3381

Yamashita, Rei-2954

Yamashita, Yoichi-3062, 3400, 3445

Yamato, Kazuki-3065, 3301

Yamauchi, Akihito-3223

Yamauchi, Katsuya-3433, Cochair Session 5pNSb (3432)

Yamauchi, Kodai-2966

Yamauchi, Saki-3174

Yamazaki, Hidekatsu-3241

Yamazaki, Mayuko-3419

Yamazaki, Toru-3159, Cochair Session 3aSA (3159)

Yan, Hanbo-3336, 3395

Yanagida, Masuzo-3381

Yanagida, Riu-2988

Yanagisawa, Emi-3439

Yanagitani, Takahiko-3149, 3204, 3205, 3387

Yang, Byunggon-3113

Yang, Chen-3307

Yang, Chih-Kai-3018

Yang, Chunxia-3373

Yang, Desen-3124

Yang, Eun Jin-3242

Yang, Jie-3232

Yang, Jing-3049, 3445

Yang, Meng-3216

Yang, Min-3144
Yang, Shaofan-3230

Yang, Shubo-3250

Yang, Shuyi-3392

Yang, Stephanie-3013

Yang, T. C.-3184

Yang, Tsih C.-3229

Yang, Wei-Cheng-3132

Yang, Xiao-3224

Yang, Xiaohu-3337, 3449

Yang, Yiing J.-3013

Yang, Yiing Jang-3012

Yang, Yiing-Jang-3013

Yankaskas, Kurt-3199

Yano, Takashi-3093, 3094, Cochair Session 2pNS (3093)

Yap, Minlee-3305

Yashinski, Melisa-3259

Yashiro, Hidetaka-3297

Yasser, Haider-3031

Yasu, Keiichi-3365, 3439, 3440

Yasuda, Tohya-3303, 3304

Yasuda, Yosuke-3385

Yasueda, Kazuya-3140

Yasuhara, Ryu-3116

Yasui, Kyuichi-3122

Yasui, Nozomiko-3380, 3381

Yasukouchi, Ayumi-3267

Yasuma, Hiroki-3065, 3301, 3303

Yatabe, Kohei-3087, 3195, 3417, 3451

Yegge, Sarah-3415

Yeh, Yi-3106

Yerkes, Breanne-3208

Yeung, Gary-3448

Yi, EunYoung-2958

Yin, JIngwei-3231, 3403

Yin, Xiaoyan-2974

Yip, Jonathan-3105

Yip, Jonathan C.-3112, Chair

Session 1aSC (2960)

Yiu, Billy Y. S.-3028, 3029

Yiu, Suki-3222

Yoas, Zachary W.-3034

Yokomori, Fumiya-3400

Yokoshima, Shigenori-3093, 3094, 3279

Yokota, Takatoshi-3127, 3257

Yokoya, Toshitaka-3145, 3198

Yokoyama, Hiroshi-3253

Yokoyama, Masao-3427

Yokoyama, Sakae-3127

Yon, Sylvain-2984

Yoneda, Yutaro-3190

Yonemoto, Kiyoshi-3445

Yonemura, Miki-3257

Yoneya, Makoto-3209

Yoneyama, Kiyoko-3282

Yonovitz, Al-3228, 3262, 3421

Yonovitz, Joshua-3228, 3421

Yoo, Hyun Ji-3394

Yoon, Eunkyung-3341

Yoon, Kiseop-3248

Yoon, Soon Joon-2979, 2980

Yoshida, Junji-2954

Yoshida, Kenji-3138, 3187

Yoshida, Kohei-2964

Yoshida, Natsuya-3338

Yoshida, Sachiko-3139, 3185, 3245

Yoshida, Shota-2954

Yoshida, Tatsuya-3161 
Yoshihisa, Koichi-3383

Yoshii, Kazuyoshi-3038, 3090

Yoshii, Minoru-3087

Yoshikawa, Hideki-3083

Yoshimizu, Takuto-3376

Yoshimura, Takaki-3365

Yoshimura, Takako-3445

Yoshimura, Taku-3139

Yoshinaga, Tsukasa-3221

Yoshinaka, Kiyoshi-3306, 3309, 3369

Yoshioka, Hisashi-3256

Yoshioka, Masahiro-3372

Yoshiura, Maiko-3451

Yoshiyama, Fuminori-3450

Yoshizawa, Shin-2979, 2983, 2984 3029, 3246, 3308, Cochair Session 2aBAc (3030)

Yost, William-3100

Yotsukura, Norishige-3066

Youn, Hongwoo-3089

Young, Jeremy-3301

Younts, Robert-3421

$\mathrm{Yu}$, Alfred-Cochair Session 2aBAb (3027)

Yu, Alfred C. H.-3028, 3029

Yu, Chengzhu-3010

Yu, Cong-3272

Yu, Hsin-Yi-3132

Yu, Jaesok-2981

Yu, Roger-3261

Yudichak, Thomas W.-3170

Yue, Yisong-3004

Yuge, Kohei-3434

Yuhara, Ryosuke-3155
Yuldashev, Petr-3082

Yuldashev, Petr V.-3082, 3310

Yumoto, Masato-3046

Yuno, Yuuki-3153

Yusa, Takashi-3066

Zabolotskaya, Evgenia A.-3310, 3433

Zabotin, Nikolay A.-3076

Zabotina, Liudmila Y.-3076

Zahorik, Pavel-3438

Zajac, David J.-3107

Zaleski, Ashley C.-3200

Zampolli, Mario-3024

Zang, Eden-3018

Zang, Eden J.-3359

Zang, Xiaoqin-3076

Zannoli, Marina-2956, 3008

Zedel, Len-3184, 3363

Zednik, Ricardo-3034

Zeh, Matthew C.-3294, 3305

Zeigler, Stephen-3422

Zellou, Georgia-3219

Zeltzer, Lonnie K.-3266

Zempo, Keiichi-3277, 3302

Zemzemi, Chadi-3373

Zeng, Juan-3012, 3065

Zeqiri, Bajram-3150

Zhang, Cao-3331

Zhang, Chen-3063

Zhang, Chuangzheng-3183

Zhang, Chuanzheng-3132

Zhang, Dong-3026, 3033, 3085, 3367, 3368

Zhang, Dongye-3191

Zhang, Haixia-3394
Zhang, Haoyang-3124

Zhang, Hong-3220

Zhang, Hui-3337

Zhang, Huiming-3155

Zhang, Jie-3225, 3395

Zhang, Junjian-3119

Zhang, Jun-Yun-3155

Zhang, Lijie G.-3025

zhang, linke-3425

Zhang, Mengna-2974

Zhang, Qianchu-3351

Zhang, Tracy-3214

Zhang, Xi-3031, 3084

Zhang, Xiang-3104

Zhang, Yan-3011

Zhang, Yanlong-3337

Zhang, Yanni-3160

Zhang, Yi-ming-3232

Zhang, Ying-3184, 3307

Zhang, Yu-3297, 3298

Zhang, Yuanyuan-3116

Zhang, Yuxiang-3165

Zhang, Yu-Xuan-3155, 3272, 3389

Zhang, Yuxuan-3390

Zhang, Zhaoyan-3395

Zhang, Zhi Y.-3407

Zhao, Chuming-3377

Zhao, Dan-3422

Zhao, He-3422

Zhao, Jing-3175

Zhao, Shengkui-3055

Zhao, Sijia-3209

Zhao, Wen Yao-3012

Zhao, Wenyao-3065
Zhao, Xiaofeng-3135, 3187

Zhao, Xueqin-3340

Zhao, Yuezhe- 3236

Zheng, Annie-3340

Zheng, Hairong-3149

Zheng, Hong-3133, 3135

Zheng, Z. C.-3119

Zhong, Pei-3307

Zhong, Xiao-li-3276

Zhou, Jinming-3078

Zhu, Chunyue-3400

Zhu, Feilong-3234

Zhu, Hongxiao-2947

Zhu, Huiling-3063

Zhu, Jie-3103

Zhu, Jinying-3211, Chair Session 5aPA (3386)

Zhu, Mengyao-3278

Zhu, Xiao-Hua-3132

Zhu, Xiaohua-3135

Zhu, Xuefeng-3049, 3103, 3104

Zhu, Yifan-3049

Zhu, Yiqing-3398

Zhu, Ze-Nan-3132

Zhu, Zhi-3271

Ziaei, Ali-3010

Zipse, Lauryn-3395

Zoidis, Ann M.-3072

Zosuls, Aleks-3298

Zotkin, Dmitry N.-2999

Zou, Xinye-3049

Zuo, Shuguang-2990

Zuo, Zhuan-3139

Zwaschka, Theresa A.-3307 


\title{
Collected Works of Distinguished Acousticians
}

\author{
Isadore Rudnick
}

The first in this series of the Collected Works of Distinguished Acousticians is that of Isadore Rudnick (May 8, 1917 - August 22, 1997). Rudnick was honored by the Acoustical Society of America (ASA) with the R. Bruce Lindsay (Biennial) Award in 1948, the Silver Medal in Physical Acoustics in 1975, and the Gold Medal in 1982. He was recognized for his acoustics research in low temperature physics with this field's most prestigious award, the Fritz London Memorial Award, in 1981 and was inducted into the National Academy of Science in 1983. Izzy's research in physical acoustics addressed boundary propagation, reciprocity calibration, high intensity sound and its biological effects, nonlinear sound propagation, and acoustics in

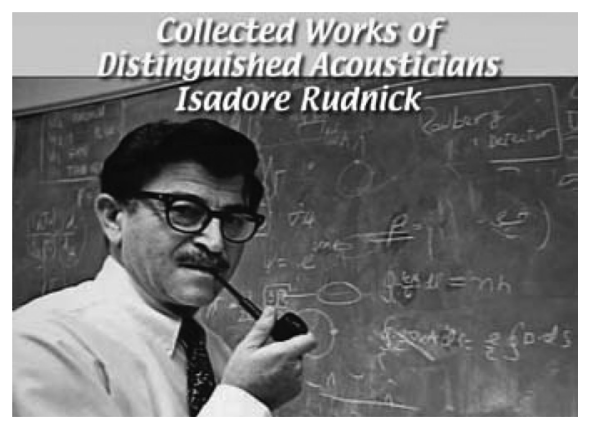
superconductors and superfluids, including critical phenomena in bulk and thin films. The first disc in this three disc set contains reprints of Rudnick's papers from scientific journals, including 26 from the Journal of the Acoustical Society of America, and 87 from other prestigious journals, as well as some consulting reports and invited papers presented at international meetings which would otherwise be difficult to obtain. The second disc includes a montage of photographs of Rudnick with colleagues and family, Rudnick's prize winning film "The Unusual Properties of Liquid Helium”, and a video of the Plenary session at the ASA's 100th meeting where Rudnick presented 90 minutes of unique and stage-sized acoustics demonstrations. While videotaped under poor conditions and of lamentable quality, the reprocessed video of acoustics demonstrations is one of the most valuable parts of this collection. The third disc is a video recording of the Memorial Session held at the 135th meeting of the ASA, which provides a comprehensive summary of Rudnick's contributions as described by former students and collaborators.

The CD was compiled by Julian D. Maynard and Steven L. Garrett of the Pennsylvania State University, State College, Pennsylvania.

\section{O R D E R F O R M}

Price: \$50-ASA members; \$60-Nonmembers. Prepayment required by check or money order in U.S. dollars drawn on a bank in the U.S. or by Visa, MasterCard or American Express credit card.

Send orders to the Acoustical Society of America, Suite 1N01, 2 Huntington Quadrangle, Melville, NY 11747-4502, Tel.: 516-576-2360; Fax: 516-576-2377; Email: asa@aip.org.

Name

Address

City State/Province Zip/Postal Code Country

Email:

Quantity Price

(a) $\$ 60.00 /$ ea.

Postage/Handling

$\$ 5.00$

Total Due and Enclosed

Exp. Date

*Security Code

\section{Signature:}

*What is this? On MasterCard and Visa cards, this is the 3-digit number printed in the signature area of the back of the card (last 3 digits AFTER the credit card number). On American Express cards it is the 4-digit card verification number on the front above the credit card number.

Due to security risks and Payment Card Industry (PCI) data security standards e-mail is NOT an acceptable way to transmit credit card information. Please use our secure web page to process your credit card payment (http://www.abdi-ecommerce10.com/asa) or securely fax this form to (516-576-2377). 


\section{wWw.pch.com/Water-Resistant-Mic}

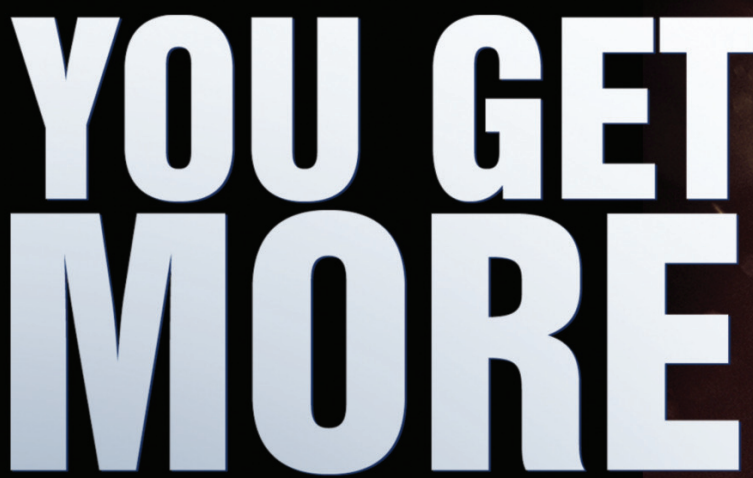

THAN JUST WHAT'S

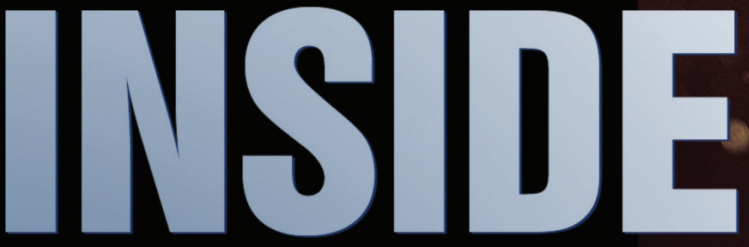

OUR MIGROPHONES

Water \& Dust Resistant Microphone Model 130A24

Make measurements where dirt, moisture

and other contaminants are a concern.

\section{NOT JUST A MIIGROPHONE}

PERFORMANGE

Inside every microphone is 40 years of sensor design and manufacturing expertise. Exceeding all expectations - our price, support, warranty and delivery are unmatched.

- UNBEATABLE PRIGE 24/7 SUPPORT BEST WARRANTY FAST SHIPMENI

\section{WPCB PIEZOTRONICS}


Melville, NY 11747-4300

\section{VIBRATON MONITORING TERMINAL - TYPE 3680}

\section{GOOD VIBRATIONS}

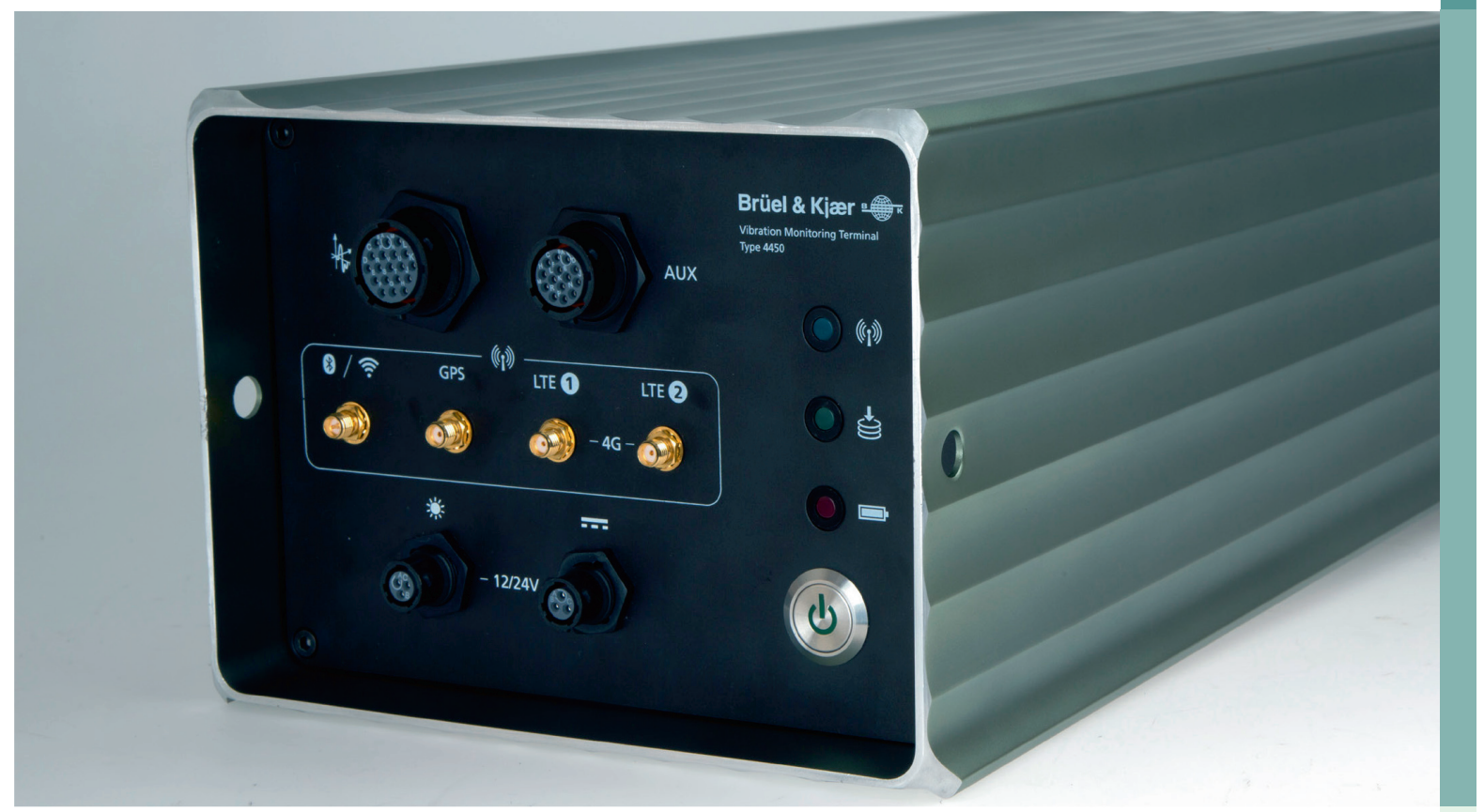

Reliably take real-time measurements with our new Vibration Monitoring Terminal Type 3860. The robust device enables you to:

- Protect against structural damage risks in construction and mining

- Assess human response to road and rail traffic vibration

- Monitor background vibration to avoid sensitive machinery disturbance

The Vibration Monitoring Terminal includes metrics for a wide range of applications. The system provides continuous, uninterrupted, real-time monitoring 24/7. Alerts are based on level and time of day. It contains a single tri-axial geophone for full coverage of vibration levels, and built-in remote access so you don't need to visit a site to retrieve data.

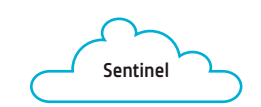

))

๙

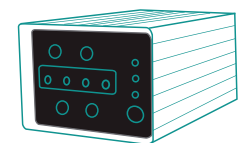

))

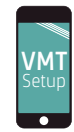

Use the unit with our Sentinel environmental monitoring service or as a stand-alone device.

See more at www.bksv.com/VMT

\section{Brüel \& Kjær}

\author{
BEYOND MEASURE
}

Bruel \& Kjaer North America Inc.

3079 Premiere Parkway, Suite 120

Duluth, GA 30097

Tel: 7702096907

bkinfo@bksv.com

www.bksv.com/VMT 